


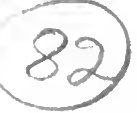

BETA- AND GAMMA-RAY SPECTROSCOPY 


\section{SERIES IN PHYSICS}

General Editors:

J. de Boer, H. Brinkman and H. B. G. Casimir

L. Rosenfeld, Nuclear Forces

E. A. Gugaenherm, Thermodynamics

J. Bouman (editor), Selected Topics in X-Ray Crystallography

L. Rosenfeld, Theory of Electrons

W. ElenbaAs, The High Pressure Mercury Vapour Discharge

S. R. DE GRoot, Thermodynamics of Irreversible Processes

J. G. Wilson (editor), Progress in Cosmic Ray Physics Volume I

J. G. Winson (editor), Progress in Cosmic Ray Physics Volume II

C. J. Gorter (editor), Progress in Low Temperature Physics

KaI Siegbahn (editor), Beta- and Gamma-Ray Spectroscopy

BøRge BAK, Elementary Introduction to Molecular Spectra

E. A. Guggenheim and J. E. Prue, Physicochemical Calculations

J. II. Burgers and H. C. vax de Hulst (editors), Gas Dynamics of Cosmic Clouds. A Symposium 


\title{
BETA- AND GAMMA-RAY SPECTROSCOPY
}

\author{
EDITED BY
}

\section{KAI SIEGBAHN}

\author{
PROFESSOR OF PHYSICS \\ UNIVERSITY OF UPPSALA
}

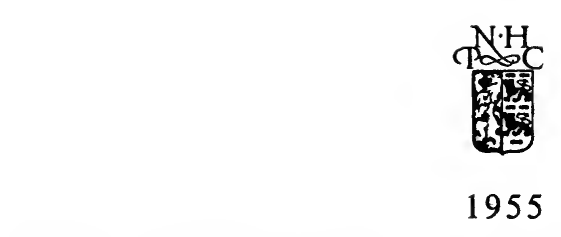

NORTH-HOLLAND PUBLISHING COMPANY A MSTER DAM 


\section{$\frac{68355.55}{20 \cdot 8 \cdot 58}$}

Sole Distributors for U.S.A.:

INTERSCIENCE PUBLISHERS INC., NEW YORK

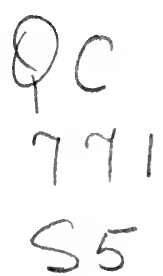




\section{PREFACE}

The field of $\beta$ - and $\gamma$-ray spectroscopy belongs to the classical domains of nuclear physics.

In view of these old traditions it is permissible to speak of a real renaissance of this field during the past ten years. This period started with developments of instruments and methods, which continued during a collecting period of rough data about nuclear levels. Then, some five years ago, came the nuclear shell model. The advancement of this theory for the first time provided nuclear spectroscopy with a really powerful theoretical tool and formed the solid foundation it had lacked before. The rapid development during the last years has clearly shown that the detailed analysis of nuclear radiation yields such information about the properties of nuclear levels, as is of basic importance for our understanding of nuclear matter. Based on such experimental information, nuclear models, in many ways surprisingly similar to those known earlier from atomic and molecular spectroscopy, have already been constructed. The analogies cannot be pressed too far but the fact that really fruitful systematic work has finally become possible indicates that we may expext a very interesting period in this field of nuclear research. No doubt the coming years will greatly extend our knowledge of the structure of nuclei in the light of the new ideas.

In order to facilitate the entry of the newcomer into this research the present volume has been written. The large amount of material is distributed in 26 chapters, and with the aim of giving an authorative account of the whole field, specialists have been chosen for each chapter. Experimental methods and theoretical discussions are presented in such a sequence that the strong connection between experiment and theory remains unbroken. The book is primarily intended for experimentalists and complete derivations of theoretical expressions have been avoided. The results of theoretical calculations have been presented in such a way that the reader will get a feeling for the theoretical starting points and the main sources of approximations. The eight appendices included at the end of the book should be useful in research work, when reworking the experimental data into the form appropriate for theoretical analysis.

In producing this volume the problem of keeping together a highly international team of not less than 42 co-workers has not been as difficult 
as one might think. This is entirely due to the patient attitude all my co-workers have shown both in the planning of the book and in the editorial work, which has caused a lot of rewriting and shortening. I take this opportunity to express my deep gratitude to my collaborators for their generous help in all respects and for their outstanding efforts to give of their best for the benefit of our common work.

The subject index has most kindly been prepared by Mr. E. G. D. Cohen, Amsterdam. and the German manuscript of Ch. I has been translated into English by Dr. J. van Kranendonk, Amsterdam. Several manuscripts written by non English speaking authors have been checked by co-authors in the book. among whom I especially would like to mention Dr D. Alburger. Brookhaven, and Dr M. E. Rose, Oak Ridge.

I should also like to express my sincere thanks to the board of the "Series in Physics", which has given me the privilege of editing this volume, and, last but not least, the Director of the North-Holland Publishing Company, Mr. M. D. Frank, who has shown an unusual personal interest in this undertaking.

KaI Siegbahn

Uppsala, January 1955. 


\section{CONTENTS}

PREFACE . . . . . . . . . . . . . . . . . . . . . . v

CONTENTS......................... . VII

LIST OF CONTRIBUTORS . . . . . . . . . . . . . . . . . . . $\mathrm{x}$

INTRODUCTION by K. Siegbahn . . . . . . . . . . . . . xviII

CHAPTER I

INTERACTION OF ELECTRONS WITH MATTER by W. Paul and $\mathrm{H}$. Steinwedel.

$\S 1$. Introduction $1-\S 2$. Elastic Scattering of Electrons by

Atomic Nuclei $1-\S 3$. Energy Loss 9

CHAPTER II

INTERACTION OF $\gamma$-RADIATION WITH MATTER by Charlotte $M$. Davisson . . . . . . . . . . . . . . . . . . .

$\S 1$ Introduction $24-\S 2$. Photoelectric Effect $26-\S 3$. Scattering $32-\S 4$. Pair Production $41-\S 5$. Total Absorption Coefficients $47-\S 6$. Absorption Methods 49

CHAPTER III

$\beta$-RAY-SPECTROMETER THEORY AND DESIGN. HIGH RESOLUTION SPECTROSCOPY by K. Siegbahn . . . . . . . . . . . . . . .

$\S 1$. Introduction. General Discussion of Spectrometers $52-\S 2$. Some Basic Relations in $\beta$-Spectroscopy $54-\S 3$. Nomenclature $56-\S 4$. The Semicircular Spectrometer $58-\S 5$. The Double Focusing Spectrometer $66-\S 6$. Other Flat Spectrometers $73-$ $\S 7$. Lens Spectrometers $77-\S 8$. Coincidence Spectrometers $86-$ §. Electrostatic Spectrometers $90-\$ 10$. Precision Spectroscopy 90

\section{CHAPTER IV}

CRYSTAL DIFFRACTION SPECTROSCOPY OF NUCLEAR $\gamma$-RAYS by J. W. M. DuMond .

$\S$ 1. Early History and Elementary Ideas Relative to Both Flat and Bent Crystal Gamma and X-Ray Spectrometers $100-\S 2$. Adaptation of the Transmission Type Bent Crystal Spectrometer Principle to the Precisıon Measurement of Very Short Wavelengths (Nuclear $\gamma$-Rays) $109-\S 3$. Typical Results Obtained with the Mark I Crystal Diffraction $\gamma$-Ray Spectrometer 123 
$\$$ I. 'Th" Sintillation Connter 133 - $\$$ :. Methods for V'arious Radlattons 1:33 - \$3. Factors Affecting Resolution $143-\$ 4$. Crystal Preparation and Mounting 147 .. 5. C'rystal Efficiency and Linearity 1.jl - $\$$ ti. Total Ahomption Gamma Spectro.

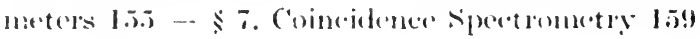

\section{('llAP'THK V'I}

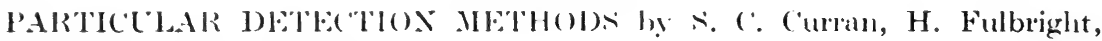
(. Bishop and R. Wilsm. . . . . . . . . . . . . . . . .

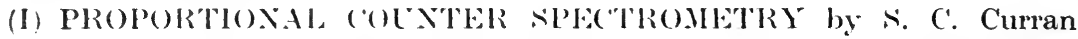
$\$$ 1. The Principle of Operation 16j - $\$$. The Cas Cain $166-$ s. The Energy Expenditure per Ion Pair and Calibration $167-$ $\$$ 4. Statistical Fluctuations in Pulse size and Resolution 168 $\$$.). Pulse Shape and Amplifier Design 172 - $\$ 6$. Geometries of sumer and Counter, Wall Effect and Magnetic Field $173-\S 7$. Photuabsorption, Fluorescence Yicld and the Escape Peak 176 \$. Pulse Amplitude Analysis 177 - \$9. Coincidences, Internal and Fxternal $17 s-\$ 10$. Proportional Counter Work at Low Intensity 179- $-\$ 11$. Notes on Recent Applications 182

(I) BETA SPECTROSCOPY BY LSE OF A HIGH PRESSURE PRO. PORTIONAL COLNTER by H. Fulbright . . . . . . . . . .

$\$ 1$. Introduction and Description of Apparatus $184-\$ 2$. Analysis of the Operation of the Counter $186-\$ 3$. Calibration $189-$ $\$$ 4. Resolving Power Corrections $190-\S 5$. Results 191

(III) SPECIAL METHODS IA (AAMMA-RAY SPECTROSCOPY by G. Bishop and R. Wilson . . . . . . . . . . . . . . . . .

$\S 1$. Photoneutron Threshold Reactions 192 - $-\$ 2$. Measurement of Phot'neutron Energies 193 - $\$ 3$. Measurement of Photoproton inergies $194-\$ 4$. Comparison with Other Methods $197-$ $\$ 5$. Polarisation of $\gamma$-Ray's $198-\$ 6$. Other Reactions 199

\section{CHAPTER VII}

THE COINCIDENCE METHOD by A. C. G. Mitchell . . . . . . .

$\S 1$. Introduction $201-\S 2$. The Disposition of Apparatus $202-$ $\S 3$. General Procedures for Coincidence Counting $204-\S 4$. The Calculation of Errors $206-\S 5$. Remarks on the Measurement of Beta-Ray Energies by Absorption $208-\S 6$. The Measurement of the Energy of $\gamma$-Rays by the Absorption of Compton-Electrons $209-\S 7$. Analysis of the Coincidence Method $211-\S 8$. Measurement of Internal Conversion Electrons $216-\S 9$. The Use of Coincidence Counting for Determining the Efficiency of $\gamma$-Counters $219-\S 10$, Coincidence Counting Using a Magnetic Spectrometer $221-\S 11$. Future Developments 223 
BETA-SPECTKOMETER MEASUREMENTS by A. C. G. Mitchell and H. Slätis

(I) INVESTIGATIONS' OF DISINTEGRATION SCHEMES - ENERGIES AND INTENSITIES OF $\beta$ - AND $\gamma$-COMPONENTS AND THEIR COUPLING by A. C. G. Mitchell . . . . . . . . . . . . . . .

$\S$ 1. Introduction $224-\S 2$. The Preparation of the Source Material $226-\S 3$. The Calibration of Instruments $226-\S 4$. The Search for $\gamma$-Rays and Measurement of their Energy $228-\S 5$. Special Considerations for Complex Spectra $233-\S 6$. The Determination of the Relative Intensities of $\gamma$-Rays $234-\S 7$. The Measurement of $\beta$-Rays $236-\S 8$. The Fermi Plot; End Points and $\beta$-Ray Groups $-\S 9$. Determination of Comparative Half-Lives-ft $245-\S 10$. Determination of Internal Conversion Coefficients and $K / L$ Ratios $246-\S 11$. Examples of Some Disintegration Schemes 251

(II) SOURCE AND WINDOW TECHNIQUE by H. Slätis . . . . . . .

$\S$ 1. Introduction $259-\$ 2$. Preparation of Foils for Backings and Windows $260-\S 3$. Estimation of Foil Thickness $263-\S 4$. Preparation of Samples $263-\S 5$. Window Absorption Correction $269-\S 6$. Acceleration of Electrons 272

CHAPTER IX

THE THEORY OF ALLOWED $\beta$-DECAY by M. E. Rose . . . . . .

$\S$ 1. Introduction $273-\S 2$. The Role of $\beta$-Decay in Nuclear Physics $274-\S 3$. The Conservation Laws and the Neutrino $274-$ $\S 4$. The $\beta$-Interaction $275-\S 5$. Allowed Transitions $277-\S 6$. The Energy Spectrum $279-\S 7$. Kurie Plots for Allowed Spectra $283-\S 8$. $f t$ Values $284-\S 9$. Orbital Electron Capture 289

\section{CHAPTER $\mathrm{X}$}

THE THEORY OF FORBIDDEN $\beta$-DECAY by E. Konopinski . . . .

$\S 1$. Introduction $292-\S 2$. The Effects Responsible for Forbidden Decays $292-\S 3$. General Selection Rules and Matrix Elements $296-\S 4$. Spectra and Half-Lives $301-\S 5$. The "Unique" For. bidden Transitions $304-\S 6$. Once-Forbidden Spectra and HalfLives $306-\S 7$. Twice-Forbidden Spectra and Half-Lives $309-$ $\S 8$. The Law of $\beta$-Decay 311 - Appendix 312

\section{CHAPTER XI}

EXPERIMENTS ON THE SHAPES OF $\beta$-SPECTRA. THE INTERACTION IN $\beta$-DECAY by C. S. Wu . . . . . . . . . . . . . . . . . . . . .

$\S 1$. Introduction $314-\S 2$. The Allowed Spectra $314-\S 3$. Fierz Interference Terms for the Allowed Transitions $319-\S 4$. Fermi and Gamow-Teller Interaction $320-\$ 5$. Rest Mass of the Neutrino $325-\S 6$. Forbidden Transitions $327-\S 7$. The 
Enique Furbidilen spectra $3: \$ \ldots \$$... Half-Lives of the Unique 'Transitions 333 -.- $\$$ 9. First Forbidden spectra $336-\$ 10$. Sipectrum of RaE and Pseurloscalar Interaction 339 - $\$ 11$. Effect of Pseudiscalar Interaction on the Shape of Allowed $\beta$-Spectra 344 - $\$ 12$. . 'Lwice Forbidlen sipectra 345 - \$13. Mu-Meson Beta spectrum 34 !

\section{C'HAP'TER XII}

NEL"TRINU RE('()IL FXPERIMENTS by O. Kofoed-Hansen. . . . .

$\$ 1$. Introluction 357-\$2. Two-Body Decays 357 - \$3. $\beta$-Decay 363 - $\$$ 4. Difticulties with Bound Mother Particle 366 - 5 . Interpretation of $\beta$-Recoil Experiments 367

\section{CHAPTER XIII}

THEORY OF MLLTIPOLE RADIATION by s. A. Moszkowski. . . . 373

$\$ 1$ Introduction $373-\$ 2$. The Multipole Radiation Field $376-$ $\S$ 3. $;$-Decay 'Transition Probabilities of a single Particle 382 $\$$ 4. Camma Decay Transition Probabilities for Configurations Consisting of Several Independent Particles 393

\section{CHAPTER XIV}

THEORY OF INTERNAL CONTERSION by M. E. Rose . . . . .

$\$ 1$. Introluction $396-\S 2$. General Theoretical Considerations $399-\$ 3$. The Calculations: I $401-\$ 4$. The Calculations: II 405 - $\$ 5$. The Internal Conversion Coefficients 406.

\section{CHAPTER XV}

THE SHELL MODEL by J. H. D. Jensen . . . . . . . . . . .

$\S 1$. Cieneral Considerations $414-\$ 2$. Magic Numbers, or Shell Numbers $415-\S 3$. Level Sequence in the Shell Model $417-\S 4$. Remarks on Spin-Orbit Coupling 422 - $\$ 5$. Parity and Orbital Angular Momentum $423-\$ 6$. Nuclei with Incompletely Filled Shells $424-\$ 7$. Pairing Energy and Level Occupation Scheme 427

\section{CHAPTER XYI}

NLCLEAR SHELL SYSTEMATICS by M. Goeppert-Mayer, M. Goldhaber and A. W. Sunyar. . . . . . . . . . . . . . . . . . .

(I) CLASSIFICATIONS OF $\beta$-TRANSITIONS by M. Goeppert-Mayer. . 433

$\S$ 1. Introduction $433-\S 2$. Nuclei of Odd $A 433-\S 3$. Nuclei with Even $A 447-\S 4$. Light Nuclei 452

(II) CLASSIFICATION OF NCCLEAR ISOMERS by M. Goldhaber and A. W. Sunyar . . . . . . . . . . . . . . . . . . .

$\S 1$. Introduction $453-\S 2$. Selection Rules $454-\S 3$. Experimental Procedures $456-\$ 4$. Some General Experimental Results 461 
COLLECTIVE NUCLEAR MOTION AND THE UNIFIED MODEL by

A. Bohr and B. R. Mottelson. . . . . . . . . . . . . . . . . . . .

$\$ 1$. Collective Oscillations of a Shell Structure $468-\$ 2$. Nuclear Coupling Scheme $470-\S 3$. Spins and Moments $475-\S 4$. Nuclear Transition Probabilities 477 - §5. Systematics of Nuclear Rotational Structure 483

CHAPTER XVIII

MEASUREMENT OF SHORT LIFETIMES OF EXCITED STATES by

R. E. Bell and K. G. Malmfors

(I) MEASUREMENT BY DELAYED COINCIDENCES. COMPARISON METHODS by R. E. Bell . . . . . . . . . . . . . . . . . .

$\S$ 1. Introduction $494-\S 2$. Fast Coincidence Techniques $496-$ $\S 3$. The Delayed Coincidence Method $505-\S 4$. The Shortest Lifetimes by Delayed Coincidence Methods $507-\S 5$. Examples of Comparison Methods for Lifetime Measurement 516

(II) RESONANT SCATTERING OF $\gamma$-RAYS by K. G. Malmfors. . . 521

$\S 1$. Resonant Scattering Experiments $521-\$ 2$. Competing Non-Resonant Scattering Processes 528

\section{CHAPTER XIX}

ANGULAR DISTRIBUTION OF NUCLEAR RADIATION by H. Frauenfelder, R. J. Blin-Stoyle, M. A. Grace and H. Halban, S. R. de Groot and H. A. Tolhoek. . . . . . . . . . . . . . . . . . . . . . . . . . . . 53

(I) ANGULAR CORRELATION by H. Frauenfelder . . . . . . . . .

$\S$ 1. Introduction 531 - Part A. Free Nuclei 534 - § 2. Naive Theory of $\gamma-\gamma$ Directional Correlation in Free Nuclei $534-\S 3$. General Theory of Angular Correlation $539-\S 4 . \gamma-\gamma$ Directional Correlation $549-\S 5$. Polarization Correlation $559-\S 6$. Directional Correlation Involving Conversion Electrons $561-\S 7 . \alpha \cdot \gamma$ Directional Correlation $564-\S 8$. $\beta$ - $\gamma$ Directional Correlation 568 - Part B. The Influence of Extranuclear Fields $572-\$ 9$. Survey $572-\S 10$. The Determination of Nuclear $g$-Factors $576-\S 11$. The Determination of the Quadrupole Coupling $581-\$ 12$. Time. Dependent Interactions $588-\S 13$. The Influence of $K$-Capture $591-\S 14$. The Determination of the True Correlation $595-$ Part C. Experimental Methods $596-\S 15$. The Treatment of Experimental Data 596

(II) EXPERIMENTS WITH ORIENTED NUCLEI by R. J. Blin-Stoyle, M. A. Grace and H. Halban. . . . . . . . . . . . . . . . . .

$\S 1$. Introduction $600-\S 2$. Production of Oriented Nuclei $600-$ $\S 3$. Properties and Detection of Oriented Nuclei 604 - $\$ 4$. Angular Distribution and Polarization of Radiation $604-\$ 5$. Experimental Results with Oriented Nuclei $607-\$ 6$. Conclusions 611 
(III) THEORY OF ANGLLAR EFFE('TS OF RADIATIONS FROM ORIENTED NUCLEI by s. R. De Groot and H. A. Tolhoek . . .

$\$ 1$. Introduction $613-\$ 2$. Orientation of Nuclei $613-\S 3$. The Angular Distribution of Nuclear Radiation 614 - \$4. The Directional Distribution of Gamma Radiation $616-\S 5$. Polarization Distribution of Gainma Radiation $617-\S 6$. The Influence of Transitions Preceding the Gamma Radiation $619-\S 7$. The Directional Distribution and Polarization of Beta Radiation 621 $\S$ 8. Other Topics 623

\section{CHAPTER XX}

SOME IN'TERNAL EFFEC'TS IN NUCLEAR DECAY by I. Bergström, R. Wilson and C. S. Wu . . . . . . . . . . . . . . . . .

(I) ALGER ELECTRONS EMITTED FROM RADIOACTIVE ATOMS by I. Bergström . . . . . . . . . . . . . . . . . . . . . . . .

$\S 1$. Introduction $624-\S 2$. Classification of Auger Electrons 625

- $\$ 3$. Energies of Auger Electrons $626-\S 4$. Intensities of Auger Electrons $628-\S 5$. Applications Depending on the Fluorescence Yield 634

(II) INTERNAL PAIR FORMATION by R. Wilson. . . . . . . . . .

$\S 1$. Introduction $636-\S 2$. Theoretical Predictions $636-\S 3$. Exact Calculations $638-\S 4$. Schrödinger Approximation $639-$ $\S 5$. Summary of Theoretical Predictions $640-\S 6$. Magnetic Spectrometer $641-\S 7$. Detection by Annihilation Radiation 644 - 8 . Counter Telescopes 646 - $\$ 9$. Cloud Chamber Measurements $647-\S 10$. Internal Pair Formation at high Energies $647-$ \$ 11. Summary 648

(III) INTERNAL BREMSSTRAHLUNG by C. S. Wu . . . . . . . .

$\$$ 1. Introduction $649-\S 2$. Energy Distribution of Internal Bremsstrahlung $650-\S 3$. Experimental Results $653-\S 4$. Angular Distribution of Internal Bremsstrahlung 655 $-\S 5$. Bremsstrahlung Due to Electron Capture Process 656

\section{CHAPTER XXI}

PARTICULAR PROBLEMS STUDIED WITH SCINTILLATION TECHNIQUES by P. E. Cavanagh, H. Fulbright, S. De Benedetti, R. E. Bell and M. Deutsch

(I) ORDINARY COMPTON EFFECT AND DOUBLE COMPTON EFFECT by P. E. Cavanagh . . . . . . . . . . . . . . . . . . . . . . . $\S$ 1. Ordinary Compton Effect $660-\S 2$. The Double Compton Effect 665

(II) USE OF A SCINTILLATION COUNTER SPECTROMETER IN A SEARCH FOR DOUBLE BETA DECAY by H. Fulbright. . . . .

$\S$ 1. Introduction $668-\S 2$. The Experimental Method $669-$ $\S 3$. Results 672 
(III) ANNIHILATION OF POSITRONS by S. De Benedetti . . . . . . 672

$\S 1$. Introduction $672-\S 2$. Two-Quanta Polarization $674-\S 3$. The Momentum of the Annihilating Pairs 675- $\$$ 4. Three. Quantum Annihilation 677

(IV) ANNIHILATION OF POSITRONS IN LIQUIDS AND SOLIDS' by R. E. Bell . . . . . . . . . . . . . . . .

(V) POSITRONIUM by M. Deutsch . . . . . . . . . . . . . . 689

\section{CHAPTER XXII}

ILLUSTRATIVE DISINTEGRATION STUDIES by R. E. Bell, D. E. Alburger, A. C. G. Mitchell and P. Preiswerk . . . . . . . . . . . . .

(I) DISINTEGRATION OF I $\mathrm{I}^{131}$ by R. E. Bell . . . . . . . . . . 696

(II) ENERGY LEVELS OF Pb ${ }^{206}$ FROM THE DECAY OF $\mathrm{Bi}^{206}$ by D. E. Alburger . . . . . . . . . . . . . . . . . .

(III) A STUDY OF THE SEQUENCE $\mathrm{I}^{130} \rightarrow \mathrm{Xe}^{130} \leftarrow \mathrm{Cs}^{130} \rightarrow \mathrm{Ba}^{130}$ AND THE LEVELS OF $\mathrm{Xe}^{130}$ by A. C. G. Mitchell . . . . . . . . . . 720

$\S 1$. Experiments on $\mathrm{I}^{130} 720-\S 2$. The Disintegration of $\mathrm{Cs}^{130} 728$

(IV) DISCUSSION OF THE SCHEME OF RADIOACTIVE DECAYS LEADING TO $\mathrm{Cd}^{111}$ by P. Preiswerk. . . . . . . . . . . . . . . 730

$\S 1$. Summary of Measurements $730-\S 2$. Interpretation 735

\section{CHAPTER XXIII}

BETA AND GAMIMA DISINTEGRATION OF SOME HEAVY RADIO. ELEMENTS $(Z>80)$ by N. Feather . . . . . . . . . . . . . . . . .

$\S 1$. Active Species of the $A=4 n$ and $A=4 n+2$ Series 740 $-\S 2$. Active Species of the $A=4 n+1$ and $A=4 n+3$ Series $756-\S 3$. Characteristics of the Capture-Active Species of Large $Z 760-\$ 4$. Miscellaneous: Series Branching; Isomerism 762

\section{CHAPTER XXIV}

GAMMA RADIATION FROM CHARGED PARTICLE REACTIONS by D. E. Alburger . . . . . . . . . . . . . . . . . . . . . . . .

$\S 1$. Introduction $767-\S 2$. Gamma Radiation from Charged Particle Bombardment $768-\S 3$. Energy and Intensity Measurements 773 - 4. Particle Gamma Coincidence and Angular Correlations $787-\S 5$. The Life times of Nuclear States $789-\S 6$. Future Developments 791

\section{CHAPTER XXV}

NEUTRON CAPTURE GAMMA RAYS by B. B. Kinsey . . . . . . .

$\S 1$. Introduction $795-\S 2$. Experimental Methods $795-\S 3$. Experimental Results $799-\$ 4$. Interpretation of tamma-Ray 
Intensities $811-\$ 5$. Intensities and Transition Probabilities of Different Multipolo Orders 813 - $\$ 6$. Absolute Emission Proba. bilities $\$ 15-\$ 7$. Total Radiation Widths 819

\section{CHAPTER XXVI}

MEASLREMENT OF DISINIEGRATION RATE by J. L. Putman . . .

$\$ 1$. Introduction $823-\S 2$. Types of Counters $825-\$ 3$. Counting Methods $827-\$ 4$. Calorimetric Methods $847-\$ 5$. Measurement of Charge Lost by a Source $849-\$ 6$. Use of Ionization Chambers 850)

APPEXIIX 1. MAMMA-RAY ABSORPTION COEFFICIENTS by Ch. M. Davision. . . . . . . . . . . . . . . . . . . 857 APPENDIX II. FERAI FLNCTIONS by M. E. Rose . . . . . . . 875 APPENIIX III. FORBIDDEN SHAPE FACTORS by M. E. Rose . . . 884 APPENDIX IV. INTERNAL CONTERSION COEFFICIENTS by M. E. Rose. . . . . . . . . . . . . . . . . . . . . . . 905

APPENDIX $\vee$ : ANGULAR CORRELATIONS by H. Frauenfelder . . 910 APPENDIX VI. ABNORPTION ENERGIES by R. D. Hill. . . . . . . 914 APPENDIX VII. Th $\left(B+\left(C^{\prime}+C^{\prime \prime}\right)\right.$ SPECTRUM by K. Siegbalın and T. R. Gerholm . . . . . . . . . . . . . . . . . 920 APPENDIX VIII. BQ vs kel bỵ T. R. Gerholm . . . . . . . . . 926 ALTHOR INDEX . . . . . . . . . . . . . . . . 933 SUBJECT INDEX. . . . . . . . . . . . . . . . . . 946 


\section{LIST OF C'ONTRIBLTORS}

D. E. Albtrger, Brookhaven National Laboratory, Upton. L.I.. N.Y., U.S.A.

P. R. Bell, Oak Ridge National Laboratory, Oak Ridge, Tenn., U.S.A.

R. E. BeLl, McGill University, Montreal, P.Q., Canada.

S. de Benedetti, Carnegie Inst. of Technology, Pittsburgh, Pa, U.S.A.

I. Bergström, The Nobel Institute of Physics, Stockholm 50, Sweden.

G. Bishop, Clarendon Laboratory, Parks Road, Oxford, England.

R. Burn-Stoyle, Clarendon Laboratory, Parks Road, Oxford, England.

A. BoHr, Institute for Theoretical Physics, Blegdamsvej 15-17, Copenhagen, Denmark.

P. Cavanagh, AERE, Harwell, Didcot, Berks., England.

S. Curran, The University of Glasgow, Glasgow W.2., Great Britain.

C. Davisson, Naval Research Laboratory, Washington 25, D.C., U.S.A.

M. Deutsch, Mass. Inst. of Technology, Cambridge, Mass., U.S.A.

J. W. M. DuMond, California Inst. of Technology, Pasadena, Calif., U.S.A.

N. Feather, University of Edinburgh, Edinburgh, Great Britain.

H. Frauenfelder, University of Illinois, Urbana, Ill., U.S.A.

H. Fulbright, University of Rochester, River Campus, Rochester, N.Y., U.S.A.

T. R. Gerholm, Institute of Physics, University of Uppsala, Uppsala, Sweden.

M. GOEPPERT-MAYER, Institute for Nuclear Studies, University of Chicago, Chicago 37, Ill., U.S.A.

M. GoldhaBer, Brookhaven National Laboratory, Upton, L.I., N.Y., U.S.A.

M. A. Grace, Clarendon Laboratory, Parks Road, Oxford, England.

S. R. DE GRoot, Institute for Theoretical Physics, University of Leiden, Holland.

H. Halban, Clarendon Laboratory, Parks Road, Oxford, England.

R. D. HILL, Univrrsity of Illinois, Urbana, Ill., U.S.A. 
J. H. I. Jexisex. Institute for Theoretical Physics, Heidelberg, Germany.

B. B. Kinsey, ('halk River Laboratory, Ont., Canada.

O. Kofoed-Hanses, Institute for Theoretical Physics, Blegdamsvej 15-17, ('openhagen. Denmark.

E. KonopIsski, Indiana University, Bloomington, Ind., U.S.A.

K. (i. Malyfors. The Nobel Institute of Physics, Stockholm 50, Sweden.

A. ('. ( i. Mitchell. Indiana University, Bloomington, Ind., U.S.A.

S. A. Moszkowski, University of California, Los Angeles 24, Calif., U.S.A.

B. R. Motrelsox, Institute for Theoretical Physics, Blegdamsvej 15-17, Copenhagen, Denmark.

W. PACL, Institute of Physics, University of Bonn, Bonn, Germany.

P. Preisiverk. CERN, Geneva 15, Switzerland.

J. L. Putman, AERE, Harwell, Dideot, Berks., England.

M. k. Rose. Oak Ridge National Laboratory, Oak Ridge, Tenn., U.S.A.

K. Si EG BAHs, Institute of Physics, University of Uppsala, Uppsala, Sweden.

H. Slätis, The Nobel Institute of Physics, Stockholm 50, Sweden.

H. Sternwedel, Institute of Physics, University of Bonn, Bonn, Germany.

A. W. Sexyar, Brookhaven National Laboratory, Upton, L.I., N.Y., U.S.A.

H. A. Tolнozk, Institute for Theoretical Physics, University of Leiden, Leiden, Holland.

R. Wrissox, Clarendon Laboratory, Parks Road, Oxford, England.

C. S. Wu, Columbia University, New York 27, N.Y., U.S.A. 


\section{INTRODUCTION}

$\beta$ - and $\gamma$-ray spectroscopy can be traced back to about forty years ago. Though the existence of $\beta$ - and $\gamma$-radiation emitted from radioactive elements became known very soon after the discovery of radioactivity, spectroscopic work did not begin until 1911. It was found by various methods that the $\beta$-radiation contained both a continuous component and discrete lines. The first type of spectra was for a long time completely unexplained, whereas the origin of line spectra was found in the beginning of 1920 to be due to the atomic core, which interacted with the decaying nucleus. The existence of long "series" of $\beta$-lines, the energy differences of which corresponded to the differences of the atomic shells of the daughter product, led to an interpretation of the $\beta$-lines in a series as photoelectrons expelled by monochromatic $\gamma$-quanta emitted from the nucleus. Later on, when the electron conversion problem was studied theoretically, it was found that this simple description did not hold. Instead conversion must be regarded as an additional mode in which nuclei decay, i.e. the excited state of a nucleus can be de-excited either by the emission of a $\gamma$-quantum or by a direct interaction with the atomic core. in which case a conversion electron from one of the atomic shells is emitted. This view has recently been confirmed by direct experimental proof, since it has been found possible to change the radioactive decay constant in the case of Tc by varying the wave function of the electrons at the nucleus by chemical means or by means of a very high pressure (see Ch. XVI). A two-step photoelectric process would leave the decay rate unaffected by such a change of the external conditions.

The conversion phenomenon has been found extremely useful in the study of the finer details of radioactive decay. Not only do the measurements of the energies of the $\beta$-lines enable one to calculate directly the corresponding energies of the nuclear transitions, which yield fundamental information on the decay scheme concerned, but a closer knowledge of the intensities of the lines also enables one to determine the quantum mechanical properties of the different nuclear states (Ch. XIV, XVI).

In 1934 the first acceptable theory for the continuous spectrum was presented. It was based on the neutrino-hypothesis and was able to account fairly well for the relation between the decay rate and maximum energy of the $\beta$-particles. Furthermore the shape of the spectrum was calculated 
(Ch. IX). The theory was soon extended to take into consideration all possible types of interactions between heavy and light particles in $\beta$-decay (Ch. X). However, the theory contained several ambiguities, which could only be removed by experiments. On the other hand the experimental methods at that time were not developed to such an extent that really fruitful work in nuclear spectroscopy was possible. It is true that some interesting information had been obtained already from the conversion spectra of natural radioactive species, but the conclusions which could be drawn from these measurements did not permit any deeper insight into the decay mechanism.

When artificially produced isotopes became available at the end of the 'thirties and the beginning of the 'forties, the domain of nuclear spectroscopy grew tremendously. Previously, the lack of adequate experimental methods and the difficulties of handling such sources resulted in a number of erroneous conclusions both as to the shapes of the spectra and as to the existence of conversion- and $\gamma$-lines and other features of the decays. During the 'forties great efforts were therefore made to meet several of the most urgent needs by developing instruments with which experimental data of higher quality could be obtained. Magnetic spectrometers of various designs were tried out (Ch. III), which, together with improved coincidence methods (Ch. VII) and more advanced radiochemical procedures, started to yield a lot of interesting data about nuclear disintegration schemes (Ch. VIII, XXII). In the numerous cases where the conversion rates were too low to be detected, the energies of the different nuclear transitions could be measured accurately by means of the photo- and compton electron secondaries, which the unconverted $\gamma$-rays expelled from thin electron converters in the spectrometer. The crystal diffraction method, earlier used for $\mathrm{X}$-ray studies, has been successfully modified to meet the difficulties encountered at short wave lengths (Ch. IV). Unconverted $\gamma$-radiation can be studied at a good resolution by this method, provided that very strong sources are available. Refined proportional counter techniques (Ch. VI) and, in particular, scintillation counter methods (Ch. V, XVIII, XXI) introduced at the end of the 'forties greatly extended experimental possibilities. The high efficiencies for counting $\gamma$-quanta and the quick response of such counters make them extremely useful in all sorts of coincidence experiments. Furthermore the energy distribution of the radiation can be studied by pulse height analyzers. Because of the statistical nature of the registration the resolution is much less than with magnetic spectrometers, being about $10 \%$ at $0.5 \mathrm{MeV}$ and then decreasing as the square root of the energy, so that one gets relative linewidths of the order of $20 \%$ or more 
at energies below $100 \mathrm{keV}^{r}$. Magnetic spectrometers, on the other hand, usually have resolving powers of the order 1 or $2 \%$ (independent of electron momentum) but require very much stronger sources, in particular when unconverted $\gamma$-rays are investigated. Under favourable conditions it is possible to push the resolving power to obtain relative linewidths of $\sim 2: 10^{4}$ (Ch. III). Already at a somewhat more moderate resolution conversion electron spectra of considerable complexity can be largely disentangled by means of magnetic spectrometers. It has been found, however, that most problems in nuclear spectroscopy cannot successfully be tackled by one single instrument or one particular method. Instead the nuclear spectroscopist has in each case to look at the problem from all angles and judge what sort of techniques or even combination of methods could be used. As an example of the latter one may recall that very efficient ways of combining magnetic spectrometers with scintillation counters as detectors and coupled in coincidence arrangements have been tried. In this way one can make use of the good resolution of a magnetic spectrometer and the high $\gamma$-efficiency or the fast response of a scintillation counter.

At higher energies $(>1 \mathrm{MeV})$ one can use the pair process for studying the $\gamma$-radiation, either in conjuction with scintillation counters or with magn tic spectrometers. Convenient coincidence arrangements for the purpcse of discriminating against other processes have in both cases been developed (Ch. XXIV, XXV, XX).

The transition probabilities in $\beta$-decay are governed by selection rules and can be determined experimentally by measuring the partial half-lives of the different branches in the decay. This can be accomplished by means of the so called Fermi analysis (Ch. VIII, IX). One can then calculate the $f t$-values of the transitions, $t$ being the partial half-life and $f$ a certain function of the transition energy and the atomic charge. The $f t$-value is proportional to the inverse square of the nuclear matrix element. Empirically the $f t$-values of various $\beta$-transitions have been grouped into different classes. Using this classification and the particular selection rules which apply to the different classes one may make conclusions regarding the changes in spin, parity and orbital angular momentum associated with the transitions (Ch. XVI).

A fundamental problem in $\beta$-decay with special bearing upon the nature of nuclear forces is the form of the Hamiltonian describing the interaction between heavy and light particles in $\beta$-decay, as was mentioned before in this introduction. This problem is best studied by making careful investigations of the shapes of the continuous spectra of forbidden transitions (Ch. X, XI). The improved experimental methods developed 
during the last years have enabled fairly decisive conclusion.s to be drawn in this respect. It has thus been possible to gradually eliminate a number of possible combinations among the different invariants. At the moment a combination of a scalar and a tensor coupling is sufficient to explain all experimental data. The possible invariants do not all yield the same selection rules. Thus the scalar and vector interactions follow the so called Fermi selection rules, stating that for allowed transitions the spin change is zero and the parity is unchanged, whereas for the tensor and the axial vector interactions the Gamow-Teller selection rules apply, in which case the spin change is either zero or one for an allowed transition, the parity again being unchanged. There is definite experimental evidence for both types of interactions. By comparing the empirically found $f t$ values of superallowed $\beta$-transitions occurring between mirror nuclei having closed shells \pm one nucleon, when the matrix elements can be well estimated by means of single particle wave functions, it can furthermore be concluded that the Fermi and Gamow-Teller couplings contribute about equally (Ch. XI).

The form of the interaction term in $\beta$-decay can also be studied by means of the angular correlation between electron and neutrino (Ch. XII). In this case also allowed transitions in principle yield the desired information. The low energies of the recoiling atoms (of the order of $100 \mathrm{eV}$ ) strongly favour the use of radioactive gases in such experiments. Only a few have been performed. They indicate among other things that a continuous neutrino spectrum is emitted in $\beta$-decay, whereas a neutrino line is emitted in $K$-capture.

The $\mu$-meson decay is of particularly great interest in connection with the interaction in $\beta$-decay. The study of the spectrum of the decay electrons would provide evidence for or against the validity of the postulate that the interaction between the four Fermi particles involved in the $\beta$-decay is a universal one applying to any four fermions (Ch. XI).

The effect of the electronic shell on the decaying nucleus manifests itself in many ways, the two most obvious ones being the electron capture and the aforementioned conversion phenomenon. Since the former varies approximately as $1 / n^{3}$ where $n$ is the principal quantum number, one usually has to consider only $K$-capture. It competes with the process of positron emission and (except for large decay energies) is favoured energetically. This follows from the fact that an electron is captured instead of emitted as in positron emission, which makes electron capture the only possible mode of decay for sufficiently low decay energies. This energy is carried away by a neutrino, the observable effect being the emission of characteristic $\mathrm{X}$ radiation or, alternatively, Auger elec- 
trons following the refilling of the atomic shell. In some cases it is possible to measure the decay energy by determining the upper energy limit of the accompanying weak continuous internal bremsstrahlung spectrum $(\mathrm{Ch} . \mathrm{XX})$. The ratio $K$-capture: positron emission can be determined from the intensity ratio of the $\mathrm{X}$-rays or Auger electrons and positrons or by means of special coincidence experiments (Ch. XX). At a given maximum positron energy this ratio increases with $Z$ and at a given $Z$ it increases with decreasing maximum positron energy (see Ch. IX). The fact that the transition probability through electron-capture, depends on the electron density at the nucleus just as in the case of internal conversion, has made it possible to change the decay constant by chemical means of another radioactive decay apart from Te, namely in the case of $\mathrm{Be}^{7}$.

Internal conversion is closely related to the process of $\gamma$-ray emission. Whereas the transition probabilities in the latter case must by necessity include more or less unknown nuclear matrix elements, these cancel in the calculations of internal conversion coefficients. The radiating nucleus can be looked upon as an oscillating electric and magnetic multipole (Ch. XIII). The contribution of the different multipoles to the radiation field is governed by selection rules. These concern the angular momentum which the quantum carries with it, and the parity. The transition probability decreases very rapidly for higher multipoles. It has so far been possible to measure only a few electric quadrupole or magnetic dipole transition rates, because of the extremely short emission times involved (say $<10^{-9} \mathrm{sec}$ ). Such experiments have been performed by means of the delayed coincidence technique in the millimicrosecond region using scintillation detectors, or by means of the nuclear resonance scattering method (Ch. XVIII). For higher multipole transitions the lifetimes are much longer and they are often classified as due to nuclear isomeric states (Ch. XVI).

It is extremely important for nuclear theory to get the spin and parity changes determined in nuclear decay. Several methods exist; but the most useful one is to measure the internal conversion coefficients. These can be calculated very accurately (Ch. XIV) for different multipole orders, energies and $Z$ values, and the fact that no nuclear matrix elements enter makes the numbers independent of any particular nuclear theory. For the lowest multipole orders the $K$-shell conversion dominates. Experimentally, it is frequently easier to measure the ratio of the conversions in different shells (e.g. the $K: L$ ratio) than the conversion rates themselves. If the conversion lines in the three subshells $L_{1} . L_{\text {II }}$ and $L_{111}$ can be resolved, one can get particularly detailed information about the 
radiation properties. such as the amount of admixture between electric and magnetic components.

For light nuclei and at high energies the conversion rates are low and difficult to determine. In such cases directional correlation studies between successively emitted $\gamma$-quanta have proved to be particularly informative in assigning spin values (Ch. XIX). When polarization correlation studies are possible, information about parity changes is obtained as well. Directional correlation studies between conversion electrons. which are much more difficult to perform. yield both quantities. $\beta \gamma$-directional correlations give information about the type of interaction in $\beta$-clecay theory discussed ahove. Investigations of angular correlations have also opened up other very interesting possibilities. It has been found that the angular correlation may be perturbed, owing to electric and magnetic fields acting on the electric and magnetic moments of the nuclei. These fields may, at least in principle, be either calculated or, in the case of a magnetic field, externally applied. By this means it has become possible to measure magnetic moments and electric quadrupole coupling energies of excited nuclear states. Magnetic moments of excited states have also been measured in a few cases by aligning the nuclei at low tempteratures (Ch. XIX).

Already by the end of the 'forties a largs amount of experimental material about disintegration schemes hac been accumulated. Admittedly this material was very incomplete and in many cases of low accuracy. Spin and parity assignments of many well-established nuclear levels had been made. in particular by the application of the selection rules for $\beta$ - and $\gamma$-transitions (using $f t$-values and $\gamma$-ray intensities) and by means of conversion data. The theoretical background for such considerations was at that time based on great uncertainties. The theory of transition probabilities of multipole radiation was not very far advanced and the calculated conversion coefficients were very approximate except for a few cases. However, by far the most serious difficulty in nuclear spectroscopy was the lack of any useful nuclear model. No really fruitfui oystematic work was therefore possible and there was only a loose connection between the results of experimental investigations and theories about nuclear structure. This picture changed completely with the advancement of the nuclear shell theory (Ch. XV, XVI, XXII), later complemented with the concept of collective nuclear motion (Ch. XVII). In order to explain the existence of the magic numbers of neutrons and protons in nuclei it was assumed that an odd nucleon could move independently in the nucleus in a potential determined by the average field of the remainder. By further assuming a strong spin orbit coupling. which splits each level into two, the further apart the higher the orbital angular momentum, it 
was found possible to get the correct sequence of quantum states in order to obtain the true magic numbers. This simple model met with considerable success in explaining the spins of many odd mass nuclei. Furthermore it accounted very satisfactorily for the phenomenon of nuclear isomerism. When applied to details in decay schemes, it also showed its great potentialities in explaining spin and parities in accordance with experimental findings. In many cases it has been found useful in predicting properties of states that were not measured before. The general"conclusion that can so far be drawn from nuclear spectroscopic work strongly indicates that the shell structure of nuclei is set beyond doubt. It is equally. obvious that the extreme single particle shell aspect of nuclear constitution is not the whole truth but must be regarded as a useful but in many respects rather crude approximation. It is sufficient in this connection to recall the fact that magnetic moments or electric quadrupole moments calculated on the basis of this extreme model are in definite clisagreement with empirical data. Though the general trends, for example among electric quadrupole moments, no doubt exhibit a pronounced correlation with the filling of nuclear shells, the numerical calculations based on the single particle approximation yield results in the regions far from closed shells which may be as much as a factor of twenty too small.

The shell model has therefore been extended in a very essential way in order to account for the abovementioned great deviations from the simple single particle estimates and also for a number of other facts, found in particular in regions far from closed shells. The collective motion of nucleons in the deformed nucleus is likely to contribute to the properties of the nuclear state. This additional degree of freedom in the excitation spectrum gives rise to rotational and vibrational levels in analogy with levels in molecular spectra. Instead of a static nuclear field one has to denl with a dynamic field acting on the single particle. In principle one should consequently consider the coupling between the collective morle of motion and that of the independent particle. Rotational levels in nuclei have been well established by means of coulomb excitation and they have also been identified in spectra from radioactive nuclei. Furthermore there exist a few examples which indicate the expected "band" structure for excited levels in $\gamma$-spectra due to combined particle and collective motion excitation. The "molecular" aspect of nuclear structure is thus a very promising one and will no doubt be the subject of a great deal of further experimental and theoretical work. 


\title{
INTERACTION OF ELECTRONS WITH MATTER
}

\author{
W. PAUL AND H. STEINWEDEL
}

\section{$\S 1$. Introduction}

Electrons penetrating matter lose energy and are deflected from their original course: they are scattered. Changes take place also in the matter that is penetrated, the constituent atoms are excited or ionized, and dissociation of molecules, changes in the lattice structure of crystals, changes in the conductivity, and many other secondary processes have been observed. These phenomena will be discussed here only if they give direct information about the energy of the electrons, as e.g. the ionization. Furthermore, we will restrict the discussion to electron energies in the range $10^{4}-10^{7} \mathrm{eV}$, i.e. to the region of the radio-active beta emitters. For these energies, the deflexion of the electrons is due almost entirely to the elastic collisions with the atomic nuclei, while the energy loss, except that due to the bremsstrahlung, which is practically negligible, results from the interaction with the atomic electrons. Therefore it is possible to treat the two phenomena separately, though of course they always occur together. A detailed review has been given by Bothe ${ }^{1}$ and by Bethe and Ashkin ${ }^{2}$, while the theoretical principles have also been discussed in detail by Sauter ${ }^{3}$. For general discussion of the theory see Mott and Massey ${ }^{4}$.

\section{$\S 2$. Elastic Scattering of Electrons by Atomic Nuclei}

The elastic scattering of electrons passing through matter can be divided roughly into four classes: 1 . Single scattering; 2 . Plural scattering: 3. Multiple scattering; 4 . Diffusion. If the thickness $d$ of the layer is very small, $d \ll 1 / \sigma N$, where $\sigma$ is the cross-section and $N$ the number of scattering atoms per $\mathrm{cm}^{3}$, we have pract:cally only single scattering, so that nearly all the scattered electrons are scattered by only one nucleus. It should be remembered, however, that the cross-section for the scattering of electrons by nuclei decreases very strongly with increasing scattering angle, so that the relation given above for the thickness of the layer shows a pronounced dependence on the angle of scattering $\Theta$. For larger values of the thickness, $d \sim 1 / \sigma N$, we get plural scattering, i.e. the probability that a given scattering angle is due to a number of successive single

1 W. Bothe, Handbuch der Physik, Yol. 22, 2 s. 1, (Berlin 1932).

2 H. A. Bethe and J. Ashkin, Experimental Nuclear Physics (E. Segré Edit.) Vol. I, (New York 1953).

3 F. Sauter in: Kosmische Strahlung (Ed. W. Heisenberg) (Berlin 1953).

4 N. F. Mott, Proc. Roy. Soc. A124, (1929) 425; N. F. Mott-H. S. W. Massey, 'Theory of Atomic Collisions, (Oxford 1948). 
scattering processes becomes appreciable. When the thickness becomes so large that the mean number $n$ of scattering processes becomes larger than about 20 , we speak of multiple scattering. The angular distribution $W(\Theta)$ of the scattered electrons is approximately Gaussian as long as the mean scattering angle is smaller than about $20^{\circ}$. For still larger values of the thickness. $d \gg 1 / \sigma \mathrm{N}$, the angular distribution becomes of the form $W(\Theta) \sim \cos ^{2} \Theta$. The mean angle of scattering $\bar{\Theta}$ then attains its maximum value $\Theta_{\max .} \approx 33^{\circ}$, and remains constant when the thickness increases still further. ("Normal" case, or "complete diffusion"). The thickness for which the normal case is reached is called the "normal thickness" $d_{n}$. Finally, electrons emerge from the foil also on the side of the incident beam. These electrons are either primary electrons which are deflected in the backward direction by single-, plural- or multiple scattering (backscattering or back-diffusion), or secondary electrons which, however, are of no interest here. The number of back-scattered electrons reaches a saturation value for a definite thickness $d_{r}$, the "thickness for saturation back-scattering", or "back-diffusion" thickness.

\section{A. Single Scattering}

The probability that an electron with kinetic energy $E$ is scattered during the passage of a foil of thickness $d$ and atomic number $Z$ through an angle $\Theta$ into the solid angle $d \Omega$ is given by

$$
W(\Theta) d \Omega=d \sigma(E, Z, \Theta) \cdot N \cdot d
$$

where $N$ is the number of scattering atoms per $\mathrm{cm}^{3}$. For the pure Coulomb field of a point charge without shielding we get, according to Mott ${ }^{4}$ :

$$
d \sigma / d \Omega=q_{\text {Mott }}=q_{\text {Ruth. }} R(E, Z, \Theta) .
$$

Here $q_{\text {Ruth. }}$ is the Rutherford cross-section

$$
q_{\text {Ruth. }}=\frac{Z^{2} r_{0}^{2}}{4 \sin ^{4} \frac{1}{2} \theta} \frac{1}{\varepsilon^{2}\left(1-1 / \varepsilon^{2}\right)^{2}}
$$

$\left(r_{0}=e^{2} / m c^{2}=\right.$ classical electron radius $\left.=2.8 \times 10^{-13} \mathrm{~cm}, \varepsilon=\frac{E+m c^{2}}{m c^{2}}\right)$. The factor $R(E, Z, \Theta)$ calculated by Mott cannot be expressed in analytic form. McKinley and Feshbach ${ }^{5}$ expanded this factor in powers of $Z \alpha(\alpha=$ fine structure constant), and the coefficients have been tabulated up to $\left(Z_{\alpha}\right)^{4}$. $R$ is different for electrons and positrons, and this difference increases with increasing energy and increasing angle of scattering and disappears in the non-relativistic limit ( $R=1) . R$ is shown in Fig. 1 for $2 \mathrm{MeV}$ electrons and various scattering angles. The expression given above represents the experimental results well for a large range of energy and scattering angle. However, the following deviations should be noted:

5 W. A. McKinley and H. Feshbach, Phys. Rev. 74, (1948) 1759. 
1. For very small scattering angles the shielding of the Coulomb field of the nucleus by the atomic electrons is no longer negligible. The resulting deviations decrease with increasing energy. They have been calculated by Molière ${ }^{7}$ on the basis of the Thomas-Fermi model, and are shown in Fig. 2, where $\Theta_{0}$ is the "shielding angle" $\Theta_{0}=\frac{Z^{1 / 3} \times 0.47}{p / m c} \sqrt{1.13+3.76(Z \alpha / \beta)^{2}}$ (degree)

2. For high energies and large scattering angles deviations occur because of the finite extension of the nucleus*. According to the recent calculations of Freese and Hain ${ }^{8}$, the resulting deviations are already appreciable for energies of about $1 \mathrm{MeV}$, cf. the measurements of Paul and Reich ${ }^{6}$ in Fig. 1.

3. For high energies we must finally take into account the quantum electrodynamic corrections, which arise from the emission of very soft light quanta which cannot be detected experimentally ${ }^{9}$.

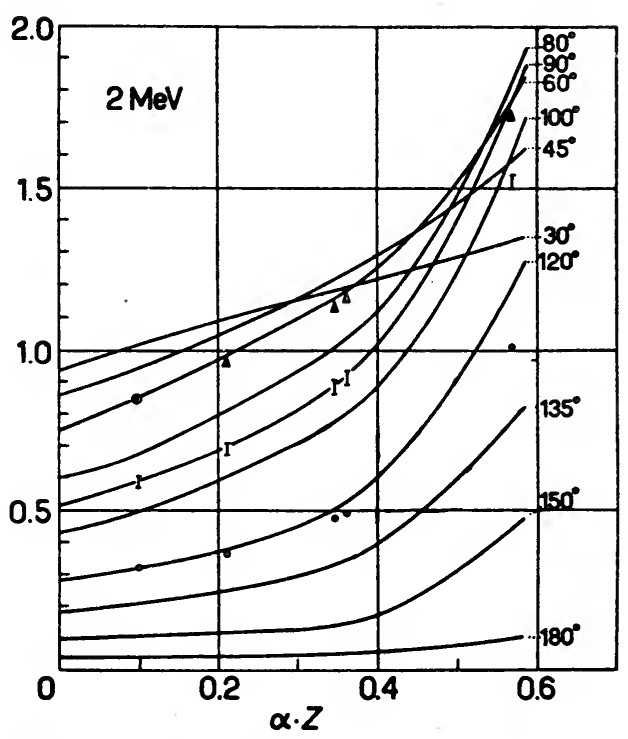

Fig. 1. The ratio $R=q_{\text {Mott }} / q_{\text {Ruth. }}$ as a function of $\alpha \cdot Z$ for various scattering angles and $2 \mathrm{MeV}^{\mathrm{T}}$ electrons. Experimental points from Paul and Reich ${ }^{6}$; the value for Al for $60^{\circ}$ is fitted to the theoretical value

Fig. 2. Influence of the shielding of the Coulomb field by the atomic electrons on the scattering crosssection for various values of the parameter $\gamma=\alpha Z / \beta$; $\Theta_{0}=$ shielding angle; $q^{\prime}=$ cross-section with shielding

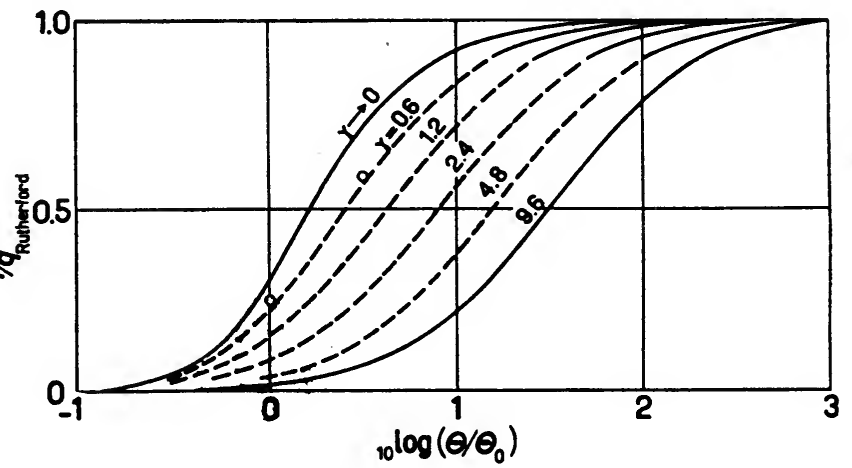

- W. Paul and H. Reich, Z. Phys. 130 (1951).

7 G. Molière, Z. Naturf. 2a, (1947) 133.

8 E. Freese and K. Hain, Z. Naturf., 9a, (1954) 456.

9 J. Schwinger, Phys. Rev., 75, (1949) 898.

* Cf. the experiments at $17 \mathrm{MeV}$ by E. M. Lym ın, A. D. Hanson and M. B. Scott. Phys. Rev. 84 (1951) 626. 


\section{B. Plural Scattering}

Not many quantitative theoretical or experimental results concerning the plural scattering are available. In principle, the plural scattering is contained in Molière's theory of multiple scattering ${ }^{10}$, but the resulting expressions are useful only if the plural scattering gives only a small correction to the multiple scattering.

\section{('. Multiple Scattering}

The multiple scattering has been investigated theoretically by Bothe ${ }^{1}$ and by Williams ${ }^{11}$, and more recently in particular by Molière ${ }^{10}$ and by Snyder and Scott ${ }^{12}$. For small values of the mean angle of scattering $\left(\bar{\Theta}<20^{\circ}\right)$ in the region of multiple scattering (mean number of collisions $n>20$ ), (cf. Fig. 3), Molière obtains relatively simple expressions for the angular

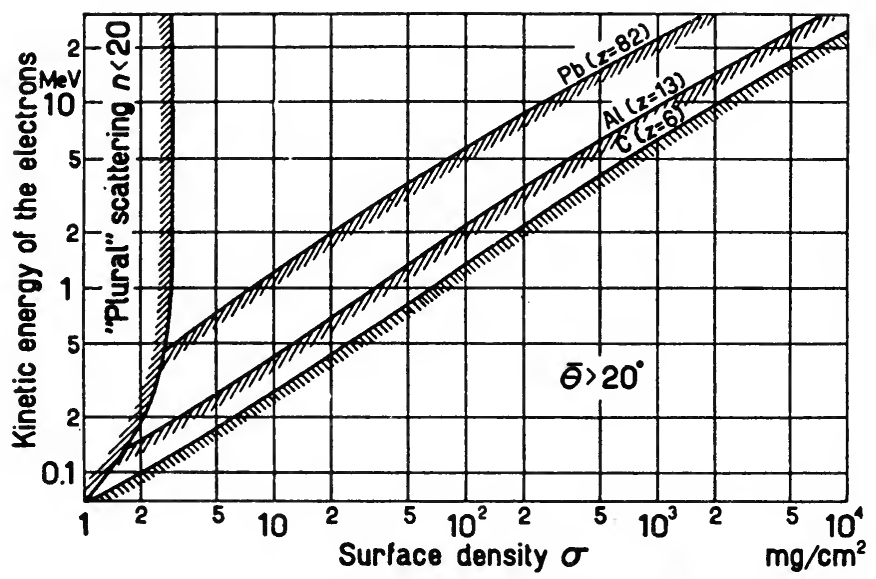

Fig. 3. Approximate range of validity of Molière's theory

distribution, which are very well verified experimentally (cf. below). According to Molière, the probability that an electron passing through a foil of thickness $d$ is scattered through an angle $\Theta$ into the solid angle $d \Omega \approx 2 \pi \Theta d \Theta$ is given by

in which

$$
W(\Theta) 2 \pi \Theta d \Theta=\left[2 e^{-\vartheta^{2}}+F_{1}(\vartheta) / B+F_{2}(\vartheta) / B^{2}+\ldots\right] \vartheta d \vartheta,
$$

$$
\vartheta=\left(\Theta|\chi|^{\prime} \bar{B}\right) ; \quad \chi=\frac{44.8 Z}{\left(\frac{E+m c^{2}}{m c^{2}}-\frac{m c^{2}}{E+m c^{2}}\right)} \mid \overline{\bar{\sigma}} \quad \text { (degree) }
$$

When we project this angular distribution on a plane parallel to the

10 G. Nolière, Z. Naturf., 3a, (1948) 78.

11 E. J. Williams, Proc. Roy. Soe. A169, (1950) 531.

12 H. S. Snyder and W. 'T. Scott, Phys. Rev., 76, (1949) 220. 
direction of the incident electron. we get for the "projected angular distribution" as a function of the projected angle $\varphi$ :

$$
W(\Phi) d \Phi=\left[\pi^{-1 / 2} e^{-\psi^{2}}+f_{1}(\varphi) / B+f_{2}(\varphi) / B^{2}+\ldots\right] d \varphi,
$$

where $\varphi=(\Phi / \chi \mid \bar{B}), Z$ is the atomic number and $A$ the atomic weight of the scattering atoms, $E$ and $m$ are the kinetic energy and the mass of the electron, and $\sigma=$ surface density in $\mathrm{g} / \mathrm{cm}^{2}$ is a measure for the thickness of the foil. $B$ depends slightly on the mean number of collisions. $n$, which according to Molière is given by

$$
{ }^{10} \log n=8.215+{ }^{10} \log \left[\frac{\sigma}{A Z^{2 / 3}} \cdot \frac{\gamma^{2}}{1.13+376 \gamma^{2}}\right],
$$

where $\gamma=\alpha Z / \beta$. $B$ is given in Table $\mathrm{I}$ for various values of $n$.

TABLE I

\begin{tabular}{c|c|c|c|c|c|c|c|c|c}
\hline \hline $10 \log n$ & 1 & 2 & 3 & 4 & 5 & 6 & 7 & 8 & 9 \\
\hline$B$ & 3,36 & 6,29 & 8,93 & 11,49 & 13,99 & 16,46 & 18,90 & 21,32 & 23,71
\end{tabular}

The functions $F_{1}, F_{2}$ and $f_{1}, f_{2}$ have been calculated and tabulated by Molière, cf. Table II.

TABLE II

\begin{tabular}{c|c|c|c|c}
\hline \hline$F_{1}(\vartheta)$ & $F_{2}(\vartheta)$ & $\vartheta, \varphi$ & $f_{1}(\varphi)$ & $f_{2}(\varphi)$ \\
\hline$+0,8456$ & $+2,49$ & 0,0 & $+0,0206$ & $+0,416$ \\
$+0,700$ & $+2,07$ & 0,2 & $-0,0246$ & $+0,299$ \\
$+0,343$ & $+1,05$ & 0,4 & $-0,1336$ & $+0,019$ \\
$-0,073$ & $-0,003$ & 0,6 & $-0,2440$ & $-0,229$ \\
$-0,396$ & $-0,606$ & 0,8 & $-0,2953$ & $-0,292$ \\
$-0,528$ & $-0,636$ & 1 & $-0,2630$ & $-0,174$ \\
$-0,477$ & $-0,305$ & 1,2 & $-0,1622$ & $+0,010$ \\
$-0,318$ & $+0,052$ & 1,4 & $-0,0423$ & $+0,138$ \\
$-0,147$ & $+0,243$ & 1,6 & $+0,0609$ & $+0,146$ \\
0,000 & $+0,238$ & 1,8 & $+0,1274$ & $+0,094$ \\
$+0,080$ & $+0,131$ & 2 & $+0,147$ & +0.045 \\
$+0,106$ & $+0,020$ & 2,2 & $+0,142$ & $-0,049$ \\
$+0,101$ & $-0,046$ & 2,4 & $+0,1225$ & $-0,071$ \\
$+0,082$ & $-0,064$ & 2,6 & $+0,100$ & $-0,064$ \\
$+0,062$ & $-0,055$ & 2,8 & $+0,078$ & $-0,043$ \\
$+0,045$ & $-0,036$ & 3 & $+0,059$ & $-0,024$ \\
$+0,033$ & $-0,019$ & 3,2 & $+0,045$ & $-0,010$ \\
$+0,0206$ & $+0,0052$ & 3,5 & $+0,0316$ & $+0,001$ \\
$+0,0105$ & $+0,0011$ & 4 & $+0,0194$ & $+0,006$ \\
$+3,82 \cdot 10^{-3}$ & $+0,836 \cdot 10^{-3}$ & 5 & $+9,14 \cdot 10^{-3}$ & $+1,98 \cdot 10^{-3}$ \\
$+1,74 \cdot 10^{-3}$ & $+0,345 \cdot 10^{-3}$ & 6 & $+5,06 \cdot 10^{-3}$ & $+0,928 \cdot 10^{-3}$ \\
$+0,91 \cdot 10^{-3}$ & $+0,157 \cdot 10^{-3}$ & 7 & $+3,12 \cdot 10^{-3}$ & $+0,482 \cdot 10^{-3}$ \\
& & & &
\end{tabular}


Thus for not too small values of $B$ the angular distribution of the scattered electrons is essentially a Gaussian distribution; the higher terms are corrections arising from the single- and plural scattering. Finally, the mean value of the scattering angle $\Theta$ is given by

$$
(2 / \tau) \bar{\Theta}=\bar{\Phi}=\chi \sqrt{B}\left\{1+0.982 / B-0.117 / B^{2}+\ldots\right\} .
$$

The multiple scattering has been investigated experimentally by Kulchitsky and Latyshev ${ }^{13}$ for a large number of elements with $2 \mathrm{MeV}$ electrons, and recently by Hanson. Lanzl. Lyman and Scott ${ }^{14}$ for $\mathrm{Be}$ and $\mathrm{Au}$ with 15.7 MeV electrons. Excellent agreement was obtained with the above expressions. In Fig. 4 the experimental results of Hanson et al. are shown.

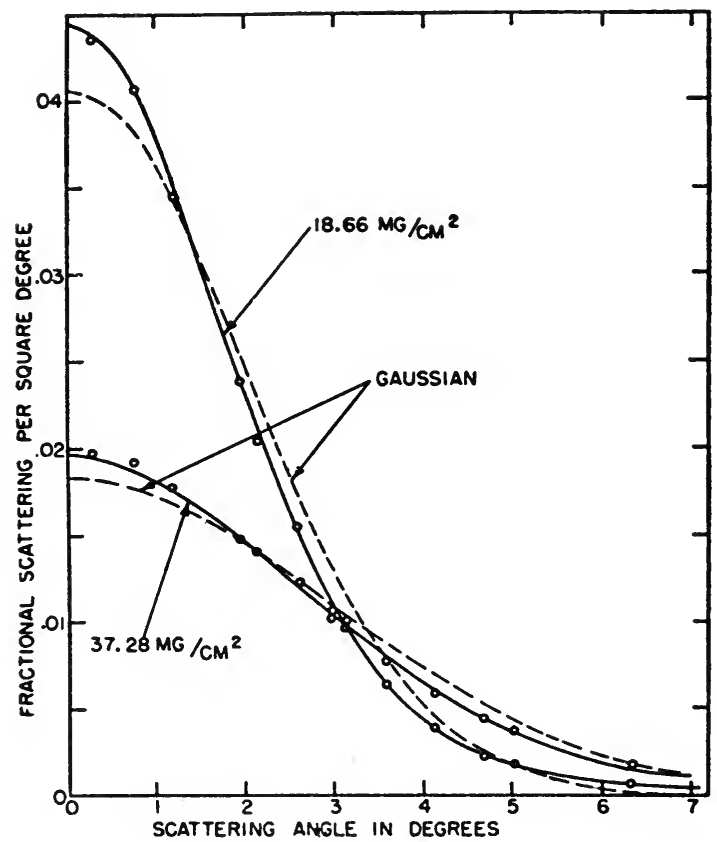

Fig. 4. Angular distribution ${ }^{14}$ for multiple scattering of 15.7 $\mathrm{MeV}$ electrons passing through gold foils $\left(18.66 \mathrm{mg} / \mathrm{cm}^{2}\right.$; $37.28 \mathrm{mg} / \mathrm{cm}^{2}$ )

\section{Diffusion}

Since no detailed theoretical investigations about the diffusion are available, we show the relevant experimental data in Figs. 5-8. In Fig. 5 the normal thickness $d_{n}$ (thickness for which the angular distribution becomes the $\cos ^{2} \Theta$ distribution). and the back-diffusion thickness $d_{r}$ (thickness for saturation back-scattering) are shown as functions of the primary energy for normal incidence of the electrons. In Fig. 6 the back-diffusion

\footnotetext{
13 L. A. Kulchitsky and G. D. Latyshev, Phys. Rev., 61, (1942) 254.

14 A. O. Hanson, L. H. Lanzl, E. M. Lyman and M. B. Scott, Phys. Rev., 84, (1951) 634 .
} 


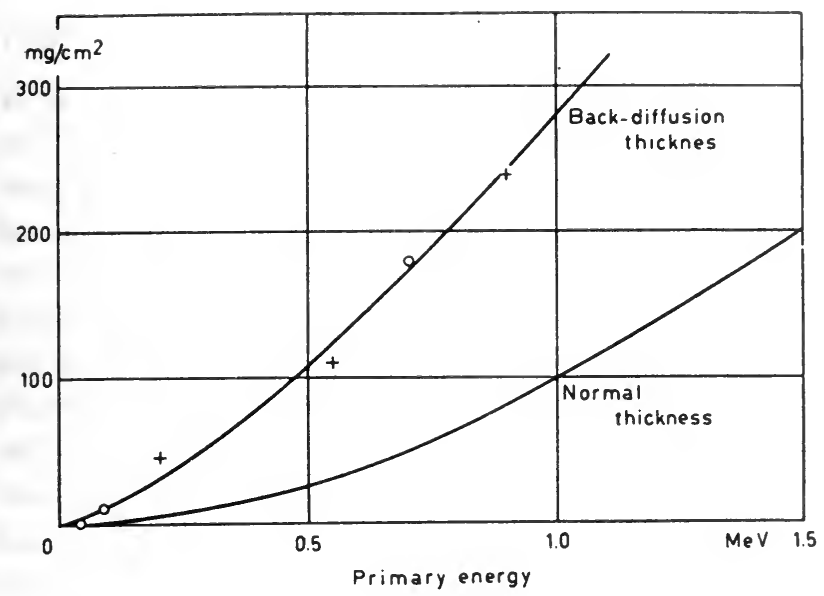

Fig. 5. Normal thickness and hack-diffusion thickness for normal incidence. Experimental points are $\mathrm{for} . \mathrm{Al} 14 a .14 b .20$

Fig. 6. Thickness for saturation backscattering, $d_{r}$, for diffuse angular distribution of the incident electrons, measured with $\mathrm{P}^{32}, \mathrm{Rh}^{106}, \mathrm{~J}^{131}, \mathrm{~S}^{35}$, $\mathrm{Co}^{60}$ and $\mathrm{C}^{14}$. Cf. ref. ${ }^{17}$
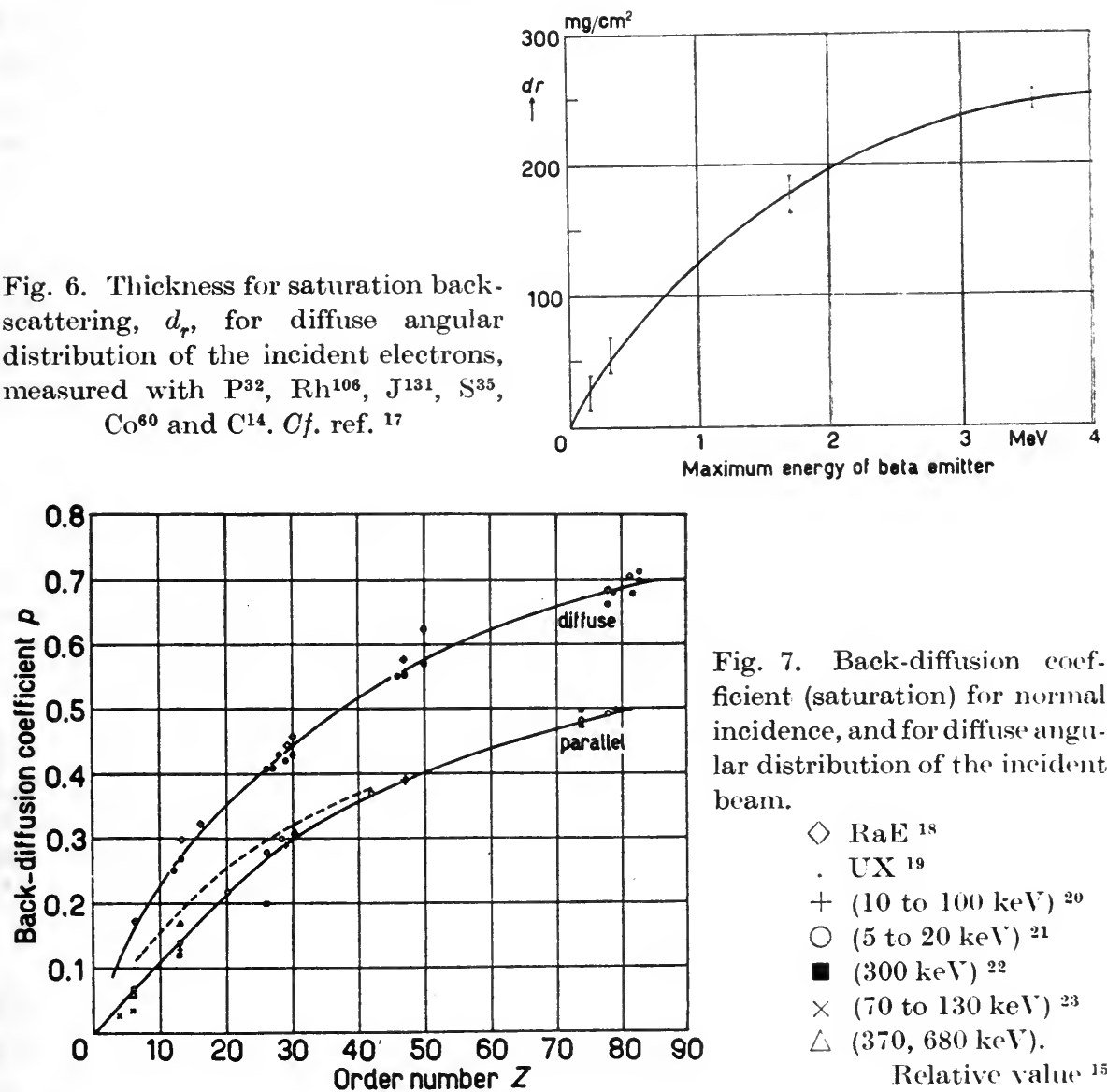

Fig. 7. Back-diffusion coefficient (saturation) for normal incidence, and for diffuse angular distribution of the incident beam.

$$
\begin{aligned}
& \diamond \operatorname{RaE}^{18} \\
& \text { UX } 19 \\
& +(10 \text { to } 100 \mathrm{keV})^{20} \\
& 0(5 \text { to } 20 \mathrm{keV})^{21} \\
& \mathbf{0}(300 \mathrm{keV})^{22} \\
& \times(70 \text { to } 130 \mathrm{keV})^{23} \\
& \triangle(370,680 \mathrm{keV}) .
\end{aligned}
$$

Relative value 15

14 W. Wilson, Proc. Roy. Soc. A87, (1912) 100, 310.

$14 b$ P. Lenard. Quantitatives über Kathodenstrahlen (Heidelberg 192s).

15 W. Botlie, Z. Naturf., 4a, (1949) it?. 
thickness for a diffuse angular distribution of the primary electrons is shown as a function of the maximum energy of the beta-spectrum. In Fig. 7 the back-scattering coefficient $p$ (= number of back-scattered electrons/number of incident primary electrons at saturation) is shown as a function of the atomic number both for parallel and for diffuse incidence.

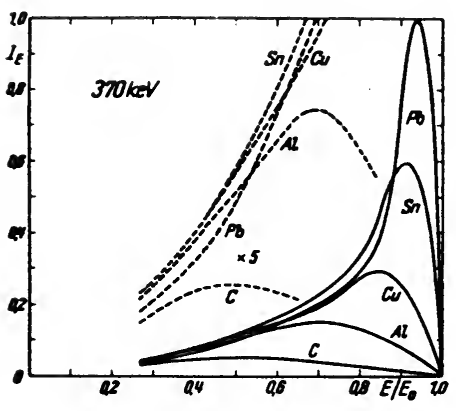

Fig. 8

Finally. in Fig. 8, the experimental data of Bothe ${ }^{15}$ on the energy distribution of the back-scattered electrons are shown. Bothe's theory ${ }^{15}$ shows that $p$ is independent of the primary energy. The back-scattering coefficient for positrons is different from that for electrons ${ }^{16}$, and this difference between electrons and positrons is due to the fact that for large scattering angles the single scattering is different for electrons and positrons (cf. above). We have $p^{-} / p^{+}=1.3$, independent of $Z$ (measured from $\mathrm{Be}$ to $\mathrm{Pb}$ ).

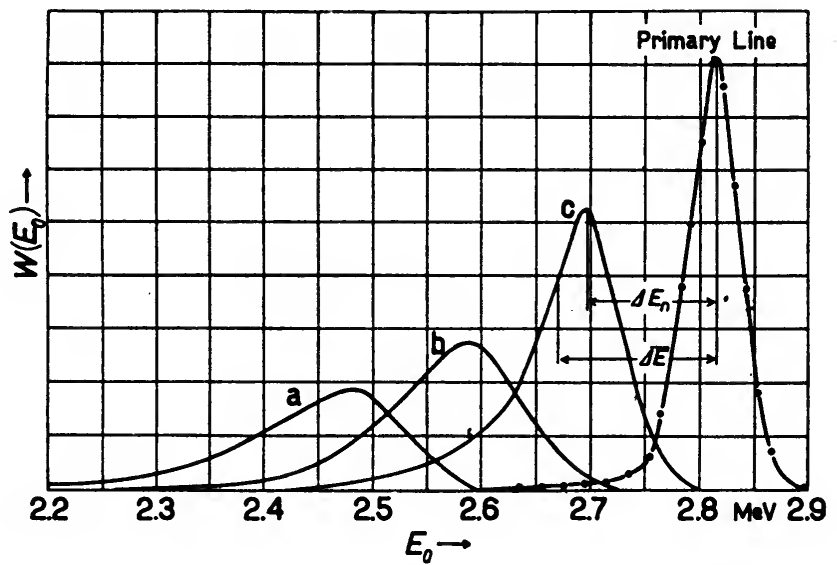

Fig. 9. Energy distribution of electrons passing through graphite according to measurements of Knop and Paul. Foil thickness for curve a $1.33 \mathrm{~mm}$; b $0.895 \mathrm{~mm}$; c $0.475 \mathrm{~mm}$

16 H. H. Seliger, Phys. Rev., 78, (1950) 491.

17 L. Yaffe and K. Justus, J. Chem. Soc., 5, (1949) 341.

18 A. F. Kovaric, Phil. Mag., 20, (1910) 849.

19 H. W. Schmidt, Ann. Phys., 23, (1907) 677.

20 B. F. J. Schonland, Proc. Roy. Soc. A108, (1925) 187.

21 P. Palluel, C. R., 224, (1947) 1492, 1551.

22 .J. G. 'Trump and R. J. van de Graaff, Phys. Rev., 75, (1949) 44.

23 H. V. Neher, Phys. Rev., 37, (1931) 655. 


\section{\$3. Energy Loss}

When electrons of a definite energy pass through a foil of matter, one observes that their energy is decreased, as is shown e.g. in Fig. 9. The energy spectrum becomes broader and unsymmetrical. Thus a well-defined energy loss does not exist. one can define only a mean energy loss $\overline{\Delta E}$, or a probable energy loss, $\Delta E_{w}$ which is equal to the maximum of the energy distribution curve. This energy loss is due to the inelastic collisions of the electrons with the atomic electrons, by which the atoms are excited or ionized, and to the emission of bremsstrahlung in the Coulomb field of the nucleus.

\section{A. Energy Loss Due to Inelastic Collisions}

The interaction of the incident electrons with the atomic electrons in the foil is characterized by the fact that the energy transferred to the atoms per collision is very small. Even for very high primary energies excitation is more probable than ionization. and the resulting secondary electrons have a mean kinetic energy of only a few el. The total energy loss after passage through a foil of thickness $x$ is therefore the result of a very large number of small energy losses. The theory was developed mainly by Bohr, Bethe and Bloch; it gives a very accurate description of the phenomena. For relatively small energies, the mean energy loss per $\mathrm{cm}$ path is given by

$$
-\frac{\overline{d E}}{d x}=\frac{4 \pi e^{4} N Z}{m v^{2}} \ln \frac{1.16 m v^{2}}{2 I} \quad ; \quad\left(\beta=\frac{v}{c}<0.5\right)
$$

$N$ is the number of atoms per $\mathrm{cm}^{3}, N=L \varrho / A$. where $L$ is the Loschmidt's number, $\varrho$ the density and $A$ the atomic weight. $I$ is the mean excitation energy of the atomic electrons. Numerically we get

$$
-\left(\overline{d E / d x)}=0.306 \varrho(Z / A) \beta^{-2} \cdot \ln (1,16 E / I) . \quad[\mathrm{MeV} / \mathrm{cm}]\right.
$$

In the relativistic region. this Bethe-Bloch expression for the energy loss becomes

$$
\begin{aligned}
& -\frac{\overline{d E}}{d x}=0.153 \cdot \varrho \frac{Z}{A} \beta^{-2}\left[\ln \frac{E\left(E+m c^{2}\right)^{2} \beta^{2}}{2 I^{2} m c^{2}}+\left(1-\beta^{2}\right)+\right. \\
& \left.\left.\quad-\left(2 \sqrt{1-\beta^{2}}-1+\beta^{2}\right) \ln 2+\frac{1}{8}\left(1-/ \overline{1-\beta^{2}}\right)^{2}-\Delta_{\mathrm{pol}}\right], \quad[\mathrm{MeV} / \mathrm{cm}] .\right)
\end{aligned}
$$

Since $Z / A$ is approximately constant, $\overline{d E / d x}$ depends practically only on the density of the matter that is penetrated. A very slight dependence on $Z$ occurs only through the excitation energy $I$ appearing in the logarithm. Bloch assumed that $I$ is proportional to $Z$, i.e. $I=K Z$, but this proportionality does not hold rigorously. From the very accurate measure- 
ments of R. R. Wilson ${ }^{24}$ on the practical ionization curve for protons in air and aluminum, the quantity $I$ has been determined with great accuracy. Other elements were investigated by Bakker and Segré ${ }^{25}$. The values of $I$ obtained in this way are given in Table III. The mean differential energy loss per $\mathrm{cm}$ path, calculated for aluminum and lead with the help of these data, is shown in Fig. 10. The energy loss at first falls sharply with increasing energy, reaches a minimum at $E \sim 1.5 \mathrm{MeV}$, and then increases very slowly logarithmically. When the density of the material is high, this increase is reduced because of the polarizability

TABLE III

Mean Excitation Potential I (eV)

\begin{tabular}{c|c|c|c|c|c|c|c|c|c|c|c|c}
\hline & $\mathrm{H}_{2}$ & $\mathrm{Li}$ & $\mathrm{Be}$ & $\mathrm{C}$ & $\mathrm{Al}$ & $\mathrm{Fe}$ & $\mathrm{Cu}$ & $\mathrm{Ag}$ & $\mathrm{Sn}$ & $\mathrm{W}$ & $\mathrm{Pb}$ & $\mathrm{U}$ \\
\hline$I$ & 15.6 & 34.0 & 60.4 & 76.4 & 150 & 243 & 279 & 422 & 472 & 630 & 737 & 853
\end{tabular}

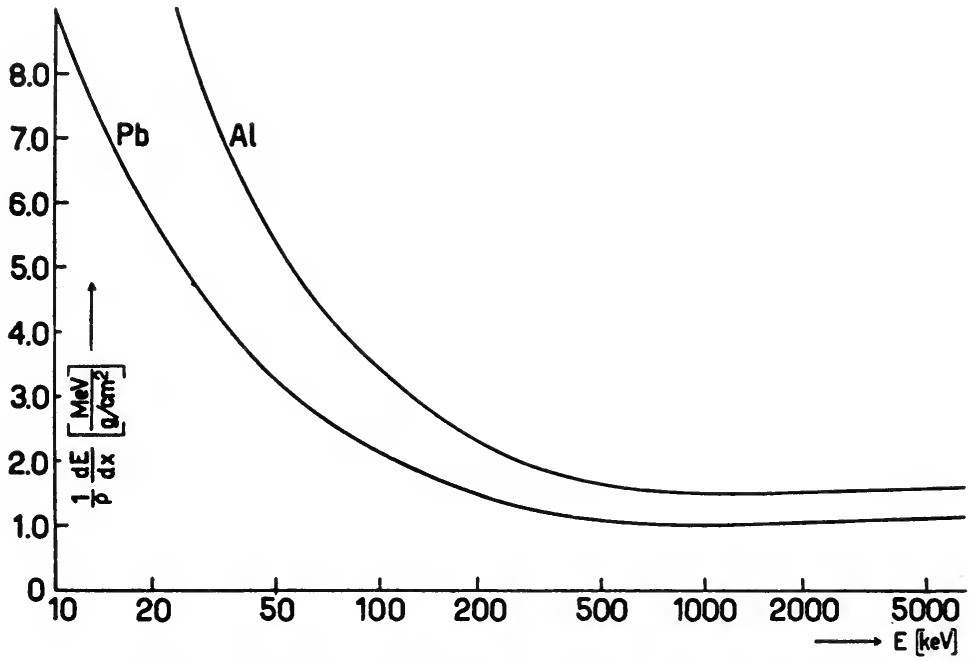

Fig. 10. Mean energy loss in aluminum and lead

of the material by which the Coulomb field of the incident electron is reduced. In Eq. (3) this effect is represented by the term $\Delta_{\text {pol }}$. The importance of this effect was first pointed out by Fermi, and numerical calculations were carried out by Halpern and Hall ${ }^{26}$ and by Sternheimer ${ }^{27}$. At $10 \mathrm{MeV}$ its contribution is a few percent.

\footnotetext{
24 R. R. Wilson, Phys. Rev., 66, (1941) 749.

25 C. Bakker and E. Segré, Phys. Rev., 81, (1951) 489.

26 O. Halpern and H. Hall, Phys. Rev., 73, (1948) 477.

27 R. M. Sternheimer, Phys. Rev. 88, (1952) 851.
} 
The experimental determination of $\overline{\Delta E / \Delta x}$ is particularly difficult for small energies, since, because of the multiple scattering. the true path length is not equal to the thickness of the foil. Because of this, most of the earlier results are $10-20 \%$ too high. The measurements of Williams ${ }^{28}$ in gases showed very good agreement with the Bethe formula. More recent measurements at higher energies. i.e. in the region of the relativistic increase, by Hanson, Goldwasser and Mills ${ }^{29}\left(E=17 \mathrm{MeV}^{r}\right)$ and by Paul, Reich and Knop ${ }^{30}$ at 2, 3 and $4 \mathrm{MeV}$ were in excellent agreement with the theory.

The experimentally determined quantity usually is the probable energy loss $\left(\overline{\Delta E_{v o} / x}\right)$. In the energy region considered here it is about $\frac{1}{3}$ less than $(\overline{\Delta E / \Delta x})$. Also here, the theory (Landau ${ }^{31}$. Blunck and Leisegang ${ }^{32}$, Williams ${ }^{11}$ ) is in excellent agreement with the experimental results. The expression for $\Delta E_{w}$ is

$$
\Delta E_{w}=a x\left[\ln \frac{m v^{2} a x}{I^{2}\left(1-\beta^{2}\right)}-\beta^{2}+K-\Delta_{\mathrm{pol}}\right], \quad\left[\mathrm{MeV}^{\prime}\right]
$$

where according to Landau $K=1.12, a=\left(2 \pi e^{4} N Z / m v^{2}\right)=0.153\left(\varrho Z / A \beta^{2}\right)$. The probable energy loss thus increases somewhat more than linearly with increasing thickness $x$, cf. e.g. Fig. 11 . To facilitate the comparison

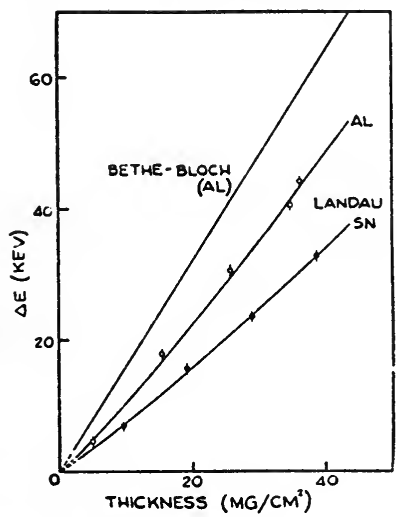

Fig. 11. Probable energy loss as a function of foil thickness for $\mathrm{Al}$ and $\mathrm{Sn}$ at a primary energy of $624 \mathrm{keV}$ according to Warshaw and Chen (Phys. Rev. 80 (1950) 97)

of (4) with the experimental results, we write (4) in the following form, $\Delta E_{w} / x=a \ln \left(x / x_{0}\right)+S, \quad$ where $S=a\left[\ln \frac{m v^{2} a x_{0}}{I^{2}\left(1-\beta^{2}\right)}-\beta^{2}+K-\Delta_{\mathrm{pol}}\right]$, $S$ is called the reduced stopping power. In Fig. 12 this quantity is compared

28 E. J. Williams, Proc. Roy. Soc., A135, (1932) 108.

29 A. O. Hanson, E. L. Goldwasser and F. S. Mills, Phys. Rev., 86, (1952) 617.

30 W. Paul and H. Reich, Z. Phys., 127, (1950) 429; W. Paul and G. Knop, Z. Naturf. to appear.

31 L. Landau, J. Phys. USSR 8, (1944) 201.

32 O. Blunck and S. Leisegang, Z. Physik, 128, (1950) 500. 
with the experimental data; the deviations are seen to be only a few percent.

It is noteworthy that the probable energy loss does not show the relativistic increase. This increase is exactly compensated by the polarization effect. For energies above $2 \mathrm{MeV}$ we get

$$
\Delta E_{u} \mid x=0.153 \varrho(Z / A)\left[\ln \left(x / x_{0}\right)+19.45\right],[\mathrm{MeV}],\left(x_{0}=1 \mathrm{~cm}\right) .
$$

Also in this energy region, the theoretical formula is in excellent agreement with the experimental results, as has been shown by Hanson et al. ${ }^{29}$ and by Paul and Knop ${ }^{30}$.

The energy spectrum of mono-energetic electrons passing through a foil has been investigated experimentally by White and Millington ${ }^{35}$,

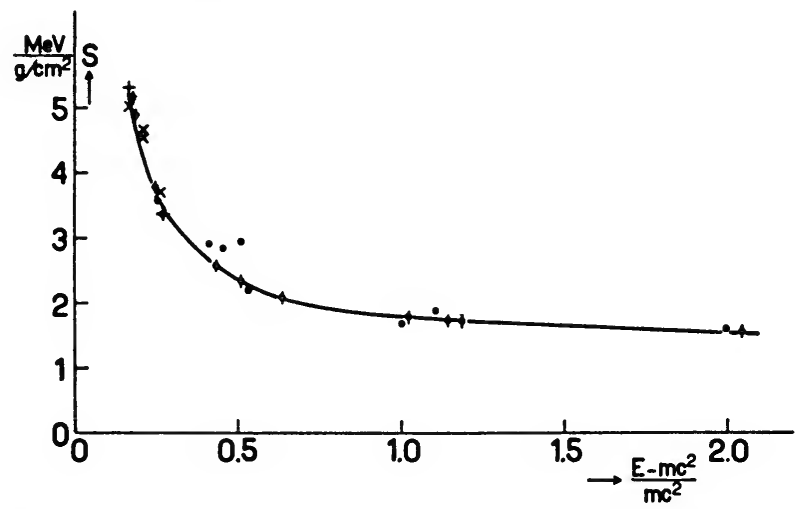

Fig. 12. Reduced stopping power $S$ in aluminium according to Chen and Warshaw, Phys. Rev. 84 (1951) 361. The solid curve represents Landau's theoretical curve

Paul and Reich ${ }^{30}$, Chen and Warshaw ${ }^{36}$ and by Hanson ${ }^{29}$. A theoretical treatment of the problem has been given by Landau. According to this theory of Landau, the half-width $\Gamma$ is given by

$$
\Gamma=3.98 \times a x=0.61 \varrho(Z / A) \beta^{-2} \cdot x, \quad[\mathrm{MeV}] .
$$

The inclusion of higher approximations, calculated by Blunck and Leisegang, gives somewhat higher values. When we compare (6) with (4) and (5), we see that the half-width is about $20-25 \%$ of the probable energy loss.

\section{B. Energy Loss Due to Bremsstrahlung}

In addition to the energy loss resulting from the excitation and ionization processes discussed above, there is an energy loss due to the emission of

35 P. White and G. Millington, Proc. Roy. Soc., A120, (1928) 701.

36 .J. J. L. Chen and S. D. Warshaw, Phys. Rev., 58, (1940) 243; 84, (1951) 361. 
bremsstrahlung, which occurs when the electron is accelerated in the Coulomb field of a nucleus. According to Heitler ${ }^{37}$, the mean energy loss per $\mathrm{cm}$ path due to this radiation is given by

$$
\left.\begin{array}{rl}
\left(\frac{\overline{d E}}{d x}\right)_{\mathrm{rad} .} & =N r_{0}^{2} \frac{Z^{2}}{137}\left(E+m c^{2}\right)\left[4 \ln \frac{2\left(E+m c^{2}\right)}{m c^{2}}-\frac{4}{3}\right]= \\
& =3.44 \times 10^{-4}\left(E+m c^{2}\right) \frac{Z^{2}}{A} \varrho \cdot[4 \ln \ldots] .
\end{array}\right\}
$$

This energy loss thus increases quadratically with increasing atomic number, while the energy loss due to ionization is proportional to $Z$. For relatively small energies, $E<m c^{2}$, the loss is independent of the kinetic energy of the electrons, above $m c^{2}$ it increases proportionally to $E$, while the loss due to ionization at first decreases sharply, and increases only logarithmically in the relativistic region. From Eqs. (7) and (3) we get for the ratio of the two effects in the relativistic region the approximation of Bethe and Heitler:

$$
\left(\frac{\overline{d E}}{d x}\right)_{\mathrm{rad} .} /\left(\frac{d E}{d x}\right)_{\mathrm{coll} .}=\frac{\left(E+m c^{2}\right) Z}{1600 m c^{2}} .
$$

The effects are equal for a critical energy $E_{c r} \approx 1600 m c^{2} / Z$. For lead the exact calculation gives $E_{c r}=6.9 \mathrm{MeV}$, and for aluminum $E_{c r}=47 \mathrm{MeV}$. For aluminum at an energy of $10 \mathrm{MeV}$ the loss due to radiation is about $16 \%$ of the loss due to the collisions with the atoms. Below $1 \mathrm{MeV}$ we may therefore neglect the radiation loss completely. It should be noted that the spread of the energy around the mean value $\Delta E$ is much larger for the radiation than for the ionisation loss. This is due to the fact that it is equally probable that for a given path length an electron loses a definite amount of energy in a single collision or in a large number of collisions. For a detailed discussion of the bremsstrahlung we refer to Heitler ${ }^{37}$ and Bethe ${ }^{2}$.

\section{Ionisation}

As shown in part A of this section, the total energy loss of an electron passing through a foil is made up of a large number of small energy losses due to excitation and ionisation of the atoms. It has been shown experimentally that for a large range of electron energies, at least from $5 \mathrm{keV}$ to $17 \mathrm{MeV}$, the energy $\varepsilon$ required to form an ion in a gas is independent of the energy of the primary electron and is only slightly different for the different elements. Some experimental results are given in Table IV. Because of this, the quantity $\varepsilon^{-1}(\overline{d E / d x})_{\text {coll. }}$ is equal to the mean number of ions formed per $\mathrm{cm}$ path. The energy dependence of this quantity, called

37 W. Heitler, Quantum Theory of Radiation (Oxford 1948). 
'TABILE IV

\begin{tabular}{|c|c|c|c|c|c|c|c|c|c|c|c|}
\hline Literature & Energy & $\mathrm{H}_{2}$ & $\mathrm{He}$ & Ne & A & $\mathbf{K r}$ & $\mathrm{Xe}$ & $\mathbf{N}_{2}$ & $\mathrm{O}_{2}$ & Air & $\mathrm{CO}_{2}$ \\
\hline 38 & $5.7 \mathrm{KeV}$ & & & & 28.5 & & & 32 & 28.8 & 31.0 & 33.5 \\
\hline $39,40,41$ & $10-1000$ & 36 & 27.8 & 27.4 & 2.5 .4 & 2.2 .8 & 20.8 & 35.3 & 32.0 & 32.3 & 31 \\
\hline 42 & 25600 & 37.2 & 26 & & 28 & & & & & 31.2 & \\
\hline 43 & & 38 & 32.5 & & 27.0 & & & & & 35 & \\
\hline
\end{tabular}

the specific (total) ionization, is therefore also given by Bethe's formula. Since the specific total ionization is not only due to the collisions of the primary electrons but also to those of the secondary electrons, we must distinguish between the specific and the spec. primary ionisation. These two quantities differ by a factor 2 to 3 , which means that on the average a secondary electron gets enough kinetic energy to form 1 to 2 secondary ions. These ions are formed in the immediate vicinity of the primary ion. The ions are therefore formed in small clusters the number of which per $\mathrm{cm}$ path is approximately equal to the specific primary ionization. The total ionization is defined as the total number of ions formed by an electron and its secondary electrons, and is equal to $N_{i}=E / \varepsilon$. Conversely, one can

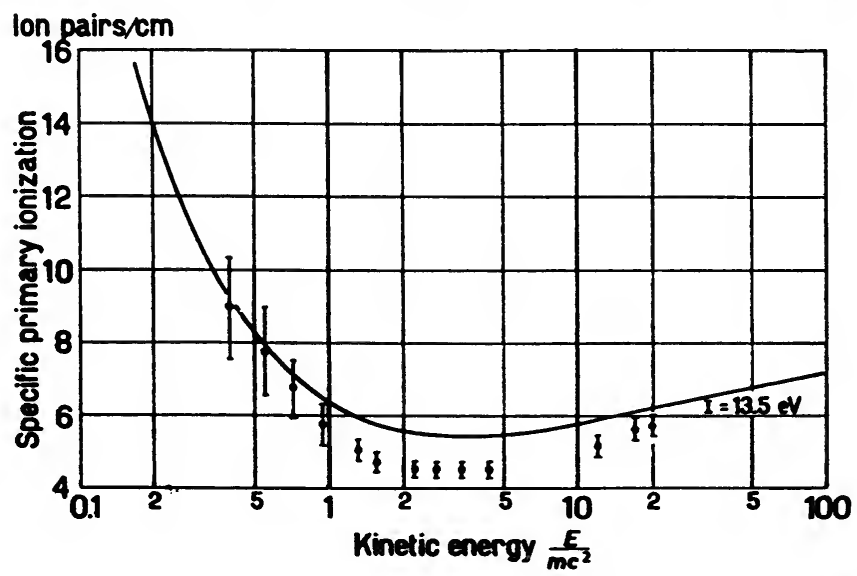

Fig. 13. Specific primary ionization according to Hereford ${ }^{23}$ Solid line Bethe's theory

38 S. C. Curran, A. L. Cockeroft and S. M. Insch, Phil. Mag., 41, (1950) 517.

39 E. Rutherford, J. Chadwick and C. Ellis, Radiation from Radioactive Substances (Cambridge 1930).

10 H. H. Compton - S. K. Allison, X-Rays in Theory and Experiment (London 1936).

41 W. Gerbes, Ann. Physik, 23, (1935) 648.

12 R. H. Frost and C. E. Nelson, Phys. Rev., 91, (1953) 864.

43 J. M. Valentine and S. C. Curran, Phil. Mag. 43, (1952) 964. 
TABLE V

SPECIFIC PRIMARY IONIZATION IN $\mathrm{H}_{2}$ AND $\mathrm{O}_{2}$, AND TOtal IONIZATION IN AIR FOR VARIOUS ENERGIES AND A PRESSURE OF $760 \mathrm{~mm} \mathrm{Hg}$.

From Klemperer: Einführung in die Elektronik (Berlin 1933) p. 159; and Curran and Craggs: Counting Tubes (London 1949) p. 16

\begin{tabular}{|c|c|c|c|c|}
\hline 9 & $\begin{array}{c}\text { Energy } \\
\mathrm{MeV}\end{array}$ & $\begin{array}{c}\mathrm{Nu} \\
\text { prir } \\
\text { pairs } \\
\mathrm{H}_{2} \\
\end{array}$ & $\begin{array}{c}r \text { of } \\
y \text { ion } \\
\text { path } \\
\mathrm{O}_{2} \\
\end{array}$ & $\begin{array}{l}\text { Total number } \\
\text { of ion pairs/ } \\
\text { cm path air }\end{array}$ \\
\hline & 0,001 & - & - & 2500 \\
\hline & 0,005 & - & - & 1700 \\
\hline & 0,010 & - & - & 1030 \\
\hline & 0,020 & - & - & 440 \\
\hline & 0,028 & - & 96,0 & - \\
\hline & 0,030 & - & - & 320 \\
\hline & 0,050 & - & - & 280 \\
\hline & 0,062 & 18,3 & - & 200 \\
\hline & 0,094 & 12,6 & 43,0 & 110 \\
\hline & 0,100 & - & - & 152 \\
\hline & 0,169 & 8,9 & 34,0 & 110 \\
\hline & 0,246 & 7,6 & 28,4 & 87 \\
\hline & 0,300 & - & - & 68 \\
\hline & 0,444 & 7,1 & 27,0 & 64 \\
\hline & 0,565 & 6,4 & - & 58 \\
\hline & 0,792 & 6,1 & 25,2 & 54 \\
\hline & 1,125 & 5,1 & - & 51 \\
\hline & 1,663 & - & 22,2 & 46 \\
\hline
\end{tabular}

determine the kinetic energy of the primary electron by measuring the number of ions. In Table $\mathrm{V}$ the experimentally determined number of primary ions and the total number of ions per $\mathrm{cm}$ path are given for a large range of energies. The specific ionization is usually measured with a proportional counter, or by counting the number of droplets in a slow cloud chamber. When one counts the number of droplet clusters before the droplets begin to diffuse, one gets the specific primary ionization (Wilson ${ }^{44}$ ). Hereford ${ }^{33}$ measured this quantity by means of the pressure dependence of the efficiency of a counting tube. His results are shown in Fig. 13. Frost and Nelson ${ }^{42}$ recently measured the specific total ionization in various gases in the energy range $0.25-0.8 \mathrm{MeV}$. Their results are in excellent agreement with the expression (2), (3) of Bethe for the energy dependence. To the minimum energy predicted by this equation there corresponds a minimum ionisation density. which is reached at an energ. of about $1 \mathrm{MeV}$. For a number of gases this minimum ionisation is given in Table VI.

44 C. T. R. Wilson, Proc. Roy. Soc. A104, (1923) 192. 
'T.ABLA VI

\begin{tabular}{l|c|c|c|c|c}
\hline & Air & He & Ar & $\mathrm{H}_{2}$ & $\left(p=760 \mathrm{~mm} ; 0^{\circ}\right.$ Cels $)$ \\
\hline ions minimum & 46.1 & 8.13 & 53.1 & 6.48 & 42 \\
cm & \pm 2.2 & \pm 0.51 & \pm 2.8 & \pm 0.34 &. \\
,$\quad E=17.5 \mathrm{MeV}$ & 64 & & 101 & 8.4 & 43
\end{tabular}

The increase of the ionization that corresponds to the increase of the energy loss in the relativistic region has been observed at first by Corson and Brode ${ }^{46}$ in a cloud chamber. Recent measurements of Ovadia and Laughlin ${ }^{45}$ at 9 and $17.5 \mathrm{MeV}$ showed an increase of $5 \%$ in this region. The values for 17.5 MeV are given in Table VI.

\section{Range of Electrons. Determination of Energy by Absorption Measurements}

When we consider the range of electrons in matter we must distinguish the following two cases:

1) The true length of the track. This can be measured only in a photographic plate or, for gases, in a cloud-chamber. As an example we show in Fig. 14 the results of $\mathrm{O}^{\prime} \mathrm{Neill}$ and $\mathrm{Scott}^{47}$ for the energy range $0.3-1 \mathrm{MeV}$.

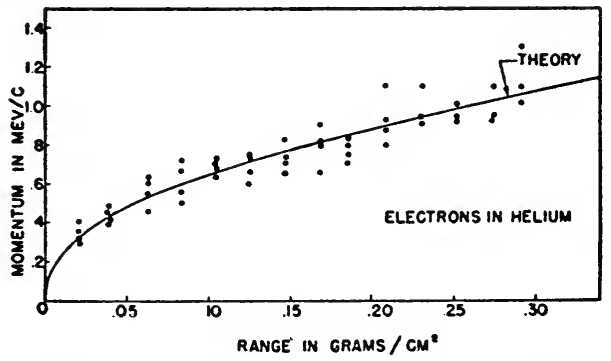

Fig. 14. Momentum-range relation between 0.3 and $1.1 \mathrm{MeV} / \mathrm{c}(=81$ and $700 \mathrm{keV}$ energy) in helium according to O'Neill and Scott (Phys. Rev. 80 (1950) 473)

The solid curve represents the lengths $R=\int_{0}^{E} d E /(\overline{d E / d x})$ calculated according to Møller, Rossi and Greisen. A collection of all available experimental data can be found in Landolt-Börnstein ${ }^{48}$.

2) On the other hand, when one measures the absorption of monoenergetic electrons passing through matter, an aluminum foil for example, i.e. when one measures the ratio of the number of transmitted electrons to the number of incident electrons, one obtains curves like those shown in

t5 J. Ovadia and J. S. Laughlin, Phys. Rev., 88, (1952) 165.

t6 D. R. Corson and R. B. Brode, Phys. Rev. 53, (1938) 773.

17 (:. F. O'Neill and W. T. Scott, Phys. Rev. 80, (1950) 473.

18 Landolt-Börnstein, Vol. I. 5, (Berlin 1952). 
Fig. 15, which correspond to various primary energies. This ratio first decreases slowly with increasing thickness of the foil, then decreases linearly, and finally merges into the background intensity. The end of the absorption curve, corrected for the background intensity, is called the

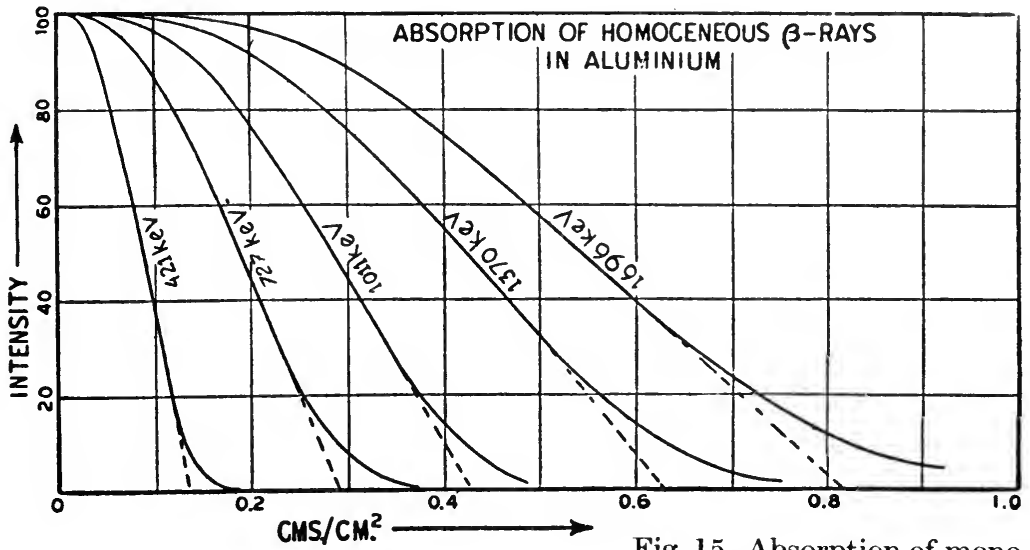

Fig. 15. Absorption of monochromatic electrons in alumi.

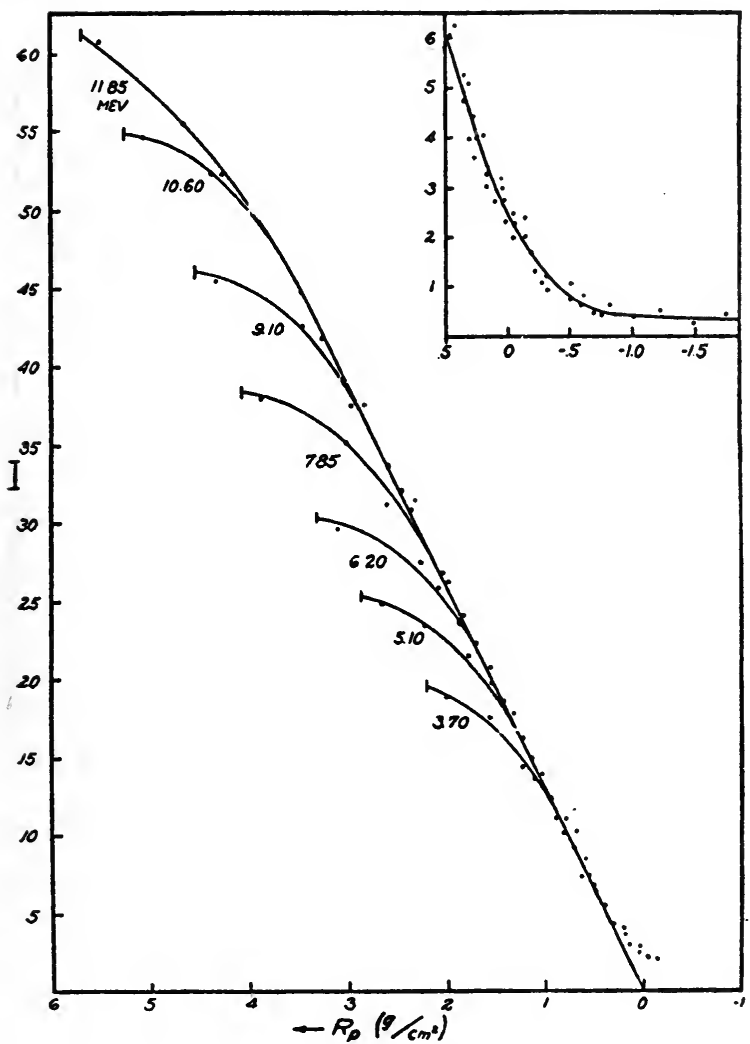
nium. From J. Marshall and A. G. Ward, Can. J. Research, A15 (1937) 39

Fig. 16. Aluminium absorption curves for various electron energies. The curves are normalized at the practical range $R_{p}$. Intensity units are -arbitrary. The insert shows the end point region on an expanded scale. From Hereford and Swann (Phys. Rev. 78 (1950) 727) 
true range. Because of the strong scattering of the very slow electrons, this range is shorter than the length of the electron path. It is evident that a unique determination of the end of the curve is not well possible. Therefore one extrapolates (Varder ${ }^{49}$ ) the linear part of the curve to zero, and the intersection with the axis is called the practical range $R_{p}$. As shown in Fig. 16. the absorption curves for different energies have the same form, and this justifies the extrapolation procedure. The initial part of the absorption curve depends on the geometry of the measuring apparatus, but the linear part is practically independent of the experimental arrangement. This is due to the fact that for thick foils there is complete diffusion, so that the electrons leave the foil without any preferred direction. The relation between the practical range and the electron energy is shown in Fig. 17 for energies between 0 and $12 \mathrm{MeV}$. Above $0.6 \mathrm{MeV}$ the rangeenergy relation can be represented very well by the linear equation:

$$
R_{p}=0.526 \frac{E}{\mathrm{MeV}}-0.094 \quad\left[\mathrm{~g} / \mathrm{cm}^{2}\right] .
$$

A number of theoretical and semi-emperical expressions have recently been given for the absolute and the practical range of mono-energetic electrons ${ }^{49 a}$.

Fig. 17. Practieal range of electrons in $\mathrm{Al}$ and $\mathrm{Gu}$ for energies between 0 and 12 $\mathrm{MeV}^{\circ}$ according to Hereford and Swann (Phys. Rev. is (1950) 727). Dotted line: ealculated values from Tow. ler et al. (Rev. Mod. Phys. 20 (1948) 236). Solid lines

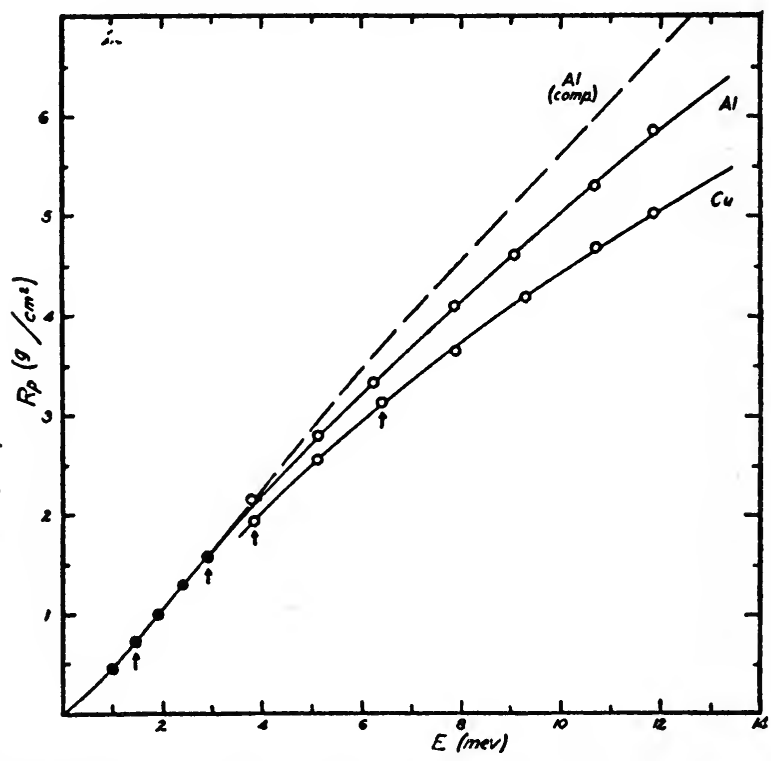
were calculated by Hereford and swann. Experimental points between 0 and $3 \mathrm{MeV}$ from Bleuler and Zünti (Helv. Phys. Acta 19 (1946) 376). The arrows indicate the normalisation points for the theoretical curves

49 Z. W. Varder, Phil. Mag. 29, (1915) 725.

$49 a$ F. I. Hereford and Ch. P. Swann, Phys. Rev., 78, (1950) 727. 


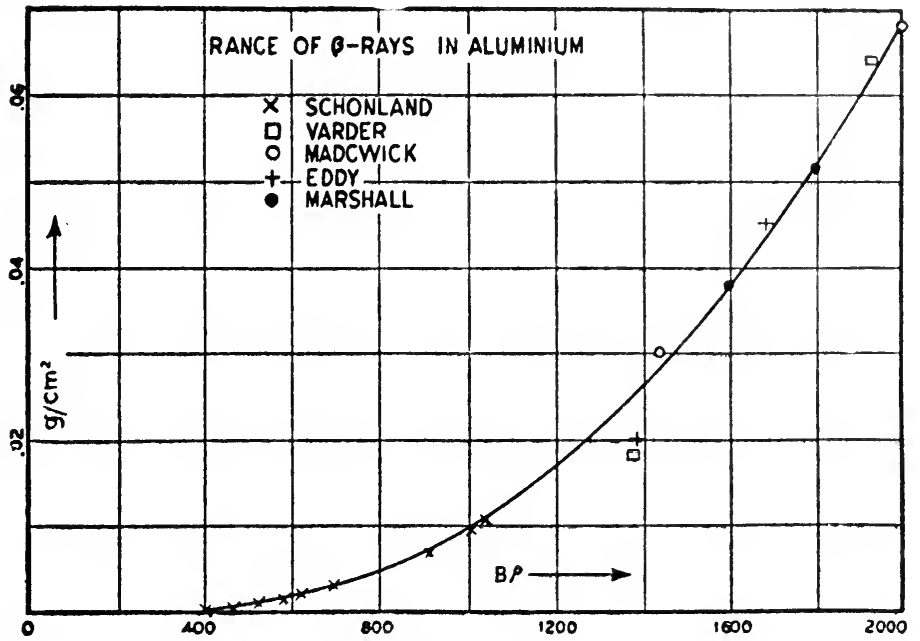

Fig. 17a

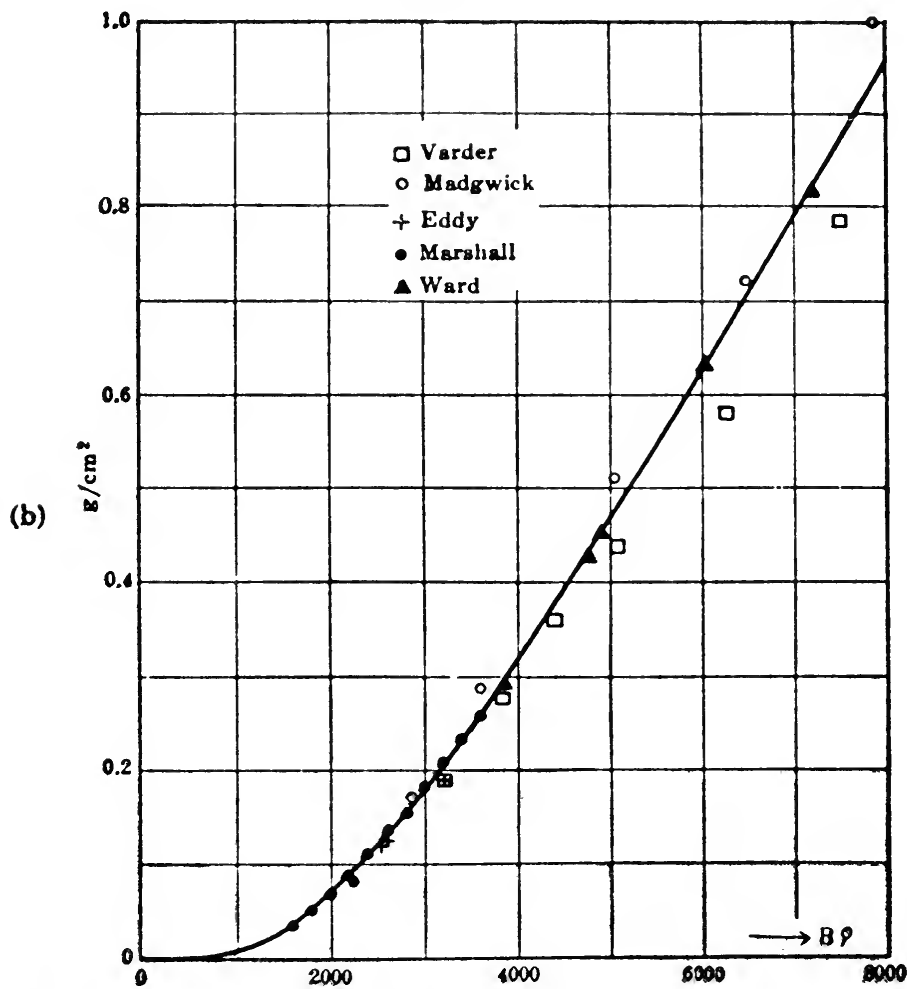

Fig. 17a, b. Practical range of electrons vs. energy $(\mathrm{B} Q=2000$ Gauss $\times \mathrm{cm} \bumpeq 277,0 \mathrm{keV} ; 8000$ Gauss $\times \mathrm{cm} \cong 1943 \mathrm{keV})$. From Marshall and Ward, Can. J. Research, A15 (1937) 40; 41 


\section{E. Absorption of Beta-Rays}

When one measures the absorption of the electrons of beta-emitters of inhomogeneous energy with an apparatus ${ }^{51}$ shown schematically in Fig. 18, one observes absorption curves an example of which is shown in Fig. 19. Here the number of electrons registered decreases initially exponentially with increasing thickness $x$ of the absorber: $N=N_{0} \exp (-\mu x / \varrho)$, where $\mu / \varrho$ is the mass absorption coefficient. When the thickness increases, the number of electrons slowly approaches to the background intensity which is here usually determined by a gamma component. For positrons, the annihilation radiation gives a contribution to the background intensity of about $1 \%$. By subtracting this background, we can obtain the maximum range $R_{\beta}$ of the beta-rays. A simple extrapolation, as used for the determination of the practical range of electron of homogeneous energy, cannot be used here.

The quantities $\mu / \varrho$ and $R_{\beta}$ are functions of the maximum energy $E_{0}$ of the beta-spectrum. They can be used for the determination of $E_{0}$, but the accuracy is small. A detailed discussion of these methods has been given by Glendenin, Gleason and Katz ${ }^{50,51,52}$. For simple beta-spectra

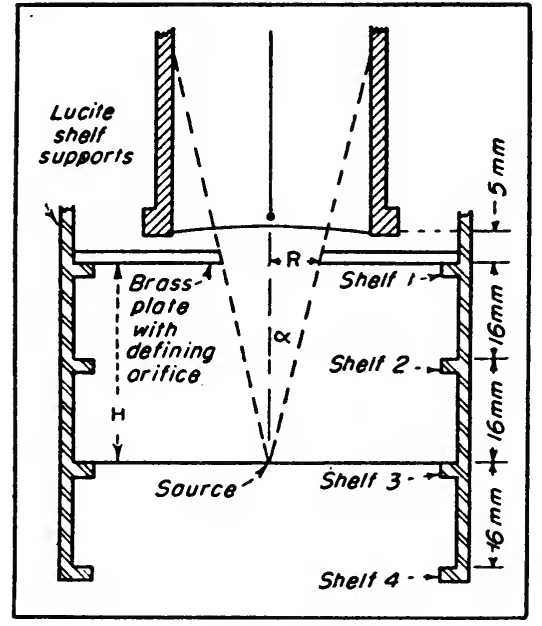

Fig. 18

Fig. 19. Transmission of beta rays from $\mathrm{Al}^{28}$ in Aluminum. From Bleuler and $\mathrm{Zünti}$, Helv. Phys. Acta 20 (1947) 197

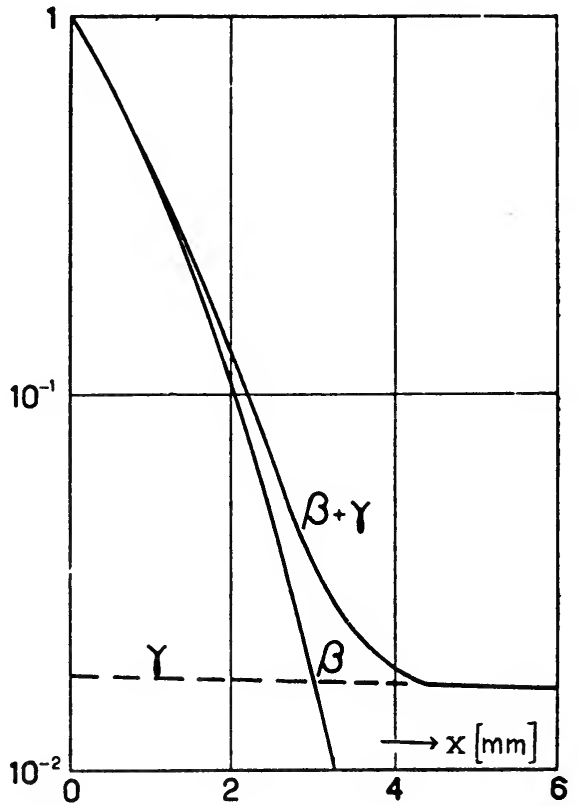

50 L. E. Glendenin, Nucleonics 2 (Jan. 1948) 12.

51 G. I. Gleason, I. D. Taylor and D. L. Tabern, Nucleonics 8, (May 1951) 12.

5. L. Katz and A. S. Penfold, Rev. Mod. Phys. 24, (1952) 30. 
the exponential law is valid so long as the foil transmits at least half of the electrons. Its range of validity is largest when the number of lowenergy electrons is large. Thus one can expect good exponential absorption curves for beta-emitters with a high atomic number, a small maximum energy or for those corresponding to highly forbidden transitions. An example, measured with the set-up menticned above. is shown in Fig. 20.

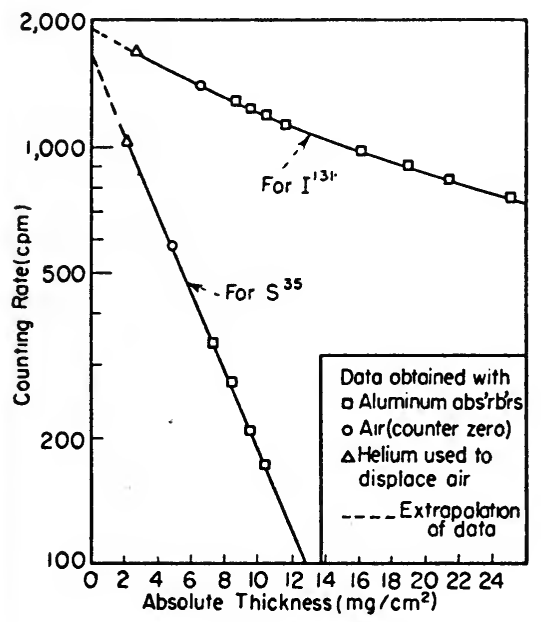

Fig. 20.

Typical beta absorption curves (from ${ }^{51}$ )

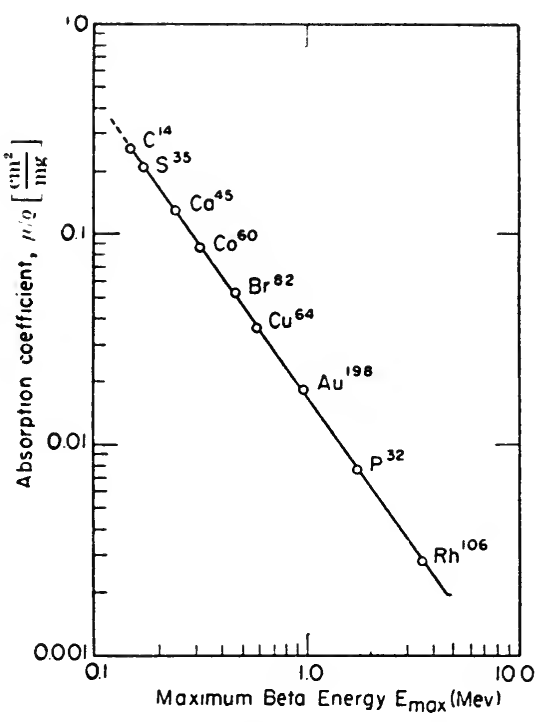

Fig. 21.

Absorption coefficient as a function of maximum beta energy ${ }^{51}$

Experimentally Gleason finds from Fig. $21 \mu / \varrho=17.0 E^{-1.43}\left[\mathrm{~cm}^{2} / \mathrm{g}\right]$. Also Seren ${ }^{53}$ measured the mass absorption coefficients for a large number of beta-emitters. It should be noted that the geometry of these experiments plays an important role, so that deviations from the relation given abuve occur easily. For the determination of the maximum energy $E_{0}$ of the betaspectrum from the range $R_{\beta}$ of the electrons, one usually uses aluminium as absorber. The empirical relation between $E_{0}$ and $R_{\beta}$ is shown in Fig. 22. Various expressions for this relation have been given by a number of authors. Feather was the first to derive such a relation.

Feather ${ }^{54}: R=0.543 E_{0}-0.160 \mathrm{~g} / \mathrm{cm}^{2} ; E_{0}>0.8 \mathrm{MeV}$.

This range-energy relation has recently been-extended to lower energies :

53 L. Seren, H. N. Friedlander and S. H. Turkel, Phys. Rev. 72, (1947) 888.

54 N. Feather, Proc. Camb. Phil. Soc. 34, (1938) 599. 


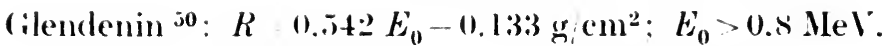

$$
R \quad\left(1.407 E_{0}^{1.38} ; \quad 0.15<E_{0}<0.8\right. \text {. }
$$

Flammersfeld 55 :

$$
R \quad 1.11\left(\sqrt{1+22.4 E_{0}^{2}}-1\right) ; 0<E_{0}<3 \mathrm{MeV} .
$$

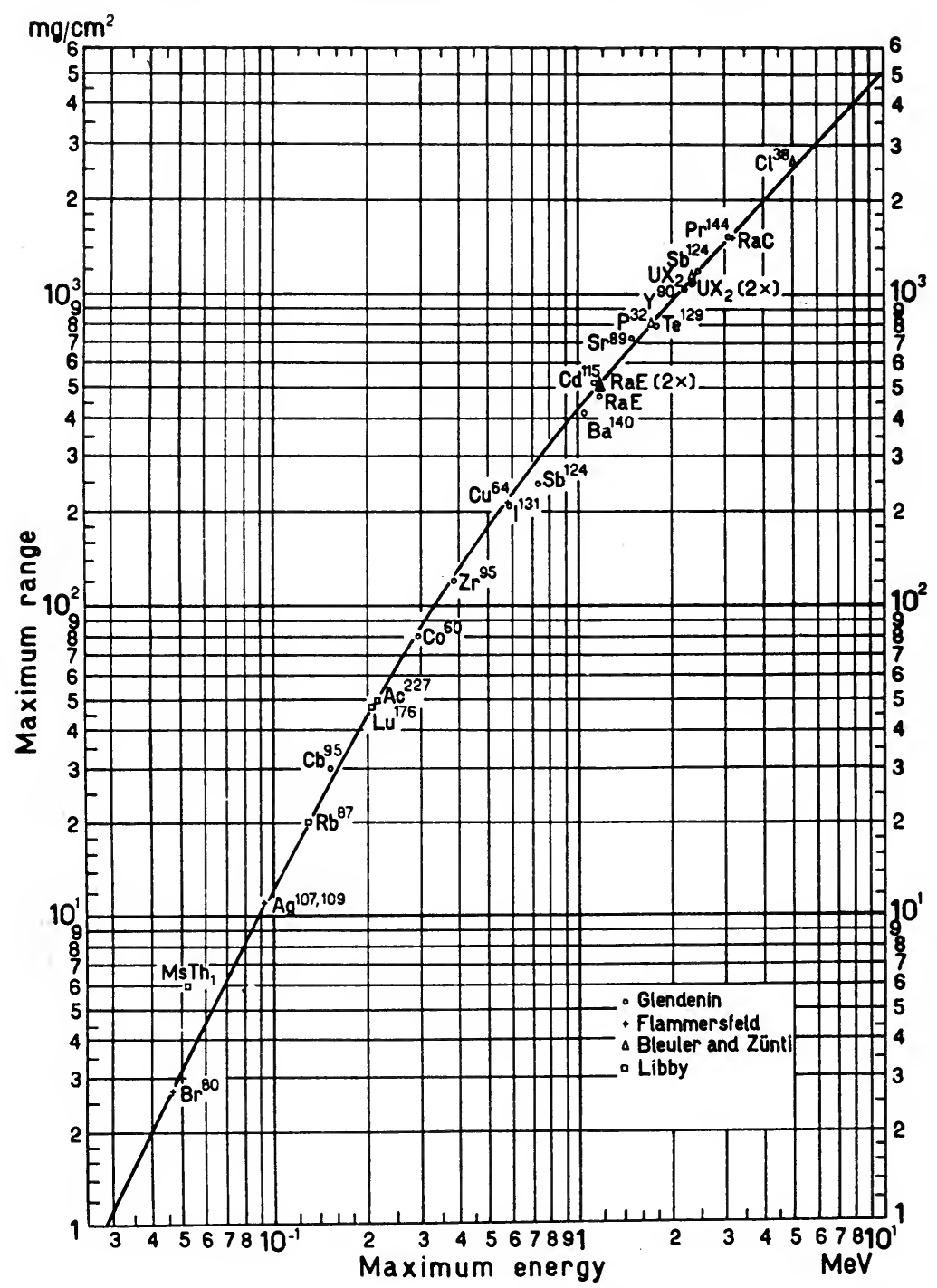

Fig. 22. Maximum range of a number of beta emitters as a function of their maximum energy

35 A. Flammersfeld, Naturw. 33, (1946) 280. 
Bleuler and Zünti ${ }^{32}$ :

$$
R=0.571 E_{0}-0.161 ; \quad E_{0}>1 \mathrm{MeV} .
$$

All the energies are expressed in $\mathrm{MeV}, R$ in $\mathrm{g} / \mathrm{cm}^{2}$.

In Fig. 22 the relation due to Flammersfeld, which has the largest range of validity, is shown graphically.

The accurate determination of $R_{\beta}$ is difficult. For simple beta-spectra, in particular if the beta-emission is accompanied by only a weak gamma radiation, this determination can be done visually. Such beta-emitters. like $\mathrm{Ra} \mathrm{E}, \mathrm{P}^{32}$ and $\mathrm{UX}_{2}$, are therefore used as standard sources. For more complicated spectra such a simple method is not practical. Feather, Bleuler and Zünti, and Katz et al. therefore worked out special methods for the determination of $R_{\beta}$. In the first two methods one compares the absorption curve with a standard absorption curve, like the one of Ra E. In the third method one assumes that the tail of the absorption curve can be represented by a power series, and one determines the coefficients. With this method it is possible to analyse complex spectra. We refer the reader for this method to the article of Katz and Penfold ${ }^{52}$. When one is very careful, the energy can be determined with an accuracy of about $5 \%$. 


\title{
INTERACTION OF $\gamma$-RADIATION WITII MATTER
}

\author{
('HARLOTTTE M. I)AVISSON
}

\section{\$. Introduction}

The interaction of $;$-radiation with matter is characterized by the fact that each $r$-ray photon is removed individually from the incident beam in a single event. Thus the number of photons removed. $\Delta B$, is proportional to the thickness traversed. $\Delta x$. and to the number of incident photons. i.e.

$$
\Delta B=-\mu B \Delta x
$$

where ", the proportionality constant. is called the absorption coefficient. If the radiation is homogeneous. $\mu$ is constant, and integration of eq. (1) yields

$$
B=B_{0} \exp (-\mu x)
$$

This shows that the number of photons remaining in the beam decreases exponentially with the distance of penetration. Since $x$ may be expressed variously as $\mathrm{cm}$. g $\mathrm{cm}^{2}$. atoms $/ \mathrm{cm}^{2}$, or electrons $/ \mathrm{cm}^{2}$. and since the product "Ix must be dimensionless. the absorption coefficient $\mu$, sometimes called the cross section. is correspondingly expressed as $\mathrm{cm}^{-1}, \mathrm{~cm}^{2} / \mathrm{g}, \mathrm{cm}^{2} /$ atom, or $\mathrm{cm}^{2}$ electron. Analysis of the interaction of $\gamma$-radiation with matter consists of determining the values of $\mu$ as a function of energy and absorbing material for the different processes by which $\gamma$-rays may be removed from the incident beam.

In the energy range being considered here.i.e. from a few $\mathrm{keV}$ to about $\therefore \mathrm{MeV}$. there are basically three processes by which $\gamma$-rays may interact with matter and be removed from an incident beam. (1) The photoelectric effect in which a photon gives all its energy to a bound electron, which uses part of the energy to overcome its binding to the atom and takes the rest as kinetic energy. This predominates at low energies. (2) Scattering by the atomic electrons. in which the photon is deflected from its original direction. At low energies when the wavelength is comparable with the dimensions of the atom. this scattering is coherent, with no change in energy. At energies just greater than the binding energies of the electrons, the scattering is incoherent and some of the photon energy is given to the electron, the amount depending on the energy and momentum of the electron within the atom. At energies much greater than the binding energies of the electrons. the photons are scattered as if the electrons were free and at rest. This is the Compton effect, and around $1 \mathrm{MeV}^{\mathrm{T}}$ it is the 
dominant mode of interaction. (3) Pair production. in which a photon. in the field of a nucleus or an electron, disappears, with the creation of an electron-positron pair whose total kinetic energy is equal to the photon energy minus the mass energy of the two particles which have been created. This can take place only when the energy of the $\gamma$-ray is equal to or greater than the mass energy of the electron-positron pair. It starts at about $\mathrm{l} \mathrm{MeV}$ and as the energy increases it becomes the dominant mode of interaction.

Since these three processes act independently of each other, we can separate the total absorption coefficient, $\mu$, into three parts, which we shall designate by $\tau$ for photoelectric effect, $\sigma$ for scattering by the atomic electrons (Compton effect), and $\varkappa$ for pair production. Thus eq. (1) can be written

and we have

$$
\Delta B=-(\tau+\sigma+\varkappa) B \Delta x
$$

$$
\mu=\tau+\sigma+\varkappa .
$$

The starting point in the theoretical analysis ${ }^{1}$ of the interaction of light with matter is the total Hamiltonian equation for the energy of the electrons, the energy of the light waves, and their energy of interaction. From this the wave equation

$$
\mathscr{H} \psi=E \psi
$$

is formed, in which the electron momenta $p_{x}$. etc. and the energy $E$ are replaced by

$$
\left\{\begin{array}{l}
p_{x}=(h / 2 \pi i)(\partial / \partial x) \\
E=-(h / 2 \pi i)(\partial / \partial t) .
\end{array}\right.
$$

The solution and analysis of eq. (5), as set up for the conditions required for each of the three processes, yields theoretical values of the absorption coefficients, as well as information concerning the energy and spatial distribution of the secondary products.

For summaries, tables, and curves, concerning $\gamma$-ray absorption the reader is also referred to R. Latter and H. Kahn, Gamma Ray Absorption Coefficients (The Rand Corporation, 1949); W. S. Snyder and J. L. Powell. Oak Ridge Natl. Laboratory, ORNL-421 (1950): G. Allen. Natl. Advisory Committee on Aeronautics, TN 2026 (1950); C. M. Davisson and R. D. Evans, Revs. Modern Phys. 24, (1952) 79; G. R. White, unpublished Natl. Bur. Standards Report No. 1003 (1952); and E. Segrè. Experimental Nuclear Physics. (John Wiley and Sons. Inc. New York 1953) Vol. I, Part II, Section 3, p. 304.

1 See e.g. W. Heitler, The Quantum Theory of Radiation, (Oxford University Press, London, 1944). 


\section{$\$$-.. Photoelectric Effect}

Fxact theoretical analysis of the photoelectric effect is difficult and tedious. since the I)irac relativistic equation for a. bound electron must be used. In the energy region of $0.35 \mathrm{MeV}^{r}$ to $2 \mathrm{MeV}$ the exact solution for the $K$-shell has been obtained by Hulme, McDougall. Buckingham, and Fowler 2 . but in other energy regions numerous approximations must be mate. Surveys of the theory have been given by Hall ${ }^{3}$. Sommerfeld ${ }^{4}$, and Heitler ${ }^{1}$.

Photoelectrons may be ejected from any of the $K . L . M . \ldots$ shells of an atom. but a free clectron cannot absorb a photon and become a photoelectron. since a third bodr. the nucleus. is necessary for conserving momentum. Therefore it is reasonable to expect. and indeed the theory shows. that the probability of photoelectric absorption increases rapidly with the tightness of hinding of the electron. so that at energies greater than the binding energies of the $K$ - and $L$-shells, the absorption due to outer shells is negligible. Most theoretical analyses deal with absorption in the $K$-shell. However, using non-relativistic quantum mechanics Stobbe ${ }^{5}$ and Hall ${ }^{3}$ have obtained equations for the photoelectric effect in the $L_{\mathrm{I}} \cdot L_{\mathrm{II}}+L_{\mathrm{II}}$, and $M$-shells as well as the $K$; and Lewis ${ }^{6}$ in a paper on oscillator strengths, gives equations and numerical values for obtaining the absorption coefficients of higher shells as well. At higher energies, in the relativistic range, a constant factor, $5 / 4$, based on experimental data ?, but roughly corroborated by theory ${ }^{3}$, has frequently been used to multiply $\tau_{k}$, the absorption coefficient for the $K$-shell, to get $\tau$, the total photoelectric absorption coefficient. Based on the results of Hall and Rarita ${ }^{8}$, Allen ${ }^{9}$ has used the factor $\{1.13+[(Z-1) / 81](0.07)\}$.

To simplify calculations and permit the use of hydrogen-like wave functions for the atomic electrons, each electron is assumed to move in the field of a nucleus of charge $Z-s_{i}$. The constants $s_{i}$ are called screening constants, and indicate the decrease in the effective nuclear charge caused by the presence of the other electrons in the atom. Slater ${ }^{10}$ has evaluated

? H. R. Hulme, J. IICDougall, R. A. Buckingham and R. H. Fowler, Proc. Roy. Sioc. (London) 149A. (1935) 131.

3 H. Hall, Revs. Modern Phys. 8, (1936) 358.

4 A. Sommerfeld, Atombau und spektrallinien, Braunschweig (1939), Vol. 2.

5 II. Ntobbe, Ann. Physik 7, (1930) 661 .

6 II. X. Jewis, unpublisherl Natl. Bur. Standards Report No. 2457, (195:3).

- E. Rutherford. J. Chadwiek and C. Ellis, Rarliations from Radioactive Sibbstances (('ambridge Cniversity Press, London, 1930) p. 464.

8 H. Hall and W. Rarita. Phys. Rev. 46, (1934) 143.

9 (i. Allen, Natl. Adrisory ('omm. on Aeronautics, TN 2026 (19.50).

10 J. C. Slater, Phys. Rov. 36, (1930) 57. 
the screening constants, $s_{i}$, for the electrons of the various shells semiempirically. Some of his values are given in Table I.

\section{TABLE I}

Sllater Screening Constants for Different Electron Groups, CARRIED THROUGH THE COMPLETED $M$-SHELL

\begin{tabular}{|c|c|c|c|c|c|}
\hline \multirow{2}{*}{$\begin{array}{l}\text { Atomic } \\
\text { Number }\end{array}$} & \multicolumn{5}{|c|}{ ELECTRON GROCPS } \\
\hline & $1 s$ & $2 s$ or $2 p$ & $3 s$ or $3 p$ & $3 d$ & $4 s$ or $4 p$ \\
\hline 2 & 0.30 & & & & \\
\hline 3 & & 1.70 & & & \\
\hline 4 & & 2.05 & & & \\
\hline 5 & & 2.40 & & & \\
\hline 6 & $\downarrow$ & 2.75 & & & \\
\hline 7 & $N$ & 3.10 & & & \\
\hline 8 & $\bar{\Xi}$ & 3.45 & & & \\
\hline 9 & $\dot{z}$ & 3.80 & & & \\
\hline 10 & $\stackrel{0}{2}$ & 4.15 & & & \\
\hline 11 & $\stackrel{\leftrightarrow}{\circ}$ & 1 & 8.80 & & \\
\hline 12 & $\dot{\vec{\theta}}$ & | & 9.15 & & \\
\hline 13 & $\uparrow$ & 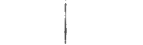 & 9.50 & & \\
\hline 14 & & $\downarrow$ & 9.85 & & \\
\hline 15 & & $N$ & 10.20 & & \\
\hline 16 & 1 & $\bar{\sigma}$ & 10.55 & & \\
\hline 17 & & $\ddot{\sigma}$ & 10.90 & & \\
\hline 18 & & 2 & 11.25 & & \\
\hline 19 & & $\stackrel{10}{-1}$ & & & 16.80 \\
\hline 20 & & $\dot{i}$ & & & 17.15 \\
\hline 21 & & 个 & & 18.00 & 18.00 \\
\hline 22 & & & $\downarrow$ & 18.35 & 18.85 \\
\hline 23 & & 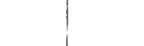 & $N$ & 18.70 & 19.70 \\
\hline 24 & & 1 & $\equiv$ & 19.40 & 21.05 \\
\hline 25 & & & 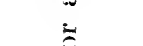 & 19.40 & 21.40 \\
\hline 26 & & & $\Xi$ & 19.75 & 22.25 \\
\hline 27 & & & 99 & 20.10 & 23.10 \\
\hline 28 & & & $\doteq$ & 20.45 & 23.95 \\
\hline 29 & & & $\uparrow$ & 21.15 & 25.30 \\
\hline 30 & & & 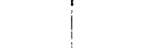 & & 25.65 \\
\hline & & & 1 & ? & \\
\hline & & & & $\downarrow$ & \\
\hline & . & & & $\stackrel{N}{=}$ & \\
\hline & & & & 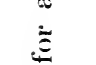 & \\
\hline & & & & $\stackrel{19}{-}$ & \\
\hline & & & & $\dot{x}$ & \\
\hline
\end{tabular}

If the energy of the photoelectron is much larger than the ionization energy of a $K$-electron, the Born approximation may be used, In this the 
attraction of the nucleus on the electron as it leaves the atom is neglected, and plane waves are used for the wave function of the photoelectron. The criterion for the validity of this approxination is that $Z e^{2} / \hbar v \ll 1$, where $v$ is the velocity of the ejected electron.

Applying the Born approximation, using non-relativistic quantum mechanics. and using hydrogen-like wave functions for the atomic electrons, the absorption coefficient for the photoelectric effect in the $K$-shell of an atom with atomic number $Z$ is found ${ }^{11}$ to be

$$
{ }_{a} \tau_{K}=\varphi_{0} Z^{5}(1 / 137)^{4}(2)^{5 / 3}\left(m c^{2} / h v\right)^{7 / 2}
$$

Here ${ }_{a} \tau_{K}$ is the cross section for photoelectric absorption in the $K$-shell, in units of $\mathrm{cm}^{2} /$ atom $; \varphi_{0}=8 \pi r_{0}^{2} / 3$, the cross section for Thomson scattering, with $r_{0}=e^{2} / m c^{2}$; and $137=h c / 2 \pi e^{2}$.

If the incident photon energy is the same order of magnitude as the ionization energy, the Born approximation is not applicable. Using hydrogen-like wave functions throughout, but disregarding the possibility that the ejected electron may receive from the radiation an angular momentum larger than $h / 2 \pi$ ("dipole transition approximation"), Stobbe ${ }^{5}$ and Hall ${ }^{3}$ have obtained an equation for ${ }_{a} \tau_{K}$ which may be written as eq. (7) multiplied by a factor ${ }^{11} f(\xi)$ which is

$$
f(\xi)=2 \pi\left(\frac{v_{1}}{v}\right)^{1 / 2} \frac{\exp \left(-4 \xi \cot ^{-1} \xi\right)}{1-\exp (-2 \pi \xi)}
$$

where $h v_{1}=(Z-0.3)^{2} \mathrm{Ry}$, is the ionization energy of the $K$-shell $(\mathrm{Ry}=$ Rydberg energy), $\xi=\left[v_{1} /\left(v-v_{1}\right)\right]^{1 / z}$, or the ratio of the initial orbital velocity of the electron to the velocity of the photoelectron. Multiplying eq. (7) by eq. (8)

$$
{ }_{a} \tau_{K}=\varphi_{0} \frac{2^{7} \pi(137)^{3}}{Z^{2}}\left(\frac{v_{1}}{v}\right)^{4} \frac{\exp \left(-4 \xi \cot ^{-1} \xi\right)}{1-\exp (-2 \pi \xi)}
$$

Hall ${ }^{3}$ has expanded eq. (9) in powers of $(1 / \xi)^{2}$ and shown that close to the absorption limit. where the energy of the photoelectron is much less than the ionization energy, i.e. $(1 / \xi) \ll 1$, the photoelectric absorption in the $K$-shell varies as $v^{3 / 3}$.

Hall $^{3}$ and Stobbe ${ }^{5}$, using the same assumptions as for eq. (9), have calculated the absorption coefficients for the two $2 s$ electrons of the $L_{\mathrm{I}}$ shell and the six $2 p$ electrons of the $L_{\mathrm{II}}$ and $L_{\mathrm{III}}$ shells. They obtain

$$
\begin{gathered}
\tau_{L_{\mathrm{I}}}=\frac{2^{12} \pi \varphi_{0}(137)^{3}}{\left(Z-s_{2}\right)^{2}}\left(\frac{v_{2}}{v}\right)^{4}\left(1+3 \frac{v_{2}}{v}\right) \frac{\exp \left(-8 \xi_{2} \cot ^{-1} \xi_{2}\right)}{1-\exp \left(-4 \pi \xi_{2}\right)} \\
\tau_{L_{\mathrm{II}}}+\tau_{L_{\mathrm{III}}}=\frac{2^{12} \pi \varphi_{0}(137)^{3}}{\left(Z-s_{2}\right)^{2}}\left(\frac{v_{2}}{v}\right)^{5}\left(3+8 \frac{v_{2}}{v}\right) \frac{\exp \left(-8 \xi_{2} \cot ^{-1} \xi_{2}\right)}{1-\exp \left(-4 \pi \xi_{2}\right)}
\end{gathered}
$$

11 Reference 1, p. 123 and 124. 
Here $v_{2}=(1 / 4)\left(Z-s_{2}\right)^{2} \mathrm{Ry}, \quad \xi_{2}=\left[v_{2} /\left(v-v_{2}\right)\right]^{1 / 2}$, or the ratio of the orbital velocity of the $L$-electron to the velocity of the photoelectron. The other symbols are the same as in previous equations.

For absorption by the 18 electrons in the $M$-shell Hall and Stobbe have obtained the equation

$$
\begin{aligned}
\tau_{M}=\frac{2^{7} \pi \varphi_{0}(137)^{3} 3^{3}}{\left(Z-s_{3}\right)^{2}}\left(\frac{v_{3}}{v}\right)^{4}(1+ & \left.8 \frac{v_{3}}{v}\right)\left\{27+136 \frac{v_{3}}{v}+20\left(8\left(\frac{v_{3}}{v}\right)^{2}\right.\right. \\
& \left.+96\left(\frac{v_{3}}{v}\right)^{3}\right) \frac{\exp \left(-12 \xi_{3} \cot ^{-1} \xi_{3}\right)}{1-\exp \left(-6 \pi \xi_{3}\right)}
\end{aligned}
$$

where $v_{3}=(1 / 9)\left(Z-s_{3}\right)^{2} \mathrm{Ry}$, and $\xi_{3}=\left[v_{3} /\left(v-v_{3}\right)\right]^{1 / 2}$.

In the relativistic region where binding energies can be neglected, calculations using the Born approximation, and making the same assumptions as were made for eq. (7), but using relativistic wave functions for the atomic electrons and the photoelectrons, have been carried out by Sauter ${ }^{12}$ and Hulme ${ }^{13}$. Sauter's result is in the form of an equation which can be written

$$
\begin{aligned}
{ }_{a} \tau_{K}=\frac{3}{2} \frac{\varphi_{0} Z^{5}}{(137)^{4}}\left(\frac{m c^{2}}{h v}\right)^{5} & \left(\gamma^{2}-1\right)^{3 / 2}\left[\frac{4}{3}+\frac{\gamma(\gamma-2)}{\gamma+1}\right. \\
& \times\left(1-\frac{1}{2 \gamma\left(\gamma^{2}-1\right)^{1 / 2}} \ln \frac{\gamma+\left(\gamma^{2}-1\right)^{1 / 2}}{\left.\left.\gamma-\left(\gamma^{2}-1\right)^{1 / 2}\right)\right]}\right.
\end{aligned}
$$

where $\gamma=1 /\left(1-\beta^{2}\right)^{1 / z}=\left(h v+m c^{2}\right) / m c^{2}$ and the other symbols are the same as in previous equations. Hulme's calculations are numerical for different energies. Neither Hulme's values nor values from Sauter's equation go smoothly into the more accurate values of Hulme, McDougall, Buckingham, and Fowler ${ }^{2}$ at higher energies. However, the fit is much improved if Sauter's eq. (13) is multiplied by Stobbe's non-relativistic correction factor, $f(\xi)$, eq. (8).

In the energy region from $0.35 \mathrm{MeV}$ to $2 \mathrm{MeV}$, Hulme, McDougall, Buckingham, and Fowler ${ }^{2}$ have carried out a rigorous numerical calculation. They assume only that each $K$-electron is independent of the other, the problem involving just a fixed nucleus and one $K$-electron, and the results being doubled to give the absorption in the $K$-shell. Their results are in the form of numerical values of absorption coefficients for the two energies, $0.354 \mathrm{MeV}$ and $1.13 \mathrm{MeV}$, and the three atomic numbers, $Z=26,50$, and 84 . These values are given in Table II. Using these values, values from Sauter's eq. (13) for $Z=0$, and values from an equation by Hall ${ }^{14}$ (eq. (14)) for $m c^{2} / h \nu=0$ and 0.194 , and multiplying ${ }_{a} \tau_{K}$ by $5 / 4$ to

\footnotetext{
12 F. Sauter, Ann. Physik 11, (1931) 454.

13 H. R. Hulme, Proc. Roy. Soc. (London) 133A, (1931) 381.

14 H. Hall, Phys. Rev. 45, (1934) 620; Revs. Modern Phys. 8, (1936) 358: Phys. Rev. 84, (1951) 167.
} 
TABLE 11

Photolectric Absorption ('oefficints for the $K$-shell calcllated BY Hulme et al. ${ }^{2}$

(in units of $\mathrm{cm}^{2} / \mathrm{atom}$ )

\begin{tabular}{c|c|c|c}
\hline$m c^{2} / h v$ & $Z=26$ & $Z=50$ & $Z=84$ \\
\hline 0.452 & $2.3 \times 10^{-26}$ & $4.6 \times 10^{-25}$ & $4.61 \times 10^{-24}$ \\
\hline 1.443 & $3.9 \times 10^{-25}$ & $7.1 \times 10^{-24}$ & $6.02 \times 10^{-23}$
\end{tabular}

get ${ }_{a} \tau$. they obtain values for ${ }_{a} \tau /\left[Z^{5}\left(m c^{2} / h \nu\right)\right]$ for different values of $Z$ and plot the results as curves.

At energies greater than $2 \mathrm{MeV}$, the difficulties of carrying out numerical calculations such as those of Hulme et al. become prohibitive. Hall ${ }^{14}$, by expanding the wave function in powers of the photon wavelength (reciprocal of photon energy) obtains the following equation which is valid for energies large compared with $m c^{2}$.

$$
\begin{aligned}
\frac{{ }_{a} \tau_{K}}{Z^{5}\left(m c^{2} / h v\right)}=\frac{(3 / 2) \varphi_{0}(137)^{-4}}{\eta^{2 \eta^{2}} \exp [\eta(\eta-2 \eta)]}\left\{k_{0}^{\prime 3} \varepsilon\left(\frac{m c^{2}}{h v}\right)^{4}\right. \\
\left.\times\left[\frac{4}{3 \varepsilon}+\frac{\varepsilon-2}{\varepsilon+1}\left(1+\frac{1}{2 \varepsilon k_{0}^{\prime}} \ln \frac{\varepsilon-k_{0}^{\prime}}{\varepsilon+k_{0}^{\prime}}\right)\right]\right\}
\end{aligned}
$$

Here $\eta$ is $Z / 137, \varepsilon$ is $\left[\left(h v / m c^{2}\right)+\left(1-\eta^{2}\right)\right]$ or the total energy of the photoelectron in units of $m c^{2}$, and $k_{0}^{\prime}$ is $\left(\varepsilon^{2}-1\right)^{1 / 3}$ or the velocity of light, $c$, times the momentum of the photoelectron, in units of $m c^{2}$. At $1.13 \mathrm{MeV}$ the values given by this equation agree with the more rigorous calculations of Hulme et al. within $8 \%$.

To obtain curves or tables of values of the total photoelectric absorption coefficient one can make calculations using the equations or numerical results appropriate for the different energy regions and adjust them graphically to join smoothly at the overlapping energies. Table I of Appendix I contains numerical values of the photoelectric absorption coefficient computèd and compiled by Gladys White ${ }^{15}$. At energies less than the $K$-edge the cross sections were calculated from eq. (10), (11), and (12) for the $L$ - and $M$-shells. The cross section for the photoelectric effect in the $K$-shell was calculated at the lower energies from the StobbeSauter combined formula, eq. (13) multiplied by eq. (8); at moderately high energies it was obtained from interpolated data taken from the Hulme et al. calculations; and at higher energies it was calculated from the Hall formula, eq. (14). The total photoelectric absorption coefficients were obtained from those for the $K$-shell by multiplying them by a ratio of $\left({ }_{a} \tau_{K}+{ }_{a} \tau_{L}+{ }_{a} \tau_{M}\right){ }_{a} \tau_{K}$ obtained from Stobbe's equations. Due to the

15 G. R. White, unpublished Natl. Bur. Standards Report No. 1003 (1952). 
slowness of variation of this ratio with both energy and atomic number, it was calculated only at the $K$-edge, and at $m c^{2} / h v=1.5$ (i.e. $0.340 \mathrm{MeV}$ ). These values are given in Table III, taken from Miss White's paper.

\section{TABLE III}

The Ratio $\left(\tau_{K}+\tau_{L}+\tau_{M}\right) / \tau_{K}$ Calculated from the Śtobbe Formulas EQS. (9), (10), (11) AND (12)

(from report by $G$. White ${ }^{15}$ )

\begin{tabular}{c|c|c|c|c|c|c|c|c|c|c|c}
\hline \hline$Z$ & 6 & 8 & 13 & 18 & 26 & 29 & 42 & 50 & 74 & 82 & 92 \\
\hline$K$ edge & 1.02 & 1.03 & 1.05 & 1.07 & 1.10 & 1.11 & 1.13 & 1.14 & 1.161 & $1.164 \mid$ & 1.167 \\
\hline$m c^{2} / h v=1.5$ & 1.01 & 1.02 & 1.03 & 1.04 & 1.06 & 1.07 & 1.09 & 1.10 & 1.124 & 1.131 & 1.138
\end{tabular}

The values of the ratio at the $K$-edge were used to multiply the values of $\boldsymbol{a}_{K}$ obtained from the Sauter-Stobbe formula for energies from the $K$-edge to $m c^{2} / h v=1.5$. The values calculated at $m c^{2} / h v=1.5$ were used to multiply the values of ${ }_{a} \tau_{K}$ obtained from Hulme et al. and Hall's equation. Finally the plot of the Sauter-Stobbe combined formula, corrected for the effect of the other shells, was adjusted graphically in the region of $m c^{2} / h v=1.5$ to join smoothly with the plot of the corrected values of Hulme et al.

Similar tables, for smaller energy ranges have been prepared by Davisson and Evans ${ }^{16}$, by Allen ${ }^{9}$ and by Latter and Kahn ${ }^{17}$. Figure 1, taken from Davisson and Evans gives the variation of ${ }_{a} \tau / Z^{5}$ with wavelength and shows that at high energies it varies linearly with the incident wavelength, (inversely with the incident energy) so that the falling off of photoelectric absorption at high energies is not as great as at low energies, with the result that for heavy elements photoelectric absorption plays a part at energies as high as $5 \mathrm{MeV}$.

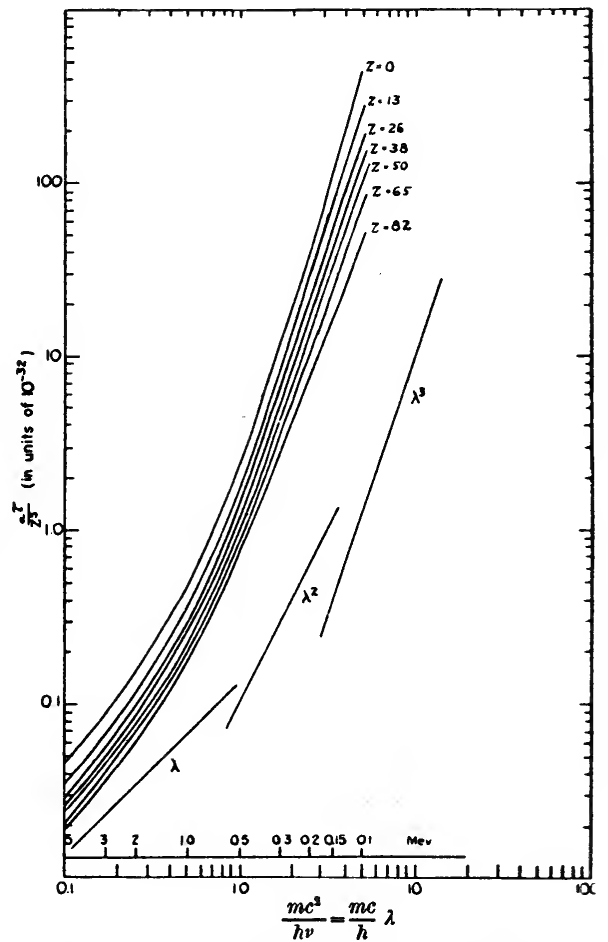

Fig. 1. Photoelectric effect. Variation of cross section with wavelength. Lines marked $\lambda, \lambda^{2}$ and $\lambda^{3}$ indicate slopes expected if $a^{\tau}$ varied as $\lambda, \lambda^{2}$, or $\lambda^{3}$

16 C. M. Davisson and R. D. Evans, Revs. Modern Phys. 24, (1952) 79.

17 R. Latter and H. Kahn, Gammn Ray Absorption Coefficients (The Rand Corporation, 1949). 
The angular distribution of photoelectrons has been studied theoretically. by Auger and Perrin ${ }^{18}$. Fischer ${ }^{19}$, Sauter ${ }^{12}$, and Hulme ${ }^{13}$. Their results have been collected and analyzed by Morette ${ }^{20}$ and by Davisson and Evans ${ }^{16}$. It is found that at low energies most of the electrons are emitted with a large component of their velocity in the direction of polarization of the incident photon, but with increasing energy the number with a large forward component of velocity increases. None, however, are emitted in the direction of propagation of the incident photon.

Very few experimental studies have been made of the photoelectric effect alone. With a magnetic spectrograph Davidson and Latyshev ${ }^{21}$ studied the photoelectrons produced by the 2.62 $\mathrm{MeV}^{\gamma} \gamma$-rays of $\mathrm{ThC}^{\prime \prime}$ in various materials, and obtained results in close agreement with theory. Most other workers obtain the photoelectric coefficient by subtracting the theoretical Compton scattering cross section from the measured total absorption coefficient. In the energy range from about $0.3 \mathrm{MeV}$ to $1 \mathrm{MeV}$, where pair production begins, this method is satisfactory, as coherent scattering is small and the theory of Compton scattering has been well checked. For the most part, the experimental values show good agreement with the theory.

\section{$\S 3 . \quad$ Scattering}

The theory of the scattering of $\gamma$-rays by electrons is most complete and satisfactory for photon energies so large compared with the electron binding energies that the electrons can be considered as free. This is the

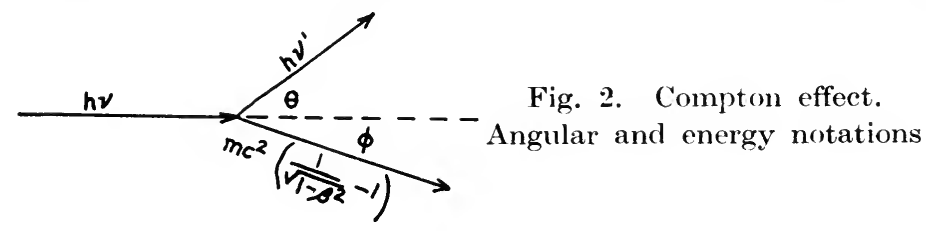

well known Compton effect. Referring to Fig. 2 and using the relativistic expressions for energy and mass, the conservation of energy and momentum in the Compton process is given by the following equations.

$$
\begin{aligned}
h v & =m c^{2}\left[\left(1-\beta^{2}\right)^{-1 / 2}-1\right]+h v^{\prime} \\
h v / c & =m c \beta\left(1-\beta^{2}\right)^{-1 / 2} \cos \varphi+\left(h v^{\prime} / c\right) \cos \theta \\
0 & =m c \beta\left(1-\beta^{2}\right)^{-1 / 2} \sin \varphi-\left(h v^{\prime} / c\right) \cos \varphi
\end{aligned}
$$

18 P. Auger and F. Perrin, Compt. rend. 180, (1925) 1742.

19 .J. Fischer, Ann. Physik 8, (1931) 821.

20 C. Morette, J. phys. et radium 7, (1946) 135.

21 Z. S. Davidson and G. D. Latyshev, J. Phys. (U.S.S.R.) 6, (1942) 15; and (i. D. Latyshev, Rers. Modern Phys. 19, (1947) 132. 
Here $\beta$ is the electron velocity in units of the velocity of light. By eliminating any two of the quantities $\beta, v^{\prime}, \varphi$, or 0 from these equations and putting $\alpha=h v / m c^{2}$ the following expressions can be obtained for the energy of the scattered photon $h v^{\prime}$, the energy of the scattered electron, $T$, and the relation between $\theta$ and $\varphi$.

$$
\begin{gathered}
h v^{\prime}=\frac{h v}{1+\alpha(1-\cos \theta)} \\
T=h \nu\left[1-\frac{1}{1+\alpha(1-\cos \theta)}\right] \\
T=h v\left[\frac{2 \alpha}{1+2 \alpha+(1+\alpha)^{2} \tan ^{2} \varphi}\right] \\
\cos \theta=1-\frac{2}{(1+\alpha)^{2} \tan ^{2} \varphi+1} \\
\cot \varphi=(1+\alpha) \tan (\theta / 2)
\end{gathered}
$$

To obtain the cross section for Compton scattering Klein and Nishina 22 have carried out a quantum-mechanical treatment of the problem using the Dirac equation for the electron. If one lets $I$ be the intensity of the scattered photons at the angle $\theta$ and distance $r$ from the scattering electron, and $I_{0}$ be the incident intensity, then

$$
I=\frac{I_{0}}{r^{2}}\left[\frac{h v^{\prime}}{h v} \frac{d_{e} \sigma}{d \Omega}\right]
$$

where $d_{e} \sigma$ is the cross section per electron for the number of photons scattered into the solid angle $d \Omega$ in the direction 0 . The analytic expression Klein and Nishina obtained for this cross section is

$$
\frac{d_{e} \sigma}{d \Omega}=\frac{r_{0}^{2}}{2}\left(\frac{h v^{\prime}}{h v}\right)^{2}\left(\frac{h v}{h v^{\prime}}+\frac{h v^{\prime}}{h v}-\sin ^{2} \theta\right)
$$

Substituting in eq. (22) the energy ratios given by eq. (16) one obtains $d_{\epsilon} \sigma$ as a function of $\theta$, i.e.

$$
\frac{d_{e} \sigma}{d \Omega}=\frac{r_{0}^{2}}{2}\left\{\frac{1}{[1+\alpha(1-\cos \theta)]^{2}}\left[1+\cos ^{2} \theta+\frac{\alpha^{2}(1-\cos \theta)^{2}}{[1+\alpha(1-\cos \theta)]}\right]\right\}
$$

For low energies, i.e. for $\alpha \leqslant 1$, this reduces to the classical Thomson equation

$$
d_{e} \sigma / d \Omega=\left(r_{0}^{2} / 2\right)\left(1+\cos ^{2} \theta\right) .
$$

Some curves for $d_{e} \sigma / d \Omega$ are given in Fig. 3. The Compton total cross section, obtained by integrating eq. (23) over all values of the angular coordinates $\theta$ and $\varphi^{\prime}$, is found to be

$$
e^{\sigma} \sigma=2 \pi r_{0}^{2}\left\{\frac{1+\alpha}{\alpha^{2}}\left[\frac{2(1+\alpha)}{1+2 \alpha}-\frac{1}{\alpha} \ln (1+2 \alpha)+\frac{1}{2 \alpha} \ln (1+2 \alpha)-\frac{1+3 \alpha}{(1+2 \alpha)^{2}}\right]\right\}
$$

$$
\text { O. Klein and Y. Nishina, Z. Physik 52, (1929) } 853 .
$$


${ }_{6} \sigma$ is the cross section for either the number of photons removed from the original beam, or for the energy removed from it, since each scattered photon diminishes the energy in the original beam by $h v$.

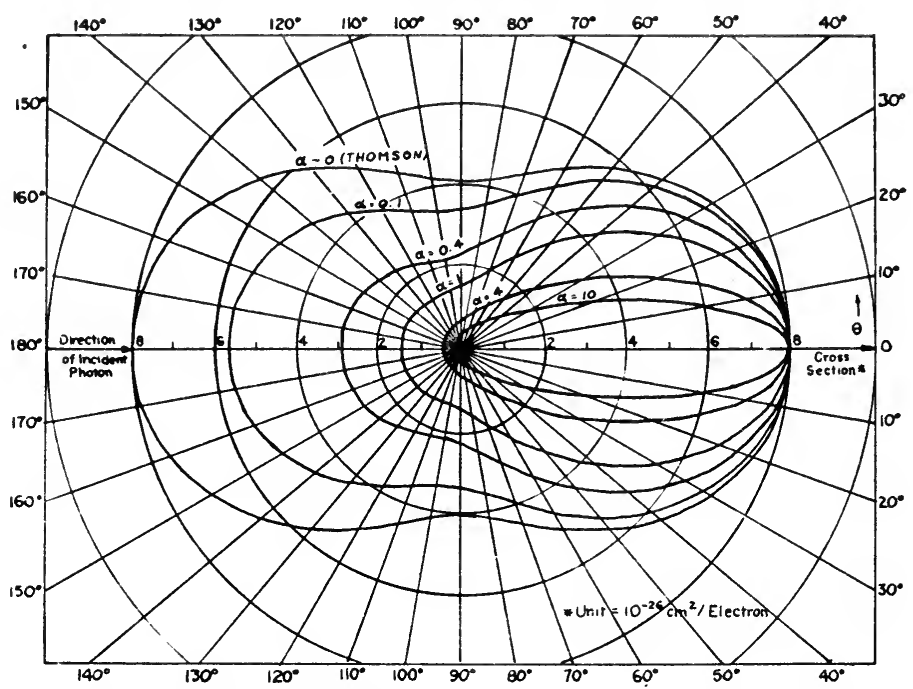

Fig. 3. Compton effect. Differential cross section per unit solid angle for the number of photons scattered at the angle $\theta$. Eq. (23)

For some problems it is of interest to know the probable amount of energy transferred to the material by the scattering process. For this purpose $e_{e} \sigma$ may be separated into ${ }_{e} \sigma_{s}$, the cross section for the amount of energy in the scattered radiation, sometimes called the Compton scattering coefficient; and ${ }_{e} \sigma_{a}$, the cross section for the amount of energy transferred to the electrons, sometimes called the Compton absorption coefficient. That is,

$$
e^{\sigma}={ }_{e} \sigma_{s}+{ }_{e} \sigma_{a}
$$

The differential cross section per unit solid angle for the scattering coefficient is the quantity shown in brackets in eq. (21). Multiplying eq. (22) by the energy ratio from eq. (16), we have

$$
\frac{d_{e} \sigma_{s}}{d \Omega}=\frac{r_{0}^{2}}{2}\left\{\frac{1}{[1+\alpha(1-\cos \theta)]^{3}}\left[1+\cos ^{2} \theta+\frac{\alpha^{2}(1-\cos \theta)^{2}}{[1+\alpha(1-\cos \theta)]}\right]\right\}
$$

Integrating this over all values of $\theta$ and $\varphi^{\prime}$ one obtains for the Compton scattering coefficient

$$
e^{\sigma_{s}}=\pi r_{0}^{2}\left[\frac{1}{\alpha^{3}} \ln (1+2 \alpha)+\frac{2(1+a)\left(2 \alpha^{2}-2 \alpha-1\right)}{a^{2}(1+2 \alpha)^{2}}+\frac{8 \alpha^{2}}{3(1+2 \alpha)^{3}}\right]
$$


Numerical values of $e_{e} \sigma_{a}$, the Compton absorption coefficient can be obtained by subtracting values of ${ }_{e} \sigma_{s}$ from ${ }_{e} \sigma$. Values of ${ }_{e} \sigma,{ }_{e} \sigma_{s}$, and $e_{e} \sigma_{a}$ for different values of $\alpha$ are given in Table IV and are plotted in Fig. 4.

TABLE IV

Cross Sections for the Compton Effect*

$$
e^{\sigma}=e^{\sigma_{a}}+e^{\sigma_{8}}
$$

(in units of $10^{-25} \mathrm{~cm}^{2} /$ electron)

\begin{tabular}{|c|c|c|c|}
\hline$\alpha=h \nu / m c^{2}$ & $e^{\sigma}$ & $e^{i \sigma_{s}}$ & $e^{\sigma_{a}}$ \\
\hline 0.025 & 6.31 & 6.31 & 0.00 \\
\hline 0.05 & 6.07 & 5.79 & 0.28 \\
\hline 0.075 & 5.83 & & \\
\hline 0.1 & 5.599 & 5.138 & 0.461 \\
\hline 0.125 & 5.409 & & \\
\hline 0.15 & 5.243 & & \\
\hline 0.20 & 4.900 & 4.217 & 0.683 \\
\hline 0.25 & 4.636 & & \\
\hline $1 / 8$ & 4.273 & & \\
\hline 0.40 & 4.032 & 3.152 & 0.880 \\
\hline 0.50 & 3.744 & & \\
\hline $2 / 3$ & 3.369 & & \\
\hline 0.80 & 3.140 & 2.158 & 0.982 \\
\hline 1.0 & 2.866 & 1.879 & 0.987 \\
\hline $1 / 8$ & 2.529 & 1.553 & 0.976 \\
\hline 1.635 & 2.303 & & \\
\hline 2.0 & 2.090 & 1.164 & 0.926 \\
\hline 2.232 & 1.979 & & \\
\hline 2.418 & 1.901 & & \\
\hline $8 / 3$ & 1.806 & & \\
\hline 3.0 & 1.696 & 0.8523 & 0.844 \\
\hline 4.0 & 1.446 & 0.6745 & 0.772 \\
\hline 5.155 & 1.246 & & \\
\hline 5.403 & 1.211 & & \\
\hline 6.0 & 1.136 & 0.4774 & 0.659 \\
\hline 8.0 & 0.9465 & 0.3700 & $0.576 . i$ \\
\hline 10.0 & 0.8168 & 0.3023 & 0.514 .5 \\
\hline 12.0 & 0.7215 & & \\
\hline 20.0 & 0.5019 & 0.1581 & (1) $3433 \times$ \\
\hline 30.0 & 0.3710 & 0.1071 & $4=213 !$ \\
\hline $100 / 3$ & 0.3424 & & \\
\hline 50.0 & 0.2498 & 0.06596 & $11.1 \cdot 38$ \\
\hline 70.0 & 0.1911 & $0.04719^{-}$ & $11 .+439$ \\
\hline 100.0 & 0.1431 & 0.03302 & 1.1101 \\
\hline
\end{tabular}

* Although in most cases four figures are given, not mu.. (hat, three can be considered significant. 
From eq. (22) by the proper substitution of quantities obtained from eq. (16) to (20), other differential cross sections can be obtained. Some which are of particular interest follow.

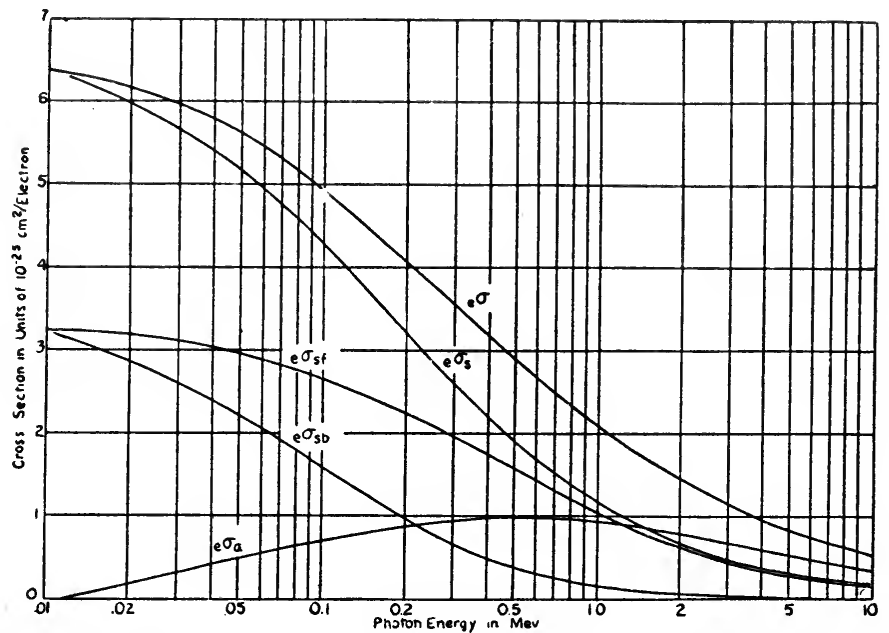

Fig. 4. Compton effect. Cross sections $v s$ energy. ${ }_{e} \sigma$ the cross section for the number of photons scattered; $e_{s}$ the cross section for the energy of the photons scattered; $e_{a} \sigma_{a}$ the cross section for the energy absorbed by the electrons; $e^{\sigma_{s f}}$ the cross section for the photon energy scattered forward; $e_{e} \sigma_{s b}$ the cross section for the photon energy scattered backward

(1) The differential cross section per unit solid angle for the number of electrons scattered in the direction $\varphi$. In terms of the analogous quantity for the scattered photons, eq. (23). this can be written

$$
\left(d_{e} \sigma / d \Omega^{\prime}\right)=\left(d_{e} \sigma / d \Omega\right)\left(d \Omega / d \Omega^{\prime}\right)
$$

where the ratio of the solid angles can be shown to be

$$
\left(d \Omega / d \Omega^{\prime}\right)=\left[(1+\alpha)^{2}(1-\cos \theta)^{2}\right] / \cos ^{3} \varphi
$$

Some representative "curves for this equation are given in Fig. 5.

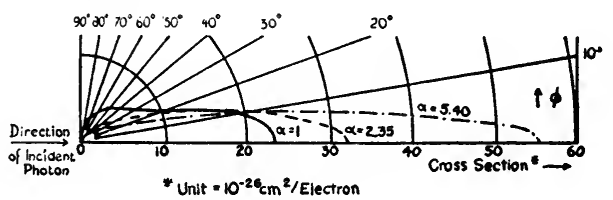

Fig. 5. Compton effect. Differential cross section per unit solid angle for the number of electrons scattered at the angle $\varphi$. Eq. (29)

(2) The differential cross section per unit solid angle for the amount of electron energy scattered in the direction $p$. In terms of eq. (29) this is

$$
\left(d_{e} \sigma_{a} / d \Omega^{\prime}\right)=(T / h v)\left(d_{e} \sigma / d \Omega^{\prime}\right) .
$$


(3) The differential cross section for the scattering of a photon of primary energy $h v$, into the energy range from $h v^{\prime}$, to $h v^{\prime}+d h v^{\prime}$. This can be shown to be

$$
\frac{d_{e} \sigma}{d h v^{\prime}}=\frac{\pi r_{0}^{2} m c^{2}}{(h v)^{2}}\left\{\frac{h v^{\prime}}{h v}+\frac{h v^{\prime}}{h v^{\prime}}-2\left(\frac{m c^{2}}{h v_{\iota}^{\prime}}-\frac{m c^{2}}{h v}\right)+\left(\frac{m c^{2}}{h v^{\prime}}-\frac{m c^{2}}{h v}\right)^{2}\right\}
$$

Representative curves of this equation for different energies are given in Fig. 6.

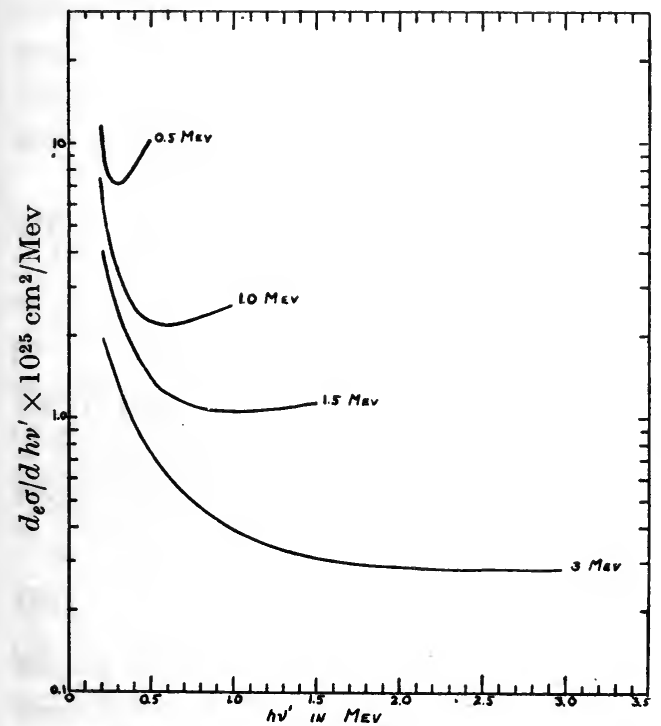

Fig. 6. Compton effect. The differential cross section for the scattering of a photon of initial energy $h v$ into the energy interval $h v^{\prime}$ to $h v^{\prime}+d h v^{\prime}$. Eq. (32)

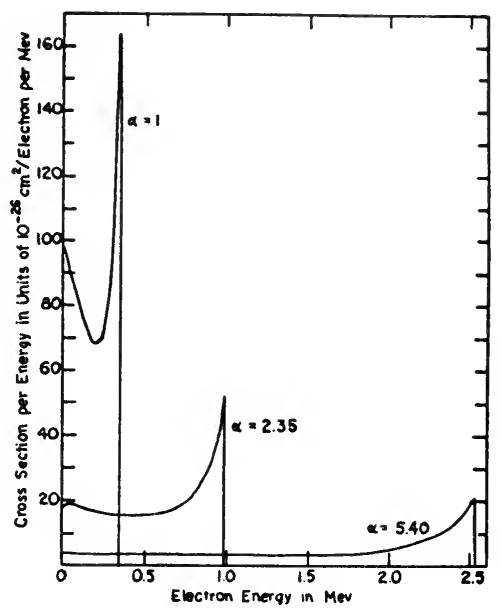

Fig. 7. Compton effect. The differential cross section for giving a free electron a recoil energy in the interval between $T$ and $T+d T$. Eq. (34)

(4) The differential cross section for giving an electron a recoil energy in the interval between $T$ and $T+d T$. In terms of eq. (23) this can be shown to be

$$
\left(d_{e} \sigma / d T\right)=\left[\left(2 \pi m c^{2}\right) /\left(h v^{\prime}\right)^{2}\right]\left(d_{e} \sigma / d \Omega\right)
$$

or in terms of only the primary energy $h v$ and the electron energy $T$ it can be written

$$
\frac{d_{e} \sigma}{d T}=\frac{\pi r_{0}^{2} m c^{2}}{(h v-T)^{2}}\left\{\left[\frac{m c^{2} T}{(h v)^{2}}\right]^{2}+2\left[\frac{h v-T}{(h v)}\right]^{2}+\frac{h v-T}{(h v)^{3}}\left\lfloor\left(T-i m c^{2}\right)^{2}-\left(m c^{2}\right)^{2}\right]\right\}
$$

Representative curves of this equation are given in Fig. 7.

For some experiments it is of interest to know the cross section for the number of photons or for the energy of the photons scattered within a 
cone of half angle $\theta_{0}$. These can be obtained by integrating eqs. (23) and (27) over $\varphi^{\prime}$ from 0 to $2 \pi$, and over $\theta$ from 0 to $\theta_{0}$. Indicating these cross sections by $e_{e} \sigma^{\theta_{0}}$ and $e_{e} \sigma_{s}^{\theta_{0}}$ respectively it can be shown that

$$
\begin{aligned}
{ }_{e} \sigma^{\theta_{0}=\pi}=\pi r_{0}^{2}\left\{\frac{1}{2 \alpha^{2}\left(1+\alpha-a \cos \theta_{0}\right)^{2}}\right. & {\left[4+10 \alpha+8 \alpha^{2}+\alpha^{3}\right.} \\
-\left(4+16 \alpha+16 \alpha^{2}+2 \alpha^{3}\right) \cos \theta_{0} & \left.+\left(6 \alpha+10 \alpha^{2}+\alpha^{3}\right) \cos ^{2} \theta_{0}-2 \alpha^{2} \cos ^{3} \theta_{0}\right] \\
& \left.+\left(\frac{\alpha^{2}-2 \alpha-2}{a^{3}}\right) \ln \left(1+\alpha-\alpha \cos \theta_{0}\right)\right\}
\end{aligned}
$$

and

$$
\begin{aligned}
e \sigma_{s}^{\theta_{0}}=\pi r_{0}^{2}\left\{\alpha^{-3}\right. & \ln \left(1+\alpha-\alpha \cos \theta_{0}\right) \\
- & {\left[6 \alpha^{2}\left(1+\alpha-\alpha \cos \theta_{0}\right)^{3}\right]^{-1}\left[6+15 \alpha+3 \alpha^{2}-12 \alpha^{3}-8 \alpha^{4}\right.} \\
& -\left(6+30 \alpha+27 \alpha^{2}-18 \alpha^{3}-24 \alpha^{4}\right) \cos \theta_{0} \\
& +\left(15 \alpha+33 \alpha^{2}-24 \alpha^{4}\right) \cos ^{2} \theta_{0} \\
& \left.\left.-\left(9 \alpha^{2}+6 \alpha^{3}-8 \alpha^{4}\right) \cos ^{3} \theta_{0}\right]\right\} .
\end{aligned}
$$

Putting $\theta_{0}=\pi$ in these equations yields ${ }_{e} \sigma$, eq. (25), and ${ }_{e} \sigma_{s}$, eq. (28). With $\theta_{0}=\pi / 2, e_{e} \sigma_{s j}$, the cross section for the energy scattered forward, which is plotted in Fig. 4 is obtained from eq. (36). ${ }_{e} \sigma_{s b}$ is ${ }_{e} \sigma_{s}-{ }_{e} \sigma_{s f}$. For small angles $\left(\ll 5^{0}\right)$ the approximation

$$
e^{\sigma^{\theta_{0}}}={ }_{e} \sigma_{s}^{\theta_{0}}=\pi r_{0}^{2} \theta_{0}^{2}
$$

can be used. (At small angles, however, coherent scattering from bound electrons is appreciable, and in any experiments, incoherent and coherent scattering from bound electrons, discussed below, should be taken into consideration.)

Curves and tables of values of quantities derived from the KleinNishina equations have been compiled by various workers 9, 17, 23. Probably the most extensive work has been done by Nelms ${ }^{23}$ who gives curves for eq. (16), (18), (23), (29), (32), and (34), from which values may be read to an accuracy of $2 \%$. All equations for the Compton cross sections given here are the cross sections per electron. To obtain the cross sections per atom for any element, they must be multiplied by the atomic number $Z$.

The scattering of radiation by free electrons discussed above is really just the limiting case, at high energies, of scattering by atomic electrons. In the more general case, the effect of the binding of the electrons to the atom, and their motions and distribution within the atom must be considered. The total scattering can be separated into coherent and incoherent scattering. In coherent scattering the energy of the scattered radiation is

23 A. T. Nelms, Natl. Bur. Standards Circular 542 (1953). 
the same as the incident energy; the electron is neither excited nor ionized; and there is a relation in phase of the scattering from different electrons so that before obtaining the total intensity of the radiation scattered by the atom, the amplitudes of that scattered by each electron must be added. In incoherent scattering the energy of the scattered radiation is less than the incident energy, the difference in the two energies being given to the scattering electron, which either jumps to an excited level of the atom or leaves the atom entirely. In this case there is no phase relation between the radiation scattered by the different electrons of an atom, so the total intensity scattered by the atom is obtained by adding the intensities scattered by each electron. In general terms we may write the total differential cross section for scattering, per atom, as

$$
d_{a} \sigma=d_{a} \sigma_{\text {coh }}+d_{a} \sigma_{\text {incoh }}
$$

Both the coherent and the incoherent differential cross sections may be expressed as the product of two factors ${ }^{15}$. The first factor concerns the probability that the photon be deflected by a certain angle and transfer to the electron a corresponding amount of momentum as though the electron were free. The second factor concerns the probability that the electron, having received this momentum, will absorb a certain amount of energy and thereby become excited or leave the atom.

For incoherent scattering the first factor may be taken as the KleinNishina cross section, eq. (22). The second factor, per electron, called in this case the incoherent scattering function, $S$, may be expressed as the square of a generalized atomic form factor summed over all excited states of the atom, integrated over the continuous spectrum, and divided by $Z .{ }^{24}$ It can also be expressed as $\left[1-(1 / Z) \sum_{1}^{z} f_{n}^{2}-C\right]$ where $f_{n}$ is the electronic scattering factor which gives the amplitude of the radiation scattered coherently by the $n$th electron in terms of that scattered coherently by a free electron, and $C$ is a corrective term which takes into account electron transitions forbidden by the exclusion principle ${ }^{24,25,26}$. The incoherent scattering per atom is then $d_{a} \sigma_{\text {incoh }}=Z d_{e} \sigma_{K-N} S$.

For coherent scattering the first factor may be taken as the cross section for Thomson scattering, eq. (24), which is what the Klein-Nishina cross section becomes after putting the ratio of the incident and scattered energies equal to each other and deleting terms which correspond to a change in the electron spin. The second factor, per atom, is the square of the atomic structure factor $F=\sum_{1}^{z} f_{n}$. That is, it is $\left(\sum_{1}^{z} f_{n}\right)^{2}$. Here $f_{n}$ is the

24 G. White, unpublished Natl. Bur. Standards Report No. 2763 (1953).

25 I. Waller and D. R. Hartree, Proc. Roy. Soc. (London) A124, (1929) 119.

26 M. H. Pirenne, The Diffraction of X-rays and Electrons by Free Molecules, (Cambridge University Press, 1946). 
electronic structure factor described in the above paragraph, and $F$, the atomic structure factor is the ratio of the amplitude of the radiation scattered by the atom to the amplitude which a single electron would scatter. We can now write eq. (38) as

$$
d_{a} \sigma=Z d_{e} \sigma_{K-x} S+d_{\epsilon} \sigma_{\text {Thomson }}\left(\sum_{1}^{z} f_{n}\right)^{2}
$$

The values which theory yields for the incoherent scattering function, $S$, and for the atomic structure factors $\sum_{1}^{z} f_{n}$, depend on the distribution of charge within the atom. One distribution ${ }^{27}$ proposed by Thomas and Fermi considers the electrons as a gas surrounding the nucleus, with a charge density which is a function only of the distance from the nucleus. Another distribution ${ }^{27}$ developed by Hartree, using the method of self-

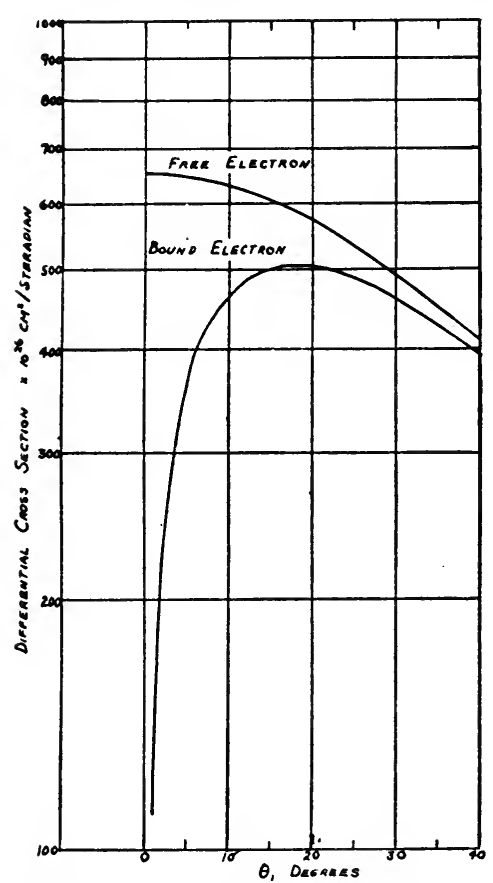

Fig. 8. Illustration of the effect of the incoherent scattering function, $S$, on the Klein-Nishina cross section for $P b$ at $h v=300 \mathrm{keV}$

consistent fields, separates the electrons into completed groups, the electron in each group having the same position probability distribution, but that for each group being different.

For the incoherent scattering function $S$, the Thomas-Fermi distribution is the easier to apply, but neither it nor modifications of it yield accurate values for all $Z$ and all recoil momenta ${ }^{24}$. Calculations using the Hartree method ${ }^{28}$ should be more accurate, but they are more difficult and require separate calculations for each atom. However, all theories agree that for small values of the recoil momentum $[\sim(1 / \lambda)$ $\sin (\theta / 2)]$ the atom is almost certain to remain in its ground state, so that $S$ becomes very small and approaches zero, while for recoil momenta much larger than the momentum of the electron in its bound state the recoiling electron is practically certain to leave the atom, and $S$ approaches 1 , so that the scattering becomes that from a free electron. (See e.g. Fig. 8). In contrast to this, coherent scattering takes place mainly at small angles,

27 A. H. Compton and S. K. Allison, X-rays in Theory and Experiment (D. Van Nostrand Company, Inc. New York, 1935) p. 145.

28 See e.g. D. R. Hartree, Reports on Progress in Physics 11, (1946-47) 113; J. C. Slater, Phys. Rev. 81, (1951) 385; and G. W. Pratt, Jr., Phys. Rev. 88, (1952) 1217. 
more than $3 / 4$ of it being scattered by less than the angle $\theta_{0}$ where ${ }^{29}$

$$
\theta_{0}=2 \sin ^{-1}\left[2.6 \times 10^{-2} Z^{1 / 3}\left(m c^{2} / h \nu\right)\right] .
$$

In obtaining the values of ${ }_{a} \sigma$ given in Table $I$ of the appendix, Miss White ${ }^{15}$ used values of $S$ calculated by Bewilogua ${ }^{30}$ from equations developed by Heisenberg ${ }^{31}$ using the Fermi-Thomas distribution. Values of $\left(\sum_{1}^{z} f_{n}\right)^{2}$ for $Z<26$ were obtained from calculations by James and Brindley ${ }^{32}$ using the Hartree distribution. For $Z>26$, the Fermi-Thomas potential values tabulated by Bewilogua ${ }^{30}$ were used. Values of the total cross section, ${ }_{a} \sigma$, were obtained from eq. (39) by numerical integration.

Franz ${ }^{33}$ has developed an integrated equation for $\sigma_{\text {coh }}$, using the ThomasFermi distribution. However, it assumes hard radiation $(<0.2 \mathrm{~A})$ and a factor of 2 was lost in the integration ${ }^{34}$.

Over quite a period of years, experiments on the angular distribution of scattered radiation and measurements of the total absorption coefficients in energy ranges where Compton scattering predominates, have indicated that the Klein-Nishina equations for scattering by free electrons are essentially correct. Moon ${ }^{29}$ and Storruste ${ }^{35}$ have studied the angular distribution of radiation scattered coherently by $0.41 \mathrm{MeV}^{\gamma}$-rays and obtain agreement with Fermi-Thomas theory within $10 \%$. In the low energy region where scattering by bound electrons becomes important, there has been no recent experimental study of absorption coefficients.

\section{$\S$ 4. Pair Production}

A theoretical analysis of pair production in the field of a nucleus has been made by Bethe and Heitler ${ }^{36}$, in the same paper in which they treat bremsstrahlung. Since pair production is the inverse process to bremsstrahlung, the electron in this case making a transition between states of positive and negative energy, the equations for pair production are similar to those for bremsstrahlung. Using the Born approximation, in which the interaction between the electron and the nucleus is considered as only a small perturbation, and neglecting the screening effects of the other electrons of the atom, the differential cross section for the creation of a

29 P. B. Moon, Proc. Phys. Soc. (London) 63A, (1950) 1189.

30

L. Bewilogua, Physik. Z. 32, (1931) 740.

W. Heisenberg, Physik. Z. 32, (1931) 737.

R. W. James and G. W. Brindley, Phil. Mag. 12, (1931) 81.

W. Franz, Z. Physik 98, (1935) 314.

Private communication from G. White.

A. Storruste, Proc. Phys. Soc. (London) 63A, (1950) 1197.

H. Bethe and W. Heitler, Proc. Roy. Soc. (London) 146A, (1934) 83. 
positron with total energy between $E_{+}$and $E_{+}+d E_{+}$, and a negative electron with total energy between $E_{-}$and $E_{-}-d E_{-}$by a $\gamma$-ray of energy $h v$, in the Coulomb field of a nucleus of charge $Z e$ is found to be

$$
\begin{aligned}
{ }_{a} \%\left(E_{+}\right) d E_{+}=\bar{\varphi} & \frac{p_{+} p_{-}}{(h v)^{3}} d E_{+}\left\{-\frac{4}{3}-2 E_{+} E_{-} \frac{p_{+}^{2}+p^{2}}{p_{+}^{2} p^{2}}\right. \\
& +m^{2} c^{4}\left(\frac{E_{+} \varepsilon_{-}}{p_{-}^{3}}+\frac{\varepsilon_{+} E_{-}}{p_{+}^{3}}-\frac{\varepsilon_{+} \varepsilon_{-}}{p_{+} p_{-}}\right) \\
& +L\left[\frac{(h v)^{2}}{p_{+}^{3} p_{-}^{3}}\left(E_{+}^{2} E_{-}^{2}+p_{+}^{2} p_{-}^{2}\right)-\frac{8}{3} \frac{E_{+} E_{-}}{p_{+} p_{-}}\right. \\
& \left.\left.-\frac{m^{2} c^{4} h v}{2 p_{+} p_{-}}\left(\frac{E_{+} E_{-}-p_{-}^{2}}{p_{-}^{3}} \varepsilon_{-}+\frac{E_{+} E_{-}-p_{+}^{2}}{p_{+}^{3}} \varepsilon_{+}+\frac{2 h v E_{+} E_{-}}{p_{+}^{2} p_{-}^{2}}\right)\right]\right\}
\end{aligned}
$$

Here $E_{-}=h v-E_{+} \cdot \varepsilon_{+}=2 \ln \left[\left(E_{+}+p_{+}\right) / m c^{2}\right]$,

$$
\varepsilon_{-}=2 \ln \left[\left(E_{-}+p_{-}\right) / m c^{2}\right], L=2 \ln \left[\left(E_{+} E_{-}+p_{+} p_{-}+m^{2} c^{4}\right) / m c^{2} h v\right],
$$

$\bar{\varphi}=Z^{2} r_{0}^{2} / 137, p_{+}$and $p_{-}$are the momenta of the electrons in energy units, and the other symbols have been defined above. Eq. (41) is limited by the condition

$$
\left(Z e^{2} / \hbar v_{+}\right) \ll 1 \quad\left(Z e^{2} / \hbar v_{+}\right) \ll 1
$$

which is the condition for the validity of the Born approximation. It also assumes that pair formation takes place in the Coulomb field of the nucleus. This is so only if

$$
137 \frac{m c^{2} h v}{2 E_{+} E_{-} Z^{1 / 3}} \gg 1
$$

The quantity on the left hand side of this equation is the radius of the atom according to the statistical model of Fermi and Thomas, i.e. $\left(137 \hbar Z^{-1 / 2}\right) / m c$, divided by the maximum value of $\hbar / q$ permissible by conservation of energy and momentum for a given radioactive process, and $\hbar / q$ is the distance from the nucleus within which pair production is most probable ${ }^{36,}{ }^{37}$.

When the distance from the nucleus at which pair production is most probable lies outside some of the electronic shells of the atom, the field in which the pairs are created is less than that of the nucleus alone, by a factor depending on the atomic form factor. Calculations taking this into consideration have been made by Bethe, using the Fermi-Thomas distribution ${ }^{38}$. He obtains as the cross section for the creation of a positive

37 E. Segré, Experimental Nuclear Physics, (John Wiley and Sons, Inc. Now York 1953) Vol. I, Part II, Section 3 p. 259.

38 H. A. Bethe, Proc. Cambridge Phil. Soc. 30, (1934) 524. 
electron with total energy between $E_{+}$and $E_{+}+d E_{+}$, the equation ${ }_{a} \chi\left(E_{+}\right) d E_{+}=\bar{\varphi} \frac{d E_{+}}{(h \nu)^{3}}\left\{\left(E_{+}^{2}+E_{-}^{2}\right)\left[\varphi_{1}(\gamma)-\frac{\xi}{3} \ln Z\right\rceil+\xi E_{+} E_{-}\left[\varphi_{2}(\gamma)-\{\ln Z]\right\}\right.$

where $\gamma=\left(100 m c^{2} h v\right) /\left(E_{+} E_{-} Z^{1 / s}\right)$ and $\varphi_{1}(\gamma)$ and $\varphi_{2}(\gamma)$ are functions of $\gamma$ whose values were obtained by numerical integration, for $\gamma$ between 0 and 2. For smaller energies $(2<\gamma<15)$ a more convenient formula ${ }^{36}$ is

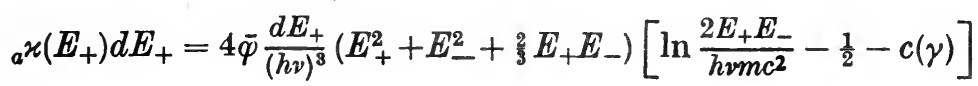

Curves of $\varphi_{1}(\gamma)$ and $\varphi_{2}(\gamma)$ and values of $c(\gamma)$ are given by Bethe and Heitler ${ }^{36}$ and by Bethe and Ashkin ${ }^{39}$. At very high energies $(\gamma \sim 0)$ screening is complete and the cross section becomes

$$
\left.a^{\varkappa(}\left(E_{+}\right) d E_{+}=4 \bar{\varphi} \frac{d E_{+}}{(h v)^{3}}\left[E_{+}^{2}+E_{-}^{2}+\frac{2}{3} E_{+} E_{-}\right) \ln \left(183 Z^{-1 / s}\right)-\frac{1}{9} E_{+} E_{-}\right]
$$

Eq. (41), (44), (45), and (46) give the differential cross section for the energy distribution of the pair electrons. The total cross section, i.e. the cross section for the production of a positive and negative electron pair, is obtained by integrating these over all possible energies of the positive electron. Analytic integration of these equations is impossible except for eq. (46), so the total cross section for a given energy must be obtained numerically from the area under the curves drawn from these equations, such as those shown in Fig.9. In order that the area under the curves be the total cross section, the ordinates of Fig. 9 are the values of $a^{\varkappa(}\left(E_{+}\right)$

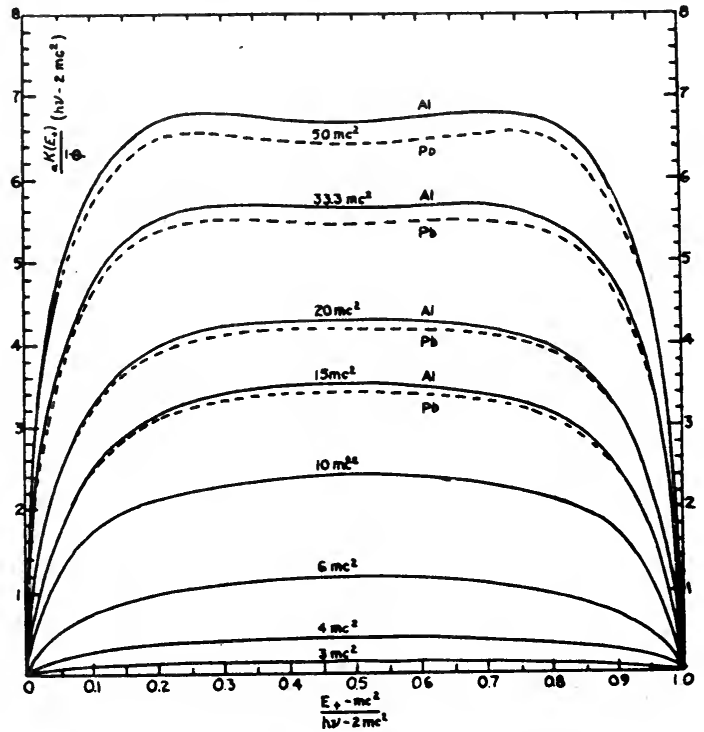

Fig. 9. Pair production. sergy distribution of pairs. For energies up to $10 m c^{2}$ Eq. (41) was used. Curves for higher energies were calculated from Eq. (44) and (45) in units of $\bar{\varphi}$, multiplied by the total kinetic energy, $h v-2 m c^{2}$, and the abscissae are the fraction of the available kinetic energy which the positron receives. 
Hough ${ }^{40}$ has given a formula for the energy distribution of pair electrons, without screening, which approximates eq. (41); is valid for $h \nu<20 m c^{2}$, and overlaps eq. (46) between 10 and $20 m c^{2}{ }^{41}$. It can be written as

$$
{ }_{a^{\varkappa}}\left(E_{+}\right) d E_{+}={ }_{a^{\varkappa}} z\left\{1+0.135\left[\left({ }_{a} \varkappa_{m} / \bar{\varphi}\right)-0.52\right] z\left(1-z^{2}\right)\right\} d E_{+}
$$

where $\left.z=2[x(1-x)]^{1 / 2}, x=\left(E_{+}-m c^{2}\right) / h v-2 m c^{2}\right)$ and $a^{\chi_{m}}$ is the differential cross section for the equal partition of energy, i.e. the value of $a_{a} x\left(E_{+}\right)$from eq. (41) when $E_{+}=E_{-}=h v / \therefore$. The second term in the curly bracket of eq. (47) should be dropped when it is negative. Values from eq. (47) agree with those from the Bethe-Heitler equations within a fraction of $1 \%$ for $0.05<x<0.95$ and $h v<10 m c^{2}$.

The equations given above for ${ }_{a} \chi\left(F_{+}\right) d E_{+}$, as can be seen in Fig. 9, show a symmetrical energy distribution between the positive and negative electrons. This is due to the use of the Born approximation which neglects the interaction between the electrons and the nucleus. At low energies, particularly for high $Z$, the interaction with the nucleus is not negligible, and because of the repulsion of the positron by the nucleus and the attraction of the negatron to the nucleus, the distribution will be asymmetric. Using Dirac wave functions for the pair electrons, Jaeger and Hulme ${ }^{42}$ and Jaeger ${ }^{43}$ have calculated values for the total pair production cross section for the photon energies of $3 m c^{2}(1.53 \mathrm{MeV})$ and $5.2 m c^{2}(2.66 \mathrm{MeV})$. Their results are shown in Table $\mathrm{V}$ where they are

TABLE V

Pair Production Cross Sections as calculated by Jaeger and Hulme 42 and Jaeger ${ }^{43}$ compared with Results from Bethe and Heitler ${ }^{36}$ who used the Born Approximation

\begin{tabular}{c|c|c|c|c}
\hline \hline & \multicolumn{3}{|c|}{$h v=3 m c^{2}$} & $h v=5.2 m c^{2}$ \\
\hline$Z$ & 50 & 65 & 82 & 82 \\
\hline$a^{x} \times 10^{24}$ & 0.17 & 0.34 & 0.67 & 3.1 \\
\hline$a^{x} \times 10^{24}$ (Born) & 0.13 & 0.21 & 0.34 & 2.6
\end{tabular}

compared with values obtained by Bethe and Heitler using the Born approximation. It is seen that for high $Z$ and low energy the Born approximation gives values of the total pair production cross section which are too low, by as much as a factor of 2 , but that this discrepancy decreases rapidly with increasing energy and decreasing atomic number. For

40 P. V. C. Hough, Phys. Rev. 73, (1948) 266.

41 H. A. Bethe and J. Ashkin, reference 37, p. 330.

42 J. C. Jaeger and H. R. Hulme, Proc. Roy. Soc. (London) 153A, (1936) 443.

43 J. C. Jaeger, Nature 137, (1936) 781 
$h v=3 m c^{2}$ Jaeger ${ }^{43}$ gives the following empirical formula for obtaining the pair production cross section for other $Z$ :

$$
a^{\varkappa} \times 10^{24}=0.95(Z / 137)^{2}+2.54(Z / 137)^{4}
$$

Experimental results at high energies $(>10 \mathrm{MeV})^{44-50}$ have indicated cross sections appreciably less than those given by the Bethe-Heitler Born approximation equations. Recent theoretical analysis by Bethe, Maximon, and Davies ${ }^{51}$, not using the Born approximation, yields results in agreement with experiment.

The production of positive and negative electron pairs is also possible in the field of an atomic electron. In this case the atomic electron may carry away an appreciable amount of the energy of the incident $\gamma$-ray. Considering the conservation of energy and momentum among the three particles involved, it can be shown ${ }^{52}$ that the threshold for this process is $4 m c^{2}$. Theoretical analysis of the process, using Dirac perturbation theory and the Born approximation has been carried out by Borsellino ${ }^{53}$, Nemirovsky ${ }^{54}$, Votruba ${ }^{55}$, and Watson ${ }^{52}$ without considering screening. Above about $20 \mathrm{MeV}$ screening must be taken into account and this has been done by Wheeler and Lamb ${ }^{56}$. Borsellino ${ }^{53}$ has analyzed the process for an energy range from 4 to $100 m c^{2}$. At the threshold, $h v \sim 4 m c^{2}$ the total cross section can be written as ${ }^{57}$

$$
a^{\varkappa_{\text {elec }}}=\frac{\bar{\varphi}}{Z} \frac{\pi V 3}{2^{2} 3^{5}}\left(\frac{h v-4 m c^{2}}{m c^{2}}\right)^{2}
$$

For intermediate energies Borsellino has made the integrations and presents

44 J. L. Lawson, Phys. Rev. 75, (1949) 433.

45 R. L. Walker, Phys. Rev. 76, (1949) 527.

46 C. D. Adams, Phys. Rev. 74, (1949) 1707.

17 J. W. DeWire, A. Ashkin and L. A. Beach, Phys. Rev. 83, (1951) 505.

48 C. R. Emigh, Phys. Rev. 86, (1952) 1028.

49 E. S. Rosenblum, E. F. Shrader and R. MI. Warner, Jr., Phys. Rev. 88, (1952) 612 .

so A. I. Berman, Phys. Rev. 90, (1953) 210.

51 H. A. Bethe and L. C..Maximon, Phys. Rev. 93, (1954) 768; and H. Davies, H. A. Bethe and L. C. Maximon, Phys. Rev. 93, (1954) 788.

s2 K. M. Watson, Phys. Rev. 72, (1947) 1060.

63 A. Borsellino, Helv. Phys. Acta 20, (1947) 136; Nuovo cimento 4, (1947) 112;

Rev. univ. Tucuman A6, (1947) 7.

s4 P. Nemirovsky, J. Phys. (U.S.S.R.) 11, (1947) 94.

55 V. Votruba, Phys. Rev. 73, (1948) 1468.

s6 J. A. Wheeler and W. E. Lamb, Phys. Rev. 55, (1939) 858.

57 This equation is from Votruba (reference 55). It is less than that given by Borsellino by a factor of 4.5 . 
the results as tabulated values of $F(\alpha)$ where

$$
a^{\chi_{\text {elec }}}=(\bar{\varphi} / Z) F(x)
$$

and $\alpha=h v / m c^{2}$. They are given in Table VI. In the energy range of radioactive $y$-rays being considered here, the contribution of pair production in the field of the atomic electrons is comparatively small. (See Tables in Appendix I.) However, it becomes more important with increasing photon energy and at extremely high energies becomes the same as that for pair production in the field of a nucleus ${ }^{58}$.

TABLF VI

Patr Production in the Field of an Electron. Values given by Borsellino ${ }^{53}$ FOR $a^{\chi_{\text {elec }}}=(\bar{\varphi} / Z) F(x)$

\begin{tabular}{c|c|c|c|c|c|c|c}
\hline$\alpha=h v / m c^{2}$ & 4 & 4.4 & 5.2 & 6 & 10 & 20 & 50 \\
\hline$F(\alpha) \cdot . \cdot . \cdot$. & 0 & 0.0044 & 0.038 & 0.102 & 0.627 & 2.05 & 4.78
\end{tabular}

The values of the cross sections for pair production in the field of a nucleus which are given in Table I of Appendix I were obtained by White in the following manner. (1) For $h v>20 m c^{2}$ calculations were made according to the Bethe-Heitler theory with screening corrections from the Fermi-Thomas model. These were corrected for errors in the Born approximation at high energies by using ratios of experimental cross sections to these calculated cross sections, as obtained from the best line drawn through a plot of the ratios, taken from the results of Lawson ${ }^{44}$, Walker ${ }^{45}$, and Adams ${ }^{46}$. Later data by DeWire, Ashkin, and Beach ${ }^{47}$, and by Emigh ${ }^{48}$ indicate that the corrections used at high $Z$ may be too large by 1 or $2 \%$. (2) For $h v<20 m c^{2}$ calculations by Davisson and Evans ${ }^{16}$ from the Bethe-Heitler theory were used. (3) At energies of $3 m c^{2}$ and $5.2 m c^{2}$ the non-Born calculations of Jaeger and Hulme ${ }^{42}$ for lead, along with the formula by Jaeger, eq. (48), for other elements were used. Graphical interpolation was made between the three energy regions to produce a smooth curve for $a^{\varkappa}$ as a function of energy.

The cross section for the production of pairs in the field of the atomic electrons was calculated from the Borsellino results. Beyond $50 \mathrm{MeV}$ an extrapolation was made, aided by the results of Wheeler and Lamb ${ }^{56}$.

Curves of the total cross section for pair production in the field of the nucleus are given in Fig. 10. It is seen that with increasing energy of the primary radiation, the probability of pair formation increases rapidly. At higher energies than those shown in Fig. 10 they approach a constant value.

68 H. A. Bethe and J. Ashkin, reference 37 " ?3? 
Most experimental studies of the pair production cross section are made by measuring the total absorption coefficient and subtracting the theoretical values for Compton effect and photoelectric effect. This is a fairly

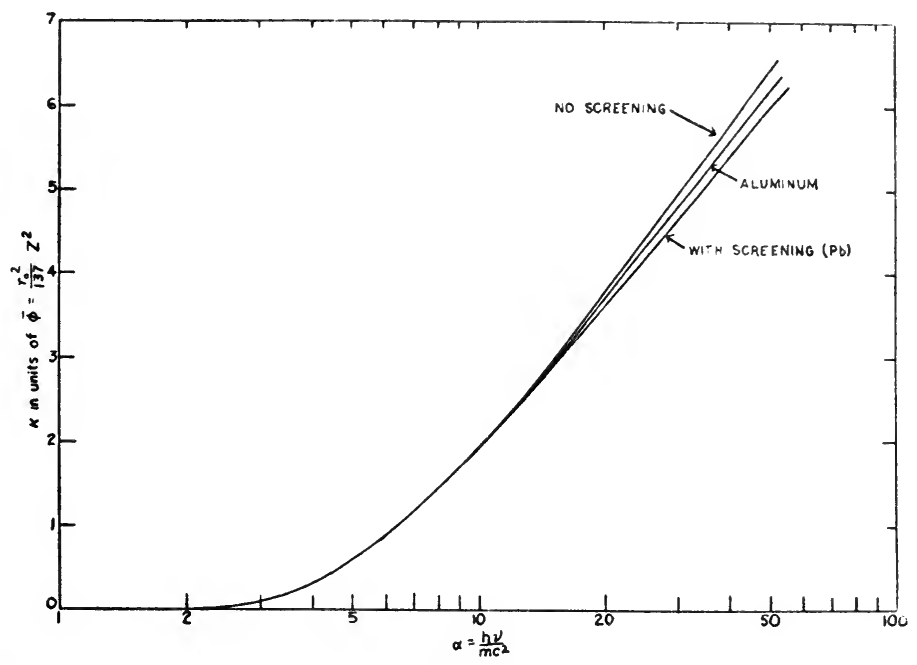

Fig. 10 Pair production. Integrated cross sections vs energy

accurate method, since the theory of the Compton effect is accurately known, and above about $2 \mathrm{MeV}$ the photoelectric effect is a comparatively small contribution. Both older experiments ${ }^{59}$ and more recent ones ${ }^{60}$ show good agreement with theory in the energy range of radioactive $\gamma$-rays.

\section{§ 5. Total Absorption Coefficients}

Values of the total absorption coefficient $\mu$, eq. (4), are given in the Tables of Appendix I in units of $\mathrm{cm}^{2} /$ atom and also in $\mathrm{cm}^{2} / \mathrm{g}$. Some curves for low $Z(\mathrm{Al})$ and high $Z(\mathrm{~Pb})$ are given in Fig. 11 and 12. It is seen that in general, at low energies the photoelectric absorption predominates, while at high energies absorption becomes completely that due to pair production. At intermediate energies there is a minimum in which the scattering by Compton effect predominates. However, the relative importance of each mode of absorption varies with the atomic number of the absorber. For $\mathrm{Al}(Z=13)$ absorption is almost entirely Compton scattering between $0.3 \mathrm{MeV}$ and $3 \mathrm{MeV}$, while for $\mathrm{Pb}(Z=82)$ all three processes are appreciable between $3 \mathrm{MeV}$ and $5 \mathrm{MeV}$.

59 See e.g. W. Gentner and J. Starkiewicz, J. phys. et radium 6, (1935) 340; and J. C. Jacobsen, Z. Physik, 103, (1936) 747.

so See e.g. S. A. Colgate, Phys. Rev. 87, (1952) 592 and review of past experimental work in reference 16. 
In the energy range from about $100 \mathrm{keV}^{\prime}$ to $10 \mathrm{MeV}^{\prime}$ most experimental measurements of the total absorption coefficients agree well with the theories, i.e. with the values given in the appendix. At lower energies, however, no recent experimental studies of total absorption coefficients have been made. Earlier measurements ${ }^{61}$ of total absorption coefficients

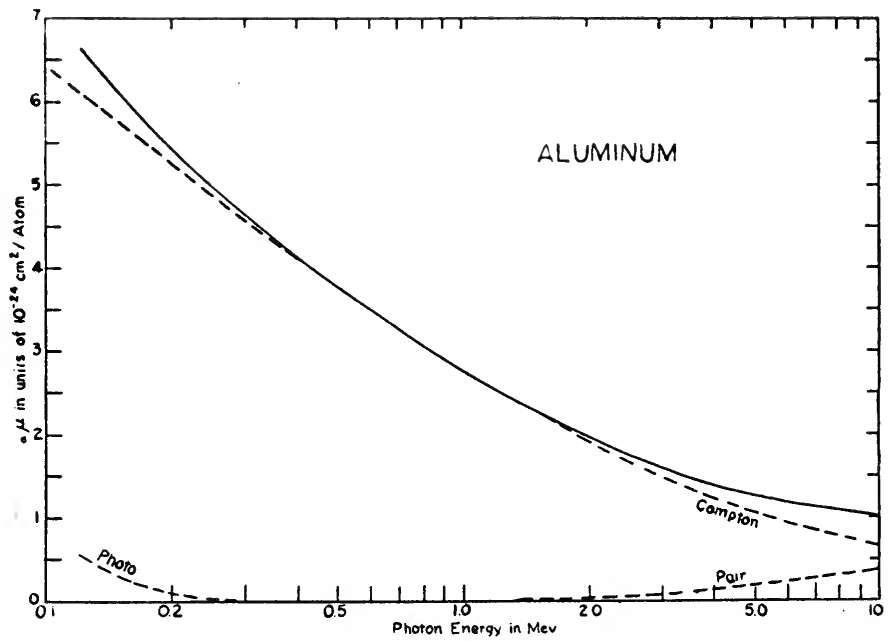

Fig. 11. Absorption coefficients is energy for $\mathrm{Al}$

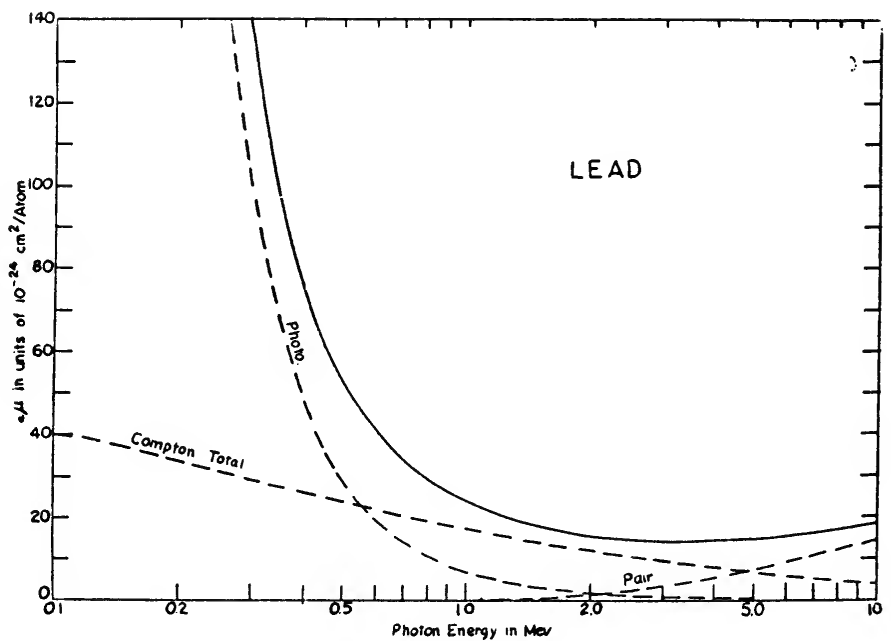

Fig. 12. Absorption cocfficients $v$ s energy for $\mathrm{Pb}$

-1 For example, S. J. M. Allen, Phys. Rev. 27, (1926) 266; K. Grosskurth, Ann. Physik 20, (1934) 197; K. Schulz, Ann. Physik 27. (1936) 1; I. Müller, Ann. Physik 32, (1938) 625; and s. Laubert, Ann. Physik, 40, (1941) 553. 
show a wide distribution of results ${ }^{62}$. For low $Z$, and in the neighborhood of $10 \mathrm{keV}$, the best curve drawn through these results indicates absorption coefficients greater than those given in Table I of Appendix I by about $10 \%$. It is hoped that more accurate measurements of total absorption coefficients in the low energy, low $Z$ region will soon be made so that the numerical results given in the appendix can be more accurately checked.

\section{§ 6. Absorption Methods}

Absorption methods can be used to measure absorption coefficients of $\mathrm{X}^{A}$ $\boldsymbol{\gamma}$-rays of known energies in order to check experimental values with those which theory predicts; or, using the absorption coefficients predicted by theory and tested experimentally, they can be used to determine the energy of a $\gamma$-ray beam. Their use for determining the energy of a $\gamma$-ray beam is at best only a rough method of measurement unless it is known in advance that only one energy is present in the beam. The method can be used, however, to give a rough idea of the degree of homogeneity of the radiation under investigation and to give some idea as to the energy region in which careful studies with more accurate methods should be made.

Basically an absorption method consists of measuring the response of a detector to a $\gamma$-ray source with and without an absorber between them. Assuming that no scattered radiation reaches the detector, the two measurements are proportional, respectively, to $B$ and $B_{0}$ of eq. (2), from which we see that

$$
\log B=-\mu x+\log B_{0}
$$

If the $\gamma$-ray source emits only one energy, then the logarithm of the measured response, $B$, when plotted against the absorber thickness, $x$, should give a straight line whose slope is the total absorption coefficient for that energy. From this measured absorption coefficient the energy of the $\gamma$-ray can be determined, provided it is known on which side of the minimum in the absorption curve the energy lies. This can be found by measuring $\mu$ with absorbers of two different materials. Most $\gamma$-rays from radioactive substances are on the low energy side of the minimum.

If a $\gamma$-ray source emits more than one energy, then a logarithmic plot of the readings will yield a curve whose slope is greater at first and decreases with increasing absorber thickness as the more highly absorbed $\gamma$-rays are filtered out. If a radioactive source emits only 2 or $3 \gamma$-rays whose energies are not too close together, and if at large absorber thicknesses the curve has become a straight line indicating that all but the highest energy has

62 For semi-empirical equations based on these results see J. A. Victoreen, J. Appl. Phys. 19, (1948) 855. 
been filtered out. then a rough determination of the absorption coefficient for each $\gamma$-ray can be made. To do this the absorption coefficient of the highest energy present is obtained from the final slope of the absorption curve. Using this slope to extrapolate back to $x=0$, and subtracting the values on this extrapolated line from those on the measured curve, for different values of $x$, a new curve can be plotted which gives the combined absorption of the two lower energies present. The final slope of this second curve is the absorption coefficient of the next highest energy present, and by repeating this procedure, the absorption coefficient of the third $\gamma$-ray can also be found. Although the results of such an analysis are not very accurate, the method can yield valuable information. For example, it was by this method that the $0.638 \mathrm{MeV} \gamma$-ray of $\mathrm{I}^{131}$ was discovered ${ }^{63}$.

For accurate measurements of absorption coefficients it is necessary to have either a source which emits $\gamma$-rays of only one energy, or a detector which can filter out or sort the low energies from the highest one. Such a detector might be a set of $\beta$-ray coincidence counters with absorbers between to filter out all but the highest energy secondary electrons, a scintillation counter biased to count only the largest impulses, or a cloud chamber.

Although the principle on which absorption measurements are based is very simple, the experimental determination of absorption coefficients is complicated by the presence of scattered radiation. Some of this may be scattered by room objects in the vicinity of the apparatus, or even by the surrounding air. ${ }^{35,64}$ If because of low specific activity the volume of the source must be large, radiation scattered within the source itself may reach the detector. The greatest amount of scattered radiation, however, is likely to be that which has been scattered in the absorber at angles small enough to reach the detector.

To prevent radiation from being scattered by room objects or air, and reaching the detector, both the source and the detector should be shielded by adequate amounts of lead or other highly absorbing material. To decrease scattering within the source itself, small sources with high specific activity should be used if possible. As to radiation scattered in the absorber, the amount which can reach the detector is a function of the solid angle within which a $\gamma$-ray from the source can be scattered in the absorber and still reach the detector. To decrease this solid angle and thus reduce the scattered radiation, distances between source and absorber, and absorber and detector should be as large as is experimentally feasible. Also the diameter of the absorber should not be larger than is necessary

63 C. M. Davisson and R. D. Evans, Phys. Rev. 81, (1951) 404.

64 C. L. Cowan, Phys. Rev. 76, (1949) 1348. 
to intercept the primary beam from the source, or else lead collimation should be placed between the absorber and the detector. For a given geometry the amount of radiation scattered in the absorber which can theoretically be expected to reach the detector can be estimated, for Compton single scattering, from the equation ${ }^{16,65}$

$$
S / B=N_{e} x_{e} \sigma^{\theta_{0}}
$$

where $S / B$ is the ratio of the number of scattered to the number of transmitted photons, $N_{e}$ is the number of electrons per unit volume in the absorber, $x$ is the absorber thickness, and $e_{e} \sigma^{\theta_{0}}$ is given by eq. (35) or (37). For coherent scattering, some idea of the amount can be gathered from eq. (40) or from the curves of Moon ${ }^{29}$ and Storruste ${ }^{35}$. Experimentally, the position of the absorber can be varied until the measured transmission is a minimum ${ }^{66}$, or the absorption coefficients can be measured for different values of the solid angle and extrapolated to zero solid angle ${ }^{60}$.

In the special case of a source which has a continuous energy distribution, or a large number of discrete energies, it can be shown theoretically ${ }^{67}$ that if no collimation is used, and secondary radiation produced in the absorber is allowed to reach the detector, then when a semi-logarithmic plot of the measured intensity versus absorber thickness yields a final straight line, the slope of this line is the absorption coefficient of the highest energy emitted by the source.

65 G. T. P. Tarrant, Proc. Cambridge Phil. Soc. 28, (1932) 475.

66 N. Rudnick, M. S. Thesis, Mass. Inst. of Tech. (May, 1953).

67 Final Report on the M.I.T. Project in High Voltage Radiography, Vol. IV. Production, Absorption, and Scattering of High Voltage X-rays. OSRD 4488 June 1, (1945) 4-3-8 seq. 


\title{
ß-RAY-SPECTROMFTER THEORY AND DESIGN HIGH RESOLUTION SPECTROSCOPY
}

\author{
K. SIEGBAHN
}

\section{$\S 1$. Introduction. General Discussion of Spectrometers}

Though the experimental work in $\beta$ - and $\gamma$-ray spectroscopy, as is evident from the contents of this book, can be performed by means of a great number of different instruments and a large variety of complimentary techniques, the $\beta$-ray spectrometer no doubt is the basic instrument, which has proved to be of the greatest general use. Combined with suitable particle detectors, magnetic or electric fields of various shapes can resolve complex nuclear spectra of particles or quanta (as secondarily produced particles) in considerable detail. A large amount of work has been devoted to the perfection of such instruments. Broadly speaking, the problem has been twofold: to collect as much as possible of the nuclear radiation into the spectrometer and furthermore to attain a maximum of energy resolution. Since all electron optical systems possess aberrations, e.g. a spherical one, it is evident that one cannot find an instrument which is perfect in both respects. The problem is still more complicated since one has to take into account a great number of other important features such as the maximum size of the emitting source, the problem of designing and shielding the detector in order to get low background, the practical difficulties of realizing a particular fieldform and of measuring the field, the power consumption and the cooling problem, the economical and workshop aspects of the construction, the flexibility of the instrument, the problem of accurate adjustments etc. It is also important to consider what type of detector can be used with a particular instrument, e.g. a photographic plate, a GM counter with a small size, very thin window, a scintillation counter etc. It may also be interesting to know whether a certain focusing principle permits ironfree construction, or if permanent magnets can be used. Some types of spectrometer can only be handled conveniently in the low energy range. (this is generally true for electrical spectrometers) and other types may be impracticable or even unreliable for high precision work in the low energy region (certain magnetic spectrometers of shaped field, containing iron). Some focusing principles which mathematically would give excellent properties as to collecting power and resolution may not be suitable for very high resolution because of practical reasons. This is true for many types of spectrometers, among them the 
lens spectrometers which are very sensitive to small deviations in the cylindrical symmetry of the fieldform.

Besides the two main characteristics of a spectrometer, namely collecting power and resolution, there are a number of other features which determine the potentialities of a particular spectrometer type. Several of these are very difficult or even impossible to formulate in mathematical language, and it is therefore understandable that one cannot select a certain spectrometer as being superior to other types only because of a more advantageous "figure of merit" based upon purely theoretical considerations. Nevertheless, such considerations have proved to be very useful and though not always decisive in the choice of a certain spectrometer type, they certainly are of basic importance in a great number of cases.

The large variety of spectrometers may for convenience be classified into magnetic and electric spectrometers, the first group being by far in greatest use. According to accepted nomenclature one further distinguishes between flat and helical spectrometers among the first group. By a "flat" spectrometer one means one where the magnetic lines of force are mainly in the direction perpendicular to the electron paths, whereas "helical" stands for all types of lens fields, i.e. where the lines of force are mainly in the direction of the electron paths.

The two basic types of focusing are those obtained by means of a homogeneous field working either perpendicular to the rays, in which case the electrons describe circles, or nearly parallel to the rays, when they describe helical paths. These two extreme cases are quite simple to treat theoretically and the focusing properties can generally be given in exact and simple form. Historically, these two types of focusing were also the first to be developed, and in particular the first mentioned type was, except for a few preliminary trials of the helical method, the only one in use before the beginning of the forties. All work on classical radioactivity was consequently made with homogeneous flat spectrometers. It is particularly illuminating to study the electron image formation in these two special cases, since many of the basic features found there are qualitatively in common with more complicated types of focusing.

The transverse homogeneous field focuses electrons only in one plane, whereas the longitudinal field is space-focusing. From this point of view the two focusing systems would correspond in optics to the cylindrical and the spherical lenses, respectively. By shaping the transverse field so as to vary as $1 / V \varrho$, $\varrho$ being the distance to the axis of symmetry, the transverse field also becomes space focusing. This so-called "double focusing" spectrometer yields an extraordinarily good theoretical figure of merit and is furthermore relatively easy to construct, both with and without iron, and 
to adjust to very high resolution. The collecting power can be made very high and the source and detection problems are easy to master. The double focusing spectrometer has therefore come into wide use.

The electron optical spherical aberrations of both flat and helical spectrometers can be greatly reduced by shaping the fields in different ways. The collecting power can be increased for flat spectrometers by using sector magnets, i.e. having the source and detector outside the field. One then introduces one more degree of freedom into the electron optical system, since the boundaries of the magnetic pole pieces can be shaped so as to accept more radiation. Several such sector magnets can be used simultaneously in order to increase the collecting power still more. The properties of lens spectrometers with or without shaped fields can be improved by using suitably placed ring focus baffles.

In following paragraphs of this chapter the different types of spectrometers will be presented and the fundamental theoretical problems discussed. There exist several good survey articles in this field. In particular one should mention those by Persico and Geoffrian ${ }^{1}$, Grivet ${ }^{2}$, Cavanagh ${ }^{3}$, Verster ${ }^{4}$ and Hayward ${ }^{5}$. For the older works performed before 1940 the reader is referred to books by Philipp ${ }^{6}$ and by Rutherford, Chadwick and Ellis?.

\section{$\S 2$. Some Basic Relations in $\beta$-Spectroscopy}

An electron (charge $e$, velocity $v$ ) moving in a homogeneous magnetic field $B$ in a plane perpendicular to the lines of force describes a circular path with a radius of curvature $=\varrho$. The motion is given by the equation:

$$
B e v=\frac{m v^{2}}{\varrho}
$$

where $m=\frac{m_{0}}{1-\frac{v^{2}}{c^{2}}}$. The momentum is:

$$
p=m v=e \cdot B \varrho .
$$

In $\beta$-ray spectroscopy it has been found convenient to express the momentum of an electron by means of its $B \varrho$-value, since this value immediately will tell what magnitude of magnetic field and apparatus

1 E. Persico and C. Geoffrian, Rev. Scient. Instr. 21, (1950) 945.

2 P. Grivet, Journal phys. radium 11, (1950) 582; 12, (1952) 1.

3 P. Cavanagh, Progress in Nuclear Physics, 1, (London 1950) 140.

4 N. Verster, Progress in Nuclear Physics, 1, (London 1952) 1.

5 R. Hayward, Advances in Electronics 5, (New York 1953) 97.

B K. Philipp, Kernspektren, Hand- und Jahrbuch d. Chemischen Physik, 9, (Leipzig 1937) 186.

7 E. Rutherford, J. Chadwick and C. Ellis, Radiations from Radioactive Substances, (Cambridge 1930). 
dimensions are required to handle the electrons in a given experiment. The use of momentum coordinates instead of energy ones in $\beta$-ray spectroscopy is partly due to the simple fact that it is the momentum of the focused electrons which is proportional to the magnetic field of the spectrometer or (approximately, if iron is used) to the exciting electric current, and not the energy. For electrostatic spectrometers however, the situation is different, i.e. the energy of the electron is proportional to the deflecting voltage, but since such spectrometers are fairly uncommon, the $B \varrho$-coordinates have been generally accepted.

Suppose that we investigate a distribution of electrons in a magnetic spectrometer, the momentum distribution being $N(p) d p$. If we instead want to plot the spectrum as an energy distribution $n(E) d E$, it is obvious that the widths $d E$ and $d p$ are different, which will influence the form of the spectrum. Since $E=\frac{p^{2}}{2 m}$ we get $d E=\frac{p}{m} d p$ and consequently, using $E$ instead of $p$ as abscissa axis, the corresponding intensities in the momentum distribution should be multiplied by $m / p$.

In a magnetic spectrometer with fixed geometry and $B$ variable the quantity $R=\frac{\Delta(B \varrho)}{B \varrho}$ is constant, where $\Delta(B \varrho)$ is a measure of the accepted momentum band. When plotting the momentum distribution it is therefore necessary to divide the number of counts $P$ at each magnetic field setting by the corresponding field in order to get the correct form of the spectrum. Table $I^{8}$ summarizes how the number of counts $P$ should be handled in the case of a magnetic spectrometer of constant field or constant radius and furthermore of a transverse electric field spectrometer. Both momentum and energy spectra are considered:

TABLE I

\begin{tabular}{|c|c|c|c|}
\hline \multirow{2}{*}{-4} & \multicolumn{2}{|c|}{ Magnetic spectrometer } & \multirow{2}{*}{$\begin{array}{l}\text { Electric spectrometer } \\
\text { Variable defl. field }\end{array}$} \\
\hline & $B=$ const. & $B=$ variable & \\
\hline Values measured directly: & & & \\
\hline Abscissa . . . . . . . . • & Radius $\varrho$ & $\begin{array}{l}\text { Magnetic } \\
\text { field } B\end{array}$ & Voltage $V$ \\
\hline 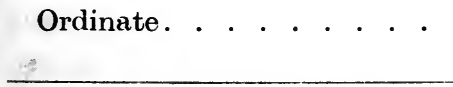 & $\begin{array}{c}\text { Number of } \\
\text { counts } P\end{array}$ & $\begin{array}{c}\text { Number of } \\
\text { counts } P\end{array}$ & Number of counts $P$ \\
\hline $\begin{array}{l}\text { Plotted as momentum distr. } \\
\text { curve: }\end{array}$ & & & \\
\hline Abscissa. . . . . . . & $\underline{g}$ & $B$ & $\begin{array}{lll}V \\
P\end{array}$ \\
\hline Ordinate. $\cdot \cdot \cdot \cdot \cdot \cdot \cdot \cdot \cdot$ & $P$ & $P / B$ & $P / / V$ \\
\hline 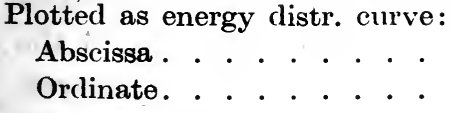 & $\stackrel{Q^{2}}{P} / \varrho$ & $\begin{array}{c}B^{2} \\
P / B^{2}\end{array}$ & $\stackrel{V}{P / V}$ \\
\hline
\end{tabular}

8 Quoted from: O. Klemperer, Electron Optics, Cambridge University Press 1953. 
Conversion lines having $B \varrho$ values extending from $\sim 1000$ to 3000 are particularly easy to observe. $50 \mathrm{keV}^{v}$ corresponds to $B \varrho \sim 800$ and $500 \mathrm{keV}$ to $B Q \sim 3000$. The relation between momentum and energy $E$ is obtained by combining (2) with

which yields

$$
E=m c^{2}-m_{0} c^{2}
$$

$$
E=\left\{\left(m_{0} c^{2}\right)^{2}+e^{2} c^{2}(B \varrho)^{2}\right\}^{1 / 2}-m_{0} c^{2} .
$$

If we express $E$ in $\mathrm{keV}$ and $B \varrho$ in Gauss $\mathrm{cm}$ we get (using DuMond and Cohen's latest adjusted values of elementary constants ${ }^{119}$ ) very accurately:

$$
E(\mathrm{keV})=(510.984 \pm 0.016)\left(l^{\prime}(3442.2 \pm 0.2) \cdot 10^{-10} \cdot(B \varrho)^{2}+1-1\right)
$$

where 510.974 is the rest mass energy of the electron expressed in $\mathrm{keV}$. If we denote this quantity by $\mu$ we can also write down another useful relationship, namely that between the fractional changes of energy and momentum:

$$
\frac{\Delta E}{E}=\left(1+\frac{\mu}{\mu+E}\right) \frac{\Delta(B \varrho)}{B \varrho}
$$

This equation may be used when an accurate interpolation in $E-B \varrho$ tables (see Appendix VIII) is needed. Furthermore, since the resolution $R$ of magnetic spectrometers is obtained as the relative line width on the momentum scale, i.e. $R=\frac{\Delta(B \varrho)}{B \varrho}$, eq. (6) may be used to obtain the corresponding energy resolution.

Frequently, momentum and energy are expressed in relativistic units, i.e.:

$$
\begin{aligned}
& \eta=\frac{p}{m_{0} c}=\frac{B \varrho}{1704.45} \\
& \varepsilon=\frac{E}{m_{0} c^{2}}=\frac{E}{510.984}
\end{aligned}
$$

If we consider also the rest mass energy we then have the simple relationship :

$$
\varepsilon^{2}=\eta^{2}+1
$$

\section{§3. Nomenclature}

In the following theoretical treatment we need a few definitions of some basic concepts.

A radioactive source generally consists of a number of radioactive atoms distributed in a much larger quantity of inactive material, for instance the carrier used in the radiochemical procedure. The number of electrons leaving the source per unit time divided by the weight of the inactive source material is a measure of the specific activity of the source. In many cases it is this quantity which sets the ultimate limit of the 
energy resolution that can be obtained, rather than the spectrometer data themselves. The total available activity itself is usually not of the same importance as the specific activity*.

When using a converter for studying $\gamma$-radiation by observing secondary electrons the important quantity is then the total activity divided by the weight of the converter, which would correspond to the specific activity of a $\beta$-source. In certain cases we may speak of a practically infinitely good specific activity, for instance in the case of active deposit of $\mathrm{Rd} T h$ (i.e. $\mathrm{Th}\left(\mathrm{B}+\mathrm{C}+\mathrm{C}^{\prime \prime}\right)$ or $\left.\mathrm{Ra}\left(\mathrm{B}+\mathrm{C}+\mathrm{C}^{\prime \prime}\right)\right)$. In such cases an electron line emitted from the radioactive species will leave the source without energy straggling and the electron line may serve as a strictly monokinetic electron source (concerning the natural line widths of conversion lines: see $\S 10$ ). These electrons are emitted in all directions but only a certain fraction of them will pass through the defining entrance baffles (or slits) of the spectrometer. This solid angle $\Omega$ of the spectrometer is usually expressed directly in percent of the total sphere. The best spectrometers in this respect have a solid angle of $\sim 10 \%$.

Not all of the monokinetic electrons passing through the entrance slit will necessarily be counted by the detector. If the detector slit is wide enough it will do so, but it may also happen that the detector is adjusted to accept only a certain fraction of the monokinetic electrons. The fraction of all monokinetic electrons leaving the source which is actually counted in the detector we may call the spectrometer transmission.

Due to the spherical aberration of the system (and other possible lens errors) the electron line when registered in the spectrometer will appear as a line of a certain finite width $\Delta(B \varrho)$. For convenience $\Delta(B \varrho)$ refers to the full width at half the height of the line ("half width"). This entity is experimentally well defined, whereas theoretical calculations usually yield with much better definition the width at the bottom of the line $\Delta^{\circ}(B \varrho)$ ("base width"). Approximately $\Delta^{\circ}(B \varrho) \sim 2 \Delta(B \varrho)$. The relative line width $R=\frac{\Delta(B \varrho)}{B \varrho}$ is a constant for a spectrometer with fixed geometry of the rays. $R$ is a good measure of the resolution or resolving power and is usually given in percent. The best resolution so far obtained in $\beta$-ray spectroscopy is $\sim 0.01 \%$. For most high transmission spectrometers the resolution is $2-4 \%$.

The ratio between the transmission and the resolving power $R$ is a good measure of the quality of a spectrometer. Since this quantity does not take into account the ability of the spectrometer to handle extended

* The problem of getting the source homogeneously distributed on the backing is treated in Ch. VIII. 
sources, which is of particular importance as soon as the specific activity is not infinitely good, a better figure of merit of a spectrometer would be to substitute in place of the transmission in the above ratio the "luminosity" $L$, defined as $L=T \cdot \sigma$, where $\sigma$ is the source area. The luminosity is expressed in $\mathrm{cm}^{2}$.

Another quantity, which is closely connected with the resolution and the relative dimensions of the spectrometer and the source, is the dispersion $\gamma$. It gives the change in position of the electron optical image if the $B \varrho$ of the electrons is slightly changed, i.e. $\gamma=d \dot{x} / d(B \varrho)$. It is for instance found (see $\S 5$ ) that the double focusing spectrometer has twice the dispersion of the homogeneous field spectrometer, which means that twice as wide sources can be used if the resolution and dimensions of the instruments are the same.

\section{§ 4. The Semicircular Spectrometer}

In 1910 the first determinations of the energies of $\beta$-particles by their deflection in a magnetic field were carried out by $\nabla$. Baeyer and Hahn ${ }^{9}$. They employed the so called "direct deflection method". $\beta$-rays emitted from a radioactive wire were allowed to pass through a narrow slit and then, after some arbitrarily chosen distance, recorded on a photographic plate placed with the surface facing the wire and the slit. The deflecting magnetic field was perpendicular to the rays. With the aid of this simple arrangement $\mathrm{v}$. Baeyer, Hahn and Meitner ${ }^{10}$ discovered the occurrence of definite lines in the energy spectrum of $\beta$-radiation. Obviously the transmission and resolving power was very poor, since no focusing of the rays was used. The first magnetic focusing device is due to Danysz ${ }^{11}$ who in 1912 suggested the so-called semicircular focusing principle, which still is of great use in $\beta$-ray spectroscopy.

Let us consider a point source which is emitting monochromatic electrons (e.g. a conversion line) in all directions. We investigate ${ }^{12}$ the electron paths in a plane perpendicular to an applied magnetic field. (See Fig. 1.) A defining slit selects a diverging pencil of electrons, all having the same radius of curvature $=\varrho$. It is easily seen that the pencil after half a revolution will reach a minimum width. The focusing is not perfect, i.e. there is a certain spherical aberration causing a finite width $\Delta x$ of the image. From the figure we notice that $\Delta x=2 \varrho-2 \varrho \cdot \cos \varphi, 2 \varphi$ being the

- O. von Baeyer and O. Hahn, Phys. Z. S. 11, (1910) 488.

10 O. von Baeyer, O. Hahn and L. Meitner, Phys. Z. S. 12, (1911) 273, 378; 13, (1912) 264.

11 J. Danysz, Le Radium 9, (1912) 1; 10, (1913) 4.

12 K. Siegbahn, Ark. f. Mat. Astr. Fys. 30A, (1944) No. 20. 
aperture. If we had started with a source the width of which was $s$, the full image width would be

$$
\Delta x=s+2 \varrho(1-\cos \varphi) .
$$

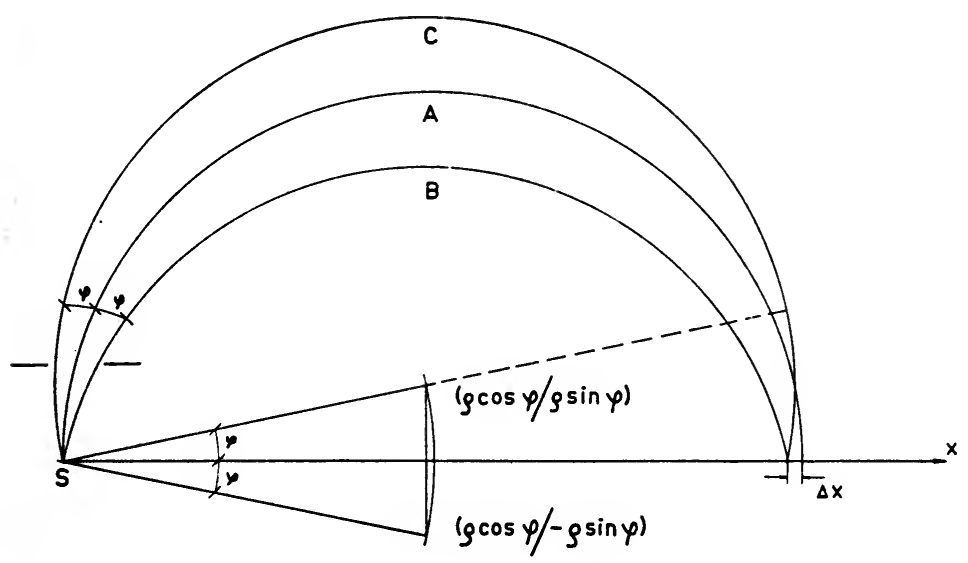

Fig. 1. The semicircular focusing principle

From this expression we can get the relative line width, e.g. the base resolving power $R^{\circ}$, considering that $\Delta x=\Delta 2 \varrho$ and that $\varphi$ is small:

$$
R^{0}=\frac{\Delta \varrho}{\varrho}=\frac{s}{2 \varrho}+\frac{\varphi^{2}}{2}
$$

The resolving power is thus made up of two terms, the first of which is dependent upon the width of the source and the radius of curvature, and the second upon the angular aperture. Only for infinitely thin sources is the resolving power independent of the spectrometer size. For sources which have a certain specific activity and which consequently must have a certain width, the first term by necessity contributes to the resolving power. For optimum conditions the two terms should give equal contributions to the resolving power, which yields the condition:

$$
\varphi=\rceil \frac{\bar{s}}{\varrho}
$$

So far we have only discussed the total width of the line, i.e. at the base. It is very instructive to study the intensity distribution within the line profile. The intensity at each point of the plate, situated along the $x$-axis, is proportional to the number of particles per unit area which strike the plate. We first study the intensity distribution from a point source. We may then write:

$$
I \sim \frac{d \varphi}{d x}
$$


Since $x=\underline{q} \cdot \cos \varphi$ we obtain

$$
I \sim \frac{1}{2 \varrho \cdot \sin \varphi} \sim \frac{1}{\sqrt{4 \varrho^{2}-x^{2}}}
$$

As a new variable we introduce the distance from the "edge" $z=2 \varrho-x$. We are only interested of the intensity distribution near the edge where $o \ll z$. We thus get

$$
I \sim \frac{1}{\sqrt{z}}
$$

which is the intensity distribution from a point source. It should be observed that the curve shows points of discontinuity at $z=0$ and $z=2 \varrho(1-\cos \varphi)$.

If we now consider the case when the source has a width $s$ we must integrate over the source, i.e.

$$
I \sim \int_{0}^{s} \frac{d s}{\sqrt{z-s}}
$$

Because of the discontinuities the integral has to be separated into three different parts:

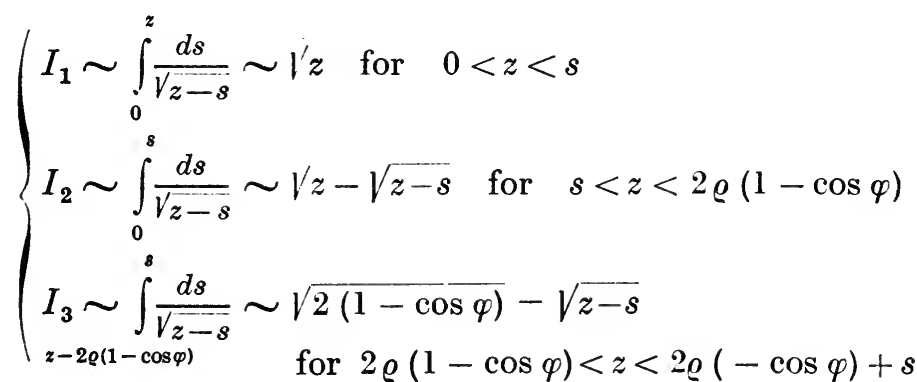

Fig. 2.

Intensity distribution of a $\beta$-line

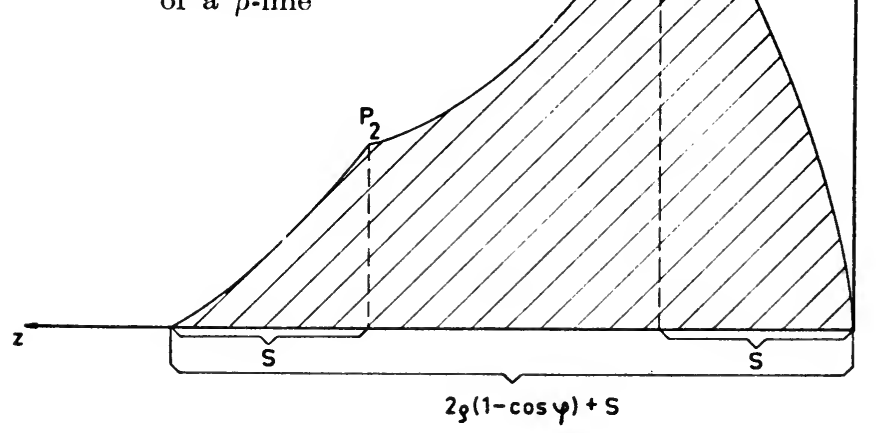


The intensity distribution so obtained is plotted in Fig. 2. The profile of the "line" has a sharp maximum (singular point) $\mathrm{P}_{1}$ at $z=s$ and a further singular point $\mathrm{P}_{2}$ at $z=20(1-\cos \varphi)$. Obviously the edge of the line at $z=0$ corresponds to the edge of the source nearest to the plate. whereas the top $\mathrm{P}_{1}$ of the line corresponds to the other $\mathrm{c}$ lge of the source. Either of these two distances corresponds to 20 , which is the quantity besides the magnetic field $B$ governed by the momentum of the electron.

In the above discussion we have made some simplifying assumptions for the sake of lucidity. In practice the active wire has a certain height and in some particular cases it may not be flat but round. Actually it turns out that the essential features of the curve given in Fig. 2 are retained while the calculations are greatly complicated ${ }^{13}, 14$.

By decreasing $\varphi$ the point $P_{2}$ approaches $P_{1}$ thus making the line sharper without affecting the intensity at $\mathrm{P}_{1}$ until $\mathrm{P}_{2}$ coincides with $\mathrm{P}_{1}$. A further reduction of $\varphi$ will result in an intensity loss at the line peak. In accordance with our previous statement optimum conditions therefore occur when

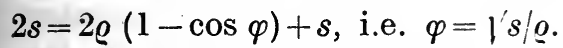

Let us further examine the optimum condition for the height $h$ of the source. An electron emitted at a distance $h / 2$ will form an angle $2 \psi$ with the medium plane if it strikes the plate at $-h / 2$. Its radius of curvature in the plane perpendicular to $B$ will be $\varrho \cos 2 \psi$. The electron will then obviously strike the plate closer to the source than the central ray. The distance along $x$ between these two points on the image will be:

$$
\Delta x=2 \varrho-2 \varrho \cos 2 \psi \text {. }
$$

Since $\psi$ is small we get

$$
\psi=\frac{h}{2 \cdot \pi \cdot \varrho}
$$

which combined with (18) yields

$$
\Delta x=\frac{h^{2}}{\pi^{2} \varrho}
$$

In analogy with the foregoing, the total height of the source should not be so great that the width of the image arising from the height exceeds that conditioned by the width $s$ of the source and the finite angular aperture $\varphi$, i.e.

$$
\frac{h^{2}}{\pi^{2} \varrho} \leqslant s
$$

As the optimum condition we thus get

$$
h \leqslant \pi / s \varrho .
$$

13 W. Woster, Proc. Roy. Soc. A114, (1927) 729.

14 K. Ii, Proc. Cambridge Phil. Soc. 33, (1937) 164. 
In many practical cases $h$ has to be made shorter than set by (22).

If the field $B$ is changed and the line is registered by means of a detector, the slit of which is $w$, the line gets correspondingly broader. We may then write down the complete expression for the base resolving power (being about twice the experimentally defined relative half width, see $\S 3$ )

The dispersion is

$$
R^{0}=\frac{1}{2 \varrho}\left\{\boldsymbol{s}+w+\varrho\left(\varphi^{2}+\psi^{2}\right)\right\}
$$

$$
\gamma=\frac{2 \varrho}{B \varrho}
$$

The transmission (or more correctly the solid angle) is given by

$$
T=\frac{\dot{\varphi} \psi}{\pi}
$$

and the luminosity by

$$
L=s h \cdot \frac{\varphi \varphi}{\pi}
$$

There are two possibilities for arranging the source, defining entrance slit and photographic plate. These are illustrated in Figs 3 and 4. In the first alternative (Fig. 3), which is the most common, the entrance slit is

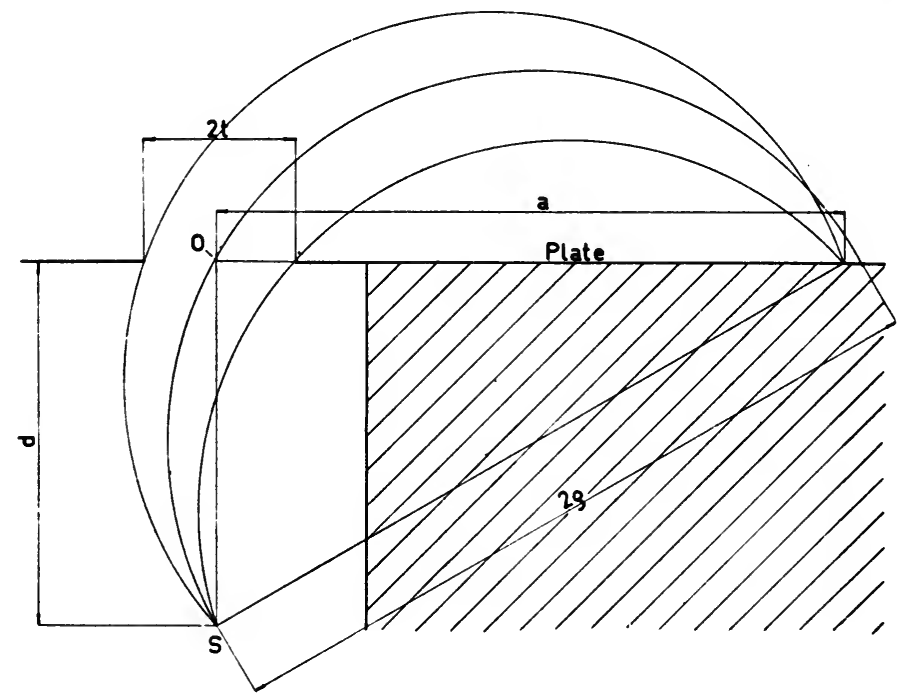

Fig. 3. Source and plate not in the plane

situated in the plane of the photographic plate, the source being situated on a perpendicular to the plane of the photographic plate going through the middle of the slit. With this mounting the ray which corresponds to the "edge" of the line ("central" ray) will pass through 0 , independently 
of $\varrho$. Thus the whole plate can be utilized without the risk that the ray forming the sharp edge of the line will be screened off at the defining slit.

In the second alternative shown in Fig. 4, the source is situated in the plane of the photographic plate and the slit is situated rather near the source and adjusted so that the central ray for a certain value of ? will

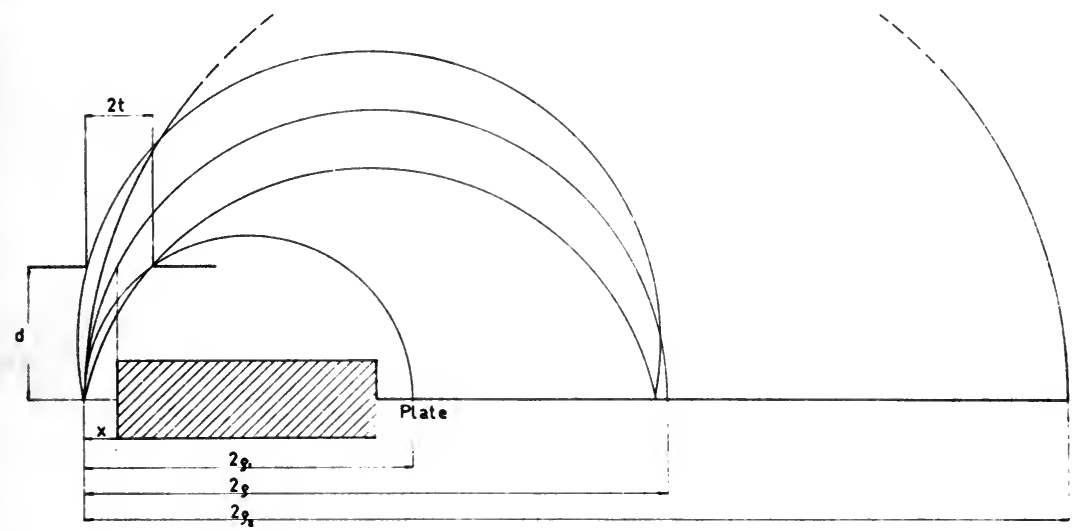

Fig. 4. Source and plate in the same plane

pass through the middle of the slit. For too large or too small $\varrho$ the "central" rays will be screened off at the defining slit. It is easy to show that this will happen when

$$
\begin{aligned}
& \varrho_{1}<\frac{d^{2} \cdot \varrho}{d^{2}+2 \cdot t \cdot \varrho} \\
& \varrho_{2}>\frac{d^{2} \cdot \varrho}{d^{2}-2 \cdot t \cdot \varrho}
\end{aligned}
$$

If $d$ is made small enough it is found that there is quite a large range in $\varrho$ which can be utilized without loss of the central rays, even at quite high resolution. This mounting has the advantage that the values of $\varrho$ and consequently the $B \varrho$-values of the lines can be accurately and simply measured, since the source as well as all the lines are situated in the same plane. Furthermore the electrons all strike the plate perpendicular to the plate surface. The rather intricate problem of measuring the $\varrho$-values in the mounting of Fig. 3 is amply discussed by Slätis ${ }^{15}$ in connection with the description of his high resolution, permanent magnet spectrograph. Fig. 5 shows a design due to Siegbahn ${ }^{12}$, where the second alternative of mounting was used. The source slit and plate are mounted on the same holder and can be adjusted and measured in a simple way in a comparator. The magnetic field is measured by means of a flip coil in this design. In

15 H. Slätis, Ark. f. Fysik. 6 Nr. 41, (1953) 415. 


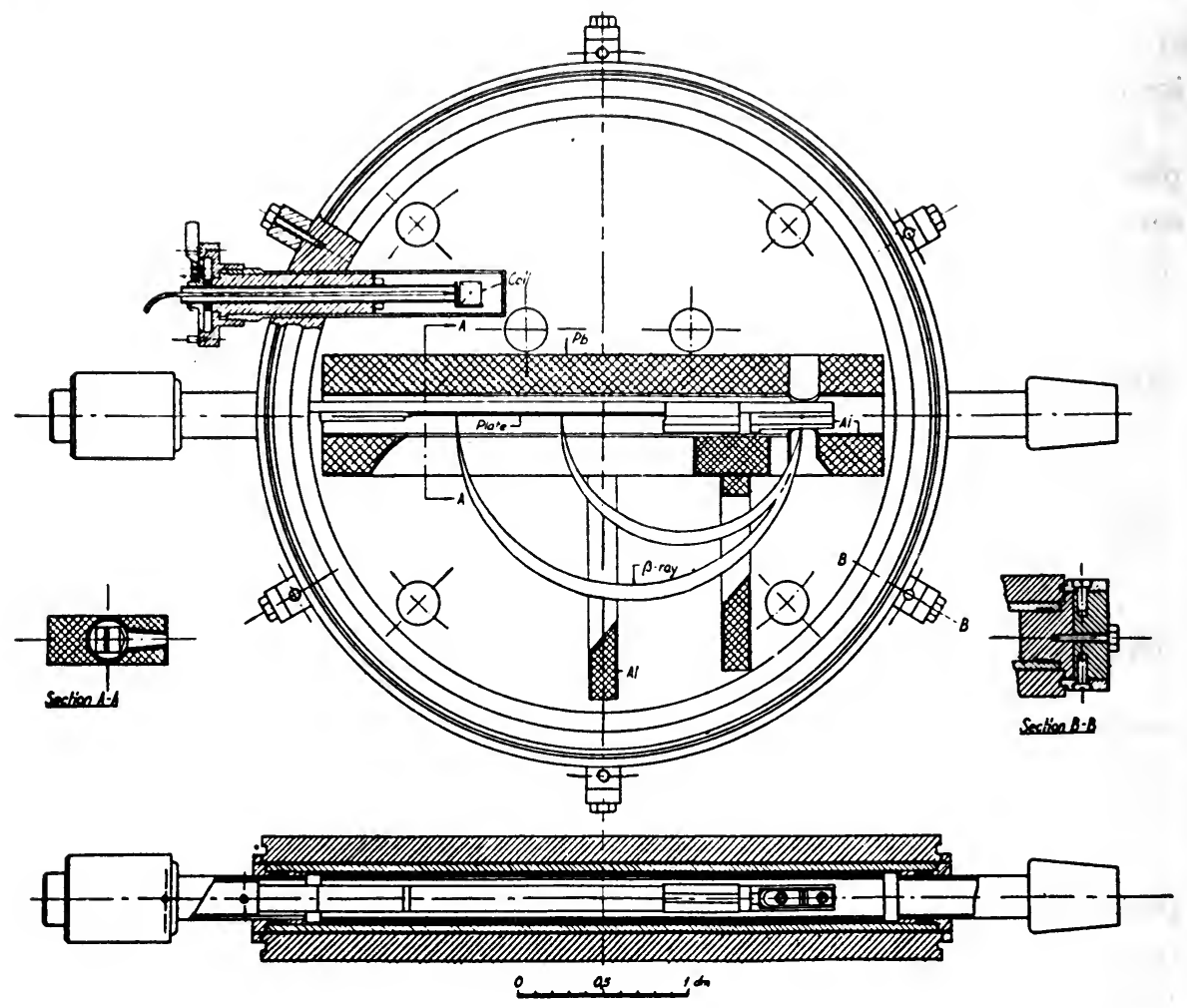

Fig. 5. Semicircular spectrograph for photographic registration due to Siegbahn

another design due to Lindström ${ }^{16}$ the accurate proton resonance method was used for the field measurement. GM counters in coincidence were used as a detector in order to reduce the background (Fig. 6).

Though the semicircular method is very inferior indeed to many other recently developed spectrometer types in regard to transmission and other properties (because of the lack of space focusing), it has some very important advantages which should be stressed here: (a) It is a simple and cheap instrument to build; (b) The magnetic field can easily be measured with great accuracy; (c) It is particularly well suited to photographic registering of a large part of the spectrum. The latter property is probably the most important, since the low transmission in this way is partly compensated for, because of the fact that a great number of unknown conversion lines can be surveyed simultaneously, which, if the resolution is set to be high, may take a long time to do with other types of spectrometers having much higher transmission. It could be noted, however,

16 G. Lindström, Ark. f. Fysik 4, Nr. 1, (1951) 1. 
CH. III - K. SiegBahn - $\beta$-Ray Spectrometer Theory and Design. High Resolution Spectroscopy

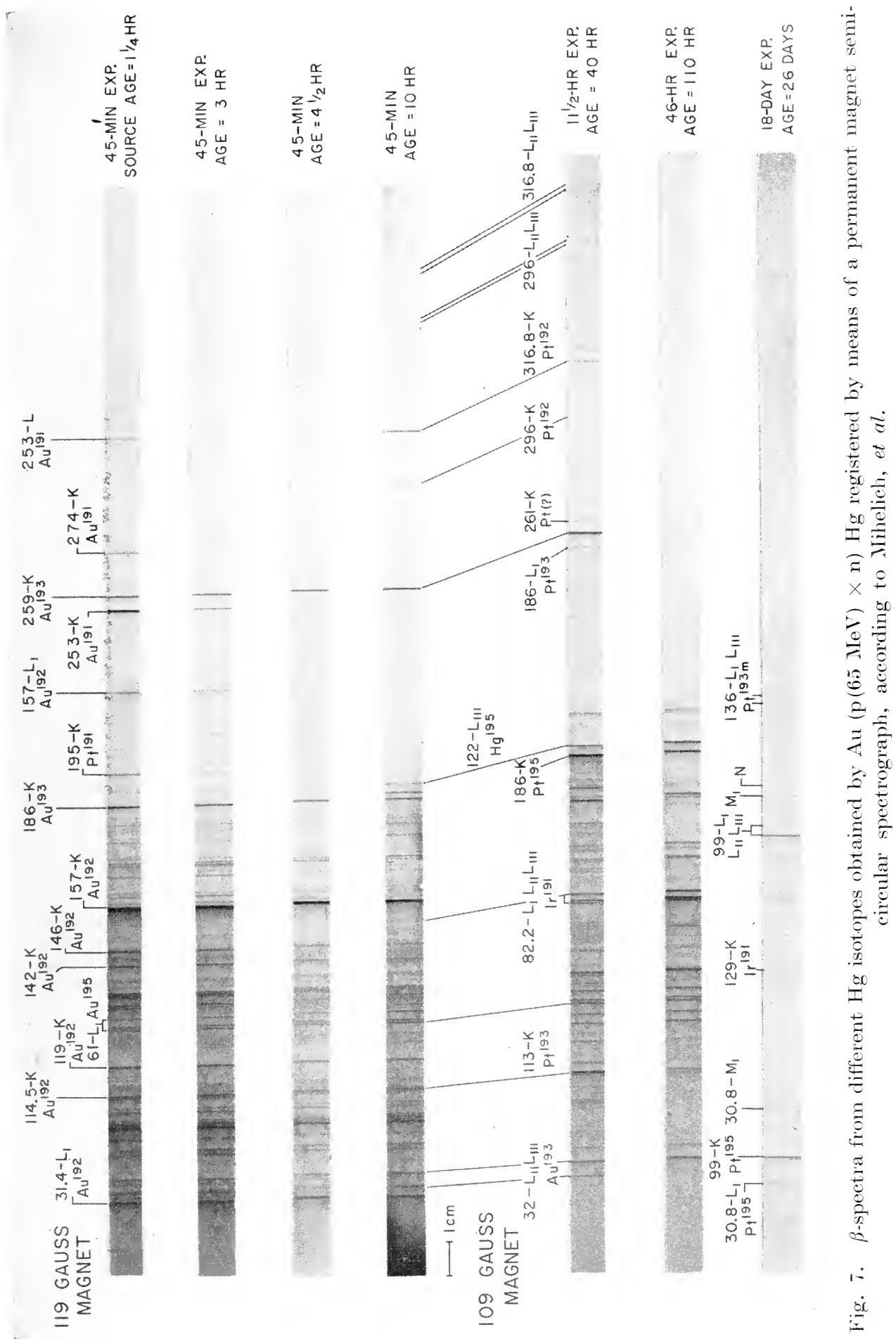




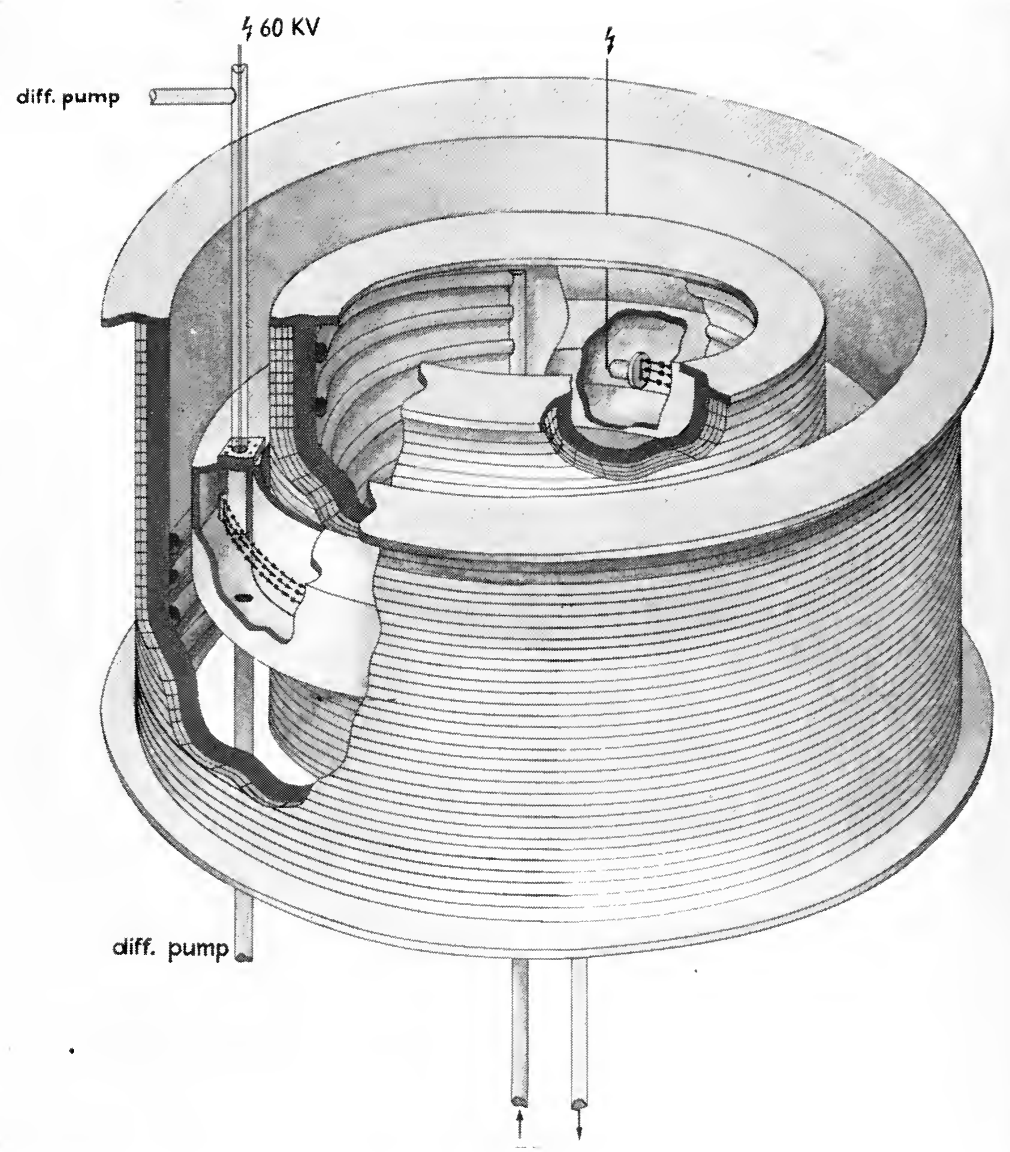

Fig. 11. Iron free double focusing spectrometer due to Siegbahn. The spectrometer is provided with an X-ray tube in order to study atomic binding energies 


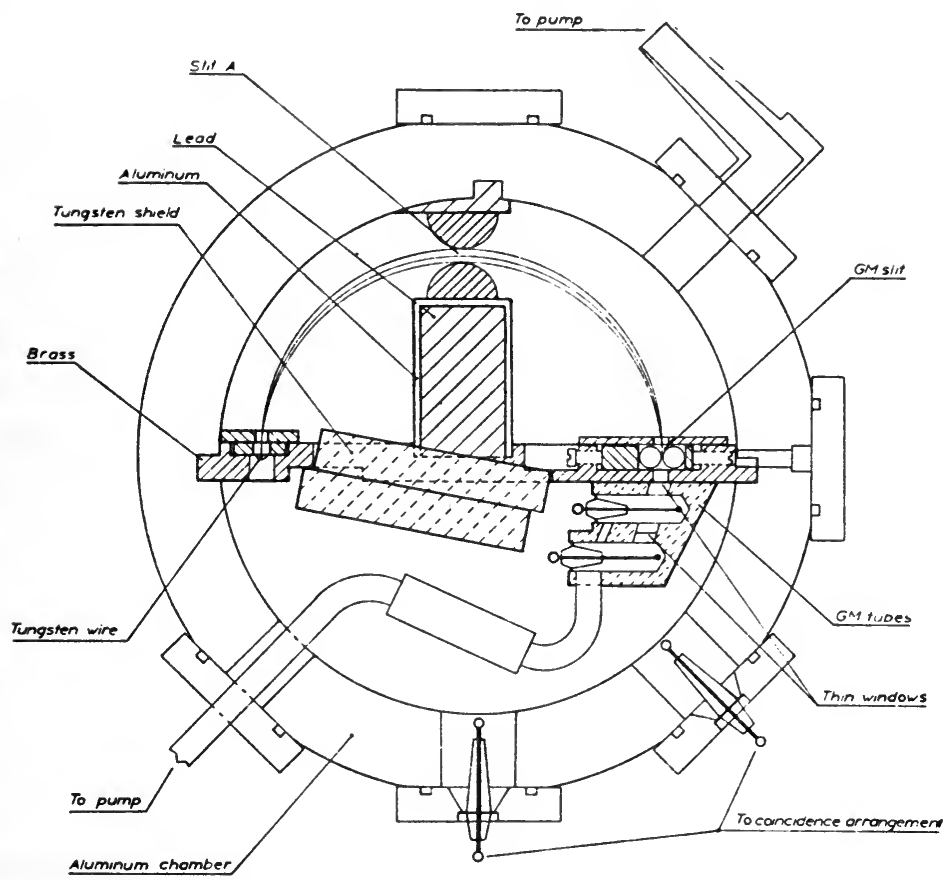

$10 \mathrm{~cm}$

Fig. 6. Semicircular spectrometer for GM registration due to Lindström

that the photographic method is not as sensitive as electric recording and that it is of somewhat limited value when dealing with the delicate problem of measuring intensities. One then has to convert photographic blackening into true intensity, which is a very tedious procedure. This problem has been discussed by several investigators and also in a recent paper by Slätis ${ }^{17}$.

In Fig. 7 is given an illustrative example of what has been discussed above. It is the complex conversion spectrum of $\mathrm{Au}(\mathrm{p}(65 \mathrm{MeV}), x \mathrm{n}) \mathrm{Hg}$ taken at successive intervals of time ${ }^{18}$. In this investigation, performed by Mihelich and co-workers, 175 spectrograms were taken and 250 electron lines analysed. In the Appendix VII is further reproduced the spectrum of Th $\mathrm{B}+\mathrm{C}+\mathrm{C}^{\prime \prime}$ recorded with Slätis' high resolution permanent magnet spectrograph.

17 H. Slätis, Ark. f. Fysik. 8, No. 43 (1954) 441.

18 L. Gillon, K. Gopalakrishnan, A. De-Shalit and J. Mihelich, Phys. Rev. 93, (1954) 124. 


\section{$\$$.) The Double Focusing Spectrometer}

The main disadvantage of the otherwise useful semicircular focusing principle is its lack of space focusing. In 1946 a device was developed 19, 20 which combines many of the features of one-dimensional semicircular focusing and two-dimensional helical or lens focusing.

It was known before from the construction of cyclotrons and betatrons ${ }^{21}$ that a magnetic field of rotational symmetry, having a gradient in the magnetic field, forces the electrons to describe oscillatory paths in the $\varrho$ as well as the $z$ coordinates of the particles. If we define an electron optical axis of the focusing device as being a circle in the symmetry plane of the magnetic field. electrons starting from a point on this optical axis and

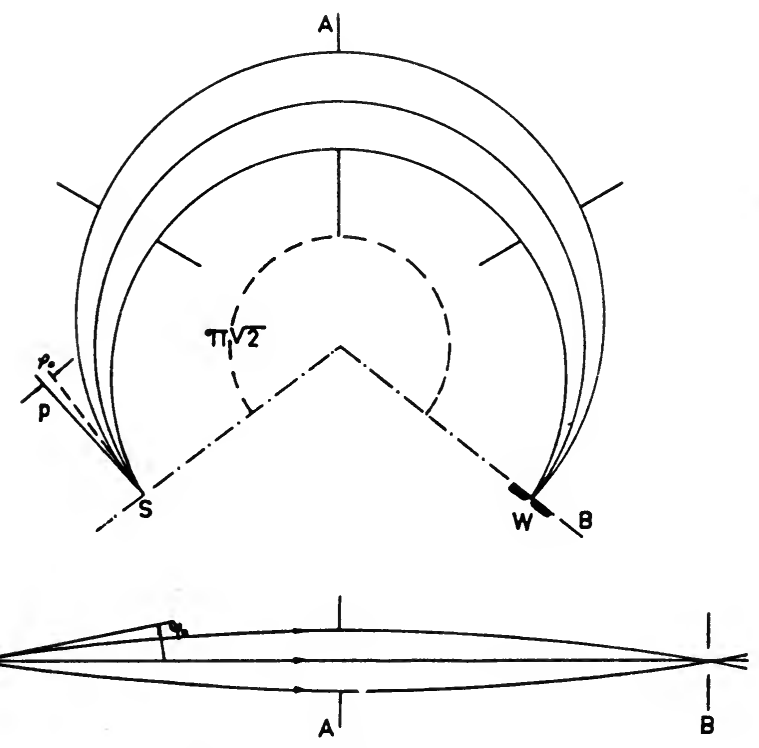

Fig. 8. Double focusing

making angles $\varphi_{0}$ and $\psi_{0}$ with this axis (see Fig. 8) will return to the axis after certain "focusing angles" $\phi_{\varrho}$ and $\phi_{z}$. For small angles $\varphi_{0}$ and $\psi_{0}$ these focusing angles are given by ${ }^{19}$

$$
\begin{aligned}
& \phi_{\varrho}=\pi\left[1+\frac{\varrho_{0} B^{\prime}\left(\varrho_{0}\right)}{B\left(\varrho_{0}\right)}\right]^{-1 / 2} \\
& \phi_{z}=\pi\left[-\frac{\varrho_{0} B^{\prime}\left(\varrho_{0}\right)}{B\left(\varrho_{0}\right)}\right]^{-1 / 2}
\end{aligned}
$$

19 N. Svartholm and K. Siegbahn, Ark. Mat. Astr. Fys. A33, (1946) No. 21.

20 K. Siegbahn and N. Svartholm, Nature 157, (1946) 872.

21 D. Kerst and R. Serber, Phys. Rev. 60, (1941) 53. 
The two focusing angles are related by

$$
\frac{1}{\phi_{Q}^{2}}+\frac{1}{\phi_{z}^{2}}=\frac{1}{\pi^{2}}
$$

The focusing system is obviously an-astigmatic if $\phi_{0}=\phi_{z}$ which gives ar condition for double focusing the following differential equation:

$$
B^{\prime}\left(\varrho_{0}\right)=-\frac{1}{2 \varrho_{0}} B\left(\varrho_{0}\right)
$$

The solution of this equation imposes on the magnetic field the condition that the field should decrease as $1 / I^{\prime} \varrho$ in the vicinity of $Q=g_{0}$ (the central path). According to (29) the double focusing takes place after an angle of $\pi / 2=255^{\circ} .56$, instead of $180^{\circ}$ as in the homogeneous field case.

These conclusions can of course also be made if one prefers to start from the expressions valid for the frequencies of oscillations in the betratron field ${ }^{21} \mathrm{~B}(\varrho)=\mathrm{B}\left(\varrho_{0}\right)\left(\varrho_{0} / \varrho\right)^{n}$. For equal frequency of the vertical $(z)$ and

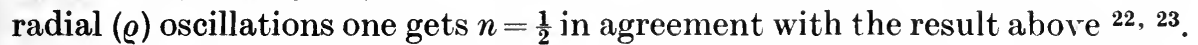

So far we have only considered "paraxial" rays $\left(\varphi_{0}\right.$ and $\psi_{0}$ small). It is of interest to see if the focusing conditions still hold for non-central rays ${ }^{24,}{ }^{25}, 26$. As one would expect this is so. In fact it is found that the $1 / V \varrho$ condition from many points of view still is the best field form to choose, though the extended analyses show that other slightly different fieldforms can also be used. The usual procedure is to consider the general fieldform

$$
B_{z}(\varrho, 0)=B_{0}\left\{1+\alpha \cdot \frac{\varrho-\varrho_{0}}{\varrho_{0}}+\beta \cdot\left(\frac{\varrho-\varrho_{0}}{\varrho_{0}}\right)^{2}+\ldots\right\}
$$

The equations of motion are solved for a particle leaving the source at a point $\varrho=\varrho_{0}+\delta \varrho, z=\delta z$, and with a direction $\varphi$ and $\psi$ with the optical axis ( $\varphi$ being in the symmetry plane perpendicular to the lines of force). It is then found, in agreement with the elementary theory given above, that an-astigmatic focusing occurs when $\alpha=-\frac{1}{2}$, i.e. after $\phi=\pi \cdot \gamma^{\prime} 2$. The particle will strike the plane $\phi=\pi \cdot / 2$ in a point, characterized by the coordinates:

$$
\begin{gathered}
\varrho^{*}=\varrho_{0}-\delta \varrho+\frac{(4 \beta-3)}{3 \varrho_{0}} \delta z^{2}+\frac{2-16 \beta}{3} \varrho_{0} \cdot \varphi^{2}+\frac{16 \beta-6}{3} \varrho_{0} \cdot \psi^{2} \\
z^{*}=-\delta z+\frac{8 \beta}{3 \varrho_{0}} \delta \varrho \delta z+\frac{32 \beta-12}{3} \varrho_{0} \psi \psi
\end{gathered}
$$

F. Kurie, J. Osoba and L. Slack, Rev. Scient. Instr: 19, (1948) 771.

A. Bartlett and K. Bainbridge, Rev. Scient. Instr. 22, (1951) 517.

Private communication by E. McMillan, 1946.

N. Svartholm, Ark. Nat. Astr. Fys. A33, (1946) No. 24.

F. Shull and D. Dennison, Phys. Rev. 71, 681; 72, (1947) 256. 
Ey. (30) determines the line width and since we have the dispersion along the $\varrho$-coordinate only, eq. (30) also will give the expression for the resolution.

It can easily be shown ${ }^{19}$ that the dispersion in the double focusing case is $\gamma=4 \varrho / B \varrho$ as compared to $2 \varrho / B \varrho$ for the homogeneous field. Introducing the source width $=s$ and height $=h$, the detector width $w$ and the defining aperture angles $\varphi_{0}$ and $\psi_{0}$ we then get from eq. (32) the base resolution, remembering the value of the dispersion:

$$
R^{0}=\frac{s}{4 \varrho}+\frac{w}{4 \varrho}+\frac{(4 \beta-3)}{48 \varrho^{2}} h^{2}+\frac{(2-16 \beta)}{12} \varphi^{2}+\frac{(16 \beta-6)}{12} \psi_{0}^{2}
$$

The $1 / \sqrt{ } \underline{Q}$ field obviously corresponds to $\beta=3 / 8$. This value of $\beta$ makes the last term in $(34)=0$, i.e. the resolution is then independent of $\psi$. If $\beta=\frac{1}{8}$ the resolution is instead independent of $\varphi_{0}$. Furthermore if $\varphi_{0}=\psi_{0}$, i.e. if we use a square entrance aperture the resolution is independent of $\beta$ (except for the term containing the source height, which in most cases is small).

The $\beta$-value at double focusing can evidently be chosen with a certain degree of freedom. Commonly used $\beta$-values are the above discussed $\beta=\frac{3}{8}$ and $\frac{1}{8}$. In the first case the resolution is set by the width of the radial shutters, whereas the axial width of the rays can be quite extended without influencing the resolution (higher order $z$ focusing). Usually there is a practical limit as to the maximum height or width of the beam. This may be set by the fixed distance between the polepieces or perhaps by the difficulties of obtaining the correct fieldform for large variations of $\varrho$. An ironfree double focusing spectrometer may be realized, as will be discussed later, by means of two concentric solenoids. In this case the natural choice of $\beta$ is $\frac{3}{8}$ since there is no limit for the field in the $z$ direction. Also the $\beta$-value $=\frac{2}{8}$ is commonly used because of the simple way one can realize this fieldform by means of iron pole pieces. In this case the fieldform is $\mathrm{B}(\varrho)=\mathrm{B}_{0} \cdot 2 \varrho_{0} /\left(\varrho+\varrho_{0}\right)$. Assuming infinite permeability of the iron this fieldform is realized by conical polepieces ${ }^{27}$. The pole distance $2 z$ is then $2 z=z_{0}\left(\varrho+\varrho_{0}\right) / \varrho_{0}$ where $z_{0}$ refers to $\varrho=\varrho_{0}$. Other $\beta$-values can be obtained, by slightly curving the pole faces. The large double focusing spectrometer ${ }^{28}\left(\varrho_{0}=50 \mathrm{~cm}\right)$ further discussed in this chapter has a $\beta$-value of $\frac{3}{8}$ which has been obtained by forming the airgap between the pole faces as a parabola $z=c \cdot V \varrho$, i.e. with the vertex at the centre of the magnet.

The condition for an-astigmatic double focusing is that $\alpha=-\frac{1}{2}$ in

27 N. Svartholm, Ark. f. Fysik 2, Nr. 14, (1949) 115.

28 A. Hedgran, K. Siegbahn and N. Svartholm, Proc. Phys. Soc. A63, (1950) 960. 
eq. (31). As stated above the value of $\beta$ may not be so critical, since it will mainly influence the choice of shutter system. However, if $\alpha$ is different from $-\frac{1}{2}$, say $-\frac{1}{2}(1+\varepsilon)$, an astigmatic error will be introduced. In the image plane $\phi=\pi \cdot \sqrt{2}$ and for $\beta=\frac{3}{8}$ this will introduce an extra linebroadening of the magnitude $\delta_{\text {ast. }}=\varrho_{0} \varphi_{0} \varepsilon \pi / 2$.

Quite generally, if the source and the detector slit are situated on a circle of radius $\varrho_{1}$, which differs by $\Delta \varrho$ from the radius $\varrho_{0}$ of the optical axis, then there is an astigmatism expressed by $\varepsilon=(3-8 \beta) \Delta \varrho / 2 \varrho_{0}$. Evidently this vanishes for $\beta=\frac{3}{8}$, which means that there is only one field form, which gives double focusing for every value of $\varrho$, namely that varying as $1 / \sqrt[V]{\text {. }}$.

A small astigmatic error can be reduced by moving the detector slit along the optic axis out of the plane $\phi=\pi \sqrt{ } 2$ until a minimum in radial width is found. If $\alpha=-\frac{1}{2}(1+\varepsilon)$ the dispersion will no longer be $4 \varrho / B \varrho$ but $\frac{4(1+\varepsilon) \varrho}{B \varrho}$. The easiest way to measure $\varepsilon$ accurately is either to determine the dispersion or to investigate the true values of $\phi_{z}$ and $\phi_{e}$.

For optimum conditions the different terms in the expression (34) for the resolving power should be made equal, just as in the semicircular case. In practice, however, the height of the source in both cases is made smaller than this condition calls for.

If we make $\varphi_{0}=\psi_{0}$ the resolution is independent of $\beta$ and we obtain:

The transmission

$$
\frac{8}{4 \varrho}=\frac{w}{4 \varrho}=\frac{2}{3} \varphi_{0}^{2}=\frac{R^{0}}{3}
$$

$$
T=\frac{4 \varphi_{0} \psi_{0}}{4 \pi}=\frac{\varphi_{0}^{2}}{\pi}
$$

We then get the simple relationship between base resolving power and transmission for a square defining shutter:

$$
R_{\square}^{0}=2 \pi \cdot T .
$$

It should be pointed out, however, that this relation between $R_{\square}^{0}$ and $T$ for square shutters does not represent a generally valid expression between the resolving power and transmission for a spectrometer having $\beta=\frac{1}{8}$ or $\frac{3}{8}$. In these cases, after having fixed the maximum allowable beam cross section (because of geometrical limitations) any linear change of the shutter system symmetrically around the optic axis from a square cross section to a rectangular one will decrease the transmission linearly but the resolving power quadratically.

If we consider the case when the source width and detector slit contribute only little to the total width as compared to the spherical aberration, we 
may write approximately, in the case of $\beta=\frac{1}{8}$ and $\beta=\frac{3}{8}$, ( $R$ now defined instead as the relative half width):

For a square shutter system in the spectrometer:

$$
R_{\square}=T_{\square} .
$$

For a variable rectangular shutter system:

$$
R=\text { const. } T^{2} \text {. }
$$

Under these conditions we thus get:

$$
R=\frac{1}{T_{\square}} \cdot T^{2} .
$$

The formation of image can be derived in identically the same way as was done in $\S 4$ for the semicircular case. It is found ${ }^{28}$ that for rectangular shutters and for $\beta=\frac{1}{8}$ and $\frac{3}{8}$ the line shape is the same as for the semicircular focusing system (Fig. 2).

The spectrometer magnetic field can be realized either by a coil system only, or by using iron for the field shaping. In the latter case the two possibilities shown in Fig. 9 offer themselves. The alternative in the upper part of the figure has the advantage that the fringing fluxes are easy to handle and have definite advantages for not too large magnets. A well designed spectrometer of this type has been made by Arbman ${ }^{29}$. $\varrho_{0}$ is $18,5 \mathrm{~cm}$, and the maximum transmission is around $4 \%$. The $\beta$-value chosen is $\frac{2}{8}$ which has enabled the use of simple conical pole faces. In Table II some data from this spectrometer are given. In all cases the source height

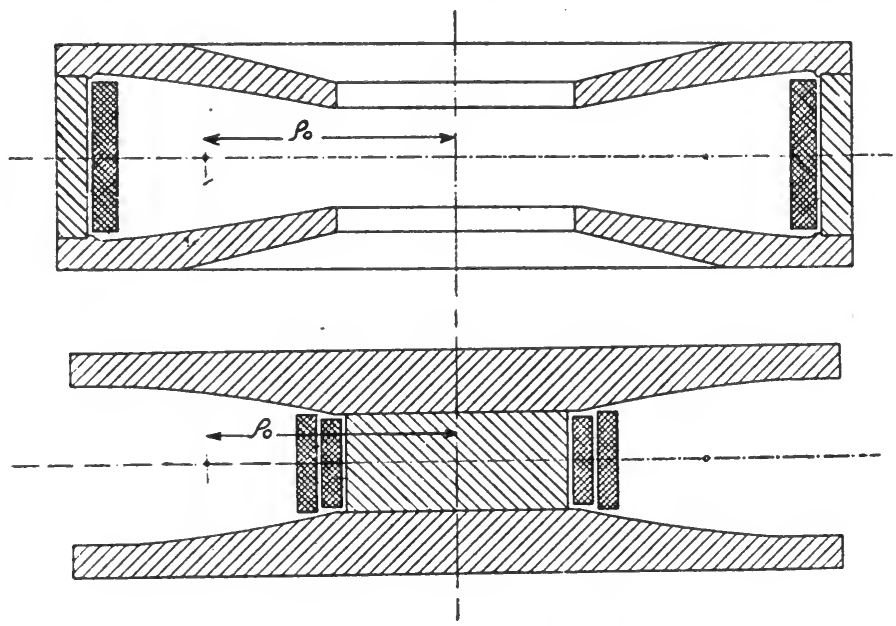

Fig. 9. Double focusing magnets

29 E. Arbman, in course of publication, Ark. f. Fysik. 
TABLE II

\begin{tabular}{|c|c|c|c|c|c|c|c|c|c|}
\hline \multirow{3}{*}{$\begin{array}{c}\text { Source } \\
\text { width } \\
\mathrm{mm}\end{array}$} & \multirow{3}{*}{$\begin{array}{l}\text { Detector } \\
\text { slit } \\
\text { mm }\end{array}$} & \multicolumn{8}{|c|}{ Relative half width in $\%$} \\
\hline & & \multicolumn{2}{|c|}{$\Omega=1 / 2 \%$} & \multicolumn{2}{|c|}{$\Omega=1 \%$} & \multicolumn{2}{|c|}{$\Omega=2 \%$} & \multirow{2}{*}{\multicolumn{2}{|c|}{$\begin{array}{c}Q=4 \% \\
\text { exp. calc. }\end{array}$}} \\
\hline & & exp. & calc. & exp. & calc. & exp. & calc. & & \\
\hline 1 & 2 & 0.44 & 0.37 & & & & & & \\
\hline 4 & 4 & 0.70 & 0.71 & 0.85 & 0.87 & & & 1.5 & 1.7 \\
\hline 8 & 8 & & & 1.3 & 1.4 & 1.6 & 1.7 & & \\
\hline 20 & 16 & 2.3 & 2.6 & & & & & 2.9 & 3.5 \\
\hline
\end{tabular}

was $20 \mathrm{~mm}$ and a circular shutter was used. Maximum resolving power used so far is $0,3 \%$ when the source width was $1 \mathrm{~mm}$ and the GM slit 2 $\mathrm{mm}$. The transmission is then $0.35 \%$.

A precision spectrometer of large radius of curvature $(\varrho=50 \mathrm{~cm})$ has been designed by Hedgran, Siegbahn and Svartholm ${ }^{28,30}$. The magnet was of the type shown in the lower part of Fig. 9, in order to make the field easily accessible for measurements. An elaborate rotating coil system was developed for the continuous field measurements in this spectrometer ${ }^{30}$. The definition of the minimum in the magnetic balancing system admits, as such, an accuracy in the field settings of about 2 parts in $10^{5}$ though the effective field is not possible to measure with that accuracy because of the iron. A schematic diagram of the arrangement is given in Fig. 10. With this spectrometer a number of $\beta$-lines. intended as reference lines in $\beta$-spectroscopic work, has been measured with an accuracy of 1 or 2 parts in $10^{4}$ (see $\S 10$ ).

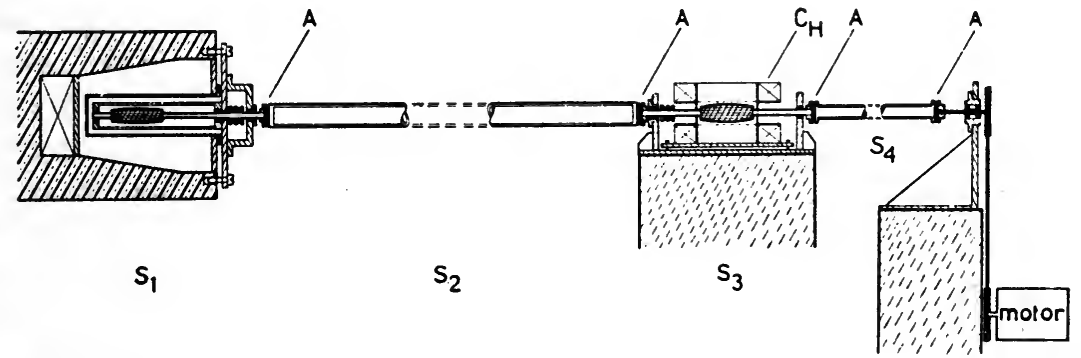

Fig. 10. Arrangement for continuous magnetic field measurement in double focusing spectrometer, according to Hedgran, et al.

It should also be mentioned that double focusing iron sector spectrometers have been used in particular in connection with heavy particle precision spectroscopy. Lauritsen et al. ${ }^{31}$ were the first to develop this

30 A. Hedgran, Ark. f. Fysik 5, Nr. 1, (1952) 1.

31 C. Snvder. S. Rubin, W. Fowler and C. Lauritsen, Rev. Scient. Inst. 21. (1950) 8.52. 
instrument and recently the $180^{\circ}$ sector field spectrometer using a $\beta$-value of $\frac{2}{8}$ (conical pole pieces) has been designed by Mileikowsky ${ }^{32}$. The main advantage of using a sector field is that the source as well as the detector are situated outside the magnetic field, which for this kind of spectroscopy has special advantages.

The ironfree double focusing spectrometer is a particularly valuable instrument for high precision work. Such a spectrometer has been designed and used by Siegbahn ${ }^{33,}{ }^{34}$ (see $\S 10$ ). It consists of two concentric coils with radii $\varrho_{1}$ and $\varrho_{2}$ and height $h$ (see Fig. 11). The space between $\varrho_{1}$ and $\varrho_{2}$ can be used for the electron optical arrangement $\left(\varrho_{0}=30 \mathrm{~cm}\right)$. It turns out that the ratio between the ampere turns through the two coils determines

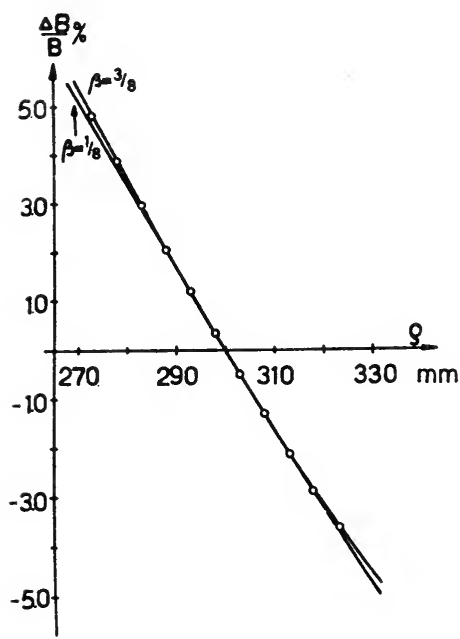

Fig. 12. Field form in iron free double focusing spectrometer the value of $\alpha$, whereas the height of the coils $h$ determines $\beta$. By means of a simple semi-empirical procedure the $1 / \sqrt[V]{\varrho}$ field could be realized over the whole region $\varrho_{2}-\varrho_{1}$ (see Fig. 12). Since there are no limiting pole shoes in this case an axially extended spectrometer tank can be used conveniently, according to the requirements of the $\beta$ value of $\frac{3}{8}$. Since the external magnetic fields (including the earth's magnetic field) is eliminated by means of a large Helmholz coil arrangement along the electron's path, the magnetic field is strictly proportional to the current through the spectrometer coils (coupled in series). Field-settings better than 1 part in $10^{5}$ are therefore achieved. With a temperature constancy better than $0.1^{\circ}$ of the different parts in the spectrometer and measuring equipments, and a careful adjustment of the spectrometer it has been possible to realize a resolving power better than 2 parts in $10^{4}$ and a precision in the $B \varrho$ measurements of 1 or 2 parts in $10^{5}$. The electron optical properties of the focusing system follow rather well what could be expected according to the theory presented above. The results of some of the measurements will be briefly discussed under $\S 10$.

Several other double focusing spectrometers of different designs have been built. So far all in use are made of iron. In particular one could mention those of Kurie, Osoba and Slack ${ }^{22}$, Bartlett and Bainbridge ${ }^{23}$,

\footnotetext{
32 C. Mileikowsky, Ark. f. Fysik 7, Nr. 3, (1953) 33.

33 K. Siegbahn, in course of publication.

34 K. Siegbahn, Physica 18, no. 12, (1952) 1043.
} 
Stoker et al. ${ }^{35}$, and Johns et al. ${ }^{36}$. An ironfree double focusing spectrometer is in the process of construction by Moussa ${ }^{37}$.

\section{§ 6. Other Flat Spectrometers}

\section{A. The Third-Order Focusing Semicircular Spectrometer}

The semicircular focusing spectrometer described in $\S 4$ is a "first order" focusing instrument in the sense that the resolving power contains only terms of higher order than the first with respect to the opening-angle $\varphi$. It is possible, however, to shape the magnetic field in such a way that the resulting resolving power only contains terms of higher orders in $\varphi$. Looking at Fig. 1 this would mean that the field is shaped so that the internal and external rays $\mathrm{B}$ and $\mathrm{C}$ are slightly moved to the right until they almost intersect the central ray A after a certain focusing angle. Naturally this would result in an improvement in resolution at a given opening angle $\varphi$. The transmission, however, will of course depend also on the type of focusing (or defocusing) taking place in the $z$-direction (compare the double focusing case). Beiduk and Konopinski ${ }^{38}$ in collaboration with Langer ${ }^{39}$ have investigated the fieldform giving a third order focusing in $\varphi$ after $180^{\circ}$. It was found that the appropriate fieldform is given by:

$$
B_{z}(\varrho, 0)=B_{0}\left\{1-\frac{3}{4}\left(\frac{\varrho-\varrho_{0}}{\varrho_{0}}\right)^{2}+\frac{7}{8}\left(\frac{\varrho-\varrho_{0}}{\varrho_{0}}\right)^{3}-\frac{9}{16}\left(\frac{\varrho-\varrho_{0}}{\varrho_{0}}\right)^{4}+\ldots\right\}
$$

With this fieldform one finds for the base resolution ${ }^{1}$ :

$$
R^{0}=\frac{s}{2 \varrho_{0}}+\frac{w}{2 \varrho_{0}}+11.86 \cdot \psi_{0}^{2}+2 \varphi_{0}^{2} \cdot \frac{s}{\varrho_{0}}+18.8 \varphi_{0} \psi_{0}^{2}+2.5 \varphi_{0}^{4}
$$

The dispersion is the same as in the semicircular case, i.e. half of the double focusing case. Apart from the lower dispersion, the transmission at a given resolution is not as favourable as in the double focusing case, if one considers the axial focusing. It is easy to see that rays outside the central ray will experience a slight $z$-focusing, whereas rays inside the central ray will be correspondingly defocused.

In Langer and Cook's spectrometer $\varrho_{0}=40 \mathrm{~cm}$ and $\varphi=32^{\circ}$. At an estimated transmission of about $0.1 \%$ the resolving power obtained with a $0.4 \mathrm{~mm}$ wide source was $0.5 \%$. The magnetic field in this spectrometer is measured by means of a rotating coil, the induced e.m.f. of which is balanced against a Helmholtz field. The accuracy is around $0.1 \%$. Another of smaller design

35 P. Stoker, Ong Ping Hok, E. de Haan and G. Sizoo, Physica 20, (1954) 337.

36 M. Johns, H. Waterman, D. McAskill and C. Cox, Can. J. of Phys. 31, (1953) 225.

37 A. Moussa, Physica 18, (1952) 1120.

38 F. Beiduk and E. Konopinski, Rev. Scient. Instr. 19, (1948) 594.

39 L. Langer and C. Cook, Rev. Scient. Instr. 19, (1948) 257. 
is due to Bruner and Scott ${ }^{40}$, having $\varrho_{0}=15 \mathrm{~cm}$. In this instrument only rays outside the central ray $\varrho_{0}=15 \mathrm{~cm}$ are used. With $\varphi=33^{\circ}$ the calculated transmission ${ }^{38}$ is $0.2 \%$. The resolving power obtained with a $1 \mathrm{~mm}$ wide source was $0.4 \%$.

Before the theory of one dimensional, third order focusing was worked out by Beiduk and Konopinski similar focusing had been tried by Korsunsky, Kelman and Petrov ${ }^{41}$ and also by Voges and Rutheman ${ }^{42}$. These approaches were semi-empirical. The improvement in radial focusing was observed though not perfected. An early investigation of the problem by Bock ${ }^{43}$ in connection with mass spectroscopy is also noted. This improvement of the semicircular focusing has been applied with great success in the electromagnetic separation of isotopes in large quantities ${ }^{44}$.

\section{B. Some Flat Sector Field Spectrometers}

There exist a great number of possibilities of arranging a focusing magnetic "prism" field, where one has the source and detector outside the magnetic field region. One example is the double focusing $180^{\circ}$ field mentioned before. Other sector fields have been considered in mass spectroscopy ${ }^{45}$. We will here only briefly discuss a few of particular interest in $\beta$-ray spectroscopy.

Bainbridge and Bender ${ }^{46,47,48}$ first designed a $\beta$-spectrometer of the prismatic type (see Fig. 13) having an inhomogeneous sector field, varying as $1 / \varrho$, where $\varrho$ is the distance to a given point in the center of the field. The pole piece boundaries were forthermore shaped so that a large fraction of the radiation was accepted by the spectrometer. The best resolution obtained was about $0.5 \%$.

A similar field form was first discussed by Richardson ${ }^{49}$, namely the case when the field varies as $1 / \varrho$ where $\varrho$ is the distance to a symmetry axis. through the source and detector. This is the field which is obtained for instance outside a linear wire, where the lines of force form circular loops around the wire. The same field may be obtained between inclined

40 J. Bruner and F. Scott, Rev. Scient. Instr. 21, (1950) 545.

4 M. Korsunsky, V. Kelman and B. Petrov, Journ. of Physics 9, (1945) 7.

42 H. Voges and G. Rutheman, Z. f. Physik 114, (1939) 709.

43 C. Bock, Rev. Scient. Instr. 4, (1953) 575.

14 J. Kistemaker, Laboratorium voor Massaspectrografie, Amsterdam.

45 See for instance K. Bainbridge's article in Experimental Nuclear Physics, edited by Segrè, 1953.

$46 \mathrm{~K}$. Bainbridge and R. Bender, private communication to Deutsch et al., Rev. Scient. Instr. 15, (1944) 178.

47 R. Bender, Thesis, Harvard 1947.

48 L. Lavatelli, Thesis, Harvard 1950.

49 H. Richardson, Proc. Phys. Soc. 59, (1947) 791. 
plane pole faces as investigated by Richardson. This field form has further been studied in the case of a sectorfield by Kofoed-Hansen. Lindhard and Nielsen ${ }^{50}$. The boundaries of the sector field are also here given such a

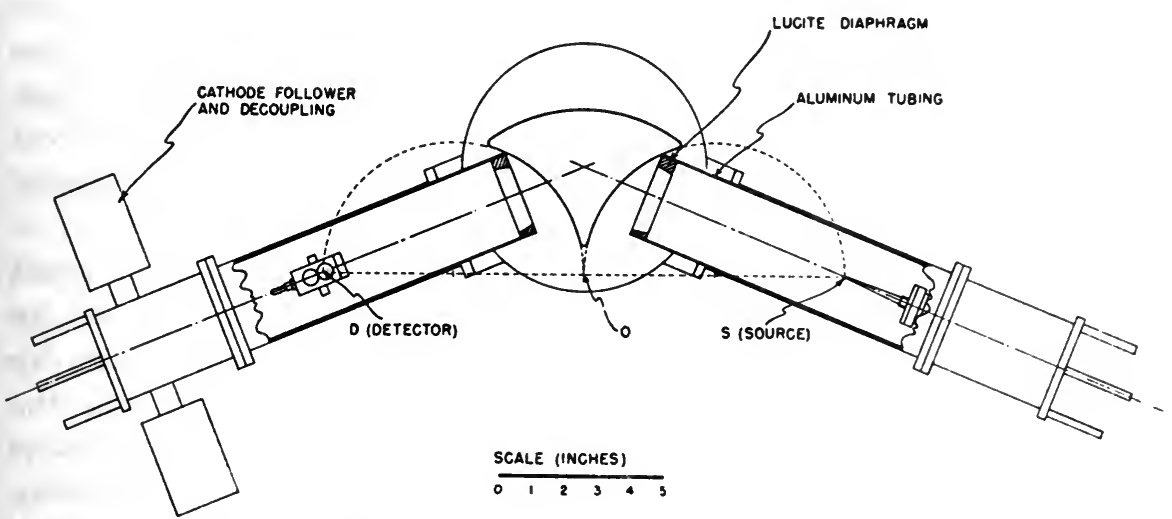

Fig. 13. Prismatic spectrometer due to Bainbridge, Benner and Lavatelli

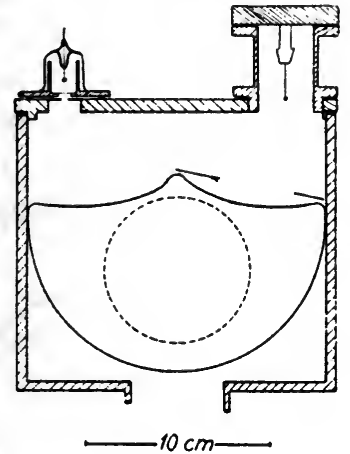

Fig. 14a.

Prismatic spectrometer due to Kofoed-Hansen. Lindhard and Nielsen

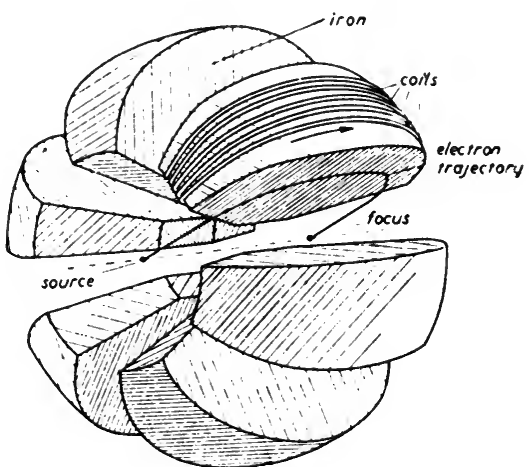

Fig. 14b.

"Orange" spectro. meter with 6 sectors

curvature that a large fraction of the radiation is accepted (see Fig. 14a). By distributing six such spectrometers in space symmetrically around a line through the source and detector a total transmission of $12 \%$ can be achieved. The resolving power obtained was $2 \%$ when the source was $5 \mathrm{~mm}$ in diameter. The fact that several sector spectrometers can be used simultaneously to accept radiation from the source explains the exceptionally high transmission of this "orange" system -(see Fig. 14b). This system is admittedly somewhat complicated to construct and perhaps to

50 O. Kofoed-Hansen, J. Lindhard and O. Nielsen, Kgl. Danske Virl. Solsk. Iled., 25. (1950) Nr. 16. 
adjust but on the other hand it yields an exceptionally high transmission. Common to the flat sector field spectrometers of prismatic type discussed above is that iron is used to form the inhomogeneous magnetic fields. The fringing fluxes and the magnetic properties of iron, in particular when used in arrays of discontinuous geometrical form, probably make the spectrometers less convenient for more precise work in the high resolution region, when the different adjustments may be rather critical and perhaps also may be dependent upon the strength of the magnetic field.

By far the highest transmission of all spectrometers so far developed is that of the so called "spiral-orbit" instrument. This was invented by Miyamoto ${ }^{51,52}$ in 1943. More recent developments has been made by Sakai ${ }^{53}$, who has studied the different possibilities of realizing the appropriate fieldform, with and without iron. Very little spectroscopic work has been done with this instrument, however, and it is therefore difficult at present to discuss its potentialities in different respects. The fact that under favourable conditions (source geometry etc.) up to $75 \%$ of the total sphere is claimed to be transmitted for focusing ${ }^{53}$ at a resolving power of $1.3 \%$ will certainly make the instrument very useful in many particular applications when extremely low activities are encountered. So far it has been used to study the continuous electron spectrum of the $\mu$-meson decay ${ }^{54}$ (see Ch. XI). Applications in mass spectroscopy ${ }^{55}$ and isotope separation ${ }^{56}$ in large quantities may also be possible.

Particles which leave a source at the center of a field which decreases symmetrically outwards will describe spiral orbits. The fieldform can be adjusted so that the particle orbits eventually converge to a definite circle, the radius of which depends upon the $B \varrho$ of the particles (see Fig. 15). A detector slit placed on the circumference of the circle will thus collect particles with a particular $B \varrho$-value. The appropriate fieldform may be

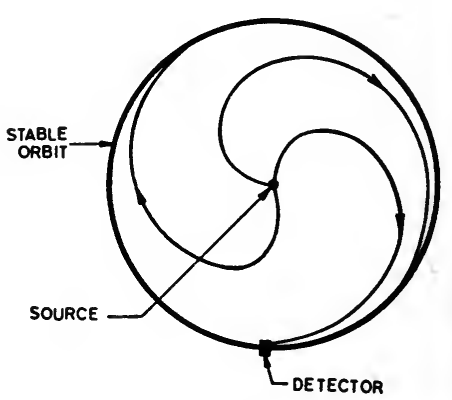

Fig. 15. Electron paths in spiral orbit spectrometer

51 G. Miyamoto, Proc. Phys.-Math. Soc. Japan (in Japanese) 17, (1943) 587.

52 R. Sagana, G. Miyamoto, K. Nakamura and Takechi, Proc. Phys.-Math. Soc. Japan 25, (1943) 274.

53 M. Sakai, Journ. Phys. Soc. Japan 5, (1950) 178; 5, (1950) 184; 6, (1951) 409 ; 6, (1951) 529 .

54 R. Sagana, W. Gardner and H. Hubbard, Phys. Rev. 82, (1951) 557.

35 V. Shipinel, Compt. Rendus (Doklady) URSS 53, (1946) 793.

58 M. Sakai and Y. Nogami, Journ. Phys. Soc. Japan 6, (1951) 409. 
chosen somewhat differently but in the main it is bell shaped with the maximum at the center of the magnet. Another form which seems to have particular advantages can be expressed by

$$
\left\{\begin{array}{l}
B(\varrho)=B_{0} \text { for } \varrho \leqslant \varrho_{0} \\
B(\varrho) \simeq \frac{B_{0} \varrho_{0}}{2 \cdot \varrho} \text { for } \varrho>\varrho_{0}
\end{array}\right.
$$

In the general case of a bell shaped field there is a strong $z$-focusing as well, which gives the particles an oscillatory movement around the symmetry plane $z=0$. The combined $\varrho$ and $z$-focusing all round the source together result in the outstandingly high transmission of this instrument. It is still in the state of development.

Other interesting types of sector field spectrometers have been described by Siday ${ }^{57}$, Korsunsky ${ }^{58}$, Jennings ${ }^{59}$, Siday and Silverston ${ }^{60}$.

High transmission instruments for the particular purpose of measuring the upper limits of spectra from extremely low activities have been designed by Libby and Lee ${ }^{61}$, Siegbahn and Slätis ${ }^{62}$ and Rae ${ }^{63}$.

\section{$\S 7$. Lens Spectrometers}

The focusing action of short and long coils has been known for a long time. Busch ${ }^{64}$ was the first, however, to point out the close analogy between ordinary light and electron optics when dealing with these "magnetic lenses". He showed that the ordinary lens fornula $1 / u+1 / v=1 / f$ could be applied also to such lenses, and was able to calculate the focal distance $f$ for a short lens. He found $\left(B_{z}(z, 0)\right.$ being the field component perpendicular to the lens along the axis):

$$
\frac{1}{f}=\frac{1}{4 \cdot(B \varrho)^{2}} \cdot \int_{-\infty}^{+\infty} B_{z}^{2}(z, 0) d z
$$

In the special case of a long lens with uniform magnetic field $B_{z}(z)=B_{0}$. It is well known that the electrons then describe helical paths. The distance between the source and the image is then given by

$$
z_{u \rightarrow v} \approx \frac{2 \pi}{B_{0}} \cdot B \varrho
$$

57 R. Siday, Proc. Phys. Soc. 59, (1947) 905.

58 M. Korsunsky, Journal of Physics (USSR) 9, (1945) 14.

59 J. Jennings, Proc. Phys. Soc. B65, (1952) 256, 265.

60 R. Siday and D. Silverston, Proc. Phys. Soc. A65,-(1952) 328.

61 W. Libby and D. Lee, Phys. Rev. 55, (1939) 245.

62 K. Siegbahn and H. Slätis, Ark. f. Mat. Astr. Fys. A36, Nr. 22, (1949).

63 E. Rae, Phil. Mag. 41, (1950) 525.

64 H. Busch, Ann. Physik 81, (1926) 974; Arch. Elektrot. 18, (1927) 583. 
For ordinary optical lenses one gets a reversed image. 'This is also true for the double focusing spectrometer. For a long uniform field lens the rotation of image $\theta$ is instead $360^{\circ}$. and for a short lens $\theta$ is given by the expression:

$$
\theta=\frac{1}{2 \cdot B \underline{Q}} \int_{-\infty}^{+\infty} B_{z}(z, 0) d z
$$

The first attempt to use the magnetic lens field for $\beta$-spectroscopy as suggested by Kapitza ${ }^{65}$ was made by Tricker ${ }^{66}$. who used a long uniform field. and later on by Klemperer ${ }^{67}$. who tried the short field. These early attempts were. however. not followed for some time by any serious efforts to perfect the instruments to such an extent that they could compete in any respect with the performance of the semicircular spectrometer, the only type of spectrometer in use at that time. The subsequent work by Deutsch et al. ${ }^{68,69}$ and by Siegbahn ${ }^{70,71}$ showed, however, the potentialities of the short lens method. A resolving power as good as $1 \%$ was achieved in these early experiments. Witcher ${ }^{72}$ simultaneously developed the uniform lens method. In particular, the short lens method, extensively used by Deutsch and his coworkers in a long series of important publications and by several others. played a dominant rôle in the rapid accumulation of spectroscopic data which followed shortly after the introduction of the lens method. The transmission could be set to be much higher than was feasible with the semi-circular spectrometers and the source geometry could be made quite favourable, which was of particular importance when working with low specific activities and secondary electron converters of the $\gamma$-radiation. It was furthermore easy to shield the counter against direct radiation from the source when secondary $\gamma$-spectra were investigated. The short lens spectrometer is very flexible in performance and easy to construct.

Though most of the spectroscopic work was performed by means of the short lens method it became apparent that much better performance data could be obtained by using other forms of lens fields. Already the long lens field, in particular when properly adjusted with ring focus slit as originally used by Witcher and later on further developed by others ${ }^{\mathbf{7 3 , 7 4 , 7 5}}$

65 P. Kapitza, Proc. Camb. Phil. Soc. 22, (1924) pt. 3.

66 R. Tricker, Proc. Camb. Phil. Soc. 22, (1924) 454.

67 O. Klemperer, Phil. Mag. 20, (1935) 545.

68 I. Deutsch, Phys. Rev. 59, (1941) 684 A.

69 M. Deutsch, L. Elliott, and R. Evans, Rev. Scient. Instr. 15, (1944) 178.

70 K. Siegbahn, Ark. f. Mat. Astr. Fys. A28, (1942) No. 17.

71 K. Siegbahn, Ark. f. Mat. Astr. Fys. A30, (1944) No. 20.

i2 C. Witcher, Phys. Rev. 60, (1941) 32.

73 S. Frankel, Phys. Rev. 73, (1948) 804. 
gives better figures of merit. Still better performance can be realized by giving the field a form reversed from that of a short lens. i.e. having a minimum midway between the source and detector ${ }^{76}$. The most startling example of this was encountered when the so-called "intermediate image" focusing was found 77. (ioing over from a short lens spectrometer with ringfocus to an intermediate image spectrometer Nichols and Jensen is recently reported a fiftyfold increase in transmission. Other strong gradient fieldforms have been used by Richardson ${ }^{79}$, I)aniel and Bothe ${ }^{80}$ and Agnew and Anderson ${ }^{81}$. Such spectrometers have. compared to the short lens type, about an order of magnitude higher transmission at the same resolving power.

\section{A. The Short Lexs Spectroneter}

As a typical example ${ }^{82}$ we may consider a short lens. having a length of $15 \mathrm{~cm}$, and internal and external diameters of $15 \mathrm{~cm}$ and $45 \mathrm{~cm}$, respectively. At $\sim 2800$ amp turns the field $B_{0}$ at the centre of the coil is 140 Gauss. The field distribution is that shown in Fig. 16. The focal

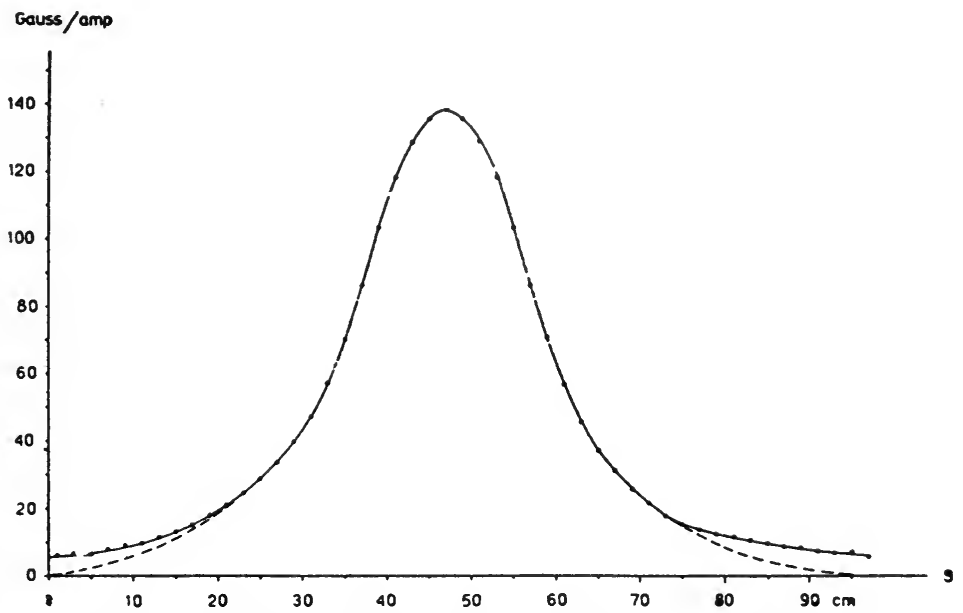

Fig. 16. Magnetic field $B(z)$ in a short magnetic lens

74 E. Persico, Rev. Sicient. Instr. 20, (1949) 191.

J. DuMond, Rev. Scient. Instr. 20, (1949) 160, 616.

K. Siegbahn, Phil. Mag. 37, (1946) 162.

H. Slätis and K. Siegbahn. Ark. f. Fysik 1, Nr. 17, (1949) 339.

R. Nichols and E. Jensen, Phys. Rev. 94, (1954) 369.

H. Richardson, Phil. Mag. 40, (1949) 233.

H. Agnew and H. Anderson, Rev. Sieient. Instr. 20, (1949) 869.

K. Niegbahn, Ark. f. Fysik 4, Nr. 10, (1952) 223 . 
length can to a fairly good approximation be calculated from

$$
f=\frac{2 a}{\pi k^{2}}
$$

where $a=$ half width of the field distribution curve and

$$
k=\frac{B_{0} \cdot a}{2 \cdot B \underline{Q}}
$$

The image rotation is obtained from:

$$
\theta=\frac{\pi k}{\sqrt{k^{2}+1}}
$$

For a distance between the source and the detector of $100 \mathrm{~cm}$ one would need about $2300 \mathrm{amp}$ turns to focus $B g=1000$. The image rotation is $\sim 80^{\circ}$. Positrons and electrons can be separated because of their different directions of rotation.

In order to calculate the theoretical resolving power one must know the spherical aberration of the system. This can be found by ray tracing, either by mathematical means (knowing the field distribution) or, preferably. experimentally which yields much more accurate results.

In order to get an estimate of the resolving power one can neglect the spherical aberration. One then can show that the geometry alone will yield a resolving power (at the base)

$$
R^{\bullet}=\frac{m s+w}{(m+1) d}
$$

where $m$ is the magnification, which is 1 for $u=v, s$ and $w$ are the radii of the source and detector slit respectively. $w$ is made equal to $m s$ in order to match the image size. $d$ is approximately the radius of tl.e internal circular slit placed at the centre of the coil. Remembering that $R^{\circ}$ in eq. 50 is the base spread, the relative half width is, if $m=1$ :

$$
R=\frac{s+w}{4 d}
$$

If $\Delta d$ is the width of the defining slit the transmission is

$$
T=\frac{d \cdot \Delta d}{2 u^{2}}
$$

The spherical aberration will make the actual resolving power of the order of $50 \%$ less favourable than given by eq. 51 . On the other hand a properly arranged ring focus slit (see B) will improve the performance by a factor of about $2^{83}$. Average figures of merits are $R \sim 2.5 \% ; T \sim 0.5 \%$ when $s \sim 3 \mathrm{~mm}$ for the instrument dimensions given above. Some good

83 W. Pratt, F. Boley and R. Nichols, Rev. Scient. Instr. 22, (1952) 92. 
designs have been described by e.g. Hornyak et al. ${ }^{84}$, Verster ${ }^{85}$, Jensen et al. ${ }^{86}$.

\section{B. The Long Lens}

So far we have only considered a "short" lens. The focal distance givein in eq. 44 could then be found by solving the well-known differential equation in electron optics valid for paraxial rays, namely

$$
\frac{d^{2} r}{d z^{2}}=\frac{e}{8 m U} B^{2}(z) \cdot r
$$

assuming that $r$ does not change very much for the passage of different rays through the lens. In that case the form of the field will not be so significant but only the total integral of the field.

In order to investigate the more general case of an extended field it is convenient to remember the fact that the field in space is completely determined by the field along the symmetry axis (optical axis) of the system. One can show that:

$$
\begin{aligned}
& B_{z}(r, z)=B(z)-\frac{1}{4} B^{\prime \prime}(z) \cdot r^{2}+\ldots \\
& B_{r}(r, z)=-\frac{1}{2} B^{\prime}(z) \cdot r+1 / 16 B^{\prime \prime \prime}(z) r^{3}-\ldots
\end{aligned}
$$

The equation of motion of the electrons is given by:

$$
\frac{d}{d t}(m \mathbf{v})=-e \cdot \mathbf{v} \times \mathbf{B}
$$

In a magnetic field $v$ is a constant and therefore also $m$. One can then give the equations of motion in the following suitable form:

$$
\begin{aligned}
& \ddot{r}=-\left(\frac{e}{m}\right)^{2}\left\{\frac{1}{4} B(z)^{2} \cdot r-\frac{1}{8} B(z) \cdot B^{\prime \prime}(z) \cdot r^{3}+\left(\frac{3}{256} B^{\prime \prime}(z)^{2}+\ldots\right) r^{5}+\ldots\right\} \\
& \ddot{z}=-\left(\frac{e}{m}\right)^{2}\left\{\frac{1}{4} B(z) \cdot B^{\prime}(z) \cdot r^{2}-\left(\frac{1}{32} B^{\prime}(z) \cdot B^{\prime \prime}(z)+\frac{11}{32} B(z) \cdot B^{\prime \prime \prime}(z)\right) r^{4} \ldots\right\}
\end{aligned}
$$

The solution of these differential equations presents in general great mathematical difficulties. For some special fieldforms such as a homogeneous or even a triangular field the solution of the equations and the elimination of $t$ may be made fairly easy.

In the case of a homogeneous field, which so far is the only one which has been treated accurately one simply has:

$$
\left\{\begin{array}{l}
\ddot{r}=-\left(\frac{e B}{2 m}\right)^{2} r \\
\ddot{z}=0
\end{array}\right.
$$

84 W. Hornyak, T. Lauritsen and V. Rasmussen, Phys. Rev. 76, (1949) 731.

85 N. Verster, Appl. Sci. Research 1, (1950) 363.

86 E. Jensen, L. Laslett, W. Pratt, Phys. Rev. 75, (1949) 458. 
The solution will then be:

where

$$
r=l) \cdot \sin \alpha \cdot \sin \zeta
$$

$$
\zeta=\frac{z}{I) \cos a} \text { and } D=\frac{2 m v}{e B}=\frac{2(B \varrho)}{B},
$$

$\checkmark$ being the angle of emission of the electron. Evidently $D$ corresponds to the diameter of a circle described by an electron emitted with $x=90^{\circ}$. In the $r z$ plane following the electron in the helical motion, the path obviously is a sine curve with the amplitude $r_{m}=D \sin x$ and the half period $\pi \cdot l) \cos v$, when the electron crosses the axis, in accordance with (45), if $\cos x \sim 1$.

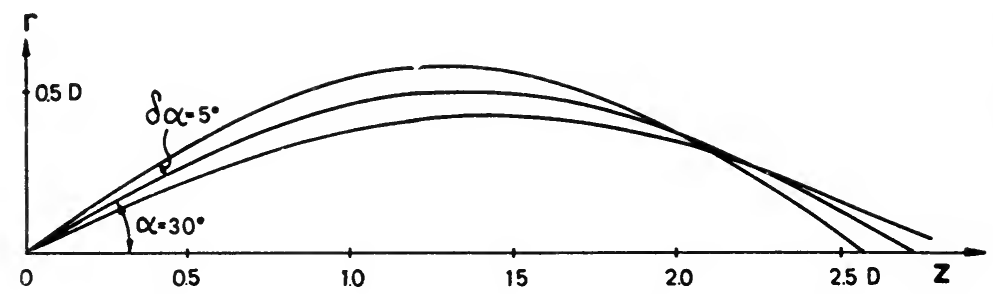

Fiy. 17. Electron paths in a homogeneous field for $\alpha=25^{\circ}, 30^{\circ}$ and $35^{\circ}$

Fig. 17 shows the electron paths for $x=25^{\circ}, 30^{\circ}$ and $35^{\circ}$. The fact that the intersection of the electron with the axis is dependent on $\alpha$ causes a spherical aberration. always with a positive sign. According to Fig. 17 the different paths intersect each other rather closely within a certain region. The condition for such a ring focus can be expressed by $d r / d \alpha=0$. From (58) we then get:

$$
\frac{\operatorname{tg} \zeta}{\zeta}=-\operatorname{tg}^{2} \alpha
$$

In order to calculate the resolving power of the system one observes that in the ring focus the width of the "image" is (neglecting higher order terms):

$$
\Delta r=\frac{\partial^{2} r}{\partial \alpha^{2}} \cdot \frac{(\Delta \alpha)^{2}}{2}
$$

where $\Delta \alpha$ is half the opening angle. Differentiating (58) once more we get using $(60)$ :

$$
\Delta r=D \cdot \frac{(\Delta \alpha)^{2}}{2} \cdot \frac{\sin \zeta}{\sin \alpha}\left(3+\cos ^{2} \alpha \cdot \operatorname{tg}^{2} \zeta\right)
$$

The resolving power $R^{0}$ is defined as $\frac{\Delta(B \varrho)}{B \varrho}$. From (59) we have:

or:

$$
\Delta r=\frac{\partial r}{\partial D} \cdot \frac{D}{(B \varrho)} \Delta(B \varrho)
$$

$$
\Delta r=\frac{\partial r}{\partial D} \cdot D \cdot R^{0}
$$


$\partial r / \partial D$ is obtained from (58) and (59):

$$
\frac{\partial r}{\partial D}=\frac{\sin \zeta}{\sin \alpha}
$$

By means of (62), (64) and (65) we finally get for a point source:

$$
R^{0}=\frac{(\Delta \alpha)^{2}}{2}\left(3+\cos ^{2} \alpha \cdot \operatorname{tg}^{2} \zeta\right)
$$

The transmission is given by

$$
T=\Delta \alpha \cdot \sin \alpha .
$$

The relation between $R^{0}$ and $T$ is then:

$$
R^{0}=\left(\frac{3}{\sin ^{2} \alpha}+\cot ^{2} \alpha \cdot \operatorname{tg}^{2} \zeta\right) \cdot \frac{T^{2}}{2}
$$

It is found that the factor enclosed in the parentheses has a minimum value at $\alpha \approx 45^{\circ}$, but this minimum is not very pronounced. If the width of the source is taken into account it is found that the optimum value of $\alpha$ is decreased to $\approx 40^{\circ}$. Since the coil has to be inconveniently large in order to accept electrons of $40^{\circ}$ one usually prefers an angle of emission of about $30^{\circ}$ without any essential loss in the performance of the spectrometer.

In a careful design due to Schmidt 87 it has been possible to realize

$$
R=8.9 \cdot T^{2}
$$

which is very near the theoretical limit. Fig. 18 shows a drawing of this instrument. The maximum transmission is set to be $5 \%$. With a source diameter of $\sim 1.5 \mathrm{~mm}$ the resolution was $0.4 \%$ and the transmission

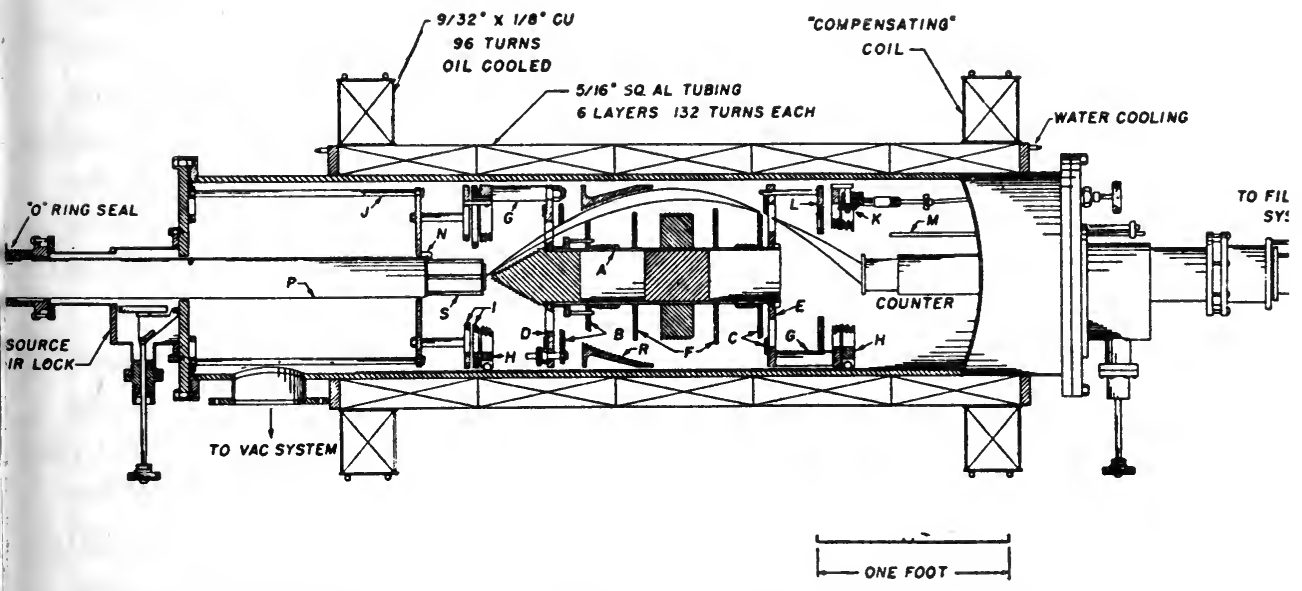

Fig. 18. Homogeneous field spectrometer with ring focus due to sclimidt

87 F. Schmidt, Rev. Scient. Instr. 23, (1952) 361. 
$2 \%$. It was furthermore verified that the resolution at constant transmission. as expected, varied linearly with the source diameter.

Another interesting design is due to DuMond et al. ${ }^{88}$. Particular care has been taken to form as uniform a field as possible in order to be able to use the proton resonance method for field measurements and to adjust the spectrometer for high resolution. A very complete system of parameters of adjustments has been worked out. The best resolution obtained so far is around $0.3 \%$.

Obviously the homogeneous field spectrometer with properly adjusted ringfocus shutters has very good figures of merit. When judging its potentialities one also has to discuss some of its limitations. When the ringfocus is set to accept a large fraction of the radiation, say several percent, the beam due to the spherical aberration spreads out considerably again after the passage of these shutters and in order to register the electrons an uncomfortably large counter has to be used, with a front window of several $\mathrm{cms}$ in diameter. Usually this window must be very thin which calls for a grid support. This support will reduce the actual transmission by as much as $30-50 \%$. It is also not very easy to get such a large thin window to be completely vacuum tight. The high background of such a large counter has to be reduced by using a shield of anticoincidence counters. It has also been tried ${ }^{88}$ to let the electrons pass into the detector through holes in the cylinder wall. The detector problem limits the maximum useful transmission to about $5 \%$ or less.

At very good resolution, say less than a few tenths of a percent other complications turn up instead. Contrary to the double focusing method, where narrow line sources are used, the lens method with ringfocus requires point sources, i.e. one dimension of the source is wasted in order to get space focusing. This seriously effects the luminosity. Furthermore the adjustments at high resolution are very critical indeed, since any small departure from the rotational symmetry of the field or of the general alignment of the system is detrimental. Again, the double focusing method is different in this respect since there the adjustment for high resolution is a fairly straight forward procedure.

Obviously, the choice of a uniform lens field is a fairly arbitrary one, apart from the fact that it is easy to handle mathematically and is the only case in which accurate magnetic field measurements are possible by the proton reconance method. Looking at eq. (54a) one would rather prefer a field which is concave upwards. For if $B^{\prime \prime}(z)$ is positive then $\frac{\partial B_{z}(r, z)}{\partial r} \approx-\frac{r}{2} B^{\prime \prime}(z)$ is negative, which means that $B_{z}$ decreases outwards

88 J. DuMond, L. Bogart, J. Kohl, D. Muller and J. Wilts, Calif. Inst. of Techn. Rep. (1952) No. 16. 
from the optical axis. This tends to counterbalance the positive spherical aberration going as $\cos \alpha$.

A long lens spectrometer with such a field form was built by Siegbahn ${ }^{76}$. The concave field form was obtained by supplying the cylindrical iron yoke with poleshoes facing the interior of the spectrometer. The spherical aberration was thus reduced. It still remained to be investigated what happened to the focusing if very strong magnetic gradients were set up. In order to do this a similar iron yoke with pole shoes was constructed by Slätis and Siegbahn ${ }^{89}$ and the exciting long solenoid subdivided into five separate coils, so that the gradient along the axis could be varied within very wide limits. A central shutter was used. It was then found that at a certain strong field gradient a pronounced increase in the transmission of the instrument occurred. By photographic ray tracing using the strong $F$-line in Th $\mathrm{B}$ as a monokinetic electron source it was found that the rays crossed each other in the middle of the spectrometer, forming an intermediate ring-formed image there. The rays are then all concentrated and collected in the small slit of the counter. The focusing action is illustrated in Fig. 19. In the upper part of the figure the rays appearing in a homogeneous field are shown. The strong spherical aber-

\section{B}

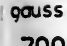

700

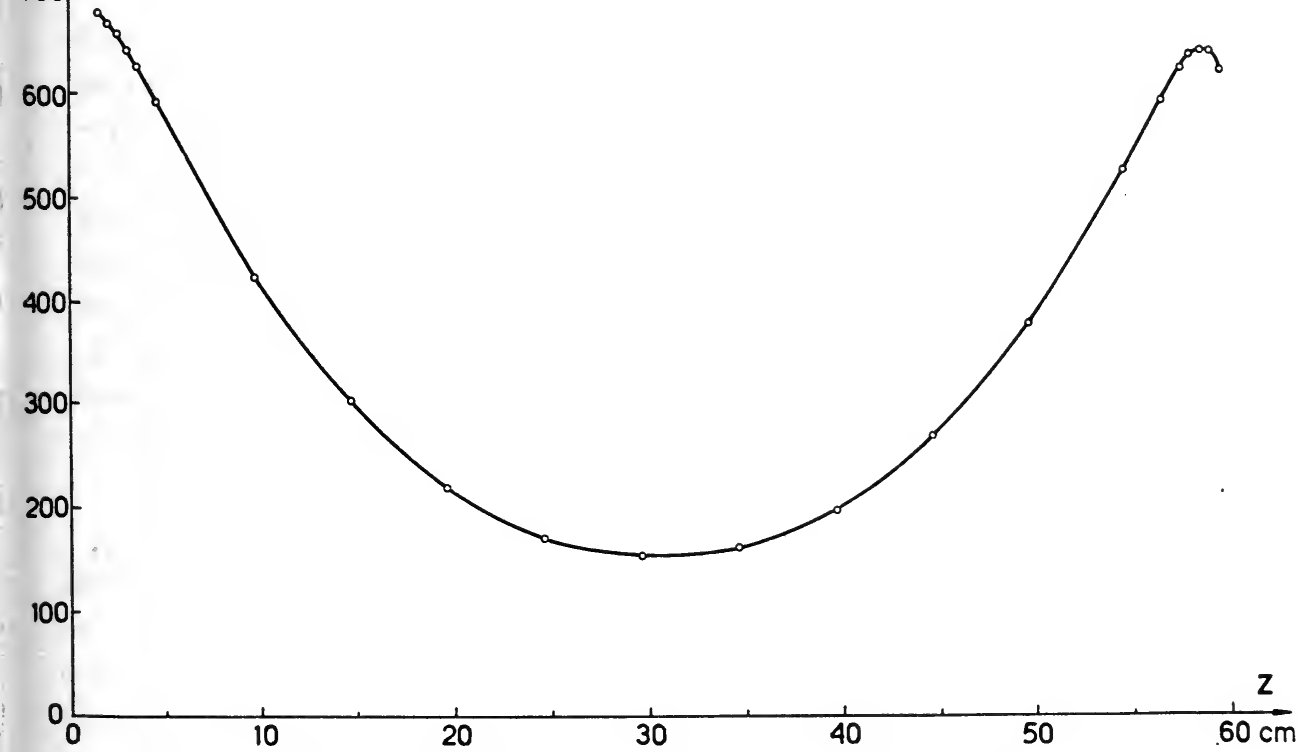

Fig. 20. Fieldform for intermediate image focusing

89 H. Slätis and K. Siegbahn, Ark. f. Fysik 1, Nr. 17, (1949) 339. 
ration. reguiring a correspondingly large counter for collecting a given solid angle of the raditation. is clearly seen. In the lower part of the figure is shown the focusing of the same solid angle of the radiation with the appropriate field-gradient for intermediate image focusing. As can be seen all ravs are gathered again in a point on the axis. Thus. an ordinary small sized counter can be used. in spite of the fact that about $8 \%$ of the total solid angle is transmitted. With a source diameter of $5 \mathrm{~mm}$ and a central shutter width of $8 \mathrm{~mm}$ the resolution is somewhat better than $4 \%$.

The field form of intermediate image focusing is given in Fig. 20. Similar field forms can easily also be realized without the use of iron (see $\mathrm{Ch}$. XXIV. Fig. 13). Unfortunately, it has been found difficult to treat this particular type of focusing theoretically. It seems resonable to assume that further refinements of the field form could improve the figures of merit still more.

Long lens spectrometers have been designed by e.g. Zünti ${ }^{90}$ (resolution $1.3 \%$. transmission $2.7 \%$, source diameter $3 \mathrm{~mm}$ ), Jensen et al. ${ }^{91}$ (intermediate image spectrometer for the particular purpose of coincidence measurements: $T=10 \%$ ), Feldman and $\mathrm{Wu}^{92}$ (homogeneous field without iron for investigations of the shapes of continuous spectra, see Ch. XI) and De Waard ${ }^{93}$ (concave field; coincidence measurements).

\section{$\S$ 8. Coincidence Spectrometers}

The first coincidence spectrometer for electrons was suggested and designed by Feather 94, 95 using the semicircular method. Other similar designs are due to Katz et al. ${ }^{96}$ and Fowler et al. ${ }^{97}$. De Waard's ${ }^{93}$ spectrometer is a suitable example of a high transmission coincidence spectrometer. Fig. 21 is a section of this instrument. The ring focus arrangement has been designed for optimum conditions by means of the photographic method of ray tracing using the $F$-line of Th $\mathrm{B}$. With a source diameter of $1 \mathrm{~mm}$ the transmission is $6.3 \%$ and the resolution $2.4 \%$. With a source diamèter of $6 \mathrm{~mm}$ the corresponding figures are $5.5 \%$ and $3.6 \%$ respectively. The scintillation counter assembly behind the source is shown in Fig. 22 .

90 W. Zünti, Helv. Phys. Acta 21, (1948) 179.

91 R. Nichols, A. Pohm, J. Talbog and E. Jensen, Ames Lab. Contr. W-7405 eng.82, (1953).

92 L. Feldman, Thesis, Columbia Univ. (1950).

93 H. de Waard, Thesis Groningen Univ. (1954).

94 N. Feather, Camb. Phil. Soc. 36, (1940) 244.

95 N. Feather, J. Kyles and R. Pringle, Proc. Phys. Soc. 61; (1948) 466.

96 R. Katz, R. Hill and M. Goldhaber, Phys. Rev. 78, (1950) 9.

97 C. Fowler and R. Schreffler, Rev. Scient. Instr. 21, (1950) 740. 


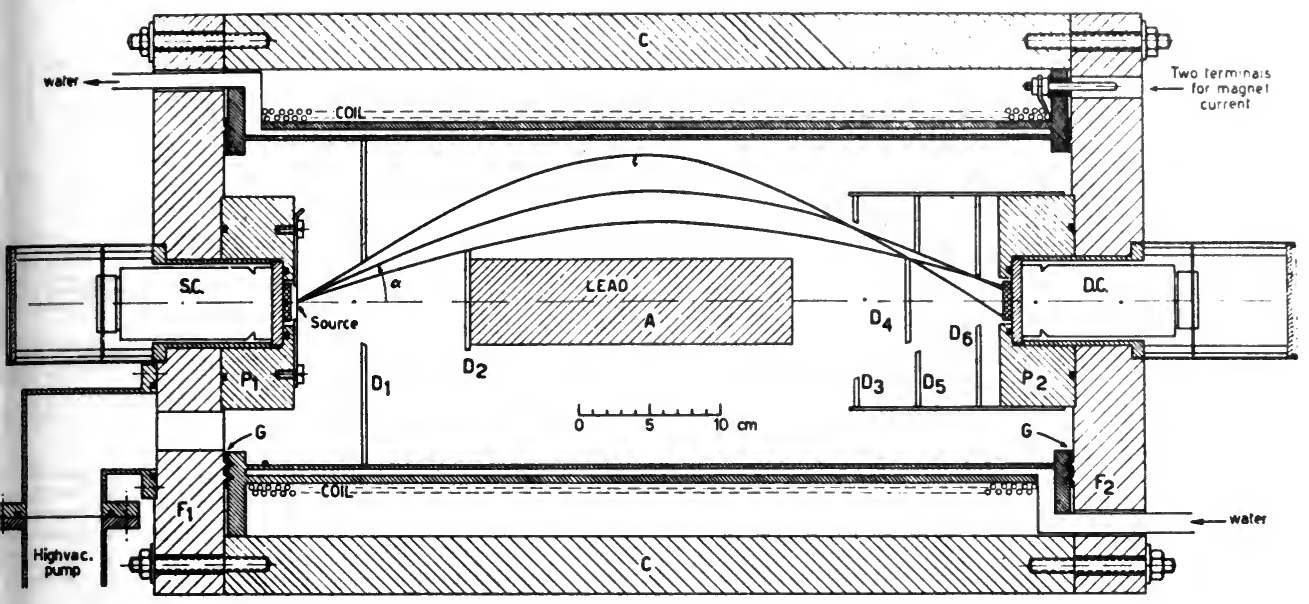

Fig. 21. Coincidence spectrometer due to de Waard

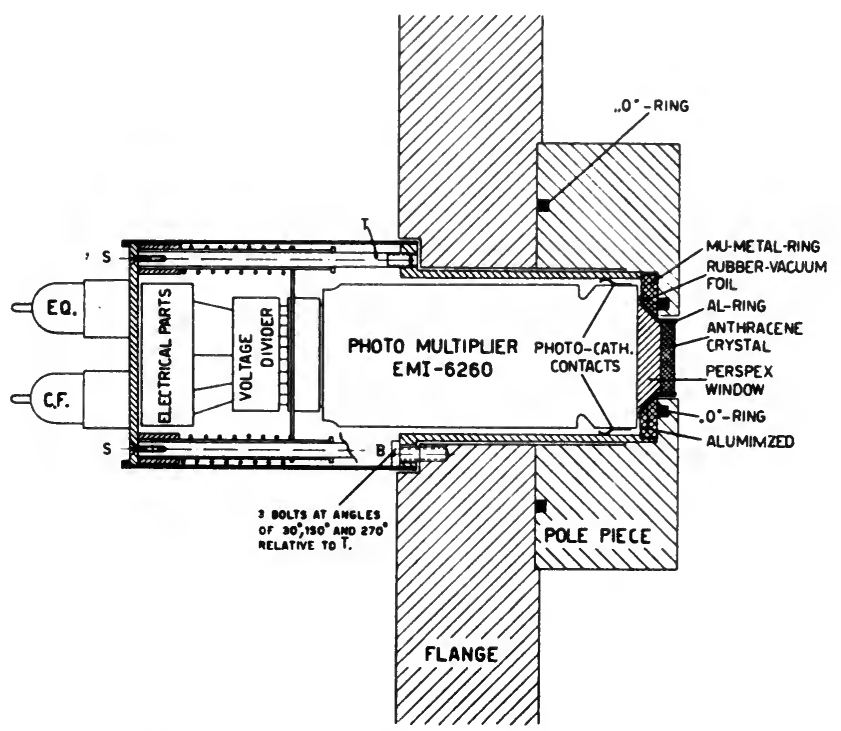

Fig. 22. Scintillation counter assembly

A $\beta$-spectrogoniometer consisting of two magnetically independent short lenses was designed by Siegbahn ${ }^{82}$. Fig. 23 shows the instrument. One of the spectrometer arms can be turned around the fixed source within a range from $180^{\circ}$ to $90^{\circ}$ relative the second arm, thus enabling the measurements of coincidences and in particular the angular correlation between successively emitted electrons of selected energies. This instrument was used by Alburger as one of the means of disentangling the complex spectrum of $\mathrm{Bi}^{206}$, as described in $\mathrm{Ch}$. XXII. The angular cor- 


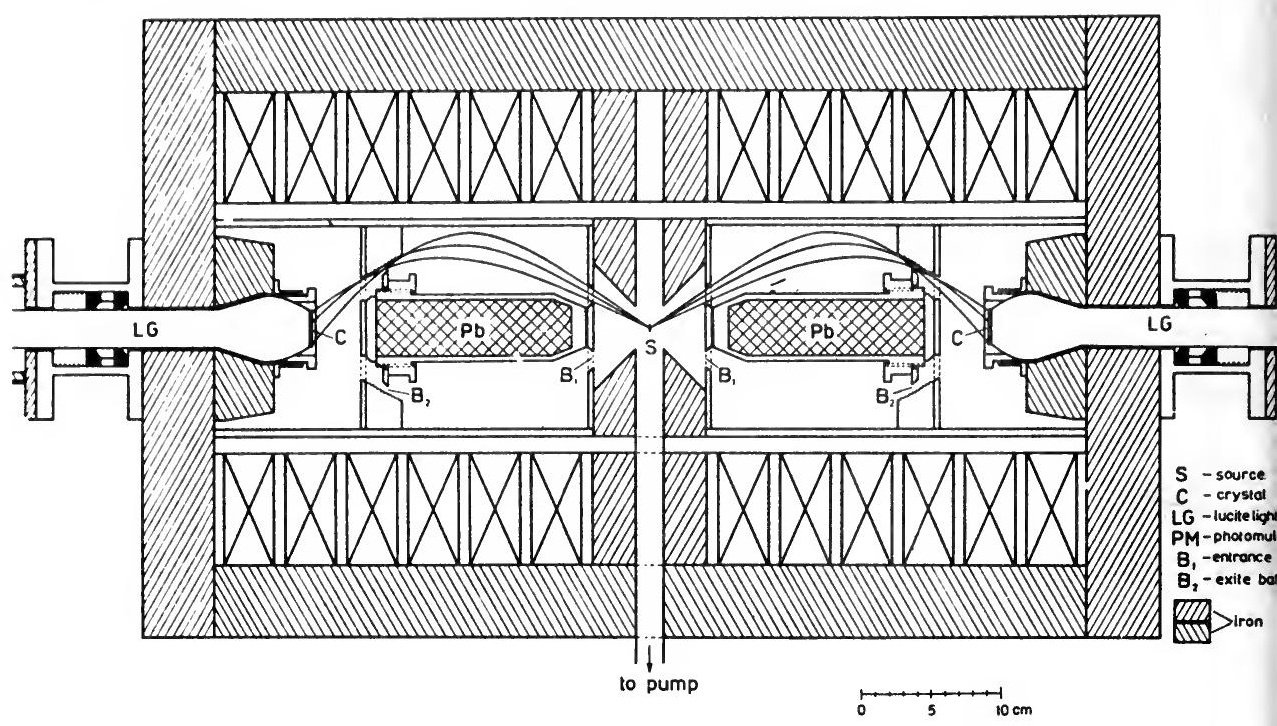

Fig. 24. Double lens coincidence spectrometer due to Gerholm

relation between electrons and positrons in the internal pair formation in $\mathrm{Na}^{24}$ has also been studied with the goniometer. (see Ch. XX)

The two short lens coincidence spectrometer by Bell et al. is described in Ch. XXI.

A double lens coincidence spectrometer of high transmission with interesting properties has recently been constructed by Gerholm ${ }^{98}$. By means of iron shields the two halves of the instrument shown in Fig. 24 are made magnetically independent of each other, but will still be able to accept electrons from the source with large solid angles. The fieldform in each half is approximately a triangular one being almost zero at the source. A theoretical treatment using the general equations of motion (55) shows that this fieldform in many respects is a more favourable one than the homogeneous field case. In particular, with the same spectrometer dimensions (the distance source-detector here being $24 \mathrm{~cm}$ ), one can use larger sources at a given transmission and resolution. The optimum angle of emission is found to be $30^{\circ}$. By a careful adjustment of the shutter system the following data have been obtained: source diameter $2 \mathrm{~mm}$, resolution $1.2 \%$, transmission $3 \%$ in each half of the instrument. The instrument can easily be converted into an "ordinary", or a $\beta \gamma$-coincidence spectrometer with the source in one end of the spectrometer. Scintillation detectors or thin window GM counters can alternatively be used in this flexible instrument. Some typical results obtained with this instrument are shown in Fig. 25.

$98 \mathrm{~T}$. Gerholm, in course of publication. 


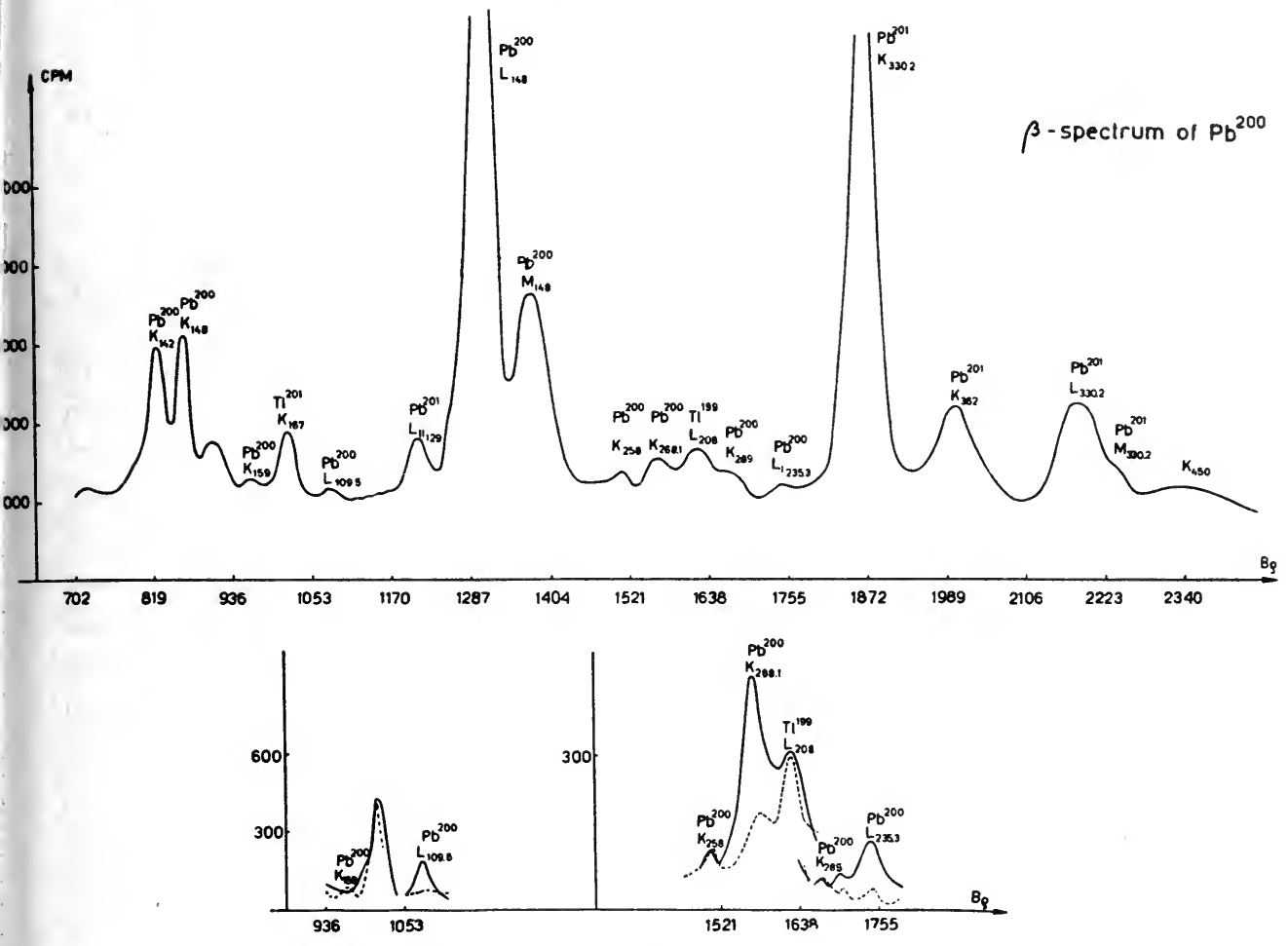

Fig. 25a

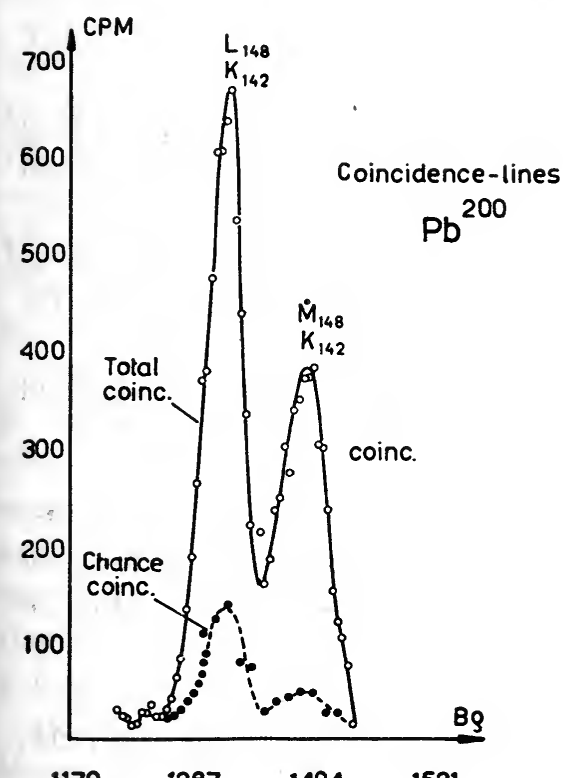

1170

Fig. $25 b$

Fig. 25a. Above: $\beta$-ray spectrum of $\mathrm{Pb}^{200}$. Below : Coincidence spectrum. One spectrometer channel (c.f. fig. 24) is scanning the spectrum when the other one is set to focus the $L_{148}$ peak. Dotted curve corresponds to chance coincidences. Fig. $25 a$ and $b$ show that the $148-\mathrm{keV} \gamma$-ray is in coincidence with the 109-, 142-, 268- and $235-\mathrm{keV}$ lines but not with the 159-, 257- and 289-keV lines.

Fig. 25b. Coincidence lines in $\mathrm{Pb}^{200}$. One channel of the spectrometer is focusing the $K_{142}$ peak, the other is scanning the spectrum over the strong $\mathrm{L}_{148}$ and $\mathrm{M}_{\mathbf{1 4 8}}$ peaks. Chance coincidences are recorded with a time delay in the $\mathrm{K}_{142}$ channel. 
The great importance of high transmission for coincidence work is further discussed in Ch. VII. Fairly good resolution is also necessary, in particular in the high $Z$-region where very line-rich spectra occur quite frequently (see $\S 10)$.

\section{\$ 9. Electrostatic Spectrometers}

So far only very little work has been done with electrostatic focusing $\beta$-ray spectrometers. In the low energy region it seems very probable, however, that such focusing devices will be much more frequently used in future than hitherto. In fact the figures of merit concerning resolution and transmission can be made very good indeed. Two cases can be considered, one using the field between two concentric cylinders (focusing after $1.27^{\circ}$ ). and the other using the field between two concentric spherical conductors (focusing after $180^{\circ}$ ). The latter case seems to offer the best advantages. It was shown by Purcell ${ }^{99}$ that the focusing in this case shows a remarkable similarity to the magnetic semicircular case with the additional advantage that it is space focusing because of the spherical symmetry. In both arrangements sector fields can be used. Contrary to the magnetic prisms, the electric fringing fields can be controlled to a high degree of precision. Unfortunately, at higher energies the relativistic mass increase of electrons starts to affect adversely the focusing properties. Cavanagh 3 has considered the case of a spherical energy analyzer for electrons having a mean diameter of $1 \mathrm{~m}$ and a gap between the spheres of $1.5 \mathrm{~cm}$. The ratio between transmission and resolving power at $0.2 \mathrm{MeV}$ is then 1.6 whereas at $0.7 \mathrm{MeV}$ it is 0.7 .

A spherical electrostatic spectrometer, primarily intended for analyzing heavy particles, has been designed by Allison and Weyl ${ }^{100}$. At a calculated transmission of $0.8 \%$ the expected energy resolving power was $0.5 \%$. Experimental data so far obtained for a $\beta$-line are $0.4 \%$ and $1.2 \%$. Because of relativity the maximum energy limit of the instrument for electrons is $1.7 \mathrm{MeV}$.

Electrostatic focusing spectrometers have further been investigated by Rogers 101 and Kobayashi ${ }^{102}$.

\section{$\S 10$. Precision Spectroscopy}

Most of the spectrometers developed during the forties were designed to be able to collect a maximum of radiation at a moderate resolution of

99 E. Purcell, Phys. Rev. 54, (1938) 918.

100 K. Allison and P. Weyl, Personal communication 1954.

101 F. Rogers Jr., Rev. Scient. Instr. 8, (1937) 22; 11, (1939) 19; 14, (1943) 216; 22. (1951) 723 .

102 Y. Kobayashi, Journ. of Phys. Soc. Japan. 1953. 
$2-4 \%$. In particular among the light elements the spacing of levels is such that this resolution for many purposes may be regarded as satisfactory. The situation changes, however, rather quickly at higher $Z$ 's. Already at $Z=36\left({ }_{36} \mathrm{Kr}^{79}\right)$ Thulin et al. ${ }^{103}$ have found a case where 15 different $\gamma$-lines are emitted within an energy region extending from the ground state up to $800 \mathrm{keV}$ excitation energy. From a shell theoretical point of view it may even be claimed that the most interesting and decisive problems are to be found among the heavier elements, where a great number of closely spaced levels frequently are excited in radioactive decay. A typical example is the decay of $\mathrm{Bi}^{206}$ studied in great detail by Alburger and discussed in Ch. XXII. In this case, which incidentally must be regarded as exhibiting a fairly normal level spacing in this region, about $28 \gamma$-lines are emitted. These lines are furthermore converted in several atomic shells and the resulting $\beta$-line spectrum is extremely line-rich and complicated to interpret. Another example is given in Fig. 7 in this chapter, studied by Mihelich et al. The above "moderate" resolution then turns out to be entirely inadequate. The situation is well illustrated by Fig. 26, showing a line group in the spectrum of $\mathrm{Bi}^{206}$. The structure is completely unresolved with a resolution of $1.2 \%$ taken in a short lens spectrometer. With the double focusing spectrometer a resolution of $0.5 \%$ is still insufficient to separate the components. At $0.22 \%$ resolution the group is completely resolved.

Another typical example is given in Fig. 27, taken by Arbman ${ }^{29}$ with his double focusing spectrometer (see $\S 5$ ) with automatic recording. It shows the energy region $55-300 \mathrm{keV}$ in the spectrum of a mixture of $\mathrm{Pb}$ isotopes (obtained by bombardment of $\mathrm{Tl}$ with $100 \mathrm{MeV} \mathrm{p}$ ) with the mass numbers 197, 198, 199. Full deflection corresponds to 100 pulses per sec. The part of the spectrum shown in Fig. 27 was registered in $38 \mathrm{~min}$. The spectrometer resolution was set to be $0.3 \%$ which is just sufficient to resolve several of the lines.

Having resolved and identified all lines in a complicated spectrum it is then of great importance to be able to determine their energies with the highest possible accuracy, say a few parts in $10^{4}$. That will enable one from pure numerical considerations to formulate a limited number of alternatives for the decay scheme and to exclude a great number of other possibilities. With that knowledge there is good reason to believe that additional coincidence spectroscopic studies on selected lines will give a fairly unique answer to the problem of the level 'scheme.

If the multipolarities of the lines can be measured the intensities of the $\beta$-lines will determine the intensities of the corresponding $\gamma$-lines and 
consequently the total intensities of the transitions. This will add further conclusive information on the decay scheme. In order to measure the multipolarities one can measure the different $K: L$ intensity ratios. If it is

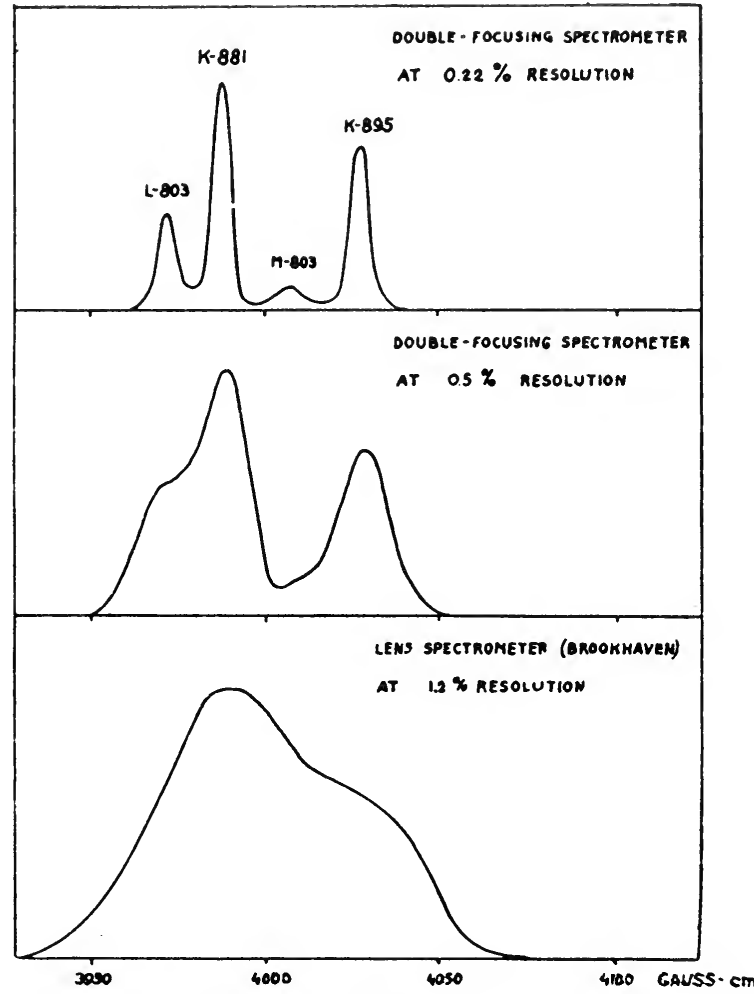

Fig. 26. A portion of the $\mathrm{Bi}^{206}(K) \mathrm{Pb}^{206}$ internal conversion spectrum showing the need for high resolution, according to Alburger

possible a better method is to measure the intensity ratios in the $L_{\mathrm{I}}, L_{\mathrm{II}}, L_{\mathrm{III}}$ triplets. This intensity ratio is a very sensitive indicator of the multipolarity of the transition concerned (see Ch. XIV and Ch. XVI). A precise determination of this quantity also yields an accurate figure for the amount of admixture between the magnetic and electric contributions to the transition probability which is of basic importance for further theoretical conclusions. Since the atomic binding energy differences between the $L_{\mathrm{I}}$, $L_{\text {II }}$ and $L_{\text {III }}$ levels are quite small very good resolution is needed in order to resolve the triplets. Fig. 28 shows the $L_{\mathrm{I}}, L_{\mathrm{II}}, L_{\mathrm{III}}$ triplet from the $279 \mathrm{keV} \gamma$-ray transition in $\mathrm{Hg}^{203}$ taken with the ironfree double focusing spectrometer (see $\S 5$ ). The source was $8 \mathrm{~mm} \times 0.5 \mathrm{~mm}$, the counter slit $0.5 \mathrm{~mm}$ wide and the transmission was set to be $0.1 \%$. The resolution 
(including natural line width) is $0.07 \%$. The triplet is completely resolved. A resolution of $0.2 \%$ would give a very poor figure for the $L_{\mathrm{I}}: L_{\mathrm{II}}$ ratio whereas a resolution of somewhat better than $1 \%$ (indicated in the figure) would give no information at all about the entire triplet structure. The situation would be better at lower energies, but on the other hand worse at smaller $Z$-values.

Evidently there are quite a few strong reasons to try to attain high resolution and good precision in energy and intensity measurements. There are several other interesting types of problems in addition to those mentioned above which no doubt will be the subject of future studies at

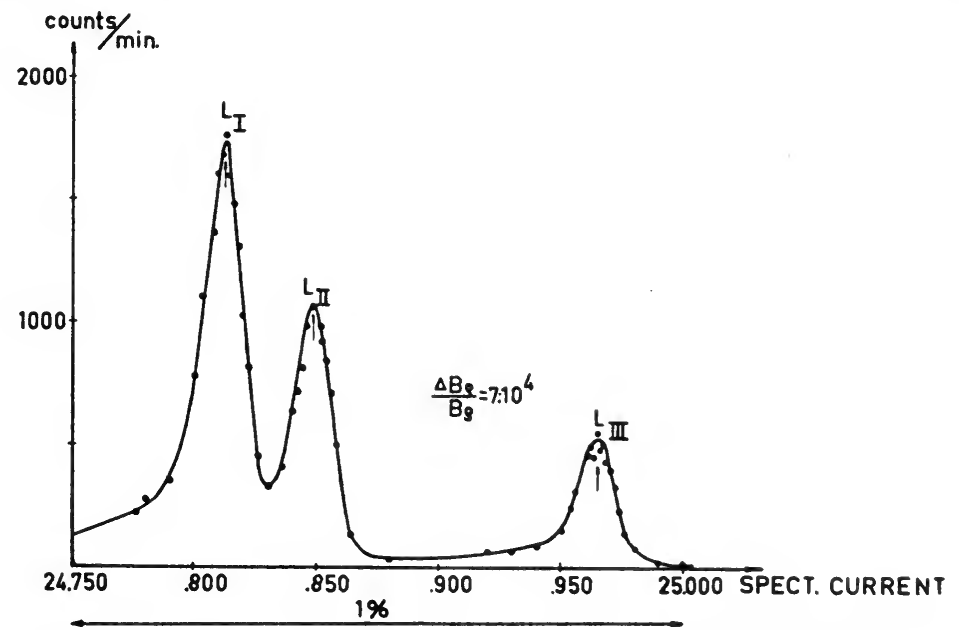

Fig. 28. $L_{\mathrm{I}}, L_{\mathrm{II}}, L_{\mathrm{III}}$ triplet from the $279 \mathrm{keV} \gamma$-line of $\mathrm{Hg}^{203}$ taken with the iron free double focusing spectrometer

very high resolution, for instance the Auger effect, discussed in Ch. XX, the binding energies of the electrons in the atomic shells etc. ${ }^{104}$ Already some work has been done in this high resolution region. This concerns e.g. measurements on natural linewidths and precision determinations of $\beta$-lines suitable as reference lines. Some of the results obtained will be briefly reviewed here.

\section{A. Natural Linewidths}

Using a $50 \mathrm{~cm}$ double focusing spectrometer Lind and Hedgran 105 investigated the Doppler width of the annihilation radiation produced by positrons stopped in copper. Since the annihilation cross-section varies as $1 / v$ the main interaction is between slow positrons and thermal electrons.

104 K. Siegbahn, Physica 18, (1952) No. 12, 1043.

105 D. Lind and A. Hedgran, Ark. f. Fysik 5, (1952) Nr. 2, 29. 
Fig. 29 shows on the left for comparison an $L_{\mathrm{III}}$ external photoline expelled from a thin $\mathrm{U}$-converter $\left(0.7 \mathrm{mg} / \mathrm{cm}^{2}\right)$ by the $411 \mathrm{keV} \gamma$-radiation of $\mathrm{Au}^{198}$ and on the right the $K$-photoline from the same U-converter expelled by the annihilation radiation. By using the left curves as "window curves" one gets the mean value of the energy spread due to Doppler effect in copper $=975 \mathrm{eV}$ (see also $\mathrm{Ch}$. XXI). It would be of some interest to study this effect in other materials too.

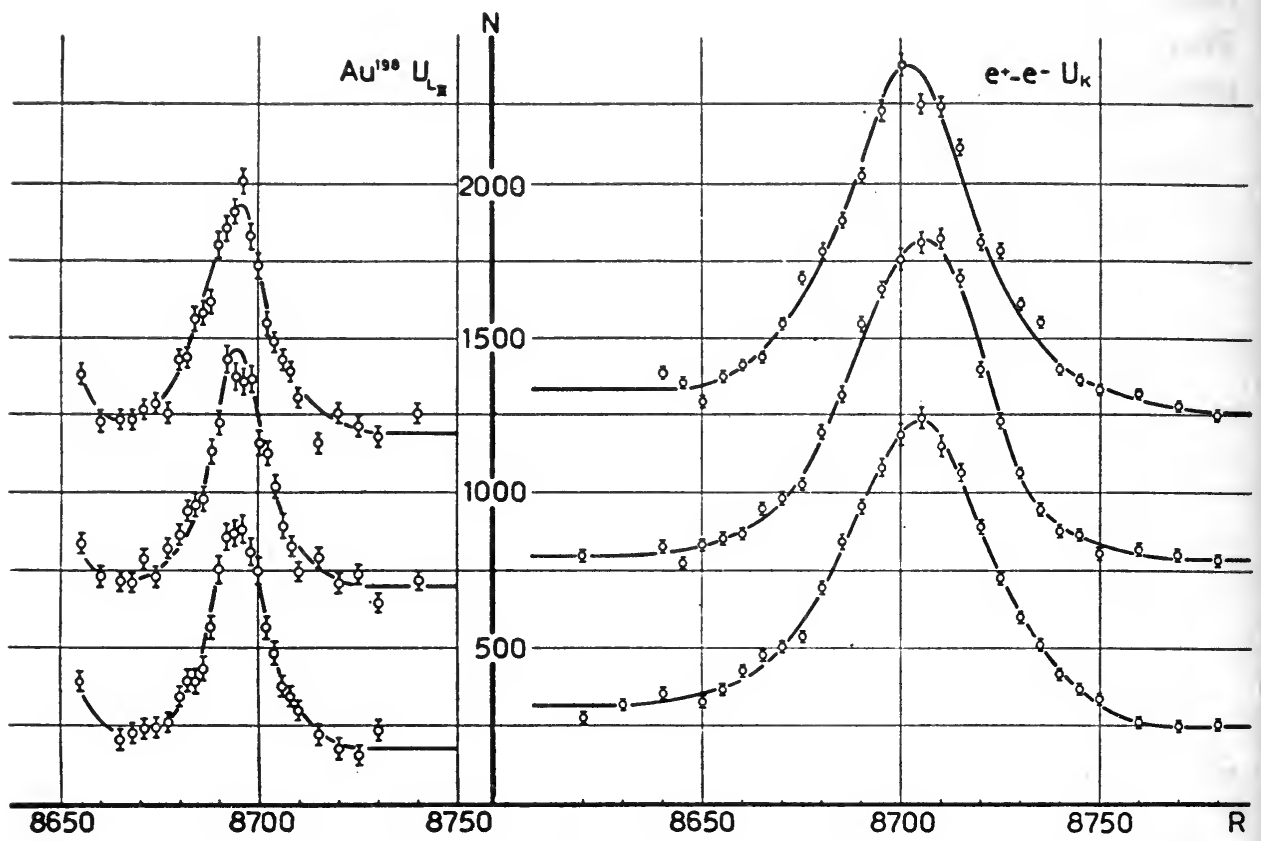

Fig. 29. Doppler width of annihilation radiation, according to Lind and Hedgran Left curves: $L_{\text {III }}$ external photolines expelled from a $0.7 \mathrm{mg} / \mathrm{cm}^{2} \mathrm{U}$ converter by the $411 \mathrm{keV} \gamma$-ray of $\mathrm{Au}^{198}$

Right curves: $K$-external photolines from the same converter expelled by the annihilation radiation

The evaporated U-converter had the dimensions $2 \mathrm{~mm} \times 20 \mathrm{~mm}$ in these experiments. At energies slightly below $0.5 \mathrm{MeV}$ a $0.7 \mathrm{mg} / \mathrm{cm}^{2} \mathrm{U}$ foil will produce almost no line broadening due to inelastic electron scattering in the foil since it can be calculated that the probability for that process at this energy and for this foil thickness is fairly small. At energies $\sim 1 \mathrm{MeV}$ a $3 \mathrm{mg} / \mathrm{cm}^{2} \mathrm{U}$ foil may be tolerated. With the above source dimensions and the transmission set to be $0.1 \%$ the resolving power measured on the U-photolines was found to be as good as $0.17 \%$.

A first indication that ordinary conversion lines may have a finite width 
(which is not caused by source scattering) was obtained by Lindström ${ }^{106}$, when he was studying the $F$ - and $I$-lines in the spectrum of Th B in his semicircular spectrometer at high resolution. This effect was further studied by Slätis ${ }^{107,} 108$ who together with Lindström was able to give definite

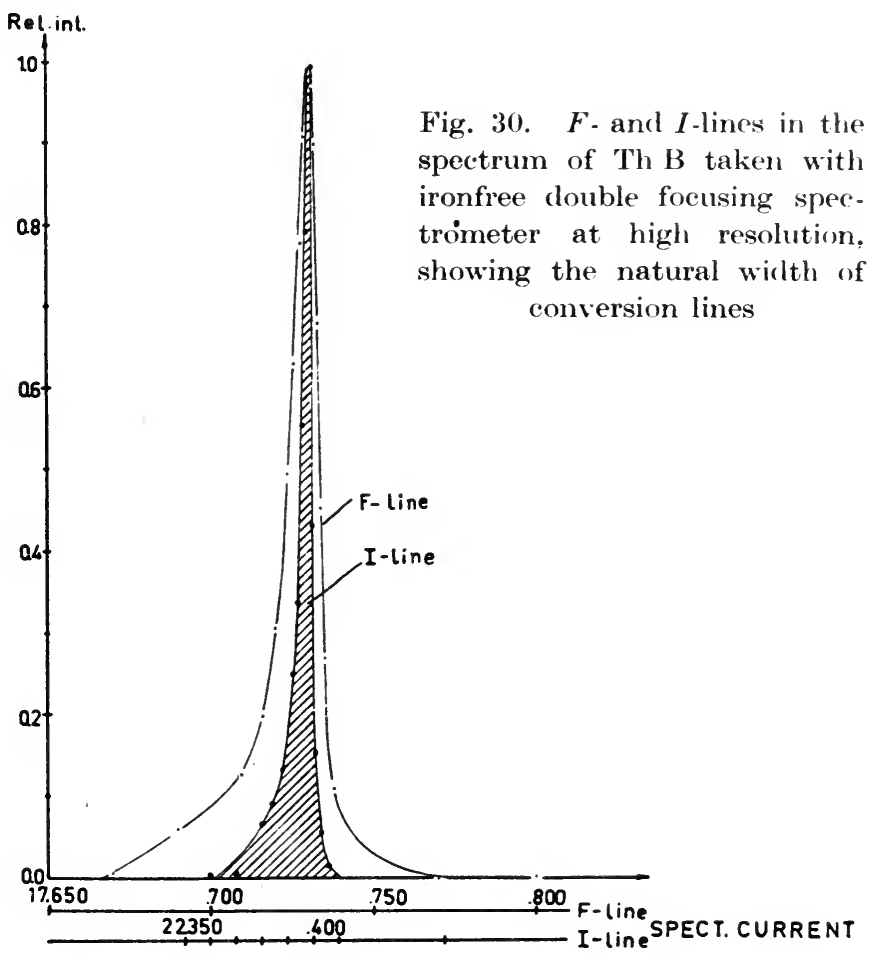

evidence that all the $K$-lines in the spectrum concerned were broader than the $L$-lines by about $80 \mathrm{eV}$. As far as these preliminary results indicate, the effect of finite linewidth of conversion lines can be explained from the widths of the corresponding atomic levels.

In order to observe the finite widths of conversion lines one has to go to extremely high resolution, of the order of a few parts in $10^{4}$. The new ironfree double focusing spectrometer is particularly well suited to this kind of investigation. With a $10 \mathrm{~mm} \cdot \times 0.01 \mathrm{~mm}$ Th $\mathrm{B}$ activated wire and with the transmission set to be $0.03 \%$ several of the conversion lines in the spectrum concerned have now. been studied in great detail ${ }^{109}$. Fig. 30

\footnotetext{
106 G. Lindström, Ark. f. Fysik 4, (1951) Nr. 1.

107 H. Slätis, Ark. f. Fysik 6, Nr. 41, (1953) 415.

108 H. Slätis and (i. Lindström, Phys. Rev. 88, (1952) 1429.

109 K. Siegbahn and K. Elvarson, in course of publication.
} 
shows the $F$ - and $I$-lines (from the $K$ - and $L_{\mathrm{I}}$-shells, respectively) registered with a GM counter in the spectrometer and plotted with equal heights and with reduced $B o$ coordinates for the sake of comparison. The relative halfwidth of the $I$-line is only $1.9: 10^{4}$ whereas the corresponding figure for the $F$-line is $5.1: 10^{4}$. Taken at this resolution this phenomenon of "natural" width of $K$ - and $L$-conversion lines evidently is a very pronounced effect.

\section{B. Precision Measurements of BQ-Values}

In principle two quantities have to be measured separately, namely $B$ and $\varrho$. If high precision is pursued the greatest difficulty concerns $B$. The ordinary flip coil method allows an accuracy of about $1: 10^{3}$. With a magnetic balance ${ }^{110}$ one can reach $1: 10^{4}$. By far the best precision is obtained, however, by means of the nuclear magnetic resonance method. Using the value $\gamma_{p}=(2.67523 \pm 0.00006) \cdot 10^{4} \mathrm{sec}^{-1}$ Gauss $^{-1}$ for the gyromagnetic ratio of the proton, as determined by Thomas et al. ${ }^{111}$, one can get a very accurate determination of the field, provided it is homogeneous. Lindström ${ }^{106}$ used this method to measure the $B \varrho$-values of the $F$-, $I$ and $L$-lines (Ellis' notation) in the spectrum of $\mathrm{Th}\left(\mathrm{B}+\mathrm{C}+\mathrm{C}^{\prime \prime}\right)$, and later on the method was applied to the $\mathrm{Th}^{\prime \prime} \mathrm{X}$-line ${ }^{112}$, the $\mathrm{Cs}^{137} \gamma$-line ${ }^{113}$ (at $661.65 \mathrm{keV}$ ) and two lines in the spectrum of $\mathrm{RaC}$ which were used as calibration lines for measurements of the well known $\mathrm{Co}^{60} \gamma$-lines ${ }^{114}$, performed in the big double focusing spectrometer. By means of this instrument Lind and Hedgran ${ }^{105,} 115$ used some of the "primary" standards of Lindström in order to obtain "secondary". standards having nearly the same accuracy, namely $\sim 2: 10^{4}$. These secondary standards are the $\gamma$-lines from $\mathrm{Au}^{198}, \mathrm{Na}^{24}, \mathrm{Co}^{60}$ and the annihilation radiation. The energy value of annihilation radiation is particularly interesting, since it affords a means of calculating the mass of the positron. As further discussed in $\mathrm{Ch}$. XXI, the mass value of the positron was found to agree within extremely narrow limits with that of the electron. The list of reference lines measured so far is given in Ch. VIII, Table I. The number of standard lines and also the accuracy is continuously being increased.

In order to use the magnetic resonance method for the field measurements the field must be very homogeneous. However, without very

110 A. Harper and N. Roberts, Proc. Roy. Soc. A178, (1941) 170.

111 H. Thomas, D. Driscoll and J. Hipple, Phys. Rev. 78, (1950) 787.

112 G. Lindström, Phys. Rev. 87, (1952) 678.

113 G. Lindström, K. Siegbahn and A. Wapstra, Proc. Phys. Soc. 66, (1953) 54.

114 G. Lindström, A Hedgrain and D. Alburger, Phys. Rev. 89, (1953) 1303.

115 A. Hedgran and D. Lind, Ark. f. Fysik 5, (1952) Nr. 13. 
Ch. III - K. Siegbahn - B-Ray Spectrometer Theory and Design. High Resolution Spectroscopy
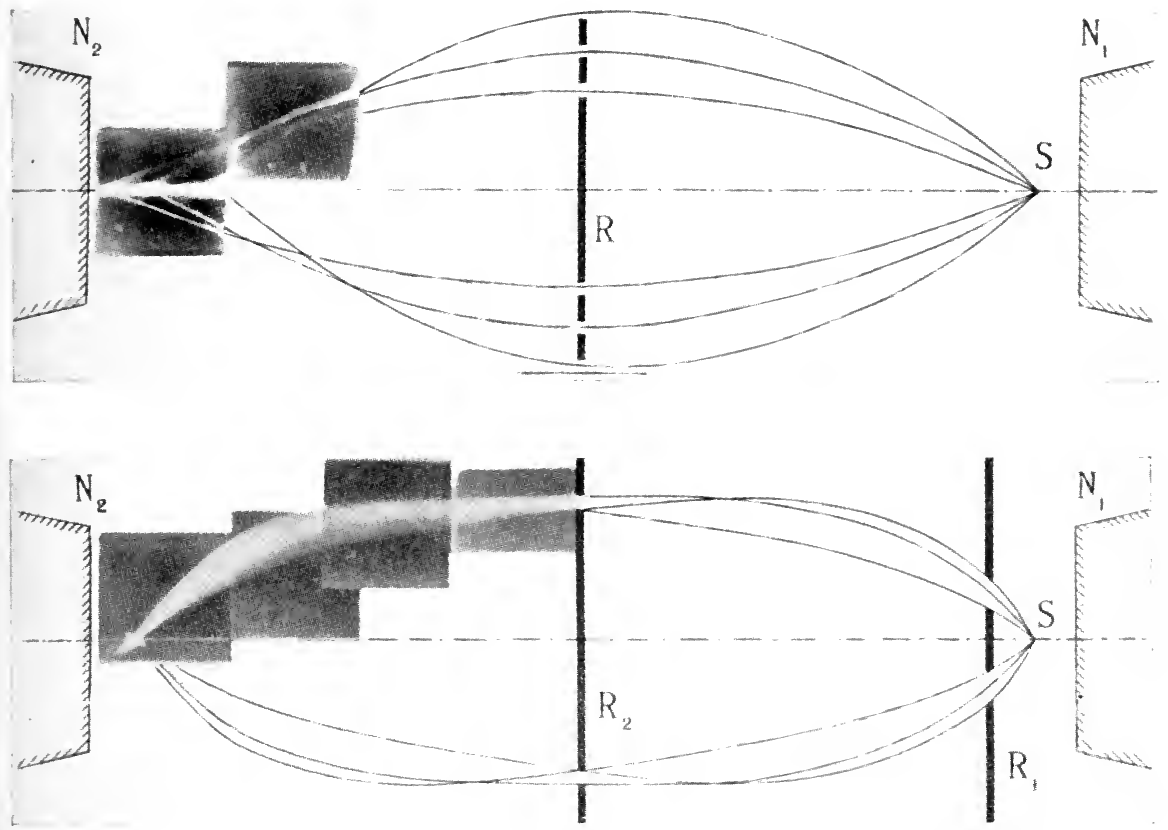

Fig. 19. Upper part: Electron paths in a homogeneous field showing the strong spherical aberration. Lower part: Intermediate image focusing, according to Slätis and Siegbahn

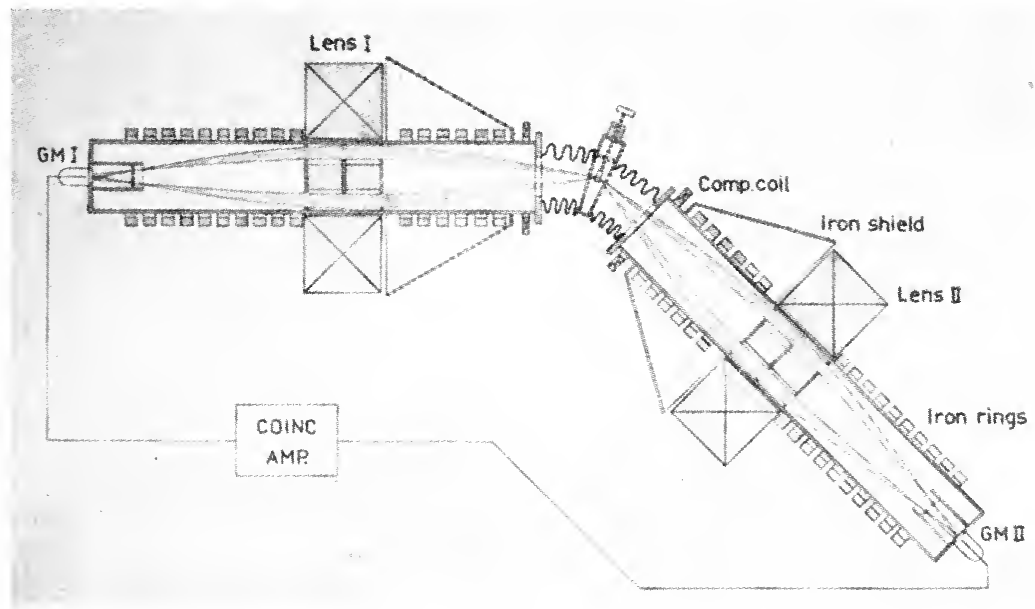

Fig. 23. $\beta$-spectrogoniometer due to Siegbahn 


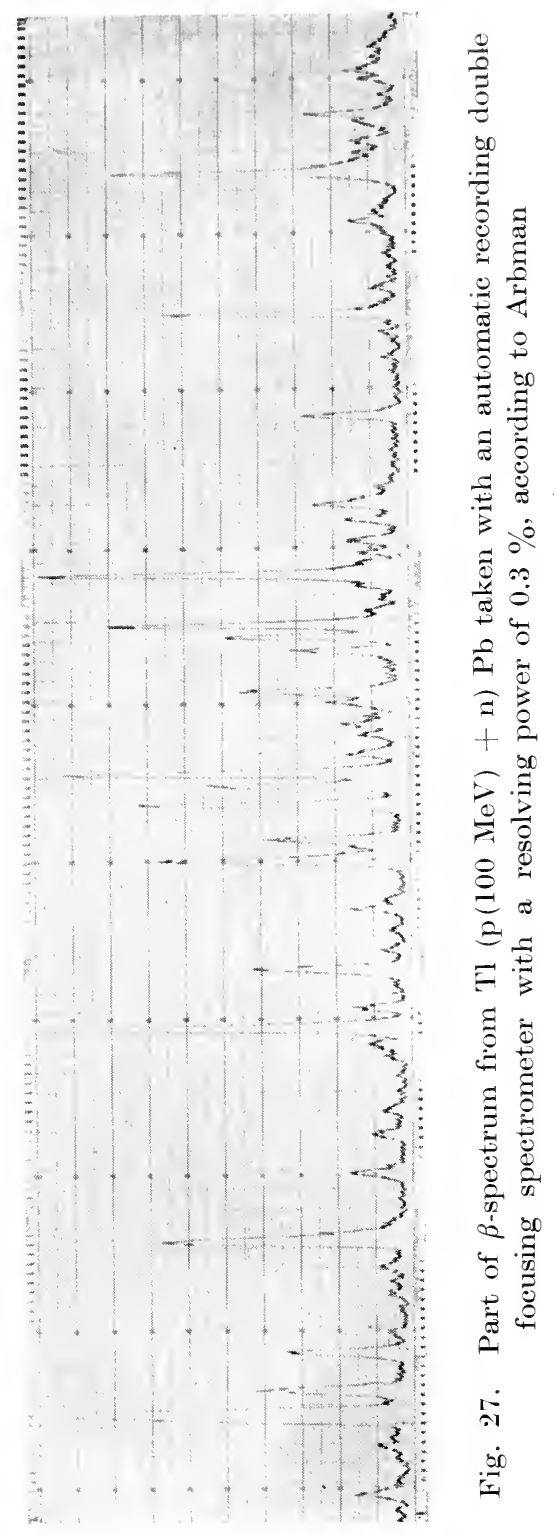

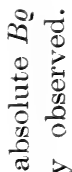

के :

志范

of 11

ㄷ. ๓ำ

$r=\frac{9}{4}$

- 10

$\geq 0$

范

ฮ)

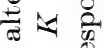
๑ . 용 ๑ 焉起量 类。

क $\bar{⿷}$ 范 क $\because \stackrel{0}{0}$ 递离 声语 के

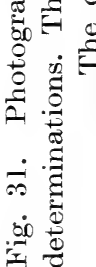


elaborate shimming of the magnet one usually has departures from the average value along the different electron paths of several parts in $10^{4}$. It is furthermore found, that the inhomogeneity over an extended region in a semi circular spectrometer depends on the magnetic pre-history. One must therefore measure the magnetic field with the proton probe along the complete path without changing the field. The departure from homogeneity is then corrected by means of a formula due to Hartree ${ }^{116}$. For very high precision there are still some intricate problems to solve, for instance to get the exact distance source-image $=2 \varrho$ which is experimentally found not to be as well defined as the theoretical treatment under idealized conditions indicates.

In order to overcome many of these difficulties a simple method was introduced by Siegbahn 117 which does not require separate absolute $B$ and $\varrho$ measurements to be made but only a determination of the ratio between two lines, both emanating from the same $\gamma$-transition but from different atomic shells. The energy difference between a pair of such $\beta$-lines can be expressed by $h v$, where $v$ is the frequency corresponding to the $\mathrm{X}$-ray transition between the two atomic levels. If $B_{2} / B_{1}=a$ is the ratio of the two fields corresponding to equivalent points on the lines (preferably the tops of the lines because of the different natural line widths) one can show that:

$$
\left\{\begin{aligned}
(B \varrho)_{1}^{2} & =U+\sqrt{U^{2}+V} \\
U & =\frac{a^{2}+1}{\left(a^{2}-1\right)^{2}} \cdot(h / e) \cdot v^{2} \\
V & =\frac{4-s^{2}}{\left(a^{2}-1\right)^{2}} \cdot(h / e)^{2} \cdot\left(e / m_{0}\right)^{-2} \cdot c^{2} \cdot v^{2} \\
s & =\frac{h}{m_{0} c} \cdot v \ll 1
\end{aligned}\right.
$$

This method was used by Lindström ${ }^{106}$ and also by Graig ${ }^{118}$ to determine the $B \varrho$ values of the $F$ - and $I$-lines in the spectrum of Th B. The agreement with the results obtained by Lindström using the proton gyromagnetic ratio is quite satisfactory. The same method was also used by Lind and Hedgran to measure the $411 \mathrm{keV} \gamma$-ray of $\mathrm{Au}^{198}$ in the double focusing spectrometer. Evidently the method has the further advantage that the spectometer field need not be homogeneous but may have any prescribed form, since only field ratios enter into the formulas.

The experimental conditions are particularly favourable when an iron-

116 D. Hartree, Proc. Camb. Phil. Soc. 21, (1923) 746.

117 K. Siegbahn, Ark. f. Mat. Astr. Fys. 28B, (1941) No. 6; 30A, (1944) No. 20.

11 H. Craig, Phys. Rev. 85, (1952) 688. 
free spectrometer is used with the average external field along the path completely compensated for. In that case the field ratio $B_{2} / B_{1}$ is simplified to be a ratio between two currents through the spectrometer coils, which, with standard procedures, can be measured with an accuracy of better than $1: 10^{5}$. By means of the iron-free double focusing instrument the $F-, I-, I_{a^{-}}, J-, A$ - and $B$-lines in the $\mathrm{Th}\left(\mathrm{B}+\mathrm{C}+\mathrm{C}^{\prime \prime}\right)$ spectrum have recently been measured with considerably increased accuracy ${ }^{109}$. In particular the relative $B \varrho$ values of these lines could be given with a probable error of $1.5: 10^{5}$. From these measurements the following absolute $B \varrho$ values were calculated:

$$
\begin{aligned}
& F=1388.44 \pm 0.10 \\
& I=1753.91 \pm 0.14 \\
& I_{a}=1757.07 \pm 0.14
\end{aligned}
$$

$$
\begin{aligned}
& J=1811.11 \pm 0.15 \\
& A=534.20 \pm 0.06 \\
& B=652.38 \pm 0.07
\end{aligned}
$$

Fig. 31 shows a photographic recording performed in the ironfree spectrometer of a "quartet" of lines, namely the $I-, F$-, $I$-, $F$-lines, alternatively. The change in current between the two $I$-lines (and the two $F$-lines) is about $1: 10^{3}$. The dispersion could then be measured accurately. A number of such quartets were taken in order to determine the $B \varrho$ values given above.

By means of the $K$-line on one hand and the $L_{\mathrm{I}}, L_{\mathrm{II}}$ and $L_{\mathrm{III}}$ lines on the other, of the $279 \mathrm{keV}$ transition in $\mathrm{Hg}^{203}$ (shown in Fig. 28) the following absolute $B \varrho$ have also been calculated (with somewhat less accuracy):

TABLE III

\begin{tabular}{l|c|c|c|c}
\hline$\beta$ line from & $I \mathrm{amp}$ & $K-L_{\mathrm{I}}$ & $\begin{array}{c}B \varrho \\
K-L_{\mathrm{II}}\end{array}$ & $K-L_{\mathrm{III}}$ \\
\hline$K$ & 20.6685 & 1618.07 & 1618.25 & 1618.00 \\
$L_{\mathrm{I}}$ & 24.8128 & 1942.51 & & \\
$L_{\mathrm{II}}$ & 24.8487 & & 1945.54 & \\
$L_{\mathrm{III}}$ & 24.9650 & & & 1954.34
\end{tabular}

With the present increased precision in the measurements of $\beta$-lines the attainable accuracy in the absolute $B \varrho$ values is in fact set by the error coming from X-ray data, being of the order of $\sim 5: 10^{5}$.

The atomic constants used are those given by DuMond and Cohen in their most recent least square adjustment ${ }^{119}$. It is of interest to note that the accuracy in the measurements of $\beta$-lines enables the evaluation of atomic constants. If one combines the results from two linepairs, e.g.

119 J. DuMond and E. Cohen, Rev. Mod. Phys. 25, (1953) 691. 
the $I: F$ ratio and the $B: A$ ratio, respectively, one can calculate the atomic constant $h / m_{0} c$. One then finds the value

$$
h / m_{0} c=(2.4262 \pm 0.0016) \times 10^{-10} \mathrm{~cm},
$$

in exact agreement with the result obtained from the wavelength determination of the annihilation radiation by DuMond et al. ${ }^{120}$, (see (h. $\mathrm{IV}^{\mathrm{r}}$ ).

It has also been suggested ${ }^{121}$ that $h / e$ can be measured by means of a direct voltage difference measurement between two $\beta$-lines, emanating from two different atomic levels, the energy difference of which is put equal to the $h v$ corresponding to the $\mathrm{X}$-ray transition in question.

120 D. Muller, H. Hoyt, D. Klein and J. DuMond, Phys. Rev. 88, (1952) 775

121 K. Siegbahn, Appl. Scient. Res. B4, (1954) 25. 


\section{CRYSTAL DIFFRACTION SPECTROSCOPY \\ OF NUCLEAR $\gamma$-RAYS}

\section{J. W. M. DUMOND}

\section{$\S$ 1. Early History and Elementary Ideas Relative to Both Flat and Bent Crystal Gamma and X-ray Spectrometers}

The earliest studies of nuclear gamma rays by direct crystalline diffraction were those of Rutherford and Andrade ${ }^{1}$ utilizing flat crystalline lamina in the arrangement schematically illustrated in Fig. 1. The shortest wavelengths measured in this early work were about 70 milliangstroms.

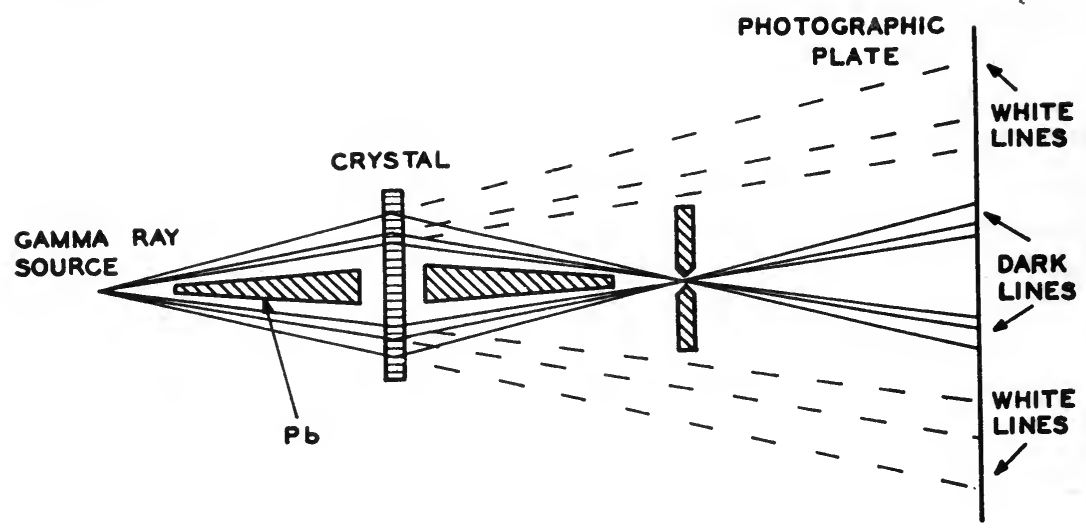

Fig. 1. Rutherford and Andrade's method for the study of nuclear gamma-ray spectroscopy utilizing a flat unstressed crystalline lamina in transmission

A photographically recording reflection type spectrometer for nuclear gamma-rays employing Bragg surface reflection at very grazing angles from a flat crystal was successfully developed by Frilley ${ }^{2}$ following a method apparently first used for such short wavelength work by Thibaud ${ }^{3}$. Frilley's shortest measured wavelengths were of order 16 milliangstroms. At such short wavelengths the chief difficulties in Frilley's instrument were, (1) low resolving power and poor wavelength accuracy, (2) impossibility of shielding the direct beam and its diffuse scattering from masking the selectively reflected spectrum, (3) indeterminacy of the "zero" of wavelength scale. The last could indeed have been obviated by

1 E. Rutherford and E. N. da C. Andrade, Phil. Mag. 27, (1914) 854; 28, (1914) 263. See also E. Rutherford, J. Chadwick and C. Ellis "Radiations from Radioactive Substances," Cambridge University Press (1930).

2 M. Frilley, Ann. phys. 11, (1929) 483.

3 J. Thibaud, Thesis, Paris (1925). 
rotating the crystal through almost $180^{\circ}$ and taking photographic spectra of the gamma-ray lines formed by reflection both to left and to right on the same negative. At the shortest wavelengths Frilley's accuracy and resolving power were very low.

$\mathrm{Y}$. Cauchois ${ }^{4}$ has taken photographic spectra of $\mathrm{X}$-rays emitted by radioactive isotopes with her transmission type curved crystal focusing spectrometer, the shortest wavelengths reached in this case being stated by her to be in the region of 160 milliangstroms. A mica crystal was used. Here again the chief difficulty in reaching the shorter wavelength domain is that of shielding the film from the directly transmitted gamma-ray beam so that it does not obscure the spectrum of selectively reflected lines.

Beginning in 1947 much shorter wavelengths than any previously referred to have been measured with considerably higher precision (from \pm 1 part in $10^{3}$ to \pm 1 in $10^{4}$ ) utilizing a transmission type curved crystal focusing spectrometer (developed by the writer at the California Institute of Technology) in which the gamma-ray source in very concentrated form is placed on the focal circle and the radiation propagates in the opposite direction to that in the Cauchois photographic instrument. In this relatively new instrument, which is in reality a monochromator of variable wavelength rather than a spectrograph, only one wavelength setting can be observed at one time. The source must explore the successive wavelength locations on the focal circle, either continuously or in small steps, and the intensity of the reflected gamma rays must be recorded as a function of source position on the focal circle relative to the crystal to delineate the profiles of the different spectral lines. Nevertheless, in the short wavelength region there are great advantages in this approach and by this method wavelengths as short as 9 milliangstroms have been measured with an absolute precision of the order of \pm 1 part in $10^{3}$, or perhaps slightly better. Because of its superior ability to yield high absolute precision in the short wavelength nuclear gamma-ray region we shall here confine our discussion of precision gamma-ray spectroscopy by direct crystal diffraction methods chiefly to this one type of instrument.

The direct spectroscopic study of gamma rays by crystal diffraction has the following advantages and disadvantages relative to the more prevalent indirect studies by means of "converted" electrons with magnetic spectrometers. The disadvantages are: (1) Much more intense sources are required (100 to 1000 times as intense) even with the present high luminosity instrument. The development of the neutron reactor has greatly diminished this difficulty. (2) Relatively small solid angles from the source have been used so that coincidence work to date is infeasible. (3) Only

- Y. Cauchois, Comptes Rendus 199, (1934) 857. 
information of one kind (wavelength or quantum energy values) is obtained whereas, by the study of converted $\beta$-rays, questions such as the multipolarity of various lines can frequently be answered by the study of the relative conversion of electrons from different atomic levels. The advantages of this direct spectroscopic crystalline diffraction method are: (1) Interpretation of the direct gamma ray spectra is much easier since the difficulty of assigning to each conversion $p$-ray the atomic level from which it was ejected, as well as the nuclear quantum energy responsible for the ejection, is not present. (2) This multiplicity of atomic levels in $\beta$-ray spectroscopy frequently results in very complicated and closely juxtaposed patterns of lines in the magnetic spectrum which are therefore difficult to resolve and to interpret. This complexity is avoided in the direct crystal diffraction method. (3) In the lower energy range the resolution of the method is much better than in the magnetic method ${ }^{5}$. (4) The lines in the direct method are very symmetrical and the difficulty of long trailing "tails" caused by retardation of the electrons within the source material is absent. (5) The problem of preparing suitable sources is much easier. (6) Over a considerable range of energies the precision ${ }^{5}$ of wavelength and energy determination is at least an order of magnitude better. This permits elimination of many proposed level schemes by the more rigorous application of the Ritz combination principle as a test of their validity.

These advantages were not all self evident at the beginning of the developments here to be described. In developing this new technique and especially in the case of the first of the three gamma-ray crystal diffraction focusing spectrometer designs which we shall describe here (the "Mark I"), the primary objective was simply to furnish a precision method of linking the absolute wavelength scale of the $X$-ray spectrum to that of the gamma-ray spectrum. Since the absolute wavelengths of $\mathrm{X}$-ray lines are now known in angstrom units to a precision of about three or four parts in $10^{5}$ (through the work of numerous physicists ${ }^{6,7,8.9}$ who have made absolute deter-

5 This statement is true of the great majority of results in magnetic $\beta$-ray spectroscopy. The recent exceptionally high precision results of K. Siegbahn and his collaborators is an outstanding exception however. By taking very exceptional precautions both as to the magnetic field and the genmetry of the source, precision entirely comparable with that of the crystal diffraction method even in the lower energy regions has been achieved.

6 J. A. Bearden, J. Appl. Phys. 12, (1941) 395.

7 E. Bäcklin, Z. Physik 93, (1935) 450.

8 II. Söderman, Nature 135, (1935) 67.

9 F. Tyrén, Z. Physik. 109, (1938) 722. 'This entire subject has also been well reviewed by R. T. Birge, Am. J. Phys. 13, (1945) 63. 
minations of the wavelengths of certain $\mathrm{X}$-ray lines using optically calibrated ruled gratings at grazing incidence), this linkage of the gammaray spectrum to the $\mathrm{X}$-ray spectrum is all that is required to place the entire range of gamma-ray quantum energies on the absolute scale of physical units. The conversion factor ${ }^{10}$ from wavelengths to quantum energies in electron volts is now known with considerably more accuracy (about 4 part in $10^{5}$ ) than necessary, i.e., than that so far attained in the measurement of gamma-ray wavelengths by crystal diffraction or in measurements of gamma-ray energies by means of converted $\beta$-rays.

In addition to the original objective of the focusing curved crystal gamma-ray spectrometer as an instrument to establish a reliable series of precision fixed points in the gamma-ray energy spectrum, the new device promises to be of more value as an exploratory instrument than was at first anticipated because of the above mentioned far greater ease of interpreting gamma-ray spectra than $\beta$-ray spectra in many cases. The gammaray: instrument must however not be thought of as a superior substitute for the $\beta$-ray method, but as a very valuable complement to the latter.

\section{A. Invention and Principles of the Curved Crystal Focusing} Spectrometer. Transmission and Reflection Types. Exact and Approximate Forms

Eighteen years elapsed between the crystal diffraction discoveries of Laue and the Braggs in 1912 and the first published enunciation ${ }^{11}$ of the principles of exact focusing with curved crystals for both reflection and transmission cases, closely followed by the first papers ${ }^{12.13 .14}$ describing the different approximate and exact realizations of that principle. During this period several suggestions coming close to the focusing curved crystal idea had been published ${ }^{15,16.17}$. In spite of these foreshadowings, however, approximately two decades elapsed after the discovery of X-ray diffraction by crystals before the first curved crystal focusing spectrometers were

10 J. DuMond and E. Cohen, Rev. Mod. Phys. 25, (1953) 691 gives the least squares adjusted "best" values of a large number of constants and conversion factors of atomic physics. The value there arrived at for the conversion factor from wavelengths in centimeters to quantum energy in electron volts is (12 $397.8 \pm 0.5) \times$ $10^{-8} \mathrm{eV}-\mathrm{cm}$.

11 J. W. M. DuMond and H. A. Kirkpatrick, Rev. Sci. Instr. 1, (1930) 88.

12 H. H. Johann, Z. Physik 69, (1931) 185.

13 Y. Cauchois, Comptes Rendus 195, (1932) 1479; Ann. phys. 1, (1934) 215.

14 T. Johansson, Z. Physik. 82, (1933) 507.

15 M. De Broglie, J. Physique 5, (1914) 265.

16 M. Gouy, Ann. phys. 5, (1916) 241.

17 R. Darbord, J. Physique 3, (1922) 218. 
actually built. The writer firmly believes that the real obstacle to creative thinking which occasioned this long delay was the fact that, for exact focusing, the Bragg law required that at every point of the curved crystal's reflecting boundary surface two apparently incompatible conditions had to be satisfied, one of which defined the position of each point of the surface while the other defined the direction of the surface at that point. So long as physicists had failed to perceive that these two conditions need not, and in fact do not, apply to the same surfaces the exact solution appeared to be insoluble.

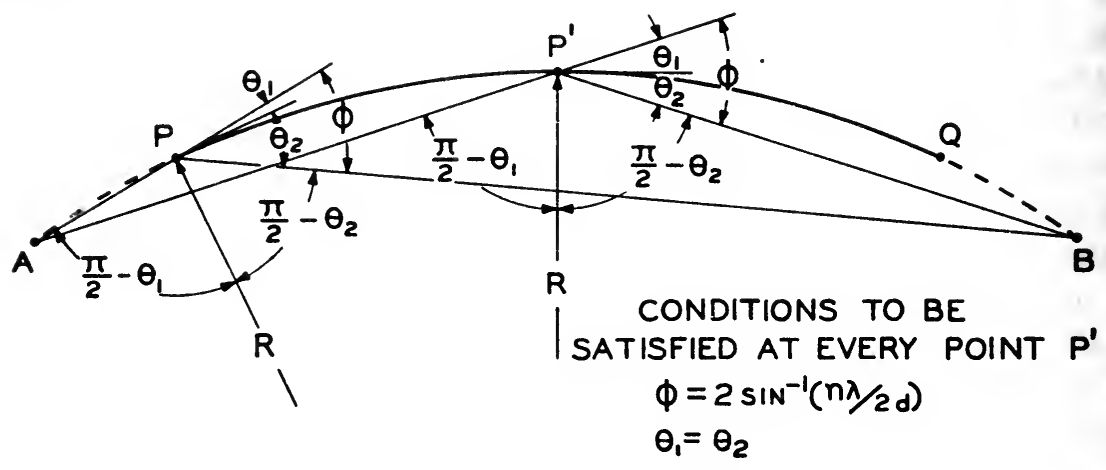

Fig. 2. To illustrate the impossibility of exactly satisfying both conditions required by Bragg reflection with a bent crystal over an extended surface if the atomic reflecting planes coincide with the reflecting boundary of the crystal and if two conjugate real foci are required

Consider the two-dimensional case shown in Fig. 2 in which $\mathrm{A}$ is a point source of composite $\mathrm{X}$-rays and $\mathrm{B}$ a point image of one narrow wavelength band, $\Delta \lambda$, of wavelength, $\lambda$, which we seek to select from the spectrum of source A by Bragg reflection at all points of the curved crystal surface. If we set ourselves the geometrical problem of finding a curve (say $y=f(x)$ ) such that the cleavage surface of a flexible crystal (mica, for example) conforming thereto would, by Bragg reflection over an extended area, take $\mathrm{X}$-radiation from $\mathrm{A}$ and focus it selectively at $\mathrm{B}$, we find the problem is in the strict sense insoluble because Bragg reflection imposes two conditions at every point of the surface $\mathrm{PQ}$ which cannot be met everywhere by any single equation $y=f(x)$. These two conditions are: (1) at all points on the surface, the angles of incidence and reflection, $\theta_{1}$ and $\theta_{2}$, referred to the reflecting atomic planes must be equal (condition for specular reflection), and (2) at all points on the surface, the angle of deviation, $\phi$, of the reflected beam must be constant and equal to

$$
\phi=2 \sin ^{-1}(n \hat{\lambda} / 2 d) .
$$


It is easy to see that these are incompatible conditions by noting that (2) is satisfied if, and only if, the curve is a circular arc passing through A and $\mathrm{B}$ of radius $R$ where

$$
R=L(2 \sin \phi)^{-1}
$$

$L$ being the distance $\mathrm{AB}$, whereas condition (1) is clearly exactly satisfied only at the midpoint of the arc $\mathrm{AB}$ and is seriously violated the nearer we approach its ends.

The writer believes that he was the first to see the way out of this apparent impasse and thus to break down the psychological obstacle which had impeded the invention of the curved crystal focusing spectrometer. He realized that the two conditions above mentioned are not in fact imposed on the same surface provided we do not insist on having the atomic reflecting planes everywhere parallel to the boundary surface of the crystal. Condition (2) dictates the position of every point on the boundary surface (the points must lie on the arc $\mathrm{AB}$ ) but demands nothing regarding the direction of the reflecting planes at that surface. Condition (1) dictates the direction of the atomic reflecting planes (they must be everywhere normal to the bisector of the angle $A P^{\prime} B$ ) but imposes no condition on the position of the boundary surface where the reflecting planes are situated.

If we arrange matters as in Fig. $3^{18}$ we have two cases, the reflection case, R, (first so-called because a "real image" was formed) or the transmission case, V (first so-called because a "virtual image" was formed). It will be evident that our ideal problem is exactly solved for either of these cases by the arrangement shown. Let us start by discussing the reflection case (case $\mathrm{R}$ ), the one which occurred to the present writer first. A crystal is shown whose reflecting boundary is a circular arc with center at $\mathrm{O}$. The atomic reflecting planes of this crystal, however, are bent so that they coincide with concentric circles centered on the circumference of the circle, $O$, at the point, $C$. For all points of the surface, condition (1) is fulfilled since the equal subtended arcs, SC and IC, now measure twice the angles of incidence and reflection, $\theta_{1}$ and $\theta_{2}$, with respect to the reflecting atomic planes whose normal is their radius of curvature, PC. Condition (2) is seen to be fulfilled since the arc SPI measures twice the angle of deviation of the beam for all points, $\mathrm{P}$. It is also evident that the locus along which the spectrum is focused lies on the circle of center, $\mathrm{O}$, and that the two conjugate foci for each wavelength lie at points on this circle symmetrically situated relative to the point $\mathrm{C}_{2}$.

The transmission case, Fig. $3 \mathrm{~V}$, was arrived at by the writer as a logical

1s Taken from the original DuMond and Kirkpatrick article. Rev. Sci. Instr. 1, (1930) 90. See Fig. 2 of that article. 
continuation of the reflection case, R. Essentially the requirements define a direction for the crystal planes at every point of the focal circle and if we inquire as to the directions in the region diametrically opposite to that occupied by the arc of crystal shown in case $R$, we automatically arrive
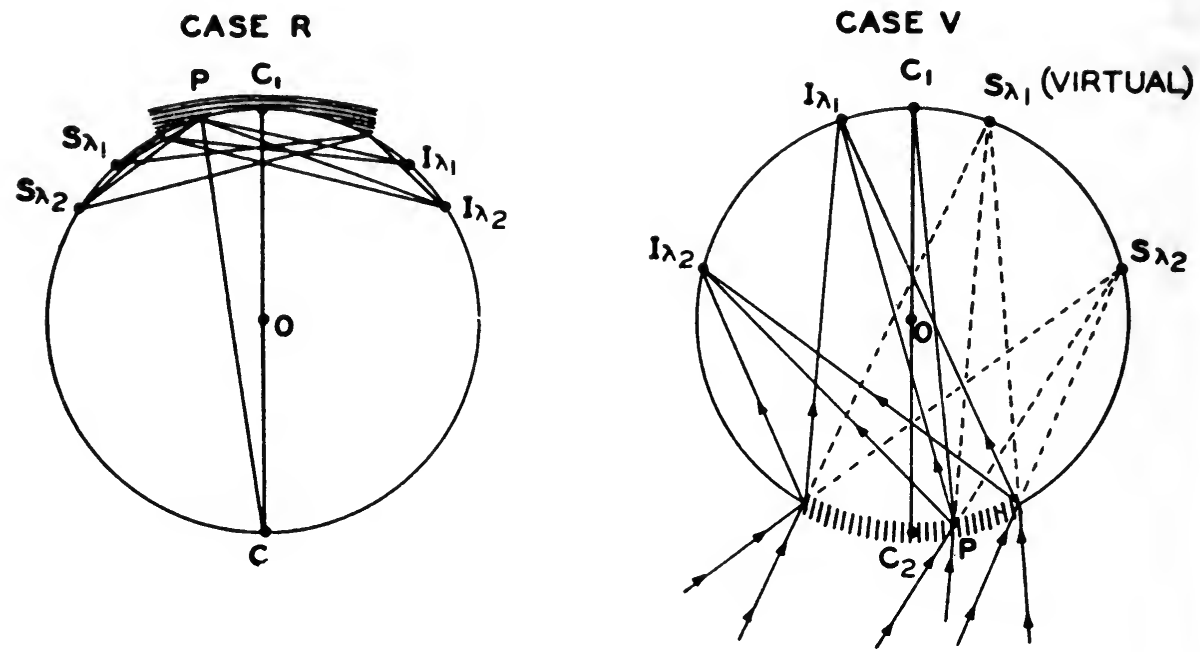

Fig. 3. Two solutions of the problem of selective X-ray focusing. This picture, giving the simple geometrical principle of the exact focusing cases for both reflection and transmission curved crystal focusing spectrometers, is taken directly from an article published by DuMond and Kirkpatrick in January 1930 (Fig. 2 of that article). The idea was conceived by Dullond in 1927

at Case V. In this case the short reflecting planes traversing the thin crystal lamina may either be thought of as normal to a radius through $\mathrm{C}_{2}$ (just as are the atomic planes in case $\mathrm{R}$ ) or as planes which, if produced, would intersect in $\mathrm{C}_{1}$, on the focal circle diametrically opposite to $\mathrm{C}_{2}$. It is clear from the figure that the transmission case has one virtual and one real focus, while the reflection case has two real conjugate foci. For rather obvious reasons the reflection case is usually better adapted to long wavelengths and large Bragg angles, while the transmission case is usually better adapted to short wavelengths and small Bragg angles.

Johann and ('auchois realized that condition (2) fixing the position of the boundary or boundaries of the crystal, was less stringent than condition (1) fixing the direction of the atomic planes so that if the arc of the focal circle covered by the crystal in not too large, the aberration of focusing resulting from violation of (2) (aln be kept sufficiently small for practical purposes without requiring the difficult anded profiling of the 
boundary surface ${ }^{19}$. Secondly, Cauchois pointed out the important new observation that, in the transmission case there is also a focusing effect through the thickness of the curved sheet as well as from side to side. When the bending stress is applied so that the crystal lamina is curved, the strain (tension) near the outside (convex) surface of the plate results in a larger grating constant than the strain-free value obtaining on the neutral axis and hence there is a slightly smaller Bragg angle reflected from these regions than the one that applies to reflection by regions of the crystal in the vicinity of its elastic neutral axis. The opposite effect occurs for reflection by regions of the crystal near the inner (concave) surface. It is easy to show that this results in exactly the required convergence of all the reflected beams over the entire thickness of the slab to give perfect focusing from front to back. This leads to the conclusion that in the transmission spectrograph it is the neutral axis of the crystalline slab which should be placed in contact with the focal circle.

Cauchois and Johann have each made careful quantitative studies of the geometrical aberrations of the respective types of instrument they developed. For gamma-ray spectroscopy we shall here be exclusively concerned with the Cauchois approximate focusing transmission type instrument. In this instrument, for simplicity of construction the crystal in its unstressed state is a plane lamina. The chief geometrical aberration then comes from the fact that the neutral axis of this lamina, after it has been elastically curved to the required radius (equal to the diameter of the focal circle) deviates at its extremities from exact coincidence with the focal circle because the latter has only half as large a radius. Fig. 4 shows how

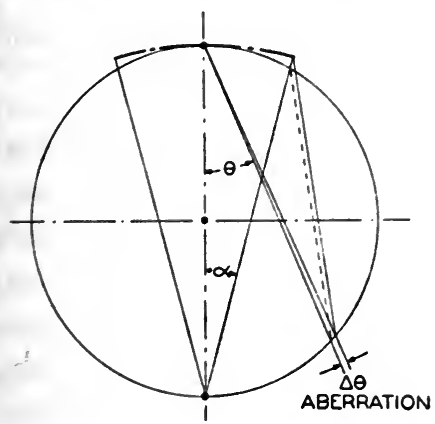

Fig. 4. Geometry determining the line broadening or aberration in the Cauchois approximate type of transmission spectrograph which results from the fact that near its extremities the neutral axis of the lamina fails to coincide with the focal circle. The aberration $\Delta \theta$ results in a corresponding aberration $4 \lambda$ given in formulae (1) and (2) of the text. For

19 Others, however, have succeeded in building curved crystal spectrometers of the exact focusing type (Case $R$ of Fig. 3) by suitably profiling the crystal lamina (in its unstressed state) to the required radius of curvature, twice the radius of the focal circle, and then bending it until the spectra focus on the focal circle, i.e., to the same radius of curvature as the latter. The first to do this in the reflection case was T. Johnnsson, Z. Physik 82, (1933) 507. Sice also R. Bozorth and F. E. Haworth, Phys. Rev. 53, (1938) 538 and A. Guinier; Ann. phys. 12, (1939) 161. 
to compute the relative instrumental line broadening, $\Delta \lambda / \lambda$, which results from this aberration. Simple geometrical considerations yield the following result,

$$
\Delta \lambda / \lambda \cong \cos \theta(1-\cos \alpha) \sec (\alpha+\theta)
$$

wherein $\theta$ is the Bragg angle and $\alpha$ the half angle of aperture of the crystal. Clearly for small Bragg angles, $\theta$, and small apertures, $\alpha$, such that $\theta<\alpha \ll 1, \Delta \lambda / \lambda$ approximates the quantity, $\alpha^{2} / 2$ and therefore rapidly becomes negligible.

The design of the first precision gamma-ray spectrometer, (shown in Fig. 5) which we shall designate as Mark I and which is still the only

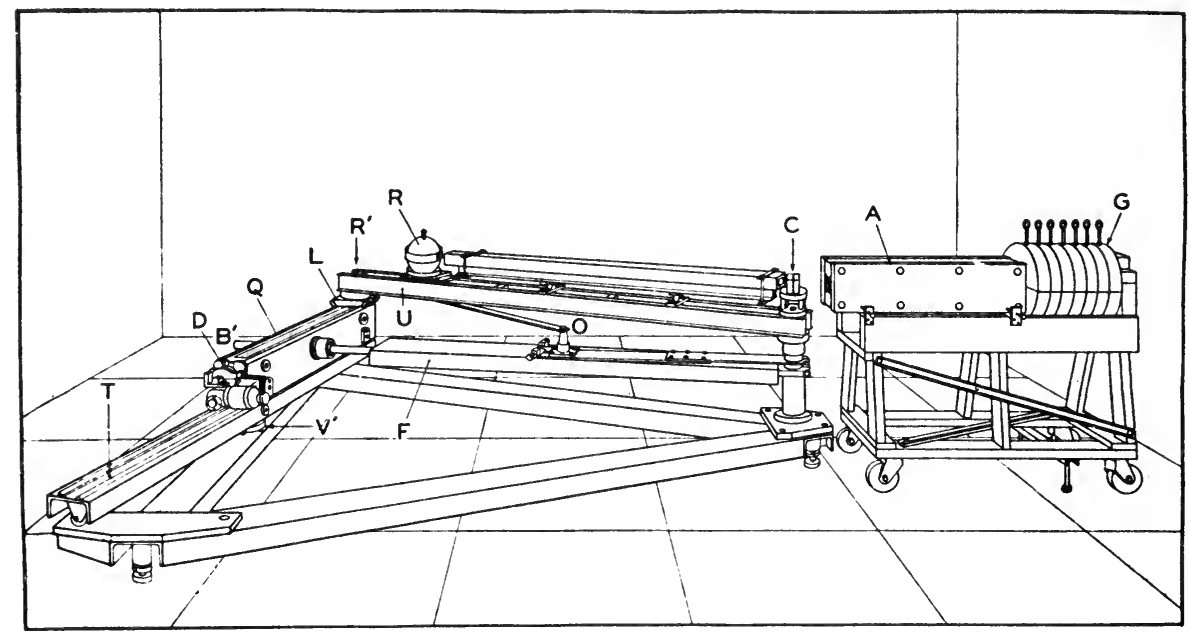

Fig 5. Perspective line drawing of the gamma-ray spectrometer showing the curved crystal at $\mathrm{C}$ and the source in its shielded holder 2 meters from it at $R$

instrument of its kind in operation, was started early in 1938 and its construction and assembly were practically completed in 1940 save for the all-important crystal and its clamping blocks and for the multicellular GM counter which was developed later in 1947 by D. A. Lind as the first detector of gamma rays used in this instrument. Unfortunately the progress on this entire technique was interrupted and the work had to be abandoned for the long war interval from 1940 to 1946 . We shall describe the Mark I instrument in the next section.

Two other designs of focusing curved crystal gamma ray spectrometer which we shall designate as Mark II and Mark III have been under very serious consideration but are not in operation at the time of writing this chapter. Mark II is a version in which the sine of the angle of rotation of the curved crystal (the Bragg angle) away from the position for zero 
gamma-ray wavelength is measured by an optical interferometric method of very high precision. Mark III is an instrument whose construction is at the date of writing well advanced at the Argonne National Laboratory. It is planned to have in this instrument a very large (probably $30 \times 30 \mathrm{~cm}$ ) bent quartz lamina some $6 \mathrm{~mm}$ thick and with a radius of curvature of 8 meters. The instrument is intended for the study of neutron-capture gamma rays below $2 \mathrm{MeV}$ quantum energy and is designed to be used for the study of radiation from an emitting source situated in a region of very high neutron flux density in a tube passing through or near the center of a large liquid chain reactor.

\section{§ 2. Adaptation of the Transmission Type Bent Crystal Spectrometer Principle to the Precision Measurement of Very Short Wavelengths (Nuclear Gamma Rays)}

\section{A. Two Possible Arrangements of Source and Crystal}

The philosophy behind the design of the Mark I instrument about to be described, was first set forth in a paper ${ }^{20}$ published in 1947. These considerations still apply at the time of writing and we shall briefly summarize them here.

The transmission type of curved crystal spectrometer, the one best adapted to the measurement of short wavelengths has, as we have pointed out above in Fig. 3, one real and one virtual focus, and hence it is not immaterial in which direction the radiation propagates. Let us designate two alternative arrangements by roman numerals I and II. Referring to Fig. 6 we may either place an extended source at A as shown in the left hand sketch (I) or a very concentrated source at the real focus $R$ as shown in the right hand sketch II. Now if we are to work with gamma radiation from artificial or natural.radioactive substances, it is clear that in order to obtain as much gamma ray line intensity as possible of some specified wavelength with the spectrometer at a fixed setting and with a fixed amount of source activity, the real focus, $R$, is the best place to put the source (arrangement II in Fig. 6) for two reasons: (1) with the source concentrated at the appropriately chosen point on the focal circle for the given wavelength, every nucleus in the sample at $R$ has a chance to radiate the specified gamma-ray wavelength into a solid angle subtended by the entire working aperture of the crystal, (2) by interposing a set of fan-shaped lead baffles as shown, between the crystal-and the counter or scintillation detector, it is possible, with arrangement II, to shield the intense unresolved direct beam of gamma rays from reaching the detector 
and completely masking the much weaker selectively diffracted monochromatic beam which it is the object of the instrument to measure. II is seen to be the arrangement in which the instrument is essentially a monochromator as we have already mentioned and the line profiles are to be delineated and the spectrum explored point by point. The arrangement $I$ shown at the left in Fig. 6 which is essentially the transmission photographic spectrometer of Cauchois is better adapted to recording a whole region of the spectrum simultaneously on a film placed on the focal circle.

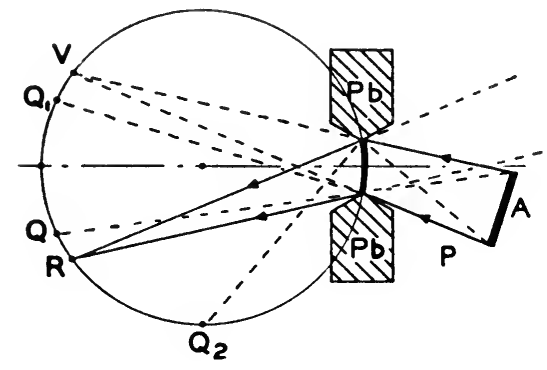

ARRANGEMENT I

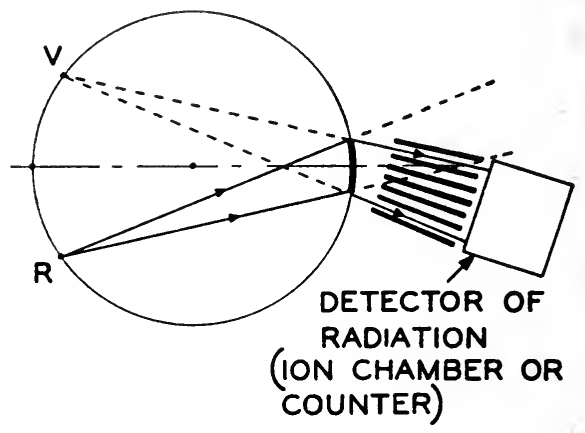

ARRANGEMENT II

Fig. 6. Two ways of using the transmission-type, curved-crystal spectrometer. An extended source may be placed at $A$, in which case spectral lines will be focused as at $\mathrm{R}$, Arrangement $\mathrm{I}$. The case is appropriate for the study of fluorescence excited in a screen placed at $\mathrm{A}$, but is uneconomical if the total intensity from the source is a limiting factor. For gamma-ray spectra the source may be placed at R, Arrangement II, and the intensity measured in an ion chamber or scintillation counter, is thus plotted as a function of the position of the source $R$ on the focal circle

Clearly an entire region between the points $Q_{1}$ and $Q_{2}$ could thus receive selectively diffracted radiation from the source in the geometry shown in the figure. As $Q$ is approached in the clockwise direction $\beta$-rays and diffusely scattered gamma rays mask the spectrum. Beyond $Q$ the unreflected radiation completely masks the spectrum to be studied. Furthermore in the arrangement, I, in Fig. 6, for an emitted gamma ray line of given wavelength, each nucleus at some point $\mathrm{P}$ in the extended source placed at A, can only make use of a very small solid angle subtended by a very tiny portion of the curved crystal where the incident radiation strikes the reflecting planes at just the right Bragg angle. This is a much more stringent restriction in short wavelength gamma ray spectroscopy than in ordinary X-ray work. Because of the very small Bragg angles involved, in gamma-ray spectroscopy only the most nearly ideally perfect crystals can profitably be used. A study with the two-crystal spectrometer using unstressed flat quartz plates ("parallel" position) has permitted us 
to establish that, for the (310) planes of quartz the selective reflection of a given wavelength is restricted to an angular region of the order of only a few seconds of arc ${ }^{21}$. When this is compared with the available horizontal angular opening of $\mathbf{1 . 5}$ degrees, for the arrangement II, Fig. 6 , in the case of the Mark I instrument, it is seen that the latter position should yield of the order of 5000 times as much intensity at a specified wavelength as the arrangement $I$.

At longer wavelengths where the problem of shielding the film from the direct beam is less severe and where the radiation is soft enough so that photographic spectra can be profitably used with reasonable exposures, the use of the curved crystal spectrometer in the arrangement $I$ as a photographic spectrograph does have advantages for exploratory work. The lines are all formed simultaneously over a considerable segment of spectrum in a single long exposure. To explore exhaustively point-bypoint the same range of spectrum using the spectrometer in arrangement II would in many cases take as long or longer than by photography with arrangement I. This statement however is far less likely to be true if one knows approximately where to look for the lines using arrangement II so that it is unnecessary to make a search run in sufficiently fine detail everywhere to insure that no line shall be passed over. Here we shall be almost exclusively concerned with the arrangement II of Fig. 6.

\section{B. The Baffle or Collinator for Arresting the Heterogenous Transmitted Beam}

In the Mark I spectrometer, if the wavelength setting is 27 milliangstroms, the angle of deviation between the reflected and directly transmitted beams is only about 0.4 of a degree. The direct beam may be easily two thousand times as intense as the diffracted beam to be studied. The means provided to arrest the transmitted beam as completely as possible while still permitting about half of the diffracted beam to reach the detector, consists of a baffle system of twenty-four tapering, die-cast, lead-tinantimony alloy partitions 31.5 inches long and 0.040 of an inch thick at the thin entry end, separated by tapering spacers clamped at top and bottom between the partitions so as to leave twenty-five open tapering channels each 31.5 inches long, 0.040 inch wide at their entry ends and 3 inches high.

Although we refer to this baffle system as the "collimator" it should be clearly understood that this element of the instrument has nothing to do with the spectral resolving power. It is only a baffle to discriminate between the reflected beam and the transmitted beam. and its angular

21 W. Watson, W. West, D. Lind and J. DuMond, Phys. Rev. 75, (1949) 505. 
resolving power is some twenty times coarser than the angular resolving power of the focusing crystal in Bragg reflection, the true factor which determines the angular and spectral resolving power of the spectrometer.

\section{Mechanical Design}

The primary objective in the Mark I was, as clearly stated, to furnish a precision device which would bridge the gap between the known wavelengths of $\mathrm{X}$-ray lines and the less accurately known wavelengths of gamma ray lines so as to link these two regions of the spectrum together with high precision. The X-ray and gamma-ray wavelengths to be measured are, in the transmission type focusing instrument, strictly proportional to the sine of the grazing angle at which the radiation is incident on the atomic planes of the crystal. The cheapest and easiest way to build a device for measuring the sines of angles appeared to be a linkage simulating a right triangle with constant hypotenuse in which a precision screw would displace a carriage in such a way as to vary the length of that leg of the triangle which was opposite to the argle whose sine was desired. We might characterize this briefly as a sine-screw arrangement. A precision sinescrew device is one of the easiest and cheapest angle measuring devices to produce-far cheaper for the same angular precision than a worm wheel and worm for instance. This is partly because precision can actually be generated in the screw by the very simple process of lapping with a long appropriately designed nut.

It is important also to provide means that will maintain the lead collimator and the diffracted monochromatic beam from the crystal always in correct alignment while the spectrum is being explored, as we have explained above. In the Mark I instrument, to avoid the necessity of moving the heavy collimator and the still more heavily shielded gamma-ray detecting components behind the collimator the instrument was designed so that the direction of the reflected beam while the spectrum is being explored, is at all times sensibly invariable. The principle of the design is schematically illustrated in Fig. 7. To accomplish the desired result both the curved crystal and the focal circle, rigidly related thereto, must rotate about a point $\mathrm{C}$ situated at the center of the neutral axis of the bent crystal slab and at the same time the source, while always remaining on the moving focal circle, must explore along its circumference in such a way that a line from the source to the point $\mathrm{C}$ on the crystal rotates at twice the angular rate at which the crystal rotates. The collimator channels will then always remain sensibly pointed at the virtual image point $\mathrm{V}$ as they should. The length of the chord, $\mathrm{CV}$, changes slightly so that, at long wavelengths and large Bragg angles, $V$ is slightly closer to the collimator 
but this introduces a quite negligible change in transmission of the reflected beam through the latter.
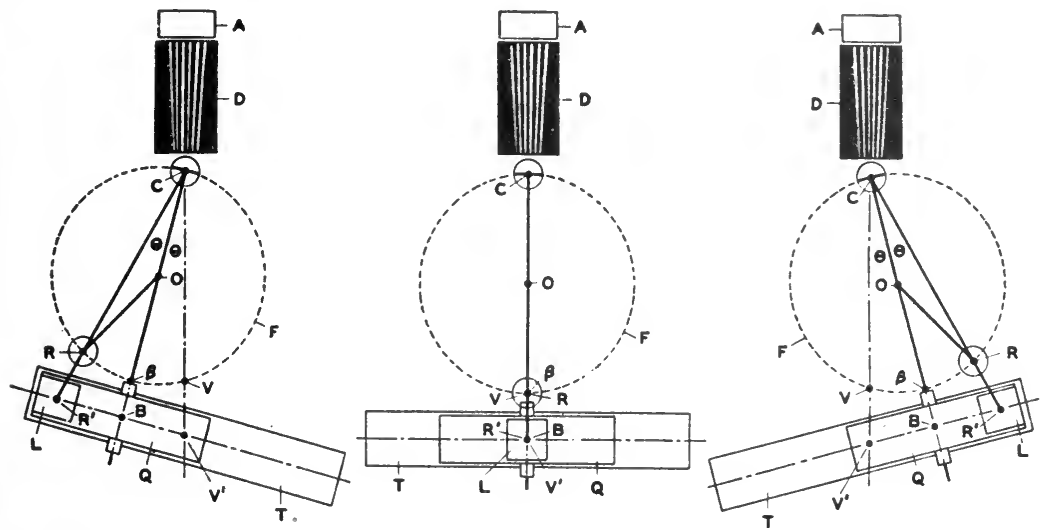

Fig. 7. To illustrate schematically the geometry of the gamma ray spectrometer. The view at center shows the instrument at the zero wavelength position, while the views to left and right show different wavelength settings for reflections to left and to right of the atomic reflecting planes. The constant distances $\mathrm{CR}^{\prime}$ and $\mathrm{CV}^{\prime}$ are made rigorously equal. Displacements of the carriages $\mathrm{L}$ and $\mathrm{Q}$, effected by means of precision screws, are proportional to the sine of the Bragg angle, $\theta$, and hence, proportional to wavelengths. The drawing is not to scale. The aperture of the crystal at $\mathrm{C}$ and the width of the collimator are exaggerated

It is also highly desirable to be able to interchange the position of $\mathrm{R}$ relative to $\mathrm{V}$ (Fig. 7) so that spectra resulting from reflection on both sides of the (310) planes of the quartz crystal can be conveniently studied. By measuring the distance (on the precision screw) between the spectra reflected from both sides of the planes, the exact position of the "zero" of the wavelength scale need not be known beforehand.

In the Mark I instrument the focal circle is approximately 2 meters in diameter. It is defined by a radius bar OR which links the source carriage on the upper beam $\mathrm{CR}^{\prime}$ to the pivot, $\mathrm{O}$, defining the center of the focal circle on the lower beam.

The entire design of the Mark I instrument was carefully planned so that it admitted of a rational procedure for measuring and adjusting all of the essential geometry of the instrument to insure its accuracy. Space limitations, however, preclude a detailed description of these many details.

It should be noted that all that is required of this instrument in order to bridge the gap reliably between the known X-ray wavelengths and the unknown gamma-ray wavelengths, is that its wavelength scale (measured in turns on the screw after correction for its errors) shall be linear with wavelength. Therefore all sources of error which would require 
corrections to the wavelength of magnitude proportional everywhere to the wavelength itself (i.e., linear corrections) can be ignored, since the calibration with $\mathrm{X}$-rays automatically takes care of these. It is only nonlinear corrections that are to be feared.

In conirast to the design of the Mark I just described, in both the Mark II and Mark III instruments the plan has been to keep the gammaray emitting source stationary and to explore the spectrum by turning the curved crystal through small angular displacements, while at the same time turning the entire assembly of the collimator and the detecting agency through angular displacements just twice as large so as to satisfy the law of specular reflection.

\section{Source axd Source Holder Desigxs}

A typical neutron activated source for the Mark I instrument might consist of a thin rectangular strip of metal, such as tantalum or gold, of dimensions $30 \mathrm{~mm} \times 5 \mathrm{~mm}$ and $0.05 \mathrm{~mm}$ thick. The strip is supported in the source holder on the beam with its long axis vertical and with its flat faces parallel to the direction of gamma ray propagation from source to crystal. In such a case the geometry of the source itself plays the role of a spectroscopic slit. To give the thin source mechanical rigidity, it is usual to compress it in an aluminum sheath (pure aluminum must be used, not alloy) which, depending on the hardness of the radiation to be studied and other considerations, may or may not be cut away on the edge where gamma rays are to emerge. The assembly of source strip and aluminum sheath is irradiated as a whole in the neutron flux in a chain reactor. The activities set-up in the aluminum are very short lived and therefore do

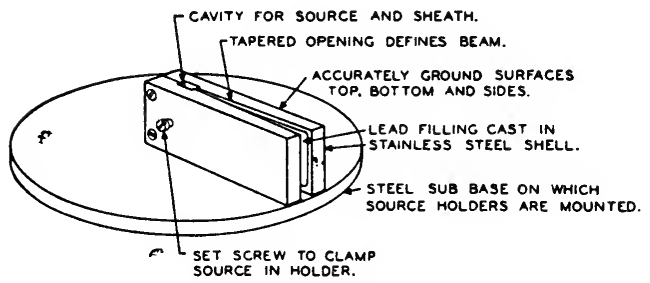

Fig. 8. Perspective line drawing of source holder of the lead-filled variety. Solid source holders of similar shape have been made of "heavimet" and also of uranium not constitute a difficulty.

The aluminum sheath is made to fit smoothly in the $30 \times 13 \times 2 \mathrm{~mm}$ cavity in one of the source holders. The source holder may be of lead, tungsten, "heavimet" (a sintered composite of copper and tungsten powder) or uranium. Its shape is pictured in Fig. 8. It provides a means of holding the aluminum sheathed source in a vertical position and of defining the utilized outgoing beam of gamma rays in the shape of a narrow wedge which just about fills the useful aperture of the crystal and no more. This is done by a long tapering opening visible in the figure. 
The source in this source holder is accurately centered over a vertical pivot which connects with the radius bar defining the focal circle. This pivot is the center of rotation of a turn table mounted on a carriage which can roll on ball bearing ways over a short longitudinal travel along the upper beam to accommodate the changing length from source to crystal as the spectrum is explored. The source holder is supported inside a spherical lead source bomb. In Fig. 11 (see p. 122) the source holder supported on the lower hemispherical half of this protective lead bomb can be clearly seen at $\mathbf{R}$. In this figure the upper hemispherical cover of the bomb has been removed. The turn table and one of the centering screws for centering the source by rotation of the turn table are clearly visible.

\section{E. The Quartz Crystal and Its Clamping Blocks}

Two general methods have been used by various experimenters to impose the requisite curvature on the focusing curved crystal lamina. In one of these which we might call the freely sprung lamina, equal and opposite torques are applied to the two ends of the lamina, which is otherwise left free to assume its circular cylindrical curved shape by reason of the play of elastic forces ${ }^{22}$ in it. The lamina must therefore be quite uniform in thickness and in elastic properties if the curvature is to be uniform and the all-important atomic reflecting planes correctly oriented. The second method, which has sometimes been called the "method of imprisonment", is the one utilized in the Mark I precision gamma-ray spectrometer and is the one which will be described here.

The quartz crystal slab, whose faces have been polished optically flat and closely parallel, is compressed between two hardened blocks of stainless steel, one with a concave, the other with a convex circular cylindrical profile, to hold the slab

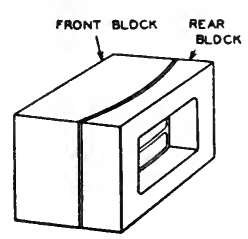

CRTSTAL CLAMPING BLOCKS VIEWED FROM EXIT SIDE.

Fig. 9. The stainless steel clamping blocks for bending the crystal. A rubber gasket is placed between the rear block and the crystal at the correct curvature. Only the convex block actually determines the

22 J. B. Borovskii, Dokl. Akad. Nauk. SSSR 72, (1950) 485; A. B. Gilvarg, Dokl. Akad. Nauk. SSSR 72, (1950) 489; G. Brogen, Ark. Fys. 3, 515 (Paper 30, 1951). Dr Thomas C. Furnas, Jr. (see hị Doctorate thesis, Massachusetts Institute of Technology, 1952) has applied the method to a point focusing monochromator for low angle diffraction studies. A method somewhat similar has been applied in a commercially manufactured X-ray spectrometer based on patents assigned to the General Electric Co., by Davicl Harker. In this case the erystal curvature varies as the spectrum is explored. 
curvature of the quartz plate since a rubber gasket is placed between the concave steel surface and the quartz plate. Fig. 9 shows the crystal clamping blocks and the ribbed window in the convex block through which the gamma rays reach the crystal.

The use of a special stainless steel ${ }^{23}$ together with the procedure for hardening and tempering it was kindly recommended to us by C. G. Peters of the U. S. National Bureau of Standards.

The all-important procedure ${ }^{24}$ for the precision profiling (to 2 meter radius in the Mark I) of the convex cylindrical stainless steel block assures an accuracy of $0.0001 \mathrm{inch}$. This is not sufficient for the extreme demands of crystal spectroscopy of gamma rays. The surfaces must be corrected still further by a lapping procedure in which a concave cast iron lap of as closely as possible the same radius as the convex crystal clamp is rubbed against the latter with an extremely fine abrasive material. If the grinding of the two surfaces has been carefully done in every respect, very little lapping will be required. The procedure is first to lap the two (concave and convex) cast iron blocks against each other until a good mirror surface is obtained. The concave cast iron lap is then tested optically by examining the image of a fine "straight-line filament" lamp (as formed by reflection in the concave surface) with a low power microscope while stopping off different regions of the mirror with paper shields.

The convex crystal block is next lapped against the concave cast iron lap until perfect contact with a mirror finished surface has been obtained. The concave cast iron lap may then be optically tested once more. If it is found to have lost its accuracy of figure somewhat it may be corrected once again by lapping the two cast iron blocks together. Then one returns to the convex crystal clamping block and gives. it a final correction of figure with the corrected concave cast iron lap. Usually this second approximation is all that is needed.

The mounting of the crystalline lamina in the clamping blocks may require considerable"patience to eliminate invisible particles of foreign matter from the interface between the quartz and the convex steel block. The lamina is clamped under pressure of the screws and springs and the colored interference fringes formed in the quartz-steel interfaces are observed. The central black fringe should occupy most of the width of

23 The material is a heat-treatable stainless steel having the fortunate property of possessing a thermal expansion coefficient which matches quite closely with that of quartz in the direction transverse to the optic axis.

24 This procedure has been described in detail in an article by J. W. M. DuMond, D. A. Lind and E. R. Cohen, Rev. Sci. Instr. 18, (1947) 617 which should be consulted by those planning to apply this method. 
the curved are over the entire working aperture of the crystal. The first colored fringes may be permitted to show in the outer ten percent of the width on either side.

After the quartz plate has been mounted in its steel clamp, its concave cylindrical surface can be tested with optical light for correctness of curvature (focus). A still more significant test however, which the writer regards as indispensable in short wavelength gamma ray spectrometers of the types here described, should be made using reflection of the $X$-rays or gamma rays themselves from the atomic planes of the curved crystal slab. We shall refer to these as "Hartmann" tests, drawing the name from analogy with the well-known Hartmann method of testing the aberrations of focus of large astronomical mirrors.

A strip of tantalum, say $0.05 \mathrm{~mm} \times 5 \mathrm{~mm} \times 30 \mathrm{~mm}$, which has been "activated" in the strong neutron flux in a chain reactor to an activity of say $500 \mathrm{mc}$, through the presence of the unstable $\beta$-emitting isotope $\mathrm{Ta}^{182}$ will emit, beside its rich series of nuclear gamma-ray lines, copious tungsten and also tantalum $K$-series $\mathrm{X}$-ray lines of ample strength for such a Hartmann test. Such a tantalum source is supported in the source holder with its long axis vertical and with its thin edge facing the crystal. The source itself then constitutes the resolving "slit". For the Hartmann test a lead shield is provided for the crystal aperture so that only one small portion of that aperture is used at one time. As many as 15 subdivisions of the total aperture have been made in this way. The Hartmann test consists in plotting for each of these isolated portions of the crystal the line profile of say the $K \alpha_{1} \mathrm{X}$-ray line of tungsten which might, in the Mark I instrument have a total width at half maximum height of $0.12 \mathrm{~mm}$ displacement of the wavelength screw carriage, L. Settings might be made every $0.02 \mathrm{~mm}$ with accumulation of counts for a chosen interval at each setting of say 2 minutes, to delineate these profiles. It might then be found that the positions of the centers of the line profiles as obtained using the several regions of the crystal might fail to coincide by some sinall fractions (say tenths) of a mm. (1 mm quite closely approximates 1 X.U. or 1 milliangstrom in the Mark I instrument.) To date the best we have been able to achieve in reducing aberrations of focus from all over an area of $20 \mathrm{~cm}^{2}$ of crystal aperature at a focal distance of 2 meters has been an aberration width of about $0.05 \mathrm{~mm}$.

Four sources of line breadth should be listed in the gamma-ray crystal spectrometer. These are: (1) The natural spectral breadths of the gammaray or X-ray lines. Most nuclear gamma ray lines are far too narrow (frequently less than 1 part per million) to have detectable breadths with this crystal diffraction instrument. X-ray natural line breadths are easily 
detected however, giving noticeably broader line profiles than gamma rays, and this serves as one valuable way of discriminating between the two types of radiation when an unknown spectrum is being examined. (2) The geometrical width of the emitting source or the limiting slit on the focal circle. The distribution may or may not be uniform across this width depending on the preparation and nature of the source. (3) The aberrations of focus from residual imperfections of the crystal and of the curvature of its neutral axis. This is the component contributory to the line breadth which the Hartmann test determines. (4) The Darwin or Ewald diffraction pattern width intrinsic to any small region of the crystal itself including any mosaic or other effect due to crystalline structure. The observed line breadths and structures are to be regarded as the "fold" ("faltung") of all four of these sources combined. Fig. 10 gives a rough idea of typical relative sizes and shapes of these contributing factors.

1. ABERRATION PATTERN (W, MIGHT BE $0.05 \mathrm{~mm}$.)

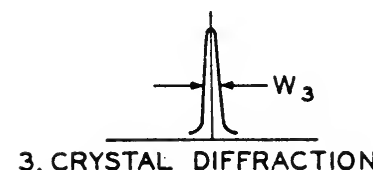

3. CRYSTAL DIFFRACTION PATTERN ( $W_{3}$ MIGHT BE $0.005 \mathrm{~mm}$.)

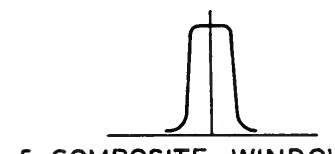

5. COMPOSITE WINDOW (FOLD OF 1. AND 3.).

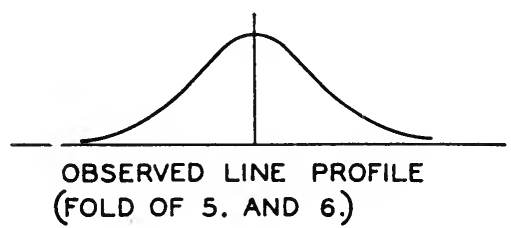

2. SOURCE PROFILE ( $W_{2}$ MIGHT BE $0.05 \mathrm{~mm}$.)

4. SPECTRAL LINE PROFILES ( $X$-RAY \& $Y$-RAY) $\left(W_{4 X}\right.$ MIGHT BE $0.02 \mathrm{~mm}$. $W_{4 Y}$ NEGLIGIBLE)

6. COMPOSITE SOURCE (FOLD OF 2.AND 4.)

D. A. Lind as his doctorate thesis ${ }^{25}$ has studied the integrated reflection coefficient for the (310) planes of quartz as a function of wavelength for

25 A published aceount has been given of these results. D. A. Lind, W. W. West, and J. W. II. Dullond, Phys. R('. 77, (1950) 475. 
curved quartz plates over the large range from $700 \mathrm{X}$-units to $9 \mathrm{X}$-units and has found that the integrated reflection coefficient is proportional to the square of the wavelength. Tests made by William W. West, on the contrary, show that for unstressed quartz plates the integrated reflection coefficient falls off with diminishing wavelength, $\lambda$, even less rapidly than the first power of $\lambda$, (roughly as $\lambda^{1 / 2}$ in fact). These results are interesting from the point of view of purely empirical facts. Their theoretical interpretation is less clear however since no "dynamical theory" of X-ray reflection in elastically curved crystals has yet been worked out either for the mosaic or perfect crystal cases.

It is worth noting that, thanks to the design in the Mark I, which permits delineation of line profiles for reflection on both sides of the crystal planes, it is possible to infer as to the cause of any asymmetry in line profile if such is observed. If the asymmetric profiles on the two sides stand in mirror.image relation to each other the asymmetry is attributable to the spectral shape (close unresolved companion lines for example). If the asymmetry of the line profile faces in the same spatial direction on both sides (i.e., toward increasing wavelengths on one side and toward decreasing wavelengths on the other) then it is a characteristic of the source distribution or of the aberrations of focusing of the crystal (i.e., it is an instrumental artifact).

\section{F. Procedure in Making Precision Wavelength Measurements.}

\section{E. Muller's Method of Superposition of Profiles}

Asymmetric profiles of the last above mentioned type, though they are infrequent, do not interfere seriously with precision wavelength determinations. This is because to compute the wavelength, we determine the interval between the right-hand and left-hand orders of reflection of a given line by ascertaining just how far (in turns and fractions of turns of the precision wavelength screw) a standard master "composite" profile must be displaced in order that it shall superpose in best register throughout its entire profile structure upon the two profiles in question. (If any difference in intensity exists in the two profiles their ordinates are first normalized to equality before effecting the superposition.) The standard master "composite" profile, the registering tool to be used in determining displacements, is prepared by taking an average of carefully selected line profiles made with the same source sample. Its shape is thus characteristic not only of the slight aberrations of focus of the crystal but of the particular distribution of activity which obtains in that source sample. If there is no reason to expect any appreciable contribution to any of the observed line profiles from the natural spectral line structure (this is 
practically always the case for $\gamma$-ray lines) then all of these profiles may be averaged together to form the master composite tool. In the case of $\mathrm{X}$-ray lines somewhat more circumspection is needed since the contribution of the natural spectral line breadth is detectable. Usually X-ray lines of the same series have quite closely the same natural breadth and one master profile will serve for all. The use of a master profile is also of value since, by its occasional failure to superpose in good register on a given profile, it may reveal that the line is multiple in character or that some unsuspected weaker line (perhaps from an impurity) is closely superposed upon its structure. It will be noted that any slight asymmetry introduced into the line profile by aberrations of curved crystal focusing and by non-uniform distribution of source activity interferes not at all with the above described method of matching profiles to determine wavelengths with high precision because both asymmetric profiles to be matched face the same way.

A quantitative analysis of the factors governing the precision with which wavelengths can be determined by the profile matching method is griven elsewhere ${ }^{26}$.

\section{(i. The Gamia-Ray Detecting and Intexsity Measuring System}

The multicellular G.M. counting system (seen in Fig. 5, Sec. 1.1) proved to be unsatisfactory and has been replaced by a scintillating crystal ${ }^{27}$ of thallium-activated sodium iodide. The sensitive element is a monocrystal, $3 \times 3 \times 1$ inches in dimensions, hermetically sealed in a rectangular thin walled glass box fitting closely around the crystal with mineral oil filling the space completely between crystal surfaces and glass. The crystal is placed so that its $3 \times 3$ inch face intercepts the diffracted gamma ray beam from the quartz crystal immediately after it issues from the collimator. Scintillations from this crystal are detected by two R.C.A. 5819 photomultiplier tubes ${ }^{28}$ making optical contact with two opposite $1 \times 3$ inch faces of the crystal through Plexiglass light conductors. These two tubes are operated in coincidence with a coincidence resolving time of about 1 microsecond, to exclude a large number of random shot noise signals from the photomultipliers. Such spurious signals would otherwise

26 D. E. Muller, H. C. Hoyt, D. J. Klein, and J. W. M. Du.Mond, Phys. Rev. 88, (1952) 775 .

27 We are much indebted to Professor Robert Hofstadter of Stanford for generously supplying us with a large fund of extremely valuable information which has been of great help in the development of our scintillation detector. See J. A. McIntyre and R. Hofstadter, Phys. Rev. 78, (1950) 617.

28 Wo have recently accuuirenl tubes manufactured by the Dumont Co., which apperar to be superior. 
produce pulses in the counting circuit indistinguishable from those produced by low energy gamma rays and thus cause a very troublesome background.

No anti-coincidence shielding counters are found necessary with this system. Instead, pulses from the coincidence circuits whose magnitudes fall within a chosen range are selected by means of adjustable upper and lower pulse-height discriminators, thus providing a means of rejecting pulses due to cosmic rays (which are generally very large) and some unwanted gamma rays. The lower bound of the pulse height discriminator eliminates many smaller pulses from tube noise and other accidental sources.

H. Detection of Minute Sources of Error from Imperfect Rigidity of Mechanism in Mark I. Calibration of Screw ERRORS

Improvements in the stability, sensitivity and counting statistics of the detecting system have made it possible to detect and correct certain faults in the linearity of the wavelength scale of the Mark I instrument which, it turns out, were due to minute flexures in different parts of the mechanism. Figs. 11 and 12 show the means adopted for a study and correction of these and other errors. The chief source of these troubles turned out to be flexures in the $1^{1 /} / 4$ inch round bar, $B^{\prime}$, Fig. 11, terminating the lower beam although flexures in the base of the instrument also played a part. The reader is referred to two published articles ${ }^{29.30}$ the first of which describes the causes of these inaccuracies and the second gives a brief account of the optical accessories which have been added to the Mark I instrument and the other precautions taken to measure the small corrections to the screw readings which must be introduced to compensate for these flexural errors.

A small elbow microscope $\mathrm{M}_{2}$ and a pair of very fine illuminated crosshairs just above it are supported near the center of the system of two horizontal rails which has been added as a superstructure to the long carriage, Q, visible in Fig. 11. Just above the curved crystal and rigidly attached to its pivot is a 6 inch concave mirror, seen in Fig. 12, which forms an image of the crosshairs in the focal plane of the microscope. In passing from right hand to left hand reflections of a given spectral line, if no flexures occurred in rod $\mathrm{B}^{\prime}$ the crystal and the mirror turning in perfect unison with the motion of carriage $Q$ would leave the image of

29 J. W. M. DuMond, Physics Today 5, (1952) 10.

30 D. E. Muller, H. C. Hoyt, D. J. Klein and J. W. M. DuMond, Phys. Rev. 88, (1952) 775 . 


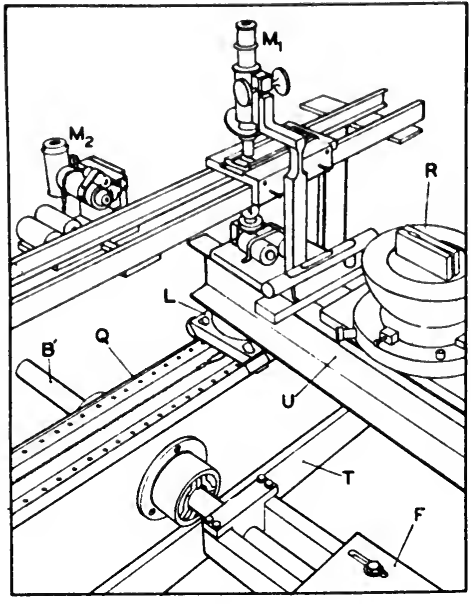

Fig. 11

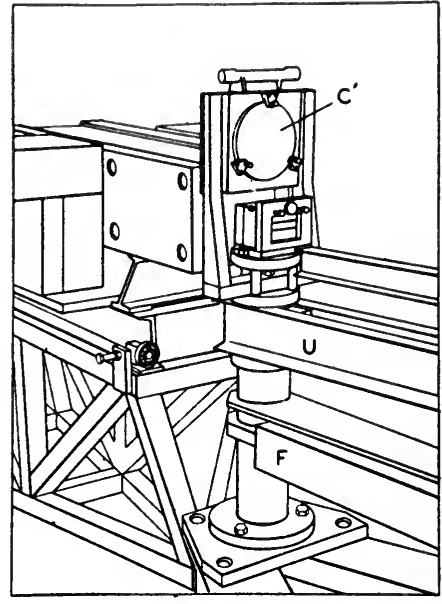

Fig. 12

Fig. 11. Arrangements for the calibration of the spectrometer. A pair of rails rigidly supported on the long screw carriage carry a small carriage provided with a Bureau of Standards calibrated precision glass scale. A vertical microscope $\mathbf{M}_{1}$, rigidly attached to the upper (source supporting) beam $U$, views the glass scale. The focus of the microscope is at the same height as the source $R$ and is directly above the pivot of the small carriage L, supporting the upper beam. The horizontal elbow microscope $\mathbf{M}_{2}$ and tiny light source used in conjunction with the 6-inch concave mirror to detect any anomalies in the motion of the crystal pivot are mounted at the center of the rails. The source holder $R$, standing on the lower half of the lead bomb used for shielding, can be seen at the right center

Fig. 12. The 6-inch precision-figured concave mirror $\mathrm{C}^{\prime}$, mounted on the crystal pivot directly above the crystal, where it served, along with the elbow microscope $\mathrm{M}_{2}$ and light source of Fig. 11 to check the fidelity with which the turning of the crystal pivot followed the motion of the long screw carriage

the illuminated crosshairs immovable in the field of the microscope. Any small motion which does occur can be read off on an eyepiece scale and correction can thus be made for this when reducing the data. This is called the "mirror correction".

Clearly visible also in Fig. 11 are the microscope $M_{1}$ rigidly attached to the upper beam $U$ and the precision calibrated Bureau of Standards glass scale supported on the superstructure rails of carriage $Q$. These are the provisions for calibrating the errors of uniformity of the precision wavelength screw. The non-linearity in the instrumental wavelength scale was first detected by comparing the apparent wavelengths of different gamma ray and $\mathrm{X}$-ray lines as computed from the positions of their profiles reflected in the first, second and third orders. It was found also ${ }^{30}$ that, when means were taken to correct for all these flexural effects, a 
slight apparent discrepancy ${ }^{31}$ between the measured value of the annihilation radiation wavelength and its value calculated from very accurate recent measurements ${ }^{10}$ of the natural constants $h, m$ and $c$ ranished completely ${ }^{30,32}$ so that agreement to 1 part in $10^{4}$ was obtained.

\section{$\S 3$. Typical Results Obtained with the Mark I Crystal Diffraction Gamma Ray Spectrometer}

The list of artificial and natural radioisotopes and the number of gamma ray lines from each which have to the date of this writing been measured with the Mark I instrument together with the references giving a descrip)tion of the latest work is given in Table I. In the case of the $y$-ray lines the isotope named in each item is the parent rather than the daughter product after $\beta$-decay or $K$-capture although in reality the gamma ray is properly to be associated with the daughter product. For the X-ray lines, on the contrary, the element of which each is characteristic is named.

As an illustrative example we show Fig. 13 which is a "bird's-eveview" of the entire spectrum of X-ray and gamma-ray lines (for reflection from one side of the crystal planes only) obtained with a source of $\mathrm{Ta}^{182}$ (the parent isotope). The spectrum for reflection from the other side of the planes is simply a mirror image of this plot, save that the center of symmetry of the two halves may be slightly shifted from the nominal zero of the wavelength scale. (Such small displacements usually occur as a result of small variations in the positioning of the source in its holder. The wavelength is determined by the distance in screw turns between each positive line position and its negative counterpart and such small asymmetries of the screw scale can therefore readily be shown to have negligible influence on the measured wavelength values). The abscissa coordinate is a linear scale of wavelength and the energy scale is therefore highly compressed toward the short wavelength end. The rising background at short wavelengths coming from the increasing scattering on the partitions of the collimator can be clearly seen. On the scale of this "bird's-eyeview", individually observed points are too close together to be depicted. Fig. 14 shows a detailed view of the line profiles for the three hardest lines in this spectrum (at 10.13, 10.44, and 11.06 milliangstroms) in which the individually observed points appear. The statistical uncertainty (square root of the total number of counts observed) on one of these points is indicated by a vertical stroke.

31 J. W. M. DuMond, D. A. Lind, W. W. Watson, Phys. Rev. 75, (1949) 1226; Arne Hedgran and D. A. Lind, Phys. Rev. 82, (1951) 126; J. W. M. DuMond, Phys. Rev. 81, (1951) 468.

32 G. Lindstrom, Phys. Rev. 83, (1951) 465; L. A. Page, P. Stehle and s. B. Ciunst, Phys. Rev. 89, (1953) 1273. 


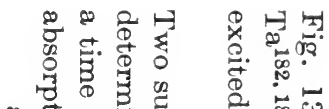

क or :

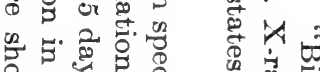

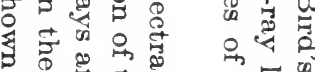

- 0 象

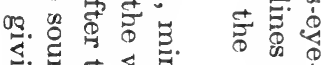

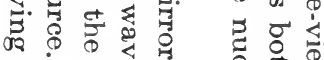

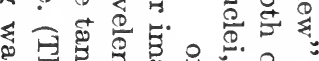

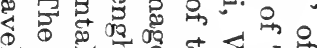

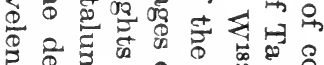

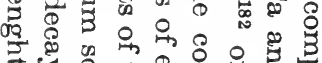

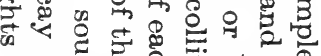

₹. कृ

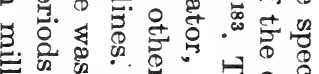

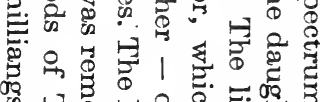

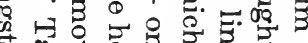

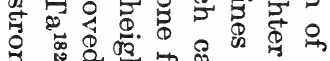

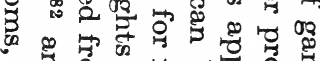

క.

$8 \mathrm{Q}$

๑

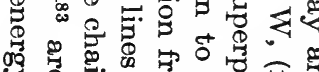

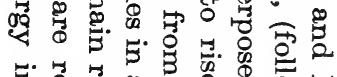
క. 每

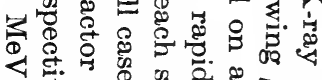

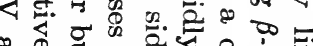

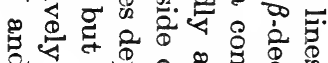
- 1 ঊ.

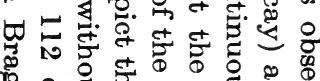

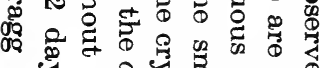

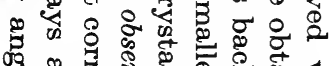

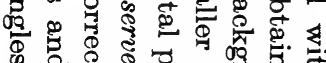

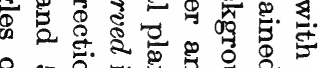

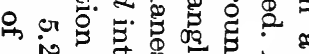

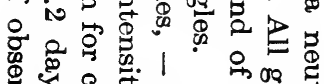

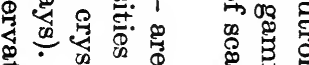
苛,

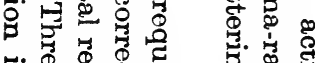

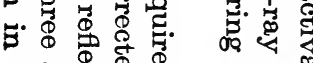
ค유: 品票过客

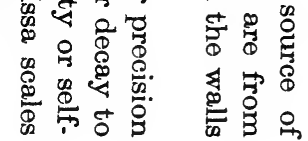

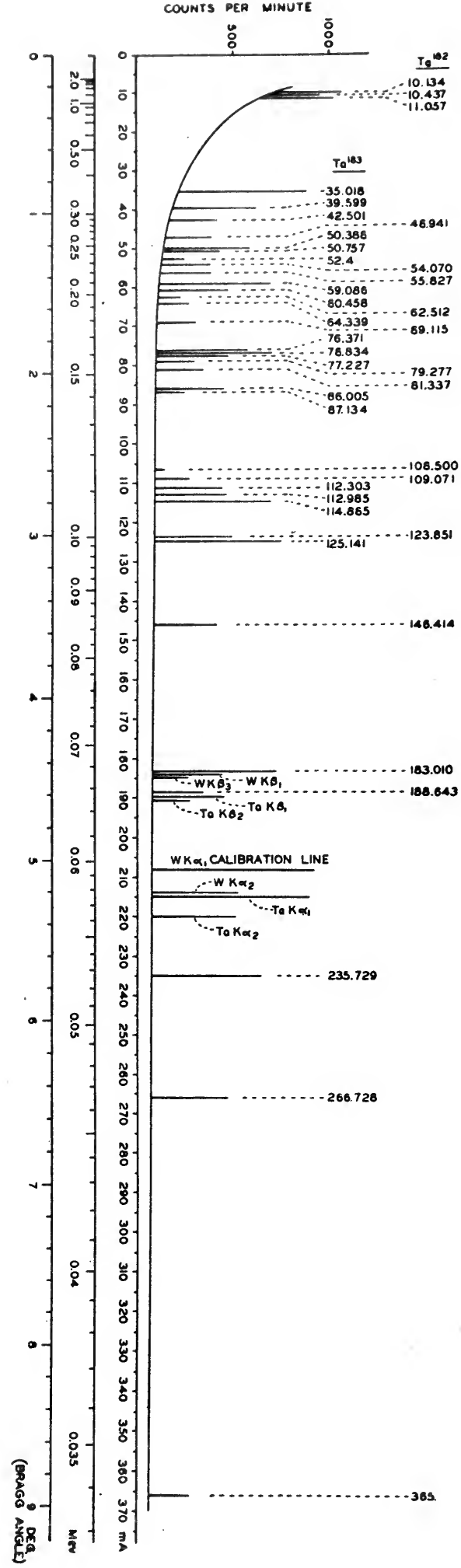


TABLE I

Measurements Made With Mark I Crystal diffraction $\gamma$-Ray Spectrometer

\begin{tabular}{l|l|c|c}
\hline \hline Isotope or Source & Kind of Radiation & $\begin{array}{c}\text { Number of } \\
\text { Lines Measured }\end{array}$ & References \\
\hline
\end{tabular}

Gamma Ray Lines: (The isotope named is the parent although the $\gamma$-ray lines are of course emitted by the daughter.)

\begin{tabular}{l|c|c|c}
\hline $\mathbf{A u}^{198}$ & $\gamma$ & 1 & $b$ \\
$\mathrm{Cu}^{84}$ & Annihil. Rad. & 1 & $b$ \\
$\mathbf{I}^{131}$ & $\gamma$ & 3 & $c$ \\
$\mathrm{Co}^{80}$ & $\gamma$ & 2 & $a, \mathrm{~g}$ \\
$\mathrm{Ta}^{182}$ & $\gamma$ & 17 & $b$ \\
$\mathrm{Ta}^{183}$ & $\gamma$ & 20 & $d$ \\
$\mathbf{W}^{187}$ & $\gamma$ & 5 & $b$ \\
$\mathrm{Ir}^{192}$ & $\gamma$ & 11 & $b$ \\
Cs$^{137}$ & $\gamma$ & 1 & $b$ \\
Radon & $\gamma$ & 5 & $b$ \\
Radio-Th. & $\gamma$ & 6 & $b$ \\
\hline
\end{tabular}

X-Ray Lines:

\begin{tabular}{l|l|l|l}
\hline Ta & K X-ray & 4 & $f$ \\
W & $K$ X-ray & 4 & $e$ \\
Ir & $K$ X-ray & 5 & $b$ \\
Po & K X-ray & 1 & $b$ \\
Os & $K$ X-ray & 4 & $b$ \\
Pt & $K$ X-ray & 6 & $b$
\end{tabular}

(a) D. A. Lind, J. R. Brown, J. W. M. DuMond, Phys. Rev. 76, (1949) 1838.

(b) D. E. Muller, H. C. Hoyt, D. J. Klein, J. W. M. DuMond, Phys. Rev. 88, (1952) 775.

(c) H. C. Hoyt and J. W. M. DuMond, Phys. Rev. 91, (1953) 1027.

(d) J. W. M. DuMond, H. C. Hoyt, P. Marmier, J. J. Murray, Phys. Rev. 92, (1953) 202.

$(e, f, g)$ To be published.

When lines are as closely adjacent (relative to their instrumental width) as the three of Fig. 14 and with as unfavorable a background as these (from the point of view both of its large value and its steep slope) an absolute wavelength determination by the method of superposition of profiles to better than about \pm 8 or $10 \%$ of the width of the lines at half maximum height is about the best that should be claimed. The lines of Fig. 14 depict about the worst example of this sort. To give an idea of about the best that can be done we show in Fig. 15 a typical pair of line profiles for the $412 \mathrm{keV}$ gamma radiation following decay of $\mathrm{Au}^{198}$. The size of the dots represents approximately the statistical uncertainty of the 


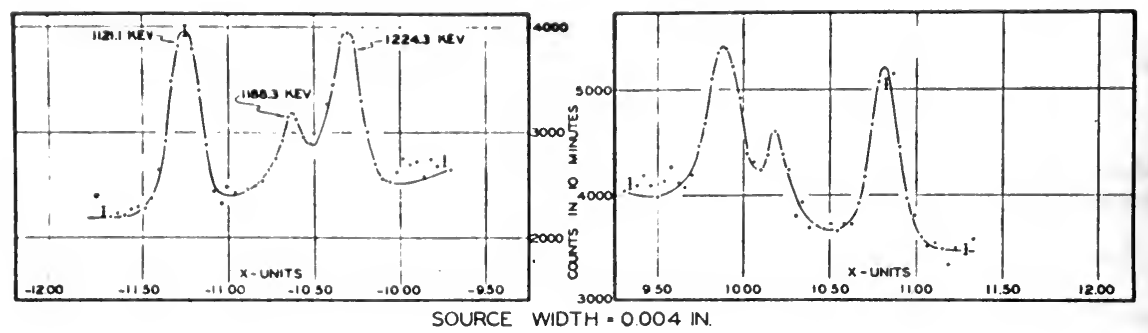

${ }_{73}$ Ta $^{102}-$ HIGH ENERGY GAMMA RADIATION
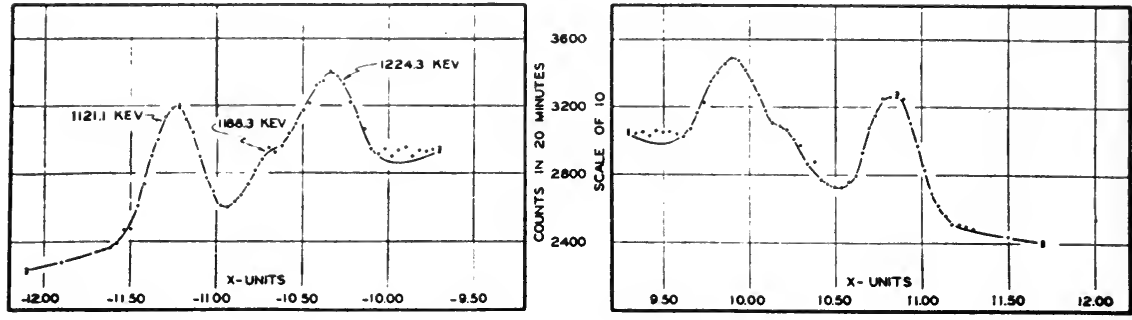

SOURCE WIDTH $=0.011$ IN

Fig. 14. Detailed spectrum of the three shortest wavelength lines in the bird'seye-view of Fig. 13. The statistical error (square root of the total number of counts observed at a given point) is shown for a few points

RUN NO. 4 - FIRST ORDER
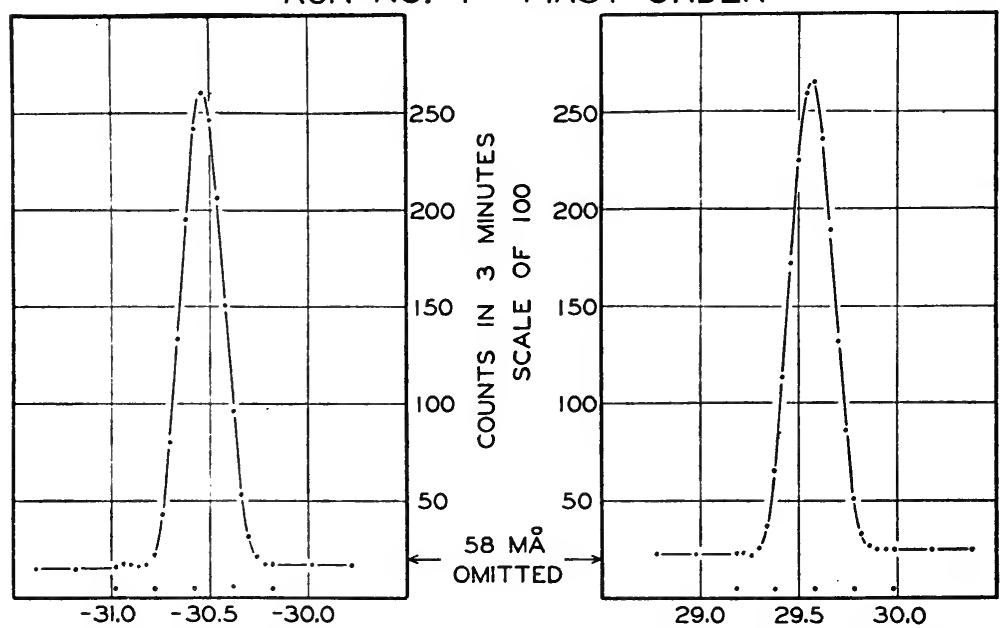

SPECTROMETER SETTING IN MILLIANGSTROMS

\section{KEV GAMMA-RADIATION FOLLOWING DECAY OF GOLD 198}

Fig. 15. A typical pair of line profiles. This pair of profiles was obtained for the $412-\mathrm{keV}$ gamma radiation following decay of gold 198. The wavelength is found by first forming a composite profile by superposition of all profiles (plotted to the same scale) obtained with the same source and then matching each profile individually to the composite profile. The difference in screw readings for the two members of a pair corresponding to a fiducial mark on the composite profile gives twice the wavelength, before corrections 
counting and these can be seen to be entirely consistent with the minute fluctuations in the otherwise level background. The method of superposition of profiles is reliable here to 2 or $3 \%$ of the width of the lines at half maximum height, i.e., to from \pm 0.008 to \pm 0.01 milliangstrom. We give in Table II the results of 13 repeated measurements on this line.

\section{TABLE II}

The wavelength and energy of the $412 \mathrm{keV}$ gamma-ray line following decay of $A u^{198}$ as obtained by D. E. Muller and H. C. Hoyt in measurements of first, second and third order reflections. The uncertainties given are standard deviations. They are given to one extra place to avoid incorrect weighting when combining these values with those of other workers. All corrections including the "mirror corrections" have been made.

\begin{tabular}{c|c|c|c|c}
\hline \hline Order & $\begin{array}{c}\text { Number of } \\
\text { Independent } \\
\text { Runs over } \\
\text { Profile }\end{array}$ & $\begin{array}{c}\text { Corrected } \\
\text { Wavelength in } \\
\text { Screw Divisions }\end{array}$ & $\begin{array}{c}\text { Wavelength in } \\
\text { Milliangstroms }\end{array}$ & $\begin{array}{c}\text { Energy } \\
\text { in keV }\end{array}$ \\
\hline 1 & 10 & $30.016 \times 0.0028$ & & \\
2 & 1 & $30.019 \times 0.0023$ & & \\
3 & 2 & $30.021 \pm 0.0016$ & \\
$\begin{array}{c}\text { Weighted } \\
\text { Mean }\end{array}$ & & $30.019 \pm 0.0012$ & $30.105 \pm 0.0026$ & $411.770+0.036$
\end{tabular}

The annihilation radiation resulting from the recombination with negative electrons of positrons emitted by the nuclei, $\mathrm{Cu}^{64}$, in a small block of ordinary copper which has been exposed to a strong slow neutron flux in a chain reactor has been studied repeatedly and with considerable attention to high precision. The use of a relatively large block of activated copper behind a fine slit $(0.2 \mathrm{~mm}$ wide) which defines the effective instrumental source width is indicated in this case because the range of the positrons emitted by $\mathrm{Cu}^{64}$ before these are decelerated to thermal velocities is about $0.3 \mathrm{~mm}$. The cross section for annihilation is very low except for thermal positrons. The slit thus utilizes the annihilation radiation formed by positrons which have been supplied from a layer of activated copper $0.3 \mathrm{~mm}$ thick on both sides of the central region and this affords a very considerable gain in intensity as compared to a source of total thickness say $0.2 \mathrm{~mm}$ which might be used instead of the defining slit.

Nine pairs of annihilation line profiles were delineated with the measured wavelengths from each pair as listed in Table III.

Table IV lists the results of the annihilation wavelength measurements by two different methods, (1) and (2), and gives also for comparison the value, $\lambda_{c}=h / m_{0} c$, computed from the November 1952 least-squares analysis 
TABLE III

The values of $2 \%$ (twice the wavelength), in screw divisions for the annihilation radiation ficm $\mathrm{Cu}^{64}$ are given. The first column gives the direct values as read off by superposition of the master profile on the line profiles. The second column gives the values after correction for the elastic flexure in the $1 \frac{1}{4}$ inch bar terminating the lower beam of the instrument. This correction, since it is made by observing the motion of the inage of illuminated cross hairs formed by a 6 inch mirror mounted above the crystal pivot, is called the "mirror correction".

\begin{tabular}{c|c|c}
\hline \multirow{2}{*}{ Run No. } & $\begin{array}{c}2 \lambda \\
\text { Before mirror } \\
\text { correction }\end{array}$ & $\begin{array}{c}2 \lambda \\
\text { After mirror } \\
\text { correction }\end{array}$ \\
\hline 1 & 48.328 & 48.383 \\
2 & 48.352 & 48.390 \\
3 & 48.338 & 48.388 \\
4 & 48.320 & 48.381 \\
5 & 48.332 & 48.385 \\
6 & 48.328 & 48.378 \\
7 & 48.326 & 48.384 \\
8 & 48.336 & 48.390 \\
9 & 48.344 & 48.392
\end{tabular}

\section{TABLE IV}

Results on annihilation radiation. The uncertainties given after each \pm are standard deviations. These are given to one extra place to avoid incorrect weighting when combining these values with those of other experimenters.

Wavelength:

1. $\lambda_{A}=24.262 \pm 0.0033 \mathrm{~mA}$

2. $\lambda_{A}=24.263 \pm 0.0033 \mathrm{~mA}$

3. $\lambda_{c}=24.26067 \pm 0.00048 \mathrm{~mA}$

$\mathrm{Cu}^{64}$ direct measurement of $\lambda_{A}$ $\mathrm{Au}^{198}$ expt. + Hedgran and Lind

DuMond and Cohen least-squares analysis

Energy:

1. $E_{A}=510.941 \pm 0.067 \mathrm{keV}$

2. $E_{A}=510.920 . \pm 0.070 \mathrm{keV}$

$\mathrm{Cu}^{64}$ direct measurement of $\lambda_{A}$

3. $m_{-} c^{2}=510.969 \pm 0.015 \mathrm{keV}$

$\mathrm{Au}^{198}$ expt. + Hedgran and Lind

DuMond and Cohen least-squares analysis

Positron mass:

1. $\left(m_{-}-m_{+}\right) / m_{-}=(1.01 \pm 1.85) \times 10^{-4}$

$\mathrm{Cu}^{64}$ direct measurement of $\lambda_{A}$

2. $\left(m_{-}-m_{+}\right) / m_{-}=(1.92 \pm 2.80) \times 10^{-4}$

$\mathrm{Au}^{198}$ expt. + Hedgran and Lind

of the values of the atomic constants by DuMond and Cohen based on entirely independent sources of data of many kinds and of very much higher precision ${ }^{10}$. The table also gives the annihilation quantum energies corresponding to each of the three wavelengths and the inferred electronpositron mass difference, which last is seen to be less than its own standard deviation and hence zero to the precision of these measurements. The items 
labelled (1) are obtained, by direct measurement of the annihilation wavelength from the $\mathrm{Cu}^{64}$ source with the Mark I spectrometer. The items labelled (2) are obtained indirectly from the crystal diffraction measurement of the $\mathrm{Au}^{198}$ wavelength combined with Hedgran and Lind's ${ }^{33}$ determination by magnetic $\beta$-ray spectroscopy of the energy difference between the annihilation radiation and the gold radiation. In Hedgran and Lind's work the energy levels from which the $\beta$-rays were ejected were so chosen that there was very little difference indeed as to $B \varrho$ in the two cases so that errors in calibration of the magnetic spectrometer were very unimportant.

Fig. 16 shows the effect of Doppler broadening on the line profile of the annihilation radiation obtained from the $\mathrm{Cu}^{64}$ source. This broadening is occasioned by the fact that the annihilating pairs are in motion and the momentum inferred from the broadening, $p=9.8 \times 10^{-3} m_{-} c$, indicates that, for the most part, only the conduction electrons of the copper take part in the annihilation process.

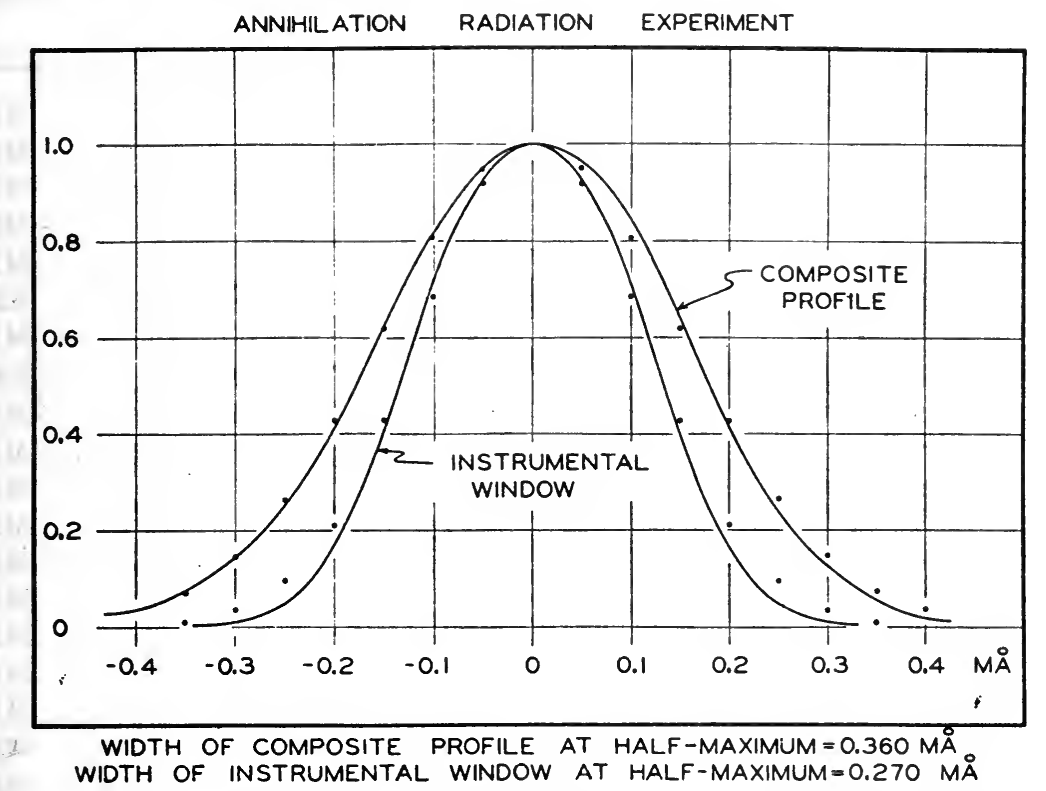

Fig. 16. Showing the effect of Doppler broadening on the observed line profile for the annihilation radiation from copper 64. The composite profile formed by superposition of all observed annihilation line profiles is compared with the calculated instrumental window. The dots represent Gaussian functions, $e^{-h^{2} x^{2}}$, having the same widths at half-maximum as the solid curves. The composite profile has not been corrected for decay of the source during the time required to obtain a single profile, since such corrections are only slight

33Arne Hedgran and D. A. Lind, Phys. Rev. 82, (1951) 126. 
The need for irradiation with high neutron flux densities to obtain sources. of high specific radioactivity for crystal gamma-ray spectrometry has led to the discovery and study of a new isotope $\mathrm{Ta}^{183}$. This nuclide is formed from natural $\mathrm{Ta}^{181}$ by two successive neutron captures during irradiation with neutrons and the initial amount produced is therefore proportional to the square of the neutron flux density. The presence of a short lived gamma line at $250 \mathrm{keV}$ (half life 5 days) in studying the spectrum of $\mathrm{Ta}^{182}$ was first noticed by $\mathrm{H}$. Hoyt in 1951 and reported in his doctorate thesis (1952). The suggestion that this line is emitted by $\mathrm{Ta}^{183}$ was first made by W. Mihelich in 1952 and this is now verified. $\mathrm{Ta}^{183}$ emits a very rich spectrum of lines of which we list 20 in Table $\mathrm{V}$. The same process of

TABLE $V$

$\gamma$-RAYS EMITTED BY A Ta ${ }^{183}$ SourCe

\begin{tabular}{|c|c|c|c|c|c|c|c|c|}
\hline \multirow{2}{*}{$\operatorname{Lin} \theta$} & \multirow{2}{*}{$\begin{array}{c}\text { Energy } \\
\text { Level }\end{array}$} & \multicolumn{2}{|c|}{ Intensity } & \multicolumn{4}{|c|}{$\begin{array}{c}\text { Absolute Conversion } \\
\text { Coefficient }\end{array}$} & \multirow{2}{*}{$\begin{array}{l}\text { Multi- } \\
\text { polarity }\end{array}$} \\
\hline & & $\gamma$-ray & $\begin{array}{c}\text { Decay } \\
\text { Frac. } \%\end{array}$ & $\alpha_{K}$ & ${ }^{x} L_{\mathrm{I}}$ & ${ }^{\alpha} L_{\Pi}$ & $a_{L_{\text {III }}}$ & \\
\hline $\mathbf{a}$ & $40.97 \pm .01$ & 1.4 & 10.3 & - & 18.2 & $(4.0)$ & 1.3 & MI \\
\hline b & $46.48 \pm .01$ & 13.5 & 61.4 & - & 11.7 & 1.7 & 0.8 & Ml \\
\hline c & $52.59 \pm .01$ & 15.0 & 41.7 & - & 6.1 & 1.4 & 0.9 & M1 \\
\hline d & $82.92 \pm .01$ & 0.7 & 2.5 & - & 2.2 & - & - & (M1) \\
\hline$\theta$ & $84.70 \pm .02$ & 4.5 & 14.2 & - & 1.7 & - & - & Ml \\
\hline f & $99.07 \pm .02$ & 13.8 & 31.9 & 2.2 & - & 2.5 & 2.3 & E2 \\
\hline g & $101.94 \pm .02$ & 0.6 & 1.55 & 9.3 & - & - & - & M1 \\
\hline h. & $103.14 \pm .02$ & 0.2 & 0.5 & - & - & - & - & (E2) \\
\hline i & $107.93 \pm .02$ & 23.6 & 45.7 & 5.7 & 0.9 & - & - & Ml \\
\hline j & $109.73 \pm .02$ & 1.7 & 2.9 & $(5)$ & $(0.9)$ & - & - & (M1) \\
\hline $\mathbf{k}$ & $120.38 \pm .02$ & 0.3 & 0.25 & 2.1 & 0.33 & - & - & Ml \\
\hline 1 & $144.12 \pm .03$ & 8.5 & 6.4 & 1.65 & 0.28 & - & - & Ml \\
\hline $\mathrm{m}$ & $160.53 \pm .04$ & 10.5 & 4.4 & 0.37 & - & (1) & (1) & Ml \\
\hline n & $161.36 \pm .04:$ & 31.0 & 18.2 & 1.15 & (1) & - & - & Ml \\
\hline $\mathbf{o}$ & $162.33 \pm .04$ & 16.5 & 9.8 & 1.12 & (1) & - & - & Ml \\
\hline$p$ & $192.64 \pm .06$ & 0.9 & 0.4 & 0.63 & - & - & - & M1 \\
\hline$q$ & $203.27 \pm .06$ & 0.9 & 0.4 & 0.65 & - & - & - & M1 \\
\hline $\mathbf{r}$ & $205.06 \pm .06$ & 3.4 & 1.45 & 0.59 & 0.13 & - & - & Ml \\
\hline s & $208.81 \pm .07$ & 2.6 & 1.15 & 0.70 & - & - & 一 & M1 \\
\hline $\mathbf{t}$ & $209.87 \pm .07$ & 16.4 & 5.4 & 0.22 & - & 0.066 & 0.031 & E2 \\
\hline u & $244.26 \pm .09$ & 34.0 & 10.0 & 0.14 & - & - & - & E2 \\
\hline $\mathrm{v}$ & $246.05 \pm .09$ & 100 & 35.6 & 0.38 & 0.07 & - & - & M1 \\
\hline $\mathbf{w}$ & $291.71 \pm .13$ & 20 & 5.4 & 0.068 & - & 0.023 & - & E2 \\
\hline $\mathbf{x}$ & $313.03 \pm .15$ & 29 & 8.8 & 0.22 & 0.036 & - & - & Ml \\
\hline $\mathbf{y}$ & $354.04 \pm .2$ & 42 & 12.0 & 0.14 & 0.025 & - & - & M1 \\
\hline $\mathrm{z}$ & $365.60 \pm .2$ & 3.4 & 0.85 & - & - & - & - & (M1) \\
\hline$\dot{\mathbf{z}}^{\prime}$ & $406.58 \pm .25$ & 3.8 & 0.95 & - & - & - & - & (E2) \\
\hline
\end{tabular}


double neutron capture has been observed earlier in the study of the spectrum of $\mathrm{Au}^{198}$ where some lines ascribable to $\mathrm{Au}^{199}$ have been observed There seems little doubt that many other similar cases remain to be found and studied.

Note added in proof: - The combination of (1) the wavelength and intensity data obtained by the crystal diffraction method with (2) the intensities of conversion $\beta$-rays obtained with a new and very luminous axial focusing magnetic spectrometer ${ }^{34,35}$ has very recently permitted

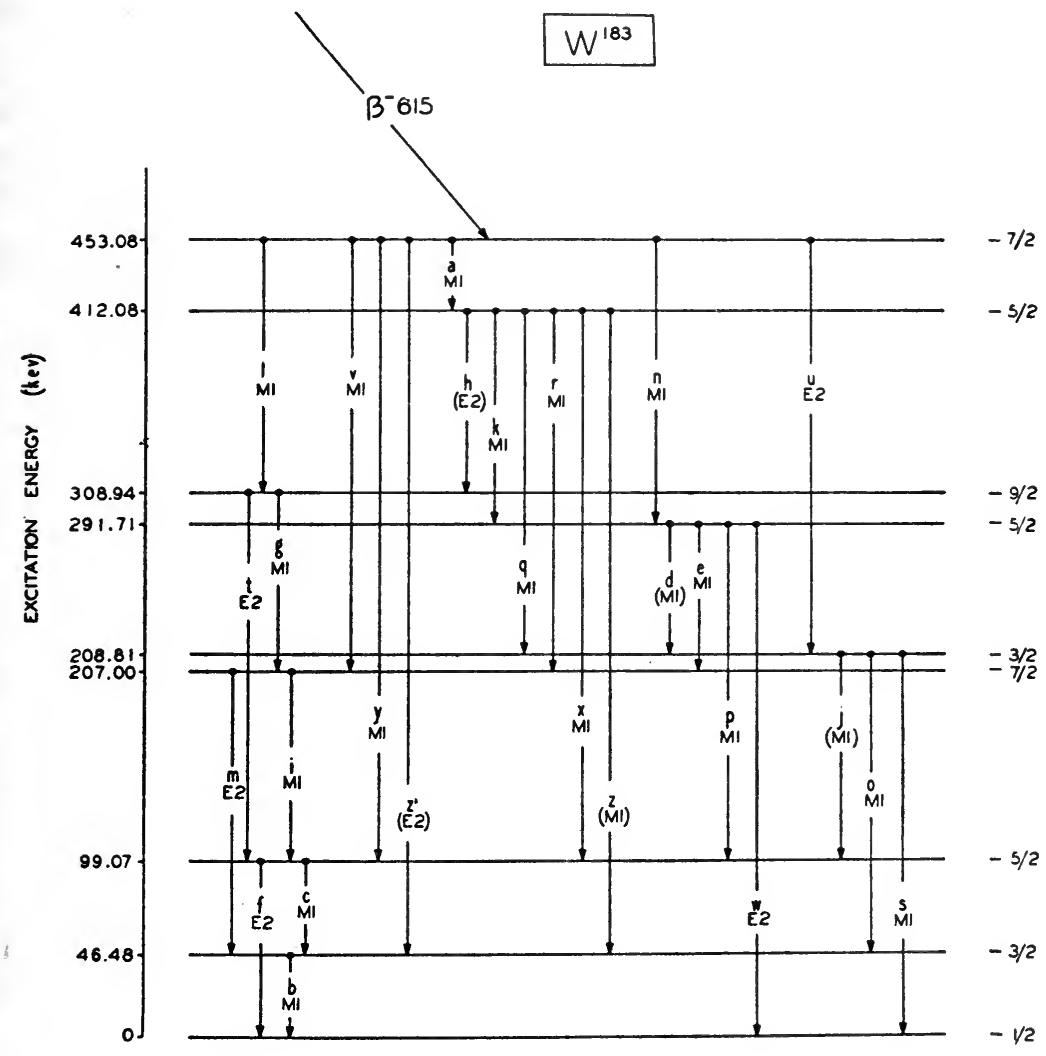

Fig. 17

84 Jesse W. M. DuMond, Rev. Sci. Instr. 20, (1949) í60.

35 Special Technical Report No. 16 to the O.N.R. "An Axial Focusing Magnetic $\beta$-Ray Spectrometer of High Luminosity, Resolving Power and Precision ?with Proton Resonance Stabilized Homogeneous Field". J. Duliond, L. Bogart, J. Kohl, D. Muller and J. Wilts, March 1952. 
J. J. Murray ${ }^{36}$ (for $W^{183}$ ) and P. Marmier and Felix Boehm ${ }^{37}$ (for $W^{182}$ ) to obtain a complete and unambiguous description of the gamma-ray decay schemes of these nuclei with unequivocal assignments of multipolarities to the lines (based on $K, L$ and $M$ conversion coefficients) and of spins and parities to all levels, (11 levels and $27 \gamma$-lines for $\mathrm{W}^{182}, 9$ levels and $27 \gamma$-lines for $W^{183}$ ). See Table $\mathrm{V}$ above and Fig. 17 for $\mathrm{W}^{183}$.

These results are of considerable interest since they furnish evidence ${ }^{38}$ corroborative of the Bohr-Mottelson theory ${ }^{39}$ of nuclear rotational levels based on the idea of collective motion of nucleons. Also the measured conversion coefficients corroborate very satisfactorily the calculated values of Rose et al. ${ }^{40}$. The combination of crystal diffraction $\gamma$-ray spectroscopy with magnetic $\beta$-ray spectroscopy thus has been shown to constitute a most powerful tool for the investigation and interpretation of nuclear spectroscopy.

36 The Decay of $\mathrm{Ta}^{183}$, Thesis, J. J. Murray, Calif. Inst. of Technology (1954).

37 F. Boehm, P. Marmier and J. W. M. DuMond, Phys. Rev. 45, (1954) 864.

38 See paper by A. Bohr, Session III, Glasgow Nuclear Physics Conference, July 14, 1954.

39 See Ch. XVII of this work, also G. Alaga, K. Alder, A. Bohr and B. R. Mottelson, Dan. Mat. Fys. Medd. (1955).

40 M. E. Rose, G. Goertzel, B. Spinrad, J. Harr and P. Strong, Phys. Rev. 83, (1951) 79 . 


\title{
THE SCINTILLATION METHOD
}

\author{
P. R. BELL
}

\section{$\S$ 1. The Scintillation Counter}

The electronic scintillation counter is a modern device. The use of a photomultiplier with a zinc sulfide screen for the detection of alpha particles was introduced by Curran and Baker in 1944, but the development of generally useful detectors did not begin until it was found by Kallmann ${ }^{1}$ that beta particles could be detected with a thick phosphor of naphthalene.

Thin phosphors for the detection of alpha particles are still occasionally used but the scintillation counter generally consists of a solid fluorescent medium with a volume from a few cubic millimeters to as large as ten cubic feet. One or many photomultipliers are coupled to the phosphor to produce current pulses from the emitted light flashes.

The development of scintillation phosphors and counter equipment is still very rapid. A review with bibliography of the subject to 1949 is given by Jordan and Bell ${ }^{2}$ and to 1952 by Jordan ${ }^{3}$. Recent books on the subject by Curran ${ }^{4}$ and by Birks ${ }^{5}$ give good summaries of many aspects of the scintillation method but the subject must be followed in current publications as it is still subject to change without notice.

\section{§ 2. Methods for Various Radiations}

\section{A. Alpha Particles and Other Highly Ionizing Radiations}

Essentially all the phosphors used in scintillation counters will detect any nuclear radiation which leads to ionization in the phosphor, but it was found in early investigations that the amount of light produced by alpha particles and electrons was very different in some phosphors although the same amount of energy was lost. In general a $5 \mathrm{MeV}$ alpha particle will produce about $1 / 10$ as much light as a $5 \mathrm{MeV}$ electron in all of the organic single crystals, the plastic phosphors, and the solution phosphors. In the inorganic phosphors the alpha particle will give from less than half to perhaps $70 \%$ as much light as the electron depending on the

1. H. Kallmann, Natur und Technik, July, 1947.

2 W. H. Jordan and P. R. Bell, Nucleonics 8, No. 4, (1949) 30.

3 W. H. Jordan, Annual Review of Nuclear Science, 1, (1952) 207.

4 S. C. Curran, Luminescence and the Scintillation Counter, Academic Press, New York (1953); Butterworth's Scientific Publications, London (1953).

5 J. B. Birks, Scintillation Counters, McGraw-Hill Co.. New York (1953); Pergamon Press, Ltd., London (1953). 
phosphor. The phosphor generally chosen for the detection of alpha particles, protons, etc., is either $\mathrm{NaI}(\mathrm{Tl})$ or $\mathrm{ZnS}(\mathrm{Ag})$. The zinc sulfide, silver activated, is stable in air but is only available as a fine crystalline powder. As a consequence it is difficult to obtain good energy resolution and the long mean life of its fluorescent light $(\sim 10 \mu \mathrm{sec})$ restricts its use to comparatively slow counting rates.

Sodium iodide activated with thallium gives excellent energy resolution but it is hygroscopic and must be protected from the atmosphere and present a clean surface to the entering particles.

\section{B. BETA RAYs}

Several factors influence the choice of a phosphor for beta ray spectrometry. The pulse height per $\mathrm{MeV}$ of energy lost in the phosphor is very important since the resolution (full width of pulse distribution at half maximum counting rate for monoenergetic electrons) is inversely proportional to the square root of the number of photoelectrons in the pulse to a first approximation. Sodium iodide activated with thallium produces the largest pulse height of presently known phosphors and among organic phosphors the largest pulse is produced by anthracene although the pulse is smaller than that from sodium iodide.

Unfortunately the scattering of fast electrons in matter is very large and increases rapidly with increasing atomic number. About $8 \%$ of the beta rays entering a flat anthracene surface from a nearby source will scatter out of the crystal before being stopped and hence will produce smaller pulses than they should. For NaI(Tl), on the other hand, $80 \%$ to $90 \%$ of the entering electrons will scatter out before stopping. It is consequently quite difficult to obtain a satisfactory beta spectrum using $\mathrm{NaI}(\mathrm{Tl})$ with an external source.

The scattering out may be reduced by collimating the entering beta rays so that fewer rays enter the surface at low angles. Electrons entering the surface near normal incidence have a greater chance of deep penetration before severe scattering. Fig. 1 shows the pulse distribution obtained with the beta rays of $\mathrm{P}^{32}$. Curve $a$ was for the uncollimated source, Curve $b$ was obtained with a pclystyrene collimator thick enough to stop all of the beta rays. The reduction of the excess small size pulses is obvious.

Even with a collimator the Fermi-Kurie plot shows considerable distortion at the lowest energy end and other devices must be employed to obtain good spectra. The split-crystal spectrometer first reported by Ketelle ${ }^{6}$ prevents any scattering-out of beta rays by surrounding the source with anthracene. Two separate crystals close together with the source between are used. The light is taken out of the crystal edges and 
led to the photomultiplier by the lucite light piper. Fig. l(c) shows the pulse distribution obtained for $\mathbf{P}^{\mathbf{3 2}}$, and Fig. 2(b) shows the resulting Kurie plot and Fig. 2(a) is the result for the flat crystal (Fig. 1(a)). The $\mathrm{P}^{32}$ sample contained some $\mathrm{P}^{33}$ giving the rapid rise below $250 \mathrm{keV}$.

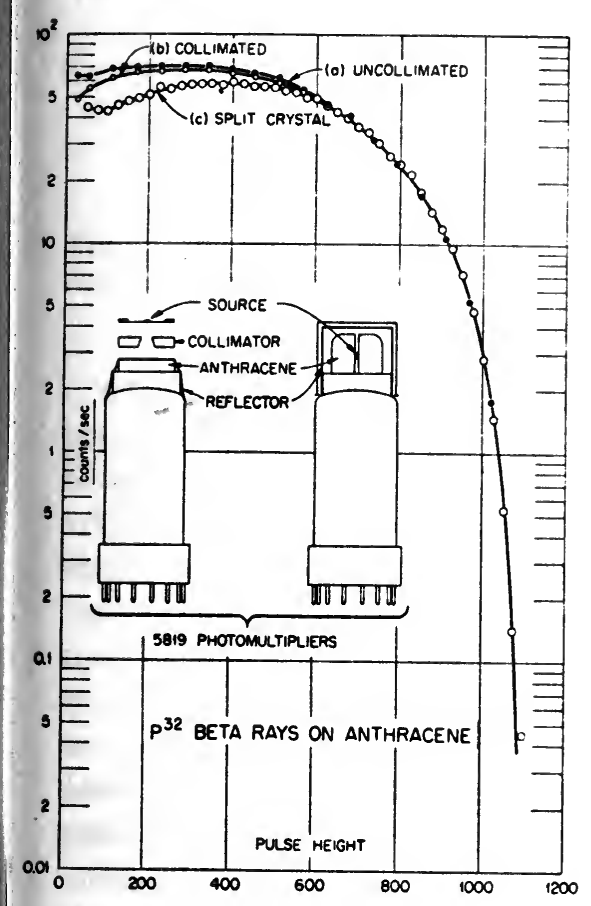

Fig. 1. Pulse height distribution from the beta rays of $\mathrm{P}^{32}$ on an anthracene detector

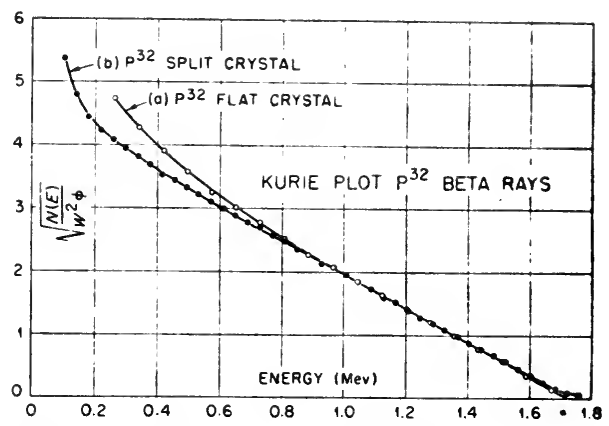

Fig. 2. Kurie Plot of $\mathrm{P}^{32}$ beta rays on anthracene

The counting efficiency for the split-crystal spectrometer is essentially $100 \%$ and very low specific activity sources may be measured. A source area of $11 / 2 \times 2 \mathrm{~cm}$ may be used with the crystals in Fig. 1 .

The high efficiency is embarrassing upon occasion since a following coincident gamma-ray transition (especially if strongly internally converted) will be detected. The photomultiplier cannot distinguish between two separate light flashes if they are simultaneous and a pulse representing the sum results. The pulse distribution will consist of a normal beta distribution plus a similar distribution to each pulse of which a constant amount (for the internal conversion electron) has been added. The gamma ray whose detection adds a continuum (Compton process), not a line, will further add to the mess. A Kurie plot of 'such a distribution will be terribly distorted but will have a more or less straight upper end intersecting the energy axis at the sum of the beta-ray transition energy and the conversion electron energy.

- B. H. Ketelle, Phys. Rev. 80, (1950) 758. 
When coincident radiation is present, the hollow crystal spectrometer ${ }^{7}$ is more suitable than the split-crystal. In this arrangement, shown in Fig. 3, the beta rays are collimated onto the bottom of a conical hole in the phosphor. The scattered beta ray's have small probability of finding their way out of the entrance hole.

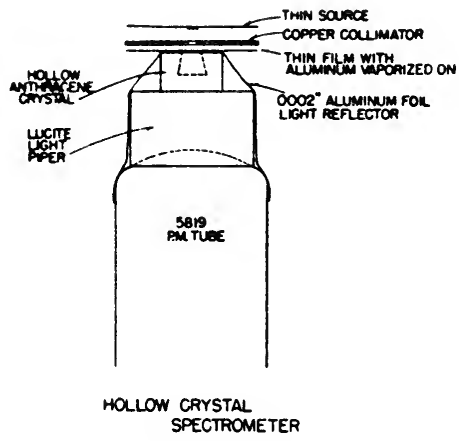

Fig. 3. Hollow crystal spectrometer

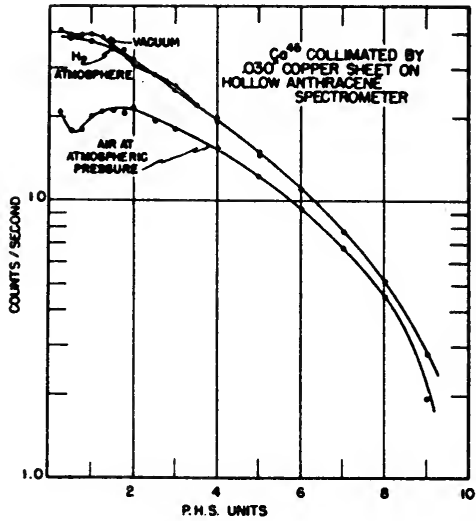

Fig. 4. Change in pulse distribution for $\mathrm{Ca}^{45}$ with various atmospheres in the scintillation counter

Fig. 4 shows the pulse distribution obtained with the hollow crystal for the beta rays of $\mathrm{C}^{45}(145 \mathrm{keV})$. The source to bottom of hole distance was about $2 \mathrm{~cm}$ and the scattering of the air in this distance was considerable. Evacuating the equipment can produced the upper curve, with air in the can the lower curve was found, and with hydrogen in the can the intermediate curve was obtained. Hydrogen is more satisfactory than vacuum since anthracene is very slowly volatile in a vacuum and the crystal surfaces become etched. In addition the Canada balsam used for the optical joint contains considerable solvent and bubbles may form.

The Fermi-Kurie plot of the hydrogen atmosphere data is shown in Fig. 5. The points give a reasonably straight line with the proper energy intercept.

Beta sources for scintillation spectrometry must be extremely thin just as for magnetic spectrometers and provision for the prevention of source charging must be made. See Ch. VIII for details of source preparation. Any reflector lying between source and detector (as it often does) must be extremely thin. Aluminum evaporated on formvar-polystyrene film is often used.

7 Physics Quarterly ORNL-940, December 1950 and Physies Quarterly ORNL1092, June 1951. 
The organic phosphors all exhibit a decrease of pulse height per $\mathrm{MeV}$ at low electron energy. This effect becomes perceptible in anthracene at about $100 \mathrm{keV}$ and must be corrected for to obtain good Kurie plots. The data of Hopkins ${ }^{8}$ can be used to provide correction.

Fig. 5. Kurie plot of $\mathrm{Ca}^{45}$ with a hollow crystal spectrometer with a hydrogen atmosphere

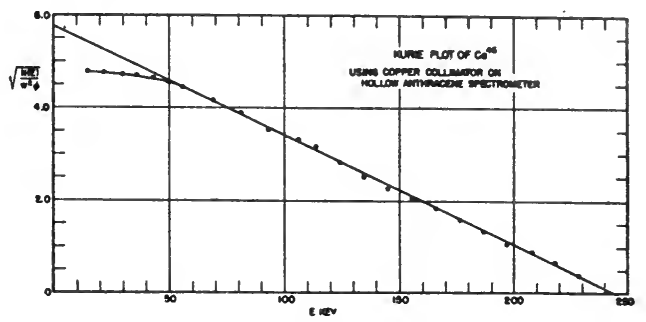

An excellent method that circumvents the undesirable scattering property of $\mathrm{NaI}(\mathrm{Tl})$ was introduced by Scharff-Goldhaber et al. and Sharpe and Stafford ${ }^{9.10}$. The radioactive species to be investigated is added to the melt from which the NaI crystals are grown. The crystal then has an internal source and the beta rays are completely absorbed (except near the surface). The surface effect is very small even in quite modest sized crystals due to the high density of NaI. The method suffers from the same defect as the split-crystal method when coincident radiations are present. A coincidence counting arrangement with another detector nearby detecting the escaped gamma ray and recording only those beta pulses coincident with a gamma-ray producing a full energy pulse in the auxiliary crystal reduces this defect. The same method may be used for the split-crystal spectrometer, of course.

\section{Gamma Rays}

For best detection of gamma rays, as with beta rays, a large pulse height per $\mathrm{MeV}$ is desirable but in contrast to the situation for beta rays, a high atomic number element in the phosphor reduces the scattering of the incoming radiation.

Gamma-rays interact with a phosphor or other matter by three principal processes-Compton scattering, photoelectric effect, and pair production (see Ch. II).

In the photoelectric process the gamma-ray energy appears as the kinetic energy of a photoelectron leaving only $\mathrm{X}$-rays from the residual atom that could escape from the crystal.

8. J. I. Hopkins, Phys. Rev. 77, (1950) 406.

- G. Scharff-Goldhaber, E. der Mateosian, M. Goldhaber, G. W. Johnson, and M. McKeown, Phys. Rev. 83, (1951) 480.

10 J. Sharpe and G. H. Stafford, Proc. Phys. Soc. A64, (1951) 211. 
Pair production creates a positron and electron pair. This creation requires $1.02 \mathrm{MeV}$ of energy and the remaining energy of the gamma ray appears as kinetic energy shared between the electron and positron. After the electron and positron are stopped, the positron annihilates with a nearby electron leaving two photons of 511 ke! gamma ray which may escape the phosphor.

The Compton process transfers some of the incident gamma-ray energy to an electron leaving the remainder as a scattered photon, sometimes of energy almost as great as the original photon. This scattered photon may escape from the phosphor or may be further absorbed.

Both the photoelectric effect and pair production increase rapidly relative to the Compton effect with increasing atomic number $(\mathrm{Z})$ of the atoms in the phosphor. A phosphor containing high $\mathrm{Z}$ elements, therefore, favors the complete absorption of gamma rays since photoelectric effect leaves only the easily absorbed $\mathrm{X}$-ray and pair production leaves only $511 \mathrm{keV}^{\top}$ photons. The Compton process, giving sometimes relatively hard photons, more readily allows some of the gamma-ray energy to escape.

In phosphors where the pulse height per $\mathrm{MeV}$ is nearly independent of electron energy (this is the case for $\mathrm{NaI}(\mathrm{Tl})$ ), any combination of processes by which the gamma ray is completely absorbed in the phosphor produces the same pulse height. For example, a photoelectric event in an iodine atom, followed by the substantially simultaneous absorption of the iodine $\mathrm{X}$-ray, produces the same pulse height as is produced when another gamma ray of the same energy is absorbed by two successive Compton scatterings followed by a photoelectric process and X-ray absorption. Such multiple processes are for this reason quite desirable and as large a phosphor as possible should be chosen. Fig. 6 shows to what extent multiple processes are effective in ordinary size NaI crystals. Curve A shows the ratio of pulses of maximum size (peak) to all pulses produced (total), for a $1 \frac{1}{2}$ in. diameter, 1 in. high crystal, while Curve $C$ is the ratio of photoelectric cross section to photoelectric and Compton + pair cross section, which would be the ratio of peak to total if no multiple processes occurred. Curve B is the ratio of peak to total for a $3 \mathrm{in}$. diameter $3 \mathrm{in}$. high crystal.

The most common arrangement for the measurement of gamma rays from a radioactive source is shown in Fig. 7. The phosphor in the form of a right circular cylinder is attached to the photomultiplier and the source is placed along an extension of the axis of the cylinder. Rectangular or cubical phosphor shapes are less satisfactory than the cylinder due to the increased edge effects near the corners.

The response of the spectrometer depends upon the source distance 


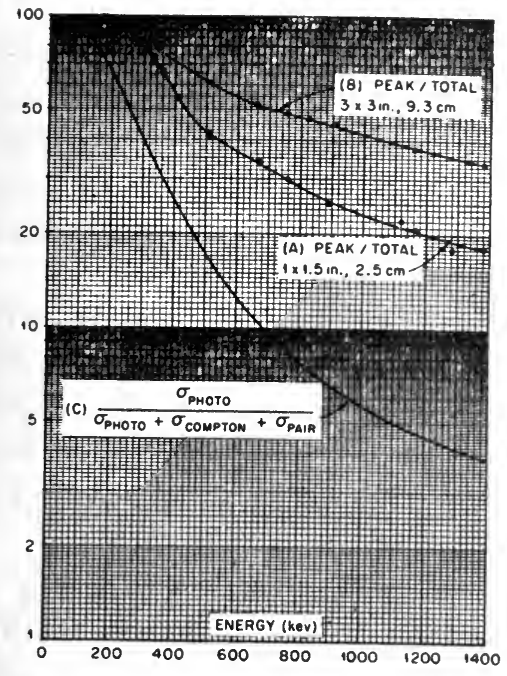

Fig. 6. Peak to total ratios for two crystal sizes and the peak to total ratio that would be found if no secondary absorption occurs

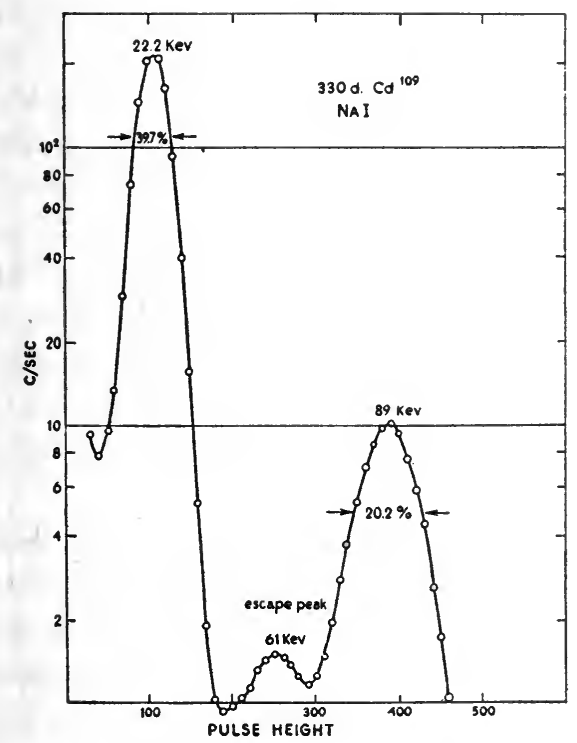

Fig. 8. The response of a $11 / 2$ "diame. ter by 1" high NaI crystal to the radiations of $\mathrm{Cl}^{109}$
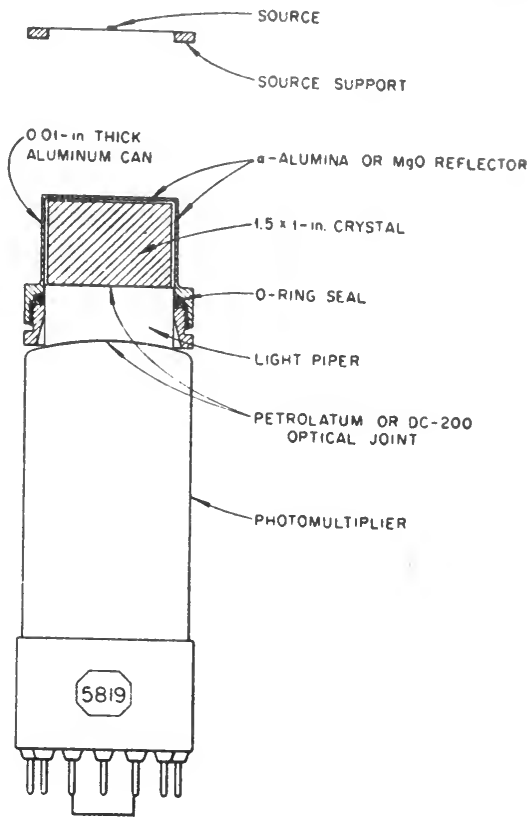

Fig. 7. Standard gamma ray scintillation counter arrangement

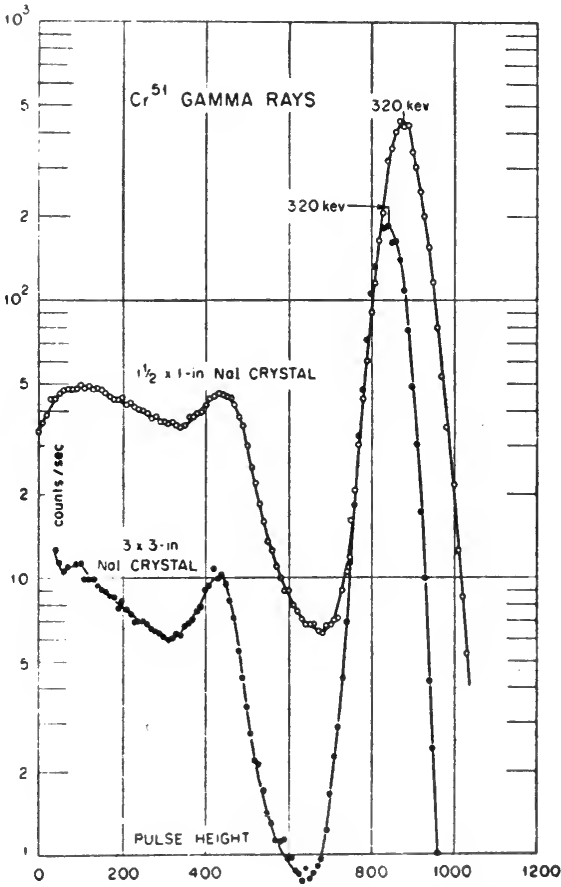

Fig. 9. The response of a $11 / 2^{\prime \prime} \times 1^{\prime \prime}$ and $3^{\prime \prime} \times 3^{\prime \prime}$ crystals to gamma rays of $\mathrm{Cr}^{51}$ 
from the phosphor. At large source distances any ray striking the phosphor will encounter a more or less uniform thickness. When the source is very close to the phosphor surface, the rays also encounter nearly constant thickness. At moderate source distances, however, the variation of thickness seen by the rays is considerable. The effect of this edge penetration can be seen in the detector efficiency curves given in $\S 5$.

Pulse distributions obtained for various energies of gamma ray with $\mathrm{NaI}(\mathrm{Tl})$ phosphors of severai sizes are shown in Figs. 8-12. The smaller crystal, $1 \frac{1}{2}$ in. diameter and 1 in. thick, is the usual size, and is used with type 5819, $6342(\mathrm{C}-7164 \mathrm{~A})$, and 6292 tubes. The 3 in. diameter, $3 \mathrm{in}$. thick crystal gives better results but is much more expensive; it was used with a Dumont $\mathrm{K}-11973 \mathrm{in}$. diameter photomultiplier.

The response of the phosphor at very low energy is shown in Fig. 8. $\mathrm{Cd}^{109}$ gives an $88.5 \mathrm{keV}$ gamma ray (strongly converted) and $\mathrm{Ag} \mathrm{X}$-rays (from both the $K$-capture and the internal conversion). The weak peak $28 \mathrm{keV}$, lower in energy than the gamma-ray line, is produced by those events in which a photoelectron is produced and an iodine $\mathrm{X}$-ray escapes the crystal. This "escape peak" must be considered for quantitative work; its magnitude is given in the section on efficiency, (§5).

The Ag X-ray is too low in energy to produce iodine $K \mathrm{X}$-rays and $L \mathrm{X}$-ray escape is negligible so no escape peak is seen. Fig. 9 shows the response to $\mathrm{Cr}^{51}$ gamma rays, $320 \mathrm{keV}$. At this energy the escape peak is small since the gamma ray penetrates more deeply before most of it is absorbed, also the escape peak has drawn into the main peak and is poorly resolved. The continuous pulse distribution extending up to 500 pulse height divisions is produced by Compton effect in the phosphor followed by escape of a scattered photon. The prominent broad peak near the large pulse height end of this continuum is partly due to the detection of gamma rays scattered from the surrounding shield but is mostly due to the escape of a backscattered photon. A photon scattered backward (hence of nearly minimum energy) will have a greater probability of escaping the phosphor than will a photon scattered forward due to the great opacity of the phosphor.

A source of $\mathrm{Cs}^{137}$ gives beta rays, gamma rays of $661 \mathrm{keV}$ energy and barium X-rays (following internal conversion). If the beta rays are absorbed by a thin polystyrene absorber, the gamma and $\mathrm{X}$-rays give the pulse distribution shown in Fig. 10. The main peak produced by complete gamma ray absorption by any process is less prominent compared to the Compton distribution than at lower gamma-ray energy. Three curves are shown in this figure. The two curves-one for a small crystal, one for the large, that were taken with the source relatively close to the phosphor- 
show only a small peak near 300 pulse height divisions. This peak is due to gamma rays that are backscattered by the Compton process from the shield surrounding both source and detector. Since the source was close to the detector and the wall distant, the backscattered photons were almost monoenergetic $(184 \mathrm{keV})$ and produced a relatively sharp peak. In the third curve the source was more distant from the detector and a smaller fraction of the direct rays reached the detector while the radiation scattered from the walls changed only slightly. The sharp single or double scattered line can be seen to be accompanied by. a broader distribution due to multiple scattering in the shield.

The shield used for the small crystal had walls of mercury (in a thin iron tank), 5 inches square inside by 15 inches high. The larger crystal was surrounded by a lead shield $14-1 / 2$ inches square and 32 inches high. The intensity of backscattering is much increased if lower atomic number shields are used and is lessened by increasing the ratio of shield to detector size.

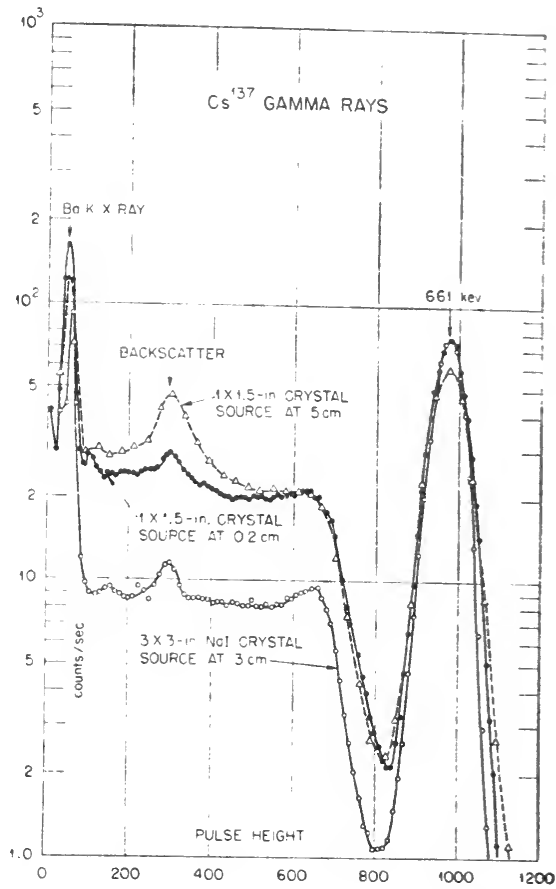

Fig. 10. The response of a $11^{1 /} \times 1^{\prime \prime}$ and $3^{\prime \prime} \times 3^{\prime \prime}$ crystals to gamma rays of ${ }^{\prime} S^{137}$ and the response of the $11^{1 / 2} \times 1^{\prime \prime}$ crystal with the source at some distance

The sharp peak at low pulse height in Fig. 10 is produced by the detection of barium $K \mathrm{X}$-rays. Just to the right of this peak in the small crystal curve can be seen a small peak produced by the detection of fluorescent mercury $\mathrm{X}$-rays formed in the shield wall by the primary radiation. This effect becomes quite annoying at low gamma ray energies and is prevented by lining the walls of the shield with a graded absorber. A liner' of 1/18 inch cadmium or tin, followed by 0.015 inch copper suppresses this $\mathrm{X}$-ray by a factor of at least 100. A thinner graded shield more suitable for lining a collimator consists of 0.008 inch tantalum, 0,020 inch cadmium and 0.005 inch copper. This also has an attenuation greater than 100 times.

Harder gamma rays produce pulse distributions with less full energy peak and more Compton distribution as can be seen from Fig. 11 which shows the response of the crystals to $\mathrm{Zn}^{65}$ gamma rays $(1.114 \mathrm{MeV})$. A small 
amount of annihilation radiation from the weak positron branch can be seen.

Above $1.5 \mathrm{MeV}$ the effect of pair production becomes apparent. Even at this energy the peaks representing the stopping of a pair in the phosphor with escape of one or both of the annihilation photons is very weak. At $2 \mathrm{MeV}$ the pair peaks are fairly prominent. The $2.76 \mathrm{MeV}$ gamma rays of $\mathrm{Na}^{24}$ produce very strong pair peaks in small phosphores. Fig. 12 shows the pulse distributions obtained. The peak at 700 pulse height divisions

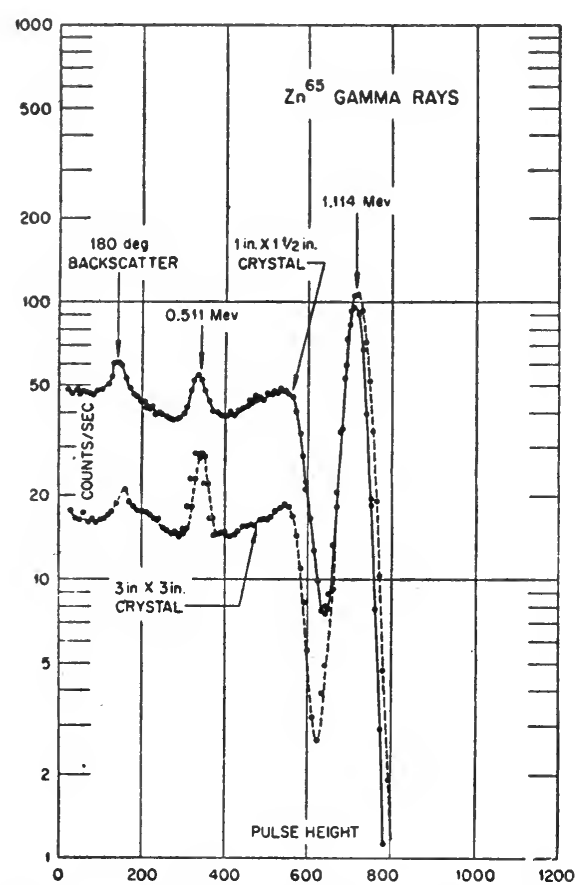

Fig. 11. The pulse height distributions for $\mathrm{Zn}^{65}$ on $1^{1} / 2^{\prime \prime} \times \mathrm{I}^{\prime \prime}$ and $3^{\prime \prime} \times 3^{\prime \prime}$ crystals

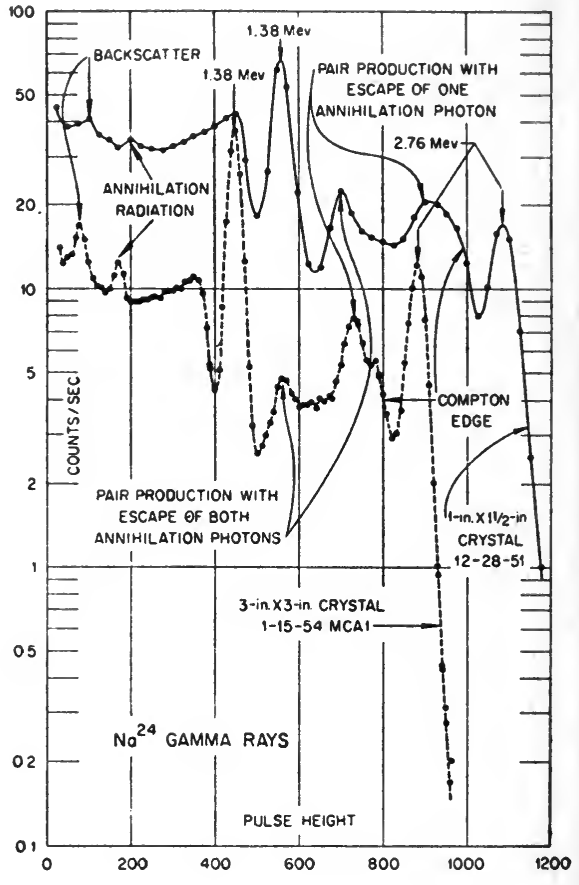

Fig. 12. The response to the two gamma rays of $\mathrm{Na}^{\text {ik }}$ for the $11^{1} \mathbf{2}^{\prime \prime} \times 1^{\prime \prime}$ and the $3^{\prime \prime} \times 3^{\prime \prime}$ crystal

in the small crystal curve is the peak produced by escape of both annihilation photons. The broad peak between 900 and 1000 divisions is composed of the peak representing escape of one annihilation photon with complete absorption of the other and the more or less sharp end of the Compton electron distribution. When high energy gamma rays are present in the source a considerable number of annihilation photons escape from the shield walls around the counter and a small peak at $0.51 \mathrm{MeV}$ is seen (at 200 divisions). The 3 inch by 3 inch crystal shows one of the advantages of a large phosphor, the lower pair peak is much less prominent and the 
full energy peak is much increased. The single escape peak (730 divisions) is quite clearly resolved from the Compton edge which begins at 780 divisions.

\section{§ 3. Factors Affecting Resolution}

A scintillation spectrometer operating under the best conditions shows a resolution limited principally by the statistical uncertainty of the number of photoelectrons emitted at the photocathode. Under good conditions a resolution (full width of peak at $1 / 2$ counting rate) of $6.0 \%$ can be obtained for the $0.661 \mathrm{MeV}$ gamma rays of $\mathrm{Cs}^{137}$. The system used was a $1.5 \mathrm{in}$. diameter 1 in. long $\mathrm{Naf}(\mathrm{Tl})$ crystal with a Dumont 6292 photomultiplier. Consideration of the following factors affecting resolution will show how best resolution may be obtained with a given counter system.

\section{A. Phosphor Fluorescent Efficiency}

The number of photons emitted by the phosphor per $\mathrm{MeV}$ of electron energy lost differs widely among phosphors. This light must be emitted in a wave length region to which the photosurface is sensitive and must be the same for every part of the phosphor.

\section{B. Light Collection Efficiency}

The emitted light must not be self-absorbed in the phosphor. Sodium iodide for example is very sensitive to small amounts of some impurities like lead or free iodine, causing flashes produced far from the photosurface to give smaller pulses than do flashes produced close to it.

Phosphors like $\mathrm{NaI}(\mathrm{Tl}), \mathrm{CaWO}_{4}$ and $\mathrm{LiI}(\mathrm{Eu})$, as well as many others, have high indices of refraction especially in the wave length region of their fluorescent emission. Although the phosphors are joined optically to the photomultiplier glass (refractive index $\sim 1.5$ ), much of the light will be critically reflected and must return to the phosphor body. This light must not be wasted but should be returned to the exit surface within the angle of critical reflection. A right circular cylinder (or rectangular parallelopiped) with polished faces will not return the light, once reflected, within this angle and it is necessary to re-direct the light. This can be accomplished by rough grinding the crystal surfaces. Light striking these diffusing surfaces has an opportunity to return to the exit surface at a suitable angle. The light leaving the other surfaces of the crystal should be returned by the most efficient reflector available. Magnesium oxide has a better reflecting power than any available specular surface and is quite effective. $\alpha$-aluminum oxide (Linde "A" abrasive) * has almost the same reflecting power and because of higher refractive index its efficiency

* Linde Air Products Company. 
is not so much reduced by traces of oils as is that of $\mathrm{MgO} . \mathrm{MgO}$ appears to react slightly with $\mathrm{NaI}$ while alumina does not.

The optical joint between the phosphor and the photomultiplier envelope is important as it reduces the amount of light that must be re-circulated in the phosphor. Since some of the re-circulated light is inevitably lost, a good joint is required. Canada balsam may be used for anthracene whose fluorescent light ends sharply at $4300 \AA$ but is not suitable for $\mathrm{NaI}(\mathrm{Tl})$ since the light emitted ends near $3200 \AA$ and Canada balsam has a broad absorption band near $3400 \AA$. Mineral oil or the high viscosity silicone oils like Dow-Corning DC-200 ( $10^{6}$ centistokes) are suitable for $\mathrm{NaI}(\mathrm{Tl})$ since they are transparent to $3000 \AA$.

\section{C. Рhotocathode Efficiency and Uniformity}

The number of photoelectrons emitted by the photosurface for each

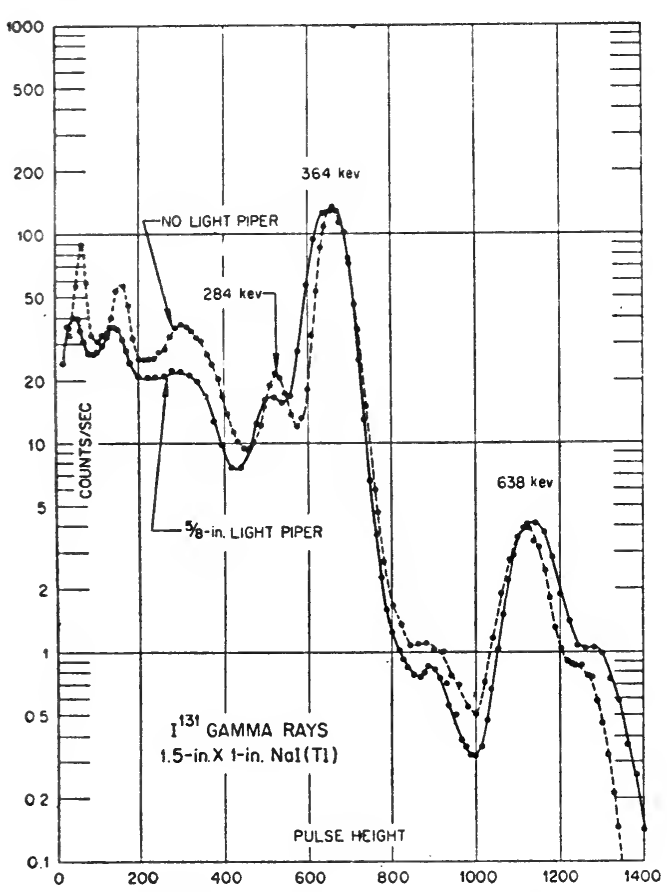

Fig. 13. The response of two $1-1 / 2{ }^{\prime \prime} \times 1^{\prime \prime}$ $\mathrm{NaI}$ crystals to the gamma rays of $I^{131}$ showing the effect of a light piper incident photon must be as large as possible. Surfaces of $\mathrm{Cs}_{3} \mathrm{Sb}$ seem to be the most satisfactory for the blue light of most of the phosphors now used. A direct correlation between photosensitivity to tungsten light and resolution would be expected and is observed.

Photomultipliers generally show considerable variation of photosensitivity across the cathode surface. This variation (often $2: 1$ ) produces a different pulse height for flashes occurring in the phosphor near photocathode regions of high and low sensitivity. The effect of cathode non-uniformity may be alleviated by inserting a short piper between crystal and tube. This permits the light from each flash to fall more nearly upon the whole cathode surface. Fig. 13 shows two spectra of the radiations of 8 day $\mathrm{I}^{131}$. Curve A was taken without a light piper and Curve B was taken with a slightly poorer tube and crystal using a $5 / 8$ in. long lucite light piper. Notice that 
although the resolution of the $284-364 \mathrm{keV}$ lines is better in the arrangement without light piper, the resolution does not improve with increasing energy as expected and the resolution of the $638-720 \mathrm{keV}$ lines is better in the arrangement with light piper despite the poorer inherent resolution of this tube and crystal. The cause of this effect is the rapid absorption of the low energy radiations in the crystal, which was 1 in. thick, causing most. of the flashes from these radiations to occur near the top of the crystal and allowing the light from all the flashes to spread over the whole photocathode. The higher energy radiations penetrate the phosphor more completely so that a considerable number of flashes originate near the bottom. Without a light piper the cathode non-uniformity causes a spread in pulse heights in excess of that expected. The square of the peak width should be proportional to gamma-ray energy and a deviation from this rule generally shows non-uniformity of the phosphor fluorescent efficiency or the effect of cathode non-uniformity. The resolution improvement due to a light piper is also reported by Swank who shows an average improvement from a resolution of $9.5 \%$ to a resolution of $8.5 \%$ for a group of Dumont type K-1186 (6292) tubes by using a $1 \mathrm{~cm}$ light piper.

\section{Photoelectron Collection Efficiency}

The photoelectrons emitted by the photocathode must reach the first dynode to be effective. The field in the photocathode-first dynode space in tubes like RCA 5819 and Dumont 6292 is weak and the electrons must be shielded from magnetic fields to prevent their being deflected away from the dynode. The earth's field is sufficient to cause a large effect. RCA 6342 (C7164A) and Dumont 6292 tubes have an auxiliary focus electrode near the dynode entrance which increases the number of photoelectrons collected and reduces the magnetic field effect somewhat.

\section{E. Multiplication Effect upon Resolution}

The multiplication of the photoelectrons at the dynodes, especially the first, increases the peak width. The analysis of Morton ${ }^{11}$ indicates that the mean square deviation of the pulse height is increased, over what is expected from the number of photoelectrons multiplied, by the factor $R /(R-1)$ where $R$ is the secondary emission coefficient at the dynodes. The secondary emission coefficient increases rapidly with voltage in the range that can be used so that it is well to keep the voltage between cathode and first dynode as high as the tube will permit without increased tube noise. We have found about $300-350$ volts satisfactory for 5819 and 6292 tubes.

11. G. A. Morton, Nucleonics 10, (1952) 3. 
F. Space ('harge Limitation

Photomultipliers deliver comparatively large peak currents but the pulses are quite short so that the largest voltage pulses delivered must be restricted if space-charge limitation and consequent non-linearity is to be avoided. For tubes like 5819,6342 and 6292 operating into a total load capacitance of $25 \mu \mu \mathrm{f}$, a pulse of about 1 volt may be obtained without serious space charge non-linearity using $\mathrm{NaI}(\mathrm{Tl})$. A pulse of 0.2 volt may be used with anthracene and about 0.05 to 0.1 volt with stilbene, the plastic phosphors and the solution phosphors.

\section{G. Amplifier Noise and Pre-Differentiator Non-Linearity}

Photomultiplier pulses as large as given in the previous paragraph do not require great amplification before analysis. It is very convenient, however, to have a considerable amplifier gain control range so that high energy $(\sim 3-4 \mathrm{MeV})$ and low energy radiation $(\sim 20-30 \mathrm{keV})$ may be examined without changing the photomultiplier voltage. Changing the voltage is undesirable since the resolution is changed with voltage and energy recalibration is necessary unless very precise voltage control is used. The noise of the amplifier becomes appreciable when a gain control range of $20: 1$ or $30: 1$ is used even using the regular linear amplifier technique. If an ordinary voltage amplifier is used with a short time constant at the input, the amplifier noise is quite serious. An input time constant of $100-300 \mu$ sec followed by some amplification (4 to 20 times) before reaching the pulse length determining differentiation line gives relatively little noise. Care should be used to insure linearity over a wide signal voltage range in the amplifier before the differentiator since the signals "pile-up" on each other at moderate to high counting rates giving a larger voltage fluctuation than expected from the individual signal sizes. Pre-differentiation nonlinearity results in reduced resolution at high counting rates.

\section{H. Рhоtomultiplier "Noise"}

The principal background of the photomultiplier consists of single photoelectron pulses from thermionic emission. Statistical fluctuations of these pulses results in some pulses as high as 8 average photoelectron pulses. At room temperature these 8 "photoelectron" pulses are fewer than 1 per sec for the 2 in. photocathode tubes and their number falls very rapidly with increasing pulse size. This size corresponds to 5 to $10 \mathrm{keV}$ gamma ray energy on an ordinary $\mathrm{NaI}(\mathrm{Tl})$ crystal or about $10-20 \mathrm{keV}$ for an anthracene crystal.

The photocathode of photomultipliers has very high resistance, some- 
times $>10^{7} \mathrm{ohms} / \mathrm{square}$, and the conduction through the glass envelope is not negligible. The current produced by connecting even as good an insulator as a 1 in. piece of glass rod $1 / 8$ in. in diameter from the center of the tube face to ground will produce very large burst pulses if the photocathode is being operated $\sim 1000$ volts from ground. A solution phosphor bridging from the envelope to ground with the cathode at high voltage produces similar burst pulses and will result in the complete failure of the photomultiplier in about 30 days.

Satellite pulses or delayed after pulses are observed in many photomultipliers. These are small ( $\sim 1-10$ photoelectron) pulses following about $1 / 2$ to $1-1 / 2 \mu$ sec after large pulses. These pulses seem to be due to gas ions in the tube. They are least numerous in tubes like RCA 6342 (C 7164A) and Dumont 6292, probably because these tubes having silvermagnesium dynodes can be outgassed more thoroughly.

\section{Fatigue Effects}

Fatigue effects have sometimes been reported for photomultiplier pulse counting equipment but careful search by the author has not discovered any fatigue effect for pulses above $10 \mathrm{keV}$ equivalent gamma energy in 5819 type tubes operated at voltages from 500 to 1100 volts. No change in pulse height was found with counting rates of $\sim 100$ counts/sec to $\sim 20,000$ counts/sec and periods of several minutes to several days*. Photomultipliers exposed to room light with voltage applied do show, however, considerable damage. The pulse height is much reduced and resolution is impaired. Some tubes so exposed recovered almost completely after some weeks of operation.

\section{§ 4. Crystal Preparation and Mounting}

\section{A. Organic Solids}

\section{Anthracene}

Anthracene may be shaped by cutting with very sharp tools. It cannot be drilled or sawed since it splits easily in one plane like mica. Fairly good results can be obtained by encasing the crystal in plaster of paris and cutting under water with a diamond cutoff wheel such as is used for glass. A thread saw using toluene or xylene as solvent may also be used.

* Although NO 5819 (RCA) has been found with a change of pulse height with counting rate, all of the tubes we have tested with silver magnesium dynode do show such a shift. The RCA 6342 and Dumont $6292\left(2^{\prime \prime}\right), 6293\left(3^{\prime \prime}\right)$ and $6364\left(5^{\prime \prime}\right)$ all show shifts of pulse height of 2 percent to 10 percent for counting rates from 100 to 10,000 counts/sec. The pulse height generally increases with increasing counting rate. The cause and cure of this sericus defect in these tubes is not known at the time of writing. 
The hole required for a hollow crystal spectrometer is usually excavated with a very sharp knife. After shaping, the surface should be polished by immersing the crystal for $1-3$ minutes in toluene. The excess toluene should be removed with absorbent paper. Some hours after solvent polishing a film of powder appears on the crystal surface and should be wiped off. Several days later any further surface film should be wiped off and no more will form.

The crystal may be attached to a lucite light piper with Canada balsam dissolved in xylene. Canada balsam may also be used for the optical joint between the light piper and the photomultiplier. No advantage has been found in roughening the crystal surface as is necessary for $\mathrm{NaI}(\mathrm{Tl})$.

The crystal and light piper should be surrounded with bright aluminum foil. The foil should fit closely but great care should be used to prevent the C'anada balsam or other material used for the optical joint from flowing up between the foil and crystal or light piper. Such an event replaces the good reflection of the crystal surface outside the critical angle with the much poorer reflection of aluminum. The resolution is much reduced. For the same reason, reflectors evaporated on the crystal surface (or on the surface of plastic phosphors) seriously reduce the resolution obtainable.

The foil over the section of the crystal into which the beta rays are to go should be removed and a very much thinner reflector attached. "Dutch leaf" aluminum sign painters foil $\left(\sim 0.2 \mathrm{mg} / \mathrm{cm}^{2}\right)$ may be used or aluminum evaporated on polystyrene-formvar film as thin as $\sim 25 \mu \mathrm{g} / \mathrm{cm}^{2}$.

For the split crystal spectrometer a closely fitting plastic cap into which silver has been evaporated gives very good results.

\section{Stilbene}

Stilbene is much more brittle than anthracene and is very sensitive to thermal shock. Surfaces may be ground on carborundum paper with water. (Anthracene may be ground similarly but must be finished by removing a thin surface layer with a sharp knife.) Solvent polishing must be done carefully and the excess removed quickly before the solvent evaporates and cools the surface enough to cause cracking.

Canada balsam is less satisfactory for attaching stilbene since the fluorescent light is shorter in wave length. Very thin layers should be used.

\section{Plastic Phosphors}

Polystyrene containing $\sim 3 \%$ p-terphenyl is sometimes used for electron counting. A somewhat better phosphor consists of polyvinyl toluene containing $4 \%$ p-terphenyl and $0.025 \%$ tetraphenyl butadiene. This phosphor gives a pulse height approximately $50 \%$ of that given by 
anthracene. These phosphors may be machined like ordinary polystyrene and polished with $\alpha$-alumina. The vapour pressure of these phosphors is negligible and they may be used in vacuum systems while anthracene or stilbene must be covered with very thin foils to aroid degrading the vacuum.

\section{B. Solution Phosphors}

Solutions of $p$-terphenyl in various solvents gives pulses of very short mean duration, $\sim 2 \times 10^{-9} \mathrm{sec}$, but of small pulse height. The best mixture to date gives a pulse height approximately $50 \%$ of that of a good anthracene crystal. The mixtures that give the best pulse height are:

1) $5 \mathrm{~g} /$ liter $\mathrm{p}$-terphenyl $+0.02 \mathrm{~g} /$ liter $1,1,4,4$ tetraphenyl-1, 3 butadiene in phenyl cyclohexane

2) $3 \mathrm{~g} /$ liter 2, 5 diphenyloxazole in toluene.

The performance of these liquids is quite sensitive to the presence of impurities in the solvent, some samples of phenyl cyclohexane giving twice as large pulses as others. In particular dissolved oxygen has a large effect on the pulse height. An increase of a factor of two is sometimes found by bubbling oxygen-free nitrogen through the phosphor ${ }^{12}$. The containers used for these liquids should be optically coupled to the photomultiplier and surrounded by diffuse reflectors not in optical contact, as would be done for other organic scintillators. The solution phosphors find their greatest use for high energy work where their lower pulse height is less of a disadvantage but they are also used for low energy work where internal sources are necessary ${ }^{13}$.

\section{Inorganic Crystals}

Sodium iodide activated with thallium is the most important inorganic phosphor. The treatment used for it may be used for some other inorganic phosphors ( $\mathrm{KBr}, \mathrm{KI}, \mathrm{CsI}, \mathrm{CsBr}$ ) except that some of them do not need to be sealed against moisture.

The crystal shape is generally cut from the ingot with a string saw. This leaves a rough surface containing water and often free iodine to a depth of $1 / 32$ to $1 / 16$ inch. This outer surface is removed by grinding the crystal dry on coarse grinding paper (No. 80 to No. 120) in a dry atmosphere. A dry box, through which dry air is blown, fitted with rubber gloves is generally used. The crystal surface can be assumed to be free of combined

12 R. W. Pringle, L. D. Black, B. L. Funt and S. Sobering, Phys. Rev. 92, (1953) 1582.

13 James R. Arnold, 4th Annual Scintillation Counter Symposium (1954) Nucleonics (to be published 1954). 
water when it does not turn chalk white when allowed to stand for $10-20$ minutes in the dry atmosphere. The crystal surface should be left rough ground on all sides. The finest grade of abrasive paper should be no finer than from No. 120 to No. 320. Grinding finer or polishing seriously reduces the resolution for moderate-sized to large crystals. C'leaved surfaces similarly give comparatively poor resolution. The face to be jointed to the light piper or photo tube is polished by some workers although we could not find any improvement by polishing this face.

Large crystals should next be attached to the light piper or to the photomultiplier by wetting the surface of the crystal with $\mathrm{DC}-200\left(10^{6}\right.$ centistokes) and pressing together strongly the surfaces to be joined for several minutes. Any excess of liquid should be removed completely from the exposed surfaces of the crystal by using grinding paper.

Alpha-alumina or $\mathrm{MgO}$ should be dusted onto the crystal surfaces. A coating of about 6 to $8 \mathrm{mg} / \mathrm{cm}^{2}$ is all that is required for crystals that are going to have a reflector of bright aluminum foil. This gives a reflectivity very near that of an indefinitely thick layer of reflector. For surfaces that are not going to be surrounded by other good reflecting material, three or four times this amount should be applied. In some cases difficulty in getting this amount to adhere can be corrected by spreading a very small amount of mineral oil on the surface and then remoring as much as

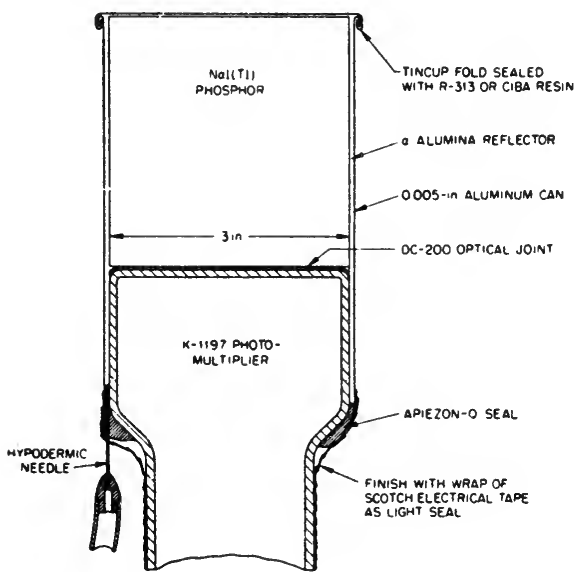

Fig. 14. Reduced pressure crystal can used for scintillation phosphors having the same diameter as the photomultiplier possible of the oil with dried absorbent tissues. The crystal should not be surrounded by thick layers of reflecting material nor by a thick container. The Compton electrons and degraded gamma rays produced in material surrounding the crystal reduces the good performance of the crystal. A can like that shown in Fig. 14 is then carefully lowered over the crystal and the open end is sealed with a ring of Apiezon $\mathrm{Q}$ or other modeling-clay-like vacuum wax. A hypodermic needle or other very small tube is inserted through the seal and some of the air is exhausted (say to $3 / 4$ or $1 / 2$ atmosphere) and the needle with-drawn while pressing down the seal. The air pressure forces the thin can down onto the crystal and holds it firmly to the phototube. Such an assembly for a 3 in. $\times 3$ in. crystal using a 0.005 in. 
thick aluminum foil can is quite rugged and may be supported by the tube in any position indefinitely with no danger.

\section{§ 5. Crystal Efficiency and Linearity}

Quantitative measurement with the scintillation spectrometer requires a knowledged of the efficiency of the system. One of the important advantages of the scintillation spectrometer is that once the efficiency of a crystal has been obtained another crystal of the same size will have exactly the same efficiency for a source at the same distance (and direction). The pulse heights may differ between the two crystals and another photomultiplier may give different resolution but the counts under the peak of a gamma spectrum or the counts in a beta distribution do not depend upon pulse height or resolution.

Beta ray efficiency of the organic phosphors is essentially unity. Very few electrons which enter the crystal will leave without producing a countable pulse. The pulse spectrum can be counted with anthracene from high energies down to $10-20 \mathrm{keV}$ without appreciable background correction and the probability of losing a count because no photoelectron was produced is negligible down to $2-4 \mathrm{keV}$. Absolute beta counting efficiency with a split-crystal spectrometer is probably as good as that obtained with $4 \pi$ proportional counters.

Corrections must be made however for the detection of gamma rays in the phosphor. An exception to this rule is found for the split-crystal spectrometer if the gamma rays are all in coincidence with the beta rays. No correction to the counting rate is required but the spectrum shape will be distorted.

The gamma-ray part of the counter response is generally obtained by inserting an absorber of low atomic number between source and detector. Polystyrene or beryllium sheets are generally used. The absorber should be no thicker than is necessary as Compton electrons are generated in the absorber and add to the gamma-ray response. The error produced is generally small but in severe cases may be reduced by an anti-coincidence absorber. Fig. 15 shows the arrangement of an anti-coincidence absorber. An absorber made from a plastic phosphor (or from sodium iodide for reduction of $\mathrm{X}$-rays or Bremsstrahlung) is interposed between source and detector. A photo tube attached to the absorber will give a pulse if a gamma ray interacts with it. The pulses from the absorber phototube are put in anti-coincidence with the spectrometer output so that any pulse in the spectrometer in coincidence with a pulse in the absorber will not be counted.

If the sample contains coincident radiation, some of the counts lost 
will be due, not to Compton scattering in the absorber, but to coincidence of separate radiations. The anti-coincidence absorber may be rotated around the source so that it is not between source and spectrometer for evaluation of the number of these coincidences.

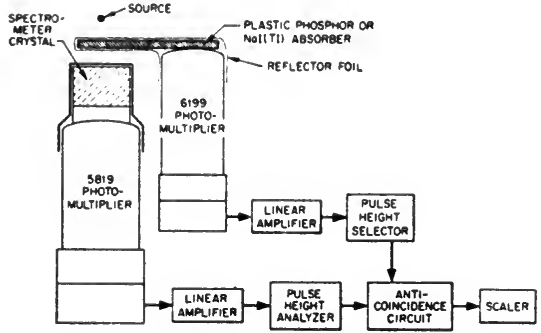

Fig. 15. General arrangement of the anti-coincidence absorber

Fig. 16. Gamma-ray absorption coefficients for anthracene

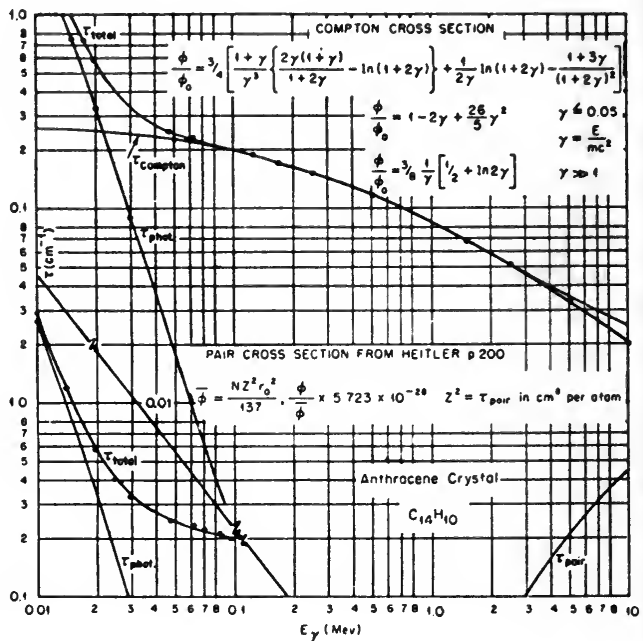

The total gamma ray efficiency for anthracene is given in Fig. 16. The counting efficiency of a crystal may be obtained by computation using this cross section curve. No correction to the counting efficiency need be made for multiple processes since the absorption of a scattered photon is essentially simultaneous with the scattering event that produced it and only one pulse is produced. The pulse will of course have a different height and the pulse spectrum will not resemble the Compton distribution except in' small crystals.

A similar cross section curve for sodium iodide is shown in Fig. 17. This curve is based upon the National Bureau of Standards Report No. 1003 with the coherent scattering cross section subtracted.

Using the values of Fig. 17 the counting efficiency of a $1 \frac{1}{2}$ in. diameter, 1 in. long vylinder of $\mathrm{Nal}$ was calculated for small sources placed at various distances from the crystal surface along the axis of the cylinder. The counting efficiencies were normalized to 1.0 at low energy (high cross section) where any photon striking the crystal surface is detected. Fig. 18 shows the relative efficiency for source distances from 0 to $1 / 2 \mathrm{~cm}$ and Fig. 19 the values from $2 \mathrm{~cm}$ to infinity. The odd behavior of the efficiency with source distance is caused by penetration of the edge of the cylinder as discussed in $\S 2$.

A gamma-ray intensity in a spectrum is much easier to obtain if it is 
only necessary to count the number of pulses in the peak rather than in the complete spectrum produced by each gamma ray. The peaks have a shape indistinguishable from a Gaussian curve and the number of counts represented may be found by subtracting a peak of the required width, position and intensity so that the remainder curve has the shape expected from the remaining components of the spectrum. To obtain the number

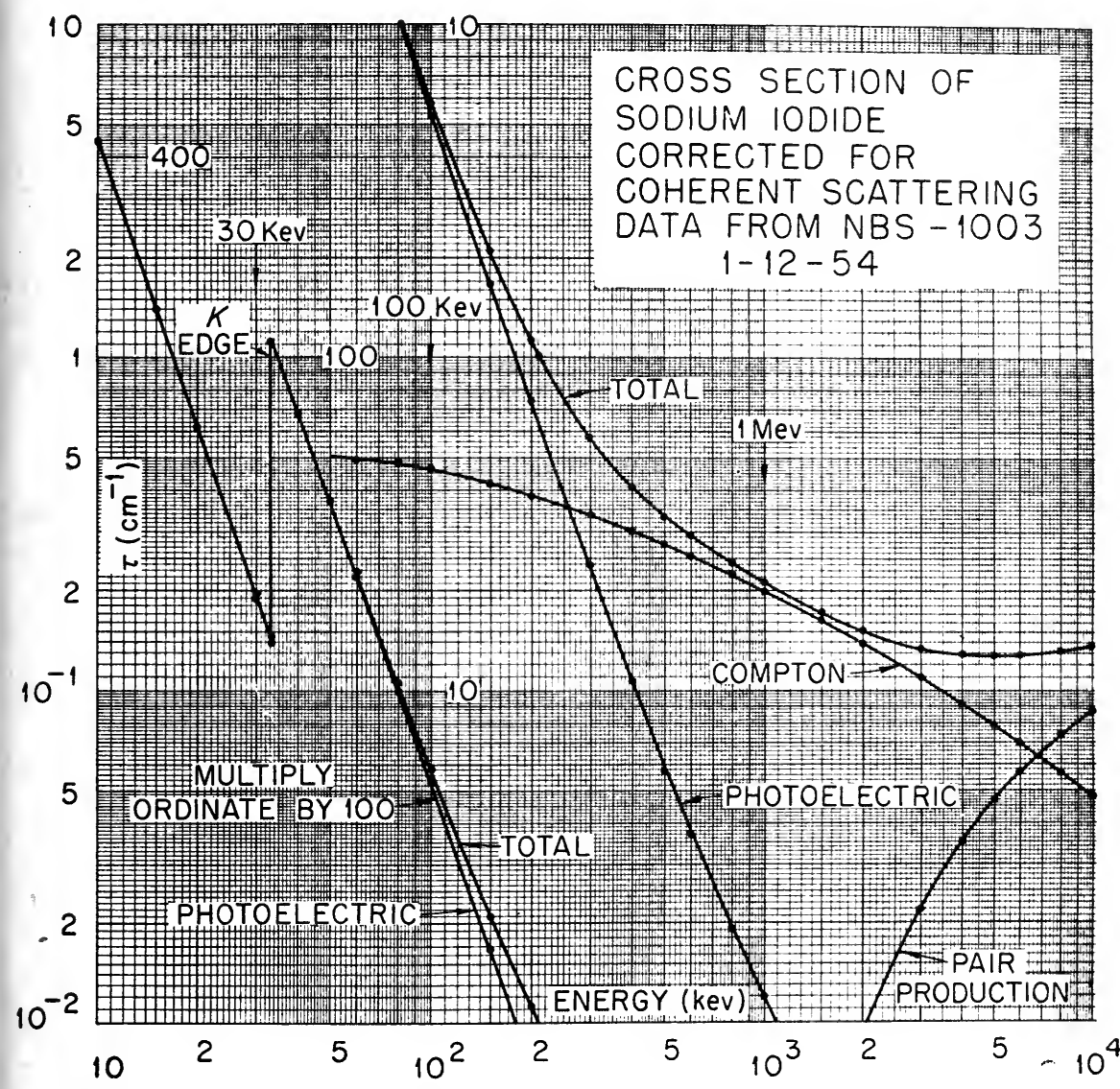

Fig. 17. Absorption coefficient of NaI for various gamma-ray energies

of photons represented by the number of pulses in the peak it is necessary to know the ratio of the number of pulses in the peak to the number of pulses in the total response (peak to total) for a single gamma ray of the same energy. For this purpose the curves of Fig. $6, \S 2$ were obtained. It should be noted that the curves shown include in the "peak" counts the $\mathrm{X}$-ray escape peak. This X-ray escape peak is $28 \mathrm{keV}$ lower in energy. than the main peak and is prominent only from $32 \mathrm{keV}$ to $\sim 100 \mathrm{kel}$. 


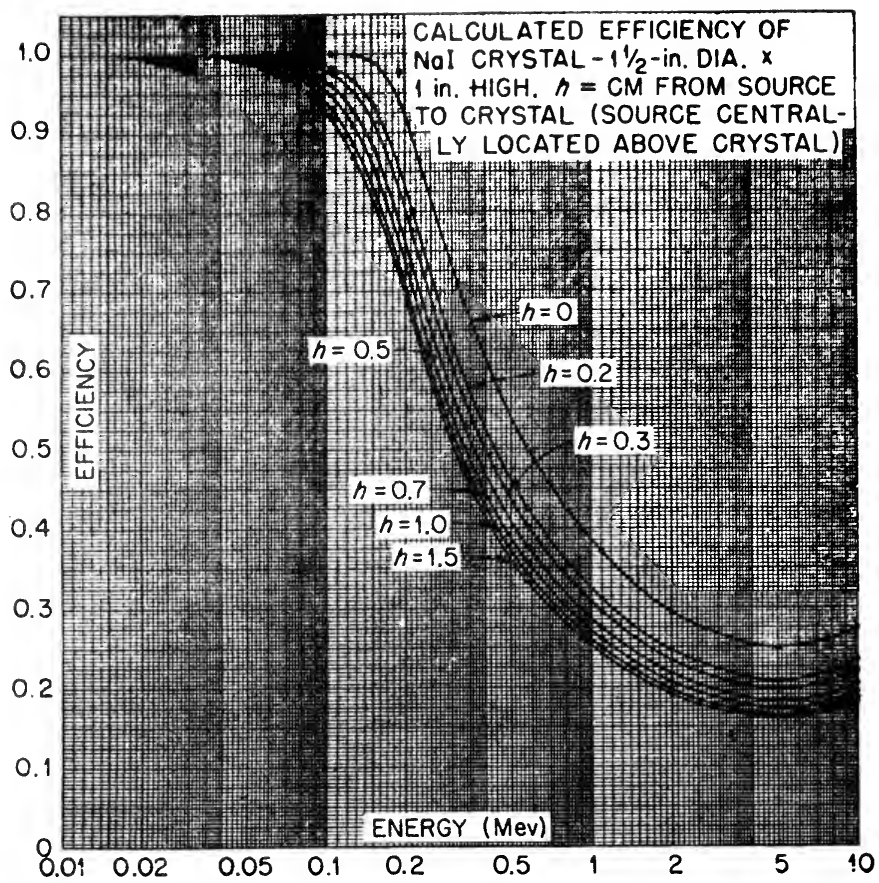

Fig. 18. Efficiency of $\mathrm{NaI}$ crystal for various gamma ray energies and source distance of 0 to $1 \frac{1}{2} \mathrm{~cm}$

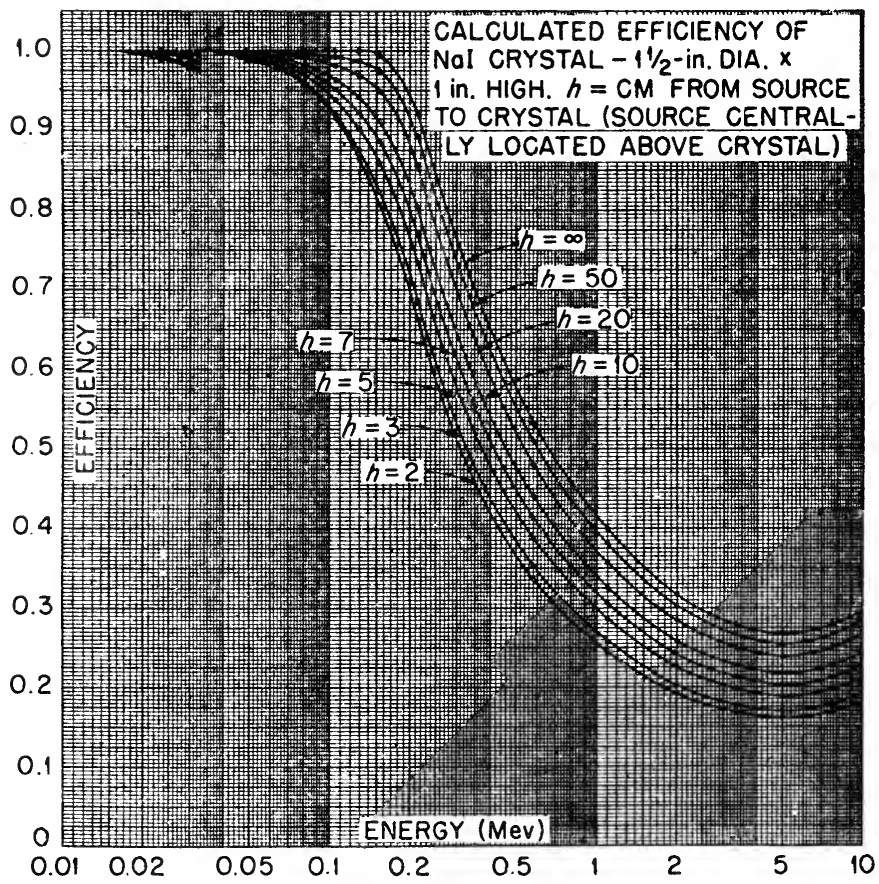

Fig. 19. Efficiency of NaI crystal for various gamma ray energies and source distance of $2 \mathrm{~cm}$ to infinity 
A curve of the calculated relative intensity of this secondary peak to the total peak for gamma rays entering perpendicular to a sodium iodide surface of infinite extent is shown in Fig. 20. Some experimental values obtained by F. K. Mckiowan for a source distance of $0.4 \mathrm{~cm}$ are also shown.

Fig. 20. Iodine X-ray escape from $\mathrm{NaI}$ with incoming radiation normal to the surface and some experimental values

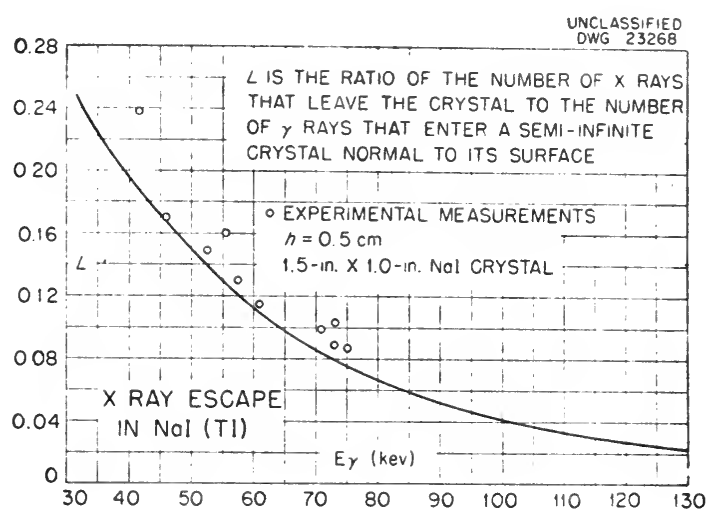

Pulse height against energy plots for scintillation counters are more or less linear. Anthracene crystals show some non-linearity for electrons below $100 \mathrm{keV}^{8}$ but above this energy give essentially linear response to at least $12 \mathrm{MeV}$. Other organic phosphors behave similarly and nonlinearity for electrons sets in at about $100 \mathrm{keV}$ for stilbene and p-terphenyl in $\mathrm{m}$-xylene.

Sodium iodide and many of the inorganic phosphors do not show such non-linearity at least at energies above $10-15 \mathrm{keV}$. It is possible however to' get non-linear energy-pulse height responses in several ways. The most common cause of non-linearity is unsatisfactory linear amplifiers or pulse height analyzers and the cure is obvious. A more subtle effect is produced by an inefficient optical reflector around the crystal. Low energy gamma rays are absorbed chiefly in the upper part of the crystal while higher energy gamma rays are more uniformly absorbed. On the average a poor reflector will get more opportunity to remove light from a flash of a low energy gamma ray than from the flash of a high energy gamma ray causing the pulse height to be disproportionally larger for higher energy. Under good conditions substantial linearity to about $20 \mathrm{MeV}$ has been observed.

\section{§ 6. Total Absorption Gamma Spectrometers}

Large sodium iodide crystals have such a high gamma-ray efficiency that gamma rays from a source inside the crystal may be almost completely absorbed. This arrangement has many obvious advantages; the 
Compton distribution accompanying the peak is greatly reduced, the efficiency can be more accurately determined since it is less dependent upon the accuracy with which the gamma-ray absorption coefficients are known, self-coincidence or sum lines are strong and permit easy determination of cascade relationships.

A large phosphor may be obtained as one single mass or by arranging several smaller phosphors together. It has not proved satisfactory to join different sodium iodide crystals and view them with one photomultiplier so that if several phosphors are used careful adjustment of the response of the different units is necessary if a good resolution is to be obtained when their outputs are summed.

A single Nal crystal equivalent to a $5^{1 / 4}$ in. diameter sphere is shown in Fig. 21. A hole $1 / 4$ in. diameter is drilled to the center and sources with or without shields may be inserted. Fig. 22 compares the response of

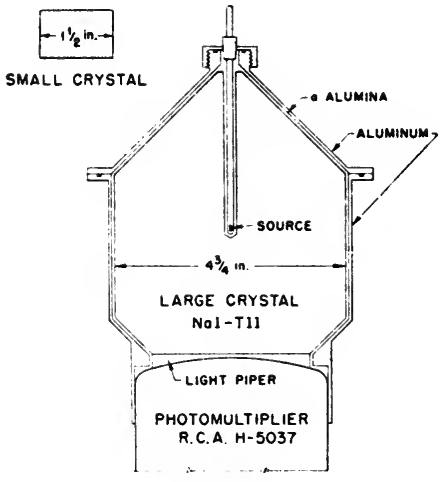

Fig. 21. Source and crystal arrangement for total absorption spectrometer

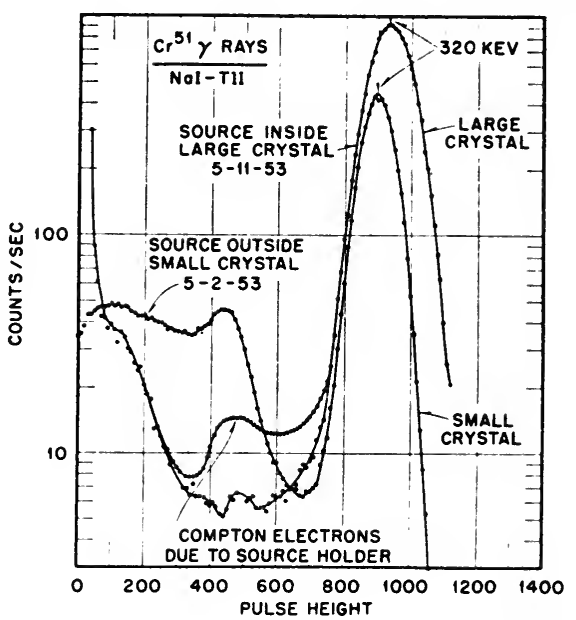

Fig. 22. Response of total absorption spectrometer and the small crystal to $\mathrm{Cr}^{51}$ gamma rays

the standard 1 in. by $1^{1 / 2}$ in. diameter crystal and the large crystal to the $320 \mathrm{keV}$ gamma ray of $\mathrm{Cr}^{51}$. When the $\mathrm{Cr}^{51}$ source was enclosed in an aluminum source holder with 0.020 in. walls a broad peak of Compton electrons was ejected from the aluminum. With a thin source these were largely removed. A considerable part of the pulse distribution below 200 pulse height divisions must be due to the inner Bremsstrahlung from the $90 \% K$-capture transition to the ground state of $V^{51}$ since only $3 \%$ of the $320 \mathrm{keV}$ gamma rays can escape.

The usual comparison source, ('s ${ }^{137}$, gives the curves of Fig. 23. The 
large reduction of the Compton distribution and the greater absorption of the $661 \mathrm{keV}$ gamma ray makes the $\mathrm{Ba} \mathrm{X}$-ray seem less intense.

A source having coincident gamma rays produces intense sum lines and the peaks representing the individual gamma rays are reduced. Fig. 24 shows the response of the large and small phosphors to the gamma rays of $\mathrm{Co}^{60}$ and it can be seen that there is almost equal probability for (1) detection of either gamma ray by complete absorption with complete escape of the other or (2) for detection of both gamma rays by complete absorption.

A more difficult case is presented by the radiations of $\mathrm{Na}^{24}$ (Fig. 25). The hard gamma ray $(2.76 \mathrm{MeV})$ produces intense pair production. The standard small crystal (see Fig. 12) gives a strong lower pair peak (complete escape of annihilation gammas) but the large phosphor with the source

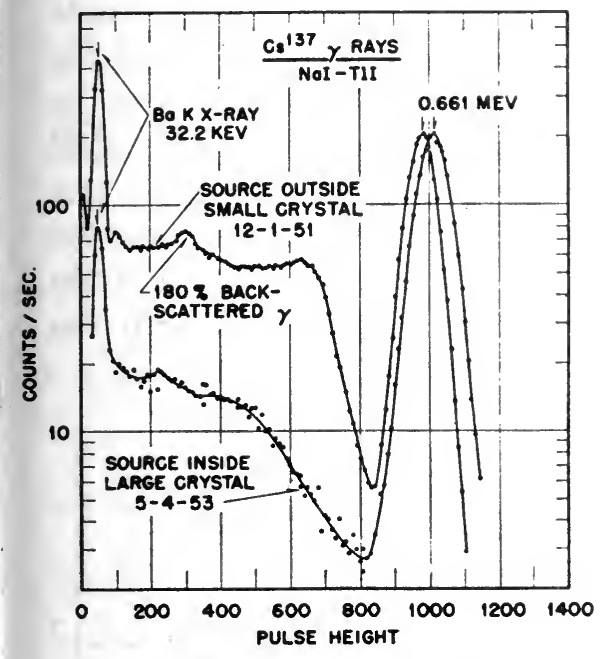

Fig. 23. Response of total absorption spectrometer and the small crystal to $\mathrm{Cs}^{137}$ gamma rays

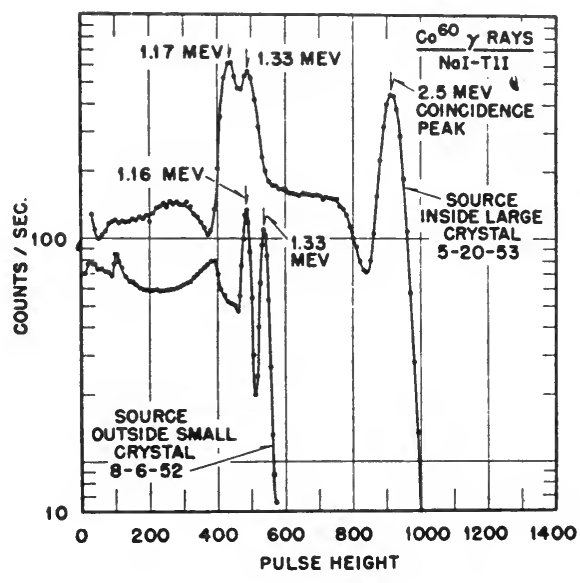

Fig. 24. Response of small crystal and total absorption spectrometer to the gamma rays of $\mathrm{Co}^{65}$ showing intense sum line

outside gives only a small peak at 200 pulse height divisions and no lower pair peak at all with the source inside. The probability that both $.51 \mathrm{MeV}$ gamma rays will escape the phosphor is about $0.8 \%$. The middle pair peak is moderately prominent with the source outside the phosphor since pair production near the entering surface is likely to send one photon out.

The sum line at $4.14 \mathrm{MeV}$ gives the total energy of the gamma-ray cascade. It would be desirable to have a still larger phosphor than the $5^{1} / 4$ in. effective diameter since the gamma-ray absorption is low enough 


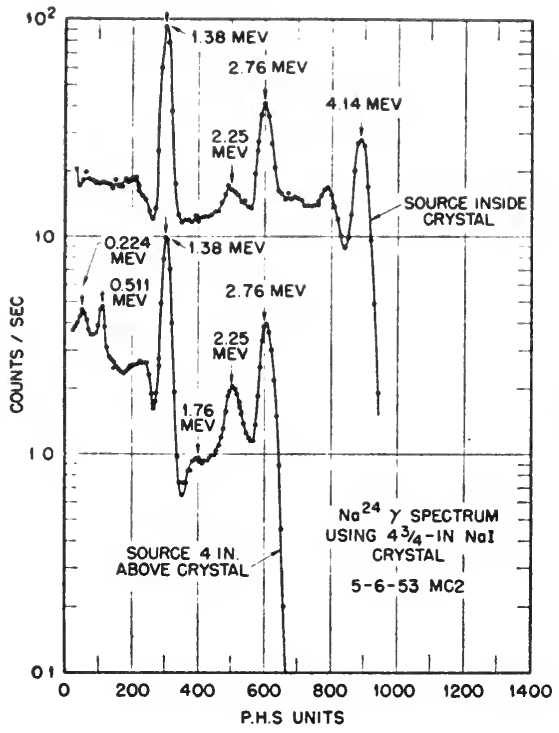

Fig. 25. Response of total absorption spectrorneter to a source of $\mathrm{Na}^{24}$ inside and outside the spectrometer

that a decay scheme containing many. gamma rays produces an inconveniently large number of peaks instead of the few expected from cascade sums. An example of this effect can be seen from Fig. 26 and Fig. 27. Figure 26 is the response of a $3 \mathrm{in}$. by 3 in. crystal to the radiations of a mixture of $\mathrm{Mn}^{54}(0.824 \mathrm{MeV})$ and $\mathrm{Mn}^{52}$. $\mathrm{Mn}^{52}$ decays by position emission and $K$-capture to a state giving a cascade of three gamma rays, $0.75 \mathrm{MeV}, 0.93$ $\mathrm{MeV}$ and $1.45 \mathrm{MeV}$. The peak at 202 $\mathrm{keV}$ is the backscatter peak. Inside the large crystal (Fig. 27) ten of the eighteen possible sum lines can be seen as well as the independent $\mathbf{M n}^{\mathbf{5 4}}$ line. With a very large phosphor this array would be reduced to three; the $.824 \mathrm{MeV}$ line, the $3.14 \mathrm{MeV}$ and the 4.15 MeV line. The smaller crystal however serves well even with the complicated pulse height distribution if care is used.

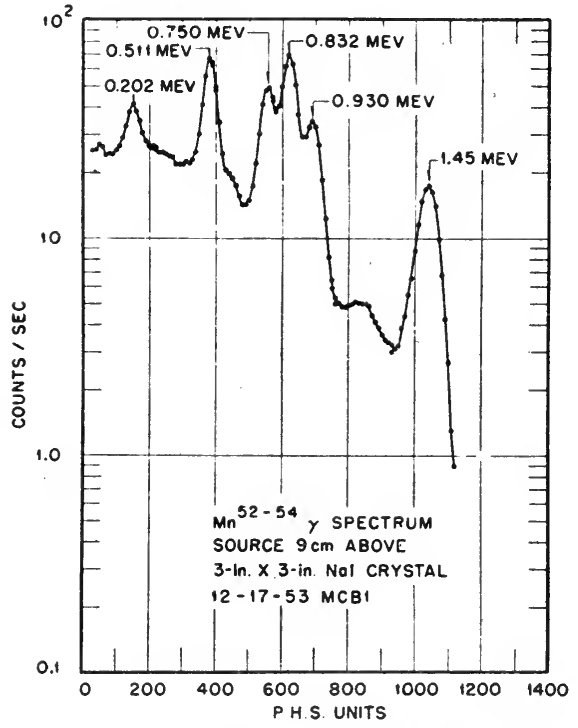

Fig. 26. Response of a $3^{\prime \prime} \times 3^{\prime \prime} \mathrm{NaI}$ crystal to a mixed source of $\mathrm{Mn}^{52}$ and $\mathrm{Mn}^{54}$

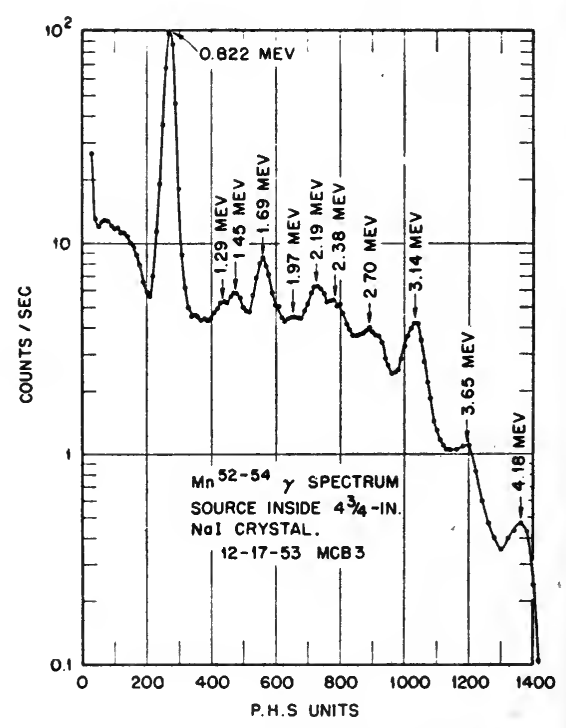

Fig. 27. Response of the total absorp. tion spectrometer to the same source as used in Fig. 26 


\section{$\S 7$. Coincidence Spectrometry}

The combination of energy proportionality, high efficiency and short pulse duration makes the scintillation detector ideally suited to coincidence spectrometry. In this section the coincidence method will be applied to spectrometry. For a general treatment of the coincidence method see Ch. VII. Coincidence methods with special emphasis upon extremely short resolving times are given in Ch. XVIII.

A block diagram of the coincidence system used at Oak Ridge is shown in Fig. 28. For use with two single channel analyzers instead of one single

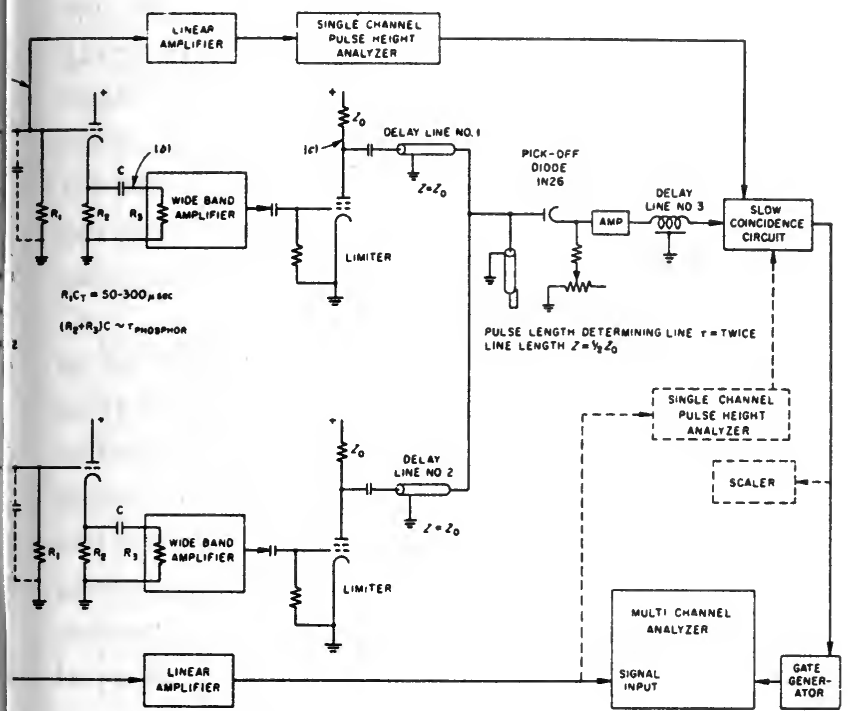

Fig. 28. Block diagram of the fast-slow coincidence system

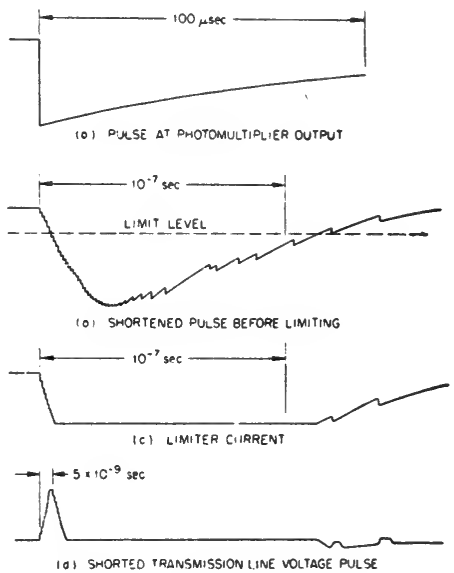

Fig. 29. Wave forms in the fast-slow coin. cidence system

channel and one multi-channel analyzer, the analyzer shown dotted is added, with its output going to the slow coincidence unit and a scaler is used to record the slow coincidence output. The multi-channel unit and its gate generator is not present. The anode of each photomultiplier is connected to an integrating circuit using the stray capacitances and a comparatively high resistor so that a time constant of 50 to $300 \mu \mathrm{sec}$ is obtained (wave form (a), Fig. 29). This pulse is fed to the linear amplifier where differentiating time (clipping time) is chosen suitable to the phosphor and the analyzer (in our case $\sim 1 \mu$ sec for $\mathrm{NaI}(\mathrm{Tl})$ or anthracene). The integrated pulse, wave form (a), is coupled by a cathode follower to a differentiating $R C$ circuit whose time constant is chosen to suit the phosphor, about $0.3 \mu$ sec for $\mathrm{NaI}(\mathrm{Tl})$, about $3 \times 10^{-8}$ sec for anthracene and about $10^{-8}$ sec for stilbene or the plastic phosphors. The shortened 
pulse obtained (wave form (b) Fig. 29) is amplified in the wide band amplifier and strongly limited in the limiter so that a current pulse with a very quick rise, a flat top and a slow decay is formed (wave form (c), Fig. 29). This current pulse is fed into a section of shorted transmission line giving a voltage pulse like wave form (d). Since this pulse is formed by reflection in the line, its rising side has the shape of the rise of the current pulse and its fall is the inverted image of this same shape. The slow fall of the current pulse gives a long low pulse of opposite sign, too small to be important. The current pulse from the other channel is fed into the same shorted line. A biased diode at the open end of the line delivers a very small lengthened pulse to the output circuit when only one pulse is present at a given time. When the two pulses overlap, in time the diode passes a comparatively large signal to the output circuit since the sum of the voltages exceeds the diode bias. This circuit is used for resolving times from $1-2 \mu \mathrm{sec}$ to as short as $10^{-9}$ sec. When resolving times longer than $2 \mu$ sec are required, a more conventional unit using univibrator pulse shapers is used.

The lengthened $(1 / 2$ to $1 \mu \mathrm{sec})$ fast coincidence pulses are delayed in delay line 3 until the slower pulse height analyzer circuits have had time to decide upon the pulse height. These delayed pulses are then placed in slow coincidence with the analyzer output. This slow coincidence selects from all the fast coincidences those that satisfy the additional requirement that one pulse (or both, in the case of the two single channel analyzers) has the desired size. The two delay lines, 1 and 2, are used to get correction for the circuit and photomultiplier delays and for obtaining delayed coincidences for short lived metastable states (see Ch. XVIII).

The resolving time usable with this arrangement depends upon the kind of phosphor and the energy of the radiation. For stilbene $\tau=10^{-9} \mathrm{sec}$ is usable down to $25 \mathrm{keV}$, for $\mathrm{NaI}(\mathrm{Tl}) \tau=3 \times 10^{-9}$ sec down to an energy of $50-100 \mathrm{keV}$ and $\tau=10^{-8} \mathrm{sec}$ down to $10 \mathrm{keV}$. These times are considerably shorter thän is usually considered usable with $\mathrm{NaI}(\mathrm{Tl})$ and are made possible by this circuit arrangement which depends for its timing upon the arrival of the first few photoelectrons after amplification in the photomultiplier. It has been shown by Morton ${ }^{14}$ that much more precise timing is possible using the first few electrons than using the mean time of the photomultiplier pulse as do the coincidence arrangements using no integration followed by limiting.

Coincidence spectrometry with high efficiency detectors brings to light many interfering effects not important with low efficiency detectors.

The spectra have the usual single and multiple Compton back scattering

14. G. A. Morton, Nucleonics 10, No. 3, (1952) p. 39. 
components found with single crystal spectrometers but in addition the Compton scattering from detector to detector produces false coincidences. Fig. 30 shows the spectrum in one NaI crystal (erystal 1) coincident with

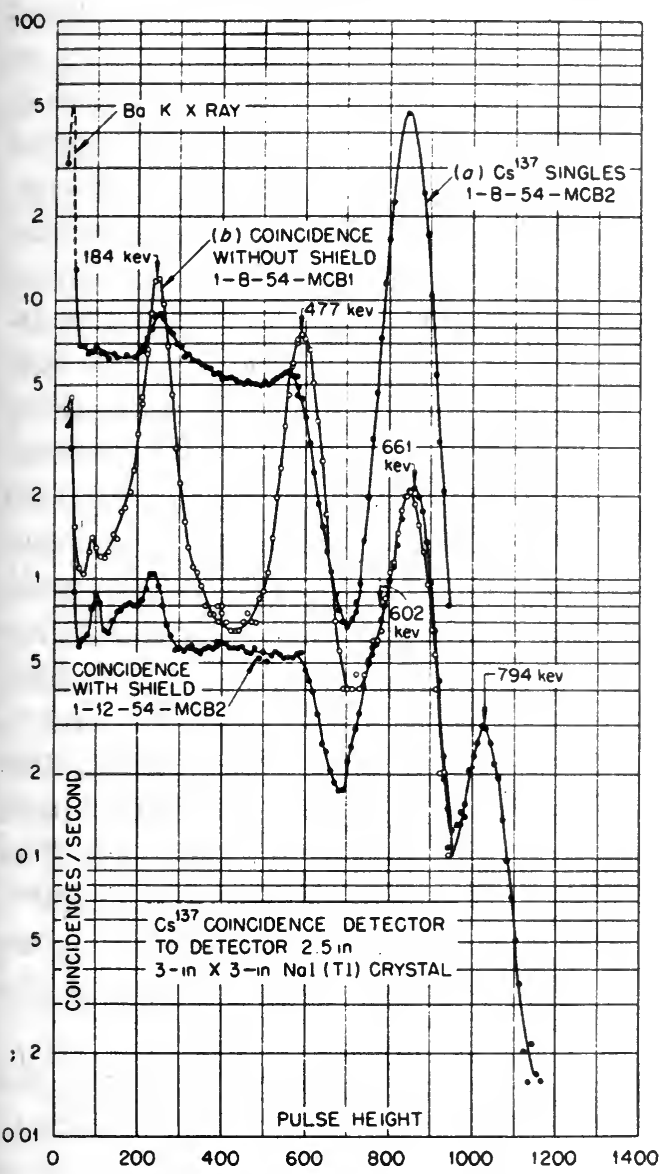

Fig. 30. The response of a coincidence spectrometer to the gamma ray's from a $\mathrm{Cs}^{137}$ source with and without an anti-Compton shield
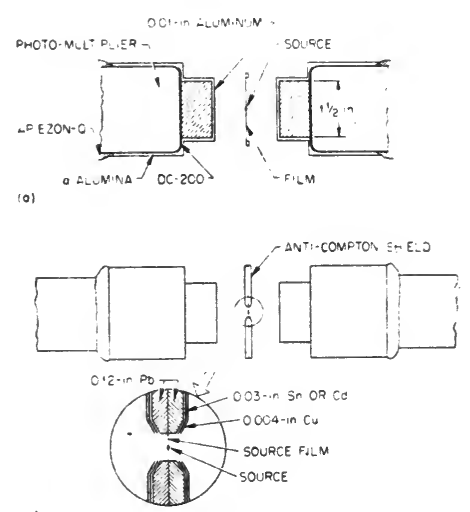

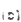

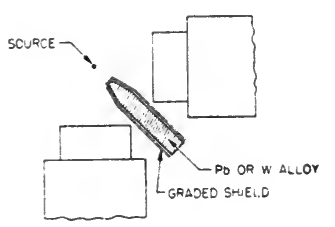

Fig. 31. Coincidence spectrometer arrangements showing the use of anti-Compton shields

any pulse in the other crystal (crystal 2). The crystal arrangement is shown in Fig. 31(a). C'urve $A$ is the single response in the same crystal. The prominent peak near $184 \mathrm{keV}$ represents the detection in crystal 1 of the back scattered photon from a Compton process in crystal 2. The peak near $477 \mathrm{keV}$ represents a Compton scattering in crystal 1 with the scattered photon being detected in crystal 2. With the crystals close together these peaks are broad and intense, as the crystals are moved further apart the peaks become weaker and much sharper due to the smaller angle 
through which a gamma ray may be scattered in one detector and still reach the other detector. The scattering situation is not improved by increasing the source to detector distance since the coincidence rate decreases as fast as the scattering. Anti-scattering shields may be placed at various locations and considerably reduce the effect. Curve C, Fig. 30 shows the improvement obtained with a lead plate with a central hole in which the source is supported as shown in Fig. 31(b). The scattered photon must strike the hole in the shield to reach the other detector. The plate may be made tapered so that a greater thickness is presented to the back-scattered photons of higher energy found at larger scattering angles near the crystal edge. The shield shown in Fig. 31(b) has a penetration transmission slightly less than the transmission through the hole for the thickness and spacings shown with an overall transmission of $\sim 4 \%$ of the scattered photons from a $1 \mathrm{MeV}$ gamma ray. Any such antiscattering shield should be faced with a graded shield to prevent lead $\mathrm{X}$-rays generated in the plate from reaching the crystal. The $\mathrm{Cs}^{137}$ source used for Fig. 30 should show no real coincidences. Although it has strong internal conversion and the beta ray will produce some Bremsstrahlung the $\mathrm{Ba}^{137}$ level from which the gamma-ray transition comes has a long metastable lifetime (2.6 min.). This isotope is a fission product and is always accompanied by a small amount of $\mathrm{Cs}^{134}$ as a contaminent. The peak at $794 \mathrm{keV}$ and the bulge on the low energy side of the $661 \mathrm{keV}$ peak are produced by the real coincidences between the $\mathrm{Cs}^{134}$ gamma rays. The $\mathrm{X}$-ray line at low energy comes from the internal conversion in $\mathrm{Cs}^{134}$. The peak at $661 \mathrm{keV}$ and the majority of the Compton distribution are random coincidences which have not been subtracted from any of the curves.

The escape of $\mathrm{X}$-ray following a photoelectric process produces the escape peak in ordinary spectrometry, but in coincidence work the escaping $\mathrm{X}$-ray falling on the other detector produces a coincidence count, as a result, coincidence spectra (especially with low energy gamma rays) show a strong $28 \mathrm{keV}$ line in coincidence with each low energy gamma ray. These false lines may be reduced by the same type of shield used for Compton scattered photons. Another method sometimes used is to surround the detectors with a graded shield designed for iodine $K \mathrm{X}$-rays. A suitable arrangement is $0.006 \mathrm{in}$. cadmium followed by 0.004 in. copper. The copper should be on the crystal side of the cadmium. The attenuation for iodine $\mathrm{X}$-rays is about 100 times.

Pair production produces a similar false coincidence line due to the production of annihilation radiation. This effect is more serious than that due to the Compton process since the harder $0.511 \mathrm{MeV}$ photons more 
easily escape the crystal and are harder to absorb in shields. The most convenient arrangement to use when gamma rays hard enough (above 1.5 $\mathrm{MeV}$ ) to cause important pair production are present is that of Fig. 31 (c). Here the detectors are placed at $90^{\circ}$ instead of the usual $180^{\circ}$. This arrangement is also necessary when positrons are present in the source. The advantage of this sristem is that a thicker detector-to-detector shield is possible.

The maximum usable source disintegration rate $(V)$ is given by the relation

$$
\frac{N_{C}}{N_{R}}=\frac{f\left(E_{1}, E_{2}\right)}{2 \tau N}
$$

where $N_{C} / N_{R}$ is the ratio of the true coincidence rate to the random coincidence rate, $\tau$ is the resolving time (duration of one of the coincidence pulses when they are equal), and $f\left(E_{1} . E_{2}\right)$ is the fraction of the pulses in the portion of the spectrum accepted by one analyzer window that is related by coincidence to the radiation being accepted by the other analyzer. This relation differs from the usual one, given in Ch. VII, because the coincidence rate varies rapidly orer the spectrum. At the peak of a coincident radiation a high disintegration rate could be used even with many interfering radiations. This could not be done without the energy selection provided. On the other hand, very low disintegration rates will be required to get the spectrum shape in deep valleys of the coincident spectrum if the random rate is not to exceed the true rate.

In practice the random coincidence spectrum is generally obtained by using the relation

$$
N_{B}(E)=2 \tau n_{1} n_{2}(E)
$$

where $n_{1}$ is the counting rate in the fixed window and $n_{2}(E)$ is the singles spectrum seen by the movable single channel analyzer or the multi-channel. The constant $2 \tau$ is obtained by adding enough delay in delay line 1 or 2 so that no true coincidences are obtained. The value of $2 \tau$ is then calculated from the observed random rate. The whole random coincidence spectrum can be obtained in this way but the first procedure is much faster.

Random sum lines in the spectrum are to be expected as in ordinary spectrometry, but a new type of difficulty, the coincident-random sum line arises whenever coincident and non-coincident radiation are both present. The random sum line of one coincident radiation and a noncoincident one is also coincident. Fig. 32 shows an example of this effect. The radiations are those of $\mathrm{I}^{131}$ in coincidence with $80 \mathrm{keV}$ and the weak coincident line near $638 \mathrm{keV}$ is the coincident-random sum of 284 and $364 \mathrm{keV}$, not a weak coincidence with the true $638 \mathrm{keV}$ gamma ray as it appears to be. 


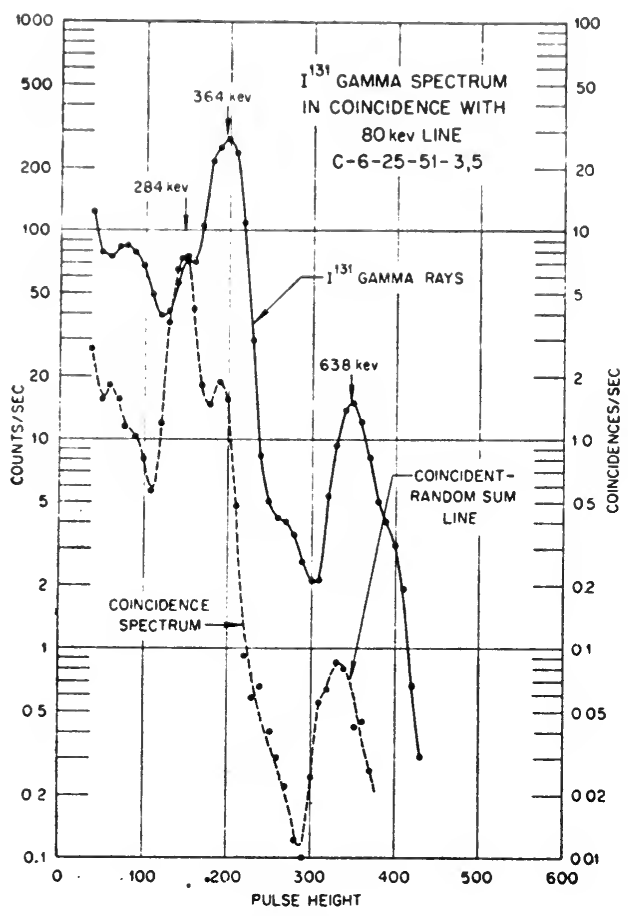

Fig. 32. Coincidence spectrum of $I^{131}$ showing a coincident-random sum line

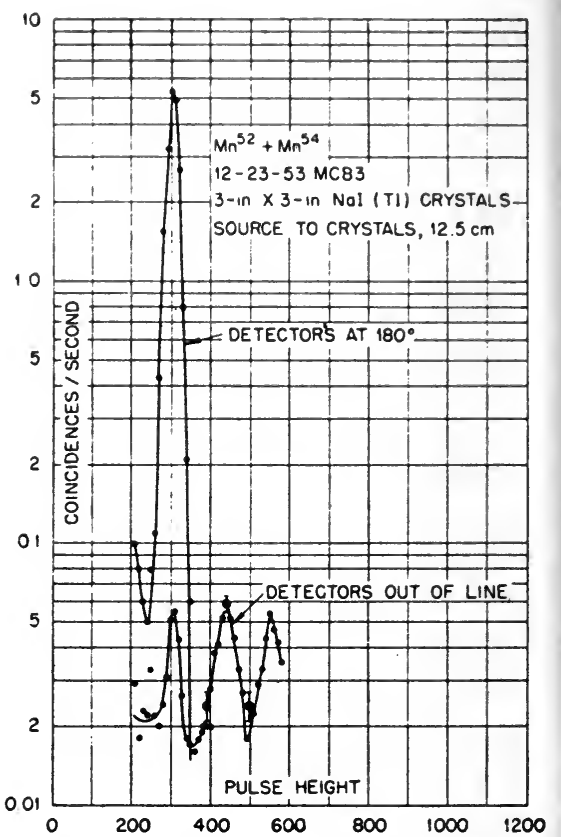

Fig. 33. An example of the coincidence spectrometer with large crystals using annihilation radiation

Coincidence work with large scintillation phosphors is extremely fast and easy; and in certain cases, the results are spectacular. Fig. 33 shows a portion of the coincidence curves obtained for the $\mathrm{Mn}^{54}-\mathrm{Mn}^{52}$ mixture whose spectrum is shown in Fig. 26. The line is the $511 \mathrm{keV}$ coincidence line and the reduction in counting rate was produced by rotating one detector so that no straight line could be drawn from detector to detector through the source." The remaining peak is due to coincidence with the Compton response of the other $\mathrm{Mn}^{52}$ gamma rays as the random rate was still much lower. The counting time required for each of the two curves was 2800 seconds. 


\title{
PARTICULAR DETECTION METHODS
}

\author{
S. C. CURRAN, H. FULBRIGHT, G. BISHOP AND R. WILSON
}

\section{(I) S. C. CURRAN - Proportional Counter Spectrometry}

\section{$\S$ 1. The Principle of Operation ${ }^{1 a_{0} b_{\Delta} c_{0} l_{0} e}$}

If an ionizing particle moves through a gas (or gases) this primary particle will liberate a number of ion pairs in the form of electrons and positive ions. Provided that the gas does not exhibit appreciable attachment the electrons can be drawn as such towards a positive electrode. Unless the primary particle is of high energy the collection of these electronic charges does not give rise to a voltage sufficiently large to be measured or indeed detected with a pulse amplifier. If, however, the electrode configuration is suitable, for example a positive wire along the axis of a cylinder, the field strength in the immediate vicinity of the wire can be made great enough to cause additional ionization by collision. A gas gain or multiplication factor is so secured and used to render the signal at the wire adequately greater than the noise peaks of the amplifier to allow detection and amplitude measurement. Fortunately this same geometry is such as to give a multiplication factor effectively independent of the location of the primary ions. This property follows from the fact that the region of collision ionization is confined to a very limited zone of high field strength near the wire. Each initiating electron passes through this region and liberates an additional number generally considerably exceeding

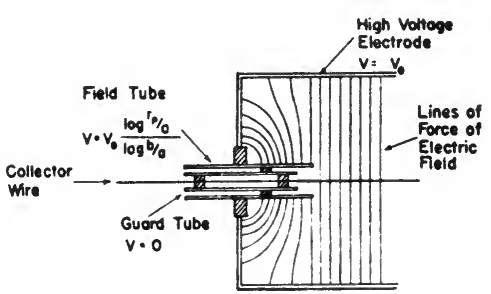

Fig. 1. Schematic arrangement of a proportional counter showing typical lines of force with field tubes set at correct voltage. The radii of wire, field tube and cylinder are $a, r_{p}$ and $b$ respectively unity. The effect of the initial ion pairs becomes negligibly small in such circumstances.

In Fig. 1 a suitable form of proportional counter is shown schematically.

$1 a$ S. A. Korff, Electron and Nuclear Counters, Van Nostrand, (1946).

$1 b$ S. C. Curran and J. D. Craggs, Counting Tubes, Butterworths; Acad. Press, (1949).

1c B. Rossi and H. Staub, Ionization Chambers and Counters, MeGraw-Hill, (1949).

1d D. H. Wilkinson, Ionization Chambers and Counters, Cambridge Press, (1950).

16 D. West, Progress in Nuclear Physics, vol. 3, (1953). 
The collector electrode, generally a wire of tungsten from 2 to $8 \mathrm{mil}$. in diameter, is taken to a pulse amplifier through a grounded guard tube. The insulating material is the finest readily available since discharges through or across the surface readily give rise to spurious pulses at the collector. A very useful, if not absolutely essential, refinement is the fieldadjusting tube ${ }^{2}$ which surrounds the guard tube. In both Geiger and proportional counters end-effect can be removed by such field-adjusting tubes. The end-effect in the proportional counter takes the form of a gradual reduction of the field strength and hence of the gas gain as we proceed towards the end of the wire from the centre. This transition region extends over a length of wire (at each end) of between a radius and diameter of the cylinder. The field tube, when adjusted at the correct potential, makes the electric field configuration near the end of the wire strictly radial. Thus the gas gain is made the same from one end of the wire to the other and the working length of the counter is accurately defined.

The positioning of the wire is not very critical, as was shown by work 3.4 with rectangular cathodes. Rossi and Staub ${ }^{10}$ have calculated that the extreme fractional difference of field $\Delta E / E$ is given by the formula

$$
\Delta E / E=4 a d / b^{2}
$$

where $a$ =wire radius, $b=$ cathode radius and $d$ is the amount of displacement from the centre. More serious effects are usually associated with variations of wire diameter and it is sometimes essential to check the constancy of this.

The impulsive charges reaching the collector are passed to an amplifier of suitable characteristics ${ }^{5}$ and subsequently counted or recorded in terms of number per chosen interval of pulse amplitude to give in effect the energy spectrum of the primary ionizing particles. Many types of "kicksorting" devices ${ }^{6}$ are available.

\section{$\S$ 2. The Gas Gain}

Each primary electron forms a miniature avalanche of electrons in the vicinity of the wire and the gas gain must be limited at a value such that no appreciable interaction occurs between the avalanches. Single electrons,

2 A. L. Cockroft and S. C. Curran, Rev. Sci. Instrum., 22, (1951) 37.

3 S. C. Curran and J. M. Reid, Rev. Sci. Instrum., 19, (1948) 67.

4 J. Becker, P. Chanson, E. Nageotte, P. Treille, B. T. Price and P. Rothwell, Proc. Phys. Soc., A65, (1952) 437.

s S. C. Curran, J. Angus and A. L. Cockroft, Phil. Mag. 40, (1949) 36.

- S. C. Curran, Luminescence and the Scintillation Counter, Butterworths; Acad. Press, (1953). 
for example photoelectrons released at the cathode 7 , may give rise to avalanches of $10^{7}$ electrons while the tube remains proportional in action but more densely ionizing particles, especially if the tracks are nearly perpendicular to the wire, may exhibit saturation effects in amplitude development at a gas gain of about $10^{4}$. The gain must, therefore, be related to the nature of the radiation involved.

Metastability makes the pure rare gases unsuitable, but mixed with methane or other organic gas or vapour to a pressure of several $\mathrm{cm}$ of $\mathrm{Hg}$, they form the most generally useful group. Argon with methane is the most widely adopted mixture, but pure methane, pure nitrogen, krypton or xenon plus methane have been used extensively. Xenon is particularly suitable for the investigation of harder electromagnetic radiations. Some investigators add a trace of carbon dioxide to the gas or gas mixture to bring about an increase in the drift velocity of electrons ${ }^{10}$. The actual material of the cathode does not, in general, seriously influence the performance of the counter and for various reasons polished aluminium has found most favour. No special preparation of the surface beyond smoothing and cleaning is required. The light elements are preferred mainly to avoid the excitation of confusing $\mathrm{X}$-radiation at the walls by electromagnetic radiations from a source. The cathode is frequently in the form of a lining for the main vessel.

The variation of the gas gain with applied voltage is close to exponential for all of the above gases or mixtures, and for a typical tube an increase of between 100 and 200 volts doubles the gain. Excellent stabilization of the supply is required and to maintain the multiplication constant to within $1 \%$ a supply constant to a few parts in $10^{4}$ is required. Wilkinson ${ }^{8}$ has described a self-adjusting device that achieves high stabilization.

\section{§ 3. The Energy Expenditure per Ion Pair and Calibration}

The number of ion pairs released by a primary particle in the gas must vary linearly with the energy of the particle if the tube has to act as a straightforward proportional device. This implies that the energy expended in forming an ion pair should be independent of the energy of the primary. Work prior to 1944 was reviewed by Gray ${ }^{9}$ but more recently ionization by particles, particularly electrons, has been explored carefully with the proportional tube itself ${ }^{5,10,11,12}$. A new and very direct series of measure-

? S. C. Curran, A. L. Cockroft and J. Angus, Phil. Mag., 40, (1949) 929.

8 D. H. Wilkinson, J. Sci. Instrum., 27, (1950) 36.

- L. H. Gray., Proc. Camb. Phil. Soc., 40; (1944) 72:

10 J. M. Valentine, Proc. Roy. Soc. A211, (1952) 75, see also Thesis, Glasgow University, (1953).

11 J. M. Valentine and S. C. Curran, Phil. Mag., 43, (1952) 964.

12 B. Pontecorvo, Helv. Physica Acta, 23, Suppl. IIT, (1950) 97. 
ments fails to reveal any clear evidence of departure from constancy, at least for some of the gases discussed above. It is probably safe to conclude that for electrons as primary particles and for energies in the range $200 \mathrm{eV}$ to at least $200 \mathrm{keV}$ the energy per ion pair is constant to within $2 \%$. The situation regarding alpha particles and protons is less definite but at least approximate constancy ${ }^{13,14,15}$ is observed. Values of the energy per ion pair are listed in Table 1, together with values relative to that of argon as

TABLE I

Energy Dissipation $W$ per Ion Pair

\begin{tabular}{|c|c|c|c|c|c|}
\hline \multirow{2}{*}{ (ias } & \multicolumn{2}{|c|}{ Electrons } & \multicolumn{2}{|c|}{ Alpha rays of polonium } & \multirow{2}{*}{$\frac{\text { Protons, } 340 \mathrm{MeV}}{W_{\text {gas }}^{\gamma} / \mathrm{W}_{\mathrm{A}}}$} \\
\hline & $W$ in eV & $W_{\text {tad }} / W_{A}$ & II in er & $W_{\text {gas }} / W_{\mathrm{A}}$ & \\
\hline A & 27.0 & 1.00 & 25.9 & 1.00 & 1.00 \\
\hline $\mathrm{He}$ & 32.5 & 1.20 & 31.7 & 1.22 & 1.17 \\
\hline $\mathrm{H}_{2}$ & 38.0 & 1.41 & 37.0 & 1.43 & 1.38 \\
\hline $\mathrm{N}_{2}$ & 3.5 .8 & 1.32 & 36.0 & 1.39 & 1.31 \\
\hline Air & 35.0 & 1.29 & 35.2 & 1.36 & 1.30 \\
\hline $\mathrm{O}_{2}$ & 32.2 & 1.19 & 32.2 & 1.24 & 1.23 \\
\hline $\mathrm{C}_{4}$ & 30.2 & 1.11 & 29.0 & 1.12 & - \\
\hline
\end{tabular}

standard. It follows from this work that the proportional counter can be calibrated with radiation of well-defined energy and the energies of other radiations deduced directly from the observed ratios of pulse amplitudes. Many sources produced by pile irradiation may be used as calibrating agent ${ }^{5.19 .20 .21}$. Isomeric tellurium $\mathrm{Te}^{125 \mathrm{~m}}$ is particularly notable as a source of homogeneous electrons, which can be prepared in carrier-free form after slow neutron irradiation of tin. A list of suitable sources is given by IVest ${ }^{10}$.

\section{$\S$ 4. Statistical Fluctuations in Pulse Size and Resolution}

In most applications of the spectrometer the total track of the primary particle is confined to the gas and the total energy of the particle is available

13 T. E. Cranshaw and J. A. Harvey, Can. J. Research, A26, (1948) 243.

14 P. R. Tunnicliffe, and A. G. Ward, Proc. Phys. Soc., A65, (1952) 233.

15 G. C. Hanna, Phys. Rev., 80, (1940) 530.

16 P. Rothwell and D. West, Proc. Phys. Soc., A63, (1950) 541.

17 G. M. Insch, Phil. Mag., 41, (1950) 857.

18 W. Bernstein, H. G. Brewer and W. Rubinson, Nucleonics 6, (1950) 39.

19 S. C. Curran, J. Angus and A. L. Cockroft, Nature, 162, (1948) 302.

20 G. C. Hanna, D. H. W. Kirkwood and B. Pontecorvo, Phys. Rev., 75, (1949) 985.

21 'T. E. Cranshaw, Thesis, Cambridge University, (1951). 
for useful ionization. Fluctuations in the number of ion pairs arise and in addition the multiplication factor fluctuates about its average value. In the proportional tube it is possible to separate these two components of the total fluctuation in the manner used by Curran et al. ${ }^{7}$. It was found that the pulse distribution due to individual photoelectrons released at the cathode, see Fig. 2, could be fitted with the curve $y=x^{1 / 3} \exp x$ and

Fig. 2. The pulse size distribu. tion for single slow photo-electrons multiplied in a counter. The full curve fits the experimental data and the broken curve represents $y=x^{1 / 2} \mathrm{e}^{-x}$

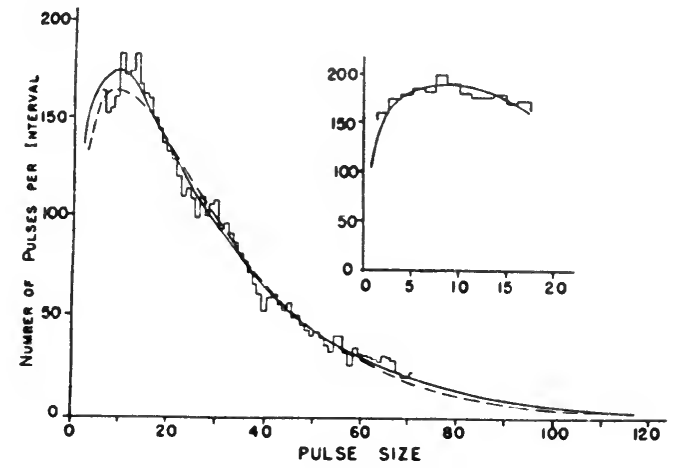

hence the line width contributed by fluctuations in the gas gain alone could be deduced for any definite number of electrons released within the volume.

Comparison of the lines deduced when gas gain fluctuation alone is involved with those observed in practice when both components contribute to the width, see Fig. 3, led to the conclusion that the components were

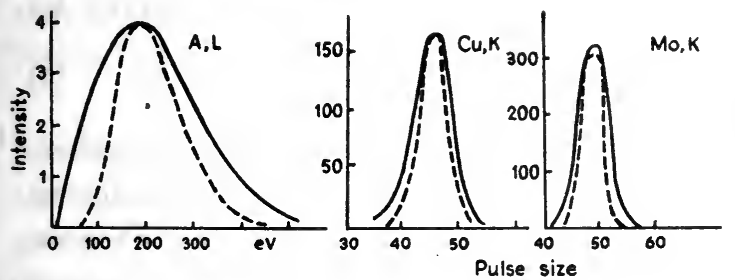

Fig. 3. The width contributed by fluctuation in gas gain (broken curve) shown as part of the total width for different energies of radiation

roughly equal in magnitude and that for each $\left(\overline{N^{2}}-\bar{N}^{2}\right) / \bar{N}^{2}=0.67$. Franzen ${ }^{22}$ has pointed out the disagreement between this and the predictions of a rough duplication theory due to Snyder ${ }^{23}$, in which it is assumed that the probability of electron duplication per unit potential traversed by an electron is $\lambda$ so that for one particle starting at $x=0$ the probability of having $N$ particles at $x$ is given by

$$
P_{N}(x)=\mathrm{e}^{-\lambda x}\left[1-\mathrm{e}^{-\lambda x}\right]^{N-1}
$$

22 W. Franzen, Theory and Use of Pulse Ionization Chambers, Unpublished Report, Dec. 1951, Princeton Univ., distributed in mimeographed form.

23 H. S. Snyder, Phys. Rev., 72, (1947) 181. 
The probability distribution $P_{N, \bar{N}}$ about the mean number $\bar{N}\left(=\mathrm{e}^{\lambda x}\right)$ is given by

$$
\left.\begin{array}{rl}
P_{N \bar{N}} & =\frac{1}{\bar{N}}\left(1-\frac{1}{\bar{N}}\right)^{N-1} \\
\text { or } P_{N \bar{N}} \cong\left(e^{-N / \bar{N}}\right) / \bar{N}
\end{array}\right\}
$$

for $\bar{N} \gg 1$. For $M$ particles starting from $x=0$ the probability of having a total number $\nu=N M$ at $x$ is given by

$$
P_{\nu}(x)=q_{M}\left\{\frac{(\nu-1) !}{(M-1) !(\nu-M) !} \mathrm{e}^{-\lambda M x}\left(1-\mathrm{e}^{-\lambda x}\right)^{\nu-M}\right\}
$$

where $q_{M}$ is the probability of having $M$ particles initially. Hence

$$
\frac{\overline{v^{2}}-\bar{v}^{2}}{\bar{v}^{2}}=\frac{\overline{M^{2}}-\bar{M}^{2}}{\overline{M^{2}}}+\frac{1}{\bar{M}}-\frac{1}{\bar{v}} \text {. }
$$

gives the relative mean square fluctuation (or variance) of the total number collected. As $v$ is generally large the last term is negligible and the variance consists of that of the original number $\left(\overline{M^{2}}-\bar{M}^{2}\right) / \bar{M}^{2}$ together with $1 / \bar{M}$ the contribution due to the multiplication process. Regarding the latter equation (2) gives $\left(\overline{N^{2}}-\bar{N}^{2}\right) / \bar{N}^{2}=1.0$ while $x^{2 / s} e^{-x}$ gives $\left(\overline{x^{2}}-\bar{x}^{2}\right) / \bar{x}^{2}=0.67$. Further experimental work and more thorough theoretical treatments are required to resolve this disagreement.

Regarding the fluctuations in the number of ion pairs liberated along the complete track Fano ${ }^{24}$ indicated how this should be less than expected from a simple Poisson distribution about a mean value. His conclusion is that the mean square deviation in the number of ion pairs is:given by

$$
\overline{\left(J^{\prime}-J_{0}\right)^{2}}=\overline{(n-\varepsilon / W)^{2}} J_{0} / \bar{n}
$$

where $J^{\prime}=$ actual number of ion pairs produced, $J_{0}=$ average number, $n=$ number of ion pairs liberated per collision, $\varepsilon=$ energy lost per collision and $W=$ average energy of formation of an ion pair. For the Poisson distribution $\overline{\left(J^{\prime}-J_{0}\right)^{2}}=J_{0}$ and the difference factor $\overline{(n-\varepsilon / W)^{2}} / \bar{n}$ is less than unity. The factor was found experimentally by Curran et al. for a $50: 50$ mixture of argon and methane. Their value of 0.67 must be regarded as a lower limit due to later experiments modifying the value of $W$ adopted in analysing the data ( $W$ was assumed to be $32 \mathrm{eV}$ but it was shown later to be $28.6 \mathrm{eV}$ ). It would appear essential that further experimental and theoretical work should be attempted.

It is assumed above that extraneous sources of amplitude fluctuation are rendered negligible. In practice it is difficult to ensure that this situation holds. Noise in the amplifier can give rise to an appreciable contribution

24 U, Fano, Phys. Rev., 70, (1946) 44; 72, (1947) 26. 
to line width and the kicksorting equipment, whatever its form, is liable to variations in channel widths. The high voltage supply and the diameter of the wire must be strictly controlled for optimum results. Long term changes in the purity of the gas have to be eliminated. Assuming careful control of these various factors it is yet obvious that at relatively low energies of the primaries $(<50 \mathrm{keV}$ say) the spectrometer is a lowresolution device. Nevertheless it is superior to its rival in this region, the scintillation spectrometer and its resolution is sufficient for the majority of investigations. For further discussion of this question, following the theoretical treatment of Frisch ${ }^{25}$, the reader is referred to West ${ }^{1 e}$.

At high energies, $\sim 0.5 \mathrm{MeV}$ say, the expected resolving power has not yet been achieved. The statistical fluctuation at about $600 \mathrm{keV}$ should be less than one percent but experimentally observed line widths indicate a value considerably exceeding this (see Fig. 4). The cause of the relatively poorer performance is still obscure, but it must be remembered that the same components of the line width that are set in evidence at high energies may be present at lower energies, except that in the latter case their effect is masked

Fig. 4. The beta spectrum of $\mathrm{Cs}^{137}$ with the $K$ and $L$ conversion lines of width exceeding theoretical expec. tation. Experimental arrangement as indicated in inset figure (from work of A. L. Cockcroft)

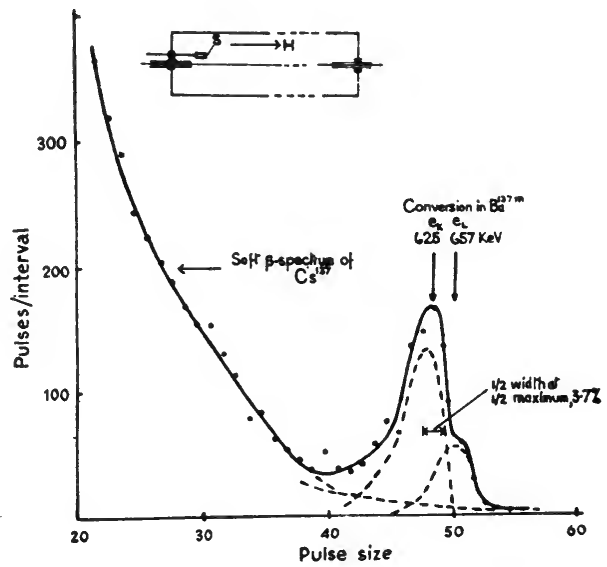

by the relatively larger statistical fluctuation discussed above. It would not appear to be caused by increased electron attachment at the higher gas pressures required in this region. The impurities causing deleterious effects can be removed by a purifier without any major change being observed. The type of purifier suitable for this work has been described by Klema and Allen ${ }^{26}$ and Klema and Barschall ${ }^{27}$. A tube containing calcium turnings which can be heated electrically is attached to the counter and the gas caused to circulate through the turnings by convection. The volume of the purifier is between $1 / 10$ and $1 / 20$ of the volume of the counter. Vacuum outgassing of the calcium is done at $400^{\circ} \mathrm{C}$ and

25 O. R. Frisch, unpublished work discussed by West, to whom the author is indebted for loan of the proof of his own work. See ref. le.

28 A. D. Klema, and J. S. Allen, Phys. Rev., 77, (1950) 661.

27 A. D. Klema and H. H. Barschall, Phys. Rev., 63, (1943) 18. 
subsequent purification at $300^{\circ} \mathrm{C}$. Above $300^{\circ} \mathrm{C}$ reactions between calcium and gases such as nitrogen may occur. Purification can proceed continuously while the counter is in use. Franzen ${ }^{20}$ has given a most useful discussion of purification techniques applicable to a number of gases.

\section{$\S 5$. Pulse Shape and Amplifier Design}

In the normal operation of the counter the multiplication factor exceeds 10 and may be as high as $10^{7}$. All of the electrons may be collected rapidly, in a time $<10^{-6}$ sec, see Fig. 5, due to English and Hanna ${ }^{28}$. The actual collection of the electrons themselves gives rise to a nearly negligible impulse at the collector but the concurrent rapid drift of the positive ions,

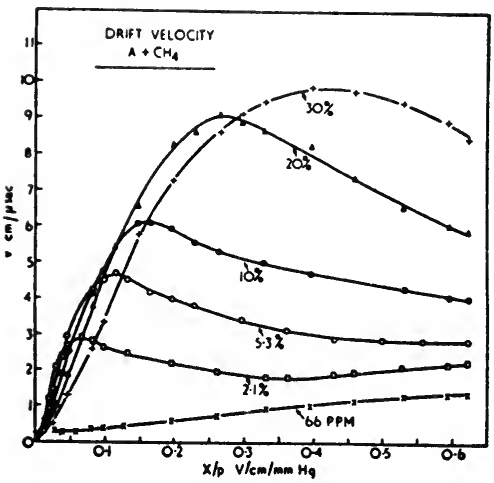

Fig. 5. The drift velocity of electrons in argon-methane mixtures for various percentages of methane as marked on the curve formed round the wire, towards the cathode leads to a rapid increase in signal strength. In the initial stages of development of the pulse the drift velocity of the positive ions is relatively very high. Let the drift velocity be denoted by $\omega$, the electric field strength by $X(r)$ where $r$ is radial distance from the axis, then

$$
\omega=d r / d t=\mu(X / p)
$$

where $\mu=$ mobility and $p=$ gas pressure. Also

so that

$$
X=V_{0} /[r \ln (b / a)]
$$

$$
\omega=\mu V_{0} /[r p \ln (b / a)]
$$

It can be shown that the signal at the wire is given by

$$
S(t)=\frac{-e}{2 C \ln (b / a)} \ln \left\{1+\left[(b / a)^{2}-1\right](t / T)\right\}
$$

and putting $t=T$, the collection time of the positive ions, the final amplitude of the signal is $S_{T}=-e / C$ while for $t=(a / b) T$ we have $S_{a T / b}=-\frac{1}{2} e / C$ approximately. With $a / b \cong 10^{-3}$ the signal is shown to reach half-maxi-

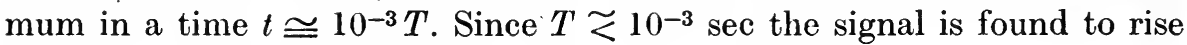
rapidly to about half its final value in $<10^{-6}$ sec.

In practice the amplifier is designed to amplify linearly the initial stages of development of the signal and to reject the later stages of slow rise to maximum. Since the pulses are essentially similar to each other in shape, such rejection does not interfere appreciably with the proportionality of output signal strength. It is usual practice to have a differentiating time

28 W. N. English and G. C. Hanna, Can. J. Physics, 31 (1953) 768. 
constant for the amplifier roughly equal to, but not less than, the integrating time constant. 'The latter must be chosen in the light of the collection times for electrons at the wire which may vary widely in some applications. The effectively short duration of the pulses means that the counter can be used with little danger of overlapping at rates exceeding $10^{3} /$ sec. A low-noise high gain electronic amplifier reduces the gas multiplication required and mutual interference of the avalanches is reduced so that it helps in operating at higher counting rates. The nature of the source and primary particles influences the value of maximum counting rate. A localised alpha source of high specific intensity gives rise to avalanches in the same region of the wire. The size of the counter and the gas pressure influence the rate of collection of electrons so that amplifier characteristics should be adjusted to suit these parameters.

\section{§ 6. Geometries of Source and Counter, Wall Effect and Magnetic Field}

The most elegant way in which to use the counter is with the radioactive material in the form of a gas or vapour. Unfortunately, the number of elements available in a suitable gaseous form is limited but the spectra of $\mathrm{H}^{3}, \mathrm{~A}^{37}, \mathrm{C}^{14}, \mathrm{~S}^{35}, \mathrm{Br}^{80}, \mathrm{Zn}^{65}, \mathrm{Kr}^{83}$, RaD and others have been analysed. Attention must be paid to the effect of the limited dimensions of the vessel on the tracks of the particles-the wall effect. It is doubtful if reliance can be placed on calculations of the wall effect except perhaps where we are concerned with alpha rays or soft $\gamma$ and X-rays. The scattering of beta particles complicates calculation and for this reason it is generally more satisfactory if an empirical procedure is adopted. Thus the spectrum can be observed at different gas pressures or with counters of different dimensions. Such changes will quickly indicate whether appreciable distortions are to be ascribed to the limitations in volume and pressure, and indeed higher gas pressure may often be a satisfactory solution of the problem. This technique has been used a great deal, large counters at about 7 atmos. of argon being employed as in the work of Angus et al. ${ }^{29}$. Where it is obvious or demonstrated that distortion is present the application of a magnetic field represents a very powerful aid to its elimination. A field parallel to the axis of the tube was shown by Rothwell and West ${ }^{30}$ to eliminate or considerably reduce the effect of the cathode itself. The passing of particles beyond the ends of the wire is not thereby prevented, but there is no serious limitation on the length of counter that can be operated. Restriction of the trajectory of the primary particle in two or in all three dimensions is possible and methods of

20. J. Angus, A. L. Cockcroft and S. C. Curran, Phil. Mag., 40, (1949) 522.

so P. Rothwell and D. West, Proc. Phys. Soc., A63, (1950) 539. 
accomplishing this have been described by Curran et al. ${ }^{\mathbf{3 1} . \mathbf{3 2}}$ and Frank ${ }^{\mathbf{3 3}}$. The most appealing technique would appear to be a suitable compromise of the different attacks, such as is represented by a long counter of fairly large diameter placed in a reasonably strong uniform field (2000 gauss say) parallel to its axis and filled to a pressure of about 7 atmospheres. The field can be produced by a long solenoid containing the counter itself.

Two points require mention in regard to the application of a magnetic field. It can easily be shown that the influence on pulse size should be very small and generally completely negligible when the whole energy of the ionizing particle is spent in the gas of the counter ${ }^{31.34}$. Secondly, the sizes of pulses due to particles leaving the wall are considerably reduced, and this can be an important adrantage in reducing the undesirable effects of $\gamma$-radiation falling on the walls or incleed of any "background" due to particles leaving the cathode.

Examples of the effect of magnetic field on the observed spectra of isomeric $\mathrm{Br}^{80 m}$ and $\mathrm{P}^{32}$ are given in Fig. 6. The examples show the great improvement effected by using suitable fields.
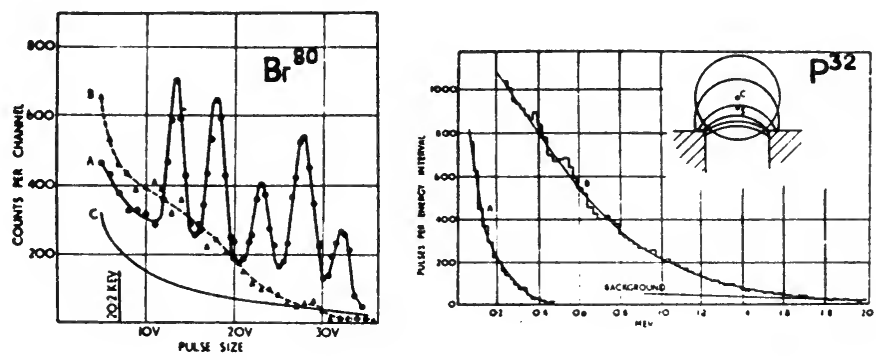

Fig. 6. The effect of a homogeneous magnetic field, parallel to the wire, on the spectrum of $\mathrm{Br}^{80}$. Curve $\mathrm{A}$ refers to field on, $\mathrm{B}$ to field off and $\mathrm{C}$ is the background due to $\mathrm{Br}^{82}$ (Rothwell and West ${ }^{30}$ ). The non-homogeneous field (inset) changes curve A to curve B when a source of $\mathrm{P}^{32}$ is examined (Curran, Cockeroft and Insch ${ }^{\mathbf{3 1}}$ )

For the investigation of sources in solid form a variety of geometries is available ${ }^{32.35 .36}$. Where the radiation is sufficiently penetrating it may be transmitted through a thin window. For the investigation of softer radiations the source can be mounted on a very thin film of nylon or other suitable support, and this can be placed at an aperture in the cathode and nearly flush with its surface. It is good practice to have the metal

31 S. C. Curran, A. L. Cockeroft, and G. M. Insch, Proc. Phys. Soc., A63, (1950) 845.

32 S. C. Curran, Atomics, 1/13, (1950) 221.

33 S. G. F. Frank, Thesis, Camb. Univ., (1952).

34 D. West, and P. Rothwell, Phil. Mag., 41, (1950) 873.

35 S. C. Curran, Advances in Physics, Phil. Mag. Suppl. 2, (1953) 411.

36 I. Bergström, "Manne Siegbahn", Uppsala 1951, p. 307. 
parts of the vessel remote from the source, as this reduces back scattering to that of the thin film and the gas. To minimise wall effect a solid source may be mounted on the end of a probe-type support running parallel to the wire and placed fairly close to it. This probe is held at a voltage appropriate to its position in the electric field, and it can be such as to place the source at one end of the counter or near the middle. A magnetic field parallel to the counter wire can be applied if required to limit the range of particles of higher energy. Finally, when radiations of low intensities are under investigation the source may be deposited on the cathode itself. In all of these geometries there are certain advantages and disadvantages, so that it is difficult to come to general conclusions. The choice will be determined largely by the nature of the particular source involved. It is assumed throughout that the source uniformity and thickness is the best that is practicable, but in some cases the use of a thick support becomes inevitable. The reflection of beta rays has been examined by Balfour and Curran to determine its influence on the data obtained with the proportional counter.

A source of isomeric $\mathrm{Te}^{125 m}$ placed within a proportional counter gave the spectra shown in Fig. 7. The main peaks correspond to conversion electron lines and the influence of sheets of different materials placed immediately behind the thin source (mounted on thin nylon) was studied. Analysis of the various spectra yielded the data summarised in the curve of Fig. 8. Here the percentage back-scattering into $2 \pi$ solid angle is given as a function of nuclear charge and energy of the particles. The work of Burtt and Yaffé ${ }^{37}$ and of Suzor and Charpak ${ }^{38}$ is pertinent here. It is particularly important to note that the use of thick supports of the very light elements does not lead to any gross effects at the low energy end of a beta spectrum. The main effect is to give rise to a "tail" on the low energy side of a line. Moreover for sources down to $\sim 10 \mu \mathrm{g} / \mathrm{cm}^{2}$ in thickness Balfour found that there is no appreciable contribution $(<2 \%)$ to the counting rate that can be ascribed to true slow secondary electrons (energy $<50 \mathrm{eV}$ say) as distinct from reflected primaries that have lost some of their energy.

- It is found that scattering can be helpful in reducing wall effect. The path of a beta particle of energy less than about $100 \mathrm{keV}$ is usually tortuous and studies in various gases ${ }^{39}$ have shown that the total distance between

37 B. P. Burtt, Conference on Absolute Beta Counting, Prelim. Report No. 8, N.R.C., 1950, p. 1., also L. Yaffé, p. 27., together with L.' Yaffé and K. Justus, Jour. Chem. Soc. 5, (Suppl. 2) (1949) 341.

38 F. Suzor and G. Charpak, J. de Phys. et le Radium, 13, (1952) 1; C. R. Acad.

Sci. (Paris), 233, (1951) 1356; 234, (1952) 720.

39 T. San-Tsiang, C. Marty and B. Dreyfus, J. de Phys. et le Radium, 8, (1947) 269. 
start and finish of the track may be much less than the complete length of the path described. For example, in argon it is 0.55 of the total path length. West et al. ${ }^{40}$ noted that for electron tracks there was no appreciable tail on the low energy side of a peak even when wall effect was large. They associated this with the fact that the tort yous nature of beta tracks implies large reductions in pulse size when wall effect is present.

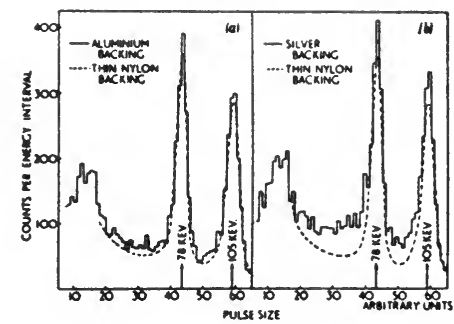

Fig. 7. The conversion electron lines of $\mathrm{Te}^{125 m}$ as observed with a source at an aperture in the cathode, (Balfour and Curran)
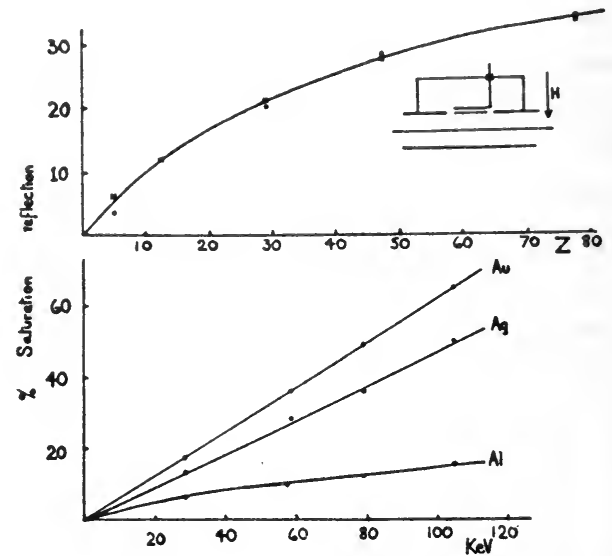

Fig. 8. The intensity of reflected electrons expresserl as a percentage of the intensity of the incident particles for saturating thicknesses of source support. Upper curve - the variation with atomic number $z$ for the electrons of $\mathrm{Te}^{125 m}$. Lower curve - the variation with the energy of the electrons for different elements (Balfour and Curran)

\section{$\S 7$. Photoabsorption, Fluorescence Yield and The Escape Peak}

In the proportional tube the measurement of the energy of incident quanta generally depends on the occurrence of photoelectric absorption in the gas of the tube and the detection efficiency is generally calculable with the help of suitable tables of absorption coefficients (see Compton and Allison ${ }^{41}$ ). For example, at an energy of $47 \mathrm{keV}$ approximately $10 \%$ of the incident radiation is absorbed in $8 \mathrm{mg} / \mathrm{cm}^{2}$ of gas, largely by photoeffect in the $K, L, M \ldots$ shells of argon. The photoelectrons so released dissipate their energy in the gas. The fluorescence yield is a measure of the probability that $\mathrm{X}$-radiation of argon will be emitted by the filling of vacancies in the $K, L, M \ldots$ shells. On the other hand, Auger electrons may be emitted, so that practically all of the energy $h v$ of the incident radiation can be directly effective in producing ionization. Indeed in the light elements, such as argon, the fluorescence yield is small $(10 \%)$. When the charac-

40 D. West, J. K. Dawson and C. J. Mandleberg, Phil. Mag., 43, (1952) 875.

41 A. H. Compton and S. K. Allison, X-rays in Theory and Experiment, 2nd Edn. (D. van Nostrand) (1935). 
teristic X-rays are emitted there is a distinct possibility of escape of such radiation from the gas and the energy expended in ionization may be less than $h v$ (possibly $=h v-h v_{E} ;=h v-h v_{L}$ where $h v_{K}, h v_{L} \ldots \ldots \ldots$ are the $K, L \ldots \ldots \ldots$ binding energies). Pulses arising in this way give rise to "escape peaks" in the observed spectrum of the incident radiation. They can be identified by the fact that they occur at constant energy intervals below the main peak. The peak corresponding to $K$-shell absorption is prominent for the heavier gases such as krypton and xenon, and readily observed even in argon. The main peak corresponds to the escape of $K_{a}$ radiation and a less intense one to escape of $K_{\beta}$. Examples due to West et al. ${ }^{34,40}$ for krypton and to Beling et al. ${ }^{42}$ for xenon are given in Fig. 9. The escape peaks may have a considerable muisance value in the analysis of gamma and X-ray spectra so that the relatively clean results observed with argon are desirable. Nevertheless, the large in-

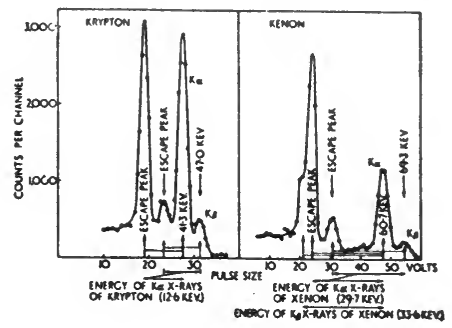

Fig. 9. Examples of escape peaks in krypton and xenon crease in photoelectric absorption coefficient with increase in $Z$-value of the absorber makes xenon a very important gas in such spectrometry. Useful curves for the photo-absorption of the important gases have been given by West ${ }^{1 e}$.

The proportional counter has been used to measure the fluorescence yields ${ }^{34,43.1 e}$ for the $K$-shell, for the gases argon, krypton and xenon, yielding $0.11 \pm 0.01,0.67 \pm 0.03$ and $0.81 \pm 0.05$ respectively.

\section{§ 8. Pulse Amplitude Analysis}

Most of the standard methods of kick-sorting ${ }^{6}$ can be employed with the proportional tube. Much work has been done by straightforward photographic registration of the pulses. The delay-line type of storage used by Hutchinson and Scarrott ${ }^{44}$ is notable in giving both electronic viewing and recording and the storage capacity and speed of the instrument is rather well suited to use with the proportional tube. For general information the reader is referred to the review of Van Rennes ${ }^{45}$.

42 J. K. Beling, J. O. Newton and B. Rose, Phys. Rev., 86, (1952) 797.

43 J. H. Kahn, O.R.N.L., (1951) 1089.

44 G. W. Hutchinson and G. C. Scarrott, Phil. Mag., 42, (1951) 792.

45 A. B. van Rennes, Nucleonics 10/7, (1952) 20; 10/8, (1952) 22; 10/9, (1952) $32 ; 10 / 10$, (1952) 50 . 


\section{$\S 9$. Coincidences, Internal and External}

\section{A. INTERNAL}

The term internal coincidence is used here to denote the simultaneous occurrence of ionizing events in the tube. The spectrometer is frequently operated at a high detection efficiency so that all but sources of the simplest type may readily give rise to simultaneous ionizing events. The output pulses are then integral in nature and a complex spectrum results, particularly when electromagnetic radiations which may escape detection are present. Rothwell and West ${ }^{30}$ illustrated the phenomenon in their study of isomeric $\mathrm{Br}^{80 m}$. Although in many instances the phenomenon adds to the difficulties of interpretation it can be turned to very real advantage, as for example by these workers in determining the conversion coefficients of gamma radiations. It is perhaps desirable to stress the fact that deliberate variations of the source geometry $(4 \pi, 2 \pi$ and small angle) and the gas pressure can be employed to facilitate interpretation. A straightforward illustration of the useful application of the integrating property is shown in the work of Wilson and Curran ${ }^{46}$ on $\mathrm{Hg}^{203}$ and of Insch et al. ${ }^{47}$ on RaD, see Fig. 10. The coincidence of the beta particles with conversion electrons is evident in the form of the spectra, and the conversion coefficients for $\mathrm{Hg}^{203}$ are deduced. With $\mathrm{RaD}$ the total transition energy, $64 \mathrm{keV}$, is observed and the very soft beta spectrum (18 $\pm 2.5 \mathrm{keV}$ for end-point energy) is carried along the energy scale by the conversion electrons. The form is found to agree with that of an allowed transition, a result confirmed by work on RaD by Bannerman and Curran ${ }^{48}$ with scintillation spectrometers, by Jaffé and Cohen ${ }^{49}$ with a gaseous source in the proportional tube and by the recent studies of $\mathrm{RaD}$ by $\mathrm{Wu}$ et $a l .{ }^{50}$, in which the many uncertainties arising from earlier studies are clarified and a relatively simple decay scheme proposed.

\section{B. External}

There has been little application of two or more proportional tubes in coincidence with each other, although they are well suited to this purpose and resolving times of about $10^{-7}$ sec could be obtained in favourable oircumstances. A gaseous source, $\mathrm{H}^{3}$, was examined by Insch and Curran ${ }^{51}$ for coincidences in a useful arrangement. The wire of a large counter was

46 H. W. Wilson and S. C. Curran, Phil. Mag., 43, (1951) 762.

47 G. M. Insch, J. G. Balfour and S. C. Curran, Phys. Rev., 85, (1952) 805.

48 R. C. Bannerman and S. C. Curran, Phys. Rev., 85, (1952) 134.

49 A. A. Jaffé and S. G. Cohen, Phys. Rev., 89, (1953) 454.

50 C. S. Wu, F. Boehm and E. Nagel, Phys. Rev., 90, (1953) 388; 91, (1953) 319.

51 G. M. Insch and S. C. Curran, Phil. Mag., 42, (1951) 892. 
split into two portions held together and insulated from each other by a small elongated glass bead. This system constitutes two counters placed end to end within one cylinder without any window between, and it is suitable for many investigations of decay schemes.
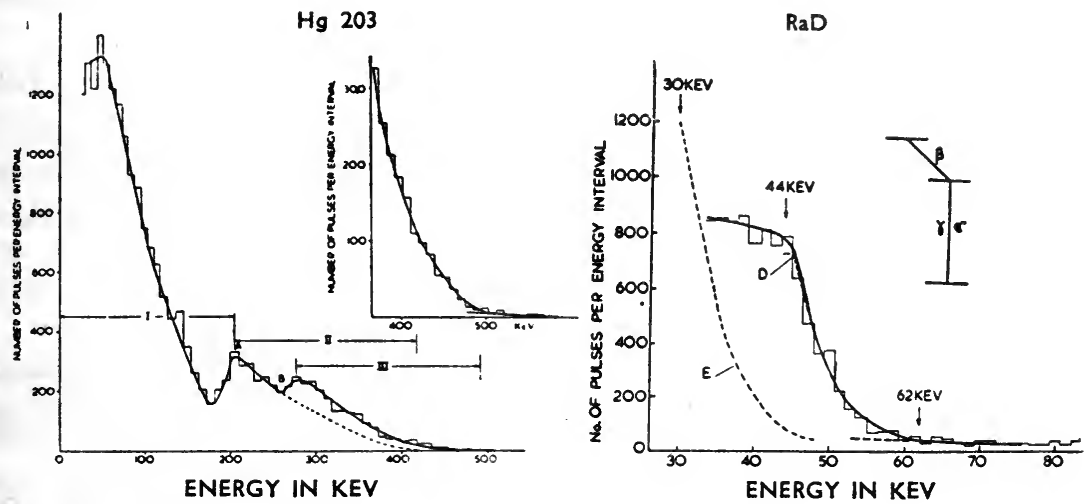

Fig. 10. The L.H. curve shows the spectrum of $\mathrm{Hg}^{203}$. Part I refers to unaccompanied beta rays, part II to beta rays coincident with $K$ conversion electrons, and part III to coincidence with $L$ conversion electrons. The R.H. curve shows the high energy part of the spectrum of $\mathrm{RaD}$ where coincidences between $M$ conversion electrons and beta particles occur

The proportional tube and scintillation spectrometer form a powerful combination, the first analysing particle radiations mainly and the latter $\mathrm{X}$ and $\gamma$-radiation. Both of these devices can, of course, be operated in conjunction with magnetic spectrometers, and each has its own peculiar advantages and disadvantages in such circumstances.

\section{$\S$ 10. Proportional Counter Work at Low Intensity}

The proportional tube can be used readily with very extended sources, so it is well adapted to the examination of sources of very low specific intensity. Furthermore, since it is both detector and spectrometer, if the radiation is approximately homogeneous the pulses due to the source may be relatively concentrated in energy'compared with those constituting the "background", since these are usually spread out in a smooth continuous manner throughout the energy range involved, say 1 to $500 \mathrm{keV}$. Even when the source gives rise to beta rays the same argument applies though with less force. The soft beta rays of several sources cover a very limited energy range and within this range the relative background is reduced. The instrument is hence more sensitive than the Geiger tube, and it can be more sensitive than the scintillation spectrometer. Gamma radiation is one of the stubborn contributions to background and anti-coincidence 
shielding systems consisting of Geiger tubes do not remove it. Freedom of the materials of the counter from radioactive impurities is fairly easily achieved with the simple substances such as iron used in building the proportional tube. The work of Libby and his collaborators ${ }^{52}$, and more recently of Kulp and Tryon ${ }^{53}$, with radio-carbon as a dating tracer element is interesting in this connection.

One of the earliest examples of the application of the proportional tube to low intensity sources was the discovery by Wilson ${ }^{54}$ of the decay of $\mathrm{Ni}^{59}$ by $K$-capture, following the analysis ${ }^{55}$ of the weak beta spectrum of $\mathrm{Ni}^{63}$. For the examination of extremely low intensity radiations a typical system as used by the writer generally includes:

1. A layer of lead all round to a thickness of about $4 \mathrm{in}$.

2. A layer of iron within this to a thickness of $1 \mathrm{in}$. (This was replaced by mercury in the work of Kulp and Tryon and definite advantages claimed.)

3. A cylindrical assembly of long Geiger tubes side by side inside the iron, arranged to operate in anti-coincidence with the proportional tube.

4. A proportional tube, inner walls lined with stainless steel.

With a tube of inner diameter $5.63 \mathrm{in}$. and of operating length $11 \mathrm{in}$. the observed back-ground counting rate was close to $18 / \mathrm{min}$ and the energy range covered by the pulses stretched to beyond $500 \mathrm{keV}$ at a pressure of $4 \mathrm{~atm}$ of argon. The pulses produced by sources spread on the cathode (useful area $=1200 \mathrm{~cm}^{2}$ ) were displayed on an oscillograph and photographed, provided they were not in coincidence with those in the shielding Geiger tubes. With rubidium as source ${ }^{56.57}$ the results given in the form of Fermi plots, Fig. 11, were obtained. This is a rather striking example of the power of the method in handling highly forbidden transitions of long half-life (here $\Delta I=3$, yes and $6.15 \times 10^{10} \mathrm{y}$ respectively).

Recent work by Dixon, McNair and Curran with the same instrument shows that isotope $\mathrm{N}^{150}$ is stable or that it has a minimum half-life of about $10^{17} \mathrm{y}$ (the precise value depending, of course, on the energy release, if any, of the transioion). Likewise the isobaric pair, $\operatorname{Re}^{187}$ and $\mathrm{Os}^{187}$ fail to show observable activity and the minimum value of the half-life of

52 W. Libby, Phys. Rev., 69, (1946) 671; E. C. Anclerson and W. Libby, Phys. Rev., 81, (1951) 64; J. R. Arnold and W. Libby, science 110, (1949) 678.

${ }_{53}$ J. L. Kulp and L. E. Tryon, Rev. Sci. Instrum., 23, (1952) 296.

54 H. W. Wilson, Phys. Rev. 79, (1950) 1932 and 82, (1951) 548.

55 H. W. Wilson and S. C. Curran, Phil. Mag., 40, (1949) 631.

56 Nuclear Data, U.S. Dept. of Comm., N.B.S. Circular 499.

57 S. C. Curran, D. Dixon and H. W. Wilson, Phys. Rev., 84, (1951) 151; Phil. Mag., 43, (1952) 82. 
either is in the region of $10^{16} \mathrm{y}$. This is in disagreement with the work of some earlier observers ${ }^{56}$. The beta spectrum of another natural radioelement, $\mathrm{Lu}^{176}$, has been examined in some detail and the intensity of superposed conversion lines due to cascade gamma transitions measured. Here again a third forbidden type of decay is involved.

Fig. 11. The Fermi plots for $R b^{87}$ showing $[N /(p W F)]^{1 / 2}$, curve $I$ and $\left[\mathrm{N} /\left(\mathrm{pWFC}_{3 \mathrm{~T}}\right)\right]^{1 / 2}$ curve II. The correction factor $\mathrm{C}_{3 \mathrm{v}}$ gives equally good linearity. Curves I and II have arbitrary scales for the ordinate

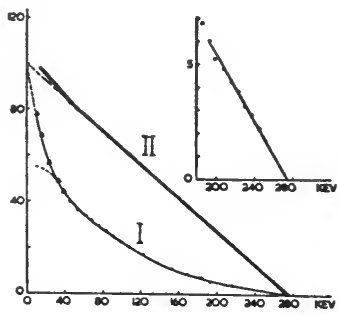

Clearly the instrument is well adapted to the study of such low intensity radiations as the above, and it lends itself readily to the radio-carbon assays. In Fig. 12 the instrument designed by Curran and Wilson for the analysis of weak gaseous sources (such as $\left({ }^{14} \mathrm{H}_{4}\right)$ is shown. A large solenoidal magnet surrounds the counter and provides a magnetic field of 4000 gauss or more in the direction of the tube axis. The counter itself is in the form of a light wire cage consisting of parallel wires of stainless steel, set up within a grounded cylinder. The magnetic field prevents

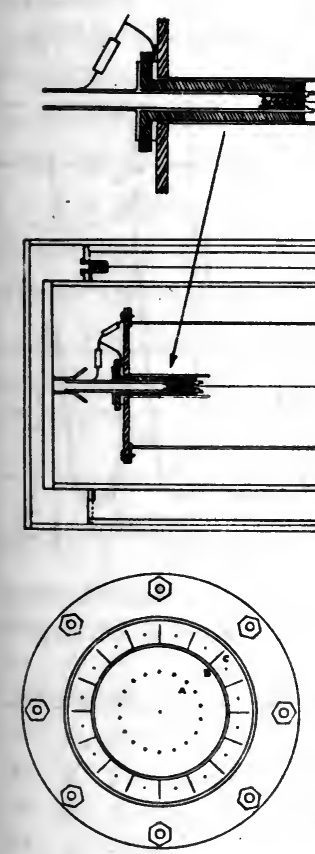

Fig. 12. A large proportional counter for the assay of low intensity sources in gaseous form. The cage counter $\mathbf{A}$ is contained within the vessel $\mathrm{B}$, part of the surrounding system of shielding by the special Geiger counters C. The stcrion $\mathbf{x}$ assembly operates within a large solenoidal magnet 
electrons such as those released by gamma rays from entering the counter itself. The reduced mass of material forming the cathode means reduced background arising from impurities in the material or interaction with gamma rays. The carbon for analysis is used as methane or other suitable gas. The anti-coincidence Geiger shield is a single multi-element unit as shown, and lead or other materials can be placed round as usual.

\section{$\S 11$. Notes on Recent Applications}

Perhaps the most notable beta spectrum examined by the spectrometer, see Fig. 13, is that of $\mathrm{H}^{3}$, measured by Curran et al. ${ }^{58}$ and later by Hanna and Pontecorvo ${ }^{59}$. This has a close connection with the determination of the rest mass of the neutrino, a question discussed in some detail by Kofoed-Hansen ${ }^{60}$. It should be noted that a possible discrepancy ${ }^{51}$ between experiment and theory found at very low energy $(<1 \mathrm{keV})$ may be due, according to very recent experiments of Moljk and C'urran, as yet unpublished, to deficiencies of kicksorting equipment in this region. Similar work at such very low energies with heavy radioelements has been discussed by Curran ${ }^{64}$ who deduces that the energy of rearrangement of the atomic shells has to be included as part of the decay energy, dividing itself between $\beta$-particle and neutrino. For an example of the most recent practice in the application of the spectrometer to gaseous sources the reader is referred to the work of Moljk and Curran ${ }^{61}$ on $\mathrm{C}^{14}$ and $\mathrm{S}^{35}$. Here the excellent agreement (better than $1 \%$ ) between theory and experiment is very

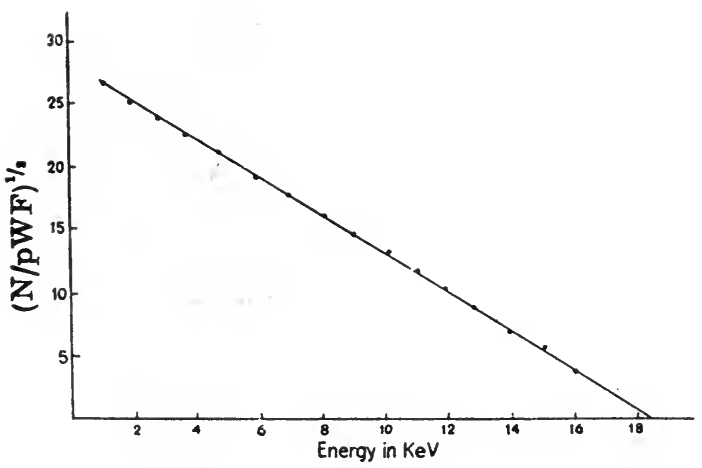

Fig. 13. The spectrum of $\mathrm{H}^{3}$ showing allowed form to below $1 \mathrm{KeV}$

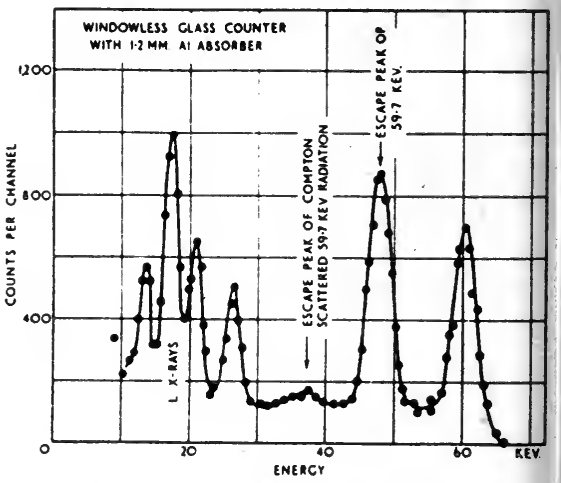

Fig. 14. Gamma and $L$-radi. ation from a source of $\mathrm{Am}^{241}$

58 S. C. Curran, J. Angus and A. L. Cockroft, Nature, 162, (1948) 302; Phil. Mag., 40, (1949) 53; Phys. Rev., 76, (1949) 853.

58 G. C. Hanna and B. Pontecorvo, Phys. Rev., 75, (1949) 983.

Bo O. Kofoed-Hansen, Phil. Mag., 42, (1951) 1448.

61 A. Moljk and S. C. Curran, Phys. Rer., 96 (1954) 395. 
notable. The results on $\mathrm{C}^{14}$ are the more interesting in view of disagreements ${ }^{62,}{ }^{63}$ previously observed.

Among recent investigations of weak sources of gamma radiation we note the work of West et al. ${ }^{40}$ on $\mathrm{U}^{233}$ and of Beling et al. ${ }^{42}$ on $\mathrm{Am}^{241}$. An example from this work, performed with a krypton filled counter, is shown in Fig. 14. Gamma rays of energies 59.7 and $26.3 \mathrm{keV}$ are observed together with $L$-radiation corresponding closely to that of neptunium. Radiation detected in this way acts as a sensitive method of examining the fine structure of an alpha particle spectrum. The radiation associated with isomeric states of nuclei has, of course," been investigated with notable success in a similar fashion. Nuclear capture of $L$-electrons was first observed by Pontecorvo et al. ${ }^{65}$ in the case of $\mathrm{A}^{37}$. Two peaks were observed, one at $2.8 \mathrm{keV}$ corresponding to $K$-capture, and one at $250 \mathrm{eV}$ which was shown to be largely due to $L$-capture.

Cook ${ }^{66}$ pointed out the importance of the proportional tube in finding $\beta^{+} \mid K$-capture ratios where the only uncertainty arises in the knowledge of fluorescence yield. As an example of this work we have the observations of Townsend ${ }^{67}$ on $\mathrm{Zn}^{65}$. He used a gaseous compound of zinc, $\mathrm{Zn}\left(\mathrm{C}_{2} \mathrm{H}_{5}\right)_{2}$ as the source and peaks corresponding to $K$ - and $L$-capture processes were superposed on the continuum formed by the positrons. The recent work of Brown et al. ${ }^{68}$ on $\mathrm{Ca}^{41}$ is a good example of work on $K$-capture.

Weak conversion peaks may be examined with high sensitivity in the proportional tube. Such conversion may be detected in the form of the photo-electrons or in the form of the related X-radiation. In the recent work of West and Dawson ${ }^{69}$ on the $L$-radiation of $\mathrm{Pu}^{239}$ an intensity of $4 \pm 1 \times 10^{-2}$ quanta per alpha particle was measured. The associated photoelectrons and Auger electrons may be observed with thin sources within the spectrometer, as in the work of Cockroft ${ }^{64}$ on $\mathrm{Cr}^{51}$, where 6 per $10^{4}$ disintegrations lead to the emission of photoelectrons. Another notable example of work on conversion electrons is that on $\mathrm{Lu}{ }^{176}$ by Dixon et al. ${ }^{70}$, mentioned in $\S 10$ above.

-2 J. Angus, A. L. Cockroft and S. C. Curran, Phil. Mag., 40, (1949) 822.

cs J. P. Mize and D. J. Zaffarano, P. 33 of Report on Indiana Conf. on Nuclear Spectroscopy and the Shell Model, Indiana Univ., May 1953., Phys. Rev. 89, (1953) 902 (A); 91, (1953) 210 (A).

64 S. C. Curran, Physica, 18, (1952) 1161.

os B. Pontecorvo, D. H. W. Kirkwood and G. C. Hanna, Phys. Rev. 75, (1949) 982.

6 C. S. Cook, Amer., J. Phys., 19, (1951) 37.

or J. Townsend, Thesis, Washington University, St. Louis, 1951.

68 F. Brown, G. C. Hanna and L. Yaffé, Phys. Rev., 84, (1951) 1243.

60 D. West and J. K. Dawson, Proc. Phys. Soc., A64, (1951) 586.

20 D. Dixon, A. MeNair and S. C. Curran, Phil. Mag. 45, (1954) 683. 


\section{H. FULBRIGHT - Beta Spectroscopy by Use of a High Pressure Proportional Counter}

\section{§ I. Introduction and Description of Apparatus}

A high pressure proportional counter has been used successfully to determine the spectral shapes of several long-lived beta emitters with $E_{0}<1.5 \mathrm{MeV} 1,2,3$. The experimental arrangement is shown in Fig. 1. The sample was mounted in the center of a very thin aluminum foil having a density of about $0.2 \mathrm{mg} / \mathrm{cm}^{2}$. The foil was held in a metal frame mounted so as to divide a rectangular cathode cylinder into two equal volumes. The volumes were $1^{\prime \prime} \times 2.7^{\prime \prime} \times 6^{\prime \prime}$ long. Wolfram anode wires 1.0 mil in diameter

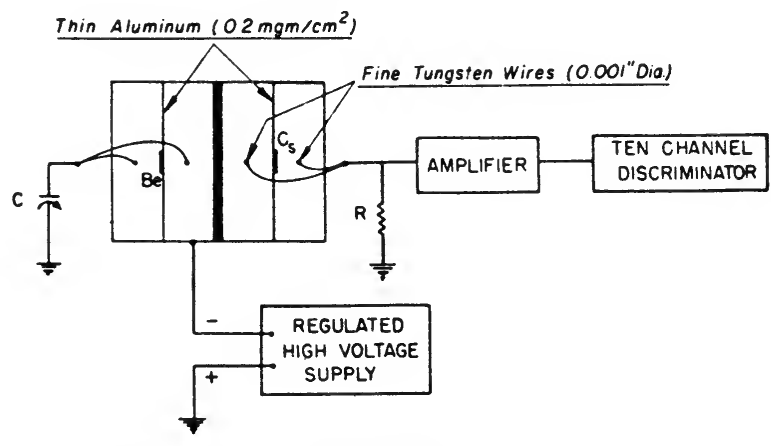

EXPERIMENTAL ARRANGEMENT

Fig. 1. The experimental arrangement $C$ was used to equalize the capacitances of the two units. The range of the particles was less than the distance from source to opposite side of Cathode

were mounted centered in each of the two volumes, and the two wires were connected together. Thus the sample was supported by the very thin common wall of two identical counter units connected in parallel. One of the chief advantages of this geometry is that primary electrons scattered backward through the foil from one unit into the other will produce proper sized pulses, providing that their energy is high enough so that a negligible amount of energy is lost in the foil.

This dual unit and a second identical unit included for calibration purposes were mounted together inside the high pressure vessel. The

1 H. W. Fulbright and J. C. D. Milton, Phys. Rer. 76, (1949) 1271.

2 J. C. D. Milton, Thesis, Princeton University, 1951.

3 H. W. Fulbright and J. C. D. Milton, Phys. Rev. 82, (1951) 274. 
calibration unit was necessary because of the sensitivity of the operating characteristics of the counter to variations in gas conditions. The amplifier input lead could be switched from one unit to the other. The calibrating source, normally left in the counter for all measurements, was $\mathrm{Cs}^{137}$, whose daughter, $\mathrm{Ba}^{137}$, is usually formed in a $662 \mathrm{keV}$ metastable state decaying to the ground state with a half life of 2.6 minutes by gamma ray or internal conversion electron emission. The conversion electrons provide a line spectrum suitable for calibration purposes. Details of the calibration procedure will be given later.

A calcium purifier was attached to the vessel by means of two tubes so that the argon could circulate by convection when the purifier was heated.

Best signal-to-noise ratio was obtained with rise time and clipping
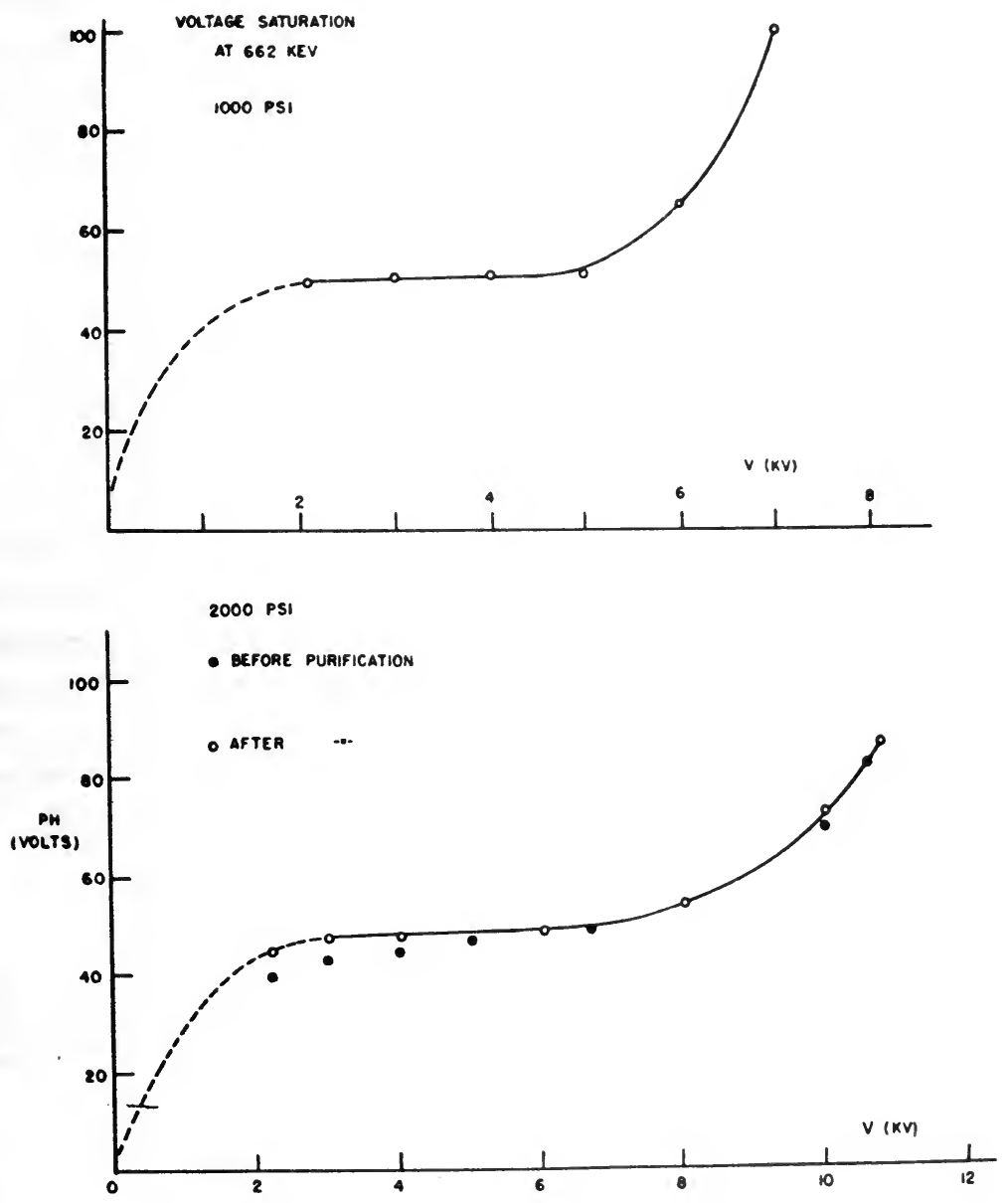

Fig. 2. Saturation curves at two pressures 
$R C$ constants set at about $20 \mu \mathrm{sec}$, but the clipping constant was increased to more than $100 \mu$ sec for most experiments.

The power supply was of the $R F$ oscillator-plus-rectifier type giving up to 20,000 rolts with regulation within $0.1 \%$.

Tank argon, either 99.6 or $99.8 \%$ pure, was used. The main difference between the two types is in the guaranteed maximum amount of $\mathrm{N}_{2}$ present. A trace of nitrogen helps rather than hurts the operation of the counter and, anyway, the autual amounts of impurities may be much lower than those guaranteed. About three cylinders out of four were satisfactory, giving proper pulses without excessive attachment trouble. The purifier was operated immediately after filling, then turned off before data were taken. With some cylinders of gas no purification was necessary. Bad gas could not be made usable although the purifier did improve the good gas somewhat. (See Fig. 2.) It is not known which impurities present in the bad cylinders caused the difficulty. An analysis performed by the supplier on an unsatisfactory cylinder of gas produced the following results:

TABLE III

Analysis of a cylinder of Argon

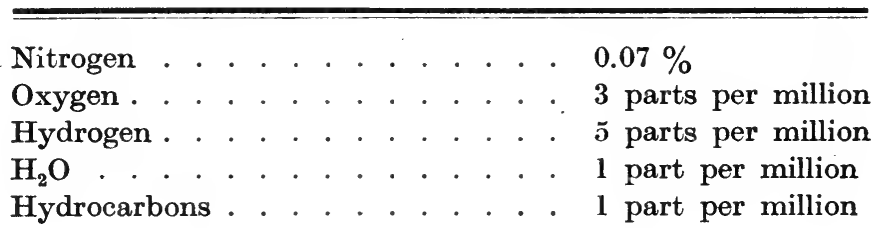

About half a percent of $\mathrm{CH}_{4}$ was often added, especially when gas pressures over 2000 pounds per square inch were involved, in order to make the gas multiplication less sensitive to voltage changes. A stainless steel cold trap was especially useful in the operation of building up the pressure in the vessel above that in the supply cylinders through a cyclic process of condensing argon from the tank by use of liquid air, then allowing the argon to evaporate into the vessel when the trap was warmed.

\section{$\S 2$. Analysis of the Operation of the Counter}

\section{A. Potential Distribution}

A calculation ${ }^{4}$ based upon the method of images shows that if end effects are neglected the potential distribution in the ion chamber is given by the expression

$$
V=\frac{A}{2} \sum_{-\infty}^{\infty}(-1)^{n} \ln \frac{\cosh \pi(x-n a)-\cos \pi y}{\cosh \pi(x-n a)+\cos \pi y}
$$

- J. C. D. Milton, Thesis, Princeton University, 1951. 
where $a=$ length of long side of rectangular cylinder $=2.7$ inches, $x$ and $y$ are distances from the wire in inches ( $x$ measured along longer side), and $A$ depends upon the geometry. In this case $A=140 V_{0}$ volts, where $V_{0}$ is in kilovolts. The series converges so rapidly that the first two terms are adequate for our purposes.

The potential near the central wire is given by

$$
V=A \ln (r / 2) .
$$

The field strength near the walls is rather small, roughly 100 volts per em for $V_{0}=1000$ volts. This is the most serious drawback of the geometry of the chamber.

\section{B. Spectrum Expected from a Moxoenergetic Source}

If an ionizing particle, say an alpha particle, leaves the source (assumed a point hereafter) and forms $\dot{a}$ straight track of ion pairs of linear density $\varrho(r)$, the fast electron pulse appearing at the collector where there is no gas multiplication, i.e. in purely ion chamber operation, will be given by

$$
Q_{\text {eff }}=e \int_{0}^{r_{0}} \varrho(r)\left(1-V(r) / V_{0}\right) d r
$$

where $V_{0}$ is the applied voltage, $V(r)$ is the potential (measured with respect to the cathode) at a distance $r$ along the track, and $r_{0}$ is the range of the track. Thus it is possible to calculate the pulse height to be expected from any given track from a knowledge of the Bragg curve and the potential distribution inside the chamber. Furthermore, the idealized spectrum

Equipotentials in a rectangular cylinder

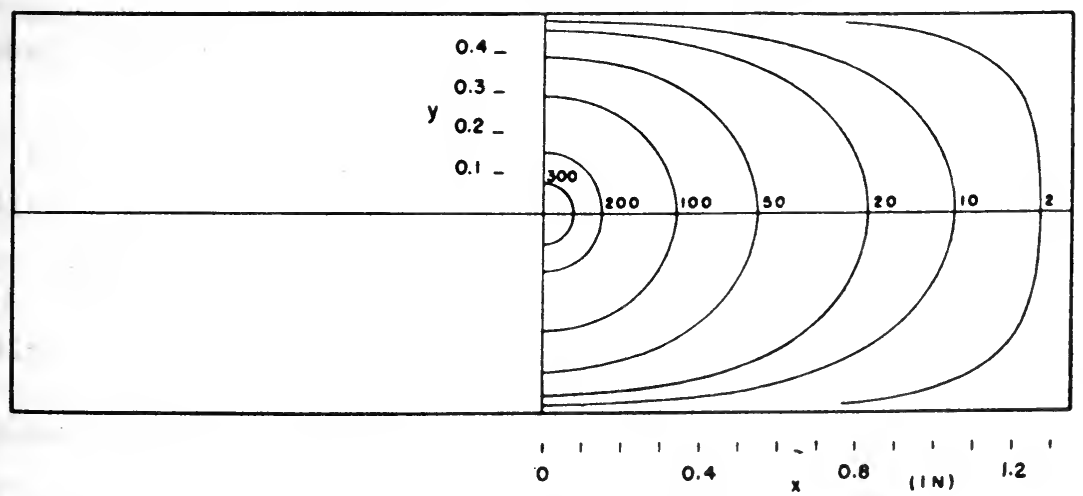

one mil centre wire at 1000 volts

$$
\mathrm{a}=2.7^{\prime \prime} \quad \mathrm{b}=1.0^{\prime \prime}
$$

Fig. 3. Potential Plot 
expected from a monoenergetic source of isotropically emitted particles can be found by a semiempirical process consisting essentially of dividing the counter space into many equal sectors of solid angle. calculating $Q_{\text {eff }}$ for a track centered in each, then assembling the results. Calculations made for $\varrho(r)=$ constant or $\varrho(r)$ given by the Bragg curve show that, very roughly speaking, there is a constant characteristic spectral shape for monoenergetic particles with straight tracks when $1 /{ }^{\prime \prime}<r_{0}<1^{\prime \prime}$ of the form

$$
P=\exp -\left(\epsilon_{0}-\epsilon\right) / c \epsilon_{0},
$$

where $\epsilon=$ idealized pulse height expected, $\epsilon_{0}=\max$. pulse height corresponding to the energy of the particles, and $c$ is a constant having an

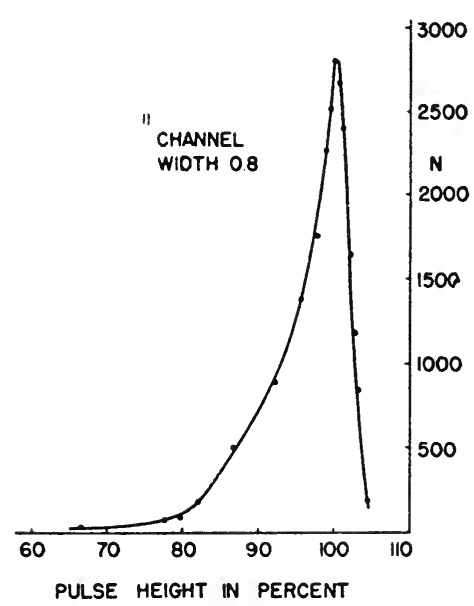

Fig. 4. Polonium alpha particle spectrum. $\pi_{0}=0.75^{\prime \prime} P \sim 2 \mathrm{~atm}$.

$V_{0}=300$ volts. Tank Argon average value of about $7 \%$. For $r_{0}<1 / 3^{\prime \prime}$ the resolving power improves rapidly with decreasing $r_{0}$. A measured spectrum of Po alpha particles observed with ion chamber operation with $r_{0}=0.75^{\prime \prime}$ is shown in Fig. 4 .

An exact resolving power calculation for electrons is obviously impossible because of scattering, but it is reasonable to suppose that the main effect of scattering is to reduce the effective range of the tracks somewhat without altering the general character of the resolving power curves.

The distribution (14) can never be realized experimentally because of attachment and recombination, straggling in ionization, and, especially, amplifier noise. The saturation curves obtained strongly suggest that gas troubles can be overcome. However, amplifier noise adds a gaussian contribution to the spectrum being amplified.

In our case a noise spectrum of the form

$$
R(\epsilon)=\mathrm{e}^{-\left(\epsilon-\epsilon^{\prime}\right)^{2} / 0^{2}}
$$

where $g$ is a constant, is superimposed upon (14) giving a spectrum

$$
S(\epsilon)=\int_{0}^{\epsilon_{0}} \mathrm{e}^{--\frac{\epsilon_{0}-\epsilon^{\prime}}{c \epsilon_{0}}} \mathrm{e}^{-\frac{\left(\boldsymbol{\epsilon}^{\prime}-\boldsymbol{\epsilon}\right)^{2}}{0^{2}}} d E^{\prime}
$$

which is the expected observable spectrum in ion chamber operation under the limitations mentioned earlier in connection with (14).

Empirically the best resolving power was found for the $\mathrm{Ba}^{137}$ line with gas multiplications, $G_{g}$, between 2 and 4, i.e. with enough gas gain to make the observed pulses 2 to 4 times their ion chamber value. 
With gas gain the width $c$ of the resolving power curve is reduced percentagewise from its ion chamber value because of the increase in pulse height, but there will be an increase in the width of the symmetrical part because of irregularities in the multiplication process. Strictly speaking. $g$ in (16) increases and is no longer a constant. Irregularities in the $1.0 \mathrm{mil}$ wolfram collector wires were no doubt responsible for some broadening. Better results might have been obtained with larger. more uniform wires and with higher gas gains. The best full width at half maximum found with $\mathrm{Ba}^{137}$ (see Fig. 5) was $10 \%$.
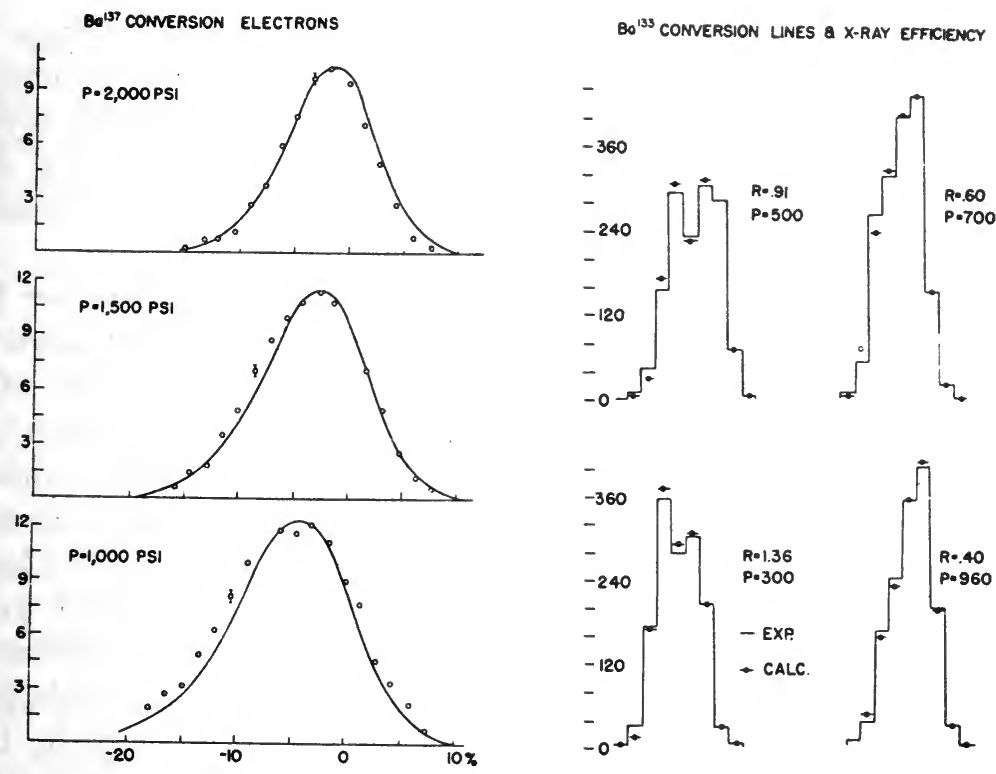

Fig. 5. $\mathrm{Ba}^{133}$ and $\mathrm{Ba}^{1 £ \overline{7}}$ conversion lines at various pressures. $G_{g}=4$ for $\mathrm{Ba}^{133}$ and 2 for $\mathrm{Ba}^{137}$

\section{§ 3. Calibration}

The $\mathrm{Ba}^{137}$ conversion line used in calibration has a structure because of the fact that in a considerable fraction of cases the $x$-radiation emitted following internal conversion is recaptured in the gas and produces additional ion pairs indistinguishable from primary pairs. The Auger effect also contributes. Of all the possible combinations of recovery of $\mathrm{X}$-ray photons possible, only two need be considered: (a) the $K \mathrm{X}$-ray escapes, but subsequent $\mathrm{X}$-radiation is captured; and (b) all $x$-radiation is captured in the gas.

A special study of the structure to be expected at various pressures was made by use of the $K$-capturer $\mathrm{Ba}^{133}$ which 'emits $276 \mathrm{keV} \gamma$-ravs and 
$239 \mathrm{keV}^{\prime} K$ conversion electrons and which has no continuum to interfere with the observations. Results for gas pressures of 300,500, 700, and 960 p.s.i. and $G_{0}=4$ are shown in Fig. 5. A double peak is seen at intermediate pressures, while at high pressures the higher energy peak predominates. Theoretical points were calculated with allowance for $L$ conversion, Auger effect ( 0.14 per $K$ conversion electron) and photoelectric recovery of $K$ X-rays. Good agreement is evident in the results. It appears that for $p>700$ p.s.i. the higher energy peak is dominant with its position not appreciably shifted by the presence of the small lower energy group.

The linearity of ionization yield in argon as a function of energy, was checked in an auxiliary experiment carried out with a special high pressure cylindrical proportional counter having a thin aluminum side window into which roughly monoenergetic beta particles selected by a short magnetic lens spectrometer could be directed. Results indicate linearity up to $1.2 \mathrm{MeV}$, the highest energy employed.

The number of ionization electrons arriving at the collector per $\mathrm{Ba}^{137}$ conversion electron was determined with some care for tank argon by measurements of absolute pulse height and input capacitance of the amplifier including ion chamber and leads. The result corresponds to an average of $W=36 \pm 3$ electron volts per ion pair compared with the value 28 obtained by Curran and Valentine ${ }^{4}$ using a method involving a long time average current measurement made with a source of known activity. In addition, Gerbes ${ }^{5}$ found 25 Volts and Nicodemus 26.9 Volts ${ }^{6}$. See also Table 1, page 000. It has been suggested that such differences may arise because of the slowness of quenching of the metastable atoms produced as a result of optical exitation by the primary particle. Using the pulse method Jesse and Sadauskis ${ }^{7}$ have found $W=36.3$ Volts per ion pair for spectroscopically pure neon and 26.1 Volts for neon with $0.12 \%$ argon. The argon presumably quenches the excited neon by becoming ionized in collisions with metastable atoms. The explanation seems plausibe in the present case because of the high ionization potential, 15.5 Volts, of $\mathrm{N}_{2}$, which is by far the most abundant impurity present.

\section{$\S 4$. Resolving Power Corrections}

The theory of Primakoff and Owen ${ }^{8}$ which shows that in the approxima-

5 W. Gerbes, Ann. D. Phys. 30, (1937) 169.

- D. Nicodemus, Thesis, Stanford University, 1946, quoted in "Ionization Chambers and Counters", B. Rossi and H. Staub, MeGraw-Hill 1949.

7 W. P. Jesse and J. Sadauskis, Phys. Rev. 88, (1952) 417.

8 G. E. Owen and H. Primakoff, Phys. Rev. 74, (1948) 1406. 
tion of eq. (16) the measured spectrum $N(\epsilon)$ should be corrected to give the implied "true" spectrum.

$$
M(\epsilon)=N(\epsilon)-c \epsilon_{0} \frac{d N}{d \epsilon}-\frac{g^{2}}{4} \frac{d^{2} N}{d \epsilon^{2}} .
$$

Since $c$ is not strictly a constant it is obviously best to use a high enough gas gain to make the first derivative term in (17) negligible. The second derivative term is usually small. It was generally found that energy calibration and resolving power corrections could be ignored together with very small error in the spectral shape, for $G_{g} \geqslant 2$.

\section{$\S 5$. Results}

The high pressure counter has reproduced accurately a variety of well known spectra having widely differing shapes, including $\mathrm{Cs}^{137}, \mathrm{Y}^{91}, \mathrm{Tl}^{204}$ $\mathrm{RaE}, \mathrm{K}^{40}$ and $\mathrm{Be}^{10}$ (Fig. 6).

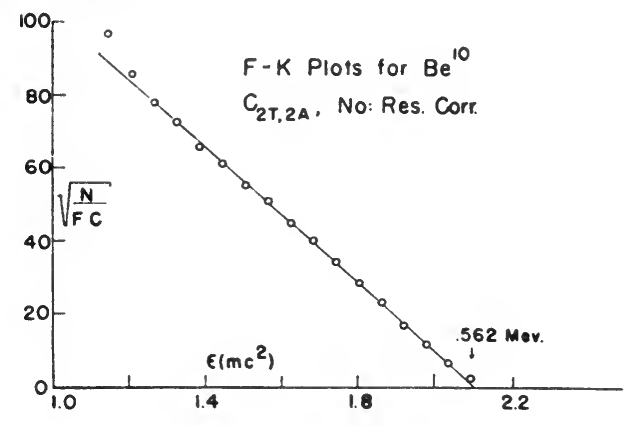

Fig. 6. $\mathrm{Be}^{10}$ results

$\mathrm{Be}^{10}$ was the first and most interesting one studied. The spectrum, which was obtained with a source too weak for conventional spectrometry, proved to be of the unique $\mathrm{D}_{2}$ type predicted by Marshak ${ }^{9}$.

In many cases spectra were examined with various gas pressures and gas gains. Excellent agreement of results was obtained. The $\mathrm{Cl}^{36}$ spectrum in particular was examined especially carefully because of the report 10 that its shape required a mixture of interactions for its explanation. The results show that the shape can be accounted for by either the $C_{2 T}$ or $C_{2 V}$ correction factor. However, there remains a question as to the exact shap of the spectrum in the low energy region ${ }^{11,3}$.

Several unsuccessful attempts were made to check the operation of the counter at 165 atmospheres using $\mathrm{P}^{32}\left(E_{0}=1.7 \mathrm{MeV}\right)$. The F-K plot was always slightly concave downward.

2 R. E. Marshak, Phys. Rev. 75, (1949) 513.

10 C. Longmire, C. S. Wu and C. H. Townes, Phys. Rev. 76, (1949) 695.

11 C. S. Wu and L. Feldman, Phys. Rev. 76, (1949) 693; C. S. Wu, Int. Conf. on Nuc. Phys., Univ. of Chicago (1951). 
(III) G. BISHOP and R. WILSON - Special Methods in Gamma-Ray Spectroscopy

\section{$\S 1$. Photoneutron Threshold Reactions}

The two reactions

$$
\begin{aligned}
& \gamma+\mathrm{D}^{2}=\mathrm{N}+\mathrm{P} \\
& \gamma+\mathrm{Be}^{9}=\mathrm{Be}^{8}+\mathrm{N}=2 x+N{ }^{\prime}
\end{aligned}
$$

have been used as threshold detectors for weak $\gamma$-rays.

When neutrons are detected from one of these reactions, we know that there must be $\gamma$-rays with an energy exceeding the binding energy of deuterium ( $2.23 \mathrm{MeV})$ or of beryllium $(1.67 \mathrm{MeV})$. "The advantage of this threshold detection is that $\gamma$-rays of lower energy cannot produce spurious effects even though they are several orders of magnitude more intense than the high energy $\gamma$-rays.

It is necessary to take a few precautions: $\gamma$-ray sources which come from a natural radioactive element such as Radium or Radiothorium, often have a natural neutron background of their own due to $\alpha-$ n reactions in the source constituents. These form a background. Also it is important that the neutron detector should not be appreciably affected by the presence of the intense $\gamma$-ray source.

\section{A. Neutrox Detector}

A high efficiency for neutron detection can be combined with an insensitivity to $\gamma$-rays by using a Szilard-Chalmers reaction. Thus Wilson ${ }^{1}$ has studied $\gamma$-ray spectra by bombarding $\mathrm{Be}^{9}$ and $\mathrm{D}^{2}$ and by slowing down the neutrons in ethyl iodide where they were captured by the iodine. The active iodine was concentrated chemically and the artivity measured with a Geiger counter. The chemical processing of this ethyl iodide reaction is now reproducible to $1 \%{ }^{2}$. A similar detector, used by Fluharty ${ }^{3}$ consisted of a solution of potassium permanganate from which the active manganese was separated and counted. On the other hand, others ${ }^{4}$ counted the neutrons directly with $\mathrm{BF}^{3}$ counters enriched in Boron 10.

Richard Wilson, Phys. Rev., 79, (1950) 1004.

P. F. D. Shaw and C. H. Collie, J. Chem. Soc., 264, (1949) 1217.

R. E. Fluharty and M. Deutsch, Phys. Rev., 76, (1949) 182.

4 E. Der Mateosian and M. Goldhaber, Phys. Rev. 78, (1950) 326.

V. Myers and A. Wattenberg, Phys. Rev. 75, (1949) 992.

B. Lissell, D. Sachs, A. Wattenberg and R. Fields, Phys. Rev. 73, (1948) 545. 
By these methods several very weak $\gamma$-rays have been found. Although the neutron energy was not determined the existence of transitions between known excited levels of nuclei was inferred merely from the presence of photoneutrons.

\section{$\S 2$. Measurement of Photoneutron Energies}

The energies from the reactions (1) are almost monochromatic if monochromatic $\gamma$-rays are used. Thus a measurement of the photoneutron energy gives a measurement of the $\gamma$-ray energy.

Two methods have been used to give an approximate measure of the neutron energy. The first is to measure the absorption coefficient of the photoneutrons in hydrogen; in the useful energy range of a few hundred $\mathrm{keV}$ neutron energy, the absorption coefficient varies rapidly with energy and is well known. The second, somewhat earlier method, is to plot the density distribution of slow neutrons when the photoneutron source is immersed in water. If $I$ is the neutron density at a distance $r$ from the source, the plot of $I r^{2}$ against $r$ has a maximum, whose position is a function of the neutron energy. In Fig. 1 the curves for several neutron

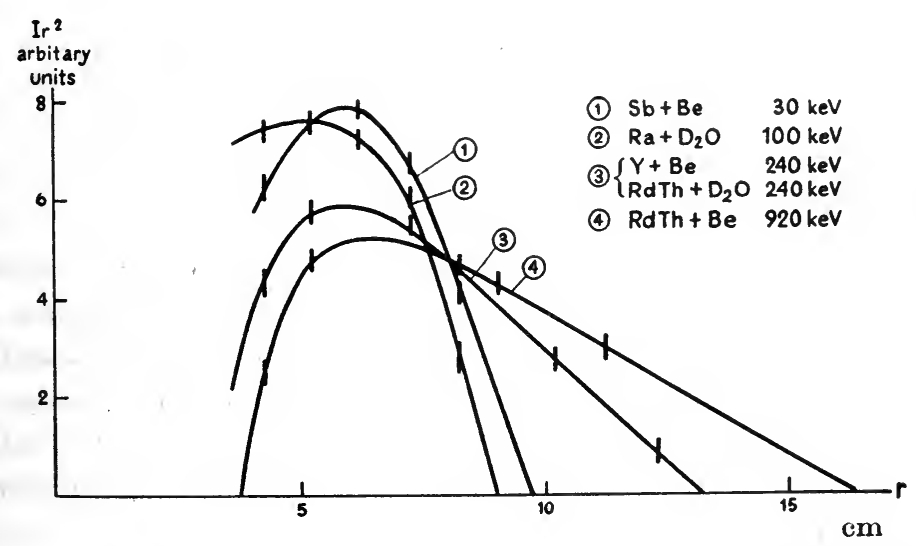

Fig. 1. Plot of $I r^{2}$ versus $r$ for different photoneutron sources; a resonance neutron detector was used. The points for $\mathrm{Y}+\mathrm{Be}$ and $\mathrm{RdTh}+\mathrm{D}_{\mathbf{2}} \mathrm{O}$ are so close that they are drawn together

energies are plotted taken from the data of several authors ${ }^{5}$. This method is practical only when strong sources of the $\gamma$-rays are available.

An inverse use of this method was made to place a lower limit on the

5 O. R. Frisch, H. Halban and J. Koch, Det. Kal. Danske Videnskaberng Selskab., XV, (1937) 10; E. Amaldi. L. R. Hafstad and M. A. Tuve, Phys. Rev. 51, (1939) 896; R. D. O’Neal, Phys. Rev. 70, (1946) 1. 
binding energy of the deuteron by use of a known $\gamma$-ray line in $\mathrm{Ga}^{72}$ $(2.230 \mathrm{MeV})^{6}$.

A list of nuclei studied by the photoneutron method is given in Table I.

TABLE I

NUClei STUDied BY THEIR PHOTONEUTRONS

\begin{tabular}{l|l|c|c|c|l}
\hline Source & Nucleus & $\begin{array}{c}\gamma \text {-ray energy } \\
\mathrm{MeV}\end{array}$ & $\begin{array}{c}\gamma \text {-ray } \\
\text { intensity }\end{array}$ & $\begin{array}{c}\text { Expected } \\
\text { crossover } \\
\text { MeV }\end{array}$ & \multicolumn{1}{|c|}{ Reference } \\
\hline $\mathrm{Ir}^{191}$ & $\mathrm{Pt}^{191}$ & $>2.2$ & $2 \times 10^{-6}$ & & Wilson \\
$\mathrm{Ir}^{194}$ & $\mathrm{Pt}^{194}$ & $1.6<h v<2.2$ & $1.4 \times 10^{-3}$ & 1.7 & Wilson \\
$\mathrm{Ag}^{110}$ & $\mathrm{Cd}^{110}$ & $>2.2$ & $<10^{-4}$ & & Wilson \\
$\mathrm{Ag}^{110}$ & $\mathrm{Cd}^{110}$ & $1.6<h v<2.2$ & $3 \times 10^{-4}$ & & Wilson, der Mateosian \\
$\mathrm{Cs}^{134}$ & $\mathrm{Ba}^{134}$ & $1.6<h v<2.2$ & $7 \times 10^{-7}$ & & Fluharty, Wilson \\
$\mathrm{Bi}^{206}$ & $\mathrm{~Pb}^{266}$ & $>1.6$ & $1 / 3$ & & der Mateosian \\
$\mathrm{Cl}^{38}$ & $\mathrm{~A}^{38}$ & $>2.2$ & $<3 \times 10^{-4}$ & 3.74 & Myers \\
$\mathrm{Co}^{60}$ & $\mathrm{Ni}^{60}$ & $>2.2$ & $<2 \times 10^{-7}$ & 2.4 & Myers, Fluharty \\
$\mathrm{Co}^{60}$ & $\mathrm{Ni}^{63}$ & $1.6<h v<2.2$ & $10^{-5}$ & & Fluharty \\
$\mathrm{Br}^{82}$ & $\mathrm{Kr}^{82}$ & 1.7 to 2.0 & $1.4 \times 10^{-3}$ & 1.9 & Myers \\
$\mathrm{Sb}^{124}$ & $\mathrm{Te}^{124}$ & $>2.2$ & $5 \times 10^{-4}$ & 2.3 & Myers \\
$\mathrm{Mn}^{56}$ & $\mathrm{Fe}^{56}$ & $\sim 2.7$ & $10^{-2}$ & 2.7 & Russell \\
$\mathrm{V}^{48}$ & $\mathrm{Ti}^{48}$ & $1.6<h v<2.2$ & $10^{-2}$ & 2.32 & Fluharty \\
$\mathrm{V}^{48}$ & $\mathrm{Ti}^{48}$ & $h v>2.2$ & $<10^{-2}$ & 2.32 & Fluharty \\
$\mathrm{Sc}^{46}$ & $\mathrm{Ti}^{46}$ & $1.6<h v<2.2$ & $1.2 \times 10^{-7}$ & 2.01 & Fluharty
\end{tabular}

\section{$\S 3$. Measurement of Photoproton Energies}

If an ionisation chamber or proportional counter is filled with deuterium gas at a high pressure it is possible to observe the photoprotons. For an adequately designed counter, the pulse amplitude is proportional to the ionisation by the proton and thus to the proton energy within certain limits. The amplitude distribution of these pulses is found with a kicksorter and the spectrum, of all $\gamma$-rays with energies greater than the binding energy, is calculated from the distribution?

If monochromatic $\gamma$-rays fall on the counter a spectrum of protons is obtained. This is because; owing to conservation of the $\gamma$-ray momentum, the energy of the photoprotons depends upon the angle which they make with the incident $\gamma$-ray direction. The energy spectrum is narrow, however, because the momentum of a $\gamma$-ray is small.

B G. R. Bishop, C. H. Collie, H. Halban and R. Wilson, Phys. Rev. 76, (1949) 683.

7 J. H. Carver and D. H. Wilkinson, Proc. Phys. Soc. A64, (1951) 199; G. R. Bishop, R. Wilson and H. Halban, Phys. Rev. 77, (1950) 416; R. Wilson, Phys. Rev. 80, (1950) 90; L. Beghian, G. R. Bishop and H. Halban, Phys. Rev. 83, (1950) 186. 
The energy of the photoproton is given by

$$
E=\frac{h \nu-\varepsilon}{2}+\mid \frac{\overline{h v-\varepsilon}}{4 M c^{2}} h v \cos \theta
$$

for $\gamma$-ray energies small compared with the rest mass of the proton. In an ionisation chamber all the photoprotons are recorded regardless of the angle $\theta$, so that the dependence of $E$ on $\theta$ leads to an expression for the energy resolution of the method. The total fractional energy spread is

$$
\frac{2 h \nu}{\sqrt{(h \nu-\varepsilon) M c^{2}}}
$$

This is about $20 \%$ for $h v=3 \mathrm{MeV}$ and $\varepsilon=2.23 \mathrm{MeV}$. The resolution curve is calculated from the angular distribution of the photoprotons about the incident $\gamma$-ray direction.

The angular distribution is of the form $a+b \sin ^{2} \theta$, where the values of $a$ and $b$ are obtained from a "best-fit" to just such a resolution curve. The resolution curve is an inverted parabola ${ }^{8}$.

The assumption is made that the ionisation produced by the photoprotons is proportional to their energy. For the instrument under consideration this assumption can be justified by two independent methods. A scale of pulse amplitude against energy is easily established by employing sources of known $\gamma$-ray energies. Thus the $\gamma$-rays of $\mathrm{Ga}^{72}(2.504 \mathrm{MeV})$, $\mathrm{ThC}^{\prime \prime}(2.615 \mathrm{MeV})$ and $\mathrm{Na}^{24}(2.758 \mathrm{MeV})$ were used ${ }^{8}$ to calibrate a deuterium filled proportional counter. The photoproton energies are $138 \mathrm{keV}, 199 \mathrm{keV}$ and $265 \mathrm{keV}$ respectively, and the pulse heights were proportional to these energies to an experimental accuracy of $1 \%$. The energy scale was extrapolated to the maximum pulse amplitude obtained with deuteron recoils from bombardment by neutrons from a $\mathrm{Rd} T \mathrm{~T}-\mathrm{Be}$ source and the energy agreed to within $2 \%$ with the expected value of $848 \mathrm{keV}$.

An alternative justification depends on the measurements of the ionisation of $\alpha$ particles in hydrogen which shows that even at small velocities the average energy loss per ion pair, $W$, is approximately constant. The ionisation in hydrogen and deuterium was found equal for protons by Crenshaw ${ }^{9}$ over the energy range $(80-300 \mathrm{keV})$. Even for $340 \mathrm{MeV}$ protons it has been shown that any deviation from the value of $W$ obtained at low energies is less than $5 \%{ }^{10}$. Thus the assumption of proportionality between ionisation and energy of heavy particles in deuterium gas is fairly safe.

8 G. R. Bishop, L. E. Beghian, H. Halban, Phys. Rev. 83, (1951) 1052.

9 C. M. Crenshaw, Phys. Rev. 62, (1942) 54.

10 C. Bakker and E. Segré, Phys. Rev. 81, (1951) 489. 
Other factors influence the resolution in deuterium filled counters recording the proton. These may be summarised

(1) Wall effect

(2) Incomplete collection of ions

(3) Statistics of the initial ionisation process

(4) Statistics of multiplication in a proportional counter

(5) Amplifier noise

(6) "Pile-up" of secondary electron pulses from $\gamma$-rays

The wall effect is the reduction in amplitude of those pulses due to protons which strike the boundaries of the sensitive volume and therefore do not lose all their energy to the gas. The fraction of pulses which do this is equal to

$$
\frac{1}{4} \times \frac{\text { surface area }}{\text { volume }} \times \text { range }
$$

to a good approximation for ionisation tracks short compared with the linear dimensions of the counter. This does not appreciably affect the utility of the counter as a $\gamma$-ray spectrometer, because such pulses merely form a continuous low energy background.

Incomplete collection is often the result of electron capture in the filling gas. Great care is needed to purify the gas. The necessary purity has been successfully obtained by passing the deuterium through a palladium leak ${ }^{11}$ or by successive distillation from a charcoal purifier at liquid air temperature ${ }^{12}$. The result of electron capture is that some of the pulses are reduced in amplitude and form a low energy tail. Only a very slight shift towards low energies should result in the peak of the spectrum.

When the number of secondary electron pulses from the $\gamma$-rays is large the effect of their "pile-up" on the distribution is to broaden it. When, as is usual, an a.c. coupled amplifier is used, there is no displacement of the peak of the distribution, because the conditions are the same as for the amplifier noise ${ }^{13}$. If the number of secondary electron pulses is small, the chance of pile up is remote and the effect on the distribution will not be noticed. In the intermediate case, some of the photoproton pulses will be coincident with the pile up pulses and produce a high energy tail to the distribution. At the same time the peak of the distribution will be displaced to a slightly lower energy by the averaging effect of the a.c. amplifier to maintain the same centre of gravity of the distribution.

11 G. H. Stafford, Nature, 162, (1948) 771.

12 R. Wilson, L. E. Beghian, C. H. Collie, H. Halban and G. R. Bishop, Rev. Sci. Inst. 21, (1950) 699.

13 R. Wilson, Phil. Mag. 41, (1950) 66. 
The other two sources merely spread the resolution curve slightly without altering its symmetry or the position of the maximum.

\section{$\S 4$. Comparison with Other Methods}

In order to compare the photodisintegration spectrometer with other methods we consider a specific example. The radioactive isotope $\mathrm{Na}^{24}$ decays by $\beta$-ray emission to $\mathrm{Mg}^{24}$ followed by a $\gamma$-ray cascade.

We wish to measure to what extent the 4.14 $\mathrm{MeV}$ level decays by $\gamma$-ray emission directly to the ground state, relative to the normal $\gamma$-cascade.

With the photoproton counter the $2.76 \mathrm{MeV}$ $\gamma$-ray gives protons of energy $265 \mathrm{keV}$. A 4.14 MeV $\gamma$-ray would produce protons of $950 \mathrm{keV}$ energy which are easily separated from the low energy ones. Moreover, if two $\gamma$-rays by chance produce photoprotons within the resolution time of the apparatus the pulse will not exceed $530 \mathrm{keV}$, which is also comfortably separable.

If a sodium iodide crystal and photomultiplier tube were used as a spectrometer, both the $1.38 \mathrm{MeV}$ and $2.76 \mathrm{MeV} \gamma$-rays could be counted. If both record simultaneously we might get a pulse of $4.14 \mathrm{MeV}$ energy, simulating a crossover $\gamma$-ray of $4.14 \mathrm{MeV}$. Moreover, the 4.14 MeV $\gamma$-rays would produce pulses principally in the "pair peak" at $3.14 \mathrm{MeV}$ which could be lost beneath the "pile-up" of $\gamma$-rays.

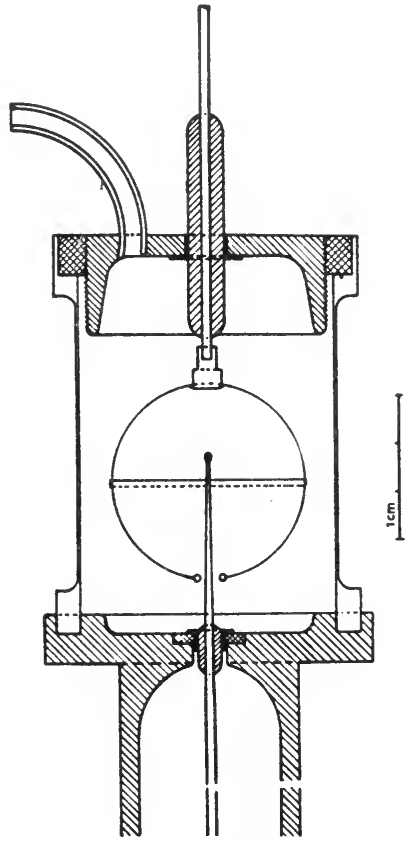

Fig. 2. Ionization chamber for study of $(\gamma p)$ processes in deuterium used by Bishop

We see that the deuterium filled photoproton counter is therefore particularly suited to a study of weak high energy $\gamma$-rays in the presence of intense low energy $\gamma$-rays.

\section{A. Photographic Plates}

It is also possible to measure the $\gamma$-ray spectra by measuring the photoprotons in a photographic plate. This has been done in a preliminary study of neutron-capture $\gamma$-rays ${ }^{14}$.

Table II is a summary of all $\gamma$-ray transitions in nuclei studied by their photoprotons.

14 B. Hamermesh, Phys. Rev. 80, (1950) 415. 
TABLE II

NLCLEI STUDIED BY THEIR PHOTOPROTONS

\begin{tabular}{|c|c|c|c|c|c|}
\hline Source & Nucleus & $\begin{array}{c}\gamma \text {-ray } \\
\text { energy } \\
\text { Mel }\end{array}$ & $\begin{array}{c}\gamma \text {-ray } \\
\text { intensity }\end{array}$ & $\begin{array}{c}\text { Expected } \\
\text { "crossover" } \\
\text { energy Mel" }\end{array}$ & Reference \\
\hline${ }^{24} \mathrm{Na}$ & ${ }^{24} \mathrm{Mg}$ & $3.8 \pm 0.2$ & $5 \times 10^{-4}$ & 4.14 & Beghian et al. \\
\hline${ }^{24} \mathrm{Na}$ & ${ }^{24} \mathrm{Mg}$ & 4.14 & $<3 \times 10^{-7}$ & 4.14 & Bishop et al. \\
\hline $56 . \mathrm{Mn}$ & ${ }^{56} \mathrm{Fe}$ & $2.7 \pm 0.2$ & 0.001 & 2.66 & Bishop .. \\
\hline${ }^{58} \mathrm{Mn}$ & ${ }^{56} \mathrm{Fe}$ & $3.0 \pm 0.1$ & 0.002 & 2.96 & Bishop ,. \\
\hline${ }^{22} \mathrm{Ga}$ & ${ }^{72} \mathrm{Ge}$ & $3.05 \pm 0.1$ & 0.0013 & 3.05 & Bishop $\quad .$. \\
\hline${ }^{72} \mathrm{Ga}$ & ${ }^{72} \mathrm{Ge}$ & $3.35 \pm 0.1$ & 0.0004 & 3.35 & Bishop \\
\hline${ }^{140} \mathrm{La}$ & ${ }^{140} \mathrm{Ce}$ & $2.55 \pm 0.05$ & 0.04 & - & Bishop \\
\hline${ }^{140} \mathrm{La}$ & ${ }^{140} \mathrm{C} \mathrm{e}$ & $2.9 \pm 0.1$ & 0.001 & - & Bishop \\
\hline Rd.'Th & ${ }^{208} \mathrm{~Pb}$ & 3.2 & $<10^{-4}$ & 3.2 & Bishop \\
\hline Ra & ${ }^{210} \mathrm{~Pb}$ & 4.0 & $<0.05$ & 4.0 & Bishop \\
\hline $\mathrm{n}+\mathrm{C}^{12}$ & $\mathrm{C}^{13}$ & 4.9 & & 4.9 & Wilson \\
\hline \multirow{2}{*}{\multicolumn{2}{|c|}{$\begin{array}{c}\mathrm{n}+\mathrm{C} a, \mathrm{n}+\mathrm{Cl} \\
\mathrm{n}+\text { several elements }\end{array}$}} & $\begin{array}{l}+ \text { impuritiess } \\
\text { spectra }\end{array}$ & & & Wilson \\
\hline & & spectra & & & Hamermesh \\
\hline $\mathrm{P}+{ }^{9} \mathrm{Be}$ & ${ }^{10} \mathrm{~B}$ & $7.39 \pm 0.15$ & & & Carver \\
\hline$P+{ }^{11} 13$ & ${ }^{12} \mathrm{C}^{\circ}$ & $12.50 \pm 0.21$ & & & Carver \\
\hline $\mathrm{P}+{ }^{13} \mathrm{C}$ & ${ }^{14} N$ & $8.14 \pm 0.08$ & & & Carver \\
\hline $\mathrm{P}+{ }^{3} \mathrm{C}$ & ${ }^{14} \mathrm{~N}$ & $5.81 \pm 0.25$ & & & Carver \\
\hline $\mathrm{P}-{ }^{19} \mathrm{~F}$ & ${ }^{20} \mathrm{Ne}$ & $12.09 \pm 0.28$ & & & Carver \\
\hline $\mathrm{P}+{ }^{7} \mathrm{Li}$ & ${ }^{8} \mathrm{Be}$ & $14.4 \pm 0.4$ & & & Carver \\
\hline$P+{ }^{15} \mathrm{Y}$ & ${ }^{12} \mathrm{C}$ & $4.45 \pm 0.04$ & & & Carver \\
\hline
\end{tabular}

\section{$\S 5$. Polarisation of Gamma Rays}

The photodisintegration of the deuteron at $\gamma$-ray energies of more than several hundred kilovolts in excess of the binding energy, is due almost entirely to the electric dipole absorption. This is confirmed by measurements of the angular distribution of the photoprotons or photoneutrons and also of the total cross section for photodisintegration as a function of the incident $\gamma$-ray energy. In the case of electric dipole absorption the theory predicts that the angular distribution of the photoprotons relative to the electric vector of the $\gamma$-rays is proportional to $\cos ^{2} \phi$, where $\phi$ is the angle of emission relative to the electric vector. This prediction has been used ${ }^{15}$ to demonstrate the polarisation of the $\gamma$-rays from the radiative capture of protons by deuterons to form $\mathrm{He}^{3}$ ( $\gamma$-ray energy $\left.6 \mathrm{MeV}\right)$. The deuterium was contained in a loaded photographic plate. Since the $\gamma$-ray emitted in this reaction is plane-polarised, it is particularly suited to detection with a nuclear emulsion, but other plane-polarised $\gamma$-rays would

15 D. H. Wilkinson, Phil. Mag. 43, (1952) 660. 
be available from cer tain nuclear reactions if selected in coincidence with a heavy particle emitted at a suitable angle to the $\gamma$-ray direction. Naturally such a coincidence method would necessitate the use of a deuterium filled counter. A possible counter might consist of a cylindrical proportional counter of length several times the diameter. This would serve to determine the plane of polarisation since when the axis of the counter is parallel to the electric vector a majority of the photoprotons expend their full energy in the counter, whereas for a rotation of $90 \%$ the majority of photoprotons collide with the wall and lead to smaller pulses. From the direction of the plane of polarisation the nature of the transition as electric or magnetic could be decided and thence the parity change in the $\gamma$-ray transition, in accordance with the well known selection rules.

The photodisintegration method for the determination of polarisation is useful for high energy $\gamma$-rays since other processes such as Compton scattering and the photoelectric effect either lose in sensitivity to the polarisation, or in cross section.

Deuterium loaded emulsions have also been used to study the polarisation of bremsstrahlung emitted from a $20 \mathrm{MeV}$ betatron ${ }^{16}$. In this case the more obvious way of detecting the polarisation, namely by the dependence of the reciprocal process of pair production on the plane of polarisation, is likely to prove difficult owing to the large background close to a betatron.

\section{§6. Other Reactions}

At high energies there is a continual search for other special reactionsparticularly those that might have a threshold, and be useful as threshold detectors. One example is the reaction ${ }^{17}$

$$
\gamma+\mathrm{C}^{12}=3 \alpha .
$$

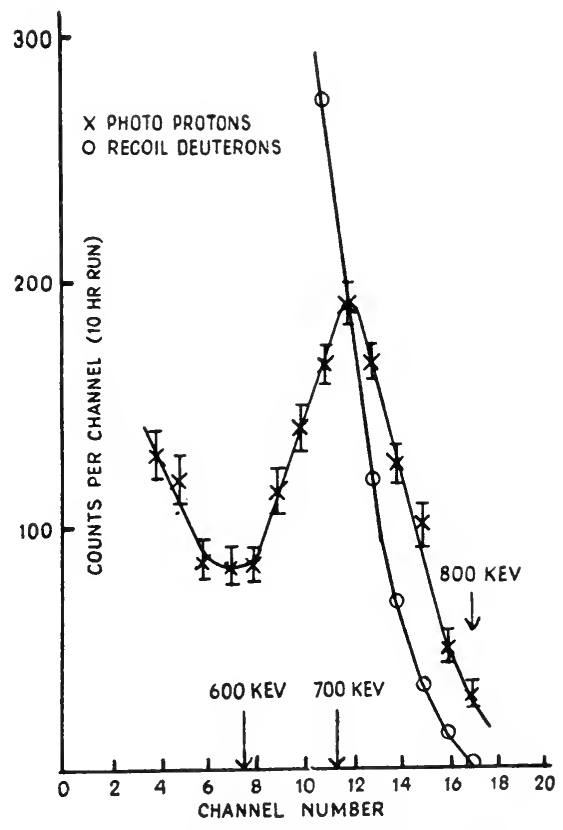

Fig. 3. Photoprotons from the 3.8 $\mathrm{MeV} \gamma$-rày line in $\mathrm{Na}^{24}$ measured in the presence of an intense background

Thus it should be possible to fill an ionisation chamber at high pressure

16 K. Phillips, Phil. Mag. 44, (1953) 169.

17 F. K. Goward and J. J. Wilkins, Proc. Roy. soc. 217, (1953) 357; V. L. Telegdi, Phys. Rev. 87, (1952) 196. 
with methane ${ }^{12}$ and to stop the $\alpha$-particles in the gas. Then the pulse height should be proportional to the difference between the $\gamma$-ray energy and the binding energy of $\mathrm{C}^{12}$. There should be no intrinsic energy spread for monochromatic $\gamma$-rays, but troubles due to incomplete collection will be worse for methane gas than for deuterium gas. A study of this reaction in a scintillation crystal instead of a high pressure counter is likely to prove unfruitful because of the non-linearity of the response of organic scintillation crystals.

If the reaction itself is the main study, it is possible to study it in a photographic plate, where an $\alpha$-particle star is easily identified. The energy of the $\gamma$-ray causing the star is given by the sum of the observed $\alpha$-particle energies as before. This relation is unlikely, however, to prove profitable as a study of $\gamma$-rays themselves. 


\title{
THE COINCIDENCE METHOD
}

\author{
A. C. G. MITCHELL
}

\section{$\S 1$. Introduction}

For many years coincidence counting techniques have been used as an aid in the determination of nuclear disintegration schemes. The information obtained by that method, when taken together with that obtained with the help of a magnetic spectrometer is at present one of the best methods of obtaining the energy levels of radioactive nuclei.

The principle of the method is to measure the "simultaneous" emission of two types of radiation, for example a beta-ray and a gamma-ray, two gamma-rays, or a heavy particle and a gamma-ray, with the help of two counters and a coincidence circuit for measuring the simultaneity of these events within a resolving time $\tau$. Since in this chapter we limit ourselves to the study of beta- and gamma-rays, Geiger-Müller counters or scintillation counters may be used. In making measurements of this type, one is interested not only in the time connection between the emission of two radiations but also in the energy distribution of the radiations which are correlated in time. For this reason it is necessary to use some kind of energy analysis before each counter. For beta particles and electrons, absorption techniques or magnetic analysis may be used. For gammarays, up until the last three years, no very suitable method of energy analysis has been available. At the present time, however, the scintillation method (see $\mathrm{Ch}$. V) provides a reasonably accurate means of energy discrimination for gamma-rays.

The greater part of the information on the determination of disintegration schemes by coincidence counting methods, accumulated over the past fifteen years, was obtained with the help of absorption techniques. This was necessary owing to the low specific activity of radioactive sources then available. A comparison of the coincidence method using absorption techniques for the determination of disintegration schemes with the magnetic analysis method (nuclear spectrograph) without time correlation (see Ch. VIII) gives a picture of the advantages and disadvantages of each.

The first attempts at applying the coincidence method to the investigation of the characteristics of radioactive decay appear to have been made by W. Bothe ${ }^{1,2,3,4}$ and his school about 1935. In the ensuing

1 W. Bothe and H. Geiger, Zeits. f. Phys. 32, (1925) 639.

2 W. Bothe and H. J. v. Bayer, Gott. Nachr. 1, (1935) 195.

3 H. J. v. Bayer, Zeits f. Phys. 95, (1935) 417.

- W. Bothe and H. Maier-Leibnitz, Zeits f. Phys. 104, (1936) 604. 
four years, this method was applied to the study of nuclear disintegration schemes by Norlin' ${ }^{5}$. Feather and Dunworth ${ }^{6}$ and Langer, Mitchell and McDaniel 7.8. A stirvey of the art as it existed in 1948 has been published by the present writer ${ }^{9}$, and also by Mandeville and Scherb ${ }^{10}$.

\section{$\S$ :. The Disposition of Apparatus}

In order to measure coincidences between beta- and gamma-rays or between two gamma-rays, Geiger-Müller counters are usually used. For measuring beta-rays, a counter having thin walls or an end window counter having a mica window of about $4 \mathrm{mg} / \mathrm{cm}^{2}$ thickness is used. To measure the gamma-rays, either a thick-walled counter or a beta counter with an external radiator is used. Amplifiers and coincidence circuits for use with (ieiger-Müller counters are now standard apparatus and are described in detail in the literature. It is possible to get resolving times of $10^{-6}-10^{-7}$ secs without much difficulty.

There are two ways in which the resolving time of the coincidence circuit may be measured. The first one relies on the principle that, if the two counters are counting at rates $N_{1}$ and $N_{2}$ per sec. respectively, then the chance rate $N_{\text {ch }}$ is given by

$$
N_{\text {ch }}=2 \tau N_{1} N_{2}
$$

where $\tau$ is the resolving time in seconds. It is, therefore, necessary to take two independent sources, place one over each counter, measure the single rates for each counter and the coincidence rate for the combination. Thus, having measured $N_{1}, N_{2}$ and $N_{\mathrm{ch}}$, the value of $\tau$ can be calculated from (1). If the single counting rate in each counter is 6000 per min or 100 per sec, the chance rate will be 7.2 per hour for $\tau=10^{-7} \mathrm{sec}$ or 72 per hour for $\tau=10^{-6}$ sec. Since the error in counting a total of $n$ particles varies as $\sqrt{n}$ it.will be seen that, in order to determine $\tau$ to $3 \%, 1000$ coincidences will have to be counted. In addition, corrections have to be made for the coincidence rate arising from cosmic rays, $N_{\text {cos }}$, which occurs when no sources are present. Such a procedure is obviously time consuming.

With the advent of new electronic devices, produced commercially, it is now possible to measure the resolving time with the help of a double pulse generator and a fast oscilloscope with calibrated sweep. Each oscillator of the double pulse generator produces a square wave of known, and usually,

5 F. Norling, Zeits f. Phys. 111, (1938) 158.

- N. Feather and J. V. Dunworth, Proc. Camb. Phil. Soc. 34, (1938) 442.

7 L. M. Langer, A. C. G. Mitchell and P. W. McDaniel, Phys. Rev. 56, (1939) 380.

8 L. M. Langer, A. C. G. Mitchell and P. W. McDaniel, Phys. Rev. 56, (1939) 422.

9 A. C. G. Mitchell, Rev. Mod. Phys. 20, (1948) 296.

10 C. E. Mandeville and M. W. Sherb, Nucleonies, Nol. 3, No. 4, (1948) 2. 
variable width and a known frequency. The set of pulses from one generator can be delayed in time with respect to the other by a measurable, and usually, variable amount. In lining up the coincidence amplifier it is only necessary to feed pulses from each of the generators into each of the amplifiers, change the delay until coincidences are counted by the apparatus, and measure the delay time on the calibrated oscilloscope. This saves considerable time in the lining up process. However, in performing any experiment it is always best to check the resolving time by the method given in the preceding paragraph.

In measuring coincidences between beta-rays and gamma-rays (betaganma coincidences) or coincidences between two gamma-rays (gammagamma coincidences) from a radioactive source, the apparatus is arranged as shown in Fig. 1. The two counters are in the same horizontal plane

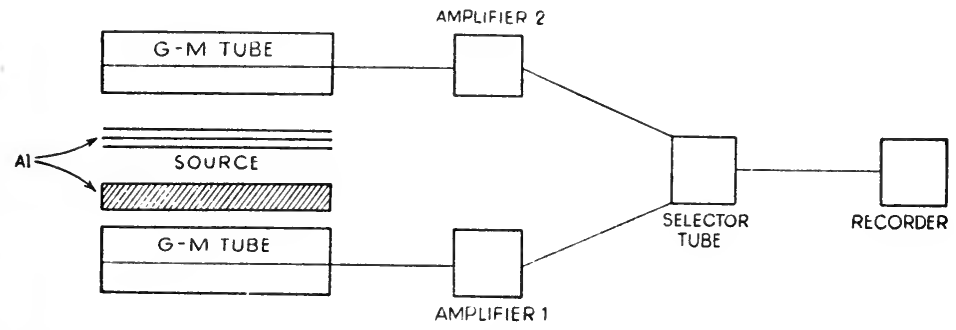

Fig. 1. Arrangement of Apparatus for Coincidence Counting

with the source placed midway between them. In Fig. 1 it is supposed that the counters are of the thin walled cylindrical type. If coincidences between gamma-rays are to be observed. enough aluminum is placed on either side of the source to prevent any beta-rays from reaching the counters. The aluminum then serves as an external radiator for measuring gamma-rays. If beta-gamma coincidences are to be measured, thin sheets of aluminum, whose thickness can be varied. are placed bet ween the source and one of the counters (beta-counter). By varying the thickness of aluminum between the source and the beta-counter, the number of betagamma coincidences can be measured as a function of the range of the beta-particles. If an end window counter is used for the beta-rays and a thick-walled counter for the gamma-rays the arrangement is the same except for the thick aluminum radiator in front of the gamma-counter.

In order to keep the chance rate small compared to the true coincidence rate and still get a sufficient number of coincidences in a reasonable time, it is best to keep the counters close together. in order to subtend as large a solid angle as possible, and to use a source of not too high specific activity. To see this, consider a source undergoing $N_{0}$ disintegrations per second 
which emits one beta-particle followed by one ganma-ray, the two being in coincidence. Let $\omega_{\gamma}$ be the solid angle subtended by the gamma-counter, $\varepsilon_{\gamma}$ be the efficiency of the gamma-counter for detecting the gamma-ray. Let $\omega_{\beta}$ be the solid angle subtended by the beta-counter and, for simplicity, suppose that all beta-particles emitted by the source into the solid angle $\omega_{\beta}$ are registered by the beta-counter. The counting rate in the betacounter will be given by

$$
N_{\beta}=N_{0} \omega_{\beta}
$$

and that in the gamma-counter by

$$
N_{\gamma}=N_{0} \omega_{\gamma} \varepsilon_{\gamma}
$$

The true beta-gamma coincidence rate will be given by

and the chance rate by

$$
N_{\beta \gamma}=N_{0} \omega_{\beta} \omega_{\gamma} \varepsilon_{\gamma}
$$

$$
N_{\mathrm{ch}}=2 \tau N_{\beta} N_{\gamma}=2 \tau N_{0}^{2} \omega_{\beta} \omega_{\gamma} \varepsilon_{\gamma}
$$

neglecting small backgrounds in each counter. The ratio of true to chance coincidences will therefore be

$$
N_{\beta \gamma} / N_{\mathrm{ch}}=\frac{1}{2 \tau N_{0}} .
$$

Thus for a given $\tau$, determined by the apparatus, $N_{0}$ must be kept as small as possible to make the true to chance rate as large as possible. The author has found that a convenient distance between the centers of the counters is about $7 \mathrm{~cm}$. It should be noted also, that the absolute source strength $N_{0}$ can be determined as a result of eq. (6) without a knowledge of solid angle or efficiencies provided that the assumptions about the decay scheme set forth above are valid.

\section{$\S$ 3. General Procedures for Coincidence Counting}

At this point, it would seem worthwhile to discuss in detail the technique used in actually determining the properties of the radiations from a radioactive source by the coincidence method. We suppose that we have the coincidence apparatus shown in Fig. 1 available and that it has been lined up initially by the procedures of $\S 2$. We wish to investigate a radioactive source whose half-life is known, but, for the sake of the argument, nothing further is known except that it emits beta-rays (no positrons or $K$-capture) and gamma rays. Suppose also, for the moment, that the half-life is reasonably long.

After making a good chemical separation of the source to be sure that it is pure, the first thing to do is to run a beta-ray absorption curve, using 
aluminum absorbers, with the help of a single counter. This procedure shows at once what the end point of the spectrum is and whether there are gamma-rays. Assuming there are gamma-rays (otherwise the coincidence technique would be of no use), the next step is to get some idea of the energy of the gamma-rays by taking an absorption curve in lead or by other procedures discussed in $\S 6$. We are now ready to carry out the coincidence measurements.

It is first necessary to determine the cosmic ray coincidence background, $N_{\text {cos }}$, by allowing the apparatus to run for a considerable time with no sources present. Next the resolving time is determined, using two independent sources as described earlier.

The source is now investigated for gamma-yamma coincidences. Assuming that two thin walled cylindrical counters are used, the source is placed midway between the two counters, and in their plane, and enough aluminum is put between the source and each counter to stop all betarays. As before, the single count is taken in each counter, the coincidence experiment performed, and finally the singles count taken again. The total coincidence rate is now made up as follows

$$
N_{T}=N_{\gamma \gamma}+N_{\text {ch }}+N_{\text {cos }}
$$

If the source decays slowly it is sufficient to use the singles counting rate in each counter at the mean time in calculating $N_{\mathrm{ch}}$. For rapidly decaying sources, the chance rate is calculated as follows.

Let $N_{1}(t)$ and $N_{2}(t)$ be the counting rate in each counter arising from radiations from the source and $B_{1}, B_{2}$ be the respective background rates. The average chance rate is then given by

$$
N_{\mathrm{ch}}=\frac{2 \tau}{T} \int_{0}^{T}\left[N_{1}(t)+B_{1}\right]\left[N_{2}(t)+B_{2}\right] d t
$$

where $T$ is the total time of the coincidence run. The result can be expressed as a function of the counting rate in each counter at the beginning and end of the coincidence run, the backgrounds, the decay constant $\lambda$, and $T$.

Knowing the counting rate due to gamma-rays in the "gamma-ray counter", the ratio $N_{\gamma \gamma} / N_{\gamma}$ can be calculated. In most cases this is of the order of magnitude of $10^{-3}$, owing to the low efficiency of counters for gamma-rays. Obviously, if $N_{\gamma \gamma}$ is zero, the source emits no gamma-rays in cascade ${ }^{10 a}$.

$10 a$ In all of the work of this nature published so far, it has been assumed that the distribution of cascade gamma-rays is isotropic. Recent work has shown that an anisotropy of up to $20-30 \%$ may exist for two ganima-rays in cascade (see ch. XIX). Since the experiments discussed in this section have been done with 
Having determined $N_{\gamma \gamma} / N_{\gamma}$ it is now possible to proceed to the investigation of beta-gamma coincidences. To do this, aluminum is removed in steps from between the source and one of the counters, called the betaray counter, and a coincidence experiment similar to the one described above is carried out for each absorber thickness until all absorbers have been removed. The thickness of each absorber is chosen in consideration of the beta-ray absorption curve taken at the outset of the series of experiments. The total coincidence counting rate at each point is given by

$$
N_{T}=N_{\beta \gamma}+N_{\gamma,}+N_{\text {ch }} \div N_{\text {cos }}
$$

In calculating $N_{\gamma \gamma}$ it is necessary to estimate the number of gamma-ray counts in the beta-counter and apply the factor $N_{\gamma \gamma} / N_{\gamma}$ already deter-

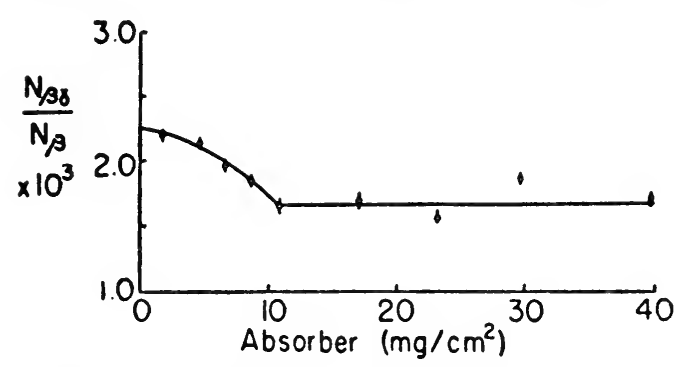

Fig. 2. Beta-gamma Coincidences in $\mathrm{C}^{134}$ mined. The value of $N_{\beta \gamma}$, for a given absorber thickness, can then be determined. It is then customary to calculate $N_{\beta \gamma} / N_{\beta}$, where $N_{\beta}$ is the number of counts due to beta-rays in the beta-counter, and to plot this ratio against the thickness of the absorber before the betacounter. Some typical results taken from some work on $\mathrm{Cs}^{134}$ are given in Table $\mathrm{I}$ and shown in Fig. 2.

\section{$\S$ 4. The Calculation of Errors}

The error in the result of a coincidence counting experiment can be calculated from the general principle that the error in counting a total of $n$ particles is $\sqrt{n}$. In general the error in the singles count may be made small compared to that of the coincidence count and may be neglected.

Consider, for simplicity, a source which gives beta-gamma coincidences only. If the total coincidence rate is $N_{T}$ then

$$
N_{T}=N_{\beta \gamma}+N_{\text {ch }}+N_{\text {cos }} \text {. }
$$

For a counting time $T$, the total number of coincidences is $N_{T} T$ and the error in the total coincidence rate is

$10 \alpha$ continued

$$
e_{T}=\left(\frac{N_{T}}{T}\right)^{1 / 2}
$$

Geiger-Mueller counters, this effect has been neglected except for cases in which annihilation radiation is present. When more precise experiments are performed with scintillation counters to determine disintegration schemes, this effect will have to be taken into consideration. 
TABLE I

Conncidence Studies of $\mathrm{Cs}^{134}$

(From Data of Meem and Maienschein*)

All rates in counts/min

\begin{tabular}{|c|c|c|c|c|c|c|c|c|c|c|}
\hline $\begin{array}{c}\text { Total } \\
\text { Absorber } \\
\mathrm{mg} / \mathrm{cm}^{2}\end{array}$ & $N_{\gamma}(\gamma)$ & $N_{T}^{\prime}(\beta)$ & $\lambda_{\gamma}(\beta)$ & $N_{\beta}(\beta)$ & $N_{T}$ & $N_{\mathrm{ch}}$ & $N_{\gamma \gamma}+N_{\cos }$ & $N_{\beta \gamma}$ & $\begin{array}{c}\Lambda_{\beta \gamma} / \Lambda_{\beta} \\
\times 10^{3}\end{array}$ & $\begin{array}{l}\text { Error } \\
\text { in } \\
N_{\beta \gamma} / N_{\beta}\end{array}$ \\
\hline 39.7 & 12010 & 4704 & 2200 & 2504 & 8.30 & 1.02 & 3.10 & 4.08 & 1.62 & 0.06 \\
\hline 29.5 & 12400 & 5254 & 2200 & 3054 & 10.11 & 1.21 & 3.25 & 5.65 & 1.85 & 0.05 \\
\hline 23.2 & 11988 & 5404 & 2200 & 3204 & 9.27 & 1.16 & 3.10 & 4.86 & 1.56 & 0.07 \\
\hline 17.1 & 12475 & 5186 & 2200 & 2986 & 9.47 & 1.16 & 3.25 & 5.06 & 1.69 & 0.07 \\
\hline 10.9 & 12443 & 5121 & 2200 & 2921 & 9.24 & 1.15 & 3.25 & 4.84 & 1.65 & 0.07 \\
\hline 8.7 & 12120 & 7185 & 2200 & 4985 & 13.90 & 1.57 & 3.15 & 9.18 & 1.84 & 0.05 \\
\hline 6.6 & 12017 & 8609 & 2200 & 6419 & 17.52 & 1.86 & 3.10 & 12.56 & 1.96 & 0.05 \\
\hline 4.2 & 12110 & 8790 & 2200 & 6590 & 18.10 & 1.91 & 3.15 & 14.64 & 2.13 & 0.05 \\
\hline$(\gamma-\gamma)$ & 12574 & - & 2200 & - & 3.75 & 0.497 & 3.25 & 3.15 & 0.25 & 0.05 \\
\hline $\begin{array}{l}N_{\cos } \\
N_{\gamma}(\gamma) \\
N_{T}(\beta) \\
N_{\gamma}(\beta) \\
N_{\beta}(\beta) \\
N_{T}, N\end{array}$ & $\begin{array}{l}=0.01 \\
- \text { cour } \\
- \text { Tot } \\
- \text { Cou } \\
- \text { Cou } \\
N^{2}\end{array}$ & $\begin{array}{l}\text { cou } \\
\text { ting } \\
\text { ting } \\
N_{y}\end{array}$ & $\begin{array}{l}\text { te in } \\
\text { ing } \\
\text { te } \mathrm{fr} \\
\text { te } \mathrm{fr} \\
\text { nd } N\end{array}$ & $\begin{array}{l}m \text { ga } \\
m \text { be } \\
\text { are }\end{array}$ & $\begin{array}{l}\text { coun } \\
\text { eta-ra } \\
\text {-rays } \\
\text { lefine }\end{array}$ & $\begin{array}{l}\text { inter. } \\
\text { in } \\
\text { bet } \\
\text { in th }\end{array}$ & $\begin{array}{l}\text { eta-count } \\
\text {-counter. } \\
\text { text. }\end{array}$ & & & \\
\hline
\end{tabular}

* For description of apparatus and results see J. L. Meem, Jr. and F. Maienschein, Phys. Rev. 76, (1949) 328.

Since the determination of $N_{\beta \gamma}$, in the case mentioned above, depends on measurements of $N_{T}, N_{\mathrm{ch}}$ and $N_{\text {cos }}$, the error in $N_{\beta \gamma}$ is

$$
\left.\begin{array}{rl}
e_{\beta \gamma} & =\left[e_{T}^{2}+e_{\mathrm{ch}}^{2}+\epsilon_{\mathrm{cos}}^{2}\right]^{1 / 2} \\
& =\frac{1}{T^{1 / 2}}\left[N_{T}+N_{\mathrm{ch}}+N_{\mathrm{cos}}\right]^{1 / 2}
\end{array}\right\}
$$

Since $N_{\mathrm{T}}$ and $T$ are measured, $N_{\text {ch }}$ can be calculated from $\tau$ and the singles rate, and $N_{\text {cos }}$ has been measured in a separate experiment, all quantities in (10) are known and $e_{\beta \gamma}$ can be evaluated.

With the help of eq. (2), (3), (4) and (5) eq. (10) can be thrown into the following useful form, originally given by Dunworth ${ }^{11}$

$$
\begin{aligned}
\frac{e_{\beta \gamma}}{N_{\beta \gamma}} & =\frac{1}{T^{1 / 2}}\left[N_{\beta \gamma}+2\left(N_{\mathrm{ch}}+N_{\mathrm{cos}}\right)\right]^{1 / 3} \frac{1}{N_{\beta \gamma}} \\
& \left.=\frac{1}{T^{1 / 2}}\left[\frac{1}{N_{0} \omega_{\beta} \omega_{\gamma} \varepsilon_{\gamma}}+\frac{2 N_{\cos }}{\left(N_{0} \omega_{\beta} \omega_{\gamma} \varepsilon_{\gamma}\right)^{2}}+\frac{4 \tau}{\omega_{\beta} \omega_{\gamma} \varepsilon_{\gamma}}\right]^{1 / 2}\right)
\end{aligned}
$$


Eq. (12) shows that, in order to obtain the smallest relative error in the quantities $T, \omega_{\beta}, \omega_{\gamma}$ and $\varepsilon_{\gamma}$ should be made as large as possible. Furthermore, if $N_{0}$ is increased, the relative error rapidly approaches

$$
\left[\frac{4 \tau}{T \omega_{\beta} \omega_{\gamma} \varepsilon_{\gamma}}\right]^{1 / \%}
$$

However, for $N_{0}=1 / 2 \tau, N_{\mathrm{ch}}$ and $N_{\beta \gamma}$ are equal and it does not pay to increase the source strength beyond this point. Thus, the best results in coincidence counting are to be obtained by the use of large solid angles, efficient counters and short resolving times.

Broadening the scope of the above cur erations to take in the case in which the source gives both beta-gamma and gamma-gamma coincidences, eq. (10) becomes

$$
e_{\beta \gamma}=\frac{1}{T^{1 / 2}}\left[N_{T}+N_{\gamma \gamma}+N_{\mathrm{ch}}+N_{\mathrm{cos}}\right]^{1 / 2} .
$$

In this case $N_{\gamma \gamma}$, for the experiment, is obtained from the counting rate of the gamma-counter. $N_{\gamma}$, and the value of $N_{\gamma \gamma} / N_{\gamma}$ determined previously from a gamma-gamma coincidence experiment. Clearly the limiting error given by (13) holds in this case.

\section{$\$ 5$. Remarks on the Measurement of Beta-Ray Energies by Absorption}

Since the end result of coincidence counting is to obtain the number of beta-gamma coincidences as a function of the energy of the beta-rays, some means of measuring the energy of the beta-rays must be employed. Since, owing to the small source strengths available in the early experiments, magnetic analysis was not possible, most experimentors relied upon the measurement of the beta-ray energy by the method of absorption in aluminum. These methods are adequately discussed in Ch. I. One method consists of inserting aluminum between source and counter until the further addition of aluminum causes no further decrease in tise counting rate. The range, $R$, thus determined is connected with the end point energy by the empirical range curves of Feather ${ }^{12}$, Bleuler and Zünti ${ }^{13}$, Glendenin ${ }^{14}$ or Evans ${ }^{15}$. The second method, employed by Feather ${ }^{12}$ and Bleuler and Zünti ${ }^{13}$, consists of determining $E_{0}$ from an analysis of the shape of an absorption curve. Thus, if the logarithm of the transmission of beta rays through aluminum is plotted as a function of

11 J. V. Dunworth, Rev. Sci. Inst., 11, (1940) 167.

12 N. Feather, Proc. Camb. Phil. Soc., 34, (1938) 599.

13 E. Bleuler and W. Zünti, Helv. Phys. Acta 19, (1946) 375.

14 L. E. Glendenin, Nucleonics, 2, (1948) 12.

15 R. D. Evans, The Science and Engineering of Nuclear Power, Vol. 1, (1947) 53. 
the thickness of aluminum, a curve is obtained which deviates somewhat from a straight line (see Fig. 3). From this curve, various values of $d_{n}$, the thickness which reduces the transmission by a factor of $2^{n}$, can be read off and compared with semi-theoretical curves relating $d_{n}$ to $E_{0}$.

From such curves it is sometimes possible to demonstrate the existence of more than one group in the beta-ray spectrum. Fig. 3 shows a plot of the beta-ray spectrum of $\mathrm{In}^{114}$. It will be seen from the figure that, in addition to the main group of beta rays, there is a highly absorbable part arising from the absorption of the internal conversion electrons of the $190 \mathrm{keV}$

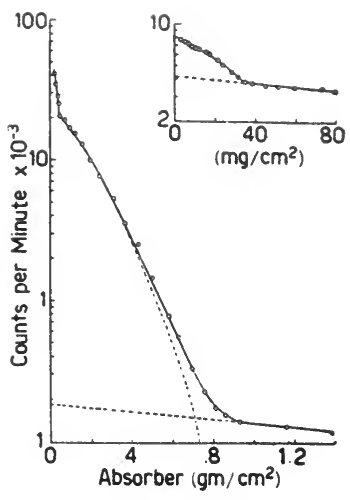

Fig. 3. Absorption of the Beta-Rays of In ${ }^{114}$ gamma-ray from the isomeric transition governing the life-time of the source.

\section{$\S 6$. The Measurement of the Energy of Gamma-Rays by the Absorption of Compton-Electrons}

The measurement of the energy of gamma-rays is carried out most accurately with the help of a magnetic spectrograph, in which the energy of photoelectrons from a radiator is measured. In those cases in which the specific activity of the source is low, certain other useful techniques are provided. One of the most useful, however, is the measurement of the range of Compton electrons produced in a radiator of low atomic number.

In order to measure the range of the Compton electrons a coincidence counting apparatus is employed. Two versions of the arrangement of apparatus are shown in Fig. $4 \mathrm{a}$ and $4 \mathrm{~b}$. In the simpler arrangement,

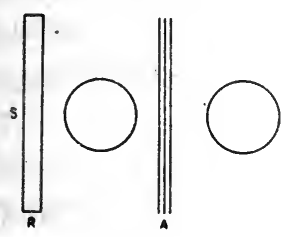

$4 a$

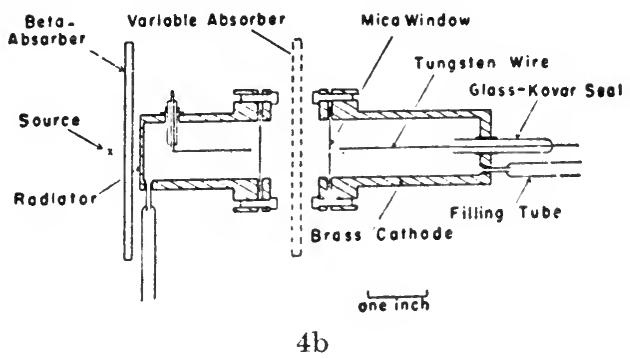

$4 \mathrm{~b}$

Fig. 4a. Arrangement of Counters for Measuring the Range of Secondary Electrons Produced by Gamma-Rays

Fig. 4b. Arrangement of Counter's for Measuring the Range of Secondary Electrons Produced by Gamma-Rays (Low Energy Radiation) 
(Fig. 4a) the source $S$ is shielded from the first counter by a sheet of aluminum $\mathrm{R}$ which is thick enough to stop all beta-rays and at the same time serves as a radiator to produce C'ompton electrons. The secondaries thus produced pass through the two counters connected in coincidence. Arrangements are made to place absorbers of aluminum at the point $\mathrm{A}$ to measure the absorption of the secondaries. An absorption curve is then taken by varying the absorber at $A$. As the absorber is increased, the number of coincidences decreases, until a constant (background) coincidence counting rate is obtained. In this manner, the range of the secondaries is measured. In using this method it should be noted that the most energetic secondary electron will be that one which is produced in the wall of the first counter nearest the source by the gamma-ray having the highest energy in the substance under investigation. It is therefore necessary, in computing the range, to add the thickness of two counter walls to the thickness of the aluminum absorber which has been added. By comparing the range thus determined with a curve published by Curran, Dee and Petrzilka ${ }^{16}$, which gives the range of Compton electrons ejected by gamma-rays of known energy, the energy of the gamma-ray in question may be found. It should be emphasized again that this method determines the energy of the most energetic gamma-ray emitted by the source.

Bleuler and Zünti have amplified the method, first introduced by Becker and Bothe ${ }^{17}$, by calculating the spectrum, $f(\varepsilon) d \varepsilon$, of secondary electrons produced by a gamma-quantum of energy $E$ in a layer $d x$ at a depth $x$ in the aluminum radiator. Combining this result with the results of their absorption experiments, they have given a group of curves from which $E$ can be found, provided the $d_{n}(n=1,2,3 \ldots)$ have been determined. Thus it is only necessary to plot the absorption curve, subtract the background, determine the absorber thicknesses $d_{n}$ for which the transmitted intensity hạs dropped to $1 /{ }_{2}{ }^{n}$ of its original value and determine $E$ from the published curves. Obviously if several gamma-rays are present this value of $E$ will not be constant for all $n$. The method is most useful in determining the energy of the highest energy gamma-ray and getting an indication of the energies of others present.

In most cases in which low energy gamma-rays are present, it is convenient to use the counter arrangement shown in Fig. $4 \mathrm{~b}$. In this arrangement both counters have thin windows so that secondaries of low energy can be counted in the second counter. In addition, the metallic wall of the counter nearest the source serves as a radiator. An absorption curve for

16 S. C. Curran, P. I. Dee, and V. Petrzilka, Proc. Roy. Soc. 169, (1940) 1107.

17 H. Becker and W. Bothe, Zeits. f. Physik. 76, (1932) 421. 
the gamma-rays of $\mathrm{Ni}^{65}$ taken by Maienschein and Meem ${ }^{18}$, using this arrangement is shown in Fig. 5.

The secondaries from the most energetic gamma-ray have a range in aluminum of $0.626 \mathrm{gm} / \mathrm{cm}^{2}$ which corresponds to an energy for the gamma-ray of $1.64 \mathrm{MeV}$. Closer investigation of the low energy region, shown in the insert of the figure. indicates a break in the curve at a gamma-ray energy of $0.3 \mathrm{MeV}$. The energy of the gamma-rays of $\mathrm{Ni}^{65}$ determined by a magnetic spectrometer are $0.37,1.12$ and $1.49 \mathrm{MeV}$.

\section{$\S 7$. Analysis of the Coincidence Method}

In discussing the analysis of a coincidence counting experiment, it will be assumed that the procedures of $\S 3$ have been carried out. The experi-

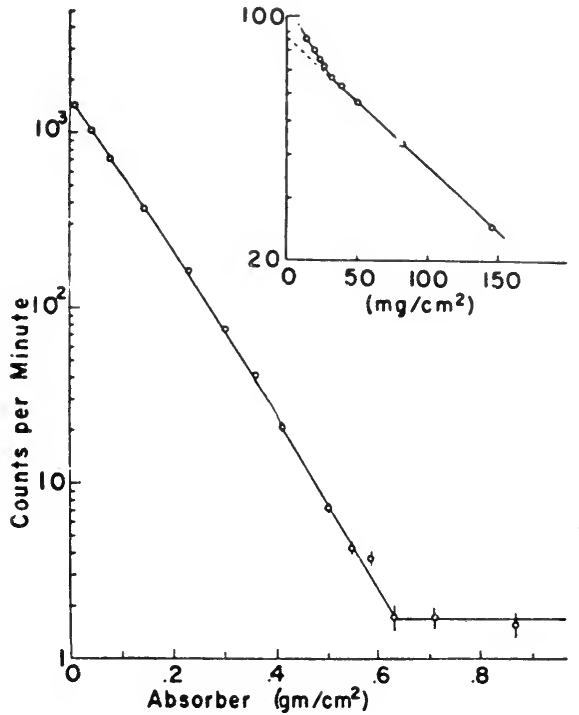

Fig. 5. Absorption of the Secondary Electrons Produced by the Gamma-Rays Emitted from $\mathrm{Ni}^{\mathbf{6 5}}$ menter will have determined the end-point of the beta-ray spectrum from an absorption experiment and will have found out as much as possible about the gamma-rays from absorption and coincidence experiments on the Compton electrons arising form the gamma-rays. Experiments on gammagamma coincidences will have determined whether there is more than one gamma-ray per disintegration and if so the value of $N_{\gamma \gamma} / N_{\gamma}$. Finally, the results of the beta-gamma coincidence experiment will have shown whether a plot of $N_{\beta \gamma} / N_{\beta}$ against the thickness of absorber gives a straight line parallel to the axis or a curve which rises as the thickness of the absorber is decreased. It will be assumed in the analysis that follows that there are no positrons emitted. The special precautions needed for positron emitters will be discussed in a separate section.

From the experimental point of view certain cases can be recognized at once. If there is only one gamma-ray, no gamma-gamma coincidences will be observed. If the plot of $N_{\beta \gamma} / N_{\beta}$ against absorber thickness is a straight line parallel to the thickness axis it implies that there is only one group of beta-rays. The analysis will be carried out for various simple cases using the notations of $\S 3$. 
A. Beta-Ray Spectrum Simple; Oxe Gamalar per Disintegration

In addition to the notation used in $\S 3$, let $F(x)$ be the fraction of betarays which are transmitted through a thickness $x$ of absorber. The following equaticns now hold for the counting rate in each counter and the betagamma coincidence rate.

$$
\begin{aligned}
& N_{\beta}=N_{0} \omega_{\beta} F(x) \\
& N_{\gamma}=N_{0} \omega_{\gamma} \varepsilon_{\gamma} \\
& N_{\beta \gamma}=N_{0} \omega_{\beta} F(x) \cdot \varepsilon_{\gamma} \cdot \omega_{\gamma} .
\end{aligned}
$$

It follows then that

$$
N_{\beta \gamma} / N_{\beta}=\varepsilon_{\gamma} \omega_{\gamma}
$$

Eq. (17) shows that $N_{\beta \gamma} / N_{\beta}$ is a constant independent of the energy of the beta-rays. In addition it will be seen that the quantity $\left(\varepsilon_{\gamma} \omega_{\gamma}\right)$ can be determined from the value of $N_{\beta \gamma} / N_{\beta}$.

The classical example of this type of disintegration was, until recently, considered to be that of $\mathrm{Au}^{198}$. The main features of the disintegration are one-beta-ray of energy $0.97 \mathrm{MeV}$ followed by a gamma-ray of energy 0.411 $\mathrm{MeV}$. Coincidence measurements, using Geiger Müller counters, have

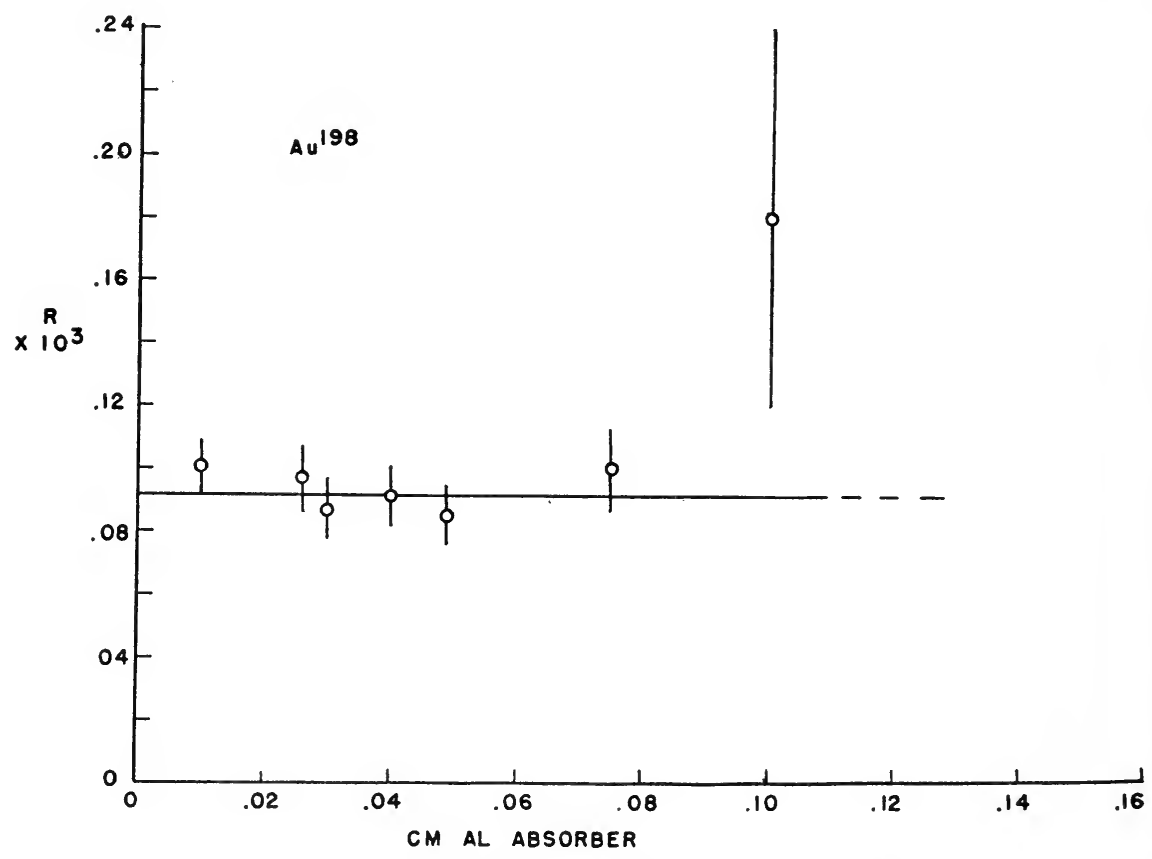

Fig. 6. Beta-Gamma Coincidences in $\mathrm{Au}^{198}$ 
been made by Norling ${ }^{19}$, Clark ${ }^{20}$ and Jurney ${ }^{21}$. All authors find that the number of beta-gamma coincidences per beta-particle is independent of the energy of the beta-particles, indicating only one group of beta-rays. The results of Jurney's measurements are shown in Fig. 6.

Recently, it has been shown that $\mathrm{Au}^{198}$ emits other beta-ray groups accompanied by gamma-rays ${ }^{22}$ but all of these are of very low intensity. This case shows at once that low intensity groups are sometimes missed by the type of coincidence work discussed here.

B. Beta-Ray Spectrum Complex, One Gamma-Ray per jisinteGRATION

If the number of beta-gamma coincidences is dependent on the energy and there are no gamma-gamma coincidences, the equations become

$$
\begin{aligned}
& N_{\beta}=N_{0} \omega_{\beta}\left[F_{1}(x)+F_{2}(x)\right] \\
& N_{\gamma}=N_{0} \omega_{\gamma} \varepsilon_{\gamma} \\
& N_{\beta \gamma}=N_{0} F_{1}(x) \omega_{\beta} \omega_{\gamma} \varepsilon_{\gamma} .
\end{aligned}
$$

Hence

$$
\begin{aligned}
& N_{\beta \gamma} / N_{\beta}=\frac{F_{1}(x)}{F_{1}(x)+F_{2}(x)} \omega_{\gamma} \varepsilon_{\gamma} \\
& N_{\beta \gamma} / N_{\gamma}=F_{1}(x) \omega_{\beta} .
\end{aligned}
$$

Here $F_{i}(x)$ is the fraction of the beta-rays of the $i^{\text {th }}$ group transmitted through a thickness $x$ of aluminum. Since there is only one gamma-ray, one of the electron groups must go to the ground state of the product. $F_{1}(x)$ is zero for a value of $x$ equal to or greater than the range of the first group, so that the end point of the lower energy group is determined from measuring $N_{\beta \gamma} / N_{\beta}$ as a function of $x$. A measurement of $N_{\beta \gamma} / N_{\gamma}$ gives the absorption curve of the lower energy group. Extrapolation of these measurements to $x=0$, and a comparison with $N_{\beta}$ at $x=0$ gives the relative intensity of the two electron groups $F_{1} /\left(F_{1}+F_{2}\right)$.

Since the energy of the gamma-ray can be determined by measuring the range of the secondary electrons emitted from aluminum, the end point of the spectrum for the most energetic group of beta-rays by absorption, and that of the lower energy group by one of the methods discussed above, the disintegration scheme can be determined.

Several cases of this type have been found. Among them are $K^{42}$,

19 F. Norling, Ark. f. Mat. Ast. och Fys. B27(3), (1941) 9.

20 A. F. Clark, Phys. Rev. 61, (1942) 242.

21 E. T. Jurney, Phys. Rev. 74, (1948) 1049.

22 National Bureau of Standards Circular No. 499. 
investigated by Siegbahn and Johansson ${ }^{23}$ and $\mathrm{Rb}^{86}$ investigated by Jurney ${ }^{21}$. A comparison of the results of the coincidence method and the spectroscopic method on $\mathrm{Rb}^{86}$ is of interest.

The absorption of the beta-rays in aluminum was determined, and the resulting data were analyzed by the method of Bleuler and Zünti, which showed two beta-rays with end points at $1.82 \mathrm{MeV}^{\mathrm{N}}(\sim 67 \%)$ and $0.56 \mathrm{MeV}$

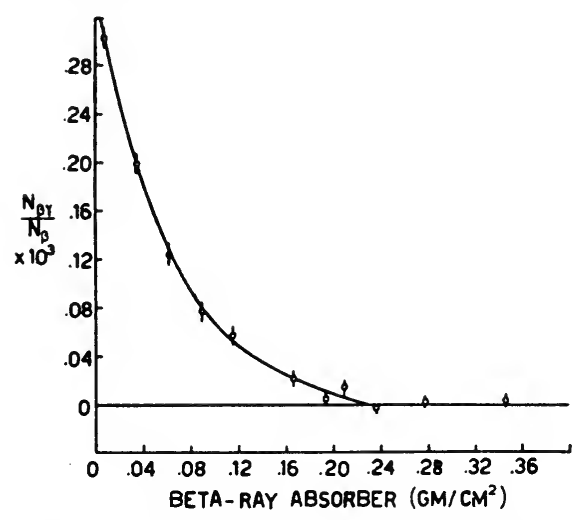

Fig. 7. Beta-Gamma Coincidences in $\mathrm{Rb}^{86}$

$(\sim 33 \%)$. The energy of the gammaray was found to be $1.12 \mathrm{MeV}$ by coincidence absorption of Compton recoils.

Fig. 7 shows the results of betagamma coincidence measurements. It is evident that no coincidences are present for thicknesses greater than $0.23 \mathrm{gm} / \mathrm{cm}^{2}$ of aluminum, which corresponds to $0.62 \mathrm{MeV}$.

The above results are to be compared with those obtained in a magnetic lens spectrograph by Zaffarano, Kern and Mitchell ${ }^{24}$ who found one gamma-ray of energy $1.081 \mathrm{MeV}$ and two beta-ray groups with end points at 1.822 and $0.716 \mathrm{MeV}$. It will be seen that the agreement between the two experiments is reasonably satisfactory.

\section{Beta-ray Spectrum Smple, More thax One Gama-Ray per Disintegration}

If gamma-gamma coincidences are present, the analysis becomes more complicated. It is desired to determine the number and energy of the gamma-rays and whether they are in cascade or in parallel. The simplest case to discuss is that in which the number of beta-gamma coincidences per disintegration is independent of absorber thickness. As an example, consider the case of $\mathrm{Na}^{24}$. This isotope was investigated first by Langer, Mitchell and McDaniel ${ }^{25}$ and more recently by Wiedenbeck ${ }^{26}$ with substantially the same results.

The beta-ray end point of the $\mathrm{Na}^{24}$ spectrum is $1.39 \mathrm{MeV}$. The coincidence experiments showed that there were both beta-gamma and gammagamma coincidences. The number of beta-gamma coincidences per recorded

23 K. Siegbahn and A. Johanson, Ark. f. Mat. Ast. och Fys. 34A, (1946) No. 10.

24 D. J. Zaffarano, B. D. Kern and A. C. G. Mitchell, Phys. Rev. 74, (1948) 682.

25 L. M. Langer, A. C. G. Mitchell and P. W. McDanicl, Phys. Rev. 56, (1939) 962.

26 II. L. Wiedenbeck, Phys. Rev. 72, (1947) 429. 
beta-count was found to be independent of the energy. This shows at once that all beta-ray transitions lead to the same excited state of the product, and the beta-spectrum is simple.

The gamma-ray spectrum of $\mathrm{Na}^{24}$ has been investigated by various observers. The most reliable measurements give two gamma-rays - one at, 1.38 $\mathrm{MeV}^{\gamma}$ and one at $2.76 \mathrm{MeV}$, of practically equal intensity. The inference to be drawn from the gamma-gamma coincidence measurements is that those two gamma-rays are in cascade. The level system which results requires that the energy of $\mathrm{Na}^{21}$ be $5.53 \mathrm{MeV}$ above the ground state.

In carrying out the analysis, it is now necessary to take into account the efficiencies of the gamma-counters for the two gamma-rays concerned. Let $\varepsilon_{1.38}$ and $\varepsilon_{2.76}$ be the efficiences for the 1.38 and $2.76 \mathrm{MeV}$ gamma-rays, respectively. The rarious counting rates are then given by

$$
\begin{aligned}
& N_{\beta}=N_{0} \omega_{\beta} F^{\prime}(x) \\
& N_{\gamma}=N_{0} \omega_{\gamma}\left(\varepsilon_{1.38}+\varepsilon_{2.76}\right) \\
& N_{\beta \gamma}=N_{0} \omega_{\beta} F(x)\left[\varepsilon_{1.38}+\varepsilon_{2.76}\right] \\
& N_{\gamma \gamma}=2 N_{0} \omega_{\gamma}^{2} \varepsilon_{1.38} \varepsilon_{2.76} .
\end{aligned}
$$

It therefore follows that

$$
\begin{aligned}
& N_{\beta \gamma} / N_{\beta}=\omega_{\gamma}\left(\varepsilon_{1.38}+\varepsilon_{2.76}\right) \\
& N_{\gamma \gamma} / N_{\gamma}=2 \frac{\varepsilon_{1.38} \cdot \varepsilon_{2.76}}{\varepsilon_{1.38}+\varepsilon_{2.76}}
\end{aligned}
$$

and the ratio

$$
R=\frac{N_{\gamma \gamma} / N_{\gamma}}{N_{\beta \gamma} / N_{\beta}}=2 \frac{\varepsilon_{1.38} \cdot \varepsilon_{2.76}}{\left(\varepsilon_{1.38}+\varepsilon_{2.76}\right)^{2}}
$$

\section{Posirrox Euitters}

If positron emitters are to be investigated essentially the same considerations apply as are given above except for the strongly correlated coincidences between annihilation radiation quanta. Consider a positron emitter, accompanied by two gamma-rays $\gamma_{1}, \gamma_{2}$, contained in a small aluminum capsule between two counters. One can observe with the two counters at $180^{\circ}$ or at $90^{\circ}$. It is easy to show that

$$
N_{\gamma \gamma} / N_{\gamma}=\frac{2 \varepsilon_{a}^{2} \delta+\omega_{\gamma}\left[\varepsilon_{\gamma_{1}} \varepsilon_{\gamma_{z}}+\varepsilon_{a}\left(\varepsilon_{\gamma_{2}}+\varepsilon_{\gamma_{2}}\right)\right]}{2 \varepsilon_{a}+\varepsilon_{\gamma_{1}} \varepsilon_{\gamma_{2}}}
$$

where $\delta=1$ for $\Theta=180^{\circ}$ and $\delta=0$ for $\Theta=90^{\circ}$. Thus the existence of yamma-gamma coincidences can be confirmed from these two measurenents. The question of positron-gamma coincidences is treated in the same way as beta-gamma coincidences, since a positron which is counted 
in the beta-counter does not produce annihilation radiation at the source and solid angle considerations suppress the effects of any annihilation radiation coincidences.

\section{$\S 8$. Measurement of Internal Conversion Electrons}

In certain cases the number of internal conversion electrons from a partially converted gamma-ray can be determined by coincidence counting. It is particularly useful when the specific activity of the source is not large enough to allow the use of magnetic analysis of the beta-ray spectrum. The method consists in measuring coincidences between internal conversion electrons and disintegration electrons, here called particle-particle coincidences. The method was first used by Norling ${ }^{19}$ and has been further developed by Mitchell, Wiedenbeck, and Mandeville. The method has been quite successful in determining the position of internal conversion lines in the spectrum but somewhat less successful in obtaining the internal conversion coefficient of a given gamma-ray.

Since internal conversion electrons are usually of low energy, it is necessary to use thin walled counters - window thickness a few $\mathrm{mg} / \mathrm{cm}^{2}$. In addition, care must be taken to eliminate scattered electrons. A convenient experimental arrangement is to mount the counters side by side with the source above as shown in Fig. 8. The counters are separated by an aluminum shield which has the purpose of preventing secondaries, produced by an electron which has been counted in one counter, from entering the other counter. In addition, thin sources must be used to prevent scattering and the production of secondaries in the source.

The principle of the method is to measure coincidences between disintegration electrons and internal conversion electrons, arising from a subsequent gamma-ray, the emission of which occurs in a short time with respect to the resolving time of the coincidence circuit. Energy discrimination between the disintegration electrons and the internal conversion electrons is accomplished by means of aluminum absorbers. The absorbers should be placed as near the counters as possible and away from the source to prevent scattering and the formation of secondaries from interfering with the experiment. Various dispositions of absorbers have been used but the author has found that a symmetrical arrangement is extremely convenient. Thus, equal thicknesses of absorber are placed over each counter simultaneously and the number of particle-particle coincidences is measured as a function of absorber thickness. The equations developed below apply to the symmetrical arrangement only. For other arrangements the reader can work out the necessary equations. Finally, in carrying out an experiment, corrections have to be applied for the 
number of gamma-gamma and beta-gamma coincidences occurring in the counters.

As an example, the case in which a source with one beta-ray group followed by one partially converted gamma-ray will be considered. Let $N_{p}$ be the number of particles per second, disintegration electrons and

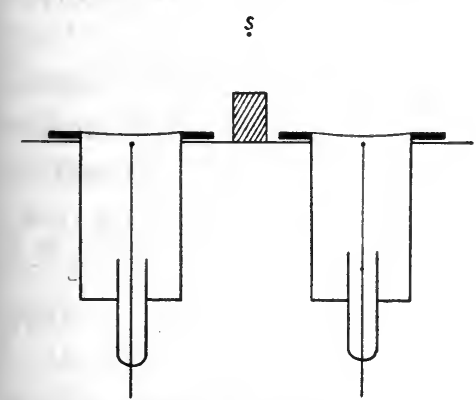

Fig. 8. Arrangement of Counters for Measuring Coincidences Between Internal Conversion Electrons and Beta-Rays

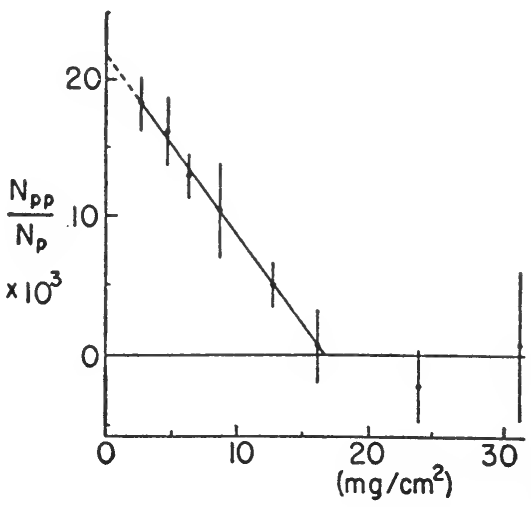

Fig. 9. Beta-Beta Coincidences in $\mathbf{A u}^{190}$

internal conversion electrons, detected in one of the counters; let $N_{p p}$ be the number of coincidences per second between disintegration electrons and internal conversion electrons. Let $\varkappa$ be the number of internal conversion electrons per disintegration. This quantity is related to the internal conversion coefficient $\alpha$ by

$$
1 / x=1+1 / \alpha .
$$

Furthermore, since the absorption coefficient in aluminum is different for the (continuous) disintegration electrons than it is for the (essentially monoenergetic) conversion electrons, let the fraction of each transmitted at absorber thickness $x$ be $F_{\beta}(x)$ and $F_{o}(x)$. It therefore follows that

$$
\begin{gathered}
N_{p}=N_{0} \omega_{\beta}\left[\varkappa F_{c}(x)+F_{\beta}(x)\right] \\
N_{p p}=2 N_{0} \omega_{\beta}^{2} \cdot \varkappa F_{c}(x) F_{\beta}(x) \\
N_{p p} / N_{p}=2 \omega_{\beta} \frac{\varkappa F_{c}(x) F_{\beta}(x)}{\varkappa F_{c}(x)+F_{\beta}(x)} .
\end{gathered}
$$

Equations (32), (33) and (34) serve two purposes. They can be used as a means of determining the energy of the internal conversion electrons. $F_{c}(x)$ is zero for values of $x$ greater than the range of the conversion electron. Thus if the range of the conversion electrons is less than the range of the disintegration electrons, a plot of $N_{p p} / N_{p}$ against the absorber thickness will give at once the range of the internal conversion electrons and hence their energy. As an example such a plot is shown in Fig. 9 for 
$A u^{199}$, taken from the work of Meem and Maienschien ${ }^{2 i}$. In this particular case the beta-ray spectrum has an end point of $0.32 \mathrm{MeV}$. The range of the internal conversion electrons shown in Fig. 9 corresponds to an energy of $0.120 \mathrm{MeV}$. Adding to this the binding energy of the $K$-electrons in $\mathrm{Hg}$, $0.083 \mathrm{MeV}$, the energy of the gamma-ray responsible for the particleparticle coincidence is found to be $0.203 \mathrm{MeV}$ in agreement with spectroscopic values.

The actual determination of the internal conversion coefficient is more interesting but also more difficult. Eq. (34) gives $N_{p p} / N_{p}$ in terms of the absorber thickness $x$. If the curve of $N_{p p} / N_{p}$ against $x$ is extrapolated to zero absorber thickness, both $F_{c}(0)$ and $F_{\beta}(0)$ are unity and eq. (34) becomes

$$
\left(N_{p p} / N_{p}\right)_{x=0}=2 \omega_{\beta} \frac{x}{1+x} .
$$

In order to find $\%$, it is necessary to determine $\omega_{\beta}$. This is most easily done by obtaining a source which gives a known number of beta-particles/ sec, measuring the number of counts/sec from it as a function of absorber thickness in the same geometry, and extrapolating this result to zero thickness. A thin source containing a weighed amount of $\mathrm{U}_{3} \mathrm{O}_{8}$ is convenient for that purpose. From these two pieces of information it is clear that $\varkappa$ can readily be determined.

Since in addition beta-gamma coincidences can also be measured, by putting enough aluminum over one of the counters to stop all particles, then for the case under consideration

Hence for $x=0$

$$
\begin{aligned}
& N_{\gamma}=N_{0} \omega_{\gamma} \varepsilon_{\gamma}(1-\varkappa) \\
& N_{\beta \gamma}=N_{0} \omega_{\beta} \omega_{\gamma} \varepsilon_{\gamma}(1-\varkappa) F_{\beta}(x) .
\end{aligned}
$$

$$
\left(\frac{N_{p p}}{N_{p}} / \frac{N_{\beta \gamma}}{N_{\gamma}}\right)_{x=0}=2 \frac{\varkappa}{\varkappa+1} \text {. }
$$

Thus, in principle "at least, $x$ can be obtained.

However, a more accurate result is obtained if the first method is employed since, in the second method, errors which arise on account of the use of both $N_{\beta \gamma}$ and $N_{p p}$ are inherently larger than if only one of these is used together, with the single count of the $\mathrm{U}_{3} \mathrm{O}_{8}$ source.

Methods for more complicated schemes can be worked out along the lines suggested in the discussion of beta-gamma coincidences.

Experiments of this nature cannot be expected to yield a very accurate value of the internal conversion coefficient. Wiedenbeck and $\mathrm{Chu}{ }^{28}$

¿7 See for example, J. L. Meem and F. Maienschein, Phys. Rev. 76, (1949) 328.

:8 M. L. Wiedenbeck and K. Y. Chu, Phys. Rev. 72, (1947). 1171. 
measured internal conversion coefficients for $\mathrm{Au}^{198}, \mathrm{Cs}^{13 t}$ and $\operatorname{Ir}^{192,} 194$ (60 days). Of these it is only possible to compare the results on $\mathrm{Au}^{198}$ with the spectroscopic value, since the later two elements emit several gammarays. The value obtained by this method for $\mathrm{Au}^{198}$ (0.411 MeV line) is $x=0.0470 \pm 0.0024$ corresponding to a total internal conversion coefficient $\alpha=0.0495 \pm 0.002$. The value quoted for the internal conversion coefficient for this line by spectroscopic means ${ }^{29}$ is $\alpha=0.0353\left(\alpha=\alpha_{K}+\alpha_{L}+\alpha_{M 1}\right)$. Thus the coincidence method can give an indication of the order of magnitude of the internal conversion coefficient but cannot give very accurate values.

\section{§ 9. The Use of Coincidence Counting for Determining the Efficiency of Gamma-Counters}

As has been pointed out in earlier sections of this chapter it is necessary to know the counting efficiency of a gamma-counter as a function of energy in working out most disintegration schemes. This problem has been investigated by various authors: on the experimental side by Dunworth ${ }^{11}$, Norling ${ }^{19}$, Bradt, Gugelot, Huber, Medicus, Preiswerk and Scherrer ${ }^{30}$, Jurney and Maienschein ${ }^{31}$; on the theoretical side by $\mathrm{v}$. Droste ${ }^{32}$. It is the purpose of this section to illustrate how the coincidence method is employed for this purpose. The details of the counters used and the results of the investigations may be looked up by the reader.

The principle of the method is to find a number of disintegration schemes which are known both from coincidence counting and magnetic spectrometer experiments. The energies of the gamma-rays chosen will have been measured in a magnetic spectrograph.

It is more convenient to determine a relative efficiency $\omega \varepsilon_{\gamma}$ consisting of the absolute efficiency $\varepsilon_{\gamma}$ times the solid angle factor $\omega$. This procedure eliminates the necessity for determining the solid angle intercepted by the counter, but requires that the solid angle be kept constant for all measurements. It is instructive to see how the method can be used for determining a few points. We follow the procedure of Jurney and Maienschein, who used a lead lined counter.

For the first point on the curve $\mathrm{Co}^{60}$ (5.3 years) is chosen. It decays by the emission of a single $0.300 \mathrm{MeV}$ beta-ray group followed by the emission of two gamma-rays in cascade whose energies are 1.1 and $1.3 \mathrm{MeV}$. Two

29 J. M. Hollander, J. Perlman and G. T. Seaborg, Rev. Mod. Phys. 25, (1953) 469.

so H. L. Bradt, P. C. Gugelot, O. Huber, H. Medicus, P. Preiswerk and P. Scherrer, Helv. Phys. Acta 19, (1946) 77.

31 E. T. Jurney and F. Maienschein, Rev. Sci. Inst. 20, (1949) 932.

32 G. v. Droste, Zeits. f. Phys. 100, (1936) 529. 
similar gamma-ray counters are arranged in a standard geometry, to be used throughout the series of experiments, and the gamma-gamma coincidence rate, together with the singles counting rate, determined. From the results of $\S 7$ it follows that

$$
N_{\gamma \gamma} / N_{\gamma}=\frac{2\left(\omega \varepsilon_{1.1}\right)\left(\omega \varepsilon_{1.2}\right)}{\omega \varepsilon_{1.1}+\omega \varepsilon_{1.2}}
$$

Since the energies of the two gamma-rays are very nearly the same, one is justified in considering this ratio to be the same as that given by two 1.2 $\mathrm{MeV}$ gamma-rays in cascade:

$$
N_{\gamma \gamma} / N_{\gamma}=\omega \varepsilon_{1.2} .
$$

The value of $\left(\omega \varepsilon_{1.2}\right)$ was found to be $\omega \varepsilon_{1.2}=(1.12 \pm 0.002) \times 10^{-3}$ for the particular counter used.

The next point investigated was that corresponding to the gamma-ray of $0.411 \mathrm{MeV}$ from $\mathrm{Au}^{198}$. To investigate this, one of the gamma-counters was removed and an end window beta-counter put in its place. Since the main mode of disintegration of $\mathrm{Au}^{198}$ is by a beta-ray followed by the emission of an internally converted gamma-ray, beta-gamma coincidences were measured. With no absorber between the source and the beta-ray counter, the ratio $N_{\beta \gamma} / N_{\beta}$ is given by

$$
N_{\beta \gamma} / N_{\beta}=\frac{(1-\varkappa)}{(1+\varkappa)} \omega \varepsilon_{0.411} .
$$

Since $\varkappa$ is known, $\left(\omega \varepsilon_{0.411}\right)$ was determined and found to be

$$
\left(\omega \varepsilon_{0.411}\right)=(0.736 \pm 0.01) \times 10^{-3} .
$$

In order to extend the measurements to higher energies a gammagamma coincidence experiment was performed on $\mathrm{Na}^{24}$ which has two gamma-rays of energy 1.38 and $2.76 \mathrm{MeV}$. In this case

$$
N_{\gamma \gamma} / N_{\gamma}=\frac{2\left(\omega \varepsilon_{1.38}\right)\left(\omega \varepsilon_{2.76}\right)}{\omega \varepsilon_{1.38}+\omega \varepsilon_{2.76}}
$$

As a first approximation, one can insert the value of $\left(\omega \varepsilon_{1.2}\right)$ for $\left(\omega \varepsilon_{1.38}\right)$ and calculate the value of $\left(\omega \varepsilon_{2.76}\right)$. Since the values for the gamma-rays whose energies are $0.411,1.2$ ard $2.76 \mathrm{MeV}$ determine a straight line, an interpolation can be made to get the correct value for $\omega \varepsilon_{1.38}$ and, using this, recompute the value for $\left(\omega \varepsilon_{2.76}\right)$. The values obtained in this manner were

$$
\left(\omega \varepsilon_{1.38}\right)=(1.22 \pm 0.02) \times 10^{-3} ;\left(\omega \varepsilon_{2.76}\right)=(1.91 \pm 0.05) \times 10^{-3} .
$$

Proceeding in this manner a complete efficiency curve can be obtained. 


\section{$\S 10$. Coincidence Counting Using a Magnetic Spectrometer}

Owing to the development of magnetic spectrometers of good resolution and large solid angle, fast concidence circuits, and scintillation counters it is now possible to do coincidence work using a spectrograph.

In order to measure beta-gamma coincidences, it is most convenient to use a magnetic lens spectrometer owing to its high transmission and the convenient arrangement of the source and detectors. A thin source of the material to be investigated is mounted as shown in Fig. 10. Behind the source is mounted the gamma-ray detector, a $\mathrm{NaI}(\mathrm{T} 1)$ scintillation counter. The crystal itself is placed as close to the source as possible in order to give a large solid angle for detection, $\omega_{\gamma}$. The light pulses from the crystal

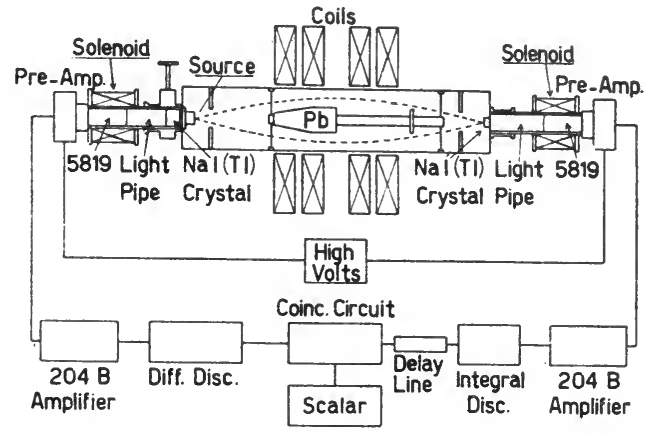

Fig. 10. Coincidence Spectrograph are led through a lucite "light-piper" to the photomultiplier tube which should be located outside of and be shielded from the magnetic field. The pulses from the photomultiplier are fed through a cathodefollower to a linear amplifier and a differential pulse height analyser to either a scalar or a coincidence circuit and scalar. The beta-rays are counted, after magnetic resolution, by either a Geiger-Müller counter or a scintillation counter using a $\mathrm{NaI}$ or an anthracene crystal. If a crystal detector is used it should be thick enough to stop all beta-rays. The arrangement of crystal, photomultiplier, and electronic components is essentially similar to that used for the gamma-ray detector except that no differential pulse height analyser is needed. The linear amplifier is used with an integral bias, set high enough to cut out noise but low enough to register true counts.

In order to use the apparatus, it is necessary to determine the coincidence efficiency of the circuit, $\eta$. This is necessary since two pulses which are actually coincident may be delayed the one behind the other, in reaching the coincidence stage, owing to the complicated circuitry involved. The best way to do this is to feed coincident pulses from a double pulse generator into each circuit and insert delay lines just ahead of the coincidence circuit until all coincidences are registered. In what follows it will be assumed that $\eta=1$.

As an example of the method, imagine that it is applied to the study of the disintegration of $\mathrm{Rb}^{86}$, which emits two beta-rays, one of energy 
1.872 $\mathrm{MeV}$ and the other of energy $0.716 \mathrm{MeV}$. the latter being followed by a gamma ray of energy $1.081 \mathrm{MeV}$. From earlier work it is known that the higher energy group has a forbidden shape. It is decided to make a more exact determination of the end point and shape of the interior group.

In this case it is not necessary to know the absolute counting efficiency of either counter, but only whether the efficiency of the beta-ray counter $\varepsilon_{\beta}(p)$ changes with energy. This can be accomplished by measuring the beta-spectrum. without coincidences, and showing that the Fermi plot has the required shape.

Before measuring the coincidence spectrum, it is necessary to measure the pulse height distribution exhibited in the gamma-ray counter. This measures the energies of the gamma-rays emitted by the source. If, as in the case in point, there is only one gamma-ray, the scintillation spectrometer should be set on the peak of the line and the window of the differential pulse height analyser opened sufficiently to cover the whole line. With this accomplished, beta-gamma coincidences can be measured for different settings of the magnetic spectrometer.

The following equations, similar to those developed in $\S 7$ apply:

$$
\begin{aligned}
& N_{\beta}=N_{0}\left(\omega_{\beta} \Delta \cdot\left\{f_{1}(p)+f_{2}(p)\right\}\right. \\
& N_{\gamma}=N_{0}\left(\omega_{\gamma} \varepsilon_{\gamma}\right. \\
& N_{\beta \gamma}=N_{0}\left(\omega _ { \beta } \Delta \cdot \{ f _ { 2 } ( p ) \} _ { 3 } \left(\omega_{\gamma} \varepsilon_{\gamma}\right.\right. \\
& \frac{N_{\beta \gamma}}{N_{\gamma}}=\omega_{\beta} \Delta \cdot\left\{f_{2}(p)\right\}
\end{aligned}
$$

Here, in addition to the usual symbols we have

$\Delta=$ resolution of the spectrometer $=\frac{\Delta B(\varrho)}{B \varrho}$

$f_{i}(p)=$ distribution function for the $i^{\text {th }}$-group.

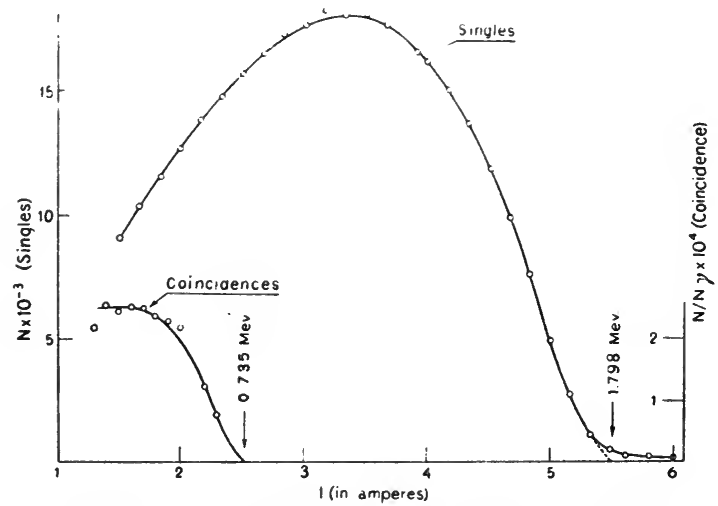

Fig. 11. Coincidence Spectrum of $\mathrm{Rb}^{86}$ 
It will be seen at once that eq. (42) gives the beta-ray distribution of the inner group. As an example the spectrum of $\mathrm{Rb}^{86}$, taken in this way, is shown in Fig. 11.

The method can be amplified to include more complicated types of spectra.

\section{$\S$ 11. Future Developments}

This chapter has described, for the most part, coincidence experiments in which mainly Geiger-Müller counters have been used. In other sections of the book the properties and use of scintillation counters are described. The reader will see at once that, since scintillation counters will resolve gamma-rays according to their energies. they can be used to measure gamma-gamma coincidences between resolved gamma-rays. In adclition, a scintillation counter, when equipped with a differential pulse height analyser, will detect and resolve beta-rays according to their energy. Figure 12 shows the beta-ray spectrum of $\mathrm{Cs}^{137}$ taken with an anthracene

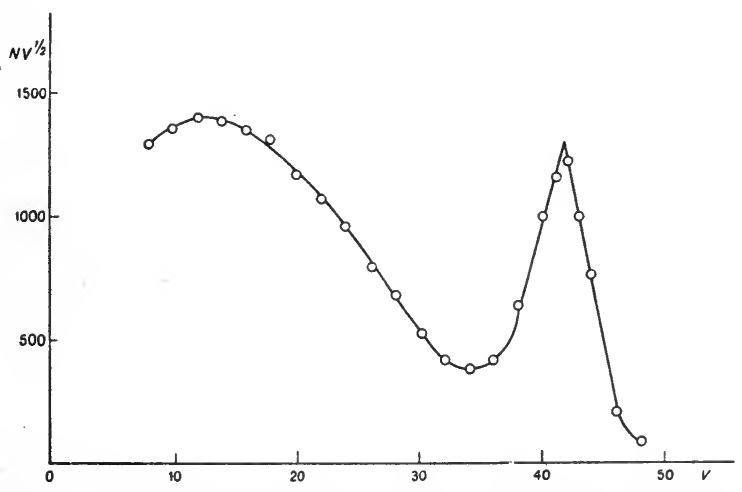

Fig. 12. $\mathrm{Cs}^{\mathbf{1 3 7}}$ Spectrum Taken with a Scintillation Spectrometer

crystal used in a scintillation spectrometer. The curve shows the internal conversion line and the spectral distribution of the beta-rays. A Fermi plot was made from this curve which shows the forbidden shape of the $\mathrm{Cs}^{137}$ spectrum. It is therefore clear that scintillation spectrometers used in coincidence, without magnetic resolution, will open up new possibilities in the use of coincidence counting for the investigations of spectra. 


\title{
$\beta$-SPECTROMETER MEASUREMENTS
}

\author{
A. C. G. MITCHELI, H. SLÄTIS
}

\section{(I) A. C. G. MITCHELL - Investigations of Disintegration Schemes - Energies and Intensities of Beta and Gamma Components and their Coupling}

\section{$\S$ 1. Introduction}

In this Chapter the general processes and procedures used in determining disintegration schemes through a study of the beta- and gamma-rays emitted will be discussed. It will be assumed that the reader is familiar with the types of magnetic spectrometers available, through a study of Chapter III, and is also familiar with the scintillation method (Chapter V), proportional counters (Chapter VI) and with the use of coincidence methods (Chapter VII).

The subject has its beginning ${ }^{1}$ in the early work of Danysz ${ }^{2}$, and Rutherford and Robinson ${ }^{3}$, who invented and used the $180^{\circ}$ magnetic spectrometer, with photographic recording, to study the energies of "beta-ray lines" - internal conversion electrons formed through the interaction of a gamma-ray of energy $E_{\gamma}$ with an electron in the $K$ or $L$-shell of the product nucleus. The energy of the electrons, which are brought to a focus and recorded on the photographic film at some position designated by a given value of $B \varrho$, is given by

$$
E_{e}=E_{\gamma}-E_{K_{i}}^{\prime} \quad\left(\text { or } E_{L}\right)
$$

It was also discovered very early in the study of radioactivity that a gamma-ray would eject an electron from the $K$ or $L$-shell of an external radiator of high atomic number (photo-effect). Thus, even in the early days of the study of radioactivity, it was possible to measure the energies of gamma-rays with reasonably good accuracy, to demonstrate the existence of a combination principle, and to show that energy levels exist in nuclei.

The discovery and investigation of the particles directly emitted from the nucleus-disintegration electrons (beta-rays)-came considerably later ${ }^{1}$. Since the beta-rays are emitted with a continuous distribution of energy, they did not lend themselves to investigation by the photographic

1 E. Rutherford, J. Chadwick and C. Ellis, Radiations from Radioactive Substance, Cambridge, 1930.

2 J. Danysz, Le Radium, 10 (1913) 4.

3 E. Rutherford and H. Robinson, Phil. Mag. 26 (1913) 717. 
plate method. For many years absorption techniques were the main methods used for the investigation of beta-rays. For a period, mostly in the decade starting with 1930 , cloud chambers were used for the investigation of the distribution of beta-ray spectra. These studies, together with those in which coincidence counting methods were used (see Chapter VII), showed that several beta-ray groups may arise in one radioactive disintegration and that in most cases the end-point energy of the various groups could be correlated with the energies of gamma-rays emitted in the disintegration. The shapes of the beta-ray distributions obtained turned out to be quite wrong. They fitted the so-called Konopinski-Uhlenbeck ${ }^{4}$ distribution instead of the Fermi ${ }^{5}$ distribution. This arose since thick sources were used and in addition since there was some scattering of betaparticles by the gas of the cloud chamber.

With the advent of strong sources from cyclotrons and piles and the development of new types of spectrometers the subject received a great impetus. Since 1940, and with the greater part of the activity coming since 1946, the spectra of a host of radioactive species have been investigated with the aid of various types of spectrographs. The large accumulation of data arising from these researches has been instrumental in laying the ground work for a systematics of nuclear spectra (see Chapter XVI).

In the present chapter, the processes involved in working out disintegration schemes will be concerned primarily with the problems arising when magnetic spectrographs with counter recording are used. It should be borne in mind, however, that experience has shown that no one technique is sufficient in working out all of the details of a complicated disintegration scheme. Each instrument has its own advantages and disadvantages. For example, weak gamma-ray lines have been missed when magnetic spectrographs using counter recording have been employed, which have been discovered with the help of scintillation spectrometers and permanent field type spectrographs using photographic recording. On the other hand, numerous erroneous disintegration schemes have been published. as a result of the use of spectrographs using photographic recording, since it is more difficult with this type of instrument to follow the decay of the various lines. In general, the most reliable results are obtained when both the gamma-rays, together with their intensities, and the beta-ray groups are determined and the results can be fitted into a satisfactory scheme.

Finally, it should be pointed out, that the principal aim of nuclear spectroscopy is to determine not only the relative positions of energy levels

4 E. J. Konopinski and G. E. Uhlenbeck, Phys. Rev. 48 (1935) 7.

5 E. Fermi, Zeits. f. Physik 88 (1934) 161. 
but also to determine the state characters (spins and parities of the various states). For this reason, in addition to measuring the energy and intensities of gamma-rays and beta-ray groups, it is necessary to determine internal conversion coefficients, $K / L$ ratios, and to do experiments on the angular correlation of the various radiations.

\section{$\S 2$. The Preparation of the Source Material}

In most cases the radioactive material, except for naturally radioactive substances, will have been prepared by nuclear bombardment with high energy particles or by exposure to neutrons, usually in a chain reacting pile. It is not enough to select materials of the highest purity, activate them, and measure them without further treatment. In deuteron bombardments in a cyclotron, for example, $(\mathrm{d}, \mathrm{n})$ and $(\mathrm{d}, \mathrm{p})$ reactions take place with comparable probabilities. In addition, material from the target backing and from protective windows placed over the target can be driven into the target and are usually found to be common contaminants. In pile irradiations, on the other hand, various unexpected activities may arise owing to the tremendous variation of neutron cross sections from element to element. In numerous cases a supposedly pure substance has been placed in a pile for the purpose of forming a radioactive element by neutron capture only to find that the activity of some unsuspected contaminant is greater than the desired activity. For these reasons, good chemical separations must be performed on the sources after irradiation. Unfortunately, the literature contains many examples in which chemical separacions were neglected with disasterous results.

The problems of radioactive chemistry are discussed in numerous books on the subject, and need not be belabored here. The main problem involved is the chemical behavior of very minute amounts of substances which would be of no consequence in ordinary chemical procedures but which are extremely important here. If the activity desired is in an element different from that bombarded, small amounts of carrier are added so that it may be separated chemically. Small amounts of impurities must be controlled in the same way. Since it is generally advantageous to have a source of as high a specific activity as possible, it is extremely useful, wherever possible, to make use of $(p, n),(d, n)$ and $(\alpha, n)$ reactions and to bring the material down with a small amount of carrier.

\section{$\S 3$. The Calibration of Instruments}

Before setting out to investigate decay schemes it is obviously necessary to calibrate the instruments to be used. In most of the discussions of this Chapter, it will be assumed that magnetic spectrographs will be employed. 
venient one since it is easily produced and has no high energy gamma-rays to produce a disturbing Compton background. The high energy edge of the line can easily be extrapolated to zero intensity, and a good calibration obtained.

\section{$\S$ 4. The Search for Gamma-Rays and Measurement of Their Energy}

In investigating disintegration schemes both the gamma-ray spectrum and the beta-ray or positron spectrum must be measured. While it makes no particular difference which is measured first, certain advantages lie in making a survey of the gamma-ray spectrum first. If the gamma-ray energies are measured first and the beta-ray spectrum later, particular care can then be taken to hunt for internal conversion lines since the energy of the gamma-rays are already known.

The measurement of the energy of gamma-rays is usually accomplished by measuring the energy of the secondary electrons produced by a) photoeffect, b) Compton effect, or c) pair production (if the energy of the gammaray is greater than $2 m_{0} c^{2}$ ). Of these three, in most cases, the photo-effect is the most advantageous since it produces a reasonably sharp line of electrons. Photoelectrons are ejected from the $K$-shell and the $L$-shell of the converter so that two lines are seen for each gamma-ray, thus affording a check on the energy. In some cases, usually for low energy gamma-rays and instruments of high resolution, the electrons ejected from the $M$-shell are also seen. The Compton effect, on the other hand, owing to poor angular definition and the thickness of the converter, manifests itself as a broad distribution. It is, of course, possible to measure the high energy edge of the Compton distribution and thus obtain the energy of the gammaray, but for complicated spectra this is an uncertain proceeding. The most convenient method therefore is to measure the $K$ and $L$ electrons ejected from a converter and to calculate the energy of the gamma-ray with the help of eq. (1).

In order to investigate a radioactive source emitting both beta-rays and gamma-rays with the help of a magnetic spectrograph, it is necessary to place the material in a container thick enough to stop all beta-rays. The front surface of the container is then covered with a thin converter of high atomic number and inserted at the source position of the instrument. The choice of converter depends somewhat on the complexity of the gamma-ray spectrum emitted by the source and by the strength of the source itself.

Since the photoelectric cross section per unit mass increases approximately as $Z^{5}$, elements of high atomic number are indicated as converters. Of these lead and gold are easily obtainable as thin foils. In many cases uranium, thorium and bismuth converters have been used. 
A typical spectrum of the photoelectrons ejected from a lead radiator is shown in Fig. 1. The substance under investigation in this case is $\mathrm{Os}^{185}$. The lines labelled $K_{3}$ and $L_{3}$ are the photoelectrons ejected from the $K$ - and $L$-shells by a gamma-ray of energy 0.648 $\mathrm{MeV}$ and those labelled $K_{4}, L_{4}$ are due to a gamma-ray of energy $0.878 \mathrm{MeV}$. To the low energy side will be seen the distribution of Compton electrons from the gamma-rays. It will be noticed also that the peaks $K_{3}$ and $L_{3}$ are superimposed on the Compton background from the higher energy gamma-ray. This is an example of a rather simple spectrum of only two gamma-rays. Many cases which arise consist of more gamma-rays, some of them quite weak, so that some of the photoelectron peaks are small prominences superimposed on an underlying Compton distribution. This immediately brings up the question of how to measure the energy of any given gamma-

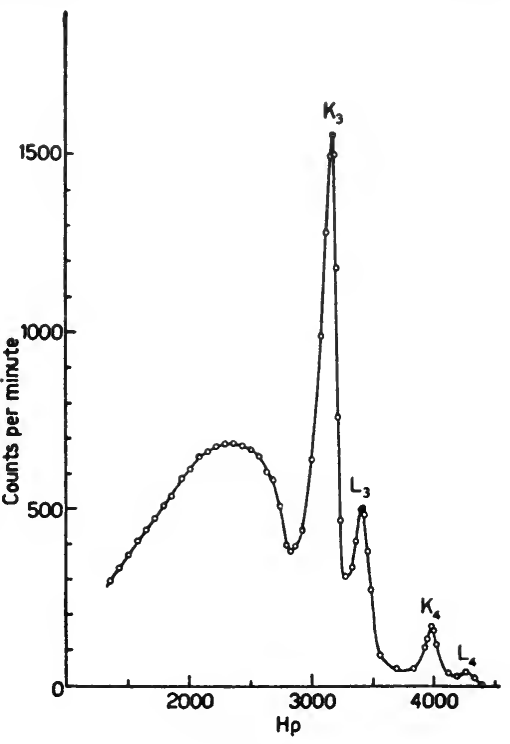

Fig. 1 ray in terms of the calibration and what thickness of radiator to use.

Suppose, for example, that the source is contained in a capsule, such as that shown in Fig. 2a, equipped with a radiator of thickness $t$. The number of photoelectrons produced in the radiator by a gamma-ray of energy $E$ proceeding from a point $a$ under an angle $\vartheta$ will be given by Fig. $2 b$

$$
N_{p} \propto \tau(E) t f(\vartheta) d \vartheta
$$

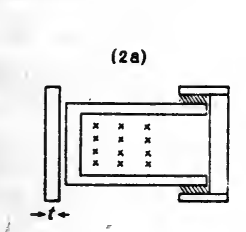

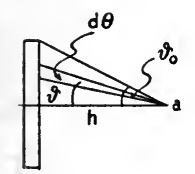

(2b)
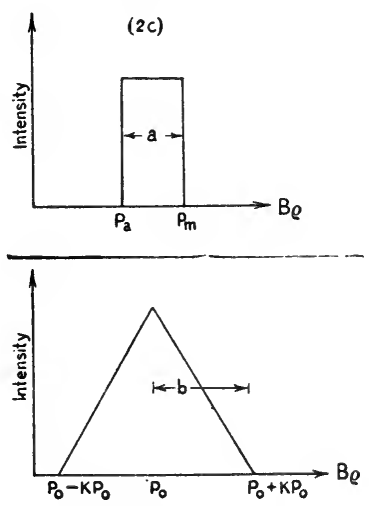

(2d)

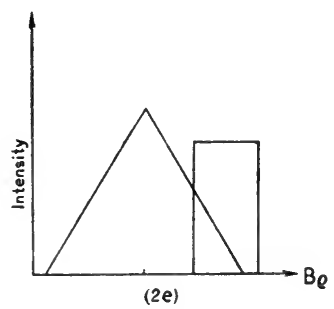

Fig. 2 
where $\tau(E)$ is the photoelectric cross section. The number of photoelectrons detected by the instrument at the momentum $B \varrho$ and in the range $\Delta(B \varrho)$ will be dependent on a number of additional factors. The number of photoelectrons ejected from a thickness $d t$ of the converter is dependent on the angle between the direction of the gamma-ray and that of the ejected photoelectron. For low energy gamma-rays, the maximum number of photoelectrons is ejected at an angle approaching $\pi / 2$ to the direction of the gamma-ray and moves in the forward direction as the energy of the gamma-ray is increased. Thus the number of photoelectrons received at the counter will depend on these factors and also the acceptance angle of the instrument. Secondly, photoelectrons of energy $E_{e}$ which are produced in a thickness $d t$ of the radiator will be slowed down in getting out of the radiator, so that they will have an energy $E_{e}-\Delta E_{e}$ and will be observed at the position $B o-\Delta(B o)$. In any practical case, these factors will be so complicated that an exact computation will be out of the question.

It will be seen from the above considerations that, in order to measure an appreciable number of photoelectrons, a converter of reasonable thickness has to be used. On the other hand, the thicker the converter the greater is the energy loss in the converter and the spread in momentum of the conversion line. In practice, it is clear that some compromise has to be reached.

An empirical approach to the problem has been made by Jensen, Laslett and Pratt ${ }^{12}$. They consider a particular instrument namely a magnetic lens spectrograph. They assume that the transmission curve of a magnetic lens spectrometer has approximately the shape of an isosceles triangle, which can be verified by making measurements on an internal conversion line from a thin source. The base of the triangle is proportional to the resolution and hence to the momentum $B \varrho$. This situation is depicted in Fig. $2 \mathrm{~d}$ in which the base of the triangle is taken as $2 b$. The half-width $b$ is taken as equal to a constant $K$ multiplied by the momentum $P_{0}$ corresponding to maximum transmission.

The photoelectrons ejected from the radiator by a given gamma-ray will have energies which depend on the depth of the point at which they originate. To a first approximation the momentum distribution will be rectangular with a width equal to the momentum loss associated with a full traversal of the foil. Let the maximum momentum of the photoelectrons be $P_{m}$ and the minimum $P_{a}$. The distribution will then be that shown in Fig. 2c, with $P_{m}-P_{a}=a$.

Consider a thin radiator for which $P_{m}-P_{a}$ is small compared to $2 b$. The process of measuring a photo line consists in shifting $P_{0}$ across the

12 E. N. Jensen, L. J. Laslett and W. W. Pratt, Phys. Rev. 75 (1949) 458. 
distribution as shown in Fig. -2e. The maximum of the distribution will occur when

$$
P_{0} \cong \frac{P_{a}+P_{m}}{2}=P_{m}-a / 2 .
$$

Thus, the effect of the radiator is to shift the peak of the line to lower energy and this effect increases as the thickness of the converter is increased.

For a thick converter, on the other hand, for which $P_{m}-P_{a} \gg 2 b$, similar considerations show that the line will be flat topped, and that the onset of the flat portion will occur at

$$
P_{m}=P_{0}(1+K)
$$

For any intermediate case it is clear that the line will be rounded off on top and that the peak will shift to lower energy with increasing thickness of converter. As an illustration Table II gives the position of the peak of the $1.10 \mathrm{MeV}$ line of $\mathrm{Zn}^{65}$ (measured in amperes) as a function of the surface density of a lead converter.

TABLE II

Relation between Position of the Peak of a

Photoelectric Line and the Thickness of the

CONVERTER

\begin{tabular}{c|c}
\hline $\begin{array}{c}\text { Thickness of Pb Converter } \\
\mathrm{mg} / \mathrm{cm}^{2}\end{array}$ & $\begin{array}{c}\text { Position of Peak } \\
\text { (amp) }\end{array}$ \\
\hline 6.4 & 4.525 \\
17.5 & 4.500 \\
24.8 & 4.490 \\
55.2 & 4.450 \\
65.9 & 4.435 \\
88.2 & 4.430 \\
147.0 & 4.425
\end{tabular}

From the above discussion it will be seen that the calibration of the instrument for gamma-ray energies and the measurement of these energies for any particular case depend on the strength of the source available and the complexity of the spectrum. For a strong source emitting a simple spectrum it is more accurate to calibrate by extrapolating the high energy edge of the line to the background, employing a source of annihilation radiation, and using the same procedure on the gamma-rays whose energies are to be measured. In this case also one can use a thin radiator made by evaporating lead, gold, bismuth or thorium onto the capsule containing the source. For complex spectra it will in general be impractical to make an extrapolation to the background and one is therefore 
forced to calibrate and measure on the peak of the gamma-ray lines. As an example of a simple spectrum we consider the spectrum of $\mathrm{Be}^{7}$ which emit' one gamma-ray of energy $474 \mathrm{keV}$. The spectrum is shown in Fig. 3 together with the calibration line of annihilation radiation from

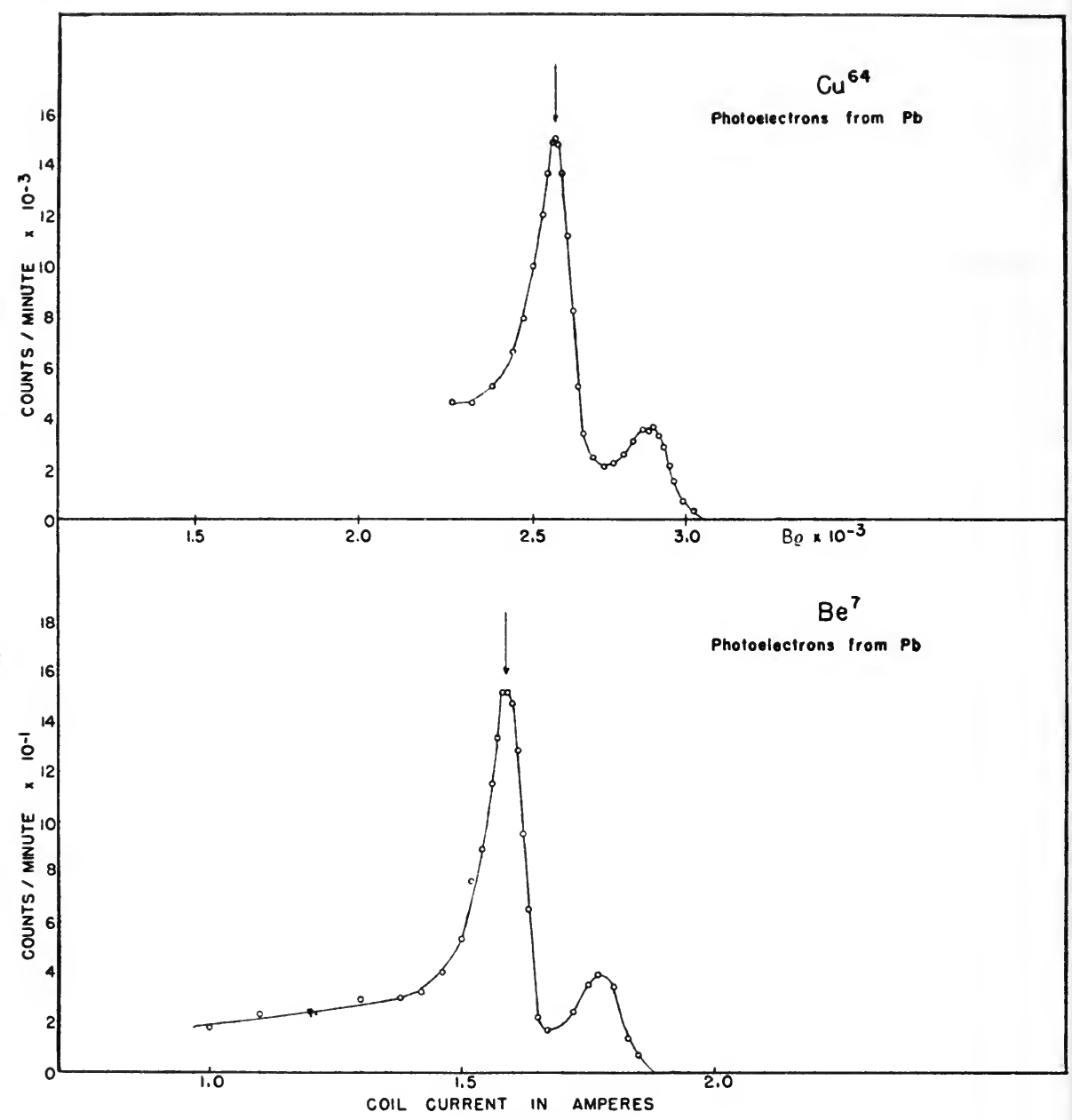

Fig. 3

$\mathrm{Cu}^{64}$. A lead radiator of surface density $21 \mathrm{mg} / \mathrm{cm}^{2}$ was used and the measurements were made in a magnetic lens. The data of Table III, illustrate the method ${ }^{13}$. In the instrument used the momentum focussed is linear with the current in the lens. Hence,

$$
(B \varrho)_{B e}=(B \varrho)_{\mathrm{ann}} \times \frac{i_{B e}}{i_{\mathrm{ann}}} .
$$

13 D. J. Zaffarano, B. D. Kern and A. C. G. Mitchell, Phys. Rev. 74, (1948) 105. 
The current at the peak and at the edge of the $K$ - and $L$-lines for both annihilation radiation and the $\mathrm{Be}^{7}$ line were measured and the energy of the Be line calculated.

TABLE III

ENERgy of THE $\mathrm{Be}^{7}$ Gamma-ray

\begin{tabular}{l|cc|c|c|c|c}
\hline \multicolumn{1}{|c|}{ Type of Radiation } & $\begin{array}{c}i \text { (Peak) } \\
(\mathrm{amp})\end{array}$ & $\begin{array}{c}i \text { (edge) } \\
(\mathrm{amp})\end{array}$ & $\begin{array}{c}\text { Bauss-cm } \\
\text { geak }\end{array}$ & $\begin{array}{c}\text { Energy } \\
\text { keV }\end{array}$ \\
\hline & & & & peak & edge \\
\hline Annihilation Radiation Run 1 & $1.685 \pm 0.002$ & $1.746 \pm 0.002$ & & & & \\
Annihilation Radiation Run 2 & $1.685 \pm 0.002$ & $1.746 \pm 0.002$ & & & & \\
Be Gamma-Ray & $1.585 \pm 0.005$ & $1.685 \pm 0.002$ & 2436 & 2457 & 470 & $\mathbf{4 7 8}$
\end{tabular}

\section{$\S$ 5. Special Considerations for Complex Spectra}

In many cases the spectra may be quite complex. Some of the lines may be so close together that, for a given converter, the $L$-photo-line from one gamma-ray may coincide with the $K$-photo-line from the neighboring gamma-ray. In other cases the spectrum may consist of some very low energy and some very high energy lines. In either case measurements with several radiators are indicated.

In those cases in which an $L$-photo-line from one gamma-ray falls under a $K$-photo-line from another, it is particularly necessary to separate them when attempting to get a measure of the relative intensity of the two gamma-rays. If lead was used as the converter in the original experiments, a second experiment can be performed using a converter such as thorium or uranium which has a different $K$ or $L$ binding energy. In this manner the lines can usually be separated.

Since the photoelectric cross section increases with $Z$ and decreases with $E_{\gamma}$, it will be seen that a uranium converter will be better than one of lead in bringing up high energy gamma-rays. If the energy of the gammaray is less than the $K$ binding energy of lead only the $L$ and $M$ lines will be seen. In order to bring out $K$ - and $L$-photo-lines a radiator of low $Z$, such as tin or indium can be used.

It will be seen from the discussion in the foregoing that the energies of the gamma-rays can be obtained from the measurements of the $K$ and $L$ photo lines. If, in addition these lines are also internally converted, another check on their energies can be obtained. As an example Table IV gives the energies of seven gamma-rays of $\mathrm{Kr}^{82}$ arising as a result of the beta-disintegration of $\mathrm{Br}^{82}$ on the one hand and that of the positron and $K^{r}$-capture disintegration of $\mathrm{Rb}^{82}$ on the other. It will be seen that quite satisfactory 
agreement is obtained between the different investigators and between the different methods of determination.

TABLE IV

ENERgies of the Gamma-RAYS OF $\mathrm{Kr}^{82}$ (FROM $\mathrm{Br}^{82}$ aND $\mathrm{Rb}^{82}$ ) FROM the Photo and Internal Conversion Line Spectrum

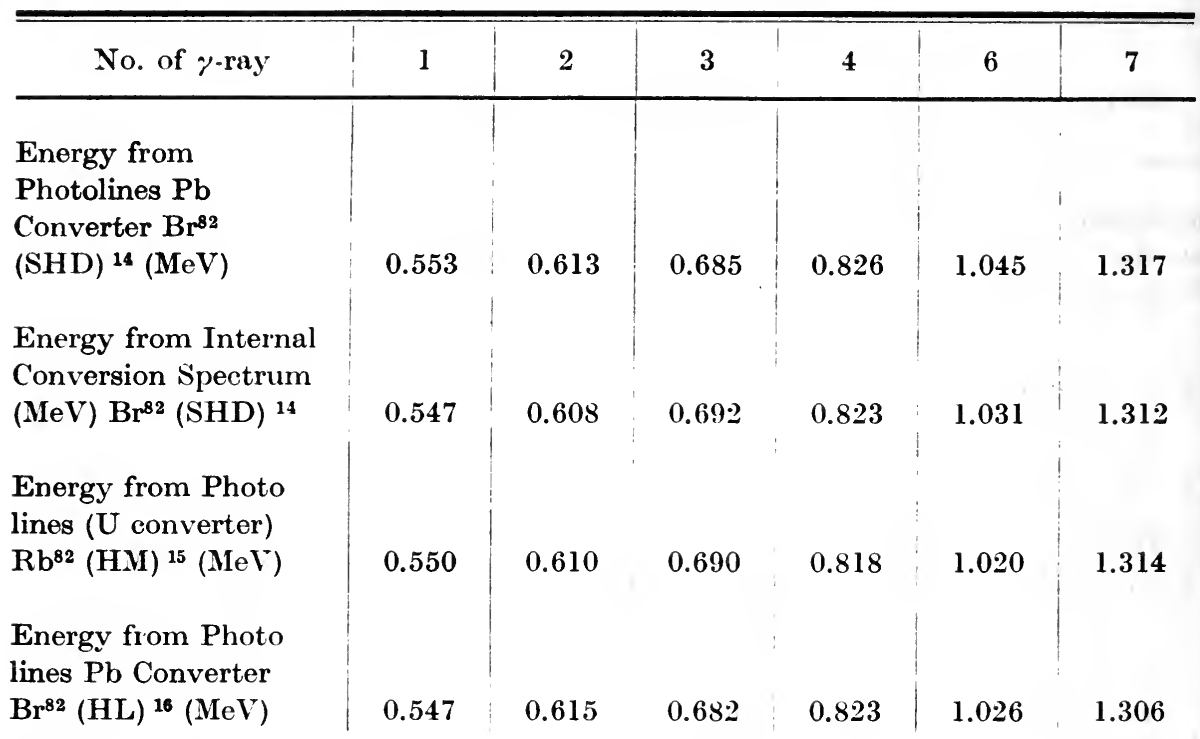

N.B. There are also additional lines in the spectrum.

\section{$\S$ 6. The Determination of the Relative Intensities of Gamma-Rays}

The question of the determination of the relative intensities of gammarays is a difficult one, just as is the similar problem in atomic line spectra. We limit ourselves in this Chapter to the determination of relative intensities by means, of magnetic spectrographs. The treatment of the problem using the scintillation method is discussed in Chapter V.

Since gamma rays are detected by the production of their secondaries, Compton electrons or photoelectrons, either process may be used for the measurement of relative intensities. In either case, the efficiency for a converter must be determined as a function of energy. Siegbahn ${ }^{17}$ has devised a method for the determination of the intensities of gamma-rays from the measurement of their Compton secondaries in a magnetic spectro-

\footnotetext{
14 K. Siegbahn, A. Hedgran and M. Deutsch, Phys. Rev. 76 (1949) 1263.

15 C. M. Huddleston and A. C. G. Mitchell, Phys. Rev. 88 (1952) 1350.

16 P. Hubert and J. Laberrigue-Frolow, Comptes Rend 232 (1951) 2420.

17 K. Siegbahn, Proc. Roy. Soc. A188 (1947) 541.
} 
graph. Rasmussen, Hornyak, Lauritsen and Lauritsen ${ }^{18}$, have given a detailed treatment of the method. However, the method is rather limited in its applicability and is not at present in general use. The method usually employed is that of measuring the number of photoelectrons ejected from a converter by a gamma-ray of given energy.

As was pointed out in $\S 4$, this method requires a knowledge of the photoelectric cross section and its variation with angle, a knowledge of the energy loss in the converter, and the transmission of the instrument. All of these factors have not, in general, been considered when estimates of relative intensities are given in papers on nuclear spectroscopy. What is obtained is an approximate answer which gives reasonably good estimates of relative intensities in most cases. A detailed treatment of the problem attempting to take into account all of the factors mentioned above has been given by Thomas ${ }^{19}$.

The assumptions made in the usual method of measuring relative intensities by means of the ejected photoelectrons are that the resolution of the instrument is good and that the converter is not too thick. Under these conditions (see $\S 4$ ), the shape of the distribution of the photoelectrons ejected from the $K$-shell, say by a gamma-ray of energy $E_{\gamma}$, will approximate the shape of the transmission curve of the instrument. If $n(B \varrho) d(B \varrho)$ is the number of photoelectrons counted in an interval $d(B \varrho)$, the total number of photoelectrons counted "under the line" will be

$$
\int_{\text {line }} n(B \underline{0}) d(B \underline{0}) \text {. }
$$

It is then assumed that this quantity is proportional to $\tau_{K}\left(E_{\gamma}\right)$, the probability for the ejection of an electron from the $K$-shell of the converter by a gamma-ray of energy $E_{\gamma}$.

The procedure, when measuring with a magnetic spectrograph, is to take a series of counts at a sufficient number of settings of the magnetic field to determine the profile of the line. Let the number of counts per unit time received when the field is $B$ be $N$. Since the momentum interval accepted by a magnetic spectrograph is proportional to $B \varrho$, it follows that

$$
n(B \varrho) d(B \varrho) \sim \frac{N}{B \varrho} d(B \varrho) .
$$

If now a plot of $N / B \varrho$ vs $B \varrho$ is made, that area under the profile of the line is just

$$
\int_{\text {line }} n(B \varrho) d(B \varrho)=\text { const } \tau_{K}\left(E_{\gamma}\right) .
$$

18 V. K. Rasmussen, W. F. Hornyak, C. C. Lauritsen, and T. Lauritsen, Phys. Rev. 77 (1950) 617.

19 R. G. Thomas, Thesis, California Institute of Technology, Pasadena (1951). 
For a radiator of high $Z$, it is obvious that in addition to the $K$-lines from the various gamma-rays that there will be $L$ and perhaps $M$-lines also visible. The $L$-line will of course be separated from the $K$-line. In addition, the $K$-lines from the lower energy gamma-rays will be superimposed upon the Compton distribution from the higher energy lines. Such a situation is illustrated in Fig. 1 for the case of Osmium 185. In this figure it should be noted that $N$ (not $N / B \varrho$ ) is plotted against $B \varrho$. To measure the relative intensities of the two lines shown there $(0.648$ and $0.878 \mathrm{MeV}$ ) it is necessary to replot the curve as $N / B \varrho$ vs $B \varrho$ and determine the area under each $K$-line (drawing in the apparent background). The area under a given line, $A_{\gamma}$, is then given by

$$
A_{\gamma}=k I_{\gamma} \tau_{\boldsymbol{K}}\left(E_{\gamma}\right) \text {. }
$$

The relative intensity of the two gamma-rays is then

$$
\frac{\left[A_{\gamma_{1}} / \tau_{K}\left(E_{\gamma_{1}}\right)\right]}{\left[A_{\gamma_{s}} / \tau_{K}\left(E_{\gamma_{s}}\right)\right]}=\frac{I_{\gamma_{2}}}{I_{\gamma_{s}}}
$$

It will be seen that his process neglects the change in angle between the direction of the photoelectron and the incident gamma-ray with the energy of the gamma-ray.

In order to get a value for $I_{\gamma_{1}} / I_{\gamma_{3}}$ from eq. (8), it is necessary to know how $\tau_{\boldsymbol{K}}\left(E_{\gamma}\right)$ varies with energy. The quantity $\tau_{\boldsymbol{K}}\left(E_{\gamma}\right)$, in $\mathrm{cm}^{2} / \mathrm{gm}$, can be derived from the atomic cross section for photoelectric emission $\sigma_{K}\left(E_{\gamma}\right)$ in $\mathrm{cm}^{2}$. The theoretical expression for $\sigma_{K}\left(E_{\gamma}\right)$ is discussed by Heitler 20 who gives the following expression

$$
\sigma_{K}\left(E_{\gamma}\right)=3 / 2 \frac{Z^{5}}{137^{4}} \sigma_{0} F\left(E_{\gamma}\right)
$$

where $\mathcal{} F\left(E_{\gamma}\right)$ is a rather complicated expression giving the energy dependence. The quantity $\tau_{\boldsymbol{K}}\left(E_{\gamma}\right)$ for lead has been determined experimentally by Gray ${ }^{21}$ and his results give an excellent fit to the theoretical curve. Thus if a lead converter is employed, Gray's curve can be used in evaluating the relative intensities. For other converters the results on lead can be modified in accordance with the $Z$ dependence of eq. (9). The reader is referred to Chapter II for further details. Examples of the results of the method will be given when various decay schemes are discussed.

\section{$\S 7$. The Measurement of the Beta-Rays}

A. Precautions concerning Source and Counter ef. p. 259

The preceeding sections have described the measurement of the gammarays emitted by a radioactive source, the determination of their energies

20 W. Heitler, The Quantum Theory of Radiation, Second Edition, Oxford 1944.

21 L. H. Gray, Proc. Camb. Phil. Soc. 27 (1931) 103. 
and relative intensities. In order to determine the complete disintegration scheme, the distribution in energy of the beta-rays (or positrons) must be measured. The purpose of measuring the beta-ray distributions, of course, is to determine the end-point energies and relative abundances of the several beta-ray groups which may occur. The beta-ray spectrum is analyzed by comparing the measurements with the results to be expected from the theory of beta-decay (('hapters IX and X). Since the spectrum from a substance emitting a single group of beta-rays extends from the maximum energy $E_{0}$ to low energies with a certain theoretical shape and since, in many disintegration schemes, several beta-ray groups are emitted, it will be clear that a determination of these groups will depend on a shape analysis. For this reason particular care must be taken in the experimental technique to prevent a distortion of the spectrum.

The most serious distortions arise at the low energy end of the spectrum and are caused by source thickness, source backing and counter window thickness. Some apparent distortion may occur at the high energy end of the spectrum unless corrections for the resolution of the instrument are properly made.

Since electrons are scattered and lose energy in traversing a material source, the source must be as thin as possible. The preparation of thin sources has been discussed by various authors ${ }^{22}$ (see also Chapter XI). One of the best ways of making a thin and uniform source is to evaporate it in vacuo onto a thin aluminum foil. This is somewhat wasteful of radioactive material, but if the source is strong enough and if a chemical compound suitable for this purpose can be found, a thin and uniform source can be prepared. It is sometimes possible to electroplate the source. Since electroplating onto aluminum is not, in general, practical, some heavier metal such as copper must be used. In many cases the source must be evaporated from solution onto a thin zapon foil. In such cases the source does not deposit uniformly and tends to form agglomerates. Langer ${ }^{23}$ has shown that such effects are almost completely eliminated by first treating the backing with a thin layer of insulin. It is sometimes necessary to use solid sources which are formed as a result of chemical precipitation. In such cases the source must be finely ground and is held on a thin backing with a drop of zapon solution. The thickness of the source which can be used depends on the nature of the spectrum to be measured.

22 L. M. Langer, R. D. Moffat and H. C. Price. Phys. Rev. 76 (1949) 1725; C. S. Cook and L. II. Langer, ibid., 73 (1948) 601; R. D. Albert and C. S. Wu, ibid., 74 (1948) 847; G. E. Owen and C. S. Cook, ibid., 76 (1949) 1726; C. S. Cook, Nucleonics, December 1953, January 1954.

23 L. M. Langer, Rev. Sici. Inst. 20 (1949) 216. 
The general rule is to make the source as thin and as uniform as possible. Source thicknesses as low as 1 microgram per square centimeter have been obtained.

The source backing must also be kept thin to avoid backscattered electrons. In most cases thin films of zapon, formvar or L.C. 600 are used. Thin aluminum of $0.0002^{\prime \prime}$ thickness is easily obtainable and especially useful for evaporated sources. Since zapon, formvar, etc. are usually very good insulators and since the electrical capacity of the source is small, it is found that, with strong radioactive deposits, the source may charge to several kilovolts with respect to the vacuum chamber. Such a charging of the source may influence the shape of the spectrum. To circumvent this, the source can be grounded to the vacuum chamber by a thin line of colloidal graphite (Aquadag) running across the film from the source to the source holder.

If the detector of the particles is a Geiger Müller counter, the window must be kept thin. The effect of the window, of course is to cut down the transmission of particles into the counter. Mica or aluminum counter windows are seldom made which transmit electrons having energies of less than 50 kilovolts. For transmission to lower energies, thin films of zapon or formvar can be made which, if supported on a grid, can transmit down to less than 1 kilovolt. The values given above are "cut off energies" corresponding to a thickness such that no electrons having energies less than $E$ are transmitted. The transmission of a given window rises gradually as the energy of the electrons is increased until $100 \%$ transmission is reached. Thus the window thickness will influence the shape of the spectrum until full transmission is reached. A good working rule is that the window thickness will not influence the shape of the spectrum above an energy which is four times the cut-off energy.

\section{B. The Measurement of the Spectrum}

After the precautions mentioned in the last section have been taken, the source is placed in the spectrograph and counts are taken for various settings of the magnetic field. After making corrections for decay, a plot of the number of counts per minute against the magnetic field is made. Usually this plot is made while the data are being taken. Such a plot, taken using a magnetic lens spectrometer in which the field is proportional to the current, is shown ${ }^{24}$ in Fig. 4 . This gives an idea of the shape of the spectrum. An internal conversion line corresponding to a gamma-ray of $0.435 \mathrm{MeV}$ is also seen. The beta-ray spectrum was shown to consist of only one group. In order to make a theoretical analysis of the shape of the

24 A. B. Smith, unpublished. 
CH. VIII (I)]

spectrum, to determine the internal conversion coefficient, or to determine the relative abundance of several beta-ray groups (if present), it is necessary to determine the number of beta-ray's per unit momentum interval. For'

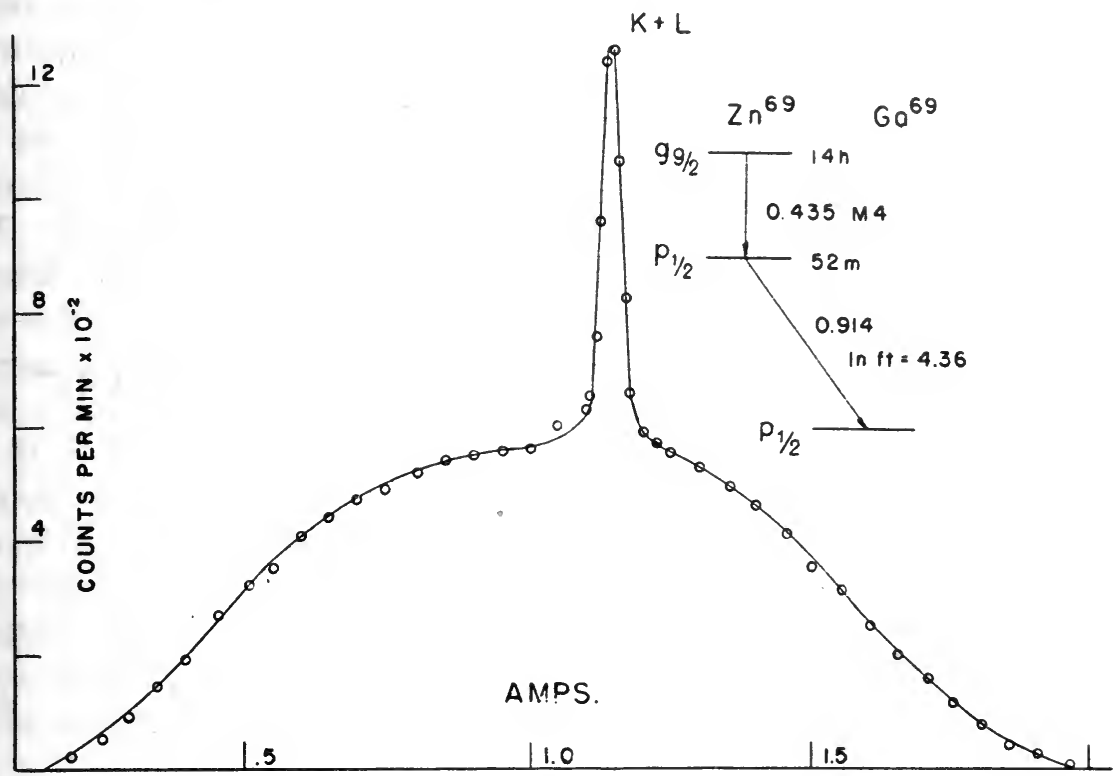

Fig. 4

this purpose one plots $N / B \varrho$ against $B \varrho$. The value of $B \varrho$ for a given value of the field is determined from the calibration of the instrument.

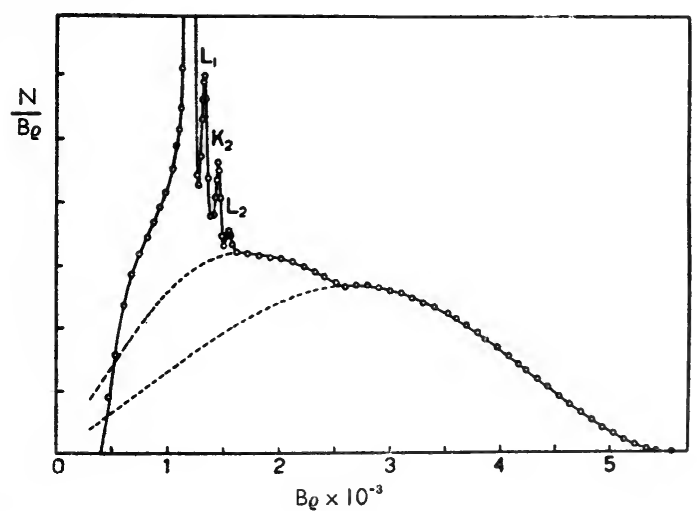

Fig. 5

As an example of a complex spectrum, Fig. 5 shows a plot of the spectrum ${ }^{25}$ of $\mathbf{M o}^{99}$. In this figure $\boldsymbol{N}^{\gamma} / B O$ is plotted against $B \varrho$. Two groups

25 M. E. Bunker, Thesis, Indiana University. 
are easily seen in the graph. In addition internal conversion lines are also seen.

An example of the spectrum of a positron emitter C $\mathrm{s}^{130}$ accompanied by $K$-electron capture is shown in Fig. 6. Here the positron spectrum and the Auger line produced by the $\mathrm{X}$-ravs arising from $K$-electron capture are clearly seen.

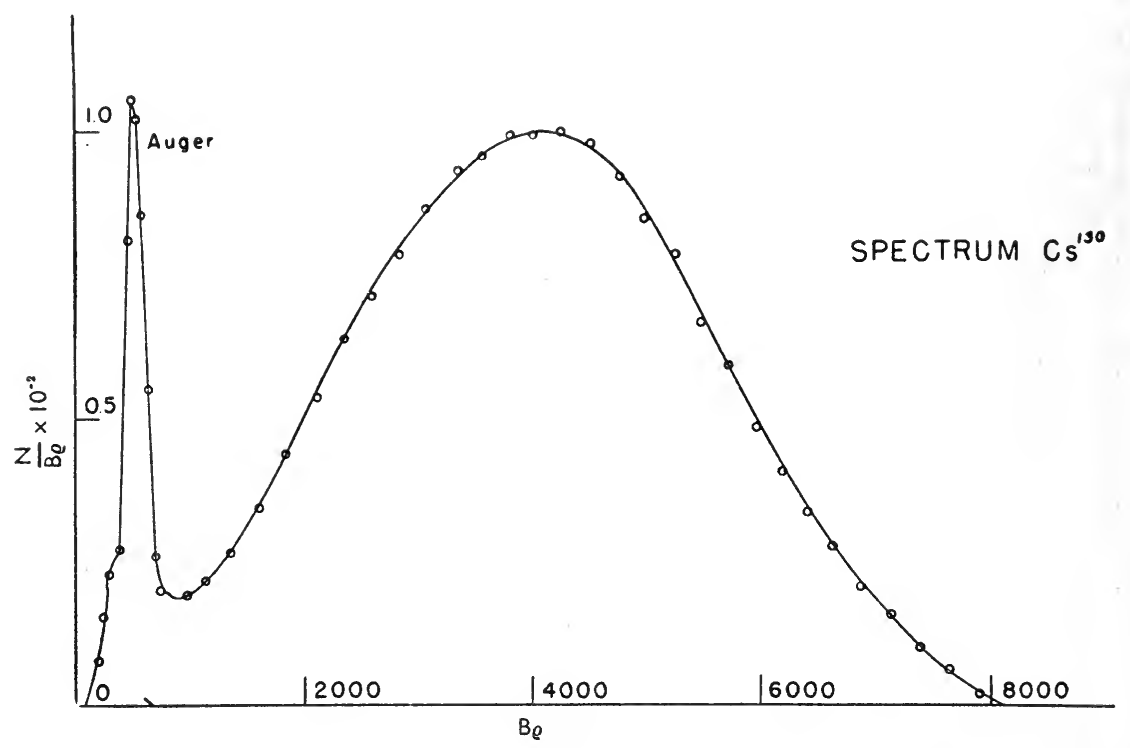

Fig. 6

With these examples in mind we turn now to the examination of the shapes of the spectra.

\section{$\S 8$. The Fermi Plot; End Points and Beta-Ray Groups}

\section{A. One Beta-Ray Group}

The distribution in energy of the beta-rays (or positrons) from a radioactive source is discussed in detail in Chapter $I X$ and $X$. Equation (10) of Chapter IX gives an expression for the number of electrons in an energy range $d W$ as a function of the energy and the atomic number of the product nucleus. If this equation is rewritten in terms of the notation of Eq. (27) Chapter $\mathrm{X}$, it becomes

$$
N_{ \pm}(W) d W=\frac{g^{2}}{2 \pi^{3}} F(\mp Z, W) p W\left(W_{0}-W\right)^{2} d W\left[S_{n}(W)\right] .
$$

In this equation the term $p W\left(W_{0}-W\right)^{2} d W^{r}$ represents the socalled statistical shape, $F\left(\mp Z, W^{*}\right)$ denotes the Coulomb factor and $S_{n}(W)$ the shape 
factor for forbidden spectra. $W$ is the total energy in $"{ }_{0} c^{2}$ units. Thus $W=E / m_{0} c^{2}+1$ where $E$ is the energy of the electron in MeV. If eq. (10) is rearranged it becomes (see eq. (12) ('hapter IX and eq. (27) ('hapter X)

$$
\left[N_{ \pm}(W) / F(\mp Z, W) p W^{1 / 2}=K\left(W_{0}-W\right)\left[S_{n}(W)\right]^{1 / s}\right.
$$

For allowed spectra $S_{n}(W)=1$. It is then clear that, for allowed spectra, if the function on the left hand side of eq. (11) is plotted against $W$ a straight line will result. The intercept of this line on the $W$ axis will be $W_{0}$, the total disintegration energy in $m_{0} c^{2}$ units, from which the endpoint energy in $\mathrm{MeV}$ can be calculated. Such a plot is called a Fermi Plot or a Kurie. Plot. In the discussion that follows we shall assume that the spectrum has an allowed shape and leave until a later section those cases in which the shape of the spectrum is clearly not allowed.

It is the problem of the experimenter to fit the data obtained from the magnetic spectrograph to eq. (11). Let us assume, first, that the spectrum is simple. As mentioned in the preceeding section, the data obtained from the spectrograph is in the form of the number of counts per minute, $N$, against the field $B$. From the calibration of the instrument the value of the momentum $B O$ in gauss-cm corresponding to the field is obtained. The number of counts per unit momentum interval is then given by

and

$$
N(p)=N / B Q
$$

$$
p=B \varrho / 1700
$$

for $p$ in relativistic units. The relation between $p$ and $W$ is

$$
W=1 \overline{1+p^{2}}
$$

Eq. (10) now becomes

$$
\left.\begin{array}{l}
\left.N_{ \pm}(p) d p=g^{2} / 2 \pi^{3} F(\mp Z, W) p^{2} d p\left[\sqrt{1+p_{0}^{2}}-\sqrt{1+p^{2}}\right]^{2}\right) \\
N_{ \pm}(p) d p=g^{2} / 2 \pi^{3} G\left({ }^{\mp} Z, P\right) W p d p\left[\sqrt{1+p_{0}^{2}}-\sqrt{1+p^{2}}\right]^{2}
\end{array}\right\}
$$

The quantity $F(Z, W)$ or $G(Z, P)$ can be calculated from eq. (9c, 9d) of Chapter IX, or determined from Appendix II. The calculation can then be carried out in a straightforward manner.

As an example, the beta-ray spectrum of $\operatorname{In}^{114}$, taken in a $180^{\circ}$ type spectrograph by Langer (unpublished), is shown in Fig. 7 . This is a typical example of an allowed spectrum. The end point of the spectrum comes at $W_{0}=4.93$ or $E_{0}=2.00 \mathrm{MeV}$. The spectrum is linear from the endpoint to about $0.35 \mathrm{MeV}$, where it bends away owing to source thickness.

Thus, in using Fermi Plots, to help in the determination of disintegration schemes it is necessary to know whether any deriation from the plot is 
caused by source thickness or by the presence of a lower energy group. There are several ways of determining this. If the curve is extrapolated below the point at which it rises above the straight line, this line can be used to obtain a distribution of $V_{\text {th }}(p)$ against $p$. ('learly one uses the

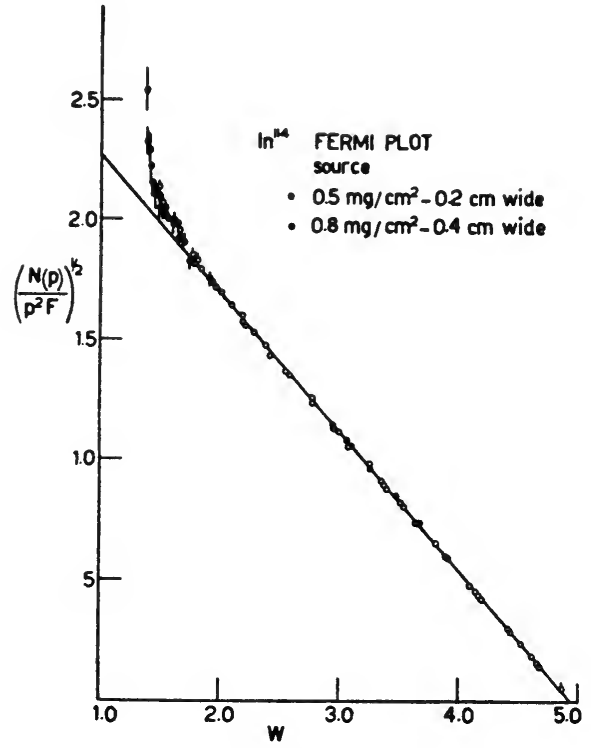

Fig. 7 reverse process to that used in making the Fermi Plot. This curve can then be compared with the original curve of $N_{\text {obs }}(p)$ against $p$.' The difference $N_{\text {obs }}(p)-N_{\text {th }}(p)=N^{\prime}(p)$ can then be used to make another Fermi Plot. If the low energy portion is due to scattering in the source the new plot obtained using $N^{\prime}(p)$ will not, in general, give a straight line. If a lower energy group exists it will be accompanied by the emission of a gamma-ray. If no gamma-ray is found corresponding to the energy difference between the end point and the point of rise away from the striaght line, it is clear that the low energy counts are due to thick source. This point can be checked by attempting beta-gamma coincidence experiments (see Chapter VII). In the case of $\operatorname{In}^{114}$ there are no gamma-rays in coincidence with beta-rays.

\section{B. More Thax One Beta-Ray Group}

If more than one group of beta-rays is present, this can sometimes be recognized immediately from a plot of $\mathrm{N} / \mathrm{B}_{\boldsymbol{Q}}$ vs $B \underline{Q}$, as is the case in $\mathbf{M o}^{99}$ (see Fig. 5). In most cases this will not be possible and the groups will only show up through an analysis of the Fermi Plot. An example of this is shown in Fig. 8 in which a Fermi Plot for the beta-rays ${ }^{26}$ of $\mathbb{I}^{187}$ is shown.

The procedure for making the Fermi Plot is the same as that mentioned in the last section. One starts at the high energy end and when a certain energy is reached the curve bends upwards from the normal straight line, indicating the presence of a second group. If $N(p) d p$ is the number of counts per unit momentum interval from the observed data and $N_{1}(p) d p$ is a similar quantity for those points which fit the high energy portion of the Fermi Plot and its low energy extropolation, a new Fermi Plot can be

26 C. L. Peacock and R. G. Wilkinson, Phys. Rev. 74 (1948) 297. 
made from the difference $N_{2}(p)=N(p)-N_{1}(p)$. The Fermi Plot for this difference is shown as the interior group on the graph. The end points of the two groups come at $1.33 \mathrm{MeV}$ and $0.63 \mathrm{MeV}$ respectively. A gamma-ray of energy $0.696 \mathrm{MeV}$ was found corresponding to the difference in energy of the two groups.

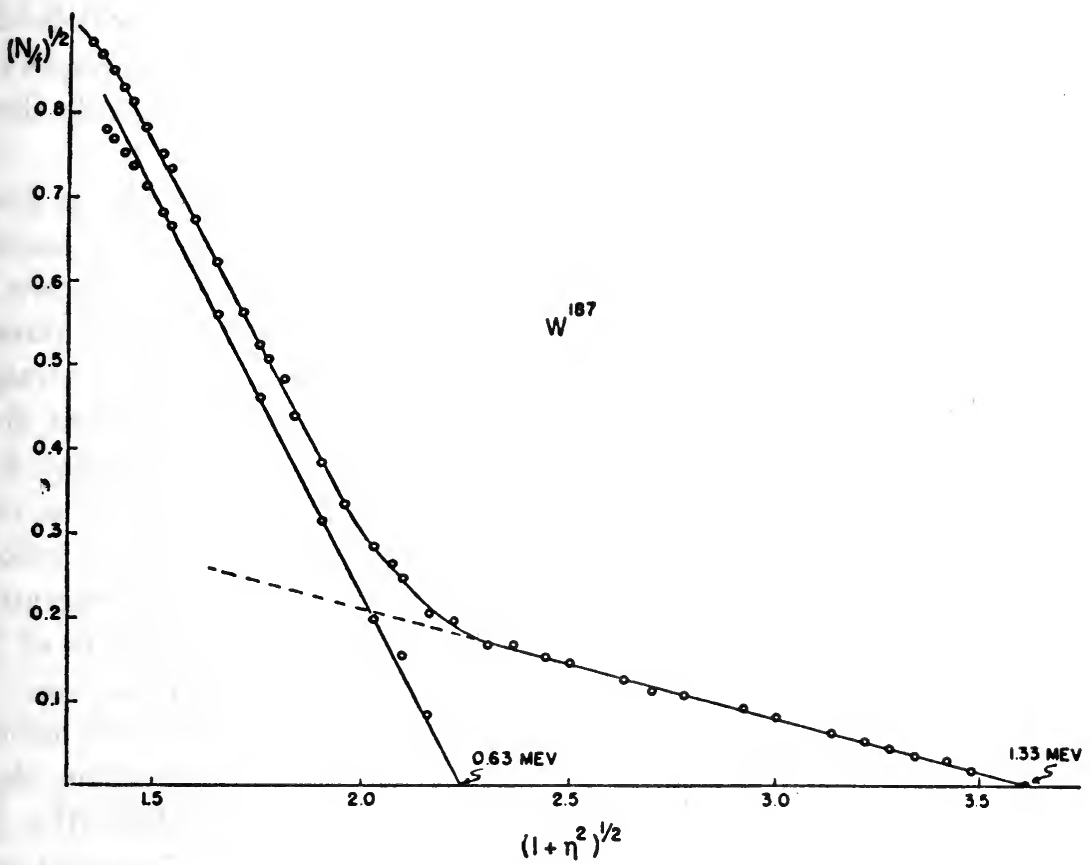

Fig. 8

If more than two groups are present, the process is repeated and the various lower energy groups obtained. For a very complicated spectrum, the end point energies of the lower energy, or interior, groups cannot be determined as exactly as those for the most energetic groups, owing to the many subtractions involved. The end point energies of the various groups will determine energy levels in the product nucleus, measured from the ground state of the parent, and these energy levels will be established by the measurements of gamma-rays.

\section{The Relative Abundances of Beta-Ray Groups}

If several beta-ray groups are present, the number of counts per unit moment interval $N_{i}(p) d p$, for the $i^{\text {th }}$ group, can be determined from the several Fermi Plots. If these points are plotted the area under each curve $\int N_{b}(p) d p$ gives the number of disintegration electrons of this group per disintegration. From this information the relative abundance of the various 
groups can be determined. The relative abundance is usually expressed as the percentage of the number of electrons in the $i^{\text {th }}$ group to the total number of electrons emitted.

\section{Spectra Having Forbidden Shapes}

The question of beta-ray spectra having forbidden shapes will be discussed in detail in Chapter XI. Nevertheless, since forbidden shapes can and do arise in complex spectra, it is well to know how to handle them, so that a few remarks here will not be out of place.

The distribution of beta-rays which has a forbidden shape will be given by eq. (10), where $S_{n}(W)$ is an energy dependent factor. For allowed transitions $S_{n}(W)=1$. Consider now a radioactive transition known to emit only one group of beta-rays. If the spectrum does not have an allowed shape and the usual Fermi Plot is made, the result will not be a straight line. With a proper choice of the shape factor $S_{n}(W)$, obtained from Chapter $\mathrm{X}$, a plot of $\left[N / F p W S_{n}\left(W^{\prime}\right)\right]^{1 / 2}$ is $W$ will give a straight line. Since the quantity $\left[N / F p W^{1}\right]^{1 / 2}$ is carried in the calculation it is only necessary to divide this by the proper choice of $\left[S_{n}\left(W^{*}\right)\right]^{1 / 2}$ to make a corrected plot. In any given case certain criteria of the disintegration, such as the end point energy of the beta-rays and the half-life. of the disintegration, can be employed to help decide which $S_{n}(W)$ to use.

As has been pointed out in Chapter $\mathrm{X}$, the deviations from the allowed shape are most pronounced in one or two eases. The forbidden shape which has been observed most commonly to date is that for which $\Delta j= \pm 2$ and there is a change of parity. This particular shape, if plotted as a normal Fermi Plot, shows a deficiency of electrons at high energies and an excess at low energies. It is clear that if such a shape should be present among the beta-ray groups of a complex spectrum, considerable difficulties would be found in obtaining the correct energies and relative abundances of the various groups, unless proper corrections are made for the shape.

A forbidden shape, occurring in a complex spectrum, can be most easily recognized when it occurs in the group of highest energy. Owing to the fact that information about the interior groups has to be obtained by differences, attempts to discover shapes in interior groups has not been satisfactory.

As an example the Fermi Plot ${ }^{27}$ for the spectrum of $\mathrm{I}^{124}$, a positron emitter, is shown in Fig. 9. It will be seen that the highest energy group does not have an allowed shape. The correction to the shape, which

27 A. C. G. Mitchell, J. Y. Mei, F. C. Maienschein and C. L. Peacock, Phys. Rev. 76 (1949) 1450. 
turned out to be that for $\Delta j= \pm 2$ and a change of parity, was made by using

$$
S_{n}(W) \cong\left(W^{2}-1\right)+\left(W_{0}-W\right)^{2}
$$

When this correction was made the three groups of positrons had energies of 2.20, 1.50 and $0.67 \mathrm{MeV}$. Other examples will be given in Chapter XI.

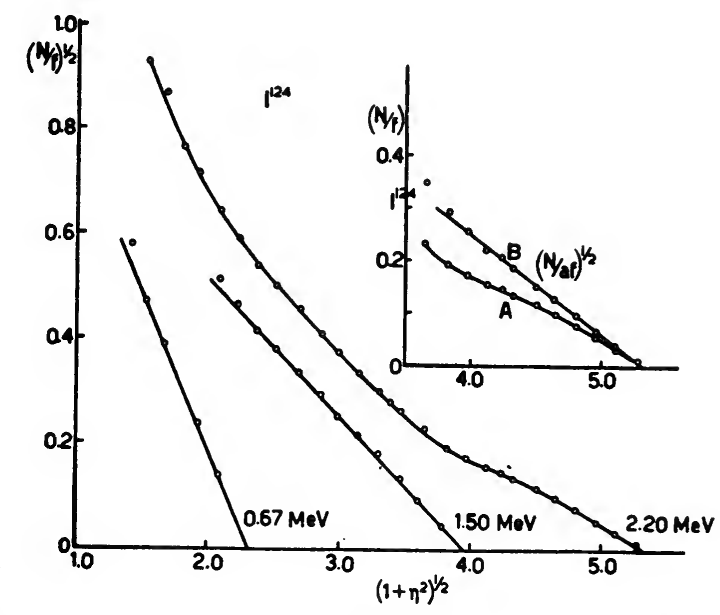

Fig. 9

\section{§ 9. Determination of Comparative Half-Lives $-\boldsymbol{f t}$}

As is pointed out in Chapters IX and X, the comparative half-life, $f t$, is a quantity which gives useful information on the nature of betadisintegration. Referring to eq. (13) and (14) Chapter IX, the total probability of decay for a given beta transition is given by

$$
\lambda=\int_{1}^{W_{0}} N(W) d W=\frac{g^{2}}{2 \pi^{3}}\left[C_{F}^{2}\left|M_{F}\right|^{2}+C_{G T}^{2}\left|M_{F T}\right|^{2}\right] f\left(Z, W_{0}\right)=\frac{\ln 2}{t}
$$

for allowed transitions, where

$$
f\left(Z, W_{0}\right)=\int_{1}^{W_{0}} p W\left(W_{0}-W\right)^{2} F(Z, W) d W
$$

For forbidden transitions eq. (33) of. Chapter $\mathrm{X}$ applies. In eq. (16), $t$ is the half-life for the transition. Thus it is seen that $f t$.is a constant of the disintegration and has a value which is characteristic of the type of disintegration, i.e., whether it is allowed or whether it has a certain degree of forbiddeness $n$. The quantity $f\left(Z, W_{0}\right)$, as defined in eq. (17), depends only on the charge of the product nucleus and the energy of the dis- 
integration. (iraphs for $f\left(Z, W_{0}\right)$ for various $Z$ and $W_{0}$ have been given by Feenberg and Trigg ${ }^{28}$ (see Ch. IX).

There is no difficulty in determining $f t$ from an experimental point of view. For a beta-emitter having only one group, $W_{0}$ and $t$ are measured and $Z$ is known. It is, therefore, only necessary to calculate $f\left(Z, W_{0}\right)$, or look it up on the graphs, and calculate $f t$. For a beta-emitter giving several groups of beta-rays it is necessary to calculate the partial half-life $t_{i}$. If $\lambda$ is the disintegration constant for the decay, determined from the observed half-life $t$, and $\lambda_{i}$ is the partial half-life of the $i^{\text {th }}$ group, it follows that

$$
\frac{\lambda_{i}}{\lambda}=A_{i}
$$

where $A_{i}$ is the relative abundance of the $i^{\text {th }}$ group. From the relation between half-life and decay constant, the partial half-life $t_{i}$ can be calculated. Since the individual end points $W_{0}^{i}$ are known, $f_{i}\left(Z, W_{0}\right)$ can be calculated for each group and hence $(f t)_{i}$ can be obtained. For positron emitters, since there is also a probability of $K$-capture, the appropriate quantity to use is $f=f_{K}+f_{+}$, which is also tabulated ${ }^{28}$. Examples will be given when individual decay schemes are discussed.

The quantity which is usually used in discussing changes in spin and parities for any transition is $\log _{10} f t$ rather than $f t$. Mayer, Moszkowski and Nordheim ${ }^{29}$ have used $\log _{10} t t$ to show the connection between nuclear shell structure and beta decay. (See also Chapter XVI).

\section{$\S 10$. Determination of Internal Conversion Coefficients and $\boldsymbol{K} / \boldsymbol{L}$ Ratios}

Having shown how to measure the energies and intensities of gammarays and how to determine the characteristics of beta-ray groups, it is appropriate at this point to consider the question of internal conversion electrons. It has been shown that the measurement of the energy of internal conversion electrons serves as a confirmation of gamma-rays measured by the photoelectric method. We wish to discuss now the determination of the number of internal conversion electrons associated with a given gamma-ray. This quantity, termed the internal conversion coefficient is defined as

$$
\alpha=N_{e} / N_{\gamma}
$$

While this discussion anticipates, to some extent, the determination of disintegration schemes discussed in the next section, it will, nevertheless, be considered here.

28 E. Feenberg and G. Trigg, Rev. Mod. Phys. 22 (1950) 399.

29 M. G. Mayer, S. A. Moszkowski and L. W. Nordheim, Revs. Mod. Phys. 23, (1951) 315 . 
The quantities which can be calculated theoretically are $\alpha_{E}, \alpha_{L}$, etc., the internal conversion coefficient from the $K$-, $L$-shell etc. (See Chapter XIV). Thus, for example $\alpha_{K}=\left(N_{e}\right)_{K} / N_{\gamma}$. An experimental determination of $\alpha_{K}$ presupposes that the $K$ - and $L$-lines can be separated by the instrument and that the number of $K$ internal conversion electrons from a given gamma-ray can, in some way, be compared to the intensity of that gammaray. The first condition merely entails the use of an instrument of resolving power sufficiently good to separate the $K$ - and $L$-lines; the second requires some means of comparison between the two measured quantities.

There are several possible methods which can be used for this purpose. One method is to make a direct comparison between the intensity of the gamma-ray, usually measured through the production of photoelectrons, with the number of internal conversion electrons produced by the same gamma-ray. A second method is to compare the number of internal conversion electrons from a source with the number of beta rays from the same source. A third is to compare the intensity of $\mathrm{X}$-rays, following the ejection of the internal conversion electrons, with the intensity of the gamma-ray itself. Other methods, dependent on coincidence counting, are described in Chapter VII. The thing which must be determined in using all three methods as a standard of comparison is the source strength, say $N_{0}$ disintegrations per sec. Of the three methods described above, the first two involve the use of magnetic spectrographs, and the third the use of scintillation counters. Since the second method is the one which is most in use we describe it first.

Consider the hypothetical disintegration scheme shown in Fig. 10a, consisting of one beta-ray group followed by one internally converted gamma-ray. The spectrum obtained from a beta-ray source in a magnetic spectrometer will look like Fig. 10d. Here $K$ and $L$ conversion lines are seen riding on the beta-spectrum. In this case, every beta transition is followed by a transition which gives either a gamma-ray quantum or an internal conversion electron. The area under the beta-ray spectrum, exclusive of the conversion lines, is proportional to the number of disintegrations per second and hence to $\left(N_{e}+N_{\gamma}\right)$. It is clear that this affords a medium of comparison between the number of internal conversion electrons and $\left(N_{e}+N_{\gamma}\right)$. The number of conversion electrons in the $K$-shell is obtained by taking the integral under the $K$ conversion line

$$
\left[\int N(p) d p\right]_{K}=\left(N_{e}\right)_{K}
$$

by a graphical method. Thus a numerical value for $\left(N_{e}\right)_{K} /\left(N_{e}+N_{\gamma}\right)$ can be obtained from which $\alpha_{K}=\left(N_{e}\right)_{K} / N_{\gamma}$ can be calculated. By comparing the area under the $K$-line to that under the $L$-line the quantity $\left(N_{e}\right)_{K} /\left(N_{e}\right)_{L}$ 

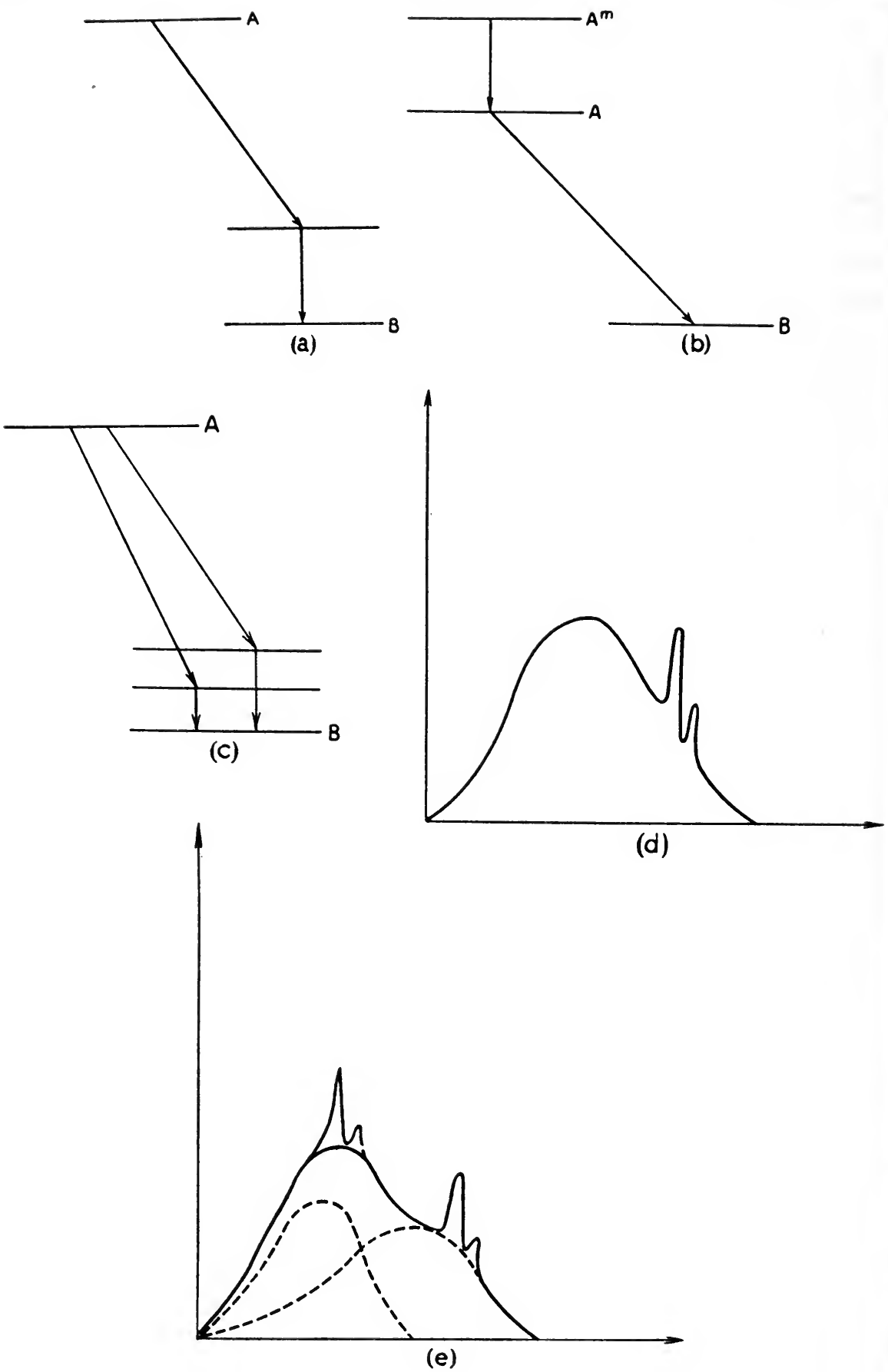

Fig. 10 
called the $K / L$ ratio can be obtained. Such cases are to be found in the main disintegration schemes of $\mathrm{Au}^{198}$ and $\mathrm{Cs}^{137}$. It is clcar that this method can be used for disintegration schemes which consist of one beta-ray group followed by a number of gamma-rays all in cascade.

The same method can be used for the case shown in Fig. 10b which represents two isomeric states of the parent nucleus such that the lower energy state has the shorter half life and is followed by a single beta-ray group. Here the internal conversion coefficient of the gamma-ray associated with the isomeric transition is measured. Such a situation is illustrated by the case of $\mathrm{Zn}^{69}$ shown in Fig. 4 . In that figure, however, the $K$ - and $L$ lines are not resolved.

For more complicated disintegration schemes the above method becomes more difficult. Figs. $10 \mathrm{c}$ and $10 \mathrm{e}$ illustrate a case in which there are two beta-ray groups each followed by a single internally converted gamma-ray. Since the relative abundance of the two beta-ray groups can be obtained, it is clear that the $\alpha_{K}$ for each gamma-ray can be determined. As a somewhat more complicated case, suppose that the scheme of Fig. 10c were modified to include two cascaded gamma-rays in parallel with the one shown. In this case the integral under the beta-ray spectrum will give the number of disintegrations per second, and that under each $K$ line the quantity $\left(N_{e}\right)_{K}$ for each gamma-ray. If the internal conversion coefficient is small, the relative intensity of the high-energy (cross over) gamma-ray to that of either one of the cascaded pair can be determined by measurement of photoelectrons. In this manner the branching ratio can be determined and hence the value of $\alpha_{K}$ for all three lines. It is clear that, for the measurement of the relative intensities of the gamma-rays, a separate source will have to be used. The medium of comparison is the integral under the beta-ray spectrum. Such a measurement, however, will be subject to the usual inaccuracies involved in the determination of the relative intensities of gamma-rays. For the more complicated schemes it becomes increasingly difficult to measure internal conversion coefficients.

Since the $K / L$ ratio does not involve a knowledge of the disintegration scheme but depends only on the use of an instrument of sufficiently good resolving power, it is clear that this quantity is relatively easily determined even for complicated schemes. The use of this quantity is discussed in detail in Chapters XIV and XVI.

The case of positron emitters provides an interesting example of the first method discussed, namely that in which the intensity of the gammaray is compared directly with the number of internal conversion electrons, the medium of comparison being annihilation radiation. Consider a positron emitter which emits two groups of positrons, one of which leads 
to the ground state and the other to an excited state which is followed by a partially internally converted gamma-ray. The number of positrons in each group and the total number which are emitted by a given source and which are detected in the acceptance angle of the spectrometer can be determined in the usual manner with the help of the Fermi analysis. The number of $K$ internal conversion electrons $\left(N_{e}\right)_{K}$ can be determined from an integration under the $K$ internal conversion line. It will be recalled that in a positron emitter, the various levels can be populated by $K$-electron capture as well as by positron emission. Hence the probability of reaching a given level is proportional to $\left(f_{+}+f_{K}\right)_{i}$. At first sight, it would appear that the quantity $\left(f_{+} / f_{K}\right)_{i}$ would have to be known in order to calculate the internal conversion coefficient. Suppose, now, that a gamma-ray source is made up of the same material and the photoelectrons produced from some converter are measured. The spectrum will show photoelectrons produced by the gamma-ray in question and also by annihilation radiation. If the internal conversion coefficient is small the intensity of the gammaray as determined from the number of photoelectrons will be proportional to $N_{\gamma}$, and

$$
I_{\gamma} / I_{a}=\left.2 N_{\gamma}\right|_{N_{+}}
$$

where $N_{+}$is the total number of positrons. Here, of course, it is assumed that corrections for the photoelectric efficiency of the converter as a function of the energy of the gamma-ray have been made. From the measurements of the particle spectrum $\left(N_{e}\right) / N_{+}$can be measured, where $N_{+}$is again the integral under the total positron spectrum.

The probability of populating the excited level $i$ is given by

$$
N_{0}\left(f_{+}+f_{K}\right)_{2} \omega_{s p}
$$

The probability of depopulating the excited state is proportional to $\left(N_{e}+N_{\gamma}\right)$. It is clear that since the ratio of the number of conversion electrons to the total positron spectrum is compared to the ratio of the gamma-ray intensities, the quantities $\left(f_{+}+f_{\Sigma}\right)_{i}$, the source strength and the solid angle of the instrument cancel out and

$$
\frac{N_{e}}{N_{\gamma}}=\frac{\left(N_{e} / N_{+}\right)}{2\left(I_{\gamma} / I_{a}\right)}
$$

This method can be extended to complicated disiniegration schemes, even those in which some of the levels are populated entirely by $K$-capture, provided that some positrons are emitted and provided that the relative intensities of the gamma-rays and their relation to annihilation radiation can be determined. 
The above method for positron emitters is a special case of that worked out for electron emitters by Waggoner, Moon and Roberts ${ }^{30}$. This method consists in comparing the number of photoelectrons ejected by a gammaray from a converter with the number of internal conversion electrons produced by the same gamma-ray from two sources of different strengths but whose relative strengths can be determined. In using the method it is necessary to know the absolute source strength $N_{0}$ of one of the sources and the absolute transmission of the instrument. Since the method appears to have no practical advantages over those already discussed the reader is referred to the original paper for details.

Finally, the internal conversion coefficient can be measured by using scintillation counters. An atom, which has lost a $K$-electron owing to the internal conversion process, will emit $K$ X-rays. Thus a source can be measured with the help of a scintillation counter and a differential pulse height analyzer. Peaks will be seen corresponding to a photoelectric peak for the gamma-ray and one for the X-ray. Using the methods of Chapter $\mathrm{V}$, it is possible to calculate the relative intensity of the gamma-ray and that of the X-ray. Thus $N_{X} / N_{\gamma}$ can be determined. For every internal conversion electron from the $K$-shell there will be emitted either an X-ray or an Auger electron.

$$
N_{e}=N_{X}+N_{A} .
$$

The fluorescent yield is defined as

Hence

$$
W_{K}=\frac{N_{X}}{N_{X}+N_{A}} \text {. }
$$

$$
\alpha_{K}=\frac{N_{e}}{N_{\gamma}}=\frac{N_{X}}{W_{K} N_{\gamma}} \text {. }
$$

Tables of the fluorescent yield have been published by the $\mathrm{X}$-ray spectroscopists *. The values of $\alpha_{K}$ obtained in this manner compare quite favorably with those obtained by the more usual methods.

\section{$\S 11$. Examples of Some Disintegration Schemes}

To illustrate the methods by which disintegration schemes are worked out, a few examples will be given in this section. The schemes are picked to illustrate the method and no attempt is made to give all the references to the various workers who have contributed to the scheme. In general the simpler schemes were done some years ago before the better techniques now available were developed and for this reason the data are not as good as could be obtained by present techniques. If the author leans rather

30 M. A. Waggoner, M. L. Moon and A. Roberts, Phys. Rev. 80 (1950) 420.

* See Chapter XX. 
heavily on the work of his own laboratory in these illustrations it is simply because the original data and figures are available to him.

\section{A. $\mathrm{Na}^{24} \rightarrow \mathrm{Mg}^{24} 15.06$ HOURS}

One of the earliest schemes to be worked out was that of $\mathrm{Na}^{24}$. The scheme was investigated by Itoh ${ }^{31}$, Elliott, Deutsch and Roberts ${ }^{32}$, and Siegbahn ${ }^{33}$. The distribution of secondary electrons taken with a lead radiator by Siegbahn is shown in Fig. 11.

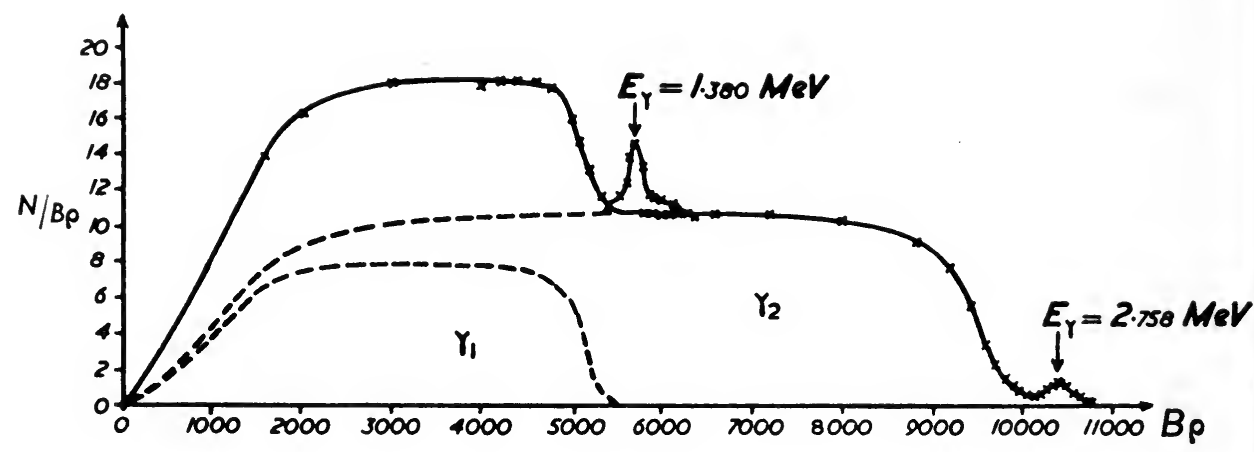

Fig. 11

The quantity $N(B \varrho) d(B \varrho)$ is plotted against $B \varrho$. Two $K$ photo-lines are shown (the resolution is not good enough to show the $L$-lines) which correspond to gamma-rays of energies 1.380 and $2.758 \mathrm{MeV}$. The distribution of Compton electrons associated with each of the gamma-rays is shown. The ratio of the $K$-photo peaks, when corrected for the variations of photoelectric efficiency with energy, is $1: 1$. The two gamma-rays are therefore assumed to be in cascade.

The Fermi Plot for the beta-ray spectrum, taken by the same author is shown in Fig. 12.

The end point of the spectrum is seen to be $1.390 \mathrm{MeV}$. The plot is straight to about " $W=1.4$ or $E \sim 0.200 \mathrm{MeV}$ where it curves upward owing to source thickness. Coincidence measurements have shown (Ch. VII) that the beta-rays are in coincidence with both gamma-rays. The disintegration scheme is as shown in Fig. 13.

The half-life is 15.06 hours or $t=5.4 \times 10^{4} \mathrm{sec}$. For $E_{0}=1.390, W_{0}=3.72$, $Z=24$ the tables give $\log f=1.52$ which gives $\log f t=6.25$. The actual value when worked out exactly is $\log f t=6.13$. The total disintegration energy is $1.390+1.386+2.758=5.528 \mathrm{MeV}$.

31 J. Itoh, Proc. Phys. Math. Soc. Japan 23 (1941) 605.

32 L. G. Elliott, M. Deutsch and A. Roberts, Phys. Rev. 63 (1943) 386.

33 K. Siegbahn, Phys. Rev. 70 (1946) 127. 


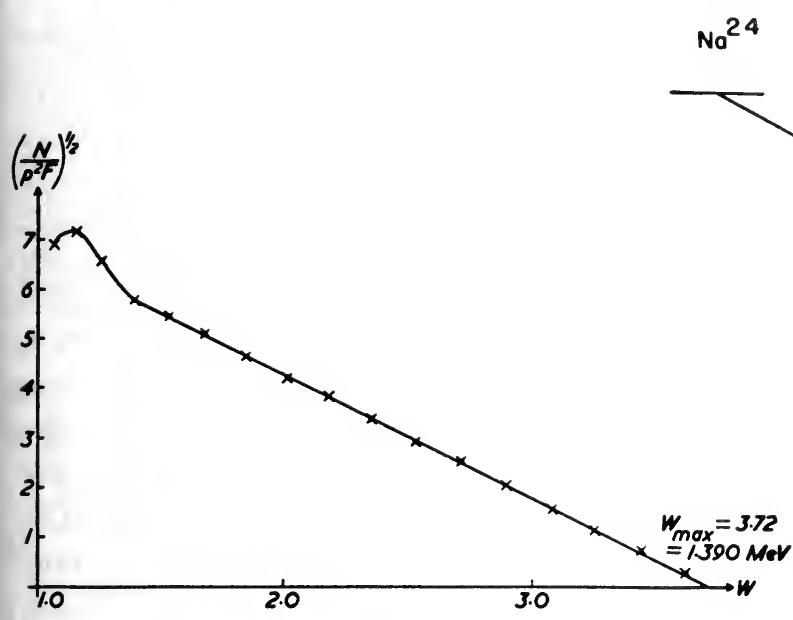

Fig. 12
$\mathrm{Mg}^{24}$

5.54

1.39

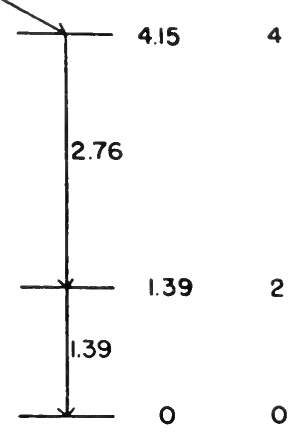

Fig. 13

B. $\mathrm{Cs}^{137} \rightarrow \mathrm{Ba}^{137} .33$ YEARS

The disintegration scheme of $\mathrm{Cs}^{137}$ is one of the most interesting in nuclear spectroscopy. It is relatively simple, but the working out of all the details has brought to light some very fundamental concepts for nuclear physics. In addition since $\mathrm{Cs}^{137}$ has a relatively long life and is procurable as a fission product source, it serves as a standard for many investigations.

$\mathrm{Cs}^{137}$ emits one gamma-ray whose energy is $661.65 \pm 0.15 \mathrm{keV}$. The beta-ray spectrum is shown in Fig. 14 in which $N(p) d p$ is plotted against $p$.

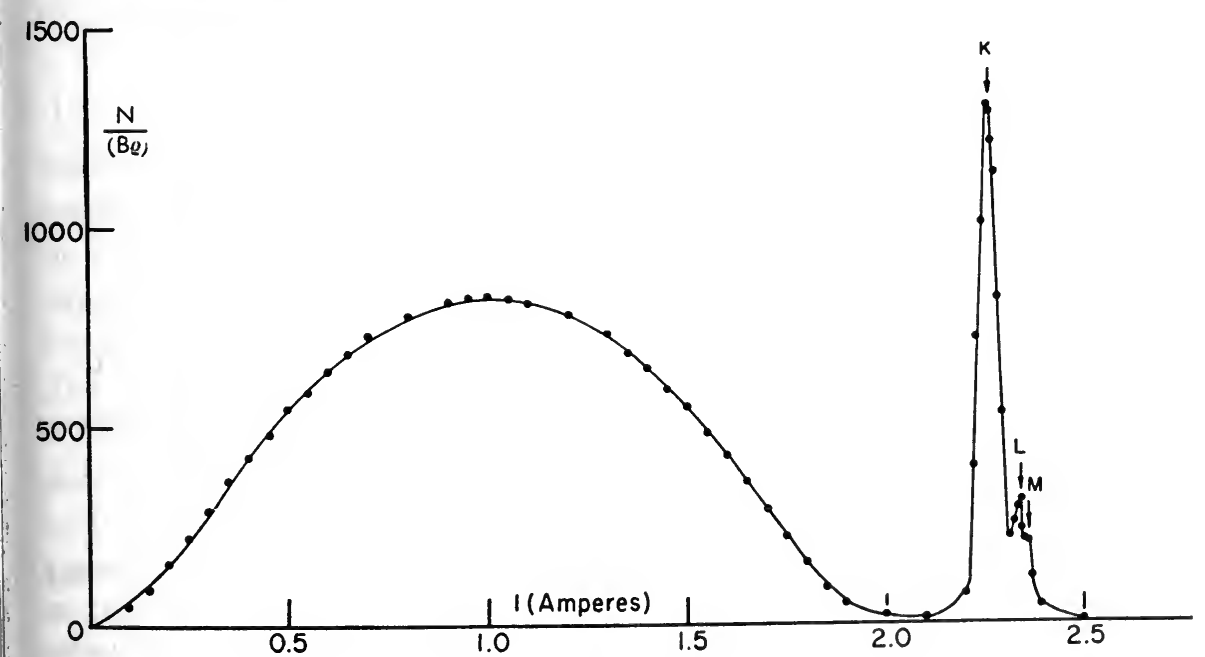

Fig. 14 
The spectrum was taken with a magnetic lens of medium resolution. The gross features of the spectrum show a single beta-ray spectrum and an internally converted gamma-ray. Since superficial inspection discloses that there is only one beta-ray group of comparable intensity to that of the ganuma-ray, it is natural to suppose that this one group feeds the gamma-ray. Beta-gamma coincidence experiments were tried but no beta-gamma coincidences were found. Since the lack of coincidences might be explained on the assumption, that the first excited state of $\mathrm{Ba}^{137}$ has a measurable half-life, a chemical separation of barium from cesium was performed and the half-life of the gamma-ray was found to be $156 \pm 3 \mathrm{sec}$.

A curve of the type of that shown in Fig. 14 allows one to estimate the $K / L$ ratio and the internal conversion coefficient in the $K$-shell, $\alpha_{K}$. The presently accepted value is that of Waggoner ${ }^{34}, x_{K}=0.097 \pm 0.005$. The value of the area under the $K$-line to that under the $L+M$-lines from the above figure gives a rough value of 4.3. Graves, Langer and Moffat ${ }^{35}$, using an instrument of higher resolution have obtained the value $K /(L+M)=4.64$.

If a Fermi Plot of the beta-ray spectrum is made, two things are apparent at once ${ }^{36}-(1)$ there is a high energy, low intensity group which

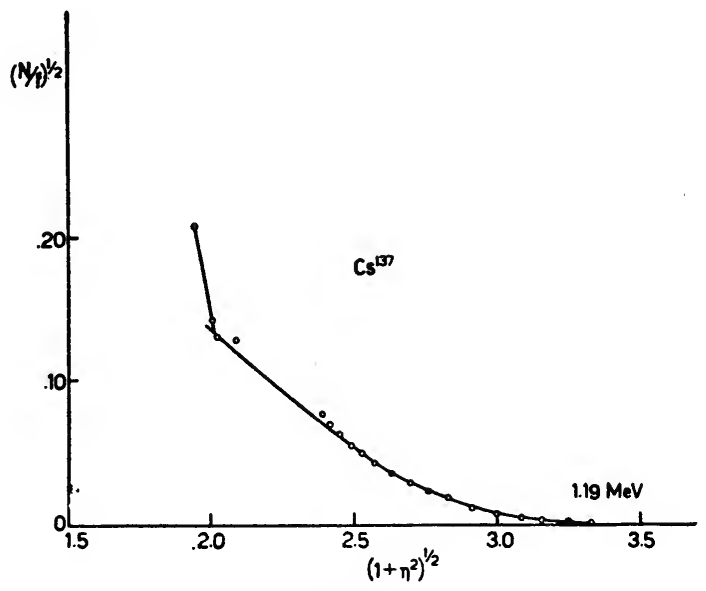

Fig. 15

goes to the ground state and (2) neither group gives a straight line Fermi Plot. The situation is illustrated in Figs. 15 and 16.

34 M. A. Waggoner, Phys. Rev. 82 (1951) 906.

35 G. A. Graves, L. M. Langer, and R. D. Moffat, Phys. Rev. 88 (1952) 344.

36 See C. L. Peacock and A. C. G. Mitchell, Phys. Rev. 75 (1949) 1272; L. M. Langer and H. C. Price, ibid, 76 (1949) 641; L. M. Langer and R. J. D. Moffat, ibid, 82 (1951) 635. 
The high energy group has an end point of $1.17 \mathrm{MeV}$ and an abundance of $8 \%$, while the lower energy group has an end-point of $0.521 \mathrm{MeV}$ and an abundance of $92 \%$. Hence an energy balance is obtained if it is assumed that the high energy group goes to the ground state and the low energy group to the excited (metastable) state and is followed by the emission of the gamma-ray. The energy level scheme is given in Fig. 17.

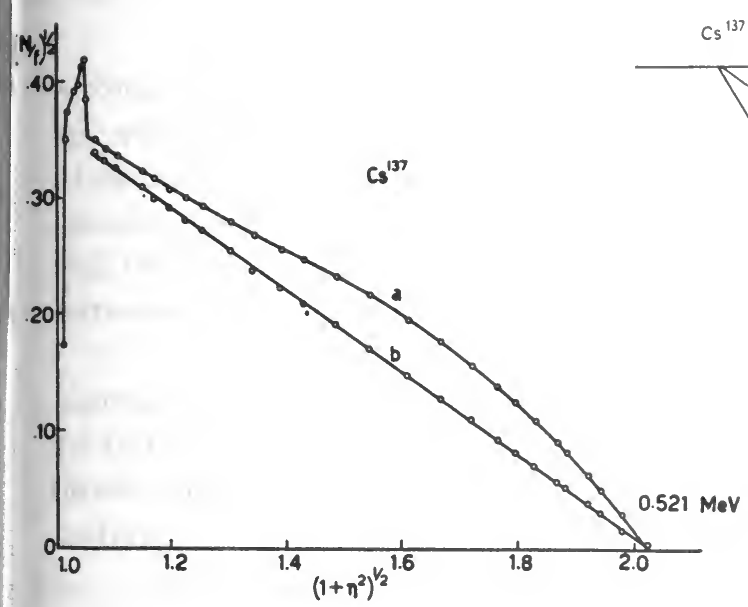

Fig. 16

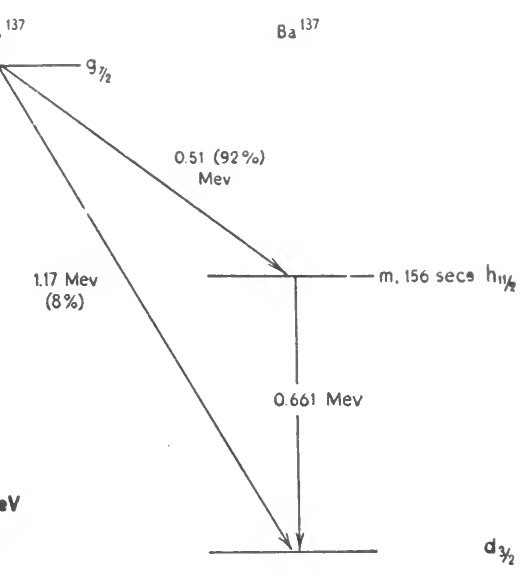

Fig. 17

Fig. 16, curve $a$, shows the Fermi Plot for the low energy group made in the usual way. Curve $b$ shows the rectified Fermi Plot using

$$
S_{n}(W) \cong\left[W^{2}-1-\left(W_{0}-W\right)^{2}\right]
$$

which is applicable if $\Delta j= \pm 2$ and there is a change of parity. In the original work various forms for $S_{n}(W)$ were tried and the best fit was given by the one listed above. Later, using a very strong source, Langer and Moffat ${ }^{36}$ measured the high energy spectrum and found that a rectified Fermi Plot could be obtained using an $S_{n}(W)$ which is characteristic of $\Delta j= \pm 2$ and no change of parity.

All of the information obtained on the disintegration of $\mathrm{Cs}^{137}$ make it possible to give a complete disintegration scheme for $\mathrm{Cs}^{137}$, assigning spins and parities to all levels concerned. The ground state spin of $\mathrm{Ba}^{137}$ has been measured and found to be $3 / 2$ with a configuration $d_{3 / 2}$. The half-life of the metastable state, the value of $\alpha_{K}$ and $K /(L+M)$ for the gamma-ray are all consistent with the assumption that the gamma-ray transition is M4 (see Chapters XIV and XVI). Since M4 radiation requires a change of 
parity and a spin change of 4 units the configuration of the first-excited state is $h_{11 /}$. The shape of the lower energy spectrum requires that the ground state of $\mathrm{Cs}^{137}$ have even parity and a spin of $7 / 2$. The configuration is thus determined as $g_{\%}$. The slope of the high energy group is consistent with this interpretation. Finally, the spin of $\mathrm{C}^{1} \mathrm{~s}^{137}$ has been measured and is $7 / 2$.

\section{C. $\quad \mathrm{Cd}^{114} \leftarrow \mathrm{In}^{114} \rightarrow \mathrm{Sn}^{114}$}

As a final example we discuss the disintegration of $\mathrm{In}^{114}$. This isotope shows two half-lives, one of 50 days and one of 72 sec and is, therefore, an isomeric transition. Lawson and Cork ${ }^{37}$ showed that the 72 sec state emits a beta-ray of $1.984 \mathrm{MeV}$ energy unaccompanied by any gammarays. The spectrum has an allowed shape as shown in Fig. 7. The 50 day state goes to the $72 \mathrm{sec}$ state under the emission of an internally converted gamma-ray, whose energy is $0.192 \mathrm{MeV}$.

Later, through coincidence counting experiments, a high energy gammaray of low intensity was discovered. The spectrum was reinvestigated by Boehm and Preiswerk ${ }^{38}$, and Mei, Mitchell and Zaffarano ${ }^{39}$ who found weak gamma-rays at $0.556,0.722$ and $1.27 \mathrm{MeV}$. Fig. 18 shows the gamma-

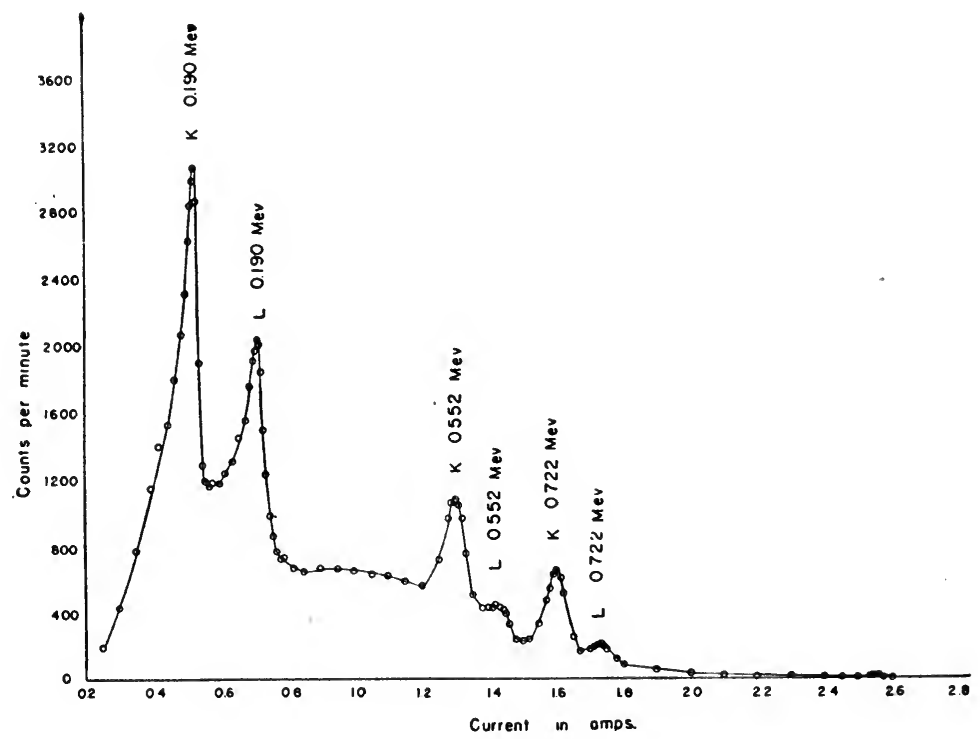

Fig. 18

37 J. L. Lawson and J. M. Cork, Phys. Rev. 57 (1940) 982.

38 F. Boehm and P. Preiswerk, Helv. Phys. Acta 22 (1949) 331.

39 J. Y. Mei, A. C. G. Mitchell, D. J. Zaffarano, Phys. Rev. 76 (1949) 1883. 
ray spectrum as exhibited by the photoelectrons from a lead radiator. The figure also shows the internally converted gamma-ray at $0.190 \mathrm{Mel}$. The weak gamma-ray at $1.27 \mathrm{MeV}$ is not shown in the figure. Recently two additional weak gamma-rays at 0.576 and $1.30 \mathrm{MeV}$ have been found ${ }^{40}$.

The relative intensities of the various gamma-rays were determined with the help of the photoelectric efficiency curve of Gray. The relative intensities are given in Table $\mathrm{V}$.

TABLE V

Energies and Intensities of the Gamma-Rays of In ${ }^{114}$

\begin{tabular}{c|c|c}
\hline \multirow{2}{*}{ Energy (MeV) } & \multicolumn{2}{|c}{ Relative Intensity } \\
& $\mathrm{MMJ}^{39}$ & JCDM ${ }^{40}$ \\
\hline 0.192 & 5.38 & 5.4 \\
0.556 & 1.00 & 1.02 \\
0.576 & 1.00 & 0.03 \\
0.722 & 1.00 \\
1.271 & $j 0.067$ & 0.008 \\
1.300 & & 0.043
\end{tabular}

The agreement in relative intensities between the two sets of experimentors is quite remarkable. In addition the lines at 0.556 and at 0.7.22 $\mathrm{MeV}$ have been shown to be in coincidence so that their intensities should be roughly equal. Aside from the weak gamma-rays which are produced in a $K$-capture process to $\mathrm{C}^{114}$ there is a very weak positron group which goes to the ground state.

Figure 19 shows the decay scheme which has finally been worked out. Since no positrons go to the excited states relative intensities of the gamma-rays in the $K$-capture branch to the gamma-ray at $0.192 \mathrm{MeV}$ can give some information as to the probability of $K$-capture to that of betaray emission, since all transitions are fed through the gamma-ray of energy of $0.192 \mathrm{MeV}$. In order to calculate the percentage of transitions which go by $K$-electron capture, it is necessary to know the ratio $N_{\mathrm{o}} /\left(N_{\mathrm{e}}+N_{\gamma}\right)$ for the internally converted $0.192 \mathrm{MeV}$ line. This has been found by various investigators to be $0.80 \pm 0.05$. The fraction of transitions which go by $K$-electron capture will be approximately

$$
\left(I_{0.722}+I_{1.27}\right) /\left(\frac{I_{0.192}}{1-0.08}\right)=0.04
$$

40 M. W. Johns, C. D. Cox, R. J. Donelly, and C. C. McMullen, Phys. Rev. 87 (1952) 1134. 
The half life of the 50 day state, the internal conversion coefficient of the $0.192 \mathrm{MeV}$ line and its $K / L+M$ ratio (4.63) establish this transition as M4. The value of $\log f t$ for the 1.984 beta-ray transition is 4.4 which establishes it as allowed. Since the spin of the ground state of $\mathrm{Sn}^{114}$ is zero, it follows that that of the 72 sec state is l-even and that of the 50 day state is 5 odd. Gamma-gamma angular correlations have been measured for the $K$-capture branch giving the spins shown in the figure.

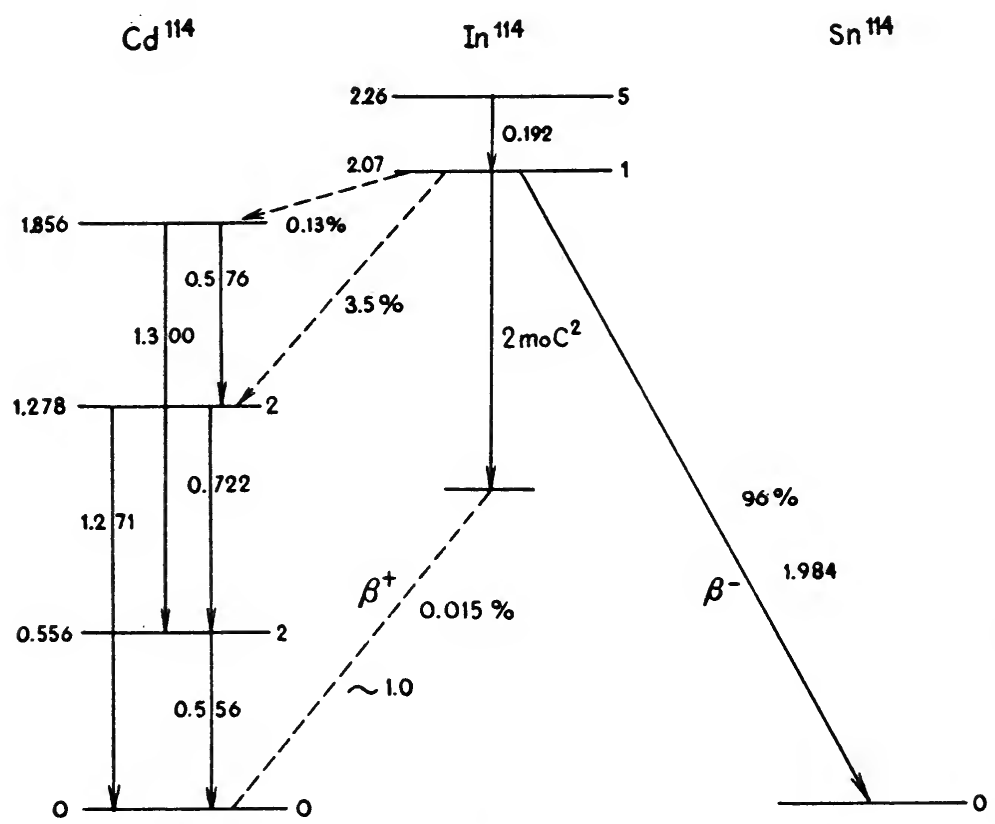

Fig. 19

The three disintegration schemes discussed above illustrate many of the main features of this type of work. More complicated schemes are worked out in the same manner but it does not seem worth while to discuss them here. The reader is referred to the voluminous literature on the subject for further details. 


\section{(II) HILDING SLÄTIS - Source and Window Technique}

\section{§ 1. Introduction}

In beta-spectroscopy thin films are used as backings for sources and as windows for Geiger-Müller counters. The source or the active material itself has to be prepared as a thin layer on the backing foil in order to diminish the influence of absorption of the beta rays in the sample and the influence of backscattering. Because of absorption the energy of the beta-particles is decreased and because of backscattering the number of low energy electrons is increased, thus producing a distortion in the experimental beta-spectrum. In order to get a picture of the importance of thin sources and backings when studying the shape of beta spectra some of the results obtained by $\mathrm{Wu}$ and Feldman ${ }^{1}$ may be mentioned. The beta-spectrum of an allowed transition as obtained from a thick source showed definite deviation from the expected straight line on the Fermi plot. Instead of a straight line the experimental curve was convex towards the energy axis. Hence, when a spectrum obtained from a thick source shows an allowed distribution, it is most likely that the true distribution is concave towards the energy axis. Thus it can happen that forbidden shapes may be masked and believed to be allowed. The beta-spectrum of $\mathrm{Cl}^{36}$ (Fig. 1) from a thin source ( $\left.\sim 0.3 \mathrm{mg} \mathrm{LiCl} / \mathrm{cm}^{2}\right)$ has been shown to follow the unique $\mathrm{D}_{2}$ spectrum (Marshak's notation) closely, but the curvature of the Kurie plot from a thick source $\left(\sim 10 \mathrm{mg} / \mathrm{cm}^{2}\right)$ is much less concave towards the energy axis and could wrongly be classified as a spectrum of the $\alpha$-type (correction factor $\left.\alpha \sim\left(p^{2}+q^{2}\right)^{2 / 3}\right)$. In the same way a surplus of low energy electrons make an analysis of a complex beta-spectrum

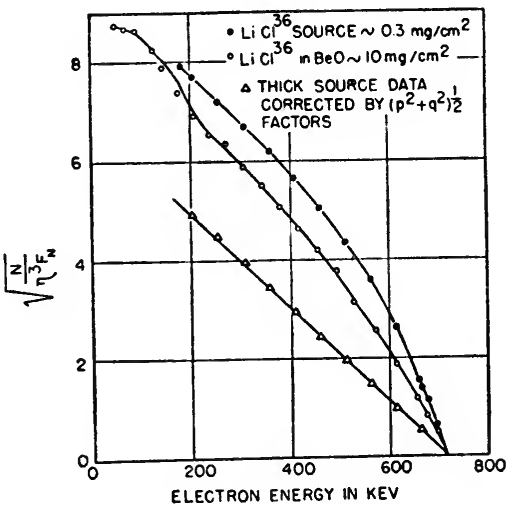

Fig. 1.

Fermi-Kurie plots of beta-spectra of $\mathrm{Cl}^{36}$ in thin and thick sources according to $\mathrm{Wu}$ and Feldman very difficult, especially as to low energy components. The surplus of low energy electrons may also give rise to a belief that soft beta components are present in cases where soft components do not exist.

1 C. S. Wu and I. Feldman, Phys. Rev. 76, (1949) 697, 698. 
The variation of the amount of backscattering with thickness and atomic weight of the backscatterer for beta radiation of several maximum energies was studied by Engelkemeir et al. ${ }^{2}$. The maximum backscattering contribution for beta spectra with maximum energies above $1 \mathrm{MeV}$ amounts to about $27 \%$ for an infinitely thick mica backing. When beryllium, aluminium, copper, cadmium, and lead were used as backscatterers, the maximum backscattering rose from 17 to $80 \%$. showing the strong dependence of backscattering on the atomic number. For very thin backings the increase in backscattering was almost proportional to the increasing thickness of the backing material. For thicker backings the backscattering increased more slowly, reaching a maximum at a thickness of about one-fifth the beta range.

From these and other investigations it is clear that backings have to be made from material with low atomic numbers. Organic compounds and aluminium are therefore commonly used.

Organic backing materials are generally good insulators. This circumstance may cause charging of the source, thereby changing the velocity of the electrons emitted ${ }^{3,4,5}$. Douglas ${ }^{4}$ found in the beta-spectrum of $\mathrm{Lu}^{177}$ for the $46.9 \mathrm{keV}$ conversion line a shift towards lower energies by as much as $19 \mathrm{keV}$, when he used an ungrounded nylon backed source. In order not to distort the beta-spectrum, the source therefore has to be electrically connected to ground through an aluminium strip, a colloidal graphite dispersion or some thin evaporated metal layer. Langer ${ }^{6}$ points out that a solution of zapon in amyl acetate can be loaded with colloidal graphite ("Aquadag"). Films made from such a solution have a certain conductivity. However, as they do not have the strength of normal zapon lacquer films, they have to be sandwiched between unloaded films. Wolfson ${ }^{7}$ prevents charging of negatron emitting sources by use of a low density electron cloud immediately behind the source.

\section{$\S$ 2. Preparation of Foils for Backings and Windows}

\section{A. Mica FoILs}

Thin layers of mica may be removed easily from large crystals by means

2 D. W. Engelkemeir, J. A. Seiler, E. P. Steinberg, L. Winsberg and T. B. Novey, paper 5 in Radiochemical Studies: The Fission Products; C. D. Coryell, N. Sugarman Editors, NNES, Div. IV, Vol. 9, Mc Graw-Hill Book Co., Inc., 1951.

3 C. H. Braden, G. E. Owen, J. Townsend, C. S. Cook and F. B. Shull, Phys. Rev. 74, (1948) 1539.

4 D. G. Douglas, Phys. Rev. 75, (1949) 1960.

5 L. M. Langer and H. C. Price, Jr., Phys. Rev. 76, (1949) 641.

- L. M. Langer, Rev. Sci. Instr. 20, (1949) 216.

7 J. L. Wolfson, Rev. Sci. Inst. 22, (1951) 280. 
of thin needles, a piece of paper or a steel foil. In order to overcome the large electrostatic forces between the sheets which are to be separated, this procedure can be carried out in water (then, of course, paper cannot be used in the detachment). The homogeneity of the mica foil obtained is nicely tested if the foil is studied between Nicol's prisms or between two polaroids. Commercial Geiger Müller tubes of bell shape have mica windows of $\sim 30 \mathrm{~mm}$ diameter and a thickness of about $2-3 \mathrm{mg} / \mathrm{cm}^{2}$. Such a window stops all electrons of energy less than $33-40 \mathrm{keV}$. Thinner mica windows can be made. Kageyama ${ }^{8}$ reports some of $0.3 \mathrm{mg} / \mathrm{cm}^{2}$ thickness.

\section{B. Zapon Lacquer Foils}

Zapon lacquer is diluted in amyl acetate and one or more drops of the solution are put on a surface of distilled water. The drops are then immediately spread by surface tension forces as a thin film over the water surface. After a few minutes the zapon film has dried and can be removed by a rectangular or circular frame made of copper wire. The area of the frame should be a little smaller than the area of the film, in order to permit the rim of the film to be folded around the wire when the film is removed from the water. In this way films ranging in thickness from several $\mu \mathrm{gs} / \mathrm{cm}^{2}$ to several $\mathrm{mgs} / \mathrm{cm}^{2}$ can be made. These zapon lacquer foils do not have the same strength as foils of nylon or formvar (see below).

\section{Collodium Films}

Collodium is dissolved or a solution of collodium in ether is diluted in amyl acetate, and several drops of the solution are put on a water surface as described for zapon lacquer foils above. $\mathrm{Wu}^{9}$ used $6-7$ layers of thin collodium films, each about $6 \mu \mathrm{g} / \mathrm{cm}^{2}$ thick, as a G M counter window. The window was supported by a grid, obtained by making a number of $3 \mathrm{~mm}$ diameter holes in a brass plate. The pressure in the counter was constant and equal to $15 \mathrm{~mm}$, the filling gas being pure alcohol vapor in equilibrium with liquid alcohol kept at a constant temperature of $+5^{\circ} \mathrm{C}$. This arrangement compensated for any possible slow leakage of gas through the window.

Langer and Moffat ${ }^{10}$ used a Cartesian Manostat in order to maintain a pressure of $2-2,5 \mathrm{~cm} \mathrm{Hg}$ automatically constant (within $0.01 \mathrm{~cm}$ ) in their thin window counters.

8 S. Kageyama, J. Phys. Soc. Japan 8, (1953) 689.

- C. S. Wu, private communication, 1949.

10 L. M. Langer and R. J. D. Moffat, Phys. Rev. 80, (1950) 651; Phys. Rev. 88 , (1952) 689 . 


\section{NyLon Films}

Nylon, in the form of chips or sheets, is dissolved in isobutyl alcohol by careful boiling in a double boiler ${ }^{11}$ for a few minutes. The concentrated solution is then poured off from the undissolved nylon and is ready to drop on water as described above for zapon lacquer solution. For best results the solution should be used within about an hour. If the distilled water is weakly acidic it should be made neutral by tri-sodium phosphate. In this way films of about $10 \mu \mathrm{g} / \mathrm{cm}^{2}$ can be made. Thicker films are obtained using several films together, thus eliminating the effect of microscopic pinholes which may be present in the individual layers.

\section{E. Formvar Films}

A solution is made of $1 \mathrm{~g}$ formvar (ethylen dichloride) in $200 \mathrm{~g}$ trichlorethylene. This solution is put into a suitable container, which permits vertical insertion of a glass plate a little larger than the film desired. The glass plate is slowly drawn out of the solution ${ }^{12}$. When the thin layer of formvar on the glass plate is dry, the plate is put into water. The water then immediately penetrates between the formvar film and the glass. By careful treatment it is possible to detach the film, which will then float on the water surface and can be removed by means of a rectangular copper wire frame as described for the other films above. The films are quite homogeneous down to a thickness of about $0.02-0.03 \mu\left(\sim 2-3 \mu \mathrm{g} / \mathrm{cm}^{2}\right)$.

\section{F. Other Special Plastic Filus}

Two way stretched rubber hydrochloride films of $5 \mu\left(\sim 0.5 \mathrm{mg} / \mathrm{cm}^{2}\right)$ and $7.5 \mu\left(\sim 0.75 \mathrm{mg} / \mathrm{cm}^{2}\right)$ thickness are commercially obtained. They are useful as backings for samples and may for that purpose be mounted by means of zapon lacquer on aluminium rings. So called Tygon films and vinyl chloride-vinyl acetate films ("Number 1060 Clear Clad" diluted in "Number 1060 Thinner") have been used as backings and windows by O'Kelley ${ }^{\mathbf{1 3}}$.

\section{G. Aluminium Förs}

Aluminium foils of about $6 \mu\left(1.6 \mathrm{mg} / \mathrm{cm}^{2}\right)$ thickness are convenient as backings, because no precautions are necessary in order to prevent charging of the sample. According to Cook ${ }^{14,15,16}$ such foils do not produce a

11 J. Brown, F. Felber, J. Richards and D. Saxon, Rev. Sci. Inst. 19, (1948) 818.

12 J. Halter, private communication. See also P. Barreau, P. Leger, J. Moreau and P. Prugne, J. de Phys. 15, (Jan. 1954) p. 4 A.

13 G. D. O'Kelley, UCRL-1243 and private communication, 1954.

14 C. S. Cook, Office of Naval Research, U.S.Navy Dept., RDB No NR 024-018, (1953).

15 G. E. Owen and C. S. Cook, Phys. Rev. 76, (1949) 1726.

16 G. E. Owen, C. S. Cook and P. H. Owen, Phys. Rev. 78, (1950) 686. 
backscattering distortion in the measured beta-spectra above $50 \mathrm{keV}$. Smith ${ }^{17}$ reports that the most convenient foil is commercially produced 0.00003 inch $\left(\sim 0.75 \mu\right.$ or $\left.0.2 \mathrm{mg} / \mathrm{cm}^{2}\right)$ aluminium. This is quite resistant to acids, and sources may be prepared from quite strong nitric acid solutions ( $\mathrm{pH} \sim 2$ ), or from very slightly acid solutions of $\mathrm{HCl}$ or $\mathrm{H}_{2} \mathrm{SO}_{4}$.

\section{H. Aluminium Oxide Foils}

Strohmaier ${ }^{16 a}$ has made aluminium oxide foils of $50-300 \mathrm{~m} \mu$ thickness. These foils are transparent, have a quite uniform thickness and are chemically and thermally more resistant than the organic films.

\section{$\S 3$. Estimation of Foil Thickness}

The foils or films made by means of the procedures described above may not be of uniform thickness. By weighing we get the mean thickness of the foil. Usually only a small part of the film is used as a counter window. Hence the value of the thickness obtained by weighing is only an approximate one.

In some cases the foil thickness may be estimated from the interference colours produced by the foils ${ }^{18}$.

Accurate values for the foil thickness expressed in light wave lengths can be obtained by means of a Michelson interferometer ${ }^{19}$.

\section{$\S$ 4. Preparation of Samples}

A number of foil preparation methods are given by Dodson et $u l .{ }^{20}$, and Hufford and Scott ${ }^{21}$. The simplest and most important methods are given briefly here.

\section{A. Evaporation of Droplets}

One of the most common methods of preparing sources for beta-ray spectroscopy consists in evaporating droplets of a solution containing the activity. By means of a pipet the droplets are brought onto a backing

162 K. Strohmaier, Z. Naturf. 6A, (1951) 508.

17 D. B. Smith, A.E.R.E. I/R 1210, Harwell (1953).

18 L. Grebe, Handb. d. Ph. Bd. XX, Berlin (1928), p. 10-20.

19 A. J. McPherson and D. G. Douglas, Rev. Sci. Inst. 20, (1949) 457.

20 R. W. Dodson, A. C. Graves, L. Helmholz, D. L. Hufford, R. M. Potter and J. G. Povelites, Chapter I in A. C. Graves and D. K. Froman, Miscellaneous Physical and Chemical Techniques of the Los Alamos Project, McGraw-Hill Book Co., Inc., (1952).

21 D. L. Hufford and B. F. Scott, Techniques for the Preparation of Thin Films of Radioactive Material, in G. T. Seaborg, J. J. Katz and W. M. Manning, The Transuranium Elements, Part II, McGraw-Hill Book Co., Inc., (1949).

22 L. M. Langer, R. J. D. Moffat, and H. C. Price, Phys. Rev. 76, (1949) 1725. 
foil with subsequent drying under an infra-red lamp. Samples made in this way are not uniform, however ${ }^{14,22}$. In fact, autoradiographs of such sources have shown variations in intensity of the radiations from different parts of the source of as much as 100 to 1. The reason for this are partly the growth of crystals and partly the ringshaped precipitation of material on the backing. Langer ${ }^{6}$ used the method of Schaefer and Harker ${ }^{23}$ for making films hydrophilic by forming a layer of a protein, such as insulin or egg albumin, on the film surface. A drop of Lilly insulin diluted with 20 parts of water is put ${ }^{6}$ on the film spot desired for the source, and the excess insulin removed with a pipet. When a drop of the radioactive solution is placed on the wetted area, it spreads uniformly over this. Afterward the source is dried by evacuation, care being taken to avoid freezing.

\section{B. Slurry Technique}

By the slurry technique a more or less uniform layer of material is produced by sedimentation of a suspension of finely divided solid ${ }^{20}$. A glass cylinder, with a cross section corresponding to the desired area of deposit, is put against a thin rubber gasket on the backing material. The slurry is precipitated in or transferred into the glass cylinder, where the sedimentation occurs. After this the superfluous water is pipetted off, the glass tube removed and the foil dried.

\section{Vacuum Methods}

Very homogeneous samples are obtained by thermal evaporation or distillation in vacuum. In this connection "radiators" may also be considered. "Radiators" are thin foils of lead, uranium or some other element with high atomic number, used for the production of photo electrons from a gamma-ray active sample (the energies of the gamma-rays are determined from the energy of the photo electrons.

An evaporation èquipment consists of a flat steel or brass plate to which a bell jar has been ground to fit and sealed with wax or other compound, a high vacuum pump, a support for the sample backing, and a heating device for the material to be evaporated. The shape of the heating device depends on the properties of the sample material. When metals such as aluminium, lead or gold are to be evaporated, small pieces are put on filaments consisting of spirals or bands of tungsten, which are electrically heated. Hedgran and Lind ${ }^{24}$ have made uranium foils in this way using a zig zag shaped $2 \mathrm{~mm}$ thick tungsten filament, with small

23 V. J. Schaefer and D. Harker, J. App. Phys. 13, (1942) 427.

24 A. Hedgran, private communication, 1954. 
pieces of uranium placed on the flattened regions at the bends of the filament. Because of the large current needed, the leads had to be artificially cooled. In other cases furnaces of carbon or graphite may be used. Where the required temperatures are not too high, the furnace may consist of a metal tube through the ground plate which is heated outside the vacuum system ${ }^{20}$. Frauenfelder's ${ }^{25}$ evaporation equipment with graphite furnaces inside the vacuum chamber is shown diagrammatically in Fig. 2. Figs. 3a and 3b show the graphite furnace, the arrangement for heating the mica foils before they receive the active deposit from the furnace, and the distribution of activity on the receiving foil.

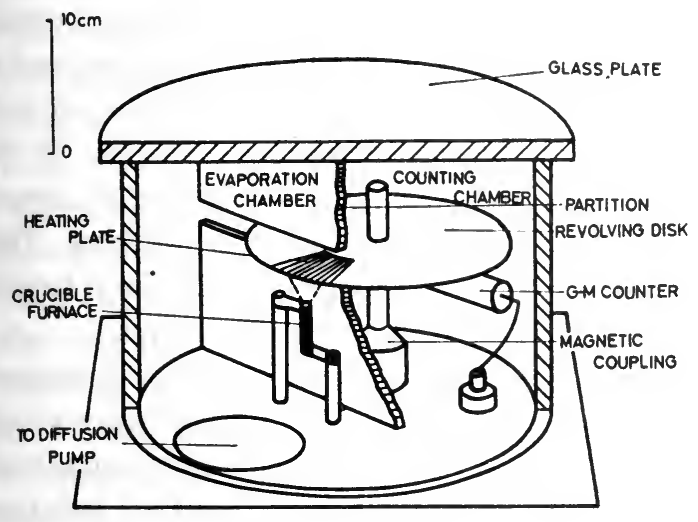

a)

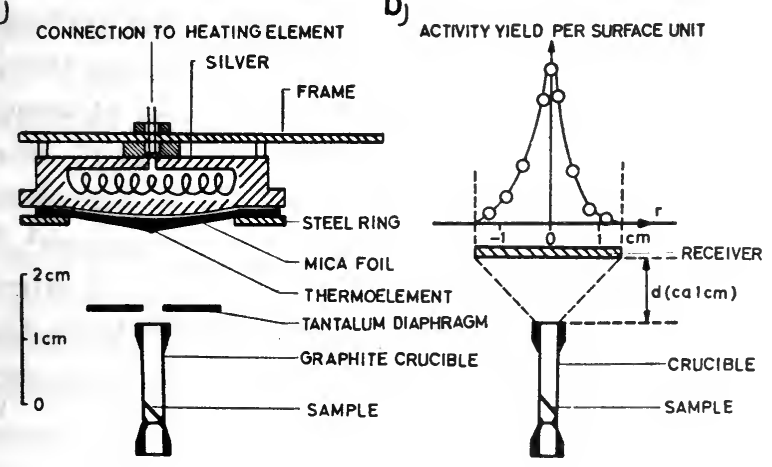

Fig. 2.

Frauenfelder's evaporation chamber, diagrammatically. Sketch simplified for clarity. The position of one of the directly heated graphite fur. naces is reproduced
Fig. 3.

a) Position of furnace and receiving foil;

b) Distribution of activity on the receiving foil (Frauenfelder)

\section{Electrodeposition Procedures}

Electroplating represents in many cases a convenient method of obtaining homogeneous active deposits on conducting backings. The equipment is similar to that used for the slurry technique: a glass cylinder

25 H. Frauenfelder, Helv. Phys. Acta 23, (1950) 347. 
(chimney), with a cross section of the same size as the desired active area, is put against a thin rubber gasket on the backing material. Thin backings can be prepared by evaporating a thin layer of copper onto a zapon or other foil backing ${ }^{14}$.

In this way Smith and Wildblood ${ }^{26}$, following a suggestion by Gray, prepared a $\mathrm{Co}^{60}$ source by electroplating from a very dilute active cobalt chloride solution on to $260 \mu \mathrm{g} / \mathrm{cm}^{2}$ aluminium foil. Using a carbon anode an almost invisible source was produced, the excess of the electrolyte being washed away to prevent crystal formation.

Stronger sources of $\mathrm{Co}^{60}$ may be electrodeposited on thin copper foils, made by evaporation copper onto aluminium foils. These copper foils are pressed against the lower end of a glass tube. The electrolysis is performed in the container formed in this way, with the copper foil as cathode and a platinum wire at the upper end of the glass tube as anode. With a current density of about $4 \mathrm{~mA} / \mathrm{cm}^{2}$ for $20-30$ hours an activity yield of $70-95 \%$ is obtained ${ }^{27}$. The electrolytic solution is prepared in the following manner ${ }^{28}$. Concentrated sulphuric acid is added to the $\mathrm{CoCl}_{3}$-solution. The solution is evaporated, the $\mathrm{HCl}$ removed by heating, and after the solution has cooled down an excess of concentrated ammonia is added.

Alburger ${ }^{29}$ has frequently used the following plating method. A copper foil is mounted on a frame and everything is painted with zapon except for the area to be plated. First of course a suitable lead-in wire is connected to the frame. The whole thing is then immersed in the plating solution together with the platinum anode and the plating is carried out.

A detailed description of electrodeposition of uranium and plutonium from different solutions is given by Dodson et al. (loc. cit.). Also thorium and neptunium have been treated. As an example the plating of uranium using a solution of $10 \mathrm{ml}$ of $0.03 \mathrm{M} \mathrm{NaF}$ and $5 \mathrm{ml}$ of uranyl nitrate $(0.2 \mathrm{mg} / \mathrm{ml})$ may be mentioned. Deposition is found to be complete in $11 / 2 \mathrm{~h}$, with a potential of 7 volts and a current density of $5 \mathrm{~mA} / \mathrm{cm}^{2}$. The anode is combined. with a stirrer, having a moderate rate of stirring, approximately $100 \mathrm{rpm}$. Uranium is precipitated as fluoride. If the cathode material is platinum, it may be ignited and the deposit thus converted into $\mathrm{U}_{3} \mathrm{O}_{8}$.

\section{E. Tetraethylene Glycol Spreading Technique}

In many cases a very thin backing is not necessary, for instance when

26 D. B. Smith and A. M. Wildblood, AERE I/M 27, Harwell 1953.

27 H. Slätis and K. Siegbahn, Arkiv. f. Fysik 4, (1952) 485.

28 D. A. Lind, private communication, 1951.

29

I. E. Alburger, private communication, 1954. 
only internal conversion lines are studied. Monoenergetic electrons, which are backscattered from a thick backing, have lost very different amounts of energy and therefore give no contribution to the shape or the size of the conversion line, only to the general background for conversion lines of lower energy. A number of different methods of preparing thin foils on platinum backings are given in the quoted work by Dodson et al. (loc. cit.). One of the simplest is the tetraethylene glycol spreading technique.

In preparing backings for a tetraethylene glycol film, flatness of the backing is of the utmost importance. The platinum plates are therefore preferably cut by means of a punch ${ }^{21}$. The circular platinum foil is ignited to remove any grease that may be on the surface and then it is fastened to a $3 \mathrm{~mm}$ glass or aluminium plate by small pieces of Scotch tape. Since the adhesive power of Scotch tape diminishes as heat is applied, a zapon ring is painted over the edge of the foil. The area inside of this painted ring receives the deposit. When dry, the tetraethylene glycol required is added. The amount depends on the deposit density desired and is 1 to 2 $\mu \mathrm{l} / \mathrm{cm}^{2}$ for deposit densities of about $0.1 \mathrm{mg} / \mathrm{cm}^{2}$. The active solution is then delivered into the glycol drop and the two phases mixed by placing the foil and support on an asbestos board which is rocked slowly. After that the aqueous portion of the solution is carefully evaporated by means of a heat lamp. Upon further heating the glycol partially polymerizes, holding the precipitated film in place. The distance between the foil and the lamp is decreased until the film is dry. Thereafter the foil is removed from the supporting plate and ignited. Thin deposits are ignited in a gas flame, thicker deposits in a muffle furnace by slowly raising the temperature. The active solutions should contain no sulphuric acid and only a minimum amount of nitric acid.

\section{F. Zapon Spreading Technique}

An alcoholic solution of the nitrate of the substance to be deposited is mixed with a dilute solution of zapon lacquer in alcohol, acetone, or zapon thinner. The resulting solution is spread over the foil backing, dried and ignited to remove organic substances and to convert the nitrate to oxide. Zapon films have been coated on aluminium, platinum, brass, copper, iron, steel, molybdenum, and nickel. For detailed description of this and the methods above see the original paper by Dodson et al. (loc. cit.).

\section{G. Samples ubtained by Means of the Electromagnetic Isotope SEPARATOR}

If the activity is obtained by separation in an electromagnetic isotope separator, the preparation of the sample offers no difficulties. The ions 
generally have a velocity corresponding to $30-60 \mathrm{keV}$, and penetrate into the collecting aluminium foil to a depth of the order of 100 atomic layers ${ }^{30,31}$. This foil is an almost ideal sample for beta-ray spectroscopy. The method is especially suitable for studying the radiations from noble gases. The activity is covered with only a few $\mu \mathrm{g} / \mathrm{cm}^{2}$ of $\mathrm{Al}$.

\section{H. Methods Applicable in Special C'ases}

In certain cases use may be made of some special properties of the elements to be investigated. For example, when gold is bombarded with deuterons the activities $\mathrm{Hg}^{195}$ and $\mathrm{Hg}^{197}$ are produced. When the bombarded gold is heated in vacuum, the active mercury evaporates and is easily absorbed in a thin gold layer kept at a lower temperature ${ }^{32}$. This gold layer may have been prepared by evaporation of gold onto a backing into which mercury will not penetrate, for instance formvar, or, if samples are made for a semicircular beta-ray spectrograph, onto a thin wire of stainless steel.

Another example concerns the preparation of a sample for studying the low energy end of the beta-spectrum of $\mathrm{C}^{14}$. Mize and Zaffarano ${ }^{33}$ have been able to transfer active $\mathrm{Li}_{2} \mathrm{CO}_{3}$ by subliming lithium metal onto a thin zapon film, converting it to the hydroxide in situ, and then surrounding it with an atmosphere of enriched $\mathrm{CO}_{2}$, of as high a specific activity as they could obtain. With this technique they made a source of $\mathrm{LiCO}_{3}$ only $10 \mu \mathrm{g} / \mathrm{cm}^{2}$ thick, with sufficient specific activity that they could get a good beta spectrum.

The most ideal sources of ThB are obtained on metal foils or wires kept at a negative potential $(500-1000 \mathrm{~V})$ in vessels containing $\mathrm{RdTh}(1.9$ years). When decaying, RdTh gives rise to $\operatorname{ThX}$, Tn, ThA, and ThB. The thoron as a noble gas diffuses throughout the vessel and decays with a half-life of $54.5 \mathrm{sec}$ to ThA, and this in turn to ThB with a half-iife of 0.14 sec. These atoms, when created, are left in an ionized state and consequently can be collected on a charged foil or wire. In this way practically monoatomic layers of ThB are obtained. ThB, having a half-life of 10.6 hours, is very often used for calibration purposes in beta-ray spectroscopy work. In a similar way ideal sources of $\mathrm{RaB}^{34}$ and $\mathrm{AcB}$ are obtained from radon and $\mathrm{RdAc}$, resp.

30 J. Koch, Nature 161, (1948) 566.

31 I. Bergström, Arkiv. f. Fysik 5, (1952) 191.

S. Thulin, Thesis to be published in Arkiv f. Fysik.

32 O. Huber, J. Halter, R. Joly, D. Maeder, and J. Brunner, Helv. Phys. Acta 26, (1953) 591.

33 J. Zaffarano, private communication, 1954.

34 See for instance M. Mladjenovié and H. Slätis, Arkiv f. Fysik 8, (1954) 65. 


\section{$\S$ 5. Window Absorption Correction}

The variation in the transmitted fraction of electrons passing through thin foils of aluminium, copper, silver, and gold as a function of the velocity of the incident electrons has been investigated by Schonland 35,36 . Transmission curves for electrons passing through glyptal-cellulose acetate foils as a function of the foil thickness have been given by Arnoult ${ }^{37}$ for the electron energies 24.5, 36, 57, 60.5 and $97 \mathrm{keV}$ (Fig. 4). Heller, Sturcken, and Weber ${ }^{38}$ studied the per cent beta-ray transmission by thin nylon and formvar windows to obtain experimental absorption corrections to be used with GM counters employing such thin windows. The results are given

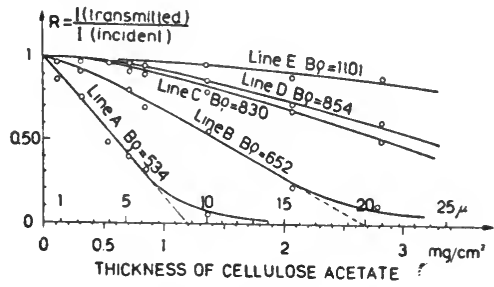

Fig. 4. Transmission of electrons through thin cellulose acetate foils according to Arnoult $\left(\mathrm{mg} / \mathrm{cm}^{2}\right.$. scale added by the author) in diagrams. The energy of the electrons used varied from 16 to $32 \mathrm{keV}$, the thickness of the foils from 0.01 to $0.22 \mathrm{mg} / \mathrm{cm}^{2}$. Saxon ${ }^{39}$ has published very useful curves for the window correction factor (Fig. 5).

It should be noticed that the transmission factors will depend on the

TABLE I

Properties of Conducting Films

\begin{tabular}{c|c|c|c}
\hline $\begin{array}{c}\text { Film } \\
\text { Number }\end{array}$ & $\begin{array}{c}\text { Average Area } \\
\text { Density }\left(\mathrm{mg} / \mathrm{cm}^{2}\right)\end{array}$ & Material * & $\begin{array}{c}E_{p} \\
(\mathrm{keV})\end{array}$ \\
\hline 1 & 1.57 & $\mathrm{Al}$ & 27.3 \\
2 & 0.39 & F-Al & 12.7 \\
3 & 0.10 & F-Al & 6.3 \\
4 & 0.091 & F-Al & 5.5 \\
5 & 0.082 & C-Al & 5.3 \\
6 & 0.068 & C-Al & 4.7 \\
7 & 0.039 & C-Al & 3.2 \\
8 & 0.032 & C-Al & 2.6 \\
9 & 0.024 & C-Al & 2.3 \\
10 & 0.023 & C-Al & 2.3 \\
11 & 0.024 & C-Al & 2.2
\end{tabular}

* Al indicates aluminium; F-Al, aluminium-coated formvar; and C-Al, aluminium-coated collodion.

35 B. F. J. Schonland, Proc. Roy. Soc. 108A, (1925) 187.

36 E. Rutherford, J. Chadwick and C. D. Ellis, Radiations from Radioactive Substances, Cambridge (1930) p. 420.

37 R. Arnoult, Ann. de Phys. 12, 241 (1939).

38 R. B. Heller, E. F. Sturcken and A. H. Weber, Rev. Sci. Inst. 21, (1950) 898.

39 D. Saxon, Phys. Rev. 81, (1951) 639. 
geometry used in the experiments. The angle of incidence, for instance,

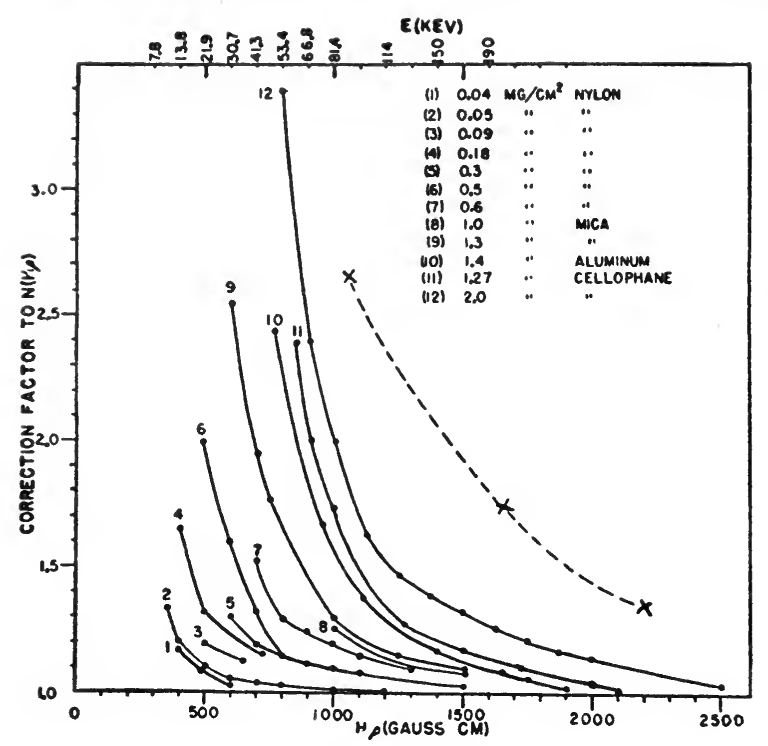

Fig. 5. Correction factor for various counter windows according to Saxon. For details (e.g. the broken line) see ref. 39 is different in different betaray spectrometers.

Lane and Zaffarano ${ }^{40}$ measured the transmission of electrons in the energy range $0-40 \mathrm{keV}$ through thin plastic and metal films of thickness $0.024-1.57 \mathrm{mg} /$ $\mathrm{cm}^{2}$. The films were coated with a thin conducting layer of aluminium. It was found, that Geiger-Müller counters equipped with formvar windows without such a conducting metal layer showed a strong hysteresis effect (Fig. $6)$. In order to get reproducible results for low energy electrons when working with GM counters the windows therefore have to be conducting. Fig. 7 shows the curves of transmission versus area density of the windows obtained by Lane and Zaffarano for

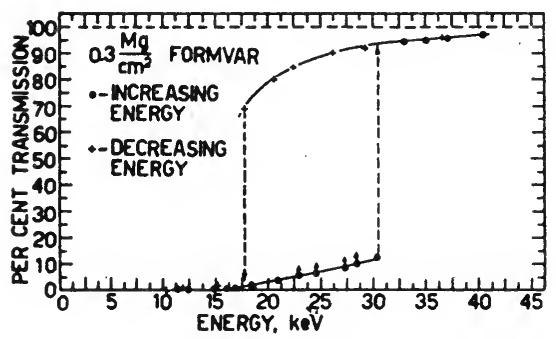

Fig. 6. Typical hysteresis loop obtained for a formvar film not coated with aluminium. Dotted lines indicate electron energies for which sudden changes in transmission occur. Short arrows on points of lower curve indicate magnitude and direction of transmission drift at these points due to charging effects (Lane \& Zaffarano)

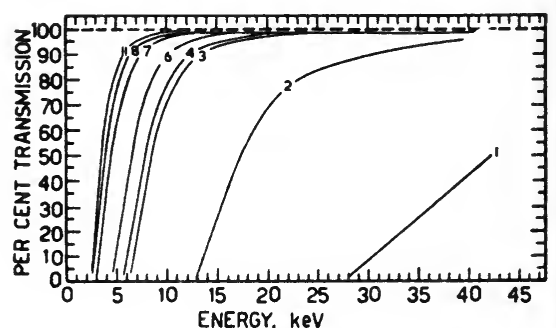

Fig. 7. Transmission curves for various films. See Table 1 for description of films and values of $E_{p}$ (intercept on energy axis of extrapolation of descending linear portion of transmission curve). For clarity, transmission curves for films 5, 9, and 10 are not shown (Lane and Zaffarano)

40 R. O. Lane and D. J. Zaffarano, LR-211, Contribution from the Institute for Atomic. Research and Department of Physics, Iowa State College, Ames, Iowa; Phys. Rev. 94, (1954) 960. 
various energies. The thickness, film materials, cut-off energies and the corresponding film numbers are given in Table I.

The curves by Lane and Zaffarano were obtained with an equipment consisting of an electron filament source, an accelerating field, and a collecting Faraday cup. This collecting cup had, however, a little smaller space angle efficiency than the GM counter used in the beta-ray spectrometer experiments, and thus it was found that the transmission curve, constructed from the measurements of the allowed beta spectrum of $\mathrm{Pm}^{\mathbf{1 4 7}}$ and the assumption of a linear Fermi plot for this spectrum, had a slightly different form than the curves obtained with the collecting cup arrangement (Fig. 8). Hence the transmission curves given by Lane and

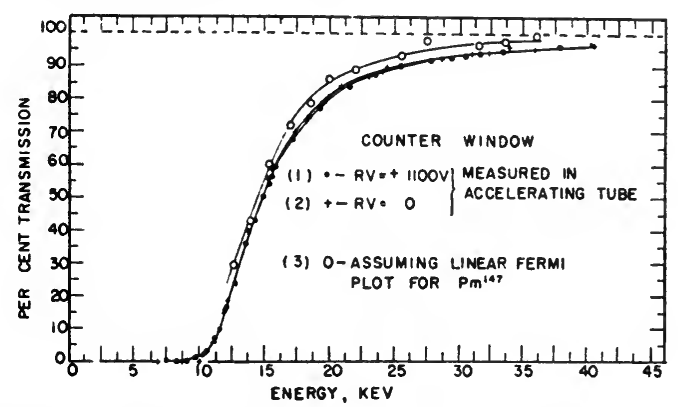

Fig. 8. Transmission curves for $\mathrm{G} M$ counter window. Window $0.3 \mathrm{mg} / \mathrm{cm}^{2}$ formvar coated with aluminium. The difference in the ordinates of curve 1 and curve 2 is proportional to the number of secondary electrons emanating from the side of the film toward the Faraday cup. The open circles represent points computed by assuming that the true Fermi plot for $\mathrm{Pm}^{147}$ has an allowed shape to $10 \mathrm{keV}$ (Lane and Zaffarano)

Zaffarano in Fig. 7 may give some over-correction when they are applied to a beta-spectrum. However, considering that the transmission curves are made by a method independent of the counter of the spectrometer, Lane and Zaffarano regard the transmission curves in Fig. 7 to be in satisfactory accord with the data obtained from the Fermi plot of $\mathrm{Pm}^{147}$. They point out that the use of $\mathrm{Pm}^{147}$ (half-life 2.6 years) beta-spectrum as a standard probably offers the simplest present method of obtaining a transmission curve for a GM counter window employed in a spectrometer above $10 \mathrm{keV}$.

The electron acceleration method may also be used in order to get window correction curves (Butt ${ }^{43}$, see below).

As a working rule, Langer and Cook $^{41}$ mention that a thin counter window has no effect on the shape of the beta-ray distribution for energies which are more than four to five times the cut-off energy.

41 L. M. Langer and C. S. Cook, Rev. Sci. Inst. 19, (1948) 257. 


\section{$\S 6$. Acceleration of Electrons}

Attempts have been made to avoid the troubles in making thin windows and the corrections to be applied for the absorption in these by giving the electrons investigated an additional energy, large enough to ensure the passage of all the incident electrons through the counter window. Thus Langer and Cook ${ }^{41}$ used a potential difference between the detecting slit and the GM counter. At potentials above $25 \mathrm{kV}$, however, the counting rate started to increase rapidly. As a compromise. Agnew and Anderson ${ }^{42}$ used only $8 \mathrm{kV}$ for acceleration of the electrons, thus being able to measure down to electrons of originally $1 \mathrm{keV}$ energy. The window thickness was $9 \mathrm{keV}$, and window corrections were considered correct down to $10 \mathrm{keV}$ electron energy. Butt ${ }^{43}$ used an accelerator for the electrons after they had been focused. The voltage was about $18 \mathrm{kV}$. No spurious counts were observed below an accelerating potential of approximately $19 \mathrm{kV}$. Above this voltage, however, the number of such counts increased very rapidly. The thickness of the counter window was $0.4 \mathrm{mg} / \mathrm{cm}^{2}$. As this thickness corresponds to the range for electrons of about $18 \mathrm{keV}$ energy, the acceleration of secondary electrons was considered as the origin of the superfluous counts.

Butt used the method of electron acceleration for determination of the transmission curves for counter windows. A source is put into the spectrometer and electrons of a certain momentum are focused. The accelerating voltage is then applied and increased in steps of $1 \mathrm{kV}$, counting being continued until a saturation counting rate is reached. At this stage all the electrons incident on the window are transmitted. From one such curve the transmission of the film for electrons of any energy may be determined.

In order to avoid the additional spurious counts Chang and Cook 44, 45 applied the voltage at the source in the spectrometer. The source material was electroplated uniformly over the forward hemisphere of the spherical source holder. Concentric with this source, and surrounding it very closely, was an electrically grounded mesh of fine wire. The accelerating field was situated between the mesh and the source, the latter being given a negative potential. The arrangement was used for determination of window transmission factors.

The causes of the increase in background counting rate and the difficulties of the electron accelerating method are discussed by Cook ${ }^{45}$.

42 H. M. Agnew and H. L. Anderson, Rev. Sci. Inst. 20, (1949) 869.

43 D. K. Butt, Proc. Phys. Soc. London, 63A, (1950) 986.

44 C. H. Chang and C. S. Cook, Nucleonics 10, No. 4, (1952) 24.

45 C. S. Cook, Office of Naval Research, U.S. Navy Dept,. RDB, No NR 024-018 (!) 953$)$. 


\section{THE THEORY OF ALLOWED $\beta$-DECAY}

M. E. ROSE

\section{§ 1. Introduction}

The first evidence for the instability of nuclei, in certain energy states, was the observation, in the closing years of the past century, of the radioactivity of the heavy elements. In addition to $\alpha$ and $\gamma$-emission, radiations consisting of negatively charged particles were observed in many cases. These particles have since been identified beyond doubt as electrons, for example by the measurement of their $e / m$ value. With the advent of artificial production of radioactive nuclei this phenomenon of $\beta$-emission has been shown to be very wide spread and there are very few mass values corresponding to which there is not at least one $\beta$-radioactive isobar.

In addition to the negative electron emission two other kinds of $\beta$-radioactive processes are known. These are positron emission and orbital electron capture. Since the only charged particle emitted, aside from the recoil nucleus, is the $\beta$-particle $\left(\mathrm{e}^{ \pm}\right)$one may represent the process of $\mathrm{e}^{ \pm}$ emission by

$$
\mathrm{Z}^{\mathrm{A}} \rightarrow(\mathrm{Z} \pm 1)^{\mathrm{A}}+\mathrm{e}^{\mp}+v
$$

where $\nu$ represents the possibility of accompanying neutral particles. On the other hand the orbital electron capture is represented by

$$
\mathrm{Z}^{\mathrm{A}}+\mathrm{e}^{-} \rightarrow(\mathrm{Z}-1)^{\mathrm{A}}+v
$$

The nature of the neutral particle(s) is discussed in section 3 below. For the moment we need only add that the lifetimes for the $\beta$-process are very long compared to any other type of radioactivity: measured half-lives range from $3 \times 10^{-2}$ sec to about $4 \times 10^{10}$ years. Longer halflives no doubt exist but the activities. would be difficult to detect and more difficult to measure. The 0.03 sec half-life of $\mathrm{B}^{12}$ is associated with an energy release of $13.4 \mathrm{MeV}$ and, as will be clear from the sequel, appreciably shorter half-lives would entail a larger energy release leading to the emission of a nucleon rather than a $\beta$-particle. The only other type of emission processes which exhibit comparatively long half-lives are those in which $\gamma$-rays (or conversion electrons) are emitted from isomeric states. However, such slow processes are associated with large angular momentum changes $(\Delta J \geqslant 3)$ between the nuclear states involved. While the angular momentum change also has a profound effect on the half-life in $\beta$-decay, (see section 5 below and especially $C h . \mathrm{X}$ ), eren in the 
most favorable cases, $\Delta J=0$ or 1 , the $\beta$-process goes much more slowly than the $\gamma$-emission (for the same $\Delta J$ ) or the emission of any other type of particle. In terms of the description presented in the following section, this means that the $\beta$-interaction responsible for the phenomenon of $\beta$-decay is comparatively weak. Thus, when a nucleus is formed in an excited state with insufficient energy for nuclear particle emission it will usually go to the ground state by radiative transitions. If the ground state is not stable a $\beta$-process will follow.

\section{$\S 2$. The Role of $\beta$-Decay in Nuclear Physics}

We are interested in the process of $\beta$-decay for two reasons. The first is the fact that by measuring the shape of the $\beta$-energy spectrum one can obtain information about the angular momentum and parity changes involved in the transition. This information constitutes an important link in the chain of reasoning, from the experimental evidence, whereby a decay scheme is constructed. The technique of $\beta-\gamma$ coincidence measurements also enables one to determine where the $\gamma$-rays occur in the level scheme. The manner in which the data is used is discussed in Ch. XVI.

The second reason for the importance of the $\beta$-decay process is that it brings to light a new type of interaction in physics. This interaction plays a role similar to that of the electromagnetic interaction in the process of emission and absorption of light quanta. However, as will be seen, it is considerably more complicated than the electromagnetic interaction. In the latter case one has the Maxwell theory and the correspondence principle as an invaluable guide. Unfortunately there is no corresponding classical counterpart for the $\beta$-interaction ${ }^{1}$.

\section{$\S 3$. The Conservation Laws and the Neutrino}

As a basic principle one requires that the process of $\beta$-decay, like all other phenomena, be subjected to the laws of conservation of energy, linear momentum, angular momentum, statistics and charge.

The facts that nuclei have well defined energy states and that the $\beta$-particles exhibit a continuous energy spectrum then demands that at least one other particle be emitted; i.e., the $(v)$ in (1) or (2). We may at once eliminate the possibility that $v$ corresponds to more than one additional particle. The only other practical possibility is two particles and the observed spectrum (see section 6 below) does not correspond to this case.

1 There is, however, another type of decay process which may very well be characterized by the same kind of interaction; namely, the decay of the charged $\mu$ meson. See J. Tiomno and J. A. Wheeler, Rev. Mod. Phys. 21 (1949) 144. 
That the linear momentum is not conserved without an additional particle is well established 2. Conservation of angular momentum $(j)$ requires that the particle in question have half-integral $j$. This follows since both isobars have either integral or half-integral angular momentum and the $j$ of the $\beta$-particle is half-integral. This implies. spin $\frac{1}{2}, \frac{3}{2}$ etc., for $v$. Only the possibilities $\frac{1}{2}$ and $\frac{3}{2}$ are left when one considers (for example) the neutron decay, $\mathrm{n} \rightarrow \mathrm{p}+\mathrm{e}^{-}+\nu$. $\left(J_{n}=J_{p}=\frac{1}{2}\right.$ and $\left.j_{e}=\frac{1}{2}\right)$. However a $\operatorname{spin} \frac{3}{2}$ for $v$ corresponds to an energy spectrum in disagreement with experiment ${ }^{3}$. The spin $\frac{1}{2}$ is left as the only possibility.

The last conclusion is in accord with the result of applying conservation of statistics. The two nuclear isobars, containing the same number of nucleons (fermions) must obey the same kind of statistics. The $\beta$-particle is a fermion. Therefore the $v$-particle is also a fermion.

Finally, as already indicated, the $v$-particle is neutral.

Essentially these arguments led Pauli to postulate the existence of a very light, neutral particle as a concomitant of every $\beta$-decay process. That the $v$-particle, called the neutrino (little neutron), has a very small or vanishing rest mass is amply confirmed by subsequent developments (Ch. XI). The failure to date to detect secondaries due to neutrino collisions points to a small or vanishing magnetic moment of this particle as well.

\section{$\S$ 4. The $\beta$-Interaction}

In constructing a theory of $\beta$-decay there are two facts which are of prime importance. The first is that one is here confronted with a situation in which particles are created (or destroyed, if one regards the positron emission as an absorption of a negative energy electron). It is therefore necessary to treat the problem in terms of a field theory. The nucleons are the source of the field and the light particles $\left(\beta^{ \pm}\right.$and $\nu$, collectively the leptons) together constitute the field particle. Secondly, one is obliged to treat the leptons relativistically - this is imperative for the neutrino, of course. This situation is precisely the one faced in the emission and absorption of electromagnetic radiation. There the interaction can be represented by a coupling energy which, per unit volume, is

$$
\varrho \Phi-\frac{1}{c} j \cdot A
$$

in which $\boldsymbol{j}$ and $\varrho$ are the source charge and current densities respectively ( $\boldsymbol{j}$, ic $\varrho$ form a four-vector). Also $\boldsymbol{A}$ and $\Phi$ are the electromagnetic potentials

2 For example, R. F. Christy et al, Phys. Rev. 72 (1947) 698 and Ch. XII.

3 In the allowed spectrum (see Eq. (10) below) one finds $\left(W_{0}-W\right)^{4}$ instead of $\left(W_{0}-W\right)^{2}$. 


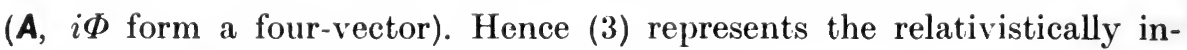
variant scalar product of two four-vectors.

For the $\beta$-decay process one needs to replace $\boldsymbol{j}$, $\varrho$ by the transition current and charge densities expressed in terms of nuclear wave functions, $\Psi$, and $\Psi_{i}$ for final and initial states respectively. Similarly $\boldsymbol{A}, i \Phi$ are replaced by the four vector current in the electron $(\psi)$ and neutrino $(\phi)$ wave functions:

$$
\boldsymbol{A} \rightarrow c \psi^{*} \alpha \phi, \Phi \rightarrow \psi^{*} \phi
$$

Thus, the energy density would be ${ }^{4}$

$$
\mathscr{H}_{V}=g\left[\left(\Psi_{j}^{*} \Psi_{i}\right)\left(\psi^{*} \phi\right)-\left(\Psi_{j}^{*} \alpha \Psi_{i}\right) \cdot\left(\psi^{*} \alpha \phi\right)\right]
$$

Here there are two Dirac $\alpha$-operators, the first acting on the (spinor) components of the nuclear wave function, the second on a lepton wave function. This interaction is just the one used by Fermi ${ }^{5}$ in his original formulation of the theory. Because a contraction of two 4-vectors is involved the interaction is referred to as the "vector" type and designated by the suffix $V$. In (4) we have also explicitly introduced the coupling constant $g$, analogous to $e$ (charge) in the electromagnetic case.

In the electromagnetic case, only the term $\varrho \Phi$ is important if the source particles are moving slowly $(\boldsymbol{j} \sim \varrho \mathbf{v}, \mathbf{v}$ the source particle velocity). Similarly, since $v / c$ is small for nucleons, only the first term of (4) will be important. The exception to this occurs when this first term vanishes due to selection rules on parity and/or angular momentum-see eq. (8).

For electromagnetic phenomena one has vector interactions for particles by virtue of their charge. If they also possess magnetic (or electric) moments there is a tensor interaction. For example, for a magnetic dipole $\mu \sim \sigma$ (where $\boldsymbol{\sigma}$ is the spin operator) the interaction in a magnetic field $\boldsymbol{H}$ is proportional to $\boldsymbol{\sigma} \cdot \boldsymbol{H}$. This is the contraction of two tensors:

and

$$
\sigma_{j}=-i \alpha_{k} \alpha_{l}
$$

$$
H_{j}=\frac{\partial A_{l}}{\partial x_{k}}-\frac{\partial A_{k}}{\partial x_{l}}
$$

$j, k, l$ cyclic. In the same way one is permitted to construct a tensor interaction $\mathscr{H}_{T}$ in $\beta$-decay. However, this is not all because so far as the

- Formally, it is necessary to insert an operator $Q$ acting on $\Psi_{i}$ which changes a neutron into a proton. Then $\Psi_{j}$ and $Q \Psi_{i}$ correspond to the same neutron and proton numbers. Also to $\mathscr{H}_{V}$ a term which is the hermitean conjugate of the right side should be added. This term would correspond to positron emission when (4) corresponds to electron emission. The $Q^{*}$ operator in the ronjugated term changes a proton into a neutron, of course.

5 E. Fermi, Zs. für Physik 88 (1934) 161. 
requirements of relativistic invariance are concerned there are three more possibilities:

$\mathrm{S}$ or scalar interaction: contraction of two scalars

A or axial vector interaction: contraction of two axial vectors

$\mathbf{P}$ or pseudoscalar interaction: contraction of two pseudoscalars.

Restricting our attention to the case in which only the non-relativistic terms in the nuclei are considered, the five interaction energy densities may be written ${ }^{4}$ :

$$
\begin{aligned}
& \mathscr{H}_{S}=g\left(\Psi_{i}^{*} \beta \Psi_{i}\right)\left(\psi^{*} \beta \phi\right) \\
& \mathscr{H}_{V}=g\left(\Psi_{f}^{*} \Psi_{i}\right)\left(\psi^{*} \phi\right) \\
& \mathscr{H}_{T}=g\left(\Psi_{f}^{*} \beta \boldsymbol{\sigma} \Psi_{i}\right) \cdot\left(\psi^{*} \beta \boldsymbol{\sigma} \phi\right) \\
& \mathscr{H}_{{ }}=g\left(\Psi_{j}^{*} \sigma \Psi_{i}\right) \cdot\left(\psi^{*} \boldsymbol{\sigma} \phi\right) \\
& \mathscr{H}_{{ }_{P}}=g\left(\Psi_{j}^{*} \beta \gamma_{5} \Psi_{i}\right)\left(\psi^{*} \beta \gamma_{5} \phi\right)
\end{aligned}
$$

In each case the operators work in nucleon and lepton spaces. We have introduced the fourth Dirac matrix $\beta$ and also $\gamma_{5}=i_{\lambda_{1} \chi_{2} x_{3} .} \mathscr{H}_{S}$ and $\mathscr{H}_{P}$ are represented in complete form. For the complete form of $\mathscr{H}_{V}, \mathscr{H}_{T}$ and $\mathscr{H}_{A}$ the reader should consult Ch. X.

The additional complication which arises, relative to electromagnetic processes, is clearly due to the absence of a classical theory which would restrict the number of possibilities. In the electromagnetic case there are two coupling constants, phenomenologically speaking: the charge and the magnetic moment. In practice the latter can ultimately be expressed in terms of the former. In the $\beta$-decay case there are five coupling constants, all different and, so far as is now known, all independent. Thus, the complete $\beta$-interaction is a linear combination of the above five:

$$
\mathscr{H}=C_{S} \mathscr{H}_{S}+C_{V} \mathscr{H}_{V}+C_{T} \mathscr{H}_{T}+C_{A} \mathscr{H}_{A}+C_{P} \mathscr{H}_{P}
$$

Since there are only five independent constants, one can define the $C_{X}$ so that $\sum_{X} C_{X}^{2}=1$. 'It has been shown that the $C_{X}$ can be taken to be real.

One of the major purposes to be served by comparison of theory and experiment is the determination of the five "charges" $g C_{X}$.

\section{$\S 5$. Allowed Transitions}

The detailed theory by which the energy spectrum is calculated involves the evaluation of matrix elements arising from

$$
\left|\sum_{k} \int \mathscr{H} \delta\left(\boldsymbol{r}-\boldsymbol{r}_{k}\right) \cdot d \tau_{k}\right|^{2}
$$

summed over all nucleons (coordinate $\boldsymbol{r}_{k}$ ) and integrated over the nuclear volume. In addition a sum over all non-observed quantities is to be performed. These will include the angular momentum quantum numbers 
or spin directions of leptons, magnetic substates of the nuclear levels and, except in the angular correlation (Ch. XIX), the directions of motion of the leptons. Without entering into these details, one may suppose that the Coulomb field acting on the $\mathrm{e}^{ \pm}$is neglected. Then both leptons are represented by plane waves, $\sim \exp i \boldsymbol{k} \cdot \boldsymbol{r}$ where $\boldsymbol{k}$ is the wave vector of the particle. If one expands $\exp i \boldsymbol{k} \cdot \boldsymbol{r}$ as

$$
\exp i \mathbf{k} \cdot \boldsymbol{r}=\sum_{0}^{\infty} \frac{1}{n !}(i \mathbf{k} \cdot \boldsymbol{r})^{n}=\sum_{0}^{\infty}(2 l+1) i^{l} j_{l}(k r) P_{l}(\cos \vartheta)
$$

where $j_{l}$ is the spherical Bessel function, then this expansion corresponds essentially to an expansion in waves of increasingly higher angular momentum ${ }^{6}$. Since $r \lesssim R$, the nuclear radius, and since $k R \ll 1$ for all practical cases, it is clear that the major contribution arises from the first term in each expansion; that is exp $i \boldsymbol{k} \cdot \boldsymbol{r}$ is replaced by unity. The only exception arises when the leading term vanishes by selection rules (see (8) below).

It will now be observed that two expansions have been made. The one discussed just above is a retardation expansion based on $k R \ll 1$ and is analogous to the expansion in the electromagnetic case into dipole, quadrupole, etc., radiation. The one discussed in the foregoing section neglects $v / c$ for the nucleons compared to unity. The grouping of the various terms must be made on the basis of selection rules and for each parity and angular moment change only the leading terms need be considered. The matrix elements arising from the higher terms in the expansion (7) and in $v / c$ cannot all coexist. For example, consider any interaction listed in (5) except the $\mathrm{P}$ interaction. Then if the $l$-values for $\beta$-particle and neutrino have an odd (even) sum the parity change $\Delta \pi$ in the nuclear transition is odd (even). That is, $\Delta \pi$ is yes (no). For the $\mathrm{P}$ interaction $\Delta \pi$ is no (yes). Similarly, the two terms of (4) imply $\Delta \pi=$ no and yes, respectively. In addition, for a given nuclear angular momentum change $J_{i} \rightarrow J_{f}$ the angular momentum of the lepton field $J$ lies between $\left|J_{i}-J_{f}\right|$ and $J_{i}+J_{f}$ while the main contribution comes from $J=\left|J_{i}-J_{f}\right|$. Only the leading terms (lowest power of $k_{\beta} R$ or $k_{v} R$ ) are taken in accordance with the retardation expansion?

- In a relativistic calculation the appropriate expansion is a little more complicated since the spin must be included. However, an expansion essentially like (7) can be made and each term corresponds to a definite angular momentum $j=\left|l \pm \frac{1}{2}\right|, l \geqslant 0$.

7 If the Coulomb field is included the arguments are unchanged in principle. The lepton wave functions are then eigenfunctions of the angular momentum, or linear combinations thereof. For increasing angular momentum, the radial parts of these wave functions, for long wave lengths, behave like a power of $k R$ which increases with $i$. 
It is clear that the transitions with the largest decay constant should be those for which the matrix elements of (5) do not vanish when the lepton wave functions are replaced by their long wave length limit. These transitions are referred to as allowed transitions. To establish the selection rules one merely need note the matrix elements involved. Writing these as $\int \Psi_{i}^{*} \Omega \Psi_{i} d \tau$ one has $\Omega=\beta, 1, \beta \sigma, \sigma$ and $\beta \gamma_{5}$ for $\mathrm{S}, \mathrm{V}, \mathrm{T}, \mathrm{A}$ and $\mathrm{P}$ respectively. For each of the first four one has $\Delta \pi=$ no. For the $\mathrm{P}$ interaction $\Delta \pi=$ yes. Therefore, unless the $\mathrm{P}$ interaction occurs alone, its matrix elements cannot coexist with those of the other interactions. There is incontrovertible evidence that the $\mathrm{P}$ interaction does not exist alone. For example, it predicts an angular momentum change $\Delta J=0$ for allowed transitions and there are several allowed transitions with $\Delta J=1$. Thus, the $\mathrm{P}$ interaction does not really belong in the list of interactions giving allowed transitions. Since we restrict the present consideration to allowed transitions, we shall henceforth ignore the $\mathrm{P}$ interaction.

For the remaining interactions one readily finds that the selection rules are

$$
\left.\begin{array}{l}
\mathrm{S}, \mathrm{V}: \Delta J=0, \Delta \pi=\text { no } \\
\mathrm{T}, \mathrm{A}: \Delta J=0, \pm 1, \text { no } 0 \rightarrow 0 ; \Delta \pi=\text { no }
\end{array}\right\}
$$

This follows from the vector character of $\beta \sigma$ and $\sigma$ and the scalar character of $\beta$ and 1 under rotations. It will be noted that the selection rules are here identical for the group $\mathrm{S}$ and $\mathrm{V}$ (Fermi interactions) and for the group $\mathbf{T}$ and $\mathbf{A}$ (Gamow-Teller interactions).

\section{§6. The Energy Spectrum}

We consider the four interactions $\mathrm{S}, \mathrm{V}, \mathrm{T}, \mathrm{A}$ and for the sake of future reference we give the probability per unit time that the $\beta$-particle is observed with total energy between $W$ and $W+d W$ and the electron and neutrino make an angle $\vartheta$ to $\vartheta+d \vartheta$. We assume zero neutrino rest mass and use units in which $\hbar=m=c=1$. The probability functions are given in reciprocal time units; i.e. $m c^{2} / \hbar=7.7 \times 10^{20} \mathrm{sec}^{-1}$. Then, the energyangular distribution is

$$
\left.\begin{array}{c}
N_{ \pm}(W, \vartheta) d W \sin \vartheta d \vartheta=\frac{g^{2}}{4 \pi^{3}} F(\mp Z, W) p W\left(W_{0}-W\right)^{2}\left[\left(C_{S}^{2}+C_{V}^{2}\right)\left|M_{F}\right|^{2}+\right. \\
\left.+\left(C_{T}^{2}+C_{A}^{2}\right)\left|M_{G T}\right|^{2}\right] \times\left[1+\frac{a p}{W} \cos \vartheta \mp \frac{b}{W}\right] d W \sin \vartheta d \vartheta
\end{array}\right\}
$$

where $p=\left(W^{2}-1\right)^{\natural}$ is the $\beta$-particle momentum, $q=W_{0}-W$ is the neutrino momentum or energy and $W_{0}$ is the energy release (in $m c^{2}$ ) involved in the nuclear transitions. 
The kinetic energy of the $\beta$-particle is $E=W-1$ and, in the units used here,

$$
p=[E(E+2)]^{\prime} \quad \text { or } \quad E=\left(1+p^{2}\right)^{4}-1
$$

Very often the momentum is measured in a magnetic field $B$ by observing the radius of curvature $\varrho$. Then

$$
p=e B \varrho / m c^{2}=5.94 \times 10^{-4} B \varrho
$$

with $B$ in gauss, $\varrho$ in $\mathrm{cm}$.

The upper sign in (9) refers to positron, the lower to electron emission. We have used the abbreviations:

$$
\begin{gathered}
a=\frac{\frac{1}{3}\left(C_{T}^{2}-C_{A}^{2}\right)\left|M_{G T}\right|^{2}-\left(C_{S}^{\prime 2}-C_{V}^{2}\right)\left|M_{F}\right|^{2}}{\left(C_{T}^{2}+C_{A}^{2}\right)\left|M_{G T}\right|^{2}+\left(C_{S}^{2}+C_{V}^{2}\right)\left|M_{F}\right|^{2}} \\
b=2 \gamma \frac{C_{S} C_{V}\left|M_{F}\right|^{2}+C_{T} C_{A}\left|M_{G T}\right|^{2}}{\left(C_{S}^{2}+C_{V}^{2}\right)\left|M_{F}\right|^{2}+\left(C_{T}^{2}+C_{A}^{2}\right)\left|M_{G T}\right|^{2}} \\
F(Z, W)=2(1+\gamma)(2 p R)^{2(\gamma-1)} \exp \pi y \frac{|\Gamma(\gamma+i y)|^{2}}{[\Gamma(2 \gamma+1)]^{2}} \\
F(0, W)=1 \\
\gamma=\left[1-(\alpha Z)^{2}\right]^{t}, \alpha=e^{2} / \hbar c \approx 1 / 137, y=\alpha Z W / p
\end{gathered}
$$

The factors $F$ and $\gamma$ represent the effect of the Coulomb field. In addition, advantage is taken of the fact that for the matrix elements involved here one can replace $\beta$ in the nucleon space by unity. Then

$$
M_{F}=\sum_{k} \int \Psi_{j}^{*} \Psi_{i} d \tau_{k}, M_{G T}=\sum_{k} \int \Psi_{j}^{*} \sigma \Psi_{i} d \tau_{k} .
$$

For the energy spectrum we obtain

$$
\left.\begin{array}{r}
N_{ \pm}(W) d W=\left(g^{2} / 2 \pi_{*}^{3}\right) F(\mp Z, W) p W\left(W_{0}-W\right)^{2}\left[\left(C_{S}^{2}+C_{V}^{2}\right)\left|M_{F}\right|^{2}+\right. \\
\left.+\left(C_{T}^{2}+C_{A}^{2}\right)\left|M_{G T}\right|^{2}\right] \times(1 \mp b / W) d W .
\end{array}\right\}
$$

This seemingly complex result actually has a simple structure and each part of it can be understood in rather simple physical terms:

(i) First consider the intrinsic features of $\beta$-emission by neglecting the Coulomb field. Then, for a pure interaction, the energy dependence is given by the factor $p W\left(W_{0}-W\right)^{2}$ which is referred to as the statistical weight. The actual interaction is almost certainly not pure (section 8 and Ch. X) but, as explained below, the interference term $b / W$ is zero or, in any case, too small to produce observable effects on the spectrum. The designation statistical weight is readily understood since the volume in 
momentum space for two particles with momenta $p$ and $q$ when the total energy $\left(W_{0}\right)$ is fixed and the energy of the first lies between $W$ and $W+d W$ is $p^{2} d p \int \delta\left(W_{0}-W-q\right) q^{2} d q=p W\left(W_{0}-W\right)^{2} d W$. This is for zero neutrino rest mass. For a neutrino mass $m_{v}$ one factor $W_{0}-W$ is replaced by the appropriate value of the neutrino momentum $\left[\left(W_{0}-W\right)^{2}-m_{v}^{2}\right]^{\ddagger}$. Present evidence is that $m_{v} \approx 0$, see Ch. $\mathrm{XI}$.

(ii) The Coulomb field factor $F(\mp Z, W)$ essentially represents the density of $\beta$-particles at the nuclear radius relative to the density at infinity. This would be an exact interpretation except for relativistic effects. Allowed transitions arise from the emission of $\beta$-particles (and neutrinos) in $s_{z}$ and $p_{z}$ states and only that part of the wave functions of these states corresponding to no spin-orbit energy enters. It will be noted that, due to relativistic effects, there is a small "centrifugal force" influence even for allowed transitions and this is contained in the factor $(2 p R)^{2(\gamma-1)}$ in (9c). However, the major influence is the Coulomb repulsion for $Z<0$, or attraction for $Z>0$, contained in the remaining factors of (9c) and as a consequence the number of slow positrons is negligible while the number of slow electrons can be quite large and for heavy emitters the maximum of the energy spectrum may not be apparent.

(iii) The remaining factors in (9) or (10) represent the coupling constant $\left(g^{2}\right)$ and an appropriately weighted mean of squares of nuclear matrix elements. For F and GT interactions the latter are analogous to electric and magnetic dipole matrix elements in the emission of light.

According to the most carefully measured spectra, $\mathrm{Cu}^{64}$ for example ${ }^{8}$, the term $b / W$ is effectively zero ${ }^{9}$. Striking this term out, one concludes that the shape of the spectrum is independent of the precise form of the $\beta$-interaction.

The Fermi function $F(Z, W)$ is important for all but the lightest elements. Even for these it should be taken into account if the experimental points which are free of errors, due to backing and source thickness distortion or to the presence of a lower energy group of $\beta$-particles, extend down to energy values for which $\alpha Z W / p \sim 0.1$. In Appendix II numerical values for the Fermi function are given for all values of $Z \leqslant 96$ for both electrons and positrons as a function of $p$ (in $m c$ units) over

8 C. S. Wu and R. D. Albert, Phys. Rev. 75 (1949) 315 and 1107; L. M. Langer, R. J. D. Moffat and H. C. Price, Phys. Rev. 76 (1949) 1725.

- The departure of the experimental points from linearity in the conventional Kurie plots (section 7) is rather insensitive to the value of $b$. Upper limits on $|b|$ were given by H. M. Mahmoud and E. J. Konopinski, Phys. Rev. 88 (1952) 1266. These upper limits $(\sim 0.2)$ are just about equal to the root mean square deviation of $b$ due to statistical errors in the measurements of the counting rate. 
a range covering any conceivable practical case ${ }^{10}$. Instead of $F$ it is somewhat more convenient to tabulate the function $G$ where

$$
G=(p / W) F
$$

since $G$ is easier to interpolate at small energies, than is $F$. The value of $Z$ given in the tables in Appendix II refers, of course, to the daughter nucleus and the nuclear radius has been taken to be $R=\frac{1}{2} \alpha A^{1 / 3}$, corresponding to $1.41 \times 10^{-13} A^{1 / 3} \mathrm{~cm}$ in ordinary units. The mass number $A$ is chosen for each $Z$ so that it represents an average of known electron or positron emitters. Actually, the $R$ or $A$ dependence is irrelevant for the shapes of allowed transitions and essentially so for the decay constant ( 8 below). It is of some importance for forbidden transitions ${ }^{10}$. In addition to the tables for the Fermi function, the shape factors, which are necessary for forbidden transitions, are tabulated in Appendix III.

The most important corrections to be applied in the analysis of $\beta$-energy spectra are those due to screening and to the finite size of the nucleus. The screening correction may be determined by using the approximate $W K B$ procedure ${ }^{11}$ according to which $F( \pm Z, W)$ is to be replaced by

$$
F\left( \pm Z, W \mp V_{0}\right)\left[\frac{\left(W \mp V_{0}\right)^{2}-1}{W^{2}-1}\right]^{k}\left(\frac{W \mp V_{0}}{W}\right)
$$

where $V_{0}$ is the shift in the potential energy near the nucleus arising from screening. Using the Thomas-Fermi model

$$
V_{0} \cong 1.13 \alpha^{2} Z^{4 / 3}
$$

Alternatively, one may use the more accurate results obtained from numerical evaluation of $\beta$-particle wave functions in a screened (ThomasFermi-Dirac) field. These have been obtained by Reitz for a few elements ${ }^{12}$. Screening corrections for electrons, diminishing the number of slow particles, are seldom larger than $2-3$ percent, except for the heaviest

10 This table is taken from the work of M. E. Rose, N. M. Dismuke, C. L. Perry and P. R. Bell, Oak Ridge National Laboratory Report No. 1222. A similar tabulation has been made by I. Feister, Tables for the Analysis of Beta Spectra, National Bureau of Standards, Applied Mathematics Series 13. A small correction to the Fermi function for allowed transitions, not included here, is to be found in Tables for the Analysis of Allowed and Forbidden Beta Transitions by M. E. Rose, C. L. Perry and N. M. Dismuke, Oak Ridge National Laboratory Report No. 1459. The latter tables also contain numerical values of all the bilinear combinations of electron, or positron, wave functions which occur in forbidden transitions through third-order forbidden, (see Ch. X) and Table in Appendix III.

11 M. E. Rose, Phys. Rev. 49 (1936) 727. See also C. Longmire and H. Brown, Phys. Rev. 75 (1949) 1102.

12 J. R. Reitz, Phys. Rev. 77 (1950) 10. 
elements. For positrons, where the number of slow particles is increased, the corrections are appreciably larger, but where the corrections are largest there are very few positrons to be expected.

Finite nuclear size corrections ${ }^{\mathbf{1 3}}$ are important only for very heavy emitters and are of somewhat minor importance for allowed transitions. Typical numerical values are given at the end of section 7 .

Finally, instrumental corrections, due to finite energy resolution, have to be applied ${ }^{14}$.

\section{$\S$ 7. Kurie Plots for Allowed Spectra}

From (10) with $b=0$ it follows that

$$
\left[N_{ \pm}(W) / F(\mp Z, W) p W\right]^{\sharp}=K\left(W_{0}-W\right)
$$

where $K$ is independent of the energy $W$. Consequently, if the observed energy distribution is used to compute the quantity on the left of (12), that is, $[N(W) / G]^{t} / W$ is plotted, an allowed spectrum will yield a straight line. Here $G=p F / W$. In any case, a curve constructed in this fashion is referred to as an allowed Kurie plot. The extrapolation of the straight line is the customary procedure for determining the maximum energy $W_{0}$. Similarly, if a momentum spectrum is measured where the number of $\beta$-particles per unit momentum range is $n=(p / W) N(W)$, the Kurie plot is obtained from the $[n / p W G]^{\frac{1}{3}}$ versus $W$-curve.

Of course, the allowed Kurie plots will show upward curvature at the low energy end due to source and backing distortion. However, if there is no lower energy group or if such groups are of very low intensity so that the subtraction there of leads to negligible error, there will be an appreciable straight portion. If such is not the case there is a strong indication that the transition is forbidden. Here the parity change and $f t$ value (section 8 ) are invaluable guides.

On the other hand the converse question cannot be answered unambiguously from the spectrum alone. That is, if the Kurie plot is straight it does not follow that the transition is allowed. Thus, most first forbidden spectra show allowed shapes, (Ch. X and XI). However, if in addition the selection rules for allowed transitions are known to be fulfilled and/or the $f t$ value (section 8 ) is in the allowed range, the conclusion that the transition is allowed would follow.

Kurie plots for forbidden transitions can also be constructed on the basis of the correction or shape factors introduced in Ch. X.

13 M. E. Rose and D. K. Holmes, Phys. Rev. 83 (1951) 190 and Oak Ridge National Laboratory Report No. 1022.

14 G. E. Owen and H. Primakoff, Phys. Rev. 74 (1948) 1406. 
As numerical examples of the procedure to be followed in constructing a Kurie plot we consider the two cases shown in Table I.

TABLE I

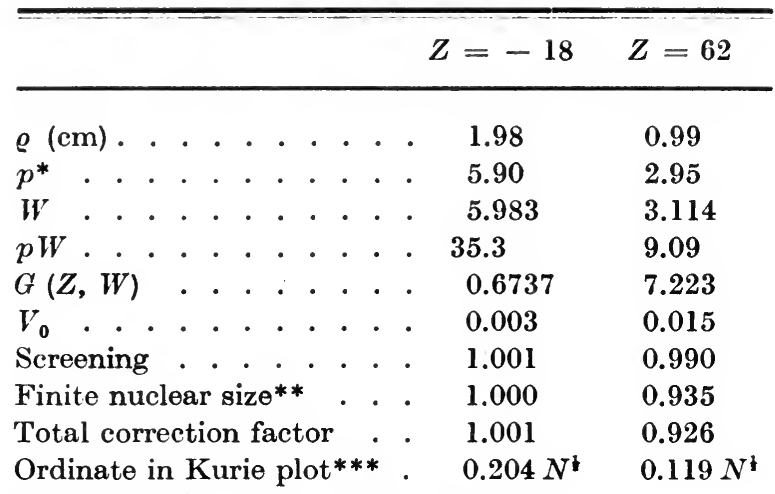

* Assuming a magnetic field of 5000 gauss.

** While this correction seems large for $Z=62$ it is very little energy dependent.

*** $\quad N$ is the measured number of electrons per unit energy interval.

It is assumed that instrumental corrections have already been made.

In constructing Table $I$ it was assumed that a momentum spectrum was measured and converted to a Kurie plot versus energy. The values of $G$ are obtained from the Tables in Appendix II by linear interpolation which is more than sufficiently accurate. The entries in rows 7 and 8 are correction factors also obtained by interpolation. Mass values have not been specified since these do not affect the shape of the spectrum.

\section{$\S 8$. ft Values}

The decay constant for the $\beta$-transition is

where

$$
\lambda=\int_{1}^{W_{0}} N(W) d W=\frac{g^{2}}{2 \pi^{3}}\left[C_{F}^{2}\left|M_{F}\right|^{2}+C_{G T}^{2}\left|M_{G T}\right|^{2}\right] f\left(Z, W_{0}\right)
$$

and

$$
\begin{gathered}
C_{F}^{2}=C_{S}^{2}+C_{V}^{2} \\
C_{G T}^{2}=C_{T}^{2}+C_{A}^{2}
\end{gathered}
$$

$$
f\left(Z, W_{0}\right)=\int_{1}^{W_{0}} p W\left(W_{0}-W\right)^{2} F(Z, W) d W
$$

Numerical values of $f\left(Z, W_{0}\right)$ have been extensively tabulated ${ }^{15}$; see Figs. 1-5.

15 E. Feenberg and G. Trigg, Rev. Mod. Phys. 22 (1950) 399. 


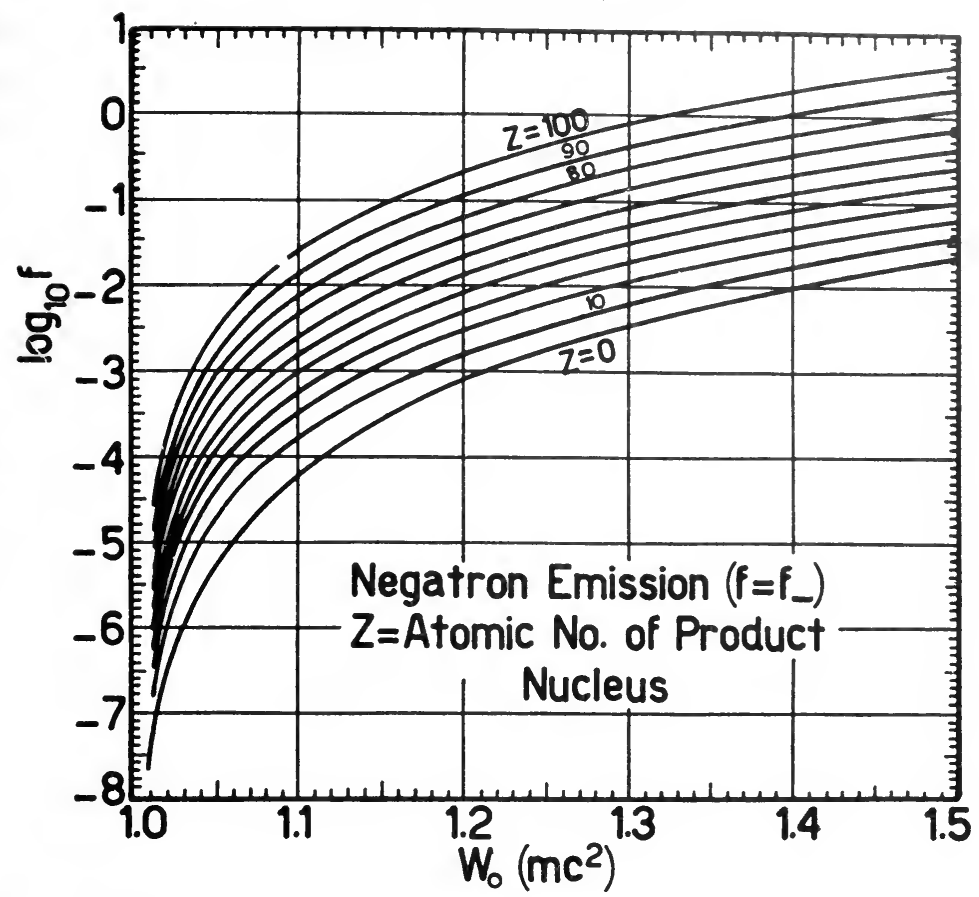

Fig. 1. $f$ for $\beta^{-}$emission, low energy scale (courtesy Feenberg and Trigg)

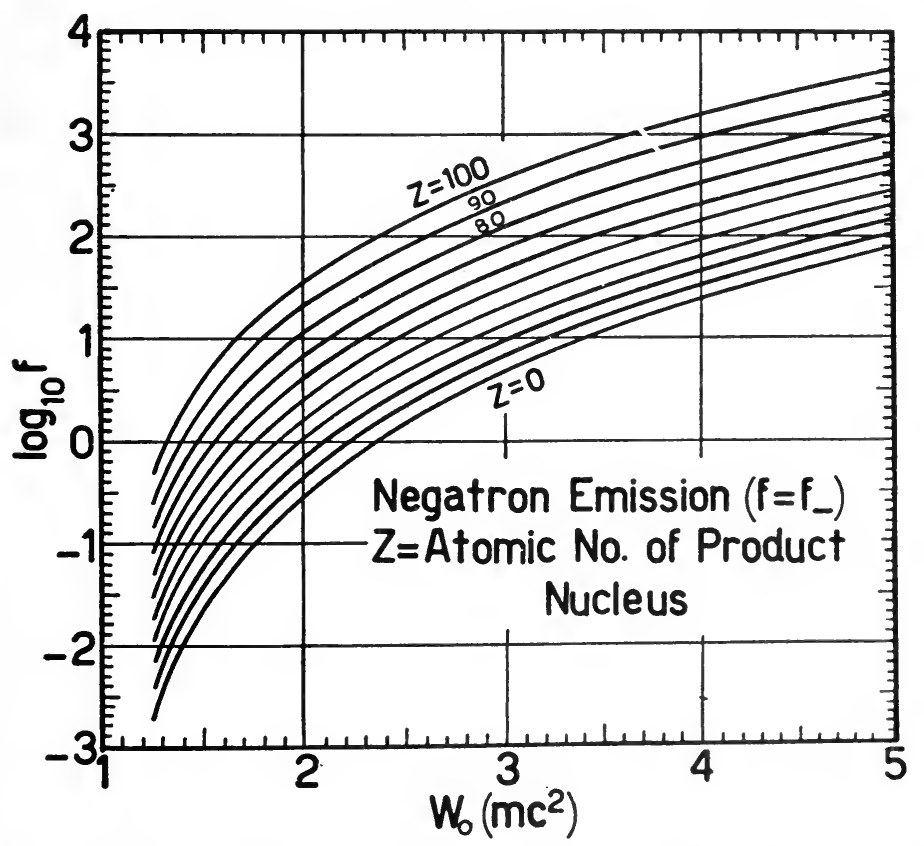

Fig. 2. $f$ for $\beta$ - emission, medium energy scale (courtesy Feenberg and Trigg) 
It is clear that the half life, $t=(\ln 2) / \lambda$, is not in itself characteristic of an allowed transition since $t$ is strongly dependent on $W_{0}\left(t \sim W_{0}^{-5}\right)$ and the half-lives of allowed transitions will show a wide variation on this account.

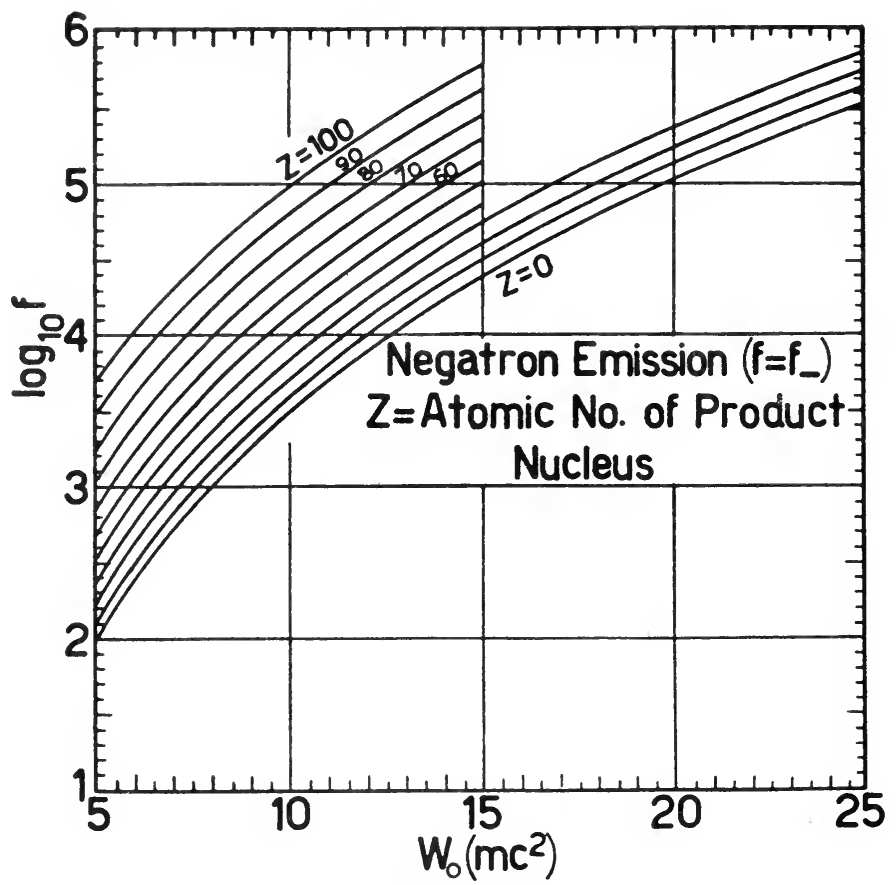

Fig. 3. $f$ for $\beta^{-}$emission, high energy scale (courtesy Feenberg and Trigg)

On the other hand the matrix elements $M_{F}$ and $M_{G T}$ should be of the same order of magnitude for all allowed transitions ${ }^{16}$. Therefore the characteristic quantity, or comparative half-life, is $f t$; that is

where, with $C_{P} \approx 0$,

$$
f t=\frac{B}{(1-x)\left|M_{F}\right|^{2}+x\left|M_{G T}\right|^{2}}
$$

$$
x=C_{G T}^{2}=1-C_{F}^{2}
$$

and

$$
B=\frac{2 \pi^{3} \ln 2}{g^{2}}=\frac{43}{g^{2}}
$$

are both universal constants. Thus, the $f t$ product may vary only because of the matrix elements.

If the matrix elements were known accurately then the measured $f t$ value determines a straight line in a $B-x$ diagram ${ }^{17}$. There would be a

16 Notable exceptions are $\mathrm{C}^{14}$ and $\mathrm{P}^{32}$, see $\mathrm{Ch}$. XI and XVI.

17 O. Kofoed-Hansen and A. Winther, Phys. Rev. 86 (1952) 428. 
separate line for each allowed transition and all these lines should intersect at a single point, giving the values of $x$ (relative strength of Gamow-Teller to Fermi interactions) and $B$ (thus $g$ ). Using the $j-j$ coupling model to evaluate the matrix elements ${ }^{17}$, it is found (see $\mathrm{Ch}$. XI) that the intersection points exhibit a spread but cluster around

$$
\left.\begin{array}{l}
x \sim 0.5-0.6 \\
g \sim 2.2 \times 10^{-49} \mathrm{erg} \mathrm{\textrm {cm } ^ { 3 }}
\end{array}\right\}
$$

where ordinary units are used for the coupling constant. Other models give somewhat larger values for $x$ but in all cases an appreciable amount of Fermi interaction is found 18 .

If the observed $f t$ values for allowed transitions are examined ${ }^{19}$ it is seen that they fall into two fairly distinct groups. The first may be referred

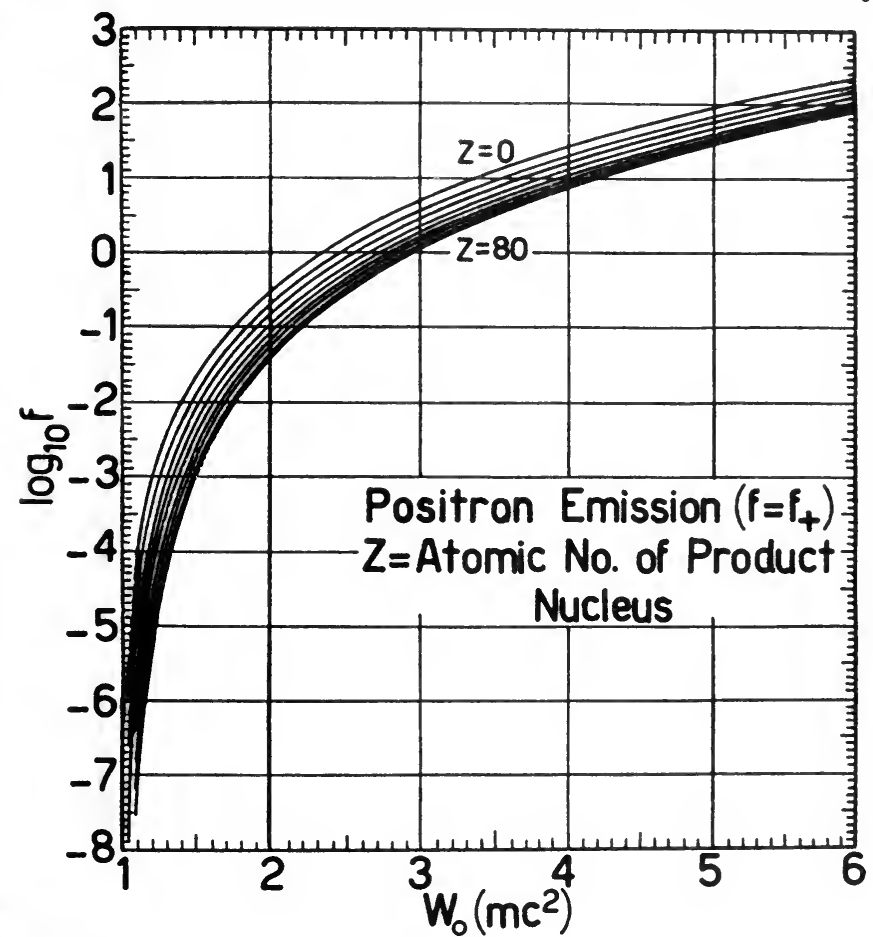

Fig. 4. $f$ for $\beta^{+}$emission, energy low scale (courtesy Feenberg and Trigg)

18 G. Trigg, Phys. Rev. 86 (1952) 506; J. M. Blatt, Phys. Rev. 89 (1953) 83.

19 M. G. Mayer, S. A. Moszkowski and L. W. Nordheim, Rev. Mod. Phys. 23 (1951) 315; L. W. Nordheim, ibid 23 (1951) 322. See also À. M. Feingold, ibid 23 (1951) 10. The so-called $l$-forbidden transitions, which otherwise conform to the allowed selection rules usually exhibit somewhat larger $f t$ values than those quoted in the text below. Due to the presence of such transitions there is some overlap of allowed and first forbidden $f t$ values. 
to as normal allowed and are characterized by $\log _{10} f t$ in the range 4.5 to 6.0 for odd mass nuclei and 4.0 to 5.7 for even mass nuclei. The second group, comprising the so-called super-allowed transitions, shows values of $\log _{10} f t$ closely clustered around 3.5. These are the mirror transitions $(N-Z= \pm \mathbf{l}$ for both initial and final nuclei, the neutron and tritium decay and the light positron emitters) and a group of transitions in which $N=Z$ for one member of the isobaric pair and $N-Z= \pm 2$ for the other. Examples of the latter group are $\mathrm{He}^{6} \rightarrow \mathrm{Li}^{6}, \mathrm{C}^{10} \rightarrow \mathrm{B}^{10 *}, \mathrm{O}^{14} \rightarrow \mathrm{N}^{14 *}, \mathrm{~F}^{18} \rightarrow \mathrm{O}^{18}$, $\mathrm{Al}^{26 *} \rightarrow \mathrm{Mg}^{26}, \mathrm{~K}^{38} \rightarrow \mathrm{A}^{38 *}$. The fact that the neutron decay belongs to

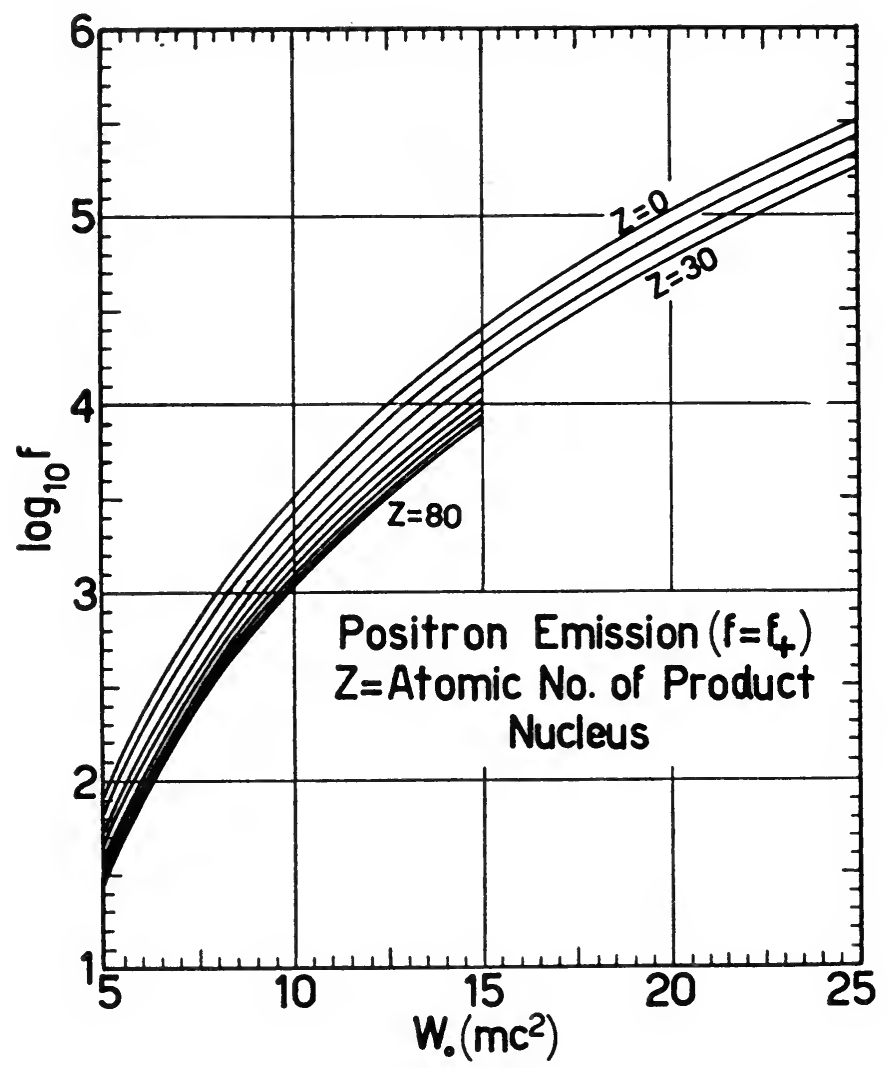

Fig. 5. $f$ for $\beta^{+}$emission, high energy scale (courtesy Feenberg and Trigg)

the super-allowed group is sufficient evidence that in this group the matrix elements have almost their maximum value; that is, the initial and final wave functions overlap strongly. This is easily understood for the light mirror nuclei as a consequence of the charge symmetry of the nuclear forces ${ }^{20}$.

20 See E. P. Wignel, Phys. Rev. 51 (1937) 106; ibid 56 (1939) 519. 
While the $f t$ value alone is not an infallible criterion on which a forbiddenness classification can be based, except in the case of super-allowed transitions, the $f t$ value plus a knowledge of the parity change should be decisive. Thus, if the parity change is "no" the ambiguity can be between only two orders of forbiddenness which differ by two units (allowed or second forbidden, say). Since the $f t$ values for normal allowed and second forbidden transitions differ by a factor of $10^{7}$ roughly, no confusion can arise. In addition, one does not generally expect to find the allowed shape spectrum for second forbidden transitions (Ch. X and XI).

\section{$\S 9$. Orbital Electron Capture}

The process of orbital electron capture may be detected by observation of the $x$-rays which are subsequently emitted. The energy release $W_{0}$ may be measured by observing the spectrum of the weak internal bremsstrahlung or by measuring $p-n$ thresholds if the residual nuclear state in

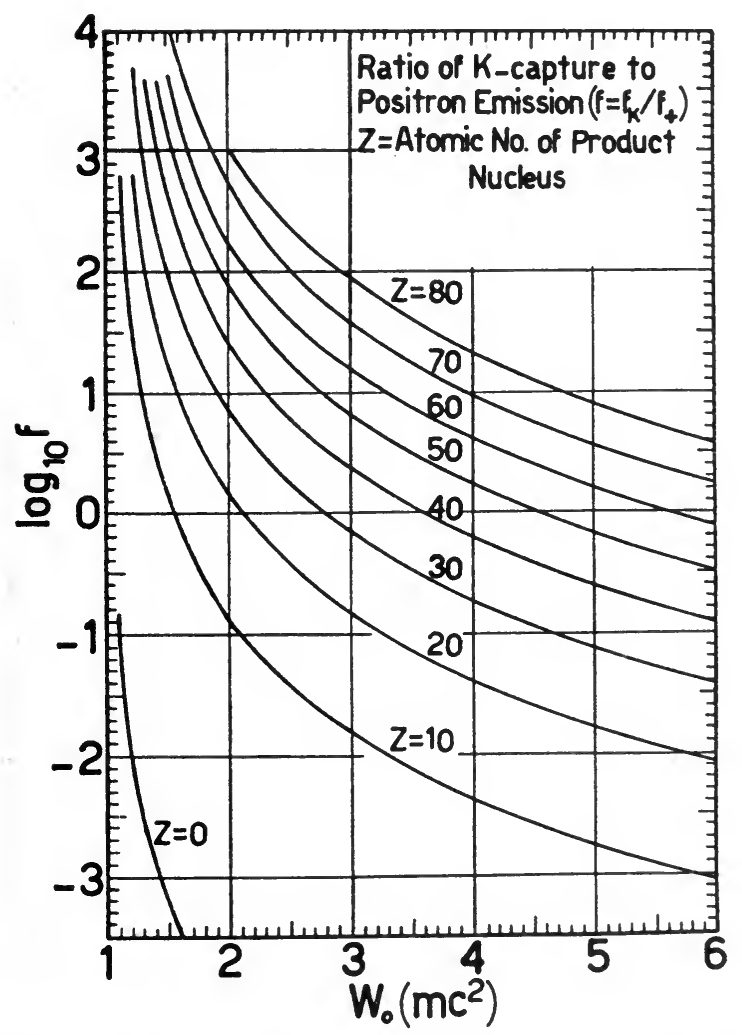

Fig. 6. $K / \beta^{+}$branching ratio, high energy scale (courtesy Feenberg and Trigg). The notation $f_{K}$ and $f_{+}$used by these authors is replaced by $\lambda_{K}$ and $\lambda_{+}$respectively in this section 
the $p-n$ reaction is the same as in the capture. Otherwise the $\gamma$-ray energy connecting the states must be added.

For allowed transitions capture of both $s_{\xi}$ or $p_{q}$ bound electrons is permitted. Of these the dominant process is $K$-capture. The decay constant for $K$-capture is

where

$$
\lambda_{K}=\frac{g^{2}}{2 \pi^{3}}|M|^{2} \frac{\pi}{2}\left(W_{0}+W_{K}\right)^{2} g_{K}^{2}(R)
$$

$$
|M|^{2}=\left(C_{S}^{2}+C_{V}^{2}\right)\left|M_{F}\right|^{2}+\left(C_{T}^{2}+C_{A}^{2}\right)\left|M_{G T}\right|^{2}
$$

and $W_{K}$ is the $K$-shell energy including rest mass; i.e., $W_{K}=1-\varepsilon_{K}$ where $\varepsilon_{K}$ is the $K$-shell binding energy. In (13) the large radial wave function $g_{K}$ occurs. If we neglect screening, which is a sufficiently good approximation for the $K$-shell

and $W_{K}=\gamma$.

$$
g_{K}^{2}(R)=\frac{1+\gamma}{2 \Gamma(2 \gamma+1)} R^{2 \gamma-2}(2 \alpha Z)^{2 \gamma+1}
$$

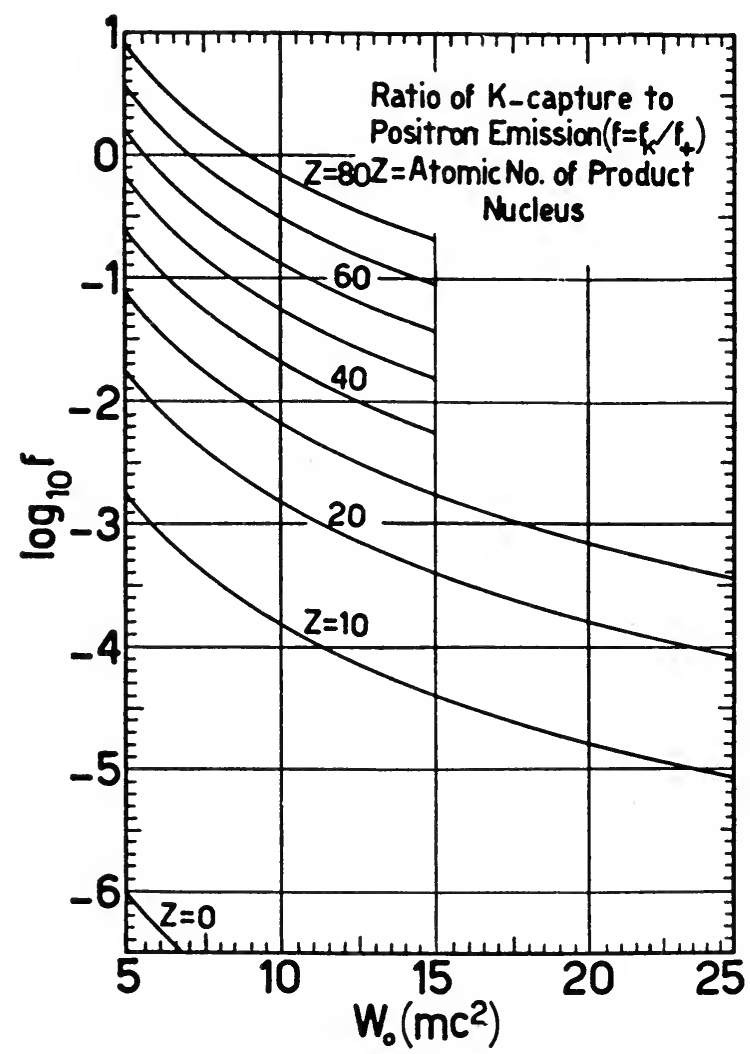

Fig. 7. $K / \beta^{+}$branching ratio, high energy scale (courtesy Feenberg and Trigg). The notation $f_{K}$ and $f_{+}$used by these authors is replaced by $\lambda_{K}$ and $\lambda_{+}$respectively in this section 
$L$-capture for allowed transitions can occur from the $L_{\mathrm{I}}$ or $L_{\mathrm{II}}$ subshells. Of these the $L_{\mathrm{I}}$ shell makes the major contribution. Of particular interest is the $L$ to $K$-capture ratio

$$
\lambda_{L} / \lambda_{K} \approx \lambda_{L_{\mathrm{I}}} / \lambda_{K}=\left(\frac{W_{0}+W_{L_{I}}}{W_{0}+W_{K}}\right)^{2} \frac{g_{L_{l}}^{2}(R)}{g_{K}^{2}(R)} .
$$

To neglect screening for the $L_{\mathrm{I}}$-subshell is not a very good approximation. Numerical results with screening taken into account have been given ${ }^{21}$ for the wave-function ratio $g_{L_{I}}^{2} / g_{K}^{2}$. In most cases $W_{0} \gg W_{K}, W_{0} \gg W_{L_{I}}$ so that this ratio can be taken as $\lambda_{L} / \lambda_{K}$.

When the atomic mass difference of initial and final states is greater than $2 m c^{2}=1.02 \mathrm{MeV}$ both positron emission and orbital capture occur. The branching ratio in this case is

$$
\lambda_{K} / \lambda_{+}=\lambda_{K} / \int_{1}^{W_{0}} N_{+}(W) d W
$$

for $K$-capture. Numerical results have been given by Feenberg and Trigg ${ }^{15}$ and these are presented in Figs. 6 and 7. The total decay constant of the initial state is obviously $\lambda=\lambda_{+}+\lambda_{K}=\lambda_{+}\left(1+\lambda_{K} / \lambda_{+}\right)$.

21 M. E. Rose and J. L. Jackson, Phys. Rev. 76 (1949) 1540. 


\title{
THE THEORY OF FORBIDDEN $\beta$-DECAY
}

\author{
E. KONOPINSKI
}

\section{$\S 1$. Introduction}

The theory of allowed $\beta$-decay was presented in Ch. IX. It was there seen that the allowed transitions can occur only between nuclear states of the same parity, and differing in spin by no more than one unit. When the energy is available for states which violate the allowed-transition selection rules, then a transition may still occur: a slower, "forbidden" transition. The allowed selection rules arose only after certain small effects were left out of consideration. These neglected effects become allimportant when their absence leads to a vanishing transition rate. They are responsible for the forbidden transitions, which are the primary concern of this chapter.

Forbidden transitions ( $\gamma$, as well as $\beta$ !) play a much more prominent role in nuclear than in atomic spectroscopy. This is principally because a large fraction of observed nuclear transitions do violate the allowed selection rules. Further, the expectations for forbidden $\beta$-transitions are more sensitive to differences in the formulation of the theory, than are those for allowed decay. Thus, decisions about the correct theory are helped by the study of the forbidden decays.

\section{$\S$ 2. The Effects Responsible for Forbidden Decays}

The small effects neglected in the allowed theory are of two kinds. The first are corrections needed to produce Lorentz invariance in the vector, tensor and pseudovector forms $\mathscr{H}_{\text {V.T,A }}$ [eq. (5), Ch. IX]. The second kind are effects arising from the variation of the lepton waves across the nucleus: the "retardation" effects, which were dropped when only the first term of the expansions in eq. (7), Ch. IX, were retained.

The complete, relativistically invariant interaction energy densities leading to negatron emission are:

$$
\begin{aligned}
& \mathscr{H}_{S}=g\left(\Psi_{f}^{*} \beta \Psi_{i}\right)\left(\psi^{*} \beta \varphi\right), \\
& \mathscr{H}_{V}=g\left[\left(\Psi_{f}^{*} \Psi_{i}\right)\left(\psi^{*} \varphi\right)-\left(\Psi_{f}^{*} \alpha \Psi_{i}\right) \cdot\left(\psi^{*} \alpha \varphi\right)\right], \\
& \mathscr{H}_{T}=g\left[\left(\Psi_{f}^{*} \beta \sigma \Psi_{i}\right) \cdot\left(\psi^{*} \beta \sigma \varphi\right)+\left(\Psi_{f}^{*} \beta \boldsymbol{\alpha} \Psi_{i}\right) \cdot\left(\psi^{*} \beta \alpha \varphi\right)\right], \\
& \mathscr{H}_{A}=g\left[\left(\Psi_{f}^{*} \sigma \Psi_{i}\right) \cdot\left(\psi^{*} \sigma \varphi\right)-\left(\Psi_{f}^{*} \gamma_{5} \Psi_{i}\right)\left(\psi^{*} \gamma_{5} \varphi\right)\right], \\
& \mathscr{H}_{P}=g\left(\Psi_{f}^{*} \beta \gamma_{5} \Psi_{i}\right)\left(\psi^{*} \beta \gamma_{5} \varphi\right),
\end{aligned}
$$

in the notation of Ch. IX. The complex conjugates of these terms should 
be added to take care of negatron absorption (or positron emission). The second terms of $\mathscr{H}_{v, T, A}$ are of order $\mathrm{v} / c$, if $\mathrm{v}$ is the nucleonic relocity, hence will be referred to as "source velocity effects". Some of their consequences can be seen immediately from comparisons with the allowed theory.

\section{A. Source Velocity Effects}

It was found that the rate of allowed decay depended on the nuclear "sources" of the $\beta$-radiation through the nuclear properties measured by the matrix elements $M_{F}$ and $M_{G T}$, of Ch. IX. By definition of selection rules, $M_{F}=M_{G T}=0$ when the allowed selection rules are violated. We shall be able to see by inspection, what new matrix elements arise when the velocity interactions are now included, and also what selection rules govern them.

Reference to $(9 \mathrm{e})$ of $\mathrm{Ch}$. IX shows that $M_{F}$ and $M_{G T}$ both have the form :

$$
M=\sum_{k} \int \Psi_{f}^{*} \Omega \Psi_{i} \equiv \int \Omega,
$$

with $\Omega=1$ in $M_{F}$ and $\Omega=\sigma$ in $M_{G T}$. The symbols like $\int \Omega$, eqs. $M_{F} \equiv \int 1$ and $M_{G T} \equiv \int \sigma$, prove conveniently economical when dealing with the many new matrix elements which it is necessary to introduce.

The origins of $\int 1 \equiv \sum \int \Psi_{f}^{*} \Psi_{i}$ and $\int \sigma \equiv \sum \int \Psi_{f}^{*} \sigma \Psi_{i}$ in the first terms of $\mathscr{H}_{V}$ and $\mathscr{H}_{A}$ respectively, are obvious from (1V) and $(1 A) . \mathscr{H}_{S}$ and $\mathscr{H}_{T}$ similarly give rise to $\int \beta \approx \int 1$ and $\int \beta \sigma \approx \int \sigma$, respectively. (The presence of the scalar $\beta$ has no effect on selection rules). Now one can expect that the velocity terms of $\mathscr{H}_{V, T, A}$ will lead, respectively, to $\int \alpha$, $\int \beta \alpha$ and $\int \gamma_{5}$. Moreover, $\mathscr{H}_{P}$, which did not contribute at all to the allowed transitions, will yield $\int \beta \gamma_{5}$, which may be expected to contribute whenever $\int \gamma_{5}$ does. It should be emphasized that the present, simple generalizations from the allowed results depend on the continued neglection, as in the allowed theory, of the retardation effects. Thus, $\int(\beta) \alpha$ and $\int(\beta) \gamma_{5}$ measure only the largest contributions of the velocity interactions.

The operator in $\int(\beta) \alpha$ is a vector as is the operator in the allowed matrix element $\int(\beta) \sigma$. This rotational covariance property is enough to determine the selection rules on the total nuclear angular momentum, J. Hence $\int(\beta) \alpha$ vanishes, just as $\int(\beta) \boldsymbol{\sigma}$ does, unless $\Delta J=0,1$, or if $J=0 \rightarrow J=0$ [see (8), Ch. IX]. However, $\alpha$ is a polar vector whereas $\sigma$ is an axial vector. The different behavior under reflection means that $\int(\beta) \alpha$ vanishes unless there is a parity change in the transition (denoted $\Delta \pi=-1$ ), whereas $\int(\beta) \sigma$ required that the parity not change $(\Delta \pi=+1)$ !! Similarly, the scalar operator in $\int(\beta)$ required $\Delta J=0, \Delta \pi=+1$, while the pseudoscalar in 
$\int(\beta) \gamma_{5}$ requires $\Delta J=0, \Delta \pi=-1$. Thus the velocity interactions may contribute to transitions which violate the allowed selection rules, and so generate forbidden transitions.

\section{B. Retardation Effects}

Next, we consider the second kind of neglection made in the allowed theory: that of the retardation effects. These will first be illustrated for the scalar coupling, $\mathscr{H}_{S}$. We omit coulomb effects on the electron since they do not affect the results here. The leptons are both represented by plane waves, so that

$$
\psi^{*} \beta \varphi \sim \exp [-i(\boldsymbol{p}+\boldsymbol{q}) \cdot \boldsymbol{r} / \hbar]
$$

where $\boldsymbol{p}$ and $-\boldsymbol{q}$ are the electron and neutrino momenta. $(+\boldsymbol{q}$ is then the momentum of the antineutrino which is ejected together with a negatron.) We consider all the terms in the power series expansion of (3) [see (7), Ch. IX], rather than only the unit first term as in the allowed theory. Now the allowed matrix element $\int \beta$ is replaced by:

$$
\left.\begin{array}{rl}
\sum_{n=0}^{\infty} \frac{1}{n !} \int \beta[-i & (\mathbf{p}+\boldsymbol{q}) \cdot \boldsymbol{r} / \hbar]^{n}= \\
& =\sum_{n} \sum_{i_{1}, i_{2}} \sum_{i_{n}=1}^{3} \frac{(-i / \hbar)^{n}}{n !}\left(p_{i_{1}}+q_{i_{1}}\right) \cdots\left(p_{i_{n}}+q_{i_{n}}\right) \int \beta x_{i_{1}} \ldots x_{i_{n}} \cdot
\end{array}\right\}
$$

We thus see that the $n$th retardation term introduces $n$ components $x_{1,2,3}$ of the moment arm vector, $r$, into the nuclear matrix element. This is accompanied by $n$ factors $(\boldsymbol{p}+\boldsymbol{q})_{1,2,3}$. The ratio of successive terms has the small order of magnitude: $|\boldsymbol{r}| /(n \hbar /|\boldsymbol{p}+\boldsymbol{q}|)$, with $|\boldsymbol{r}|$ limited by the nuclear radius.

\section{Once-Forbidden Transitions}

It is now clear that the largest retardation effect $(n=1)$ is governed by the matrix elements $\int \beta \boldsymbol{r}$ and $\int \boldsymbol{r}$, with the $\mathrm{S}$ and $\mathrm{V}$ interactions, respectively. These are analogous to the electric dipoles of electromagnetic theory. The T- and A-couplings yield instead: $\int \beta \sigma_{i} x_{j}$ and $\int \sigma_{i} x_{j}$, respectively. $\boldsymbol{r}$ is a polar vector like $\alpha$ and will lead to the same selection rules, already known for the electric dipole transitions of electromagnetic theory: $\int(\beta) r$ and $\int(\beta) \alpha$ both vanish unless $\Delta J=0,1 ; \Delta \pi=-1$ (and also if $J=0 \rightarrow J=0)$. The selection rules for $\int(\beta) \sigma_{i} x_{i}$ require further discussion.

A general product of two vectors like $\sigma$ and $\boldsymbol{r}$ has nine independent components $\sigma_{i} x_{j}$. Four of these are grouped as the scalar and vector products, $\boldsymbol{\sigma} \cdot \boldsymbol{r}$ and $\boldsymbol{\sigma} \times \boldsymbol{r}$. The remaining 5 form a symmetrical tensor of zero trace: $B_{i j}=\int(\beta)\left[\sigma_{i} x_{j}+\sigma_{j} x_{i}-(2 / 3) \delta_{i j}^{-} \sigma \cdot r\right]$, which has only 5 independent 
components because $B_{j i}=B_{i j}$ and $\sum B_{i i}=0 . \sigma \cdot \boldsymbol{r}$ is a pseudo-scalar, hence $\int(\beta) \boldsymbol{\sigma} \cdot \boldsymbol{r}$ obeys the same selection rules as $\int(\beta) \gamma_{5}(\Delta J=0, \Delta \pi=-1) \cdot \boldsymbol{\sigma} \times \boldsymbol{r}$ is a polar vector hence $\int(\beta) \boldsymbol{\sigma} \times \boldsymbol{r}$ obeys the same selection rules as $\int(\beta) \boldsymbol{r}$ and $\int(\beta) \alpha$ above. New rules govern $B_{i j}$; as might be expected, $B_{i j}=0$ unless: $\Delta J=0,1,2 ; \Delta \pi=-1$. Further, $B_{i j}=0$ for $0 \rightarrow 0, \frac{1}{2} \rightarrow \frac{1}{2}, 1 \leftrightarrow 0$ transitions. A mnemonic aid for the latter exceptions is to conceive the operation in $B_{i j}$ as adding an angular momentum vector of 2 units to the initial angular momentum, in accordance with quantum rules.

We now stress the fact that the largest velocity interactions and the largest retardation effects are active in the same class of transitions: $\Delta J=0,1 ; \Delta \pi=-1$. Nioreover, the matrix elements which measure the magnitudes may be of the same order: $v / c$ vs. $|\boldsymbol{r}| /(\hbar /|\boldsymbol{p}+\mathbf{q}|)$. The transitions governed by the matrix elements of this order are called once-forbidden. This includes $B_{i j}$, which also contributes to $\Delta J=2$ transitions and therefore $\Delta J=2$ also in once-forbidden transitions. The $\Delta J=2$ cases are sometimes treated separately as the first "unique" forbidden transitions.

We summarize the once-forbidden selection rules in relation to the matrix elements which determine the magnitudes:

$$
\begin{aligned}
& \begin{array}{ll}
\mathrm{S}, \mathrm{V}: & \int(\beta) \boldsymbol{r} \\
\mathrm{T}, \mathrm{V}: & \int(\beta) \boldsymbol{\alpha}
\end{array} \Delta J=0,1 ; \Delta \pi=-\mathrm{l} \\
& \begin{array}{ll}
\text { T, A: } & \int(\beta) \boldsymbol{\sigma} \times \boldsymbol{r}
\end{array} \quad(=0 \text { for } 0 \rightarrow 0) \\
& \mathrm{T}, \mathrm{A}: \quad B_{i j} \quad ; \quad \Delta J=0,1,2 \quad ; \quad \Delta \pi=-1 \\
& \text {. }\left(=0 \text { for } 0 \rightarrow 0, \frac{1}{2} \rightarrow \frac{1}{2}, 0 \leftrightarrow 1\right) \\
& \left.\begin{array}{ll}
\text { T, A: } & \int(\beta) \boldsymbol{\sigma} \cdot \boldsymbol{r} \\
\mathrm{P}, \mathrm{A}: & \int(\beta) \gamma_{\mathbf{5}}
\end{array}\right\} \quad \Delta J=0 \quad ; \quad \Delta \pi=-1
\end{aligned}
$$

Of course, $\beta$ enters into all the matrix elements due to $\mathrm{S}, \mathrm{T}, \mathrm{P}$-couplings and into none due to $\mathrm{V}$, A-couplings.

\section{Twice-Forbidden Transitions}

The retardation effects should also be applied to the velocity interactions, but will then yield more highly forbidden (intrinsically slower) transitions, than the once-forbidden ones. The largest retardation effect $(n=1)$ on the velocity interactions produces twice-forbidden transitions. Each successive retardation term of (4) raises the "degree of forbiddenness" by one.

New points are still to be learned before treating the general, $n$-times forbidden, case in the next section. They are adequately illustrated by twice-forbidden transitions generated by tensor coupling. 
Twice forbidden matrix elements are formed by inserting two powers of $x_{1.2 .3}$ into the allowed matrix element, $\int \beta \sigma$, and one power into the once-forbidden, velocity matrix element, $\int \beta \alpha$. The latter process yields:

$$
\int \beta \boldsymbol{\alpha} \cdot \boldsymbol{r}, \int \beta \boldsymbol{\alpha} \times \boldsymbol{r} \text { and } A_{i j}=\int \beta\left[\alpha_{i} x_{j}+\alpha_{j} x_{i}-(2 / 3) \delta_{i j}(\alpha \cdot \boldsymbol{r})\right] .
$$

The tensor $\int \beta \sigma_{i} x_{j} x_{k}$ is reducible into

and

$$
\begin{aligned}
& \int \beta \boldsymbol{\sigma} r^{2}, \int \beta(\boldsymbol{\sigma} \cdot \boldsymbol{r}) \boldsymbol{r}, \\
& T_{i j}^{\prime}=\int \beta\left[(\boldsymbol{\sigma} \times \boldsymbol{r})_{i} x_{j}+(\boldsymbol{\sigma} \times \boldsymbol{r})_{j} x_{i}\right],
\end{aligned}
$$

$$
B_{i j k}=\int \beta\left[\sigma_{(i i} x_{j} x_{k)}-\frac{1}{\delta} \delta_{(i j} x_{k)} \sigma \cdot r-\frac{q}{5} \delta_{(i j} \sigma_{k)} r^{2}\right] .
$$

Enclosure of the subscripts in parentheses signifies that there is a term for each permutation of the subscripts.

To be noticed now is that the new axial vectors $\int \beta \alpha \times r, \int \beta \sigma r^{2}$ and $\int \beta(\sigma \cdot r) r$ can only contribute under allowed selection rules, i.e. when the axial vector $\int \beta \sigma$ does. Hence, these new contributions will only be negligible corrections to the allowed transitions and may be discarded.

The case for discarding $\int \beta \boldsymbol{\alpha} \cdot \boldsymbol{r}$ is not as straightforward because it yields $0 \rightarrow 0$ transitions for which $\int \beta \sigma$ vanishes. However, the Fermi coupling (S or $\mathrm{V}$ ) is known (section 8) to be about as strong as the Gamow-Teller coupling $(\mathrm{T}$ or $\mathrm{A})$. Then the $0 \rightarrow 0$ transitions via the allowed $\mathrm{S}$ or $\mathrm{V}$ matrix element, $\int(\beta)$, overwhelm the $\int \beta \boldsymbol{\alpha} \cdot \boldsymbol{r}$ contribution.

We thus need to consider, for twice-forbidden transitions generated by the T-coupling, only the matrix elements labelled $A_{i j}, T_{i j}$ and $B_{i j k}$. $A_{i j}, T_{i j}$ vanish unless $\Delta J=0,1,2$ and $\Delta \pi=+1$; also in $0 \rightarrow 0, \frac{1}{2} \rightarrow \frac{1}{2}$, $0 \leftrightarrow 1$ transitions. $B_{i j k}$ requires $\Delta J=0,1,2,3$ and $\Delta \pi=+1$; it also vanishes for $0 \leftrightarrow 0, \frac{1}{2} \rightarrow \frac{1}{2}, 0 \leftrightarrow 1,0 \leftrightarrow 2,1 \rightarrow 1$ and $\frac{1}{2} \leftrightarrow \frac{3}{2}$ transitions. The $\Delta J=0,1$ cases are allowed transitions to which $A_{i j}, T_{i j}$ and $B_{i j k}$ make only negligible corrections. These matrix elements are actually needed only for the twiceforbidden transitions, $\Delta J=2,3$ and $\Delta \pi=+1$. It must be kept in mind that $B_{i j k}=0$ for $0 \leftrightarrow 2$ transitions even though $\Delta J=2$.

\section{§ 3. General Selection Rules and Matrix Elements}

The selection rules for the general, $n$-times forbidden transition, as well as the matrix elements"determining its magnitude, can now be presented.

First, it is clear that the successive degrees of forbiddenness alternate as to the requirement on the parity change. $\Delta \pi=(-)^{n}$ for an $n$-times forbidden transition.

Second, the Fermi couplings ( $\mathrm{S}$ or $\mathrm{V}$ ) contribute to $n$-times forbidden transitions with $\Delta J \leqslant n$; the Gamow-Teller couplings ( $\mathrm{T}$ or $\mathrm{A}$ ) for $\Delta J \leqslant n+1$; the pseudoscalar $(\mathrm{P})$ coupling for $\Delta J \leqslant n-1$. However, the 
matrix elements responsible are actually needed only for the largest of the spin changes here. None of them are needed for $\Delta J \leqslant n-2$ if $n>1$. They are then negligible corrections to the matrix elements responsible for $(n-2)$-times forbidden transitions. They are also not needed for $\Delta J=n-1$ if $n>1$. This is now obvious when the Gamow-Teller coupling acts. If only Fermi couplings existed. then the $n$th order matrix elements would also be needed for $\Delta J=n-\mathrm{l}$ in view of the parity rules. However. the existence of a large Gamow-Teller coupling is well-established (section $\$$ ). Accordingly, there are $n$-times forbidden transitions only tor

$$
\left.\begin{array}{ll}
\Delta J=n, n+1 ; \Delta \pi=(-)^{n} & {[n \neq 1]} \\
\Delta J=0,1,2 ; \Delta \pi=-1 & {[n=1]}
\end{array}\right\}
$$

The singularity of the $n=1$ case is of course due to the fact that no $(n-2)$ times forbidden transitions exist for $n=1$, hence no transitions to overwhelm the $\Delta J=n-\mathrm{l}=0$ contribution. The $\Delta J=n+\mathrm{l}$ cases are frequently referred to as the "unique" forbidden transitions.

The rules (6) comprise insufficient information. One also needs to know just which matrix elements contribute to a given case. We proceed to list them according to the interaction possibilities $S, T, P, V, A$. The present list will be complete for any $n$-times forbidden transition except $n=1$. The additional $n=1$ matrix elements are shown in the complete summary of the once-forbidden matrix elements in (5).

S-Coupling. This interacts with a unique nuclear moment at each degree of forbiddenness. The moment is formed by inserting $n$ powers of the moment arm components $x_{1,2,3}$ into the allowed matrix element $\int \beta$ ( $\equiv R_{0}$ here):

$$
R_{i_{1} i_{2} \ldots i_{n}}=\int \beta x_{i_{1}} x_{i_{3}} \ldots x_{i_{n}}-\ldots \quad\left(i_{s}=1.2 .3\right) .
$$

The unspecified terms - . . are those needed to insure that the traces will vanish. $R_{i}=\int \beta x_{i}$ is the $i$ th component of $\int \beta r$ in the $n=1$ list, (5).

$T$-Coupling. This acts on the following nuclear moments in an $n$-times forbidden transition:

$$
\begin{aligned}
B_{i_{1} \ldots i_{n+1}} & =\int \beta \sigma_{\left(i_{1}\right.} x_{i_{2}} \ldots x_{\left.i_{n+1}\right)}-\ldots \\
T_{i_{1} \ldots i_{n}} & =\int \beta[\boldsymbol{\sigma} \times \boldsymbol{r}]_{\left(i_{1}\right.} x_{i_{2}} \ldots x_{i_{n}}-\ldots, \\
A_{i_{1} \ldots i_{n}} & \left.=\int \beta \boldsymbol{x}_{\left(i_{1}\right.} x_{i_{2}} \ldots x_{i_{n}}\right) \ldots
\end{aligned}
$$

For $n=0, A_{0}=T_{0}=0$ and $B_{i}=\int \beta \sigma_{i}$. the allowed matrix element. For $n=1, A_{i}=\int \beta \alpha_{i}, T_{i}=\int \beta[\sigma \times \boldsymbol{r}]_{i}^{\prime}$; thus together with $B_{i j}$. the list is complete except for $\int \beta \boldsymbol{\sigma} \cdot \boldsymbol{r}$ [see (5)]. One should also remember that $B_{i_{1} \ldots i_{n+1}}=0$ for $J=0 \leftrightarrow J=n$ even though $\Delta J=n$. 
P-Coupling. The pseudoscalar coupling gives only negligible contributions in all but once-forbidden transitions, for which see (5). This peculiarity arises because the general $n$th degree matrix element, $\int \beta \gamma_{5} x_{i_{1}} \ldots x_{i_{n-1}}-\ldots$, vanishes unless $\Delta J=n-1$. It is then a negligible correction to Gamow-Teller contributions, which are only $(n-2)$-times forbidden if $n>1$. The consequence is that one can hope to detect the presence of P-coupling only in once-forbidden transitions with $\Delta J=0$.

$V$-Coupling. The polar vector coupling acts on the moments: $R_{i_{1} \ldots i_{n}}$ and $A_{i_{1} \ldots i_{n}}$, with $\beta \rightarrow 1$ [see $(7 \mathrm{~S})$ and $(7 \mathrm{~T})$ ].

$A$-Coupling. The axial vector coupling acts on: $S_{i_{1} \ldots i_{n+1}}$ and $T_{i_{1} \ldots i_{n}}$, with $\beta \rightarrow 1$ see (7T). The velocity interaction (1A) is effective only in once-forbidden transitions $\left[\int \gamma_{5}\right.$, see (5)], for the same reason that the P-coupling is detectable only for $n=1$.

\section{A. Spherical Representations}

Since the advent of the shell model, it has become important to express the matrix elements in terms of spherical harmonics. For this, it is significant that a reduced, symmetrical matrix element tensor of rank $n$ has just $2 n+1$ components, as does the spherical harmonic

$$
\boldsymbol{Y}_{n m}(m=0, \pm 1, \pm 2, \ldots, \pm n) .
$$

The relation between a given tensor component and the spherical harmonics forms an unwieldy expression, in general. We take advantage of the fact that, in actual results comparable to measurements, only certain spatially invariant combinations of tensors can occur. The invariants are formed by contracting two tensors of equal rank.

$$
\sum_{i_{1} \ldots i_{n}-1}^{3}\left|R_{i_{1} \ldots i_{n}}\right|^{2}
$$

will occur, as well as similar sums of squares formed from $S_{i_{1} \ldots i_{n+1}}, T_{i_{1} \ldots i_{n}}$, $A_{i_{1} \ldots i_{n}}$. There will also be terms proportional to invariants like

$$
\sum_{i_{1} \ldots i_{n}} R_{i_{1} \ldots i_{n}}^{*} T_{i_{1} \ldots i_{n}}+\text { c.c. }
$$

due to interference between radiations arising from the various nuclear moments. Only pairs formed from $R_{i_{1} \ldots i_{n}}, T_{i_{1} \ldots i_{n}}$ and $A_{i_{1} \ldots i_{n}}$ can interfere, since $B_{i_{1} \ldots i_{n+1}}$ has a different rank. The latter engages in no interferences which are not negligible.

When all the vectors which build up a tensor matrix element are identical, like the moment arm vectors $\boldsymbol{r}$ in (8), (7S), then the relation- 
ship to the usual, normalized spherical harmonics $Y_{l m}(y, \varphi)$ is simple:

$$
\sum\left|R_{i_{1} \ldots i_{n}}\right|^{2}=\frac{4 \pi n !}{(2 n+1) ! !} \sum_{m=-n}^{+n}\left|\int \beta r^{n} \boldsymbol{Y}_{m n}\right|^{2}
$$

if $(2 n+1) ! ! \equiv 1 \cdot 3 \cdot 5 \ldots(2 n+1)$.

The other matrix elements contain vectors $\boldsymbol{\sigma}$ or $\boldsymbol{\alpha}$, as well as $\boldsymbol{r}$. To handle these we follow the lead of Uhlenbeck and Falkoff ${ }^{1}$ by introducing the solid spherical harmonics:

$$
\mathscr{Y}_{l m}(\boldsymbol{r}) \equiv r^{l} \boldsymbol{Y}_{l m}(\vartheta, \varphi) \text {. }
$$

and also others formed from these by the process known as "polarization". This is merely the systematic substitution of a new vector, such as $\sigma$, for one of the $l$ powers of $\boldsymbol{r}$ implicit in $\mathscr{Y}_{l m}(\boldsymbol{r})$. We define:

$$
\mathscr{Y}_{l m}(\boldsymbol{\sigma})=(i / l \hbar) \boldsymbol{\sigma} \cdot \boldsymbol{p} \mathscr{Y}_{l m}(\boldsymbol{r})
$$

where $\boldsymbol{p}=(\hbar / i) \boldsymbol{\nabla}$ is the momentum operator. Obviously, the operation $\sigma_{i} \partial / \partial x_{i}$ removes $x_{i}$ from $\mathscr{Y}_{l m}(\boldsymbol{r})$ and substitutes $\sigma_{i}$ in its place. A few examples are:

$$
\begin{aligned}
& \mathscr{Y}_{00}(r)=(4 \pi)^{-t} \\
& y_{00}(\sigma)=(4 \pi)^{-1} \\
& \mathscr{Y}_{10}(\boldsymbol{r})=(3 / 4 \pi)^{\natural} z \\
& \mathscr{Y}_{10}(\sigma)=(3 / 4 \pi)^{\sharp} \sigma_{z} \\
& \mathscr{Y}_{1 \pm 1}(\boldsymbol{r})=\mp(3 / 2 \pi)^{\mathbf{t}} x_{ \pm} \\
& \mathscr{Y}_{1 \pm 1}(\sigma)=\mp(3 / 2 \pi)^{k} \sigma_{\text {土土 }} \\
& \mathscr{Y}_{20}(\boldsymbol{r})=(5 / 16 \pi)^{\mathfrak{1}}\left(3 z^{2}-r^{2}\right) \\
& \mathscr{Y}_{20}(\boldsymbol{\sigma})=(5 / 16 \pi)^{\ddagger}\left(3 \sigma_{z} z-\boldsymbol{\sigma} \cdot \boldsymbol{r}\right) \\
& \mathscr{Y}_{2 \pm 1}(\boldsymbol{r})=\mp(15 / 2 \pi)^{\sharp} z x_{ \pm} \\
& \mathscr{Y}_{2 \pm 1}(\sigma)=\mp(15 / 8 \pi)^{\sharp}\left(\sigma_{z} x_{ \pm}+\sigma_{ \pm} z\right) \\
& \mathscr{Y}_{2 \pm 2}(\boldsymbol{r})=(15 / 2 \pi)^{\mathbf{t}} x_{ \pm}^{2} \\
& \mathscr{Y}_{2 \pm 2}(\sigma)=(15 / 2 \pi)^{\sharp} \sigma_{ \pm} x_{ \pm}
\end{aligned}
$$

Here, $x_{ \pm} \equiv \frac{1}{2}(x \pm i y)$ and $\sigma_{ \pm} \equiv \frac{1}{2}\left(\sigma_{x} \pm i \sigma_{y}\right)$.

Now, one can write:

$$
\begin{gathered}
\sum\left|B_{i_{1} \ldots i_{n+1}}\right|^{2}=\frac{4 \pi[(n+1) !]^{3}}{(2 n+3) ! !} \sum_{m=-4 n+1)}^{n+1}\left|\int \beta \mathscr{Y}_{n+1, m}(\boldsymbol{\sigma})\right|^{2}, \\
\sum\left|T_{i_{1} \ldots i_{n}}\right|^{2}=\frac{4 \pi[n !]^{3}}{(2 n+1) ! !} \sum_{m=-n}^{n}\left|\int \beta \mathscr{Y}_{n m}(\boldsymbol{\sigma} \times \boldsymbol{r})\right|^{2}, \\
\sum\left|A_{i_{1} \ldots i_{n}}\right|^{2}=\frac{4 \pi[n !]^{3}}{(2 n+1) ! !} \sum\left|\int \beta \mathscr{Y}_{n m}(\boldsymbol{\alpha})\right|^{2}, \\
\sum T_{i_{1} \ldots i_{n}}^{*} A_{i_{1} \ldots i_{n}}=\frac{4 \pi[n !]^{3}}{(2 n+1) ! !} \sum\left[\int \beta \mathscr{Y}_{n m}(\boldsymbol{\sigma} \times \boldsymbol{r})\right]^{*}\left[\int \beta \mathscr{Y}_{n m}(\boldsymbol{\alpha})\right], \\
\sum R_{i_{1} \ldots i_{n}}^{*} A_{i_{1} \ldots i_{n}}=\frac{4 \pi[n !]^{2}}{(2 n+1) ! !} \sum\left[\int \beta \mathscr{Y}_{n m}(\boldsymbol{r})\right]^{*}\left[\int \beta \mathscr{Y}_{n m}(\boldsymbol{\alpha})\right] . \\
\sum R_{i_{1} \ldots i_{n}}^{*} T_{i_{1} \ldots i_{n}}=\frac{4 \pi[n !]^{2}}{(2 n+1) ! !} \sum\left[\int \beta \mathscr{Y}_{n m}(\boldsymbol{r})\right]^{*}\left[\int \beta \mathscr{P}_{n m}(\boldsymbol{\sigma} \times \boldsymbol{r})\right] .
\end{gathered}
$$

1 D. L. Falkoff and G. E. Uhlenbeck. Phys. Rev. 79 (1950) 3:2:3. 
To form $\mathscr{Y}_{l m}(\sigma \times \boldsymbol{r})$, one replaces the vector $\sigma$ in (12) with $\sigma \times \boldsymbol{r}$. It then follows that

$$
\mathscr{Y}_{l m}(\boldsymbol{\sigma} \times \boldsymbol{r})=(i / l \hbar) \boldsymbol{\sigma} \cdot \boldsymbol{L} \mathscr{Y}_{l m}(\boldsymbol{r}),
$$

where $L=[\boldsymbol{r} \times \boldsymbol{p}]$ is the orbital angular momentum operator, hence $\boldsymbol{\sigma} \cdot \boldsymbol{L}$ yields spin-orbit coupling.

In actual calculations of nuclear matrix elements for given nuclear states, it is useful to relate $\mathscr{Y}_{l m}(\sigma)$ and $\mathscr{Y}_{l m}(\alpha)$ to the ordinary spherical harmonics. One has:

$$
\left.\begin{array}{r}
\mathscr{Y}_{l m}(\boldsymbol{\sigma})=\frac{r^{l-1}}{l} \mid \frac{\overline{2 l+1}}{2 l-1}\left\{l^{\prime}\left(l^{2}-m^{2}\right) Y_{l-1, m} \sigma_{z}-l^{\prime}(l+m)(l+m-1) Y_{l-1, m-1} \sigma_{+}\right. \\
\left.+V^{\prime}(l-m)(l-m-1) Y_{l-1, m+1} \sigma_{-}\right\} .
\end{array}\right\}
$$

The coefficients here are just the Clebsch-Gordon coefficients which are needed to express states with a given total angular momentum $\boldsymbol{j}=\boldsymbol{L}+\mathbf{s}$ and spin (s) one-half: $\psi_{j m}^{l}$. Actually, if one operates with $\mathscr{Y}_{l m}(\sigma)$ on a nonrelativistic spin state $\chi_{ \pm 1}$ (initial orbital angular momentum zero):

$$
\mathscr{Y}_{l m}(\sigma) \chi_{ \pm \downarrow}= \pm l^{-1} V^{\prime}(l \mp m)(2 l+1) r^{l-1} \psi_{l-1, m \pm l}^{l-1} \text {. }
$$

The only final state not orthogonal to this is $\psi_{l-1, m \pm k}^{l-1}$, hence $(l-1)$ units of orbital momentum are carried off by the leptons because of the interaction represented by $\mathscr{Y}_{l m}(\sigma)$.

For $\mathscr{Y}_{l m}(\alpha)$, one merely substitutes $\alpha$ for $\sigma$ in $(20)$. $\mathscr{Y}_{l m}(\sigma \times r)$ is perhaps in its most useful form when written:

with $s^{2}=3 \hbar^{2} / 4$.

$$
\mathscr{Y}_{l m}(\boldsymbol{\sigma} \times \boldsymbol{r})=\left(i r^{l} / l \hbar^{2}\right)\left(j^{2}-L^{2}-s^{2}\right) Y_{l m}(\vartheta, \varphi),
$$

We finally summarize ${ }^{2}$ the nuclear moments responsible for $n$-times forbidden transitions, together with the spin changes $\Delta J$ for which each moment is not negligible. The summary is incomplete for $n=1$, for which case the complete summary (5) should be consulted.

$$
\left.\begin{array}{c}
\mathrm{S}, \mathrm{V}: \int(\beta) \mathscr{Y}_{n m}(\boldsymbol{r}) \quad ; \Delta J=n \\
\mathrm{~T}, \mathrm{~V}: \int(\beta) \mathscr{Y}_{n m}(\boldsymbol{\alpha}) ; \Delta J=n \\
\mathrm{~T}, \mathrm{~A}: \int(\beta) \mathscr{Y}_{n m}(\boldsymbol{\sigma} \times \boldsymbol{r}) ; \Delta J=n \\
\mathrm{~T}, \mathrm{~A}: \int(\beta) \mathscr{Y}_{n+1 . m}(\boldsymbol{\sigma}) ; \Delta J=n, n+1 \\
=0 \text { for } J=0 \leftrightarrow J=n \\
{[P: n=1 \text { only, see }(5)]}
\end{array}\right\}
$$

The operator $\beta$ is to be omitted in all $\mathrm{V}$ and $\mathrm{A}$ moments.

2 For comparison with previous tabulations, the following relationships among notations are useful. Our tensor notation followed closely the original one of G. E. Uhlenbeck and E. J. Konopinski, Phys. Rev. 60 (1941) 308. However, we have written $B_{i j k}$ in place of their $S_{i j k}$. E. Greuling, Phys. Rev. 61 (1942) 568, writes: $R_{i_{1} \ldots i_{n}}=[n !]^{-1} Q_{n}\left(\beta_{\boldsymbol{r}}, \boldsymbol{r}\right), S_{i_{1} \ldots i_{n}}=Q_{n}(\beta \boldsymbol{\sigma}, \boldsymbol{r}), T_{i_{1} \ldots i_{n}}=Q_{n}(\beta \boldsymbol{\sigma} \times \boldsymbol{r}, \boldsymbol{r}), A_{i_{1} \ldots i_{n}}=Q_{n}(\beta \boldsymbol{\alpha}, \boldsymbol{r})$. 
The presence of the operator $\beta$ in the nuclear matrix elements will make a practical difference only in those which also contain $\alpha$ or $\gamma_{5}$, with which $\beta$ does not commute. In all others, a non-relativistic treatment of the nucleons is adequate, and then $\beta \approx 1$.

For later economies of presentation, it will be advantageous to introduce the real ${ }^{3}$ parameters $\xi_{n}, \eta_{n}$ defined by:

$$
\left.\begin{array}{l}
\int(\beta) \mathscr{Y}_{n m}(\boldsymbol{r})=-i \xi_{n} \int(\beta) \mathscr{Y}_{n m}(\boldsymbol{\sigma} \times \boldsymbol{r}), \\
\int(\beta) \mathscr{Y}_{n m}(\boldsymbol{\alpha})=\eta_{n}(\alpha Z / 2 \varrho) \int(\beta) \mathscr{Y}_{n m}(\boldsymbol{\sigma} \times \boldsymbol{r}),
\end{array}\right\}
$$

where $\alpha Z / \varrho$ is the coulomb potential energy (units of $\mathrm{mc}^{2}$ ) of an electron at the nuclear radius $\varrho$ (units of $\hbar / m c$ ). It has been shown ${ }^{3}$ that $\xi_{n}, \eta_{n}$ are independent of $m$.

\section{$\S$ 4. Spectra and Half-Lives}

\section{A. Spectra}

The electron (positron or negatron) spectrum for an $n$-times forbidden transition can always be written:

$$
N_{ \pm}(W) d W=\left(g^{2} / 2 \pi^{3}\right) F_{0}(\mp Z, W) p W\left(W_{0}-W\right)^{2} \cdot S_{n}(W)
$$

in the notation ${ }^{4}$ used for the allowed spectrum, eq. (10) of Ch. IX. $S_{n}$ will be called 5 the "shape factor" for reasons which appear immediately.

Comparison with the allowed spectrum shows that

$$
S_{0}=\frac{1}{2}(1+\gamma)\left[C_{S, V}^{2}\left|\int 1\right|^{2}+C_{T, A}^{2}\left|\int \sigma\right|^{2}\right],
$$

if we take $C_{S} C_{V}=C_{T} C_{\Delta}=0$, as indicated by the experiments (see Ch. IX and also section 8). Whenever $S_{n}$ happens to be independent of the energy $W$, as in (26), then the spectrum has the "statistical shape" $\sim p W\left(W_{0}-W\right)^{2}$, modified only by the Coulomb effect $\sim F(\mp Z, W)$. In general, $S_{n}$ will depend on the energy and a departure from the statistical shape will be expected.

The statistical shape is associated with a linear Kurie plot [eq. (12) of Ch. IX]. In general, however, one expects deviation from linearity according to

$$
\left[N_{ \pm}(W) / F_{0}(\mp Z, W) p W\right]^{k} \sim\left(W_{0}-W\right)\left[S_{n}(W)\right]^{\ddagger} .
$$

s Shown by considerations such as those of C. L. Longmire and A. M. L. Messiah, Phys. Rev. 83 (1951) 464.

- Because of existing tabulations (see footnote 7), $F_{0}^{-}$here is defined as the $\dot{F}(\mp Z, W)$ of $\mathrm{Ch}$. IX divided by the usually trivial factor $(1+\gamma) / 2\left(\approx L_{0}\right)$.

5 Called merely "correction factor" in much of the literature. Moreover the symbol $C_{n}$ is more frequently used. We change to $S_{n}$ in order to avoid possible confusion with the coupling coefficients $C_{S, V, T, A, P}$ introduced in Ch. IX. 
The usual procedure in testing a measured spectrum $N_{ \pm}(W)$, as to whether it is governed by a given shape factor $S_{n}$, is to plot

$$
\left[N_{ \pm}(W) / S_{n} F_{0} p W\right]^{k} \sim\left(W_{0}-W\right)
$$

i.e. one tries to linearize the spectrum with an appropriate choice of theoretical shape factor.

We shall present in detail only the shape factors resulting from combinations of S, T, P-couplings. The experimental evidence indicates that $C_{V}=C_{A}=0$ (Section 8). Remarks about the V, A-couplings will nevertheless. be added, in subsequent sections, in order to make the evidence understandable. Details of the V, A-couplings can be found in the original papers ${ }^{2.6}$.

The general, $n$-times forbidden shape factor can be written as the sum of two partial ones:

$$
S_{n}=S_{n}^{(n)}+S_{n}^{(n+1)}
$$

The first of these contributes to $\Delta J=n$ but not $\Delta J=n+1$ transitions. The second of these is the total shape factor for the "unique" forbidden transitions, $\Delta J=n+1$. Its presentation will be delayed to the next section, where the "unique" transitions will be discussed in detail. $S_{n}^{(n+1)}$ contributes to $\Delta J=n$ transitions also (except $J=0 \rightarrow J=n$ ), but as we shall see in section 5 , its contribution is negligible if the coulomb energy $\alpha Z / \varrho$ is much greater than the maximum kinetic energy, $W_{0}-1$. The latter condition is fulfilled for almost all the observed cases of forbidden transitions.

Further, (29) is incomplete for once-forbidden transitions; it must then be supplemented with $S_{1}^{(0)}$, which contributes only to the $\Delta J=0$, parity change transitions and will be presented in section 6 . Here we present only $S_{n}^{(n)}$, which is primarily responsible for the $\Delta J=n$ transitions :

$$
\left.\begin{array}{l}
S_{n}^{(n)}=[4 \pi n ! /(2 n+1) ! !] \sum\left|\int \beta \mathscr{Y}_{n m}(\boldsymbol{\sigma} \times \boldsymbol{r})\right|^{2} \times \\
\times\left\{C_{T}^{2} P_{n}(\boldsymbol{\sigma} \times \boldsymbol{r})+\eta_{n}^{2}(\alpha Z / 2 \varrho)^{2} C_{T}^{2} P_{n}(\boldsymbol{\alpha})+\xi_{n}^{2} C_{S}^{2} P_{n}(\boldsymbol{r})\right. \\
\left.+\eta_{n}(\alpha Z / \varrho) C_{T}^{2} I_{n}(\boldsymbol{\sigma} \times \boldsymbol{r}, \boldsymbol{\alpha}) \mp 2 \xi_{n} C_{S} C_{T} I_{n}(\boldsymbol{\sigma} \times \boldsymbol{r}, \boldsymbol{r}) \mp \xi_{n} \eta_{n}(\alpha Z / \varrho) C_{S} C_{T} I_{n}(\boldsymbol{\alpha}, \boldsymbol{r})\right\} .
\end{array}\right\}
$$

As in Ch. IX, the upper sign refers to positron, the lower to negatron, emission. The functions $P_{n}$ represent radiations by the individual, 'pure'

6 D. L. Pursey, Phil. Mag. 42 (1951) 1193 and A. Smith, Phys. Rev. 82 (1951) 955 present the interference effects. Smith's version of the TP interference is the correct one. 
moments, whereas the functions $I_{n}$ are the results of interference between the radiations.

$$
\begin{aligned}
& P_{n}(\alpha)=\sum_{v=0}^{n} B_{n v} q^{2(n-v)-2}\left\{(n-v)(2 n-2 v+1) L_{v}\right\} . \\
& P_{n}(\boldsymbol{r})=\quad " \quad\left\{(n-v)(2 n-2 v+1) M_{v}+2(n-v) q N_{v}+\frac{v+1}{2 v+1} q^{2} L_{v}\right\} . \\
& \left.P_{n}(\sigma \times \boldsymbol{r})=\quad, \quad \quad \quad \quad \quad-2(n-v) q N_{v}+\frac{n(v+1)-v}{(n+1)(2 v+1)} q^{2} L_{v}\right\} . \\
& I_{n}(\boldsymbol{\sigma} \times \boldsymbol{r}, \boldsymbol{r})=, \quad, \quad\left\{\quad-\frac{v}{2 v+1} q^{2} L_{v}\right\} . \\
& I_{n}(\alpha, \boldsymbol{r})=\quad, \quad\left\{(n-v)(2 n-2 v+1) N_{\nu}+(n-v) q L_{\nu}\right\} . \\
& I_{n}(\boldsymbol{\sigma} \times \boldsymbol{r}, \boldsymbol{\alpha})=\tilde{I}_{n}(\boldsymbol{\alpha}, \boldsymbol{r}) \text {, i.e. } I_{n}(\boldsymbol{\alpha}, \boldsymbol{r}) \text { with the sign of } q \text { changed. }
\end{aligned}
$$

The constants $B_{n v}$ are Greuling ${ }^{2}$ coefficients:

$$
B_{n v}=(2 v+1) ! 2^{n-2 v} /(v !)^{2}(2 n-2 v+1) !
$$

The formidability of evaluating these expressions has been practically eliminated by the work of Rose, Perry and Dismuke ${ }^{7}$, who have constructed exhaustive tables of the functions $L_{v}, M_{\nu}$ and $N_{v}$ for $v=0,1,2,3$. They are functions of $Z$ and $p$ only. These tables are included in the Appendix III.

The influence of $S_{n}^{(n)}$ on spectral shapes is not easily seen from the formal expression (30). A fair idea can be obtained when $\alpha Z / \varrho \gg W_{0}-1$ can be assumed. Then:

$$
\left.\begin{array}{r}
S_{n}^{(n)} \approx[4 \pi n ! /(2 n+1) ! !] \sum\left|\int \beta \oiint_{n n}(\sigma \times \mathbf{r})\right|^{2} \cdot 2^{n}(x Z / 2 Q)^{2} \times \\
\left.\times \sum_{v=0}^{n} \frac{(n-v)\left[C_{T}-(v+1) \eta_{n} C_{T} \mp \xi_{n} C_{s}\right]^{2}}{(2 n-2 v) !(2 v+1) !(v+1)^{2}} q^{2(n-v)-2}\right)^{2 v} .
\end{array}\right\}
$$

Thus, the energy dependence becomes simply a homogeneous polynomial, of degree $(n-1)$, in $p^{2}=W^{2}-1$ and $q^{2}=\left(W_{0}-W\right)^{2}$.

\section{B. HaLf-Lives}

The decay constant $\lambda$ and the half-life $t$ are in general given by:

$$
\lambda=(\ln 2) / t=\left(g^{2} / 2 \pi^{3}\right) \int_{1}^{W_{0}} d W F_{0} p W\left(W_{0}-W^{\prime}\right)^{2} S_{n}(W) .
$$

For $S_{n} \rightarrow \frac{1}{2}(1+\gamma)$, the integral here becomes the standard function $f\left(\mp Z, W_{0}\right)$ presented in (14), Ch. IX. Thus the "comparative half-life", $f t$, is in general:

$$
(f t)_{n}=\left(2 \pi^{3} \ln 2 / g^{2}\right)\left\langle S_{n}\right\rangle^{-1}
$$

7 M. E. Rose, C. L. Perry and N. M. Dismuke, Oak Ridge Nat. Lab., Report No. 1459 (1953). 
where

$$
\left\langle S_{n}\right\rangle=f^{-1} \int_{1}^{W_{0}} d W F_{0} p W\left(W_{0}-W^{\prime}\right)^{2} S_{n}
$$

is the statistical spectrum average of $S_{n}(W)$. The shape factor $S_{n}$ is usually the most sensitive to the degree of forbiddenness, $n$. Hence, one expects $f t$-values which are calculated from experimental energies and half-lives to be roughly separable into groups according to $n=0,1,2,3 \ldots$ More refined examinations of experimental $f t$-values. which consider individual nuclear moments, can be found in C'h. XVI.

\section{$\S 5$. The „Unique” Forbidden Transitions}

\section{A. Spectra}

The transitions with $\Delta J=n+1$, which arise only from Gamow-Teller coupling $\mathrm{T}$ (or $\mathrm{A}$ ), each depend on just one nuclear moment, $\int(\beta) \mathscr{Y}_{n+1, m}(\boldsymbol{\sigma})$, according to (23). A consequence is that the shape factor $S_{n}^{(n+1)}$ then contains the moment merely as a proportionality factor, and an unambiguous energy dependence is predictable.

The origin of the energy dependence is readily understood. The responsible moment was formed, as seen in sections 2 and 3 , by inserting $n$ powers of the moment arm $\boldsymbol{r}$ into the allowed matrix element $\int(\beta) \boldsymbol{\sigma}$. This comes from the $n$th retardation term (4). Hence, the moment will be multiplied by $n$ powers of the lepton momentum $(\boldsymbol{p}+\boldsymbol{q})$. Now, it is not surprising that $S_{n}^{(n+1)} \sim a_{n}=\left\langle\mid \boldsymbol{p}+\mathbf{q}^{\mid 2 n}\right\rangle$, where $a_{n}$ is the indicated average over all directions of the electron and neutrino momenta $\boldsymbol{p}, \boldsymbol{q}$.

$$
S_{n}^{(n+1)} \sim a_{n}=\sum_{\nu=0}^{n} \frac{(2 n+1) !}{(2 v+1) !\left(2 n-21^{\prime}+1\right) !} p^{2 v} q^{2(n-v)},
$$

where $q=W_{0}-W$ is the neutrino energy (= momentum in the present units). This is a homogeneous polynomial, of degree $n$, in $p^{2}$ and $q^{2}$ [compare (31)].

About 20 cases of $\Delta J=2$ transitions with parity change have been found, all having measured spectra which could be linearized [see (28)] with the help of

$$
a_{1}=p^{2}+q^{2} \text {. }
$$

The spectrum of $\mathrm{Be}^{10}$ ( $\Delta J=3$ and no parity change) was found to be linearized by

$$
a_{2}=p^{4}+q^{4}+(10 / 3) p^{2} q^{2} .
$$

Finally, $\mathrm{K}^{\mathbf{4 0}}(\Delta J=4, \Delta \pi=-1)$ yielded a linear Kurie plot after correction by

$$
a_{3}=p^{6}+q^{6}+7 p^{2} q^{2}\left(p^{2}+q^{2}\right) .
$$

Thus the "unique" forbidden spectra have been checked for $n=1,2,3$. This is one of the two strongest legs of evidence for the existence of 
Gamow-Teller coupling; the other consists of the short (i.e. allowed) lives of many $\Delta J=1$ transitions with no parity change.

The expressions (34) for the shape factor are modified somewhat when the coulomb effect on the electron is taken into account:

$$
a_{n}=\sum_{v=0}^{n} \frac{(2 n+1) !(2 v+1) !}{2^{22}\left(v^{2} !\right)^{2}(2 n-2 v+1) !} q^{2(n-v)} L_{\nu}(p, \mp Z)
$$

where $L_{v}$ is a tabulated ${ }^{7}$ function. The coulomb effect here is far less important than in the $\Delta J=n$ transitions. This is indicated by the absence of the factor $(\alpha Z / \varrho)^{2}$ in (34), contrasting with its presence in the usually largest terms of $S_{n}^{(n)}(31)$; yet (34), like (31) is valid when the coulomb effect is strongest. This circumstance is due to the extra unit of orbital momentum which is radiated in the $\Delta J=n+1$ transitions [see eq. (21), and (7) of Ch. IX]. The consequent centrifugal force helps keep the electron out of the range of the strongest coulomb effects.

\section{B. Half-Lives}

The proportionality $S_{n}^{(n+1)} \sim a_{n}$ is adequate for discussions of energy dependence but the factor of proportionality is needed for the half-lives:

$$
S_{n}^{(n+1)}=C_{T(A)}^{2} \cdot \frac{4 \pi(n+1) !}{(2 n+3) ! !} \cdot \frac{2^{n}}{(2 n+1) !} \cdot \sum_{m}\left|\int \beta \mathscr{Y}_{n+1, m}(\sigma)\right|^{2} \cdot a_{n}
$$

The statistical spectrum average $\left\langle a_{n}\right\rangle$ will be needed for the half-life, according to (33). The general expression for $\left\langle a_{n}\right\rangle$ is extremely unwieldy and it is easier to evaluate it by numerical integration for any particular case. A very rough value is $\left\langle a_{n}\right\rangle \approx\left(p_{0}^{2} / 2\right)^{n} \equiv\left(W_{0}^{2}-1\right)^{n} / 2^{n}$.

One can now say from (33), that experimental values of $\left(W_{0}^{2}-1\right)^{n} f t$ should show more constancy for a given $n$ than do the $f t$-values themselves. This is well borne out by the known group of once-forbidden cases $(n=1)$. For those, $f t$ varies by roughly a factor 500 , whereas $\left(W_{0}^{2}-1\right) f t$ varies by only a factor 30 or so. Exceptions to both ranges of variation are not correlated. Of course, absolute constancy cannot be expected because variations in the individual nuclear moments may occur, aside from the rough value of $\left\langle a_{1}\right\rangle$ being used. Even in the allowed $(n=0)$ case one has variations of $f t$ by a factor 60 or so among normal (unfavored) transitions.

It is also interesting to see whether the huge variation with $n$, predicted by (36), is borne out. To treat this we make the reasonable assumption that $\left.\sum \mid \int(\beta) \mathscr{Y}_{n+1, m} \sigma\right)\left.\right|^{2} \sim \varrho^{2 n}$, with $\varrho$ the nuclear radius, is the main factor by which the moments vary. With this,

$$
(f t)_{n} /(f t)_{0} \approx[(2 n+1) !(2 n+3) ! ! / 3(n+1) !]\left(p_{0} \varrho\right)^{-2 n} .
$$


Experimentally, $\log \eta_{0}^{2}(f t)_{1}-\log (f t)_{0}$ is roughly 3.6 to 6.5 . This formula yields about 5 for the same number. Comparing $\operatorname{Be}^{10}(n=2)$ against typical $n=1$ cases gives an experimental ratio $(f t)_{2} / p_{0}^{2}(f t)_{1} \approx 10^{4}$. The above formula gives $\approx 3(10)^{5}$, which is amended to $4(10)^{4}$ when cognizance is taken of the weight factor $\left(2 J_{i}+1\right)$ which applies when $J_{i}=0$ as in $\mathrm{Be}^{10}$. For $\mathrm{K}^{40}(n=3), \quad(f t)_{3} / p_{0}^{2}(f t)_{1} \approx 10^{8}$ from measured values. The above formula gives $\sim 1.4(10)^{8}$. About a factor 4400 of this comes from the numerical constant in (37). Hence, even though the evaluations here are very crude, a strong dependence on $n$ as indicated by (37), seems to be definitely required by the evidence.

\section{$\S 6$. Once-Forbidden Spectra and Half-Lives}

\section{A. Shape Factors}

We examine now the $\Delta J=0$ or 1 transitions with parity change. The moments contributing to these were summarized in (5). They lead to the shape factor

$$
S_{1}=S_{1}^{(0)}+S_{1}^{(1)}+S_{1}^{(2)}
$$

as already mentioned in section 4. $S_{1}^{(1)}$ arises from the spatial vector moments (5a) and is given by formula (30). $S_{1}^{(2)}$ arises from the moment $B_{i j}$ of $(5 \mathrm{~b})$ and is given by (36). The partial shape factor $S_{1}^{(0)}$ contributes only to the $\Delta J=0$ transitions and is the entire shape factor for $0^{-} \leftrightarrow 0^{+}$. It arises from the spatial pseudoscalar moments (5c): $\int \beta \sigma \cdot r$ and $\int \beta \gamma_{5}$. It is helpful to define the real parameter $\zeta$ by:

Then

$$
\int \beta \gamma_{5}=i_{\zeta}(\alpha Z / 2 o) \int \beta \boldsymbol{\sigma} \cdot \boldsymbol{r} .
$$

$$
\left.\begin{array}{c}
S_{1}^{(0)}=\left|\int \beta \boldsymbol{\sigma} \cdot \boldsymbol{r}\right|^{2} \\
\left\{C_{T}^{2}\left(\frac{1}{9} q^{2} L_{0}+M_{0}+\frac{2}{3} q N_{0}\right)+C_{P}^{2} \zeta^{2}(\alpha Z / 2 \varrho)^{2} L_{0}\right. \\
\left.\mp C_{P} C_{T} \zeta(\alpha Z / \varrho)\left(\frac{1}{3} q L_{0}+N_{0}\right)\right\} .
\end{array}\right\}
$$

It is obviously independent of the S-coupling $\left(C_{S}\right)$ like $S_{1}^{(2)}$, while $S_{1}^{(1)}$ and $S_{1}^{(2)}$ are both indepeñdent of P-coupling.

To enable discussion, we look at approximations valid for $\alpha Z \ll 1$ (and $W_{0^{-1}}$ not necessarily small) which are actually fairly good except for the heaviest nuclei:

$$
S_{1}^{(0)}=\left|\int \beta \boldsymbol{\sigma} \cdot \boldsymbol{r}\right|^{2}\left\{\left[\left(C_{T} \pm \zeta C_{P}\right)(\alpha Z / 2 \varrho)+\frac{1}{3} C_{T}\left\{\left(p^{2} / W\right)-q\right\}\right]^{2}+C_{T}^{2}(p / 3 W)^{2}\right\},
$$

and, with $(4 \pi / 3) \sum\left|\int \beta \mathscr{Y}_{l m}(\boldsymbol{\sigma} \times \boldsymbol{r})\right|^{2} \equiv\left|\int \beta \boldsymbol{\sigma} \times \boldsymbol{r}\right|^{2}$ in (30),

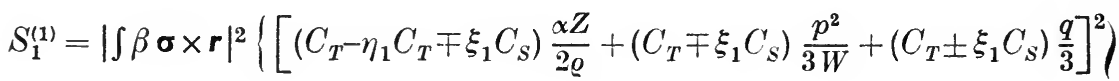

$$
\begin{aligned}
& \left.+\left(\frac{C_{T} \mp \xi_{1} C_{S}}{3} \frac{p}{W}\right)^{2}+\frac{\left(C_{T} \pm 2 \xi_{1} C_{S}\right)^{2}}{18} p^{2}+\frac{\left(C_{T} \mp \xi_{1} C_{S}\right)^{2}}{18} q^{2}\right\} \text {. }
\end{aligned}
$$


The corresponding approximation for $S_{1}^{(2)}$ is given by (36) with $\left(34^{\prime}\right)$. To be noticed in $S_{1}^{(0)}$ and $S_{1}^{(1)}$ is the large coulomb energy term $\sim(\alpha Z / \varrho)^{2}$, which does not appear in $S_{1}^{(2)}$, as discussed in the preceding section.

\section{B. Observed Spectra}

For all the once-forbidden spectra measured so far, the coulomb energy $\alpha Z / \varrho$ is much larger than the maximum kinetic energy $W_{0}-1$. In consequence

$$
\begin{aligned}
& S_{1}^{(0)} \approx\left|\int \beta \boldsymbol{\sigma} \cdot \boldsymbol{r}\right|^{2}\left(C_{T} \pm \zeta C_{P}\right)^{2}(\alpha Z / 2 \varrho)^{2} \\
& S_{1}^{(1)} \approx\left|\int \beta \boldsymbol{\sigma} \times \boldsymbol{r}\right|^{2}\left(C_{T}-\eta_{1} C_{T} \mp \xi_{1} C_{S}\right)^{2}(\alpha Z / 2 \varrho)^{2},
\end{aligned}
$$

are practically independent of the electron energy, $W$, and statistical shapes should be observed for the once-forbidden spectra with $\Delta J=0,1$. Only one exception (RaE) to this behavior has been found so far among the numerous adequately measured cases. Very accurate statistical shapes have been found just for cases in which the condition $\alpha Z / \varrho \gg W_{0}-1$ is best fulfilled. However, more accurate work on higher energy spectra should reveal small departures from the statistical shape; such work has been hindered by the prevalence of obscuring transitions to excited states, in nuclei having sufficient energy for the test.

It may be noticed that $S_{1}^{(2)}$ does not share the property now discussed, yet contributes to many of the $\Delta J=0,1$ transitions. However, as will be more fully described below, $S_{1}^{(2)}$ becomes a negligible addition to $S_{1}^{(0)}$ and $S_{1}^{(1)}$ just because it does not contain terms $\sim(\alpha Z / \varrho)^{2}$.

The singular case of $\mathrm{RaE}$ appears to demonstrate a circumstance under which a once-forbidden, $\Delta J=0$ or 1 , spectrum may depart substantially from the statistical shape even though the condition $\alpha Z / \varrho \gg W_{0}-1$ is well fulfilled. $\mathrm{RaE}$ seems to be a $1^{-} \rightarrow 0^{+}$transition in which the ST interference is nearly completely destructive, i.e. $C_{T}\left(1-\eta_{1}\right)+\xi_{1} C_{S} \approx 0$ in (44), hence the energy dependent terms of (42) come into play. This may come about if the nuclear moments concerned have the particular relation so implied. A consequence is that the nominally largest term of (42) almost cancels out and the transition becomes substantially slower. Indeed, $\mathrm{RaE}$ is slower by about the expected factor, $\sim 10^{2.5}$, than otherwise comparable transitions $\left(\mathrm{Tl}^{206}, \mathrm{Hg}^{205}, \mathrm{~Pb}^{206}\right)$.

\section{V, A-Couplings}

The once-forbidden spectra provide the only evidence available so far that the Fermi coupling is given by the $S$ rather than the $V$ form, in the correct law of $\beta$-decay. This is based on the character of the shape 
factor $S_{1}^{(1)}$ which follows from the replacement of the ST combination in (44), with VT components. In place of (44), one then has ${ }^{8}$ :

$$
\left.\begin{array}{c}
S_{1}^{(1)}(\mathrm{VT}) \approx \mid \int \beta \sigma \times \boldsymbol{r}^{2}(\alpha Z / 2 \varrho)^{2}\left[C_{T}^{2}\left(1-\eta_{1}\right)^{2}+C_{V}^{2}\left(\xi_{1}-\eta_{1}\right)^{2}-\right. \\
\left.-2\left(1-\eta_{1}\right)\left(\xi_{1}-\eta_{1}\right) C_{V} C_{T}^{\prime} / W\right] .
\end{array}\right\}
$$

The striking result here is the energy dependence $\sim C_{V} C_{T} / W$, like that of the term $\mp b / W$ which was eventually eliminated from the allowed spectrum, eq. (10) of Ch. IX, by the experimental evidence. Such a term leads to substantial departures from the statistical shape even when $\alpha Z / \varrho \gg W_{0}-1-$ a behavior which is contradicted by the measured onceforbidden spectra. A similar behavior follows from the replacement of the ST combination in $S_{1}^{(1)}$ with VA or SA; this is not crucial since there is independent evidence (section 8 ) that the $\mathrm{T}$ component is essential. Further, the replacement of TP in $S_{1}^{(0)}$ with AP also yields terms $\sim 1 / W$ even for $\alpha Z / \varrho \gg W_{0}-1$. However, there appear to be no sufficiently well measured once-forbidden, $\Delta J=0$ spectra to check this point about the $S_{1}^{(0)}$ shape factor. Again, this is not as crucial as it might be, because of the independent evidence for the $\mathrm{T}$ component.

\section{Half-Lives}

If the once-forbidden shape factors for $\Delta J=1$ are well-approximated by (44), as asserted above, then one should expect the comparative halflives to show a trend with nuclear size:

$$
f t \sim(\alpha Z / \varrho)^{-2}
$$

according to (33). None such has so far been distinguished, but this is not surprising. Practically all the observed once-forbidden transitions are confined to $35 \lesssim Z \lesssim 80$, hence the trend in question should be a variation by less than a factor $\sim 4$. On the other hand, the $f t$-values show local (near a given $Z$ ) variations by factors of 5 , and even 30 .

A trend toward shorter half-lives with increasing $Z$ is reported ${ }^{9}$ for $\Delta J=0$, once-forbidden transitions. Since it is a trend not shared by the $\Delta J=1$ transitions, and, moreover, the $\Delta J=0 \mathrm{ft}$-values in general tend to lie lower than $\Delta J=1$ values, Nordheim prefers to attribute this to the incidence of $\mathrm{P}$-coupling, which is ineffective in the $\Delta J=1$ cases.

We next discuss our assertion above (see "Observed Spectra") that the partial shape factor $S_{1}^{(2)}$ becomes negligible with respect to $S_{1}^{(0)}$ or $S_{1}^{(1)}$ for the $\Delta J=0,1$ cases in which $\alpha Z / \varrho \gg W_{0}-1$. The experimental facts bear

8 See references in footnotes 2 and 6 for more accurate versions.

- L. Nordheim, Conf. on Nuc. Spectroscopy, Indiana Univ., (1953). 
this out immediately. They show the size of $S_{1}^{(2)}$ in the "unique", $\Delta J=2$ transitions, when that shape factor is the entire one: $p_{0}^{2}(f t)^{(2)} \approx 6(10)^{9}$ for $Z \approx 37$. The $\Delta J=1$ transitions near $Z=37$ have $(f t)^{(1)} \approx(4$ to 20$) 10^{6}$ and are therefore roughly 300 to 1500 times as fast. One can estimate whether just this ratio is to be expected by supposing that all the moments concerned have roughly the same magnitude. Then

$$
p_{0}^{2}(f t)^{(2)} /(f t)^{(1)}=p_{0}^{2}\left\langle S_{1}^{(1)}\right\rangle /\left\langle S_{1}^{(2)}\right\rangle \approx(15 / 4)(\alpha Z / \varrho)^{2} \approx 900 .
$$

There is at least no inconsistency, on the present evidence.

Finally, we may make similar estimates comparing the once-forbidden, $\Delta J=0,1 \mathrm{ft}$-values above with allowed transitions: $(f t)_{0} \approx(1$ to 4$) 10^{5}$ near $Z=37$, hence $(f t)_{1} /(f t)_{0} \approx 10$ to 200 is about the best one can say from the data. Our methods of estimation yield: $(f t)_{1} /(f t)_{0} \approx(2 / \alpha Z)^{2} \approx 55$. Again, one can at least say that no inconsistency shows up.

\section{$\S 7$. Twice-Forbidden Spectra and Half-Lives}

\section{A. Spectra}

Accurate measurements have been made on several transitions with $\Delta J=2$ and no parity change. We discuss the theoretical expectations for these, but in detail only for ST-coupling. The P-coupling is expected to have no effect (section 3 ). Remarks about the V-coupling will be added.

The shape factor is in general [see (29)]:

$$
S_{2}=S_{2}^{(2)}+S_{2}^{(3)}
$$

but it is evident that for the observed cases $S_{2}^{(3)}$ is negligible, as in the similar situation for the once-forbidden transitions. $S_{2}^{(2)}$ is given accurately by $(30)$; it will suffice to discuss the practical case: $\alpha Z / \varrho \gg W_{0}-1$. Then, from (31),

$$
S_{2}^{(2)} \approx(2 \pi / 45) \sum\left|\int \beta \mathscr{Y}_{2 m}(\boldsymbol{\sigma} \times \boldsymbol{r})\right|^{2}(\alpha Z / 2 \varrho)^{2}\left(C_{T}-2 \eta_{2} C_{T} \mp \xi_{2} C_{S}\right)^{2}\left(p^{2}+\lambda q^{2}\right),
$$

with

$$
\lambda=4\left(C_{T}-\eta_{2} C_{T} \mp \xi_{2} C_{S}\right)^{2} /\left(C_{T}-2 \eta_{2} C_{T} \mp \xi_{2} C_{S}\right)^{2} .
$$

$S_{2}^{(3)}$, given by $(36)$ with $\left(34^{\prime \prime}\right)$, does not contain the factor $\sim(\alpha Z / \varrho)^{2}$, whence follows its neglibility in all cases so far known.

Several measured spectra have been identified as due to $\Delta J=2$, no parity change transitions. They can be adequately fitted with a shape factor $\sim p^{2}+\lambda q^{2}$, when $\lambda$ is treated as a free parameter.

There is interest also in the shape factor $S_{2}^{(2)}$ which would follow from a VT-coupling, in place of the ST combination used for (47): 


$$
\left.\begin{array}{r}
S_{: 2}^{(2)}(\mathrm{VT})=(2 \pi / 4 \overline{5}) \sum \mid \int \beta \mathscr{Y}_{2 m}(\boldsymbol{\sigma} \times \boldsymbol{r})^{2}{ }^{2}(\alpha Z / 2 \varrho)^{2}\left[(A+B / W) p^{2}+\right. \\
\left.+(C+D / W) q^{2}\right],
\end{array}\right\}
$$

with

$$
\begin{array}{ll}
A=\left(1-2 \eta_{2}\right)^{2} C_{T}^{2}+\left(\xi_{2}-2 \eta_{2}\right)^{2} C_{V}^{2}, \quad B=2\left(1-2 \eta_{2}\right)\left(\xi_{2}-2 \eta_{2}\right) C_{V} C_{T}, \\
C^{\prime}=4\left[\left(1-\eta_{2}\right) C_{T}^{2}+\left(\xi_{2}-\eta_{2}\right)^{2} C_{V}^{2}\right], \quad D=8\left(1-\eta_{2}\right)\left(\xi_{2}-\eta_{2}\right) C_{V} C_{T} .
\end{array}
$$

One sees, as in the VT result for once-forbidden transitions, the incidence of terms $\sim C_{V} C_{T} / W$. The unknown group of parameters here are so many, however, that the twice-forbidden spectra do not give the opportunity for as clear-cut a test of the alternative theories as do the once-forbidden spectra.

\section{B. Half-Lives}

The comparative half-lives of the twice-forbidden, $\Delta J=2$ transitions lie in the range log $f t=12.2$ to 13.5. $S_{2}^{(2)} \sim(\alpha Z / \varrho)^{2}\left(p^{2}+\lambda q^{2}\right)$, hence $\left\langle S_{2}^{(2)}\right\rangle \sim(\alpha Z / \varrho)^{2} p_{0}^{2}$ roughly. Thus, according to (33), $(\alpha Z / \varrho)^{2} p_{0}^{2} f t$ should be more constant than is $f t$ itself. Indeed, the range of variation is greatly narrowed, $\log (\alpha Z / \varrho)^{2} p_{0}^{2} f t=15.7$ to 15.9 , if one excludes $\mathrm{Cl}^{36}$, which gives 16.2. The latter is a far lighter nucleus than the others of the group (all of which have $Z \geqslant 43$ ), so the $\mathrm{Cl}^{36}$ discrepancy would have been greater without the help of the factor $(\alpha Z / \varrho)^{2}$. Since $\mathrm{Cl}^{36}$ is also the only even nucleus of the group, it is perhaps not too surprising that its moments should be $\sim 2.5$ times smaller than the norms of the others.

One finally ought to check whether the $f t$-values quoted above for the twice-forbidden, $\Delta J=2$ transitions have the correct order of magnitude as -a class. We may compare them against the once-forbidden $(\Delta J=0$ or 1$)$ $f t$-values if we treat the moments as before i.e. assume:

$$
\int \beta \mathscr{Y}_{2 m}(\boldsymbol{\sigma} \times \boldsymbol{r}) / \int \beta \mathscr{Y}_{1 m}(\boldsymbol{\sigma} \times \boldsymbol{r}) \approx \varrho,
$$

the nuclear radius. Then it follows from (33) that the ratio of the twiceforbidden to once forbidden $f t$ values is roughly:

$$
(f t)_{2} /(f t)_{1} \approx 60 /\left(p_{0} \varrho\right)^{2}
$$

when $p_{0}=\left(W_{0}^{2}-1\right)^{1}$ is the maximum momentum in the twice forbidden transition. The twice-forbidden $f t$-values show much the narrower range of variation, hence they would lead to a relatively restricted range of $(f t)_{1}$ values by way of this formula: $\log (f t)_{1} \approx 7.9$ to 8.6. These are distinctly larger than the $(f t)_{1}$-values actually observed: $\log (f t)_{1} \approx 6$ to 8 . The conclusion seems to be that the twice-forbidden moments are consistently smaller, by at least a factor 10 or so, than our crude estimation would make them. 


\section{$\S 8$. The Law of $\beta$-Decay}

Here we summarize very briefly the character of the evidence that the correct law of $\beta$-decay is a combination of $\mathrm{S}$ - and T-couplings, with some chance that a $\mathrm{P}$-coupling exists. This can be done conveniently, now that all the pertinent formulas have been developed. The "phenomenological derivation" here will vary slightly from that of the MahmoudKonopinski ${ }^{10}$ paper, because of later evidence which makes the same conclusions more straightforward.

First, it seems necessary to include both a Gamow-Teller type (T or A), and a Fermi type (S or V), of coupling. The evidence ${ }^{11}$ for the GamowTeller coupling has already been mentioned immediately following equation $\left(34^{\prime \prime \prime}\right)$. The evidence for Fermi coupling rests on the existence of allowed $0 \rightarrow 0$ transitions [see eq. (8), Ch. IX] and also on the relation of the comparative half-lives, $f t$, to $\left|\int \boldsymbol{\sigma}\right|^{2}$ as deduced from the magnetic moments of the mirror nuclei [Ch. XVI, and eq. (15) of Ch. IX]. There is general agreement from such evidence that $C_{S, V}^{2} / C_{T, A}^{2}>30 \%$ at least [see eq. (16), Ch. IX].

Electron-neutrino angular correlation measurements on $\mathrm{He}^{6}$ [Ch. XII and (9a) of Ch. IX] show that it is the T, rather than A, interaction which is responsible for the Gamow-Teller coupling. The simultaneous existence of a small amount of A-coupling is however not excluded by this difficult experiment.

The observed statistical shapes of allowed spectra require that $C_{A, T}^{2} / C_{T, A}^{2} \lesssim 4 \%$ and $C_{V, S}^{2} / C_{S, V}^{2} \lesssim 4 \%$ [see footnote 10 , Ch. IX]. These are the maximum percentages which have resulted from several evaluations of the best data. A theory including such small components in a fundamental law of interaction is difficult to defend. The highly probably conclusion is that $C_{A}$ or $C_{T}=0$ and $C_{V}$ or $C_{S}=0$.

Up to this point we are left with two alternatives: an STP combination or a VTP combination. $C_{P}=0$ is still possible but $C_{T} \neq 0$ and $C_{S, V}^{2} / C_{T}^{2}>30 \%$ are necessary.

When one tries to explain the observed statistical shapes of onceforbidden, $\Delta J=1$ spectra with a VTP law [see (45)], then it is found that $C_{V}^{2} / C_{T}^{2} \lesssim 1 \%$. Lack of knowledge concerning the momental ratios $\xi_{1}$ and $\eta_{1}$ does not interfere with this result ${ }^{10}$. However, uncertainties in the correct evaluation of the coulomb effect may actually make the limit quoted here somewhat more uncertain than the limits discussed above for the allowed spectra. Nevertheless, one has here the great advantage

10 H. M. Mahmoud and E. J. Konopinski, Phys. Rev. 88 (1952) 1266.

11 To avoid bulk, we leave all references to experimental work to the experimental chapters of this book. 
of knowing that $C_{V}^{2} / C_{T}^{2}>30 \%$ is necessitated by the known existence of Fermi coupling. Such a large ratio would have led to easily perceptible deviations of the once-forbidden spectra from the statistical shape. Any evaluations of the coulomb effect which would destroy this conclusion would also leave the statistical shapes unexplainable. We thus arrive at the conclusion that the ST components of the law are necessary and that $C_{A}=C_{V}=0$.

Finally, there is fragmentary evidence that also $C_{P} \neq 0$. Its character was mentioned in connection with Nordheim's conclusions concerning the once-forbidden half-lives in section 6 .

It should be mentioned that there have been proposed ${ }^{12}$ methods of evaluating the nuclear moment $\int \beta \gamma_{5}$, on which the P-coupling acts, which would alter the once-forbidden, $\Delta J=0$ shape factor $S_{1}^{(0)}$ quite radically. These methods make the magnitude of $\int \beta \gamma_{5}$ negligible for any reasonable value of $C_{P}$. This makes doubtful the value of incorporating these modifications in the present treatise. The formulas for the P-coupling contributions as given in this treatise are the ones expected to be valid if theories of nuclear forces arise which make the P-coupling appreciable.

\section{Appendix}

The shape factor for the VT interaction will be given here. It remains as the alternative to $\mathrm{ST}(\mathrm{P})$ as the law of $\beta$-decay if one accepts only evidence based on the simpler allowed transitions. It has further the attractive feature that it is consistent with the Tolhoek-de Groot ${ }^{13}$ symmetry principle, and the $\mathrm{ST}(\mathrm{P})$ combination is not. The principle asserts that there should be no distinction between positron and negatron emission in the absence of a coulomb effect [see the alternative signs in (30)].

$$
\begin{aligned}
S_{n}^{(n)}(\mathrm{VT}) & =[4 \pi n ! /(2 n+1) ! !] \sum\left|\int \beta \mathscr{Y}_{n m}(\boldsymbol{\sigma} \times \boldsymbol{r})\right|^{2} \times \\
& \times\left\{C_{T}^{2} P_{n}(\boldsymbol{\sigma} \times \boldsymbol{r})+\left[\eta_{n}^{2} C_{T}^{2}+y_{n}^{2} C_{V}^{2}\right](\alpha Z / 2 \varrho)^{2} P_{n}(\boldsymbol{\alpha})+\xi_{n}^{2} C_{V}^{2} \tilde{P}_{n}(\boldsymbol{r})\right. \\
& +\left[\eta_{n} C_{T}^{2}+\xi_{n} y_{n} C_{V}^{2}\right](\alpha Z / \varrho) \tilde{I}_{n}(\boldsymbol{\alpha}, \boldsymbol{r})-2 \xi_{n} C_{\boldsymbol{V}} C_{T} J_{n}(\boldsymbol{\sigma} \times \boldsymbol{r}, \boldsymbol{r}) \\
& \left.+\left[\xi_{n} \eta_{n}+y_{n}\right](\alpha Z / \varrho) C_{V} C_{T} J_{n}(\boldsymbol{\alpha}, \boldsymbol{r})+2 \eta_{n} y_{n}(\alpha Z / 2 \varrho)^{2} C_{\boldsymbol{V}} C_{T} I_{n}(\boldsymbol{\alpha}, \boldsymbol{\alpha})\right\} .
\end{aligned}
$$

The notation is the same as in (30) but new functions have been added: $J_{n}(\boldsymbol{\sigma} \times \boldsymbol{r}, \boldsymbol{r})=\sum_{n=0}^{v} B_{n v} q^{2(n-v)-2}\left\{(n-v)(2 n-2 v+1) Q_{v}+2(n-v) q R_{v}-\frac{v}{2 v+1} q^{2} P_{v}\right\}$ $J_{n}(\boldsymbol{\alpha}, \boldsymbol{r})=\quad, \quad\left\{(n-v)(2 n-2 v+1) R_{v}-(n-v) q P_{v}\right\}$. $I_{n}(\alpha, \alpha)=\quad, \quad \cdot\left\{(n-v)(2 n-2 v+1) P_{v}\right\}$.

12 Originally, V. B. Berestetsky and I. Y. Pomeranchuk, J. Exp. \& Theo. Physics, U.S.S.R., 19 (1949) 756. Most lately, M. E. Rose, Phys. Rev. 93 (1954) 1315.

13 H. A. Tolhoek and S. R. de Groot, Phys. Rev. 84 (1951) 150; Physica 16 (1950) 4.56. 
The functions $P_{v}, Q_{v}, R_{v}$ have also been tabulated ?. The real parameter $y_{n}$ is defined by:

$$
\int \mathscr{Y}_{n m}(\boldsymbol{\alpha})=y_{n}(\alpha Z / 2 \varrho) \cdot \int \beta \mathscr{Y}_{n m}(\boldsymbol{\sigma} \times \boldsymbol{r}) .
$$

[Compare the definition of $\eta_{n}$ in (24)].

Of course $S_{n}^{(n+1)}$ as given in (36) applies also when the interaction is a VT-coupling. $S_{1}^{(0)}(\mathrm{VT})$ is obtained from (40) merely by setting $C_{P}=0$. 


\title{
EXPERIMENTS ON THE SHAPES OF $\beta$-SPECTRA
}

\section{THE INTERACTION IN $\beta$-DECAY}

\author{
C. S. WU
}

\section{§ 1. Introduction}

Two decades ago when the rresent theory of $\beta$-decay was first proposed ${ }^{1}$, a hypothetical new particle named the neutrino had to be introduced and a heretofore totally unknown type of interaction between the nucleon and the two light particles (electron and neutrino) had to be defined and determined. Furthermore, at that iime our knowledge of the properties of the nuclear states was very limited.

The task was difficult at the beginning. However, due to the recent advance in the experimental study of the $\beta$-process and the aid given by the nuclear shell model, a great stride forward in the understanding of $\beta$-decay has been made. At present, hundreds of cases of $\beta$-decay have been investigated. The accurate half-lives and maximum $\beta$-energies thus obtained together with the assigned spins and parities provide us with information on the pertinent properties of nuclear states. Some $\beta$-decays have had their spectra precisely measured and compared with various theoretical predictions. This latter study provides the best source of conclusive evidence for a definite form of the $\beta$-interaction.

\section{$\S$ 2. The Allowed Spectra}

In Ch. IX it has been pointed out that the energy distribution of the $\beta$-particles for allowed transitions is independent of the precise form of the interaction and is determined by the statistical term and the Fermi function alone. Therefore no information on the choice of the interaction term could be derived from its study. However, its confirmation is required as the first step to establish the Fermi theory of $\beta$-decay. Early investigations of the $\beta$-spectra reported considerable discrepancies between the experimental results and the theory. This was mostly cleared away in 1939 by the work of Lawson and Cork ${ }^{2}$ on $\mathrm{In}^{114}$ and of Tyler ${ }^{3}$ on $\mathrm{Cu}^{64}$. By using comparatively thin sources $\left(\sim\right.$ a few $\left.\mathrm{mg} / \mathrm{cm}^{2}\right)$ the Kurie plots of the spectra which they obtained were linear over a large part of the energy distribution. While the major portion of a spectrum followed the

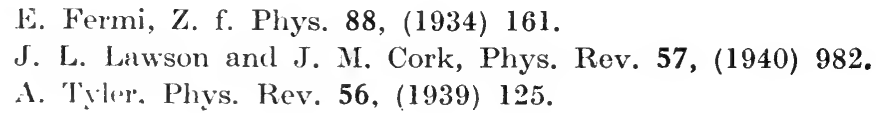


Fermi distribution closely, an excess of electrons in the very low energy region ${ }^{4}$ was always present. In particular it seemed that the deviation remained even when seemingly thin sources were used. Had these discrepancies at the very low energy region remained real, it would have been necessary to revise Fermi's theory of $\beta$-decay.

The investigation of very low energy electrons involves many difficulties. Of these the absorption and scattering effect in the finite and non-uniform source thickness and its backing material is most serious of all. It was observed by $\mathrm{Wu}$ and Albert in their investigation of the $\beta$-spectra of $\mathrm{S}^{355}$ and $\mathrm{Cu}^{646}$ that the excess of particles at low energies was a function of source thickness. The thinner and more uniform the source became, the lower and less became the deviation from linearity of the Kurie plot. Figures 1 and 2 show the Kurie plots of $\mathrm{S}^{35}$ and $\mathrm{Cu}^{64}$ which were obtained with thin sources. The deviation at the low energy region is reduced by a factor of four as compared with the excess reported previously. $\mathrm{Cu}^{64}$ is a

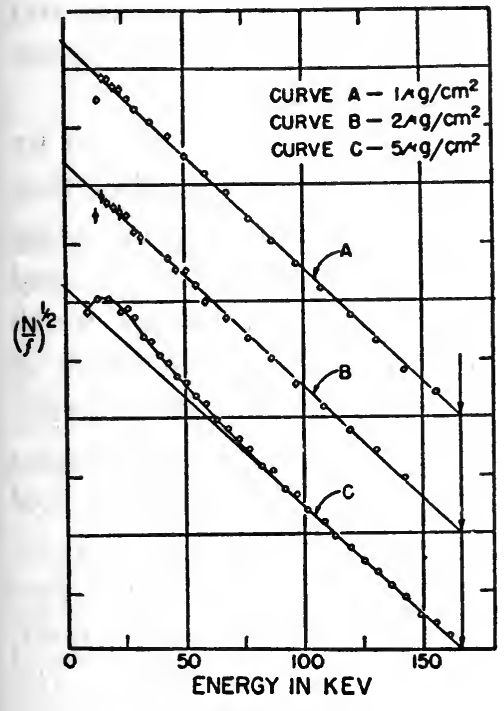

Fig. 1. Kurie plots of the $\beta$-spectrum of $\mathrm{S}^{35}$. A, B, C are plots for various source thicknesses.

(See note 5)

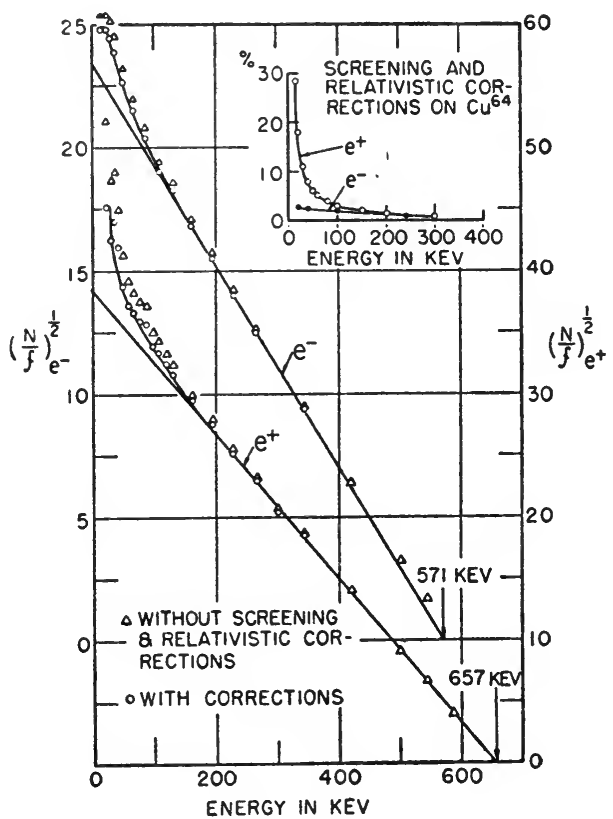

Fig. 2. Kurie plots of $\mathrm{Cu}^{64}$ negatron and positron spectra. (See note 6)

- J. Backus, Phys. Rev. 68, (1945) 59; C. S. Cook and L. M. Langer, Phys. Rev. 73, (1948) 601 .

5 R. D. Albert and C. S. Wu, Phys. Rev. 75, (1948) 847.

B C. S. Wu and R. D. Albert, Phys. Rev. 75, (1949) 315; 75, (1949) 1107. 
particularly interesting case. Because it emits both an electron and a positron spectrum of approximately equal intensity and about the same maximum energy, it offers a good test of the Fermi theory. As any elastic

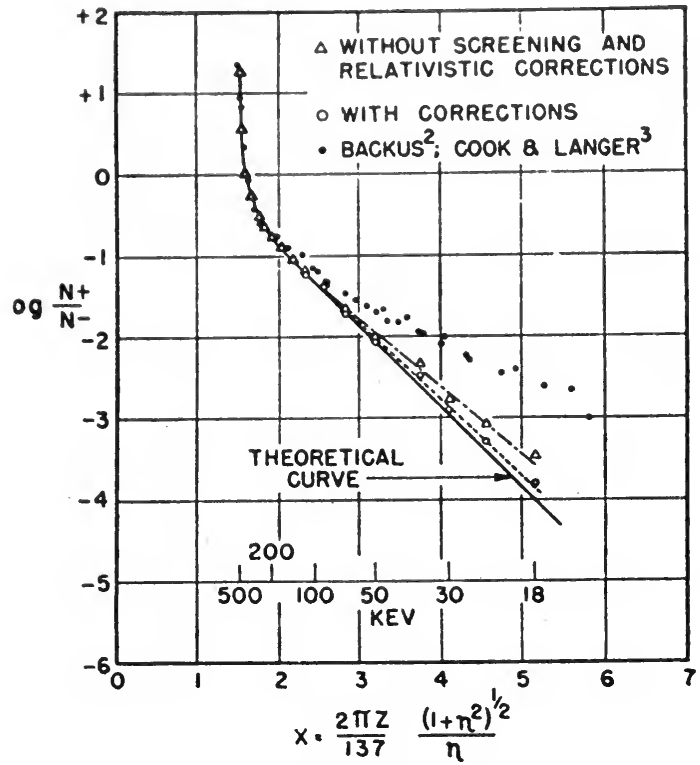

Fig. 3. The ratio of the number of positrons to the number of negatrons as a function of energy. (See note 6) back-scattering effect on the $\mathrm{e}^{-}$ will be presumably the same on the $\mathrm{e}^{+}$of the same source, one therefore expects to observe the ratio of $\mathrm{Ne}^{+} / \mathrm{Ne}^{-}$unaffected. In Fig. 3 the $\log \mathrm{Ne}^{+} / \mathrm{Ne}^{-}$is plotted against

$$
x=\frac{2 \pi Z}{137} \frac{\left(1+\eta^{2}\right)^{1 / 2}}{\eta} .
$$

The theoretical curve is as indicated. The experimental data corrected by taking into account the screening effect are represented by the open circles and they are in excellent agreement with the theory.

Furthermore, it was shown by the autoradiographic method that a source prepared by evaporating a drop of solution can have a local variation in thickness as much

as 100 to 1 due to crystallization. Naturally the average thickness of a non-uniform source of $100 \mu \mathrm{g} / \mathrm{cm}^{2}$ is rather meaningless. When carrierfree or microscopically uniform sources prepared by the vacuum evaporation method were used, the linearity extended to the extremely low energy region where the absorption effect of the window set in. Fig. 4 shows the Kurie plots of the $\beta^{+}$and $\beta^{-}$spectra of $\mathrm{Cu}^{64}$ prepared by evaporating the active $\mathrm{Cu}^{64}$ metal onto a thin film in vacuum. The autoradiograph of the source showed complete uniformity. The Kurie plots of the data obtained from such thin and uniform sources ranging from a few $\mu \mathrm{g} / \mathrm{cm}^{2}$ to $75 \mu \mathrm{g} / \mathrm{cm}^{2}$ show no deviation from that expected from the Fermi theory for all energies above $50 \mathrm{keV} 7,8$. In the very low energy region the screening effect due to the atomic electrons ${ }^{9}$ on the ordinary Fermi function must be taken into account. This is in

7 L. Langer, R. Moffat and H. Price, Phys. Rev. 76, (1949) 1725.

8 G. E. Owen and C. S. Cook, Phys. Rev. 76, (1949) 1726.

9 M. E. Rose, Phys. Rev. 49, (1936) 727; C. Longmire and H. Brown, Phys. Rev. 75, (1949) 264; J. R. Reitz, Phys. Rev. 77, (1950) 10. 
particular more important for the positrons than for the electrons. Fig. 5 shows the screening effect vs energy with several values of $Z$ on both electrons and positrons.

Further confirmation of this point is given by the linear Kurie plots of $\mathrm{Pm}^{147} 10$ and $\mathrm{S}^{35}$ 10, 11 which extended all the way to $8 \mathrm{keV}$. It seems quite conclusive that there is no real deviation from the Fermi distribution in the very low energy region.

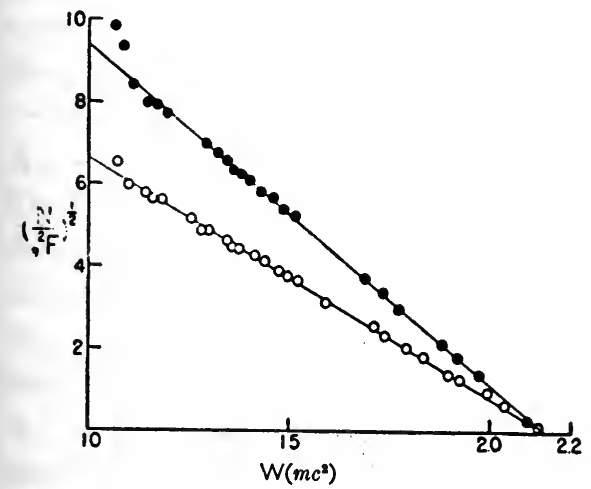

Fig. 4. Kurie plot of the negatron. spectrum of $\mathrm{Cu}^{64}$. Solid circles are for data obtained with the $75 \mu \mathrm{g} / \mathrm{cm}^{2}$ sources backed by $0.18 \mathrm{mg} / \mathrm{cm}^{2} \mathrm{Al}$ leaf. The open circles are for data obtained 'with the less than $5 \mu \mathrm{g} / \mathrm{cm}^{2}$ source backed by $15 \mu \mathrm{g} / \mathrm{cm}^{2}$ zapon. (See note 7).
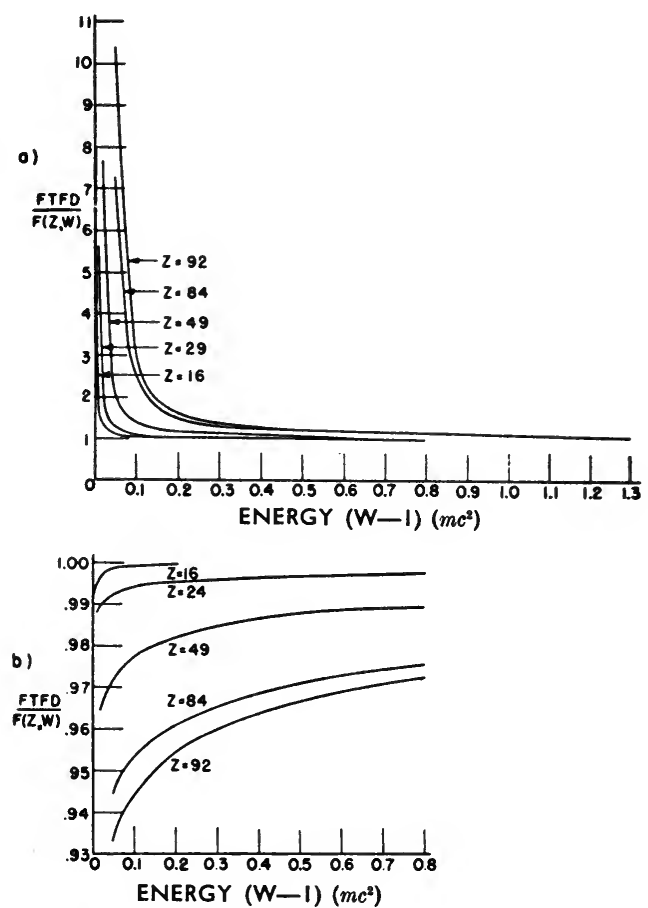

Fig. 5. Screening correction factors $F_{T F D} / F(Z, W)$ to allowed $\beta$-spectra, a) for positrons b) for electrons. $F_{T F D}$ is calculated from Thomas. Fermi-Dirac wave functions. From Reitz, Phys. Rev. 77, p. 10

To investigate electrons of energy below $10 \mathrm{keV}$, a method using a proportional counter with the radioactive gas introduced inside the counter has been developed. Curran, Angus, and Cockcroft ${ }^{12}$ of Glasgow and Hanna and Pontecorvo ${ }^{13}$ of Chalk River investigated tritium, in this manner and found that the Kurie plot is a straight line from its maximum energy $18.6 \mathrm{keV}$ to $0.5 \mathrm{keV}$ (Fig. 6).

There have been several reports from investigators using a proportional

10 H. Price, H. Motz and L. Langer, Phys. Rev. 77, (1950) 743; Phys. Rev. 77, (1950) 798.

11 L. Gross and D. R. Hamilton, Phys. Rèv. 78, (1950) 318.

12 S. Curran, J. Angus and A. Cockroft, Phil. Mag. 50, (1949) 53; Nature 162, (1948) 302.

13 G. C. Hanna and B. Pontecorvo, Phys. Rev. 75, (1949) 983. 
counter and from others using a cloud chamber that the Kurie plots of $\mathrm{RaF}^{14}{ }^{14} \mathrm{~S}^{35}{ }^{15}, \mathrm{C}^{14} 16$ and $\mathrm{H}^{3}{ }^{17}$ all deviate downwards from the linear Kurie plots at the very low energy region. The radioactive sources used in the proportional counter were prepared in the gaseous form in order to avoid non-un:formity and finite source thickness. However there are disturbing effects due to the non-uniform sensitivity at the counter ends which must be corrected for. Recently Zaffarano ${ }^{18}$ prepared extremely uniform and thin sources of $\mathrm{Pm}^{\mathbf{1 4 7}}$ and $\mathrm{S}^{35}$ and introduced the sources directly inside of a proportional counter. The end effects in this case are greatly minimized.

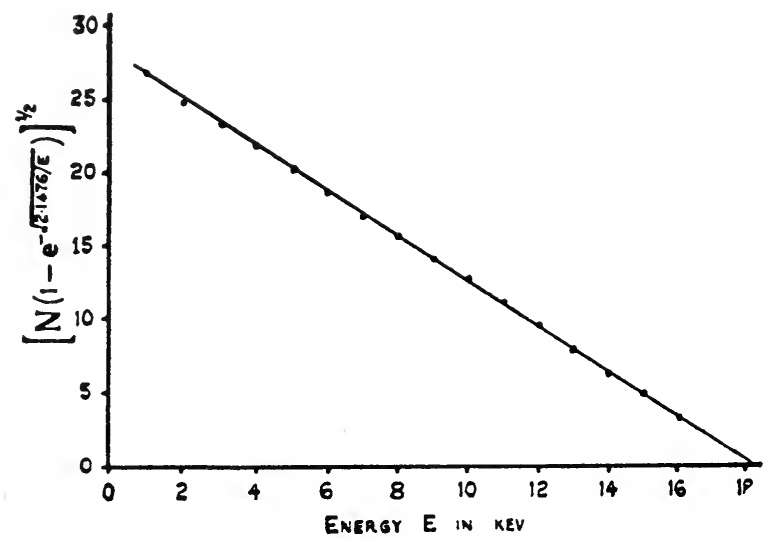

Fig. 6. Kurie plot for $\mathrm{H}^{3}$. (See note 12)

The Kurie plots thus obtained are linear all the way down to $\sim 10 \mathrm{keV}$ and are in good agreement with the results from the magnetic spectrometers. However, the latest work done on the tritium gas $\left(\mathrm{H}^{3}\right)$ inside of a proportional counter revealed a kind of "hole" 17 in the energy spectrum of electrons below $1 \mathrm{keV}$. Since this energy region is beyond the capability of any other type of $\beta$-spectrometer, a further confirmation will probably come from the further investigation of the characteristics of a proportional counter in the ver'y low energy region $* \dagger$.

14 A. Walter and F. T. Rogers Jr., Phys. Rev. 74, (1948) 699.

15 A. L. Cockroft and G. M. Insch, Phil. Mag. 40, (1949) 1014.

16 J. Angus, A. L. Cockroft and S. C. Curran, Phil. Mag. 40, (1949) 522.

17 S. C. Curran, Physica 18, (1952) 1161.

18 J. P. Mize and D. J. Zaffarano, Phys. Rev. 91, (1953) 210 A.

* An increase in the mean energy necessary to produce an ion pair at low energy was reported in the case of $\mathrm{Cl} L_{\mathrm{I}}$ peak $(\sim 280 \mathrm{keV})$ of $\mathrm{A}^{37}$. B. Pontecorvo, D. H.W. Kirkwood and G. C. Hanna. Phys. Rev. 75, (1949) 982.

$\dagger$ The latest investigation on the low energy spectra of $\mathrm{S}^{35}$ and $\mathrm{C}^{14}$ by A. Moljk and S. C. Curran concluded no more deviation from the linear Kurie plot (to within $1 \%$ ) down to $\sim 2 \mathrm{keV}$ (Glasgow International Nuclear Physics Conference July 1954). 


\section{$\S 3$. Fierz Interference Terms ${ }^{19}$ for the Allowed Transitions}

The confirmation of the linearity of the Kurie plots from allowed spectra such as $\mathrm{B}^{12}{ }^{20}, \mathrm{He}^{6}{ }^{21}, \mathrm{~S}^{35}, \mathrm{Cu}^{64}$ etc. minimizes the contribution of the Fierz interference term $b / w$ in eq. $(9-10)$. Owing to the slowly varying nature of the term $1 / w$ it is rather difficult to establish experimentally the Fierz's condition $C_{S} C_{V}=C_{A} C_{T}=0$. Mahmoud and Konopinski ${ }^{22}$ have, in a systematic study, estimated the largest value that the Fierz interference term could have and yet be consistent with the observed spectra. For this purpose, $\mathrm{Cu}^{64}, \mathrm{~N}^{13}$ and $\mathrm{S}^{35}$ were chosen (see Table I). These isotopes have simple decay schemes and their spectra have been measured reliably to very low energies and therefore offer the best possibility of revealing a Fierz term.

They drew the conventional Kurie plot $K_{n} \sim\left(W_{0}-W\right)$ and the corresponding Fierz plot $K_{n}^{\prime} \sim\left(W_{0}-W\right)(1 \pm b / w)^{1 / 2}$ the deviation of the Fierz plot from linearity is judged from straight line drawn through those points on the high energy side of the intensity maximum. The conventional Kurie plot has a random deviation from linearity due to the statistics involved. The root-mean-squire deviation of the Kurie plot $\varepsilon$ was taken as a measure of experimental accuracy. The upper limit of $b$ was obtained when the systematic mean deviation in the Fierz plot just exceeded the random deviation $\varepsilon$ in the Kurie plot. The upper limit of $b$ thus found is $\cong 0.2$ which is about equal to the root-meansquare deviation of Kurie plot (see Fig. 7). This implies that the following strong inequalities exist

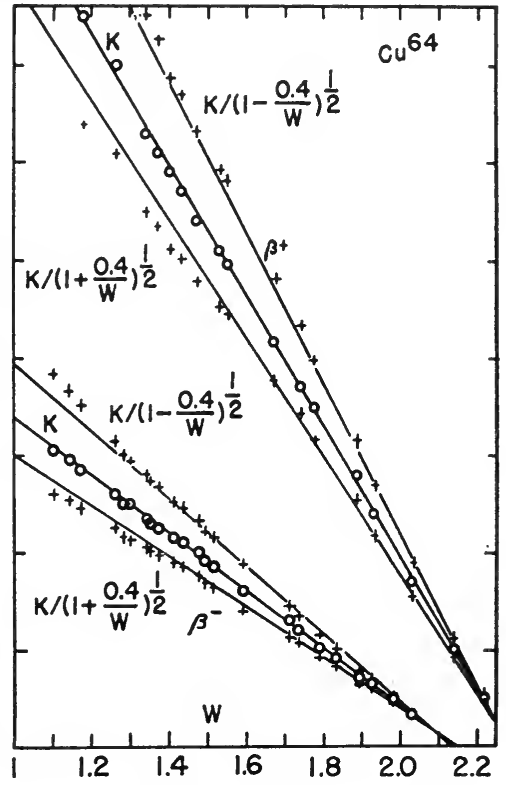

Fig. 7. The Kurie plots and the Fierz plots for $b= \pm 0.4$ of the positrons and electrons of $\mathrm{Cu}^{64}$. (See note 22)

$$
2 C_{S} C_{V} \ll C_{S}^{2}+C_{V}^{2} \quad 2 C_{T} C_{A} \ll C_{T}^{2}+C_{\boldsymbol{A}}^{2} .
$$

Davidson and Peaslee ${ }^{23}$ studied a similar group of allowed spectra but

19 M. Z. Fierz, Physik 104, (1937) 553.

20 W. F. Hornyak and T. Lauritsen, Phys. Rev. 77, (1950) 160.

21 C. S. Wu, B. M. Rustad, V. Perez-Mendez and L. Lidofsky, Phys. Rev. 87, (1952) 1140.

${ }_{22}$ H. M. Mahmoud and E. J. Konopinski, Phys. Rev. 88, (1952) 1266.

23 .J. P. Davidson Jr. and D. C. Peaslee, Phys. Rev. 91, (1953) 1232. 
adopted a liberal criterion in analyzing the data. They concluded the even smaller value: $b<.08$ for GT interaction and $b \leqslant .20$ for Fermi interaction.

In conclusion, it seems that the Fierz interference term $b / w$ is effectively zero and can be neglected in most applications. Nevertheless. an accurate and precise $\beta$-spectrum of $\mathrm{He}^{6}$ will provide more convincing evidence.

TABLE I

Fierz-Type Interferexce in Allowed Spectra

\begin{tabular}{lcc|c}
\hline \multicolumn{2}{c|}{ Radiation $W_{0}$ in $m_{0} c^{2}$} & $b$ \\
\hline $\mathrm{N}^{13}$ & $\beta+$ & 3.44 & $-0.08<b<0.18$ \\
$\mathrm{~S}^{35}$ & $\beta-$ & 1.33 & $-0.24<b<0.24$ \\
$\mathrm{C}^{1}{ }^{64}$ & $\beta-$ & 2.119 & $-11.12<b<0.16$ \\
$\mathrm{C}^{\prime}{ }^{64}$ & $\beta+$ & 2.285 & $-0.20<b<0.06$ \\
From Reference 22 &
\end{tabular}

\section{$\S$ 4. Fermi and Gamow-Teller Interaction}

One of the most outstanding arguments for the Gamow-Teller interaction is obtained from the decay of $\mathrm{He}^{6} \rightarrow \mathrm{Li}^{6}, \Delta J=1$ (no) which is not allowed by the Fermi selection rules. Its " $f t$ " value of 815 sec classifies it as a super allowed transition. Another strong argument for this interaction is given by the existence of the group of unique forbidden spectra which are given by pure Gamow-Teller interaction.

On the other hand, consider the two positron emitters ${ }^{24 a}$

$$
0^{14} \stackrel{\beta^{+}}{\longrightarrow} \mathrm{N}^{14 *} \text { and } \mathrm{C}^{10} \stackrel{\beta^{+}}{\rightarrow} \mathrm{B}^{10 * *}
$$

The $\mathrm{O}^{14}$ nucleus decays through an allowed positron transition to an excited state of $\mathrm{N}^{14}$ at $2.30 \pm 0.03 \mathrm{MeV}$ (see Fig. 8). The assumption of
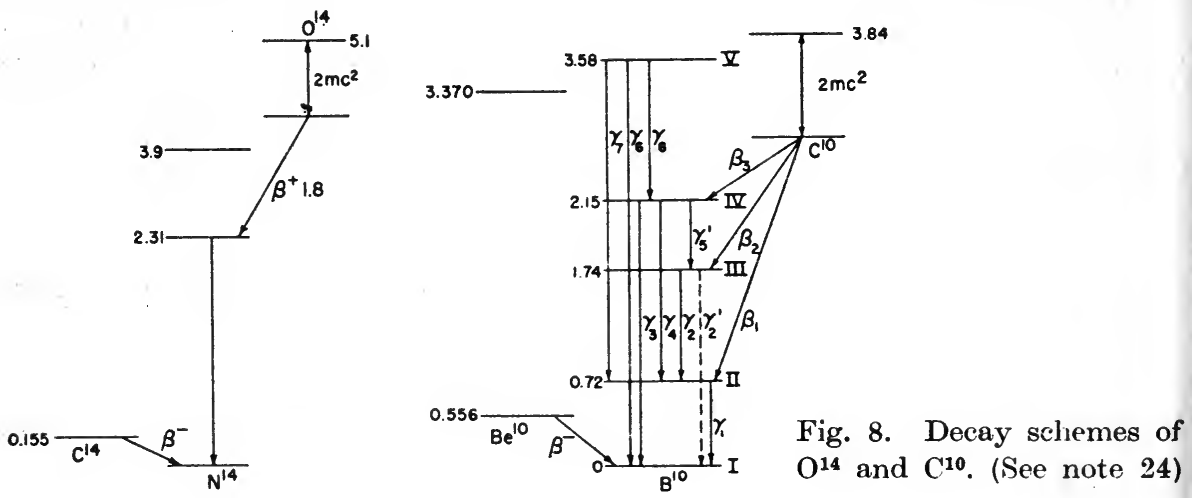

$24 a$ B. Sherr and J. B. Gerhart, Phys. Rev. 91, (1953) 909.

J. B. Gerhart Phys. Rev. 95, (1954) 288. 
charge independence leads to the expectation that the ground states of $\mathrm{C}^{14}$ and $\mathrm{O}^{14}$ and one of the excited states of $\mathrm{N}^{14 *}$ form a $T=1$ charge multiplet. Now, the measured energy of the excited state in $\mathrm{N}^{14}$ agrees closely with the calculated energy for the $T=1$ charge multiplet state in $\mathrm{N}^{14}$. The absence ${ }^{25}$ of $\alpha$ and scattered deuterons from the 2.31 excited level of the $\mathrm{O}^{16}(\mathrm{~d}, \alpha) \mathrm{N}^{14}$ and $\mathrm{N}^{14}\left(\mathrm{~d}, \mathrm{~d}^{1}\right) \mathrm{N}^{14}$ reactions, also lends weight to the identification of this $\mathrm{N}^{14 *}$ as the analog state of $T=1$. Since the spin of the ground state of $\mathrm{C}^{14}$ was measured to be zero, this leads to the conclusion that $\mathrm{O}^{14} \stackrel{\beta+}{\rightarrow} \mathrm{N}^{14 *}$ is undergoing a $0 \longrightarrow 0$ (no) $\beta$-transition which is strictly forbidden by the Gamow-Teller selection rulès and allowed only by the Fermi interaction. This was the first definite indication of the necessary presence of the Fermi interaction as well as the GT interaction.

The same reasoning applies to $\mathrm{C}^{10} \stackrel{\beta+}{-\rightarrow} \rightarrow \mathrm{B}^{10} 24 \mathrm{c}$.

Recently, the $\beta^{+}$-transition from the ground state of (' 134 to that of $\mathrm{S}^{34}$ has also been identified as $0 \longrightarrow 0$ (no) transition ${ }^{24^{\mathrm{b}}}$.

Since both Fermi and GT interactions have shown their presence in beta processes, what is the relative contribution of each of these two interactions? Several attempts had been made to evaluate the relative contributions of these two interactions, but most of them failed to obtain a consistent picture. Recently. Winther and Kofoed-Hansen ${ }^{26}$, Trigg ${ }^{27}$ and Bouchez and Nataf ${ }^{28}$ independently analyzed the problem with the aid of the nuclear shell model and arrived at the same conclusion that the Fermi interaction is approximately of the same strength as the GamowTeller interaction.

Kofoed-Hansen and.Winther used the expression for the comparative half-life

$$
f t=\frac{B}{(1-x)\left|M_{F}\right|^{2}+x\left|M_{G-T}\right|^{2}}
$$

where $x$ is the relative contribution of the GT interaction.

If the matrix elements $\left|M_{F}\right|^{2}$ and $\left|M_{G-T}\right|^{2}$ for a $\beta$-transition can be evaluated, then a straight line of $B$ vs $x$ can be drawn with the measured $f t$ value for the $\beta$-decay. A number of such cases will give a set of straight

24b W. Arber and P. Stähelin. Helv. Phys. Acta 26, (1953) 433.

24c D. Green and J. R. Richardson, Bull. Am. Phys. Soc. 29 Abstract L7, 1954.

25 C. Bockelman, C. Browne, A. Sperduto and W. Buechner, Phys. Rev. 90, (1953) 340; R. F. Adair, Phys. Rev. 87, (1952) 1041.

26 O. Kofoed-Hansen and A. Winther, Phys. Rev. 86, (1952) 428; Physica 18, (1952) 1079; Dan. Mat. Fys. Medd. 27, no. 14 (1953).

27 G. Trigg, Phys. Rev. 86, (1952) 506.

28 R. Bouchez et R. Natof, Comptes rendus, Ac. Sc. Paris 234, (1952) 86; Physica 18, (1952) 1013; J. Phys. Radium 14, (1953) 15. 
lines. A common intersection point of these lines will represent the fixed value of $B$ and $x$ in which we are interested.

The matrix elements $\left|M_{F}\right|^{2}$ and $\left|M_{G-T}\right|^{2}$ involved in the transitions between a pair of mirror nuclei or members of isobaric triads can be evaluated if the nuclear orbital states are known. These evaluations are based on Wigner's supermultiplet model. However, the most reliable cases would be those mirror transitions which take place between nuclei with closed shells in both neutrons and protons \pm one nucleon. This view is supported by the fact that only these mirror nuclei exhibit the magnetic moments which are not far away from the predicted Schmidt lines of the single particle model.

Choosing nuclei with closed shells \pm one nucleon, the results are shown in Fig. 9. A least square fit of the data yields

and

$$
B=2600 \pm 85
$$

$$
x=0.50 \pm 0.05 \text {. }
$$

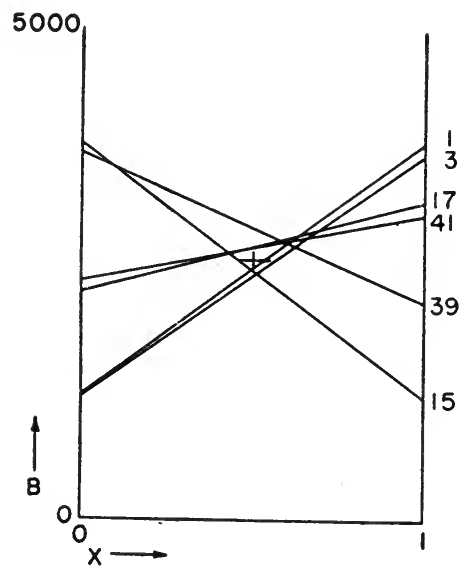

Fig. 9. Plot of $B=f t\left\{(1-x)\left|\int 1\right|^{2}+x\left|\int \sigma\right|^{2}\right\}$ against $x$ for various values of $A$ which are shown on right of diagram.

(See note 26)

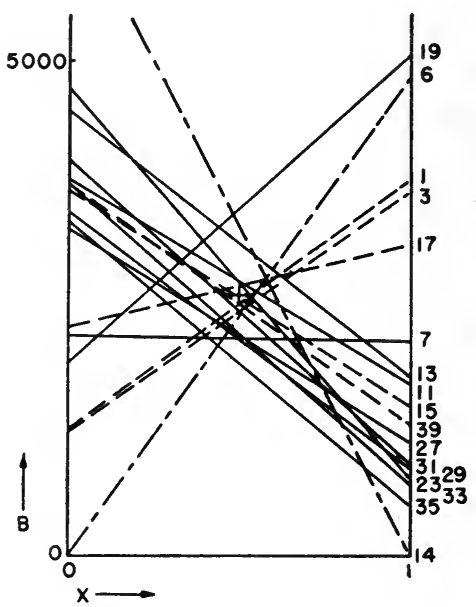

Fig. 10. $B(x)$ lines for mirror transitions with adjusted matrix elements and for $\mathrm{He}^{6}$ and $\mathrm{O}^{14}$ decay. Mass numbers are indicated.

(See note 26)

...... closed shell \pm 1 transitions other mirror transitions

$-\cdot-\cdot-\mathrm{He}^{6}$ and $\mathrm{O}^{14}$ decay

However, when this simple method of analysis is applied to the rest of the mirror nuclei, the lines begin to diverge and all the lines no longer intersect within a small region. To explain this discrepancy Winther and Kofoed-Hansen assumed that the angular momentum was shared between the single particle and the other particles in the nucleus, as would be consistent with the deviations of the magnetic moments from the Schmidt 
limits. Using Aage Bohr and B. Mottelson's model 29, an approximate formula for the $\left|M_{G \sim T}\right|^{2}$ may be written as

$$
\begin{aligned}
& \left|M_{G-T}\right|^{2}=4 \frac{(J+1)}{J}\left|<S_{Z}>\right|^{2} \approx 4 \frac{J+1}{J} \frac{\left(\mu-g_{l} J\right)^{2}}{\left(g_{s}-g_{l}\right)^{2}} \\
& \left|M_{F}\right|^{2}=1 \text { for } \Delta I=0
\end{aligned}
$$

TABLE II

Trigg's Analysis of the " $f t$ " values of Mirror Nuclei

\begin{tabular}{|c|c|c|c|c|c|c|c|}
\hline Transition & I & $\begin{array}{c}a^{2} \\
(S-M W)\end{array}$ & $\begin{array}{c}a^{2} \\
\text { (ad- } \\
\text { justed) }\end{array}$ & $\left|\int 6\right|^{2}$ & $|M|^{2}$ & $\begin{array}{l}r=0.5 \\
f t|M|^{2}\end{array}$ & $\begin{array}{c}r=1 \\
f t|M|^{2}\end{array}$ \\
\hline${ }^{1} \mathrm{n} \rightarrow{ }^{1} \mathrm{H}$ & $1 / 2$ & 1 & & 3 & 3.5 & 4900 & 5600 \\
\hline${ }^{3} \mathrm{H} \rightarrow{ }^{3} \mathrm{He}$ & $1 / 2$ & 1 & 0.95 & 2.61 & 3.11 & 3400 & 3970 \\
\hline${ }^{7} \mathrm{Be} \rightarrow{ }^{7} \mathrm{Li}$ & $3 / 2$ & 0.85 & 0.85 & 0.96 & 1.46 & 3350 & 4410 \\
\hline${ }^{11} \mathrm{C} \rightarrow{ }^{11} \mathrm{~B}$ & $3 / 2$ & 0.76 & 0.68 & 0.40 & 0.90 & 3500 & 5460 \\
\hline${ }^{13} \mathrm{~N} \rightarrow{ }^{13} \mathrm{C}$ & $1 / 2$ & $\mathbf{0}$ & 0.05 & 0.21 & 0.71 & 3400 & 5808 \\
\hline${ }^{15} \mathrm{O} \rightarrow{ }^{15} \mathrm{~N}$ & $1 / 2$ & 0 & 0 & 0.33 & 0.83 & 3100 & 4920 \\
\hline${ }^{17} \mathrm{~F} \rightarrow{ }^{17} \mathrm{O}$ & $5 / 2$ & 0.99 & 0.95 & 1.17 & 1.67 & 3800 & 4880 \\
\hline${ }^{19} \mathrm{Ne} \rightarrow{ }^{19} \mathrm{~F}$ & $1 / 2$ & 0.95 & 0.90 & 2.35 & 2.85 & 5700 & 6100 \\
\hline${ }^{21} \mathrm{Na} \rightarrow{ }^{21} \mathrm{Ne}$ & $3 / 2$ & - & 0.70 & 0.45 & 0.95 & 3400 & \\
\hline${ }^{21} \mathrm{Na} \rightarrow{ }^{21} \mathrm{Ne}$ & $(5 / 2)$ & - & 0.75 & 0.46 & 0.96 & 3400 & 5220 \\
\hline${ }^{23} \mathrm{Mg} \rightarrow{ }^{23} \mathrm{Na}$ & $3 / 2$ & 0.65 & 0.60 & 0.22 & 0.72 & 3300 & 5610 \\
\hline${ }^{25} \mathrm{Al} \rightarrow{ }^{25} \mathrm{Mg}$ & $5 / 2$ & 0.80 & 0.80 & 0.59 & 1.09 & 3300 & 4700 \\
\hline${ }^{27} \mathrm{Si} \rightarrow{ }^{27} \mathrm{Al}$ & $5 / 2$ & 0.78 & 0.73 & 0.40 & 0.90 & 3300 & 5180 \\
\hline${ }^{29} \mathrm{P} \rightarrow{ }^{29} \mathrm{Si}$ & $(1 / 2)$ & - & 0.52 & 0.39 & 0.89 & 3400 & \\
\hline${ }^{29} \mathrm{P} \rightarrow{ }^{29} \mathrm{Si}$ & $(3 / 2)$ & - & 0.07 & 0.40 & 0.90 & 3400 & 5280 \\
\hline${ }^{31} \mathrm{Si} \rightarrow{ }^{31} \mathrm{P}$ & $1 / 2$ & 0.45 & 0.48 & 0.28 & 0.78 & 3400 & 5504 \\
\hline${ }^{33} \mathrm{Cl} \rightarrow{ }^{33} \mathrm{~S}$ & $3 / 2$ & 0.20 & 0.10 & 0.32 & 0.82 & 2900 & 4620 \\
\hline${ }^{35} \mathrm{~A} \rightarrow{ }^{35} \mathrm{Cl}$ & $3 / 2$ & 0.22 & $0.12^{\mathrm{b}}$ & 0.28 & 0.68 & 2700 & 4350 \\
\hline${ }^{37} \mathrm{~K} \rightarrow{ }^{37} \mathrm{~A}$ & $(3 / 2)$ & - & 0.00 & 0.60 & 1.10 & 3000 & 4320 \\
\hline${ }^{39} \mathrm{Ca} \rightarrow{ }^{39} \mathrm{~K}$ & $3 / 2$ & 0.08 & 0.08 & 0.33 & 0.83 & 3300 & 5370 \\
\hline${ }^{41} \mathrm{Sc} \rightarrow{ }^{41} \mathrm{Ca}$ & $(7 / 2)$ & - & 0.88 & 0.79 & 1.29 & 3300 & 4570 \\
\hline${ }^{7} \mathrm{Be} \rightarrow{ }^{7} \mathrm{Li}$ & $3 / 2 \rightarrow 1 / 2$ & $x\left({ }^{2} P_{3 / 2}\right.$ & $\left.{ }^{2} P_{1 / 2}\right)$ & $4 / 3 x$ & $4 / 3 x$ & $4800 x^{\mathrm{c}}$ & \\
\hline${ }^{6} \mathrm{He} \rightarrow{ }^{6} \mathrm{Li}$ & $0 \rightarrow 1$ & ${ }^{1} S_{0}$ & ${ }^{3} S_{1}$ & 6 & -6 & 3300 & 4890 \\
\hline${ }^{10} \mathrm{C} \rightarrow{ }^{10} \mathrm{~B}$ & $(0 \rightarrow 1)$ & $y\left({ }^{1} S_{0}-\right.$ & $\left.\rightarrow{ }^{3} S_{1}\right)$ & $6 y$ & $6 y$ & $12000 y^{d}$ & \\
\hline${ }^{10} \mathrm{C} \rightarrow{ }^{10} \mathrm{~B}$ & $(0 \rightarrow 0)$ & ${ }^{1} S_{0}$ & ${ }^{1} S_{0}$ & 0 & 1 & - & \\
\hline${ }^{14} \mathrm{O} \rightarrow{ }^{14} \mathrm{~N}$ & $(0 \rightarrow 0)$ & ${ }^{1} S_{0}$ & $\rightarrow{ }^{1} S_{0}$ & 0 & 1 & 3300 & 6300 \\
\hline${ }^{18} \mathrm{~F} \rightarrow{ }^{18} \mathrm{O}$ & $(1 \rightarrow 0)$ & $z\left({ }^{3} S_{1}\right.$ & $\left.\rightarrow{ }^{1} S_{0}\right)$ & $2 z$ & $2 z$ & $7400 z^{\mathrm{e}}$ & \\
\hline
\end{tabular}
( $a^{2}$ adjusted for best fit, $r=\left|C_{F}\right|^{2} /\left|C_{G-T}\right|^{2}$

The last column is added for $r=1$. ( ) assumed value.

a, $\mathrm{b}-\mathrm{a}$ good fit would require excessively large adjustment.

c $-x \sim 0.7$ consistent with $85 \%{ }^{2} P_{3 / 3}$ and $85 \%{ }^{2} P_{1 / 3}$.

d $-y \sim 0.3$ consistent with $30 \%{ }^{3} S_{1}$ in the linear combination ${ }^{3} S_{1}+{ }^{3} D_{1}+{ }^{1} P_{1}$. e $-z \sim 0.45$ consistent with $45 \%{ }^{3} S_{1}$ in the linear combination ${ }^{3} S_{1}+{ }^{3} D_{1}+{ }^{1} P_{1}$. 
where $\mu$ is the magnetic moment of the daughter nucleus and the $g$ 's are the usual $g$-factors.

The improved $B-x$ plots thus obtained are shown in Fig. 10. The spread of the intersecting region although greater than the experimental uncertainties, could be due to the approximate nature of the formula for $\left|M_{G-T}\right|^{2}$.

Trigg also made a detailed analysis of all the available data on the super-allowed transitions. First he investigated the matrix elements of the light nuclei assuming a mixture of $L=J \pm 1 / 2$ and $L^{\prime}=J \mp 1 / 2$. Then he evaluated the amount of admixture from the deviation of the observed magnetic moments from spin-orbit coupling (Schmidt) limits. In this way the best ratio of $\left.\left|\mathrm{C}_{F}\right|^{2}|| \mathrm{C}_{G-T}\right|^{2}$ is found to be around 1 (Table II). Nataf and Bouchez arrived at a similar conclusion in their analysis of the data of the super allowed transitions. $W u^{30}$ and Konopinski and Langer ${ }^{31}$ compared the $f t$ values and the matrix elements of the $\mathrm{He}^{6}$ and $\mathrm{H}^{3}$ decays and obtained the ratio of the two interactions to be $\leqslant 1.4_{-0.7}^{+1.0}$. Blatt ${ }^{32}$ used the $\mathrm{O}^{14}$ and $\mathrm{H}^{3}$ decays and obtained the ratio of the two interactions equal to $0.54_{-0.25}^{+0.5}$. Sheer and Gerhart ${ }^{24}$ calculated the ratio $\left.\left|\mathrm{C}_{F}\right|^{2}|| \mathrm{C}_{G-T}\right|^{2}$ from their latest data on $\mathrm{C}^{10}$. They found a value $0.79_{-0.13}^{+0.27}$ for $L-S$ coupling and $0.44_{-0.07}^{+0.15}$ for $j-j$ coupling. To obtain more accurate value of the ratio of $\left|\mathrm{C}_{F}\right|^{2} /\left|\mathrm{C}_{G-T}\right|^{2}$, a more accurate determination of the $f t$ value and a better knowledge of the nuclear wave functions are required.

However, the nuclear matrix element for the $0 \rightarrow 0(\Delta T=0$ no $)$ transition is given in terms of the isotopic spin quantum numbers $T$ and $T_{Z}$.

$$
\left|\int 1\right|^{2}=T(T+1)-T_{z} T_{z}^{\prime}=2 .
$$

This calculation of the matrix elements depends solely on the assumption of charge independence of nuclear forces and needs no further assumption about nuclear structure. Gerhart ${ }^{24}$ recently measured the $\beta^{+}$spectrum of $\mathrm{O}^{14}$ and redetermined its half-life. The accurate $f t$ value of $\mathrm{O}^{14}$ is $3275 \pm 75 \mathrm{sec}$. From this $f t$ value, he obtained the Fermi coupling constant to be $g_{F}=1.374$ $\pm 0.016 \ldots 10^{-49} \mathrm{ergs} \mathrm{cm}^{3}$.

In comparison with the $f t$ value of neutron decay, he obtained $R=\left|\frac{C_{G T}}{C_{F}}\right|^{2}=1.37_{-0.30}^{+0.40}$. The entire uncertainty in $R$ is due to the uncertainty in the neutron half-life. Therefore it emphasizes the importance of an accurate determination of the half-life of neutron.

29 A. Bohr and B. Mottelson, Dan. Mat. Fys. Medd. 27, no. 16 (1953).

30 C. S. Wu, Physica 18, (1952) 989.

31 E. J. Konopinski and L. M. Langer, Annual Review of Nuclear Science 1953.

32 J. Blatt, Phys. Rev. 89, (1953) 83, 86. 
The $f t$ values of the other member of the group of $0^{+} \rightarrow 0^{+}$transitions ${ }^{24 b}, 24 \mathrm{c}$ are

$\begin{array}{lllll}\mathrm{C}^{10} & \mathrm{O}^{14} & \mathrm{Al}^{26} & \mathrm{Cl}^{26} & \mathrm{~K}^{38}\end{array}$

$5900 \pm 2400 \quad 3275 \pm 75 \quad 3312 \pm 857 \quad 3220 \pm 200 \quad 2238 \pm 390$

The $f t$ va!ue of $\mathrm{K}^{38}$ seems to be definitely out of line in comparison with the others.

\section{$\S 5$. Rest Mass of the Neutrino}

All experimental evidence indicates that the rest mass of the neutrino must be very small. Therefore, it is justified to assume zero rest mass of neutrino for simplicity and obtain the statistical factor of $\beta$-distribution for allowed transition as

$$
\begin{aligned}
N_{ \pm}(W) d W & \sim P W P_{v} W_{v} d W \\
& \sim P W\left(W_{0}-W\right)^{2} d W
\end{aligned}
$$

Based on this assumption of zero neutrino mass, one obtains the maximum $\beta$-energy $W_{0}$ in the allowed $\beta$-transition by extrapolating the straight Kurie plot as

$$
\left[\frac{N_{ \pm}(W)}{F(\mp Z, W) P W}\right]^{1 / 2}=K\left(W_{0}-W\right)
$$

Actually, the precise shape of the allowed $\beta$-spectrum in the vicinity of its upper limit depends on the rest mass of the neutrino. If the rest mass of neutrino is different from zero and equal to $m_{\nu} C^{2}$, the statistical factor becomes

$$
\left.N_{ \pm}(W) d W \sim P W\left(W_{0}+m_{v} C^{2}-W\right) \times\left[\left(W_{0}-m_{\nu} C^{2}-W\right)^{2}-m_{\nu} C^{2}\right)^{2}\right]^{\xi} d W
$$

Near the upper limit of the $\beta$-spectrum, the factors $P$ and $W$ may be regarded as constant as $W \rightarrow W_{0}$. The factor $\left(W_{0}+m_{v} C^{2}-W\right)$ which is the total energy of the neutrino may also be considered as constant as long as $W_{0}-W \ll m_{v} C^{2}$. However, the last term which represents the momentum of the neutrino may be rewritten as

$$
\left[2 m_{v} C^{2}\left(W_{0}-W\right)\right]^{1 / 2} \text {. }
$$

This is the non-relativistic expression of the momentum of neutrino. Therefore at its upper limit the energy spectrum would go to zero with a vertical tangent as $\left(W_{0}-W\right)^{2 / 2}$, as contrasted with the curve for a zero neutrino rest mass which would have a horizontal tangent as it approaches zero as $\left(W_{0}-W\right)^{2}$.

The general evidence as based on the best measured $\beta$-spectra ruled out the vertical approach of the upper end of the spectrum to the energy 
axis. Nevertheless, to set an upper limit to the rest mass of neutrino, one must carefully compare the upper portion of the experimentally observed Kurie plot with theoretically predicted ones of various assumed neutrino masses.

Futhermore, for finite neutrino mass, the energy distribution of an allowed spectrum is determined not by the statistical factor alone but also by a relativistic correction factor ${ }^{33}$ which arises from the summation over spins of the emitted particles. The correction factor is represented by

$$
\left[1+b \frac{m_{\nu} C^{2} m_{e} C^{2}}{W\left(W_{0}+m_{\nu} C^{2}-W\right)}\right]
$$

where $b$ is a constant which may vary between -1 and +1 according to the definite combinations of $\beta$-interactions assumed. However, when the value of $b$ is not known, one can only set an upper limit on $m_{\nu} C^{2}$ for a possible value of $b$ varying from -1 to +1 . If the mass of a neutrino could be determined by some other methods, the analysis of the spectrum near its maximum energy could yield a value of $b$.

The most suitable $\beta$-spectra which could be used for evaluating the mass of neutrino would be those of low maximum energies. The $\beta$-spectrum of $\mathrm{S}^{35}$ was the first one used for this evaluation and an upper limit of $5 \mathrm{keV}{ }^{34}$ for the neutrino mass was thus obtained. The $\beta$-spectrum of $\mathrm{H}^{3}$ has an upper energy of $18.6 \mathrm{keV}$ so that a precise measurement of the upper end of its spectrum offers the best case for this determination.

Two independent groups ${ }^{35}$ investigated the $\mathrm{H}^{3} \beta$-spectrum by proportional counter methods at about the same time and gave an upper limit of the neutrino mass less than $1 \mathrm{keV}$. This $1 \mathrm{keV}$ is the maximum deviation of the Kurie plot from a straight line that could be allowed from the data. Because of the arbitrariness in assigning the value to the constant $b$, the observed deviation may represent only $1 / 5 m_{v}$ and therefore the upper limit may be raised to $5 \mathrm{keV}$ as suggested by Kofoed-Hansen ${ }^{36}$.

Recently the $\mathrm{H}^{\grave{3}} \beta$-spectrum was investigated in a high resolution magnetic $\beta$-spectrometer ${ }^{37 *}$. That portion of the spectrum close to the

33 J. R. Pruett, Phys. Rev. 73, (1948) 219.

34 C. S. Cook, L. M. Langer and H. C. Price, Phys. Rev. 73, (1948) 1395.

35 G. C. Hanna and B. Pontecorvo, Phys. Rev. 75, (1949) 983.; S. C. Curran, J. Angus and A. L. Cockroft, Nature 162, (1948) 302; Phil. Mag. 50, (1949) 53; Phys. Rev. 76, (1949) 853 L.

36 O. Kofoed-Hansen, Physica 18, (1952) 1287.

37 L. M. Langer and R. J. D. Moffat, Phys. Rev. 88, (1952) 689.

* The maximum energy limit obtained in this investigation is $17.95 \pm .10 \mathrm{keV}$. The $f t$ value of $\mathrm{H}^{8}$ thus calculated is $1014 \pm 20 \mathrm{sec}$ which does not agree with the common intersection point of the $B-x$ diagram (See note 26). 
upper limit of the energy was extensively studied (Fig. 11). Because of the high resolution used on the magnetic spectrometer compared with that from the proportional counter the upper limit of the neutrino mass obtained in this case is reduced to $250 \mathrm{eV}$. However, a limit of $1 \mathrm{keV}$ should be adopted because of the uncertainty in the value of $b$.

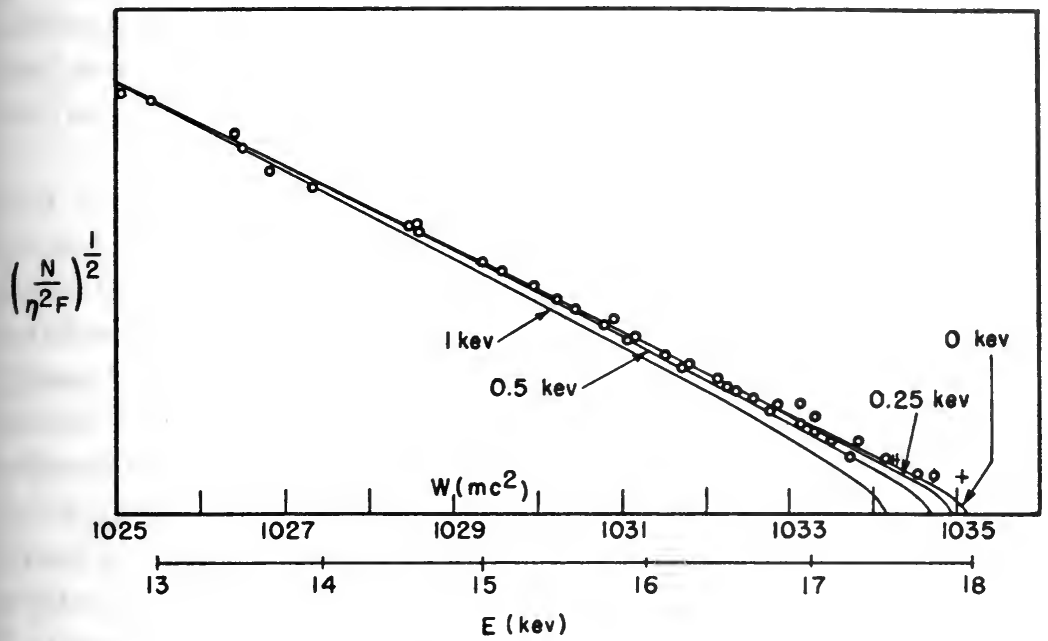

Fig. 11. Kurie plot of $\mathrm{H}^{3}$ for various values of the rest mass of the neutrino. Circles which are experimental points lie on theoretical curve corresponding to zero rest mass. (See note 37 )

In principle, there is a direct method of determining the rest mass of a neutrino. That is to measure separately the available energy $\Delta M$ in the $\beta$-transition and the maximum $\beta$-energy $W_{0}$ and then make use of the energy balance

$$
m_{y} C^{2}=\Delta M-W_{0} .
$$

Unfortunately the best method of determining $W_{0}$ is by extrapolating the straight Kurie plot which involves the assumption of zero neutrino mass. Therefore this method is intrinsically unsuitable for this determination ${ }^{38}$.

\section{§6. Forbidden Transitions}

Until 1949, all the $\beta$-spectra investigated had an allowed shape except the $\beta$-spectrum of RaE, which yielded the only known deviation from the allowed shape. The reason for this uniformity in shape is now rather well understood (Ch. X). As most of the forbidden spectra investigated 
before 1949 belonged to the first forbidden $\Delta J=0$ or 1 transition, no deviations from the allowed shape should be expected if the $\beta$-interaction involves only certain linear combinations.

Ever since long life radioactive isotopes of high specific activity could be prepared in a nuclear reactor of high neutron flux and the isotopes of low abundance isotopically enriched by the electromagnetic separation method large numbers of $\beta$-spectra of higher order of forbiddenness have been carefully studied. As predicted by the theory definite deviations from allowed shape have been found in most of them.

The most striking group of forbidden $\beta$-spectra is that obtained from the unique transitions where $\Delta J=n+1$, known as the parity favored group. Spectra of this group with $n=1,2$ and 3 have all been observed and identified. The shape of this group of spectra is unique and follows the theoretical predictions with surprising accuracy. There is also the group of parityforbidden spectra of $n=2$ or 3 . Although all of them showed definite deviations from the allowed shape they are not unique in form. To interpret spectra of this type, great caution must be exercised. As shown in eq. $(\mathrm{X}-30)$, several matrix elements whose magnitudes are unknown coexist and each of them exerts a different influence on the shape of the spectrum. One could adjust the ratio of the various unknown matrix elements to make the resultant spectrum fit the observed one. This procedure might help to eliminate a few less flexible single $\beta$-interactions such as the scalar or axial vector. It might also have led to erroneous conclusions were not a systematic study of the comparative half-lives, selection rules, and the nuclear recoil experiments made at the same time. As will be shown in section 12, the analysis of this group of spectra has nevertheless yielded valuable information on the $\beta$-interaction.

As to the first forbidden spectra of allowed shape, it has turned out that they have provided some evidences for the elimination of certain possible linear combinations in the $\beta$-interaction.

\section{$\S 7$. The Unique Forbidden Spectra}

The energy distribution of the $\beta$-spectra from the transitions with $\Delta J=n+1$ was theoretically predicted to be unique since just one nuclear matrix element fails to vanish for it. Furthermore, it could be realized only through the Gamow-Teller interaction. The eventual observation of abundant examples of unique first forbidden spectra together with representative cases of the unique second and third forbidden spectra gave convincing evidence that the present approach to the theory of $\beta$-decay was correct. It provides additional evidence for the existence of the Gamow-Teller interaction; the well known argument concerning the 
$\beta$-decay of the $\mathrm{He}^{6}$ as a super-allowed transition also requires the existence of a GT interaction.

According to eq. (X.34) when the Coulomb effect on the electron is neglected, the unique shape factors for various degrees of forbiddenness can be simplified as

\section{Unique Shape Factor}

$$
\begin{aligned}
& \text { lst } \quad a_{1}=\left(W^{2}-1\right)+\left(W_{0}-W\right)^{2} \\
& \text { 2nd } a_{2}=\left(W^{2}-1\right)^{2}+\left(W_{0}-W\right)^{4}+10 /{ }_{3}\left(W^{2}-1\right)\left(W_{0}-W\right)^{2} \\
& \text { 3rd } a_{3}=\left(W^{2}-1\right)^{3}+\left(W_{0}-W\right)^{6}+7\left(W^{2}-1\right)\left(W_{0}-W\right)^{2} \\
& {\left[\left(W^{2}-1\right)+\left(W_{0}-W\right)^{2}\right]}
\end{aligned}
$$

The energy dependence of these factors strongly emphasizes the contribution of high energy $\beta$-particles; therefore the energy distribution of the unique $\beta$-spectra is readily distinguishable from an allowed one. To illustrate the characteristics of each of these factors, $a_{1}, a_{2}$ and $a_{3}$ are plotted vs energy for a $\beta$-transition of maximum total energy $W_{0}=3 m c^{2}$ as shown in Fig. 12. All three factors decrease rapidly with decreasing energy at the high energy end. The rate of decrease becomes smaller in the low

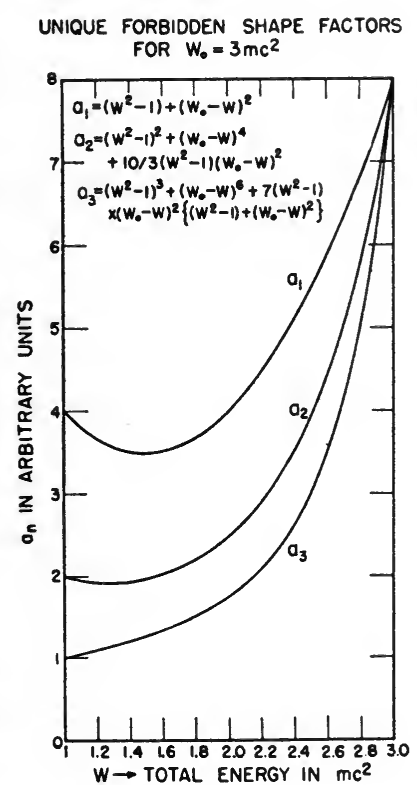

Fig. 12.

Unique forbidden shape factors for $W_{0}=3 m c^{2}$
UNCORRECTED KURIE PLOTS

UNIQUE Ist, 2 nd, AND 3 rd FORBIDDEN TRANSITIONS

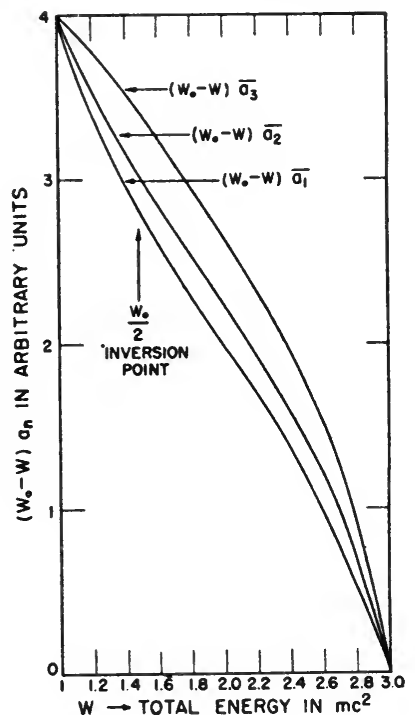

Fig. 13.

Uncorrected Kurie plots for unique 1st, 2nd, and 3rd forbidden transitions 
energy region. For $a_{1}$, the factor reaches a minimum at an energy equal to $W_{0} / 2$ if $W_{0}>2 m c^{2}$. Then the curve swings up again below that energy. This effect makes the uncorrected Kurie plot bulge upward at high energy and also curve upward at low energy and thus produces an inversion point at $W_{0} / 2$ (Fig. 13). For comparison, the momentum distributions of an allowed spectrum and of the unique forbidden ones are exhibited in Fig. 14. (For simplicity no Coulomb effect is taken into account.) A pronounced shift to the high energy end for the unique forbidden spectra can be easily seen.

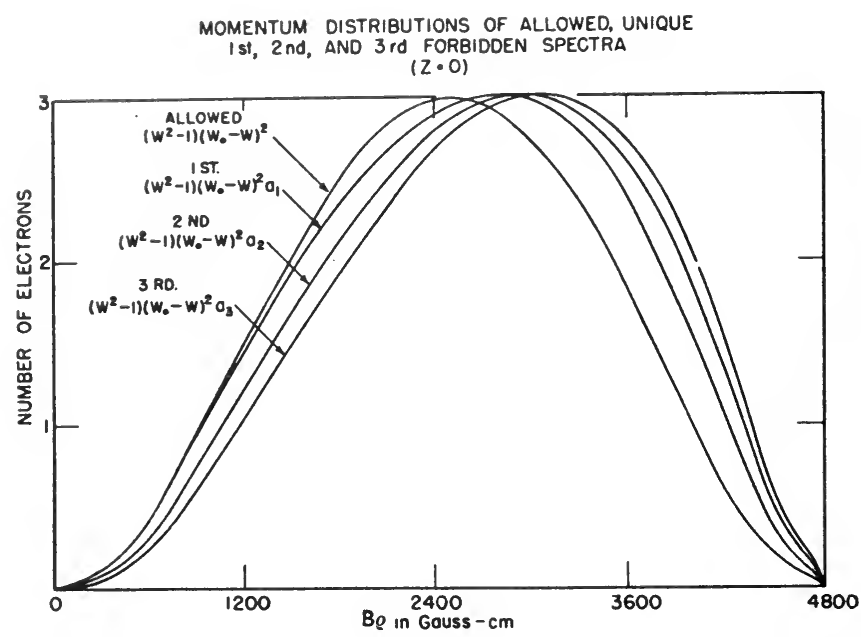

Fig. 14. Momentum distributions of allowed, unique 1st, 2nd and 3rd forbidden spectra with $Z=0$

Although the existence of the unique forbidden spectra was theoretically predicted as early as 1941, its confirmation was not reported until 1949. Langer and Price ${ }^{39}$ found that the $\beta$-spectrum of $\mathrm{Y}^{91}$ distinctly deviated from the allowed shape. According to its comparative half-life $\left(f_{0} t=5 \times 10^{8}\right.$ sec), this transition should be classified as second forbidden. But from shell model consideration, this transition should involve a total momentum change of two units and a parity change of ${ }_{39} \mathrm{Y}^{91}\left(p_{1 / 2}\right.$ odd $) \rightarrow{ }_{40} \mathrm{Z}^{91}\left(d_{5 / 3}\right.$ even). Therefore it is a unique first forbidden transition $(\Delta J=1$, yes). When the Kurie plot was divided by the corresponding unique shape factor $\left(a_{1}\right)^{1 / 3}$, all the curvatures were removed and an exceedingly striaght line resulted. (See Fig. 15.)

In deriving the formula (X.34) for the unique shape factor, $a_{n}$, the Coulomb effect on the electron is neglected. The unique shape factor $a_{n}$ is

39 L. M. Langer and H. C. Price Jr., Phys. Kev. 75, (1945) 1109; 76, (1949) 641. 
relatively insensitive to the Coulomb forces as contrasted with most types of forbidden spectra where the Coulomb effect on the shape factor is quite considerable. The inadequacy of the simplified formula for $S_{n}^{n+1}$ (X.34)

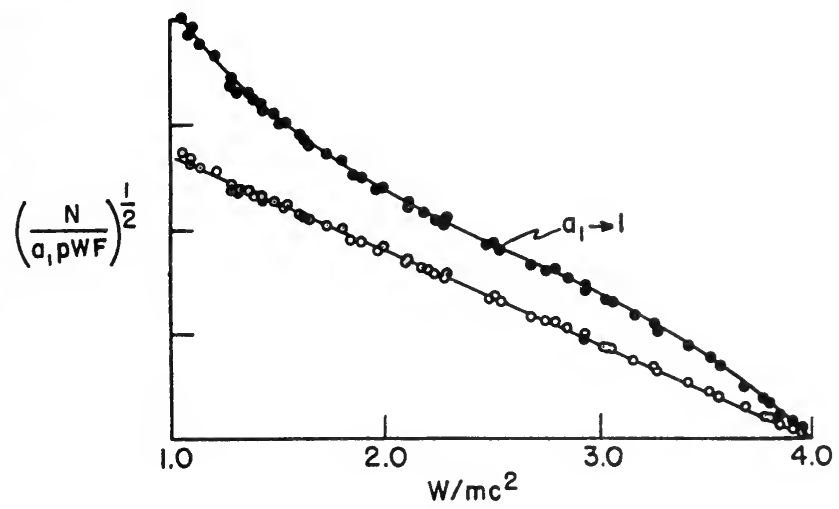

Fig. 15. The Kurie plot of $\mathrm{Y}^{91}$ using the unique lst forbidden correction factor $a$. (See note 39)

was detected for the case of $\mathrm{Tl}^{204}{ }^{40}$ where $Z$ is equal to 81 . Fig. 16 shows the three Kurie plots of $\mathrm{Tl}^{204} \beta$-spectrum.

The bottom one is the uncorrected Kurie plot $\left(a_{1}=1\right)$. The middle curve is corrected by the shape factor $a_{1}$ of the low $Z$ approximation (X.34). The linear plot starts to deviate upward around $350 \mathrm{keV}$. The top curve is corrected by the exact shape factor as represented by (X.35). It linearizes the plot all the way down to $100 \mathrm{keV}$. The deviation observed below $100 \mathrm{keV}$ could be due to finite source thickness.

There is only one case definitely observed for each of the unique forbidden spectra of $n=2$ and $n=3$. These are the $\mathrm{Be}^{10}$ and $\mathrm{K}^{40}$ for the second and third forbidden transition respectively. The shape of the $\beta$ spectrum of $\mathrm{Na}^{22}$ is investigated and also favors a unique second forbidden transition. $\mathrm{Be}^{10}$ is an even-even nucleus and is assumed

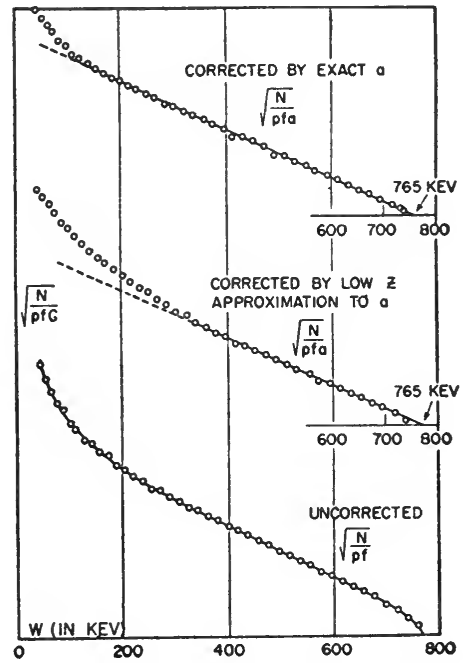

Fig. 16. The Kurie plots of the $\mathrm{Tl}^{204} \beta$-spectrum. (See note 40) to have zero spin, whereas the spin of $\mathrm{B}^{10}$, the product nucleus, has been found to be three. Thus the spin change involved in the transition is 
three. Furthermore, the shell model of the nucleus predicts no parity change for the transition. Thus $\Delta J=3$ (no). These are the conditions for the unique second forbidden transition given by the GT selection rules.

Experimental evidence that the spectrum shape of $\mathrm{Be}^{10}$ deviated from the alluwed shape was first reported by Bell et al. ${ }^{41}$ using a scintillation spectrometer. Wu and Feldman ${ }^{42}$ first inferred the unique shape factor $a_{2}$ of the $\mathrm{Be}^{10}$ spectrum by comparing the spectrum shape of thick sources of radioactive $\mathrm{BeO}\left(\sim 10 \mathrm{mg} / \mathrm{cm}^{2}\right)$ to that of the thick sources of inactive $\mathrm{BeO}$ containing activities of $\mathrm{Cu}^{64}$ and $\mathrm{Cl}^{36}$. Material of high specific activity was finally prepared by irradiating Be metal in the nuclear reactor and by further isotopically enriching it by electromagnetic separation ${ }^{43}$.

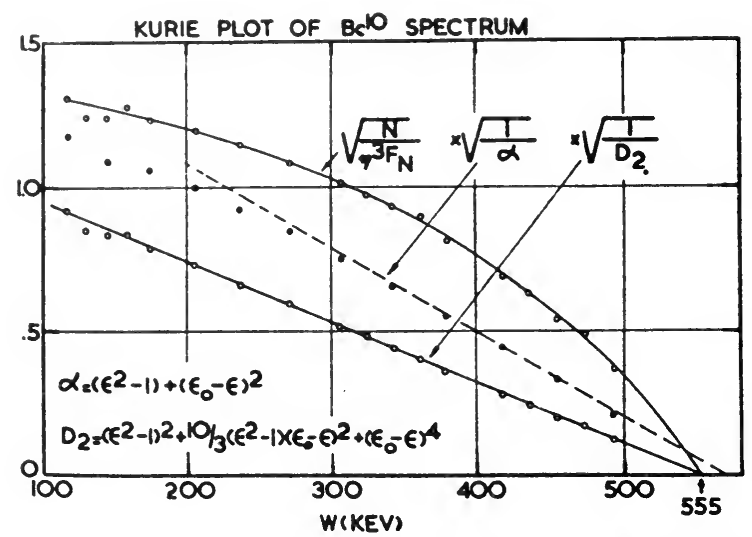

Fig. 17. Kurie plots of the $\mathrm{Be}^{10} \beta$-spectrum. From Rev. of Mod. Phys. 22, (1950) 386

When thin sources of less than $0.5 \mathrm{mg} / \mathrm{cm}^{2}$ were finally used the $\beta$-spectrum of $\mathrm{Be}^{10}$ obtained had the predicted unique shape from the upper energy end down to $120 \mathrm{keV}$. (See Fig. 17.) The results from various laboratories ${ }^{44}$ are in essential agreement with these results except for differences in the energy at which the low energy deviation sets in. This can be explained for the most part by the difference in uniformity and thickness of the sources used. This is certainly a great triumph for the theory of $\beta$-decay.

In the case of $\mathrm{K}^{40}$, the spin change is 4 units. The parity should change

41 P. R. Bell and J. M. Cassidy, Phys. Rev. 76, (1949) 183.

42 C. S. Wu and L. Feldman, Phys. Rev. 76, (1949) 698; L. Feldman and C. S. Wu, Phys. Rev. 76, (1949) 697.

43 C. P. Keim, Annual Review of Nuclear Science (1952).

44 H. W. Fulbright and J. C. D. Milton, Phys. Rev. 76, (1949) 1271 ; D. E. Alburger, D. J. Hughes and C. Eggler, Phys. Rev. 78, (1950) 318; P. R. Bell and J. M. Cassidy, Phys. Rev. 77 (1950) 301; L. Feldman and C. S. Wu, Phys. Rev. 78, (1950) 318. 
according to the shell model prediction. Therefore $K^{40}$ represents the unique third forbidden transition. Unfortunately $\mathrm{K}^{40}$ has a half-life of $2.7 \times 10^{9}$ years and the natural abundance of $\mathrm{K}^{40}$ is only $0.016 \%$. To obtain an undistorted spectrum of $\mathrm{K}^{40}$, a highly enriched $\mathrm{K}^{40}$ source must be used. Some $\mathrm{KCl}$ enriched to $\sim 10 \%$ in $\mathrm{K}^{40}$ was prepared by electromagnetic separation at Oak Ridge under the supervision of C. P. Keim ${ }^{43}$. Feldman and $\mathrm{Wu}{ }^{44,65}$ prepared uniform sources of $2.5 \mathrm{mg} / \mathrm{cm}^{2}$ and investigated its $\beta$-spectrum in the solenoid spectrometer of a momentum resolution of $9 \%$. The uncorrected Kurie plot is definitely forbidden. The unique third forbidden shape factor $a_{3}=p^{6}+7 p^{4} q^{2}+7 p^{2} q^{4}+q^{6}$ provides a good fit to the data from the end point $1325 \pm 15 \mathrm{keV}$ down to $500 \mathrm{keV}$ with the deviation increasing towards lower energies. The deviation is readily explained by the excessive thickness of the source. These results are in general agreement with thick source $\left(\sim 2.5 \mathrm{mg} / \mathrm{cm}^{2}\right)$ spectrum measurements of $\mathrm{K}^{40}$ reported by other laboratories ${ }^{45}$. Alburger ${ }^{45}$ using a thin lens with a resolution of about $17 \%$ found agreement with shape factor $a_{3}$ down to about $500 \mathrm{keV}$. Bell, Weaver and Cassidy ${ }^{45}$ using a scintillation spectrometer found the deviation to begin at about $700 \mathrm{keV}$.

\section{$\S 8$. Half-lives of the Unique Transitions}

The comparative half-life of a $\beta$-decay is defined as the product of the observed half-life " $t$ " multiplied by a function $f_{n}$ where $f_{n}$ is given by

$$
f_{n}=\frac{g^{2}}{2 \pi} \int_{1}^{W_{0}} S_{n} F_{0}(W, \mp Z) p w\left(W_{0}-W\right)^{2} d W .
$$

The $S_{n}$ inside the integral is the shape factor. For allowed transition $S_{0}=1 / 2(1-\gamma)\left[C_{F}{ }^{2}\left|\int 1\right|^{2}+C_{G T}{ }^{2}\left|\int \sigma\right|^{2}\right](10-26)$. The integral thus obtained is usually denoted by $f_{0}$ (eq. IX - 14). One expects that the values of $f_{0} t$ for allowed transitions should be relatively constant for a given class of nuclei. The numerical values of $f_{0}\left(Z, W_{0}\right)$ for various values of $Z$ and $W_{0}$ has been calculated and presented by a set of formulas and curves by Feenberg and Trigg ${ }^{46}$. For a forbidden spectrum, the shape factor $S_{n}$ is energy dependent. To evaluate $f_{n}$, one must first know the exact formula for the shape factor which fits the observed spectrum then substitute it in for $S_{n}$ and carry out the integration. In general, it is easier to evaluate it by numerical integration for any particular case. However, for unique forbidden transition of $Z=0$, the shape factors are simple and exact. Davidson ${ }^{47^{\circ}}$ has shown

45 P.R. Bell, B. Weaver and J.M. Cassidy, Phys. Rev.77, (1950) 399 ; D. E. Alburger, Phys. Rev. 79, (1950) 236; L. Feldman and C. S. Wu, Phys. Rev. 87, (1952) 1091.

46 E. Feenberg and G. Trigg, Rev. Mod. Phys. 22, (1950) 399.

47 J. P. Davidson Jr., Phys. Rev. 82, (1951) 48. 
that one could express $f_{n}$ for $Z=0$ in terms of $\bar{C}_{n} f_{0}$ where $\bar{C}_{n}$ are given approximately by:

$$
\begin{aligned}
& 12 \bar{C}_{1}\left(W_{0}\right)=6 / 10\left(W_{0}^{2}-1\right)-1 / 5\left(W_{0}-1\right)^{*} \\
& \text { first forbidden } \\
& 5.6^{3} \bar{C}_{2}\left(W_{0}\right)=3 / 7\left(W_{0}^{2}-1\right)-\left({ }^{26} / 105\right)\left(W_{0}^{2}-1\right)\left(W_{0}-1\right)-2 / 105\left(W_{0}-1\right)^{2} \\
& \text { twice forbidden } \\
& 70.72^{2} \bar{C}_{3}\left(W_{0}\right)=1 / 3\left(W_{0}^{2}-1\right)^{3}-9 / 35\left(W_{0}^{2}-1\right)^{2}\left(W_{0}-1\right)-(2 / 35) \\
& \left(W_{0}^{2}-1\right)\left(W_{0}-1\right)^{2}+8 / 105\left(W_{0}-1\right)^{3} \quad \text { third forbidden. }
\end{aligned}
$$

For any value of $Z$, Davidson derived the expression for

$$
f_{1}=f_{0}\left[a(Z)\left(W^{2}-1\right)+b(Z)\left(W_{0}-1\right)\right] .
$$

The function $a(Z)$ and $b(Z)$ are average values determined empirically and are plotted against $Z$ as shown in Fig. 19.

Using Feenberg and Trigg's curve for $f_{0}$ and the formula and curves of Davidson, one obtains the $\log f_{1} t$ value for the unique first forbidden transition as listed in Table III. It is significant to note that the variation

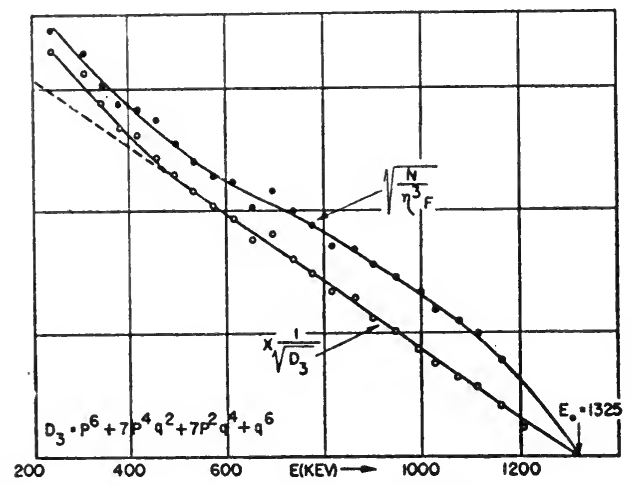

Fig. 18. Kurie plots of $K^{40} \beta$-spectrum. - From Rev. of Mod. Phys, 22, (1950) 386

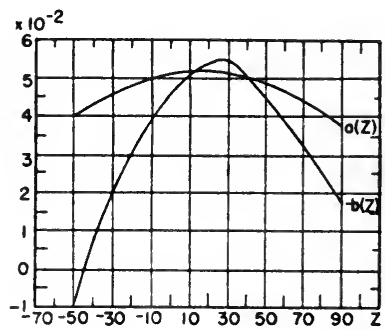

Fig. 19.

Davidson's curve: an approximate value for $a$ and $b$ in the equation $f_{1} / f_{0}=a\left(W_{0}^{2}-1\right)+b\left(W_{0}-1\right)$. (See note 47)

of the $f_{0} t$ value throughout the list is more than a factor of 300 while the constancy of $f_{n} t$ values is improved to within a factor of 30 .

The $\log f_{n} t$ values of the first forbidden unique transitions are all between $7-9$, while the $\log f_{0} t$ values of the first forbidden parity unfavored transitions are between $6-8$. The ratio of the $(f t)_{n}$ values between the various degrees of forbiddenness are in accord with theoretical predictions. $(\S 5$, Ch. X).

* For more accurate $f_{1} t$ values the expression $f_{1}=f_{0}\left[a(Z)\left(W_{0}^{2}-1\right)+b(Z)\left(W_{0}-1\right)\right]$ is preferred then the expression for $\bar{C}_{1}$. 
TABLE III

First Forbidden Unique Transitions

\begin{tabular}{|c|c|c|c|c|}
\hline Odd $A$ & $W_{0}\left(m c^{2}\right)$ & $t_{1 / 2}$ & $\log f_{0} t$ & $\log f_{n} t$ \\
\hline$A^{30}$ & 2.11 & 265 & 9.85 & 9.03 \\
\hline$* * A^{41}$ & 5.89 & $109 \mathrm{~m} \quad(1 \%)$ & 8.47 & 8.67 \\
\hline $\mathbf{K} \mathbf{r}^{\mathbf{8 5}}$ & 2.36 & 9.4 y $\quad(99 \%)$ & 9.09 & 8.30 \\
\hline $\mathrm{Sr}^{88}$ & 3.93 & 54 d & 8.57 & 8.34 \\
\hline $\mathrm{Sr}^{01}$ & 6.22 & $9.7 \mathrm{~h} \quad(26 \%)$ & 7.91 & 8.16 \\
\hline$Y^{91}$ & 4.02 & $61 \mathrm{~d}$ & 8.52 & 8.37 \\
\hline $\mathrm{Sn}^{123 m}$ & 3.78 & $136 \quad d$ & 8.88 & 8.60 \\
\hline $\mathrm{Sn}^{125 \mathrm{~m}}$ & 5.57 & $9.4 \mathrm{~d} \quad(95 \%)$ & 8.86 & 8.96 \\
\hline $\mathrm{Cs}^{137}$ & 2.02 & 33 y $\quad(92 \%)$ & 9.41 & 8.55 \\
\hline Even $A$ & $W_{0}$ & $t_{1 / 2}$ & $\log f_{0} t$ & $\log f_{n} t$ \\
\hline $\mathrm{Cl}^{38}$ & 10.43 & $37.3 \mathrm{~m} \quad(53 \%)$ & 7.44 & 8.15 \\
\hline$K^{42}$ & 8.02 & $12.44 \mathrm{~h}(80 \%)$ & 8.02 & 8.49 \\
\hline $\mathrm{As}^{72}$ & 7.54 & 26 h $(19 \%)$ & 8.21 & 8.63 \\
\hline${ }_{A 074} \beta-$ & 3.66 & $17.5 \mathrm{~d} \quad(27 \%)$ & 8.29 & 8.04 \\
\hline $\mathrm{As}^{74} \beta+$ & 3.99 & $17.5 \mathrm{~d} \quad(5 \%)$ & 8.44 & 8.28 \\
\hline $\mathrm{As}^{76}$ & 6.95 & $26.8 \mathrm{~h} \quad(60 \%)$ & 8.20 & 8.56 \\
\hline$R b^{84}$ & 4.19 & 34.0 d $(7 \%)$ & 8.64 & 8.56 \\
\hline $\mathrm{Rb}^{86}$ & 4.57 & $19.5 \mathrm{~d} \quad(80 \%)$ & 8.51 & 8.43 \\
\hline $\mathbf{R} b^{88}$ & 11.37 & $17.8 \mathrm{~m}(78 \%)$ & 7.25 & 8.04 \\
\hline $\mathrm{Sr}^{90}$ & 2.04 & $19.9 \mathrm{y}$ & 9.06 & 8.29 \\
\hline$Y^{90}$ & 5.40 & $61 \mathrm{~h}$ & 8.03 & 8.11 \\
\hline $\mathrm{Y}^{92}$ & 8.05 & $3.6 \mathrm{~h}$ & 7.64 & 8.07 \\
\hline $\mathrm{Sb}^{122}$ & 4.795 & $2.8 \mathrm{~d} \quad(25 \%)$ & 8.28 & 8.30 \\
\hline$I^{124}$ & 5.305 & $4.5 \mathrm{~d} \quad(15 \%)$ & 7.77 & 7.88 \\
\hline$I^{126}$ & 3.482 & 13 d $(27 \%)$ & 8.58 & 8.22 \\
\hline $\mathrm{Tl}^{204}$ & 2.51 & $3.5 \mathrm{y}$ & 9.60 & 8.85 \\
\hline $\operatorname{Pr}^{142}$ & 5.21 & $19.2 \mathrm{~h}$ & 7.39 & 7.42 \\
\hline
\end{tabular}

Twice Forbidden Unique Transitions

\begin{tabular}{l|l|l|l|l}
\hline $\mathrm{Be}^{10}$ & 2.10 & $2.5 \times 10^{6} \mathrm{y}$ & 14.50 & 12.08 \\
$\mathrm{Na}^{22}$ & 4.7 & $2.6 \mathrm{y}(.06 \%)$ & 12.7 & 11.9 \\
\hline
\end{tabular}

Third Forbidden Unique Transition

\begin{tabular}{l|l|l|l|l}
\hline $\mathrm{K}^{40}$ & 3.64 & $1.3 \times 10^{9} \mathrm{y}$ & 18.46 & 15.60 \\
\hline
\end{tabular}

$\mathrm{Cl}^{38}$ L. M. Langer, Phys. Rev. 77, (1950) 50.

$\mathrm{K}^{42}$ Shull and Feenberg, Phys. Rev. 75, (1949) 1768.

$\mathbf{A s}^{72}$ Mei, Mitchell and Huddleston. Phys. Rev. 79, (1950) 19.

$\mathrm{As}^{74}$ Johansson, Cauchois, and Siegbahn, Phys. Rev. 82, (1951) 275.

As $^{76}$ Tomlinson and Ridgeway. Phys. Rev. 88, (1952) 170.

$\mathrm{Rb}^{84}$ C. M. Huddleston and A.C. G. Mitchell, Phys. Rev. 88, (1952) 1352.

$\mathrm{Rb}^{86}$ Zaffarano, Kern and Mitchell, Phys. Rev. 74, (1948) 682.

** Shape has not been verified experimentally. 


\section{$\S 9$. First Forbidden Spectra}

When a $\beta$-transition involves a change of spin of $\Delta J=0, \pm 1$ and a change of parity, the main nuclear matrix elements vanish. However, the matrix elements of magnitude of one order smaller than the main ones must be taken into consideration. They yield $\beta$-decays of much slower rates. These $\beta$-decays are called first forbidden transitions and are characterized by a $\log f t$ value between $6-8$.

These second order matrix elements are classified according to their origin: (see Ch. X).

(1) The group due to the source velocity effects consist of $\int \alpha \quad \int \beta \alpha$ and $\int \gamma_{5}$.

(2) Those of retardation effects are

$$
\frac{\alpha Z}{2 R} \int r, \quad \frac{\alpha Z}{2 R} \int \boldsymbol{\sigma} \cdot \boldsymbol{r} \quad \frac{\alpha Z}{2 R} \int \boldsymbol{\sigma} \times \boldsymbol{r} \text { and } S_{i j} .
$$

The shape factors due to the first group of matrix elements are energy

$\mathrm{Rb}^{88}$ Bunker, Langer and Moffat, Phys. Rev. 81, (1951) 30.

$\mathrm{Sr}^{90}$ Braden, Slack and Shull, Phys. Rev. 75, (1949) 1964.

Y ${ }^{90} \quad$ Braden, Slack and Shull, Phys. Rev. 75, (1949) 1964.

$\mathrm{Y}^{92}$ D. P. Ames and M. E. Bunker,' (unpublished).

$\mathrm{Sb}^{122} \mathrm{Wu}$, Intern. Conf. on Nucl. Phys., Univ. of Chicago, (1951).

I'124 Mitchell, Mei, Maienschein and Peacock, Phys. Rev. 76, (1949) 1450.

I 126 Stevenson and Deutsch, Phys. Rev. 84, (1951) 1071.

Wu, private communication.

Pr142 Jensen, Laslett and Zaffarano, Phys. Rev. 80, (1950) 865.

$\mathrm{Tl}^{204}$ Lidofsky, Macklin and Wu, Phys. Rev. 87, (1952) 391.

Be $^{10}$ L. Feldman and C. S. Wu, Phys. Rev. 87, (1952) 1091; Alburger, Hughes and Eggler, Phys. Rev. 78, (1950) 318; H. W. Fulbright and J. C. D. Milton, Phys. Rev. 76, (1949) 1271.

$\mathrm{Na}^{22}$ B. Wright, Phys. Rev. 90, (1953) 159.

K ${ }^{40}$ L. Feldman aǹd C. S. Wu, Phys. Rev. 87, (1952) 1091; D. E. Alburger, Phys. Rev.79, (1950) 236; Bell, Weaver and Cassidy, Phys. Rev. 77, (1950) 399.

$\mathrm{A}^{39} \quad$ Brosi, Zeldes and Ketelle, Phys. Rev. 79, (1950) 902.

$\mathrm{A}^{41} \quad$ L. G. Elliot, Phys. Rev. 85, (1952) 942.

$\mathrm{Kr}^{85}$ Zeldes, Ketelle and Brosi, Phys. Rev. 79, (1950) 901.

$\mathrm{Sr}^{91}$ Jensen and Laslett, Phys. Rev. 75, (1949) 1949.

Ames, Bunker and Langer, Phys. Rev. 89, (1953) 903.

$\mathrm{Sr}^{89}$ L. M. Langer and H. C. Price Jr., Phys. Rev. 76, (1949) 641; Slack, Braden and Shull, Phys. Rev. 75, (1949) 1965.

$Y^{91} \quad$ Langer and Price, Phys. Rev. 76, (1949) 641.

$\mathrm{Sn}^{125 \mathrm{~m}}$ Ketelle, Nelson and Boyd, Phys. Rev. 79, (1950) 242 (A).

$\mathrm{Sn}^{125 \mathrm{~m}}$

Cs ${ }^{137}$ Mitchell and Peacock, Phys. Rev. 75, (1949) 197.

Langer and Moffat, Phys. Rev. 82, (1951) 635. 
independent, so they will not alter the linear character of the Kurie plot. The shape factor associated with the matrix elements of the retardation effects usually has two terms. One is energy dependent and of the form $\left(p^{2}+q^{2}\right)$. The other is the Coulomb term which is independent of the energy. The energy dependent terms are usually overwhelmed by the Coulomb terms which are proportional to $Z e^{2} / 2 R$ (except $S_{i j}$ ). Therefore, if one considers only a single interaction at a time one would expect that most of the first forbidden spectra should show an allowed shape, particularly $\beta$-transitions from elements of high atomic number.

However, since recent experimental evidence has dictated the adoption of a linear combination of interaction terms, it has been brought to light that a Fierz type of interference term existed in the first forbidden spectra ${ }^{22}$ just as in the allowed transitions. This $1 / W$ dependent character is the result of the interference of interaction terms involving the operator $M$ with terms involving the operator $\beta M .(\mathrm{V}, \mathrm{T})(\mathrm{S}, \mathrm{A})$ and $(\mathrm{A}, \mathrm{P})$ show such energy dependent terms but not $(\mathrm{S}, \mathrm{T})$ or $(\mathrm{V}, \mathrm{A})$. The allowed shape of the first forbidden spectra therefore provides a strong argument that the contribution from the combination of $(\mathrm{V}, \mathrm{T})(\mathrm{S}, \mathrm{A})$ or $(\mathrm{A}, \mathrm{P})$ must be trivial. However, there are possibilities that energy dependent terms may cancel each other in the forbidden shape factor giving again the shape of an allowed spectrum. This can be seen from the shape factors of (S, A) and $(V, T)$.

$$
\begin{aligned}
& S_{1}^{1}(\mathrm{~S}, \mathrm{~A}) \approx\left|\int \sigma \times \boldsymbol{r}\right|^{2}\left(\frac{\alpha Z}{2 R}\right)^{2}\left[C_{A}^{2}+C_{S}^{2} \xi_{1}^{2}-2 \xi_{1} \frac{C_{A} C S}{W}\right] \\
& S_{1}^{1}(\mathrm{~V}, \mathrm{~T}) \approx\left|\int \beta \sigma \times \boldsymbol{r}\right|^{2}\left(\frac{\alpha Z}{2 R}\right)^{2}\left[C_{T}^{2}\left(1-\eta_{1}\right)^{2}+C_{V}^{2}\left(\xi_{1}-\eta_{1}\right)^{2}\right. \\
& \left.-2\left(1-\eta_{1}\right)\left(\xi_{1}-\eta_{1}\right) \frac{C_{V} C_{T}}{W}\right] \text {.) }
\end{aligned}
$$

The allowed shape of the first forbidden spectra conclusively excludes the $(\mathrm{S}, \mathrm{A})$ combination since the estimated value of $\xi_{1} \approx 1$ and the only other possibility for an energy independent shape factor of $S_{1}^{1}(\mathrm{~S}, \mathrm{~A})$ is that $C_{A} C_{S}=0$. This is in agreement with the conclusion based on the evidence from the second forbidden spectra of $\Delta J=2$ (no) and also the $\mathrm{He}^{6}$ neutrino recoil experiment which gives evidence for a preponderant tensor interaction. The case of a $(\mathrm{V}, \mathrm{T})$ combination is more involved. One could expect an energy independent shape factor of $S_{1}^{1}(\mathrm{~V}, \mathrm{~T})$ for $C_{V} C_{T} \neq 0$ if either $\left(1-\eta_{1}\right)$ or $\left(\xi_{1}-\eta_{1}\right)$ is zero but not both. From the estimation of the matrix elements

$$
\xi_{1}=-\eta_{1} \text { so } \xi_{1}-\eta_{1} \neq 0
$$

but the value of $\eta_{1}$ is very close to 1 . This may fortuitously give the observed allowed shape of the first forbidden spectra. Nevertheless it 
is rather unlikely that the $\left(1-\eta_{1}\right)$ terms of all the first forbidden transitions investigated exactly cancel out. Therefore the combination of $(\mathrm{V}, \mathrm{T})$ is rather improbable.

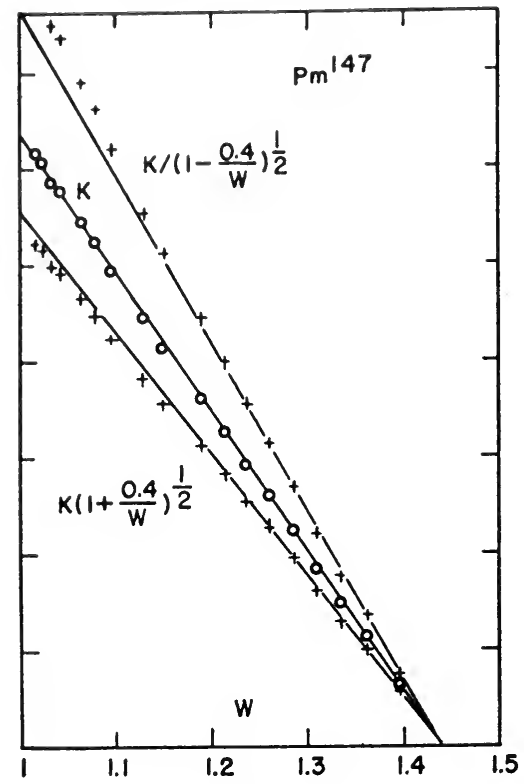

Fig. 20.

Mahmoud and Konopinski ${ }^{22}$ compared the Kurie plots of a few well measured first forbidden spectra with the Fierz plots of $(1 \pm \gamma / W)^{2 / 2}$ (Fig. 20). The spectra selected are $\operatorname{Pm}^{147}, \mathrm{~W}^{185}$, and $\operatorname{Pr}^{143}$ (Table IV). The upper limit of $|\gamma|$ found for the first forbidden spectra is $\leqslant 0.2$ thereby supporting the exclusion of (S, A) and $(\mathrm{V}, \mathrm{T})$ combinations.

But the known cases of simple first forbidden transitions are only few. There are many first forbidden transitions coexisting with concurrent decays from same radioactive parent. Under those conditions, only the upper portion of the spectrum with the highest transition energy can be analyzed in a conventional spectrometer. However, if the complex decay scheme is not too complicated, coincidence counting between a gamma detector located near the The Kurie plot of $\mathrm{Pm}^{147}$ corrected by the Fierz term. (See note 22) source and the beta detector inside of a spectrometer could be employed to investigate the spectrum of the lower energy group. The electrons of the high energy group will not be registered in the coincidence counting if it is a ground to ground transition, such as:

The coincidence spectrometer has been very successfully applied to the investigation of the $\beta$-spectrum of the low energy group of $\mathrm{Rb}^{86}{ }^{48,49}, \mathrm{Sb}^{122}{ }^{48,49}$ and $\mathrm{Tm}^{170}{ }^{50,51}, \mathrm{I}^{126}$ and $\mathrm{K}^{42} \quad(\mathrm{Wu}$, Macklin, Lidofsky and Koerts, unpublished results).The low energy transitions all give $\beta$-spectra of allowed shape. (The investigations did not extend

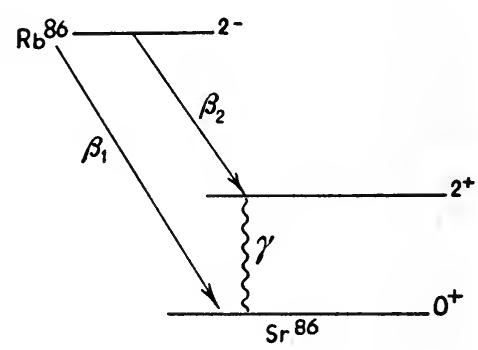

48 C. S. Wu, P. Macklin and L. Lidofsky, Phys. Rev. 82, (1951) 334; Int. Conf. on Nuclear Physics, U. of Chicago (1951).

49 H. R. Nuether and S. L. Ridgeway, Phys. Rev. 80, (1950) 750.

50 R. Graham, J. Wolfson and P. R. Bell, Cam. J. Phys. 30, (1952) 459.

51 R. 'T. Nichols et al., AEC - ISC (Iowa State College) 345, (May 15, 1953). 
to very low energy region.) The $2^{-} \rightarrow 2^{+}$transitions form a special group among the first forbidden transitions with the $f t$ values centered around 7.5. This is an order of magnitude larger than the usual $f t$ values for $\Delta I=0$ but is an order of magnitude smaller than that for $\Delta I=2$, yes (see Table III). It was estimated by King and Peaslee ${ }^{518}$ that the shell model specifications of individual orbitals are $90 \%$ valid in these cases. Therefore the $2-\rightarrow 2+$ spectra should contain an average of only $10 \%$ the $a_{1^{-}}$ type unique spectrum and show very little deviation from an allowed one.

TABLE IV

Fierz-Type Interference in Once-Forbidden Spectra

\begin{tabular}{lll|c}
\hline \multicolumn{2}{c|}{ Radiation $W_{0}$ in $m_{0} c^{2}$} & $\gamma$ \\
\hline $\operatorname{Pm}^{147}$ & $\beta-$ & 1.415 & $-0.06<\gamma<0.2$ \\
$\mathrm{~W}^{185}$ & $\beta-$ & 1.84 & $-0.18<\gamma<0.28$ \\
$\operatorname{Pr}^{143}$ & $\beta-$ & 2.82 & $-0.26<\gamma<0.18$
\end{tabular}

From Reference 22

\section{$\S 10$. Spectrum of RaE and Pseudoscalar Interaction}

The spectrum of $\mathrm{RaE}$ is the only first forbidden spectrum that showed definite deviation from the allowed shape. ${ }_{83} \mathrm{RaE}^{210}$ has 83 protons and 127 neutrons. The shell model predicts that the extra proton is $h_{\% / z}, f_{\% /,}$ or $p_{\% / \mathrm{s}}$ and that the extra neutron is $i_{11 / 2}, g_{\% / 2}$ or $d_{\mathrm{z} / \mathrm{z}}$. The shell model prediction that the parity of $\mathrm{RaE}$ is odd is therefore quite certain. The $\beta$-transition must involve a parity change as the parity of the final even-even Po nucleus must be even. The previous fitting of $\mathrm{RaE}^{52}$ to second forbidden tensor interaction must therefore be discarded.

As was pointed out above for the first forbidden transitions of $\Delta J=0, \pm 1$ (yes), all spectra resulting from a single interaction alone yield an allowed shape. Thus in this special case, a linear combination must be resorted to, unless it turns out that the RaE spectrum is a complex one instead of a simple one. A weak $\gamma$-ray of $85 \mathrm{keV}$ with an intensity of 1 in $10^{3}$ disintegrations and its internal conversion electrons of $66 \mathrm{keV}$ were reported ${ }^{53}$. But recent extensive investigations with a $\mathrm{NaI}$ crystal and $\beta$-spectrometer in various laboratories ${ }^{54}$ failed to confirm the existence of such a weak $\gamma$-ray and its internal conversion electrons. Only the theoretically

51a R. W. King and D. C. Peaslee, Phys. Rev. 94, (1954) 1284.

52 E. J. Konopinski and G. E. Uhlenbeck, Phys. Rev. 60, (1941) 308.

s3 A. S. Zavelski, et al., J. Exp. Th. Phys. U.S.S.R. 19, 1136.

54 F. Boehm and C. S. Wu, Phys. Rev. 90, (1953) 339; Phys. Rev. 93, (1954)

518; D. G. E. Martin and G. Parry, Phil. Mag. 44, (1953) 344. 
expected characteristic X-rays ${ }^{55}$ near the region of $80 \mathrm{keV}$ from the ionization of the atom due to $\beta$-decay with an intensity of $1.2 \times 10^{-4}$ per disintegration was found to be superposed on the continuous background of the internal Bremsstrahlung.

Petschek and Marshak ${ }^{56}$ investigated the possibilities of fitting the RaE $\beta$-spectrum with the various linear combinations. The forbidden shape factor was calculated using the formulae of Konopinski and Uhlenbeck ${ }^{52}$ and the interference formula of Smith ${ }^{57}$. The finite radius corrections were taken from the paper by Rose and Holmes ${ }^{58}$. No corrections were applied for screening by the atomic electrons. Because the RaE spectrum is peculiar in that it gives a great abundance of low energy electrons (Fig. 21), none of the linear combinations $(\mathrm{S}, \mathrm{T})(\mathrm{S}, \mathrm{A})(\mathrm{V}, \mathrm{T})$ and $(\mathrm{V}, \mathrm{A})$ could give a good fit. Only the $(\mathrm{T}, \mathrm{P})$ combination for a $(0-0)$ yes transition with its large energy independent terms concelling out can. give a satisfactory fit. This can be seen from the shape factor for $0 \rightarrow 0$ (yes) transition:

$S_{1}^{0}=\left|\int \beta \boldsymbol{\sigma} \cdot \boldsymbol{r}\right|^{2}\left\{\left[\left(C_{T} \pm \zeta C_{p}\right) \frac{\alpha Z}{2 R}+\frac{1}{3} C_{T}\left(\frac{p^{2}}{W}-q\right)\right]^{2}+C_{T}^{2}\left(\frac{p}{3 W}\right)^{2}\right\}$.

To have the terms containing $\alpha Z / 2 R$ cancelled out, one must first have the condition $C_{T} \pm \zeta C_{p}=0$ or the ratio

$$
\frac{C_{p}}{C_{T}}=\frac{i(\alpha Z / 2 R) \int \beta \sigma \cdot r}{\int \beta \gamma_{5}} . \quad \text { For } \operatorname{RaE} \frac{\alpha Z}{2 R} \approx 13 \text {. }
$$

In the actual fitting, Petschek and Marshak used a ratio

$$
P=-i \frac{C_{p} \int \beta \gamma_{5}}{C_{T} \int \beta \sigma \cdot r} \approx 13
$$

Ahrens, Feenberg and Primakoff ${ }^{59}$ have approximately estimated the nuclear matrix elements for the pseudoscalar interaction and found that

$$
\left|\frac{C_{P}}{C_{T}}\right| \sim 1836 \times \frac{A^{1 / s}}{Z}=133
$$

is necessary for the pseudoscalar interaction to contribute appreciably to $\Delta J=0$ (yes) transition. Independently, Alaga, Kofoed-Hansen and Winther 60a used the Foldy-Wouthuysen transformation with a simple

55 A. Migdal, U.S.S.R., J. of Phys. 4, (1941) 449; E. L. Feinberg, U.S.S.R., J. of Phys. 4 (1941) 423.

56 A. Petschek and R. Marshak, Phys. Rev. 85, (1952) 698.

57 A. Smith, Phys. Rev. 82, (1951) 955 (L.)

58 M. E. Rose and D. K. Holmes, Phys. Rev. 83, (1951) 190.

59 I. Ahrens, E. Feenberg and H. Primakoff, Phys. Rev. 87, (1952) 663.

${ }^{802}$ G. Alaga, O. Kofoed-Hansen and A.Winther, Dan. Mat. Phys. Medd. 28 no. 3 (1953). 
potential well and found

$\frac{i \int \beta_{5}^{\nu}}{\int \beta \sigma \cdot r} \approx \frac{E^{2}}{6 M}$ where $E$ is the kinetic energy of the decaying nucleon.

This leads to $\mathrm{C}_{P} / \mathrm{C}_{T} \approx 150$. This large ratio $\mathrm{C}_{P} / \mathrm{C}_{T}$ implies the presence of a disproportionately large amount of pseudoscalar interaction in the combination.

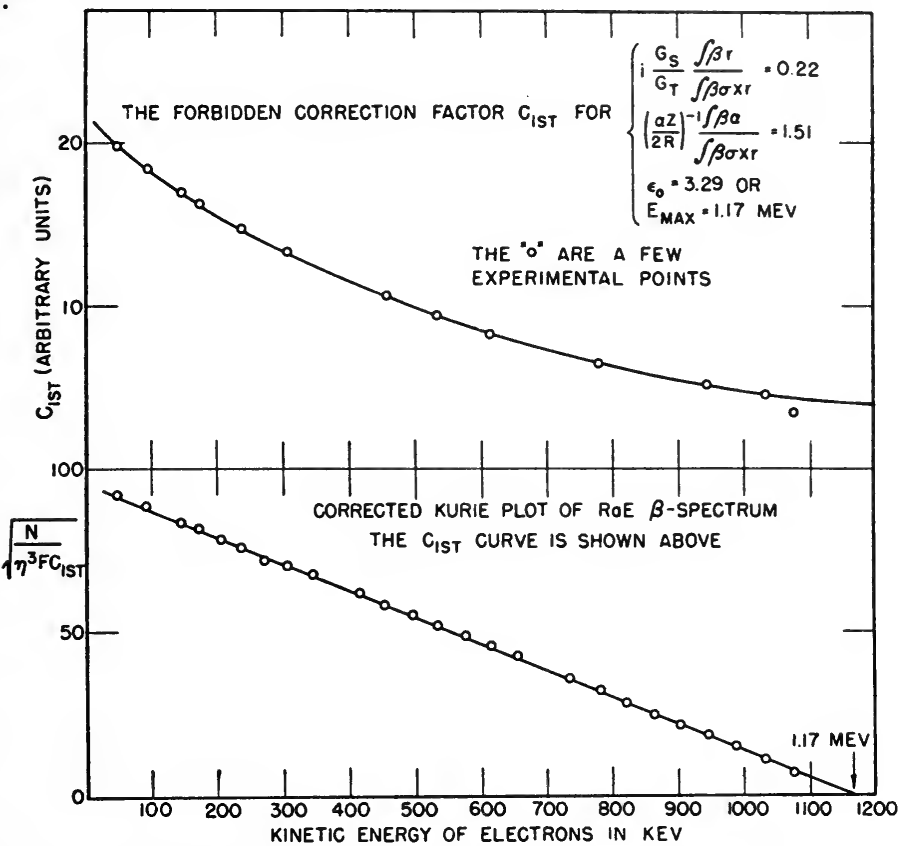

Fig. 21. The forbidden correction factor curve and the Kurie plot of RaE $\beta$-spectrum. (Wu, Lidofsky, Macklin and Benczer)

When the existence of a pseudoscalar interaction in $\beta$-decay was suggested, immediate interest was aroused and as a result the correctness of the conventional treatment of pseudoscalar interaction was questioned. $\left({ }^{59,60 a, b, c}\right)$

As the $\beta \gamma_{5}$ operator in P-interaction is an odd Dirac operator which connects large Dirac components of the nucleon wave function to small ones, in a non-relativistic approximation for the nucleons, one can express the small components $v$ in terms of the large ones $u$ by

where

$$
\begin{gathered}
v=\frac{f \sigma \cdot p u}{2 M} \\
f=\left[i \mathrm{i}+\frac{E-U}{2 M}\right]^{-1} \approx 1
\end{gathered}
$$

$U$ is the nuclear potential. Consequently ${ }^{60}$

6ob M. E. Rose and K. Osborn, Phys. Rev. 93, (1954) 1315.

600 E. J. Konopinski, Phys. Rev. 94, (1954) 492. 


$$
\int \beta \gamma_{5} L=\frac{1}{2 M} \int u_{f}^{*} \sigma \cdot \nabla(f L) u_{i}=\frac{1}{2 M} \int u_{f}^{*} \sigma \cdot[L \nabla f+f \nabla L] u_{i}
$$

where $L$ is the lepton covariant. It implies that the variation of $f$ as well as the variation of the lepton waves over the nucleus should be considered. This is contrary to the conventional way of treating the forbidden theory of $\beta$-decay where one assumes the nuclear matrix elements as certain numbers independent of the parameters describing the lepton fields.

Rose and Osborn ${ }^{60^{\mathrm{b}}}$ considered the variation of the nuclear potential $U$ over the nucleus small and took into account only the term of the variation of the lepton waves over the nucleus. They found that the correction factor for this term is very different from that used by Petschek and Marshak. Applying this new correction factor to the RaE spectrum, he found no satisfactory fit for the combination of $(\mathrm{T}, \mathrm{P})$ 0-0 any more.

On the other hand, Yamada ${ }^{60 d}$ re-investigated the $\beta$-spectrum of RaE by taking into account the finite de Broglie wave length effect ${ }^{600}$, it turned out that the $\mathrm{S}, \mathrm{T}(1-0)$ and $\mathrm{VA}(1-0) \dagger$ fit the experimental data as well as the $\mathrm{TP}(0-0)$. The definitions of the finite radius correction by Rose and Holmes ${ }^{58}$ and the finite de Broglie wave length effect by Rose, Perry and Dismuke ${ }^{56}$ are as follows. The finite radius correction is to take account of the effect of the finite nuclear radius on the behaviour of certain of the electron wave functions at the nuclear radius. In actual calculations, the factors arising from the electron wave functions evaluated at the nuclear radius are obtained from the first term or two in the powers series expansion of $(p R)$ of the radial wave functions $(p$ : electron momentum, $R$ : nuclear radius). The validity of this procedure rests on the fact that the electron de Broglie wave length is large compared to the nuclear radius. However, it was pointed out by Rose, Perry and Dismuke that when the $p$ and $Z$ are large, the above approximation is insufficient. The only safe way to obtain the required numerical results is to calculate the radial wave functions with an essentially exact procedure. This computation was carried out on the Whirlwind I and is now available in book form. ${ }^{60}$ The good fitting of the $\beta$-spectrum of $\mathrm{RaE}$ by $(\mathrm{S}, \mathrm{T})(1 \rightarrow 0)$ combination has been definitely confirmed by the Columbia group and Plassmann and Langer $\dagger \dagger$ with a vacuum evaporated $\mathrm{RaE}$ source. The required shape factors and the

6od M. Yamada, Int. Conf. of Theo. Phys., Japan (1953). Prog. of Theo. Phys. 10, (1953) 252.

60 i. E. Rose and C. L. Perry, Phys. Rev. 90, (1953) 479.

M. E. Rose, C. L. Perry and N. M. Dismuke, Oak Ridge National Lab. report 1459 (1953).

$\dagger \mathrm{VA}(1-0)$ can be excluded on the evidence from the $\mathrm{He}^{7}$ recoil experiment.

†† The author is indebted to Dr. Langer for the pre-print of their paper on the $\beta$-spectrum of RaE. 
corrected Kurie plot of the RaE $\beta$-spectrum are shown in Fig. 21 together with the adjusted parameters.

An attempt to determine the spin of RaE by its hyperfine spectrum has been reported recently ${ }^{61}$. The $\lambda 3607$ line was found to be a single sharp one. From the width of the line, it was assumed that the nuclear magnetic moment must be less than 0.35 N.M. Its interpretation is consistent with the spin assignment of RaE of 1 as well as 0 . However, K. Smith $\dagger$ has very recently determined the spin of RaE by atomic beam method and found it to be 1 . Therefore the single direct evidence for the existence of a pseudoscalar in $\beta$-decay as offered by the shape of $\operatorname{RaE} \beta$-spectrum is definitely invalidated.

That the spin of RaE is unity was also predicated by the following arguments ${ }^{63,60 \mathrm{~b}}$ :

(1) A group of highly favored first forbidden transitions ( $\log f t=5-6)$ between nuclei near the double magic $(82-126)$ shell exists and is characterized by $\Delta I=0$. These are
(a) $\quad 0^{-} \stackrel{\Delta I=0}{\rightarrow} 0^{+}$
$\log f t$
(b) not $\begin{gathered}\Delta I=0 \\ 0 \rightarrow 0\end{gathered}$
$\log f t$
$\mathrm{Tl}^{206} \rightarrow \mathrm{Pb}^{206}$
5.18
$\mathrm{Hg}^{205} \rightarrow \mathrm{Tl}^{205}$
5.52
$\mathrm{Pb}^{210} \rightarrow \mathrm{Bi}^{210}$
$5-6$
$\mathrm{Tl}^{207} \rightarrow \mathrm{Pb}^{207}$
5.16
$\mathrm{Pb}^{212} \rightarrow \mathrm{Bi}^{212+}$
5.17
$\mathrm{Pb}^{209} \rightarrow \mathrm{Bi}^{209}$
5.59

(2) On the other hand, the $\log f t$ values of the first forbidden transition in similar nuclei of $\Delta I=1$ are grouped around 7.5.

(3) In the decay scheme of $\mathrm{RaD}-\mathrm{RaE}-\mathrm{RaF}^{62}$, the spins of the $46.5 \mathrm{keV}$ state and the ground state of $\mathrm{RaE}$ should be different; i.e. either 0 and 1 respectively or 1 and 0 . The $\log f t$ value for the transition from the $0_{+}$state of $\mathrm{RaD}$ to the excited state of $\mathrm{RaE}$ is $5-6$ and that from the ground state of RaE to RaF is 8 . A spin of unity for the ground state of $\mathrm{RaE}$ again gives a consistent picture.

The indirect evidence for the existence of a pseudoscalar interaction in $\beta$-decay has been suggested recently by King and Peaslee ${ }^{63}$ and is based on the difference of the $f t$ values between $\Delta I=0$ and $\Delta I=1$ of first forbidden transitions. They interpret the difference to be partly due to a substantial contribution from the pseudoscalar term for $\Delta I=0$ and partly due to the cancellation of the large factors for $\Delta I=1$. In order to have the contribution

61 M. Fred, F. S. Tomkins and R. F. Barnes, Phys. Rev. 92, (1953) 1324 L.

62 C. S. Wu, F. Boehm and E. Nagel, Phys. Rev. 91, (1953) 319.

63 D. C. Peaslee, Phys. Rev. 91, (1953) 1447; R. W. King and D. C. Peaslee, Phys. Rev. 94, (1954) 795 (A).

$\dagger$ The author is indebted to Dr.K. Smith of Cambridge University for communicating to her the final result of the determination of the spin of RaE. 
from the pseudoscalar tern comparable with that from other interactions, $C_{P} / C_{T}$ must be large (50) or the presence of pseudoscalar-coupled forces in the nucleus must be assumed ${ }^{64}$. Therefore the role played by the pseudoscalar interaction in the $\beta$-decay is still rather uncertain at the present moment.

\section{$\S 11$. Effect of Pseudoscalar Interaction on the Shape of Allowed $\beta$-Spectra}

It can be shown that the shape of allowed $\beta$-spectra will be affected by including a large portion of pseudoscalar interaction ${ }^{60^{a}}$. For $\Delta J=1$ (no) transitions, the spectrum distribution obtained using a plane wave approximation becomes ${ }^{60}$ :

$$
\begin{aligned}
P(W) d W= & \frac{1}{2 \cdot \pi^{3}} q^{2} p W d W\left(\frac{C_{T}}{T}\left|\int \beta \sigma\right|^{2}\right\} 1+\left[\frac{p^{2}+q^{2}}{3}-\frac{2}{9} \frac{p^{2} q}{W}\right] \\
& \left(\frac{C_{P}}{C_{T}} / 2 M\right)^{2}+\frac{2}{3}\left(\frac{p^{2}}{W}-q\right) \frac{C_{P}}{C_{T}} \mid 2 M !
\end{aligned}
$$

The distortion effect due to the inclusion of pseudoscalar interaction will be mostly from the $\left(\frac{P^{2}}{W}-q\right) \frac{C_{P}}{C_{T}} / 2 M$ term and will be more pronounced for $\beta$-spectra of large maximum energies.

By assuming $\frac{C_{P}}{C_{T}} / 2 M= \pm \frac{1}{20}$, the deviation of the Kurie plot of the $\mathrm{He}^{6} \beta$-spectrum from linearity is quite noticeable ${ }^{60^{\mathrm{a}}}$. From the comparison

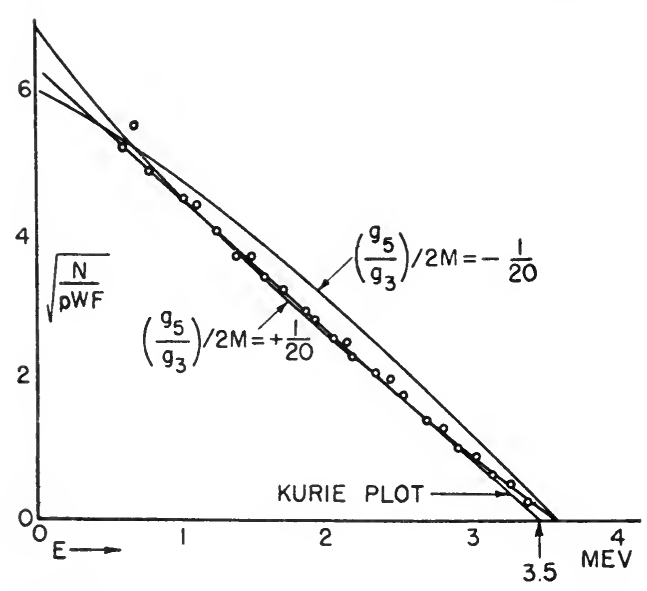

Fig. 22. Comparison of the theoretically expected $\beta$-spectrum of the $\mathrm{He}^{6}$ with the inclusion of the term $\frac{C_{P}}{C_{T}} / 2 M= \pm \frac{1}{20}$ to the experimentally obtained data. (See notes $60^{\mathrm{a}}$ and 21) with the measured $\mathrm{He}^{6}$ (Fig. $22)$ and $\mathrm{B}^{12} \beta$-spectra, the upper limit of $C_{P} / C_{T}$ is set at $<100$ for $\mathrm{He}^{6}$ and $<50$ for $\mathrm{B}^{12}$. Fujita and Yamada ${ }^{64^{\mathrm{a}}}$ also obtained the two limits of the ratio $C_{P} / C_{T}$ as $-52<$ $<C_{P} / C_{T}<19$ from the $\beta$ spectra of $\mathrm{He}^{6}$ and $\mathrm{B}^{12}$. Since the above equation is independent of the amount of pseudoscalar coupling in the nuclear forces, a good measurement of the $\mathrm{He}^{6}$ spectrum would put an upper limit on $C_{P} / C_{T}$. independent of the question of pseudoscalar forces.

64 M. Ruderman, Phys. Rev. 89, (1953) 1227.

${ }^{64 a}$ S. Nakamura, Int. Conf. of Theo. Phys., Japan (1953). 


\section{$\S 12$. Twice Forbidden Spectra}

Since all the unique forbidden spectra are discussed in $\S 7$, we will be concerned here only with the second order parity-forbidden transitions, that is $\Delta J=2$ and no parity change. There are five known beta-decays in this group, namely $\mathrm{Cl}^{36}{ }^{65}, \mathrm{Tc}^{99}{ }^{65}, \mathrm{Cs}^{13566}, \mathrm{Cs}^{137} 67$ and $\mathrm{I}^{129}{ }^{68}$. Except for $\mathrm{C}^{137}$ they all have simple beta spectra. $\mathrm{Cs}^{137}$ decays in two branches. The high energy group which we are interested in is $9 \%$ of the other. Three more $\beta$-decays of this group which have been recently reported are $\mathrm{Fe}^{5969}$, $\mathrm{Co}^{6070}$, and $\mathrm{Se}^{46}{ }^{70}$. They all give spectra similar to that of Cs group. However, the extremely weak activities as compared with the main transitions (a few thousandths) and the large Compton background due to a high energy gamma-radiation make the investigation of their spectrum shapes very difficult.

The half-lives of these radioactive decays are all greater than $10^{5}$ years. Special activation methods were devised for each of them to increase the specific activity. $\mathrm{I}^{129}$ and $\mathrm{Tc}^{99}$ are fission products. They were prepared nearly isotopically pure from fission fragments. $\mathrm{Cs}^{135}$ source was the daughter product of a 40 Curie short life $\mathrm{Xe}^{135}$ activity ${ }^{71}$ which was collected from the "water boiler" nuclear reactor. Cl ${ }^{36}$ was obtained through the $\mathrm{Cl}^{35}(\mathrm{n}, \gamma) \mathrm{Cl}^{36}$ reaction.

All of them have been investigated by magnetic spectrometers and their spectra show definite forbidden shapes. The $\beta$-spectrum of $\mathrm{Cl}^{36}$ was also investigated in a high pressure proportional counter ${ }^{72}$. It is interesting to note that the three groups of transitions of different initial and final level configuration show marked differences in their forbidden shapes (see Fig. 23, 24, 25) and can be grouped as shown in Table V.

TABLE V

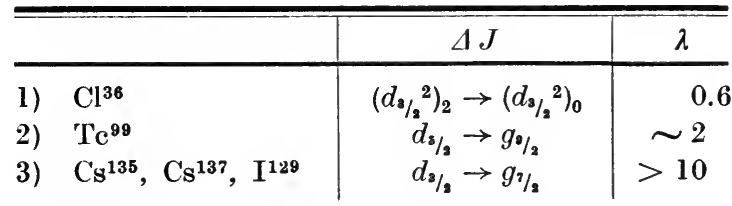

65 L. Feldman and C. S. Wu, Phys. Rev. 87, (1952) 1091.

66 L. Lidofsky, E. Alperovitch and C. S. Wu, Phys. Rev. 90, (1953) 387.

67 A. Mitchell and C. Peacock, Phys. Rev. 75, (1949) 197; L. M. Langer and R. J. D. Moffat, Phys. Rev. 82, (1951) 635.

68 E. der Mateosian and C. S. Wu, Phys. Rev. 91 (1953) 497.

69 F. R. Metzger, Phys. Rev. 88, (1952) 1360.

70 G. L. Keister and F. H. Schmidt. The author is indebted to them for letting her read their manuscript prior to its publication.

71 N. Sugarman, Phys. Rev. 75. (1949) 1473.

72 H. Fulbright and J. Milton, Phys. Rev. 82, (1951) 274. 


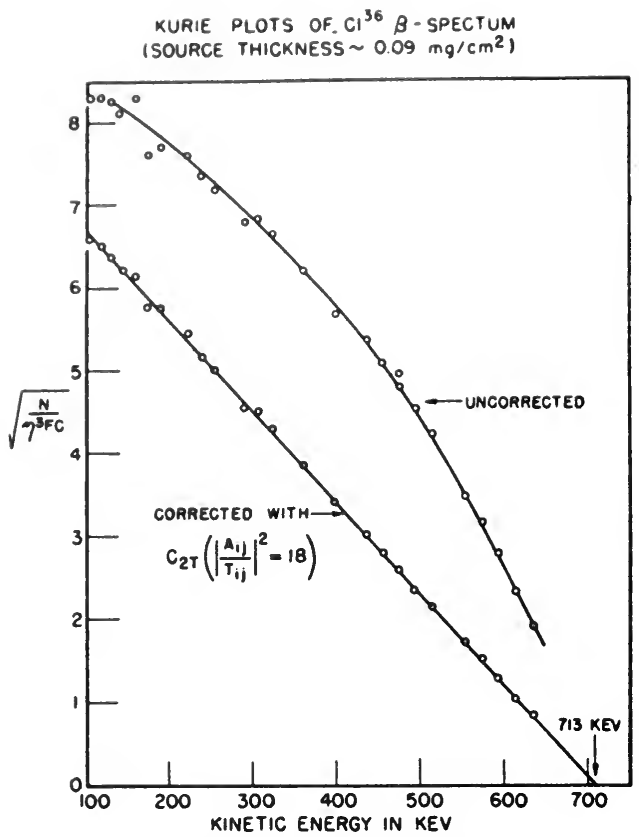

Fig. 23. Kurie plots of $\mathrm{Cl}^{36} \beta$-spectrum (source thickness $\sim 0.09 \mathrm{mg} / \mathrm{cm}^{2}$ ). (See note 65)

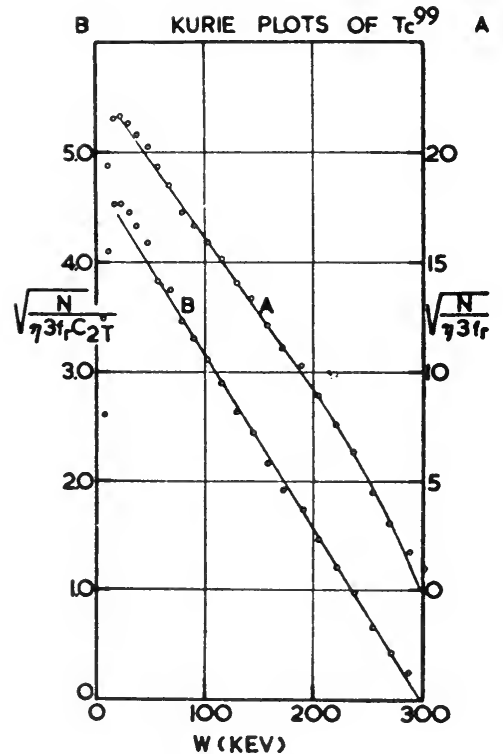

Fig. 24. Kurie plots of $\mathrm{Tc}^{99}$. (See note 30)
KURIE PLOTS OF $\mathrm{Cs}^{135}$ AND $\mathfrak{l}^{129} \beta$-SPECTRUM

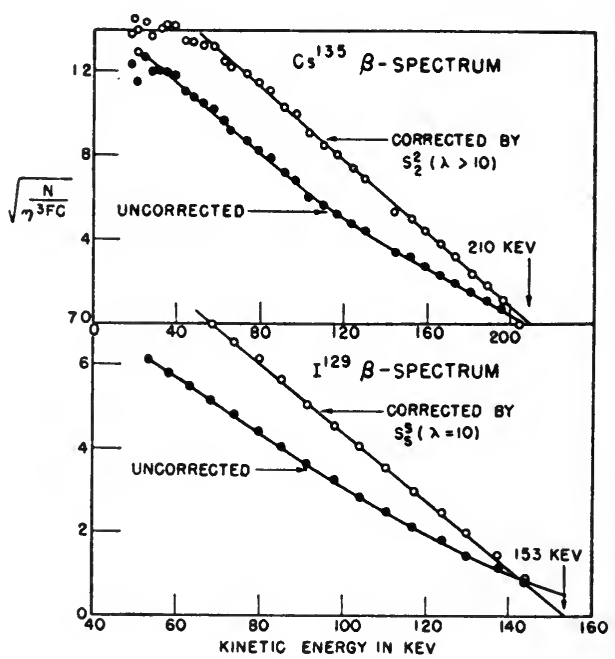

Fig. 25. Kurie plots of $\mathrm{Cs}^{135}$ and $\mathbf{I}^{129}$ $\beta$-spectrum. (See notes 66 and 68) 
Prior to the time when linear combinations had to be taken seriously, one usually started out to fit the observed spectrum by using one single interaction at a time. In so doing, one has been able to fit the parity unfavored twice forbidden $\beta$-spectra by tensor or polar vector alone but not by axial vector or scalar.

However, recent analysis of the super allowed transitions has shown that the contributions from the Gamow-Teller and Fermi interactions in the $\beta$-decay processes are comparable. This means that a linear combination of approximately equal parts of GT and Fermi interactions must be used in the analysis.

The shape factors of various linear combinations have been calculated by Smith ${ }^{57}$, Good ${ }^{73}$, and Pursey ${ }^{74}$. They are expressed in terms of $L_{n}, M_{n}, N_{n}$, etc., which were the notations used in the original paper on forbidden spectra by Konopinski and Uhlenbeck.

From these detailed calculations, a much simplified shape factor ${ }^{63}$ in terms of either of the four linear combinations ST, VA, SA and VT for the twice forbidden spectra of $\Delta J=2$ has been obtained by using the low $Z$ approximation $(\alpha Z / R) \gg W_{0}-1$

$$
S_{2}^{2} \sim p^{2}+\lambda q^{2}=\left(W^{2}-1\right)+\lambda\left(W_{0}-W\right)^{2}
$$

where

$$
\lambda=\left[\frac{2 \frac{\alpha Z}{2 R}\left(C+\frac{t}{2}\right)-b}{\frac{\alpha Z}{2 R}\left(C+\frac{t}{2}\right)-b}\right]^{2}
$$

$b$ represents

\begin{tabular}{ccccc}
\hline \hline & $(\mathrm{S}, \mathrm{T})$ & $(\mathrm{V}, \mathrm{A})$ & $(\mathrm{S}, \mathrm{A})$ & $(\mathrm{V}, \mathrm{T})$ \\
\hline$b$ & $a$ & $C a^{\prime}$ & $o$ & $a+C a^{\prime}$
\end{tabular}

where $t, a$ and $a^{\prime}$ are defined by

$C$ is $C_{F} / C_{G-T}$.

$$
\begin{aligned}
& \int T_{i j}=i t \int R_{i j} \\
& \int \beta A_{i j}=i a \int R_{i j} \\
& \int A_{i j}=i a^{\prime} \int R_{i j}
\end{aligned}
$$

From the simplified shape factor $S_{2}^{2}$, one could see at a glance that the parameter $\lambda$ of $(S, T)$ or $(V, A)$ combination each has two independent parameters $(t, a)$ or $\left(t, a^{\prime}\right)$. Therefore they are equally_flexible in explaining forbidden shapes. The $\lambda$ of $(\mathrm{S}, \mathrm{A})$ combination is practically fixed at 4 . This inflexibility greatly limits its possibility of fitting observed spectra.

73 R. H. Good Jr. Phys Rev. 86, (1952) 620 (A).

74 D. L. Pursey, Phil. Mag. 42, (1951) 1193. 
This may explain why $(\mathrm{S}, \mathrm{A})$ combination can not explain the $\mathrm{Cl}^{36} \beta$ spectrum where $\lambda=0.6$ but roughly fits the $\mathrm{Tc}^{99} \beta$-spectrum where the value of $\lambda$ obtained in fitting the experimental data is equal to $\sim 2$. Since $\lambda$ of $(V . T)$ has three independent parameters $\left(t, a, a^{\prime}\right),(\mathrm{V}, \mathrm{T})$ should be capable of fitting any shape of spectra. Therefore one should not rely on the fit as conclusive evidence for $(\mathrm{V}, \mathrm{T})$.

From this simplified shape factor one will also realize at once why these spectra had been successfully fitted by tensor or polar vector interaction alone. Applying the tensor interaction alone, where one has the ratio $C=C_{F} / C_{G-T}=0$, one has only to choose another value of " $a$ " so that it will give the same value of $\lambda$ as it was given by the $(\mathrm{S}, \mathrm{T})$ combination where the ratio of $C=C_{F} / C_{G-T}$ is assumed to be \pm 1 .

To see the connection between the old way of interpreting the spectra by the tensor interaction alone where the ratio $\left.\left|A_{i j}\right| T_{i j}\right|^{2}$ is determined and the new method of analysis of spectra by (ST) linear combinations by finding a suitable $\lambda$, one could express the parameter $\lambda$ in terms of $\left.\left|A_{i j}\right| T_{i j}\right|^{2}$ directly.

$$
\lambda=\left[\frac{2 \frac{\alpha Z}{2 R} C+\frac{t}{2}-a}{\frac{\alpha Z}{2 R}\left(C+\frac{t}{2}\right)-a}\right]^{2}=\left[\frac{2 \frac{\alpha Z}{2 R}\left(-i R_{i j}+1 / 2 T_{i j}\right)-A_{i j}}{\frac{\alpha Z}{2 R}\left(-i R_{i j}+1 / 2 T_{i j}\right)-A_{i j}}\right]^{2} .
$$

For considering tensor interaction alone, $R_{i j}=0$.

$$
(\lambda)_{T}=\frac{\left(\frac{\alpha Z}{2 R} T_{i j}-A_{i j}\right)^{2}}{\left(\frac{\alpha Z}{2 R} / 2 T_{i j}-A_{i j}\right)^{2}}=4 \frac{\left(\frac{\alpha Z}{2 R}-\frac{A_{i j}}{T_{i j}}\right)^{2}}{\left(\frac{\alpha Z}{2 R}-\frac{2 A_{i j}}{T_{i j}}\right)^{2}} .
$$

This is the direct relation between $\left|A_{i j}\right| T_{i j} \mid$ and the value of $\lambda$. The value of $\lambda_{T}$ calculated from the value of $+A_{i j} / T_{i j}$ is very close to the value of $(\lambda)_{S T}$ derived from the experimental curves directly (see Table VI).

TABLE VI

\begin{tabular}{l|c|c|c}
\hline & $\mathrm{Cl}^{36}$ & $\mathrm{Tc}^{99}$ & $\mathrm{Cs}^{135}$ \\
\hline$(\lambda)_{S . T}$ & 0.6 & 2 & $>10$ \\
\hline$\left(\frac{A_{i j}}{T_{i j}}\right)_{T}$ & +4.2 & +6.7 & +2.7 \\
\hline$(\lambda)_{T}$ & $\sim 0.7$ & $\sim 2$ & $\sim 10$
\end{tabular}

If the matrix elements involved could be calculated exactly, one could determine the ratio $C=C_{F} / C_{G-T}$ directly from fitting the spectra. Unfortunately our knowledge of the nuclear wave functions is not yet ready for such detailed calculations. Nevertheless a crude estimate of the ratio of some of the matrix elements can be made and a comparison with the 
observed spectra may shed some more light on the question of the linear combinations.

Peaslee ${ }^{63}$ estimated the value of the parameters $t, a$ and $a^{\prime}$ according to the shell model, using a method similar to those of Ahrens and Feenberg. Pseudoscalar nuclear forces were taken into oonsideration as suggested by Ruderman in his solution to the dilemma in RaE.

Even though considerable variations in the estimated values of the parameters are allowed, one can still draw the following conclusions:

1) From the spectrum of $\mathrm{Cl}^{36}$, the combination of $(\mathrm{V}, \mathrm{A})$ is excluded in favor of $(\mathrm{S}, \mathrm{T})$. This is strongly supported by the recent $\beta-v$ correlation experiments in $\mathrm{He}^{6}$.

2) The relative sign of the $(\mathrm{S}, \mathrm{T})$ term is $(\mathrm{S}-\mathrm{T})$ for negatron emission. This is deduced from the experimentally fitted value $\lambda$ for the spectrum of $\mathrm{Tc}^{99}$ and of Cs group and the estimated value of $t$. This is also supported by the deduction from a recent analysis of the $\beta$-spectra of $\mathrm{Cs}^{137}$ and $\mathrm{Rb}^{87}$ by Yamada, Morita and Fujita ${ }^{64^{2}}$.

3) A substantial correction for pseudoscalar coupled forces in the nucleus is required in estimating the matrix elements.

\section{$\S 13 . \mu$-Meson $\beta$-Spectrum *}

The $\mu$-meson is an unstable particle. It decays at rest to an electron and some other neutral particles with a mean life of $\tau_{\mu}=2.22 \times 10^{-6} \mathrm{sec}$. Early measurements on the energy distribution of the electrons from $\mu$-meson decay ${ }^{75}$ indicated that the electrons did not all possess the same energy according to the conservation laws. The continuous spectrum observed can be accounted for by a three particle decay process; an electron and two neutrinos are most generally agreed upon. The early identification ${ }^{76}$ of the charged secondary particle from $\mu$-decay as an electron is obtained from the penetration of the charged particles from $\mu$-decays through various absorbers. The bremsstrahlung associated with the charged decay particles is measured. The intensity so determined requires that the mass of the charged decay particle be of the order of magnitude of the electronic mass. Further substantiation of the positron nature of the minimum ionization particle from $\mu$-meson decay was obtained from the correct order of magnitude of the measured annihilation

75 R. Leighton, C. Anderson and A. Seriff, Phys. Rev. 75, (1949) 1432.; J. Steinberger, Phys. Rev. 75, (1949) 1136.

78 E. Hincks and B. Pontecorvo, Phys. Rev. 77, (1950) 102; 73, (1948) 257.

* The author wishes to express her thanks to Dr L. Michel for his extensive advice on this section. 
length ${ }^{84}$. As to the nature of the neutral particles one deduces that their masses must be negligible in comparison with the $\mu$-meson mass because the observed maximum energy of the electron is nearly the maximum amount available $\frac{1}{2} m_{\mu} \cdot{ }^{*}$ Furthermore, the possibility of a photon being one of the neutral particles is excluded 77 because of the negative results of a search for coincidences between the decay electrons and materialized $\gamma$-quanta. Therefore one writes

$$
\mu^{ \pm} \rightarrow \mathrm{e}^{ \pm}+2 \nu
$$

The continuous electron spectrum has a maximum energy given by the energy of the electron when the two neutrinos fly out in the same direction and opposite to that of the electrons

$$
W_{\max }=\frac{m_{\mu}^{2}+1}{2 m_{\mu}} \quad \text { (Total energy) }
$$

If the rest mass of the electron is neglected in comparison with the mass of the $\mu$-meson $\left(m_{\mu}=207 m_{e}\right)$ then the maximum total energy $W_{\max }$ of the $\beta$-spectrum of the $\mu$-meson will be $\left(207 m_{e}\right) / 2$ equal to $52.9 \mathrm{MeV}$.

So far, no satisfactory explanations of the $\mu$-meson decay have been obtained by considering the indirect interaction through any known virtual particles. For example, an indirect coupling through a virtual $\pi$ meson $(\mu v)-\pi-(\mathrm{e} v)$ would require a life time for $\mu$-meson much too long to be compatible with the measured half-life. On the other hand, the apparent analogy between the $\beta$-decay of the nucleus and that of $\mu$-meson is further enhanced by the observed nearly identical interaction strength $G \sim 10^{-49} \mathrm{erg} \mathrm{cm}^{3}$. This suggests ${ }^{78}$ the direct interaction between the four fermions $\mu$, e, $v$ and $v$ analogous to that occurring between $\mathrm{p}, \mathrm{n}, \mathrm{e} v$ in $\beta$-decay.

Michel ${ }^{79}$ and Tiomno and Wheeler ${ }^{80}$ were the first ones to apply the same coupling $\mathrm{S}, \mathrm{V}, \mathrm{T}, \mathrm{A}$, and $\mathrm{P}$ used in the ordinary $\beta$-decay to the $\mu$-meson decay. In comparing these two phenomena one must assume which particles play" corresponding roles in these two processes, as a single interaction term in one set of particles can be written as a linear com-

77 E. Hincks and B. Pontecorvo, Phys. Rev. 73, (1948) 257; R. Sard and E. Althaus, Phys. Rev. 74, (1948) 1364.

78 O. Klein, Nature 161, (1948) 897.

79 L. Michel, Nature 163, (1949) 959; Proc. Phys. Soc., 63A (1950) 514; Phys. Rev. 86, (1952) 814; Progress in Cosmic Ray Physics (1952) edited by Wilson; Thesis, University of Paris, 1953.

80 J. Tiomno, and J. Wheeler, Rev. Mod. Phys. 21, (1949) 144.

84 H. J. Bramson, A. H. Seifert and W. W. Havens, Jr., Phys. Rev. 88, (1952) 304.

* The upper limit of the mass of the two neutral particles is estimated around $15 m_{e}$ each from the most accurate $\mu$-meson $\beta$-spectrum. 
bination of the five forms with a different correspondence of particles. However, in the case of $\mu$-meson decay, all the products are light particles, therefore there is no way to tell whether the $\mu$ transforms to e or $v$. Furthermore, there is the question whether the two neutrinos created are distinguishable or indistinguishable. There are all together twenty-four possible permutations of the order of the four particles in the Hamiltonian. However, they fall into three essentially different classes represented by

(a) $\quad \begin{aligned} & \mathrm{p} \mathrm{n} \text { e } v \\ & v \mu \mathrm{e} v \\ & \mathrm{p} \mathrm{n} \text { e } v \\ & \mu \nu \mathrm{e} v \\ & \mathrm{p} \mathrm{n} \text { e } v \\ & \mu \mathrm{e} v \nu\end{aligned}$

Michel ${ }^{79}$ calculated the energy distribution of the electrons from $\mu$-meson decay for a more general case in which a linear combination of all five interactions is used and also for both distinguishable or indistinguishable neutrinos. If the rest mass of the electron is negligible in comparison with its total energy, as is certainly true for the high energy region of the spectrum, the distribution of the $\beta$-spectrum may be written as follows

$$
\tau_{\mu} P(E) d E=\frac{4 E^{2}}{W_{\max }^{4}}\left[3\left(W_{\max }-E\right)+2 \varrho\left({ }^{4} / 3 E-W_{\max }\right)\right] d E .
$$

When one uses the condition

$$
\int_{1}^{W_{\max }} P(E) d E=\frac{1}{\tau_{\mu}} .
$$

The parameter $\varrho$ is a function of the five coupling constants and depends on the nature of the neutrino and also the assumed "one to one" correspondences between the two sets of four fermions in the $\beta$-decay and $\mu$-decay. However, it can be generally represented by three essentially different modes of decay (see Table VII). When $\varrho=0$ the spectrum goes to zero at the upper energy limit. The parameter $\varrho$ has been defined by Michel so that $0 \leqslant \varrho \leqslant 1$ for distinguishable neutrinos; then one finds $0 \leqslant \varrho \leqslant 3 / 4$ only for indistinguishable neutrinos (see Fig. 26).

The general agreement between the observed spectra and the theoretical ones is good. However, a precise determination of its shape and therefore the value of $\varrho$ may yield some information on the possible combinations of the couplings or the mode of decay. This is particularly interesting because of the possibility of a universal Fermi interaction ${ }^{78}$ among any four of the five fermions. 


$$
\begin{aligned}
& \begin{array}{l}
\text { (a) } \quad\left\{\begin{array}{l}
\neq=\frac{(2 / 3)\left[C_{S}^{2}+4 C_{V}^{2}+6 C_{T}^{2}+4 C_{A}^{2}+C_{P}^{2}\right]}{C_{S}^{2}+C_{V}^{2}+3 C_{T}^{2}+3 C_{A}^{2}} \\
\text { p n e } v
\end{array}\right\} \quad \varrho=\frac{(3 / 4)\left[\left(C_{S}-C_{T}\right)^{2}+C_{T}-C_{P}\right)^{2}+2\left(C_{V}-C_{A}\right)^{2}}{C_{S}^{2}+4 C_{V}^{2}+6 C_{T}^{2}+4 C_{A}^{2}+C_{P}^{2}}
\end{array} \\
& \begin{array}{l}
\nu \operatorname{e} v \\
C_{S}^{2}+C_{V}^{2}+3 C_{T}^{2}+3 C_{A}^{2}
\end{array} \lambda=\frac{(1 / 12)\left\{\left(C_{S}+6 C_{T}+C_{P}\right)^{2}+16\left(C_{V}+C_{A}\right)^{2}+2\left[C_{S}+C_{T}+2\left(C_{V}-C_{A}\right)\right]^{2}\right\}}{3 C_{S}} \\
& \equiv \varrho=\frac{3\left[C_{S}-C_{P}+2\left(C_{V}-C_{A}\right)\right]^{2}}{2\left\{\left(C_{S}+6 C_{T}+C_{P}\right)^{2}+16\left(C_{V}+C_{A}\right)^{2}+2\left[C_{S}+C_{T}-2\left(-C_{V}+C_{A}\right)\right]^{2}\right\}} \\
& \text { (b) } \quad \neq \varrho=\frac{(3 / 4)\left[\left(C_{S}+C_{T}\right)^{2}+\left(C_{T}+C_{P}\right)^{2}+2\left(C_{V}+C_{A}\right)^{2}\right]}{C_{S}^{2}+4 C_{V}^{2}+6 C_{T}^{2}+4 C_{A}^{2}+C_{P}^{2}}
\end{aligned}
$$

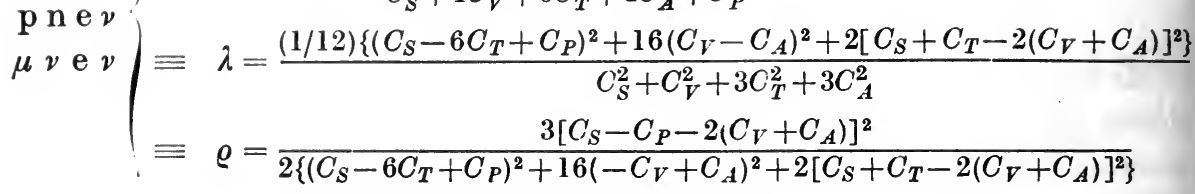

$$
\begin{aligned}
& \left.\begin{array}{l}
\text { (c) } \\
\text { p ne } v
\end{array}\right\} \quad \begin{array}{l}
\neq=\frac{(2 / 3)\left[C_{S}^{2}+4 C_{V}^{2}+6 C_{T}^{2}+4 C_{A}^{2}+C_{P}^{2}\right]}{C_{S}^{2}+C_{V}^{2}+3 C_{T}^{2}+3 C_{A}^{2}} \\
C_{S}^{2}+4 C_{V}^{2}+6 C_{T}^{2}+4 C_{A}^{2}+C_{P}^{2}
\end{array} \\
& \mu \text { e } v v \mid \equiv \lambda=\frac{(2 / 3) C_{S}^{2}+4 C_{A}^{2}+C_{P}^{2}}{C_{S}^{2}+C_{V}^{2}+3 C_{T}^{2}+3 C_{A}^{2}} \\
& \equiv \varrho=\frac{3 C_{A}^{2}}{C_{S}^{2}+4 C_{A}^{2}+C_{P}^{2}}
\end{aligned}
$$

" $\neq$ " distinguishable neutrinos; " $\equiv "$ identical neutrinos.

This table is from Dr L. Michel's Thesis, University of Paris, 1953.

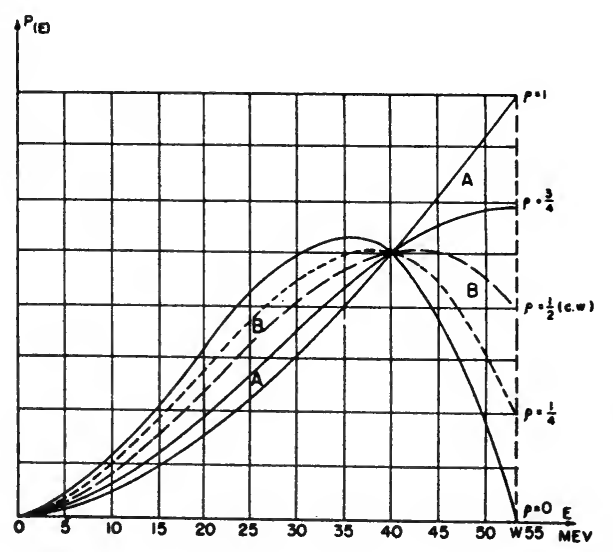

Fig. 26. The energy spectrum of the electrons from the $\mu$ meson decays as function of the energy $W$ given for various values of $\varrho$ where $\varrho$ is a function of the five coupling constants 
TABLE VIII

\begin{tabular}{l|c|c|c|c|c|c|c}
\hline \hline & Year & $\begin{array}{c}\text { No. of } \\
\text { events }\end{array}$ & Source & Method & $\varrho$ & $\begin{array}{c}m_{\mu}=207 m_{e} \\
\varrho_{1}\end{array}$ & Remarks \\
\hline $\begin{array}{l}\text { 1. Leighton, } \\
\text { Anderson and } \\
\text { Seriff } 91\end{array}$ & 1949 & 75 & cosmic & $\begin{array}{l}\text { cloud } \\
\text { chamber }\end{array}$ & $.075 \pm 20$ & & \\
2. Davies, \\
$\begin{array}{l}\text { Lock and } \\
\text { Muirhead 92 }\end{array}$
\end{tabular}

The $\beta$-spectra of $\mu$-decay were investigated mostly by photographic plates or by the cloud chamber method. The only magnetic spectrometer work was done in a spiral type spectrometer ${ }^{81}$. Table VIII summarizes the results to date. The most important information obtained from the analysis of the spectrum is the value of $\varrho$. Owing to the statistical nature of the limited number of events and also the uncertainty associated with the energy determination of each event, the interpretation of the data requires special methods of analysis. Lagarrigue and Peyrou ${ }^{82}$ noticed that at energy $E=3 W_{\max } / 4$, the term containing $\varrho$ vanishes or $\left(3 W_{\max } / 4\right)$ is independent of the value of $\varrho$. However, the theoretical ratio of events before

81 R. Sagana, W. P. Gardner and H. W. Hubbard, Phys. Rev. 82, (1951) 557.

82 A. Lagarrigue and C. Peyrou, C. R. Acad. Sci., Paris, 233 (1951) 478; J. Phys. Radium 12, (1951) 848; C. R. Acad. Sci., Paris, 234, (1952) 1873. 
and after this $E$ is a linear function of $\varrho$ and one may determine the value of $\varrho$ by comparing the experimental ratio to the theoretical ones. In so doing, one only utilizes the data in the immediate neighborhood of $E=\left(3 \mathrm{H}^{-}\right) / 4$, and does not use to its fullest extent the shape of the entire spectrum. Hubbard ${ }^{83}$ takes advantage of the relation that $P(E) / E^{2}$ is linear in $E$ and finds $\varrho$ as the slope of a least squares fit. The application of least square fit in this ease is rather questionable as the errors are not given by a gaussian distribution. Furthermore, the weak point of this least square fitting method is the undue influence exercised by those points of extreme energy which happen to be the least accurate ones. A different and improved approach to evaluating $\varrho$ and its corresponding standard error employed by Bramson ${ }^{84}$ and Vilain and Williams ${ }^{85}$ was the method of moments or the method of maximum likelihood ${ }^{86}$. These are the statistical methods of estimating the parameters of a distribution by means of a set of sample values.

The determination of $\varrho$ is extremely sensitive to the rest mass of $\mu$-meson (Fig. 27). The new value of the $\mu$-meson mass $\mu=207.0 \pm 0.6 m_{e}{ }^{87}$ will raise the value of $\varrho$ in Bramson's work from 0.41 to .48 and Lagarrigue and

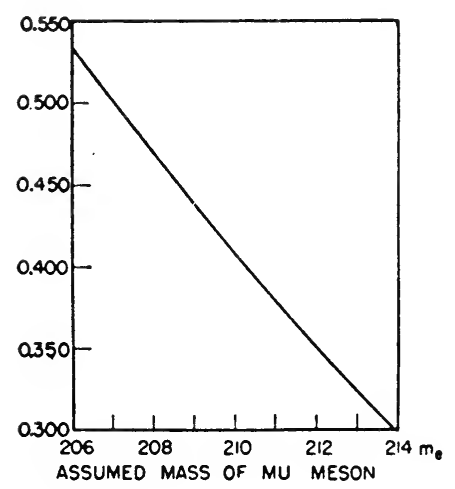

Fig. 27. Dependente of estimate of $\varrho$ with $\mu$-meson mass assumed. (See note 84 )
Peyron's value of $\varrho$ from 0.23 to 0.43 using their empirival formula of $\varrho=\left(58-W_{\max }\right) / 12$. The $\beta$-spectrum obtained by Sagana is the only one which goes to zero at the upper limit. However, using the new meson mass and applying the corrections for the radiation loss of the electron, the $\beta$-spectrum of $\mu$ meson decay as observed by Sagana also yields non zero cut off and is no longer in contradiction with the latest value of $\varrho \sim 1 / 2{ }^{*}$.

The two most recent results were obtained by Vilain and Williams ${ }^{85}$ and Sargent ${ }^{88}$ et $a l$. Both investigations have been carried out in a cloud chamber. The first one analyzed 830 tracks and found 280 tracks satisfying their criteria. The value of $\varrho$ is $0.50 \pm 0.12$. The latter analyzed 500 tracks

83 H. W. Hubbard, U. C. R. L. No. 1623.

H. Cramer, Mathematical Methods of Statistics. Princeton University Press. F. M. Smith et al., Phys. Rev. 91, (1953) 765.

Sargent, Rinehart, Lederman and Rogers, Private communication.

The latest result from the spiral magnetic spectrometer is $\varrho=0.23_{-.05}^{+.03}$ R. Sagana, W. Dudjiak and J. Vedder, Phys. Rev. 95, (1954) 863. 
and found a value of $\varrho \sim 0.64 \pm .10$. This latter investigation was carried out in a continuous diffusion cloud chamber filled with high pressure hydrogen gas, so the correction for radiation loss of the electron is very small.

It seems quite certain that the $\beta$-spectrum of $\mu$-decay does not go to zero at its upper limit. The most probable value of $\varrho$ from the various experiments is around $(0.5-0.6)$. The value of $\varrho=0.5$ is the one predicted for Critchfield and Wigner's combination $(\mathrm{S}-\mathrm{A}-\mathrm{P})$ which is obtained by considering all four particles on the same footing and is invariant under any permutations of the order of the four fermions. In other words, the particular order of the particles in the Hamiltonian is immaterial. This seems rather appropriate for $\mu$-decay because all four particles involved are light particles. However, the evidence from nuclear $\beta$-decay does not agree with the $(\mathrm{S}-\mathrm{A}-\mathrm{P})$ combination. On the other hand, it is attractive to entertain the possibility of a universal interaction among any four fermions. The present experimental evidence from $\beta$-decay strongly favors the presence of $S, T$ and possibly $P$, (there is no direct evidence of the presence of $\mathrm{P}$ at the present moment) but is not accurate enough to give the proportion of $\mathrm{S}, \mathrm{T}$ and $\mathrm{P}$ and their relative signis uniquely. Therefore there is ample flexibility to adjust the coupling constants to reach an agreement between the $(\mathrm{S}, \mathrm{T}, \mathrm{P})$ combination and the observed $\varrho \sim(0.5-$ $0.6)$ and $\lambda \sim 1.0-1.3$. Michel and Wightman $89 *$ have investigated the general possibilities $( \pm \mathrm{S}+\mathrm{T}+p \mathrm{P})$ and obtained very good fits for $\varrho$ and $\lambda$ for certain cases. For example; in the case when the two neutrinos are distinguishable for $|p|<1 / 2$, for (a) $-\mathrm{S}+\mathrm{T}$ or (b) $+\mathrm{S}+\mathrm{T}$, one obtains $\varrho=0.54$ and $\lambda=1.17$. For two indistinguishable neutrinos, $|p| \sim 3.5$ and for (a) $+\mathrm{S}+\mathrm{T}-3.5 \mathrm{P}$ or (b) $-\mathrm{S}+\mathrm{T}+3.5 \mathrm{P}, \varrho=0.58$ and $\lambda=1.10$ are reasonable. $\lambda$ is a parameter introduced by Michel which is related with the ratio of $f t$ values for $\mu$ - and $\beta$-decays.

$$
\lambda=\frac{8}{15} \frac{(f t)_{n}}{(f t)_{\mu}}=2^{9} \frac{B}{(1+2 x)} \times \frac{1}{m_{\mu}^{5} \tau_{\mu} \log _{\mathrm{e}} 2} .
$$

If $\tau_{\mu}=(2.22 \pm 0.02) \times 10^{-6} \mathrm{sec}$ for $\mu$-meson decay,

$$
B=\frac{2 \pi^{3} h^{7} 12}{g^{2} m^{5} c^{4}}=2650 \pm 10 \%
$$

for the rate constant of $\beta$-radioactivity and $x=0.50 \pm 0.05$ (see $\S 4$ ) are used; $\lambda$, thus calculated, is equal to $1.16 \pm 0.12$.

89 L. Michel and A. Wightman, Letter to the Editor. Phys. Rev. in Press.

* The author is deeply indebted to Dr. Michel and Dr. Wightman for communicating to her the results of their investigation prior to its publication. 
There have been several unique interactions proposed by various authors ${ }^{90}$ based on certain symmetry principles. These interactions can be summarized by the expressions:

or

$$
\left.\begin{array}{l} 
\pm \mathrm{S}+\mathrm{T} \pm \mathrm{P} \\
\pm \mathrm{S}+\mathrm{T} \mp \mathrm{P}
\end{array}\right\} \equiv
$$

Using Michel's expression of $\varrho$ and $\lambda$ as listed in Table VII, it can be shown that the fits which could be obtained with such combinations are not satisfactory. Particularly if the neutrinos are considered to be indistinguishable ( $\equiv$ ) and only the case (b) is considered then the proposed unique interactions are definitely excluded.

90 E. Caianiello, Nuovo Cim. 10, (1953), 43. D. Pursey, Physica 18, (1952) 1017. D. C. Peaslee, Phys. Rev. 91, (1953) 1447. E. J. Konopinski, and H. M. Mahmoud, Phys. Rev. 92, (1953) 1045; R. Finkelstrein and P. Kaus, Phys. Rev. 92, (1953) 1316.

${ }^{91}$ R. B. Leighton, C. D. Anderson and A. J. Seriff, Phys. Rev. 75, (1949) 1432.

92 L. H. Davis, W. O. Lock and H. Muirhead, Phil. Mag. 40, (1949) 1250.

93 R. Levi-Setti and G. Tomasino, Nuovo Cim. 8, (1951) 994, a (1952) 1244.

94 C. O'Ceallaigh, Communication at the Congress of Copenhagen, July 1951. Phil. Mag. 42, (1951) 1032. 


\section{NEUTRINO RECOIL EXPERIMENTS}

\section{O. KOFOED-HANSEN}

\section{§ 1. Introduction}

Neutrino recoil experiments are often carried out in order to demonstrate that momentum is missing if the existence of the neutrino is not postulated. This has been very clearly proved by many investigations, of which some will be mentioned below. Furthermore, from neutrino recoil experiments it is possible to obtain measurements of the angular correlation between the particles participating in $\beta$-decay. This type of information is very helpful in deciding among the different possible theoretical interactions between the nucleons and the leptons. In the following a survey of the results so far obtained is given; we shall discuss in what sense such measurements contribute to our knowledge about the interaction in $\beta$-decay.

Originally many $\beta$-decay processes were successfully investigated in order to show that conservation of momentum necessitates the neutrino hypothesis. Nevertheless, the most clear-cut results for this demonstration are provided by the study of two-body decays. In such cases the particles after the disintegration are a neutrino and its recoil. The processes in question are $K$-capture, $\pi-\mu$ meson decay and $\mu$ meson capture. We shall discuss experiments with two-body decays in section 2 and $\beta$-recoil experiments in section 3 .

When the radioactive material applied in recoil experiments is deposited on a surface or forms part of a molecule the recoil phenomena are very complicated for small energies. We shall return to this question in section 4. In sections 2 and 3 we shall, however, confine ourselves to a discussion of experiments with monatomic gases and meson reactions. In the case of meson reactions the energies involved are sufficiently large so that substrata do not play any important role.

Finally the interpretation of recoil experiments with $\beta$-decay will be dealt with in section 5 .

\section{$\S 2$. Two-Body Decays}

Recoil experiments with $K$-capture of inert gases have been carried out with $\mathrm{A}^{37}$. This isotope decays by $92 \% \mathrm{~K}$-capture and $8 \% \mathrm{~L}$-capture to $\mathrm{Cl}^{37}$. The half life is $35^{\mathrm{d}} 1$. The $\mathrm{Cl}^{37}$ recoil is primarily electrically neutral

1 Cf. e.g. J. M. Hollander, J. Perlman and G. T. Seaborg, Rev. Mod. Phys., 25 (1953) 469. 
but formed with a vacancy in the $K$ (or $L$ ) shell. This vacancy is to a large extent restored by successive Auger effects. Also the abrupt shaking of the electronic core from nuclear charge 18 to 17 gives rise to ionization. All these effects take place within very short times (of the order $10^{-14} \mathrm{sec}$ ). In practice, therefore, we need consider only primarily highly charged recoils. The average charge has been found to be $3.41 \pm 0.14^{2}$.

Two different methods have been applied. These methods are also characteristic of the possibilities for making $\beta$-recoil investigations.

Rodeback and Allen ${ }^{3}$ have used an instrument which is shown in Fig. 1. Delayed coincidences between the fast Auger electrons and the recoil ions

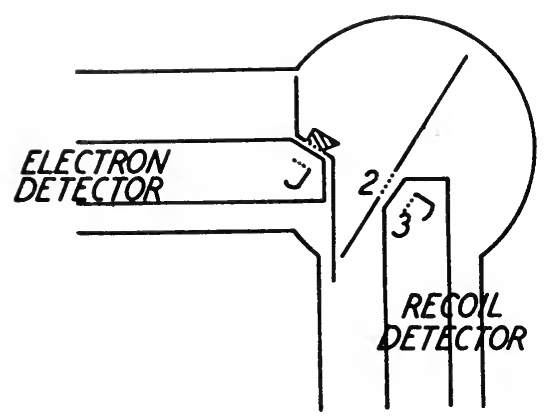

Fig. 1. The neutrino recoil instrument used by Rodeback and Allen are recorded. The distance travelled and the delay time give the velocity of the recoils. The Auger electrons are recorded by the electron detector, which is an Allen type multiplier. The recoils are accelerated between grid 2 (0 potential) and grid 3 ( -4500 volt) and detected by a multiplier. The shaded trapezoidal cross section in Fig. 4 is the effective source volume. This volume is defined by baffles and also by the region seen simultaneously by both detectors. All shields, baffles, etc., are maintained at zero potential. The pressure in the instrument is $\sim 10^{-5} \mathrm{~mm} \mathrm{Hg}$. This corresponds to a mean free path $\lambda_{z}$ of about $20 \mathrm{~m}$ for singly charged ions. The mean free path for capture of electrons $\lambda_{z}$ may vary considerably with $z$, where $z$ is the ionic charge. Therefore collisions cannot be entirely avoided. The observed time of flight distribution is shown in Fig. 2. The $\mathrm{A}^{37}$, of course, spread out everywhere inside the instrument. Therefore, one of the complications of the instrument is the rather poor geometry which can be obtained when a reasonable ratio of chance to true coincidences is desired. The thermal motion of the $\mathrm{A}^{37}$ atoms causes a spread of $\sim 7 \%$ in the velocity distribution. The time of flight distribution must be corrected for this effect, which corresponds to the tail on the distribution from $\sim 7.5$ to $\sim 9 \mu$ sec. Thus the maximum time of flight, i.e., the time spent by an ion in travelling the maximum distance $6 \mathrm{~cm}$, is $\sim 7.5 \mu \mathrm{sec}$. Furthermore, one has to take into account the fact that the electrons spend some time being accelerated between grid 2 and 3 and that the field between these grids penetrates grid 2 to a certain extent. Thereby the time

2 M. L. Perlman and J. A. Miskel, Phys. Rev. 91 (1953) 899.

3 G. W. Rodeback and J. S. Allen, Phys. Rev. 86 (1952) 446. 
of flight is shortened by $\sim 1.4 \mu$ sec. for three-fold charged ions. When all these corrections are calculated a maximum time of flight of $8.9 \pm 0.9 \mu$ sec. is obtained. This gives a neutrino momentum times the velocity of light $c$ equal to $0.8 \pm 0.1 \mathrm{MeV}$, in good agreement with the $Q$ value $816 \pm 4 \mathrm{keV}$ for the $\mathrm{Cl}^{37}(\mathrm{p}, \mathrm{n}) \mathrm{A}^{37}$ reaction ${ }^{1}$. The origin of the short time coincidences in this experiment (see Fig. 2) is not yet understood. The experiment has clearly demonstrated the momentum peak from the recoils after neutrino emission.

The application of a gaseous source necessarily leads to a rather complicated geometry. The author has tried to take this fact into account.

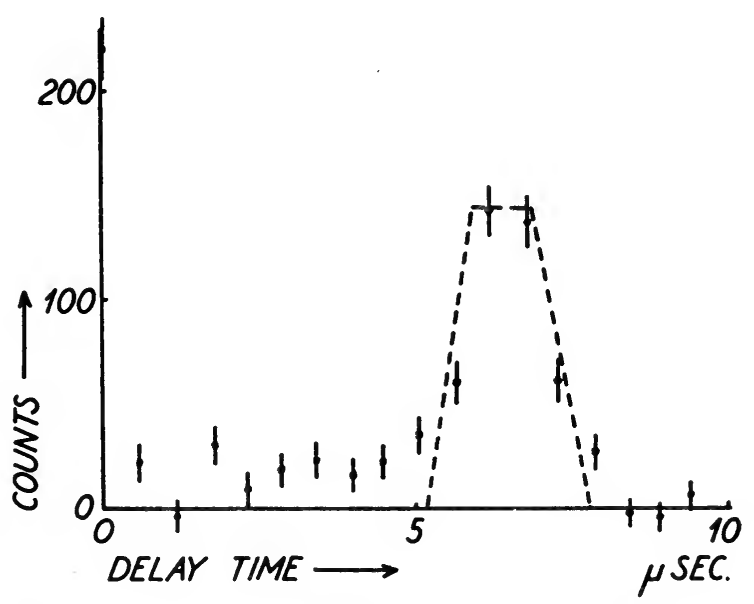

Fig. 2. The time of flight distribution of $\mathrm{Cl}^{37}$ recoils from $\mathrm{A}^{37} \mathrm{~K}$-capture. The dotted curve is the predicted resolution of the instrument

Instead of using usual spectrometers with poor geometry, one can build instruments with as simple a geometry as possible in such a manner that one is able to control the geometry in a nearly perfect way. The inert radioactive gas fills the space between the plates of a plane parallel condenser. The central part of one of the plates is used as a collector plate, the rest as a guard ring. The current of recoils and electrons going to the collector is measured when the condenser is placed in a magnetic field. The magnetic lines of force should be parallel to the plates of the condenser. When the motion of the charged particles is studied in crossed electric and magnetic fields and at various pressures, one is able to obtain information about the momentum and charge of the recoil ions by measuring the current to the collector ${ }^{4 \mathrm{a}}$.

At zero pressure and zero electric potential across the condenser, the current $i$ of mono-energetic recoils with charge $z e$ and momentum $p$ is given as a function of the magnetic field $B$ by ${ }^{4}$

4 O. Kofoed-Hansen, Dan. Mat. Fys. Medd. 26 (1951) no. 8.

4a O. Kofoed-Hansen, Phys. Rev. in press, O. Kofoed-Hansen and A. Nielsen, Dan. Mat. Fys. Medd. in press. The charge spectrum for the recoils and information about the Auger electrons was obtained after the present paper was written. 


$$
i B= \begin{cases}(B N z e / 4)\left\{\left[1-(B z e a / c p)^{2}\right]^{1 / 2}\right. & \\ \left.+(c p / B z e a) \sin ^{-1}(\text { Bzea } / c p)\right\} & \text { for Bzea } \leqslant c p \\ N \pi c p / 8 a & \text { for Bzea } \geqslant c p .\end{cases}
$$

$N$ is the total number of disintegrations, $a$ is half the distance between the condenser plates. For a distribution of charges and momenta, eq. (1) has to be integrated over these distributions. When the pressure becomes larger some of the recoils spiralling up along the field will capture electrons, and the expression for the currents becomes more involved. However, if the following conditions are fulfilled:

$$
l / \lambda_{z} \gg 1 \text { and } \varrho_{z} / \lambda_{z} \ll 1 \text { (for } z>1 \text { ), }
$$

the expression for $i B$ is approximately equal to a constant plus a constant times the expression (1) taken for $z=1$ and in the region $2 B e a \geqslant c p$. In

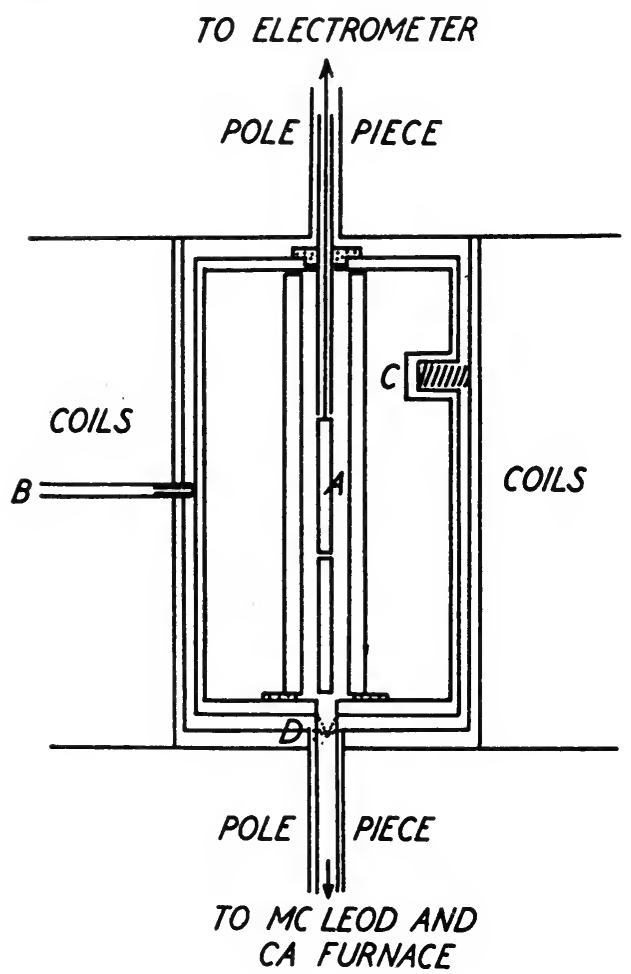

Fig. 3. A recoil instrument utilizing crossed electric and magnetic fields. The distance $2 a$ between the plates is $7 \mathrm{~mm}$ eq. (2) $l$ is the length of the instrument parallel to $B$ and $\varrho_{z}$ is the radius of curvature in an orbit perpendicular to $B$.

The instrument is shown in Fig. 3. The collector is placed between two plates, giving a double condenser. This is only done for convenience in the construction; the two outer plates are maintained at the same electric potential. The collector is the central part of plate A. The collector is insulated from the rest of $\mathrm{A}$ but kept at the same potential as $\mathbf{A}$ and connected to a vibrating reed electrometer. The electrometer acts as a zero indicator in connection with an additional condenser and a potentiometer. In order to measure the currents, rather large amounts of activity are needed, and thereby it is hardly possible to obtain sufficiently low pressures with long lived activities. The argon must of course not be pumped off, and therefore the pumping is effected by means of a calcium furnace kept at $\sim 400^{\circ} \mathrm{C}$. 
$\sim 810 \mathrm{keV}$ for the momentum of the neutrino. A closer analysis is given in Fig. 6, where the experimental points are compared with theoretical curves obtained by integrating (1) together with the momentum distribution obtained from thermal motion, Auger effect, and

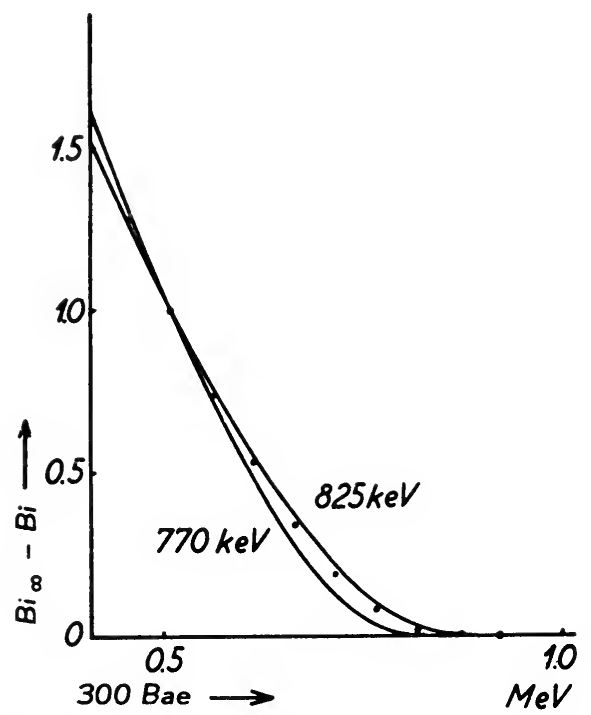

Fig. 6. The difference between the $B i$ value for $300 \mathrm{Bae}>900 \mathrm{keV}^{\prime}$ and that for $B i$ smaller than this value as a function of 300 Bae compared with theoretical curves for neutrino momenta $770 \mathrm{keV}$ and $825 \mathrm{keV}$. The curves are normalized so as to go through a common point assumed neutrino energies. The result is $(806 \pm 8) \mathrm{keV}$ for the neutrino momentum.

The most clear-cut demonstration of the existence of a neutral particle with small rest mass is perhaps obtained in the $\pi-\mu$ meson decay. This decay shows that the $\mu$ meson energy spectrum has the characteristic line property for a two body decay ${ }^{5}$. The total energy available is the $\pi$ meson mass $(276 \pm 1) m_{e}{ }^{5}$. The kinetic energy $T_{\mu}$ of the $\mu$ meson is $4,15 \mathrm{MeV}^{5}$. Thus the momentum is $p_{\nu}=58 m_{e} c$, since the $\mu$ meson mass is $(210 \pm 2) m_{e}{ }^{5}$. The energy of the neutral particle is thus

$E_{\nu}=m_{\pi} c^{2}-m_{\mu} c^{2}-T_{\mu}=(: 5 \pm \pm 2) m_{e} c^{2}$

Consequently the mass of the neutral particle can be found from

$$
m_{\nu}^{2} c^{4}=E_{\nu}^{2}-p_{\nu}^{2} c^{2} .
$$

This leads to $m_{v}<14 m_{e}$. Of course, this determination of $m_{\nu}$ is very poor. This small magnitude occurs squared and is found as the difference between two much larger and nearly equal numbers. The situation is more favourable when the $\mathrm{A}^{37}$ data are used, but still the same general argument holds, and recoil measurements cannot compete with the determination of $m_{v}$ from the shape of the $\mathrm{H}^{3}$ spectrum ${ }^{6}$.

Since the $\mu$-meson follows the usual laws for $\beta$-radioactivity and emits a $\beta$-particle and two neutrinos, the $\mu$-meson capture may be assumed to

5 Cf e.g. W. Heisenberg, Vorträge über Kosmische Strahlung, Springer 1953.

- S. C. Curran, J. Angus and A. L. Cockeroft, Phil. Mag. 40 (1949) 36 and 53. G. C. Hanna and B. Pontecorvo, Phys. Rev. 75 (1949) 983. D. R. Hamilton, W. P. Alford and L. Cross, Phys. Rev. 83 (1951) 215. O. Kofoed-Hansen, Phys. Rev. 71 (1947) 451 and Phil. Mag. 42 (1951) 1448. 
be similar to $K$-capture. Momenta from this type of neutrino recoil have been measured by Fry ${ }^{7}$, who finds results in general agreement with the above assumption. The decay is in this case complicated by the subsequent break up of the recoil nucleus, leading to small cosmic ray stars.

In Table I most of the investigations of neutrino recoils from two body decays are summarized. Some of the investigations with bound mother nuclei will be discussed in $\S 4$.

\section{§ 3. $\beta$-Decay}

The most important results in $\beta$-decay have been obtained with $\mathrm{He}^{6}$. This $\beta$-emitter decays with a maximum energy of $3.50 \mathrm{MeV}$ and a half life of $.823 \mathrm{sec}$. The spin change is $\Delta I=1$, and the recoii correlation can therefore be used to distinguish between the GT interactions. The maximum recoil energy is found from

$$
\begin{aligned}
& T_{R}^{\operatorname{mux}}=(533 / A) \\
& \left(T_{\beta}^{\max }+1.022\right) T_{\beta}^{\max }, \hat{l}
\end{aligned}
$$

where $T_{R}^{\max }$ is measured in $\mathrm{eV}$ and the maximum kinetic energy $T_{\beta}^{\max }$ of the $\beta$-particles is measured in $\mathrm{MeV}$. The result for $\mathrm{He}^{6}$ is $\sim 1400 \mathrm{eV}$, which is the highest neutrino recoil energy dealt with in nuclear physics. Rustad and Ruby ${ }^{8}$ have examined the angular correlation between recoil and $\beta$-particle from this decay. Fig. 7 shows their experimental arrangement. The effective source volume is defined by the space seen simultaneously by the two counters and by the pumping diaphragm, which ensures that the spread of $\mathrm{He}^{6}$ in the instrument is smaller than that of $\mathrm{A}^{37}$ in Rodeback and Allen's experiment (Fig. 1). The recoils are accelerated between grid

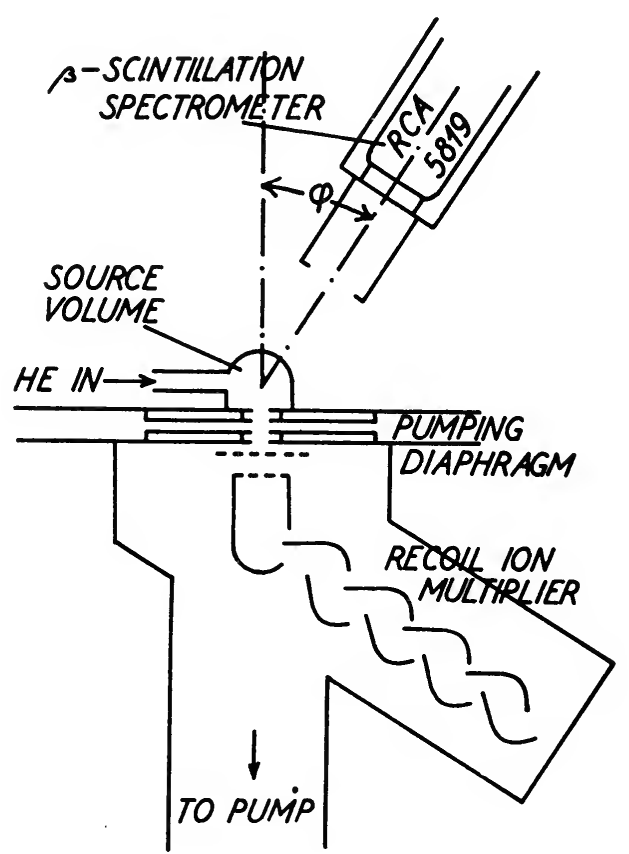

Fig. 7. The angular correlation instrument used by Rustad and Ruby in their investigation of $\mathrm{He}^{6}$ 1 and grid 2 and detected in a multiplier. The electrons are detected in a crystal counter and the pulse height from these particles is analyzed.

7 W. F. Fry, Phys. Rev. 90 (1953) 999.

8 B. M. Rustad and S. L. Ruby, Phys. Rev. 89 (1953) 880. The author is indebted to Dr. Rustad for sending unpublished material concerning his experiments. 
Delayed coincidences are selected between 0.08 and $1.15 \mu$ sec. after the arrival of the $\beta$-particle. In this instrument the angular correlation can be measured for different intervals of the $\beta$-energy, or the energy distribution of the $\beta$-particles can be found for a certain angular interval. Examples of these two types of curves are shown in Figs. 8 and 9.

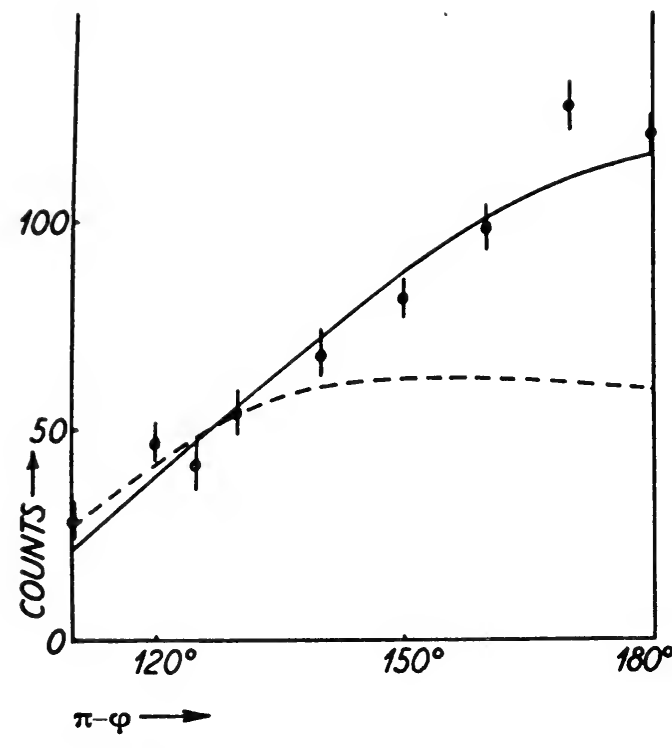

Fig. 8. Rustad and Ruby's electron recoil angular correlation for $\mathrm{He}^{6} \beta$-rays in the range $2.5 \mathrm{mc}^{2}<E_{\beta}<4.0 \mathrm{mc}^{2}$ and for recoils arriving between 0.08 and $1.15 \mu$ sec. after the $\beta$-particle. The full drawn curve represents the theoretical predictions for tensor interaction and the dotted curve represents the axial vector interaction

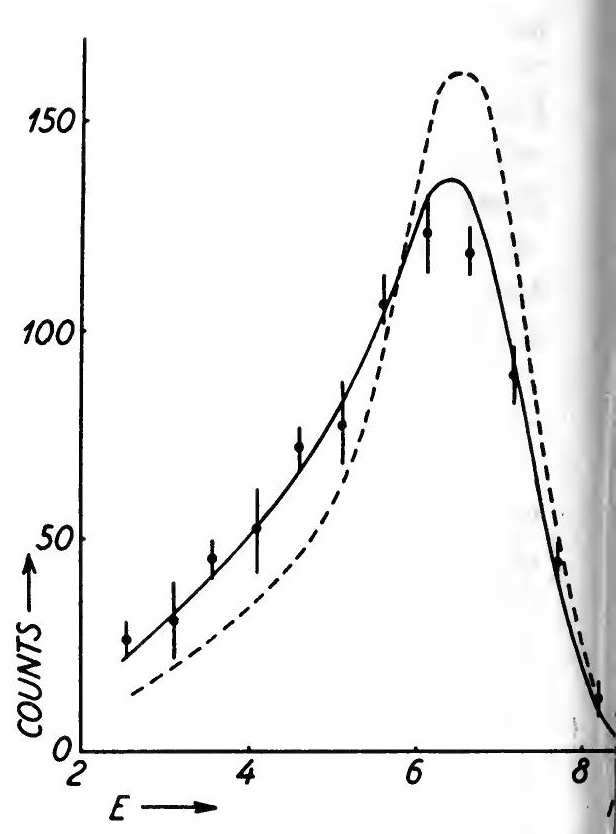

Fig. 9. Rustad and Ruby's $\mathrm{He}^{6} \beta$-ray spectrum with $1 \mathrm{mc}^{2}$ channel width and $\varphi=0^{\circ}$ between the electron and the recoil. Full drawn curve predicted for tensor interaction, dotted curve for axial vector interaction

The experimental results are compared with theoretical expectations corrected for the finite resolution as regards $\beta$-energy, recoil energy and angle. The results strongly favour the tensor interaction.

A somewhat similar experiment has been carried out by Allen and Jentschke ${ }^{9}$. Their experimental arrangement selects recoils going in a direction forming an average angle of $120^{\circ}$ relative to that of the electrons, but apart from this the instrument is similar to that shown in Fig. 1. The time of flight distribution for the recoils is measured. The results are

- J. S. Allen and W. K. Jentschke, Phys. Rev. 89 (1953) 902. The author is indebted to Dr. Allen for sending unpublished material concerning his experiment. 
shown in Fig. 10. A least square fit is said to favour the tensor interaction in agreement with the results obtained by Rustad and Ruby.

The neutron decays by $782 \mathrm{keV}$ electrons with a half life of $(12.8 \pm 2.5)^{m}$. The disintegration has been observed by recoil investigations ${ }^{10}$. The decay goes by $\sim 25 \%$ Fermi interaction and $\sim 75 \%$ GT interaction ${ }^{11}$. A careful study of the recoil spectrum or the recoil electron angular correlation should therefore permit a distinction between the Fermi interactions when the GT interaction is known. Such experiments are not yet completed.

It may be mentioned that for $\beta$-transitions the rnotion of the recoils in electric and magnetic fields can also be utilized to give information about the angular correlation. When recoils are collected in a plane parallel condenser with an electric potential $V$ across the condenser the number $N_{+}$of recoils that

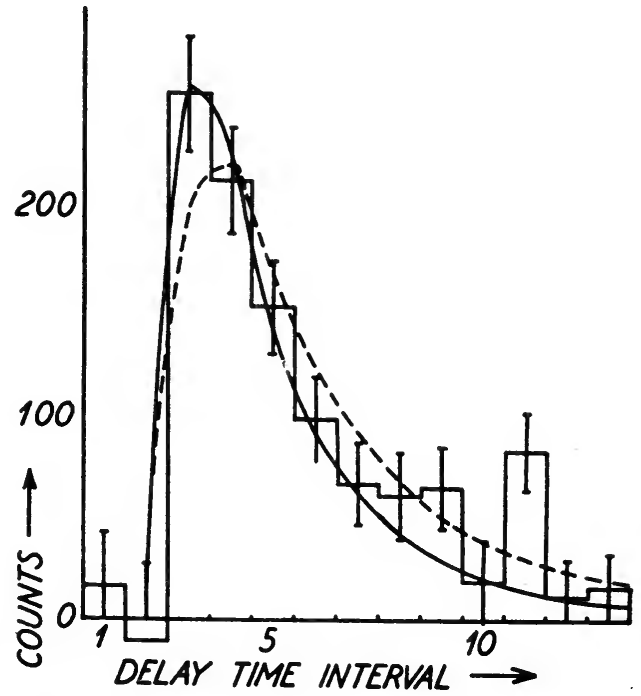

Fig. 10. The time of flight spectrum of the recoils from $\mathrm{He}^{6}$ measured by Allen and Jentschke. Full drawn curve represents expectations for the tensor interaction and dotted curve corresponds to axial vector interaction are collected on the positive plate relative to the total number, $N_{+}+N_{-}$, is, for monoenergetic particles of charge $z$ and energy $T$, given by ${ }^{4}$

$$
6 V e N_{+} /\left(N_{+}+N_{-}\right)= \begin{cases}3 V e-2 V e(V e z / T)^{1 / 2} & \text { for } T>V e z \\ T / z & \text { for } T<V e z .\end{cases}
$$

$\mathrm{Kr}^{88}$ and $\mathrm{Kr}^{89}$ have radioactive daughter substances and therefore $N_{+}$ and $N_{-}$can be found by the tracer method ${ }^{12}$. The results of the $\mathrm{Kr}^{89}$ investigation are shown in Fig. 11. For a distribution of charges and energies, (6) has again to be integrated over this distribution, and it is immediately apparent that the average value of $T / z$ equals the constant

10 A. H. Snell and L. C. Miller, Phys. Rev. 74 (1948) 1217. A. H. Snell, F. Pleasonton and R. V. McCord, Phys. Rev. 78 (1950) 310. J. M. Robson, Phys. Rev. 78 (1950) 311 and 83 (1951) 349.

11 cf. e.g. O. Kofoed-Hansen and A. Winther, Phys. Rev. 86 (1952) 428.

12 J. C. Jacobsen and O. Kofoed-Hansen, Phys. Rev. 73 (1948) 675. O. Kofoed. Hansen and P. Kristensen, Dan. Mat. Fys. Medd. 26 (1951) no. 6. 
value of $6 V N_{+} /\left(N_{+}+N_{-}\right)=(58 \pm 2)$ volt obtained at $V>105$ volt. The decay schemes of $\mathrm{Kr}^{88}$ and $\mathrm{Kr}^{89}$ are very complicated, and very little can therefore be concluded from these experiments. For the simplest case, $\mathrm{Kr}^{89}$, one can conclude that the electron and neutrino are emitted preferably with small angles between their momenta. This agrees with theoretical predictions for a forbidden decay.

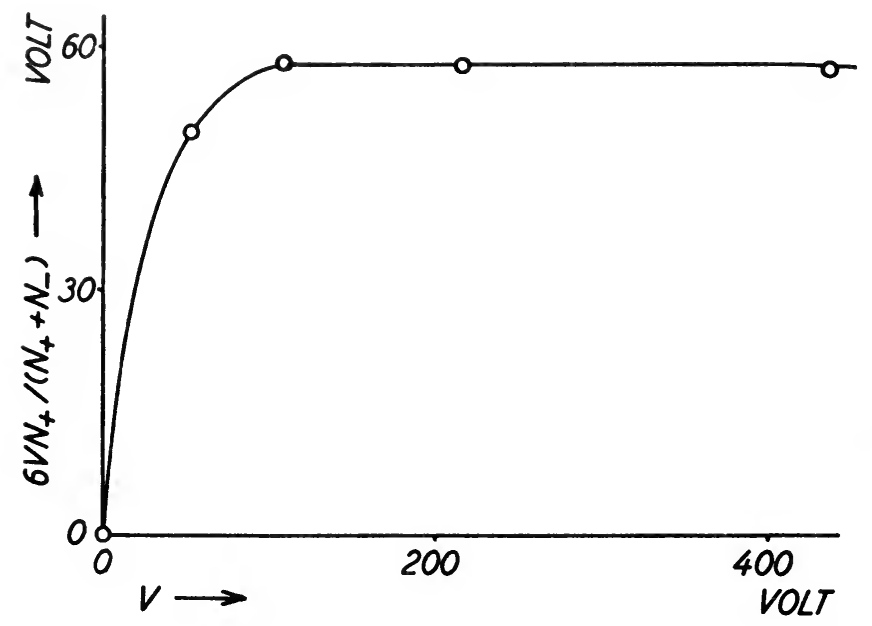

Fig. 11. $6 V N_{+} /\left(N_{+}+N_{-}\right)$as a function of $V$ for $\mathrm{Kr}^{89}$

In Table II most of the investigations of neutrino recoils from $\beta$-decay are summarized.

\section{$\S 4$. Difficulties with Bound Mother Particle}

In Tables I and II many investigations are summarized where the mother nucleus has formed part of a molecule or been deposited on some substrata. In such investigations essentially two difficulties appear.

Firstly, the momentum of the recoil is more or less shared with other particles; it should be remembered that close collisions between the partners of the molecule are very probable when the decay takes place and the molecule breaks up. That the results are greatly distorted is most easily seen from the investigations of $\mathrm{Be}^{7}$ carried out by Davis jr. ${ }^{13}$. The observed momentum peak shows a very pronounced tail towards low energies and furthermore is broadened by at least a factor 10 and smeared out towards the low energy side as compared with the theoretically calculated resolution curve for the instrument ${ }^{14}$.

Secondly, the problem of determining which partner of the molecule gets the charge in the break up is very complicated. If some of the stable

13 R. Davis jr., Phys. Rev. 86 (1952) 976.

14 O. Kofoed-Hansen, Physica 18 (1952) 1287. 
atoms get the charge they will be observed and of course give rise to a very false picture of the momentum distribution.

In contrast to this the experiments with free mother nuclei, i.e. essentially noble gases, follow the theoretically predicted resolution curves very well, as illustrated in Figs. 2 and 6. Furthermore, when an inert gas decays by $\beta^{-}$emission stable positive alkali ions are formed; when it decays by $\beta^{+}$emission stable negative halogen ions are formed. This adds to the advantages in using inert gases for the study of recoil phenomena.

Of course, when the behaviour of the recoils is known bound mother nuclei can be used to many interesting investigations of molecular properties and surface effects.

\section{$\S$ 5. Interpretation of $\boldsymbol{\beta}$-Recoil Experiments}

As already mentioned in connection with eq. (4), very little information can be gained about the neutrino rest mass from recoil experiments.

In two-body decays one is able to obtain information about the $Q$ values in the reactions. Also information about the charge distribution after Auger processes and processes connected with the decay can be found. Furthermore, the often highly charged recoil ions offer a convenient tool for the study of electron capture cross section.

In $\beta$-decays the important thing is to decide among the different possibilities for the angular correlation between the directions of the momentum $p$ of the electron and that $q$ of the neutrino. For allowed transitions this correlation is given by ${ }^{15}$

$$
P(E, \Theta) d E d \Omega_{\Theta}=F(Z, E) p E q^{2}[1+(b / E)+(a p / E) \cos \Theta] d E d \Omega_{\Theta},
$$

$E$ is the $\beta$-energy, $\Theta$ is the angle between $p$ and $q$ and $d \Omega_{\Theta}$ is a solid angle interval around $\Theta . F$ is the Coulomb correction and $Z$ is the nuclear charge of the recoil. The constant $a$ is given by

$$
\left.\begin{array}{r}
a=\left[\left(g_{V}^{2}-g_{S}^{2}\right)\left|\int 1\right|^{2}+(1 / 3)\left(g_{T}^{2}-g_{A}^{2}\left|\int \sigma\right|^{2}\right] /\left[\left(g_{S}^{2}+\right.\right.\right. \\
\left.\left.+g_{V}^{2}\right)\left|\int 1\right|^{2}+\left(g_{T}^{2}+g_{A}^{2}\right)\left|\int \sigma\right|^{2}\right]
\end{array}\right\}
$$

where the $g$ 's are the coupling constants for scalar (S), vector $(\mathrm{V})$, tensor $(\mathrm{T})$, and axial vector (A) couplings, respectively. $\left|\int 1\right|^{2}$ is the square of the nuclear matrix element for the Fermi interactions and $\left|\int \sigma\right|^{2}$ that for GT interactions. Finally, $b$ is given by

$$
\left.\begin{array}{r}
b=2\left(1-(\alpha Z)^{2}\right)^{1 / 2}\left[g_{S} g_{V}\left|\int 1\right|^{2}+g_{T} g_{A}\left|\int \sigma\right|^{2}\right] /\left[\left(g_{S}^{2}+\right.\right. \\
\left.\left.+g_{V}^{2}\right)\left|\int 1\right|^{2}+\left(g_{T}^{2}+g_{A}^{2}\right)\left|\int \sigma\right|^{2}\right]
\end{array}\right\}
$$


where $\alpha$ is the fine-structure constant. The expression (7) is mathematically convenient since $E$ and $\Theta$ may, vary independently inside the intervals $m c^{2} \leqslant E \leqslant E^{\max }$ and $0 \leqslant \Theta \leqslant \pi$, respectively, and since $E$ and $\Theta$ completely determine, the momentum triangle apart from orientation in space.

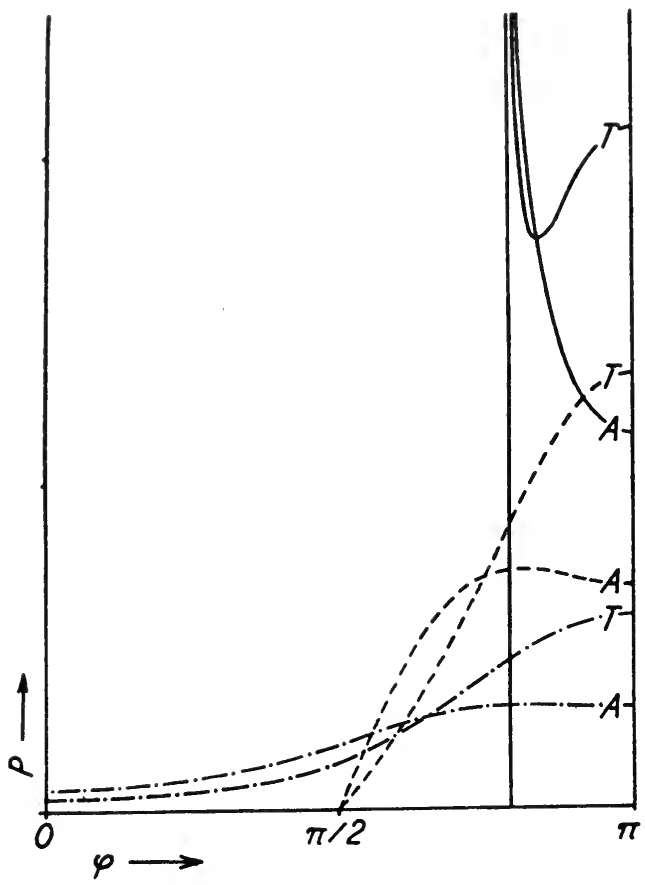

Fig. 12. Relative angular correlations for tensor and axial vector interactions per unit solid angle interval for $E^{\max }=8 \mathrm{mc}^{2}$ and $Z=0$

$$
\begin{aligned}
& \text {-. - . - - . . - - }-E=2.79 \\
& -\cdots-\cdots-\cdots--E=4.06 \\
& E=5
\end{aligned}
$$

(For suggestions of angular correlation measurements on oriented $\beta$-emitters see note 15 ). Physically, however, (7) is less adequate because it refers to the neutrino. On the other hand, (7) can easily be transformed into the distribution function for any two of the three observable variables: the electron momentum $p$, the recoil momentum $r$, and the angle $q$ between $p$ and $r^{16}$. Although the constant $a$ does not enter into the shape of the $\beta$-spectrum, it enters in any of the above mentioned correlation functions. In Fig. 12 are shown the distributions for a specific numerical example and for different values of $E$ for pure tensor interaction and for pure axial vector interaction. In these cases $b=0$, and it is seen how the angular correlations depend on $a$. Also, the recoil energy distribution or the average values of the recoil energy or momentum are rather sensitive to $a$, so that measurements of these variables are sufficient to determine $a$.

On the other hand, $a$ in itself is not the final magnitude which one wants to determine, but so far the aim is to determine the magnitudes of the $g$ 's. Here it should be mentioned that from observations of the shapes of $\beta$-spectra and comparison of $f t$-values with theoretical nuclear matrix elements, one of the $g_{F}$ 's and one of the $g_{G-T}$ 's are rather pure and nearly equal to each other ${ }^{11}{ }^{17}$. Since the investigation of Rustad and Ruby ${ }^{8}$

16 O. Kofoed-Hansen, Dan. Mat. Fys. Medd. 28 (19.54) no. 9.

17 D. C. Peaslee, Phys. Rev, 91 (1953) 1447. 
TABLE I

NEUTRINO RECOIL EXPERIMENTS WITH TWO-BODY DECAYS

BOUND MOTHER NUCLEUS

\begin{tabular}{|c|c|c|c|c|c|c|}
\hline Author & Periodical & Decay & $\begin{array}{l}\text { Sub- } \\
\text { strata }\end{array}$ & $\begin{array}{c}\text { Particle } \\
\text { detection }\end{array}$ & $\begin{array}{c}\text { Energy } \\
\text { deter- } \\
\text { mination }\end{array}$ & Results \\
\hline $\begin{array}{l}\text { J. S. Allen } \\
\text { suggested by } \\
\text { K. C. Wang }\end{array}$ & $\begin{array}{l}\text { Phys. Rev. } \\
61 \text { (1942) } 692 \\
\text { Phys. Rev. } \\
61 \text { (1942) } 97\end{array}$ & $\mathrm{Be}^{7}$ & $\begin{array}{l}\text { pla- } \\
\text { tinum }\end{array}$ & $\begin{array}{l}\text { multi- } \\
\text { plier }\end{array}$ & $\begin{array}{l}\text { retard- } \\
\text { ing } \\
\text { field }\end{array}$ & $\begin{array}{l}\text { recoil } \\
\text { momen- } \\
\text { tum exixts } \\
\text { not mono- } \\
\text { energetic }\end{array}$ \\
\hline $\begin{array}{l}\text { P. B. Smith and } \\
\text { J. S. Allen }\end{array}$ & $\begin{array}{l}\text { Phys. Rev. } \\
77 \text { (1950) } 747 \text {; } \\
81 \text { (1951) } 381\end{array}$ & $\mathrm{Be}^{7}$ & $\begin{array}{l}\text { tan- } \\
\text { talum }\end{array}$ & $\begin{array}{l}\text { multi- } \\
\text { plier }\end{array}$ & $\begin{array}{l}\text { retard- } \\
\text { ing } \\
\text { field }\end{array}$ & $\begin{array}{l}\text { agreement } \\
\text { with max- } \\
\text { imum en- } \\
\text { ergy, not } \\
\text { mono- } \\
\text { energetic }\end{array}$ \\
\hline H. Frauenfelder & $\begin{array}{l}\text { Helv. phys. } \\
\text { Acta } 23(1950) \\
347\end{array}$ & $\mathrm{Cd}^{107}$ & & & & $\begin{array}{l}\text { investi- } \\
\text { gation of } \\
\text { surface } \\
\text { properties }\end{array}$ \\
\hline R. Davis Jr. & $\begin{array}{l}\text { Phys. Rev. } \\
86 \text { (1952) } 976\end{array}$ & $\mathrm{Be}^{7}$ & $\begin{array}{l}\text { wol- } \\
\text { fram }\end{array}$ & $\begin{array}{l}\text { multi- } \\
\text { plier }\end{array}$ & $\begin{array}{l}\text { el.-stat. } \\
\text { analyser }\end{array}$ & $\begin{array}{l}\text { single } \\
\text { energy } \\
\text { peak ob- } \\
\text { served }\end{array}$ \\
\hline
\end{tabular}

MONATOMIC GASES

\begin{tabular}{|c|c|c|c|c|c|}
\hline Author & Periodical & Decay & $\begin{array}{c}\text { Particle } \\
\text { detection }\end{array}$ & $\begin{array}{c}\text { Energy } \\
\text { deter- } \\
\text { mination }\end{array}$ & Results \\
\hline $\begin{array}{l}\text { G. W. Rodeback } \\
\text { and J. S. Allen }\end{array}$ & $\begin{array}{l}\text { Phys. Rev. } \\
81 \text { (1951) } 318 ; \\
83 \text { (1951) } 215 \\
86 \text { (1952) } 446\end{array}$ & $A^{37}$ & multiplier & $\begin{array}{l}\text { time of } \\
\text { flight }\end{array}$ & $\begin{array}{l}\text { monoenergetic } \\
E=(0.8 \pm 0.1 \\
\mathrm{MeV}\end{array}$ \\
\hline $\begin{array}{l}\text { O. Kofoed. } \\
\text { Hansen }\end{array}$ & $\begin{array}{l}\text { preliminary } \\
\text { unpublished } \\
\text { results }\end{array}$ & $A^{37}$ & current & $\begin{array}{l}\text { magnetic } \\
\text { field }\end{array}$ & $\begin{array}{l}E 806 \pm 8 \mathrm{keV} \\
\text { charge distri- } \\
\text { bution Auger } \\
\text { probabilities }\end{array}$ \\
\hline
\end{tabular}

MESON DECAYS

C. F. Powell

W. F. Fry

Rep. Progr.
Phys. 13
(1950) 350
Phys. Rev.
90 (1953) 999

$\pi-\mu$
$\mu$-cap-
ture

photographic emulsions

photographic emulsions monoenergetic

agreement with neutrino hypothesis 
TABLE II

NeUtrino recoll experiments With $\beta$-Radioactive substances BOUND MOTHER NUCLEUS

\begin{tabular}{|c|c|c|c|c|c|c|}
\hline Author & Periodical & Decay & $\begin{array}{l}\text { Sub- } \\
\text { strata }\end{array}$ & $\begin{array}{c}\text { Particle } \\
\text { detection }\end{array}$ & $\begin{array}{c}\text { Energy } \\
\text { deter- } \\
\text { mination }\end{array}$ & Results \\
\hline A. J. Leipunski & $\begin{array}{l}\text { Proc. Camb. } \\
\text { phil. Soc. } \\
32 \text { (1936) } 301\end{array}$ & $\mathrm{C}^{11}$ & $\begin{array}{l}\text { con- } \\
\text { dended } \\
\mathrm{CO}_{2}\end{array}$ & $\begin{array}{l}\text { "multi- } \\
\text { plier" }\end{array}$ & $\begin{array}{l}\text { retard- } \\
\text { ing } \\
\text { field }\end{array}$ & $\begin{array}{l}\text { disagree } \\
\text { with no } \\
\text { neutrino }\end{array}$ \\
\hline $\begin{array}{l}\text { H. R. Crane and } \\
\text { J. Halpern }\end{array}$ & $\begin{array}{l}\text { Phys. Rev. } \\
53(1938) 789 \\
56(1939) 232\end{array}$ & $\mathrm{Cl}^{38}$ & $\begin{array}{l}\text { ethy- } \\
\text { lene } \\
\text { dichlo- } \\
\text { ride }\end{array}$ & $\begin{array}{l}\text { cloud } \\
\text { chamber }\end{array}$ & $\begin{array}{l}\text { droplet } \\
\text { counting }\end{array}$ & $\begin{array}{l}\text { disagree } \\
\text { with no } \\
\text { neutrino }\end{array}$ \\
\hline $\begin{array}{l}\text { R. F. Christy } \\
\text { E. R. Cohen } \\
\text { W. A. Fowler } \\
\text { C. C. Lauritsen } \\
\text { and T. Lauritsen }\end{array}$ & $\begin{array}{l}\text { Phys. Rev. } \\
72 \text { (1947) } 698\end{array}$ & $\mathrm{Li}^{8}$ & $\begin{array}{l}\mathrm{LiOH} \\
\text { on foil }\end{array}$ & $\begin{array}{l}\text { cloud } \\
\text { chamber }\end{array}$ & & $\begin{array}{l}\text { disagree } \\
\text { with no } \\
\text { neutrino }\end{array}$ \\
\hline C. W. Sherwin & $\begin{array}{l}\text { Phys. Rev. } \\
73 \text { (1948) } 216 ; \\
73(1948) 1219 \\
75(1949) 1799 ; \\
82(1951) 52\end{array}$ & $\mathrm{p}^{32}$ & $\begin{array}{l}\text { vari- } \\
\text { ous }\end{array}$ & $\begin{array}{l}\text { multi- } \\
\text { plier }\end{array}$ & $\begin{array}{l}\text { time of } \\
\text { flight }\end{array}$ & $\begin{array}{l}(1+(v / c) \\
\cos \Theta)\end{array}$ \\
\hline C. W. Sherwin & $\begin{array}{l}\text { Phys. Rev. } \\
73(1948) 1173 \\
76(1949) 183\end{array}$ & $Y^{90}$ & $\begin{array}{l}\text { wol- } \\
\text { fram }\end{array}$ & $\begin{array}{l}\text { multi- } \\
\text { plier }\end{array}$ & $\begin{array}{l}\text { time of } \\
\text { flight }\end{array}$ & $\begin{array}{l}(1-(v / c) \\
\cos \Theta)^{2} \\
\text { tentative }\end{array}$ \\
\hline
\end{tabular}


TABLE II (continued)

Free mother nUCLeUS

\begin{tabular}{|c|c|c|c|c|c|}
\hline Author & Periodical & Decay & $\begin{array}{c}\text { Particle } \\
\text { detection }\end{array}$ & $\begin{array}{c}\text { Energy } \\
\text { deter- } \\
\text { mination }\end{array}$ & Results \\
\hline $\begin{array}{l}\text { J. C. Jacobsen } \\
\text { and O. Kofoed- } \\
\text { Hansen }\end{array}$ & $\begin{array}{l}\text { Dan.Mat.Fys. } \\
\text { M. 23, no. } 12 \\
\text { (1945); Phys. } \\
\text { Rev. } \\
70 \text { (1948) } 789 \text {; } \\
73 \text { (1948) } 675\end{array}$ & $\mathrm{Kr}^{88}$ & $\begin{array}{l}\text { tracer } \\
\text { method }\end{array}$ & $\begin{array}{l}\text { retard- } \\
\text { ing } \\
\text { field }\end{array}$ & $\begin{array}{l}E^{\max }=(52 \pm 2) \\
\mathrm{eV} \\
\left\langle E_{R} \mid z\right\rangle= \\
(29 \pm 1) \mathrm{eV} \\
\text { forward } \\
\text { neutrino }\end{array}$ \\
\hline $\begin{array}{l}\text { J. S. Allen } \\
\text { H. R. Paneth and } \\
\text { A. H. Morrish }\end{array}$ & $\begin{array}{l}\text { Phys. Rev. } \\
74 \text { (1948) } 1224 ; \\
75 \text { (1949) } 570\end{array}$ & $\mathrm{He}^{6}$ & multiplier & $\begin{array}{l}\text { retard- } \\
\text { ing } \\
\text { field }\end{array}$ & $\begin{array}{l}(1-(v / 3 c) \cos \Theta) \\
\text { tentativ } \Theta\end{array}$ \\
\hline $\begin{array}{l}\text { A. H. Snell and } \\
\text { L. C. Miller }\end{array}$ & $\begin{array}{l}\text { Phys. Rev. } \\
74 \text { (1948) } 1217\end{array}$ & $\mathbf{n}$ & & & $\begin{array}{l}\text { recoil protons } \\
\text { observed }\end{array}$ \\
\hline $\begin{array}{l}\text { A. H. Snell } \\
\text { F. Pleasonton } \\
\text { and R. V.McCord }\end{array}$ & $\begin{array}{l}\text { Phys. Rev. } \\
78 \text { (1950) } 310\end{array}$ & $\mathbf{n}$ & & & $\begin{array}{l}p-\theta \text { coin- } \\
\text { cidences, } t\end{array}$ \\
\hline J. M. Robson & $\begin{array}{l}\text { Phys. Rev. } \\
78 \text { (1950) } 311 ; \\
83 \text { (1951) } 349\end{array}$ & $\mathbf{n}$ & multiplier & & $\begin{array}{l}\beta \text {-spectrum, } \\
E^{\max }=(782 \pm \\
13) \mathrm{keV} ; t= \\
12,8 \pm 2,5) \mathrm{min}\end{array}$ \\
\hline $\begin{array}{l}\text { O. Kofoed- } \\
\text { Hansen and } \\
\text { P. Kristensen }\end{array}$ & $\begin{array}{l}\text { Dan.Mat.Fys. } \\
\text { M. } 26 \text { (1951) 6; } \\
\text { Phys. Rev. } \\
82 \text { (1951) } 96\end{array}$ & $\mathrm{Kr}^{89}$ & $\begin{array}{l}\text { tracer } \\
\text { method }\end{array}$ & $\begin{array}{l}\text { retard- } \\
\text { ing } \\
\text { field }\end{array}$ & $\begin{array}{l}E_{R}^{\max }=(115 \pm 5) \\
\mathrm{eV} \cdot\left\langle E_{R} / z\right\rangle= \\
(58 \pm 2) \mathrm{eV} \\
\text { forward } \\
\text { neutrino }\end{array}$ \\
\hline $\begin{array}{l}\text { B. M. Rustad and } \\
\text { S. L. Ruby }\end{array}$ & $\begin{array}{l}\text { Phys. Rev. } \\
89 \text { (1953) } 880\end{array}$ & $\mathrm{He}^{6}$ & see text & see text & $\begin{array}{l}\text { tensor inter- } \\
\text { action predo- } \\
\text { minant }\end{array}$ \\
\hline $\begin{array}{l}\text { J. S. Allen and } \\
\text { W. K. Jentschke }\end{array}$ & $\begin{array}{l}\text { Phys. Rev. } \\
89 \text { (1953) } 902\end{array}$ & $\mathrm{He}^{6}$ & see text & see text & $\begin{array}{l}\text { tensor inter- } \\
\text { action gives } \\
\text { best fit. }\end{array}$ \\
\hline
\end{tabular}


shows that $g_{G-T}=g_{T}$, one can visualize the result of a precise measurement $a_{G-T}$ in Fig. 13, which shows that when $g_{A}$ is small $a$ is rather insensitive to $g_{A} / g_{T}$, whereas $b$ is very sensitive to this ratio.

For allowed decays with $\Delta I=1$ or $\Delta T \not \neq 0$, where $T$ is the isotopic spin, one has pure $\mathrm{G}-\mathrm{T}$ interaction, and for $0 \rightarrow 0$ (no) transitions one has pure Fermi interaction. These cases are of primary interest for the study of $a$ and thereby a study of the coupling constants. For complicated decays where both $g_{F}$ and $g_{G-T}$ are present, $a$ can be used to give ratios of nuclear matrix elements. Summing up, we may conclude that so far the results show that $g_{G-T}=g_{T}$, and that one still would like to

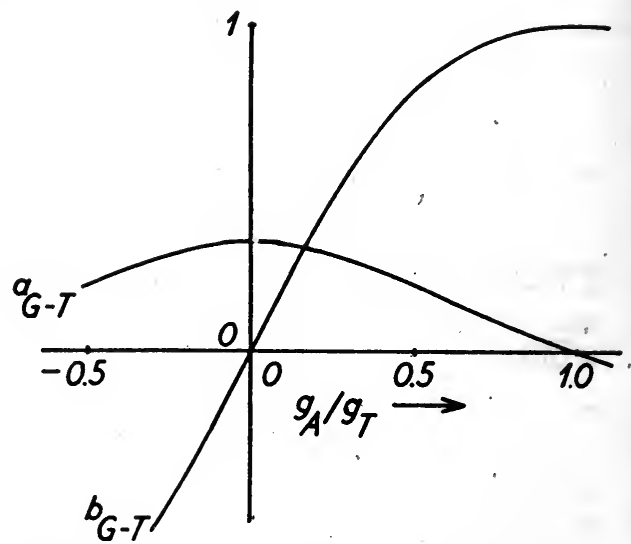

Fig. 13. The constants $a_{G-T}$ and $b_{G-T}$ as functions of $\mathrm{g}_{A} / \mathrm{g}_{T}$

determine $g_{F}$ from recoil experiments. This will be most easy to do with a $0 \rightarrow 0$ (no) transition, and it is hoped that there exists a noble gas $A^{34} 18$ which shows this type of decay.

18 O. Kofoed-Hansen, Phys. Rev. 92 (1953) 1075. 


\title{
THEORY OF MULTIPOLE RADIATION
}

\section{XIII}

\author{
S. A. MOSZKOWSKI
}

\section{§ 1. Introduction}

In 1921, Hahn ${ }^{1}$ discovered a radioactive substance UZ which was both isotopic (same charge) and isobaric (same mass) with the well known substance $\mathrm{UX}_{2}$ but had different radioactive properties. This pair of nuclei was the first, and for a long time, the only known example of nuclear isomerism. After the discovery of artificial radioactivity in 1934, new isomers were found at an ever increasing rate. At present, well over 100 examples of isomerism are known.

According to von Weizsäcker ${ }^{2}$, nuclear isomerism may occur whenever the angular momentum of a low-lying excited state of a nucleus differs from the angular momentum of any lower state by several units of $\hbar$. An isomeric (metastable)state is conventially defined as an excited state of measurable life-time. With present techniques, life-times as short as $10^{-10}$ seconds have been measured. Practically all excited states of nuclei are subject to decay to lower states with simultaneous emission of electromagnetic radiation (gamma-rays). Of course, other modes of decay; e.g. internal conversion, beta-decay, may also occur, depending on the particular state.

We shall find it convenient to classify electromagnetic radiation by multipole orders $L^{3}$, according to the angular momentum $L$ (in units of $\hbar$ ) carried off by each quantum. For each multipole order, there are two possible classes of radiation: electric $2^{L}$ pole (EL) and magnetic $2^{L}$ pole (ML), which differ with respect to parity. Classically EL and ML refer to the radition emitted by a vibrating electric or magnetic $2^{L}$ pole ${ }^{4}$. The term "multipolarity" is commonly used to specify the kind of radiation as to both class and multipole order. The conservation of angular momentum and parity for the system of nucleus plus gamma rays imposes selection rules on the possible multipolarities of a gamma transition between two states of specified angular momenta $\left(J_{i}, J_{f}\right)$ and parities $\left(\pi_{i}, \pi_{f}\right)$ :

$$
\left|J_{i}-J_{f}\right| \leqslant L \leqslant J_{i}+J_{f}
$$

1 O. Hahn, Chem. Berichte, 54 (1921) 1131.

2 C. F. von Weizsäcker, Naturwiss. 24 (1936) 813.

3 W. Heitler, Proc. Camb. Phil. Soc. 32 (1936) 112.

4 J. M. Blatt and V. F. Weisskopf, Theoretical Nuclear Physics (J. Wiley and Sons, New York, 1952), Chapter 12 and Appendix B. 
and

$$
\begin{aligned}
\Delta \pi \equiv \pi_{i} / \pi_{f} & =(-1)^{L} \text { for } \mathrm{EL} \text { radiation } \\
& =(-1)^{L-1} \text { for } \mathrm{ML} \text { radiation. }
\end{aligned}
$$

Here,

$$
\begin{aligned}
& \Delta \pi=+1 \text { denotes no parity change } \\
& \Delta \pi=-1 \text { denotes parity change. }
\end{aligned}
$$

An additional requirement, a consequence of the transverse nature of electromagnetic waves, is that no radiation of multipole order zero can occur.

The transition probability of an excited nuclear state for gammaradiation depends on the multipolarity, the energy of the gamma rays, and the wavefunctions of the nuclear states involved in the transition. As will be seen later in detail, the transition probability for gamma-decay decreases rapidly with increasing multipole order, regardless of the details of the nuclear wavefunctions. This, of course, is subject to the selection rules, and also to the requirement that the radius of the nucleus is small compared to the wave-length of the gamma-ray. The latter condition is satisfied for most known nuclear gamma-transitions. One would therefore expect that most radiative transitions in nuclei proceed primarily with emission of gamma-rays of the lowest multipole order permitted by the selection rules, $\left(L=\left|J_{i}-J_{f}\right|\right.$ in most cases), or perhaps with a mixture of the lowest two multipole orders, (but of opposite class because of parity considerations).

An excited nuclear state can decay to a lower state by internal conversion; viz. by ejection of an electron from an atomic orbit instead of emission of a gamma-ray. The number of electrons ejected from a particular atomic orbit per quantum emitted for a radiative transition is called the internal conversion coefficient for that orbit. Values of internal conversion coefficients depend on the energy of the corresponding gamma rays, the multipolarity of the transition, the particular atomic orbit, and the wavefunction of the electrons involved, but not on the nuclear wavefunctions. Thus conversion coefficients can be calculated to great accuracy when the relevant electronic wavefunctions are known. They have now been calculated for a large number of cases involving $K$-shell and $L$-shell electrons ${ }^{5}$.

Comparisons of experimental and theoretical conversion coefficients have already proven of great value for deducing multipolarities of gamma transitions ${ }^{6}$. Information about angular momenta and parities of nuclear

5 Ch. XIV and Appendix IV; M. E. Rose, G. Goertzel, B. I. Spinrad, J. Harr and P. Strong. Phys. Rev. 83, (1951) 79; J. R. Reitz, Phys. Rev. 77, (1950) 10; H. Gellmann, B. A. Giriffith and J. P. Stanley, Phys. Rev. 85, (1952) 944.

6 Ch. XVI; M. Goldhaber and A. W. Sunyar, Phys. Rev. 83, (1951) 906; J. W. Mihelich, Physs. Rev. 87, (1952) 646. 
states can be obtained in many cases by one or more of the following: direct measurements ${ }^{7}$, scattering and nuclear reaction experiments ${ }^{8}$, electric coulomb excitation ${ }^{9}$, interpretation of beta transitions ${ }^{10}$, angular correlations ${ }^{11}$, and shell model considerations ${ }^{12}$.

Before 1949, considerable progress had already been made in the interpretation of isomeric transitions ${ }^{13}$ in spite of the lack of empirical knowledge regarding angular momenta and parities of nuclear states at that time. However, the discovery of the nuclear shell model (independent particle model with strong spin-orbit coupling) provided a strong impetus to the understanding of isomerism. A striking success of the shell model is that is accounts ${ }^{14}$ for the known existence of the "islands of isomerism" ${ }^{15}$; viz. the frequent occurrence of long-lived isomers in nuclei for which either the number of odd protons or the number of odd neutrons is just below one of the "magic numbers".

The gamma-decay transition probabilities between two nuclear states depend sensitively on the wavefunctions of these states. Because of this dependence, significant information regarding nuclear wave-functions can be obtained from a comparison of experimental gamma-decay transition probabilities with theoretical values calculated on basis of specific models of the nucleus.

In the following sections of this chapter, we calculate transition probabilities of nuclear excited states for gamma-decay on the basis of a number of simplified models. Section 2 contains the basic quantum theory of multipole radiation, which is independent of any particular nuclear

7 P. F. A. Klinkenberg, Revs. Modern Phys. 24 (1952) 63.

8 F. Ajzenberg and T. Lauritsen, Revs. Modern Phys. 24 (1952) 321; W. E. Burcham, Prog. Nuclear Phys. 2 (1952) 174.

9 T. Huus and C. Zupančič, Kgl. Danske Videnskab. Selskab. Mat.-fys. Medd. 28, (1953) No. 1.

10 Ch. IX, X, XI and XVI; M. G. Mayer, S. A. Moszkowski and L. W. Nordheim, Revs. Modern Phys. 23, (1951) 315 ; L. W. Nordheim, Revs. Modern Phys. 23, 322 ; R. W. King, Ph. D. dissertation (Washington University, St. Louis, Missouri, (1952) unpublished.

11 See Ch. XIX; Also H. Frauenfelder, Ann. Revs. Nuclear Sci. 2, (1953), 129;

L. C. Biedenharn and M. E. Rose, Revs. Modern Phys. 25, (1953) 729.

12 See Ch. XV and XVI. Also M., G. Mayer, Phys. Rev. 78 (1950) 16; O. Haxel, J. H. D. Jensen and H. Suess, Z. Physik 128 (1950) 301 ; B. H. Flowers, Prog. Nuclear Phys. 2 (1952) 235.

13 For a review of the status of nuclear isomerism in 1949, see E. Segré and A. C. Helmholtz, Revs. Modern Phys. 21 (1949) 271; also see P. Axel and S. M. Dancoff, Phys. Rev. 76 (1949) 892.

14 M. Goldhaber and R. D. Hill, Revs. Modern Phys. 24 (1952) 179.

15 E. Feenberg, Phys. Rev. 75 (1949) 320. 
model 4.16. In Section 3, we calculate gamma-decay transition probabilities for a very simple version of the shell model, namely a single particle model ${ }^{17.18,19}$. Section 4 contains calculations of transition probabilities for some configurations consisting of several independent particles. Gammadecay transition probabilities calculated with use of single particle or independent particle wavefunctions are, in general, only in fair agreement with experimental values.

The effect of cooperative ruclear motions on gamma-decay transition probabilities is discussed in Ch. XVII. The collective model, an extension of the shell model which takes into account coherent motions of nucleons ${ }^{20.21}$, shows promise of being able to account quantitatively for many aspects of nuclear structure.

\section{§ 2. The Multipole Radiation Field}

The electromagnetic field of gamma rays can be derived from a vector potential $\boldsymbol{A}$ which satisfies the wave-equation in free space:

$$
\Delta \mathrm{A}-\frac{1}{c^{2}} \frac{\partial^{2} \boldsymbol{A}}{\partial t^{2}}=0 .
$$

If the time dependent part of the scalar potential is eliminated by a gauge transformation ${ }^{22}$, then the transversality of electromagnetic waves is expressed by the divergence condition:

$$
\operatorname{div} \mathbf{A}=0
$$

The electric and magnetic fields are given as follows:

$$
\begin{aligned}
& \boldsymbol{E}=-\frac{1}{c} \frac{\partial \boldsymbol{A}}{\partial t} \\
& \boldsymbol{H}=\operatorname{curl} \boldsymbol{A}
\end{aligned}
$$

\section{A. Quantization of the Radiation Field}

To quantize the radiation field it is first necessary to expand the vector potential as a linear combination of eigen-solutions of the above equations

16 W. Franz, Z. Physik 127 (1950) 363.

17 V. F. Weisskopf, Phys. Rev. 83 (1951) 1073.

18 B. Stech, Z. Naturforschung 7a (1952) 401.

19 S. A. Moszkowski, Phys. Rev. 89 (1953) 474.

20 Ch. XVII; A. Bohr and B. R. Mottelson, Physics 18, (1952) 1066; Kgl. Danske Videnskab. Selskab. Mat-fys. Medd. 27, (1953) No. 16.

31 D. L. Hill and J. A. Wheeler, Phys. Rev. 89 (1953) 1102. K. W. Ford, Phys. Rev. 90 (1953) 29.

22 Although the use of this particular gauge is very convenient for the treatment of multipole radiation, it is not necessary. For problems of internal conversion (Ch. XIV), it is in fact preferable to use a different gauge. 
$(3,4)$. We confine the radiation field in a specified volume ${ }^{3.23}$, and impose certain boundary conditions. This enables one to write any vector potential which obeys these boundary conditions as a linear combination of an infinite but denumerable number of orthogonal waves:

$$
\mathbf{A}=\sum_{\lambda} q_{\lambda}(t) \mathbf{A}_{\lambda}(\boldsymbol{r})+q_{\lambda}^{*}(t) \mathbf{A}_{\lambda}^{*}(\boldsymbol{r})
$$

The index $\lambda$ denotes the various quantum numbers which characterize each wave. (The asterisk denotes complex conjugate.) It is convenient to normalize $\boldsymbol{A}_{\lambda}$ so that:

$$
\int_{V} \boldsymbol{A}_{\lambda^{\prime}}^{*}(\boldsymbol{r}) \cdot \boldsymbol{A}_{\lambda}(\boldsymbol{r}) d V=4 \pi c^{2} \delta_{\lambda^{\prime}, \lambda}
$$

where the integration extends over the enclosed volume $V$. The $\boldsymbol{A}_{\lambda}$ and their complex conjugates are solutions of the vector Helmholtz equation:

$$
\Delta \boldsymbol{A}_{\lambda}+k_{\lambda}^{2} \boldsymbol{A}_{\lambda}=0
$$

[where $k_{\lambda}$ is the wave number (an eigenvalue) characterizing $A_{\lambda}$ ], and of the divergence condition:

$$
\operatorname{div} \boldsymbol{A}_{\lambda}=0
$$

Equations (8) and (9) can be combined to give a single "divergenceless vector Helmholtz equation":

$$
\text { curl curl } \boldsymbol{A}_{\lambda}-k_{\lambda}^{2} \boldsymbol{A}_{\lambda}=0
$$

Any solution of eq. (10) automatically satisfies both of the two previous equations, as can be seen by taking the divergence of both terms in eq. (10). The solutions of eq. (10) depend on the boundary conditions and will be discussed below. The field amplitudes $q_{\lambda}$ (and $q_{\lambda}^{*}$ ) satisfy the harmonic oscillator equation:

$$
\frac{d^{2} q_{\lambda}}{d t^{2}}+\omega_{\lambda}^{2} q_{\lambda}=0
$$

where $\omega_{\lambda}$ is the frequency, equal to $c k_{\lambda}$.

Apart from a multiplicative constant and a harmonic time factor, the $q_{\lambda}$ and $q_{\lambda}^{*}$ represent annihilation and creation operators respectively. It is convenient to normalize these quantities so that the commutation rules obeyed by them are:

$$
\begin{aligned}
& q_{\lambda} q_{\lambda^{\prime}}-q_{\lambda^{\prime}} q_{\lambda}=0 \\
& q_{\lambda}^{*} q_{\lambda^{\prime}}^{*}-q_{\lambda^{\prime}}^{*} q_{\lambda}^{*}=0 \\
& q_{\lambda} q_{\lambda^{\prime}}^{*}-q_{\lambda^{\prime}}^{*} q_{\lambda}=\left(\hbar / 2 \omega_{\lambda}\right) \delta_{\lambda^{\prime} \lambda} .
\end{aligned}
$$

The matrix elements of $q_{\lambda}$ and $q_{\lambda}^{*}$ taken between an initial state containing

23 W. Heitler, The Quantum Theory of Radiation (Oxford University Press, London, 1947), Second Edition, Chapter 2. 
$n_{i}$ quanta of type $\lambda$ and a final state containing $n_{f}$ quanta of type $\lambda$ are given by:

$$
\begin{aligned}
& \left\langle n_{f, \lambda}\left|q_{\lambda}\right| n_{i, \lambda}\right\rangle=\left(n_{i} \hbar / 2 \omega_{\lambda}\right)^{\dagger} \delta_{n_{f, n_{i}-1}} \exp \left(-i \omega_{\lambda} t\right) \\
& \left\langle n_{f, \lambda}\left|q_{\lambda}^{*}\right| n_{i, \lambda}\right\rangle=\left[\left(n_{i}+1\right) \hbar / 2 \omega_{\lambda}\right]^{k} \delta_{n_{f, n_{i}+1}} \exp \left(i \omega_{\lambda} t\right) .
\end{aligned}
$$

The Hamiltonian of the radiation field is given by the well known equation:

$$
\mathscr{H}=(8 \pi)^{-1} \int_{V}|\boldsymbol{E}|^{2}+|\boldsymbol{H}|^{2} d V
$$

With the aid of eqs. (5), (6), and (7) this can be put into the form:

$$
\mathscr{H}=\sum_{\lambda} 2 \omega_{\lambda}^{2} q_{\lambda}^{*} q_{\lambda} .
$$

The energy eigenvalues $E_{n}$ are given by

$$
E_{n}=\sum_{\lambda} n_{\lambda} \hbar \omega_{\lambda}
$$

The order of the field amplitudes $q_{\lambda}$ and $q_{\lambda}^{*}$ in eq. (15) has been chosen in such a way to give zero energy when no quanta are present.

In this chapter we expand the vector potential in a series of standing spherical waves with a certain point 0 as center. As will be șhown presently, this results from choosing a sphere of large radius $R_{0}$ as volume of integration, with the boundary condition that the tangential components of $\boldsymbol{A}$ vanish at the surface of the sphere. This is equivalent to confining the radiation field inside a sphere (of radius $R_{0}$ ) with a perfectly reflecting surface. The vector potential for each spherical wave decreases as $r^{-1}$ for large distances $r$ from the center, but remains finite throughout the volume of integration and at the center of the sphere. Each (standing) spherical wave has a definite angular momentum $L \hbar$ and corresponds to the (outgoing) spherical wave emitted by a suitable oscillating classical $2^{L}$ pole located at $0^{4}$. We could instead have quantized the radiation field into running plane waves ${ }^{23}$ by confining it within a cube and imposing the boundary condition that $\boldsymbol{A}$ and its derivatives have the same values on opposite planes of this cube. However this approach, while simpler for most radiation problems of interest, is not well suited for a study of multipole radiation since plane waves do not carry a unique angular momentum, and since they do not correspond directly to the radiation emitted by a vibrating multipole (Section 3 ).

\section{B. Solutions of the Scalar Helmholtz Equation}

It is of interest to consider first a somewhat simpler problem; viz. the non-singular solutions of the scalar Helmholtz equation:

$$
\Delta u+k^{2} u=0 \text {. }
$$


It can be shown that functions of the form:

$$
u_{L M}=j_{L}\left(k_{\lambda} r\right) Y_{L M}(\theta, \phi)
$$

(with $L$ an integer $\geqslant 0$, and $|M| \leqslant L$ ) satisfy eq. (17).

(The $u_{L M}$ are, of course, also characterized by $k_{\lambda}$, but this is not indicated explicitly).

The $Y_{L M}(\theta, \phi)$ are the well-known normalized spherical harmonics. The $j_{L}\left(k_{\lambda} r\right)$ are spherical Bessel functions:

$$
j_{L}\left(k_{\lambda} r\right)=\left(\pi / 2 k_{\lambda} r\right)^{\sharp} J_{L+\xi}\left(k_{\lambda} r\right)
$$

where $J$ is an ordinary Bessel function of half-integer order.

Asymptotic values of the $j_{L}$ are:

where

$$
\begin{aligned}
& j_{L}\left(k_{\lambda} r\right) \underset{k_{\lambda} r \rightarrow 0}{\longrightarrow} \frac{\left(k_{\lambda} r\right)^{L}}{(2 L+1) ! !} \\
& j_{L}\left(k_{\lambda} r\right) \underset{k_{\lambda^{r} \rightarrow \infty}}{\longrightarrow} \frac{\sin \left[k_{\lambda} r-(L \pi / 2)\right]}{k_{\lambda} r}
\end{aligned}
$$

$$
(2 L+1) ! ! \equiv 1 \times 3 \times 5 \times \ldots \times(2 L+1)
$$

If the boundary condition $u\left(R_{0}\right)=0$ is imposed, it is necessary to have $j_{L}\left(k_{\lambda} R_{0}\right)=0$. This requires that $k_{\lambda}=\pi\left(n+\frac{L}{2}\right) / R_{0}$, where $n$ is a positive integer, provided $R_{0} \gg L \pi / 2 k_{\lambda}$.

The functions $u_{L M}$ have several interesting characteristics:

1) They form a complete orthogonal set of non-singular solutions to the scalar Helmholtz equation. The "completeness" means that any function which does not have a singularity at the origin and which vanishes for $r=R_{0}$, can be expressed as some linear combination of the $u_{L M}$. The orthogonality condition:

$$
\int_{V}^{*} u_{L M}^{*} u_{L M} d V=\left(R_{0} / 2 k_{\lambda}^{2}\right) \delta_{L^{\prime} L} \delta_{M^{\prime} M} \delta_{k_{\lambda^{\prime}} k_{\lambda}}
$$

is a consequence of the orthogonality of spherical harmonics, and of the spherical Bessel functions. In eq. (22) $V$ denotes the volume of integration.

2) If one applies to the $u_{L M}$ the orbital angular momentum operator:

$$
\boldsymbol{L}=-i \boldsymbol{r} \times \operatorname{grad}
$$

then the following relations are satisfied:

$$
\begin{gathered}
L_{z} u_{L M}=M u_{L M} \\
L^{2} u_{L M}=L(L+1) u_{L M}
\end{gathered}
$$

i.e. the $u_{L M}$ are eigenfunctions of the orbital angular momentum operators. These relations are exactly the equations satisfied by the wavefunction of a particle with orbital angular momentum $L, z$-component of angular 
momentum $M$, and no intrinsic spin. (In this Chapter all angular momenta are expressed in units of $\hbar$ ).

3) The $u_{L M}$ satisfy the equation

$$
u_{L M}(-\boldsymbol{r})=(-1)^{L} u_{L M}(\boldsymbol{r})
$$

Thus the $u_{L M}$ have even $(+1)$ or odd $(-1)$ parity according to whether $L$ is an even or an odd integer.

\section{Solution of the Divefanceless Vector Helmholtz Equation}

The standing wave solutions to the divergenceless vector Helmholtz eq. (10), with boundary condition $\boldsymbol{A}_{\text {tang }}\left(R_{0}\right)=0^{3}$, can be expressed in terms of the solutions $u_{L M}$ to the scalar Helmholtz equation. However, for each $u_{L M}$ we can choose two linearly independent solutions $\boldsymbol{A}_{L M}^{\sigma}$, where $\sigma$ is given the index " $E$ " or " $M$ " denoting electric or magnetic multipole fields (which correspond closely to the fields of a vibrating electric or magnetic multipole).

These solutions can be written as follows ${ }^{18.18}$ :

$$
\begin{aligned}
& \boldsymbol{A}_{L M}^{E}=k_{\lambda}^{-1} C_{L} \operatorname{curl} \boldsymbol{L} u_{L M} \\
& \boldsymbol{A}_{L M}=+i C_{L} \boldsymbol{L} u_{L M}
\end{aligned}
$$

where $C_{L}$ is an arbitrary constant, and where

$$
\left[\frac{\partial}{\partial r}\left(\boldsymbol{r} u_{L M}\right)\right]_{R_{0}}=0 .
$$

Each solution is understood to be also characterized by $a k_{\lambda}$. Note that the solutions of (26) vanish identically for $L=0$. This is a consequence of the transverse nature of electromagnetic waves.

The $\boldsymbol{A}_{L M}^{\sigma}$ have properties similar to the $u_{L M}$ :

$1^{\prime}$ ) They form a complete orthogonal set of non-singular solutions to the divergenceless vector Helmholtz equation. Thus any vector function which does not have a singularity at the origin and whose tangential components vanish for $r=R_{0}$, can be expressed as some linear combination of the $A_{L M}^{\sigma}$.

The orthogonality condition for this case is:

$$
\int_{V}\left(\boldsymbol{A}_{\left.L^{\prime} M^{\prime}\right)^{\prime}}\right)^{*} \cdot \boldsymbol{A}_{L M}^{\sigma} d V=\frac{L(L+1)}{2 k_{\lambda}^{2}} R_{0} C_{L}^{2} \delta_{\sigma^{\prime} \sigma} \delta_{L^{\prime} L} \delta_{M M^{\prime} M} \delta_{k_{\lambda^{\prime}} k_{\lambda}}
$$

The normalization condition (7) is satisfied if

$$
C_{L}=\left[\frac{8 \pi \omega_{\lambda}^{2}}{L(L+1) R_{0}}\right]^{k}
$$

$\left.2^{\prime}\right)$ The solutions $\boldsymbol{A}_{L M}^{\sigma}$ are eigenvectors of angular momentum operators. (Strictly speaking, each of the three components of $\boldsymbol{A}_{L M}^{\sigma}$ is an eigen- 
function). However, for the vector case considered here it is necessary to assume that the total angular momentum is the vector sum of orbital angular momentum $L$ and an intrinsic spin $S$ (of the radiation).

Thus

$$
J=L+S
$$

where $L$ is defined as above, and

$$
S_{z} \mathbf{A}=i \boldsymbol{\epsilon}_{z} \times \mathbf{A}
$$

Here $\epsilon_{z}$ is a unit vector in the $z$-direction, while $S_{z}$ is the component of intrinsic spin in the $z$-direction. Equations identical in form to (31) hold for $S_{x} A$ and for $S_{y} A$.

The angular momentum operators $\boldsymbol{L}, \boldsymbol{S}$, and $\boldsymbol{J}$ satisfy the required commutation relations; for example:

$$
\mathbf{L} \times \mathbf{L}=i \mathbf{L}
$$

The following equations can be derived: ${ }^{4,16}$

$$
\begin{aligned}
J_{z} \boldsymbol{A}_{L M}^{\sigma} & =M \boldsymbol{A}_{L M}^{\sigma} \\
\boldsymbol{J}^{2} \boldsymbol{A}_{L M^{\sigma}} & =L(L+1) \boldsymbol{A}_{L M^{\sigma}} \\
\boldsymbol{S}^{2} \boldsymbol{A}_{L M^{\sigma}} & =S(S+1) \boldsymbol{A}_{L M}^{\sigma}=2 \boldsymbol{A}_{L M^{\sigma}}{ }^{\sigma} .
\end{aligned}
$$

However $\boldsymbol{A}_{L M}{ }^{\sigma}$ is not an eigenfunction of the operators

$$
L_{z}, L^{2} \text { and } S_{z^{*}}
$$

These relations have the following physical interpretation:

The electromagnetic field which corresponds to a vector potential $\boldsymbol{A}_{L M}{ }^{\sigma}$ contains total angular momentum $L$ with component $M$ in the $z$-direction. The radiation is also characterized by an "intrinsic spin" of value 1 .

Indeed, it is easily shown from eqs. (5), (26) and (29) that the $z$-component of angular momentum:

$$
(4 \pi \hbar c)^{-1} \int_{V} r \times(E \times H) d V
$$

of a single light quantum is equal to $M$, while the square of the angular momentum is $L(L+1)$.

$\left.3^{\prime}\right)$ A feature of the vector potential $\boldsymbol{A}_{L M M}{ }^{\circ}$, but not of the functions $u_{L M}$, is the additional division into electric and magnetic multipoles. The electric and magnetic fields, $\boldsymbol{E}$ and $\boldsymbol{H}$, are related to the vector potentials by:

$$
\begin{aligned}
& \boldsymbol{E}_{L M}^{\sigma}=i k_{\lambda} \boldsymbol{A}_{L M}^{\sigma} \\
& \boldsymbol{H}_{L M}^{\sigma}=\operatorname{curl} \boldsymbol{A}_{L M}^{\sigma} .
\end{aligned}
$$

(The time dependent part of these fields has been factored out just as was done for the vector potential). 
Note that:

$$
\begin{aligned}
& \boldsymbol{E}_{L M}^{E}=+\boldsymbol{H}_{L M}^{M}=i C_{L} \operatorname{curl} \boldsymbol{L} u_{L M} \\
& \boldsymbol{E}_{L M}^{M}=-\boldsymbol{H}_{L M}=-k_{\lambda} C_{L} \boldsymbol{L} u_{L M} .
\end{aligned}
$$

These nields satisfy the following equations:

$$
\boldsymbol{r} \cdot \boldsymbol{E}_{L M}^{M}=\boldsymbol{r} \cdot \boldsymbol{H}_{L . M}^{E}=\mathbf{0}
$$

i.e. an electric multipole field has non-vanishing radial components of the electric field $\boldsymbol{E}$ but not of the magnetic field $\boldsymbol{H}$, while a magnetic multipole field has non-vanishing radial components of $\boldsymbol{H}$, but not of $\boldsymbol{E}$.

The two solutions $\boldsymbol{A}_{L, M}{ }^{E}$ and $\boldsymbol{A}_{L, Y}^{M}$ have opposite parity, namely $(-1)^{L-1}$ and $(-1)^{L}$, respectively. It is seen later that the parity of the radiation field itself is opposite to the parity of the vector potential. Thus an EL or ML radiation field carries parity $(-1)^{L}$ or $(-1)^{L-1}$, respectively. In any case, a knowledge of $k_{\lambda}, L, M$ and parity of the vector potential $\boldsymbol{A}_{L M}{ }^{\circ}$ uniquely specifies its form.

\section{$\S$ 3. $\gamma$-Decay Transition Probabilities of a Single Particle}

The quantum mechanical transition probability $T_{i \rightarrow f}$ for any process is given by the well known equation ${ }^{24}$ from perturbation theory:

$$
T_{i \rightarrow f}=\frac{2 \pi}{\hbar}|<f| \mathscr{H}^{\prime}|i>|^{2} \frac{d N}{d E}
$$

where $\mathscr{H}^{\prime}$ is the perturbing interaction. $\left\langle f\left|\mathscr{H}^{\prime}\right| i\right\rangle$ is the first order matrix element of the interaction taken between the wavefunction of initial state $i$ and final state $f . d N / d E$ denotes the number of possible final states per unit energy interval.

\section{A. Single Proton in a Central Velocity-Independent Potential}

We consider first the spontaneous emission of radiation by a single non-relativistic proton which is moving in a potential due to the other particles in the nucleus. It is assumed, at this point, that the potential is central, independent of the proton velocity, and independent of time. This is, of course, a very oversimplified picture of the nucleus. However transition probabilities for other more realistic nuclear models can be conveniently expressed in terms of the results of the present assumptions.

The total non-relativistic Hamiltonian of proton and electromagnetic field can be written as follows ${ }^{24}$ :

$$
\mathscr{H}=\frac{(\boldsymbol{p}-(e / c) \boldsymbol{A})^{2}}{2 m}+V(\boldsymbol{r})-\mu_{P} \frac{e \hbar}{2 m c} \boldsymbol{\sigma} \cdot \boldsymbol{H}+\sum_{\lambda} n_{\lambda} \hbar \omega_{\lambda},
$$

24 W. Heitler, op. cit., Chapter 3. 
Here $m$.denotes mass of the proton, $\mu_{P}(e \hbar / 2 m c)$ is its magnetic moment, $\boldsymbol{H}$ is the magnetic field, $\sigma$ is the Pauli spin vector, and $p$ is the momentum operator -ih grad.

The interaction between proton and field in lowest order is given by the following Hermitean operator:

$$
\mathscr{H}^{\prime}(\boldsymbol{A})=-\frac{e}{m c} \frac{\boldsymbol{p} \cdot \boldsymbol{A}+\boldsymbol{A} \cdot \boldsymbol{p}}{2}-\mu_{P} \frac{e \hbar}{2 m c} \boldsymbol{\sigma} \cdot \boldsymbol{H} .
$$

The interaction term $\left(e^{2} A^{2}\right) /\left(2 m c^{2}\right)$ in eq. (39) is neglected here, as are second order perturbation terms. Both of these give rise to double quantum emission ${ }^{25}$, for which the transition probability is usually several orders of magnitude smaller than the transition probability for single quantum emission.

The perturbing interaction (40) is proportional to the first power of the vector potential $\boldsymbol{A}$. The matrix element of $\mathscr{H}^{\prime}(\boldsymbol{A})$ for single quantum emission is to be taken between two states one of which, viz. the initial state, contains a proton in state $i$ and no quanta while the other, the final state, contains a proton in state $f$ and one quantum in state $\lambda$. As a consequence of eq. (6) and (13) this matrix element satisfies the following relation:

where

$$
\left\langle f, \mathbf{1}_{\lambda}\left|\mathscr{H}^{\prime}(\mathbf{A})\right| i, 0\right\rangle=(\hbar / 2 \omega)^{1 / 2}\left\langle f\left|\mathscr{H}^{\prime}\left(\boldsymbol{A}_{\lambda}^{*}\right)\right| i\right\rangle
$$

$$
\omega=\left(E_{i}-E_{f}\right) / \hbar
$$

The matrix element on the right hand side of (41) does not involve the operators $q_{\lambda}$, or $q_{\lambda}^{*}$, or time dependent factors, and it is taken between initial and final states $i$ and $f$ of the proton.

The number of possible states per unit energy in a spherical volume of large radius $R_{0}$ (for radiation of specified $\sigma, L$ and $M$ ) is given by ${ }^{18}$ :

$$
\frac{d N}{d E}=\frac{R_{0}}{\pi \hbar c} \text {. }
$$

As consequence of eqs. (38), (41) and (43), we obtain the following expression for the transition probability:

$$
T_{i \rightarrow f}^{(\lambda)}=\frac{R_{0}}{\hbar c \omega}\left|\left\langle f\left|\mathscr{H}^{\prime}\left(\boldsymbol{A}_{\lambda}^{*}\right)\right| i\right\rangle\right|^{2} .
$$

From eqs. (26) and (40), it follows that:

and

$$
\mathscr{H}^{\prime}\left(\boldsymbol{A}_{L M}{ }^{*}\right)=-\frac{C_{L} e}{m \omega} \boldsymbol{p} \cdot\left(\operatorname{curl} \cdot \mathbf{L} u_{L M}\right)^{*}-\frac{C_{L} \mu_{P} e \hbar}{2 m c^{2}} \omega \sigma \cdot\left(L u_{L M}\right)^{*}
$$

$$
\mathscr{H}^{\prime}\left(\boldsymbol{A}_{L M}^{M *}\right)=+\frac{C_{L} i e p \cdot\left(L u_{L M}\right)^{*}}{m c}+\frac{C_{L} i \mu_{P} e \hbar}{2 m c} \sigma \cdot\left(\operatorname{curl} L u_{L M}\right)^{*} .
$$

25 M. G. Mayer, Annalen d. Physik 9 (1931) 273. R. G. Sachs, Phys. Rev. 57 (1940) 194. 
To evaluate the expression on the right hand side of eqs. (45), we make the approximation that the radius of the nucleus, denoted by $a$, is small compared to the wavelength of the radiation; i.e. that $(\omega a / c) \ll 1$. Thus, over the region where the nuclear wavefunctions do not vanish, we have from (18) and (20a):

$$
u_{L M I}=\frac{(\omega r / c)^{L}}{(2 L+1) ! !} Y_{L M}(\theta, \phi) .
$$

Also, it can be readily shown ${ }^{18}$ that:

and that

$$
\left(\operatorname{curl} L u_{L M}\right)^{*}=-i(L+1)\left(\operatorname{grad} u_{L M}\right)^{*}
$$

for $\omega r / c \ll 1$.

$$
\left\langle f\left|\mathbf{p} \cdot\left(\operatorname{grad} u_{L M}\right)^{*}\right| i\right\rangle=-i m \omega\left\langle f\left|u_{L M} *\right| i\right\rangle
$$

We now introduce new quantities $\mathscr{M}_{L M}^{\sigma}$ as follows, using eqs. (46) to (48):

where

$$
\left\langle f\left|\mathscr{H}^{\prime}\left(\boldsymbol{A}_{L M}{ }^{* *}\right)\right| i\right\rangle=C_{L} \frac{\dot{L}+1}{(2 L+1) ! !}\left(\frac{\omega}{c}\right)^{L}\left\langle f\left|\mathscr{M}_{L M}{ }^{\sigma}\right| i\right\rangle
$$

and 26

$$
\mathscr{M}_{L M}^{E}=e r^{L} Y_{L M}^{*}-\frac{i \mu_{P} e \hbar \omega}{2 m c^{2}}(L+1)^{-1} \sigma \times \boldsymbol{r} \cdot\left[\left(\operatorname{grad}\left(r^{L} Y_{L M}\right)\right]^{*}\right.
$$

$$
\mathscr{M}_{L M}^{M I}=\frac{e \hbar}{m c} \frac{1}{L+1} L \cdot\left[\operatorname{grad}\left(r^{L} Y_{L M}\right)\right]^{*}+\frac{e \hbar}{2 m c} \mu_{P} \sigma \cdot\left[\operatorname{grad}\left(r^{L} Y_{L M}\right)\right]^{*} .
$$

One can rewrite equation (44) with the help of eq. (29) to obtain:

$$
T_{i \rightarrow f}^{(\sigma L M)}=\frac{8 \pi(L+1)}{L[(2 L+1) ! !]^{2}} \frac{1}{\hbar}\left(\frac{\omega}{c}\right)^{2 L+1}\left|\left\langle f\left|\mathscr{M}_{L M}^{\sigma}\right| i\right\rangle\right|^{2} .
$$

The $\mathscr{M}_{L M}^{E}$ and $\mathscr{M}_{L M}^{M}$ are the electric and magnetic multipole operators for a single proton. The first term in each of the two expressions (50a) and (50b) denotes the contributions due to orbital motions of the proton, while the second term arises from intrinsic proton spin. To consider two simple examples: the dipole matrix elements with $M=0$ are given as follows:

$$
\begin{aligned}
\ddot{\mathscr{M}}_{10}^{E} & =\sqrt{\frac{3}{4 \pi}} e z-i \sqrt{\frac{3}{4 \pi}} \mu_{P} \frac{e \hbar}{4 m c^{2}} \omega(\sigma \times \boldsymbol{r})_{z} \\
\mathscr{M}_{10}^{M} & =\sqrt{\frac{3}{4 \pi}} \frac{e \hbar}{2 m c}\left(L_{z}+\mu_{P} \sigma_{z}\right) .
\end{aligned}
$$

Before evaluating the multipole matrix elements let us examine the general conditions for which these quantities do not vanish. It is readily seen from eqs. (52) that the three electric dipole matrix elements $\mathscr{M}_{1 M}{ }^{E}$ transform among themselves under space rotations and inversion through the origin in the same way as the three components of a vector; i.e., a

26 In the following equation the operator $L$, which is outside the brackets, operates on all terms to its right, including the wavefunction of the initial state. 
tensor of rank 1 and of odd parity. The magnetic dipole matrix elements $\mathscr{H}_{1 M}^{M}$ transform as the components of a pseudovector, i.e., a tensor of rank 1 and of even parity. Similarly, it is easy to show from eqs. (50), that the $2 L+1$ components of $\mathscr{M}_{L M}^{E}$ or $\mathscr{M}_{L M}^{M}$ transform among themselves as the components of a tensor of rank $L$ and parity $(-1)^{L}$ or $(-1)^{L-1}$, respectively. These remarks are in agreement with the following considerations: a) any $2^{L}$ pole multipole radiations field carries angular momentum $L$ and $z$-component of angular momentum $M$ (Section 2); b) the parity of a multipole field is $(-1)^{L}$ or $(-1)^{L-1}$ for EL or ML radiation, respectively. It follows from the conservation of angular momentum that $2^{L}$ pole radiation can occur only between states whose angular momenta $\left(j_{i}, j_{f}\right)$ obey the selection rules:

$$
\left|j_{i}-j_{f}\right| \leqslant L \leqslant j_{i}+j_{f}
$$

The conservation of angular momentum about the $z$-axis requires:

$$
M=m_{i}-m_{f}
$$

Note that radiative transitions between two states both of which have zero angular momentum are absolutely forbidden ${ }^{4}$. From conservation of parity, we have:

$$
\begin{aligned}
\Delta \pi \equiv \pi_{i} / \pi_{f} & =(-1)^{L} & & \text { for EL radiation } \\
& =(-1)^{L-1} & & \text { for ML radiation }
\end{aligned}
$$

where $\pi_{i}$ and $\pi_{f}$ are the parities of the nuclear states involved in the transitions. In (2), $\Delta \pi=+1$ means that the initial and final states have the same parity, (no parity change), while $\Delta \pi=-1$ signifies a parity change.

For the case of a particle moving in a central velocity-independent potential there is an additional selection rule on orbital angular momenta $\left(l_{i}, l_{f}\right)$, as can be seen from eqs. $(50)$.

$$
\left|l_{i}-l_{f}\right| \leqslant L \leqslant l_{i}+l_{f}
$$

A transition between two states of specified angular momentum and parity can, in principle, involve radiation of any of the multipolarities permitted by the selection rules. However, if $(\omega a / c) \ll 1$, then it can be readily shown that transitions for a single proton will proceed essentially by the lowest multipole order possible.

This can be seen from the following crude estimates for the order of magnitudes of the multipole operators $(50)$ :

$$
\begin{aligned}
\mathscr{M}_{L M}^{E} & =0\left(e a^{L}\right) \\
\mathscr{M}_{L M}^{M} & =0\left[\left(\frac{\hbar}{m c a}\right)\left(e a^{L}\right)\right] .
\end{aligned}
$$


The transition probabilities have the following forms:

$$
\begin{aligned}
& T_{\leftrightarrow \rightarrow \prime}^{(E L)}=0\left[\omega\left(\frac{e^{2}}{\hbar c}\right)\left(\frac{\omega a}{c}\right)^{2 L}\right] \\
& T_{i \rightarrow j}^{(M L)}=0\left[\omega\left(\frac{e^{2}}{h c}\right)\left(\frac{\hbar}{m c a}\right)^{2}\left(\frac{\omega a}{c}\right)^{2 L}\right] .
\end{aligned}
$$

Thus for a radiative transition involving $\left|j_{i}-j_{f}\right| \leqslant L(\geqslant 1)$ and $\Delta \pi=(-1)^{\boldsymbol{L}}$, the ratio of the lowest two terms is:

$$
\frac{T^{[M(L+1)]}}{T^{(E L)}}=0\left[\left(\frac{\hbar \omega}{m c^{2}}\right)^{2 n}\right] \text {. }
$$

For a transition involving $\left|j_{i}-j_{f}\right|=L(\geqslant 1)$ and $\Delta \pi=(-1)^{L-1}$, we have:

$$
\frac{T(E(L+1)]}{T^{(M L)}}=0\left[\left(\frac{m a^{2} \omega}{\hbar}\right)^{2}\right] \text {. }
$$

Both of the ratios (57), are expected to be small compared to unity for most isomeric transitions.

Radiative transition probabilities are often derived by an alternative method; namely by expansion of the radiative field into plane waves. Thus we have

where

$$
\mathbf{A}_{\lambda}^{*}=N_{L} \in e^{-i \mathbf{k} \cdot \mathbf{r}}
$$

$\boldsymbol{k}$ is the propagation vector of the quantum

$\epsilon$ is the unit vector in the direction of polarization

$N_{L}$ is the normalization constant, which is given by

$\left(4 \pi c^{2} / V\right)^{2}$ for a single quantum in an enclosure of volume $V$.

The radiative transition probability between two states is given by ${ }^{10}$ :

$$
T_{i \rightarrow f}^{(\lambda)}=\frac{\omega V}{8 \pi^{2} \hbar c^{3}} \int_{4 \pi} d \Omega \sum_{\boldsymbol{\epsilon}}\left|\left\langle f\left|\mathscr{H}^{\prime}\left(\boldsymbol{A}_{\lambda}^{*}\right)\right| i\right\rangle\right|^{2}
$$

where $\Sigma_{\epsilon}$ denotes the sum over two perpendicular directions of polarization, and $d \Omega$ denotes solid angle for direction of emission.

Consider an interaction $\mathscr{H}^{\prime}$ of the form (40), and with the quantum emitted along the $z$ axis and polarized along the $x$ axis.

Then we have:

$$
\left.\mathscr{H}^{\prime}\left(\boldsymbol{A}_{\lambda}^{*}\right)=-N_{L}\left[\frac{e}{m c}\left(\boldsymbol{p} \cdot \boldsymbol{\epsilon}_{x}\right) e^{-i k z}\right)+\frac{e \hbar}{2 m c} \mu_{\boldsymbol{P}} \boldsymbol{\sigma} \cdot \operatorname{curl}\left(\boldsymbol{\epsilon}_{x} e^{-i k z}\right)\right] .
$$

One can, by a rather lengthy calculation ${ }^{4}$, expand the vector (58) potential as a series of standing spherical waves. This is made possible by the following wellknown expansion of the exponential:

$$
e^{i k z}=\sum_{L=0}^{\infty} i^{L}(4 \pi)^{\xi}(2 L+1)^{\xi} j_{L}(k r) Y_{L 0}(\theta, \phi) .
$$


Terms of all possible multipolarities appear in this expansion of the vector potential. However, all terms have $M= \pm 1$, since a quantum carries angular momentum unity about the direction of emission.

We could have instead expanded the exponential in powers of $\mathrm{kz}$. Thus

$$
\boldsymbol{A}_{\lambda}^{*}=N_{L} \boldsymbol{\epsilon}_{x} e^{-i k z}=N_{L} \sum_{n=1}^{\infty}\left(\frac{-i \omega}{c}\right)^{n-1} \frac{\epsilon_{x} z^{n-1}}{(n-1) !}
$$

Each term in this expansion can be broken into parts as follows ${ }^{27}$ :

$$
\epsilon_{x} z^{n-1}=\frac{\operatorname{grad}\left(x z^{n-1}\right)}{n}+\frac{n-1}{n} \varepsilon_{y} \times r z^{n-2} .
$$

The first term on the righthand side of (63) gives a "reducitie En moment", $(L=n)$, the second term gives a "reducible $M(n-1)$ moment", $(L=n-1)$.

With the aid of (48), we can write the matrix element (60) of the interaction in the following form:

$$
\left\langle f\left|\mathscr{H}^{\prime}\left(\boldsymbol{A}_{\lambda}^{*}\right)\right| i\right\rangle=-N_{L} \sum_{L=1}^{\infty}(-i \omega / c)^{L}\left\langle f\left|\mathscr{M}_{L}^{\prime E}+\mathscr{M}_{L}^{\prime M}\right| i\right\rangle
$$

where the reducible multipole operators are defined as follows:

$$
\begin{gathered}
\mathscr{M}_{L}^{\prime E}=\frac{e x \frac{z^{L-1}}{L !}}{\therefore} \\
\mathscr{M}_{L}^{\prime M}=\frac{L}{(L+1) !} \frac{e \hbar}{2 m c}\left[\left(L_{v}+\frac{\mu_{P}(L+1)}{2} \sigma_{v}\right) z^{L-1}\right]+\text { herm. conj. }
\end{gathered}
$$

If $(\omega a / c) \ll 1$, the term of lowest order (for a transition of given $\left|j_{i}-j_{f}\right|$ and $\Delta \pi$ ) will give the largest matrix element. The radial dependence of any reducible multipole operator (65) and of the corresponding pure multipole operator $(50)$ is the same only for small $r$.

The reducible E1, M1 and E2 multipole operators have the same angular momentum and parity relations as the corresponding pure multipoles. However, all other reducible multipole operators (M2, E3, etc.) also contain terms of lower order.

For $(\omega a / c) \ll 1$, and $L=\left|j_{i}-j_{f}\right| \geqslant 1$, the dominant term to the matrix element of $\mathscr{H}^{\prime}$ is given by a reducible moment of order $L$. Only terms of the highest order, viz. the pure multipole terms of order $L$, will contribute to this matrix element, because of the selection rules.

The expansion of the vector potential into plane wave is unsuited to cases for which $\omega a / c$ is not small compared to unity. For problems of this kind, it is necessary to use spherical waves. The farm of the multipole moments is then different ${ }^{28}$ from (50), since it is no longer correct to replace $j_{L}$ by its asymptotic form (20a).

As will be seen later, according to more detailed_nuclear models one would expect to find some transitions which involve significant mixtures

27 R. G. Sachs and N. Austern, Phys. Rev. 81 (1951) 705.

28 J. G. Brennan and R. G. Sachs, Phys. Rev. 88 (1952) 824. 
of the lowest two permitted multipole orders. Examples of such mixtures have recently been found experimentally ${ }^{6}$.

Table I lists multipolarities to be expected for a gamma transitions between two states of specified angular momenta and parities. The lowest two multipole orders are given; the second is in parenthesis to denote that it is usually not significant. It has been assumed in the table that the condition $L \leqslant j_{i}+j_{f}$ is satisfied.

TABLE I.

Possible Multipolarities for Gamma Radiations

(Two lowest possible multipole orders, $L<j_{i}+j_{f}$ )

\begin{tabular}{r|cccccc}
\hline $\begin{array}{c}\text { Parity Change } \\
\Delta \pi\end{array}$ & \multicolumn{5}{|c}{ Change of Angular Momentum $\left|j_{i}-j_{f}\right|$} \\
\hline No & M1(E2) & E2(M3) & M3(E4) & E4(M5) & M5(E6) \\
& Yes & E1(M2) & M2(E3) & E3(M4) & M4(E5) & E5(M6)
\end{tabular}

For EL transitions involving a single proton, the main contribution to $\mathscr{M}_{L M}^{E}$ is provided by the orbital term. The spin term is not expected to have an appreciable effect for this case. However, for ML transitions the spin term is expected to be actually somewhat more important than the orbital term.

If the proton moves in a central potential, each of the wavefunctions $\psi_{i}, \psi_{j}$ can be written as a product of $a$ ) a radial function $\mathscr{R}_{i}, \mathscr{R}_{f}$, which depends on the details of the potential, and $b$ ) a two-component function $\Theta_{l_{i}: i_{i}}^{m_{i}}, \Theta_{l_{j} . j_{j}}^{m_{f}}$, which contains angle and spin variables but is independent of details of the potential. Thus electric multipole matrix element can be written as follows:

$$
\left\langle f\left|\mathscr{M}_{L M}^{E}\right| i\right\rangle=e \int_{0}^{\infty} \mathscr{R}_{f} r^{L} \mathscr{R}_{i} r^{2} d r \int_{4 \pi} \Theta_{l_{f}, j_{f}}^{m_{f} *} Y_{L M}{ }^{*} \Theta_{l_{i}, j_{i}}^{m_{i}} d \Omega
$$

where $d \Omega$ refers to integration over all angles and sum over spins. The small contribution to $\mathscr{M}_{L M}^{E}$ due to intrinsic spin has been neglected in (66). This term is of the same order of magnitude as the magnetic multipole matrix element of order $(L+1)$.

The transition probability summed over $m$-substates of the final state $f$ and averaged over $m$-substates of the initial state $i$, denoted $T_{i \rightarrow j}^{(\sigma L)}$ is given by the expression:

$$
T_{i \rightarrow f}^{(\sigma L)}=\left(2 j_{i}+1\right)^{-1} \sum_{m_{i}} \sum_{m_{j}} \sum_{M} T_{i \rightarrow f}^{(\sigma L M)}
$$


To evaluate this transition probability, it is convenient to define a "statistical factor" $S$, as follows ${ }^{29}$ :

$$
S\left(j_{i}, L, j_{f}\right)=4 \pi\left(2 j_{i}+1\right)^{-1} \sum_{m_{i}} \sum_{m_{f}} \sum_{M}\left|\int_{4 \pi} \Theta_{l_{f}, i_{f}}^{m_{f}^{*}} Y_{L M}^{*} \Theta_{l_{i_{i}}, j_{i}}^{m_{i}} d \Omega\right|^{2}
$$

A general expression for $S$ has been derived ${ }^{30}$ :

$$
\left.\begin{array}{rl}
S\left(j_{i}, L, j_{f}\right)= & \left(2 l_{i}+1\right)\left(2 l_{f}+1\right)(2 L+1)\left(2 j_{f}+1\right) \times \\
& \times\left|V\left(l_{i} l_{f} L ; 000\right)\right|^{2}\left|W\left(l_{i} j_{i} l_{f} j_{f} ; \frac{1}{2}, L\right)\right|^{2}
\end{array}\right\}
$$

where the quantities $V^{\mathbf{3 1}}$ are closely related to Clebsch-Gordan coefficients, and the quantities $W 31$ are Racah coefficients. For a given $j_{i}, L, j_{f}$, of the four possible kinds of transitions, $\left(l_{i}=j_{i} \pm \frac{1}{2}, l_{f}=j_{f} \pm \frac{1}{2}\right)$, two have the parity change $(-1)^{L}$ required for an EL radiation; viz. those transitions for which $l_{i}-l_{f}-L$ is an even integer. It can be shown ${ }^{18,19}$ that $S$ is the same for these two transitions and thus $S$ is a fraction of $j_{i}, L$, and $j_{f}$ alone.

The expression for $S$ takes a comparatively simple form for the special case that $\left|j_{i}-j_{f}\right|=L$.

For $j_{f}=j_{i}-L, l_{f}=l_{i}-L$, we have:

In particular,

$$
S\left(j_{i}, L, j_{f}\right)=\frac{\left(j_{i}-\frac{1}{2}\right) !}{\left(2 j_{i}\right) ! !} \times \frac{(2 L+1) ! !}{L !} \times \frac{\left(2 j_{f}\right) ! !}{\left(j_{f}-\frac{1}{2}\right) !} .
$$

$$
S\left(L+\frac{1}{2}, L, \frac{1}{2}\right)=1
$$

For transitions where $j_{f}>j_{i}, S$ is given in terms of the $S$ for the reverse transition by the principle of detailed balance:

$$
\frac{S\left(j_{f}, L, j_{i}\right)}{S\left(j_{i}, L, j_{f}\right)}=\frac{2 j_{i}+1}{2 j_{f}+1}
$$

Values of $S\left(j_{i}, L, j_{f}\right)$ are listed in Table II for transitions for which $\left|j_{i}-j_{f}\right|=L$.

The EL transition rate for a single proton (non-relativistic and in a central velocity-inaiependent potential), is obtained from eq. (51), (66), (67), and (68):

$$
T_{i \rightarrow f}^{(E L)}=\frac{2(L+1)}{L[(2 L+1) ! !]^{2}} \omega\left(\frac{e^{2}}{\hbar c}\right)\left(\frac{\omega a}{c}\right)^{2 L}\left(\int_{0}^{\infty} \mathscr{R}_{f}\left(\frac{r}{a}\right)^{L} \mathscr{R}_{i} r^{2} d r\right)^{2} S\left(j_{i}, L, j_{f}\right) .
$$

29 The corresponding quantity $C\left(j_{>}, j_{<}\right)$defined by Bohr and Mottelson ${ }^{20}$ equals

$$
\left(2 j_{f}+1\right)^{-1}\left(2 j_{<}+1\right) S\left(j_{i}, L, j_{f}\right)
$$

$\left(j_{<}\right.$and $j_{>}$refer to the larger and smaller respectively of $j_{i}$ and $\left.j_{f}\right)$. The quantity $\overline{F\left(j_{i}, j_{f}\right)^{2}}$ defined by $\operatorname{Stech}^{18}$ is equal to $\frac{2}{2 j_{f}+1} S\left(j_{i}, L, j_{f}\right)$.

so J. M. Blatt and V. F. Weisskopf, op. cit. p. 627.

31 G. Racah, Phys. Rev. 62 (1942) 438. 
TABLE II

Coffricients $S\left(j_{i}, L, j_{f}\right)$ for $L=\left|j_{i}-j_{f}\right|$

\begin{tabular}{rc|ccccccc}
\hline$j_{1}$ & $j_{i}$ & $1 / 2$ & $3 / 2$ & $5 / 2$ & $7 / 2$ & $9 / 2$ & $11 / 2$ & $13 / 2$ \\
\hline $1 / 2$ & 1 & 1 & 1 & 1 & 1 & 1 & 1 \\
$3 / 2$ & 2 & & $6 / 5$ & $9 / 7$ & $4 / 3$ & $15 / 11$ & $18 / 13$ \\
$5 / 2$ & 3 & $9 / 5$ & & $9 / 7$ & $10 / 7$ & $50 / 33$ & $225 / 143$ \\
$7 / 2$ & 4 & $18 / 7$ & $12 / 7$ & & $4 / 3$ & $50 / 33$ & $700 / 429$ \\
$9 / 2$ & 5 & $10 / 3$ & $50 / 21$ & $5 / 3$ & & $15 / 11$ & $225 / 143$ \\
$11 / 2$ & 6 & $45 / 11$ & $25 / 33$ & $25 / 11$ & $18 / 11$ & & $18 / 13$ \\
$13 / 2$ & 7 & $63 / 13$ & $525 / 143$ & $1225 / 429$ & $315 / 143$ & $21 / 13$ &
\end{tabular}

The matrix element $\mathscr{M}_{L M}^{M}$ has a simple form for the special case $\left|j_{i}-j_{f}\right|=L,\left|l_{i}-l_{f}\right|=L-1$. For this case it follows from eq. (50b) that:

$$
\left\langle f\left|\mathscr{M}_{L M}^{M}\right| i=\frac{e \hbar}{2 m c}\left(\mu_{P}-\frac{1}{L+1}\right)\left\langle f\left|\sigma \cdot \operatorname{grad}\left(r^{L} Y_{L M}^{*}\right)\right| i\right\rangle .\right.
$$

From eqs. (51), (67), (68) we obtain the following result:

$$
\left.\begin{array}{r}
T_{i \rightarrow f}^{(M L)}=\frac{2(L+1)}{L[(2 L+1) ! !]^{2}} \omega \frac{e^{2}}{\hbar c}\left(\frac{\omega a}{c}\right)^{2 L}\left(\frac{\hbar}{m c a}\right)^{2} \cdot \\
\cdot\left(\mu_{P} L-\frac{L}{L+1}\right)^{2}\left(\int_{0}^{\infty} \mathscr{R}_{f}\left(\frac{r}{a}\right)^{L-1} \mathscr{R}_{i} r^{2} d r\right)^{2} S\left(j_{i}, L, j_{f}\right) .
\end{array}\right\}
$$

The radial integrals can be evaluated explicitly on the basis of various simple assumptions regarding nuclear wavefunctions. Perhaps the simplest possible picture of the nuclear wavefunctions is provided by the assumption ${ }^{4.17}$ that the radial wavefunctions $\mathscr{R}$ for both initial and final states are constant throughout the interior of the nucleus (for $r<a$ ) and vanish outside (for $r>a$ ). On the basis of this constant density picture, the values of the radial integrals are given as follows:

$$
\int_{0}^{\infty} \mathscr{R}_{f}(r / a)^{L} \mathscr{R}_{i} r^{2} d r=3(L+3)^{-1}
$$

A more detailed calculation of radial integrals, which uses the radial wavefunctions of a single proton in a spherical square well for several cases of interest gives, practically the same results ${ }^{19}$, except for M1 transitions.

The magnetic dipole operator for a proton in a velocity - independent central potential has been given above; e.g., for the component with $M=0$, we have

$$
\mathscr{M}_{10}^{M}=\mid \overline{\frac{3}{4 \pi}}\left(\frac{e \hbar}{2 m c}\right)\left(L_{z}+\mu_{P} \sigma_{z}\right) .
$$

This operator connects only states of the same orbital angular momentum and the 
same radial quantum number. However, such states are degenerate for the potential assumed here. Therefore, Ml transitions cannot occur at all for a proton in a velocityindependent central potential. Magnetic transitions of higher multipole order are not forbidden, provided $\left|l_{i}-l_{f}\right| \leqslant L-1$.

With the aid of estimate (76), the gamma-decay transition probability for a single proton, [eqs. (73), (75)], can be rewritten in the following convenient form ${ }^{4,17}$ :

$$
\left.\begin{array}{r}
T_{S P}^{(E L L)}=\frac{4.4(L+1)}{L[(2 L+1) ! !]^{2}}\left(\frac{3}{L+3}\right)^{2}\left(\frac{\hbar \omega}{197 M e V}\right)^{2 L+1}\left(a \text { in } 10^{-13} \mathrm{~cm}\right)^{2 L} \times \\
S\left(j_{i}, L, j_{f}\right) \times 10^{21} \mathrm{sec}^{-1}
\end{array}\right\}
$$

and

$$
\left.\begin{array}{r}
T_{S P}^{(M L)}=\frac{0.19(L+1)}{L[(2 L+1) ! !]^{2}}\left(\frac{3}{L+2}\right)^{2}\left(\mu_{P} L-\frac{L}{L+1}\right)^{2}\left(\frac{\hbar \omega}{197 \mathrm{MeV}}\right)^{2 L+1} \times \\
\left(a \text { in } 10^{-13} \mathrm{~cm}\right)^{2 L-2} S\left(j_{i}, L, j_{f}\right) \times 10^{21} \mathrm{sec}^{-1} .
\end{array}\right\}
$$

\begin{tabular}{|c|c|c|c|c|}
\hline Multipolarity & \multicolumn{4}{|c|}{$T_{S P}$ in $\mathbf{s e c}^{-1}$} \\
\hline El & $1.5 \times$ & $<10^{14}$ & $\mathbf{A}^{2 / 3}$ & $\mathrm{E}_{\gamma}{ }^{3} \mathrm{~S}$ \\
\hline M1 & $2.8 \times$ & $<10^{13}$ & & $\mathbf{E}_{\gamma^{3}} \mathbf{S}$ \\
\hline E2 & $1.6 \times$ & $10^{8}$ & $\mathbf{A}^{4 / 3}$ & $\mathbf{E}_{\gamma^{5}} \mathrm{~S}$ \\
\hline M2 & $1.2 \times$ & $<10^{8}$ & $\mathbf{A}^{2 / 3}$ & $\mathrm{E}_{\gamma}{ }^{5} \mathrm{~S}$ \\
\hline E3 & $1.1 \times$ & $<10^{2}$ & $\mathbf{A}^{2}$ & $\mathrm{E}_{\gamma}^{\gamma} \mathrm{S}$ \\
\hline M3 & $1.8 \cdot x$ & $<10^{2}$ & $A^{4 / 3}$ & $\mathrm{E}_{\gamma}^{\gamma} \mathrm{S}$ \\
\hline E4 & $5.0 \times$ & $\times 10^{-5}$ & $A^{8 / 3}$ & $\mathrm{E}_{\gamma} \otimes \mathrm{S}$ \\
\hline M4 & $1.5 \times$ & $<10^{-4}$ & $A^{2}$ & $\mathrm{E}_{\gamma}^{\gamma}{ }^{9} \mathrm{~S}$ \\
\hline E5 & $1.6 \times$ & $10^{-11}$ & $A^{10 / 3}$ & $\mathrm{E}_{\gamma}^{11} \mathrm{~S}$ \\
\hline M5 & $7.5 \times$ & $<10^{-11}$ & $A^{8 / 3}$ & $\mathrm{E}_{\gamma}^{11} \mathrm{~S}$ \\
\hline
\end{tabular}

The estimates (77) for various multipolarities are listed in Table III.

TABLE III

Estmates (77) for the Transition Probability of a Single Proton

In Figure 1, $T_{S P}$ is plotted as function of energy for all multipolarities of interest for the special conditions: $A=100, S=1$ (it has been assumed that $a=1.45 \times 10^{-13} A^{1 / 3} \mathrm{~cm}$ and $\mu_{P}=2.79$ ).

It must be emphasized again that the above theoretical estimates are crude and are not expected to represent accurately transitions in actual nuclei. Nevertheless, these estimate provide at least a starting point for the interpretation of known results. The "experimental" gamma-decay transition probability ${ }^{32}$ of an isomeric state is given in terms of the

32 The gamma-decay lifetime, $\tau_{\gamma}$, which has been used by some authors 6.19 equals $T^{-1}$. 


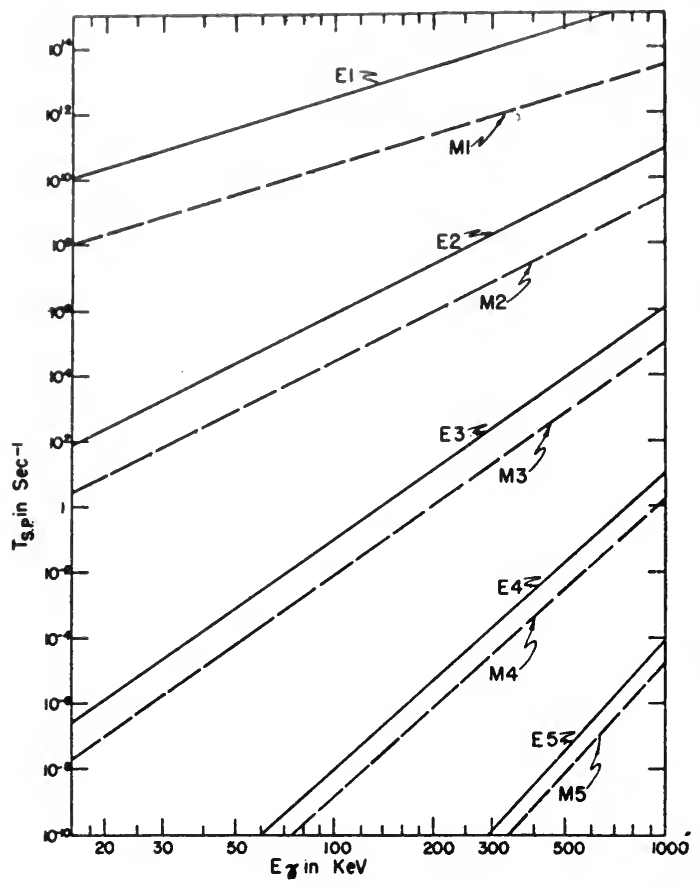

experimental half-life $\tau_{1 / 2}$ of the isomeric state by means of the equation:

$$
T_{\text {exp. }}=\frac{0.693}{\tau_{1 / 2}(1+\alpha)}
$$

The factor $(1+\alpha)$ accounts for the effect of internal conversion in speeding up transitions. (If additional branching, e.g., $\beta$-decay, occurs, this must also be corrected for.)

Fig. 1. Gamma-decay transition probability (in $\mathrm{sec}^{-1}$ ) for a single proton (Eqs. 77) as function of gamma-ray energy (in $\mathrm{MeV}$ ) for various multipolarities.

$$
\begin{gathered}
\left(a=1.45 \times 10^{-1 / 3} \mathrm{~A}^{1 / 3} \mathrm{~cm},\right. \\
\left.\mathrm{A}=100, \quad \mathrm{~S}=1, \quad \mu_{P}=2.79\right)
\end{gathered}
$$

\section{B. Transitions of a Single Neutron}

It is also of interest here to consider electromagnetic transitions of a single neutron. Because of the absence of electric charge, one would expect no interaction between orbital motions of neutrons and the electromagnetic field. However, the orbital motion of the rest of the nucleus around the center of mass of the whole nucleus will give a matrix element $\mathscr{M}_{L M}^{E}$ for electric transitions which is smaller than the matrix element for a single proton in the corresponding transition by a factor $\left(Z / A^{L}\right)$; this factor is small except for El transitions. An additional but also small contribution to the electric multipole matrix element comes from the interaction of the neutron spin with the electromagnetic field. The electric multipole matrix element due to this effect has the same order of magnitude as the magnetic multipole matrix element of one higher order.

According to a strict single particle picture, magnetic transition probabilities in odd-neutron nuclei are expected to be slightly smaller than the corresponding magnetic transition probabilities in odd proton nuclei, the ratio being

$$
\left[\frac{\mu_{N}}{\mu_{P}-(L+1)^{-1}}\right]^{2} \text {. }
$$

Many M4 transitions in nuclei of odd mass number are known ${ }^{6,14}$. 
Transition probabilities for gamma-decay are in qualitative agreement with the crude estimates (77), being larger than those estimates by factors ranging from 5 to 20 in most cases. There is no clear evidence for any favoring of M4 transitions in odd proton nuclei relative to odd neutron nuclei. According to (79) one would expect such a favoring, (viz. by a factor 1.8).

\section{Effect of Velocity-Dependent Interactions}

The presence of velocity-dependent interactions ${ }^{27.33}$ (e.g., spin orbit coupling, exchange forces, etc.) alters some of the above conclusions about transition probabilities since the interaction is no longer given by (40). Thus, the special rule which forbids M1 transitions is relaxed, and magnetic transitions which involve change of orbital angular momentum $\left|l_{i}-l_{f}\right|=L+1$ can occur.

The forms of the magnetic multipole moments (50b), (65b), depend strongly on the interaction. However, because of gauge invariance, the forms of the electric multipole moments (50a), (65a), are independent ${ }^{27}$ of the interaction, provided $(\omega a / c) \ll 1$.

\section{§ 4. Gamma Decay Transition Probabilities for Configurations Consisting of Several Independent Particles}

It is of interest to evaluate transition probabilities between states containing several particles, since such calculations are expected to be more realistic, for actual nuclei, than calculations based on a singleparticle model (Section 3).

In this section, we consider transitions between states consisting of several independent particles. The nuclear wavefunctions are then appropriate linear combinations of products of single particle wavefunctions. Now a state of several particles is characterized by more quantum numbers than a single-particle state. The multipole matrix element for a transition is a sum of matrix elements, each of which involves only one particle $p$ :

$$
\sum_{p} \mathscr{M}_{L M}^{\sigma}(p) \text {. }
$$

It is seen that the selection rules on angular momenta apply not only to the single particle making the transition, but also to the nucleus as a whole; i.e., neither $\left|J_{i}-J_{f}\right|$ of the nucleus nor $\left|j_{i}-j_{f}\right|_{p}$ of any particle can exceed $L^{34}$.

33 N. Austern and R. G. Sachs, Phys. Rev. 81 (1951) 710. J. H. D. Jensen and M. G. Mayer, Phys. Rev. 85 (1952) 1040.

34 For comparison of radiative transition probabilities with theory it is very 
It is apparent from the form (80) for the multipole operators, that transitions which involve change of wavefunction for more than one particle, (many particle transitions) cannot occur, if the particles are independent. This includes any case where the orbit of more than particle changes, as well as transitions in which only one particle changes its orbit, but the remaining particles change from being paired (coupled to spin zero) to being lined up in any other way,

$$
\text { \{for example, } \left.\left[p_{1 / 3}\left(g_{\% / 2}\right)_{0}^{2}\right]_{1 / 3} \rightarrow\left(g_{\% / 2}\right)_{/ / 3}^{3}\right\} \text {. }
$$

If the wavefunctions of the states are close to independent particle wavefunctions, but with some "impurities", then the selection rules are expected to break down to a slight extent ${ }^{35}$. The small E3 transition probabilities for a number of isomers can be accounted for in this manner (Ch. XVI, XVII).

Some transitions, even though they are not forbidden, may still be expected to have reduced transition probabilities, on basis of an independent particle model. The transition probability between states of partially filled orbits is less than that between corresponding single particle states. The reduction arises from the fact that a fraction of the $m$-substates, which would be available to the particle in the corresponding transition between single particle states, are not available in this case to the jumping particle because they are already occupied.

The ratio $\varrho$ of the transition probability between two states of "seniority" (number of unpaired particles) unity, divided by the transition probability between corresponding single particle states $j_{i} \rightarrow j_{j}$, has the following values:

For

$$
\left.\begin{array}{c}
{\left[\left(j_{i}\right)^{2 s_{i}+1}\left(j_{f}\right)^{2 s_{f}}\right]_{j_{i}} \rightarrow\left[\left(j_{i}\right)^{2 s_{i}}\left(j_{j}\right)^{2 s_{f}+1}\right]_{i_{f}}} \\
\varrho=\frac{j_{i}+\frac{1}{2}-s_{i}}{j_{i}+\frac{1}{2}} \times \frac{j_{1}+\frac{1}{2}-s_{f}}{i_{i}+\frac{1}{2}} .
\end{array}\right\}
$$

For

$$
\left.\begin{array}{c}
{\left[\left(j_{i}\right)^{2 s_{i}-1}\left(j_{f}\right)^{2 s_{f}}\right]_{j_{i}^{\prime}}^{\prime} \rightarrow\left[\left(j_{i}\right)^{2 s_{i}}\left(j_{f}\right)^{2 s_{f}-1}\right]_{j_{f}}} \\
\varrho=\frac{s_{i}}{j_{i}+\frac{1}{2}} \times \frac{s_{f}}{j_{f}+\frac{1}{2}} .
\end{array}\right\}
$$

convenient to define a "reduced transition probability" $B\left(\sigma L, J_{i} \rightarrow J_{f}\right)$ as follows ${ }^{20}$ :

$$
B\left(\sigma L, J_{i} \rightarrow J_{f}\right)=\frac{1}{2 J_{i}+1} \sum_{m_{i}} \sum_{m_{f}} \sum_{M}\left|\left\langle f\left|\sum_{p} \mathscr{M}_{L M^{\sigma}}(p)\right| i\right\rangle\right|^{2} .
$$

According to Eq. 51, the transition probability, which depends on the detailed nuclear structure only through $B$, is then given by:

$$
T_{i \rightarrow f}^{(\sigma L)}=\frac{8 \pi(L+1)}{L[(2 L+1) ! !]^{2}} \frac{1}{h}\left(\frac{\omega}{c}\right)^{2 L+1} B\left(\sigma L, J_{i} \rightarrow J_{f}\right) .
$$

35 A. De Shalit and M. Goldhaber, Phys. Rev. 92 (1953) 1211. 
We briefly mention two selection rules on radiative transitions which are valid if "isotopic spin" is a good quantum number for nuclei ${ }^{8}$.

a) Gamma-transitions between two states whose isotopic spin differs by more than unity are forbidden.

b) El transitions between two states of the same isotopic spin in a selfconjugate $(N=Z)$ nucleus are essentially forbidden ${ }^{36}$. There is experimental evidence that the latter selection rule is operative in a number of light nuclei ${ }^{37}$.

s6 L. A. Radicati, Phys. Rev. 87 (1952) 521.

37 M. Gell-Mann and V. L. Telegdi, Phys. Rev. 91 (1953) 169. 


\title{
THEORY OF INTERNAL CONVERSION
}

\author{
M. E. ROSE
}

\section{§ 1. Introduction}

One of the most useful procedures for the classification of nuclear energy levels is the method of internal conversion. A nucleus in an excited state, with excitation energy less than the binding energy" of a nuclear particle, will almost always make a transition to a lower state by either one of two competing processes. These are (a) the emission of a gamma ray (cf. Ch. XIII), or (b) the ejection of one of the orbital electrons *. The ratio of transition probability, $N_{e}$, for process (b) to that for process (a), $N_{q}$, is referred to as the internal conversion coefficient. The conversion coefficient will depend very strongly on the following parameters:

(i) The transition energy, $k m c^{2}$.

(ii) The atomic number $(Z)$ of the emitter.

(iii) The shell or subshell from which the electron is ejected.

(iv) The multipolarity $L$, or angular momentum of the radiated field, i.e., of the competing $\gamma$-radiation.

(v) The character of the nuclear transition, electric or magnetic, which, in turn, uniquely determines the nuclear parity change once $L$ is fixed, Ch. XIII.

The primary nuclear information is obtained from a comparison of observation and accurate theoretical values of the conversion coefficients, whence the parity change and angular momentum change of the nucleus may be deduced. In this chapter consideration is confined to the theoretical aspects of the problem. Application of the theory is illustrated in Ch. XXII.

Except for very low $Z$ (< about 20 ) and large $k(\gtrsim 5$, i.e. $2.5 \mathrm{MeV}$ ) the conversion coefficients are usually large enough to measure. In some cases, $k$ near the threshold and especially for the larger $L$ values $\left(2^{4}\right.$ or $2^{5}$-poles),

1 M. E. Rose, Phys. Rev. 76, (1949) 678; Phys. Rev. 78, (1950) 184.

* The alternative possibility of emitting an electron-positron pair when the transition energy $>1.02 \mathrm{MeV}$, takes place with a frequency $10^{-4}$ to $10^{-3}$ relative in gamma ray emission. Of course, this process is readily distinguishable from the conversion process. For $0 \rightarrow 0$ transitions no conversion or $\gamma$-emission is possible. In other cames the internal pair emission provides information where conversion is intrecuent, that is. at high energies and/or low $Z$, see reference 1 and Chapter $\mathrm{XX}$. 
the conversion coefficients may become so large that it is difficult to observe any $\gamma$-radiation. In such cases the requisite information is forthcoming from relative conversion coefficients for different shells or subshells. Thus, one refers to the $K / L$ ratio which is

where

$$
\frac{K}{L}=\frac{N_{e}(K)}{N_{e}(L)}
$$

$$
N_{e}(L)=N_{e}\left(L_{\mathrm{I}}\right)+N_{e}\left(L_{\mathrm{II}}\right)+N_{e}\left(L_{\text {III }}\right) .
$$

Designating the conversion coefficients by $\alpha(K)$ etc. ${ }^{*}$, this is obtained from the theory as

$$
\frac{K}{L}=\frac{\alpha(K)}{\alpha\left(L_{\mathrm{I}}\right)+\alpha\left(L_{\mathrm{II}}\right)+\alpha\left(L_{\mathrm{III}}\right)}
$$

When $K$-conversion is energetically impossible the ratios of the various $L$-subshell coefficients are most useful. It will be found that quite often this is a more sensitive indication of the type of transition involved than the $K / L$ ratio. Which ratio is of greatest interest depends on the resolution of the measurement (and therefore, to some extent on $Z$ ) and also on the value of $L$ and parity change, since there are cases in which $L$-conversion takes place almost entirely in two of the three subshells. In any event, the required theoretical ratios are readily obtained from the appended tables, (see Appendix III).

It should be strongly emphasized that for the theoretical values of $\alpha$ it is necessary to use the rigorous Dirac theory of the electron. Comparison of results of calculations using various types of approximation with the exact values shows that the former may be in sufficiently great error as to lead to erroneous classifications. With the use of the Dirac representation of the electron wave functions it is of especial interest to realize that the results are virtually exact. In particular, as long as the nucleus is regarded as a point, which is a very good approximation, no details of nuclear structure enter the theory. The nucleus merely serves as a source of a (virtual) electromagnetic field of given angular momentum and parity.

Until 1954 the only existing calculations which were sufficiently exact were the following:

(1) The $K$-shell results of Rose et al. ${ }^{2}$ These results gave the conversion coefficients for $\mathrm{EL}$ and $\mathrm{ML}$ radiation with $\mathrm{L}=1 \ldots 5$, for six values

2 M. E. Rose, G. H. Goertzel, B. I. Spinrad, J. Harr and P. Strong, Phys. Rev. 83, (1951) 79.

* In general, since $\alpha$ depends on só many parameters of interest, we will explicitly express only those which are of interest for the discussion on hand. Later we distinguish between electric and magnetic conversion coefficients by using the symbol $\beta$ for the latter. 
of $k$ in the range $0.3 \leqslant k \leqslant 5.0$ and 12 values of $Z$ in the range $10 \leqslant Z \leqslant 96$. No screening was included and the work of Reitz, ${ }^{3}$ as well as the most recent calculations presented here, shows that this omission was fully justified.

(2) More extensive tabulation of the $K$-shell coefficients was presented by Rose, Goertzel and Perry. ${ }^{4}$ These results were based on interpolation of the foregoing work of Rose et al. ${ }^{2}$ The range of parameters was therefore the same. In all about 3600 coefficients were obtained in this way. The most serious lack, insofar as $K$-coefficients are concerned, was the nonavailability of low energy $(k<0.3)$ values. Reitz' ${ }^{3}$ results (which include screening) are mainly in this region but only about 50 coefficients (for 3 values of $Z$ and $\mathrm{E} 1, \mathrm{E} 2$ and M1 transitions) were calculated. The results discussed below, together with additional results which should be available in the near future, will fill this gap. Threshold values for the $K$-conversion coefficients were calculated by Spinrad and Keller. ${ }^{5}$

(3) The only previously existing $L$-shell results were those of Gellman, Griffiths and Stanley. ${ }^{6}$ These were calculated for 3 values of $Z(49,84$, 92 ), $3 k$ values, 3 multipoles (E1, E2 and $\mathrm{M} 1$ ) and for the $3 L$-subshells. The necessity for a more extensive calculation is evident from the extreme sensitivity of the coefficients with $k$ (especially in the interesting low energy region) and also with $Z$. As a result interpolation and extrapolation is very uncertain. In addition, one needs coefficients for the higher multipoles.

The present results include an extension of the $K$-shell calculations toward low energy ( $k$ less than the minimum values considered in reference 2 ), and conversion coefficients for the three $L$-subshells. Both the $K$ - and $L$-subshell coefficients include the effect of screening. Altogether the present results comprise $160 \mathrm{~K}$-shell and $920 \mathrm{~L}$-shell coefficients covering the range $15 \leqslant Z \leqslant 85,0.05 \leqslant k \leqslant 2.0$ and $L=1 \ldots 5$ for both electric and magnetic multipoles. ${ }^{*} \mathrm{It}$ is to be noted that the ranges in $Z$ and $k$ are not covered as uniformly as would be most desirable. Moreover, at present, results for the three $L$-subshells are not equally extensive. Further work is in progresı̈ for the purpose of obtaining twice as many $K$ - and $L$-shell coefficients. In the meantime the present results should prove quite useful.

3 J. Reitz, Phys. Rev. 77, (1950) 10.

4 M. E. Rose, G. H. Goertzel and C. L. Perry, Oak Ridge National Laboratory Report No. 1023.

5 B. I. Spinrad and L. B. Keller, Phys. Rev. 84, (1951) 1056.

6 H. Gellman, B. A. Griffith and J. P. Stanley, Phys. Rev. 85, (1952) 944.

* These results, which are presented in Tables A, B, and C of Appendix IV, are taken from unpublished work of M. E. Rose and G. H. Goertzel. The numerical work was carried out on the Standards Eastern Automatic Computer (National Bureau of Standards, Washington, D.C.). 


\section{$\S 2$. General Theoretical Considerations}

In the interest of brevity the general theory which forms the basis of the calculation of the internal conversion coefficients will be presented in outline form. The primary motivation is to emphasize the physical ideas without presenting detailed calculations. The same remark applies to the treatment of the actual conversion coefficient calculations as given in sections 3 and 4.

To begin with, it is important to recognize that the internal conversion process, consisting, as it does, in the interaction between two particles (one of the nucleons and the orbital electron ${ }^{*}$ ), is a second order process. That is to say, one envisages an initial state in which the nucleus is excited with energy $E$ above the ground state (or state of lower excitation) and the electron is in its ground state. In the final state the electron is in an excited (continuum) state with energy $W$ above its ground state and the nucleus has lost the energy $E$. The number of quanta present is zero in both initial and final states. Clearly, the mechanism for this process involves a set of intermediate states in which one or the other particle, but not both, has made the transition to its final state. In this intermediate state a photon of frequency $\omega$ exists. The state in which both particles have their final energy is reached by the mechanism of absorption of the photon by particle 1 (or 2 ) if it was originally emitted by particle 2 (or 1). Thus, there are actually two sets of intermediate states.

The dynamical description of this double transition leading to the expected correspondence-principle result for the internal conversion coefficient, see Eq. (1) below, has been given by Tralli and Goertzel. ${ }^{7}$ The only modification in their procedure which is worth mentioning is that it is actually unnecessary to specify the nature of the interaction between the nucleons and the electromagnetic field in the point-nucleus limit. This has been explicitly demonstrated. 8

The result for the internal conversion, to which the standard perturbation theory leads, is

$$
\alpha_{L}(e \text { or } m)=\frac{N_{e}}{N_{q}}=2 \pi^{3} k \mathfrak{S}\left|\left(f\left|\mathscr{H}\left(\mathbf{B}_{L}^{M}\right)\right| i\right)\right|^{2}
$$

Here the last factor is the square modulus of the matrix element, between initial $(i)$ and final $(f)$ electron states, of the perturbation $\mathscr{H}$, representing

7 N. Tralli and G. H. Goertzel, Phys. Rev. 83, (1954) 399.

8 M. E. Rose, Multipole Fields, John Wiley and Sons, New York (1955).

* The fact that all the nucleons in the emitting nucleus are involved is taken care of by a summation over all nucleons. However, for $\alpha=N_{e} / N_{q}$ this fact is irrelevant as long as small corrections due to the finite size of the nucleus are ignored. 
the coupling with the radiation field. This perturbation depends on the electromagnetic multipole potentials and is different for EL and ML transitions, see Eqs. (4) and (6) below. These potentials are normalized to a flux of

$$
N_{q}=1 / \pi^{2} k
$$

photons per second. Here, and throughout, we take $m=c=\hbar=1$. The operation represented by $\mathfrak{S}$ is a summation over all unobserved degrees of freedom of the radiation, a summation over final and an average over initial magnetic substates of the electron. It is this summation and averaging process which removes interference terms when the transition has mixed character [ML and $E(L+1)$, say]. In such cases the conversion coefficient is a weighted average of the electric and magnetic conversion coefficients and the weight factors are the relative intensities (oscillator strengths) of the electric and magnetic multipole $\gamma$-rays which are emitted as competing radiations. The evaluation of this mixture ratio by comparison of observed and calculated coefficients is also feasible and constitutes an item of interesting information.

We give the following description of the field without derivation. Full details are given in reference 8 ; see also Ch. XIII. The coupling term in the matrix element of (1) is

$$
\mathscr{H}=e(\boldsymbol{\alpha} \cdot \mathbf{B}+\phi)
$$

where $\alpha$ is the usual Dirac matrix vector (velocity operator) and B, $\phi$ are respectively the vector and scalar potentials of an outgoing wave solution of the Maxwell equations. In these units the charge $e=\sqrt{\alpha} \cong 1 / \sqrt{137}$. For a $2^{L}$ pole field the vector potential is an eigenfunction of $J^{2}$ (where $J$ represents the angular momentum operator of the radiation field) with eigenvalue $L(\mathrm{~L}+1)$, and of $J_{z}$ with eigenvalue $M$. Also, under coordinate inversion the magnetic field $\boldsymbol{H}=\operatorname{curl} B$ has the parity properties that it is multiplied by $(-)^{L+1}$ for magnetic radiation and $(-)^{L}$ for electric. For magnetic radiation

since, in general

$$
\begin{aligned}
& \mathbf{B}=\left[\frac{2}{\pi} \frac{1}{L(L+1)}\right]^{\frac{1}{2}} h_{L} L Y_{L}^{M} \\
& \phi=0
\end{aligned}
$$

or

$$
\operatorname{div} B+\frac{\partial \phi}{\partial t}=\mathbf{0}
$$

$$
\phi=-\frac{i}{k} \operatorname{div} B
$$

That is, $B$ as given by (4) is in the solenoidal gauge. In (4) $L=-i r \times \nabla$ 
is the orbital angular momentum operator of the radiation field, $Y_{L}^{M}$ is a normalized spherical harmonic and

$$
h_{L}=\left(\frac{\pi}{2 k r}\right)^{\frac{1}{2}} H_{L+1}^{(1)}(k r)
$$

is the spherical Hankel function (of the first kind).

For the electric multipoles the situation is somewhat more complex. The most important point of difference, as compared to the magnetic multipole case, lies in the fact that one cannot use the solenoidal gauge if the simple result (1) is used. In fact a commitment to a particular gauge, the so-called "conventional" gauge, has been made. * This is not to say that one cannot preserve gauge invariance. However, as discussed in reference 8 , if one uses any other gauge it is necessary to add to (1) certain terms arising from contributions on the nuclear surface. The restriction to the conventional gauge, described by Eq. (6) below is made in the interest of simplicity. In this gauge a $2^{L}$ pole is described by

and

$$
\mathbf{B}=\left[\frac{2}{\pi} \frac{1}{L(L+1)}\right]^{\frac{1}{2}} h_{L-1}\left(r \nabla+\frac{L r}{r}\right) Y_{L}^{M}
$$

$$
\phi=i\left[\frac{2}{\pi} \frac{L}{L+1}\right]^{\frac{1}{2}} h_{L} Y_{L}^{M}
$$

\section{$\S 3$. The Calculations: I.}

While it is not our purpose to present a detailed description of the somewhat involved calculations, it is necessary to present sufficient information concerning notation so that the results may be understood. At the same time sufficient detail is presented so that the reader may be able to reproduce these results.

The electron states are most economically described, for present purposes, by a single quantum number $\varkappa$. This number gives both the angular momentum $j$ by $j=|x|-\frac{1}{2}$ and the parity $(-)^{l+1}$ by

$$
\begin{array}{ll}
l=x & x>0 \\
=-x-1 & x<0
\end{array}
$$

* In general, for electric multipoles $B$ is a linear combination of two terms, one with $h_{L+1}$ and one with $h_{L-1}$. Either of these terms can be removed by a gauge transformation, which automatically renormalizes the other term. In the conventional gauge only the singular term $h_{L-1}$ is present - see reference 8 . As emphasized there, the origin of the confusion concerning gauge transformation in internal conversion arises from the fact that this type of transformation cannot be made on outgoing wave fields, which never occur in a Hamiltonian, but only on standing wave fields which do. In the perturbation theory leading to (1) the summation over $\omega$, the intermediate state photon energies, converts a standing wave into an outgoing wave. 
Thus, $j=l-\frac{1}{2} S_{\varkappa}$ where $S_{\varkappa}$ is the sign of $\varkappa$. In general, $x \neq 0$ and $\varkappa=-1,1,-2 \ldots$ for $s_{\xi}, p_{\xi}, p_{2 /,} \ldots$ states. We designate the final state by the aforementioned symbols and use primes for the initial state.

As is well known, a Dirac central field wave function is easily constructed from two Pauli spin-angle functions $\chi_{ \pm x}^{\mu}$ by adjoining appropriate radial functions. Thus, for the final state

$$
\psi_{f}=\left(\begin{array}{c}
-i f_{x} \chi_{-*}^{\mu} \\
g_{x} \chi_{x}^{\mu}
\end{array}\right)
$$

Here $\mu$ is the $z$-component of the angular momentum and $f_{x}, g_{x}$ are radial functions defined in reference 9 . The spin-angle functions are

$$
\chi_{\varkappa}^{\mu}=\sum_{\tau}\left(l \frac{1}{2} j \mu \mid l \frac{1}{2} \mu-\tau \tau\right) \chi_{t}^{\tau} Y_{l}^{\mu-\tau}
$$

where $\tau= \pm \frac{1}{2}$ and the Condon-Shortley ${ }^{10}$ notation for vector addition coefficients is used. With one exception, noted below, Eq. (11), one does not need numerical values of these coefficients and only the fact that they are elements of a unitary matrix is used. In (7) $\chi_{\neq}^{\tau}$ is a simple spin function.

$$
\chi_{t}=\left(\begin{array}{l}
1 \\
0
\end{array}\right) ; \chi_{i}^{-t}=\left(\begin{array}{l}
0 \\
1
\end{array}\right)
$$

For the initial state one replaces $\varkappa, \mu$ by $\varkappa^{\prime}, \mu^{\prime}$. The bound state wave functions are given elsewhere. 11

Since the Dirac matrix vector $\alpha$ is

$$
\alpha=\left(\begin{array}{ll}
0 & \sigma \\
\sigma & 0
\end{array}\right)
$$

where $\sigma$ is the Pauli spin operator, one encounters the following quantities:

$$
\begin{gathered}
(\sigma \cdot L+1) \chi_{x}^{\mu}=-\varkappa \chi_{x}^{\mu} \\
\sigma_{r} \chi_{x}^{\mu}=-\chi_{-\varkappa}^{\mu}
\end{gathered}
$$

We also note that"the spin-angle functions are orthonormal

and

$$
\left(\chi_{\varkappa}^{\mu}, \chi_{x^{\prime}}^{\mu^{\prime}}\right)=\delta_{x x^{\prime}} \delta_{\mu \mu^{\prime}}
$$

$$
\left(\chi_{*}^{\mu}|\Omega| \chi_{\varkappa^{\prime}}^{\mu^{\prime}}\right)=\left(\chi_{-\varkappa}^{\mu}|\Omega| \chi_{-x^{\prime}}^{\mu^{\prime}}\right)
$$

if the operator $\Omega$ commutes with $\sigma_{r}$.

With this information one may evaluate the conversion coefficients in

9 M. E. Rose, Phys. Rev. 57, (1937) 484.

10 E. U. Condon and G. H. Shortley, Theory of Atomic Spectra (Cambridge University Press, Cambridge, 1935). Chapter III.

11 H. A. Bethe, Handbuch der Physik XXIV/1. p. 1366. (Julius Springer, Berlin, 1933). 
terms of radial integrals. For ML transitions, where the conversion coefficient will be denoted by $\beta_{L}$, one finds

$$
\left.\begin{array}{rl}
\beta_{L} & =\frac{\pi k\left(2 j^{\prime}+1\right)\left(2 l^{\prime}+1\right)}{137 L(L+1)(2 L+1)} \sum_{x}(2 j+1)(2 \bar{l}+1)\left(\varkappa+\varkappa^{\prime}\right)^{2} \\
& \times\left(\bar{l} l^{\prime} L 0 \mid \bar{l} l^{\prime} 00\right)^{2} W^{2}\left(j \bar{l} j^{\prime} l^{\prime} ; \frac{1}{2} L\right)\left|R_{1}+R_{2}\right|^{2}
\end{array}\right\}
$$

Here $\bar{l}=-x$ if $\varkappa<0$ and $\bar{l}=\varkappa-1$ if $\varkappa>0$. The sum over final states is restricted by the conservation rules that $j, j^{\prime}$ and $L$ must form a triangle, $\left[\left|j-j^{\prime}\right| \leqslant L \leqslant j+j^{\prime}\right]$, as must $\bar{l}, l^{\prime}$ and $L$. In addition, $\bar{l}+l+L=$ even integer or $l+l^{\prime}+L$ is odd which constitutes the parity selection rule for the conversion electrons. Table I lists the possible final states for $K, L_{\mathrm{I}}, L_{\mathrm{II}}$ and $L_{\mathrm{III}}$ as initial states. In addition this table gives the values of the square of the Racah coefficient ${ }^{12}\left(W^{2}\right)$ corresponding to each final state.

\section{TABLE I}

Data for Magnetic Transitions

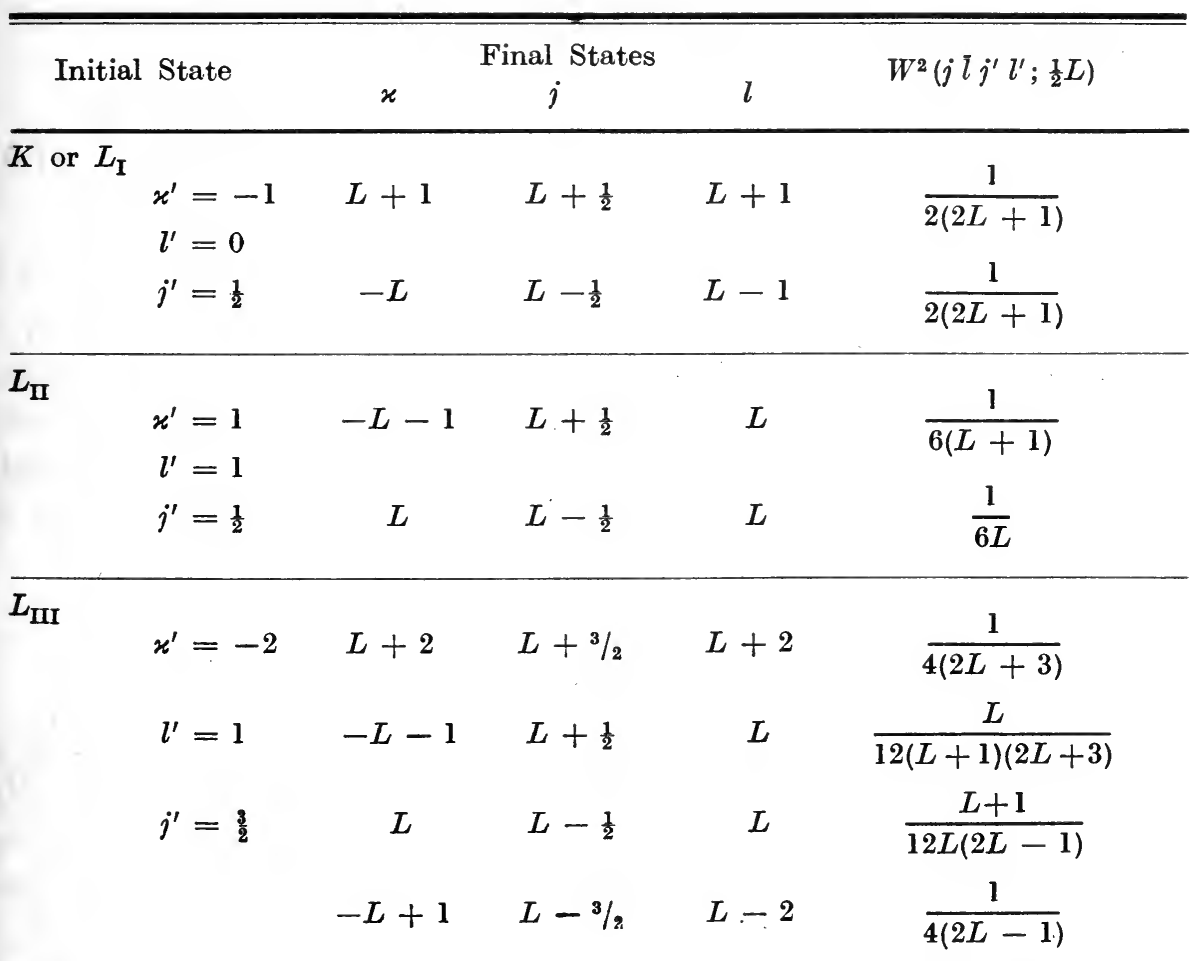

12 G. Racah, Phys. Rev. 62, (1942) 438; also L. C. Biedenharn, J. M. Blatt and M. E. Rose, Revs. Mod. Phys. 25, (1952) 249. 
The square of the vector addition coefficient is

$$
\left.\begin{array}{rlrl}
\left(\bar{l} l^{\prime} L 0 \mid \bar{l} l^{\prime} 00\right)^{2} & =1 & & \text { if } l^{\prime}=0 \\
& =\frac{l+1}{2 l+1} & & \text { if } l^{\prime}=1, L=\bar{l}+1 \\
& =\frac{l}{2 l+1} & & \text { if } l^{\prime}=1, L=\bar{l}-1
\end{array}\right\}
$$

Finally, $R_{1}$ and $R_{2}$ are the radial integrals

$$
\begin{aligned}
& R_{1}=\int_{0}^{\infty} f_{x} h_{L} g_{x^{\prime}} r^{2} d r \\
& R_{2}=\int_{0}^{\infty} g_{x} h_{L} f_{x^{\prime}} r^{2} d r
\end{aligned}
$$

The electric conversion coefficients are calculated in the same way The spin-angular matrix elements are evaluated by aid of the identity

$$
r \boldsymbol{\sigma} \cdot \boldsymbol{\nabla}=\sigma_{r}\left(r \frac{\partial}{\partial r}-\sigma \cdot L\right)
$$

The final result is

$$
\left.\begin{array}{rl}
\alpha_{L}= & \frac{\pi}{137} \frac{k L\left(2 j^{\prime}+1\right)(2 l+1)}{(L+1)(2 L+1)} \sum_{x}(2 l+1)(2 j+1)\left(l l^{\prime} L 0 \mid l l^{\prime} 00\right)^{2} \\
& W^{2}\left(j l j^{\prime} l^{\prime} ; \frac{1}{2} L\right)\left|\frac{\varkappa^{\prime}-\varkappa}{L}\left(R_{5}+R_{6}\right)+R_{6}-R_{5}+R_{3}+R_{4}\right|^{2}
\end{array}\right\}
$$

The selection rules are:

$$
\begin{aligned}
& j, j^{\prime} \text { and } L \text { form a triangle } \\
& l, l^{\prime} \text { and } L \text { form a triangle } \\
& l+l^{\prime}+L=\text { even integer. }
\end{aligned}
$$

The additional radial integrals are

$$
\left.\begin{array}{l}
R_{3}=\int_{0}^{\infty} f_{x} h_{L} f_{x^{\prime}} r^{2} d r \\
R_{4}=\int_{0}^{\infty} g_{x} h_{L} g_{x^{\prime}} r^{2} d r \\
R_{5}=\int_{0}^{\infty} f_{x} h_{L-1} g_{x^{\prime}} r^{2} d r \\
R_{6}=\int_{0}^{\infty} g_{x} h_{L-1} f_{x^{\prime}} r^{2} d r
\end{array}\right\}
$$

The possible final states and corresponding numerical factors are listed in Table II. Eq. (11) again gives the vector addition coefficient. 
TABLE II

Data for Electric Transitions

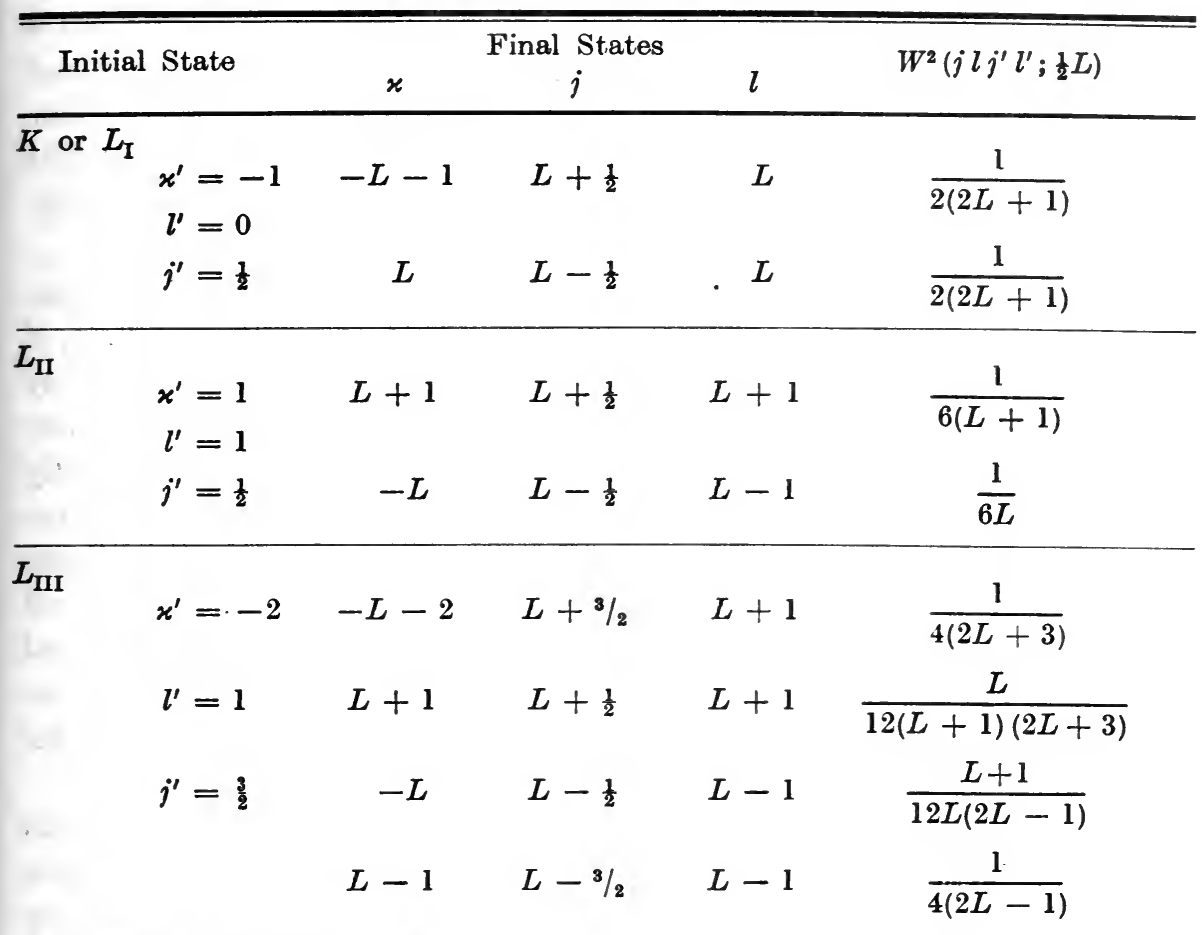

\section{§ 4. The Calculations: II.}

It is clear from the results of the last section that by far the major effort in a program designed to obtain numerical values of conversion coefficients is devoted to evaluation of the radial integrals $R_{1} \ldots R_{6}$. The radial wave functions which are contained in these integrals are solutions of the differential equations

$$
\left.\begin{array}{l}
\frac{d f_{x}}{d r}=\frac{\varkappa-1}{r} f_{x}-(W-1-V) g_{x} \\
\frac{d g_{x}}{d r}=(W+1-V) f_{x}-\frac{\varkappa+1}{r} g_{\varkappa}
\end{array}\right\}
$$

where $W$ is the total energy (in $m c^{2}$ units) and $V$ is the (central) potential. The initial state wave functions fulfill similar equations. Of course, in both initial and final state the usual boundary conditions at the origin and at infinity must be applied.

To take screening into account one may adopt the Thomas-Fermi-Dirac model according to which

$$
V=-\frac{\alpha Z}{r} \Phi(r), \quad \alpha \cong \frac{1}{137}
$$


where $\Phi$ is the well-known screening function ${ }^{13}$. This is used in the initial state. For the final state a small correction is applied corresponding to the fact that the electron in the continuum sees not a neutral atom but a partly ionized one: the degree of ionization at $r$ is given by the amount of charge. due to the ejected electron. contained in a sphere of this radius. This model for the screening should be highly adequate in view of the fact that screening effects influence the conversion coefficients by only a comparatively small amount even for the $L$-shell.

An entirely different kind of consideration is the possibility that conversion occurs in partially filled shells. This would occur, for example, if following $K$-capture, the $K$-shell were not filled by Auger and X-ray transitions before the conversion took place. It is easy to see, however, that the latter type of transitions will always occur much more rapidly than the conversion transitions and such corrections are entirely negligible.

Another source of correction would arise from the fact that the large distance behavior of the wave functions is not precisely represented because the converting atom is not isolated but exists in some chemical environment. This effect has been observed ${ }^{14}$ and, as expected, the influence of chemical environment is only a few tenths of a percent. This then represents the limits of accuracy of the results.

So far as the procedures for numerical evaluation of the wave functions are concerned, mention should be made of the fact that a very accurate eigenvalue for the bound state is needed. This may be determined by integrating (15) from a large value of $r$ inwards toward an intermediate point $r_{i}$; than an integration from $r=0$ to $r=r_{i}$ is made. A smooth join of $f / g$ at $r_{i}$ (where the value of $r_{i}$ is immaterial) constitutes a condition which fixes the eigenvalue. This procedure can be made iterative so that, starting with a rough initial value of the energy, it is rapidly improved. The only distinction between the $K$ - and $L_{\mathrm{I}}$-shells lies in the choice of this initial trial eigenvalue.

\section{$\S 5$. The Internal Conversion Coefficients}

The numerical results for the $K$-shell and for the three subshells of the $L$-shell are presented in Tables A, B and C of Appendix IV. The coefficients given in Table A refer to the $K$-shell and are taken from reference 2 . The effect of screening, which is not included in Table A, can be determined by comparison with the low energy $K$-shell coefficients given in Table $\mathrm{B}$, where entries in these two tables overlap. For interpolated values obtained from the entries of Table $A$ the compilation given in reference 4 should be

13 K. Umeda, Journ. Fac. Sci., Hokkaidô Imperial University ; II-3, (1942) 171.

14 K. T. Bainbridge, M. Goldhaber and E. Wilson, Phys. Rev. 84, (1951) 1260. 
consulted. The screening corrections for either $K$ - or $L$-shell coefficients seldom exceed $15 \%$ and are often appreciably less. *

Fig. 1 shows the $K$-shell coefficients as a function of $k$ for $Z=64$. This was chosen as a fairly typical case to illustrate the dependence on energy

Fig. 1. Electric $\left(\alpha_{L}\right)$ and magnetic $\left(\beta_{L}\right)$ conversion coefficients for the $K$-shell and for $Z=64$. These curves are constructed from the values given in Table A, Appendix IV

Fig. 2. Electric and magnetic conversion coefficients for the $L_{\mathbf{I}}$ subshell and for $Z=55$. For clarity the electric and magnetic curves have been separated by using the separate energy scales given at the bottom and top of the figure
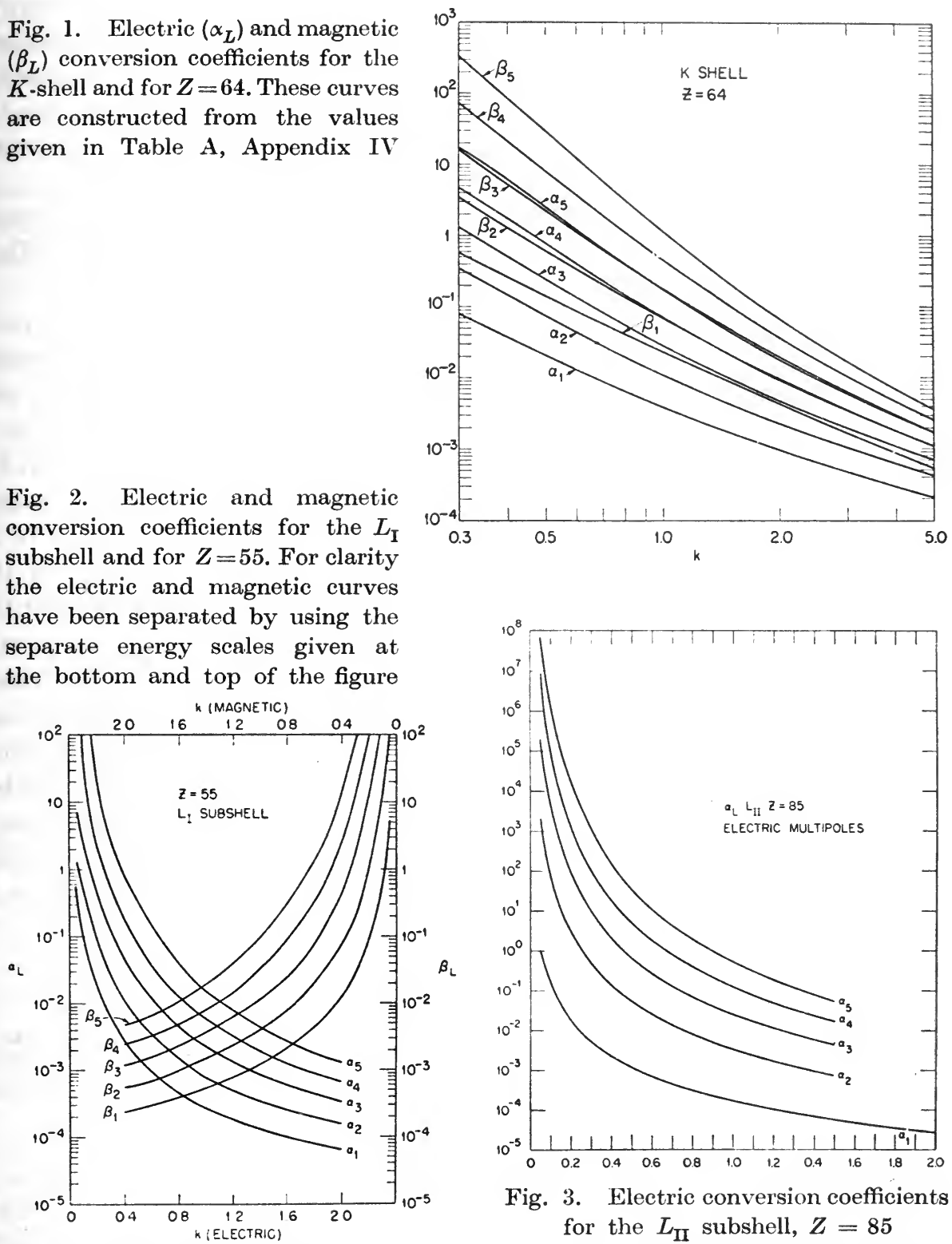

Fig. 3. Electric conversion coefficients for the $L_{\text {II }}$ subshell, $Z=85$

* For the $L$-shell this statement follows from a comparison of the results of Table C with those of Gellman et al., ref. 6 . 
and multipolarity. The rapid rise toward low energies, which is continued up to the threshold, is characteristic of the conversion coefficients for all cases considered; for example, see Fig. 2, 3 and 4 which show the behavior of the co-efficients for the $L_{\mathrm{I}}, L_{\mathrm{II}}$ and $L_{\mathrm{III}}$ subshells.

The very sensitive dependence of the conversion coefficients on energy for low values of $k$, particularly evident for the higher multipoles, implies that some care should be exercised in interpolation between the tabulated values. This interpolation should be carried out with the results given in the tables and it is preferable to change both the conversion coefficients and $k$ values to logarithmic scales.

In all cases one finds the same type of dependence on multipolarity: $\alpha_{L}$ and $\beta_{L}$ increasing fairly rapidly with $L$, especially at low energies. This is also evident from Fig. 1-4.

For interpolation purposes it is of interest to note that while the con-

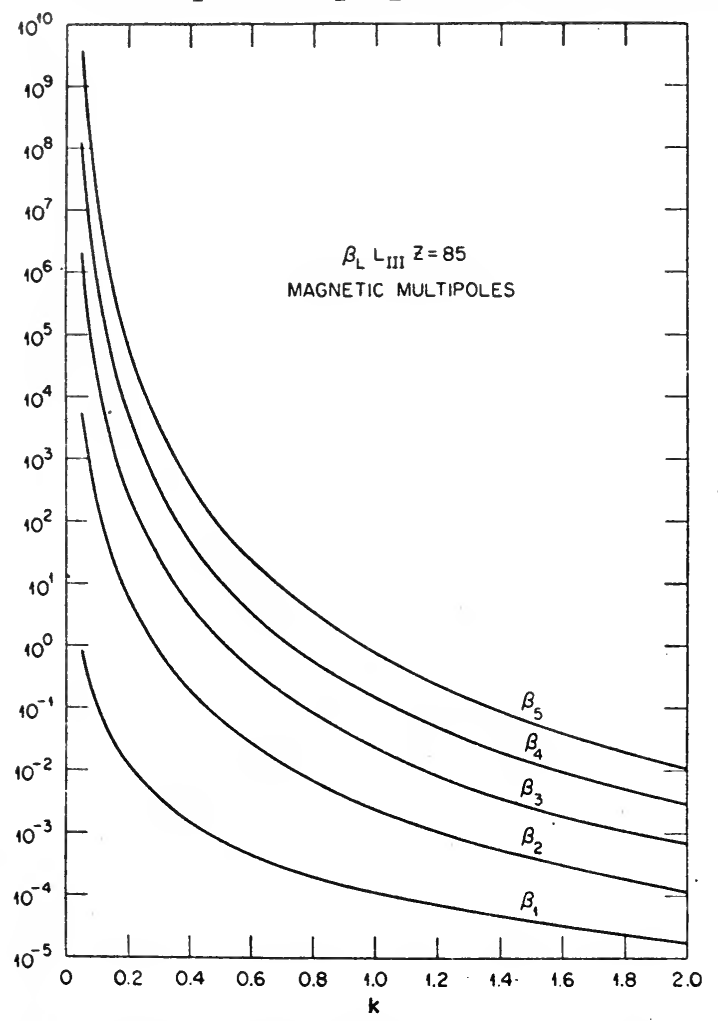

version coefficients generally increase with $Z$ this is not always the case. ${ }^{*}$ This is a question of some concern for the $L$-shell where, at present, only a limited number of $Z$ values are included in the results; for the $K$-shell the number of $Z$-entries is sufficiently large so that interpolation should be very accurate. ${ }^{4}$ In this connection it is to be remembered that for the $K$-shell the magnetic coefficients increase more rapidly with $Z$ than do

Fig. 4. Magnetic conversion coefficients for the $L_{\mathrm{III}}$ subshell,

$$
Z=85
$$

* There is a weak maximum for the high order multipoles at low energies in the $K$-shell coefficients. The same deviations from a monotonic increase with $\mathrm{Z}$ appears in the $L_{1}$-shell for the electric multipoles $(L>1)$. This result shows that, at least for electric multipoles, the conversion does not occur in the immediate vicinity of the nucleus. 
the electric coefficients. Thus, while for low $Z, \alpha_{L} \sim \beta_{L}$, as $Z$ increases the group of $\beta_{L}$ curves separate from the $\alpha_{L}$ curves until for the larger $Z$ values $\alpha_{5} \sim \beta_{1}$. In the case of the $L_{\mathrm{I}}$ and $L_{\mathrm{II}}$ subshells there is an effect of this kind but it is not so marked.

Of perhaps greater interest than the absolute conversion coefficients are the $K / L$ ratios. These are illustrated by the $Z=85$ case in Fig. 5. * It will be seen that there is a considerable degree of coincidence between curves belonging to different multipoles. In the event that a knowledge of the parity change and some upper or lower limits on the angular momentum change are known from independent data, this overlap of the $K / L$ curves should not result in ambiguity. However, it is more often desirable that the internal conversion data furnish this information in a

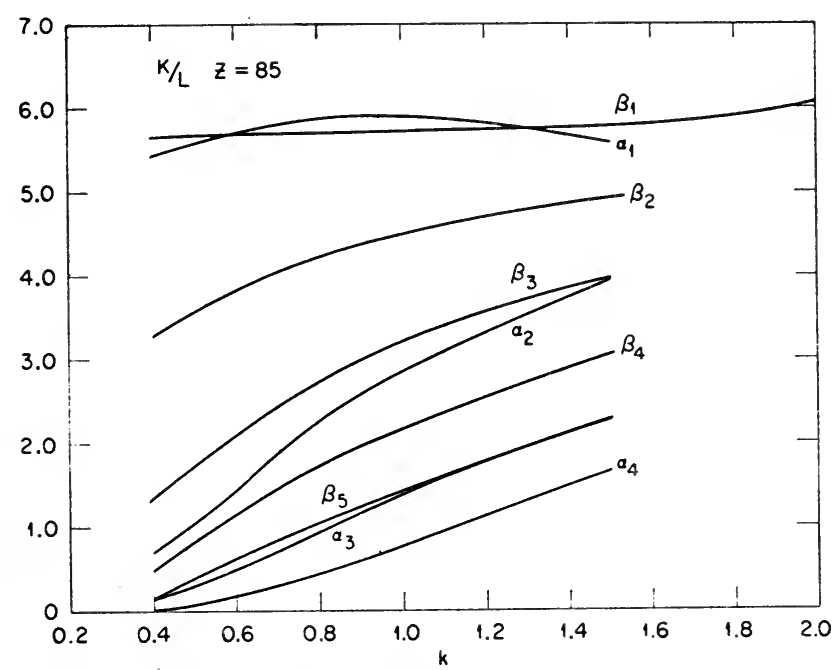

Fig. 5. $K / L$ ratio for electric and magnetic conversion, $Z=85$. Note the overlap between some of the electric and magnetic multipole curves

fairly unambiguous manner. Inasmuch as the $K / L$ ratios shown in Fig. 5 would apply without very major changes for a range of $Z$ values of about 10 units around $Z=85$ one concludes that for heavy elements a measurement of the $K / L$ ratio may not suffice without corroborative evidence. ${ }^{* *}$

This statement together with an examination of the ratios of conversion

* While it has been customary to plot $K / L$ versus $Z^{2} / E$, where $E=511 k$ is the transition energy in keV, this has not been done in Figs. 5 and 6 and a plot in terms of $k$ is used. Unlike the results of the approximate calculations, the $K / L$ ratios are not very well represented in terms of a universal curve with $Z^{2} / E$ as independent variable.

** One also notes that the $K / L$ ratio cannot be used to determine the E2 + M3 mixture ratio near the crossing point of the $\alpha_{2}$ and $\beta_{3}$ curves. 
coefficients for the $L$-subshells leads to the conclusion that $K / L$ ratios should be supplemented by, if not replaced with, measurement of either the $L_{\mathrm{I}} / L_{\mathrm{II}}$ or $L_{\mathrm{III}} / L_{\mathrm{I}}$ ratio. It is to be understood that this includes a measurement of equivalent ratios such as $L_{\mathrm{II}} / L_{\mathrm{III}}$ or $L_{\mathrm{III}} /\left(L_{\mathrm{I}}+L_{\mathrm{II}}\right)$ ete. Since this implies a need for greater resolution than is generally employed, it is fortunate that for lighter elements, where the improved resolution may be difficult to attain, the value of the $K / L$ ratio is more decisive. This is seen in Fig. 6 where the $K / L$ ratios for $Z=55$ are given.

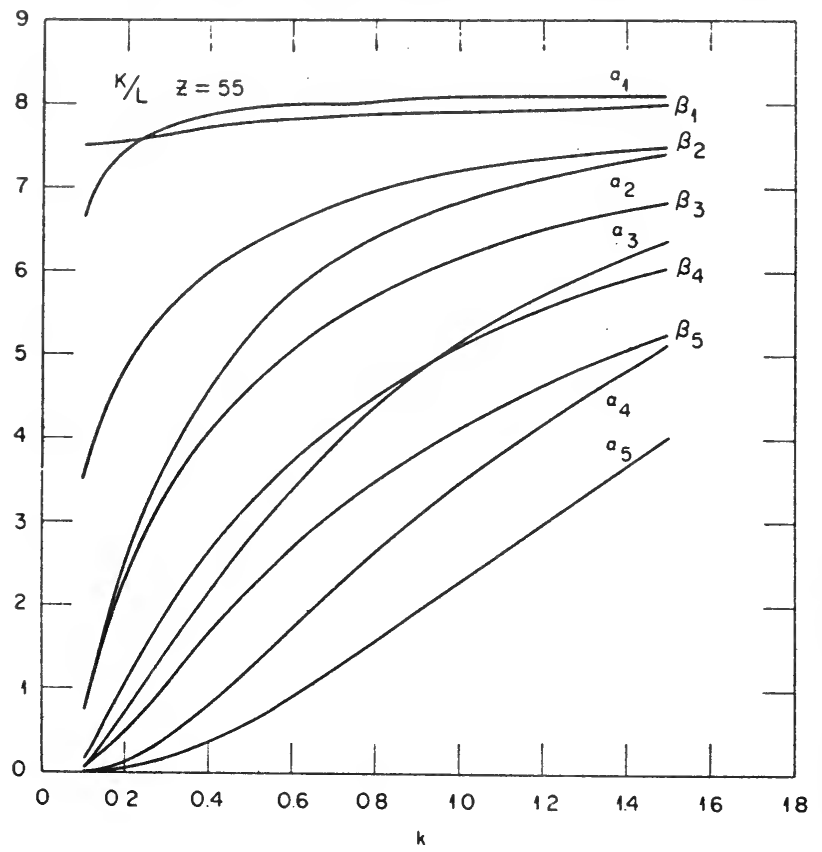

Fig. 6. $K / L$ ratio for electric and magnetic conversion, $Z=55$. Compared to $Z=85$, see Fig. 5 , there would be less ambiguity in assignment of multipolarity for this $Z$

In Fig. 7 the $L_{\mathrm{I}} / L_{\mathrm{II}}$ ratio for magnetic multipoles, $Z=85$, is shown. Here, for reasons explained below, we have used both $Z^{2} / E$ and $E$ as independent variables, although since $Z$ is constant and a logarithmic scale is used the distinction between the two abscissa scales is one of convenience. The results of Fig. 7 show that $\beta\left(L_{\mathrm{I}}\right)>\beta\left(L_{\mathrm{II}}\right)$ in all cases and especially so for dipole radiation. In fact it is characteristic of Ml radiation that

$$
\beta_{1}\left(L_{\mathrm{I}}\right) \gg \beta_{1}\left(L_{\mathrm{II}}\right) \gg \beta_{1}\left(L_{\mathrm{II}}\right) .
$$

One expects $L_{\mathrm{III}}$ conversion to be unobservable and the $L_{\mathrm{II}}$ line to be about 0.1 compared to the $L_{\mathrm{I}}$ line almost independently of $k$. As an example of the wide range of application of this result Fig. 8, showing the corresponding ratios for $Z=55$, may be consulted. For the higher multipoles, see Fig. 7 and 8 , the dominance of $L_{\mathrm{I}}$ conversion is not so great 


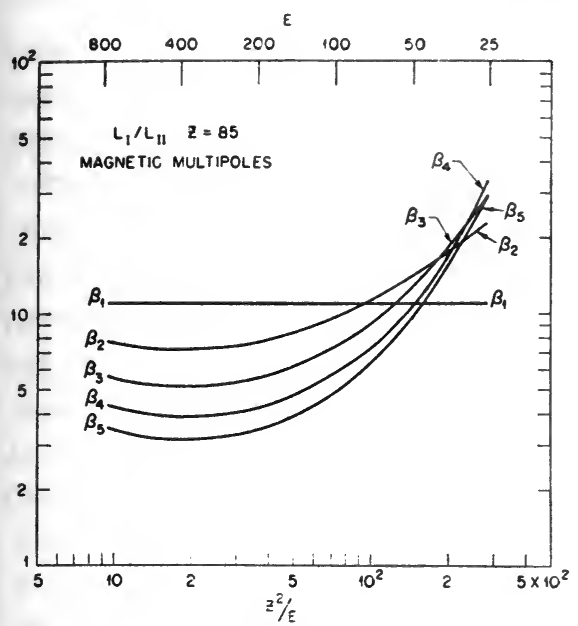

Fig. 7

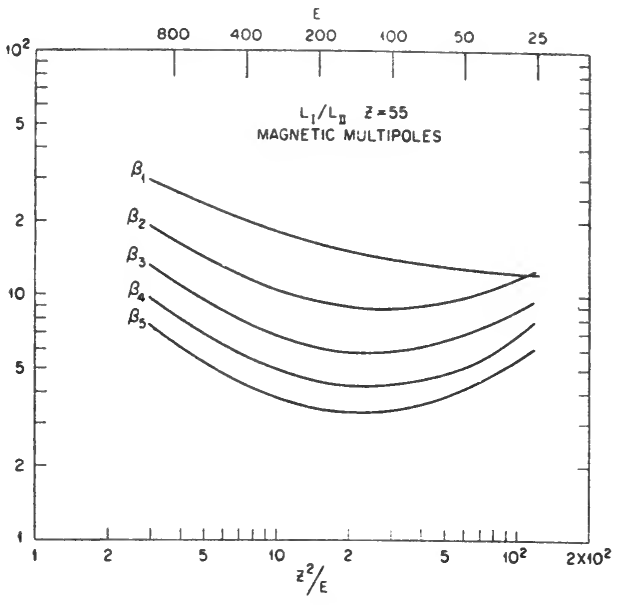

Fig. 8

Fig. 7. $L_{\mathrm{I}} / L_{\mathrm{II}}$ ratio for magnetic multipoles, $Z=85$. The lower abscissa scale is logarithmic in $Z^{2} / E$, where $E=511 k$ in the transition energy in $\mathrm{keV}$. In this case this is essentially a logarithmic scale in $E$ but for convenience values of $E$ can be read directly from the upper abscissa scale

Fig. 8. $L_{\mathrm{I}} / L_{\mathrm{II}}$ ratio for magnetic multipoles, $Z=55$. The abscissa scales are as in Fig. 7

and it diminishes steadily with increasing $L$. The apparent ambiguity between the various magnetic multipoles for $Z=85$ at low energies is resolved by noting that the $L_{\mathrm{III}} / L_{\mathrm{I}}$ conversion ratio is fairly strongly $L$ dependent in this energy region. This is evident from Fig. 9.

Fig. 9. $L_{\mathrm{III}} / L_{\mathrm{I}}$ ratio for magnetic multipoles, $Z=85$. The lower abscissa scale is linear in $Z^{2} / E$, $\left(E=511^{\circ} k\right)$. The upper scale gives $E$ directly. The curve for $\beta_{1}$ lies below $10^{-2}$

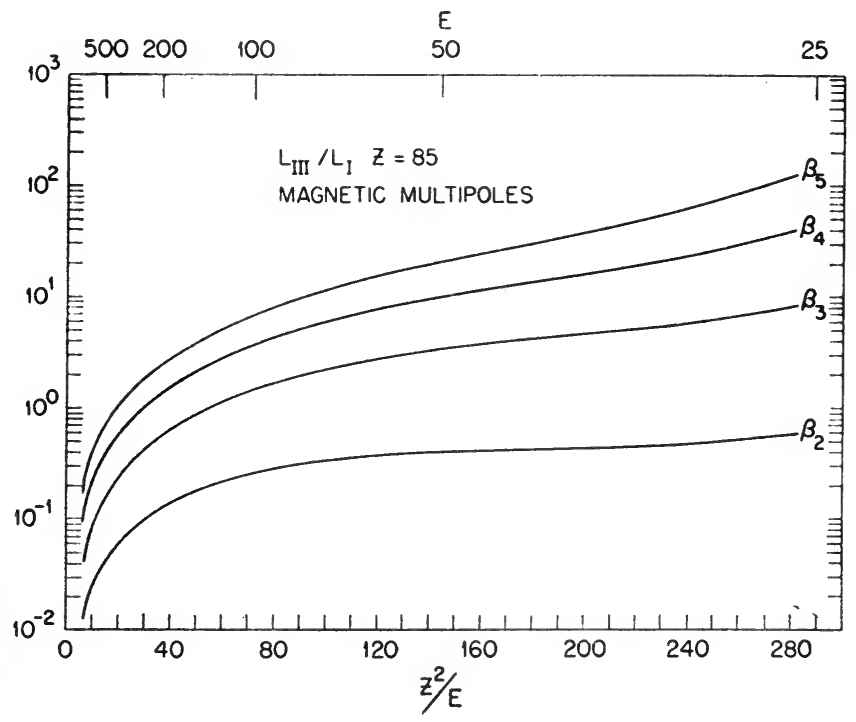


The sensitivity of the $L_{\mathrm{I}} / L_{\mathrm{II}}$ conversion ratios is not so satisfactory for electric multipoles emitted by heavy elements. This is illustrated by the results for $Z=85$ shown in Fig. 10. Although the El ratio is sharply differentiated from those of the other electric radiations, the $L_{\mathrm{I}} / L_{\mathrm{II}}$ ratio would not seem to be very useful for higher multipoles, especially for $E \sim 70 \mathrm{keV}$. In this case a measurement of the $L_{\mathrm{III}} / L_{\mathrm{r}}$ ratio would be helpful. The calculated results are shown in Fig. 11 for $Z=85$. Of course, $L_{\mathrm{III}} / L_{\mathrm{II}}$ and/or $L_{\mathrm{III}} /\left(L_{\mathrm{I}}+L_{\mathrm{II}}\right)$ ratios, which may be more convenient in some cases for experimental purposes, are easily obtained from these curves or from Table C in Appendix IV.

As an example of a simple application of these results one may consider the $L_{\mathrm{I}} / L_{\mathrm{II}}$ ratio for $Z=85$. If this ratio is appreciably less than 3 the transition must be pure electric or at best, only a small magnetic admixture would be permitted. Conversely, for an $L_{\mathrm{I}} / L_{\mathrm{II}}$ ratio greater than 3 pure magnetic radiation is indicated in most cases. The only exception is for dipole transitions above $100 \mathrm{keV}$. This possibility of making a division between electric and magnetic transitions can often be helpful in itself. When combined with further conversion data it should be possible to make unique assignments of multipolarity and parity.

Finally, mention is made of the fact that while the conversion process is rather complex from the point of view of the theory it is possible to summarize the competition between the $L$-subshells in terms of a simple physical parameter. This parameter may be chosen to be the ratio of the binding energy to the transition energy. This ratio will be designated by $R_{E}$. Roughly speaking, this is proportional to $Z^{2} / E$. An alternative parameter is $R_{l}$, the ratio of the (reduced) wave length to the shell radius. For the $L$ shell $R_{E} \sim \frac{1}{4} \alpha Z R_{l}$ and, since we are particularly interested in

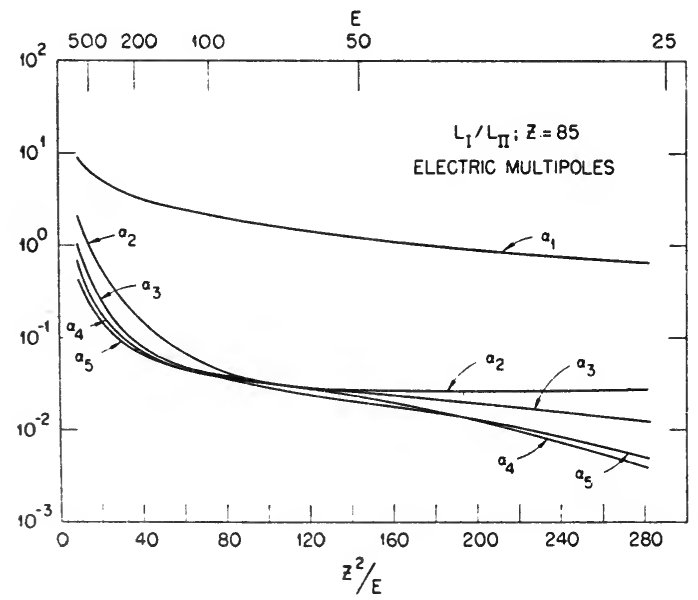

Fig. 10. $L_{\mathrm{I}} / L_{\mathrm{II}}$ ratio for electric multipoles, $Z=85$. The abscissa scales are as in Fig. 9 
CH. XIV]

heavy elements, no further distinction is made between these two parameters. It is for this reason that the parameter $Z^{2} / E$ was introduced in Fig. 7-11.

From an examination of Fig. 7-11, or better the results of Table $\mathrm{C}$ in Appendix IV, one is led to the following conclusions: whenever $R_{E}$ is small the $L_{\mathrm{I}}$ subshell dominates the others in $L$ conversion. On the other hand for $k$ approaching the threshold energy or for light elements, where $R_{E}$ approaches its maximum value of unity, either $L_{\mathrm{II}}$ or $L_{\mathrm{III}}$ conversion is most important. For ML radiation $(L>1)$ it is $L_{\mathrm{III}}$ which dominates at sufficiently low energies so that $\beta_{L}\left(L_{\mathrm{III}}\right)>\beta_{L}\left(L_{\mathrm{I}}\right)>\beta_{L}\left(L_{\mathrm{II}}\right)$. The energy for which the $L_{\mathrm{III}} / L_{\mathrm{I}}$ ratio $=1$ is higher the greater $L$ and/or the lower $Z$.

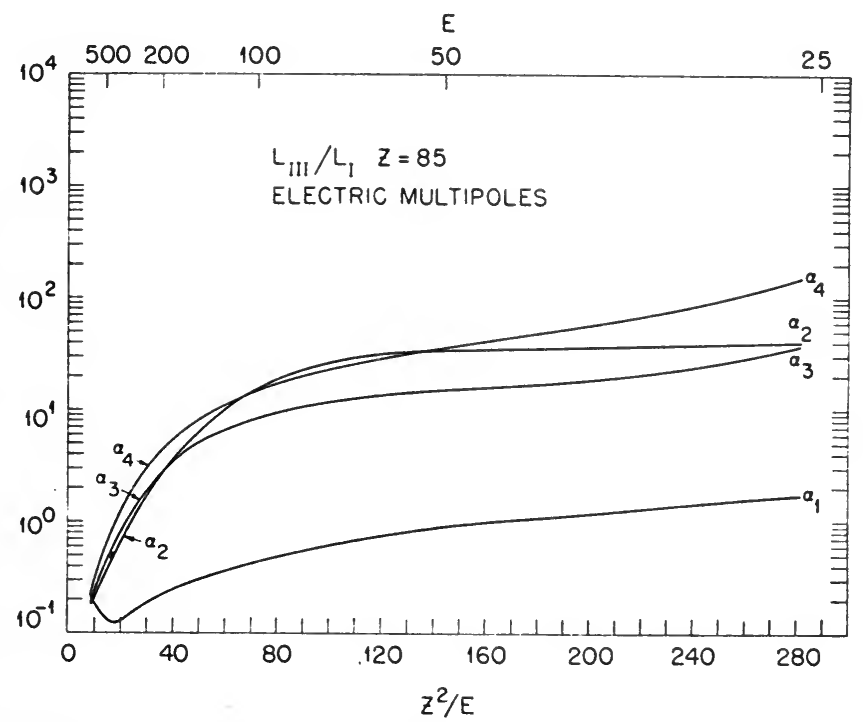

Fig. 11. $L_{\mathrm{III}} / L_{\mathrm{I}}$ ratio for electric multipoles, $Z=85$. The abscissa scales are as in Fig. 9

For EL radiation $L_{\mathrm{II}}$ is somewhat more important than $L_{\mathrm{III}}$ conversion. Specifically, for heavy elements with their large binding (or small $L$-shell radius), $L_{\mathrm{I}}$ conversion is most important for El radiation only above 200 $\mathrm{keV}$. * Below $200 \mathrm{keV}$ all three subshells convert about equally for El transitions. For EL $(L>1) L_{\mathrm{I}}$ conversion is important only for energies at the upper end of the range considered, and is rather unimportant below $200 \mathrm{keV}$. For these higher multipoles neither $L_{\text {II }}$ nor $L_{\text {III }}$ conversion is negligible: in general, $0.15 \lesssim \alpha_{L}\left(L_{\mathrm{III}}\right) / \alpha_{L}\left(L_{\mathrm{II}}\right) \lesssim 1$ with the smaller values appearing at the higher energies.

* The values given apply for $Z=85$ but should not be much different for $Z \gtrsim 75$. 


\title{
THE SHELL MODEL
}

\author{
J. H. D. JENSEN
}

\section{$\S 1$. General Considerations}

The complex problem of nuclear constitution can at present be treated theoretically only by very crude approximations. The reasons for this lie partly in the inherent complexity of all many particle structures, and partly in our very scanty information about the forces between nucleons. Only very little is known beyond some general features: short range, charge independence, and probably a predominant exchange character. By charge independence it is understood that the forces between a proton and a neutron are the same as between two neutrons or between two protons, except for the Coulomb interaction between the latter.How. ever, Pauli's exclusion principle is supposed to hold separately for all protons and for all neutrons, while there are no antisymmetry conditions for neutrons and protons. This is the reason why, in spite of charge independence of nuclear forces, there exist only one, or very few stable isobars for a given mass number, and why, apart from Coulomb effects, nuclei with about as many protons as neutrons are energetically favoured. The exchange character of nuclear forces was originally postulated in order to explain the saturation phenomena (total binding energy and nuclear volume roughly proportional to the number of nucleons). By now the exchange character is to some extent corroborated by the study of collision processes. Whether it is sufficient to explain saturation satisfactorily is still an open question. It is quite probable that essential non-additivity of the nuclear forces is required, which means, that if three nucleons $(1,2,3)$ are close together, their interaction: $V(1,2,3)$ may be quite different from the interactions of the three pairs: $V(12)+V(23)+$ $+V(31)$.

In view of our very poor knowledge of the nuclear forces it was rather surprising that a very simple nuclear model turned out to be quite successful in accounting qualitatively, and, to some extent quantitatively for a great variety of experimental nuclear data. This approximate description of nuclear structure is usually called "nuclear shell model", or more precisely "model of individual orbits", or "Hartree model". It is quite analogous to the successful and well known orbital description of the electronic shells of the atom. Its main feature is the following: We consider one arbitrarily chosen nucleon in the nucleus, and make the assumption that the actions of all other nucleons on the one under consideration can, 
in a good approximation, be averaged to a static potential. Then the internal motion of the nucleus can be described by the superposition of all quasi independent motions of the individual nucleons. Each nucleon orbital can be characterised by individual quantum numbers, which determine an individual nucleon energy level. According to Pauli's principle these levels are filled successively with protons and with neutrons independently. Whenever two successive levels are widely separated, the complete filling of the lower one, either with protons or with neutrons, corresponds to a shell closure, just as is the case with the rare gases in the electronic shells.

For the atom, the validity of such an approximate solution of the many particle problems can be conclusively justified by applying straightforward perturbation methods in higher approximations, because the forces which govern the atomic structure are well known. In nuclear theory such a procedure is badly limited by our ignorance concerning the nuclear forces. At the moment the justification of any approximate model lies in its success in correlating independent experimental data, rather than in theoretical considerations. We will therefore simply discuss the consequences of the shell model, without commenting on the attempts to justify it theoretically ${ }^{1}$. It may only be stressed that the model can at best account for the properties of the groundstates and low exited states. It will certainly break down for highly exited nuclei, where the exclusion principle does no longer put sharp restrictions on the individual particle motions and the coupling between them makes features of collective motion predominant.

\section{§ 2. Magic Numbers, or Shell Numbers}

The empirical evidence for the occurrence of closure of neutron shells and proton shells at the neutron (resp. proton-) numbers 2, 8, 20 (28), 50, $82,126^{2}$ has been substantially increased during the last five years ${ }^{1}$. Here we shall discuss only the evidence obtained from $\beta$-energy systematics ${ }^{3}$.

In order to avoid all complications connected with the "pairing energy"

1 For references see the forthcoming monograph, Mayer and Jensen, "Elementary Theory of Nuclear Shell Structure"; Wiley, New York 1955.

2 This phenomenon was so surprising from the point of view of current ideas about nuclear constitution, that the designation "magic numbers" was adopted by many authors for these numbers. The name "shell numbers" is probably more characteristic. We shall use both names indiscriminately.

3 L. Kowarski, Phys. Rev. 78, (1950) 477; H. E. Suess, Phys. Rev. 81, (1951) 447, and Report Inst. f. Nuclear Studies, Chicago, Feb. 19 (1951); H. E. Suess and J. Hans D. Jensen, Arkiv. f. Fysik (Stockholm), 3 (1951) 577. 
in even-even nuclei (see below, $\S 7$ ), let us consider such $\beta$ - processes in which an odd neutron transforms into an odd proton under $\beta$ - emission or vice versa an odd proton transforms into an odd neutron under $\beta+$ emission or orbital electron capture:

$$
{ }_{Z} \mathrm{X}_{N} \underset{\beta^{+}}{\stackrel{\beta^{-}}{\longrightarrow}}{ }_{Z}^{*} \stackrel{*}{\mathrm{X}}_{N}^{*}
$$

(X and $\mathrm{X}^{*}$ chemical symbol, $Z$ even, $N$ odd $; \stackrel{*}{Z}=Z+1$ odd, $\stackrel{*}{N}=N-1$ even). In any nuclear model which does not imply shell structures, the energy which is liberated (or required) in such a transformation should be a smooth function of $Z$ and $N$. Or, if one keeps the neutron excess $I=N-Z=\stackrel{*}{N}-\stackrel{*}{Z}+2$ fixed, it should vary smoothly with $A$. This is indeed the case for the odd $A$ mirror nuclei $(N=Z+1, \stackrel{*}{N}=\stackrel{*}{Z}-1, I=1$ in our notation) ${ }^{4}$. For greater values of the neutron excess $I$ the energy curves show characteristic breaks just at the shell numbers, see Fig. 1. While the curves are indeed nearly straight lines between the shell numbers, they are dislocated upwards at $N=50$, and 82 , and downwards at the same $Z$ values. The obvious interpretation is that the $51^{\text {st }}$ and $83^{\text {rd }}$ neutron is particularily loosely bound, and the decrease in binding energy appears as increase of $\beta^{-}$-energy when the loosely bound neutron transforms into a normally bound proton. The sudden decrease of the $\beta^{-}$energy, if the resulting proton is the $51^{\text {st }}$ or $83^{\text {rd }}$, finds the same explanation in the particularly low binding energy of these protons. Note that the upward dislocation of the $\beta$-decay curves always appears at $N=50$, whatever the associated proton numbers are ${ }^{5}$, and correspondingly at the other neutron or proton shell edges. This is the strongest argument in favour of the basic assumption of the shell model, that protons and neutrons are filling their shells independently of each other. It is evident that the analogy with atomic shell closure lies in the low ionisation potential of the alkaline atoms rather than in the exceptionally high stability of the rare gas atoms. It is easy to find arguments to explain this difference

4 The well known curve can be found in practically every text book or review article on nuclear binding energy; see for example the quotations in footnote 1. The smoothness of this curve, in spite of the definite existence of shells at 2, 8 and 20 , is caused by the fact that for these nuclei the neutron and proton shells coincide, and the discontinuities which appear at the curves for different neutron excess, cancel each other in the mirror nuclei curve.

5 The exception are the mirror nuclei, where the proton number is simultaneously "magic" (see footnote 4), and also the neutron excess curve $I=N-Z=45$ at the point ${ }_{82} \mathrm{~Pb}_{127}^{209} \stackrel{\beta^{-}}{\longrightarrow}{ }_{83} \mathrm{Bi}_{126}^{209}$ where both the $127^{\text {th }}$ neutron and the $83^{\text {rd }}$ proton are just outside closed shells. 
of behaviour from the different types of forces which govern the atomic and the nuclear structure.

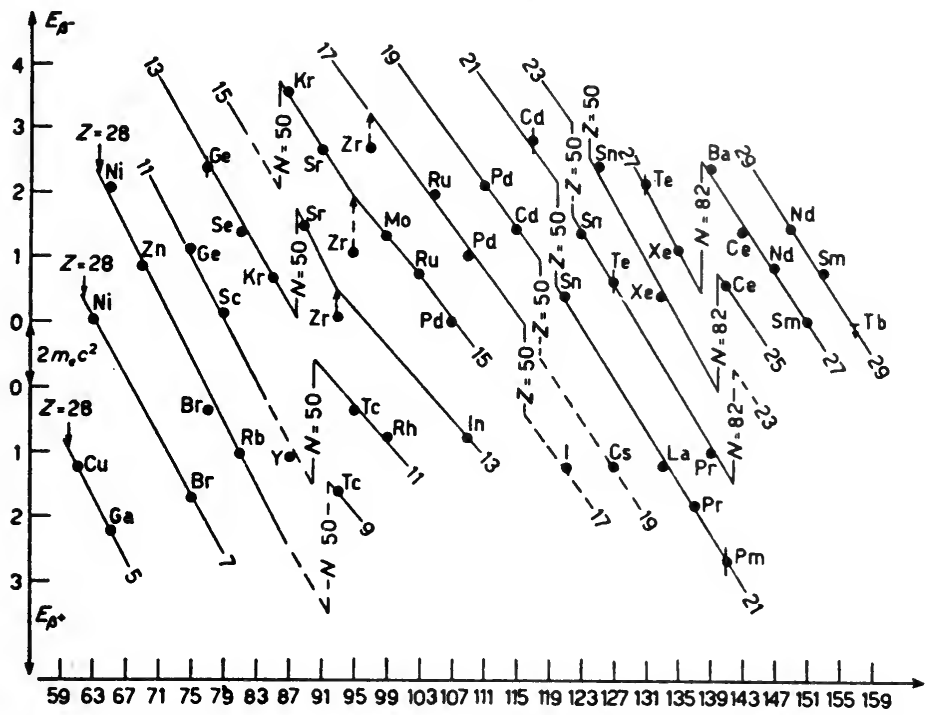

Fig. 1. $\beta$-transformation energy for transition of an odd neutron into an odd proton,

$$
{ }_{Z} \mathrm{X}_{N} \underset{\beta^{+}}{\stackrel{\beta-}{Z}} \stackrel{*}{Z} \mathrm{X}_{N^{*}}^{*} . \quad Z \text { even; } N \text { odd } ; \stackrel{*}{Z}=Z+1 \text { odd, } \stackrel{*}{N}=N-1 \text { even. }
$$

Abscissa: atomic number $A$. Ordinate: total transformation energy $=$ kinetic energy of electron or positron (plus subsequent $\gamma^{\prime} s$ if $\beta$ transition does not lead to the groundstate). The $\beta^{-}$region is separated from the $\beta^{+}$region by a band of 2 $\mathrm{mc}^{2}=$ rest masses of electron and positron. In this region the nuclei would lie which can only transform by electron cupture. No energy values are inserted in this region because neutrino energy is not known. Solid lines connect nuclei with the same neutron excess $I=N-Z=\stackrel{*}{N}-{ }^{*}+2$. The value of $I$ is written at the end of the lines. The chemical symbol is always that of the unstable nucleus ( $Z$ for $\beta^{-}$ emitters, $\stackrel{*}{Z}$ for $\beta^{+}$emitters).

Notice the nearly linear variation with $A$ of the $\beta$-transformation energy, and the sudden dislocations upwards at $N=50$ and 82 , and downwards at $Z=50$. The same behaviour is exhibited at the other magic numbers, (see footnote 3 ). The comparatively small $\beta$ - energies of the zirconium isotopes are as yet not well understood. The author is reluctant to ascribe this to a "magicity" of the proton number $Z=\mathbf{4 0}$.

\section{§ 3. Level Sequence in the Shell Model}

In the Hartree model of the atom the auxilary independent particle potential which is obtained by averaging the action of all other particles on the one under consideration, is well determined through the method of 
finding the self-consistent field. This is possible because the forces between the electrons are well known. In the nuclear case such a prcodure is inhibited not only by the difficulty of solving the equations, but it is also hardly conclusive because one does not know the nuclear forces and their saturation properties in the many nucleon problem sufficiently well. We only can make plausible assumptions about the auxiliary potential $V(\boldsymbol{r})$ from general features of the nuclear forces: No singularity at the center of gravity, $\partial V / \partial r$ must even be zero at $r=0$; a rapid decrease of the potential at the nuclear boundary (which is defined well enough for our purposes); and spherical symmetry ${ }^{6}$. The potential thus being fixed, one calculates the general order of the level sequence, and finally one has to determine the more precise relative position from experimental information. The spherical symmetry of the auxiliary potential implies the conservation of orbital angular momentum for each individual nucleon, which determines the quantum number $l,-$ and the $(2 l+1)$ fold degeneracy of an $l$-level in energetically equivalent substates with magnetic quantum numbers $|m| \leqq l$. Without spin orbit interaction the degeneracy is $2 \cdot(2 l+1)$ fold because of the two orientations of the individual particle spin. For a given $l$, the spherical potential provides a unique sequence of levels with increasing energy, characterised by the number of radial nodes of the eigenfunction. In nuclear physics it has become customary to write this radial quantum number $n_{r}$ in front of the symbols $s, p, d, f, g, h, i$ for the angular momentum ${ }^{7}$; for example, $3 d$ means the third level with angular momentum 2.

The properties of the potential listed in the preceding paragraph are practically sufficient to determine uniquely the ordering of levels (not their spacing ${ }^{1}$ ), as long as one is concerned only with first dozen of lowest levels. Two simply practicable potentials, the isotropic harmonic oscillator and the square well with infinite walls, give the level sequence on the left hand, resp. right hand side of Fig. 2. Numerical calculations for the square well with finite walls give the same level order and a level spacing which is intermediate between the extremes of Fig. 2. Any other monotonic potential which fulfils the conditions listed above, leads to a very similar

- The assumption of spherical symmetry of the auxiliary potential has important consequences. For the nucleus it is certainly not as well justified as in the Hartree approximation for the electronic cloud. In Ch. XVII Bohr and Mottelson discuss the modifications of the shell model which result from abandoning this basic simplifying assumption.

? The habit of writing $\left(n_{r}+l\right)$ in front of the $l$ symbol, - so that $3 d$ would mean the first level with angular momentum 2, - is, even in atomic spectroscopy, only advantageous for hydrogen-like spectra, because of the accidental level degeneracy in the Coulomb field. 
pattern. One has to make very drastic and hardly plausible changes in the spherical potential ${ }^{8}$ in order to alter the level pattern substantially. We may therefore consider the level order in the middle of Fig. 2 which is obtained by interpolation between those extremes, as typical for the level pattern in a spherically symmetric static nuclear potential.

Fig. 2. Schematic representation of level sequence in the spherically symmetric potential of the isotropic harmonic oscillator of frequency $\omega$ (left side), and the potential well with infinite walls at radius $R$ (right side). Energy units are $\hbar \omega$ (oscillator) and $2 \hbar^{2} / M R^{2}$ (well); $M=\mathrm{nu}$ cleon mass. Radial quantum number and symbol for the angular momentum like $2 p$, are written besides the level. Numbers in round brackets, like (6), give the degeneracy of the level; numbers in square brackets, like [20], give the number of protons (or neutrons) which find place in all levels up to the one under consideration (inclusively) = "total occupation number". In the middle of Fig. 2 the level sequence intermediate between these extremes is inserted by schematic interpolation.

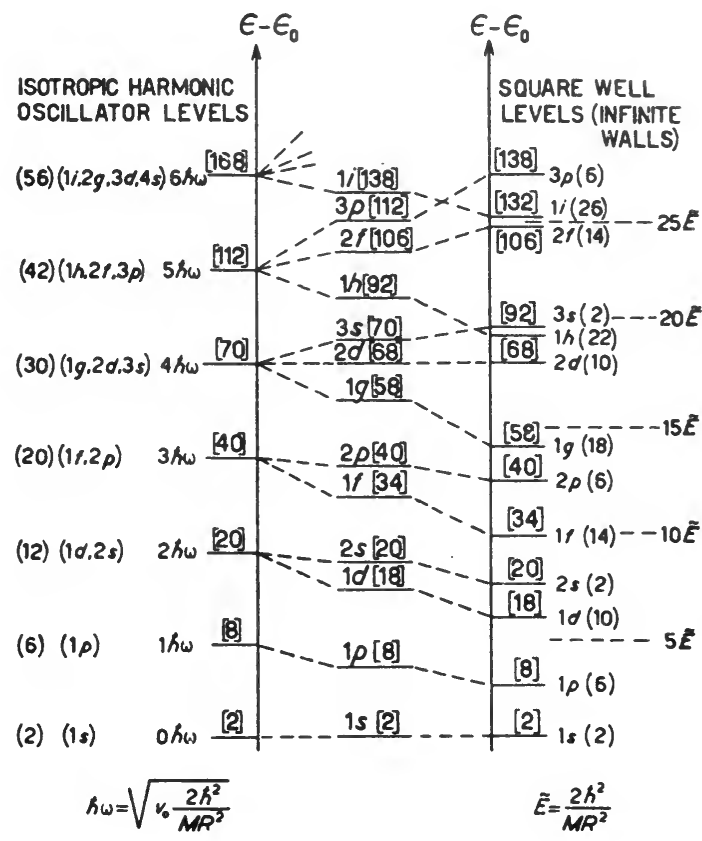

It is seen that at the total occupation numbers 2, resp. 8, resp. 20, the $1 s$ level resp. the $1 p$ level, resp. the $2 s$ level, is widely separated from the next higher one. This gives the long known shell model explanation 9,10 for the magic numbers $2,8,20$. However the pattern fails completely to account for the other magic numbers 50, 82, 126. Only with artificial assumptions about the shape of the potential ${ }^{11}$ and its changes with increasing nuclear size, a level order could be obtained which might account for the higher magic numbers.

8 For example an extreme "wine bottle shape", in which the bottom of the bottle is nearly prolapsing through the neck.

- W. M. Elsasser, J. Phys. Radium, 4, (1933) 549; 5, (1934) 635; E. Wigner, Phys. Rev. 51, (1937) 947.

10 For more complete references see H. A. Bethe and R. F. Bacher, Rev. Mod. Phys. 8, (1936) 82; O. Haxel, J. H. D. Jensen and H. E. Suess, Ergebnisse der Exakt. Naturwiss., XXVI, (1952) 244.

11 W. M. Elsasser, l.c.; E. Feenberg and K. C. Hammack, Phys. Rev. 75,(1949) 320. 1877. 
Then it turned out ${ }^{12}$ that a straightforward change in the level pattern which at once gave a unique explanation of shell closures at all observed magic numbers could be obtained by assuming a spin-orbit coupling term of about fifteen times the magnitude of the electromagnetic spin orbit coupling, in addition to the main static spherical potential which was hitherto considered. Let us first examine the effect of such a term, and afterwards discuss the arguments for its justification. By spin-orbit coupling every $l$ level splits into two levels ${ }^{13}$ with total angular momenta $j=l \pm 1 / 2$. The distance between the two levels is proportional ${ }^{14}$ to $2 l+1$, which means that the splitting increases with $l$. If for neutrons as well as

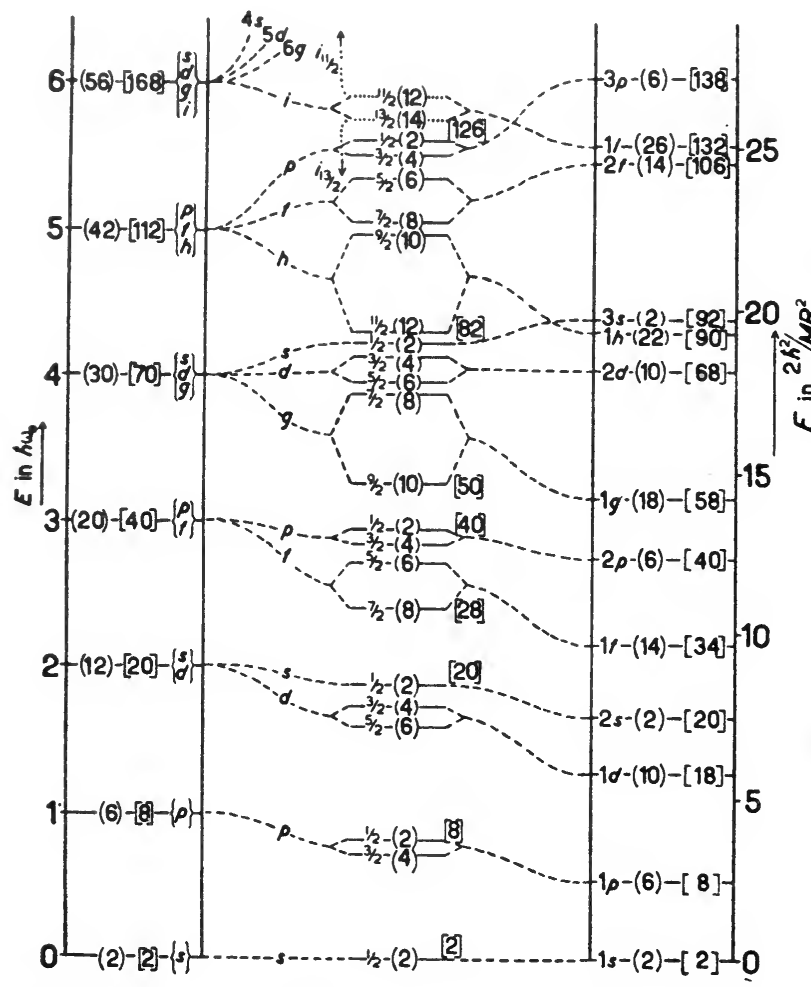

Fig. 3. Same as Fig. 2. In the middle part the level splitting brought about by spin orbit interaction, is indicated schematically. The resulting angular momentum value $j=l \pm 1 / 2$ is written at the level. Round brackets give the degeneracy $2 j+1$. Square brackets as in Fig. 2. Both Fig. 2 and Fig. 3 are to be understood as schematic representations. With increasing $A$ the energy scales are decreasing because in the square well the radius $R$ increases, and in the isotropic oscillator $\hbar \omega$ is decreasing because the potential parabola becomes flatter. For lighter nuclei the oscillator limit, for heavy nuclei the square welllimitlies probably closer to the actual situation

12 M. Goeppert-Mayer, Phys. Rev. 75, (1949) 1969; O. Haxel, J. H. D. Jensen and H. E. Suess, Phys. Rev. 75, (1949) 1766, see also footnote 1.

13 We shall characterise the two levels by writing the value $j$ of the resulting angular momentum after the symbol for the orbital momentum; for example the $3 d$ level is split into $3 d_{5 / 2}$ and $3 d_{0 / 2}$. Each level has the multiplicity $2 j+1$ corresponding to all half integral magnetic quantum numbers $\left|m_{j}\right| \leqq j$. From an $s$ level there originates only the one $s_{z} / 2$ level with multiplicity 2 .

14 This is a consequence of the expression of spin orbit interaction, see below, equation (2); $2 l+1$ is the difference of the eigen values of $I . s$ for the two states with $j=l \pm 1 / 2$. 
for protons the sign of the spin orbit interaction is chosen such that the $j=l+1 / 2$ level is the lower one, a level pattern of the type of Fig. 3 is obtained. For the sake of clarity the splitting is chosen in such a way that no level crossings occur. The typical change of the level spacings become clearly visible: the occurrence of gaps in the level sequence after occupation numbers $2,8,20,(28), 50,82$ and 126 , and a crowding of levels between these occupation numbers. The great level spacings at the wrong places

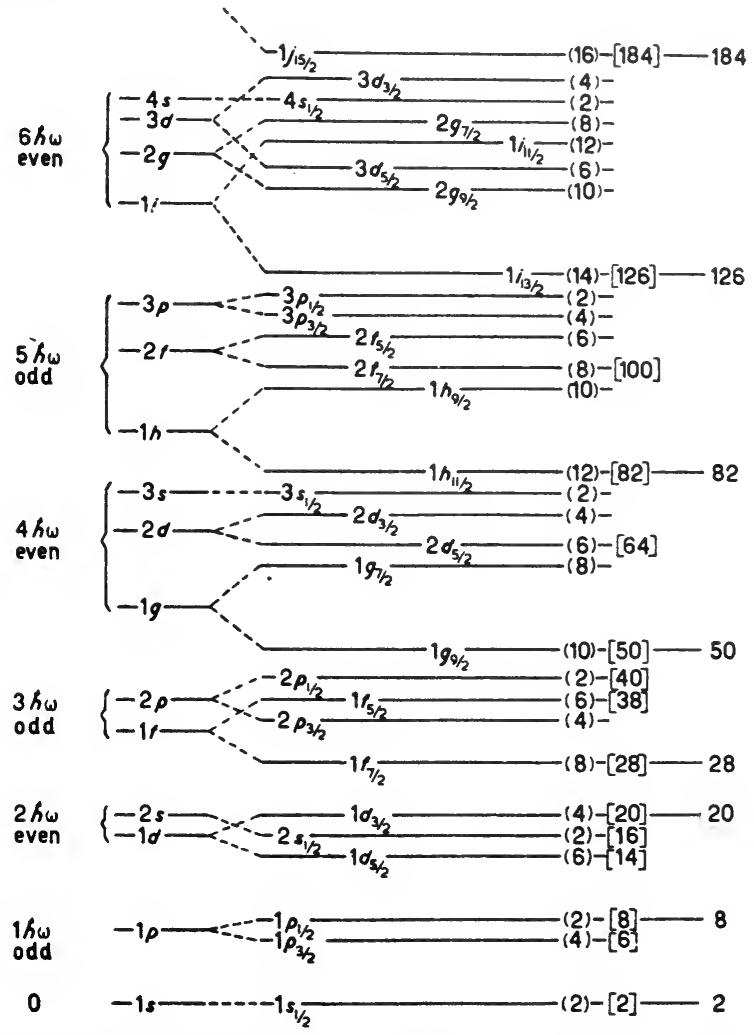

Fig. 4. Approximate level pattern for protons. The schematic spin-orbit splitting of Fig. 3 is adjusted in such a way that the empirical level sequence is represented. For convenience the oscillator level grouping and the parities of these groups are indicated at the left side of Fig. 4. Round brackets (2), (4), etc. and square brackets [2], [6] etc. indicate the level degeneracies and the total occupation numbers, in the same way as in Figs 2 and 3. In the $6 \hbar \omega$ oscillator group the splittings are not drawn in a proper scale, the $3 d$ splitting is too large. A more accurate drawing would have confused the picture too much.

For neutrons the level pattern is practically the samie as for protons, up to $N=50$. From then on typical changes take place. Low neutron angular momenta are less disfavoured than low proton angular momenta. The $2 d_{5 / 2}$ level is below the $1 g / \mathrm{s}$ level, the $3 s_{1 / \mathrm{s}}$ level is below the $2 d_{3 / \mathrm{s}}$ level, the $2 f_{1 / 2}$ level is very close to the $1 \hbar_{0 / 2}$ level, and probably also the $3 p_{\mathrm{s} / \mathrm{s}}$ level is below the $2 f_{\mathrm{s} / \mathrm{a}}$ level. 
in the oscillator model (at 40, 70, 112) or in the square model (at 34, 58, 92) disappear completely. As we will learn from empirical information about nuclei with incompletely filled shells, ( $\$ 6$ and 7 ), the effect of spin orbit coupling is still more drastic. A pattern which describes the empirical data more closely is given in Fig. 4. It is seen that, for example, the splitting of the $1 d$ level is sufficient to bring the $2 s_{1 / 2}$ between the $1 d_{s / 2}$ and $1 d_{s / 2}$ level, the $2 p_{2 / s}$ falls below the $1 f_{s / s}$ level, etc.

But even Fig. 3 has still to be understood as a schematic representation. It is quite reasonable that the relative position of, say, two adjacent proton levels is influenced by the nuclear size and the number of neutrons present in the nucleus. Direct experimental evidence for such a behaviour is obtained for example from the study of level spacing in isomeric states of isotopes, (see Ch. XVI). Another example is the change in the groundstate configuration from $\left(p_{3 / 2}\right)^{-1}$ in ${ }_{37} \mathrm{Rb}_{48}^{85}$, to $\left(f_{\mathrm{s} / \mathrm{2}}\right)^{-1}$ in ${ }_{37} \mathrm{R}^{{ }^{1}}{ }_{50}^{87}$ (see below). Also the configuration $\left(d_{s_{2}}\right)^{-2}\left(s_{1_{2}}\right)^{1}$ in ${ }_{48} \mathrm{Cd}_{63}^{111}$ indicates the lowering of the $s_{1 / 3}$ neutron level with increasing nuclear size.

\section{§ 4. Remarks on Spin-Orbit Coupling}

The spin-orbit coupling, represented by a term

$$
f(r)(\boldsymbol{I} \cdot \mathbf{s})=f(r)([\mathbf{r} \times \mathbf{p}] \cdot \mathbf{s})
$$

(with $r, p, s$, and $I$ individual nucleon coordinate, momentum, spin and orbital angular momentum vectors resp. and $f(r)$ an arbitrary function of the distance from the center of gravity), - was originally just an hypothesis to explain the magic numbers. In recent years it found some experimental confirmation. The phase shift analysis of neutron or proton scattering on highly symmetric nuclei, such as $\mathrm{He}^{4}, \mathrm{C}^{12}$, and $\mathrm{O}^{16}$, showed that resonance scattering of the $p_{3 / 2}$ and $p_{1 / 2}$ components of the incident plane wave occurs at different energies which are separated by several $\mathrm{MeV}$. The same holds for the $d_{s / 2}$ and $d_{3 / 3}$ components. This interpretation of the scattering experiments was conclusively confirmed by the observation of a high degree of polarisation of the beam through repeated resonance scattering of protons on helium ${ }^{15}$. Also the most recent experiments on polarisation of very high energy protons scattered on nuclei seem to indicate ${ }^{16}$ the existence of spin orbit coupling of the same type even at these high energies.

The operator of the square of the orbital angular momentum, $\boldsymbol{r}^{2}$, commutes with the energy term (2). Therefore $l$ remains a good quantum

15 M. Heusinkveld and H. Freier, Phys. Rev., 85, (1952) 80. See also the discussion in footnote 1 .

16 E. Fermi, Nuovo Cimento, 11 (1954) 407, in press. 
number, even when the energy of the levels is decisively determined by the quantum number $j$. This consequence of the spin orbit coupling hypothesis found a surprisingly strong confirmation from the deuteron stripping experiments at low energies ${ }^{1}$.

A spin orbit interaction of the type (2) of sufficient magnitude to explain the magic numbers, and of the same sign both for protons and neutrons, cannot be caused by electromagnetic effects. Also relativistic effects which were considered by Inglis, Breit, and others ${ }^{17}$, are too small in magnitude. The coupling must be brought about directly through the mesonic field of force between the nucleons. At present, meson theories of nuclear forces are not sufficiently developed to predict or to exclude such a term convincingly.

\section{$\S 5$. Parity and Orbital Angular Momentum}

The nuclear Hamiltonian is certainly invariant under the transitjon from a right hand to a left hand coordinate system (or inversion of the coordinate system at the origin). Therefore the wave function of any nuclear state must be either invariant under such a transformation (even parity) or just change its sign (odd parity). Consequently the parity has to be a unique quantum number in any approximation. In the Hartree model the parity of every individual particle state is uniquely determined by the orbital angular momentum, (odd if $l$ is odd, even if $l$ is even). The parity of the whole nucleus is simply the product of all individual parity quantum numbers ${ }^{18}$.

It follows that all levels filled with an even number of particles, in particular all completely filled $j$ levels, have even parity. Because there is only a choice between the two values of parity, one may safely expect the parity prediction of the shell model to be quite reliable, even if the Hartree "Ansatz" were only a poor approximation of the nuclear wave function. This is quite important because the change of parity governs the probabilities of $\beta$-transitions, as well as multipole order and -type of radiative transitions between nuclear states decisively. The experimental determination of spins and parities provides a sensitive test of shell model level assignments, because it is a peculiarity of the shell model level pattern that two levels with the same $j$ but different parities always belong to widely separated shells. This is seen immediately from the schematic Fig. 3 or Table I.

17 See the discussion of this problem in L. Rosenfeld, Nuclear Forces (1948), Ch. XV, 22.

18 Even parity is characterized by the quantum number +1 , and odd parity by the quantum number -1 . 
TABLE I

Spins and Parities in Shells and Oscillator Levels

\begin{tabular}{|c|c|c|c|c|c|c|c|c|}
\hline Shell & I & II & II I & & IV & V & VI & \\
\hline $\begin{array}{c}\text { Occupation } \\
\text { Numbers } \\
j \\
\text { Parity }\end{array}$ & $\begin{array}{l}1-2 \\
1 / 2 \\
+\end{array}$ & $\begin{array}{l}3-8 \\
3 / 21 / 2 \\
--\end{array}$ & $\mid \begin{array}{c}9-20 \\
5 / 23 / 21 / 2 \\
++++\end{array}$ & $\begin{array}{c}21-28 \\
7 / 2 \\
-\end{array}$ & $\mid \begin{array}{c}29-50 \\
5 / 23 / 21 / 29 / 2 \\
---+\end{array}$ & $\left|\begin{array}{c}51-82 \\
7 /{ }^{5} /^{3}{ }^{3} 1 / 2^{11} / 2 \\
+++++-\end{array}\right|$ & $\begin{array}{c}83-126 \\
9 / 2{ }^{7}{ }^{5} 2^{3} / 21 / 2^{13} / 2 \\
-----+\end{array}$ & $\begin{array}{l}127 \rightarrow \\
11 / 2 \%= \\
++\end{array}$ \\
\hline $\begin{array}{l}\text { Oscillator } \\
\text { level } \hbar \omega=\end{array}$ & 0 & 1 & 2 & & 3 & 4 & 5 & 6 \\
\hline
\end{tabular}

Note that every major shell contains nearly all half integral spins up to a highest value, but that no spin value occurs more than once. It reoccurs in the next or second but next shell with opposite parity. It is easy to memorize the spin and parity distribution by observing that the level pattern is essentially governed by the oscillator levels: up to 20, each shell is simply identical with one oscillator level; beyond 20 , the level with highest angular momentum is brought down into the shell from the next oscillator level. The sequence of oscillator levels has alternating parity.

The determination of the parity is the most important consequence of the assignment of a definite orbital angular quantum number $l$ to any individual particle $j$ state. However, the physical significance of the $l$ assignment appears to reach much further. This can be inferred from the deuteron stripping experiments and from the definite separation of the magnetic moment values into two distinct groups. As the discussion of these questions lies beyond the scope of this book, we must refer to the monograph quoted in footnote 1.

\section{§6. Nuclei with Incompletely Filled Shells}

The shell model makes unique predictions about the properties of those nucleonic configurations in which either one proton or one neutron is present outside otherwise closed neutron and proton shells, or in which just one nucleon is missing from a closed shells configuration. In these cases angular momentum, parity, etc., are that of the missing, resp. extra, nucleon. For all such nuclei the shell model predictions are in agreement with the experimental findings. The same holds to a wide extent also in the much greater number of nuclei where not a shell, but only a subshell, that is one of the $j$ levels is completely filled. Here however, level crossings connected with different pairing energies must be taken into account (see below § 7).

For the majority of nuclei there are several protons and (or) neutrons outside closed shells or closed subshells. In these cases the resultant angular momenta and more model-sensitive properties like electric and 
magnetic moments etc., can be predicted from the shell model only with additional "coupling rules". In principle the several nucleons can couple their angular momenta in many different ways to a resultant total angular momentum, even if Pauli's exclusion principle is observed. This gives rise to different eigenfunctions of the whole nucleus from which the expectation values of the other nuclear properties are calculated. In the strict shell model Hartree-Fock approximation all these states with different total angular momentum have the same energy. The selection of one of them as the ground state can be achieved only by taking account of the deviation of the nuclear forces from the Hartree potential in perturbation calculations, in the same way as the problem is treated for the electronic configurations of the atom. But for the nucleus such calculations are again on poor footing, because of our ignorance concerning the exact nuclear forces. Such calculations can therefore at best serve to give confidence in the empirical coupling rules which are stated below, or reversely they can be used as a test for the assumed nuclear forces.

Even if one of the possible states of a given configuration is found to be the groundstate, the rest of them should occur as the lowest excited states if the Hartree model were a very good approximation. The fact, that in many cases the first excited states can be interpreted ${ }^{19}$ as single particle excitations rather than states of different coupling in the groundstate configuration, shows that the splitting of the states of a pure shell model configuration is often larger than the distance between adjacent single particle levels ${ }^{20}$.

This raises the question whether the spin orbit coupling of the individual particles is sufficiently strong to justify the assumption that each particle is still in a definite $l, j$ state when several particles are present outside closed shells, - which is the basis for the $i j$ coupling description of the groundstate of such nuclei. For heavier nuclei, where the $(I \cdot s)$ splitting governs the shell closures exhibited by the magic numbers 50, 82, and 126 , such a description is certainly justified. It is doubtful whether it is as good for the nuclei with proton or neutron numbers below twenty, where the shell structure arises essentially from the classification of the individual particle states by their orbital angular momenta. In this region one might expect that Russels Saunders coupling ${ }^{21}$, (which implies mixing of indi-

19 See especially Ch. XVI on nuclear isomerism.

20 In the discussion of excited nuclear states in terms of the shell model, it should always be borne in mind that both types of excitations can be encountered, and that at higher energies the occurrence of mixtures of such different excited states (with the same parity and angular momentum) is to be expected.

21 First vectioral addition of the orbital angular momenta $\boldsymbol{I}_{i}$ of the particles outside closed shells, to a total orbital momentum $L$, and independently vectorial 
vidual particle $j=l \pm 1 / 2$ states), could be a better starting point. Howerer, the analysis of the experimental data as well as theoretical calculations suggest that even for nuclei below oxygen, pure $j j$ coupling is at least as good a starting point as pure $L S$ coupling, although for such light nuclei the actual situation certainly lies intermediate between these extremes. In order to keep the discussion homogeneous for all nuclei, pure $j j$ coupling will be assumed in the remaining part of this chapter as well as in Ch. XVI on $\beta$-decay systematics.

The following simple coupling rules for the groundstate have been abstracted from the experimental data; they are also in general agreement with calculations performed on the basis of conventional nuclear forces ${ }^{22}$ and trial wave functions.

\section{A. Coupling Rules}

I) Even-even, odd-even, and even-odd nuclei ${ }^{26}$

Extra protons and extra neutrons in different levels:

I, 1) $Z$ and $N$ even: Neutrons and protons independently of each other couple their angular momenta to zero.

This rule is fulfilled in all even-even nuclei; it results also theoretically from all reasonable assumptions about nuclear forces.

I, 2) $Z$ odd, $N$ even: Neutrons couple their spins to zero; in nearly all cases the protons in the incompletely filled $j$ levels couple their angular momenta to a resulting total $J$ which is equal to $j$. There are a few cases in which $J=j-1$.

These latter cases of "anomalous coupling" have been discussed theoretically in great detail from different points of view in perturbation calculations ${ }^{23,24}$. It appears that the general rule, as well as the occurrence of the exceptional $J=j-1$, has a good theoretical foundation. The "normal case", $J=j$ is equivalent to the statement, that only the odd nucleon contributes to the angular momentum of the nucleus, while all

addition of the spins $\boldsymbol{s}_{i}$ to a total $\boldsymbol{S}$, and finally vectorial addition $\boldsymbol{L}+\boldsymbol{S}=\boldsymbol{J}=$ total angular momentum of the nucleus.

22 Strength, rage and exchange character chosen such that the deuteron properties, or nucleon scattering on Helium etc., are obtained consistently. Also tensor forces or spin orbit coupling between pairs of particles have been considered; ses the references in footnote 1 .

23 M. Goeppert-Mayer, Phys. Rev., 78, (1950) 15; Report International Conference Japan 1953; D. Kurath, Phys. Rev., 80, (1950) 98; 88, (1952) 804; 91, (1953) 1430; A. Bohr, and B. R. Mottelson, Kgl. Danske Vidensk. Selskap. Math. Phys. Meddeleser, 26, (1952) 14; 27, (1953) 16. For more references see footnote 1.

24 A careful survey of this problem has been given by B. H. Flowers, Phil. Mag. VII, 25, (1954) 329 . 
paired nucleons are spherically distributed with mutually compensated angular momenta.

I, 3) $Z$ even, $N$ odd: The same rule as I, 2 applies if the words neutrons and protons are systematically interchanged.

II) Even-even, even-odd, and odd-even nuclei

Extra protons and neutrons in the same level:

The coupling rules are effectively the same as in the cases I: Even-even nuclei have zero angular momentum; for even-odd - and for odd-even nuclei $J=j$, or in cases of anomalous coupling $J=j-1$.

In the construction of eigenfunctions, and in the calculations of expectation values and transition matrix elements, however, neutrons and protons cannot be treated independently. As far as Coulomb perturbations can be disregarded ${ }^{25}$, the states must be eigenstates of the total isobaric spin. Therefore the last remark after rule I 2 does not apply. The groundstate has usually the lowest possible isobaric $\operatorname{spin} T=\left|T_{z}\right|=|N-Z| / 2$.

\section{III) Odd-odd nuclei}

All nuclei of this type, except $\mathrm{H}^{2}, \mathrm{Li}^{6}, \mathrm{~B}^{10}$, and $\mathrm{N}^{14}$, are $\beta$-unstable. The experimental determinations of spins and parities of such nuclei are at present essentially based on applications of $\beta$ decay selection rules. The conclusions are not always unique. The "experimental data" for these nuclei are therefore less complete. The majority of cases is covered by Nordheims coupling rules, which are discussed in Ch. XVI on $\beta$-decay systematics.

These simple coupling rules together with the individual particle level pattern of Fig. 4, are sufficient to account for the very large field of experimental findings. This has been considered as the basic justification of the shell model.

\section{§ 7. Pairing Energy and Level Occupation Scheme}

Because the concept of "pairing energy" is very useful for the discussion of nuclei with incompletely filled shells, we must insert a short section on this topic. It is well known from the most elementary discussion of nuclear binding energy, that nuclei which contain protons as well as neutrons in pairs, are energetically favoured compared with even-odd, odd-even, and odd-odd nuclei ${ }^{26}$. We shall start with an empirical definition of the

25 The Coulomb effect is small in such nuclei where the extra neutrons and protons are in the same level.

${ }^{26}$ Odd-even nucleus means odd $Z$, even $N$; even-odd nucleus means even $Z$, odd $N$. 
"pairing energy" in the following way. Consider a $Z$ even, $N$ even nucleus and successively add two protons to the nucleus. The pairing energy of the added proton pair is then defined as the binding energy of both protons minus twice the binding energy of the $(Z+1)^{\text {st }}$ proton. Empirically as far as these binding energies have been determined, the pairing energy thus defined is: (1) Always positive. (2) In its order of magnitude it is independent of the number of neutrons present. (3) In terms of the shell model, the pairing energy is increasing with the angular momentum of the orbit in which the protons are added to the even-even nucleus. (4) When protons and neutrons are in different orbits the proton pairing energy defined in the same way for an even-odd nucleus, is nearly the same as that of the adjacent even-even nuclei. In a crude approximation this statement also holds when protons and neutrons are in the same shell.

For a neutron pair the pairing energy is defined correspondingly. The pairing energy is of the order of magnitude of $1-3 \mathrm{MeV}$ and the difference for different angular momentum exceeds in some cases $1 \mathrm{MeV}$. Therefore it is nearly as important for the discussion of shell effects as the spacing of single particle levels, and for example for the magic number 28 it appears to be predominant.

The theoretical justification for such an empirical definition depends widely on the assumptions about the forces between the nucleons. For attractive forces $\sum_{i \neq k} V_{i k}$ of $\delta$-function type, first order perturbation calculations with antisymmetrized shell model eigenfunctions give ${ }^{27}$ a perturbation energy which has precisely the properties of the pairing energy which we have stated empirically. For forces of finite range the statements would hold approximately ${ }^{1}$ as long as the forces were reasonably short ranged and had saturation character.

The magnitude of the pairing energy determines the successive filling of closely adjacent levels. This will be exemplified by the two levels $2 p_{3 /,}$ and $1 f_{\mathrm{s} / \mathrm{a}}$. The $2 p_{\mathrm{s} / \mathrm{a}}$ level is the lower one of the two because the $29^{\text {th }}$ proton as well as the $29^{\text {th }}$ neutron is bound in a $p_{3 / 3}$ orbit, in ${ }_{29} \mathrm{Cu}_{34}^{63},{ }_{29} \mathrm{Cu}_{36}^{65}$, and in ${ }_{24} \mathrm{Cr}_{29}^{53}$.

The $2 p_{s / s}$ level would be completely filled with the $32^{\text {nd }}$ proton (resp. neutron), and without considering pairing energy effects, one would expect to find the $33^{\text {rd }}$ proton (resp. neutron) in the $1 f_{s}$, state. However both ${ }_{33} \mathrm{As}_{42}^{75}$ and ${ }_{28} \mathrm{Ni}_{33}^{61}$ have the properties of a $p_{2 / 3}$ state. This is simply explained in terms of the pairing energy. Because in the $f_{5 / 3}$ state the pairing energy is larger than in the $p_{3 /,}$ state, energy is gained when a $2 p_{3 / 3}$ pair is broken and one of the nucleons of the pair is raised to the 
$1 f_{\mathrm{s} / \mathrm{s}}$ level, where it forms a pair with the one nucleon already present in that level. The energetically lowest $28+5$ nucleons configuration has therefore one hole in the $2 p_{3 / \mathrm{s}}$ level and one pair in the $1 f_{\mathrm{s} / \mathrm{a}}$ level. For a more detailed discussion of such situations we refer to ${ }^{1}$.

In many cases such level assignments can be tested by the measured sign of the nuclear quadrupole moments. For an odd $Z$ nucleus the quadrupole moment calculated from the properly antisymmetrized wave functions, has always a negative sign if the level is less than half filled, and a positive sign if the level is more than half filled. From the positive quadrupole moment of ${ }_{33} \mathrm{As}_{42}^{75}$ the alternative proton configuration $\left(2 p_{3 / 3}\right)^{1}\left(1 f_{s / 3}\right)^{4}$ is ruled out and the assignment $\left(2 p_{s / 3}\right)^{3}\left(1 f_{s / 3}\right)^{2}$ is unique.

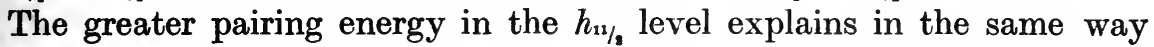
the repeated reoccurrence of the $3 s_{1 / 2}$ level in all odd $N$ nuclei with $N$ between 65 and 75. It also explains, why the $h_{11 / 2}$ level does not occur as the groundstate of any odd $A$ nucleus.

For the influence of the pairing energy on $\beta$-energy systematics see ${ }^{3}$ and ${ }^{1}$.

In Table II we give a survey of the groundstate configurations of the stable odd $A$ nuclei with $Z$ and $N$ up to 82 . For a detailed analysis of the experimental data which confirm the shell model assignments and predictions, we must refer to ${ }^{1}$. Except for very few doubtful cases ${ }^{28}$ the agreement is excellent. Especially the parity predictions have been confirmed in all investigated cases. The existence of the predicted subshells (definite $l, j$ levels) is accentuated by the signs of all measured quadrupole moments.

Up to odd $Z$ or odd $N=40$, the level sequence and spacings are approximately the same for protons and neutrons. Between 40 and 50, the penetrating $2 p_{1 / 2}$ orbit has apparently a slightly lower energy for neutrons than for protons. This can easily be understood from the Coulomb repulsion, and from the larger size of odd $\mathrm{Z}$ nuclei, in comparison to nuclei with the same odd $N$. These effects which disfavour low angular momentum values for protons more than for neutrons, become more pronounced beyond the shell number 50. Here the level ordering is definitely different

28 For ${ }_{9} \mathrm{~F}_{10}^{19}$ one would have expected $a^{5} / 2+$ or a $3 / 2+$ groundstate, even if one takes into account that the $9^{\text {th }}$ proton and the $9^{\text {th }}$ and $10^{\text {th }}$ neutron are all in the same level. The $3 / 2+$ state of ${ }_{65} \mathrm{~Tb}_{94}^{159}$ and especially the $1 / 2+$ state of ${ }_{69} \mathrm{Tm}_{100}^{169}$ require some stressing of the arguments on the relative positions of the levels and pairing energies. If the positive quadrupole moment of ${ }_{77}$ Ir is confirmed, a $\left(d_{8 / 2}\right)^{3}\left(h_{11 / 2}\right)^{-2}$ assignment for the groundstate would require some squeezing of the level pattern. It is quite possible that the great deformability of the nuclei in this mass number region is closely connected with the breakdown of a simple occupation number pattern for these nuclei. 
TABLE II

\section{ODD PARTIClES CONFIGURATIONS}

The exponent gives the number of particles in the level. Negative exponent indicates a hole in the otherwise filled level. Completely filled levels are not listed. In cases of anomalous coupling the resulting angular momentum is given as a subscript. Radial quantum numbers are omitted, they can be taken from Fig. 4

\begin{tabular}{|c|c|c|c|c|c|c|}
\hline $\begin{array}{c}\text { Odd } Z \\
\text { Nucleus }\end{array}$ & $\begin{array}{l}\text { Spin } \\
\text { and } \\
\text { Parity }\end{array}$ & $\begin{array}{c}\text { Proton } \\
\text { Configuration }\end{array}$ & Remiarks & $\begin{array}{l}\text { Odd } N \\
\text { Nucleus }\end{array}$ & $\begin{array}{c}\text { Spin } \\
\text { and } \\
\text { Parity }\end{array}$ & $\begin{array}{c}\text { Neutron } \\
\text { Configuration }\end{array}$ \\
\hline${ }_{3} \mathrm{Li}_{2.4}^{5.7}$ & $3 / 2-$ & $\left(p^{3} / 2\right)^{1}$ & $\leftarrow\left\{\begin{array}{c}\mathrm{Li}^{5} \text { and } \mathrm{He}^{5} \text { from } \\
\text { scattering data }\end{array}\right\} \rightarrow$ & ${ }_{2} \mathrm{He}_{3}^{5}$ & $3 / 2-$ & $\left(p^{3} / 2\right)^{1}$ \\
\hline${ }_{5} \mathrm{~B}_{6}^{11}$ & $3 / 2-$ & $\left(p^{3} / 2\right)^{3}$ & & ${ }_{4} \mathrm{Be}_{5}^{9}$ & $3 / 2-$ & $\left(p^{3} / 2\right)^{3}$ \\
\hline${ }_{7} \mathrm{~N}_{8}^{15}$ & $1 / 2-$ & $\left(p^{1} / 2\right)^{1}$ & & ${ }_{6} \mathrm{C}_{7}^{13}$ & $1 / 2-$ & $\left(p^{1} / 2\right)^{1}$ \\
\hline${ }_{9} \mathrm{~F}_{8}^{17}$ & $5 / 2+$ & $\left(d^{5} / 2\right)^{1}$ & & ${ }_{8} \mathrm{O}_{9}^{17}$ & $5 / 1+$ & $\left(d^{5} / 2\right)^{1}$ \\
\hline${ }_{9} \mathrm{~F}_{10}^{19}$ & $1 / 2+$ & $\left(s^{1} / 2\right)^{1} ?$ & $\leftarrow$ \{configuration not clear $]$ & & & \\
\hline${ }_{11} \mathrm{Na}_{12}^{23}$ & $3 / 2+$ & $\left(d^{5} / 2\right)_{3 / 2}^{3}$ & $\leftarrow$ anomalous coupling $\} \rightarrow$ & ${ }_{10} \mathrm{Ne}_{11}^{21}$ & $3 / 2+(?$ & )$\left(d^{5} / 2\right)_{2 / 2}^{3}$ \\
\hline${ }_{13} \mathrm{Al}_{14}^{27}$ & $5 / 2+$ & $\left(d^{5} / 2\right)^{5}$ & & ${ }_{12} \mathrm{Mg}_{13}^{25}$ & $5 / 2+$ & $\left(d^{5} / 2\right)^{5}$ \\
\hline${ }_{15} \mathrm{P}_{16}^{81}$ & $1 / 2+$ & $\left(s^{1} / 2\right)^{1}$ & & ${ }_{14} \mathrm{Si}_{15}^{29}$ & $1 / 2+$ & $\left(s^{1} / 2\right)^{1}$ \\
\hline${ }_{17} \mathrm{Cl}_{18,20}^{35.37}$ & $3 / 2+$ & $\left(d^{3} / 2\right)^{1}$ & & ${ }_{16} \mathrm{~S}_{17}^{33}$ & $3 / 2+$ & $\left(d^{3} / 2\right)^{1}$ \\
\hline${ }_{19} \mathrm{~K}_{20.22}^{38.41}$ & $3 / 2+$ & $\left(d^{3} / 2\right)^{3}$ & & ${ }_{16} S_{19}^{35}$ & $3 / 2+$ & $\left(d^{3} / 2\right)^{3}$ \\
\hline${ }_{21} \mathrm{Sic}_{24}^{45}$ & $7 / 2-$ & $\left(f^{7} / 2\right)^{1}$ & & & & \\
\hline${ }_{23} Y_{28}^{51}$ & $7 / 2-$ & $\left(f^{7} / 2\right)^{3}$ & & & & \\
\hline${ }_{25} \mathrm{Mn}_{30}^{55}$ & $5 / 2-$ & $\left(f^{7} / 2\right)_{6 / 2}^{5}$ & $\leftarrow$ \{anomalous coupling] & ${ }_{22} \mathrm{Ti}_{25}^{47}$ & $7 / 2-$ & $\left(j^{7} / 2\right)^{5}$ \\
\hline${ }_{27} \mathrm{Co}_{32}^{59}$ & $7 / 2-$ & $\left(f^{7} / 2\right)^{7}$ & & ${ }_{22} \mathrm{Ti}_{27}^{49}$ & $7 / 2-$ & $\left(f^{7} / 2\right)^{7}$ \\
\hline${ }_{29} \mathrm{C} u_{34,36}^{63.65}$ & $3 / 2-$ & $\left(p^{3} / 2\right)^{1}$ & & ${ }_{24} \mathrm{Cr}_{29}^{53}$ & $3 / 2-$ & $\left(p^{3} / 2\right)^{1}$ \\
\hline${ }_{31} \mathrm{Ga}_{38.40}^{69.71}$ & $3 / 2-$ & $\left(p^{3} / 2\right)^{3}$ & - & ${ }_{26} \mathrm{Fe}_{31}^{57}$ & $3 / 2-$ & $\left(p^{3} / 2\right)^{3}$ \\
\hline${ }_{33} \mathrm{As}_{42}^{75}$ & $3 / 2--$ & $\left(p^{3} / 2\right)^{-1}\left(f^{5} / 2\right)^{2}$ & $\leftarrow\left\{\begin{array}{c}\text { possibly configur. } \\
\text { not pure }\end{array}\right\} \rightarrow$ & ${ }_{28} \mathrm{Ni}_{33}^{61}$ & $3 / 2-$ & $\left(p^{3} / 2\right)^{-1}\left(f^{5} / 2\right)^{2}$ \\
\hline${ }_{35} \mathrm{Br}_{44.46}^{79.81}$ & $3 / 2-$ & $\left(p^{3} / 2\right)^{-1}\left(f^{5} / 2\right)^{4}$ & & & & \\
\hline${ }_{37} \mathrm{Rb}_{48}^{85}$ & $3 / 2-$ & $\left(p^{3} / 2\right)^{-1}\left(f^{5} / 2\right)^{6}$ & & & & \\
\hline${ }_{37} \mathrm{Rk}_{50}^{87}$ & $5 / 2-$ & $\left(f^{5} / 2\right)^{-1}$ & & ${ }_{30} \mathrm{Zn}_{37}^{67}$ & $5 / 2-$ & $\left(f^{5} / 2\right)^{-1}$ \\
\hline${ }_{39} \mathrm{Y}_{50}^{89}$ & $1 / 2-$ & $\left(p^{1} / 2\right)^{1}$ & 2 and 1 & ${ }_{30} \mathrm{Zn}_{39}^{69}$ & $1 / 2-$ & $\left(p^{1 / 2}\right)^{1}$ \\
\hline${ }_{41} \mathrm{Nb}_{52}^{93}$ & $9 / 2+$ & $\left(p^{1} / 2\right)^{2}\left(g^{9} / 2\right)^{1}$ & & ${ }_{32} \mathrm{Ge}_{41}^{73}$ & $9 / 2+$ & $\left(p^{1} / 2\right)^{2}\left(g^{9} / 2\right)^{1}$ \\
\hline${ }_{43} \mathrm{Tc}_{56}^{99}$ & $9 / 2+$ & $\begin{array}{l}\left(p^{1} / 2\right)^{2}\left(g^{9} / 2\right)^{3} \text { or } \\
\left(p^{1} / 2\right)^{0}\left(g^{9} / 2\right)^{5}\end{array}$ & & ${ }_{34} \mathrm{Se}_{43}^{77}$ & $1 / 2-$ & $\left(p^{1} / 2\right)^{1}\left(g^{9} / 2\right)^{4}$ \\
\hline
\end{tabular}


TABLE II (continued)

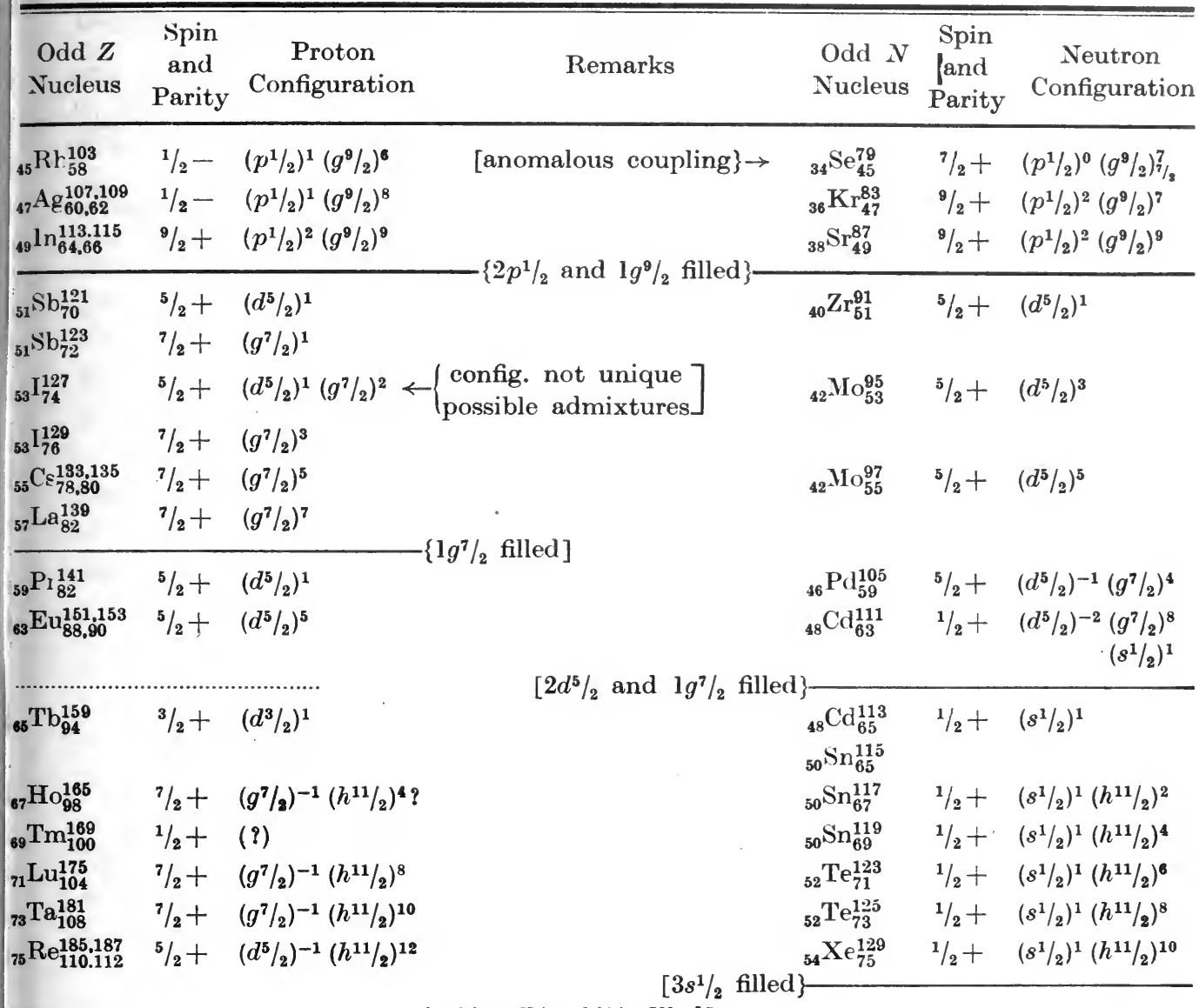

$\left\{1 g^{7} / 2 \quad 2 d^{5} / 2 \quad 1 h^{11} / 2\right.$ filled]

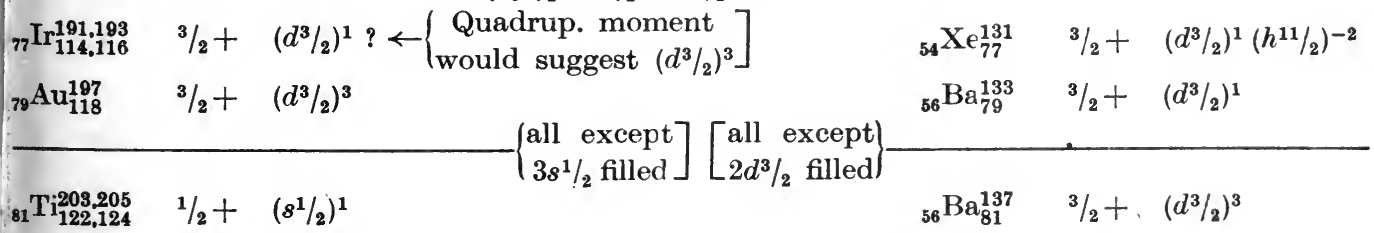


for neutrons and protons ${ }^{29}$ (see the remarks below Fig. 4).

For odd $N$ between 82 and 126, and for nuclei beyond lead, the experimental information is still too meager to allow for systematic detailed configuration assignments. In the experimentally well investigated cases a configuration assignment can generally be given ${ }^{1}$, which reasonably fits into the shell model level pattern ${ }^{30}$. The statements below Fig. 4, concerning the odd neutron level order are experimentally well confirmed.

29 The fact that $s_{1 / 2}$ occurs so frequently as groundstate for odd $N$ nuclei, while for the corresponding odd $Z$ nuclei the groundstate is $\left(g_{7 / 2}\right)^{-1}$ or $\left(d_{t / 2}\right)^{-1}$, gives a trivial reason why isomerism is encountered only for odd $N$, and not for odd $Z$. nuclei for this "island" (see Ch. XVI).

30 Only the spin $1 / 2$ of ${ }_{70} \mathrm{Yb}_{101}^{171}$ and spin $5 / 2$, negative magnetic moment, positive $Q$-moment for ${ }_{70} \mathrm{Y}_{103}^{173}$ are in serious disagreement with all possible shell model assignments, see ${ }^{1}$. 


\section{NUCLEAR SHELL SYSTEMATICS}

M. GOEPPERT-MAYER, M. GOLDHABER AND A. W. SUNYAR

\section{(I) M. GOEPPERT-MAYER - Classifications of $\beta$-Transitions}

\section{§ 1. Introduction}

The theory of $\beta$-decay has been discussed in greater detail in Chapters IX and $\mathrm{X}$. We shall here summarize the results which are pertinent for the ensuing discussion.

The lifetimes of nuclei which are unstable with respect to $\beta$-decay depend sensitively on the endpoint energy. This dependence of the transition probability is given by a known function $f(E)$, of the endpoint energy and the nuclear charge. Thus the product, $f t$, of this function times the halflife $t$, is a measure of the specific nuclear behaviour in the process. The quantity $f t$ is proportional to the inverse sqare of the nuclear matrix element.

The transition probabilities, or, more correctly, the values of the nuclear matrix elements, are governed by selection rules. They are listed in this chapter again, without making a distinction between the type of fundamental interaction from which they arise.

$\begin{array}{lcc} & \text { Change of parity } & \text { Spin change } \\ \text { allowed } & \text { no } & 0, \pm 1 \\ \text { first forbidden } & \text { yes } & 0, \pm 1, \pm 2 \\ \text { second forbidden } & \text { no } & \pm 2, \pm 3\end{array}$

One expects an increase of a factor "of at least one hundred in going from one degree of forbiddenness to the next. Since the $f t$ values are large, and since we are interested in their order of magnitude, we find in $\log f t$ a measure of the degree of forbiddenness.

\section{§ 2. Nuclei of Odd $A$}

In the process of $\beta$-decay, the spins of the parent nucleus are, as a rule, not measured. Those of the daughter nuclei are also often unknown. The shell model claims to be able to predict spins and parity, at least for nuclei of odd $A$, with some confidence. Thus it is of interest to compare shell model predictions with the experimental $f t$ values. Such comparison has been published previously, but is here brought up to date ${ }^{1}$.

1 M. Goeppert Mayer, S. Moszkowski and L. W. Nordheim,.Rev. Mod. Phys. 23, (1951) 322 . 
For nuclei with odd $A$, the spin and parity is, as a rule, that of the wavefunction containing an odd number of particles. The scheme (see (h. XV) of nuclear levels thus determines directly the spins of the whole nucleus. Occasionally, three or more identical nucleons in a level of given $j$ couple their spin, to a resultant $J=j-1$. In some regions of $Z$ or $N$ one is faced by an ambiguity, since two levels may be competing for the groundstate. However, at least the parity is definitely determined.

Table I contains experimental data of the $\beta$-decays of nuclei of odd $A$, up to $A=141$. The data were all taken from Hollander, Perlman and Seaborg ${ }^{2}$. They have been ordered according to the mass number $A$ in order to bring out clearly that the assignment of spins and parities has been done consistently. $\beta^{-}$and $\beta^{+}$decays have not been distinguished since the specification of the parent and daughter nucleus in Columns 2 and 3 definitely show which process is considered. The orbit of the last odd nucleon of the daughter nucleus is given in Column 4, and is underlined if the spin has been directly measured. In the case where it is known or suspected that three or more nucleons couple their spin to one unit less than that of the odd particle, the configuration symbols have been abbreviated: $\left(d_{b / 2}\right)_{3 / 2}^{3}$ by $D_{3 / 2},\left(f_{v_{1 / 2}}\right)_{s / 2}^{3}$ by $F_{s / 2}$, ete. Next the half-life is given; then the branching ratio, when it is known. A branching ratio of $100 \%$ means that the decay is not complex. The next columns list the maximum energy of the $\beta$-rays emitted, and the log $f t$ value $^{3}$. In cases where there is only an indication that the decay is complex (for instance because of the occurrence of $\gamma$-rays, but branching ratio's have not been measured), only a lower limit of $\log f t$ can be obtained. The orbit of the initial and the final odd nucleon, obtained from the shell model is given. Level assignments for excited states been entered only in those cases where they are fairly obvious, or corroberated by information obtained from $\gamma$-ray spectroscopy. In the following column the order of forbiddenness, as infered from these orbits is listed. The symbol a means allowed, $\mathrm{m}$ is added if the transition takes place between mirror nuclei. lst stands for first forbidden, etc.

It was first pointed out by Nordheim ${ }^{4}$ that in addition to the usual selection rules the independent particle model introduces additional ones, since not only parity and spin but also the orbital angular momentum is a quantum number. The matrix elements for allowed transitions do not contain an operator which changes the orbital angular momentum. Consequently, changes of spin by 1 , allowed by GT rules, are possible only

2 J. M. Hollander, I. Perlman, G. T. Seaborg, UCRL 1928, December 1952.

3 Values from R. W. King, N. M. Dismuke, K. Way, ORNL 1450.

4 L. W. Nordheim, Rev. Mod. Phys. 23, (1951) 322. 
if they are due to a spin flip, as in a transition $p_{3 / 2}$ to $p_{2 / 2}$. However, a transition $p_{3 / 2}, f_{s / 2}$ has a vanishing matrix element, although it involves no change of parity. We refer to transitions of this type as $l$ forbidden, and write $a, l$. In this same way, first forbidden transitions involving a change of $l$ by 3 , for example $g_{1 / 2}$ to $p_{3 / 2}$, are additionally $l$ forbidden; we denoted these by 1st, $l$. The last column headed "remarks" contains information about the spectrum shape. If the spectrum has been found to have the unique shape corresponding to $J= \pm 2$, change of parity, this is indicated by $J=2$. Besides, an asterisk is placed in this column for the cases in which the values of $\log f t$ and the shell model assignment for the decay disagree. The occurrence of $\gamma$-rays, indicating that the decay may go to an excited state, is occasionally marked by $\gamma$. The symbol $\mathrm{e}$ ? is entered in cases where the shell model would predict a decay to an excited state, but where no information about $\gamma$-rays exists.

For $K$-capture the energy is, as a rule, not known. In cases where the decay takes place to several levels of the daughter nucleus we have used the symbol $g$ for the unknown energy of the groundstate transition.

The agreement between the predictions of the shell model and the experimental $f t$ values is excellent. The transitions which are assumed as "allowed" fall into two rather distinct groups, with values of $\log f t$ about 5 , and with $\log f t$ about 3 . This latter class of transitions is usually referred to as "favoured" or "superallowed". Superallowed transitions occur only among the light nuclei, and only for special types of decay. For odd $A$, only the transitions between mirror nuclei are favoured. In mirror nuclei the groundstates of parent and daughter nucleus are completely alike, except that neutrons and protons are interchanged. Thus one expects a particularly good overlap of the wave functions of initial and final nucleus, a particularly large matrix element, and hence a low $f t$ value. The 21 mirror decays have values of log $f t$ ranging from 3.03 to 3.67 . The favoured transitions are discussed in greater detail in $\S 4$ on light nuclei.

Disregarding the few discrepancies which are marked with an asterisk, there are 49 decays, which, according to the shell model, are normal allowed decays. Their values of $\log f t$ vary from 4.4 to 5.9 . The $13 l$-forbidden decays have in the average only slightly higher $f t$ values.

The 45 first forbidden transitions all have values of log $f t$ larger than 6 . Those with a spin change of zero or one have mostly log $f t \sim 7$. The decays assigned to a spin change of 2 have higher values, namely log $f t$ about 8 or 9 , and even up to 10.9 . It should be kept in mind that for these latter transitions $f t$ is not directly a measure for the matrix element. When correction is made for the expected shape the scatter of the $f t$ values is much reduced. 
There are 5 cases of transitions which are second forbidden. For these, $\log f t$ varies between 11.6 and 13.4. The one third forbidden transition has $\log f t=17.76$, and finally the only known fourth forbidden decay, of $\mathrm{In}^{155}$, has $\log f t=23.02$.

In addition, there are 44 cases in which the shell model assigns the groundstate transitions as forbidden, and the decay takes place to excited levels only.

Among the 195 entries in Table I there are 16 in which experimental $f t$ values and the level assignment do not agree. In 11 of these cases, the experimental half-life is shorter than that predicted. This discrepancy would be resolved if the decay took place to an excited state. In many of these cases information about $\gamma$-rays is lacking so that the decay may go to excited states.

The Table has been discontinued at $A=141$. For nuclei heavier than this all decays between groundstates should be at least first forbidden, since they involve a change of parity. Between $A=143$ and $A=208$ the protons are in the shell between 50 and 82 , the neutrons between 82 and 126. Thus the proton and neutron levels have opposite parity (except for the highest spin states, $h_{11 / 2}$ and $i_{13 / 2}$, which do not tend to occur as groundstate levels). At $A=208$, the proton number crosses 82 , and simultaneously the neutron number 126, so that proton and neutron are again in different shells. Experimentally for nuclei with $A>141$ all $\beta$-decays to the groundstate of the daughter nucleus are at least first forbidden, with only three exceptions. These are the decay of ${ }_{30} \mathrm{Hg}_{\varepsilon_{125}^{205}}$ to ${ }_{81} \mathrm{Tl}_{124}^{205}$, the decay of ${ }_{81} \mathrm{Tl}_{12}^{207}$ to ${ }_{82} \mathrm{~Pb}_{125}^{207}$ and of ${ }_{82} \mathrm{~Pb}_{127}^{209}$ to ${ }_{83} \mathrm{Bi}_{126}^{209}$. The $\log f t$ values are 5.52, 5.16 and 5.53 respectively, while the shell model definitely predicts a change of parity.

The main information derived from the $\beta$-decays of heavier nuclei is the difference of parity between parent and daughter nucleus. Detailed assignment of the levels involved becomes somewhat ambiguous for heavier nuclei. 
TABLE I

Beta Decay of Nuclei with Odd $A$

\begin{tabular}{|c|c|c|c|c|c|c|c|c|c|c|}
\hline$A$ & Parent & $\begin{array}{l}\text { Daugh- } \\
\text { ter }\end{array}$ & $\begin{array}{l}\text { Spin of } \\
\text { Daughter }\end{array}$ & $\begin{array}{l}\text { Half } \\
\text { Life } t\end{array}$ & $\begin{array}{l}\text { Branching } \\
\text { Ratio \% }\end{array}$ & $E_{\beta}$ & $\log f t$ & \multicolumn{2}{|c|}{ Shell Model } & Remarks \\
\hline 1 & ${ }_{0} n_{1}$ & ${ }_{1} \mathrm{H}_{0}$ & $s_{1 / 2}$ & $12.8 \mathrm{~m}$ & .100 & 0.782 & 3.13 & $s_{1 / 2} \quad s_{1 / 2}$ & $s_{1 / 2} \quad a, m$ & \\
\hline 3 & ${ }_{1} \mathrm{H}_{2}$ & ${ }_{2} \mathrm{He}_{1}$ & $\overline{s_{1 / 2}}$ & $12.4 \mathrm{y}$ & 100 & 0.0194 & 3.03 & $s_{1 / 2} \quad s_{1 / 2}$ & $s_{1 / 2} \quad a, m$ & \\
\hline 7 & ${ }_{4} \mathrm{Be}_{3}$ & ${ }_{3} \mathrm{Li}_{4}$ & $\overline{\frac{p_{1 / 2}}{1 / 2}}$ & $52.93 \mathrm{~d}$ & $\begin{array}{l}89 \\
11\end{array}$ & $\begin{array}{l}K(0.863) \\
K(0.385)\end{array}$ & $\begin{array}{l}3.37 \\
3.53\end{array}$ & $\begin{array}{ll}p_{3 / 2} & p_{3 / 2} \\
p_{3 / 2} & p_{1 / 2}\end{array}$ & $\begin{array}{ll}p_{3 / 2} & \mathrm{a}, \mathrm{m} \\
p_{1 / 2} & \mathrm{a}\end{array}$ & \\
\hline 9 & ${ }_{8} \mathrm{Li}_{6}$ & $\mathrm{Be}_{5}$ & $\underline{p_{1 / 2}}$ & $0.168 \mathrm{~s}$ & & & & $p_{2 / 2} \quad p_{2 / 2}$ & $b_{2 / 2} \quad \mathrm{a}, \mathrm{m}$ & \\
\hline 11 & ${ }_{6} \mathrm{C}_{5}$ & ${ }_{6} \mathrm{~B}_{6}$ & $\underline{p_{1 / 2}}$ & $20.5 \mathrm{~m}$ & $\begin{array}{l}100 \\
K\end{array}$ & 0.99 & 3.09 & $p_{\mathrm{a} / \mathrm{z}} \quad p_{\mathrm{3} / \mathrm{s}}$ & $b_{3 / y} \quad \mathbf{a}, \mathbf{m}$ & \\
\hline 13 & ${ }_{7} \mathrm{~N}_{6}$ &. $\mathrm{C}_{7}$ & $\underline{p_{1 / 2}}$ & $9.93 \mathrm{~m}$ & 100 & 1.202 & 3.67 & $p_{1 / 2} \quad p_{1}$ & $b_{1 / 2} \quad a, m$ & \\
\hline 15 & {$\left[{ }_{6} \mathrm{C}_{8}\right]$} & {$\left[{ }_{7} \mathrm{~N}_{8}\right]$} & $\overline{p_{1 / 2}}$ & [2.4s] & {$[<100]$} & {$[\geqslant 8.8]$} & $>5.26$ & $s_{1 / 2} \quad p_{1}$ & $p_{1 / 2} \quad$ lst & \\
\hline & ${ }_{8} \mathrm{O}_{7}$ & ${ }_{7} \mathrm{~N}_{8}$ & $\overline{p_{1 / 2}}$ & $118.0 \mathrm{~s}$ & 100 & 1.683 & 3.59 & $p_{1 / 2} \quad p_{1 /}$ & $b_{1 / 2} \quad \mathrm{a}, \mathrm{m}$ & \\
\hline 17 & ${ }_{7} \mathrm{~N}_{10}$ & ${ }_{8} \mathrm{O}_{9}$ & $\overrightarrow{d s / 2}$ & $4.14 \mathrm{~s}$ & $\begin{array}{r}0 \\
100\end{array}$ & $\begin{array}{l}(8.8) \\
3.7\end{array}$ & 3.8 & $p_{1 / 2} \quad d_{b /}$ & $d_{5 / 2} \quad$ lst & \\
\hline & ${ }_{9} \mathrm{~F}_{8}$ & ${ }_{8} \mathrm{O}_{9}$ & $d_{8 / 2}$ & $66 \mathrm{~s}$ & 100 & 1.72 & 3.36 & $d_{s / 2} \quad d_{s /}$ & $d_{5 / 2} \quad$ a,m & \\
\hline 19 & ${ }_{8} \mathrm{O}_{11}$ & ${ }_{2} F_{10}$ & $s_{1 / 2}$ & $29.4 \mathrm{~s}$ & $\begin{array}{l}30 \\
70\end{array}$ & $\begin{array}{l}4.5 \\
2.9\end{array}$ & $\begin{array}{l}5.57 \\
4.34\end{array}$ & $D_{2 / 2}$ & $s_{1 / 2} \quad a, l$ & \\
\hline & ${ }_{10} \mathrm{Ne}_{9}$ & ${ }_{9} F_{10}$ & $s_{1 / 2}$ & $18.2 \mathrm{~s}$ & 100 & 2.18 & 3.23 & $s_{1 / 2} \quad s_{1}$ & $s_{1 / 2} \quad a, m$ & \\
\hline 21 & ${ }_{11} \mathrm{Na}_{10}$ & ${ }_{10} \mathrm{Ne}_{11}$ & $\overline{D_{2 / 2}}$ & $22.8 \mathrm{~s}$ & 100 & 2.50 & 3.61 & $D_{3 / 2} \quad D_{3}$ & $D_{3 / 2} \quad a, m$ & \\
\hline 23 & ${ }_{10} \mathrm{Ne}_{13}$ & ${ }_{11} \mathrm{Na}_{12}$ & $\overline{D_{2 / 2}}$ & $40.2 \mathrm{~s}$ & $\begin{array}{r}93 \\
7\end{array}$ & $\begin{array}{l}4.21 \\
1.18\end{array}$ & $\begin{array}{l}5.09 \\
3.83\end{array}$ & $d_{8 / 2} \quad D_{3}$ & $D_{3 / 2} \quad a$ & \\
\hline & ${ }_{12} \mathrm{Mg}_{11}$ & ${ }_{11} \mathrm{Na}_{12}$ & $\underline{D_{3 / 2}}$ & $11.9 \mathrm{~s}$ & 100 & 2.99 & 3.64 & $D_{\mathrm{s} / \mathrm{a}} \quad D_{\mathrm{a}}$ & $D_{a / 2} \quad a, m$ & \\
\hline 25 & ${ }_{11} \mathrm{Na}_{14}$ & ${ }_{12} \mathrm{Mg}_{13}$ & $d_{0 / 2}$ & $58.2 \mathrm{~s}$ & $\begin{array}{l}55 \\
45\end{array}$ & $\begin{array}{l}3.7 \\
2.7\end{array}$ & $\begin{array}{l}5.28 \\
4.76\end{array}$ & $D_{3 / 2}$ & $d_{s / 2} \quad a$ & \\
\hline & {$\left[{ }_{13} \mathrm{Al}_{12}\right]$} & {$\left[{ }_{12} \mathrm{Mg}_{18}\right]$} & $d_{t / 2}$ & {$[7.3 \mathrm{~s}]$} & & $(3.1)$ & $(3.47)$ & $d_{s / 2}$ & $d_{b / 2} \quad a, m$ & \\
\hline 27 & ${ }_{12} \mathrm{Mg}_{15}$ & ${ }_{13} \mathrm{Al}_{14}$ & $d_{s / 2}$ & $9.45 \mathrm{~m}$ & $\begin{array}{r}0 \\
80 \\
20 .\end{array}$ & $\begin{array}{l}2.65 \\
1.81 \\
0.79\end{array}$ & $\begin{array}{l}-\bar{y} \\
4.71 \\
3.9\end{array}$ & $s_{1 / 2}$ & $d_{\mathrm{s} / \mathrm{a}} \quad 2 \mathrm{nd}$ & \\
\hline & ${ }_{14} \mathrm{Si}_{18}$ & ${ }_{13} \mathrm{Al}_{14}$ & $d_{s / 2}$ & $4.9 \mathrm{~s}$ & & 3.48 & 3.65 & $d_{5 / 2} \quad d_{5}$ & $d_{\delta / 2} \quad \mathbf{a}, \mathbf{m}$ & \\
\hline 9 & ${ }_{13} \mathrm{Al}_{16}$ & ${ }_{14} \mathrm{Si}_{15}$ & $s_{1 / 2}$ & $6.56 \mathrm{~m}$ & $\begin{array}{c}0 \\
70 \% \\
30 \%\end{array}$ & $\begin{array}{l}(3.7) \\
2.5 \\
1.4\end{array}$ & $\begin{array}{c}- \\
5.25 \\
4.61\end{array}$ & $d_{6 / 2}$ & $s_{1 / 2} \quad 2 \mathrm{nd}$ & \\
\hline & ${ }_{15} \mathrm{P}_{14}$ & ${ }_{14} \mathrm{Si}_{15}$ & $s_{1 / 2}$ & $4.6 \mathrm{~s}$ & $\leqslant 100$ & 3.6 & $\geqslant 3.54$ & $s_{1 / 2}$ & $s_{1 / 2} \quad a, m$ & \\
\hline 31 & ${ }_{14} \mathrm{Si}_{17}$ & ${ }_{15} \mathrm{P}_{16}$ & $s_{1 / 2}$ & $2.64 \mathrm{~h}$ & & 1.471 & 5.51 & $d_{2 / 2}$ & $s_{1 / 2} \quad a, l$ & \\
\hline & ${ }_{16} \mathrm{~S}_{15}$ & ${ }_{15} \mathrm{P}_{16}$ & $s_{1 / 2}$ & $3.18 \mathrm{~s}$ & & 3.85 & 3.50 & $s_{1 / 2}$ & $s_{1 / 2} \quad$ a,m & \\
\hline 33 & ${ }_{15} \mathbf{P}_{18}$ & ${ }_{10} \mathrm{~S}_{17}$ & $d_{2 / 2}$ & $24.8 \mathrm{~d}$ & 100 & 0.27 & 5.05 & $\boldsymbol{s}_{1 / 2} \quad d_{2}$ & $d_{2 / 2} \quad a, b$ & \\
\hline & ${ }_{17} \mathrm{Cl}_{16}$ & ${ }_{16} \mathrm{~S}_{17}$ & $\underline{d / 2}$ & $2.8 \mathrm{~s}$ & & 4.13 & 3.53 & $d_{2 / 2} \quad d$ & $d_{\mathrm{a} / \mathrm{a}} \quad$ a.m & \\
\hline 35 & ${ }_{16} \mathrm{~S}_{19}$ & ${ }_{17} \mathrm{Cl}_{18}$ & $d x$ & $87.1 d$ & 100 & 0.1670 & 4.98 & $d_{2 / 2} \quad d_{2}$ & $d_{2 / 2} \quad a$ & \\
\hline
\end{tabular}


TABLE I (continued)

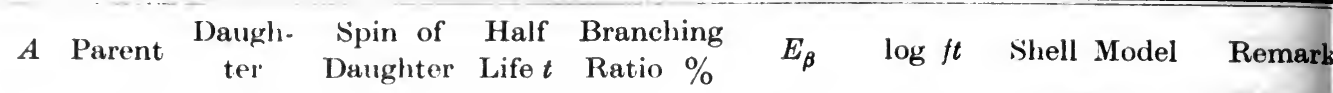

\begin{tabular}{|c|c|c|c|c|c|c|c|c|c|c|c|}
\hline & ${ }_{18} A_{17}$ & ${ }_{17}\left(\mathrm{Cl}_{18}\right.$ & $d_{3 / 2}$ & $1.88 \mathrm{~s}$ & & 4.38 & 3.53 & $d_{2 / 2}$ & $d_{2 / 2}$ & $\mathrm{a}, \mathrm{m}$ & \\
\hline \multirow[t]{4}{*}{37} & ${ }_{16} i_{21}$ & ${ }^{\prime \prime} l_{20}$ & $d_{3 / 2}$ & $5.04 \mathrm{~m}$ & 10 & 4.3 & 7.07 & $f_{7 / 2}$ & $d_{2 / 2}$ & lst & \\
\hline & & & & & 90 & 1.6 & 4.23 & & & & \\
\hline & ${ }_{18} A_{19}$ & ${ }_{17}\left(\mathrm{C}^{\prime} \mathrm{I}_{20}\right.$ & $\underline{d_{3 / 2}}$ & $34.1 \mathrm{~d}$ & & $K 0.815$ & 5.00 & $d_{2 / 2}$ & $d_{2 / 2}$ & $\mathbf{a}$ & - \\
\hline & ${ }_{10} \mathrm{~K}_{18}$ & ${ }_{18} \mathrm{~A}_{19}$ & $d_{3 / 2}$ & $1.3 \mathrm{~s}$ & & 4.6 & 3.40 & $d_{3 / 2}$ & $d_{2 / 2}$ & $\mathrm{a}, \mathrm{m}$ & \\
\hline \multirow[t]{5}{*}{39} & ${ }_{17} \mathrm{Cl}_{22}$ & ${ }_{18} A_{21}$ & $f_{2 / 2}$ & $55.5 \mathrm{~m}$ & 0 & $(3.31)$ & - & $d_{3 / 2}$ & $f_{1 / 2}$ & lst & \\
\hline & & & & & 7 & 2.96 & 7.56 & & & & \\
\hline & & & & 2354 & 93 & 1.65 & 5.31 & & & & \\
\hline & ${ }_{18} A_{21}$ & ${ }_{19} \mathrm{~K}_{20}$ & $d_{3 / 2}$ & $2.35 y$ & & 0.565 & 9.85 & $f_{1 / 2}$ & $d_{3 / 2}$ & $1 \mathrm{st}$ & 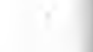 \\
\hline & ${ }_{20} \mathrm{C}^{\prime} \mathrm{a}_{19}$ & ${ }_{19} \mathrm{~K}_{20}$ & $d_{3 / 2}$ & $1.06 \mathrm{~s}$ & & 5.1 & 3.6 & $d_{2 / 2}$ & $d_{3 / 2}$ & $a, m$ & \\
\hline \multirow[t]{4}{*}{41} & ${ }_{18} \mathrm{~A}_{23}$ & ${ }_{19} \mathrm{~K}_{22}$ & $d_{3 / 2}$ & $109 \mathrm{~m}^{\circ}$ & .07 & 2.55 & 8.56 & $f_{1 / 3}$ & $d_{3 / 2}$ & lst & $\Delta \mathrm{J}=2$ \\
\hline & & & & & 99.3 & 1.25 & 5.10 & $f_{1 / 2}$ & $f_{\eta / 2}$ & a & \\
\hline & ${ }_{20} \mathrm{Ca}_{21}$ & ${ }_{19} \mathrm{~K}_{22}$ & $\underline{d_{3 / 2}}$ & $1.2 \times 10^{5} \mathrm{y}$ & $K$ & & - & $f_{1 / 3}$ & $d_{3 / 2}$ & lst & \\
\hline & ${ }_{21} \mathrm{Sc}_{20}$ & ${ }_{20} \mathrm{Ca}_{21}$ & $f_{2 / 8}$ & $0.87 \mathrm{~s}$ & & 4.94 & 3.39 & $f_{2 / 2}$ & $f_{1 / 2}$ & $a, m$ & \\
\hline \multirow[t]{6}{*}{43} & ${ }_{19} \mathrm{~K}_{24}$ & ${ }_{20} \mathrm{C}^{\prime} \mathrm{a}_{23}$ & $f_{1 / 2}$ & $22.4 \mathrm{~h}$ & 0 & $(\sim 1.3)$ & - & $d_{3 / 2}$ & $f_{2 / 2}$ & lst & 1 \\
\hline & & & & & $(80)$ & 0.83 & 5.59 & & & & \\
\hline & & & & & $(20)$ & 0.24 & 4.31 & & & & \\
\hline & ${ }_{21} \mathrm{Sc}_{22}$ & ${ }_{20} \mathrm{Ca}_{23}$ & $f_{7 / 3}$ & $3.92 \mathrm{~h}$ & & 1.1 & $\geqslant 4.77$ & $f_{2 / 2}$ & $f_{i / 2}$ & a & \\
\hline & & & & & & 0.4 & $\geqslant 3.56$ & & & & \\
\hline & ${ }_{22} \mathrm{Ti}_{21}$ & ${ }_{21} \mathrm{Sc}_{22}$ & $f_{2 / 2}$ & $0.6 \mathrm{~s}$ & & $(5.4)$ & $(3.4)$ & $f_{1 / 2}$ & $f_{2 / 2}$ & $\mathrm{a}, \mathrm{m}$ & \\
\hline \multirow[t]{3}{*}{45} & ${ }_{20} \mathrm{Ca}_{25}$ & ${ }_{21} \mathrm{Sc}_{24}$ & $f \because / 2$ & $152 \mathrm{~d}$ & 100 & 0.254 & 5.94 & $f_{7 / 2}$ & $f_{1 / 2}$ & $\mathbf{a}$ & \\
\hline & ${ }_{22} \mathrm{Ti}_{23}$ & ${ }_{21} \mathrm{Sc}_{24}$ & $\underline{f_{1 / 2}}$ & $3.09 \mathrm{~h}$ & 96 & 1.02 & 4.59 & $f_{1 / 2}$ & $f_{1 / 2}$ & a & \\
\hline & & & & & 4 & 0.57 & 4.93 & & & & \\
\hline \multirow[t]{2}{*}{47} & $21^{\mathrm{S}} \mathrm{c}_{26}$ & ${ }_{22} \mathrm{Ti}_{25}$ & $\underline{F_{s / 2}}$ & $3.43 \mathrm{~d}$ & $\begin{array}{l}34 \\
66\end{array}$ & $\begin{array}{l}0.622 \\
0.435\end{array}$ & $\begin{array}{l}6 \\
5.2\end{array}$ & $f_{1 / 2}$ & $\begin{array}{c}F_{3 / 2} \\
f_{1 /,}\end{array}$ & $\begin{array}{l}a \\
a\end{array}$ & \\
\hline & ${ }_{23} V_{24}$ & ${ }_{22} \mathrm{Ti}_{25}$ & $\underline{F_{5 / 5}}$ & $33 \mathrm{~m}$ & $<100$ & 1.7 & $>4.64$ & $f_{\eta / 2}$ & $F_{6 / 2}$ & a & \\
\hline \multirow[t]{5}{*}{49} & ${ }_{20} \mathrm{Ca}_{29}$ & ${ }_{21} \mathrm{Sc}_{28}$ & $f_{7 / 2}$ & $8.5 \mathrm{~m}$ & 0 & g & - & $p_{2 / 3}$ & $f_{\eta / 2}$ & 2nd & 1 \\
\hline & & & & & 100 & 2.7 & 5.45 & & & & \\
\hline & ${ }_{21} \mathrm{Sc}_{28}$ & ${ }_{22} \mathrm{Ti}_{27}$ & $f_{7 / 2}$ & $57 \mathrm{~m}$ & 100 & 1.8 & 5.06 & $f_{1 / 2}$ & $f_{1 / 2}$ & $\mathbf{a}$ & \\
\hline & ${ }_{23} V_{28}$ & ${ }_{22} \mathrm{Ti}_{27}$ & $f_{7 / 2}$ & $600 \mathrm{~d}$ & 100 & $K$ & & $f_{2 / 2}$ & $f_{2 / 2}$ & $\mathbf{a}$ & \\
\hline & ${ }_{24} \mathrm{Cr}_{25}$ & ${ }_{23} V_{26}$ & $f_{2 / 3}$ & $41.9 \mathrm{~m}$ & 100 & 1.45 & $>4.50$ & $f_{\eta / 2}$ & $f_{1 / 2}$ & a & \\
\hline \multirow[t]{4}{*}{51} & ${ }_{22} \mathrm{Ti}_{29}$ & ${ }_{23} \mathrm{~V}_{28}$ & $f_{7 / 2}$ & $6 \mathrm{~m}$ & 0 & $(1.92)$ & - & $p_{3 / 3}$ & $f_{2 / 2}$ & 2nd & 1 \\
\hline & & & & & $<100$ & 1.60 & $>4.34$ & & & & \\
\hline & ${ }_{24} \mathrm{Cr}_{27}$ & ${ }_{23} \mathrm{~V}_{28}$ & $f_{2 / 2}$ & $26.5 \mathrm{~d}$ & & $K$ & & $f_{1 / 2}$ & $f_{1 / 2}$ & $\mathrm{a}$ & \\
\hline & ${ }_{25} \mathrm{Mn}_{26}$ & ${ }_{24} \mathrm{Cr}_{27}$ & $f_{1 / 2}$ & $44.3 \mathrm{~m}$ & & 2.4 & 5.44 & $f_{1 / 2}$ & $f_{\eta / 2}$ & a & \\
\hline 53 & ${ }_{26} \mathrm{Fe}_{27}$ & ${ }_{25} \mathrm{MIn}_{28}$ & $f_{2 / 3}$ & $8.9 \mathrm{~m}$ & 100 & 2.5 & 4.89 & $f_{\eta / 2}$ & $f_{2 / 2}$ & $\mathbf{a}$ & \\
\hline
\end{tabular}

1 Energy systematics indicate that the transition does not go to the groundstate. 
TABLE. I (continued)

\begin{tabular}{|c|c|c|c|c|c|c|c|c|c|c|c|}
\hline$A$ & Parent & $\begin{array}{c}\text { Daugh- } \\
\text { ter }\end{array}$ & $\begin{array}{c}\text { Spin of } \\
\text { Daughter }\end{array}$ & $\begin{array}{l}\text { Half } \\
\text { Life } t\end{array}$ & $\begin{array}{c}\text { Branching } \\
\text { Ratio \% }\end{array}$ & $E_{\beta}$ & $\log f t$ & \multicolumn{3}{|c|}{ Shell Model } & Remark \\
\hline \multirow[t]{4}{*}{55} & ${ }_{26} \mathrm{Fe}_{29}$ & ${ }_{25} \mathrm{Mn}_{30}$ & $F_{s / 2}$ & $2.94 \mathrm{y}$ & 100 & $K$ & & $p_{3 / 2}$ & $F_{8 / 2}$ & $a, l$ & \\
\hline & ${ }_{27} \mathrm{Co}_{28}$ & ${ }_{26} \mathrm{Fe}_{29}$ & $p_{z / 2}$ & $18.2 \mathrm{~h}$ & 0 & $(2.44)$ & - & $f_{1 / 2}$ & $p_{3 / 2}$ & 2nd & \\
\hline & & & & & 30 & 1.50 & 6.44 & & & & \\
\hline & & & & & 30 & 1.01 & & & & & \\
\hline \multirow[t]{6}{*}{57} & ${ }_{27} \mathrm{Co}_{30}$ & ${ }_{26} \mathrm{Fe}_{31}$ & $p_{\mathbf{z} / \mathbf{z}}$ & $270 \mathrm{~d}$ & 0 & $(0.39)$ & - & $f_{1 / 2}$ & $p_{2 / 8}$ & 2nd & \\
\hline & & & & & $\begin{array}{r}0 \\
<100\end{array}$ & $\begin{array}{l}(0.27) \\
0.26\end{array}$ & $\overline{6.94}$ & & & & \\
\hline & & & & & $K$ & & & & & & \\
\hline & ${ }_{28} \mathrm{Ni}_{29}$ & ${ }_{27} \mathrm{CO}_{30}$ & $f_{1 / 2}$ & $36 \mathrm{~h}$ & 0 & $(2.22)$ & - & $p_{2 / 2}$ & $f_{\eta / 8}$ & 2nd & \\
\hline & & & & & 50 & 0.84 & 5.66 & & & & \\
\hline & & & & & & & & & & & \\
\hline \multirow[t]{3}{*}{;9 } & ${ }_{26} \mathrm{Fe}_{33}$ & ${ }_{27} \mathrm{Co}_{32}$ & $\underline{f_{1 / 2}}$ & $46 d$ & 0.3 & 1.56 & 10.9 & $p_{\mathbf{z} / \mathbf{s}}$ & $t_{\eta / 8}$ & 2nd & $\Delta \mathrm{J}=2$ \\
\hline & & & & & 53.9 & 0.460 & 6.72 & & & & \\
\hline & & & & & 45.8 & 0.257 & 5.86 & & & & \\
\hline \multirow[t]{5}{*}{31} & ${ }_{27} \mathrm{Co}_{34}$ & ${ }_{28} \mathrm{Ni}_{33}$ & $p_{2 / 2}$ & $99 \mathrm{~m}$ & 55 & 1.42 & 5.67 & $f_{1 / 2}$ & $f_{s / 2}$ & a & \\
\hline & & & & & 45 & 1.00 & 5.19 & & & & . \\
\hline & ${ }_{29} \mathrm{Cu}_{32}$ & ${ }_{28} \mathrm{Ni}_{33}$ & $p_{2 / 2}$ & $3.33 \mathrm{~h}$ & 65 & 1.205 & 4.94 & $p_{3 / 9}$ & $f_{8 / 2}$ & $\mathbf{a}, l$ & \\
\hline & & & & & 3 & 0.55 & 4.93 & & & & \\
\hline & & & & & 32 & & & & & & \\
\hline \multirow[t]{5}{*}{13} & ${ }_{28} \mathrm{Ni}_{35}$ & ${ }_{29} \mathrm{Cu}_{34}$ & $p_{z / 9}$ & $85 y$ & & 0.067 & 6.56 & $f_{5 / 2}$ & $p_{3 / 2}$ & $\mathbf{a}, \mathbf{l}$ & \\
\hline & ${ }_{30} \mathrm{Zn}_{33}$ & ${ }_{28} \mathrm{Cu}_{34}$ & $p_{\mathrm{a} / \mathrm{s}}$ & $38.3 \mathrm{~m}$ & 85 & 2.36 & 5.40 & $p_{2 / 3}$ & $p_{3 / 2}$ & $\mathbf{a}$ & \\
\hline & & & & & 7 & 1.40 & 5.47 & & & & \\
\hline & & & & & 1 & 0.47 & 4.31 & & & & \\
\hline & & & & & 7 & $K$ & & & & & \\
\hline \multirow[t]{4}{*}{5} & ${ }_{28} \mathrm{Ni}_{37}$ & ${ }_{29} \mathrm{Cu}_{36}$ & $\underline{p_{3 / 2}}$ & $3.564 \mathrm{~h}$ & 57 & 1.49 & 6.56 & $f_{s / 2}$ & $p_{3 / 2}$ & $a, l$ & \\
\hline & & & & & 14 & 1.12 & 5.93 & & & & \\
\hline & & & & & 29 & 0.37 & 4.78 & & & & \\
\hline & ${ }_{30} \mathrm{Zn}_{35}$ & ${ }_{29} \mathrm{Cu}_{36}$ & $\underline{p_{1 / 2}}$ & $250 \mathrm{~d}$ & 2.5 & $\begin{array}{l}3.25 \\
K\end{array}$ & 7.34 & $f_{3 / 2}$ & $p_{2 / 2}$ & $\mathrm{a}, \mathrm{l}$ & \\
\hline \multirow[t]{3}{*}{7} & ${ }_{29} \mathrm{Cu}_{38}$ & ${ }_{30} \mathrm{Zn}_{37}$ & $f_{2 / 2}$ & $61 \mathrm{~h}$ & 33 & 0.57 & 5.95 & $p_{2 / 2}$ & $f_{8 / 2}$ & $\mathbf{a}, \mathbf{l}$ & \\
\hline & t & & & & 67 & 0.38 & 5.3 & & & & \\
\hline & ${ }_{31} \mathrm{Ga}_{36}$ & ${ }_{30} \mathrm{Zn}_{37}$ & $\underline{f_{s i}}$ & $77.9 \mathrm{~h}$ & & $K$ & & $p_{\mathrm{s} / \mathrm{s}}$ & $f_{3 / 2}$ & $\mathbf{a}, l$ & e \\
\hline \multirow[t]{5}{*}{9} & ${ }_{30} \mathrm{Zn}_{39}$ & ${ }_{31} \mathrm{Ga}_{38}$ & $p_{z / 2}$ & $57 \mathrm{~m}$ & 100 & 1.0 & 4.37 & $p_{1 / 2}$ & $p_{2 /,}$ & $\mathbf{a}$ & \\
\hline & ${ }_{32} \mathrm{Ge}_{37}$ & ${ }_{31} \mathrm{Ga}_{38}$ & $p_{3 / s}$ & $39.6 \mathrm{~h}$ & 29 & 1.215 & 6.70 & $f_{3 / 2}$ & & $a, l$ & 2 \\
\hline & & & & & 3.3 & 0.610 & 6.41 & & & & \\
\hline & & & & & 0.7 & 0.220 & 5.09 & & & & \\
\hline & & & & & 67 & $K$ & & & & & \\
\hline
\end{tabular}

2 Energy systematics indicates that the decay goes to the groundstate. 
TABLE I (continued)

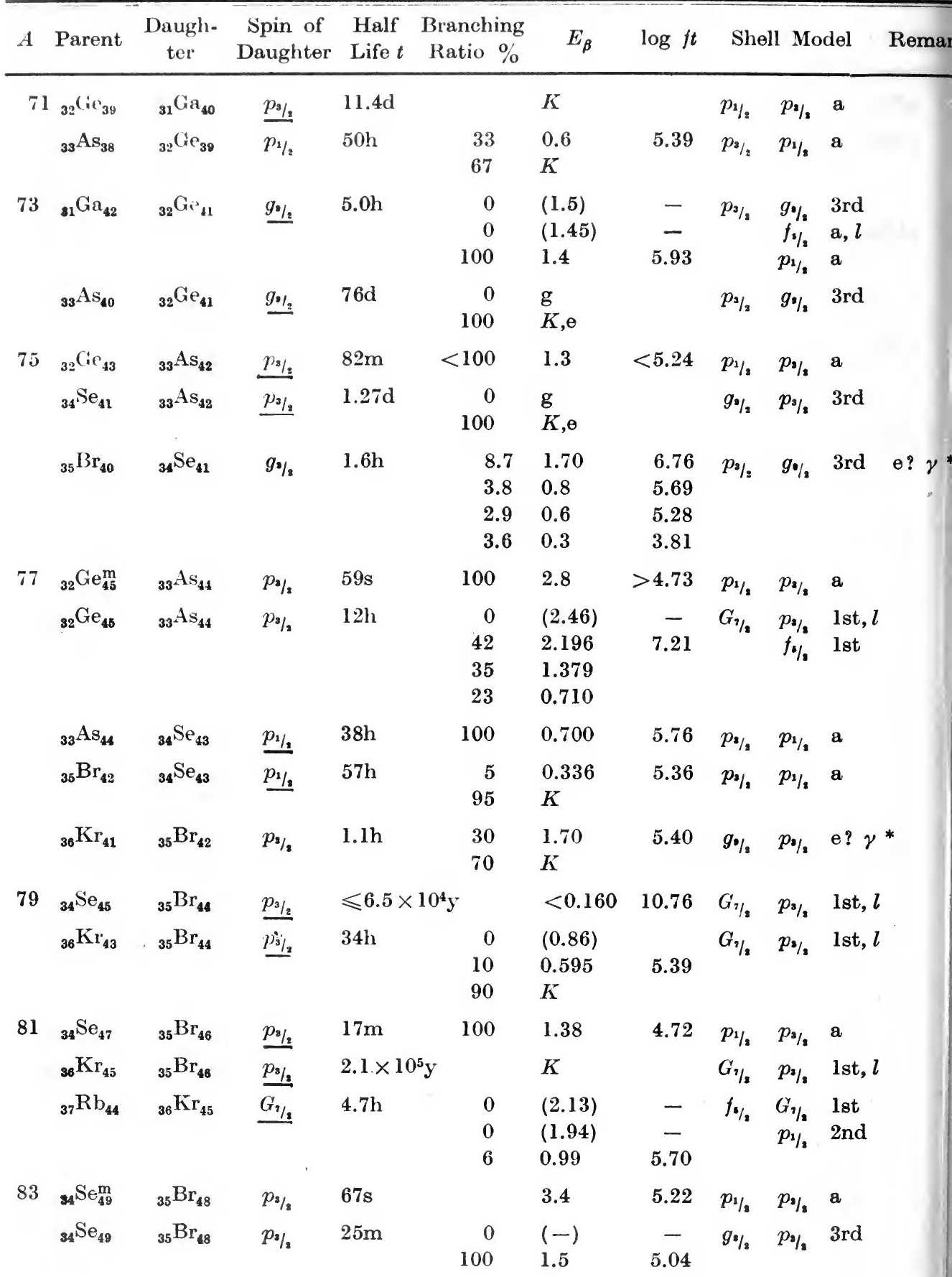


TABLE I (cotinnued)

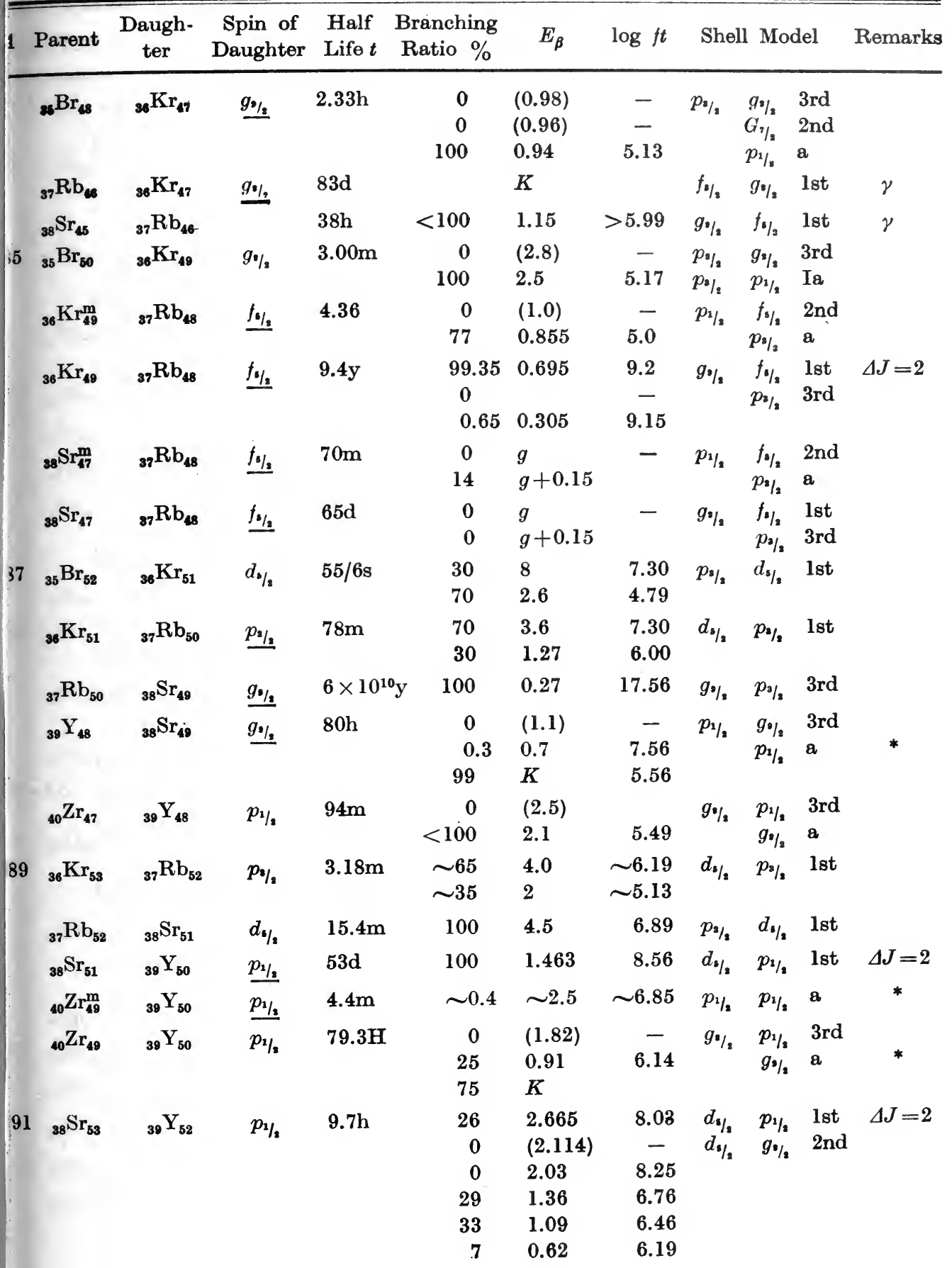


TABLE I (continued)

$A$ Parent $\begin{gathered}\text { Daugh- } \\ \text { ter }\end{gathered} \begin{gathered}\text { Spin of } \\ \text { Daughter }\end{gathered}$ Lafe $t \quad \begin{gathered}\text { Branching } \\ \text { Ratio } \%\end{gathered} \quad E_{\beta} \quad \log f t$ Shell Model Rem

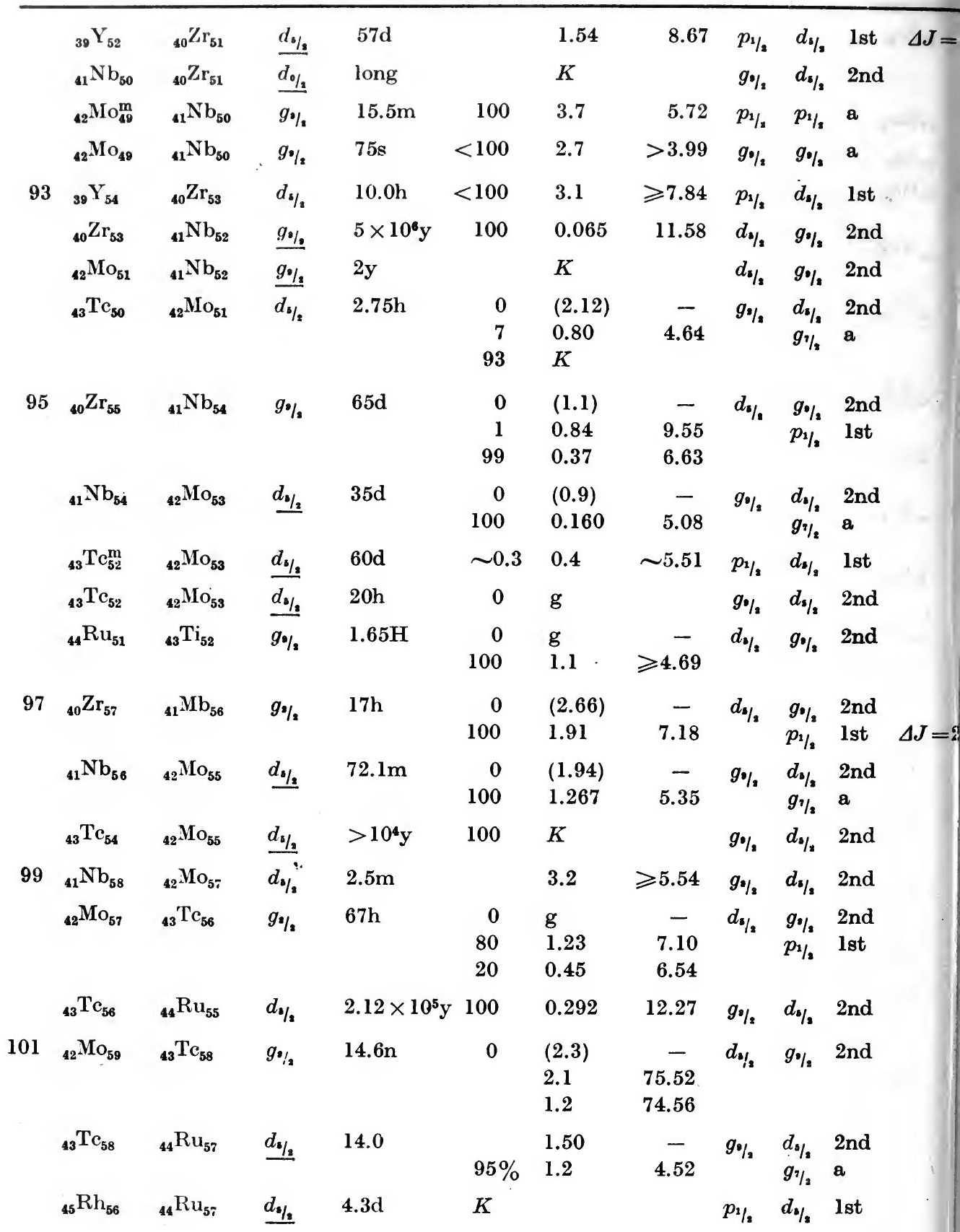


TABLE I (continued)

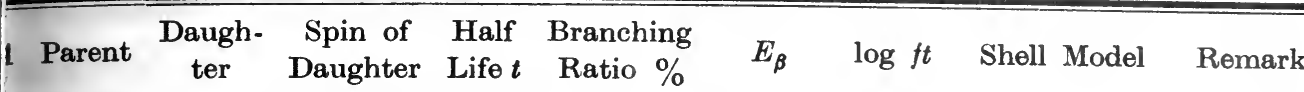

\begin{tabular}{|c|c|c|c|c|c|c|c|c|c|c|c|}
\hline & ${ }_{46} \mathrm{Pd}_{55}$ & ${ }_{45} \mathrm{Rh}_{56}$ & $p_{1 / 2}$ & $8 \mathrm{~h}$ & $\begin{array}{l}10 \% \\
90 \%\end{array}$ & $\begin{array}{l}2.3 \\
K\end{array}$ & 7.33 & $d_{s / 2}$ & $p_{1 / 2}$ & lst & no $\gamma$ \\
\hline 3 & ${ }_{44} \mathrm{Ru}_{59}$ & ${ }_{45} \mathrm{Rh}_{58}$ & $\underline{p_{1 / 2}}$ & $39.8 \mathrm{~d}$ & $\begin{array}{r}0 \\
\sim 5 \\
\sim 95\end{array}$ & $\begin{array}{l}(.702) \\
.698 \\
.217\end{array}$ & $\begin{array}{l}- \\
8.5 \\
5.67\end{array}$ & $d_{\Delta / 2}$ & $\begin{array}{l}p_{1 / 2} \\
G_{1 / 2}\end{array}$ & $\begin{array}{l}\text { lst } \\
\text { a, } l\end{array}$ & \\
\hline 5 & ${ }_{4} \mathrm{Ru}_{61}$ & ${ }_{45} \mathrm{Rh}_{60}$ & $G_{1 / 2}$ & $4.5 \mathrm{~h}$ & $\begin{array}{r}0 \\
0 \\
100\end{array}$ & $\begin{array}{l}(2.01) \\
(1.88) \\
1.15\end{array}$ & 5.80 & $d_{t / 2}$ & $\begin{array}{l}G_{\eta / 2} \\
p_{1 / 2}\end{array}$ & $\begin{array}{l}a, l \\
\text { lst }\end{array}$ & \\
\hline & ${ }_{45} \mathrm{Rh}_{60}$ & ${ }_{46} \mathrm{Pd}_{59}$ & $\underline{d_{1 / 2}}$ & $36.2 \mathrm{~h}$ & $\begin{array}{r}95 \\
\sim 5\end{array}$ & $\begin{array}{l}0.570 \\
0.25\end{array}$ & $\begin{array}{l}5.64 \\
5.74\end{array}$ & $G_{1 / 2}$ & $\begin{array}{l}d_{8 / 2} \\
g_{7 / 2}\end{array}$ & $\begin{array}{l}\mathrm{a}, l \\
\mathbf{a}\end{array}$ & \\
\hline 17 & $\left({ }_{44} R u_{63}\right)$ & $\left({ }_{45} \mathrm{Rh}_{62}\right)$ & $p_{1 / 2}$ & $(4 \mathrm{~m})$ & & $(\sim 4)$ & $\sim 6.2$ & $d_{s / 2}$ & $p_{1 / 2}$ & lst & \\
\hline & ${ }_{45} \mathrm{Rh}_{62}$ & ${ }_{46} \mathrm{Pd}_{61}$ & $d_{s / 2}$ & $26 \mathrm{~m}$ & & 1.2 & 4.86 & $p_{1 /,}$ & $d_{8 / 2}$ & lst & $e ?^{*}$ \\
\hline & ${ }_{46} \mathrm{Pd}_{61}$ & ${ }_{17} \mathrm{Ag}_{60}$ & $\underline{p_{1 / 2}}$ & $\sim 7 \times 10^{7} \mathrm{y}$ & 100 & 0.04 & $\sim 10.94$ & $d_{5 / 2}$ & $p_{1 / 2}$ & lst & \\
\hline & ${ }_{48} \mathrm{Cd}_{59}$ & ${ }_{47} \mathrm{Ag}_{60}$ & $\underline{p_{1 / 2}}$ & $67 \mathrm{~h}$ & $\begin{array}{l}0 \\
0.31 \\
K\end{array}$ & $\begin{array}{l}(.41) \\
.32\end{array}$ & $\overline{4.88}$ & $g_{\eta / \mathrm{s}}$ & $\begin{array}{l}p_{1 / 2} \\
G_{1 / 2}\end{array}$ & $\begin{array}{l}\text { 3rd } \\
\text { a }\end{array}$ & \\
\hline & ${ }_{49} \operatorname{In}_{58}$ & ${ }_{48} \mathrm{Cd}_{59}$ & $g_{1 / \mathrm{s}}$ & $30 \mathrm{~m}$ & & $\sim 2$ & $>4.99$ & $g_{\% / 2}$ & $g_{\imath / 2}$ & a & \\
\hline 19 & ${ }_{16} \mathrm{Pd}_{63-}$ & ${ }_{47} \mathrm{Ag}_{62}$ & $\underline{p_{1 / 2}}$ & $13.6 \mathrm{~h}$ & $\begin{array}{r}0 \\
100\end{array}$ & $\begin{array}{l}(1.05) \\
0.961\end{array}$ & $\overline{6.00}$ & $d_{s / s}$ & $\begin{array}{l}p_{1 / 2} \\
G_{7 / 2}\end{array}$ & $\begin{array}{l}\text { lst } \\
\mathrm{a}, l\end{array}$ & \\
\hline & ${ }_{48} \mathrm{Cd}_{61}$ & ${ }_{47} \mathrm{Ag}_{62}$ & $\underline{p_{1 / 2}}$ & $470 d$ & $\begin{array}{r}0 \\
100\end{array}$ & $\begin{array}{l}\mathrm{g} \\
\mathrm{e}\end{array}$ & & $d_{3 / 2}$ & $\begin{array}{l}p_{1 / 2} \\
G_{1 / 2}\end{array}$ & $\begin{array}{l}\text { lst } \\
\text { a, } l\end{array}$ & \\
\hline & ${ }_{49} \mathrm{In}_{60}$ & ${ }_{48} \mathrm{Cd}_{61}$ & $d_{1 / 2}$ & $4.3 \mathrm{~h}$ & $\begin{array}{r}0 \\
11 \\
K\end{array}$ & $\underset{\sim 2}{\sim .75}$ & $>\overline{5.03}$ & $g_{\bullet / 2}$ & $d / \mathrm{z}$ & 2nd & \\
\hline 1 & ${ }_{47} \mathrm{Ag}_{64}$ & ${ }_{48} \mathrm{Cd}_{63}$ & $s_{1 / 2}$ & $7.6 \mathrm{~d}$ & $\begin{array}{r}91 \\
1 \\
6\end{array}$ & $\begin{array}{l}1.04 \\
0.80 \\
0.70\end{array}$ & $\begin{array}{l}7.34 \\
8.86 \\
7.76\end{array}$ & $p_{1 / 1}$ & $\begin{array}{l}s_{1 / 2} \\
d_{3 / 2}\end{array}$ & $\begin{array}{l}\text { lst } \\
\text { lst }\end{array}$ & \\
\hline & ${ }_{40} \operatorname{In}_{62}$ & ${ }_{48} \mathrm{Cd}_{63}$ & $\underline{s_{1 / 2}}$ & $2.84 \mathrm{~d}$ & $\begin{array}{c}K 0 \\
0.01 \\
\sim 100\end{array}$ & $\begin{array}{l}g \\
g-0.393 \\
g-0.419\end{array}$ & - & $g_{1 / 2}$ & $\begin{array}{r}s_{1 / 2} \\
h_{11 / 2} \\
g_{1 / 2}\end{array}$ & $\begin{array}{l}4 \text { th } \\
\text { lst } \\
a\end{array}$ & \\
\hline & ${ }_{50} \mathrm{Sn}_{61}$ & ${ }_{49} \operatorname{In}_{62}$ & $g_{\% / 2}$ & $35.0 \mathrm{~m}$ & & 1.51 & 4.69 & $g_{\eta / 2}$ & $g_{\bullet / 2}$ & a & \\
\hline 13 & ${ }_{47} \mathrm{Ag}_{66}$ & ${ }_{48} \mathrm{Cd}_{65}$ & $\underline{s_{1 / 2}}$ & $5.3 \mathrm{~h}$ & 100 & 2.1 & 6.95 & $p_{1 / 2}$ & $s_{1 / 2}$ & lst & \\
\hline & $\begin{array}{l}{ }_{{ }_{8}} \mathrm{Cd}_{85}^{m} \\
{ }_{{ }_{8}} \mathrm{Cd}_{85}^{m}\end{array}$ & $\begin{array}{l}{ }_{49} I_{64} \\
{ }_{49} I_{64}\end{array}$ & $\frac{g_{0 / 2}}{\underline{g / / 2}}$ & $\begin{array}{l}5.1 y \\
\text { stable? }\end{array}$ & 100 & 0.59 & 8.75 & $\begin{array}{l}h_{11 / 2} \\
s_{1 / 2}\end{array}$ & $\begin{array}{l}g_{\% / 2} \\
g_{\% / 2}\end{array}$ & $\begin{array}{l}\text { lst } \\
\text { 4th }\end{array}$ & \\
\hline 15 & ${ }_{47} \mathrm{Ag}_{68}$ & ${ }_{48} \mathrm{Cd}_{67}$ & $s_{1 / 2}$ & $20 \mathrm{~m}$ & 100 & $\sim 3$ & $\sim 6.4$ & $p_{1 / 2}$ & $s_{1 / 2}$ & lst & \\
\hline & ${ }_{48} \mathrm{Cd}_{67}^{m}$ & ${ }_{49} \mathrm{In}_{66}$ & $g / /$ & $43 d$ & $\begin{array}{r}\sim 98 \\
\sim 2\end{array}$ & $\begin{array}{l}1.61 \\
0.7 \\
0.3\end{array}$ & $\begin{array}{r}\sim 8.79 \\
\sim 9.04 \\
>8.27\end{array}$ & $h_{11 / 2}$ & $g_{\%}$ & lst & \\
\hline
\end{tabular}


TABLE I (continued)

A Parent Daugh- Spin of Half Branching ter Daughter Life $t$ Ratio \%

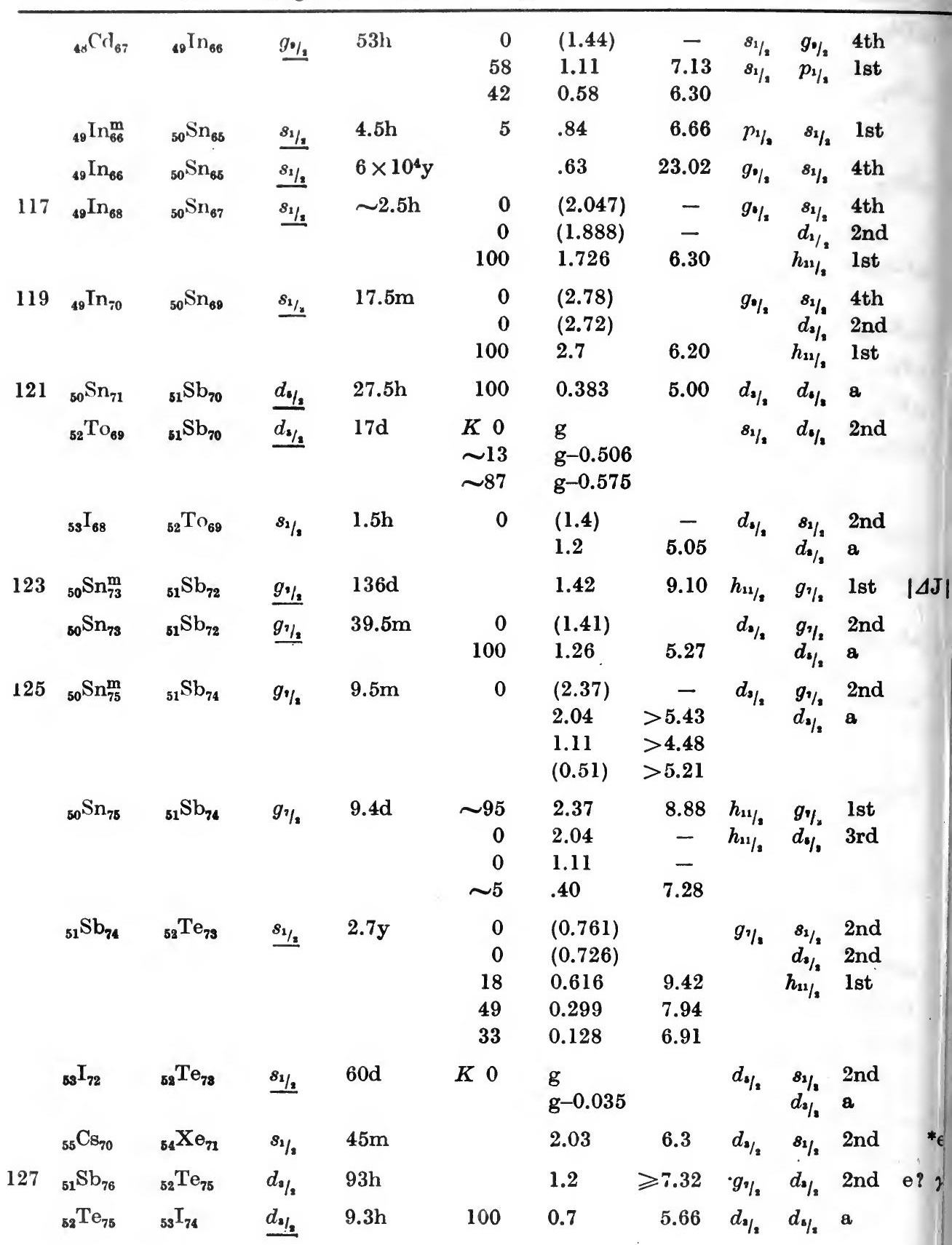


TABLE I (continued)

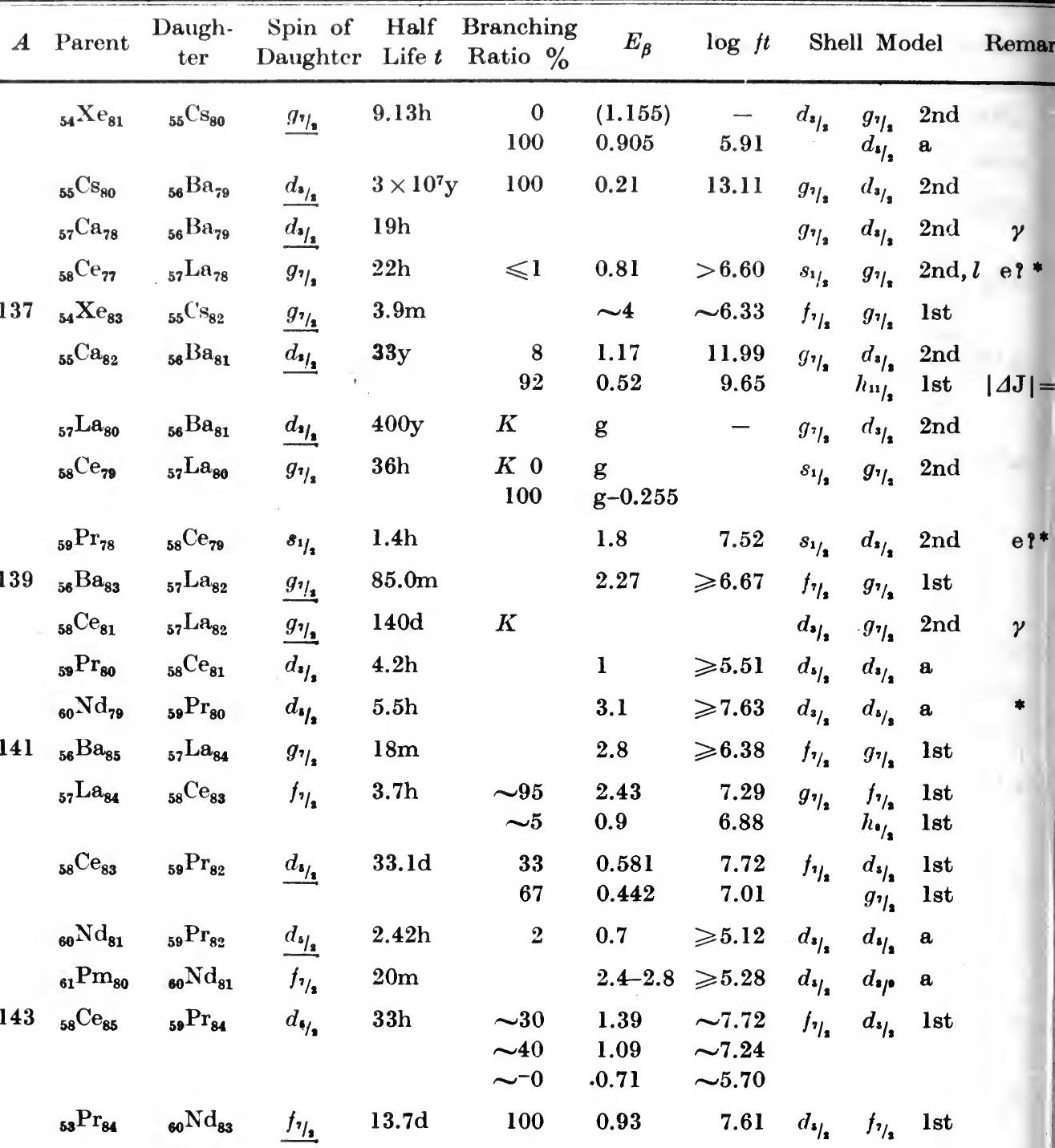




\section{§ 3. Nuclei with Even $\boldsymbol{A}$}

For even $A, \beta$-decay leads from even-even to odd-odd nuclei. Even-even nuclei have even parity and spin zero in the groundstate and usually spin 2 in the first excited state. For the lighter odd-odd nuclei the shell model is capable to determine the parity unambiguously, namely, by defining the level of the odd neutron and the odd proton. However, the shell model is not capable of predicting the spin. No clearcut coupling rule for the spin of an odd neutron to an odd proton exists. The study of $\beta$-decay halflives enables us to get some information about these coupling rules.

From investigations of $\beta$-decay Nordheim ${ }^{5}$ has been able to formulate empirical rules which seem to describe the facts in many cases. These rules can be expressed simplest by the statement that the intrinsic spins of neutron and proton tend to align. In detail, Nordheim's rules are:

I. If both proton and neutron are in levels in which intrinsic spin and orbital angular momentum are parallel, that is $j_{n}=l_{n}+\frac{1}{2}, j_{p}=l_{p}+\frac{1}{2}$, or if both are in levels in which spin and orbital angular momentum are antiparallel, $j_{n}=l_{n}-\frac{1}{2}, j_{p}=l_{p}-\frac{1}{2}$, the angular momenta $j_{n}$ and $j_{p}$ tend to add, although not necessarily to the highest possible value $J=j_{n}+j_{p}$.

II. If one of the nucleons is in a level with intrinsic spin and orbital angular momentum parallel, the other in one where they are antiparallel, that is, either $j_{n}=l_{n}+\frac{1}{2}, j_{p}=l_{p}-\frac{1}{2}$ or $j_{n}=l_{n}-\frac{1}{2}, j_{p}=l_{p}+\frac{1}{2}$, the total angular momentum of the groundstate is the smallest possible one, $J=\left|j_{n}-j_{p}\right|$.

Rule I, which only predicts a high spin, but not its exact value is often referred to as Nordheim's "weak" rule, in contrast to Rule II, Nordheim's "strong" rule, which predicts the exact value.

Among light nuclei, there are many exceptions from these rules known. Nevertheless, for heavier nuclei Nordheim's rules seem to be rather successful.

Some data about $\beta$-decay of heavier nuclei with even $A$ are given in Table II. These data show the degree of ambiguity, and agreement encountered. No attempt has been made to make the table complete. We have simply taken all the data given by Nordheim from mass 88 to mass 124. Nordheim's $f t$ values have been replaced by newer ones ${ }^{6}$ and the spin and parity assignments of the excited levels of the even-even

5 L. W. Nordheim, Rev. Mod. Phys. 23, (1951) 322.

- R. W. King, N. M. Dismuke and K. Way, ORNL 1450. 
daughter nuclei have been added. Columns 4 and 5 give the state of the odd proton and the odd neutron. The symbols a, 1st, etc. specify character of the transition which follows from the level assignments, as allowed, first forbidden, etc. The last column contains information about the spectrum for the cases in which the unique shape corresponding to $\Delta J=2$, change of parity, has been observed.

The first case in the table, ${ }_{37} R b_{51}^{88}$, is one in which the shape of the spectrum uniquely determines the groundstate as $2-$. Negative parity is to be expected. The level assignment given, $f_{3 / 3}$ for the 37 th proton, $g_{7 / 2}$ for the 5lst neutron, is the only one which agrees with Nordheim's rules. However, this assignment is not very reasonable. The first few neutrons beyond $N=50$ usually occupy the $2 d_{b / 2}$ level, in nuclei of odd $A$ as well as in even $A$, as the later entries in the table show. The configuration $\left(\pi f_{v / 2}\right)\left(v d_{3 / 2}\right)$ would be expected. The observed spin $2-$ is then in contradiction to Nordheim's strong rule which predicts $J=0$. The next two entries contain the two odd-odd Yttrium isotopes, ${ }_{39} \mathrm{Y}_{49}^{88}$ and ${ }_{80} \mathrm{Y}_{51}^{90}$. These are two cases of excellent agreement with the coupling rules. The 39th proton should be in the level $p_{1 / 2}$ in both cases, as in the nucleus ${ }_{30} \mathbf{Y}_{50}^{80}$. The 49th neutron, one less than the magic number 50 , in all cases of nuclei of odd $A$ has been found in the state of highest spin of the shell, $g_{\% / 2}$, with intrinsic spin and orbital angular momentum parallel. Thus the configuration for $\mathrm{Y}^{88}$ must be $\left(\pi p_{1_{1 / 3}}\right)\left(v g_{\vartheta_{2}}\right)$, with odd parity. If the individual spins subtract, according to Nordheim's rules, the resultant total angular momentum is $J=4$. This is in complete agreement with the decay scheme. In $\mathrm{Y}^{90}$, the neutron number is 51. The $g_{\% / 2}$ level is filled at 50, and the first level which is filled by the neutrons is $d_{s / 2}$, spin and orbit parallel. Again the spins of the $p_{1 / 3}$ proton and the $d_{5 / 2}$ neutron should subtract, giving a resultant $J=2$, with odd parity. Since the decay of $\mathrm{Y}^{90}$ to the $0+$ groundstate of ${ }_{40} \mathrm{Zr}_{50}^{90}$ has the unique spectrum shape corresponding to $\Delta J=2$, change of parity, the experiments definitely establish the groundstate of $\mathrm{Y}^{90}$ as $2-$, as predicted.

The 12 cases in which allowed $\beta$-transitions to the groundstate with very low values of $\log f t$ are observed, from mass number $A=104$ to $A=118$ inclusive, are the strongest confirmation of Nordheim's rules. No other configuration assignment than the one given, a $g_{\% /,}$ proton and $g_{\nu / \text {, neutron, }}$ can be made. Since the decays are allowed, the spin of the odd-odd nucleus can only be 0 or 1 , and the parity even. Among all the possible levels, only the combination of $g_{\eta / 3}$ with $g_{\% / 2}$ can fulfill these requirements.

The assignments just discussed show that the last odd neutron in oddodd nuclei is not necessarily in the same level as the last odd neutron in even-odd nuclei. The last two entries in the table, ${ }_{51} \mathrm{Sb}_{71}^{122}$ and ${ }_{53} \mathrm{I}_{71}^{124}$, have 
TABLE II

Decay of Nuclei of Even $A$

4 Parent Daughter Odd Odd $J \quad$ Spectrum Proton Neutron Initial Final $\log f t \quad \begin{gathered}\text { Spectrum } \\ \text { Shape }\end{gathered}$

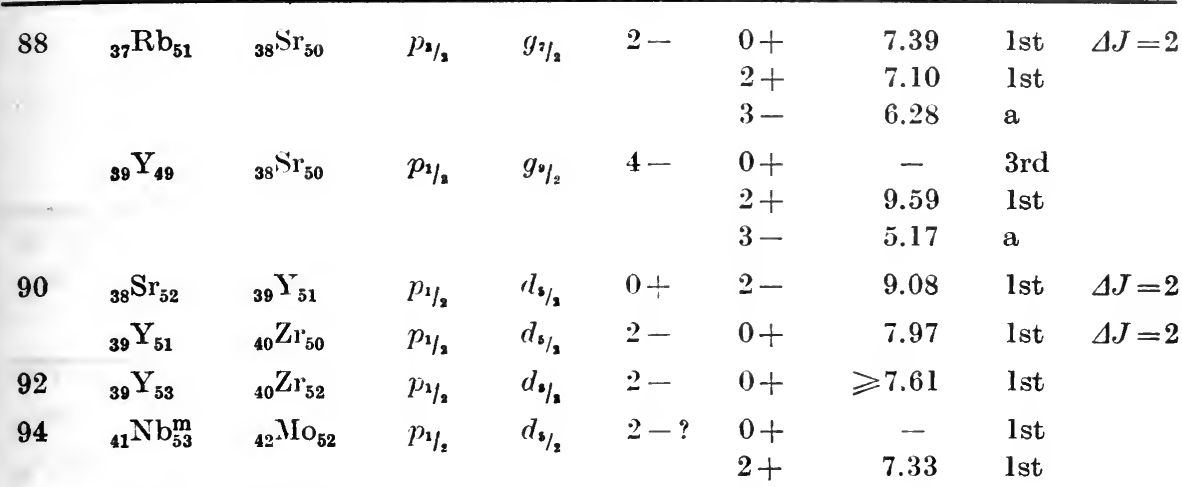

$96 \quad{ }_{41} \mathrm{Nb}_{55} \quad{ }_{42} \mathrm{M}_{54} \quad g_{\% / 2} \quad d_{6 / 2} \quad$ high cascade of $\gamma$ 's.

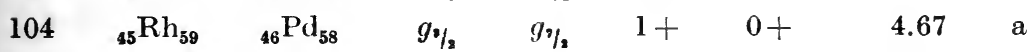

$106 \quad{ }_{44} \mathrm{Ru}_{62} \quad{ }_{45} \mathrm{Rh}_{61} \quad g_{\% / 2} \quad g_{\% / 2} \quad 0+\quad 1+\quad 4.25 \quad$ a ${ }_{45} \mathrm{Rh}_{61} \quad{ }_{46} \mathrm{Pd}_{60} \quad g_{0 / 2} \quad g_{\%}, \quad 1+\quad 0+\quad \begin{array}{llll}5.26 & \mathrm{a}\end{array}$

$2+\quad 5.82 \quad a$

$0+\quad 5.35 \quad \mathrm{a}$

$(2+) \quad 5.55 \quad \mathrm{a}$

${ }_{47} \mathrm{Ag}_{59} \quad{ }_{46} \mathrm{Pd}_{60} \quad g_{0 /} \quad g_{\% / 2} \quad 1+\quad 0+\quad \sim 4.79 \quad$ a

$108 \quad{ }_{47} \mathrm{Ag}_{61} \quad{ }_{48} \mathrm{Cd}_{60} \quad g_{\% / 2} \quad g_{\% / 2} \quad 1+\quad 0+\quad \begin{array}{r}4.42 \quad \mathrm{a} \\ 110\end{array}$

$110 \quad{ }_{47} \mathrm{Ag}_{63} \quad{ }_{48} \mathrm{Cd}_{62} \quad g_{\%} \quad g_{\%} \quad 1+\quad 0+\quad \begin{array}{lllll}5.05 & \text { a }\end{array}$ ${ }_{49} \mathrm{In}_{61} \quad{ }_{68} \mathrm{Cd}_{62} \quad g_{0 / 2} \quad g_{\%} \quad 1+? \quad 0+\quad \ldots \quad$ a

$112 \quad{ }_{46} \mathrm{Pd}_{68} \quad{ }_{47} \mathrm{Ag}_{65} \quad g_{\%}, \quad g_{1 / 2} \quad 1+\quad 2+\quad 3.93 \quad$ a

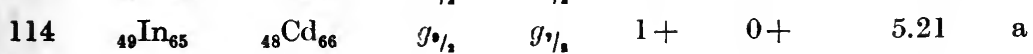
${ }_{49} \mathrm{In}_{63} \quad{ }_{50} \mathrm{Sn}_{64} \quad g_{\%}, \quad g_{\% / 2} \quad 1+\quad 0+\quad 4.47 \quad \mathrm{a}$

$116 \quad{ }_{49} \mathrm{In}_{67} \quad{ }_{30} \mathrm{Sn}_{66} \quad g_{\% / 2} \quad g_{\% / 2} \quad 1+\quad 0+\quad 4.45 \quad$ a

$118 \quad{ }_{49} \mathrm{In}_{68} \quad{ }_{50} \mathrm{Sn}_{68} \quad g_{\%} \quad g_{\%} \quad 1+\quad \begin{array}{llllll}0+ & 4.55 & \text { a }\end{array}$

$122 \quad{ }_{51} \mathrm{Sb}_{71} \quad{ }_{52} \mathrm{Te}_{70} \quad g_{\%} \quad h_{11} \quad 2-\quad 0+\quad 8.61 \quad$ lst

$2+\quad 7.66 \quad 1$ st

$2+\quad 7.68 \quad$ lst

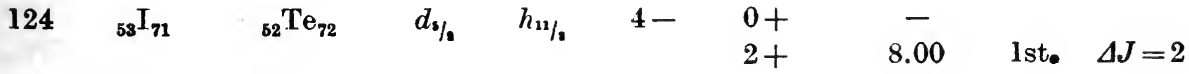


groundstates of negative parity. Since all levels between 50 and 82 are even, with the single exception of $h_{21 / 8}$, this must be taken as evidence that the $h_{31 / s}$ levels can be occupied by an odd number of neutrons in the groundstates of odd-odd nuclei.

TABLE III

Matrix Elements for Decay

A) Mirror Nuclei

\begin{tabular}{|c|c|c|c|c|c|c|}
\hline \multirow{3}{*}{ Parent } & \multirow{3}{*}{ Daughter } & \multirow{3}{*}{$(f t)^{-1} \times 10^{4}$} & \multicolumn{3}{|c|}{$M^{2}$} & \\
\hline & & & Scalar & \multicolumn{2}{|c|}{ Tensor } & \\
\hline & & & & $j j$ & $L S$ & \\
\hline${ }_{0} n_{1}^{1}$ & ${ }_{0} \mathrm{H}_{1}^{1}$ & 7.4 & 1 & 3 & 3 & \\
\hline${ }_{1} \mathrm{H}_{2}^{3}$ & ${ }_{2} \mathrm{He}_{1}$ & 9.3 & 1 & 3 & 3 & \\
\hline${ }_{4} \mathrm{Be}_{8}^{7}$ & ${ }_{8} \mathrm{Li}_{4}^{7}$ & 4.3 & 1 & 0.9 & 1.67 & \\
\hline $\mathrm{C}^{11}$ & $B^{11}$ & 2.5 & 1 & 1.67 & 1.67 & \\
\hline$N^{18}$ & $\mathrm{C}^{13}$ & 2.1 & 1 & 0.33 & 0.33 & \\
\hline $\mathrm{O}^{16}$ & $N^{15}$ & 2.6 & 1 & 0.33 & 0.33 & \\
\hline$F^{17}$ & $\mathrm{O}^{17}$ & 4.4 & 1 & 1.4 & 1.4 & \\
\hline$N_{\theta}{ }^{10}$ & $\mathrm{~F}^{10}$ & 5.9 & 1 & 3 & 3 & $\left(8_{1} /\right)^{3}$ \\
\hline & & & 1 & 1.6 & 0 & $\left(d_{s / 2}\right)^{3}$ \\
\hline $\mathrm{Na}^{21}$ & $\mathrm{Ne}^{21}$ & 2.5 & 1 & 0.19 & 0.6 & $2 / 8$ \\
\hline & & & 1 & 0.92 & 1.4 & $5 / 2$ \\
\hline $\mathbf{M g} \dot{g}^{23}$ & $\mathrm{Na}^{28}$ & 2.3 & 1 & 0.19 & 0.6 & \\
\hline $\mathrm{Si}^{27}$ & $\mathrm{Al}^{27}$ & 2.8 & 1 & 1.4 & 1.4 & \\
\hline $\mathrm{P}^{29}$ & $\mathrm{Si}^{29}$ & 2.9 & 1 & 3 & 3 & \\
\hline $\mathrm{Si}^{31}$ & $\mathbf{P}^{31}$ & 3.2 & 1 & 3 & 3 & \\
\hline $\mathrm{Cl}^{33}$ & $\mathrm{~S}^{33}$ & 2.9 & 1 & 0.6 & 0.6 & \\
\hline$A^{85}$ & $\mathrm{Cl}^{35}$ & 3.0 & 1 & 0.32 & 0.6 & \\
\hline $\mathrm{K}^{37}$ & $A^{87}$ & 4.0 & 1 & 0.32 & 0.6 & \\
\hline $\mathrm{Ca}^{39}$ & $\mathrm{~K}^{39}$ & 2.5 & 1 & 0.6 & 0.6 & \\
\hline $\mathrm{Sc}^{41}$ & $\mathrm{Ca}^{41}$ & 4.1 & 1 & 1.28 & 1.28 & \\
\hline $\mathrm{Ti}^{43}$ & $\mathrm{Sc}^{43}$ & & 1 & 0.40 & 1.28 & \\
\hline
\end{tabular}


TABLE III (continued)

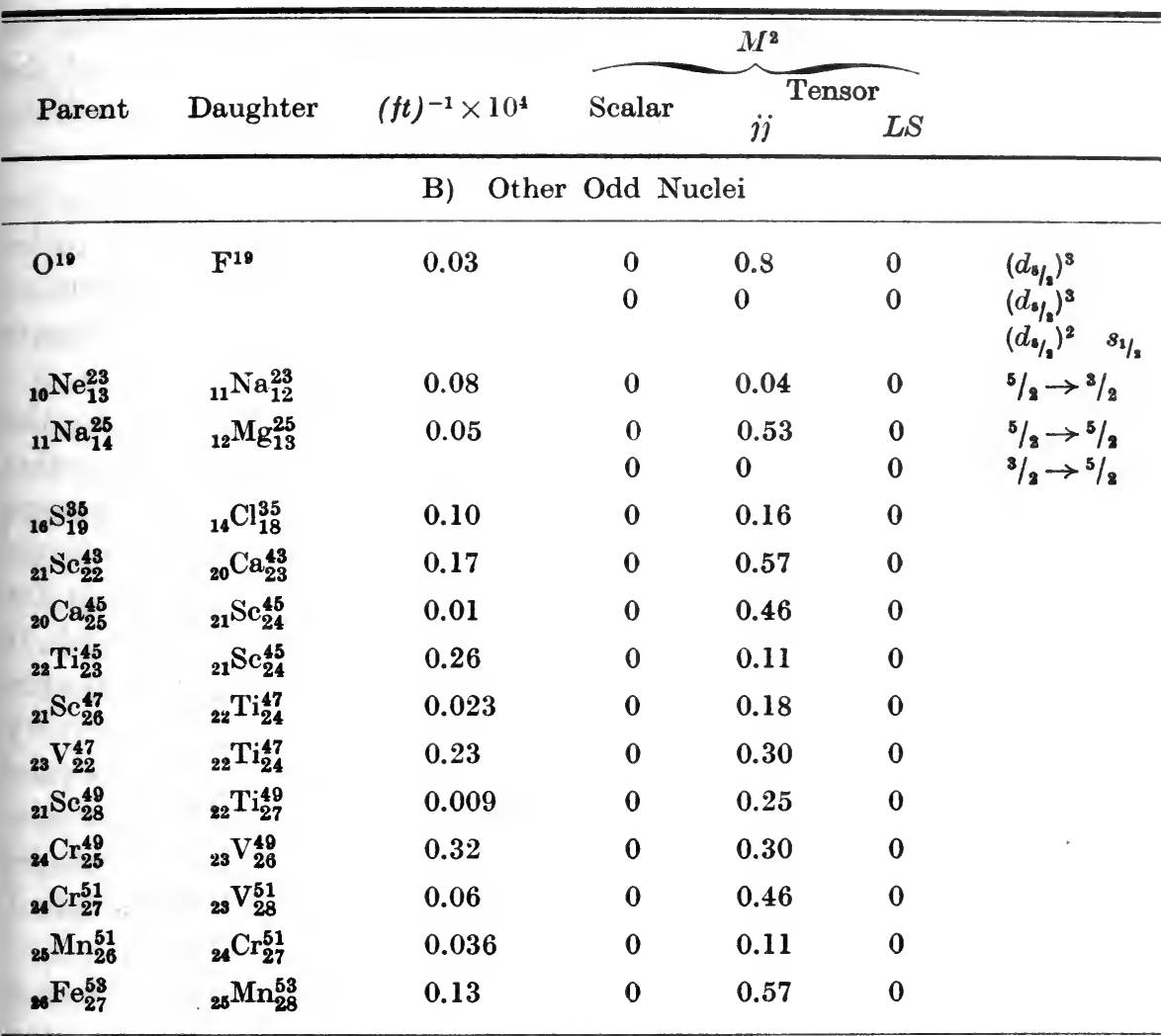

C) Even $A$

\begin{tabular}{|c|c|c|c|c|c|c|}
\hline${ }_{8} \mathrm{He}_{4}^{6}$ & ${ }_{8} \mathrm{Li}_{3}^{6}$ & 11.5 & 0 & 3.3 & 6 & $0 \rightarrow 1$ \\
\hline \multirow[t]{2}{*}{. $\mathrm{C}_{4}^{10}$} & ${ }_{5} B_{5}^{10 *}$ & 4.79 & 0 & 3.3 & 6 & $0 \rightarrow 1$ \\
\hline &. $\mathrm{B}_{5}^{10 * *}$ & 1.7 & 2 & 0 & 0 & $0 \rightarrow 0$ \\
\hline${ }_{6} B_{7}^{12}$ & ${ }_{6} \mathrm{C}_{6}^{12}$ & 0.68 & 0 & 1.8 & 0 & $1 \rightarrow 0$ \\
\hline${ }_{7} \mathrm{~N}_{5}^{12}$ & ${ }_{.} \mathrm{C}_{6}^{12}$ & 0.50 & 0 & 1.8 & 0 & $1 \rightarrow 0$ \\
\hline${ }_{6} \mathrm{C}_{8}^{14}$ & ${ }_{7} \mathrm{~N}_{7}^{14}$ & $10^{-5}$ & 0 & 0.67 & 6 & $0 \rightarrow 1$ \\
\hline \multirow[t]{2}{*}{${ }_{8} \mathrm{O}_{6}^{14}$} & ${ }_{7} \mathrm{~N}_{7}^{14}$ & 0 & 0 & 0.67 & 6 & $0 \rightarrow 1$ \\
\hline & ${ }_{7} \mathrm{~N}_{7}^{14 *}$ & 3.00 & 2 & 0 & 0 & $0 \rightarrow 0$ \\
\hline. $\mathrm{F}_{9}^{18}$ & ${ }_{8} \mathrm{O}_{10}^{18}$ & 2.34 & $\begin{array}{l}0 \\
0\end{array}$ & $\begin{array}{l}2 \\
0.93\end{array}$ & $\begin{array}{l}2 \\
2\end{array}$ & $\begin{array}{l}\left(\delta_{1 / 2}\right)^{2} \\
\left(d_{s / 8}\right)^{2}\end{array}$ \\
\hline${ }_{18} \mathrm{Al}_{13}^{26}$ & ${ }_{12} \mathrm{Mg}_{14}^{26}$ & 5.50 & 0 & 0.93 & 2 & $1 \rightarrow 0$ \\
\hline${ }_{15} P_{15}^{80}$ & ${ }_{14} \mathrm{Si}_{16}^{30}$ & 0.11 & 0 & 2 & 2 & $1 \rightarrow 0$ \\
\hline${ }_{17} \mathrm{Cl}_{17}^{34}$ & ${ }_{16} \mathrm{Si}_{18}^{34}$ & 3.77 & 2 & 0 & 0 & $0 \rightarrow 0$ \\
\hline${ }_{10} K_{19}^{38}$ & ${ }_{18} A_{20}^{38 *}$ & 0.09 & 0 & 0.24 & 0 & 3 \\
\hline
\end{tabular}




\section{$\S 4$. Light Nuclei}

So far, we merely discussed the overall selection rules depending on change of spin and parity. For light nuclei, where, on the basis of the single particle model, the warefunction of the nucleus can be written explicitly, more detailed calculations can be made.

For allowed transitions, two types of $\beta$-ray interactions enter into the decay propability, the scalar and the vector interaction. For both interactions, the transition probability, or $f t$ value, depends on the interaction strength and on a nuclear matrix element. These nuclear matrix elements can be computed for light nuclei.

The matrix elements for the scalar interaction (which leads to the Fermi selection rules (Ch. IX and $\mathrm{X})$ ) is particularly simple. Its value does not depend on any model, but merely on the assumption of charge independence of the nuclear forces. Under this assumption, the isotopic spin of a nucleus is a good quantum number. The matrix element for allowed transitions for scalar interaction is a simple overlap integral. It vanishes for all transitions in which the isotopic spin, or the nuclear spin changes. Thus the selection rules obtained for scalar interaction are very stringent. Among nuclei of odd $A$, only transitions between mirror nuclei are allowed, with a matrix element 1 . For nuclei of even $A$, transitions between a self-mirrored $[N=Z]$ nucleus in a state with spin 0 , isotopic spin 1 and the groundstate of an even-even nucleus, spin 0 , isotopic spin 1 are allowed. The square of the matrix element is 2 .

No rule of equal generality applies to the matrix elements for tensor interaction. The results of calculations made for a nuclear model assuming $L S$ coupling ${ }^{7}$ and one assuming $j j$ coupling are not, in general, the same. The selection rules obtained from the independent particle model with $L S$ coupling are much more stringent than those obtained for the $i j$ coupling model.

Table III summarizes matrix elements for scalar interaction and for tensor interaction, calculated under the assumption of $j j$ coupling as well as $L S$ coupling. As experimental comparison, the values of $(f t)^{-1}$ are listed. The detailed agreement is rather poor.

7 E. Wigner, Phys. Rev. 51, (1937) 95. 


\section{(II) M. GOLDHABER and A. W. SUNYAR - Classification of Nuclear Isomers}

\section{$\S 1$. Introduction}

The last few years have seen a gradual shift of emphasis and interest in the study of isomers : no longer are isomers only an important and integral part of the extensive semi-empirical field of radioactivity; they are also playing an increasingly significant role in the understanding of the details of nuclear structure. The interest has now proceeded from questions of lifetimes of isomers to questions of the multipole character and the matrix elements of $\gamma$-ray transitions, and since the electromagnetic field is well understood theoretically, the interaction of electromagnetic radiations with nuclei forms a particularly reliable "tool" for the study of finer questions of nuclear structure.

Isomeric states are distinguished from ordinary excited states of nuclei by the fact that they have lifetimes measurable with present techniques, a property which has made their study particularly fascinating. A relatively long lifetime of a nuclear state provides a possibility for a more detailed and direct investigation of many of the intrinsic properties of the state (e.g. spin, magnetic moment, etc.), as well as of its modes of production and decay. This is perhaps similar to the situation where a new element appears more fascinating to a chemist than a new isotope, because it allows new "manipulations". We must remember, however, that the "isomeric" property of a state is not absolute but only relative with respect to properties of states of lower energy. It occurs usually when the state has a spin which differs considerably from those of all lower lying states. If, therefore, we redefine isomeric transitions to mean transitions of known multipole character and more specifically of known (or delimited or determinable) matrix element, we widen the field in a very natural way. There are many other ways of deducing matrix elements of $\gamma$-ray transitions besides a direct lifetime measurement (e.g. Coulomb excitation, partial widths of neutron, proton, etc. capture resonances, competition of $\gamma$-rays with other processes like $\alpha$-emission, resonant absorption and scattering of $\gamma$-rays, photonuclear effects, etc.).

From the multipole character and matrix elements of $\gamma$-ray transitions many valuable conclusions can be drawn to check existing nuclear models and to obtain hints for necessary modifications. Thus, e.g., the establishment of the remarkable uniformity of the matrix elements of M4 transi- 
tions 1 (see below, Fig. 10) has strengthened the "single particle model" as an excellent approach in many cases. On the other hand, the realization that most first excited states of even-even nuclei have spin 2 and even parity, and that the transitions from these states are often too fast to be accounted for by a single particle model, has led us to postulate a "cooperative" phenomenon ${ }^{1}$, which has been re-interpreted by Bohr and Mottelson 2 as "collective" or "rotational"' motion of the nucleus. Their formula for rotational states has had considerable success in predicting the energies and spins of further excited states of even-even, as well as odd $A$ nuclei; in regions where the proton and neutron numbers are far removed from magic numbers. Generally speaking, one may say that to the branches of nuclear science which deal with properties of ground states of nuclei (measurements of masses, spins, magnetic and electric moments, etc.) new, related branches have been added. They extend our knowledge in a natural way, and to a considerable degree, through the study of excited states and their transition probabilities. The close connection of these fields of study becomes more apparent if we use the language of quantum mechanics: The study of the diagonal matrix elements (which describe e.g. magnetic dipole moments, electric quadrupole moments, etc.) has been extended to a study of the off-diagonal matrix elements, which in many cases teach us related facts and allow a considerable extension of our knowledge to higher moments, hitherto inaccessible to observation as long as ground states alone were studied.

\section{$\S 2$. Selection Rules}

Gamma-ray transitions of different multipole character can take place between two states of a nucleus, the initial and final states, characterized by spins $I_{i}$ and $I_{f}$ (in units of $\hbar$ ), and parities $\pi_{i}$ and $\pi_{f}$ (odd [-] or even $[+])$ respectively. The spin change $\Delta I$, where $\left|I_{i}-I_{j}\right| \leqslant \Delta I \leqslant\left|I_{i}+I_{f}\right|$, and the parity change (yes or no), are "carried away" by the photon. The smallest possible spin difference carried away by the photon is 1 , since electromagnetic waves, because of their "transverse" character, cannot have zero angular momentum relative to the emitter. Therefore, when $I_{i}=I_{f}=0$, no photon emission can take place; in this case only internal conversion electrons or internal electron pairs can be emitted. Since these are fairly slow processes, the lifetimes of such transitions have in some cases been found directly measurable $\left(\mathrm{O}^{16}\right.$ (6.1 MeV internal electron pair,

1 M. Goldhaber and A. W. Sunyar, Phys. Rev. 83, (1951) 906.

2 A. Bohr and B. R. Mottelson, Chapter XVII. 
$\left.T_{1 / 2}=5 \times 10^{-11} \mathrm{sec}\right)^{3}, \mathrm{Ge}^{72}\left(0.7 \mathrm{MeV}\right.$ internal conversion electron, $T_{1 / 2}=$ $\left.0.3 \mu \mathrm{sec})^{4}\right)$.

Apart from the $0+\rightarrow 0+$ transitions, the multipole characters of all electromagnetic transitions which have so far been observed are sum. marized in the following table:

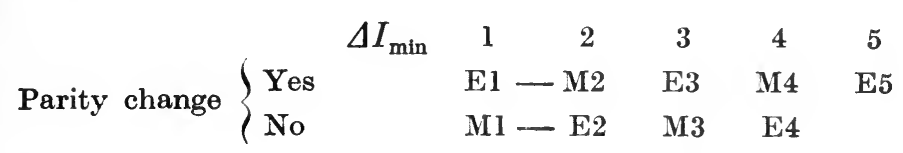

Where admixtures of multipole character higher than the minimum one have been observed they are indicated by a connecting line. An appreciable admixture is usually noticeable only when the matrix element representing the lowest possible multipole is relatively small, or that representing the next higher multipole is relatively large. Nevertheless, because of the strong dependence of $\gamma$-ray transition probabilities on the multipole character, the contribution to the $\gamma$-ray intensity from the second term is usually considerably less than from the first, with the most important exceptions occurring for $\mathrm{M} 1+\mathrm{E} 2$ and $\mathrm{E} 1+\mathrm{M} 2$ mixtures *. These exceptions may be connected with further selection rules (e.g. $l$-forbidden transitions ${ }^{5}$, transitions involving many particles or changes in the core shape or direction ${ }^{2}$, isotopic spin selection rules ${ }^{6}$, seniority selection rules ${ }^{7}$ ). No example of a mixed multipole radiation where a term higher than the second is of any importance is known, and as the lifetime formulae ${ }^{5}$ indicate, these higher admixtures may be usually expected to be negligibly small. When $I_{i}=I_{f}=1 / 2$, or when only one of the states has spin 0 (e.g. $\left.I_{i}>0, I_{f}=0\right), \Delta I$ has only one possible value; this means that the multipole character of the transition is necessarily pure $\mathrm{El}$ or $\mathrm{Ml}$ for the first case, and $\mathrm{E} I_{i}$ or $\mathrm{M} I_{i}$ in the second case, if

3 S. Devons, G. Goldring, and G. R. Lindsey, Proc. Phys. Soc. A67, (1954) 134.

4 J. C. Bowe, M. Goldhaber, R. D. Hill, W. E. Meyerhof and O. Sala, Phys. Rev. 73, (1948) 1219 (A).

5 S. Moszkowski, Chapter XIII:

- D. H. Wilkinson, Nature 172, (1953) 576.

7 G. Racah, Phys. Rev. 62, (1942) 438; A. De Shalit and M. Goldhaber, Phys. Rev. 92, (1953) 1211; J. Kraushaar and M. Goldhaber, Phys. Rev. 89, (1953) 1081.

* There have been reports of isolated cases of other mixtures, e.g. M4 + E5 for $\mathrm{Pb}^{204 m}$ (H. Frauenfelder, J. S. Lawson, W. Jentschke, and G. DePasquali, Phys. Rev. 92, (1953) 1241), but the older interpretation of this transition as pure E5 (A. W. Sunyar, D. E. Alburger, G. Friedlander, M. Goldhaber and G. ScharffGoldhaber, Phys. Rev. 79, (1950) 181) cannot be exluded. Recently, evidence for a possible M3 + E4 mixture, has been found in $\mathrm{Os}^{191 \mathrm{~m}}(\mathrm{~J}$. W. Mihelich and M. Goldhaber, Phys. Rev. (to be published)). 
$\left.I_{f}=0\right)$. For the theoretically possible $0+\rightarrow 0-$ transition, which can take place only by the emission of two photons, two internal conversion electrons. or one photon and one internal conversion electron ${ }^{8}$, no illustrative example has yet been found ${ }^{9}$. While pairs of states with spin differences $\Delta I>5$ are known for many nuclei, no isomeric transition between such states has thus far been observed. This can be explained by the low transition probability to be expected for multipole transitions with $\Delta I>5$ : some other process (e.g., an $\alpha$ or $\beta$-transition, or a $\gamma$-transition to another state "nearer in spin") is usually so much more probable that the transition of high multipole order is too weak to be detected by present methods.

While $\gamma$-transition probabilities can be obtained in absolute units $\left(\mathrm{sec}^{-1}\right)$, matrix elements have a meaning only within the framework of a definite model (e.g. the single-particle model). Even the relative values of matrix elements are not independent of a model, since the dependence of a transition probability on nuclear radius and energy is given by a function which is different for different models. We shall therefore follow here the convention ${ }^{1}$ of giving relative (or absolute) squares of matrix elements, or comparative $\gamma$-ray lifetimes, by means of the functional dependence on radius and energy given by the single particle model, and the constants which follow from this model for odd proton transitions. The use of "single proton" units is a valuable, but perhaps in some cases only a temporary, expedient.

\section{$\S 3$. Experimental Procedures}

A $\gamma$-ray transition between two states of energy $E_{i}$ and $E_{f}$, of spin $I_{i}$ and $I_{f}$, and parity $\pi_{i}$ and $\pi_{f}$, is completely characterized if besides these spins and parities we know from experiment the following: the (partial) $\gamma$-ray lifetime (or its inverse, the $\gamma$-ray transition probability) and the relative amount of admixture, if not negligible, of higher multipole order, as well as its relative phase $\left(0^{\circ} \text { or } 180^{\circ}\right)^{10}$. Often only part of this information is available, e.g. only the multipolarity or the lifetime.

To obtain absolute transition probabilities for $\gamma$-ray emission, the observed lifetimes of nuclear states have to be corrected for competing

8 R. G. Sachs, Phys. Rev. 57, (1940) 194; M. L. Goldberger, Phys. Rev. 73, (1948) 1119.

9 The continuum of photons reported for $\operatorname{Ir}^{192 m}$ (1.5 min) (M. Goldhaber, C. O. Muehlhause and R. Turkel, Phys. Rev. 71, (1947) 372; E. der Mateosian and M. Goldhaber, Phys. Rev. 82, (1951) 115) has been recently explained by J. P. Mize, J. W. Starner and M. E. Bunker, Phys. Rev. 95, (1954) 627 as due to bremsstrahlung accompanying the internal conversion electrons.

10 See chapter on angular correlation, H. Frauenfelder. Chapter XIX. 
processes, the most common of these being internal conversion. All competing processes shorten the lifetime of a nuclear state. In the case of internal conversion, the observed lifetime, $\tau_{\text {obs }}=\tau_{\gamma}(1 / 1+\alpha)$, where $\tau_{\gamma}$ is the lifetime for $\gamma$-ray emission alone, and $\alpha$ is the total internal conversion coefficient, i.e. the sum of the conversion coefficients for all electron shells. The competition from internal conversion depends on the exact nature of the electronic environment, which can be altered to only a slight extent. A noticeable change in lifetime of a nuclear state has been demonstrated in one case, that of $\mathrm{Tc}^{99 \mathrm{~m}}(6 \mathrm{~h})$, where the lifetime of the isomeric state has been shown ${ }^{11}$ to change as different chemical compounds of Tc are used (see Fig. 1). This is of considerable fundamental interest, as it illustrates most directly the fact that internal conversion competes with, rather than follows, $\gamma$-ray emission:

Fig. 1. The difference in intensity of two sources of $\mathrm{Tc}$ in different chemical states is shown. From K. T. Bainbridge, M. Goldhaber and E. Wilson, Phys. Rev. 90, (1953) 430

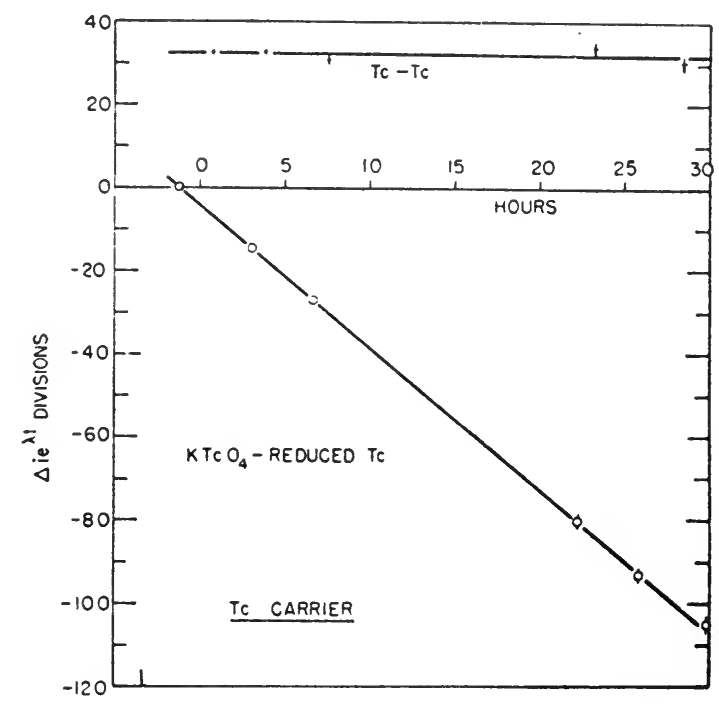

There are many ways of determining multipolarities. One of the most widely applicable methods consists of measuring the internal conversion coefficient for a particular atomic shell, e.g. the $K$-shell, and comparing it with the conversion coefficients computed by Rose et al. ${ }^{12}$. The degree of accuracy to which the experimental determination has to be made depends on the difference between the $K$ conversion coefficients of different (likely) multipole orders (compatible with other information, e.g. $K / L$ ratio, $L_{\mathrm{I}} / L_{\mathrm{III}} / L_{\mathrm{III}}$ ratio, lifetime). The method often also requires some knowledge

11 K. T. Bainbridge, M. Goldhaber and E. Wilson, Phys. Rev. 84, (1951) 1260.

12 M. E. Rose, G. H. Goertzel, B. I. Spinrad, J. Harr and P. Strong, Phys. Rev. 83, (1951) 79. See Appendix IV. 
of the "purity" of the multipole character, i.e. the percentage admixture of higher multipoles.

Since a knowledge of the internal conversion coefficient is essential for correct evaluation of radiative decay probabilities, we shall briefly discuss the experimental methods for measuring conversion coefficients, of which there are essentially three.

1) The absolute determination of the number of internal conversion electrons $N_{e}$, i.e. $\left(N_{e}\right)_{\text {tot }}$ or $\left(N_{e}\right)_{K},\left(N_{e}\right)_{L} \ldots$, and of the number of unconverted $\gamma$-rays, $N_{\gamma}$, leading to

$$
\varepsilon_{K, L \ldots \text { or tot }}=\frac{\left(N_{e}\right)_{K, L_{0} \ldots \text { or tot }}}{N_{\gamma}} .
$$

Such measurements often consist of comparing the number of conversion electrons emitted by a source into a definite solid angle to the number of unconverted $\gamma$-rays emitted by the same source into the same solid angle, as in magnetic spectrometer measurements utilizing external photoelectron conversion for the determination of the $\gamma$-ray intensity. In such a determination one must have an accurate knowledge of the conversion efficiency of the $\gamma$-rays into photo electrons under the particular experimental conditions employed. A similar type of measurement can be made with a scintillation spectrometer.

2) The measurement of the ratio of $N_{e}$ or $N_{\gamma}$ relative to a preceding or following radiation $(\alpha, \beta$, or $\gamma)$ when the decay scheme is completely known.

To illustrate, let us assume that a nuclear transition follows $\beta$-emission. Let $N_{\beta}$ be the total number of $\beta$-rays, $N_{e}=N_{K}+N_{L}+\ldots$ the number of conversion electrons for the transition in question, and $N_{\gamma}$ the number of unconverted $\gamma$-rays emitted per unit time. Let $f_{1}$ denote the fraction of $\beta$-disintegrations which populate the state in question. This includes those $\beta$-disintegrations which lead directly to the state and in addition those $\beta$-disintegrations to more highly excited states which ultimately decay to the state in question by $\gamma$-ray emission. Once the state is formed, it may decay via a number of competing transitions. Let $f_{2}$ denote the fraction of decays of this state which proceed via the transition under investigation. Then, since $f_{1} f_{2} N_{\beta}=N_{\gamma}+N_{e}$ and since

we find

$$
\varepsilon_{K, L_{\text {. } \ldots \text { or tot }}} \equiv\left(N_{e}\right)_{K, L_{\text {. } \ldots \text { or tot }}} / N_{\gamma} \text {, }
$$

$$
\frac{1}{\varepsilon_{K, L} \ldots \text { or tot }}=\frac{f_{1} f_{2} N_{\beta}}{\left(N_{e}\right)_{K_{0}, L_{1} \ldots \text { or tot }}}-1 \text {. }
$$

The ratio $N_{\beta} /\left(N_{e}\right)_{K, L} \ldots$ or tot can be determined experimentally in a magnetic spectrometer from the momentum distribution of electrons emitted by the source ${ }^{13}$. It is clear from the above relation that when $f_{1} f_{2} N_{\beta} / N_{e} \approx 1$,

13 M. A. Waggoner, M. L. Moon and A. Roberts, Phys. Rev. 80, (1950) 420; M. Deutsch and K. Siegbahn, Phys. Rev. 77, (1950) 680. 
unavoidable experimental errors render the determination of $\varepsilon$ subject to considerable uncertainty. The method is most useful in those cases where $\varepsilon$ is small and the decay scheme relatively simple (e.g. $f_{1}=f_{2}=1$ ). If the preceding radiation consists of a highly internally converted $\gamma$-transition (as e.g. in the two-step isomeric transitions ${ }^{14}$ in $\mathrm{Te}^{121}$ and $\mathrm{Te}^{123}$ ) the ratio of electron intensities gives the internal conversion coefficient for the second step in a straightforward manner.

When two or more $\gamma$-rays are emitted in cascade, a comparison of relative $\gamma$-ray intensities with a calibrated scintillation spectrometer gives a means of determining the total conversion coefficient for one of the transitions in the sequence, provided a) the total conversion coefficient for another transition in the sequence is either known or is negligibly small, and b) all features of the decay scheme are known. This method is quite useful when the conversion coefficient to be determined is reasonably large, provided that the unconverted $\gamma$-rays are still present in detectable quantity.

3) A determination of the number of $K \mathrm{X}$-rays, $N_{K}$, which accompany internal conversion in the $K$-shell, relative to the number, $N_{\gamma}$, of unconverted $\gamma$-rays.

Such measurements are customarily made with scintillation spectrometers ${ }^{15,16}$. The instrument must be calibrated for detection efficiency as a function of $\gamma$-ray energy. In addition, the decay scheme must be known, so that the number of $K \mathrm{X}$-rays arising from $K$-shell conversion of the transition in question can be determined. Coincidence techniques are often of considerable use in this respect. Assuming that the proper ratio $N_{K} / N_{\gamma}$ can be experimentally determined, a correction for the $K$-shell fluorescence yield ${ }^{17}$ must be applied. $\mathrm{NaI}(\mathrm{Tl})$ is the most widely used phosphor for this type of measurement. If the energy of the $K \mathrm{X}$-ray in question exceeds the $K$ work function of iodine, the escape of iodine $K \mathrm{X}$-rays from the crystal subtracts events from the $K \mathrm{X}$-ray photo peak. Such events reappear in the so-called "escape peak", and the escape peak intensity must, of course, be included in the evaluation of $N_{K} / N_{\gamma}$. The determination of $N_{\gamma}$ is also often complicated by the distribution of pulse heights arising from a single energy $\gamma$-ray. Calibration of the spectrometer in terms of photo peak intensities is particularly useful when the pulseheight distributions of several $\gamma$-rays are superimposed.

In order to evaluate radiative lifetimes for isomeric states, it is necessary

14 R. Katz, R. D. Hill and M. Goldhaber, Phys. Rev. 78, (1950) 9.

15 A. W. Sunyar, Phys. Rev. 83, (1951) 864.

16 F. K. McGowan, Phys. Rev. 93, (1954) 163.

17 R. M. Steffen, O. Huber and F. Humbel, Helv. Phys. Acta 22, (1949) 167. 
to apply the total conversion correction to the experimentally determined lifetime. The total conversion correction is obtained from one or more of the following procedures:

1) Absolute $K$ conversion coefficients computed by Rose et al. ${ }^{12}$ are used where applicable.

2) In a number of cases, reliable experimental values for $K$ conversion coefficients are available.

3) Conversion in outer shells is deduced from experimental $K / L / M \ldots$ ratios when these are known.

4) Empirical $K / L$ and $L_{\mathrm{I}} / L_{\mathrm{II}} / L_{\text {III }}$ ratio curves are used ${ }^{1,18}$.

5) For low energy and high multipole order transitions, where $L$ conversion is dominant, the partial tables of $L$-shell conversion coefficients of Rose, Goertzel and Swift ${ }^{19}$ have been used to estimate $L$ conversion, in conjunction with empirical $L_{\mathrm{I}} / L_{\mathrm{II}} / L_{\mathrm{III}}$ conversion ratios.

6) For low energy and low multipole order transitions, interpolations based on the $L$-shell computations of Gellman et al. ${ }^{20}$ and the low energy $K$ conversion computations of Reitz ${ }^{21}$ are of considerable aid in estimating total conversion coefficients.

The semi-empirical approach to the conversion correction which is often found necessary implies that some of the radiative lifetimes deduced are to be treated as tentative. However, it is unlikely that many drastic changes will be necessary when complete theoretical tables of $L$-shell and low energy $K$-shell conversion coefficients become available.

In deducing the spin difference between two states from internal conversion measurements, errors can arise in the following cases:

1) It is in principle possible to have a situation where the lowest multipole radiation compatible with the initial and final spins is so highly forbidden that only the next highest is observed. Under such circumstances the spin difference between two states would be overestimated by one unit if only internal conversion measurements were used.

2) The transition studied may be preceded or followed by a "hidden" transition, i.e. a transition of low energy which may be overlooked unless

18 J. W. Mihelich, Phys. Rev. 87, (1952) 646; G. A. Graves, L. M. Langer and R.J.D. Moffat, Phys. Rev. 88, (1952) 344; J. B. Swan and R. D. Hill, Aust. Journ. of Physics 6, (1953) 371; I. Bergström, K. Nybö, S. Thulin, A. H. Wapstra and B. Åström, A series of 8 papers, Arkiv För Fysik 7, (1954) 239.

19 M. E. Rose, G.H. Goertzel and C.J.Swift, private communication. See Ch. XIV.

20. H. Gellman, B. A. Griffith and J. P. Stanley, Phys. Rev. 85, (1952) 944.

21 J. R. Reitz, Phys. Rev. 77, (1950) 10. 
its existence is suspected. Under such circumstances the spin difference between two states might be underestimated by one or more units. It is therefore desirable either to measure spins of metastable states directly, as was recently done for ${ }^{22} \mathrm{Cs}^{134 \mathrm{~m}}$, where the existence of a "hidden" low energy transition was subsequently detected ${ }^{23}$ (see Fig. 2), or to obtain confirmation on spin assignments by other means, e.g. angular correlation experiments ${ }^{10}$ or deuteron stripping reactions ${ }^{23 a}$.

Fig. 2. Decay scheme of $\mathrm{Cs}^{134 m}$, as given by A. W. Sunyar, J. W. Mihelich and M. Goldhaber, Phys. Rev. 95, (1954) 571. The most plausible configurations for the odd proton and odd neutron leading to the observed spins and compatible with the measured magnetic moments are shown

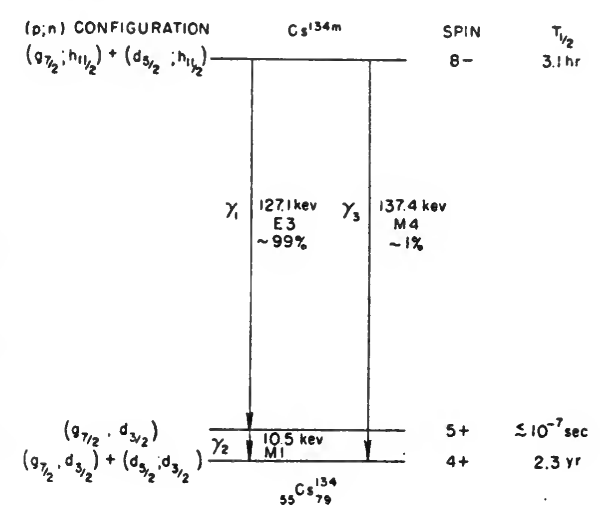

\section{§ 4. Some General Experimental Results}

The most widely studied and best understood isomers are the long lived ones found in odd $A$ nuclei. They occur in "islands" just before the number of protons or neutrons, whichever is odd, reaches a magic number (see Fig. 3, which is an up-to-date version of a figure given by Goldhaber and Hill ${ }^{24}$ ). Only two examples are known just beyond the two "magic barriers", at 50 and 126 neutrons: $\mathrm{Mo}^{93 \mathrm{~m}}{ }^{25,}{ }^{26}$ and $\mathrm{Po}^{211 \mathrm{~m}}{ }^{27}$, and they are probably due to the existence of complex excited states in which the odd neutron as well as the even protons or the core take part ("core isomerism" $\left.{ }^{25}\right)$. In $\mathrm{Mo}^{93 \mathrm{~m}}(7 \mathrm{~h})$ the energy of the isomeric state is extraordinarily high $(2.43 \mathrm{MeV})$. The transition to the ground state takes place in three steps ${ }^{28}$.

22 V. W. Cohen and D. A. Gilbert, Phys. Rev. 95, (1954) 569; L. S. Goodman and S. Wexler, Phys. Rev. 95, (1954) 570.

${ }^{23}$ A. W. Sunyar, J. W. Mihelich and M. Goldhaber, Phys. Rev. 95, (1954) 570.

${ }^{23 a}$ F. S. Eby, R. D. Hill and W. K. Jentschke, Phys. Rev. 93, (1954) 925.

24 M. Goldhaber and R. D. Hill, Revs. Mod. Phyṣ. 24, (1952) 179.

25 M. Goldhaber, Phys. Rev. 89, (1953) 1146.

26 J. J. Kraushaar, Phys. Rev. 92, (1953) 318.

27 F. N. Spiess, Phys. Rev. 94, (1954) 1292.

23 The spin sequence ${ }^{23} / 2+\stackrel{\mathrm{E} 4}{\longrightarrow} 15 / 2+\stackrel{\mathrm{E} 2}{\longrightarrow}{ }^{11} / 2+\stackrel{\mathrm{E} 2}{\longrightarrow} 7 / 2+$ is consistent with the evidence from angular correlation and internal conversion. However, as was pointed 
Below the magic numbers the longlived isomeric transitions are of the M4 or E3 type. The M4 and some E3 transitions can be accounted for by the strong spin orbit coupling model, assuming the excitation of a single odd particle. The existence of $\mathrm{E} 3$ transitions below $Z$ or $N=50$ requires the assumption of a $7 / 2+$ state, probably resulting from the coupling of several $g_{\% / s}$ states ${ }^{1,5}$.

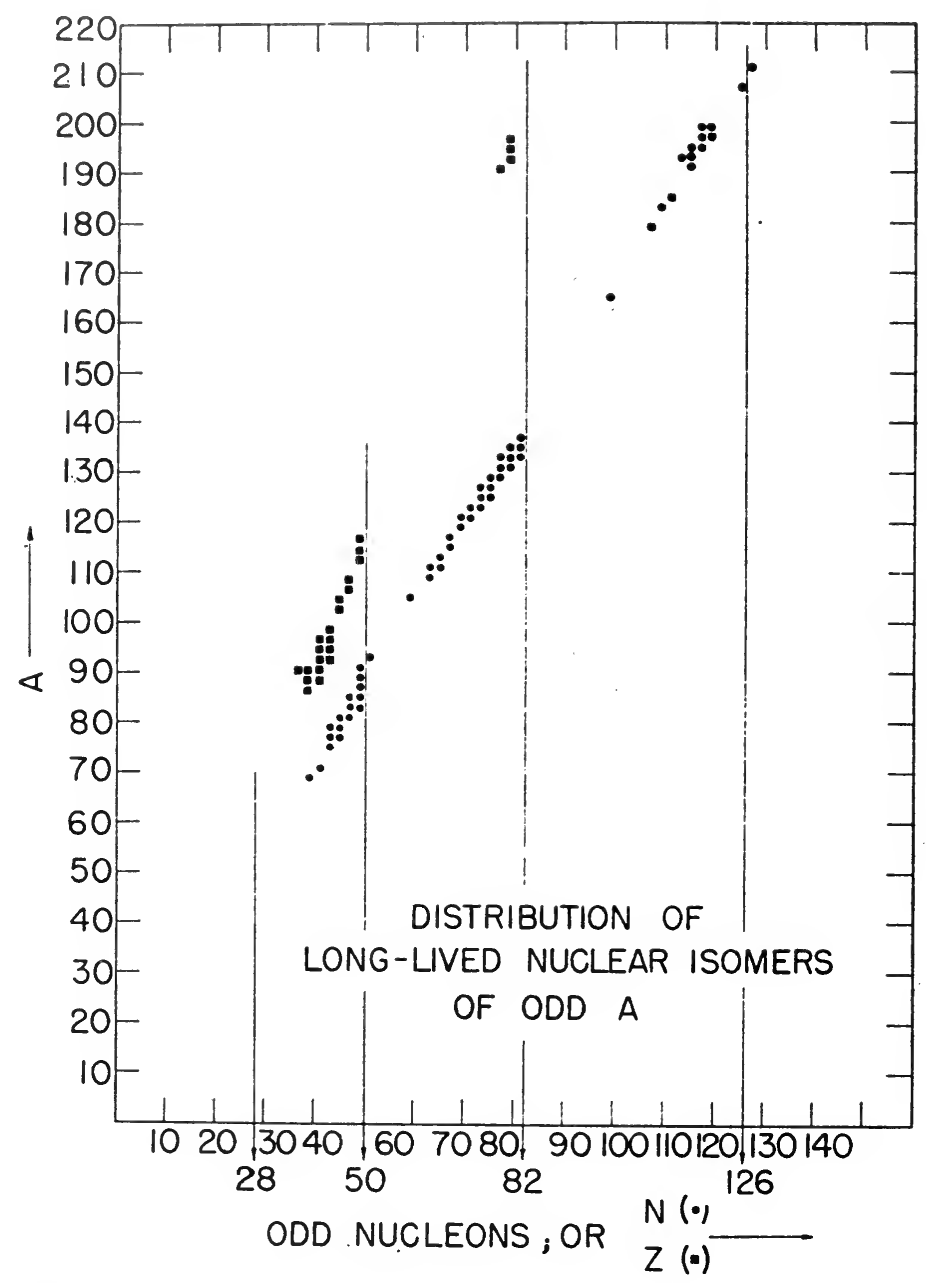

Fig. 3. The distribution of longlived isomers of odd mass number $A$, plotted against the number of odd nucleons $(N$ or $Z$ ). Based on a figure first given by Goldhaber and Hill (see ref. 24)

out by G. Scharff-Goldhaber and J. Weneser (private communication), the spin sequence $19 / 2+\mathrm{E}_{4} \rightarrow 11 / 2+\stackrel{\mathrm{E} 2}{\rightarrow}{ }^{7 / 2}+\mathrm{M} 1_{1}+\mathrm{E2}_{2} \rightarrow 5 / 2+$ is also compatible with existing evidence within the present experimental errors. 
The observed transitions (E1 ... E5; M1 ... M4) are found to have rates approximately equal to, or smaller, than expected theoretically for single proton transitions, with the exception of a large number of $\mathrm{E} 2$ transitions, especially in even-even nuclei far removed from magic numbers, which have considerably higher transition probabilities $1,29$.

The known radiative lifetimes (deduced from the observed lifetimes by the corrections mentioned above) are shown in Fig. 4 and some of the successive figures. For electric transitions the values of

$$
\log \left[\tau_{\gamma} A_{-\varsigma}^{\frac{2 \Delta I}{3}} E_{\gamma}^{2 \Delta I+1}\right]
$$

and for magnetic transitions the values of

$$
\log \left[\tau_{\gamma} A^{\frac{2 \Delta I-2}{3}}: E_{\gamma}^{2 \Delta I+1}\right]
$$

are plotted against neutron number, in order to bring out possible shell effects. Apart from statistical weight corrections, which cannot be made for all isomers, the points obtained can be compared directly with the theoretical predictions for single proton transitions ${ }^{5}$, represented by lines which are horizontal on such a plot. The E1 transitions shown in Fig. 4

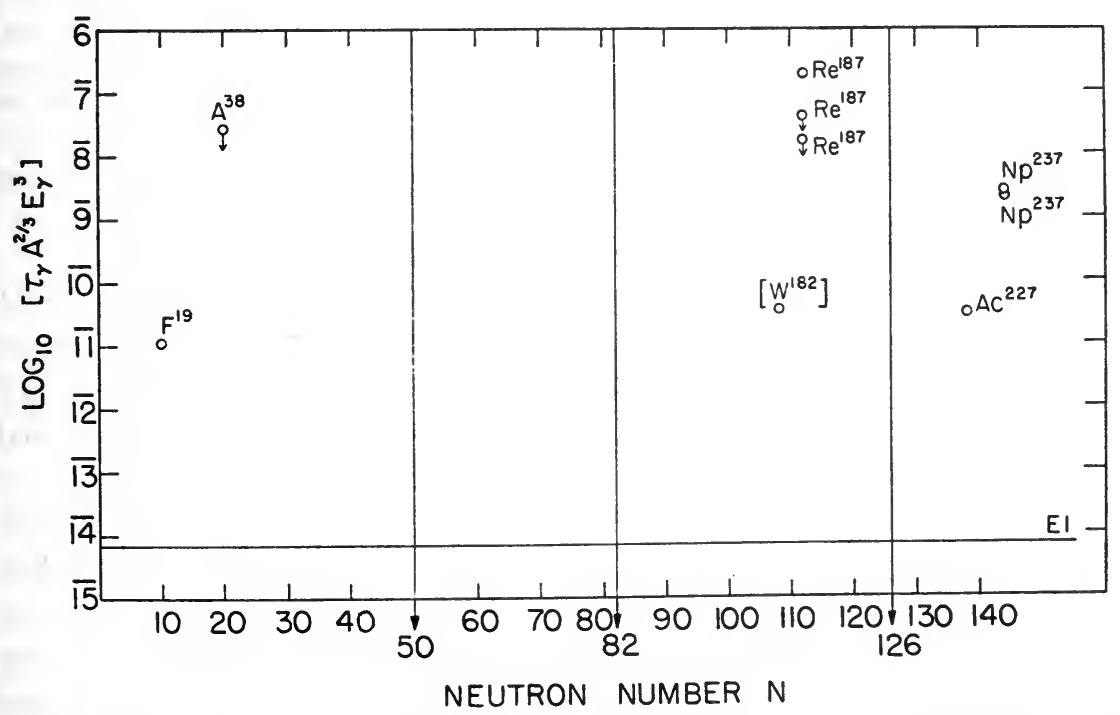

Fig. 4. Comparative lifetimes for low energy El transitions; plotted against neutron number $N$. The theoretical line corresponds to matrix elements equal to unity for single proton transitions

2. A. W. Sunyar, Phys. Rev. 95, (1954) 626. 
are seen to be from $10^{3}$ to $10^{7}$ times slower than expected for single proton transitions. This seems not unexpected, since on the shell model, most low energy El transitions are likely to be transitions involving at least one rather complex state. In light nuclei, high energy transitions exist $((p, \gamma)$ resonances) which have nearly the theoretical single particle speed ${ }^{30}$ (not shown in Fig. 4).

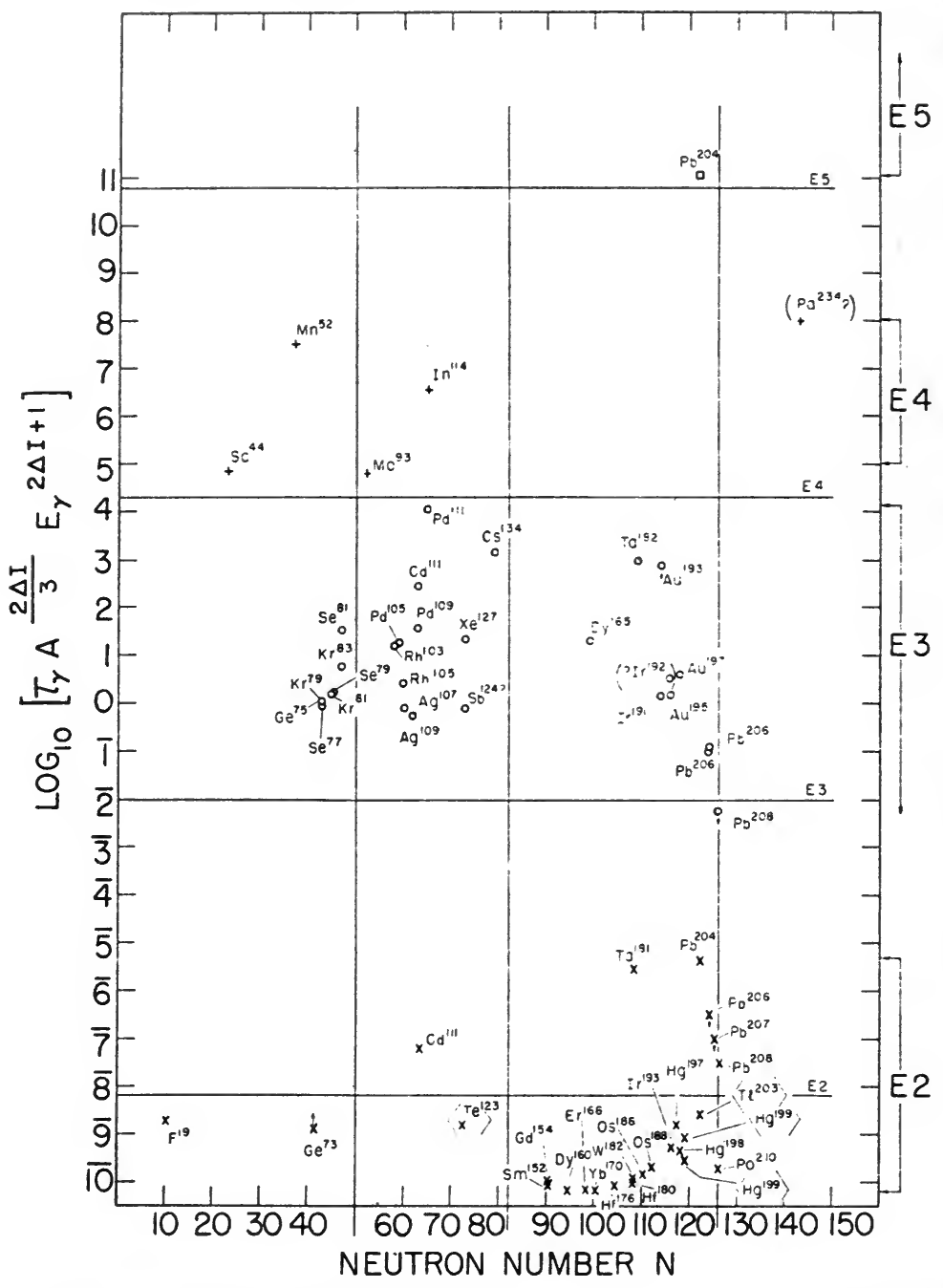

Fig. 5. Comparative lifetimes for E2, E3, E4 and E5 transitions, plotted against neutron number $N$. The theoretical lines, corresponding to matrix elements equal to unity for single particle proton transitions, are also shown. Points marked \langle\rangle are based on mixed transitions 
The E2, E3, E4 and E5 transitions are shown in Fig. 5. The following rules emerge: The E2 transitions are unique in being, with few exceptions, considerably faster than expected for single proton transitions. Also, the matrix elements vary fairly smoothly as a function of neutron number, being largest in the "middle" between magic numbers. Clearly, we are dealing here with a "cooperative" phenomenon ", interpreted by Bohr and Mottelson" as a "rotational" transition. The E2 transitions for eveneven nuclei are shown separately in Fig. $6{ }^{29}$.

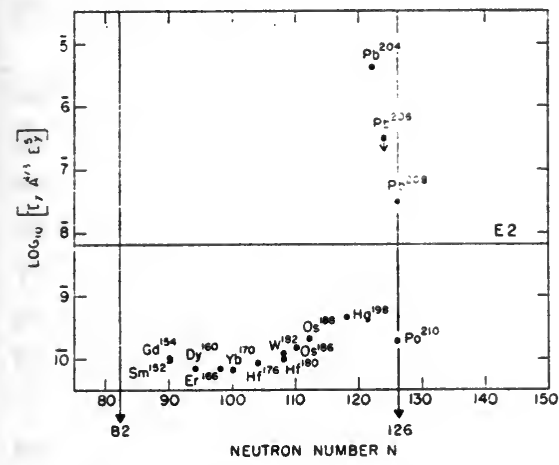

Fig. 6. Comparative lifetimes for E2 transitions in even-even nuclei, plotted against neutron number $N$. The theoretical line, corresponding to matrix elements equal to unity for single particle proton transitions, is also shown

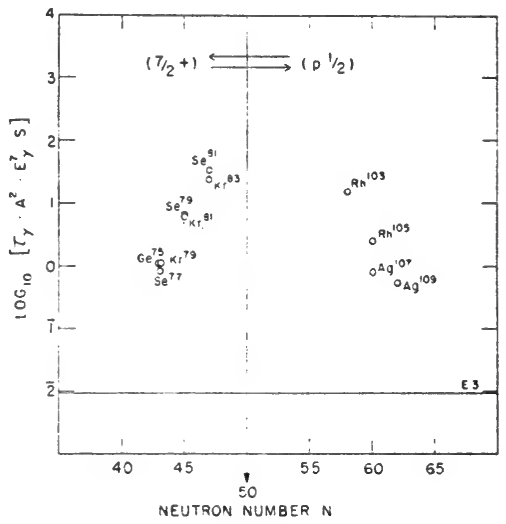

Fig. 7. Comparative lifetimes of $7 / 2+\stackrel{\text { E3 }}{\longrightarrow} p_{1 / 2}$ transitions, plotted against neutron number, $N$. The statistical weight correction was made as though we were dealing with single particle transitions

For E3 transitions, different kinds of shell structure effects show up around $N=50$, as well as for $N=126$ (Pb isotopes) where $Z$ is also magic. The region around $N=50$ is shown in more detail for the $7 / 2+\leftrightarrow p_{1 / 2}$ transitions in Fig. 7 , where a correction for the statistical weight ${ }^{5}$, as if we were dealing with single particle transitions, is included. It is seen that the lifetime increases in a regular manner for each element as one approaches $N=50$. This regularity was first noticed by Hill ${ }^{31}$. It may be connected with the increase in purity of the wavefunctions as one approaches the magic number. If the $7 / 2+$ states can be ascribed to $\left(g_{\%} /\right)_{7 / \mathrm{s}}^{3}$ configurations, the transitions $7 / 2+\leftrightarrow p_{1 / 3}$ are forbidden as one particle transitions and may therefore be slower as the wavefunctions become purer at the end of the shell. The apparent "speed up" of E3 transitions for $Z=82$ may be connected with the "enhancement" of (electric) transi- 
tions at closed shells which has been pointed out by Lane and Radicati ${ }^{32}$ and Wilkinson ${ }^{33}$.

Figure 8, brought up-to-date from a figure given by Goldhaber and Hill ${ }^{24}$, shows the interesting regular variations of the energy difference

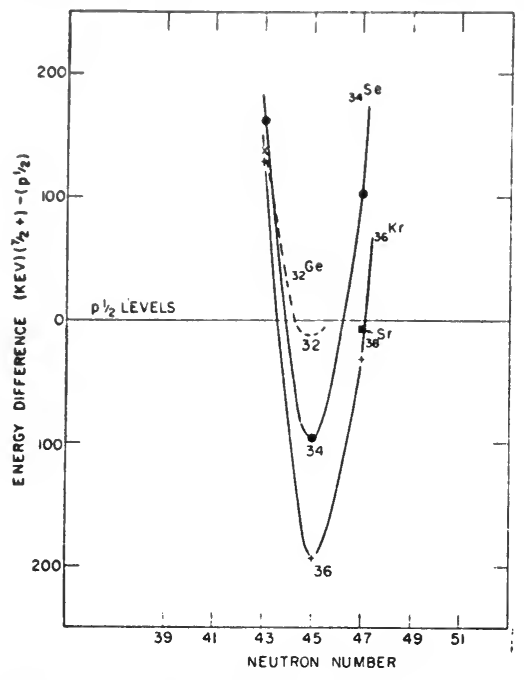

Fig. 8. The energy difference between $7 / 2+$ and $p_{1 / 2}$ levels for odd $N$ nuclei, plotted against neutron num. ber. Based on a figure given by

Goldhaber and Hill (ref. 24) between the $7 / 2+$ and $p_{1 / \text { s }}$ states. Such regularities are of great help in finding new isomers and are a challenge to a more detailed theory. Many similar regularities have been reported previously for odd $A$ nuclei ${ }^{24}$ and for even-even nuclei ${ }^{34}$.

Figure 9 gives a summary of the comparative lifetimes known for all magnetic transitions, except for some high energy Ml transitions in the light elements, which are discussed by Wilkinson ${ }^{30}$. It can be seen that the matrix elements for M1, M2 and M3 transitions scatter considerably, whereas for the M4 transitions they are remarkably uniform. This is brought out more clearly for odd $A$ nuclei in Fig. 10, where the statistical weight correction given by Moszkowski ${ }^{5}$ has been made. It is interesting to note that, contrary to theoretical expectations ${ }^{5}$, odd proton and odd neutron transitions exhibit no systematic difference. Shell effects are not very large. In order to establish the existence of such effects better experimental data and more conclusive spin assignments are required in some cases.

\footnotetext{
32 A. M. Lane and L. A. Radicati, Proc. Phys. Soc. A67, (1954) 167, 410A.

33 D. H. Wilkinson, Report at Photo-Nuclear Conference at Philadelphia, May 1954.

34 G. Scharff-Goldhaber, Phys. Rev. 90, (1953) 587; P. Preiswerk and P. Stähelin, Nuovo Cimento 10, (1953) 1219.
} 


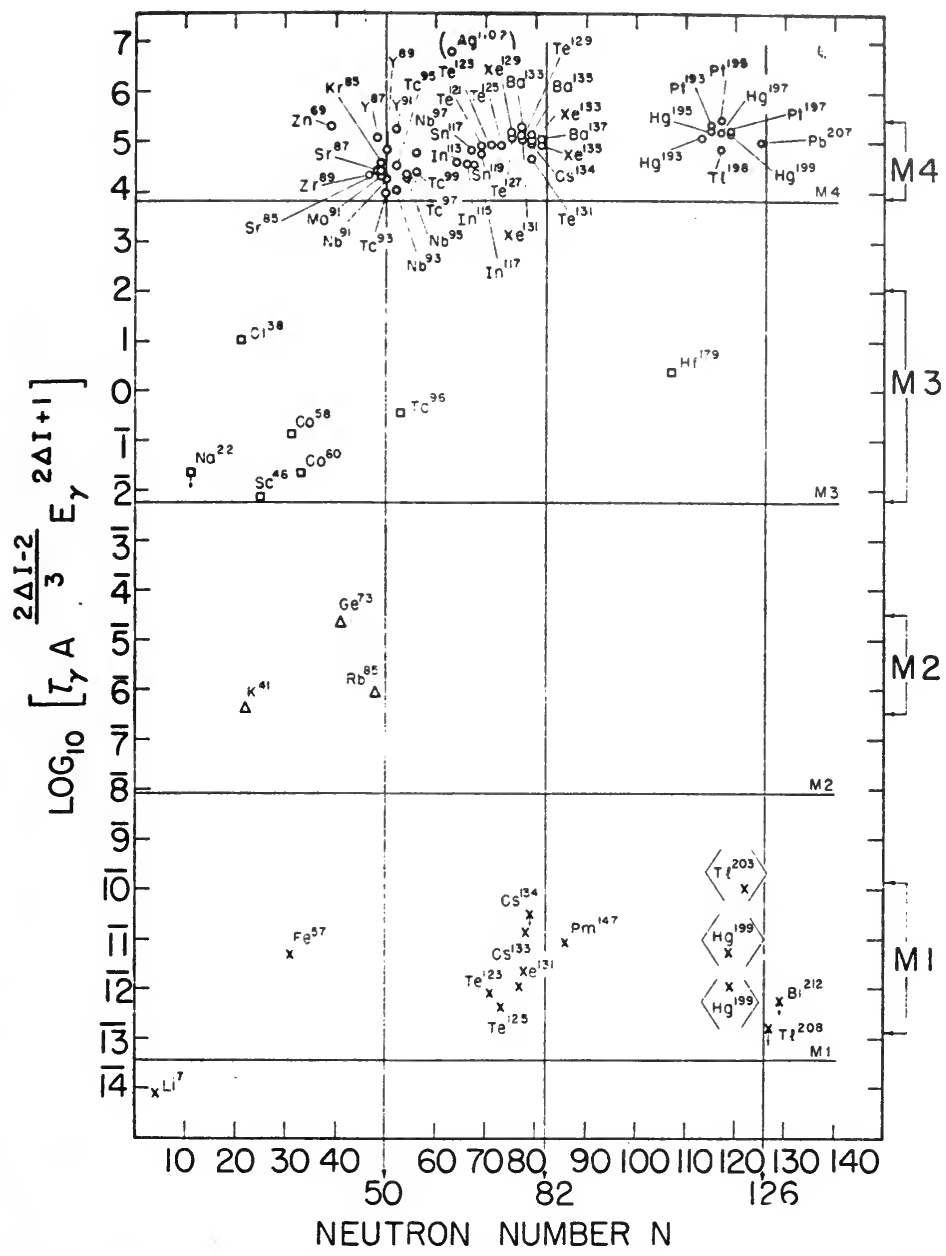

Fig. 9. Comparative lifetimes for M1, M2, M3 and M4 transitions plotted against neutron number, $N$. The theoretical lines corresponding to matrix elements equal to unity for single proton transitions are also shown. Points marked $\langle>$ are based on mixed transitions

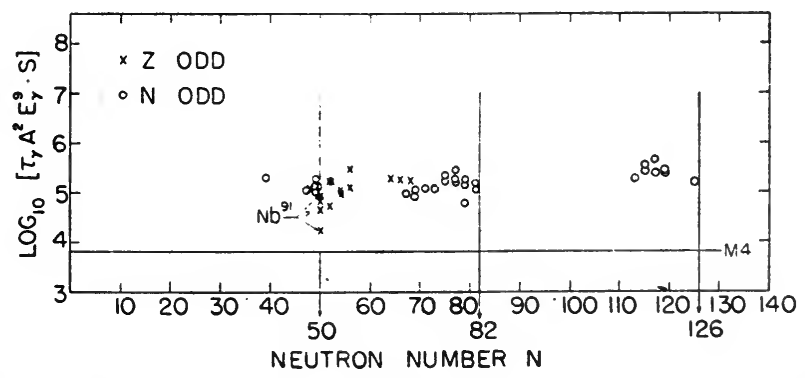

Fig. 10. Comparative lifetimes for $\mathbf{M} 4$ transitions occurring in odd $A$ nuclei. Statistical weight corrections have been applied 


\section{A. BOHR aNd B. R. MOTTELSON}

\section{$\S 1$. Collective Oscillations of a Shell Structure}

While a large body of empirical data has revealed the existence of a nuclear shell structure (cf. Ch. XV and XVI of this book), other features of the nuclear phenomena exhibit the influence of collective nuclear motion ${ }^{1}$. Indeed, it is to be expected from very general arguments that a system of particles held together by their mutual attraction can perform collective oscillations which resemble the degrees of freedom possessed by matter in bulk ${ }^{2}$.

The generalization of shell models to include collective motion can be effected in a straightforward manner. In fact, the shell model operates with an average field in which the particles more and which is generated collectively by all the particles. In the usual shell models, this field is considered a static quantity, but a more complete description must take into account the variation of the field associated with collective oscillations.

In the atomic shell model, this aspect of the structure is of relatively minor significance due to the stabilizing influence of the heavy central nucleus. However, the lack of such an external stabilization of the nuclear field implies that the collective degrees of freedom are of fundamental significance for the nuclear dynamics.

Since the collective oscillations involve variations in the average nuclear field, they are strongly coupled to the motion of the individual nucleons. The unified model thus describes the nucleus in terms of a coupled system of particle and collective degrees of freedom. In many respects, the dynamics of such a system is similar to that of molecules, where one has a coupled system of electronic motion and collective rotations and vibrations of the structure as a whole.

The most important collective degrees of freedom for the low energy nuclear properties are expected to be those associated with oscillations in shape with approximate preservation of the nuclear volume. For a nucleus of constant density, these oscillations have the character of surface waves.

1 Cf., e.g., N. Bohr and J. A. Wheeler, Phys. Rev. 56, (1939) 426; J. Rainwater, Phys. Rev. 79, (1950) 432; A. Bohr, Phys. Rev. 81, (1951) 134 and Dan. Mat. Fys. Medd. 26, (1952) no. 14 ; D. L. Hill and J. A. Wheeler, Phys. Rev. 89, (1953) 1102 ; A. Bohr and B. R. Mottelson, Dan. Mat. Fys. Medd. 27, (1953) no. 16. The latter reference will be denoted by $\mathrm{BM}$ in the following and its sections and equations designated by BII $\S I I b$, iii and BII VII, 19, etc.

${ }^{2}$ N. Bohr and F. Kalckar, Dan. Mat. Fys. Medd. 14, (1937) no. 10. 
Oscillations of compressive character (sound waves) and those involving a motion of the neutrons with respect to the protons (dipole oscillations) are generally of appreciably higher frequency and are thus of lesser importance for the low energy nuclear properties considered in this chapter.

For small amplitudes of oscillation, the normal modes of vibration are approximately of the harmonic oscillator type, and can be classified by their multipole order $(\lambda, \mu)$, corresponding to an expansion of the nuclear shape in spherical harmonics. In general, the oscillations in shape of lowest order, $\lambda=2$, are of primary importance. A deformation of order $\lambda=1$ simply corresponds to a displacement of the whole system.

The frequencies of oscillation depend on the structure of the collective flow and on the nuclear binding forces. While a quantitative determination in terms of the interactions in the system is difficult, an orientation in the magnitudes involved may be obtained by considering the simplified case of an incompressible uniformly charged nucleus (hereafter referred to as the incompressible model ${ }^{3}$ ) and relating the nuclear deformability to the surface tension as deduced from the empirical binding energies. The quanta of the shape oscillations are referred to as phonons and their energies estimated from the incompressible model are shown in Fig. 1.

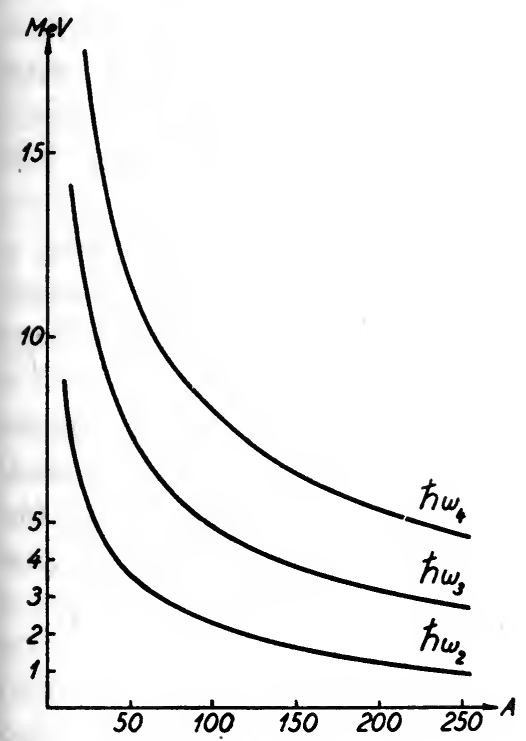

Fig. 1. Phonon energies

The quantum energies $\hbar \omega_{\lambda}$ for nuclear shape oscillations, of multipole orders $\lambda=2,3$ and 4 , are shown as functions of the mass number $A$. The above estimates refer to the incom. pressible model, and the parameters are the same as used in BM, §II $a$, ii. The actual oscillation energies are expected to depend to some extent on the nucleonic configuration and to be somewhat larger than this average estimate in the case of closed shell configurations.

The coupling between particle and collective motion reflects the dependence of the particle energy on the nuclear deformation and in turn implies

3 In BM, this simplified model was referred to as "the hydrodynamical approximation". 
a tendency of the particle structure to produce a deformation in the nuclear field ${ }^{4}$. The properties of the nucleus depend in an essential way on the magnitude of this deformation.

In the next paragraph. we shall discuss the nuclear coupling scheme and the excitation spectrum arising from the coupled motion of individual particles and collective oscillations. In subsequent paragraphs, we discuss the implications of this unified model for various nuclear properties of interest in connection with investigations of nuclear $\beta$ - and $\gamma$-radiation. A more comprehensive discussion of the model and of the available empirical evidence has been presented elsewhere (cf. BM, which also contains references to other papers on this subject).

\section{$\S 2 . \quad$ Nuclear Coupling Scheme}

\section{A. Regios of C'losed Shells}

For a nucleus in which both neutrons and protons form closed shells, the spherical shape represents a stable equilibrium. In the immediate neighbourhood of such a configuration, the deformations are therefore small, and one retains many of the features of the static shell model. The system possesses, however, the additional degrees of freedom associated with the oscillations in shape. These degrees of freedom are expected to manifest themselves in the nuclear energy spectrum as excitation quanta with spin $\lambda$, parity $(-)^{\lambda}$, and energy $\hbar \omega_{\lambda}$. These states would be characterized, among other things, by enhanced electric transition probabilities (cf. $\S 4 \mathrm{~B}, \mathrm{i}$, below).

The coupling between the particles and the nuclear shape implies a slight interweaving of these vibrational modes with the particle motion. The resulting modifications in the nuclear properties can be studied by means of a perturbation calculation.

Already for configurations involving a few particles or holes outside of closed shells, the nuclear states involve appreciable interweaving between particle and collective motion. Solutions of the coupled equations in this region are usually difficult to obtain, but some exploratory studies have been made ${ }^{5}$.

\section{B. Stroxgly Deformed Nuçlei}

For configurations sufficiently far removed from closed shells, the nucleus acquires a large equilibrium deformation resulting from the

4 J. Rainwater, Phys. Rev. 79, (1950) 432.

5 Cf. L. L. Foldy and F. J. Milford, Phys. Rev. 80, (1950) 751 ; BM, IIb, i and iii; A. K. Kerman, Phys. Rev. 92, (1953) 1176; D. C. Choudhury, Dan. Mat. Fys. Medd. 28, (1954) no. 4; F. J. Milford, Phys. Rev. 93, (1954) 1297. 
deforming effects of many nucleons; the nuclear coupling scheme then acquires a relative simplicity resembling that of a linear molecule. Thus, one obtains an approximate solution by considering first the relatively fast motion of the particles with respect to the deformed nuclear field, considered as fixed in space, and subsequently the relatively slow vibration and rotation of the entire system.

The equilibrium shape of the nuclear field is determined by considering the energy of the particles and of the field as a function of the deformation, considered as fixed, and minimizing this energy.

For most configurations, the equilibrium shape possesses axial symmetry so that orbits of the individual particles may be characterized by their component of angular momentum, $\Omega_{p}$, along the symmetry axis; the total angular momentum of a particle is in general not a constant of the motion for such non-spherical fields. The quantum numbers $\Omega_{p}$ take on half integer values, positive or negative. States differing only in the sign of $\Omega_{p}$ are degenerate since they are identical, except for the opposite sense of rotation about the axis. The $\Omega_{p}$ of the individual particles add up to a total component, $\Omega$, of particle angular momentum along the nuclear symmetry axis.

The ground state of a nucleus is obtained by filling the particles pairwise in states of opposite $\Omega_{p}$. Thus, in an even-even nucleus, one obtains a total $\Omega=0$, while in an odd $A$ nucleus the total $\Omega$ is equal to the $\Omega_{p}$ of the last odd particle. In an odd-odd nucleus, the last odd neutron and proton each contribute an $\Omega_{p}$ and the total is either the sum or difference of the two.

The next step in the solution of the equations of motion for strongly deformed nuclei involves the consideration of the oscillations around the equilibrium shape. A necessary condition for the validity of the solution is that the amplitudes of these vibrations are small compared to the total magnitude of the deformation. If we restrict ourselves to quadrupole deformations, there are two types of normal vibrations. The first preserves cylindrical symmetry and only implies variations in the eccentricity of the spheroidal shape. The second leads to deviations from cylindrical symmetry. For small oscillations about the equilibrium shape, these modes are expected to be approximately harmonic and we denote their quantui.. numbers by $n_{\beta}$ and $n_{\gamma}$, respectively. The excitation energies are of the order of magnitude of $\hbar \omega_{2}$ (cf. Fig. 1), but should depend somewhat on the configuration of the nucleons. An anomaivusly low energy associated with $n_{\gamma}$ may result for special particle configurations which do not strongly prefer deformations possessing cylindrical symmetry ${ }^{6}$.

- Cf. e.g., BM, Ap. III, ii; B. Segall, Phys. Rev. 95, (1954) 605 (A); M. Jean, to be published. 
Finally, the system as a whole may rotate with the preservation of the shape and the internal structure. This motion gives rise to a spectrum of the same type as that of rotating rigid bodies, although the collective motion that generates the nuclear rotation is essentially different from that of a rigid body and may best be pictured as a wave travelling around the nucleus (cf. Fig. 2). The moment of inertia associated with such a

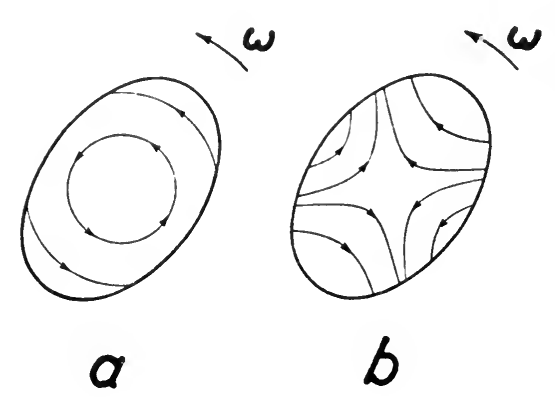

Fig. 2. Velocity fields for collective rotations Fig. $2 a$ illustrates the rotation of a rigid body, while in Fig. $2 b$ the velocity field for the wave-like rotation of an irrotational fluid is shown. While the former motion refers to a system in which each particle is located near an equilibrium position, the latter type of rotation is characteristic of a shell structure, like the nucleus, in which the individual particles freely traverse the entire volume of the system.

wave-like rotation is small compared to the total moment characterizing the rigid rotation; it is proportional to the square of the amplitude of the wave, i.e. to the square of the nuclear deformation with respect to the axis of rotation.

For a nucleus with axial symmetry, the spectrum is that of a symmetric top and the energy of a state with total angular momentum $I$ is given by

$$
E_{\mathrm{rot}}=\frac{\hbar^{2}}{2 \jmath} I(I+1)
$$

where the moment of inertia is

$$
J=\frac{2}{5} M A(\Delta R)^{2}
$$

for a spheroidal deformation of the incompressible model. In this expression, $\Delta R$ represents the difference between the major and minor semiaxis of the spheroid, which is assumed to be small compared to the mean radius $R_{0}{ }^{7}$. The nucleon mass is denoted by $M$, and $A$ is the nuclear mass number.

The rotations of the symmetric nucleus are characterized by the three quantum numbers $I, K, M$, where $K$ and $M$ are the components of $I$ along the intrinsic symmetry axis and a space fixed axis, respectively.

The coupling scheme for strongly deformed nuclei is illustrated in Fig. 3. The wave function for such a system can be constructed in analogy to that for diatomic molecules and consists of a product of three parts

7 In BM, the quantity $\beta=4 / 3 \sqrt{\pi / 5}\left(\Lambda R / R_{0}\right)$ has been employed to describe the magnitude of the deformation. 
representing the particle motion, the collective vibration, and rotation, respectively.

In the nuclear ground state, $K=\Omega$, and since the moment of inertia about an axis of symmetry vanishes, changes in $K$ involve the excitation of asymmetric vibrations characterized by the quantum number $n_{\gamma}{ }^{8}$. The value of $I$ must be at least equal to the numerical value of $K$.

Fig. 3. Coupling scheme for strongly deformed nuclei

For a strongly deformed nucleus possessing axial symmetry, the individual particles are coupled separately to the symmetry axis in states characterized by the projection $\Omega_{p}$ of their angular momentum on the symmetry axis $S$. The sum of the individual $\Omega_{p}$ gives the resultant $\Omega$, which for the lowest rotational band is equal to the projection $K$ of the total angular momentum $I$ on $S$. In this case, the collective rơtational angular momentum $R$ is perpendicular to $S$. The component of $I$ on a fixed $Z$-axis is denoted by $M$.

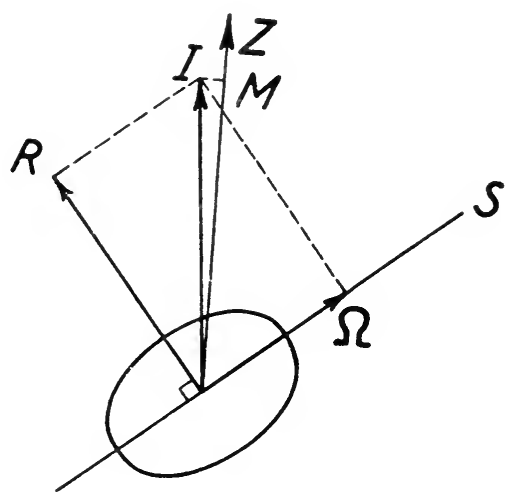

The possible quantum states of the nucleus are restricted by the reflection symmetry of the deformation, which implies that states labelled by $(K, \Omega)$ must be combined in a definite way with those labelled by $(-K,-\Omega)^{9}$. Moreover, the reflection symmetry implies that the collective motion has even parity, and the parity of a nuclear state is, therefore, determined by that of the particle structure.

Thus, for an even-even nucleus, in which the ground state has $K=\Omega=0$, the lowest rotational band is given by (1) with spins and parities

$$
I=0,2,4,6 \ldots \text { even parity. }
$$

The odd values of $I$ are excluded by the above mentioned symmetry condition, which requires the wave function to be invariant to a rotation of $180^{\circ}$.

In an odd- $A$ or an odd-odd nucleus (or in an even-even nucleus with an excited particle structure with $\Omega \neq 0$ ), the ground state has normally $I_{0}=K=\Omega$ and a rotational spectrum given by (1) with

$$
I=I_{0}, I_{0}+1, I_{0}+2, \ldots \text { same parity as ground state. }
$$

A special case occurs for an odd- $A$ nucleus with $\Omega=1 / 2$; the intrinsic spin

8 Cf. BM, § VIc, iv.

$\rightarrow$ Cf. BM, II, 15. 
of the particle is then partly decoupled from the rotational motion, which implies a modified rotational spectrum

with

$$
E_{\mathrm{rot}}=\frac{\hbar^{2}}{2 J}\left[I(I+1)+a(-)^{I+1 / 2}(I+1 / 2)\right]
$$

$$
a=\sum_{j}(-)^{1-1 / 2}(j+1 / 2)\left|c_{j}\right|^{2}
$$

where $\left|c_{j}\right|^{2}$ represents the probability that the last odd particle has an angular momentum $j$. This correction term leads to an anomalous rotational spectrum and may also yield a ground state spin $I_{0} \neq \Omega$.

Thus, the spectrum for a strongly deformed nucleus is expected to exhibit three distinct types of excitation. The first corresponds to a change in the particle motion with respect to the deformed field and is characterized by a change in the quantum numbers $\Omega_{p}$; the energies involved in such excitations are determined by the position of particle levels in a spheroidal field ${ }^{10}$, which have an average spacing of about $100 \mathrm{keV}$ in a heavy nucleus. The second mode of excitation is associated with the collective vibrations characterized by the quantum numbers $n_{\beta}$ and $n_{\gamma}$, and for a heavy nucleus the vibrational energies are expected to be about 1 or $2 \mathrm{MeV}$ (cf. Fig. 1). The third mode of excitation is the nuclear rotation with preservation of shape and is characterized by the quantum number $I$; the rotational energies depend on the nuclear deformation and become very much smaller than the vibrational energies for the strongly deformed nuclei.

This simple separation of the spectrum represents a limiting case; for less deformed nuclei, the more rapid rotational motion will somewhat distort the shape of the nucleus and will also perturb the particle structure due to the inability of the particles to follow the rotations completely adiabatically. While the latter effect depends specifically on the particle configurations in question, the former, which is analogous to the rotationvibration interaction in molecules, can be expressed in terms of the vibrational energies. The resulting correction term is, to leading order, given by

$$
\Delta E=-2\left[\frac{3}{\left(\hbar \omega_{\beta}\right)^{2}}+\frac{1}{\left(\hbar \omega_{\gamma}\right)^{2}}\right] \frac{\hbar^{2}}{J} E_{\mathrm{rot}}^{2},
$$

where $\hbar \omega_{\beta}$ and $\hbar \omega_{\gamma}$ are the vibrational quanta associated with the quantum numbers $n_{\beta}$ and $n_{\gamma}$, respectively: The rotational energy, $E_{\text {rot }}$, in (6) is given by (1) for $\Omega \neq 1 / 2$ and by (5) for $\Omega=1 / 2$.

While the details of the expressions (6) depend on the assumptions of the incompressible model, the dependence of the vibration-rotation interaction on the spin, $I$, is expected to be more general.

10 For a calculation of the single-particle level spectrum in a spheroidal potential, ef. S. G. Nilsson, to be published in Dan. Mat. Fys. Medd. 


\section{$\S$ 3. Spins and Moments}

\section{A. Ground State Spirs}

The ground state spin is determined in general by the competition of the direct forces between particles outside of closed shells and the forces coupling the particles to the nuclear deformation. For small deformations, as near major closed shells, the former forces may dominate, coupling the individual particle angular momenta together to a resultant $J$. The particular $J$ value favoured depends somewhat on the range of the forces and their exchange character (cf. Ch. XV of this book). With increasing deformation the interaction of the particles with the nuclear shape dominates, and the particles become coupled independently to the nuclear symmetry axis in states characterized by $\Omega_{p}$. The nuclear ground state spin then has the value $I_{0}=\Omega=\sum \Omega_{p}$.

The various couplings have been studied in some detail for only certain especially simple configurations involving a few particles outside of closed shells and on the assumption that the particles can be characterized by a definite $j^{100}$.

For a single particle, one finds $I_{0}=j$, irrespective of the nuclear deformation.

For two equivalent particles, both particle forces and the coupling to the deformation favour the ground state spin $I_{0}=0$.

For three equivalent particles, two-body forces of the expected range produce $I_{0}=j$, while the three lowest $\Omega_{p}$ values are $\Omega_{p}= \pm j, j-1$, which yield $I_{0}=\Omega=j-1{ }^{11}$. In this case, the resulting ground state spin is expected to depend on the magnitude of the deformation. The observed spin values for these configurations exhibit both values $I_{0}=j$ and $j-1$, and the trends may be interpreted in terms of the influence of the deformation ${ }^{12}$.

For more complicated many-particle configurations, the effect of particle forces and their competition with the coupling to the nuclear deformation may present very difficult problems, especially since the rather strongly deformed shapes encountered for these configurations imply that the particle orbitals are essentially different from those in a spherical potential.

100 More general calculations of the coupling scheme for interacting particles moving in a spherical potential have been made for certain light nuclei; cf. e.g., D. Inglis, Rev. Mod. Phys. 25 (1953) 390 and B. H. Flowers, reported at Glasgow Conference, July 1954.

11 The coupling to the collective motion can also be studied for small deformations by the perturbation method and yields the ground state spins: $I_{0}=5 / 2$ for $(j=5 / 2)^{3}$; $I_{0}=8 / 2$ for $(j=7 / 2)^{3} ; I_{0}=7 / 2$ for $(j=8 / 2)^{3}$. We are indebted to Drs. I. Talmi and C. Schwartz for pointing out an error in these results as quoted in BM, p. 35 . 12 Cf. BM, § III, iii. 
However. for sufficiently large deformations, the problem again simplifies, since the particles become coupled individually to the nuclear axis. Thus, for any even-even nucleus, the total $\Omega$ vanishes and one obtains the ground state spin $I_{0}=0$. For an odd- $A$ nucleus. the ground state spin equals the $\Omega_{p}$ of the last odd particle and could thus be obtained from the single-particle level scheme in the deformed field. (Note, however, the anomalous case of $\Omega=1 / 2$; cf. p. 473 above).

In an odd-odd nucleus, the total $\Omega$ of the lowest states may equal either the sum or the difference of the $\Omega_{p}$ 's of the last odd proton and last odd neutron. One thus obtains two close lying states of the particle structure.

\section{B. Quadrupole Moments}

The occurrence of nuclear quadrupole moments an order of magnitude larger than those associated with single-proton orbits gives some of the most direct evidence for the cooperative behaviour of the nucleons. At the same time, the nuclear shell structure manifests itself in the signs and the variations in magnitude of the quadrupole moments ${ }^{13}$. The moments are small in the region of closed shells and become larger with the addition of more particles. It is possible to account for these trends in terms of the tendency of the particle structure to produce a collective deformation of the nucleus ${ }^{14}$.

While the spectroscopically determined quadrupole moment, $Q$, characterizes the asymmetry in the averaged charge density in the nuclear state, the shape of the nucleus is more directly described in terms of the intrinsic quadrupole moment with respect to the nuclear symmetry axis. For the incompressible model, the intrinsic quadrupole moment is

$$
Q_{0}=\frac{4}{5} Z R_{0}^{2} \frac{\Delta R}{R_{0}}
$$

The relationship between $Q$ and $Q_{0}$ depends on the nuclear coupling scheme. For the strongly deformed nuclei, one has

$$
Q=\frac{I_{0}}{I_{0}+1} \frac{2 I_{0}-1}{2 I_{0}+3} Q_{0}
$$

for the ground state $\left(I_{0}=K\right)$. This reduction of $Q$ as compared with $Q_{0}$ is a consequence of the quantum fluctuations in the direction of the nuclear axis.

13 C. H. Townes, H. M. Foley and W. Low, Phys. Rev. 76, (1949) 1415.

14 J. Rainwater, Phys. Rev. 79, (1950) 432; for references to more detailed discussions, ef. BM, Chapter V. 


\section{Magnetic Monests}

In the unified model, the magnetic moments result in part from the collective motion and in part from the motion of the particles. The sharing of angular momentum between particle and collective motion, and the modification of the particle orbitals resulting from motion in a nonspherical field, both cause appreciable shifts of the magnetic moments from those corresponding to single-particle motion in a spherical potential.

For strongly deformed nuclei, the magnetic moment has an especially simple structure and, for a nuclear ground state with $I_{0}=\Omega \neq 1 / 2$,

$$
\mu=\frac{I_{0}^{2}}{I_{0}+1} g_{\Omega}+\frac{I_{0}}{I_{0}+1} g_{R},
$$

where $g_{\Omega}$ is the $g$-factor characterizing the state of the last odd particle in the deformed potential, and where $g_{R}$ is the $g$-factor for the collective rotational motion, given by

$$
g_{R}=\frac{Z}{A}
$$

for the incompressible model.

\section{$\S 4$. Nuclear Transition Probabilities}

As discussed in $\S 2$, one expects, according to the unified model, in the region of closed shells as well as for the strongly deformed nuclei, to be able to distinguish between particle excitations, which are associated with a change in the quantum state of the particle structure, and collective excitations, which involve vibrations or rotations of the nucleus as a whole, leaving the particle structure unaffected. In an intermediate region with relatively small deformations, however, the particle and collective motion may be interwoven in a more complex manner.

The measurement of transition probabilities provides an important tool for distinguishing these two modes of excitation. Such evidence comes from the $f t$-values of $\beta$-transitions and from the reduced transition probability for $\gamma$-decay ${ }^{15}$. More recently, the Coulomb excitation reaction has provided an alternative method for determining electromagnetic transition probabilities ${ }^{16}$.

15 For the definition of the reduced transition probability $B\left(L ; I_{i} \rightarrow I_{f}\right)$, cf., e.g., Cl. XIII.

18 The Coulomb excitation cross-section can be written as a product of two factors, of which the first depends only on the bombarding particles and on the nuclear charge and excitation energy, while the second is identical with the reduced transition probability which characterizes the radiative transition of corresponding multipole order. For a recent review of the theory and applications of the Coulomb excitation reaction, cf. K. Alder, A. Bohr, T. Huus, B. R. Mottelson, A. Winther and C. Zupančič, to be submitted to the Rev. Mod. Phys. 


\section{A. Particle Transitions}

The spin and parity of the lowest particle configurations, of a given nucleus, are in many cases, especially in the region of closed shells, unaffected by the deformation, and it is thus often possible to classify particle transitions in terms of the shell model.

Striking examples are the interpretation of the M4 and E3 isomeric transitions (cf. Ch. XVI) and the classification of the $\beta$-decays into orders of forbiddenness (cf. Ch. XVI).

Even where such a shell model classification is possible, however, the nuclear deformations may influence the quantitative estimate of transition probabilities in a number of ways.

\section{i) The Unfavoured Factor}

Since the combining states in a particle transition will in general have somewhat different shapes, one expects a reduction in the transition probability associated with this lack of overlap (cf. the Franck-Condon principle in molecules). The effect is difficult to estimate quantitatively; it depends exponentially on the difference in the nuclear shapes and may become very large when this difference exceeds the amplitude of the zeropoint oscillations. Further, the modification of the particle wave functions, implied by the non-spherical potential, in general reduces the transition probability (at the same time as it breaks down selection rules on $l$ and $j$ (cf. § $4 \mathrm{~A}$, iii)).

An additional reduction in the transition probability may result when more than one particle participates in the transition ${ }^{17}$.

The isomeric particle transitions and $\beta$-decays are observed to be in general retarded by a factor in the range of 5 to 100 (the unfavoured factor) as compared with single-particle estimates ${ }^{17}$.

Valuable information on the origin of the observed unfavoured factors would be provided by a determination of the variation of transition probabilities with the trends of nuclear deformations. The interpretation of the unfavoured factor would be further clarified by the measurement of branching ratios to different vibrational states associated with the same particle structure.

In special cases, the combining states are expected to have almost identical shapes, in which case the transition matrix elements depend only on the particle structure. Examples of such unhindered transitions are the $\beta$-decays between mirror nuclei. Another case should be provided by the $\gamma$-transitions between the two close lying particle states char-

17 Cf., e.g., S. A. Moszkowski, Phys. Rev. 89, (1953) 474; BM, VIId, ii and VIIIc, ii and iv. 
acterized by $\Omega=\mid \Omega$ (proton) $\pm \Omega$ (neutron) $\mid$ in strongly deformed odd-oddnuclei.

\section{ii) Branching Ratios to Rotational States}

For the very deformed nuclei which possess excited states that can be described in terms of a rotational motion of the whole nucleus, the transition probabilities to states of the same rotational family obey simple relations similar to the intensity rules encountered in the multiplets of atomic spectra ${ }^{18}$. Thus, for electromagnetic transitions of multipole order $L$, from a state characterized by $I_{i}, K_{i}=\Omega_{i}$ to members of a rotational sequence characterized by $K_{f}=\Omega_{f}$ and spins $I_{f}, I_{f}^{\prime}$ etc. (cf. Fig. 4), the reduced transition probabilities satisfy the relation

$$
\frac{B\left(L ; I_{i} \rightarrow I_{f}\right)}{B\left(L ; I_{i} \rightarrow I_{f}^{\prime}\right)}=\left(\frac{\left\langle I_{i} L K_{i} K_{f}-K_{i} \mid I_{i} L I_{f} K_{f}\right\rangle}{\left\langle I_{i} L K_{i} K_{f}-K_{i} \mid I_{i} L I_{f}^{\prime} K_{f}\right\rangle}\right)^{2},
$$

where the quantities on the right are Clebsch-Gordon coefficients for the addition of angular momenta ${ }^{19,20}$. The same relation holds for the ratio of inverse $f t$-values for $\beta$-transitions associated with a tensor operator of multipole order $L$ (cf. $\mathrm{Ch}$. $\mathrm{X}$ of this book). ${ }^{21}$

Fig. 4. Branching ratios to rotational states

The figure illustrates the quantum num. bers involved in a branching of a $\beta$ - or $\gamma$-decay to two members of a rotational family. An example of the intensity rules for such transitions is provided by the $\beta$-decay of ${ }_{60} \mathrm{Tm}^{170}$ to the ground state $\left(I_{f}=K_{f}=\Omega_{f}=0\right)$ and first excited

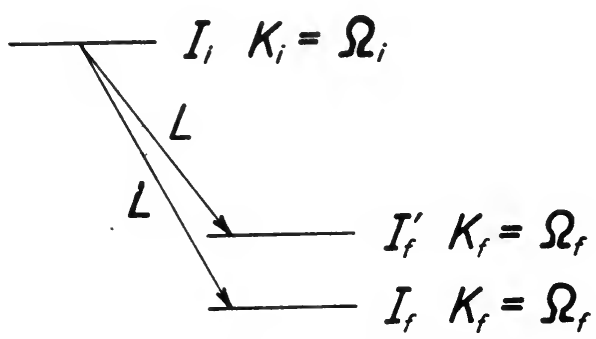
state $\left(I_{f}^{\prime}=2 ; K_{f}=\Omega_{j}=0\right)$ of ${ }_{70} \mathrm{Yb}^{170}$. The observed ratio of the $f t$-values for the transitions $\left(I_{f}=2\right)$ and $\left(I_{f}=0\right)$ is $1.9 \pm 0.2$ (R. L. Graham, J. L. Wolfson and R. E. Bell, Can. J. Phys. 30, (1952) 459), which agrees with the value 2.0 calculated from (11), assuming $I_{i}=K_{i}=\Omega_{i}=1$ and $L=1$.

18 Cf. e.g., E. U. Condon and G. H. Shortley, The Theory of Atomic Spectra. The University Press, Cambridge (1935), pp. $241 \mathrm{ff}$.

19 We use a notation similar to that of Condon and Shortley, loc. cit., pp. $76 \mathrm{ff}$., in which $\left\langle j_{1} j_{2} m_{1} m_{2} \mid j_{1} j_{2} j m\right\rangle$ is the coefficient for addition of the angular momentum vectors $\boldsymbol{j}_{\mathbf{1}}$ and $\boldsymbol{J}_{\mathbf{g}}$ to form a resultant $\boldsymbol{j}$ with component $m$.

20 The appearance of components with $(-K,-\Omega)$ as well as $(K, \Omega)$ in the nuclear wave function (cf. p. 473) may lead to additional terms in (11), but only in the rather unusual case of $L \geqslant K_{i}+K_{f}$ with neither $K_{i}$ nor $K_{f}$ equal to zero; cf. reference 21.

21 For a more detailed discussion of intensity rules for $\beta$ - and $\gamma$-transitions to nuclear rotational states, cf. G. Alaga, K. Alder, A. Bohr, and B. R. Mottelson, Dan. Mat. Fys. Medd., in press. Similar intensity rules apply also to the $\alpha$-decay fine 
Such simple rules governing branching ratios in nuclear transitions provide a useful tool in the classification of nuclear states ${ }^{21}$. Examples illustrating these intensity rules are discussed in $\S 5$ below (cf. also caption to Fig. 4 and reference 21 ).

\section{iii) Selection Rules}

While very general selection rules on the multipole order of $\gamma$-transitions and degree of forbiddenness of $\beta$-transitions are associated with the total angular momentum, $I$, and the parity of the combining states (Ch. X and XIII), additional partial selection rules may result from the existence of other angular momentum quantum numbers in the nuclear states. Since these latter quantum numbers are not exact constants of the motion, the corresponding selection rules have the effect of retarding the transitions without entirely forbidding them.

In the region of closed shells, where the nuclei are essentially spherical, the angular momenta, $l$ and $j$, of the individual particles may be such approximate constants of the motion. Reductions in transition probabilities have been observed in certain transitions and ascribed to such $l$ - and $j$-forbiddenness ${ }^{212}$. With increasing nuclear deformation these selection rules are expected to become less effective.

For the very deformed nuclei, where the coupling scheme again simplifies, partial selection rules are expected to result from the rotational constant of the motion $K$ (and $\Omega$ ). Especially for transitions to high members of a rotational band, where $I_{f}$ is very different from $K_{f}$, such a $K$-forbiddenness may be very effective (for an example, cf. p. 487 below).

\section{iv) Polarization of the Nucleus by Particle Transitions}

In the same way as a static quadrupole moment is induced in the nuclear shape by the mass quadrupole moment of the particle state, a particle transition of multipole order $L$ induces a corresponding oscillating moment in the nuclear shape. Even in cases where the induced deformation is small, the corresponding electric moment may be large compared with that of the particle. Since the induced moment is proportional to the mass multipole moment of the particle transition, the transition matrix element is simply multiplied by a factor that depends on the coupling between particle and collective motion and on the nuclear deformability. Estimates of this effect 22 indicate that electric transitions in general proceed primarily structure; cf. A. Bohr, P. Fröman, and B. R. Mottelson, Dan. Mat. Fys. Medd., in press.

21a Cf., e.g., L. Nordheim, Rev. Mod. Phys. 23, (1951) 322; BM § VIId, ii and $\S$ VIIc, iii.

22 BM, §VIIb, iii. 
by means of collective radiation. An important consequence is that the electric radiation of a neutron transition is expected to be of the same order of magnitude as for a proton transition (cf. the $870 \mathrm{keV} \mathrm{E} 2$ transition in $\mathrm{O}^{17}{ }^{22 \mathrm{a}}$ and the electric transitions in $\mathrm{Pb}^{206}{ }^{22 \mathrm{~b}}$ ). In the region of closed shells, such polarization effects may give rise to transitions which are appreciably faster than pure particle transitions in a spherical field (cf. the $\mathrm{E} 3$ radiative decays of the 3 -states in $\mathrm{O}^{16}{ }^{23}$ and $\mathrm{Pb}^{208}{ }^{24}$ ). Further away from closed shells, such enhancement of the electric radiation may be obscured by the unfavoured factor (cf. $\S 4 \mathrm{~A}, \mathrm{i}$ ), but is expected to manifest itself in the occurrence of mixed multipole transitions, especially of the $\mathrm{M} 1+\mathrm{E} 2$ type ${ }^{21}$.

For El transitions, there is no directly corresponding effect due to the lack of surface oscillations with $\lambda=1$. However, the coupling of the particle motion to the collective dipole oscillations ${ }^{24 a}$, which manifest themselves in the nuclear photo-effect, is expected to have an important influence on the particle transition probabilities. Since the coupling is such as to cause the neutron density to follow the motion of a proton and vice versa, it implies a reduction in the transition probabilities.

\section{B. Collective $\gamma$-Transitions}

\section{i) Phonon Transitions}

The radiation emitted by free oscillations of the nuclear shape is of electric multipole type of the same multipole order, $\lambda$, as the nuclear deformation. For the decay of a one-phonon to a non-phonon state, the reduced transition probability is given for the incompressible model by

$$
B(\mathrm{E} \lambda)=\frac{3 \lambda}{8 \pi} Z^{2} e^{2} R_{0}^{2 \lambda} \frac{\hbar^{2}}{A M R_{0}^{2}} \frac{1}{\hbar \omega_{\lambda}} .
$$

The cooperative nature of such a transition, as expressed in the appearance of the factor $Z^{2}$ in (12), in general leads to a much faster decay than for a corresponding particle transition.

\section{ii) Vibrational Transitions}

For the strongly deformed nuclei, the collective transitions are of vibrational and rotational type. While the $\mathrm{E} 2$ transition probabilities of

22a J. Thirion and V. L. Telegdi, Phys. Rev. 92, (1953) 1253.

22b D. E. Alburger and M. H. L. Pryce, in press.

23 S. Devons, reported at the Glasgow Conference, July 1954.

24 L. G. Elliott, R. L. Graham, J. Walker and J. L. Wolfson, Phys. Rev. 93, (1954) 356.

24a M. Goldhaber and E. Teller, Phys. Rev. 74, (1948) 1046; H. Steinwedel and J. H. D. Jensen, Z. f. Naturforsch. 5a, (1950) 413. 
the latter depend on the static $\lambda=2$ deformation and become much larger than (12), those of the former remain of order (12). For the transition from a member of the $n_{\beta}=1$ rotational band with spin $I_{i}$ to a member of the $n_{\beta}=0$ band with spin $I_{f}$, one has

$$
B\left(\mathrm{E} 2 ; I_{i} \rightarrow I_{f}\right)=B(\mathrm{E} 2)_{\mathrm{ph}}\left\langle I_{i} 2 K 0 \mid I_{i} 2 I_{f} K\right\rangle^{2},
$$

where $B(\mathrm{E} 2)_{\mathrm{ph}}$ is the reduced transition probability for the $\lambda=2$ phonon transition (12). For the corresponding transitions from $n_{\gamma}=1, K_{i}=K \pm 2$ to $n_{\gamma}=0, K_{f}=K$, one has

$$
B\left(\mathrm{E} 2 ; I_{i} \rightarrow I_{f}\right)=B(\mathrm{E} 2)_{\mathrm{ph}} \begin{cases}\left\langle I_{i} 2, K \pm 2, \mp 2 \mid I_{i} 2 I_{f} K\right\rangle^{2} & K \neq 0 \\ 2\left\langle I_{i} 2,2,-2 \mid I_{i} 2 I_{f} 0\right\rangle^{2} & K=0 .\end{cases}
$$

For vibrational transitions, magnetic radiation is forbidden, even though $\Delta I$ may be zero or one.

\section{iii) Rotational Transitions}

Transition probabilities within a rotational band may be related to the static electric and magnetic moments of the nuclear states. Thus, for E2 transitions from a rotational state with $\left(I_{i} K\right)$ to a state with $\left(I_{f} K\right)$, the reduced transition probability becomes

$$
B\left(\mathrm{E} 2 ; I_{i} \rightarrow I_{f}\right)=\frac{5}{16 \pi} e^{2} Q_{0}^{2}\left\langle I_{i} 2 K 0 \mid I_{i} 2 I_{f} K\right\rangle^{2}
$$

in terms of the intrinsic quadrupole moment of the nuclear shape (cf. $\S 3 \mathrm{~B}$, above).

For the ground state rotational band in an even-even nucleus, the consecutive states have $\Delta I=2$, and the transitions proceed by a cascade of pure E2 radiation. In an odd-A nucleus, however, the consecutive states have $\Delta I=1$, and the radiation will in general be a mixture of $\mathrm{M} 1+\mathrm{E} 2$. The reduced transition probability for the $M 1$ radiation can be expressed in terms of the gyromagnetic ratios $g_{\Omega}$ and $g_{R}$, appearing in the static magnetic moments (cf. (9)),

$$
B\left(\mathrm{M} 1 ; I_{i} \rightarrow I_{f}\right)=\frac{3}{4 \pi}\left(\frac{e \hbar}{2 M c}\right)^{2}\left(g_{\Omega}-g_{R}\right)^{2} \Omega^{2}\left\langle I_{i} 1 K 0 \mid I_{i} 1 I_{f} K\right\rangle^{2} .
$$

In the special case of rotational states with $\Omega=K=1 / 2$, which possess an anomalous spectrum (cf. (5)), there are correction terms to (16), which depend on the details of the particle structure. However, (15) remains valid even in this case.

For pure particle transitions of such low energy, M1 radiation would strongly predominate over the E2; however, the strong enhancement of the $\mathrm{E} 2$ radiation in these collective transitions may lead to an appreciable 
admixture of $\mathrm{E} 2$ in the $\Delta I=1$ transitions, and to frequent $\Delta I=2$ cross-over transitions.

\section{$\S 5$. Systematics of Nuclear Rotational Structure}

In recent years, a considerable body of data has revealed the existence of a rotational structure in the nuclear excitation spectrum ${ }^{25,26}$. The simple rotational motion is characteristic of the strongly deformed nuclei

25 A. Bohr and B. R. Mottelson, Physica 18, (1952) 1066; Phys. Rev. 89, (1953) 316; Phys. Rev. 90, (1953) 717; BM, § VIc, ii; K. W. Ford, Phys. Rev. 90, (1953) 29; F. Asaro and I. Perlman, Phys. Rev. 91, (1953) 763; Phys. Rev. 93, (1954) 1423; Phys. Rev. 94, (1954) 381 ; F. Asaro, S. G. Thompson and I. Perlman. Phys. Rev. 92, (1953) 694; F. Asaro, F. Stephens and I. Perlman, Phys. Rev. 92, (1953) 1495; T. Huus and C. Zupančič, Dan. Mat. Fys. Medd. 28, (1953) no. 1; J. O. Newton and B. Rose, Phil. Mag. 45, (1954) 58; cf. also J. O. Newton, Nuclear Properties of Very Heavy Elements, contribution to "Progress in Nuclear Physics", vol. 4, ed. by O. R. Frisch; J. O. Rasmussen, Ark. f. Fysik 7, (1954) 185; R. S. Caird and A. C. G. Mitchell, Phys. Rev. 94, (1954) 412; J. W. Mihelich, G. Scharff-Goldhaber and M. McKeown, Phys. Rev. 94, (1954) 794 (A); J. R. Arnold, Phys. Rev. 93, (1954) 743; T. Huus and J. Bjerregård, Phys. Rev. 92, (1953) 1579; G. Temmer and N. P. Heydenburg, Phys. Rev. 93, (1954) 351 and 906; E. L. Church and M. Goldhaber, Phys. Rev. 95, (1954) 626 (A); C. L. McClelland, H. Mark and C. Goodman, Phys. Rev. 93, (1954) 904; G. M. Temmer and N. P. Heydenburg, Phys. Rev. 94, (1954) 1399 ; J. T. Eisinger, C. F. Cook and C. M. Class, Phys. Rev. 94, (1954) 735 and Phys. Rev., in press; P. Falk-Vairant, J. Teillac, G. Valladas and P. Benoist, C. R. 238, (1954) 1409 and C. R., in press; J. Milsted, S. Rosenblum and M. Valadares, C. R. 239, (1954) 259; F. Boehm, P. Marmier and J. W. M. DuMond, Phys. Rev. 95, (1954) 864; T. Huus and A. Lundén, Phil. Mag., 45, (1954) 966; R. F. Christy, B.A.P.S. 29, (1954) no. 6 (L6); N. P. Heydenburg and G. M. Temmer, Phys. Rev. 95, (1954) 861; W. I. Goldburg and R. M. Williamson, Phys. Rev. 95, (1954) 767. For a recent discussion of the theory and a review of the experimental data, cf. A. Bohr, Rotational States of Atomic Nuclei, Ejnar Munksgaard, Copenhagen (1954).

26 The excitation spectra for even-even nuclei have also been considered in terms of the recoupling of particles outside of closed shells:

H. Horie, M. Umezawa, Y. Yamaguchi and S. Yoshida, Progr. Theor. Phys. 6, (1951) 254; B. H. Flowers, Phys. Rev. 86, (1952) 254 ; P. Preiswerk and P. Stähelin, Nuovo Cimento 10, (1953) 1219; A. de Shalit and M. Goldhaber, Phys. Rev. 92, (1953) 1211.

It has been shown that, for an even group of equivalent particles, two-body forces of the expected character lead to a ground state with spin 0 and a first excited state of spin 2. This result of the particle couplings may play an important role in influencing the spins in the regions of closed shells. A similar analysis of excitation spectra further away from closed shells would require the inclusion of configuration mixings (cf. de Shalit and Goldhaber, loc. cit.). Moreover, the necessity of including excitations of the closed shell core is implied by the magnitude of the observed quadrupole moments. The existence of spectra of the rotational type corresponds to the possibility of obtaining simple solutions to the cquations of motion by introducing appropriate collective coordinates. 
(cf. § 2 B) and therefore especially well defined in regions far removed from closed shells. The rotational structure has been studied most extensively for the heavy elements $(A>140)$, where the distances between shell-closings are great and where the deformations are also known to be especially large.

\section{A. Even-Even Nuclei}

\section{i) Energies of First Excited States}

In even-even nuclei, the lowest particle configuration has $\Omega=0$ and the rotational spectrum is given by (3). This sequence of states is in accordance with the well-known empirical rule that even-even nuclei have a ground state spin $I_{0}=0$, and also with the more recent findings that the first excited state has $I=2$ and even parity ${ }^{27}$. Only very few exceptions to this latter rule have been encountered, all referring to essentially spherical nuclei, for which (3) does not apply.

The rotational excitation energy (1) with the moment of inertia (2) is expected to decrease as one moves away from closed shells, corresponding to the increasing nuclear deformation produced by the increasing number of nucleons outside of closed shells.

The observed energies of the first excited states of even-even nuclei are found to exhibit a marked regularity just corresponding to the expected trends. For the heavy elements $(A>140)$, the data are shown in Fig. 5. In the regions away from closed shells, the energies become very small, but inversions of the $0+$ and $2+$ levels are never encountered, attesting to the intimate relation between the two levels. In these regions, especially for $155<A<185$ and $A>225$, the slowness of the rotational motion as compared with other frequencies in the system, makes the rotational description very accurate. Thus, correction terms of the type (6) contribute less than one per cent of the rotational energy.

From the observed energies, one can estimate the moment of inertia, $J$, which is a measure of the deformation (cf. (2)). The intrinsic quadrupole moment, $Q_{0}$, also depending on the deformation (cf. (7)), should exhibit similar trends as $J$, and a correlation has been observed ${ }^{28}$. The quantitative comparison of $J$ and $Q_{0}$ shows systematic deviations from the

27 M. Goldhaber and A. W. Sunyar, Phys. Rev. 83, (1951) 906; H. Horie, M. Umezawa, Y. Yamaguchi and S. Yoshida, Progr. Theor. Phys. 6 (1951) 254; P. Preiswerk and P. Stähelin, Helv. Phys. Acta 24, (1951) 623; Physica 18, (1952) 1118; Nuovo Cimento 10, (1953) 1219; G. Scharff-Goldhaber, Physica 18, (1952) 1105; Phys. Rev. 90, (1953) 587; F. Asaro and I. Perlman, Phys. Rev. 87, (1952) 393 ; S. Rosenblum and M. Valadares, C. R. 235, (1952) 711 ; A. H. Wapstra, Physica 18, (1952) 799.

28 K. W. Ford, Phys. Rev. 90, (1953) 29. 
relationship given by (2) and (7), which may be associated with the occurrence of higher multipole moments in the nuclear shape or the inadequacy of the incompressible model. Especially significant as a test of the former possibility would be the observation in the Coulomb excitation process of collective transitions of higher order (e.g., E4 transitions).

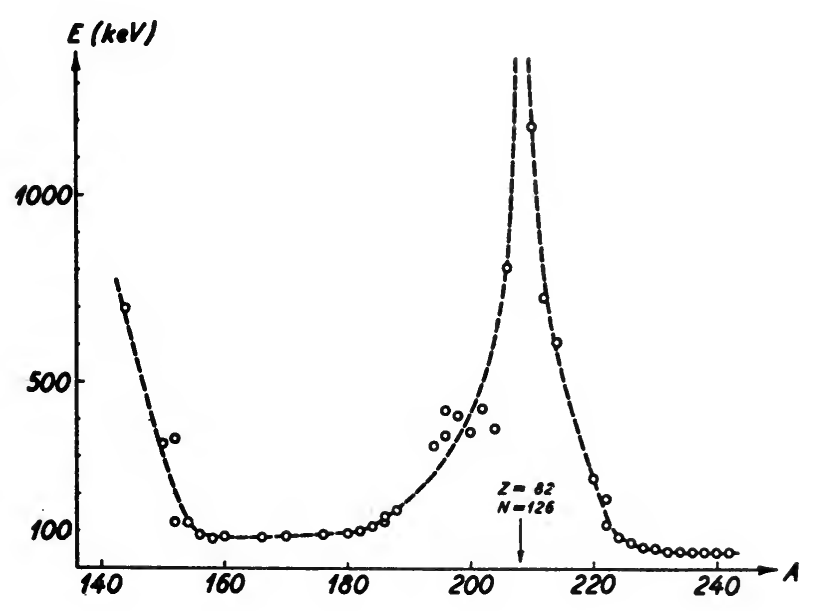

Fig. 5. Energies of first excited states in even-even nuclei with $A>140$

The data are taken from the compilations of G. Scharff-Goldhaber (ref. 27), P. Stähelin and P. Preiswerk (ref. 27), J. M. Hollander, I. Perlman and G. T. Seaborg, Rev. Mod. Phys. 25, (1953) 469. For W182. 184, ef. T. Huus and J. Bjerregård, Phys. Rev. 92, (1953) 1579 and C. McClelland, H. Mark and C. Goodman, Phys. Rev. 93, (1954) 904 ; for $\mathrm{Po}^{210}$, cf. Hoff, UCRL 2325, Berkeley (1953).

Similar curves, extending also to the region of lower $A$, have been given by G. Scharff-Goldhaber (loc. cit.) and by P. Ștähelin and P. Preiswerk (loc. cit.).

With the approach to closed shells, the rotational motion becomes more rapid and distortion terms increase in importance. In the vicinity of closed shells, the excitation spectrum changes completely (cf. $\S 2 \mathrm{~A}$ ). In these regions, the first excited states may represent particle excitations, collective vibrations, or combinations of both, and the energy may vary less regularly. The nature of a particular state reveals itself especially in the transition probability to the ground state. Thus, the relatively long lifetime of the first excited state of $\mathrm{Pb}^{204}{ }^{29}$ identifies it as an excitation of the particle structure $^{30}$. In these regions, and especially for nuclei possessing closed shells in both neutrons and protons, particle excitations may give

29 Cf., e.g., V. E. Krohn and S. Raboy, Phys. Rev. 95, (1954) 1354.

30 The recent measurement of the magnetic moment of this state (H. Frauenfelder, J. S. Lawson Jr., and W. Jentschke, Phys. Rev. 93, (1954) 1126) is in agreement with this interpretation. 
rise to first excited states having spins other than $2+$, as are indeed observed for ${ }_{8} \mathrm{O}^{16}{ }^{31},{ }_{32} \mathrm{Ge}^{72}{ }^{31}$, and ${ }_{82} \mathrm{~Pb}^{208} 32$.

Similar regularities in the energies of the first excited states of eveneven nuclei, as illustrated in Fig. 5, have been found in the lighter nuclei. While, in the heavy elements, the energy is approximately a function of $A$ only, due to the simultaneous filling of neutron and proton shells at $\mathrm{Pb}^{208}$, the trends are somewhat more complicated in the lighter elements and must be considered a function of the two variables $N$ and $Z^{33}$. In the regions between closed shells, the energies often become rather small compared with the expected vibrational energies (cf. Fig. 1), and a rotational structure is expected, although not with as great accuracy as in the heavy elements.

\section{ii) Higher Rotational States}

The analysis of the first excited states in even-even nuclei is confirmed by the observation of the expected higher members of the rotational sequence. Thus, in regions where the energy of the first excited state, $E_{2}$,

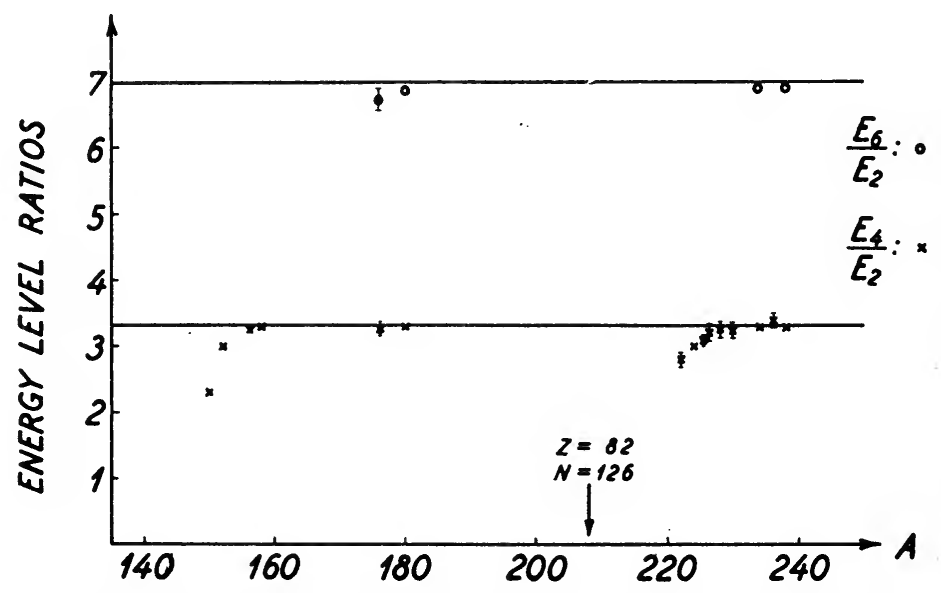

Fig. 6. Energy ratios for rotational states in even-even nuclei with $A>140$ The horizontal lines give the theoretical ratios $E_{4}: E_{2}$ and $E_{6}: E_{2}$, obtained from(1) for the limit of large deformations. The estimated uncertainties in the measured energy ratios are indicated in the figure; where no error is shown, the uncertainty is believed to be less than one per cent. For reference to the empirical data, cf. footnote 25. A figure similar to this was first given by F. Asaro and I. Perlman, Phys. Rev. 91, (1953) 763.

31 Cf., e.g., G. Scharff-Goldhaber, Phys. Rev. 90, (1953) 587.

32 L. G. Elliott, R. L. Graham, J. Walker and J. L. Wolfson, Phys. Rev. 93, (1954) 356.

33 Cf. especially G. Scharff-Goldhaber, loc. cit., and P. Preiswerk and P. Stähelin, Nuovo Cimento 10, (1953) 1219. 


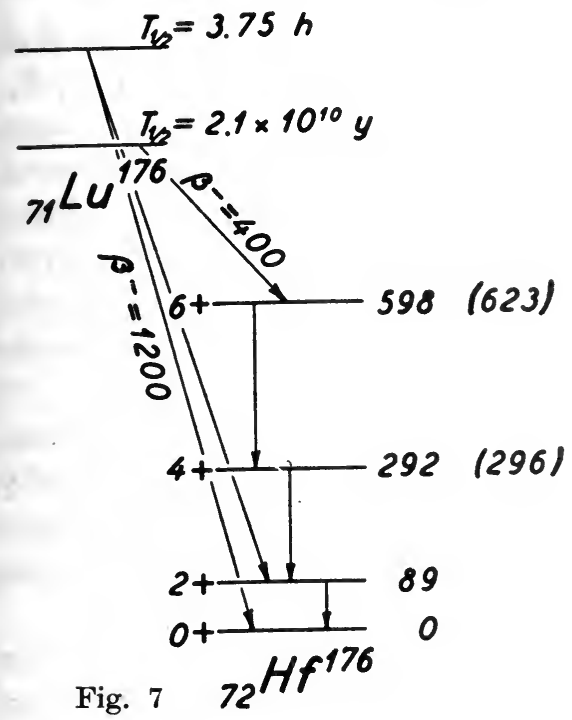

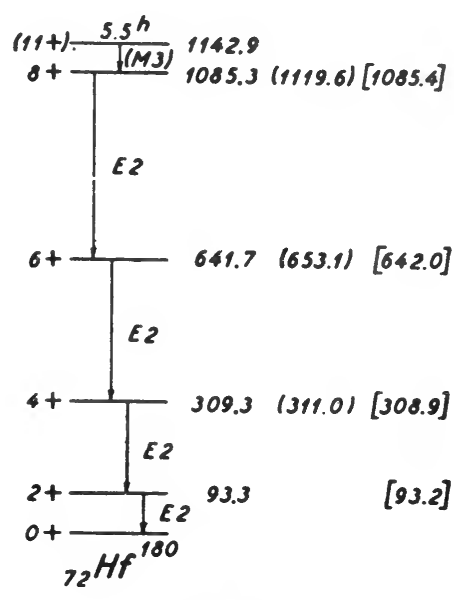

Fig. 8

Fig. 7. Excitation of rotational states in the $\beta$-decay of $\mathrm{Lu}^{178}$

The data for the decay of the $\mathrm{Lu}^{176}$ ground state are taken from J. R. Arnold (ref. 25). The energies in parenthesis for the $4+$ and $6+$ rotational states in $\mathrm{Hf}^{176}$ are obtained from the energy of the $2+$ state by means of (1). The deviations between the observed and calculated energies are of the order of the experimental uncertainty, but from the systematics of the rotational spectra in this region (cf. Fig. 8) one expects deviations of the order of $-1 \%$ and $-2 \%$ for the $4+$ and $6+$ states, resulting from the rotation-vibration interaction (cf. (6)). The $400 \mathrm{keV} \beta$-transition has a $\log f_{0} t$-value of 18.5 ; the appreciable retardation, resulting from the $K$-forbiddenness (cf. $\$ 4 \mathrm{~A}$, iii) of this transition may imply a second forbidden transition $(\Delta I=2$ or $3 ; \mathrm{no})$, despite the large $f_{0} t$-value.

For the decay of the $3.75 \mathrm{~h} \mathrm{La176}$, ef. M. Goldhaber and R. D. Hill, Rev. Mod. Phys. 24, (1952) 179. If the spin of this state is 1 , one expects about $75 \%$ of the transitions to go to the ground state and $25 \%$ to the first excited state (cf. (11) and Fig. 4). A greater intensity of the branch to this latter state would indicate a 2 - assignment for the $\mathrm{Lu}^{176}$ isomeric state and imply a weak $\beta$-transition to the 290 $\mathrm{keV} 4+$ level in $\mathrm{Hf}^{176}$ (cf. the similar case of $\mathrm{Ho}^{166}$ discussed in Alaga et al. (ref. 21)).

The $\gamma$-decay of the $89 \mathrm{keV}$ level in $\mathrm{Hf}^{176}$ (F. K. McGowan, Phys. Rev. 87, (1952) 542 ) is strongly enhanced (cf. Table I).

Fig. 8. Decay of $5.5 h$ isomeric state of $\mathrm{Hf}^{180}$

The $\gamma$-energies following the decay of $5.5 \mathrm{~h} \mathrm{Hf}^{180}$ are taken from Mihelich et al. (ref. 25). The numbers in parenthesis are those obtained from (1) with the moment of inertia fitted to the first excited state. The observed minor deviations from these energies show a systematic trend and are of the sign and order of magnitude expected from the rotation-vibration interaction (6). The energies in square brackets are calculated rotational energies with the inclusion of the rotation-vibration effect. The vibrational energies in (6) have been adjusted to give the best fit to the observed levels, and are found to be about $3 \mathrm{MeV}$, in agreement with the expected order of magnitude (cf. Fig. 1). 
is very low, corresponding to a large deformation, one expects to find higher excited states with spins $4+, 6+$, etc. and energies $E_{4}=\frac{10}{3} E_{2}$, $E_{6}=7 E_{2}$, etc. (cf. (1) and(3)). States with these characteristics have been found to occur systematically in the expected regions, and the observed energy ratios are shown in Fig. 6. It is seen that, in regions far from closed shells, the rotational energy ratios are rather accurately realized. Moreover, the small deviations observed show a systematic trend increasing with the approach to closed shells and have the sign and order of magnitude expected from the rotation-vibration effect (cf. (6)).

The rotational states are populated in a variety of nuclear processes; in particular, the states up to $6+$ have been found to occur systematically in the $\alpha$-spectra of the heavy elements ${ }^{34}$. High rotational states may also be excited in the $\beta$ - or $\gamma$-decay of a nuclear state with high spin value (cf. Figs. 7 and 8).

The expected occurrence of rotational structures in lighter elements in regions removed from closed shells finds some support in the identification of $4+$ states with energies approximating those expected. (Cf., e.g., $\mathrm{Mg}^{24}, \mathrm{Fe}^{56}, \mathrm{Xe}^{130}$ ).

Apart from the rotational band associated with the ground state, one expects additional bands for each intrinsic excitation of the nucleus (particle or vibrational excitation). For examples of such excited rotational bands, cf., e.g., the level structure of $W^{182}{ }^{34 a}$.

\section{iii) Transition Probabilities}

A number of E2 transition probabilities have been determined for the transition between the ground state and the first excited, $2+$, state in even-even nuclei. The data are listed in Table I and are obtained partly from lifetime determinations and partly from Coulomb excitation crosssections.

The intensity of the transitions is indicated by the quantity, $F$, in column 3, which represents the ratio of the observed transition probability to that expected for a corresponding particle transition. The largeness

34 F. Asaro and I. Perlman, Phys. Rev. 91, (1953) 763.

342 G. Alaga, K. Alder, A. Bohr, and B. R. Mottelson, Dan. Mat. Fys. Medd., in press.

Continuation caption Fig. 8

The long-lived $57 \mathrm{keV}$ transition has been tentatively classified as M3 on the basis of its lifetime and conversion data (Mihelich et al., loc. cit.). The high degree of $K$. forbiddenness (cf. $\S 4 \mathrm{~A}$, iii) of this transition implies, however, a strong retardation, which may suggest an M2 transition, having an unfavoured factor of $F \sim 10^{-8}$; the conversion data appear also to be consistent with such a classification (G. ScharffGoldhaber, private communication). 
of the $F$-factors clearly exhibits the collective nature of the transitions.

From the observed transition probability the intrinsic quadrupole moment can be obtained from (15). The values thus derived are listed in column 4 and show the expected trend, decreasing regularly with the approach to closed-shell configurations.

The nuclear quadrupole moments obtained from E2 transition probabilities may be compared with the static moments obtained from spectroscopic measurements in neighbouring odd- $A$ nuclei (cf. column 5). While the two quadrupole moment determinations give values of the same order of magnitude, the latter are somewhat larger than the former, and also fluctuate more. The difference, however, may not be significant in view of the considerable uncertainty that attaches to most of the spectroscopic determinations.

TABLE I

Rotational tRansitions IN EVEN-EVEN NUCLEI

\begin{tabular}{|c|c|c|c|c|}
\hline \multirow{2}{*}{ Nucleus } & \multirow{2}{*}{$E(\mathrm{keV})$} & \multirow{2}{*}{$F$} & \multicolumn{2}{|c|}{$Q_{0}\left(10^{-24} \mathrm{~cm}^{2}\right)$} \\
\hline & & & transition & spectroscopic \\
\hline${ }_{60} \mathrm{Nd}^{148}$ & 300 & 10 & 2.6 & $3\left({ }_{63} \mathrm{Eu}^{153}\right)$ \\
\hline${ }_{60} \mathrm{Nd}^{150}$ & 131 & 30 & 4 & \\
\hline${ }_{62} \mathrm{Sm}^{152}$ & 122 & 60 & 6 & $7\left({ }_{63} \mathrm{Eu}^{153}\right)$ \\
\hline${ }_{68} \mathrm{Dy}{ }^{160}$ & 85 & 80 & 7 & \\
\hline${ }_{68} \operatorname{Er}^{168}$ & 80 & 100 & 8 & $\sim 20\left({ }_{68} \operatorname{Er}^{167}\right)$ \\
\hline${ }_{70} \mathrm{Yb}^{170}$ & 84 & 100 & 8 & $11\left({ }_{70} \mathrm{Yb}^{173}\right)$ \\
\hline${ }_{72} \mathrm{Hf}^{176}$ & 89 & 90 & 8 & $14\left({ }_{71} \mathrm{Lu}^{175}\right)$ \\
\hline${ }_{72} \mathrm{Hf}^{180}$ & 93 & 80 & 7 & \\
\hline${ }_{74} W^{182}$ & 100 & 80 & 7 & $14\left({ }_{73} \mathrm{Ta}^{181}\right)$ \\
\hline${ }_{74} W^{184}$ & 113 & 80 & .7 & \\
\hline${ }_{74} \mathrm{~W}^{186}$ & 124 & 80 & 7 & $8\left({ }_{75} \operatorname{Re}^{185}\right)$ \\
\hline${ }_{76} \mathrm{Os}^{186}$ & 137 & 50 & 6 & $7\left({ }_{75} \operatorname{Re}^{187}\right)$ \\
\hline${ }_{76} \mathrm{Os}^{188}$ & 155 & 40 & 5 & $\sim 10\left({ }_{76} \mathrm{Os}^{189}\right)$ \\
\hline${ }_{90} \mathrm{Th}^{232}$ & 50 & 170 & 9 & $\sim 20\left({ }_{92} \mathrm{U}^{235}\right)$ \\
\hline${ }_{92} U^{238}$ & 44 & 110 & 8 & \\
\hline
\end{tabular}

For references to the empirical data, cf. A. Bohr (ref. 24, table I) and A. W. Sunyar, Phys. Rev. 95, (1954) 627 (A); B. E. Simmons, D. M. van Patter, K. F. Famularo, and R. V. Stuart, Phys. Rev., in press; N.. P. Heydenburg and G. Temmer, Phys. Rev. 93, (1954), 351 and 906, and private communication; K. L. V. Sluis and J. R. McNally, J. Opt. Soc. Am. 44, (1954), 87. The $F$-factor represents the ratio of the observed transition probability to that estimated for a corresponding particle transition. The $Q_{0}$-values in column four are intrinsic nuclear quadrupole moments calculated from the observed transition probabilities (cf. (15)), while those in column five are derived from spectroscopic data in neighbouring odd- $A$ nuclei by means of (8). 


\section{B. Odd-A Nuclei}

Recently, evidence has become available, principally from the Coulomb excitation studies, for the systematic occurrence of rotational spectra in odd- $A$ isotopes in the region of strongly deformed nuclei. These states are characterized, as are rotational states in even- $A$ isotopes, by the regularities in their energies and by their enhanced E2 transition probabilities.

In Table II are listed the energy levels in odd- $A$ nuclei in the region $150<A<190$, which have been identified by means of their large Coulomb excitation cross-sections as rotational excitations. The energy of the first rotational state, $E^{(1)}$, is listed in column 3 and that of the second rotational member, $E^{(2)}$, appears in column 4 . The value $E^{(2)}$ calculated from (1) and (3) by using the moment of inertia implied by $E^{(1)}$ is given in parenthesis. Columns 5 and 6 compare the observed moment of inertia with that of neighbouring even-even nuclei. The quantity listed, $3 \hbar^{2} / J$, represents

\section{Fig. 9. Rotational Spectrum of $\mathrm{Ta}^{181}$}

The best studied rotational spectrum of an odd- $A$ nucleus is that of ${ }_{73} \mathrm{Ta}^{181}$. By means of the Coulomb excitation process (double arrows in the figure), the two first rotational states have been excited. The ground state spin of $\mathrm{Ta}^{181}$ is known from spectroscopic data to be $I=7 / 2$ (cf., e.g., J. E. Mack, Rev. Mod.

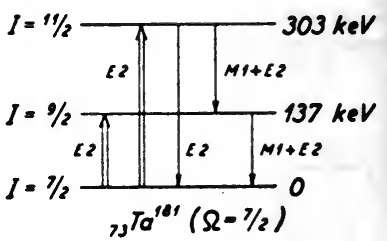
Phys. 22, (1950) 64) and the first and second rotational states are thus expected to have $I=9 / 2$ and $11 / 2$, respectively. These spin assignments have been confirmed by angular correlation measurements (F. K. McGowan, Phys. Rev. 93, (1954) 471; J. T. Eisinger, C. Cook and C. M. Class, Phys. Rev., 94, (1954) 735.

The energies and radiative decays of the rotational states are shown in the figure. The study of these transitions by the Coulomb excitation process ( $T$. Huus and C. Zupančič, Dan. Mat. Fys. Medd. 28, no. 1 (1953); T. Huus and J. Bjerregård, Phys. Rev. 92, (1953) 1579), has provided a number of tests of the theory of rotational states and yielded various information on the intrinsic nuclear properties. We list the quantities on which empirical evidence is available.

1) $E_{12 / 2}: E_{\% / 2}=2.21 \pm 0.02$ (theor. values 2.22 ; cf. (1)).

2) $E_{0 / 2}=137 \mathrm{keV}$ (thèor. estimate, using J of $\mathrm{Hf}^{180}$, gives $E_{\mathrm{s} / \mathrm{s}} \approx 140 \mathrm{keV}$; cf. Fig. 8).

3) $B(\mathrm{E} 2 ; \gamma / 2 \rightarrow \% / 2): B\left(\mathrm{E} 2 ; \gamma^{7} / 2 \rightarrow 11 / 2\right)=3.8 \pm 0.7$ (theor. value 3.89 ; cf. (11)).

4) $B\left(\mathrm{E} 2 ; \% / 2 \rightarrow \% / 2\right.$ ) yields $\left|Q_{0}\right| \approx 7 \times 10^{-24} \mathrm{~cm}^{2}$ (cf. (15)), in accordance with the deformation expected from the data in Table I; spectroscopic determinations (T. Schmidt, ZS. f. Phys. 121, (1943) 63; B. M. Brown and D. H. Tomboulian, Phys. Rev. 88, 1050 and 91, 1580, 1952) have led to an average $Q_{0}$ value of about $14 \times 10^{-24} \mathrm{~cm}^{2}$, but with a rather large uncertainty.

5) $B(\mathrm{Ml} ; 11 / 2 \rightarrow 9 / 2): \mathrm{B}(\mathrm{Ml} ; 9 / 2 \rightarrow 7 / 2)=2.5 \pm 1.0 *$ (theor. value 1.53 ; cf. (11)).

6) $B(\mathrm{Ml} ; 11 / 2 \rightarrow 9 / 2)$ together with $\mu=2.1$ (B. M. Brown and D. H. Tomboulian, loc. cit.) yield $g_{\Omega} \approx 0.75$ and $g_{R} \approx 0.25$; cf. (9) and (16).

* A recent reinterpretation of the angular correlation data of McGowan (loc. cit.) yields for this ratio the smaller value of about 0.7 , but again with a considerable uncertainty (Alaga et al., ref. 21). 
the excitation energy of the $(2+)$ rotational state in an even-even nucleus.

The observed energy ratios, $E^{(2)}: E^{(1)}$, agree well with the rotational value. The comparison between the moments of inertia in odd- $A$ and even-even elements seems to indicate a tendency for the former to somewhat exceed the latter, which may imply larger deformations in odd- $A$ nuclei. The largest difference occurs for $\mathrm{Eu}^{153}$ with neutron number $N=90$, in which region the nuclear deformations are known to vary rapidly (cf. the ratio of quadrupole moments of $\mathrm{Eu}^{151}$ and $\mathrm{Eu}^{153}$, and the excitation energies in the even-even isotopes of $\mathrm{Nd}, \mathrm{Sm}$, and $\mathrm{Gd}$ ).

The ground states of $\mathrm{Tm}^{169}$ and $\mathrm{W}^{183}$ have $\Omega=1 / 2$ and therefore the anomalous spectrum (5). The observed levels determine the moment of inertia as well as the coefficient $a$ (given in column 5 of Table II). The expected higher members of these rotational spectra appear to agree with observed $\gamma$-transitions following $K$-capture in $\mathrm{Yb}^{169} 35$ and the $\beta$ decay of $\mathrm{Ta}^{183}{ }^{36}$.

The E2 transition probabilities for the levels listed in Table II, as found from the Coulomb excitation cross-sections, exceed those estimated for single-particle transitions by factors of the order of one hundred, and the derived values of $Q_{0}$ (cf. (15)) are comparable with those in neighbouring even-even isotopes (cf. Table I).

The radiative decay of the rotational states in odd- $A$ nuclei also contains Ml radiation, the intensity of which can be related to the gyromagnetic ratios of the particle and collective motion (cf. (16)). The best studied case is that of $\mathrm{Ta}^{181}$, the analysis of which is discussed in Fig. 9.

In the region well beyond $\mathrm{Pb}^{208}$, rotational bands are again identified in the spectra of the odd $-A^{37}$ as well as of the even-even elements. The $\alpha$-decay process provides an especially convenient means of studying such sequences in this region of elements. The simple coupling scheme for the particle motion in these strongly deformed nuclei (cf. $\S 2 \mathrm{~B}$ ) also makes possible an interpretation of many features of the observed intensity relations in the $\alpha$-decay fine structure ${ }^{38}$.

The study of elements lighter than those included in Table II, by means of the Coulomb excitation process, has also revealed the existence of

35 D. S. Martin Jr., E. N. Jensen, F. J. Hughes and R. T. Nichols, Phys. Rev. 82, (1951) 579 .

${ }_{36}$ J. W. M. DuMond, H. C. Hoyt, P. E. Marmier and J. J. Nurray, Phys. Rev. 92, (1954) 202; J. J. Murray, P. Snelgrove, P. E. Marmier and J. W. M. DuMond, B.A.P.S. 29, (1954) no. 6 (L5); R. F. Christy, B.A.P.S. 29, (1954) no. 6 (L6).

${ }_{37}$ J. O. Rasmussen, Ark. f. Fysik 7, (1954) 185; F. Asaro and I. Perlman, Phys. Rev. 93, (1954) 1423; J. Milsted, S. Rosenblum and M. Valadares, C'. R. 239, (1954) 259.

38 A. Bohr, P. O. Fröman and B. R. Mottelson, Dan. Mat. Fys. Medd.. in press. 
rotational excitations in other regions of the periodic table. Interesting examples are afforded by the isotopes of $\mathrm{Ag}$ and $\mathrm{Rh}$, which exhibit the pattern expected for $\Omega=1 / 2$ nuclei ${ }^{39}$.

In still lighter elements, low lying collective excitations have been identified by means of their strongly enhanced E2 transition probabilities ${ }^{40}$. In some of these latter cases, the rotational motion appears to be coupled to certain intrinsic modes with a resulting more complex excitation spectrum.

39 N. P. Heydenburg and G. Temmer, Phys. Rev. 95, (1954) 861 ; T. Huus and A. Lundén, Phil. Mag., 45, 966 (1954)

40 N. P. Heydenburg and G. Temmer, Phys. Rev., in press.

TABLE II

Rotational States IN ODD-A NUClei

\begin{tabular}{|c|c|c|c|c|c|c|}
\hline Nucleus & $I_{0}$ & $E^{(1)}$ & $E^{(2)}$ & $3 \hbar^{2} / J$ & $\begin{array}{c}3 \hbar^{2} / J \\
\text { (even-even) }\end{array}$ & References \\
\hline${ }_{63} \mathrm{Eu}^{153}$ & $5 / 2$ & 84 & $194(192)$ & 72 & 122 & $(2),(3),(4)$ \\
\hline${ }_{65} \mathrm{~Tb}^{159}$ & $3 / 2$ & 57 & 138 (137) & 69 & 79 & $(2),(4)$ \\
\hline${ }_{66} \mathrm{Dy}_{163}^{161}$ & $2 / 2$ & 76 & $166(169)$ & 50 & 85 & $(2),(3),(7)$ \\
\hline${ }_{67} \mathrm{Ho}^{165}$ & $7 / 2$ & 93 & 205 (207) & 63 & 76 & $(2),(3)$ \\
\hline${ }_{68} \operatorname{Er}^{167}$ & $7 / 2$ & 79 & $174(175)$ & 53 & 80 & $(2),(3),(8)$ \\
\hline${ }_{69} \mathrm{Tm}^{169}$ & $1 / 2$ & 10 & 120 & $\begin{array}{c}76 \\
(a=-0.74)\end{array}$ & 80 & $(2),(4)$ \\
\hline${ }_{70} \mathrm{Yb}^{173}$ & $5 / 2$ & 81 & $180(185)$ & 69 & & $(2),(3)$ \\
\hline${ }_{71} \mathrm{Lu}^{175}$ & $7 / 2$ & 113 & $251(251)$ & 75 & 82 & $(2),(3),(4)$ \\
\hline${ }_{72} \mathrm{Hf}_{179}^{177}$ & $(7 / 2)$ & 112 & $240(249)$ & 75 & 89 & $(2),(3),(4),(9)$ \\
\hline${ }_{73} \mathrm{Ta}^{181}$ & $2 / 2$ & 137 & $303(304)$ & 91 & 93 & (1) \\
\hline${ }_{74} W^{183}$ & $1 / 2$ & 46 & 99 & $\begin{array}{c}78 \\
(a=+0.19)\end{array}$ & 100 & $(4),(6)$ \\
\hline${ }_{75} \mathrm{Re}^{185}$ & $5 / 2$ & 123 & $(281)$ & 105 & 113 & $(2),(4)$ \\
\hline${ }_{75} \operatorname{Re}^{187}$ & $5 / 2$ & 134 & $(306)$ & 114 & 123 & $(2),(4)$ \\
\hline${ }_{77} \mathrm{Ir}^{191}$ & $3 / 2$ & 129 & $(310)$ & 155 & & $(2),(4),(10)$ \\
\hline${ }_{77} \mathrm{Ir}^{193}$ & $3 / 2$ & 138 & (331) & 166 & & $(2),(4),(10)$ \\
\hline${ }_{79} \mathrm{~A} u^{197}$ & $3 / 2$ & 279 & $555(670)$ & 335 & 358 & $(2),(5)$ \\
\hline
\end{tabular}

The Table lists the states in the region $150<A<200$, identified from their large Coulomb excitation cross-sections, as rotational excitations. 'The two first rotational states are denoted by $E^{(1)}$ and $E^{(2)}$, and the calculated value of $E^{(2)}$ obtained from (1) is listed in parenthesis; all energies are in $\mathrm{keV}$. The spin assignments of the rotational band are based on the ground state spins (cf. (4)) listed in column two; they are taken from J. E. Mack, Rev. Mod. Phys. 22, (1950) 64, except where additional references are listed. The two last columns compare the moments of inertia, J, with those of the neighbouring even-even nuclei, lacking the odd particle. The quantity listed, $3 \hbar^{2} / J$, represents the excitation energy of the $(2+)$ rotational state in an eveneven nucleus (cf. Fig. 5). $\mathrm{Tm}^{169}$ and $\mathrm{W}^{183}$ with $I_{0}=1 / 2$ have the anomalous spectrum 
characteristic of a nucleus with $\Omega=1 / 2$. From the energies of the first two rotational states, one may determine the moment of inertia as well as the parameter, $a$ (cf. (5)).

\section{REFERENCES TO TABLE II}

(1) C. L. McClelland and C. Goodman, Phys. Rev. 91, (1953) 760;

T. Huus and C. Zupančič, Dan. Mat. Fys. Medd. 28, (1953) no. 1;

T. Huus and J. Bjerregård, Phys. Rev. 92, (1953) 1579.

(2) G. Temmer and N. P. Heydenburg, Phys. Rev. 93, (1954) 351, 906.

(3) G. Temmer and N. P. Heydenburg, Phys. Rev. 94, (1954) 1399.

(4) Private communication from T. Huus.

(5) J. Eisinger, C. F. Cook, and C. M. Class, Phys. Rev. 95, (1954) 628 (A) and Phys. Rev., in press.

W. I. Goldburg, S. A. Cox and R. M. Williamson, Phy: Rev. 95, (1954) 628 (A) and Phys. Rev., in press.

(6) C. L. McClelland, H. Mark and C. Goodman, Phys. Rev. 93, (1954) 904.

(7) K. Murakawa and T. Kanei, Phys. Rev. 92, (1953) 325.

(8) B. Bleaney and H. E. D. Scovil, Proc. Phys. Soc. 644, (1951) 204.

(9) The ground state spin, $I_{0}=7 / 2$, listed for the odd Hf isotopes, is that indicated by the observed rotational energies. The spectroscopic evidence, although uncertain, has been tentatively interpreted as indicating $I_{0}=1 / 2$ or $3 / 2$ (E. Rasmussen, Naturwiss. 23, (1935) 69).

(10) K. Murakawa and S. Suwa, Phys. Rev. 87, (1952) 1048. 


\author{
R. E. BELL, K. G. MALMFORS
}

\title{
(I) R. E. BELL - Measurement by Delayed Coincidences. Comparison Methods
}

\section{§ 1. Introduction}

In this Chapter we shall review the main methods of directly measuring short nuclear lifetimes. The word short will be taken to mean less than $10^{-4}$ seconds. The word lifetime is used here to denote qualitatively the duration of an excited state. When precise numerical quantities are mentioned, the terms used will be mean life, $\tau=1 / \lambda$, where $\lambda$ is the decay constant, and half-life, $T_{1 / 2}=0.693 / \lambda$. In the past, theoretical workers have usually employed the mean life, $\tau$, and experimental results have usually been quoted in terms of the half-life, $T_{1 / 3}$. The notation of the original workers in each case will be followed here.

Generally speaking, nuclear lifetimes that are short according to the above definition occur only for gamma-ray transitions. (In alpha decay a few cases of short lifetimes are known, but except in the historical sense they lie outside the scope of the present volume.) Though it is customary to speak loosely of the lifetime of a gamma ray, it must be emphasized that in every case it is really the lifetime of the gamma-emitting excited state that is measured. If, for example, nuclei in a given excited state are de-excited by the emission of two different gamma rays, then both these gamma rays will necessarily have the same observed half-life, viz., that of the parent excited state. From the observed ratio of the intensities of the two gamma transitions, partial half-lives can be derived for each of them, but these must still be looked upon as properties of the states between which the transitions occur and not merely of the gamma rays.

The most systematically applicable method of measuring short nuclear lifetimes is the delayed coincidence method, discussed in $\S 3$. There are in addition a number of cases where short lifetimes have been measured by various comparison methods, as described in $\S 5$. Gamma-transition probabilities can also be measured indirectly by measurements on the inverse transition, i.e., by the Coulomb excitation or resonant nuclear scattering processes. These measurements are complementary to the delayed coincidence method because they are most suitable for large transition probabilities, corresponding to short lifetimes.

Experience has shown that most gamma rays emitted by radioactive substances are of E2 or M1 character, a fact that is consistent with the nuclear shell model. The lifetimes of this numerous M1 and E2 group 
fall into the range of interest of this chapter. A rough prediction of the mean lives to be expected is shown in Fig. 1, for two values of $Z, 10$ and 82. Fig. I was calculated from the lifetime estimates of Ch. XIII, together with the internal conversion data of $\mathrm{Ch}$. XIV. In addition, use has been made in the Ml curves of the recently discovered fact that many Ml transitions appear to be about 100 times longer in mean life than the estimate of $\mathrm{Ch}$. XIII ${ }^{1}$. The curves of Fig. 1 should be used

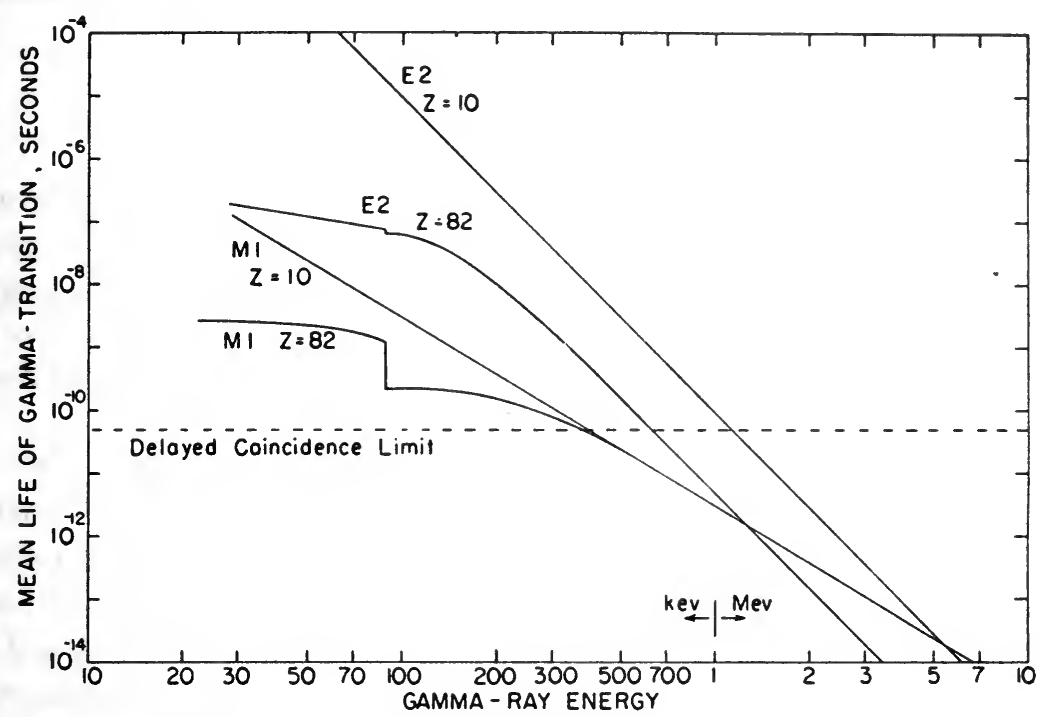

Fig. 1. Expected mean lives of M1 and E2 gamma transitions as a function of energy, for $Z=10$ and $Z=82$, estimated by the methods of Ch. XIII. The internal conversion correction has been included, the sudden breaks in the $Z=82$ curves occurring at the gamma-ray energy above which $K$ conversion is energetically possible

only as a very rough guide, because large deviations from the mean life estimates are to be expected, as mentioned in Ch. XIII. The mean lives for gamma transitions are seen in Fig. 1 to range from $10^{-4}$ seconds for low energies to $10^{-13}$ seconds for energies near $3 \mathrm{MeV}$. We will see in $\S 4$ that a practical lower limit for the mean life that can be measured with present day fast coincidence technique is about $5 \times 10^{-11}$ seconds; a horizontal broken line in Fig. 1 indicates this limit, and shows that we may expect to be able to measure the mean lives of a majority of Ml and E2 transitions with energies up to about $400 \mathrm{keV}$ by the delayedcoincidence method.

Of the large number of $\mathrm{M} 1$ and E2 gamma transitions available for

1 R. L. Graham and R. E. Bell, Can. J. Phys. 31, (1953) 377. 
lifetime measurements only a few have actually been measured, owing chiefly to the relative complexity of the fast coincidence techniques involved. It is to be hoped that a large enough body of data will be accumulated in the next few years to cnable a detailed comparison of the experimental material with nuclear theories. Even in the present early stage, the results on E2 lifetimes have stimulated the discussion of cooperative motion in the nucleus, while results on $\mathbf{M l}$ lifetimes have been used as evidence for the existence of exchange magnetic moments in nuclei ${ }^{2}$.

\section{§ 2. Fast Coincidence Techniques}

Before we can proceed to the delayed coincidence method of measuring short lifetimes, it is necessary to outline the fast coincidence techniques used therein. The term fast coincidence techniques is meant to suggest only that the resolving time of the coincidence circuit is short. For the sake of brevity, the reader will be assumed to have a reasonable familiarity with electronic pulse techniques.

A coincidence circuit can be defined as a non-linear circuit with two input terminals and one output terminal, whose nature is such that an output signal is produced only when both input terminals have received a pulse within a short time of each other. There are a number of nearly equivalent ways of measuring or defining this snall time, called the resolving time of the coincidence circuit. It is one of the main objects of the design of fast coincidence circuits to reduce the resolving time as far as possible. Such a step not only permits the measurement of short intervals of time, but also reduces the number of chance coincidences occurring between causally unrelated pulses. The quantitative treatment of resolving time will be deferred until $\S 4$.

In practice the input pulses are derived from some type of radiation counter, such as a Geiger, proportional, or scintillation counter, and it is found that the attainable time resolution in a coincidence circuit is usually limited by the speed of response of the counters. For example, Geiger counters of ordinary dimensions show a variable delay of the order of $10^{-7}$ seconds between the time of passage of the ionizing particle and the time of appearance of the pulse on the output terminal. For scintillation counters the delay times involved are 100 to 1000 times shorter. In either case, it is comparatively easy to build an electronic arrangement capable of resolving times as short as is justified for the fastest counters presently available. We therefore conclude, first, that any coincidence circuit should 
be taken to include the counters connected to it, and second, that the recent progress in shortening the resolving time of coincidence circuits is almost entirely due to the development of high-speed scintillation counters.

The earliest coincidence circuits in the present sense of the term were described by Bothe ${ }^{3}$ and Rossi ${ }^{4}$ in 1930 . The basic circuit configuration due to Rossi is still in use, usually in modified form, and is shown in Fig. 2(a). Negative pulses from two Geiger counters (not shown) are applied

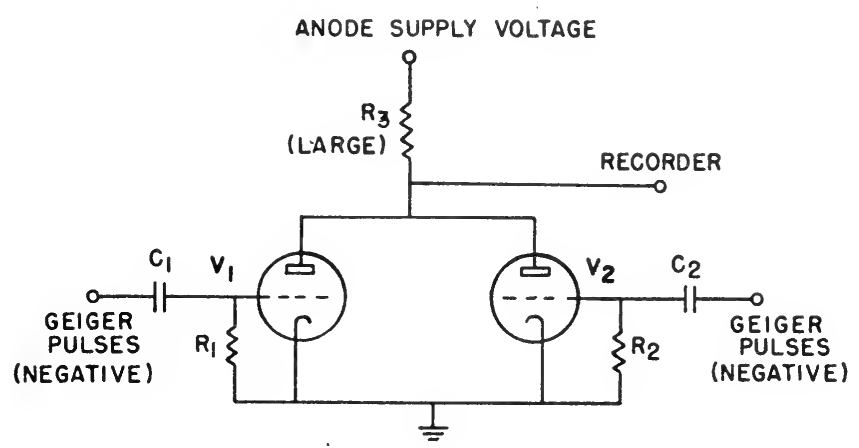

(a)

Fig. $2 a$ Original Rossi coincidence circuit

to the grids of the two triodes $V_{1}$ and $V_{2}$, whose plate load is the resistance $R_{3}\left(\sim 10^{6} \mathrm{ohms}\right)$. In the quiescent state both $V_{1}$ and $V_{2}$ are conducting heavily and their common anode voltage is low. When a large negative pulse is applied to the grid of $V_{1}$, the anode current of $V_{1}$ is cut off for the duration of the pulse. The anode voltage rises only a small amount, since the equivalent $d-c$ resistance of the anode of $V_{2}$ to ground is still low compared with $\mathrm{R}_{3}$. When simultaneous pulses arrive at the grids of $V_{1}$ and $V_{2}$, however, the current in the anode circuit is entirely interrupted, and the anode potential rises to that of the supply. This corresponds to a very large positive output pulse, perhaps several hundred volts. The recorder connected to the anode circuit detects only the large pulses corresponding to overlapping input pulses. Thus in the Rossi circuit the two triodes of Fig. 2(a) constitute the main non-linear circuit element in which the coincidences are detected, the recorder furnishing only a mild discriminating action.

The resolving time of this arrangement depends on the duration of the pulses applied to the two grids, and in Rossi's original circuit was perhaps

3 W. Bothe, Zeits. f. Phys. 59, (1930) 1.

4 B. Rossi, Nature 125, (1930) 636. 
$10^{-3}$ seconds. However, the resolving time in the circuit of Fig. 2(a) cannot be shortened indefinitely by shortening the input pulses, because the time required for the common anode potential to rise is of the order of $R_{3} C$, where $C$ is the capacity to ground of the anodes of $\mathrm{V}_{1}$ and $\mathrm{V}_{2}$ and of the recorder input. Taking, for example, $R_{3}=4 \times 10^{6}$ ohms and $C=25 \mu \mu \mathrm{f}$, we get $R_{3} C=100 \mu$ sec. Thus a pair of coincident grid pulses each of duration $1.0 \mu \mathrm{sec}$ would cause an output of only about $1 \%$ of the full value. If we now attempt to avoid this limitation by reducing $R_{3}$ by a factor of 100 or more, we will find that $R_{3}$ is no longer large compared with the equivalent resistance of the triodes. In the limit of small $R_{3}$, a pair of coincident input pulses produces an output signal only double that produced by a single pulse, instead of the large ratio obtained previously.

In one sense, then, making $R_{3}$ small converts the circuit of Fig. 2(a) into a linear circuit (response to two input pulses twice the response to one pulse), and in order for coincidences to be detected, a non-linear circuit must be added to the output of Fig. 2(a) to distinguish between single-size and double-size pulses. In Fig. 2(b) we show a modified circuit,

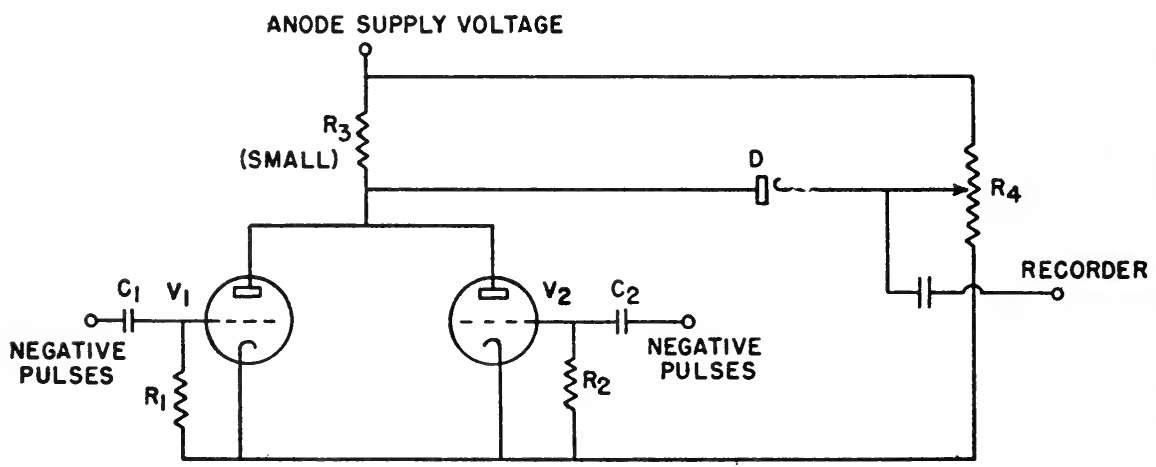

(b)

Fig. 2b. Modified Rossi circuit for shorter resolving time ("fast Rossi circuit")

the non-linear element in this case being a diode $\mathrm{D}$, biased in a reverse direction by means of the potentiometer $R_{4}$ so that a positive pulse across $R_{3}$ whose magnitude is less than this diode bias will not be transmitted to the recorder. The value of the bias is so adjusted that the single-size pulses are rejected and the double-size pulses are passed to the recorder. We will follow current practice by referring to circuits of the type of Fig. 2(b) as fast Rossi circuits, though their action is different from that of the original Rossi circuit. The actual non-linear coincidence-detecting element in 
Fig. 2(b) is the diode $D$, the pair of triodes now serving merely as pulse size limiters. When $R_{3}$ is small, the maximum possible magnitude of the positive pulse at the common anodes of $V_{1}$ and $V_{2}$, Fig. 2(b), is $R_{3} i$ for a single input pulse, and $2 R_{3} i$ for a coincident pair of input pulses, where $i$ is the standing current in each of $V_{1}$ and $V_{2}$. This amplitude-limiting process is necessary in this type of circuit to ensure that no single pulse is recorded as a coincidence. Provided $R_{3}$ is made small enough, the circuit of Fig. 2(b) will operate with resolving times in the $10^{-9}$ second range. When $R_{3}$ is made very small, it is necessary to insert an amplifier between diode $\mathrm{D}$ of Fig. 2(b) and the recorder. This amplifier need not have as fast a response as the coincidence circuit to which it is connected, unless for some reason it is desired to record with small delay the fact that a coincidence has occurred. The speed of response of a coincidence counting assembly need only be maintained up to and including the non-linear element in which the coincidences are detected, and it is for this reason that this element should be correctly identified in the circuit, and should be placed as early in the coincidence assembly as possible.

The fast Rossi circuit of Fig. 2(b) has appeared in many forms. Among recent fast coincidence circuits, it has perhaps been most usual to use miniature pentodes or germanium diodes for $V_{1}$ and $V_{2}$, and a germanium diode for $D$. The essential elements of the fast Rossi circuit are a pair of elements in which the input pulses are limited and equalized in amplitude $\left(V_{1}\right.$ and $V_{2}$ in Fig. $\left.2(b)\right)$, an element in which the equalized pulses are added in amplitude $\left(R_{3}\right.$ in Fig. $\left.2(b)\right)$, and a non-linear element by which the double-size pulses are selected (D in Fig. 2(b)).

- In the coincidence circuit of Bothe ${ }^{3}$, the detecting element was a multigrid vacuum tube whose anode current could be cut off by either of two grids. With both grids held at negative voltages sufficient to cut off the anode current, the anode current can flow only when simultaneous positive pulses are applied to the two grids. Thus a signal is produced across a resistance in the anode circuit only for coincident input pulses of sufficient magnitude. We may look upon Bothe's scheme as a pair of switches in series, both of which must be closed if any current is to flow; the Rossi circuit reduces to a pair of switches in parallel, both of which must be opened if current is to be fully interrupted. The series type of coincidence circuit has seen less application than the parallel, Rossi-type circuit, and will not be discussed further. Nevertheless, a successful series coincidence circuit of short resolving time has been described recently by Fischer and Marshall 5 .

In recent years a number of alternatives to the fast Rossi circuit have

5 J. Fischer and J. Marshall, Rev. Sci. Instruments 23, (1952) 417. 
been proposed as high-speed coincidence elements. These circuits usually take the form of a bridge arrangement containing one or more non-linear elements, in which the necessity for limiting the amplitudes of the pulses from the individual counters is avoided by making each single pulse from one counter cancel itself, and so give zero output. The cancelling action is upset by the presence of a simultaneous pulse from the other counter, so that a finite output signal is obtained only when two input pulses coincide. Circuits of this kird have not been as widely used as the fast Rossi type, because it is usually difficult to maintain the balance of a bridge containing non-linear elements over a wide range of input pulse amplitudes. This fact means that there is a danger that an exceptionally large single input pulse may give sufficient output from the imperfectly balanced bridge to be recorded as a coincidence. When germanium diodes are used as the non-linear elements, the bridge type circuit can be used at a very low pulse amplitude level, and advantage can be taken of this fact to place the bridge early in the coincidence assembly. The fear of the single pulse effect mentioned above has perhaps unduly hampered. the application of these otherwise useful circuits. Examples of the bridge type of circuit may be found in several published papers ${ }^{6}$.

In all the circuits described so far, the resolving time has been determined mainly by the duration of the individual pulses applied to the detecting element. Bay ${ }^{7}$, however, has recently shown how to make the resolving time smaller than the durations of the input pulses. The method involves two non-linear detectors, between which a small fixed delay is inserted; the action is that of a bridge circuit involving a time balance. This "differential" coincidence circuit has not yet seen wide application.

Mention should also be made of a coincidence detecting system in which the pulses of one counter are applied to the vertical deflection plates of an oscilloscope tube, and those of the other counter to the horizontal plates. Single counts then give either vertical or horizontal deflections, while two coincident pulses produce a diagonal deflection. With proper masks over the face of the oscilloscope tube, photoelectric cells can be used to record the single counts and coincidences. This method began with the work of Jacobsen ${ }^{8}$, who used two moving-iron oscillographs

6 R.H. Dicke, Rev. Sci. Instruments 18, (1949) 907; G. A. Morton and Robinson, Nucleonics 4, No. 2, (1949) 25; Meyer, K. Baldinger, and Huber, Helv. Phys. Acta 22, (1949) 420; E. F. Shrader, Rev: Sci. Instruments 21, (1950) 883; Z. Bay, Rev. Sci. Instruments 22, (1951) 397.

7 Z. Bay, Phys. Rev. 83, (1951) 242; Z. Bay, Meijer, and G. Papp, Nucleonics 10, No. 3, (1952) 39.

8 J. C. Jacobsen, Nature 128, (1931) 185 and Nature 133, (1934) 565; H. K. Ticho, Rev. Sci. Instruments 18, (1947) 271 ; Pocza and Farago, Jour. Sci. Instruments 
with crossed deflections and recorded the results photographically. Many elaborations of this method, using the modern cathode ray oscilloscope, have been described ${ }^{8}$.

Using Geiger counters and faster coincidence circuits, experimenters were able to reduce the duration of their input pulses, and hence their resolving times, by passing the pulses from the counters through sharpening or "differentiating" circuits, usually consisting of amplifiers with short $R C$ coupling constants. Thus in 1938 Feather and Dunworth ${ }^{9}$ used a resolving time of $6 \times 10^{-6}$ seconds, and in 1939 Dunworth ${ }^{10}$ had reduced this to a few times $10^{-7}$ seconds, the limitation then lying in the random time delays found with Geiger counters. The pulses from Geiger counters have, however, the advantage of being all very much of the same size and shape. The introduction of pulse ionization chambers, proportional counters, and scintillation counters reduced the time lags, but brought with it the difficulty that the pulses from a single detector could be very non-uniform in amplitude, thus complicating the action of the coincidence circuit.

A circuit arrangement designed to overcome this effect, and at the same time to provide pulses at the input of the coincidence circuit whose durations are well determined, is shown in Fig. 3, in the form of a block diagram. Such arrangements are capable of resolving times down to $10^{-7}$ seconds or less, and have appeared many times in the literature ${ }^{11}$.

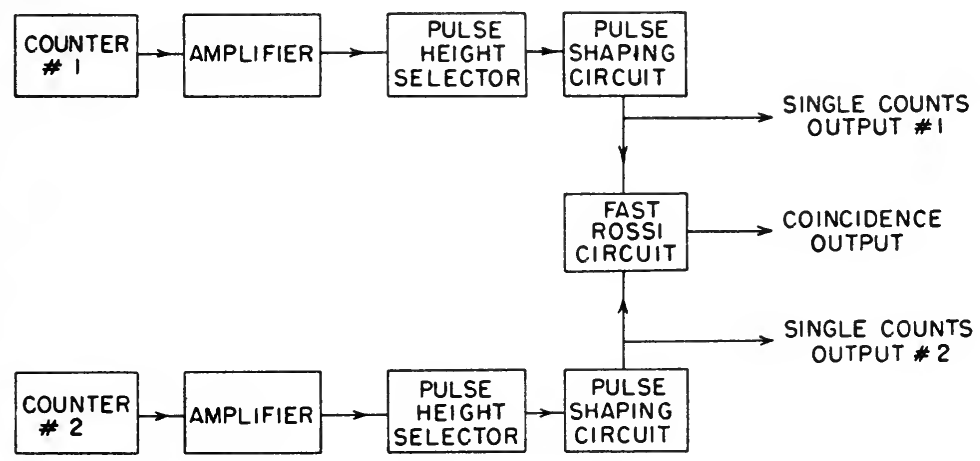

Fig. 3. Block diagram of a coincidence assembly suitable for resolving times of about $10^{-7}$ seconds or longer

25, (1948) 399; H. Kallmann and C. A. Accardo, Rev. Sci. Instruments 21, (1950) 48; Hofstadter and J. A. McIntyre, Rev. Sci. Instruments 21, (1950) 52.

- N. Feather and J. V. Dunworth, Proc. Roy. Soc. (London) A168, (1938) 566.

10 J. V. Dunworth, Nature 144, (1939) 152.

11 J. Rotblat, Proc. Roy. Soc. (London) A177, (1941) 260; H. Schultz and R. Beringer, Rev. Sci. Instruments 19, (1948) 424; H. Schultz and E. Pollard, Rev. Sci. Instruments 19, (1948) 617; F. K. McGowan, S. De Benedetti, and J. E. Francis, Phys. Rev. 75, (1949) 1761. 
As Fig. 3 shows, the pulses from the individual counters are amplified, selected in amplitude, and converted to standard size and shape in the conventional manner. The standardized pulses are then applied to a coincidence circuit, for example that of Fig. 2(b). The arrangement of Fig. 3 has the advantage that the pulses applied to the coincidence circuit are all of the same size and shape, making the operation of the Rossi circuit stable, and yielding a well-defined resolving time. In addition, the counting rates of the individual counters are well defined, and correspond exactly to the yield of coincidences observed at the coincidence output. This kind of arrangement is undoubtedly the best for general coincidence counting, where short resolving times are not necessary, and is in constant use.

The defect in the circuit of Fig. 3, from the fast coincidence point of view, is that the fast Rossi circuit is placed very late in the assembly, and it is difficult to keep the speed of response of all the elements of the circuit commensurate with a short resolving time. In particular the process of pulse height selection imposes a variable delay in the pulse output, depending on the size and shape of the pulses on which it operates; the magnitude of this time spread is at least of the order of magnitude of the rise time of the pulses from the counter and amplifier. Stable high gain linear amplifiers of rise time much less than $10^{-7}$ seconds are difficult to construct, so that assemblies of the type of Fig. 3 are restricted in normal practice to resolving times greater than $10^{-7}$ seconds. Thus we see that the process of pulse height selection has transformed the original spread of pulse heights into a spread in pulse delay. Some form of pulse height selection is necessary for any type of counter which produces pulses having a large spread of pulse sizes, and particularly for scintillation counters where effects due to thermal noise pulses must be eliminated.

The solution to this problem lies in performing the coincidence selection and the pulse height selection in entirely separate channels, and combining the results of these operations in a separate, relatively slow coincidence circuit. This system was described by Bell and Petch ${ }^{12}$ in 1949, and is illustrated in the block diagram of Fig. 4. The coincidence circuit is designed to operate at a low pulse level, accepting the pulses directly from the counters, which are usually scintillation counters. (If the counter pulses are small, fast amplifiers are used following the counters, as suggested in brackets in Fig. 4; these amplifiers need not be particularly linear or stable.) All pulses are fed to the coincidence circuit without amplitude selection, so that the number of circuit elements before the

12 R. E. Bell and H. E. Petch, Phys. Rev. 76, (1949) 1409. 
coincidence-determining element is kept to a minimum. The number of coincidence output pulses formed at this stage may greatly exceed the number which it is desired to record; for example, chance coincidences caused by photomultiplier noise may be present in large numbers. The coincidence pulses are amplified, and any small spurious pulses are removed by a pulse height selector in the usual way. In the meantime the upper

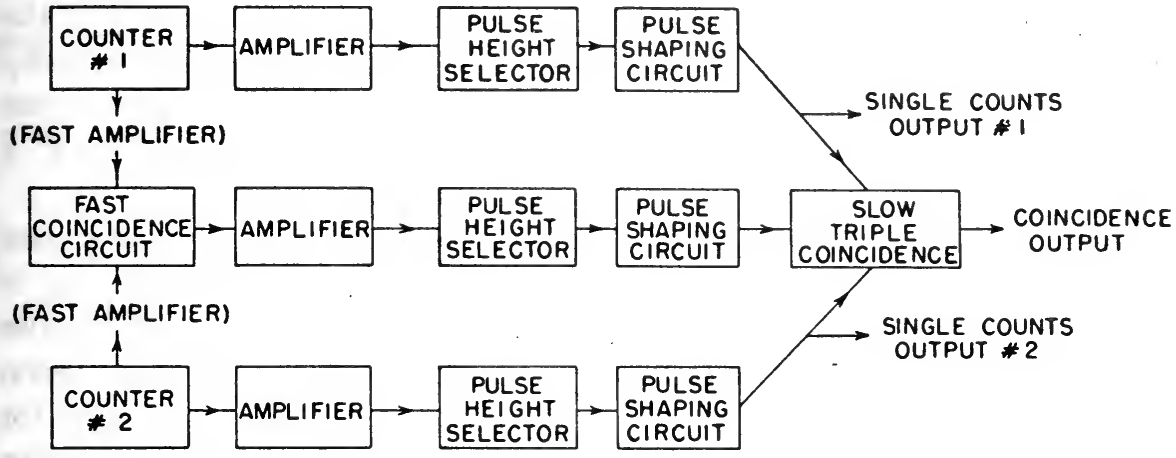

Fig. 4. Block diagram of a "fast-slow" coincidence assembly suitable for the shortest resolving times

and lower channels of Fig. 4 accept untreated pulses from the counters, amplify them, and select their height, exactly as in Fig. 3. At the end of the chain the slow triple coincidence unit selects, out of all the fast coincidences originally formed, only those corresponding to the pulse heights selected by the upper and lower channels of Fig. 4. In this way the full speed of the counters is preserved, and all amplification and pulse height selection is performed with circuits which need not have a particularly fast response. This "fast-slow" type of assembly, now in common use ${ }^{13}$, can be used with any type of fast coincidence circuit, and the pulse height selectors may be as complex as desired. Thus coincidence scintillation spectrometry can be carried on, using the fastest coincidence circuits.

A complete coincidence assembly contains means for determining the length of the pulses applied to the coincidence element, in order to fix the resolving time. Mention has already been made of the $R C$ "differentiation" of pulses, and of the use of standard pulse shaping circuits. For the shortest pulse durations, delay-line clipping ${ }^{14}$ is commonly used. In this

13 F. K. McGowan, ORNL Report No. 865, (1950); H. E. Petch and M. Johns, Phys. Rev. 80, (1950) 478; A. Lundby, Rev. Sci. Instruments 22, (1951) 324; Lewis and Ferguson, Phil. Mag. 44, (1953) 1011.

14 O. R. Frisch, quoted by D. R. Corson and Wilson, Rev. Sci. Instruments 19, (1948) 207. 
method a pulse having a steep leading edge is fed down a section of transmission line whose far end is short-circuited. The pulse reflected from the short circuit is of the same size and shape as the input pulse, but of opposite sign, and thus cancels all of the input pulse except that part occurring within the first $2 p$ seconds, where $p$ is the one-way transit time of the section of transmission line. Another way to look at this action is to regard the transmission line as providing a delayed short circuit on the input pulse. As in $R C$ differantiation, the method works well only if the rise time of the original pulse is short compared with $p$, and if the decay time of the original pulse is long compared with $p$. The transmission line may be of the lumped-constant artificial type, or for the fastest work, a section of coaxial cable ${ }^{12}$.

In the delayed coincidence method it is necessary to delay the pulses from each counter in turn by accurately known amounts. This delay is provided either by the use of pulse shaping circuits of variable pulse length, or by passing the pulses through measured lengths of transmission line of known propagation velocity. The former method is suitable for delays of the order of $10^{-7}$ seconds or longer, and the latter for shorter delays. Bell, Graham, and Petch ${ }^{15}$ have described a helically-coiled coaxial line which provides a variable delay. between two counters of up to $6.4 \times 10^{-8}$ seconds, with a resetting accuracy of a few times $10^{-12}$ seconds.

We conclude this section by showing in Fig. 5 the complete diagram of a modern coincidence circuit of short resolving time, that of Bell, Graham, and Petch 12.15. Only those parts of the circuit concerned with the fast pulses are shown in detail. Negative pulses from the anodes of the type.1P21 photomultipliers $V_{1}$ and $V_{4}$ are applied to the grids of pentodes $V_{2}$ and $V_{3}$, cutting off their standing anode current. These pentodes correspond to the triodes $V_{1}$ and $V_{2}$ of Fig. 2(b), and the germanium diode $V_{5}$ corresponds to the diode $D$ of that figure. Cables $L_{1}$ and $\mathrm{L}_{3}$ are of impedance $100 \mathrm{ohms}$, terminated at their outer ends, and they are varied in length to provide the inserted delays. The clipping cable $\mathrm{L}_{2}$ is of impedance 50 ohms, so that pulses reflected from its shortcircuited end pass into the two parallel cables $\mathrm{L}_{1}$ and $\mathrm{L}_{3}$ without further reflection and are absorbed in the $100 \mathrm{ohm}$ resistors at $V_{2}$ and $V_{3}$ anodes. Since $L_{1}$ and $L_{3}$ have no function other than that of providing delay, we may for the moment assume that their length is zero: it then becomes clear that this is a fast Rossi circuit, the equivalent value of the common anode load resistance of $V_{2}$ and $V_{3}$ being $25 \mathrm{ohms}$, composed of two $100 \mathrm{ohm}$ resistors and the $50 \mathrm{ohm}$ cable $\mathrm{L}_{2}$ in parallel. The bias on diode $\mathrm{V}_{5}$ is

15 I. E. Bell, R. L. Graham, and H. E. Petch, Can. Jour. Phys. 30, (1952) 35. 
adjusted to pass only double-size pulses. In this circuit the photomultipliers $V_{1}$ and $V_{4}$ are run at high gain (applied voltage $\sim 2000 \mathrm{~V}$, gain $\sim 10^{8}$ ) so as to make the circuit react to the first photoelectron of any pulse released at their photocathodes. The remainder of Fig. 5 is identical

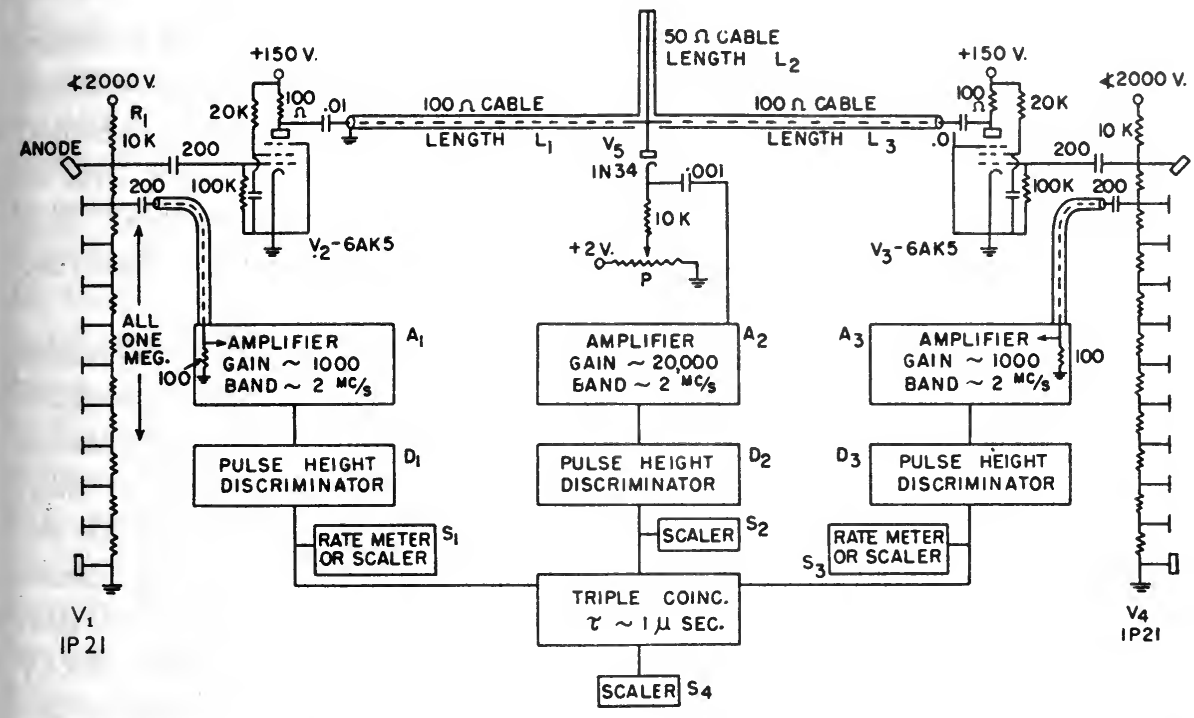

Fig. 5. Diagram of a coincidence circuit capable of resolving times near $10^{-9}$ seconds, after ref. 15

with Fig. 4, whose action has already been explained. With stilbene phosphors this circuit yields resolving times in the $10^{-9}$ second region, the limit being set by the properties of the phosphors, as described in $\S 4$.

\section{$\S$ 3. The Delayed Coincidence Method}

Consider a hypothetical disintegration scheme in which beta-ray emission from nuclide $A$ leads to the excited state $B^{\prime}$ of nuclide $B$, the excited state decaying to the ground state with a mean life $\tau$ which we wish to measure. If one counter detects only the beta rays, and a second counter only the gamma rays, then the distribution of pulses with time delay $t$ between the firing of counter 1 and the firing of counter 2 will be of the form $\exp (-t / \tau)$. We can measure this time distribution in two ways:

(1) The "integral" delayed coincidence method, in which the pulses from counter 1 are extended to a known duration ${ }^{-} T$, while those from counter 2 are kept relatively short. The two trains of pulses are then impressed on a suitable coincidence circuit, and the coincidence counting rate is measured as $T$ is changed. At any one pulse length $T$ we detect all 
the counts from counter 2 that occur within a time $T$ after the firing of counter 1 . The form of the curve of coincidence counting rate as a function of $T$ is then

$$
(1 / \tau) \int_{0}^{T} \exp (-t / \tau) d t=1-\exp (-T / \tau)
$$

The original form of the distribution can be reconstructed by differentiating the measured curve; in practice this means taking differences between successive readings. The same experimental result is obtained if both counter pulses are lengthened to the duration $T$, except that in this case the number of chance coincidences is somewhat increased. This method is therefore sometimes called the method of variation of resolving time.

(2) The "differential" delayed coincidence method, in which the pulses from counters 1 and 2 are kept fixed in length, but the pulses from counter 1 are delayed by a known time $x$ before being impressed on the coincidence circuit. We now detect those pulses from counter 2 which occur later than those in counter 1 by a time $x$, the range of delays near $x$ over which the pulses are detected being set by the resolving time of the coincidence circuit. The form of the curve of coincidence counting rate as a function of $x$ is then $\exp (-x / \tau)$, which measures directly the form of the decay curve of the state $\mathrm{B}^{\prime}$. The method presents no problems so long as the resolving time can be kept much smaller than the mean life to be measured. The differential method measures directly the quantity actually sought, while the integral method can yield this information only by the subtraction of successive readings. The differential method is thus statistically superior to the integral one. The modern practice is to use the differential method, so that the phrase "delayed coincidence method" now almost automatically means the differential method.

The earliest lifetime measurement by the delayed coincidence method was that of the alpha emitter $\mathrm{Po}^{214}\left(\mathrm{RaC}^{\prime}\right)$ by Jacobsen ${ }^{8}$ in 1934. The apparatus involved the two moving-iron oscillographs already mentioned, in what was in effect the differential method. Jacobsen's result was $T_{1 / 3}=2 \times 10^{-4}$ seconds. For the next few years the integral method was developed by Feather and Dunworth ${ }^{9,10,16}$, Rotblat ${ }^{17}$, Ward ${ }^{18}$, and Bradt and Scherrer ${ }^{19}$, all devoted to measuring the half-lives of members of the short-lived alpha-emitting series $\mathrm{Po}^{216}\left(T_{1 / 2}=0.16 \mathrm{sec}\right)$ to $\mathrm{Po}^{212}$ $\left(T_{1 / 2}=3 \times 10^{-7} \mathrm{sec}\right)$. The serious use of the integral delayed coincidence

16 J. V. Dunworth, Rev. Sci. Instruments 11, (1940) 167.

17 J. Rotblat, Nature 144, (1939) 248; Proc. Roy. Soc. (London) A177, (1941) 260.

18 A. G. Ward, Proc. Roy. Soc. (London) A181, (1942) 183.

19 H. Bradt and P. Scherrer, Helv. Phys. Acta 16, (1943) 251 and 259. 
method practically ends with the measurement of the half-life of $\operatorname{Po}^{213}\left(T_{1 / 2}=4.2 \times 10^{-6} \mathrm{sec}\right)$ by Jelley ${ }^{20}$ in 1948 .

The differential delayed coincidence method as we now know it was introduced by Jacobsen and Sigurgeirsson ${ }^{21}$ in 1943 in another experiment on Po ${ }^{214}$. These workers used a univibrator (one-shot multivibrator or flip-flop) circuit of variable pulse length to insert the required delay time in series with the pulses from their Geiger counters, and for the $\mathrm{Po}^{214}$ measurements they used a resolving time of $75 \mu \mathrm{sec}$. The differential method was taken up by De Benedetti and McGowan in 1946, and applied to a search for short gamma-ray half-lives in radioactive substances ${ }^{22}$. The earliest gamma-ray half-life to be correctly measured was $22 \mu$ sec for the $481 \mathrm{keV}$ gamma ray of $\mathrm{Ta}^{181}$, by these workers. By 1948 De Benedetti and McGowan ${ }^{22}$ had surveyed over 60 radioactive species, finding measurable half-lives in $\operatorname{Tm}^{171}(2.5 \mu \mathrm{sec}), \operatorname{Tm}^{169}$ (1 $\left.\mu \mathrm{sec}\right)$ and $\operatorname{Re}^{187}$ $(0.65 \mu \mathrm{sec})$. Their apparatus was similar to that of Jacobsen and Sigurgeirsson ${ }^{21}$, using Geiger counters and a range of delays from 0.75 to $975 \mu \mathrm{sec}$, with resolving times ranging from 0.75 to $70 \mu \mathrm{sec}$. Many of De Benedetti and McGowan's results have been verified by Bunyan, Lundby, and Walker ${ }^{23}$. In 1947 Rowlands ${ }^{24}$ introduced the method of having counter 1 trigger a linear oscilloscope sweep, the output of counter 2 being displayed on the sweep. Pulses from 2 occurring later than pulses from 1 are thus detected, and their delays may be measured on a photograph of the sweep. Multi-channel delay analyzers have also been built; one of high precision covering the range 1 to $11 \mu \mathrm{sec}$ in $1 \mu \mathrm{sec}$ channels has been described by Bell and Hincks ${ }^{25}$.

As an example of the result of a delayed coincidence experiment, we show in Fig. 6 the measured decay curve of the $22 \mu$ sec excited state of $\mathrm{Ta}^{181}$, as recorded by De Benedetti and McGowan ${ }^{22}$. The observed coincidence counting rate is plotted on a logarithmic scale as a function of the inserted delay time, and the half life is read from the curve in the same way as for any radioactive decay curve.

\section{$\S 4$. The Shortest Lifetimes by Delayed Coincidence Methods}

The progressive reduction of the half-life measurable by the delayed

20 J. V. Jelley, Can. J. Research A26, (1948) 255.

21 J. C. Jacobsen and Th. Sigurgeirsson, Dan. Mat. Fys, Medd. 20, (1943) No. 11.

22 S. De Benedetti and F. K. MeGowan, Phys. Rev. 70, (1946) 569; Phys. Rev. 74, (1948) 728 .

${ }_{23}$ D. Bunyan, A. Lundby, and D. Walker, Proc. Phys. Soc. (London) A62, (1949) 253.

24 S. Rowlands, Nature 160, (1947) 191.

25 W. E. Bell and E. P. Hincks, Phys. Rev. 88, (1952) 168. 
coincidence method has proceeded in two ways; first, and most important, by reducing the resolving times of coincidence circuits, and second by improving the analysis of the results of delayed coincidence experiments.

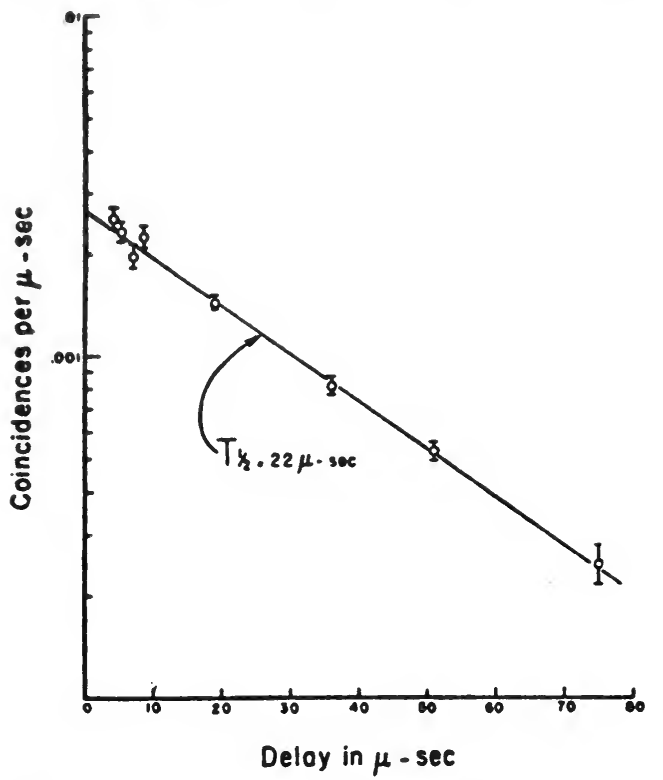

Fig. 6. Decay curve of the $22 \mu$ sec excited state of $\mathrm{Ta}^{181}$, as recorded by the delayed coincidence method by De Benedetti and McGowan (ref. 22)
The latter step enables us to measure half lives that are actually much smaller than the smallest available resolving time.

Advances in coincidence techniques, permitting the use of shorter resolving times, came rapidly after the introduction of counters which did not depend on the collection of ionization in gases; i.e. electron multipliers and scintillation counters. In 1948 Bay and Papp ${ }^{26}$ showed that electron multipliers were capable of resolving times of a few times $10^{-9}$ seconds, and Papp ${ }^{26}$ in effect applied the delayed coincidence method to a study of the counters themselves, a technique now considered indispensable in delayed coincidence work ${ }^{15}$. The introduction of scintillation counters with fast anthracene and stilbene phosphors enabled the resolving time to be progressively reduced. Meyer, Baldinger and Huber ${ }^{27}$ described coincidence systems in 1948 with resolving time around $10^{-8}$ seconds, and Schultz and Beringer ${ }^{28}$ used both scintillation counters and proportional counters in a circuit of resolving time a few times $10^{-8}$ seconds. By 1949 Bell and Petch ${ }^{12}$ were using a resolving time of $5 \times 10^{-9}$ seconds, later reduced to $2 \times 10^{-9}$ seconds, and such resolving times are now common. In the meantime theoretical work on the interpretation of delayed coincidence results by Bay ${ }^{29}$ and Newton ${ }^{30}$ extended the power of the method,

26 Z. Bay and G. Papp, Nature 161, (1948) 59; Rev. Sci. Instruments 19, (1948) 565 ; G. Papp, Rev. Sci. Instruments 19, (1948) 568.

27 K. Meyer, P. Huber, and K. Baldinger, Helv. Phys. Acta. 21, (1948) 188.

28 H. Schultz and R. Beringer, Rev. Sci. Instruments 19, (1948) 424.

29 Z. Bay, Phys. Rev. 77, (1950) 419.

30 T. D. Newton, Phys. Rev. 78, (1950) 490. 
and a calculation by Post and Schiff ${ }^{31}$ enabled the role of the decay time of the scintillation phosphor in limiting the available resolving time to be evaluated. (With the phosphor effect eliminated by using Cerenkov counters, Bay ${ }^{32}$ in 1952 demonstrated a resolving time less than $10^{-9}$ seconds.) These results were collected and experimentally illustrated by Bell, Graham, and Petch ${ }^{15}$, who also showed how to measure the shortest possible lifetimes with the resolving times now available. The following paragraphs are based largely on their paper.

We first define the term resolving time as applied in delayed coincidence work. Suppose two ideal counters, 1 and 2, are each arranged to produce perfectly rectangular pulses of duration $\tau_{0}$ sec, and that these pulses are impressed on an ideal coincidence circuit which detects any overlap, however small, between pulses from 1 and 2 . If the counters are counting unrelated events at rates $N_{1} \mathrm{sec}^{-1}$ and $N_{2} \mathrm{sec}^{-1}$ respectively, the rate of recording chance coincidences is $N_{c}=2 \tau_{0} N_{1} N_{2} \sec ^{-1}$. Suppose now that the same two counters are made to count only promptly coincident events; a curve can be plotted of the recorded coincidence rate as a function of an artificial delay time inserted in series with each counter in turn, delay in series with one counter being the equivalent of negative delay in series with the other. Such a curve is called a prompt resolution curve, and in the present case would be a rectangular curve, of full width $2 \tau_{0}$ seconds. Thus we may define the resolving time $2 \tau_{0}$ of an ideal coincidence circuit as the full width of the prompt resolution curve, or alternatively as $2 \tau_{0}=N_{c} / N_{1} N_{2}$. When the counters and the coincidence circuit are no longer ideal, the prompt resolution curve will be rounded rather than rectangular, but we can still define the resolving time $2 \tau_{0}$ as the effective width of the prompt resolution curve, i.e. its area divided by its maximum height. For the shape of prompt resolution curve usually found in practice, this figure is approximately equal to the full width of the curve at half maximum. In addition, we can perform the chance coincidence experiment as before, and define $2 \tau_{0}=N_{c} / N_{1} N_{2}$. Provided the departures of the apparatus from the ideal are not too great, all three definitions of resolving time are approximately equivalent. Bay ${ }^{29 .}{ }^{33}$ has considered the various definitions of resolving time, and finds that the three definitions of resolving time given here are not equivalent in cases where the random time delays of the counter pulses are long compared with the resolving time. Such a case is seldom encountered in practice.

Numerical values of resolving times have often been quoted in terms

31 R. F. Post and L. I. Schiff, Phys. Rev. 80, (1950) 1113.

32 Z. Bay, Phys. Rev. 87, (1952) 901.

Z. Bay, Phys. Rev. 87, (1952) 194. 
of $\tau_{0}$ rather than $2 \tau_{0}$, as used here; however, the use of $2 \tau_{0}$ is to be preferred because the full width at half maximum of the prompt resolution curve has a clear meaning when the curve is not symmetrical, and because the resolving powers of other devices (e.g. beta-ray spectrometers) are usually quoted in this way. The prompt resolution curve in the non-ideal case is in large part a test of the counters used, and therefore an essential test of a coincidence assembly is the measurement of its prompt resolution curve under the actual conditions of use.

It is of interest to estimate the ultimate resolving times possible with one of the best available scintillation phosphors, trans-stilbene. Suppose that neither photomultiplier tube introduces any transit-time spread in its pulses, and that the coincidence circuit is of sufficient sensitivity to be actuated by the pulse due to a single photoelectron produced at the photocathode. The resolving time of such an arrangement is limited only by the uncertainty in the time of ejection of the first photoelectron from the photocathode after the excitation of the phosphor by the particle being counted, and no refinements in the circuit can shorten the resolving time below this limit. Suppose the total number of photoelectrons produced during a pulse is $R$. Post and Schiff ${ }^{31}$ have shown that the mean time delay for the appearance of the first photoelectron is

$$
\bar{t}=(\tau / R)(1+1 / R), R \gg 1,
$$

where $\tau$ is the mean life of the light flash from the phosphor, about $8 \times 10^{-9} \mathrm{sec}$ for trans-stilbene. Thus the action of the phosphor is to give each counter a built-in mean life for the appearance of the first photoelectron equal to $\bar{t}$. In order to have a probability of 0.95 of observing the first photoelectron, we must wait a time roughly equal to $3 \bar{t}$ after the excitation of the phosphor by the particle being counted. An otherwise perfect coincidence circuit having resolving time $2 \tau_{0}=6 \bar{t}$ would then have a coincidence efficiency of $(0.95)^{2}=0.90$, that is it would miss only $10 \%$ of the true coincidences occurring, when set on the peak of the prompt resolution curve. With RCA-1P21 photomultipliers and careful attention to light collection, we may expect $R \sim E / 4$ for stilbene, where $E$ is the electron energy in kiloelectron volts expended in the phosphor. We can now write down the theoretical minimum resolving time for $90 \%$ coincidence efficiency:

$$
\left.2 \tau_{0} \text { (theor. } \min .\right)=6 \bar{t}=1.9 \times 10^{-7}(1+4 / E) / E \text { sec }
$$

It must be emphasized that this equation gives the limit due to the phosphor alone, assuming no contribution to the resolving time from transit-time spread in the photomultipliers or inadequacies in the rest 
of the coincidence circuit. Equation (3) suggests a minimum resolving time for $100 \mathrm{keV}$ pulses of $2 \times 10^{-9}$ seconds. At large delays the sides of the prompt resolution curve should drop off exponentially with mean life $\bar{t}$, that is, the observed coincidence rate should drop by a factor of two for every $0.693 \bar{t}$ of inserted delay, in this case about $2 \times 10^{-10}$ sec. The experimental verification of these predictions is shown in Fig. 7, which

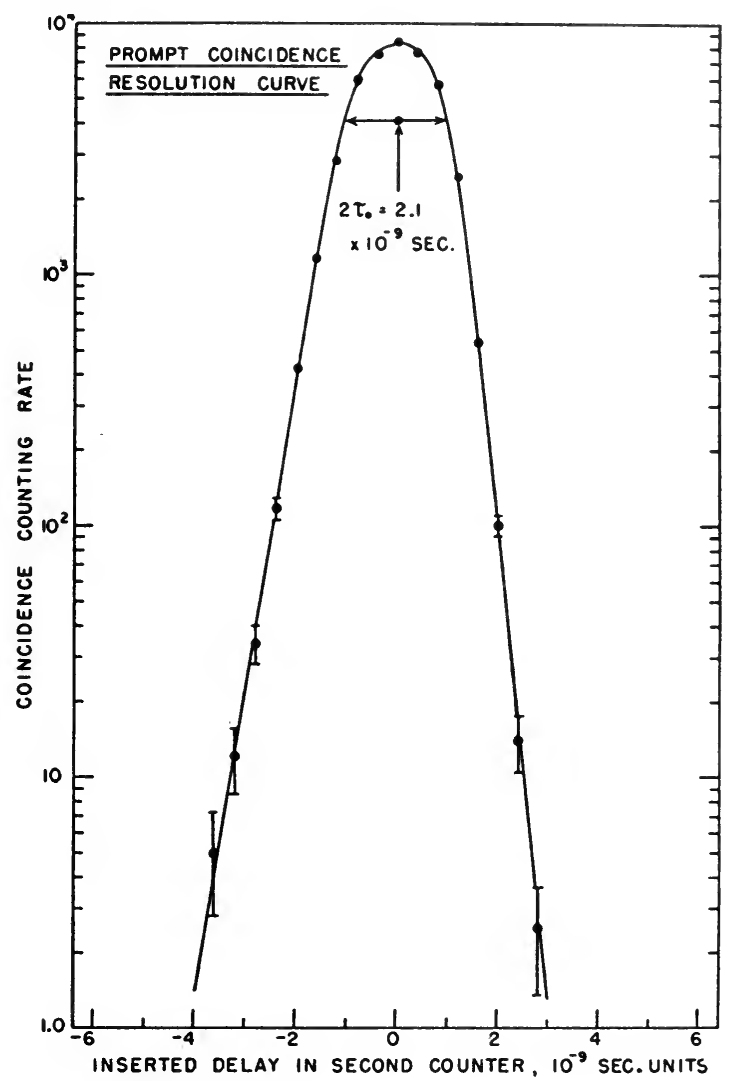

Fig. 7. Prompt coincidence resolution curve observed in testing the coincidence circuit of Fig. 5. The observed coincidences were produced by passing hard betarays through the thin stilbene crystal of the first counter into the stilbene crystal of the second counter. (After ref. 15)

is a prompt resolution curve recorded with the circuit of Fig. 5 for the case when hard beta particles from a radioactive source were passed through the thin stilbene crystal of one counter into the stilbene crystal of the second counter. The pulse size from the thin crystal was equal to that produced by electrons of about $100 \mathrm{keV}$. The curve of Fig. 7 shows a resolving time $2 \tau_{0}=2.1 \times 10^{-9} \mathrm{sec}$. The slopes of the sides of the curve 
are such that the coincidence rate falls by a factor of two in $2.4 \times 10^{-10} \mathrm{sec}$ (left side of curve) or $1.6 \times 10^{-10} \mathrm{sec}$ (right side of curve). The slight asymmetry of the curve of Fig. 7 is attributable to the fact that the pulses from the first counter, whose crystal is traversed by the beta particles, are more nearly uniform in size than those from the second counter. The resolution curve of Fig. 7 was recorded with a pulse clipping cable of airequivalent length $15 \mathrm{~cm}$ and hence a transit time $p$ of $5 \times 10^{-10} \mathrm{sec}$. The expected resolving time $2 \tau_{0}$ is therefore $4 p=2 \times 10^{-9} \mathrm{sec}$, in good agreement with the observed value. The coincidence efficiency for this case was about $85 \%$. If the resolving time is progressively increased, the resolution curve approaches the ideal rectangular shape, with the flat top representing $100 \%$ coincidence efficiency. In order to understand the shape of the curve of Fig. 7 in detail, Bell ${ }^{34}$ had to assume variable delay due to transittime effects in the photomultiplier tubes (type 1P21) of about $2 \times 10^{-10}$ seconds, in addition to the random delay $(\bar{t})$ caused by the phosphor. Measurements by Bay ${ }^{32}$ have verified the magnitude of this effect for the 1P21 photomultiplier. Photomultiplier tubes of larger dimensions may be expected to be worse in this respect. Transit time effects of this magnitude are not serious at a resolving time of $2 \times 10^{-9}$ seconds, but they constitute a limit on the possible time resolution when the phosphor effect is absent, as in the use of scintillation counters based on Cerenkov radiation.

It is clear from these results that when measurements are being made near the limit of resolving time, care must be taken to see that the influence of the electron energy dissipated in the phosphor is correctly taken into account. Another inference is that the best coincidence results will be obtained when electrons of a single energy are incident on the phosphor.

Bell, Graham, and Petch have applied these ideas, together with the theoretical analyses of Newton ${ }^{30}$ and Bay ${ }^{29}$, to permit the measurement of lifetimes short compared with the available resolving times. They employed a pair of lens beta-ray spectrometers placed end to end, the two stilbene counters of the coincidence circuit acting as detectors for the two spectrometers. A radioactive source was mounted on a thin film at the centre of the pair of spectrometers so that time correlations between independently focused radiations. from the same source could be investigated. This arrangement is useful for coincidence beta-ray spectroscopy as well as for lifetime measurements. The advantage of the use of the pair of beta-ray spectrometers for lifetime measurements is that the desired radiations can be selected free of interference by unwanted radiations of different lifetime, or by prompt coincidences caused by radiations scattered between

R. E. Bell, unpublished calculations (1952). 
the two counters. This freedom from interference is especially important when the shortest lifetimes are to be'measured.

The method consists of a careful comparison between the delayed coincidence resolution curve observed with the source under investigation, and the prompt resolution curve observed with radiations of similar energy whose lifetime is known to be very short. An example of this kind of measurement is shown in Fig. 8. Here $F(x)$ is the delayed coincidence

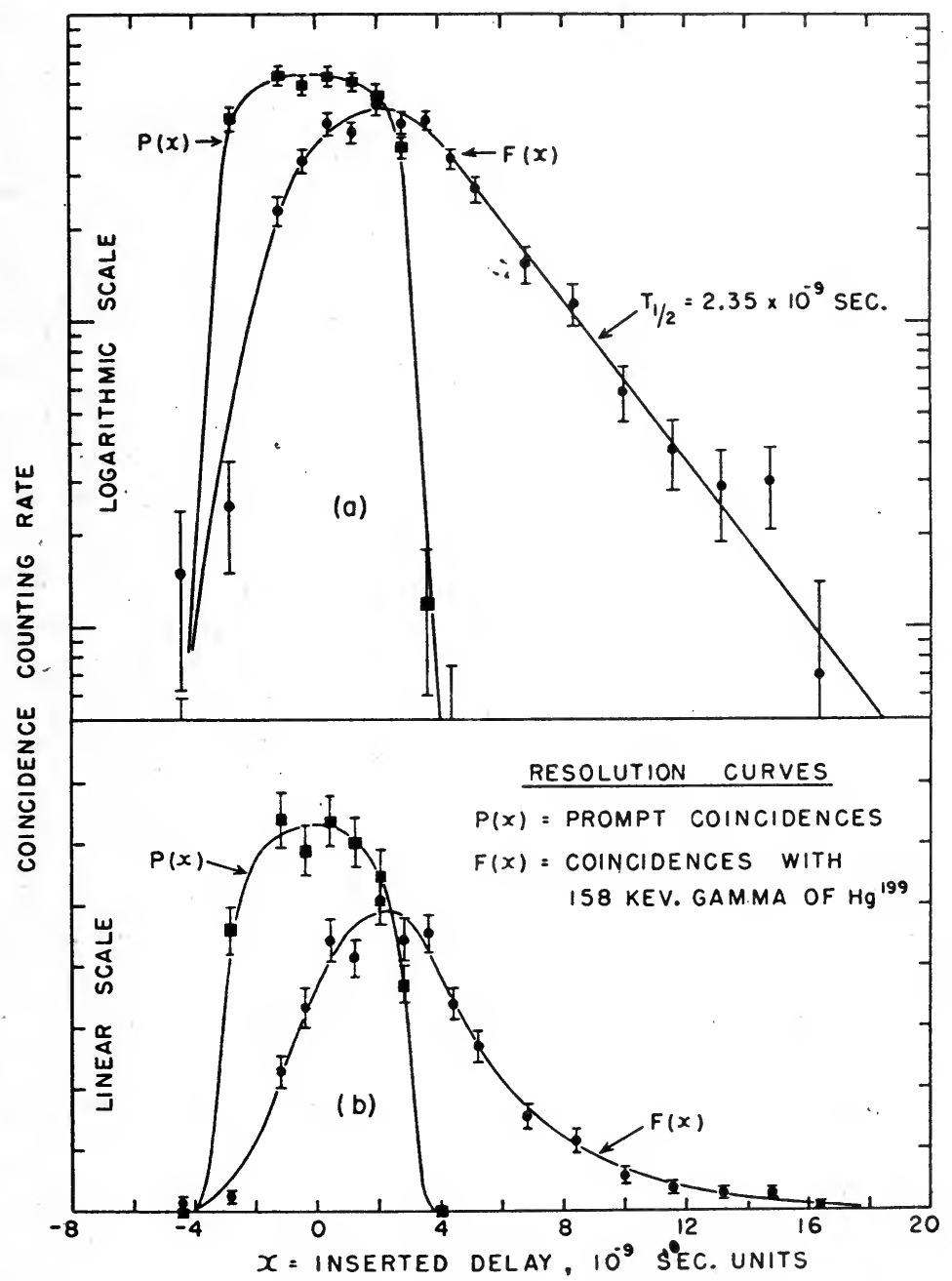

Fig. 8. $P(x)$ prompt and $F(x)$ delayed coincidence resolution curve for a radiation of $\mathrm{Hg}^{199}$ having a half life of $2.35 \times 10^{-9}$ seconds. Diagram $(a)$ logarithmic scale, diagram (b) linear scale. Chance coincidences have been subtracted.. 
resolution curve observed when one spectrometer was focused on the $145 \mathrm{keV} L$-conversion peak of the $158 \mathrm{keV}$ gamma ray emitted by $\mathbf{H g}^{199}$ following the beta decay of $\mathrm{Au}^{199}$; the second spectrometer was focused at a point on the continuum of nuclear beta rays from $\mathrm{Au}^{199}$. The prompt resolution curve $P(x)$ was observed when the $\mathrm{Au}^{199}$ source was replaced by a ThB source, the coincidences then observed being due to the $147 \mathrm{keV}$ $F$-line of ThB in one counter and beta rays in the other, as before. The half-life of the ThB $F$-line has been shown to be less than $2 \times 10^{-11} \mathrm{sec}$, so that it is effectively a prompt radiation ${ }^{1}$. In Fig. 8 , positive values of the inserted delay $x$ correspond to delaying the pulses from the counter recording the beta rays of the continuum. The chance coincidence background observed at very large delays has been subtracted from both $P(x)$ and $F(x)$, and the two curves have been plotted to the same included area in Fig. $8(b)$; Fig. $8(a)$ shows the same data on a logarithmic scale. Newton 30 has shown that, when the data are treated in this way, $F(x)$ and $P(x)$ should intersect at the maximum of $F(x)$, and that at delays great enough so that $F(x) \gg P(x), F(x)$ falls off as $\exp (-x / \tau)$, where $\tau$ is the mean life of the radiation being measured. Thus the half life of the $158 \mathrm{keV}$ gamma ray of $\mathrm{Hg}^{199}$ can be read off from Fig. 8(a) in the usual way; a least squares fit of the present data yields $T_{1 / 2}=(2.26 \pm 0.12) \times 10^{-9}$ sec. In addition, Bay ${ }^{29}$ has shown that the centroid of $F(x)$ is delayed relative to the centroid of $P(x)$ by the mean life $\tau$, so that the mean life can be read from the displacement of the centroids in Fig. 8(b). A numerical integration procedure for determining the centoids of $F(x)$ and $P(x)$ yields in this case $T_{1 / 2}=(2.43 \pm 0.12) \times 10^{-9}$ sec. From these two analyses, Bell, Graham and Petch gave the half-life of the $158 \mathrm{keV}$ gamma transition in $\mathrm{Hg}^{199}$ as $(2.35 \pm 0.20) \times 10^{-9}$ sec.

As an example of the technique of measuring the shortest half-lives, we take the $412 \mathrm{keV}$ gamma ray of $\mathrm{Hg}^{198}$ emitted following the beta disintegration of $\mathrm{Au}^{198}$ : For this case a more sensitive method of detecting short half-lives was used, the so-called "self-comparison" method, in which the resolution curve for the $\mathrm{Hg}^{198}$ conversion electrons was compared, not with a prompt resolution curve, but with its own inverse ${ }^{15}$. The shift between the centroids of the two resolution curves is then $2 \tau=2 T_{1 / 3} / 0.693=2.89 T_{1 / 3}$. The method is as follows: A thin source of $\mathrm{Au}^{198}$ on a thin aluminium foil is placed at the center of the pair of lens beta-ray spectrometers mentioned previously. Let the two spectrometers be called the north and the south spectrometer. For the first run, the north spectrometer is focused on the beta-ray continuum just below the $K$ conversion line of the $412 \mathrm{keV}$ gamma ray, and the south spectrometer is focused on the line. For the second run, both spectrometers have their 
currents increased by the same small percentage, so that the north spectrometer is now on the line, and the south spectrometer is on the beta-ray continuum just above the $K$-conversion line. Thus all effects on the coincidence resolution curve arising from changes in the electron energy or momentum between the runs are small, and in the same direction for both spectrometers. At the same time, each counter is counting only electrons of a single energy at any one time, the condition for the best resolution curve.

The pair of resolution curves found in this experiment is shown in Fig. 9. The curve resulting from the second run (lower curve in Fig. 9) should lie to the right of the curve resulting from the first run (upper curve) by $2.89 T_{1 / 2}$. Applying the centroid calculation to the points of Fig. 9 we find for the $412 \mathrm{keV}$ gamma ray of $\mathrm{Hg}^{198}, T_{1 / 3}=(1.0 \pm 1.7) \times 10^{-11}$ sec. The standard deviation here is the statistical one only. Graham and Bell ${ }^{1}$ have discussed the systematic errors likely to be encountered in such an experiment, and conclude that they will be around $10^{-11}$ seconds. Thus the half life of the $412 \mathrm{keV}$ gamma ray cannot be more than a few times $10^{-11}$ seconds. The initial results of Moon ${ }^{35}$ by the resonant nuclear scattering method had already shown that this half life was possibly a few times $10^{-11}$ seconds, and certainly not much shorter. The combined results imply that the half life is a few times $10^{-11}$ seconds. This result has since been verified by further resonant nuclear scatter-

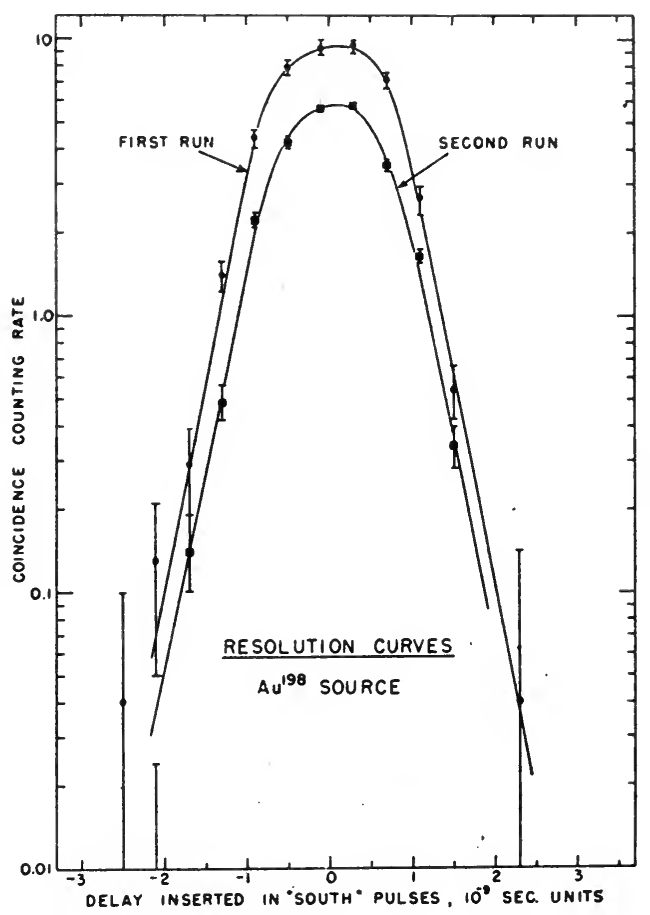

Fig. 9. A coincidence resolution curve ("first run") and its inverse ("second run") for the $412 \mathrm{keV}$ gamma ray of $\mathrm{Hg}^{198}$. These curves enable us to put a limit on the half life of the $412 \mathrm{keV}$ excited state of $\mathrm{Hg}^{198}$ by the self-comparison method. (After ref. 15) ing work, and the half life is now known to be about $2 \times 10^{-11}$ seconds. The above considerations are the basis for the statement made earlier, that in the present development of the delayed coincidence method, the 
shortest measurable mean life is likely to be about $5 \times 10^{-11}$ seconds, even in a favorable case. The lowering of this limit calls for a distinct shortening of the available coincidence resolving times, a goal that does not seem to be in sight at the present time. The measurement of shorter half lives must depend on indirect methods or on comparison methods such as those described in the next section.

\section{$\S$ 5. Examples of Comparison Methods for Lifetime Measurement}

Historically, the first rough experimental information on the lifetimes of low lying excited states of nuclei was provided by a comparison method involving the long range alpha particles emitted by $\mathrm{ThC}^{\prime}$ and $\mathrm{RaC}^{\prime}$. The examples to be presented here are a revision, in the light of more recent knowledge, of those given by Bethe ${ }^{36}$. Consider the chain of disintegrations ThC $(\beta) \mathrm{ThC}^{\prime}(\alpha)$ ThD. At least two excited states of $\mathrm{ThC}^{\prime}$ are formed in the beta-disintegration of $\mathrm{ThC}$, and each state decays either by gamma transitions to lower states of $\mathrm{ThC}^{\prime}$, or by alpha emission to ThD. The alpha particles emitted in low intensities by these excited states are the well known long range alphas. Choosing a particular excited state, let the intensity of the long range alpha group emitted from the state be $N_{\alpha}$, and the intensity of the gamma ray from the same state be $N_{\gamma}$ : then if the corresponding transition probabilities are $\lambda_{\alpha}$ and $\lambda_{\gamma}$, we have for the branching ratio,

$$
N_{\alpha} / N_{\gamma}=\lambda_{\alpha} / \lambda_{\gamma}=\tau_{\gamma} / \tau_{\alpha}
$$

whence

$$
\tau_{\gamma}=\tau_{\alpha}\left(N_{\alpha} / N_{\gamma}\right)
$$

In this equation, $N_{\alpha}$ and $N_{\gamma}$ are the experimentally measured intensities, and $\tau_{\alpha}$, the mean life of the state against alpha decay, can be estimated from the systematices of alpha decay: in this way an estimate of $\tau_{\gamma}$ is obtained. Unfortunately for the accuracy of this procedure, the experimental quantity $N_{\gamma}$ is not very accurately known, and the theoretical estimate of $\tau_{\alpha}$ is probably uncertain by a factor of at least 5 . Thus the result for $\tau_{\gamma}$ has a probable uncertainty of a factor of 10 or so.

'A similar but more complicated case occurs in $\mathrm{RaC}^{\prime}$, where there are 12 groups of long range alphas; only two of these will be used here to estimate the corresponding values of $\tau_{\nu}$.

The results are given in Table $\mathrm{I}$ for two excited states in $\mathrm{ThC}^{\prime}$ and two in $\mathrm{RaC}^{\prime}$. The quantities $N_{\gamma}$ and $N_{\alpha}$ have been taken from sources

${ }_{36}$ H. A. Bethe, Revs. Mod. Phys. 9, (1937)69, Sections 67, 68, 69 and 88. 
quoted in Nuclear Data ${ }^{37}$. The values of $\tau_{\alpha}$ have been calculated following the methods of Perlman et al. ${ }^{38}$ on the systematics of alpha decay.

TABLE I

\begin{tabular}{l|c|c|c|c|c|c}
\hline \hline Nucleus & $\begin{array}{c}\text { Level } \\
\text { Energy, } \\
\mathrm{MeV}\end{array}$ & $N_{\gamma}$ & $N_{\alpha}$ & $E_{\alpha}, \mathrm{MeV}$ & $\tau_{\alpha}, \mathrm{sec}$ & $\tau_{\gamma}, \mathrm{sec}$ \\
\hline $\mathrm{ThC}^{\prime}$ & 0.72 & 0.185 & $3.4 \times 10^{-5}$ & 9.674 & $5.1 \times 10^{-9}$ & $0.9 \times 10^{-12}$ \\
$\left(\mathrm{Po}^{212}\right)$ & 1.81 & 0.07 & $1.9 \times 10^{-4}$ & 10.745 & $5.6 \times 10^{-11}$ & $0.15 \times 10^{-12}$ \\
& & & & & \\
$\mathrm{RaC}^{\prime}$ & 0.61 & 0.089 & $4.3 \times 10^{-7}$ & 8.437 & $3.3 \times 10^{-6}$ & $16 \quad \times 10^{-12}$ \\
$\left(\mathrm{Po}^{214}\right)$ & 1.41 & 0.0031 & $2.2 \times 10^{-5}$ & 9.242 & $4.7 \times 10^{-8}$ & $0.31 \times 10^{-9}$
\end{tabular}

The mean lives in Table I apply to isotopes of Po $(Z=84)$ and can therefore be compared, with little error, with the mean life curves for $Z=82$ in Fig. 1. When this is done, the first three values of $\tau_{\gamma}$ in Table I are seen to compare fairly well with the values predicted by Fig. 1, averaging about ten times smaller than those expected for E2 radiations. Actually these three gamma rays are all believed to be of the E2 type ${ }^{39}$.

The fourth entry in Table I, disagreeing with E2 lifetimes, refers to the well known excited state of $\mathrm{RaC}^{\prime}$ at $1.414 \mathrm{MeV}$ whose only mode of decay is by internal conversion; no gamma-ray photons are observed. The accepted explanation for this behaviour is that the excited state is of zero angular momentum and even parity; the ground state of this eveneven nucleus should also have zero angular momentum and even parity. Direct gamma-ray photon emission in transitions between the two states therefore does not occur, but internal conversion can still take place, as well as internal pair production to a much smaller extent. Drell ${ }^{40}$ has considered this case, as well as some other 0-0 transitions, and concludes that the mean life found in this way fits reasonably well with what theory is available.

Our second example of the comparison methods for measuring short lifetimes concerns another 0-0 transition, occurring in $\mathrm{O}^{16}$. In the nuclear reaction $\mathrm{F}^{19}(\mathrm{p}, \alpha) \mathrm{O}^{16}$, two groups of alpha particles appear, among others, whose energies correspond to leaving the $\mathrm{O}^{16}$ nucleus in its ground and

37 K. Way, L. Fano, M. R. Scott, and K. Thew, Nuclear Data, N.B.S. Circular 499, Washington (1950).

38 I. Perlman, A. Ghiorso, and G. T. Seaborg, Phys. Rev. 77, (1950) 26.

39 D. G. E. Martin and H. O. W. Richardson, Proc. Phys. Soc. (London) A53, (1950) 233; G. D. Latyshev, Revs. Mod. Phys. 19, (1947) 132.

40 S. D. Drell, Phys. Rev. 81, (1951) 656. 
first excited states, respectively. The first excited state at $6.05 \mathrm{MeV}$ decays to the ground state only by the emission of electron-positron pairs ${ }^{41}$. If we assume that the first excited state of $\mathrm{O}^{16}$ has zero angular momentum and even parity, like the ground state, emission of gamma-ray photons is forbidden, and only internal conversion or internal pair production can occur. In the case of $\mathrm{O}^{16}$, the large energy available and the low $Z$ both greatly favor internal pair production, which is therefore the dominant process.

Devons, Hereward, and Lindsey ${ }^{42}$ have measured the mean life of the 6.05 $\mathrm{MeV}$. state by the following method: In the reaction $\mathrm{F}^{\mathbf{1 9}}(\mathrm{p}, \dot{\alpha}) \mathrm{O}^{\mathbf{1 6}}$, the break-up of the compound nucleus $\mathrm{Ne}^{20}$ into an alpha particle and excited $\mathrm{O}^{16}$ imparts to the $\mathrm{O}^{16}$ nucleus a recoil velocity of $2.6 \times 10^{8} \mathrm{~cm} \mathrm{sec}^{-1}$. Devons et al. bombarded a thin target of calcium fluoride with protons, and observed the pairs emitted by the recoiling $\mathrm{O}^{16}$ nuclei through a set of baffles so arranged that pairs were detected only from nuclei that had recoiled in vacuum more than a measured distance away from the surface of the target. By measuring the variation in the number of recorded pairs with this distance, they were able to measure the mean distance travelled by an excited $\mathrm{O}^{16}$ nucleus before emitting its pair. The result was $0.18 \mathrm{~mm}$, which yields immediately by comparison with the known recoil velocity,

$$
\tau=7 \times 10^{-11} \mathrm{sec} .
$$

Devons, Hereward, and Lindsey estimate the probable error of this measurement as $\pm 10 \%$. Again this mean life agrees with the estimates of Drell ${ }^{40}$.

Our final example of comparison methods describes the measurement of a gamma-ray lifetime by comparing it with the time required for a charged particle to slow down in a solid.

When $\mathrm{B}^{10}$ is bombarded with slow neutrons, the reaction $\mathrm{B}^{10}(\mathrm{n}, \alpha) \mathrm{Li}^{7}$ takes place, and in $93 \%$ of the cases the resultant $\mathrm{Li}^{7}$ nucleus is left in an excited state with an energy $479 \mathrm{keV}$ above the ground state. The excited state decays to the ground state by the emission of a $479 \mathrm{keV}$ gamma ray. The mean life, $\tau$, of this excited state was measured by Elliott and Bell ${ }^{43.44}$ by a method that also provided the first example of the direct observation of the Doppler broadening of a gamma-ray line.

The reaction $\mathrm{B}^{10}(\mathrm{n}, \alpha) \mathrm{Li}^{7}$ is exothermic by $2.8 \mathrm{MeV}$, so that in the case

41 V. K. Rasmussen, W. F. Hornyak, C. C. Lauritsen, and T. Lauritsen, Phys. Rev. 77, (1950) 617 .

42 S. Devons, H. G. Hereward, and G. R. Lindsey, Nature 164, (1949) 586.

43 L. G. Elliott and R. E. Bell, Phys. Rev. 74, (1948) 1869.

44 I. G. Elliott and R. E. Bell, Phys. Rev. 76, (1949) 168. 
when the $\mathrm{Li}^{7}$ nucleus is left in its excited state, it receives a recoil kinetic energy equal to $(4 / 11)(2.8-0.48) \mathrm{MeV}=0.85 \mathrm{MeV}$. The initial velocity, $V_{0}$ of the recoiling excited $\mathrm{Li}^{7}$ nucleus is therefore $4.8 \times 10^{8} \mathrm{~cm} \mathrm{sec}^{-1}$, corresponding to $V_{0} / c=0.016$; this velocity is quickly lost by ionization as the charged $\mathrm{Li}^{7}$ particle traverses the target material. If the excited $\mathrm{Li}^{7}$ particles emit their gamma rays before slowing down, an observer will see a gamma-ray line Doppler broadened by $\pm 1.6 \%$. If, however, the $\mathrm{Li}^{7}$ particles do not emit their gamma rays until after slowing down, a sharp gamma-ray line will be seen. Intermediate cases are of course possible. If the Doppler broadening can be detected, we have here a method of comparing the mean life of the $\mathrm{Li}^{7}$ excited state with the slowing down time of $\mathrm{Li}^{7}$ particles in the material of the target.

The actual measurement proceeded as follows. A lens beta-ray spectrometer was set up for observing photoelectron lines ejected from a thin uranium radiator by gamma-ray sources, in the usual manner. The source of gamma rays in this case was a small cylinder of boron compound continuously irradiated with thermal neutrons from the Chalk River nuclear reactor, and continuously emitting the $\mathrm{Li}^{7}$ gamma rays. Three different boron compounds, of widely differing densities, were used as neutron targets in order to vary as much as possible the slowing down time of the $\mathrm{Li}^{7}$ particles; the compounds were $\mathrm{LiBH}_{4}(\varrho=0.35), \mathrm{B}_{4} \mathrm{C}(\varrho=2.5)$ and $\mathrm{FeB}(\varrho=7.2)$. The shape of the $K$ photoelectron line ejected from the radiator by the $479 \mathrm{keV}$ gamma ray was measured using each substance for a source of the $\mathrm{Li}^{7}$ gamma rays in turn, and also using a comparison gamma ray of similar energy, the $468 \mathrm{keV}$ gamma ray emitted from a

Fig. 10. Diagram showing Doppler broadening of the $479 \mathrm{keV}$ gamma ray of $\mathrm{Li}^{7},(a)$ Comparison gamma ray. (b) (c) (d) $\mathrm{Li}^{7}$ gamma rays from three targets of progressively decreasing density

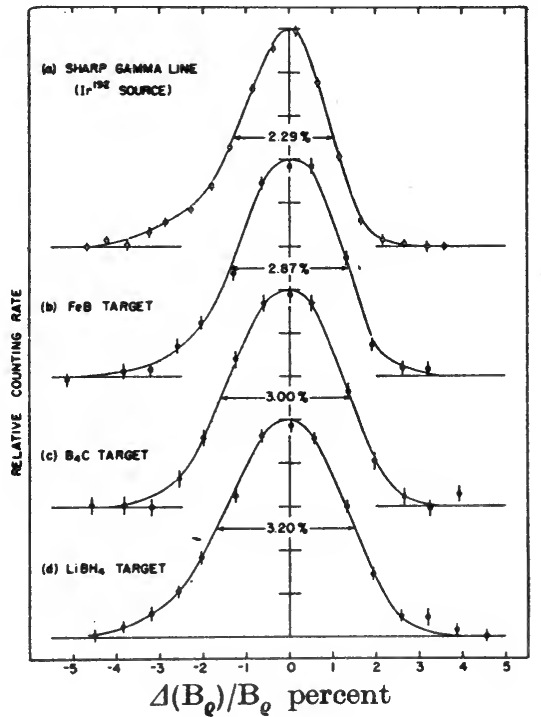


source of radioactive $\operatorname{Ir}^{192}$. The results are shown in Fig. 10, where each photoelectron line is shown with its background subtracted, and with the Ir $^{192}$ comparison line shifted so that its centre coincides with that of the $\mathrm{Li}^{7}$ lines. Both the Doppler broadening and its variation with the stopping power of the target material are clearly shown.

The analysis of the results of Fig. 10 to give the actual value of $\tau$ is shown in Fig. 11. A characteristic slowing down time, $R / V_{0}$, can be assigned to each of the three target materials, where $R$ is the range of the $\mathrm{Li}^{7}$ particles in each material. For any one value of $\tau$ we compute a curve of expected spectrometer line width as a function of $R / V_{0}$. The three measured points must all lie on one such curve corresponding to the actual value of $\tau$. Fig. 11 shows that the measured points agree with the curve computed for $\tau=0.75 \times 10^{-13}$ seconds, with probable error $0.25 \times 10^{-13}$ seconds. This value of $\tau$ is consistent with lifetime estimates for M1 radiation (Ch. XIII).

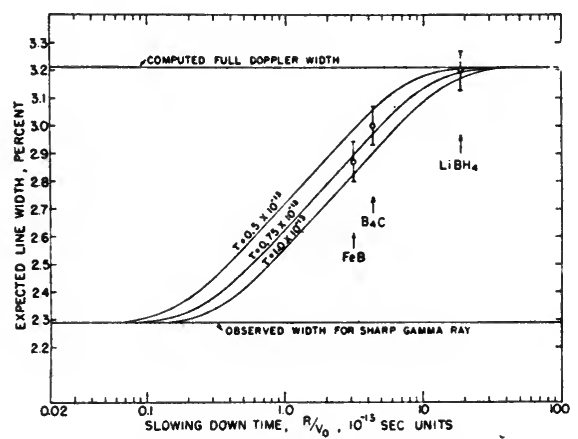

Fig. 11. Computed variation of spectrometer line width with characteristic slowing down time $R / V_{0}$, for three different assurned values of $\tau$. The experimental points show that the mean life of the $\mathrm{Li}^{7}$ first excited state is $(0.75 \pm 0.25) \times 10^{-13}$ seconds 


\section{(II) K. G. MALMFORS - Resonant Scattering of $\gamma$-Rays}

\section{§ 1. Resonant Scattering Experiments}

Resonant scattering of light is a well-known phenomenon which is observed when the energy of an incident photon coincides with the energy difference between an excitation level and the ground state of the scattering atom. The incident photon can originate either from an atom of the same kind as the scattering atom or from a continuous spectrum in which case resonant scattering gives rise to absorption lines. According to electrodynamical theory the cross-section for resonant scattering is given by the following equation:

$$
\sigma=g \cdot \frac{\lambda^{2}}{8 \pi} \cdot \frac{\Gamma^{2}}{\left(E-E_{0}\right)^{2}+\frac{1}{4} \Gamma^{2}}
$$

where $E$ is the energy and $\lambda$ the wavelength of the incident ray and $E_{0}$ is the energy and $\Gamma$ the width of the actual excitation level. $g$ is a factor which takes into account the multiplicities of the ground state and the excited level.

$$
g=\frac{1+2 J_{e}}{1+2 J_{\sigma}},
$$

$J_{e}$ and $J_{g}$ are the spins of the excited level and the ground state respectively.

Since resonance effects play such a great role in the scattering and absorption of low energy quanta which arise from transitions in the electron shells of atoms it has been realized for a long time that the analogous effect ought to be expected in the region of gamma-rays associated with transitions between energy levels in nuclei. Several experiments have been made since Kuhn in 1929 first drew attention to the problem but not until recently has it been possible to observe resonantly scattered $\gamma$-rays. Before discussing the different experiments we shall consider briefly the general case in which a gamma-ray is scattered by a nucleus of the same kind as that from which it is emitted. A gamma-ray emitted from a nucleus initially at rest always has an energy which is slightly less than the energy difference between those levels which correspond to the transition because the emitting nucleus takes a small portion of the available energy for the recoil. If $M$ is the mass of the emitting atom, conservation of energy and momentum leads to: $h v=E_{0}-\left(E^{2} / 2 M c^{2}\right)$. The same amount of kinetic energy is transferred to the nucleus which is excited by the gamma-ray so that the total energy displacement amounts to: $\Delta E=\left(E^{2} / M c^{2}\right)$. If this energy difference is large compared to the width of the level, the system is out of resonance and the cross-section for resonant scattering becomes extremely small. The width of a level can be calculated from the life-time $\tau$ by means of the uncertainty relation $\Gamma \cdot \tau \simeq \hbar$. The 
order of magnitude of $\tau$, which depends strongly on the type of transition, can be estimated from Weisskopf's formula (see Ch. XIII). Such calculations show that, even in case of $\mathrm{M} 1$ and $\mathrm{E} 2$ transitions, the recoil energy is several orders of magnitude larger than the level width. The energies are comparable only in the case of electric dipole radiation, a type of transition which has never been observed. Such considerations explain why no resonantly scattered radiation was observed in the early experiments.

Kuhn ${ }^{1}$ and Meitner and Hupfeld ${ }^{2}$ were able to show that the gammaradiation from $\mathrm{ThC}^{\prime \prime}$ was not more strongly absorbed in lead than in adjacent elements and thus an upper limit could be estimated for the cross-section of resonant absorption. Zuber ${ }^{3}$ repeated these early experiments with an improved apparatus and also studied the scattering in paraffin of the $4.3,11.8$ and $16.6 \mathrm{MeV}$ gamma-rays from the reaction $\mathrm{B}^{11}(\mathrm{p}, \gamma) \mathrm{C}^{12}$. In neither case could any effect due to resonant scattering be detected.

Already Kuhn had pointed out that the resonance condition might be restored by a preceding beta- or gamma-emission. This argument was discussed in detail and studied experimentally by Pollard and Alburger ${ }^{4}$. A nucleus emitting a beta-particle obtains a recoil velocity in the opposite direction and if the subsequent emission of the actual gamma-ray takes place within such a short time that the nucleus has not lost its velocity the energy shift may be compensated by the Doppler-effect. The resonance condition is exactly restored if the emitting nucleus approaches the scatterer with the velocity $u=(E / M c)$. By measuring coincidences between the betaparticles and the scattered gamma-rays one might expect to find an increased counting rate when the beta-particles are measured at such an angle that the recoiling nucleus has the appropriate velocity component towards the scatterer. The same argument holds for a nucleus decaying by two succesive gamma-rays. Pollard and Alburger attempted an experiment according to this principle using the gamma-activity from $\mathrm{Na}^{24}$ but owing to the insufficient resolution of their counters for this type of coincidence measurement they found it necessary to arrange the experiments in another way, namely by studying large angle scattering. In this case they also studied the gamma-rays from $\mathrm{Na}^{24}$ which decays to $\mathrm{Mg}^{24}$ by a $1.39 \mathrm{MeV}$ beta-ray followed by two gamma-rays in cascade having energies of $2.76 \mathrm{MeV}^{\prime}$ and $1.38 \mathrm{MeV}$. The energy displacement which has to be compensated by the recoil from preceding emission is of

1 W. Kuhn, Phil. Mag. 8, (1929) 625.

2 L. Meitner, and H. H. Hupfeld, Z. f. Phys. 67, (1930) 147.

3 K. Zuber, Helv. Phys. Acta, 16, (1943) 407 and 429.

4 E. Pollard, and D. E. Alburger, Phys. Rev. 74, (1948) 926. 
the order of $100 \mathrm{eV}$. The experiment was made with an automatic apparatus capable of detecting small effects, i.e. a small difference in counting rate when the $\mathrm{Mg}$-scatterer was substituted by Al. No resonant scattering was observed and it could be concluded that the width of the actual level was less than 1/100 eV. In an other series of experiments $\mathrm{Mn}^{56}$ and $\mathrm{Fe}^{56}$ were used as source and scatterer.

A similar type of investigation was made by Metzger ${ }^{5}$ who studied the resonant scattering of the last gamma-ray in the decay of $\mathrm{Tc}^{96}$ which at that time was suspected to be an electric dipole transition. The radiation was scattered in $\mathrm{Mo}^{96}$ into which $\mathrm{Tc}^{96}$ decays. Owing to preceding gammaemission it was expected that the resonance condition should be fulfilled in a small but still observable fraction of the disintegrations. The negative result showed that the width of the level was less than $1 / 100 \mathrm{eV}$ and consequently that the transition to the ground state is not an electric dipole radiation.

Another method of restoring the resonance-by mechanical motionwas proposed by Moon ${ }^{6.7}$ and the experiments performed were the first which gave a positive result. As discussed earlier the energy shift is compensated by the Doppler-effect if the emitting nucleus moves towards the scatterer with the velocity $u=(E / M c)$. If the nucleus is a heavy one and the gamma-energy not too high this velocity can be obtained at the edge of a high speed rotor. ( $E=400 \mathrm{keV}$ and $M=200$ gives $u=640 \mathrm{~m} / \mathrm{s}$ ). Moon derived an expression for the effective cross-section of resonant scattering taking into account the mechanical velocity of the gamma-source and the thermal velocities of the nuclei in the source and the scatterer, which will be reproduced here. It is supposed that the thermal velocities of the nuclei are distributed as in a gas at temperature $T$. The probability that a source nucleus and a scattering nucleus shall have thermal velocity components in the direction of the gamma-ray that differ by $v \mathrm{~cm} / \mathrm{sec}$ is then given by:

$$
P(v) d v=\sqrt{\frac{M}{4 \pi k T}} \cdot e^{-\frac{M v^{2}}{4 k T}} d v .
$$

If at the same time the source as a whole moves towards the scatterer with the velocity $u \mathrm{~cm} / \mathrm{sec}$, the Doppler effect will add the amount $(v+u) \cdot(E / c)$ to the energy of the gamma-ray. The effective cross-section for resonant scattering is obtained by replacing $E-E_{0}$ in equation (1) by

$$
\frac{E}{c}(v+u)-\frac{E^{2}}{M c^{2}}
$$

5 F. R. Metzger, Phys. Rev. 83, (1951) 842.

6 P. B. Moon, Proc. Phys. Soc. 63, (1950) 1189.

7 P. B. Moon, Proc. Phys. Soc. 64, (1951) 76. 
multiplying by $P(v)$ and integrating with respect to $v$.

$$
\sigma=g \cdot \frac{\lambda^{2}}{8 \pi} \int_{-\infty}^{+\infty} \frac{P(v) \cdot \Gamma^{2} d v}{\frac{E^{2}}{c^{2}}\left(u+v-\frac{E}{M c}\right)^{2}+\frac{1}{4} \Gamma^{2}}
$$

In this integral the integrand has a sharp maximum around $v=(E / M c)-u$ and little error will be made by replacing $P(v)$ by $P((E / M c)-u)$. It then follows that

$$
\sigma=g \cdot \frac{h^{2} \cdot c^{2} \cdot \Gamma}{4 E^{3}} \cdot \mid \frac{\overline{M c^{2}}}{4 \pi k T} \cdot e^{-\frac{M(u-(E / M c))^{2}}{4 k T}} .
$$

The resonant scattering experiments in Moon's laboratories have been made with the $411 \mathrm{keV}$ gamma-radiation of $\mathrm{Hg}^{198}$, which follows the 2.7 day beta-decay of $\mathrm{Au}^{198}$. The activated gold source. was electroplated to the tips of a steel rotor (diam. $6^{\prime \prime}$ ) and could be given any velocity up to $8 \cdot 10^{4} \mathrm{~cm} / \mathrm{sec}$. Fig. 1 shows one of the geometrical arrangements used ${ }^{8}$. Gamma-rays leaving the source in the forward direction fell upon a scatterer of liquid mercury and the scattered radiation was detected either with a Geiger-counter or with a $\mathrm{NaI}(\mathrm{Tl})$ scintillation counter biased for counting elastically scattered radiation only. The isotopic abundance of $\mathbf{H g}^{198}$ is $10 \%$ which reduces the effective cross-section as calculated from equation (4) by a factor of 10. Control experiments were made with lead replacing the mercury scatterer. Fig. 2 which is the result of the most recent experiments ${ }^{9}$, shows that the counting rate goes through a maximum when the velocity is about $7 \cdot 10^{4} \mathrm{~cm} / \mathrm{sec}$. The curve fitting the experimental points is calculated for a quadrupole transition between a ground state of spin 0 and an excited level of spin 2 having a width of $2.1 \cdot 10^{-5} \mathrm{eV}$. The crosssection for resonant scattering has been measured relative to that of nonresonant elastic scattering which has been taken as $1.32 \cdot 10^{-26} \mathrm{~cm}^{2} / \mathrm{sterad}$, a matter which will be discussed below. The intrinsic width corresponds to a half-life of $(2.2 \pm 0.5) \cdot 10^{-11} \mathrm{sec}$ which is in agreement with the delayedcoincidence value $(1.0 \pm 1.7) \cdot 10^{-11}$ sec reported by Graham and Bell ${ }^{10}$. The theoretical curve is based on the assumption that the nuclei are free to recoil and that the beta-recoil is completely dissipated before the gamma-ray is emitted. The experiments seem to verify this fundamental basis of the theory.

The thermal motion is a factor which in Moon's case reduces the effective cross-section of resonant scattering. But if no mechanical motion is applied, an increased temperature of the source or of the scatterer or of

\footnotetext{
18 P. B. Moon and A. Storruste, Proc. Phys. Soc. 66, (1953) 585.

9 P. B. Moon and W. G. Davey, Proc. Phys. Soc. 66, (1953) 956.

10 R. L. Graham and R. E. Bell, Phys. Rev. 84, (1951) 380.
} 


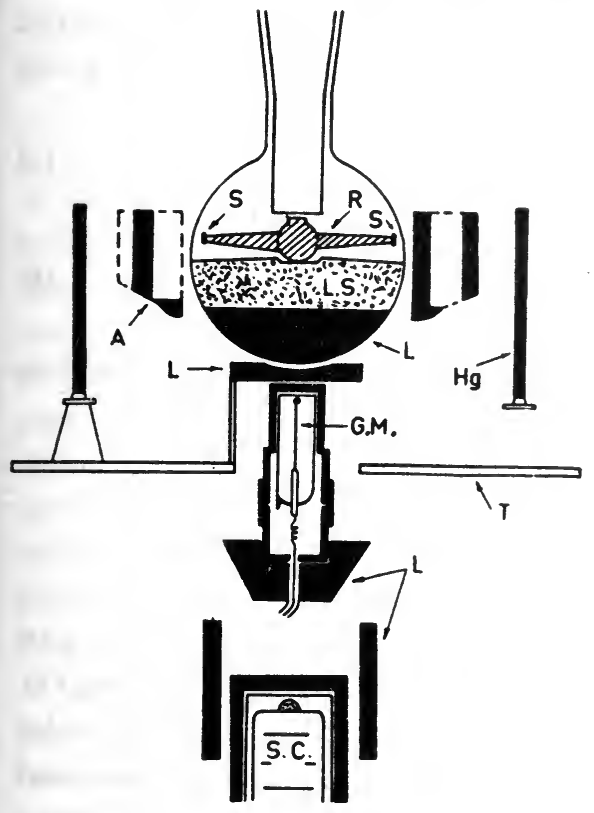

VERTICAL SECTION

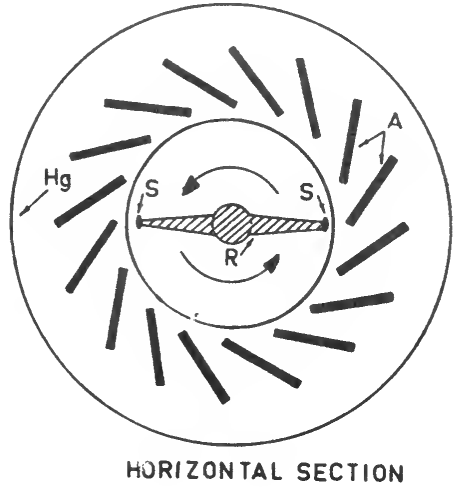

R ROTOR

$S$ SOURCES

A COLLIMATOR

L.S. LEAD SHOT

L SOLID LEAD

Hg MERCURY

GM. GEIGER MÜLLER COUNTER

T TABLE

S.C. SCINTILLATION COUNTER

Fig. 1. Rotor arrangement (Moon and Storruste, 1953)

both will result in a broadened energy distribution which increases the probability for two nuclei having the appropriate relative velocities for compensating the recoil. This possibility was pointed out and verified experimentally by Malmfors ${ }^{11}$. If we start from equation (4) we have to

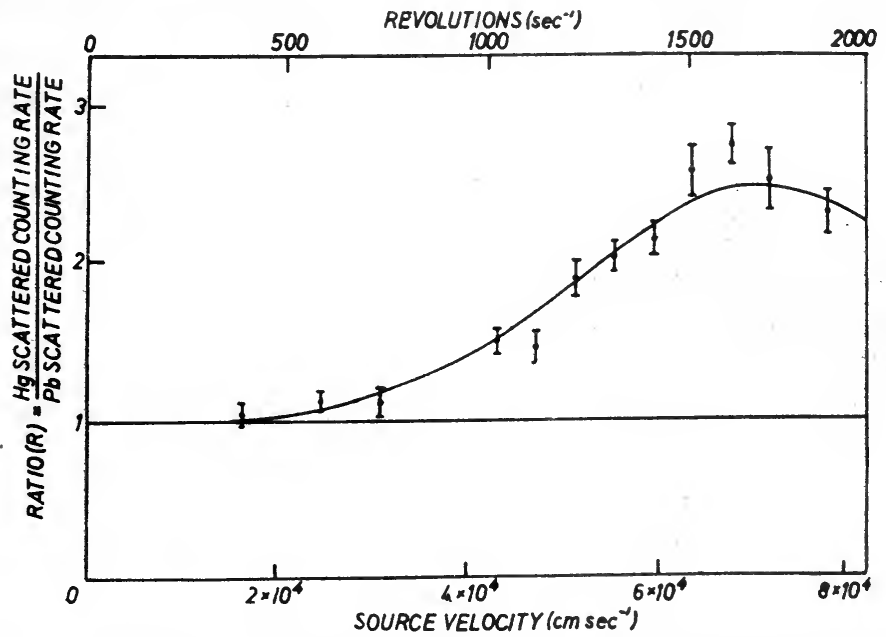

Fig. 2. Results from rotor experiment (Moon and Davey, 1953)

K. G. Malmfors, Ark. f. Fysik 6, (1952) 49. 
put $u=0$ since there is no mechanical motion and replace $T$ by $\left(T_{1}+T_{2}\right) / 2$ where $T_{1}$ and $T_{2}$ are the temperatures of the source and the scatterer. By the substitution

there results:

$$
\frac{E^{2}}{M c^{2}} \cdot \frac{1}{2 k\left(T_{1}+T_{2}\right)}=p^{2}
$$

$$
\sigma=g \cdot \frac{h^{2} c^{4}}{4 \sqrt{\pi}} \cdot \frac{M}{E^{4}} \cdot \Gamma \cdot p \cdot \mathrm{e}^{-p^{2}} .
$$

In the experiments the $411 \mathrm{keV}$ transition in $\mathrm{Hg}^{198}$ was studied and in this case there applies:

$$
\begin{aligned}
& \sigma=7 \cdot 10^{-21} \cdot \Gamma \cdot p \cdot \mathrm{e}^{-p^{2}} \\
& p=\frac{73.3}{\sqrt{T_{1}+T_{2}}} \quad(\Gamma \text { in } \mathrm{eV}) .
\end{aligned}
$$

If both source and scatterer are at room temperature, the factor $p \cdot \mathrm{e}^{-p^{2}}$ is very small. But if the gold-source is heated to its melting point, $1063^{\circ} \mathrm{C}$, the factor is more than 200 times larger, 0.065 , and since $\Gamma$ is of the order $10^{-5} \mathrm{eV}$ the effective cross-section for resonant scattering can be estimated as $\sim 5 \cdot 10^{-27} \mathrm{~cm}^{2}$. This is a few percent of the effect due to competing elastic scattering processes. In the experiments it could be verified that the heating of the gamma-source, a gold sphere (diam. $2.7 \mathrm{~mm}$ ), resulted in an increased intensity of scattered raditation. As a check experiment the mercury scatterer was replaced by lead. In this case the heating also gave a slight increase due to lowered self-absorption in the gamma-source. After correcting for this effect the relative increase in counting rate was determined as $3 \%$, which corresponds to an intrinsic width $\Gamma=8 \cdot 10^{-6} \mathrm{eV}$. The angular distribution of resonantly scattered raditation depends on the type of transition and can be calculated in each separate case. In the evaluation of Malmfors' results this distribution was assumed to be isotropic while in the calculation of Moon's most recent value the deviation from the isotropic distribution has been taken into account. If the same correction is applied, Malmfors' value should be changed to: $\Gamma=1.3 \cdot 10^{-5} \mathrm{eV}$, which corresponds to a half-life of $3.5 \cdot 10^{-11} \mathrm{sec}$.

A repetition of this experiment with improved technique by Metzger and Todd ${ }^{12}$ gave for the first excited state in $\mathrm{Hg}^{198}$ a half-life of $(2.2 \pm 0.2) \cdot 10^{-11} \mathrm{sec}$ which is in good agreement with Davey and Moon's value. Metzger ${ }^{13}$ also has applied the method of heating the source to the case of the $209 \mathrm{keV}$ transition in $\mathrm{Hg}^{199}$ and to the $280 \mathrm{keV}$ transition in $\mathrm{Tl}^{203}$. The lifetimes were found to be $(3 \pm 1) \cdot 10^{-10} \mathrm{sec}$ and $(9 \pm 4) \cdot 10^{-10} \mathrm{sec}$

12 F. R. Metzger and W. B. Todd, Phys. Rev. 95, (1954) 853.

13 Report at the Glasgow conference, 1954. 
respectively. In the latter case the methods of mechanical motion and beta-recoil were also successfully applied.

Recently very promising experiments have been reported by Moon and Ilakovac ${ }^{14,15}$. As in the experiments by Pollard and Alburger and in those of Metzger the recoil from a preceding $\beta$-emission is used for restoring the resonance. By using a liquid gamma-source they were able to observe resonantly scattered radiation. The difference between the emission mechanism in a liquid and a solid gamma-source is due to the fact that the recoiling nucleus loses its velocity more slowly in a liquid body which makes it more probable that the velocity persists at the actual moment of emission of the gamma-ray. The effect is expected to be even more pronounced if the scattering of the gamma-radiation from a gaseous source is studied. Successful experiments were made with a source of $\mathrm{Zn}^{63}$ which decays into $\mathrm{Cu}^{63}$. When a liquid source was used a copper scatterer was $3.6 \pm 0.9$ times more effective than a similar scatterer of iron, while in the case of a solid source used in the same geometrical arrangement the corresponding figure was $1.6 \pm 0.7$. The analysis of these data gave for the mean life of the $0.96 \mathrm{MeV}$ level in $\mathrm{Cu}^{63}: \tau=6 \cdot 10^{-13}$ sec.

Similar experiments were made with the gamma-radiation produced in the decay of $\mathrm{Mn}^{56}$. From the absence of any observable resonant scattering the mean life of the $0.84 \mathrm{MeV}$ level in $\mathrm{Fe}^{56}$ was estimated to be longer than $8 \cdot 10^{-12} \mathrm{sec}$. In the same apparatus aluminum and magnesium scatterers were used in the hope of observing resonant scattering of 0.84 $\mathrm{MeV}$ gamma-rays from $\mathrm{Mg}^{27}$ on aluminium. Since no effect was observed the mean life must be longer than $10^{-12}$ sec. This limit is not as good as that obtained for $\mathrm{Fe}^{56}$ due mainly to the rather short half-life of $\mathrm{Mg}^{27}$.

Quite another type of resonant scattering experiment has been discussed by Schiff ${ }^{16}$ who analyzed the possibility of using a continuous gammaspectrum, for instance from a betatron. According to this discussion it might be possible to observe resonance lines in the scattered spectrum. Gaerttner and Yeater ${ }^{17}$ investigated the problem with a $100 \mathrm{MeV}$ betatron, studying the radiation scattered in copper and carbon, but in neither case was any measurable effect detected.

The measurement of the cross-section for resonant scattering is in fact a direct determination of the level width $\Gamma$ and an indirect way of finding the mean life $\tau$ which is related to $\Gamma$ by the uncertainty relation. This method of determining mean lives provides an important complement to

14 P. B. Moon and K. Ilakovac, Phys. Rev. 93, (1954) 254.

15 K. Ilakovac, Proc. Phys. Soc. 67, (1954) 601.

16 L. I. Schiff, Phys. Rev. 70, (1946) 761.

17 E. R. Gaerttner, and M. L. Yeater, Phys. Rev. 76, (1949) 363. 
the delayed coincidence technique which is capable of measuring delays longer than $\sim 10^{-10}$ sec. The resonant scattering method is more easily applied the shorter the mean life and, as shown in the case of $\mathrm{Hg}^{198}$, the regions of applicability are overlapping for mean lives of the order of $10^{-10}$ ser. The following table is a summary of those cases where lifetimes have been measured by the resonant scattering method.

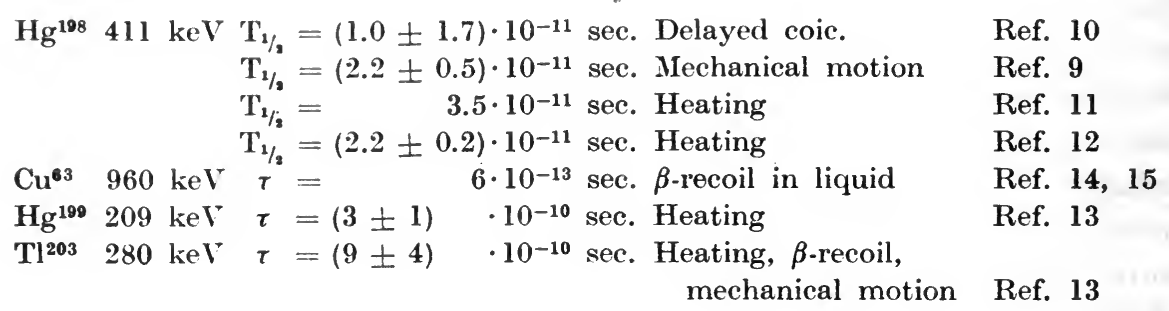

\section{$\S$ 2. Competing Non-Resonant Scattering Processes}

The study. of resonant scattering requires accurate knowledge of competing scattering processes of which the following have to be considered:

a) Compton scattering

b) Rayleigh ,"

c) Thomson ,"

d) Delbruck ",

Compton scattering does not need much discussion in this connection since it is inelastic and can be filtered out or discrimnated against by the use of an energy-selective gamma-detector which measures elastically scattered quanta only. This is more easily arranged the larger the scattering angle. Considerable complications due to Compton scattering arise, however, if strong gamma-radiation of higher energy than that to be measured is present.

The other three processes give elastically scattered gamma-rays which are not distinguishable energetically but possibly could be analyzed according to their different angular distributions.

Rayleigh scattering, which is due to interaction with bound electrons, is well known in the $\mathrm{X}$-ray region and is the basic process in the crystal spectroscopy of gamma-rays. Calculations by Franz ${ }^{18}$ lead to the following equation for the cross-section:

$$
\sigma_{R}=\frac{8.67 \cdot 10^{-33}}{\sin ^{3} \frac{1}{2} \Theta} \cdot\left(\frac{Z \cdot E_{0}}{E}\right)^{3} \cdot \frac{1+\cos ^{2} \Theta}{2} \mathrm{~cm}^{2} / \text { sterad }
$$

18 W. Franz, Z. f. Phys. 98, (1935) 314. 
where $Z$ is the atomic number of the scattering nucleus, $E_{0}$ the selfenergy of the electron (511 keV), $E$ the energy of the gamma-ray and $\Theta$ the scattering angle.

The nuclear charge as a whole gives rise to Thomson scattering and the cross-section can be calculated from an equation analogous to Thomson's classical equation for the scattering of $\mathrm{X}$-rays by free electrons

$$
\sigma_{T}=2.39 \cdot 10^{-32} \cdot\left(\frac{Z}{A}\right)^{4} \cdot \frac{1+\cos ^{2} \Theta}{2} \mathrm{~cm}^{2} / \text { sterad }
$$

Rayleigh and Thomson scattering should be coherent with each other and have the same phase.

The cross-section of Delbruck scattering, which results from virtual pair production in the nuclear Coulomb field ${ }^{19.20 .21}$ is not calculated completely. Since the cross-section increases rapidly with increasing gamma-ray energy it is expected that this type of scattering should predominate at high energies.

The elastic scattering of $411 \mathrm{keV}$ gamma-rays in lead has been measured by Storruste 22. 23. The results reproduced in Fig. 3 show good agreement with the curve calculated according to equation (7). (At the energy in question Thomson scattering is negligible compared to Rayleigh scattering.) Such measurements have been extended to higher energies (1.3 $\mathrm{MeV}$ and

Fig. 3. Differential crosssection for the elastic scattering of $411 \mathrm{keV}$ gammarays from lead. (Storruste, 1950)

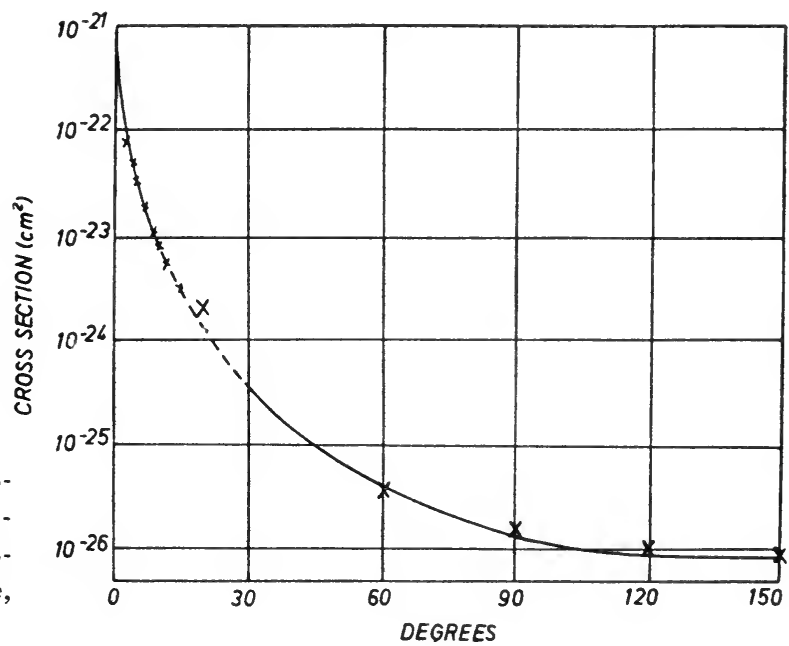

19 M. Delbruck, Z. f. Phys. 84, (1933) 144.

20 F. Rohrlich and R. L. Gluckstern, Phys. Rev. 86; (1952) 1.

21 H. A. Bethe and F. Rohrlich, Phys. Rev. 86, (1952) 10.

22 A. Storruste, Proc. Phys. Soc. 63, (1950) 1197.

23 A. Storruste, Thesis, University of Oslo. 
2.7 MeV) by Wilson ${ }^{24.25}$ and by Davey ${ }^{26}$. As Fig. 5 shows the experimental points are a little higher than the theoretical curve. If this discrepancy is not attributable to experimental errors due to secondary effects it is still an open question whether it indicates that equation (7), which is nonrelativistic and based on the Thomas-Fermi electron distribution, is incorrect at such high energies or if the discrepancy is due to Delbruck scattering.

Fig. 4. Differential cross-section for the elastic scattering of $1.33 \mathrm{MeV}$ gamma-rays from lead (Davey, 1953)
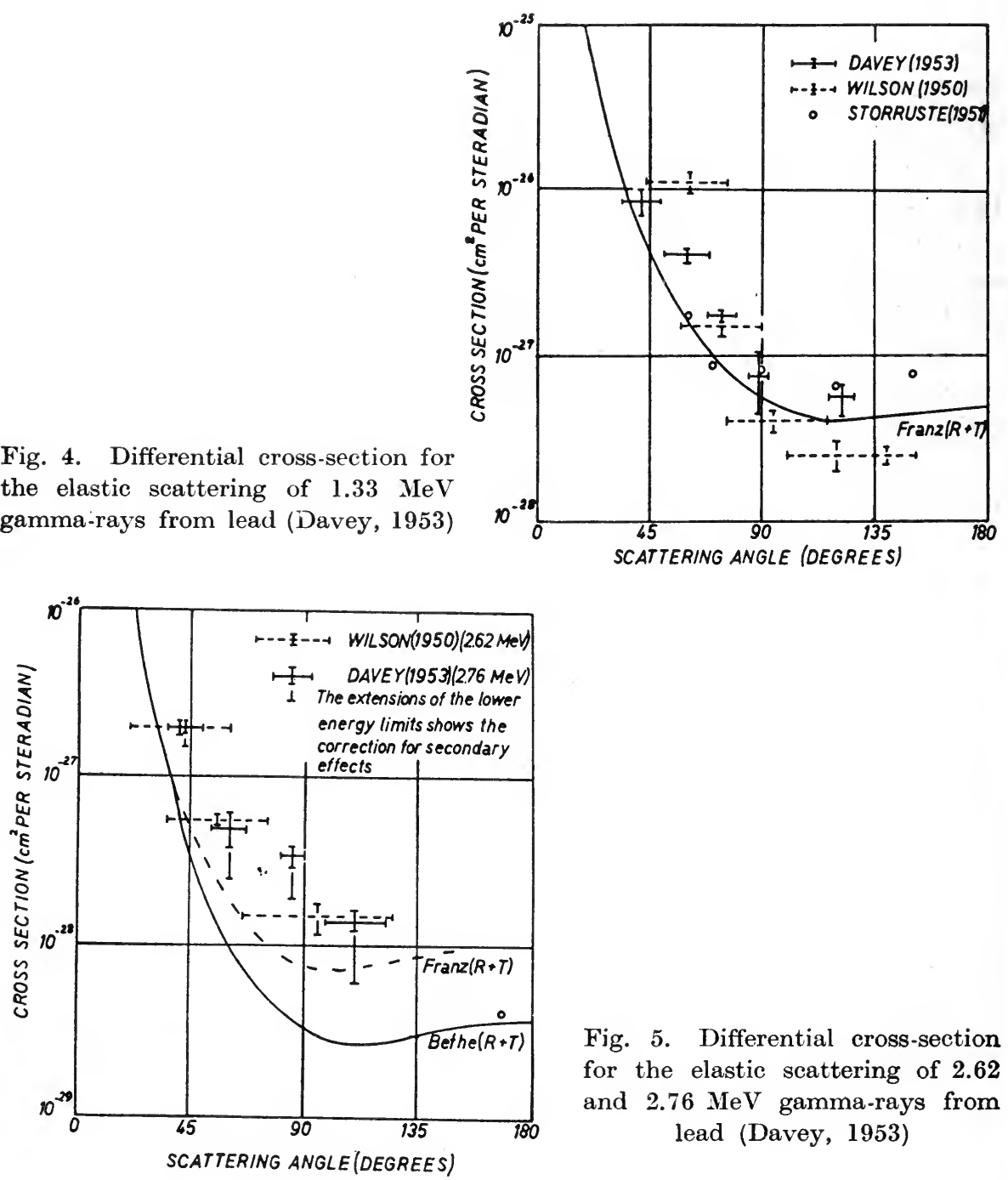

Fig. 5. Differential cross-section for the elastic scattering of $\mathbf{2 . 6 2}$ and $2.76 \mathrm{MeV}$ gamma-rays from lead (Davey, 1953)

24 R. R. Wilson, Phys. Rev. 82, (1951) 295.

25 R. R. Wilson, Phys. Rev. 90, (1953) 720.

26 W. G. Davey, Proc. Phys. Soc. 66, (1953) 1059. 


\title{
ANGULAR DISTRIBUTION OF NUCLEAR RADIATION
}

\author{
H. FRAUENFELDER; R. J. BLIN-STOYLE, M. A. GRACE AND \\ H. HALBAN; S. R. DE GROOT ANd H. A. TOLHOEK
}

\section{(I) H. FRAUENFELDER - Angular correlation}

\section{$\S 1$. Introduction}

A. The probability of emission of a particle or quantum by a radioactive nucleus depends in general on the angle between the nuclear spin axis and the direction of emission. Under ordinary circumstances, however, the total radiation from a radioactive sample is isotropic because the nuclei are randomly oriented in space. An anisotropic radiation pattern can be observed only from an ensemble of nuclei which are not randomly oriented.

One method of arriving at such an ensemble consists in placing the radioactive sample at a very low temperature in a strong magnetic field or electric field gradient, thereby orienting or aligning the nuclei, and then measuring the angular distribution of the emitted radiation with respect to the direction of the applied field. This topic will be dealt with in the last parts of this Chapter (see p. 600).

Another method, the subject of the present survey, consists in picking out only those nuclei whose spins happen to lie in a preferred direction. This case can be realized if the nuclei decay through successive emission of two radiations $R_{1}$ and $R_{2}$. The observation of $R_{1}$ in a fixed direction selects an ensemble of nuclei which has a nonisotropic distribution of spin orientations. The succeeding radiation $\mathrm{R}_{2}$ then shows a definite angular correlation $\dagger$ with respect to $R_{1}$.

B. In angular correlation work, a basic situation is as follows: a nucleus emits in rapid succession two radiations $R_{1}$ and $R_{2}$. We ask for the relative probability $W(\theta) d \Omega$ that $\mathbf{R}_{2}$ is emitted into the solid angle $d \Omega$ at an angle $\theta$ with respect to $R_{1}$. The theoretical expression for the correlation function $W(\theta)$ has been worked out for almost all cases of interest. Experimentally, one records the number of coincidences between $R_{1}$ and $R_{2}$ as a function of the angle $\vartheta$ subtended by the axes of the two counters. Because of the finite solid angles of the counters these numbers $C(\vartheta)$ are averages of the true correlation $W(\theta)$ over angles $\theta$ distributed around $\vartheta$ (cf. Fig. 1).

$\dagger$ We use the term angular correlation as comprising directional correlation and polarization correlation. In directional correlation only the directions of the two radiations are observed; in polarization correlation one determines also the polarization of one or both of the radiations. 
Hence the $C(\vartheta)$ must be properly corrected and normalized (see Part C) to yield $W_{\text {exp }}(\theta)$. The comparison of $W_{\text {exp }}(\theta)$ with theory finally provides the desired information about the properties of the nuclear levels and the radiations.

THE DECAY

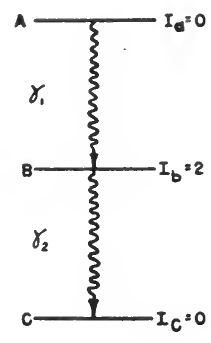

THE APPARATUS

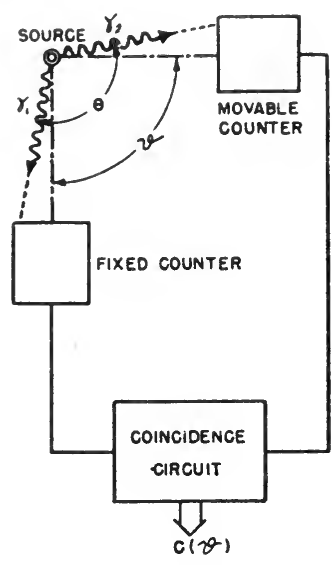

THE RESULT

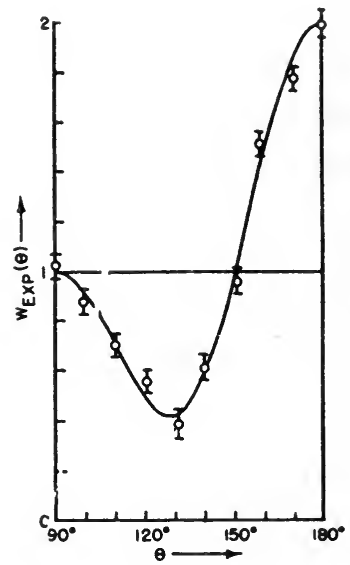

Fig. 1. Example of a directional correlation measurement

C. The information which can be obtained from angular correlation work depends on the type of radiation observed $\left(\alpha, \beta, \gamma, \mathrm{e}^{-}\right)$, on the properties which are singled out by the experiment (direction, polarization, energy),' and on the extranuclear fields acting on the nucleus.

In Part $\mathrm{A}$, we assume that the decaying nuclei are free, i.e., that no extranuclear fields act on the nucleus and disturb its orientation in the intermediate state. To the extent that this can be realized in practice, angular correlation measurements provide information about the properties of the nuclear levels involved and the angular momenta carried away by the radiations. To be more precise, $\alpha-\gamma$ and $\gamma-\gamma$ directional correlation yield the spins of the nuclear levels, but not the parities. The relative parities can be determined, however, if one observes in addition to the direction also the polarization of the $\gamma$-rays, or if one measures the directional correlation between conversion electrons $\left(\mathrm{e}^{-}\right)$. The directional correlation of a $\beta-\gamma$ cascade depends not only on the nuclear spins, but also on the type of interaction involved in $\beta$-decay.

In Part B, we discuss the information which can be obtained from the influence of extranuclear fields on the nuclear angular correlation. Very often, one can determine the quadrupole coupling $Q \cdot \partial^{2} V / \partial z^{2}$ from the change of the correlation due to the extranuclear fields. (The calculation of the quadrupole moment $Q$ from the value of $Q \cdot \partial^{2} V / \partial z^{2}$ is extremely 
difficult because very little is known about the electric field gradient $\partial^{2} V / \partial z^{2}$ in solids and liquids.) In a few cases, one can measure the $g$-factor of an excited nuclear state by observing the directional correlation as a function of an external magnetic field. From the $g$-factor one gets the magnetic moment if the spin of the nuclear state is known, e.g. from the unperturbed directional correlation.

D. The pioneer work on the theory of directional correlation was done in 1940 by Hamilton ${ }^{1}$. In the following years, some attempts were made to verify experimentally the predictions of Hamilton's theory, but they were unsuccessful. In 1946, Goertzel investigated theoretically the influence of extranuclear fields on the directional correlation ${ }^{2}$. Such an interaction could have explained the negative experimental results in at least some cases. However, the first successful measurement, performed by Brady and Deutsch ${ }^{3}$ in 1947, showed that inadequate experimental techniques, and not extranuclear fields, were responsible for the earlier failures.

These first experiments of Brady and Deutsch were done with Geiger counters. A major improvement was the introduction of the scintillation counter into angular correlation work in 1948 (Brady and Deutsch 4, 5, Metzger and Deutsch ${ }^{6,7}$ ). It is difficult today, only seven years later, to realize how tedious were $\gamma-\gamma$ correlation measurements with Geiger counters. A run which now takes a day would have taken more than ten years of continuous work with Geiger counters.

The introduction of the scintillation counter started a rapid development of the field of angular correlation. This development is witnessed by the fact that more than 250 theoretical and experimental papers have appeared since 1948. Today, angular correlation measurements form a wellestablished tool in nuclear spectroscopy. Moreover, the sensitivity of the correlation function to extranuclear fields $8,9,10$ offers some promise for the investigation of such fields in liquids and solids.

1

2

3

4

5

6

Metzger and M. Deutsch, Phys. Rev 78 (1950)

8 H. Aeppli, A. S. Bishop, H. Frauenfelder, M. Walter and W. Zünti, Phys. Rev. 82 (1951) 550.

9 H. Aeppli, H. Albers-Schönberg, H. Frauenfelder and P. Scherrer, Helv. Phys. Acta 25 (1952) 339.

10 H. Albers-Schönberg, F. Hänni, E. Heer, T. B. Novey and P. Scherrer, Phys. Rev. 90 (1953) 322. 


\section{PART A. FREE NUCLEI}

Throughout Part A (i.e. $\S \S 2$ to 8 ), we assume that the nuclei are not disturbed by extranuclear fields. This assumption is justified if the mean life $\tau_{b}$ of the intermediate nuclear state is much shorter than the "interaction time" $\hbar / \Delta E$, where $\Delta E$ is the energy of interaction between the nucleus and the extranuclear fields. For very short-lived states $\left(\tau_{b}<10^{-11}\right.$ sec), this condition is always fulfilled. For states with longer lifetimes, perturbations are possible. In order to observe the unperturbed correlation (maximum correlation) one must find a nuclear environment where the extranuclear fields are very small (e.g. a regular lattice site in a cubic crystal) or where their time average vanishes (e.g. certain liquids). Except for occasional remarks, we postpone until Part B all questions concerning how one finds such an environment.

\section{$\S 2$. Naive Theory of $\gamma-\gamma$ Directional Correlation in Free Nuclei}

A. The general theory of angular correlation has been highly developed in the last few years and anyone interested in the theory can find among the various approaches one that suits his taste (e.g. $\left.{ }^{1,2,11-20}\right)$. We give in the present $\S$ a treatment of $\gamma-\gamma$ directional correlation which is simple but which, nevertheless, provides some insight into the correlation mechanism ${ }^{21}$, 22. In $\S 3$, we proceed to outline the general theory of angular correlation. In $\S \S 4$ through 8 , we discuss the main results of the theory, together with pertinent experiments.

B. We investigate first a single $\gamma$-transition, of angular momentum $L$, between two nuclear levels $B$ and $C$ with spins $I_{b}$ and $I_{c}$. For conservation of angular momentum, we have $\boldsymbol{I}_{\mathbf{b}}=\boldsymbol{I}_{\mathbf{c}}+\boldsymbol{L}$. $L_{z}$ shall denote the projection of $\boldsymbol{L}$ on the arbitrary axis of quantization (z-axis). The emitted $\gamma$-ray is then characterized by the angular momentum quantum number or multipolarity $L$, and the magnetic quantum number $M ; L^{2}=L(L+1) \hbar^{2}, L_{z}=M \hbar$.

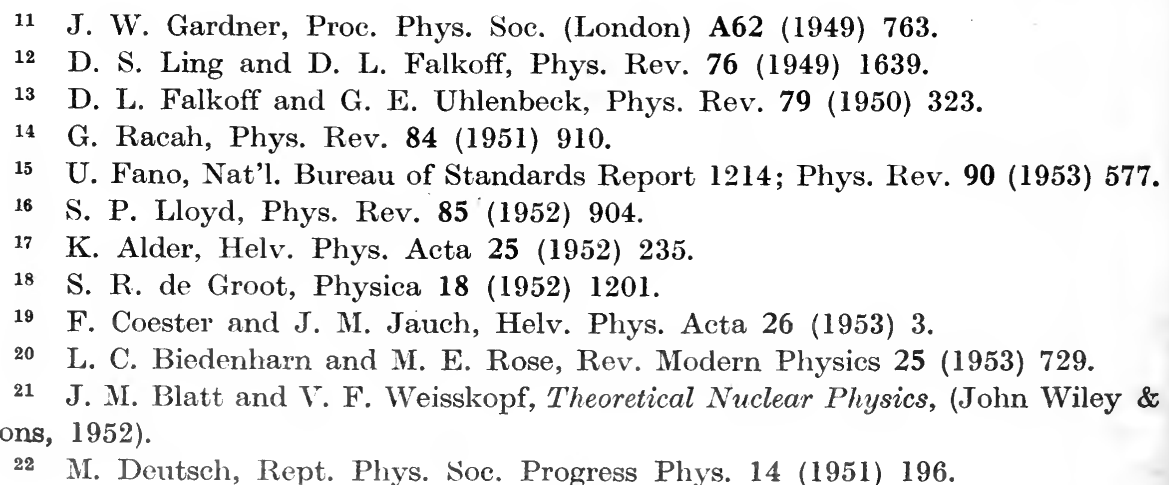


The quantum numbers of the states $\mathrm{B}$ and $\mathrm{C}$ are given by $I_{b}, m_{b}$ and $I_{c}, m_{c}$. Clearly, $m_{b}=m_{c}+M$.

Each component $* m_{b} \rightarrow m_{c}$ between specified magnetic sublevels ( $m$-states) possesses a characteristic directional distribution $F_{L}^{M}(\theta)$, which is independent of $I_{b}$ and $I_{c}$. Here $\theta$ denotes the angle between emitted $\gamma$-ray and $z$-axis. For $\gamma$-rays, the distribution function $F_{L}^{M}(\theta)$ can be found by calculating the energy flow (Poynting vector) as a function of $\theta$ for multipole radiation characterized by the quantum numbers $L$ and $M$ 12, 21, 23. For dipole radiation $(L=1)$, one obtains ${ }^{23}$
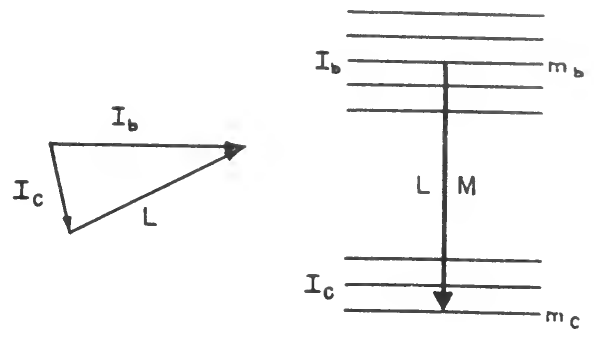

อ

b.

Fig. 2. Single $\gamma$-transition

$$
\left.\begin{array}{l}
F_{1}^{0}(\theta)=3 \sin ^{2} \theta=2-2 P_{2}(\cos \theta), \\
F_{1}^{ \pm 1}(\theta)=\frac{3}{2}\left(1+\cos ^{2} \theta\right)=2+P_{2}(\cos \theta) .
\end{array}\right\}
$$

The first of these functions, $F_{1}^{0}(\theta)$, is the familiar radiation pattern from a dipole lying in the $z$-axis.

In atomic spectroscopy, one observes the individual components (1) and their directional distributions in the Zeeman effect: the different $m$ states of $\mathrm{B}$ and $\mathrm{C}$ split up in a magnetic field and the components associated with $F_{1}^{1}, F_{1}^{0}$, and $F_{1}^{-1}$ thus differ in energy sufficiently to be observed individually. Usually, one selects only the two positions $\theta=0^{\circ}$ and $\theta=90^{\circ}$ and calls the corresponding observations longitudinal and transverse Zeeman effect. In nuclear spectroscopy the Zeeman effect is unobservable, because the splitting of the nuclear energy levels even in very strong magnetic fields is only of the order of $10^{-8} \mathrm{eV}$.

In nuclear physics, it is thus impossible to separate the components and one always observes the unresolved line $\mathrm{B} \rightarrow \mathrm{C}$. In order to calculate its directional distribution $F_{L}(\theta)$, we must know not only the functions $F_{L}^{M}(\theta)$, but also the relative population $P\left(m_{b}\right)$ for each sublevel $m_{b}$, and the relative transition probability $G\left(m_{b} m_{c}\right)$ for each component $m_{b} \rightarrow m_{c}$. The directional distribution $F_{L}(\theta)$ of the total line then is given by

$$
F_{L}(\theta) \sim \sum_{m_{b} m_{c}} P\left(m_{b}\right) G\left(m_{b} m_{c}\right) F_{L}^{M}(\theta),
$$

23 E. U. Condon and G.H. Shortley, The Theory of Atomic Spectra (Cambridge 1951).

* We follow Condon and Shortley ${ }^{23}$ in designating the radiation resulting from a transition between a particular pair of $m$-states as a component. The total radiation associated with all possible transitions between two levels is called a line. 
where

$$
M=m_{b}-m_{c} .
$$

The absolute transition probability for a component $m_{b} \rightarrow m_{c}$ can be written as a product of a "nuclear" factor and a "geometrical" factor. The nuclear factor depends on specific nuclear properties, but not on $m_{b}$ and $m_{c}$, and enters into Eq. (2) only as a constant factor. The geometrical factor depends explicitly on $m_{b}$ and $m_{c}$ and yields the desired relative transition probability $G\left(m_{b} m_{c}\right)$.

The factoring into a geometrical and a nuclear part can be seen most easily in the case of the matrix element $z_{c b}$ for the radiation from an electric dipole lying in the $z$-axis:

$$
z_{c b}=\int \psi_{c}^{*} z \psi_{b} d \tau
$$

Assuming wavefunctions of the form $\psi=R(r) Y_{I m}(\vartheta, \varphi)$, where $Y_{I m}(\vartheta, \varphi)$ denotes a normalized spherical harmonic, and setting $z=r \cos \vartheta, z_{c b}$ becomes the product of two integrals. One depends only on the radial factor of $\psi$ and cannot be evaluated further without specific assumptions about $R(r)$, the other yields the geometrical factor and can be calculated explicitly. A more appropriate derivation can be given by using the properties of the Clebsch-Gordan coefficients *. We write $z=r \cos \vartheta=r(4 \pi / 3)^{\ddagger} Y_{10}^{*}$, and assume as before $\psi=R(r) Y_{I m}$. The functions $Y_{10}^{*}, Y_{I_{b} m_{b}}$, and $Y_{I_{c} m_{c}}$ are all eigenfunctions of the operators $I^{2}$ and $I_{z}$. Moreover, $\boldsymbol{I}_{\boldsymbol{b}}=\boldsymbol{I}_{\boldsymbol{c}}+\boldsymbol{L}$, where $L=1$. We can thus, after inserting the expressions for $z$ and $\psi$ into Eq. (3), expand the product $Y_{I_{c} m_{c}}^{*} Y_{10}^{*}$ in terms of $Y_{I_{b} m_{b}^{\prime}}^{* \prime}$ by using Eq. (5). Because of the orthogonality of the spherical harmonics, all integrals vanish except that with $I_{b}^{\prime}=I_{b}$, and $m_{b}^{\prime}=m_{b}$. The matrix element $z_{c b}$ therefore becomes proportional to the

* We give here a short definition of the Clebsch-Gordan coefficients. Let $u\left(I_{b}, m_{b}\right)$ be one of an orthonormal set $u\left(I_{b}^{\prime} m_{b}^{\prime}\right)$ of eigenfunctions of the operators $I_{b}^{2}$ and $I_{b z}$, with eigenvialues $I_{b}\left(I_{b}+1\right) h^{2}$ and $m_{b} \hbar$; let $v\left(I_{c}, m_{c}\right)$ and $v(L, M)$ be similar eigenfunctions for the vectors $I_{c}$ and $L$. Further assume $I_{b}$ to be the vector sum of $I_{c}$ and $L: I_{b}=I_{c}+L, m_{b}=m_{c}+M$. Then the Clebsch-Gordan coefficients are the coefficients in the expansion of $u\left(I_{b}, m_{b}\right)$. in terms of products $v\left(I_{c}, m_{c}\right) v(L, M)$ :

$$
u\left(I_{b}, m_{b}\right)=\sum_{m_{c} M} v\left(I_{c}, m_{c}\right) v(L, M)\left(I_{c} L m_{c} M \mid I_{b} m_{b}\right) .
$$

The Clebsch-Gordan coefficients $\left(I_{c} L m_{c} M \mid I_{b} m_{b}\right)$ are the elements of a unitary matrix. The phases of the eigenfunctions are so chosen that the Clebsch-Gordan coefficients are real numbers and hence the matrix is orthogonal. The inversion of Eq. (4) thus yields

$$
v\left(I_{c}, m_{c}\right) v(L, M)=\sum_{I_{b}^{\prime} m_{b}^{\prime}}\left(I_{c} L m_{c} M \mid I_{b}^{\prime} m_{b}^{\prime}\right) u\left(I_{b}^{\prime}, m_{b}^{\prime}\right) .
$$


Clebsch-Gordan coefficient $\left(I_{c} 1 m_{c} 0 \mid I_{b} m_{b}\right)$, and the relative transition probability is proportional to its square.

The outlined calculation of the relative transition probability was performed with single particle wave functions and for electric dipole radiation only. The result, however, is independent of the first assumption and can easily be generalized for all multipole orders. We can thus define the relative transition probability to be equal to the square of the Clebsch-Gordan coefficient for the vector addition $I_{b}=I_{c}+L, m_{b}=m_{c}+M$ :

$$
G\left(m_{b} m_{c}\right)=\left(I_{c} L m_{c} M \mid I_{b} m_{b}\right)^{2} .
$$

We will derive Eq. (6) in $\S 3$. (The most general derivation of Eq. (6) is based on group theoretical methods; see ${ }^{24,}{ }^{25}$ ).

The relative populations $P\left(m_{b}\right)$ depend, in contrast to $F_{L}^{M}(\theta)$ and $G\left(m_{b} m_{c}\right)$, on the energies of the different $m_{b}$-states and on the way in whi ? level $\mathbf{B}$ was created. If the nuclei are randomly oriented, i.e. if all $m_{b}$-states are equally populated for any choice of the $z$-axis, then $F_{L}(\theta)$ is independent of $\theta$, i.e. the distribution is isotropic. This result can be confirmed by evaluating Eq. (6) for equally populated $m_{b}$-states.

C. We now assume that a nucleus decays through the cascade $\mathrm{A} \rightarrow \mathrm{B} \rightarrow \mathrm{C}$ by successive emission of two $\gamma$-rays of multipolarity $L_{1}$ and $L_{2}$, and we ask for the directional correlation function $W(\theta)$ between the two $\gamma$-rays.

In order to simplify the calculation, we let the direction of the quantization axis coincide with the direction of emission of the first $\gamma$-ray. The directional correlation $W(\theta)$ between the two $\gamma$-rays then becomes identical with the directional distribution $F_{L}(\theta)$ of the second $\gamma$-ray with respect to the $z$-axis. It can thus be calculated from Eq. (2) and (6) once each relative population $P\left(m_{b}\right)$ is known. This, however, is given by. the sum of all transitions $m_{a} \rightarrow m_{b}$ leading into the state $m_{b}$. Assuming all $m_{a}$-states equally populated, we have, with $M_{1}=m_{a}-m_{b}$ :

$$
P\left(m_{b}\right) \sim \sum_{m_{a}} G\left(m_{a} m_{b}\right) F_{L_{1}}^{M_{1}}(\theta=0) .
$$

The special choice of the $z$-axis introduces a second simplification. A photon propagating in a definite direction can carry in its direction of motion only the angular momentum $+\hbar$ or $-\hbar^{21,23}$. In our case, the first $\gamma$-ray travels along the $z$-axis and thus $M_{1}$ is restricted to the two values \pm 1. Only the functions $F_{L_{1}}^{1}(0)$ and $F_{L_{1}}^{-1}(0)$ then appear in Eq. (7). For

24 C. Eckart, Revs. Modern Phys. 2 (1930) 305.

25. E. P. Wigner, Gruppentheorie (F. Vieweg \& Sohn, Braunschweig 1931).

${ }^{26}$ W. Heitler, Quantum Theory of Radiation (Oxford University Press, 1944, 1954) Appendix I. 
the special case of dipole radiation, this can be seen from Eq. (1): $F_{1}^{0}$ vanishes for $\theta=0$.

The desired directional correlation function $W(\theta)$, which for physical reasons must be independent of our special choice of the $z$-axis, finally follows from the combination of Eq. (2), (6) and (7), with $M_{2}=m_{b}-m_{c}$ :

$$
W(\theta) \sim \sum_{m_{b} m_{c} m_{a}}\left(I_{b} L_{1} m_{b} \pm 1 \mid I_{a} m_{a}\right)^{2} F_{L_{1}}^{ \pm 1}(0)\left(I_{c} L_{2} m_{c} M_{2} \mid I_{b} m_{b}\right)^{2} F_{L_{2}}^{M_{a}}(\theta) .
$$

We still have to justify one point in the derivation of Eq. (8). The summation over $m_{b}$ in Eq. (8) has been done incoherently. In other words, the various possible ways $m_{a} \rightarrow m_{b} \rightarrow m_{c}$ in which a nucleus can decay from a given initial state $m_{a}$ into a given final state $m_{c}$ are assumed to be independent, and thus no interference terms appear. This simplification is due to the special choice of the $z$-axis, which allows only the values \pm 1 for $M_{1}=m_{a}-m_{b}$. Each of these two values describes one mode of circular polarization, as one can most easily verify by discussing the longitudinal Zeeman effect. In principle, we can now observe the circular polarization of each individual photon $\gamma_{1}$, in addition to its direction. Such a polarization measurement would yield a definite -value of $M_{1}$, either +1 or -1 . Starting from a given state $m_{a}$, therefore, only one state $m_{b}$ is reached (either $m_{a}-1$ or $m_{a}+1$ ) and no interference occurs ${ }^{27,}{ }^{28}$. The fact that in the actual experiment one does not measure the circular polarization, but averages over the two modes, does not change the conclusions. It is the possibility of performing the measurement, and the fact that the observation would yield a sharp value of $M_{1}$, which destroys the interference. This possibility, however, is lost if one determines in addition to the direction also the linear polarization of the photons. In this case, interference terms appear.

In simple cases, $W(\theta)$ can be obtained from Eq. (8) without excessive labor. As an example, we have evaluated three cascades, using the $F_{L}^{M}(\theta)$ from Eq. (1) and taking the Clebsch-Gordan coefficients from reference ${ }^{23}$. The cascades and the resulting directional correlation functions are given in Fig. 3.

Hamilton first derived Eq. (8) by second order damping theory ${ }^{29}$ and evaluated it for all possible cascades involving pure dipole and quadrupole $\gamma$-rays ${ }^{1}$. He used the expansion

$$
W(\theta)=1+a_{2} \cos ^{2} \theta+a_{4} \cos ^{4} \theta
$$

and gave explicit expressions for $a_{2}$ and $a_{4}$. For many years, experimental

27 B. A. Lippmann, Phys. Rev. 81 (1951) 162.

28 O. Halpern and B. A. Lippmann, Phys. Rev. 87 (1952) 1128.

29 V. F. Weisskopf, Ann. der Physik 9 (1931) 23. 
physicists used Hamilton s results to compare their measurements with theory. However, Hamilton's method of evaluating (8) involves tedious summations over the magnetic quantum numbers and its extension to transitions higher than quadrupole is extremely cumbersome.
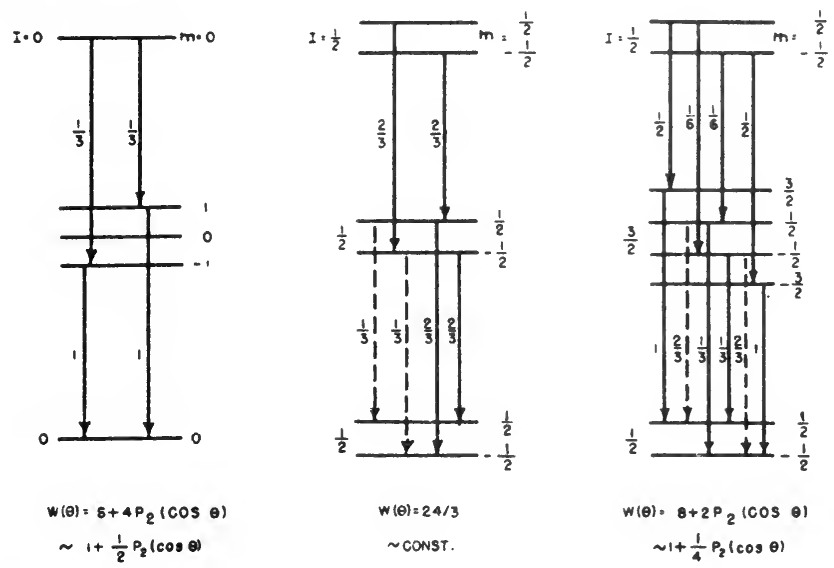

Fig. 3. Directional correlation of simple cascarles. The first $\gamma$-ray of each cascade is observed in the quantization axis. Therefore, only transitions with $M= \pm 1$ occur (solid lines). The second $\gamma$-ray is detected in an arbitrary direction, thus no such restriction exists and transitions with $M= \pm 1$ (solid lines) and $M=0$ (dotted lines) are allowed for dipole radiation. The relative intensities for the components are shown in the figure, their angular distributions are given by Eq. (1).

In the further development of angular correlation theory (see references ${ }^{2}, 11-20,30-32$ ), not only was this difficulty overcome, but the theory was generalized and extended to radiations other than $\gamma$-rays and to cases where the nucleus is disturbed in its intermediate state.

\section{§ 3. General Theory of Angular Correlation*}

In the last $\S$ we gave a simple theory of the directional correlation of successive $\gamma$-rays and mentioned some of its limitations. Here, we outline a more general theory, which overcomes these limitations and is also valid for particles other than $\gamma$-rays. The treatment given here is not elementary, but the later $\S \S$ can be understood without it.

A. The progress in the theory of angular correlation after Hamilton's work is largely due to the use of three tools: i) group theory, ii) Racah algebra, and iii) density matrix formalism.

30 A. Abragam and R. V. Pound, Phys. Rev. 92 (1953) 943.

31 K. Alder, H. Albers-Schönberg, E. Heer and T. B. Novey, Helv. Phys. Acta 26 (1953) 761.

32 F. Coester, Phys. Rev. 93 (1954) 1304.

* This $\S$ was written in cooperation with F. Coester. 
i) In the approach discussed in $\S 2$, the quantization axis coincides with the direction of emission of one particle. This special choice eliminates interference terms and facilitates the description of this particle. However, the description of the second particle is not simplified and the equivalence of the twc radiations is ignored. Lloyd ${ }^{16,33}$, Alder ${ }^{17}$ and Racah ${ }^{14}$ developed the theory further by taking advantage of this symmetry rather than destroying it through the special choice of the $z$-axis. For each of the two radiations $R_{1}$ and $R_{2}$, a separate coordinate system is introduced. The $z$-axis of each system coincides with the direction of emission $\Omega_{i}$ of $\mathbf{R}_{i}$; the direction of polarization can be taken as $x$-axis. The connection between the two radiations is established through the introduction of an arbitrary quantization axis by using the transformation properties of the eigenfunctions of $R_{1}$ and $R_{2}$. The eigenfunction of $R_{i}$ describes a system with total angular momentum $L_{i}$ and thus transforms according to the $\left(2 L_{i}+1\right)$ dimensional irreducible representation $D^{L}$ of the three-dimensional rotation group ${ }^{25}$. Using this fact, the eigenfunctions of $R_{1}$ and $R_{2}$, quantized along the arbitrary $z$-axis, are expressed in terms of the functions quantized along the directions of emission $\Omega_{1}$ and $\Omega_{2}$. These eigenfunctions, however, are much simpler because they correspond to plane waves propagating along the directions of emission, i.e. to the emission of $R_{1}$ and $R_{2}$ with intrinsic angular momentum only.

This approach leads naturally to an expansion of the correlation function $W(\theta)$ in terms of representations $D^{L}$ (which reduce to Legendre polynomials $P_{L}(\cos \theta)$ for directional correlation). The coefficients in such an expansion can be expressed in closed form and break up into independent factors for the separate transitions of the cascade.

ii) For physical reasons, one would expect that the sums over the magnetic quantum numbers in Eq. (8) could be expressed in closed form: the $m$-states are no more than a means of describing the orientation of a nucleus in space, and they are in no way connected with nuclear dynamics. They are thus essentially geometrical in character and do not appear in the final result. Gardner ${ }^{11}$ first showed that the sums can indeed be written in closed form, independent of $m$, by using the Racah algebra ${ }^{34,35}$. Gardner's treatment was restricted to conversion electrons, but his method was generalized by Lloyd, Alder, and Racah.

iii) From a correlation experiment, one gains only incomplete knowledge about the nuclear system and the radiations involved. Usually, the initial nuclear level is uniformly populated and the radiations can be 
mixed (i.e. they can contain more than one value of $L$ ). We thus know only statistically how the intermediate and final states of the nuclei are populated. The quantum mechanical system with which we are dealing is therefore in an impure state. Moreover, our system does not consist of one nucleus only, but it is an ensemble of nuclei decaying statistically.

This situation, a statistical ensemble with impure states, can be handled best by using the density matrix (e.g. ${ }^{36,37,37 a)}$. Fano ${ }^{15}$ and Coester and Jauch 19, 32 introduced this tool into angular correlation theory and thereby simplified many aspects. Here, we use the density matrix for the derivation of the starting formula only and refer to the above mentioned papers for a complete treatment.

B. After these preparatory remarks, we turn now to the explicit construction of the angular correlation function $W\left(\Omega_{1} \Omega_{2}\right)$, where $W\left(\Omega_{1} \Omega_{2}\right)$ $d \Omega_{1} d \Omega_{2}$ is defined as the probability that a nucleus, decaying through the cascade $\mathrm{A} \rightarrow \mathrm{B} \rightarrow \mathrm{C}$, emit the two radiations $\mathrm{R}_{1}$ and $\mathrm{R}_{2}$ in the directions $\Omega_{1}$ and $\Omega_{2}$ into the solid angles $d \Omega_{1}$ and $d \Omega_{2}$.

The following expression for $W\left(\Omega_{1} \Omega_{2}\right)$ is used as a starting point for most theoretical investigations:

or

$$
W\left(\Omega_{1} \Omega_{2}\right)=\mathscr{S} \sum_{m_{a} m_{c}}\left|\sum_{m_{b}}\left(c\left|\mathscr{H}_{2}\right| b\right)\left(b\left|\mathscr{H}_{1}\right| a\right)\right|^{2}
$$

$$
W\left(\Omega_{1} \Omega_{2}\right)=\mathscr{S} \sum_{m_{a} m_{c} m_{b} m_{b}^{\prime}}\left(c\left|\mathscr{H}_{2}\right| b\right)\left(b\left|\mathscr{H}_{1}\right| a\right)\left(a\left|\mathscr{H}_{1}\right| b^{\prime}\right)\left(b^{\prime}\left|\mathscr{H}_{2}\right| c\right) .
$$

Eq. (10) was first derived by Hamilton for a $\gamma-\gamma$ cascade ${ }^{1}$, but it is used for other radiations also. (See, however, the remark at the end of the derivation of Eq. (11) below). In Eq. (10) and (11), $\mathscr{S}$ means a summation over all unmeasured radiation properties. The matrix element $\left(b\left|\mathscr{H}_{1}\right| a\right)$ stands for $\left(I_{b} m_{b} \Omega_{1} \sigma_{1}\left|\mathscr{H}_{1}\right| I_{a} m_{a}\right)$. The other matrix elements have corresponding meanings. $\mathscr{H}_{1}$ is the interaction Hamiltonian for the emission of $\mathrm{R}_{1}$ into the direction $\Omega_{1}$ with polarization $\sigma_{1}$. The polarization $\sigma_{1}$ is given by the component of the spin of $R_{1}$ in the direction of propagation. In going from Eq. (10) to Eq. (11), we used the assumption that $\left(b\left|\mathscr{H}_{1}\right| a\right)^{*}=$ $=\left(a\left|\mathscr{H}_{1}\right| b\right)$; i.e. that the matrix is Hermitian. Angle-independent factors are omitted in Eqs. (10) and (11).

The usual derivation of Eq. (11) is based on the following idea. A nucleus emits two radiations in succession and one observes, after the decay is completed, the directions of the two radiations. This two-step process is described by second-order damping theory. On the other hand,

36 W. Pauli, Handbuch der Physik (Second Edition, Vol. 24/1, Berlin 1933).

37 R. C. Tolman, The Principles of Statistical Mechanics (Oxford 1938) p. 327.

$37 a$ W. H. McMaster, Am. J. Physics 22 (1954) 351. 
we sketch below a derivation of Eq. (11) which corresponds to the descrip. tion used in $\S 2$ : a nucleus decays first from the randomly populated level A, described by the density matrix $\mathscr{P}_{a}$, to the level $\mathrm{B}$; the emitted radiation $\mathrm{R}_{1}$ is observed in the direction $\Omega_{1}$. The perturbation theory in first order describes this process and yields the density matrix $\mathscr{P}_{b}$. Through the density matrix $\mathscr{P}_{b}$, the state $\mathrm{B}$ is now described. The second step of the cascade, $\mathrm{B} \rightarrow \mathrm{C}$, is treated in the same manner, differing only in that the density matrix $\mathscr{P}_{b}$ is not known a priori, but is the result of the preceding transition. First-order perturbation theory and the density matrix together thus provide an adequate description of the correlation process.

The difference between the two descriptions becomes more apparent when one takes external fields into account. In the first method, the extranuclear fields split the degenerate states of the intermediate nuclear level and this splitting causes a perturbation of the angular.correlation. In the second method, the density matrix $\mathscr{P}_{b}$, describing the end product of the first transition, is no longer identical with the density matrix $\mathscr{P}_{b}^{\prime}$ for the initial state of the second transition. The extranuclear fields cause transitions between the different $m$-states of $\mathrm{B}$ and thus produce a change $\mathscr{P}_{b} \rightarrow \mathscr{P}_{b}^{\prime}$.

In order to derive the correlation function (11), we first consider a transition $\mathrm{A} \rightarrow \mathrm{B}$. The distribution of the nuclei over the states $\alpha, \alpha^{\prime}, \ldots$ of level $\mathrm{A}$ is described by a density matrix $\mathscr{P}_{a}$ with elements $\left(\alpha\left|\mathscr{P}_{a}\right| \alpha^{\prime}\right)$; the distribution over the states $\beta, \beta^{\prime}, \ldots$ of level $\mathrm{B}$ is similarly given by a density matrix $\mathscr{P}_{b}$. The density matrix $\mathscr{P}_{a}$ does not change in time since all states $\alpha, \alpha^{\prime}, \ldots$ decay with the same half-life. The transition rate for a transition $\alpha \rightarrow \beta$ is proportional to $|(\beta|\mathscr{H}| \alpha)|^{2}$. The proportionality constant is independent of $\alpha$ and $\beta$ and thus of no interest to us. We assume now that the states $\alpha, \alpha^{\prime}, \ldots$ are chosen such that $\mathscr{P}_{a}$ is diagonal; the distribution over the states $\alpha, \alpha^{\prime}, \ldots$ then is completely described by the population probability $\mathscr{P}_{a}(\alpha)\left[\equiv\left(\alpha\left|\mathscr{P}_{a}\right| \alpha\right)\right]$. Since $\mathscr{P}_{a}(\alpha)$ is constant in time and the population of the states $\beta, \beta^{\prime}, \ldots$ is due entirely to the decay $\mathrm{A} \rightarrow \mathrm{B}, \mathscr{P}_{b}(\beta)$ is proportional to the transition rate to the state $\beta$.

$$
\mathscr{P}_{b}(\beta)=\text { const } \sum_{\alpha}|(\beta|\mathscr{H}| \alpha)|^{2} \mathscr{P}_{a}(\alpha) \text {. }
$$

In general, the density matrix $\mathscr{P}_{a}$ is not diagonal and the density matrix $\mathscr{P}_{b}$ is given by

$$
\left(\beta\left|\mathscr{P}_{b}\right| \beta^{\prime}\right)=\text { const } \sum_{\alpha \alpha^{\prime}}(\beta|\mathscr{H}| \alpha)\left(\alpha\left|\mathscr{P}_{a}\right| \alpha^{\prime}\right)\left(\alpha^{\prime}|\mathscr{H}| \beta^{\prime}\right) .
$$

We can easily see that Eq. (12') is fully equivalent to Eq. (12) by choosing the states $\alpha, \alpha^{\prime}, \ldots$ and $\beta, \beta^{\prime}, \ldots$ such that $\mathscr{P}_{a}$ and the right-hand side of Eq. $\left(12^{\prime}\right)$ are diagonal. 
We now apply Eq. $\left(12^{\prime}\right)$ to the second transition of the cascade $A \rightarrow B \rightarrow C$ by setting $\alpha=b, \beta=c$. We remember that $c$ stands for the quantum numbers $I_{c}, m_{c}, \Omega_{2}, \sigma_{2}$. However, we do not observe the spin orientation of the nuclei in the final level $\mathrm{C}$ and thus sum over $m_{c}$ :

$$
W\left(\Omega_{1} \Omega_{2}\right)=\text { const } \sum_{m_{c}} \sum_{m_{b} m_{b}^{\prime}}\left(c\left|\mathscr{H}_{2}\right| b\right)\left(b\left|\mathscr{P}_{b}\right| b^{\prime}\right)\left(b^{\prime}\left|\mathscr{H}_{2}\right| c\right) \text {. }
$$

We write $W\left(\Omega_{1} \Omega_{2}\right)$ in this expression, because the density matrix $\mathscr{P}_{b}$ depends on the direction $\Omega_{1}$ of the first radiation. In order to find $\mathscr{P}_{b}$, we apply Eq. $\left(12^{\prime}\right)$ also to the first transition $A \rightarrow B$, noting that the density matrix for a uniformly populated level is given by $\left(a\left|\mathscr{P}_{a}\right| a^{\prime}\right)=$ $=\left(2 I_{a}+1\right)^{-1} \delta_{m_{a} m_{a}^{\prime}}$ :

$$
\left(b\left|\mathscr{P}_{b}\right| b^{\prime}\right)=\text { const } \sum_{m_{a}}\left(b\left|\mathscr{H}_{1}\right| a\right)\left(a\left|\mathscr{H}_{1}\right| b^{\prime}\right)
$$

By inserting expression (14) into (13), and setting the angle-independent constants equal to unity, we immediately get the desired correlation function $W\left(\Omega_{1} \Omega_{2}\right)$ in the form of Eq. (11).

The derivation given above shows that Eq. (11) needs only a minor modification in cases where perturbation theory is not applicable (e.g., in $\alpha-\gamma$ correlation). The transition probability $\mathrm{A} \rightarrow \mathrm{B}$ is still timeproportional, but the transition matrix is no longer Hermitiail. One replaces $(\beta|\mathscr{H}| \alpha)$ in Eq. (12') by a general transition matrix element $(\beta|\mathscr{R}| \alpha)$ and corresponding changes occur in Eq. (11).

The correlation function $W\left(\Omega_{1} \Omega_{2}\right)$ as given in Eq. (11) can be rewritten in the following form:

where

$$
W\left(\Omega_{1} \Omega_{2}\right)=\sum_{m_{b} m_{b}^{\prime}}\left(b\left|E^{(1)}\right| b^{\prime}\right)\left(b\left|E^{(2)}\right| b^{\prime}\right)^{*},
$$

$$
\begin{aligned}
\left(b\left|E^{(1)}\right| b^{\prime}\right) & =\mathscr{S} \sum_{m_{a}}\left(b\left|\mathscr{H}_{1}\right| a\right)\left(a\left|\mathscr{H}_{1}\right| b^{\prime}\right) \\
\left(b\left|E^{(2)}\right| b^{\prime}\right) & =\mathscr{S}_{2} \sum_{m_{c}}\left(b\left|\mathscr{H}_{2}\right| c\right)\left(c\left|\mathscr{H}_{2}\right| b^{\prime}\right) .
\end{aligned}
$$

This form of the correlation function allows one to divide the construction of a more explicit correlation function into three steps. In the first step (in $\S 3 \mathrm{C}$, below), we investigate a single matrix element $\left(b\left|\mathscr{H}_{1}\right| a\right)$; in the second step (in $\S 3 \mathrm{D})$, we study a matrix element $\left(b\left|E^{(1)}\right| b^{\prime}\right)$ as given by Eq. (16). The third step (in $\S 3 \mathrm{E}$ ) consists in transforming Eq. (15) to get $W\left(\Omega_{1} \Omega_{2}\right)$ in a form suitable for explicit evaluation and comparison with experiment.

C. Here, we investigate and transform a typical matrix element $\left(b\left|\mathscr{H}_{1}\right| a\right) \equiv\left(I_{b} m_{b} \Omega_{1} \sigma_{1}\left|\mathscr{H}_{1}\right| I_{a} m_{a}\right)$. This matrix element is represented 
in terms of the nuclear spins and the direction $\Omega_{1}$ and polarization $\sigma_{1}$ of the emitted radiation. This representation is natural for the description of the observed radiation properties, but it does not provide us with the desired information about the nucleus. Instead of linear momentum $(\Omega)$ and polarization $(\sigma)$, we would like to know the angular momentum and the parity of the radiation. These properties, described by the quantum numbers $L, M$ and $\pi$, yield the desired data about the nuclear transition, since $I_{a}-I_{b}=L_{1}, \pi_{a} \pi_{b}=\pi_{1}$.

The transformation from the quantum numbers $\Omega_{1}, \sigma_{1}$ to $L_{1}, M_{1}, \pi_{1}$ is provided by a unitary matrix $\left(\Omega_{1} \sigma_{1} \mid L_{1} M_{1} \pi_{1}\right)$. The connection between the "observed" matrix element $\left(I_{b} m_{b} \Omega_{1} \sigma_{1}\left|\mathscr{H}_{1}\right| I_{a} m_{a}\right)$ and the "desired" matrix element $\left(I_{b} m_{b} L_{1} M_{1} \pi_{1}\left|\mathscr{H}_{1}\right| I_{a} m_{a}\right)$ is then given by

$$
\left(I_{b} m_{b} \Omega_{1} \sigma_{1}\left|\mathscr{H}_{1}\right| I_{a} m_{a}\right)=\sum_{L_{1} M_{1} \pi_{1}}\left(\Omega_{1} \sigma_{1} \mid L_{1} M_{1} \pi_{1}\right)\left(I_{b} m_{b} L_{1} M_{1} \pi_{1}\left|\mathscr{H}_{1}\right| I_{a} m_{a}\right) .
$$

The sum over the parity $\pi_{1}$ actually consists of one term only because of the conservation of parity; henceforth we omit the sum over the parity. The coefficients $(\Omega \sigma \mid L M \pi)$ of the unitary transformation (18) describe the eigenfunction of the radiation $R$ corresponding to the eigenvalues $L, M$ and $\pi$ of the operators of angular momentum, $z$-component of angular momentum and parity, respectively. We can establish a connection with the naive treatment in $\S 2$ by remarking that ${ }^{13,17,20}$

$$
F_{L}^{M}(\Omega) \sim \sum_{\sigma}(\Omega \sigma \mid L M \pi)^{*}(\Omega \sigma \mid L M \pi)
$$

At this point, we use the transformation properties of $(\Omega \sigma \mid L M \pi)$. The transformation coefficients $(\Omega \sigma \mid L M \pi)$ are defined with respect to the quantization coordinate system $x-y-z$, whose origin coincides with the source, but which is otherwise completely arbitrary. In this coordinate system, the direction of emission of the radiation $R$ is characterized by the polar angles $\vartheta$ and $\varphi: \Omega \equiv(\vartheta, \varphi)$. As stated earlier, the eigenfunctions $(\Omega \sigma \mid L M \pi)$ are especially simple if the quantization coordinate system coincides with that of the radiation. We denote these particular eigenfunctions with $(0 \sigma \mid L \mu \pi)$.

We now use the fact that the eigenfunctions belonging to an angular momentum $L$ transform according to the representation ${ }^{*} D^{L}$ and write the eigenfunctions $(\Omega \sigma \mid L M \pi)$ for the arbitrary system in terms of the $(0 \sigma \mid L \mu \pi)$ :

$$
(\Omega \sigma \mid L M \pi)=\sum_{\mu}(0 \sigma \mid L \mu \pi)\left(\mu\left|D^{L}\left(\Re^{-1}\right)\right| M\right) .
$$

The argument $\Re^{-1}$ denotes the inverse of that rotation $\mathfrak{R}$ which carries

* Instead of using the notation $D_{\mu M}^{L}$, we write a matrix element of $D^{L}$ as $\left(\mu\left|D^{L}\right| M\right)$. 
the quantization system over into the coordinate system of the radiation. The Euler angles of this rotation are $\varphi, \vartheta$, and $\chi$.

We proceed by simplifying also the second factor in Eq. (18), the matrix element $\left(I_{b} m_{b} L_{1} M_{1} \pi_{1}\left|\mathscr{H}_{1}\right| I_{a} m_{a}\right)$. We have already mentioned in $\S 2$ that matrix elements of this form can be factored into a Clebsch-Gordan coefficient ("geometrical" factor) and a reduced matrix element ("nuclear" factor). We verify this theorem here for the matrix element given above by first remarking that it corresponds to the vector addition $I_{a}=I_{b}+L_{1}$ $m_{a}=m_{b}+M_{1}$. By use of Eq. (5), we get

$$
\left(I_{b} m_{b} L_{1} M_{1} \pi_{1}\left|\mathscr{H}_{1}\right| I_{a} m_{a}\right)=\sum_{I_{a}^{\prime} m_{a}^{\prime}}\left(I_{b} L_{1} m_{b} M_{1} \mid I_{a}^{\prime} m_{a}^{\prime}\right)\left(I_{a}^{\prime} m_{a}^{\prime} I_{b} L_{1} \pi_{1}\left|\mathscr{H}_{1}\right| I_{a} m_{a}\right) .
$$

We now use the fact that $\mathscr{H}_{1}$ for physical reasons is invariant under rotations. The matrix element $\left(I_{a}^{\prime} m_{a}^{\prime} I_{b} L_{1} \pi_{1}\left|\mathscr{H}_{1}\right| I_{a} m_{a}\right)$ then is different from zero only if $I_{a}^{\prime}=I_{a}, m_{a}^{\prime}=m_{a}$, and it is furthermore independent of $m_{a}$ (e.g. $\left.{ }^{23,38}\right)$. We denote the resulting reduced matrix element by $\left(I_{b}\left\|L_{1}\right\| I_{a}\right)$, and get from Eq. (21):

$$
\left(I_{b} m_{b} L_{\mathbf{1}} M_{1} \pi_{1}\left|\mathscr{H}_{1}\right| I_{a} m_{a}\right)=\left(I_{b} L_{1} m_{b} M_{1} \mid I_{a} m_{a}\right)\left(I_{b}|| L_{1} \| I_{a}\right) .
$$

Combining Eq. (18), (20), and (22), we get the final expression for a typical matrix element:

$\left(b\left|\mathscr{H}_{1}\right| a\right)=\sum_{L_{1} M_{1} \mu_{1}}\left(0 \sigma_{1} \mid L_{1} \mu_{1} \pi_{1}\right)\left(\mu_{1}\left|D^{L_{1}}\right| M_{1}\right)\left(I_{b} L_{1} m_{b} M_{1} \mid I_{a} m_{a}\right)\left(I_{b}\left\|L_{1}\right\| I_{a}\right)$.

D. We turn now to the expression for a matrix element $\left(b\left|E^{(1)}\right| b^{\prime}\right)$. Incidentally we note that $\left(b\left|E^{(1)}\right| b^{\prime}\right)$ is, apart from a normalization factor, just the density matrix $\left(b\left|\mathscr{P}_{b}\right| b^{\prime}\right)$ of Eq. (14).

After inserting the matrix elements of the form (23) into Eq. (16), $\left(b\left|E^{(1)}\right| b^{\prime}\right)$ becomes a somewhat unwieldy expression. However, it can be brought into a convenient form by using some properties of the reduced matrix elements and the representations $D^{L}$, and by applying the Racah technique. We proceed by describing these steps.

The reduced matrix elements which appear in $\left(b|E| b^{\prime}\right)$ may always be chosen real:

$$
\left(I_{b}\left\|L_{1}\right\| I_{a}\right)^{*}=\left(I_{b}\left\|L_{1}\right\| I_{a}\right) \text {. }
$$

This result follows from the fact that the interaction Hamiltonian commutes with the operator of time reversal ${ }^{20,39,40}$. The relative phases of reduced matrix elements in a multipole mixture thus is always 0 or $\pi$

38 E. Feenberg and G. E. Pake, Quantum Theory of Angular Momentum (AddisonWesley, Cambridge Mass. 1953) p. 31.

39 S. P. Lloyd, Phys. Rev. 81 (1951) 161.

40 F. Coester, Phys. Rev. 89 (1953) 619. 
(cf. $\S 4 \mathrm{D})$. This is true only when perturbation theory is applicable. The reduced matrix elements of a non-Hermitian transition are not real.

The products $\left(\mu\left|D^{L}\right| M\right)\left(\mu^{\prime}\left|D^{L^{\prime}}\right| M^{\prime}\right)$ which appear in $\left(b|E| b^{\prime}\right)$ can be reduced by applying the two following relations ${ }^{2}$ ).

$$
\begin{gathered}
\left(\mu\left|D^{L}\right| M\right)^{*}=(-1)^{M-\mu}\left(-\mu\left|D^{L}\right|-M\right), \\
\left(\mu\left|D^{L}\right| M\right)\left(\mu^{\prime}\left|D^{L^{\prime}}\right| M^{\prime}\right)=\sum_{\nu} \sum_{\rho^{\tau}}\left(L L^{\prime} \mu \mu^{\prime} \mid \nu \tau\right)\left(L L^{\prime} M M^{\prime} \mid \nu \varrho\right)\left(\tau\left|D^{\nu}\right| \varrho\right) .
\end{gathered}
$$

Here, we have used the abbreviations $\mu+\mu^{\prime} \equiv \tau$ and $M+M^{\prime} \equiv \varrho$; the summation index $v$ runs from $\left|L-L^{\prime}\right|$ to $L+L^{\prime}$.

Through Eq. (26), the Clebsch-Gordan coefficient $\left(L L^{\prime} M M^{\prime} \mid v \varrho\right)$ is introduced into the expression for $\left(b|E| b^{\prime}\right)$. This coefficient now makes the application of Racah's technique possible: the sum over $m_{a}, M_{1}$, and $M_{1}^{\prime}$ of the product of the three coefficients $\left(I_{b} L_{1} m_{b} M_{1} \mid I_{a} m_{a}\right)$, $\left(I_{b} L_{1}^{\prime} m_{b}^{\prime} M_{1}^{\prime} \mid I_{a} m_{a}\right)$ and $\left(L_{1} L_{1}^{\prime} M_{1}-M_{1}^{\prime} \mid \nu \varrho\right)$ can be evaluated by using the formula ${ }^{35}$ :

$\sum_{\beta \in \delta}(a b \alpha \beta \mid e \varepsilon)(e d \varepsilon \delta \mid c \gamma)(b d \beta \delta \mid f \phi)=(2 e+1)^{\sharp}(2 f+1)^{\ddagger}(a f \alpha \phi \mid c \gamma) W(a b c d ; e f)$.

Here, $a, b, \ldots$ are angular momenta; $\alpha, \beta, \ldots$ are the corresponding magnetic quantum numbers; $W(a b c d$; ef) is the so-called Racah coefficient, which is independent of magnetic quantum numbers and which can be calculated explicitly ${ }^{20,35}$. The application of Eq. (27) involves some shuffling of the arguments of the Clebsch-Gordan coefficients in $\left(b|E| b^{\prime}\right)$. (This shuffling can be avoided by using the $\Gamma$-coefficients, see ${ }^{19}, \mathbf{3 2}$ ).

Before writing down the final expression for $\left(b|E| b^{\prime}\right)$, we introduce with Racah ${ }^{14}$ the following radiation parameters:

$$
c_{\nu \tau}\left(L L^{\prime}\right)=\mathscr{S} \sum_{\mu \mu^{\prime}}(-1)^{L^{\prime}-\mu^{\prime}}\left(L L^{\prime} \mu-\mu^{\prime} \mid \nu \tau\right)(0 \sigma \mid L \mu \pi)^{*}\left(0 \sigma^{\prime} \mid L^{\prime} \mu^{\prime} \pi^{\prime}\right) .
$$

These parameters $c_{v \tau}\left(L L^{\prime}\right)$ are characteristic of the emitted radiation through the eigenfunctions $(0 \sigma \mid L \mu \pi)$, and of the experimental arrangement through the averaging process designated by $\mathscr{S}$. Formally, the $c_{v \tau}$ are eigenfunctions of the operators for the total angular momentum and the $z$-component of the angular momentum with eigenvalues $v$ and $\tau$. However, no physical significance is attached to $v$ and $\tau$; they are convenient summation indices.

The symbol $\mathscr{S}$ in Eq. (28) has a definite meaning, which is expressed best through the efficiency matrix ${ }^{19} \varepsilon$. We introduce $\varepsilon$ here in a somewhat specialized form: the sensitivity (efficiency) of the detector for a radiation with polarization $\sigma$ shall be given by $\varepsilon_{\sigma}$. We may consider $\varepsilon_{\sigma}$ to be the eigenvalues of an efficiency matrix $\varepsilon$. In general, the symbol $\mathscr{S}$ can then be replaced by

$$
\mathscr{S} \rightarrow \sum_{\sigma \sigma^{\prime}}\left(\sigma|\varepsilon| \sigma^{\prime}\right) \text {. }
$$


For the special case of polarization insensitive detectors we simply have $\left(\sigma|\varepsilon| \sigma^{\prime}\right)=\delta_{\sigma \sigma}$. For a counter which responds only to $\gamma$-rays of a definite circular polarization, we would have $\varepsilon=\left(\begin{array}{ll}1 & 0 \\ 0 & 0\end{array}\right)$. If the detector responds only to photons linearly polarized in the $x$-direction then $\varepsilon=(1 / \sqrt{2})\left(\begin{array}{rr}1 & -1 \\ -1 & 1\end{array}\right)$.

In general, $\varepsilon$ can always be chosen real. This choice fixes the third Euler angle $\chi$. The eigenfunctions $(0 \sigma \mid L \mu \pi)$ in Eq. (28) are real and the radiation parameters $c_{v \tau}\left(L L^{\prime}\right)$ are then also real. For a given kind of particle and a given experimental arrangement, the $c_{v z}\left(L L^{\prime}\right)$ can be calculated explicitly.

By pooling all the information contained in the Eqs. (16), (23)-(29), we find for the matrix element $\left(b\left|E^{(1)}\right| b^{\prime}\right)$ of the first transition:

$$
\left.\begin{array}{rl}
\left(b\left|E^{(1)}\right| b^{\prime}\right) & =\sum_{L_{1} L_{1}^{\prime}} \sum_{v_{1} \tau_{1}} \sum_{\varrho_{1}}(-1)^{I_{b}-m_{b}^{\prime}-v_{1}}\left(2 I_{a}+1\right)\left(I_{b}\left\|L_{1}\right\| I_{a}\right)\left(I_{b}\left\|L_{1}^{\prime}\right\| I_{a}\right) \\
& \times\left(I_{b} I_{b} m_{b}-m_{b}^{\prime} \mid v_{1} \varrho_{1}\right) W\left(I_{b} I_{a} v_{1} L_{1}^{\prime} ; L_{1} I_{b}\right) \\
& \times c_{v_{1} \tau_{1}}\left(L_{1}^{\prime} L_{1}\right)\left(\tau_{1}\left|D^{\nu_{1}}\left(\Re_{1}^{-1}\right)\right| \varrho_{1}\right)^{*}
\end{array}\right\}
$$

and similarly

$$
\left.\begin{array}{rl}
\left(b\left|E^{(2)}\right| b^{\prime}\right) & =\sum_{L_{2} L_{2}^{\prime}} \sum_{v_{2} \tau_{2}} \sum_{\varrho_{2}}(-1)^{I_{b}-m_{b}^{\prime}}\left(2 I_{b}+1\right)\left(I_{c}\left\|L_{2}\right\| I_{b}\right)\left(I_{c}\left\|L_{2}^{\prime}\right\| I_{b}\right) \\
& \times\left(I_{b} I_{b} m_{b}-m_{b}^{\prime} \mid v_{2} \varrho_{2}\right) W\left(I_{b} I_{c} v_{2} L_{2}^{\prime} ; L_{2} I_{b}\right) \\
& \times c_{v_{2} \tau_{2}}\left(L_{2} L_{2}^{\prime}\right)\left(\tau_{2}\left|D^{v_{2}}\left(\Re_{2}^{-1}\right)\right| \varrho_{2}\right) .
\end{array}\right\}
$$

E. The expressions $(30)$ and $\left(30^{\prime}\right)$ can now be inserted into Eq. (15) for $W\left(\Omega_{1} \Omega_{2}\right)$. The resulting formula is simplified by using the orthogonality of the Clebsch-Gordan coefficients:

$$
\sum_{m_{b} m_{b}^{\prime}}\left(I_{b} I_{b} m_{b}-m_{b}^{\prime} \mid v_{1} \varrho_{1}\right)\left(I_{b} I_{b} m_{b}-m_{b}^{\prime} \mid v_{2} \varrho_{2}\right)=\delta_{v_{1} v_{2}} \delta_{\varrho_{1} \varrho_{2}} .
$$

With $v_{1}=v_{2} \equiv v$ and $\varrho_{1}=\varrho_{2} \equiv \varrho$, it then becomes possible to combine the two representations $D^{\nu_{1}}$ and $D^{\nu_{2}}$ :

$$
\left.\begin{array}{rl} 
& \sum_{\varrho}\left(\tau_{2}\left|D^{\nu}\left(\Re_{2}^{-1}\right)\right| \varrho\right)\left(\tau_{1}\left|D^{v}\left(\Re_{1}^{-1}\right)\right| \varrho\right)^{*} \\
= & \left.\sum_{\varrho}\left(\tau_{2}\left|D^{y}\left(\Re_{2}^{-1}\right)\right| \varrho\right)\left(\varrho\left|D^{y}\left(\Re_{1}\right)\right| \tau_{1}\right)=\left(\tau_{2}\left|D^{y}\left(\Re_{2}^{-1} \Re_{1}\right)\right| \tau_{1}\right) \cdot\right\}
\end{array}\right\}
$$

The rotation $\mathfrak{R}_{2}^{-1} \Re_{1}$, with Euler angles $\phi, \theta, X$, carries the coordinate system of the second radiation into that of the first. Thus $\theta$ denotes the angle between $\Omega_{1}$ and $\Omega_{2}$. Using the Eq. (31) and (32), we write the final expression for the angular correlation between two successive radiations:

$$
\left.\begin{array}{rl}
W\left(\Omega_{1} \Omega_{2}\right) & =\sum(-1)^{v}\left(I_{b}\left\|L_{1}\right\| I_{a}\right)\left(I_{b}\left\|L_{1}^{\prime}\right\| I_{a}\right)\left(I_{c}\left\|L_{2}\right\| I_{b}\right)\left(I_{c}\left\|L_{2}^{\prime}\right\| I_{b}\right) \\
& \times W\left(I_{b} I_{a} v L_{1}^{\prime} ; L_{1} I_{b}\right) W\left(I_{b} I_{c} v L_{2}^{\prime} ; L_{2} I_{b}\right) \\
& \times c_{v \tau_{1}}\left(L_{1}^{\prime} L_{1}\right) c_{v \tau_{2}}\left(L_{2} L_{2}^{\prime}\right)\left(\tau_{2}\left|D^{\nu}\left(\Re_{2}^{-1} \Re_{1}\right)\right| \tau_{1}\right) .
\end{array}\right\}
$$


The summation here is extended over $L_{1}, L_{1}^{\prime}, L_{2}, L_{2}^{\prime}, \tau_{1}, \tau_{2}$ and $\nu$. This form of the angular correlation function was derived independently by Racah; Lloyd, and Alder.

A number of conclusions can be drawn from Eq. (33). We mention here only the most important ones and refer for all details to the review article by Biedenharn and Rose 20 .

It is apparent from (33) that the "natural" expansion of the angular correlation function is in terms of the representations $D$ ". The expansion coefficients then break up into independent factors and the interpretation as well as the calculation is facilitated.

For pure multipole radiations $\left(L_{1}=L_{1}^{\prime}, L_{2}=L_{2}^{\prime}\right)$, the reduced matrix elements in Eq. (33) can be omitted and the sums over $L_{1}, L_{1}^{\prime}, L_{2}$, and $L_{2}^{\prime}$ all degenerate into one term. For mixed radiations, $L$ and $L^{\prime}$ vary independently over the range of allowed angular momenta.

The correlation functions for two cascades $I_{a}\left(L_{1}\right) I_{b}\left(L_{2}\right) I_{c}$ and $I_{c}\left(L_{2}\right) I_{b}\left(L_{1}\right) I_{a}$ are identical. This symmetry, which follows from Eq. (11) by the Hermitian character of the matrix elements, is often useful in calculations.

The Racah coefficients $W$ in Eq. (33) yield some information as to the form of the correlation function. A Racah coefficient $W(a b c d ;$ ef) vanishes unless each of the vector triples $(a b e),(c d e),(a c f)$, and $(b d f)$ forms a triangle. Applying these restrictions to Eq. (33), we find selection rules for $v$ :

$$
0 \leqslant v \leqslant \operatorname{Min}\left(2 I_{b}, 2 L_{1}, 2 L_{2}\right) \text {. }
$$

Yang has shown that these selection rules follow directly from the invariance of the correlation processes under rotation and inversion ${ }^{\mathbf{4 1}}$.

The summation index $v$ is an even integer as long as the measurements are restricted to the directions and the linear polarizations of the radiations. In the case of the observation of circular polarization of $\gamma$-rays (experimentally not yet realized), odd integers will occur also. We restrict our formulas in the following to even integers only.

F. We turn now to the experimentally most important case, the directional correlation. Because only the directions of the radiations are observed, the correlation function must be independent of the Euler angles $\chi_{1}$ and $\chi_{2}$, which denote rotations about the directions of propagation. Only the functions $c_{\nu 0}$ then appear in $W\left(\Omega_{1} \Omega_{2}\right)$, as can be seen immediately: $c_{\nu_{1}}$ is an eigenfunction of the operator $L_{z}=-i \hbar\left(\partial / \partial \chi_{1}\right)$, with eigenvalue $\tau_{1}$. Independence of $\chi_{1}$ thus means $\tau_{1}=0$. The same argument applies to $\tau_{2}$.

With $\tau_{1}=\tau_{2}=0$, the representations $D^{\nu}$ become Legendre polynomials, $\left(0\left|D^{\nu}(\phi \theta X)\right| 0\right)=P_{\nu}(\cos \theta)$, and expression (33) can be written as

$$
W\left(\Omega_{1} \Omega_{2}\right)=W(\theta)=\sum_{\nu \text { even }} A_{v} P_{v}(\cos \theta) .
$$

41 C. N. Yaing, Phys. Rev. 74 (1948) 764. 
This form of the directional correlation function will be used in the following. One sees immediately from Eq. (33), that the coefficients $A_{\text {, }}$ break up into two factors, each factor depending on only one transition of the cascade. This fact simplifies the tabulation of numerical data for the directional correlation (cf. § 4).

\section{§ 4. $\gamma-\gamma$ Directional Correlation}

We present in $\S 4 \mathrm{~A}$ to $4 \mathrm{D}$ those theoretical results which are needed in the evaluation of the data from $\gamma-\gamma$ directional correlation measurements. In $\S 4 \mathrm{E}$ to $4 \mathrm{H}$, we discuss some selected experiments.

A. We consider first a $\gamma-\gamma$ cascade $\mathrm{A} \rightarrow \mathrm{B} \rightarrow \mathrm{C}$, in which both $\gamma$-rays, of multipole orders $L_{1}$ and $L_{2}$, respectively, are pure. Such a cascade will be denoted by $I_{a}\left(L_{1}\right) I_{b}\left(L_{2}\right) I_{c}$. According to Eq. (35), the most convenient form of describing the directional correlation $W(\theta)$ between $\gamma_{1}$ and $\gamma_{2}$ is

$$
W(\theta)=1+A_{2} P_{2}(\cos \theta)+\ldots+A_{v \max } P_{\nu \max }(\cos \theta) .
$$

The highest term in this expansion is determined by the selection rules (34):

$$
v_{\max }=\operatorname{Min}\left(2 I_{b}, 2 L_{1}, 2 L_{2}\right) \text {. }
$$

The explicit calculation of the coefficients $A_{v}$ is greatly facilitated by the fact that they can be broken up into two factors, each factor depending on only one transition of the cascade. We denote these factors by $F_{v}$ (no confusion with the $F_{L}^{M}(\theta)$ of $\S 2$ should arise) and write:

$$
A_{v}=F_{v}\left(L_{1} I_{a} I_{b}\right) F_{v}\left(L_{2} I_{c} I_{b}\right)
$$

We derive here, as an example of the application of the general formula (33), an explicit expression for the functions $F_{v}$. Omitting angle-independent factors, and assuming pure $\gamma$-rays, we get from Eq. (33) and (34):

$$
F_{v}\left(L I_{a} I_{b}\right)=W\left(I_{b} I_{a} v L ; L I_{b}\right) c_{v 0}(L L) .
$$

The radiation parameters $c_{\nu 0}(L L)$ for polarization-independent detectors follow from Eq. (28) and (29):

$$
c_{\nu 0}(L L)=\sum_{\mu}(-1)^{L-\mu}(L L \mu-\mu \mid \nu 0) \sum_{\sigma}(0 \sigma \mid L \mu \pi)^{*}(0 \sigma \mid L \mu \pi) .
$$

With Eq. (19), this can be written as

$$
c_{\nu 0}(L L) \sim \sum_{\mu}(-1)^{L-\mu}(L L \mu-\mu \mid \nu 0) F_{L}^{\mu}(0) .
$$

The sum here extends over the two values $\mu= \pm 1$ only (cf. $\S 2 \mathrm{C}$ ). $F_{L}^{\mu}(0)$ is proportional to the function $Z_{L \mu}(0)$ of Blatt and Weisskopf (page 594 of reference ${ }^{21}$ ); it can also be calculated by using the vector potential $\boldsymbol{A}_{L \mu}$ of Ch. XIII. We thus find 


$$
\begin{aligned}
& F_{L}^{1}(0)=F_{L}^{-1}(0)=(1 / 2)\left[Y_{L 0}(0)\right]^{2}=(2 L+1) / 8 \pi ; \text { and } \\
& c_{\nu 0}(L L) \sim(-1)^{L-1}(2 L+1)(L L 1-1 \mid \nu 0) .
\end{aligned}
$$

The coefficients $F_{v}$ then are given, apart from a normalization factor, by

$$
F_{v}\left(L I_{a} I_{b}\right) \sim(-1)^{L-1}(2 L+1)(L L 1-1 \mid \nu 0) W\left(I_{b} I_{a} \nu L ; L I_{b}\right) .
$$

Numerical values and formulas for the Clebsch-Gordan and Racah coefficients are available ${ }^{42,43}$ and hence the coefficients $F_{v}$ can be calculated for any desired case.

The coefficients $F$, have been calculated numerically by Biedenharn and Rose ${ }^{20,43^{\mathrm{a}}}$; we include their results in Appendix $V^{*}$. As an example of the use of these tables, we calculate the coefficients $A_{2}$ and $A_{4}$ for the cascade $4(2) 2(2) 0$ :

$$
\begin{array}{ccc}
F_{0}=1 & F_{0}=1 & A_{0}=1 \\
F_{2}(2,4,2)=-0.1707 & F_{2}(2,0,2)=-0.5976 & A_{2}=0.1020 \\
F_{4}(2,4,2)=-0.0085 & F_{4}(2,0,2)=-1.069 & A_{4}=0.0091 .
\end{array}
$$

Experimentally, one determines the coefficients $A_{v}$ by assuming an appropriate value for $v_{\max }$ (usually 4) and then making a least square fit of Eq. (36) to the values of $W_{\text {exp }}(\theta)$ measured at several angles. Very often, however, one restricts the observations to the two angles $\theta=90^{\circ}$ and $\theta=180^{\circ}$. This experiment then determines the anisotropy

$$
A \equiv \frac{W\left(180^{\circ}\right)-W\left(90^{\circ}\right)}{W\left(90^{\circ}\right)}
$$

If we assume the highest coefficient in the expansion (36) to be $A_{4}$, then the anisotropy $A$ is related to the coefficients $A_{2}$ and $A_{4}$ by

$$
A=\frac{1+A_{2}+A_{4}}{1-(1 / 2) A_{2}+(3 / 8) A_{4}}-1 .
$$

B. The experimental investigation of a $\gamma-\gamma$ cascade usually yields the two constants $A_{2}$ and $A_{4}$. (Higher terms have not yet been observed: the energies which normally are available in radioactive decays are such that multipoles with $L>2$ possess a half life $>10^{-6}$ sec. It is experimentally difficult or even impossible to measure a directional correlation function of a cascade involving an intermediate state with so long a half-life.) The

42 A. Simon, J. H. Van der Sluis and L. C. Biedenharn, Oak Ridge National Laboratory Report 1679. (1954).

43 A. Simon, Oak Ridge National Laboratory Report 1718 (1954).

43a A more complete table can be found in H. Ferentz and N. Rosenzweig, Argonne National Laboratory Report 5324 (1954).

* In order to use these tables, the following changes in notation must be made: $I_{a} \rightarrow j_{1}, I_{b} \rightarrow j, I_{c} \rightarrow j_{1}$. 
sonstants $A_{2}$ and $A_{4}$ depend on five parameters: the multipole orders $L_{1}$ and $L_{2}$ of the $\gamma$-rays and the spins $I_{a}, I_{b}$, and $I_{c}$ of the nuclear states. Thus, the directional correlation measurement alone never allows a complete determination of all nuclear parameters involved. Only in conjunction with other methods is it possible to establish a complete decay scheme.

The fact that the directional correlation function $W(\theta)$ does not depend on the parities of the nuclear states can be understood classically. Electric and magnetic radiations of the same multipole order $L$ are related by the transformation $\boldsymbol{E} \rightarrow \boldsymbol{H}, \boldsymbol{H} \rightarrow-\boldsymbol{E}$. This transformation leaves the Poynting vector and therefore the angular distribution of the radiation unaltered. It is thus impossible to distinguish between electric and magnetic radiation by means of a directional correlation measurement.

C. We have assumed so far that the nuclear cascade consists of two successive $\gamma$-rays (double cascade). In many decays, more than two $\gamma$-rays are in cascade and one can for example observe the correlation functions $W\left(\gamma_{1}, \gamma_{2}\right), W\left(\gamma_{2} \gamma_{3}\right)$ and $W\left(\gamma_{1} \gamma_{3}\right)$. Biedenharn, Arfken, and Rose ${ }^{44,} 45$ have extended the theory to this case (see also $\left.{ }^{46}\right) . W\left(\gamma_{1} \gamma_{3}\right)$ can be written in the form (36), and values for the coefficients $A_{v}$ are given in Table XI of reference ${ }^{20}$. In some instances, the observation of $W\left(\gamma_{1} \gamma_{3}\right)$ can be used to decide on the order of emission of the $\gamma$-rays. In other cases, however, the correlation function is independent of the order of transition ${ }^{47,48}$.

D. The directional correlation for double cascades in which one of the two $\gamma$-rays is mixed, i.e. must be described by more than one value $L$ of the angular momentum, was first treated by Ling and Falkoff ${ }^{12}$. Their calculations were restricted to dipole and quadrupole radiation. The further development of the theory (cf. $\S 3$ ) yielded formulas which are easier to evaluate. We describe here the main results ${ }^{20}$.

Suppose the first transition in a cascade to consist of a mixture of $L$ and $L^{\prime}>L$, with a mixing ratio $\delta$. We define $\delta$ as the ratio of the reduced matrix elements

$$
\delta \equiv\left(I_{b}\left\|L^{\prime}\right\| I_{a}\right) /\left(I_{b}\|L\| I_{a}\right)
$$

The ratio of the total (i.e. angle-integrated) intensity of the $L^{\prime}$ pole to that of the $L$ pole is then equal to $\delta^{2}$. (This can be seen from Eq. (22) by using the orthogonality relations of the Clebsch-Gordan coefficients). We

44 L. C. Biedenharn, G. B. Arfken, and M: E. Rose, Phys. Rev. 83 (1951) 586.

45 G. B. Arfken, L. C. Biedenharn, and M. E. Rose, Phys. Rev. 86 (1952) 761.

4 G. R. Satchler, Phys. Rev. 94 (1954) 1304.

17 J. Weneser and D. R. Hamilton, Phys. Rev. 92 (1953) 321.

48 E. J. Hellund and J. M. Jauch, Phys. Rev. 92 (1953) 203. 
have mentioned in $\S 3 \mathrm{I}$ ) that the reduced matrix elements are chosen to be real; thus the mixing ratio $\delta$ is real ${ }^{39}$. For a given intensity ratio $\delta^{2}$, the mixing ratio $\delta$ can have either a positive or negative sign, depending on the relative phase of the reduced matrix elements. However, the sign depends on the definition of the reduced matrix elements. This fact must be kept in mind when one compares the sign of $\delta$ as determined from a correlation experiment with that calculated on the basis of a certain nuclear model. (See the discussion by Biedenharn and Rose, reference ${ }^{20}$, section II-C, D and F.; and reference ${ }^{48 \mathrm{a}}$.)

The directional correlation for the cascade $I_{a}\left(L_{1} L_{1}^{\prime}\right) I_{b}\left(L_{2}\right) I_{c}$ can now be written as

$$
W(\theta)=W_{\mathrm{I}}+\delta^{2} W_{\mathrm{II}}+2 \delta W_{\mathrm{III}} .
$$

$W_{\mathrm{I}}$ and $W_{\mathrm{II}}$ are the correlation functions for the pure cascades $I_{a}\left(L_{1}\right) I_{b}\left(L_{2}\right) I_{c}$ and $I_{a}\left(L_{1}^{\prime}\right) I_{b}\left(L_{2}\right) I_{c}$, and can be calculated from Eq. (36) and (37). $W_{\text {III }}$ is the contribution due to the interference between $L_{1}$ and $L_{1}^{\prime}$ and is given by

with

$$
W_{\mathrm{III}}=\sum_{\substack{\nu \neq 0 \\ \text { even }}} A_{\nu}^{\mathrm{III}} P_{\nu}(\cos \theta)
$$

and

$$
A_{v}^{\mathrm{III}}=F_{v}\left(L_{1} L_{1}^{\prime} I_{a} I_{b}\right) F_{\nu}\left(L_{2} I_{c} I_{b}\right)
$$

$F_{\nu}\left(L_{1} L_{1}^{\prime} I_{a} I_{b}\right)=(-1)^{I_{b}-I_{a}-1}\left[\left(2 I_{b}+1\right)\left(2 L_{1}+1\right)\left(2 L_{1}^{\prime}+1\right)\right]^{t} G_{\nu}\left(L_{1} L_{1}^{\prime} I_{a} I_{b}\right)$.

The function $G_{v}\left(L L^{\prime} I_{a} I_{b}\right)$ has been evaluated for the case $L^{\prime}=L+1$ by Biedenharn and Rose ${ }^{20}$; their results are included as Appendix V. Tables of the function $F_{v}\left(L_{1}, L_{1}^{\prime}, I_{a}, I_{b}\right)$ are given in reference ${ }^{43 a}$. Formally, $W(\theta)$ can again be written as in Eq. (36).

The correlation function for cascades in which both transitions are mixed has been calculated by Rose ${ }^{49}$.

E. Among the large number of $\gamma-\gamma$ cascades investigated to date, we select three typical examples and show how the considerations of $\S 4 \mathrm{~A}$ to $\S 4 \mathrm{D}$ apply.

As our first example, we choose an even-even nucleus, $\mathrm{Ni}^{60} *$ (Fig.4). Even-even nuclei are especially well suited for angular correlation measurements. They presumably have spin 0 in their ground state and the second transition $I_{b}\left(\gamma_{2}\right) 0$ must therefore be pure. Only the first $\gamma$-ray can be mixed and this reduces considerably the number of possible interpretations of a measured correlation.

48a G. R. Satchler, to be published in Proc. Phys. Soc.

49 M. E. Rose, Phys. Rev. 93 (1954) 477.

* We denote $\gamma-\gamma$ cascades by the nuclide in which the cascade takes place, e.g. $\mathrm{Ni}^{60}$ and not $\mathrm{Co}^{60} . \beta-\gamma$ and $\alpha-\gamma$ cascades are named after the parent nucleus. 
The directional correlation of $\mathrm{Ni}^{60}$ was first investigated successfully by Brady and Deutsch 3, 4, 5. On the basis of their correlation data and of earlier measurements ${ }^{50}$, they concluded that the $\gamma-\gamma$ cascade probably occurs between spins 4,2 , and 0 . An accurate determination of the conversion coefficients ${ }^{51}$ confirmed their conclusions. (A recent investigation ${ }^{52}$ casts some doubt on the consistency of the complete decay scheme $\mathrm{Co}^{60} \rightarrow \mathrm{Ni}^{60}$. However, the assignment $4(2) 2(2) 0$ can nevertheless be considered correct.)

Since the first report by Brady and Deutsch, a large number of publications contain data on $\mathrm{Ni}^{60}$, and this cascade has become a "standard cascade" for directional correlation work. At a certain time there seemed to be a definite discrepancy between the theoretical and the experimental correlation ${ }^{53}$. Later measurements, however, showed that the discrepancy was entirely due to insufficient correction of the experimental data ${ }^{54,55}$. We have already calculated the coefficients $A_{2}$ and $A_{4}$ for the cascade $4(2) 2(2) 0$ in $\S 4 \mathrm{~A}$. Using those values, we get for the anisotropy $A=0.1667$ and for the correlation function $W(\theta)=1+0.1020 P_{2}(\cos \theta)+0.0091 P_{4}$ $(\cos \theta)$. The main features of the decay $\mathrm{Co}^{60} \rightarrow \mathrm{Ni}^{60}$ and the directional correlation function measured by Steffen ${ }^{56}$ are shown in Fig. 4. The

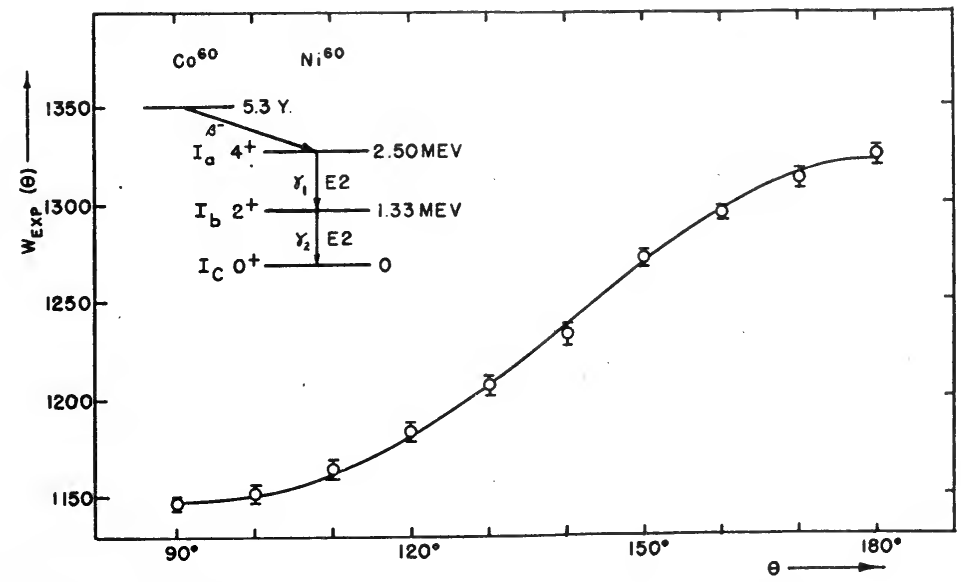

Fig. 4. Decay of $\mathrm{Co}^{60}$ and directional correlation of the $\mathrm{Ni}^{60} \gamma-\gamma$ cascade (R. M. Steffen). The solid line is the theoretical function $W(\theta)$ for the cascade $4(2) 2(2) 0$.

50 M. Deutsch, L. G. Elliott, and A. Roberts, Phys. Rev. 68 (1945) 193.

51 M. A. Waggoner, M. L. Moon, and A. Roberts, Phys. Rev. 80 (1950) 420.

52 G. L. Keister and F. H. Schmidt, Phys. Rev. 93 (1954) 140.

53 H. Aeppli, H. Frauenfelder, E. Heer and R. Rüetschi, Phys. Rev. 87 (1952) 379.

54 E. D. Klema and F. K. McGowan, Phys. Rev. 91 (1953) 616.

55 J. S. Lawson and H. Frauenfelder, Phys. Rev. 91 (1953) 649.

56 R. M. Steffen, to be published in Am. J. Physics. 
theoretical curve and the experimental values agree very well. The comparison of the experimental anisotropy $A_{\text {exp }}=0.167 \pm 0.001$ with the theoretical value $A=0.1667$ shows that directional correlation measurements are capable of a rather high precision. The fact that no perturbation by extranuclear fields is observed is not surprising since the lifetime of the intermediate state of the $\mathrm{Ni}^{60}$ cascade is shorter than $10^{-11} \mathrm{sec}^{57}$.

F. For some years after the pioneer work of Brady and Deutsch, only nuclei emitting two $\gamma$-rays in cascade could be investigated with a fair degree of accuracy. The extension of directional correlation measurements to cascades of more than two $\gamma$-rays failed because one could not unambiguously single out the desired $\gamma$-rays. Today, most of the directional correlation work is performed by using scintillation spectrometers as counters and it is thus possible to attack even very complicated decays.

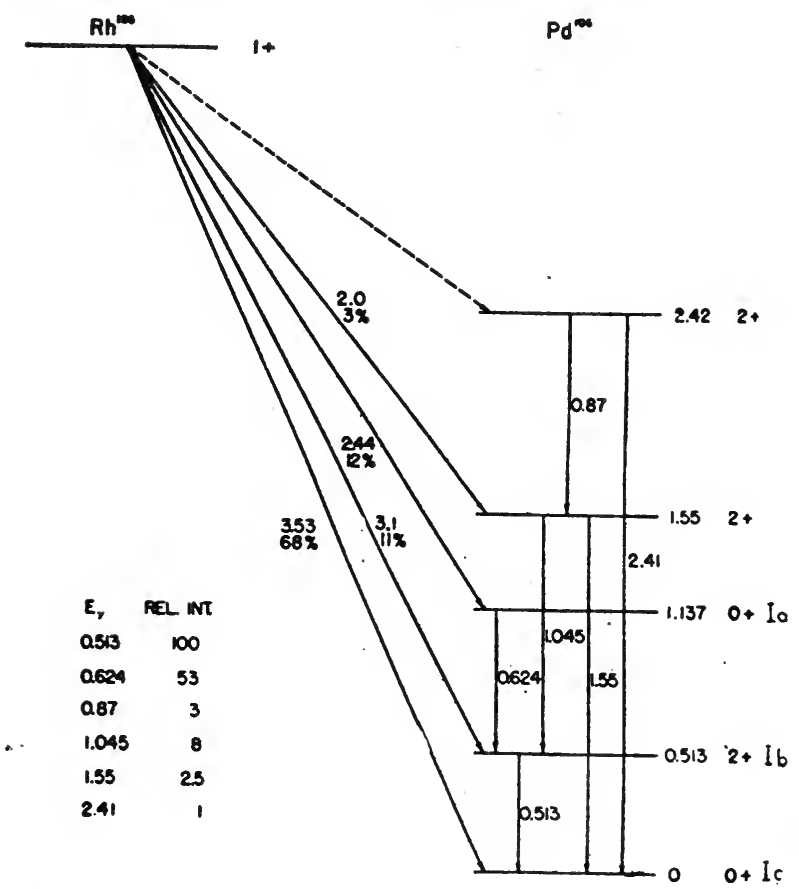

Fig. 5.

The decay $\mathrm{Rh}^{106} \rightarrow \mathrm{Pd}^{106}$. (D. E. Alburger, reference ${ }^{69}$ )

The investigation of the triple cascades in $\mathrm{Ba}^{134}$ and $\mathrm{Ce}^{\mathbf{1 4 0}}$ by Robinson and Madansky ${ }^{58}$ constitutes one of the first successful applications of scintillation spectroscopy to directional correlation. In addition to selecting

67 Z. Bay, V. P. Henri, and F. McLernon, Phys. Rev. 94 (1954) 780.

38 B. L. Robinson and L. Madansky, Phys. Rev. 84 (1951) 604, Phys. Rev. 84 (1951) 1067.

so D. E. Alburger, Phys. Rev. 88 (1952) 339. 
the $\gamma$-ray energies, they used a third counter with a very large solid angle to detect beta particles in (triple) coincidence with the $\gamma$-rays. This methodaccepting only $\gamma$-ray coincidences which are in coincidence with a beta branch of a given energy - may be very useful for further correlation work.

The work of Klema and McGowan ${ }^{60}$ on $\mathrm{Pd}^{106}$ (cf. Fig. 5) well exemplifies the highly refined technique of directional correlation measurements. The directional correlation of $\mathrm{Pd}^{106}$ was a long-standing puzzle. Brady and Deutsch ${ }^{5}$ first measured the correlation of the $0.624 \mathrm{MeV}-0.513 \mathrm{MeV}$ cascade and found that the form of $W(\theta)$ agreed with that expected for a 0-2-0 cascade, but that the coefficients $A_{2}$ and $A_{4}$ were only about half as large as theory predicted. Many explanations were advanced, but none proved to be completely satisfactory. Klema and NicGowan finally found the solution by using a highly-developed apparatus. The $\gamma$-spectrum of $\mathrm{Pd}^{106}$ as seen by their detectors is given in Fig. 6. By measuring $W(\theta)$ for each of the energy ranges indicated by $A, B, \ldots$ in Fig. 6 and by suitably combining the resulting data, Klema and McGowan obtained the following coefficients $A_{2}$ and $A_{4}$ :

$0.624 \mathrm{MeV}-0.513 \mathrm{MeV}$ cascade: $A_{2}=0.346 \pm 0.008, A_{4}=1.109 \pm 0.012$. $1.045 \mathrm{MeV}-0.513 \mathrm{MeV}$ cascade: $A_{2}^{\prime}=0.092 \pm 0.003, A_{4}^{\prime}=0.035 \pm 0.009$.

Fig. 6.

$\gamma$-spectrum of $\mathrm{Rh}^{106} \rightarrow \mathrm{Pd}^{108}$, measured by Klema and Mc Gowan ${ }^{80}$. The limits indicated by $\mathrm{A}, \mathrm{B}, \mathrm{C}$ etc. show the positions of the windows (i.e. the energy ranges) of the two scintillation spectrometers used as detectors. One counter was fixed at position $\mathrm{A}$, the other counter was set at the various other positions and the directional correlation function was determined for each setting.

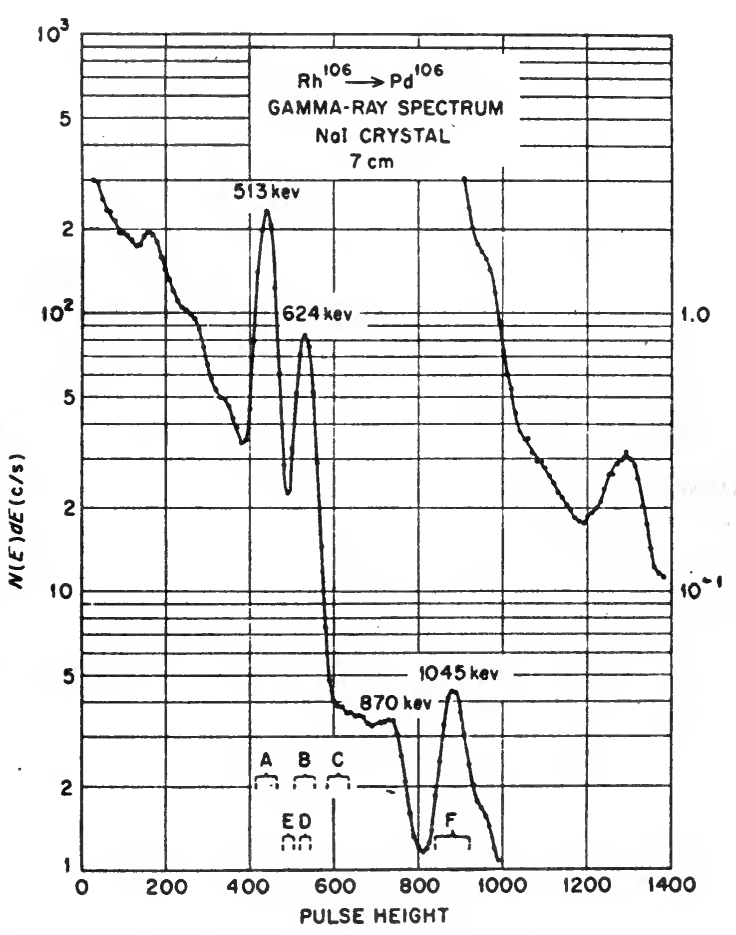

E. D. Klema and F. K. McGowan, Phys. Rev. 92 (1953) 1469. 
The interpretation of these coefficients is best made by using the spin assignments which were deduced by Alburger ${ }^{59}$ from measurements of the beta spectrum of $\mathrm{Rh}^{106}$ and from conversion data. These spin assignments are shown in Fig. 5. The $0.624 \mathrm{MeV}-0.513 \mathrm{MeV}$ cascade occurs between spins $I_{a}=0, I_{b}=2, I_{c}=0$. If this spin assignment is correct, then both $\gamma$-rays must be pure quadrupoles, and the theoretical coefficients are $A_{2}=0.3571, A_{4}=1.143$. The agreement between these coefficients and the ones determined by Klema and McGowan is good, and it confirms the assumed spin assignment. Moreover, the old puzzle is solved by this measurement: in the earlier work the true correlation was masked by competing cascades.

The measured coefficients for the $1.045-0.513 \mathrm{MeV}$ cascade exclude pure dipole radiation, because $A_{4}^{\prime} \neq 0$. However, the theoretical coefficients for a cascade $2(2) 2(2) 0, A_{2}^{\prime}=-0.0766$ and $A_{4}^{\prime}=0.3265$, are in clear disagreement with the experimental values given above. As long as one assumes that both $\gamma$-rays are pure multipoles, no other spin assignment fits the experimental data. One is thus forced to assume that the $1.045 \mathrm{MeV}$

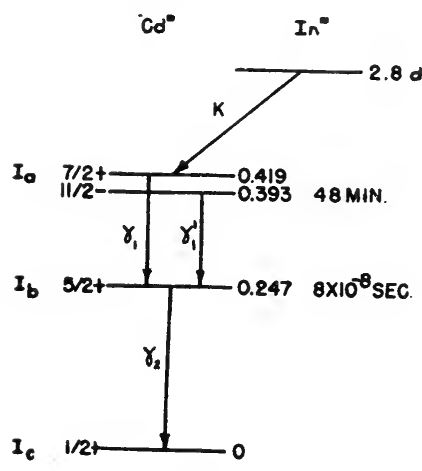

Fig. 7.

The decay $\operatorname{In}^{111} \rightarrow \mathrm{Cd}^{111}$ $\gamma$-ray between the two states with spin 2 must be a mixture of M.1 and E2.

G. In the past few years, a number of cascades have been investigated in which at least one of the $\gamma$-rays was found to be mixed. As an example, we discuss the main $\gamma-\gamma$. cascade in $\mathrm{Cd}^{111}$.

The energy levels of $\mathrm{Cd}^{111}$ have been very carefully investigated and an unambiguous spin assignment (Fig. 7) can be constructed without the help of directional correlation data. (See Ch. XXII for a detailed discussion and for references). The main $\gamma-\gamma$ cascade observed in the decay of $\operatorname{In}^{111}$ occurs between states $I_{a}=7 / 2, I_{b}=5 / 2$ and $I_{c}=1 / 2$. The measured conversion coefficients of $\gamma_{1}$ and $\gamma_{2}$ indicate that $\gamma_{1}$ is an $\mathrm{Ml},{ }^{*}, \gamma_{2}$ an $\mathrm{E} 2$ radiation and that both $\gamma$-rays involve less than $15 \%$ admixture of higher multipoles. From Appendix $\mathrm{V}$ we find the coefficient $A_{2}$ for the cascade ${ }^{7} / 2(1)^{5} / 2(2){ }^{1} / 2: A_{2}=-0.0714$; the anisotropy then becomes $A=-0.1034$. The first experimental determination of the anisotropy ${ }^{61}$ yielded $A_{\text {exp }}=-0.07 \pm 0.04$ and thus confirmed this decay scheme.

61 F. Boehm and M. Walter, Helv. Phys. Acta 22 (1949) 378.

**** Following the usual notation, we denote a magnetic (electric) $L$ pole with ML (EL). M should not be confused with the magnetic quantum number. 
However, the investigation ${ }^{8}$ of the influence of extranuclear fields on the directional correlation of $\mathrm{Cd}^{111}$ revealed the surprising result that the magnitude of the anisotropy could be made considerably larger than the theoretical value 0.103 by embedding the radioactive atoms in a suitable environment. In some sources, anisotropies as large as $A=-0.18 \pm 0.01$ were found. This value was in marked disagreement with the theoretical predictions and necessitated a reinvestigation of the $\mathrm{Cd}^{111}$ decay ${ }^{62}$.

We repeat here briefly the discussion ${ }^{62}$ of the $\mathrm{Cd}^{111}$ decay, but use the latest value ${ }^{56} A=-0.245 \pm 0.015$ for the "unperturbed correlation". The spin and parity assignments are unambiguous and one must thus assume that at least one of the $\gamma$-rays is mixed. An admixture of M3 to E2 in $\gamma_{2}$ is very unlikely (see Ch. XIII and XVI). Much more probable is the mixing of $\mathbf{E} 2$ and $\mathbf{M 1}$ in $\gamma_{1}$. We thus assume that $\gamma_{1}$ consists of a mixture of E2 and M1, with an intensity ratio $\delta^{2}=\operatorname{Int}(\mathrm{E} 2) / \mathrm{Int}$ (M1). Using Eq. (41)-(44) and the Tables in Appendix V, we find for the coefficients $A_{2}$ and $A_{4}$ as function of the mixing ratio $\delta$ :

$$
A_{2}=-\left(0.0714+0.7424 \delta+0.1734 \delta^{2}\right) /\left(1+\delta^{2}\right) ; A_{4}=-0.0726 \delta^{2} /\left(1+\delta^{2}\right) \text {. }
$$

Using these expressions for $A_{2}$ and $A_{4}$, we calculate the anisotropy $A$ (Eq. (39)) as a function of $\delta$. In Fig. 8b, the anisotropy $A$ is plotted vs. $|\delta|$ for each of the two signs of $\delta$. Fig. 8a shows the conversion coefficient $\alpha_{K}$ as a function of $|\delta|$. (The conversion coefficient for a mixed transition is the weighted sum of the individual conversion coefficients, i.e. no interference terms appear ${ }^{63}$.) The experimental values of $\alpha_{K}{ }^{64}$ and of $A$ are also shown in Fig. 8.

Fig. 8 shows immediately that of the two mixing ratios allowed by the measured value of the anisotropy, II can be excluded because of the measured conversion coefficient. (Moreover, inserting the value of $\delta$ corresponding to II into the expression for $A_{4}$ yields $A_{4}=-0.07$. The experimental correlation function completely excludes so large a value of $\left.A_{4}\right)$. We thus conclude that the $(7 / 2) \rightarrow(5 / 2), \gamma$-transition in $\mathrm{Cd}^{111}$ must be a mixture of $\mathrm{E} 2$ and $\mathrm{M} 1$, with a mixing ratio $\delta=+0.145 \pm 0.015$. This corresponds to an intensity ratio E2/M1 of $\delta^{2}=0.021$; i.e. the first transition is composed of $2 \% \mathrm{E} 2$ and $.98 \% \mathrm{M} 1$, with a positive sign of $\delta$.

H. The examples discussed in the last three sections show three effects which may cause difficulties in the interpretation of measured correlation data:

62 H. Aeppli, H. Frauenfelder and M. Walter, Helv. Phys. Acta 24 (1951) 335.

63 H. B. G. Casimir, On the Interaction Between Atomic Nuclei and Electrons.

(De Erven F. Bohn N.V. Haarlem 1936.)

64 C. L. McGinnis, Phys. Rev. 81 (1951) 734. 
i) Multipole mixtures, even with a small mixing ratio, can alter the correlation function to a great extent and can even change the signs of the coefficients $A_{v}$.

ii) Extranuclear fields may appreciably attenuate the correlation. However, the form of the attenuation is well known (Part B) and con-
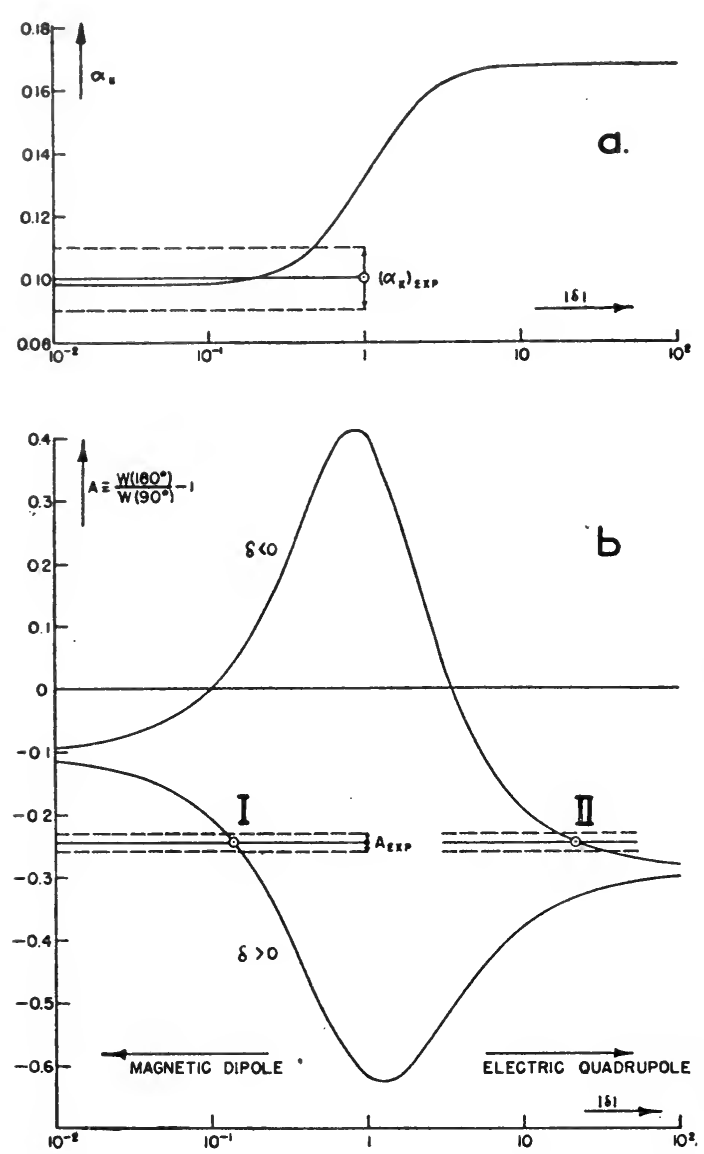
clusions as to the multipole order of the $\gamma$-rays and the nuclear spins often are still possible.

iii) Competing cascades can mask the desired correlation function. These three effects should always be considered carefully in the choice of the apparatus, the preparation of the sources, and the evaluation and discussion of the experimental results.

Fig. 8. Decay of $\mathrm{Cd}^{111}$. The first $\gamma$-ray of the cascade $\gamma_{1}-\gamma_{2}$ (see Fig. 7) is assumed to be a mixture of E2 and M1, with an intensity ratio $\delta^{2}=\operatorname{Int}(\mathrm{E} 2) / \operatorname{Int}(\mathrm{M} 1)$. This figure shows as a function of $|\delta|$ a) the $K$-conversion coefficient $\alpha_{K}$ of $\gamma_{1}$, and b) the anisotropy $A$ of the cascade $\gamma_{1}-\gamma_{2}$. Each one of the two curves in b) corresponds to a definite sign of $\delta$. The mixing ratio which is compatible with the measured conversion coefficient and the measured anisotropy is designated by $I$.

Finally, we add some references which can be consulted for further insight into $\gamma-\gamma$ directional correlation: papers concerning the directional correlation of pure and mixed cascades ${ }^{65-69}$, directional correlation of

65 J. J. Kraushaar and M. Goldhaber, Phys. Rev. 89 (1953) 1081.

66 D. Schiff and F. R. Metzger, Phys. Rev. 90 (1953) 849.

67 F. R. Metzger and W. B. Todd, Phys. Rev. 92 (1953) 904.

68 F. K. McGowan, Phys. Rev. 93 (1954) 471.

69 R. M. Steffen, to be published in Phys. Rev. 


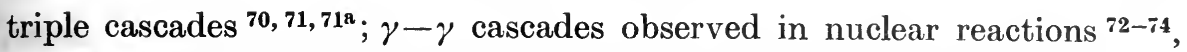
angular distribution of nuclear resonance fluorescence radiation ${ }^{74 \mathrm{a}}$.

\section{$\S$ 5. Polarization Correlation}

A. In the last $\S$ we saw that $\gamma-\gamma$ directional correlation measurements allow the determination of the angular momentum of each $\gamma$-ray, but not of the parity. The latter property, and consequently the nuclear parity change in the transition, can be found if one determines in addition to the directional correlation also the direction of the electric vector of the $\gamma$-ray, i.e. its polarization. Pertinent calculations were suggested by Madansky and performed by Falkoff ${ }^{75}$, Hamilton ${ }^{76}$, Zinnes ${ }^{77}$, Lloyd ${ }^{33}$, and Biedenharn and Rose ${ }^{20}$. The first polarization correlation measurement was done by Deutsch and Metzger 6, ?.

B. We consider first the case where one measures the direction of one $\gamma$-ray and the direction and polarization of the other. We denote with $X$ the angle between the polarization vector (electric vector) and the normal to the plane of the two $\gamma$-rays. $W_{1}(\theta, X)$ designates the polarization correlation as a function of the angles $\theta$ and $X$; the subscript 1 indicates that the polarization of $\gamma_{1}$ only is determined.

We assume that the coefficients $A_{v}$ for the directional correlation $W(\theta)$ have already been obtained. The theory then shows that the parity of $\gamma_{1}$ can be found from the two values $W_{1}\left(\theta=90^{\circ}, X=0^{\circ}\right)$ and $W_{1}\left(\theta=90^{\circ}\right.$, $\left.X=90^{\circ}\right)$. Actually it is sufficient to know, besides the $A_{v}$, the sign of $\left[W_{1}\left(90^{\circ}, 0^{\circ}\right)-W_{1}\left(90^{\circ}, 90^{\circ}\right)\right]$. In practice, both $\gamma$-rays enter the polarizationsensitive detector and are then counted with different sensitivities. The observed function $W(\theta, X)$ is the weighted sum of the two functions $W_{1}(\theta, X)$ and $W_{2}(\theta, X)$. Biedenharn and Rose ${ }^{20}$ give the formulas (with $\theta \equiv \beta, X \equiv \varphi$ ) which are necessary to determine the character of the $\gamma$-transitions from $W\left(90^{\circ}, 0^{\circ}\right), W\left(90^{\circ}, 90^{\circ}\right)$, the coefficients $A_{v}$, and the detector sensitivities.

70 C. E. Whittle and P. S. Jastram, Phys. Rev. 92 (1953) 205.

71 J. J. Kraushaar, Phys. Rev. 92 (1953) 318.

71a J. W. Mihelich, G. Scharff-Goldhaber and M. McKeown, to be published in Phys. Rev.

72 T. P. Hubbard, E. B. Nelson and J. A. Jacobs, Phys. Rev. 87 (1952) 378.

73 G. A. Jones and D. H. Wilkinson, Phys. Rev. 88 (1952) 423; and to be published.

74 S. M. Shafroth and S. S. Hanna, Phys. Rev. 95 (1954) 86.

${ }^{74 a}$ F. R. Metzger, to be published.

75 D. L. Falkoff, Phys. Rev. 73 (1948) 518.

76 D. R. Hanilton, Phys. Rev. 74 (1948) 782.

77 I. Zinnes, Phys. Rev. 80 (1950) 386. 
C. The determination of $W(\theta, X)$ requires a "polarimeter", i.e. an instrument sensitive to the polarization of $\gamma$-rays. A polarimeter for low energy $\gamma$-rays can be based on the photoeffect ${ }^{78}$ or the Compton effect ${ }^{79,}{ }^{80}$; for high energy $\gamma$-rays, one can use pair-production ${ }^{81}$ or the photodisintegration of the deuteron ${ }^{82,83}$.

Most polarimeters for angular correlation work are constructed after the prototype first used by Metzger and Deutsch ${ }^{7}$, which operates as follows (see Fig. 9a): some of the $\gamma$-rays which enter the scatterer B are Compton-scattered; a certain fraction of these scattered $\gamma$-rays hit the

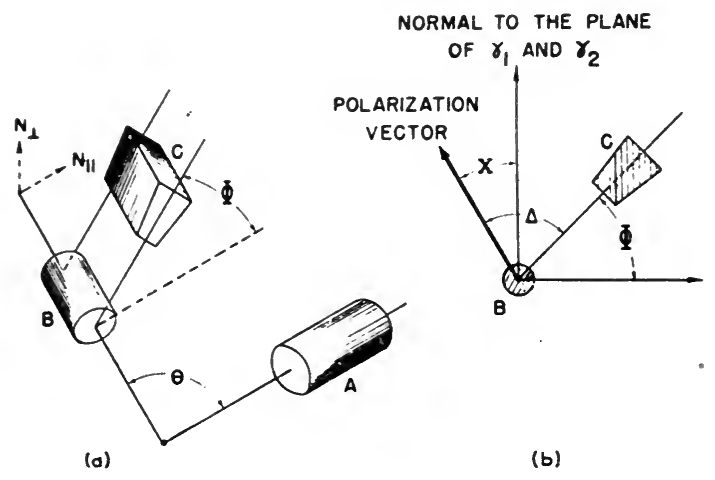

Fig. 9. a) Schematic diagram of a polarization-direction correlation measurement (Metzger and Deutsch). A, B, and C are scintillation counters.

b) Schematic cross section through the polarimeter $\mathrm{B}-\mathrm{C}$.

detector $\mathrm{C}$ and are there counted. The cross section for Compton scattering depends on the angle $\Delta$ between the polarization vector and the direction of the scattered $\gamma$-ray (Fig. 9b) ${ }^{26}$. The number of counts in detector $\mathrm{C}$ as a function of the angle $\Phi$ thus yields information about the polarization. In practice, one uses as scatterer $\mathrm{B}$ a scintillation crystal, records coincidences between $\mathrm{B}$ and $\mathrm{C}$, and thus reduces the background. The determination of the polarization correlation now consists in measuring triple coincidences $N(\theta, \phi)$ among the three counters $\mathrm{A}, \mathrm{B}$, and $\mathrm{C}$, as a function of the angles $\theta$ and $\phi$. Usually, one determines only the two values $N\left(90^{\circ}, 0^{\circ}\right) \doteq N_{\|}$and $N\left(90^{\circ}, 90^{\circ}\right) \equiv N_{\perp}$.

A qualitative relation between the experimental data $N_{\|}$and $N_{\perp}$ and the theoretical function $W(\theta, X)$ can be established by remarking that Compton scattering occurs predominantly perpendicular to the plane containing the incident $\gamma$-ray and the vector of polarization. The sign of $\left[W\left(90^{\circ}, 0^{\circ}\right)-W\left(90^{\circ}, 90^{\circ}\right)\right]$ is thus the sign of $\left[N_{11}-N_{\perp}\right]$. For a detailed

78 F. L. Hereford and J. P. Keuper, Phys. Rev. 90 (1953) 1043.

79 A. Wightman, Phys. Rev. 74 (1948) 1813.

80 N. R. Steenberg, Canadian J. Phys. 31 (1953) 204.

81 M. M. May, Phys. Rev. 84 (1951) 265.

82 D. H. Wilkinson, Phil. Mag. 43 (1952) 659.

83 L. W. Fagg and S. S. Hanna, Phys. Rev. 92 (1953) 372. 
comparison between theory and experiment, the sensitivity of the polarimeter as a function of the angle $\Delta$ must be determined. We refer to references ${ }^{7,65}$ for a detailed discussion of all the related problems.

D. As an example, we show in Fig. 10 the polarization-direction correlation of $\mathrm{Ti}^{46}$, as determined by Metzger and Deutsch ${ }^{7}$. The decay $\mathrm{Sc}^{46} \rightarrow \mathrm{Ti}^{46}$ is very similar to the decay $\mathrm{Co}^{60} \rightarrow \mathrm{Ni}^{60}$ (cf. Fig. 4 and reference ${ }^{52}$ ) and the directional correlation data ${ }^{6}$ indicate that the two $\gamma$-rays are quadrupole radiation. The theoretical curves for the four possibilities E2-E2, M2-M2, E2-M2, M2-E2 are shown in Fig. 10 together with the experimental points. (The measurement was performed for various angles $\theta$ in order to show that the experimental result really agrees with the theory. For the parity determination, only the values for $\theta=90^{\circ}$ and $\theta=180^{\circ}$ would have been necessary.) Fig. 10 shows that both $\gamma$-rays are E2. The three nuclear levels of $\mathrm{Ti}^{46}$ therefore have the same parity.

Fig. 10. Polarization-direction correlation for $\mathrm{Ti}^{46}$, as measured by Metzger and Deutsch ?. The three curves correspond to the different parity assingments $(\mathrm{E} 2 \equiv \mathrm{EQ}$,

$$
\mathrm{M} 2 \equiv \mathrm{MQ}) \text {. }
$$

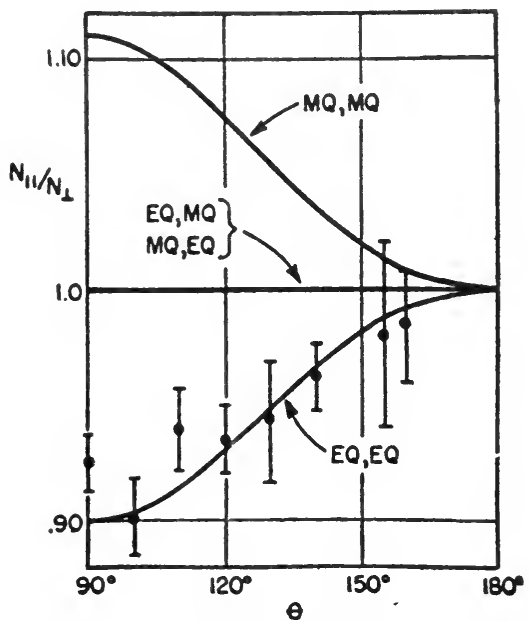

E. The method of determining the relative parities of nuclear levels by measuring the $\gamma$-ray polarization has not been restricted to $\gamma-\gamma$ cascades, but has been applied also to $\beta-\gamma$ correlation ${ }^{84},{ }^{85}, \alpha-\gamma$ correlation ${ }^{86}$, and nuclear reactions ${ }^{82,83,87}$. It is especially useful in the case of high energy $\gamma$-rays, where the accurate measurement of the conversion coefficient is difficult. In one case, the polarization of both $\gamma$-rays in a $\gamma-\gamma$ cascade was measured 88 .

\section{$\S$ 6. Directional Correlation Involving Conversion Electrons}

A. Instead of measuring the directional correlation between successive $\gamma$-rays, one can detect the corresponding conversion electrons and measure

84 R. Stump, Phys. Rev. 86 (1952) 249.

85 D. R. Hamilton, A. Lemonick, and F. M. Pipkin, Phys. Rev. 92 (1953) 1191.

86. J. S. Fraser and J. C. D. Milton, Phys, Rev. 94 (1954) 795.

87 A. P. French and J. O. Newton, Phys. Rev. 85 (1952) 1041.

88 B. L. Robinson and L. Madansky, Phys. Rev. 88 (1952) 1065. 
their correlation. This becomes feasible if at least one of the $\gamma$-rays is moderately converted (conversion coefficient $\alpha \sim 1$ ). The simultaneous determination of $\gamma-\gamma$ correlation and conversion correlation then allows a check on the spin and multipole assignment (e.g. $\left.\mathrm{Cd}^{111}, \mathrm{~Pb}^{207}\right)$. In the case where at least one of the $\gamma$-rays is very strongly converted $(\alpha \gg 1)$, it may even be impossible to measure the $\gamma-\gamma$ correlation and only the conversion correlation yields the desired information (e.g. $\mathrm{Te}^{121}, \mathrm{Te}^{123}$ ).

In contradistinction to $\gamma-\gamma$ correlation, the conversion correlation depends not only on the spins of the nuclear levels and the multipole orders of the transitions, but also on the parity changes and the energies of the converted transitions and on the nuclear charge. The conversion correlation thus may yield more information than the $\gamma-\gamma$ directional correlation.

B. The relativistic theory of the directional correlation involving conversion electrons from the $K$-shell is due to Rose, Biedenharn, and Arfken ${ }^{20,89}$. Suppose the $\gamma-\gamma$ directional correlation of a given cascade to be given by Eq. (36). Then for the cascade in which one of the $\gamma$-rays is replaced by a conversion electron, the correlation function is obtained by multiplying each coefficient $A_{v}$ by a particle parameter $b_{v}\left(e^{-}\right)$:

$$
W_{\gamma-e^{-}}(\theta)=1+b_{2}\left(e^{-}\right) A_{2} P_{2}(\cos \theta)+b_{4}\left(e^{-}\right) A_{4} P_{4}(\cos \theta)+\ldots
$$

If both $\gamma$-rays are replaced by conversion electrons, each coefficient $A_{v}$ is multiplied by two particle parameters: $A_{v} \rightarrow b_{v}\left(e_{1}^{-}\right) b_{\nu}\left(e_{2}^{-}\right) A_{v}$. Numerical values for the particle parameters $b_{\nu}\left(e^{-}\right)$for conversion in the $K$-shell have been calculated by Rose, Biedenharn, and Arfken. Curves for the coefficients $b_{2}\left(e^{-}\right)$are given in reference ${ }^{89}$, numerical values are tabulated in reference ${ }^{20}$. The higher coefficients $b_{4}, b_{6} \ldots$ can readily be obtained from $b_{2}$ by a recurrence formula ${ }^{20,89}$. The coefficients $b_{2}\left(e^{-}\right)$are, except for dipole radiation, always positive and larger than one.

In the high-energy limit, the particle parameters $b_{v}$ approach the value unity, hence conversion electrons for pure multipoles in this limit give the same correlation function as do the corresponding $\gamma$-rays.

So far, we have considered only cascades with pure multipoles. Eq. (45) is also valid if the unconverted $\gamma$-ray is mixed, provided the $A_{\nu}$ have been calculated for the $\gamma-\gamma$ cascade with the mixed multipole. The case where the converted transition is mixed is somewhat more complicated. Biedenharn and Rose tabulate particle parameters for this case also ${ }^{\mathbf{2 0}}$. (Note that conventionally the mixing ratio refers to the intensity of the $\gamma$-rays, and not to the intensity of the total transitions.)

89 II. E. Rose, L. C. Biedenharn, and G. B. Arfken, Phys. Rev. 85 (1952) 5. 
C. In the first conversion correlation experiments, Geiger counters acted as detectors for the electrons. A crude energy selection was performed by using absorbers (e.g. $\left.{ }^{90}\right)$. However, a comparison with theory is possible only if the $K$-electrons are singled out unambiguously. To achieve this goal, one can use a beta spectrometer ${ }^{91-91}$, or a scintillation spectrometer ${ }^{95}$, or can observe the conversion electrons in a cloud chamber ${ }^{96}$.

The main difficulty in conversion correlation experiments consists in avoiding excessive scattering of the electrons in the source and in the air between source and electron detector. The scattering in the air can be avoided either by placing source and detector in a vacuum ${ }^{90-94}$ or by displacing the air between source and counter by $\mathrm{H}_{2}$ gas ${ }^{95}$. The scattering in the source can often be reduced to acceptable values only with considerable experimental difficulties. No complete theory for the scattering of electrons in the source and the source backing is available and the allowable thicknesses for the source and the source backing therefore are somewhat uncertain. For estimates, one can use Frankel's treatment ${ }^{97}$ or one can obtain the most probable deflection angle of the electrons after passing through a given source thickness from a nomogram presented by Walter ${ }^{98}$. A discussion of the preparation of thin sources can be found in Ch. VIII and in the literature (e.g. ${ }^{93,}{ }^{94}$ ).

The requirement of having very thin sources can cause an additional difficulty. If the lifetime of the intermediate nuclear state is long enough to allow perturbations, one is forced to embed the radioactive atoms in a suitable environment in order to obtain the true correlation (cf. Part B). This is very difficult if the source must be extremely thin. In the case of $\mathrm{In}^{111} \rightarrow \mathrm{Cd}^{111}$, the Zürich group has overcome the obstacle by an ingenious method ${ }^{93}$ which may work also in other cases: they evaporate the carrierfree radio-isotope $\mathrm{In}^{111}$ together with a small amount of gallium onto a thin backing (e.g. mica). The gallium remains liquid even at room temperature and the $\operatorname{In}^{111}$ atoms thus are contained in a very thin liquid film $\left(50-300 \mu \mathrm{g} / \mathrm{cm}^{2}\right)$ which does not evaporate in a vacuum.

D. The results so far obtained with conversion electrons are very

90 H. Frauenfelder, M. Walter and W. Zünti, Phys. Rev. 77 (1950) 557.

91 D. T. Stevenson and M. Deutsch, Phys. Rev. 83 (1951) 1202.

92 K. Siegbahn, Arkiv för Fysik, 4 (1952) nr. 10.

93 F. Gimmi, E. Heer and P. Scherrer, Z. Physik 138 (1954) 394.

94 N. Goldberg and S. Frankel, Phys. Rev. 93 (1954) 1425, and to be published in Phys. Rev.

95 F. K. McGowan, Phys. Rev. 92 (1953) 524.

96 R. R. Roy and M. L. Goes, Comptes rendus 238 (1954) 581.

97 S. Frankel, Phys. Rev. 83 (1951) 673.

98 M. Walter, O. Huber and W. Zünti, Helv. Phys. Acta 23 (1950) 697. 
encouraging and the method may be of considerabe importance for further investigations (cf. ${ }^{99}$ ).

In two cases, $\mathrm{Pb}^{207}$ and $\mathrm{Cd}^{111}$, it was possible to measure both the $\gamma-\gamma$ and the $\gamma-\mathrm{e}^{-}$correlation. In $\mathrm{Pb}^{207}$, the two $\gamma$-rays have energies of 1.055 MeV and $0.555 \mathrm{MeV}$ and are, according to lifetime and shell theory considerations, to be associated with M4 and E2 transitions between the levels $i_{1 / \mathrm{s}} \rightarrow f_{\% / \mathrm{s}} \rightarrow p_{\mathrm{r} / \mathrm{s}}$. The $\gamma-\gamma$ directional correlation and internal conversion coefficient measurements confirm these assignments ${ }^{100}$. The directional correlation between the $K$-conversion electrons from the M4 $(1.055 \mathrm{MeV})$ transition and the $0.555 \mathrm{MeV} \gamma$-rays was determined by McGowan with a source of $300 \mu \mathrm{g} / \mathrm{cm}^{2}$ thickness deposited on a $100 \mu \mathrm{g} / \mathrm{cm}^{2}$ formvar film ${ }^{95}$. The experimentally obtained particle parameters $b_{2}=1.09 \pm 0.02$ and $b_{4}=1.20 \pm 0.42$ agree with the theoretical values $b_{2}=1.0487$ and $b_{4}=1.2760$ and thus confirm the proposed spin and parity assignments.

In $\mathrm{Cd}^{111}$ (cf. Fig. 7), Gimmi, Heer, and Scherrer measured all the combinations $\gamma-\gamma, \gamma-\mathrm{e}^{-}, \mathrm{e}^{-}-\gamma, \mathrm{e}^{-}-\mathrm{e}^{-}$between the 0.173 and the $0.247 \mathrm{MeV}$ transition and found excellent agreement with theory ${ }^{93}$.

In $\mathrm{Pb}^{207}$ and $\mathrm{Cd}^{111}$ the conversion correlation has yielded no new data concerning the decay. The investigation of $\mathrm{Te}^{121}$ and $\mathrm{Te}^{123}$ by Goldberg and Frankel, however, provided new information ${ }^{94}$. $\mathrm{Te}^{121}$ and $\mathrm{Te}^{123}$ both are isomers decaying through a cascade $h_{11 / 8} \rightarrow d_{3 / 3} \rightarrow s_{1 / 3}$. Goldberg and Frankel measured the combinations $e_{K}-\gamma, e_{L}-\gamma$ and $e_{K}-e_{K}$ with sources less than $1 \mu \mathrm{g} / \mathrm{cm}^{2}$ on a backing of $\leqslant 10 \mu \mathrm{g} / \mathrm{cm}^{2}$. They showed that the transitions $d_{\% / \mathrm{s}} \rightarrow s_{1 / \mathrm{s}}$ in both nuclides consist of a mixture of M1 and E2 radiation and determined mixing ratios and phases.

\section{§ 7. $\alpha-\gamma$ Directional Correlation}

A. The directional correlation between an $\alpha$-particle and a $\gamma$-ray depends, like the $\gamma-\gamma$ correlation, on the spins of the three nuclear states and on the angular momenta of the two radiations.

Before going into details, wè mention one essential difference between correlations involving $\alpha$-decay and those involving or following $\beta$-decay or an isomeric transition. The perturbation of a cascade following one of the latter processes usually results from a static crystalline field present at the regular lattice site. The recoil of the nucleus due to the first decay occurs with an energy of the order of $10 \mathrm{eV}$ and does not dislocate the atom from its regular position. Hence the correlation is unperturbed if the field gradient at the regular lattice site is small. In an $\alpha$-decay, on the 
other hand, the atom recoils with an energy of the order of $100 \mathrm{KeV}$, travels many atomic radii through the source material, and may encounter regions of considerable electric field gradients. More important, the atom will rarely come to rest in a regular lattice site and hence will be subjected to field gradients even in cubic crystals. Accordingly, most correlations following or involving $\alpha$-decay show signs of a perturbation.

B. The naive theory of $\alpha-\gamma$ directional correlation can easily be developed along the lines of $\S 2$. The fact that the $\alpha$-particle is spinless simplifies the considerations, because an $\alpha$-particle emitted along the $z$-axis cannot carry away angular momentum about that axis. The sum over $m_{a}$ in Eq. (7) and (8) thus reduces to one term. The angular distribution function $F_{L}^{M}(\theta)$ in Eq. (2) and (8) is proportional to the square of the spherical harmonic: $F_{L}^{M}(\theta) \sim\left|Y_{L M}(\theta, \varphi)\right|^{2}$. For simple cases, one can immediately write down $W(\theta)$ from Eq. (8).

For the explicit calculation of $W(\theta)$, one again introduces particle parameters $b_{v}$ (cf. $\S 6 \mathrm{~B}$ ). Suppose the $\alpha$-particles are emitted with only a single value of the angular momentum $L$. (This is always ensured if the spin $I_{a}$ of the $\alpha$-particle emitting state is 0 or $1 / 2$ ). Then the particle parameters $b_{v}(L ; \alpha)$, normalized to $b_{0}=1$, are given by ${ }^{20,101}$ :

$$
b_{v}(L ; \alpha)=\frac{2 L(L+1)}{2 L(L+1)-v(v+1)} .
$$

In case the $\alpha$-particle can carry away more than one value of the angular momentum $L$, the theory can be extended by using the formalism of Biedenharn and Rose ${ }^{20}$ (cf. also ${ }^{102}$ ). The parity selection rule permits mixtures among values $L,-L+2, L+4, \ldots$ If the angular momentum selection rule also allows such a mixture (i.e. if $I_{a} \geqslant 1, I_{b} \geqslant 1$ ), then the theory of $\alpha$-decay shows that the contribution of higher $L$-values can be appreciable ${ }^{103,104}$.

We restrict the discussion here to the case where the $\alpha$-particle can carry away two angular momenta $L_{1}$ and $L_{1}^{\prime}=L_{1}+2$, and denote the intensity ratio $\left(L_{1}^{\prime} / L_{1}\right)$ by $\delta^{2}$. As in the case of mixed $\gamma-\gamma$ cascades, $\delta$ must be real. First we calculate $W(\theta)=W_{\mathrm{I}}+\delta^{2} W_{\text {II }}+2 \delta W_{\text {III }}$ for a $\gamma-\gamma$ cascade $I_{a}\left(L_{1}, L_{1}+2\right) I_{b}\left(L_{2}\right) I_{c}$ by using the formalism described in $\S 4 \mathrm{D} *$. (Note that the coefficients $G_{v}$ for $W_{\text {III }}$ with $L_{1}^{\prime}=L_{1}+2$ are not tabulated in the Appendix.) Then we multiply the coefficients $A_{v}^{\mathrm{I}}$ of $W_{\mathrm{I}}$ with $b_{v}\left(L_{1} ; \alpha\right), A_{v}^{\mathrm{II}}$

101 S. P. Lloyd, Phys. Rev. 83 (1951) 716.

102 J. Seed and A. P. French, Phys. Rev. 88 (1952) 1007.

103 N. Feather, Nuclear Stability Rules (Cambridge University Press, 1952) p. 53.

104 J. J. Devaney, Phys. Rev. 91 (1953) 587.

* Numerical values for the required coefficients $F_{v}\left(L_{1}, L_{1}+2, I_{a}, I_{b}\right)$ are given in reference ${ }^{432}$. 
of $W_{\text {II }}$ with $b_{v}\left(L_{1}^{\prime} ; \alpha\right)$ and $A_{v}^{\mathrm{UI}}$ of $W_{\text {III }}$ by $b_{v}\left(L_{1}, L_{1}^{\prime} ; \alpha\right)$. The particle parameters $b_{v}\left(L_{1} ; \alpha\right)$ and $b_{v}\left(L_{1}^{\prime} ; \alpha\right)$ are given by Eq. (46), $b_{v}\left(L_{1}, L_{1}^{\prime} ; \alpha\right)$ by

$$
b_{v}\left(L, L^{\prime} ; \alpha\right)=\frac{2\left[L(L+1) L^{\prime}\left(L^{\prime}+1\right)\right]^{\prime}}{L(L+1)+L^{\prime}\left(L^{\prime}+1\right)-v(\nu+1)} \cos \left(\sigma_{L^{\prime}}-\sigma_{L}\right) .
$$

Here $\sigma_{L}$ is the phase shift due to the Coulomb potential. If the $\alpha$-particle energie is well below the Coulomb barrier height, the phase difference becomes ${ }^{102,105}$

$$
\sigma_{L+2}-\sigma_{L}=\tan ^{-1}(\eta /(L+2))+\tan ^{-1}(\eta /(L+1)),
$$

with $\eta=2 Z e^{2} / \hbar v$. Here $Z$ is the charge of the daughter nucleus and $v$ is the velocity of the $\alpha$-particle $\left(\eta \approx 0.63 Z / E_{\alpha}^{\ddagger}(\mathrm{MeV})\right)$.

C. Various arrangements have been used to measure $\alpha-\gamma$ directional correlations. The $\gamma$-rays are usually detected with a scintillation counter, the $\alpha$-particles observed with a proportional counter ${ }^{106}$ or a scintillation counter ${ }^{107}$ (Fig. 11a). The selection of the desired $\alpha$-particle energy is performed either by analyzing the pulses from the counter or by introducing a magnetic analyzer between source and detector ${ }^{102}$.

Recently a French group has introduced a very efficient technique 108 (Fig. 11b) which uses the fact that the size of the pulses from an ionization chamber depends on the angle $\varphi$ between ionizing particle and electric axis of the chamber ${ }^{109}$. The coincidences of the pulses from the ionization chamber with the $\gamma$-ray pulses, recorded as a function of the size of the $\alpha$-pulses and properly corrected, yield the correlation function $W(\theta)$. This method has two advantages: the solid angle for the $\alpha$-particles, including angles $\theta$ to $\theta+\Delta \theta$ with the direction of the $\gamma$-rays, is much larger than in a conventional set-up, and many points of the correlation curve can be measured simultaneously by using a multichannel analyzer after the ionization chamber.

D. Some of the first extensive experiments ${ }^{110,111}$ on $\alpha-\gamma$ directional correlation were done with $\mathrm{Th}^{228}$. They yielded pronounced correlation curves, but no satisfactory agreement with the theory could be obtained. The first completely successful measurement was performed by DeBene-

105 I. Bloch, M. M. Hull, A. A. Broyles, W. G. Bouricus, B. E. Freeman and G. Breit, Rev. Modern Phys. 23 (1951) 147.

108 W. R. Arnold, Phys. Rev. 80 (1950) 34.

107 G. M. Temmer and J. M. Wykoff, Phys. Rev. 92 (1953) 913.

108 G. Valladas, J. Teillac, P. Falk-Vairant and P. Benoist, to be published in J. Physique.

109 H. H. Staub, Detection Methods, in E. Segré Experimental Nuclear Physics (J. Wiley \& Sons, New York 1953) p. 24.

110 J. K. Beling, B. T. Feld, and I. Halpern, Phys. Rev. 84 (1951) 155.

111 J. Battey, L. Madansky, and F. Rasetti, Phys. Rev. 89 (1953) 182. 
detti and Minton ${ }^{112}$ on the $\alpha$-decay of $\mathrm{Po}^{210}$ into the first excited level of $\mathrm{Pb}^{206}$. They found that their $\alpha-\gamma$ correlation curve agreed well with a pure $\sin ^{2} 2 \theta$-distribution. Such a distribution follows from the theory for the cascade $0 \stackrel{\alpha}{\rightarrow} 2 \stackrel{\gamma}{\rightarrow} 0$. The assignment $2^{+}$to the first excited state of $\mathrm{Pb}^{206}$ was later confirmed by more detailed investigations (cf. the discussion of $\mathrm{Bi}^{206}$ in Ch. XXII).

The discrepancy between the experiments and the theory in the case of Th ${ }^{228}$ was explained by Abragam and Pound ${ }^{113}$ as being due to the interaction of the nuclear quadrupole moment with the electric field gradients of the surroundings. The same effect was later found in a number of $\alpha-\gamma$

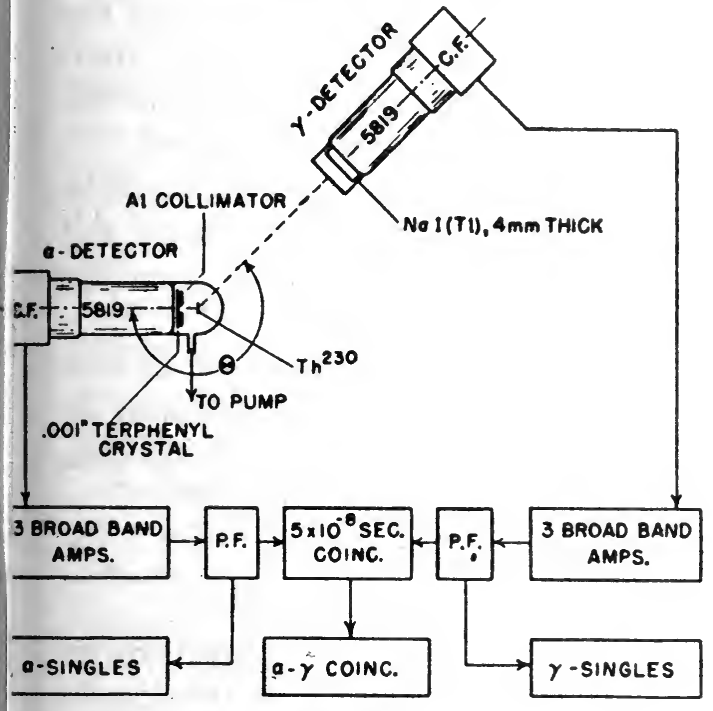

Fig. $11 a$

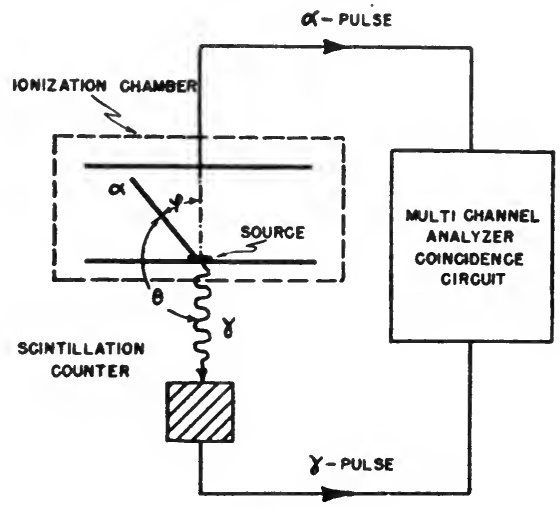

Fig. $11 b$

Fig. 11. Experimental methods for the measurement of $\alpha-\gamma$ directional correlation. a) Conventional set-up (Temmer and Wykoff ${ }^{107}$ ). b) An ionization chamber is used for the determination of the angle $\theta$ between $\alpha$-particle and $\gamma$-ray (Valladas, Teillac, Falk-Vairant and Benoist ${ }^{108}$ ).

correlations and its absence in the case of $\mathrm{Po}^{210}$ can be explained by the short lifetime of the excited state of $\mathrm{Pb}^{208}$.

The $\alpha$-decay of $\mathrm{Am}^{241}$ into the $60 \mathrm{keV}$ level of $\mathrm{Np}^{237}$ offers some evidence for a mixed $\alpha$-transition. This decay probably occurs through the cascade $(5 / 2 \stackrel{\alpha}{\rightarrow}(5 / 2) \stackrel{\nu}{\rightarrow}(5 / 2) 114,115$. The $\alpha-\gamma$ correlation is considerably perturbed by extranuclear fields. However, Fraser and Milton ${ }^{86}$ measured the correlation

112 S. DeBenedetti and G. H. Minton, Phys. Rev. 85 -(1952) 944.

113 A. Abragam and R. V. Pound, Phys. Rev. 89 (1953) 1306.

114 J. O. Rasmussen, Arkiv för Fysik 7 (1953) 185.

115 I. Perlman and F. Asaro, Phys. Rev. 93 (1954) 1423. 
as a function of the delay time between the $\alpha$-particle and the $\gamma$-ray. The extrapolation to zero delay then yields the unperturbed correlation, with an anisotropy $A=-0.15$. Novey confirmed this result by measuring the $\alpha-\gamma$ correlation with thin liquid sources ${ }^{116}$; his value of the anisotropy is $A=-0.16 \pm 0.02$. (Both methods of finding the true anisotropy depend on the assumption that the perturbation stems from the static field of the irregular position of the nucleus in the lattice. The time of flight of a recoiling nucleus is of the order of $10^{-11} \mathrm{sec}$. Hence the assumption is justified if the electric field gradients encountered during the flight are not extremely large and the recoil does not excite the atomic shell.) The anisotropy $A=-0.16$ can be explained only if one assumes that the $\alpha$-transition is mixed ${ }^{116,117}$; the mixture occurs probably between transitions with angular momenta $L=0$ and $L=2$. This assignment, however, is not unique and more work is necessary to clear up all aspects.

Besides the three decays discussed here, some other $\alpha-\gamma$ cascades have been investigated (e.g. ${ }^{107,108,118,119}$ ) and $\alpha-\gamma$ directional correlation and polarization correlation experiments will be of increasing importance, especially in the study of heavy nuclei.

Successful $\alpha-\gamma$ correiation experiments were also performed in connection with nuclear reactions. The directional correlation between an outgoing $\alpha$-particle and a subsequent $\gamma$-ray was measured in the reactions $\mathrm{F}^{19}(\mathrm{p}, \alpha \gamma) \mathrm{O}^{16}{ }^{102,}{ }^{106}, \mathrm{~N}^{15}(\mathrm{p}, \alpha \gamma) \mathrm{C}^{12}{ }^{120}$, and $\mathrm{Na}^{23}(\mathrm{p}, \alpha \gamma) \mathrm{Ne}^{20} \cdot{ }^{120^{a}}$

\section{$\S 8 . \quad \beta-\gamma$ Directional Correlation}

The correlation between a $\beta$-particle and a subsequent $\gamma$-ray involves problems much more complex than those which we have encountered in the previous $\S$. We discuss in A the reasons for this complexity; in B, we outline some of the theoretical results; in $\mathrm{C}$, we survey the experimental data.

A. In the first transition of a $\beta-\gamma$ cascade, a $\beta$-particle and a neutrino are emitted simultaneously. In a correlation experiment one measures only the direction of the electron, while the neutrino escapes unobserved. The theoretical calculation of the directional correlation thus necessitates an averaging over all neutrino directions and over the spins of neutrino and electron. Formally, this is done by treating the process as if an antineutrino enters the nucleus and an electron is emitted ${ }^{20}$.

\footnotetext{
118 T. B. Novey, Phys. Rev. 96 (1954) 547.

117 J. O. Rasmussen and B. Segall, to be published.

118 F. Stephens, F. Asaro and I. Perlman, Phys. Rev. 96 (1954) 1568.

119 J. C. D. Milton and J. S. Fraser, Phys. Rev. 95 (1954) 628.

120 J. Seed and A. P. French, Phil. Mag. 43 (1952) 1214.

$120 a$ J. Seed, Phil. Mag. 44 (1953) 921.
} 
In the theory of the directional correlation of conversion electrons and $\gamma$-rays, the interaction Hamiltonian and the coupling constant $(e)$ are well known, because the nuclear transitions are induced by the electromagnetic field. In $\beta$-decay, however, one deals with a point interaction between the light particles (electron and neutrino) and the nucleons, and the type and magnitude of the coupling are not completely known. The interaction must be some combination of the five relativistically invariant "pure" forms S, V, T, A, P (see Ch. IX, X and XI). Very probably more than one of these pure interactions is responsible for $\beta$-decay. As a result, the $\beta-\gamma$ correlation function contains interference terms.

As in the case of $\gamma$-decay, one can classify the $\beta$-transitions according to their order $L$, i.e. the angular momentum $L$ carried away by the electron-neutrino pair. In $\gamma$-decay, there exists for each order $L$ only one operator. In $\beta$-decay, however, several operators can exist for a given order $L$ even if one considers pure interactions only (cf. table IX of reference ${ }^{20}$ ). The matrix elements of these operators may interfere.

Interference can also occur between matrix elements belonging to different values of $L$, usually $L$ and $L+1$. This type of interference is similar to that occurring in $\gamma$-transitions. The two other modes of interference have no analogue in $\gamma$-decay.

Summing up, we say that three modes of interference are possible in a $\beta-\gamma$ correlation. The first two-interference between different pure interactions and between different matrix elements belonging to the same pure interaction-can occur between transitions of the same multipole order $L$ and can be called 121 "quasi-interferences". These quasi-interferences appear not only in the directional correlation, but also in the $\beta$-transition probability and in the energy distribution of the $\beta$-spectrum. The third mode-"true" interference between different multipole orders $L$-shows up only in the angular distribution and leaves the total radiated energy unaltered.

It is apparent from this discussion that the difficulties in the interpretation of a measured $\beta-\gamma$ correlation function are caused by the possibility of three types of interference and by the fact that one is still uncertain as to which interactions participate in $\beta$-decay.

B. The theory of $\beta-\gamma$ correlation was first developed by Falkoff and Uhlenbeck ${ }^{122}$. Their work is based on three important restrictions: a zero nuclear charge $Z$, pure interactions only, and only one nuclear matrix

121 M. Fuchs, Ph. D. dissertation (University of Michigan, 1951). 
element responsible for the $\beta$-transition. Later calculations by Fuchs ${ }^{121}$, by Morita, Yamada, and Fujita ${ }^{123-127}$ and by Biedenharn and Rose ${ }^{20}$ overcame these restrictions. However, the results are still not in a form in which they can be applied as easily as those discussed in earlier sections. Each decay needs a separate treatment in which the correlation data are worked out in connection with other information gained from the $\beta$ - and $\gamma$-decay. We therefore mention here only the most important features of $\beta-\gamma$ correlation.

We have already stated that the $\beta-\gamma$ correlation depends on the $\beta$-interaction Hamiltonian and on the particular matrix elements responsible for the $\beta$-transition. Of course, it also depends on the spins of the three nuclear states, the parity change in the $\beta$-transition, and the multipole order $L_{\gamma}$ of the $\gamma$-ray. Moreover, it is a function of the energy of the $\beta$-particle. The $\beta-\gamma$ correlation for $\beta$ particles in the energy range $E$ to $E+d E$ is called the differential correlation $W(\theta, E)$. The integral correlation $W(\theta)$ corresponds to the observation of $\beta$-particles of all energies. $W(\theta, E)$ has in general its maximum anisotropy for the maximum $\beta$-energy, and always becomes isotropic for $E \rightarrow 0$.

The correlation function $W(\theta, E)$ can be represented by the expansion (35). The highest term $v_{\max }$ is limited by the order of the spherical harmonic of the electron wave function and by $I_{b}$ and $L_{\gamma}$. All allowed and some additional matrix elements cause isotropic distributions. If the shape of the energy spectrum is strictly identical with that of an allowed transition, $W(\theta, E)$ is also isotropic (see ${ }^{121,}{ }^{20}$ ).

For matrix elements giving rise to anisotropic correlations, there is a close connection between the behavior of the correlation and that of the correction factor $C(Z, E)$ by which an allowed spectrum has to be multiplied to give the desired forbidden distribution ${ }^{121}$. This connection, however, can be masked if true interference occurs between two or more matrix elements.

C. The experimental technique for determining a $\beta-\gamma$ directional correlation is very similar to that for $\mathrm{e}^{-}-\gamma$ correlation and we refer to $\S 6 \mathrm{~B}$ for a discussion and for references. In addition to the problems mentioned there, one more appears in $\beta-\gamma$ correlation experiments. The inner bremsstrahlung emitted in $\beta$-decay displays a pronounced directional

M. Yamada and M. Morita, Progr. Theor. Phys. 8 (1952) 431.

M. Morita and M. Yamada, Progr. Theor. Phys. 8 (1952) 449, 10 (1953) 111.

M. Morita, Progr. Theor. Phys. 10 (1953) 363.

M. Morita and M. Yamada, Progr. Theor. Phys. 10 (1953) 641.

J. Fujita, M. Morita and M. Yamada, Progr. Theor. Phys. 11 (1954) 219. 
correlation with respect to the $\beta$-particles ${ }^{128,}{ }^{129}$. This effect may interfere strongly with the $\beta-\gamma$ directional correlation ${ }^{130}$.

Most of the $\beta-\gamma$ cascades which have been investigated so far show an isotropic distribution. A list of these decays and of relevant references is contained in reference ${ }^{131}$, page 156 . In almost all cases, the isotropy is explained because the $\beta$-transition is allowed or the $\beta$-spectrum has an allowed shape.

Anisotropic directional correlations have been found in the following nuclides: $\mathrm{K}^{42}{ }^{132}, \mathrm{As}^{76}{ }^{133}, \mathrm{Rb}^{86}{ }^{91}, \mathrm{Sb}^{122}{ }^{134}, \mathrm{Sb}^{124}$ 91, 135, $\mathrm{I}^{126}{ }^{132}, \mathrm{Tm}^{170}{ }_{136},{ }_{137}$. In all these cases the differential correlation has been measured.

A very detailed discussion has so far been presented only for the $\beta-\gamma$ correlation of $\mathrm{Sb}^{124}$ and $\mathrm{Tm}^{170}$. Morita and Yamada ${ }^{124,}{ }^{126}$ find that the decay $\mathrm{Sb}^{124} \rightarrow \mathrm{Te}^{124}$ occurs between states $3^{-} \stackrel{\beta}{\rightarrow} 2^{+} \stackrel{\nu}{\rightarrow} 0^{+}$, and that the $\beta$-transition is first forbidden, requires the interactions $S$ and $T$, and contains the matrix elements $\mathscr{M}(\beta \boldsymbol{r}), \mathscr{M}(\beta \boldsymbol{\alpha}), \mathscr{M}(\beta \boldsymbol{\sigma} \times \boldsymbol{r})$, and $\mathscr{M}\left(B_{i j}^{\beta}\right)$. (See the chapters on $\beta$-decay for the meaning of these abbreviations.) They discuss the various ratios of these matrix elements which fit the experimental function $W(\theta, E)$ as well as the $\beta$-spectrum. The decay $\mathrm{Tm}^{170} \rightarrow \mathrm{Yb}^{170}$ is explained ${ }^{127}$ as occurring between states $1^{-} \stackrel{\beta}{\rightarrow} 2^{+} \stackrel{\gamma}{\rightarrow} 0^{+}$. Here again, a mixture of $S$ and $T$ interaction with matrix elements $\mathscr{M}(\beta \boldsymbol{r}), \mathscr{M}(\beta \boldsymbol{\alpha})$, and $\mathscr{M}(\beta \boldsymbol{\sigma} \times \boldsymbol{r})$ fits the $\beta-\gamma$ correlation and the $\beta$-spectrum. However, one puzzle is still left in this decay. Siegbahn found an isotropic directional correlation between the $\beta$-particles and the $L$-conversion electrons of the $\gamma$-transition ${ }^{92}$. A further investigation is needed to explain this unexpected isotropy.

The extension of $\beta-\gamma$ directional correlation measurements to other nuclides and to $\beta^{+}$-emitters may help to solve some of the problems in $\beta$-decay. Even more important is a higher accuracy in the determination of the differential correlation $W(\theta, E)$, because the form of $W(\theta, E)$ is rather sensitive to the ratios of the various competing matrix elements.

128

T. B. Novey, Phys. Rev. 89 (1953) 672.

P. Bolgiano, L. Madansky and F. Rasetti, Phys. Rev. 89 (1953) 679.

T. B. Novey, Phys. Rev. 84 (1951) 145.

H. Frauenfelder, Ann. Rev. Nucl. Science 2 (1953) 129.

D. T. Stevenson and M. Deutsch, Phys. Rev. 84 (1951) 1071.

H. Rose, Phil. Mag. 44 (1953) 739.

I. Shaknov, Phys. Rev. 82 (1951) 833.

E. K. Darby and W. Opechowski, Phys. Rev. 83 (1951) 676.

T. B. Novey, Phys. Rev. 78 (1950) 66.

H. Rose, Phil. Mag. 43 (1952) 1146. 


\section{PART B. THE INFLUENCE OF EXTRANUCLEAR FIELDS}

In the following $\S$, we emphasize the information which is of importance in nuclear physics and only occasionally mention the possibilities for the investigation of solids and liquids. We make no attempt to give a complete account of either theory or experiment, but present some of the main results of the theory in connection with pertinent experiments. For the derivation and the detailed discussion of the various formulas, we refer to the excellent papers by Goertzel ${ }^{2}$, Alder and co-workers ${ }^{17},{ }^{31}$, Abragam and Pound ${ }^{30,113,138}$, and Coester ${ }^{32}$. These papers constitute an adequate basis for the understanding and the quantitative evaluation of most of the experimentally observed facts. More details about the experimental results can be found in the series of papers by the Zürich group (e.g. ${ }^{9},{ }^{139-142}$ ) and in a review by Steffen ${ }^{56}$.

Many of the considerations of Part B are not restricted to cascade decays, but apply also to radiations from aligned nuclei ${ }^{143}$ and to nuclear reactions. Among nuclear reactions, Coulomb excitation seems especially promising for work along the lines discussed here; evidence for a perturbation by extranuclear fields has been found in two investigations 144,145 . Excitation experiments using external magnetic fields and evaporated "single crystal" films (e.g. ${ }^{146,147}$ ) may provide information about the magnetic dipole and the electric quadrupole moments of excited states of nuclei, or about the undisturbed directional distribution of the de-excitation $\gamma$-rays.

\section{$\S 9$. Survey}

A. The considerations of Part A apply only if the decaying nuclei are free. The angular correlation of a cascade $\mathrm{A} \rightarrow \mathrm{B} \rightarrow \mathrm{C}$ will generally be attenuated as soon as the nuclei in their intermediate level $B$ are subject to torques, due to the interaction of either the magnetic dipole moment $\mu$ with an extranuclear magnetic field $B$, or of the electric quadrupole

\footnotetext{
138 R. V. Pound and A. Abragam, Phys. Rev. 90 (1953) 993.

139 E. Heer, Physica 18 (1952) 1215.

140 E. Heer, to be published in J. Physique.

141 H. Albers-Schönberg, E. Heer, T. B. Novey, and P. Scherrer, Helv. Phys. Acta 27 (1954) 547.

142 H. Albers-Schönberg, E. Heer, and P. Scherrer, to be published in Helv. Phys. Acta.

143 N. R. Steenberg, Phys. Rev. 95 (1954) 982.

144 R. F. Christy, Phys. Rev. 94 (1954) 1077.

145 W. I. Goldburg and R. M. Williamson, Phys. Rev. 95 (1954) 767.

146 J. H. van der Merve, Disc. Faraday Soc. 5 (1949) 201.

147 H. Raether, Erg. Ex. Naturwissenschaften 24 (1951) 114.
} 
moment * $Q$ with electric field gradients $\partial^{2} V / \partial z^{2}$. In the semiclassical picture, these interactions produce a precession of the nuclei around the symmetry axis. The changing nuclear orientation results in an altered angular correlation. In the quantum mechanical language of $\S 2$, where the quantization axis was chosen to coincide with the direction of the first radiation, the interactions cause transitions among the $m_{b}$-states. The second radiation is emitted from a level with an altered population distribution and this change is responsible for the attenuation of the correlation. In the language of $\S 3$, the transitions among the $m_{b}$-states are described by a time dependence in the density matrix $\mathscr{P}_{b}$.

The semiclassical picture of a precessing nucleus adequately describes the situation if the extranuclear fields are static. However, there also exist time-dependent fields which produce a change in the correlation function. In this case, realized for example in certain viscous liquids, the fluctuating electric field gradients at the site of the nucleus induce transitions among the $m_{b}$-states.

One major difference between static and time-dependent interactions can be described as follows. Consider first a static interaction in an axially symmetric single crystal. The populations of the $m_{b}$-states quantized along the symmetry axis remain constant, hence the correlation is undisturbed if the symmetry axis points in the direction of one counter (i.e. coincides with the direction of emission of one radiation). If it points in any other direction, then the populations of the $m_{b}$-states quantized along the direction of emission change in time and this change causes an attenuation of the angular correlation. In a polycrystalline source, this description applies for each microcrystal and ultimately for each nucleus. The angular correlation in a polycrystalline source is never wiped out completely because a certain fraction of nuclei experience the static field in such a direction that their correlation is unperturbed or only slightly attenuated. Static interactions in polycrystalline sources thus never reduce the correlation below the "hard core" value ( $\$ 11 \mathrm{~A})$. Time-dependent interactions, however, can wipe out the correlation completely. This is due to the fact that the direction of the field at each nucleus changes continuously in a random manner. No quantization axis exists for which the populations of the $m_{b}$-states remain constant. Eventually, all $m_{b}$-states are equally populated for any choice of the quantization axis and the directional correlation is isotropic.

* Actually, the electric quadrupole moment and the electric field gradient are tensors 21. 148. 149. For the sake of simplicity, we assume here axial symmetry of the field gradients and express the interaction in terms of the "scalar" quadrupole moment $Q$ and the magnitude $\partial^{2} V / \partial z^{2}$ of the field gradient along the field axis.

148 R. V. Pound, Phys. Rev. 79 (1950) 685.

149 H. G. Dehmelt, Am. •J. Physics 22 (1954) 110. 
B. When will a particular cascade be influenced by an extranuclear field? It is clear that the perturbation depends primarily on the magnitude of the interaction and on the length of time it can act, i.e. on the mean life $\tau_{b}$ of the intermediate level B.

For static perturbations, the magnitude can be described by a precession frequency $\omega$. For magnetic interactions, $\omega$ is equal to the Larmor frequency $\omega_{L}$, i.e. proportional to $\mu$ and $B$. In the quadrupole case, $\omega$ is proportional to $Q$ and $\partial^{2} V / \partial z^{2}$. Time-dependent perturbations can be characterized by an inverse time constant $\lambda$ : the $m_{b}$-states approach a uniform population exponentially and $\lambda$ is the corresponding relaxation constant ${ }^{30}$.

The angular correlation of a cascade is perturbed if at least one of the following crude criteria holds: $\omega \tau_{b} \gtrsim 1, \lambda \tau_{b} \geqslant 1$. A more refined criterion depends on the sensitivity of the experimental arrangement to alterations in the correlation function. A detailed discussion ${ }^{56}$ leads to the conclusion that with the present experimental techniques the limits are given by $\omega \tau_{b}>0.1, \lambda \tau_{b}>0.05$.

For a large number of solids and liquids, precession frequencies have been measured by microwave and nuclear resonance techniques (see reference ${ }^{150}$, Appendix, Table A 6). For quadrupole interaction, values for $\omega / 2 \pi$ as large as $3000 \mathrm{Mc} / \mathrm{sec}$ have been found. According to our criterion for perturbation $\left(\omega \tau_{b}>0.1\right)$, cascades with a lifetime $\tau_{b}>10^{-11}$ sec can thus be measurably perturbed in extreme cases. The magnetic interaction can be extremely strong in free atoms ${ }^{151}$. In solids, however, the orbital contribution to the magnetic moment of the electron shell is quenched (e.g. ${ }^{152}$ ) and the magnetic interaction can usually be neglected in comparison with the quadrupole interaction.

C. Here, we compare briefly some of the possibilities and difficulties of the angular correlation method with those of the microwave and nuclear resonance techniques.

The magnetic and electric interaction between the nucleus and its environment are well known from studies of the hyperfine structure of spectral lines, both by opticàl means ${ }^{151}$ and with microwaves ${ }^{150}$, and from nuclear resonance absorption work $148,149,153-155$. These conventional methods have beside the much higher accuracy and much broader range of applicability two main advantages over angular correlation: i) By

150 W. Gordy, W. V. Smith, and R. F. Trambarulo, Microwave Spectroscopy (J. Wiley \& Sons, New York, 1953).

151 H. Kopfermann, Kernmomente (Akad. Verlagsgesellschaft, Leipzig, 1940).

152 C. Kittel, Solid State Physics (J. Wiley \& Sons, New York, 1953).

153 G. E. Pake, Am. J. Physies 18 (1950) 438; 18 (1950) 473.

154 R. V. Pound, Progr. Nuclear Phys. 2 (1952) 21.

155 N. F. Ramsey, Nuclear Moments (J. Wiley \& Sons, New York, 1953). 
choosing a nucleus with spin $1 / 2$, one can eliminate the quadrupole interaction and study the magnetic effects alone. This separation of the interactions is impossible in angular correlation work, for in order to observe an anisotropic correlation, the spin of the intermediate nuclear level must be at least one (cf. Eq. (34)) and hence the nucleus can possess a quadrupole moment. ii) In the conventional methods, the environment of the nucleus under investigation is in its ground state. In angular correlation work, the nuclear decay leading to the cascade (or the first radiation of the cascade itself) very often excites the atomic shell. The nuclear environment is thus in an unknown state. This situation is especially pronounced in a cascade preceded by a $K$-capture or an $\alpha$-decay.

Fortunately, angular correlation offers some possibilities which compensate for these disadvantages. We have already mentioned the possibility of finding the $g$-factor and the quadrupole moment of short-lived excited nuclear levels. The internal fields in solids and liquids can be investigated even if they are so large that they wash out nuclear resonance lines. It is also possible to work with metal crystals without difficulties arising from the skin effect. Moreover, the changes in the atomic shell due to $K$-capture can be studied.

D. It is clear that the measured correlation function for a given decay depends on the physical and chemical state of the radioactive source and on the time delay between the two channels of the coincidence circuit. Two different approaches are thus possible:

i) One can use a given source state and change the delay between the two channels. By measuring $W(\theta)$ as a function of the delay, one gets information about the time dependence of the perturbation. (The finite resolving time of the coincidence circuit causes a smearing-out of the time dependence, so one must choose a resolving time as short as possible.) This approach ("delayed coincidences") was first proposed by Lundby 156 and the corresponding theory was constructed by Lloyd ${ }^{157}$. Fraser and Milton performed the first completely successful experiment ${ }^{86}$.

ii) The second, more widely-used method consists in keeping the time delay and the resolving time constant and varying the physical state of the source. This approach was first tried by Brady and Deutsch ${ }^{5}$ in the case of the $\mathrm{Ni}^{60} \gamma-\gamma$ cascade. However, the lifetime $\tau_{b}$ of $\mathrm{Ni}^{60}$ is so short that no influence is to be expected and none was found.

A positive effect was first found with the $\gamma-\gamma$ cascade of $\mathrm{Cd}^{111}$ by the Zürich group ${ }^{8,158}$. By embedding the radioactive nuclei in different

156 A. Lundby, Phys. Rev. 76 (1949) 993.

157 S. P. Lloyd, Phys. Rev. 82 (1951) 277.

158 H. Frauenfelder, Phys. Rev. 82 (1951) 549. 
media, anisotropies ranging from zero to -0.18 were found. Since this first unambiguous evidence for the influence of the nuclear environment on angular correlation, many experiments have been performed with the $\gamma-\gamma$ cascade of $\mathrm{Cd}^{111}$ and the investigations have been extended to other nuclides also. Most of the experiments are in satisfactory agreement with theory and the main aspects of the interaction of the nucleus with its environment are fairly well understood. However, there still remain some unsolved problems, mostly connected with the processes following $K$ capture and $\alpha$-decay. Also, since no reliable way of determining the magnitude of the electric field gradient has been found, the magnitudes of the quadrupole moments as measured by angular correlation are very uncertain.

\section{$\S 10$. The Determination of Nuclear $\mathbf{g}$-Factors}

A. Brady and Deutsch ${ }^{5}$ first pointed out that the $g$-factor of the intermediate nuclear level in a $\gamma-\gamma$ cascade can be determined by observing the effect of an external magnetic field on the directional correlation. Alder ${ }^{17,}{ }^{159}$ and Lloyd ${ }^{157}$ later developed the pertinent theory. The first measurement was performed by the Zürich group ${ }^{9,160}$, which determined magnitude and sign of the $g$-factor of the first excited state of $\mathrm{Cd}^{111}$. Since this first successful experiment, two more $g$-factors have been measured: $\mathrm{Pb}^{204}{ }^{161},{ }^{162}$ and $\mathrm{Ta}^{181}{ }^{163}$. The method has also been extended to $\alpha-\gamma$ cascades; Krohn, Novey and Raboy ${ }^{163 a}$ thus determined the $g$-factor of the first excited level in $\mathrm{Np}^{237}$.

The angular correlation method of determining $g$-factors of excited levels is at present limited to nuclear levels with a lifetime $\tau_{b}$ between $10^{-8} \mathrm{sec}$ and $10^{-5} \mathrm{sec}$. The lower limit, $10^{-8} \mathrm{sec}$, is set by available magnetic field strengths. The upper limit, $10^{-5} \mathrm{sec}$, is determined by the circumstance that an optimum measurement of the attentuation requires a resolving time of the coincidence circuit which is comparable to $\tau_{b}$. The number of accidental coincidences, however, is proportional to the resolving time and outweighs the true coincidences for $\tau_{b}>10^{-5} \mathrm{sec}$. Further improvements in experimental technique, i.e. the use of very strong, pulsed magnetic fields and simultaneously gated amplifiers, may bring the lower limit in the region of $10^{-9} \mathrm{sec}$, where a number of new cases can be investigated.

159. K. Alder, Phys. Rev. 84 (1951) 369.

${ }_{160}$ H. Aeppli, H. Albers-Schönberg, A. S. Bishop, H. Frauenfelder, and E. Heer, Phys. Rev. 84 (1951) 370.

161 V. Krohn and S. Raboy, Phys. Rev. 95 (1954) 608; and to be published in Phys. Rev.

162 H. Frauenfelder, J. S. Lawson, and W. Jentschke, Phys. Rev. 93 (1954) 1126.

183 S. Raboy and V. Krohn, Phys. Rev. 95 (1954) 1689.

163a V. Krohn, T. B. Novey and S. Raboy, to be published in Phys. Rev. 
B. We discuss here the physical picture which underlies the determination of the sign and magnitude of the $g$-factor of an intermediate state in a $\gamma-\gamma$ cascade. We assume that the cascade takes place in a nucleus which is not subject to extranuclear fields other than an external magnetic field which is applied perpendicular to the plane of the two counters. Each of the two counters shall be sensitive to only one $\gamma$-ray of the cascade. During the time $t$ which elapses between the emission of $\gamma_{1}$ and that of $\gamma_{2}$, the nucleus precesses in the field $B$ by an angle $\omega_{L} t$, where $\omega_{L}$ is the Larmor frequency $\left(\omega_{L}=\mu B / I_{b} \hbar=g \mu_{N} B / \hbar ; \mu_{N} \equiv\right.$ nuclear magneton). Instead of the correlation $W(\theta)$, we observe $W\left(\theta-\omega_{L} t\right)$. Suppose now that the coincidence circuit registers coincidences only if $\gamma_{2}$ is emitted within the time interval $\tau_{1}$ to $\tau_{2}$ after the emission of $\gamma_{1}$. The measured correlation function $W(\theta, B)$ then is given by

$$
W(\theta, B) \sim \int_{\tau_{1}}^{\tau_{2}} \mathrm{e}^{-t / \tau_{b}} W\left(\theta-\omega_{L} t, B=0\right) d t .
$$

For the sake of simplicity, we assume now that the highest term in the $\gamma-\gamma$ correlation function (36) is given by $\nu=2$. Instead of using (36), we choose an equivalent expansion which is more appropriate to the symmetry of the problem ${ }^{17}: W(\theta)=1+B_{2} \cos 2 \theta$. One can easily establish the relation between the coefficients $B_{2}$ and $A_{2}: B_{2}=3 A_{2} /\left(4+A_{2}\right)$. Moreover, we assume that the resolving time of the coincidence circuit is long compared to the mean life $\tau_{b}$, and that no delay is present. Thus we can set $\tau_{1}=0$ and $\tau_{2}=\infty$ and we get from Eq. (49) after some calculation:

$$
W(\theta, B)=\frac{B_{2}}{\sqrt{1+\left(2 \omega_{L} \tau_{b}\right)^{2}}} \cos 2(\theta-\Delta \theta) \text { where } \Delta \theta=\frac{1}{2} \tan ^{-1} 2 \omega_{L} \tau_{b} .
$$

This formula clearly shows the influence of the external magnetic field. The directional correlation pattern is attenuated and simultaneously rotated by the angle $\Delta \theta$. For small angles, $\Delta \theta$ is approximately equal to the classical angle of precession $\omega_{L} \tau_{b}$. The magnitude of the $g$-factor can be determined from the attenuation of the correlation or from the rotation of the correlation pattern. The sign of $g$ follows from the direction of rotation. (Provoked by our own experience, we add the remark that the correlation pattern rotates in the same direction as would a magnetic dipole in the applied field, if the fixed counter detects $\gamma_{1}$. It rotates in the opposite direction if $\gamma_{1}$ is observed in the movable counter).

Eq. (50) is based on four assumptions:

i) The highest term in the correlation function $W(\theta)$ is $\nu=2$. This restriction simplified the derivation of Eq. (50), but is easy to remove. All required formulas are given in references $17,20,31,159$.

ii) The resolving time $\tau_{R}$ is long compared with the lifetime $\tau_{b}$. This 
assumption is usually not justified. If a delay $\tau_{D}$ is inserted after counter $\mathbf{1}$, then coincidences in the time interval $\tau_{D}-\tau_{R}$ to $\tau_{D}+\tau_{R}$ are recorded and the correlation function must be calculated correspondingly. All necessary formulas are contained in a paper by Alder and co-workers ${ }^{31}$.

iii) Each counter is sensitive to one $\gamma$-ray only. In many cases, it is possible to realize this condition experimentally either by using pulse height selection or by inserting a delay $\tau_{D}>\tau_{R}$ after one counter. If, on the other hand, both counters are equally sensitive, then the correlation function is a superposition of two functions of the form (50), describing rotations in opposite directions. One no longer observes a rotation of the correlation pattern, but only an attenuation

$$
W(\theta, B)=\frac{B_{2}}{1+\left(2 \omega_{L} \tau_{b}\right)^{2}} \cos 2 \theta \text {. }
$$

Such an experiment allows the determination of only the magnitude of the $g$-factor.

iv) No perturbations by extranuclear fields are present. This condition is not easy to fulfill experimentally. Even in a carefully selected and prepared source, lattice imperfections (and other demons) may cause field gradients large enough to affect the determination of the $g$-factor. Abragam and Pound have treated the most important cases of the simultaneous influence of electric and magnetic interaction ${ }^{30}$. We refer to their paper for a detailed discussion and mention here only one interesting conclusion.

Suppose that the source is a polycrystalline solid or a liquid. The determination of the $g$-factor from a measurement of the attenuation then is impossible if the quadrupole coupling is large compared to the magnetic coupling. However, the calculation of $g$ from the rotation of the correlation pattern is still possible, provided the condition $\omega_{L} \tau_{R} \ll 1$ is fulfilled, cf. also ${ }^{164}$.

C. We present here briefly the determination of the $g$-factor of the first excited state of $\mathrm{Cd}^{111}$ by the Zürich group ${ }^{9,160}$ (cf. $\S 4 \mathrm{G}$, Fig. 7 and Fig. 8). The measurements with an external magnetic field were performed with the radioactive In $^{111}$ embedded in silver, because an earlier investigation $^{8}$ had shown that the anisotropy of the $\operatorname{Cd}^{111} \gamma-\gamma$ cascade assumes its largest value in this medium. Both counters were equally sensitive. Fig. 12 shows the coefficient $B_{2}(B)=B_{2} /\left[1+\left(2 \omega_{L} \tau_{b}\right)^{2}\right]$ as a function of the magnetic field strength $B$.

A least square fit to the experimental points with the theoretical correlation function $W(\theta, B)$ of Eq. (51), properly modified for the finite resolving time, yielded a value for $|g| \cdot \tau_{b}$. The magnitude of $g$ then followed immediately, because $\tau_{b}$ was known: $\tau_{b}=(1.23 \pm 0.06) \times 10^{-7}$ sec. The sign of $g$ was measured with counters of different efficiencies. The 
final result, $g=-(0.28 \pm 0.05)$, together with the known spin of the intermediate state, $I_{b}=5 / 2$, yielded the magnetic moment $\mu\left(\mathrm{Cd}^{111}, d_{b / 2}\right.$ level $)$ $=-(0.70 \pm 0.12) \mu_{N}$. (Improved experiments ${ }^{141,164,164 \mathrm{a}}$ give a value $g=-(0.76 \pm 0.03) \mu_{N}$. It thus seems certain that the magnetic moment of the first excited state of $\mathrm{Cd}^{111}$ is closer to the Schmidt limit than that of the ground state, $\mu\left(s_{1 / 2}\right)=-(0.59492 \pm 0.00008) \mu_{N}$.)

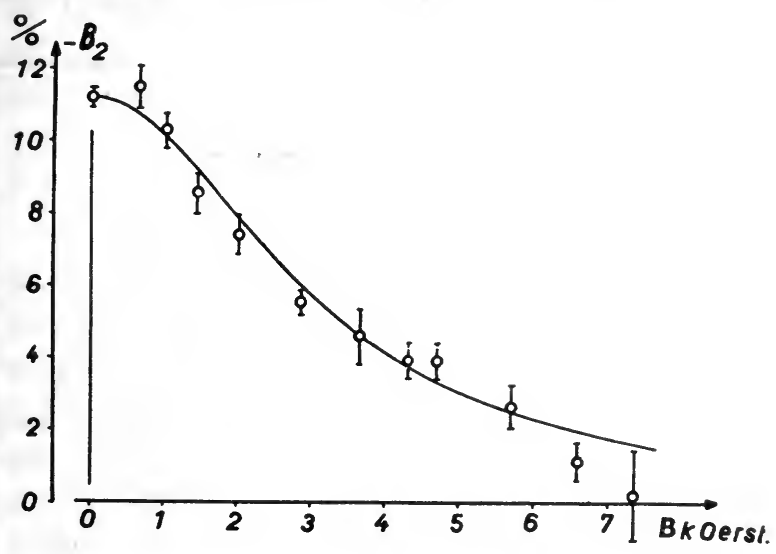

Fig. 12. The directional correlation of the $\mathrm{Cd}^{111} \gamma-\gamma$ cascade as a function of a magnetic field $B$, applied perpendicular to the plane of the two equally-sensitive counters (Zürich group ${ }^{\circ}$ ). The solid line is a least square fit to the experimental points with the function

The calculation of $g$ as outlined here assumes that no quadrupole interaction is present in the source. A correction must be applied if this assumption is not justified ${ }^{30}$; this correction increases the magnitude of $g$.

D. The experiments of the Zürich group with $\mathrm{Cd}^{111}$ have furnished an example of the determination of $|g|$ from the attenuation of the correlation function by a magnetic field $B$. Here, we discuss the $\gamma-\gamma$ cascade of $\mathrm{Pb}^{204}$ and present an example of the rotation of the correlation pattern.

The decay and the directional correlation of $\mathrm{Pb}^{204}$ were first investigated by the Brookhaven group ${ }^{165}$. The decay scheme ${ }^{166}$ which resulted from their work was used as a basis for the later directional correlation measurements ${ }^{167}$ and for the determination of the $g$-factor of the first excited state ${ }^{161,162}$. The decay was thought to consist of two $\gamma$-rays in cascade. Recently, Krohn and Raboy discovered that actually three $\gamma$-rays are in cascade, two of them with nearly the same energy ${ }^{168}$. A tentative decay

164 E. Heer, private communication.

164a W. Zobel and R. M. Steffen, to be published in Phys. Rev.

165 A. W. Sunyar, D. Alburger, G. Friedlander, M. Goldhaber, and G. ScharffGoldhaber, Phys. Rev. 79 (1950) 181.

166 M. Goldhaber and R. D.' Hill, Rev. Modern Phys. 24 (1952) 179.

${ }_{167}$ H. Frauenfelder, J. S. Lawson, W. Jentschke, and G. DePasquali, Phys. Rev. 92 (1953) 1241.

168 V. E. Krohn and S. Raboy, Phys. Rev. 95 (1954) 1354. 
scheme, based on Krohn and Raboy's results, is given in Fig. 13a. The energy values are taken from Bergström ${ }^{169}$; the order of the $\gamma$-rays $\gamma_{2}$ and $\gamma_{3}$ is based on the fact that $\mathrm{Tl}^{204}$ decays entirely to the ground state of $\mathrm{Pb}^{204}$. The tentative spin and parity assignments are derived from conversion data ${ }^{166}$, from the lifetimes (the lifetime of the first excited state is less than $5 \times 10^{-10} \mathrm{sec}^{170}$, that of the second excited state is $2.6 \times 10^{-7} \mathrm{sec}^{165,168}$ ), and from analogy with the decays of $\mathrm{Pb}^{202}$ and $\mathrm{Pb}^{206}$.

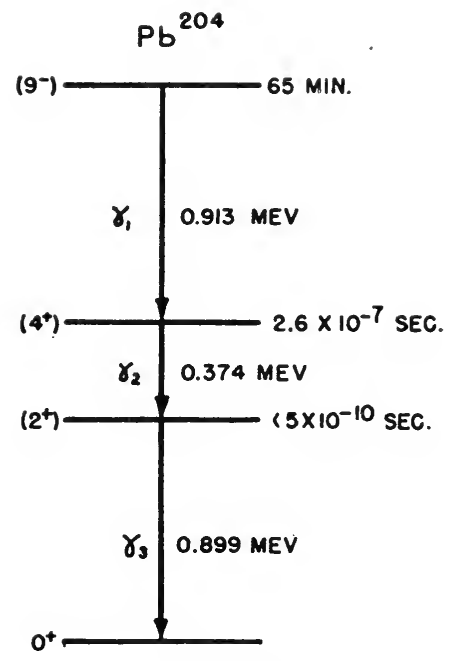

Fig. 13a

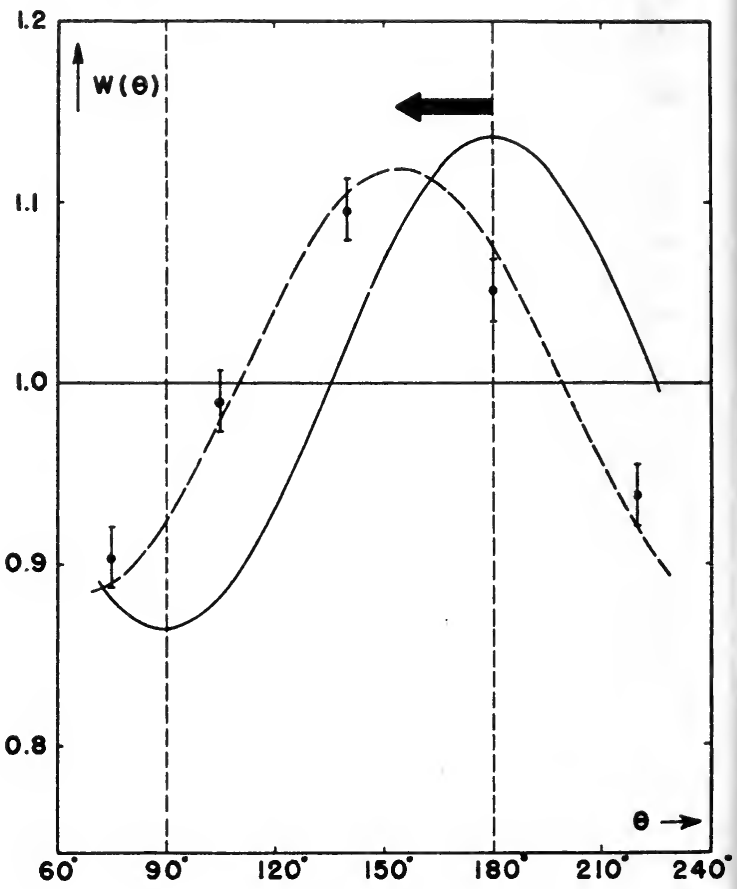

Fig. 13b

Fig. 13. a) Tentative decay scheme of $\mathrm{Pb}^{204}$. b) Rotation of the directional correlation pattern of the $\mathbf{P b}^{204} \gamma_{1}-\left(\gamma_{2} \gamma_{3}\right)$ cascade in an external magnetic field $B$. The solid line represents the zero field correlation, the dotted line is the theoretical curve for $g=+0.07$ and $B=4300$ oersteds. The arrow indicates the classical precession angle of a magnetic dipole (Illinois group ${ }^{162}$ ).

There remain, however, some inconsistencies in this $\mathrm{Pb}^{204}$ decay scheme.

The measurements of the $g$-factor of the $2.6 \times 10^{-7} \mathrm{sec}$ level were performed prior to the revision of the decay scheme. Nevertheless, the main features are still correct. Sign and magnitude of the $g$-factor were independently determined by Krohn and Raboy ${ }^{161,} 168$ and by the Illinois

169 I. Bergström, private communication.

170 D. Alburger, G. Friedlander and A. W. Sunyar, private communication. 
group ${ }^{162}$. The former found a value $g=+0.054 \pm 0.005$, the latter $g=+0.07 \pm 0.03$. Assuming that the spin assignment of Fig. 13a is correct, the magnetic moment is given by

$$
\mu\left(\mathrm{Pb}^{204}, 4^{+} \text {level }\right)=+(0.22 \pm 0.02) \mu_{N} .
$$

Fig. 13b shows the rotation of the directional correlation pattern ${ }^{162}$.

Raboy and Krohn have also measured the $g$-factor of the $10^{-8}$ sec state in $\mathrm{Ta}^{181}{ }^{163}$. They used both methods described above (attenuation and rotation). However, the assignments in the decay of $\mathrm{Ta}^{181}$ are even less certain than for $\mathrm{Pb}^{204}$ and more work is necessary to clear up all difficulties.

\section{$\S 11$. The Determination of the Quadrupole Coupling}

A. The static quadrupole interaction can, in analogy with the magnetic interaction, be explained as causing a precession of the nucleus. The magnetic and electric interactions differ, however, in two respects. i) In a magnetic field, a nucleus precesses in one direction only and the sign of the magnetic moment can be determined from the sense of rotation. With quadrupole interaction, both directions of precession are equally probable, no rotation of the correlation pattern occurs, and the sign of the quadrupole moment cannot be determined. (This statement is true for unpolarized nuclei. The application of the methods discussed below in B and $\mathrm{C}$ to polarized nuclei can yield the sign of the quadrupole moment. Thus far, no pertinent experiments have been performed.) ii) The energy differences between adjacent $m$-states, split up by a magnetic field, are equal and the nuclei all precess at the same frequency, the Larmor frequency $\omega_{L}$. The quadrupole interaction causes a non-equidistant splitting of the energy levels and a spectrum of frequencies exists (e.g. for $I=5 / 2: \pm \omega, \pm 2 \omega, \pm 3 \omega)$.

For the discussion of some of the results of the theory ${ }^{30,31}$, we distinguish two cases:

I. A preferred direction exists in the radioactive source. In this case, realized for instance in an axially symmetric single crystal, the angular correlation depends not only on the angle $\theta$ between the directions $\Omega_{1}$ and $\Omega_{2}$ of the two radiations, but also on the orientation of $\Omega_{1}$ and $\Omega_{2}$ with respect to the symmetry axis. The expression for the correlation function becomes rather involved ${ }^{31}$.

II. No preferred direction exists in the source considered as a whole. If the unperturbed correlation be given by Eq. (36), then the perturbed correlation can be written ${ }^{17}$.

$$
W(\theta)=1+G_{2} A_{2} P_{2}(\cos \theta)+\ldots+G_{v \max } A_{v \max } P_{v^{\prime} \max }(\cos \theta) .
$$

The attenuation factors $G_{\text {, }}$ contain the whole influence of the extra- 
nuclear fields; explicit expressions for them are available for most of the interesting cases 17, 30,31. We mention here some general properties of the $G_{v}$.

i) The attenuation factors $G_{v}$ depend on the interaction in the intermediate nuclear state (i.e. on $I_{b}, \tau_{b}, Q, \partial^{2} V / \partial z^{2}$, and other parameters) and on the time characteristics $\left(\tau_{D}, \tau_{R}\right)$ of the coincidence circuit. They do not depend on the parameters describing the initial and final levels of the cascade nor on the radiations. The latter independence has been verified experimentally by the Zürich group ${ }^{93,171}$. Their results are shown in Fig. 14 .

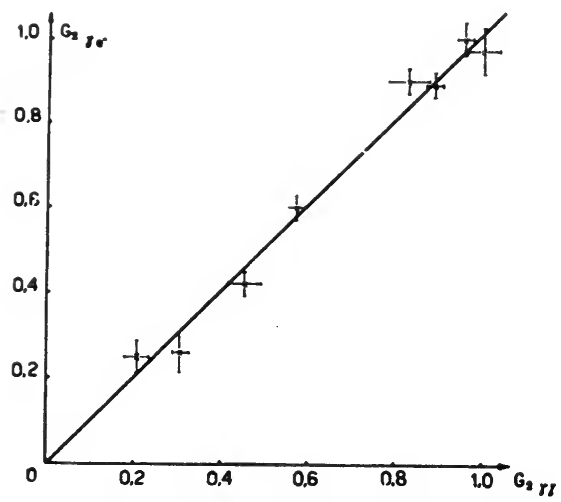

Fig. 14. Evidence that the attenuation factor $G_{2}$ does not depend on the type of radiation: $G_{2}$ was measured by the Zürich group in sources with different attenuation; for each source, the $\gamma-\gamma$ and the $\gamma-e^{-}$ directional correlations were determined. As expected, the coefficients $G_{2}(\gamma-\gamma)$ are equal to the corresponding $G_{2}\left(\gamma-\mathrm{e}^{-}\right)$.

ii) Eq. (52) is valid for both static and time-dependent processes, provided the source as a whole is isotropic. However, the $G_{v}$ behave quite differently for these two cases. For time-dependent interactions, they can vanish completely, i.e. the correlation function can become isotropic. Static interactions never reduce the correlation to zero: for very large coupling, the attenuation factors approach a minimum ${ }^{17}$ or hard core ${ }^{30}$ value $G_{v}(\lim )$. For axial symmetry, these are ${ }^{\mathbf{3 0}}$

$$
G_{v}(\lim )=1 /(2 \nu+1) \text {. }
$$

If one finds experimental values for $G_{v}$ which are smaller than predicted by Eq. (53), one must assume that at least one of the two assumptions (static interactions, isotropic source) is violated.

iii) In the case of a magnețic interaction, the higher terms in Eq. (52) are always more attenuated than the lower ones, i.e. $G_{v}<G_{v}$ for $\boldsymbol{\nu}^{\prime}>\boldsymbol{v}$. This relation is not always true for electric quadrupole interaction. An experimental result $G_{4}>G_{2}$ thus unambiguously proves the existence of a quadrupole interaction.

171 H. J. Gerber, F. Gimmi, E. Heer, and P. Scherrer, Helv. Phys. Acta 27 (1954) 180. 
This convincing argument was first pointed out by Abragam and Pound ${ }^{113}$ in discussing the $\mathrm{Th}^{228} \alpha-\gamma$ correlation. The measured $\alpha-\gamma$ directional correlation of $\mathrm{Th}^{228}$ is characterized by ${ }^{110,111} A_{2, \text { exp }}=0.30$ and $A_{\text {4, exp }}=-0.86$. The most plausible spin assignment, $0(\alpha) 2(\gamma) 0$ leads to $A_{2, \text { theor }}=5 / 7$ and $A_{4, \text { theor }}=\rightarrow^{12} / 7$. The resulting attenuation factors $G_{2}=0.42$ and $G_{4}=0.50$ suggest very strongly that the attenuation is mostly (or entirely) due to quadrupole interaction.

B. The beautiful experiments of the Zürich group with metal single crystals constitute the most convincing proof of the existence of the quadrupole interaction and, at the same time, allow the most accurate determination of the quadrupole coupling. The basic idea of these experiments is as follows. The electric field gradients in solids possess the right order of magnitude to influence cascades with mean lives $\tau_{b}$ between $10^{-5} \mathrm{sec}$ and $10^{-9}$ sec. Instead of varying the field strength, as is done in the magnetic case $(\S 10)$, one varies the orientation of the crystal axis with respect to the detectors and observes the directional correlation as a function of this orientation.

Various possible experimental arrangements for the determination of the quadrupole coupling have been treated theoretically by Alder, Albers-Schönberg, Heer, and Novey ${ }^{31}$. We discuss here their results for one especially important case. The arrangement is shown in Fig. 15a. The symmetry axis, i.e. the c-axis of the axially symmetric crystal, lies in the plane determined by the direction $\Omega_{1}$ to the fixed counter and the normal to the plane of the two counters. Denoting by $\vartheta$ the angle between c and $\Omega_{1}$, the anisotropy becomes

$$
A(\vartheta)=\sum_{n} d_{n} \cos ^{n} \vartheta / \sum_{n} e_{n} \cos ^{n} \vartheta
$$

The coefficients $d_{n}$ and $e_{n}$ are tabulated in reference ${ }^{31}$; they are functions of the product $Q\left(\partial^{2} V / \partial z^{2}\right) \tau_{b}$. In order to determine the quadrupole coupling, one measures $A(\vartheta)$, makes a least square fit to the experimental points with the function (54), and then calculates $Q\left(\partial^{2} V / \partial z^{2}\right)$ from the coefficients $d_{n}$ and $e_{n}$ and from $\tau_{b}$ and $I_{b}$.

Such an experiment was first performed by the Zürich group ${ }^{10}$ with the $\gamma-\gamma$ cascade of $\mathrm{Cd}^{111}$. They used metallic indium single crystals containing the radioactive $\mathrm{In}^{111} \rightarrow \mathrm{Cd}^{111}$. Metallic In is face-centered tetragonal and it is reasonable to assume that the crystalline $c$-axis is identical with the symmetry axis of the electric field gradient. The c-axis was determined with $\mathrm{X}$-rays. A typical result is shown in Fig. $15 \mathrm{~b}$. The evaluation of this and later experiments ${ }^{141},{ }^{172}$, together with the known

172 H. Albers-Schönberg, K. Alder, O. Braun, E. Heer, and T. B. Novey, Phys. Rev. 91 (1953) 1287. 
spin $\left(I_{b}=5 / 2\right)$ and mean life $\left[\tau_{b}=(1.23 \pm 0.06) \times 10^{-7} \mathrm{sec}\right]$, yielded the quadrupole coupling $(e Q / h)\left(\partial^{2} V / \partial z^{2}\right)=(6.86 \pm 0.4) \mathrm{Mc} / \mathrm{sec}$, or $Q\left(\partial^{2} V / \partial z^{2}\right)=$ $=(2.84 \pm 0.18) \times 10^{-8}$ volt .

At the present time, no reliable estimate of the field gradient $\left(\partial^{2} V / \partial z^{2}\right)$ in metallic indium exists and it is thus impossible to calculate the quadrupole moment $Q$.

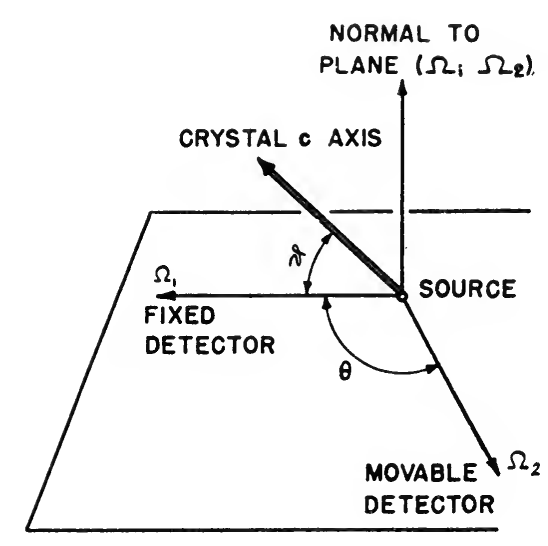

Fig. 15a

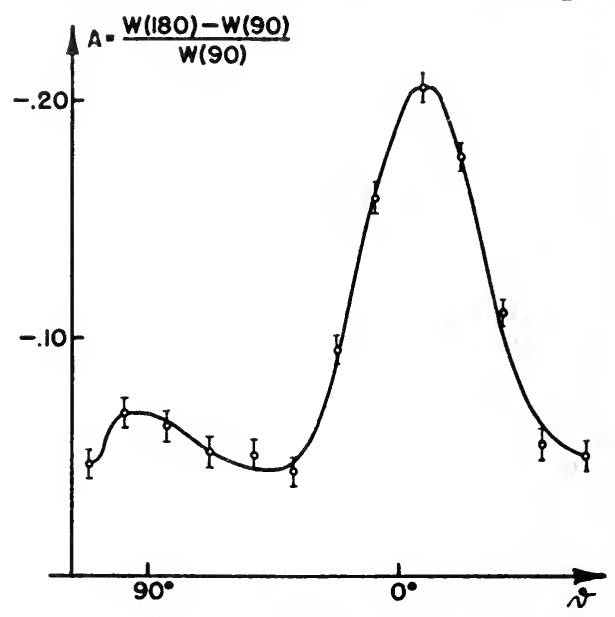

Fig. 15b

Fig. 15. Determination of the quadrupole coupling in single crystals by the Zürich group ${ }^{10,171}$. The directional correlation $W(\theta, \vartheta)$ is measured as a function of the angle $\vartheta$ between the c-axis of a single crystal and the direction $\Omega_{1}$. a) Schematic diagram of the arrangement. b) The measured anisotropy $A$ as a function of $\vartheta$.

A major limitation to this method of determining the quadrupole coupling is set by the time which is necesssary to grow and orient a single crystal. Only radioactivities with lifetimes of the order of hours or longer can be investigated at present.

C. An interesting effect occurs if a magnetic and an electric interaction are simultaneously present. Alder and coworkers ${ }^{31}$ treated theoretically the special case of a magnetic field parallel to the symmetry axis of an axially symmetric electric field gradient. The behavior of the correlation function can be understood qualitatively by a semiclassical picture ${ }^{31}$. The quadrupole interaction causes the nuclei to precess around the symmetry axis with both directions of precession equally probable. The magnetic interaction gives rise to a unidirectional precession and hence cancels part of the influence of the quadrupole interaction. The anisotropy as a function of the strength of the applied magnetic field thus first increases with increasing field, reaches a maximum (for $\omega_{\mathrm{magn}}=\omega_{\mathrm{el}} / 2$ ) and then decreases. This effect was actually observed by the Zürich group ${ }^{141,173}$ with an indium

${ }_{173}$ H. Albers-Schönberg, K. Alder, E. Heer, T. B. Novey, and P. Scherrer, Proc. Physical Soc. A66 (1953) 952. 
single crystal. In principle, such an experiment allows the simultaneous determination of $g$ and $Q\left(\partial^{2} V / \partial z^{2}\right)$. In practice, however, two independent measurements of these quantities are more advantageous.

D. The angular correlation measured with polycrystalline sources is expected to be undisturbed if the individual microcrystals are cubic and if the radioactive atoms occupy regular lattice sites. A perturbation should result if the crystal structure possesses a symmetry lower than cubic. For an isotropic source, one can express the perturbed correlation in the form (52) and determine the quadrupole coupling from the attenuation factors $G_{v}$.

As an example of this method of determining the quadrupole coupling, we again take the $\mathrm{Cd}^{111} \gamma-\gamma$ cascade. The anisotropy of this cascade in a polycrystalline indium source was measured independently by the Zürich group ${ }^{174}$ and by Steffen ${ }^{175}$. The former found $A^{Z}=-0.095 \pm 0.005$, the latter $A^{S}=-0.085 \pm 0.003$. Assuming for the unperturbed correlation an anisotropy $A=-0.245 \pm 0.015$, or $A_{2}=-0.175 \pm 0.010$, one finds $G_{2}^{Z}=0.37 \pm 0.03$ and $G_{2}^{S}=0.33 \pm 0.02$. Taking a mean value, $G_{2}=0.35 \pm 0.02$, and using the formulas of Abragam and Pound ${ }^{30}$ or of Alder and coworkers ${ }^{31}$ to determine the quadrupole coupling from $G_{2}, \tau_{b}$, and $I_{b}$, one finds $(e Q / h)\left(\partial^{2} V / \partial z^{2}\right)=(6.2 \pm 0.8) \mathrm{Mc} / \mathrm{sec}$. This value agrees with that from the single crystal experiment (§ 11B).

The influence of crystal symmetry on angular correlation can be seen directly in some experiments with $\mathrm{Pb}^{204}$ (cf. $\S 9$ and Fig. 13a). The directional correlation of $\mathrm{Pb}^{204}$ in two different $\mathrm{Pb}-\mathrm{Tl}$ alloys has been studied by the Illinois group ${ }^{176}$. The first alloy, produced by irradiating $\mathrm{Tl}$ in the cyclotron with deuterons, constitutes a $\mathrm{Pb}-\mathrm{Tl}$ crystal with vanishingly small $\mathrm{Pb}$ content. Such a crystal possesses a hexagonal close-packed lattice below $235^{\circ} \mathrm{C}$ and a body-centered cubic lattice between $235^{\circ} \mathrm{C}$ and the melting point $303^{\circ} \mathrm{C}$. The second type $(\sim 40 \% \mathrm{Tl})$, prepared by adding $\mathrm{Pb}$ to the irradiated $\mathrm{Tl}$, shows the face-centered cubic structure of $\mathrm{Pb}$. The anisotropies $A$ as a function of temperature are shown in Fig. 16. The data show clearly the influence of the crystal symmetry.

Besides the two measurements which we have treated here, many more experiments have been performed so far (e.g. ${ }^{8}, 56,68,131,139,142,162,177-179$ ).

174 H. Albers-Schönberg, E. Heer, T. B. Novey, and R. Rüetschi, Phys. Rev. 91 (1953) 199.

175 R. M. Steffen, to be published.

176 H. Frauenfelder, J. S. Lawson, W. Jentschke, and G. DePasquali, Phys.

Rev. 92 (1953) 513.

177 R. M. Steffen, Phys. Rev. 90 (1953) 1119.

178 J. J. Kraushaar and R. V. Pound, Phys. Rev. 92 (1953) 522.

179 J. C. D. Milton and J. S. Fraser, to be published. 
From these investigations, the following general conclusions concerning polycrystalline sources can be drawn.

i) The determination of the quadrupole coupling is much less accurate with polycrystalline sources than with single crystals. Moreover, experiments with the $\mathrm{Cd}^{111} \gamma-\gamma$ cascade in indium polycrystals have shown that these sources always tend to crystallize in preferred directions and
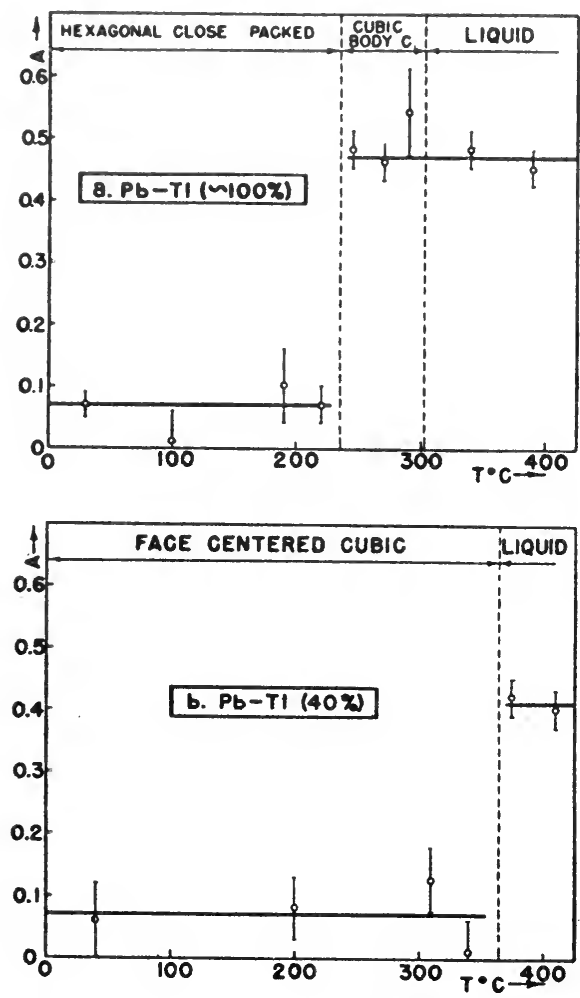

Fig. 16. Anisotropy of the $\mathrm{Pb}^{204} \gamma_{1}-\left(\gamma_{2} \gamma_{3}\right)$ directional correlation in $\mathrm{Pb}-\mathrm{Tl}$ alloys as a function of the source temperature $T$ (Illinois group ${ }^{176}$ ). a) Alloy with $1 \mathrm{C0} \%$ $\mathrm{Tl}$, b) alloy with $60 \% \mathrm{~Pb}-40 \% \mathrm{Tl}$. that it is extremely difficult to produce isotropic samples ${ }^{180}$. (This fact may explain why values below the hard core have been found in polycrystalline metal sources of $\mathrm{Pb}^{204} 176$ and $\mathrm{Ta}^{181}{ }^{68}$.) The isotropy of the anisotropy should always be checked experimentally by determining the correlation function for various source orientations.

ii) The directional correlation is unperturbed when measured with the radioactive atoms in positions of cubic symmetry. This fact has been demonstrated for the $\gamma-\gamma$ cascades of $\mathrm{Cd}^{111}$ and $\mathrm{Pb}^{204}$ and is displayed in Fig. $16 \mathrm{a}$, where the anisotropy assumes a high value in the bodycentered cubic lattice. Fig. $16 \mathrm{~b}$, however, shows that the "overall" cubic symmetry of a crystal is not sufficient to ensure an unperturbed correlation. Even though the $60 \%$ $\mathrm{Pb}-40 \% \mathrm{Tl}$ alloy possesses a facecentered cubic lattice, considerable field gradients must be present at the sites of the $\mathrm{Pb}$ nuclei. These gradients very probably are due to the fact that each $\mathrm{Pb}$ atom is surrounded by both $\mathrm{Pb}$ and $\mathrm{Tl}$ atoms. (Similar effects have been observed in nuclear resonance work ${ }^{181}$.) Electric field gradients can also be caused by lattice deformations and imperfections ${ }^{182}$.

E. The directional correlation of $\alpha-\gamma$ cascades has been measured in

180 Illinois group, unpublished results.

181 N. Bloembergen and T. J. Rowland, Acta Metallurgica 1 (1953) 731.

182 G. D. Watkins and R. V. Pound, Phys. Rev. 89 (1953) 658. 
various cases (cf. § 7), mostly by using thin polycrystalline sources. We have already mentioned in $\S 11 \mathrm{~A}$ that the attenuation found in the $\alpha-\gamma$ cascade of $\mathrm{Th}^{228}$ can be explained by assuming pure quadrupole interaction. Most of the other $\alpha-\gamma$ directional correlation measurements can be evaluated in the same manner. Fig. 17 shows the result of an investigation by Milton and Fraser ${ }^{119}$. The solid lines are the theoretical attenuation coefficients $G_{2}$ and $G_{4}$ for a spin $I_{b}=2$ as a function of $\omega \tau_{b}{ }^{30,113}$. The values of the experimental attenuation coefficients for $\mathrm{Ra}^{224}, \mathrm{Ra}^{226}$, and $\mathrm{Pu}^{238}$ show that all three cases can be explained by pure quadrupole interaction. No detailed comparison with a nuclear model is yet possible because the field gradients are unknown. Incidentally, one can see from Fig. 17 that this method of determining the quadrupole interaction is very insensitive for values $\omega \tau_{b}>2$.

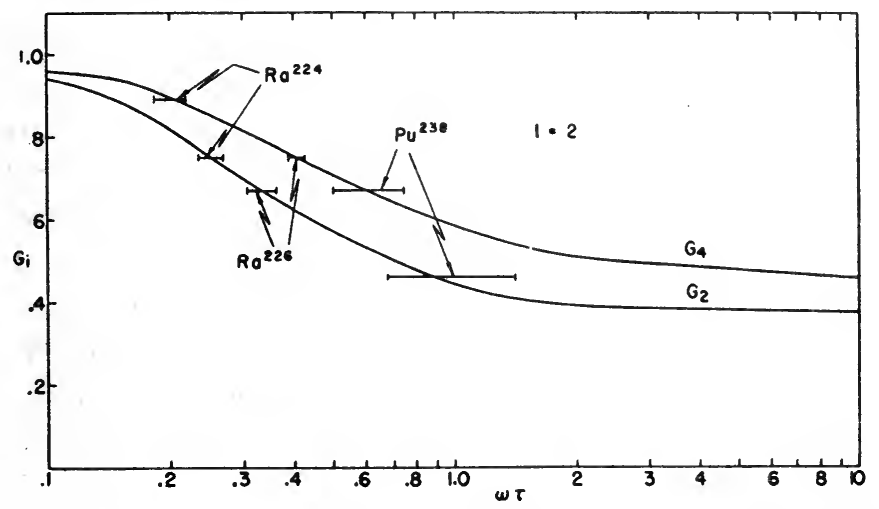

Fig. 17. The attenuation coefficients $G_{2}$ and $G_{4}$ for the $\alpha-\gamma$ directional correlations in $\mathrm{Ra}^{224}, \mathrm{Ra}^{228}$, and $\mathrm{Pu}^{238}$, as determined by Milton and Fraser ${ }^{119}$. The solid lines are the attenuation coefficients for pure quadrupole interaction, as calculated from the theory of Abragam and Pound ${ }^{30,113}$ for the spin $I_{b}=2$.

A very interesting and promising work was performed by a French group ${ }^{108}$ on the $\alpha-\gamma$ decay in $\mathrm{Th}^{230}$ (Io). $\mathrm{Th}^{230}$ decays by $\alpha$-emission into several excited levels of $\mathrm{Ra}^{226}$. The most probable spin and parity assignments to the first two excited levels of $\mathrm{Ra}^{226}$ are $2+$ and $4+$; the ratio of their excitation energies $(67.8 \mathrm{keV}$ and $210 \mathrm{keV})$ characterizes them as ratational levels (cf. Ch. XVII and reference ${ }^{183}$ ). By measuring the $\alpha-\gamma$ directional correlation for the $\alpha$-branches leading to these two levels, the French workers were able to determine attenuation coefficients for both cascades. From these attenuation coefficients, they in turn calculated the products $\omega \tau_{b}$. For the ratio $R \equiv\left(\omega \tau_{b}\right)^{\prime} /\left(\omega \tau_{b}\right)$, they found $R_{\text {exp }}=0.13$, with an uncertainty of less than $40 \%$. This ratio $R$ is indepen-

183 A. Bohr and B. R. Mottelson, Dan. Mat. Fys. Medd. 27, no. 16 (1953). 
dent of the electric field gradient if we assume that the nuclear environment is the same for both levels; it depends only on $Q_{b}^{\prime} / Q_{b}, \tau_{b}^{\prime} / \tau_{b}$, and the spins $I_{b}^{\prime}$ and $I_{b}$. The theory of Bohr and Mottelson (Ch. XVII and ${ }^{183}$ ) yields the ratio $Q_{b}^{\prime} \mid Q_{b}$ in terms of the spins, and $\tau_{b}^{\prime} / \tau_{b}$ in terms of the spins and energies of the two levels. With the known spin and energy values, the theoretical value for the ratio $R$ is then $R_{\text {theor }}=0.11 \pm 0.04$, the error being due to uncertainties in the $\gamma$-ray energies and the conversion coefficients. The agreement between the theoretical and experimental value is thus good and provides new confirmation of the theory of Bohr and Mottelson. (One can reverse the argument and use the theoretical value of the quadrupole moments to get an estimate for the value of the electric field gradient.)

\section{$\S 12$. Time-Dependent Interactions}

A. Kluyver and Deutsch ${ }^{184}$ found that $\mathrm{Cd}^{111}$ in a liquid source (a dilute $\mathrm{In}_{2}\left(\mathrm{SO}_{4}\right)_{3}$ solution) gives an anisotropy of $A=-0.19$, whereas the same chemical compound as a solid shows very nearly an isotropic directional correlation. The observation that a liquid source generally gives a larger anisotropy than a solid one was subsequently confirmed in many other cases, mostly with the $\mathrm{Cd}^{111} \dot{\gamma}-\gamma$ cascade ${ }^{174}, 176,177$, but also with other cascades, e.g. $\operatorname{Cd}^{111}\left(\gamma_{1}^{\prime}-\gamma_{2}\right)^{178}, \operatorname{Er}^{166}(\gamma-\gamma)^{179}, \operatorname{Ta}^{181}(\gamma-\gamma)^{68}$, $\mathrm{Pb}^{204}(\gamma-\gamma)^{167},{ }^{176}$ (cf. Fig. $16 \mathrm{~b}$ ), $\mathrm{Cd}^{111}\left(\gamma-\mathrm{e}^{-}\right)^{93}$, and $\mathrm{Am}^{241}(\alpha-\gamma)^{116}$.

B. Abragam and Pound treated theoretically the influence of extranuclear fields in liquids ${ }^{30,138}$ and found that the attenuation factors $G_{v}$ (Eq. (52)) are of the form

and

$$
G_{v}(t)=e^{-\lambda_{\nu} t}
$$

$$
G_{v}=\frac{1}{\tau_{b}} \int_{0}^{\infty} e^{-t / \tau_{b}} G_{v}(t) d t=\frac{1}{1+\lambda_{v} \tau_{b}} .
$$

$G_{v}(t)$ is the differential attenuation coefficient which applies to an experiment where only those nuclei are observed which stay in level $\mathrm{B}$ for a time between $t$ and $t+d t$ after. the emission of the first radiation. This case is realized by inserting a delay $\tau_{D}(=t)$ and using a resolving time $\tau_{R}(=d t) \ll \tau_{b}$. The integrated coefficients $G_{v}$ apply when all nuclei are observed, regardless of the time spent in the intermediate state $B$, i.e. when $\tau_{D}=0$ and $\tau_{R} \geqq \tau_{b}$. The relaxation constant $\lambda_{\nu}$ ' depends on $\nu$ and $I_{b}$, and is proportional to the correlation time $\tau_{c}$ of the liquid, to the square of the quadrupole moment, and to the average of the square of the electric field gradient, $\left\langle\left(\partial^{2} V / \partial z^{2}\right)^{2}\right\rangle_{\mathrm{AV}}$. In order to define the correlation time $\tau_{c}$, one assumes that liquids possess at any instant

184 J. C. Kluyver and M. Deutsch, Phys. Rev. 87 (1952) 203. 
local configurations similar to local configurations in solids (see e.g. ${ }^{185}$ ). However, these local configurations change continuously, in a random manner. The correlation time $\tau_{c}$ now is loosely defined as the time during which a local configuration undergoes an appreciable change. The Eq. (55) and (56) are derived under the assumption that $t \gg \tau_{c}$. In the practical application, this means $\tau_{b} \gg \tau_{c}$ and $\tau_{D} \gg \tau_{c}$.

Several conclusions can be drawn from the form (55) or (56) of the attenuation coefficients. i) The attenuation factors $G_{v}$ and $G_{v}(t)$ depend on the product $Q^{2}\left\langle\left(\partial^{2} V / \partial z^{2}\right)^{2}\right\rangle_{A V} \tau_{b} \tau_{c}$. In order to find the quadrupole moment $Q$, one must know the correlation time $\tau_{c}$ in addition to $\tau_{b}$ and $\left(\partial^{2} V / \partial z^{2}\right)$. Thus it is difficult to determine $Q$ from the attenuation of the directional correlation in liquids. ii) No hard core exists for the timedependent interactions; the directional correlation in liquids can become isotropic. iii) For correlation times $\tau_{c}$ so small that $\lambda_{v}$ (which is proportional to $\tau_{c}$ ) is small compared with $t^{-1}$ (or $\tau_{b}{ }^{-1}$ ), the directional correlation is unperturbed. This explains the high anisotropy found with dilute solutions.

The Eq. (55) and (56) do not apply if $\tau_{c} \gg \tau_{b}$. This case, however, corresponds to a crystalline powder and hence the conclusions of $\S 11$ are applicable. We notice in particular that the correlation cannot be wiped out completely. The directional correlation as a function of the correlation time thus should behave as follows. For very short times $\tau_{c}\left(\lesssim 10^{-11} \mathrm{sec}\right)$ the correlation is unperturbed. With increasing $\tau_{c}$, the correlation is attenuated according to Eq. (55) or (56) and it can even be wiped out completely. In the vicinity of $\tau_{c} \sim \tau_{b}$, the anisotropy starts rising again and assumes for $\tau_{c} \gg \tau_{b}$ the value corresponding to that in a crystalline powder.

C. The correlation time $\tau_{c}$ in a liquid is roughly proportional to its

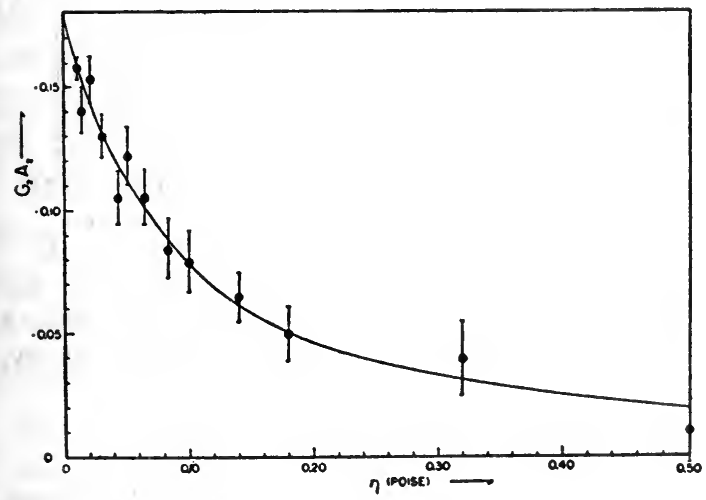

Fig. 18. Attenuation of the $\mathrm{Cd}^{111} \gamma-\gamma$ directional correlation in water-glycerin mixtures as a function of the viscosity $\eta$. The solid line represents the theoretical curve eq. (56) for $A_{2}=-0.18$ and $\lambda_{2}=25 \eta$ (where $\eta$ is measured in poise). From Hemmig and Steffen ${ }^{186}$

185 B. R. T. Frost, Progress in Metal Physics 5 (1954) 96.

188 P. B. Hemmig and R. M. Steffen, Phys. Rev. 92 (1953) 832. 
viscosity 187,188 ; hence the investigation of the directional correlation in a liquid as a function of its viscosity permits a test of the foregoing considerations. Such experiments were carried out by the Zürich group 189 and by Hemmig and Steffen ${ }^{186}$ with the $\mathrm{Cd}^{111} \gamma-\gamma$ cascade. The radioactive sources consisted of $\mathrm{InCl}_{3}$ in water-glycerin solutions. In order to change the viscosity, either the ratio of glycerin to water or the temperature of the source was varied. The investigations of both groups clearly displayed the expected dependence of the anisotropy on the viscosity. Some of the results of Hemmig and Steffen are shown in Fig. 18.

D. Abragam and Pound's theory ${ }^{30}$ predicts that the time behavior of the differential attenuation coefficients $G_{v}(t)$ for a time-dependent interaction is quite different from that for a static interaction. In the former case, $G_{v}(t)$ approaches zero exponentially (Eq. (55)). In the latter case, the $G_{v}(t)$ vary periodically with time; no true "loss of memory" is connected with static interactions.

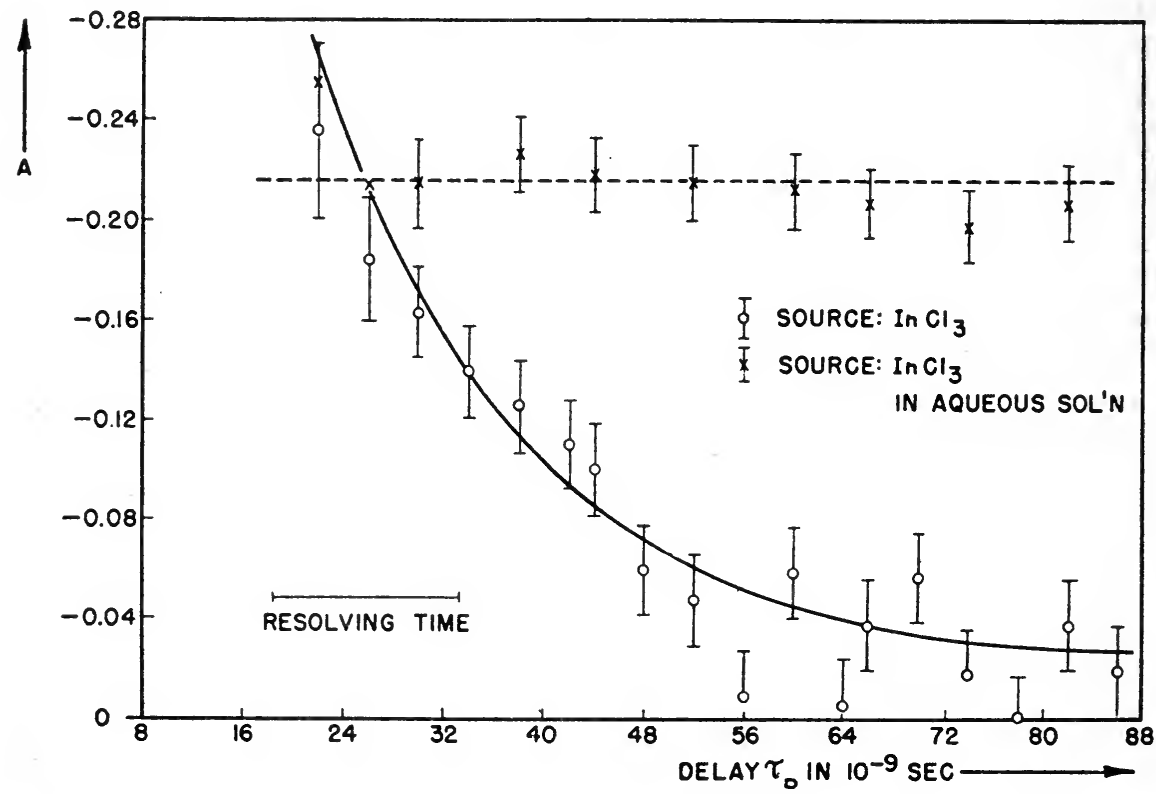

Fig. 19. The anisotropy $A$ of the $\mathrm{Cd}^{111} \gamma-\gamma$ cascade as a function of the delay $\tau_{D}$, as measured by Fraser and Milton' ${ }^{86}$. The aqueous solution gives, as expected, a nearly constant anisotropy. The solid source shows a rapid décay of the anisotropy, but does not display the predicted periodic behavior.

187 P. Debye, Polar Molecules (Dover Publications, New York, 1945).

188 N. Bloembergen, E. M. Purcell, and R. V. Pound, Phys. Rev. 73 (1948) 679.

188 H. Albers-Schönberg, E. Heer, F. Gimmi and T. B. Novey, Helv. Phys. Acta 26 (1953) 599. 
Experiments with delayed coincidences were performed by Fraser and Milton ${ }^{86}$ and by the Zürich group ${ }^{164}$. The $\alpha-\gamma$ cascade of $\mathrm{Am}^{241}$ and the $\gamma-\gamma$ cascade of $\mathrm{Cd}^{111}$ were investigated in various liquids and solids. The liquid sources show qualitatively the expected behavior: in aqueous solution, where $\tau_{c}$ is very small, the anisotropy is nearly independent of the delay time. An example, $\mathrm{InCl}_{3}$ in aqueous solution, is shown in Fig. 19. Viscous solutions display the exponential decay of the anisotropy as predicted by Eq. (55). The anisotropies measured with solid sources do not show the expected periodicity (Fig. 19). This deviation from the theory could be due to the $K$-capture in $\mathrm{Cd}^{111}$ and the $\alpha$-decay in $\mathrm{Am}^{241}$, or it could be due to experimental inadequacies. Further investigations, especially with $\gamma-\gamma$ cascades which start from isomeric levels, are needed to complete the picture.

\section{$\S 13$. The Influence of $K$-Capture}

A. Suppose we are measuring the directional correlation of a $\gamma-\gamma$ cascade $\mathrm{A} \rightarrow \mathrm{B} \rightarrow \mathrm{C}$, which is initiated by a $K$-capture. The $K$-capture, by creating a hole in the $K$-shell, causes the atomic shell to be in an excited state. (In addition, $K$-capture can also excite or eject other electrons of the inner shells. Calculations indicate that the probability with which such "shake-up" processes occur is small 190. However, even if they do occur to an appreciable extent, they do not affect the present considerations.) After the $K$-capture, shell and nucleus tend to reach their ground states: the nucleus by decaying through the $\gamma-\gamma$ cascade, the atomic shell by setting free the excitation energy through emission of Auger electrons and $\mathrm{X}$-rays. We are interested in the magnetic field and the electric field gradient which are present at the nucleus during the time the nucleus is in level $\mathrm{B}$. A knowledge of these quantities would allow the calculation of the attenuated angular correlation. No accurate data are yet available, but some quantitative considerations are nevertheless possible.

B. We consider first the processes occurring in a free atom. After the $K$-capture, the atom initially remains neutral. The emission of Auger electrons then causes the atom to become charged ${ }^{191}$. Experimental investigations show that the average charge of an atom after $K$-capture is quite large ${ }^{192-194}$. From the measured values, one expects for $\mathrm{Cd}$, for

190 H. Primakoff and F. T. Porter, Phys. Rev. 89 (1953) 930.

191 E. P. Cooper, Phys. Rev. 61 (1942) 1.

192 M. L. Perlman and J. A. Miskel, Phys. Rev. 91 (1953) 899.

193 S. Wexler, Phys. Rev. 93 (1954) 182.

194 O. Kofoed-Hansen, Phys. Rev. 96 (1954) 1045. 
instance, an average charge of approximately $7 e$; individual $\mathrm{Cd}$ atoms may acquire much higher charges ${ }^{194}$. The perturbation of the directional correlation now depends on how far the atomic cascade has proceeded when the nucleus reaches the level B; i.e. it depends on the mean life $\tau_{a}$ of level $\mathrm{A}$ and on the transition probabilities for $\mathrm{X}$-ray and Auger emission in the various shells,

A hole in the $K$-shell decays predominantly through radiation; the Auger effect is not very important, especially for heavy atoms. The lifetime is of the order of $10^{-15} \mathrm{sec}$ for argon and $10^{-17} \mathrm{sec}$ for gold. The transition probability for radiation is proportional to the third power of the transition energy. As the hole moves outward, its lifetime against radiation thus becomes larger. In the outermost shell, it is of the order of $10^{-8} \mathrm{sec}$ (optical transitions). However, the probability for Auger transitions is nearly energy-independent and hence the Auger effect becomes more and more predominant as the hole moves out. In fact, it almost entirely determines the transition probabilities in all except the $K$-shell ${ }^{195,}{ }^{196}$. The total time required for a hole to move out to the outermost shell is of the order of $10^{-14} \mathrm{sec}$ for argon and $10^{-15} \mathrm{sec}$ for gold. However, once the hole is in the outermost shell, no electrons are available for Auger effect and the lifetime is given by that of the radiation. Thus the picture after a $K$-capture is as follows. A hole in the $K$-shell moves very rapidly to the outermost shell. This movement occurs mainly through Auger effects and hence more holes are created and also move outward. Once these holes have reached the outermost shell, they can decay only slowly and hence can persist long enough to cause very strong perturbations of the angular correlation. Moreover, the correlation can be attenuated below the hard core value: For a given configuration in the outermost shell, the correlation can be reduced to nearly the hard core value. Then a transition in the atomic shell can change the configuration while the nucleus is in level B and the correlation will be further attenuated.

C. Measurements with free atoms are hard to perform and hence the discussion above is somewhat academic. Usually, the radioactive atoms are embedded in a solid or a liquid and the whole picture is changed in two respects. Firstly, the nucleus now is subject also to extra-atomic fields. We have already discussed the resulting effects in $\S 11$ and 12. Secondly, the transition times in the outermost shell of the atom depend strongly on the atomic environment. For these reasons the attenuation of the correlation is influenced by the choice of the surroundings. We consider here three environments: a metal, an insulator, and a liquid.

195 E. G. Ramberg and F. K. Richtmeyer, Phys. Rev. 51 (1937) 913.

198 R. Rubenstein and J. N. Snyder, to be published. 
If the radioactive atom is embedded in the proper metal, it returns to its ground state in a very short time (less than $10^{-12} \mathrm{sec}$ ). Hence the nucleus is not perturbed by fields originating within its atomic shell, and the correlation is unperturbed if the atom is embedded in a lattice site of cubic symmetry. (Two steps are involved in the fast return of the radioactive atom to its ground state. The transitions to the conduction band occur radiationless, through a sort of Auger effect involving the electrons in the bands ${ }^{196 a}$. Hence the holes move out to the conduction band in a very short time. Once they are there, they flow away rapidly and leave the radioactive atom in its ground state.)

The radioactive atom embedded in an insulator (e.g. an ionic crystal) can act as an impurity center. Impurity centers in general have long decay times in the outermost shell $\left(\gg 10^{-8} \mathrm{sec}\right)$. (The excited electron orbits have a great radial extension ${ }^{197}$; therefore the matrix element for the transition to the ground state is small, the lifetime large.) Thus the directional correlation with an "insulator source" should be attenuated.

In a liquid, the transition times in the outermost shell should not differ vastly from those in the corresponding solid. For instance, in a molten metal, the shell of the decaying atom returns very fast to its ground state and the $K$-capture has no influence on the directional correlation. In a liquid "insulator" (e.g. in a molten ionic crystal or a solution) the influence of the $K$-capture depends on the correlation time and the interactions between the molecules (or ions) in the liquid. If the molecule containing the decaying nucleus rotates very fast (i.e. if the interaction is strong and the correlation time very short) the correlation will be unperturbed, even if the atomic shell is not in its ground state. In such a situation it is not possible to decide whether the $K$-capture exerts an influence or not. Such a decision, however, is possible if one increases the correlation time to values $\tau_{c} \gg \tau_{b}$. In contrast to the interactions discussed in $\S 12$, the directional correlation can then stay below the hard core value, if the influence due to $K$-capture is strong.

D. The foregoing considerations ${ }^{158}$ were first applied by the Zürich group to the $\mathrm{Cd}^{111}$ cascade $^{8}$. As expected, the anisotropy was very large when the $\mathrm{In}^{111}$ was embedded in silver, while ionic crystal sources displayed a nearly isotropic distribution. This latter result, attenuation below the hard core value, is good evidence for an influence of the $K$-capture.

196a A similar effect, involving atoms at a metallic surface, has been treated by H. D. Hagstrum, Phys. Rev. 96 (1954) 336.

197 N. F. Mott and R. W. Gurney, Electronic Processes in Ionic Crystals (The Clarendon Press, Oxford, England, 1940) p. 275. 
Kraushaar and Pound ${ }^{178}$ later confirmed indirectly the fact that $K$ capture plays an important role in reducing the correlation function below the hard core value. Their work was performed with the $\gamma-\gamma$ cascade which starts from the $48 \mathrm{~min}$ isomeric level in $\mathrm{Cd}^{111}$. This cascade, which is designated by $\gamma_{1}^{\prime}-\gamma_{2}$ in Fig. 7 , possesses the same intermediate state as the "standard cascade" $\gamma_{1}-\gamma_{2}$ which follows the $K$-capture in In"11. Because the cascade $\gamma_{1}^{\prime}-\gamma_{2}$ starts from an isomeric level and the recoil due to $\gamma_{1}^{\prime}$ is very small, no complications due to atomic excitation are to be expected and all attenuation coefficients should be larger than the hard core value Eq. (53). The correlation function for the unperturbed $\gamma_{1}^{\prime}-\gamma_{2}$ cascade is given by $A_{2}=0.1786,\left|A_{4}\right| \ll A_{2}$; the hard core value is $G_{2}(\lim )=1 / 5$. Some of the results of Kraushaar and Pound are shown in Table I. For comparison, we include attenuation factors for the cascade $\gamma_{1}-\gamma_{2}$ in sources with similar environments.

\section{TABLE I}

Experimental attenuation coefficients $G_{2}$ for the two $\gamma-\gamma$ cascades in $\mathrm{Cd}^{111}$. The cascade $\gamma_{1}-\gamma_{2}$ follows $K$-capture in $\operatorname{In}^{111}, \gamma_{1}^{\prime}-\gamma_{2}$ starts from the $48 \mathrm{~min}$ isomeric level in $\mathrm{Cd}^{111}$. The $G_{2}$ values for the cascade $\gamma_{1}-\gamma_{2}$ are calculated under the assumption that the unperturbed correlation is given by $A=-0.245$. This anisotropy may be somewhat too large, hence the $C_{2}$ may be somewhat too small.

\begin{tabular}{|c|c|c|c|}
\hline \multirow{2}{*}{ Source } & \multicolumn{2}{|c|}{ Attenuation factor $G_{2}$} & \multirow{2}{*}{ References } \\
\hline & Cascade $\gamma_{1}-\gamma_{2}$ & $\gamma_{1}^{\prime}-\gamma_{2}$ & \\
\hline Molten metal $\left\{\begin{array}{l}\text { Cd } \\
\text { In }\end{array}\right.$ & $\begin{array}{l}0.86 \pm 0.07 \\
0.86 \pm 0.05\end{array}$ & $\begin{array}{c}0.97 \pm 0.08 \\
-\end{array}$ & $\begin{array}{l}178,180 \\
174.177\end{array}$ \\
\hline Solid metal $\left\{\begin{array}{l}\mathrm{Cd} \\
\text { In }\end{array}\right.$ & $\begin{array}{l}0.32 \pm 0,04 \\
0.35 \pm 0.02\end{array}$ & $\begin{array}{c}0.40 \pm 0.07 \\
-\end{array}$ & $\begin{array}{l}128,180 \\
56.174\end{array}$ \\
\hline Aq. solution $\left\{\begin{array}{l}\mathrm{CdCl}_{2} \\
\mathrm{InCl}_{3}\end{array}\right.$ & $0.90 \pm 0.02$ & $\begin{array}{c}0.88 \pm 0.05 \\
-\end{array}$ & $\begin{array}{l}178 \\
177\end{array}$ \\
\hline Solid $\left\{\begin{array}{l}\mathrm{CdCl}_{2} \\
\mathrm{InCl}_{3}\end{array}\right.$ & $0.04 \pm 0.02$ & $\begin{array}{c}0.19 \pm 0.06 \\
-\end{array}$ & $\begin{array}{l}178 \\
177\end{array}$ \\
\hline
\end{tabular}

Tab. I shows that all attenuation factors $G_{2}$ for the $\gamma_{1}^{\prime}-\gamma_{2}$ cascade are indeed above the hard core value $1 / 5$. The attenuation factors in similar environments for the two cascades agree very well, except for the "insulator source". There, the influence of the $K$-capture is very clearly displayed.

The main features of the processes following a $K$-capture seem to be explained by the considerations given here. More experiments, however, are required to clear up all the details. 


\section{$\S 14$. The Determination of the True Correlation}

In several earlier sections, we mentioned source conditions under which one expects the angular correlation to be unperturbed. We summarize here these conditions and list the references where details can be found.

i) Cubic metal crystals (cf. $\S 11 \mathrm{D}$ and references $167,174,176$. The radioactive atoms must sit in regular lattice sites and the environment of each radioactive atom must possess cubic symmetry. Moreover, imperfections in the crystal should be avoided as much as possible. The recoil of the decaying atom can displace it from a regular lattice site $(\alpha-\gamma$ correlations thus are attenuated even in cubic crystals).

ii) Noncubic single crystals (cf. $\S 11 \mathrm{~B}$ and references ${ }^{10,141}$ ). Such crystals yield the unperturbed correlation if the radioactive atoms sit in regular lattice positions and the symmetry axis of the crystal points toward the fixed counter.

iii) Liquids (cf. $\S 12 \mathrm{~A}$ and $\S 13 \mathrm{C}$, and references ${ }^{93}, 116,142,174,177$ ). Liquids generally yield the unperturbed correlation if the correlation time is of the order of $10^{-11} \mathrm{sec}$ or smaller. Molten metals are often very suitable for determining the maximum correlation. The observation of the anisotropy as a function of the viscosity of a liquid and a fit of the resulting data with the theoretical curve may yield a good value for the unperturbed correlation (cf. Fig. 18 and references ${ }^{56,198}$ ).

iv) Delayed coincidences ( $\$ 12 \mathrm{D}$ and references ${ }^{119,}{ }^{142}$ ). The determination of the anisotropy as a function of the delay time allows an extrapolation to the unperturbed value (cf. Fig. 19).

v) Magnetic decoupling (references ${ }^{2,10,20,30,177}$ ). Goertzel ${ }^{2}$ suggested that a strong magnetic field, applied to the source in the direction of the fixed counter, should "decouple" the nucleus from the atomic field and yield the unperturbed correlation. However, this method works only in some special cases ${ }^{30}$, and experiments have so far failed to show the effect unambiguously ${ }^{10,177 .}$

None of the methods $i$ ) $-\mathrm{v}$ ) is guaranteed to yield the unperturbed correlation. It is thus advisable to use more than one method to obtain a reliable value for the true angular correlation function, if the lifetime of the intermediate state is longer than about $10^{-10}$ sec. Caution is especially necessary when one works with cascades following or involving $K$-capture, $\alpha$-decay, and conversion electrons. 


\section{PART C. EXPERIMENTAL METHODS}

In various $\S \S$ of Part $A$, we have briefly discussed some of the experimental arrangements used in angular correlation work. These arrangements are basically the same as those used in conventional coincidence work, with the exception that the angle $\vartheta$, subtended at the source by the axes of the two counters, can be varied. We refer to the pertinent chapters for experimental details (counters, electronic equipment, source preparation, determination of the resolving time, stability checks, energy selection) and discuss here only those problems which are specific to angular correlation work.

\section{$\S 15$. The Treatment of Experimental Data}

A. The dependence of the coincidence rate on the position of the movable detector corresponds to the theoretical correlation function $W(\theta)$ only under the assumption of centered point sources, point detectors, no scattering, and no disturbing radiations. In order to compare experimental and theoretical results, one corrects the experimental data for the various deviations from such an ideal arrangement. In order to outline these corrections, we discuss briefly a $\gamma-\gamma$ directional correlation measurement and its evaluation.

B. One of the first problems to be considered in angular correlation work is scattering. Effects caused by scattering can mask the true correlation. If the radiation under investigation is scattered in the source, the correlation function is falsified in a manner treated below in $f$. Moreover, scattering of any radiation in or near the counters can give rise to spurious coincidences ${ }^{98}$. These in general are not isotropic and therefore simulate a correlation. One way of avoiding such spurious coincidences consists in shielding the counters frontally and laterally with lead. The necessary wall thicknesses for a given $\gamma$-ray energy can be found in reference ${ }^{131}$. If the lead shields are formed as cones which accept only $\gamma$-rays from a point source in the center, a slight maladjustment of the source changes the solid angle markedly. Thus the lead shields should be so constructed that the size of the source and slight decentering do not affect the solid angle. A more refined protection against scattered $\gamma$-rays and spurious coincidences consists in using scintillation spectrometers as detectors and accepting only the photopeaks of the desired $\gamma$-rays (cf. Fig. 6 and reference ${ }^{60}$ ).

The effectiveness of the protection against spurious coincidences can be checked by using a strong source and preventing its $\gamma$-rays from entering one counter directly by placing heavy shielding between the 
source and this counter. All measured true coincidences are then spurious.

C. Before a run, resolving time $\left(\tau_{R}\right)$ and background $\left[N_{f}^{0}(\vartheta), N_{m}^{0}(\vartheta)\right.$, and $C^{0}(\vartheta)$ ] are determined. (We denote with $N$ and $C$ single counts and coincidences, respectively, per unit time; the index $f$ refers to the fixed counter, $m$ to the movable counter.)

A source of proper strength is prepared. The maximum source strength is determined by the ratio of accidental to true coincidences. For accurate measurements, this ratio should be smaller than about 0.2 ; under unfavorable conditions, one may be forced to make it as high as unity.

The source is then "centered" to about $1 \%$; i.e. the counting rate $N_{m}(\vartheta)$ of the movable counter as a function of the angle $\vartheta$ should be constant to about $1 \%$.

After the source is centered, the coincidence rate and the single count rates are measured for various angles. The angle should be changed frequently. The choice of the angles depends on the form of the correlation function. In general, it is best to use a few angles only (e.g. $90^{\circ}, 120^{\circ}, \ldots$, $270^{\circ}$ ) and to obtain at each angle a large number of coincidences.

From the measured data, one obtains the "true" values $C^{t}(\vartheta)$ and $N^{t}(\vartheta)$ by subtracting background, accidental coincidences $\left[=2 \tau_{R} N_{m}(\vartheta) N_{f}(\vartheta)\right]$ and, if necessary, contributions due to disturbing radiations. The coincidence ratio $R_{c}(\vartheta) \equiv C^{t}(\vartheta) / N_{m}^{t}(\vartheta)$ is then formed. This ratio is independent of the decay of the source. Moreover, small errors in the centering of the source are corrected in first order.

The coincidence ratios $R_{c}(\vartheta)$ now are fitted by

$$
R_{c}(\vartheta)=\text { const }\left\{1+A_{2}^{\prime} P_{2}(\cos \vartheta)+\ldots+A_{v \max }^{\prime} P_{v \max }(\cos \vartheta)\right\} \text {. }
$$

(All correlation functions measured to date can be fitted by this formula with $v_{\max }=4$; cf. $\S 4 \mathrm{~B}$ ). Rose ${ }^{199}$ has exhaustively treated the determination of the coefficients $A_{v}^{\prime}$ by a least square fit, and also the calculation of the errors in $A_{v}$ and the determination of $v_{\max }$. An example of the application of Rose's formulas is given in reference ${ }^{54}$. (Frequently, the constant in Eq. (57) has been chosen so that the fitted curve passes through the experimental point at $90^{\circ}$. This method lends infinite weight to the measurement at $90^{\circ}$ and is clearly not correct.)

D. The next step consists in correcting the coefficients $A_{v}^{\prime}$ for the finite solid angle of the counters. Using a method first developed by Frankel ${ }^{97}$, and neglecting the correction for the extension of the source, we write the corrected coefficients $A_{v}$ as ${ }^{55}$

$$
A_{v}=\left(Q_{0} / Q_{v}\right) A_{v}^{\prime} .
$$


In order to determine the solid angle correction factors $Q_{v}$, we assume here that the efficiency $\varepsilon$ of the detectors is a function only of the angle $\alpha$ between the counter axis and the direction of propagation of the incident particle. We call such a detector cylindrically symmetric or circular (most scintillation counters fall in this category). The correction formulas for arbitrary detectors have been worked out by Feingold and Frankel ${ }^{200}$. For a circular detector, the $Q_{v}$ can be determined in various ways:

i) As an approximation ${ }^{98}$, one can use the coefficients $\left(Q_{0} / Q_{2}\right)=(1-3 \omega)^{-1}$ and $\left(Q_{0} / Q_{4}\right)=(1-10 \omega)^{-1}$. Here $\omega=\Omega / 2 \pi$, where $\Omega$ is the solid angle of one of the two identical detectors; the efficiency $\varepsilon(\alpha)$ is assumed to be constant over the whole counter. This approximation, which is valid for $\omega \ll 1$, may be useful for quick estimates.

ii) The angular efficiencies $\varepsilon(\alpha)$ of the two detectors are determined experimentally by using a well collimated $\gamma$-ray beam. A numerical integration then yields $Q_{0}, Q_{2}, \ldots$ (cf. reference ${ }^{55}$ ).

iii) The correction factors can be calculated if the shape of the scintillation crystals and the distances from the source to the crystals are known (cf. reference ${ }^{199}$ ). This approach works well if the scintillation crystals are perfect and not shielded. It has been shown experimentally ${ }^{60}$ that both methods ii) and iii) then yield identical results. If the crystals are shielded, however, the solid angle is enlarged because some of the $\gamma$-rays scattered in the shielding are also counted. Method ii) then must be applied.

Both methods ii) and iii) are applicable regardless of the energy of the $\gamma$-rays, provided the coefficients $Q_{v}$ are properly calculated ${ }^{55}$.

iv) If the energies of the $\gamma$-rays are not very different from that of annihilation radiation, one can measure the angular resolution of the counters with annihilation radiation ${ }^{201}$. The coefficients $Q_{v}$ are found by a numerical integration ${ }^{55}$.

E. In most correlation experiments, the source can be so chosen that no correction for source size is necessary. In some cases (e.g. very small specific activities) one may be forced to use large sources and the following consideration then applies $55,98,200$.

The only source size correction which has been treated rigorously is that for an "axial" source, i.e. a line source located at the origin and oriented perpendicular to the plane of the two counters. Thus in experimental work, one best prepares the source as a long thin cylinder and

200 A. M. Feingold and S. Frankel, to be published in Phys. Rev.

201 E. L. Church and J. J. Kraushaar, Phys. Rev. 88 (1952) 419. 
corrects only for the axial extension. All necessary formulas are given in reference ${ }^{200}$.

F. After having corrected the coefficients $A_{v}$ for finite solid angle and finite source extension, one is left with the problem of correcting them for scattering in the source. Unfortunately, this correction is more complex and more difficult to perform than the others.

Suppose the activity to be dispersed uniformly in a cylindrical source. Two effects then mask the correlation function: i) Some of the $\gamma$-rays are deflected before being counted. This effect, caused by Compton scattering, tends to "smear out" the correlation, i.e. it always decreases the magnitude of the coefficients $A_{v}$.ii) The $\gamma$-ray pairs which give coincidences in the $90^{\circ}$ position are on the average less absorbed than those which give coincidences in the $180^{\circ}$ position. Thus one misses more coincidences at $\theta=180^{\circ}$ than at $\theta=90^{\circ}$. A correlation function with negative anisotropy is enhanced, one with positive anisotropy is attenuated. The absorption occurs mainly through the photoeffect. However, the Compton effect also contributes to the. "absorption", because the $\gamma$-rays can lose so much energy that they no longer are counted. The absorption is very pronounced for low $\gamma$-ray energies and sources with high atomic number $Z$.

If the radioactive source itself is very thin, but surrounded by scattering material, only effect $i$ ) is present and the correlation is attenuated.

The unperturbed correlation function can be found experimentally by measuring the anisotropy for various source diameters and plotting it against the probability that a $\gamma$-ray pair escape from the source without undergoing either photoeffect or Compton effect.

G. We have now discussed all steps which are necessary to derive the coefficients $A_{v}$ from the measured data. The coefficients $A_{v}$ finally can be compared with theory.

Before attacking an unknown correlation function, it is advisable to check the correct working of the whole apparatus by determining the coefficients $A_{v}$ and the anisotropy $A$ for well known cascades. $\mathrm{Ni}^{60}$ and $\mathrm{Pd}^{106}$ have suitable properties (cf. $\S 4 \mathrm{E}$ and $\S 4 \mathrm{~F}$ ). 


\section{§ 1. Introduction}

The spins and parities of atomic nuclei, as well as their magnetic moments and electric quadirupole moments, are of great interest for testing and improving nuclear models, and a variety of methods have been evolved for their study. In the following we will review those methods which depend on orienting nuclei along a fixed direction in the laboratory system at low temperatures.

Given a nucleus with spin (total angular momentum) $j$ in a field free space, there is a $2 j+1$ degeneracy, which can be removed by the application of a magnetic field. If the direction of the magnetic field is chosen as the $z$-direction we can interpret the $2 j+1$ "magnetic substates" as different orientations of the spin $j$, such that its $z$-component, the magnetic quantum number $m$, varies from $-j$ to $j$, in unit steps.

In a statistical assembly of many such nuclei, with spins oriented at random, the populations $a_{m}$ of the different substates are independent of $m$. An assembly in which $a_{m}$ varies with $m$ is said to be oriented with respect to the $z$-axis. Such ordering of the spins can be defined completely in terms of a set of parameters $f_{k}(j)$ (see following article) which are functions of the $a_{m}$. For our purposes, however, it is sufficient to separate the types of ordering into two groups according as all $f_{k}(j)$ with odd $k$ do or do not vanish. In the latter case, the nuclei are so oriented that. there is a resultant spin along the axis of symmetry and they are said to be polarized, whereas in the former case (in particular when $a_{m}=a_{-m}$ ) there is no resultant spin and they are said to be,alined.

\section{$\S 2$. Production of Oriented Nuclei}

By the application of magnetic or inhomogeneous electric fields to the magnetic or electric multipole moment of the nucleus each substate $m$ is given a different energy $E(m)$. The equilibrium populations of these states will then be governed by a Boltzmann function $a_{m}=A \mathrm{e}^{-E(m) / k T}$. At temperatures where $\beta=[E(m)-E(m+1)] / k T$ is small the populations are closely the same and the nuclear orientations are random. However, when $\beta$ is of the order of unity these populations will be unequal and there is consequently some degree of nuclear orientation.

Apart from the application of external fields there are a number of ways in which use is made of ionic and crystalline fields. For their descrip- 
tion it is convenient to use the spin Hamiltonian of Abragam and Pryce ${ }^{1}$. They have shown that the energy levels of an ion in a paramagnetic crystal possessing axial symmetry about the $z$-axis, in the presence of both crystalline fields and an external magnetic field applied along this axis, may be represented by a Hamiltonian of the following form:

$$
\begin{aligned}
\mathscr{H}=\beta_{B}[ & \left.g_{/ /} B_{z} S_{z}+g_{\perp}\left(B_{x} S_{x}+B_{y} S_{y}\right)\right]+D\left[S_{z}^{2}-1 /{ }_{3} S(S+1)\right] \\
& +\left[A S_{z} j_{z}+B\left(S_{x} j_{x}+S_{y} j_{y}\right)\right]+Q\left[j_{z}^{2}-{ }^{1} /{ }_{3} j(j+1)\right]-(\mu / j) \mathbf{B} \cdot \mathbf{j} .
\end{aligned}
$$

$\beta_{B}=$ Bohr magneton.

The first term represents the splitting of the electronic levels by the external field $B$ and is formulated in terms of an effective electron spin $S$ defined by setting the multiplicity of the electronic levels equal to $2 S+1$. $g_{/ /}$and $g_{1}$ are the atomic $g$-factors parallel and perpendicular to the $z$-axis. The term in $D$ represents the splitting of the electronic levels by the crystalline electric field. $j$ is the nuclear spin and the $A$ and $B$ terms represent the h.f.s. splitting due to the interaction between the nuclear magnetic moment and the unfilled electron shells which give rise to the magnetic properties of a paramagnetic ion. The $Q$ term is the nuclear electric quadrupole splitting in the crystalline electric field gradient. $\left(Q=3 e \frac{\partial^{2} V}{\partial z^{2}} \times \frac{1}{4 j(2 j-1)} \times\right.$ (nuclear electric quadrupole moment) where $V$ is the electric field potential.) The last term corresponds to the direct coupling between the external magnetic field and the nuclear magnetic dipole moment $\mu$.

Four methods have been suggested for orienting nuclei (see for instance Simon, Rose and Jauch ${ }^{2}$ ). For their discussion it is convenient to suppose that in (1) some of the terms are zero.

(i) Direct interaction of the nuclear magnetic moment $3,4,5$ with a large external magnetic field causes a splitting $\Delta E=\mu B / j$ between neighbouring magnetic substates: this is characterized by the term $(\mu / j) \mathbf{B} \cdot \mathbf{j}$ of the spin Hamiltonian.

The population of individual states will then be proportional to

$$
a_{m}=\mathrm{e}^{m \beta} / \sum_{m} \mathrm{e}^{m \beta} \quad \text { where } \beta=\mu B / k T j .
$$

To achieve appreciable polarization, $\beta$ must be of the order of unity, which means very high field and very low temperature. For instance, if $j=2$ and $\mu=1$ values of $B / T \sim 5 \times 10^{7}$ are required; thus one would need,

A. Abragam and M. H. L. Pryce, Proc. Roy. Soc. A205, (1951) 135.

A. Simon, M. E. Rose and J. M. Jauch, Phys. Rev. 84, (1951) 1155.

C. J. Gorter, Phys. Z. 35, (1934) 923.

N. Kurti and F. E. Simon, Proc. Roy. Soc. A149, (1935) 152.

5 F. E. Simon, C. R. Cong, Sur le magnetisme, Strasbourg 3, (1939) 1. 
for example, 50,000 Gauss at $0.001^{\circ} \mathrm{K}$. Such experiments are very difficult, and no successful attempt has yet been reported.

(ii) A method which avoids the need for such intense magnetic fields has been proposed independently by Gorter ${ }^{6}$ and Rose ${ }^{7}$. Their suggestion is based on the fact that the unfilled electron shell produces a magnetic field at the nucleus of a paramagnetic ion which is known from data on hyperfine structure to be of the order of $10^{5}$ to $10^{6}$ gauss. At sufficiently low temperatures each nucleus will orient itself with respect to the electronic field in an energetically most favourable direction. Nuclear orientation will follow automatically if the electronic magnetic moments are oriented, and a small external field of the order of a few hundred gauss suffices for this at the low temperatures at which the nuclear orientation will become ordered. This method is described by the $A, B$ and $g$ terms. It leads to nuclear polarization. The cooling is produced either by a separate paramagnetic salt (Gorter) or by the substance to be investigated acting as its own cooling agent (Rose). Ambler et al. ${ }^{8}$ have given an account of an experiment in which this method has been shown to work.

Two other methods have been put forward by Pound ${ }^{9}$ and Bleaney ${ }^{10,11}$ in which the orientation of the nucleus results directly or indirectly from the action of local electric fields. The direction of these fields is fixed with respect to the crystallographic axes, and to obtain spatial ordering a single crystal (or several with their axes parallel) must be used. Another significant difference from the foregoing methods is that a nuclear alinement results with no resultant polarization.

(iii) The method suggested by Pound ${ }^{9}$ makes use of the hyperfine structure associated with the nuclear electric quadrupole moment. The degeneracy associated with the orientation of the nucleus is resolved through the interaction of the nuclear electric quadrupole moment with an electric field gradient. In general such gradients must be at least of the order $10^{15}$ e.s.u. to give a h.f.s. of the required magnitude, and they can be produced only by an asymmetrical distribution of the electron cloud immediately around the nucleus. Such a distribution can be set up in homopolar bonds, and if these bonds have a parallel orientation for all similar nuclei, as may be the case in a suitable organic compound, nuclear orientation will set in at sufficiently low temperatures. No experiment with this method has as yet been successful.

(iv) The method due to Bleaney ${ }^{10,11}$ like method (iii) is based on the

6 C. J. Gorter, Physica 14, (1948) 504.

7 M. E. Rose, Phys. Rev. 75, (1949) 213.

8 E. Ambler, M. A. Grace, H. Halban, N. Kurti, H. Durand, C. Johnson and H. R. Lemmer, Phil. Mag. 44, (1953) 216.

9 R. V. Pound, Phys. Rev. 76, (1949) 1410. 
fact that the different orientations of the nuclear moments relative to a crystal axis may have different energy. In this method this energy difference is due to the interaction between the inhomogeneous crystalline electric field and the electronic magnetic moments through the intermediary of the electronic orbits. The same mechanism is responsible for the anisotropy of the magnetic susceptibility. The strong electric field set up by the neighbouring ions in the crystal in the absence of external magnetic fields defines an axis of quantization. The interaction is often so strong that already at comparatively high temperatures $\left(10^{\circ}-100^{\circ} \mathrm{K}\right)$ only the lowest levels of quantization will be occupied. If the responsible electric fields have axial symmetry alinement of electronic magnetic moments relative to the axis of a single crystal will take place. This will lead at sufficiently low temperatures to nuclear alinement through h.f.s. coupling. Temperatures of about $0.01^{\circ} \mathrm{K}$ are required. They are most conveniently reached by adiabatic demagnetization of the paramagnetic single crystal. In this method $B=0$ and only the $D, A$ and $B$ terms contribute to the spin Hamiltonian. Successful experiments making use of this effect have been described by both the Oxford group 12, 13, 14, 15, 16 and the Leiden group 17, 18, 19, 20.

Although in these ideal cases certain terms vanish, in most practical cases their contribution will be significant, and thus the degree of nuclear orientation will deviate from the limiting case where the interaction is simple.

Such are the processes which have been suggested as possible methods of obtaining oriented nuclei. Some of the experimental conditions which distinguish these different mechanisms are summarized in Table $I$.

10 B. Bleaney, Proc. Phys. Soc. A64, (1951) 315.

11 B. Bleaney, Phil. Mag. 42, (1951) 441.

12 J. M. Daniels, M. A. Grace and F. N. H. Robinson, Nature, Lond., 168, (1951) 780.

13 B. Bleaney, J. M. Daniels, M. A. Grace, H. Halban, N. Kurti and F. N. H. Robinson, Phys. Rev. 85, (1952) 688.

14 J. M. Daniels, M. A. Grace, H. Halban, N. Kurti and F. N. H. Robinson, Phil. Mag. 43, (1952) 1297.

15 M. A. Grace and H. Halban, Physica 18, (1952) 1227.

16 B. Bleaney, J. M. Daniels, M. A. Grace, H. Halban, N. Kurti, F. N. H. Robinson and F. E. Simon, Proc. Roy. Soc. A221, (1954) 170.

17 C. J. Gorter, O. J. Poppema, M. J. Steenland and J. A. Beun, Physica 17, (1951) 1050.

18 C. J. Gorter, H. A. Tolhoek, O. J. ${ }_{0}$ Poppema, M. 'J. Steenland and J. A. Beun, Physica 18, (1952) 135.

19 O. J. Poppema, J. A. Beun, M. J. Steenland and C. J. Gorter, Physica 18, (1952) 1235.

20 O. J. Poppema, Thesis, Groningen 1954. 
TABLE I

\begin{tabular}{|c|c|c|c|c|}
\hline \multicolumn{2}{|r|}{ Method } & Temp. & Applied field & Specimen \\
\hline (i) & $\begin{array}{l}\text { External field } \\
\text { (Polarization) }^{3,4}\end{array}$ & $0.01^{\circ} \mathrm{K}$ & $10^{5}-10^{6}$ gauss & $\begin{array}{l}\text { Non-magnetic: } \\
\text { external cooling }\end{array}$ \\
\hline (ii) & $\begin{array}{l}\text { Magnetic h.f.s. } \\
\text { (Polarization) } 6,7\end{array}$ & $0.01^{\circ} \mathrm{K}$ & $10^{2}$ gauss & $\begin{array}{l}\text { Paramagnetic crystals, } \\
\text { isotropic h.f.s. }\end{array}$ \\
\hline (iii) & $\begin{array}{l}\text { Electric h.f.s. } \\
\text { (Alinement) }^{9}\end{array}$ & $0.001^{\circ} \mathrm{K}$ & none & $\begin{array}{l}\text { Single crystal, nuclei with } \\
\text { E.Q. moment, suitable } \\
\text { internal electric fields }\end{array}$ \\
\hline (iv) & $\begin{array}{l}\text { Magnetic h.f.s. } \\
\text { (Alinement) } 10,11\end{array}$ & $0.01^{\circ} \mathrm{K}$ & none & $\begin{array}{l}\text { Single crystal, internal elec- } \\
\text { tric fields acting on para- } \\
\text { magnetic ion }\end{array}$ \\
\hline
\end{tabular}

\section{$\S 3$. Properties and Detection of Oriented Nuclei}

A number of methods are available for the detection of oriented nuclei. If the nuclei are radioactive the angular distribution of the radiation, whether particle or electromagnetic, will depend on both the degree of orientation of the parent nuclei and the angular momenta involved in the transition. Thus the detection of an anisotropic distribution of radiation demonstrates the existence of oriented nuclei. Associated with this angular distribution will be a polarization of the radiation.

Another method of detection is to study the interaction of the nuclei with beams of particles or $\gamma$-rays ${ }^{21}$. This method has been tried using thermal neutrons by Poppema 20, 22. However no significant effect was obtained. More recently Bernstein et al. ${ }^{23}$ using polarized thermal neutrons and polarized $\mathrm{Min}^{55}$ nuclei obtained an effect of $3.4 \%$.

For completeness it should be mentioned that the method of nuclear paramagnetic resonance might also be applied. Radiofrequency radiation in resonance will change the population of the magnetic sublevels. This process could in principle be detected either by a change in the angular distribution of nuclear radiation or by standard radiofrequency methods. (See § 4.)

\section{$\S 4$. Angular Distribution and Polarization of Radiation}

A theoretical investigation of the angular distribution of radiation from radioactive nuclei has been made by Spiers ${ }^{24}$ and later extended by Steen-

21 H. Halban, Nature, Lond. 140, (1937) 425.

22 O. J. Poppema, Helv. Phys. Act. 23, (Supplement 3) (1950) 187.

23 S. Bernstein, J. W. J. Dubbs, L. D. Roberts, A. K. Snell, C. P. Stanford and T. E. Stephenson. Private communication.

24 J. A. Spiers, Nature, Lond. 161, (1948) 807; Directional Effects in Radioactivity, N.R.C.C. publication. 
berg ${ }^{25}$ and by Tolhoek and Cox ${ }^{26}$ to cases where several radiations are emitted in cascade. In the following article a detailed summary of this work is given and closed forms for the angular distribution functions $W(\Theta)$ are obtained in terms of the $f_{k}(j)$ and parameters dependent on the type of transition considered.

When all the $a_{m}$ are equal, corresponding to random orientation of the nuclei, $W(\Theta)=$ constant and an isotropic distribution of radiation is found. In cases where the angular distribution is not spherically symmetrical, $W(\Theta)$ involves only Legendre functions of even order and can therefore be expanded in even powers of $\cos \Theta$. The maximum power of $\cos \Theta$ appearing will be $2 l$ where $l \leqslant L$ and $j$; here $L$ is the maximum value of the orbital angular momentum carried away by an emitted particle or the multipolarity of a $\gamma$ transition. Thus:

$$
W(\Theta)=1+a \cos ^{2} \Theta+b \cos ^{4} \Theta+\ldots+\lambda \cos ^{2 l} \Theta
$$

where the coefficients $a, b \ldots$ will be functions of the angular momenta and the initial populations. When the initial substates are equally spaced by an amount $\mu B / j$ (e.g. arising from the interaction between the nuclear magnetic moment and an external magnetic field) the population function

$$
a_{m}=\mathrm{e}^{m \beta} / \sum_{m} \mathrm{e}^{m \beta}
$$

where $\beta=\mu B / k T j$ and therefore the coefficients $a, b \ldots$ will depend on the temperature through this parameter $\beta$.

Where there are a number of radiations in cascade the general form of the angular distribution is again

$$
W(\Theta)=1+a \cos ^{2} \Theta+b \cos ^{4} \Theta+\ldots+\lambda \cos ^{2 i} \Theta
$$

both for each member of the cascade and for the radiation as a whole. The maximum value of $l$ appearing will be governed by the values of $j$ and $L$ occurring in the transitions and again the coefficients $a, b \ldots$ will involve the angular momenta together with the Boltzmann parameter $\beta$.

In cases where the spacing of nuclear levels is unequal or the levels are no longer pure, due to admixtures of other nuclear states, more detailed calculations are necessary.

It is here assumed that the time between successive emission processes is so short that the populations of intermediate nuclear states are governed only by the initial equilibrium distribution and the character of the

25 N. R. Steenberg, Phys. Rev. 84, (1951) 1051; Proc. Phys. Soc. A65, (1952) 791 ; Proc. Phys. Soc. A66,(1953) 391; Proc. Phys. Soc. A 66,(1953) 399; Can. J. Phys. 31, (1953) 204; Thesis, Oxford (1953).

26 H. A. Tolhoek and J. A. M. Cox, Physica 18, (1952) 357; Physica 18, (1952) 1257; Physica 18, (1952) 1262; Physica 18, (1952) 359; Physica 19, (1953) 101. 
preceding emission processes in the cascade. When this time is not short disturbance to the populations of the states may occur through such processes as the interaction of the nuclear spin with the thermal vibrations of the lattice; recoil of the nucleus from its lattice position through regions of different fields; changes in magnetic or electric field gradient at the nucleus following a $\beta$-process due to the atomic rearrangement or to ionisation or excitation of the atom. Maximal information will only be gained from a nuclear orientation experiment when the absence of disturbance has been established or its magnitude determined.

The following conclusions may be drawn:

No angular distribution different from isotropic can be expected from states preceded in a cascade by a state of angular momentum 0 .or $1 / 2$.

Particle emission is isotropic if $L$, the orbital angular momentum of the particles emitted, is zero. If $L$ is non-zero the angular distribution will characterize the angular momenta of the nuclear levels and the initial substate populations.

At temperatures where complete orientation of the nuclear spins $\left(j_{\text {inttial }}\right)$ is obtained the angular distribution of the particles will depend only on $L$ and the angular momenta $j_{\text {initial }}, j_{\text {fnal }}$ of the levels: thus a single measurement of the angular distribution at these temperatures may give $j_{\text {initial }}, j_{\text {enal }}$ and $L$. At higher temperatures where nuclear sublevels other than the lowest are occupied, the angular distribution will yield information on their relative populations. Thus when these are equally spaced due, for instance, to magnetic h.f.s. coupling the Boltzmann factor, $\beta=\mu B / k T j_{\text {initial }}$ may be found: if $B$ and $T$ are known a value for $\mu$ the nuclear magnetic moment of the initial state may be inferred.

Similar conclusions may be drawn from observations of $\gamma$-radiation from oriented nuclei whether or not they are preceded by a previous emission process. In cases where previous emission has occurred, the angular distribution will involve the angular momenta of the previous processes. Thus in the observation of $\gamma$-radiation following a $\beta$-process it may be possible to determine the angular momentum carried away in the $\beta$-process. This gives information on the relative contributions of Fermi and Gamow-Teller type interactions in the $\beta$-decay process. Such information is not easily accessible for allowed $\beta$-transitions by more conventional methods. Because. only even powers of $\cos \Theta$ occur in these angular distributions nuclear alinement is sufficient to produce anisotropy (see, however, below).

Associated with this anisotropic distribution of radiation there will be a partial or total transverse polarization of the $\gamma$-radiation ${ }^{25,26}$. The plane of the electric vector will depend on the direction of observation, the 
multipolarity of the transition and the electric or magnetic character of the transition. It is thus possible to determine not only the angular momenta involved in a transition (from the angular distribution of the radiation) but also the parity change.

All phenomena characteristic of alinement are observed with polarization. There are, however, additional effects associated specifically with polarization. The $\gamma$-radiation from polarized radioactive nuclei will in general contain circularly polarized components ${ }^{13}, 25,26$. The sense of this polarization will depend particularly on the sign of the nuclear magnetic moment. In $\beta$-radioactive nuclei the spins of the $\beta$-particles may be polarized. The theory of this process has been examined by Tolhoek and de Groot ${ }^{27}$ (see following article) who relate the polarization to the character of the $\beta$-transition. Electron spin polarization has been produced and detected by a double scattering process. Although only single scattering would have to be achieved the application of this method to polarized nuclei presents formidable difficulties.

\section{$\S 5$. Experimental Results with Oriented Nuclei}

\section{A. Cobalt 60}

The bulk of experimental results on oriented nuclei is confined to the observation of $\gamma$-radiation from radioactive sources. The earliest results were reported by Daniels et al. ${ }^{12}$ who obtained alined nuclei by method (iv). A single crystal of $\mathrm{CuSO}_{4} \cdot \mathrm{Rb}_{2} \mathrm{SO}_{4} \cdot 6 \mathrm{H}_{2} \mathrm{O}$, in which some of the copper had been replaced by cobalt 60 and the whole diluted with zinc, was cooled to about $0.01^{\circ} \mathrm{K}$ by adiabatic demagnetization, the copper ions acting as principal cooling agent. In this crystal Bowers ${ }^{28}$ had shown that for the cobalt ions $A \gg B$ and at these temperatures considerable nuclear alinement should be obtained. The disintegration scheme ${ }^{29}$ of cobalt 60 (Fig. 1) shows that two $\gamma$-rays are emitted in cascade following a $\beta$-transition. If the anisotropy of the $\gamma$-radiation $\varepsilon$ is defined as $[W(\pi / 2)-$ $-W(0)] / W(\pi / 2)$ where $W(\Theta)$ is the intensity of the radiation observed at an angle $\Theta$ with the axis of alinement it would be expected that for this disintegration scheme $\varepsilon=1$ at complete saturation of the nuclear spins. However, in this crystal there are two cobalt ions in each unit cell with their axes of symmetry at $74^{\circ}$ and the maximum value of $\varepsilon$ will be $\cos ^{4} 37^{\circ}=0.41$. Values of $\varepsilon$ up to 0.3 were obtained and their variation with temperature was in satisfactory agreement with theory. It. was necessary to apply a number of corrections involving some degree of uncertainty, notably for

27 H. A. Tolhoek and S. R. de Groot, Physica 17, (1951) 81.

28 K. D. Bowers, Thesis, Oxford (1953).

29 M. Deutsch and G. S. Goldhaber, Phys. Rev. 83, (1951) 1059. 
the scattering of the $\gamma$-rays in the low temperature cryostat and for the difference between absolute temperature and that determined by susceptibility measurements. The theoretical curve (Fig. 2) chosen for fitting the points was that corresponding to $j_{\beta}=5$ where $j_{\beta}$ is the spin of $\mathrm{Co}^{60}$ : from measurements on the decay scheme (Fig. 1) this seemed the most probably assignment: this theoretical curve was the same for each $\gamma$-ray of the

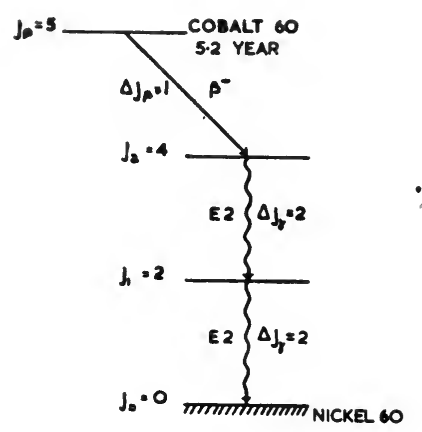

Fig. 1. Decay scheme of cobalt 60

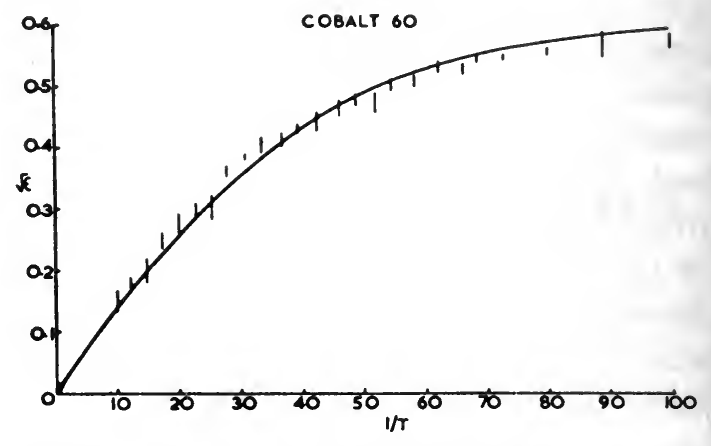

Fig. 2. Variation of anisotropy with temperature showing experimental points and theoretical curve for $J_{\beta}=5 \Delta J_{\beta}=1$

cascade. Sufficient precision was not available to distinguish between this spin assignment and other less likely choices. The correlation between $\beta$ and $1 / T$ gave a value for $\mu$ the magnetic moment in terms of the h.f.s. field which had been determined by paramagnetic resonance measurements on the stable nucleus. A value for $\mu$ of $3.5 \pm 0.5$ nuclear magnetons was obtained ${ }^{13}, 14,16$. Correction was made for the inequality of level spacing in the h.f.s. and the mixing of nuclear states introduced by the finite value of $B$.

This result was confirmed by the Leiden group $18,19,20$ using a crystal of dilute $\mathrm{Co}\left(\mathrm{NH}_{4}\right)_{2}\left(\mathrm{SO}_{4}\right)_{2} 6 \mathrm{H}_{2} \mathrm{O}$. In this case two types of experiment were performed. In one the sample was demagnetized from a known value of $B / T$ using stable cobalt as cooling agent and the Boltzmann factor $\beta$ of the cobalt 59 calculated from the known amount of entropy removed: (the sample was screened from heat leakage by embedding it in chrome alum). $\beta$ for the cobalt 60 was deduced from $\varepsilon$ for the $\gamma$-radiation. Comparison of the two values of $\beta$ gave an approximate value of $\mu$ for cobalt 60 of 3.9 nuclear magnetons ${ }^{20}$. In the second case the sample in the absence of chrome alum was demagnetized using copper as cooling agent and the dependence of $W(\Theta)$ on the magnetic temperature $T^{*}$ found for a number of values of $\Theta$ (see Fig. 3). Comparison of this with theoretical dependence of $W(\Theta)$ on $\beta$ in the region $\left(\sim 0.07^{\circ} \mathrm{K}\right)$ where the $T / T^{*}$ correction is small $(1.5 \%)$ gave a value for $\mu\left(\mathrm{Co}^{60}\right)$ of $4.3 \pm 0.2$ nuclear magnetons. 
Experiments by Bishop et al. ${ }^{30}$ on the transverse polarization of the quadrupole $\gamma$-radiation from cobalt 60 confirmed that the transitions are electric in character.

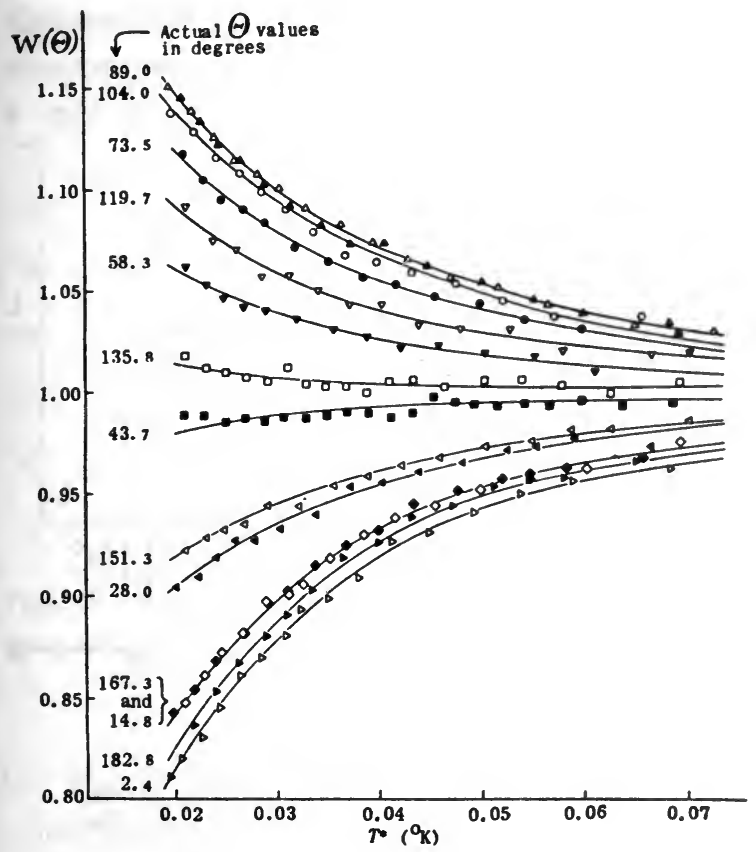

Fig. 3. Normalized intensity $W(\Theta)^{\dagger}$ of $\gamma$-radiation from $\mathrm{Co}^{60}$ in single crystals as a function of $T^{*}$, for different values of $\Theta$. Open points refer to one counter and solid points to another: readings on both counters were obtained at $\Theta=89.0^{\circ}$

Ambler et al. ${ }^{8}$ have described an experiment in which nuclear polarization has been obtained by method (ii) (magnetic h.f.s. polarization). A mixed crystal of $\mathrm{Ce}_{2}\left(\mathrm{NO}_{3}\right)_{6} \cdot \mathrm{Mg}_{3}\left(\mathrm{NO}_{3}\right)_{6} \cdot 24 \mathrm{H}_{2} \mathrm{O}$ was demagnetized to a temperature of about $0.004^{\circ} \mathrm{K}$. In this crystal some of the $\mathrm{Mg}$ was replaced by $\mathrm{Co}^{60}$. Paramagnetic resonance experiments by Trenam ${ }^{31}$ have shown that for two of the three Co ions in each unit cell $A$ and $B$ are approximately equal, i.e. they possess isotropic h.f.s. Thus in the absence of a polarizing field these nuclei should show isotropic distribution of radiation. The results show that in the presence of a polarizing field of about 500 gauss values of $\varepsilon$ up to 0.5 are obtained. No detailed interpretation of these

30 G. R. Bishop, J. M. Daniels, G. Goldschmidt, H. Halban, N. Kurti and F. N. H. Robinson, Phys. Rev. 88, (1952) 1432.

31 R. S. Trenam, Proc. Phys. Soc. A66, (1953) 118.

+ Again in this crystal there are two axes of alinement. The angle $\Theta$ in this figure is different from that used in the text; it is the angle between the direction of emission of the $\gamma$-ray and the internal bisector of the angle between these two axes of alinement. Thus it is equal to $\cos ^{-1}\left(\cos \Theta / \cos 33^{\circ}\right)$. 
results has been made owing to the unknown influence of the third ion of the unit cell in which $A$ is much greater than $B$.

\section{B. Cobalt 58}

Using their earlier method Daniels et al. ${ }^{14}$ have carried out nuclear alinement experiments with cobalt 58, a case where spin assignments were uncertain. Thus starting with the decay scheme shown in Fig. 4

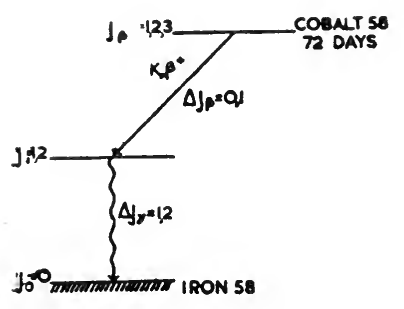

Fig. 4. Decay scheme of cobalt 58

Fig. 5. Variation of anisotropy with $\beta$ (theory) for cobalt 58

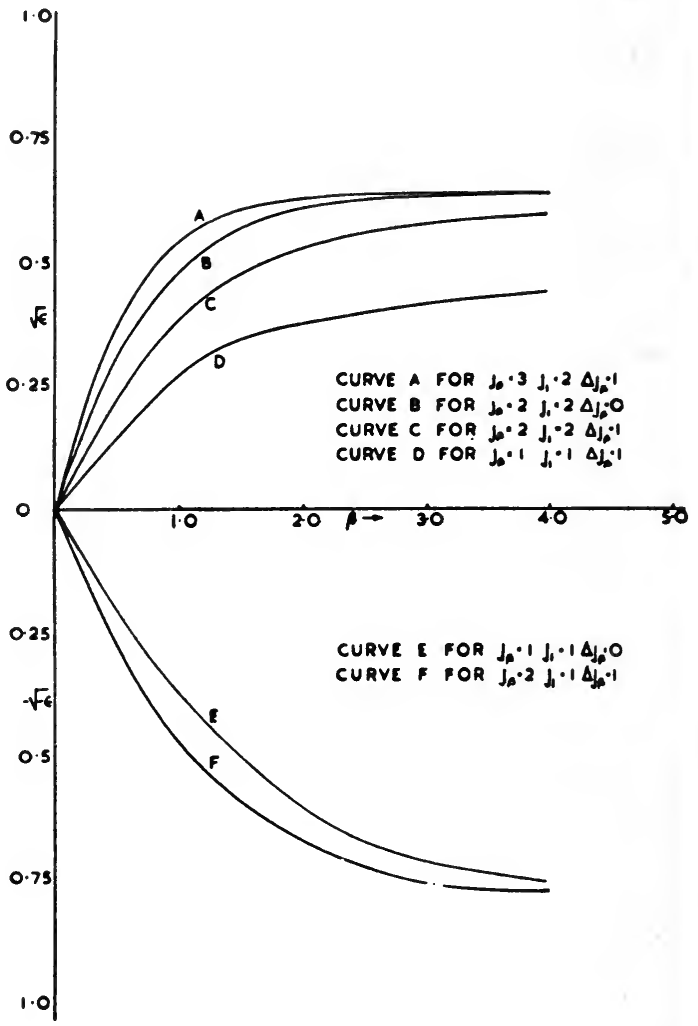

since $j_{0}=0$ (even-even nucleus), it was concluded that $j_{1}$ must be 1 or 2 since the lifetime of the state was $10^{-7} \mathrm{sec}$ or less. From the theoretical and observed angular distributions (Figs. 5 and 6) it was shown that the radiation could not be dipole and therefore $j_{1}=\Delta j_{\gamma}=2$. By determining the parameters $a, b$ of the angular distribution

$$
W(\Theta)=1-a \cos ^{2} \Theta-b \cos ^{4} \Theta
$$

it was shown from the dependence of $b$ on temperature that the most satisfactory agreement was obtained for $j_{\beta}=2$ and $\Delta j_{\beta}=1$ (Fig. 7). If no parity change occurred in the $\beta$-transition (this was suggested by the comparative half-life of the transition) it was concluded that an axial- 
vector or tensor interaction was predominant. A value for $\mu$ of $3.5 \pm 0.3$ nuclear magnetons was found.

Measurements of the plane of polarization of this $\gamma$-radiation by Bishop et al. ${ }^{30}$ showed that this transition is electric in character and therefore the excited state of iron 58 has even parity.

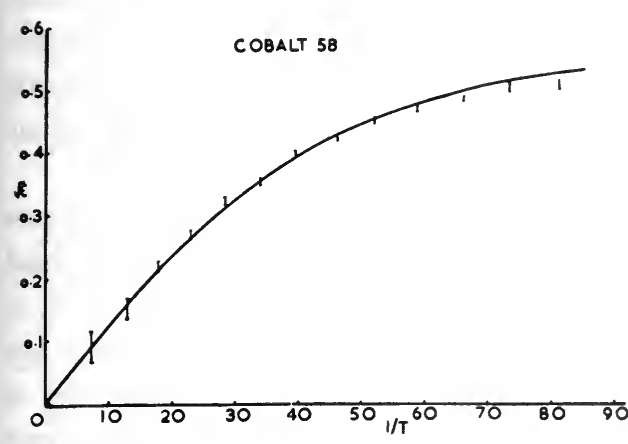

Fig. 6. Variation of anisotropy with temperature showing experimental points and theoretical curve for $J_{\beta}=2 \quad J_{1}=2 \Delta J_{\beta}=1$

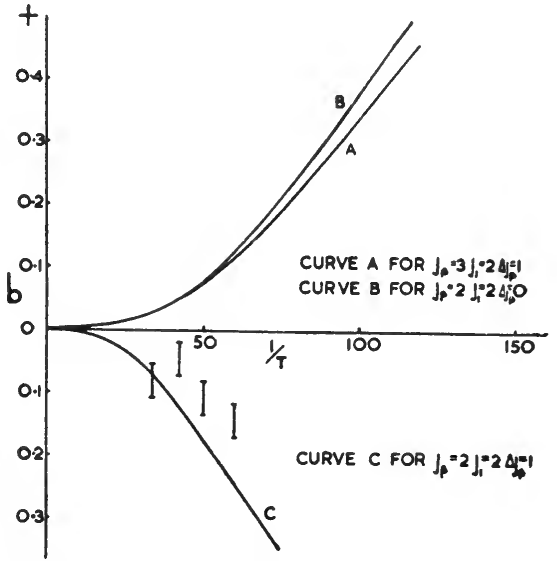

Fig. 7. Showing the variation of $b$ with temperature for different spin assignments in the decay of cobalt 58

\section{Manganese $\mathbf{5 4}$}

Method (ii) has also been applied by the Oxford group ${ }^{32}$ to the polarization of radioactive $\mathrm{Mn}^{54}$ in a double nitrate crystal. $K$-capture in this nucleus leads to the excitation of the $0.845 \mathrm{MeV}$ level in $\mathrm{Cr}^{54}$ which decays by a single $\gamma$-ray to the ground state. With polarizing fields up to 1000 gauss values for $\varepsilon$ up to 0.82 were observed and this indicates that approximately $90 \%$ of the nuclei were in the lowest substate. Measurements on the angular distribution and polarization of the $\gamma$-rays by the Oxford group showed the transition to be E2. Since $\mathrm{Cr}^{54}$ is an even-even nucleus its ground state is presumably $0^{+}$and therefore these experiments have shown that the $0.845 \mathrm{MeV}$ state is $2^{+}$.

\section{$\S 6$. Conclusions}

In the present chapter methods of orienting nuclei and the knowledge that can be derived from the observation of radiations emitted by them have been discussed. The number of successful experiments in this field is not yet considerable. It is however already possible to indicate how these new methods will fit into the general system of modern tools that are giving information on excited and radioactive nuclei.

32 Unpublished material. 
The discussion in this chapter shows that a considerable number of data can be obtained from a system of oriented nuclei of one species by determining the angular distribution of the $\gamma$-radiation at a number of temperatures, its plane of polarization and the sense of the circular polarization. However, this will even in the most favourable cases strain the precision required to the practical possible limit, even if the problems of reorientation in crystalline and atomic fields mentioned in $\S 4$ have been avoided or accounted for. It will in general be more desirable to combine information obtained from nuclear orientation work with other measurements. Nuclear orientation work will of course be most usefully applied in cases where all other methods fail.

Outstanding amongst those would be the case of an isomeric nucleus emitting a single $\gamma$-ray of high energy. The only other available method for the determination of the angular momentum and parity in such a transition is the study of internal conversion which becomes increasingly difficult and ambiguous with higher energy. In nuclear orientation a single measurement for $\beta>1$ of the angular distribution of the single $\gamma$-ray emitted by an isomer will give the change of angular momentum. Observation of the plane of polarization will determine the electric or magnetic character of the transition. Together with $L$ this gives the change of parity in the transition. A second measurement of the angular distribution at a temperature where $\beta \sim 1$ will then enable $\mu / j$ to be determined.

If the oriented nucleus emits a $\beta$-ray followed by $\gamma$-radiation the situation is more complicated since the angular distribution will not be a function of the multipole character of the $\gamma$-radiation alone but will also depend on the total angular momentum carried away in the $\beta$-transition. In some cases (cobalt 58) it will be possible to obtain all data of interest by the measurement of angular distribution alone. In other cases it might be essential to obtain supplementary information by an experiment of different type, for instance $\mu$ and $j$ from an atomic beam experiment.

Generally the determination of the plane of polarization, particularly in the case of the emission of a single $\gamma$-ray, will be the least ambiguous experimental result to be obtained since it is of the "yes or no" type.

A considerable simplification of the interpretation of results might in some cases be expected from nuclear resonance experiments. In this case the anisotropy of $\gamma$-ray distribution would merely serve as a detector permitting one to ascertain the frequency at which nuclear resonance is achieved. Such experiments could truly supplement ordinary angular pistribution measurements with oriented nuclei. They would yield values of $\mu / j$ which would be independent of the detailed angular distribution. 
(III) S. R. DE GROOT and H. A. FOLHOEK - Theory of Angular Effects of Radiations from Oriented Nuclei

\section{$\S 1$. Introduction}

By means of very low temperatures (e.g., $0.01^{\circ} \mathrm{K}$ ) and making use of the interaction of the nuclear magnetic moment (or possibly the nuclear electric quadrupole moment) with the surroundings, it is possible to orient nuclei.

If the nucleus is radioactive the emitted $\alpha, \beta$ or $\gamma$-radiation may show angular effects, which do not exist when the nuclear spins are distributed equally in all directions. These angular effects consist of two kinds, viz., a directional distribution which differs from spherical symmetry and polarization effects. The theoretical study of these phenomena is somewhat analogous to the theory of the angular correlation of nuclear radiations. To a certain extent the analysis is simpler and the evaluation of the data more straightforward since each nuclear radiation can be studied separately.

\section{$\S 2$. Orientation of Nuclei}

An adequate description of the orientation of an ensemble of nuclei is necessary for a simple theory of the angular effects. Suppose we have an ensemble of nuclei (with spin $j$ ) with rotational symmetry about a certain axis. We call $m(=-j, \ldots,+j)$ the magnetic quantum numbers with respect to this axis. The orientation of the nuclei is then completely characterized by the relative populations $a_{m}$ of the magnetic sublevels. Of these parameters $2 j$ are independent if we normalize according to $\sum_{m=-j}^{m=+j} a_{m}=1$. In the calculations the "moments" $\sum_{m} m^{v} a_{m}$ of these populations occur, making it useful to introduce $2 j$ independent linear combinations $f_{k}(j)$ of these moments, which are completely defined by the conditions ${ }^{1}$ )

$$
\begin{gathered}
f_{k}(j)=\sum_{v=0}^{k} \alpha_{k, v} \sum_{m} m^{v} a_{m}, \\
f_{k}(j)=0, \text { if } a_{m}=\sum_{p=0}^{k-1} A_{p} m^{p} \text {, for arbitrary values of the } A_{p}, \\
\alpha_{k_{s} k}=j^{-k} \text { (normalization). }
\end{gathered}
$$

We shall call $f_{k}(j)$ the degree of orientation of order $k$. If an arbitrary orientation is given by $a_{m}$, the dependence of $a_{m}$ on $m$ can always be expressed by a polynomial of degree $2 j: a_{m}=\sum_{p=0}^{2 j} A_{p} m^{p}$. Hence we obtain the

1 H. A. Tolhoek and J. A. M. Cox, Physica 19, (1953) 101. 
following property directly from definition (1): all $f_{k}$ with $k \geqslant 2 j+1$ vanish identically. An explicit expression for the $f_{k}(j)$ can be derived from (1)

$$
f_{k}(j)=\left(\begin{array}{c}
2 k \\
k
\end{array}\right)^{-1} j^{-k} \sum_{m} \sum_{v=0}^{k}(-1)^{\nu} \frac{(j-m) !(j+m) !}{(j-m-v) !(j+m-k+v) !}\left(\begin{array}{l}
k \\
v
\end{array}\right)^{2} a_{m} .
$$

(For examples see $\S \S 4$ and 5).

For totally oriented nuclei $\left(a_{m}=\delta_{m j}\right) f_{k}(j)$ has the value

$$
f_{k}(j)=\left(\begin{array}{c}
2 k \\
k
\end{array}\right)^{-1} j^{-k} \frac{(2 j) !}{(2 j-k) !} .
$$

A nomenclature ${ }^{2}$, which is now most often used, is to speak of nuclear polarization if one or more $f_{k}$ with odd $k$ is different from zero (regardless of the values of the other degrees of orientation) and to use the term nuclear alignment for a situation in which one or more "even" $f_{k} \neq 0$ and all "odd" $f_{k}=0$. For the general case in which one or more $f_{k} \neq 0$ (for arbitrary orders $k \geqslant 1$ ) the expression nuclear orientation is used. The degrees of orientation $f_{k}(j)$ have been introduced in the way described here by Tolhoek and $\operatorname{Cox}^{1}$. They are proportional to the statistical tensors introduced by Fano ${ }^{3}$, when we specialize for ensembles of nuclei with rotational symmetry. For rotational symmetry Fano's definition reduces to

$$
\langle\mid(j j) k 0\rangle=\sum_{m} a_{m}(-1)^{j-m}\langle j m j-m \mid j j k 0\rangle .
$$

The $\langle j m j-m \mid j j k 0\rangle$ are Clebsch Gordan coefficients (transformation coefficients for the addition of angular momenta).

Using $F_{k}(j)$ as an abbreviation for this quantity, we have

$$
f_{k}(j) / F_{k}(j)=w_{k}(j)=\left(\begin{array}{c}
2 k \\
k
\end{array}\right)^{-1} j^{-k}\left[\frac{(2 j+k+1) !}{(2 k+1)(2 j-k) !}\right]^{k} .
$$

\section{$\S 3$. The Angular Distribution of Nuclear Radiation 1, 5, 6, 7}

The subject of angular distribution can be divided into two parts viz., directional distribution and polarization distribution, depending on whether or not one ignores polarization effects.

The most elegant and general way to treat these problems is with the aid of the techniques developed by Racah ${ }^{4}$ and Fano ${ }^{3}$. We start by

2 S. R. de Groot, Physica 18, (1952) 1201.

3 U. Fano, Nat. Bur. Stand. Rep. no. 1214, 1951.

4 G. Racah, Phys. Rev. 84, (1951) 910.

5 H. A. Tolhoek and J. A. M. Cox, Physica 18, (1952) 357.

6 N. R. Steenberg, Proc. Phys. Soc. A65, (1952) 791.

7 J. A. Spiers, Nature 161, (1948) 807, and Nat. Res. Council Canada report no. 1925, Chalk River, Ontario, 1949. 
expanding the interaction Hamiltonian for emission of a $p$ article in the direction of the axis of quantization according to

$$
\bar{H}=\Sigma_{L M} \alpha_{L M}(\boldsymbol{c}) T_{M}^{L}\left(\boldsymbol{X}_{i}\right),
$$

where $c$ is a vector characterizing the polarization of the emitted particle. $\alpha_{L M}(c)$ is a function of the polarization variable $\boldsymbol{c}$ for the emitted radiation. $T_{M}^{L}\left(\boldsymbol{X}_{i}\right)$ is an irreducible tensor operator of degree $L$ formed with vectors $\boldsymbol{X}_{\boldsymbol{i}}$ operating on the nuclear wave functions. The Hamiltonian for emission of a particle at an angle $\vartheta$ with the axis of quantization is obtained by considering the transformation of $T_{M}^{L}$ for a rotation with Euler angles $(0, \vartheta, 0)$

$$
H=\Sigma_{L M \mu} \alpha_{L M}(\mathbf{c}) T_{\mu}^{L} D_{\mu M}^{L}(0, \vartheta, 0) .
$$

$D_{\mu_{M}}^{L}$ characterizes the $(2 L+1)$-dimensional representation of the group of rotations (Jacobi polynomial). If only one value of $L$ is of importance for the emitted radiation no summation over $L$ is needed.

The probability for emission of radiation in the direction $k$, with polarization given by $c$, while the axis of quantization is $\eta$, may be written as $((\boldsymbol{k} \cdot \eta)=\cos \vartheta)$

$$
W(\vartheta, c)=W(\mathbf{k}, \mathbf{c}, \eta)=\sum_{m_{i} m_{f}} a_{m_{i}}\left|\left\langle j_{i} m_{i}|H| j_{f} m_{f}\right\rangle\right|^{2} .
$$

Substituting (3.2) in (3.3) we obtain

$$
W(\vartheta, c)=\Sigma_{m_{i} m_{f} M M^{\prime} \mu \mu^{\prime}} a_{m_{i}} \alpha_{L M^{\prime}}^{*} \alpha_{L M^{\prime}}\left\langle j_{i} m_{i}\left|T_{\mu}^{L}\right| j_{f} m_{f}\right\rangle^{*}\left\langle j_{i} m_{i}\left|T_{\mu^{\prime}}^{L}\right| j_{f} m_{f}\right\rangle D_{\mu M^{\prime}}^{L *} D_{\mu^{\prime} M^{\prime}}^{L} \text {. }
$$

The matrixelements of $T_{\mu}^{L}$ can be factorized as products of an invariant and a Clebsch-Gordan coefficient. The product of the two $D$-factors in (3.4) can be written as a sum of products of two Clebsch-Gordan coefficients and one $D$-factor. It is possible ${ }^{4,8}$ to perform a summation over magnetic quantum numbers by introducing the Racah coefficients $W(a b c d ; e f)$ and the degrees of orientation $F_{k}\left(j_{i}\right)$ of $\S 1$. One obtains the following result in a closed form

with

$$
W(\vartheta, c)=\Sigma_{k \sigma}(-1)^{k} c_{k \sigma}(L L) W\left(j_{f} j_{i} L k ; L j_{i}\right) F_{k}\left(j_{i}\right) D_{0 \sigma}^{k}(0, \vartheta, 0),
$$

and

$$
c_{k \sigma}(L L)=\Sigma_{M M^{\prime}}(-1)^{L-M} \alpha_{L M}^{*}(\mathbf{c}) \alpha_{L M^{\prime}}(\mathbf{c})\left\langle L L k \sigma \mid L-M L M^{\prime}\right\rangle,
$$

$$
D_{0 \sigma}^{k}=(4 \pi)^{\ddagger}(2 k+1)^{-\dagger} Y_{k}^{\sigma}(\vartheta)
$$

(5) is the sum of products of which the factors are: $c_{k \sigma}(L L)$ which needs a special evaluation for each kind of radiation, the Racah coefficient $W\left(j_{1} j_{i} L k ; L j_{i}\right)$ characterizing the recoupling of angular momenta, $D_{0 \sigma}^{k}(0, \vartheta, 0)$ which is the only function of the direction of emission, which occurs in the product.

8 G. Racah, Phys. Rev. 62, (1942) 438. 


\section{$\S$ 4. The Directional Distribution of Gamma Radiation 1, 5, 6, 7, 9}

We can characterize a plane electromagnetic wave, which is propagated in the direction $\boldsymbol{k}(|\boldsymbol{k}|=2 \pi / \lambda)$, by the complex vector potential $\boldsymbol{A}$

$$
\boldsymbol{A}=\left(c_{1} \mathbf{e}_{1}+c_{2} \mathbf{e}_{2}\right) A, \quad A=\hat{A} \exp [i(\mathbf{k} \cdot \mathbf{r}-\omega t)] .
$$

Here $k /|k|, e_{1}$ and $\mathbf{e}_{2}$ are mutually perpendicular unit vectors. We assume $c_{1}$ and $c_{2}$ to be normalized to $\left|c_{1}\right|^{2}+\left|c_{2}\right|^{2}=1$. The state of polarization is characterized by the complex vector $c=\left(c_{1}, c_{2}\right)$.

If we consider the quantities $\alpha_{L M}$ from (3.2) for $\gamma$-radiation, only $\alpha_{L 1}$ and $\alpha_{L-1}$ are different from zero and are expressed by $c$ as follows

$\alpha_{L 1}=-\left(c_{1}-i c_{2}\right) / \sqrt{2}, \alpha_{L-1}=\left(c_{1}+i c_{2}\right) / \sqrt{2}$ (electric $2^{L}$ pole radiation),

$\alpha_{L 1}=-\left(c_{2}+i c_{1}\right) / \sqrt{2}, \alpha_{L-1}=\left(c_{2}-i c_{1}\right) / \sqrt{2}$ (magnetic $2^{L}$ pole radiation).

From (4.2) and (4.3) we can calculate the quantities $c_{k \sigma}(L L)$ according to (3.6).

By taking the sum over two orthogonal states of polarization we obtain the result for the directional distribution of $\gamma$-radiation (without observation of the polarization). It is found that

$$
W(\vartheta)=\sum_{k \text { even }} 2\langle L L k 0 \mid L 1 L-1\rangle W\left(j_{j} j_{i} L k ; L j_{i}\right) F_{k}\left(j_{i}\right)(2 k+1)^{-t} Y_{k}^{0}(\vartheta) \text {. }
$$

It is often convenient to normalize $W(\vartheta)$ such that $\int W(\vartheta) d \Omega=8 \pi$. The expression for $W(\vartheta)$, according to (4.4) is not normalized in this way, but the normalization is easily obtained by calculating $\int W(\vartheta) d \Omega$ for (4.4).

Results for the directional distribution of $2^{L}$-pole radiation (valid as well for electric as magnetic $2^{L}$-pole radiation). We give specializations of (4.4) in a form immediately suitable for numerical calculations. (Similar explicit forms for mixed dipole and quadrupole radiation with $\Delta j=0, \pm 1$ and for octupole radiation $(L=3)$ with $\Delta j= \pm 2, \pm 3$ are given in ${ }^{9}$.)

Dipole Radiation $(L=1)$

$$
\begin{array}{lll}
j_{f}=j_{i}-1 & , \quad W(\vartheta)=2\left[1+3 /{ }_{2} N_{2} f_{2} P_{2}(\cos \vartheta)\right] \\
j_{f}=j_{i} & , \quad W(\vartheta)=2\left[1-3 /{ }_{2} K_{2} f_{2} P_{2}(\cos \vartheta)\right] \\
j_{f}=j_{i}+1 & , \quad W(\vartheta)=2\left[1+3 /{ }_{2} M_{2} f_{2} P_{2}(\cos \vartheta)\right]
\end{array}
$$

QuadRupole Radiation ( $L=2$ )

$$
\begin{array}{ll}
j_{f}=j_{i}-2 & , \quad W(\vartheta)=2\left[1-{ }_{15} /{ }_{7} N_{2} f_{2} P_{2}(\cos \vartheta)-5 N_{4} f_{4} P_{4}(\cos \vartheta)\right] \\
j_{f}=j_{i}+2 & , \quad W(\vartheta)=2\left[1-15 / 7 H_{2} f_{2} P_{2}(\cos \vartheta)-5 M_{4} f_{4} P_{4}(\cos \vartheta)\right]
\end{array}
$$

9 Chr. D. Hartogh, H. A. Tolhoek and S. R. de Crroot, Physica (in course of publication). (Some explicit formulae for the angular distribution and polarization of gamma radiation emitted by oriented nuclei.) 
CH. XIX (III)] THEORY OF RADIATIONS FROM ORIENTED NUCLEI

In these formulae the symbols have the following meaning

$$
\begin{gathered}
P_{2}(\cos \vartheta)=3 / 2\left(\cos ^{2} \vartheta-1 / 3\right) \\
P_{4}(\cos \vartheta)={ }^{35} / 8\left(\cos ^{4} \vartheta-6 /{ }_{7} \cos ^{2} \vartheta+3 / 35\right) \\
N_{2}=j_{i} /\left(2 j_{i}-1\right) \quad, N_{4}=j_{i}^{3} /\left(j_{i}-1\right)\left(2 j_{i}-1\right)\left(2 j_{i}-3\right), \\
M_{2}=j_{i}^{2} /\left(j_{i}+1\right)\left(2 j_{i}+3\right), M_{4}=j_{i}^{4} /\left(j_{i}+1\right)\left(j_{i}+2\right)\left(2 j_{i}+3\right)\left(2 j_{i}+5\right) \\
K_{2}=j_{i} /\left(j_{i}+1\right), \\
f_{2}=\left(1 / j_{i}^{2}\right)\left[\sum_{m_{i}} m_{i}^{2} a_{m_{i}}-1 /{ }_{3} j_{i}\left(j_{i}+1\right)\right] \\
f_{4}=\left(1 / j_{i}^{4}\right)\left[\sum_{m_{i}} m_{i}^{4} a_{m_{i}}-1 /{ }_{7}\left(6 j_{i}^{2}+6 j_{i}-5\right) \sum_{m_{i}} m_{i}^{2} a_{m_{i}}+3 /{ }_{35} j_{i}\left(j_{i}-1\right)\left(j_{i}+1\right)\left(j_{i}+2\right)\right] .
\end{gathered}
$$

The functions $W(\vartheta)$ are all normalized to

$$
\int W(\vartheta) d \Omega=8 \pi \text {. }
$$

\section{$\S$ 5. Polarization Distribution of Gamma Radiation 1, 5, 9, 10}

In $\S 3$ we characterized the state of polarization by $c=\left(c_{1}, c_{2}\right)$. It is often more convenient to use a real three dimensional unit vector $\xi$ for this purpose ${ }^{1,11}$. $\xi$ is expressed by $c$ according to

$$
\xi_{1}=\left|c_{1}\right|^{2}-\left|c_{2}\right|^{2}, \quad \xi_{2}=c_{1} c_{2}^{*}+c_{1}^{*} c_{2}, \quad \xi_{3}=i\left(c_{1} c_{2}^{*}-c_{1}^{*} c_{2}\right) .
$$

A partially polarized beam must be described by a statistical matrix. This matrix can always be split into a fraction $(1-P)$ of unpolarized radiation and a fraction $P$ of polarized radiation,

$$
\varrho=\left\|\begin{array}{ll}
\varrho_{11} & \varrho_{12} \\
\varrho_{21} & \varrho_{22}
\end{array}\right\|=(1-P)\left\|\begin{array}{cc}
\frac{1}{2} & 0 \\
0 & \frac{1}{2}
\end{array}\right\|+P\left\|\begin{array}{cc}
\left|c_{1}\right|^{2} & c_{1} c_{2}^{*} \\
c_{1}^{*} c_{2} & \left|c_{2}\right|^{2}
\end{array}\right\| \text {. }
$$

$P$ is called the degree of polarization and $c$, or the corresponding $\xi$, the way of polarization (three independent real parameters). According to quantum mechanics the probability that a detector, set to detect a polarization $c^{d}$ (or $\xi^{d}$ ), will respond for a beam given by (5.2) is

$$
\operatorname{Tr}\left[\varrho \varrho\left(c^{d}\right)\right]=\frac{1}{2}\left[1+P \xi \cdot \xi^{d}\right] \text {. }
$$

It will be convenient to express $\xi$ with the aid of its components in 3 directions in the following way

$$
\xi=\xi_{1} \chi_{/ /}+\xi_{2} \chi_{1}+\xi_{3} \chi_{c}
$$

The unit vectors $\chi_{/ /}, \chi_{\perp}$ and $\chi_{c}$ are taken to represent linear polarization with $\boldsymbol{E}$ in the plane through the direction of propagation and the axis $\vartheta=0$, linear polarization turned over $45^{\circ}$ with respect to the preceding, and left circular polarization respectively.

10 N. R. Steenberg, Proc. Phys. Soc. A66, (1952) 391.

11 U. Fano, J. Opt. Soc. Am. 39, (1949) 859. 
From (5.3) it follows that we may write

$$
W\left(\vartheta, \xi^{d}\right)=\frac{1}{2} W(\vartheta)\left(1+P \xi \cdot \xi^{d}\right) .
$$

The first term of (5.5) is known according to (4.4). We will use the notation with $P$ and $\xi$ to express the polarization distribution for $\gamma$-radiation. This follows from (3.5) when calculating the $c_{k \sigma}(L L)$ according to (3.6) with the aid of (4.2), (4.3). The result expressed with $P$ and $\xi$ is

$$
\left.\begin{array}{rl}
W(\vartheta) P \xi= & \left.\mp \sum_{k \text { even }} 2\langle L L k 2 \mid L 1 L 1\rangle W\left(j_{f} j_{i} L k ; L j_{i}\right) F_{k}\left(j_{i}\right)(2 k+1)^{-t} Y_{k}^{2}(\vartheta, 0) \chi_{/ /}+\right\} \\
& +\sum_{k \text { odd }} 2\langle L L k 0 \mid L 1 L-1\rangle W\left(j_{f} j_{i} L k ; L j_{i}\right) F_{k}\left(j_{i}\right)(2 k+1)^{-i} Y_{k}^{0}(\vartheta, 0) \chi_{c^{\bullet}}
\end{array}\right\}
$$

The circular and linear polarization terms contain odd and even orientation coefficients $F_{k}$ respectively. The upper sign is valid for electric and the lower for magnetic multipole radiation. Hence observation of the linear polarization will provide information on the electric or magnetic character of the radiation.

\section{A. Results for the polarization of $\gamma$-RAdiation}

We give explicit formulae for $W(\vartheta) P \xi$, which are valid for electric dipole and quadrupole radiation. For the case of magnetic radiation the sign of $\chi_{1 /}$ is changed in the corresponding formulae for the electric case, while the sign of $\chi_{c}$ remains unaltered. These formulae are special cases of (5.6). (Similar explicit expressions for the polarization for mixed dipole and quadrupole radiation with $\Delta j=0, \pm 1$ are given in ${ }^{9}$.)

Electric Dipole Radiation $(L=1)$

$$
\begin{aligned}
& j_{i}=j_{i}-1, W(\vartheta) P \xi=-9 / 2 N_{2} f_{2}\left(1-\cos ^{2} \vartheta\right) \chi_{/ /}+3 N_{1} f_{1} \cos \vartheta \chi_{c} \\
& j_{f}=j_{i}, W(\vartheta) P \xi=9 / 2 K_{2} f_{2}\left(1-\cos ^{2} \vartheta\right) \chi_{1 /}+3 K_{1} f_{1} \cos \vartheta \chi_{c} \\
& j_{f}=j_{i}+1, W(\vartheta) P \xi=-9 / 2 M_{2} f_{2}\left(1-\cos ^{2} \vartheta\right) \chi_{/ 1}-3 M_{1} f_{1} \cos \vartheta \chi_{c}
\end{aligned}
$$

Electric Quadrupole Radiation $(L=2)$

$$
\begin{aligned}
j_{1}=j_{i}-2, W(\vartheta) P \xi= & {\left[5 /{ }_{7} N_{2} f_{2}\left(\cos ^{2} \vartheta-1\right)+{ }^{25} /{ }_{4} N_{4} f_{4}\left(-7 \cos ^{4} \vartheta+8 \cos ^{2} \vartheta-1\right)\right] \chi_{11}+} \\
& +\left[2 N_{1} f_{1} \cos \vartheta+5 N_{3} f_{3}\left(-5 \cos ^{3} \vartheta+3 \cos \vartheta\right)\right] \chi_{c} \\
j_{f}=j_{i}+2, W(\vartheta) P \xi= & {\left[55 / 7 M_{2} f_{2}\left(\cos ^{2} \vartheta-1\right)+{ }^{25} / M_{4} M_{4} f_{4}\left(-7 \cos ^{4} \vartheta+8 \cos ^{2} \vartheta-1\right) \chi_{1 /}-\right.} \\
& -\left[2 M_{1} f_{1} \cos \vartheta+5 M_{3} f_{3}\left(-5 \cos ^{3} \vartheta+3 \cos \vartheta\right)\right] \chi_{c}
\end{aligned}
$$

The quantities, which have not yet been given in $\S 4$ are

$$
\begin{aligned}
& N_{1}=1 \quad, N_{3}=j_{i}^{2} /\left(j_{i}-1\right)\left(2 j_{i}-1\right), K_{1}=1 /\left(j_{i}+1\right), \\
& M_{1}=j_{i} /\left(j_{i}+1\right), M_{3}=j_{i}^{3} /\left(j_{i}+1\right)\left(j_{i}+2\right)\left(2 j_{i}+3\right), \\
& f_{1}=\left(1 / j_{i}\right) \sum_{m_{i}} m_{i} a_{m_{i}}, \\
& f_{3}=\left(1 / j_{i}^{3}\right)\left[\sum_{m_{i}} m_{i}^{3} a_{m_{i}}-1 / 5\left(3 j_{i}^{2}+3 j_{i}-1\right) \sum_{m_{i}} m_{i} a_{m_{i}}\right] .
\end{aligned}
$$


$\S$ 6. The Influence of Transitions Preceding the Gamma Radiation 5, 6, 12

For experiments in which radioactive nuclei are oriented the lifetimes must be rather large. Except for the isomeric transitions most half-lifes for $\gamma$-emission are very short. However, there are no, or very few, isomeric nuclei which have a suitable half-life, a suitable energy (in addition the $\gamma$-radiation must not be entirely converted) and which can be oriented by the present experimental methods. Hence the experimental situation will generally be the following: a) the nuclei, which are oriented will be $\beta$-radioactive with a sufficiently long lifetime, b) the $\beta$-transition will be followed by a $\gamma$-transition (Fig. 1) or possibly by two or more $\gamma$-transitions in cascade (Fig. 2). In Figure 1 and 2 we have indicated by $j_{0}, j_{i}, j_{e}, j_{f}$ the angular momenta of the different levels which may occur.

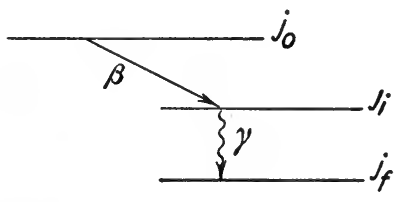

Fig. 1

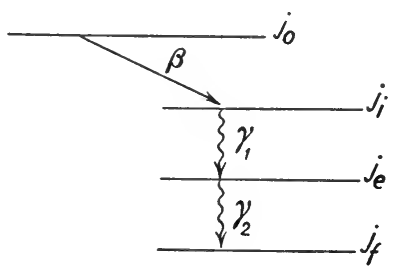

Fig. 2

The mechanism of the orientation determines the population of the levels of the nucleus with spin $j_{0}$. For a complete discussion of the phenomena we need the formulae relating the initial orientation with the orientation after the $\beta$ - or $\gamma$-transitions, which precede the observed $\gamma$-radiation.

For the derivation of these relations, we assume again that there is an axis $\eta$ of rotational symmetry. The orientation of the nuclei with spin $j_{0}$ is completely characterized by the relative populations $a_{m_{0}}$ of the sublevels $m_{0}$. After a $\beta$-transition (or a $\gamma$-transition) $j_{0} \rightarrow j_{i}$ the probabilities $a_{m_{i}}$ are connected with $a_{m_{0}}$ by

with

$$
a_{m_{i}}=\sum_{m_{0}} a_{m_{0}} P_{m_{0} m_{i}}
$$

$$
\sum_{m_{i}} P_{m_{0} m_{i}}=1 \text {. }
$$

$P_{m_{0} m_{i}}$ is the partial transition probability for the transition $\left(j_{0} m_{0}\right) \rightarrow\left(j_{i} m_{i}\right)$. If the radiation carries an angular momentum $L$ from the system, $P_{m_{0} m_{i}}$ has the expression

$$
P_{m_{0} m_{i}}^{(L)}=\left|\left\langle j_{i} m_{i} L M \mid j_{i} L j_{0} m_{0}\right\rangle\right|^{2} .
$$

We specialize to some simple cases

$$
L=0 \quad, \quad P_{m_{0} m_{i}}^{(0)}=\delta_{m_{0} m_{i}} .
$$


This formula is valid for

a) allowed $\beta$-transitions with matrix elements $\left|\int 1\right|^{2}$ or $\left|\int \beta \gamma_{5}\right|^{2}$,

b) forbidden $\beta$-transitions with scalar matrix elements.

$$
L=1 \quad, \quad P_{m_{0} m_{i}}^{(1)}=\left|\left\langle j_{i} m_{i} 1 M \mid j_{i} 1 j_{0} m_{0}\right\rangle\right|^{2} .
$$

This is valid for

a) allowed $\beta$-transitions with matrix element $\left|\int \sigma\right|^{2}$,

b) first forbidden $\beta$-transitions with matrix elements $\left|\int \boldsymbol{\sigma} \wedge \boldsymbol{r}\right|^{2},\left|\int \boldsymbol{\alpha}\right|^{2},\left|\int \boldsymbol{r}\right|^{2}$ or $\left|\int \gamma_{5} \mathbf{r}\right|^{2}$

c) $\gamma$-transitions with electric or magnetic dipole character.

$$
L=2 \quad, \quad P_{m_{0} m_{i}}^{(2)}=\left|\left\langle j_{i} m_{i} 2 M \mid j_{i} 2 j_{0} m_{0}\right\rangle\right|^{2} .
$$

This formula applies to

a) first forbidden $\beta$-transitions with matrixelement $B_{i j}$,

b) second forbidden $\beta$-transitions with matrixelements $R_{i j}, A_{i j}, T_{i j}$ or $R_{i j}^{\gamma}$,

c) $\gamma$-transitions with electric or magnetic quadrupole character.

Using the expression (6.3) for $P_{m_{0} m_{i}}$ a relation between the degrees of orientation before and after the transition may be derived with the aid of the Racah algebra ${ }^{12}$.

$$
f_{k}\left(j_{0}\right)=w_{k}\left(j_{i}\right) w_{k}\left(j_{0}\right)^{-1}\left(2 j_{0}+1\right) W\left(j_{i} L k j_{0} ; j_{0} j_{i}\right) f_{k}\left(j_{0}\right)
$$

Let us now consider a few special cases.

For transitions $j_{0} \rightarrow j_{i}=j_{0}-L,(6.7)$ can be written as

$$
N_{k}\left(j_{i}\right) f_{k}\left(j_{i}\right)=N_{k}\left(j_{0}\right) f_{k}\left(j_{0}\right) \text {. }
$$

For transitions $j_{0} \rightarrow j_{i}=j_{0}+L,(6.7)$ can be written as

$$
M_{k}\left(j_{i}\right) f_{k}\left(j_{i}\right)=M_{k}\left(j_{0}\right) f_{k}\left(j_{0}\right) \text {. }
$$

The quantities $N_{k}$ and $M_{k}$ are given by

$$
\left.\begin{array}{l}
N_{k}(j)=b_{k} j^{k}(2 j-k) ! /(2 j) ! \quad, \quad M_{k}(j)=b_{k} j^{k}(2 j+1) ! /(2 j+k+1) !, \\
b_{k}=2^{t k} \text { if } k \text { is even; } \quad b_{k}=2^{i(k+1)} \text { if } k \text { is odd. }
\end{array}\right\}
$$

(The quantities $N_{k}$ and $M_{k}$ were already used in the explicit formulae in $\S \S 3$ and 4$)$.

For $j_{0} \rightarrow j_{0}$ with total angular momentum quantum number of the emitted radiation $L=1$ we find

$$
f_{k}\left(j_{i}\right)=\left[1-k(k+1) / 2 j_{0}\left(j_{0}+1\right)\right] f_{k}\left(j_{0}\right) \text {. }
$$

(Similar explicit expressions for the change of the $f_{k}$ by preceding radi- 
ations for mixed dipole and quadrupole radiation with $\Delta j=0, \pm 1$ and octupole radiation $(L=3)$ with $\Delta j= \pm 2$ are given in ${ }^{9}$.)

It should be noted that formula (6.3) for $P_{m_{0} m_{i}}$ is valid if the emitted radiation has a well determined $L$, meaning that all the matrix elements contributing appreciably to the transition probability must have the same tensorial character. This is not always true, for example $P_{m_{0} m_{i}}$ for allowed $\beta$-transitions with $j_{0}=j_{i}$ will in general be a "mixture" of $P_{m_{0} m_{i}}^{(0)}$ and $P_{m_{0} m_{i}}^{(1)}$. This gives some complication of the discussion of the experiments, but may provide a means to obtain information on the character of the $\beta$-transition ${ }^{12}$.

In all these considerations it is assumed that there is no appreciable spin precession after the $\beta$-transition ( $\beta^{+}$or $\beta^{-}$emission or $K$-capture) and before the $\gamma$-transition. Recently the influence of spin precession on the angular distribution of the radiations has received some interest $13,14,15$. These influences are similar to the influence on angular correlations, but the experimental difficulty is that the sources must be contained in special crystals in order to obtain orientation of the nuclei, while the source material for angular correlation experiments can be varied arbitrarily.

\section{$\S$ 7. The Directional Distribution and Polarization of Beta Radiation}

Although the influence of nuclear orientation is more difficult to observe for beta radiation from an experimental point of view, let us survey the effects which should be expected.

For allowed $\beta$-transitions the effects were studied by Tolhoek and de Groot ${ }^{16}$. The result is that the directional distribution remains spherically symmetric for allowed transitions with any nuclear orientation.

However, a polarization of the emitted $\beta$-radiation must be expected if the orientation parameter $f_{1} \neq 0$. The degree of polarization of electrons may even be unity, for completely polarized nuclei. Let $\eta$ be the axis of orientation, $p$ the momentum of the emitted electron and $E$ its energy (including its rest mass). We put $\boldsymbol{p} \cdot \boldsymbol{\eta}=p \cos \vartheta$. The probability for observing the emission of an electron in direction $\vartheta$, while the polarization detector is set for the polarization $\zeta^{d}$ can be expressed as

13 M. A. Grace and H. Halban, Physica 18, (1952) 1227; J. M. Daniels, M. A. Grace, H. Halban, N. Kurti and F. N. H. Robinson, Phil. Mag. 43, (1952) 1297.

14 M. A. Grace, Colloque Intern. C.N.R.S. no. 54 (1954); H. A. Tolhoek, Chr. D. Hartogh and S. R. de Groot, Colloque Intern. C.N.R.S. no. 54 (1954). (Communications in the Proceedings of the Conference on "Le rôle du cortège électronique dans les phénomènes radioactifs"'):

15 N. R. Steenberg, Phys. Rev. 95 (1954) 982.

16 H. A. Tolhoek and S. R. de Groot, Physica 17, (1951) 1, 17, 81. 


$$
W_{E}\left(\vartheta, \zeta^{d}\right)=W_{E}(\vartheta) \frac{1}{2}\left(1+P \zeta \cdot \zeta^{d}\right) \text {. }
$$

The degree of polarization $P(0 \leqslant P \leqslant 1)$ and the unit vector $\zeta$ (direction of electron spin) characterize completely the polarization of the emitted electrons. The calculated polarization, (if we have pure tensor or axial vector interaction) can be expressed as

$$
P \zeta=R f_{1}[(\eta / E)+\boldsymbol{P} p \cos \vartheta / E(E+1)],
$$

with

$$
R=\left\{\begin{array}{ll}
N_{1}, & \text { if } j_{i} \rightarrow j_{f}=j_{i}-1 . \\
K_{1}, & \text { if } j_{i} \rightarrow j_{f}=j_{i} . \\
-M_{1}, & \text { if } j_{i} \rightarrow j_{f}=j_{i}+1 .
\end{array}\right\}
$$

$\left(N_{1}=1, K_{1}=1 /\left(j_{i}+1\right), M_{1}=j_{i} /\left(j_{i}+1\right)\right.$ as in $\left.\S 5\right)$.

From (7.2) we can conclude, that the degree of polarization for electrons emitted perpendicularly to $\eta$ is given by $R f_{1}$.

For an arbitrary "mixture" for the Hamiltonian of the $\beta$-interaction, the result is given in ${ }^{16}$. (In (7.2) relativistic units are used for which $\left.\hbar=1, c=1, m_{e}=1\right)$. The form of this general result for the mixture of interactions is of interest in connection with possible symmetry properties of the beta-interaction ${ }^{16,17}$.

In the forbidden beta-transitions of oriented nuclei, we may have as well as a polarization effect, a directional distribution which differs from spherical symmetry ${ }^{18}$. However, this deviation from spherical symmetry will occur only for those forbidden $\beta$-transitions which have a $\beta$-spectrum with a shape different from the "allowed" shape (that is for the same $\beta$-transitions for which the $\beta-\gamma$ directional correlation function may differ from isotropy if the $\beta$ is followed by a $\gamma^{19}$ ). The best known group of $\beta$-transitions with this property is the group of first forbidden $\beta$-transitions with $\Delta j= \pm 2$ and change of parity ${ }^{20}$. The directional distribution of the radiation for this group of $\beta$-transitions can be calculated along the lines of 1 and 19 and is

$$
\begin{aligned}
& j_{i} \rightarrow j_{1}=j_{i}-2, \quad \frac{1}{2} W(\vartheta)=1-3 \mu N_{2} f_{2} P_{2}(\cos \vartheta) \\
& j_{i} \rightarrow j_{t}=j_{i}+2, \quad \frac{1}{2} W(\vartheta)=1-3 \mu M_{2} f_{2} P_{2}(\cos \vartheta)
\end{aligned}
$$

$N_{2}, M_{2}$ and $f_{2}$ have the same meaning as in $\S 4$.

17 S. R. de Groot and H. A. Tolhoek, Physica 16, (1950) 456; S. R. de Groot, Physica 18, (1952) 1011.

18 H. A. Tolhoek, Physica 18, (1952) 1257.

19 D. L. Falkoff and G. E. Uhlenbeck, Phys. Rev. 79, (1950) 334.

20 C. S. Wu, Rev. Mod. Phys. 22, (1950) 386; M. G. Mayer, S. A. Moszkowski and L. W. Nordheim, Rev. Mod. Phys. 23, (1951) 315; L. W. Nordheim, Rev. Mod. Phys. 23, (1951) 322. 
$\mu=p^{2} /\left(p^{2}+q^{2}\right)=\left(E^{2}-1\right) /\left[E^{2}-1+\left(E_{0}-E\right)^{2}\right]$, where $p$ is the electron momentum, $q$ the neutrino momentum, $E$ the energy of the electron, $E_{0}$ the maximum energy of the $\beta$-spectrum. The factor $\mu$ varies from $\mu=0$ for $E=1$ to $\mu=1$ for $E=E_{0}$, hence we see that the anisotropy of the directional distribution of the electrons depends on their energy and is most pronounced for electrons with energy near to maximum.

\section{$\S 8$. Other Topics}

The discussions of the preceding sections may be generalized in several ways :

Although we assumed up till now that the ensemble of nuclei admits an axis of rotational symmetry, this is not a necessity. When nuclei are oriented with the aid of anisotropic crystals, this rotational symmetry may be absent, although up till now it has not been necessary to assume these conditions in real experiments. The directional distributions of radiations from ensembles of nuclei without rotational symmetry have been calculated by Cox and de Groot ${ }^{21}$.

If two $\gamma$ 's are emitted by a nucleus, we may study how their angular correlation is changed if the nuclei are oriented. This is expressed by a rather complicated function of the direction of the axis of orientation $\eta$ and the directions of emission $\boldsymbol{k}_{1}$ and $\boldsymbol{k}_{2}$ of both $\gamma$ 's. Calculations of this effect were made by Cox and Tolhoek ${ }^{22}$.

If it would become possible to orient nuclei showing $\alpha$-disintegration, the directional distribution of the $\alpha$-particles may be studied ${ }^{7}$. Conclusions concerning the angular momentum of the $\alpha$-particles would be possible.

21 J. A. M. Cox and S. R. de Groot, Physica, 19, (1953) 683.

22 J. A. M. Cox and H. A. Tolhoek, Physica 18, (1952) 359; J. A. M. Cox, Physica 18, (1952) 1262; J. A. M. Cox and H. A. Tolhoek, Physica 19, (1953) 1178. 


\title{
SOME INTERNAL EFFECTS IN NUCLEAR DECAY
}

\author{
I. BERGSTRÖM, R. WILSON, C. S. WU
}

\section{(I) I. BERGSTRÖM - Auger Electrons Emitted from Radioactive Atoms}

\section{$\S$ 1. Introduction}

Both electron capture and internal conversion of $\gamma$-ray transitions produce vacancies in the atomic shells which are filled again by electrons from outer shells. The energy then released may be carried away by an X-ray quantum but it can also be transferred to one of the outer electrons which is ejected from the atom. Such an electron is called an Auger electron ${ }^{1}$. Emission of $\mathrm{X}$-rays and Auger electrons are therefore alternative processes resulting from vacancies in the electron orbits of the atom. The process may be compared with the internal conversion of $\gamma$-rays although in the latter case the radiation field has its origin in the center of the nucleus which may be considered as a point charge. The fraction of the number of primary vacancies of a certain shell leading to the emission of X-rays is called the fuorescence yield $(w)$. This quantity was introduced in X-ray spectroscopy ${ }^{1}$. The X-ray emission observed in this case was a secondary effect due to vacancies having been produced by incident X-rays, which explains the introduction of the quantity fluorescence yield. In the same way the Auger yield (a) may be defined as the fraction leading to the emission of Auger electrons. If the fluorescence or Auger yields are known, it is evident that measurements of the intensity of X-rays or Auger electrons can give information as to the relative number of primary vacancies in the atomic shell. This has a special interest in the field of radioactivity when the vacancy has been produced by electron capture or internal conversion.

$\mathrm{X}$-rays emitted from radioactive atoms have been studied previously much more than Auger electrons. It is well known for example that critical absorption measurements of X-rays may be used in order to determine the atomic number. Ratios of $\mathrm{X}$-ray and $\gamma$-ray intensities also give results of interest such as the intensity ratio of $K$-capture to positron emission between the same nuclear states. The reason why Auger electrons have not been investigated more frequently in connection with problems in radio-activity is the fact that only during the last five years has the rapid development of $\beta$-spectroscopy made a thorough study of low energy electrons possible. As can be seen from Table I Auger electrons in most cases have very low energies.

1 E. H.S. Burhop, "The Auger Effect and other Radiation less Transitions", (Cambridge University Press, London, 1952). This book gives references to earlier works. 
CH. XX (I)] AUGER ELECTRONS EMITTED FROM RADIOACTIVE ATOMS

The main purpose of this section is to show how measurements of energies and intensities of Auger electrons may be used in order to obtain information about a radioactive decay. The reader is referred to the book by E. H. S. Burhфp ${ }^{1}$ for more complete information about Auger electrons.

\section{$\S 2$. Classification of Auger Electrons}

In most cases of Auger electron emission two electrons and three electron orbits are involved. In the initial state of the atom there is one vacancy in a certain shell, the $V_{o}$ shell, and in the final state there are two vacancies in shells which will be denoted $X_{p}$ and $Y_{q}$. The indices $o, p$, and $q$ denote subshells. A certain Auger transition is therefore written as $V_{o} \rightarrow X_{p} Y_{q}$, a relation which gives information about the ionization conditions in the initial and final states of the atom. The symbol $V_{o} X_{p} Y_{q}$ may now be used in order to denote unambigously a certain Auger electron. $V_{o}$ gives the primary vacancy filled by an electron from the $X_{p}$ shell, the energy released being transferred to an electron in the $Y_{q}$ shell which leaves the atom and goes to a free energy state. X-rays due to a primary $K$-vacancy are called $K \mathrm{X}$-rays. In the same way $K$ Auger electrons refers to those which result from a primary $K$ vacancy; for example $K L_{\mathrm{I}} L_{\mathrm{III}}$, $K L_{\mathrm{II}} M_{\mathrm{I}}, K L_{\mathrm{II}} N_{\mathrm{v}}$ etc. $L, M \ldots$ Auger electrons are thus due to primary $L, M \ldots$ vacancies. In $\beta$-spectroscopy the $K$ Auger electrons are most frequently observed. The $L$ Auger electrons have such a low energy that they can be observed only for high atomic numbers (cf. Table I).

There is a large number of discrete $K X_{p} Y_{q}$ electron lines. If $X$ and $Y$ represent different shells the number of $K X_{p} Y_{q}$ electron lines is given by the product of the number of subshells in the $X$ and $Y$ shells. If $X$ and $Y$ are the same shells for example $K L_{p} L_{a}\left(K M_{p} M_{q}, K N_{p} N_{q}\right.$ etc. $)$ there will be fewer corresponding $K$ Auger electron lines due to the fact that the

TABLE I

Energies (keV) of Auger electron lines calculated according to the APPROXIMATIVE FORMULA $K X_{p} X_{q}=K-x_{p}-X_{q}^{*}$

\begin{tabular}{c|c|c|c|c|c|c|c}
\hline$Z$ & $K L_{I} L_{I}$ & $K L_{I} M_{I}$ & $K L_{I} N_{I}$ & $K M_{I} M_{I}$ & $K M_{I} N_{I}$ & $K N_{I} N_{I}$ & $L_{I} M_{I} M_{I}$ \\
\hline 20 & 3.2 & 3.6 & & 3.9 & & & 0.31 \\
30 & 7.3 & 8.3 & & 9.4 & & & 0.93 \\
40 & 12.9 & 15.0 & 15.4 & 17.13 & 17.5 & 17.9 & 1.67 \\
50 & 20.3 & 23.9 & 24.6 & 27.4 & 28.2 & 28.9 & 2.71 \\
60 & 29.3 & 34.9 & 36.1 & 40.4 & 41.7 & 42.9 & 3.97 \\
70 & 39.9 & 48.0 & 49.9 & 56.5 & 58.4 & 60.4 & 5.67 \\
80 & 53.4 & 64.7 & 67.4 & 76.0 & 78.8 & 81.5 & 7.72 \\
90 & 68.9 & 84.2 & 88.0 & 99.5 & 103.3 & 107.2 & 10.13
\end{tabular}

* In the following bold types are used in order to denote energies (cf. Eqs. (1) and (4)). 
electrons $K L_{p} L_{q}\left(K M_{p} M_{q}, K N_{p} N_{q}\right.$ etc.) and $K L_{q} L_{p}\left(K M_{q} M_{p}, K N_{q} N_{p}\right.$ etc. $)$ have equal energies. In both cases the initial states are the same ( $K$ vacancy) and in both cases the final states are the same, i.e. vacancies in the $L_{p}\left(M_{p}, N_{p}\right.$ etc. $)$ and $L_{q}\left(M_{q}, N_{q}\right.$, etc. $)$ shells. Conservation of energy then requires that these Auger electrons must have the same energies. The fact that in one case the $L_{p}$ electron fills the $K$-shell, the $L_{q}$ electron being expelled and vice versa in the other case can never be checked experimentally since electrons are identical particles. For example, in the $K L_{p} L_{q}$ group which has been studied frequently there are six lines; on the other hand there are 15 lines in the $K L_{p} M_{q}$ group, 21 in $K L_{p} N_{q}, 15$ in $K M_{p} M_{q}, 35$ in $K M_{p} N_{q}$ and 28 lines in the $K N_{\nu} N_{q}$ group. Up to and including the $N$-shell there are altogether 120 lines in the $K$ Auger group. If extremely high resolution could be used the Auger lines would thus exhibit a very complicated pattern.

\section{$\S 3$. Energies of Auger Electrons}

There are several cases in $\beta$-spectroscopy where a knowledge of the energies of Auger electrons may give useful information. The energies of Auger electrons are most generally derived by using the principle of conservation of energy. The system considered is a nucleus and a certain number of electrons. In the initial state of the system the atom is singly ionized and all the electrons are bound. In the final state of the system the atom is doubly ionized but one electron is now in a free energy state having the kinetic energy $\boldsymbol{V}_{\mathbf{0}} \boldsymbol{X}_{\boldsymbol{p}} \boldsymbol{Y}_{\mathbf{q}}$. Assume that the energy of the neutral atom is $\boldsymbol{E}_{\mathbf{N}}$. The energy of the atom singly ionized in the $V_{0}$ shell is then $-\boldsymbol{E}_{\mathbf{N}}+\boldsymbol{V}_{\mathbf{0}}$ : and the energy of the atom ionized in the $X_{p}$ and $Y_{q}$ shells is $-E_{N}+X_{p}+$ $+Y_{q}\left(X_{p}\right)$. Here $V_{o}$ and $X_{p}$ are the binding energies in the $V_{o}$ and $X_{p}$ shells of a neutral atom. However, $Y_{q}\left(X_{p}\right)$ is the binding energy in the $Y_{q}$ shell when there is a vacancy in the $X_{q}$ shell. Since the energy of the initial state of the system is equal to the energy of the final state we obtain:

$$
-E_{N}+V_{0}=-E_{N}^{\prime} X_{p}+Y_{q}\left(X_{p}\right)+V_{0} X_{p} Y_{q} \quad V_{0} X_{p} Y_{q}=V_{0}-X_{p}-Y_{q}\left(X_{p}\right) .
$$

[It is evident that:

$$
V_{0} X_{p} Y_{q}=V_{0} X_{q} Y_{q}
$$

As a consequence the relation

always holds].

$$
X_{p}-X_{p}\left(Y_{q}\right)=Y_{q}-Y_{q}\left(X_{p}\right)
$$

Consider now the $K L_{p} L_{q}$ electrons, which have been studied frequently. Their energies are

$$
K L_{p} L_{q}=K-L_{p}-L_{q}\left(L_{p}\right) \text {. }
$$

Fig. 1A shows the Auger electrons in $\operatorname{Ag}^{107}(Z=47)$ obtained by Johnson and Foster ${ }^{2}$ in a study of the $K$ capturing $\mathrm{Cd}^{107}$. Fig. 1B shows Auger 
Сн. XX (I) Bergström - Auger Electrons Emitted from Radioactive Atoms
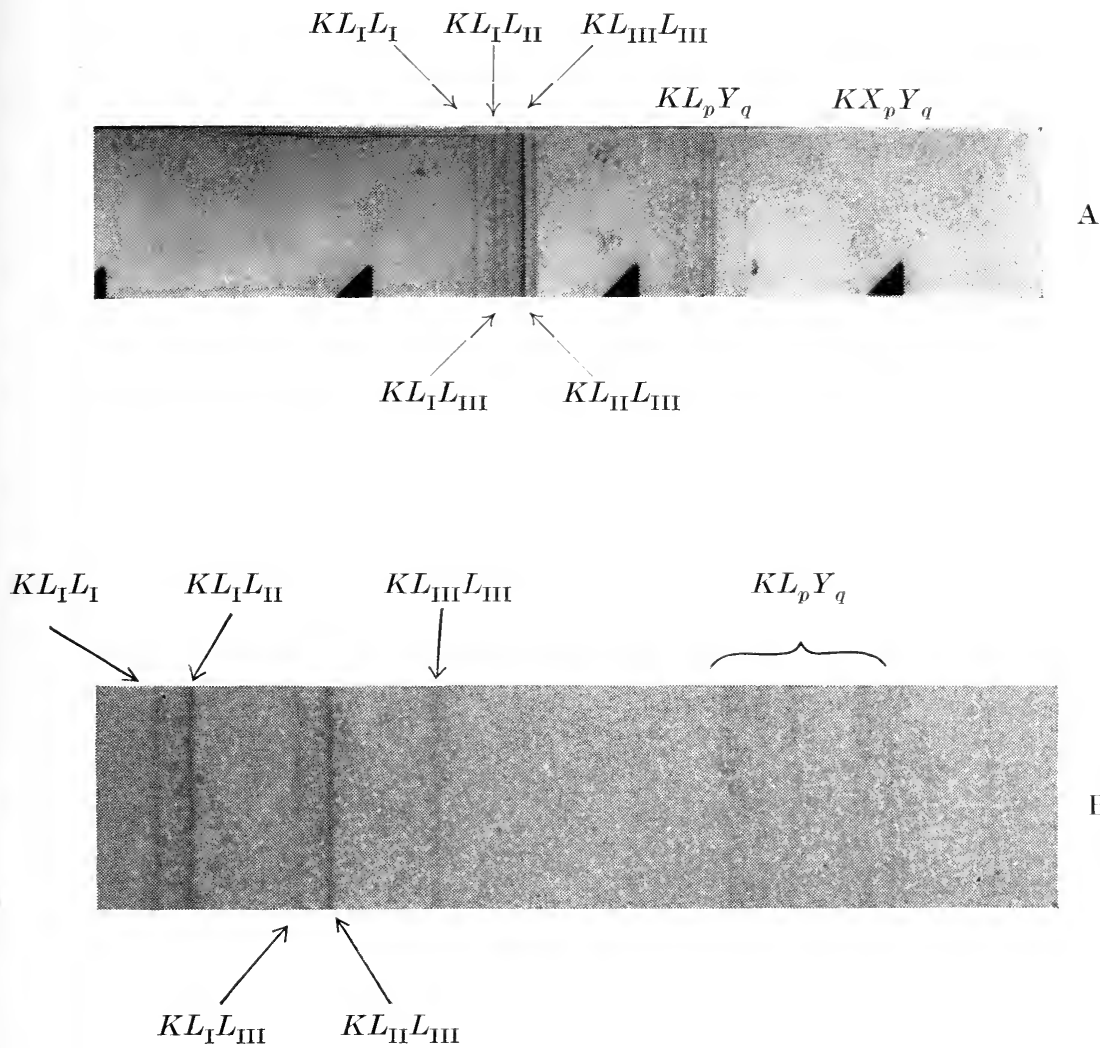

Fig. 1. A. $K$ Auger electrons in Ag (Johnson and Foster ${ }^{2}$ );

B. $K$ Auger electrons in $\mathrm{Bi}$ (Slätis and Mladjenović ${ }^{2}$ ) 
electrons for $Z=83$ in a measurement by Slätis and Mladjenović ${ }^{2}$. Semicircular $\beta$-spectrographs were used in both cases.

$\boldsymbol{K}-\boldsymbol{L}_{\mathbf{p}}$ may be obtained with high accuracy from $\mathrm{X}$-ray emission data (for example $K-L_{\Pi I}=K_{\boldsymbol{\alpha}_{\mathrm{I}}}$ ). If $\boldsymbol{L}_{q}\left(L_{p}\right)$ were known with high precision it would be possible to predict the Auger electron energy with a high accuracy. On the other hand it can be seen from $(1,4)$ that a precision measurement of the Auger electron energy may yield accurate values for the binding energies in doubly ionized shells. Bergström and Hill ${ }^{3}$ for example measured $L_{q}\left(L_{p}\right)$ in mercury. They found that within the limits of error $(1: 1000)$

$$
\boldsymbol{L}_{\mathbf{q}}\left(L_{\mathrm{I}}\right)=\mathbf{L}_{\mathbf{q}}\left(L_{\mathbf{I}}\right)=\boldsymbol{L}_{\mathbf{q}}\left(L_{\mathrm{III}}\right) .
$$

Experimentally it is found and it is clearly consistent with the picture of the Bohr atom that $L_{q}<L_{q}\left(L_{p}\right)$. The numerical evaluation of $L_{q}\left(L_{p}\right)$, although theoretically straightforward, is tedious because of having to use empirically obtained electronic wave functions, and except for light atoms ${ }^{4}$ this calculation has not been carried out. There might be a possibility of obtaining $L_{q}\left(L_{p}\right)$ from emission X-ray data. $K$ X-ray satellites ${ }^{5}$ have been observed in large number of elements, but accurate calculations of the energies of the doubly ionized shells are in general lacking. It should also be pointed out that where identification of the initial and final states has been carried out without accurate evaluation the energy differences derived from $\mathrm{X}$-ray satellites may not give directly the energy differences involved in an Auger transition ${ }^{3}$. Due to these difficulties Bergström and Hill preferred to write eq. (4) in another form more suitable for practical purposes. They defined an effective incremental charge $\Delta Z$, by considering a linear increase of binding energy with atomic number

$$
\begin{gathered}
\Delta Z=\frac{L_{q}\left(L_{p}\right)-\left(L_{q}\right)_{Z}}{\left(L_{q}\right)_{Z+1}-\left(L_{q}\right)_{Z}} \\
K L_{p} L_{q}=\left(K-L_{p}\right)_{Z}-\left(L_{q}\right)_{Z+\Delta Z} .
\end{gathered}
$$

For mercury Bergström and Hill found $\Delta Z=0.55$ for the $L_{\mathrm{I}}$ and $L_{\mathrm{II}}$ shells and $\Delta Z=0.76$ for the $L_{\mathrm{III}}$ shell. These results have recently been confirmed by Slätis and Mladjenović ${ }^{2}$ who found $\Delta Z=0.52$ for the $L_{\mathrm{I}}$ and $L_{\mathrm{II}}$ shells

2 F. A. Johnson and J. S. Foster, Can. J. of Physics 31, (1953) 469; H. Slätis and M. Mladjenovič, to be published in Arkiv för Fysik.

3 I. Bergström and R. D. Hill, Arkiv för Fysik 8, 2, (1954), 21.

- H. C. Wolfe, Phys. Rev. 43, (1953) 221; E. H. Kennard and E. Ramberg, Phys. Rev. 46, (1934) 1040.

5 Y. Cauchois and C. R. Hulubei: "Longeurs d'onde des emissions $\mathrm{X}$ et des discontinuités d'absorption X", Paris, Hermann, 1947; C. R. Hulubei, Acad. Sci. Paris 224, (1947) 773; Y. Cauchois, J. Phys. Radium 5, (1944) 1; L. Groven and J. Morlet, Bull. Acad. Roy. Belg. 37, (1951) 630. 
and $\Delta Z=0.76$ for the $L_{\mathrm{III}}$ shell in bismut. From an investigation of copper by Robinson and Cassie ${ }^{6} \Delta Z=0.75$ can be calculated for the $L_{\mathrm{I}}$ shell. There are several other measurements indicating values ${ }^{2,7}$ of $\Delta Z$ between 1 and 0 , but most of them are not accurate. If therefore $\Delta Z=0.54$ for the $L_{\mathrm{I}}$ and $L_{\mathrm{II}}$ shells and 0.76 for the $L_{\mathrm{III}}$ shell is used in formula (4) any $K L_{p} L_{Q}$ energy would be predictable with an accuracy of about $1: 1000$. However, it has to be proved that this relation also holds for lower atomic numbers.

Formula (6) (and similar expressions for other electrons) may be of some use for calibration of $\beta$-spectrographs in the low energy region. In the future there will certainly be many low energy gamma rays which can be used for calibration with higher accuracy, but in many radioactive samples the Auger electrons are already present and therefore give a convenient energy check.

Many times in the past Auger electrons have been interpreted as conversion lines. The use of formula (4) should eliminate similar misinterpretations. There are also cases where (4) can be used in the same way as $\mathrm{X}$-ray energies in order to obtain the atomic number of a radioactive nucleus, the simplest case being that of pure $K$-capture.

In some cases the pattern of the $K L_{p} L_{q}$ group may give useful information. As mentioned earlier there are six different $K L_{p} L_{q}$ lines corresponding to a given atomic number. If for some reason $K$-conversion vacancies have been produced in adjacent atomic numbers due to a certain radioactivity, the Auger $K L_{p} L_{a}$ pattern will be complicated. Let us consider a hypothetical case where there is an isomeric state for atomic number $Z$, decaying both by electron capture and $\beta$ and $\gamma$-emission to levels in the daughter nuclei. Due to $K$-capture and $K$ internal conversion there may now be $K$ vacancies in the atoms $Z+1, Z$, and $Z-1.18 K L_{p} L_{q}$ lines from three different atomic numbers may be observed if it were possible to resolve all the lines. The same thing may happen for successive $\beta$-decay or $K$-capture.

\section{$\S 4$. Intensities of Auger Electrons}

In some cases specific features of the radioactive nucleus cannot be obtained in any other way than by intensity measurements of X-rays or Auger electrons. It is then important to know the fluorescence or Auger yields, which for the $K$-shell are defined as:

$\omega_{K}=\frac{X_{K}}{V_{K}}=\frac{X_{K}}{A_{K}+X_{K}}$

$$
a_{K}=\frac{A_{K}}{A_{K}+X_{K}}
$$

$$
a_{K}+\omega_{K}=1 .
$$

$V_{K}=$ Number of primary vacancies in the $K$-shell

$X_{K}=$ Number of $K$-vacancies resulting in $K$ X-rays

$A_{K}=$ Number of $K$-vacancies resulting in $K$ Auger electrons.

6 H. R. Robinson and A. M. Cassie, Proc. Roy. Soc. A113, (1926) 282.

7 C. D. Ellis, Proc. Roy. Soc. A139, (1933) 336; J. W. Mihelich, Phys. Rev. 88, (1952) 415 . 
Similar constants may of course be defined for the $L, M$..., Auger electrons but measurements of these constants are very scarce and uncertain ${ }^{1}$.

$a_{K}$ and $\omega_{K}$ are functions of the atomic number. Wentzel ${ }^{8}$ was first to treat the Auger electron problem theoretically although no numerical calculations were made. In this case the Auger transition probability was independent of the atomic number. Since the allowed X-ray transitions are electric dipole transitions their intensities should approximately be proportional to $Z^{4}$.

The $K$-Auger yield and the $K$-fluorescence yield could then be written:

$$
\begin{aligned}
a_{K}(Z) & =\frac{c_{1}}{c_{1}+c_{2} Z^{4}}=\frac{1}{1+b Z^{4}} \\
\omega_{K}(Z) & =\frac{c_{2} Z^{4}}{c_{1}+c_{2} Z^{4}}=\frac{1}{a Z^{-4}+1}
\end{aligned}
$$

$c_{1}, c_{2}, a$ and $b$ are constants.

There is a large number of possible ways of measuring $a_{K}$ and $\omega_{K}$, most of which are described in the book by Burhop ${ }^{1}$. Knowing the relative number of primary $K$-vacancies measurements of the $K$ X-ray intensity or $K$-Auger intensity give $\omega_{K}$ or $a_{K}$. X-ray measurements are complicated and involve several corrections. This type of measurement seems to agree up to approximately $Z=40$ but at higher $Z$ the uncertainties are large. Recently, however, Roos ${ }^{8^{a}}$ has measured the $K$-fluorescence yield for $Z=40-50$, using a new more accurate $X$-ray method where the $X$-rays were detected with a NaI scintillation counter. From the atomic number $Z>30$ the $K$-Auger electron energies are large enough so (cf. Table 1) that their intensities may be measured with good precision in a $\beta$-spectrometer. This method undoubtly gives the most accurate values of $a_{K}$ and $\omega_{K}$. The most suitable case is a pure isomeric transition where the gamma ray is strongly converted ${ }^{9}$. Here the $K$-conversion intensity is a direct measure of the number of primary $K$ vacancies $\left(V_{K}\right)$. Thus a comparison of the area under the $K$-Auger electrons and the $K$-conversion line gives $a_{K}$ directly as can be seen from Eq. (8).

The latest survey of $\omega_{K}(Z)$ was compiled by Broyles et al. ${ }^{10}$. They point out that there is not a pure $Z^{4}$ dependence. Steffen et al. ${ }^{11}$ earlier found a $Z^{3.5}$ dependence. In Fig. $2 \omega_{K}$ is plotted as a function of $Z$. For $Z>40$ only the values measured by Roos and $\beta$-spectrometer values have been included. For $Z<40$ there is a large number of independent measure-

8 G. Wentzel, Z. Physik 43, (1927) 524.

8a C. E. Roos, Phys. Rev. 93, (1953) 401.

9 I. Bergström, Arkiv för Fysik, 5, 14, (1952) 191.

10 C. D. Broyles, D. A. Thomas and S. Haynes, Phys. Rev. 89, (1953) 715.

11 R. Steffen, O. Huber and F. Humbel, Helv. Phys. Acta, 22, (1949) 167. 
ments ${ }^{10}$. The points in the figure for $Z<40$ are mean values. There are still lacking measurements in the rare earth region. It is believed that this plot is representative for the present knowledge of $\omega_{K}(Z)$. A serious discrepancy, however, occurs for the values $a_{K}=0.11$ for $Z=83^{12}$ and 0.05 for $Z=80,81$, and $82^{2,10,13}$. There is no theoretical reason for such a sudden drop. More $\beta$-spectrometer measurements with high precision are strongly needed, due to the importance of $a_{K}$ and $f_{K}$ and to the great difficulties in the numerical calculations of $\omega_{K}(Z)$.

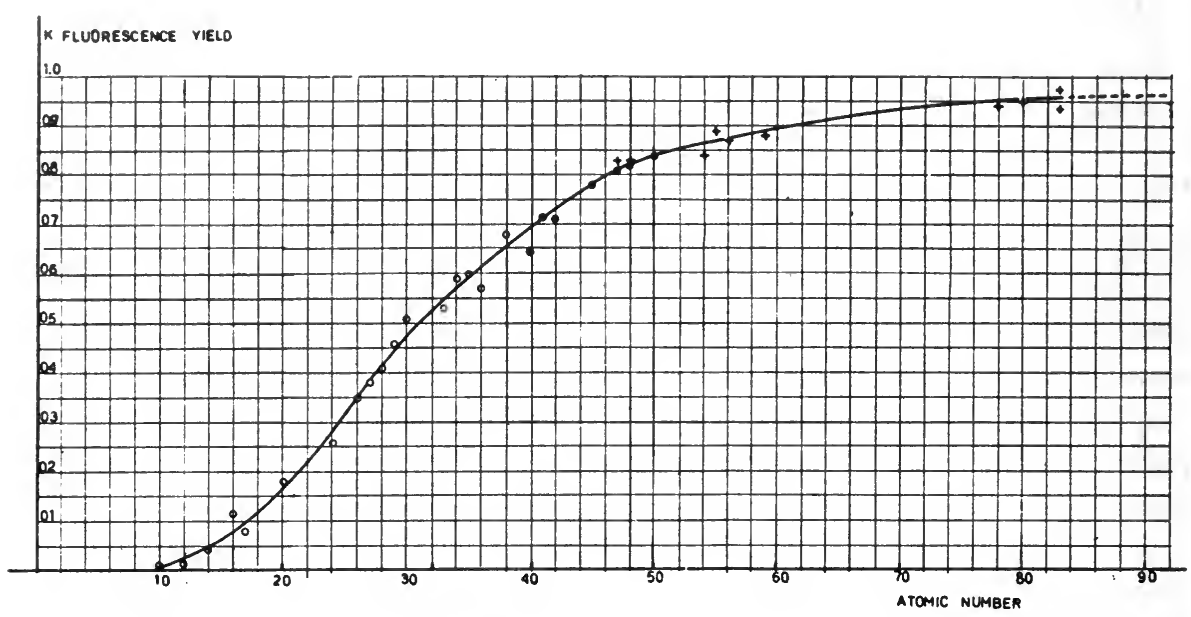

Fig. 2. $K$ fluorescence yield as a function of the atomic number. Above $Z=40$ only $\beta$-spectrometer and the recent $\mathrm{X}$-ray values by $R_{0 o s}{ }^{24}$ have been included. Below $Z=40$ the mean values of the measurements compiled by Broyles et al. ${ }^{10}$ have been plotted

In an Auger electron transition only two electrons are involved, one electron filling a vacancy and the other being expelled from the atom. First order perturbation theory may therefore give a reasonable result for the Auger transition probability. The perturbing energy is the electrostatic potential betwieen the two electrons. The presence of the other electrons may be taken into account by the use of appropriate shielding constants. In the nonrelativistic case (low $Z$ ), the probability $P_{A_{K}}$ for the ejection of $K$-Auger electrons would therefore be given by

$$
P_{A_{K}}=\text { constant } \cdot \sum\left|\iint \chi_{f}^{*} \psi_{f}^{*} V\left(\boldsymbol{r}_{1} \boldsymbol{r}_{2}\right) \chi_{i} \psi_{i} d \boldsymbol{r}_{1} d \boldsymbol{r}_{2}\right|^{2} .
$$

The sum is taken over all possible Auger transitions due to a primary $K$-vacancy. In principle the calculation of $P_{A_{K}}$ is simple, involving only

12 L. S. Germain, Phys. Rev. 80, (1950) 937.

13 A. H. Wapstra, D. Maeder, G. J. Nijgh and L. Th. M. Ornstein, Physica (in print 1954). A. H. Wapstra, Arkiv för Fysik, 7, 22, (1953) 283. 
the electrostatic interaction $V\left(\boldsymbol{r}_{1}, \boldsymbol{r}_{2}\right)$ and the initial and final wavefunctions of the electrons. However, there are several difficulties involved. $P_{A_{K}}$ decreases for electrons expelled in outer shells. In order to calculate $a_{K}$ accurately it is probably necessary to include also the transitions $K N_{p} N_{q}$. As pointed out earlier, stopping with the $N$-shell there are 120 transitions. Thus even if $V\left(\boldsymbol{r}_{1}, \boldsymbol{r}_{2}\right)$ and the wavefunctions were known the calculation would still be very tedious.

Pincherle ${ }^{14}$ has made the most complete calculations of Auger transition probabilities considering interactions of electrons in the $L, M$, and $N$ shells. However, he used unscreened hydrogen wave functions in which case the transition probability is independent of the nuclear charge. This is a rough approximation as may be seen from Fig. 3 where the relative intensities of $K L_{p} L_{q}$ and $K L_{p} Y_{q}\left(Y_{q} \neq L_{p}\right)$ electrons is plotted for different atomic numbers. As can be seen the relative intensity of these groups changes with the atomic number. This also seems to be the case for relative intensities inside the $K L_{p} L_{q}$ group (cf. Table II and Fig. 1). Burhop ${ }^{15}$ used the more accurate screened hydrogenlike wave functions in the calculation of the intensities of the $K L_{p} L_{q}$ electrons for $\mathrm{Ag}$, the screening being accounted for by the application of Slater's rules. However, even these calculations do not agree with the experiments (cf. Table II). Ramberg and Richtmeyer ${ }^{16}$ calculated the intensities of the $K L_{p} L_{\varrho}$ electrons in $\mathrm{Au}$, using the more accurate Fermi-Thomas field and Hartree electron wave functions. The only relativistic calculation has been

TABLE II

COMPARISON OF EXPERIMENTAL AND THEORETICAL VALUES OF THE $K L_{p} L_{q}$ INTENSITIES

\begin{tabular}{|c|c|c|c|c|c|c|c|c|c|c|c|}
\hline Reference & 14 & $*$ & 2 & 16 & 17 & 19 & 7 & 3 & 7 & 2 & 2 \\
\hline$Z$ & any & 47 & 47 & 79 & 79 & 80 & 79 & 80 & 83 & 83 & 84 \\
\hline . & $\begin{array}{l}\text { Theor. } \\
\text { nonrel. }\end{array}$ & $\begin{array}{l}\text { Theor. } \\
\text { nonrel. }\end{array}$ & Exp. & $\begin{array}{l}\text { Theor. } \\
\text { nonrel. }\end{array}$ & $\begin{array}{c}\text { Theor. } \\
\text { rel. }\end{array}$ & Theor. & Exp. & Exp. & Exp. & Exp. & Exp. \\
\hline$L_{\mathbf{I}} L_{\mathbf{I}}$ & 1.0 & 1.0 & 1.0 & 1.0 & 1.0 & 1.0 & 1.0 & 1.0 & 1.0 & 1.0 & 1.0 \\
\hline$K L_{\mathbf{I}} L_{\mathrm{II}}$ & $\int_{359}$ & 1.13 & 1.3 & 1.16 & 5.5 & 1.02 & 1.70 & 1.2 & 1.83 & 1.76 & 1.5 .5 \\
\hline$K L_{\mathbf{I}} L_{\mathrm{III}}$ & & 2.26 & 1.3 & 2.20 & 5.3 & 1.65 & 1.20 & 0.70 & 1.30 & 1.10 & 0.90 \\
\hline$K L_{\mathrm{II}} L_{\mathrm{II}}$ & 75 & 0.38 & 0.5 & & & 0.23 & 0.30 & 0.20 & 0.20 & 0.30 & 0.54 \\
\hline$K L_{\mathrm{II}} L_{\mathrm{III}}$ & & 4.03 & 3.2 & & & 1.46 & 1.40 & 1.40 & 2.3 & 1.55 & 1.44 \\
\hline$K L_{\text {IIII }} L_{\text {III }}$ & 8.98 & 2.30 & 1.8 & & & 0.81 & 0.80 & 0.60 & 1.30 & 0.78 & 0.58 \\
\hline
\end{tabular}

* Private communication from Prof. E. H. S. Burhop to Prof. H. Slätis (1954).

14 L. Pincherle, Nuovo Cimento, 12, (1935) 81.

15 E. H. S. Burhop, Proc. Roy. Soc. (London) A148, (1935) 272.

16 E. C. Ramberg and F. K. Richtmeyer, Phys. Rev. 51, (1937) 913. 
carried out by Massey and Burhop ${ }^{17}$ for the $K L_{\mathrm{I}} L_{\mathrm{I}}, K L_{\mathrm{I}} L_{\mathrm{II}}$ and $K L_{\mathrm{I}} L_{\mathrm{III}}$ electrons in Au. As can be seen from Table II these calculations deviate still more from the experiments than the nonrelativistic values. Rose and Goertzel 18 have recently investigated the possibility of computing Auger transition probabilities with modern calculating machines, but because of the tedious nature of the computations no numerical results have yet been obtained.

Hill ${ }^{19}$ tried quite a different way of estimating the intensities of the $K L_{p} L_{q}$ electrons. The emission of Auger electrons might be looked upon as internal conversion of $\mathrm{X}$-rays. If this is so it should be possible to define an internal conversion coefficient $\alpha_{V_{o} x_{p} Y_{q}}$, for each Auger transition. In the case of the $K L_{p} L_{q}$ electrons:

$$
\alpha_{K L_{p} L_{q}}=\frac{A_{K L_{p} L_{q}}}{X_{L_{p} \rightarrow K}}
$$

$A_{K L_{p} L_{q}}=$ intensity of $K L_{p} L_{q}$ electrons

$X_{L_{p} \rightarrow K}=$ intensity of $L_{p} \rightarrow K$ X-rays.

If $\alpha_{K L_{p} L_{q}}$ and the relative intensities for different $L_{p} \rightarrow K$ transitions were known, it would then be possible to calculate the relative intensities of $K L_{p} L_{q}$ electrons. The conversion coefficients for $\gamma$-rays converted in the $L$-shell have been calculated in a few cases by Gellman et al. ${ }^{20}$. These calculations although approximate, have proved to be consistent with experiments ${ }^{21}$. Hill then assumed that it would be possible to use the same conversion coefficients for X-rays converted in the $L$-shell. It was emphasized that this approximation would be better for $L_{p} \rightarrow K \mathrm{X}$-rays and also for higher atomic numbers where the $K$-shell is closer to the nucleus. A difficulty arises now because the magnetic dipole transition $L_{\mathrm{I}} \rightarrow K$ is forbidden. Nevertheless $K L_{\mathrm{I}} L_{\mathrm{I}}$ electrons of appreciable intensity are observed. Since $X_{L_{\mathrm{I}} \rightarrow K}$ is very small, $\alpha_{K L_{\mathrm{I}} L_{q}}$ has to be very large in order to obtain a reasonable value of $A_{K L_{\mathrm{I}} L}$. Gellman's calculations also give an extremely high $L_{\mathrm{I}}$ conversion coefficient for magnetic dipole transitions of low energy and high $Z$. Only a few years ago Groven and Morlet $^{5}$ observed a satellite line which could be due to the transition $L_{\mathrm{I}} \rightarrow K$. From their measurements the relative intensity of the $L_{\mathrm{I}} \rightarrow K$ transitions was estimated. In Table II Hill's values of the relative intensities

17 H. Massey and E. H. S. Burhop, Proc. Roy. Soc. (London) A153, (1936) 661 .

18 M. E. Rose and G. Goertzel, Oak Ridge National Laboratory, private communication. (1953).

19 R. D. Hill, Phys. Rev. 91, (1953) 770.

20 H. Gellman, B. A. Griffith and J. P. Stanley, Phys. Rev. 85, (1952) 944.

21 J. B. Swan and R. D. Hill, Australian Journal of Physies, 6, 4, (1953) 371. 
are included and as can be seen there is a reasonable agreement. Already in 1925 Bothe ${ }^{22}$ treated the Auger electron process as an internal absorption of X-rays. Later this explanation was abandoned because of the difficulty discussed above. However, Hill's calculations indicate that it may not be too unreasonable to consider the Auger process as an internal conversion of $\mathrm{X}$-rays, even if the use of nuclear $L$ conversion coefficients may be debatable.

Sometimes there is interest in knowing the relative intensities of Auger groups rather than separate lines. Pincherle ${ }^{14}$ for example gave the intensity ratios $K L_{p} L_{q}: K L_{p} Y_{q}: K L_{p} Y_{q}=1: 0.716: 0.103$. (When $K L_{p} L_{q}$ $K L_{p} Y_{a} K X_{p} Y_{a}$ denote intensities $X_{p}$ and $Y_{q}$ refer to shells outside the $L$-shell). This should be compared with the values given in Fig. 3 where there seems to be a definite $Z$ dependence. Experimental values of the intensity ratio $K L_{p} L_{q}: K X_{p} Y_{q}$ are too inaccurate to allow a definite statement as to the dependence of the atomic number. Fig. 4 shows the $K L_{p} L_{a}, K L_{p} Y_{a}$ and $K X_{p} Y_{q}$ Auger lines for $Z=81$.

Fig. 3. The $Z$ dependence of the intensity ratio $K L_{p} Y_{q}: K L_{p} L_{q}$. Most probably the curve does not increase linearly for higher $Z$, since the measurements 16 and 17 seem to be rather accurate

\section{References to Fig. 3.}

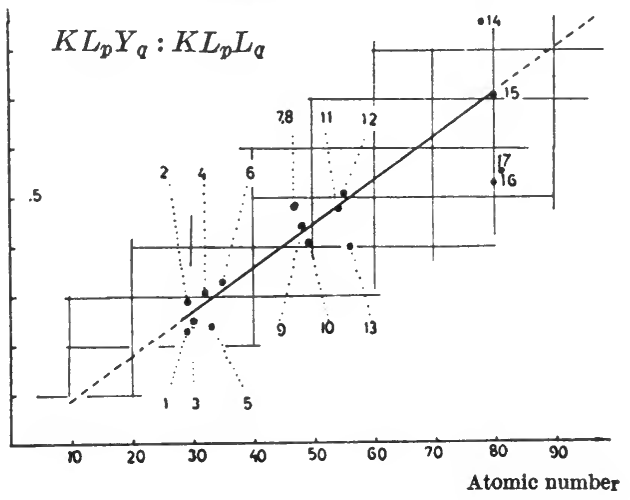

1. E. Plassman and F. R. Scott, Phys. Rev. 84, (1951) 156.

2. J. F. Perkins and S. Haynes, Phys. Rev. 92, (1953) 687.

3. T. Yusa, Physica 18, (1952) 1267.

4. M. Ference, Phys. Rev. 51, (1937) 720.

5. F. R. Scott, Phys. Rev. 84, (1954) 659.

6. S. Thulin (private communication).

7. O. Huber et al., Helv. Phys. Acta 25, (1952) 3.

8. O. Huber et al., Helv. Phys. Acta 24, (1952) 627.

9. O. Huber èt al., Helv. Phys. Acta 25, (1952) 3.

10. C. D. Broyles, Phys. Rev. 89, (1953) 715.

11. I. Bergström, Ark. f. fysik, 5, 14 (1952) 191.

12. I. Bergström, Ark. f. fysik, 5, 14 (1952) 191.

13. C. D. Broyles et al., Phys. Rev. 89, (1953) 715.

14. R. Steffen et al., Helv. Phys. Acta 22, (1949) 107.

15. C. D. Broyles et al., Phys. Rev. 89, (1953) 715.

16. T. O. Passel et al., Phys. Rev. 95, (1954).

17. See ref. 23. 
As mentioned previously Auger lines have been interpreted several times as $\gamma$-conversion lines. However, by knowing not only the energies but also the relative intensities of Auger lines or groups, such misinterpretations can be avoided. Fig. 5 shows an example. The $K L_{p} Y_{q}: K L_{p} L_{q}$ ratio in the case of $\mathrm{Xe}^{127}$ was found to be 0.33 as compared with the expected value 0.50 (see Fig. 3). This indicated the presence of a conversion line superimposed on the $K L_{p} L_{a}$ group. This proved to be a $K$ conversion line since a faint line was found at the corresponding $L$-line position ${ }^{9}$.

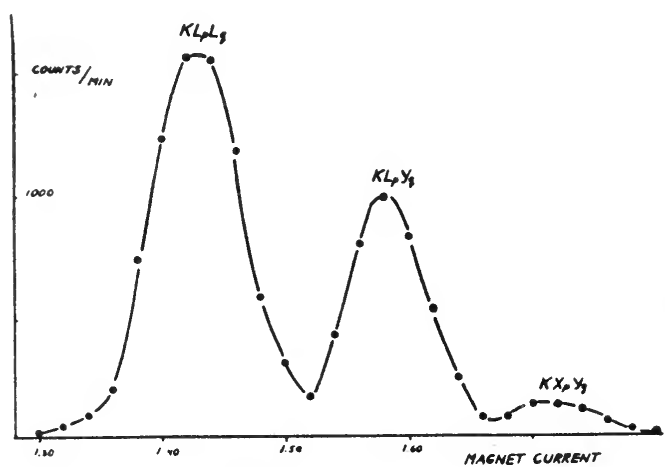

Fig. 4

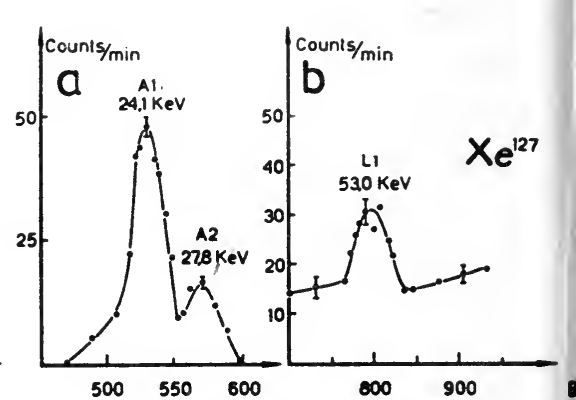

Fig. 5

Fig. 4. The $K$ Auger groups for $Z=81$ (Herrlander ${ }^{23}$ )

Fig. 5. A $K$-conversion line coinciding with the $K L_{p} L_{q}(A 1)$ Auger electrons. The presence of the $K$-conversion line was proved by the appearance of the corresponding $L$-conversion line

\section{$\S 5$. Applications Depending on the Fluorescence Yield}

There are many cases in $\beta$ - and $\gamma$-spectroscopy where $\mathrm{X}$-ray and Auger electron measurements and a knowledge of $a_{K}$ or $\omega_{K}$ give interesting features of the radioactive decay. In the following are summarized only a few cases which may illustrate the importance of $a_{K}$ or $\omega_{K}$.

1. Intensity ratio of $K$-capture to positron emission. The transition probabilities of $K$-capture and beta-ray emission involve unknown nuclear matrix elements. Therefore measurements of decay energies and half-lives cannot be compared directly with theoretical transition probabilities However, in the case where $K$-capture and positron emission occur between the same nuclear states the nuclear matrix elements are the same. The ratio $P_{K C} / P_{\beta^{+}}$of the transition probability for $K$-capture and positron emission is therefore independent of nuclear matrix elements.

23 C. J. Herrlander, Nobel Institute of Physics, Stockholm. Private communication. (1954). 
Measurement of $P_{K C} / P_{\beta^{+}}$thus affords a possibility of checking features in the $\beta$-decay theory independent of nuclear matrix elements and wave functions. From the expression

$$
\frac{P_{K C}}{P_{\beta^{+}}}=\frac{X_{K C}}{\omega_{K} \beta^{+}}=\frac{A_{K C}}{a_{K} \beta^{+}}
$$

it is clear the $P_{K C} / P_{\beta^{+}}$is obtained by comparing $K \mathrm{X}$-ray and $K$ Auger intensities. As discussed in a paper by Bouchez ${ }^{24} X_{K C} / \beta^{+}$can be measured in several ways, for example:

a) $\mathrm{X}_{K C} / \beta^{+}$may be obtained by absolute counting measurements of $\mathrm{X}$-rays and positrons.

b) Intensities of $\mathrm{X}$-rays and annihilation radiation may be compared with scintillation crystals.

c) Auger electrons and positron intensities may be compared in a $\beta$ spectrometer.

d) Coincidence measurements may in special cases give $P_{K C} / P_{\beta^{+}}$.

2. Proof of electron capture: In the case of a pure $K$-capture the $K$ Auger electrons give direct evidence for $K$-capture (like $K \mathrm{X}$-rays). If $\gamma$-rays internally converted in the $K$-shell are also present the following relation proves the presence of $K$-capture ${ }^{9}$

$$
\frac{\text { area under } K \text { Auger lines }}{\text { area under all } K \text {-conversion lines }}>a_{K} \text {. }
$$

3. Determination of the $K$-conversion coefficient $\varepsilon_{K}$ : Comparison of $\gamma$ - and X-ray intensities in a scintillation crystal of known efficiency gives ${ }^{25}$

$$
\varepsilon_{K}=\frac{K}{\gamma}=\frac{X_{K}}{\omega_{K} \gamma} .
$$

4. Determination of $K / L$ ratios: In some cases the $K$-conversion line has such a low energy that it cannot be measured accurately. $\mathrm{Xe}^{125}$ for example decays by $K$-capture to a $35 \mathrm{keV}$ level in $\mathrm{Te}^{125}$; neglecting $L$ capture we obtain ${ }^{9}$

$$
\frac{K}{L+M+N+\ldots}=\left(\frac{A_{K}}{(L+M+\ldots) a_{K}}-1\right):\left(\frac{1}{\varepsilon_{K}}+2\right) .
$$

On the other hand knowing $K /(L+M+N+\ldots)$, it is possible to calculate the $K$-conversion coefficient $\varepsilon_{K}$.

24 R. Bouchez, Physica XVIII, 12, (1952) 1171.

25 I. Bergström, S. Thulin, A. H. Wapstra and B. Åström, Arkiv för Fysik, 7, 22, (1953) 255. 


\section{(II) R. WILSON - Internal Pair Formation}

\section{§ 1. Introduction}

The internal conversion coefficient discussed in Chapter XIV is small for low atomic numbers and for high gamma-ray energies. An alternative process which can yield information about gamma-ray transitions is available for gamma-ray energies above $1 \mathrm{MeV}$. This is by the emission of an electron-positron pair instead of the usual emission of the gamma ray, called variously internal pair production and internal pair formation. This process, linlike the internal conversion, does not depend upon the atomic electron shells, and therefore the dependence upon the atomic number is slight. Fortunately, also, the internal pair formation coefficient increases with increase of gamma-ray energy, where the internal conversion coefficient decreases with increasing energy. It is in this region of low atomic number $-Z<40$ - and of high gamma-ray energy that the presently arailable theoretical calculations are the most reliable.

\section{$\S$ 2. Theoretical Predictions}

In the same way as the atomic internal coefficient may be considered as an internal photo-electric effect, so the internal pair formation can be considered as an extension of the external pair production. The calculations follow the calculations of the external pair production.

When-it is possible, it is most convenient to calculate the internal pair formation coefficient by using the Born approximation ${ }^{1}$.

This approximation assumes that $2 \pi Z e^{2} / h v=Z \alpha /(v / c) \ll 1$, where $v$ is the velocity of either the electron or the positron. The most complete calculations are those of Rose. He found it impossible to obtain an analytical formula for the total pair formation coefficient, but an analytic formula was found for the differential internal pair formation coefficient for formation of pairs with a definite energy division of the internal pairs and a definite angle between them. From this one may readily obtain, by numerical integration, the angular distribution of the pairs by numerical integration over the energy division the energy distribution of the positrons by integration over an angle, and the total internal pair formation coefficient by integration over both energy and angle. Rose has performed

1 J. R. Oppenheimer and L. Nedelski, Phys. Rev. 44, (1933) 948; M. E. Rose and G. E. Uhlenbeck, Phys. Rev. 48, (1935) 211 ; M. E. Rose, Phys. Rev. 76, (1949) 678; G. K. Horton, Proc. Phys. Soc. 60, (1943) 457. 
such integrations, and presents some of the results in graphical form. Rose presents graphs showing the total internal pair formation coefficient as a function of gamma ray energies for electric and magnetic $2^{l}$ transitions. He has also calculated the ratio of the intensities, for all energies of the pairs, at $0^{\circ}$ and $90^{\circ}$ between the pairs. Horton ${ }^{1}$ has calculated the complete angular distribution for various energy division between the electron and the positron. Experimentally, the important conclusion from these calculations is that the differential cross section for production of pairs with a small angular seperation is almost independent of multipole order, whereas the differential crosssection for production of pairs with a large angular seperation depends critically upon multipole order. Thus it is important to measure at large angles.

A special case of internal pair formation which is of great interest arises from the decay of excited states of nuclei which have spin 0. A gamma-ray transition from a spin 0 level to another spin 0 level is forbidden. In a nucleus of high atomic number, the state would decay by internal conversion and this would become the dominant process. For light nuclei or for high energies, however, internal pair formation becomes more probable. Internal pair formation from such a monopole transition is also forbidden if there is a parity change. This selection rule could only be broken by a specific electron nuclear interaction. Oppenheimer ${ }^{2}$ has calculated the energy and angular distribution of pairs from such a monopole transition with no parity change. In the absence of a gamma ray, the internal pair formation coefficient no longer has a meaning, but it is possible to calculate a lifetime of the pair emitting state.

For low positron or electron energies the Born approximation may not be valid.

An approximate correction to the differential coefficient-giving the energy distribution of the positrons - can be found. We assume that the principal correction to the Born approximation value comes from the normalization of the wave functions of the electron and the positron.

The correction to be applied is then the normalization factors for the wave functions. These factors are simple if non relativistic wave functions are assumed ${ }^{3 *}$.

The differential cross section should be multiplied by the factor

$$
F=\frac{4 \pi Z^{2} \alpha^{2} c^{2} / v_{+} v_{-}}{\left(e^{2} \pi \alpha Z c / v_{+-1}\right)\left(1-e^{-2 \pi u Z c / v-)}\right.}
$$

2 J. R. Oppenheimer, Phys. Rev. 60, (1941) 164.

3 W. Heitler, Quantum Theory of Radiation O.U.P. (1936) 167.

* This has been further justified by G. K. Horton and E. Phibbs, Phys. Rev. 96, (1954) 1066. 
The Born approximation gives an energy distribution which is symmetrical as between the positron and the electron. For the case of high $Z$ or low $k$ we are considering, the positron will be appreciably repelled by the ('oulomb field of the nucleus and will consequently be produced preferentially with a high energy. The good agreement with the experimental positron distribution, to be described later, (see Fig. 4) shows that the factor $F$ may be used to correct for the shape of the positron spectrum. However, use of the factor $F$ will overcorrect for the effect on the total pair formation coefficient; the "exact" calculations show that the total pair formation coefficient changes little for large changes in the positron spectrum.

Dalitz ${ }^{4}$ has studied in detail the corrections to the Born approximation for the experimentally important case of the pairs emitted by the $6 \mathrm{MeV}$ monopole transition in $\mathrm{O}^{16}$. He has included a consideration of radiative corrections, and shows that even the corrections to the differential crosssection are only of the order of one or two $\%$. For these energies the Born approximation may therefore be used with some confidence.

Goldring ${ }^{5}$ has extended the Born approximation calculations to cases where the gamma rays are not emitted isotropically. These arise when the nucleus is excited by proton capture or as a result of nuclear alinement.

In most cases there is no interference when different radiations (e.g. electric quadrupole and magnetic dipole) are mixed, so that a linear combination of the pair formation coefficients may be taken. In anisotropic emission, however, mixing can often occur, unless measurements are made at appropriate angles.

\section{$\S 3$. Exact Calculations}

Exact calculations have been made using the exact Coulomb wave function solutions of the Dirac equation ${ }^{6}$, but neglecting the radiative corrections.

The numerical computation necessary is very tedious, so that only a few cases were calculated. Jaeger and Hulme calculated the energy distribution, and by numerical integration the total pair formation coefficient, for $Z=0$, and $Z=84$, and $k$ taking the successive values 3,5 , and 7 ; only electric dipole and electric quadrupole were considered.

As might be expected, the results for $Z=0$ are in agreement, to within computational error, with the Born approximation results. For $Z=84$, the total pair formation coefficient was as expected, less than the Born

4 R. H. Dalitz, Proc. Roy Soc. A206, (1951) 521.

5 G. Goldring, Proc. Phys. Soc. (Lond.) A66, (1953) 341.

6 J. C. Jaeger and H. R. Hulme, Proc. Roy. Soc. 148, (1935) 708; M. H. Wang, Nature 162, (1948) 264; \$. A. S. Brimberg, Phys, liev. 87, (1952) 150. 
approximation value, and the energy distribution assymmetric. The energy distribution for the electric dipole for $k=3$ is shown in Fig. 2 for $Z=0$ and $=84$, together with the distribution for the Born approximation and the Schrödinger approximation value discussed in the next section.

Since the change in the total coefficient is small between $Z=0$ and $Z=84$, it is tempting to interpolate linearly for intermediate $Z$. Brimberg has made some exact calculations for $Z=29$ and $k=2.57$. He finds that the interpolation is not even approximately linear. A good approximation, valid for low $k$ and high $Z$ would be useful to avoid the tedium of exact calculations. Such an approximation is the Schrödinger approximation.

Wang extended the calculations of Jaeger and Hulme to include magnetic dipole radiation. He first makes an order of magnitude estimate, based upon the values of certain integrals, to show that $\Gamma$ (Mag. dipole) $>$ $\Gamma$ (Elec. dipole) $>\Gamma$ (Elec. quad.) independent of $Z$. He then obtains exact values for $Z=84$. Wang's conclusions are in direct contradiction with the Born approximation calculations of Rose and of Horton. Since the Born approximation calculations are relatively simple to perform and have been made by the two authors independently, we must assume that there is a numerical error in Wang's results. In order to find a more accurate result for the magnetic dipole than is given by the Born approximation, we must assume that the change of $Z$ affects all multipoles in a similar way, and use the exact calculations for electric dipole. This assumption is, of course, implicit in the correction factor $F$ which was derived earlier to make approximate corrections.

\section{$\S$ 4. Schrödinger Approximation}

Rose and Uhlenbeck have calculated the internal pair formation coefficient using the so called Schrödinger approximation to the Dirac equation. Like the Born approximation, the assumption is made that $Z e / \hbar c \ll 1$ but in distinction to the Born approximation they assume that $v / c \ll 1$ for both the positron and the electron.

TABLE I

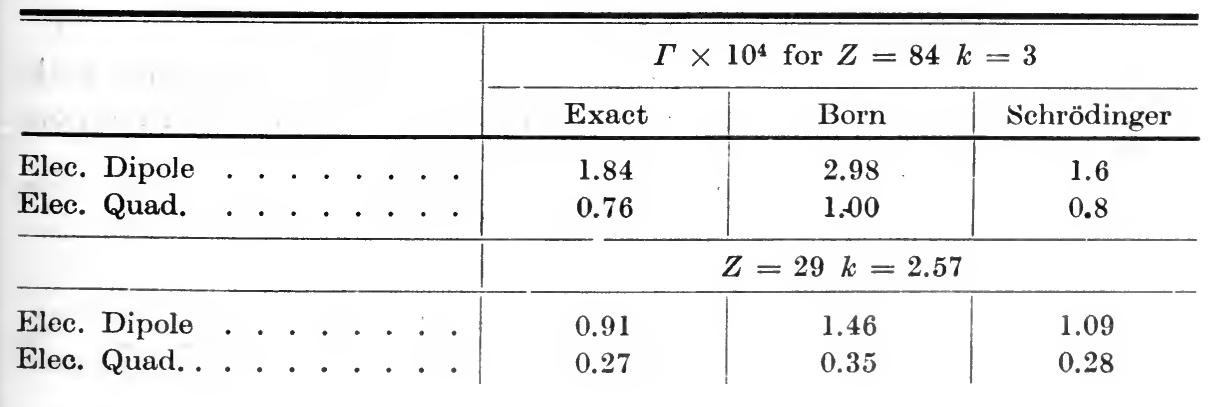


A calculation of the energy distribution for the electric dipole is shown in Fig. 2. Table I shows a comparison of the numerical values for the total coefficient for two cases where exact calculations are available, $Z=84$ and $k=3$, and for $Z=29$ and $k=2.57$.

It will be seen that the Schrödinger approximation is adequate to obtain a multipolarity assignment for $k<3$ and for all $Z$.

\section{$\S 5$. Summary of Theoretical Predictions}

The formulae for the energy distribution of the positrons are here presented. For the angular distribution the reader is referred to the original papers of Horton and Rose.

For the monopole transition with no parity change:

$$
d \Gamma_{0}\left(W_{+}, \cos \theta\right) \propto d W_{+} d(\cos \theta) P_{+} P_{-}\left(W_{+} W_{-}-1+P_{+} P_{-} \cos \theta\right) .
$$

For electric transition of polarity $l$ :

$$
\left.\begin{array}{rl}
\Gamma_{l}\left(W_{+}\right)=\{ & \left.\alpha / \pi(l+1) k^{3}\right\}\left\{(l / 2) k^{2} J_{l+1}\right. \\
+ & {\left[2 l W_{+} W_{--}-1 / 4(7 l+1) k^{2}\right] J_{l}} \\
+ & {\left[l\left({ }^{3} / 2\left(W_{+}^{2}+W_{-}^{2}\right)+1\right)+1+W_{+} W_{-}\right] J_{l-1}} \\
& \left.-1 / 4(l-1)\left(W_{+}-W_{-}\right)^{2} J_{l-2}\right\} .
\end{array}\right\}
$$

For the magnetic transition of polarity $l$ :

$$
\Gamma_{l}\left(W_{+}\right)=\alpha / \pi k^{3}\left\{\left(1+W_{+} W_{-}\right) J_{l}-\left(k^{2} / 4\right)\left(J_{l+1}-x_{1} x_{2} J_{l-1}\right)\right\}
$$

where the $J_{l}$ are the elementary integrals

$$
\begin{gathered}
J_{l}=\int_{x_{1}}^{x_{2}} x^{l}(1-x)^{-2} \\
x_{1}=\left(P_{+}-P_{-}\right)^{2} / k^{2}, x_{2}=\left(P_{+}+P_{-}\right)^{2} / k^{2} .
\end{gathered}
$$

The total pair formation coefficient is then given by

$$
\Gamma_{l}=\int_{1}^{k-1} d W_{+} \Gamma_{l}\left(W_{+}\right)
$$

All the above are Born approximation calculations. For $k<3$ the Schrödinger approximation is recommended and gives for electric dipole:

$$
\Gamma\left(W_{+}\right) d W_{+}=d W_{+} \frac{48 \pi \alpha^{3} Z^{2} W_{+} W_{-}}{k^{5}\left(e^{2 \pi \alpha Z / P}+-1\right)\left(1-e^{-2 \pi a Z / P}-\right)}
$$

for electric quadrupole:

$$
\Gamma\left(W_{+}\right) d W_{+}=d W_{+} \frac{20 \pi \alpha^{3} Z^{2}}{3 k^{5}}\left(1-\frac{4}{k}+\frac{12}{k^{2}}\right) \frac{W_{+} W_{-}\left(P_{+}^{2}+P_{-}^{2}+2 \alpha^{2} Z^{2}\right)}{\left(e^{2 \pi a Z / P}+-1\right)\left(1-e^{-2 \pi \alpha Z / P}-\right)}
$$


and for magnetic dipole:

$$
\Gamma\left(W_{+}\right)(\text {M.D. })=3 / 5 \Gamma\left(W_{+}\right) \text {(E.Q.). }
$$

Figures 1, 2, and 3 show numerical computations from these formulae together with the "exact" calculations.

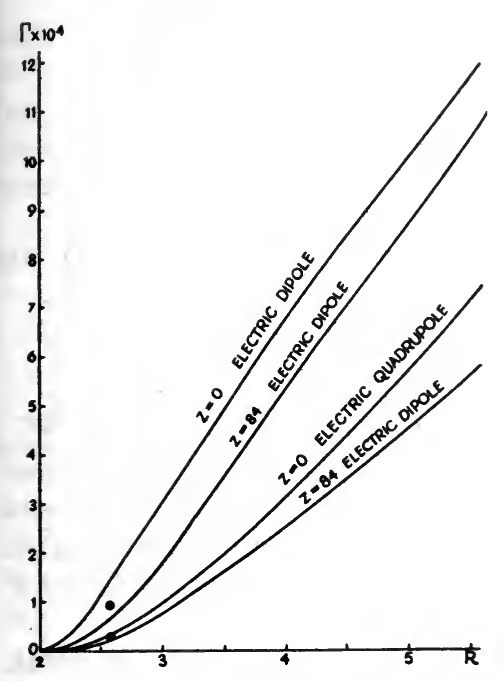

Fig. 1. Exact calculations of internal pair formation coeff. cients. The curve for magnetic dipole $Z=0$ falls almost exactly on top of the curve for electric quadrupole $Z=84$ and has not been drawn. The circles are Brimberg's points for $Z=29$

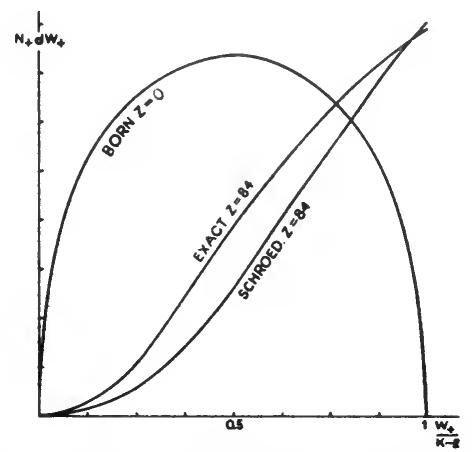

Fig. 2. Energy distribution of positrons for $k=3$ and electric dipole transitions accord. ing to exact theory $Z=84$, Schrodinger approximation $Z=84$ and Born approximation

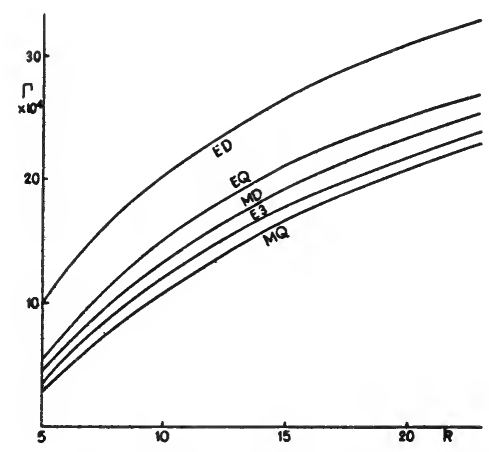

Fig. 3. Calculations of internal pair formation coefficients according to Rose using the Born approximation

\section{§ 6. Magnetic Spectrometer}

If a magnetic spectrometer can be made to count positrons, to the exclusion of electrons, the positron spectrum from excited nuclei may be studied. When a radioactive nucleus is stable against $\beta^{+}$emission, the only source of positrons is from pairs, either internal, or created externally by a high energy gamma ray. The external pairs can be reduced to negligible proportions by using thin sources. The same spectrometer may 
be used to measure electrons from the same source so that the internal pair formation coefficient may be calculated. If a radioactive nucleus is used, these electrons will be the $\beta$-rays which accompany each transition, or if $p-\gamma$ reactions are being studied, the electrons may be photo electrons ejected from a lead radiator by an accompanying gamma ray.

Alichanow ${ }^{7}$ and Rae ${ }^{8}$ have used semi circular focussing spectrometers, and Slätis ${ }^{9}$, Bloom ${ }^{10}$ and Dougherty ${ }^{11}$ have used lens spectrometers fitted with helical baffles to distinguish between positrons and electrons.

The most accurate of these investigations is that of Bloom who used a studied the internal pairs from the excited states of $\mathrm{Mg}^{24}$ with a source of $\mathrm{Na}^{24}$. He obtained an excellent agreement with the theoretical positron distribution (see Fig. 4).

When more than one gamma-ray transition occurs, the positron spectrum becomes very complex, and it is usually difficult to make an unambiguous assignment. Such assignments as can be made are summarised in Tables II and III.

Siegbahn ${ }^{12}$ has used two lens type $\beta$-ray spectrometers in coincidence $(\beta$ goniometer) to measure the angular separation of the internal pairs pro-

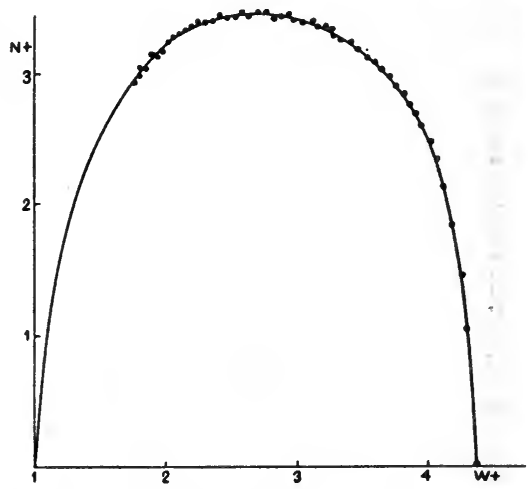

Fig. 4. The positron spectrum from $\mathrm{Mg}^{24}$ according to Bloom. The solid curve is the theoretical curve for E.Q. radiation weighted by the factor $F$ duced by the excited states of $\mathrm{Mg}^{24}$. Although the accuracy is not great, the results agree with the usual electric quadrupole assignment.

During a more detailed recent study of the positron spectra from thorium and radium active deposit sources, the Russian group discovered an effect which is somewhat allied to internal pair formation. In a radioactive decay of a heavy nucleus, the product nucleus is often left with a vacant $K$-shell. When this occurs, it is possible for an electron positron pair to be formed, with the electron filling this vacant $K$-shell. When this

7 G. D. Latyshev, Rev. Mod. Phys. 19, (1947) 132.

$\checkmark$ E. R. Rae, Phil. Mag. 40, (1949) 1155.

9 H. Slätis and K. Siegbahn, Ark. Fys. 4, No. 32, (1952) 485.

10 S. D. Bloom, Phys. Rev. 88, (1952) 312.

11 Dougherty, W. F. Hornyak, C. C. Lauritsen and V. K. Rasmussen, Phys. Rev. 74, (1948) 712; V. K. Rasmussen, W. F. Hornyak, C. C. Lauritsen and T. Lauritsen, Phys. Rev. 77, (1948) 617; R. Thomas and T. Lauritsen, Phys. Rev. 88, (1952) 969; R. J. Mackin, Phys. Rev. (in press).

12 K. Siegbahn, Ark. Fys. 4, No. 10, (1952) 223. 
TABLE II

Total Internal Pair Formation Coefficient

Internal Pair Coefficient $\times 10^{4}$

\begin{tabular}{|c|c|c|c|c|c|c|c|}
\hline \multirow{2}{*}{ Author } & \multirow{2}{*}{ Source } & \multirow{2}{*}{$\begin{array}{c}\gamma \text { ray } \\
\text { energy } \\
\mathrm{MeV}\end{array}$} & \multirow{2}{*}{$\begin{array}{l}\text { Accepted } \\
\text { Polarity }\end{array}$} & \multirow{2}{*}{$\begin{array}{l}\text { Experi- } \\
\text { mental }\end{array}$} & \multicolumn{3}{|c|}{ Theoretical } \\
\hline & & & & & E.D. & E.Q. & M.D. \\
\hline \multirow[t]{6}{*}{ Alichanow } & ThC-C" & 2.615 & E3 & 4 to 5 & $9.3(b)$ & $4.7(b)$ & \\
\hline & & 2.20 & $?$ & 5.9 & $6.3(b)$ & $3.05(b)$ & \\
\hline & & 1.80 & $?$ & 1.6 & $3.5(b)$ & $1.6(b)$ & \\
\hline & & 1.60 & $?$ & 0.9 & $2.3(b)$ & $0.95(b)$ & \\
\hline & & 1.50 & $?$ & 1.4 & $1.65(b)$ & $0.65(b)$ & \\
\hline & & 1.35 & $?$ & 0.7 & $0.9(b)$ & $0.28(b)$ & \\
\hline Bradt & ThC-C" & 2.615 & E3 & $<6.1$ & $9.3(b)$ & $4.7(b)$ & \\
\hline Rae & $\mathrm{Mg}^{24}$ & 2.758 & E.Q. & $11.6 \pm 1$ & $11.2(a)$ & $6.95(a)$ & $5.36(a)$ \\
\hline Mims & $\mathrm{Mg}^{24}$ & 2.758 & E.Q. & $7.8 \pm 1$ & $11.2(a)$ & $6.95(a)$ & $5.36(a)$ \\
\hline Cleland & $\mathrm{Mg}^{24}$ & 2.758 & E.Q. & $6.7 \pm 1$ & $11.2(a)$ & $6.95(a)$ & $5.36(a)$ \\
\hline \multirow[t]{2}{*}{ Bloom } & $\mathrm{Mg}^{24}$ & 2.758 & E.Q. & $7.1 \pm 0.2$ & $11.2(a)$ & $6.95(a)$ & $5.36(a)$ \\
\hline & $\mathrm{Mg}^{24}$ & 2.758 & E.Q. & $0.6 \pm 0.1$ & $1.52(c)$ & $0.43(c)$ & \\
\hline \multirow[t]{5}{*}{ Slätis } & ThC-C" & 2.615 & E3 & 4.3 & $9.3(b)$ & $4.7(b)$ & \\
\hline & $\mathrm{Mg}^{24}$ & 2.758 & E.Q. & $8 \pm 0.5$ & $11.2(a)$ & $6.95(a)$ & $5.36(a)$ \\
\hline & $\mathrm{Mg}^{24}$ & 1.380 & E.Q. & 0.3 & $1.52(c)$ & $0.43(c)$ & \\
\hline & $\mathrm{Fe}^{56}$ & 2.13 & M.D. + E.Q. ? & $4.6 \pm 1$ & $7.2(a)$ & $3.4(a)$ & \\
\hline & $\mathrm{Fe}^{56}$ & 1.81 & M.D. ? & $5.6 \pm 0.8$ & $5.0(a)$ & $2.1(a)$ & \\
\hline Lindenfeld & $\begin{array}{l}\pi^{+}+\mathbf{H} \\
\pi^{\circ} \text { decay }\end{array}$ & $\begin{array}{r}130 \\
70\end{array}$ & $\begin{array}{l}\text { E.D. } \\
-\end{array}$ & $\{80 \pm 16$ & & 63 & \\
\hline
\end{tabular}

(a) indicates Born approximation calculation

(b) indicates calculation for $Z=84$

(c) indicates Schrödinger approximation calculation

occurs, a positron of unique energy is produced, the energy being greater than the maximum of the continuous positron spectrum by the binding energy of the $K$-shell. A similar group of positrons can be found corresponding to the electron entering the $L$-shell. Latyshev ${ }^{13}$ has found these groups.

Attempts have been made to make numerical estimates of the probability of this process by Gie and by Sliv ${ }^{14}$. So far, however, the theory is only approximate, so that only a rough agreement has been obtained.

It is interesting to note that Alichanow found positrons from a ThC" source above the limit of $1.615 \mathrm{MeV}$ given by internal pair formation from the gamma ray $2.615 \mathrm{MeV}$. This was interpreted in the original paper

13 G. D. Latyshev, Gie, Bashilov and Barchuk, C. R. Acad. Sci. U.S.S.R. 63, (1948) 511.

14 Gie, G. D. Latyshev and Pasechnik, Bull. Sec. U.R.S.S. Physics Series 12 Sect. 6 (1948); Sliv. C. R. Acad. Sci. U.R.S.S. 64, (1949) 321. 
TABLE III

OTHER POLARITy ASSIGNMENTS BY INTERNAL PAJR FORMATION

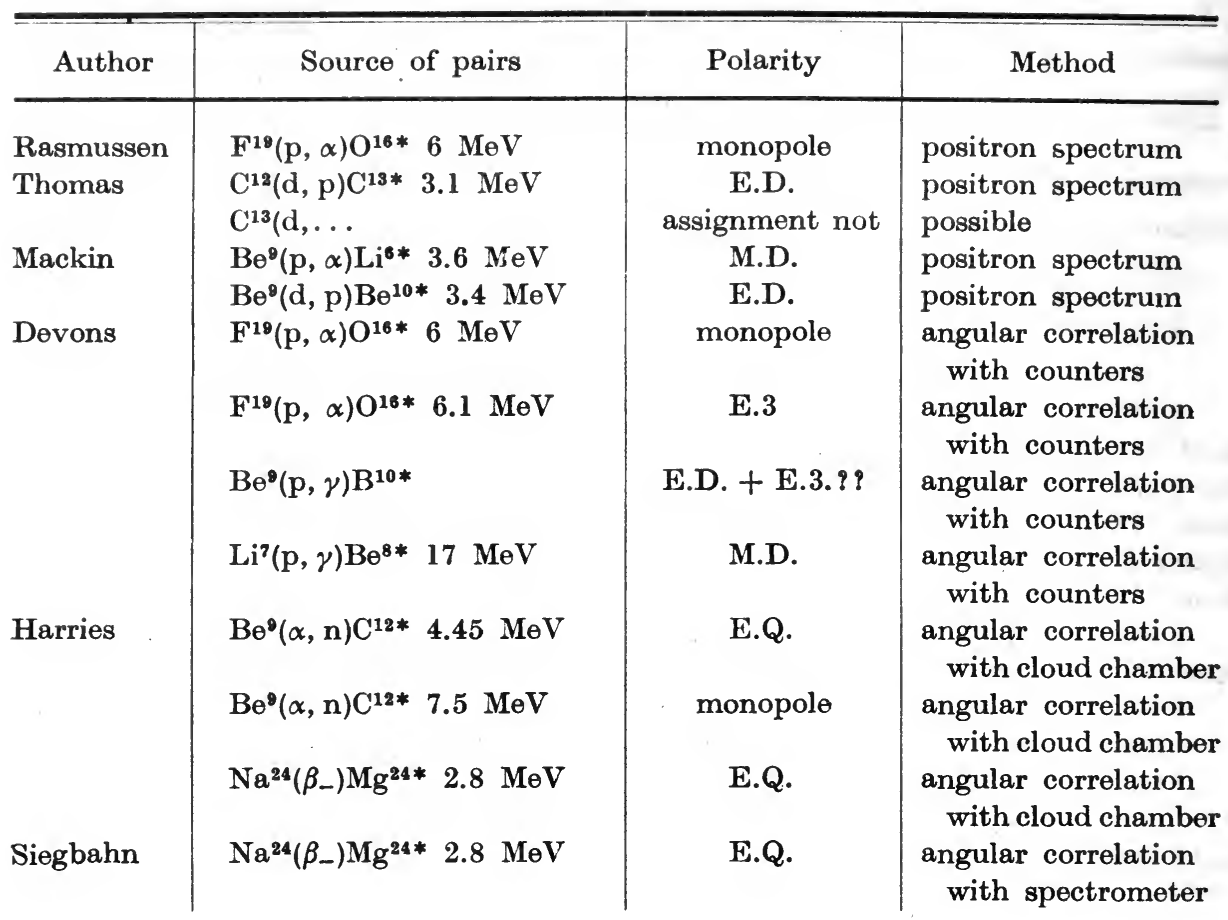

as conversion of a gamma ray of energy 3.2 $\mathrm{MeV}$ suggested by the decay scheme of ThC". Several searches ${ }^{15}$ for this $\gamma$-ray have failed, and an upper limit to its intensity may be given as $2 \times 10^{-4}$ per disintegration. Searches for internal conversion electrons, however, do not exclude the interesting possibility that there is a monopole transition with an intensity $10^{-4}$ per disintegration. It is difficult to exclude the explanation of an instrumental background.

\section{$\S 7$. Detection by Annihilation Radiation}

Mims ${ }^{16}$ measured the internal pair formation coefficient of the $2.8 \mathrm{MeV}$ gamma ray from $\mathrm{Mg}^{24 *}$ by detecting the annihilation radiation. Cleland ${ }^{16}$ performed a similar experiment. This method has the advantage of simplicity but is limited in its application to cases where only one or two gamma rays produce pairs.

The source is mounted on a thin foil, and surrounded by two thin sheets

15 e.g. G. R. Bishop, R. Wilson and H. Halban, Phys. Rev. 77, (1950) 416.

18 W. Mims, H. Halban and R. Wilson, Nature 166, (1950) 1027; M. R. Cleland, J. Townsend and A. L. Hughes, Phys. Rev. 84, (1951) 298. 
of beryllium, of sufficient thickness to stop the internal positrons. Each positron is then annihilated, and emits two gamma rays in opposite directions. These two gamma rays are highly correlated in angle, the separation angle always falling within the range $180 \pm 0.5^{\circ}$. The two gamma rays are then recorded by two scintillation counters which are in coincidence.

The efficiency for counting positrons is measured by inserting a $\mathrm{Na}^{22}$ source in place of the $\mathrm{Na}^{24}$ source used to provided the $\mathrm{Mg}^{24}$. The $\mathrm{Na}^{22}$ emits a positron for every disintegration, so that all that is now necessary is the relative calibration of the sources. (The source calibration used by Mims is believed to be in error by $5 \%$. The figure in Table II is the published figure corrected by this amount which is in any case well within the quoted error.)

Since $\mathrm{Na}^{24}$ emits two gamma rays in cascade, it is possible to obtain coincidences between the two scintillation counters from direct counting of the gamma rays as well as from internal pair formation. Since the annihilation radiation has a high angular correlation, whereas that of the two cascade gamma rays is comparitively weak, this background is reduced by increasing the distance between the scintillation counters. Then we may remove the counters till the angle subtended by the source is $0.5^{\circ}$, and solid angle $\Omega \simeq 10^{-5}$.

If the efficiency of each counter to gamma rays is the source strength $N$ disintegrations per second, and the internal pair formation coefficient $\Gamma$ we then have

$$
\begin{array}{ll}
\text { Wanted coincidence rate } & G=N \epsilon^{2} \Omega \Gamma . \\
\text { Unwanted coincidence rate } & B=N \in^{2} \Omega^{2} . \\
G / B=\Gamma / \Omega . &
\end{array}
$$

The chance coincidence rate $C=2 N^{2} \in^{2} \Omega^{2} T$ where $T$ is the resolving time of the coincidence set. So that

$$
G / C=\Gamma /(2 N \Omega T)=\epsilon^{2} \Gamma^{2} /(2 G T) .
$$

If we accept an effect to background ratio of $1 / 1$ we can measure internal pair coefficients down to $10^{-5}$ without background from coincident gamma rays. For a gamma counting efficiency of $30 \%$ and $T=10^{-9}$ minutes, we will have to reduce the counting rate to 1 per hour, so that data will be tedious to accumulate. Improvement in this apparatus can be made by reducing $T$ to $2 \times 10^{-11}$ minutes which is about the limit of present techniques. Alternatively the scintillation detectors can be made sensitive only to annihilation radiation by pulse amplitude discrimination. This should increase the efficiency of counting annihilation radiation by a 
factor of 10 relative to other gamma rays, which would mean a factor 100 increase in sensitivity, but the resolving time could not simultaneously be decreased.

Both sources of background are simply measured by displacing the source form the line between the two counters.

A further background arises from formation of pairs in the beryllium absorber by the gamma rays emitted. For low energy gamma rays the internal pair formation coefficient is reduced but so is the cross-section for pair production externally, in roughly the same proportion. Moreover at low energies a thinner absorber suffices: It is estimated by placing two additional sheets of beryllium outside and extrapolating to zero thickness. For $3 \mathrm{~cm}$ diameter counters, the method is limited to gamma-ray energies below $3 \mathrm{MeV}$ by the diffusion of the positrons through the beryllium absorber. The limit could be raised with larger counters.

\section{$\S 8$. Counter Telescopes}

Some early abortive attempts were made with radiactive sources to detect triple coincidences between $\beta$-rays and a subsequent electronpositron pair, using Geiger Counters ${ }^{17}$. A search was also made for double coincidences from proton bombardment of various nuclei ${ }^{18}$. The use of two double coincidence telescopes by Devons has enabled great success to be achieved.

Devons ${ }^{19}$ makes his counters with thin walls, to minimise electron

Fig. 5. Angular correlation of electronproton pairs from the first excited state of $\mathrm{O}^{16}$ together with the theoretical curve (Born approx.) for a monopole transition. Two curves are shown for two different geometrical arrangements (from Dévons)

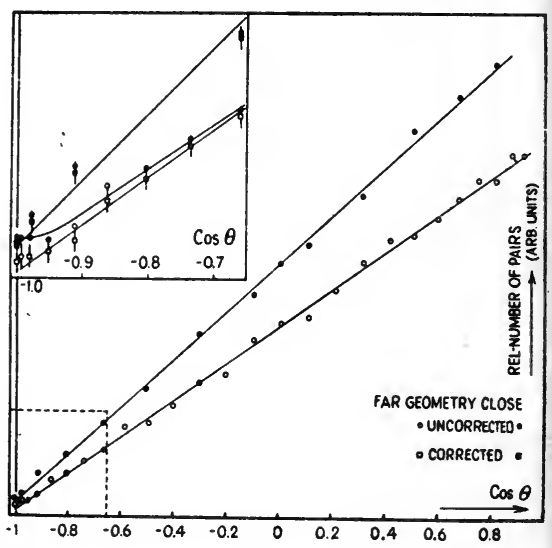

17 H. L. Bradt, J. Halter, H. G. Heine and P. Scherrer, Helv. Phys. Acta. 19, (1946) 431. W. Mims, H. Halban and R. Wilson, Nature 166, (1950) 1027.

18 Philips, Cowie and Heydenberg, Phys. Rev. 83, (1951) 1049.

19 S. Devons, H. G. Hereward and G. R. Lindsey, Nature 164, (1949) 586 ; S. Devons and G. R. Lindsey, Nature 164, (1949) 539 ; S. Devons and G. Goldring, Proc. Phys. Soc. (Lond.) A67, (1954) 134; S. Devons and G. Goldring, Proc. Phys. Soc. A67, (1954) 413. 
scattering, and reduces the material in the surrounding vacuum tank. Fig. 5 shows Devons' measurements on the angular correlation of the pairs from $\mathrm{O}^{16}$ together with the theoretical predictions. The method is being used to study other transitions, as summarized in Table III.

\section{§ 9. Cloud Chamber Measurements}

Harries and Davies ${ }^{20}$ have made a study of internal pair formation using a cloud chamber. Using a source of $\mathrm{Na}^{24}$ a search was made for electron positron pairs whose energy added to $1.76 \mathrm{MeV}$. The angular correlation between these pairs was in agreement with the usual electric quadrupole assignment of this gamma ray.

From bombardment of beryllium by $\alpha$-particles excited states of $\mathrm{C}^{12}$ are formed. Harries measured the angular correlation between pairs from the excited level at $4.45 \mathrm{MeV}$ and showed that the transition was electric quadrupole. In the course of this study of pairs from the $4.45 \mathrm{MeV}$ level in $\mathrm{C}^{12}$ some pairs were found with a total energy of $7.0 \pm 0.6 \mathrm{MeV}$. Since no $7 \mathrm{MeV} \gamma$-ray has been found the conclusion is that the level has spin 0 with positive parity and that competitive decay occurs via the $4.45 \mathrm{MeV}$ excited state.

Cloud chamber measurements with thin sources would seem to be ideally suited to the measurement of the angular distribution of internal pairs; counters have too high an efficiency for counting gamma rays for the simple experimental arrangement of Bradt or Mims to be successful, and special arrangements such as that of Devons are necessary. A cloud chamber method has not this difficulty, because the electrons and the positrons may be traced to the source.

Moreover the cloud chamber method obviates another serious defect of all the other methods mentioned. None of them work satisfactorily for a complicated gamma-ray spectrum. When using a cloud chamber it is possible to add together the energies of the electron and the positron, and therefore to discover to which gamma transition they belong. It is possible to increase the usual efficiency of taking photographs by triggering the cloud chamber either by a bank of counters immediately outside the cloud chamber or else by an internal counter.

\section{$\S 10$. Internal Pair Formation at High Energies}

A test of the theoretical formula at high energies may be found from

20 G. Harries and W. T. Davies, Proc. Phys. Soc. (Lond.) A65, (1952) 564; G. Harries, Proc. Phys. Soc. (Lond.) A67, (1954) 153. 
the internal pair formation of the gamma rays produced by meson capture in hydrogen.

The reactions are $\pi^{+}+\mathrm{p} \rightarrow \gamma+\mathrm{n}$

$$
\begin{aligned}
\rightarrow \mathrm{e}^{+}+\mathrm{e}^{-} & +\mathrm{n} \\
\rightarrow \pi^{\circ}+\mathrm{n} & \rightarrow \gamma+\gamma+\mathrm{n} \\
& \rightarrow \mathrm{e}^{+}+\mathrm{e}^{-}+\gamma+\mathrm{n}
\end{aligned}
$$

The photons of the reaction (1) are electric dipole and have energy of $130 \mathrm{MeV}$ : those of the reaction (3) have no definite multipole and have an energy about $70 \mathrm{MeV}$. In the decay of the $\pi^{\circ}$ meson no definite multipolarity can be assigned to the gamma rays. Dalitz ${ }^{21}$ has calculated the formula to be expected in this case. The high energy formulae for internal pair formation, valid for $k>100$, is

$$
\Gamma=(2 \alpha / 3 \pi) \log (2 k-C)
$$

where $C=3 / 5$ for electric dipole radiation $61 / 30$ for electric quadrupole radiation and 2.45 for $\pi^{0}$ meson decay. Since there are two gamma rays in the $\pi^{0}$ meson decay the ratio of reactions (4) to (3) is $2 \Gamma$.

At these high energies the conversion coefficients were measured by counting the electrons in a telescope of three scintillation counters, both with and without a lead radiator in front, to convert the gamma rays ${ }^{22}$.

The electrons from the meson decay may be separated from the others by measuring the second gamma ray in coincidence. The result for the average value of $\Gamma$ is $0.0080 \pm 0.0016$. The theoretical average value is 0.0069 . Direct production of electron positron pairs from cosmic ray stars and high energy meson stars have been attributed to internal pair formation in meson decay by photographic plate studies ${ }^{23}$.

\section{$\S 11$. Summary}

A table of all measured total pair formation coefficients is presented in Table II and other measurements in Table III. It will be seen that there is agreement with other multipole assignments so that internal pair formation may be used as a reliable method of measuring the multipolarity of a gamma-ray transition.

21 R. H. Dalitz, Proc. Phys. Soc. A64, (1951) 667.

22 P. Lindenfeld, A. Sachs and J. Steinberger, Phys. Rev. 89, (1953) 531.

23 R. R. Daniel, J. H. Davies, J. H. Hulvey and D. H. Perkins, Phil. Mag. 43, (1952) 753; J. J. Lord, J. Fainberg, D. M. Haskin and M. Schein, Phys. Rev. 87, (1952) 538; M. Schein, J. Fainberg, D. M. Haskin and R. G. Glasser, Phys. Rev. 91, (1953) 973. 


\section{(III) C. S. WU - Internal Bremsstrahlung}

\section{§ 1. Introduction}

Most $\beta$-disintegration is accompanied by the emission of $\gamma$-radiation. These $\gamma$-rays are highly monochromatic and result from the deexcitation processes in the nucleus before or after the process of $\beta$-decay. Under certain conditions, the deexcitation processes may take place partially or completely through the emission of internal conversion electrons. If that is the case, one observes a continuous $\beta$-spectrum with a few monochromatic lines superposed on it. However, there are certain $\beta$-emission nuclei which not only give a normal continuous electron spectrum, but also emit no monochromatic $\gamma$-rays. Nevertheless, there are always fairly weak inhomogeneous $\gamma$-rays accompanying this kind of disintegration (Fig. 1). This was first observed by Aston ${ }^{1}$ in his measurements on RaE. His $\gamma$-ray measurements on $\mathrm{RaE}^{1}$ showed that on the average energy equal to $10 \mathrm{keV}$ is emitted as $\gamma$-radiation for every $\beta$-disintegration. Bramson ${ }^{2}$ made some more accurate measurements of the $\gamma$-radiation of $\mathrm{RaE}$ and estimated roughly that 1.6 quanta were emitted per hundred electrons of disintegration. The most probable interpretation of this inhomogeneous $\gamma$-radiation which gives the right order of magnitude for the effect is the one in which the radiation is considered as secondary in origin, emitted by the changing dipole moment of the atom when the electronic charge is suddenly shifted from the nucleus to a region outside, by the emission of a

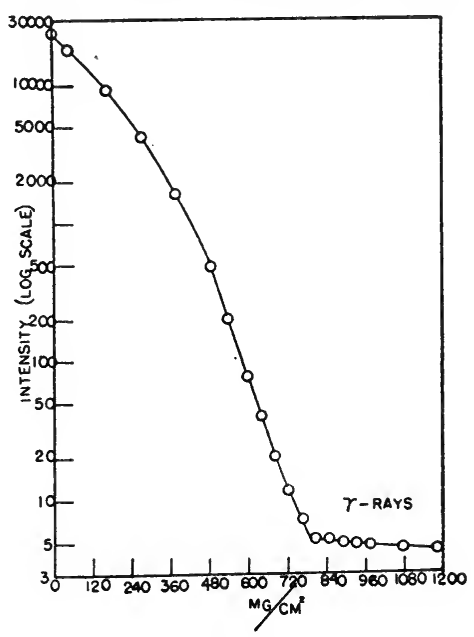

Fig. 1. Absorption curve of the radiations from a $\mathbf{5 . 0}$ me $\mathbf{P}^{32}$ source. The portion marked " $\gamma$-rays" is the bremsstrahlung radiation. (See footnote 9) $\beta$-particle. The above radiation, known as internal bremsstrahlung, is to be contrasted with the external bremsstrahlung produced due to the deflection of $\beta$-particle in the electrostatic field of a nucleus other than its own. 


\section{$\S 2$. Energy Distribution of Internal Bremsstrahlung}

Theoretical calculations on the internal bremsstrahlung spectrum have been made, first by Knipp and Uhlenbeck ${ }^{3}$ and by Bloch ${ }^{4}$ for allowed $\beta$-transitions with a polar vector interaction. Chang and Falkoff ${ }^{5}$ and Madansky ${ }^{6}$ extended these calculations to first and second forbidden $\beta$-transitions and different types of interactions.

In evaluating the production of internal bremsstrahlung, one usually treats the overall process in two steps:

$$
\mathrm{n}_{0} \rightarrow \mathrm{p}+\nu+\mathrm{e}^{\prime} \rightarrow \mathrm{p}+\nu+\mathrm{e}+\gamma
$$

1) The transition from initial to intermediate state with the transformation of the nucleon and the creation of a pair of light particles. 2) The transition of the electron from the intermediate state to the final state with the simultaneous emission of a light quantum of energy $k$.

The joint probability of the overall process of emission of a quantum of energy $k$ can be calculated with the second order perturbation method. The expression for the total probability $S(k) d k$ for the emission of a $\gamma$-quantum with energy between $k$ and $k+d k$ is

$$
\left.\begin{array}{rl}
S(k) d k= & \frac{1}{(2 \pi)^{8}} d k k^{2} \int d \Omega_{e} \int d \Omega_{v} \int d \Omega_{k} d W_{e} \times\left(W_{0}-W_{e}\right)^{2} \\
\left(W_{e}-k\right)\left\{\left(W_{e}-k\right)^{2}--1\right]^{1 / 2} \times\left|\sum_{e} \frac{\left\langle f\left|H_{\gamma}\right| 1\right\rangle\left\langle 1\left|H_{\beta}\right| 0\right\rangle}{W_{e}-W_{0}}\right|^{2}
\end{array}\right\}
$$

where $W_{0}$ is the available energy. $W_{v}$ is the energy of the antineutrino. $W_{e}=W_{0}-W_{v}=W+k$ is the energy with which the electron is born. The integrations over $\Omega_{e}, \Omega_{v}, \Omega_{k}$ are integrations over the directions of the momenta of the electron, anti-neutrino and the light quantum and summations over the polarizations of these particles respectively. $H_{\beta}$ is the interaction term between the heavy particle and the electron-neutrino field and is the cause of the first step of transition. The exact form is expressed according to the $\beta$-coupling assumed. In the original work, the polar-vector coupling was used as it was the original $\beta$-coupling used in the Fermi theory of $\beta$-decay. $H_{\gamma}$ is the interaction term between the electron and the electromagnetic radiation field and is responsible for the second step of the transition.

However, Knipp and Uhlenbeck ${ }^{3}$ introduced an alternate method in which the joint probability for the overall process was simply the product of the probabilities of the two steps. This simplification could be justified

3 J. K. Knipp and G. E. Uhlenbeck, Physica 3, (1936) 425.

4 F. Bloch, Phys. Rev. 50, (1936) 272.

5 C. S. W. Chang and D. L. Falkoff, Phys. Rev. 76, (1949) 365.

6 L. Madansky, F. Lipps, P. Bolgiano and T. H. Berlin, Phys. Rev. 84, (1951) 596. 
if the two processes were independent of each other. Although there is no basis for assuming these processes as independent, the result obtained with this simple approach for the allowed $\beta$-transition turns out to be exactly the same as that from the more rigorous second order perturbation method. Therefore, the acceptance of its use for the allowed transition invoked no arguments.

The question was then raised as to the validity of the application of this simple method to forbidden transitions. It was pointed out by Morrison and Schiff ${ }^{7}$ that the close agreement between the calculations by second order and by first order perturbation method for allowed transitions is due to the fact that the electron neutrino coupling does not depend explicitly on the momenta of these particles. This is certainly not true in forbidden cases. Chang and Falkoff ${ }^{5}$ carried out calculations with both the second order perturbation method and the simple method for first and second forbidden transitions of scalar interactions. They found that although the results from these two methods are not exactly the same, the close agreement between them justifies the adoption of the simple method. They also concluded that both the ratio of $\gamma$-intensity to $\beta$ intensity and the shape of the bremsstrahlung spectrum are almost the same for forbidden transitions as for allowed transitions, irrespective of the type of interactions (Allowed: scalar or tensor; first forbidden: scalar or tensor $(\Delta I=2$ yes); second forbidden: scalar). See Fig. 2a and $2 \mathrm{~b}$.

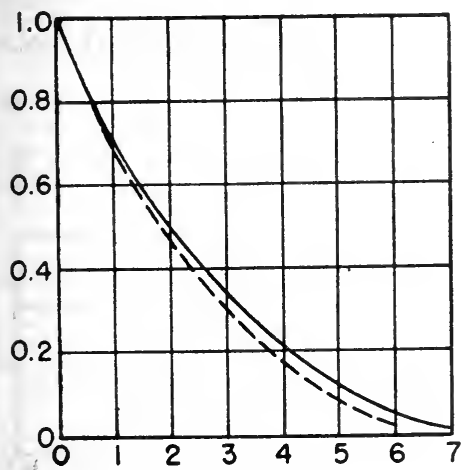

Fig. $2 a$

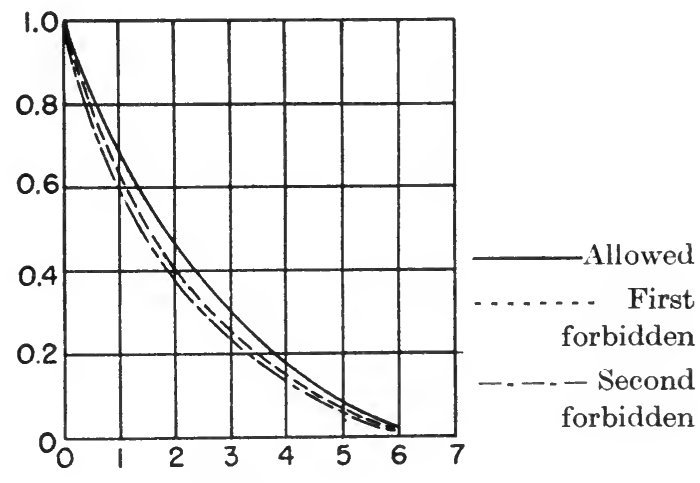

Fig. $2 b$

Fig. 2a. Energy distribution of the $\gamma$-rays following allowed $\beta$-transitions, scalar and vector theory, for $W_{0}=10 \mathrm{in}$ relativistic units. Ordinate $k S(k)$ normalized to unity for $k=0$. Abscissa $k$ measured in relativistic units. Full line-exact.

Broken line approximate $W_{0}$ large. (See footnote 5 )

Fig. 2b. Energy distribution of $\gamma$-rays following $\beta$-transitions of different degree of forbiddenness, scalar theory. $W_{0}=10$. Ordinate $k S(k)$ normalized to unity for $k=0$, abscissa $k$ measured in relativistic units. (See footnote 5 )

7 P. Morrison and L. Schiff, Phys. Rev. 58, (1940) 24. 
Under the assumption of independence of these two processes, the probability for the emission of $\gamma$-quantum with energy $k$ can be expressed as

$$
S(k)=\int_{1+k}^{W_{0}} d W_{e} P\left(W_{e}\right) \Phi\left(W_{e} k\right)
$$

where $P\left(W_{e}\right) d W_{e}$ is the distribution of the $\beta$-spectrum and $\Phi\left(W_{e}, k\right)$ is the probability of an electron coming from the nucleus with energy $W_{e}$ emitting a quantum of enersy $k$. The function $\Phi\left(W_{e}, k\right)$ was calculated to be

$$
\Phi\left(W_{e}, k\right)=\frac{\alpha P}{\pi P_{e} k}\left\{\frac{W_{e}^{2}+W^{2}}{W_{e} P} \log (W+P)-2\right\}
$$

" $W_{e}$ " and " $W$ " and " $P_{e}$ " and " $P$ " are the energies and momenta of the electron before and after the emission of the photon $k$. It can be seen that one obvious advantage of using the simple expression is that one may use the experimentally measured $\beta$-spectrum for $P\left(W_{e}\right) d W_{e}$ thereby avoiding the difficulty of dealing with the lack of uniqueness in the type of interaction in $\beta$-decay. The function $\Phi\left(W_{e}, k\right)$ is roughly proportional to $1 / k$, therefore it becomes very large for low energy quanta. When $k \Phi$ is plotted vs photon energy $k$ for various initial energies of the electrons $W_{e}$, the intensity curves start at certain values for soft radiation then gradually decrease to zero as $k$ approaches to its upper limit $W_{e}-1$. (Fig. 3.) The total energy spectrum of the internal bremsstrahlung from $\beta$-decay of an allowed transition of $W_{0}=3.6$ is shown in Fig. 4. To obtain the number of quanta of energy greater than $k$ per electron $N(k)$ one integrates the following integral:

$$
N(k)=\int_{k}^{W_{0}-1} S(x) d x .
$$
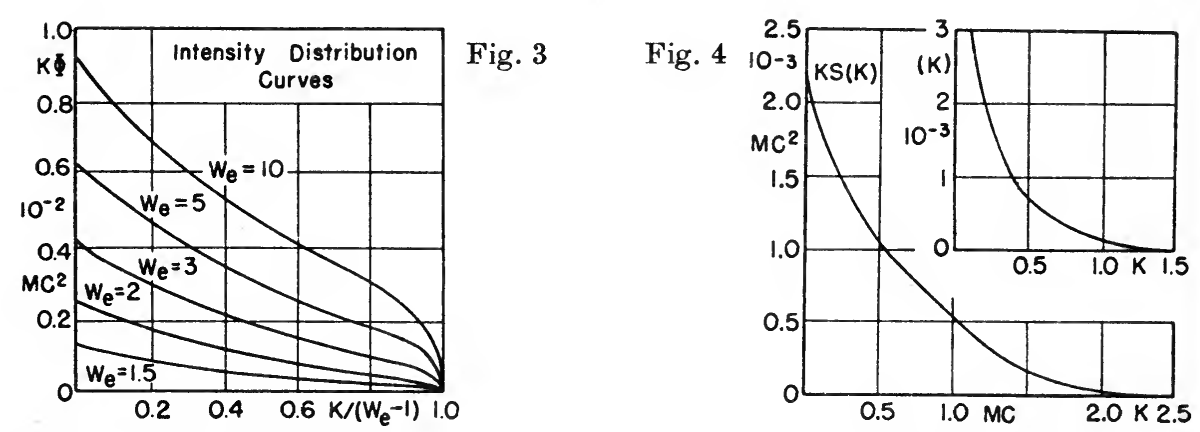

Fig. 3. Theoretical intensity distribution of internal bremsstrahlung $k \Phi$ vs $k / W_{e}-1$ as function of energy of the electron $W_{e}$. (See footnote 3)

Fig. 4. Total energy of bremsstrahlung radiation as obtained in an "allowecl" nuclear transition with $W_{0}=3.6$ and $Z=0$. Inset shows $N(k)$ vs $k$ where $N(k)$ equals the number of gammas with energy greater than $k$. (See footnote 3 ) 
The relation between $N(k)$ and $k$ for a continous $\beta$-spectrum with upper limit of $3.6 \mathrm{mc}^{2}$ is shown as an insert in the upper right corner of Fig. 4. From this figure one can read that there are only three quanta having energy greater than $50 \mathrm{keV}$ per thousand $\beta$-disintegrations.

\section{§ 3. Experimental Results}

Both the emission probability and the energy distribution of the internal bremsstrahlung found experimentally are in good agreement with the theoretical predictions. Prior to the days of the NaI scintillation spectrometer, most of the investigations were done with the ionization chamber ${ }^{8}$. Therefore, no detailed analysis of the energy distribution of the bremsstrahlung could be made. However, $\mathrm{Wu}^{9}$ and Stahel and Guillessen ${ }^{10}$ investigated the absorption curves of the bremsstrahlung from $\mathrm{P}^{32}$ and $\mathrm{RaE}$, calibrated the sensitivity of the ionization chamber and found that the total yield of internal bremsstrahlung thus calculated is in good accord with that predicted. The most difficult part of the investigation is to minimize the amount of external bremsstrahlung excited in the finite source thickness and surrounding materials getting into the detector. ,The internal bremsstrahlung is relatively independent of $Z$ and is only a small fraction of the total external bremsstrahlung excited in materials of high atomic number as the latter is proportional to the square of the atomic number. Fig. 5 shows the intensity of external bremsstrahlung

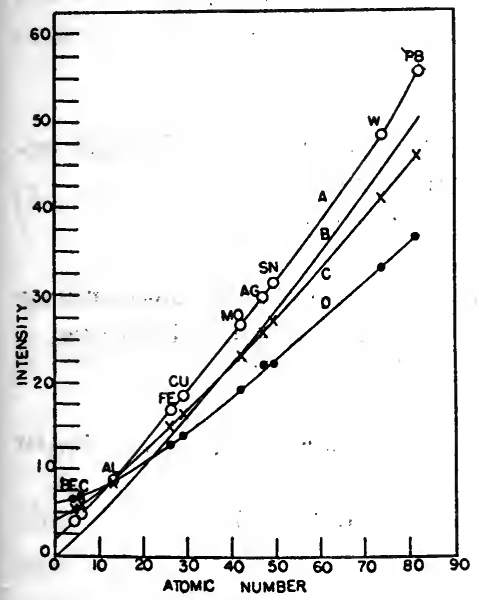

Fig. 5. The intensity of X-rays excited in different metals plotted against the atomic number.

Curve A - Extrapolated curve for an infinitely thin sample;

B - theoretically calculated curve;

C - actually measured curve for a sample of thickness of $40 \mathrm{mg} / \mathrm{cm}^{2}$;

D - actually measured curve for a sample of thickness $80 \mathrm{mg} / \mathrm{cm}^{2}$.

(See footnote 9)

8 S. Bramson, J. de phys. et rad. 66, (1930) 721 ; E. Stahel and D. J. Conmore, Physica 2, (1935) 707; G. J. Sizoo, and D. J. Conmore, 47, (1936) 921 ; E. McMillan, Phys. Rev. 47, (1935) 801; G. V. Droste, Zeits. f. Physik 100, (1936) 529; G. J. Sizoo, C. Eickman and P. Green, Physica 6, (1939) 1057.

- C. S. Wu, Phys. Rev. 59, (1941) 481.

10 E. Stahel and J. Guillessen, J. de Phys. et Rad. 1, (1940) 12. 
excited in different metals by the $\beta$-particles of $\mathrm{P}^{32}\left(W_{0}=1.67 \mathrm{MeV}\right)$. From curve $A$ one can read that the internal bremsstrahlung is about $0.00 .0 \mathrm{mc}^{2}$ per disintegration electron and the external bremsstrahlung excited in aluminum and in lead are $0.0082 \mathrm{mc}^{2}$ and $0.056 \mathrm{mc}^{2}$ per electron respectively.

Recently, Novey ${ }^{11}$, Bolgiano et al. ${ }^{12}$ and Boehm and $\mathrm{Wu}{ }^{13},{ }^{15}$ have reported good agreement with the theory of the shape of the bremsstrahlung spectrum by using a $\mathrm{NaI}$ scintillation spectrometer. The $\beta$-radioactive substances used in these investigations were $\mathrm{P}^{32}, \mathrm{Y}^{91}, \mathrm{RaE}^{210}$, $\mathrm{Pm}^{147}$, and $\mathrm{S}^{35}$. The internal bremsstrahlung spectra of $\mathrm{P}^{32}, \mathrm{Y}^{\mathbf{9 1}}$ and $\mathrm{Pm}^{\mathbf{1 4 7}}$ are shown in Fig. 6, 7 and 8. The experimental $\gamma$-ray energy per $\beta$-particle for $\mathrm{P}^{32}$, $\mathrm{Y}^{91}$ and $\mathrm{RaE}$ as compared with theoretical predictions are listed in Table I. Recently, Renard 14 investigated the internal bremsstrahlung from $\mathrm{P}^{32}$ in an energy region of $3-30 \mathrm{keV}$ in a proportional counter and

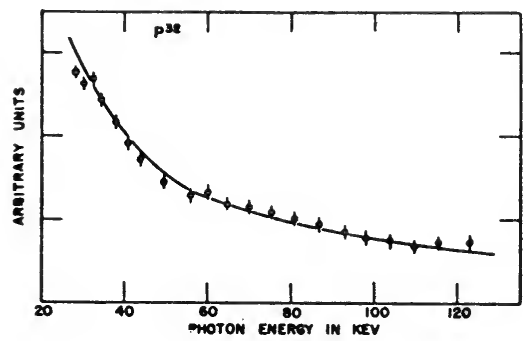

Fig. 6

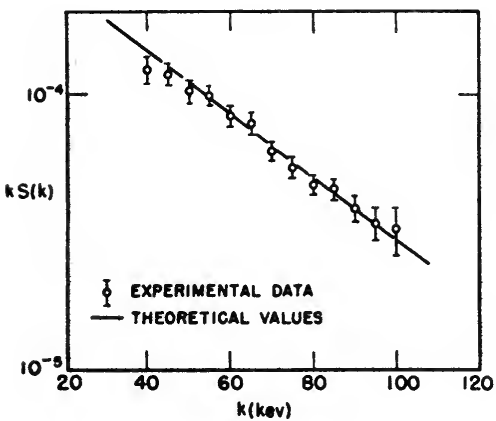

Fig. 8

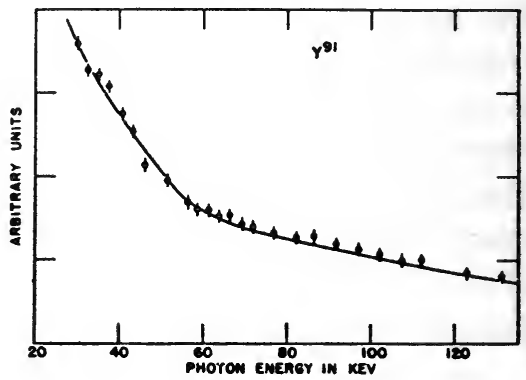

Fig. 7

Fig. 6. Theoretical spectrum for $\mathrm{P}^{32}$ and experimental data. (See footnote 12)

Fig. 7. Theoretical internal bremsstrahlung spectrum for $\mathrm{Y}^{21}$ and experimental data.

(See footnote 12)

Fig. 8. Internal bremsstrahlung from $\mathrm{Pm}^{\mathbf{1 4 7}}$ $\beta$-decay.

(See footnote 15)

11 T. B. Novey, Phys. Pev. 89, (1953) 672.

12 P. Bolgiano, L. Madansky and F. Rasetti, Phys. Rev. 89, (1953) 679; L. Madansky and F. Rasetti, Phys. Rev. 83, (1951) 187.

13 F. Boehm and C. S. Wu, Phys. Rev. 90, (1953) 369.

14 G. Renard, Le Journal de Physique et le Radium 14, (1953) 361.

is F. Boehm and C. S. Wu, Phys. Rev. 93, (1954) 518. 
also obtained good accord with the theory. The excellent agreement. between the theory and the experimental findings may imply that the simplification (first order perturbation) and approximation ( $Z$ independent) introduced in the theoretical calculations have very little effect on the distribution or yield of the bremsstrahlung.

TABLE I

GAMMA-RAY ENERGY PER BETA PARTICle $I_{\gamma} / I_{\beta}$ (See footnote 12)

\begin{tabular}{l|c|c|c}
\hline Source & $\begin{array}{c}\text { Max. } \beta \text {-Energy } \\
\text { Mev. }\end{array}$ & $\begin{array}{c}I_{\gamma} / I_{\beta} \text { in Units of } \mathrm{mc}^{2} \\
\text { Theory } \\
\text { (Allowed transition) }\end{array}$ & Experimental \\
\hline $\mathrm{P}^{32}$ & 1.72 & $2.38 \times 10^{-3}$ & $2.32 \times 10^{-3}$ \\
$\mathrm{Y}^{91}$ & 1.5 & $1.97 \times 10^{-3}$ & $1.90 \times 10^{-3}$ \\
$\mathrm{RaE}$ & 1.17 & $1.11 \times 10^{-3}$ & $0.84 \times 10^{-3}$
\end{tabular}

\section{§ 4. Angular Distribution of Internal Bremsstrahlung}

In eq. 2 the integral $\int d \Phi_{k}$ is meant to extend over all directions of the quanta. If one is interested in the angular distribution of the internal bremsstrahlung, then the integration should not be carried out. Let us introduce " $\theta$ " as the angle between the final direction of the electron and that of the quantum $k,(\boldsymbol{k}, \mathbf{p})$; the differential probability that a beta particle emitted with energy $W_{e}$ will radiate a quantum of energy $k$ per unit photon energy interval at an angle $\theta$ between $\boldsymbol{k}$ and $\boldsymbol{p}$ is given by

$$
d \Phi\left(W_{e}, k, \theta\right)=\frac{\alpha p}{4 \pi^{2} p_{e} k}\left[\frac{W_{e}^{2}+W^{2}}{W_{e}(W-p \cos \theta)}-\frac{1}{(W-p \cos \theta)^{2}}-1\right] d \Omega_{k} .
$$

On the assumption that $k$ is small and thus that $W_{e}=W, p_{\theta}=p$ and

$$
\frac{p}{W}=\beta \text { and } W^{2}=\frac{1}{1-\beta^{2}}
$$

it has been shown by Wang Chang and Falkoff ${ }^{5}$ that

$$
\frac{d \Phi}{d \Omega}=\frac{\alpha}{4 \pi^{2} k} \frac{\beta^{2} \sin ^{2} \theta}{(1-\beta \cos \theta)^{2}} .
$$

The above expression can also be directly derived by a purely classical method. The angular distribution thus obtained has a sharp forward peak and is insensitive for low energy photons to the degree of forbiddenness or type of interaction involved in the $\beta$-decay ${ }^{6}$. Novey ${ }^{11}$ investigated the angular distribution of internal bremsstrahlung from $\mathrm{P}^{32}$ and $\mathrm{RaE}$ by measuring the angular correlation between the electron and the internal bremsstrahlung quantum with a coincidence circuit. The electron detector was a stilbene crystal scintillator and could detect electrons of energy as 
low as $50 \mathrm{keV}$. The gamma detector was a NaI (Tl) crystal scintillator, its detecting efficiency dropping off at $40 \mathrm{keV}$ and also at the high energy region. The resolving time used was around $0.1 \mu \mathrm{sec}$. The experimental data (circles) for $\mathrm{P}^{32}$ and RaE are shown in Fig. 9 and 10, together with the theoretical curves shown in solid lines which are calculated by integrating the approximation formula, normalized at $45^{\circ}$

$$
\frac{d \Phi}{d \Omega}=\frac{\alpha}{4 \pi^{2}} \int_{W_{\min }}^{W_{\max }} \int_{k_{\min }}^{W} \frac{P(W) \beta^{2} \sin ^{2} \theta}{k(1-\beta \cos \theta)^{2}} d k d W .
$$

The agreement between the experimental angular correlation and that predicted from theory is excellent.

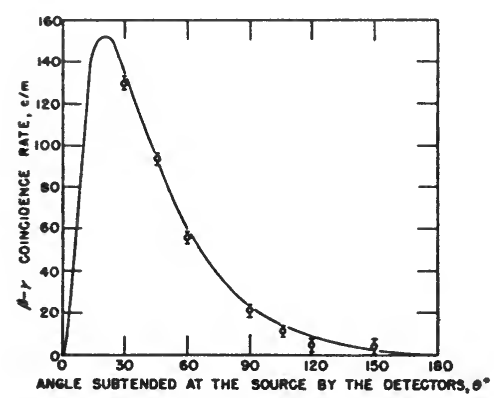

Fig. 9

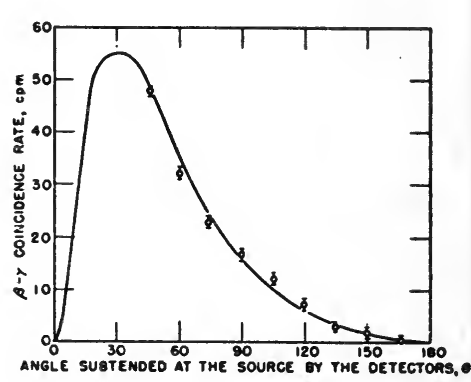

Fig. 10

Fig. 9. Beta-internal bremsstrahlung angular correlation in $\mathrm{P}^{32}$. Comparison of experiment (circles) with theory (solid lines) normalized at $45^{\circ}$. (See footnote 11)

Fig. 10. Beta-internal bremsstrahlung angular correlation in RaE. Comparison of experiment (circles) with theory (solid lines) normalized at $45^{\circ}$. (See footnote 11)

\section{$\S 5$. Bremsstrahlung Due to Electron Capture Process}

A case analogous to the emission of the internal bremsstrahlung due to $\beta$-decay is the production of bremsstrahlung when a nucleus captures an atomic electron and falls to a stable state. The origin of this secondary radiation is due to the sudden change of charge from atom to nucleus. The theoretical aspect of this process was first investigated by Morrison and Schiff ?.

Although the probability of production of the photon in the case of the electron capture process is much smaller than that in the $\beta$-decay, there is no external bremsstrahlung spectrum to mask the effect. In the $\beta$-decay the upper limit of the continuous bremsstrahlung spectrum is given by the maximum energy of the $\beta$-particles. In the electron capture process the upper energy limit $W_{0}$ is given by the transition energy $\Delta E$ which is the 
energy difference between the isobaric nuclei $\Delta E=E_{Z}-E_{Z-1}$, plus the energy gained in capturing the $K$-shell electron which is equal to the rest mass minus the ionization potential of the $K$-shell electron

$$
W=\Delta E+\left(m_{0} c^{2}-E_{k}\right)=M(Z)-M(Z-1)-E_{k}
$$

where $M(Z)$ and $M(Z-1)$ are the atomic masses. Therefore the determination of the upper limit of the bremsstrahlung spectrum provides a method to determine the transition energy in an electron capture process.

The energy distribution of the bremsstrahlung from $K$-capture process of an allowed transition was derived by Morrison and Schiff ${ }^{7}$ and by Jauch ${ }^{16}$ as

$$
N(k) d k=C(k) \frac{\alpha}{\pi m_{0}^{2} c^{4}} \frac{k}{W_{0}^{2}}\left(W_{0}-k\right)^{2} d k
$$

$N(k) d k$ is the number of photons in the energy interval between $k$ and $k+d k . W_{0}$ is the upper limit of the photon energy. The factor $C(k)$ is a complicated function that is slowly varying except at very low energies. If the factor $C(k)$ is considered as a constant $C$ then

$$
\left[\frac{N(k)}{C k}\right]^{1 / 2}=K\left(W_{0}-k\right)
$$

where $K$ is a constant.

To obtain the upper limit of the photon energy $W_{0}$ one plots $\left[\frac{N(k)}{C k}\right]^{k}$ vs $k$ as one does in making "Kurie plot" for $\beta$-spectrum. The interception of the linear plot on the energy axis should therefore give the upper limit $W_{0}$.

The bremsstrahlung from electron capture was first observed by Bradt ${ }^{17}$ et al. in $\mathrm{Fe}^{55}$. It is about $3 \times 10^{-5}$ quantum per disintegration and the average energy estimated is around $70 \mathrm{keV}$ which yields a transition energy of $150 \mathrm{keV}$.

Using a NaI scintillation spectrometer, Maeder and Preiswerk ${ }^{18}$ and Bell, Jauch and Cassidy ${ }^{19}$ investigated the energy distribution of the bremsstrahlung from $\mathrm{Fe}^{55}$. Fig. 11 and 12 show the $\gamma$-ray spectrum and its corresponding linearized plot. Owing to the presence of impurities and the poor resolution of the scintillation spectrometer, the low energy portion of the spectrum was not investigated in detail. However, the upper energy portion of the spectrum is in fair agreement with that predicted theoretically: The upper limit $W_{0}$ obtained in this manner is $206 \pm 20 \mathrm{keV}$. The transition energy of the electron capture $\mathrm{Fe}^{55}+\mathrm{e}^{-} \rightarrow \mathrm{Mn}^{55}$ can be

16 J. Jauch, ORNLS 1102.

17 H. Bradt, et al. Helv. Phys. Acta 19, (1946) 222.

18 D. Maeder and P. Preiswerk, Phys. Rev. 84, (1951) 595.

19 P. R. Bell, J. Jauch and J. M. Cassidy. Science 115. (1952) 12. 

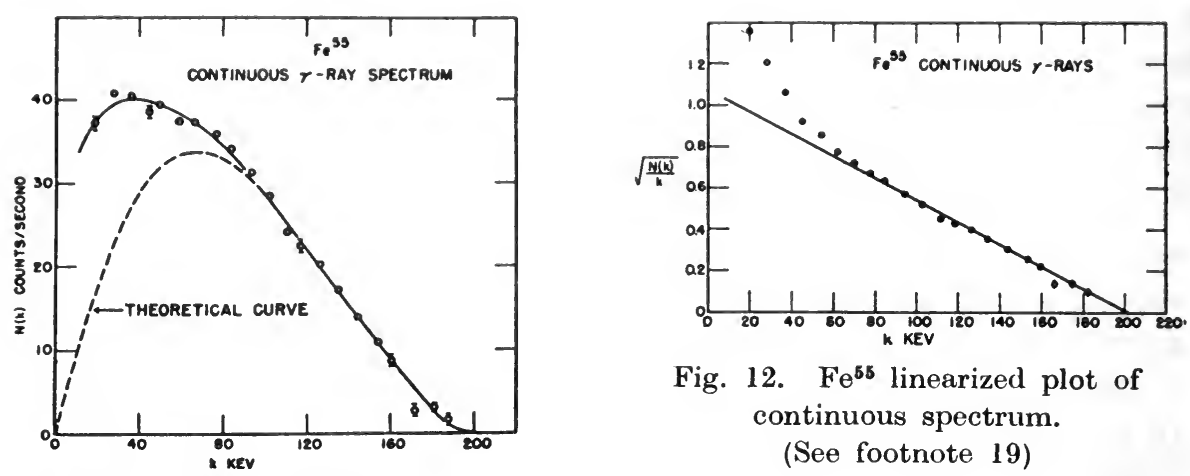

Fig. 12. $\mathrm{Fe}^{55}$ linearized plot of continuous spectrum.

(See footnote 19)

Fig. 11. $\mathrm{Fe}^{55}$ continuous $\gamma$-ray

spectrum. (Sec footnote 19)

obtained also through the $Q$ value of the reaction

$$
\mathrm{Mn}^{55}+\mathrm{p} \rightarrow \mathrm{Fe}^{55}+\mathrm{n} .
$$

Using the value of $Q=-1.006 \mathrm{MeV}$ as reported by Stelson and Preston ${ }^{20}$, the $W_{0}$ value is $217 \pm 10 \mathrm{keV}$. It is in good agreement with that determined from the bremsstrahlung spectrum.

Recently the investigation on the internal bremsstrahlung from electron capture process of $\mathrm{Fe}^{55}$ was repeated again by Madansky and Rasetti *. It was found that the portion of the spectrum above $60 \mathrm{keV}$ indeed corresponded fairly closely to the theoretical distribution of Morrison and Schiff, but a large excess of low energy photons below $50 \mathrm{keV}$ was definitely present. B. Saraf ${ }^{21}$ investigated the internal bremsstrahlung spectrum from $\mathrm{Cs}^{131}$ and found its distribution in disagreement with that predicted by the simple theory.

The Morrison and Schiff's calculation is for the $s$ electron capture in allowed transitions only. The electron capture process of $\mathrm{Fe}^{55}$ which is known to be a first: forbidden transition ( $\log f t=7.1)$ may yield a bremsstrahlung spectrum different from that expected for the allowed transition. Furthermore, no radiative capture of $p$-electrons was taken into the theoretical consideration; its contribution to the spectrum at the low energy portion may be important. Furthermore, the plane wave approximation used in the previous calculations should be improved by the

20 P. H. Stelson and W. M. Preston, Phys. Rev. 83, (1951) 469.

21 B. Saraf, Bulletin of A. P. S. Jan., 1954.

* The author is indebted to Dr L. Madansky and F. Rasetti for communi. cating the result prior to its publication. 
correct Coulomb wave function. It is obvious that more theoretical calculation on this subject is needed. $\dagger$

The ratio of the total number of bremsstrahlung photons to the total number of $K$-capture is given by

$$
\int_{0}^{k} \frac{N(k) d k}{N_{c}}=\frac{\alpha}{12 \pi}\left(\frac{W_{0}}{m_{0} c^{2}}\right)^{2}
$$

It is proportional to $W_{0}^{2}$; therefore it is easier to detect the bremsstrahlung from an electron capture process of high $W_{0}$ value. $\mathrm{A}^{37}$ decays mostly by $K$-capture process $(\sim 92 \%)^{22}$ in an allowed transition and has a value of $W_{0}$ as high as $816 \mathrm{keV}$. The bremsstrahlung spectrum of $\mathrm{A}^{37}$ observed by Anderson, Wheeler and Watson ${ }^{23}$ by using a NaI scintillation spectrometer agrees roughly with the theoretical predictions $\uparrow \dagger$, but no detail was given for the low energy portion of the spectrum.

Even in the cases where electron capture is accompanied by $\beta$-decay or $\gamma$-radiation, one might be still able to determine the transition energy by investigating its bremsstrahlung through the application of coincidence counting between the characteristic $\mathrm{X}$-ray of the daughter nucleus and the bremsstrahlung quantum. E. der Mateosian ${ }^{24}$ used this method to determine the upper limit of the bremsstrahlung spectrum of $\mathrm{Tl}^{204}$ as $250 \mathrm{keV}$ and therefore the transition energy of $335 \mathrm{keV}$ which is in fair agreement with the value of the transition energy evaluated from the branching ratio.

22 B. Pontecorvo, D. H. W. Kirkwood and G. C. Hanna, Phys. Rev. 75, (1949) 982.

23 C. E. Anderson, G. W. Wheeler and W. W. Watson, Phys. Rev. 87, (1952) 668.

24 E. der Mateosian, Phys. Rev. 88, (1952) 1186.

$\dagger$ A theoretical paper dealing with these factors has been presented by R. J. Glauber and P. C. Martin, Phys. Rev. 95, (1954) 572. The radiative capture of $p$-electrons seems to be significant in the low energy region.

if The contribution from the Compton electrons was overlooked. 


\title{
XXI \\ PARTICULAR PROBLEMS STUDIED WITH SCINTILLATION TECHNIQUES
}

\author{
P. E. CAVANAGH, H. FULBRIGHT, \\ S. DE BENEDETTI, R. E. BELL, M. DEUTSCH
}

\section{(I) P. E. CAVANAGH - Ordinary Compton Effect and Double Comp- ton Effect}

\section{$\S$ 1. Ordinary Compton Effect}

\section{A. Introduction}

No one has seriously doubted the validity of the quantum theory of the scattering of radiation by free electrons since about 1936. In that year, Shankland ${ }^{1}$, repeating earlier experiments of Compton and Simon ${ }^{2}$, with improved techniques, found no evidence for conservation of energy and momentum in the individual scattering process. If energy and momentum were to be conserved it must be in some statistical manner involving an average over many events. For a short time this led to the revival of a theory initially advanced by Bohr, Kramers, and Slater ${ }^{3}$, which required conservation to exist in just such a manner. Although Shankland's results were quickly disproved they nevertheless served a useful purpose in leading to a critical re-examination of the basis of the theory. The experiments which were performed subsequently gave essentially two pieces of information: first that there is no time interval between the appearance (and detection) of the recoil electron and the scattered quantum greater than $10^{-4}$ seconds, and second, that for an incident quantum of given energy, the angle of ejection of the recoil electron is uniquely determined by the quantum scattering angle. Since geiger counters were used for detecting both quanta and electrons, and very thin scattering foils were necessary in order to avoid multiple scattering of electrons, it was not possible to verify this second consequence of the conservation laws to any high degree of accuracy. In fact the angles could not be defined to better than $30 \%$.

With the advent of the new techniques for the detection of $\gamma$-rays it has been found possible not only to repeat these experiments with much greater accuracy, but also to verify further detailed predictions of the theory.

1 R. S. Shankland, Phys. Rev. 49 (1936) 8.

2 A. H. Compton and A. Simon, Phys. Rev. 26 (1925) 289.

8 N. Bohr, H. A. Kramers and J. C. Slater, Phil. Mag. 47 (1924) 785. 


\section{B. The Conservation of Energy and Momentum in Compton ScatTering}

According to the quantum theory and the conservation laws the angle of recoil of the electron, $\phi$, and the quantum scattering angle, $\theta$, are related uniquely by the equation

$$
\cot \phi=(1+\alpha) \tan \frac{1}{2} \theta
$$

where $\alpha$ is the incident quantum energy in $m c^{2}$ units. So that, if we have a parallel beam of $\gamma$-rays falling on a thin scatterer, and the counter, which detects only those quanta scattered through $\theta$, is placed in coincidence with an electron counter, then coincidences will only be observed when the latter is at angle $\phi$ given by the above equation, and coplanar with scatterer and quantum counter. This statement must of course be modified when account is taken of the angular resolution of the counters and of multiple scattering of recoil electrons in the scatterer. It is this last consideration which makes these experiments so difficult; such thin foils have to be used to avoid excessive multiple scattering that very low coincidence rates obtain, and background effects become important. Bothe and Maier-Leibnitz ${ }^{4}$ avoided the necessity of making background measurements at successive angular positions of the electron counter by adopting a different arrangement, in which the counters were fixed and the foil was moved axially. For the $2.62 \mathrm{MeV} \gamma$-rays from $\mathrm{ThC}^{\prime \prime}$, which they used, almost symmetrical positions for the quantum and electron counters of $30^{\circ}$ and $31.6^{\circ}$, respectively, will give coincidences.

Cross and Ramsey ${ }^{5}$ used a similar arrangement. $\gamma$-rays from a $195 \mathrm{mg}$ Ra equivalent of RdTh were filtered through $2 \mathrm{cms}$ of lead and collimated, so that a beam of predominantly $2.62 \mathrm{MeV} \gamma$-rays fell on a $13.8 \mathrm{mg} / \mathrm{cm}^{2} \mathrm{Be}$ scatterer. Electrons were swept from the beam with a magnet, scattering at the mouth of the collimator was minimized, while gas-scattering was reduced by a factor of seven by replacing air by helium. Both electrons and scattered quanta were detected by means of anthracene crystals, the latter having an efficiency of about $15 \%$ for the mean energy of the scattered quanta. IP21 photomultipliers were used, the output pulses being amplified and passed through discriminators to a diode coincidence circuit with resolving time $0.3 \mu$ sec.

The plot of coincidence rate against scatterer position is given in Fig. 1, and shows a well defined maximum in the zero position. A more accurate measurement in which the electron counter was moved through small

4 W. Bothe and H. Maier-Leibnitz, Zeits f. Phys. 102 (1936) 143; Phys. Rev. 50 (1936) 187.

- W. G. Cross and N. F. Ramsey, Phys. Rev. 80 (1950) 929. 
angles about $31.6^{\circ}$, the scatterer remaining fixed, gave this as the maximum, within about $1^{\circ}$. A similar experiment showed that the condition of coplanarity must also be observed. The calculated angular distribution

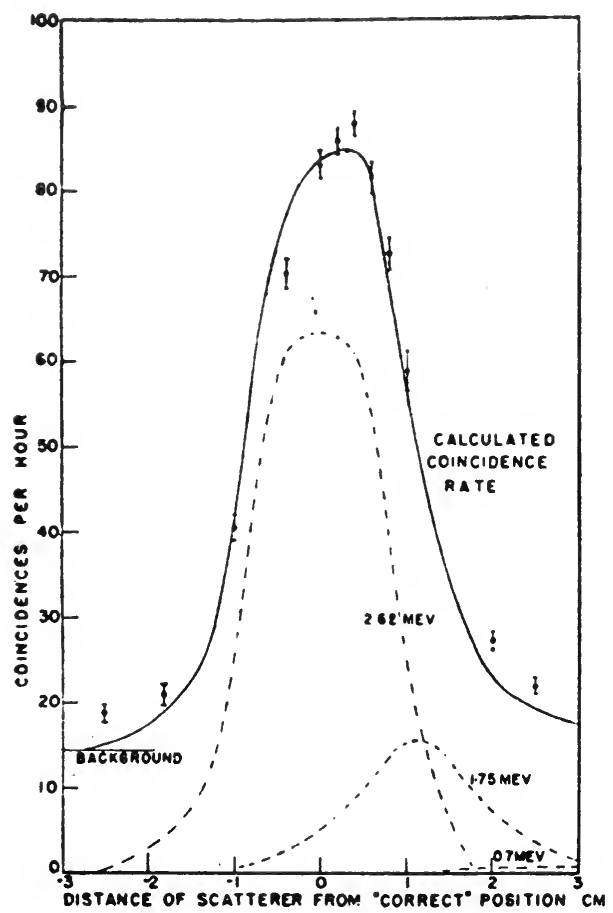

Fig.. 1. Angular Distribution of Coincidences in Horizontal Plane was a good fit to the experimental points. The main contributions to the half-width of $14^{\circ}$ arose from the angles subtended by the counters at the scatterer, contributing $\sim 9^{\circ}$, multiple electron scattering, and the presence of lower energy components in the beam. The coinci: dence rate at maximum was also in agreement with calculations based on the Klein-Nishina formula within about $10 \%$; the principal error occurring in the determination of the incident beam intensity.

\section{Simultaneity}

The quantum theory of the Compton effect requires that the scattered quantum and recoil electron should appear together within a time interval of $\sim 10^{-20}$ seconds. This is far below the present limits of observation; however several groups of workers in recent years have reduced the upper limit of $\sim 10^{-4}$ seconds obtained in 1936 by several orders of magnitude.

In all these experiments a narrow beam of $\mathrm{Co}^{60} \gamma$-rays has been directed onto a scintillator which acted also as a scatterer, while a second scintillator detected quanta scattered by the first. Bell and Graham ${ }^{6}$, and Cross and Ramsey ${ }^{5}$ used IP21 photomultipliers working direct into fast crystal diode coincidence circuits, which are now familiar, and were originally described by Bell and Petch ?. By introducing artificial delays between the two counters so as to vary the coincidence rate Cross and Ramsey were able to place an upper limit of $1.5 \times 10^{-8}$ seconds between the detection of the electron and scattered quantum. More careful examination of the slopes of the edges of the delay curves has enabled Bell and

6 R. E. Bell, private communication.

7 R. E. Bell and H. E. Petch, Phys. Rer. 76 (1949) 1409. 
Graham to reduce this upper limit to $5 \times 10^{-10}$ seconds. A variant of this method used by Hofstadter and McIntyre ${ }^{8}$, in which the two pulses were displayed on a fast triggered oscilloscope with an artificial delay of $0.16 \mu$ secs introduced between them, also gave an upper limit of $1.5 \times 10^{-8}$ seconds.

\section{The Angular Distribution of Scattered Quanta}

Few attempts have been made to verify accurately the angular distributions of recoil electron and scattered quantum predicted by Klein and Nishina ${ }^{9}$, for energies in the $\gamma$-ray region. The most successful has been that of Hofstadter and McIntyre ${ }^{10}$ who directed a beam of $\mathrm{Co}^{60} \gamma$-rays onto a half inch cube of stilbene, which acted as a sce.tterer, a second block of stilbene $\frac{1}{2} \times \frac{1}{2} \times 1$ inch serving to detect the scattered quanta. IP21 photomultipliers cooled to dry-ice temperatures were used, and brought into coincidence by an oscilloscope method. The coincidence counting rate was determined for a number of different angular positions of the scattered quantum counter, spaced at about $10^{\circ}$ intervals up to $90^{\circ}$. Measurements made with increasing gain of the photomultipliers showed that all coincidences were being recorded, except at $15^{\circ}$ and $20^{\circ}$, where plateaux were not obtained, presumably because of the low energy, $\sim 100 \mathrm{keV}$, of the recoil electron.

The results were corrected for the variation with energy in detection efficiency of the quantum counter, and also for the effects of absorption both in the scatterer and in the counter housings. The incident beam

Fig. 2. Comparison of Experimental and Theoretical Curves, for Angular Distribution of Scattered Quanta

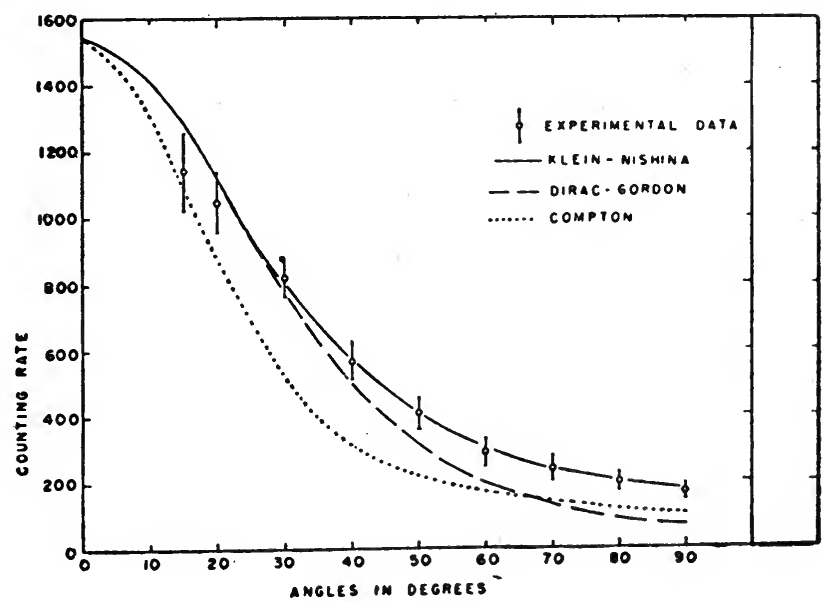

B R. Hofstadter and J. A. McIntyre, Phys. Rev. 78 (1950) 24.

9 O. Klein and Y. Nishina. Z. Phys. 52 (1929) 853.

10. R. Hofstadter and J. A. McIntyre, Phys. Rev. 76 (1949) 1269. 
strength was not known to better than $15 \%$, so the Klein-Nishina formula was normalized to fit the experimental data for scattering angles of $30^{\circ}$ and greater. It is evident from Fig. 2 that the fit is very much better than would be obtained by either of the older theories.

\section{E. Polarization Effects in Compton Scattering}

The azimuthal angle of ejection of the recoil electron and scattered quantum should depend on the direction of the electric vector in the incident radiation. Such polarization effects are well established in the $\mathrm{X}$-ray region, and have recently been put to good use by Deutsch ${ }^{11}$ and others in the $\gamma$-ray region to establish direction-polarization correlation in successive $\gamma$-ray transitions, from nuclei excited in radioactive decay. The differential scattering cross-section as a function of the angle $\Theta$ between the polarization directions of the incident and scattered quanta has been given by Klein and Nishina,

$$
k(\theta, \Theta)=\frac{r_{0}^{2}}{4} \frac{\alpha^{2}}{\alpha_{0}^{2}}\left[\frac{\alpha_{0}}{\alpha}+\frac{\alpha}{\alpha_{0}}-2+4 \cos ^{2} \Theta\right]
$$

where $r_{0}=e^{2} / m c^{2}, \alpha_{0}$ is the incident quantum energy in $m c^{2}$ units, and $\alpha$ is the scattered quantum energy given by $\alpha=\alpha_{0} /\left\{1+\alpha_{0}(1-\cos \theta)\right\}$.

At low energies $\alpha=\alpha_{0}$, and for polarized incident radiation the scattered radiation is completely polarized. At higher energies the degree of polarization at large scattering angles decreases, until at high relativistic energies, even for polarized incident radiation, the large angle scattered radiation is completely unpolarized.

The degree of polarization in the scattered radiation, where the incident beam has been partially polarized by a previous Compton scattering, has been calculated recently by Wightman ${ }^{12}$, using a method similar to that of Heitler ${ }^{13}$. The first and second scattering angles, $\theta_{1}$ and $\theta_{2}$, may be chosen so as to make the polarization ratio a maximum, its magnitude decreasing from 3.85 at $0.5 \mathrm{MeV}$ to 2.14 at $1 \mathrm{MeV}$, and 1.17 at $5 \mathrm{MeV}$.

Excellent agreement with theory was obtained by the experiments of Hoover, Faust and Dohne ${ }^{14}$. They directed a collimated beam of $\mathrm{Co}^{60}$ $\gamma$-rays from a 5 curie source on to a copper scatterer. The first and second scattered quanta were detected by $\mathrm{NaI}(\mathrm{Tl})$ crystals 1 inch square and 2 to $4 \mathrm{~mm}$ thick, crystal $\mathrm{C}_{1}$, (Fig. 3) acting as a Compton scatterer. Single scattered radiation from the copper block was prevented from striking

11 M. Deutsch and F. Metzger, Phys. Rev. 74 (1948) 1542.

12 A. Wightman, Phys. Rev. 74 (1948) 1813.

13 W. Heitler. The Quantum Theory of Radiation (Oxford Univ. Press 1944) p.146

14 J. I. Hoover, W. R. Faust and C. F. Dohne, Phys. Rev. 85 (1952) 58. 
the crystal $\mathrm{C}_{2}$ by interposing a $5 \mathrm{~cm}$ thick lead shield. The solid angles subtended mutually by the crystals, and also between $\mathrm{C}_{1}$ and the copper block, were maintained constant at about 0.06 steradians, while $\mathrm{C}_{2}$ was rotated about $\mathrm{C}_{1}$. 5819 photomultipliers were used, followed by conventional amplifier and trigger circuits, and feeding into a $0.147 \mu$ sec resolving time coincidence circuit. Coincidences were recorded for four values of $\phi$, the angle of rotation of $\mathrm{C}_{2}$ about $\mathrm{C}_{1}$, and compared with the value obtained with $\mathrm{C}_{2}$ in the same plane as $\mathrm{C}_{1}$ and the copper scatterer. These measurements were repeated for two values of $\theta_{1}$, the first scattering angle, of $83^{\circ}$ and $50^{\circ}$, (the second scat-

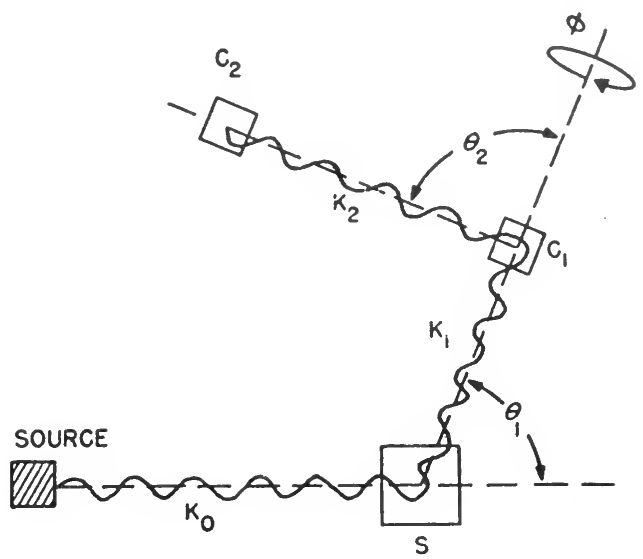

Fig. 3. Successive Compton Scatterings

tering angle, $\theta_{2}$, remained fixed at $\left.90^{\circ}\right)$. The results were compared with calculations based on Wightman's formulae and agreement obtained on an average within one and a half times the statistical error, which was about $5 \%$.

This experiment suffers from the usual difficulty associated with double scattering experiments, that of low intensity. Only one coincidence was recorded for every $\sim 10^{9}$ quanta falling on the first scatterer, and this necessitated long counts $\sim 100$ hours.

\section{$\S 2$. The Double Compton Effect}

In 1934 Heitler and Nordheim ${ }^{15}$ predicted the occurrence of higher order Compton processes in which, as a result of the interaction of an incident quantum and a free electron, two or more scattered quanta are produced in the same elementary act. The cross-section for such processes, compared with the normal Compton cross-section decreases with increasing number, $n$, of scattered quanta as $(1 / 137)^{n-1}$. The experimental work to be described was undertaken by the author ${ }^{16}$ on the basis of this prediction. The effects have assumed further importance at the present time as a result of the calculations of Feynman ${ }^{17}$ and others of the radiative correction to the Klein-Nishina formula. These corrections, due to processes in which

15 W. Heitler and L. Nordheim, Physica 1 (1934) 1059.

16 P. E. Cavanagh, Phys. Rev. 87 (1952) 1131.

17 L. M. Brown and R. P. Feynman, Phys. Rev. 85 (1952) 231. 
virtual quanta are emitted and reabsorbed by the electron, are in fact divergent for small values of the energy of the virtual quantum. The divergencies can however be removed by adding to the cross-section that for the double and higher order Compton effects.

A detailed theory has been given by Mandl and Skyrme ${ }^{18}$ using the methods developed by Feynman. It is difficult to display the properties of the general expression for the differential cross-section since it is a function of four variables in addition to the incident quantum energy. A number of special cases have therefore been worked out, the most important being where one of the scattered quanta is assumed to be very soft, since this represents the most probable splitting of the scattered quantum energy.

The energy spectrum for quanta scattered at right angles to each other and to the direction of the incident quantum has also been calculated, this being a case which approximates to the experimental arrangement. This condition was, however, considerably modified by the large solid angles ( $\sim 0.5$ steradian) subtended by each detector at the scatterer. A $200 \mathrm{mc}$ $\mathrm{Co}^{60}$ source was used. A biconical lead collimator, designed to minimize scattering, defined a $\gamma$-ray beam 0.9 inches in diameter, which fell on a thin foil placed perpendicular to it. Secondary electrons were removed from the beam with a magnet. The scattered quanta were detected by means of two $\mathrm{NaI}(\mathrm{Tl})$ crystals $2 \frac{3}{4}$ inches in diameter and just under 1 inch thick with $20 \mathrm{gm} / \mathrm{cm}^{2}$ of lead placed between them to eliminate crossscattering. EMI 5045 photomultipliers were used, followed by conventional amplifiers and discriminators and feeding into a coincidence cricuit with $0.5 \mu \mathrm{sec}$ resolving time.

It was quickly found that secondary processes could easily obscure the primary effect which was sought. The only important secondary effect occurred when a recoil electron, resulting from a normal Compton process, scattered in the foil, produced a bremsstrahlung, which was then counted together with the "scattered quantum in coincidence. Two methods of separating off the effects of secondary processes suggested themselves. The magnitude of the coincidence rate from the double Compton effect should go up linearly with the scatterer thickness, $t$, whereas that from secondary processes should vary as $t^{2}$. By plotting the coincidence rate divided by some measure of the number of electrons per $\mathrm{cm}^{2}$ in the scatterer, against $t$, a straight line should result, the intercept representing the double Compton effect, and the slope, bremsstrahlung processes. Moreover the slope should depend on the atomic number of the scatterer material.

18 F. Mandl and T. H. R. Skyrme. Proc. Roy. Soc. A215 (1952) 497. 
Coincidence measurements were made with scatterers of varying thickness up to $400 \mathrm{mg} / \mathrm{cm}^{2}$, of $\mathrm{Be}, \mathrm{Al}, \mathrm{Cu}$, and $\mathrm{Ag}$. The results shown in Fig. 4 indicate that the intercept is independent of atomic number, as it should be for an effect depending only on scattering from free electrons. The straight line plots have a slope $\propto Z^{2.24}$, the increase in the value of the exponent over that appropriate to bremsstrahlung production is probably due to the increased path length of electrons brought about by scattering.

A rough estimate, with certain simplifying assumptions regarding angular distributions and detection efficiencies, gave the total double Compton crosssection, integrated over the energy range $80-530 \mathrm{keV}$, to be $3 \times 10^{-3}$ of the single Compton cross-section. Mandl and Skyrme's result integrated over the same energy range, and assuming $100 \%$ detection efficiencies, gave a value for the coincidence rate per recorded quantum of $0.4 \times 10^{-4}$ compared with the experimental value of $1 \times 10^{-4}$.

The principal difficulty in this experiment was that not only were low

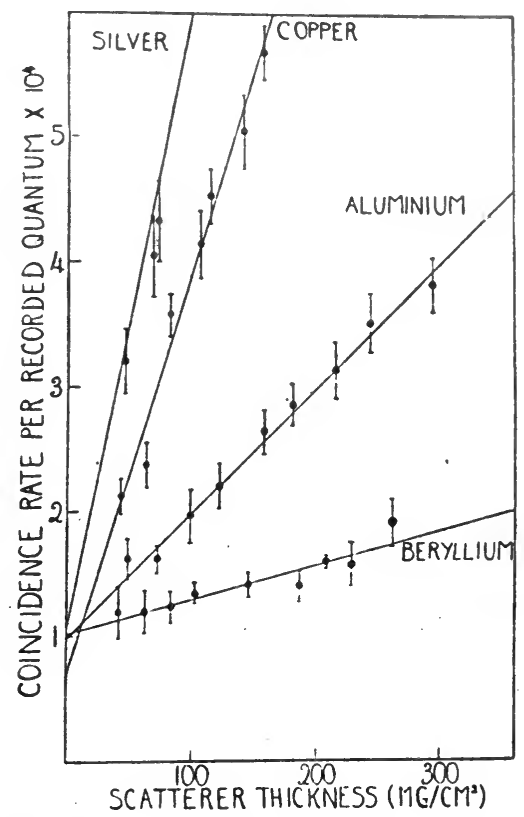

Fig. 4. Coincidence Rate per Recorded Quantum versus Scatterer Thickness coincidence rates involved, of as little as 0.01 per second, but that these were comparable with or smaller than the background coincidence rate of 0.04 per second. In further work, with a better defined geometry, reported at the Birmingham Conference July 1953, the scatterer was replaced by a thin crystal of anthracene, which also served to count the recoil electrons. Triple coincidences were recorded, and this had the effect of reducing the background to completely negligible proportions, as well as decreasing the accidental rate considerably. The quantity of interest in this case was the ratio of the triple coincidence rate to the sum of the quantum-electron double coincidence rates. This, when corrected for bremsstrahlung effects gives a value $0.76 \times 10^{-4}$. The theoretical value obtained by Mandl for the ratio of, the differential cross-sections integrated over the energy range accepted by the counters, is $0.48 \times 10^{-4}$. This calculation does not take into account the variation in the angular distribution over the angle of acceptance of the counters. Until this very laborious integration over 
angles has been performed a more accurate comparison with experiment, in which account is taken of the intrinsic quantum detection efficiency, is not justified.

An attempt to detect the double Compton effect has also been made by Boekelheide ${ }^{19}$ using $663 \mathrm{keV} \gamma$-rays from $\mathrm{Cs}^{137}$. He took advantage of the fact that to a close approximation, considering scattering in the plane perpendicular to the incident direction, the sum of the energies of the quanta in the double Compton effect is equal to the energy of the single scattered quantum. He restricted himself to detecting in coincidence only those quanta which were about half the energy of the latter. In this way a large degree of discrimination was obtained against single scattered quanta, and what is probably more important, against secondary coincidences produced by bremsstrahlung. This permitted the use of a thick scatterer, and with small solid angles of $\sim 0.05$ steradians, subtended by the $\mathrm{NaI}(\mathrm{Tl})$ crystals, a reasonable value of the coincidence rate could still be obtained. However, it was only one tenth the size of the accidental rate, which was difficult to estimate accurately because of varying single channel rates. A positive result of the right order of magnitude was found but its actual size is subject to very considerable uncertainties.

I. F. Boekelheide. Dissertation for Ph. D. State University of Iowa (1952).

\section{(II) H. FULBRIGHT - Use of a Scintillation Counter Spectrometer in a Search for Double $\beta$-Decay}

\section{$\S 1$. Introduction}

Studies of beta-ray spectral shapes and of the angular correlations between beta particles and recoiling nuclei have confirmed the neutrino hypothesis and have given some information concerning the properties of the neutrino. The neutrino is neutral electrically, it has spin $1 / 2$, and its mass is less than $1 / 200 m_{e}$. But the question as to whether it is a Dirac particle ${ }^{1}$, therefore occurring in two forms, neutrino and antineutrino, analogous to the electron and the positron, or whether instead it is a symmetrical particle occurring,in only one form as postulated by Majorana ${ }^{2}$ cannot be settled by observations of ordinary beta decay.

A higher order process which offers the possibility of an experimental test of the question is double beta decay, the virtually simultaneous

1 "The Principles of Quantum Mechanics", P.A.M. Dirac, Oxford University Press (1935).

2 E. Majorana, Nuovo Cimento 14 (1937) 171. 
emission of two electrons by a nucleus $Z$ which becomes the nucleus $Z+2$. If the neutrino is a Dirac particle two antineutrinos must be emitted along with the two electrons. M. Goeppert-Mayer has calculated ${ }^{3}$ that the lifetime in this case would be of the order of magnitude of $10^{23}$ years. If the neutrino is a Majorana particle neutrinos do not have to be emitted because in that case absorption is the same as emission; thus the first electron can be thought of as being emitted along with a neutrino which is quickly reabsorbed as the second electron is emitted. Furry ${ }^{4}, \mathrm{Sliv}^{5}$, and Primakoff ${ }^{6}$ have calculated that the lifetime in this case would be in the order of magnitude of $10^{13}-10^{18}$ years. Thus an experimental observation of double beta decay with a lifetime of less than, say, $10^{20}$ years would shown that the neutrino is a Majorana particle.

Experiments to test the matter are, of course, quite difficult because of the long lifetime. Several attempts have been made to detect the process by indirect means ${ }^{7.8}$. In addition, direct attempts have been made by use of coincidence counters ${ }^{9,10}$, cloud chambers ${ }^{11}{ }^{12}$, scintillation counters ${ }^{13.14}$, and photographic plates ${ }^{15}$, but as yet there is no satisfactory answer to the question, although the scintillation spectrometer studies of J. A. McCarthy ${ }^{16.17}$, to be described below, may have detected double beta decay in $\mathrm{Zr}^{96}$.

\section{$\S 2$. The Experimental Method}

McCarthy's experiment made use of the fact that in neutrinoless double beta decay all the energy must be carried away by the two electrons

3 M. Goeppert-Mayer, Phys. Rev. 48 (1935) 512.

4 Wendell H. Furry, Phys. Rev. 56 (1939) 1184.

5 L. A. Sliv, Zhur. Eksp. Teoret. Fiz. U.S.S.R., 20 (1950) 1039.

- H. Primakoff, Phys. Rev. 85 (1951) 888.

7. M. Ingraham and S. H. Reynolds, Phys. Rev. 78 (1950) $822, \mathrm{Te}^{130}$.

8 C. Levine, A. Ghiorso, and G. T. Seaborg, Phys. Rev. 77 (1950) 296, Us8,

- Edward L. Fireman, Phys. Rev. 75 (1949) 323, Sn ${ }^{124}$.

10 Marvin I. Kalkstein and Willard F. Libby, Phys. Rev. 85 '(1952) 368, Sn ${ }^{124}$.

11 R. G. Winter, Thesis, Dept. of Physics, Carnegie Institute of Technology, Pittsburgh, Pa. Pd ${ }^{108}, \mathrm{Pd}^{110}$.

12 E. L. Fireman and D. Schwartzer, Phys. Rev. 85 (1952), Sn ${ }^{124}$.

13 R. M. Pearce and E. K. Darby, Phys. Rev. 86 (1952) 1049, Sn ${ }^{124}$.

14 J. A. McCarthy, Thesis, University of Rochester (1952). Also Phys. Rev.

$\mathrm{Zn}^{96}, \mathrm{Sn}^{124}$. This work has also been reported elsewhere?

15 H. W. Fulbright, Physica, XVIII, (1952) 1026.

16 J. H. Fremlin and M. C. Walters, Proc. Phys. Soc. (London) A65 (1952) 911, $\mathrm{Ca}, \mathrm{Mo}, \mathrm{Sn}, \mathrm{Sn}, \mathrm{Te}, \mathrm{Ba}, \mathrm{W}, \mathrm{Os}, \mathrm{Pt}$ and Mo.

17 Jean-Francis Detouef, Raymond Moch and Jean Salmon, Journal de Physique et le Radium, 13 (1952) 618. 
(neglecting nuclear recoil), so the total energy spectrum is a line. A scintillation spectrometer with trans stilbene crystals was set up as shown

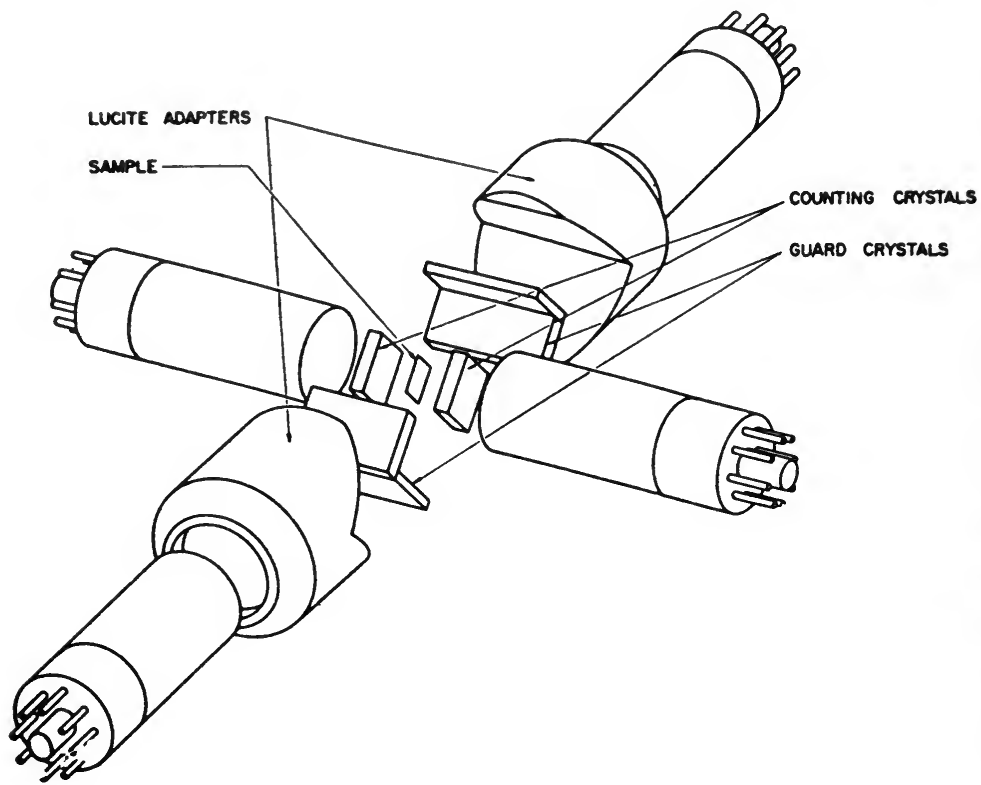

Fig. 1. Exploded diagrammatic view of counter arrangement. The counting crystals $(2.5 \times 2.5 \times 1.1 \mathrm{~cm})$ were covered on five sides with thin light tight Al foil. The guard counters were used in anticoincidence with the others to reduce the background. The counter unit rested on a sheet of cadmium placed on a concrete floor. It was covered with $1 / 4$ inches of steel, 4 inches of lead and 1 inch of borax.

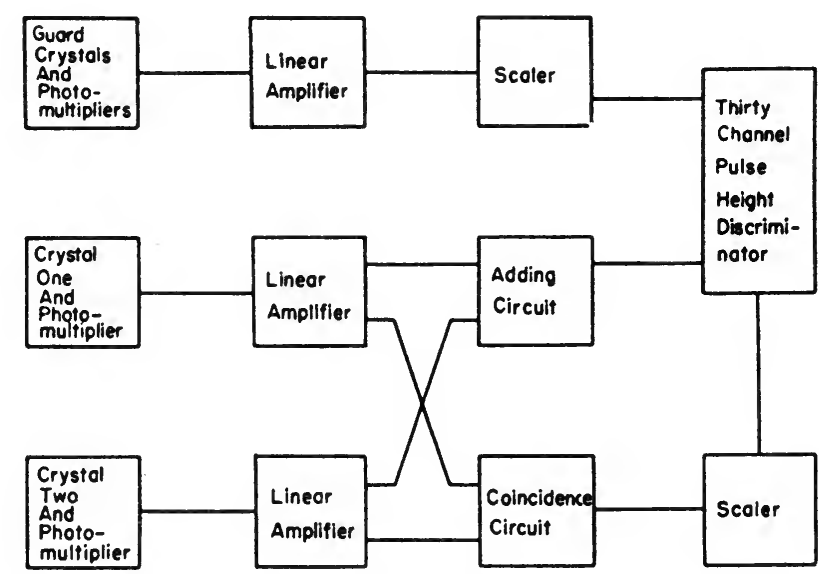

Fig. 2. Block diagram of electronic equipment. The coincidence circuit was turned off when total activity was measured. 
in Figures 1 and 2. The resolving power was $15 \%$, so double beta decay would be observed as a broad line superimposed upon a continuous background spectrum.

ATOMIC MASS DIFFERENCES ABOVE MOST

STABLE NUCLIDE OF ISOBARIC SERIES A.96

Fig. 3. Atomic masses for $A=96$. Notice that the relative positions of $\mathrm{Zr}^{96}$ and $\mathrm{Nb}^{96}$ may be shown incorrectly here, because no activity attributable to the decay of $\mathrm{Nb}^{96}$ was observed

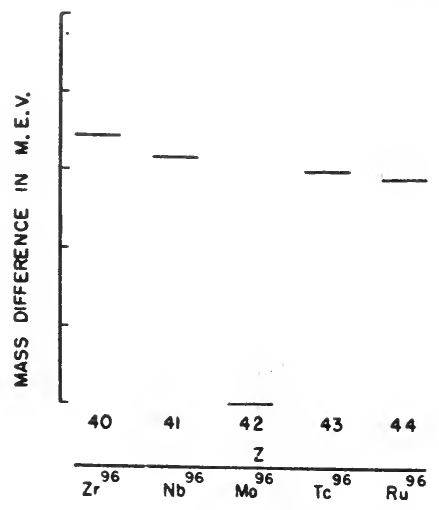

The enriched sample studied was $\mathrm{Zr}^{96}$ in the form of $\mathrm{ZrO}_{2}$ containing $52 \mathrm{mg}$ of $\mathrm{Zr}, 89.5 \% \mathrm{Zr}^{96}$. The background counting sample was similar, but enriched to $97.79 \%$ in $\mathrm{Zr}^{94}$. Duckworth's latest mass spectrographic studies show that $3.4 \pm 0.3 \mathrm{MeV}$ should be available for double beta decay. See Fig. 3. This was therefore a favorable case to investigate.

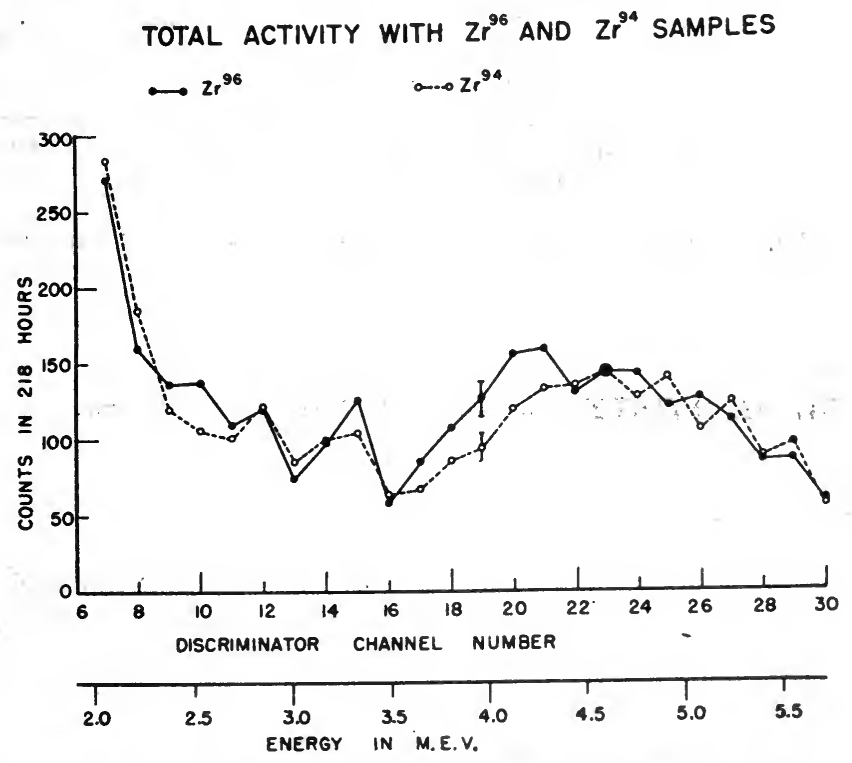

Fig. 4. Total activity with $\mathrm{Zr}^{96}$ and $\mathrm{Zr}^{94}$ samples 


\section{$\S 3$. Results}

Data were taken with and without a coincidence requirement between the two counting crystals because of the possibility ${ }^{6}$ of a fairly strong angular correlation between the two electrons. Results shown in Figures 4 and 5 show a slight excess counting rate in the region between 3.3 and 4.3 MeV. The excess is consistent with the assumption that $\mathrm{Zr}^{98}$ undergoes neutrinoless beta decay with a lifetime of $6.2 \pm 2 \times 10^{16}$ years. However, the result should only be accepted with reservations until it has been checked. Experiments along these lines are continuing both at M.I.T. and Rochester.

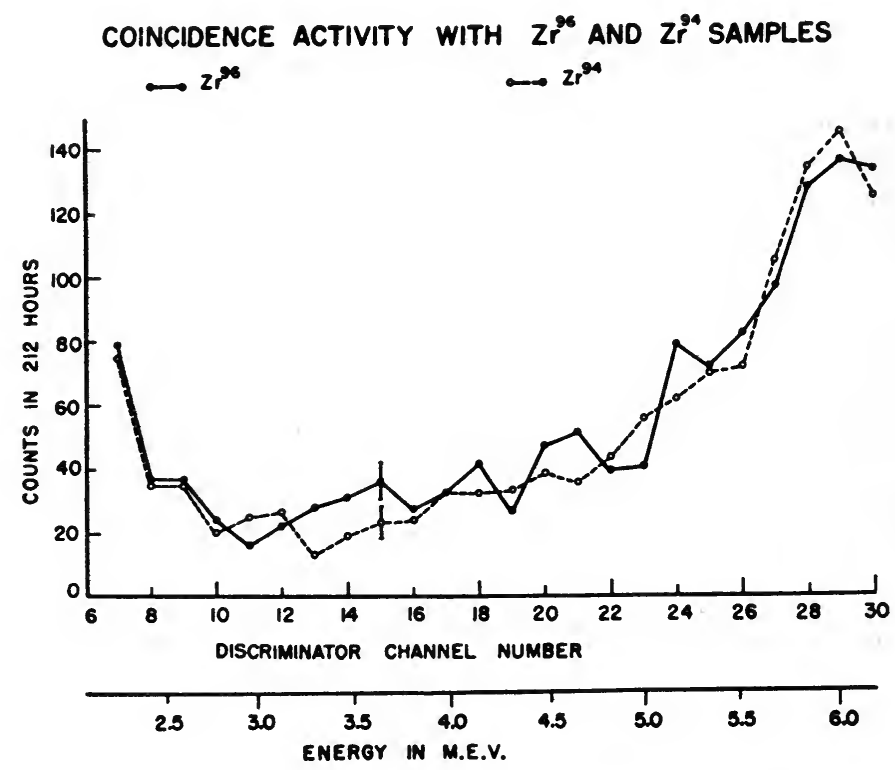

Fig. 5. Coincidence activity with $\mathrm{Zr}^{98}$ and $\mathrm{Zr}^{94}$ samples

\section{(III) S. DE BENEDETTI - Annihilation of Positrons}

\section{§1. Introduction}

After the discovery of positive electrons ${ }^{1}$ and the proof that these particles were produced in cosmic ray showers ${ }^{2}$, several investigations were undertaken in order to prove that they exhibited the annihilation

1 C. D. Anderson, Phys. Rev. 43 (1933) 491.

2 P. M. S. Blackett and G. P. S. Occhialini, Proc. Roy. Soc. 139 (1933) 699. 
properties predicted by Dirac theory ${ }^{3}$. According to this, the rest masses of a pair of positive and negative electrons can be radiated in the form of electromagnetic energy, as a direct consequence of the interaction of electrons and electromagnetic field. Conservation of momentum requires that at least two quanta should be emitted; since most positrons stop before annihilating, the two quanta will be in opposite directions, each carrying an energy $m c^{2}$.

The pioneering experimental work was done by Thibaud ${ }^{4}$, using positrons produced by the $\gamma$-rays of $\mathrm{ThC}^{\prime \prime}$ and separated by a trochoidal method. This author found quanta of about $0.5 \mathrm{MeV}$ (absorption method) originating from the absorber where the positrons came to rest, and identifiable with the expected radiation of annihilation. Joliot ${ }^{5}$ obtained similar results with positrons emitted by artificial radioactive sources showing that the particles of $\beta^{+}$decay are of the same nature as those resulting from the process of pair production.

The first work proving that two simultaneous $\gamma$-rays are produced, was a coincidence experiment by Klemperer ${ }^{6}$. This was improved by Alichanian and others ${ }^{7}$, and by Beringer and Montgomery ${ }^{8}$ who proved that the quanta are emitted in opposite directions within one degree.

In recent years, progress in the field of instrumentation has made possible a much more careful study of the annihilation process.

Using the big double focusing $\beta$-ray spectrometer in Stockholm together with a homogeneous field spectrometer for calibration purposes Hedgran, Lind and Lindström ${ }^{9}$ first showed that the energy of the annihilation radiation $\left(E_{\gamma}=510.96 \mathrm{keV}\right)$ within the experimental errors of 3 parts in $10^{4}$ agreed with that calculated from the elementary constants $m c^{2}$. The Compton wave length was furthermore studied with DuMond's crystal spectrometer by Muller et al. ${ }^{10}$ and the results $(\lambda=24.262 \pm 0.0033 \mathrm{~m} \AA)$ ) are in excellent agreement with the calculated value $\lambda=24.2625 \pm 0.0006$ $\mathrm{m} \AA$ from elementary constants.

One can deduce from these measurements that the mass of the positron is the same as that of the electron within a few parts in $10^{4}$.

3 See for instance: W. Heitler, The Quantum Theory of Radiation, Oxford, 1944.

4 J. Thibaud, C. R. 197 (1933) 1629; Phys. Rev. 45 (1934) 781.

5 F. Joliot, C. R., 197 (1933) 1622; C. R. 198 (1934) 81.

- O. Klemperer, Proc. Camb. Phil. Soc. 30 (1934) 347.

7 A. I. Alichanian, A. I. Alichanow, and L. A. Arzimovich, Nature 137 (1936) 703, and C. R. (Doklady) 1 (1936) 287.

8 R. Beringer and C. G. Montgomery, Phys. Rev. 61 (1942) 222.

- G. Lindström, Festskrift Manne Siegbahn (1951).

10 D. E. Muller, H. C. Hoyt, D. J. Klein, and J. W. M. DuMond, Phys. Rev. 88 (1952) 775. 


\section{$\S 2$. Two-Quanta Polarization}

We have considered thus far only the features of the two-quantum annihilation process which can be derived as a simple consequence of the conservation of energy and momentum. Taking into account conservation of angular momentum, one can predict some of the polarization properties of the annihilation radiation.

Two quanta traveling in opposite direction carry either two or zero units of angular momentum according to whether they are circularly polarized in the same or in the opposite sense. Assuming that the positron and the electron possess no relative orbital angular momentum before annihilation, it follows that they must be in a singlet $S$ state and that an experiment designed to measure simultaneously the sense of circular polarization of the two annihilation photons will find this sense to be opposite.

An experiment to investigate this point has been performed by Clay and Hereford ${ }^{11}$. Their work is based upon the fact that the cross-section for forward Compton scattering of circularly polarized radiation depends upon the orientation of the electron spin relative to the direction of propagation. Two magnetized iron foils were used as scatterers. The difference in coincidence counting rates for parallel or antiparallel magnetization of these foils, was found to be $3.5 \pm 0.75$ percent, somewhat larger than the two percent difference theoretically predicted for such experiment ${ }^{12}$.

From the experimental point of view plane polarization is easier to study than circular polarization. Theoretically one predicts in this regard that the two $\gamma$-rays of annihilation should be plane polarized in perpendicular directions. This is a consequence of conservation of parity and of the fact that, according to Dirac theory, a particle and its anti-particle have opposite parities, making the initial $S$ state an odd one ${ }^{13}$.

The perpendicular direction of polarization of the $\gamma$-rays of annihilation has been verified by several investigators ${ }^{14}, 15,16,17.18$, all using essentially the same experimental arrangement. In the experiment of $\mathrm{Wu}$ and Shaknov (Fig. 1), a $\mathrm{Cu}^{64}$ source was situated at the center of a heavy

11 F. P. Clay and F. L. Hereford, Phys. Rev. 85 (1952) 675.

12 O. Halpern, Nature 168 (1951) 782.

13 C. N. Yang, Phys. Rev. 77 (1950) 242.

14 E. Bleuler and H. L. Bradt, Phys. Rev. 73 (1948) 1398.

15 G. C. Hanna, Nature 162 (1948) 332.

16 C. S. Wu and I. Shaknov, Phys. Rev. 77 (1950) 136.

17 N. A. , Vlasov, Izv. Akad. Nauk. S.S.S.R. Fiz 14 (1950) 337.

18 F. L. Hereford, Phys. Rev. 81 (1951) 482. 
lead block provided with a long through hole from which the two oppositely directed annihilation rays emerged. These impinged upon Al scatterers, and the Compton scattered radiation was detected, at $90^{\circ}$, by means of two anthracene scintillation counters in coincidence. The

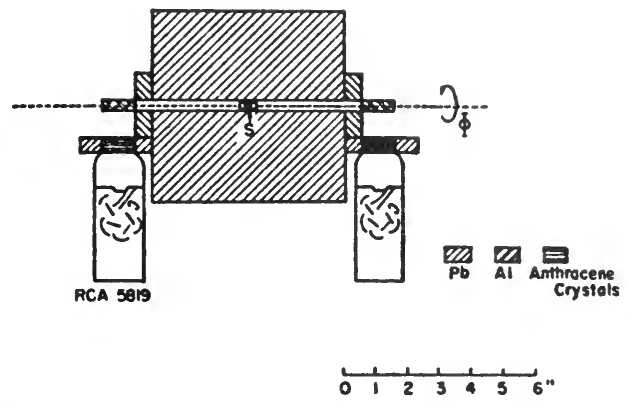

Fig. 1. Schematic diagram of experiment

counters could be rotated around an axis defined by the hole and were alternatively located either both coplanar with the hole, or in such a way that one of them would be $90^{\circ}$ away from the plane of the hole and of the other counter. The first arrangement responds preferentially to parallel polarized rays, the second to perpendicular polarized ones. The observed ratio of counting rates was $2.04 \pm 0.08$ in favor of perpendicular polarization. When one takes into account the geometry of the experiment and the anisotropy of the Compton scattering crosssection, one concludes that this result is in complete agreement with perpendicular polarization of the annihilation quanta which predicts a counting rate ratio of 2.00 .

\section{$\S 3$. The Momentum of the Annihilating Pairs}

We have thus far assumed that the annihilating positron and electron are at rest. In a strict sense, however, this can hardly be the case since, even neglecting the positron kinetic energy, the electrons of matter have momenta at least of the order of $m c / 137$, corresponding to kinetic energies of $\sim 13$ electron volts. It follows that the $\gamma$-rays of annihilation should have an angular spread of about $1 / 137$ radian around $180^{\circ}$, and that the annihilation line should exhibit a width of the order of $10 \mathrm{eV}$ at least.

These qualitative expectations are corroborated by the experiment. In the first experiment ${ }^{19}$ to point out a departure from perfect anti-

19 S. DeBenedetti, C. Cowan, W. R. Konneker and H. Primakoff, Phys. Rev. 77 (1950) 205. 
parallelism of the two annihilation quanta a thin source of $\mathrm{Cu}^{64}$ was surrounded by a gold absorber in such a way that most positrons stopped in a region a few tenths $\mathrm{mm}$ in thickness. Two anthracene counters in coincidence were located on opposite sides, at a distance of $120 \mathrm{~cm}$ from the source. It was found that the number of coincidences did not vanish immediately when the counters and the source were out of alinement; instead, it decreased exponentially being reduced of $1 / \mathrm{e}$ for an angle of $0.6 / 137 \mathrm{rad}$. When the finite thickness of the counters is taken into account, this is shown to correspond to an average momentum of the annihilating pair of $1.2 \mathrm{mc} / 137$.

The order of magnitude of the mean momentum obtained with the method of angular correlation is in agreement with a determination of the Doppler width of the annihilation line obtained with the curved crystal $\gamma$-ray spectrometer ${ }^{10,20}$. For positrons stopping in $\mathrm{Cu}$, this corresponds to an average momentum of $1.1 \mathrm{mc} / 137$ (1949 data), or $1.35 \mathrm{mc} / 137$ (1952 data). Hedgran and Lind ${ }^{21}$ have investigated the width of the annihilation line with a $\beta$-ray spectrometer finding for the average momentum of the annihilating pairs $0.52 \mathrm{mc} / 137$, a value somewhat lower than those reported above.

At any rate, these measurements are in order of magnitude agreement and seem to support the following points:

1) The positrons reach very low (possibly thermal) energy, so that only the electrons contribute to the observed momentum.

2) Only the external atomic electrons (the free electrons in the case of a conductor) participate in the annihilation.

A somewhat detailed theoretical discussion based on these points ${ }^{19}$ leads to results in sufficient agreement with the observation, the agreement being better for the lowest experimental results.

The experiments. mentioned thus far, however, are not sufficient to decide whether the slow positrons end their life wandering in matter as free particles (in a manner reminiscent of the diffusion of thermal neutrons), or whether some bound state is formed prior to annihilation. The failure of an attempt to measure the "diffusion length" of slow positrons ${ }^{22}$, favors the second viewpoint which is also corroborated by the measurements of positron half life to be discussed in another section of this chapter. Measurements of angular correlation of annihilation radiation have

20 J. W. M. DuMond, D. A. Lind and W. W. Watson, Phys. Rev. 75 (1949) 1226 .

21 A. Hedgran and D. A. Lind, Ark. f. Fysik, 5 (1952) no 2.

22 L. Madansky and F. Rasetti, Phys. Rev. 75 (1950) 397. 
been extended to several substances because of their connection with electronic wave functions, and of their possible interest in solid state studies 23. 24. Though these measurements show some interesting trends, more extensive and systematic work is required before deciding whether positrons can be used as a tool for the investigation of electrons in solids.

\section{§4. Three-Quantum Annihilation}

In the conventional perturbation treatment of the interaction of electrons with the electromagnetic field, the phenomenon of annihilation with the emission of two $\gamma$-rays is treated as a second order process. One can expect that the higher order process, annihilation with emission of three quanta, will occur in about $1 / 137$ of the times. A more exact theoretical study ${ }^{25}$ shows that, if positrons and electrons meet with randomly oriented spins, the annihilation will occur with the emission of three quanta in $1 / 370$ of the cases. Three-quantum annihilation, however, will result only from triplet collisions, while two quanta will arise only from singlet $S$ pairs.

It is possible to remove the random distribution in the relative orientation of the spins of positron and electron by producing the stable system of the positronium atoms ${ }^{26}$. All the singlet atoms of positronium decay promptly (mean life $1.25 \times 10^{-10} \mathrm{sec}$ ) with the emission of two quanta while the triplet ones decay much more slowly (mean life $1.4 \times 10^{-7} \mathrm{sec}$ ) with the emission of three. As a result of their longer life, triplet $S$ states are much more abundant in a positronium sample than in a source where positrons and electrons collide freely.

The discussion of positronium and its properties will be presented in another part of this chapter; here, we want to describe only some of the physical characteristics of the phenomenon of three quantum annihilation.

A first experimental study of three-quantum annihilation is due to Rich ${ }^{27}$ who presented some evidence of the existence of the effect. The work was followed by experiments conducted at the Carnegie Institute of Technology ${ }^{28}$ where three $\mathrm{NaI}(\mathrm{Tl})$ scintillation counters were used in coincidence for the detection of the three quanta emitted in the annihilation of positrons from a $\mathrm{Na}^{22}$ source. The total momentum of the quanta being practically zero, the three photons must be coplanar; thus the three

23 J. B. Warren and G. M. Griffiths, Can. Journ. Phys. 29 (1951) 325.

24 H. Maier-Leibnitz, Z. Naturforsch. 6a (1951) 663.

25 A. Ore and J. L. Powell, Phys. Rev. 75 (1949) 1696.

28 M. Deutsch, Phys. Rev. 82 (1951) 455.

27 J. A. Rich, Phys. Rev. 81 (1951) 140.

28 S. DeBenedetti and R. Siegel, Phys. Rev. 85 (1951) 371. 
counters were located in the same plane as the source (Fig. 2) for the measurement of the effect, and one of them was removed from this plane (without varying its distance from the source) for the measurement of the background. In this manner it was possible to detect the coincidences due
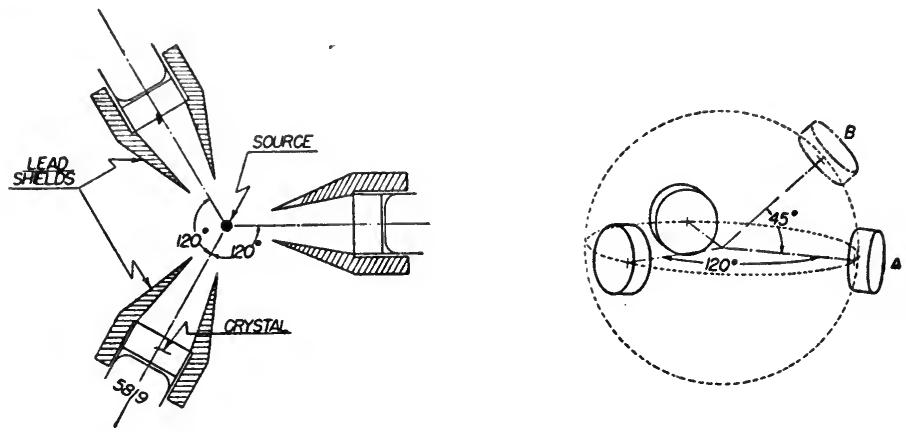

Fig. 2. Arrangement of the counters for detection of three-quantum annihilation (a) Top view, (b) Perspective of the crystals. Position $B$ used for background measurement.

to the three quantum annihilation both for positrons stopped in solid absorbers (where the spin orientations are presumably statistically distributed) and, in considerably higher number, from gaseous absorbers where positronium was formed.

A study of the distribution in height of the coincident pulses provided a check on the energy of the quanta proving unambiguously that they arose from the annihilation process ${ }^{29}$. The results are shown in Fig. 3. The energy résponse of the three counters was calibrated using the two quantum annihilation line (upper curves) and the pulse height in triple coincidence was measured for various geometrical arrangements. The agreement between the position of the photopeaks and the computed values (vertical lines) is satisfactory in all cases.

After geometrical computations and experimental determination of the efficiency of the counters, it was possible to compare the number of triple coincidences observed with an $\mathrm{Al}$ absorber with the theoretical ratio $1 / 370$ between 2 and 3-quanta annihilation events. The experimental result $(.92 \pm .1$ coinc $/ \mathrm{min})$ was somewhat lower than the theoretical expectation (1.24 $\pm .16 \mathrm{coinc} / \mathrm{min})$, but, owing to the difficulty in the evaluation of some of the parameters entering in the comparison, the difference can hardly be regarded as significant.

The interpretation of the data in terms of angular distribution is also affected by errors due to the poor knowledge of counter efficiency as a

29 S. De Benedetti and R. Siegel, Phys. Rev. 94 (1954) 955. 


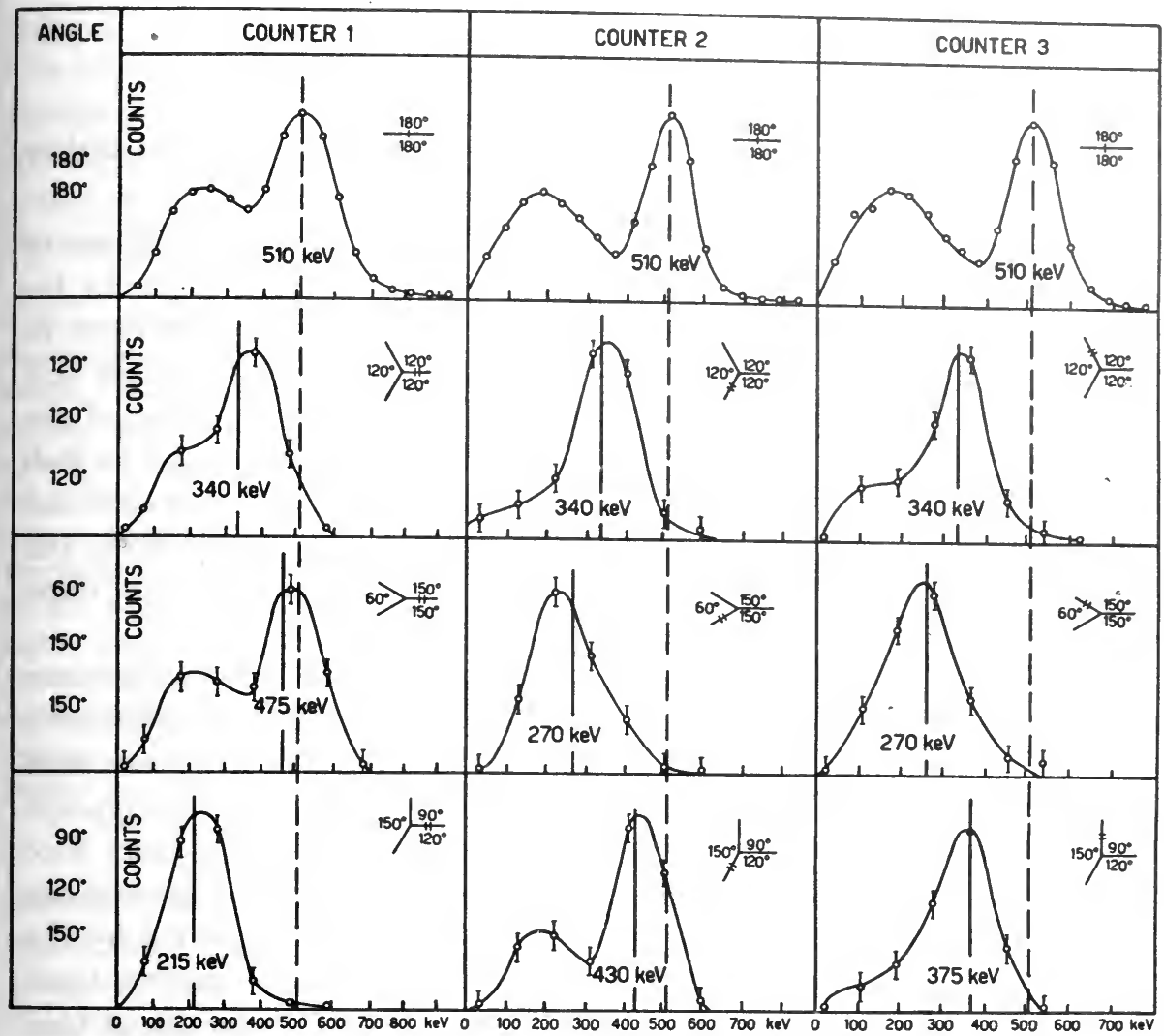

Fig. 3. Pulse height spectra of three photon annihilation at various angles

function of energy. One has, however, the impression that the experiment confirms the rather flat angular dependence predicted by the theory ${ }^{25}$.

Finally, a measurement of the polarization of the three quantum annihilation radiation was performed ${ }^{30}$. It was chosen to measure the polarization of one of the three rays, in a symmetrical arrangement, relative to the plane of emission of the three photons. The polarization was detected as usual by the Compton scattering method. The theory predicts ${ }^{31}$ a ratio $3 / 1$ in favor of rays plane polarized with the electric vector perpendicular to the plane of the 3 photons. When applied to our experimental arrangement this leads to a theoretical ratio of coincidences $1.80 \pm 0.15$ in favor of rays scattered in the plane of emission. The experimentally observed ratio, $1.87 \pm .23$, is in satisfactory agreement.

30 L. Leipuner, R. Siegel and S. DeBenedetti, Phys. Rev. 91 (1953) 198.

31 R. M. Drisko, Personal Communication. 


\section{(IV) R. E. BELL - Annihilation of Positrons [in Liquids and Solids}

The work of Deutsch and his collaborators on the annihilation of positrons in gases (see following Section) has led several groups to investigate this process in liquids and solids. The lifetimes of positrons in liquids and solids are expected to lie in the $10^{-9}$ to $10^{-10} \mathrm{sec}$ range and hence are more difficult to measure than in the case of gases. Most of the results secured so far are due to DeBenedetti and Richings ${ }^{1}$ and to Bell and Graham ${ }^{2}$. The latter authors have found that in certain solids and liquids positrons decay with a complex decay curve having two mean life components, strongly indicating the formation of positronium in these materials.

DeBenedetti and Richings made comparison measurements of positron lifetimes using a pair of stilbene-5819 scintillation counters in a coincidence circuit of resolving time $2 \tau_{0}=4 \times 10^{-9}$ seconds. The two counters were pointed at a source of $\mathrm{Na}^{22}$, which emits positrons accompanied by prompt nuclear gamma rays of energy $1.28 \mathrm{MeV}$. The biases on the counters were so adjusted that a coincidence ras recorded only if a $1.28 \mathrm{MeV}$ gamma ray was detected by one of the counters and an annihilation gamma by the other. Thus one counter recorded the instant of birth of the positron, and the other its instant of death. The $\mathrm{Na}^{22}$ source $\mathrm{S}$ was deposited on thin aluminum foil and sandwiched between the substances $A$ and $B$ for which the lifetime was to be compared. With the sandwich arranged BASAB, the positrons emerging from the source were annihilated in substance $A$, and with the sandwich arranged ABSBA, they were annihilated in $\mathbf{B}$. The substance $A$ was usually aluminum, and the result of a pair of runs was the difference in positron lifetime between aluminum and the substance $B$. The results showed that the difference in annihilation mean life between aluminum and the metals $\mathrm{Li}, \mathrm{Na}, \mathrm{K}, \mathrm{Cu}, \mathrm{Ag}$, and $\mathrm{Pb}$ was $(0 \pm 0.7) \times 10^{-10}$ seconds. Similar results were secured for graphite, $\mathrm{NaCl}$ crystal, $\mathrm{AgCl}, \mathrm{PbO}, \mathrm{Al}_{2} \mathrm{O}_{3}$, sulfur crystals, and $\mathrm{H}_{2} \mathrm{O}_{2}$. The mean life observed for some other substances was apparently longer than that in aluminum, usually by about $3 \times 10^{-10}$ seconds; these substances were $\mathrm{H}_{2} \mathrm{O}$, methyl alcohol, benzene, paraffin, polystyrene, teflon (polytetrafluoroethylene $\left.\left(\mathrm{CF}_{2}\right)_{n}\right)$, and quartz.

A series of more extensive measurements was made by Bell and Graham ${ }^{2}$,

1 S. DeBenedetti and H. J. Richings, Phys. Rev. 85 (1952) 377.

2 R. E. Bell and R. L. Graham, Phys. Rev. 90 (1953) 644. 
using a method which gave the absolute value of the positron lifetime. The experimental arrangement is shown in Fig. 1. A pair of lens spectrometers placed end to end was used to select a beam of positrons which had already passed through a thin stilbene crystal at the entrance to the first spectrometer. Each of these positrons had therefore registered the instant of its entry into the apparatus. The pair of spectrometers was so

Fig. 1. Pair of identical end-to-end $\beta$-ray spectrometers which are operated with the coils in series. Positrons emerging through the stilbene crystal of the left scintillation counter, shown enlarged in (b), are selected in energy and trajectory by the left spectrometer. Positrons which pass through the hole in the central diaphragm into the right spectrometer can strike only the sample placed in front of the right hand, $\gamma$-sensitive scintillation counter, shown enlarged in $(c)$, or the diphenylacetylene crystal of the counter directly if the sample is removed. The scintillation counters are con. nected to a coincidence circuit (not shown) of resolving time $2 \tau_{0}=2 \times 10^{-9} \mathrm{sec}$

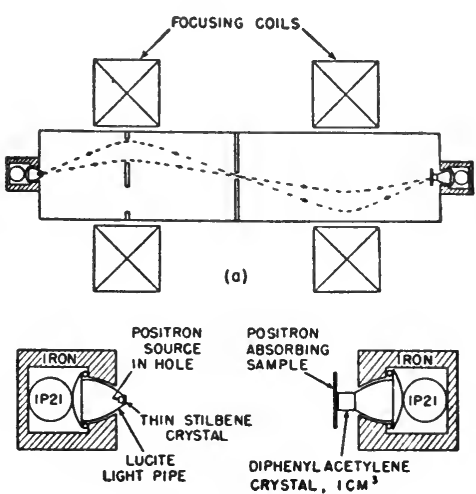

(b)

(c)

arranged that positrons passing into the right hand spectrometer could strike only the sample material, which was placed immediately over a $1 \mathrm{~cm}^{3}$ crystal of diphenylacetylene. The thin stilbene crystal and the $1 \mathrm{~cm}^{3}$ diphenylacetylene crystal were viewed by two $1 \mathrm{P} 21$ photomultipliers (Fig. 1) connected to a coincidence circuit of resolving time $2 \tau_{0}=2 \times 10^{-9}$ seconds. With the sample removed, the positrons focused by the spectrometers fell directly on the diphenylacetylene crystal, and the coincidence circuit received prompt coincidences. When the sample was in place, the positrons were annihilated in it, and the diphenylacetylene crystal then counted a fraction of the annihilation quanta, delayed by the annihilation lifetime. A measurement of the positron annihilation lifetime properties of any sample consisted of a comparison of sample-out (prompt) and sample-in (delayed) coincidence resolution curves in the usual manner (see Chapter XVIII).

The results for an aluminum sample are shown in Fig. 2. The delayed curve (solid circles) was obtained with the sample in place, and the prompt curve $P(x)$ was obtained with the sample removed. The displacement of the aluminum curve to the right of the prompt curve (whose center determines the zero of the delay scale) shows that the mean life of positrons in aluminum is $(1.5 \pm 0.3) \times 10^{-10}$ seconds. Similar results were obtained with other metals, with perhaps a slight tendency for denser metals to 
show a shorter mean life. This tendency was hardly outside the limit of experimental error, and the definite results on metals were, first, a surprisingly short annihilation mean life, about $1.5 \times 10^{-10}$ seconds (see discussion to follow), and second, a surprising constancy in this value from metal to metal, in agreement with DeBenedetti and Richings. Other simple crystalline substances such as $\mathrm{NaCl}, \alpha$-quartz, graphite, diamond, and monoclinic sulfur crystals, gave results qualitatively similar to those for the metals but with slightly longer mean lives. A typical result, that for crystalline quartz, is shown in Fig. 3.

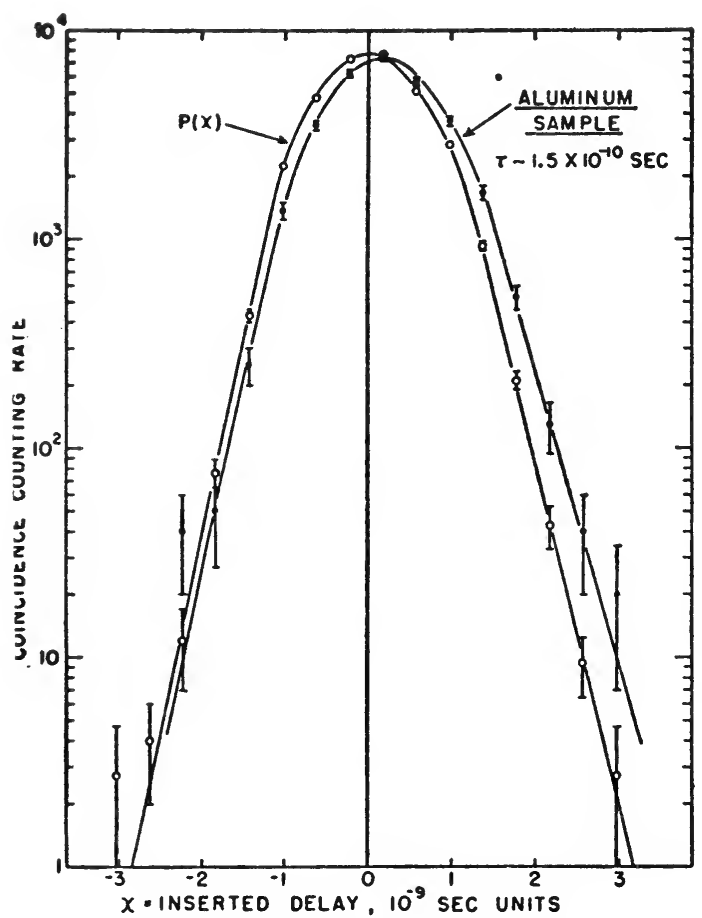

Fig. 2. Coincidence resolution curves. The delayed curve (solid circles) was obtained when the positrons were absorbed in a sample of aluminum, and the prompt curve $P(x)$ was obtained when the sample was removed and positrons were detected directly by the crystal of the right counter in Fig. 1

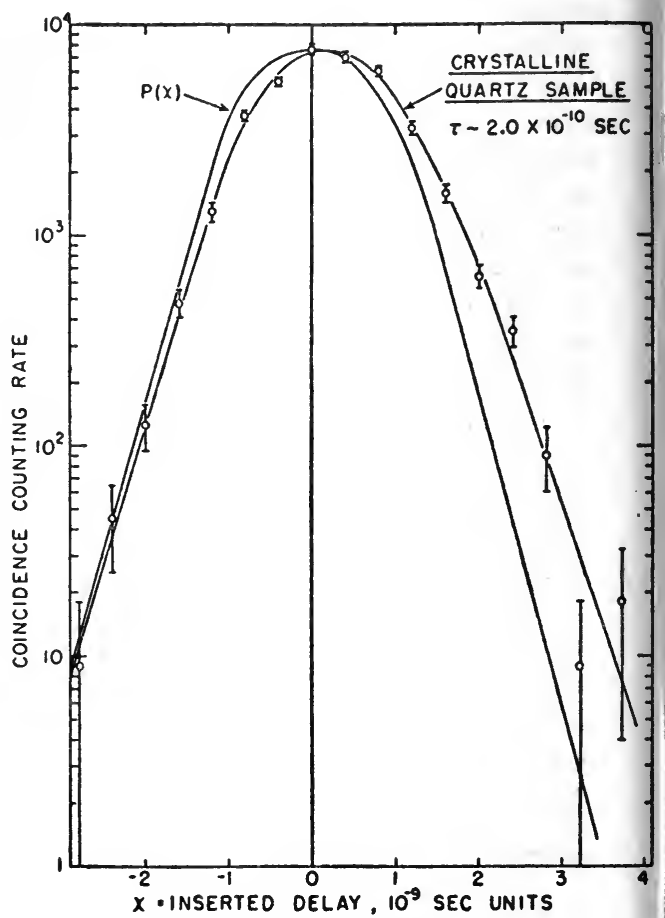

Fig. 3. Coincidence resolution curve obtained with a sample of crystalline $\alpha$-quartz with a prompt curve $P(x)$ for comparison. Since the prompt curve was always better determined statistically than the delayed curve, the experimental points have been omitted

from $P(x)$ here and in Fig. 4

Upon replacing the sample of crystalline quartz with one of fused quartz, the curve of Fig. 4 was found. Here there are two mean lives present, as shown by the analysis of the delayed resolution curve into 
two components indicated by dashed lines. The areas under the two dashed curves give the relative intensities of the two mean life components. The results for fused quartz are $\tau_{1}=3.5 \times 10^{-10} \mathrm{sec}$ (intensity $71 \%$ ) and $\tau_{2}=1.8 \times 10^{-9} \sec (29 \%)$. Results of this kind were found for many

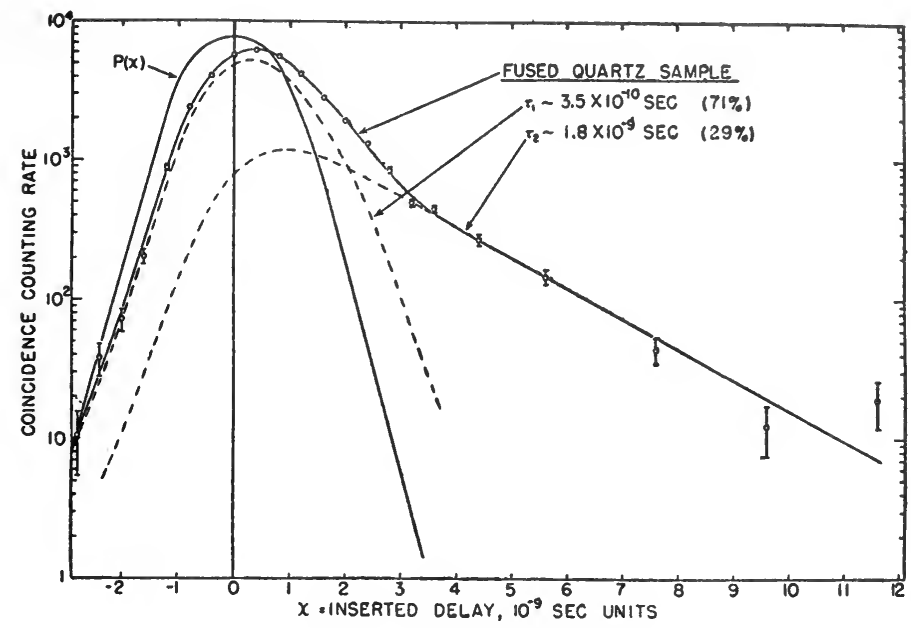

Fig. 4. Coincidence resolution curve obtained with a sample of fused quartz, with a prompt curve $P(x)$ for comparison. The composite nature of the positron decay in fused quartz is evident

"amorphous" solids, including polystyrene, paraffin wax, cellulose nitrate, polyethylene, teflon, vitreous borax $\left(\mathrm{Na}_{2} \mathrm{~B}_{4} \mathrm{O}_{7}\right)$, pyrex glass, and probably plastic sulfur. All these substances showed about the same relative intensity for the longer lived component, about $1 / 3$, but the value of the longer lifetime, $\tau_{2}$, varied from $\sim 4.5 \times 10^{-10} \mathrm{sec}$ for plastic sulfur to $3.5 \times 10^{-9}$ sec for teflon.

Having established this double lifetime effect, Bell and Graham reverted to an experimental arrangement like that of DeBenedetti and Richings, and studied liquids (which would have been difficult to insert into the vacuum of the spectrometer), and temperature effects. The longer lifetime was absent in liquid mercury, but present in water and in a saturated solution of $\mathrm{NaCl}$ in water. Freezing the water sample to ice did not eliminate the $\tau_{2}$ component, but did lower the value of $\tau_{2}$ from $1.7 \times 10^{-9}$ to $1.2 \times 10^{-9}$ seconds, the intensity remaining fairly constant. Cooling the ice to $-196^{\circ} \mathrm{C}$ reduced $\tau_{2}$ to $0.85 \times 10^{-9}$ seconds, again without large change in the intensity of the $\tau_{2}$ component. These results showed that some crystals may have the longer lifetime effect; another such example was crystalline borax $\left(\mathrm{Na}_{2} \mathrm{~B}_{4} \mathrm{O}_{7} \cdot 10 \mathrm{H}_{2} \mathrm{O}\right)$ with $\tau_{2}=0.9 \times 10^{-9}$ seconds. 
Cooling fused quartz from room temperature to $-196^{\circ} \mathrm{C}$ did not markedly affect either its value of $\tau_{2}$ or the intensity of the $\tau_{2}$ component. On the other hand teflon showed a marked dependence of $\tau_{2}$ on temperature, the values in seconds being $3.5 \times 10^{-9}\left(20^{\circ} \mathrm{C}\right), 2.5 \times 10^{-9}\left(-78^{\circ} \mathrm{C}\right)$, and $1.6 \times 10^{-9}\left(-196^{\circ} \mathrm{C}\right)$, again without much change in intensity; these results are shown in Fig. 5. Bell and Graham then added a NaI scintillation

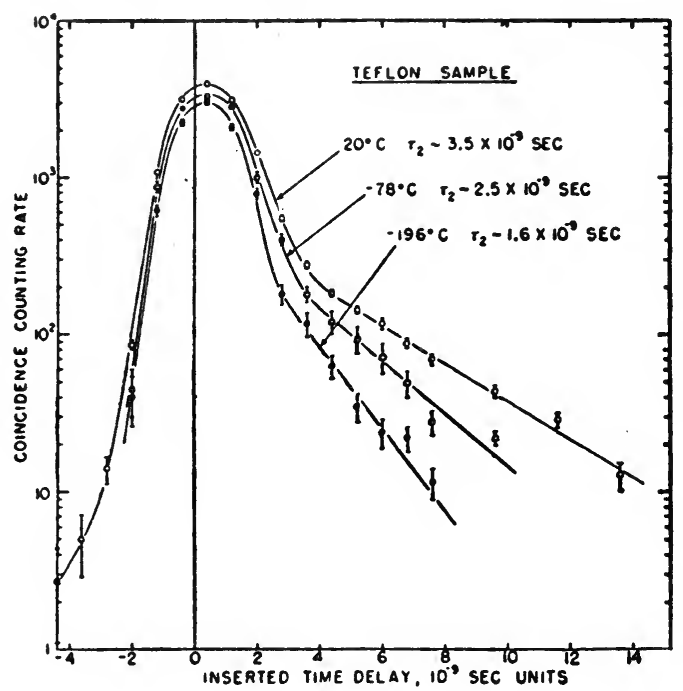

Fig. 5. Comparison of the coincidence resolution curves obtained with a sample of teflon at three temperatures, showing the variation of $\tau_{2}$ with temperature

spectrometer to their apparatus, arranged to measure the pulse height spectrum of the $180^{\circ}$ partner of the annihilation photon whose delay was being measured by the fast coincidence apparatus. The results showed that the delayed annihilation photons ( $\tau_{2}$ component) from a polystyrene sample had the same energy as the more prompt photons from an aluminum sample, to an accuracy of $\pm 3 \%$. The yield of photon partners was also the same for the two cases, showing that the delayed radiation consists mainly of pairs of photons strongly correlated at $180^{\circ}$, like the more prompt component. Thus the long delayed component of the annihilation radiation appears to be similar to ordinary annihilation radiation in everything, except mean life; however, an admixture of a small percentage of three-quantum annihilation would not have been detected in the polystyrene measurement.

The simple picture of positrons annihilating with the valence electrons (or "free" electrons) of a metal is unable to explain either the shortness of the mean life in metals, or the relative constancy from metal to metal. 
The Dirac cross section for annihilation of a slow positron by a free electron is

$$
\sigma=\pi r_{0}^{2} c / v \mathrm{~cm}^{2},
$$

where $r_{0}$ is the classical electron radius and $v$ is the relative velocity of positron and electron. This cross section varies as $1 / v$, so that the mean life of slow positrons in a medium of available electron density $D$ would be

$$
\tau=\left[\pi r_{0}^{2} c D\right]^{-1} \text { sec. }
$$

Both the angular correlation of annihilation quanta near $180^{\circ}$ and the Doppler spread in the energy of the annihilation radiation suggest that only electrons of a few volts energy participate appreciably in the annihilation process. In a solid metal there will be about one of these electrons per atom, so that the available electron density becomes $D=N_{0} \varrho / A \mathrm{~cm}^{-3}$, where $N_{0}$ is Avogadro's number, $\varrho$ is the mass density, and $A$ is the atomic weight. The expected lifetime then is

$$
\tau=\left[\pi r_{0}^{2} c N_{0} \varrho / A\right]^{-1} \mathrm{sec}=2.2 \times 10^{-10} \mathrm{~A} / \varrho \text { sec. }
$$

Putting in values for aluminum $(A=27, \varrho=2.7)$, we get $\tau=22 \times 10^{-10} \mathrm{sec}$, a value about fifteen times the observed one. The value of $A / \varrho$ varies widely among metals, being 5.0 for beryllium and 23.7 for sodium, for example. The above simple analysis thus fails completely to explain either the magnitude or the constancy of the observed positron lifetimes in metals.

These speculations make it appear that the former picture of the slowing down and annihilation of positrons in metals is in need of revision. Positrons were formerly supposed to slow down to thermal energies and spend some time diffusing through the metal before being annihilated ${ }^{3}$. The mean time for slowing down to thermal energies has been calculated by DeBenedetti et al. ${ }^{3}$ as $3 \times 10^{-10}$ sec for metallic gold. Both the fact that the total mean life of fast positrons in gold is only about $1.2 \times 10^{-10} \mathrm{sec}$, and the fact of the nonappearance of thermal positrons in the experiment of Madansky and Rasetti ${ }^{4}$ suggest that most of the positrons are annihilated in a metal before they have slowed down to thermal energies. Garwin $^{5}$ has recently suggested a mechanism by which the positron thermalization time could be much shorter than the figure given by DeBenedetti et al. ${ }^{3} \mathrm{He}$ remarks, however, that his result still fails to explain the lifetimes in metals. It seems likely that fast positrons in a metal slow down rapidly to energies of a few $\mathrm{eV}$, where they have a high

3 S. DeBenedetti, C. Cowan, W. R. Konneker and H. Primakoff, Phys. Rev. 77 (1950) 205.

4 L. Madansky and F. Rasetti, Phys. Rev. 79 (1950) 397.

s R. L. Garwin, Phys. Rev. 91 (1953) 1571. 
probability of forming singlet positronium, whose free space mean life is $1.25 \times 10^{-10}$ sec. In support of this idea, no sample has shown an experimental mean life shorter than $1.25 \times 10^{-10} \mathrm{sec}$ within experimental error. A possible mechanism by which this could occur is suggested by the phenomenon of electron capture and loss by protons and alpha particles in passing through matter. The electron capture cross section is large $\left(\sim 10^{-16} \mathrm{~cm}^{2}\right)$ for protons with velocities of the order of $e^{2} / \hbar$, the Bohr orbit velocity ${ }^{6}$; in metals the loss cross section is smaller than the capture cross section ${ }^{7}$ for velocities lower than $e^{2} / \hbar$. For particles of a given charge, these cross sections depend mainly on the velocity of the particle ${ }^{6}$. A positron with velocity $e^{2} / \hbar$ has an energy of $13.6 \mathrm{eV}$, and will be capturing and losing electrons at a rate of perhaps $10^{16} \mathrm{sec}^{-1}$. Positrons with energies of a few $\mathrm{eV}$ will therefore spend a large fraction of their time with an electron attached, this fraction increasing as the positrons lose energy. Ultimately a positron has insufficient energy to continue the capture and loss process, at which time it is likely to be found bound with an electron.

The existence of a longer mean life component in complex and "amorphous" substances also strongly suggests the formation of positronium in these materials. The effect is similar to that observed by Deutsch, in which a fraction of the positrons absorbed in a gas forms triplet positronium decaying with a mean life of $1.4 \times 10^{-7} \mathrm{sec}$ (extrapolated to zero gas pressure). This fraction has been measured by Pond ${ }^{8}$ for various gases, with results varying from $0.16\left(\mathrm{~N}_{2}\right)$ to $0.26\left(\mathrm{H}_{2}\right)$, when corrected for the magnetic field in which his measurements were made ${ }^{8}$. These amounts are slightly less than the intensity of the long-lived component $(\sim 1 / 3)$ found by Bell and Graham. Deutsch found that in the gas freon 12 $\left(\mathrm{CCl}_{2} \mathrm{~F}_{2}\right)$ the mean life of triplet positronium could be represented by

$$
\tau=\left(6.8 \times 10^{6}+0.3 \times 10^{6} p\right)^{-1} \mathrm{sec}
$$

where $p$ is the gas pressure in atmospheres, and the second term of (4) represents a shortening of the mean life due to collisions with gas atoms. If we now regard one of our amorphous condensed materials as equivalent to a gas at, say, 1000 atmospheres, the value deduced from (4) is

$$
\tau \sim 3 \times 10^{-9} \mathrm{sec}
$$

agreeing in order of magnitude with the values found for $\tau_{2}$.

- N. Bohr, Dan. Matt. Fys. Medd. 18 (1948) No. 8, Chapter 4.

7 T. Hall, Phys. Rev. 79 (1950) 504.

8 T. A. Pond, Phys. Rev. 85 (1952) 489; 'T. A. Pond and R. H. Dicke, Phys. Rev. 85 (1952) 489. 
A possible explanation of the $\tau_{2}$ component found in the experiments is then as follows. Positrons slowing down in the sample form triplet positronium in some fraction of the cases, according to our results about $1 / 3$. This triplet positronium is destroyed mainly by conversion to the short-lived singlet state $\left(\tau=1.25 \times 10^{-10} \mathrm{sec}\right)$ in collisions with atoms of the sample material. The triplet positronium thus has its own lifetime shortened, as suggested by (4) and (5), by a factor of 50 or more. On this view, less than $2 \%$ of the $\tau_{2}$ component observed in the experiments would consist of three-photon annihilations, a fraction which would have escaped detection in any of the experiments reported so far. The absence of an observable $\tau_{2}$ component in metals and some crystals might then be explained by assuming that electrons in energy bands determined by the crystal structure are effectively free so far as positrons or positronium are concerned. These electrons would rapidly convert the triplet positronium to the singlet state by collision, in much the same way that the odd electron in NO destroys triplet positronium in a gas.

There seems to be a connection between shortness of $\tau_{2}$ and the regularity or simplicity of structure of the sample, simple regular crystals not showing an observable $\tau_{2}$. Generally speaking, lowering the temperature of a substance increases its regularity of structure, and this step shortened $\tau_{2}$ in the experiments reported above. Ice, for example, is believed to be regular in the position of its molecules but random in their orientation, but presumably is more nearly regular at lower temperatures. The observation of the $\tau_{2}$ component in a complex crystal like $\mathrm{Na}_{2} \mathrm{~B}_{4} \mathrm{O}_{7} \cdot 10 \mathrm{H}_{2} \mathrm{O}$ is consistent with this idea. Experiments using positrons may some day be applied as a tool in the experimental study of the solid and liquid state.

Bell and Graham ${ }^{2}$ remarked that if the analysis given above is correct, a sample showing a long $\tau_{2}$ would yield substantially more three-quantum annihilations than one not showing an appreciable $\tau_{2}$. The three-quantum events would arise from the small fraction of the triplet positronium that decays by three-quantum annihilation before being converted to the singlet state by collisions. The relative yield of three-quantum events from teflon at room temperature would be about

$$
1 / 3\left(3.5 \times 10^{-9}\right) /\left(1.4 \times 10^{-7}\right)=0.8 \%
$$

This effect was sought unsuccessfully by Pond ${ }^{9}$, using an indirect method, but has been found by Graham and Stewart ${ }^{10}$, using a triple coincidence method similar to one used earlier by DeBenedetti and Siegel ${ }^{11}$. Three

9 T. A. Pond, Phys. Rev. 91 (1953) 455.

10 R. L. Graham and A. T. Stewart, Can. J. Phys. 32 (1954) 678.

11 S. DeBenedetti and R. Siegel, Phys. Rev. 85 (1952) 371. 
$\mathrm{NaI}$ scintillation counters were spaced at $120^{\circ}$ intervals in a plane around a source of positrons sandwiched between pieces of the specimen material. For $120^{\circ}$ spacing, the energy of each of the three quanta is $2 / 3 m c^{2}=341 \mathrm{keV}$, and pulse height selection was used on all three counters to ensure that the quanta detected had energies near this value. Spurious triple coincidences (due to Compton scattering, etc.) were observed separately by lifting one of the three counters out of the plane of the source, and subtracted from the observed result. The results showed that different metals gave a low and constant yield of triple coincidences, but that fused quartz, polystyrene, and teflon gave a much larger effect, of the correct order of magnitude. The largest yield was that from teflon, as expected from the value: found for $\tau_{2}$. The variation of the triple coincidence yield from teflon was measured as a function of temperature, and found to vary with temperature in the same way as the value of $\tau_{2}$, as expected from equation (6). These results provide a strong support for the mechanism postulated above.

\section{Note}

T. A. Pond has repeated the experiment referred to in ref. 9, and has now found a three-quantum effect of about the expected magnitude in teflon, polystyrene, and fused quartz. Pond detects the three-quantum annihilation in these substances by measuring the small defect in their two-quantum annihilation rate as compared with that of a metal. He has also demonstrated the suppression of the three-quantum effect in liquid benzene by the addition of a few percent of a free radical, in complete analogy with the action of NO in gases demonstrated by Deutsch. (T.A. Pond, Phys. Rev. 93 (1954) 478). 


\section{(V) M. DEUTSCH - Positronium}

The rate of annihilation of slow positrons in matter is given by the equation ${ }^{1}$

$$
1 / \tau=\lambda=C_{s} \psi_{s}^{2}+C_{t} \psi_{t}^{2} .
$$

Here $\psi_{s}^{2}$ is the density (i.e. square of the wave function) at the average position of the positron, of electrons with "antiparallel" spin direction, i.e. forming a singlet state and $\psi_{t}^{2}$ is the same quantity for triplet spin orientation. $C_{s}$ and $C_{t}$ are ${ }^{2}$ fundamental interaction rates in the singlet and triplet states respectively.

$$
\begin{aligned}
C_{s}=4 \pi c\left(\frac{e^{2}}{m c^{2}}\right)^{2}= & 3 \times 10^{-14} \mathrm{~cm}^{3} / \mathrm{sec} ; \\
& C_{t}=4 \pi c\left(\frac{e^{2}}{m c^{2}}\right)^{2} \frac{4 \alpha}{9 \pi}\left(\pi^{2}-9\right)=\frac{1}{1120} C_{s}=2.7 \times 10^{-17} \mathrm{~cm}^{3} / \mathrm{sec} .
\end{aligned}
$$

If one neglects all effects of the coulomb forces, it is obvious that $\psi_{s}^{2}=n_{e} / 4$, $\psi_{t}^{2}=3 n_{e} / 4$, where $n_{e}$ is the number of electrons per cubic centimeter of matter. If coulomb forces are considered but it is still assumed that the positrons move freely through the medium, these expressions remain valid, but $n_{e}$ is now an "effective" number of electrons. An explicit calculation of the $\psi$ 's is difficult ${ }^{3.4}$. The situation in solids is discussed elsewhere in this chapter by R. E. Bell. In the case of gases near atmospheric pressure, eq. 1 predicts decay rates of the order of $10^{7} \mathrm{sec}^{-1}$. In any case, for "free" collisions $\lambda$ should be proportional to the gas pressure and the term in eq. 1, giving rise to three-photon annihilation should be $1 / 370$ of the first term (cf. DeBenedetti elsewhere in this chapter).

In 1949 Shearer and Deutsch ${ }^{5,6}$ performed measurements of $\lambda$ in a variety of gases by means of the apparatus shown in Fig. 1. A source of $\mathrm{Na}^{22}$ is mounted on the face of gamma counter $\mathrm{A}$ which serves to detect the 1.3 MeV nuclear gamma ray emitted practically simultaneously with the positron. Positrons leaving the source stop in the gas or at the wall of the container, and the resulting annihilation gamma rays are registered

1 P.A.M. Dirac - Proc. Camb. Phil. Soc. 26 (1930) 361.

2 A. Ore and J. L. Powell, Phys. Rev. 75 (1949) 1696.

3 S. DeBenedetti, C. Cowan, W. R. Konneker and H. Primakoff, Phys. Rev. 11 (1950) 205.

4 I. Pomeranchuk, Dokl. Akad. Nauk. S.S.S.R. 60 (1948) 213.

5 J. W. Shearer and M. Deutsch, Phys. Rev. 76 (1949) 642.

6 M. Deutsch, Phys. Rev. 82 (1951) 455; ibid. 83866. 
by counter B. The distribution of time intervals between counts in $\mathrm{A}$ and in $\mathrm{B}$ is analyzed by means of a multi-channel delayed-coincidence circuit.

The experimental results showed that the decay is more complex than anticipated, in particular that the decay rate is not proportional to the gas pressure and contains several components. Fig. 2 illustrates the

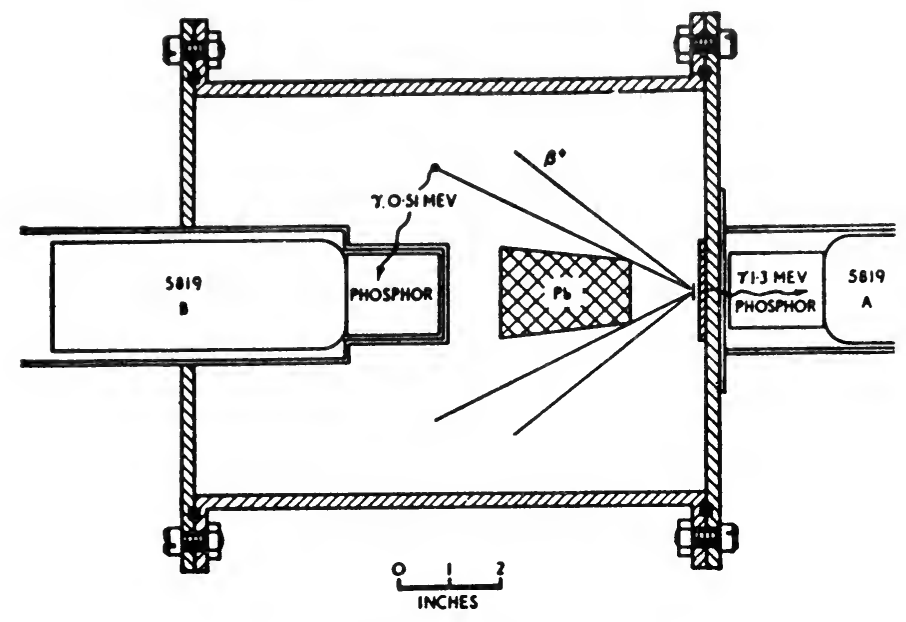

Fig. 1. Apparatus to measure the life-time of positrons in gases

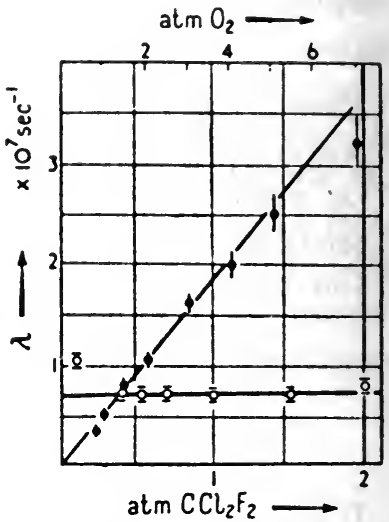

Fig. 2. Pressure dependence of the decay rates of positrons in two gases

dependence on pressure of the decay rate for positrons surviving beyond $0.1 \mu$ sec in two gases. We see that while $\mathrm{O}_{2}$ shows the behavior expected for "free" collisions, in freon at least some of the positrons must be annihilated in a bound system in which $\psi^{2}$ does not depend on the number of gas atoms present. Further experiments demonstrated that this system consists simply of a positron bound to a single, otherwise free electron.

The possibility of such a system was first suggested by Mohorovicic? and the name postronium was coined by Ruark ${ }^{8}$. Its theory was discussed by 'Wheeler ${ }^{9}$ and, in more detail by Pirenne ${ }^{10}$, Berestetzky ${ }^{11}$, and Ferrell ${ }^{12}$. The fine structure was evaluated to order $\alpha^{5}$ (including effects of the order of the Lamb shift) by Karplus and Klein ${ }^{13}$ and by Fulton and Martin ${ }^{14}$.

7 S. Mohorovićić, Astron. Nachr. 253 (1934) 94.

8 E. Ruark, Phys. Rev. 68 (1945) 278.

9 J. A. Wheeler, Ann. N. Y. Acad. Sci. 48 (1946) 219.

10 J. Pirenne, Arch. Sci. Phys. et Natur. 29 (1947) 121, 207.

11. V. B. Berestetzky, Zhurn. Eksp. Teor. Fiz. S.S.S.R. 19 (1949) 673, 1139.

12 R. A. Ferrell, Phys. Rev. 84 (1951) 858; Also Thesis, Princeton, 1951.

13 R. Karplus and A. Klein, Phys. Rev. 87 (1952) 848.

14 T. Fulton and P. C. Martin, Phys. Rev. 93 (1954) 903. 
The gross structure of positronium states is exactly like that of hydrogen except for the effect of the different reduced mass of the electron, which reduces all energies by a factor 2 compared with a hydrogenic atom with an infinitely heavy nucleus. Thus the energy of the $n$ 'th state is

$$
E_{n}=-e^{4} m_{0} / 4 \hbar^{2} n^{2}
$$

the ionization potential is 6.8 volt, the energy of the first exited state is $5.1 \mathrm{eV}$, the Lyman $\alpha$-line has a wavelength of about $2400 \AA$ etc. The electron wave functions in the center-of-mass system are, in this approximation, the same as for hydrogen. The life-times of excited states against optical de-excitation are twice as great as those of corresponding hydrogen states.

The $1 S$ ground state is split into a lower singlet (para) and higher triplet (ortho) level by the magnetic interaction and by radiative terms. The splitting is ${ }^{13}$

$\Delta W=\left(\mu_{0}^{2} / a^{3}\right)\left[\frac{7}{3}-\left(\frac{32}{9}+2 \ln 2\right) \frac{\alpha}{\pi}\right]=2.0337 \times 10^{5} \mathrm{Mc} / \mathrm{sec}=8.3462 \times 10^{-4} \mathrm{eV}$

Radiative transitions between these states are very slow since they are of magnetic dipole character. We see (cf. eq. 1) that, for the singlet state $\psi_{t}^{2}=0, \psi_{s}^{2}=1 / \pi a^{3}$ where $a=2 \times \hbar^{2} / m e^{2}$. This yields a decay rate (eq. 1) of $\lambda_{s}=0.804 \times 10^{10} \mathrm{sec}^{-1}$, entirely by two-quantum annihilation. For the triplet state $\psi_{s}^{2}=0, \psi_{t}^{2}=1 / \pi a^{3}$ and $\lambda_{t}=7.2 \times 10^{6} \mathrm{sec}^{-1}$, entirely by threequantum annihilation, yielding a continuous gamma ray spectrum. The result shown in Fig. 2 for freon, which is fairly representative of most gases may be understood by assuming that positronium is formed in a time short compared with $10^{-7} \mathrm{sec}$. One quarter of these atoms are in the singlet state and decay within about $10^{-10}$ sec. The remaining three quarters show the characteristic decay rate of the triplet state. In $\mathrm{O}_{2}$ the triplet is rapidly destroyed in gas collisions.

The fact that the long period is accompanied by a continuous gammaray spectrum characteristic of three-quantum annihilation was first demonstrated by Deutsch ${ }^{6}$ by means of a scintillation spectrometer. DeBenedetti and Siegel ${ }^{15}$ and Wheatley and Halliday ${ }^{16}$ demonstrated the triple coincidences and Pond ${ }^{17}$ showed that the number of collinear $510 \mathrm{keV}$ quanta in double coincidence is reduced when positronium is formed. Lewis and Ferguson ${ }^{18}$ measured the annihilation gamma ray

S. DeBenedetti and R. Siegel, Phys. Rev. 85 (1952) 371; ibid. 87, 335.

J. Wheatley and D. Halliday, Phys: Rev. 87 (1952) 235; ibid. 88, 424.

T. A. Pond, Phys. Rev. 85 (1952) 489.

G. M. Lewis and A. V. Ferguson, Phil. Mag. 44 (1953) 1011. 
spectrum of those positrons which survived after $10^{-7}$ sec in freon by means of a delayed coincidence scintillation spectrometer and found good agreement with the theoretical spectrum ${ }^{2}$ within the rather large experi-

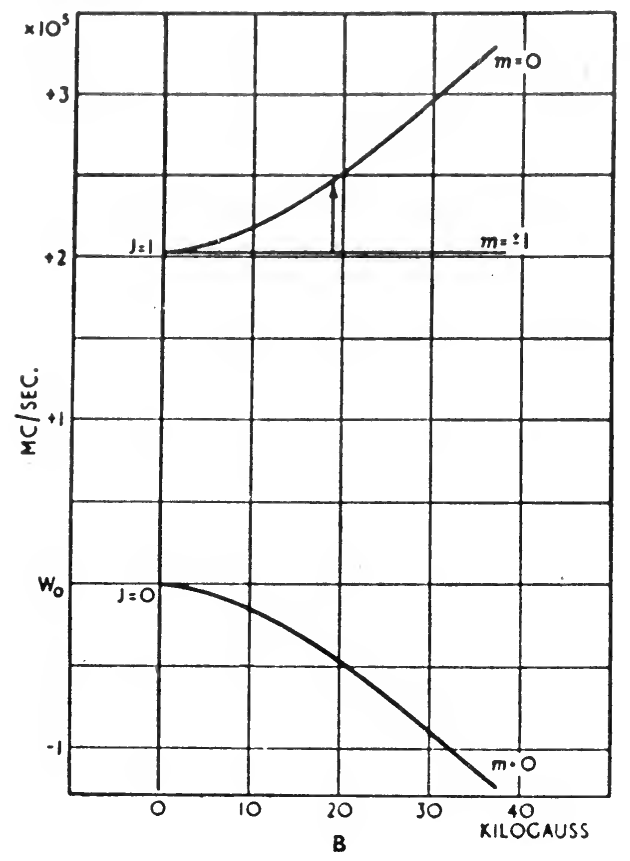

Fig. 3. Zeeman splitting of the ground state of positronium mental uncertainty.

The states of positronium show no first-order (linear) Zeeman effect. There is, however, a secondorder effect, due to the mixing of singlet and triplet components in a magnetic field. In particular, the situation for the ground state is illustrated in Fig. 3. The two substates with $m=0$ are displaced by a field $B$ with respect to the unperturbed position by an amount

$$
h v= \pm \frac{\Delta W}{2}\left[\left(1+x^{2}\right)^{1 / 2}-1\right]
$$

where $x=4 \mu_{e} B / W, \mu_{e}$ being the magnetic moment of the electron. At the same time, the $m=0$ triplet state contains an admixture of singlet to the extent

$$
\begin{aligned}
& \frac{a^{2}}{1+a^{2}}, \text { with } \\
& \qquad a=\frac{1}{x}\left[\left(1+x^{2}\right)^{1 / 2}-1\right] \simeq \frac{2 \mu_{e} B}{\Delta W} .
\end{aligned}
$$

Due to this, admixture, the state can now decay by two-photon annihilation, the applied magnetic field absorbing the unbalanced angular momentum. The relative probability of this induced two-photon decay is given by $a^{2} /\left(\lambda_{t} / \lambda_{s}+a^{2}\right)$ where $\lambda_{t}$ and $\lambda_{s}$ are the annihilation rates of the unperturbed ortho- änd para-states. One should therefore observe a partial quenching of the three-photon process by the magnetic field. If all three photons are observed in the plane perpendicular to the magnetic field ${ }^{\mathbf{1 6}}$, just half of the triple coincidences are due to the $m=0$ state. If one observes the quenching by the increase in two-quantum annihilation ${ }^{19}$ one-third of the ortho-positronium is affected and very nearly the same is true if the overall spectrum is observed at right angles to the field ${ }^{20}$.

Let $f_{0}$ be the contribution of the $m=0$ state in the particular detection scheme chosen. Then the fraction of the observed three-photon process

19 T. A. Pond and R. H. Dicke, Phys. Rev. 85 (1952) 489.

20 M. Deutsch and E. Dulit, Phys. Rev. 84 (1951) 601. 
remaining in the presence of the field is

$$
\phi(B)=\frac{\lambda_{t} / \lambda_{3}+a^{2}\left(1-f_{0}\right)}{i a^{2}+\lambda_{t} / \lambda_{8}} .
$$

In a very strong field $a^{2} \gg \lambda_{t} / \lambda_{s}$ we have $\phi(B) \rightarrow\left(1-f_{0}\right)$.

The magnetic quenching was first observed by Deutsch and Dulit ${ }^{20}$ who assumed $\lambda_{t} / \lambda_{s}=1 / 1120$ and interpreted the result, illustrated in Fig. 4 as a rough measurement of the fine structure splitting $\Delta W$ of the ground state. The result was confirmed by Pond and Dicke ${ }^{19}$. Wheatley and Halliday ${ }^{16}$

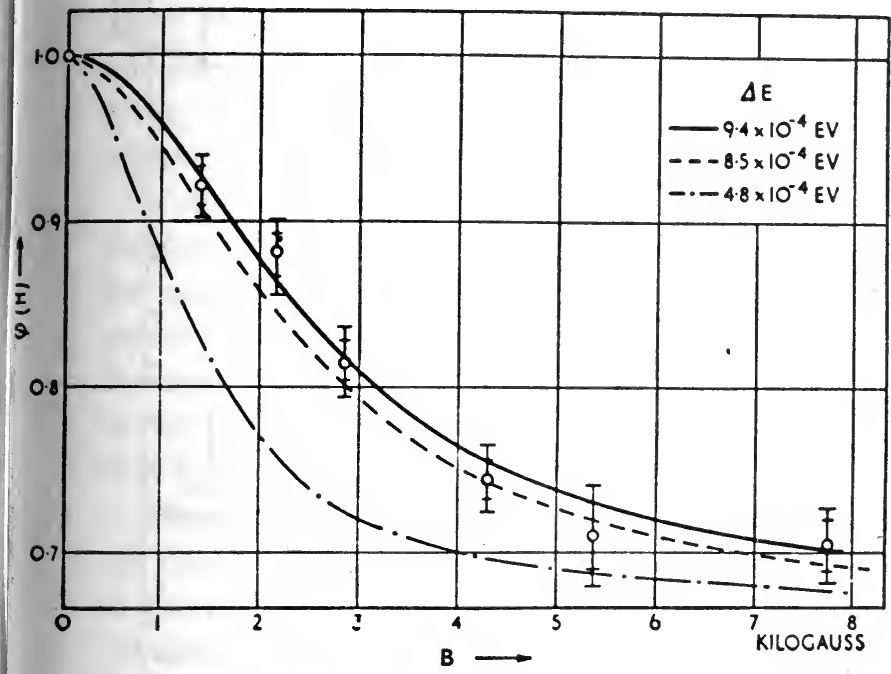

Fig. 4. Quenching of three-photon annihilation by a magnetic field

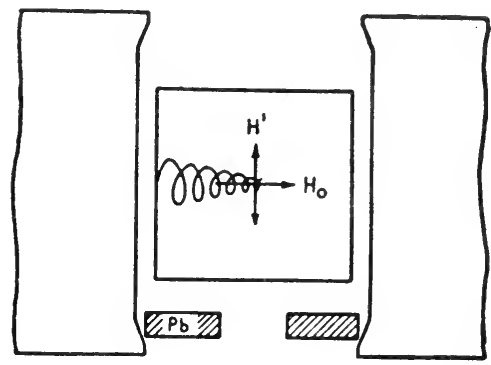

บ110
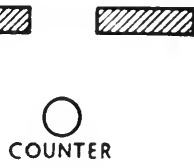

Fig. 5.

Schematic arrangement for observing the Zeeman effect of positronium

applied a careful analysis to similar data, including a correction for the effect of collisions in the gas. Using the value of $\Delta W$ measured by Deutsch and Brown ${ }^{21}$, they interpret their results as a measurement of $\lambda_{t} / \lambda_{s}=$ $=1 /(1050 \pm 140)$, in good agreement with the theoretical value. Deutsch and Brown ${ }^{21}$ measured the Zeeman splitting (eq. 2) by inducing radio frequency transitions from the $m= \pm 1$ states to the $m=0$ state (cf. Fig. 3). The transition is detected by a reduction of the relative intensity of the continuous three-quantum spectrum since the $m=0$ state decays almost entirely by two-quantum emission in the presence of a field of about 10,000 gauss. The experimental arrangement is shown schematically in Fig. 5. The source is located at one end of a resonant cavity excited by a magnetron oscillator in a mode in which the radiofrequency magnetic field is perpendicular to the steady field. Careful lead collimation assures

21 M. Deutsch and S. C. Brown, Phys. Rev. 85 (1952) 1047. 
that only annihilation quanta originating in the gas are registered in the scintillation spectrometer. In Fig. 6 we show a typical curve of the intensity of the continuous radiation vs. magnetic field for constant radiofrequency. This curve has recently been obtained by R. Weinstein at M.I.T. The ordinate represents readings on a proton resonance gauss meter and the full width of the graph is about $1.5 \%$. The ground state splitting deduced from

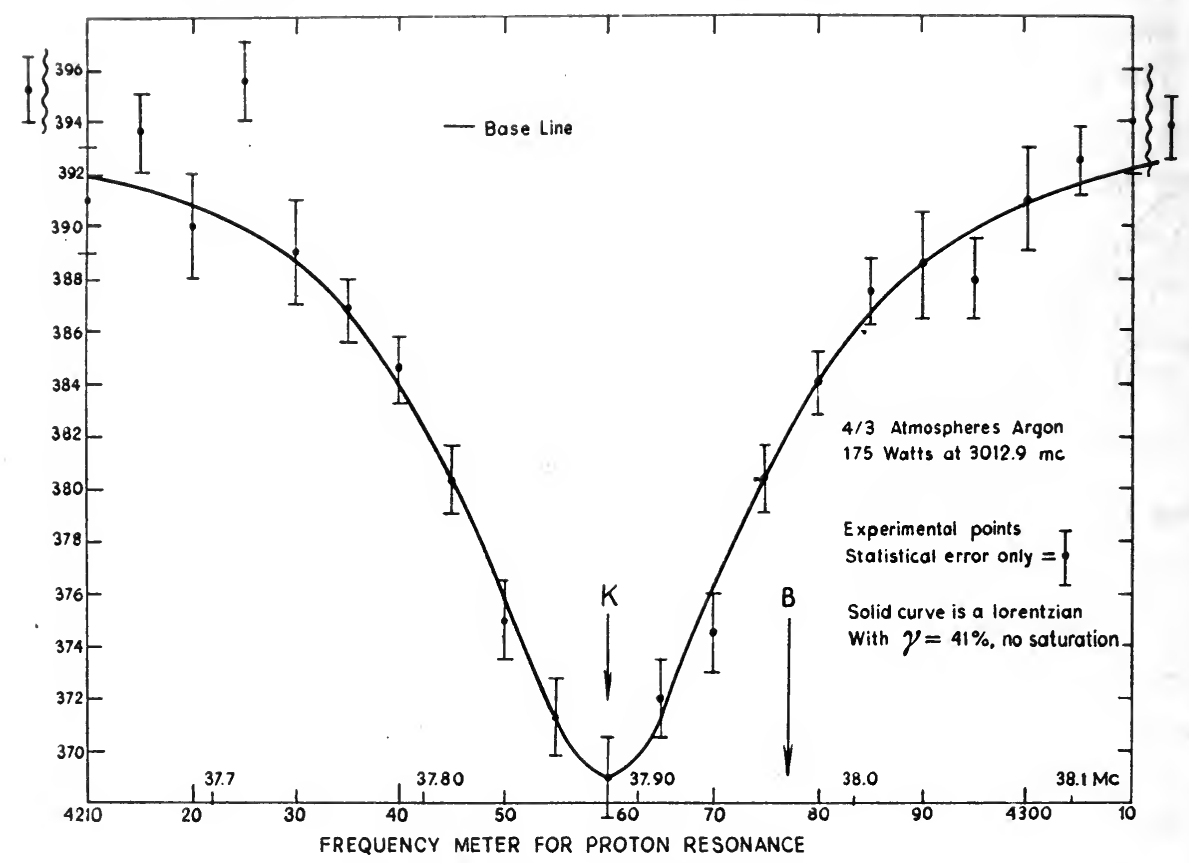

Fig. 6. Quenching of three-quantum annihilation due to Zeeman transition. Arrow marked $\mathrm{K}$ indicates position of the line from eqs. 2 and 3. B marks the line position expected without radiative corrections

this and many similar measurements through eq. 3 is $\Delta W=2.0335 \pm$ $0.0004 \times 10^{5} \mathrm{Mc} / \mathrm{sec}$, in excellent agreement with the theoretical value of 2.0337. For comparison we indicate by an arrow marked $B$ the location of the line expected in the absence of radiative corrections to $\Delta W$.

Several experiments have been performed to study the production and quenching of ortho-positronium in various gases ${ }^{6,15,17}$.

There is general agreement that the fraction of positrons which form positronium is the same to about $25 \%$ in most gases, notably $\mathrm{He}, \mathrm{A}, \mathrm{H}_{2}$, $\mathrm{N}_{2}, \mathrm{CO}_{2}, \mathrm{CF}_{2} \mathrm{Cl}_{2}$, and $\mathrm{SF}_{6}$. The relative yields in the several gases reported by different investigators differ somewhat probably due to effects of small impurities. Pond ${ }^{17}$ estimates that about one-third of all positrons form positronium. 
This estimate is supported by an observation of Deutsch and Brown ${ }^{21}$ It was found that in argon the application of a strong radio-frequency electric field more than doubled the amount of positronium formed. Recent work of Dulit and Deutsch (unpublished) showed the same effect in the case of a static electric field. The phenomenon can be understood as follows. Positrons which fail to form positronium before their energy is reduced below $\left(V_{i}-6.8\right) \mathrm{eV}$-about $9 \mathrm{eV}$ in argon-will normally be annihilated in collisions. In argon these positrons can, however, readily regain sufficient energy from an applied electric field since the first excited state of the gas atoms is at $11.6 \mathrm{eV}$, too high to interfere by inelastic collisions. The addition of a small amount of a polyatomic gas with lowlying excited states prevents this energy gain and suppresses the enhancing effect of the electric field on positronium formation.

With increasing electric field the enhancement rises until the time for acceleration to the required energy is short compared with the mean time for annihilation by collision. In fields of this magnitude virtually all positrons should form positronium and no further enhancement is possible. Preliminary results of Dulit and Deutsch indicate that this saturation occurs for static fields of about $500 \mathrm{~V} / \mathrm{cm}$ in argon of commercial purity at atmospheric pressure.

The stability of ortho-positronium in various gases has been investigated by observing the relative amount of three-quantum annihilation ${ }^{15.18}$; or the decay rate ${ }^{6}$ as a function of gas pressure. Published results show that in $\mathrm{CF}_{2} \mathrm{Cl}_{2}$ and $\mathrm{SF}_{6}$ the effective molecular cross section for annihilation in a collision is of the order of $10^{-21} \mathrm{~cm}^{2}$. Similar great stability is indicated in other gases for which positronium formation has been investigated. For $\mathrm{CO}, \mathrm{N}_{2} \mathrm{O}, \mathrm{SO}_{2}, \mathrm{H}_{2} \mathrm{O}, \mathrm{CH}_{3} \mathrm{OH}, \mathrm{CH}_{3} \mathrm{I}, \mathrm{CHCl}_{3}$ and $\mathrm{CCl}_{4}$ only an upper limit of $10^{-19} \mathrm{~cm}^{2}$ has been set for the quenching cross section. In $\mathrm{O}_{2}$ a rather high value of $4 \times 10^{-19} \mathrm{~cm}^{2}$ is found. As a consequence ortho-positronium is largely quenched in partial oxygen pressures above about $1 / 2 \mathrm{~atm}$.

The quenching cross section of the NO molecule-presumably due to ortho-paraconversion by spin exchange-is estimated to be about $10^{-16} \mathrm{~cm}^{2}$ from the fact that a partial pressure of $0.3 \mathrm{~mm}$ reduces the intensity of three-quantum annihilation by a factor of two. Similar high efficiency for quenching ortho-positronium is found for $\mathrm{NO}_{2}$ and for the halogens $\mathrm{Cl}_{2}, \mathrm{Br}_{2}$ and probably $\mathrm{I}_{2}$. The halogens do not have a free electron spin so that a different mechanism must be. responsible, possibly the formation of positronium compounds. Estimates of the energy balance for the reaction $\mathrm{Cl}_{2}+\mathrm{e}^{+} \mathrm{e}^{-} \rightarrow \mathrm{Cl}+\mathrm{Cl}^{-} \mathrm{e}^{+}$indicate that it probably cannot take place at thermal energies. More accurate calculations and further experiments are required to clarify the quenching mechanism. 


\title{
ILLUSTRATIVE DISINTEGRATION STUDIES
}

\author{
R. E.BELL, D. E. ALBURGER, A.C.G. MITCHELL, P. PREISWERK
}

\section{(I) R. E. BELL - Disintegration of $\mathbf{I}^{131}$}

The 8.05-day beta-ray emitter $\mathrm{I}^{131}$ was discovered in 1938 by Livingood and Seaborg ${ }^{1}$. The element iodine plays an important part in animal metabolism, and the convenient half life and radiation energies of $\mathbf{I}^{\mathbf{1 3 1}}$ have made it the most frequently used iodine radioisotope in medical research and practice. $I^{131}$ is produced commercially in iarge quantities in nuclear reactors either by fission of uranium or by irradiation of tellurium with neutrons using the following reactions:

$$
\begin{aligned}
& \mathrm{Te}^{130}(n, \gamma) \mathrm{Te}^{131} \\
& \mathrm{Te}^{131} \underset{25 \mathrm{~min}}{\stackrel{\beta_{-}}{\longrightarrow}} \mathrm{I}^{131}
\end{aligned}
$$

The $\mathrm{I}^{131}$ produced in either way is separated carrier-free from the target material.

Because of the wide applications of $\mathrm{I}^{131}$, its radiations have perhaps been studied more frequently than those of any other radioactivity. Nevertheless the disintegration of $\mathbf{I}^{131}$ is sufficiently complex so that there have been frequent disagreements among different experimenters about the exact details of the disintegration scheme, and it is only recently that reasonable agreement has been secured. In the present review, only a few of the many papers on $I^{131}$ can be referred to. Slight inconsistencies will be noticed in the quoted energies of some of the radiations, representing the differences found by different observers.

The first systematic study of the disintegration of $I^{131}$ was that of Metzger and Deutsch ${ }^{2}$ in 1948, following earlier work by Downing, Deutsch, and Roberts ${ }^{3}$. These workers used a lens beta-ray spectrometer, and studied both the beta spectrum of $I^{131}$ and the secondary electron spectrum ejected from a lead radiator by the gamma rays following the beta decay. By Fermi plot analysis of the beta-ray spectrum they found partial beta-ray spectra of end-point energies $600 \mathrm{keV}$ (intensity $85 \%$ ) and $315 \mathrm{keV}$ (intensity $15 \%$ ). From internal conversion lines in the beta

J. J. Livingood and G. T. Seaborg, Phys. Rev. 54 (1938) 775.

F. Metzger and M. Deutsch, Phys. Rev. 74 (1948) 1640.

3 I. R. Downing, M. Deutsch and A. Roberts, Phys. Rev. 61 (1942) 686. 
spectrum and from the secondary electron spectrum they found gamma rays of $80 \mathrm{keV}(\sim 6 \%), 283 \mathrm{keV}(6 \%), 363 \mathrm{keV}(79 \%)$ and $638 \mathrm{keV}$ $(15 \%)$. These results, together with coincidence measurements, led Metzger and Deutsch to propose the disintegration scheme of Fig. 1. It is a tribute to these workers that their scheme differs from the presently

$$
{ }_{53} \mathrm{I}^{131}
$$

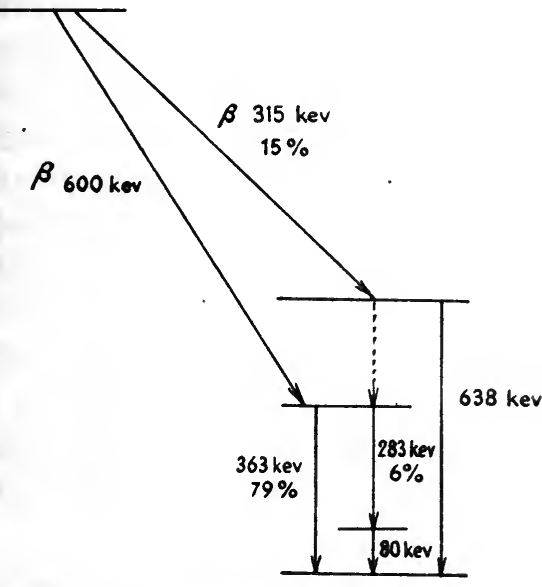

Fig. 1. Disintegration scheme of $I^{131}$ proposed by Metzger and Deutsch (ref. 2). Except for a fow weak radiations not detected by Metzger and Deutsch, this scheme agrees with the currently accepted one (Fig. 5). The gamma transition indicated by a broken line was not definitely detected by Metzger and Deutsch, and has not been found by subsequent observers

accepted disintegration scheme only by slight changes in the energies and intensities of the radiations, and by the omission of a few weak radiations now known to be present.

Almost simultaneously with Metzger and Deutsch, Owen, Moe, and Cook ${ }^{4}$ published a different disintegration scheme which, although consistent with the radiation energies known at the time, was not capable of explaining the intensities of those radiations. These workers, however, were the first to observe the weak $K$ and $L$ internal conversion lines in the beta spectrum corresponding to a gamma transition of energy $163 \mathrm{keV}$. Accurate measurements of the energies of three of the gamma rays emitted by sources of $\mathrm{I}^{131}$ were made by Lind, Brown, Klein, Muller, and DuMond ${ }^{5}$, using the crystal diffraction method, in 1949. The disintegration scheme of Metzger and Deutsch (Fig. 1) had proposed that the 80 and $283 \mathrm{keV}$ gamma rays formed a cascade pair in parallel with the $363 \mathrm{keV}$ gamma ray. This proposal received strong support from the results of Lind et al., who gave the energies of the three gamma rays as 80.133, 284.13 , and $364.18 \mathrm{keV}$ respectively; the sum of the first two energies is equal to the third within 1 part in 4500 .

4 G. E. Owen, D. Moe and C. S. Cook, Phys. Rev. 74 (1948) 1879; 75 (1949) 1270.

5 D. A. Lind, J. Brown, D. Klein, D. Muller and J. DuMond, Phys. Rev. 75 (1949) 1544. 
Doubt was thrown on the analysis of Metzger and Deutsch in 1949 by the findings of Kern, Mitchell, and Zaffarano ${ }^{6}$. These workers made a Fermi analysis which showed an inner beta-ray group of endpoint energy $250 \mathrm{keV}$ instead of the $315 \mathrm{keV}$ of Metzger and Deutsch. They therefore suggested a disintegration scheme in which the $638 \mathrm{keV}$ gamma ray followed a $250 \mathrm{keV}$ beta spectrum, and led to the $80 \mathrm{keV}$ excited state of $\mathrm{Xe}^{131}$ rather than to the ground state (see Fig. 1). The discrepancy was not resolved by the work of Feister ${ }^{7}$, whose Fermi analysis lay between the two results already mentioned. The two disintegration schemes can be differentiated by the fact that in Kern et al.'s scheme the $638 \mathrm{keV}$ and $80 \mathrm{keV}$ gamma rays are in cascade, whereas in Metzger and Deutsch's scheme they are not. In an attempt to use this fact to resolve the difficulty, P. R. Bell, Cassidy, and Kelley ${ }^{8}$ made coincidence measurements with two NaI scintillation counters which apparently showed coincidences between the 638 and $80 \mathrm{keV}$ gamma rays, thus supporting Kern et al.'s scheme. On the other hand, on the Kern scheme the total intensity of the $80 \mathrm{keV}$ gamma transition would have to be equal to the sum of the intensities of the 284 and $638 \mathrm{keV}$ gamma transitions. Careful absorption measurements by Emery ${ }^{9}$ at about this time showed that the intensity of the $80 \mathrm{keV}$ transition favored the Metzger-Deutsch scheme.

In the meantime Zeldes, Brosi, and Ketelle ${ }^{10}$ and Cork, Rutledge, Stoddard, Branyan, and Childs ${ }^{11}$ had discovered a weak gamma ray of energy about $720 \mathrm{keV}$. Since this gamma ray differed by about $80 \mathrm{keV}$ in energy from the $638 \mathrm{keV}$ gamma ray, the former authors suggested that it was a crossover transition in parallel with the supposed $638-80 \mathrm{keV}$ cascade apparently found by $\mathrm{P}$. R. Bell et al., thus supporting the Kern scheme. However, Thulin ${ }^{12}$ immediately showed that the difference in energy between the 638 and $720 \mathrm{keV}$ gamma rays was about $86 \mathrm{keV}$ and could not be equal to $80 \mathrm{keV}$, so that the crossover suggestion of Zeldes et al. was incorrect. In spite of this finding, Thulin's other results favored the Kern scheme. Measurements by Verster, Nijgh, Van Lieshout, and Bakker ${ }^{13}$ in 1951 supported a scheme similar to that of Metzger and

- B. D. Kern, A. C. G. Mitchell and D. J. Zaffarano, Phys. Rev. 76 (1949) 94.

7 I. Feister, Phys. Rev. 78 (1950) 179.

8 P. R. Bell, J. M. Cassidy and G. G. Kelley, Phys. Rev. 82 (1951) 103.

- E. W. Emery, Phys. Rev. 83 (1951) 679.

10 H. Zeldes, A. R. Brosi and B. H. Ketelle, Phys. Rev. 81 (1951) 642.

11 J. M. Cork, W. C. Rutledge, A. E. Stoddard, C. E. Branyan and W. J. Childs, Phys. Rev. 81 (1951) 482.

12 S. Thulin, Phys. Rev. 83 (1951) 860.

13 N. F. Verster, G. J. Nijgh, R. Van Lieshout and C. J. Bakker, Physica 17 (1951) $637 ; 17$ (1951) 658. 
Deutsch, but were unable to account for the $720 \mathrm{keV}$ gamma ray. The evidence now seemed to be about equally divided between the two schemes.

In the meantime, work had been going forward on a small additional feature of the $\mathrm{I}^{131}$ disintegration. In 1949 Brosi, De Witt, and Zeldes ${ }^{14}$ found that a 12 day Xenon activity grows from $\mathrm{I}^{131}$, the yield being something less than $1 \%$. This $\mathrm{Xe}$ activity was definitely assigned to $\mathrm{Xe}^{131}$ by the use of a mass separator by Bergström ${ }^{15}$, who also showed that the radiations of the 12 day $\mathrm{Xe}^{131}$ were just the $K, L$, and $M$ conversion lines of the $163 \mathrm{keV}$ gamma transition already observed in some cases in the beta spectrum of $\mathrm{I}^{131}$. The place of the $163 \mathrm{keV}$ excited level of $\mathrm{Xe}^{131}$ in the disintegration scheme of $\mathbf{I}^{131}$ remained in doubt until Zeldes, Brosi, and Ketelle ${ }^{10}$ showed that there was a weak high-energy beta group in the $\mathrm{I}^{\mathbf{1 3 1}}$ beta spectrum whose energy $(812 \mathrm{keV})$ and intensity $(\sim 0.7 \%)$ were correct to lead to the formation of the $163 \mathrm{keV} \mathrm{Xe}{ }^{131}$ isomeric state. This part of the $\mathrm{I}^{131}$ disintegration scheme is now considered settled.

The uncertainty about the main part of the disintegration scheme stemmed from the fact that the energies and intensities of the partial beta

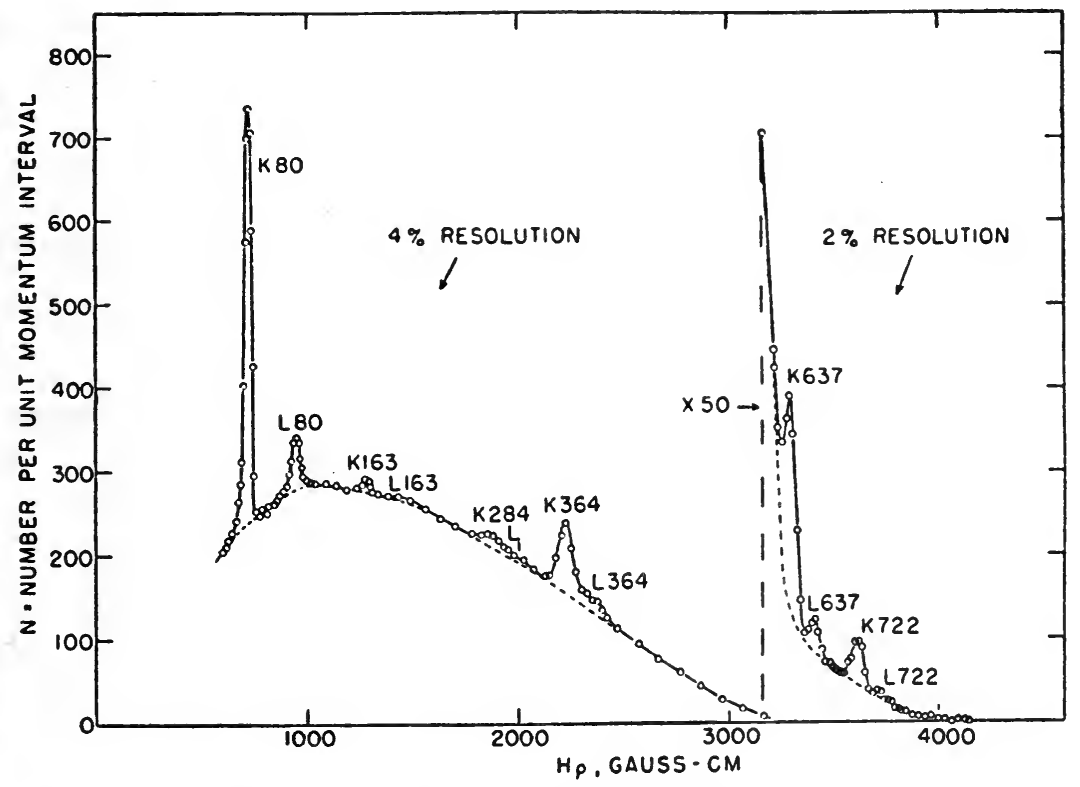

Fig. 2. Beta-ray spectrum of $I^{131}$ recorded by Bell and Graham (ref. 16). The statistical standard deviations of the points are everywhere smaller than the radii of the circles

14 A. R. Brosi, T. W. De Witt and H. Zeldes, Phys. Rev. 75 (1949) 1615.

15 I. Bergström, Phys, Rev. 80 (1950) 114. 
spectra of $\mathrm{I}^{131}$ were determined only by Fermi analysis of the observed spectrum shape. Such analyses are critically dependent on the excellence of the beta-ray source and on the properties of the beta-ray spectrometer at low energies; they also rest on the assumption that all the partial spectra present in appreciable intensity have the allowed shape. Only detailed measurements of the partial beta spectra in coincidence with each gamma ray in turn could resolve the difficulty.

With this aim in mind, R. E. Bell and Graham ${ }^{16}$ re-examined the whole of the $I^{131}$ disintegration process. They used a pair of lens beta-ray spectrometers placed end to end, with a single source of $I^{131}$ on a thin film at the center. The various radiations of $\mathbf{I}^{131}$ could then be focused independently in the two spectrometers, whose stilbene scintillation counters were connected to a coincidence circuit of resolving time. $2 \tau_{0}=2 \times 10^{-9}$ seconds (see Chapter XVIII). The beta-ray spectrum recorded by Bell and Graham in one of their spectrometers is shown in Fig. 2. The intensities of the various conversion lines were measured as a fraction of the area under the total beta spectrum in the usual way. A Fermi analysis of the beta continuum is shown in Fig. 3, where partial beta spectra of energies

Fig. 3. Fermi analysis of the $I^{131}$ beta spectrum of Fig. 2. For the part below $570 \mathrm{keV}$ the conversion lines were omitted as indicated by broken lines in Fig. 2. Where the statistical standard deviations of the points are greater than the radii of the circles, they are shown as vertical bars. Since the errors of the points of the subcomponents ending at 335 and $250 \mathrm{keV}$ are probably chiefly systematic, no attempt has been made to indicate standard deviations for them. (After Bell and Graham, ref. 16)

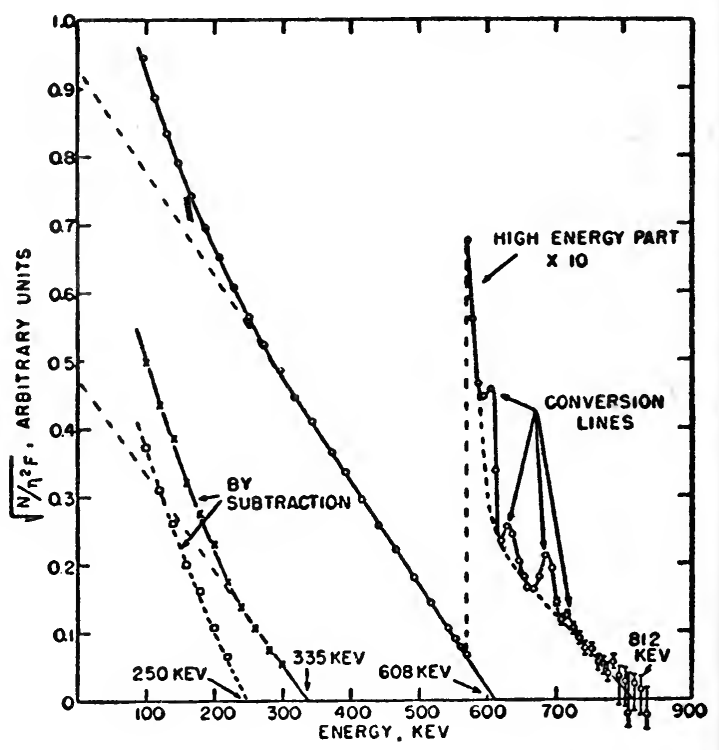

$812 \mathrm{keV}$ (intensity $0.7 \%$ ), $608 \mathrm{keV}(80.5 \%$ ), $335 \mathrm{keV}(9.4 \%)$, and $250 \mathrm{keV}(9.4 \%)$ are apparently present. From this analysis alone it is difficult to be sure that the $250 \mathrm{keV}$ component artually exists, and in 
any case its intensity has almost certainly been exaggerated by source thickness effects.

Bell, Graham, and Petch ${ }^{17}$ had already shown by coincidence measurements on focused conversion lines that the 80 and $284 \mathrm{keV}$ gamma rays were in cascade, verifying the strong presumption to that effect made by Lind et al. ${ }^{5}$. They also showed that the $80 \mathrm{keV}$ gamma ray follows nuclear beta rays with a half-life of $4.8 \times 10^{-10}$ seconds, whereas the $364 \mathrm{keV}$ gamma ray is prompt within $10^{-10}$ seconds. These results prove that the $80 \mathrm{keV}$ gamma ray follows the $284 \mathrm{keV}$ gamma ray, and that the arrangement of these three gamma rays is exactly that of Fig. 1.

Using the pair of spectrometers, Bell and Graham next focused one spectrometer on the $K$ conversion peak of the $80 \mathrm{keV}$ gamma ray, and used the other spectrometer to scan the beta spectrum in coincidence with this peak. The result is shown in Fig. 4 in the form of a Fermi plot of the beta spectrum in coincidence with the $80 \mathrm{keV}$ gamma ray. The coincidence spectrum consists only of the $608 \mathrm{keV}$ beta-ray component, with a slight rise at low energies attributable to source thickness effects. The expected shape of this coincidence spectrum on the scheme of Kern, Mitchell, and Zaffarano ${ }^{6}$ is shown in Fig. 4 as a broken line rising sharply

Fig. 4. Coincidence Fermi plots for $I^{131}$. Upper curve, spectrum of coincidences between betarays and $80 \mathrm{keV}$ gamma-rays; the broken line shows what would be expected on the disintegration scheme of Kern et al. (ref. 6). Middle curve, spectrum of coincidences between betarays and $637 \mathrm{keV}$ gamma-rays. Lower curve, spectrum of coincidences between beta-rays, and an equal mixture of 637 and $722 \mathrm{keV}$ gamma-rays. The standard deviations of the points are shown by vertical bars. (After

Bell and Graham, ref. 16)

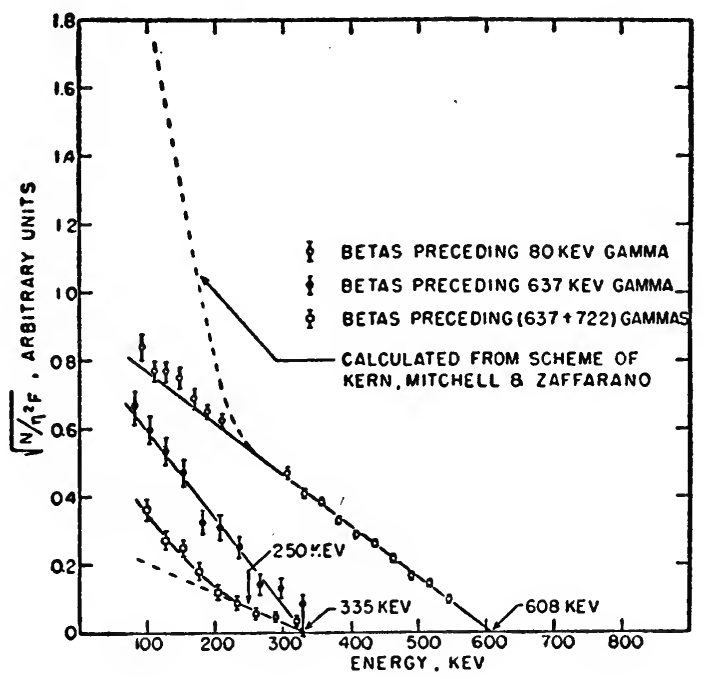

at low energies, because on this scheme every beta particle of the low energy spectrum is followed by an $80 \mathrm{keV}$ gamma transition. The coincidence spectrum of Fig. 4 thus strongly contradicts the scheme of Kern et al. Bell and Graham next placed a thorium secondary electron radiator 
behind their $\mathrm{I}^{131}$ source, so that one spectrometer could observe photoelectron lines from the 637 and $722 \mathrm{keV}$ gamma rays. The $L$ photoelectron peak from the $637 \mathrm{keV}$ radiation was not resolved from the $K$ photoelectron peak of the $722 \mathrm{keV}$ radiation, so that this spectrometer could observe effects of the $637 \mathrm{keV}$ gamma ray alone, or of the $637+722 \mathrm{keV}$ gamma rays mixed about equally. Spectra of the beta rays in coincidence with these radiations are also shown in Fig. 4 in the form of Fermi plots. The beta rays preceding the $637 \mathrm{keV}$ gamma ray form a spectrum of end point $335 \mathrm{keV}$, and those preceding the $722 \mathrm{keV}$ gamma ray form a spectrum of end point wivout $250 \mathrm{keV}$. Similar results have been secured by Ketelle, Zeldes, Brosi, and Dandl ${ }^{18}$, using a single magnetic spectrometer in coincidence with a $\mathrm{NaI}$ scintillation spectrometer, and by Cavanagh ${ }^{18}$, using organic scintillators with coincidence absorption techniques. In addition, the gamma-gamma coincidence experiment of P. R. Bell et al. ${ }^{8}$ has been repeated by Johansson and Almquist ${ }^{19}$, with results now agreeing with those reported here.

These results lead unambiguously to the disintegration scheme of Fig. 5, in which are shown four partial beta-ray spectra of energies 812 ,

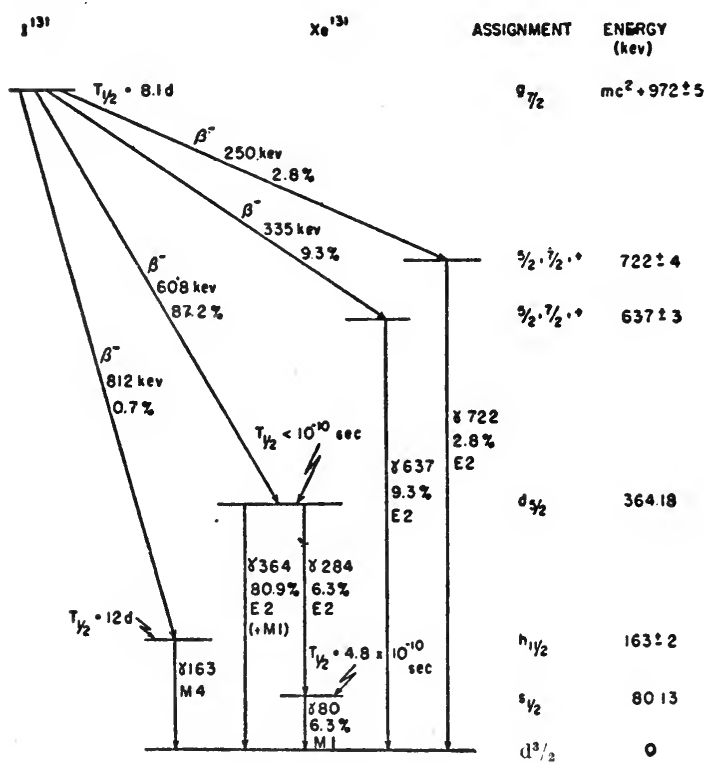

Fig. 5. Disintegration scheme of $\mathrm{I}^{131}$. The detailed properties of the beta-components and gamma-rays are explained in the text and listed in Tables I and II. The orbital assignments are made from experimental results and are consistent with the nuclear shell model. Most of the published observations on $I^{131}$ can be reconciled with this scheme. (After Bell and Graham, ref. 16)

608,335 , and $250 \mathrm{keV}$. (A scheme of this kind had been tentatively suggested earlier by Emery ${ }^{9}$, on the grounds that it would account for

18 B. H. Ketelle, H. Zeldes, A. R. Brosi and R. A. Dandl, Phys. Rev. 84 (1951) 585; P. E. Cavanagh, Phil. Mag. 43 (1952) 221.

19 S. A. E. Johansson and S. Almquist, Ark. f. Fys. 5 (1952) 427. 
most of the experimental evidence known at that time.) Most of the Fermi analyses previous to those of Bell and Graham had detected some average of the 250 and $335 \mathrm{keV}$ spectra, and thus yielded end points lying between these two limits.

Having identified all the beta components with their corresponding gamma rays, Bell and Graham assigned intensities to the beta components by measuring the gamma-ray intensities. This procedure is justifiable only if the identification has been made; otherwise the measured betaand gamma-ray intensities (as well as energies) must be compared as a means of identification. This reversal of the customary procedure permitted the assignment of intensities to the beta components by gamma-ray measurements alone, so that the beta-ray intensities were measured independent of source thickness effects and spectrum shapes. The gammaray intensities were measured on a relative basis by observing photoelectron peaks ejected from a thin thorium radiator by the $284,364,637$, and $722 \mathrm{keV}$ gamma rays. Use was then made of the fact that, by Fig. 5, exactly one of these gamma rays follows each beta ray (except those in the weak $812 \mathrm{keV}$ beta spectrum) to assign absolute intensities to these four gamma rays. The intensity of the $80 \mathrm{keV}$ gamma ray was measured in the following way. In their examination of the total beta spectrum of $I^{131}$, Bell and Graham had measured the intensities of all the conversion peaks present. The sum of the $K$ conversion peak intensities, multiplied by the $\mathrm{X}$-ray fluorescent yield, is just the intensity of $K \mathrm{X}$-rays emitted by the $I^{131}$ source, and is known. The quantum intensity of the $80 \mathrm{keV}$ gamma ray was measured by comparison with the known intensity of the $K \mathrm{X}$-rays in a NaI scintillation spectrometer. The thickness of the NaI crystal used, $1.0 \mathrm{~cm}$, was great enough so that the counting efficiencies both for the $80 \mathrm{keV}$ gamma rays and for the $30 \mathrm{keV} K \mathrm{X}$-rays were nearly $100 \%$.

A summary of all the measurements on the gamma rays is given in Table I. In this table, energies are based on Lind et al.'s measurements, and intensities are given in percent of total disintegrations. The total intensity of a gamma transition, $I_{\text {total }}$ (col. 2), is the sum of the intensities of the emitted photons, $I_{\gamma}$ (col. 3 ) and the intensities of the internal conversion lines, $I_{K}$ (col. 4) and $I_{L+M+N}$. The $K$ internal conversion coefficient for any gamma ray, $\alpha_{K}$, (col. 5) is the ratio $I_{K} / I_{\gamma}$. The measured total intensities of the 80 and $284 \mathrm{keV}$ gamma transitions were $6.6 \%$ and $5.6 \%$ respectively: since these two radiations have been shown to be in cascade, and no other branching radiations were found, their true intensities have been taken as equal. The average taken, $6.3 \%$, gives somewhat more weight to the more accurately measured $80 \mathrm{keV}$ intensity. The 
TABLE I

Properties of the $\mathrm{Xe}^{131}$ gamma transitions following the beta disintegration of $\mathrm{I}^{\mathbf{1 3 1}}$. Intensities are given per 100 beta disintegrations. The $K / L$ values have been calcu. lated assuming that 0.8 of any $L+M+N$ peak is due to $L$ conversion. Probable errors for cols. $2,3,4,5$ and 6 range from $3 \%$ for the most intense radiations to $30 \%$ for the least intense

\begin{tabular}{c|c|c|c|c|c|c|c}
\hline $\begin{array}{c}(1) \\
\text { Gamma Energy } \\
\mathrm{keV}\end{array}$ & $\begin{array}{c}(2) \\
I_{\text {total }}\end{array}$ & $\begin{array}{c}(3) \\
I_{\gamma}\end{array}$ & $\begin{array}{c}(4) \\
I_{K}\end{array}$ & $\begin{array}{c}(5) \\
\alpha_{K}\end{array}$ & $\begin{array}{c}(6) \\
\mathrm{K} / L\end{array}$ & $\begin{array}{c}(7) \\
\text { Multi- } \\
\text { polarity }\end{array}$ & $\begin{array}{c}(8) \\
\text { Half Life, } \\
\text { sec }\end{array}$ \\
\hline $\mathrm{s} 0.13$ & 6.3 & 2.2 & 3.75 & 1.7 & 7 & $\mathrm{M} 1$ & $4.8 \times 10^{-10}$ \\
$163 \pm 2$ & 0.70 & - & - & - & - & $\mathrm{M} 4$ & $1.04 \times 10^{6}$ \\
284.1 & 6.3 & 5.3 & 0.24 & 0.047 & 5 & $\mathrm{E} 2$ & $<0^{-10}$ \\
364.2 & 80.9 & 80.0 & 0.40 & 0.018 & 8 & $\mathrm{E} 2(+\mathrm{M} 1)$ & $<10^{-10}$ \\
$637 \pm 3$ & 9.3 & 9.3 & 0.034 & 0.0037 & 9 & $\mathrm{E} 2$ & - \\
$722 \pm 4$ & 2.8 & 2.8 & 0.008 & 0.0028 & 8 & $\mathrm{E} 2$ & -
\end{tabular}

intensity of the $163 \mathrm{keV} 12$ day isomeric transition in $\mathrm{Xe}^{131}$ is taken as equal to that of the weak $812 \mathrm{keV}$ beta spectrum which it is assumed to follow, since the intensity actually observed in an $I^{131}$ source will depend on the age of the source and on its retentivity for Xe atoms. The multipolarity assignments (col. 7) were made by comparison with the theoretical internal conversion data of $M$. Rose et al. ${ }^{20}$, and the empirical $K / L$ ratios of Goldhaber and Sunyar ${ }^{21}$, and are consistent on these grounds. Both Verster et al. ${ }^{13}$ and D. Rose, Hinman, and Lang ${ }^{22}$ had measured the internal conversion coefficient of the $637 \mathrm{keV}$ gamma ray, and both gave values consistent with $\mathrm{E} 1$ radiation, rather than $\mathrm{E} 2$ as given in Table $\mathrm{I}$. This question was re-examined with great care by Wolfson ${ }^{23}$, who concluded that the conversion coefficient was in close agreement with the theoretical value for E2 radiation, in agreement with Table I.

The properties of the four partial beta spectra are listed in Table II, their intensities being taken from the total intensities of the corresponding gamma rays in Table I. The end point energies of the two low energy spectra were only roughly determined from Figs. 3 and 4, and the values given in Table II are deduced from the corresponding gamma-ray energies and the fact that the total disintegration energy of the $\mathrm{I}^{131}$ nucleus is $\left[m c^{2}+(608 \pm 5)+364\right] \mathrm{keV}=\left[m c^{2}+972 \pm 5\right] \mathrm{keV}$. This procedure is only permissible because of the coincidence results, and so cannot be applied to the weak $812 \mathrm{keV}$ beta spectrum. Table II also includes $\log f_{0} t$ values

20 M.E. Rose, G. Goertzel, B. Spinrad, J. Harr and P. Strong, Phys. Rev. 83 (1951) 74.

21 M. Goldhaber and A. W. Sunyar, Phys. Rev. 83 (1951) 906.

22 D. Rose, G. Hinman and L. G. Lang, Phys. Rev. 86 (1952) 863.

23 J. L. Wolfson, Can. J. Phys. 30 (1952) 715. 
TABLE II

Properties of the separate components of the beta-ray spectrum of $\mathrm{I}^{131}$. Intensities are given per 100 beta disintegrations. Entries are made (in brackets) for two beta spectra which were not observed but which are energetically possible according to Fig. 5

\begin{tabular}{|c|c|c|c|}
\hline 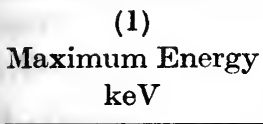 & $\begin{array}{c}(2) \\
\text { Intensity }\end{array}$ & $\begin{array}{c}(3) \\
\log f_{0} t\end{array}$ & $\begin{array}{c}(4) \\
\text { Spectral classification }\end{array}$ \\
\hline $\begin{array}{l}812 \pm 15 \\
608 \pm 5 \\
335 \pm 6 \\
250 \pm 7 \\
(892) \\
(972)\end{array}$ & $\begin{array}{r}0.7 \pm 0.2 \\
87.2 \pm 3.0 \\
9.3 \pm 1.4 \\
2.8 \pm 0.7 \\
(<0.2) \\
(<0.2)\end{array}$ & $\begin{array}{c}9.2 \\
6.6 \\
6.7 \\
6.9 \\
(>\quad 9.9) \\
(>10.0)\end{array}$ & $\begin{array}{l}\text { first forbidden, } \alpha \text { type } \\
\text { allowed ( } l \text {-forbidden) } \\
\text { allowed } \\
\text { allowed } \\
\text { second or higher forbidden } \\
\text { second or higher forbidden }\end{array}$ \\
\hline
\end{tabular}

(col. 3) calculated from the observed energies and intensities of the partial spectra, including those for two possible beta spectra leading to the ground and $80 \mathrm{keV}$ excited states of $\mathrm{Xe}^{131}$; these two spectra were not observed, and must have intensities less than $0.2 \%$.

Single-particle orbital assignments have been made for the nuclear states involved in Fig. 5 by Bergström ${ }^{15}$, Verster et al. ${ }^{13}$, and Bell and Graham ${ }^{16}$, in complete agreement. The ground state of $\mathrm{I}^{131}$ is assigned $g_{\gamma_{2}}$ by analogy with the measured spin and magnetic moment of $I^{129}$. The ground state of $\mathrm{Xe}^{131}$ is $d_{3 / 2}$ by its measured spin and magnetic moment. The $80 \mathrm{keV}$ state of $\mathrm{Xe}^{131}$ can be only $s_{1 / 3}$ or $d_{3 / \mathrm{s}}$, because the $80 \mathrm{keV}$ gamma ray is identified as $\mathrm{Ml}$ by its conversion coefficient, $K / L$ ratio, and lifetime ${ }^{24}$. The assignment $s_{1 / 3}$ is favored both by the absence of beta disintegrations to this state from the $g_{\gamma_{2}}$ ground state of $\mathrm{I}^{131}$, and by the observation by Schiff ${ }^{25}$ that there is no directional correlation in the 284-80 keV gamma-ray cascade. The $364 \mathrm{keV}$ excited state of $\mathrm{Xe}^{131}$ is connected by E2 gamma transitions both to the $d_{s / s}$ ground state and the $s_{1 / 2} 80 \mathrm{keV}$ state, and must therefore be $d_{s / 2}$. The $608 \mathrm{keV}$ beta transition from $I^{131}$ to this state therefore has $\Delta I=1, \Delta l=2$, no, and so falls into the allowed but " $l$-forbidden" classification ${ }^{26}$, consistent with its $\log f_{0} t$ value of 6.6. Less can be said about the 637 and $722 \mathrm{keV}$ excited states of $\mathrm{Xe}^{131}$; they are connected to the $d_{x / 2}$ ground state by E2 radiations, and to the $\mathrm{I}^{131}$ ground state by beta spectra whose $\log f_{0} t$ values seem slightly high for allowed transitions, but which cannot be first forbidden

24 R. L. Graham and R. E. Bell, Can. J. Phys. 31 (1953) 377.

25 D. Schiff, Phys. Rev. 85 (1952) 727.

26 R. Bouchez, Compt. Rend. 231 (1950) 139; M. G. Mayer, S. A. Moszkowski, and L. W. Nordheim, Revs. Mod. Phys. 23 (1951) 315. 
on account of the parities involved. The spins and parities of these two states must therefore be $5 / 2+$ or $7 / 2+$. The 12-day isomeric state of $\mathrm{Xe}^{131}$ has been classified by Goldhaber and Sunyar ${ }^{21}$ as emitting M4 radiation, and the systematics of M4 isomers give $h_{12 /,}$ as the orbital assignment for this isomeric state. This assignment makes the $812 \mathrm{keV}$ beta spectrum leading to this state a first forbidden, $\alpha$-type spectrum $\left(\Delta I=2\right.$, yes), consistent with the observed value of $\log \left(W_{0}^{2}-1\right) f_{0} t=9.9$. Most of this spectrum is obscured by intense spectra of lower energy, so that there does not appear to be any experimental way of determining whether it in fact has the $\alpha$ shape $\left(p^{2}+q^{2}\right.$ correction factor). The level assignments made here are shown on the disintegration scheme, Fig. 5.

$\mathrm{Xe}^{131}$ has 77 neutrons, and the available states near nucleon number 77 on the shell model are $d_{2 / 8}, d_{3 / 2}, s_{1 / 2}$ and $h_{11 / 2}$, in agreement with those already assigned. The exact order in which these levels occur is not predicted by the shell model. Goldhaber and Hill ${ }^{27}$ have plotted the observed energy separations of some of these states as a function of neutron number, and have shown that these separations (and hence the order in which the levels occur) vary in a complex but systematic way. The $\mathrm{Xe}^{131}$ level assignments given here are consistent with their curves. The fact that the two highest states of $\mathrm{Xe}^{131}$ shown in Fig. 5 have spins $5 / 2+$ or $7 / 2+$ is is also satisfactory from the shell model point of view; one of these states could, for example, be assigned $g_{\eta / 2}$. Further support for the level assignments of Fig. 5 is obtained from the fact that no gamma transitions other than those found here occur with appreciable intensity, and none would be expected. (We define "appreciable" intensity as one which would upset seriously the intensities adopted in Fig. 5). The assignment $g_{r / s}$ for the ground state of $\mathrm{I}^{131}$ ( 53 protons) is also satisfactory, according to the shell model.

The discrepancies between different observers about the details of the $\mathrm{I}^{131}$ disintegration scheme have arisen mainly from the interpretation of experimental data, and not from the data themselves. It is not difficult to reconcile all the experiments with the scheme given in Fig. 5. The $I^{131}$ scheme can therefore take its place as one of the best determined disintegration schemes known at present.

27 M. Goldhaber and R. D. Hill, Revs. Mod. Phys. 24 (1952) 179. 
(II) D. E. ALBURGER - Energy Levels of $\mathrm{Pb}^{206}$ from the Decay of $\mathrm{Bi}^{206}$

The success of the shell model in predicting many nuclear phenomena such as ground state spins and the occurrence of isomers has led naturally to extensions of the theory to finer details of nuclear structure. In 1952 M. H. L. Pryce ${ }^{1}$ used shell-theoretical considerations in an attempt to predict the positions of low-lying energy levels in certain nuclei close to the double magic number nucleus $\mathrm{Pb}^{208}$. For nuclei differing from $\mathrm{Pb}^{208}$ by one proton or neutron, calculations giving the order of one-particle levels were made in a straightforward manner by treating the extra nucleon (or hole) as moving in the equivalent potential of the core.

Those nuclei differing by two particles from the closed shell were considered next. Here the calculations are more difficult because the mutual interaction of the two extra nucleons must be taken into account as a perturbation. As a guide it is helpful if the levels in the corresponding single-odd-particle nucleus have been determined experimentally. A case in point examined by Pryce is that of the nuclei $\mathrm{Pb}^{207}$ and $\mathrm{Pb}^{206}$ which may be thought of as closed shells with one and two neutron holes respectively. The experimental evidence on the levels in $\mathrm{Pb}^{207}$, although not complete, was sufficient to serve as a basis for preliminary calculations on the positions of the $\mathrm{Pb}^{206}$ levels. But, unfortunately, a detailed comparison of the latter with experiment could not be made owing to the lack of information on $\mathrm{Pb}^{206}$. The nucleus $\mathrm{Pb}^{206}$ can be formed by the radioactive decays $\mathrm{Po}^{210}(\alpha) \mathrm{Pb}^{206}$ (138-day), $\mathrm{Tl}^{206}(\beta) \mathrm{Pb}^{206}$ (5-min), and $\mathrm{Bi}^{206}(K) \mathrm{Pb}^{206}$ (6.4 days). In the first of these processes the well-known gamma ray of $803 \mathrm{keV}$ is excited, in the second beta decay takes place only to the ground state and no gamma radiation appears, while in the last an exceedingly complex spectrum of internal conversion lines, not all resolved, had been observed ${ }^{2}$. Only the fact that the first excited state of $\mathrm{Pb}^{206}$ occurs at $803 \mathrm{keV}$ had been well established by experiments. The theoretical approach based on such inconclusive data was admittedly exploratory and to quote Pryce - "an elaborate deductive structure had been erected on extremely flimsy foundations".

A more detailed study of $\mathrm{Bi}^{206}$ appeared to be a way in which further information about the interesting nucleus $\mathrm{Pb}^{206}$ could be obtained. $\mathrm{By}$ means of some of the more advanced techniques of beta spectroscopy

1 M. H. L. Pryce, Proc. Phys. Soc. A65, (1952) 773.

2 D. E. Alburger and G. Friedlander, Phys. Rev. 81 (1951) 523. 
experiments on $\mathrm{Bi}^{206}$ have now been carried out adding considerably to our knowledge of the $\mathrm{Pb}^{206}$ energy levels. Although the reader may refer to the original papers of Alburger and Pryce ${ }^{3}$ for more details, the stepby-step manner in which the work was carried out and the interpretations (or sometimes initial misinterpretations) of the data were made will be presented here as an illustration of a coöperative effort between theorist and experimentalist and as a guide for future work on similar problems. It has been possible to determine a set of energies, spins, and parities of 10 excited states in $\mathrm{Pb}^{206}$ and the probable energies, spins and parities of two additional states consistent with the experimental data. Relative transition probabilities, including the occurrence of two isomeric transitions, fit into the picture and the agreement of the level arrangement with approximate calculations based on the shell model can be considered as very satisfactory. At the present writing the decay scheme of $\mathrm{Bi}^{206}$ is probably the most complex for which our detailed knowledge is so complete.

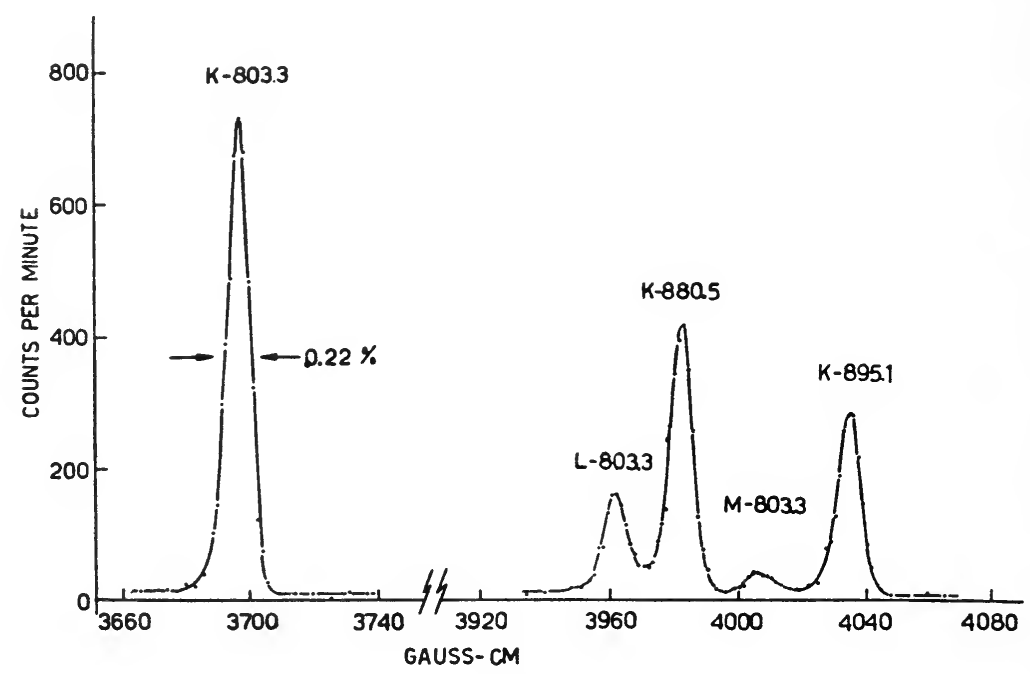

Fig. 1. Part of the $\mathrm{Bi}^{206}$ internal conversion spectrum observed with the doublefocusing spectrometer at $0.22 \%$ resolution

The first stage of the experiments on $\mathrm{Bi}^{206}$ consisted of making energy and intensity measurements on the internal conversion lines in the $50 \mathrm{~cm}$ radius double-focusing spectrometer ${ }^{4}$ at the Nobel Institute of Physics. Typical of the data obtained is a portion of the spectrum given in Fig. 1.

3 D. E. Alburger and M. H. L. Pryce, Phys. Rev. 92 (1953) 514; Phys. Rev. 95 (1954) 1482.

4 A. Hedgran, K. Siegbahn and N. Svartholm, Proc. Phys. Soc. A63 (1950) 960. 
One of the advantages of high resolution $(0.22 \%)$ is beautifully illu strated here by the clean separation of the $L$ and $M$ electron lines of the $803.3 \mathrm{keV}$ transition from two neighboring $K$-lines of 880.5 and $895.1 \mathrm{keV}$ transitions. The right hand group of 4 lines appeared as one broad peak in the earlier lens spectrometer investigation ${ }^{2}$ at $1.2 \%$ resolution.

The energies of 20 conversion lines, all of which were thought at first to be $K$-lines, were determined by comparing their extrapolated momentum edges with those of the three well-known standards $\mathrm{ThF}, \mathrm{Cs}^{137}$, and $\mathrm{Co}^{60}$. These measurements could be made with an accuracy of about 5 parts in 10,000 because of a number of convenient features of the $\mathrm{Bi}^{206}$ sources. Among these were the 6-day half-life, high specific activity and uniform source thickness made possible by electroplating carrier-free material, and the absence of a beta-ray continuum background. The transition energies are given in Table I.

TABLE I

Precision energy values of the strongest $\mathrm{Bi}^{206}$ gamma rays

\begin{tabular}{c|c|c|c}
\hline \hline Gamma No. & Energy $(\mathrm{keV})$ & Gamma No. & Energy (keV) \\
\hline 1 & 184.1 & $11 *$ & 600.6 \\
2 & 234.3 & 12 & 620.6 \\
3 & 262.8 & 13 & 632.2 \\
4 & 343.4 & 14 & 803.3 \\
5 & 386.0 & 15 & 880.5 \\
6 & 398.1 & 16 & 895.1 \\
7 & 497.1 & 17 & 1018.8 \\
8 & 516.1 & 18 & 1098.6 \\
9 & 537.5 & 19 & 1596.3 \\
$10^{*}$ & 588.6 & 20 & 1719.7
\end{tabular}

* Shown from later experiments and analysis to be non-existent.

As a first approach to analysing the data cascade crossover relationships involving three or more gamma rays were examined. It was assumed (wrongly) at the beginning that the two most energetic lines were caused by transitions to the ground state and that only those sums less than $1720 \mathrm{keV}$ need be considered. Restricting the discussion to pairs of gamma rays, there were 146 cases in this category of which the 14 in Table II warranted further inspection. It was observed that the first eleven of these sums agreed with measured gamma-ray energies within the combined probable errors while the last three, $l, m$, and $n$ were just outside the errors. An idea of the importance of precision determinations when dealing with 
highly complex spectra can be gained from the fact that relations $l, m$, and $n$ could be rejected in spite of energy differences of less than $0.3 \%$. Relation $k$ was grouped with the improbable cases since it was noticed that $i$ and $k$ both contain gammas 7 and 18 and could not be satisfied simultaneously. Relation $k$ was chosen as being more doubtful than $i$ because its difference energy was closer to the sum of the probable errors than any of the first 10 relations in the table.

Cascades of three gamma rays were also considered but here there were more than 300 combinations adding up to less than $1720 \mathrm{keV}$. Since there was greater likelihood with these many possibilities that chance additions would fall within the errors of measurement triple cascades were not taken into account in the early analysis.

\section{TABLE II}

Cascade crossover relationships for pairs of lines. Gamma-ray number from Table I. Improbable cases are grouped separately at the end of the Table

\begin{tabular}{l|c|c|c|c}
\hline & Gamma No. & Sum $(\mathrm{keV})$ & $\begin{array}{r}\text { Possible Crossover } \\
\text { Gamma No. }\end{array}$ & Difference (keV) \\
\hline$a$. & $2+3$ & 497.1 & 7 & 0.0 \\
$b$. & $2+5$ & 620.3 & 12 & 0.3 \\
$c$. & $2+6$ & 632.4 & 13 & 0.2 \\
$d$. & $3+13$ & 895.0 & 16 & 0.1 \\
$e$. & $4+9$ & 880.9 & 15 & 0.4 \\
$f$. & $5+13$ & 1018.2 & 17 & 0.6 \\
$g$. & $6+7$ & 895.2 & 16 & 0.1 \\
$h$. & $6+12$ & 1018.7 & 17 & 0.1 \\
$i$. & $7+18$ & 1595.7 & 19 & 0.6 \\
$j$. & $12+18$ & 1719.2 & 20 & 0.9 \\
& & & & 18 \\
$k . *$ & $7+11$ & 1097.7 & 18 closest & 1.4 \\
$l$. & $1+12$ & 804.7 & 15 closest & 2.9 \\
$m$. & $3+12$ & 883.4 & 15 closest & 2.6 \\
$n$. & $5+7$ & 883.1 & &
\end{tabular}

* Later eliminated by showing gamma 11 to be non-existent.

Another way of studying the $\mathrm{Bi}^{206}$ scheme was to find out which transitions were in prompt cascade by detecting coincidences between various $K$ conversion lines. Experiments of this type were performed with a double lens spectrometer or so-called spectrogoniometer ${ }^{5}$. When a source is placed at the center of this instrument coincidences between electrons of

5 K. Siegbahn, Arkiv f. Fysik 4 (1952) 223. 
separately selected energy, such as two $K$ conversion lines, can be measured. The number of cases which could be examined in $\mathrm{Bi}^{206}$ was limited either by the $3 \%$ spectrometer resolution or by low conversion intensities. Among the results given in Table III comparison shows that relations $e$ and $g$ of Table II are checked by the coincidence data. The non-coincident cases of Table III are only those for which a coincidence yield could have been expected on the basis of relative line intensities. From the point of view of analysing the decay scheme they are just as important as the coincident pairs.

With the amount of information accumulated thus far one might have believed that a tentative level scheme could be suggested. Indeed some of the transitions fell naturally into groups yet all efforts to fit in every gamma ray failed. In particular there seemed to be no place at all for gammas number 10 and 11 of Table I. Up to this point, however, two incorrect assumptions had been made, firstly that the high energy lines were ground state transitions and secondly that no delayed states existed in $\mathrm{Pb}^{206}$. The latter assumption was based in part on the early theoretical analysis ${ }^{1}$ by Pryce that all of the $\mathrm{Pb}^{206}$ transitions should be electric quadrupoles or magnetic dipoles and therefore should not have life-times destroying coincidence effects, and in part on experiments in which lifetimes between $10^{-7}$ and $10^{-4}$ sec and above 1 sec were searched for but not found ${ }^{2}$. The general absence of isomeric states in even-even nuclei also influenced these considerations.

\section{TABLE III}

$\mathrm{Bi}^{206}$ coincident and non-coincident transitions from measurements on pairs of $K$-lines in the spectrogoniometer. Gamma energies in $\mathrm{keV}$

\begin{tabular}{l|c}
\hline Coincident pairs & No coincidences detected \\
\hline $184.1-262.8$ & $184.1-343.4$ \\
$184.1-398.1$ & $184.1-516.1$ \\
$184.1-497.1$ & $184.1-537.5$ \\
$184.1-880.5$ and/or 895.1 & $184.1-803.3$ \\
$184.1-1018.8$ & $343.4-398.1$ \\
$343.4-497.1$ & $343.4-880.5$ \\
$343.4-516.1$ & $343.4-895.1$ \\
$343.4-537.5$ & $398.1-516.1$ \\
$343.4-803.3$ & $398.1-537.5$ \\
$398.1-497.1$ & $537.5-880.5$ \\
$537.5-803.3$ & $537.5-895.1$
\end{tabular}

In casting about for a solution to this perplexing problem it began to 
be suspected that one or both of the above assumptions might be wrong. At the same time theoretical reconsiderations resulted in the idea that the uppermost level occurring in $\mathrm{Bi}^{206}$ decay might be at a greater energy than previously thought and that the energetic transitions could be taking place between states of high excitation.

To make a test of this it was decided to look for coincidences between the high energy gamma rays and various $K$ conversion lines. The experiments were done in the Slätis-Siegbahn intermediate-image spectrometer ${ }^{6}$ which had been modified by the installation of a NaI gamma detector at the source end of the instrument. In spite of the great complexity of the spectrum it will be observed from Table I that gammas 19 and 20 are about $50 \%$ higher in energy than their nearest neighbors. Thus even though the NaI gamma detector has a comparatively poor resolving power, it is quite adequate here for observing the two energetic gamma rays, unresolved from each other, at the exclusion of the many at lower energies. With the pulse height discriminator set for these conditions a search was made for gamma-electron coincidences between the $\mathrm{NaI}$ detector and the intermediate-image spectrometer counter when various $K$-lines were focused. The summary of results listed in Table IV showed that the suspicion about the high position of one or both of the energetic lines was confirmed.

TABLE IV

Coincidence effects between the 1596.3 and/or $1719.7 \mathrm{keV}$ gamma rays and the $K$ lines of other transitions as measured in a modified intermediate-image spectro. meter. Gamma energies in keV

\begin{tabular}{c|c}
\hline \hline Coincidences Observed & No Detectable Coincidences \\
\hline 343.4 & 184.1 \\
537.5 & 262.8 \\
$803 . \ddot{3}$ & 497.1 \\
880.5 and/or 895.1 & 516.1 \\
& $588.6 *$ \\
& 1018.8
\end{tabular}

* Non-existent gamma ray. The line studied here later proved to be $(L+M)$ of the 516.1 gamma.

These data, considered together with the coincidence results of Table III, at once placed the uppermost level at least as high as $803.3+880.5+$ $+(1596.3$ or 1719.7$)$ or at about $3.4 \mathrm{MeV}$.

- H. Slätis and K. Siegbahn, Arkiv f. Fysik 1 (1949) 339. 
Summarizing the results thus far there were 41 independent bits of information (10 cascade crossover relations, 22 coincident or non-coincident pairs of $K$-lines, and 9 coincident or non-coincident gamma-electron effects) to be satisfied by the level arrangement. One case in Table IV has been omitted because later work showed that the $588.6 \mathrm{keV}$ gamma was spurious. Nevertheless a decay scheme still could not be formulated unless allowance was made for the possibility of a delayed state. When this was done all of the transitions excepting No. 10 and No. 11 of Table I fell into a consistent picture (see Fig. 2) in which the $516.1 \mathrm{keV}$ line was assigned as the isomeric transition. It was even possible from theoretical estimates to assign tentatively the $516.1 \mathrm{keV}$ transition as an electric octupole in which case its conversion electron ratios would be abnormal and its lifetime might be measurable.

A re-examination of the conversion electron data was then made and it was found that the line which had been assigned as $K-588.6$ actually fit very well as $L_{\mathrm{I}}+L_{\mathrm{II}}-516.1$ and the line called $K-600.6$ corresponded to $M-516.1$. The incorrect interpretations of these lines as $K$-electrons was natural because of their large amplitude compared with $K-516.1$ and the idea existing then that no isomers (or low $K / L$ ratios) could be present. Further examination substantiated these new assignments when the $L$-line corresponding to the supposed $588.6 \mathrm{keV}$ transition turned out to have an energy differing from $L-588.6$ by $2.8 \mathrm{keV}$ or little more than $1 / 2 \%$. In the first survey runs its position and intensity appeared about right to be $L-588.6$ but in these later and more careful ehecks this line was re-assigned as the $K$-line of a $657.3 \mathrm{keV}$ gamma ray. Furthermore the $L$-line of the supposed $600.6 \mathrm{keV}$ gamma, at first thought to be too weak for observation, could not have been missed without making the $K / L$ ratio of the $600.6 \mathrm{keV}$ transition abnormally large (i.e. $>20$ ). These new assignments resulted in $K: L: M$ ratios of the $516.1 \mathrm{keV}$ isomeric transition which were consistent with $\mathrm{E} 3$ radiation. The initial misinterpretations of the $516.1 \mathrm{keV} L$ - and $M$-lines might have been avoided if a careful energy measurement of $K-657.3$ had been made to begin with. Perhaps this may serve as an example of the importance of tying down every line, no matter how seemingly unimportant, in a complex conversion spectrum.

Proof of the non-existence of gammas number 10 and 11 of Table I was fortunate in that these were the on!y two transitions which did not fit into a term diagram, although the question of the new $657.3 \mathrm{keV}$ transition was now left open. Removal of gamma number $1+$ at once resolved the only conflict in any of the data, namely that the previously mentioned cascade crossover relations $i$ and $k$ of Table II could not both be satisfied. Things were now definitely looking brighter. Except for inability to 
place the $657.3 \mathrm{keV}$ transition a tentative level scheme could be drawn which satisfied all of the cascade crossover and coincidence data and whose only ambiguity lay in the position of the second excited state. (See Fig. 2.) At this point there seemed to be no way of establishing the emission order of the 343.4 and $537.5 \mathrm{keV}$ gamma rays.

Having obtained strong indications that the $516.1 \mathrm{keV}$ transition was isomeric the next step consisted in attempts to establish that a metastable state exists in $\mathrm{Pb}^{206}$ and to measure its life-time. A glance at the empirical curves published by Goldhaber and Sunyar ${ }^{7}$ suggested that the half-life of a state decaying by $516.1 \mathrm{keV}$ electric octupole radiation might be of the order of milliseconds. This difficult region for life-time measurements was not examined in the earlier work ${ }^{2}$. The technique adopted ${ }^{3}$, a rather simple but infrequently used method, consisted of looking for delayed pulses with a triggered oscilloscope. A weak source of $\mathrm{Bi}^{206}\left(\sim 5 \times 10^{-4}\right.$ microcurie) was placed close to a NaI crystal detector and every pulse, excluding tube noise, was allowed to enter the trigger circuit of the scope trace. Delayed events were searched for by placing a slotted mask on the scope face at various distances from the origin pulse and counting by eye the number of pulses appearing in the slot. Using a 50 microsecond per cm sweep these rough measurements showed, after subtraction of an easily calculated random background, that the number of pulses as a function of distance from the origin pulse decreased exponentially. The method was then refined by recording the pulses with an optical lens and photomultiplier and by making suitable calibration and trace linearity corrections. The half-life of $145 \pm 15$ microseconds so obtained seemed shorter than expected of an E3 transition but did not correspond as well with any other multipole order. This value is just above the $10^{-7}$ to $10^{-4}$ sec region previously investigated but at the same time it is well below one millisecond where such a measurement would have been much more difficult.

One might wonder how a single delayed state could be detected in the presence of more than 28 gamma rays corresponding to at least 12 excited states. For an answer to this an inspection of the decay scheme shows that a number of the strong transitions directly or indirectly feed the isomeric level and a number follow it. Many of the pulses corresponding to gamma rays which are prompt with respect to $K$-capture can start a useful scope sweep. The probability of detecting delayed pulses is enhanced by the presence of five strong transitions following the isomeric state. Of course those sweeps started by pulses from the energetic gammas which by-pass the isomeric state or by pulses from the delayed gammas themselves are not useful.

7 M. Goldhaber and A. W. Sunyar, Phys. Rev. 83 (1951) 906. 
In the final phases of the $\mathrm{Bi}^{206}$ investigation closer examination with a very strong source of the regions between the main conversion lines in the double-focusing spectrometer and a study of the very low energy region in the intermediate-image spectrometer revealed a number of new and very weak lines of which those assigned in the scheme are listed as numbers 20 through 28 in Table V. Referring now to Fig. 2 all of the states except those at 1998.1, 2525.5 and $3124.7 \mathrm{keV}$ had been established by the experiments and analysis already described. Two of the new transitions, of energies 123.6 and $816.3 \mathrm{keV}$, fitted between levels already established. Three more, of energies 202.5, 313.6 , and $1405.2 \mathrm{keV}$, together with the previously unplaced gamma ray of $657.3 \mathrm{keV}$ were consistent with a level at $1998.1 \mathrm{keV}$. Oneimmediate consequence of this was to remove the only ambiguity up to this point concerning the second excited state whose position was now fixed by the 657.3 $\mathrm{keV}$ transition.

The $202.5 \mathrm{keV}$ gamma was especially interesting because its low $K / L$ ratio $(\sim 0.1)$ suggested that it was an E3 isomeric tran-

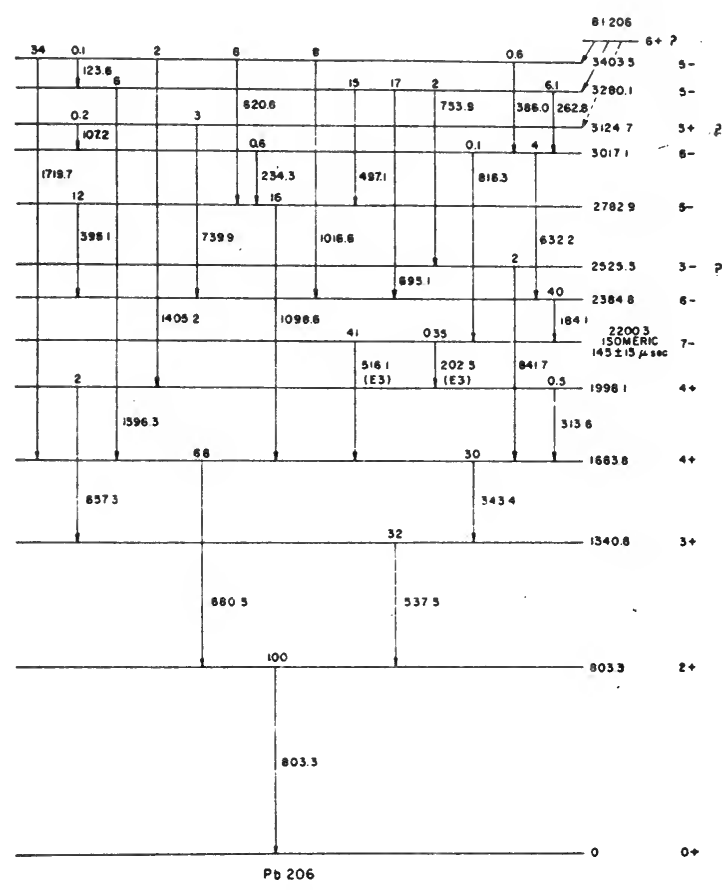

Fig. 2. Energy level diagram of $\mathrm{Pb}^{206}$. The num. ber at the head of each transition is its intensity in percent per disintegration

sition in weak competition with the $516.1 \mathrm{keV}$ line. This led to the spin and parity assignment of $4+$ to the $1998.1 \mathrm{keV}$ state. Thus not only was $\mathrm{Pb}^{206}$ one of the rare cases of isomerism in an even-even nucleus but two isomeric transitions were found. The life-time is determined almost entirely by the $516.1 \mathrm{keV}$ transition whose radiation probability is much larger than that of its $202.5 \mathrm{keV}$ competitor.

Twenty-four gamma rays and 10 excited states had. now been assigned with fair certainty. Four more gammas, numbers 23, 24, 26, and 28 in Table $\mathrm{V}$, formed the basis for assigning probable levels at $2525.5 \mathrm{keV}$ and $3124.7 \mathrm{keV}$ as shown in Figs. 2 and 3. Inclusion of these resulted from the 
shell model predictions to be discussed later. Altogether the term diagram of Fig. 2 contains 28 transitions and satisfies all of the experimental information cited thus far. Agreement of this scheme with the data of Tables II, III, and IV is obvious from inspection in most cases but it should perhaps be pointed out that the $343.4-497.1$ coincidences in Table III arise from feeding of the $1683.8 \mathrm{keV}$ state by the $1098.6 \mathrm{keV}$ transition.

In the light of this decay scheme one can obtain a measure of how far cascade crossover relationships can be relied upon for analysis, at least for this amount of complexity. If all 28 gamma rays had been known at the start there would have been 256 pairs of lines adding to less than $1720 \mathrm{keV}$. Twenty-eight sums agree with measured gamma energies within the limits of error and of these 20 are consistent with the decay scheme while 8 are not and must be considered as chance additions. It was fortunate that none of the original 10 relations given in Table II was spurious.

Not only must the positions of the gamma rays fit together in such a way that the coincidence and cascade crossover data are satisfied, but the spins and parities assigned to the various states should result in a reasonable agreement between the expected relative transition intensities and those calculated from the conversion lines. In addition the observed $K / L$ ratio of each-transition should be consistent with the multipole order according to the parities and spins adopted for these states. A set of spins and parities satisfying these requirements, but not necessarily unique, was deduced and they are included in Fig. 2. It was, of course, assumed that the ground state of this even-even nucleus is $0+$ while the fact that the first excited state is $2+$ had been established earlier ${ }^{8}$ from the $\alpha-\gamma$ angular correlation of $\mathrm{Po}^{210}$. Some of the other low-lying levels in Fig. 2 were assigned spins and parities more on the basis of sholl model considerations than on experimental evidence. A list of the gamma energies, together with multipole orders, relative measured $K$-line intensities, and adopted transition:intensities of the 28 gamma rays of Fig. 2 is given in the first 5 columns of Table V. In some cases the estimated intensity is only approximate because of the possibility that a mixture of radiations has affected the observed $K$-line intensity. $K / L$ ratios are not given here but insofar as comparison could be made with other empirical information they, too, were found to be consistent with the scheme ${ }^{3}$.

After the above analysis had been completed, further experiments were undertaken at Brookhaven to measure the relative intensities of the strongest $\mathrm{Bi}^{206}$ gamma rays using a $\mathrm{NaI}$ scintillation spectrometer. Nine photo peaks were observed, several of which contained two or three

8 S. DeBenedetti and G. H. Minton, Phys. Rev. 85 (1952) 944. 
TABLE V

Transitions and Intensities in the Decay of $\mathrm{Bi}^{206}$

\begin{tabular}{|c|c|c|c|c|c|}
\hline Gamma & $\begin{array}{c}\text { Energy } \\
(\mathrm{keV})\end{array}$ & Multipole & $\begin{array}{c}K \text {-line Intensity } \\
\text { (electrons per } \\
100 \text { dis- } \\
\text { integrations) }\end{array}$ & $\begin{array}{c}\text { Transition } \\
\text { Intensity } \\
\text { adopted from } \\
K \text {-line intensities } \\
(803.3=100)\end{array}$ & $\begin{array}{l}\text { Transition } \\
\text { Intensity from } \\
\text { gamma meas. }\end{array}$ \\
\hline 1 & 184.1 & M1 & 28 & 40 & 45 \\
\hline 2 & 234.3 & M1 & 0.24 & 0.6 & \\
\hline 3 & 262.8 & Ml & 2.1 & 6.1 & \\
\hline 4 & 343.4 & M1 & 6.6 & 30 & 25 \\
\hline 5 & 386.0 & M1 & 0.13 & 0.6 & \\
\hline 6 & 398.1 & $\mathrm{M} 1(+\mathrm{E} 2)$ & 1.76 & 12 & \\
\hline 7 & 497.1 & $\mathrm{M} I(+\mathrm{E} 2)$ & 1.37 & 15 & \\
\hline 8 & 516.1 & E3 & 1.95 & $41\}=88$ & 76 \\
\hline 9 & 537.5 & $\mathrm{M} 1(+\mathrm{E} 2)$ & 2.08 & $32)$ & \\
\hline 10 & 620.6 & $\mathrm{Ml}(+\mathrm{E} 2)$ & 0.27 & 6 & \\
\hline 11 & 632.2 & $\mathrm{M} 1(+\mathrm{E} 2)$ & 0.21 & 4 & \\
\hline 12 & 657.3 & $M 1(+E 2)$ & 0.084 & 2 & \\
\hline 13 & 803.3 & E2 & 0.85 & 100 & 100 \\
\hline 14 & 880.5 & E2 & 0.48 & 68) & \\
\hline 15 & 895.1 & $\mathrm{M} 1(+\mathrm{E} 2)$ & 0.32 & $17\}=85$ & 65 \\
\hline 16 & 1018.8 & $\mathrm{M}(+\mathrm{E} 2)$ & 0.11 & 8 & 8 \\
\hline 17 & 1098.6 & El & 0.029 & 16 & 14 \\
\hline 18 & 1596.3 & El & 0.0054 & 6 & 5.5 \\
\hline 19 & 1719.7 & El & 0.029 & 34 & 32 \\
\hline 20 & 123.6 & $\mathrm{M} 1(+\mathrm{E} 2)$ & 0.069 & 0.1 & \\
\hline 21 & 202.5 & E3 & 0.020 & 0.35 & \\
\hline 22 & 313.6 & $\mathrm{M} 1(+\mathrm{E} 2)$ & 0.14 & 0.5 & \\
\hline 23 & 739.9 & E1 & 0.011 & 3 & \\
\hline 24 & 753.9 & $\mathrm{E} 2$ & 0.0074 & 2 & \\
\hline 25 & 816.3 & $M 1(+E 2)$ & 0.0025 & 0.1 & \\
\hline 26 & 841.7 & $\mathrm{E} 1$ & 0.0050 & 2 & \\
\hline 27 & 1405.2 & E1 & 0.0020 & 2 & \\
\hline 28 & 107.2 & El & 0.23 & 0.2 & \\
\hline
\end{tabular}

unresolved lines. The relative transition intensities derived from these measurements are listed in the last column of Table V. It is seen that in the cases of those lines accessible to the experiments, the intensities check the values derived from the conversion data, thus confirming many of the adopted spins and parities.

The level diagram of Fig. 2 appears to be very complex but it actually does not contain as many levels as expected from the theoretical calcu- 
lations. This is illustrated in Fig. 3 which shows the observed levels and transitions of $\mathrm{Pb}^{206}$ (solid lines) together with additional calculated levels (dashed lines) all separated according to spin value. As the basis for these calculations the levels in the one-hole nucleus $\mathrm{Pb}^{207}$ were taken from experimental information, some unpublished, to have the following energies (in $\mathrm{MeV}$ ) and configurations - $0.00, p_{1 / \mathrm{s}} ; 0.57, f_{\mathrm{s} / \mathrm{g}} ; 0.87, p_{\mathrm{s} / \mathrm{s}} ; 1.63, i_{\mathrm{ux} / \mathrm{z}}$, and 2.35, $h_{\%}$. Levels in $\mathrm{Pb}^{206}$ were derived by assigning one of the $\mathrm{Pb}^{207}$ levels to each neutron hole. From each such pair of $\mathrm{Pb}^{207}$ levels a number

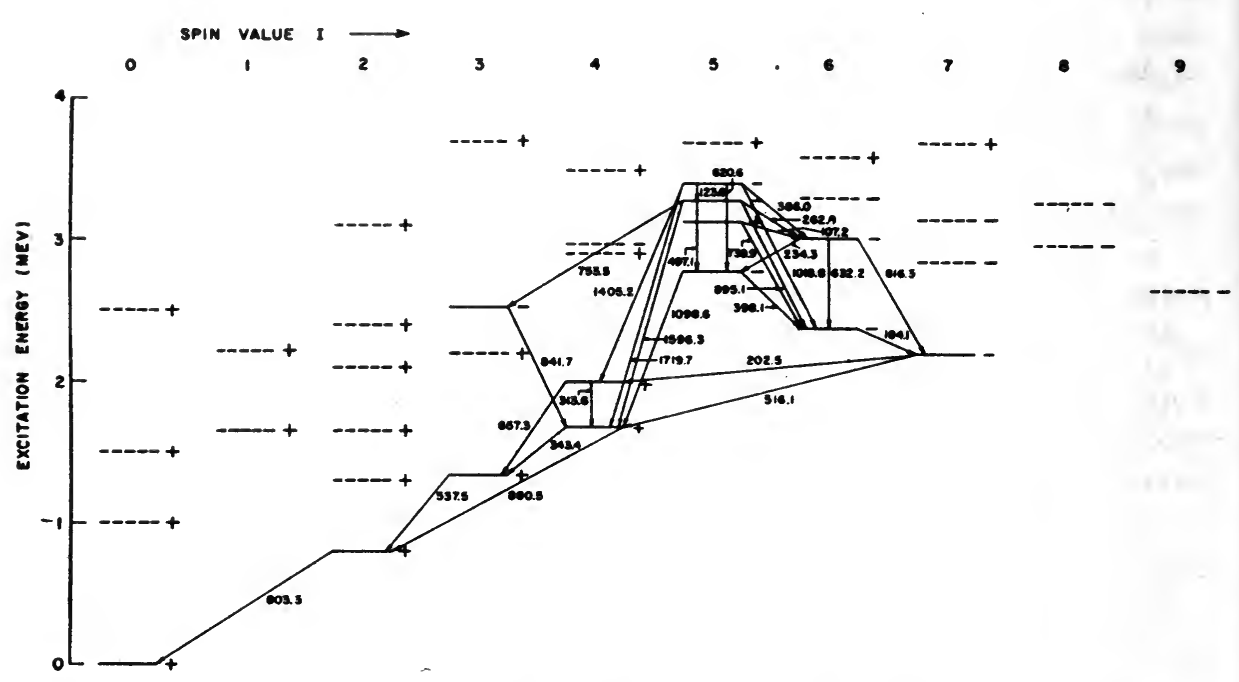

Fig. 3. The energy levels of $\mathbf{P b}^{208}$ separated according to spin value. Solid lines - levels and transitions determined experimentally. Dashed lines - additional levels expected from theoretical calculations. Accuracies in the positions of calculated levels are $1 \mathrm{MeV}$ for the $0+$ levels and $0.3 \mathrm{MeV}$ for all others

of energy levels of "different spins were constructed for $\mathrm{Pb}^{206}$ according to the ordinary rules of vector coupling. As an example the configuration $p_{1 / 3}-f_{\% /,}$ contains two levels $(2+$ and $3+)$. If there were no interaction between the holes the energies of the levels derived from a given pair would be just the sum of the corresponding $\mathrm{Pb}^{207}$ levels and all such levels from the same configuration would be degenerate. However, because of interaction the levels are split, some being unshifted and others being depressed by an amount estimated by comparison with the experimental data. Except for the $0+$ levels the calculated positions are accurate to about $0.3 \mathrm{MeV}$. Within this accuracy all of the observed levels agree with the calculations. 
One of the most significant results of the calculations is that the lowest level of spin 7 lies below the lowest spin 5, 6 and 8 levels. Electron capture in $\mathrm{Bi}^{206}$ takes place to either of the upper two 5 - levels of $\mathrm{Pb}^{206}$. From the shell model the most desirable assignment for the ground state of $\mathrm{Bi}^{206}$ would be $6+$, although $5+$ could also be a possibility. De-excitation of $\mathrm{Pb}^{206}$ by gamma emission in many instances carries the nucleus to higher spin values, eventually leading to the $7-$ state. The appearance of delayed radiation, first suggested by the calculations, arises from the fact that the nucleus then has no other choice but to undergo an E3 transition to ore of the $4+$ levels. It will be noted that cascading to the ground state generally proceeds via the lowest level of each spin value and that more than half of the calculated levels are not excited because of unfavorable transition probabilities. However, the general appearance of Fig. 3 suggests that several of these levels should be observable.

In addition to the 28 transitions listed in Table $\mathrm{V}$ several more weak lines were observed. Some of these occurred at energies as low as $10 \mathrm{keV}$ but as yet the assignments of any transitions derived from them are uncertain. It is not unreasonable from Fig. 3 that they result from weak excitation of other $\mathrm{Pb}^{206}$ levels: Further investigation of these lines might be profitable.

Except for the unassigned lines there is now enough information about the levels of $\mathrm{Pb}^{206}$ to serve as the basis for further theoretical considerations. However, it should not be concluded that the present experimental information is sufficiently complete. Thus, one would like to know where the weak transitions mentioned above fit in, whether the dashed levels in Fig. 3 really exist and what their positions are. Inasmuch as these levels are simple, in the sense that they correspond to excitation of the two neutron holes, there also remains a question as to whether complex levels, expected as low as $2.5 \mathrm{MeV}$ from excitation of the core, are present. For the answers to these problems other methods will have to be employed such as magnetic analysis of the triton groups from the $\mathrm{Pb}^{207}(\mathrm{~d}, \mathrm{t}) \mathrm{Pb}^{206}$ reaction. In this process (ground state $Q$-value $-\overline{0.4} \mathrm{MeV}$ ) one might expect excitation of many low-lying levels in $\mathrm{Pb}^{206}$ that cannot be reached in the decay of $\mathrm{Bi}^{206}$. Harvey has made ${ }^{9}$ range measurements on this reaction and has obtained rough indications of several $\mathrm{Pb}^{206}$ levels. A further study using high resolution techniques might reveal additional details of the $\mathrm{Pb}^{206}$ level structure.

- J. A. Harvey, Can. J. Phys. 31 (1953) 278 


\section{(1II) A. C. G. MITCHELL - A Study of the Sequence $\mathrm{I}^{130} \rightarrow \mathrm{Xe}^{130} \leftarrow \mathrm{Cs}^{130} \rightarrow \mathrm{Ba}^{130}$ and the Levels of $\mathrm{Xe}^{130}$}

As an additional example of the working out of complex decay schemes and their relations to theoretical models the sequence $\mathrm{I}^{130} \rightarrow \mathrm{Xe}^{\mathbf{1 3 0}} \leftarrow$ $\leftarrow \mathrm{Cs}^{130} \rightarrow \mathrm{Ba}^{130}$ will be discussed. As a result of recent experiments of Caird and Mitchell ${ }^{1}$ a considerable amount of information has been obtained on the mode of disintegration of $\mathrm{I}^{130} \rightarrow \mathrm{Xe}^{130}$. In carrying out these experiments, the above investigators made use of a conventional magnetic lens spectrometer, a coincidence lens, scintillation counters, and scintillation counters in coincidence. The spectrum of $I^{\mathbf{1 3 0}}$ was originally studied by Roberts, Elliott, Downing, Peacock and Deutsch ${ }^{2}$ in the early days of nuclear spectroscopy. They showed that $\mathbf{I}^{\mathbf{1 3 0}}$ decays by the emission of two beta-ray groups of energies 1.03 and $0.61 \mathrm{MeV}$ together with four gamma-rays whose energies are 0.417, 0.537, 0.667, $0.714 \mathrm{MeV}$. According to these authors the position of the gamma-ray at $0.417 \mathrm{MeV}$ is established from the energies of the two beta-ray groups but the remaining three gamma-rays are all in cascade and their arrangement -is arbitrary. Smith, Mitchell and Caird ${ }^{3}$ measured the spectrum of $\mathrm{Cs}^{\mathbf{1 3 0}}$, which also goes to $\mathrm{Xe}^{130}$, with the hope of establishing the order of the gamma-rays in cascade, but found that these gamma-rays were not excited in the disintegration of $\mathrm{Cs}^{130}$.

\section{$\S 1$. Experiments on $I^{130}$}

$\complement^{130}$ was prepared by bombarding tellurium with deuterons. The sample was dissolved in $\mathrm{HNO}_{3}$ with some $\mathrm{KI}$, to serve as a carrier, and the iodine was distilled off, extracted by $\mathrm{CCl}_{4}$ and eventually precipitated as AgI for use as sources. The $\mathrm{I}^{130}$ (half-life 12.6 hours) was accompanied by other iodine activities and corrections for these had to be made in doing the experiments. In order to keep unwanted activities to a minimum, separated $\mathrm{Te}^{130}$ was used as a target for some experiments.

\section{A. The Gamma-Rays}

In order to investigate the gamma-rays, the spectrum of the photoelectrons ejected from either a lead or a uranium radiator was measured in a magnetic lens spectrograph. The main curve of Fig. 1 shows the

1 R. S. Caird and A. C. G. Mitchell, Phys. Rer. 94 (1954) 412.

2 A. Roberts, L. G. Elliott, J. R. Downing. W. C. Peacock and M. Deutsch, Phys. Rer. 64 (1943) 268.

3 A. B. 'Smith, A. C. G. Mitchell and R. S. Caird, Phys. Rev. 87 (1952) 454. 
CH. XXII (III)] SEQUENCE $\mathrm{I}^{130} \rightarrow \mathrm{Xe}^{130} \leftarrow \mathrm{Cs}^{130} \rightarrow \mathrm{Ba}^{130}$ AND LEVELS OF $\mathrm{Xe}^{130}$

number of photoelectrons per unit momentum interval for a lead radiator of $16 \mathrm{mg} / \mathrm{cm}^{2}$ surface density plotted against the current in the lens. The peaks labelled $K_{1}, L_{1}$, to $K_{4}, L_{4}$ denote the $K$ and $L$ photoelectric peaks arising from four different gamma-rays emitted by $\mathrm{I}^{130}$. The peak labelled $K_{4}+L_{3}$ contains the $K$ photoelectrons for a line at $0.744 \mathrm{MeV}$ and the $L$ peak for a line at $0.660 \mathrm{MeV}$. A similar experiment with a uranium radiator resolved the $K_{4}$ from the $L_{3}$ peak but caused the overlapping of other lines. The energies of these lines are given in Table I.

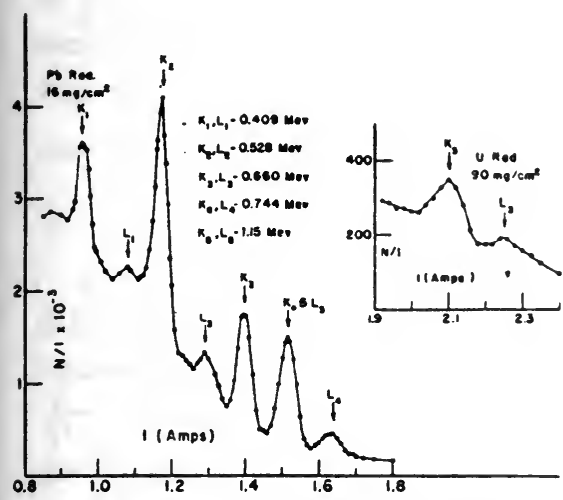

Fig. 1. Photoelectrons Ejected by Gamma-rays from $\mathrm{I}^{130}$

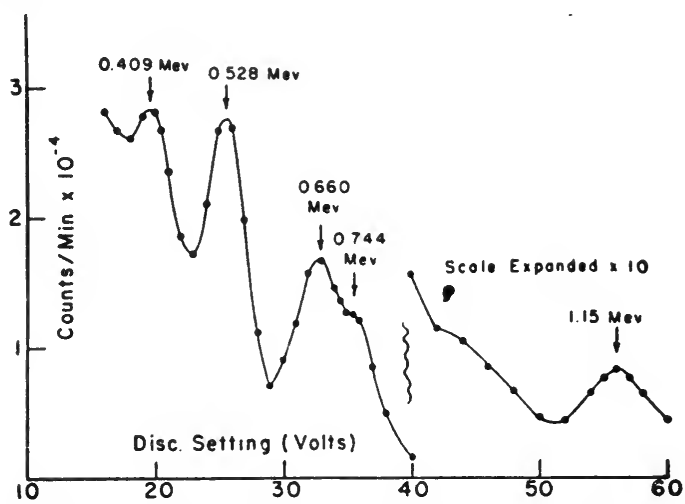

Fig. 2. Scintillation Spectrum of Gamma. rays from $I^{130}$

The gamma-rays were also measured using a $\mathrm{NaI}(\mathrm{Tl})$ scintillation spectrometer and the results are shown in Fig. 2. In this figure, the two low energy lines shown in Fig. 2 are clearly resolved but the line at $0.660 \mathrm{MeV}$ shows some overlapping with that at $0.744 \mathrm{MeV}$. In addition a higher energy line of energy about $1.15 \mathrm{MeV}$ is seen. Experiments were performed to be sure that this line was not due to "pulse pile up". The spectrum was then reinvestigated in the magnetic" lens spectrometer employing a strong source and a uranium radiator. The result is shown in the insert of Fig. 1 where a $K$ and an $L$ photo-peak will be seen corresponding to a line at $1.15 \mathrm{MeV}$.

TABLE I

Gamma-rays from $I^{130}$

\begin{tabular}{c|c|c}
\hline \hline Gamma-ray & $\begin{array}{c}\text { Energy of Gamma-ray } \\
\mathrm{MeV} .\end{array}$ & Relative Intensity \\
\hline 1 & 0.409 & 0.3 \\
2 & 0.528 & 1.00 \\
3 & 0.660 & 0.9 \\
4 & 0.744 & 0.8 \\
5 & 1.15 & 0.4
\end{tabular}


Estimates of the relative intensities of the gamma-rays were obtained by determining the area under the several $K$ photo-peaks involved and applying the corrections for the variation of photoelectric efficiency with energy. Since the $K$ photo-peak for the line at 0.744 and the $L$ photo-peak for the line at 0.660 coincide, a correction had to be made for the intensity of the latter. This was accomplished by measuring the $K$ and $L$ photopeaks for the line at $0.530 \mathrm{MeV}$ and using this to compute the contribution of the $L$-peak for the line at $0,660 \mathrm{MeV}$. The intensity of the line at $1.15 \mathrm{MeV}$ was determined by comparing the $K$ photo-peak produced by it in a uranium radiator with those of the other lines produced by the same source using a uranium radiator. The results shown in Table I, are not felt to be known to better than $20 \%$.

\section{B. The Beta-ray Spectrum}

The disfribution of beta-rays and internal conversion electrons was investigated with the help of the magnetic lens spectrometer. Fig. 3 shows that part of the spectrum containing the internal conversion lines. All lines are internally converted. A Fermi analysis of the beta-ray spectrum was made and it was found to be resolvable into two groups with

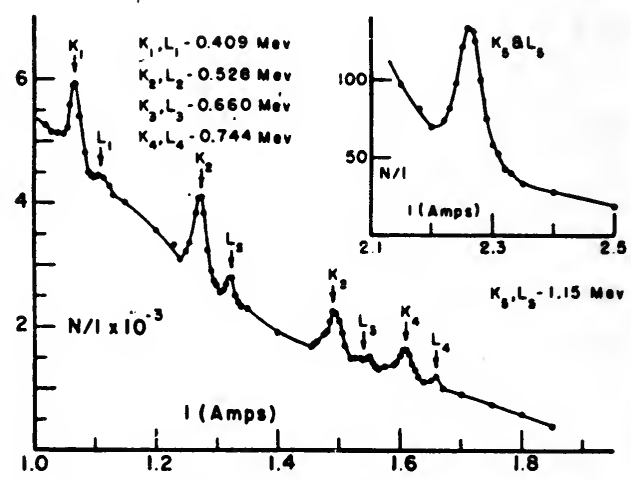

Fig. 3. Internal Conversion Lines from $I^{130}$ end point energies of 1.02 and $0.597 \mathrm{MeV}$, in agreement with Roberts et al. No groups of higher energy than that at $1.02 \mathrm{MeV}$ were found. Table II gives the characteristics of the beta-ray groups.

TABLE II

Beta-rays from $I^{130}$

\begin{tabular}{c|c|c}
\hline $\begin{array}{c}E \\
\mathrm{MeV}\end{array}$ & $\begin{array}{c}\text { Rel. Abundance } \\
\%\end{array}$ & $\log f t$ \\
\hline 1.02 & 46 & 6.5 \\
0.597 & 54 & 5.5
\end{tabular}

The number of internal conversion electrons per disintegration were obtained by taking the area under the $K$ internal conversion peaks, and comparing this to the area under the beta-ray spectrum. For the line at $1.15 \mathrm{MeV}$ the $L$-line was not resolved from the $K$-line. The results are given in Table III together with estimates of the $K / L$ ratios. 
CH. XXII (III)] SEQUENCE $\mathrm{I}^{130} \rightarrow \mathrm{Xe}^{130} \leftarrow \mathrm{Cs}^{130} \rightarrow \mathrm{Ba}^{130}$ AND LEVELS OF $\mathrm{Xe}^{130}$

TABLE III

Internal Conversion Data for $\mathrm{I}^{130}$

\begin{tabular}{l|c|c|c|c}
\hline \hline$E_{\gamma}$ & $\begin{array}{c}K \text {-Internal } \\
\text { Conversion Electrons } \\
\text { per Disintegration }\end{array}$ & $K / L$ & $\alpha_{K}$ (obs) & $\alpha_{K}$ (theor) \\
\hline 0.409 & $4.4 \times 10^{-3}$ & 11 & $1.6 \times 10^{-2}$ & - \\
0.528 & $5.5 \times 10^{-3}$ & 8 & $5.5 \times 10^{-3}$ & $6.8 \times 10^{-3}(\mathrm{E} 2)$ \\
0.660 & $3.2 \times 10^{-3}$ & 16 & $3.2 \times 10^{-3}$ & $3.8 \times 10^{-3}(\mathrm{E} 2)$ \\
0.744 & $2.1 \times 10^{-3}$ & 4 & $2.7 \times 10^{-3}$ & $2.9 \times 10^{-3}(\mathrm{E} 2)$ \\
1.15 & $\left(8.8 \times 10^{-5}\right)^{*}$ & - & $2.5 \times 10^{-4}$ & $4.7 \times 10^{-4}(\mathrm{E} 1)$
\end{tabular}

* The number in parenthesis denotes that this is the total number of internal conversion electrons per disintegration.

\section{Coincidence Experiments}

Two scintillation counters using $\mathrm{NaI}(\mathrm{Tl})$ crystals and equipped with differential pulse height analysers were used to measure coincidences between certain gamma-ray lines. The resolving time of the coincidence

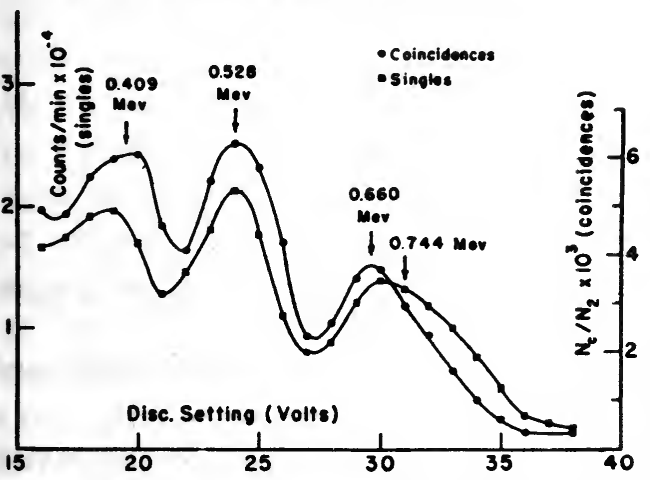

Fig. 4. Gamma-Gamma Coincidence Experiment. (Channel 2 Set on the Line at $0.744 \mathrm{MeV}$ ) circuit was 0.2 micro-sec.

Two sets of experiments were carried out. In the first one, Channel 2 was set on the high side of the peak arising from the $0.660+0.744 \mathrm{MeV}$ lines, and Channel 1 was swept through the range of the spectrum. Fig. 4 shows the result of the experiment.

It will be seen that the coincidence curve shows a peak at $0.660 \mathrm{MeV}$, somewhat to the lower energy side of the composite peak shown in the singles spectrum. Peaks are also seen for the 0.528 and $0.409 \mathrm{MeV}$ lines in the coincidence spectrum.

A similar experiment was carried out in which Channel I was set on the line at $1.15 \mathrm{MeV}$ and Channel 2 swept across the spectrum. The results show that the $1.15 \mathrm{MeV}$ line is in coincidence with those at 0.660 and $0.528 \mathrm{MeV}$ but not with those at 0.409 and $0.744 \mathrm{MeV}$.

\section{Discussion of the Results on $\mathrm{I}^{130}$}

The experiment showed that, in addition to the ganma-ray lines established by Roberts et al., there is an additional gamma-ray having an 
energy of $1.15 \mathrm{MeV}$. The position of the line at $0.409 \mathrm{MeV}$ is fixed by the difference in energy of the two beta-ray groups and is therefore placed at the top of the disintegration scheme in agreement with previous work. The existence of the line at $1.15 \mathrm{MeV}$ and the gamma-gamma coincidence experiments involving this line show that it must be in parallel with those at 0.409 and $0.744 \mathrm{MeV}$, which definitely fixes the position of the $0.744 \mathrm{MeV}$ line. The other two lines are placed in cascade at the bottom of the scheme. Relative intensity determinations, although not very accurate, tend to confirm this assignment. The proposed decay scheme is shown in Fig. 5 .

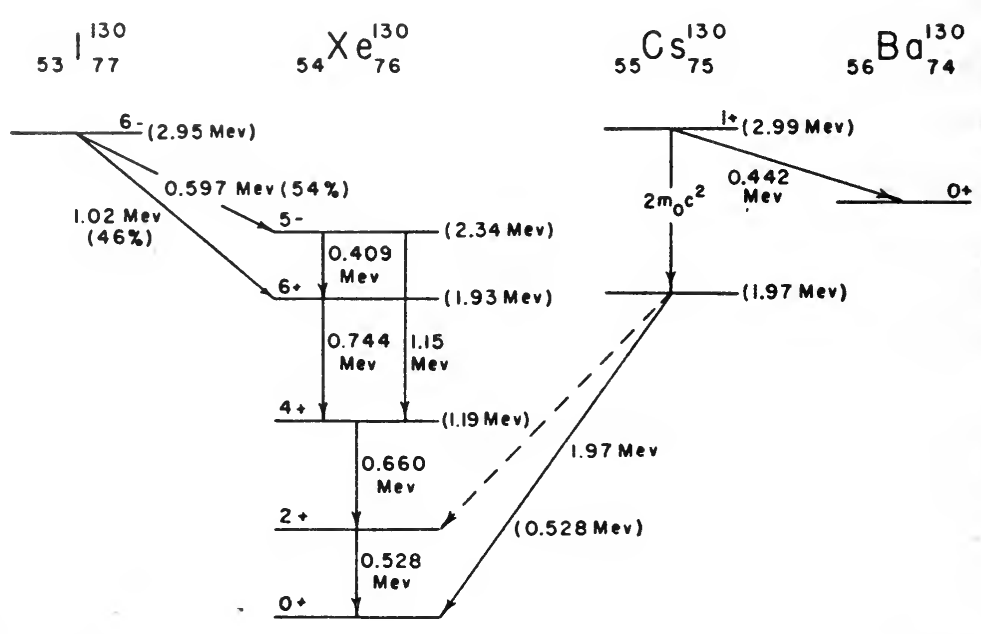

Fig. 5. Disintegration Schemes for the Sequence $\mathrm{I}^{130} \rightarrow \mathrm{Xe}^{130} \leftarrow \mathrm{Cs}^{130} \rightarrow \mathrm{Ba}^{130}$

The line at $0.528 \mathrm{MeV}$ is placed below that at $0.660 \mathrm{MeV}$ from considerations involving the collective model.

In order to obtain some idea of the spins and parities of the states involved, the internal conversion coefficients of the various lines have been calculated and compared with the values given by Rose, Goertzel and Perry ${ }^{4}$. Since the lines at 0.528 and $0.660 \mathrm{MeV}$ are in cascade and follow all beta and all other gamma-transitions, the calculation is straightforward. In order to calculate the internal conversion coefficients for the other lines, the number of transitions per disintegration for the line in question must be calculated making use of the assumed disintegration scheme, the relative abundance of the two beta-ray groups, and the branching ratio for the gamma-rays emitted from the state at $2.34 \mathrm{MeV}$. The branching ratio

$$
X=\frac{I_{0.409}}{I_{0.409}+I_{1.15}}
$$

4. M. Rose, G. H. Goertzel and C. L. Perry, ORNL 1023, 1951, June 25. 
OH. XXII (III)] SEQUENCE $\mathrm{I}^{130} \rightarrow \mathrm{Xe}^{130} \leftarrow \mathrm{Cs}^{130} \rightarrow \mathrm{Ba}^{130}$ AND LEVELS OF $\mathrm{Xe}^{130}$

can be calculated either from the observed relative intensities of the lines at 0.409 and $1.15 \mathrm{MeV}$ or from the relative intensities of the 0.409 and $0.744 \mathrm{MeV}$ line taking into consideration the relative abundance of the two beta-ray groups. The value for the branching ratio is $X=0.47 \pm 0.04$. The number of transitions per disintegration involving the line at $0.744 \mathrm{MeV}$ is therefore

$$
\frac{N_{0.744}}{N_{\mathrm{dis}}}=0.54 X+0.46=0.79 .
$$

Taking into account the number of $K$-internal conversion electrons per disintegration given in Table III, the internal conversion coefficient can be calculated. A similar procedure was used for the other lines. The results together with the theoretical values are shown in Table III. The internal conversion coefficients for lines at $0.528,0.660$ and $0.744 \mathrm{MeV}$ all agree with the theoretical prediction for $\mathrm{E} 2$ radiation. The internal conversion coefficient of the 0.409 and $1.15 \mathrm{MeV}$ lines are much less certain since their determination is very sensitive to the branching ratio $X$. The line at $1.15 \mathrm{MeV}$ appears to be reasonably consistent with $\mathrm{El}$ radiation, while that at $0.409 \mathrm{MeV}$ might be E1 $\left(4.2 \times 10^{-3}\right)$, E2 $\left(4.0 \times 10^{-2}\right)$ or Ml $\left(1.74 \times 10^{-2}\right)$. Since the ground state of $\mathrm{Xe}^{130}$ has zero spin and even parity, the E2 character of the lines at $0.528,0.660$ and $0.744 \mathrm{MeV}$, suggests spins of 2, 4, 6 and even parity for the first three excited states. This is in agreement with what is to be expected from either the single particle model for an even-even nucleus or from the collective model. The value of $\log f t$ for the $1.02 \mathrm{MeV}$ beta group would imply $\Delta I=0$, yes for this transition ${ }^{5}$. Combining this information with that obtained for the first three excited states of $\mathrm{Xe}^{130}$, the spin and parity of the ground state of $\mathrm{I}^{130}$ is 6 , odd. The value of $\log f t$ for the $0.597 \mathrm{MeV}$ group shows that it is allowed and is consistent with $\Delta I=1$, no. The spin and parity of the state in $\mathrm{Xe}^{130}$ at $2.34 \mathrm{MeV}$ would then have to be 5, odd which is consistent with the $\mathrm{E} 1$ character of the $1.15 \mathrm{MeV}$ line. The internal conversion coefficient and relative intensity of the line at $0.409 \mathrm{MeV}$ is still somewhat of an anomaly.

Since neither the parent nor the product nucleus corresponds to a case in which the proton or the neutron number is one less or one greater than a closed shell, the single particle model is not strictly applicable. It is interesting to compare the present results with what would be expected on the basis of the collective model of Bohr and Mottelson ${ }^{6.7}$. They poirt

5 L. W. Nordheim, Rev. Mod. Phys. 23 (1950) 322.

- A. Bohr and B. R. Mottelson, Dan. Mat. Fys. Medd. 27 (1953) No. 16.

7 A. Bohr and B. R. Mottelson, Phys. Rev. 90 (1953) 717. 
out that in the region between closed shells, the strong coupling collective model gives good results for the ratio of the energies of the lower excited states. The closer one approaches closed shells (magic numbers for protons or neutrons) the less appropriate the strong coupling theory becomes. In the present instance both the parent and product are reasonably close to closed shells (50 protons and 82 neutrons). Nevertheless, these experiments may serve as a test of the collective model.

For even-even nuclei with sufficiently great deformation, the collective model predicts a rotational spectrum of levels with energies ${ }^{6}$ :

$$
E_{I}=\left(\hbar^{2} / 2 J\right) I(I+1)+\Delta E_{I} ; I=0,2,4, \ldots,
$$

in which $J$ is the effective moment of inertia, and $\Delta E_{I}$, the first order correction to the strong-coupling formula, is given approximately by:

$$
\Delta E_{I}=-2(\hbar \omega)^{-2}\left(\hbar^{2} / J\right)^{3} I^{2}(I+1)^{2} .
$$

Here $\hbar \omega$ is the surface phonon excitation energy, about $2.2 \mathrm{MeV}$ at $A=130$. Eq. (2) is the sum of Eq. (VI 3a) and (VI $3 \mathrm{~b}$ ) of reference 6, in which $\omega_{\beta}$ and $\omega_{\gamma}$ have been replaced by limiting strong-coupling values.

One may check the rotational interpretation of the low states of $\mathrm{Xe}^{130}$ qualitatively in several ways: (1) the level order is consistent with the predicted order $0+, 2+, 4+, 6+$. (2) One can calculate the nuclear deformation from the energy of the first excited state using the relation 8

$$
\beta^{2}=171 / A^{8 / 8} E_{2}(\mathrm{MeV}) \text {. }
$$

The result is $\beta=0.310$, which fits very well into the systematic trend of the other $\mathrm{Xe}$ isotopes ${ }^{8}$, suggesting a regularly decreasing nuclear deformation as the closed neutron shell at $N=82$ is approached. (3) The ratios of the energies of the excited states, $\mathrm{E}_{4} / \mathrm{E}_{2}$ and $\mathrm{E}_{6} / \mathrm{E}_{2}$ deviate from the limiting strong coupling ratios in the expected direction, but the deviation is too large to receive quantitative explanation in terms of the first order correction given by Eq. (2). The comparison of theoretical and experimental ratios is shown in Table IV. It seems reasonable to interpret the

TABLE IV

Energy Ratios for Excited States in $\mathrm{Xe}^{130}$

\begin{tabular}{lc|c|c}
\hline \multirow{2}{*}{ Experiment } & \multicolumn{2}{|c}{ Theory } \\
\cline { 3 - 4 } & Without $\Delta E_{1}$ & With $\Delta E_{1}$ \\
\hline & 2.25 & 3.33 & 1.94 \\
$\mathrm{E}_{1} / \mathrm{E}_{2}$ & 3.66 & 7.00 & - \\
$\mathrm{E}_{6} / \mathrm{E}_{2}$ & & &
\end{tabular}

8 K. W. Ford, Phys. Rev. 90 (1953) 29. 
$\mathrm{Xe}^{130}$ levels as collective rotations, but the nuclear deformation is not great enough to lend quantitative validity to the strong coupling approximation which works so well in the rare earth region and the region near Uranium.

Ford ${ }^{9}$ has calculated the values of $\beta_{1}$ from the already existing data on the first excited states of $\mathrm{Xe}^{126}, \mathrm{Xe}^{128}$ and $\mathrm{Xe}^{132}$ with the help of Eq. (3). The value obtained for $\beta_{1}$ using the $0.528 \mathrm{MeV}$ line of $\mathrm{Xe}^{130}$ is 0.310. The results for the four even isotopes of $\mathrm{Xe}$ are given in Table $\mathrm{V}$.

TABLE V

Values of $\beta_{1}$ for Isotopes of $\mathrm{Xe}$

\begin{tabular}{c|l|c}
\hline \hline Isotope & $E_{1}(\mathrm{MeV})$ & $\beta_{1}$ \\
\hline${ }_{54} \mathrm{Xe}_{72}^{126}$ & 0.382 & 0.374 \\
${ }_{54} \mathrm{Xe}_{74}^{128}$ & 0.428 & 0.351 \\
${ }_{54} \mathrm{Xe}_{78}^{130}$ & 0.528 & 0.310 \\
.${ }_{54} \mathrm{Xe}_{78}^{132}$ & 0.68 & 0.271
\end{tabular}

The values of $\beta_{1}$ for a considerable number of isotopic species have been obtained by Ford and are discussed in his paper.

A word is in order about the character of the ground state of $I^{130}$. The experiments establish rather convincingly that the spin is 6 and the parity odd. Having leaned heavily on the collective model in discussing the product, we turn hopefully to the shell model for guidance in regard to the parent. From the point of view of the extreme single particle model, the 53rd proton should be in a $g_{7 / \mathrm{s}}$ or $d_{5 / \mathrm{s}}$ state, the energies of the two being approximately equal. The 77th neutron should be in a $d_{3 / 2}$ or $h_{11 / 2}$ state. Since the parity has been established by the experiment to be odd, the neutron would be presumed to be in an $h_{11 / \mathrm{s}}$ state. The question of the spin is somewhat more difficult. Following Nordheim's rule, for the extreme single particle formalism, one would expect spins of 2 or $>3$ for the configurations $\left(d_{3 / 3}, h_{11 / 3}\right)$ or $\left(g_{1 / 2} h_{11 / 3}\right)$ respectively. On the other hand, we are not dealing with a single particle or a single hole for either the protons or the neutrons so that the single particle picture is oversimplified. The collective model would predict that, since the $h_{11 / \mathrm{s}}$ shell is more than halffilled, the action of the neutron may be looked upon as that of a "hole" in the $h_{11 / 2}$ shell. The theory would then predict that the spin of $11 / 2$ would line up with the symmetry axis of the deformed nucleus and the interaction with the proton would give a resultant spin of $11 / 2 \pm 1 / 2$. Such a

- The author is indebted to Dr K. W. Ford for making this calculation. 
prediction would be in agreement with experiment. This collective interpretation also considers only the last odd proton and last odd neutron, however. Neither model can make a really satisfactory prediction about the spin because of our ignorance of the detailed configurations of all the nucleons cutside closed shells.

\section{$\S$ 2. The Disintegration of $\mathbf{C s}^{130}$}

As mentioned earlier, the disintegration of $\mathrm{Cs}^{130}$ has been investigated by Snith, Mitchell and Caird. The half-life is $30 \mathrm{~min}$. The particle spectrum was measured in a magnetic lens spectrometer and is shown in Fig. 6 of Chapter VIII. On making the Fermi Plot it was discovered that the points fall into two groups, one characteristic of positrons having an end point of $1.97 \mathrm{MeV}$ and the other of electrons having an end point of $0.442 \mathrm{MeV}$. Other experiments, in which the electrons were separated from the positrons confirmed the analysis. In addition the particle distribution shows Auger electrons arising from $K$-electron capture. It is clear, therefore, that $\mathrm{Cs}^{130}$ goes to $\mathrm{Xe}^{130}$ by positron emission and to $\mathrm{Ba}^{130}$ by betaray emission. The characteristics of the decay are given in Table VI.

TABLE VI

Characteristics of the Decay of $\mathrm{Cs}^{130}$

\begin{tabular}{ll|c|c|c}
\hline \multicolumn{1}{c|}{ Energy $\mathrm{MeV}$} & $\begin{array}{c}\% \\
\text { Abundance }\end{array}$ & $\log f t$ & Auger/Positron \\
\hline $\mathrm{Cs}^{130} \rightarrow \mathrm{Xe}^{130}(\mathrm{e}+)$ & 1.97 & 96.5 & 5.08 & 0.045 \\
$\mathrm{Cs}^{130} \rightarrow \mathrm{Ba}^{130}(\mathrm{e}-)$ & 0.442 & 3.5 & 4.96 & -
\end{tabular}

A search was made for gamma-rays using a scintillation counter and pulse height analyzer. A peak corresponding to annihilation radiation was seen. No gamma-rays at 0.660 or $0.744 \mathrm{MeV}$ could be found. The lines at 0.528 and $0.409 \mathrm{MeV}$ would be hard to detect on account of the strong peak from the annihilation radiation. The annihilation peak was not broadened appreciably and it is felt that at least the line at $0.409 \mathrm{MeV}$ is also absent. In addition an X-ray of energy $29.5 \mathrm{keV}$ was found arising from the $K_{a}$ radiation from Xenon. Finally no internal conversion electrons were seen.

The log $f t$ values, shown in Table VI, indicate that the decay to both $\mathrm{Xe}^{130}$ and $\mathrm{Ba}^{130}$ is allowed. Assuming that the disintegration of $\mathrm{Cs}^{130}$ takes place to the ground state of both $\mathrm{Ba}^{130}$ and $\mathrm{Xe}^{130}$, the validity of which will be considered further below, it follows, from the allowed character of the decay and from the fact that $\mathrm{Xe}^{130}$ and $\mathrm{Ba}^{130}$ have zero spin and 
even parity, that $\mathrm{Cs}^{130}$ has even parity and spin 1 or 0 , with spin 1 preferred.

The energy available for the disintegration of $\mathrm{Cs}^{130}$ to $\mathrm{Xe}^{130}$ is $1.97+2 m_{0} c^{2} \mathrm{MeV}$. Thus one could expect the higher states of $\mathrm{Xe}^{130}$ to be excited by $K$-electron capture. However, since these states have configurations $4+, 6+$ and $5-$, it is clear that transitions from a $1+$ state of $\mathrm{Cs}^{130}$ to any of these would be highly forbidden, which explains why the lines at $0.409,0.744$ and $0.660 \mathrm{MeV}$ are not seen.

It remains to investigate the transition probability to the 0 and $2+$ states of $\mathrm{Xe}^{130}$ assuming that the character of the ground state of $\mathrm{Cs}^{130}$ is $1+$. As a first approximation, let us assume that the matrix element for the transition to the 0 and $2+$ states of $\mathrm{Xe}^{130}$ are the same, since both transitions should be allowed. One can compute, from the tables, the values of $\left(f_{+}+f_{\Sigma}\right), f_{\Sigma} / f_{+}$for the two transitions involved by using the two decay energies $1.97+2 m_{0} c^{2} \mathrm{MeV}$ and $1.43+2 m_{0} c^{2} \mathrm{MeV}$. The expected positron branching ratio, $\left(f_{+}\right)_{2} /\left[\left(f_{+}\right)_{0}+\left(f_{+}\right)_{2}\right]=0.15$. However on the basis of the strong coupling collective model, the matrix element for the transition to the $2+$ state will be lowered compared to that to the ground state. This comes about owing to the quantization of the particle motion along the axis of the deformed nucleus. On computing this effect, the branching ratio to be expected ${ }^{9}$ is $\lambda_{2} /\left[\lambda_{0}+\lambda_{2}\right]=0.07$. Thus, one would have to detect a positron group whose energy is $1.47 \mathrm{MeV}$ and whose relative abundance is $7 \%$. Such a group was not detected although a weaker electron group was seen. The presence of a $1.47 \mathrm{MeV}$ positron group cannot be excluded since, owing to its relatively high end-point, the group would be much more spread out and more difficult to detect than the much lower energy electron group. The problems connected with the measurement of a substance of 30 min half-life considerably enhance the difficulty of finding weak groups.

The additional information obtained from the experiments on $\mathrm{Cs}^{130}$ is shown in the decay scheme of Fig. 5. In addition to the discussion of the character of the states, it is obvious that the masses of all isotopes in the sequence can be determined relative to one of the members, say $\mathrm{Xe}^{\mathbf{1 3 0}}$.

From a naive single particle point of view the character of the ground state of $\mathrm{Cs}^{130}$ can be explained if the 55th proton is assumed to be in a $d_{x / 2}$ state and the 75th neutron in a $d_{z / 2}$ state. Such a configuration could give a spin 1 and even parity. At the moment it is difficult to explain why $I^{130}$ has a configuration $6-$ and $\mathrm{Cs}^{130}$ a configuration $1+$ except as a result of the phenomenological description already given. However, the best way to gain enough experimental information so that a logical theoretical explanation may be forthcoming is to measure sequences like the one discussed here. 


\section{(IV) P. PREISWERK - Discussion of the Scheme of Radioactive Decays Leading to Cd111}

The level scheme of C'd11 is one of those best studied. Special techniques have been applied which delivered data not, or not yet, available in other cases. First we shall summarize very roughly the elements which have been measured and the way in which the conclusions for the disintegration scheme have been drawn. Secondly. we shall discuss how far our understanding of the phenomena has evolved.

The scheme of disintegration of the two neighbouring isobars ${ }_{47} \mathbf{A g}^{111}$ and ${ }_{49} \mathrm{In}^{111}$ and of the decay of the 48 minutes isomer of $\mathrm{Cd}^{111}$, which is generally admitted to be correct, is reproduced in Figure 1.

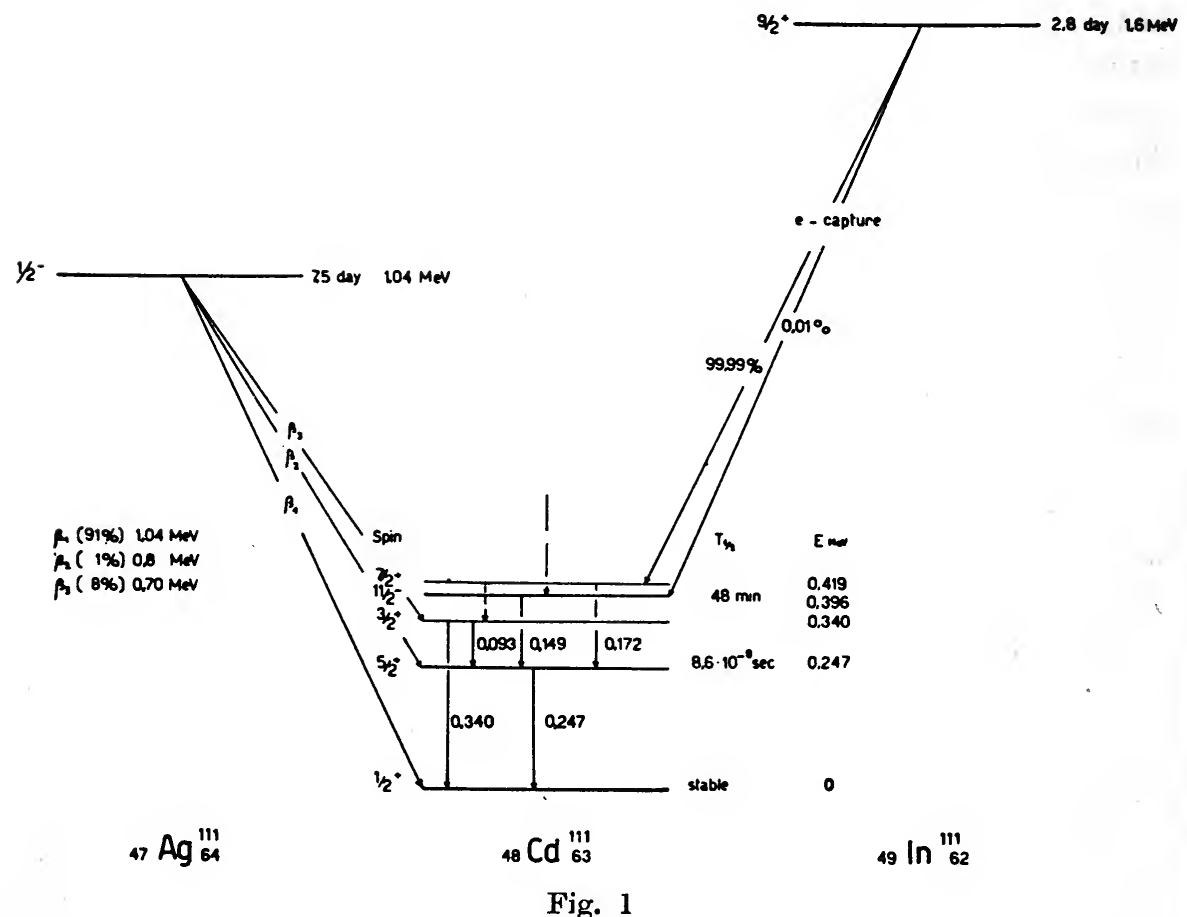

\section{$\S$ 1. Summary of Measurements}

\section{A. $\beta$-DECAY of $\operatorname{Ag}^{111}(7,5 \mathrm{~d})$}

The Fermi plot and the presence of the $\gamma$-lines in unequal intensity in the decay of $\mathrm{Ag}^{111}$, shows that the $\beta$-spectrum is complex and has three components. 
TABBLE I

Partial- $\beta$-transitions

\begin{tabular}{l|c|c|c}
\hline & Intensity & Maximum Energy & $\log f t$ \\
\hline 1 & $91 \%$ & $1.04 \mathrm{MeV}$ & 7.30 \\
2 & $1 \%$ & $0.80 \mathrm{MeV}$ & 8.85 \\
3 & $8 \%$ & $0.70 \mathrm{MeV}$ & 7.78
\end{tabular}

For the determination of the $f t$ values of the partial spectra, Johansson 1 had to have recourse to the relative intensity of the $\gamma$-rays which follow the $\beta$-transitions. The low relative intensity of the second partial spectrum prevented decomposition with the help of the Fermi-Kurie plot and prevented the determination of the form of the spectrum even more which is - on account of the high $f t$ value-expected to deviate from the allowed form.

\section{B. 48 Minutes Isomer of $\mathrm{Cd}^{111}$}

The 48 minutes isomer can only be formed by highly forbidden transitions from $\mathrm{Ag}^{111}$ and $\mathrm{In}^{111}$. It is produced in different reactions for example by the $\operatorname{Cd}^{111}(n, n) C^{111}$ reaction.

\section{Electron Capture of In ${ }^{111}$}

No positron emission in the $\mathrm{In}^{111}$ decay could be detected ${ }^{2}$. The desintegration energy (the value is needed at least for the evaluation of the $f t$ value) could be determined from the threshold energy $(2.35 \pm 0.2) \mathrm{MeV}$ of the ${ }_{48} \mathrm{Cd}^{111}(\mathrm{p}, \mathrm{n})_{49} \mathrm{In}^{111}$-reaction. The observation of a low intensity partial branch is still more difficult in electron capture than in $\beta$-desintegration. In particular, if one intends to prove that no direct transitions to the ground state of $\mathrm{Cd}^{111}$ occur, one has to compare the $\mathrm{X}$-rays and $\gamma$ rays intensities. The measurement of the continuous $\gamma$-ray spectrum accompanying electron capture would in principle give the desired information, but due to the low intensity this method most often is not feasible in practice. It is found that most of the $\mathrm{In}^{111}$ goes to the $419 \mathrm{keV}$ level of $\mathrm{Cd}^{111}$. No other transition is appreciable, only $0.01 \%$ decays to the $396 \mathrm{keV}$ level. The presence of the $93 \mathrm{keV}$ line in weak intensity indicates a weak, not yet detected $\gamma$-transition most probably from the $419 \mathrm{keV}$ level to the $340 \mathrm{keV}$ level.

\section{D. $\gamma$-Transitions}

The $\gamma$-lines following the mentioned decays were measured with normal

1 S. Johansson, Phys. Rev. 79 (1950) 896.

2 C. L. Mc Ginnis, Phys. Rev. 81 (1951) 734. 
accuracy by measuring photo and conversion electrons. The values indicated by different authors agree within few keV. From a higher precision measurement one would not be able to draw more information unless a splitting is expected, but a higher resolution in order to separate the $L$-lines would be desirable as a suitable supplementary check of the radiation character. The actual limitation for the resolution is mainly determined by the difficulty to make sufficiently thin samples.

TABLE II

$\gamma$-Lines

\begin{tabular}{|c|c|c|c|c|c|c|}
\hline \multirow[b]{2}{*}{ Decay } & \multirow[b]{2}{*}{$\begin{array}{c}\text { Energy } \\
\text { keV }^{\top}\end{array}$} & \multicolumn{2}{|c|}{ Conversion } & \multirow{2}{*}[\frac{(N_{e})_{K}}{N_{\gamma}}]{$_{\exp }$} & \multirow{2}{*}[\frac{(N_{e})_{K}}{N_{\gamma}}]{$_{\text {theor. }}$} & \multirow{2}{*}{$\begin{array}{l}\text { Multipole } \\
\text { character }\end{array}$} \\
\hline & & $\frac{\left(N_{e}\right)_{K}}{\left(N_{e}\right)_{L}}$ & $\frac{N_{e}}{N_{\gamma}}$ & & & \\
\hline $\mathbf{A g}^{111}$ & $\begin{array}{l}243 \pm 2 \\
340 \pm 2\end{array}$ & & $\begin{array}{l}<0.08 \\
\sim 0.015\end{array}$ & & & $\begin{array}{l}\mathrm{E} 2 \text { or } \\
\mathrm{E} 2+\mathrm{M} 1\end{array}$ \\
\hline $\begin{array}{l}48^{\prime} 6 \min - \\
\mathrm{Cd}^{111}\end{array}$ & $\begin{array}{l}149.6 \pm 0.3 \\
246 \pm 2\end{array}$ & $\begin{array}{l}1.99 \pm 0.3 \\
5.12 \pm 0.22\end{array}$ & $\begin{array}{l}2.3 \\
\text { (indirect } \\
\text { determi- } \\
\text { nation) }\end{array}$ & & & E3 \\
\hline $\operatorname{In}^{111}$ & $\begin{array}{c}172.1 \pm 0.5 \\
246.6 \pm 0.7 \\
330 \pm 10 \\
93 \pm 10\end{array}$ & $\begin{array}{l}6.6 \pm 0.4 \\
5.19 \pm 0.01\end{array}$ & $\begin{array}{l}0.12 \\
0.06\end{array}$ & $\begin{array}{l}0.10 \pm 0.010 \\
0.054 \pm 0.005\end{array}$ & $\begin{array}{l}0.0982 \pm 0.0013 \\
0.0544 \pm 0.0008\end{array}$ & $\begin{array}{l}\mathrm{M} 1+\mathrm{E} 2 \\
\mathrm{E} 2\end{array}$ \\
\hline
\end{tabular}

In the whole scheme the $\gamma$-cascade $(172 \mathrm{keV}-247 \mathrm{keV})$ is the crucial part and many measurements are made by using it as a reference.

For these two $\gamma$-lines, the $K$ conversion coefficient was measured accurately ${ }^{2}$ by the method based on the measurement of the $\mathrm{e}^{-}-\mathrm{e}^{-}$-coincidences. Figure 2 shows a measurement of the conversion electron lines and the respective $\mathrm{e}^{-}-\mathrm{e}^{-}$-coincidences ${ }^{3}$ (Electron lines of $\mathrm{In}^{114}$ are superimposed).

The result is accurate enough to fix the $172 \mathrm{keV}$ transition as Ml with eventually only small admixtures of $\mathrm{E} 2$, and the $247 \mathrm{keV}$ transitions as pure E2. The half-life of the 247 level was measurable by delayed coincidences $\left(8.6 \times 10^{-8} \mathrm{sec}\right)$. More sensitive to small admixtures is the directional $\gamma-\gamma$-correlation. From such a measurement it can be concluded that in the first cascade partner $0.9 \% \mathrm{E} 2$ radiation is admixed to the M1 radiation (if an order of levels $7 / 2,5 / 2,1 / 2$ is assumed) 4 .

3 O. Huber, F. Humbel, H. Schneider and A. de Shalit, Helv. Phys. Acta 25 (1952) 3.

4 H. Aeppli, H. Frauenfelder and M. Walter, Helv. Phys. Acta, 24 (1951) 335. 


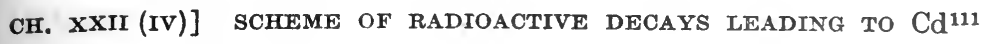

For the other transitions the conversion coefficients are obtained in a less direct way and are less acurate. For instance, for the $149 \mathrm{keV}$ transition (half-life $48 \mathrm{~min}$ ) which is also in cascade with the $247 \mathrm{keV}$ transition, the $\mathbf{e}^{-}-\mathrm{e}^{-}$-coincidence measurement was not sufficiently accurate. The second electron line for which the coincidence rate would have to be detected is too different in intensity. Instead of this, the $\gamma$-intensity of the $149 \mathrm{keV}$ transition relative to that of the $247 \mathrm{keV}$ transition was measured. For the $247 \mathrm{keV}$ transition the ratio of conversion electrons to $\gamma$-rays was already known. And as in the cascade both partners have the same intensity, the intensity of the conversion electrons of the $149 \mathrm{keV}$ transition can be derived and the conversion coefficient follows. So it was possible to establish the E3-character of the isomeric $149 \mathrm{keV}$ transition ${ }^{5}$. For the determination of the conversion coefficient of the $340 \mathrm{keV}$ transition the relative intensity of the $\beta$-spectra were used. The value obtained limits the possibilities to $\mathrm{E} 2$ or $\mathrm{E} 2+\mathrm{M} 1$ radiation.

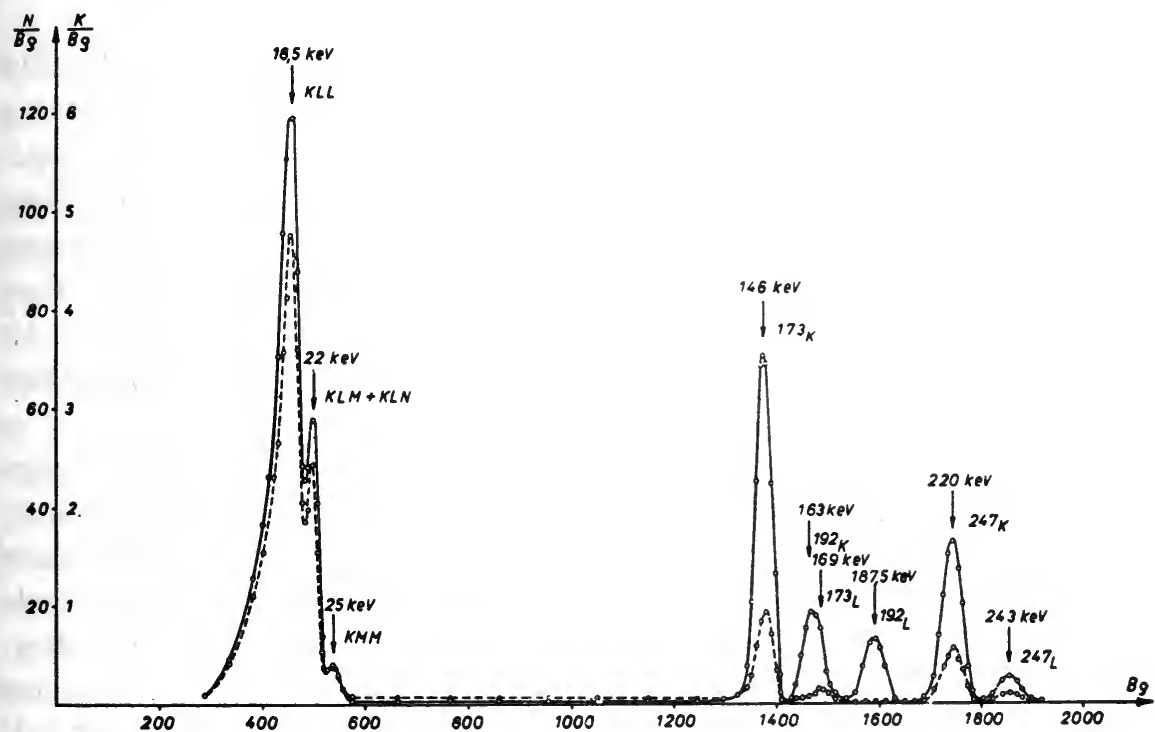

Fig. 2

\section{E. Magnetic Moment}

The magnetic moment of the ground state

$$
\mu(1 / 2+)=-(0.59492 \pm 0.00008) \mu_{k}
$$

is well determined by classical methods (nuclear induction). In the case of the first excited level at $247 \mathrm{keV}$, it was possible for the first time to

s C. L. Mc Ginnis, Phys. Rev. 83, (1951) 686. 
measure the value and sign of the $g$ factor for an excited short lived level ${ }^{6}$. This level has a suitable life-time, so that the disturbance of the directional correlation by the interaction of the magnetic moment with an external magnetic field became measurable

$$
g\left(\mathrm{Cd}^{111}-247 \mathrm{keV} \text { level }\right)=-(0.28 \pm 0.05) .
$$

\section{F. Quadrupole Moment}

It has been shown for the same cascade that the external inhomogeneous electric field of the material surrounding the decaying atoms may disturb the directional $\gamma-\gamma$-correlation by an interaction with the quadrupole moment of the intermediate state. The quadrupole moment of $\mathrm{Cd}^{111}$ in its excited state at $247 \mathrm{keV}$ could be derived?

$$
Q\left(\mathrm{Cd}^{111}-247 \mathrm{keV} \text { level }\right)=5.10^{-25} \mathrm{~cm}^{2} .
$$

\section{G. Spin and Parity Assignment}

Besides the spin of the ground state, which has been measured directly as $1 / 2$, the assignment for the excited levels has to be based on data coming from conversion and directional correlation measurements, on semiempirical classification of transition probabilities and on some assumptions deduced from the shell model. Though we have no special reason for not believing in the deduction used, we nevertheless want to remember that several fundamental assumptions are not yet directly verified. An "abnormal" matrix element may occur and a wrong class of transition probability may be simulated.

The ascription of even parity to the ground state is based on the sign of the $g$-factor and the classification of the magnetic moments. The parity of the other levels follows immediately from the parity of the ground state and the multipole character of the $\gamma$-and $\beta$-transitions. The multipole character of the $172 \mathrm{keV}$ and the $247 \mathrm{keV}$ transitions leaves open two alternatives for the spinss of the $419 \mathrm{keV}$ and $247 \mathrm{keV}$ levels. The E2 character of the $247 \mathrm{keV}$ transition to the ground state $1 / 2+$ allows for the $247 \mathrm{keV}$ level an assumption of the values $5 / 2+$ or $3 / 2+$ for the spin. Following the Ml character of the $172 \mathrm{keV}$ transition $7 / 2+$ would be the spin of the $419 \mathrm{keV}$ level in the first case and $5 / 2+$ in the second. Arguments against the second alternative are that a crossover transition from the $419 \mathrm{keV}$ level would be possible with E2 and is not observed, and secondly the

6 H. Aeppli, H. Albers-Schönberg, H. Frauenfelder and P. Scherrer, Helv. Phys. Acta, 25 (1952) 339.

${ }^{7}$ H. Albers-Schönberg, K. Alder, O. Braun, E. Heer and T. B. Novey, Phys. Rev. 91 (1953) 1287. 
negative sign of the $g$-value of the $247 \mathrm{keV}$ level is only compatible with a $d_{\% /,}+$ state and not a $d_{\% /,}+$ state. From the E3 character of the isomeric $149 \mathrm{keV}$ transition, leading to the $247 \mathrm{keV}^{5} / 2+$ level, $11 / 2$ - follows for the spin of the $396 \mathrm{keV}$ level. The spin $9 / 2$ - for the $396 \mathrm{keV}$ level would allow M2 radiation which is not indicated by the conversion coefficient. The uncertainty of an eventual $\mathrm{Ml}$ admixture in the $340 \mathrm{keV}$ radiation leaves open for the $340 \mathrm{keV}$ level the spin possibilities $3 / 2+$ and $5 / 2+$. A decision can be reached with the help of the $\beta$-transitions. $f t$ values classify the transitions $\beta_{1}$ and $\beta_{3}$ as first forbidden transitions $(\Delta l=1, \Delta J=0$ or 1$)$ ard $\beta_{2}$ as forbidden $(\Delta l=1, \Delta J=2)$ transition. From $\beta_{1}$ and the spin of the ground state of $\mathrm{Cd}^{111}$ follows that $\mathrm{Ag}^{111}$ must have therefore either spin $1 / 2-$ or $3 / 2-$. From $\beta_{2}$ and the spin assignment of $5 / 2+$ to the first level, follows that either $1 / 2$ - or $9 / 2$ - would be possible for the spin of $\mathrm{Ag}^{111}$. Only the spin $1 / 2$ - satisfies therefore both conditions. From this value with help of $\beta_{3}$ the spin $3 / 2+$ can be concluded for the $340 \mathrm{keV}$ level.

\section{§ 2. Interpretation}

\section{A. Spin and Parity}

The shell model has proved to be very useful for the interpretation of nuclear states, and it is obvious that the discussion of the disintegration scheme has to be made in the light of this model. Indeed, the first striking

N

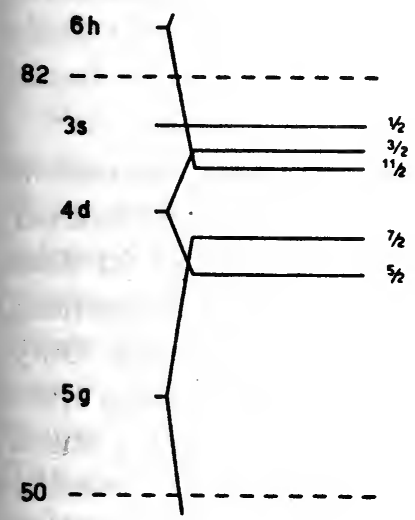

Fig. 3 fact is that the lowest levels of $\mathrm{Cd}^{111}$ have the spins and parities expected from the single particle shell model, and no second level with the same spin or levels with a different spin are present.

$\mathrm{Cd}^{111}$ was the first example where a unique correspondence between so many excited states and the levels of the single particle shell model seemed to exist. This behaviour encouraged to ascribe to the level the quantum number of the orbit of the single particle besides the good quantum numbers spin and parity. But we hesitate to do it here although it is normally done and it may be even useful. It is well known that quite a number of important measurable facts contradict such a simple description. It is now established that pure or nearly pure single particle states occur seldom, and that mixed configurations are the norm ${ }^{8}$. We could make the ascription of one con-

- P. Stähelin and P. Preiswerk, Il Nuovo Cimento, 10 (1953) 1219.

A de Shalit and M. Goldhaber, Phys. Rev. 92 (1953) 1211. 
figuration in the sense that we consider this "pure single particle state" as a first approximation to which the other possible states with same spin and parity are mixed, but then we should always have in mind that the mentioned admixed part may be the determinant factor for certain qualities.

\section{B. Energy of Levels and their Spacing}

For nuclei with particle rumber comprised between the same closed shells the spacing of the levels and even their order vary from nucleus to nucleus. The variation depends on the range of forces. The reversion of the level order cannot be taken a priori as a contradiction to the expectations of the single particle model. In several cases systematic shifts of levels are revealed. E.g. in $\mathrm{Cd}^{113}$ the $1 / 2+$ level is followed by a $11 / 2-$. In $\mathrm{Cd}^{111}$ between these levels both $5 / 2+$ and $3 / 2+$ levels are interposed. A possible reason is that the two more neutrons in $\mathrm{Cd}^{113}$ will fill out the two subshells $g_{7 / 2}$ and $d_{s / 3}$. But especially the low spacing of $93 \mathrm{keV}$ of the $5 / 2+$ and the $3 / 2+$ level of $\mathrm{Cd}^{111}$ attracts the attention. A spacing of the order of $1 \mathrm{MeV}$ would be expected for the spacing of a $d_{s / 3}$ and a $d_{3 / 2}$ level from the comparison of the single particle model with the known binding energies. We may see here a consequence of the presence of mixed configurations. As soon as we drop the restriction of single particle states, we have to consider a great number of possibilities of configurations of the 13 neutrons outside the closed particle shell which could be responsible for the small level spacing by the influence of their interaction energy.

\section{Magnetic Moment}

As already mentioned we know the value of the magnetic moment in the case of $\mathrm{Cd}^{111}$, not only for the ground state, but also for the first excited level. The values are about the same. The accuracy obtained so far does not allow to recognize a definite difference. It would be very interesting to know to what extent a change in the magnetic moment occurs. Only the sign of the magnetic moment corresponds to the expectations from the single particle model. The deviation from the Schmidt values is about the same as in other cases for nuclei with odd neutrons. The deviation indicates, as it is well known, the necessity to take into account cooperation properties, as it has been tried in different ways.

\section{Quadrupole Moment}

In the case of the quadrupole moment the situation is quite similar to that described for the magnetic moment. In the special case of $\mathrm{Cd}^{111}$, the quadrupole moment of the first excited level has been measured. The 
value of the measured moment is of the order expected from the empirical known regularities, which are known to be quite larger than what the single particle model could explain. Since $\mathrm{Cd}^{111}$ has spin $1 / 2$ in the groundstate, the quadrupole moment in this state must be 0 .

\section{E. Transition Probabilities}

The semi-empirical classification of transition probabilities ${ }^{9}$ has proved to be extremely useful for the rough interpretation of the nuclear levels. We are however still far from the exact knowledge of the nuclear wave functions involved in $\mathrm{Cd}^{111}$. Since the transition probabilities are very sensitive to the wave functions of the involved nuclear states, they have to be used as criteria for our approach in the description of nuclear states. Further guidance may be found in the comparative studies of the transition probabilities.

\section{a. $\gamma$-Transitions}

Following the single particle model, only the odd neutron would be affected in the transitions of $\mathrm{Cd}^{111}$ In that case electric radiation may occur due to a relativistic term arising from the magnetic moment of the neutron.11 It is also known that the proton and neutron transitions have often, even for electric multipole radiation, the same order of transition probabilities. This fact may be due to the existence of mixed configurations.

Among the $\gamma$-transition in $\mathrm{Cd}^{111}$ there are mainly three for which further characterizing data than energy and multipole character have been determined.

For the $149 \mathrm{keV}$ transition between the $11 / 2$ and $5 / 2$ levels, the E3 character was revealed from the conversion coefficient. The half-life of the $11 / 2$ state is 48 minutes. The observed transitions probabilities are often referred to theoretical values of the Blatt-Weisskopf ${ }^{10}$ formula. For practical reasons this is often done even in cases where the premises of the formula are not fullfilled. If one follows this practice-having in mind this reservation-the $149 \mathrm{keV}$ transition appears to be rather slow. The transition probability is smaller than the reference value by a factor $10^{4}$. The slowness was explained as due to the neutron transition, while the reference value is derived for a charged particle-transition. This fact will not allow the conclusion that in the $149 \mathrm{keV}$ transitions relatively pure

- See e.g. L. W. Nordheim, Phys. Rev. 78 (1950) 294; Rev. mod. Phys. 53 (1951) 315. M. G. Mayer, S. A. Moszowski and L. W. Nordheim, Rev. mod. Phys. 53 (1951) 315. M. Goldhaber and A. W. Sunyar, Phys. Rev. 83 (1951) 906.

10 J. M. Blatt and V. F. Weisskopf, Theoretical Nuclear Physics 1952.

11 B. Stech, Z. Naturforsch., 9a (1954) 1. 
single neutron states are involved, since we know that proton (E3)transitions may occur with the same slowness.

In the case of the $247 \mathrm{keV}$ transition which is a pure E2 transition as it is seen from the directional correlation measurement, the slowness is less pronounced. The mean life-time differs only by a factor of 10 from the reference value.

For the $172 \mathrm{keV}$ transition between the $7 / 2$ and $5 / 2$ states the directional correlation measurements gi ze the ratio of mixture of E2 and M1. The value of the intensity ratio is $|\beta|_{\mathrm{E} 2}^{2}:|\alpha|_{\mathrm{M} 1}^{2}=0.01 \pm 0.003$ and the phase $180^{\circ}$.

If we want to try to explain the intensity ratio, we must have in mind that between the pure $g_{\% / \mathrm{s}}$ and $d_{\% / \mathrm{s}}$ neutron states, E2 radiation would be forbidden. The already mentioned M1 transition would be allowed from a $g_{\% / \mathrm{s}}$ to a $d_{\% / \mathrm{s}}$ state only with nuclear forces depending on the velocity.

\section{b. $\quad \beta$-Transitions}

For the spin assignment to the different excited levels a rough semiempirical classification of the $f t$-value has already been used. Now we want to try if we can get more details out of a finer comparison of $f t$ values inside the different classes.

In the decay of $\operatorname{In}^{111}$ the $f t$ value could be determined for the $\operatorname{In}(9 / 2)^{--}$ $\mathrm{Cd}\left(\gamma_{2}\right)$ transition, but the transition energy is not known very accuratly. This $\operatorname{In}\left({ }^{9} / 2^{+}\right)-\mathrm{Cd}\left({ }^{7} / 2^{+}\right)$transition is interpreted as an allowed one. The log $f t$ value 6.7 is high compared with "normal" values. Goldhaber and de Shalit ${ }^{12}$ have given examples of abnormal values near closed shell in their discussion of mixed configurations in nuclei. $\operatorname{In}^{115}$ is one of these.

The $f t$ values of $\beta_{1}$ and $\beta_{3}$ decay of $\mathrm{Ag}^{111}$ to $\mathrm{Cd}^{111}$ are also relatively high. The $\log f t$ value for the first forbidden transitions with 7.1 and 7.8 are higher than normal ones which are between 6.2 and 7.2. The $\log f t$ value respectively the $\log f_{1} t$ value 13 of 8.1 of $\beta_{2}$ is "normal" for a $(\Delta l=1, \Delta J=2)$-transition.

It is possible to consider only the initial and the final state of the particle involved, if we havè to deal with pure single particle states. As soon as this premise no more holds, we have to include in our considerations the initial and the final states of the proton and neutron configurations. The case of $\mathrm{Cd}^{111}$ is very instructive in this sense. In the picture of the single particle, we would explain the $9 / 2$ and $7 / 2$ transitions as follows:

Initial state

Final state

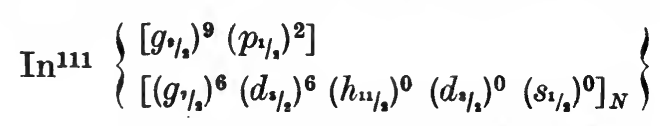

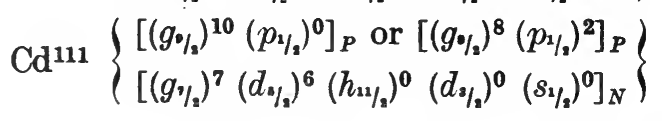

12 A. de Shalit and M. Goldhaber, Phys. Rev. 92 (1953) 1211.

18 J. P. Davidson, Phys. Rev. 82, 48, 51. 
In the first alternative of the final proton configuration, the transition would be forbidden, as three particles are involved. In the second we would see no reason, why the transition should have such a high $f t$ value. But as soon as we do no more consider the final proton states as pure, the assumption of the mixture of the two indicated proton configurations could give the explanation. A reduction of the $f t$ value could naturally also come from the impurity of the neutron configuration. In the case of the proton configurations the possible mixtures are limited since in $\operatorname{In}^{111}$ only one particle is missing for the completion of the 50 particle shell. For the neutron configurations, the number of possibilities is greater. One may arrive to a closer limitation of the possibilities by taking into account the other data as the $\gamma$-transition probabilities. Most probably the problem cannot be solved from considerations based only on one particular disintegration scheme.

\section{Conclusion}

The single particle brought an extremely valuable starting point into the very difficult task to describe nuclear states. The approximation of pure single particle states is far from satisfying. The task of finding out which are the most important admixtures of other configurations, is the next step in nuclear spectroscopy. 


\title{
$\beta$ - AND $\gamma$-DISINTEGRATION OF SOME HEAVY RADIOELEMENTS $(Z>80)$
}

\author{
N. FEATHER
}

\section{$\S 1$. Active Species of the $A=4 n$ and $A=4 n+2$ Series}

\section{A. $\gamma$-Radiations of the $\alpha$-ACtive $\left(\begin{array}{l}e \\ e\end{array}\right)$ Species}

It is an interesting reflection on the increase in sensitivity of experiinental methods that whereas the first 50 years' study of classical radioactivity produced only four examples of $\gamma$-emitting $\alpha$-active $\left(\begin{array}{l}e \\ e\end{array}\right)$ species $\left({ }_{80} \mathrm{Io}^{230},{ }_{90} \mathrm{RdTh}^{228},{ }_{88} \mathrm{Ra}^{226}\right.$ and $\left.{ }_{84} \mathrm{Po}^{210}\right)$, four others, previously overlooked, were similarly identified during the following period of 5 years. And, during the same period, along with these four ${ }_{{ }_{92}} \mathrm{U}^{238},{ }_{92} \mathrm{U}^{234},{ }_{90} \mathrm{Th}^{232}$ and $\left.{ }_{88} \mathrm{ThX}^{224}\right)$, at least nine $\left({ }_{96} \mathrm{Cm}^{244},{ }_{96} \mathrm{Cm}^{242},{ }_{94} \mathrm{Pu}^{240},{ }_{94} \mathrm{Pu}^{238},{ }_{94} \mathrm{Pu}^{236},{ }_{92} \mathrm{U}^{236}\right.$, ${ }_{92} \mathrm{U}^{232},{ }_{92} \mathrm{U}^{230}$ and ${ }_{90} \mathrm{Th}^{226}$ ) of the more recently discovered "artificiallyproduced" species were added to the list. For this spectacular change in the extent of our knowledge the development of the pulse ionisation chamber method of $\alpha$-particle energy spectrometry, and the use of $\beta$ sensitive photographic emulsions in $\alpha$-particle-conversion-electron correlation studies, are mainly responsible.

As will readily be appreciated, the two methods of investigation just mentioned yield complementary results-and neither is very precise. In the former the precision in energy is that appropriate to the (small) differences between the pairs of (large) energy values which individually constitute the primary data; for the latter track-length or grain-count distributions yield the energies of the conversion electrons, and so the $\gamma$-ray energies, but again with rather large relative errors and poor resolution. Relative intensities are in each case subject to normal statistical uncertainty - in the latter case not inconsiderable. In spite of this the overall picture which the collected results provide is one of some simplicity; it represents the most obvious feature of regularity by which present-day information concerning the disintegration modes of the heavy elements is characterised.

Table I contains the experimental results suitably condensed. In column 2 differences in disintegration energy $(\eta)$ between the ground-to-ground state mode $(0)$ and ground-to- $r^{\text {th }}$ excited state mode $(r)$ are given for the various $\alpha$-active species listed in column 1 , and in column 3 the percentage intensities of the various disintegration modes are quoted. Column 4 lists the quantum energies $\left(E_{\gamma}\right)$ of the $\gamma$-radiations, deduced from conversion electron energies or otherwise, and column 5 the intensities of conversion 
electron emission, again expressed as percentages. Wherever possible an estimate of probable error is included. For sake of convenience column 6 gives the daughter species in each case.

TABLE I

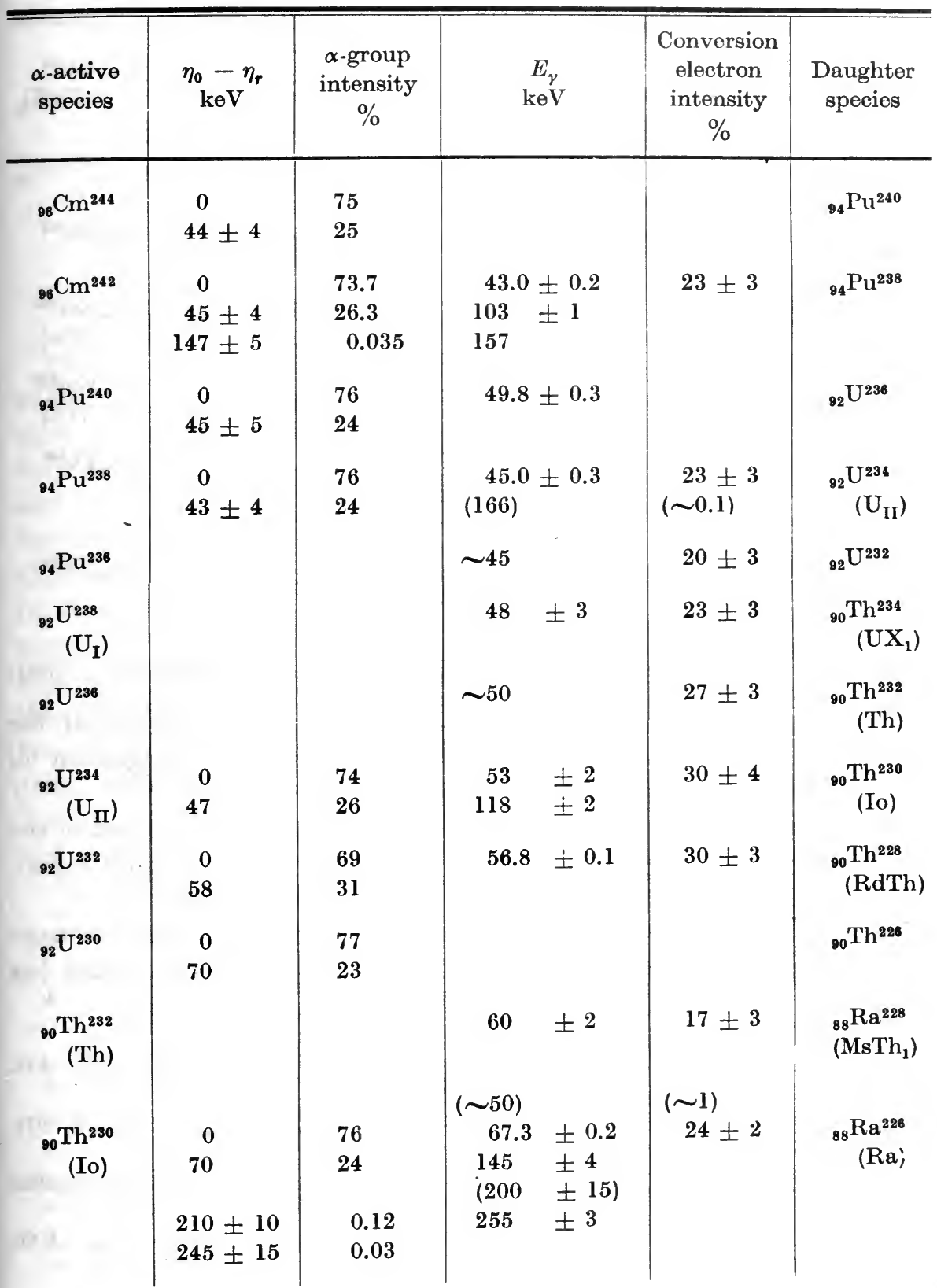


TABLE I (continued)

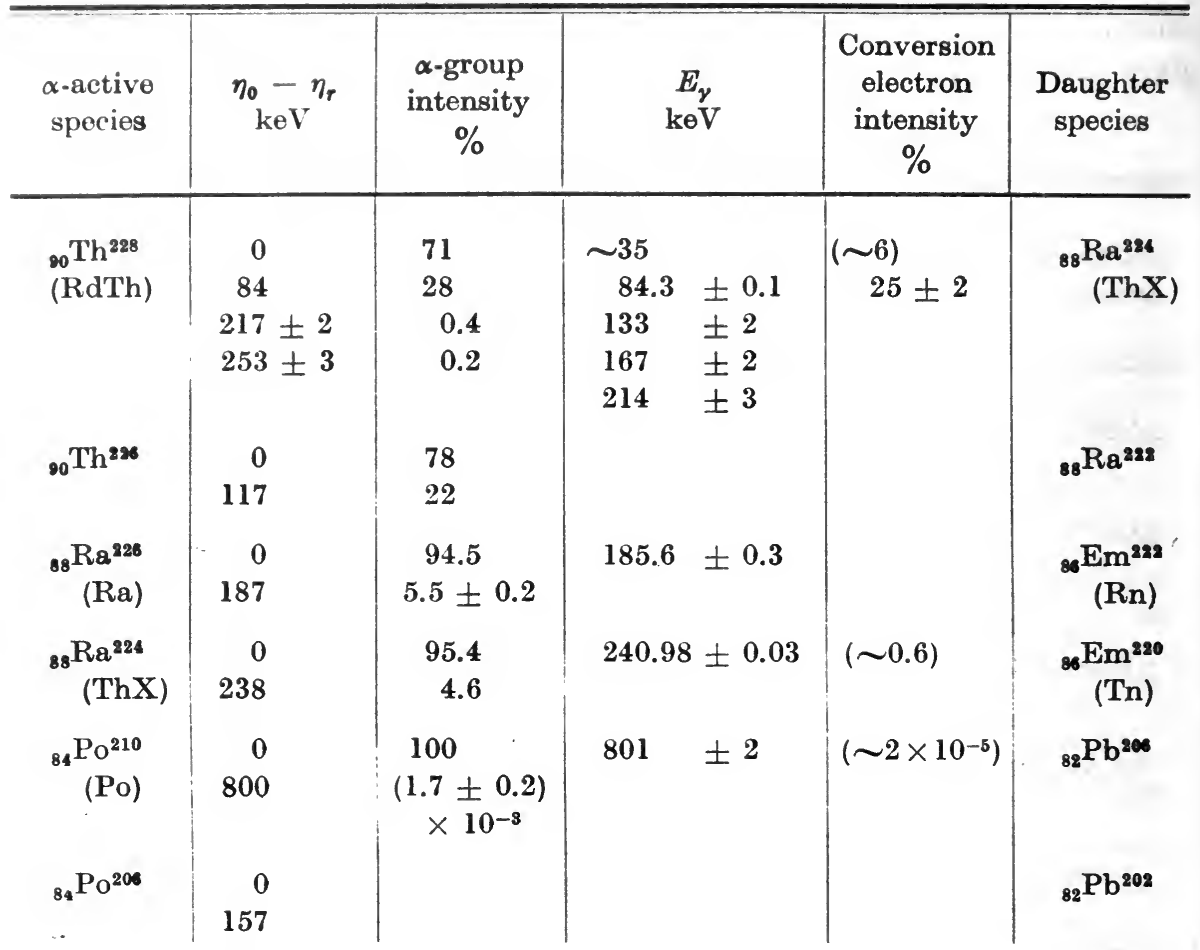

Notes on individual entries in Table $I$ are as follows (see Note on p. 766): ${ }_{96} \mathrm{Cm}^{242}$ : energies of the $\gamma$-rays are deduced from the energies of the conversion electrons emitted following the $\beta$-disintegration of ${ }_{93} \mathrm{~Np}^{238}$, which also has ${ }_{24} \mathrm{Pu}^{238}$ as daughter species ${ }^{1,2}$.

${ }_{94} \mathrm{Pu}^{238}$ : the $\gamma$-ray energy $45.0 \mathrm{keV}$ is as given by West et al. ${ }^{3}$, that of the higher energy $\gamma$-ray as reported by Reed ${ }^{4}$ in a circulated report. ${ }_{92} \mathrm{U}^{234}: \gamma$-ray energies taken from an unpublished report ${ }^{5}$.

${ }_{92} \mathrm{U}^{232}: \gamma$-ray energy deduced from the energies of the conversion electrons emitted following the $\beta$-disintegration of ${ }_{89} \mathrm{Ac}^{228}$, which also has ${ }_{90} \mathrm{Th}^{228}$ as daughter species ${ }^{6}$.

1 M. S. Freedman, A. H. Jaffey and F. Wagner, Phys. Rev. 79, (1950) 410.

J. W. Mihelich, Phys. Rev. 87, (1952) 646.

D. West, J. K. Dawson and C. J. Mandleberg, Phil. Mag. 43, (1952) 875.

4 G. W. Reed, ANL report 4679 (1951).

5 P. R. Beil, R. C. Davis, J. E. Francis and J. M. Cassidy, American declassified report D 72 (1951).

6 J. Kyles, J. G. Campbell and W. J. Henderson, Proc. Phys. Soc. A 66, (1953) 519. 
${ }_{90} \mathrm{Th}^{232}$ : very different values of $55 \mathrm{keV}$ and $75 \mathrm{keV}$ have been published 7.8 for the $\gamma$-ray energy in this case. The value quoted is from preliminary unpublished work of Dr M. A. S. Ross (Edinburgh). ${ }_{90} \mathrm{Th}^{228}$ : fairly conclusive evidence for the low-energy $\gamma$-ray is provided by preliminary work of Jarvis ${ }^{8 a}$. However the position regarding intensity is still in considerable doubt. The weak $\alpha$-particle groups have recently been reported by Asaro et $a l .{ }^{8 b}$.

${ }_{88} \mathrm{Ra}^{224}: \gamma$-ray energy as measured by Muller et al. ${ }^{9}$, though not identified by them as belonging to this body.

${ }_{84} \mathrm{Po}^{210}$ : the conversion electron intensity is a rough estimate ${ }^{10}$ based on the magnetic spectrum of Bothe ${ }^{11}$. Values based on total secondary electron emission ${ }^{12}$ or on fluorescent $\mathrm{X}$-ray intensities are now known to be too high because of ionisation effects produced by the outgoing $\alpha$-particle ${ }^{13}$ 14.

The outstanding feature of regularity which the results of Table I exhibit concerns the energy and degree of excitation of the first excited state of the product nucleus. In the range of mass number $230 \leqslant A \leqslant 240$ both excitation energy and excitation probability are sensibly constant, and, as the mass number decreases, the energy of the first excited state increases-at an increasing rate as the "doubly-magic" number 208 is approached ${ }^{15,16.17}$ - and the excitation probability falls in consequence. In those cases in which the internal conversion spectrum of the deexcitation radiation has been studied in detail it is clear that the radiation type is E2. There is every indication that this classification is applicable throughout the series, and that the spin and parity characteristics of the first states are $2+$ throughout. This result is part of a wider regularity, valid, so it would appear, for $\left(\begin{array}{l}e \\ e\end{array}\right)$ nuclei of whatever complexity ${ }^{18}$.

7 D. C. Dunlavey and G. T. Seaborg, Phys. Rev. 87, (1952) 165.

8 G. Albouy, J. Physique 13, (1952) 309.

sa C. J. D. Jarvis, Proc. Phys. Soc. A 66, (1953) 1074.

${ }^{8 b}$ F. Asaro, F. Stephens and I. Perlman, Phys. Rev. 92, (1953) 1495.

9 D. E. Muller, H. C. Hoyt, D. J. Klein and J. W. M. DuMond, Phys. Rev. 88, (1952) 775 .

10 N. Feather, Phys. Rev. 70, (1946) 88.

11 W. Bothe, Z. Physik 96, (1935) 607.

12 M.A. Grace, R. A. Allen, D. West and H. Halban, Proc. Phys. Soc. A 64, (1951) 493.

13 'W. C. Barber and R. H. Helm, Phys. Rev. 86, (1952) 275.

14 M. Riou, J. Physique 13, (1952) 487.

15 P. Stähelin and P. Preiswerk, Hèlv. Phys. Acta 24, (1951) 623; Nuovo Cim. 10, (1953) 1219.

16 G. Scharff-Goldhaber, Phys. Rev. 87, (1952) 218; Phys. Rev. 90, (1953) 587.

17 F. Asaro and I. Perlman, Phys. Rev. 87, (1952) 393.

18 M. Goldhaber and A. W. Sunyar, Phys. Rev. 83, (1951) 906. 
As regards the characteristics of the second excited states, Table I obviously contains some information, but we shall postpone discussion of this until further information from $\beta$-disintegration studies has been presented in $\S 1 \mathrm{~B}$.

\section{B. Paired $\beta$-disintegrations of the Type $\left(\begin{array}{l}e \\ e\end{array}\right) \rightarrow\left(\begin{array}{l}o \\ 0\end{array}\right) \rightarrow\left(\begin{array}{l}e \\ e\end{array}\right)$}

That the $\beta$-active species in the three disintegration series of classical radioactivity occur in associated pairs was pointed out-and that this fact was genuinely significant was suggested - as long ago as 1921 by Meitner ${ }^{19}$ and Harkins ${ }^{20}$. In 1943 Feather ${ }^{21}$ ascribed further significance to the fact that the first member of each such pair is an $\left(\begin{array}{l}\mathrm{e} \\ \mathrm{e}\end{array}\right)$ species. Feather and Richardson ${ }^{22}$ later indicated the basic simplicity underlying this pairing, namely that both the initial and the final states involved in the compound process are likely to be states of zero spin and even parity, and discussed various special cases on this well-grounded assumption. The discussion was extended to include two cases of $\beta$-capture branching in $\left(\begin{array}{l}0 \\ 0\end{array}\right)$ species by Feather ${ }^{23}$. In this section we shall be chiefly concerned with $\beta-\beta$ pairs, and with bringing up to date and extending the discussion of Feather and Richardson regarding individual cases. In the process further information regarding the excited states of the $\left(\begin{array}{c}e \\ e\end{array}\right)$ nuclei which are the ultimate products of the paired disintegration processes will emerge, and this and other similar information will be considered together with that already collected in $\S 1 \mathrm{~A}$ at the end of the section.

Amongst active species of mass type $A=4 n(Z>80)$ only three $\beta-\beta$ pairs are known which satisfy our present specification. They are the pairs ${ }_{92} \mathrm{U}^{240},{ }_{93} \mathrm{~Np}^{240}, \mathrm{MsTh}_{1}, \mathrm{MsTh}_{2}$ and $\mathrm{ThB}$, ThC.

Precise information regarding the first of these pairs has only very recently been published ${ }^{24}$. Plotted on the Sargent diagram, the representative points for the presumed ground-to-ground state modes of ${ }_{92} \mathrm{U}^{240}$ and ${ }_{93} \mathrm{~Np}^{240}$ fall the one in the allowed and the other in the first-forbidden band, according to the identification of Feather and Richardson. If this is the correct interpretation then the latter disintegration mode involves a change of parity whereas the former does not-an impossibility for successive transitions leading directly from one $0+$ state to another.

19 L. Meitner, Z. Physik 4, (1921) 146.

20 W. D. Harkins, Nature (Lond.) 107, (1921) 202.

21 N. Feather, British declassified report $\mathrm{Br} 312$ (1943).

22 N. Feather and H. O. W. Richardson, Proc. Phys. Soc. 61, (1948) 452.

23 N. Feather, Proc. Roy. Soc. Edin. A 63, (1952) 242.

24 J. D. Knight, M. E. Bunker, B. Warren and J. W. Starner, Phys. Rev. 91, (1953) 889. 
Clearly, the apparent contradiction may be removed either by assuming that the experimental results are incomplete, which they almost certainly are, or by modifying the scheme of Feather and Richardson, assuming in

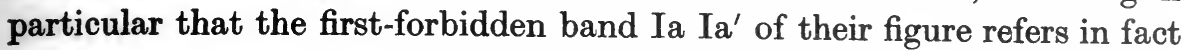
to "allowed but $L$-forbidden" transitions. That this modification might lead to the removal of difficulties in other cases has already been suggested by Kyles, et al. ${ }^{6}$. In the present case there is however a further difficulty connected with the non-appearance of transitions to the first $(44 \mathrm{keV})$ excited state of ${ }_{94} \mathrm{Pu}^{240}$ (see Table I) in the disintegration of ${ }_{93} \mathrm{~Np}^{240}$. A precisely similar difficulty arises with $\mathrm{UX}_{2}$ and will be discussed more fully below.

In respect of the pair $\mathrm{MsTh}_{1}, \mathrm{MsTh}_{2}$ much new information has been obtained since the previous discussion. It is now clear that the principal (probably the only important) mode of $\beta$-disintegration of $\mathrm{MsTh}_{1}$ is by an allowed transition of less than $15 \mathrm{keV}$ energy, also that with $\mathrm{MsTh}_{2}$ the ground-to-ground state transition is unobserved and thus highly forbidden. From these two conclusions we derive a third: that the $\beta$-disintegration of $\mathrm{MsTh}_{1}$ leads to an excited-not to the ground-state of $\mathrm{MsTh}_{2}$. To the ground state Kyles et al. ${ }^{6}$ assign spin and parity characteristics 4 -; the excited state is probably a $1+$ state. If our understanding of the situation is incomplete it is chiefly because the expected de-excitation radiation. from this state is so little in evidence. No $\gamma$-rays have been detected ${ }^{25}$ $\left(E_{\gamma}>10 \mathrm{keV}\right)$ and the intensities of the low energy conversion electrons ${ }^{26}$ and the $L$-X-rays ${ }^{25}$ which have been observed are consistent with no more than $15 \% L$-shell ionisation per disintegration. We are almost forced to conclude that the main de-excitation is by a cascade of at least two radiations, each of less than the $L_{\text {III }}$ ionisation energy.

In contrast with the case just considered there is not much of recent discovery to report regarding the $\beta-\beta$ pair ThB, ThC. Ground-to-ground transitions are observed in each case and are of the same degree of forbiddenness, as would be expected. The principal disintegration mode of ThB is certainly by an allowed transition, and the position of the representative point on the Sargent diagram strongly suggests that the spin change is $+1{ }^{27}$. Measurements of relative intensity of $L_{\mathrm{I}}$ and $L_{\mathrm{II}}$ internal conversion of the $238 \mathrm{keV}$ de-excitation radiation ${ }^{28,29}$ make it almost certain that the transition is pure Ml, though the absolute value of the $K$-internal

M. Lecoin, M. Perey and M. Riou, J. Physique 10, (1949) 390.

M. Lecoin, M. Perey and J. Teillac, J. Physique 10, (1949) 33.

N. Feather, Nuclear Stability Rules, C.U.P. (1952) p. 98.

N. V. Forafontov and V. S. Shipinel, Dokl. Akad. Nauk, SSSR, 72, (1950) 49.

D. A. Silverston, Proc. Phys. Soc. A 65, (1952) 344. 
conversion coefficient is significantly different from the currently accepted theoretical value ${ }^{30}$. On balance it would appear that, on the basis of these results, the suggestion 22 that the ground state of $\mathrm{ThC}$ is a $0+$ state has more to commend it than the only admissible alternative possibilities, namely that it is a 0 - or a 1 - state. From this initial state three ${ }^{31}$ longrange $\alpha$-particle emitting states of $\mathrm{ThC}^{\prime}$ are excited by $\beta$-transition, and the experimentally determined excitation probabilities allow us to assume that the characteristics of the three states in question are $728 \mathrm{keV}, 2+$; $1.67 \mathrm{MeV}, 1-; 1.80 \mathrm{MeV}, 1-$. This assignment, together with the natural assumption that the ground state of $\mathrm{ThC}^{\prime}$ is a $0+$ state, is consistent with the result ${ }^{30}$ that the intensity of internal conversion of the $728 \mathrm{keV}$ de-excitation radiation points to its characterisation as $\mathrm{E} 2$.

Amongst the active species of mass-type $A=4 n+2$ five $\beta-\beta$ pairs are at present known for which $Z>80$ and the other specifications of this section are fulfilled, but our knowledge of radiation characteristics is very scanty for two of these pairs, ${ }_{88} \mathrm{Ra}^{230},{ }_{89} \mathrm{Ac}^{230}$ and $\mathrm{RaA},{ }_{85} \mathrm{At}^{218}$.

The three more intensively studied $\beta-\beta$ pairs of mass type $A=4 n+2$ are the pairs $\mathrm{UX}_{1}, \mathrm{UX}_{2}(\mathrm{UZ}), \mathrm{RaB}, \mathrm{RaC}$ and $\mathrm{RaD}, \mathrm{RaE}$. In each case difficulties of interpretation arise which only further experiment can resolve. So far as the first pair is concerned the recent very detailed investigation of Stoker et al. ${ }^{32}$ certainly has not provided a full resolution. By a close study of the conversion electron line spectrum in the low-energy region these authors have made it reasonably certain that neither the $45.0 \mathrm{keV}$ state nor any other low-lying state of the daughter nucleus ${ }_{92} \mathrm{U}^{234}$ (see Table I) is excited in more than a few per cent. of the disintegrations of $\mathrm{UX}_{2}$; these go almost entirely to the ground state. This in itself introduces a difficulty. Whether the high-energy mode of $\beta$-disintegration of $\mathrm{UX}_{2}$ is effectively single (as it thus appears to be), or consists in reality of two competing modes of very nearly the same energy and comparable intensities, the transition (transitions) is (are) allowed, and $1+$ is the only acceptable assignment for the initial $\beta$-emitting state of ${ }_{91} \mathrm{~Pa}^{234}$ which is $\mathrm{UX}_{2}$. However, with this assignment we should expect ${ }^{33,34}$ transition to the first $(2+)$ excited state, rather than to the ground $(0+)$ state, of ${ }_{92} \mathrm{U}^{234}$ to óccur in about $60 \%$ of the high-energy disintegrations. As already mentioned, the experimental upper limit appears to be less than $1 / 20$ of this.

D. G. E. Martin and H. O. W. Richardson, Proc. Phys. Soc. A 63, (1950) 223.

A. Rytz, C. R. Acad. Sci. (Paris) 233, (1951) 790.

P. H. Stoker, M. Heerschap and P. H. Ong, Physica 19, (1953) 433.

R. E. Marshak, Phys. Rev. 61, (1942) 431.

C. Strachan, Proc. Phys. Soc. 61, (1948) 481. 
Comparing now the disintegrations of $\mathrm{UX}_{1}$ and $\mathrm{UX}_{2}$, we note that the principal (higher-energy) mode of $\beta$-disintegration of $\mathrm{UX}_{1}$ is, empirically, significantly more forbidden than the ground-to-ground state mode of $\mathrm{UX}_{2}$. We can only retain the "allowed" specification (no parity change) for the $\mathrm{UX}_{1}$ disintegration in this case by invoking the "allowed but $L$ forbidden" classification already referred to in respect of the pair ${ }_{92} \mathrm{U}^{240}$, ${ }_{93} \mathrm{~Np}^{240}$ discussed above. (It should be mentioned here that to have introduced this classification for the pair ThB, ThC would have made the interpretation of the experimental results more rather than less difficult in that case.) The $93.1 \mathrm{keV} \gamma$-ray of $\mathrm{UX}_{1}$ is internally converted as an M1 radiation. This involves no difficulty of interpretation so long as the two $\beta$-modes of this body are equally forbidden-as they seem to be. As regards the high-energy $\gamma$-rays of $\mathrm{UX}_{2}$ the most significant-and difficult-comparison is with the $\gamma$-rays of the ground-state isomer UZ, and with those of the capture-active ${ }_{93} \mathrm{~Np}^{234}$ which also has ${ }_{92} \mathrm{U}^{234}$ as daughter species.

The principal mode of disintegration of UZ involves $\beta$-emission followed by two or more $\gamma$-rays in cascade. Here it might be tempting to suppose that this cascade is essentially the same as the $\gamma$-ray cascade involved in the capture disintegration of ${ }_{93} \mathrm{~Np}^{234}$, which has been studied in some detail recently ${ }^{35,36}$. In point of fact a recent preliminary publication by Bouissières et al. ${ }^{59}$ renders this supposition extremely unlikely. Very few states appear to be common to the two disintegrations. Finally it may be remarked that if the nucleus ${ }_{92} \mathrm{U}^{234}$ possesses relatively low-lying states of high spin (e.g. $4+$ or $6+$ states) then the absence of a strong high-energy component in the $\beta$-spectrum of $\mathrm{UZ}$ (and the probable absence of the corresponding capture mode with ${ }_{93} \mathrm{~Np}^{234}$ ) presents an unresolved difficulty (see below).

For the pair $\mathrm{RaB}, \mathrm{RaC}$, the ground-to-ground state transition $\mathrm{RaC} \rightarrow \mathrm{RaC}^{\prime}$ is at least second- (probably third-) forbidden and the corresponding transition $\mathrm{RaB} \rightarrow \mathrm{RaC}$ is unobserved. This is as it should be. On the other hand, on the assumption that with RaB the principal modes of $\beta$-disintegration (of maximum energies 0.67 and $0.73 \mathrm{MeV}$ ) excite states of 0.352 and $0.295 \mathrm{MeV}$, respectively, in $\mathrm{RaC}^{37}$, each by an allowed transition, and that the first excited state of this nucleus, assumed to lie at $53 \mathrm{keV} 9$, becomes involved only in the de-excitation process, we encounter grave difficulties in assigning spins and parities to these states and to the ground state, without seriously disregarding the evidence from

35 D. A. Orth, UCRL report 1059 (1951).

36 G. D. O'Kelley, UCRL report 1243 (1951).

37 E. E. Berlovich, Izvest. Akad. Nauk (Fiz.), SSSR, 16, (1952) 314. 
the relative intensities of $L$-shell conversion of the $\gamma$-rays ${ }^{38,}$, the $K / L$ conversion ratios or the absolute values of $K$-shell internal conversion coefficients.

Empirically, the three transitions to the ground state ( $\gamma$-rays of energy 352,295 and $53 \mathrm{keV}$, respectively) are M1 transitions, though absolute values of internal conversion coefficients are somewhat less than accepted theoretical values, and the cross-over transition of energy $242 \mathrm{keV}$ shows similar characteristics. If thes? assignments are accepted, together with the assumption that the exciting $\beta$-transitions are allowed transitions, we have to conclude that the ground state of $\mathrm{RaC}$ is of even parity and spin 0 , 1 or 2 . None of these assignments is acceptable: if either of the first two were chosen, an allowed $\beta$-transition would be expected from $\mathrm{RaB}$ to the ground state of $\mathrm{RaC}$, and with the third an allowed transition to the $2+$ first excited state of $\mathrm{RaC}^{\prime}$ would occur (see below). If all three are rejected, the only tolerable agreement (and then quite good agreement) in respect of total internal conversion coefficient follows from the assignment 3 - The $53 \mathrm{keV}$ state has then to be considered as a $3-$ state, also, (the de-excitation radiation remains classified as M1) and the two upper states regarded as $0+$ states (classifying the higher energy $\gamma$-rays as E3). We shall adopt this assignment here, recognising the serious difficulties regarding $K / L$ conversion ratios which attach to it (see Addenda).

For the disintegration $\mathrm{RaC} \rightarrow \mathrm{RaC}^{\prime}$ we are still confronted with a great deal of conflicting evidence, in spite of recent researches. The coincidenceabsorption experiments of Wapstra ${ }^{39}$ appear to show conclusively that the ground state is $\beta$-excited (in about $18 \%$ of the disintegrations), but comparative estimates of intensities of the quantum radiations of $\mathrm{Ra}(\mathrm{B}+\mathrm{C})$ would imply that the $609 \mathrm{keV}$ radiation of $\mathrm{RaC}$ is emitted to the extent of approximately 1 quantum per disintegration ${ }^{9}$. Obviously the two results are mutually contradictory. Similarly, $\gamma-\gamma$ coincidence experiments by Waipstra ${ }^{39}$ and Demichelis and Malvano ${ }^{40}$ have formed the basis of equally conflicting conclusions. Demichelis and Malvano have concluded that the $1.76 \mathrm{MeV}-1.12 \mathrm{MeV} \gamma$-ray cascade is a prominent feature of the disintegration; Wapstra has concluded that it is not. In each case of disagreement we shall here follow Wapstra's interpretation: to do otherwise would appear to involve very grave difficulties indeed ${ }^{22}$. Likewise a considerable degree of uncertainty remains regarding energies and intensities in general, in spite of the extended surveys of the whole

38 K. Siegbahn, Arkiv. Mat. Astron. Fys. A 30, (1944) 20.

39 A. H. Wapstra, Physica 18, (1952) 1247; Thesis (1953), Amsterdam.

40 F. Demichelis and R. Malvano, Nuovo Cim. 10, (1953) 405. 
$\gamma$-ray spectrum of $\mathrm{RaC}^{\prime}$ which have been made ${ }^{41,42,43,44}$ in recent years in order to try to resolve the differences which the earlier work of Ellis and Aston ${ }^{45}$ and Latyshev ${ }^{46}$ revealed.

The one line of investigation which has not recently been re-opened, possibly because the original survey ${ }^{47}$ is unlikely to be easily improved upon even with modern equipment, is that concerning the energy spectrum of the long-range $\alpha$-particles of $\mathrm{RaC}^{\prime}$ which are emitted in competition with the $\gamma$-rays. As is well known, this spectrum gives directly a part of the spectrum of excitation energies of the emitting nucleus. Assuming $0+$ as the character of the ground state of the daughter nucleus RaD, the limitation is as follows. Including the $\alpha$-emitting ground state which is a $0+$ state, the levels concerned are expected to be of the type $(2 n)+$ or $(2 m+1)-$, and so to de-excite to the ground state by transitions which are predominantly electric multipole in character (or completely for bidden as radiative transitions when $n=0)$. Levels of the types $(2 n)-$ and $(2 m+1)+$ should not show up in $\alpha$-emission ${ }^{48}$.

In Table II are given the excitation energies of the twelve long-1 ange $\alpha$-emitting states and of eight other states postulated to account for the $\gamma$-rays. This level system - like many others advanced by previous writers -is put forward in the knowledge that it can be no more than a speculative approximation to the truth, and quite arbitrarily, though other levels must be involved, excitations are made to total $100 \%$ (column 2). How far this is in error is not known, but at least the $39 \gamma$-rays accommodated in the scheme include all those definitely observed in emission, and nine others the intensities of which, listed as $0 \%$ in column 6 , are presumed to be less than $0.2 \%$ each. The mean excitation energy per disintegration is $1.55 \mathrm{MeV}$ on the basis of the excitations given in Table II, and is in satisfactory agreement with direct determinations of $\gamma$-ray heating effect and total ionisation. Column 3 of Table II gives spin and parity assignments, column 4 the predicted degrees of forbiddenness of the $\beta$-transitions (from the 3 - ground state of $\mathrm{RaC}$ ) which would excite the several states,

\footnotetext{
41 K. C. Mann and M. J. Ozeroff, Canadian J. Res. A 27, (1949) 164.

42 M. Miwa and S. Kageyama, J. Phys. Soc. (Japan) 5, (1951) 416.

43 S. Kageyama, J. Phys. Soc. (Japan) 7, (1952) 93.

44 M. Mladjenovic and A. Hedgran, Physica 18, (1952) 1242.

45 C. D. Ellis and G. H. Aston, Proc. Roy. Soc. A 129, (1930) 180.

46 G. D. Latyshev, Rev. Mod. Phys. 19, (1947) 132.

47 Lord Rutherford, W. B. Lewis and B. V. Bowden,-Proc. Roy. Soc. A 142, (1933) 347.

48 This restriction is absolute, even if the general possibility of $\alpha$-emission by more than one 'spin channel' is admitted (see Devaney ${ }^{70}$ ), so long as the character of the final state is $0+$.
} 
TABLE II

\begin{tabular}{|c|c|c|c|c|c|}
\hline $\begin{array}{l}\text { Exci- } \\
\text { tation } \\
\text { energy } \\
\text { kel }\end{array}$ & $\begin{array}{l}\text { Per- } \\
\text { centage } \\
\text { exci- } \\
\text { tation }\end{array}$ & $\begin{array}{l}\text { Assign- } \\
\text { ment }\end{array}$ & $\begin{array}{c}\text { Exciting } \\
\beta \text {-tran- } \\
\text { sition }\end{array}$ & $\begin{array}{c}\alpha \text {-particle } \\
\text { de-exci- } \\
\text { tation } \\
\times 10^{7}\end{array}$ & $\begin{array}{c}\text { Energies ( } \mathrm{keV}^{\top} \text { ) } \\
\text { (and percentage intensities) } \\
\text { of de-exciting } \gamma \text {-rays }\end{array}$ \\
\hline 2880 & 0.5 & $3-$ & 0 & 2.3 & $610(0.5), 270(0)$ \\
\hline 2697 & 0.5 & $3-$ & 0 & 11.2 & $497(0), 427(0.5)$ \\
\hline 2610 & 0.7 & $2-$ & 0 & - & $2610(0.5), 1329(0.2)$ \\
\hline 2506 & 0 & $0+$ & III & 3.8 & $2506(0), 236(0)$ \\
\hline 2440 & 2.5 & $2+$ & I & 16.7 & $2440(2.5)$ \\
\hline 2270 & 0 & $1-$ & II & 3.6 & $2270(0), 989(1)$ \\
\hline 2200 & 7.5 & $1+$ & $\mathbf{I}$ & - & $2200(6), 1591(0.5), 570(1)$ \\
\hline 2142 & 2.5 & $4+$ & I & $10.6 ?$ & $2142(0), 1533(1), 764(1.5)$ \\
\hline 2110 & 7.8 & $3-$ & 0 & ? & $2110(1.5), 1501(3), 480(3.3)$ \\
\hline 2012 & 0 & $0+$ & III & 3.5 & $2012(0), 250(0)$ \\
\hline 1847 & 14 & $2+$ & $\mathrm{I}$ & 13.5 & $1847(2.5), 1238(6), 469(5.5)$ \\
\hline 1762 & 20.5 & $1+$ & $\mathbf{I}$ & - & $1762(19.4), 1153(0.6), 829(0.5)$ \\
\hline 1729 & 20 & $1+$ & $\mathbf{I}$ & - & $1729(2.5), 1120(17.5)$ \\
\hline 1672 & 4.5 & $2+$ & $\mathrm{I}$ & 3.81 & $1672(1.5), 391(3)$ \\
\hline 1630 & 1 & $1-$ & II & $? !$ & $1630(2.3), 697(3)$ \\
\hline 1416 & 0 & $0+$ & III & $220\}$ & $1416(0)$ \\
\hline 1378 & 0 & $1-$ & II & $?\}$ & $1378(7)$ \\
\hline 1281 & 0 & $1-$ & II & 4.5 & $1281(4.2)$ \\
\hline 933 & 0 & $1+$ & I & - & $933(3.5)$ \\
\hline 609 & 0 & $2+$ & I & 4.3 & $609(28.6)$ \\
\hline 0 & 18 & $0+$ & III & - & - \\
\hline
\end{tabular}

column 5 the intensities of the long-range $\alpha$-particle transitions from the states, and column 6 the energies and intensities of the $\gamma$-rays of deexcitation: Concerning these last intensities certain remarks should be made. In the first place the intensities of almost all the prominent $\gamma$ radiations are somewhat less than previous estimates, that of the $609 \mathrm{keV}$ $\gamma$-ray markedly so. But if the intensities of the internal conversion electron groups are regardèd as correctly given by Ellis and Aston ${ }^{45}$, the newlyassigned intensity of the $609 \mathrm{keV}, \gamma$-ray in emission leads to an "experimental" internal conversion coefficient in good agreement with the theoretical value ${ }^{49}$ for $\mathrm{E} 2$ radiation. Almost equally satisfactory agreement oktains for the $1630,1501,1378$ and $1281 \mathrm{keV} \gamma$-rays (predominantly $\mathrm{E} 1$ ), and for the 2110 and $764 \mathrm{keV}$ radiations (predominantly E3). On the other hand, for the $\gamma$-rays involved in transitions between pairs of states one of which is $\alpha$-emitting and the other not (or between pairs of states of the same spin and parity), and for which predominantly M1

49 M. E. Rose, G. H. Goertzel, B. I. Spinrad, J. Harr and P. Strong, Phys. Rev. 83, (1951) 79 . 
characteristics would be expected, no such agreement emerges. By contrast internal conversion coefficients are consistently $40-50 \%$ of the MI values only, and the radiation character is empirically to be described as $\mathrm{E} 2+\mathrm{M} 1$. The $\gamma$-rays in this group are those of energy 2200, 1762, 1729, 1238, 1120 and $933 \mathrm{keV}$. Finally, the experimental internal conversion coefficients of the expectedly E2 radiations of 2440,1847 and $1672 \mathrm{keV}$ energy are all some $30 \%$ less than the theoretical values. Clearly this deficit cannot be explained by any admixture of magnetic multipole radiation in these cases. So far as the primary excitation by $\beta$-transition is concerned, consideration of columns 2 and 4 of Table II brings to light no glaring discrepancy except the lack of excitation of the first and second states (609 and $933 \mathrm{keV}$ ). This discrepancy-at least in relation to the $609 \mathrm{keV}$ state-cannot be removed without drastic modification of the whole scheme and loss of much of its present content; we can only tentatively relate the difficulty to the similar difficulties encountered with $\mathrm{UX}_{2}$ and ${ }_{93} \mathrm{~Np}^{240}$ (see above).

It is not necessary to write at length concerning the pair $\mathrm{RaD}, \mathrm{RaE}$ since the position has recently been re-examined and critically discussed by $\mathrm{Wu}$ et $a l .{ }^{50}$. Hitherto, from the experimental side, the difficulty has been in reaching agreement concerning the details of the disintegration process with $\mathrm{RaD}$ - a difficulty originating primarily in the smallness of the total disintegration energy $(\sim 64 \mathrm{keV})-$ and, from the theoretical side, in providing an acceptable interpretation of the relatively wellestablished facts regarding the effectively single mode of transformation of $\mathrm{RaE}$. Now, as a result of the investigations of $\mathrm{Wu}$ et al. ${ }^{50}$, Jaffe and Cohen ${ }^{51}$, Ewan and Ross ${ }^{52}$, Butt and Brodie ${ }^{53}$, Cork et al. ${ }^{54}$ and Cranberg ${ }^{55}$, it appears that neither in emission nor through internal conversion is there evidence for the excitation of more than one state (of energy $46.52 \mathrm{keV}^{52}$ ) in the transition $\mathrm{RaD} \rightarrow \mathrm{RaE}$, at least in significant intensity, though it must be admitted that the most recent results ${ }^{50}$ still require the occurrence of the ground-to-ground state disintegration mode in some $8 \pm 5 \%$ of the disintegrations. Direct experimental observation of this mode is still lacking. Empirically, the chief $\beta$-disintegration mode (maximum energy $17 \pm 2 \mathrm{keV} 51.56$ is an allowed mode and the $46.5 \mathrm{keV}$ de-

50 C. S. Wu, F. Boehm and E. Nagel, Phys. Rev. 91, (1953) 319.

51 A. A. Jaffe and S. G. Cohen, Phys. Rev. 89, (1953) 454.

52 G. T. Ewan and M. A. S. Ross, Nature (Lond.) 170, (1952) 760.

53 D. K. Butt and W. D. Brodie, Proc. Phys. Soc. A 64, (1951) 791.

54 J. M. Cork, C. E. Branyan, A. E. Stoddard, H. B. Keller, J. M. Le Blanc and W. J. Childs, Phys. Rev. 83, (1951) 681.

55 L. Cranberg, Phys. Rev. 77, (1950) 155.

56 G. M. Insch, J. G. Balfour and S. C. Curran, Phys. Rev. 85, (1952) 805. 
excitation radiation is $M 1$. If these classifications are accepted at their face value the most natural spin and parity assignments for the product states are: $46.5 \mathrm{keV}, 1+$; ground state (RaE), $2+^{\circ}$. Earlier discussions of the form of the $\mathrm{RaE} \beta$-spectrum had also tended towards the conclusion that $2+$ was appropriate for the latter state, but more recently it has been common to suppose that the shell model requires the parity to be odd, and to assume that the best solution to the difficult problem of classifying the $\beta$-disintegration $\mathrm{RaE} \rightarrow \mathrm{RaF}$ is contained in the form $0 \rightarrow \rightarrow 0+$. The only possibility of maintaining any difference in degree of forbiddenness as between the $17 \pm 2$ and $64 \pm 2 \mathrm{keV}$ modes of $\mathrm{RaD}$ then lies in reclassifying the $46.5 \mathrm{keV} \gamma$-radiation as $\mathrm{E} 1+\mathrm{M} 2$. This is in any case an uncommon mixture, and it is doubtful whether the experimental $L_{\mathrm{I}}: L_{\mathrm{II}}: L_{\text {III }}$ ratios could be satisfactorily explained on the proposed classification. Alternatively, it is again necessary to appeal to the shell model-on this occasion to provide a plausible reason why a $\beta$-transition of the type $0+\rightarrow 1-$ is particularly favoured (though the type $0+\rightarrow 0-$ is not) for a nucleus containing near-magic numbers of protons and neutrons. Obviously no thoroughly convincing solution is available as yet, though a recent preliminary investigation of the spectrum of RaE in the ultra-violet favours spin 0 rather than 2 for the ground state ${ }^{56 a}$ (see Addenda).

Having discussed in detail the various $\beta-\beta$ pairs we are now in a position to take up again the general problem of the excited states of the $\left(\begin{array}{l}\mathbf{e} \\ \mathbf{e}\end{array}\right)$ nuclei at the point where it was left in $\S 1 \mathrm{~A}$. Table III collects the information from $\alpha$-disintegrations already discussed, from $\beta$-disintegrations some of which have been considered in this section, and from certain capture-disintegrations. Bracketed values refer to states the identification of which may still be held in some doubt. With this presentation the trend of first excitation energies with $A$ and $Z$ becomes strikingly obvious. We now see that, for $Z$ constant, the first excitation energy decreases smoothly with increasing $A$ for $Z=86,88$ and 90 , the rate of decrease itself decreäsing as $Z$ increases in this range. For $Z=92$ and $Z=94$ there is some slight evidence that a minimum has been reached and the opposite variation has set in, while for $Z=82$, and values of $A$ less than the "doubly magic" 208, the first excitation energy increases rapidly as $A$ increases. Apart from the appearance of a slight anomaly with $Z=84$, the whole picture is coherent.

Various interpretations of the uniform character of the $(2+j$ first excited states of $\left(\begin{array}{l}e \\ e\end{array}\right)$ nuclei have recently been given. Of these that of Bohr and Mottelson ${ }^{57}$ has been elaborated in the greatest detail. On this

56a M. Fred, F. S. Tomkins and R. F. Barnes, Phys. Rev. 92, (1953) 1324.

57 A. Bohr and B. R. Mottelson, Dan. Mat. Fys. Medd. 27, (1953) 16. 
interpretation these generally low-lying states are of a special rotational type involving oscillations of the nuclear surface, and the variation of excitation energy with $A$ and $Z$ is plausibly connected with the variation in degree of coupling of individual-particle and collective motions as the number of nucleons outside closed shells changes in a series of increasing $A$. It is a special feature of this interpretation that it predicts the occurrence, in nuclei with several extra-shell nucleons, of a series of even-spin states,

TABLE III

\begin{tabular}{l|l}
\hline Nucleus & \multicolumn{1}{|c}{ Excitation energies $(\mathrm{keV})$} \\
\hline${ }_{94} \mathrm{Pu}^{240}$ & $44 \pm 4,(\sim 560),(\sim 900)$ \\
${ }_{94} \mathrm{Pu}^{238}$ & $43.0 \pm 0.2,146 \pm 1$, others \\
${ }_{94} \mathrm{Pu}^{236}$ & $(150 \pm 2)$ \\
${ }_{92} \mathrm{U}^{236}$ & $49.8 \pm 0.3$ \\
${ }_{92} \mathrm{U}^{234}$ & $45.0 \pm 0.3,803$, others \\
${ }_{92} \mathrm{U}^{232}$ & $\sim 45,(\sim 210),(\sim 560),(\sim 920)$ \\
${ }_{90} \mathrm{Th}^{234}$ & $48 \pm 3$ \\
${ }_{90} \mathrm{Th}^{232}$ & $\sim 50$ \\
${ }_{90} \mathrm{Th}^{230}$ & $53 \pm 2,(171 \pm 3)$ \\
${ }_{90} \mathrm{Th}^{228}$ & $56.8 \pm 0.1,184.4 \pm 0.3,393$, many others \\
${ }_{90} \mathrm{Th}^{226}$ & $\sim 70$ \\
${ }_{88} \mathrm{Ra}^{228}$ & $60 \pm 2$ \\
${ }_{88} \mathrm{Ra}^{226}$ & $67.3 \pm 0.2,212 \pm 4,255 \pm 5$ \\
${ }_{88} \mathrm{Ra}^{224}$ & $84.3 \pm 0.1,(167 \pm 2), 214 \pm 2$ \\
${ }_{88} \mathrm{Ra}^{222}$ & $117 \pm 5$ \\
${ }_{86} \mathrm{Em}^{222}$ & $185.6 \pm 0.3$ \\
${ }_{86} \mathrm{Em}^{220}$ & $241.0 \pm 0.1$ \\
${ }_{84} \mathrm{Po}^{214}$ & $609,(933), 1281$, many others \\
${ }_{84} \mathrm{Po}^{212}$ & $728,1670,1800$ \\
${ }_{82} \mathrm{~Pb}^{208}$ & 2615,3197, others \\
${ }_{82} \mathrm{~Pb}^{206}$ & 801, many others \\
${ }_{82} \mathrm{~Pb}^{204}$ & 374,1280 \\
${ }_{82} \mathrm{~Pb}^{202}$ & 157 \\
\end{tabular}

$2+, 4+, 6+, \ldots$, which in the limit of strong coupling have excitation energies in the "classical rotator" ratios $3: 10: 21: \ldots$ De-excitation from an upper state of such a system should clearly be by means of a cascade of E2 transitions without cross-over. That there is some evidence in Table III in line with this prediction is undeniable: the ratio of second to first excitation energies is $3.40 \pm 0.03$ with ${ }_{94} \mathrm{Pu}^{238}, 3.23 \pm 0.13$ with ${ }_{90} \mathrm{Th}^{230}, 3.25 \pm 0.01$ with ${ }_{90} \mathrm{Th}^{228}$ and $3.15 \pm 0.06$ with ${ }_{88} \mathrm{Ra}^{226}$-decreasing as it is predicted to do as the number of extra-shell nucleons decreasesand the ratio of third to first excitation energies is 6.93 with ${ }_{90} \mathrm{Th}^{228}$, relatively closer to the ideal 7.00 than the second-to-first excitation 
energy ratio is to the theoretical 3.33 for this nucleus. According to Asaro and Perlman ${ }^{58}$, there are other examples of third-to-first excitation energy ratios in the neighbourhood of 7 . Obviously Table III is much too incomplete to allow of a statement, one way or the other, regarding the appearance of the classical rotator energy ratios $3: 10: 21: \ldots$ in the energy spectra of excited states of all $\left(\begin{array}{l}e \\ e\end{array}\right)$ nuclei having, for example, $A \geqslant 226$, but one statement may certainly be made which has a definite bearing on the general suggestion of Bohr and Mottelson. It is that even when the excitation energy ratios have the predicted values, the predicted spin and parity assignments $2+, 4+, 6+, \ldots$ are frequently inappropriate - except that the first state is invariably a $2+$ state, as already mentioned. Thus, with ${ }_{90} \mathrm{Th}^{228}$, cross-over transitions from the second excited state to the ground state, and from the third excited state to the first, certainly occur, and with ${ }_{88} \mathrm{Ra}^{226}$ the second-to-ground state cross-over is found. Moreover, if a low-lying $4+$ state were to exist with ${ }_{90} \mathrm{Th}^{228}$ this would be expected to be the terminus of a favoured (almost single-mode) $\beta$-disintegration $\mathrm{MsTh}_{2} \rightarrow \mathrm{RdTh}$, whereas the facts are very different: many disintegration modes occur and none of those feeding the low-lying states is an intense mode. A similar difficulty has already been remarked concerning possible $4+$ and $6+$ states in ${ }_{92} \mathrm{U}^{234}$. If these were present they wowld be expected to show up in one or more high-energy favoured modes in the disintegration $\mathrm{UZ} \rightarrow \mathrm{U}_{\mathrm{II}}$. Such modes do not occur. If spin and parity predictions are not borne out for many nuclei, including ${ }_{92} \mathrm{U}^{234}$, it is interesting to note that just with this nucleus there is perhaps the most extensive evidence (approximate, only, as yet) for the validity of the classical-rotator energy formula. The cascade $\gamma$-rays predicted by this formula (taken in the reverse order) have energies in the ratios $3: 7: 11: 15: 19: \ldots$ Preliminary results of Bouissières et al. ${ }^{59}$ for the disintegration $\mathrm{UZ} \rightarrow \mathrm{U}_{\mathrm{II}}$ have been interpreted by these authors in terms of $\gamma$-rays of $104,126,161,233,277,600, \cdot 758$ and $855 \mathrm{keV}$ energy (accuracy about $1.5 \%$ ). Omitting only the second of these values (representing a rather weak conversion electron line), and making the plausible, but quite untested, assumption that the de-excitation radiation of the first excited state of ${ }_{92} \mathrm{U}^{234}$ is also involved in the $\mathrm{UZ}$ disintegration, we have the following series of relative energy values, $3: 6.9: 10.7: 15.5: 18.5: \ldots$ The numerical agreement is impressive, but until further information regarding intensities, and concerning the relation between the highenergy $\gamma$-rays and the $\gamma$-rays of this series, is obtained, it would obviously be unwise to attempt to say more (see Note p. 766).

58 F. Asaro and I. Perlman, Phys. Rer. 91. (1953) 763.

59 G. Bouissières, N. Marty and J. Teillac, C. R. Acad. Sci. (Paris) 237, (1953) 324. 


\section{C. $\gamma$-Radiations of the $\alpha$-Active $\left(\begin{array}{l}0 \\ 0\end{array}\right)$ Species}

Although some $17 \alpha$-active species of this type are known, very little information is available concerning the associated $\gamma$-radiation. Only with ThC has the internal conversion spectrum been studied in detail, and only in this disintegration and in those of ${ }_{91} \mathrm{~Pa}^{228},{ }_{87} \mathrm{Fr}^{212}$, ${ }_{85} \mathrm{At}^{210}$ and $\mathrm{RaC}$ has definite evidence of $\alpha$-particle fine-structure been obtained. Clearly this scanty information does not provide a very adequate basis on which to discuss possible regularities in the spacing and the spin and parity characteristics of the excited states of the $\left(\begin{array}{l}0 \\ 0\end{array}\right)$ daughter species concerned. As in the cases considered in $\S 1 \mathrm{~A}$, however, we should be able to extend this information to some extent by taking into consideration the evidence from the $\beta$ - or capture-disintegration of $\left(\begin{array}{l}e \\ e\end{array}\right)$ species. In fact nothing of significance arises from present knowledge of capture-disintegrations of this type, but the $\beta$-disintegrations of the first members of the $\beta-\beta$ pairs discussed in $\S 1 \mathrm{~A}$ reveal portions of the level structure of five additional $\left(\begin{array}{l}0 \\ 0\end{array}\right)$ nuclei. All the information derivable from the $\alpha$ - and $\beta$-disintegrations is collected in Table IV.

TABLE IV

\begin{tabular}{|c|c|}
\hline Nucleus & Excitation energies (keV) \\
\hline${ }_{91} \mathrm{~Pa}^{234}$ & 93.1, others (possibly of lower energy) \\
\hline${ }_{89} \mathrm{Ac}^{228}$ & probably two below $50 \mathrm{keV}$ \\
\hline${ }_{89} \mathrm{Ac}^{224}$ & $\sim 250$ \\
\hline${ }_{85} \mathrm{At}^{208}$ & 22,71 \\
\hline${ }_{83}^{8} \mathrm{Bi}^{214}$ & $53.2,295.2,352.0$, others \\
\hline${ }_{83} \mathrm{Bi}^{212}$ & 238.6, others \\
\hline${ }_{83}^{6} \mathrm{Bi}^{210}$ & 46.5 \\
\hline${ }_{83}^{80} \mathrm{Bi}^{206}$ & 83,167 \\
\hline${ }_{81} \mathrm{Tl}^{210}$ & $50 \pm 5,190 \pm 5$ \\
\hline${ }_{81} \mathrm{Tl}^{208}$ & $40,328,473,492,608$ \\
\hline
\end{tabular}

No obvious regularities emerge from a simple consideration of the excitation energies listed in Table IV. It may be pointed out that in almost all cases the first excited state is low-lying, and that there is no suggestion at all that the energy of this state is shell-closure sensitive. In order to recognise any regularity in the situation we have to appeal to information regarding the characteristics of the $\gamma$-rays emitted in de-excitation. Then it does appear that in all cases investigated the de-excitation of the first excited state is by a transition which is M1. This holds for the nuclei ${ }_{91} \mathrm{~Pa}^{234},{ }_{83} \mathrm{Bi}^{214},{ }_{83} \mathrm{Bi}^{212},{ }_{83} \mathrm{Bi}^{210}$ and ${ }_{81} \mathrm{Tl}^{208}$ in Table IV. Whether or not there is significance in the fact that the first excited state of a heavy $\left(\begin{array}{l}0 \\ 0\end{array}\right)$ nucleus 
has so frequently the same parity as the ground state is for the theorists to investigate.

\section{$\S 2$ Active Species of the $A=4 n+1$ and $A=4 n+3$ Series}

\section{A. $\gamma$-Radiations of the $\alpha$-Active $\left(\begin{array}{l}\mathrm{e} \\ 0\end{array}\right)$ ANd $\left(\begin{array}{l}0 \\ \mathrm{e}\end{array}\right)$ Species}

In this group of $\alpha$-emitters we find many examples of ground-to-ground state transitions which are of extremely feeble intensity - such that they might easily pass unrecognised in any but the most careful examination of the $\alpha$-particle spectrum. This was a situation not seriously envisaged when it was thought that partial $\alpha$-disintegration probabilities, for a given active species, could be explained in terms of energy release and spin change only. On this view, unless a low-lying isomeric state of the product nucleus were in question, it was not to be thought that the ground-to-ground state $\alpha$-transition would be of such small intensity as to be overlooked. Yet this is precisely what did happen, at first, in respect of the disintegration ${ }_{95} \mathrm{Am}^{241} \rightarrow{ }_{93} \mathrm{~Np}^{237}$ - and it is now clear from other evidence that the ground-to-ground state transformations ${ }_{96} \mathrm{Cm}^{243} \rightarrow{ }_{94} \mathrm{Pu}^{239}$ and ${ }_{95} \mathrm{Am}^{243} \rightarrow{ }_{93} \mathrm{~Np}^{239}$ have still not been observed. These facts show the need for considerable caution in the interpretation of experimental results as they stand at any particular time. (It is entirely another consideration that the sheer complexity of the $\alpha$-particle spectrum may defeat the experimentalist - thus the new analysis ${ }^{60,60 a}$ of the fine-structure spectra of RdAc and AcX is, in detail, quite different from the previous analysis ${ }^{61}$ offered by the same workers.) Having remarked on the need for caution, we shall now discuss the $\alpha$ - and following $\gamma$-modes of ${ }_{95} \mathrm{Am}^{241},{ }_{96} \mathrm{Cm}^{243}$ and ${ }_{95} \mathrm{Am}^{243}$ in further detail.

With ${ }_{95} \mathrm{Am}^{241}$ the disintegration energies (in $\mathrm{MeV}$ ) and intensities of the $\alpha$-modes are $5.640(0.23 \%), 5.628(0.34 \%), 5.596(0.21 \%), 5.568(84.2 \%)$, $5.525(13.6 \%)$ and $5.470(1.4 \%)^{62}$, and $\gamma$-rays of energy $59.8,42$ and $26.4 \mathrm{keV}$ have been observed in emission ${ }^{63,64,65} \cdot \gamma$-rays of the same energies, and others of higher energy, have been reported ${ }^{66}$ following the

60 S. Rosenblum, M. Perey, M. Valadares and M. Guillot, private communi • cation, quoted in UCRL report 1928 (revised) (1952).

60a M. Frilley, S. Rosenblum, M. Valadares and G. Bouissières, J. Physique 15, (1954) 45 .

61 S. Rosenblum, M. Guillot and M. Perey, C. R. Acad. Sci. (Paris) 204, (1937) 175.

62 F. Asaro, F. L. Reynolds and I. Perlman, Phys. Rev. 87, (1952) 277.

63 J. K. Beling, J. O. Newton and B. Rose, Phys. Rev. 86, (1952) 797; 87, (1952) 670.

64 M. S. Freedman, F. Wagner and D. W. Engelkemeir, Phys. Rev. 88, (1952) 1155.

65 J. O. Newton and B. Rose, Phys. Rev. 89, (1953) 1157.

${ }^{66}$ F. Wagner, M. S. Freedman, D. W. Engelkemeir and J. R. Huizenga, Phys. Rev. 89, (1953) 502. 
$\beta$-disintegration of ${ }_{92} \mathrm{U}^{237}$, both disintegrations leading to the same product nucleus ${ }_{93} \mathrm{~Np}^{237}$. Clearly, $\alpha$-excited states of this nucleus of energies 11,44 , 71,115 and $170 \mathrm{keV}$ provide full possibilities of accounting for the $\gamma$-rays of ${ }_{95} \mathrm{Am}^{241}$ - and their observation in emission (Dunlavey and Seaborg ? give the total internal conversion electron intensity as only $0.56 \pm 0.05$ per disintegration) indicates that there is no large difference of spin as between the $71 \mathrm{keV}$ state of ${ }_{93} \mathrm{~Np}^{237}$ and the states of $44 \mathrm{keV}$ and $11 \mathrm{keV}$ energy. If this conclusion is set against the fact that $\alpha$-excitation of the $71 \mathrm{keV}$ state is 400 times more probable than $\alpha$-excitation of the $44 \mathrm{keV}$ state, and 250 times more probable than that of the $11 \mathrm{keV}$ state, it is evident that the concept of a variable "hindrance factor" is necessary to explain the results. It is evident, too, that this hindrance factor is not a "difficulty of $\alpha$-formation" factor in any naive sense of the term: in this case very different hindrance factors apply when the same initial and different final nuclear states are in question. In point of fact the principal mode of $\alpha$-disintegration of ${ }_{95} \mathrm{Am}^{241}$ shows no hindrance at all ${ }^{67}$, when compared with the $\alpha$-disintegration modes of neighbouring $\left(\begin{array}{l}\mathrm{e} \\ \mathrm{e}\end{array}\right)$ nuclei. There must be some important but subtle difference in character, as yet imperfectly understood, between the $71 \mathrm{keV}$ state and the lower excited states of the nucleus ${ }_{93} \mathrm{~Np}^{237}$. Spin and parity considerations alone cannot explain this difference so long as the general possibility of $\alpha$-emission by more than one "spin-channel" is admitted 70, the prior necessity, for a transition to be forbidden, being then that the spin of one of the two states involved in the process should be zero. With an odd- $A$ nucleus this condition cannot be met. It should be added that none of the states of ${ }_{93} \mathrm{~Np}^{237}$ identified through the $\alpha$-disintegration of ${ }_{95} \mathrm{Am}^{241}$ is excited appreciably in the $\beta$-disintegration of ${ }_{92} \mathrm{U}^{237}$.

With ${ }_{96} \mathrm{Cm}^{243} \alpha$-disintegration energies and intensities (expressed as above) are $6.085(6.4 \%), 5.874(80.5 \%)$ and $5.828(13.1 \%)$, and $\gamma$-rays of energies 226 and $277 \mathrm{keV}$ have been observed in coincidence with the $\alpha$-particles of the second group ${ }^{68}$. These facts in themselves are enough to prove that the $\alpha$-particles of the first group are not emitted in a groundto-ground state transition. Clarification of the situation is provided by the observations of Graham and Bell ${ }^{69}$ that in the $\beta$-disintegration ${ }_{93} \mathrm{~Np}^{239} \rightarrow{ }_{94} \mathrm{Pu}^{239}$, also, $\gamma$-rays of 227 and $276 \mathrm{keV}$, not in cascade with one another, are emitted in coincidence with $\beta$-particles of the same partial spectrum, and that the $\gamma$-cascade of energies 210 and $67 \mathrm{keV}$ belongs to

67 N. Feather, Nuclear Stability Rules, C.U.P. (1952) p. 45.

68 F. Asaro, S. G. Thompson and I. Perlman, Phys. Rev. 92, (1953) 694.

69 R. L. Graham and R. E. Bell, Phys. Rev. 83, (1951) 222.

70 J. J. Devaney, Phys. Rev. 91, (1953) 587. 
the same disintegration mode. All these results fall into line if excitation energies of 49, 67, 277 and $324 \mathrm{keV}$ are assumed for the nucleus ${ }_{94} \mathrm{Pu}^{239}$. It will be noted that, as yet, investigations of the $\alpha$-disintegration of ${ }_{96} \mathrm{Cm}^{243}$ have not been sensitive enough for the detection either of the weak $\alpha$-particle group corresponding to the excitation of the first excited state, or of that exciting the ground state of ${ }_{94} \mathrm{Pu}^{239}$. As with ${ }_{93} \mathrm{~Np}^{237}$, the third excited state of this nucleus is the particularly favoured (or not particularly unfavoured) state for $\alpha$-excitation. On the other hand there is merely a difference of one degree of forbiddenness as between the $\beta$-disintegration (of ${ }_{93} \mathrm{~Np}^{239}$ ) which feeds the ground state and that which feeds the third excited state of ${ }_{94} \mathrm{Pu}^{239}$.

With ${ }_{95} \mathrm{Am}^{243} \alpha$-disintegration energies are $5.355(84 \%), 5.312(13 \%)$ and $5.257(\sim 3 \%)$, and $\gamma$-rays of energy $75 \mathrm{keV}$ have been observed in coincidence with the $\alpha$-particles of the highest-energy group ${ }^{71} \cdot \gamma$-rays of energy $73.6 \mathrm{keV}$ are known to be emitted in the principal mode of $\beta$-disintegration of ${ }_{92} \mathrm{U}^{239}$. Clearly the first excited state of ${ }_{93} \mathrm{~Np}^{239}$ is at $74 \mathrm{keV}$, and the second excited state at $117 \mathrm{keV}$, on this evidence-and the ground-to-ground state transition is of negligible intensity in the $\alpha$.disintegration of ${ }_{95} \mathrm{Am}^{243}$. Again, the favoured $\alpha$-transition (to the $74 \mathrm{keV}$ state) appears as unhindered, and that to the ground state-because the spin-change cannot on the $\gamma$-ray evidence be very different-must be characterised by a hindrance factor of the order of 100 or more.

Examples of unhindered ground-to-ground state $\alpha$-transitions are almost certainly provided by the disintegrations of ${ }_{94} \mathrm{Pu}^{239}$ and ${ }_{92} \mathrm{U}^{233}$. The only possibility of a misinterpretation of results in these cases would arise if the true ground state for each product nucleus were to lie within an energy range less than the corresponding $L_{\mathrm{III}}$ ionisation energy of the highly $\alpha$-excited state which is here presumed to be the ground state. Measurements of the total $L$-X-ray intensities ${ }^{3}$ lead unambiguously to this conclusion. Disregarding this possibility, the $\gamma$-rays observed in these disintegrations can"be explained in terms of the level schemes based on the $\alpha$-particle fine-structure spectra, with excitations as follows: ${ }_{94} \mathrm{Pu}^{239} \rightarrow{ }_{92} \mathrm{U}^{235}$; ground state, $69 \%$; $14 \mathrm{keV}, 20 \%$; $53 \mathrm{keV}, 11 \%$; ${ }_{92} \mathrm{U}^{233} \rightarrow{ }_{90} \mathrm{Th}^{229}$; ground state, $83 \% ; 43 \mathrm{keV}, 15 \% ; 99 \mathrm{keV}, 2 \%$. In each case the cross-over $\gamma$-ray is observed.

Beyond what has already been said regarding the disintegration modes of the five odd- $A \alpha$-emitters discussed above, there is not much which can be added with confidence concerning transformations of the type we are now considering. Investigations of $\alpha$-particle fine structure and of $\gamma$-rays have been made for seven other odd- $A \alpha$-emitters $\left({ }_{92} \mathrm{U}^{235},{ }_{91} \mathrm{~Pa}^{231}\right.$,

71 F. Asaro and I. Perlman, Phys. Rev. 93, (1954) 1423. 
${ }_{90} \mathrm{Th}^{227},{ }_{88} \mathrm{Ra}^{223},{ }_{87} \mathrm{Fr}^{221},{ }_{86} \mathrm{Em}^{219}$ and $\left.{ }_{83} \mathrm{Bi}^{211}\right)$ and of $\alpha$-particle fine structure only for another five species $\left({ }_{97} \mathrm{Bk}^{245},{ }_{97} \mathrm{Bk}^{243},{ }_{90} \mathrm{Th}^{229},{ }_{86} \mathrm{Em}^{211} \text { and }{ }_{84} \mathrm{Po}^{211}\right)_{;}$ Information concerning $\gamma$-rays only is on record for ${ }_{94} \mathrm{Pu}^{241}$ and ${ }_{93} \mathrm{~Np}^{237}$. An earlier conclusion ${ }^{72}$, that the ground-to-ground state $\alpha$-mode with ${ }_{88} \mathrm{Ra}^{223}$ ( $\mathrm{AcX}$ ) had not then been observed, has been shown ${ }^{62}$ to have been well-founded - and the observation ${ }^{7}$ of low-energy conversion electrons in association with $80 \pm 5 \%$ of the $\alpha$-particles of ${ }_{93} \mathrm{~Np}^{237}$ is sufficient to make it extremely unlikely that the directly determined $\alpha$-disintegration energy of this body $(4.85 \pm 0.03 \mathrm{MeV})$ is the energy of disintegration, ground state to ground state. It is not impossible that the $\alpha$-particle spectrum of ${ }_{93} \mathrm{~Np}^{237}$ is very similar in general structure to that of ${ }_{95} \mathrm{Am}^{241}$, its immediate parent in the series.

\section{B. Some $\beta$-Active Species of Odd $A$}

The first case for discussion is that of ${ }_{91} \mathrm{~Pa}^{233}$, of interest more for the amount of detailed work ${ }^{73.74,75}$ done on it than for the finality of the conclusions reached. This is one of the few disintegrations which have so far been studied ${ }^{74}$ by the method ${ }^{76}$ of line-to-line coincidences. Some $13 \gamma$-rays have been identified, and the total number of electrons (of energy $>4 \mathrm{keV}$ ) in the line spectrum is $1.0 \pm 0.15$ per disintegration. The $K$-conversion electrons of the $313 \mathrm{keV} \gamma$-ray alone number 0.34 per disintegration.

Secondly, two $\beta$-disintegrations of low energy are of interest for that fact alone. The disintegration ${ }_{94} \mathrm{Pu}^{241} \rightarrow{ }_{95} \mathrm{Am}^{241}$ appears as an allowed transition of $20.5 \mathrm{keV}$ energy ${ }^{64}$ proceeding by a single $\beta$-mode with no $\gamma$-radiation. By contrast, the other disintegration, ${ }_{89} \mathrm{Ac}^{227} \rightarrow{ }_{90} \mathrm{Th}^{227}$, known for a much longer time, is much less thoroughly understood. Radiations reported in the published literature 77 amount only to low-energy electrons ( 0.15 per disintegration), $L$-X-radiation $(0.05 \pm 0.01$ quanta per disintegration) and a $\gamma$-radiation of $36.8 \pm 0.7 \mathrm{keV}$ energy $(\sim 0.002$ quanta per disintegration)-together with the $\alpha$-particles of the competing mode (0.0125 per disintegration) ${ }^{78}$. If the $37 \mathrm{keV}$ transition is regarded as associated with a $\beta$-mode, being highly converted ( $L$-conversion coefficient $\sim 100$ ) the total disintegration energy cannot well be less than $50 \mathrm{keV}$,

72 N. Feather, Phil. Mag. Supp. 2, (1953) 141.

73 H. B. Keller and J. M. Cork, Phys. Rev. 79, (1950) 1030.

74 L. G. Elliott and A. B. Underhill, A.E.R.E. report HaR 761, (1952).

75 W. D. Brodie, Proc. Phys. Soc. A 67, (1954) 397.

76 N. Feather, Proc. Camb. Phil. Soc. 36, (1940) 224.

77 M. Lecoin, M. Perey, M. Riou and J. Teillac, J. Physique 11, (1950) 227.

78 S. Peterson and A. Ghiorso, American desclassified report MDDC 1743 (1948). 
and failure to observe the main $\beta$-component of this maximum energy is a real difficulty. The difficulty might be somewhat reduced by regarding the $\gamma$-radiation as an $\mathrm{E} 1$ radiation associated with an $\alpha$-mode of disintegration ${ }^{79}$. On this hypothesis two components of $\beta$-radiation would still be required, but maximum energies of $\sim 10 \mathrm{keV}$ and $\sim 30 \mathrm{keV}$ could be admitted. Only further experiments with sources of higher specific activity can resolve the present uncertainty.

The $\beta$-disintegration of ${ }_{90} \mathrm{Th}^{231}$ (UY) is interesting for two reasons. In the first place, the large-scale separation of the uranium isotopes in a state of high purity has for the first time made it possible to examine the radiations of this body without interference ${ }^{81}$ from the more energetic radiations of $\mathrm{U}\left(\mathrm{X}_{1}+\mathrm{X}_{2}\right)$ with which it is inevitably associated when prepared from natural uranium ${ }^{80}$. The second point of interest is that the analysis of this more detailed investigation postulates the occurrence of a low-energy $\beta$-mode characterised by an $f t$ value about five times smaller than any other $f t$ value for a heavy $\beta$-emitter ${ }^{82}$. If this does not represent an error in interpretation, it poses a very particular problem in respect of the isobaric pair ${ }_{90} \mathrm{Th}^{231},{ }_{91} \mathrm{~Pa}^{231}$.

\section{$\S 3$. Characteristics of the Capture-Active Species of Large $Z$}

In the range of atomic numbers $80<Z<98$ between 70 and 80 species are known.which are capture active, and for some 30 of them total disintegration energies are known on the basis of closed-cycle calculations. All these species are potentially $\alpha$-active, and $\alpha$-/capture- branching is an observed effect complicating the experimental situation for some 40 of them. In this section we shall discuss the characteristics of only a few of these species in detail. Moreover, any case which is of interest chiefly as an example of radioactive branching or of isomerism (or both) will be dealt with in $\S 4$ rather than here.

We take, then, as first examples, the three neptunium isotopes ${ }_{93} \mathrm{~Np}^{238}$, ${ }_{93} \mathrm{~Np}^{235}$ and ${ }_{93} \mathrm{~Np}^{234}$. For each of these a very considerable proportion of $L$-electron capture has been established-about $65 \%$ for ${ }_{93} \mathrm{~Np}^{236}{ }^{84}$, more than $90 \%$ for ${ }_{93} \mathrm{~Np}^{23585}$, and of the order of $50 \%$ for ${ }_{93} \mathrm{~Np}^{234}{ }^{35}$. These results are generally understandable in view of the fact ${ }^{83}$ that the main

79 N. Feather, Nucleonics 5, (1949) 22.

80 P. H. Stoker, P. H. Ong and G. J. Sizoo, Physica 17, (1951) 164.

81 M. S. Freedman, A. H. Jaffey, F. Wagner and J. May, Phys. Rev. 89, (1953) 302 .

82 N. Feather, Nuclear Stability Rules, C.U.P., (1952) p. 99.

83 N. Feather, Physica 18, (1952) 1023.

84 D. A. Orth and G. D. O'Kelley, Phys. Rev. 82, (1951) 758.

85 R. James, A. Ghiorso and D. A. Orth, Phys. Rev. 85, (1952) 369. 
disintegration mode in each case is a forbidden transition, and of the further fact that with ${ }_{93} \mathrm{~Np}^{235}$ the disintegration energy is small enough to provide an additional factor of the order of 4 favouring $L$ - as against $K$-electron capture. The complexity of the $\gamma$-radiation following the capture process with ${ }_{93} \mathrm{~Np}^{234}$ has already been referred to $(\S 1 \mathrm{~B})$.

The next case of interest is that of ${ }_{92} \mathrm{U}^{231}$. This body and the $\beta$-active ${ }_{90} \mathrm{Th}^{231}$ (UY) have ${ }_{91} \mathrm{~Pa}^{231}$ as common daughter product. Yet of the three $\gamma$-radiations reported ${ }^{86}$ for ${ }_{92} \mathrm{U}^{231}$ only one can plausibly be identified, on the basis of determined energy, with one of the $\gamma$-rays of UY-and this $\gamma$-ray has been assumed ${ }^{81}$ to arise in a transition between excited states of $231 \mathrm{keV}$ and $167 \mathrm{keV}$ in the product nucleus. There is a lack of coherence in the two disintegration schemes which should prompt further investigation.

Four capture-active isotopes of bismuth have been studied in some detail, each possessing a $\gamma$-ray spectrum extending to high energies. With ${ }_{83} \mathrm{Bi}^{207}, 8 \gamma$-rays have been observed ${ }^{87}$, the most energetic being of $2.49 \mathrm{MeV}$, and three others having quantum energies in excess of $2 \mathrm{MeV}$. The indications are of a group of four closely-spaced excited states in ${ }_{82} \mathrm{~Pb}^{207}$ in the energy range 2 to $2.5 \mathrm{MeV}$. The most intense excitation appears to be of the $1.63 \mathrm{MeV}$ metastable $(0.82 \mathrm{sec})$ state, but the $0.87 \mathrm{MeV}$ state-the only one known to be excited in the $\beta$-disintegration of ${ }_{81} \mathrm{Tl}^{207}$ $\left(\mathrm{AcC}^{\prime \prime}\right)$ - is not appreciably excited (see Note p. 766 ). The $\gamma$-ray spectrum of ${ }_{83} \mathrm{Bi}^{206}$ is still more complex, $15 \gamma$-rays having been reported ${ }^{88}$. Accepting the conclusion that the first excited state of the daughter nucleus ${ }_{82} \mathrm{~Pb}^{206}$ is at $801 \mathrm{keV}(\S 1 \mathrm{~B})$, we must again assume several closely-spaced states, in the energy range above this value (see Note p. 766). With ${ }_{83} \mathrm{Bi}^{205}$, a $\gamma$-ray of $1.84 \mathrm{MeV}$ and four others of quantum energies between 0.4 and $0.8 \mathrm{MeV}$ have been observed ${ }^{89}$. It seems possible that the level system of the daughter nucleus is somewhat simpler in this case $\left({ }_{82} \mathrm{~Pb}^{205}\right)$, the $1.84 \mathrm{MeV}$ radiation arising in a cross-over transition bracketing a three-member cascade. With all three bismuth isotopes, however, it is a striking feature that capture transitions to the lower-lying states of the product nuclei of lead have every appearance of being highly forbidden. It is a question worthy of consideration whether this forbiddenness is to be described entirely in terms of the ordinary spin and parity changes involved, or whether some factor of "hindrance", analagous to that required for the description of the $\alpha$-disintegrations of the bismuth

G. D. O'Kelley and F. L. Reynolds, quoted in UCRL report 1928 (revised) (1952).

H. M. Neumann and I. Perlman, Phys. Rev. 81, (1951) 958.

D. E. Alburger and G. Friedlander, Phys. Rev. 81, (1951) 523.

D. G. Karraker and D. H. Templeton, Phys. Rev. 81, (1951) 510. 
isotopes, is necessary to complete the picture. With ${ }_{83} \mathrm{Bi}^{204}$, on the other hand, the situation is not so extreme, the first excited state of ${ }_{82} \mathrm{~Pb}^{204}$ is highly excited, though there is some excitation of the (second) $1.28 \mathrm{MeV}$ state, or possibly of a state at $1.50 \mathrm{MeV}^{90}$, also.

Of the capture-active lead isotopes, only ${ }_{82} \mathrm{~Pb}^{203}$ is of interest for the present survey. Three $\gamma$-rays have been observed ${ }^{36}$, of energies 153,269 and $422 \mathrm{keV}$, obviously representing a two-member cascade with crossover radiation. The interesting fact is the discrepancy (see Note p. 766) between the energy value $269 \mathrm{keV}$ and $279 \pm 1 \mathrm{keV}$, which is the value obtained for the first excitation energy of the product nucleus ${ }_{81} \mathrm{Tl}^{203}$ as observed in the $\beta$-disintegration of ${ }_{80} \mathrm{Hg}^{203}$ (see, for example, Cork et al. ${ }^{91}$ ).

Our last example of a capture-active species of interest is ${ }_{81} \mathrm{Tl}^{199}$. The disintegration modes of this species, those of the $\beta$-active ${ }_{79} \mathrm{Au}^{199}$, which also has ${ }_{80} \mathrm{Hg}^{199}$ as daughter species, and the modes of isomeric transition in ${ }_{80} \mathrm{Hg}^{199}$ itself, have recently been studied by Israel et al. ${ }^{92}$. The first excited state (at $159 \mathrm{keV}$ ) is involved in all three processes, the second excited state (at $209 \mathrm{keV}$ ) in the $\beta$ - and capture-transformations, and two further states (at 454 and $491 \mathrm{keV}$ ) only when excited in the capturedisintegration of ${ }_{81} \mathrm{Tl}^{199}$. In this last disintegration two low-energy $\gamma$-rays are also emitted the placing of which in the disintegration scheme is as yet indefinite.

\section{$\S 4$. Miscellaneous: Series Branching; Isomerism}

As already mentioned in $\S 3$, all species for which $Z>82$ are potentially $\alpha$-active, and as there are some 180 such species known'at present, and as less than 50 of these are pure $\alpha$-active species ${ }^{93}$, possibilities in respect of series branching are widespread. Only a few cases will be considered in this section-first a group of 8 species for each of which all three modes, $\alpha-, \beta$ - and capture-disintegration are possible energetically. These are the "intermediate" species corresponding to the 8 pairs of even- $A$ isobars $(A>210)$ for which" $\Delta Z=2$. The species in question are ${ }_{95} \mathrm{Am}^{242},{ }_{93} \mathrm{~Np}^{238}$, ${ }_{93} \mathrm{~Np}^{236},{ }_{91} \mathrm{~Pa}^{232},{ }_{91} \mathrm{~Pa}^{230},{ }_{89} \mathrm{Ac}^{226},{ }_{89} \mathrm{Ac}^{224}$ and ${ }_{85} \mathrm{At}^{216}$. Of these species ${ }_{85} \mathrm{At}^{216}$ is known only as an $\alpha$-emitter (disintegration energy $7.94 \mathrm{MeV}$ ), and ${ }_{93} \mathrm{~Np}^{238},{ }_{91} \mathrm{~Pa}^{232}$ and ${ }_{89} \mathrm{Ac}^{226}$ only as $\beta$-emitters (estimated $\alpha$-disintegration energies 5.0, 4.5 and 5.5 MeV, respectively); $\beta$-/capture- branching has been observed with ${ }_{93} \mathrm{~Np}^{236}$, but no $\alpha$-activity (estimated disintegration

90 M. Goldhaber and R. D. Hill, Rev. Mod. Phys. 24, (1952) 179.

91 J. M. Cork, D. W. Martin, J. M. LeBlanc and C. E. Branyan, Phys. Rev. 85, (1952) 386.

92 H. I. Israel, C. H. Pruett and R. G. Wilkinson, private communication, quoted in UCRL report 1928 (revised) (1952). 
energy 5.0 MeV), and $\alpha$-/capture- branching with ${ }_{89} \mathrm{Ac}^{224}$, but no $\beta$-activity. Only with ${ }_{95} \mathrm{Am}^{242}$ and ${ }_{91} \mathrm{~Pa}^{230}$ have all three modes been found, though in each case the $\alpha$-mode is of such low intensity that no direct measurement of disintegration energy has been made. Estimates are $5.40 \pm 0.05 \mathrm{MeV}$ and $5.4 \pm 0.2 \mathrm{MeV}$, respectively. These results, regarded as a fair sample of the whole, are in rough qualitative agreement with the conclusion ${ }^{93}$ that generally the observation of $\alpha-\beta$ - or $\alpha$-/capture- branching is limited to those cases in which the energy of $\alpha$-disintegration lies between $5 \mathrm{MeV}$ and $7 \mathrm{MeV}$. A ninth "intermediate" species for which $Z>80$ is ${ }_{81} \mathrm{Tl}^{204}$. In this case the observation of $\alpha$-activity is clearly not in question, but recently ${ }^{94,} 95$ the occurrence of $\beta$-/capture- branching has been established. Because no $\gamma$-rays are emitted, it must be concluded ( $\S 1 \mathrm{~B})$ that the $\beta$ - and capture- modes are equally forbidden.

Recent work on the natural actinium series has provided results of considerable interest. Hyde and Ghiorso ${ }^{96}$ have shown that ${ }_{87} \mathrm{Fr}^{223}$ (AcK), itself produced from ${ }_{89} \mathrm{Ac}^{227}$ in a $1.2 \% \alpha$-mode, exhibits $\alpha$ - $/ \beta$-branching in approximate ratio $5 / 10^{5}$, and that ${ }_{85} \mathrm{At}^{219}$, its $\alpha$-produced daughter, does the same $(\alpha / \beta$ ratio, $97 / 3)$. Thus ${ }_{85} \mathrm{At}^{219}$ and ${ }_{83} \mathrm{Bi}^{215}$ are newly recognised as short-lived members of the natural series (half-value periods $\sim 1 \mathrm{~min}$ and $\sim 8$ min, respectively). Somewhat earlier, Avignon ${ }^{97}$ had shown that ${ }_{85} \mathrm{At}^{215}$ is also a member of this series, formed in a rare $\beta$-mode from ${ }_{84} \mathrm{Po}^{215}$ (AcA).

Having considered examples of recently investigated branching disintegrations, it is worth while drawing attention to two cases in which, surprisingly, branching has so far not been observed, or shows unusual features. The species here in question are ${ }_{84} \mathrm{Po}^{209}$ and ${ }_{84} \mathrm{Po}_{0}{ }^{208}$. The former is a predominantly $\alpha$-active body of half-value period of the order of $100 \mathrm{y}$. The energy available for capture-disintegration is $1.82 \pm 0.12 \mathrm{MeV}^{72}$, yet the capture- $/ \alpha$ - branching ratio is less than $1 / 10$. With ${ }_{84} \mathrm{Po}^{208}$, an $\alpha$-active body of lifetime $2.93 \mathrm{y}$, no capture activity has been detected. In this case $1.03 \pm 0.22 \mathrm{MeV}$ energy is available ${ }^{72}$, and as in the other case an effective lifetime for capture of at least $1000 \mathrm{y}$ must apply. Obviously connected with this fact is the conclusion, from failure to observe the activity, that the lifetime of ${ }_{83} \mathrm{Bi}^{208}$ for capture is also long, though the energy available is considerably greater, $2.93 \pm 0.07 \mathrm{MeV}{ }^{72}$. Pryce ${ }^{98}$ has suggested that the spin of ${ }_{83} \mathrm{Bi}^{208}$ in the ground state is 5 units: this would

93 N. Feather, Nuclear Stability Rules, C.U.P. (1952) p. 83.

94 L. Lidofsky, P. Macklin and C. S. Wu, Phys. Rev̀. 87, (1952) 391.

95 A. C. G. Mitchell and R. S. Caird, Phys. Rev. 87, (1952) 388.

96 E. K. Hyde and A. Ghiorso, UCRL report 2019 (1952).

97 P. Avignon, J. Physique 11, (1950) 521.

98

M. H. L. Pryce, Proc. Phys. Soc. A 65, (1952) 773. 
explain its long lifetime-and that of ${ }_{84} \mathrm{Po}^{208}$, if there is no state of small spin amongst the closely-spaced low-lying states predicted for ${ }_{83} \mathrm{Bi}^{208}$. It should be remembered, however, that we have already drawn attention (§ 3 ) to the long lifetimes for capture of the bismuth isotopes of mass numbers 207, 206 and 205. We must now add ${ }_{83} \mathrm{Bi}^{208}$ to this list.

As concerns isomerism, 10 species are known for which $Z>80$ each having one isomeric state of lifetime of the order of $1 \mathrm{sec}$ or longer. One of these species, ${ }_{91} \mathrm{~Pa}^{234}$, provides the classical example of the phenomenon ${ }^{99}$. Although the isomeric excitation energy in this case is still in doubt 100. 32. 100a, there can be no doubt that the prior $\beta$-disintegration (of $\mathrm{UX}_{1}$ ) excites the metastable state $\left(\mathrm{UX}_{2}\right)$, and not the ground state, and that transition to the latter (UZ) is characterised by an effective lifetime of about $13 \mathrm{~h}$.

Four of the remaining 9 isomeric states of our survey belong to isotopes of lead. Mass numbers and exitation energies are $201,670 \mathrm{keV} ; 202,890 \mathrm{keV}$; $204,1.28 \mathrm{MeV}$; 207, 1.63 MeV. Arranged in this order, the energies constitute a monotonic sequence, also there is strong evidence for the conclusion that the long-lived state is not the first excited state in any case. With ${ }_{82} \mathrm{~Pb}^{207}$ and ${ }_{82} \mathrm{~Pb}^{294}$ the matter has been studied in some detail, the "delayed" transitions being to intermediate states at $565 \mathrm{keV} 101$ and $374 \mathrm{keV} \mathrm{102}$, respectively; with ${ }_{82} \mathrm{~Pb}^{202}$, though the delayed transition is probably to the ground state ${ }^{103}$, the existence of an intermediate state at $157 \mathrm{keV}$ is known from other evidence ${ }^{60}$-and with ${ }_{82} \mathrm{~Pb}^{201}$ preliminary results appear to indicate an intermediate state at $250 \mathrm{keV}$ (or $420 \mathrm{keV}$ ). with delayed modes both to this state and to ground ${ }^{103}$.

Two of the reported cases of isomerism for which $Z>80$ have not been examined in sufficient detail for comment to be profitable here. The species concerned are ${ }_{83} \mathrm{Bi}^{201}$ and ${ }_{85} \mathrm{At}^{208}$. This leaves the three cases - of ${ }_{83} \mathrm{Bi}^{210}$, ${ }_{84} \mathrm{Po}^{211}$ and ${ }_{95} \mathrm{Am}^{242}$-for further discussion. As $\mathrm{RaE}$ and $\mathrm{AcC}^{\prime}$ the first two species in this group are well-known members of the classical radioactive series; their isomerism, however, was discovered only when they were produced "artificially". Natural RaE had already been shown to be the parent of the $\beta$-active ${ }_{81} \mathrm{Tl}^{206}$ in a rare mode of $\alpha$-disintegration ${ }^{104}$, when Neumann et al. ${ }^{105}$ discovered a long-lived $\alpha$-activity, also producing

99

100

$100 a$

101

102

103 N. J. Hopkins, Phys. Rev. 88, (1952) 680.

104 E. Broda and N. Feather, Proc. Roy. Soc. A 190 (1947) 20.

105 H. M. Neumann, J. J. Howland and I. Perlman, Phys. Rev. 77, (1950) 720. 
${ }_{81} \mathrm{Tl}^{206}$ as daughter product, in neutron-irradiated bismuth. At first this activity was interpreted as arising in a state of ${ }_{83} \mathrm{Bi}^{210}$ somewhat higher in energy than the state which is $\mathrm{RaE}$-though grave difficulties were seen to lie in that interpretation ${ }^{106}$. More recently, however, a redetermination of the $\alpha$-particle energy ${ }^{107}$ suggests that the long-lived state is the ground state, and $\mathrm{RaE}$ the isomeric state of this nucleus, and that the excitation energy is $30 \pm 30 \mathrm{keV}$. It seems almost impossible that the order of these two states could be otherwise, since the lifetime of the artificially-produced state is estimated as $(2.6 \pm 0.8) \times 10^{6} \mathrm{y}^{108}$. If this interpretation is accepted we are at present left without direct evidence whether there is significant production of the ground-state product by isomeric transition in natural RaE. One per cent production would almost certainly have escaped detection hitherto: the most straightforward approach would be through the isolation of bismuth from an unchanged uranium mineral, after the manner of Widdowson and Russell ${ }^{109}$ but using modern pulse-chamber technique (see Notep. 766). From the viewpoint of the one-particle (nuclear shell) model, Pryce ${ }^{98}$ has shown that there is no insuperable difficulty in explaining the existence of close lowlying states in the ${ }_{83} \mathrm{Bi}^{210}$ nucleus with a spin difference of as much as 6 units.

${ }_{84} \mathrm{Po}^{211}\left(\mathrm{AcC}^{\prime}\right)$ was first obtained "artificially" ${ }^{110}$ as the daughter product of the capture-mode of disintegration of ${ }_{85} \mathrm{At}^{211}$ produced by $\alpha$-particle bombardment of bismuth. With material so obtained, weak fine-structure components of the $\alpha$-radiation were detected ${ }^{111}$ and the half-value period was measured ${ }^{112}$. Then it was discovered that a longer-lived polonium $\alpha$-emitter is formed directly in the $\alpha$-particle bombardment both of ${ }_{83} \mathrm{Bi}^{209}$ and of ${ }_{82} \mathrm{~Pb}^{208}$. The energy of $\alpha$-disintegration of this species was found to be $300 \pm 50 \mathrm{keV}$ less than that of $\mathrm{AcC}^{\prime}$, and, although there was no sign of its production from $\mathrm{AcC}^{\prime}$ directly, it was concluded on all the available evidence that it represents the ground state, and $\mathrm{AcC}^{\prime}$ the isomeric state, of the nucleus ${ }_{84} \mathrm{Po}^{211}{ }^{113}$. Empirically, the $\alpha$-disintegrations of $\mathrm{AcC}^{\prime}$ and the ground-state isomer appear to be forbidden to approximately the same extent. In view of the large difference of spin which must characterise the two $\alpha$-emitting states, it is likely that in one case the smallness of the

N. Feather, Phil. Mag. 42, (1951) 568.

H. B. Levy and I. Perlman, Phys. Rev. 85, (1952) 758.

D. J. Hughes and H. Palevsky, Phys. Rev. 92, (1953) 1206.

W. P. Widdowson and A. S. Russell, Phil. Mag. 46, (1923) 915.

D. R. Corson, K. R. MacKenzie and E. Segrè, Phys. Rev. 58, (1940) 672.

H. M. Neumann and I. Perlman, Phys. Rev. 81, (1951) 958.

R. F. Leininger, E. Segrè and F. N. Spiess, Phys. Rev. 82, (1951) 334.

F. N. Spiess, Phys. Rev. 94, (1954) 1292. 
disintegration probability is to be attributed to spin-forbiddenness, in the other to "hindrance" of another origin 114.

The $\alpha$ - $/ \beta$ - /capture- branching disintegration of ${ }_{95} \mathrm{Am}^{242}$ has already been discussed $(\S 3)$, but without consideration of the fact that this species exhibits nuclear isomerism as well. In order to complete the picture it should now be stated that an upper state of $35 \mathrm{keV}$ excitation energy and $16 \mathrm{~h}$ lifetime decays in the approximate ratios $6: 2: 2$ by $\beta$-emission, electron capture, and isomeric transition to ground ${ }^{36}$, and that the ground state (lifetime $\sim 100$ y) decays principally by $\beta$-emission, with an $\alpha$-mode of about $1 \%$ intensity ${ }^{115}$. The one puzzling feature of these observations is the apparent absence of $K$-X-radiation ${ }^{116}$. The naive conclusion that capture proceeds almost entirely from the $L$-shell is difficult to reconcile with considerations of available energy and lifetime. The capture process cannot possibly be of a higher degree of forbiddenness than two.

114 N. Feather, Phil. Mag. 43, (1952) 476.

115 K. Street, A. Ghiorso and G. T. Seaborg, Phys. Rev. 79, (1950) 530.

116 G. D. O'Kelley, G. W. Barton, W. T. Crane and I. Perlman, Phys. Rev. 80, (1950) 293.

\section{NOTE}

To take account of recent publications necessitating revision of certain data and opinions included above has been impossible in page proof. To remedy the situation as far as possible the following additional references (noted in the text) are now appended.

Table I (page 742): H. Diamond, L. B. Magnusson, J. F. Mech, C. M. Stevens, A. M. Friedman, H. M. Studier, P. R. Fields and J. R. Huizenga, Phys. Rev. 94, (1954) 1083.

F. Asaro and I. Perlman, Phys. Rev. 94, (1954) 381.

H. Slätis, J. O. Rasmussen and H. Atterling, Phys. Rev. 93, (1954) 646.

RaB (page 748): M. Mladjenovic and H. Slätis, Arkiv Fys. 8, (1954) 65.

RaE (page 752): M. Yamada, Prog. Theor. Phys. (Japan) 10, (1953) 252.

M. E. Rose and R. K. Osborn, Phys. Rev. 93, (1954) 1315.

R. W. Kirg and D. C. Peaslee, Phys. Rev. 94, (1954) 795.

UZ (page 754): P. H. Ong and G. J. Sizoo, Physica 19, (1953) 1205.

S. A. E. Johansson, American report ISC 462 (1954).

${ }_{83} \mathrm{Bi}^{207}$ (page 761): A. H. Wapstra, Arkiv Fys. 7, (1953) 279.

D. E. Alburger, Phys. Rev. 92, (1953) 1257.

J. R. Prescott, Proc. Phys. Soc. A 67, (1954) 540.

${ }_{83} \mathrm{Bi}^{200}$ (page 761): D. E. Alburger and M. H. L. Pryce, Phys. Rev. 92, (1953) 514. ${ }_{82} \mathrm{~Pb}^{203}$ (page 762): J. R. Prescott, Proc. Phys. Soc. A 67, (1954) 254.

J. Varma, Phys. Rev. 94, (1954) 1688.

RaE isomer (page 765): H. B. Levy and I. Perlman, Phys. Rev. 94, (1954) 152. 


\title{
$\gamma$-RADIATION FROM CHARGED PARTICLE REACTIONS
}

\section{XXIV}

\author{
D. E. ALBURGER
}

\section{§ 1. Introduction}

Although the nuclear energy levels occurring in beta activity have been discussed at length in previous chapters it is well to point out that the total decay energy available, in the vast majority of cases, limits the number of levels which can be observed to those within a few $\mathrm{MeV}$ of the ground state. Alpha decay usually takes place with greater energy release than electron emission or capture but the strong dependence of barrier penetration probability on energy prevents the population of highly excited states. Since the spacing of low lying nuclear levels is generally greater than $100 \mathrm{keV}$ and is often of the order of $1 \mathrm{MeV}$ it is characteristic that only a few states, perhaps 3 on the average, are excited in radioactivity. There are, of course, exceptions such as the complex decay schemes discussed in Ch. XXII where 10 or more levels have been established.

On the other hand the binding energy of a nucleon is $6-8 \mathrm{MeV}$ and it is known that not only do discrete bound levels exist this far up but that well-defined virtual levels can be observed at considerably higher energies. Gamma radiation from levels close to the binding energy formed by slow neutron capture is discussed in Ch. XXV. It is theoretically expected and experimentally observed that the density of levels increases with energy above the ground state. Insofar as the study of the systematics of level positions, spacings, and other relevant properties is concerned it is clear that radioactive decay processes are rather limited and that they are unable to expose a rich region of excitation where nuclear configurations might be of great interest. Even at low excitation beta decay may fail to reveal all of the energetically possible states as a consequence of selection rules. A case in point is the $\mathrm{Pb}^{206}$ level arrangement described in Ch. XXII.

One approach to the gamma-ray spectroscopy of nuclear levels has been pursued to a lesser extent than others already described in earlier chapters. This consists of bombarding nuclei with heavy particles and simultaneously observing the gamma radiation emitted as the excited nucleus undergoes transitions between states. From the experimental point of view the subject is most conveniently divided into charged particle and fast neutron excitation, as discussed in the present chapter, and slow neutron capture reactions described in the next chapter inasmuch as the former 
require accelerators and the latter reactors. The lag of gamma-ray. spectroscopy in nuclear reactions behind its counterpart in radioactivity is understandable in view of the more difficult nature of the research and the need for more suitable measuring techniques. Many excellent instruments have been developed for radioactivity which can be constructed at relatively low cost and used with sources prepared elsewhere in a reactor or cyclotron. But in charged particle nuclear reactions where the entire process of excitation and de-excitation can almost always be considered as taking place instantaneously one must work with a continuous beam from an expensive and complex accelerator. The beam must have a sufficient energy to make the desired reaction go and it must meet the requirements of small physical size, sufficient intensity, and reasonably low energy spread. Background gamma rays from the accelerator itself must not obscuie those from the target. Even if these conditions have been satisfied there are other problems which are not encountered in radioactivity studies. The target material should be stable under bombardment and should not contain other elements or isotopes which might cause confusion. Accelerators rarely have the required constancy of beam intensity to allow one to do experiments without monitoring either the beam current or the yield of the reaction. There are also problems, to be discussed in the next section, of identifying the reaction from which the gamma rays arise and sorting out the radiations from several competing reactions.

The lack of experimental techniques has to do with the expected nature of gamma-ray spectra from nuclear reactions. Because of the high excitations a wide range of energies will generally result, but measuring devices above $3 \mathrm{MeV}$ are definitely poorer than those for lower energies. At present no instrument exists with which precision measurements (accuracies of parts in $10^{4}$ ) can be made on energetic gamma radiation from charge particle reactions. Even the development of pair spectrometers suitable for accelerators and operating with $2-3 \%$ resolution, comparable with the ordinary photoelectric lens spectrometer at low energy, has only just begun.

\section{$\S 2$. $\gamma$-Radiation from Charged Particle Bombardment}

Gamma radiation resulting from the bombardment of a target nucleus with charged particles can arise from two general types of processes. One of these, known as Coulomb excitation, was first predicted ${ }^{1}$ theoretically $^{-}$ in 1938 by Weisskopf, but was not observed experimentally until 1953

1 V. F. Weisskopf, Phys. Rev. 53 (1938) 1018. 
when Huus and Zupančič ${ }^{2}$ and McClelland and Goodman ${ }^{3}$ independently found evidence for this effect. As the name implies the nucleus is excited through the interaction between its own electric field and that of a closely passing bombarding particle. The energy to charge ratio of the latter is chosen to be low enough so that it cannot enter the potential barrier of the target nucleus. Bohr and Mottelson have related ${ }^{4}$ the levels thus excited to modes of surface oscillations involving the cooperative motion of many nucleons. Since these oscillations are thought of as waves moving around the surface of a non-spherically shaped nucleus the levels are not rotational in the sense of those occurring for a rigid body. Excitation of this type of state takes place more readily if the target nucleus is easily deformable and one may infer from this that the appearance of these levels is more apt to occur in regions away from the closed shells. It is in these same regions where nuclear distortion can be correlated with large electric quadrupole moments. $\mathrm{Ta}^{181}$, having 73 protons and 108 neutrons, both of which lie between magic numbers $(50,82,126)$, and having a ground state spin of $7 / 2$, was the first case discovered by the above mentioned experimenters. Rotational levels can also be observed in beta activity and since they are discussed elsewhere in this volume it suffices to say here that the theory relates the energy of a state $E_{J}$ to the moment of inertia of the nucleus $I$ and the angular velocity of the surface wave $\omega$ according to the relation

$$
E_{J}=\frac{1}{2} I \omega^{2}=\frac{\hbar^{2}}{2 I}\left[J(J+1)-J_{0}\left(J_{0}+1\right)\right]
$$

where $J_{0}$ is the spin of the ground state and $J$ takes on the values $J=J_{0}$, $J_{0}+1, J_{0}+2$, etc. for odd- $A$ nuclei. In the case of even-even nuclei the $J_{0}\left(J_{0}+1\right)$ term is absent and $J$ can have the values $0,2,4$, etc.

The first experimental observations ${ }^{2,3}$ of the rotational levels in $\mathrm{Ta}^{181}$ were made by allowing a beam of low energy protons to strike a Ta target and then measuring the gamma ray spectrum with a NaI scintillation spectrometer. Later, Huus and Bjerregaard arranged ${ }^{5}$ a beta spectrometer of $0.7 \%$ resolution to examine the conversion electrons resulting from transitions excited in a thin Ta target by Coulomb excitation. Two gamma rays of energies 137 and $166 \mathrm{keV}$ were observed. Their energy ratio, $2.21 \pm 0.02$, agrees very well with the ratio $20 / 9$ predicted from the

2 T. Huus and C. Zupančič, Kgl. Danske Videnskab. Selskab, Mat.-fys. Medd. 28 (1953) No. 1.

8 C. McClelland and C. Goodman, Phys. Rev. 91 (1953) 760.

4 A. Bohr and B. R. Mottelson, Phys. Rev. 89 (1953) 316; Dan. Mat. Fys. Medd. 27 (1953) No. 16.

5 T. Huus and J. H. Bjerregaard, Phys. Rev. 92 (1953) 1579. 
above formula by giving $J_{0}$ the value $7 / 2$ and $J$ the values $9 / 2$ and $11 / 2$. Huus and Bjerregaard also observed gamma rays of 102,113 and $124 \mathrm{keV}$ resulting from the Coulomb excitation of tungsten and they assigned these to the first rotational levels of $W^{182}, W^{184}$ and $W^{186}$ respectively. The measured energy separations are consistent with inserting in the above formula the relative nuclear moments of inertia $I$ for the three isotopes according to empirical mass curves. $I$ is known to decrease as the number of neutrons approaches a closed shell. In general the gamma energies expected to occur in Coulomb excitation are mostly in the range of $50-500 \mathrm{keV}$. Alpha particles result in a more favorable yield ratio of nuclear gamma rays to $\mathrm{X}$-rays but they excite higher rotational levels much less readily than do protons.

The second type of process resulting in gamma radiation is the true nuclear reaction where the bombarding particle is given sufficient energy

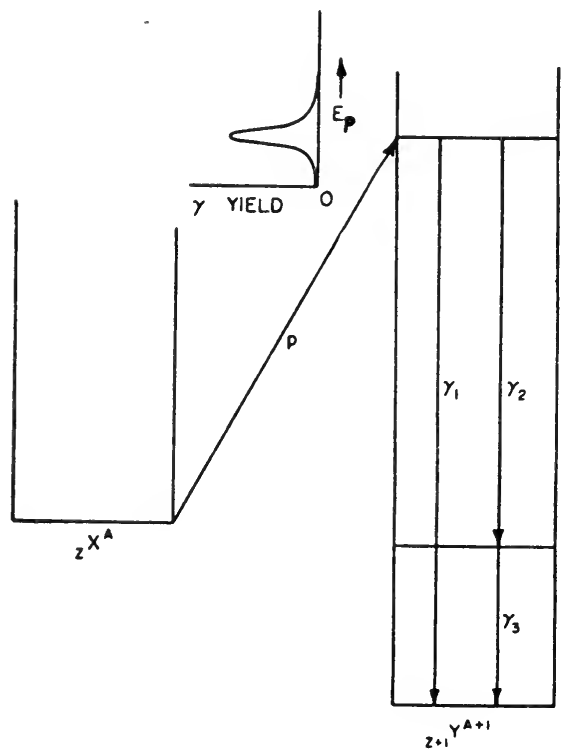

Fig. 1. Energy levels diagram for a radiative proton capture reaction. to enter the target nucleus. These reactions may be divided further into. two classes depending on whether secondary particles are emitted or not. The latter case is designated as radiative capture and is analogous to the neutron capture discussed in the next chapter. Fig. 1 gives an energy level diagram for proton capture of the sort commonly used for illustration of nuclear reactions ${ }^{6}$. An incident proton of energy $E_{p}$ will be able to enter the target nucleus ${ }_{Z} \mathrm{X}^{A}$ of charge $Z$ and atomic weight $A$ and form a product nucleus ${ }_{Z+1} \mathrm{Y}^{A+1}$ only if $E_{p}$ is such that the total energy of the system is close to one of the energy levels of $\mathrm{Y}$. If the levels of $\mathrm{Y}$ are spaced far enough apart and if the incident particle energy is well-defined then the phenomenon of resonance can be observed as a variation of gamma-ray yield with beam energy. A sharp resonance peak such as the one included in Fig. 1 will be obtained only when the target is thin enough to avoid excessive energy spreading. Within a very short time after the nucleus $\mathrm{Y}$ is formed it decays to the ground state 
directly or by cascade through other levels with the emission of gamma radiation.

The proton radiative capture reaction may be written $\mathrm{X}^{A}(\mathrm{p}, \gamma) \mathrm{Y}^{A+1}$ or according to the equation

$$
\mathrm{X}^{A}+\mathrm{p}=\mathrm{Y}^{A+1}+Q .
$$

$Q$ is the reaction or binding energy and it is equivalent to the excitation above the ground state the nucleus $\mathrm{Y}$ would have if $\mathrm{p}$ were able to enter $\mathrm{X}$ at $E_{p}=0$. In contrast to slow neutron capture charged particles can never enter at zero energy because of the Coulomb barrier and in all cases the gamma rays to the ground state, if any, will be greater in energy than $Q$. In Fig. $1 Q$ is equal to the vertical distance between the ground state of $\mathrm{Y}$ and the zero of the gamma yield curve. The slanting line marked $p$, not generally included in energy level diagrams, represents the addition of an energy to the ground state of $\mathrm{X}$ equalling the mass defect energy of the proton plus its kinetic energy $E_{\mathrm{cm}}$ in the center-ofmass system. Protons have a mass defect energy of $7.581 \mathrm{MeV}$ and in Fig. 1 this is equal to the vertical distance between the ground state of $\mathrm{X}$ and the zero of the gamma yield curve. The quantity $E_{\mathrm{cm}}$ can be calculated from the energy in laboratory co-ordinates $E_{p}$ according to the relationship

$$
E_{\mathrm{cm}}=\left(1-\frac{m}{M}\right) E_{p}=\frac{\text { mass of init. nucl. }}{\text { mass of comp. nucl. }} \times E_{p}
$$

where $m$ is the mass of the bombarding proton and $M$ is the mass of the compound nucleus. For proton capture $Q$ is seldom less than $6 \mathrm{MeV}$ which means that gamma rays of high energy can occur.

Resonance levels are always virtual in the sense that they are located above the binding energy of nucleons. Capture can take place with deuterons or alpha particles as well as protons and there can, of course, be emission of particles from a resonance level rather than gamma rays. Resonances are important because they represent unique states whose individual properties can be studied from such information as the level widths or shapes, angular correlations, or the relative energies and intensities of gamma rays emitted by the product nucleus.

In the second type of nuclear reaction the incident particle enters the target nucleus in the same way as described above but in this case another particle is emitted before the compound nucleus can decay by gamma-ray transitions. A single state in the intermediate nucleus can be formed, particularly for proton bombardment of a thin target at resonance, but in deuteron reactions the excitation of the compound nucleus is usually much higher than nucleon binding energies and several broad overlapping 
levels may be involved. The yield of secondary particles or of gamma radiation from the product nucleus will then be relatively insensitive to bombarding energy. Fig. 2 illustrates a $(d, p)$ reaction in which a deuteron

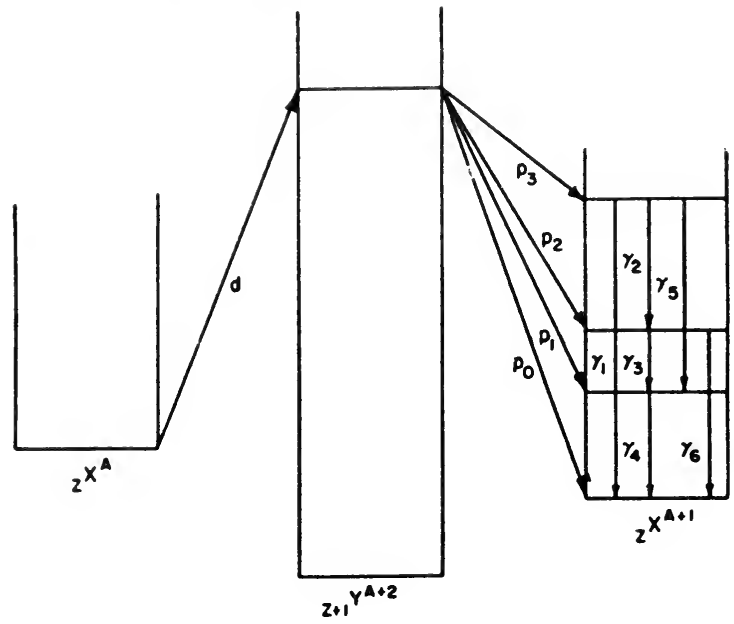

Fig. 2. Energy level diagram for a $(d, p)$ nuclear reaction leading to excited states in the product nucleus.

enters the target nucleus ${ }_{Z} \mathrm{X}^{A}$ to form a compound nucleus ${ }_{Z+1} \mathrm{Y}^{A+2}$. The upper horizontal line in the nucleus ${ }_{z+1} \mathrm{Y}^{A+2}$ does not necessarily correspond to a single state at that energy. Proton groups are then emitted leaving the product nucleus ${ }_{Z} \mathrm{X}^{A+1}$ in the ground state or in excited states which decay by gamma-ray transitions. This reaction may be written as $\mathrm{X}^{A}(\mathrm{~d}, \mathrm{p}) \mathrm{X}^{A+1}$ or according to the equation

$$
\mathrm{X}^{A}+\mathrm{d}=\mathrm{X}^{A+1}+\mathrm{p}+Q
$$

where each proton group has a unique $Q$-value associated with it. If one were to imagine the reaction as taking place at zero deuteron kinetic energy then $Q_{0}$, the ground state $Q$-value, is equal to the vertical separation $\mathrm{X}^{A}-\mathrm{X}^{A+1}$ in Fig." 2 plus the difference $(\mathrm{d}-\mathrm{p})$ between the deuteron and proton mass defects. The mass defect of the deuteron is $13.721 \mathrm{MeV}$. Energy levels of the final nucleus are most often of interest and their positions can be inferred either from the energy differences $\left(p_{0}-p_{1}\right)$, $\left(p_{0}-p_{2}\right)$, etc. as measured in a proton spectrometer, or from the gammaray spectrum. Other important properties of the levels are their spins and parities. Angular distributions of the particles sometimes can yield this information. Just as in beta decay the relative gamma-transition intensities are also useful for this purpose provided one can determine the population of each level from the intensities of particle groups. Whereas in beta decay the population of states can be obtained from the relative intensities of 
various beta ray components observed at a single angle the same is by no means true with particle groups. In this case strong angular dependence is often present so that one must find the state populations by measuring the yield of each group at a number of angles and integrating the results. If such information can be obtained then the analysis of gamma transition probabilities is similar to that in complex beta decay. A discussion will be given in a later section as to how coincidence studies can be used to evaluate transition probabilities without having to know the particle group angular distributions.

All of the above discussion of course applies to reactions involving protons, neutrons, deuterons, tritons, alpha particles, etc. in a wide variety of combinations as bombarding and emitted particles. While charged bombarding particles are almost always provided directly by an accelerator fast neutron reactions such as $(n, \alpha)$ or $(n, p)$ require an intermediate target from which the neutrons are produced by a $(d, n)$ or a $(\mathrm{p}, \mathrm{n})$ reaction.

Study of the gamma radiation resulting from charged particle bombardment in many instances has a characteristic difficulty which is almost never found in the beta decay of a single isotope or in slow neutron capture. This arises from competition between various possible reactions. As an example, the proton bombardment of $\mathrm{Li}^{7}$ can lead to the following processes:

$$
\begin{array}{ll}
\mathrm{Li}^{7}(\mathrm{p}, \mathrm{n}) \mathrm{Be}^{7} & Q=-1.6 \mathrm{MeV} \\
\mathrm{Li}^{7}(\mathrm{p}, \mathrm{p}) \mathrm{Li}^{7} & Q=0 \\
\mathrm{Li}^{7}(\mathrm{p}, \gamma) \mathrm{Be}^{8} & Q=17.2 \mathrm{MeV}
\end{array}
$$

Excited states can ke produced in all three product nuclei. Sorting out the gamma rays belonging to each of a number of competing reactions often requires other information such as knowledge of level positions derived from particle groups or separate experiments in which the excitation functions for various components in a given gamma spectrum are determined and evaluated on the basis of known $Q$-values or resonances. In the above example reaction (1) will not take place at low bombarding energies because of the negative $Q$-value, gammas in (2) can be identified by relating them to inelastic proton scattering data, and (3) is the only reaction in which very high energy gamma rays can occur because of the large $Q$-value.

\section{$\S 3$. Energy and Intensity Measurements}

Gamma rays from charged particle reactions have been investigated for many years but it is only since about 1948 that magnetic spectrometers 
have been applied to their measurement. Prior to that time the techniques were comparatively crude in view of the complexities now known to exist. The determination of lead absorption coefficients was used but it is actually rather unsuitable at the higher energies often encountered because of the insensitive dependence of the coefficient on energy above $2 \mathrm{MeV}$. At best the absorption measurements could give only some of the broad features of the de-excitation modes of an excited nucleus. For example, if it were known from particle groups that the average level spacing was $1 \mathrm{MeV}$ or less one could tell from the gamma absorption curve whether the decay took place by cascades or by direct transitions to the ground state?

Analysis of Compton recoil electrons from a thin radiator located in a cloud chamber was another of the early methods. By placing the chamber in a magnetic field and measuring the curvature of the electron tracks one could determine the Compton spectrum and from this find the energies of the gamma rays. Gas scattering effects make the cloud chamber a poor resolution device and the accumulation of data is very slow.

Another way of measuring Compton recoils is by means of coincidence absorption ${ }^{8}$. The electrons from a converter pass through two Geiger counters connected in coincidence and the electron energies are found by means of absorbers placed between the counters. The coincidence yield curve obtained for a single gamma ray decreases roughly logarithmically with absorber thickness and has an end point sufficiently well defined to allow the maximum Compton recoil energy to be measured with an accuracy of about $5 \%$ using the range-energy relation for electrons. If several components are present the ability to detect them is more favorable than by ordinary lead absorption but this can be done only if the number of gammas is small and if their energies are widely separated. In such a case the curve exhibits a break at the absorber value corresponding to the end point of each Compton group ${ }^{9}$.

Modern research on the complex gamma-ray spectra found in nuclear reactions requires instruments which will yield sharp lines. The magnetic or scintillation spectrometers already described in earlier chapters are useful for this purpose mainly at energies below $3 \mathrm{MeV}$. Almost any type of magnetic spectrometer will do provided the accelerator beam can be brought into the instrument in such a way as to strike a target located close to the normal source position. Generally the effect of the spectrometer magnetic field on the heavy incoming particles will be slight, but

7 E. Pollard and D. E. Alburger, Phys. Rev. 72 (1947) 1196.

8 W. Fowler, C. C. Lauritsen, and T. Lauritsen, Rev. Mod. Phys, 20 (1948) 236.

9 D. E. Alburger, Phys. Rev. 75 (1949) 51. 
on the other hand the magnetic field of a cyclotron or of a beam analysing magnet may constitute a serious disturbance to the spectrometer operation unless suitable precautions are taken. If a thin target is used the internal conversion electrons sometimes can be observed as they are with thin sources of radioactive materials. Otherwise arrangements must be provided to measure photoelectrons from a converter foil made of a heavy element such as lead or uranium. In either case the usual energy measuring techniques of ordinary beta spectroscopy apply except for the source strength monitoring problem to be discussed below.

Many of the first accurate measurements on reaction gamma rays were made by the Pasadena group using an electron lens spectrometer ${ }^{10}$. Fig. 3 illustrates the set-up for similar experiments reported ${ }^{11}$ from

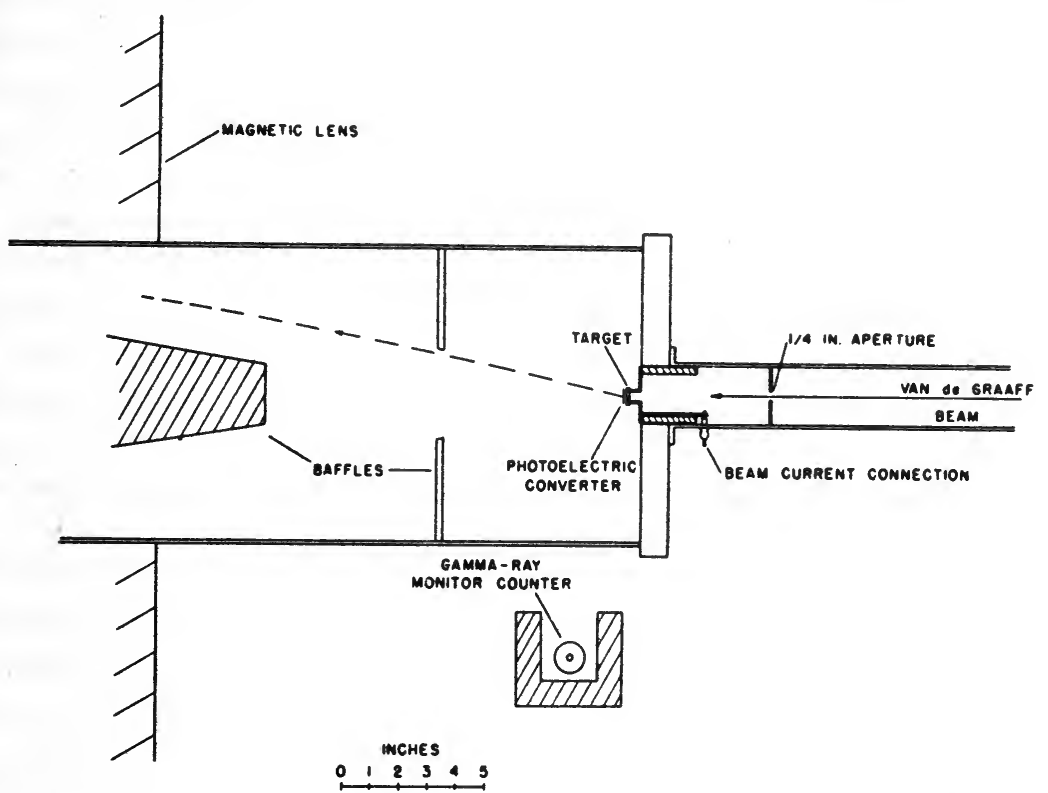

Fig. 3. Experimental arrangement for lens spectrometer measurement of gamma radiation from a bombarded target.

Brookhaven where a thin lens spectrometer was employed. A beam of protons or deuterons from a Van de Graaff accelerator is brought through an extension tube and defined by a small aperture so as to strike the target. Photoelectrons from a converter attached to the target are focused and measured by the electron lens. There are two ways of normalizing the spectrometer yield at each momentum setting with respect to a standard

10 W. F. Hornyak, T. Lauritsen, and V. Rasmussen, Phys. Rev. 76 (1949) 731.

11 D. E. Alburger, Phys. Rev. 83 (1951) 184. 
number of reactions taking place in the target. If the target is electrically insulated and thick enough to stop the beam the total charge accumulated can be found using a current integrator which shuts off the spectrometer counter automatically after a fixed quantity of charge has been collected. When tha target and converter are thin enough to allow the incident particles through the latter may be collected in an insulated Faraday cup attached to the central lead baffle. In either case precautions must be taken against false current readings resulting from secondary electron emission. This normalization technique will work well only if the beam energy and target stability are such that the gamma-ray yield is strictly proportional to the beam current. There may be difficulties if the radiation is from a thin target bombarded at a resonance whose width is comparable with the voltage regulation accuracy of the accelerator. Slow disintegration of the target material with a consequent change in gamma yield will not show up in current integration and results may not be reproducible.

The second and perhaps more reliable normalizing method is to use a monitor gamma ray counter shielded against radiation except from the direction of the target as shown in Fig. 3. This may be connected to a scalar which automatically shuts off the spectrometer scalar after a fixed number of monitor counts has registered. Because of the monitor's proximity to the target its counting rate can be made higher than that of the spectrometer detector and the statistical error of the monitor count will be comparatively small. Variations in gamma yield resulting from beam energy drift or disintegration of the target material are corrected for by the monitor. Other ways of monitoring the reaction are possible, i.e. a heavy particle counter can be placed near the target to detect reaction products. Any such device must of course give a yield which is not sensitive to the beta spectrometer field setting. One limitation to gamma-ray monitoring arises if there is built up in the target a radioactive gamma emitter whose half-life is comparable with the duration of the experiment. A large and varying gamma background from the accelerator itself or from the collimating slits may also lead to non-reproducible yields in the spectrometer. Since effects of this kind are often present the taking of data must usually be carried out more carefully than in beta activity measurements. The best spectrum will be obtained when the counting intervals are short, the various momentum points are taken in a random manner, and the data of numerous runs are averaged together. In this way the effects of unknown fluctuations will be minimized. Such considerations of course apply to taking data in any type of accelerator experiment.

It is often possible to obtain a more favorable geometry in photo- 
electron conversion measurements of this sort than in work with radioactive sources particularly if the following conditions are satisfied: 1) no appreciable beta activity is produced by the bombardment, 2) the target is thin, and 3) the size of the beam spot is smaller than the converter diameter. The second condition is usually satisfied because of the relatively short range of heavy particles in matter while the third can be accomplished by collimation. By placing the converter in contact with the target solid angles subtended by the converter may be as high as 30 or $40 \%$ of a sphere. A further advantage of such an arrangement is that a relatively low yield of Compton electrons results because of the small amount of material present and this allows the photo peaks to be seen more clearly. If on the other hand an interfering beta activity is produced the target material must be placed in a container having walls thick enough to stop the beta rays and having an open end for the beam to enter. The solid angle subtended by a photo converter attached to the container will then be less favorable and each gamma will produce a strong Compton distribution.

An example of data obtained with the lens spectrometer and target arrangement of Fig. 3 is the photoelectron spectrum resulting from the deuteron bombardment of $\mathrm{Be}^{9}$. The curve in Fig. 4 was taken using a

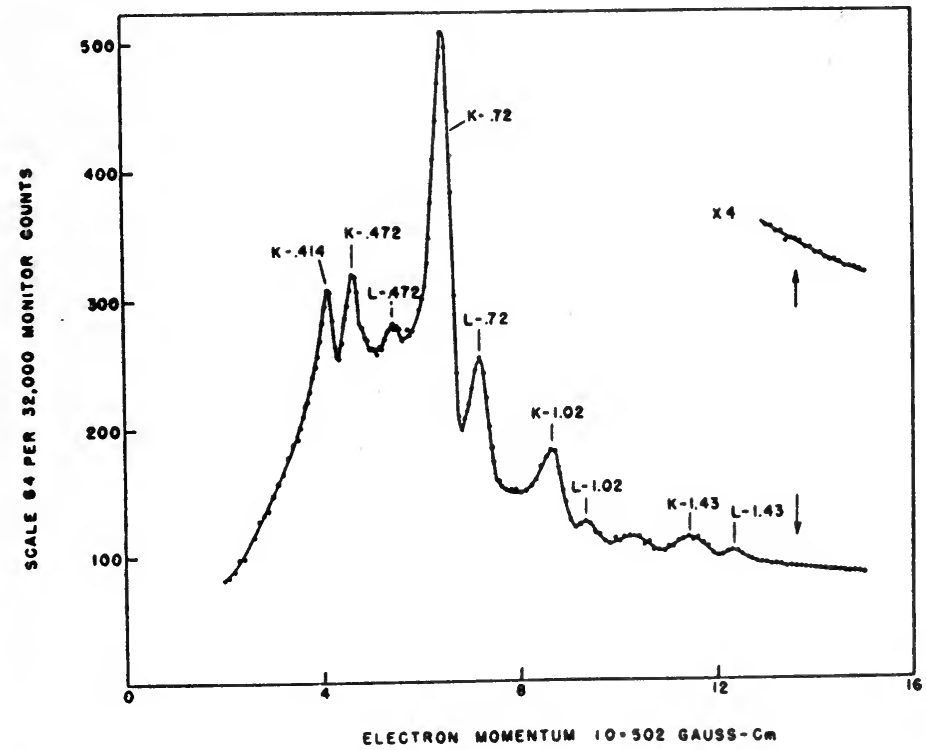

Fig. 4. Spectrum of photoelectric conversion lines from a uranium converter as observed in a lens spectrometer. Gamma rays were produced in a beryllium target bombarded with 1.2 MeV deuterons. (Reproduced from Phys. Rev. 83 (1951) 184 
0.005 inch thick Be target with a $44 \mathrm{mgm} / \mathrm{cm}^{2}$ uranium foil attached. Most of the peaks are $K$ - or $L$-shell photoelectrons corresponding to gamma rays from the $\mathrm{Be}^{9}(\mathrm{~d}, \mathrm{n}) \mathrm{B}^{10}$ reaction excepting the $472 \mathrm{keV}$ gamma which is associated with the $\mathrm{Be}^{9}(\mathrm{~d}, \alpha) \mathrm{Li}^{7}$ reaction. A search was being made for a peak indicated by the arrows representing a ground state transition from the second level in $\mathrm{B}^{10}$. In spite of the presence of the five gammas in the figure and a number of others at higher energy the Compton electron intensity is not excessive in this target-converter geometry. Just as in beta spectroscopy the relative gamma intensities may be obtained from the $K$ photoelectron amplitudes if the efficiency function of the converter is known.

The sodium iodide scintillation spectrometer has become increasingly useful for low energy reaction gamma rays especially in those cases where the yields are too small for magnetic spectrometers to be practicable. Evidently the advantages of this device are its high efficiency and the large solid angle achieved by placing the crystal close to the target. But one also has poorer resolution than magnetic spectrometers and sometimes there is difficulty in the interpretation of complex spectra. For energies higher than the pair threshold as many as four peaks can be associated with each gamma ray energy. In addition to the photoelectric and Compton lines there can be pair production with the escape of one or both annihilation quanta from the crystal resulting in lines 0.51 and $1.02 \mathrm{MeV}$ lower in energy than the photo peak. Pair escape peaks are more noticeable in small crystals and will become increasingly pronounced at higher gamma energies.

Experimental set-ups using a $\mathrm{NaI}$ detector must be made with good shielding against background radiation and for the reasons already discussed it is necessary to monitor either the beam current or the reaction yield. A spectrum is generally run with a differential pulse height analyser but if in addition the pulses from the crystal are fed to a second discriminator, integral or "differential, then the detector can serve as its own monitor. The counting rate of the monitor discriminator, maintained at a fixed setting, will be proportional to the reaction yield.

An example of a differential pulse height spectrum using a sodium iodide detector is given in Fig. 5. This shows ${ }^{12}$ the gamma rays below $2 \mathrm{MeV}$. from the proton bombardment of $\mathrm{Be}^{9}$ leading to levels in $\mathrm{B}^{10}$ according to the $\mathrm{Be}^{9}(p, \gamma) \mathrm{B}^{10}$ reaction. Inasmuch as these gamma rays are from the same product nucleus as that formed in the $\mathrm{Be}^{9}(\mathrm{~d}, \mathrm{n}) \mathrm{B}^{10}$ reaction one can obtain a comparison from Figs. 4 and 5 of magnetic lens and scintillation spectrometer data for approximately the same gamma

12 W. F. Hornyak and T. Coor, Phys. Rev. 92 (1953) 675. 
ray spectrum. The $472 \mathrm{keV}$ gamma of Fig. 4 is of course not present in the $(p, \gamma)$ reaction.

Internal conversion coefficients and photoelectron converter efficiency both decrease rapidly with energy and it is well known that magnetic spectrometer measurements on these secondary electrons usually are not feasible above $3 \mathrm{MeV}$. In a similar way the efficiency of a scintillation spectrometer also limits this device to the same energy region insofar as photo peaks are concerned. Other means are therefore to be desired for investigating higher energy nuclear reaction gamma rays.

Fig. 5. Pulse height spectra from a NaI scintillation spec. trometer when bombarding beryllium with protons of several different energies. (Reproduced from Phys. Rev. 92 (1953) 675 by permission).

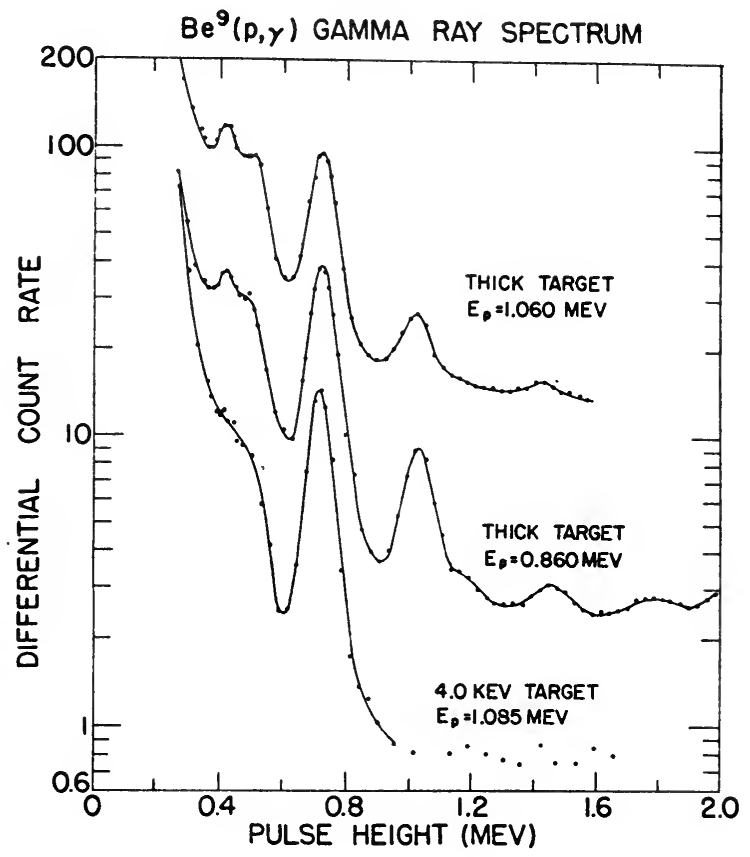

Up to about $6 \mathrm{MeV}$ the Compton effect is still appreciable and it can be employed to yield line spectra in a way developed by the Pasadena group ${ }^{13}$. This depends on the use of thin targets and light element converters resulting in a Compton distribution observed in the forward direction which is highly peaked near the maximum recoil energy. Magnetic analysis of the Compton spectrum is carried out with a lens spectrometer. Each peak is accompanied by a long low energy tail but the effective width of the line can be as small as $5 \%$ at $6 \mathrm{MeV}$. The gamma energy is found from the upper edge of the Compton peak by adding to this energy the calculated value of the back scattered quantum.

13 V. Rasmussen, W. F. Hornyak, C. C. Lauritsen and T. Lauritsen, Phys. Rev. 77 (1950) 617. 
Recently the trend has been toward the use of the pair production effect for the measurement of higher energy gamma rays. Pairs can arise from external conversion of gamma rays passing through a foil but they may also be produced in two other ways called internal and nuclear pair production. An internal pair is formed in the field of the same nucleus emitting the gamma ray whereas a nuclear pair can be considered as being emitted directly by the nucleus as it makes a transition between states. The latter will occur mainly in high energy transitions where the direct emission of a gamma ray is forbidden. The external pair cross section increases with the square of $Z$ and in lead at $3 \mathrm{MeV}$ it is equal to that of the photoelectric effect although still less than half the Compton cross section. But in contrast to the latter two processes the pair cross section increases with energy. Because of the special properties of pairs more ingenious means must be used to make measurements on them. The total energy of the pair is a fixed quantity equal to $E_{\gamma}-2 \mathrm{mc}^{2}$, where $m$ is the rest mass of the electron and $2 \mathrm{mc}^{2}$ is the $1.022 \mathrm{MeV}$ creation energy. In general energy is not equally shared between the two components but each has a continuous distribution extending from zero to $E_{\gamma}-1.022 \mathrm{MeV}$. Any pair spectrometer which is to give a spectrum of lines, one corresponding to each gamma ray present, must record the two pair components at the same time and measure the sum of their energies.

Probably the first such instrument applied to reaction gamma rays was used ${ }^{14}$ in 1937 by Delsasso, Fowler and Lauritsen in their investigation of the 14 and $17 \mathrm{MeV}$ gamma radiation resulting from the proton bombardment of $\mathrm{Li}^{7}$. This consisted of a cloud chamber placed in a uniform magnetic field with a thin foil at the center in which pairs were produced by gamma rays originating from an accelerator target located outside of the chamber. The total energy of each pair was determined from the radii of curvature of the positron and electron tracks recorded photographically. The arrangement was similar to that of Anderson when he discovered 15 the positron in 1932. Little further use of the cloud chamber pair spectrometer has been made at low energies because of the effect of gas scattering on resolution and the cumbersome accumulation of data.

When magnetic analysis and counter techniques are employed the measurement of positron-electron pairs is considerably hindered by a further characteristic of the pair effect, namely the angular distribution. The angle $\theta$ each component makes with the initial quantum direction can have a wide range of values such that the average angle depends on the electron energy $E$ according to the relation $\theta_{a v} \cong \mathrm{mc}^{2} / \mathrm{E}$. Even at $10 \mathrm{MeV}$,

14 L. A. Delsasso, W. Fowler and T. Lauritsen, Phys. Rev. 51 (1937) 391.

15 C. D. Anderson, Phys. Rev. 43 (1933) 491. 
which can be considered as favorable for pair measurements on reaction gamma rays, the angle between pair components in the majority of events will be of the order of $10^{\circ}$. This is greater than the effective acceptance angle of most magnetic spectrometers. Thus, even for those pairs which have a suitable sharing of energy the probability that both will pass through the spectrometer and reach their detectars is small.

In spite of these difficulties several successful types of magnetic pair spectrometer have been designed. One of these is the uniform field spectrometer suggested ${ }^{\mathbf{1 6}}$ by McDaniel, von Dardel, and Walker and further developed ${ }^{17,18,19}$ at several laboratories. All of the experimental results described in Ch. XXV were obtained with such a device. In this instrument, illustrated in Fig. 6, the gamma rays from a reactor source or an accelerator target are first collimated so that their angular divergence is small and they are then allowed to strike a thin foil radiator. The foil is located at the center of the spectrometer such that a uniform magnetic field bends the two pair components in opposite directions into detectors. A coincidence circuit records the arrival of a pair and from the positions of the corresponding two counter slits the sum of radii of curvature is known. To a first approxi-

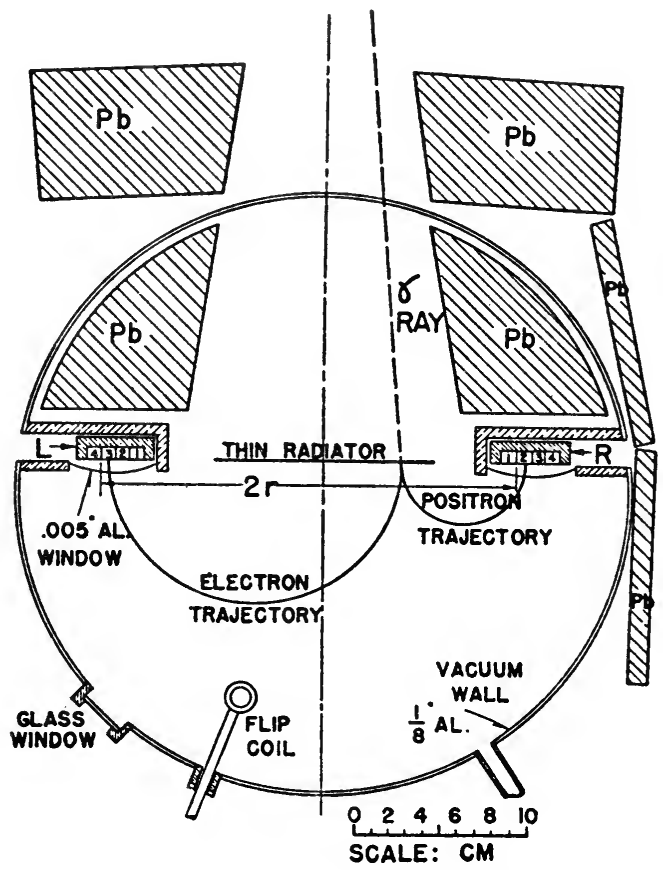

Fig. 6. Schematic diagram of the semicircular pair spectrometer of Walker and McDaniel. The magnetic field direction is normal to the plane of the paper. (Reproduced from Phys. Rev. 74 (1948) 315 by permission). mation this sum is constant for a given gamma-ray energy and magnetic field setting, hence it is not necessary to know the point in the converter from which the pair originates and rather large converters can be used. Although the distributions of positrons and electrons along the line

16 B. McDaniel, G. von Dardel and R. Walker, Phys. Rev. 72 (1947) 985.

17 R. Walker and B. McDaniel, Phys. Rev. 74 (1948) 315.

18 B. B. Kinsey, G. A. Bartholomew and W. Walker, Phys. Rev. 77 (1950) 723.

19 J. Terrell, Phys. Rev. 80 (1950) 1076. 
containing the counter slits and the converter are both continuous those events in coincidence will occur only for a fixed $2 r$ depending on the magnetic field setting and the total pair energy. By varying the magnetic field and thus the value of $2 r$ a fraction of the coincidence events are "swept" across a given pair of counter slits resulting in a "line" when coincidences are plotted against magnetic field setting. In Walker and McDaniel's instrument (Fig. 6) a total of 8 counters is used allowing 7 momentum points to be recorded simultaneously. The position of the line will give the total pair energy if the instrument is calibrated with a gamma ray of known energy or if the magnetic field and the distance between counter slits are both measured.

A number of effects contribute to the line width observed in the semicircular pair spectrometer. Among these are the finite divergence of the incident gamma ray beam, scattering in the converter, and the size of the slits in front of the detectors. Also in higher orders of approximation the sum of electron-positron orbit diameters varies with their ratio making the line width increase with converter size. Scattering in the converter seems to be the most serious difficulty. Evidently the instrument is "baffle-less" in contrast to other magnetic spectrometers and there is essentially no rejection of those electrons which suffer scattering before leaving the converter. To minimize line broadening resulting from scattering the converter must be sufficiently thin and be made of a light element such as beryllium, aluminum, or copper. These requirements somewhat offset the advantage of large converter size.

The line shape is similar to that observed with an ordinary semicircular beta spectrometer in that the high energy edge is steep. This allows the extrapolated edge to serve as an accurate measure of the total pair momentum. Bartholomew and Kinsey have developed ${ }^{20}$ a way of determining energies in which they have found both experimentally and theoretically that a small energy correction must be applied to the extrapolated end point. Their pair. lines are generally about $130 \mathrm{keV}$ wide but at high energies they have been able to make measurements accurate to $0.1-0.2 \%$ (i.e. $\sim 10 \mathrm{keV}$ ) using their end point analysis. They have also obtained the efficiency function for their instrument and have shown that at $3 \mathrm{MeV}$ the efficiency increases with the fifth power of the gamma ray energy. Terrell has found ${ }^{19}$ that the efficiency of his semicircular instrument varies from 1 pair count per $8 \times 10^{9}$ gamma rays emitted by a source of $2.62 \mathrm{MeV}$ radiation to 1 count per $7 \times 10^{7}$ gamma rays at $4.45 \mathrm{MeV}$, an increase by a factor of 110 . His sources were comparatively close

20 G. A. Bartholomew and B. B. Kinsey, Phys. Rev. 86 (1952) 605; Can. Jour. Phys. 31 (1953) 537. 
to the spectrometer and the width of the $4.45 \mathrm{MeV}$ line was $12 \%$.

The uniform field pair spectrometer is well suited to reactor measurements on neutron capture gamma rays where extremely strong sources can be produced by inserting the material into a pile. Collimation requirements on the gamma ray beam are met because of the large distance between the source material and the spectrometer converter imposed by the thickness of the reactor shielding wall. It is this collimation feature which makes the instrument more limited when studying gamrna rays produced by accelerator bombardment. Although Walker and McDaniel's results were obtained in cyclotron induced reactions it is not as often possible to produce gamma ray fluxes of sufficient intensity, particularly with Van de Graaff generators. Perhaps a more clear-cut disadvantage of the instrument for accelerator usage is that it is entirely restricted to the observation of external pairs. A source of internal or nuclear pairs placed in the converter position would not meet the requirement of a well-defined direction of the total pair momentum which one obtains with good beam collimation and external conversion. Internal pairs are preferable to external pairs in many instances because the thickness of a target yielding a given number of internal pairs can be considerably less than the thickness of an external converter necessary for an equal pair intensity. Occasionally there is transition resulting in nuclear pairs, such as the one of $6.05 \mathrm{MeV}$ in $\mathrm{O}^{16}$, which cannot be studied at all in the semicircular instrument.

Other efforts in pair spectrometer design have been directed towards the use of axial focusing beta spectrometers. It did not occur to anyone for a long time that instruments of this type were practical for detecting pairs, the reason being that the positron and electron images ordinarily coincide. In the usual arrangement, such as the magnetic lens, the focusing properties are identical for either sign of charge except that the senses of particle rotation are opposite. It is just the rotational feature which led Siegbahn and Johansson to devise the first successful lens type pair spectrometer ${ }^{21}$. Their scheme was to move the source of pairs several $\mathrm{cm}$ away from the optical axis of the lens and to depend upon the opposite senses of rotation to separate the positron and electron images. Since each particle turns $90^{\circ}$ in their instrument the two images lie on opposite sides of the axis. Counters are placed at the corresponding image positions and these are operated in coincidence. Only when the field is adjusted to focus electrons and positrons each having an energy close to $\frac{1}{2}\left(E_{\gamma}-1.02 \mathrm{MeV}\right)$, neglecting energy loss in the source or converter, is it possible for both components of a pair to get through the spectrometer at the same time.

21 K. Siegbahn and S. Johansson, Rev. Soi. Inst. 21 (1950) 442. 
This amounts to selecting a narrow band at the center of the positron and electron distributions corresponding to very nearly equal sharing of the available energy. By varying the magnetic field strength a coincidence line will be observed having a width determined by the instrument resolution, source size, and energy spread in the source. The fact that the instrument uses baffles and makes the energy selection in this way means that it is not necessary to collimate the source gamma rays as in the semicircular instrument. External, internal, or nuclear pairs can all be observed with a very favorable source or converter geometry. In the original tests by Siegbahn and Johansson external pair lines from a lead converter foil were observed using the 2.62 and $2.76 \mathrm{MeV}$ lines in $\mathrm{Na}^{24}$ and $\mathrm{THC}^{\prime \prime}$ respectively. Bame and Baggett have applied ${ }^{22}$ the device to the measurement of gamma rays as high as $9 \mathrm{MeV}$ occurring in the deuteron bombardment of $\mathrm{B}^{10}$. As in all types of pair spectrometer the angular distribution of pairs has a strong influence. The latter group found that the efficiency of this instrument, governed largely by the angular dependence, increased a factor of 6 in going from $4.4 \mathrm{MeV}$, where the resolution was $6.8 \%$ and one pair count was recorded per $1.3 \times 10^{8}$ gamma rays emitted by the target, to $8.9 \mathrm{MeV}$ where there was 1 count per $2.3 \times 10^{7}$ gamma rays. Of necessity the off-axis method limits the detection system to point focusing whereas it is commonly known that ring focus results in greater transmission at a given resolution. A further disadvantage of the off-axis scheme is that the inherent asymmetry of the source and detector geometry with respect to the instrument baffles might be a limitation in attempts to improve the resolution.

Two independent developments of another type of lens pair spectrometer have been made by Bame and Baggett ${ }^{22}$ and by Alburger ${ }^{23}$. This device holds considerable promise because it not only retains the focusing symmetry of the lens but allows ring focus to be used as well. It is based on the principle of statistical separation as illustrated in Fig. 7. Pairs are produced in a source or converter centered on the optical axis and these are focused through a common annular aperture and enter a common detecting region. Just as in the off-axis method described above only those pairs of nearly equal energy can be focused at the same time. But here the positron and electron images coincide and there will be virtually no correlation between the positions in the detecting region where the pair components arrive. If the detection circle is divided in two then in half the cases the partikles will enter different halves of the circle, and in the remaining cases both particles will enter the same half circle. By using

22 S. J. Bame and L. M. Baggett, Phys. Rev. 84 (1951) 891.

23 D. E. Alburger, Rev. Sci. Inst. 23 (1952) 671. 
semicircular shaped crystals and photomultiplier detectors connected in coincidence a pair line can be observed for each gamma ray as the field is varied. A small absorber is placed between the crystals and sufficient pulse height discrimination must be used in the detectors to avoid recording false

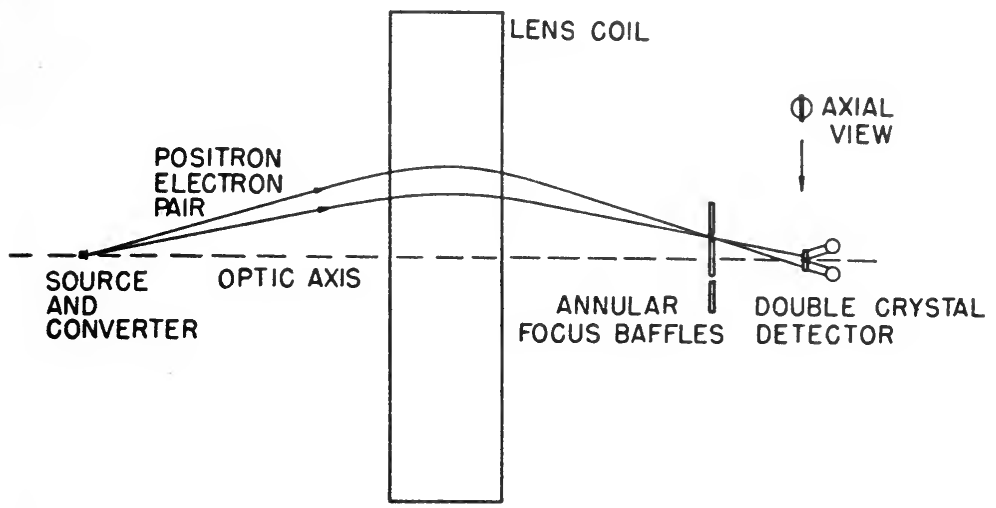

Fig. 7. Schematic diagram of the lens-type statistical separation pair spectrometer. (Reproduced from Rev. Sci. Inst. 23 (1952) 671 by permission).

coincidences resulting from the multiple scattering of electrons from one crystal to the other. In comparison with the off-axis method symmetry and ring focus have been gained at the expense of losing a little more than half of the pairs which pass through the spectrometer. But the transmission per electron using ring focus is more favorable than point focus by a factor of 2 or 3 . Since two particles are involved and taking into account that a greater spectrometer acceptance angle is more advantageous when the angular distribution of pairs is considered the statistical separation method actually has a much greater overall efficiency. Bame and Baggett obtained ${ }^{22}$ an efficiency of 1 pair count per $7.5 \times 10^{5}$ quanta at $4.45 \mathrm{MeV}$ where a 0.005 inch thick thorium converter was used and the line width was $19 \%$. An increase in efficiency by a factor of 60 between 2.6 and 4.5 $\mathrm{MeV}$ was observed ${ }^{23}$ by Alburger.

An example of data taken with this instrument at $7.5 \%$ resolution is given in Fig. 8. This shows the external pair lines from a uranium converter resulting from gamma rays occurring in the $F^{19}(\mathrm{p}, \alpha) \mathrm{O}^{16}$ reaction. The best resolution of the statistical separation pair spectrometer reported thus far is $2.8 \%$ obtained ${ }^{24}$ by Bent, Bonner and Sipple in the study of the nuclear pairs from the $6.05 \mathrm{MeV}$ state in $\mathrm{O}^{16}$.

Another possible way of using a lens to measure pairs in an axially symmetric ring focus arrangement would be to replace the statistical

24 R. D. Bent, T. W. Bonner and R. F. Sipple, Phys. Rev. 91 (1953) 472. 
separation detector of Fig. 7 with a single energy sensitive element such as a scintillation crystal. The simultaneous arrival of both pair components at the detector should result in a pulse which is distinguishable from those produced by single electrons. This scheme was suggested independently by Hornyak ${ }^{25}$ and by Freedman ${ }^{26}$ and although it appears simple in principle no results have been published at the present writing based on the use of this idea. A discussion of the difficulties is given elsewhere ${ }^{23}$.

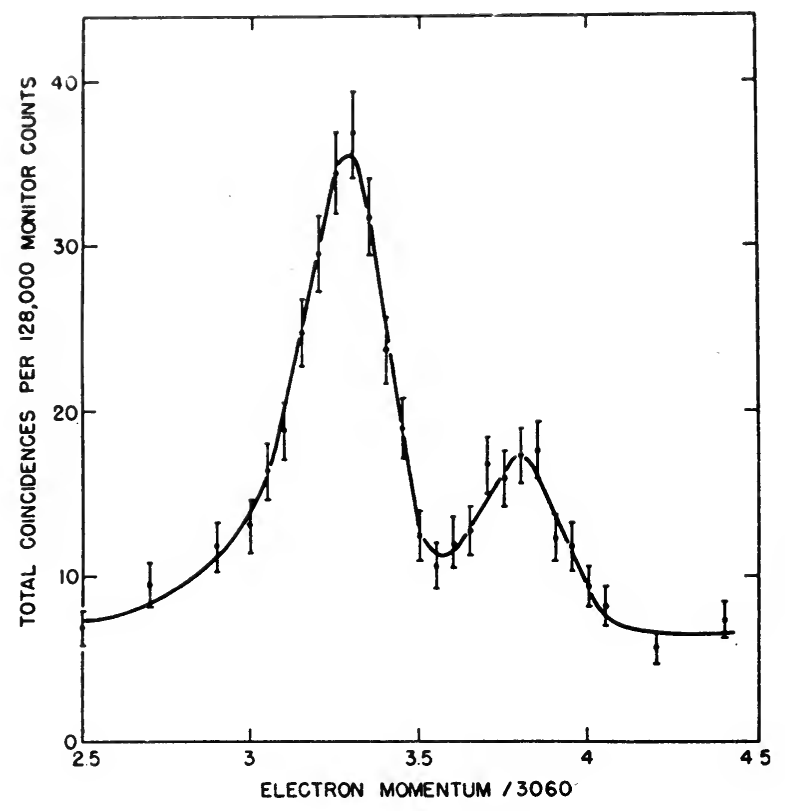

Fig. 8. Pair spectrum resulting from 6.19 and 7.13 $\mathrm{MeV}$ gamma rays occurring in the $\mathrm{F}^{19}(\mathrm{p}, \alpha) \mathrm{O}^{16}$ reaction as observed in the lens pair spectrometer of Fig. 7. (Reproduced from Rev. Sci. Inst. 23 (1952) 671 by per. mission).

As mentioned earlier the NaI scintillation spectrometer will yield pair peaks at high gamma ray energies. These are often difficult to use because of underlying background distributions of Compton electrons and furthermore there will usually be three pair lines corresponding to absorption of the pair kinetic energy combined with the following possibilities: absorption of both annihilation quanta, escape of one quantum from the crystal, or escape of both quanta. At very high energies the three peaks may be unresolved. To avoid these difficulties Hofstadter and McIntyre suggested ${ }^{27}$ the so-called three crystal pair spectrometer which was subsequently developed ${ }^{28}$ independently by Johansson and by Bair and

25 W. F. Hornyak, private communication (Dec. 1951).

26 M. S. Freedman, Rev. Sci. Inst. 23 (1952) 643.

27 R. Hofstadter and J. A. McIntyre, Phys. Rev. 79 (1950) 389.

${ }_{28}$ J. K. Bair and F. C. Maienschein. Rev. Sci. Inst. 22 (1951) 343; S. A. E. Jo. hansson, Nature 166, 794 (1950). 
Maienschein. By placing three crystals in line, operated in triple coin. cidence, and allowing the outer two to detect the oppositely directed 0.5 MeV annihilation quanta only the two-quanta escape peak mentioned above will be observed in the central crystal. Since all Compton and photoelectrons are rejected because of their inability to produce triple coincidences the instrument will record for each gamma ray present a single line having an energy $E_{\gamma}-1.02 \mathrm{MeV}$. Bair and Maienschein's tests, made with the $2.62 \mathrm{MeV}$ gamma rays of $\mathrm{THC}^{\prime \prime}$, indicated an efficiency of $0.25 \%$ (pairs detected per gamma ray falling on the center crystal) which is much higher than possible with any magnetic spectrometer devised thus far. An excellent 3-crystal pair spectrum resulting from the 1.38 and 2.76 MeV gamma rays of $\mathrm{Na}^{24}$ has been published ${ }^{29}$ by Mann, Meyerhof, and West. Both lines were observed against a very small background and the half width of the higher energy one was $6.5 \%$. The relatively high efficiency of the three crystal pair spectrometer and the possibility of operating it with a resolution of about $5 \%$ at higher energies using recently improved techniques in scintillation spectroscopy may make this instrument valuable for investigating reaction gamma rays from charged particle reactions. Little has been done thus far along these lines.

\section{§ 4. Particle-Gamma Coincidence and Angular Correlations}

It was mentioned previously that the relative intensities of gamma rays from a nuclear reaction in which particle groups are emitted may be of limited significance unless the state populations can be determined from the particle group strengths integrated over all angles. If a spectrum is complex there may also be difficulty in knowing where a given gamma transition is taking place in the level scheme of the product nucleus. Particle-gamma coincidence techniques are useful for handling such problems. Referring to Fig. 2, if an energy selection of the particles is made and if one looks only at the gamma rays associated with a particular group then the de-excitation of the product nucleus can be studied beginning with a defined state. For example the gamma ray from the first excited state can be identified at once since it is the only one in coincidence with $\mathrm{p}_{1}$. The angular distribution of particles does not affect the manner in which the final nucleus decays although the coincidence rate may depend on the angles of observation selected. When groups corresponding to higher states are chosen more gamma rays will occur in the coincidence spectrum. The advantage of this type of investigation is that a more clearcut knowledge of the relative transition probabilities may be of assistance in establishing spins and parities.

29 L. G. Mann, W. E. Meyerhof and H. J. West, Phys. Rev. 92 (1953) 1481. 
Just as in any coincidence experiment a good yield depends on observing the particles and gamma rays with high efficiency. Ideally one would like to use magnetic analysis of both the particle groups and secondary electrons resulting from the gamma radiation but then the coincidence rate would be low because of the small transmission of the spectrometers. Sufficiently high intensities may be obtained with devices such as scintillation crystals or other types of detector which can be arranged to accept a large fraction of the radiations emitted by a target. Successful results along these lines have been achieved ${ }^{30}$ by a group at Yale using the arrangement shown in Fig. 9. Energy selection of proton groups is made by means of aluminum

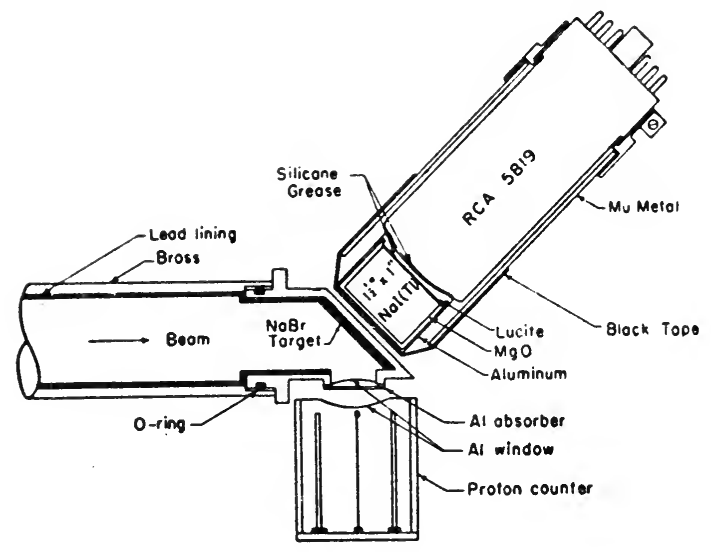

Fig. 9. Arrangement for measuring the spectrum of gamma rays ( $\mathrm{NaI} 5819$ counter) in coincidence with a proton group of selected energy (proportional counter). (Reproduced from Phys. Rev. 84 (1951) 1203 by permission).

absorbers with a proportional counter in which a "peaking" technique detects only those particles coming to rest within the counter volume. When the proton counter is set to respond to a single energy the spectrum of coincident gamma rays is examined in the $\mathrm{NaI}-5819$ scintillation spectrometer. It is clear from the geometry of the experimental arrangement that a favorable coincidence rate is possible.

Angular correlation experiments have been of great value in establishing the properties of nuclear states formed in beta decay (see Ch. XIX). In a similar but more complicated way correlations can be observed in particle reactions and these may lead to useful information. The effectiveness of the method is illustrated by the work ${ }^{31}$ of Seed and French on the $\mathrm{F}^{19}(\mathrm{p}, \alpha) \mathrm{O}^{16}$ reaction. This process may be considered as taking place in three steps; firstly, the compound nucleus $\mathrm{Ne}^{20}$ is formed in a particular state by bombarding a thin target at a resonance energy; secondly, the

30 R. C. Allen, J. E. May and W. Rall, Phys. Rev. 84 (1951) 1203; J. E. May and B. P. Foster, Phys. Rev. 90 (1953) 243.

31 J. Seed and A. P. French, Phys. Rev. 88 (1952) 1007. 
compound nucleus decays by emission of an alpha particle of fixed energy to a unique state in the product nucleus $\mathrm{O}^{16}$; and finally, a gamma ray is emitted by the $\mathrm{O}^{16}$ nucleus in making a transition to the ground state. The correlations $(p, \alpha),(p, \gamma)$, and $(\alpha, \gamma)$ can all be observed in principle. Interpretation of the results will require that the spins and parities of the initial and final nuclei in their ground states are known and that allowance is made for the various possible orbital momenta of the incoming protons and outgoing alpha particles. The theory for this special problem is discussed ${ }^{31}$ in detail by Seed and French.

In their experiments the $(\alpha, \gamma)$ correlations were studied, as illustrated in Fig. 10, by observing the alpha particles in a magnetic spectrometer

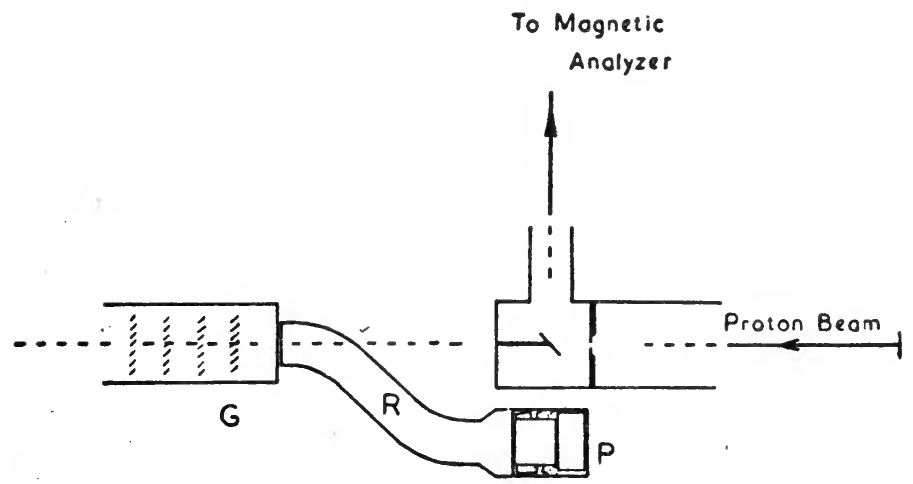

Fig. 10. Experimental arrangement for $(\alpha, \gamma)$ angular correlation studies of the $F^{19}(p, \alpha \gamma) \mathrm{O}^{16}$ reaction. (Reproduced from Phys. Rev. 88 (1952) 1007 by permission).

(transmission $4 \pi / 1500$ steradians) at a fixed angle of $90^{\circ}$ to the incoming proton beam and by rotating a NaI gamma detector $\mathrm{P}$ around the target. Magnetic analysis of the alpha particles was necessary in order to resolve the three groups investigated. To avoid changes in gain which might result if the photomultiplier $G$ were to be moved relative to the alpha particle spectrometer magnet the tube was kept in a fixed position and only the crystal holder $\mathrm{P}$ and light pipe $\mathrm{R}$ were rotated. The experiments were carried out at four resonant bombarding energies and magnetic selection of alpha groups corresponding to three excited states in $\mathrm{O}^{16}$ was made. Spins and parities of all seven states were deduced by comparing the observed angular correlations with theoretical curves.

\section{$\S 5$. The Life Times of Nuclear States}

In contrast to beta spectoscopy very few measurements on the lifetimes of excited states induced by nuclear reactions have been made. This is 
understandable because the life times ordinarily expected are very short and difficult to measure. Much of the charged particle reaction work has been done on light elements where metastable states usually do not exist and in these cases the dipole or quadrupole radiations are frequently of high energy and are therefore very fast. Nevertheless, life times represent important nuclear information which one would like to know for comparison with theories involving transition probabilities. Delayed coincidence techniques discussed elsewhere in this volume are limited by the inherent speed of scintillators to the measurement of life times greater than about $10^{-10}$ seconds. On the other hand according to theoretical formulae, this is longer than one expects for a low multipole order transition at energies above $0.5 \mathrm{MeV}$.

Other means of determining very short life times have been suggested in the literature. Elliot and Bell studied ${ }^{32}$ the life time of the $478 \mathrm{keV}$ state in $\mathrm{Li}^{7}$ (excited in the $\mathrm{B}^{10}(\mathrm{n}, \alpha) \mathrm{Li}^{7}$ reaction) by analysing the Doppler broadening of the gamma-ray (see Ch. XVIII). A distribution in velocities of the excited $\mathrm{Li}^{7}$ nuclei will result from the recoil induced by the energetic alpha particles. If the $\mathrm{Li}^{7}$ nucleus emits its $478 \mathrm{keV}$ gamma before coming to rest a Doppler-broadened gamma ray line is observed. Measurement of the broadening in various materials where the slowing down time of the recoil $\mathrm{Li}^{7}$ can be estimated could lead to a half life value. In Elliott and Bell's experiment they were able to place an upper limit of $2 \times 10^{-13} \mathrm{sec}$ on the life time of the $478 \mathrm{keV}$ state.

A scheme used ${ }^{33}$ by Thirion and Telegdi consisted of measuring the distance an excited recoil nucleus moves before it emits a gamma ray. In their experiment an accelerator beam produces a reaction in a very thin target such that excited nuclei can recoil in the forward direction with a reasonably well defined velocity. A gamma collimator and counter are arranged so as to examine at right angles a limited space in front of the target. If the life time is long enough the excited nuclei will decay at a measurable distanee from the target governed by the recoil velocity and the life time of the state. The measurement consists of moving the counter or target along a line parallel with the beam and noting the change of counting rate. From the shape of the observed distribution and other parameters of the experiment the gamma half life can be determined. A life time of $2.5 \times 10^{-10} \mathrm{sec}$ was found for the $870 \mathrm{keV}$ state in $0^{17}$ produced by the $\mathrm{O}^{16}(\mathrm{~d}, \mathrm{p}) \mathrm{O}^{17}$ reaction. At a beam energy of $1.3 \mathrm{MeV}$ almost all of the recoil $\mathrm{O}^{17}$ nuclei emit their gamma rays within a distance of $1 \mathrm{~mm}$ from the target.

32 L. G. Elliott and R. E. Bell, Phys. Rev. 74 (1948) 1869.

33 J. Thirion and V. L. Telegdi, Phys. Rev. 92 (1953) 1253. 


\section{§ 6. Future Developments}

Gamma ray spectroscopists working in the field of low energy charged particle reactions will be faced in the future with a common general problem consisting largely of determining the positions and properties of nuclear energy levels. Although the difficulties will frequently be formidable it is encouraging that already some progress has been achieved in relating experiments to the shell theory or to the surface oscillation concept proposed by Bohr and Mottelson. Certain striking similarities in level structure have shown up in some of the light element isobars and these levels can be discussed in terms of the isotopic spin concept. But of course there is still no theory which can make accurate predictions of the complete behavior of all nuclei. This ultimate aim may never be reached.

Many of the techniques already described will certainly form a basis for future experiments but the room for improvement and the needs for development of new instruments are perhaps more apparent than in the field of radoactivity. In fact many of the excellent existing devices for radioactivity have not yet been applied to the lower energy gamma-ray components of nuclear reactions. For example one could make precision measurements by means of the double-focusing spectrometer ${ }^{34}$ of Hedgran, Siegbahn, and Svartholm, or if sufficient source intensities were available the DuMond bent crystal spectrometer ${ }^{35}$ could also be applied for this purpose. Low yield reactions could be studied with the high transmission intermediate-image spectrometer ${ }^{34}$ of Slätis and Siegbahn. Very few results using the three-crystal pair spectrometer on reaction gammas have been reported thus far.

The powerful techniques of particle-gamma coincidences and angular correlations will be useful tools of investigation which will be employed not only in the manner described earlier in this chapter but in new arrangements. For example, the conversion electrons of gamma transitions associated with particle groups in a nuclear reaction might be studied in a high transmission magnetic spectrometer. Either the coincidence conversion spectrum or the angular correlation of a particle group and a conversion line could be examined.

In addition to novel arrangements of existing spectrometers or other devices it is to be hoped that new instruments and techniques for gamma ray studies will be developed. At present it appears that one of the greatest needs is for pair spectrometers to measure gamma radiation in the energy range $3-15 \mathrm{MeV}$. There are three instruments for this purpose which

34 See Ch. III.
35 See Ch. IV. 
have been proposed or which are under construction and a brief description of these may be useful.

The pair spectrometer developed by Walker and McDaniel and discussed previously requires intense and distant sources in order to allow a collimated keam of gamma rays to strike the pair converter. W. F. Hornyak has suggested ${ }^{36}$ a modification of this instrument which would allow the

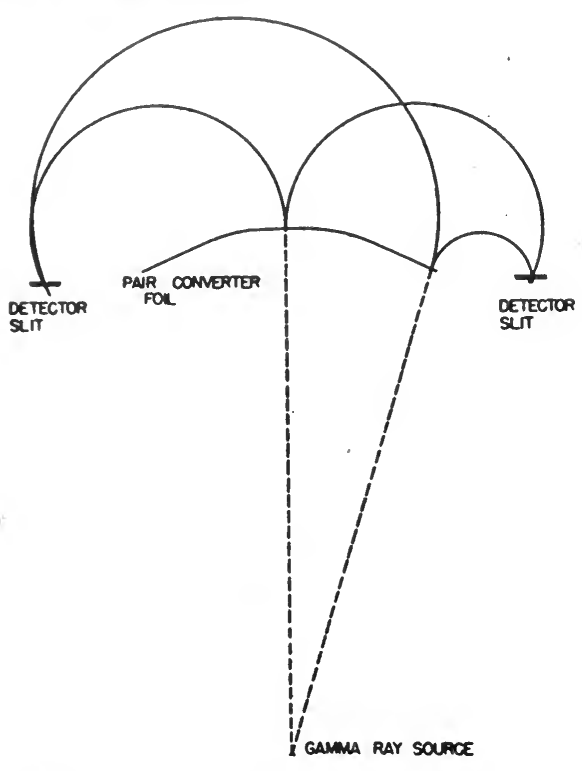

Fig. 11. A uniform field pair spectrometer proposed by W. F. Hornyak. (Figure made with permission of Dr. Hornyak). gamma ray source to be located closer to the pair converter. This is achieved by changing the converter from a flat to a curved surface and by ensuring that the dimensions of the gamma source are small. If the shape of the converter is properly selected according to the source-toconverter distance Hornyak has shown by ray tracing that the conditions for detecting pair lines are suitable even though the gamma ray beam incident on the converter is highly divergent. A cross section of the arrangement and some typical pair trajectories are illustrated in Fig. 11. The factor of improvement in overall efficiency of this device over the Walker and McDaniel spectrometer would be roughly in proportion to the inverse square ratio of source-to-converter distance assuming that unknown difficulties were not to arise. An instrument of this type might be useful in Van de Graaff experiments where the size of the source can be made small.

A beta spectrometer giving up to $15 \%$ transmission at a resolution of $2 \%$ has been developed in Copenhagen by O. B. Nielsen. This is known as "orange" spectrometer and is described in Ch. III. Nielsen has proposed ${ }^{37}$ that the instrument could be adapted to the measurement of positron-electron pairs as shown in Fig. 12. This diagram illustrates the outline of a pole piece for one section of an orange spectrometer which

36 The author is indebted to Dr Hornyak for permission to quote this unpublished information.

37 The author is indebted to Dr Nielsen for permission to quote his unpublished results. 
could have up to 5 gaps. The source and image points are in field free regions and some positron-electron paths are indicated. Exit slits have not been included but the instrument could be arranged to accomodate 4 pairs of counters. Under these conditions the overall efficiency at a gamma ray energy of $5 \mathrm{MeV}$ is expected to be $\sim 2 \times 10^{-6}$.

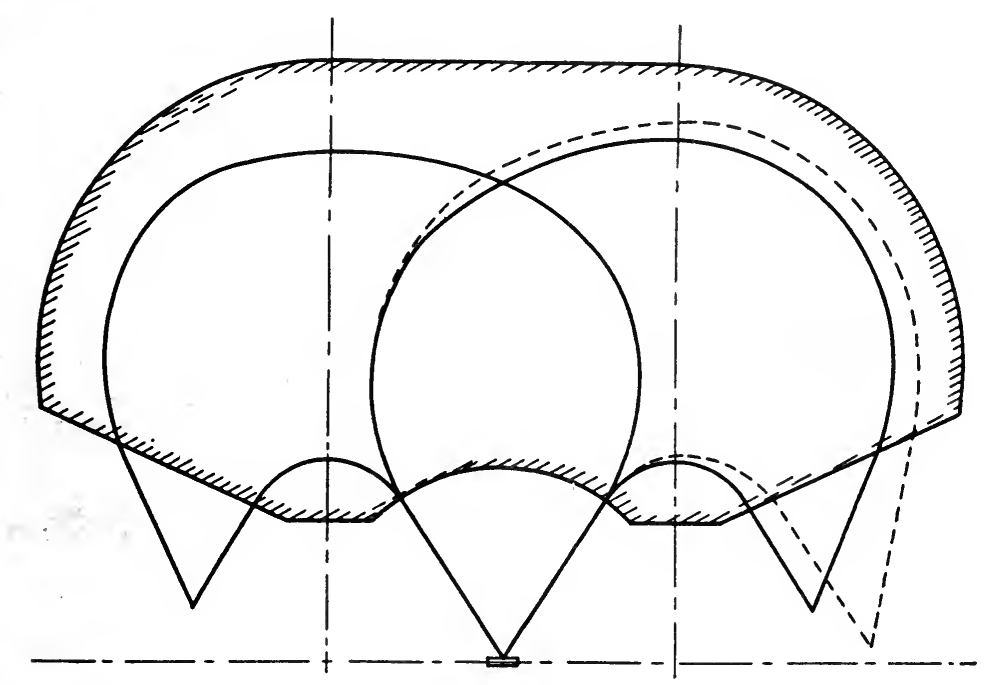

Fig. 12. Pole-face outline of one section of an "orange" pair spectrometer proposed by O. B. Nielsen (courtesy of Dr Nielsen).

A new pair spectrometer ${ }^{38}$ is now being constructed at Brookhaven for use with the Van de Graaff accelerator in the study of reaction gamma rays. This will consist of an intermediate-image spectrometer modified to use the statistical separation technique described earlier in this chapter. It has been demonstrated ${ }^{34}$ by Slätis and Siegbahn that the intermediate-image spectrometer operates with more than a factor of ten greater transmission than an ordinary thin lens. The high power dependence of the yield of pairs on transmission strongly suggests that such a spectrometer, if successfully modified to detect pairs, would be very much more effective than the thin lens pair spectrometer. Professor.W. Bothe and Dr. H. Daniel have shown ${ }^{39}$ that intermediate-image focusing can also be achieved with an iron-free spectrometer consisting of two air-core copper coils with the source located at the center of one, the detector at the center of the other, and the annular focus in bètween. Since no iron 
is used their instrument possesses the advantages of a linear field-current relationship, a field distribution independent of magnitude, and therefore an accurately constant transmission. Access to the source and detecting regions are facilitated by not having the iron jacket and pole pieces of the Slätis-Siegbahn spectrometer. Daniel and Bothe have obtained a resolution of $1.1 \%$ at a transmission of $4.6 \%$ of $4 \pi$ with a point source (0.1 $\mathrm{mm}$ diameter).
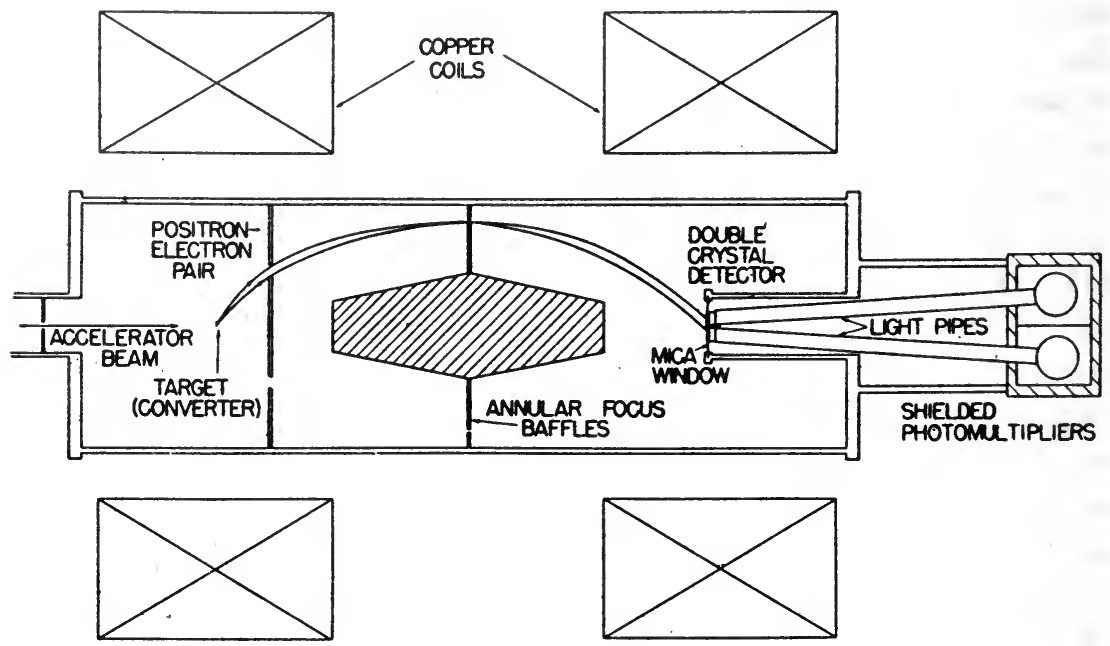

Fig. 13. Intermediate-image statistical separation pair spectrometer under design at Brookhaven. (Reproduced from Rev. Sci. Inst. 25 (1954) 1025 by permission).

The proposed modification of the Daniel and Bothe instrument to the statistical separation measurement of pairs is shown schematically in Fig. 13. Since the detecting region is at maximum magnetic field intensity it will be necessary to pipe the light pulses from the detecting crystals to a weak field region:and provide shielding to further reduce the magnetic intensity experienced by the photomultipliers. Outputs of the photo tubes will be fed to a coincidence circuit whose short resolving time $\left(\sim 5 \times 10^{-9}\right.$ sec) will minimize the random coincidence rate. It is hoped that this device will be useful for the pair measurement of gamma rays at a resolution of $3 \%$ or better. 


\title{
NEUTRON CAPTURE $\gamma$-RAYS
}

\author{
B. B. KINSEY
}

\section{§ 1. Introduction}

The product of the radiative capture of a thermal neutron by a nucleus of mass $M_{A}$ is a nucleus of mass $M_{A+1}$ in a highly excited state. In a very few instances (which include lithium, boron, and nitrogen) the product nucleus may disintegrate with the emission of a proton or an $\alpha$-particle. In most other instances the emission of a charged particle is not energetically possible, or is retarded by the Coulomb barrier when the disintegration energy is insufficient to overcome it. The most probable mode of decay is then the emission of $\gamma$-radiation. The energy balance $Q$ in the reaction.

$$
M_{A}+M_{n}=M_{A+1}+Q
$$

is the binding energy of the last neutron in the nucleus with mass $M_{A+1}$ It usually lies between 5 and $10 \mathrm{MeV}$. The degree of excitation of the product nucleus is therefore higher than that which usually results from. radioactive decay. The energy $Q$ is dissipated as radiation which is emitted in direct and cascade transitions to the ground state. In a heavy nucleus, with a large number of levels, there are many possible transitions and consequently the $\gamma$-ray spectrum may be very complex.

\section{§ 2. Experimental Methods}

The methods employed for the study of neutron capture $\gamma$-rays are essentially similar to experimental methods described in preceding chapters of this book, in particular, in Ch. XXIV. In Table I we list the authors who have experimented with neutron capture $\gamma$-rays together with the methods they have used.

Neutron capture $\gamma$-rays were first observed by Lea ${ }^{1}$ who appears to have detected the $\gamma$-ray produced by capture of neutrons in hydrogen, and by Amaldi and his associates ${ }^{2}$, who detected capture in other elements. Preliminary attempts to measure neutron capture radiation were made by Rasetti ${ }^{3}$, and by Fleischmann ${ }^{3}$. Other investigations were

1 D. E. Lea, Nature, 133, (1934) 24.

2 E. Amaldi, O. d'Agostino, E. Fermi, B. Pontecorvo, F. Rasetti, and E. Segrè, Ricerca Sc. 2, (1934) 467; Proc. Roy. Soc. (London) A149, (1935) 522.

3 F. Rasetti, Zeit. f. Phys. 97, (1935) 64; R. Fleischmann, Zeit. f. Phys. 97, (1935) 242; 97, (1935) 265; 103, (1936) 113. 
TABLE I

Experimental Methods Used in Neutron Capture $\gamma$-Rays

\begin{tabular}{|c|c|c|c|c|}
\hline Authors & Date & Neutron Source & Method of Detection & $\begin{array}{c}\text { Refer } \\
\text { ence }\end{array}$ \\
\hline . . . . & 1934 & $\mathrm{RaBe}$ & Geiger counters & 1 \\
\hline Amaldi et al. . . & 1934 & $\mathrm{RaBe}$ & Geiger counters & 2 \\
\hline Rasetti . . . & 1935 & $\mathrm{RaBe}$ & Geiger counters & 3 \\
\hline Kikuchu et al. . & 1939 & $\mathrm{D}^{2}(\mathrm{~d}, \mathrm{n}) \mathrm{He}^{\mathrm{s}}$ & Geiger counters & 4 \\
\hline Aoki. . . . . . & 1937 & $\mathrm{D}^{2}(\mathrm{~d}, \mathrm{n}) \mathrm{He}^{3}$ & Geiger counters & 5 \\
\hline Fleischmann . . & 1938 & $\mathrm{RaBe}$ & Geiger counters & 6 \\
\hline Pringle et al. . . & 1951 & $\mathrm{RaBe}$ & Single crystal Scint. & 7 \\
\hline $\begin{array}{l}\text { Meyerhof, Rode- } \\
\text { rick and Mann }\end{array}$ & 1951 & $\mathrm{Be}^{9}(d, n) B^{10}$ & Single crys & 9 \\
\hline Reardon et al. . & 1953 & $\mathrm{Be}^{9}(\mathrm{~d}, \mathrm{n}) \mathrm{B}^{10}$ & Two crystal Scint. & 10 \\
\hline Hamermesh . . & 1949 & Pile, neutron beam & $\mathrm{D}_{2} \mathrm{O}$ plates & 12 \\
\hline Millar et al.. . . & 1950 & Pile, neutron beam & $\mathrm{D}_{2} \mathrm{O}^{\prime}$ plates & 14 \\
\hline Wilson. . . . . & 1950 & Pile, neutron beam & $\mathrm{D}_{2} \mathrm{O}$ ionization chamber & 15 \\
\hline $\begin{array}{l}\text { Bell and Elliott } \\
\text { Kubitschek and }\end{array}$ & 1950 & Pile, neutron beam & Magnetic lens & 24 \\
\hline Dancoff . . . & 1949 & Pile, neutron beam & Geiger counters & 11 \\
\hline Hamermesh and & & & & \\
\hline Hummel . . & 1951 & Pile, neutron beam & Single crystal Scint. & 16 \\
\hline Moak and Dabbs & 1949 & Pile, neutron beam & Cloud chamber & 17 \\
\hline Braid . . . . & 1952 & Pile, $\gamma$-ray beam & Two crystal Scint. & 18 \\
\hline $\begin{array}{l}\text { Motz. . . . } \\
\text { Kinsey and }\end{array}$ & 1953 & Pile, $\gamma$-ray beam & Photoelectric spect. & 19 \\
\hline Bartholomew & 1953 & Pile, $\gamma$-ray beam & Pair spectrometer & 27 \\
\hline
\end{tabular}

subsequently reported by Kikuchi and his associates ${ }^{4}$, and by Griffiths and Szilard ${ }^{4}$. The most notable attempts to measure the yield of capture $\gamma$-rays were made by Aoki ${ }^{5}$, and by Fleischmann ${ }^{6}$. These authors used $\mathrm{Ra}-\mathrm{Be}$ or $\mathrm{Po}-\mathrm{Be}$ sources of neutrons, or neutrons produced by the $\mathrm{D}^{2}(\mathrm{~d}, \mathrm{n}) \mathrm{He}^{3}$ reaction, the neutrons being slowed down in hydrogenous material. They employed coincidence apparatus to select the capture radiation from that produced by the source and attempted to analyse the results by the study of absorption curves obtained when absorbers were placed between coincident Geiger counters. These experimenters suffered from the disadvantage of weak neutron sources and correspondingly feeble sources of capture radiation. With such sources it is not possible

4 S. Kikuchi, K. Husimi, and H. Aoki, Proc. Phys.-Math. Soc. Japan, 18, (1936) 188; S. Kikuchi, E. Takeda and J. Itoh, Proc. Phys.-Math. Soc. Japan, 19, (1937) 43 ; J. H. E. Griffiths and L. Szilard, Nature, 139, (1937) 323.

5 H. Aoki, Proc. Phys.-Math. Soc. Japan, 19, (1937) 799.

6 R. Fleischmann, Zeit. f. Phys. 111, (1938) 281. 
to use magnetic spectrometers, which have far better resolution. These methods of detection, moreover, do not lend themselves easily to the determination of the shape of the $\gamma$-ray spectrum nor to the determination of the $\gamma$-ray energies: they can give little more than an estimate of the energy of the most energetic $\gamma$-ray emitted. However, more recently, much better results have been obtained with weak sources by Pringle and his associates ${ }^{7.8}$ using the single crystal scintillation spectrometer (a much more sensitive detector), and similar measurements have been made by Meyerhof, Roderick and Mann ${ }^{9}$. Reardon and his associates ${ }^{10}$ have been successful with a two crystal scintillation spectrometer using the Compton effect.

The precision methods which are required for study of the complex spectra produced by neutron capture appear to be feasible at the present time only with the aid of a nuclear reactor. The very high neutron fluxes generated by the reactor can be used to produce strong sources of capture radiation easily isolated from the radiation generated in the reactor itself. Two possible arrangements present themselves: (1) A neutron beam is allowed to pass through a hole in the radiation shield of the reactor and impinge on a sample of the material to be examined. The spectrometer is then placed as close as possible to the target. (2) The material to be investigated is itself placed in a region of high neutron flux within the reactor and the radiation emitted is analysed by a spectrometer placed outside the reactor and located so as to view the sample through a hole in the radiation shield. The $\gamma$-rays produced by the reactor itself are easily eliminated by placing a bismuth block in the hole, between the sample and the reactor. Such a bismuth block emits a negligible amount of capture radiation. In method (1) it acts as a source of thermal neutrons relatively free from $\gamma$-radiation, and in (2) it screens the spectrometer from direct radiation from the reactor.

The neutron beam method (1) has been used by Kubitschek and Dancoff ${ }^{11}$ to estimate the average number of $\gamma$-rays emitted per capture. Using a coincidence absorption method similar to that employed by the earlier experimenters, they measured the $\gamma$-ray yield of a large number of elements relative to that of gold. Hamermesh ${ }^{12.13}$. and Millar et al. ${ }^{14}$

7 R. Pringle, H. Taylor, and K. Roulston, Phys. Rev. 87, (1952) 1016.

8 R. W. Pringle and G. Isford, Phys. Rev. 83, (1951) 467.

- W. Meyerhof, H. Roderick and L. Mann, Phys. Rev. 83, (1950) 203.

10 W. Reardon, R. Krone and R. Stump, Phys. Rev: 91, (1953) 334.

11 H. E. Kubitschek and S. M. Dancoff, Phys. Rev. 76, (1949) 531.

12 B. Hamermesh, Phys. Rev. 76, (1949) 182; 80, (1950) 415.

13 B. Hamermesh, Phys. Rev. 81, (1951) 487.

14 C. Millar, A. Cameron and M. Glicksman, Can. J. Res. A28, (1950) 475. 
have attempted to measure the spectrum of $\gamma$-rays emitted by a number of elements using $\mathrm{D}_{2} \mathrm{O}$-loaded photographic plates as detectors. In general their results are in poor agreement with spectrometer measurements. The origin of this discrepancy is not clear. Wilson ${ }^{15}$, however, using a high pressura deuterium-filled ionization chamber has obtained results in better agreement. More recently, Hamermesh and Hummel ${ }^{16}$ have used a single crystal scintillation spectrometer. At low energies, their measurements are in good agreement with the known energies of transitions between low lying states: Finally, $\gamma$-rays from a $\mathrm{Cd}$ target in a neutron beam have been measured by Moak and Dabbs ${ }^{17}$, by a study of the pairs produced in a thin lead radiator in a cloud chamber.

The high intensity of the $\gamma$-ray beam method (2) and the excellent collimation make this method very suitable for magnetic spectrometers. At least two such instruments are required for no one type is adequate for the study of $\gamma$-rays over the whole energy range. The low energy $\gamma$-rays (up to 2 or $3 \mathrm{MeV}$ ) can be studied with magnetic lens spectrometers working with the photoelectric or with the Compton effect or with crystal spectrometers. Such instruments, however, are unsuitable for the study of the high energy $\gamma$-rays (from 2 or $3 \mathrm{MeV}$ to the neutron binding energy) and these are best measured with some kind of pair spectrometer.

A preliminary survey of the low energy $\gamma$-rays emitted in neutron capture (from $300 \mathrm{keV}$ to $3 \mathrm{MeV}$ ) has been made by Braid ${ }^{18}$, using a double crystal Compton spectrometer. After determining the energy variation of the counting efficiency of the spectrometer (using $\gamma$-rays of known strength from radioactive sources), the intensities of capture radiations were found by comparison of the intensities with that of the hydrogen capture $\gamma$-ray obtained from weighed mixtures of a hydrogenous compound and the material examined. In this method it is necessary to know the capture cross section of the target material and of hydrogen. In some instances, e.g., Al, the absolute intensities of the capture radiations could be found from the strength of the $\gamma$-ray emitted by the radioactive product nucleus, when equilibrium was established between the rate of disintegration and the neutrons producing it.

Another method with better resolution has been developed at Brookhaven and used by Motz ${ }^{19}$ to measure the low energy capture $\gamma$-rays produced by $\mathrm{Na}$, 'Ti, and $\mathrm{Cd}$. In this method the collimated $\gamma$-ray beam is

15 R. Wilson, Phys. Rev. 80, (1950) 90.

16 B. Hamermesh and V. Hummel, Phys. Rev. 83, (1951) 663; 88, (1952) 916.

17 C. K. Moak and J. W. T. Dabbs, Phys. Rev. 75, (1949) 1770.

18 T. H. Braid, to be published.

19 W. Thornton, E. der Mateosian, H. T. Motz and M. Goldhaber, Phys. Rev. 86, (1952) 604; H. T. Motz, Phys. Rev. 90, (1953) 355; 93, (1954) 925. 
allowed to pass through the uranium radiator of a magnetic lens spectrometer. The background of Compton electrons which usually confuse the spectrum of secondary electrons in such instruments is eliminated by recording coincidences between the $K$-radiation produced by the photoelectric effect in the radiator and the photoelectrons detected at the other end of the spectrometer.

The high energy $\gamma$-rays have been studied by Kinsey and Bartholomew using a magnetic pair spectrometer. With this instrument the energies of strong $\gamma$-rays have been measured to an accuracy of a few keV. The intensity measurements were made by a comparison method ${ }^{20}$ relative to the intensity of the $2.75 \mathrm{MeV} \gamma$-ray of $\mathrm{Na}^{24}$. Whereas in Braid's apparatus, which depends on the Compton effect, the experimental determination of the energy variation of the counting efficiency presented no great difficulties, the energy variation of the efficiency of the magnetic pair spectrometer was very difficult to determine. The efficiency increases very rapidly with the energy (near $3 \mathrm{MeV}$ approximately as the fifth power), and weak $\gamma$-rays with energies below $3 \mathrm{MeV}$ are undetectable. The counting efficiency of this instrument is very low. At $7 \mathrm{MeV}$ it is only $10^{-7}$ coincidences per photon incident on the radiator: the use of this instrument is therefore possible only where very high neutron fluxes are available. No attempt has yet been made to study neutron capture radiations with a triple crystal coincidence pair spectrometer ${ }^{21}$ as described in Ch. XXIV.

The emission of internal conversion electrons by materials capturing neutrons was first detected by Hoffman and Bacher, and by Amaldi and Rasetti ${ }^{22}$. So far, the study of the internal conversion of capture $\gamma$-rays has not received the attention which it deserves. Of the few measurements which have been made, the most noteworthy are those of Muehlhause and his collaborators ${ }^{23}$, who have examined the conversion of low energy $\gamma$-rays in four elements, $\mathrm{Cd}, \mathrm{Sm}, \mathrm{Dy}$, and $\mathrm{Hg}$. They used the neutron beam method and a semicircular magnetic spectrometer. No attempt has yet been made to study the production of internal conversion pairs by neutron capture radiation.

\section{§ 3. Experimental Results}

The $\gamma$-rays produced by neutron capture in all stable nuclei have not yet been examined, for the contribution to the total neutron cross section

20 B. B. Kinsey and G. A. Bartholomew, Can. J. Phys. 31, (1953) 537.

21 S. A. E. Johansson, Phil. Mag. 43, (1952) 249.

22 J. G. Hoffman and R. F. Bacher, Phys. Rev. 54, (1938) 644; E. Amaldi and F. Rasetti, Ricerca Sc. 10, (1939) 115.

${ }_{23}$ C. O. Muehthause, Phys. Rev. 83, (1951) 235; C. T. Hibdon and C. O. Muehlhause, Phys. Rev. 83, (1951) 235; 87, (1952) 222. 
of many stable isotopes is too low to permit $\gamma$-ray measurements. Moreover, the origin of many observed $\gamma$-rays has not yet been established, for identification depends on independent data on the neutron binding energy or the positions of excited states.

In even-charge nuclei, the product nucleus may be of the even-odd or of the even-even type, i.e., the proton number $Z$ is even, and the neutron number $N$ may be odd or even. On the other hand, most stable oddcharge nuclei are odd-even, and the product nuclei, therefore, are odd-odd types. With the exception of $\mathrm{N}^{15}$, which is as yet the sole example of an odd-even nucleus for which the neutron capture spectrum is known, we have no means of comparing radiations emitted by odd-even with even-odd types. However, the high energy spectrum produced by odd-odd nuclei are very different from those of even-even or even-odd nuclei. With few exceptions, the spectra of odd-odd nuclei are much more complicated than those of even-charge nuclei of comparable atomic weight, and the intensities of individual $\gamma$-rays are lower. The weakness of these $\gamma$-rays is to be expected when it is remembered that the higher energy radiations are emitted mainly from the compound state, at (or near) the neutron binding energy, in competition with each other: the greater the number of transitions which can occur, the lower will be the intensity of any one of them.

The capture $\gamma$-ray spectra differ markedly from one element to another and we shall give here only a brief description of the experimental results, referring the reader to the original papers for details.

\section{A. Light Elements}

We arbitrarily define as light elements those for which the atomic number is less than that of zinc. The energies and intensities of the $\gamma$-rays leading directly to the ground states of the product nuclei are presented in Table II; these $\gamma$-rays will be called the "ground state $\gamma$-rays". The energies of the ground state $\gamma$-rays are equal to the $Q$ values, i.e., the neutron binding energies, less small corrections for nuclear recoil, which, in all nuclei except the lightest, are negligible. In the first group of elements $(\mathrm{H}$ to $\mathrm{N}, \S 3 a)$ the $\gamma$-ray spectra consist of one $\gamma$-ray only (the ground state $\gamma$-ray) or of relatively few $\gamma$-rays. Above oxygen $(\S 3 b)$ the $\gamma$-ray spectra become much more complex.

\section{a. Elements Lighter than Oxyg n}

The first accurate measurement of the energy of the hydrogen capture $\gamma$-ray was made by Bell and Elliott ${ }^{24}$, using thermal neutrons from a reactor and a magnetic lens spectrometer. Their measurement of the

24 R. E. Bell and L. G. Elliott, Phys. Rev. 79, (1950) 282. 
binding energy of the deuteron, $2.230 \pm 0.007 \mathrm{MeV}$, is somewhat, but not significantly, higher than that deduced from recent measurements by other methods. The intensity of this $\gamma$-ray does not appear to have been directly measured. Its intensity is probably one quantum per capture for no bound excited state of the deuteron is known to exist. A suggestion that other $\gamma$-rays ${ }^{25}$ might be emitted has not been sustained ${ }^{26}$.

The $\gamma$-ray produced by neutron capture in deuterium $(6.24 \mathrm{MeV})$ has been detected as a constituent of the radiation emitted by a heavy water reactor ${ }^{27}$ and its intensity also appears to be unity. Radiation produced by capture in $\mathrm{H}^{3}$ and $\mathrm{He}^{3}$ does not appear to have been looked for. Capture radiation from $\mathrm{H}^{3}$ should not exist if $\mathrm{H}^{4}$ is unstable, and that from $\mathrm{He}^{3}$ is probably very weak because of the high cross section of the competing $(\mathrm{n}, \mathrm{p})$ reaction.

Of the light nuclei up to oxygen only the $\gamma$-rays in ${ }^{28 .}{ }^{29} \mathrm{Be}^{10}$, in ${ }^{15.28 .29}$ $\mathrm{C}^{13}$ and ${ }^{29} \mathbf{N}^{15}$ have been studied. Strong ground state $\gamma$-rays are emitted in the first two of these nuclei, and a rather weak one in the last nucleus. A curious feature of the $\mathrm{N}^{15} \gamma$-rays is the large contribution of radiative capture (about $10 \%$ ) to the total capture cross section of nitrogen, most of which is caused by the (n, p) process. Since the probability of emission of radiation is so much lower than that of heavy particles one would not expect the emission of radiation to compete so successfully with proton emission when the latter is energetically possible.

The quantity of radiation to be expected from such targets as $\mathrm{Li}^{6}$ and $\mathrm{B}^{10}$ is relatively very small for the radiative process in these nuclei is in competition with the much more probable $(\mathrm{n}, \alpha)$ reactions. Capture radiation from $\mathrm{Li}^{7}$, although looked for, has not been found ${ }^{30}$. Capture radiation from $\mathrm{Li}^{8}, \mathrm{~B}^{12}$, and $\mathrm{C}^{14}$, has not yet been studied. No $\gamma$-rays have been detected from oxygen. This is to be expected for the capture cross sections of the oxygen isotopes are extremely low.

\section{b. Light Elements: Fluorine to Zinc}

Above oxygen, with the exceptions of the noble gases, Ne and A, the high energy capture $\gamma$-rays in all elements up to calcium have been measured 18. 31. 32. Low energy $\gamma$-rays have been measured in Na by Motz ${ }^{19}$,

25 S. Nakagawa, J. Sc. Research Inst. Tokyo 43, (1948) No. 1185; (1949) 1196.

${ }_{26}$ T. H. Braid, see reference 18; A. Bracci, U. Facchini, and A. Malvicino, Phys. Rev. 90, (1953) 162.

27 B. B. Kinsey and G. A. Bartholomew, Phys. Rev. 80, (1950) 918.

28 G. A. Bartholomew and B. B. Kinsey, Can. J. Phys. 31, (1953) 49.

29 B. Kinsey, G. Bartholomew, and W. Walker, Can. J. Phys. 29, (1951) 1,

30 B. B. Kinsey and G. A. Bartholomew, unpublished.

31 Kinsey, Bartholomew and Walker, Phys. Rev. 83, (1951) 519.

32 Kinsey, Bartholomew and Walker, Phys. Rev. 85, (1952) 1012. 
and in $\mathrm{Na}, \mathrm{Mg}, \mathrm{Al}$, and $\mathrm{S}$, by Braid ${ }^{18 .}{ }^{33}$. Excepting $\mathrm{F}^{20}$ and $\mathrm{Al}^{28}$, the ground state $\gamma$-rays in these elements are all weak (a few per cent per capture) and most of the energy is radiated in cascade transitions. The energies of the excited states determined by $\gamma$-ray measurements are usually in good agreement with that obtained from the $(d, p)$ reaction.

Above calcium, the high energy $\gamma$-ray spectra obtained with the pair spectrometer from the three odd-odd nuclei ${ }^{34} \mathrm{~V}^{52}, \mathrm{Mn}^{56}$, and $\mathrm{Co}^{60}$, present a striking contrast to the less dense $\gamma$-ray spectra produced in the evencharge nuclei ${ }^{35}$ (cf. Figs. 1 and 2). Some high energy $\gamma$-rays in the evencharge nuclei $\mathrm{Ca}^{41}$ and $\mathrm{Ti}^{49}$ have been measured. In neither is the ground state $\gamma$-ray detectable and most of the energy is radiated in very intense $\gamma$-rays leading to the first and second excited states. On the other hand, equally strong ground state $\gamma$-rays (about $50 \%$ per capture) are emitted

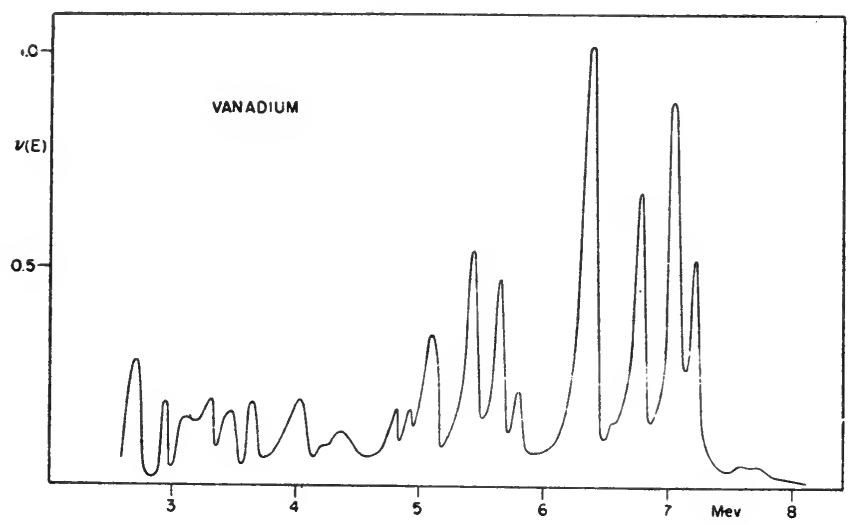

Fig. 1. High energy spectrum from $\mathrm{V}^{52}$, see ref. 34

Fig. 2. High energy spectrum from titanium, see ref. 35

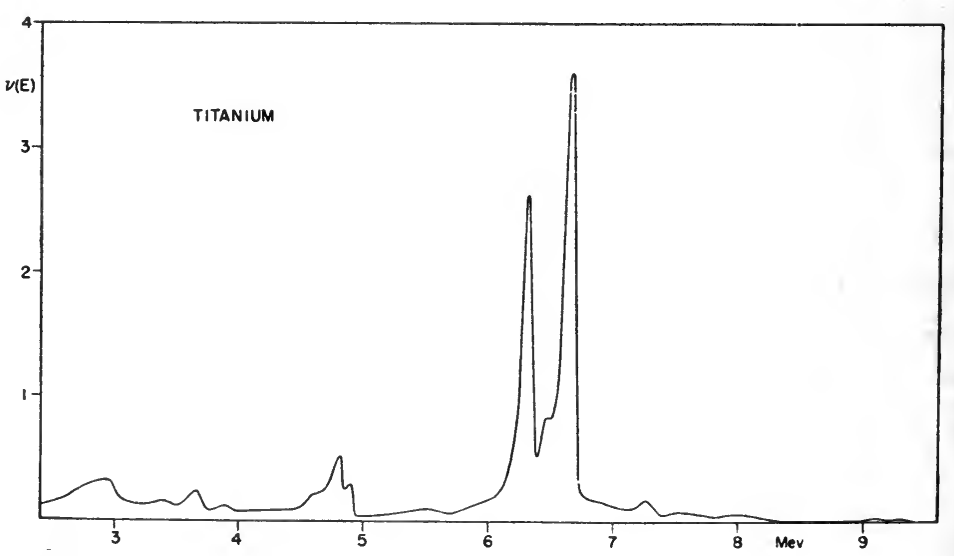

34 G. A. Bartholomew and B. B. Kinsey, Phys. Rev. 89, (1953) 386.

35 B. B. Kinsey and G. A. Bartholomew, Phys. Rev. 89, (1953) 375. 
from the nuclei $\mathrm{Cr}^{53}, \mathrm{Fe}^{55}, \mathrm{Fe}^{57}$, and $\mathrm{Ni}^{59}$, and the same is true of the oddcharge nuclei $\mathrm{Cu}^{64}$ and $\mathrm{Cu}^{66}$. Both low and high energy capture $\gamma$-rays from elements in this region have been studied by Hamermesh and Hummel 16, using a single crystal scintillation spectrometer.

A study of Table II will show that the ground state $\gamma$-rays are strong in

TABLE II

\section{Energites and Intensities of Ground State $\gamma$-Rays in Light Elements}

Where necessary, these energies are corrected for nuclear recoil. Values in parentheses are those obtained from the $Q$ of the $(\mathrm{d}, \mathrm{p})$ reaction and the binding energy of the deuteron 2.225 $\pm 0.002 \mathrm{MeV}$ (Li, Whaling, Fowler and Lauritsen, Phys. Rev. 83, (1951) 512). The third and fourth columns give references where the details of the $\gamma$-ray spectra may be found.

\begin{tabular}{|c|c|c|c|c|c|c|c|}
\hline \multirow{2}{*}{$\begin{array}{l}\text { Product } \\
\text { Nucleus }\end{array}$} & \multirow{2}{*}{\multicolumn{3}{|c|}{$\begin{array}{c}Q \\
\mathrm{MeV}\end{array}$}} & \multirow{2}{*}{\multicolumn{2}{|c|}{$\begin{array}{l}\text { Intensity of ground } \\
\text { state } \gamma \text {-ray in photons } \\
\text { per } 100 \text { captures }\end{array}$}} & \multicolumn{2}{|c|}{ References } \\
\hline & & & & & & $\begin{array}{l}\text { High } \\
\text { energy }\end{array}$ & $\begin{array}{c}\text { Low } \\
\text { energy }\end{array}$ \\
\hline $\mathrm{H}^{2}$ & $2.230 \pm$ & 0.007 & (a) & 100 & (b) & & \\
\hline $\mathbf{H}^{3}$ & 6.251 & 0.008 & (c) & 100 & $(c)$ & & \\
\hline $\mathrm{Li}^{7}$ & $(7.244$ & 0.007 ) & (d) & no & ected & & \\
\hline $\mathrm{Be}^{10}$ & 6.816 & 0.006 & (e) & 75 & $(f)$ & $(f)$ & \\
\hline $\mathrm{C}^{13}$ & 4.949 & 0.008 & (e) & 70 & $(f)$ & $(f)$ & \\
\hline$N^{15}$ & 10.832 & 0.008 & (e) & & & $(g)$ & \\
\hline
\end{tabular}

Not yet examined: $\mathrm{He}^{4}, \mathrm{Li}^{8}, \mathrm{~B}^{11}{ }^{12}, \mathrm{C}^{14}, \mathrm{~N}^{16}, \mathrm{O}^{17},{ }^{18}{ }^{19}$.

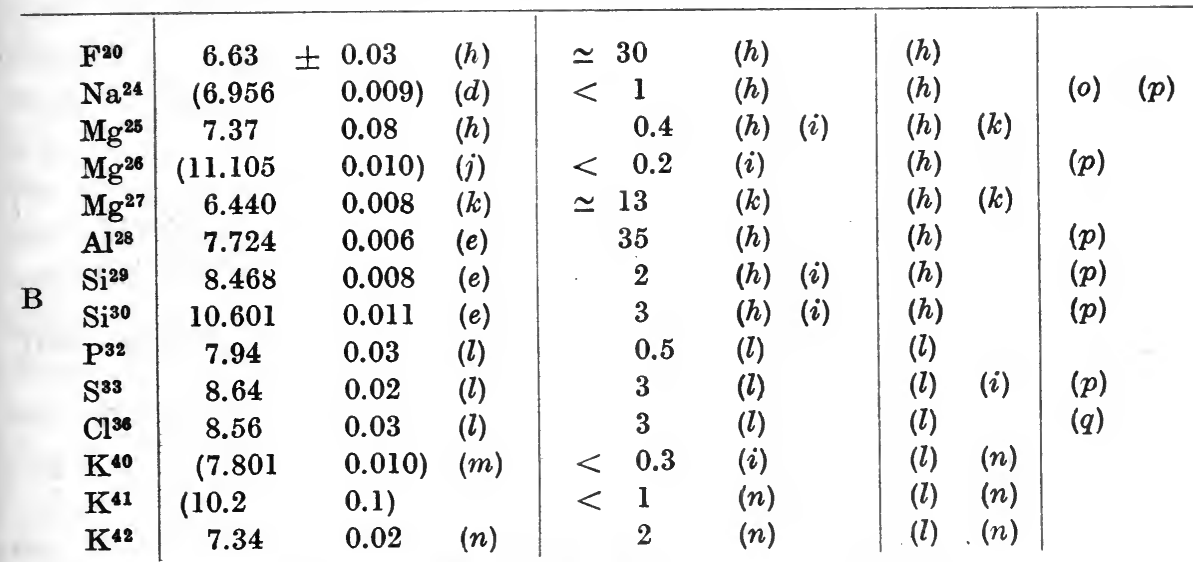

Not yet measured: $\mathrm{Si}^{31}, \mathrm{~S}^{34},{ }^{35},{ }^{37}, \mathrm{Cl}^{38}$ and isotopes of $\mathrm{Ne}, \mathrm{A}$.

\begin{tabular}{ll|ccl|lll|l|l|l}
\hline & $\mathrm{Ca}^{41}$ & $(8.40$ & \pm & $0.05)$ & $(r)$ & $<$ & 0.2 & $(i)$ & $(l)$ & $(p)$ \\
& $\mathrm{Ti}^{49}$ & $(8.04$ & $0.05)$ & $(s)$ & $<$ & 0.2 & $(t)$ & & $(t)$ & $(p)$ \\
$\mathrm{C}$ & $\mathrm{V}^{51}$ & $(10.95$ & $0.11)$ & $(u)$ & & & & & $(v)$ & \\
& $\mathrm{V}^{52}$ & 7.305 & 0.007 & $(v)$ & & & \\
& $\mathrm{Cr}^{51}$ & $(9.1$ & $0.1)$ & & $<$ & 1 & $(h)$ & & $(v)$ & \\
& & & &
\end{tabular}


TABLE II (continued)

\begin{tabular}{|c|c|c|c|c|c|c|c|}
\hline \multirow{2}{*}{$\begin{array}{l}\text { Product } \\
\text { Nucleus }\end{array}$} & \multirow{2}{*}{\multicolumn{3}{|c|}{$\begin{array}{c}Q \\
\mathrm{MeV}\end{array}$}} & \multirow{2}{*}{\multicolumn{2}{|c|}{$\begin{array}{l}\text { Intensity of ground } \\
\text { state } \gamma \text {-ray in photons } \\
\text { per } 100 \text { captures }\end{array}$}} & \multicolumn{2}{|c|}{ References } \\
\hline & & & & & & $\begin{array}{l}\text { High } \\
\text { energy }\end{array}$ & \multirow[t]{2}{*}{$\begin{array}{c}\text { Low } \\
\text { energy }\end{array}$} \\
\hline $\mathrm{Cr}^{53}$ & 7.929 & 0.008 & $(t)$ & 40 & $(t)$ & $(t)$ & \\
\hline $\mathrm{Cr}^{54}$ & 9.716 & 0.007 & $(t)$ & 13 & $(t)$ & $(t)$ & \\
\hline $\mathrm{Mn}^{56}$ & 7.261 & 0.006 & $(v)$ & 12 & $(v)$ & $(v) \quad(w)$ & $(w) \quad(q)$ \\
\hline $\mathrm{Fe}^{55}$ & 9.298 & 0.007 & $(t)$ & 40 & $(t)$ & $(t)$ & $(q)$ \\
\hline $\mathrm{Fe}^{57}$ & 7.639 & 0.004 & $(t)$ & 50 & $(t)$ & $(x)$ & $(q) \quad(x)$ \\
\hline $\mathrm{Fe}^{58}$ & 10.16 & 0.04 & $\cdot(t)$ & $\mathbf{5}$ & $(t)$ & $(t)$ & $(q)$ \\
\hline $\mathrm{Co}^{80}$ & 7.486 & 0.006 & $(v)$ & 3 & $(v)$ & $(v)$ & \\
\hline $\mathrm{Ni}^{50}$ & 8.997 & 0.005 & $(t)$ & 50 & $(t)$ & $(t)$ & \\
\hline$N i^{61}$ & $(8.31$ & $0.34)$ & $(y)$ & $?$ & $(t)$ & $(t)$ & \\
\hline $\mathrm{Cu}^{84}$ & 7.914 & 0.006 & $(v)$ & 24 & $(v)$ & $(v) \quad(x)$ & \\
\hline $\mathrm{Cu}^{68}$ & 7.634 & 0.006 & $(v)$ & 50 & $(v)$ & $(v)$ & \\
\hline $\mathrm{Zn}^{65}$ & 7.876 & 0.007 & $?(t)$ & $40 ?$ & $(t)$ & $(t)$ & \\
\hline $\mathrm{Zn}^{88}$ & 9.51 & 0.03 & $?(t)$ & & $(t)$ & $(t)$ & \\
\hline
\end{tabular}

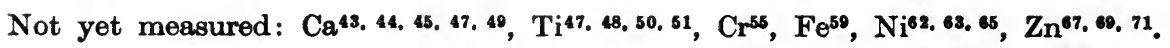

(a) See ref. (24).

References to Table II

(b) See ref. (26).

(c) See ref. (27).

(d) E. Strait, D. Van Patter, W. Buechner, and A. Sperduto, Phys. Rev. 81. (1951 747.

(e) See ref. (20).

(f) See ref. (28).

(g) See ref. (29).

(h) Seo ref. (31).

(i) See ref. (44).

(j) P. Endt, J. Haffner, and D. Van Patter, Phys. Rev. 86, (1952) 518.

(k) B. B. Kinsey and G. A. Bartholomew, Can. J. Phys. 31, (1953) 901.

(l) See ref. (32).

(m) W. Buechner, A. Sperduto, C. Browne, and C. Bockelman, Phys. Rev. 91, (1953) 1502.
(n) G. A. Bartholomew and B. B. Kinsey, Can. J. Phys. 31, (1953) 927.
(o) See ref. (19).
(p) See ref. (18).
(q) See ref. (10) and (16).
(r) V. L. Sailor, Phys. Rev. 75, (1949) 1836.
(s) G. F. Pieper, Phys. Rev. 88, (1952) 1299.
(t) Seo ref. (35).
(u) W. H. Johnson, Phys. Rev. 87, (1952) 166 and ref. (y).
(v) See ref. (34).
(w) See ref. (8).
(x) See ref. (9).
(y) From T. L. Collins, A. O. Nier, and W. H. Johnson, Phys. Rev. 86, (1952) 408. 
even-charge elements lighter than oxygen and in those in the iron group of elements, and that they are weak or absent for nuclei with intermediate masses. It will be shown in $\S 5$ that these regularities are related to shell structure and demonstrate the high transition probability of El radiation.

\section{B. Heavy Elements}

The high energy neutron capture $\gamma$-rays have been investigated with the pair spectrometer, so far, only in the following heavy elements: Even-charge ${ }^{36}$ : Se, Sr, Zr, Mo, Cd, Sn, Ba, Sm, Gd, W, Pt, Hg, and $\mathrm{Pb}$ Odd-charge ${ }^{37}$ : As, Nb, Rh, Ag, In, Sb, Pr, Ta, Au, Tl, and Bi.

Both low and high energy $\gamma$-rays have been studied with the single crystal scintillation spectrometer ${ }^{16}$, in some of these elements, and, in addition, in $\mathrm{Pd}, \mathrm{Te}, \mathrm{I}, \mathrm{Cs}$, and La. Low energy $\gamma$-rays have been studied in detail only in ${ }^{19} \mathrm{Cd}$.

The characteristic feature of the spectra of the heavy nuclei is their complexity. In many nuclei the number of individual $\gamma$-rays produced is so large that the greater part of the spectrum is too complex to be resolved by instruments now available. However, at the high energy end and at the low energy end of the $\gamma$-ray spectrum, individual $\gamma$-rays can usually be resolved. The high energy $\gamma$-rays are caused by transitions to the ground state and to low lying states, and the low energy $\gamma$-rays by transitions from these states to one another and to the ground state. A typical spectrum obtained with the pair spectrometer is shown in Fig. 3. This spectrum is resolved only partially at the high energy end. From $6 \mathrm{MeV}$ downwards the spectrum is unresolved and rises rapidly to the instrumental cutoff near $3 \mathrm{MeV}$. If the low energy end of the spectrum could be examined with the same resolution, we should expect to find that the unresolved spectrum passes through a maximum (near 1 or $2 \mathrm{MeV}$ ) near the point where the $\gamma$-rays produced by transitions between the strongly excited low lying states can be separately distinguished. This region, of course, lies below the range of the pair spectrometer.

A complete study of the unresolved spectrum is of considerable theoretical interest for it might throw light on the energy distribution of the level density. The shape of the spectrum depends on two factors: (1) the dependence of the transition probability on the energy of the $\gamma$-rays and (2) the density of the levels. The former favours the emission of high energy $\gamma$-rays leading to low lying states and the latter favours the pro-

36 B. B. Kinsey and G. A. Bartholomew, Can. J. Phys. 31, (1953) 1051.

37 G. A. Bartholomew and B. B. Kinsey, Can. J. Phys. 31, (1953) 1025. 
duction of levels at high excitation. Except for $\mathrm{Cd}$, there is, as yet, no experimental data from which the entire spectrum of a heavy nucleus can be constructed. Some nuclei (e.g., $\mathrm{Ag}$ and $\mathrm{Cd}$ ) show indications of a peak near $2.5 \mathrm{MeV}$. If the maximum in the $\mathrm{Cd}$ spectrum were genuine it should

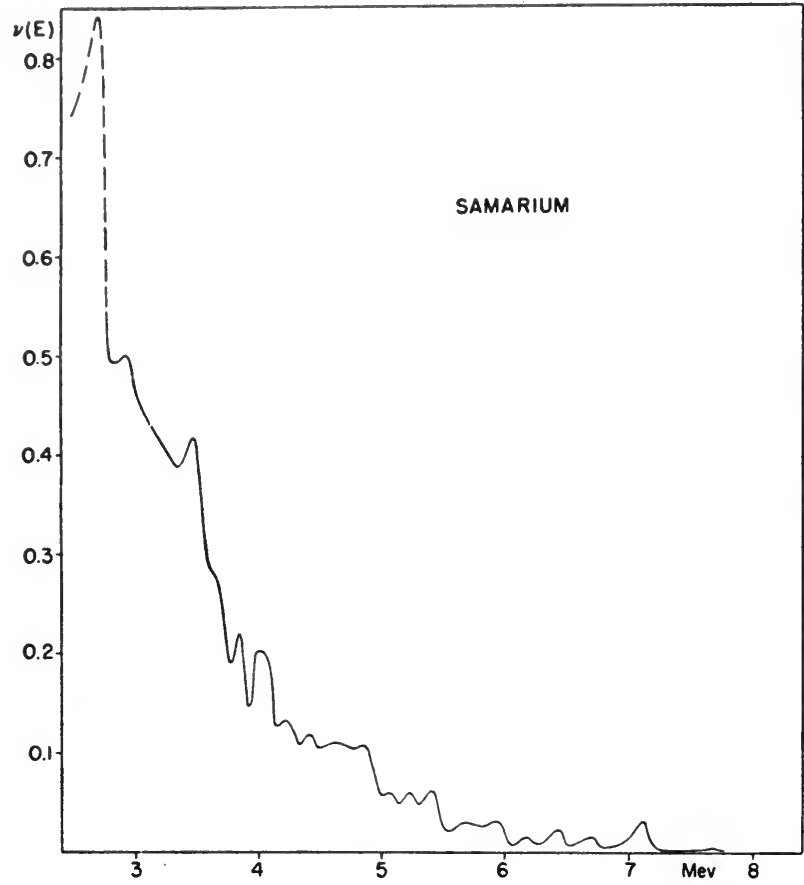

Fig. 3. High energy spectrum from samarium, see ref. 36

have been apparent in the cloud chamber measurements of Moak and Dabbs ${ }^{17}$ but these authors found no evidence for it. A few nuclei (e.g., $\mathrm{Au}^{198}, \mathrm{Hg}^{200}$, and $\mathrm{Tl}^{204}$ ) show evidence of a maximum above $3 \mathrm{MeV}$. This would appear to indicate a thinning out of the levels associated with the closing of the neutron shell at 126 neutrons and is discussed further in $\S 3 \mathrm{D}$.

The energies and intensities of the ground state $\gamma$-rays of heavy nuclei measured by the pair spectrometer are listed in Table III. Most of the even-charge nuclei listed in this Table are of even-even type because these nuclei usually have the highest binding energy and therefore the most easily recognizable ground state $\gamma$-rays. For the same reason, the ground state $\gamma$-ray of the nucleus with lowest mass in a pair of odd-odd isotopes can usually be distinguished (provided it is not forbidden) even though the density of the $\gamma$-rays in the high energy end of the spectrum is much greater than in those of even-charge nuclei. Disregarding the effect of 
TABLE III

Energies and Intensities of Ground State $\gamma$-Rays in Heavy Elements $Q$-values in parentheses are those obtained from the $(d, p)$ reaction and the binding energy of the deuteron, $2.225 \pm 0.002 \mathrm{MeV}$ (see caption to Table II) or from other sources.

\begin{tabular}{|c|c|c|c|c|c|c|c|c|}
\hline \multirow{2}{*}{$\begin{array}{l}\text { Product } \\
\text { Nucleus }\end{array}$} & \multirow{2}{*}{\multicolumn{2}{|c|}{$\begin{array}{c}Q \\
\mathrm{MeV}\end{array}$}} & & & \multirow{2}{*}{\multicolumn{2}{|c|}{$\begin{array}{l}\text { Intensity of ground } \\
\text { state } \gamma \text {-ray in photons } \\
\text { per } 100 \text { captures }\end{array}$}} & \multicolumn{2}{|c|}{ References } \\
\hline & & & & & & & $\begin{array}{l}\text { High } \\
\text { energy }\end{array}$ & $\begin{array}{l}\text { Low } \\
\text { energy }\end{array}$ \\
\hline $\mathrm{Se}^{77}$ & $7.416 \pm$ & 0.009 & & $(a)$ & 3.5 & $(a)$ & $(a)$ & \\
\hline $\mathrm{Se}^{78}$ & 10.483 & 0.014 & & $(a)$ & 0.3 & $(a)$ & (a) & \\
\hline $\mathrm{As}^{76}$ & 7.30 & 0.04 & & (b) & 0.5 & (b) & (b) & \\
\hline $\mathrm{Sr}^{87}$ & $(8.52$ & $0.2)$ & & (c) & & & (a) & \\
\hline $\mathrm{Sr}^{88}$ & (11.15 & $0.2)$ & & $(d)$ & & & (a) & \\
\hline $\mathrm{Zr}^{92}$ & 8.66 & 0.04 & & $(a)$ & 1.5 & $(a)$ & (a) & \\
\hline $\mathrm{Nb}^{94}$ & 7.19 & 0.03 & $?$ & (b) & 0.4 & (b) & (b) & \\
\hline $\mathrm{Mo}^{96}$ & 9.15 & 0.05 & & (a) & 0.04 & (a) & (a) & \\
\hline $\mathrm{Rh}^{104}$ & 6.792 & 0.014 & & (b) & 0.2 & (b) & (b) & \\
\hline $\mathrm{Ag}^{108}$ & 7.27 & 0.02 & & (b) & 1.4 & (b) & (b) & \\
\hline $\mathrm{Cd}^{114}$ & 9.046 & 0.008 & & $(a)$ & 0.14 & $(a)$ & (a) & $(e)$ \\
\hline $\operatorname{In}^{116}$ & $(6.59$ & $0.2)$ & & $(c)$ & & & (b) & $(f)$ \\
\hline $\mathrm{Sn}^{118}$ & 9.35 & 0.08 & & $(a)$ & 0.4 & $(a)$ & (a) & \\
\hline $\mathrm{Sb}^{122}$ & 6.80 & 0.04 & & (b) & 1 & (b) & (b) & \\
\hline $\mathrm{Ba}^{138}$ & 9.23 & 0.07 & $?$ & (a) & 0.3 & $(a)$ & $(a)$ & \\
\hline $\operatorname{Pr}^{142}$ & 5.83 & 0.03 & & (b) & 2 & (i) & (b) & \\
\hline $\mathrm{Sm}^{150}$ & & & & & & & (a) & $(g)$ \\
\hline $\mathrm{Gd}^{156,158}$ & & & & & & & (a) & $(g)$ \\
\hline Dy ${ }^{165}$ & & & & & & & & $(g)$ \\
\hline $\mathrm{Ta}^{182}$ & 6.07 & 0.03 & & $(b)$ & 0.7 & $(b)$ & (b) & \\
\hline$W^{183}$ & 6.182 & 0.008 & $?$ & $(a)$ & 13 & (a) & $(a)$ & \\
\hline$W^{184}$ & 7.42 & 0.02 & & (a) & 3 & $(a)$ & (a) & \\
\hline$W^{187}$ & 5.3 & 0.1 & & (a) & 5 & $(a)$ & (a) & \\
\hline $\mathrm{Pt}^{196}$ & 7.920 & 0.012 & & (a) & 0.3 & $(a)$ & (a) & \\
\hline $\mathrm{Au}^{198}$ & 6.494 & 0.008 & $?$ & (b) & 1.5 & $(b)$ & (b) & \\
\hline $\mathrm{Hg}^{200}$ & & & & & 0.1 & $(a)$ & (a) & $(g)$ \\
\hline $\mathrm{Tl}^{204}$ & 6.54 & 0.03 & & $(b)$ & 5 & (b) & (b) & \\
\hline $\mathrm{Pb}^{207}$ & 6.734 & 0.008 & & $(g)$ & 100 & (h) (i) & (h) & \\
\hline $\mathrm{Pb}^{208}$ & 7.380 & 0.008 & & $(g)$ & 100 & $(h) \quad(i)$ & (h) & \\
\hline $\mathrm{Bi}^{210}$ & 4.170 & 0.015 & $?$ & $(g)$ & & (h) & (h) & \\
\hline
\end{tabular}

References to Table III

(a) See ref. (36).

(b) See ref. (37).

(c) J. A. Harvey, Phys. Rev. 81, (1951) 353.

(d) R. Sher, J. Halpern, and A. Mann, Phys. Rev. 84, (1951) 387.

(e) See ref. (7), (10), and (17).

(f) See ref. (16).

(g) See ref. (23).

(h) See ref. (41).

(i) See ref. (42). 
the multipole order on the intensities of the $\gamma$-rays, it will be seen that, except for the isotopes of $\mathrm{Pb}$ and for nuclei of comparable atomic weight, the observed intensities are lower than for light nuclei. This is to be expected, for, as explained above, an increasing complexity of a $\gamma$-ray spectrum, in general, must be accompanied by a decreasing intensity of individual $\gamma$-rays. The elements $\mathrm{Cu}, \mathrm{W}$, and $\mathrm{Au}$, provide an illustration of this effect, for in them the highest energy $\gamma$-rays appear to be of the same multipole order. The greater density of levels in the two heavy nuclei is associated with a tenfold lower intensity for individual $\gamma$-rays. In $\mathrm{Pb}$ there is no evidence for an unresolved spectrum and, as in the hydrogen isotopes, the energy of excitation seems to be dissipated in the emission of the ground state $\gamma$-rays. This anomalous behaviour is related to shell structure $(\S 3 \mathrm{D})$.

\section{Multiplicity of Capture Radiation}

The multiplicity of capture radiation is defined as the average number of $\gamma$-rays emitted per capture. The first measurements of this quantity were made by Aoki ${ }^{5}$ and by Fleischmann ${ }^{6}$, who used Geiger counters to detect the capture radiation. These counters favour selectively the detection of high energy radiation and therefore it is probable that the results obtained are too low. Recently, multiplicities have been measured by Muehlhause ${ }^{38}$ using a coincidence method free from the above objection. He used anthracene crystals for detectors, and found that, over a range of 1 to $2 \mathrm{MeV}$, these detectors were of approximately constant sensitivity. His multiplicities and those of Aoki are listed in Table IV.

There are probably not enough measurements of the multiplicities of even-odd nuclei to give statistically significant results. However, the multiplicities of the even-even nuclei seem to be definitely higher than that of odd-odd nuclei. One would expect that a large multiplicity is associated with a complex level system. The obvious inference to be drawn from these results" is that the level systems of odd-odd nuclei are less complex than those of even-even nuclei. However, this inference cannot be correct for it is in conflict with other experimental evidence ${ }^{39.40}$.

A possible explanation of the discrepancy derives from the fact that the spins of the ground states of even-even nuclei are zero and the spins of the first excited states are 2 units. This may be seen from the following argument. If the spin of the initial state is low (e.g., 1 unit in $\mathrm{Cd}^{114}$ ) the excitation remaining after the emission of three or four successive $\gamma$-rays

38 C. O. Muehlhause, Phys. Rev. 79, (1950) 277.

39 S. Harris, C. Muehlhøuse, and G. Thomas, Phys. Rev. 79, (1950) 11.

t0 D. Hughes, R. Garth, and J. Levin, Phys. Rev. 91, (1953) 1423. 
will be about equally distributed between states with spins between 0 and 3 or 4 . Since the emission of low energy El radiation is usually highly forbidden, the subsequent decay of these states will be mainly determined by the emission of M1 radiation. Therefore only those with spin 1 can decay directly to the ground state and the remainder, some $60 \%$ of the total, will decay to the first excited state with spin 2 . If the

TABLE IV

MUlTiplicities OF CAPTURE $\gamma$-RAYS

\begin{tabular}{|c|c|c|c|}
\hline . & Product Nuclei & Muehlhause $(a)$ & Aoki $(b)$ \\
\hline \multicolumn{4}{|c|}{ Even-odd nuclei: } \\
\hline & $\mathrm{Cr}^{53}$ & 2.3 & \\
\hline 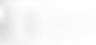 & $\mathrm{Fe}^{5 ?}$ & 1.7 & \\
\hline & $\mathrm{Ni}^{59}$ & & 2 \\
\hline 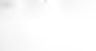 & Dy ${ }^{165}$ & 3.7 & \\
\hline \multicolumn{4}{|c|}{ Even-even nuclei: } \\
\hline & $\mathrm{Ge}^{74}$ & 4 & \\
\hline & $\mathrm{Cd}^{114}$ & 4.1 & 3 \\
\hline & $\mathrm{Sm}^{150}$ & 5.6 & \\
\hline & $\mathrm{Gd}^{158}$ & 3.9 & \\
\hline 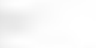 & $\mathrm{Hf}^{178}$ & 3.8 & \\
\hline & $\mathrm{Hg}^{200}$ & 3.3 & 2 \\
\hline$\sqrt{1}$ & & Mean 4.1 & \\
\hline \multicolumn{4}{|c|}{ Odd-odd nuclei: } \\
\hline & $\mathrm{Na}^{24}$ & 2 & \\
\hline 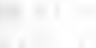 & $\mathrm{Al}^{28}$ & 2 & \\
\hline 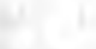 & $\mathrm{Cl}^{36}$ & 3.1 & \\
\hline 4 - & $V^{52}$ & 2.5 & \\
\hline 4 & $\mathrm{Mn}^{56}$ & 2.6 & \\
\hline & $\mathrm{Cu}^{64,68}$ & 2.6 & \\
\hline & $\mathrm{As}^{78}$ & 2.7 & \\
\hline 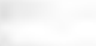 & $\mathrm{Br}^{80,82}$ & 3.4 & \\
\hline 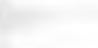 & $\mathrm{Nb}^{94}$ & 2.6 & \\
\hline$w_{1}$ & $\mathrm{Ag}^{108,110}$ & 2.9 & \\
\hline 8 & $\operatorname{In}^{116}$ & 3.3 & \\
\hline 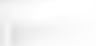 & $\mathrm{La}^{140}$ & 2.7 & \\
\hline & $\mathrm{Eu}$ & 3.8 & \\
\hline & $\operatorname{Re}^{188,188}$ & 3.2 & \\
\hline$=$ & Ir & 3.6 & \\
\hline 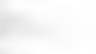 & $\mathrm{Au}^{198}$ & 3.5 & \\
\hline & & Mean 2.9 & \\
\hline
\end{tabular}

(a) See ref. (38).

(b) See ref. (5). 
spin of the initial state is high (as in $\mathrm{Sm}^{150}$ and $\mathrm{Gd}^{158}$ ) all but a negligible fraction will decay to the first excited state. Therefore the cascade process ends effectively in the first excited state. This is unlikely to occur in evenodd and odd-odd nuclei if the difference in the spins of the initial and ground states is not too different, because the spins of the ground states in these nuclei are seldom zero. Consequently three of the four or five states excited with different spins by the cascade process will decay to the ground state. If then the erergy distribution of the density of states is similar, the multiplicity of the $\gamma$-ray cascade in even-even nuclei will be greater than in even-odd or odd-odd nuclei by one unit, and we should expect to find that the first states in even-even nuclei are very strongly excited. As pointed out in $\S 3 \mathrm{~B}$, in the heavy nuclei, low energy $\gamma$-rays have been studied in detail only in $\mathrm{Cd}^{114}$. According to Motz ${ }^{19}$, a very strong $\gamma$-ray with an energy of $550 \mathrm{keV}$ is emitted by this nucleus. Its intensity is roughly one photon per capture and there seems to be no doubt that it is emitted by the first excited state.

\section{Effect of Shell Structure}

The low level density in nuclei with closed shells of neutrons or in nuclei for which the neutron configuration differs very little from that of closed shells, has been demonstrated by Hughes and his collaborators ${ }^{40}$ from studies of the capture cross sections of fast neutrons. This low level density should also have its influence on the multiplicity of the capture $\gamma$-rays. Muehlhause ${ }^{38}$ appears to have found such an effect in $\mathrm{La}^{\mathbf{1 4 0}}$.

Some capture $\gamma$-ray spectra show evidence for this low level density near the closed neutron shells of 50 and 82 neutrons ${ }^{36.37}$ but there is as yet no evidence for it in tin, where the shell of 50 protons is closed. The closing of the shell of 126 neutrons appears to have a marked effect on the $\gamma$-ray spectrum. The spectra of $\mathrm{Au}^{198}, \mathrm{Hg}^{200}$, and $\mathrm{Tl}^{204}(119,120$, and 123 neutrons respectively) appear unduly concentrated at the high energy end (Figs. 4 and 5): An upward shift of this kind should correspond to a general decrease in the density of the levels. This seems to be a satisfactory explanation of the spectra of $\mathrm{Hg}^{200}$ and $\mathrm{T}^{204}$, but is inadequate to account for the general shape of the spectrum of $\mathrm{Au}^{198}$, for the density of states near the ground state in this nucleus is certainly no less than normal; it is, in fact, some 10 times greater than that of $\mathrm{Hg}^{200}$ and $\mathrm{T}^{204}$.

The most marked effect of shell structure is shown in the very simple capture $\gamma$-ray spectrum emitted ${ }^{41}$ by lead. Of the four stable isotopes of $\mathrm{Pb}$, only $\mathrm{Pb}^{206}$ and $\mathrm{Pb}^{207}$ contribute appreciably to the total thermal

41 B. Kinsey, G. Bartholomew, and W. Walker, Phys. Rev. 78, (1950) 77; 82, (1951) 380 . 
ally the lighter even-charge nuclei), thermal neutron capture is not resonant and many levels must contribute to it. A given $\gamma$-ray, therefore, may be derived from a large number of initial states, and the measured intensity cannot be used to determine the matrix element of a unique transition even if the multipole orders of the various possible transitions are identical. At best, a matrix element found in this way must be an average over a large number of transitions. Moreover, the $\gamma$-rays emitted in non-resonant capture are often mixtures of different multipole orders and the observed intensities are not characteristic of any one of them. However, the scattering and absorption of thermal neutrons occasionally indicates that the thermal neutron capture is derived mainly from the state represented by the nearest known resonance. In some nuclei (e.g., $\mathrm{Mn}^{56}, \mathrm{~V}^{52}$ ) this resonance is the lowest with positive energy, in others, (e.g., $\mathrm{C}^{36}, \mathrm{Hg}^{200}$ ) it is a resonance at negative energy. In such nuclei, then, the intensities of the $\gamma$-rays observed are determined by the resonance responsible, and in the main, are representative of unique transitions. However, except in the rare cases when the thermal energy is equal to the resonance energy, or when the resonance energy is much less than the spacing between resonances, the contribution of other states to the observed $\gamma$-ray spectrum cannot be excluded.

Since the capture of thermal neutrons occurs only for $s$-waves, it follows that the spin of the compound state produced (whether defined by a resonance or not) differs from that of the target nucleus by $1 / 2$ and that the parity is the same. If the spin of the ground state of the target nucleus is $J_{t}$, and that of the compound state is $J^{\prime}$, than $J^{\prime}=J_{t} \pm 1 / 2$. If the spin of the final state of the product nucleus is $J$, the angular momentum of the photon emitted can have any value between $\left|J-J^{\prime}\right|$ and $\left|J+J^{\prime}\right|$, where $J^{\prime}$ can take either of the two values just quoted. For even-even target nuclei, $J_{t}=0, J^{\prime}$ can have only one value, $1 / 2$, and there will be no ambiguity in the lowest multipole order which can be emitted, whether the capture is resonant or not. For all other nuclei, $J_{t} \neq 0$, and the $\gamma$-rays produced by non-resonant capture will consist of mixtures of the $\gamma$-rays emitted in transitions from the various states which contribute to the thermal neutron capture cross section. The spins of these states are $J_{t} \pm 1 / 2$. The lowest multipole orders that can occur therefore will be $\left|J-J_{t}+1 / 2\right|$ and $\left|J-J_{t}-1 / 2\right|$; if the former is an electric radiation, the latter must be magnetic or vice-versa, and the lowest multipole order of the electric or the magnetic series will differ by one unit. For example, if the former corresponds to E2 radiation, the latter is $\mathrm{M} 1$ and the observed intensity of the $\gamma$-ray cannot be identified as characteristic of either, for it will consist of an undetermined mixture of both. 
The ambiguity in the interpretation of the measurement is resolved only when, as already mentioned, the spin of the ground state of the target nucleus, $J_{t}$, is zero, or when $J_{t}$ and the spin of the final state in the product nucleus, $J$, differ by $1 / 2$. In the latter event, the lowest multipole order is El or M1, depending on whether or not a parity change has occurred.

\section{$\S$ 5. Intensities and Transition Probabilities of Different Multipole Orders}

In the absence of measurements on pair internal conversion or on angular and polarization correlations of successive $\gamma$-rays, the multipole orders of high energy neutron capture $\gamma$-rays can be deduced only when the spins and parities of the initial and final states in the target nucleus are known from independent evidence, and when there is no ambiguity in interpretation, as discussed in the last paragraph. Even-charge nuclei, especially the lighter elements, best satisfy these conditions. In particular the ground state configurations of the even-odd nuclei are quite reliably given by current theories of shell structure.

In even-charge nuclei with masses less than that of zinc, strong ground state $\gamma$-rays are found only when the configurations of product even-odd nuclei are those of $p$-states ${ }^{43}$. These states have spins of $1 / 2$ and $3 / 2$ and odd parity and are the only ones in even-odd nuclei which are accessible by the emission of $\mathrm{E} 1$ radiation from the initial state with spin $\frac{1}{2}$. The intensities of the ground state $\gamma$-rays are listed in Table $V$ where the evenodd nuclei (for which the neutron capture $\gamma$-rays have been studied) are divided into groups according to the configuration of their ground states. It will be seen that strong ground state $\gamma$-rays occur only in Groups 1 and 4. The ground states of the nuclei in both these groups are $p$-states and the ground state $\gamma$-rays therefore are E1. The weaker ground state $\gamma$-rays in Groups 2 and 3 correspond to the emission of M1 or E2 radiation, and of $\mathrm{M} 2$ or $\mathrm{E} 3$ radiation respectively. The latter are not detected.

In an earlier paragraph we have pointed out that the absolute intensities of capture radiations derived from the initial compound state depend on both the transition probability of the $\gamma$-ray in question and, because of the competition between the $\gamma$-rays emitted by the initial state, also on the density of states accessible in direct transitions. The high intensity of E1 $\gamma$-rays, therefore, while suggesting a high transition probability, could also be accounted for by an abnormally low density of states accessible by emission of $\mathrm{E} 1$ radiation. That $\mathrm{E} 1$ radiation, is, in fact, far more probable than M1, or higher orders, was shown by a study ${ }^{44}$ of the compe- 
tition between $\mathrm{M} 1$ and E2 ground state $\gamma$-rays and the far more intense El $\gamma$-rays producing highly excited states in the nuclei $\mathrm{Mg}^{25}, \mathrm{Si}^{29}$, and $\mathrm{S}^{33}$. When allowance is made for the variation of the transition probability with the cube of the energy, the ratio of the transition probabilities (Ml : E1) can be calculated. The ratios found in these three nuclei differ very little from one another and average ${ }^{44}$ about $0.6 \%$. This value is in good agreement with Weisskopf's estimate ${ }^{45}$, viz. $10(\hbar / M c R)^{2}$ (about $2 \%$ ), where $\hbar$ and $c$ have their usual connotation, $M$ is the nuclear mass and $R$ the radius.

TABLE $V$

INTENSITIES OF GROUND STATE $\gamma$-RAYS IN EVEN-ODD NUCLEI

\begin{tabular}{|c|c|c|c|c|c|}
\hline Group & $\begin{array}{l}\text { Number of } \\
\text { Neutrons }\end{array}$ & $\begin{array}{l}\text { Configu- } \\
\text { ration }\end{array}$ & Nucleus & $\gamma$-ray & $\begin{array}{l}\text { Intensity in photons } \\
\text { per } 100 \text { captures }\end{array}$ \\
\hline \multirow[t]{3}{*}{1} & 7 & $p_{1 / 2}$ & $\mathrm{C}^{13}$ & $\mathbf{A}$ & 70 \\
\hline & 13 & $d_{8 / 2}$ & $\mathrm{Mg}^{25}$ & $\mathrm{C}$ & 0.4 \\
\hline & 15 & $s_{1 / 2}$ & $\mathrm{Mg}^{27}$ & $F_{1}$ & 13 \\
\hline \multirow{3}{*}{2} & 15 & $s_{1 / 2}$ & $\mathrm{Si}^{29}$ & B & 2 \\
\hline & 17 & $d_{3}$ & $\mathrm{Si}^{31}$ & & $<15$ \\
\hline & 17 & $d_{3 / 2}$ & $\mathrm{~S}^{33}$ & A & 1 \\
\hline \multirow{4}{*}{3} & 21 & $f_{2 / 2}$ & $\mathrm{Ca}^{41}$ & & $<\quad 0.2$ \\
\hline & 23 & $f_{\%}$ & $\mathrm{Ca}^{43}$ & & $<1$ \\
\hline & 27 & $f_{7 / 2}^{10}$ & $\mathrm{Ti}^{49}$ & & $<\quad 0.2$ \\
\hline & $2 \tau$ & $f_{7 / 2}$ & $\mathrm{Cr}^{51}$ & & $<1$ \\
\hline \multirow{4}{*}{4} & 29 & $p_{2 / 2}$ & $\mathrm{Cr}^{53}$ & $\mathrm{D}$ & 40 \\
\hline & 29 & $p_{2 / 2}$ & $\mathrm{Fe}^{55}$ & B & 50 \\
\hline & 31 & $p_{2 / 2}$ & $\mathrm{Fe}^{57}$ & $\mathbf{E}$ & 40 \\
\hline & 31 & $p_{2 / 2}$ & $\mathrm{Ni}^{59}$ & $\mathbf{A}$ & 50 \\
\hline
\end{tabular}

A comparison of the transition probabilities of $\mathrm{E} 2$ and $\mathrm{E} 1$ radiation was made in $\mathrm{Mg}^{25}$. At $7 \mathrm{MeV}$, it was found that the transition probability of the E2 $\gamma$-ray was only $0.05 \%$ of that of the El $\gamma$-ray. Unfortunately, it has not yet been possible to compare the intensities of $\mathrm{E} 2$ radiation against $\mathrm{E} 1$ or $\mathrm{M} 1$ radiation in other nuclei, for although $\mathrm{E} 2 \gamma$-rays are known to occur, they are usually diluted with M3 radiation. The above value for the ratio of the transition probabilities, however, does not seem to be unrepresentative ${ }^{44}$. No higher order $\gamma$-rays have yet been detected in competition with $\mathrm{E} 1$ or M1 radiation. In Group 3 of Table V, the ground state $\gamma$-rays emitted are E3 or M2 radiations. Since these $\gamma$-rays were not 
detected it is obvious that their transition probabilities must be very low.

The evidence discussed above is derived entirely from even-odd nuclei. No similar comparison of transition probabilities has yet been reported from other types of nuclei. As yet there is no reason to suppose that the same selection rules do not hold. However, it is important to note that the $\mathrm{Ml}$ radiation compared with $\mathrm{El}$ radiation in the even-odd nuclei mentioned above all violate the single particle selection rules. They represent forbidden transitions between $s$-states or between $s$ - and $d$-states. So far, no direct comparison of $\mathrm{El}$ with allowed $\mathrm{Ml}$ radiation has been made.

\section{§ 6. Absolute Emission Probabilities}

Except in nuclei which can give rise to the emission of charged particles, the capture of a neutron must be followed by its re-emission or by the emission of radiation. It is well known that the lifetime of a state is related to its width through the relation $\tau=\hbar / \Gamma_{t}$ where $\Gamma_{t}$ is the total width. The total width is the sum of the partial widths corresponding to these two processes, $\Gamma_{\gamma}$, and $\Gamma_{n}$, which separately measure the probability of emission of $\gamma$-rays and neutrons. The quantity $\Gamma_{\gamma}$ is the sum of the emission rates of all the $\gamma$-rays emitted by this state. The emission rate $\left(\Gamma_{r}\right)$ of a particular $\gamma$-ray (denoted by the suffix $r$ ) emitted by the initial state is related to the observed absolute intensity $I_{r}$ through the relation $\Gamma_{r}=\Gamma_{\gamma} I_{r}$ where $I_{r}$ is measured in photons per capture. Using this relation we can determine the lifetimes of some nuclear states for $\gamma$-ray emission from measurements of $\gamma$-ray intensities and the parameters of neutron resonances.

It is well known that, in the heavy elements, the greater part of the width of low energy neutron resonances is due to radiation. The numerical value of $\Gamma_{\gamma}$ is nearly constant; it is about $0.1 \mathrm{eV}$ and varies very little from one heavy nucleus to another. For lighter nuclei (with masseś below 100) the neutron width is usually larger than the radiation width and is responsible for the greater part of the total width. In some cases, the radiation width can be found from resonance activation and in others by measurements of the ratios of resonance absorption and scattering integrals. In many nuclei, however, where the product nucleus is stable, or, if radioactive, its activity is difficult to detect, radiation widths have not been measured.

Now we have seen that the absolute intensities of $\gamma$-rays of a particular multipole order decrease with increasing atomic weight. It follows that the partial widths corresponding to the emission of these $\gamma$-rays decrease 
with increasing complexity of the $\gamma$-ray spectrum. For example, the partial width for the emission of the (7.72 $\mathrm{MeV}) \mathrm{Ml}$ ground state $\gamma$-ray in $\mathrm{Al}^{28}$ is about $2 \mathrm{eV}$; in $\mathrm{Cd}^{114}(9.05 \mathrm{MeV})$ it is $0.00016 \mathrm{eV}$. It is obvious therefore that some relation exists between the radiation width and the complexity of the nucleus or of the $\gamma$-ray spectrum which it emits.

Theoretical estimates for the radiation widths based on the individual particle model have been made by Weisskopf ${ }^{45}$. If $\Gamma_{t h}$ is the theoretical radiation width, in $\mathrm{eV}$, and if the energy, $E$, is expressed in $\mathrm{MeV}$, and $R$, the nuclear radius, in units of $10^{-13} \mathrm{~cm}$, then:

$$
\begin{aligned}
& \Gamma_{t h}(\mathrm{E} 1)=0.047 E^{3} R^{2} \\
& \Gamma_{t h}(\mathrm{Ml})=0.021 E^{3} .
\end{aligned}
$$

For a nucleus of mass 100 and for $7 \mathrm{MeV}$, these expressions give $\Gamma_{t h}(\mathrm{E} 1)=760 \mathrm{eV}$, and $\Gamma_{t h}(\mathrm{M} 1)=7.2 \mathrm{eV}$. In very light elements, and to an order of magnitude, Wilkinson ${ }^{46}$ has shown that these formulae do indeed give substantially the correct radiation widths for high energy proton capture radiations. For neutron capture in heavy elements, where, as we have noted, the total radiation width is close to $0.1 \mathrm{eV}$, it is obvious that these formulae give values which are far too high.

If there exists a mixing of states, Blatt and Weisskopf ${ }^{47}$ have shown that the theoretical radiation width should be lower than that given above by a factor proportional to the spacing of those states near the initial state which combine with the lower state with the emission of radiation of the same multipole order. A better theoretical estimate of the radiation width, therefore, is $\Gamma_{t h} D / D_{0}$, where $D$ is the spacing appropriate to the initial state and $D_{0}$ is some spacing appropriate to the ground state. According to these authors, an order of magnitude estimate for $D_{0}$ is $0.5 \mathrm{MeV}$. We shall now show that, with this modification, the theoretical estimates are in surprisingly good agreement with the experimental results, and that the reduction of the total radiation width with increasing complexity of the $\gamma$-ray spectrum is very largely accounted for by this factor depending on the spacing. The ratio of the observed to the corrected theoretical width, $\Gamma_{r} /\left(\Gamma_{t h} D / D_{0}\right)$, will be denoted by $|M|^{2}$; it is a measure of the square of the matrix element of the transition.

The results of a comparison of theory and experiment are summarized in Table VI for $\mathrm{E} 1$ radiation and in Table VII for Ml radiation. The derivations of the radiation widths used in these tables are discussed elsewhere ${ }^{44}$. In both tables the second column gives the resonance responsible for thermal

46 D. H. Wilkinson, Phil. Mag. 44, (1953) 450.

47 J. M. Blatt and V. F. Weisskopf, Theoretical Nuclear Physics, John Wiley and Sons, New York, 1952. 
TABLE VI

Emission Probabilities of El Radiation

\begin{tabular}{|c|c|c|c|c|c|c|}
\hline $\begin{array}{c}1 \\
\text { Nucleus }\end{array}$ & $\begin{array}{c}2 \\
\text { Reso- } \\
\text { nance }\end{array}$ & $\begin{array}{c}3 \\
\gamma \text {-ray } \\
\text { Energy }\end{array}$ & $\begin{array}{c}4 \\
D(\mathrm{eV})\end{array}$ & $\begin{array}{c}5 \\
\Gamma_{r} \\
\text { milli-eV }\end{array}$ & $\begin{array}{c}6 \\
\Gamma_{\tau} / \Gamma_{t h}\end{array}$ & $\begin{array}{c}7 \\
|M|^{2}\end{array}$ \\
\hline \multicolumn{7}{|l|}{ Even-charge } \\
\hline $\mathrm{Si}^{29}$ & $170 \mathrm{keV}$ & 3.54 & $5 \times 10^{5}$ & 8400 & 0.18 & 0.18 \\
\hline $\mathrm{S}^{33}$ & 108 & 5.43 & $3 \times 10^{5}$ & 16000 & 0.09 & 0.15 \\
\hline$W^{183}$ & & $6.18(?)$ & 10 & 9 & $1.1 \times 10^{-5}$ & 0.6 \\
\hline$W^{184}$ & & 7.42 & 40 & $\gg 3.6$ & $>2.5 \times 10^{-6}$ & $\ngtr 0.03$ \\
\hline$W^{187}$ & & $5.24(?)$ & 100 & 9 & $1.8 \times 10^{-5}$ & 0.09 \\
\hline & & & & & & 0.2 \\
\hline \multicolumn{7}{|l|}{ Odd:charge } \\
\hline$V^{52}$ & $2700 \mathrm{eV}$ & 7.31 & $2 \times 10^{4}$ & 70 & $1.3 \times 10^{-4}$ & 0.003 \\
\hline $\mathrm{Mn}^{56}$ & 345 & 7.26 & $2 \times 10^{3}$ & 70 & $1.2 \times 10^{-4}$ & 0.03 \\
\hline $\mathrm{Co}^{60}$ & 120 & 7.48 & $1 \times 10^{3}$ & 8 & $1.2 \times 10^{-5}$ & 0.006 \\
\hline $\mathrm{Cu}^{64}$ & & 7.91 & $1 \times 10^{4}$ & 50 & $6 \times 10^{-5}$ & 0.003 \\
\hline $\mathrm{Cu}^{66}$ & & 7.63 & $1 \times 10^{4}$ & 100 & $1.3 \times 10^{-4}$ & 0.007 \\
\hline $\mathrm{Rh}^{104}$ & 1.3 & 6.79 & $\sim 100$ & 0.3 & $4 \times 10^{-7}$ & 0.002 \\
\hline $\mathrm{Ag}^{108}$ & & 7.27 & 50 & 1.4 & $1.5 \times 10^{-6}$ & 0.015 \\
\hline $\mathrm{Ag}^{110}$ & 5.2 & $6.67(?)$ & 50 & $\gtrless 0.5$ & $₹ 6 \times 10^{-7}$ & $\gtrless \underline{0.006}$ \\
\hline & & & & & & 0.01 \\
\hline
\end{tabular}

TABLE VII

Emission Probabilities of Ml Radiation

\begin{tabular}{|c|c|c|c|c|c|c|}
\hline $\begin{array}{c}1 \\
\text { Nucleus }\end{array}$ & $\begin{array}{c}2 \\
\text { Reso- } \\
\text { nance }\end{array}$ & $\begin{array}{c}3 \\
\gamma \text {-ray } \\
\text { Energy }\end{array}$ & $\begin{array}{c}4 \\
D(\mathrm{eV})\end{array}$ & $\begin{array}{c}5 \\
\Gamma_{r} \\
\text { milli-eV }\end{array}$ & $\begin{array}{c}6 \\
\Gamma_{r} / \Gamma_{t h}\end{array}$ & $\begin{array}{c}7 \\
|M|^{2}\end{array}$ \\
\hline Even-charge & & & & & & \\
\hline $\mathrm{Si}^{29}$ & $170 \mathrm{keV}$ & 8.47 & $5 \times 10^{5}$ & 280 & 0.02 & 0.02 \\
\hline $\mathrm{Si}^{29}$ & $170 \mathrm{keV}$ & 7.19 & $5 \times 10^{5}$ & 1100 & 0.14 & 0.14 \\
\hline $\mathbf{S}^{33}$ & 108 & 8.65 & $3 \times 10^{5}$ & 300 & 0.02 & 0.04 \\
\hline $\mathrm{S}^{33}$ & 108 & 7.78 & $3 \times 10^{5}$ & 400 & 0.04 & 0.08 \\
\hline $\mathrm{Cd}^{114}$ & $0.17 \mathrm{eV}$ & 9.05 & $\sim 100$ & 0.16 & $1.0 \times 10^{-5}$ & 0.05 \\
\hline $\mathrm{Cd}^{114}$ & $0.17 \mathrm{eV}$ & 8.48 & 100 & 0.25 & $2.0 \times 10^{-5}$ & 0.10 \\
\hline & & & & & & 0.07 \\
\hline Odd-charge & & & & & & \\
\hline$F^{20}$ & & 6.63 & $4 \times 10^{5}$ & 3000 & 0.5 & 0.6 \\
\hline $\mathrm{Al}^{28}$ & & 7.72 & $3 \times 10^{5}$ & 1700 & 0.2 & 0.3 \\
\hline $\mathrm{Al}^{28}$ & & 6.77 & $3 \times 10^{5}$ & 70 & 0.01 & 0.02 \\
\hline $\mathrm{Cl}^{36}$ & & 8.56 & $4 \times 10^{4}$ & 9 & $7 \times 10^{-4}$ & $\begin{array}{l}0.009 \\
0.2\end{array}$ \\
\hline
\end{tabular}


neutron capture (where it is known); column 3 , the energy of the $\gamma$-ray in question; column 4, the estimated value of the spacing appropriate to $s$-resonances at the neutron binding energy (this is equal to the observed spacing for even-even target nuclei, and twice this observed spacing in all other nuclei); column $\tilde{5}$, the partial width for the emission of the $\gamma$-ray in milli-eV; column 6 , the ratio of the observed to the theoretical widths: and column 7 , the value of $|M|^{2}$. It will be seen that the figures in the last column are remarkably constant in view of the large range in spacing. For even-charge nuclei the matrix elements of El radiation give rough agreement (to a factor of 10) with Weisskopf's estimate. For oddcharge nuclei they seem to be lower; the data, however, are hardly sufficient to establish a significant difference. For M1 radiation there is no obvious differences between the matrix elements of odd and evencharge nuclei and both are about ten times lower than Weisskopf's estimate. It is probable that in general the estimates of $D$ are too high, for, as more neutron transmission measurements are made with improved resolution, more resonances are discovered. The error in $D$ is difficult to estimate: it is probably nowhere less than a factor of 2 and in some nuclei where experimental data are scanty (e.g., $\mathrm{V}^{52}$ ) it may be much higher. The effect of the spacing on the radiation widths is shown graphically in Fig. 6, where the radiation widths, normalized to $7 \mathrm{MeV}$, are plotted against $D$.

Tables VI and VII confirm the predicted effect of the level spacing on the radiation width and show that there is some agreement between the experimental widths and those estimated theoretically. There remains one further check on the matrix elements of Table VI; this is the calculation of the total radiation width which will be discussed in the next section.

Although the matrix elements of high energy E1 radiation for heavy odd-charge nuclei appear to be low, nevertheless the transition probabilities of $\mathrm{M} 1$ and $\mathrm{E} 1$ radiation probably differ by at least a factor of 10 , in rough agreement with the theoretical expectations, of the individual particle model. This suggests that that model is indeed adequate to account for the experimental facts when allowance is made for the effect of the spacing of the levels. There is no clear indication here for the suppression of E1 radiation to be expected of transitions in certain collective models of the nucleus. Moreover, we find little evidence for that suppression of E2 radiation which should occur if the neutron alone was responsible for the radiative process nor do we find much evidence for the operation of the selection rules of the individual particle model in so far as they apply to the forbidding of Ml radiation. The ratio of the transition probabilities of M1 and E1 radiation and the dependence on level spacing suggest that the mixing of the highly excited states is such that there always exists a 
single-particle component of any appropriate multipole order in a given transition to a lower level of suitable spin and parity. The retardation of the radiative process represented by the factor depending on the lerel spacing is the quantitative expression of the mixing of the states.

$(2 J+1) \Gamma(7 / E)^{3}$

Fig. 6. Partial radiation widths of $\mathrm{El}$ and Ml radiation, corrected to $7 . \mathrm{MeV}$, plutted against the spacing of $s$-wave resonances near the neutron binding energy. Full points: evencharge; open points: odd-charge nuclei

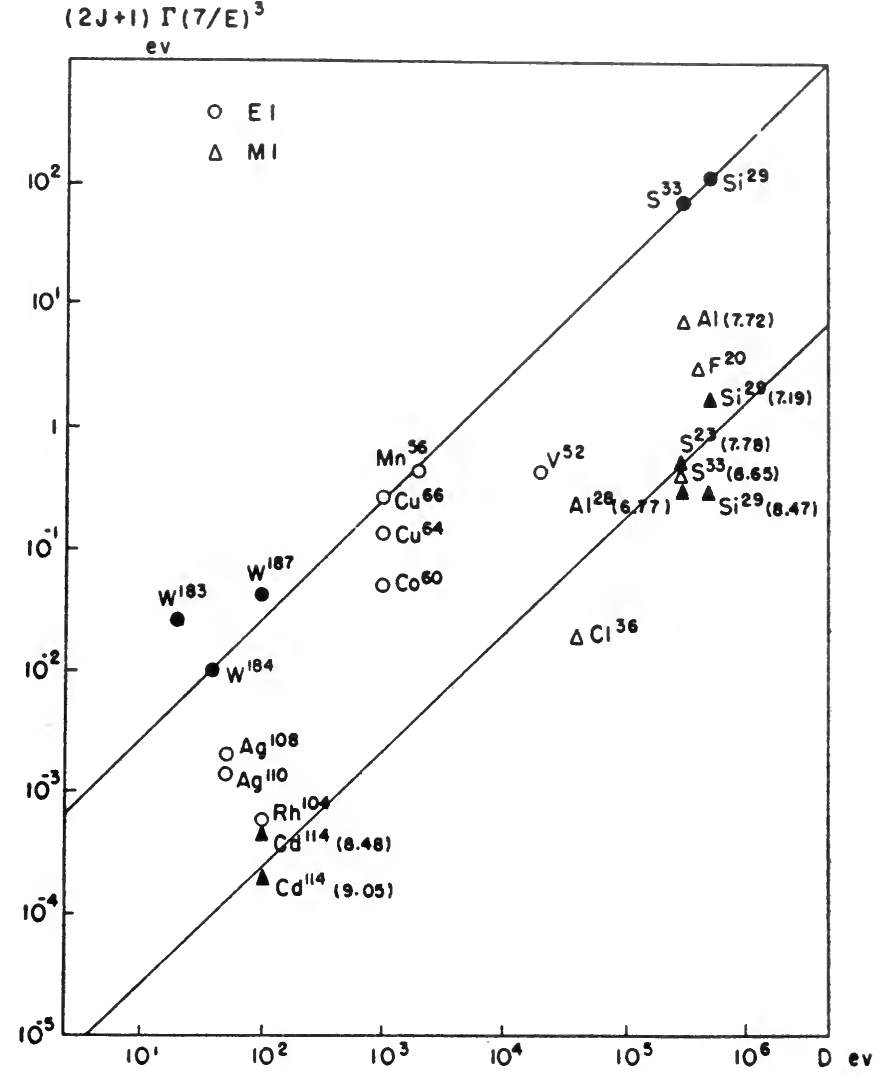

\section{$\S 7$. Total Radiation Widths}

The approximately constant value of the total radiation width of neutron resonances in the heavy elements has already been referred to. There are few accurate measurements of radiation widths; a list of the most recent results has been published by Hughes and Harvey ${ }^{48}$. These authors show that (1) there is little difference between the radiation widths of nuclei of different type; (2) there is no apparent dependence of the width on the spin of the resonances; (3) the widths of different resonances in the same isotope do not differ much one from another; and (4) the widths, as a whole, show a tendency to decrease with increasing atomic weight.

48 D. J. Hughes and J. A. Harvey, Nature, 173, (1954) 942. 
The constancy of the radiation widths may be explained as follows. First, it should be remembered that the total width is the sum of all the partial widths due to $\gamma$-rays leading to lower levels. In a nucleus such as $\mathrm{Sm}^{150}$, the arerage number of these transitions is of the order of $10^{4}$. The sum of the partial widths of such a large number of transitions therefore is quite independent of fluctuations in the matrix elements of individual transitions. Let $\Gamma_{0}$ be the unretarded average partial width of a $\gamma$-ray of unit energy. Then, assuming that dipole radiation only contributes to the capture spectrum, the average width of a $\gamma$-ray of energy $(B-E)$ is

$$
\Gamma_{0}(B-E)^{3} \cdot D(B) / D_{0}
$$

where $B$ is the neutron binding energy, and $D(B)(=1 / \varrho(B))$ is the spacing of levels at this energy. The total width is therefore:

$$
\Gamma_{\gamma}=\Gamma_{0} D(B) / D_{0} \cdot \int_{0}^{B}(B-E)^{3} \varrho(E) d E
$$

where $\varrho(E)$ is the density of levels at energy $E$.

In this expression the spacing $D(B)$ refers to states with the same parity as' the target nucleus but the term $\varrho(E)$ refers to states of the same or opposite parity according to whether the $\gamma$-ray is even or odd. Disregarding this point for the moment, we assume that the level density is given by the well-known theoretical distribution, $\exp \left(a E^{1 / 2}\right)$, and that the level spacing is $1 \mathrm{MeV}$ at the ground state and $10 \mathrm{eV}$ at the neutron binding energy $(7 \mathrm{MeV})$. The numerical value of the above expression is then found to ke very insensitive to changes in the level density; in fact, an increase in density by a factor of 10 at the binding energy reduces the width by a factor of two. When the results listed by Hughes and Harvey are adjusted to take account of variations in the neutron binding energy and in the level density ${ }^{49}$, the apparent dependence of the width on the atomic weight is eliminated.

The quantity $\Gamma_{0} D(B) / D_{0}$ can be equated to the average value of the ratio of the partial width of a $\gamma$-ray to the cube of its energy. This quantity, averaged over as many of the higher energy $\gamma$-rays as can be separately resolved, together with the integral term in the above expression, gives a value for the total radiation width which can be compared with experiment. The results of such a calculation are shown in Table VIII, where only those elements are considered for which the $\gamma$-ray intensities have been obtained by direct measurement; which are monoisotopic; or in which only one isotope contributes appreciably to the thermal neutron capture cross section. The second column contains the neutron binding energy, $B$, the

49 B. B. Kinsey and G. A. Bartholomew, to be published. 
-third, the radiation width of the lowest resonance, the fourth, the mean value of the partial width per unit energy. The latter were obtained from the experimental radiation widths in the third column and the observed intensities of the highest energy $\gamma$-rays. The level density was assumed to increase according to the theoretical law, which was adjusted to the observed spacing of the high energy $\gamma$-rays near $0.5 \mathrm{MeV}$ (fifth column) and the observed spacing of neutron resonances with a given spin near the neutron binding energy (sixth column). The calculated widths are listed in column 7 . For the lighter nuclei, $\mathrm{V}^{52}$ to $\mathrm{Cu}^{64}$, the calculated widths are too high; this is to be expected for the intensities of the unresolved parts of the spectra produced by these nuclei are lower than those anticipated from the theoretical density distribution. In the heavier nuclei, there is good agreement for $\mathrm{Rh}^{104}$ and $\mathrm{Ag}^{108}$ and poor agreement for $\mathrm{W}^{184}$ and $\mathrm{Au}^{198}$. Too high a value for $\mathrm{Au}^{198}$ is to be expected for the spectrum of this nucleus has an anomalous shape. On the other hand the results for $\mathrm{Cd}^{114}$ and $\mathrm{Sm}^{150}$ are too low. The Cd result is important for this nucleus is the only one for which the highest energy $\gamma$-rays are MI.

TABLE VIII

COMPARISON OF OBSERVED AND CALCULATED RADIATION WIDTHS

\begin{tabular}{|c|c|c|c|c|c|c|}
\hline $\begin{array}{c}1 \\
\text { Nucleus }\end{array}$ & $\begin{array}{c}2 \\
B(\mathrm{MeV})\end{array}$ & $\begin{array}{c}3 \\
\Gamma_{\gamma}(\mathrm{eV})\end{array}$ & $\mid \begin{array}{c}4 \\
\text { Mean } \Gamma_{r} / E_{r}^{3} \\
\text { eV per } \mathrm{MeV}^{3}\end{array}$ & $\begin{array}{c}\tilde{5} \\
D(0.5) \\
(\mathrm{keV})\end{array}$ & $\begin{array}{c}6 \\
D(B)(\mathrm{eV})\end{array}$ & $\begin{array}{c}7 \\
\Gamma_{\gamma}(\text { calc }) \\
(\mathrm{eV})\end{array}$ \\
\hline$V^{52}$ & 7.31 & $1.0 \quad(a)$ & $2.3 \times 10^{-4}$ & $\mathbf{2 5 0}$ & $10^{4}$ & 1.8 \\
\hline $\mathrm{Mn}^{56}$ & 7.26 & $0.6 \quad(a)$ & 0.9 & 300 & $2 \times 10^{3}$ & 1.3 \\
\hline $\mathrm{Co}^{60}$ & 7.49 & $0.28 \quad(a)$ & 0.4 & 200 & $10^{3}$ & 0.9 \\
\hline $\mathrm{Cu}^{64}$ & 7.91 & $0.2 \quad(a)$ & 0.7 & 500 & $10^{4}$ & 0.3 \\
\hline $\mathrm{Rh}^{104}$ & 6.79 & $0.155(b)$ & 0.03 & 200 & $\sim 100$ & 0.20 \\
\hline $\operatorname{Ag}^{108}$ & 7.27 & $0.10 \quad(c)$ & 0.07 & 170 & 50 & 0.11 \\
\hline $\mathrm{Cd}^{114}$ & 9.05 & $0.112(b)$ & 0.005 & 500 & $\sim 100$ & 0.025 \\
\hline $\mathrm{Sm}^{150}$ & 7.9 & $0.066(b)$ & 0.002 & 500 & 10 & 0.03 \\
\hline$W^{184}$ & 7.42 & $0.10 \quad(c)$ & 0.08 & 400 & 40 & 0.4 \\
\hline$A u^{198}$ & 6.5 & $0.15 \quad(b)$ & 0.19 & 100 & 60 & 1.9 \\
\hline
\end{tabular}

(a) See ref. (44).

(b) See ref. (48).

(c) Assumed value.

The total radiation width is the product of two independent quantities, (1) the partial unretarded width at unit energy,.$\Gamma_{0}$, and (2) a term depending only on the level density and the neutron binding energy. Thus the constancy of the widths determined experimentally shows that both these terms are constant from one nucleus to another. The constancy of 
the first term, the partial width at unit energy, implies that either E1 or Ml radiation, whichever has the greater emission probability, is the origin of the widths in heavy elements. As we have shown ( $\$ 5$ and 6) there is good reason to suppose that this must be El radiation. Since $\Gamma_{\gamma}$ is about $0.1 \mathrm{eV}$, numerical calculation shows that $\Gamma_{0}$ is about $0.01 \mathrm{eV}$. From this quantity we may deduce a mean value for the matrix element for $\mathrm{E} 1 \mathrm{radiation}$. We find $|M|^{2} \simeq 0.01$, in rough agreement with the results of Table VI. If $\mathrm{El}$ radiation then is responsible for the observed radiation widths, the constancy of the second term shows that the density of states of opposite parity do not differ. 


\title{
MEASUREMENT OF DISINTEGRATION RATE
}

\section{XXVI}

\author{
J. L. PUTMAN
}

\section{§ 1. Introduction}

\section{A. Need for Absolute Measurements}

Many applications of radioactive materials require no precise knowledge of the rate of disintegration of sources. In tracer experiments, for example, only relative disintegration rates need generally be known accurately. Even therapeutic doses need only be expressed in terms of universally accepted standards, whose physiological effects are known, but whose true disintegration rates are of secondary importance.

When the properties of the individual radiations are studied, however, such as their efficiency in producing ionization or initiating secondary reactions it may be necessary to know the total number of radiations emitted. Similarly when the number $N$ of radioactive nuclei in a sample is required (for example as an index of the neutron flux to which a sample has been exposed ${ }^{1}$ ) this can be deduced from a knowledge of the disintegration rate $(d N / d t)$ and the decay constant $\lambda$ :

$$
d N / d t=N \lambda
$$

The same relation is used in determining the decay constant of such long lived radioisotopes as $\mathrm{C}^{14}$. In this the measured specific activity $d N / d t$ per gram of a sample is compared with the concentration of mass 14 atoms, $N$ per gram, as measured with a mass spectrometer ${ }^{2}$.

\section{B. Relation with $\beta$ aNd $\gamma$-SPECTROMETRY}

Spectroscopic measurements of disintegration schemes are mainly concerned with the proportion of the total disintegrations which occur in a given manner. Thus the absolute efficiency of detectors is of secondary importance, although their relative efficiency for different types and energies of radiations must be accurately known.

On the other hand, an accurate knowledge of the disintegration scheme is usually essential to the reliable measurement of disintegration rate, so that absolute measurements of activity depend on the results of $\beta$ - and $\gamma$-ray spectroscopy.

Further, it will be seen that the comparison of other measurements with those of total disintegration rate can be used to supplement and cross

1 D. J. Littler, Proc. Phys. Soc. A64 (1951) 638.

2 G. G. Manov and L. F. Curtiss, Jour. Res. Nat. Bur. Stds. 46 (1951) 328. 
check some results of $\beta$ - and $\gamma$-ray spectroscopy. For example the mean energy per disintegration of a source can be found by comparing the disintegration rate with a calorimetric measurement of the rate of energy dissipation.

\section{Basic Methods of Measurement \\ a. Counting Methods}

The measurement of disintegration rate depends on the detection and assessment by various means of the radiations emitted by a source. The most direct approach is to count the radiations which are emitted by the source in a given time, and counting methods are generally accepted as the most reliable means of determining disintegration rate. The activities which can conveniently be measured by counting methods are usually small, however - of the order of a few microcuries in general. Thus the measurement of larger sources involves dilution, sometimes by large factors. Moreover it is difficult to ensure the long term stability of most counters to better than 1 or $2 \%$. so that counters are not very suitable for maintaining standards.

Partly for these reasons and partly because of the additional information they can supply, other methods of measurement are sometimes used to supplement counting measurements of disintegration rate.

\section{b. Calorimetric Methods}

If the mean energy per disintegration is known from spectroscopic data, the disintegration rate can be deduced from calorimetric measurements of the rate of energy dissipation by a source. Thus if $E$ (mean) is the mean energy, expressed in $\mathrm{MeV}$ and $C$ is the disintegration rate expressed in curies, and the rate of energy dissipation is easily shown to be:

$$
J=\tilde{5} .93 \times 10^{-3} C E(\text { mean }) \text { Watts }
$$

It may be noted that calorimetric measurements are not subject to losses due to absorption of radiation in the source material.

\section{c. Loss of Charge}

The rate of loss of charge by a source is a measure of the rate of emission of charged particles. and this can be used as a measure of disintegration rate.

\section{d. Ionization Chamber Methods}

The most important subsidiary method by which the disintegration rate can be determined is the measurement of ionization produced by the 
radiations in air or other gases. This measurement is of more direct interest to the radiotherapist than is the disintegration rate.

Continuous current ionization chambers are used, in which all the charges produced by the radiations in a given gas volume are collected by two electrodes. The resulting current, in equilibrium conditions, is equal to the rate of production of charge by ionization. The disintegration rate can be deduced provided that the ionizing efficiency of the radiations is known.

\section{$\S 2$. Types of Counters}

Complete descriptions of the many designs of counters for activity measurements are given in specialised books on the subject ${ }^{3-7}$ and it will be assumed that the reader is already familiar with the types available and their principles of operation. It will be convenient to review here the properties of Geiger-Müller counters, proportional counters and scintillation counters which directly affect their use for absolute measurements.

\section{A. Geiger-Müller COUnters}

These counters have been found very suitable for absolute measurements of $\beta$-particles, because a $\beta$-particle which traverses the sensitive volume of the counter has almost a $100 \%$ chance of being counted. The efficiency for positrons is similar, of course. That for $\gamma$-rays is much lower ${ }^{8}$, (generally $1 \%$ or less) because the counting of these depends on the release of secondary electrons mostly from the walls of the counter.

These counters are convenient to use, with a minimum of electronic apparatus, and are relatively stable in operation against small fluctuations in supply potentials. Their limited life, generally between $10^{8}$ and $10^{9}$ counts, is of little disadvantage except in very long experiments, or when calibrated counters are used to maintain standards. In these applications, halogen quenched counters ${ }^{9}$ are sometimes preferable, as these have much longer life, although their intrinsic efficiency is slightly lower than $100 \%$.

The recording of a radiation in a Geiger-Müller counter is followed by

3 S. C. Curran and J. D. Craggs, Counting Tubes: Butterworth, London 1949.

4 D. H. Wilkinson, Ionization Chambers and Counters, Cambridge University Press, 1950.

5 B. B. Rossi and H. H. Staub, Ionization Chambers and Counters, McGraw Hill, New York, 1949.

- S. C. Curran. Luminescence and the Scintillation Counter, Butterworth, London 1953.

7 J. B. Birks, Scintillation Counters, Pergamon Press, London 1953.

8 R. D. Evans and R. A. Mugele, Rev. Sci. Instr. 7 (1936) 411.

9 E. Franklin and W. R. Loosemore, Proc. I.E.E. Part II, 98 (62), (1951) 237. 
an insensitive period, or dead time during which the counter is insensitive to other radiations. This dead time is generally of about $100 \mu$ s duration and is followed by a recovery time of about 200 to $300 \mu \mathrm{s}$, during which pulses of reduced size are delivered. In all accurate counting experiments a correction is necessary for counts lost in the dead time, and counting rates are limited to 300 or 400 counts per second for reliable operation. Hole has shown ${ }^{10}$ that the observed counting rate $N$ is related to the true rate of passage of ionizing radiations $N_{0}$ and the dead time $t$ by the relation:

whenee:

$$
N=N_{0} /\left(1+N_{0} t\right)
$$

$$
N_{0}=N /(1-N t)
$$

Unfortunately both the dead time and the recovery time of a counter depend both on the applied potential, and on the sensitivity of recording circuits and must be measured under the true conditions of operation. The most general practice is to apply from external circuits, an artificial dead time of constant length sufficient to allow the counter to recover after each pulse. This ensures an accurately known correction.

An external quenching unit proposed by the present author 11 and designed by Cooke-Yarborough et al. ${ }^{12}$ applies a fixed dead-time (generally about $400 \mu \mathrm{s}$ ) and also eliminates spurious counts which cause a slope in the plateau of the counter. This unit also improves the temperature stability of the counter ${ }^{11}$. It is widely used and is commercially available.

\section{B. Proportional Counters 3.4,5}

Proportional counters operate at potentials lower than the GeigerMüller region and deliver much smaller pulses. For the present purposes their chief advantage is their practical absence of dead time. This enables them to be used at very high counting rates, of the order of $10^{5}$ counts per second. Proportional counters can also be operated with internal gas pressures (of methane, for example) of one atmosphere or more, and continuous flow counters ${ }^{13}$, to which gas is continuously supplied as it leaks slowly out of the counter, are found convenient for the insertion of samples into the counter itself.

\section{Scintmllation Counters ${ }^{3: 6.7}$}

These counters are described in Chapter V. Their high $\gamma$-ray efficiency

10 N. Hole, Ark. Mat. Ast. Fys. A33 (1946) No. 11.

11 J. L. Putman, Proc. Phys. Soc. 61 (1948) 312.

12 E. H. Cooke-Yarborough, C. D. Florida and C. N. Davey, Jour. Sci. Instr. 26 (1949) 124.

13 J. A. Simpson (Jr.), Rev. Sci. Instr. 18 (1947) 884. 
as compared with that of a Geiger counter is an advantage, especially in coincidence experiments, and the short resolution times associated with suitable phosphors enables them to record counting rates as high as $10^{5}$ counts per second without appreciable loss of counts.

Liquid phosphors such as terphenyl in xylene, in which sources can be immersed, have been used to obtain $4 \pi$ geometry in measuring $\beta$ particle sources.

\section{$\S$ 3. Counting Methods}

\section{A. Defined Solid Angle Counting}

The radiations from a radioactive source are isotropic except to the extent that they are modified by absorption and scattering. Thus the rate of emission in a given solid angle $\Omega$ is:

$$
f(\Omega)=\frac{\Omega}{4 \pi} F
$$

Where $F$ is the total rate of emission in all directions. If a detector of known sensitivity, is used to count the radiations which traverse it in a given time, the total disintegration rate of a source can be deduced from the solid angle which it subtends to the source. This method was one of the earliest used ${ }^{14}$ to determine the disintegration rate of $\beta$-particle sources. Although it is subject to more corrections and inaccuracies than some other methods to be described, it is still popular, especially in the less specialised laboratories, because it requires a minimum of subsidiary equipment and can be operated with commercially available counters.

In the arrangement shown in Fig. 1, an end-window $\beta$-counter is used in air to measure the radiations from a pure $\beta$-particle source.

Geometry. After correction for dead time losses and for background rate, the

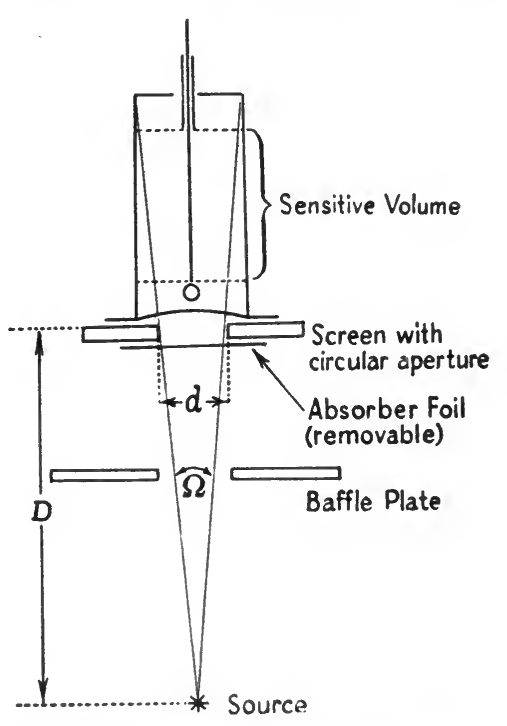

Fig. 1. Defined Solid Angle Method for $\beta$-Particles observed counting rate is equal to the rate at which $\beta$-particles traverse the sensitive volume of the counter. This is not bounded by the counter window, but ends near a plane through the free end of the centre wire.

L. F. Curtiss and B. W. Brown, Jour. Res. Nat. Bur. Stds. 37 (1946) 91, 
This boundary of the sensitive volume varies a little with the potential applied to the counter. Further the shortness of path length of those particles which pass through its outer edges makes the exact limits of the boundary rather ill defined, for particles with very short paths in the sensitive volume are not counted with $100 \%$ efficiency. A circular aperture smaller than the window defines the solid angle $\Omega$ more accurately. It can readily be shown that if $d$ is the diameter of the aperture and $D$ the distance from the plane of its top surface to a source on the axis of the counter, the solid angle $\Omega$ is given by:

$$
\frac{\Omega}{4 \pi}=\frac{1}{2}\left\{1-\frac{1}{\sqrt{\left(1+d^{2} / 4 D^{2}\right)}}\right\}
$$

or approximately if $d \ll D$ :

$$
\frac{\Omega}{4 \pi} \doteqdot \frac{1}{16} \frac{d^{2}}{D^{2}}\left\{1-\frac{3}{16} \frac{d^{2}}{D^{2}}\right\}
$$

The correction for the loss of radiations in the bead at the end of the centre wire is usually small. Further modifications of equations (5) and (6) apply to sources which are displaced from the axis of the counter ${ }^{15}$ and strictly these apply to any source of finite area, but the corrections are generally very small if the source diameter is of the order of a few $\mathrm{mm}$.

Window and Air Absorption. A correction is necessary for the loss of particles by absorption in the window of the counter and in the air between the window and the source. The window correction is generally made by introducing additional thicknesses of material in front of the window to obtain a conventional absorption curve and by extrapolating the absorption curve back by a thickness equivalent to that of the window (see Fig. .2).

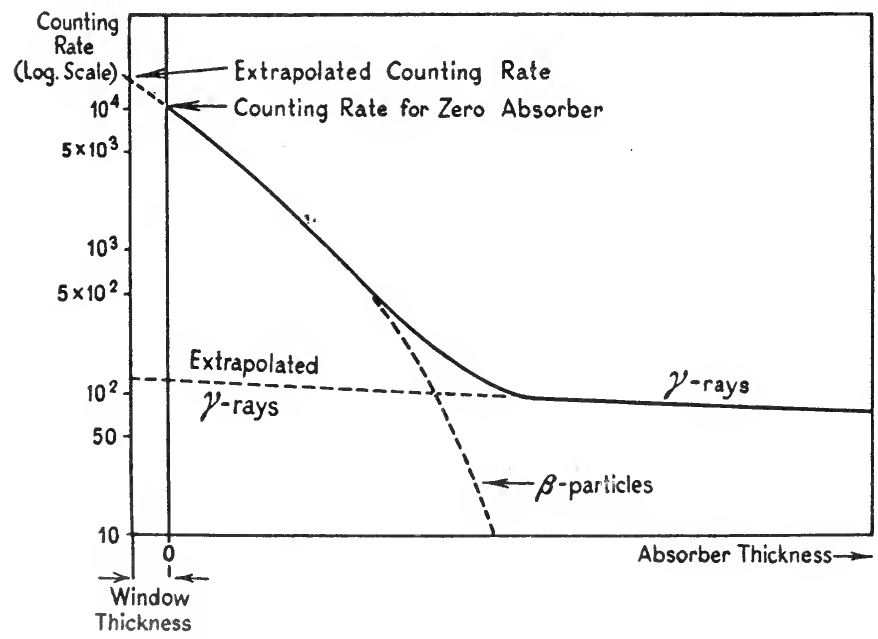

Fig. 2. Correction for Window Absorption

15 G. B. Cook and J. F. Duncan, J. Chem. Soc. Supplement 2, (1949) page S. 369. 
The material used for the absorbers should ideally be the same as the window material.

A correction for air absorption is often made in a similar manner, by adding the air thickness in $\mathrm{mg} / \mathrm{cm}^{2}$ to that of the window for extrapolating the absorption curve. This method of making the air correction is inaccurate, however, because the effects of air scattering of the radiations is neglected. It can only be used reliably if the air absorption is very small. Zumwalt ${ }^{16}$ estimated the effects of air absorption and scattering by using a series of equally spaced thin absorbers interposed between the source and the counter. The most direct method for assessing the air effect is to change the pressure of the air between the source and counter. At low pressures, however, this results in changes in the shape of the counter window, which is normally under tension through the external atmospheric pressure.

Scattering and Back-scattering. A correction is also necessary for those radiations originally outside the solid angle $\Omega$ which are scattered into the counter. The best results are obtained when the source and counter are supported by open frameworks well away from other objects, to reduce the low-angle scattering to a minimum. Baffle plates with circular apertures are sometimes used, so that radiation cannot be directly scattered into the counter (see Fig. 1) and these also reduce air scattering effects. It is almost impossible, however, when using baffles of this kind, to avoid multiple scattering which leads to an unknown proportion of radiation eventually being scattered into the counter. A demountable windowless counter has also been used for absolute measurements ${ }^{17}$.

Back-scattering from the source support increases with the thickness of the support and with its atomic number. This can result in an increase up to $80 \%$ for saturation back-scattering from a lead backing and a correction factor must be applied for backing materials of appreciable thickness 16.18.19. By mounting the source on a very thin foil of low atomic number, $10 \mathrm{~cm}$ or more clear of any backing material the effects of back-scattering can be reduced to less than $1 \%$ for most nuclides. Foils of cellulose acetate, formvar or nylon 20 to $30 \mu \mathrm{g} / \mathrm{cm}^{2}$ thick are often used.

Self-absorption and Self-scattering. If the source has appreciable thickness, some radiations are absorbed and some scattered by the source material itself. Corrections for these effects are difficult to estimate accurately but an empirical correction can be made by using several sources of

17 J. L. Putman, Brit. Jour. Radiol. 23 (1950) 46.

18 B. P. Burtt, Nucleonics 5, (No. 2) (1949) 28.

19 L. Yaffe and K. M. Justus, J. Chem. Soc., Supplement 2, page S. 341 (1949). 
known relative activity but different thickness. The apparent specific activity of the source material is then plotted as a function of source thickness and the results are extrapolated to zero thickness. The method is subject to errors due to non-uniformity of source thicknesses.

For absolute measurements, the source should therefore be made as thin as possible (preferably less than 0.01 of the half thickness of the beta particles). The error involved in estimating self-absorption and selfscattering corrections is then small and for very thin sources the two effects tend to neutralise one another.

Correction for $\gamma$-rays. When $\beta-\gamma$ emitters are measured in a defined solid angle arrangement, a correction is necessary for the counting rate due to $\gamma$-rays from the source.

The usual method of correction is to shield the counter from the source with an absorber just thick enough to stop all $\beta$-particles and to measure the residual counting rate due to $\gamma$-rays plus background. This is then subtracted from the total counting rate obtained without the absorber. It may generally be assumed that the absorption of $\gamma$-rays by the absorber is negligible, but a correction can be made for this by plotting an absorption curve for the $\gamma$-rays (see Fig. 2).

This method of correction is sufficiently accurate for most purposes. It is open to two objections however.

First, the counting rate due to all $\gamma$-rays is subtracted, whereas a proportion $\Omega / 4 \pi$ of the $\gamma$-rays (neglecting angular correlation) accompany $\beta$-particles which would have been recorded in the absence of the screen. Since a single disintegration cannot be recorded twice, counts due to these $\gamma$-rays should not really have been deducted from the total counting rate. The error involved is small provided that the solid angle factor $\Omega / 4 \pi$ is small.

Secondly, the insertion of an absorber in front of the counter window may appreciably increase the sensitivity of the counter for $\gamma$-rays, by virtue of the additional secondary electrons produced in the absorber.

Fortunately the $\gamma$-ray correction is usually a small one, except for nuclides such as $\mathrm{Co}^{60}$ and $\mathrm{Br}^{82}$ which emit low energy $\beta$-particles (subject to large absorption) accompanied by high energy $\gamma$-rays. For these the $\gamma$-ray background can be found by magnetic deflection of the $\beta$-particles, but the method is cumbersome and liable to interfere with the operation of the counter.

\section{B. $4 \pi$ COUnters}

The most direct, and for many nuclides the most accurate method of determining the disintegration rate is to record the total rate of emission 
of particles in all directions. If this can be done, a number of difficulties disappear. For example $\gamma$-rays emitted almost simultaneously with a $\beta$-particle by a $\beta-\gamma$ emitter produce no effect, for only one pulse is recorded. Similarly the annihilation radiations arising when positrons are stopped produce no additional counts. The detector must completely surround the source, and as little absorption as possible should occur between the source and the detector.

Geiger-Müller Types. In an early $4 \pi$ Geiger-Müller counter by Simpson ${ }^{20}$, the source was introduced between two separate D-shaped counters and radiations passed through thin foils into the two counters. A closer approach to $4 \pi$ geometry was obtained by introducing the source, mounted on a very thin foil, into the counter itself ${ }^{21}$, which consisted essentially of two Dshaped halves, each with its own anode wire (see Fig. 3). The two halves are identical in shape and have a common gas filling. They operate at the same potential and the anode wires are connected together externally. Several de-

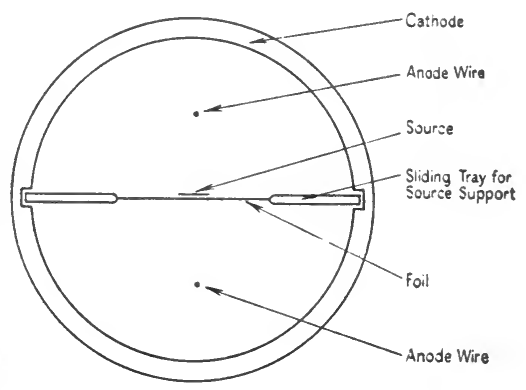

Fig. 3. Essentials of a $4 \pi$ Counter signs are in operation ${ }^{17.22,23}$, differing mainly in the method of introducing the source and in the shape of the half-counter tubes. Some typical designs are shown in Fig. 4. All of these have to be evacuated and re-filled whenever a fresh source is to be counted.

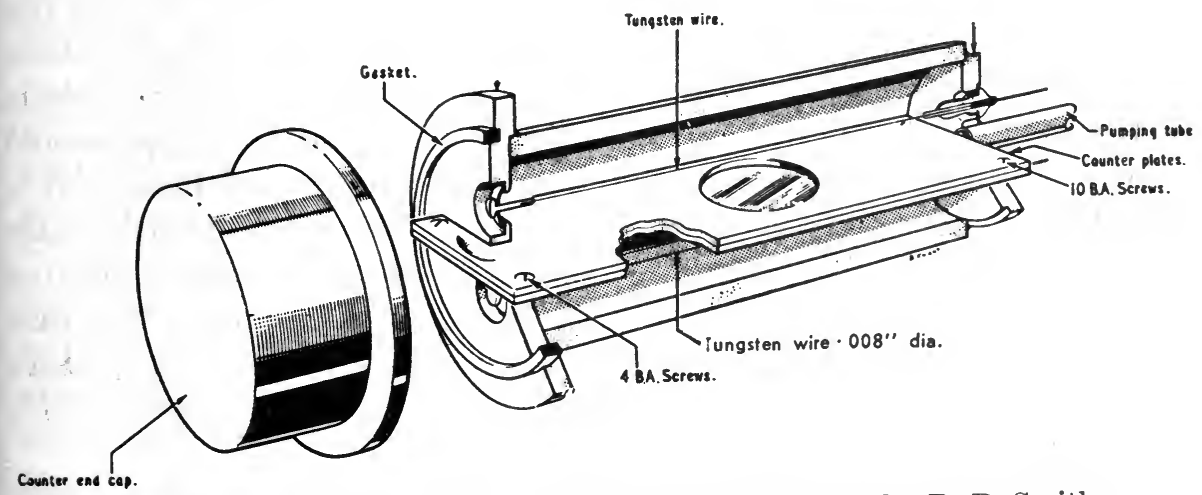

Fig. 4a. $4 \pi$ Counters. From AERE Report I/R 1210, by D. B. Smith.

20 J. A. Simpson (Jr.), Rev. Sci. Instr. 15 (1944) 119.

R. Cohen, Comptes Rendus 229 (1949) 356.

D. B. Smith, A.E.R.E. Report I/R. 1210. 
The most satisfactory method of filling is from a large flask of readymixed gas and vapour (e.g. a $9 / 1$ mixture of Argon and Alcohol), as there is then no need to wait for uniform diffusion of the mixture through the counter before operation. Very reproducible operating conditions are obtained if care is taken to use pure gases and vapours, to pump out the counter at least to $10^{-4} \mathrm{~mm} \mathrm{Hg}$ pressure before filling, and to fill to an accurately measured pressure. Beta-particles which enter the counter volume are counted with almost $100 \%$ efficiency in argon-alcohol counters at pressures above $6 \mathrm{~cm} \mathrm{Hg}$. The use of $\mathrm{CCl}_{4}$ for cleaning should be avoided, for we have found that very small traces of $\mathrm{CCl}_{4}$ vapour in the counter reduce its sensitivity very considerably.

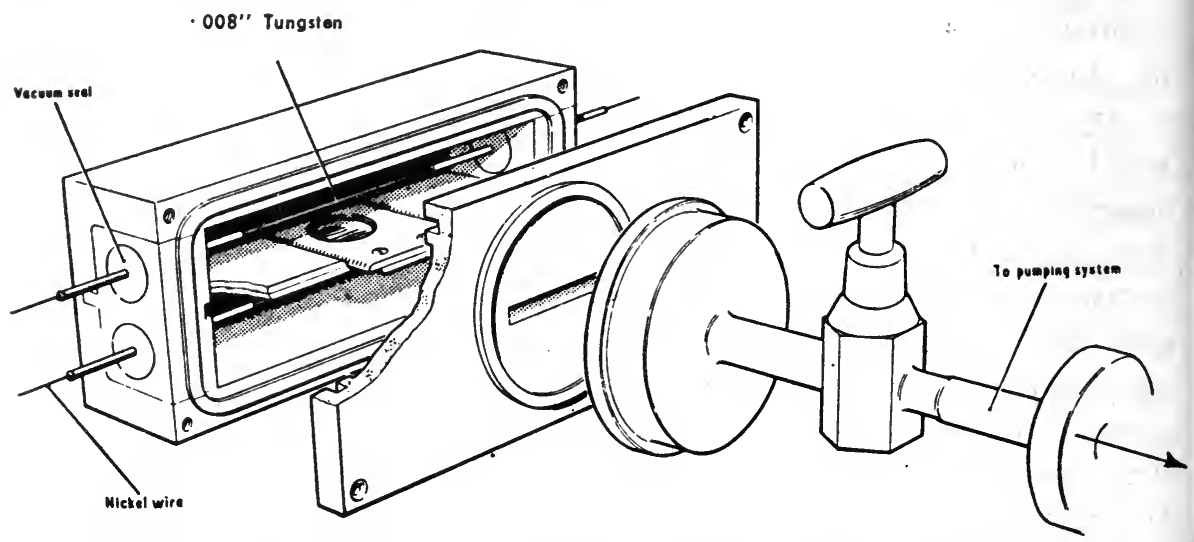

Fig. 4b. $4 \pi$ Counters. From AERE Report I/R 1210, by D. B. Smith.

In a well designed counter it is not difficult to obtain a very flat plateau region of 100 to 200 volts long, with a starting potential of about 800 volts. The use of an external quench circuit 12 to avoid spurious counts further reduces any residual slope and the resulting plateau slope should always be less than $0.02 \%$ per volt. If any residual slope is present, it is usual to plot the frrst and flattest portion of the plateau of the counter and to extrapolate this back to the starting potential to obtain the true counting rate. This rather arbitrary procedure is based on the fact that spurious counts are least. likely at the lowest potentials of the plateau region, and has been checked experimentally using $4 \pi$ counters with different plateau slopes.

Proportional Types. Proportional counters of various designs have also been used as $4 \pi$ counters. Hawkings ${ }^{24}$ used a system comprising two hemispherical counters whose anodes were loops of fine wire. Provided

24 R. C. Hawkings, W. F. Merritt and J. H. Craven, National Physical Laboratory Symposium, Teddington, May 1951. 
that sufficiently high amplification is used, a plateau region can be found, in which, over a range of applied potentials, all particles entering the sensitive volume are counted.

Source Mounting. A proportion of the particles emitted by the source is lost by absorption in the source support and by self-absorption in the source itself. This proportion is not easy to assess accurately and must be made as small as possible.

The source should be mounted on as thin a foil as possible. In GeigerMüller types of $4 \pi$ counter this must be electrically conducting, or erratic counting results. In proportional $4 \pi$ counters a non-conducting plastic film is sometimes used, but although proportional counters will operate in this way, the region of zero field which now exists near the foil results in a loss of counts.

Thin aluminium foils are available commercially with thicknesses as low as $0.85 \mu\left(230 \mu \mathrm{g} / \mathrm{cm}^{2}\right)$ and these are convenient for use with the higher energy $\beta$-emitters such as $P^{32}$. A method of clamping such a foil in the removable partition between the two halves of a $4 \pi$ counter is shown in Fig. 5. For lower energies, thin plastic films coated on both sides with evaporated metal are used.Zapon, formvar, nylon or cellulose acetate films a few thousand Ångstroms thick and weighing from 10 to $20 \mu \mathrm{g} / \mathrm{cm}^{2}$ are not difficult to produce. Coatings of evaporated gold, copper or aluminium are used, on both sides, making a total mass of 30 to $40 \mu \mathrm{g} / \mathrm{cm}^{2}$.

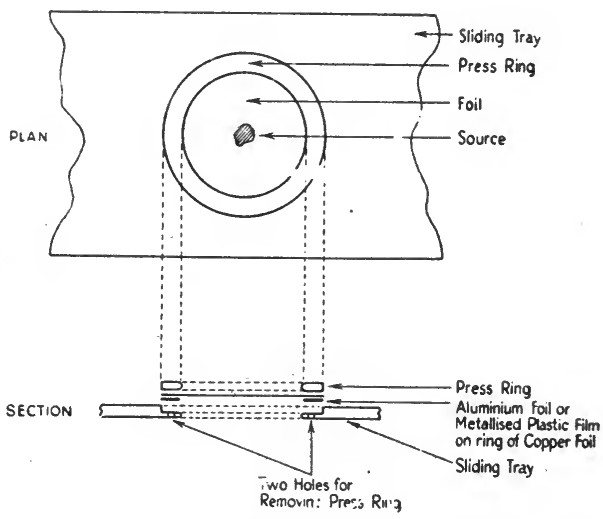

Fig. 5. Method of Source Support in a $4 \pi$ Counter

Absorption and Scattering by the Source Support. It is found that the counting rates in the two halves of the counter are not equal. Some radiations are scattered back by the supporting foil into the half-counter on the same side as the source. These radiations are obviously lost from the other half-counter, as also are those which are totally absorbed in the foil. Some particles are counted in both halves of the $4 \pi$ counter, partly through scattering from one half into the other, and partly, it is believed, because of low energy recoil electrons ${ }^{25}$. from the supporting foil. However, when both halves of the counter are connected together, these various effects tend to be eliminated. A single count is obtained whether a particle is 
counted in one or both halves of the counter. Also particles which are lost in one half-counter by scattering in the supporting foil are recorded in the other half. Only those which are totally absorbed in the foil are missed.

Houtermans et al. ${ }^{26}$ and Smith ${ }^{23}$ have shown that for the thinnest foils, the difference in counting rate between the two half-counters is entirely attributable to scattering in the foil, and that the effects of foil absorption are at first negligible in comparison. For thicker foils (and for low energy $\beta$-particles), absorption corrections have to be made empirically.

A popular method of correction has been to insert on top of the source a second foil exactly similar to the one on which it is mounted. It is then assumed that the total counting losses are doubled by the addition of the second foil. Smith ${ }^{23}$ has shown experimentally that this method overestimates the correction which should be applied for the mounting foil. The most reliable technique, wherever possible is to use a supporting foil or film so thin that absorption errors can be neglected.

Self-Absorption. Losses by self-absorption depend very largely on the uniformity of the source thickness. For high energy $\beta$-particles the source can generally be made thin enough to make the self-absorption correction negligible, but for low energies, the absorption by discrete crystals of an evaporated source becomes appreciable. In these circumstances, the progressive reduction of solid content in a solution to be evaporated is of little avail, since the resulting deposit consists of discrete crystals whose spacing increases, but whose size decreases only slowly as the solid content is reduced.

Self-absorption of this kind begins to become significant for $\beta$-particles with maximum energies below about $0.5 \mathrm{MeV}$ and special precautions are necessary in depositing the sources. We shall discuss these precautions in more detail later.

$4 \pi$ Ścintillation Counters. Scintillation counters, employing a liquid phosphor in which a source can be immersed, have also been used to provide a $4 \pi$ geometry for absolute counting. For this purpose, Belcher ${ }^{27}$ has used solutions of $p$-terphenyl or of $\alpha, \alpha^{\prime}$-dinaphthyl in xylene. The standard concentration used is $5 \mathrm{~g}$ solute per litre of solution. A slow deterioration of efficiency with time occurs, which Belcher attributes to radiation-induced decomposition of the solutions, and he recommends renewing these about once in two weeks.

For absolute $\beta$-counting, the source is sealed between two thin layers of cellulose acetate foil, which is available commercially in thicknesses

26 F. G. Houtermans, L. Meyer-Schützmeister and D. H. Vincent, Zeits. f. Physik 134 (1952) 1 and 9.

27 E. H. Belcher, J. Sci. Instr. 30 (1953) 286. 
from 0.001 inch upwards and is not effected by immersion in the phosphor solutions.

A resolving time of $200 \mu$-seconds is imposed in the recording circuit to eliminate satellite pulses in the photomultiplier and counting rates are corrected for losses in this effective dead time.

The use of a pulse amplitude discriminator to reject thermal noise pulses from the photomultiplier also has the effect of rejecting some $\beta$-particles at the low energy end of the $\beta$-spectrum. A correction is made for these by taking measurements with several settings of the discriminator and extrapolating to zero discriminator level.

Correction for absorption in the enclosing foils is made by repeating the measurements with samples prepared from a common solution mounted on different thickness of foil, and extrapolating the apparent activity per gramme of solution to zero foil thickness.

The two extrapolations limit the accuracy of the method for $\beta$-spectra with maximum energies below about $1 \mathrm{MeV}$. Good agreement with other counting methods has been obtained in measurements of higher energy $\beta$-particles.

General Observations. Absolute measurements with $4 \pi$ counters are almost independent of disintegration scheme provided that at least one highly ionizing particle is emitted per disintegration. Corrections for $\gamma$-rays are unnecessary except in the relatively rare examples of delayed $\gamma$-ray emission (e.g. $\mathrm{Cs}^{137} \rightarrow \mathrm{Ba}^{137}$ ). Positron emitters can also be measured without correction for annihilation radiations.

The Geiger-Müller types can be operated with a minimum of electronic equipment and have very stable performance, but they require a pumping and gas filling equipment. Proportional types need high gain stable amplifiers, but can be operated without special vacuum apparatus by using continuous flow counters operating just above atmospheric pressure.

For energies above $1 \mathrm{MeV}$, all types of $4 \pi$ counter are capable of an accuracy of $2 \%$ or better. At energies below $0.5 \mathrm{MeV}$, the scintillation type is unreliable and in the Geiger-Müller and proportional types the results are strongly dependent on self-absorption in the source.

\section{C. $\beta-\gamma$ Coincidence Method (see also Chapter VII)}

Let us consider a source which undergoes $N$ disintegrations per second, each involving the emission of a $\beta$-particle and one or more $\gamma$-rays. Suppose that this source is measured simultaneously with two counters, one able to detect only $\beta$-particles and one detecting only $\gamma$-rays, see Fig. 6 , if $E$ is the overall efficiency of the $\beta$-counter, it will record:

$$
A=N E \text { counts/second }
$$


and if $F$ is the overall efficiency of the $\gamma$-counter, it will record

$$
B=N F \text { counts/second }
$$

Now out of the disintegrations $A$ per second which are detected by the $\beta$-counter a fraction will be recorded also by the $\gamma$-counter, and if the chances of detection by the two counters are quite independent (e.g. there
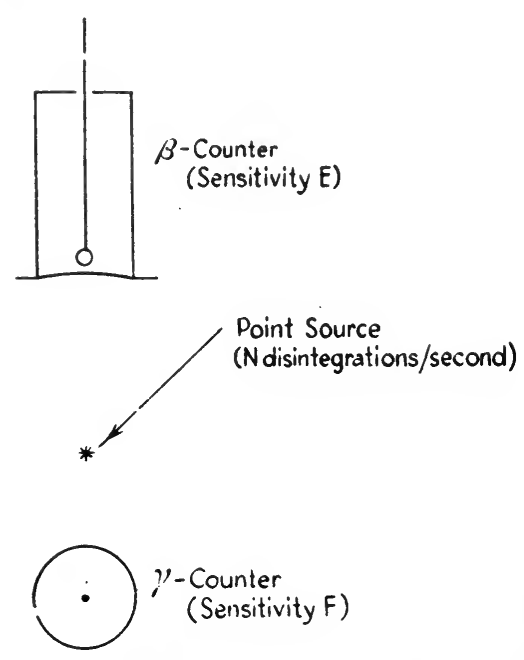

Fig. 6. $\beta-\gamma$ Coincidence Method (Diagrammatic) is no $\beta-\gamma$ angular correlation) this fraction will be $F$, the overall efficiency factor for the gamma-counter. Thus a number of coincidences:

$$
C=N E F \text { per second }
$$

occur, in which disintegrations are recorded simultaneously by both counters.

It is evident that:

$$
A B / C=N
$$

This is the essence of the $\beta-\gamma$ coincidence method, first used by Dunworth 28 to determine disintegration rates, although the principle had been employed by early nuclear physicists to find the efficiency of observers for recording scintillations in a zinc sulphide screen. The method has been reviewed more recently by Barnothy and Forro ${ }^{29}$.

Electronic circuits by which coincidences can be recorded will only be considered functionally here (see Fig. 7). A circuit consists of two separate channels, each carrying pulses from one of the counters. The pulses are

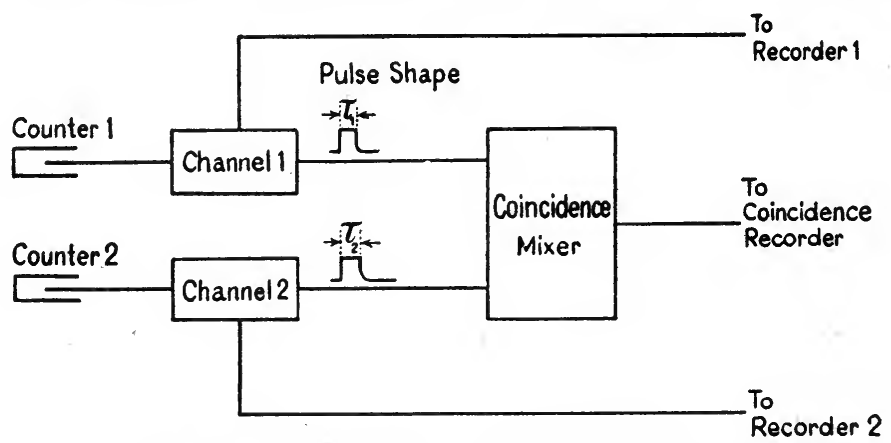

Fig. 7. Coincidence Circuit (Schematic)

28 J. V. Dunworth, Rev. Sci. Instr. 11 (1940) 167.

29 J. Barnothy and M. Forro, Rev. Sci. Instr. 22 (1951) 415. 
fed into a mixer, which delivers an output pulse when pulses arrive simultaneously in both channels.

Resolution Time and Random Coincidences. In practice, each channel continues to operate the mixer for a fixed time after the arrival of a pulse. This is known as the resolution time for the channel and may be denoted as $\tau_{1}$ for channel 1 and $\tau_{2}$ for channel 2 .

A coincidence is recorded whenever a pulse in channel 2 follows a pulse in channel 1 within time $\tau_{1}$, or whenever a pulse in channel 1 follows one in channel 2 within time $\tau_{2}$. The coincidence circuit cannot determine whether pulses correspond to the same disintegration or not. It follows that a number of coincidences will be recorded purely by chance, and it can readily be shown that if $A_{1}$ and $B_{1}$ are the rates of arrival of pulses in channels 1 and 2 respectively, the total random coincidence rate is:

$$
C \text { random }=A_{1} B_{1}\left(\tau_{1}+\tau_{2}\right)
$$

$\left(\tau_{1}+\tau_{2}\right)$ is often called the total resolution time. Very few of these coincidences correspond to observations of the same disintegrations and the random coincidence rate must be subtracted from the observed rate to find the true coincidence rate $C$ of equation 9 . The resolution times $\tau_{1}$ and $\tau_{2}$ are made as small as possible. They must, however, be long enough to. accommodate electronic delays in the separate counters, or true coincidences are lost. Typical values for use with small argon-alcohol filled Geiger-Müller counters are $\tau_{1}=\tau_{2}=0.6 \mu \mathrm{s}$. Much larger values may be necessary for larger counters, or those operating at high gas pressures (e.g. about $5 \mu$ s is necessary when using helium near atmospheric pressure, a common filling for some thin-window counters). The resolution times can be measured by using electronic test gear to introduce pulses. with adjustable spacing into the separate channels. Alternatively the total resolution time can be found from the coincidence rate obtained when the counters are operated with independent sources, or when an artificial delay is introduced into one channel to eliminate true coincidences. Given a reliable circuit, the resolution time should not vary appreciably with counting rate, and only periodic checks are necessary.

General Corrections to be Applied. In a practical arrangement the observed counting rates in the separate channels must be corrected for dead time losses and background counting rates to obtain the true rates $A$ and $B$ from which the disintegration rate can be found. The "background rate" of the $\beta$-counter must include the contribution of $\gamma$-rays from the source and correction is generally made by screening the counter as already described. This method of correction is open to the same objections we have discussed before, but the errors involved are usually 
small and are partly compensated by similar corrections to the coincidence rate. Spurious counts are to be avoided at all cost and external quenching circuits should always be used with (ieiger-Müller counters.

The coincidence rate must also be corrected and the order in which corrections are made is important. The random coincidence rate is subtracted first because it depends only on the recorded rates in the two channels and is not subject to dead time correction. If equation (11) is used for correction, it should be remembered that a few true coincidences may be lost in the dead times following random coincidences. This loss can usually be neglected, especially if the random rate is a small proportion of the total coincidence rate, but may become significant if the random rate is high.

Since the loss of a pulse in either channel can result in a lost coincidence, the dead time correction factor to be applied for coincidence losses is equal to the product of the factors for the two channels, thus:

$$
C_{o}{ }^{\prime} C_{r}=1 /\left(1-A_{r} t_{1}\right)\left(1-B_{r} t_{2}\right)
$$

where $A_{r}, B_{r}$ are the recorded rates in the two channels and $t_{1}, t_{2}$ their respective dead times.

Lastly the coincidence rate is corrected for background. When only one $\gamma$-ray follows the emission of a $\beta$-particle, the background coincidence rate is purely due to cosmic ray coincidences, and can be measured in the absence of the source. If more than one $\gamma$-ray is emitted per disintegration, a further unwanted contribution to the coincidence rate occurs by virtue of $\gamma-\gamma$ coincidences between the two counters. Correction for this is made as before, by screening the $\beta$-counter, and the error involved partly compensates the error in correcting the $\beta$-rate by this method.

Correction for Source Decay during Measurement. When shortlived nuclides are measured, a significant decay in activity may occur during the measuring period. The rates $A, B$ and $C$ of equations 7 to 10 each decay exponentially and if $A_{T}, B_{T} . C_{T}$ are the corrected total numbers of counts, which occur in a period $T$, then the product $A_{T} B_{T}{ }^{\prime} C_{T}$ represents the total number of disintegrations, from which the disintegration rate at any time can be found. Similarly:

$$
A_{0}\left(B_{T} / C_{T}\right)=B_{0}\left(A_{T}^{\prime} C_{T}\right)=N_{0}
$$

where $A_{0}, B_{0}$ are the corrected counting rates at the beginning of the experiment and $N_{0}$ is the initial disintegration rate.

The corrections now become more complicated, however, for the percentage corrections for dead time losses and for the random coincidence rate are changing with time. 
Validity of the Coincidence Method. The coincidence method is only strictly valid for nuclides with simple disintegration schemes, that is those which decay by a single $\beta$-transition followed by $\gamma$-rays. For if two or more alternative modes of disintegration are possible, the efficiencies of both counters will be different for the different modes. Thus rewriting the equations for counting rates:

$$
\begin{aligned}
& A=N\left(E+E^{\prime}+E^{\prime \prime}+\ldots\right) \\
& B=B\left(F+F^{\prime}+\ldots\right) \\
& C=N\left(E F+E^{\prime} F^{\prime}+E^{\prime \prime} F^{\prime \prime} \ldots\right) \\
& A B / C \neq N
\end{aligned}
$$

and

Modifications of the coincidence method have been proposed for nuclides with alternative breakdown schemes. Wiedenbeck ${ }^{30}$ suggested the use of extrapolated absorption curves to correct for window thickness in the $\beta$-counters, thereby making $E, E^{\prime}, \ldots$ nearly equal in equation (14), but the effects of scattering on $\beta$-particles of different energies tend to vitiate this method. The present author ${ }^{31}$ has developed expressions in which relative sensitivities of the counters to $\beta$-particles and $\gamma$-rays are used and their effect on the coincidence rate is deduced. This method is only reliable for small percentage branches, and relies heavily on the accuracy of spectroscopic data.

Provided that only one $\beta$-transition occurs, however, the coincidence method is valid. Complexity in the $\gamma$-emission only affects the mean efficiency of the $\gamma$-counter for disintegrations.

Loss of $\gamma$-rays by internal conversion affects both the $\gamma$-ray counting rate $B$ and the coincidence rate $C$ in equal proportions, and does not affect the resulting value $A B / C$, but conversion electrons can be counted in the $\beta$-counter, producing an artificially high count rate $A$. When the internal conversion coefficient is small, a correction can be made, using an estimated efficiency of the $\beta$-counter for the conversion electrons.

Source Distribution. In all of the above, we have tacitly assumed that each of the counters is equally sensitive to disintegrations occurring in all parts of the source. It can be shown that the relation (9) is true only if one or other of the counters satisfies this condition ${ }^{17,31}$.

It is usually found most convenient to make the $\gamma$-counter the one which is equally sensitive throughout the source, as the self-absorption of $\gamma$-rays in the source can usually be neglected. When tubular Geiger counters are used, the condition is easily fulfilled by evaparating the source on a

so M. L. Wiedenbeck, Phys. Rev. 72 (1947) 974.

J. L. Putman, A.E.R.E. Memo I/M. 26 (1953). 
foil which is then wrapped around the middle of the counter tube. This method also provides the maximum possible $\gamma$-sensitivity. The arrangement has the enormous advantage that the absorption, self-absorption and scattering of the $\beta$-particles has no effect on the final determination of $N$.

Typical Results using Geiger-Müller Counters. When using GeigerMüller counters, the individual counting rates are limited by dead time considerations to about 300 counts/second.

The coincidence rate is then limited by the sensitivity obtainable in the less sensitive counter. This is always the $\gamma$-counter.

For $\mathrm{Co}^{60}$ sources, wrapped around a copper-walled $\gamma$-counter a net efficiency of $\frac{3}{4} \%$ per disintegration is all that can be attained, so that, if the $\beta$-counter is now adjusted to record 300 counts/second, the true coincidence rate not likely to exceed $2 \frac{1}{4}$ counts/second (see equation 9). Evidently more than an hour is necessary to obtain 10,000 counts for a standard statistical error of $\pm 1 \%$.

The optimum activity of the source is that which will result in a counting rate of about 300 counts/second in the $\gamma$-counter. In the above conditions for $\mathrm{Co}^{60}$ it is just over 1 microcurie.

If the total resolution time is $2 \mu \mathrm{s}$, and the counting rates are 300 per second in each channel, the random coincidence rate is 0.18 counts/second, or less than $10 \%$ of the true coincidence rate for $\mathrm{Co}^{60}$. If the $\gamma$-counter is less efficient (as it is for $\mathrm{Au}^{198}$, for example), and if the same counting rates are arranged in the separate counters, then the true coincidence rate is lower, but the random coincidence rate is unchanged.

Use of Other Detectors. When using Geiger-Müller counters, a long counting period is necessitated by the low coincidence rate. In an attempt to speed this up, some workers have used proportional counters as detectors, or even Geiger-Müller counters operating at reduced potentials. In the absence of dead-time, counting rates of the order of $10^{4}$ counts/second or more can be accommodated and few, if any, spurious counts occur. Very stable power supplies and amplifiers are essential for reliable results. The sensitivity of the $\gamma$-counter is still low, and to gain any advantage over Geiger-Müller counters, both the $\beta$ - and $\gamma$-counters have to be operated at comparable rates (for the coincidence rate is limited by the lower efficiency). Thus the random coincidence rate, which is proportional to the product of these rates: becomes much higher than the true coincidence rate and corrections for it tend to be less accurate.

Scintillation counters, with their much higher $\gamma$-ray efficiency can be used to detect the $\gamma$-rays, with great improvements in the coincidence rates obtainable. These counters can also be operated at very high counting rates when suitable phosphors are used, but the user should then beware 
of secondary pulses in the photomultiplier ${ }^{27.6}$. The best results are obtained for high energy $\gamma$-rays, for which the thermal noise pulses in the multiplier can be biassed out. Scintillation counters can be used for both $\beta$ - and $\gamma$-ray detectors by using thin phosphors for the $\beta$-particles.

Advantages and Limitations of the Method. The $\beta-\gamma$ coincidence method has the advantage that it can be used without specially designed counters and involves no prior assumptions about their sensitivities. It is relatively independent of the absorption of $\beta$-particles in the source, especially if the $\gamma$-counter is made equally sensitive to all parts of it.

The main limitation of the method is that it is more or less restricted to isotopes with a simple $\beta$-decay scheme.

\section{D. $\gamma-\gamma$ Coincidence Method}

Nuclides which emit two $\gamma$-rays of nearly equal energies in cascade can also be measured by using the coincidences between two $\gamma$-counters. To do this, two identical $\gamma$-counters are used so that their relative efficiencies to $\gamma$-rays of different energies are equal. Let the efficiencies for the separate $\gamma$-rays be $F_{1}, F_{2}$ in one counter and $G_{1}, G_{2}$ in the other and let

$$
\frac{F_{2}}{F_{1}}=\frac{G_{2}}{G_{1}}=(1-\delta) \text {. }
$$

Now if the counting rates in the separate counters are $X, Y$ respectively:

$$
\begin{aligned}
& X=N\left(F_{1}+F_{2}\right)=N F_{1}(2-\delta) \\
& Y=N\left(G_{1}+G_{2}\right)=N G_{1}(2-\delta)
\end{aligned}
$$

Where $N$ is the disintegration rate of the source. The coincidence rate is now :

and

$$
Z=N\left(F_{1} G_{2}+F_{2} G_{1}\right)=2 N F_{1} G_{1}(1-\delta)
$$

$$
\frac{X Y}{Z}=N(2-\delta)^{2} / 2(1-\delta)=2 N\left(1+\delta^{2} / 4\right)
$$

It is seen that if $\delta$ is small, the error involved in assuming $X Y \mid Z=2 N$ is very small. The method has been used successfully for $\mathrm{Co}^{60}$.

In using this method, care is necessary to avoid scattering of $\gamma$-rays from one counter into the other by the Compton process, as this results in spurious coincidences. If scintillation counters are used, pulses due to the lower energy scattered $\gamma$-ray photons can be eliminated by biasing.

Correction is also necessary for angular correlation between successive $\gamma$-rays, and measurements are sometimes made with two dispositions with different angles (e.g. $180^{\circ}$ and $90^{\circ}$ ) between the directions of the $\gamma$-rays to be measured in coincidence. 


\section{E. Counters for Radioactive Gases}

For measurements of very low energy $\beta$-particles the radioactive material is sometimes introduced into the counter itself in gaseous or vaporous form. The gas must be suitable for operation in a Geiger-Müller or proportional counter, or must be compatible with some other satisfactory counter filling. There is now no loss of particles by absorption, each disintegration in the sensitive volume of the counter ideally results in a count.

Filling Mixtures. Argon-alcohol filled Geiger-Müller counters have been operated successfully by Engelkemeir and Libby ${ }^{32}$, with small additions of radioactive gas, to measure the absolute activity of Auger electrons from $\mathrm{A}^{37}$ and $\beta$-particles from $\mathrm{Kr}^{85}$. J. F. Cameron in unpublished work has measured tritium in a similar way.

Geiger-Müller counters filled with a mixture of $\mathrm{CO}_{2}$ and $\mathrm{CS}_{2}$ have been found satisfactory ${ }^{33,34}$ for measurements of $\mathrm{C}^{14}$. A typical filling comprising $20 \mathrm{~cm}$ pressure $(\mathrm{Hg})$ of $\mathrm{CO}_{2}$ with $2 \mathrm{~cm}$ of $\mathrm{CS}_{2}$ can be used at operating potentials around 3000 volts. The $\mathrm{CS}_{2}$ is not an efficient quenching agent and an external quench circuit is necessary to prevent spurious counts. Butane has been used ${ }^{35}$ as a filling for Geiger-Müller counters to measure tritium.

Bernstein and Ballentine ${ }^{36}$ have discussed the measurement of $\mathrm{C}^{14}$, $\mathrm{S}^{35}$ and $\mathrm{H}^{3}$ in proportional counters, using a series of gas fillings, particularly a filling of $\mathrm{H}_{2} \mathrm{~S}-\mathrm{CH}_{4}-\mathrm{CO}_{2}$, suitable for counting either $\mathrm{C}^{14}$ as $\mathrm{CO}_{2}$ or $\mathrm{S}^{35}$ as $\mathrm{H}_{2} \mathrm{~S}$.

Craythorn ${ }^{37}$ and Loosemore have used $\mathrm{C}_{2} \mathrm{H}_{2}$ for measuring $\mathrm{C}^{14}$ in proportional counters. The presence of two carbon atoms per molecule gives higher count rates at comparable pressures. Counters filled with $\mathrm{C}_{2} \mathrm{H}_{2}$ at pressures from 1 to $1 \frac{1}{2}$ atmospheres have been operated at potentials from 6000 to 7000 volts. The method is being developed for the measurement of very low specific activities of $\mathrm{C}^{14}$ in age determinations. A method of preparing pure acetylene from carbon samples has been described by Barker ${ }^{38}$.

Tritium can also be counted in a proportional counter either as hydrogen

32 A. G. Engelkemeir and W. F. Libby, Rev. Sci. Instr. 21 (1950) 550.

33 S. C. Brown and W. W. Miller, Rev. Sci. Instr. 18 (1947) 496.

34 R. C. Hawkings, R. F. Hunter and W. B. Mann, Canad. J. Res. B27 (1949) 555 .

35 R. F. Glascock, Radioisotope Techniques, Vol. I, H.M.S.O. (1953) p. 388, and Nucleonics 9, (No. 5), (1951) 28.

36 W. Bernstein and R. Ballentine, Rev. Sci. Instr. 21 (1950) 158.

37 A. R. Craythorn, Nature 172 (1953) 632.

38 H. Barker, Nature 172 (1953) 631. 
gas or methane. White and others ${ }^{39}$ have described a quantitative method for preparing methane from tritiated samples.

Various filling techniques are described by Bernstein and Ballentine ${ }^{36}$. The most convenient fillings, especially for absolute measurements, are those which can be condensed in a liquid air trap. In filling the typical gas counter shown in Fig. 8, the closed glass tube known as "cold finger" is immersed in liquid air. The filling gases, in quantities measured outside the counter, can now be admitted one by one through the tap in the other end of the counter. Each is condensed in the cold finger. The liquid air trap is then removed after isolating the counter with the tap.

Sensitivity and the Sensitive Volume. The sensitive volume of a gas counter is approximately that enclosed by the cathode if the centre wire passes right through it. In some designs, as in Fig. 8, its length may be limited to the exposed length of the centre wire. End effects, mainly due to the inhomogeneity of fields at the counter ends, make it difficult to estimate the length of the sensitive portion precisely. Also some $\beta$-particles which enter the sensitive volume from outside the ends make the effective length a function of $\beta$-particle energy. Corrections for end effects are best made empirically, by using two counters of equal diameter and identical end design, but of different lengths. The difference in counting rates,

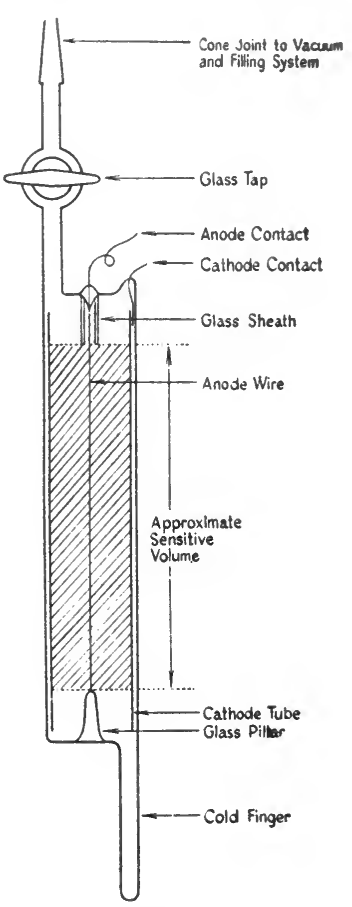

Fig. 8.

Typical Gas Counter obtained with samples of a radioactive gas at the same pressure, corresponds to a length equal to the difference in length of the two counters, and the effect is thus eliminated.

A loss of counts also occurs near the walls of the cathode, corresponding to those $\beta$-particles which escape without producing ionization in the counter. This also depends on the $\beta$-particle energy, being small for lower energy $\beta$-particles because of their higher specific ionization. Engelkemeir and Libby ${ }^{32}$, in an investigation of end and wall corrections, showed by using counters of different diameters that the percentage "wall loss" is inversely proportional to the cathode diameter $d$. This is to be expected, since the wall loss is a surface phenomenon which is proportional to $d$, whereas the total counting rate is proportional to $d^{2}$ at a given pressure. 
Wall losses found for a typical counter were approximately $5 \%$ for $\mathrm{C}^{\mathbf{1 4}}$ and $10 \%$ for $\mathrm{Kr}^{85}$ under similar operating conditions.

Having determined the effective sensitive volume $v_{s}$ for a given nuclide, the activity per unit volume of a sample can be deduced at once from the measured counting rate $r$ (corrected for dead time losses and background). To find the total disintegration rate $A$ in the counter, the total volume $v_{T}$ must be measured as well, then:

$$
A=r v_{T} / v_{s}
$$

Carbon Age Measurements with $\mathrm{C}^{14}$. Libby and his co-workers have established ${ }^{40}$ that the residual $\mathrm{C}^{14}$ activity in timber can be used to estimate its age in terms of the half-life of $\mathrm{C}^{\mathbf{1 4}}$. In new wood the specific activity is 15.3 disintegrations per minute per gram of carbon and of course in ancient samples it is much lower.

The first essential in measuring such low specific activities is to reduce the background of the detector, whilst maintaining a high efficiency for the low energy $\beta$-particles from $\mathrm{C}^{14}$. Normal backgrounds have been reduced by factors of 15 to 20 as follows: The cosmic ray background is reduced by surrounding the detector with a ring of cosmic ray counters in anticoincidence with it. The $\gamma$-ray background is reduced by heavily screening the arrangement with iron: iron is preferable to lead because it contains less radioactive impurities.

The $\mathrm{C}^{14}$ detector itself may either be a gas counter or one in which the carbon is spread thinly around the inside of the cathode. Libby has used both types and employed $\mathrm{CO}_{2}$ for gas counting. Craythorn ${ }^{37}$ and Loosemore have used acetylene, as already described, with advantages in sensitivity of the gas counter. An alternative method of detection has been developed recently by Arnold ${ }^{41}$ in which a scintillation counter is the detector, using ethanol, synthesised from the $\mathrm{C}^{14}$-bearing samples as the phosphor.

In all of these measurements, the material to be counted need not be prepared quantitatively from the wood samples, since only its specific activity is required.

\section{F. Preparation of Sources for Absolute Calibration}

The form of a source and the activity most convenient for absolute measurements are seldom the form or the activity required in a radioactive standard. It is usual therefore to prepare sources for absolute

40 W. F. Libby, Radiocarbon Dating, Univ. of Chicago Press (1952).

41 J. R. Arnold, "Scintillation counting of natural radiocarbon" Paper at 4th Scintillation Counter Symposium, Washington (Jan. 1954). 
measurement from measured quantities of a solution which can then be used for dispensing standard sources. Uniform distribution of the radioactive material is then easily ensured, dilutions are simple and small masses of dissolved material can be dispensed accurately by measuring relatively large quantities of solution.

The solutions should be sterile and a trace of formaldehyde is sometimes added ${ }^{42}$. The chemical form must be such that the radioactive material stays in solution without precipitation or adsorption to the walls of containing vessels. To avoid adsorption losses from carrier free solutions it is usual to add as much inactive carrier material as can be tolerated without introducing appreciable self-absorption into the dry sources prepared from the solutions. It is desirable to provide at least enough carrier material to form a few monomolecular layers over all contacting surfaces. The $\mathrm{pH}$ of solutions is also adjusted to keep the radioactive ions in solution. Whenever possible, all containing vessels should be rinsed before use with a sample of the radioactive solution to be used in them, and the rinsings should be thrown away. This permits the establishment of a surface equilibrium before the solution to be measured is introduced, and reduces adsorption losses.

Samples of the radioactive solutions are best dispensed by weighing rather than by volumetric measurement. The weighing method has the advantage of being independent of the specific gravity of the solution and of its temperature. A pyknometer of capacity 1 or $2 \mathrm{ml}$ like the one illustrated in

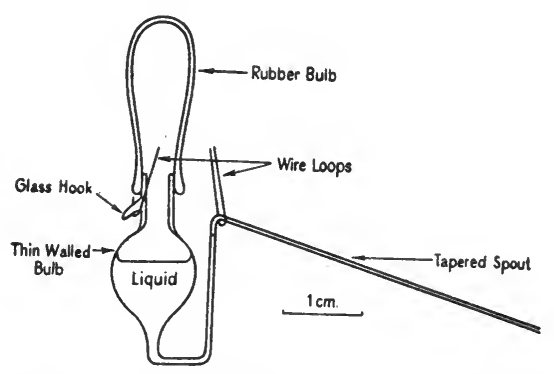

Fig. 9. Pyknometer for Dispensing Radioactive Solutions

Fig. 9 can be made in a few minutes from a piece of hard glass tube and can be thrown away after use to avoid contamination. Dilutions are also usually made by weighing.

The simplest method of depositing a dry radioactive source is by evaporation under a hot lamp. This method is generally quite satisfactory if the $\beta$-particle energy is high. For lower $\beta$-energies, self-absorption in crystals of the source may become important and microscopic examination of the deposited source should be made. The deposition of a small quantity of insulin before applying the solution for evaporation has been found by some workers ${ }^{43}$ to improve the distribution of the source.

For some sources the radioactive material is deposited as an insoluble

42 A. M. Wildblood, Nature (News and Views), 171 (1953) 1141.

43 L. M. Langer, Rev. Sci. Instr. 20 (1949) 216. 
precipitate by adding a reagent before evaporation. This is especially necessary if the radioactive solute is volatile. The chief drawback of precipitation lies in the additional solid content introduced into the source. This is sometimes more than compensated by the greater uniformity of the souice as compared with directly evaporated sources.

Very uniform thin metallic sources can be prepared by electrolysis but it is difficult to ensure that the deposition is quantitative.

Freeze drying is sometimes applied to produce low crystal sizes and even deposition. When a solution is freeze-dried on to a very thin supporting film, however, the film is sometimes strained and broken, as seen by microscopic examination of the dried source. To avoid this, Houtermans et al. ${ }^{26}$ have developed a method in which partial evaporation in a desiccator at reduced pressure is followed by freeze drying at a lower pressure just before eraporation is completed.

\section{G. Choice of Counting Method}

The preferred method of counting for measuring the activity of a particular nuclide depends on the type and energies of its radiations.

Pure $\beta$-emitters and Positron Emitters whose radiations have maximum energies above $0.5 \mathrm{MeV}$ are best measured with $4 \pi$ counters. Source preparation is generally a simple matter, especially for energies above $1 \mathrm{MeV}$.

When the maximum $\beta$-particle or positron energy is below $0.5 \mathrm{MeV}$, $4 \pi$ counters can still be used reliably for energies down to about $0.15 \mathrm{MeV}$, but increasing precautions are necessary ín source and foil preparation as the energy is reduced. In this range of energies internal gas counters compete for favour with $4 \pi$ counters, and for still lower energies they are to be preferred if suitable radioactive gases can be prepared. Wall corrections become small at the lower energies, whilst in $\mathbf{4} \pi$ counters, absorption corrections become large.

$\beta-\gamma$ emitters with simple decay schemes are suitable for measurement by the $\beta-\gamma$ coincidence method. As this can be made almost independent of self-absorption, sources are very easily prepared. Accurate corrections must be made for internal conversion of the $\gamma$-rays.

If low percentage branches in the breakdown scheme exist, corrections can be made to enable the coincidence method to be used. For nuclides with complex $\beta-\gamma$ breakdown schemes, coincidence methods are best avoided.

Decay by Electron Capture is probably the most difficult type of disintegration to measure absolutely. At the time of writing, methods are still being studied. The characteristic X-rays which are emitted after 
electron capture can be detected with $\mathrm{X}$-ray counters of the proportional type or with Geiger-Müller counters, but with low efficiency. The X-ray energies are generally too low for the reliable use of scintillation counters. The Auger electrons associated with the $\mathrm{X}$-rays can be measured with $100 \%$ efficiency in internal gas counters and, within the limits of knowledge of the Auger electron yield, this enables the disintegration rate in radioactive gases to be deduced. Auger electrons can be detected in $4 \pi$ counters, but self absorption losses, even in the thinnest sources, are too unreliable to allow the use of this method for absolute determinations.

Electron capture nuclides which also emit $\gamma$-rays can be calibrated by $\mathrm{X}-\boldsymbol{\gamma}$ or $\mathrm{e}^{-}-\boldsymbol{\gamma}$ coincidence methods.

\section{$\S$ 4. Calorimetric Methods}

The first calorimetric measurements of radioactivity were made in 1903 , when Curie and Laborde ${ }^{44}$, used a Bunsen ice calorimeter, to measure the rate of energy release from radium. Calorimeters have since been designed to measure the rate of energy dissipation by $\alpha$-particles ${ }^{45}, \beta$ particles ${ }^{46}$ and $\gamma$-rays ${ }^{47}$. Existing methods have been reviewed by Myers ${ }^{48}$ and a book by Swietoslawski ${ }^{49}$ explains the principles of measurement for low rates of heat dissipation.

Calorimetric measurements are mainly of two types. In the isothermal method the calorimeter is maintained at a constant temperature and the heat evolved is measured by changes of state which it produces, e.g. by evaporation of a liquid. In the adiabatic method the calorimeter is isolated as well as possible from its surroundings, so that heat transfer cannot take place, and changes in its temperature are recorded.

\section{A. Isothermal Calorimeters}

Curie and Dewar ${ }^{50}$ laid the foundations of modern isothermal methods in 1904, when they used the evaporation of liquid nitrogen and liquid hydrogen to measure the heat dissipated by $\alpha$ - and $\beta$-particles from radium. The essentials of their apparatus are shown in Fig. 10.

Modern isothermal measurements are generally refinements of Curie and Dewar's technique. We would mention particularly the microcalorimeter developed by Cannon and Jenks ${ }^{46}$ for $\beta$-emitters. The calorimeter

44 P. Curie and A. Laborde, Comptes Rendus 136 (1903) 673.

45 J. W. Stout and W. M. Jones, Phys. Rev. 71 (1947) 582.

46 C. V. Cannon and G. H. Jenks, Rev. Sci. Instr. 21 (1950) 236.

47 C. V. Cannon and G. H. Jenks, U.S.A.E.C., Mon. R. 261 (1947).

48 O. E. Myers, Nucleonics 5, (No. 5), (1949) 37.

49 W. Swietoslawski, Microcalorimetry, Reinhold, New York, (1946).

50 P. Curie and J. Dewar, Proc. Roy. Inst. 17 (1904) 389 and 581. 
flask was immersed in a closed Dewar ressel of liquid nitrogen in which a constant temperature was maintained by careful control of the pressure. The whole was immersed in a third flask of liquid nitrogen exposed to the atmosphere. The volume of gas evolved in the calorimeter flask was measured by the displacement of a mercury pellet in a horizontal capillary tube at constant temperature and pressure.

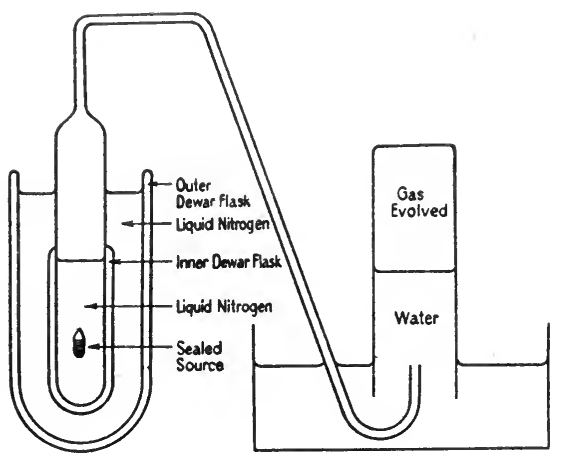

Fig. 10. Liquid Nitrogen Calorimeter of Curie and Dewar

Fig. 11. Bayly's Differential Calorimeter

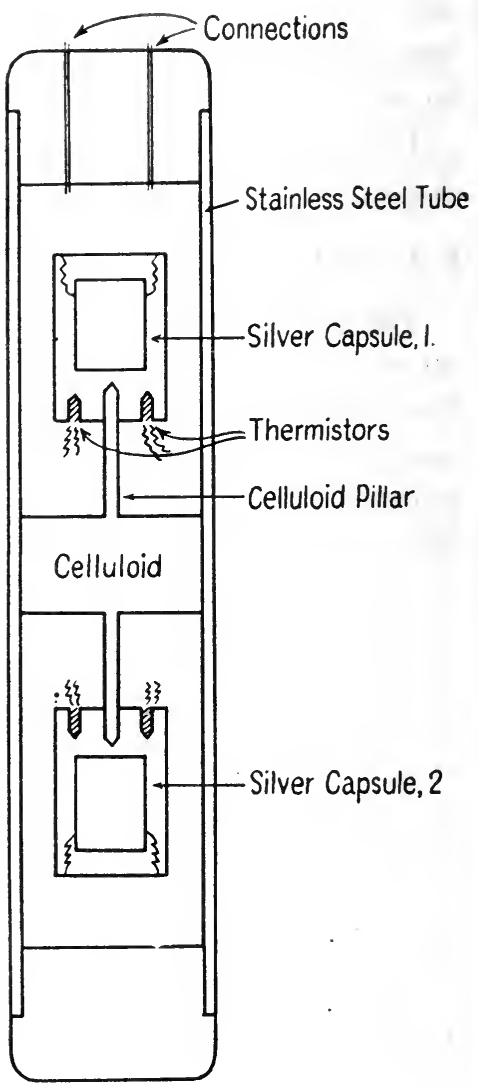

The rate of gas evolution was calibrated with a controlled source of energy. The radioaictive source in the inner calorimeter was replaced by a resistor through which a measured electrical current was passed (the wires feeding the resistor were permanently fixed so that heat leakage along them was common to all measurements). The potential developed across the resistor was also measured and the heat dissipated in watts was deduced. By this means Cannon and Jenks calibrated the calorimeter from $2 \times 10^{-5}$ to $2.5 \times 10^{-4}$ watts. They found that $24.0 \mathrm{mC}$ of $\mathrm{P}^{32}$ dissipated heat at the rate of $9.75 \times 10^{-5}$ watt and claimed an accuracy better than $1.5 \%$ in this measurement. The disintegration rate could thus be 
found to within \pm 2 or $3 \%$, allowing for errors in determining the mean energy per disintegration. After making a correction of $1.3 \%$ for the estimated loss of energy by Bremsstrahlung escaping from the calorimeter, the source activity deduced agreed within these limits with that found by counting methods ${ }^{51}$.

\section{B. Differential Calorimeter}

A different type of calorimeter developed by Bayly ${ }^{52}$ is shown in Fig. 11. In this the source is enclosed in one of two small silver capsules about $1.2 \mathrm{~cm}$ in diameter, symmetrically arranged on celluloid pillars in two separate enclosures in a stainless steel tube. Controlled electrical heating is applied to the other capsule.

The temperatures of both capsules now rise slightly above their surroundings, and the difference between their temperatures is indicated by thermistors mounted in the bodies of the two silver capsules. Each capsule carries two thermistors, which are connected to form the opposite arms of a resistance bridge.

The power supplied to the second calorimeter is adjusted until the bridge is balanced, indicating equal temperatures in the two capsules. A control experiment with electrical heaters in both silver capsules is used to correct for unbalance in characteristics of the capsules.

In operation, the calorimeter is shielded from sudden changes in temperature by surrounding it with two concentric cylinders of water, lagged with rock wool. Since the measurement is a differential one, the actual temperature of the water is not critical, nor is the result seriously affected by slow changes in the temperature of the surroundings.

The sensitivity limit of the calorimeter is stated to be less than $10^{-5}$ watt. A $\mathrm{P}^{32}$ source of activity $116 \mathrm{mC}$ was measured in the calorimeter and the estimated disintegration rate agreed to within experimental error $( \pm 2 \%)$ with measurements by counting methods.

\section{$\S 5$. Measurement of Charge Lost by a Source}

A source which emits $\beta$-particles or electrons loses the negative electronic charge $1.602 \times 10^{-19}$ coulombs for each particle emitted. Thus a 1 millicurie $\beta$-source, assumed to lose one $\beta$-particle per disintegration, loses negative charge at the rate of:

$$
\begin{aligned}
I & =3.7 \times 10^{7} \times 1.602 \times 10^{-19} \text { coulombs/second ? } \\
& =5.93 \times 10^{-12} \mathrm{amps}
\end{aligned}
$$

51 L. R. Zumwalt, C. V. Cannon, G. H. Jenks, W. C. Peacock and L. M. Gunning, Science 107 (1948) 47.

52 J. G. Bayly, Canad. J. Res. A28 (1950) 520. 
By measuring the rate of loss of charge, a direct measurement of the disintegration rate is obtained which is quite independent of counting techniques.

The chief difficulty of the method lies in the emission of large numbers of secondary electrons which are ejected by collisions of the $\beta$-particles with atoms of the source. These secondary electrons are mainly of low energy (below $200 \mathrm{eV}$ ), but increase the loss of charge very considerably unless they are prevented from leaving the source.

A method has been developed by Failla and others ${ }^{53}$ by which the charge loss can be measured reliably.

The source is supported on an aluminium foil 2.5 microns thick, which is mounted on a wire ring. This is suspended inside a large metal drum by a conducting quartz fibre which is well insulated from the drum. The drum is maintained at earth potential and the current is measured with a Lindemann electrometer, using a null method, so that the source assembly is kept at the same potential.

Just inside the drum, and insulated from it, a grid is mounted which can be biased to a negative potential to repel secondary electrons back into the source. Failla and his co-workers found that a potential of -300 volts was sufficient to do this, and such a potential would have a negligible effect on the emission of primary $\beta$-particles.

There is a small correction, which is difficult to evaluate, for the secondary electrons which are released by the collision of $\beta$-particles with the grid and which return to the source by virtue of the applied bias. Secondary electrons formed by collisions with the outer cylinder are prevented by the grid potential from reaching the source.

The whole assembly is evacuated for measurements, to avoid currents due to air ionization. A high vacuum is not necessary. Activities of the order of $0.1 \mathrm{mC}$ can be measured by this means, and the accuracy is estimated at $\pm 5 \%$ for $\mathrm{P}^{32}$.

\section{$\S 6$. Use of Ionization Chambers}

The continuous current chambers used to measure the ionization produced by radiations are of two main types, designed for measurements with $\beta$ - and $\gamma$-radiation respectively. The design and operation of ionization chambers has been described in detail by Rossi and Staub ${ }^{5}$ and an account of their use for absolute measurements of sources has been given by Gray ${ }^{54}$.

53 G. Failla, H. H. Rossi, R. K. Clark and N. Baily, A.E.C.D. 2142.

54 L. H. Gray, Brit. Jour. Radiol. 22 (1949) 677. 
A. $\gamma$-Ray Ionization Chambers

Gamma ray chambers are generally used to measure "quantities of radiation" in röntgens, or can be calibrated in these units.

The röntgen was defined in 1950 as "the quantity of $\mathrm{X}$ - or $\gamma$-radiation such that the associated corpuscular emission per 0.001293 gram of air produces, in air, ions carrying 1 electrostatic unit of quantity of electricity of either sign": that is 1 röntgen produces 1 e.s.u. of positive and of negative charge by ionization in $1 \mathrm{~cm}^{3}$ of dry air at $0^{\circ} \mathrm{C}$ and $760 \mathrm{~mm}$ $(\mathrm{Hg})$ pressure.

The quantity of radiation or "dose" measured in this way depends both on the number of radiations passing through a specified gas volume and on their ionizing power. The "source strength", that is the $\gamma$-ray dose rate produced by the source at a given distance, can be measured directly and is usually quoted in rhm (röntgens per hour at 1 metre from the source). This is a measure of the effectiveness of the source and includes the effects of self absorption of $\gamma$-rays within it.

If corrections are made for absorption effects, however, the source strength is directly related to the disintegration rate and the quantum energy, and Marinelli, Quimby and Hine ${ }^{55}$ have shown how it can be calculated. Fig. 12 shows the source strength in rhm per curie for various $\gamma$-ray energies from a point source (that is one without self-absorption).

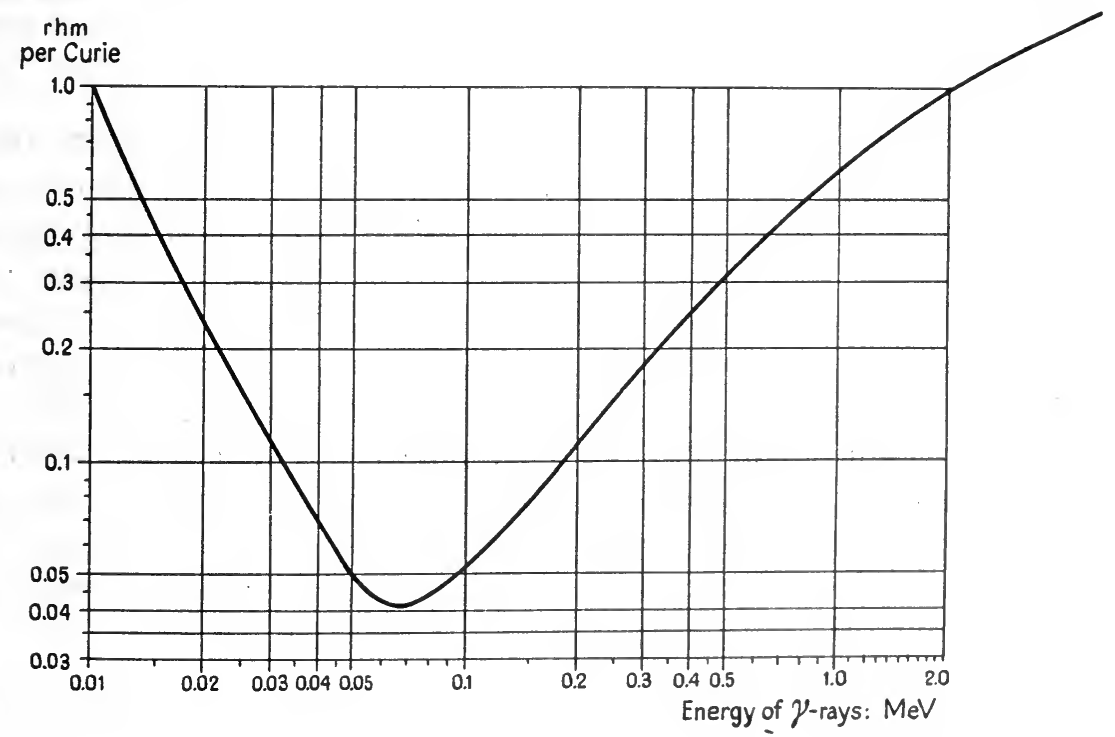

Fig. 12. Source Strength per Curie for $\gamma$-ray Sources (adapted from I. D. Marinelli, E. H. Quimby and G. J. Hine ${ }^{55}$ ) 
A factor sometimes quoted is $k$, the dose rate in röntgen/hour at $1 \mathrm{~cm}$ from $1 \mathrm{mC}$. The numerical value of $k$ for single $\gamma$-rays is 10 times the source strength in $\mathrm{rhm} /$ curie (e.g. $k \doteqdot 6$ for $1 \mathrm{MeV} \gamma$-rays). Provided that the disintegration scheme and the $\gamma$-ray energies of a nuclide are accurately known, the disintegration rate of a source can now be found from a measurement of source strength. Alternatively if the disintegration 'rate is found by other measurements, the $k$ factor can be found experimentally by measuring the source strength. Values of $k$ for a number of nuclides are quoted by Perry ${ }^{56}$ and by Sinclair ${ }^{57}$ and others.

Absolute determinations of dose rate in röntgens can be made by using the free air chamber, which is shown diagrammatically in Fig. 13. A narrow

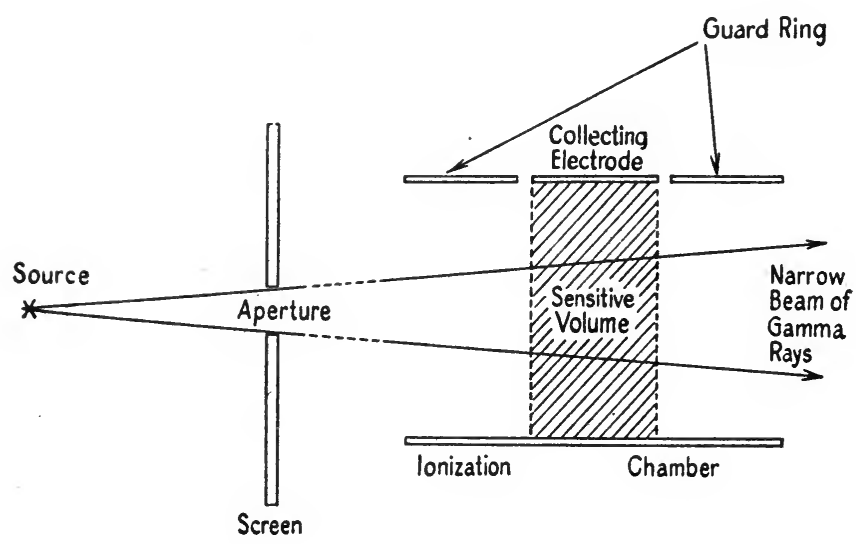

Fig. 13. Essentials of Free Air Cham. ber (Not to Scale)

pencil of radiation from the source is defined by a small aperture and is arranged to pass between the electrodes of an ionization chamber in such a way that no primary or secondary radiations can strike the electrodes. It is a necessary condition that the $\gamma$-rays shall be in equilibrium with the secondary electrons they produce in air, and this condition, together with the avoidance of scattered radiations from the edges of the aperture, makes the dimensions of the equipment necessarily large. Taylor and Singer ${ }^{58}$, by using a pressure of 10 atmosphere of air have been able to reduce the dimensions and have used the modified system to find the $k$-factor for radium, which they measured as $8.2 \mathrm{r} / \mathrm{hr}$ at $1 \mathrm{~cm}$ from $1 \mathrm{mg}$. Various figures have been quoted, from 8.2 to 8.5. In reviewing the work, Gray ${ }^{54}$ adopts a mean figure of 8.4 .

\footnotetext{
56 IV. E. Perry, Standardisation of Radioactive Isotopes, N.P.L. (Brit.) Report (1953).

57 W. K. Sinclair, N. G. Trott and E. H. Belcher, Brit. Jour. Radiol., 27, (1954) 565.

58 L. S. Taylor and G. Singer, Amer. Jour. Röntgenol. 44 (1940) 428.
} 
For routine determinations of source strength much smaller chambers, often called thimble chambers, are used. These usually have graphite walls and electrodes, although "air-wall" chambers have been made, whose wall materials have a mean atomic number approaching that of air. The wall thickness is made sufficient for equilibrium with secondary radiations to be established. The first thimble chamber, due to Gray ${ }^{59}$ contained an air volume of about $75 \mathrm{~mm}^{3}$. The current obtained in such a chamber for a given dose rate depends upon the gas volume and pressure, the mean energy $W$ dissipated per ion pair formed in air ${ }^{60,61,62}(=32.5 \mathrm{eV}$ approximately), and the mass stopping power $S$ of the walls for electrons, relative to that of the air filling.

The usual practice is to calibrate the thimble chamber by comparing the current obtained from a source with that from a standard radium sample. The measurement of the source then depends on the activity and $k$ factor assumed for the radium but is independent of corrections for geometry, chamber volume and air pressure. The measurement is almost independent of the mass stopping power of the walls, but is only completely so if the wall material is equivalent to air in mean atomic number. The corrections to be applied on this account have been discussed in detail by Gray ${ }^{54}$.

For reliable measurements with $\gamma$-ray ionization chambers, activities of the order of a few millicuries are necessary.

\section{B. $\beta$-Particle Ionization Chambers}

Ionization chambers can also be used to find the rate of energy dissipation in a medium from a uniformly distributed source of $\beta$-particles. The measurement depends on Gray's principle of equivalence ${ }^{63}$ which states essentially that the distribution of fast electrons in a medium is not disturbed by the introduction of a small cavity in it.

Thus if a $\beta$-emitting source is uniformly distributed through a liquid or solid medium with a small air cavity, the relative energy dissipations in the medium and in the air cavity will be in proportion to their stopping power for electrons. The energy dissipated in the cavity can be found by building it in the form of a small ionization chamber. The ionization current is then a measure of the energy dissipation, for each ion pair formed in the cavity corresponds to the dissipation of about $32.5 \mathrm{eV}$ of energy ${ }^{60,61,62}$.

L. H. Gray, Proc. Roy. Soc: A156 (1936) 578.

W. Binks, Reports Prog. Phys. 3 (1936) 347.

S. C. Curran, A. L. Cockeroft and G. M. Insch, Phil. Mag. 41 (1950) 517.

J. M. Valentine, Proc. Roy. Soc. A211 (1952) 75.

L. H. Gray, Proc. Roy. Soc. A122, (1928) 647. 
Gray ${ }^{51}$ has used an ionization chamber with a gelatin lining over the inside of the walls and on the central conductor. The gelatin is made up with the solution whose activity is to be measured and the lining is cast in two halves in an alkathene shell. In order to eliminate errors in the estimated volume of air enclosed in the chamber, this is calibrated by measuring the ionization current produced by a radium source.

An alternative arrangement due to Failla ${ }^{53}$ uses a solution of the source in water as one electrode of an ionization chamber. A flat metal collecting electrode with a guard electrode is mounted above the solution, parallel to its surface, and the spacing between the solution and the metal plate is found from a measurement of their electrostatic capacity. If the upper electrode could be made of water, the ionization current measured would be exactly half of that which would correspond to a cavity in a continuous medium. In practice the collecting electrode is made of thick aluminium, and a correction is necessary for the difference in back-scattering between aluminium and water. This can be made experimentally by a separate measurement: it was found to be $15 \%$ for $\mathrm{P}^{32}$.

Measurements of disintegration rate by these means are of secondary importance, since their accuracy is limited by knowledge of the mean energy of the $\beta$-particles and by the accuracy of the value assumed for $W$, the mean energy per ion pair. They are useful, however, in providing independent information as to the mean $\beta$-energy of nuclides when the disintegration rate is determined by other means. 
APPENDICES 



\title{
$\gamma$-RAY ABSORPTION COEFFICIENTS
}

\author{
CHARLOTTE M. DAVISSON
}

(Cf. Сн. II)

\section{TABLE I}

\section{Absorption Coefficients of Various Elements}

The absorption coefficients given in this set of tables have for the most part been calculated from theories concerning the interaction of $\gamma$-radiation with matter, as discussed in Chapter.II. Due to approximations in the theories, and the applicability of different theories to different ranges of energy and atomic number, the accuracy of the values tabulated here is not the same throughout. Interpolations and extrapolations made between different ranges of energy and atomic number have been such that all the values in the table fall on a smooth curve within $1 \%$. However, in some ranges, particularly low energy and low $Z$, the true accuracy may not be better than $10 \%$.

The values in this table can be compared with experimental values to determine the energy of a $\gamma$-ray, or to check the accuracy and validity of the theories if the $\gamma$-ray energy is known. They can also be used in shielding problems to find the amount by which a given absorber will reduce the intensity of the primary beam.

All such measurements or calculations are based on eq. (2) of Ch. II which can be rewritten as

$$
\log B=-\mu x+\log B_{0}
$$

Since experimentally $B$ and $B_{0}$ are proportional respectively to the response of a $\gamma$-ray detector with and without an absorber between source and detector, the logarithm of the measured response, plotted against the absorber thickness should yield a straight line whose slope is the absorption coefficient for that particular absorber. Such a plot will not yield a straight line if $\gamma$-rays of different energies are emitted by the source and counted by the detector, or if $\gamma$-rays scattered by surrounding objects, from within the source, or from the absorber, can reach the detector. Experimental measurements must either eliminate or account for such $\gamma$-rays ${ }^{2}$.

1 Tables are from G. R. White, unpublished Natl. Bur. Standards Report No. 1003 (1952).

2 See e.g. S. A. Colgate, Phys. Rev. 87, (1952) 592; C. M. Davisson and R. D. Evans, Phys. Rev. 81, (1951) 404 and Revs. Modern Phys. 24, (1952) 79; and N. Rudnick, M. S. Thesis, Mass. Inst. Tech. (May 1953). 
In the tables the cross sections are given in units of $\mathrm{cm}^{2} /$ atom and $\mathrm{cm}^{2} / \mathrm{g}$. These can be converted to $\mathrm{cm}^{2} /$ electron $\mathrm{or}^{-1}$ by the use of the following relationships.

$$
\begin{aligned}
a^{\mu} & =Z_{e} \mu \\
\mu / \varrho & =N(Z / A)_{e} \mu \\
\mu & =\varrho N(Z / A)_{e} \mu
\end{aligned}
$$

Here $e^{\mu}$ is the value in $\mathrm{cm}^{2} /$ electron, ${ }_{a} \mu$ is in $\mathrm{cm}^{2} /$ atom, $\mu / \varrho$ is in $\mathrm{cm}^{2} / \mathrm{g}$, and $\mu$ is in $\mathrm{cm}^{-1}$. $Z$ is the atomic number, $A$ the atomic weight, $N$ Avogadro's number, and $\varrho$ the density in $\mathrm{g} / \mathrm{cc}$. 


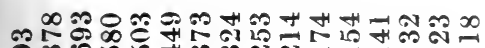

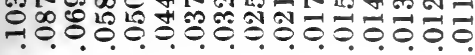

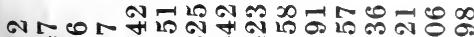

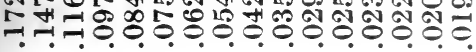

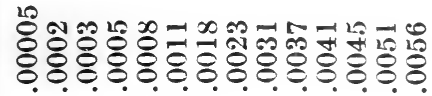

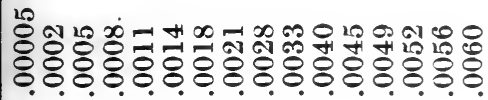

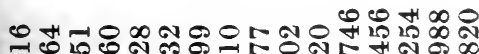

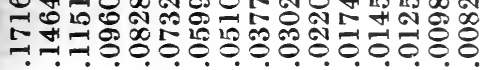

L 000000

-

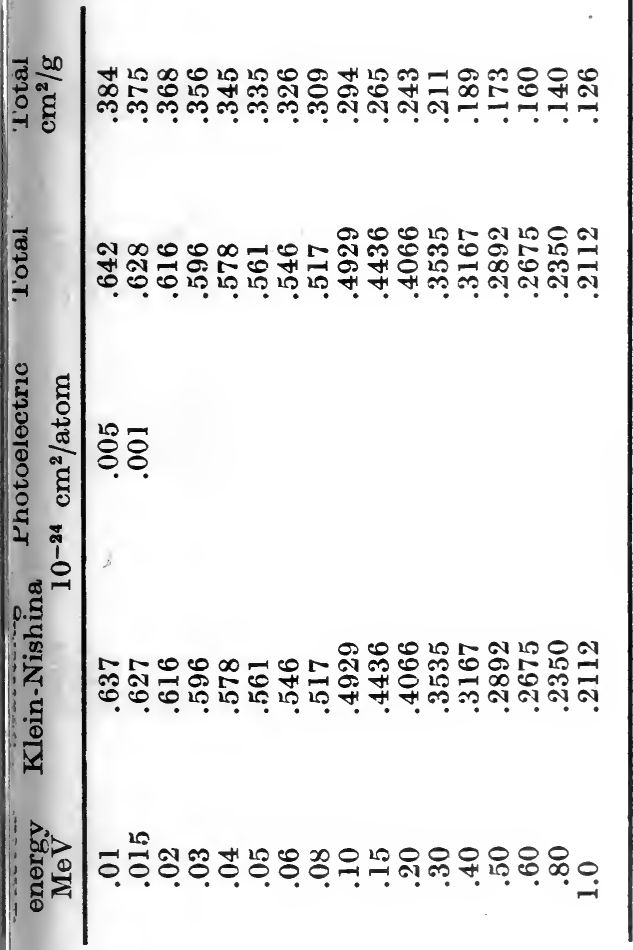

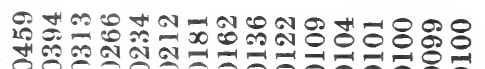

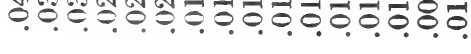

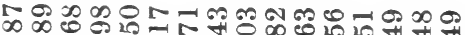

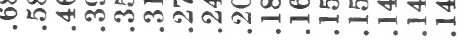

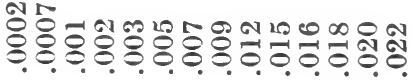

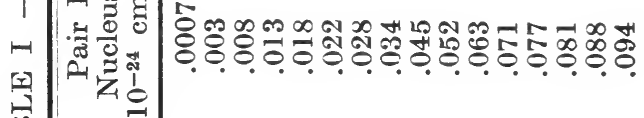

$\sum_{-1}^{\infty}$

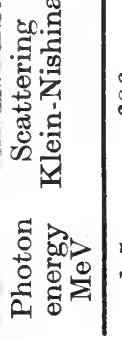

๘ 我

$\log 0.000$

-

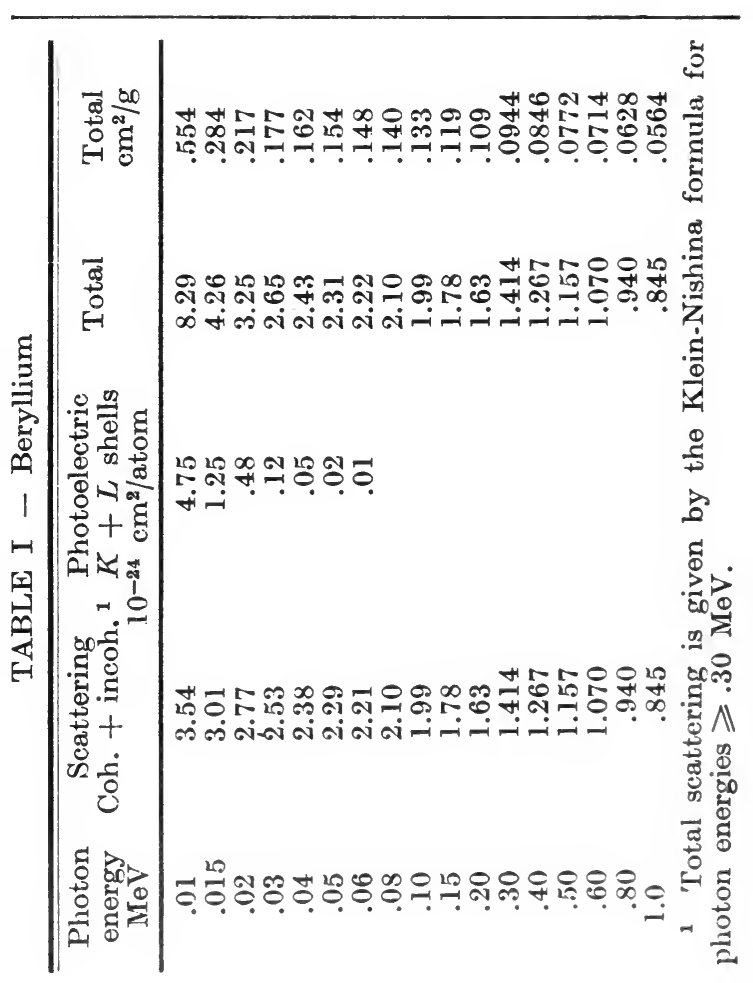




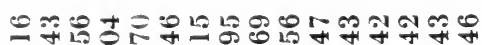

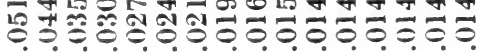

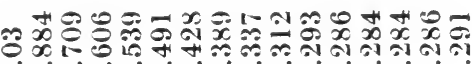

$\frac{1}{0}$

\section{을}

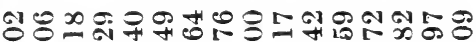

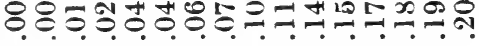

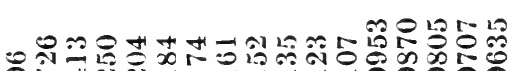

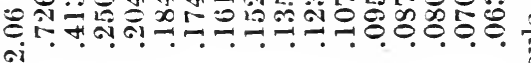

$\dot{5}$

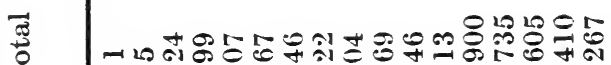

$-$

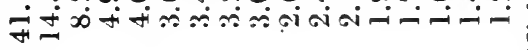




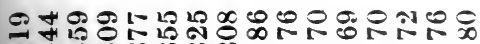

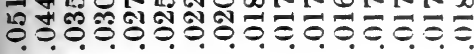

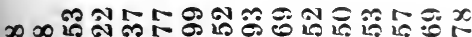

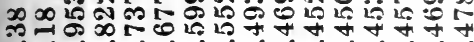
-i-i

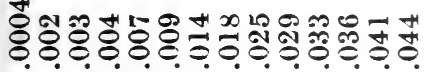

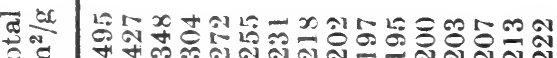
है ?

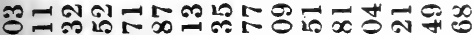

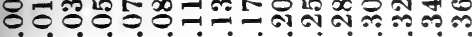

ต

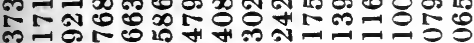
i

in 000000

穴

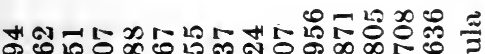
स艹 $10-1$

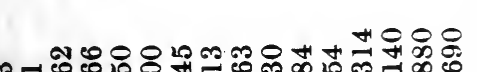
$\stackrel{\varpi}{\Xi}$

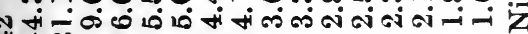
स स ล

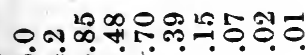

욤

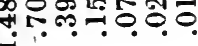

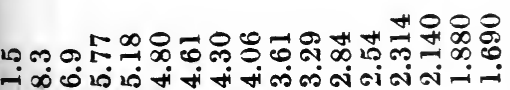



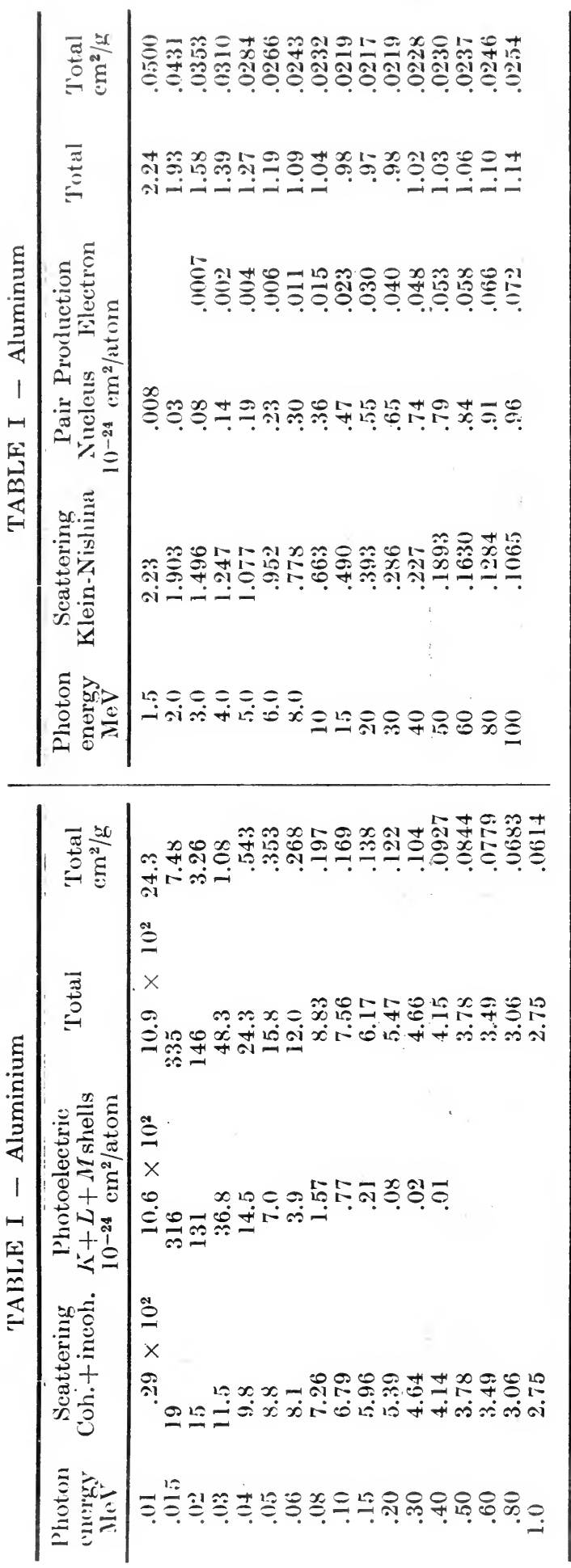
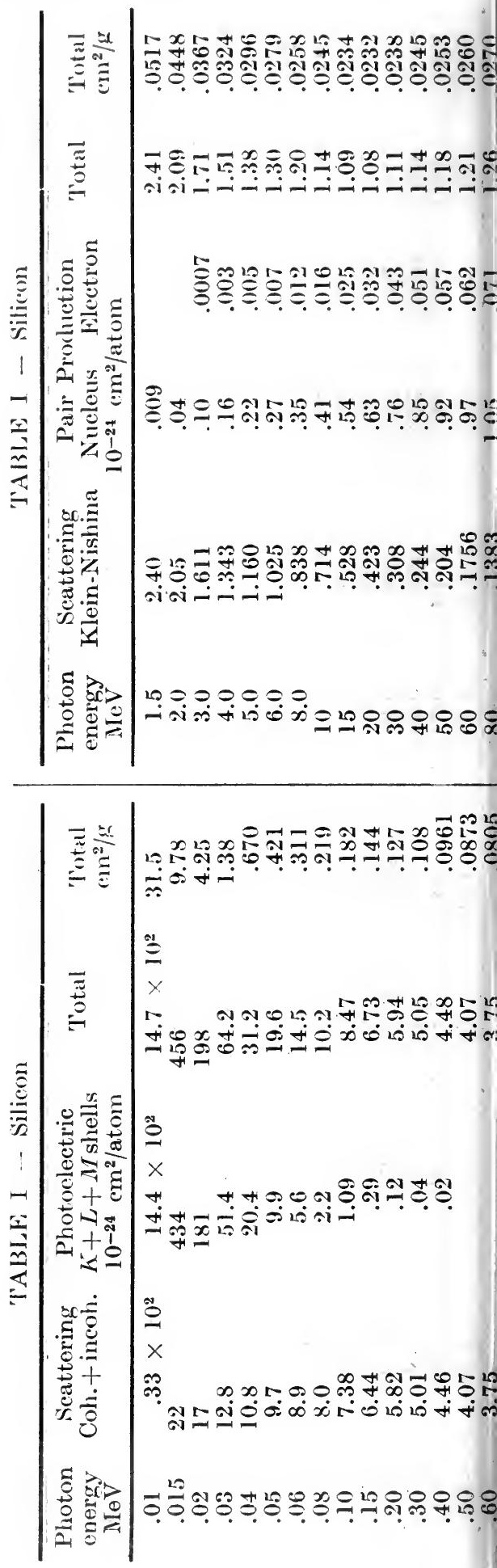


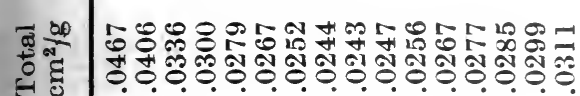

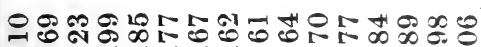

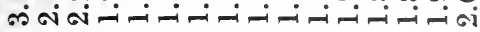

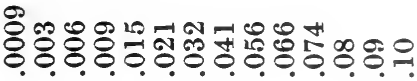

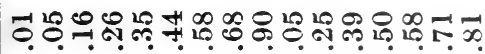

$n-\infty \infty \infty 0+\infty \pi+\infty$

○ म

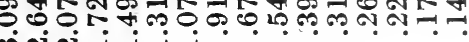

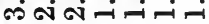

20000000

-

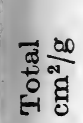

5

要

$\frac{1}{2}$
L2

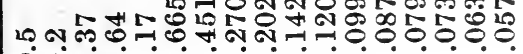
$\dot{0} \rightarrow \infty$ i

จำ

XX

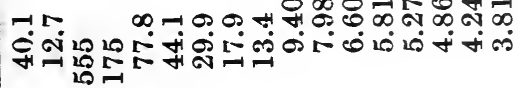

\section{ㅇํㅇำ}

$x \times$

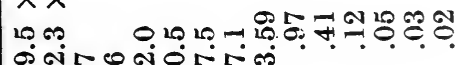

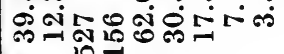

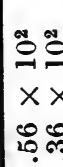

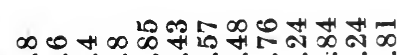

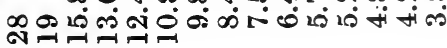

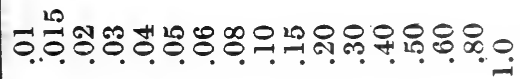

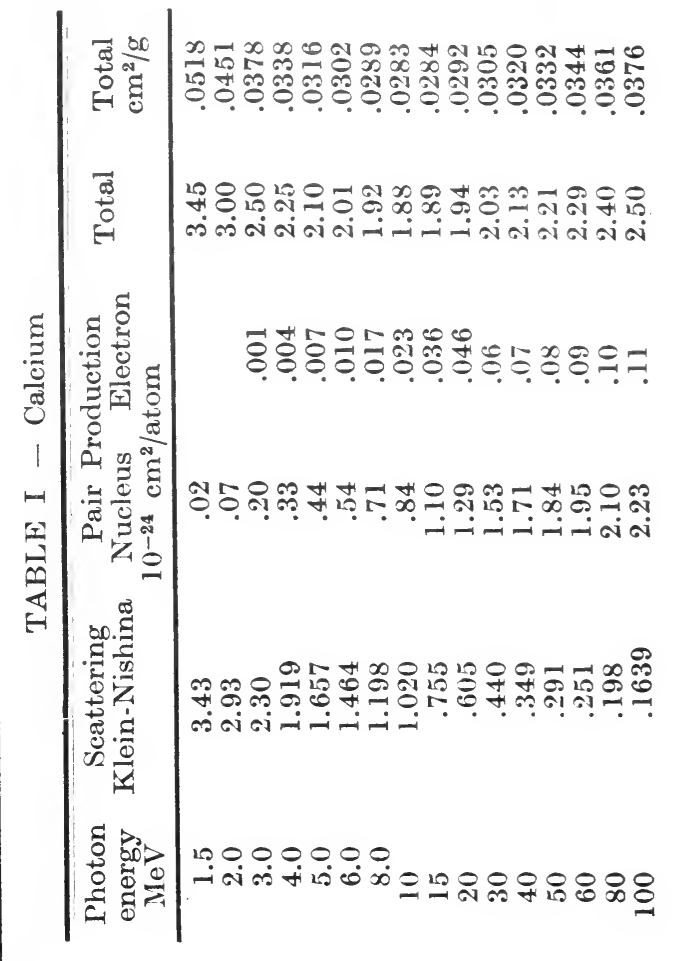

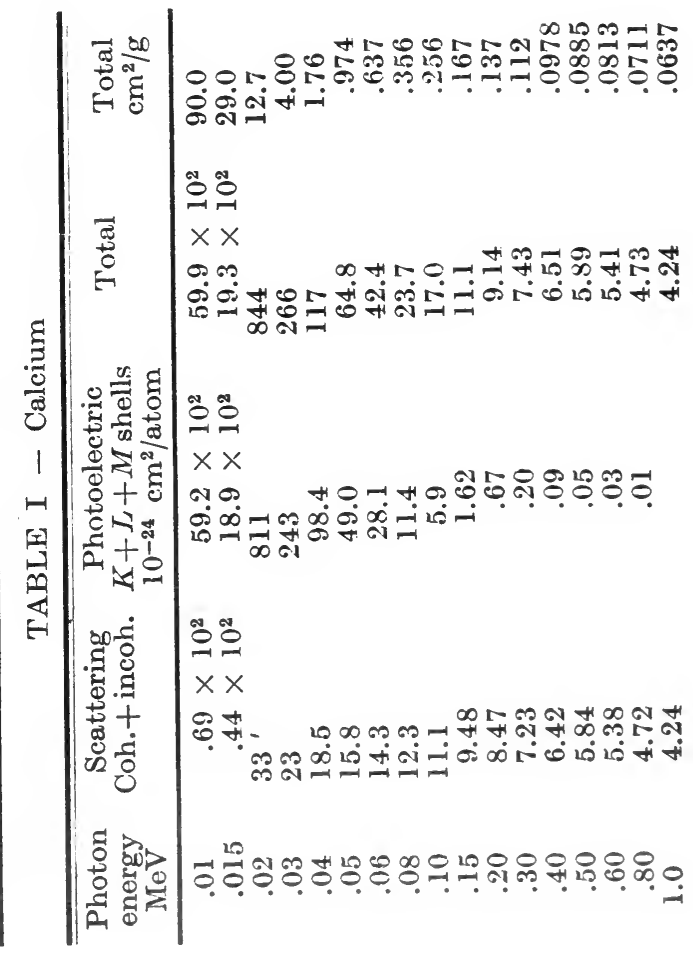



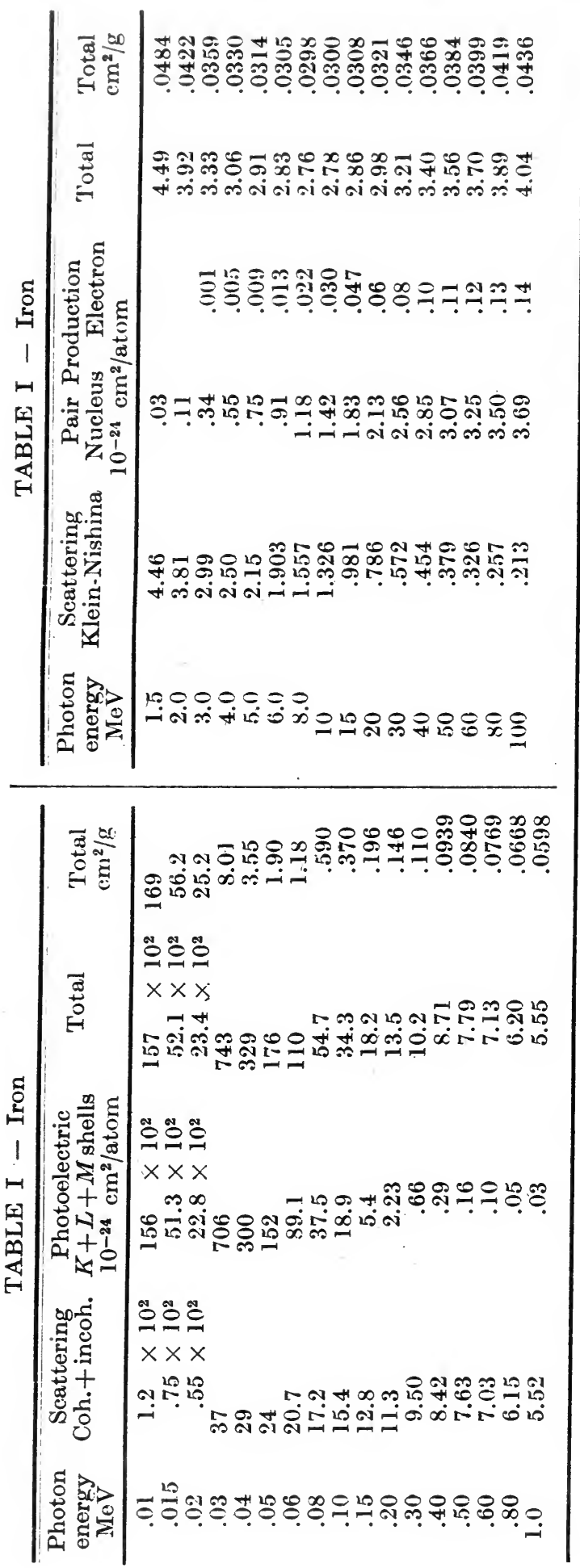
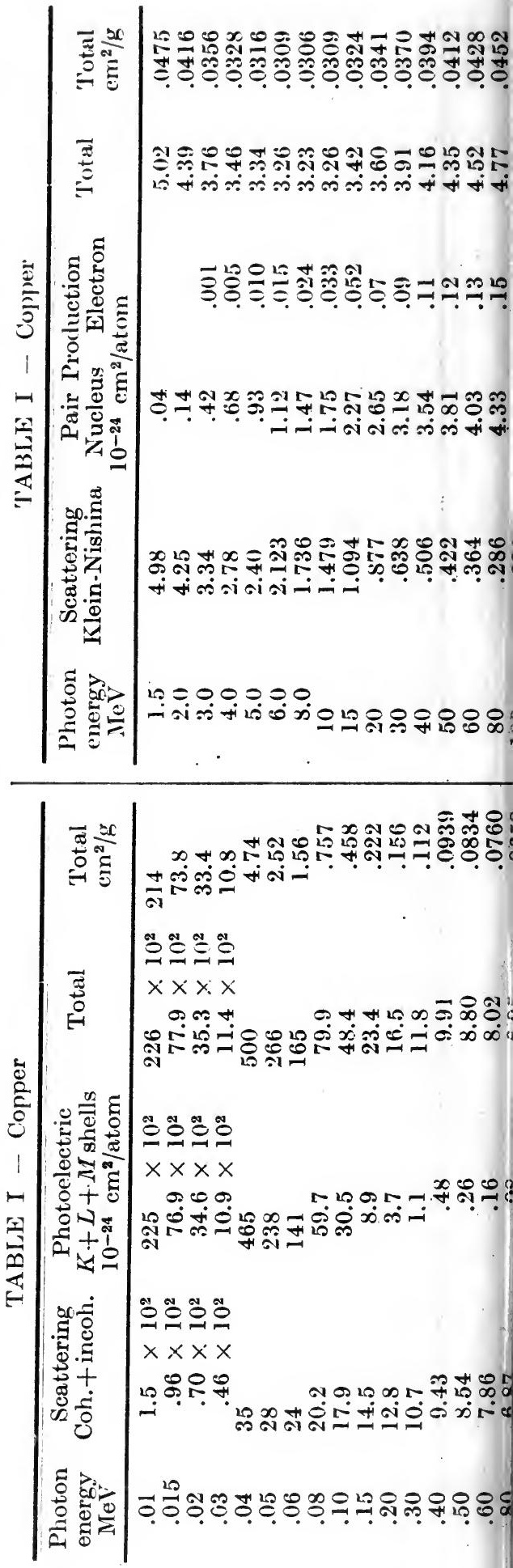
|

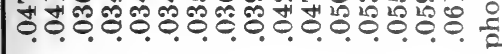

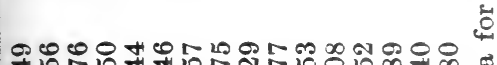
$\therefore$ ○

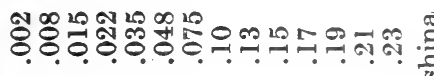

었⿻

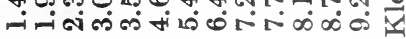

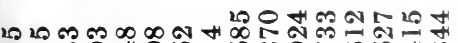

ผ

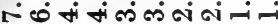

눙은

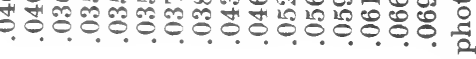

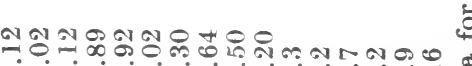

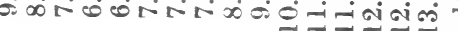

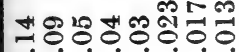

$\ln 0.00 .00$

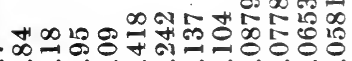

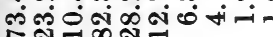

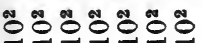

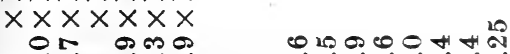

듬

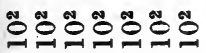

$\times \times \times \times \times \times \times$

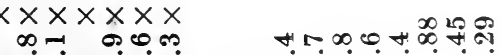
सं

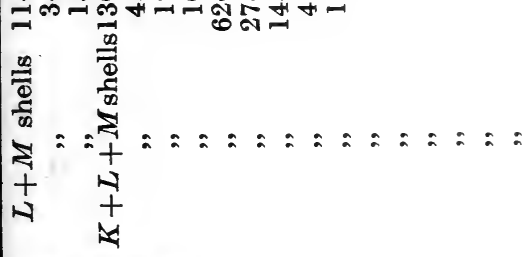

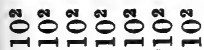

\section{$\times \times \times \times \times \times$}

\#

लं

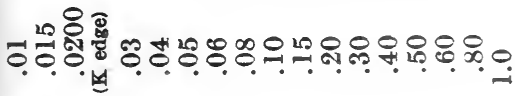




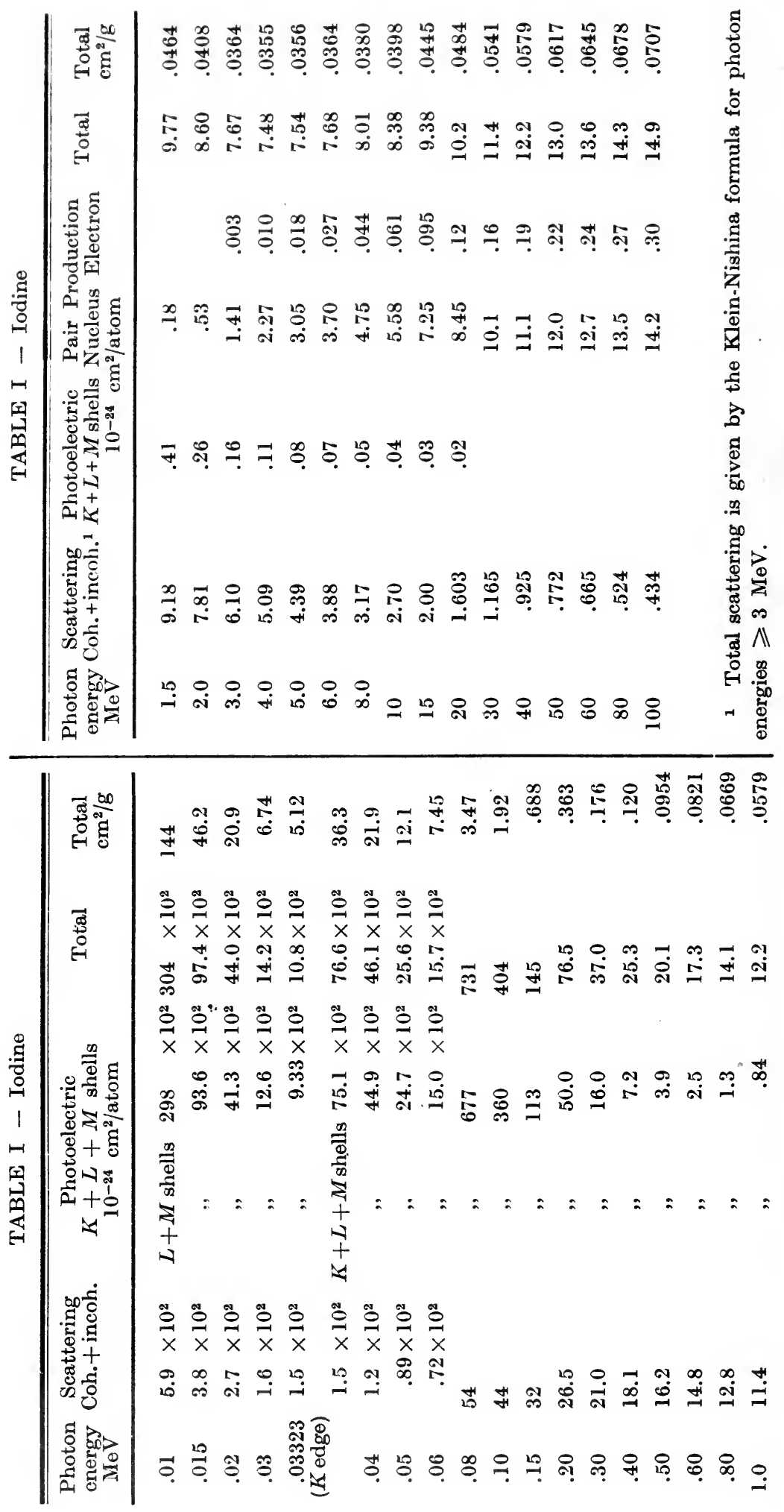




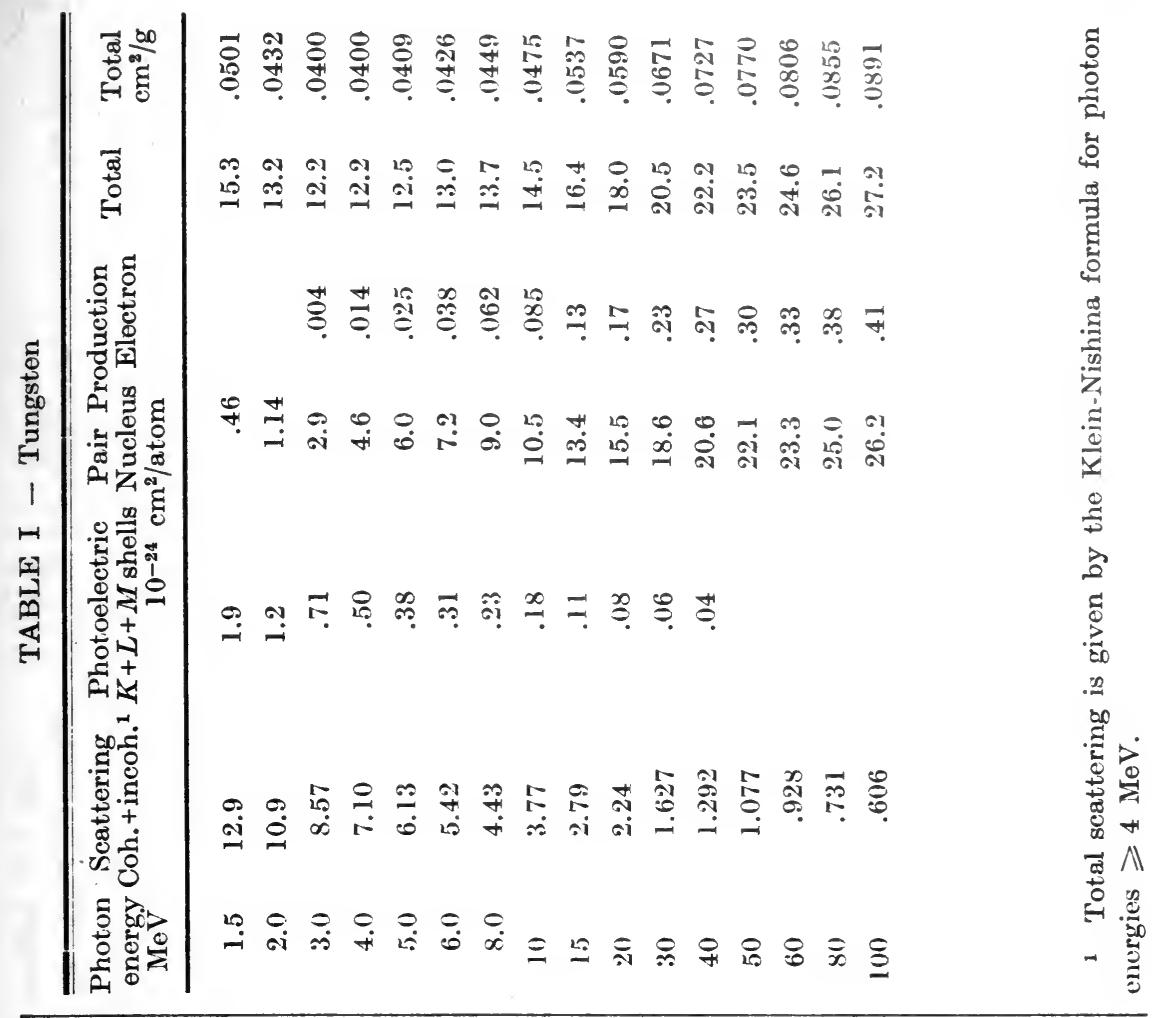

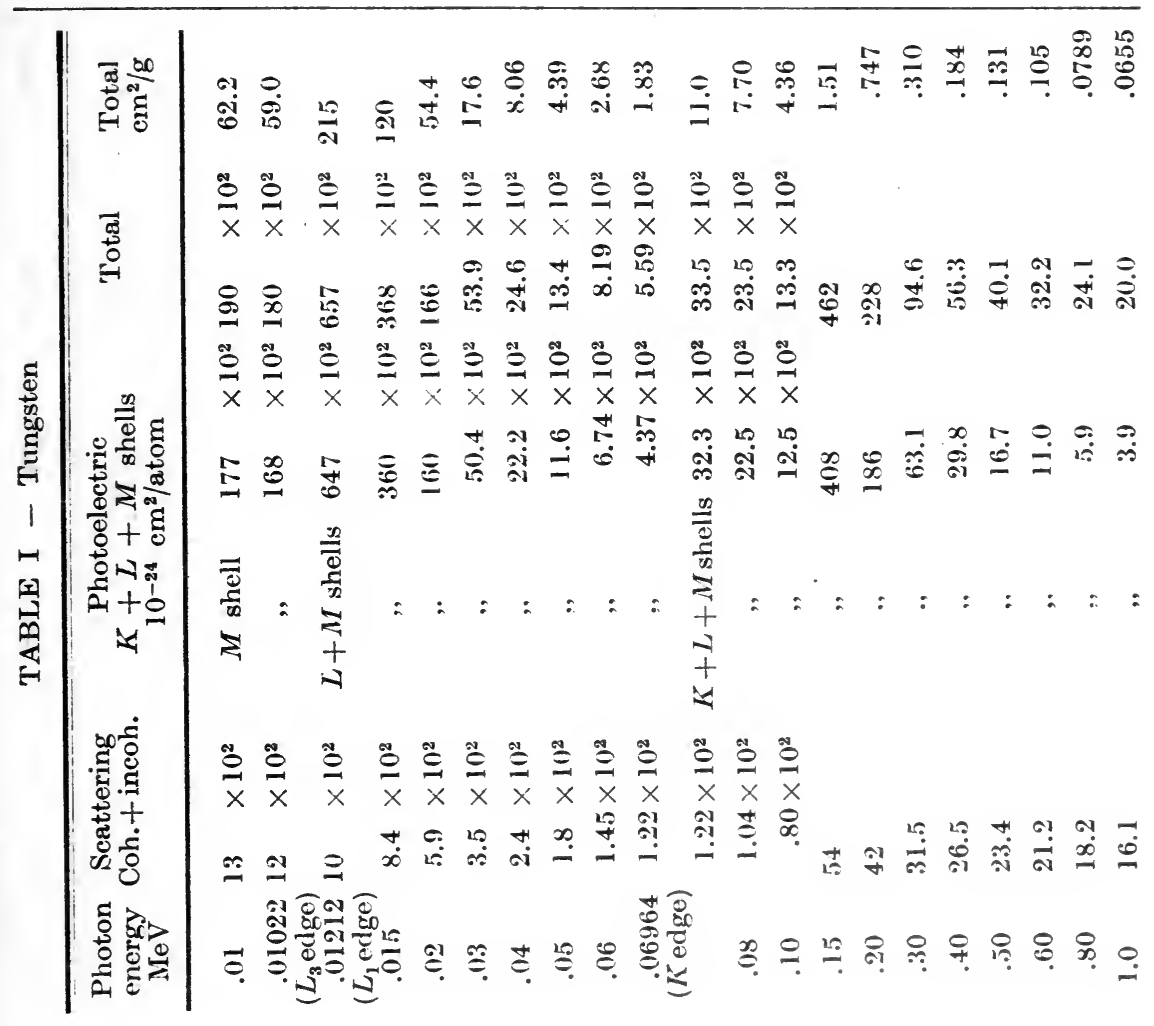




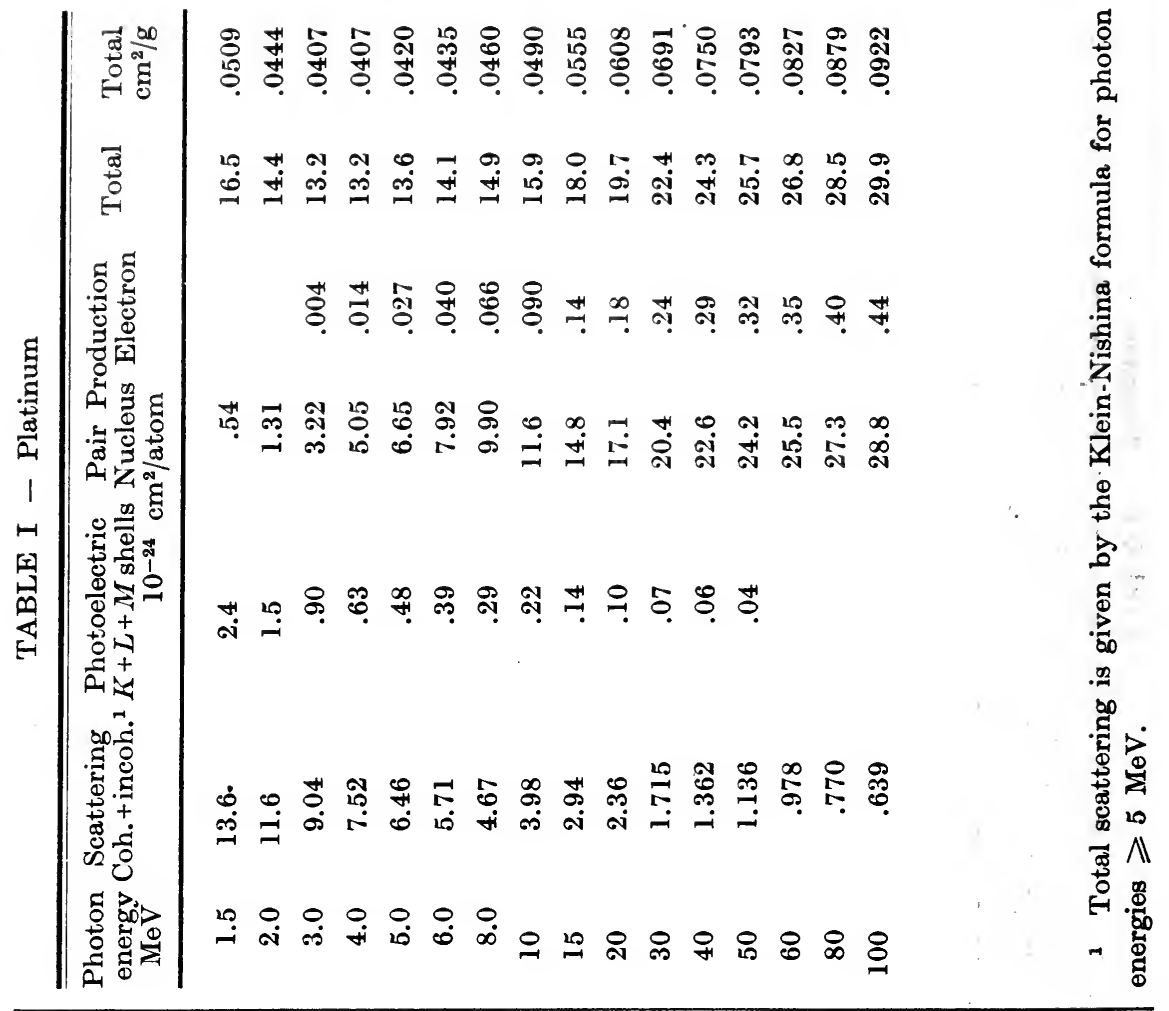

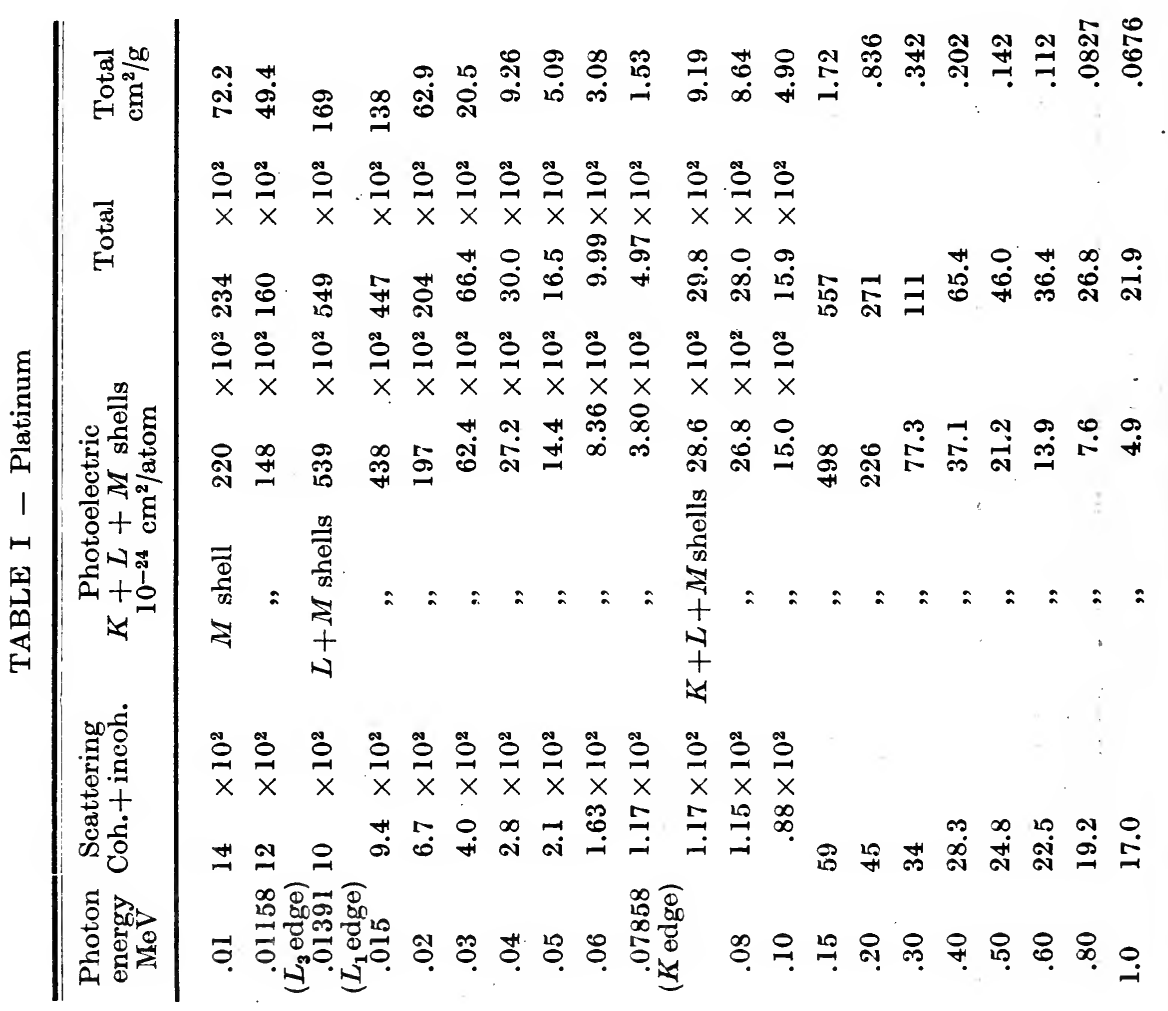




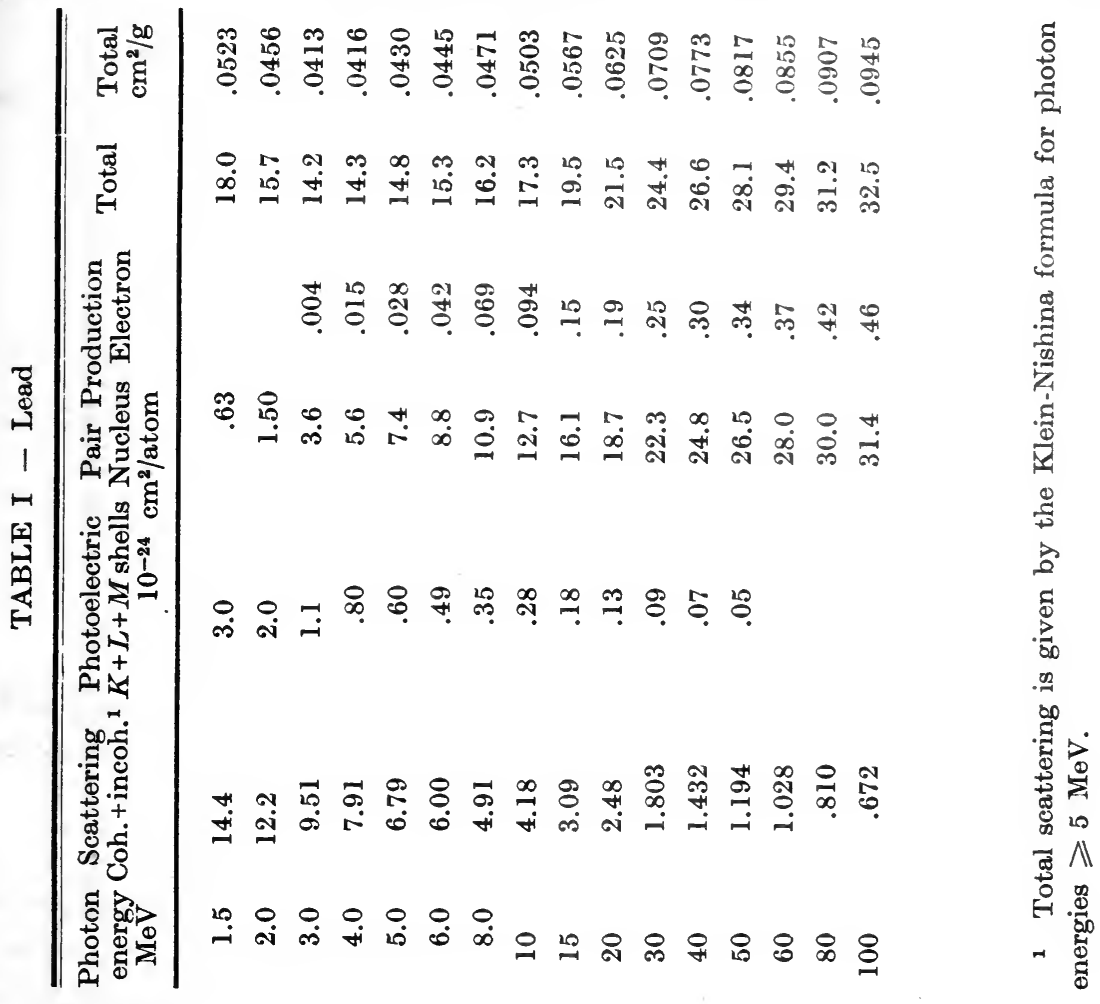

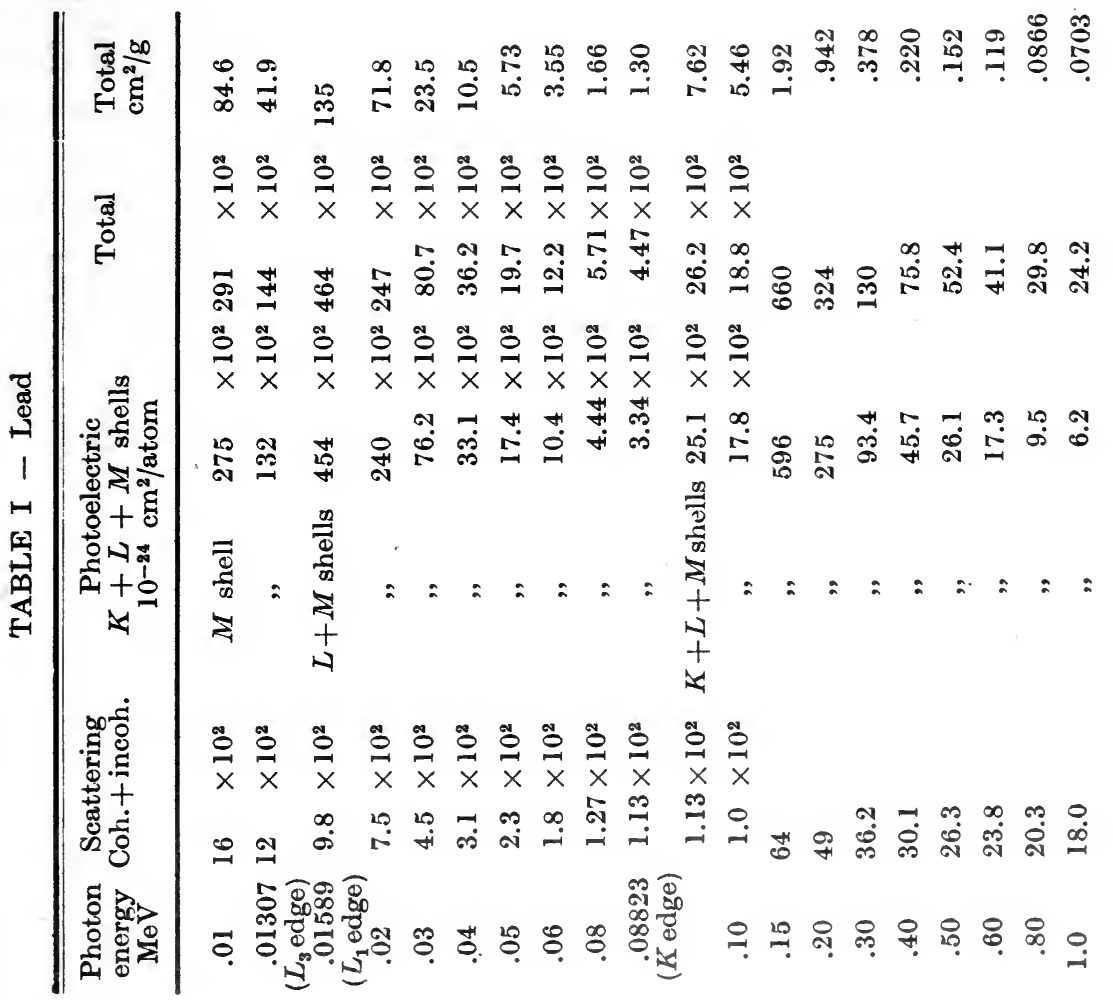




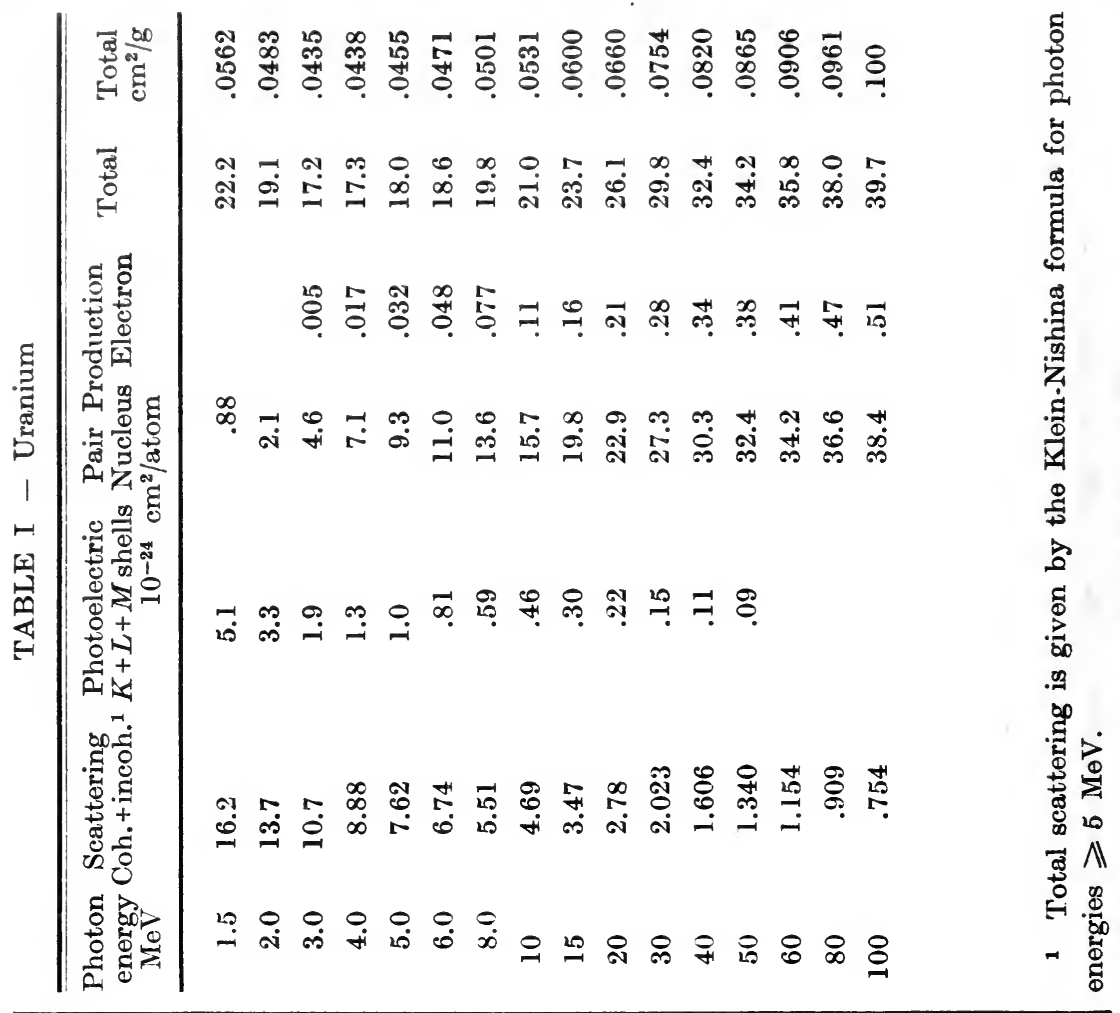

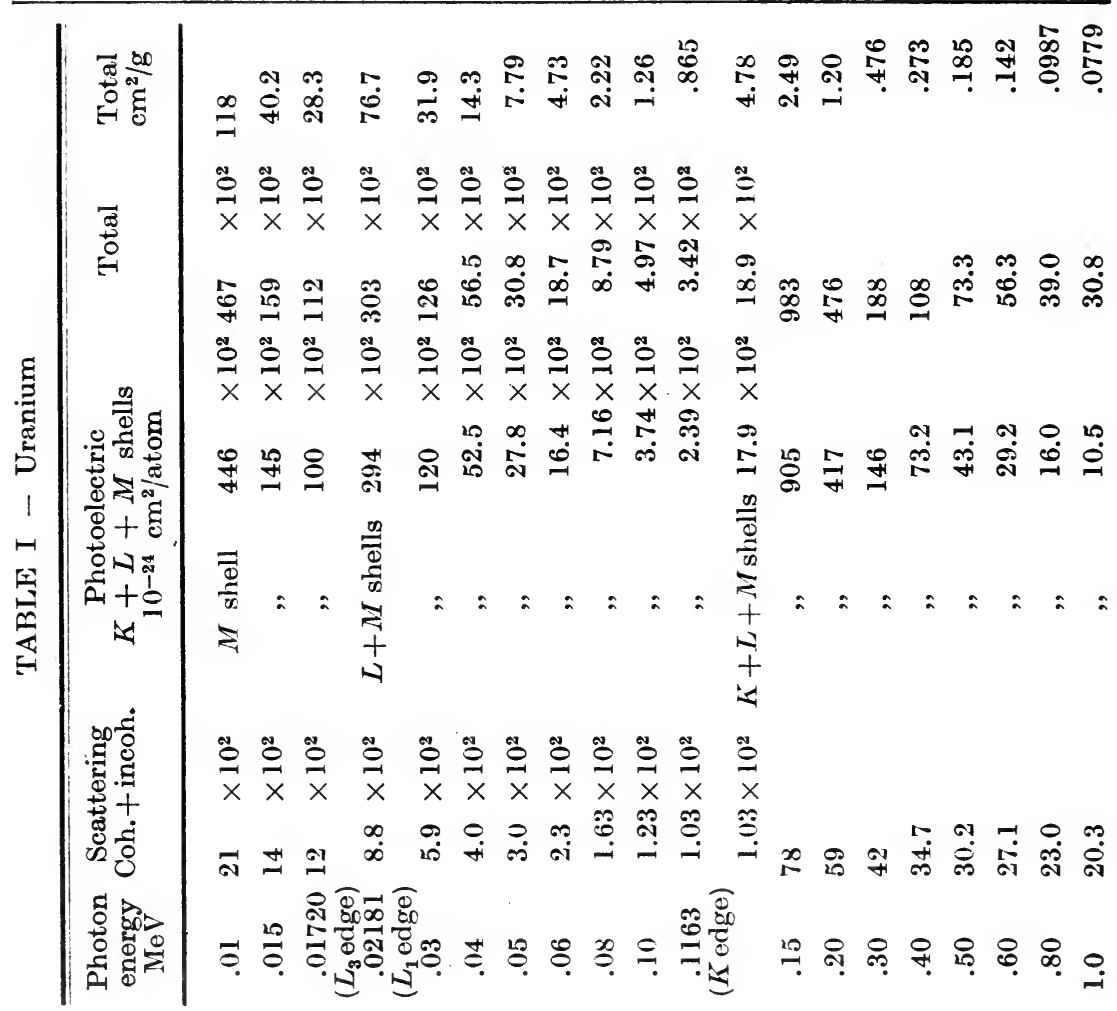




\section{TABLE II}

\section{Absorption Coefficients of Various Substances}

The total absorption coefficients given in this set of tables were obtained from those of the elements in Table I from the equation

$$
\mu / \varrho=(\mu / \varrho)_{1} a_{1}+(\mu / \varrho)_{2} a_{2}+(\mu / \varrho)_{3} a_{3}+\ldots
$$

where $(\mu / \varrho)_{i}$ is the absorption coefficient in $\mathrm{cm}^{2} / \mathrm{g}$ and $a_{i}$ is the fractional amount by weight, of the $i$ th element in the mixture or compound. The linear absorption coefficient $\mu$, in $\mathrm{cm}^{-1}$, is obtained from this through multiplication by the total density in $\mathrm{g} / \mathrm{cc}$.

The "true" absorption coefficients were obtained in a similar manner, from values of $e_{e} \sigma_{a}$ the Compton absorption cross section in $\mathrm{cm}^{2} /$ electron, ${ }_{a} \tau$ the photoelectric cross section in $\mathrm{cm}^{2} /$ atom, and ${ }_{a^{\chi}}$ the cross section for pair production in $\mathrm{cm}^{2} /$ atom, $(\mu / \varrho)_{i}$ being obtained from the equation

$$
(\mu / \varrho)_{i}=\left(N / A_{i}\right)\left(Z_{i_{e}} \sigma_{a}+{ }_{a} \tau_{i}+{ }_{a} \varkappa_{i}\right)
$$

where $N$ is Avogadro's number, $Z_{i}$ the atomic number and $A_{i}$ the atomic weight of the $i$ th element in the mixture or compound. 


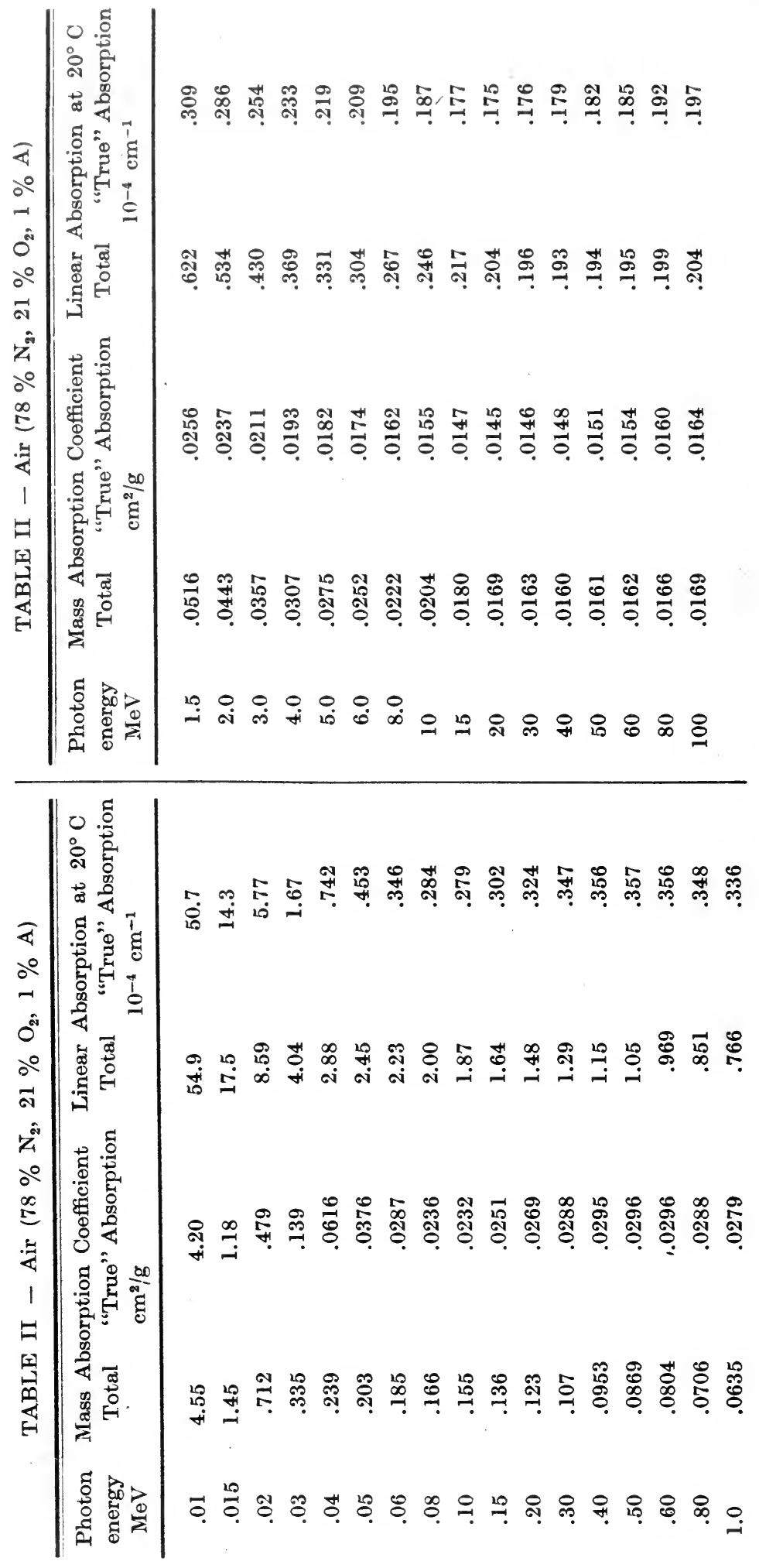




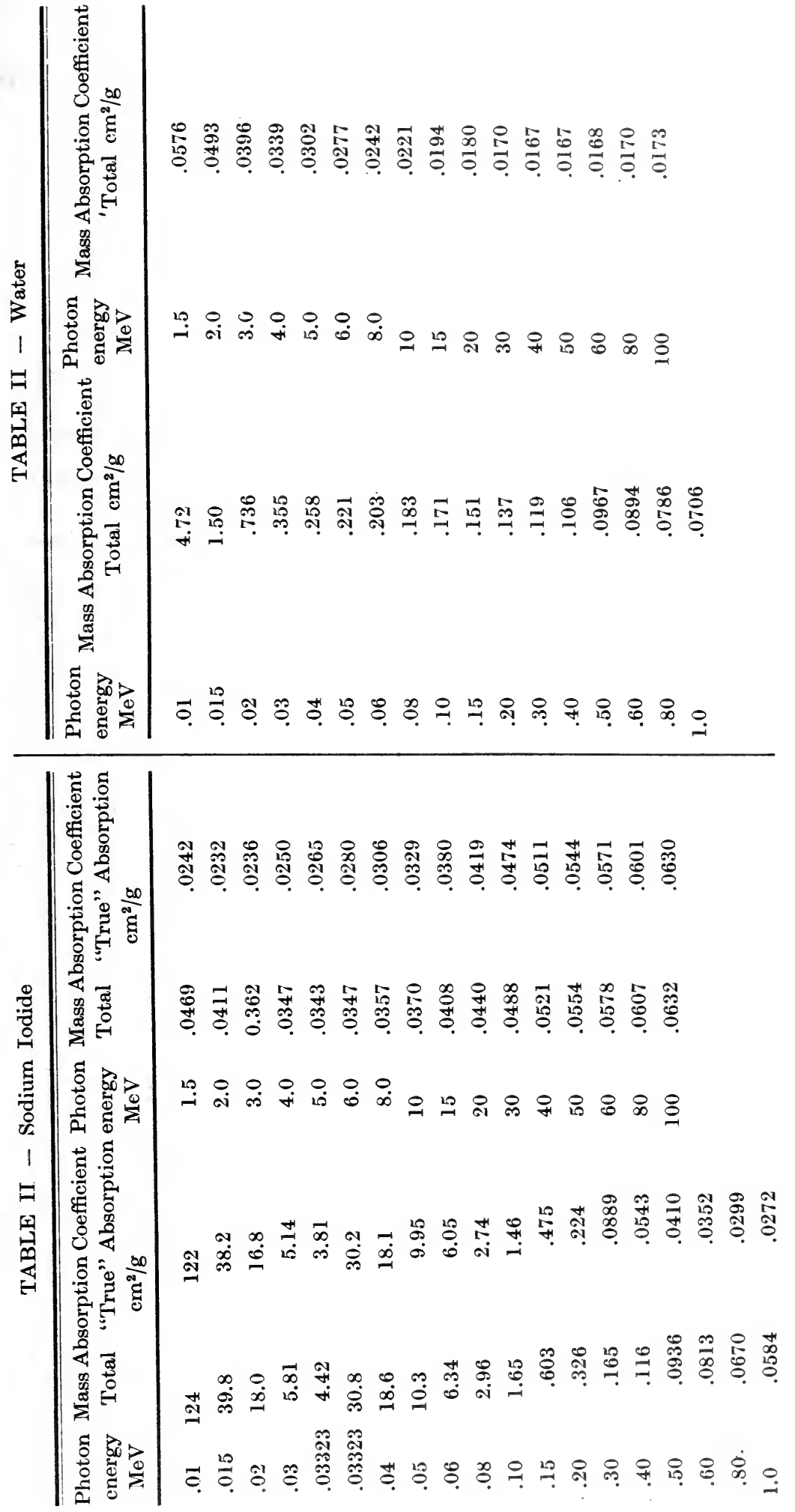




\section{TABLE II - Concrete *}

(.56 \% $\mathrm{H}_{2}, 49.56 \% \mathrm{O}_{2}, 31.35 \% \mathrm{Si}, 4.56 \% \mathrm{Al}, 8.26 \% \mathrm{Ca}, 1.22 \% \mathrm{Fe}$, $.24 \% \mathrm{Mg}, 1.71 \% \mathrm{Na}, 1.92 \% \mathrm{~K}, .12 \% \mathrm{~S})$

\begin{tabular}{|c|c|c|c|}
\hline $\begin{array}{c}\text { Photon } \\
\text { energy } \\
\mathrm{MeV}\end{array}$ & $\begin{array}{c}\text { Mass Absorption Coefficient } \\
\text { Total } \mathrm{cm}^{2} / \mathrm{g}\end{array}$ & $\begin{array}{c}\text { Photon } \\
\text { energy } \\
\text { MeV }\end{array}$ & $\begin{array}{c}\text { Mass Absorption Coefficient } \\
\text { Total } \mathrm{cm}^{2} / \mathrm{g}\end{array}$ \\
\hline .01 & 24.6 & 1.5 & .0517 \\
\hline .015 & 7.68 & 2.0 & $\therefore 0445$ \\
\hline .02 & 3.34 & 3.0 & .0363 \\
\hline .03 & 1.10 & 4.0 & .0317 \\
\hline .04 & .542 & 5.0 & .0287 \\
\hline .05 & .350 & 6.0 & .0268 \\
\hline .06 & .267 & 8.0 & .0243 \\
\hline .08 & .197 & 10.0 & .0229 \\
\hline .10 & .169 & 15 & .0214 \\
\hline .15 & .139 & 20 & .0209 \\
\hline .20 & .124 & 30 & .0209 \\
\hline .30 & .107 & 40 & .0213 \\
\hline .40 & .0954 & 50 & .0217 \\
\hline .50 & .0870 & 60 & .0222 \\
\hline .60 & .0804 & 80 & .0230 \\
\hline .80 & .0706 & 100 & .0237 \\
\hline 1.0 & .0635 & & \\
\hline
\end{tabular}

* Coherent scattering is not included in the calculations. 


\section{TABLES OF FERMI FUNCTIONS}

ACCORDING TO

M. E. ROSE, N. M. DISMUKE, C. L. PERRY AND P. R. BELL *

M. E. ROSE

(Cf. Сн. IX)

Modified Fermi function $G=(p / W) F$ [see Eq. (9c)] as a function of $p$, the $\beta$-particle momentum. The first half of the table refers to electron emission, the second half to positron emission. Each entry is given as a four digit number $m(0.1 \leqslant m<1.0)$ followed by an integer $r$. This is to be read as $m \times 10^{r}$. The number in parentheses following the value of $Z$ at the column hands, is the corresponding value of $A$.

* Oak Ridge National Laboratory Report No. 1222. 


\section{ELECTRONS}

$\begin{array}{llllllllllllllllllllll}\mathrm{p} / \mathrm{Z} & 2 & (3) & 3 & (8) & 4 & (10) & 5 & (12) & 6 & (14) & 7 & (16) & 8 & (18) & 9 & (20) & 10 & (22) & 11 & (25)\end{array}$

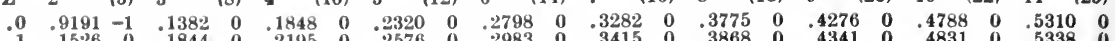

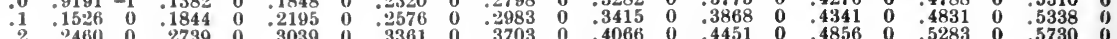

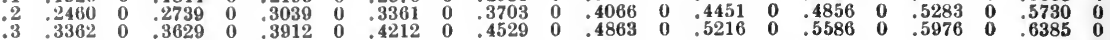

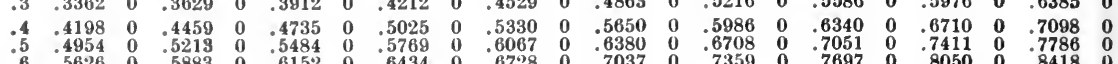

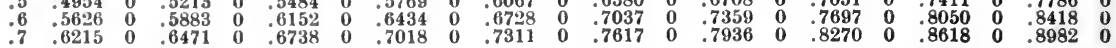

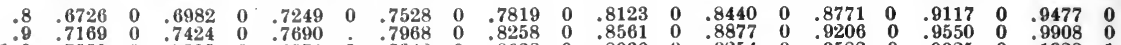

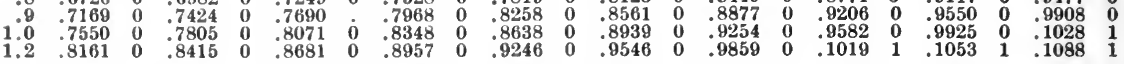
$\begin{array}{lllllllllllllllllllll}1.4 & .8616 & 0 & .8870 & 0 & .9135 & 0 & .9411 & 0 & .9699 & 0 & .9998 & 0 & .1031 & 1 & .1063 & 1 & .1097 & 1 & .1132 & 1\end{array}$

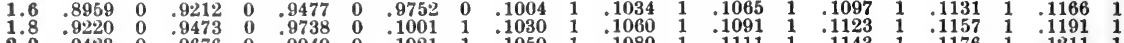

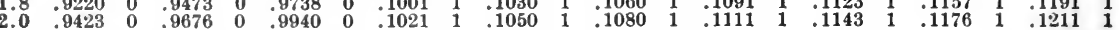

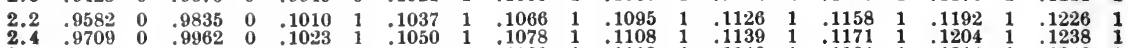

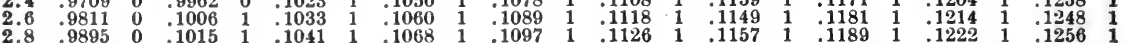

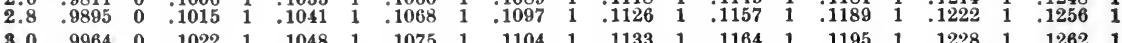

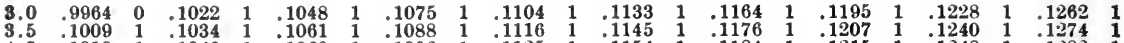

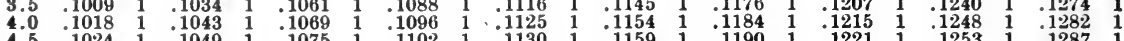

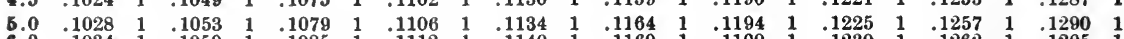

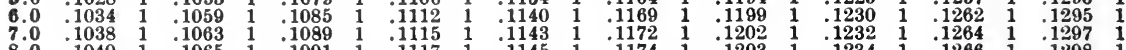

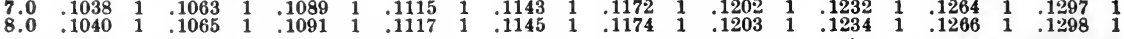
$\begin{array}{rrrrrrrrrrrrrrrrrrrrrr}9.0 & .1041 & 1 & .1066 & 1 & .1092 & 1 & .1119 & 1 & .1147 & 1 & .1175 & 1 & .1205 & 1 & .1235 & 1 & .1266 & 1 & .1299 & 1\end{array}$

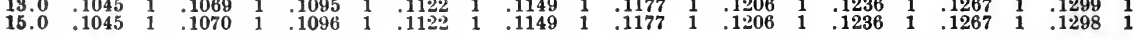

$\begin{array}{lllllllllllllllllllll}\mathrm{D} / \mathrm{Z} & 12 & (27) & 13 & (29) & 14 & (31) & 15 & (33) & 16 & (35) & 17 & (37) & 18 & (41) & 19 & (43) & 20 & (43) & 21 & (48)\end{array}$

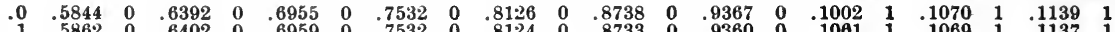

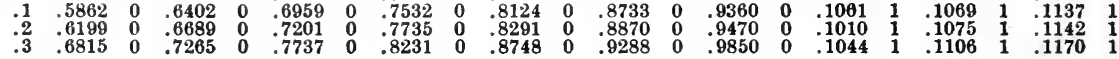

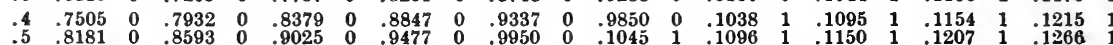

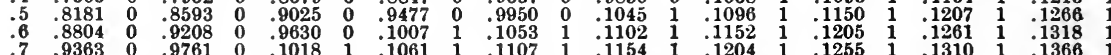

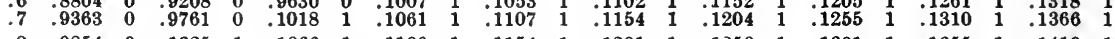
$\begin{array}{rrrrrrrrrrrrrrrrrrrrr}.8 & .9854 & 0 & .1025 & 1 & .1066 & 1 & .1109 & 1 & .1154 & 1 & .1201 & 1 & .1250 & 1 & .1301 & 1 & .1355 & 1 & .1410 & 1 \\ .9 & .1028 & 1 & .1067 & 1 & .1108 & 1 & .1151 & 1 & .1195 & 1 & .1242 & 1 & .1290 & 1 & .1341 & 1 & .1394 & 1 & .1449 & 1\end{array}$

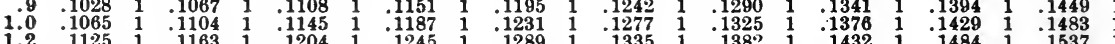

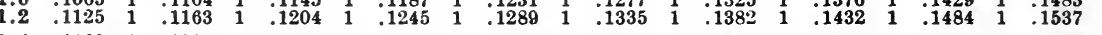

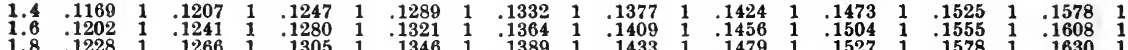

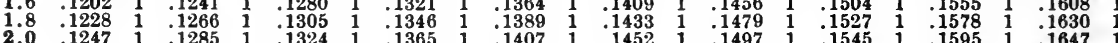

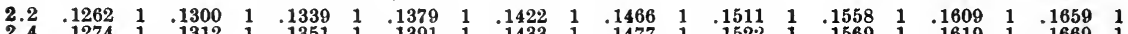

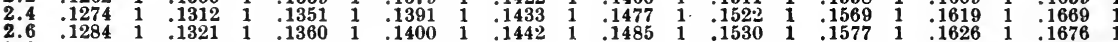

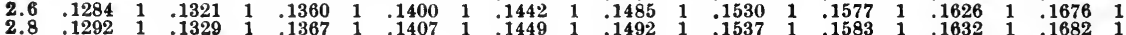

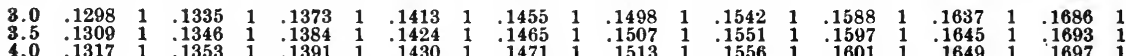

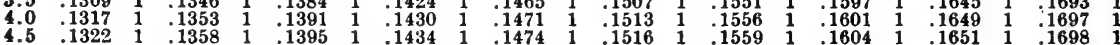

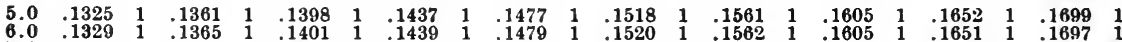

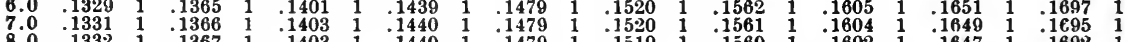

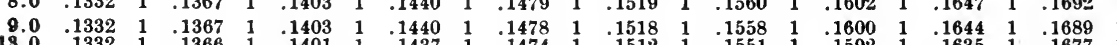

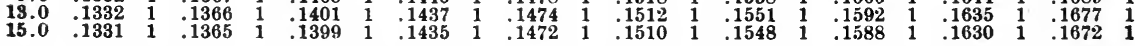

$\begin{array}{llllllllllllllllllllll}\mathrm{p} / \mathrm{Z} & 22 & (48) & 23 & (52) & 24 & (53) & 25 & (56) & 26 & (57) & 27 & (59) & 28 & (61) & 29 & (65) & 30 & (67) & 31 & (72)\end{array}$

$\begin{array}{lllllllllllllllllllll}.0 & .1211 & 1 & .1285 & 1 & .1363 & 1 & .1443 & 1 & .1527 & 1 & .1614 & 1 & .1705 & 1 & .1798 & 1 & .1896 & 1 & .1997 & 1\end{array}$

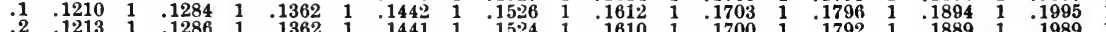

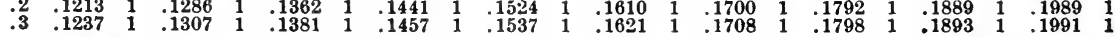

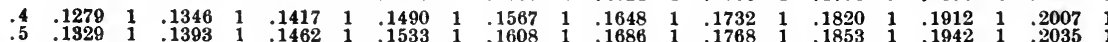

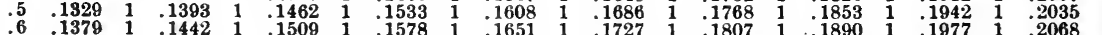

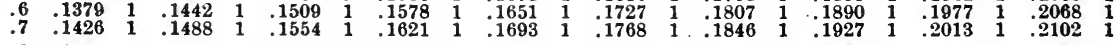

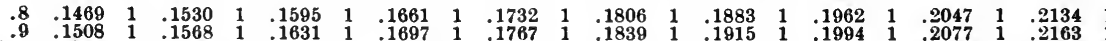

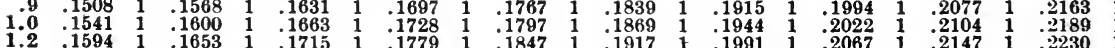

$\begin{array}{lllllllllllllllllllll}1.4 & .1634 & 1 & .1692 & 1 & .1753 & 1 & .1816 & 1 & .1883 & 1 & .1952 & 1 & .2024 & 1 & .2099 & 1 & .2178 & 1 & .2259 & 1\end{array}$

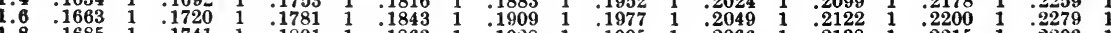

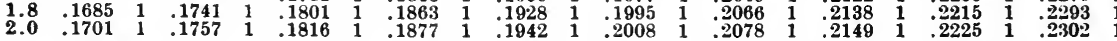

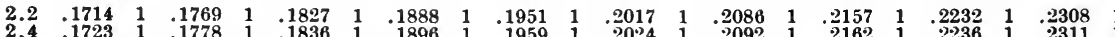

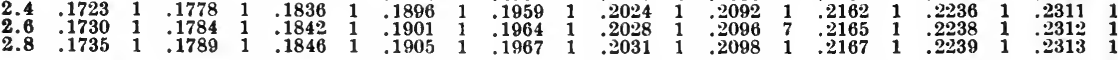

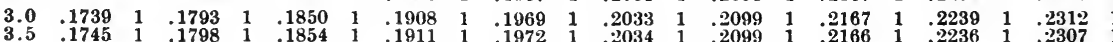

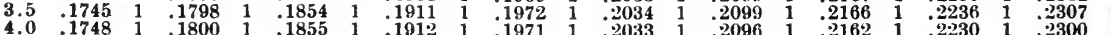

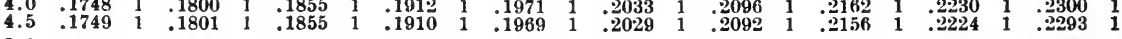

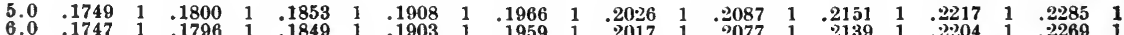

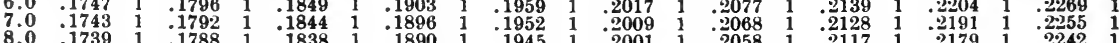

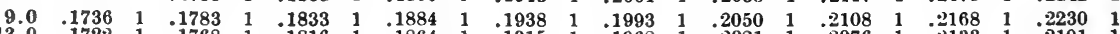

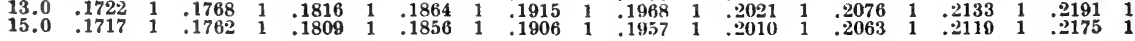




\section{ELECTRONS}

$\begin{array}{lllllllllllllllllllll}\mathrm{p} / \mathrm{Z} & 32 & (73) & 33 & (77) & 34 & (78) & 35 & (83) & 36 & (85) & 37 & (89) & 38 & (90) & 39 & (91) & 40 & (93) & 41 & (96)\end{array}$

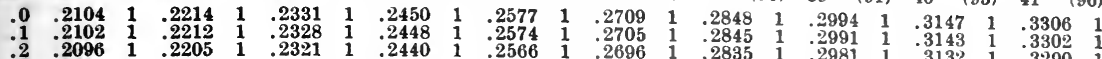

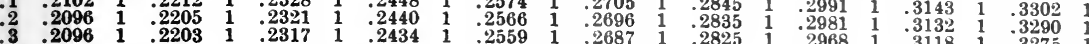

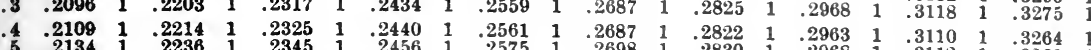

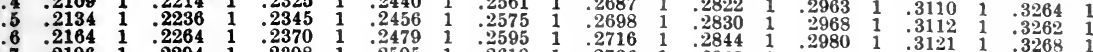

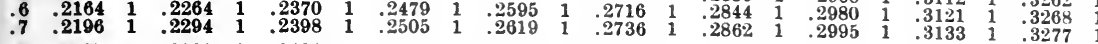

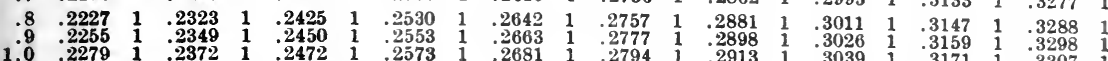

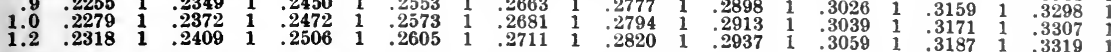
$\begin{array}{lllllllllllllllllllll}\mathbf{1 . 4} & \mathbf{. 2 3 4 6} & \mathbf{1} & \mathbf{. 2 4 3 5} & 1 & .2530 & 1 & .2627 & 1 & .2730 & 1 & .2837 & \mathbf{1} & .2951 & 1 & .3070 & 1 & .3195 & 1 & .3324 & 1\end{array}$

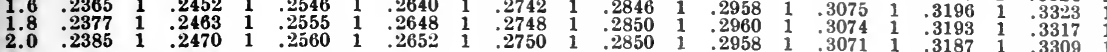

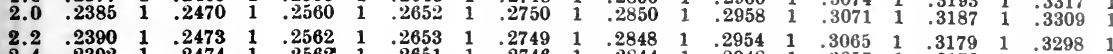

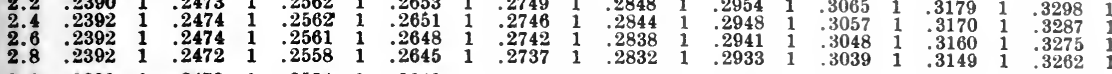

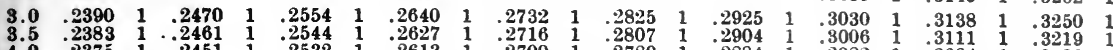

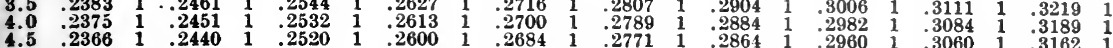

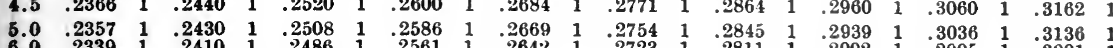

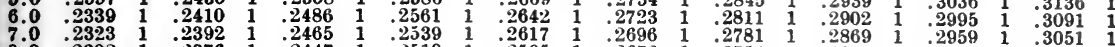

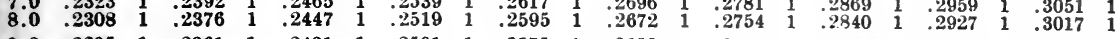

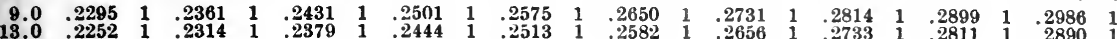

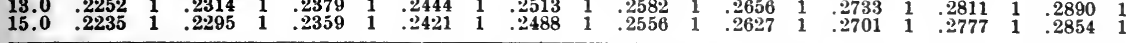

$\begin{array}{llllllllllllllllllllll}\mathrm{p} / \mathrm{Z} & 42 & (97) & 43 & (101) & 44 & (102) & 45 & (107) & 46 & (107) & 47 & (112) & 48 & (113) & 49 & (120) & 50 & (122) & 51 & (124)\end{array}$

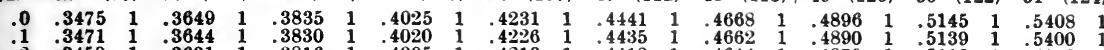

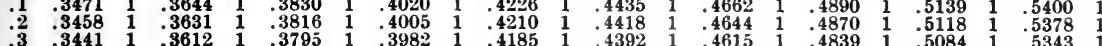

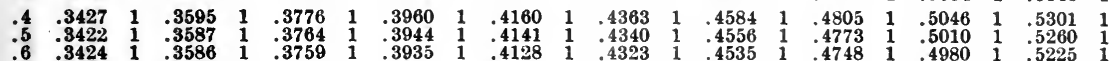

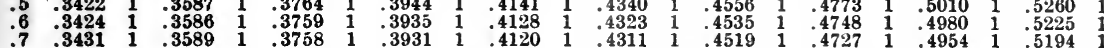

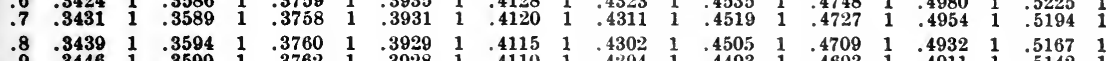

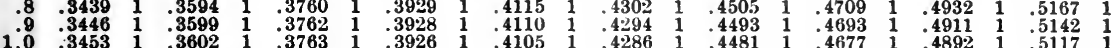

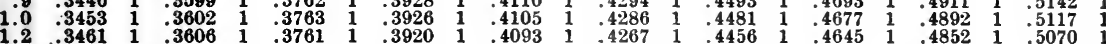

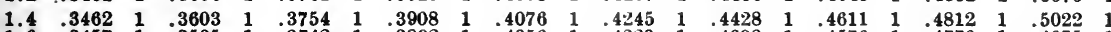

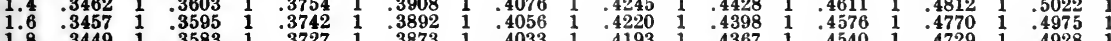

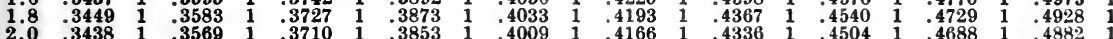

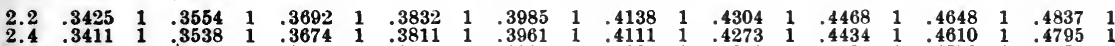

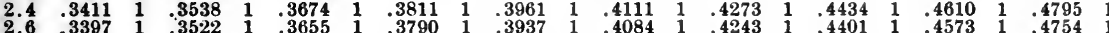

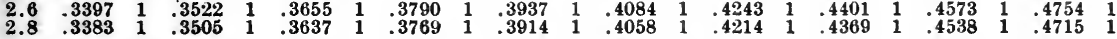
$\begin{array}{lllllllllllllllllllll}3.0 & .3368 & 1 & .3489 & 1 & .3618 & 1 & .3748 & 1 & .3891 & 1 & .4033 & 1 & .4187 & 1 & .4338 & 1 & .4504 & 1 & .4678 & 1\end{array}$

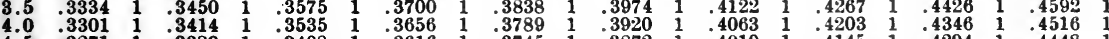

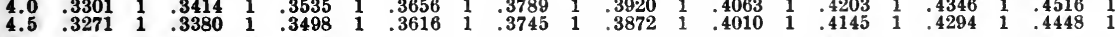

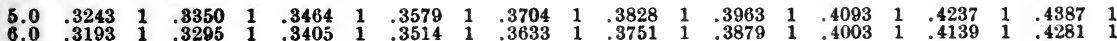

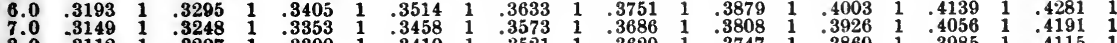

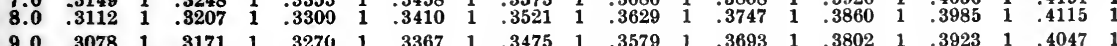

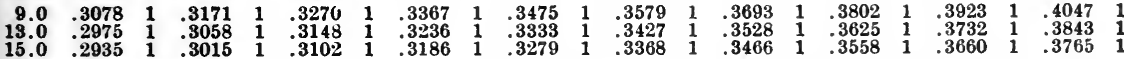

\begin{tabular}{llllllllllllllllllllll}
\hline $\mathrm{p} / \mathrm{Z}$ & 52 & $(131)$ & 53 & $(133)$ & 54 & $(135)$ & 55 & $(138)$ & 56 & $(140)$ & 57 & $(142)$ & 58 & $(144)$ & 59 & $(146)$ & 60 & $(148)$ & 61 & $(150)$
\end{tabular} $\begin{array}{llllllllllllllllllllll}.0 & .5674 & 1 & .5964 & 1 & .6270 & 1 & .6591 & 1 & .6933 & 1 & .7293 & 1 & .7673 & 1 & .8076 & 1 & .8501 & 1 & .8951 & 1\end{array}$

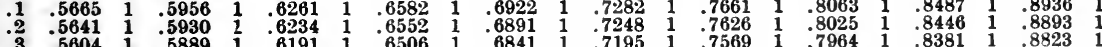
$\begin{array}{lllllllllllllllllll} & .6506 & 1 & .6841 & 1 & .7195 & 1 & .7569 & 1 & .7964 & 1 & .8381 & 1 & .8823 & 1\end{array}$

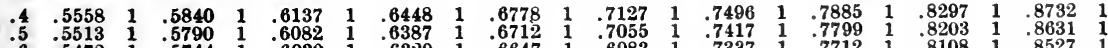

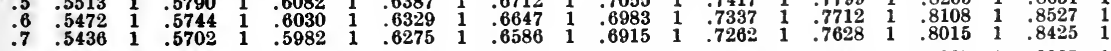
$\begin{array}{lllllllllllllllllllll}.8 & .5404 & 1 & .5664 & 1 & .5938 & 1 & .6224 & 1 & .6529 & 1 & .6850 & 1 & .7190 & 1 & .7548 & 1 & .7927 & 1 & .8327 & 1\end{array}$

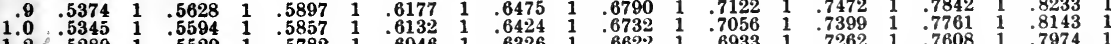

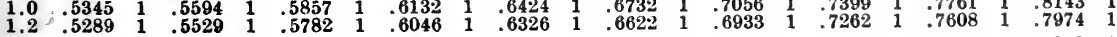
$\begin{array}{llllllllllllllllllllll}1.4 & .5233 & 1 & .5466 & 1 & .5710 & 1 & .5964 & 1 & .6235 & 1 & .6519 & 1 & .6819 & 1 & .7134 & 1 & .7467 & 1 & .7818 & 1\end{array}$

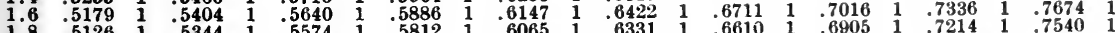

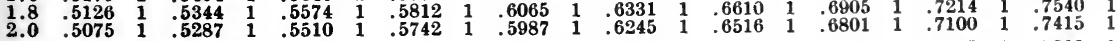
$\begin{array}{llllllllllllllllllllll}2.2 & .5026 & 1 & .5233 & 1 & .5450 & 1 & .5675 & 1 & .5914 & 1 & .6165 & 1 & .6428 & 1 & .6704 & 1 & .6995 & 1 & .7300 & 1\end{array}$

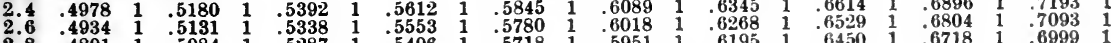

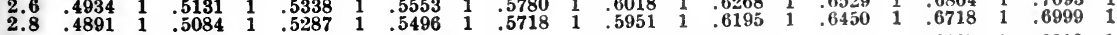
$\begin{array}{lllllllllllllllllllll}3.0 & .4850 & 1 & .5040 & 1 & .5238 & 1 & .5443 & 1 & .5661 & 1 & .5888 & 1 & .6126 & 1 & .6376 & 1 & .6637 & 1 & .6912 & 1\end{array}$

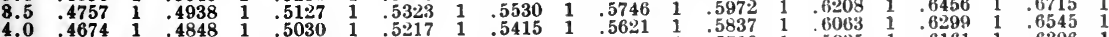

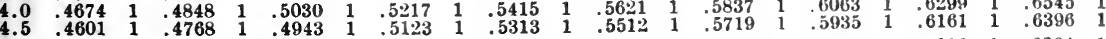
$\begin{array}{llllllllllllllllllllll}\mathbf{5 . 0} & \mathbf{. 4 5 3 4} & 1 & .4696 & \mathbf{4} & .4865 & 1 & .5039 & 1 & .5223 & 1 & .5414 & 1 & .5613 & 1 & .5821 & 1 & .6038 & 1 & .6264 & 1\end{array}$

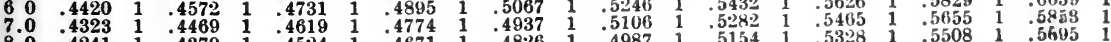

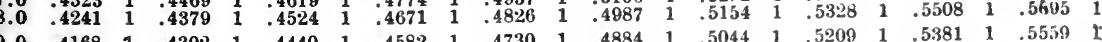

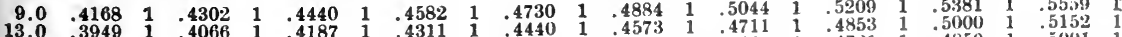

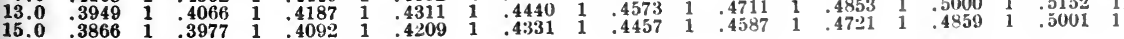


ELEC'TRONS

$\begin{array}{lllllllllllllllllllll}\nu / Z & 62 & (152) & 63 & (154) & 64 & (158) & 65 & (161) & 66 & (163) & 67 & (166) & 68 & (168) & 69 & (171) & 70 & (173) & 71 & (176)\end{array}$

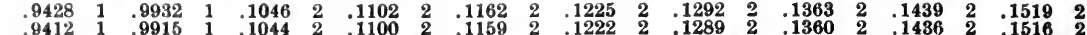

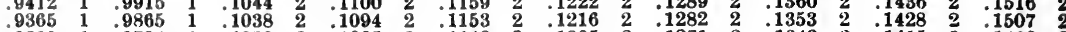

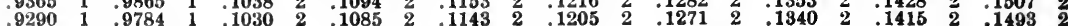

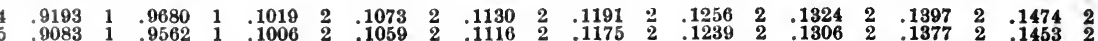

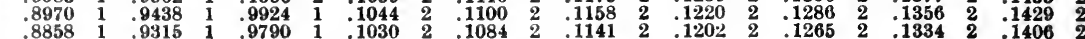

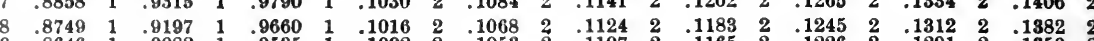

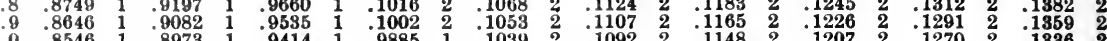

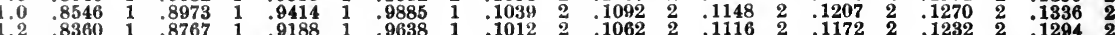

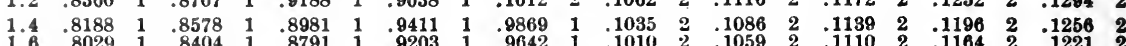

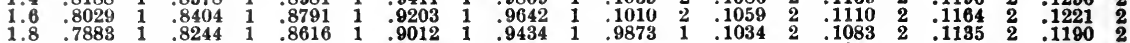

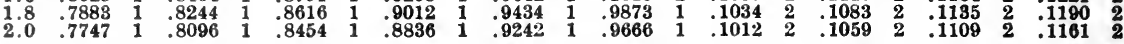

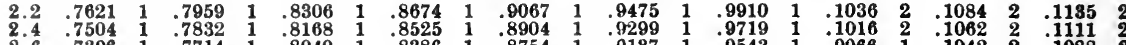

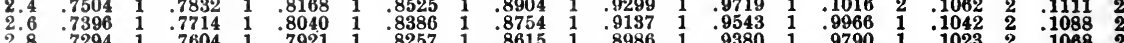

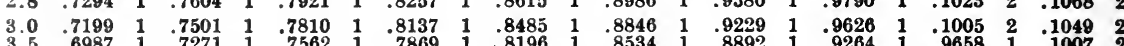

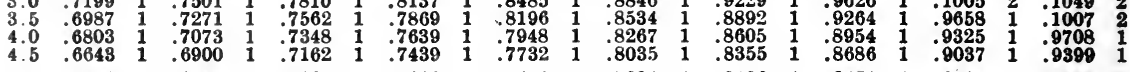

$\begin{array}{lllllllllllllllllllll}5.0 & .6500 & 1 & .6747 & 1 & .6997 & 1 & .7262 & 1 & .7542 & 1 & .7831 & 1 & .8136 & 1 & .8451 & 1 & .8784 & \mathbf{1} & .9128 & 1 \\ 6.0 & .6258 & 1 & .6487 & 1 & .6718 & 1 & .6963 & 1 & .7221 & 1 & .7486 & 1 & .7767 & 1 & .8055 & 1 & .8359 & 1 & .8673 & 1\end{array}$

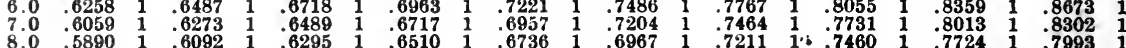

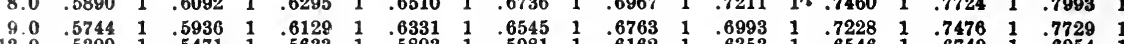

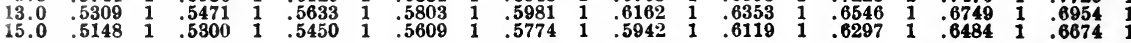

$\begin{array}{llllllllllllllllllllll}\mathrm{p} / \mathrm{Z} & 72 & (178) & 73 & (181) & 74 & (183) & 75 & (187) & 76 & (189) & 77 & \text { (193) } & 78 & (195) & 79 & (199) & 80 & (200) & 81 & (204)\end{array}$

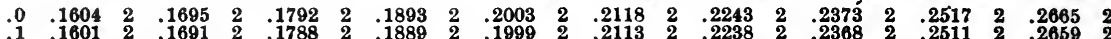

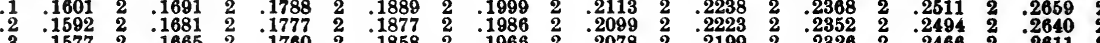

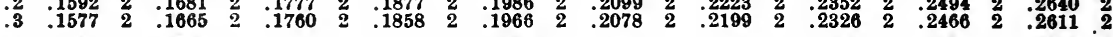

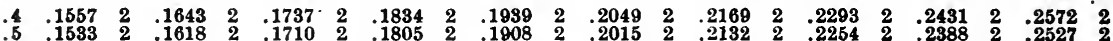

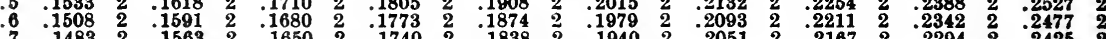

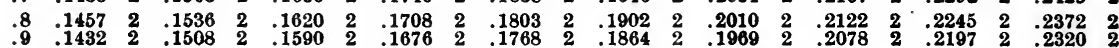

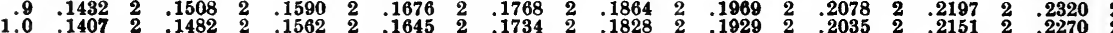

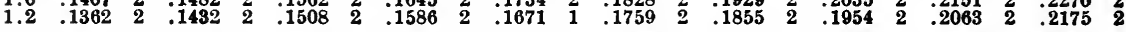

$\begin{array}{lllllllllllllllllllll}1.4 & .1320 & 2 & .1387 & 2 & .1459 & 2 & .1533 & 2 & .1613 & 2 & .1696 & 2 & .1787 & 2 & .1880 & 2 & .1983 & 2 & .2088 & 2 \\ 1.6 & 1289 & 2 & .1346 & 2 & .1414 & 2 & .1485 & 2 & 1561 & 2 & .1640 & 2 & .1725 & 2 & .1814 & 2 & .1911 & 2 & .010 & 2\end{array}$

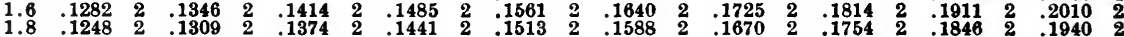

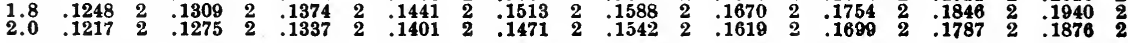

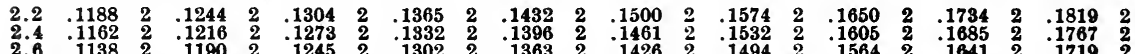

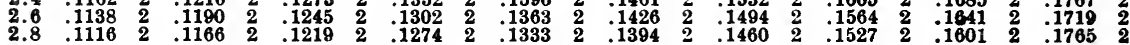

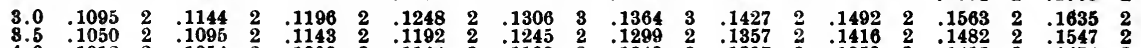

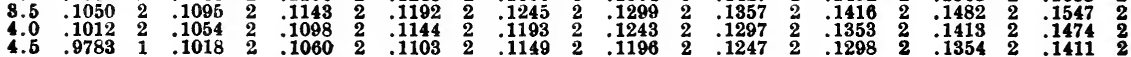

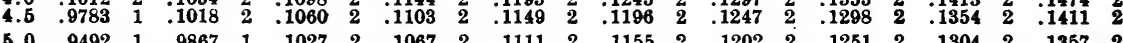

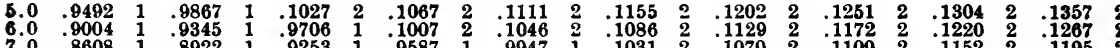

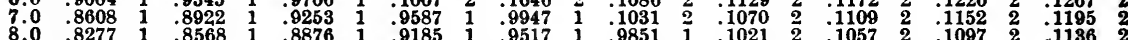

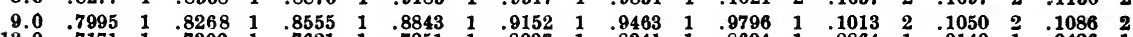

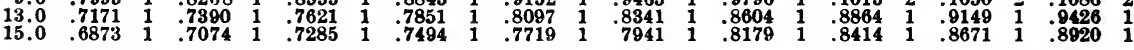

$\begin{array}{lllllllllllllllllllll}\mathrm{p} / \mathrm{Z} & 82 & (207) & 83 & (211) & 84 & (212) & 85 & (215) & 86 & (217) & 87 & (220) & 88 & (223) & 89 & (226) & 90 & (228) & 91 & (232)\end{array}$

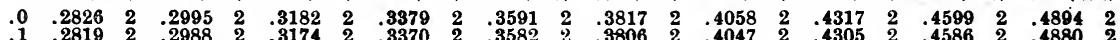

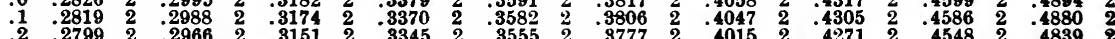

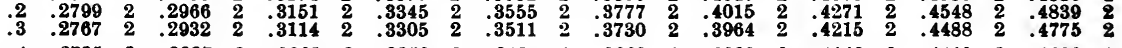

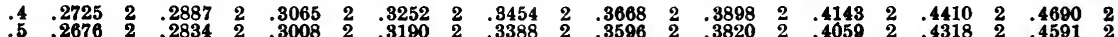

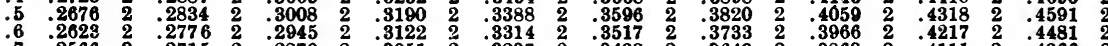

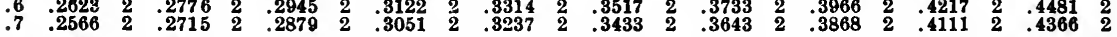

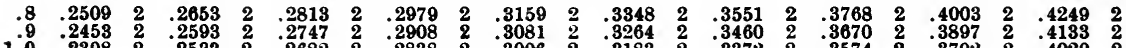

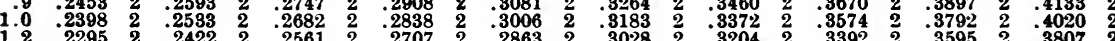

$\begin{array}{lllllllllllllllllllll}1.4 & .2201 & 2 & .2320 & 2 & .2451 & 2 & .2587 & 2 & .2734 & 2 & .2888 & 2 & .3053 & 2 & .3228 & 2 & .3417 & 2 & .3614 & 2\end{array}$

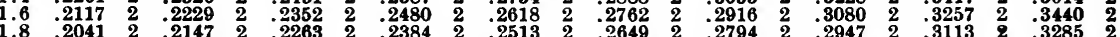

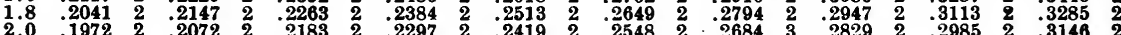

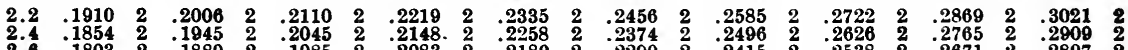

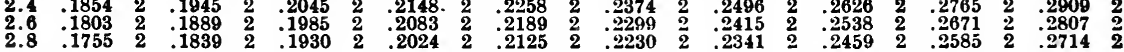

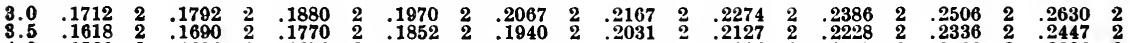

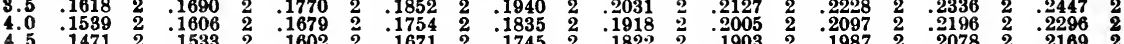

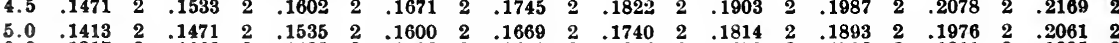

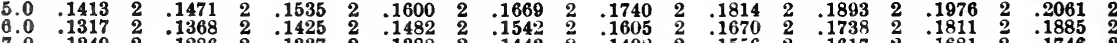

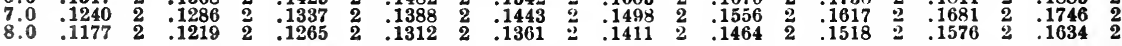

\begin{tabular}{lllllllllllllllllll}
9.0 & .1124 & 2 & .1162 & 2 & .1205 & 2 & .1247 & 2 & .1293 & 2 & .1338 & 2 & .1386 & 2 & .1436 & 2 & .1488 & 2 \\
\hline
\end{tabular}

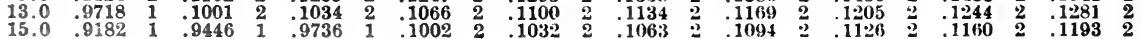




\section{ELECTRONS}

\begin{tabular}{|c|c|c|c|c|c|c|c|c|c|c|}
\hline & & & & & & & 80 & & 96 & \\
\hline . & $\begin{array}{l}.5219 \\
.5204 \\
.5160 \\
.5080\end{array}$ & $\begin{array}{l}\mathbf{2} \\
\mathbf{2} \\
\mathbf{2} \\
\mathbf{2}\end{array}$ & $\begin{array}{r}.5561 \\
.5545 \\
.5496 \\
.5420\end{array}$ & $\begin{array}{l}2 \\
2 \\
2 \\
2\end{array}$ & $\begin{array}{r}.5838 \\
.5920 \\
.5867 \\
.5784\end{array}$ & $\begin{array}{l}\dot{\mathbf{2}} \\
\mathbf{2} \\
\mathbf{2} \\
\mathbf{2}\end{array}$ & $\begin{array}{l}.6344 \\
.6324 \\
.6267 \\
.6177\end{array}$ & $\begin{array}{l}\mathbf{2} \\
\mathbf{2} \\
\mathbf{2} \\
\mathbf{2}\end{array}$ & $\begin{array}{l}.6777 \\
.6756 \\
.6693 \\
.6595\end{array}$ & \\
\hline & $\begin{array}{l}.4997 \\
.4890 \\
.4771 \\
.4646\end{array}$ & $\begin{array}{l}\mathbf{2} \\
\mathbf{2} \\
\mathbf{2} \\
\mathbf{2}\end{array}$ & $\begin{array}{r}.5320 \\
.5203 \\
.5074 \\
.4939\end{array}$ & $\begin{array}{l}\mathbf{2} \\
\mathbf{2} \\
\mathbf{2} \\
\mathbf{2}\end{array}$ & $\begin{array}{l}.5676 \\
.5549 \\
.5409 \\
.5262\end{array}$ & $\begin{array}{l}2 \\
2 \\
2 \\
2\end{array}$ & $\begin{array}{l}.60 \\
.58 \\
.57 \\
.56\end{array}$ & $\begin{array}{l}2 \\
2 \\
2 \\
2\end{array}$ & $\begin{array}{l}.6467 \\
.6316 \\
.6151 \\
.5977\end{array}$ & \\
\hline & $\begin{array}{r}.4519 \\
.4898 \\
.4271 \\
.4039\end{array}$ & $\begin{array}{l}2 \\
2 \\
2 \\
2\end{array}$ & $\begin{array}{r}.4802 \\
.4665 \\
.4532 \\
.4281\end{array}$ & $\begin{array}{l}\mathbf{2} \\
\mathbf{2} \\
\mathbf{2} \\
\mathbf{2}\end{array}$ & $\begin{array}{r}.5112 \\
.4964 \\
.4819 \\
.4546\end{array}$ & $\begin{array}{l}2 \\
2 \\
2\end{array}$ & $\begin{array}{l}.5446 \\
.5285 \\
.5127 \\
.4831\end{array}$ & $\begin{array}{l}2 \\
2 \\
2 \\
2\end{array}$ & $\begin{array}{l}.5 \\
.5 \\
.5 \\
.5\end{array}$ & \\
\hline & $\begin{array}{l}.3829 \\
.3641 \\
.3472\end{array}$ & $\begin{array}{l}2 \\
2 \\
2 \\
2\end{array}$ & $\begin{array}{r}.4053 \\
.3849 \\
.3667 \\
.3504\end{array}$ & $\begin{array}{l}\mathbf{2} \\
\mathbf{2} \\
\mathbf{2} \\
\mathbf{2}\end{array}$ & $\begin{array}{r}.4299 \\
.4078 \\
.3880 \\
.3704\end{array}$ & $\begin{array}{l}2 \\
2 \\
2\end{array}$ & $\begin{array}{l}.4 \\
.4 \\
.4 \\
.3\end{array}$ & $\begin{array}{l}2 \\
2 \\
2 \\
2 \\
2\end{array}$ & $\begin{array}{l}.4840 \\
.4580 \\
.4347 \\
.4140\end{array}$ & \\
\hline & $\begin{array}{l}.3187 \\
.3065 \\
.2955 \\
.2856\end{array}$ & $\begin{array}{l}2 \\
2 \\
2 \\
2\end{array}$ & $\begin{array}{r}.8359 \\
.3227 \\
.3109 \\
.3001\end{array}$ & $\begin{array}{l}2 \\
2 \\
2 \\
2\end{array}$ & $\begin{array}{l}.3546 \\
. \mathbf{3 4 0 4} \\
.3276 \\
. \mathbf{3 1 5 9}\end{array}$ & $\begin{array}{l}2 \\
2 \\
2\end{array}$ & $\begin{array}{l}.3746 \\
.3592 \\
.3453 \\
.3328\end{array}$ & $\begin{array}{l}2 \\
2 \\
2 \\
2\end{array}$ & $\begin{array}{l}.3955 \\
.3789 \\
.3639 \\
.3504\end{array}$ & \\
\hline & $\begin{array}{l}.2764 \\
.2567 \\
.2105 \\
.2269\end{array}$ & $\begin{array}{l}\mathbf{2} \\
\mathbf{2} \\
\mathbf{2} \\
\mathbf{2}\end{array}$ & $\begin{array}{r}.2903 \\
.2691 \\
.2517 \\
.2371\end{array}$ & $\begin{array}{l}2 \\
2 \\
2\end{array}$ & $\begin{array}{r}.3053 \\
.2825 \\
.2638 \\
.2481\end{array}$ & $?$ & $\begin{array}{r}.3213 \\
.2967 \\
.2766 \\
.2597\end{array}$ & $\begin{array}{l}2 \\
2 \\
2 \\
2\end{array}$ & $\begin{array}{l}.3 \\
.3 \\
.2 \\
.2\end{array}$ & \\
\hline & $\begin{array}{l}.2153 \\
.1964 \\
.1816 \\
.1697\end{array}$ & $\begin{array}{l}2 \\
2 \\
2\end{array}$ & $\begin{array}{r}.2246 \\
.2044 \\
.1887 \\
.1759\end{array}$ & $\begin{array}{l}2 \\
2 \\
2\end{array}$ & $\begin{array}{r}.2348 \\
.2132 \\
.1963 \\
.1827\end{array}$ & 2 & $\begin{array}{l}.2454 \\
.2223 \\
.2043 \\
.1898\end{array}$ & $\begin{array}{l}\mathbf{2} \\
\mathbf{2} \\
\mathbf{2} \\
\mathbf{2}\end{array}$ & $\begin{array}{l}.2564 \\
.2317 \\
.2125 \\
.1971\end{array}$ & \\
\hline & $\begin{array}{l}.1597 \\
.1322 \\
.1228\end{array}$ & 2 & $\begin{array}{l}.1654 \\
.1362 \\
.1263\end{array}$ & 2 & $\begin{array}{l}.1715 \\
.1406 \\
.1301\end{array}$ & & $\begin{array}{r}.1779 \\
.1451 \\
.1340\end{array}$ & $\begin{array}{l}2 \\
2 \\
2\end{array}$ & $\begin{array}{l}.1843 \\
.1496 \\
.1378\end{array}$ & \\
\hline
\end{tabular}


POSITRONS

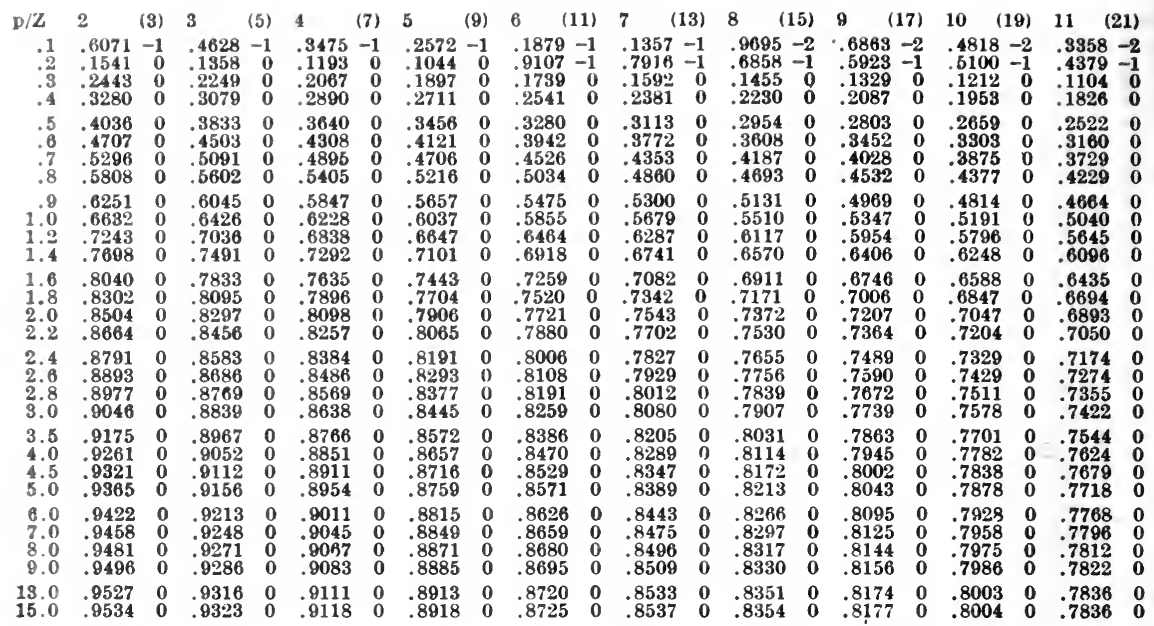

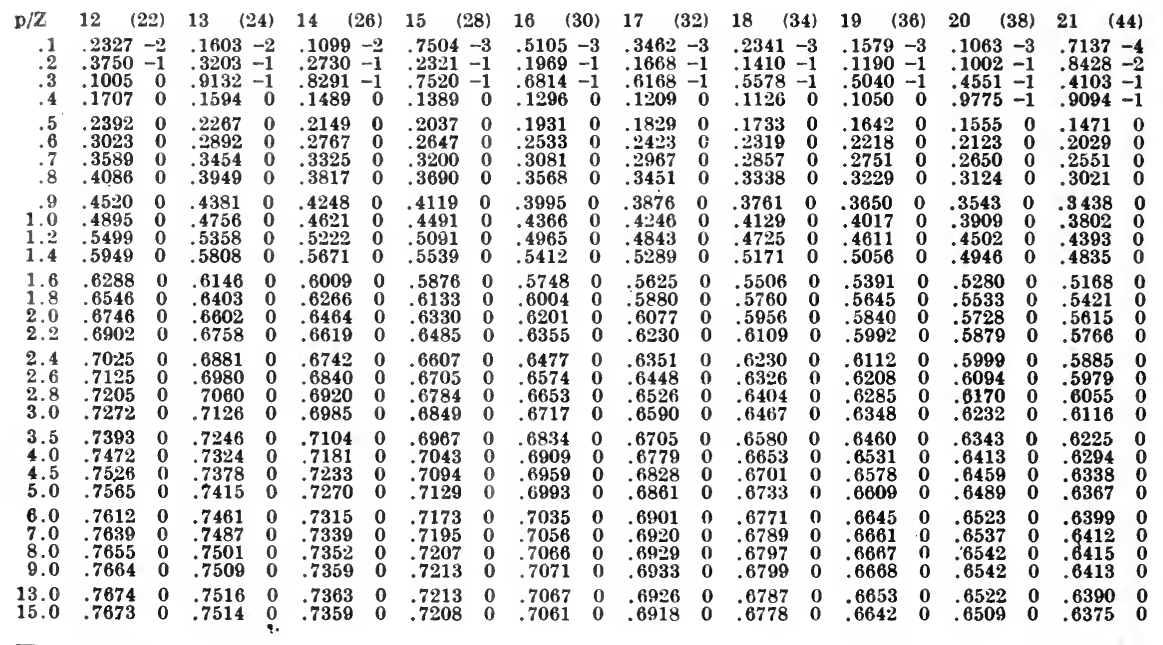

$\begin{array}{lllllllllllllllllllllll}\mathrm{p} / \mathrm{Z} & 22 & (47) & 23 & (49) & 24 & (51) & 25 & (53) & 26 & (55) & 27 & (57) & 28 & (59) & 29 & (62) & 30 & (65) & 31 & (67)\end{array}$

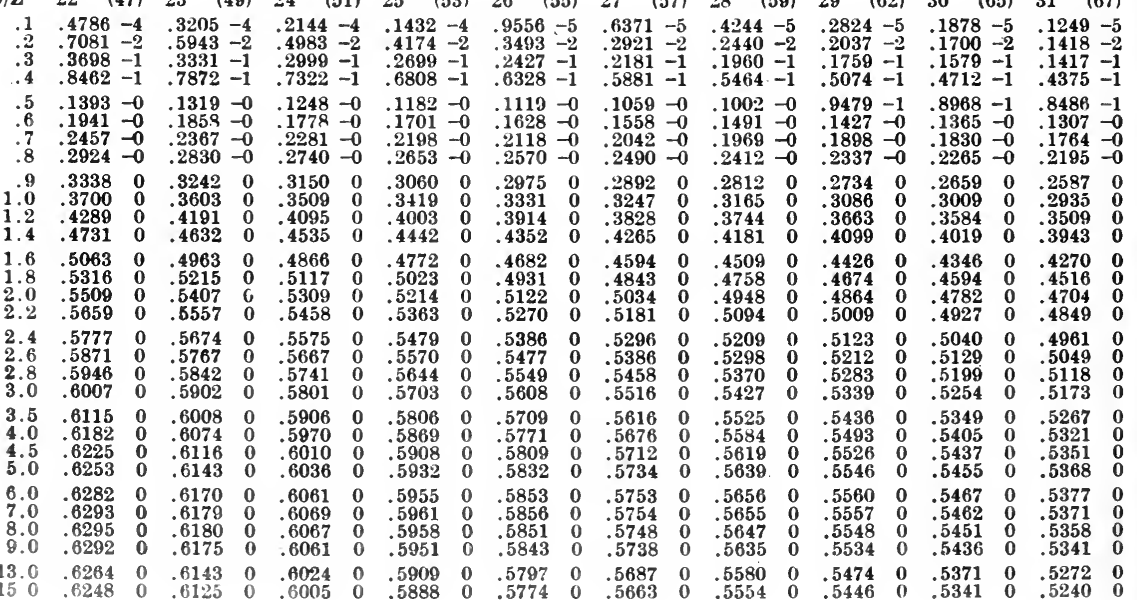




\section{POSITRONS}

$\begin{array}{lllllllllllllllllllll}\mathrm{p} / \mathrm{Z} & 32 & (71) & 33 & (72) & \mathbf{3 4} & (76) & 35 & (78) & 36 & (81) & 37 & (83) & 38 & (86) & 39 & (87) & 40 & (90) & 41 & (92)\end{array}$

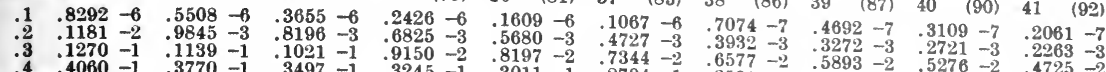

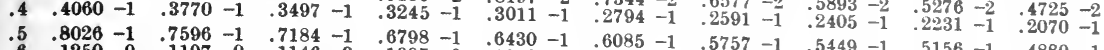

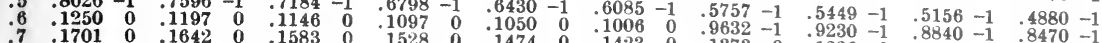

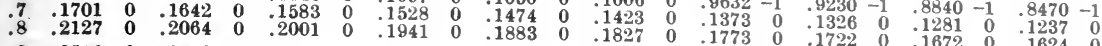

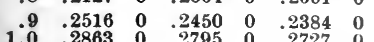

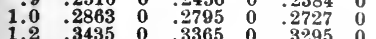

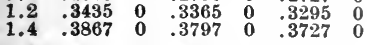

$\begin{array}{lllllll}1.6 & .4193 & 0 & .4123 & 0 & .4051 & 0 \\ 1.8 & .4440 & 0 & .4369 & 0 & .4296 & 0\end{array}$ $\begin{array}{lllllll}1.8 & .4440 & 0 & .4369 & 0 & .4296 & 0 \\ \mathbf{2 . 0} & .4627 & \mathbf{0} & . \mathbf{4 5 5 5} & \mathbf{0} & . \mathbf{4 4 8 3} & 0 \\ \mathbf{2 . 2} & \mathbf{. 4 7 7 1} & \mathbf{0} & \mathbf{. 4 6 9 9} & \mathbf{0} & . \mathbf{4 6 2 5} & 0\end{array}$ $\begin{array}{lllllll}2.4 & .4882 & 0 & .4810 & 0 & .4736 & 0 \\ 2.6 & .4969 & 0 & .4896 & 0 & .4821 & 0\end{array}$ $\begin{array}{lllllll}2.6 & .4969 & 0 & .4896 & 0 & .4821 & 0 \\ 2.8 & .5038 & 0 & .4964 & 0 & .4889 & 0\end{array}$ $\begin{array}{lllllll}\mathbf{3 . 0} & .5092 & \mathbf{0} & . \mathbf{4 9 6 4} & \mathbf{0} & . \mathbf{4 8 8 9} & 0 \\ & \mathbf{0} & .5018 & \mathbf{0} & \mathbf{. 4 9 4 1} & 0\end{array}$ $\begin{array}{lllllll}\mathbf{3} .5 & .5184 & \mathbf{0} & .5108 & \mathbf{0} & .5030 & \mathbf{0} \\ \mathbf{4 . 0} & .5236 & \mathbf{0} & .5159 & \mathbf{0} & .5079 & \mathbf{0}\end{array}$ $\begin{array}{lllllll}\mathbf{4 . 0} & .5236 & \mathbf{0} & .5159 & 0 & .5079 & 0 \\ \mathbf{4 . 5} & .5266 & \mathbf{0} & .5186 & \mathbf{0} & .5105 & 0 \\ \mathbf{5 . 0} & .5281 & \mathbf{0} & .5200 & \mathbf{0} & .5118 & \mathbf{0}\end{array}$ $\begin{array}{lllllll}6.0 & .5288 & 0 & .5205 & 0 & .5120 & 0\end{array}$ $\begin{array}{lllllll}\mathbf{7} .0 & .5279 & 0 & .5194 & 0 & .5107 & 0 \\ 8.0 & .5264 & 0 & .5177 & 0 & .5089 & 0\end{array}$ $\begin{array}{lllllll}8.0 & .5264 & 0 & .5177 & 0 & .5089 & 0 \\ 9.0 & .5246 & 0 & .5158 & 0 & .5068 & 0\end{array}$ $\begin{array}{rrrrrr}.2321 & 0 & .2260 & 0 & .2202 & 0\end{array}$ $\begin{array}{llllll}2603 & 0 & .2600 & 0 & .2540 & 0\end{array}$ $\begin{array}{llll}3230 & 0 & .3165 & 0 \\ 3660 & 0 & .3595 & 0\end{array}$

$\begin{array}{llllll}3984 & 0 & 3918 & 0 & .3533 & 0\end{array}$ $\begin{array}{llllll}.4229 & 0 & .4163 & 0 & .3856 & 0 \\ .4100 & 0\end{array}$ $\begin{array}{llllll}. \mathbf{4 4 1 5} & 0 & \mathbf{. 4 3 4 8} & \mathbf{0} & \mathbf{. 4 2 8 4} & \mathbf{0} \\ . \mathbf{4 5 5 7} & \mathbf{0} & \mathbf{. 4 4 8 9} & 0 & \mathbf{4 4 2 5} & 0\end{array}$ $\begin{array}{llllll}.4666 & 0 & .4598 & 0 & .4534 & 0\end{array}$ $\begin{array}{llllll}.4752 & 0 & .4683 & 0 & .4617 & 0 \\ .4818 & 0 & .4748 & 0 & .4682 & 0\end{array}$ $\begin{array}{rrrrrr}.4818 & 0 & .4748 & 0 & .4682 & 0 \\ .4870 & 0 & .4800 & 0 & .4733 & 0\end{array}$ $\begin{array}{llllll}.4957 & 0 & .4885 & 0 & .4817 & 0 \\ .5005 & 0 & .4931 & 0 & .4861 & 0\end{array}$ $\begin{array}{llllll}.5005 & 0 & .4931 & 0 & .4861 & 0 \\ 5029 & 0 & .4954 & 0 & .4882 & 0 \\ 5040 & 0 & .4964 & 0 & .4891 & 0\end{array}$ $\begin{array}{llllll}.5040 & 0 & .4961 & 0 & .4885 & 0\end{array}$

$\begin{array}{llllll}5025 & 0 & .4944 & 0 & .4867 & 0 \\ 5005 & 0 & .4922 & 0 & .4842 & 0 \\ 4982 & 0 & .4898 & 0 & .4816 & 0\end{array}$

$\begin{array}{lllllll}13.0 & .5172 & 0 & .5079 & 0 & .4984 & 0 \\ 15.0 & .5139 & 0 & .5044 & 0 & .4947 & 0\end{array}$

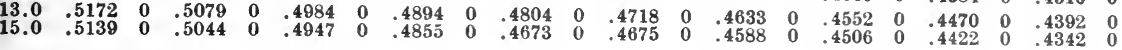
2485200

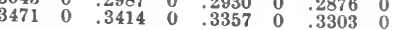
$\begin{array}{llllllll}3794 & 0 & .3737 & 0 & .3679 & 0 & .3624 & 0\end{array}$

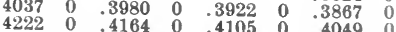
$\begin{array}{rrrrrrrr}4362 & 0 & .4164 & 0 & .4105 & 0 & .4049 & 0 \\ .4303 & 0 & .4244 & 0 & .4188 & 0\end{array}$ $\begin{array}{llllllll}4470 & 0 & .4410 & 0 & .4350 & 0 & .4294 & 0\end{array}$ $\begin{array}{llllllll}.4553 & 0 & .4493 & 0 & .4432 & 0 & .4375 & 0 \\ 4617 & 0 & .4557 & 0 & .4495 & 0 & .4437 & 0\end{array}$ $\begin{array}{llllllll}\mathbf{4 6 6 7} & 0 & . \mathbf{4 5 5 7} & 0 & .4495 & 0 & .4437 & 0 \\ & \mathbf{4 6 0 6} & \mathbf{4} & \mathbf{4 4 5 4 4} & \mathbf{0} & \mathbf{. 4 4 8 5} & \mathbf{0}\end{array}$ $\begin{array}{llllllll}.4749 & 0 & .4686 & 0 & .4622 & 0 & .4562 & 0\end{array}$ $\begin{array}{llllllll}.4791 & 0 & .4727 & 0 & .4661 & 0 & .4599 & 0\end{array}$ $\begin{array}{llllllll}.4811 & 0 & . \mathbf{4 7 4 5} & 0 & .4678 & \mathbf{0} & . \mathbf{4 6 1 5} & \mathbf{0} \\ \mathbf{4 8 1 8} & 0 & \mathbf{4 7 5 1} & \mathbf{0} & \mathbf{4 6 8 2} & \mathbf{0} & \mathbf{4 6 1 7} & \mathbf{0}\end{array}$ $\begin{array}{llllllll}4810 & 0 & .4741 & 0 & .4669 & 0 & .4602 & 0\end{array}$ $\begin{array}{llllllll}4789 & 0 & .4717 & 0 & .4644 & 0 & .4574 & 0\end{array}$ $\begin{array}{llllllll}.4736 & 0 & .4660 & 0 & .4584 & 0 & .4510 & 0\end{array}$

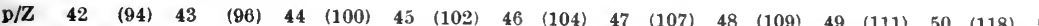

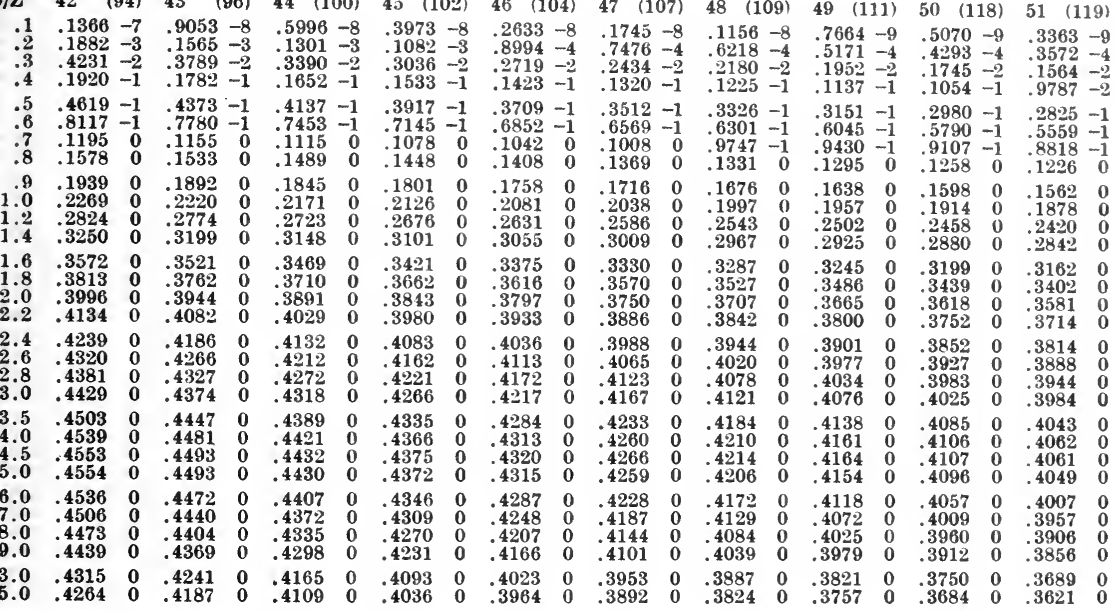

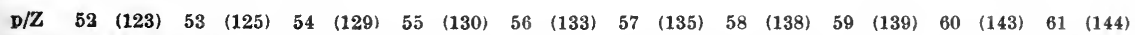
$\begin{array}{lllllllllllll}.1 & .2228 & -9 & .1477 & -9 & .9790-10 & .6497-10 & .4308-10 & .2859-10 & .1897-10 & .1260-10 & .8357-11 & .5553-11\end{array}$

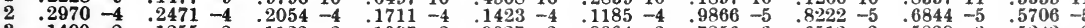

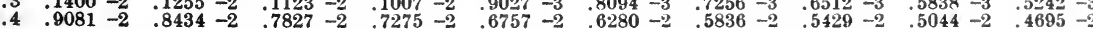

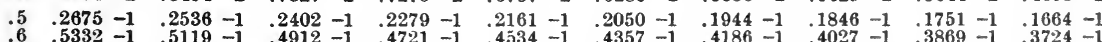

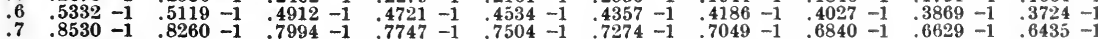

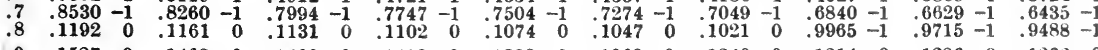
$\begin{array}{rrrrrrrrrrrrrrrrrrrrrrr}.9 & .1527 & 0 & .1493 & 0 & .1460 & 0 & .1429 & 0 & .1398 & 0 & .1369 & 0 & .1340 & 0 & .1314 & 0 & .1286 & 0 & .1262 & 0 \\ 1.0 & .1840 & 0 & .1805 & 0 & .1770 & 0 & .1738 & 0 & .1706 & 0 & .1676 & 0 & .1646 & 0 & 1618 & 0 & 1589 & 0 & .1564 & 0\end{array}$

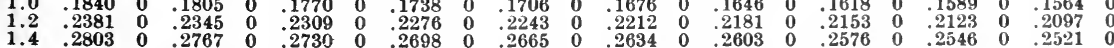
$\begin{array}{lllllllllllllllllllll}1.6 & .3122 & 0 & .3087 & 0 & .3050 & 0 & .3018 & 0 & .2985 & 0 & .2954 & 0 & .2924 & 0 & .2897 & 0 & .2867 & 0 & .2843 & 0\end{array}$

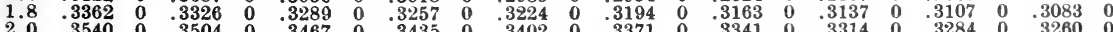

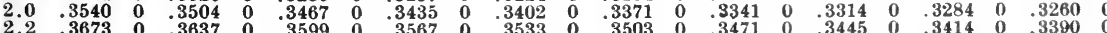

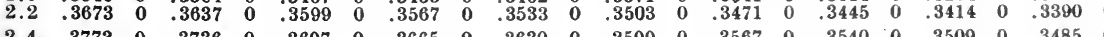

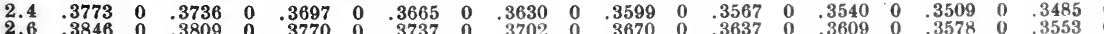

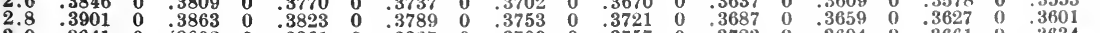

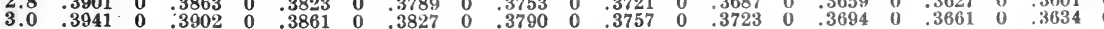
$\begin{array}{llllllllllllllllllllll}3.5 & .3997 & 0 & .3956 & 0 & .3914 & 0 & .3877 & 0 & .3839 & 0 & .3803 & 0 & .3767 & 0 & .3736 & 0 & .3701 & 0 & .3673 & 0\end{array}$

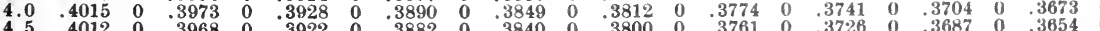

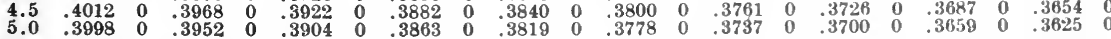
$\begin{array}{lllllllllllllllllllll}6.0 & .3953 & 0 & .3905 & 0 & 3854 & 0 & .3809 & 0 & .3763 & 0 & .3719 & 0 & .3675 & 0 & .3635 & 0 & .3591 & 0 & .3554 & 0\end{array}$

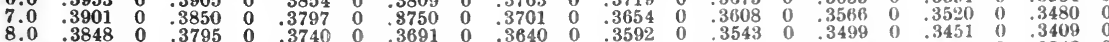

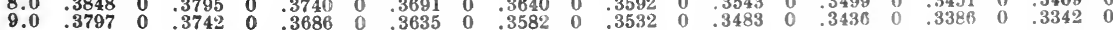
$\begin{array}{lllllllllllllllllllll}13.0 & .3625 & 0 & .3565 & 0 & .3503 & 0 & .3448 & 0 & .3390 & 0 & .3335 & 0 & .3280 & 0 & .3229 & 0 & .3174 & 0 & .3125 & 0 \\ 15.0 & .3555 & 0 & .3493 & 0 & .3430 & 0 & .3373 & 0 & 3313 & 0 & .3257 & 0 & .3200 & 0 & .3147 & 0 & .3011 & 0 & .3040 & 0\end{array}$ 


\section{POSITRONS}

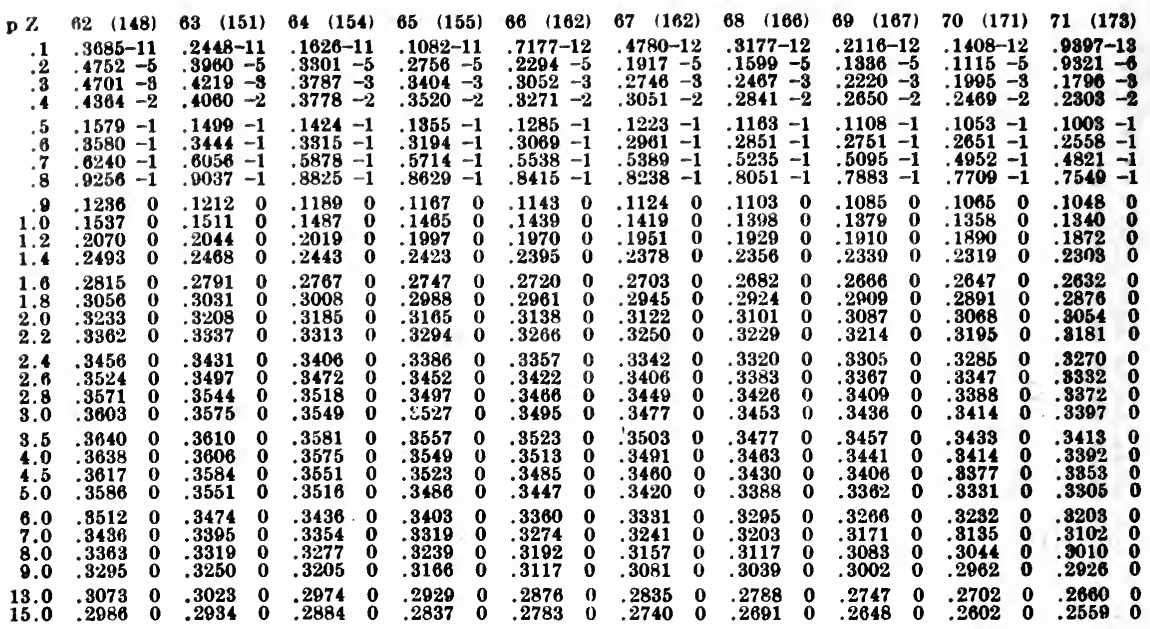

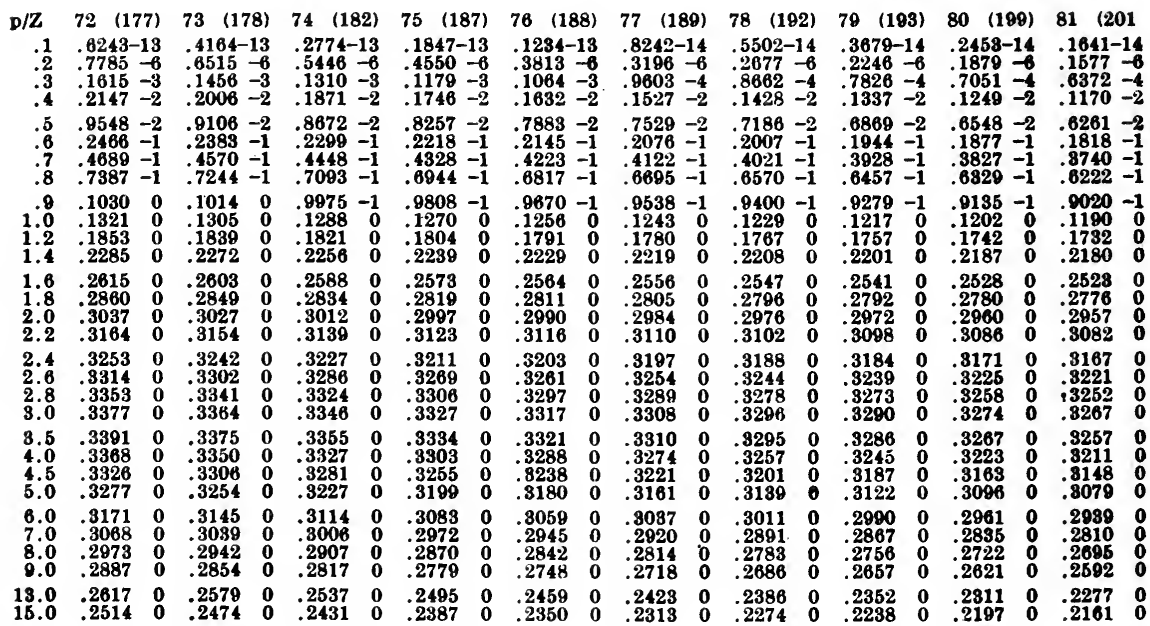

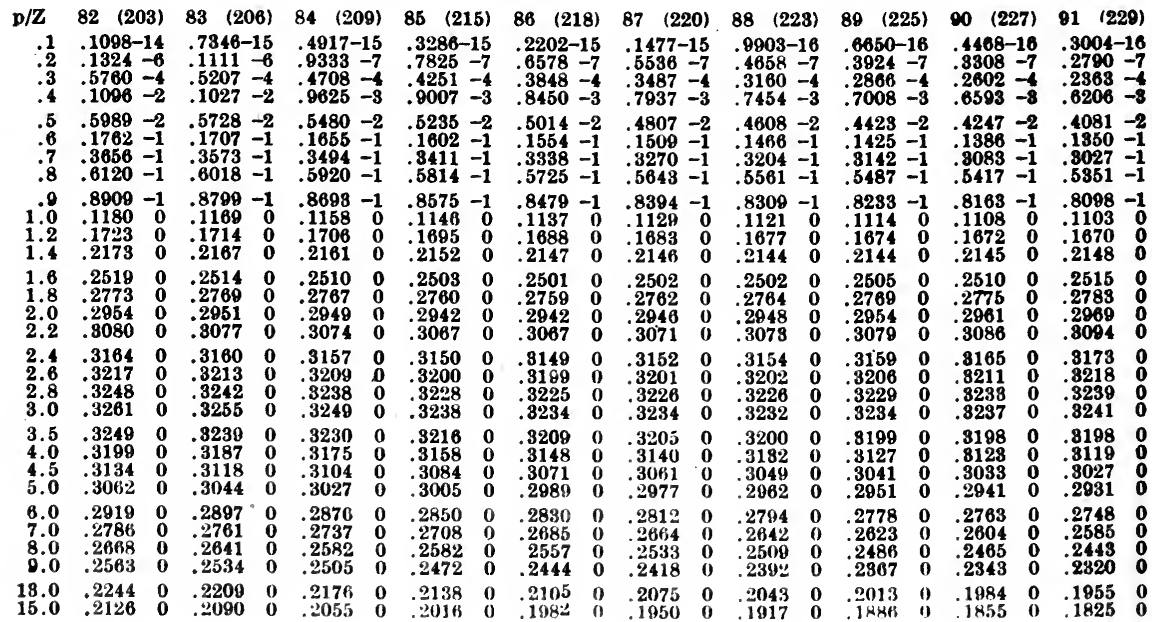




\section{POSITRONS}

$\begin{array}{lllllllllll}p / 2 & 92 & \text { (232) } & 93 & \text { (234) } & 94 & \text { (238) } & 95 & \text { (240) } & 96 & \text { (24:2) }\end{array}$

\begin{tabular}{|c|c|c|c|c|c|c|c|c|}
\hline $\begin{array}{l}.2019- \\
.2858 \\
.2146 \\
.5841\end{array}$ & $\begin{array}{l}-16 \\
-7 \\
-4 \\
-3\end{array}$ & $\begin{array}{l}.1359-1 \\
.1987 \\
.1951 \\
.5504\end{array}$ & $\begin{array}{l}16 \\
-7 \\
-4 \\
-3\end{array}$ & $\begin{array}{l}.1676 \\
.1773 \\
.5182\end{array}$ & & 8 & $\begin{array}{r}-17 \\
-7 \\
-4 \\
-3\end{array}$ & $\begin{array}{r}.4152 \\
.1199 \\
.1470 \\
.4616\end{array}$ \\
\hline $\begin{array}{l}.8920 \\
.1313 \\
.2972 \\
.5284\end{array}$ & $\begin{array}{l}-2 \\
-1\end{array}$ & $\begin{array}{r}.8771 \\
.1280 \\
.2921 \\
.5225\end{array}$ & $\begin{array}{l}-2 \\
-1 \\
-1 \\
-1\end{array}$ & $\begin{array}{l}.3624 \\
.1246 \\
.2868 \\
.5162\end{array}$ & & $\begin{array}{l}.3 \\
.1 \\
.2 \\
.5\end{array}$ & $\begin{array}{l}-2 \\
-1 \\
-1 \\
-1\end{array}$ & \\
\hline & -1 & $\begin{array}{l}.7975 \\
.1093 \\
.1668 \\
.2154\end{array}$ & $\begin{array}{r}-1 \\
0 \\
0 \\
0\end{array}$ & $\begin{array}{l}.78 \\
.10 \\
.16 \\
.2\end{array}$ & & $\begin{array}{l}.7 \\
.1 \\
.1 \\
.2\end{array}$ & -1 & \\
\hline & $\begin{array}{l}0 \\
0\end{array}$ & $\begin{array}{l}.2528 \\
.2799 \\
.2988 \\
.3113\end{array}$ & $\begin{array}{l}\mathbf{0} \\
\mathbf{0} \\
\mathbf{0} \\
\mathbf{0}\end{array}$ & & 0 & & 0 & \\
\hline & 0 & $\begin{array}{l}.3190 \\
.3233 \\
.3251 \\
.3250\end{array}$ & $\begin{array}{l}\mathbf{0} \\
\mathbf{0} \\
\mathbf{0} \\
\mathbf{0}\end{array}$ & $\begin{array}{l}.3197 \\
.3239 \\
.3255 \\
.3253\end{array}$ & 0 & $\begin{array}{l}.3211 \\
.3251 \\
.3266 \\
.3262\end{array}$ & $\begin{array}{l}0 \\
0\end{array}$ & \\
\hline .318 & 0 & $\begin{array}{l}.3200 \\
.3113 \\
.3013 \\
.2912\end{array}$ & $\begin{array}{l}\mathbf{0} \\
\mathbf{0} \\
\mathbf{0} \\
\mathbf{0}\end{array}$ & $\begin{array}{l}.3198 \\
.3108 \\
.3004 \\
.2900\end{array}$ & 0 & $\begin{array}{l}.3203 \\
.3108 \\
.3001 \\
.2893\end{array}$ & 0 & \\
\hline $\begin{array}{l}.2732 \\
.2566 \\
.2421 \\
.2205\end{array}$ & 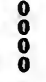 & $\begin{array}{l}.2719 \\
.2548 \\
.2401 \\
.2272\end{array}$ & $\begin{array}{l}\mathbf{0} \\
\mathbf{0} \\
\mathbf{0} \\
\mathbf{0}\end{array}$ & $\begin{array}{l}.2702 \\
.2528 \\
.2377 \\
.2247\end{array}$ & 0 & $\begin{array}{l}.2690 \\
.2512 \\
.2358 \\
.2225\end{array}$ & $\begin{array}{l}0 \\
0 \\
0 \\
0\end{array}$ & \\
\hline & 0 & $\begin{array}{l}.1898 \\
.1765\end{array}$ & 0 & $\begin{array}{l}.1867 \\
.1733\end{array}$ & & $\begin{array}{r}1840 \\
.1705\end{array}$ & 0 & $\begin{array}{l}.1813 \\
.1677\end{array}$ \\
\hline
\end{tabular}




\title{
TABLES OF FORBIDDEN $\beta$-DECAY FUNCTIONS
}

ACCORDING TO M. E. ROSE, C. L. PERRY AND N. M. DISMUKE

\author{
M. E. ROSE
}

(Cf. Ch. IX)

The functions given in the following tables are certain bilinear combinations of electron wave functions evaluated at the nuclear radius, $\varrho \approx 1.4 \times 10^{-13} \mathrm{~A}^{1 / 3} \mathrm{~cm}$. The wave functions are Dirac functions for a Coulomb field so that screening and finite nuclear size corrections should be made where necessary. The latter type of correction is important only for the lower $v$ values and only for $M_{v}, N_{v}, Q_{v}$ and $R_{v}$. The definitions of the six types of functions are in accord with those used in the literature. 1 With $\varrho=$ nuclear radius, $f_{x}$ and $g_{x}$ the Dirac radial functions normalized to one particle in a sphere of unit radius, $p$ the electron momentum in $m c$ units and $F_{0}$ the Fermi function, one has

$$
\begin{aligned}
& L_{k-1}=\left(2 p^{2} F_{0}\right)^{-1} \varrho^{2-2 k}\left(g_{-k}^{2}+f_{k}^{2}\right) \\
& M_{k-1}=\left(2 p^{2} F_{0}\right)^{-1} \varrho^{-2 k}\left(g_{k}^{2}+f_{-k}^{2}\right) \\
& N_{k-1}=\left(2 p^{2} F_{0}\right)^{-1} \varrho^{1-2 k}\left(f_{-k} g_{-k}-f_{k} g_{k}\right) \\
& P_{k-1}=\left(2 p^{2} F_{0}\right)^{-1} \varrho^{2-2 k}\left(g_{-k}^{2}-f_{k}^{2}\right) \\
& Q_{k-1}=\left(2 x^{2} F_{0}\right)^{-1} \varrho^{-2 k}\left(g_{k}^{2}-f_{-k}^{2}\right) \\
& R_{k-1}=\left(2 p^{2} F_{0}\right)^{-1} \varrho^{1-2 k}\left(f_{-k} g_{-k}+f_{k} g_{k}\right)
\end{aligned}
$$

Here $k=v+1$ is the parameter giving the electron angular momentum according to $j=k-\frac{1}{2}(k \geqslant 1) .{ }^{2}$ In the tables the heading gives $\mathrm{Z}$ and the designation "electron" or "positron". The value of $v$ is also given and the value of $\mathrm{A}$ is chosen as an average for known electron (or positron) emitters. A small change in the value of $\mathrm{A}$ is generally not important, and in view of some uncertainty in nuclear radius, not worth making in most cases. For values of $p$ listed in the first column, the values of the quantities $\mathrm{L}, \mathrm{P}, \mathrm{M}, \mathrm{Q}, \mathrm{N}, \mathrm{R}$ are given in succeeding columns. Interpolation for values of $p$ other than those given can be carried out with considerable accuracy. It will be recognized that the values given in the tables are more accurate than those obtained from the analytical representations given in reference $1 .{ }^{3}$

1 E. J. Konopinski and G. E. Uhlenbeck, Phys. Rev. 60, 303 (1941); E. Greuling, Phys. Rev. 61, 568 (1942); D. L. Pursey, Phil. Mag. 42, 1193 (1951).

2 For further details see M. E. Rose, Phys. Rev. 93, 1321 (1954).

3 See further, M. E. Rose and C. L. Perry, Phys. Rev. 90, 479 (1953). 
The entries in this table, sometimes referred to as shape factor functions for forbidden transitions, permit the analysis of spectra through third order forbidden when used in conjunction with the table of the Fermi function, see preceding table. These entries are in the form of a four digit number $m(0.1 \leqslant m<1.0)$ followed by an integer $r$. This is to be read as $m \times 10^{r}$. The results given in this table constitue a part of the more extensive tabulation given by M. E. Rose, C. L. Perry and N. M. Dismuke, Oak Ridge National Laboratory Report, No. 1459. For notation see E. Greuling, Phys. Rev. 61, 568 (1942) and D. L. Pursey, Phil. Mag. 42, 1193 (1951); see also Chapter X. 


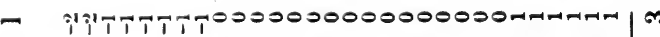

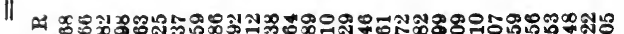

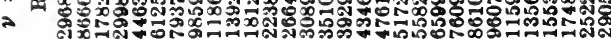
iiiiiiiiiiiiiiiiiiiiiii

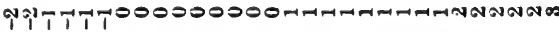

乙 品 iiiiiiiiiiiiiiiiiiiiii

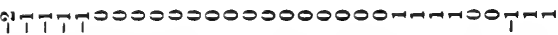

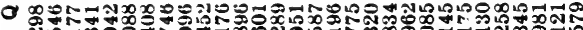

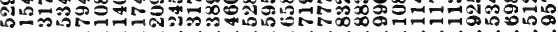
iliiiliiliiliiliiiil

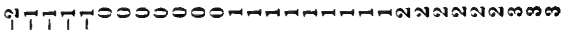

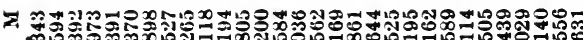

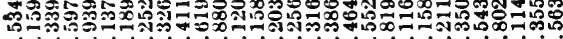

FYYTTTTTTT000000000000000000

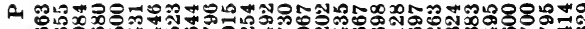
Q

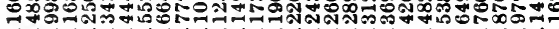

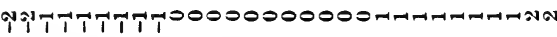

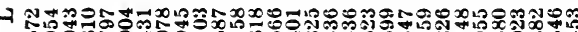

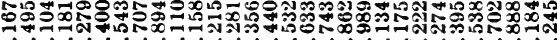

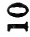

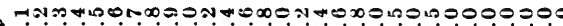

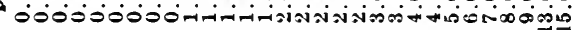
$\mathbf{N}$

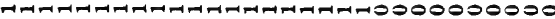

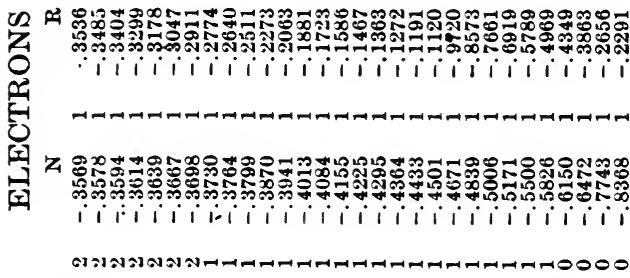

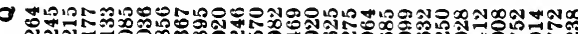

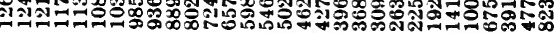
iiliiiliiilifiiiiiii

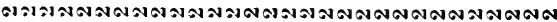

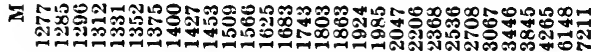
0000000000000000000000000004

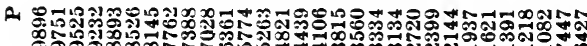

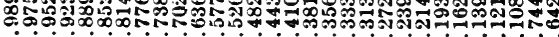

000000000000000000000000000000

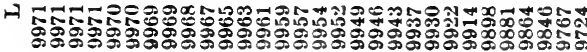

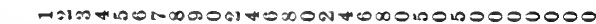
ค 000000்

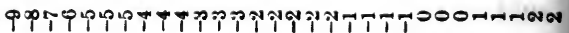

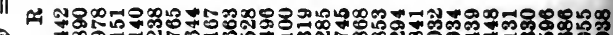
- * iiiiiiiiiiiiiiiiiiiii

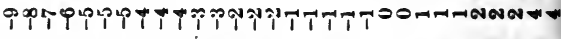
乙 ำ

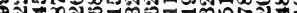
iiiliiiliiiliiiiiliiii PoT

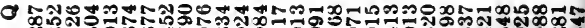

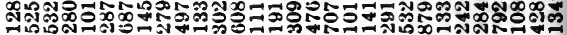
iiliiliiliiliiliiili

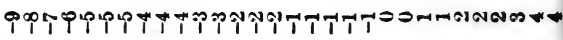

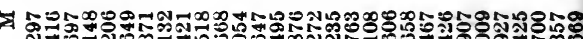

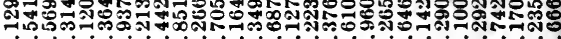

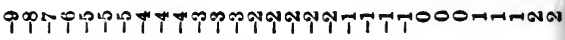

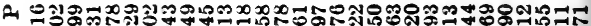

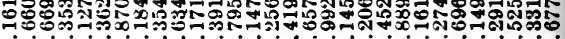

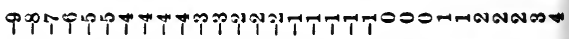

H

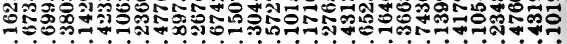

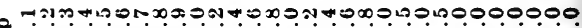

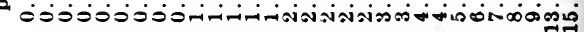

PY 션 . iiliiiiiliiiliiiiiiiii

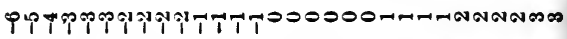

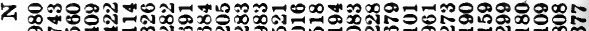

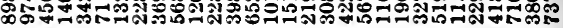
iiiliiiliiiiiliiiiiiiii

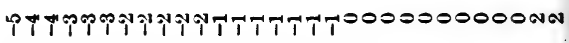

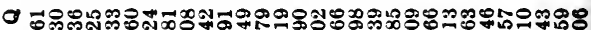

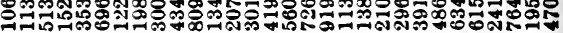
iiiiiiiiiiiiiiiiiii

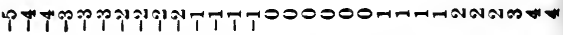

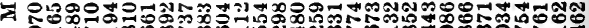

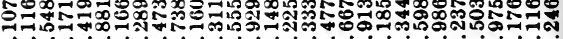

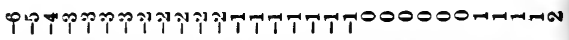

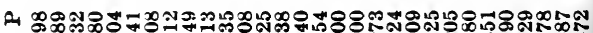
ㄲ.

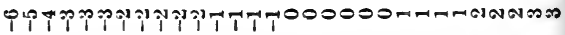

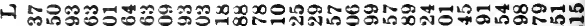
ค.

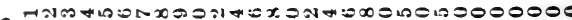
a 00000000- 


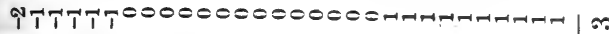

4 สุํำ

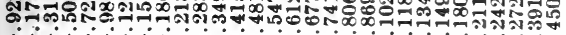
$1111111111111111111 i 1 i 1 i 1 i 1$

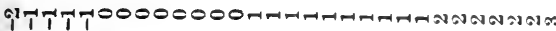

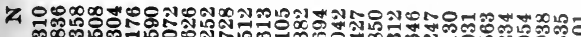

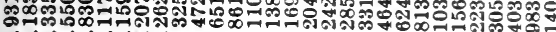
iiiiiiiiliiiiiiiiiiiii

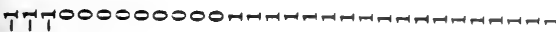

○ สุด iliiiliiiiliililiiiiii

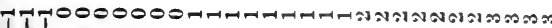

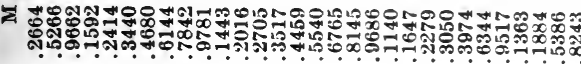
พTTTTTTTTTT00000000000000000H

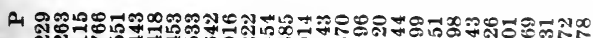
$\stackrel{4}{*}$

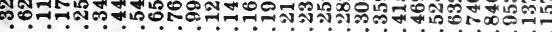

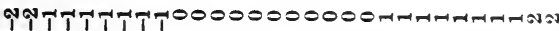

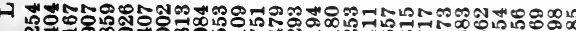
ติษ -

คิ

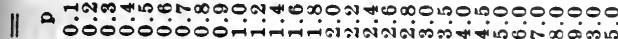
$\mathbf{N}$

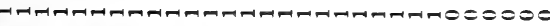

을

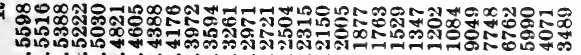

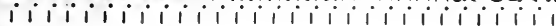

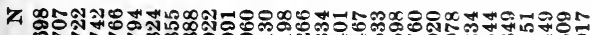

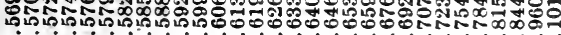
iliiiiiiiiiiiiiiliiiliili

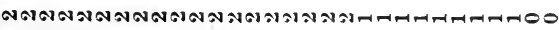

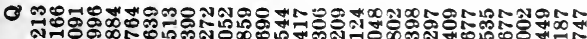
สెल

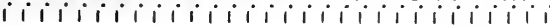

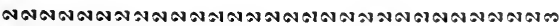

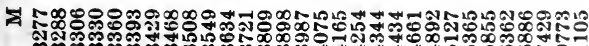

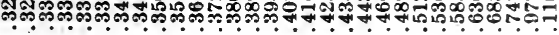

$0000000000000000000000000000 T T$ 4.

000000000000000000000000000000

구유.

0

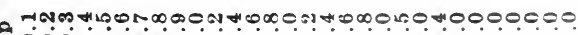

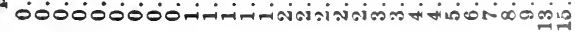

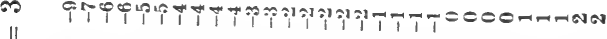

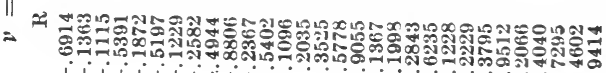
i i iliiiiiiiiiiiiiiiiiii

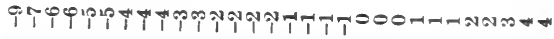
乙

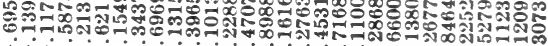
ii ii iiiiiiiiiiiiiiiiiii

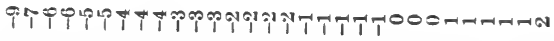

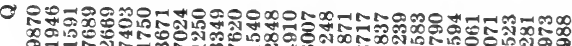

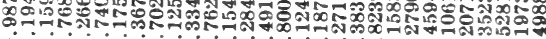
ii ii ii iiiiiiiiiiiiiiii

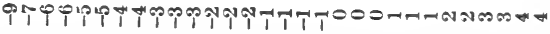

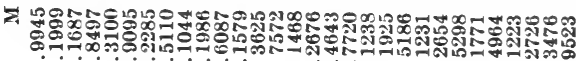

PXT TिP 거

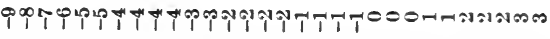

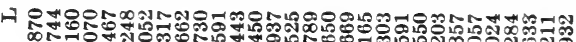

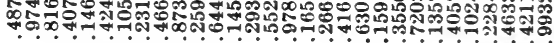

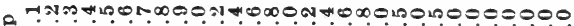

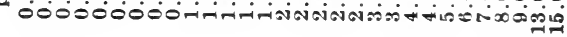

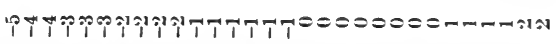

ณ กำ iiiiiiiiiliiiliiiiiiiii

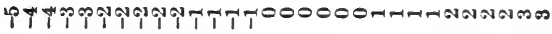

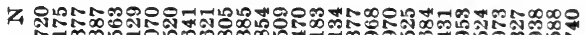

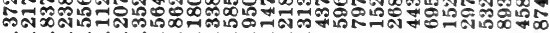
iliiliiliiliiliiliiliiil

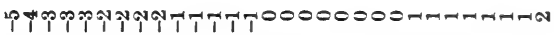

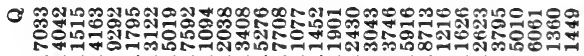
iiiiiiiiiiiiiiiiiiiiiiii 1P

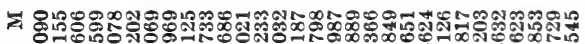

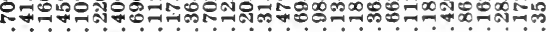

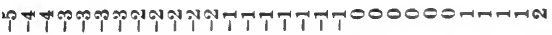

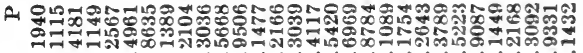

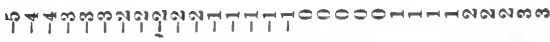

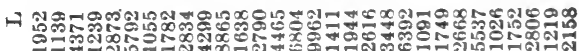
Q

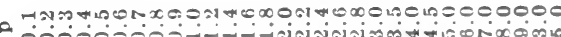
a c00000000 


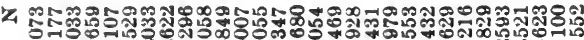
อิที่

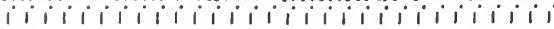

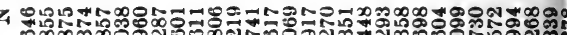

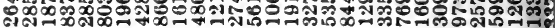
iiiiiiiiliiiliiiliiii

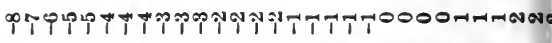

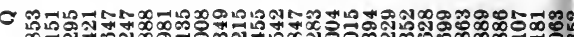

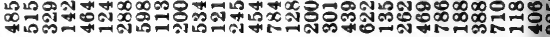
iiiiiiiiiiiiiiiiiiii

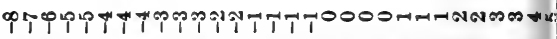

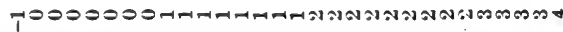

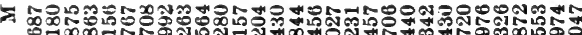
象:

அ认TTTTTTTTT00000000000000000

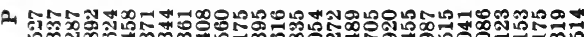

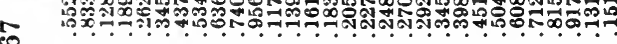

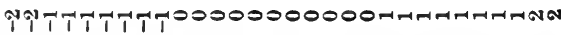

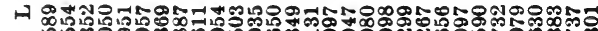
คำ.

ค

$\mathbf{N}$

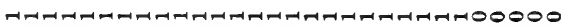

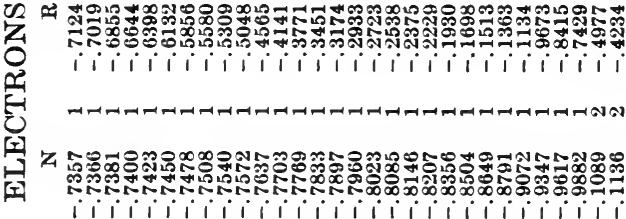

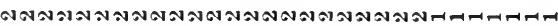

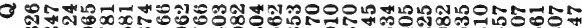
ตำ iiliiiiiiiiiiiliiiiiiii

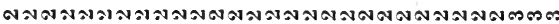

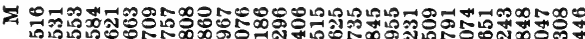
1月.

$0000000000000000000000000004 T 1$

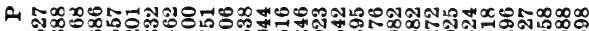

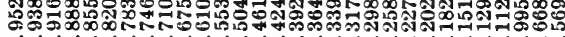

000000000000000000000000000000

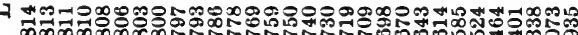

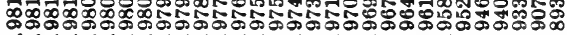

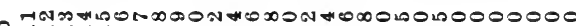

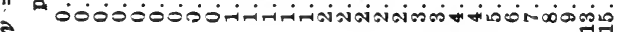

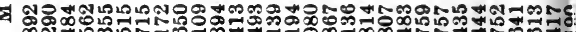
\%

PTT TP P

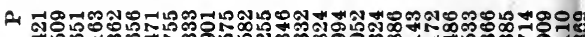

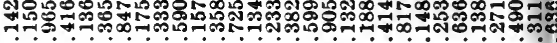

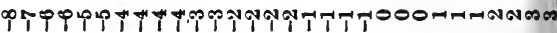
எ హ.

HNmWnN

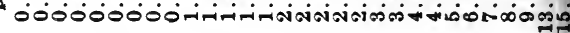

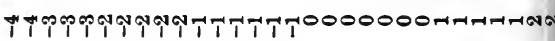

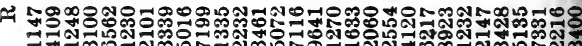

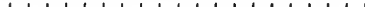

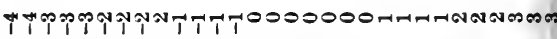

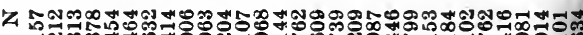

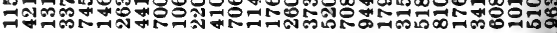
iiiiiiiiiiiiiiiiiiiiii

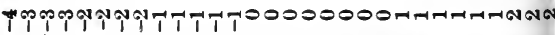

O

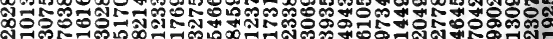

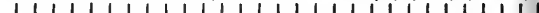

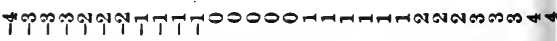
는

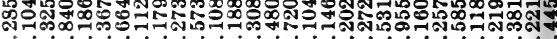

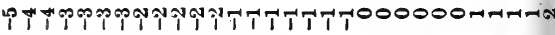
A

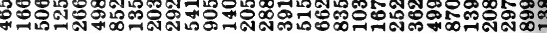

१F

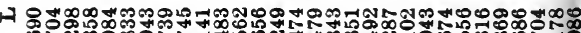

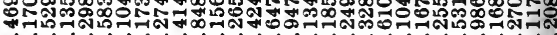

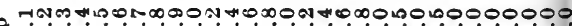

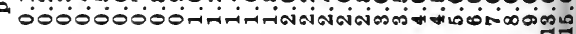


- TTTT0000000000000

ม ศొ ల. iliiiiiiiiiiiliiiliiii

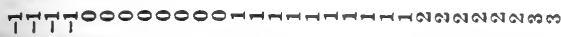

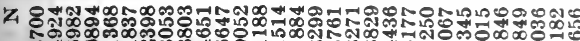
ద హ. iliiliiiliiliiliiiliii

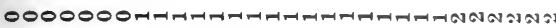

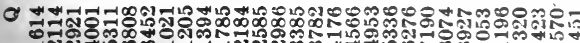
iiiiiiiiiiiiiiiiiiiiii

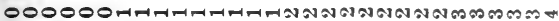

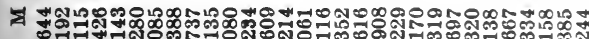

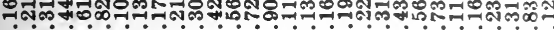

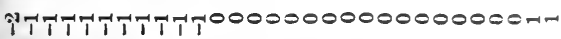

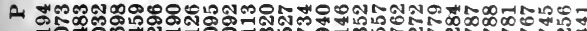
r ๓

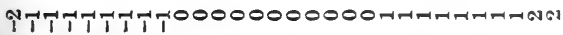

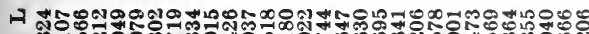
๓

F

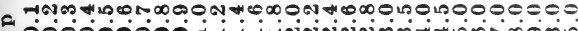

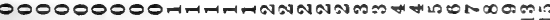

$\mathbf{N}$

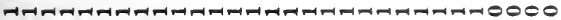

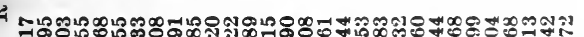
๓0 iliiiiliiiiiliiiliiilii - 01010001301

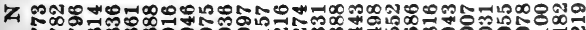
ందం iliiiliiiliiliiliiiliiili

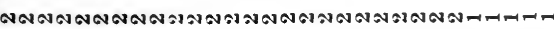

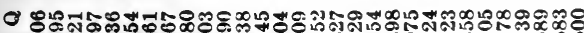

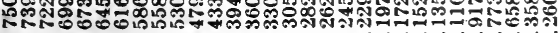
ii ii iiiiiiiiiiiiiiiiiiii

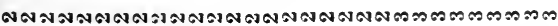

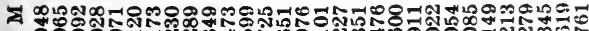

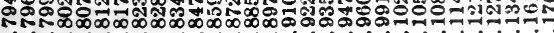

000000000000000000000000000711

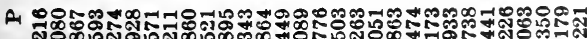

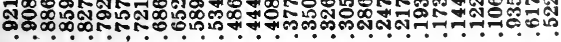

000000000000000000000000000000

\section{ㄱ.

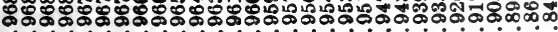

C

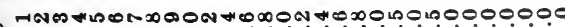

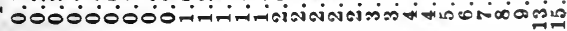

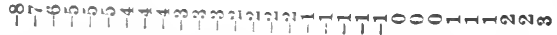

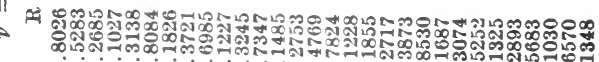
i i i i i i i i i i i i i iiiiiii?

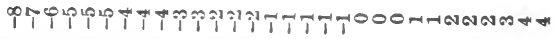
ব

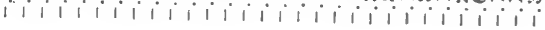

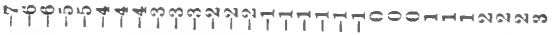

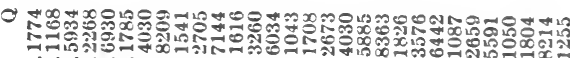
iiliiiliiiiiiiiiiiiiiii

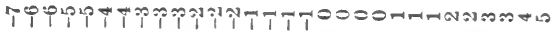

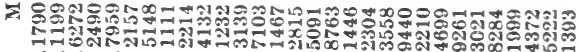
૧T TP

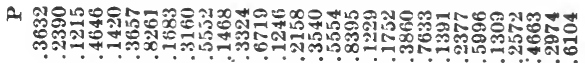

૧T T.T М

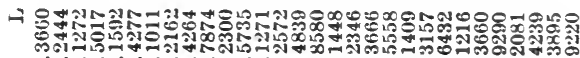

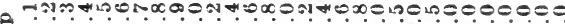

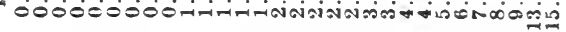

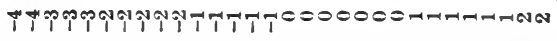

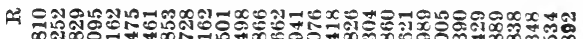

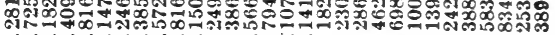
iliiliiiiiiliiiliiiiiii

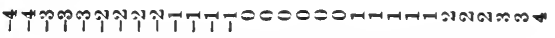
乙 웅ํำ iliiiiiiiiiiiiiiiliiiiji

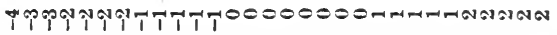
○ iliiiiiiiiiiiiiiiiiiiiiil

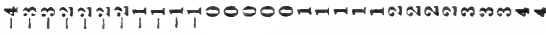

ำ

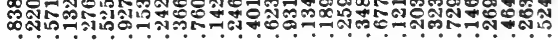

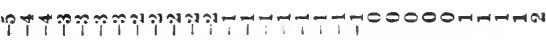

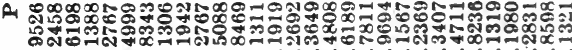

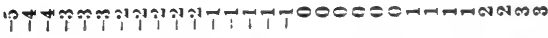

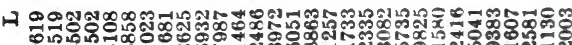
๑ง

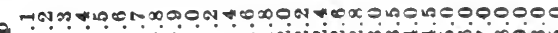

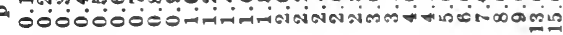




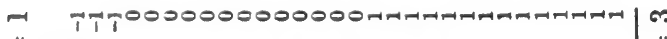

1|

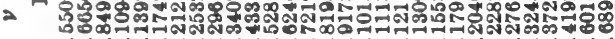
iiliiliiliiliiliiliili

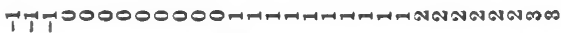

彳 ₹

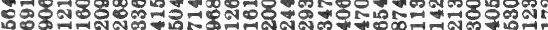
iiliiiiiiiliiiiiiiiiiiiii

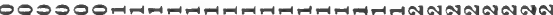

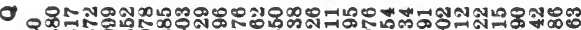

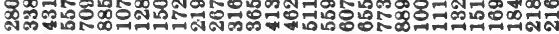
iliiiiiiiiiiiiiiiiiiiii

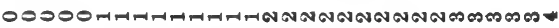

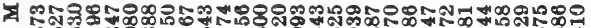
ง.

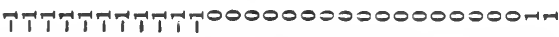

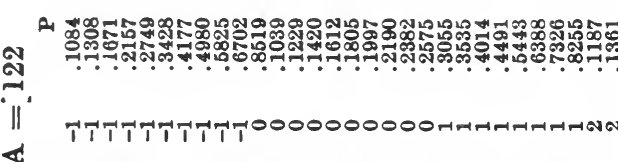

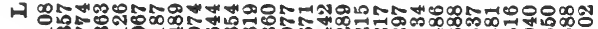

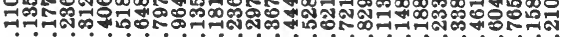

?

$\|$

HNm $\mathbf{D}$

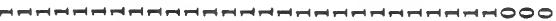

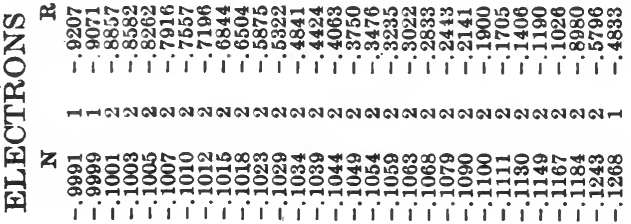

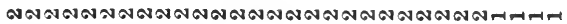

(

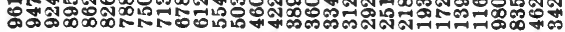
iiliiliiiliiliiliiliiiii क⿻上丨冖n

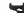

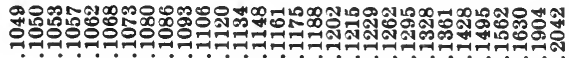

00000000000000000000000000 THT

2. $\infty m \infty$ m

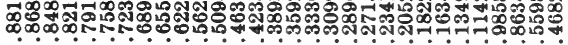
000000000000000000000000000000

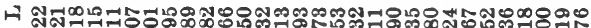
:

a HNM

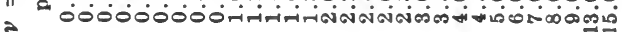

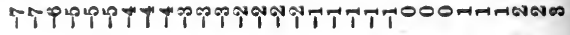
소 봉ำ

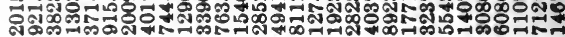
iiliiiiiiiiiiiiiiiiiiii

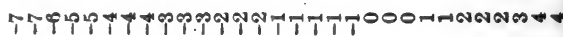
乙 iiiiiiiiiiiiiiiiiiiiiiii TPQP ○ ర్య iiliiiiiiiiiiiiiiiiiiiii

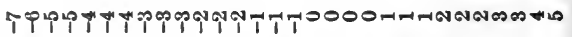

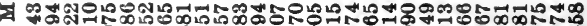

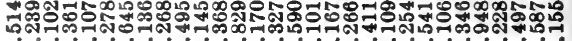

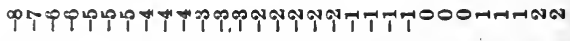

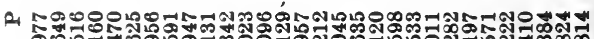

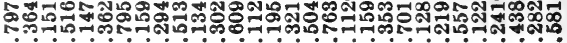

PT PQP P

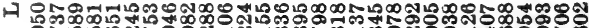

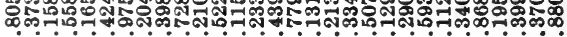

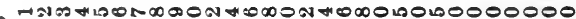

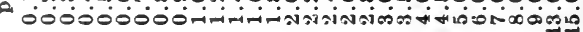

F゙ P

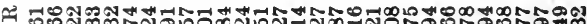
6. iiliiiiiiliiliiliiiiiii FPPPNGTYTTTTTT000000

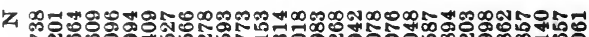

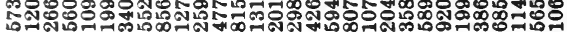
iiliiiiiiiiiiliiiiiiiii

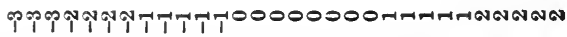

ช 유ำ

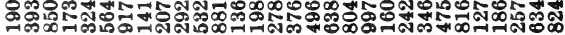
iliiliililililililiilii

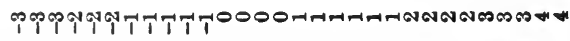

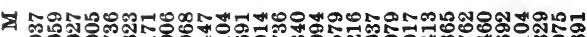

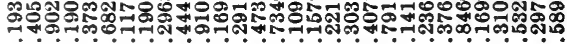

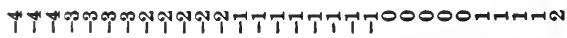

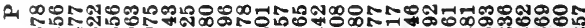

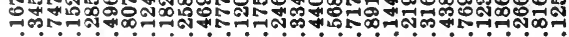

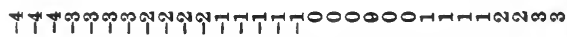
고근 ๑ొన ( )

H:Im \#

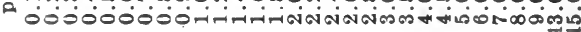


TT00000000000000

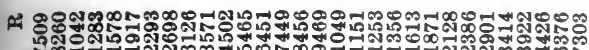
ii i i i i i i i i i i i i † Н000000000

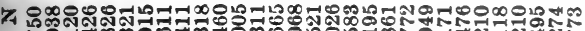

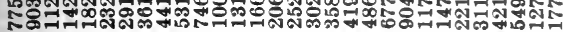

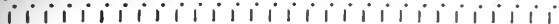

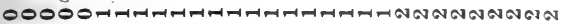

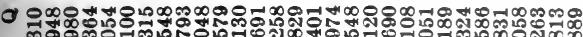

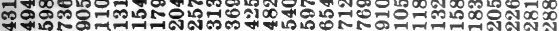
iliiliiiliiiiiiiliiiiiij

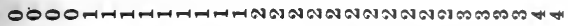

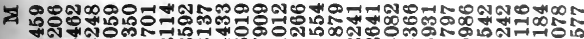

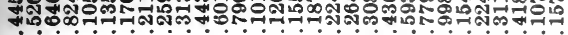

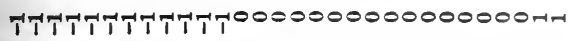

4 等

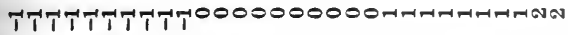

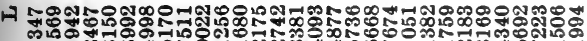

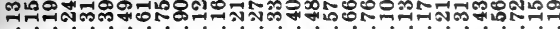

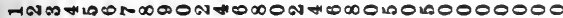

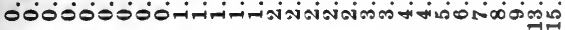

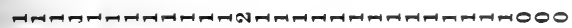

ค4 โ์

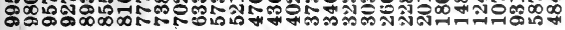
iliiiliiliiliiiliiliilii

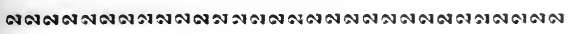

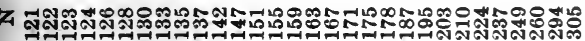
iiiiiiiiiiiiiiiiiiiiii

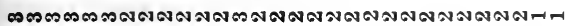

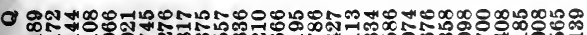

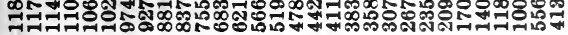
iliiiliiiliiiliiiliiili

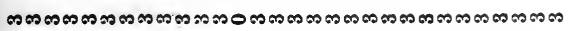
ఝำ:

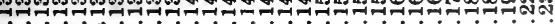

00000000000000000000000000 TTTT

4 ํㅠ꿍유

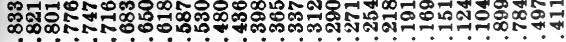

000000000000000000000000000000

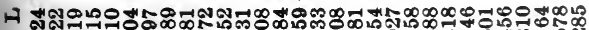

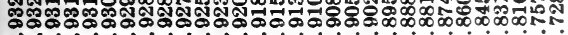

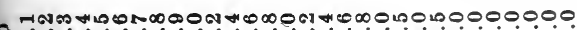
000000000-1- -

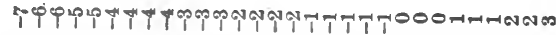
น

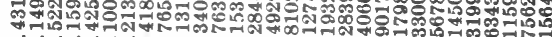
ililililiililiiliiliili

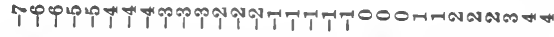
乙 אิ0

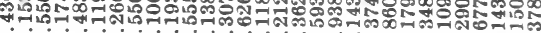
iliiliiiliiiliililiilii

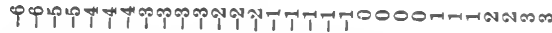

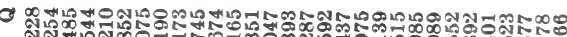

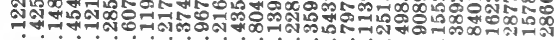
iliiiiiiiliiiliiiliiiiii १९९P

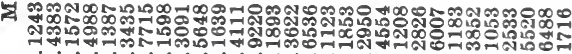

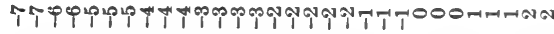
4 พ ค1.

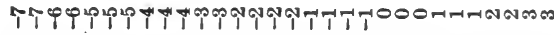
月 ต.尸.

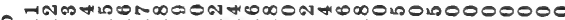

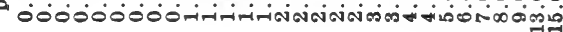

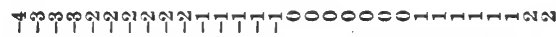

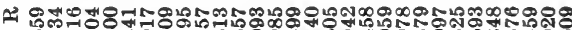
Dㄷำ iं.iiliiiliiliiliiliiili

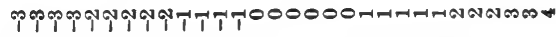

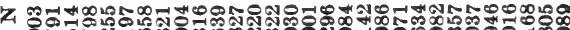

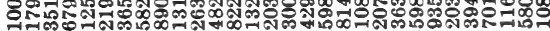

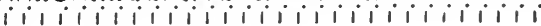

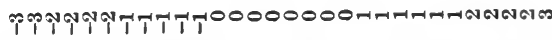

○ ผ iliiiliiiliiliiliiiliili

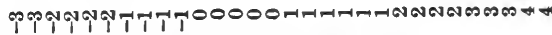
\

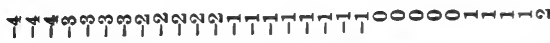

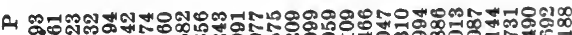
ผ.

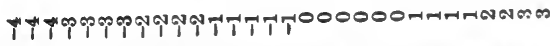

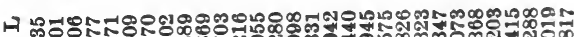
สำสำผ oi

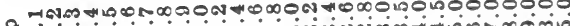

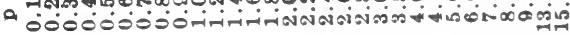




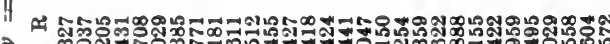

2 \%

i i i i i i i i i i i i i i i i i i i

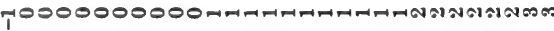

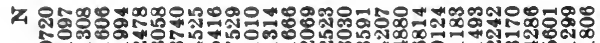
వ. 0000

HลNNNNNNNGN

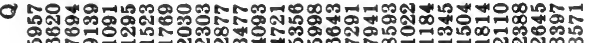
iiiiiiiiiiiiiiiiiiiiiiii

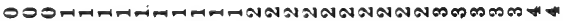

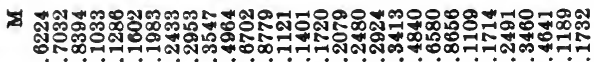

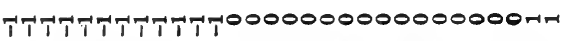

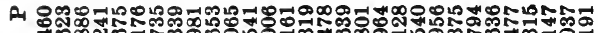

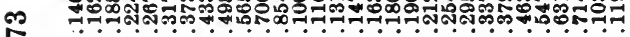

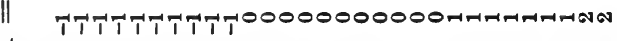
4

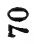

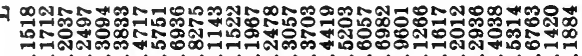

a TNM II

$\mathbf{N}$

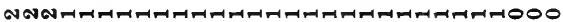

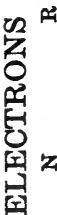

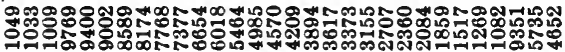
iiliiiliiiiiliiiiiiiiiii

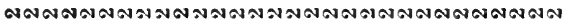

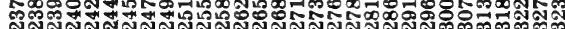

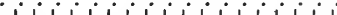

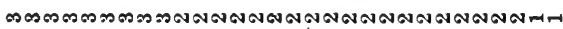

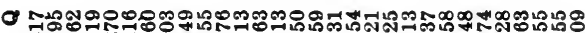

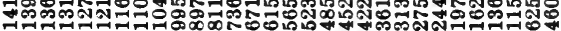
iiiiiiiiiiiiliiiiiiiii

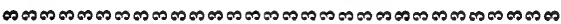
궁

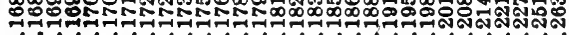
0000000000000000000000000 TTTTT

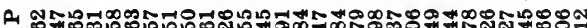

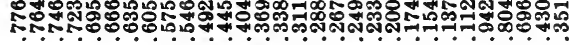

000000000000000000000000000000

月

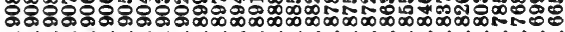

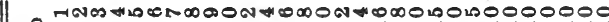
a

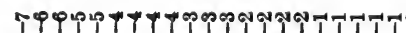

II

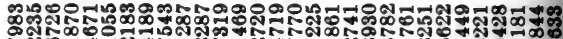

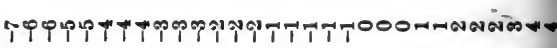

z

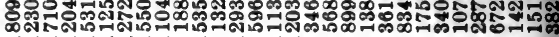
iiiiiii iiliiiiiiiiiiiiii

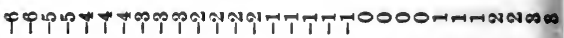
○ ヘ นี유. iiiiiiiiiiiiiiiiiiiiiii

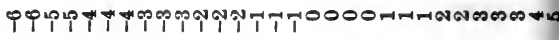
\#

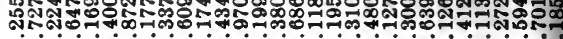

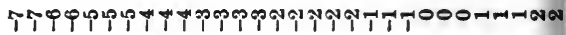

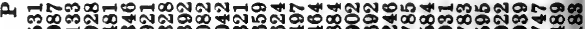
ง

TT 붕 దิส

HNmWnONDOON

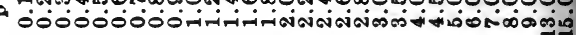

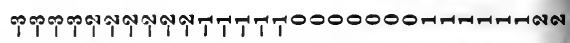
น

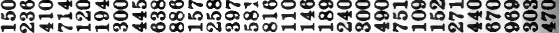
ii iiiiiiiliiiiiiiiiiii

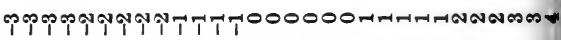

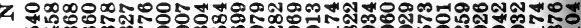
బ iliiliiiliiliiliiiliii

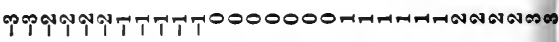
๑ 수누

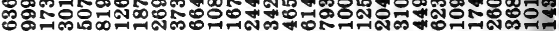
iiiiiiiiiiiiiiiiiiiii

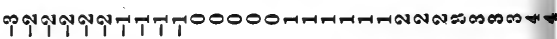

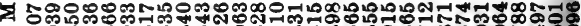

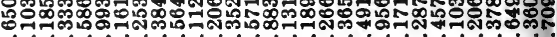

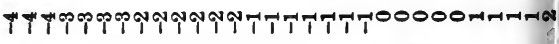
p คొด โ

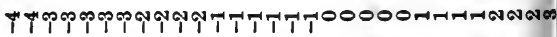

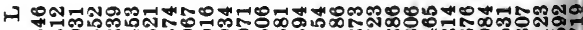

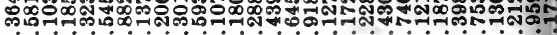

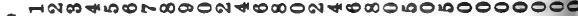

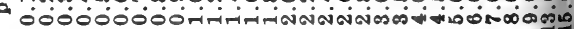




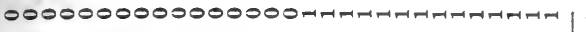

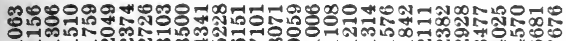

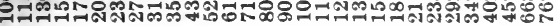
iiliiiiiiiiiiiiiiiiiiiiiii

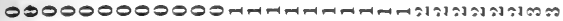

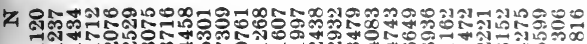

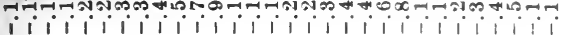
00

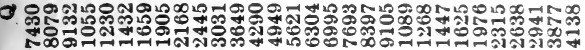
iiiiiiiiiiliiiiiiiiiiiii

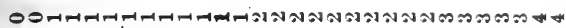

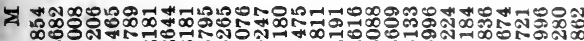
恶

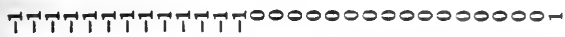

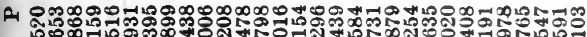

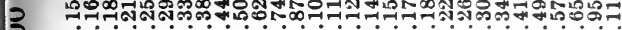

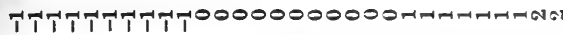

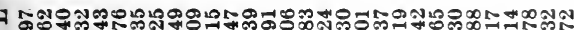

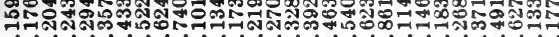

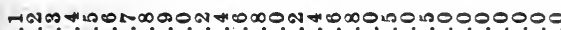

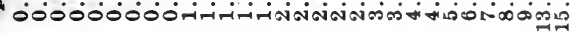

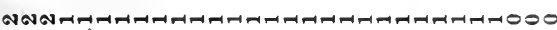

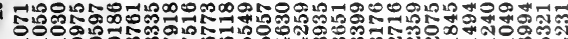
iiiiiiiiiiiiiiiiiiiiiiii

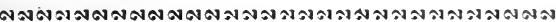

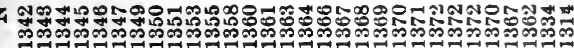
ii ii iiiiiiiiiiiiiiiiiii

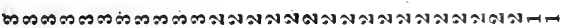

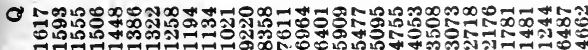
iiliiiiiiiiiiiiiiiiiiii

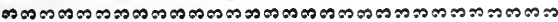

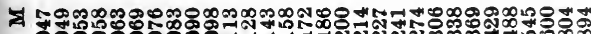

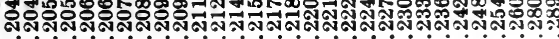

000000000000000000000000 THTH

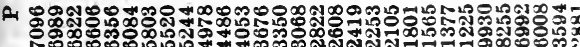
000000000000000000000000000000

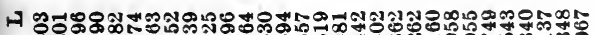

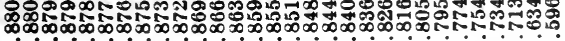

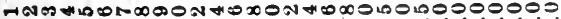
0ं0்000்0.

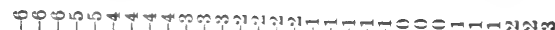
« N i i i i i i i i i i i i i i i i i p pip 乙 $1111 i 1 i 1 i 1 i 1 i i i i i i i i j i i i i$

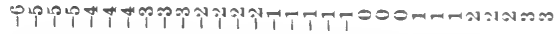

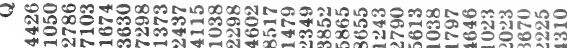
iiiiiiiiiiiiiiiiiiiiiiii

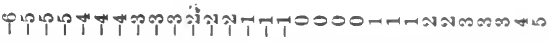
Z +.

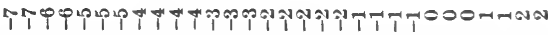

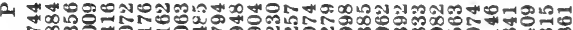

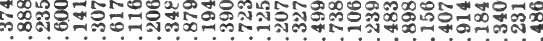

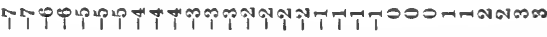

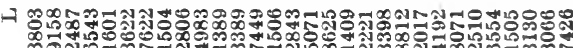

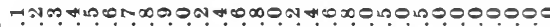
- 0000000்-

Pp

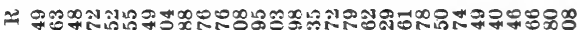

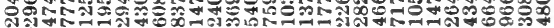
$i$ i i i i i i i i i i i i i i i i i i Pִp

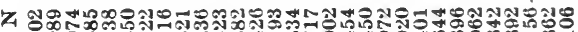
ส iiiiiiiiiiiiiiiiiiiiiii

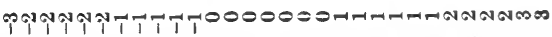

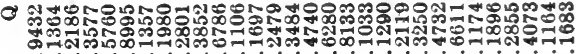
ii i i iiiiiiiiiiiiiiiiiii

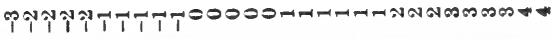
క

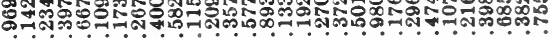

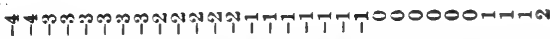

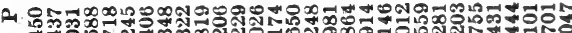

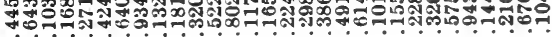

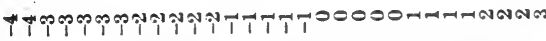
A 6)

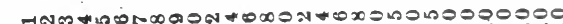

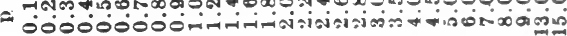




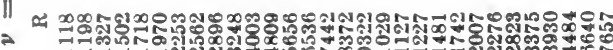
iiiiiiiiiiiiiiiiiiiiiiiiii 00000000000 GกNNGMT

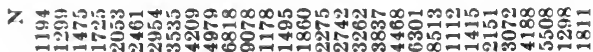
iiiiiiiiiiiiiiiiiiiiiiii

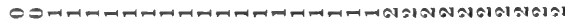

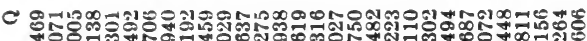

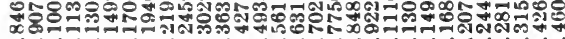
iiiiiiiiiiiiiiiiiiiiiii

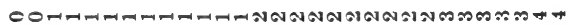

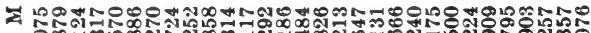
ᄋ에

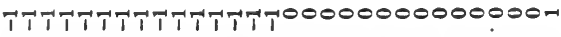

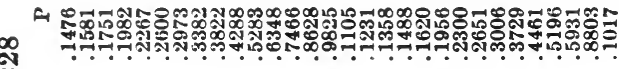
Qิ

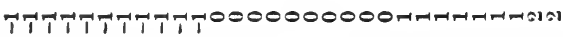
เు

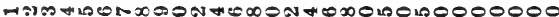
N

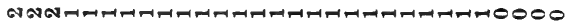

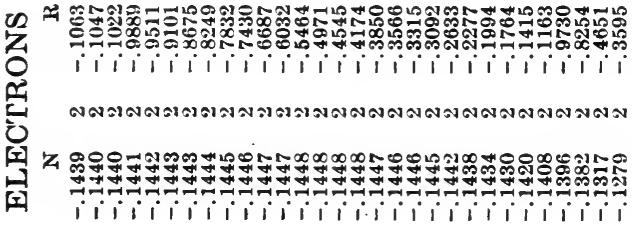

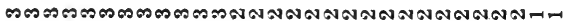

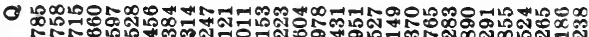

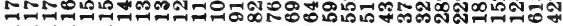
iiliiiliijiiiiiiliiiiiii

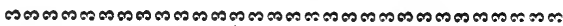

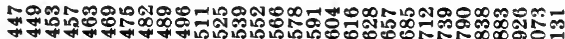

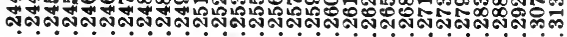

$000000000000000000000000 \uparrow T H T T T$

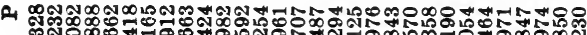

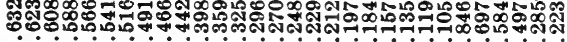

000000000000000000000000000000

0

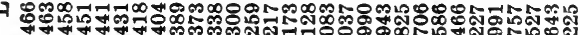

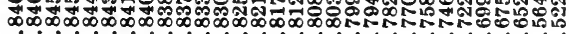

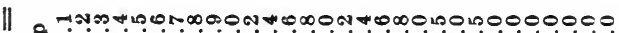

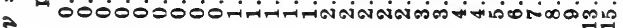

m 9Y9

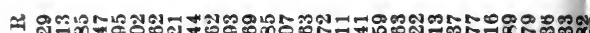

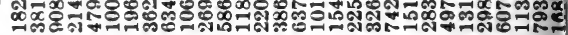
iiiiiiiiiiiliiiliiiiii

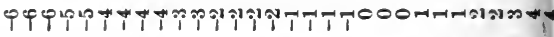

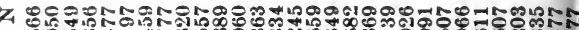

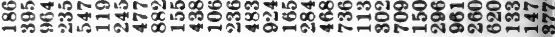
ii iiiiiiiiiiiiiiiiiiiii

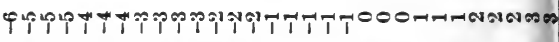
우 W. iiiiiiiliiiliiiiiiiiiii फ़िए

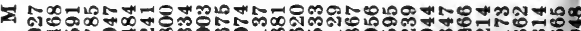

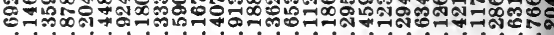

TPYYYTYP 4

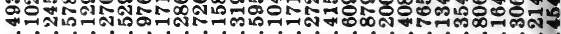

TPY९९P

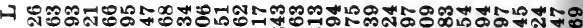
응ำ

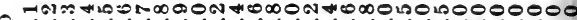

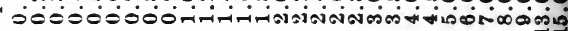
Prpp

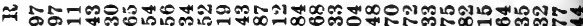

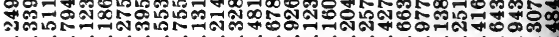
iiliiiliiliiliiliiliii

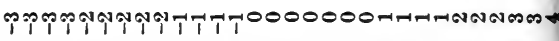

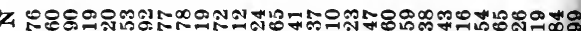

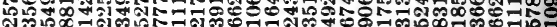
iiiiiiiliiiiiiiiiiiiiii

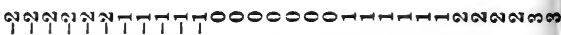

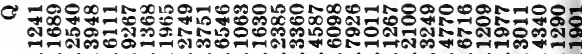
iiiliiiliiiiiiiiiiiiiii

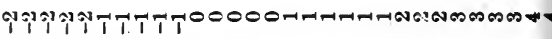

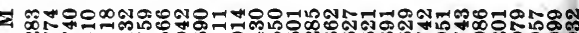

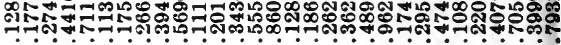

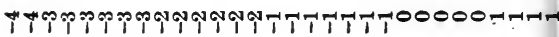
2.

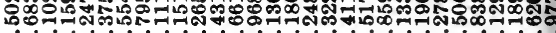

† -

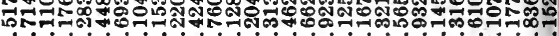
a

TNDW

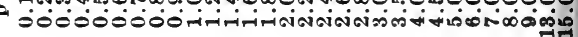


- TTTTT000000000000000

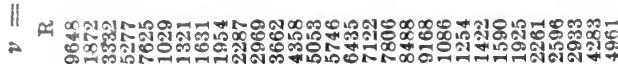
१ิTTT000000000

ห 110

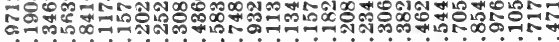

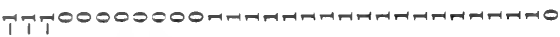

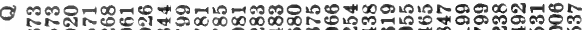
品 iliiliiiiliiiliiliilil

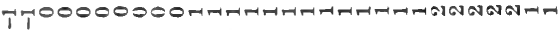

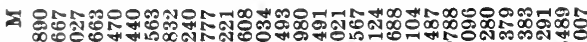
ตุด

9भTTTTTTTTT00000000000000000

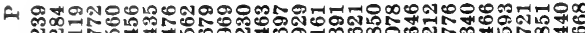
$\infty$ ஸ่

พTTTTTTTT00000000000HAHAHAHANA

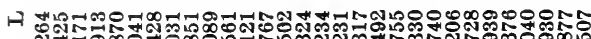
ผิ

@)

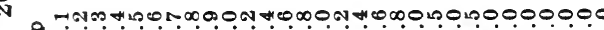

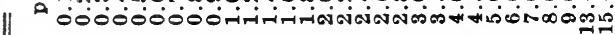
$\mathbf{N}$

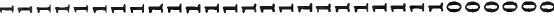

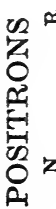

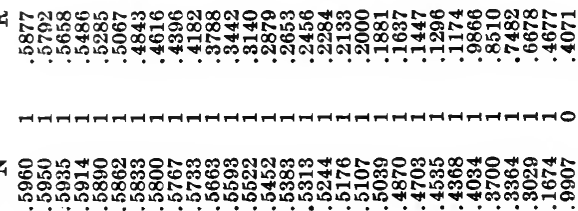

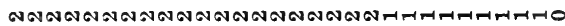

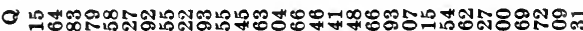
ตึพ iliiiiiiiiiiiiiiliiiiii

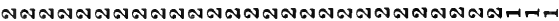

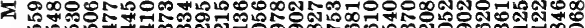

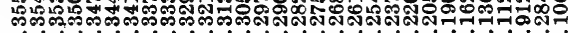

$0000000000000000000000000000 T T$

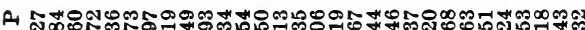

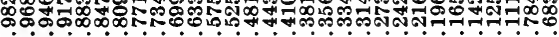

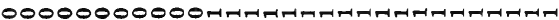

시:

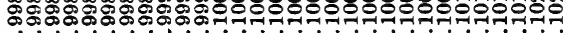

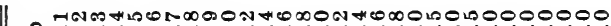

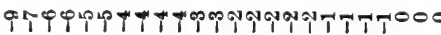

廿สำ

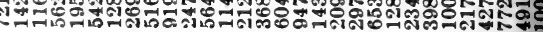

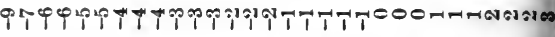

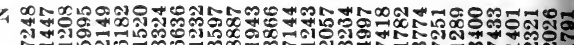

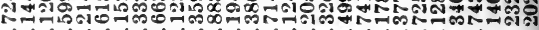

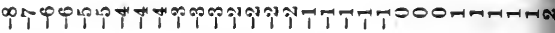
ช 규유. iiliiliiiliiiiiiiiiiii

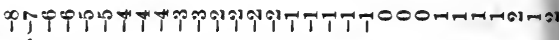
국

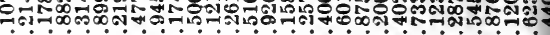

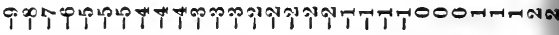

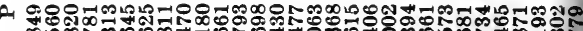

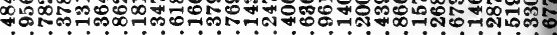

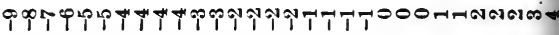

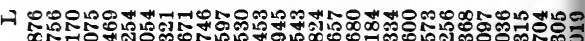

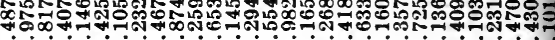

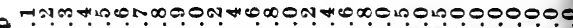

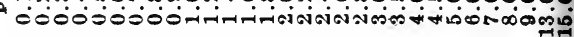

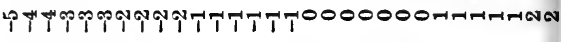
ค

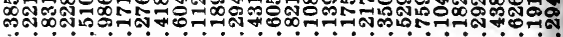

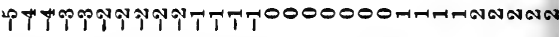

々 Кำ

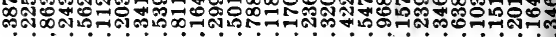

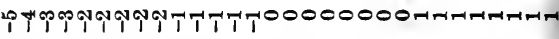

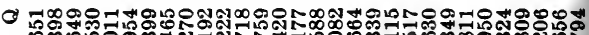
유. iliiliiliiiliiliiliii

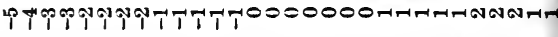
క ․ㄱㄱㅜำ.

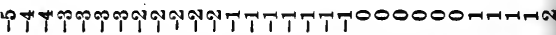

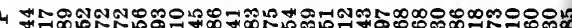
๑ี

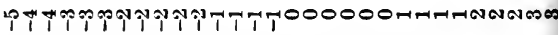

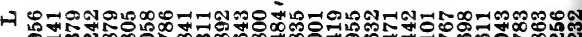

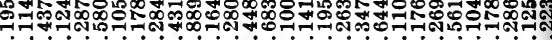

TNMHLONDOONH 000000000-1- 
TTTTT0000000000000

డำ สิศ స్థ

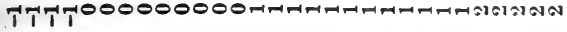

乙 ชิ่สี สุติำ.

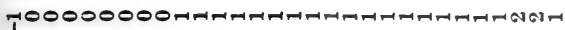

ด

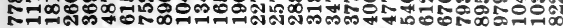

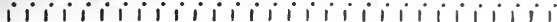

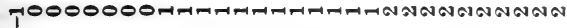

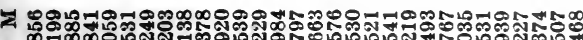

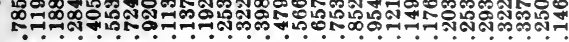

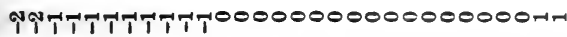

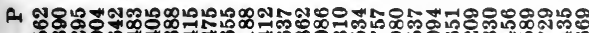
10

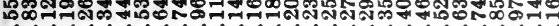

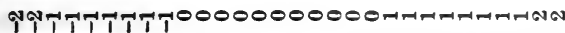

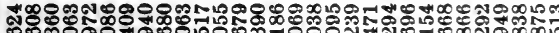
ํ.

คำ

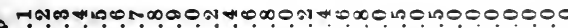

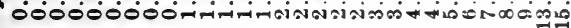
N

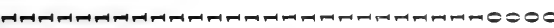

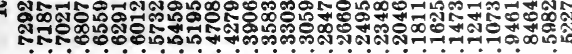

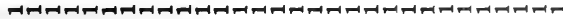

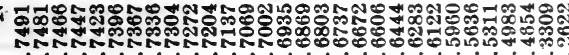

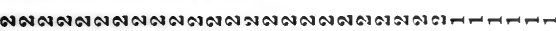

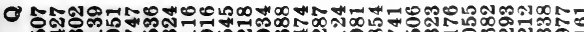
象

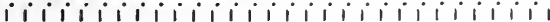

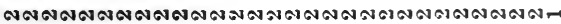

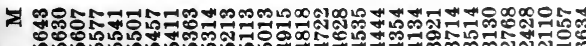

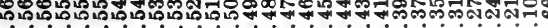

$00000000000000000000000000004 T$

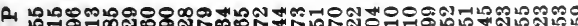
๑ัต

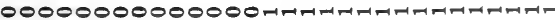

\section{-}

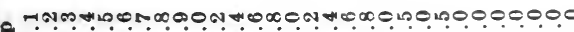

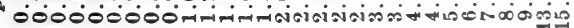

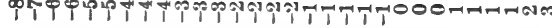

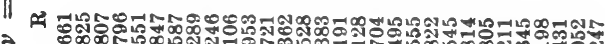
a ถ้ำ

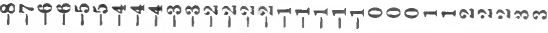
乙 우유. जั:

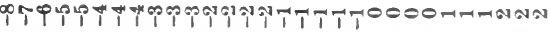

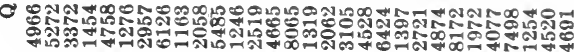
iiiiiiiiiiiiiiiiiiiiiiii

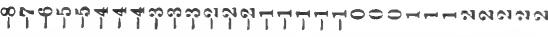

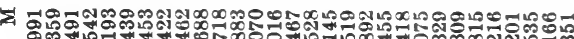

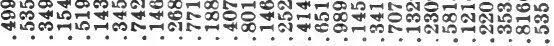

PTTYPLP

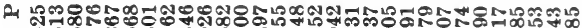
सี

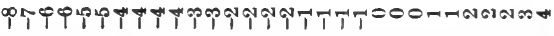

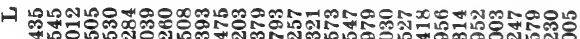

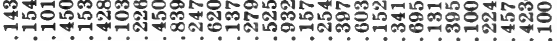

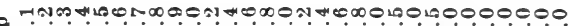

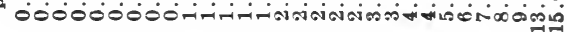

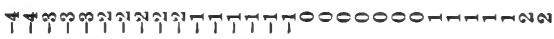

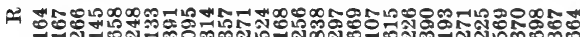
ส

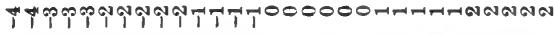

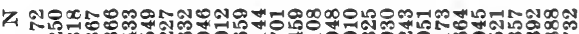
"

TPPPQY

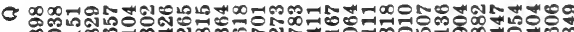
ง iiiiiiiiiiiiiiiiiiiiiiiii

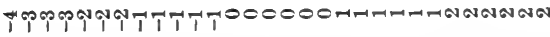

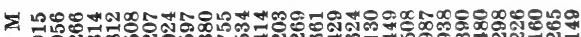

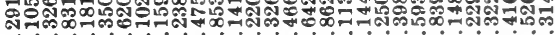

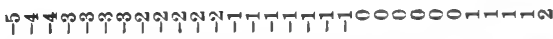

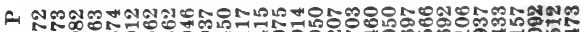
ㅇํㅇㅇㅠ

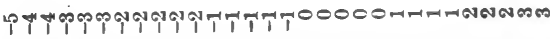
ఎ a)

Thm

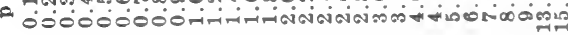




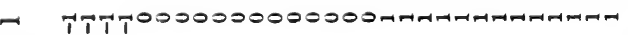
|| - స్థ

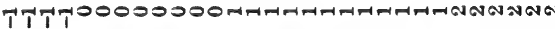

乙 ผ

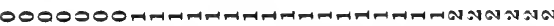

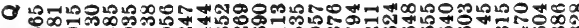
국 iiliiiiiiiliiiiiiiiiiii

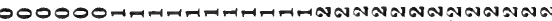

ม స్య 슈.

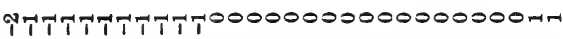

4. 유 М유유

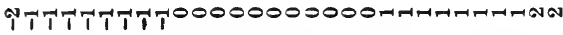

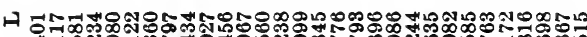

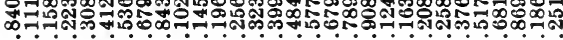

$\underset{7}{+1}$

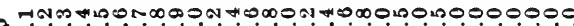

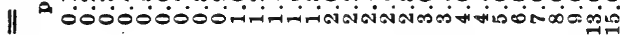
$\mathbf{N}$

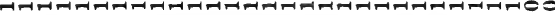

20

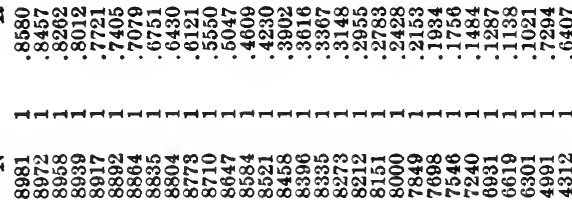

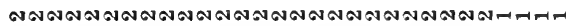

எ

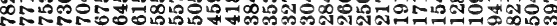
iiiiiiiiii iqiiiiiliiii

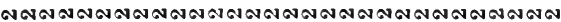

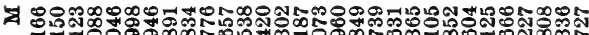
రందు

$00000000000000000000000000007 T$

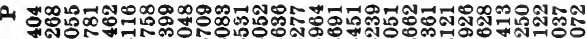

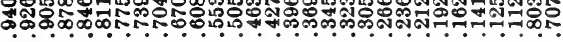

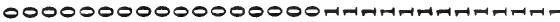

사애

0

II a r.y. a n p ||

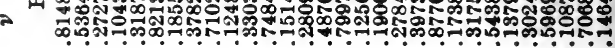

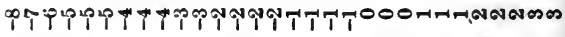

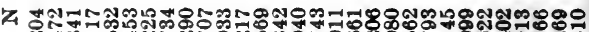
ํํำ

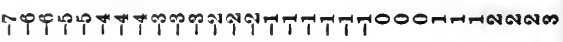

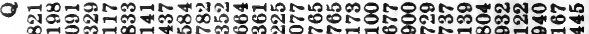

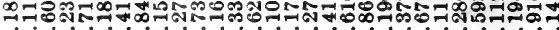
iiliiiliiliiliiliiiiiiii TPYP 그ำำ 萌

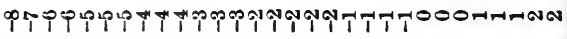

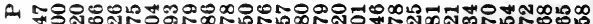

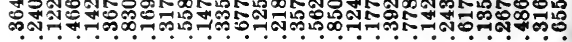

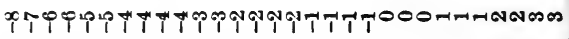

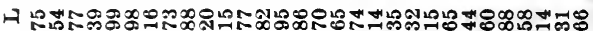

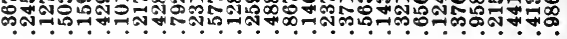

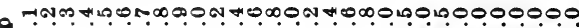

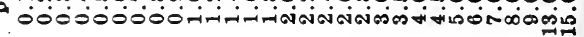

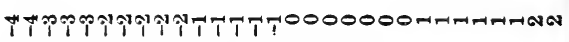

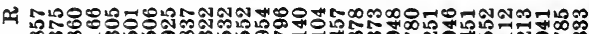
งฺุ.

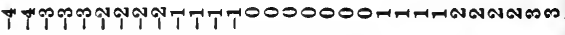

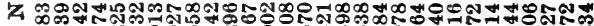

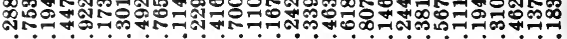

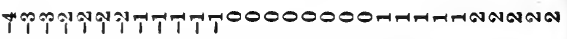

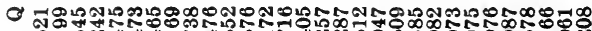

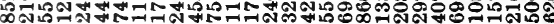
iiliiiiiiiiiiiiiiiiiii

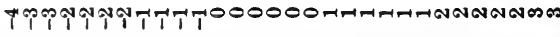

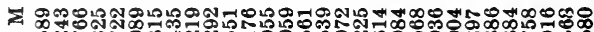

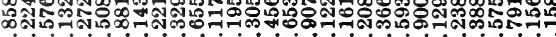

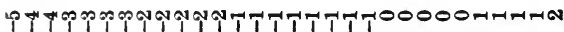

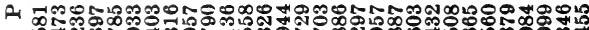
๑

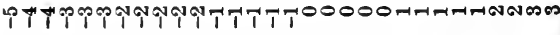

1

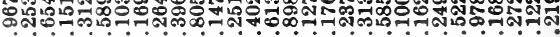

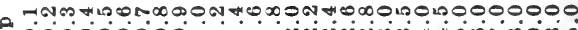

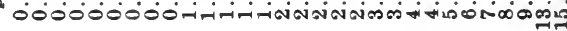




\section{$\|$ of}

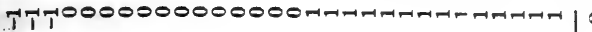

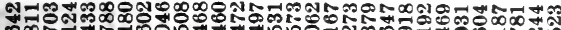
:0

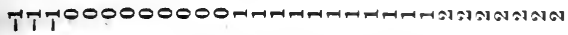

々 โิ

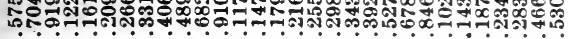

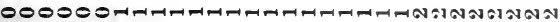

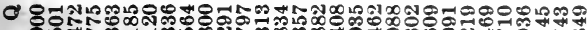
สำ iiiiiiiiliiliiiiiiiiiiii

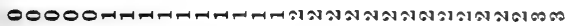

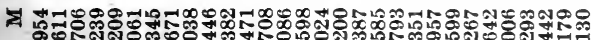

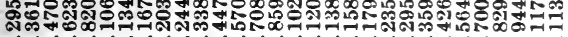

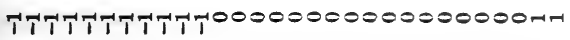

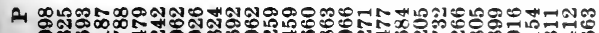

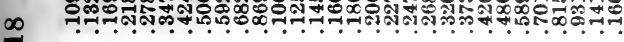

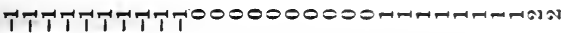

สำ

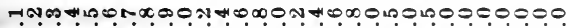

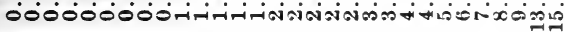



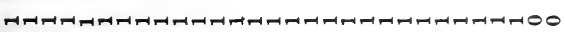

뚜

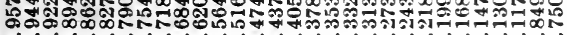

NลNNNลNNNNN

4

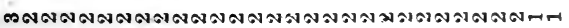

ช تีด

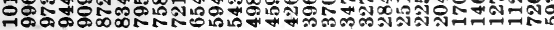
iiiiiiiiiiiiiiiiiiiiiii ๓

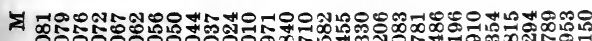

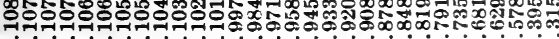

$00000000000000000000000000004 T$

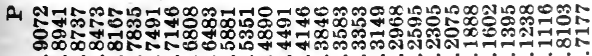

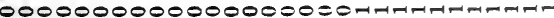

月

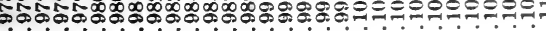

H?m\#n

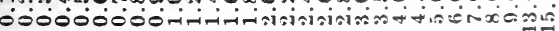

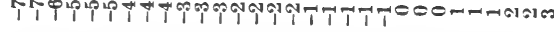

น

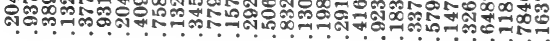

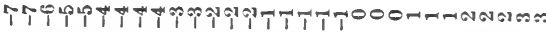

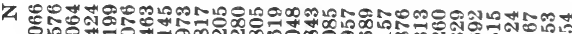

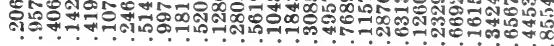

个PQ⿱ ○ iiiiiiiiiiiiiiiiiiiiiii

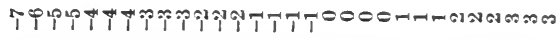

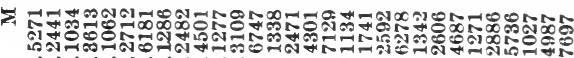

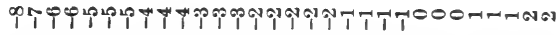

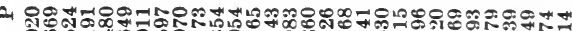

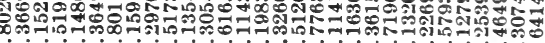

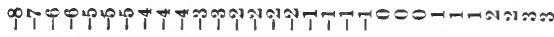

H

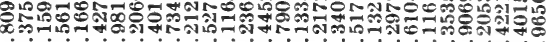

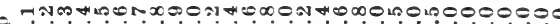
- 0000000்-1

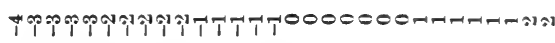

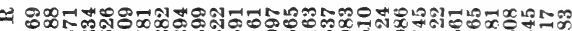
ค月-

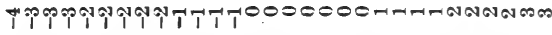

乙 מה

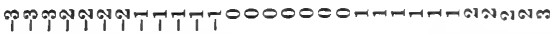

○

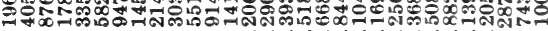

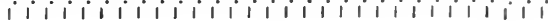

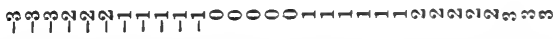

숙

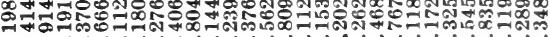

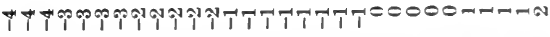

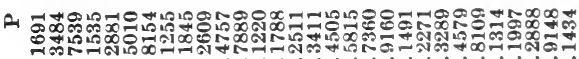

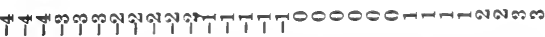
구욤ำ o

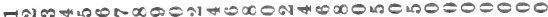

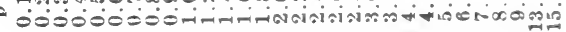




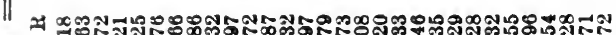

a N及

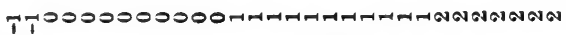

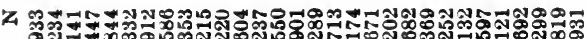
。ำ

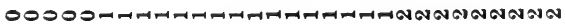

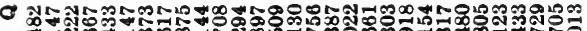
พี่ iीilililililililil

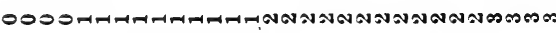

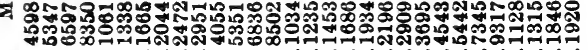

TTTTTTTTTTTT0000000000000000-4

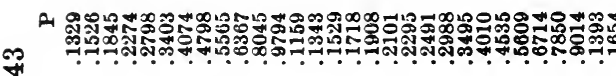

II

$$
\varangle
$$

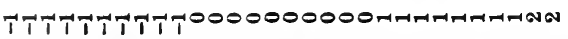

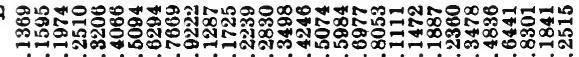

\section{8}

$\mathbf{N}$

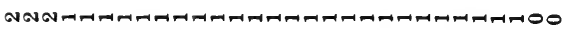

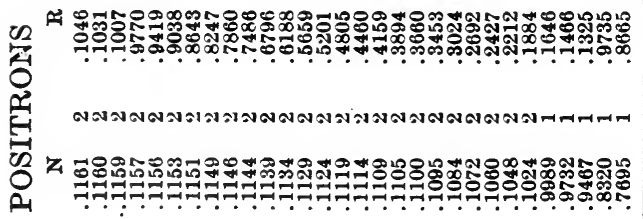

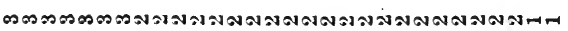

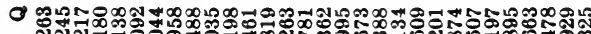

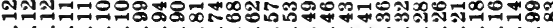

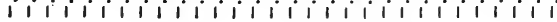

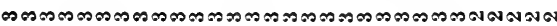

\

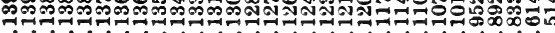

000000000000000000000000000071

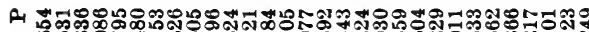
ळ.0.

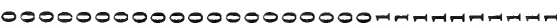

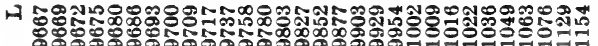

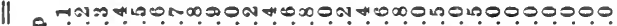

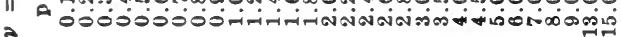

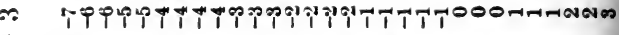

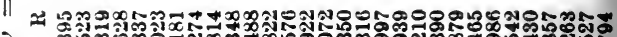
- F

T९QP

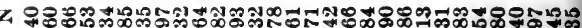
+十

PQP

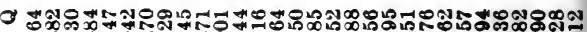

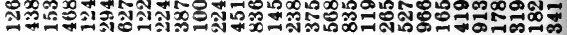
iiiiiiiiiiiiliiiliiiii

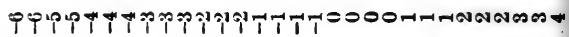
Z 유

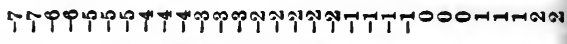
24 셕.

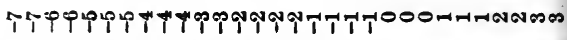
-

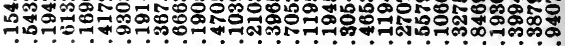

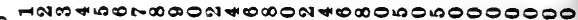

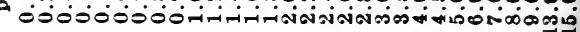

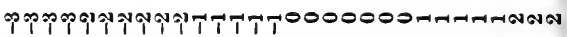

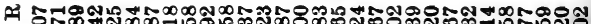

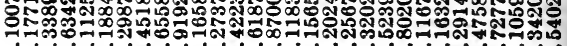

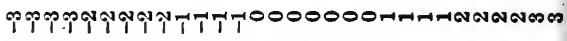

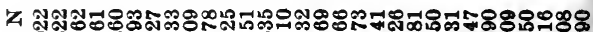
:?

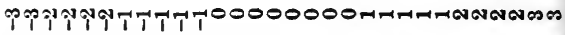

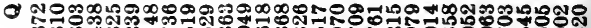

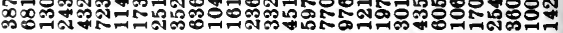
iiliiiiiliiiliiiiiiiiii

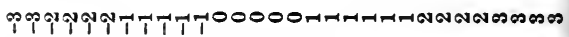

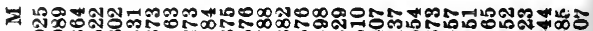

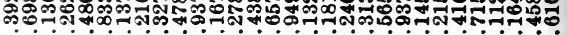

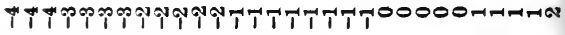

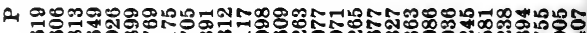

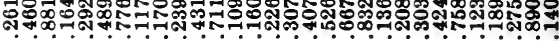

THTPP

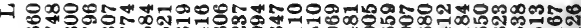

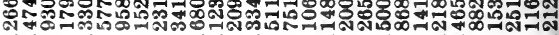

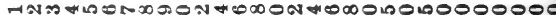

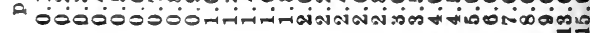




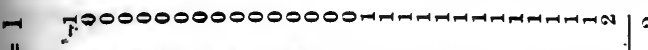
|| ผ ำดำำ

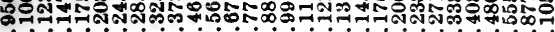

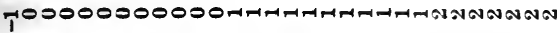

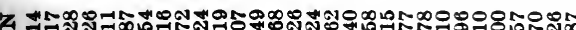

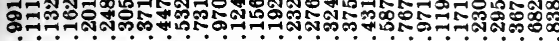

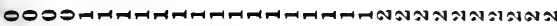

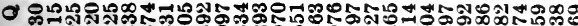

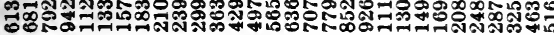
iiliiiiiiiiiiiiiiiiiiii

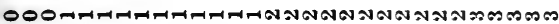

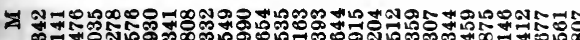

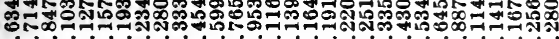

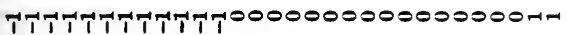

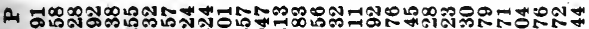

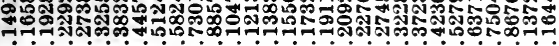

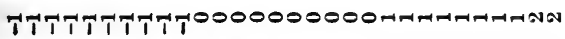

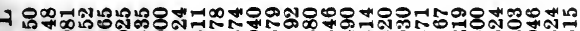

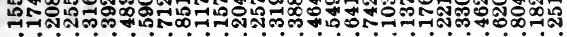

HN

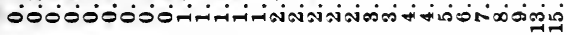

$\mathbf{N}$

Nลง

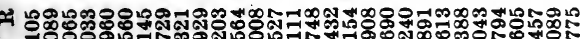
-

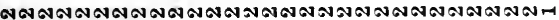

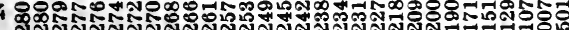

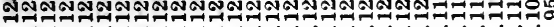

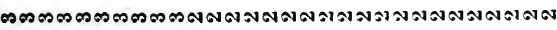

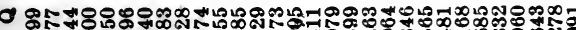
₹ ii i ii iiiiiiiiiiiiiiii कr⿻

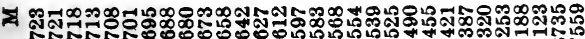

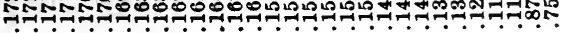
000000000000000000000000000041

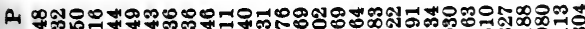

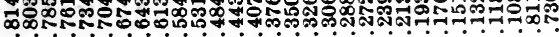

000000000000000000000मMAMAMMAM

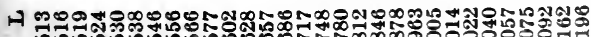
คำ

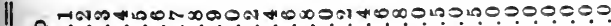

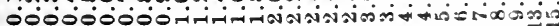

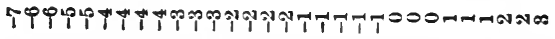

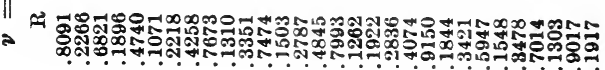

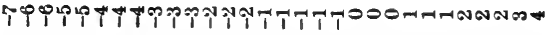

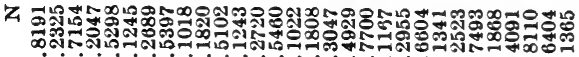

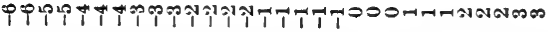

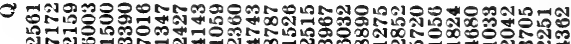
iiiiiiiiiiiiiliiiiiiiiii М์ క

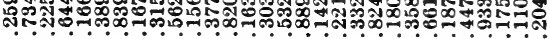

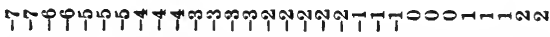

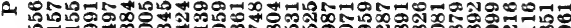

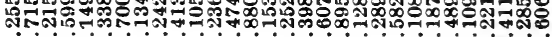

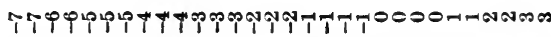

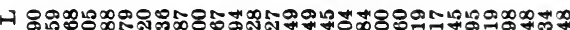

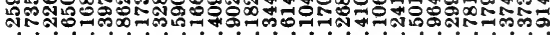

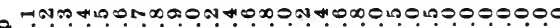

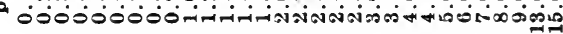

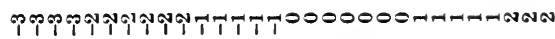

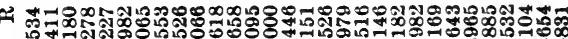
吕

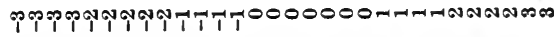
么

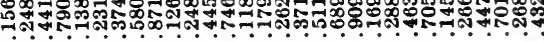
PพYTYT ○ โ

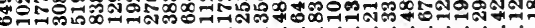
iliili1i1i11111111111111111

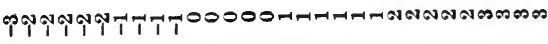

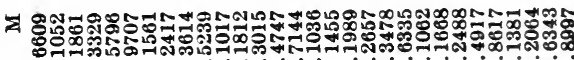

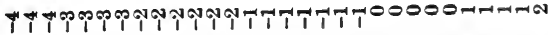

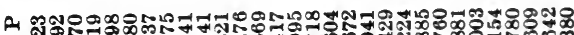

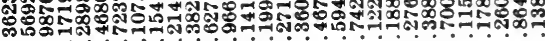

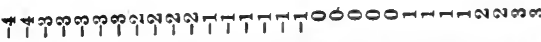
븐

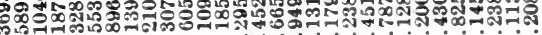
of

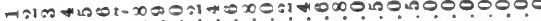

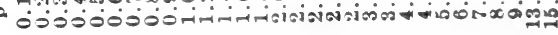




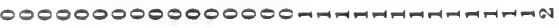

น 서유ำ $=10 \infty$ ก

000000000000

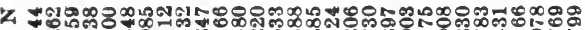
"

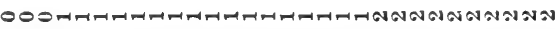

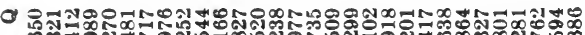

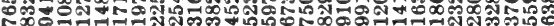

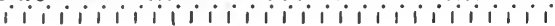

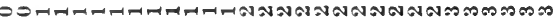

Z

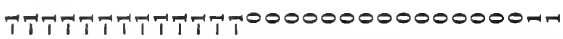

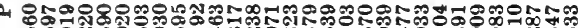
అ.

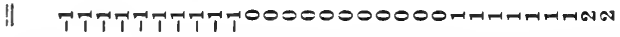
4

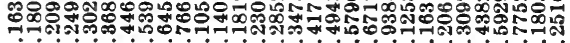
$\mathbf{N}$

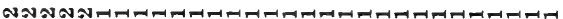

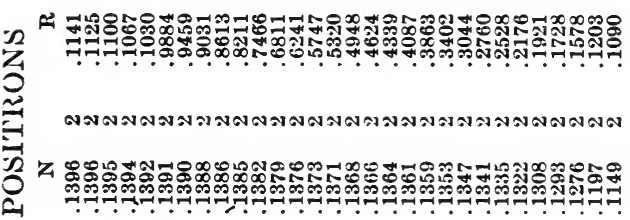

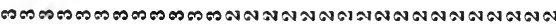

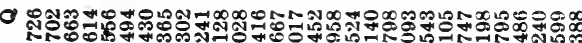

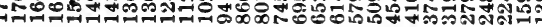
iiliiliilililiiliiliili

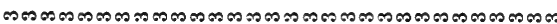

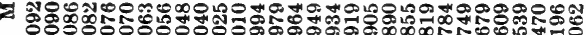

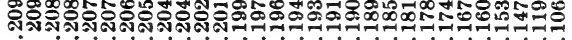

$00000000000000000000000000007 T$

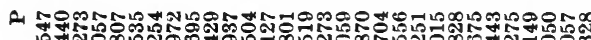
군유:

0000000000000000000000 HAHAHAH

거 ○ ๘๐

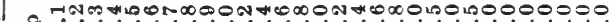
a

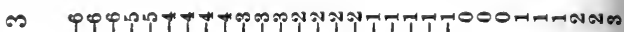

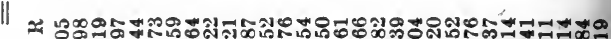

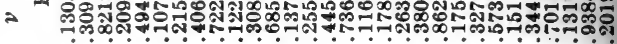

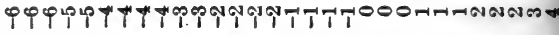
乙

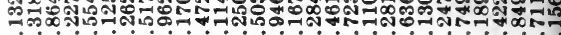

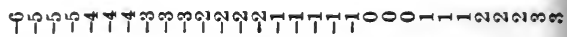

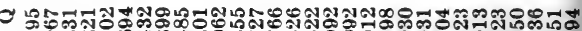

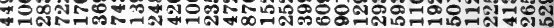

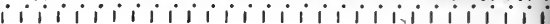

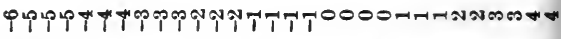

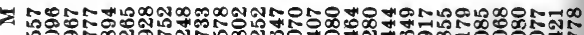

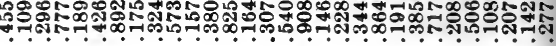

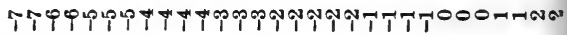

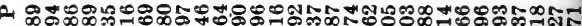
ஸ્.

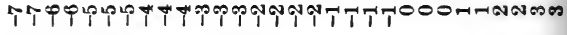

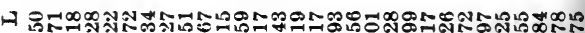
๑ัง

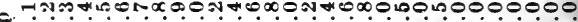

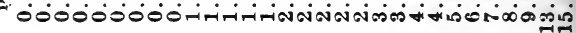

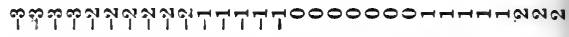

น

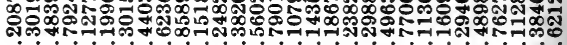

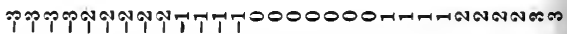

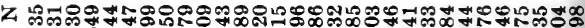

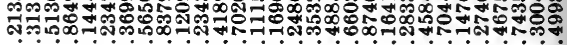

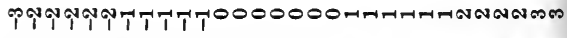

ช งิำ ఏ్స iiliiiliiliiliiiliiili

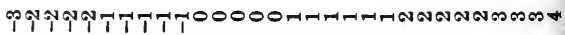

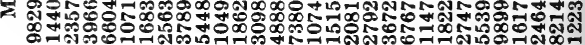

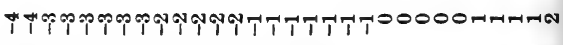

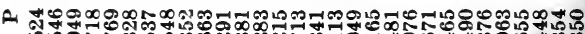

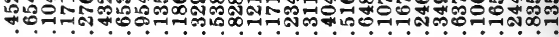

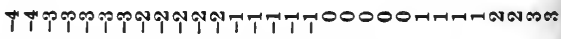

\% o

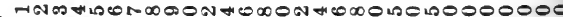

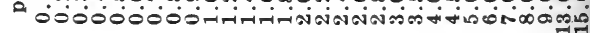


APP. III]

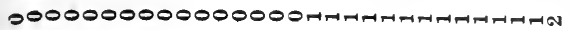

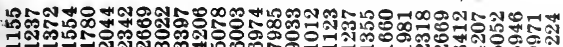

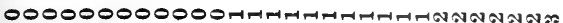

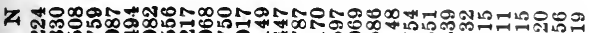

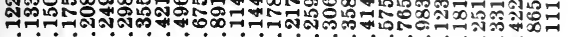

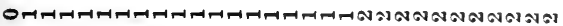

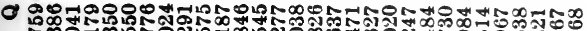

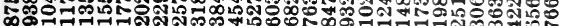
iiiiiiiiiiiiiiiiiiiiiii

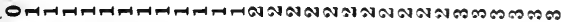

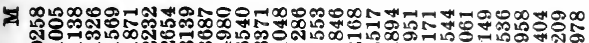

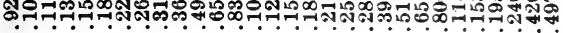

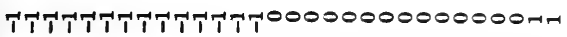

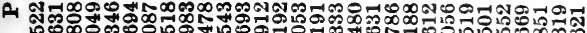

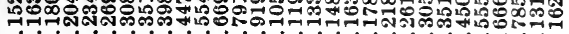

Q

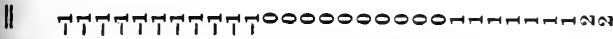

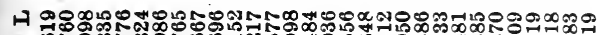

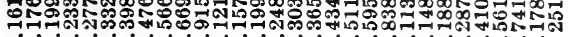

8

a HNM II

N

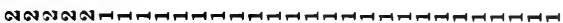

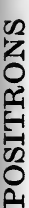

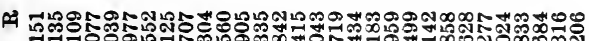

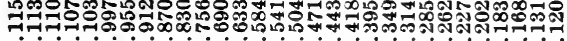

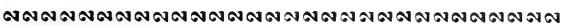

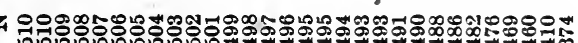
गी

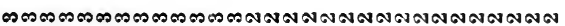

-

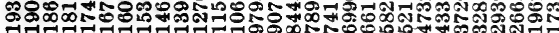
iiiiiiiiiiiiiiiiiiiii

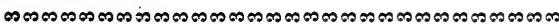

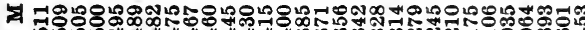
ค่ง

000000000000000000000000000041

1 ๑ొ

0000000000000000000000 HAHAHAHA

\section{十

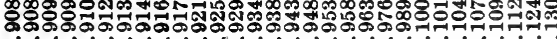

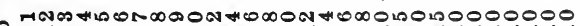

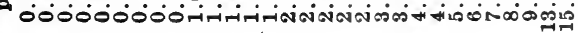

の

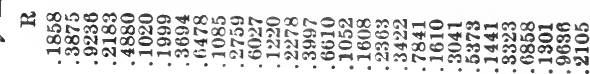

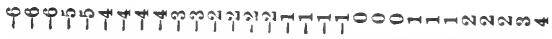

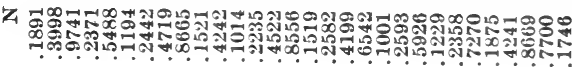

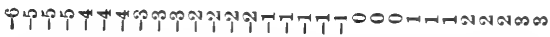
○ -

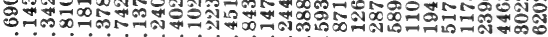
iiiiiiiiiiiiiiiiiiiiiiii PLP

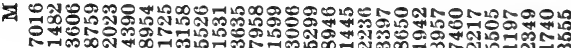
TPY TіP A

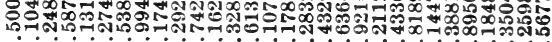

TPQY H

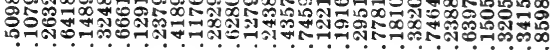

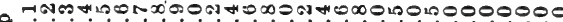
- 0000000-

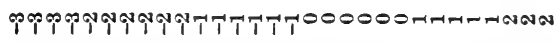

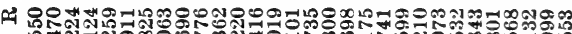

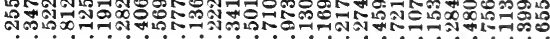

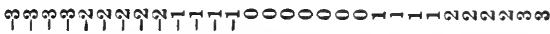

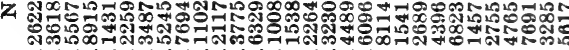

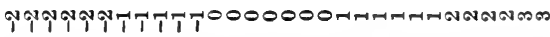

ช ్ㅜㄱำ

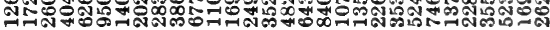
iiliiiliiliiliiliiiii

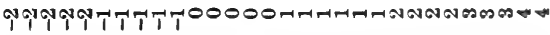

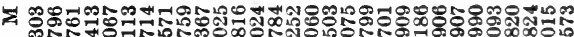
ตำสำสำ.

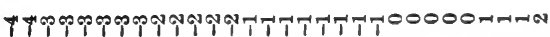

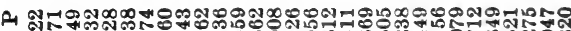
นิ

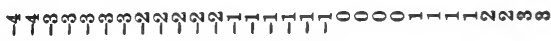
부ำ Aง

N

TกN

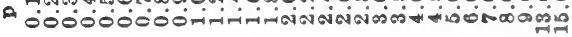




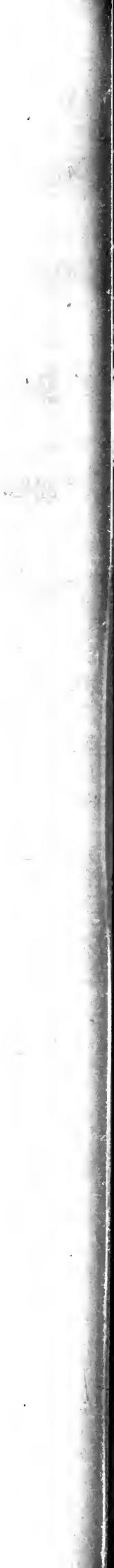




\title{
TABLES OF INTERNAL CONVERSION COEFFICIENTS
}

\author{
M. E. ROSE \\ (Cf. Ch. XIV)
}

The following three tables give the internal conversion coefficients for $K$-shell and the three $L$-subshells. The electric and magnetic conversion coefficients for $2^{L}$ pole transitions are designated by $\alpha_{L}$ and $\beta_{L}$ respectively. Throughout the tables $\alpha_{L}$ and $\beta_{L}$ are represented in the form $n(r)$ which is to be read as $n \times 10^{r} ; 1.0 \leqslant n<10$ and $r$ is a positive or negative integer. The energy of the transition is $k m c^{2}=k \times 511 \mathrm{keV}$. For each table $L=1 \ldots 5$. The ranges of the remaining parameters are as follows:

Table A. $K$-shell coefficients (without screening). $Z=10,20,30,40,54$, $64,72,78,83,88,92$ and 96 . For $Z<83, k=0.3,0.5,1.0,1.8,3.0$ and 5.0. For $Z \geqslant 83, k=0.3$ is omitted. These values of $\alpha_{L}$ and $\beta_{L}$ provide the basis for the more extensive tabulation (obtained by interpolation) given in reference $4, \mathrm{Ch} . \mathrm{XIV}$.

Table B. Low energy $K$-shell coefficients (with screening). These and the $L$-shell coefficients given in Table C represent that part of an extensive tabulation * which is now available. Values for $Z=25,35,55$ and 85 are given. The $k$-values are $0.05,0.10,0.15,0.20,0.40$ and (for $Z=85$ ) 0.60 . Of course, the lowest $k$-value is determined by the $K$-threshold.

Table C. $L$-shell coefficients (with screening). See remarks above under Table B. Here results are given for $Z=15\left(L_{\mathrm{I}}\right), 25\left(L_{\mathrm{I}}, L_{\mathrm{II}}\right)$; $55\left(L_{\mathrm{I}}, L_{\mathrm{II}}, L_{\mathrm{III}}\right) ; 75\left(L_{\mathrm{I}}\right) ; 85\left(L_{\mathrm{I}}, L_{\mathrm{II}}, L_{\mathrm{III}}\right)$. In most cases ten values of $k$ are given in the range $0.05 \leqslant k \leqslant 2.0$.

It is recommended that interpolation for other energy values be carried out in terms of the logarithms of the conversion coefficients versus $\log k$.

* M. E. Rose and G. H. Goertzel, additional results to be published. 


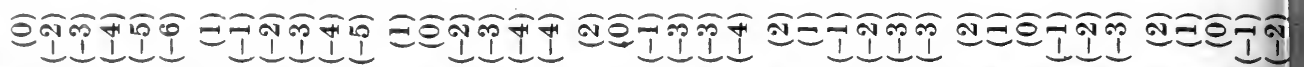
๔

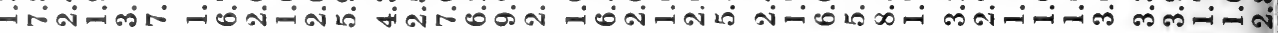

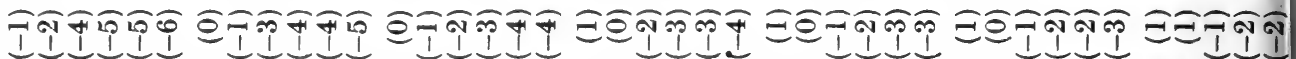

a)

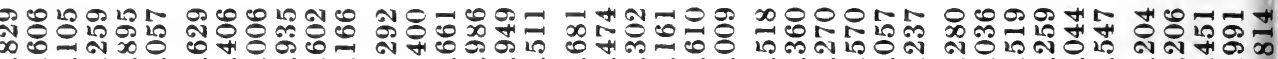

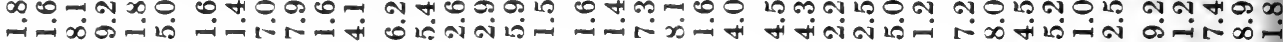

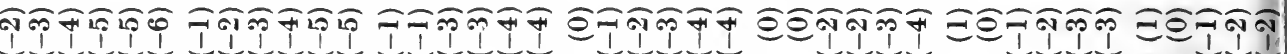

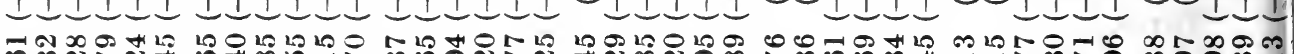

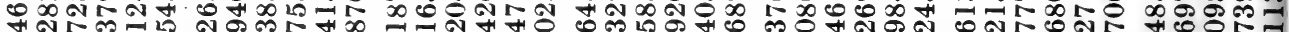

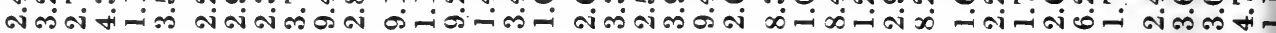

จิㅁำ

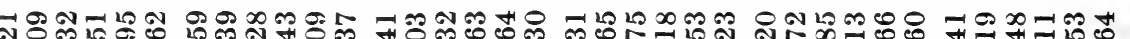

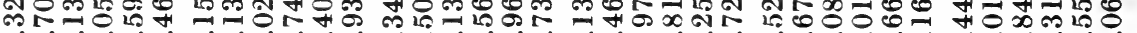

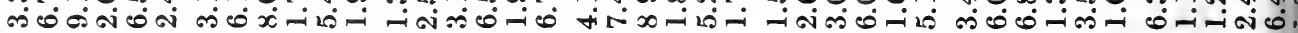

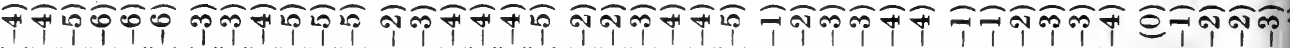
I-110 印 স-

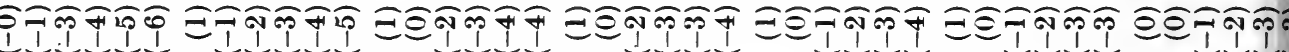
는 -

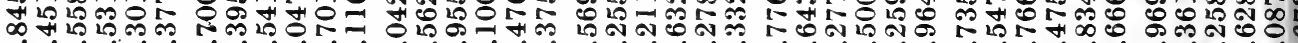

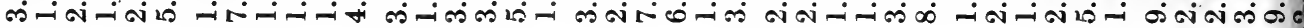

วลศ

อิสิธติด

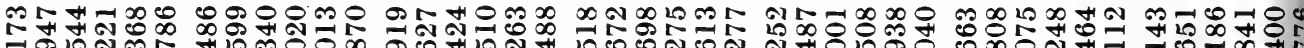

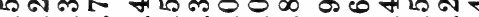
खी

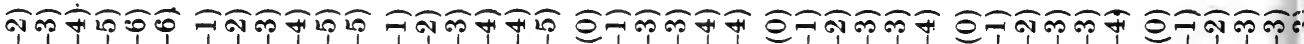
N

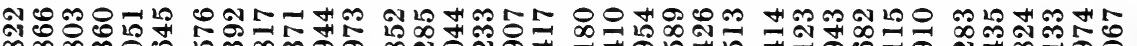

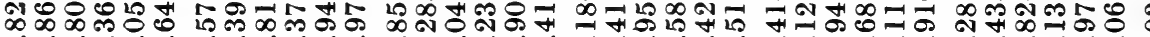
ம் 1111.

ชิธง

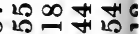

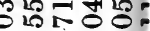

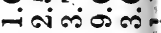

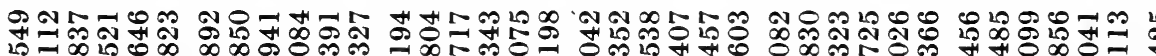

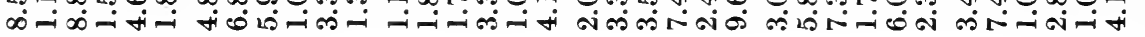

ลิกิติที

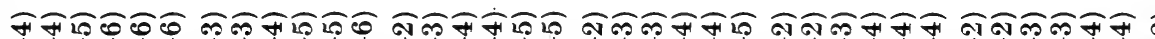

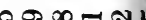

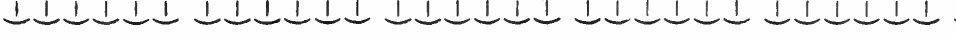

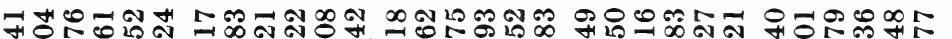

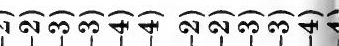

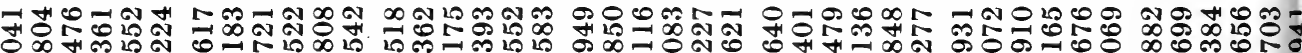

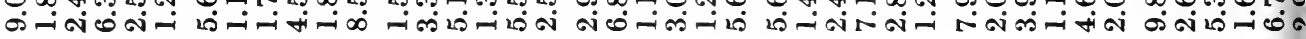

m 10000 m 100000 m 100000 m 100000 m 100000 m

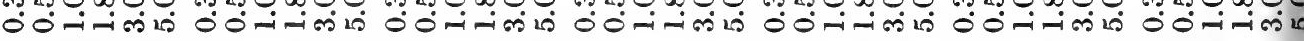
우 


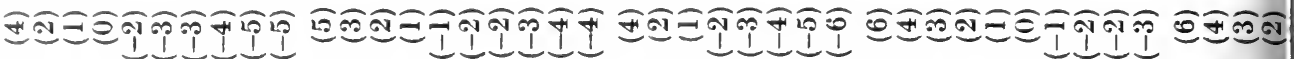

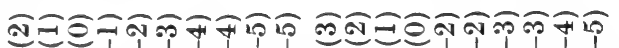

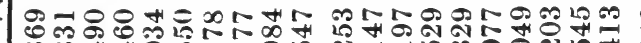

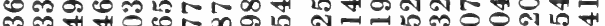

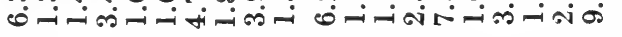

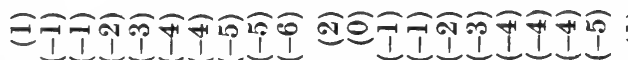

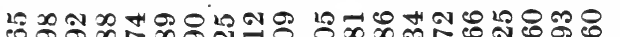
ఈ \& -

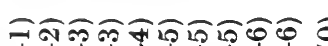

$\infty^{20}$ 1

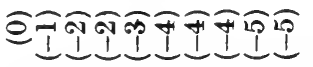

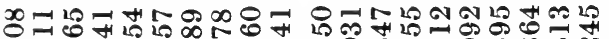

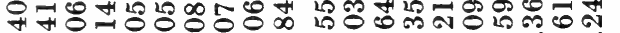

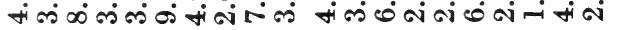

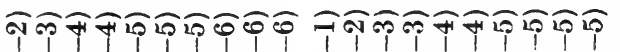

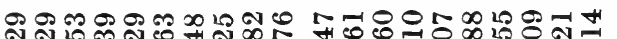

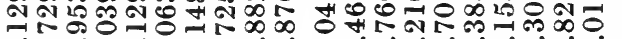

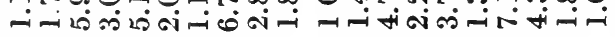

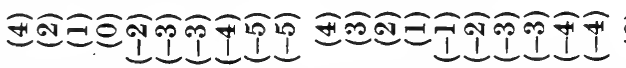

ఇ 넌 ०र

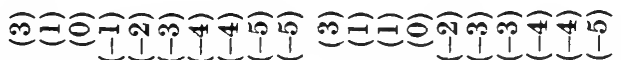

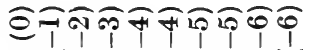

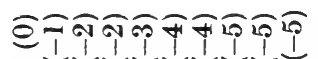

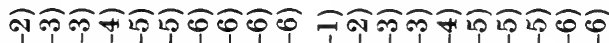

IIIIIIIII I I I I I I I I I

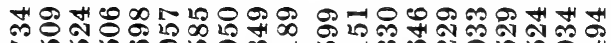

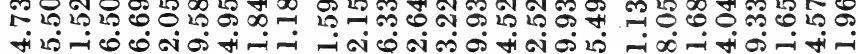

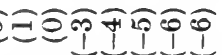

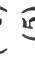

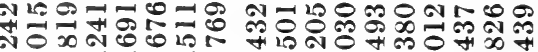

-ioi-i

Li

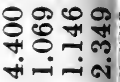

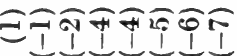

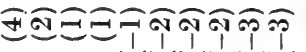

ติํํำ

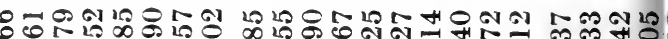
은

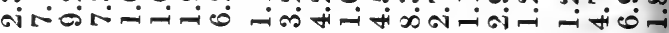

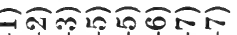

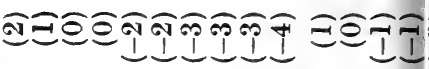
ำ 8ㄴ.

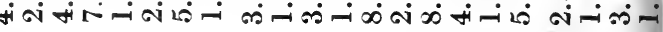

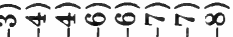

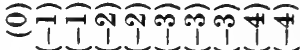
สิิงิึ $60 \infty$ H 1000

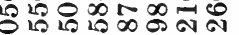

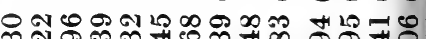

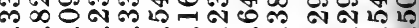

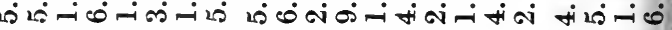

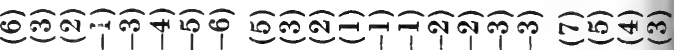

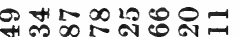

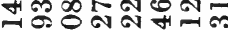
$00 \pi 0$ N inisini

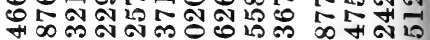

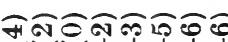

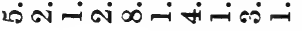
में

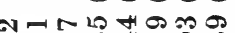

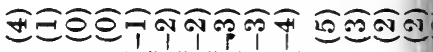

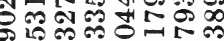

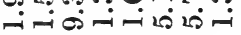
10 的

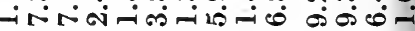

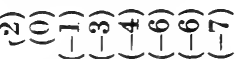

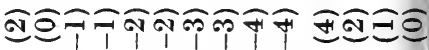

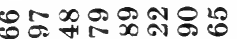

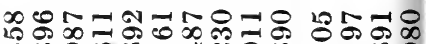

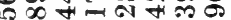

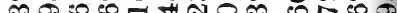
बं

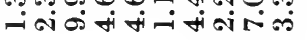

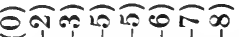

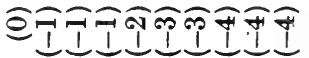
ลิำ ด 인 สำ ง

irici

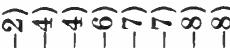

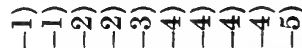

ีิงิึ

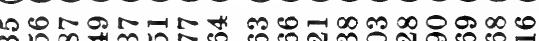
$\infty 000$

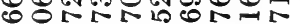
00

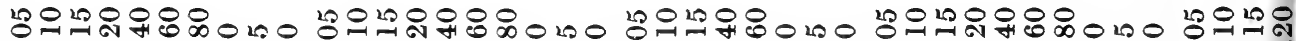

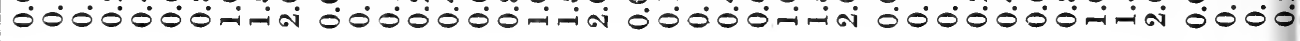




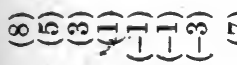

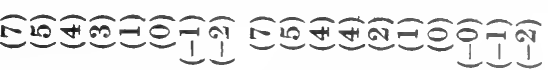

10

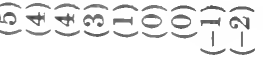
ケำ

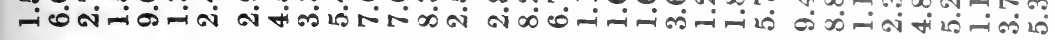

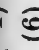

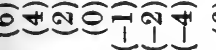

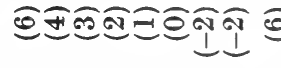

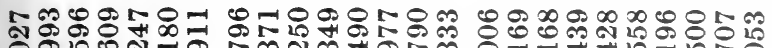
Oด.

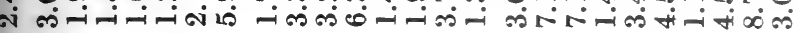

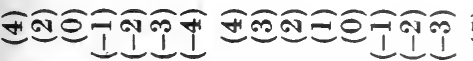

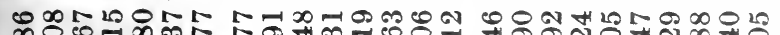

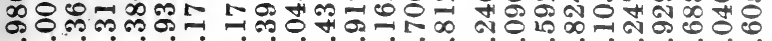

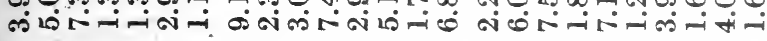
อิ-1 1.

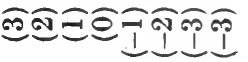

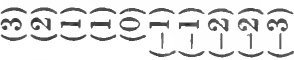

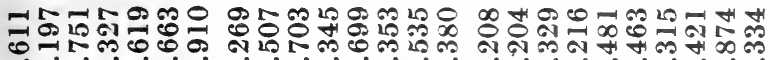

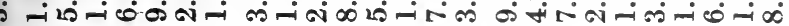

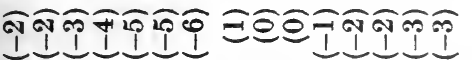

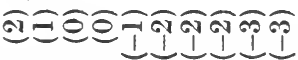

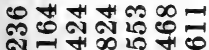
की

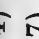

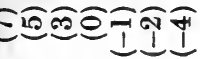

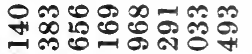

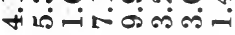

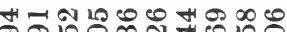
כ. Li -i + oi

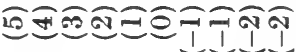
INi

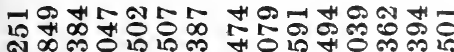

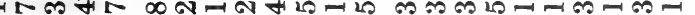

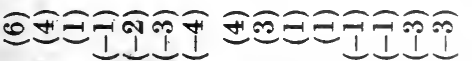

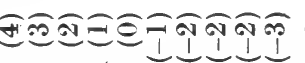

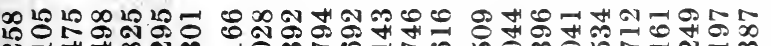
-

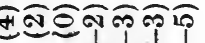

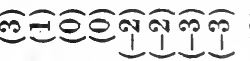

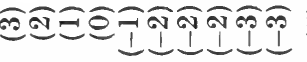

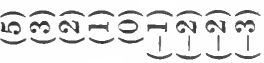

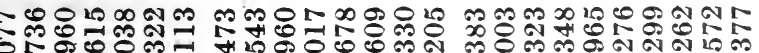
- -

स년영 $\infty 下 \infty$ O -

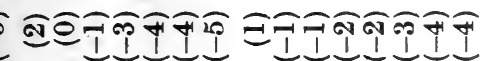

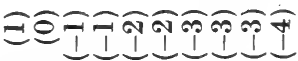

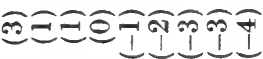
๓ー

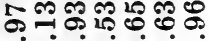

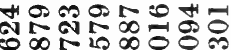

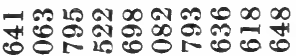
ㅂํㅇำㅇํㅇำ - O०

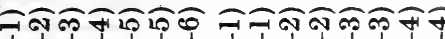

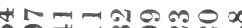

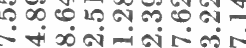

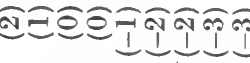
2习요 ti si 0 ov

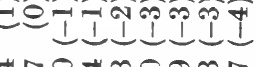

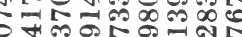

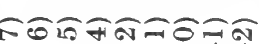

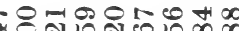
4กำ IIIITIITS

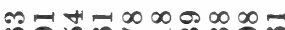

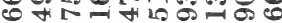
$\infty$ कें

कอ

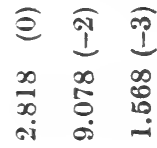

\section{D ए}

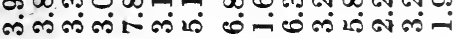

\section{mㅇำ}

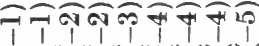
$\rightarrow 0+10000-10$

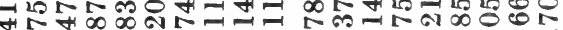
की- सं -

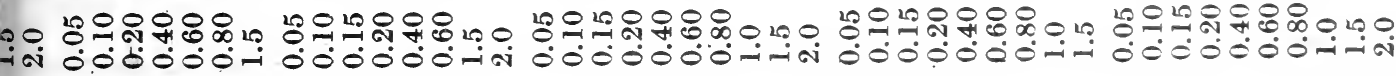

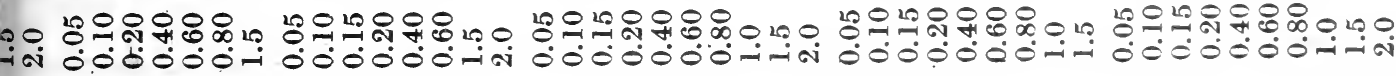

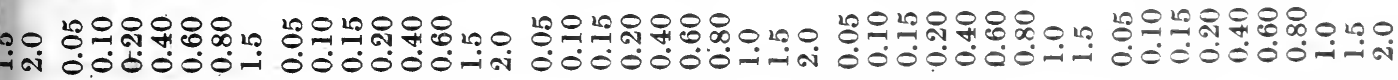
志

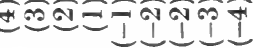

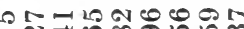

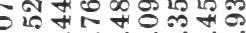
in-i

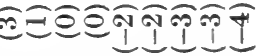

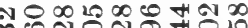

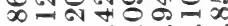

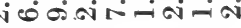

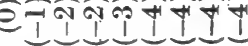
000080080 चर

ச் $0 \dot{000} \dot{0}$ 


\title{
TABLES OF DIRECTIONAL CORRELATIONS
}

\author{
H. FRAUENFELDER
}

(Cf. Ch. XIX)

\section{Directional Correlation of Pure $\gamma$-rays}

From L. C. Biedenharn and M. E. Rose, Rev. Mod. Phys. 25, 746 (1953). The use of these tables is discussed in $\S 4 \mathrm{~A}$ of Chapter XIX. Tables covering a more extended range of values have been prepared by Ferentz and Rosenzweig (Argonne National Laboratory Report ANL-5324 (1954)).

TABLE I(a). $F_{2}\left(1 j_{1} j\right)$ for integer spins

\begin{tabular}{lccccc}
\hline \hline$j^{j_{1}}$ & 0 & 1 & 2 & 3 & 4 \\
\hline 1 & 0.7071 & -0.3536 & 0.0707 & 0 & 0 \\
2 & 0 & 0.4183 & -0.4183 & 0.1195 & 0 \\
3 & 0 & 0 & 0.3464 & -0.4330 & 0.1443 \\
4 & 0 & 0 & 0 & 0.3134 & -0.4387 \\
5 & 0 & 0 & 0 & 0 & 0.2944
\end{tabular}

TABLE I(b). $F_{2}\left(2 j_{1} j\right)$ for integer spins

\begin{tabular}{lcccrc}
\hline \hline$j j_{1}$ & 0 & 1 & 2 & 3 & 4 \\
\hline 1 & 0 & -0.3535 & 0.3535 & -0.1010 & 0 \\
2 & -0.5976 & -0.2988 & 0.1281 & 0.3415 & -0.1707 \\
3 & 0 & -0.4949 & -0.1237 & 0.2268 & 0.3093 \\
4 & 0 & 0 & -0.4477 & -0.0448 & 0.2645 \\
5 & 0 & 0 & 0 & -0.4206 & 0
\end{tabular}

TABLE $I(c) . F_{\triangleleft}\left(2 j_{1} j\right)$ for integer spins

\begin{tabular}{lccrrr}
\hline \hline$j \backslash j_{2}$ & 0 & 1 & 2 & 3 & \multicolumn{1}{c}{4} \\
\hline 2 & -1.069 & 0.7127 & -0.3054 & 0.0764 & -0.0085 \\
3 & 0 & -0.4467 & 0.6700 & -0.4467 & 0.1489 \\
4 & 0 & 0 & -0.3044 & 0.6087 & -0.4981 \\
5 & 0 & 0 & 0 & -0.2428 & 0.5665
\end{tabular}

TABLE I(d). $F_{2}\left(3 j_{1} j\right)$ for integer spins

\begin{tabular}{cccccc}
\hline \hline$j_{1}$ & 0 & 1 & 2 & 3 & 4 \\
\hline 1 & 0 & 0 & -0.4243 & 0.5303 & -0.1768 \\
2 & 0 & -0.7171 & -0.1793 & 0.3287 & 0.4482 \\
3 & -0.8660 & -0.6495 & -0.2742 & 0.1443 & 0.4330 \\
4 & 0 & -0.7835 & -0.4701 & -0.0855 & 0.2678 \\
5 & 0 & 0 & -0.7360 & -0.3680 & 0.0170
\end{tabular}

TABLE I(e). $F_{4}\left(3 j_{1} j\right)$ for integer spins

\begin{tabular}{lcccrr}
\hline \hline$j_{1}$ & 0 & 1 & 2 & 3 & \multicolumn{1}{c}{4} \\
\hline 2 & 0 & 0.0891 & -0.1336 & 0.0891 & -0.0297 \\
3 & 0.2132 & 0.0355 & -0.1066 & -0.0355 & 0.1044 \\
4 & 0 & 0.1453 & -0.0484 & -0.1012 & 0.0132 \\
5 & 0 & 0 & 0.1159 & -0.0773 & -0.0852
\end{tabular}

TABLE I(f). $F_{b}\left(3 j_{1} j\right)$ for integer spins

\begin{tabular}{llllrr}
\hline \hline$\backslash^{j_{1}}$ & 0 & 1 & 2 & 3 & \multicolumn{1}{c}{4} \\
\hline 3 & 1.3056 & -0.9792 & 0.5440 & -0.2176 & 0.0593 \\
4 & 0 & 0.4214 & -0.7585 & 0.6895 & -0.3831 \\
5 & 0 & 0 & 0.2420 & -0.6049 & 0.6979
\end{tabular}

TABLE I(g). $F_{2}\left(4 j_{1} j\right)$ for integer spins

\begin{tabular}{lccccr}
\hline \hline$j j_{1}$ & 0 & 1 & 2 & 3 & \multicolumn{1}{c}{4} \\
\hline 1 & 0 & 0 & 0 & -0.4293 & 0.6010 \\
2 & 0 & 0 & -0.7257 & -0.0726 & 0.4288 \\
3 & 0 & -0.8763 & -0.5258 & -0.0956 & 0.2995 \\
4 & -0.9687 & -0.8234 & -0.5554 & -0.2101 & 0.1447 \\
5 & 0 & -0.9099 & -0.6825 & -0.3787 & -0.0437
\end{tabular}

TABLE I(h). $F_{4}\left(4 j_{1} j\right)$ for integer spins

\begin{tabular}{lccccr}
\hline \hline$j{ }^{j} j_{1}$ & 0 & 1 & \multicolumn{1}{c}{2} & 3 & \multicolumn{1}{c}{4} \\
\hline 2 & 0 & 0 & 0.1718 & -0.3436 & 0.2811 \\
3 & 0 & 0.4112 & -0.1371 & -0.2866 & 0.0374 \\
4 & 0.6034 & 0.3017 & -0.0901 & -0.2860 & -0.1408 \\
5 & 0 & 0.4814 & 0.0802 & -0.2239 & -0.2592
\end{tabular}

TABLE I(i). $F_{\mathrm{a}}\left(4 j_{1} j\right)$ for integer spins

\begin{tabular}{lcccrr}
\hline$j \backslash^{j_{1}}$ & 0 & 1 & 2 & 3 & \multicolumn{1}{c}{4} \\
\hline 3 & 0 & 0.0218 & -0.0392 & 0.0356 & -0.0198 \\
4 & 0.0674 & -0.0034 & -0.0346 & 0.0104 & 0.0243 \\
5 & 0 & 0.0387 & -0.0290 & -0.0190 & 0.0242
\end{tabular}

TABLE I(j). $F_{8}\left(4 j_{1} j\right)$ for integer spins

\begin{tabular}{lcrrrr}
\hline \hline$j{ }^{j_{1}}$ & 0 & 1 & \multicolumn{1}{c}{2} & \multicolumn{1}{c}{3} & \multicolumn{1}{c}{4} \\
\hline 4 & -1.4809 & 1.1847 & -0.7539 & 0.3770 & -0.1450 \\
5 & 0 & -0.3893 & 0.7785 & -0.8384 & 0.5989
\end{tabular}


TABLE I(k). $F_{2}\left(1 j_{1} j\right)$ for half-integer spins

\begin{tabular}{rccccccc}
\hline \hline$j\rangle_{1}$ & $1 / 2$ & $3 / 2$ & $5 / 2$ & $7 / 2$ & $9 / 2$ & $11 / 2$ & $13 / 2$ \\
\hline $3 / 2$ & 0.5000 & -0.4000 & 0.1000 & 0 & 0 & 0 & 0 \\
$5 / 2$ & 0 & 0.3742 & -0.4276 & 0.1336 & 0 & 0 & 0 \\
$7 / 2$ & 0 & 0 & 0.3273 & -0.4364 & 0.1528 & 0 & 0 \\
$9 / 2$ & 0 & 0 & 0 & 0.3028 & -0.4404 & 0.1651 & 0.4425 \\
$11 / 2$ & 0 & 0 & 0 & 0 & 0.2876 & -0.4738 \\
$13 / 2$ & 0 & 0 & 0 & 0 & 0.2774 & -0.4437
\end{tabular}

TABLE I(1). $F_{2}\left(2 j_{1} j\right)$ for half-integer spins

\begin{tabular}{lcccccrr}
\hline \hline$j_{1}$ & $1 / 2$ & $3 / 2$ & $5 / 2$ & $7 / 2$ & $9 / 2$ & $11 / 2$ & $13 / 2$ \\
\hline $3 / 2$ & -0.5000 & 0 & 0.3571 & -0.1429 & 0 & 0 & 0 \\
$5 / 2$ & -0.5345 & -0.1909 & 0.1909 & 0.3245 & -0.1909 & 0 & 0 \\
$7 / 2$ & 0 & -0.4676 & -0.0779 & 0.2494 & 0.2962 & -0.2182 & 0 \\
$9 / 2$ & 0 & 0 & -0.4325 & -0.0197 & 0.2752 & 0.2752 & -0.2359 \\
$11 / 2$ & 0 & 0 & 0 & -0.4109 & 0.0158 & 0.2890 & 0.2596 \\
$13 / 2$ & 0 & 0 & 0 & 0 & -0.3962 & 0.0396 & 0.2972
\end{tabular}

TABLE I(m). $F_{4}\left(2 j_{1} j\right)$ for half-integer spins

\begin{tabular}{rccccrrr}
\hline \hline$j j_{1}$ & $1 / 2$ & $3 / 2$ & $5 / 2$ & $7 / 2$ & $9 / 2$ & $11 / 2$ & $13 / 2$ \\
\hline $5 / 2$ & -0.6172 & 0.7054 & -0.3968 & 0.1176 & -0.0147 & 0 & 0 \\
$7 / 2$ & 0 & -0.3581 & 0.6367 & -0.4775 & 0.1736 & -0.0253 & 0 \\
$9 / 2$ & 0 & 0 & -0.2684 & 0.5857 & -0.5124 & 0.2102 & -0.0338 \\
$11 / 2$ & 0 & 0 & 0 & -0.2237 & 0.5505 & -0.5309 & 0.2359 \\
$13 / 2$ & 0 & 0 & 0 & 0 & -0.1970 & 0.5254 & -0.5418
\end{tabular}

TABLE $I(n) . F_{2}\left(3 j_{1} j\right)$ for half-integer spins

\begin{tabular}{rccccccr}
\hline \hline$j j_{1}$ & $1 / 2$ & $3 / 2$ & $5 / 2$ & $7 / 2$ & $9 / 2$ & $11 / 2$ & $13 / 2$ \\
\hline $3 / 2$ & 0 & -0.6000 & 0.1500 & 0.5000 & -0.2500 & 0 & 0 \\
$5 / 2$ & -0.8018 & -0.4410 & 0.0267 & 0.4009 & 0.4009 & -0.3341 & 0 \\
$7 / 2$ & -0.8183 & -0.5455 & -0.1637 & 0.2182 & 0.4474 & 0.3273 & -0.3819 \\
$9 / 2$ & 0 & -0.7569 & -0.4129 & -0.0275 & 0.3028 & 0.4541 & 0.2753 \\
$11 / 2$ & 0 & 0 & -0.7191 & -0.3319 & 0.0522 & 0.3476 & 0.4504 \\
$13 / 2$ & 0 & 0 & 0 & -0.6934 & -0.2774 & 0.1040 & 0.3744
\end{tabular}

TABLE I(o). $F_{d}\left(3 j_{2} j\right)$ for half-integer spins

\begin{tabular}{rlcccrrr}
\hline \hline$j j_{1}$ & $1 / 2$ & $3 / 2$ & $5 / 2$ & $7 / 2$ & $9 / 2$ & $11 / 2$ & $13 / 2$ \\
\hline $5 / 2$ & 0.1543 & -0.0772 & -0.0772 & 0.1029 & -0.0444 & 0.0070 & 0 \\
$7 / 2$ & 0.1709 & -0.0190 & -0.1076 & -0.0069 & 0.1019 & -0.0627 & 0.0121 \\
$9 / 2$ & 0 & 0.1281 & -0.0660 & -0.0932 & 0.0278 & 0.0935 & -0.0735 \\
$11 / 2$ & 0 & 0 & 0.1067 & -0.0849 & -0.0778 & 0.0469 & 0.0850 \\
$13 / 2$ & 0 & 0 & 0 & 0.0940 & -0.0940 & -0.0653 & 0.0585
\end{tabular}

TABLE I(p). $F_{6}\left(3 j_{1} j\right)$ for half-integer spins

\begin{tabular}{rccccrrr}
\hline$j j^{j_{1}}$ & $1 / 2$ & $3 / 2$ & $5 / 2$ & $7 / 2$ & $9 / 2$ & $11 / 2$ & $13 / 2$ \\
\hline $7 / 2$ & 0.6528 & -0.8704 & 0.6528 & -0.3165 & 0.0989 & -0.0183 & 0.0015 \\
$9 / 2$ & 0 & 0.3077 & -0.6714 & 0.6994 & -0.4304 & 0.1614 & -0.0344 \\
$11 / 2$ & 0 & 0 & 0.1998 & -0.5533 & 0.6916 & -0.4918 & 0.2075 \\
$13 / 2$ & 0 & 0 & 0 & 0.1498 & -0.4793 & 0.6741 & -0.5287
\end{tabular}

TABLE I(q). $F_{2}\left(4 j_{2} j\right)$ for half-integer spins

\begin{tabular}{rcccccrr}
\hline \hline$j j_{1}$ & $1 / 2$ & $3 / 2$ & $5 / 2$ & $7 / 2$ & $9 / 2$ & $11 / 2$ & $13 / 2$ \\
\hline $3 / 2$ & 0 & 0 & -0.6071 & 0.2429 & 0.5409 & -0.3091 & 0 \\
$5 / 2$ & 0 & -0.8113 & -0.3245 & 0.1711 & 0.4898 & 0.3924 & -0.4130 \\
$7 / 2$ & -0.9274 & -0.6889 & -0.3397 & 0.0482 & 0.3734 & 0.5059 & 0.2867 \\
$9 / 2$ & -0.9358 & -0.7444 & -0.4558 & -0.1155 & 0.2127 & 0.4466 & 0.4861 \\
$11 / 2$ & 0 & -0.8890 & -0.6326 & -0.3175 & 0.0122 & 0.3001 & 0.4770 \\
$13 / 2$ & 0 & 0 & -0.8573 & -0.5572 & -0.2266 & 0.0934 & 0.3521
\end{tabular}


TABLE I(r). $F_{4}\left(4 j_{1} j\right)$ for half-integer spins

\begin{tabular}{cccccrrr}
\hline \hline$j j_{1}$ & $1 / 2$ & $3 / 2$ & $5 / 2$ & $7 / 2$ & $9 / 2$ & $11 / 2$ & $13 / 2$ \\
\hline $5 / 2$ & 0 & 0.2976 & -0.2976 & -0.1082 & 0.2976 & -0.1602 & 0.0291 \\
$7 / 2$ & 0.5128 & 0.0733 & -0.2597 & -0.2131 & 0.1235 & 0.2474 & -0.2188 \\
$9 / 2$ & 0.5322 & 0.1693 & -0.1762 & -0.2786 & -0.0800 & 0.2065 & 0.1893 \\
$11 / 2$ & 0 & 0.4434 & 0.0171 & -0.2509 & -0.2363 & 0.0087 & 0.2372 \\
$13 / 2$ & 0 & 0 & 0.3906 & -0.0651 & -0.2744 & -0.1916 & 0.0668
\end{tabular}

TABLE I(s), $F_{\mathrm{B}}\left(4 j_{1} j\right)$ for half-integer spins

\begin{tabular}{lccccrrr}
\hline$j j^{j}$ & $1 / 2$ & $3 / 2$ & $5 / 2$ & $7 / 2$ & $9 / 2$ & $11 / 2$ & $13 / 2$ \\
\hline $7 / 2$ & 0.0435 & -0.0348 & -0.0071 & 0.0317 & -0.0259 & 0.0110 & -0.0025 \\
$9 / 2$ & 0.0492 & -0.0213 & -0.0269 & 0.0195 & 0.0172 & -0.0292 & 0.0167 \\
$11 / 2$ & 0 & 0.0320 & -0.0326 & -0.0123 & 0.0264 & 0.0061 & -0.0284 \\
$13 / 2$ & 0 & 0 & 0.0240 & -0.0348 & -0.0024 & 0.0275 & -0.0014
\end{tabular}

TABLE I(t). $F_{8}\left(4 j_{1} j\right)$ for half-integer spins

\begin{tabular}{rcccccrr}
\hline \hline$j j_{1}$ & $1 / 2$ & $3 / 2$ & $5 / 2$ & $7 / 2$ & $9 / 2$ & $11 / 2$ & $13 / 2$ \\
\hline $9 / 2$ & -0.6623 & 0.9633 & -0.8429 & 0.5187 & -0.2316 & 0.0741 & -0.0162 \\
$11 / 2$ & 0 & -0.2624 & 0.6460 & -0.8075 & 0.6460 & -0.3533 & 0.1330 \\
$13 / 2$ & 0 & 0 & -0.1497 & 0.4791 & -0.7336 & 0.6904 & -0.4315
\end{tabular}

\section{Directional Correlation of Mixed $\gamma$-rays}

From L. C. Biedenharn and M. E. Rose, Rev. Mod. Phys. 25, 749 (1953).

These tables are discussed in $\S 4 \mathrm{D}$ of Chapter XIX. More extensive tables have been prepared by Ferentz and Rosenzweig (Argonne National Laboratory Report ANL-5324 (1954)).

TABLE II(a). $G_{2}\left(l_{1} j\right)$ for integer spins

\begin{tabular}{ccccc}
\hline \hline$j$ & 1 & 2 & 3 & 4 \\
\hline 1 & 0.1581 & 0.0707 & 0 & 0 \\
2 & -0.1080 & 0.0707 & 0.0756 & 0 \\
3 & 0 & -0.0926 & 0.0423 & 0.0704 \\
4 & 0 & 0 & -0.0809 & 0.0289 \\
5 & 0 & 0 & 0 & -0.0725
\end{tabular}

TABLE II(b). $G_{2}\left(2 j_{1} j\right)$ for integer spins

\begin{tabular}{ccccc}
\hline \hline$j j_{1}$ & 1 & 2 & 3 & 4 \\
\hline 1 & 0 & -0.0617 & -0.0369 & 0 \\
2 & -0.0404 & 0.0432 & -0.0132 & -0.0382 \\
3 & 0.0296 & -0.0378 & 0.0279 & 0 \\
4 & 0 & 0.0298 & -0.0322 & 0.0195 \\
5 & 0 & 0 & 0.0284 & -0.0279
\end{tabular}

TABLE II(c). $G_{\triangleleft}\left(2 j_{1} j\right)$ for integer spins

\begin{tabular}{ccccc}
\hline \hline$j_{1}$ & 1 & 2 & 3 & 4 \\
\hline 2 & 0.0753 & 0.0604 & 0.0246 & 0.0047 \\
3 & -0.0667 & 0 & 0.0472 & 0.0332 \\
4 & 0 & -0.0507 & -0.0171 & 0.0346 \\
5 & 0 & 0 & -0.0409 & -0.0224
\end{tabular}

TABLE II(d). $G_{9}\left(3 j_{1} j\right)$ for integer spins

\begin{tabular}{ccccc}
\hline$j j_{1}$ & 1 & 2 & 3 & \multicolumn{1}{c}{4} \\
\hline 1 & 0 & 0 & 0.0326 & 0.0223 \\
2 & 0 & 0.0246 & -0.0213 & 0.0027 \\
3 & 0.0145 & -0.0203 & 0.0193 & -0.0105 \\
4 & -0.0114 & 0.0168 & -0.0180 & 0.0141 \\
5 & 0 & -0.0125 & 0.0161 & -0.0155
\end{tabular}

TABLE II (e). $G_{s}\left(3 j_{1} j\right)$ for integer spins

\begin{tabular}{lcrrr}
\hline \hline$j j_{1}$ & 1 & 2 & 3 & \multicolumn{1}{c}{4} \\
\hline 2 & 0 & -0.0331 & -0.0382 & -0.0199 \\
3 & -0.0386 & 0.0180 & 0.0194 & -0.0190 \\
4 & 0.0317 & -0.0242 & -0.0033 & 0.0221 \\
5 & 0 & 0.0295 & -0.0138 & -0.0106
\end{tabular}

TABLe II(f). $G_{6}\left(3 j_{1} j\right)$ for integer spins

\begin{tabular}{lcrrr}
\hline$j \backslash^{j_{1}}$ & 1 & 2 & 3 & 4 \\
\hline 3 & 0.0429 & 0.0429 & 0.0259 & 0.0101 \\
4 & -0.0429 & -0.0153 & 0.0206 & 0.0286 \\
5 & 0 & -0.0288 & -0.0236 & 0.0071
\end{tabular}


TABLE II $(\mathrm{g}) . G_{2}\left(1 j_{1} j\right)$ for half-integer spins

\begin{tabular}{rccccccc}
\hline$j j_{1}$ & $1 / 2$ & $3 / 2$ & $5 / 2$ & $7 / 2$ & $9 / 2$ & $11 / 2$ & 13,2 \\
\hline $3 / 2$ & -0.1118 & 0.1000 & 0.0764 & 0 & 0 & 0 & 0 \\
$5 / 2$ & 0 & -0.1000 & 0.0535 & 0.0732 & 0 & 0 & 0 \\
$7 / 2$ & 0 & 0 & -0.0863 & 0.0345 & 0.0677 & 0 & 0 \\
$9 / 2$ & 0 & 0 & 0 & -0.0764 & 0.0246 & 0.0628 & 0 \\
$11 / 2$ & 0 & 0 & 0 & 0 & -0.0691 & 0.0187 & 0.0586 \\
$13 / 2$ & 0 & 0 & & 0 & 0.0635 & 0.0148
\end{tabular}

TABLE II(h). $G_{2}\left(2 j_{1} j\right)$ for half-integer spins

\begin{tabular}{lcccccrr}
\hline \hline$j j_{1}$ & $1 / 2$ & $3 / 2$ & $5 / 2$ & $7 / 2$ & $9 / 2$ & $11 / 2$ & $13 / 2$ \\
\hline $3 / 2$ & 0 & 0.0535 & -0.0286 & -0.0391 & 0 & 0 & 0 \\
$5 / 2$ & 0.0261 & -0.0405 & 0.0344 & -0.0049 & -0.0366 & 0 & 0 \\
$7 / 2$ & 0 & 0.0302 & -0.0349 & 0.0231 & 0.0031 & -0.0332 & 0 \\
$9 / 2$ & 0 & 0 & 0.0292 & -0.0299 & 0.0168 & 0.0066 & -0.0305 \\
$11 / 2$ & 0 & 0 & 0 & 0.0275 & -0.0262 & 0.0129 & 0.0082 \\
$13 / 2$ & 0 & 0 & 0 & 0 & 0.0259 & -0.0234 & 0.0102
\end{tabular}

TABLE II(i). $G_{4}\left(2 j_{1} j\right)$ for half-integer spins

\begin{tabular}{cccccccc}
\hline \hline$j j_{1}$ & $1 / 2$ & $3 / 2$ & $5 / 2$ & $7 / 2$ & $9 / 2$ & $11 / 2$ & $13 / 2$ \\
\hline $5 / 2$ & -0.0753 & 0.0225 & 0.0551 & 0.0308 & 0.0070 & 0 & 0 \\
$7 / 2$ & 0 & -0.0578 & -0.0111 & 0.0402 & 0.0339 & 0.0096 & 0 \\
$9 / 2$ & 0 & 0 & -0.0452 & -0.0205 & 0.0300 & 0.0334 & 0.0109 \\
$11 / 2$ & 0 & 0 & 0 & -0.0375 & -0.0235 & 0.0233 & 0.0320 \\
$13 / 2$ & 0 & 0 & 0 & 0 & -0.0322 & -0.0243 & 0.0187
\end{tabular}

TABLE II(j). $G_{2}\left(3 j_{1} j\right)$ for half-integer spins

\begin{tabular}{rccccrrr}
\hline \hline$j \backslash j_{1}$ & $1 / 2$ & $3 / 2$ & $5 / 2$ & $7 / 2$ & \multicolumn{1}{c}{$9 / 2$} & $11 / 2$ & $13 / 2$ \\
\hline $3 / 2$ & 0 & 0 & -0.0298 & 0.0119 & 0.0234 & 0 & 0 \\
$5 / 2$ & 0 & -0.0195 & 0.0225 & -0.0149 & -0.0020 & 0.0215 & 0 \\
$7 / 2$ & -0.0094 & 0.0165 & -0.0193 & 0.0164 & -0.0074 & -0.0063 & 0.0193 \\
$9 / 2$ & 0 & -0.0122 & 0.0166 & -0.0167 & 0.0123 & -0.0035 & -0.0079 \\
$11 / 2$ & 0 & 0 & -0.0126 & 0.0156 & -0.0145 & 0.0095 & -0.0013 \\
$13 / 2$ & 0 & 0 & 0 & -0.0124 & 0.0145 & -0.0127 & 0.0076
\end{tabular}

TABLE II(k). $G_{\triangleleft}\left(3 j_{1} j\right)$ for half-integer spins

\begin{tabular}{lcccccrr}
\hline \hline$j_{1}$ & $1 / 2$ & $3 / 2$ & $5 / 2$ & $7 / 2$ & $9 / 2$ & $11 / 2$ & $13 / 2$ \\
\hline $5 / 2$ & 0 & 0.0405 & 0.0078 & -0.0282 & -0.0238 & -0.0068 & 0 \\
$7 / 2$ & 0.0295 & -0.0317 & 0.0045 & 0.0222 & -0.0122 & -0.0241 & -0.0092 \\
$9 / 2$ & 0 & 0.0310 & -0.0183 & -0.0078 & 0.0208 & -0.0037 & -0.0223 \\
$11 / 2$ & 0 & 0 & 0.0280 & -0.0104 & -0.0122 & 0.0178 & 0.0009 \\
$13 / 2$ & 0 & 0 & 0 & 0.0251 & -0.0057 & -0.0137 & 0.0150
\end{tabular}

TABLE II(1). $G_{6}\left(3 j_{1} j\right)$ for half-integer spins

\begin{tabular}{rcccccrr}
\hline \hline$j j_{1}$ & $1 / 2$ & $3 / 2$ & $5 / 2$ & $7 / 2$ & $9 / 2$ & $11 / 2$ & $13 / 2$ \\
\hline $7 ! 2$ & -0.0526 & 0 & 0.0312 & 0.0292 & 0.0142 & 0.0039 & 0.0005 \\
$9 / 2$ & 0 & -0.0347 & -0.0213 & 0.0128 & 0.0268 & 0.0178 & 0.0060 \\
$11 / 2$ & 0 & 0 & -0.0244 & -0.0242 & 0.0030 & 0.0227 & 0.0188 \\
$13 / 2$ & 0 & 0 & 0 & -0.0187 & -0.0236 & -0.0023 & 0.0190
\end{tabular}




\title{
X-RAY ABSORPTION ENERGIES
}

\author{
R. D. HILL
}

For the purpose of obtaining gamma-ray energies from spectroscopically observed internal conversion electron energies, absolute values of the binding energies of electrons in the various shells of atoms are required. The radiating atoms of the source material are generally involved in a complicated manner with the chemical and solid states of the material, and consequently the absolute energies of the electron levels may be affected to a small, but not insignificant, extent as compared with atoms in the ideal free state.

At present, in so far as beta- and gamma-ray spectroscopy are concerned, the energy values obtained from critical absorption $\mathrm{X}$-ray data are probably reliable to an accuracy of the order of 10 volts. This table has been derived directly from data originally assembled by M. Siegbahn ${ }^{1}$, who used X-ray emission lines to obtain the energy differences between atomic energy levels and $\mathrm{X}$-ray absorption edges to fix their absolute values.

For the reason that the relative energy level values are derived from accurate emission line data, Siegbahn's data are probably accurate, relatively, to within a higher order of accuracy, of the order of 1 volt. This fact is often of some importance as, for example, when determining Auger electron energies ${ }^{2}$ or when identifying the atomic number of the radiating atoms from the measured differences of the $K-L$ or $L_{\mathrm{I}}-L_{\mathrm{II}}-L_{\mathrm{III}}$ internal conversion electrons.

Subsequent to Siegbahn's compilation of the table of atomic level energies, a brilliant determination of the electronic charge was made in Siegbahn's laboratory by X-ray methods. This redetermination brought to light the significant difference between $\mathrm{X}$-units and grating units of $\mathrm{X}$-ray wavelengths, $\because$ and it is this difference which is mainly responsible for the present revision of Siegbahn's table of critical absorption energies.

This table is reprinted, with the permission of the Review of Scientific Instruments, from the paper by Hill, Church and Mihelich ${ }^{3}$. The absolute X-ray critical absorption energies, $E(\mathrm{keV})$, were derived from Siegbahn's $(v / R)$ values, using the following conversion equation:

$$
E(\mathrm{keV})=(v / R)_{\text {Siegbahn }} \times(1.35761 \pm 0.00004) \times 10^{-2} .
$$

1 M. Siegbahn, Spektroskopie der Röntgenstrahlen (Julius Springer, Berlin, 1931), second edition, pp. 278-306.

2 I. Bergström, Chapter XX (I). 
The value of the numerical constant in this equation was obtained in the manner described in the original paper ${ }^{3}$, from the tables of physical constants of DuMond and Cohen ${ }^{4}$. The value using the latest ${ }^{5}$ constants is $(1.35770 \pm .00010) \times 10^{-2}$, but the change does not warrant modification of the critical energies within the absolute or relative significance of their values in the present table.

3 R. D. Hill, E. L. Church and J. W. Mihelich, Rev. Sci. Inst. 23, (1952) 523.

4 J. W. M. DuMond and E. R. Cohen, Phys. Rev. 82, (1951) 4, 555.

5 J. W. M. DuMond and E. R. Cohen, Rev. Mod. Phys. 25, (1953) 691. 
C'RItical X-RAY absorption Fnergies in keV

\begin{tabular}{|c|c|c|c|c|c|c|c|c|c|}
\hline & $K$ & $L_{1}$ & Ln & Lin & $M i$ & $M \mathbf{n}$ & $M \mathrm{~m}$ & $M r v$ & $M v$ \\
\hline $3 \mathrm{Li}$ & $(0.0547)$ & & & & & & & & \\
\hline $4 \mathrm{Be}$ & $*(0.112)$ & & & & & & & & \\
\hline $5 \mathrm{~B}$ & $*(0.187)$ & & & & & & & & \\
\hline $6 \mathrm{C}$ & $(0.284)$ & & & & & & & & \\
\hline $7 \mathrm{~N}$ & $(0.400)$ & & & & & & & & \\
\hline 80 & $(0.532)$ & & & & & & & & \\
\hline $9 \mathrm{~F}$ & $*(0.692)$ & & & & & & & & \\
\hline $\begin{array}{l}10 \mathrm{Ne} \\
11 \mathrm{Na}\end{array}$ & $*(0.874)$ & & & & & & & & \\
\hline $11 \mathrm{Na}$ & ${ }^{*}(1.08)$ & & & & & & & & \\
\hline $12 \mathrm{Mg}$ & 1.303 & $(0.0628)$ & & & & & 07 & & \\
\hline $13 \mathrm{Al}$ & 1.559 & $(0.0870)$ & & & & & 05 & & \\
\hline $14 \mathrm{Si}$ & 1.838 & $*(0.118)$ & & & & & 07 & & \\
\hline $15 \mathrm{P}$ & 2.142 & $*(0.153)$ & & & & & 07 & & \\
\hline $16 \mathrm{~S}$ & 2.469 & $*(0.193)$ & 0.163 & 0.162 & & & 05 & & \\
\hline $17 \mathrm{Cl}$ & 2.822 & $*(0.238)$ & 0.202 & 0.201 & $(0.0297)$ & & 07 & & \\
\hline $18 \mathrm{~A}$ & 3.200 & $*(0.287)$ & ${ }^{*} 0: 246$ & $* 0.244$ & $*(0.035)$ & & 11 & & \\
\hline $19 \mathrm{~K}$ & 3.606 & $*(0.341)$ & 0.295 & 0.292 & $*(0.041)$ & & 16 & & \\
\hline $20 \mathrm{Ca}$ & 4.038 & *(0.399) & 0.350 & 0.346 & $*(0.047)$ & & 26 & & \\
\hline $21 \mathrm{Sc}$ & 4.496 & $*(0.462)$ & 0.411 & 0.407 & $*(0.054)$ & & 37 & & - \\
\hline $22 \mathrm{Ti}$ & 4.966 & *(0.530) & 0.462 & 0.456 & $*(0.061)$ & & 35 & & \\
\hline $23 \mathrm{~V}_{i}$ & 5.467 & $*(0.604)$ & 0.523 & 0.515 & $*(0.068)$ & & 41 & & \\
\hline $24 \mathrm{Cr}$ & 5.988 & $*(0.679)$ & 0.584 & 0.574 & $*(0.076)$ & & 42 & & \\
\hline $25 \mathrm{Mn}$ & 6.542 & $*(0.762)$ & 0.656 & 0.644 & $*(0.085)$ & & 52 & & \\
\hline $26 \mathrm{Fe}$ & 7.113 & 0.849 & 0.722 & 0.709 & 0.094 & & & & \\
\hline $27 \mathrm{Co}$ & 7.713 & *0.929 & 0.798 & 0.783 & 0.103 & & & & \\
\hline $28 \mathrm{Ni}$ & 8.337 & 1.015 & 0.877 & 0.858 & 0.111 & & & & \\
\hline $29 \mathrm{Cu}$ & 8.982 & 1.100 & 0.954 & 0.935 & 0.121 & & & & \\
\hline $30 \mathrm{Zn}$ & 9.662 & 1.200 & 1.047 & 1.024 & 0.137 & & 91 & & 12 \\
\hline $31 \mathrm{Ga}$ & 10.394 & $* 1.30$ & 1.168 & 1.142 & 0.182 & & 29 & & 43 \\
\hline $32 \mathrm{Ge}$ & 11.100 & $* 1.42$ & 1.244 & 1.212 & 0.175 & & 19 & & 24 \\
\hline 33 As & 11.866 & 1.529 & 1.358 & 1.322 & 0.202 & & 40 & & 41 \\
\hline $34 \mathrm{Se}$ & 12.653 & $* 1.66$ & 1.472 & 1.431 & 0.227 & & 57 & & 53 \\
\hline $35 \mathrm{Br}$ & 13.476 & $* 1.79$ & 1.599 & 1.552 & 0.259 & & 85 & & 73 \\
\hline $36 \mathrm{Kr}$ & $* 14.32$ & *1.92 & $* 1.73$ & $* 1.67$ & $* 0.289$ & $* 0.215$ & $* 0.210$ & & \\
\hline $37 \mathrm{Rb}$ & 15.197 & 2.064 & 1.863 & 1.803 & $* 0.323$ & 0.247 & 0.236 & & 10 \\
\hline $38 \mathrm{Sr}$ & 16.101 & 2.212 & 2.004 & 1.937 & 0.356 & 0.277 & 0.266 & & 32 \\
\hline $39 Y$ & 17.053 & 2.387 & 2.171 & 2.096 & 0.411 & 0.329 & 0.316 & & 76 \\
\hline $40 \mathrm{Zr}$ & 17.998 & 2.533 & 2.308 & 2.224 & 0.432 & 0.345 & 0.331 & 0.183 & 0.181 \\
\hline $41 \mathrm{Nb}$ & 18.986 & 2.700 & 2.467 & 2.372 & 0.471 & 0.381 & 0.365 & 0.209 & 0.206 \\
\hline $42 \mathrm{Mo}$ & 20.003 & 2.869 & 2.630 & 2.525 & 0.509 & 0.414 & 0.396 & 0.235 & 0.232 \\
\hline $\mathrm{Tc}$ & $* 21.05$ & $* 3.05$ & $* 2.80$ & $* 2.68$ & $* 0.546$ & $* 0.449$ & $* 0.428$ & $* 0.259$ & $* 0.256$ \\
\hline $44 \mathrm{Ru}$ & 22.117 & 3.227 & 2.968 & 2.839 & 0.584 & 0.485 & 0.463 & 0.285 & 0.281 \\
\hline $45 \mathrm{Rh}$ & 23.210 & 3.404 & 3.139 & 2.995 & 0.619 & 0.513 & 0.4 & 0.304 & 0.299 \\
\hline $46 \mathrm{Pd}$ & 24.356 & 3.614 & 3.338 & 3.181 & 0.677 & 0.569 & 0.5 & 0.348 & 0.342 \\
\hline $47 \mathrm{Ag}$ & 25.535 & 3.828 & 3.547 & 3.375 & 0.743 & 0.626 & 0.593 & 0.396 & 0.391 \\
\hline $48 \mathrm{Cd}$ & 26.712 & 4.019 & 3.731 & 3.541 & 0.775 & 0.652 & 0.616 & 0.414 & 0.407 \\
\hline 49 In & 27.929 & 4.226 & 3.929 & 3.732 & 0.823 & 0.691 & 0.653 & 0.447 & 0.445 \\
\hline $50 \mathrm{Sn}$ & 29.182 & 4.445 & 4.139 & 3.911 & 0.868 & 0.739 & 0.695 & 0.477 & 0.467 \\
\hline Sb & 30.497 & 4.708 & 4.391 & 4.137 & 0.950 & 0.823 & 0.775 & 0.543 & 0.532 \\
\hline $52 \mathrm{Te}$ & 31.817 & 4.953 & 4.621 & 4.347 & 1.013 & 0.882 & 0.831 & 0.591 & 0.577 \\
\hline & 33.164 & 5.187 & 4.855 & 4.559 & 1.074 & 30 & 0.874 & 0.634 & 0.620 \\
\hline $\mathrm{Xe}$ & *34.55 & $* 5.45$ & $* 5.10$ & $* 4.78$ & $* 1: 14$ & $* 0.992$ & ${ }^{*} 0.931$ & $* 0.686$ & *0.672 \\
\hline $55 \mathrm{Cs}$ & 35.974 & 5.706 & 5.360 & 5.014 & 1.215 & 1.056 & 0.9 & 0.741 & 0.728 \\
\hline $56 \mathrm{Ba}$ & 37.432 & 5.995 & 5.629 & 5.250 & 1.297 & 1.143 & 1. & 0.800 & 0.783 \\
\hline $57 \mathrm{La}$ & 38.923 & 6.264 & 5.902 & 5.490 & 1.371 & 1.201 & 1.120 & 0.857 & 0.839 \\
\hline $58 \mathrm{Ce}$ & 40.43 & 6.556 & 6.169 & 5.728 & 1.439 & 1.279 & 1.191 & 0.906 & 0.888 \\
\hline & 41.99 & 6.837 & 6.446 & 5.968 & 1.514 & 1.339 & 1.245 & 0.956 & 0.934 \\
\hline $60 \mathrm{Nd}$ & 43.57 & 7.134 & 6.728 & 6.215 & 1.583 & 1.413 & 1.305 & 1.007 & 0.984 \\
\hline $61 \mathrm{Pm}$ & $* 45.2$ & *7.43 & $* 7.02$ & $* 6.46$ & $* 1.65$ & $* 1.48$ & $* 1.36$ & $* 1.06$ & $* 1.03$ \\
\hline $62 \mathrm{Sm}$ & 46.85 & 7.742 & 7.316 & 6.720 & 1.727 & 1.545 & 1.424 & 1.111 & 1.083 \\
\hline Eu & 48.51 & 8.059 & 7.624 & 6.984 & 1.808 & 1.622 & 1.488 & 1.169 & 1.138 \\
\hline Gd & 50.23 & 8.383 & 7.942 & 7.251 & 1.8 & & & 1.226 & 1.191 \\
\hline & & 8.713 & 8.258 & 7.520 & 1.9 & & & & 1.245 \\
\hline & 53.77 & 9.053 & 8.587 & 7.795 & 2.036 & 1.846 & 1.679 & 1.339 & 1.301 \\
\hline
\end{tabular}


Critical X-ray absorption energies in $\mathrm{keV}$

\begin{tabular}{|c|c|c|c|c|c|c|c|}
\hline$N_{\mathrm{I}}$ & $N_{\text {II }}$ & $N_{\text {III }}$ & $N$ rv & $N \mathrm{v}$ & $O_{\mathrm{I}}$ & $O_{\text {II }}$ & OIII \\
\hline
\end{tabular}

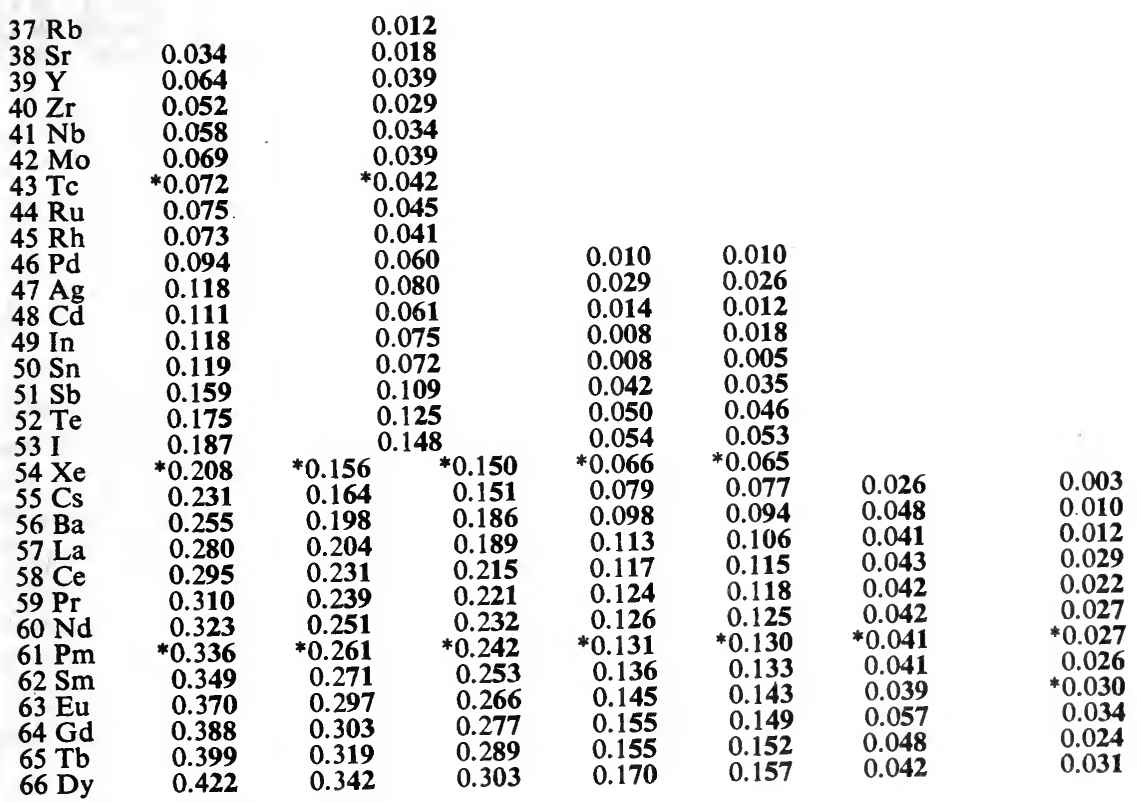


Critical X-ray absorption energies in keV

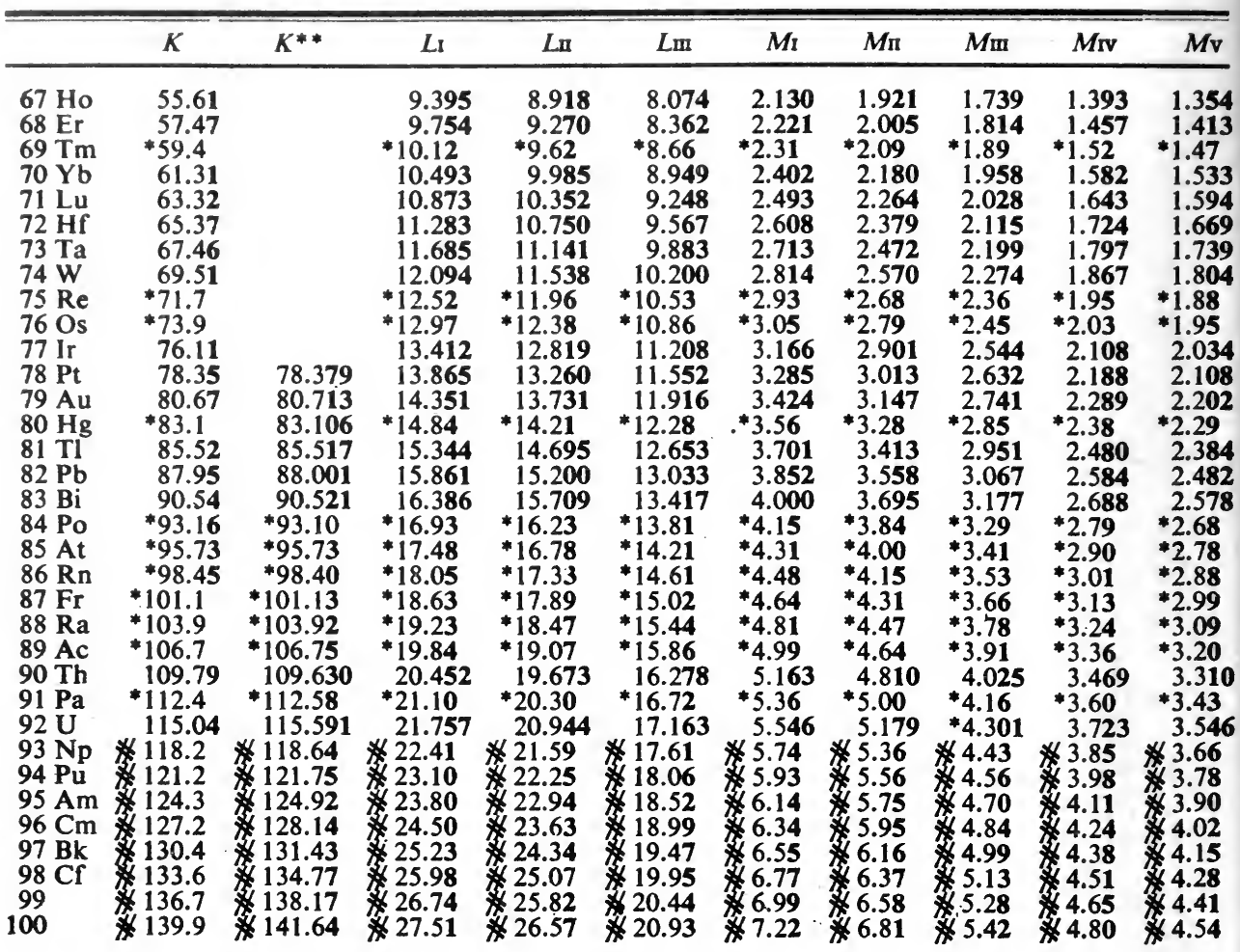


Critical X-ray absorption energies in keV

\begin{tabular}{|c|c|c|c|c|c|c|c|c|c|c|c|}
\hline & $N_{\mathrm{I}}$ & $N$ II & Nin & $N$ IV & $N v$ & $N \mathrm{vI}$ & $N$ vII & $O_{1}$ & On & $O$ III & $O \mathrm{rv}, \mathrm{v}$ \\
\hline 67 & 0 & 0.349 & 0.308 & 0.170 & 0.163 & & & $* 0.048$ & \multicolumn{3}{|c|}{0.020} \\
\hline & & 0.371 & 0.323 & 0.189 & 0.171 & & & 0.054 & \multirow{2}{*}{\multicolumn{3}{|c|}{0.038}} \\
\hline $69 \mathrm{Tm}$ & $* 0.471$ & $* 0.384$ & $* 0.336$ & $* 0.197$ & $* 0.181$ & & & ${ }^{*} 0.064$ & \multirow{2}{*}{\multicolumn{3}{|c|}{$\begin{array}{r}* 0.032 \\
0.026\end{array}$}} \\
\hline & 0.491 & 0.399 & 0.350 & 0.204 & 0.193 & & & 0.075 & & & \\
\hline$u$ & 0.5 & 0.417 & 0.365 & 0.213 & 0.198 & & & 0.058 & \multicolumn{3}{|c|}{0.031} \\
\hline $72 \mathrm{Hf}$ & 0.544 & 0.444 & 0.388 & 0.234 & 0.221 & 0.027 & 0.024 & 0.076 & \multicolumn{3}{|c|}{0.046} \\
\hline 73 & 72 & 0.468 & 0.409 & 0.247 & 0.234 & 0.031 & 0.029 & 0.075 & \multicolumn{3}{|c|}{0.043} \\
\hline & 0.588 & 0.489 & 0.421 & 0.254 & 0.239 & 0.031 & 0.027 & 0.073 & \multicolumn{3}{|c|}{0.039} \\
\hline & $* 0.619$ & $* 0.516$ & $* 0.442$ & $* 0.271$ & $* 0.256$ & $* 0.040$ & $* 0.035$ & ${ }^{*} 0.078$ & \multicolumn{3}{|c|}{$* 0.042$} \\
\hline & $* 0.650$ & $* 0.542$ & $* 0.464$ & $* 0.288$ & $* 0.272$ & $* 0.048$ & $* 0.043$ & $* 0.084$ & \multicolumn{3}{|c|}{$* 0.044$} \\
\hline & 0.683 & 0.570 & 0.486 & 0.307 & 0.291 & 0.058 & 0.053 & 0.090 & \multicolumn{3}{|c|}{0.046} \\
\hline & 0.710 & 0.597 & 0.506 & 0.318 & 0.303 & 0.061 & 0.058 & 0.087 & \multicolumn{3}{|c|}{0.045} \\
\hline & 0.758 & 0.642 & 0.542 & 0.350 & 0.333 & 0.084 & 0.079 & 0.106 & \multirow{2}{*}{\multicolumn{3}{|c|}{$\begin{array}{r}0.056 \\
* 0.063\end{array}$}} \\
\hline $\mathrm{Hg}$ & *0.799 & $* 0.677$ & $* 0.572$ & $* 0.376$ & $* 0.358$ & $* 0.100$ & $* 0.096$ & ${ }^{*} 0.116$ & & & \\
\hline & 0.842 & 0.717 & 0.605 & 0.403 & 0.381 & 0.118 & 0.114 & 0.126 & \multirow{2}{*}{\multicolumn{2}{|c|}{$\begin{array}{l}0.072 \\
0.087\end{array}$}} & \\
\hline & 0.893 & 0.760 & 0.642 & 0.437 & 0.414 & 0.143 & 0.136 & 0.148 & & & 0.022 \\
\hline & 0.938 & 0.805 & 0.676 & 0.464 & 0.440 & 0.162 & 0.156 & 0.157 & 0.119 & 0.092 & 0.024 \\
\hline & $* 0.986$ & $* 0.848$ & $* 0.712$ & $* 0.493$ & ${ }^{*} 0.468$ & $* 0.181$ & $* 0.176$ & ${ }^{*} 0.171$ & ${ }^{*} 0.130$ & $* 0.111$ & $* 0.029$ \\
\hline & $* 1.04$ & $* 0.895$ & $* 0.748$ & ${ }^{*} 0.526$ & $* 0.497$ & $* 0.202$ & $* 0.195$ & $* 0.185$ & $* 0.143$ & $* 0.118$ & $* 0.035$ \\
\hline & $* 1.09$ & $* 0.943$ & $* 0.787$ & $* 0.558$ & $* 0.529$ & $* 0.224$ & $* 0.217$ & $* 0.200$ & $* 0.155$ & $* 0.125$ & $* 0.041$ \\
\hline & $* 1.14$ & $* 0.992$ & $* 0.826$ & $* 0.591$ & $* 0.561$ & $* 0.246$ & $* 0.240$ & $* 0.215$ & $* 0.169$ & $* 0.132$ & $* 0.047$ \\
\hline & $* 1.20$ & $* 1.04$ & $* 0.867$ & ${ }^{*} 0.627$ & $* 0.593$ & $* 0.270$ & $* 0.264$ & $* 0.232$ & $* 0.182$ & $* 0.140$ & ${ }^{*} 0.054$ \\
\hline & $* 1.25$ & $* 1.10$ & ${ }^{*} 0.908$ & ${ }^{*} 0.663$ & *0.627 & $* 0.296$ & $* 0.288$ & $* 0.249$ & *0.196 & $* 0.148$ & $* 0.062$ \\
\hline & & 1.147 & $\begin{array}{r}0.944 \\
* 0.992\end{array}$ & $\begin{array}{r}0.692 \\
* 078\end{array}$ & $\begin{array}{r}0.656 \\
* 0600\end{array}$ & $\begin{array}{r}0.322 \\
* 0.353\end{array}$ & $\begin{array}{r}0.315 \\
* 0.343\end{array}$ & 0.266 & $\begin{array}{r}0.212 \\
* 020\end{array}$ & 0.156 & $\begin{array}{r}0.069 \\
* 0.082\end{array}$ \\
\hline & 1. & 1. & 1.040 & 0.781 & $\begin{array}{l}0.699 \\
0.737\end{array}$ & 0.387 & $\begin{array}{r}0.343 \\
0.375\end{array}$ & $\begin{array}{r}0.294 \\
0.322\end{array}$ & 0.248 & 0.189 & 0.095 \\
\hline
\end{tabular}

The values listed are the absolute $\mathrm{X}$-ray critical absorption energies in $\mathrm{keV}$. Figures which are not bold, or figures rounded-off to this extent, are probably of absolute significance for beta-ray spectroscopy.

Bold figures are probably only of relative significance.

Values printed between columns, e.g. $L_{\mathrm{I}}-L_{\mathrm{II}}$, represent some average for those sub-shells.

Values with an asterisk, e.g. *12.3, have been computed by interpolation.

Values with a number sign, e.g. 12.3 , have been computed by extrapolation.

Values in parentheses, e.g. (12.3) have been obtained from the tables of $Y$. Cauchois and H. Hulubei, Tables de Constantes et Données Numériques, 1. (Herman et Cie, Paris, (1947)).

Values printed in the column headed $K^{* *}$ have been obtained from the data of Y. Cauchois, J. Phys. et radium 13, (1952) 113. 


\title{
$\beta$-RAY SPECTRUM OF Th $\left(B+C+C^{\prime \prime}\right)$
}

\author{
K. SIEGBAHN AND T. R. GERHOLM
}

(Cf. Ch. III)

The thorium deposit technique (cf. Ch. III), offers a simple way to obtain strong sources of high specific activity, convenient for $\beta$-ray spectroscopic work. Fig. 1 illustrates the genetic relationships between those members of the thorium series that are under discussion. The complex $\beta$-ray spectrum of the Th $\left(\mathrm{B}+\mathrm{C}+\mathrm{C}^{\prime \prime}\right)$ is shown in Fig. 2 and Fig. 3 , and the various conversion lines are summarized in the Table.

Some of the strongest lines, frequently used as reference lines for calibration purposes, have been recently measured with increased precision by Siegbahn and Edvarson ${ }^{1}$, namely

\begin{tabular}{|c|c|c|}
\hline & $\begin{array}{c}B \varrho \\
\text { gauss } \mathrm{cms}\end{array}$ & $\begin{array}{c}\text { Electron energy } \\
\mathrm{keV}\end{array}$ \\
\hline $\mathrm{A}$ & $\dot{5} 34.20 \pm 0.06$ & $24.509 \pm 0.005$ \\
\hline B & $652.38 \pm 0.07$ & $36.150 \pm 0.007$ \\
\hline $\mathrm{F}$ & $1388.44 \pm 0.10$ & $148.08 \pm 0.02$ \\
\hline I & $1753.91 \pm 0.14$ & $222.22 \pm 0.03$ \\
\hline Ia & $1757.07 \pm 0.14$ & $222.90 \pm 0.03$ \\
\hline$J$ & $1811.11 \pm 0.15$ & $234.61 \pm 0.03$ \\
\hline
\end{tabular}

while Lindström ${ }^{2}$ has given for the lines

$$
\text { Th } \begin{array}{rl}
\mathrm{L} & 2607.17 \pm 0.30 \\
\mathrm{X} & 9986.7 \pm 1.5
\end{array}
$$$$
422.84 \pm 0.08
$$$$
2526.3 \pm 0.5
$$

These figures are given in bold type in the Table. All other B@-data are taken from Surugue ${ }^{3}$, but his original values have been adjusted to match neighbouring standard lines. The photometrically determined intensities correspond only roughly to the true intensities. In the low energy region (around the $\mathrm{A}$ and $\mathrm{B}$ lines) the true intensities are much higher than the figures given in the Table. See Fig. 3. The assignments (three last columns) are according to Surugue, and the notation is that introduced by Ellis ${ }^{4}$.

1 K. Siegbahn and K. Edvarson, to be published (see Ch. III).

2 G. Lindström, Arkiv för Fysik 4, (1951) 1. Phys. Rev. 87, (1952) 687.

3 J. Surugue, Ann. phys. 8, (1937) 484. J. Phys. et radium 9, (1938) 438.

4 C. D. Ellis, Proc. Roy. Soc. (Iondon) 138A, (1932) 318. Proc. Roy. Soc. (London) 143A, (1934) 350 . 
Apr. VII - K. Siegbahn and T. Gerholm -- Th $(\mathrm{B}+\mathrm{C}-\mathrm{C}$ ) i hertm

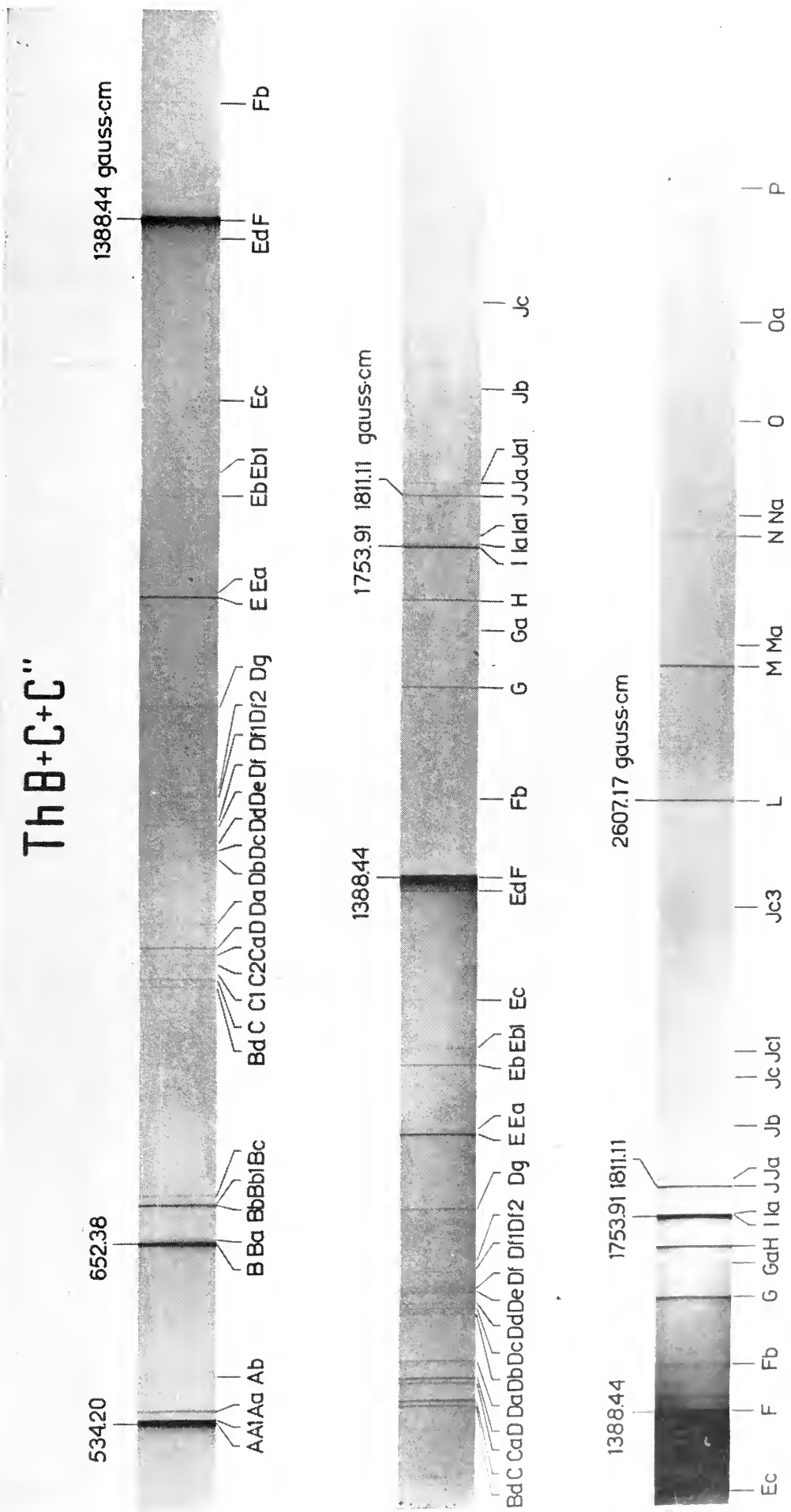

Fig. 2. The $\beta$-ray spectrum of Th $\left(\mathrm{B}+\mathrm{C}^{\prime}+\mathrm{C}^{\prime \prime}\right)$ recended in a high resolution permanent magnet spectrograph by H. Slitis 
$\beta$-RAY SPECtrum of $\mathrm{Th}\left(\mathrm{B}+\mathrm{C}+\mathrm{C}^{\prime \prime}\right)$

\begin{tabular}{|c|c|c|c|c|c|c|}
\hline Line & $B \varrho$ & $\mathrm{keV}$ & $\begin{array}{l}\text { Rel. int. } \\
\text { (photo- } \\
\text { metric) }\end{array}$ & Origin & $\gamma$-line & Shell \\
\hline $\mathbf{A}$ & 534.20 & 24.509 & 6.0 & $\mathrm{C} \rightarrow \mathrm{C}^{\prime \prime}$ & $\gamma \mathrm{A}$ & L I \\
\hline A 1 & 536 & 24.7 & 0.2 & $\mathrm{C} \rightarrow \mathrm{D}$ & $\gamma \mathbf{A}_{1}$ & L I \\
\hline Aa & 541 & 25.1 & 0.6 & $\mathrm{C} \rightarrow \mathrm{C}^{\prime \prime}$ & $\gamma \mathrm{A}$ & L II \\
\hline $\mathrm{Ab}$ & 563 & 27.2 & 0.1 & $\mathrm{C} \rightarrow \mathrm{C}^{\prime \prime}$ & $\gamma \mathrm{A}$ & L III \\
\hline $\mathbf{B}$ & 652.38 & 36.150 & 4.0 & $\mathrm{C} \rightarrow \mathrm{C}^{\prime \prime}$ & $\gamma \mathrm{A}$ & M I \\
\hline $\mathrm{Ba}$ & 655 & 36.4 & 0.1 & $\mathrm{C} \rightarrow \mathrm{C}^{\prime \prime}$ & $\gamma \mathrm{A}$ & M II \\
\hline $\mathrm{Bb}$ & 678 & 38.9 & 1.0 & $\mathrm{C} \rightarrow \mathrm{C}^{\prime \prime}$ & $\gamma \mathrm{A}$ & N I \\
\hline $\mathrm{Bb} 1$ & 680 & 39.2 & $<0.1$ & $\mathrm{C} \rightarrow \mathrm{C}^{\prime \prime}$ & $\gamma \mathrm{Bbl}$ & $\mathbf{K}$ \\
\hline $\mathrm{Bc}$ & 684 & 39.6 & 0.2 & $\mathrm{C} \rightarrow \mathrm{C}^{\prime \prime}$ & $\gamma \mathbf{A}$ & $\mathrm{O}$ \\
\hline $\mathrm{Bd}$ & 829 & 57.2 & 0.6 & $\mathrm{~B} \rightarrow \mathrm{C}$ & - & - \\
\hline C & 835 & 58.0 & 1.4 & $\mathrm{~B} \rightarrow \mathrm{C}$ & - & - \\
\hline C 1 & 840 & 58.7 & - & - & - & $\mathbf{K}$ \\
\hline C 2 & 845 & 59.3 & 0.2 & $\left\{\begin{array}{l}\mathrm{C} \rightarrow \mathrm{C}^{\prime \prime} \\
\mathrm{C}^{\prime \prime} \rightarrow \mathrm{D}\end{array}\right.$ & $\{\gamma \mathrm{C} 2$ & - \\
\hline $\mathrm{Ca}$ & 851 & 60.1 & 0.6 & $\mathrm{~B} \rightarrow \mathrm{C}$ & - & - \\
\hline $\mathrm{D}$ & 857 & 61.0 & 1.4 & $\mathrm{~B} \rightarrow \mathrm{C}$ & - & - \\
\hline $\mathrm{Da}$ & 873 & 63.1 & 0.8 & $\mathrm{~B} \rightarrow \mathrm{C}$ & - & - \\
\hline $\mathrm{Da} 1$ & 881 & 64.2 & $<0.1$ & - & - & - \\
\hline $\mathrm{Db}$ & 920 & 69.7 & 0.3 & $\mathrm{~B} \rightarrow \mathrm{C}$ & - & - \\
\hline Dc & 922 & 70.0 & 0.2 & $\mathrm{~B} \rightarrow \mathrm{C}$ & - & - \\
\hline $\mathrm{Dd}$ & 929 & 71.0 & 0.2 & $\mathrm{~B} \rightarrow \mathrm{C}$ & - & - \\
\hline $\mathrm{De}$ & 941 & 72.7 & 0.4 & $\mathrm{~B} \rightarrow \mathrm{C}$ & - & - \\
\hline $\mathrm{Df}$ & 948 & 73.7 & 0.3 & $\mathrm{~B} \rightarrow \mathrm{C}$ & $\gamma \mathrm{Df}$ & $\mathbf{K}$ \\
\hline Df 1 & 964 & 76.1 & 0.1 & $\mathrm{~B} \rightarrow \mathrm{C}$ & - & - \\
\hline Df 2 & 972 & 77.3 & 0.1 & $\mathrm{C} \rightarrow \mathrm{C}^{\prime \prime}$ & $\gamma \mathrm{Df} 2$ & $\mathbf{K}$ \\
\hline $\mathrm{Dg}$ & 1030 & 86.1 & 0.6 & $\mathrm{~B} \rightarrow \mathrm{C}$ & $\gamma \mathrm{Dg}$ & $\mathbf{K}$ \\
\hline $\mathrm{Dh}$ & 1096 & 96.5 & 0.1 & $\mathrm{~B} \rightarrow \mathrm{C}$ & $\gamma \mathrm{Dh}$ & $\mathrm{L} \mathbf{I}$ \\
\hline $\mathrm{E}$ & 1110 & 98.8 & 3.4 & $\mathrm{~B} \rightarrow \mathrm{C}$ & $\gamma \mathrm{E}$ & L I \\
\hline $\mathrm{Ea}$ & 1114 & 99.5 & 0.2 & $\mathrm{~B} \rightarrow \mathrm{C}$ & $\gamma \mathrm{E}$ & L II \\
\hline $\mathrm{Ea} 1$ & 1170 & 108.8 & 0.1 & $\mathrm{C} \rightarrow \mathrm{C}^{\prime \prime}$ & $\gamma \mathrm{Bbl}$ & L I \\
\hline $\mathrm{Eb}$ & 1183 & 111.0 & 0.6 & $\mathrm{~B} \rightarrow \mathrm{C}$ & $\gamma \mathrm{E}$ & MI \\
\hline Eb 1 & 1202 & 114.3 & 0.2 & $\mathrm{~B} \rightarrow \mathrm{C}$ & $\gamma \mathrm{E}$ & N I \\
\hline $\mathrm{Eb} 2$ & $\grave{1} 226$ & 118.5 & 0.1 & $\mathrm{~B} \rightarrow \mathrm{C}$ & $\gamma \mathrm{Eb} 2$ & $\mathbf{K}$ \\
\hline Ec & 1253 & 123.2 & 0.4 & $\mathrm{C}^{\prime \prime} \rightarrow \mathrm{D}$ & $\gamma \mathrm{Ec}$ & $\mathrm{K}$ \\
\hline Ec 1 & 1295 & 130.8 & 0.1 & - & - & - \\
\hline Ec 2 & 1323 & 135.9 & $<0.1$ & - & - & - \\
\hline Ed & 1372 & 145.0 & 0.2 & $\mathrm{C}^{\prime \prime} \rightarrow \mathrm{D}$ & $\gamma \mathrm{Ed}$ & $\mathbf{K}$ \\
\hline $\mathbf{F}$ & 1388.44 & 148.108 & 200.0 & $\mathrm{~B} \rightarrow \mathrm{C}$ & $\begin{cases}\gamma & \mathrm{F} \\
\gamma & \mathrm{Df}\end{cases}$ & $\left\{\begin{array}{l}\mathrm{K} \\
\mathrm{L} \mathbf{I}\end{array}\right.$ \\
\hline F 1 & 1436 & 157.2 & 0.1 & - & - & - \\
\hline F 2 & 1439 & 157.8 & $<0.1$ & $\mathrm{~B} \rightarrow \mathrm{C}$ & - & - \\
\hline $\mathrm{Fa}$ & 1452 & 160.3 & 0.4 & $\mathrm{~B} \rightarrow \mathrm{C}$ & $\begin{cases}\gamma & \mathrm{Fa} \\
\gamma & \mathrm{Dg} \\
\gamma & \mathrm{Df}\end{cases}$ & $\left\{\begin{array}{l}\mathrm{K} \\
\mathrm{L} I \\
\mathrm{M} I\end{array}\right.$ \\
\hline
\end{tabular}


$\beta$-RAy Spectrum of $\mathrm{Th}\left(\mathrm{B}+\mathrm{C}+\mathrm{C}^{\prime \prime}\right)$

\begin{tabular}{|c|c|c|c|c|c|c|}
\hline Line & $B \varrho$ & $\mathrm{keV}$ & $\begin{array}{l}\text { Rel. int. } \\
\text { (photo- } \\
\text { metric) }\end{array}$ & Origin & $\gamma$-line & Shell \\
\hline $\mathrm{Fb}$ & 1472 & 164.2 & 0.6 & $\mathrm{C}^{\prime \prime} \rightarrow \mathrm{D}$ & $\gamma \mathrm{Fb}$ & $\mathrm{K}$ \\
\hline Fb 1 & 1476 & 165.0 & 0.1 & $\mathrm{C}^{\prime \prime} \rightarrow \mathrm{D}$ & - & - \\
\hline G & 1596 & 189.0 & 4.0 & $\mathrm{C}^{\prime \prime} \rightarrow \mathrm{D}$ & $\gamma \mathrm{G}$ & $\mathrm{K}$ \\
\hline G 1 & 1599 & 189.7 & 0.1 & - & - & - \\
\hline G 2 & 1625 & 195.0 & 0.1 & $\mathrm{C}^{\prime \prime} \rightarrow \mathrm{D}$ & $\gamma \mathrm{Ec}$ & L I \\
\hline $\mathrm{Ga}$ & 1659 & 202.1 & 0.4 & $\mathrm{C} \rightarrow \mathrm{C}^{\prime \prime}$ & $\gamma \mathrm{Ga}$ & $\mathrm{K}$ \\
\hline $\mathrm{H}$ & 1697 & 210.1 & 6.0 & $\mathrm{~B} \rightarrow \mathrm{C}$ & $\gamma \mathrm{H}$ & $\mathrm{K}$ \\
\hline H 1 & 1721 & 215.2 & 0.1 & $\mathrm{C}^{\prime \prime} \rightarrow \mathrm{D}$ & $\gamma \mathrm{Hl}$ & $\mathrm{K}$ \\
\hline $\mathrm{H} 2$ & 1731 & 217.3 & 0.1 & $\mathrm{C}^{\prime \prime} \rightarrow \mathrm{D}$ & $\gamma \mathrm{Ed}$ & L I \\
\hline I & 1753.91 & 222.21 & 22.0 & $\mathrm{~B} \rightarrow \mathrm{C}$ & $\gamma \mathrm{F}$ & L I \\
\hline Ia & 1757.07 & 222.90 & 1.4 & $\mathrm{~B} \rightarrow \mathrm{C}$ & $\gamma \mathrm{F}$ & L II \\
\hline Ia 1. & 1766 & 224.8 & 0.1 & $\mathrm{~B} \rightarrow \mathrm{C}$ & $\gamma \mathrm{F}$ & L III \\
\hline Ia 2 & 1787 & 229.4 & 0.1 & - & - & - \\
\hline $\mathbf{J}$ & 1811.11 & 234.61 & 6.0 & $\mathrm{~B} \rightarrow \mathrm{C}$ & $\begin{cases}\gamma & \mathrm{F} \\
\gamma & \mathrm{Fa}\end{cases}$ & $\begin{cases}M & I \\
L & I\end{cases}$ \\
\hline $\mathbf{J} 1$ & 1814 & 235.2 & $<0.1$ & $\mathrm{~B} \rightarrow \mathrm{C}$ & $\gamma \mathrm{Fa}$ & L II \\
\hline $\mathrm{J} 2$ & 1818 & 236.1 & $<0.1$ & $\mathrm{C}^{\prime \prime} \rightarrow \mathrm{D}$ & $\gamma \mathrm{Fb}$ & L I \\
\hline $\mathrm{Ja}$ & 1824 & 237.4 & 1.8 & $\mathrm{~B} \rightarrow \mathrm{C}$ & $\left\{\begin{array}{l}\gamma \mathrm{F} \\
\gamma \mathrm{Fa}\end{array}\right.$ & $\begin{cases}N & \text { I } \\
\text { L } & \text { III }\end{cases}$ \\
\hline Ja 1 & 1827 & 238.1 & $<0.1$ & $\mathrm{~B} \rightarrow \mathrm{C}$ & $\gamma \mathrm{F}$ & $\mathrm{O}$ \\
\hline $\mathrm{Ja} 2$ & 1845 & 242.0 & 0.1 & $\begin{array}{l}\mathrm{C} \rightarrow \mathrm{C}^{\prime \prime} \\
\mathrm{C}^{\prime \prime} \rightarrow \mathrm{D}\end{array}$ & $\begin{array}{l}\gamma \mathrm{Ja2} \\
\gamma \mathrm{Ja2}\end{array}$ & $\begin{array}{l}\mathrm{K} \\
\mathrm{K}\end{array}$ \\
\hline Ja 3 & 1909 & 256.2 & $<0.1$ & - & - & - \\
\hline Ja 4 & 1921 & 258.9 & $<0.1$ & - & - & - \\
\hline $\mathrm{Jb}$ & 1934 & 261.9 & 0.6 & $\mathrm{C}^{\prime \prime} \rightarrow \mathrm{D}$ & $\gamma \mathrm{G}$ & L I \\
\hline Jb 1 & 1980 & 272.3 & 0.2 & $\mathrm{C} \rightarrow \mathrm{C}^{\prime \prime}$ & $\gamma \mathrm{Ga}$ & $\mathrm{L} 1$ \\
\hline $\mathrm{Jb} 2$ & 1983 & 272.9 & 0.2 & $\mathrm{C}^{\prime \prime} \rightarrow \mathrm{D}$ & $\gamma \mathrm{G}$ & MI \\
\hline Jb 3 & 1997 & 276.1 & $<0.1$ & $\mathrm{C}^{\prime \prime} \rightarrow \mathrm{D}$ & $\gamma \mathrm{G}$ & N I \\
\hline $\mathrm{Jb} 4$ & 2009 & 278.9 & $<0.1$ & - & - & - \\
\hline $\mathrm{Jc}$ & 2027 & 283.0 & 0.6 & $\left\{\begin{array}{l}\mathrm{B} \rightarrow \mathrm{C} \\
\mathrm{C} \rightarrow \mathrm{C}^{\prime \prime}\end{array}\right.$ & $\begin{array}{l}\gamma \mathrm{H} \\
\gamma \mathrm{Ga}\end{array}$ & $\left\{\begin{array}{l}L I \\
M I\end{array}\right.$ \\
\hline Je 1 & 2030 & 283.7 & $<0.1$ & - & - & - \\
\hline Jc 2 & 2080 & 295.2 & 0.2 & $\mathrm{~B} \rightarrow \mathrm{C}$ & $\gamma \mathrm{H}$ & M I \\
\hline Jc 3 & 2094 & 298.5 & $<0.1$ & $\mathrm{~B} \rightarrow \mathrm{C}$ & $\gamma \mathrm{H}$ & $\mathrm{N}$ \\
\hline Jc 4 & 2297 & 346.5 & $<0.1$ & $\mathrm{C} \rightarrow \mathrm{C}^{\prime \prime}$ & $\gamma \mathrm{Jc4}$ & $\mathrm{K}$ \\
\hline Jc 5 & 2380 & 366.6 & $<0.1$ & $\mathrm{C} \rightarrow \mathrm{C}^{\prime \prime}$ & $\gamma \mathrm{Jc5}$ & $\mathbf{K}$ \\
\hline Jc 6 & 2455 & 385.0 & $<0.1$ & $\mathrm{C} \rightarrow \mathrm{C}^{\prime \prime}$ & $\gamma \mathrm{Jc} 6$ & $\mathrm{~K}$ \\
\hline Jc 7 & 2493 & 394.4 & $<0.1$ & - & - & - \\
\hline Jc 8 & 2537 & 405.3 & $<0.1$ & $\mathrm{C} \rightarrow \mathrm{C}^{\prime \prime}$ & - & - \\
\hline $\mathbf{L}$ & 2607.17 & 422.84 & 1.4 & $\mathrm{C}^{\prime \prime} \rightarrow \mathrm{D}$ & $\gamma \mathrm{L}$ & $\mathrm{K}$ \\
\hline $\mathbf{M}$ & 2891 & 495.1 & 1.4 & $\mathrm{C}^{\prime \prime} \rightarrow \mathrm{D}$ & $\left\{\begin{array}{l}\gamma \mathrm{M} \\
\gamma \mathrm{L}\end{array}\right.$ & $\left\{\begin{array}{l}\mathrm{K} \\
\mathrm{L} \mathrm{I}\end{array}\right.$ \\
\hline $\mathrm{Ma}$ & 2938 & 507.3 & $<0.1$ & $\mathrm{C}^{\prime \prime} \rightarrow \mathrm{D}$ & $\gamma \mathrm{L}$ & $\begin{array}{l}\text { M I } \\
\mathrm{K}\end{array}$ \\
\hline Ma 1 & 3026 & 530.2 & $<0.1$ & $\mathrm{C}^{\prime \prime} \rightarrow \mathrm{D}$ & $\gamma \mathrm{Mal}$ & \\
\hline
\end{tabular}



$\beta$-RAY SpeCtRUM of Th $\left(\mathrm{B}+\mathrm{C}+\mathrm{C}^{\prime \prime}\right)$

\begin{tabular}{|c|c|c|c|c|c|c|}
\hline Line & $B \varrho$ & $\mathrm{keV}$ & $\begin{array}{l}\text { Rel. int. } \\
\text { (photo-) } \\
\text { metric) }\end{array}$ & Origin & $\gamma$-line & Shell \\
\hline $\mathrm{N}$ & 3169 & 567.8 & 0.3 & $\mathrm{C}^{\prime \prime} \rightarrow \mathrm{D}$ & $\gamma \mathrm{M}$ & L I \\
\hline $\mathrm{Na}$ & 3215 & 579.9 & $<0.1$ & $\mathrm{C}^{\prime \prime} \rightarrow \mathrm{D}$ & $\gamma \mathrm{M}$ & M I \\
\hline $\mathrm{O}$ & 3420 & 634.6 & 0.2 & $\mathrm{C} \rightarrow \mathrm{C}^{\prime \prime}$ & $\gamma O$ & $\mathbf{K}$ \\
\hline O 1 & 3539 & 666.6 & $<0.1$ & - & - & - \\
\hline $\mathrm{O} 2$ & 3567 & 674.2 & $<0.1$ & - & - & - \\
\hline O 3 & 3613 & 686.7 & $<0.1$ & - & - & - \\
\hline $\mathrm{Oa}$ & 3623 & 689.4 & 0.1 & - & - & - \\
\hline Oa 1 & 3713 & 713.8 & $<0.1$ & - & - & - \\
\hline $\mathrm{Oa} 2$ & 3753 & 724.7 & $<0.1$ & $\mathrm{C}^{\prime \prime} \rightarrow \mathrm{D}$ & $\gamma \mathrm{Oa} 2$ & $\mathbf{K}$ \\
\hline Oa 3 & 3895 & 763.6 & $<0.1$ & - & - & - \\
\hline $\mathbf{P}$ & 3931 & 7735 & 0.2 & - & - & - \\
\hline $\mathrm{Pa}$ & 4014 & 796.4 & $<0.1$ & $\mathrm{C}^{\prime \prime} \rightarrow \mathrm{D}$ & $\gamma \mathrm{Oa} 2$ & $\mathbf{L} \mathbf{I}$ \\
\hline $\mathrm{Pa} 1$ & 4199 & 847.6 & $<0.1$ & - & - & - \\
\hline $\mathrm{Pa} 2$ & 4228 & 855.7 & $<0.1$ & - & - & - \\
\hline $\mathrm{Pa} 3$ & 4279 & 869.9 & $<0.1$ & - & - & - \\
\hline $\mathbf{x}$ & 9986.7 & 2526.3 & 0.1 & $\mathrm{C}^{\prime \prime} \rightarrow \mathrm{D}$ & $\gamma \mathrm{X}$ & $\mathrm{K}$ \\
\hline $\mathrm{Xa} 1$ & 10244 & 2602.3 & $<0.1$ & $\mathrm{C}^{\prime \prime} \rightarrow \mathrm{D}$ & $\gamma \mathrm{X}$ & L I \\
\hline $\mathrm{Xa} 2$ & 10284 & 2614.1 & $<0.1$ & $\mathrm{C}^{\prime \prime} \rightarrow \mathrm{D}$ & $\gamma \mathrm{x}$ & M I \\
\hline
\end{tabular}





\section{$B \rho$ vs $\mathrm{keV}$}

\section{TABLES}

\section{T. R. GERHOLM}

The electron energies are calculated from the well known relation

$$
E=E_{0}\left\{\sqrt{\left(\frac{e}{m_{0}} \frac{B \varrho}{c}\right)^{2}+1}-1\right\}
$$

where the values of the fundamental constants are the least squares adjusted output values by DuMond and Cohen, Rev. Mod. Phys. 25, (1953)691 :

$$
\begin{aligned}
E_{0} & =m_{0} c^{2}=(510.984 \pm 0.016) \mathrm{keV} \\
\frac{e}{m_{0}} & =(1.75888 \pm 0.00005) \cdot 10^{7} \mathrm{emu} \mathrm{g}-1 \\
c & =(2.997929 \pm 0.000008) 10^{10} \mathrm{~cm} \mathrm{sec}-1
\end{aligned}
$$

Differentiating (1) one obtains the expression

$$
d E=\frac{E}{B \varrho} \cdot \frac{E+2 E_{0}}{E+E_{0}} \cdot d(B \varrho)=F(B \varrho) \cdot d(B \varrho)
$$

to be used for the interpolation.

In the tables below, covering the $B \varrho$-region $100-100000$ gauss $\mathrm{cm}$ $(0.9 \mathrm{keV}-30 \mathrm{MeV})$, the tabulated $B \varrho$-values are obtained by multiplying the BQ-factor by the decade factor. For each tabulated $B \varrho$-value the corresponding values of the energy and of the interpolation function $F(B \varrho)$ are given.

The electron energy, that corresponds to a given $B \varrho$-value, or vice versa, is then easily obtained, to the first order approximation, from the simple formula

$$
\begin{aligned}
& E=E^{0}+F^{0}\left(B \varrho-B \varrho^{0}\right) \\
& B \varrho=B \varrho^{0}+\frac{E-E^{0}}{F^{0}}
\end{aligned}
$$

where $E^{0}$ is the energy value corresponding to the nearest tabulated $B Q^{-}$ value, i.e. $B \varrho^{0}$, and $\mathrm{F}^{0}$ is the value of the interpolation function corresponding to $B \varrho^{0}$.

\section{ACCURACY}

The values derived in this simple way are of sufficient accuracy for all present purposes, even in the case of very high precision measurements. The interpolation error, which is always less than 3 parts in $10^{5}$, is smaller than the uncertainty due to the limits of error given for the fundamental constants. 


\section{EXAMPLES}

\section{BQ-value to energy}

The $B Q$-value of the ThC B-line is $652.38 \pm 0.07$ gauss $\mathrm{cm}^{1}$. What is the corresponding energy?

Nearest tabulated value 650.000 gauss $\mathrm{cm}=35.8956 \mathrm{keV}$. According to (3) we get

$$
35.8956+0.10682 \cdot 2.380=36.1498
$$

Experimental errors

$$
\pm 0.10682 \cdot 0.07 \sim \pm 0.007
$$

Thus we obtain from the table

$$
\text { Energy of ThC B-line }=36.150 \pm 0.007 \mathrm{keV} \text {, }
$$

while the "correct" value, i.e. the value calculated directly from the formula (1), is $36.1502 \mathrm{keV}$. The interpolation error is then $-0.0004 \mathrm{keV}$ $\left(1\right.$ part in $10^{5}$ ) and obviously much less than the experimental errors and the uncertainty due to the fundamental constants.

\section{Energy to BQ-value}

The $B \varrho$-value of the ThB F-line (K-conversion line) is determined to be 1388.44 gauss $\mathrm{cm}^{1}$. What will the $B \varrho$-value of the ThB I-line $\left(L_{I}\right.$ conversion line) be, if we know from $\mathrm{X}$-ray data ${ }^{2}$ that the energy difference $\left(K-L_{I}\right)$ should be $74.1379 \pm 0.004 \mathrm{keV}$ ?

From the table we get

And thus

$$
\text { Energy of ThB F-line }=148.079 \mathrm{keV}
$$

Energy of ThB I-line $=148.079+74.138=222.217 \mathrm{keV}$

Nearest tabulated value 1760.00 gauss $\mathrm{cm}=223.526 \mathrm{keV}$, and then from $(3)^{\prime}$ we get

$$
B Q \text {-value } 1760.00-\frac{223.526-222.217}{0.21536}=1753.92 \text { gauss } \mathrm{cm} \text {. }
$$

Uncertainty in the $B \varrho$-value due to errors in the $\mathrm{X}$-ray data

$$
\pm \frac{0.004}{0.21536} \sim \pm 0.02 \text { gauss } \mathrm{cm}
$$

Thus we obtain from the table for the ThB I-line $1753.92 \pm 0.02$ gauss $\mathrm{cm}$. The experimentally found value is $1753.91 \pm 0.14$ gauss $\mathrm{cm}^{1}$.

The table has been computed in a BESK-machine by the Swedish Board for Computing Machinery. Our thanks are due to Dr S. Comét for his kind cooperation.

1 K. Siegbahn and K. Edvarson, to be published.

2 Y. Cauchois et H. Hulubei, Tables de constantes et données numériques, Paris 1947. 


\begin{tabular}{|c|c|c|c|c|c|c|}
\hline \multirow[b]{2}{*}{$\begin{array}{c}B \varrho \\
\text { factor } \\
\text { gauss } \mathrm{cm}\end{array}$} & \multicolumn{2}{|c|}{$\begin{array}{l}\text { Decade factor } \\
10^{2}\end{array}$} & \multicolumn{2}{|c|}{$\begin{array}{c}\text { Decade factor } \\
\qquad 10^{3}\end{array}$} & \multicolumn{2}{|c|}{$\begin{array}{c}\text { Decade factor } \\
10^{4}\end{array}$} \\
\hline & $\underset{\text { keV }}{\text { Energy }}$ & $\begin{array}{l}\text { Interpol. } \\
\text { gauss cm } \\
\frac{\mathrm{keV}}{}\end{array}$ & $\begin{array}{c}\text { Energy } \\
\text { keV }\end{array}$ & $\begin{array}{l}\text { Interpol. } \\
\text { gauss cm } \\
\frac{\mathrm{keV}}{}\end{array}$ & $\underset{\mathrm{keV}}{\text { Energy }}$ & $\begin{array}{l}\text { Interpol. } \\
\text { gauss cm } \\
\frac{\mathrm{keV}}{}\end{array}$ \\
\hline $\begin{array}{l}1.00000 \\
1.01000 \\
1.02000 \\
1.03000 \\
1.04000 \\
1.05000 \\
1.06000 \\
1.07000 \\
1.08000 \\
1.09000\end{array}$ & $\begin{array}{l}0.878686 \\
0.896332 \\
0.914153 \\
0.932149 \\
0.950320 \\
0.968666 \\
0.987187 \\
1.00588 \\
1.02475 \\
1.04380\end{array}$ & $\begin{array}{l}0.017559 \\
0.017734 \\
0.017909 \\
0.018084 \\
0.018258 \\
0.018433 \\
0.018608 \\
0.018783 \\
0.018958 \\
0.019133\end{array}$ & $\begin{array}{l}81.4523 \\
82.9750 \\
84.5089 \\
86.0539 \\
87.6099 \\
89.1769 \\
90.7547 \\
92.3433 \\
93.9426 \\
95.5525\end{array}$ & $\begin{array}{l}0.15171 \\
0.15283 \\
0.15395 \\
0.15505 \\
0.15615 \\
0.15724 \\
0.15832 \\
0.15940 \\
0.16046 \\
0.16152\end{array}$ & $\begin{array}{l}2530.19 \\
2559.74 \\
2589.31 \\
2618.88 \\
2648.46 \\
2678.05 \\
2707.65 \\
2737.25 \\
2766.86 \\
2796.48\end{array}$ & $\begin{array}{l}0.29553 \\
0.29561 \\
0.29569 \\
0.29577 \\
0.29585 \\
0.29592 \\
0.29599 \\
0.29606 \\
0.29613 \\
0.29619\end{array}$ \\
\hline $\begin{array}{l}1.10000 \\
1.11000 \\
1.12000 \\
1.13000 \\
1.14000 \\
1.15000 \\
1.16000 \\
1.17000 \\
1.18000 \\
1.19000\end{array}$ & $\begin{array}{l}1.06302 \\
1.08241 \\
1.10198 \\
1.12173 \\
1.14165 \\
1.16174 \\
1.18201 \\
1.20245 \\
1.22307 \\
1.24386\end{array}$ & $\begin{array}{l}0.019308 \\
0.019482 \\
0.019657 \\
0.019832 \\
0.020007 \\
0.020181 \\
0.020356 \\
0.020531 \\
0.020705 \\
0.020880\end{array}$ & $\begin{array}{l}97.1729 \\
98.8037 \\
100.445 \\
102.096 \\
103.758 \\
105.430 \\
107.111 \\
108.803 \\
110.505 \\
112.216\end{array}$ & $\begin{array}{l}0.16256 \\
0.16360 \\
0.16463 \\
0.16566 \\
0.16667 \\
0.16768 \\
0.16867 \\
0.16966 \\
0.17064 \\
0.17162\end{array}$ & $\begin{array}{l}2826.10 \\
2855.73 \\
2885.36 \\
2915.00 \\
2944.65 \\
2974.30 \\
3003.96 \\
3033.62 \\
3063.29 \\
3092.97\end{array}$ & $\begin{array}{l}0.29626 \\
0.29632 \\
0.29638 \\
0.29644 \\
0.29650 \\
0.29655 \\
0.29661 \\
0.29666 \\
0.29671 \\
0.29676\end{array}$ \\
\hline $\begin{array}{l}1.20000 \\
1.21000 \\
1.22000 \\
1.23000 \\
1.24000 \\
1.25000 \\
1.26000 \\
1.27000 \\
1.28000 \\
1.29000\end{array}$ & $\begin{array}{l}1.26483 \\
1.28597 \\
1.30729 \\
1.32878 \\
1.35045 \\
1.37228 \\
1.39430 \\
1.41649 \\
1.43885 \\
1.46139\end{array}$ & $\begin{array}{l}0.021054 \\
0.021229 \\
0.021404 \\
0.021578 \\
0.021753 \\
0.021927 \\
0.022102 \\
0.022276 \\
0.022450 \\
0.022625\end{array}$ & $\begin{array}{l}113.937 \\
115.668 \\
117.408 \\
119.157 \\
120.916 \\
122.685 \\
124.462 \\
126.249 \\
128.045 \\
129.849\end{array}$ & $\begin{array}{l}0.17258 \\
0.17354 \\
0.17449 \\
0.17543 \\
0.17637 \\
0.17729 \\
0.17821 \\
0.17912 \\
0.18003 \\
0.18092\end{array}$ & $\begin{array}{l}3122.65 \\
3152.33 \\
3182.02 \\
3211.71 \\
3241.41 \\
3271.11 \\
3300.82 \\
3330.53 \\
3360.24 \\
3389.96\end{array}$ & $\begin{array}{l}0.29681 \\
0.29686 \\
0.29691 \\
0.29696 \\
0.29700 \\
0.29704 \\
0.29709 \\
0.29713 \\
0.29717 \\
0.29721\end{array}$ \\
\hline $\begin{array}{l}1.30000 \\
1.31000 \\
1.32000 \\
1.33000 \\
1.34000 \\
1.35000 \\
1.36000 \\
1.37000 \\
1.38000 \\
1.39000\end{array}$ & $\begin{array}{l}1.48410 \\
1.50699 \\
1.53005 \\
1.55328 \\
1.57669 \\
1.60028 \\
1.62403 \\
1.64797 \\
1.67207 \\
1.69635\end{array}$ & $\begin{array}{l}0.022799 \\
0.022974 \\
0.023148 \\
0.023322 \\
0.023497 \\
0.023671 \\
0.023845 \\
0.024019 \\
0.024193 \\
0.024368\end{array}$ & $\begin{array}{l}131.663 \\
133.486 \\
135.317 \\
137.157 \\
139.005 \\
140.863 \\
142.728 \\
144.602 \\
146.484 \\
148.375\end{array}$ & $\begin{array}{l}0.18181 \\
0.18269 \\
0.18356 \\
0.18443 \\
0.18529 \\
0.18614 \\
0.18698 \\
0.18782 \\
0.18865 \\
0.18947\end{array}$ & $\begin{array}{l}3419.69 \\
3449.41 \\
3479.14 \\
3508.88 \\
3538.62 \\
3568.36 \\
3598.10 \\
3627.85 \\
3657.60 \\
3687.36\end{array}$ & $\begin{array}{l}0.29725 \\
0.29729 \\
0.29733 \\
0.29736 \\
0.29740 \\
0.29743 \\
0.29747 \\
0.29750 \\
0.29753 \\
0.29756\end{array}$ \\
\hline $\begin{array}{l}1.40000 \\
1.41000 \\
1.42000 \\
1.43000 \\
1.44000 \\
1.45000 \\
1.46000 \\
1.47000 \\
1.48000 \\
1.49000\end{array}$ & $\begin{array}{l}1.72081 \\
1.74544 \\
1.77024 \\
1.79522 \\
1.82037 \\
1.84569 \\
1.87119 \\
1.89686 \\
1.92271 \\
1.94873\end{array}$ & $\begin{array}{l}0.024542 \\
0.024716 \\
0.024890 \\
0.025064 \\
0.025238 \\
0.025412 \\
0.025586 \\
0.025760 \\
0.025934 \\
0.026108\end{array}$ & $\begin{array}{l}150.274 \\
152.181 \\
154.096 \\
156.019 \\
157.949 \\
159.888 \\
161.834 \\
163.789 \\
165.750 \\
167.720\end{array}$ & $\begin{array}{l}0.19028 \\
0.19109 \\
0.19189 \\
0.19269 \\
0.19347 \\
0.19426 \\
0.19503 \\
0.19580 \\
0.19656 \\
0.19731\end{array}$ & $\begin{array}{l}3717.12 \\
3746.88 \\
3776.64 \\
3806.41 \\
3836.18 \\
3865.95 \\
3895.73 \\
3925.51 \\
3955.29 \\
3985.07\end{array}$ & $\begin{array}{l}0.29760 \\
0.29763 \\
0.29766 \\
0.29769 \\
0.29772 \\
0.29774 \\
0.29777 \\
0.29780 \\
0.29782 \\
0.29785\end{array}$ \\
\hline $\begin{array}{l}1.50000 \\
1.52000 \\
1.54000 \\
1.56000 \\
1.58000 \\
1.60000 \\
1.62000 \\
1.64000 \\
1.66000 \\
1.68000\end{array}$ & $\begin{array}{l}1.97493 \\
2.02784 \\
2.08144 \\
2.13575 \\
2.19074 \\
2.24643 \\
2.30282 \\
2.35990 \\
2.41767 \\
2.47614\end{array}$ & $\begin{array}{l}0.026282 \\
0.026629 \\
0.026977 \\
0.027324 \\
0.027672 \\
0.028019 \\
0.028366 \\
0.028713 \\
0.029060 \\
0.029407\end{array}$ & $\begin{array}{l}169.696 \\
173.672 \\
177.678 \\
181.712 \\
185.774 \\
189.864 \\
193.981 \\
198.125 \\
202.295 \\
206.491\end{array}$ & $\begin{array}{l}0.19806 \\
0.19953 \\
0.20098 \\
0.20241 \\
0.20381 \\
0.20518 \\
0.20653 \\
0.20786 \\
0.20917 \\
0.21045\end{array}$ & $\begin{array}{l}4014.86 \\
4074.44 \\
4134.03 \\
4193.63 \\
4253.23 \\
4312.85 \\
4372.48 \\
4432.11 \\
4491.75 \\
4551.40\end{array}$ & $\begin{array}{l}0.29788 \\
0.29793 \\
0.29797 \\
0.29802 \\
0.29806 \\
0.29811 \\
0.29815 \\
0.29819 \\
0.29823 \\
0.29826\end{array}$ \\
\hline
\end{tabular}




\begin{tabular}{|c|c|c|c|c|c|c|}
\hline \multirow[b]{2}{*}{$\begin{array}{c}\text { Be } \\
\text { factor } \\
\text { gauss } \mathrm{cm} \\
\end{array}$} & \multicolumn{2}{|c|}{$\begin{array}{c}\text { Decade factor } \\
10^{2}\end{array}$} & \multicolumn{2}{|c|}{$\begin{array}{c}\text { Decade factor } \\
10^{3}\end{array}$} & \multicolumn{2}{|c|}{$\begin{array}{c}\text { Decade factor } \\
10^{4}\end{array}$} \\
\hline & $\underset{\mathrm{keV}}{\text { Energy }}$ & $\begin{array}{l}\text { Interpol. } \\
\text { gauss cm } \\
\frac{\mathrm{keV}}{}\end{array}$ & $\begin{array}{c}\text { Energy } \\
\text { keV }\end{array}$ & $\begin{array}{l}\begin{array}{l}\text { Interpol. } \\
\text { gauss cm }\end{array} \\
\mathrm{keV} \\
\end{array}$ & $\underset{\text { keV }}{\text { Energy }}$ & $\begin{array}{l}\begin{array}{l}\text { Interpol. } \\
\text { gauss cm }\end{array} \\
\frac{\mathrm{keV}}{}\end{array}$ \\
\hline $\begin{array}{l}1.70000 \\
1.72000 \\
1.74000 \\
1.76000 \\
1.78000 \\
1.80000 \\
1.82000 \\
1.84000 \\
1.86000 \\
1.88000\end{array}$ & $\begin{array}{l}2.53530 \\
2.59515 \\
2.65570 \\
2.71694 \\
2.77887 \\
2.84149 \\
2.90481 \\
2.96881 \\
3.03351 \\
3.09890\end{array}$ & $\begin{array}{l}0.029753 \\
0.030100 \\
0.030446 \\
0.030793 \\
0.031139 \\
0.031485 \\
0.031831 \\
0.032177 \\
0.032522 \\
0.032868\end{array}$ & $\begin{array}{l}210.713 \\
214.960 \\
219.231 \\
223.526 \\
227.845 \\
232.187 \\
236.552 \\
240.940 \\
245.349 \\
249.781\end{array}$ & $\begin{array}{l}0.21171 \\
0.21295 \\
0.21416 \\
0.21536 \\
0.21653 \\
0.21768 \\
0.21882 \\
0.21993 \\
0.22103 \\
0.22210\end{array}$ & $\begin{array}{l}4611.06 \\
4670.72 \\
4730.39 \\
4790.07 \\
4849.75 \\
4909.44 \\
4969.13 \\
5028.83 \\
5088.54 \\
5148.25\end{array}$ & $\begin{array}{l}0.29830 \\
0.29833 \\
0.29837 \\
0.29840 \\
0.29843 \\
0.29846 \\
0.29849 \\
0.29852 \\
0.29854 \\
0.29857\end{array}$ \\
\hline $\begin{array}{l}1.90000 \\
1.92000 \\
1.94000 \\
1.96000 \\
1.98000 \\
2.00000 \\
2.02000 \\
2.04000 \\
2.06000 \\
2.08000\end{array}$ & $\begin{array}{l}3.16498 \\
3.23175 \\
3.29922 \\
3.36737 \\
3.43621 \\
3.50574 \\
3.57596 \\
3.64687 \\
3.71847 \\
3.79076\end{array}$ & $\begin{array}{l}0.033213 \\
0.033558 \\
0.033903 \\
0.034248 \\
0.034593 \\
0.034938 \\
0.035283 \\
0.035627 \\
0.035971 \\
0.036315\end{array}$ & $\begin{array}{l}254.233 \\
258.707 \\
263.201 \\
267.715 \\
272.250 \\
276.804 \\
281.377 \\
285.968 \\
290.579 \\
295.207\end{array}$ & $\begin{array}{l}0.22316 \\
0.22420 \\
0.22522 \\
0.22622 \\
0.22721 \\
0.22817 \\
0.22913 \\
0.23006 \\
0.23098 \\
0.23188\end{array}$ & $\begin{array}{l}5207.97 \\
5267.69 \\
5327.41 \\
5387.14 \\
5446.88 \\
5506.62 \\
5566.36 \\
5626.11 \\
5685.86 \\
5745.62\end{array}$ & $\begin{array}{l}0.29859 \\
0.29862 \\
0.29864 \\
0.29867 \\
0.29869 \\
0.29871 \\
0.29873 \\
0.29875 \\
0.29877 \\
0.29879\end{array}$ \\
\hline $\begin{array}{l}2.10000 \\
2.12000 \\
2.14000 \\
2.16000 \\
2.18000 \\
2.20000 \\
2.22000 \\
2.24000 \\
2.26000 \\
2.28000\end{array}$ & $\begin{array}{l}3.86373 \\
3.93739 \\
4.01174 \\
4.08678 \\
4.16250 \\
4.23892 \\
4.31601 \\
4.39380 \\
4.47227 \\
4.55142\end{array}$ & $\begin{array}{l}0.036659 \\
0.037003 \\
0.037347 \\
0.037690 \\
0.038034 \\
0.038377 \\
0.038720 \\
0.039063 \\
0.039406 \\
0.039748\end{array}$ & $\begin{array}{l}299.854 \\
304.518 \\
309.200 \\
313.898 \\
318.613 \\
323.345 \\
328.093 \\
332.857 \\
337.636 \\
342.431\end{array}$ & $\begin{array}{l}0.23277 \\
0.23364 \\
0.23450 \\
0.23535 \\
0.23617 \\
0.23699 \\
0.23779 \\
0.23858 \\
0.23935 \\
0.24011\end{array}$ & $\begin{array}{l}5805.38 \\
5865.15 \\
5924.91 \\
5984.68 \\
6044.46 \\
6104.24 \\
6164.02 \\
6223.80 \\
6283.59 \\
6343.38\end{array}$ & $\begin{array}{l}0.29881 \\
0.29883 \\
0.29885 \\
0.29886 \\
0.29888 \\
0.29890 \\
0.29891 \\
0.29893 \\
0.29894 \\
0.29896\end{array}$ \\
\hline $\begin{array}{l}2.30000 \\
2.32000 \\
2.34000 \\
2.36000 \\
2.38000 \\
2.40000 \\
2.42000 \\
2.44000 \\
2.46000 \\
2.48000\end{array}$ & $\begin{array}{l}4.63126 \\
4.71178 \\
4.79299 \\
4.87488 \\
4.95746 \\
5.04072 \\
5.12466 \\
5.20929 \\
5.29460 \\
5.38059\end{array}$ & $\begin{array}{l}0.040091 \\
0.040433 \\
0.040775 \\
0.041117 \\
0.041459 \\
0.041801 \\
0.042142 \\
0.042484 \\
0.042825 \\
0.043166\end{array}$ & $\begin{array}{l}347.241 \\
352.065 \\
356.904 \\
361.758 \\
366.626 \\
371.507 \\
376.403 \\
381.311 \\
386.233 \\
391.168\end{array}$ & $\begin{array}{l}0.24086 \\
0.24160 \\
0.24232 \\
0.24304 \\
0.24374 \\
0.24442 \\
0.24510 \\
0.24577 \\
0.24642 \\
0.24707\end{array}$ & $\begin{array}{l}6403.17 \\
6462.97 \\
6522.77 \\
6582.57 \\
6642.37 \\
6702.18 \\
6761.99 \\
6821.80 \\
6881.62 \\
6941.43\end{array}$ & $\begin{array}{l}0.29897 \\
0.29899 \\
0.29900 \\
0.29901 \\
0.29903 \\
0.29904 \\
0.29905 \\
0.29906 \\
0.29908 \\
0.29909\end{array}$ \\
\hline $\begin{array}{l}2.50000 \\
2.52000 \\
2.54000 \\
2.56000 \\
2.58000 \\
2.60000 \\
2.62000 \\
2.64000 \\
2.66000 \\
2.68000\end{array}$ & $\begin{array}{l}5.46726 \\
5.55462 \\
5.64265 \\
5.73137 \\
5.82076 \\
5.91084 \\
6.00159 \\
6.09303 \\
6.18514 \\
6.27794\end{array}$ & $\begin{array}{l}0.043507 \\
0.043847 \\
0.044188 \\
0.044528 \\
0.044868 \\
0.045208 \\
0.045548 \\
0.045887 \\
0.046227 \\
0.046566\end{array}$ & $\begin{array}{l}396.116 \\
401.076 \\
406.049 \\
411.034 \\
416.031 \\
421.039 \\
426.059 \\
431.091 \\
436.134 \\
441.188\end{array}$ & $\begin{array}{l}0.24770 \\
0.24833 \\
0.24894 \\
0.24954 \\
0.25014 \\
0.25072 \\
0.25130 \\
0.25186 \\
0.25242 \\
0.25297\end{array}$ & $\begin{array}{l}7001.25 \\
7061.07 \\
7120.90 \\
7180.72 \\
7240.55 \\
7300.38 \\
7360.21 \\
7420.04 \\
7479.88 \\
7539.71\end{array}$ & $\begin{array}{l}0.29910 \\
0.29911 \\
0.29912 \\
0.29913 \\
0.29914 \\
0.29915 \\
0.29916 \\
0.29917 \\
0.29918 \\
0.29919\end{array}$ \\
\hline $\begin{array}{l}2.70000 \\
2.72000 \\
2.74000 \\
2.76000 \\
2.78000 \\
2.80000 \\
2.82000 \\
2.84000 \\
2.86000 \\
2.88000 \\
2.90000\end{array}$ & $\begin{array}{l}6.37141 \\
6.46556 \\
6.56038 \\
6.65589 \\
6.75207 \\
6.84892 \\
6.94646 \\
7.04466 \\
7.14355 \\
7.24311 \\
7.34334\end{array}$ & $\begin{array}{l}0.046905 \\
0.047244 \\
0.047583 \\
0.047921 \\
0.048259 \\
0.048597 \\
0.048935 \\
0.049273 \\
0.049611 \\
0.049948 \\
0.050285\end{array}$ & $\begin{array}{l}446.252 \\
451.328 \\
456.414 \\
461.510 \\
466.617 \\
471.733 \\
476.860 \\
481.996 \\
487.142 \\
492.297 \\
497.462\end{array}$ & $\begin{array}{l}0.25351 \\
0.25404 \\
0.25456 \\
0.25507 \\
0.25558 \\
0.25608 \\
0.25657 \\
0.25705 \\
0.25753 \\
0.25800 \\
0.25846\end{array}$ & $\begin{array}{l}7599.55 \\
7659.39 \\
7719.23 \\
7779.08 \\
7838.92 \\
7898.77 \\
7958.62 \\
8018.47 \\
8078.32 \\
8138.18 \\
8198.03\end{array}$ & $\begin{array}{l}0.29920 \\
0.29921 \\
0.29922 \\
0.29922 \\
0.29923 \\
0.29924 \\
0.29925 \\
0.29926 \\
0.29926 \\
0.29927 \\
0.29928\end{array}$ \\
\hline
\end{tabular}




\begin{tabular}{|c|c|c|c|c|c|c|}
\hline \multirow[b]{2}{*}{$\begin{array}{c}\text { Bo } \\
\text { factor } \\
\text { gauss } \mathrm{cm}\end{array}$} & \multicolumn{2}{|c|}{$\begin{array}{c}\text { Decade factor } \\
10^{2}\end{array}$} & \multicolumn{2}{|c|}{$\begin{array}{c}\text { Decade factor } \\
10^{3}\end{array}$} & \multicolumn{2}{|c|}{$\begin{array}{c}\text { Decade factor } \\
10^{4}\end{array}$} \\
\hline & $\underset{\mathrm{keV}}{\text { Energy }}$ & $\begin{array}{l}\text { Interpol. } \\
\text { gauss cm } \\
\mathrm{keV}\end{array}$ & $\underset{\mathrm{keV}}{\text { Energy }}$ & $\begin{array}{c}\text { Interpol. } \\
\text { gauss cm } \\
\text { keV }\end{array}$ & $\underset{\mathrm{keV}}{\text { Energy }}$ & $\begin{array}{c}\text { Interpol. } \\
\text { gauss cm } \\
\mathrm{keV}\end{array}$ \\
\hline $\begin{array}{l}2.92000 \\
2.94000 \\
2.96000 \\
2.98000\end{array}$ & $\begin{array}{l}7.44425 \\
7.54583 \\
7.64808 \\
7.75101\end{array}$ & $\begin{array}{l}0.050622 \\
0.050959 \\
0.051295 \\
0.051632\end{array}$ & $\begin{array}{l}502.635 \\
507.818 \\
513.010 \\
518.210\end{array}$ & $\begin{array}{l}0.25891 \\
0.25936 \\
0.25980 \\
0.26023\end{array}$ & $\begin{array}{l}8257.89 \\
8317.74 \\
8377.60 \\
8437.46\end{array}$ & $\begin{array}{l}0.29928 \\
0.29929 \\
0.29930 \\
0.29930\end{array}$ \\
\hline $\begin{array}{l}3.00000 \\
3.05000 \\
3.10000 \\
3.15000 \\
3.20000 \\
3.25000 \\
3.30000 \\
3.35000 \\
3.40000 \\
3.45000\end{array}$ & $\begin{array}{c}7.85461 \\
8.11654 \\
8.38268 \\
8.65300 \\
8.92750 \\
9.20617 \\
9.48901 \\
9.77602 \\
10.0672 \\
10.3625\end{array}$ & $\begin{array}{l}0.051968 \\
0.052807 \\
0.053645 \\
0.054482 \\
0.055318 \\
0.056152 \\
0.056985 \\
0.057816 \\
0.058647 \\
0.059475\end{array}$ & $\begin{array}{l}523.419 \\
536.478 \\
549.589 \\
562.748 \\
575.955 \\
589.207 \\
602.504 \\
615.843 \\
629.223 \\
642.643\end{array}$ & $\begin{array}{l}0.26066 \\
0.26170 \\
0.26270 \\
0.26367 \\
0.26460 \\
0.26550 \\
0.26636 \\
0.26720 \\
0.26800 \\
0.26878\end{array}$ & $\begin{array}{l}8497.32 \\
8646.98 \\
8796.65 \\
8946.32 \\
9096.01 \\
9245.69 \\
9395.39 \\
9545.09 \\
9694.79 \\
9844.51\end{array}$ & $\begin{array}{l}0.29931 \\
0.29933 \\
0.29934 \\
0.29936 \\
0.29937 \\
0.29938 \\
0.29939 \\
0.29941 \\
0.29942 \\
0.29943\end{array}$ \\
\hline $\begin{array}{l}3.50000 \\
3.55000 \\
3.60000 \\
3.65000 \\
3.70000 \\
3.75000 \\
3.80000 \\
3.85000 \\
3.90000 \\
3.95000\end{array}$ & $\begin{array}{l}10.6619 \\
10.9655 \\
11.2732 \\
11.5850 \\
11.9010 \\
12.2210 \\
12.5451 \\
12.8734 \\
13.2057 \\
13.5420\end{array}$ & $\begin{array}{l}0.060303 \\
0.061129 \\
0.061953 \\
0.062776 \\
0.063597 \\
0.064417 \\
0.065236 \\
0.066053 \\
0.066868 \\
0.067682\end{array}$ & $\begin{array}{l}656.101 \\
669.596 \\
683.126 \\
696.691 \\
710.289 \\
723.920 \\
737.582 \\
751.273 \\
764.994 \\
778.743\end{array}$ & $\begin{array}{l}0.26953 \\
0.27026 \\
0.27096 \\
0.27164 \\
0.27229 \\
0.27292 \\
0.27354 \\
0.27413 \\
0.27470 \\
0.27526\end{array}$ & $\begin{array}{c}9994.22 \\
10143.9 \\
10293.7 \\
10443.4 \\
10593.1 \\
10742.9 \\
10892.6 \\
11042.4 \\
11192.1 \\
11341.9\end{array}$ & $\begin{array}{l}0.29944 \\
0.29945 \\
0.29946 \\
0.29947 \\
0.29948 \\
0.29948 \\
0.29949 \\
0.29950 \\
0.29951 \\
0.29951\end{array}$ \\
\hline $\begin{array}{l}4.00000 \\
4.05000 \\
4.10000 \\
4.15000 \\
4.20000 \\
4.25000 \\
4.30000 \\
4.35000 \\
4.40000 \\
4.45000\end{array}$ & $\begin{array}{l}13.8825 \\
14.2270 \\
14.5755 \\
14.9281 \\
15.2847 \\
15.6454 \\
16.0101 \\
16.3787 \\
16.7514 \\
17.1281\end{array}$ & $\begin{array}{l}0.068494 \\
0.069305 \\
0.070114 \\
0.070922 \\
0.071728 \\
0.072532 \\
0.073334 \\
0.074135 \\
0.074934 \\
0.075732\end{array}$ & $\begin{array}{l}792.520 \\
806.323 \\
820.152 \\
834.005 \\
847.883 \\
861.784 \\
875.708 \\
889.654 \\
903.621 \\
917.609\end{array}$ & $\begin{array}{l}0.27580 \\
0.27632 \\
0.27683 \\
0.27732 \\
0.27779 \\
0.27825 \\
0.27870 \\
0.27913 \\
0.27955 \\
0.27996\end{array}$ & $\begin{array}{l}11491.6 \\
11641.4 \\
11791.2 \\
11940.9 \\
12090.7 \\
12240.5 \\
12390.3 \\
12540.0 \\
12689.8 \\
12839.6\end{array}$ & $\begin{array}{l}0.29952 \\
0.29953 \\
0.29953 \\
0.29954 \\
0.29955 \\
0.29955 \\
0.29956 \\
0.29956 \\
0.29957 \\
0.29957\end{array}$ \\
\hline $\begin{array}{l}4.50000 \\
4.55000 \\
4.60000 \\
4.65000 \\
4.70000 \\
4.75000 \\
4.80000 \\
4.85000 \\
4.90000 \\
4.95000\end{array}$ & $\begin{array}{l}17.5087 \\
17.8934 \\
18.2819 \\
18.6745 \\
19.0710 \\
19.4714 \\
19.8758 \\
20.2841 \\
20.6963 \\
21.1124\end{array}$ & $\begin{array}{l}0.076528 \\
0.077322 \\
0.078114 \\
0.078904 \\
0.079693 \\
0.080480 \\
0.081265 \\
0.082049 \\
0.082830 \\
0.083610\end{array}$ & $\begin{array}{c}931.616 \\
945.644 \\
959.690 \\
973.755 \\
987.838 \\
1001.94 \\
1016.06 \\
1030.19 \\
1044.34 \\
1058.50\end{array}$ & $\begin{array}{l}0.28036 \\
0.28074 \\
0.28112 \\
0.28148 \\
0.28183 \\
0.28218 \\
0.28251 \\
0.28284 \\
0.28315 \\
0.28346\end{array}$ & $\begin{array}{l}12989.4 \\
13139.2 \\
13289.0 \\
13438.8 \\
13588.6 \\
13738.4 \\
13888.2 \\
14038.0 \\
14187.8 \\
14337.6\end{array}$ & $\begin{array}{l}0.29958 \\
0.29958 \\
0.29959 \\
0.29959 \\
0.29960 \\
0.29960 \\
0.29960 \\
0.29961 \\
0.29961 \\
0.29962\end{array}$ \\
\hline $\begin{array}{l}5.00000 \\
5.05000 \\
5.10000 \\
5.15000 \\
5.20000 \\
5.25000 \\
5.30000 \\
5.35000 \\
5.40000 \\
5.45000\end{array}$ & $\begin{array}{l}21.5324 \\
21.9562 \\
22.3840 \\
22.8156 \\
23.2511 \\
23.6904 \\
24.1336 \\
24.5806 \\
25.0314 \\
25.4860\end{array}$ & $\begin{array}{l}0.084388 \\
0.085164 \\
0.085938 \\
0.086711 \\
0.087481 \\
0.088250 \\
0.089017 \\
0.089781 \\
0.090544 \\
0.091305\end{array}$ & $\begin{array}{l}1072.69 \\
1086.88 \\
1101.09 \\
1115.31 \\
1129.55 \\
1143.80 \\
1158.07 \\
1172.34 \\
1186.63 \\
1200.93\end{array}$ & $\begin{array}{l}0.28376 \\
\ddot{u} .28405 \\
0.28433 \\
0.28461 \\
0.28488 \\
0.28514 \\
0.28540 \\
0.28565 \\
0.28589 \\
0.28613\end{array}$ & $\begin{array}{l}14487.4 \\
14637.2 \\
14787.0 \\
14936.8 \\
15086.6 \\
15236.5 \\
15386.3 \\
15536.1 \\
15685.9 \\
15835.7\end{array}$ & $\begin{array}{l}0.29962 \\
0.29962 \\
0.29963 \\
0.29963 \\
0.29963 \\
0.29964 \\
0.29964 \\
0.29964 \\
0.29964 \\
0.29965\end{array}$ \\
\hline $\begin{array}{l}5.50000 \\
5.55000 \\
5.60000 \\
5.65000 \\
5.70000 \\
5.75000\end{array}$ & $\begin{array}{l}25.9445 \\
26.4067 \\
26.8727 \\
27.3424 \\
27.8160 \\
28.2932\end{array}$ & $\begin{array}{l}0.092064 \\
0.092821 \\
0.093576 \\
0.094329 \\
0.095081 \\
0.095830\end{array}$ & $\begin{array}{l}1215.24 \\
1229.57 \\
1243.90 \\
1258.25 \\
1272.60 \\
1286.97\end{array}$ & $\begin{array}{l}0.28636 \\
0.28658 \\
0.28680 \\
0.28702 \\
0.28723 \\
0.28743\end{array}$ & $\begin{array}{l}15985.6 \\
16135.4 \\
16285.2 \\
16435.1 \\
16584.9 \\
16734.7\end{array}$ & $\begin{array}{l}0.29965 \\
0.29965 \\
0.29965 \\
0.29966 \\
0.29966 \\
0.29966\end{array}$ \\
\hline
\end{tabular}




\begin{tabular}{|c|c|c|c|c|c|c|}
\hline \multirow[b]{2}{*}{$\begin{array}{c}\begin{array}{c}B e \\
\text { factor } \\
\text { gauss } \mathrm{cm}\end{array} \\
\end{array}$} & \multicolumn{2}{|c|}{$\begin{array}{c}\text { Decade factor } \\
10^{2}\end{array}$} & \multicolumn{2}{|c|}{$\begin{array}{c}\text { Decade factor } \\
10^{3}\end{array}$} & \multicolumn{2}{|c|}{$\begin{array}{c}\text { Decade factor } \\
10^{4} \\
\end{array}$} \\
\hline & $\underset{\mathrm{keV}}{\text { Energy }}$ & $\begin{array}{l}\text { Interpol. } \\
\text { gauss cm } \\
\frac{\mathrm{keV}}{}\end{array}$ & $\begin{array}{c}\text { Energy } \\
\mathrm{keV}\end{array}$ & $\begin{array}{l}\text { Interpol. } \\
\frac{\text { gauss cm }}{\mathrm{keV}}\end{array}$ & $\begin{array}{c}\text { Energy } \\
\text { keV }\end{array}$ & $\begin{array}{l}\text { Interpol. } \\
\frac{\text { gauss } \mathrm{cm}}{\mathrm{keV}}\end{array}$ \\
\hline $\begin{array}{l}5.80000 \\
5.85000 \\
5.90000 \\
5.95000\end{array}$ & $\begin{array}{l}28.7743 \\
29.2590 \\
29.7475 \\
30.2397\end{array}$ & $\begin{array}{l}0.096577 \\
0.097322 \\
0.098065 \\
0.098806\end{array}$ & $\begin{array}{l}1301.35 \\
1315.73 \\
1330.13 \\
1344.53\end{array}$ & $\begin{array}{l}0.28763 \\
0.28783 \\
0.28802 \\
0.28820\end{array}$ & $\begin{array}{l}16884.5 \\
17034.4 \\
17184.2 \\
17334.0\end{array}$ & $\begin{array}{l}0.29966 \\
0.29967 \\
0.29967 \\
0.29967\end{array}$ \\
\hline $\begin{array}{l}6.00000 \\
6.05000 \\
6.10000 \\
6.15000 \\
6.20000 \\
6.25000 \\
6.30000 \\
6.35000 \\
6.40000 \\
6.45000\end{array}$ & $\begin{array}{l}30.7355 \\
31.2351 \\
31.7384 \\
32.2453 \\
32.7559 \\
33.2701 \\
33.7880 \\
34.3095 \\
34.8346 \\
35.3633\end{array}$ & $\begin{array}{l}0.099545 \\
0.10028 \\
0.10102 \\
0.10175 \\
0.10248 \\
0.10321 \\
0.10394 \\
0.10466 \\
0.10538 \\
0.10610\end{array}$ & $\begin{array}{l}1358.95 \\
1373.37 \\
1387.80 \\
1402.24 \\
1416.69 \\
1431.15 \\
1445.62 \\
1460.09 \\
1474.57 \\
1489.06\end{array}$ & $\begin{array}{l}0.28838 \\
0.28856 \\
0.28873 \\
0.28890 \\
0.28907 \\
0.28923 \\
0.28939 \\
0.28954 \\
0.28970 \\
0.28984\end{array}$ & $\begin{array}{l}17483.9 \\
17633.7 \\
17783.6 \\
17933.4 \\
18083.2 \\
18233.1 \\
18382.9 \\
18532.8 \\
18682.6 \\
18832.4\end{array}$ & $\begin{array}{l}0.29967 \\
0.29967 \\
0.29968 \\
0.29968 \\
0.29968 \\
0.29968 \\
0.29968 \\
0.29969 \\
0.29969 \\
0.29969\end{array}$ \\
\hline $\begin{array}{l}6.50000 \\
6.55000 \\
6.60000 \\
6.65000 \\
6.70000 \\
6.75000 \\
6.80000 \\
6.85000 \\
6.90000 \\
6.95000\end{array}$ & $\begin{array}{l}35.8956 \\
36.4315 \\
36.9710 \\
37.5141 \\
38.0607 \\
38.6108 \\
39.1645 \\
39.7217 \\
40.2824 \\
40.8466\end{array}$ & $\begin{array}{l}0.10682 \\
0.10754 \\
0.10825 \\
0.10897 \\
0.10968 \\
0.11038 \\
0.11109 \\
0.11179 \\
0.11249 \\
0.11319\end{array}$ & $\begin{array}{l}1503.56 \\
1518.06 \\
1532.57 \\
1547.09 \\
1561.61 \\
1576.14 \\
1590.68 \\
1605.22 \\
1619.77 \\
1634.32\end{array}$ & $\begin{array}{l}0.28999 \\
0.29013 \\
0.29027 \\
0.29041 \\
0.29054 \\
0.29067 \\
0.29080 \\
0.29092 \\
0.29105 \\
0.29117\end{array}$ & $\begin{array}{l}18982.3 \\
19132.1 \\
19282.0 \\
19431.8 \\
19581.7 \\
19731.5 \\
19881.4 \\
20031.2 \\
20181.1 \\
20330.9\end{array}$ & $\begin{array}{l}0.29969 \\
0.29969 \\
0.29969 \\
0.29970 \\
0.29970 \\
0.29970 \\
0.29970 \\
0.29970 \\
0.29970 \\
0.29970\end{array}$ \\
\hline $\begin{array}{l}7.00000 \\
7.10000 \\
7.20000 \\
7.30000 \\
7.40000 \\
7.50000 \\
7.60000 \\
7.70000 \\
7.80000 \\
7.90000\end{array}$ & $\begin{array}{r}41.4144 \\
42.5602 \\
43.7199 \\
44.8934 \\
46.0805 \\
47.2811 \\
48.4953 \\
49.7229 \\
50.9638 \\
52.2179\end{array}$ & $\begin{array}{l}0.11389 \\
0.11528 \\
0.11666 \\
0.11803 \\
0.11939 \\
0.12074 \\
0.12209 \\
0.12342 \\
0.12475 \\
0.12607\end{array}$ & $\begin{array}{l}1648.89 \\
1678.02 \\
1707.19 \\
1736.37 \\
1765.58 \\
1794.80 \\
1824.04 \\
1853.30 \\
1882.58 \\
1911.88\end{array}$ & $\begin{array}{l}0.29128 \\
0.29151 \\
0.29173 \\
0.29194 \\
0.29214 \\
0.29234 \\
0.29253 \\
0.29271 \\
0.29288 \\
0.29305\end{array}$ & $\begin{array}{l}20480.8 \\
20780.5 \\
21080.2 \\
21379.9 \\
21679.6 \\
21979.3 \\
22279.0 \\
22578.8 \\
22878.5 \\
23178.2\end{array}$ & $\begin{array}{l}0.29970 \\
0.29971 \\
0.29971 \\
0.29971 \\
0.29971 \\
0.29972 \\
0.29972 \\
0.29972 \\
0.29972 \\
0.29972\end{array}$ \\
\hline $\begin{array}{l}8.00000 \\
8.10000 \\
8.20000 \\
8.30000 \\
8.40000 \\
8.50000 \\
8.60000 \\
8.70000 \\
8.80000 \\
8.90000\end{array}$ & $\begin{array}{l}53.4851 \\
54.7654 \\
56.0586 \\
57.3648 \\
58.6837 \\
60.0153 \\
61.3595 \\
62.7162 \\
64.0853 \\
65.4668\end{array}$ & $\begin{array}{l}0.12738 \\
0.12868 \\
0.12997 \\
0.13125 \\
0.13253 \\
0.13379 \\
0.13505 \\
0.13629 \\
0.13753 \\
0.13876\end{array}$ & $\begin{array}{l}1941.19 \\
1970.52 \\
1999.87 \\
2029.23 \\
2058.60 \\
2087.99 \\
2117.39 \\
2146.80 \\
2176.23 \\
2205.67\end{array}$ & $\begin{array}{l}0.29321 \\
0.29337 \\
0.29352 \\
0.29367 \\
0.29381 \\
0.29394 \\
0.29407 \\
0.29420 \\
0.29432 \\
0.29444\end{array}$ & $\begin{array}{l}23477.9 \\
23777.7 \\
24077.4 \\
24377.1 \\
24676.9 \\
24976.6 \\
25276.3 \\
25576.1 \\
25875.8 \\
26175.5\end{array}$ & $\begin{array}{l}0.29973 \\
0.29973 \\
0.29973 \\
0.29973 \\
0.29973 \\
0.29973 \\
0.29973 \\
0.29974 \\
0.29974 \\
0.29974\end{array}$ \\
\hline $\begin{array}{l}9.00000 \\
9.10000 \\
9.20000 \\
9.30000 \\
9.40000 \\
9.50000 \\
9.60000 \\
9.70000 \\
9.80000 \\
9.90000\end{array}$ & $\begin{array}{l}66.8605 \\
68.2664 \\
69.6844 \\
71.1144 \\
72.5562 \\
74.0099 \\
75.4753 \\
76.9523 \\
78.4409 \\
79.9409\end{array}$ & $\begin{array}{l}0.13998 \\
0.14120 \\
0.14240 \\
0.14359 \\
0.14478 \\
0.14595 \\
0.14712 \\
0.14828 \\
0.14943 \\
0.15057\end{array}$ & $\begin{array}{l}2235.12 \\
2264.58 \\
2294.05 \\
2323.53 \\
2353.03 \\
2382.53 \\
2412.04 \\
2441.57 \\
2471.10 \\
2500.64\end{array}$ & $\begin{array}{l}0.29456 \\
0.29467 \\
0.29478 \\
0.29488 \\
0.29498 \\
0.29508 \\
0.29518 \\
0.29527 \\
0.29536 \\
0.29545\end{array}$ & $\begin{array}{l}26475.3 \\
26775.0 \\
27074.7 \\
27374.5 \\
27674.2 \\
27974.0 \\
28273.7 \\
28573.5 \\
28873.2 \\
29173.0\end{array}$ & $\begin{array}{l}0.29974 \\
0.29974 \\
0.29974 \\
0.29974 \\
0.29974 \\
0.29975 \\
0.29975 \\
0.29975 \\
0.29975 \\
0.29975\end{array}$ \\
\hline 10.00000 & 81.4523 & 0.15171 & 2530.19 & 0.29553 & 29472.7 & 0.29975 \\
\hline
\end{tabular}




\section{AUTHOR INDEX}

Abragam, A. 539, 567, 572,601

Accordo 501

Adair, R. F. 321

Adams, C. D. 45

Aeppli, H. 533, 553, 557, $576,732,734$

Agnew, H. 79, 272

Ahrens, I. 340

Ajzenberg, F. 375, 770

Alaga, G. 132, 341, 479, $487,488,490$

Albers-Schönberg, H. 533, 539, 572, 576, 583, 584, $585,590,734$

Albert, R. D. 237, 281, 315

Albouy, G. 743

Alburger, D. 96, 227, 266 , $332,333,336,455,481$, 522, 554, 579, 580, 707, 708, 761, 764, 766, 767, 774, 775, 784, 793

Alder, K. 132, 477, 479, 488, 534, 539, 576, 583, 584, 734

Alford, W. P. 362

Alichanian, A. I. 673

Alichanow, A. I. 673

Allen, G. 26

Allen, J. S. 171, 358, 364

Allen, R. A. 743

Allen, R. C. 788

Allen, S. J. M. 48

Allison, K. 90

Allison, S. K. 14, 40, 176

Almquist, S. 702

Alperovitch, E. 345

Althaus, E. 350

Amaldi, E. 193, 795, 799

Ambler, E. 602

Ames, D. P. 336

Anderson, C. D. 349, 356, 673,780
Anderson, C. E. 659

Anderson, E. C. 180

Anderson, H. 79, 272

Andrade, E. N. da C. 100

Angus, J. 166, 167, 168, $173,182,183,317,318$, 326,362

Aoki, H. 796, 809

Arber, W. 321

Arbman, E. 70

Arfken, G. B. 551, 562

Arnold, J. R. 149, 180, $483,487,844$

Arnold, W. R. 566

Arnoult, R. 269

Arzimovich, L. A. 673

Asaro, F. 483, 484, 488, $491,567,568,743,754$, $756,757,758,766$

Ashkin, A. 45

Ashkin, J. 1, 43, 44, 46

Aston, G. H. 649, 749

Åström, B. 460, 635

Atterling, H. 91, 766

Auger, P. 32

Austern, N. 387, 393

Avignon, P. 763

Axel, P. 375

Bacher, R. F. 419, 799

Bäcklin, E. 102

Backus, J. 315

Baggett, L. M. 784

Baily, N. 850

Bainbridge, K. 67, 72, 74, 406, 456

Bair, J. K. 786

Bakker, C. 10, 195, 698

Baldinger, K. 500, 508

Balfour, J. G. 178, 751

Ballentine, R. 842

Bame, S. J. 784

Bannerman, R. C. 178

Barber, W. C. 743
Barchuk, 643

Barker, H. 842

Barnes, R. F. 343, 752

Barnothy, J. 836

Barreau, P. 262

Barschall, H. H. 171

Bartholomew, G. A. 781, $782,799,801,802,804$, $805,807,810,811,813$, 820

Bartlett, A. 67, 72

Barton, G. W. 766

Bashilov, 643

Battey, J. 566

Bay, Z. 500, 508, 509, 554

Bayly, J. G. 849

Beach, L. A. 45

Bearden, J. A. 102

Becker, H. 210

Becker, J. 166

Beghian, L. 194, 195, 196

Beiduk, F. 73

Belcher, E. H. 834, 852

Beling, J. K. 177, 566, 756

Bell, P. R. 132, 133, 282, $332,333,336,338,657$, 698,742

Bell, R. E. 494, 495, 504, $512,518,524,662,680$, $696,700,701,705,757$, $790,800,804$

Bender, R. 74

Benoist, P. 483566

Bent, R. D. 785

Berestetsky, V. B. 312, 690

Bergström, I. 174, 268, $460,680,624,627,629$, $633,635,699,914$

Beringer, R. 508, 673

Berlin, 650

Berlovich, E. E. 747

Berman, A. I. 45

Bernstein, S. 604 
Bernstin, II. 165, 542 Bethe. H. A. 1, 41, 42, 43, 44. 4.5. 46, 410.2, 419, ilti. i29

13.1411. I. A. 60103

Brwilengua, L. 41

Bievenharn, L. ( 9375 , $4113,534, .5411,5.811,551$, . $66^{\circ}, 910,91: 2$

links, II. $\$ .33$

Birge, R. T. 102

Birks, .J. 13. 133, \$25

Bishop, A. ‥ 533, 576

Bishep, (i. R. 192, 194, $19.5,196,644$

Bjorregarel. J. 4\$3, 490, 493,769

Black, 1. 1). 149

Blackett. 1'. II. S. 673

Blatt, J. 2:57, 3:4, 373, 38!), 403. .734, .540, 737, S16

Bleaney, B. 493, (6)13

Bleuler, F. 208. 675

Blin-Storle, R. J. 600

Bloch, F. 6.50

Bloch, I. .566

Bloembergen, N. 586. 589

Bloom, S. D. 642

Blunck, O. 11

Bock, C. 74

Bockelman, C'. 321, 504

Boehm, F. 131, 178, 256, $339 ; 343,483,556,654$, 751

Boekelheide, I. F. 668

Bogart, L. 84, 131

Bohr, A. 132, 324, 376, $426,454,468,477,479$, $480,483,488,489,491$, $587,725,752,769$

Bohr, N. 468, 660, 686

Boley, F. 80

Bolgiano, P. 571, 650, 654

Bonner, T. W. 785

Borovskii, J. B. 115

Borsellino, A. 45

Bothe,W.1, 7, 79, 201, 210, $497,633,661,743,793$
Bonthez, R. 321, 635, 705

Bouissières, $(\mathrm{x}, 754,756$

Bouricus, IV. G. 566

Bowden, B. V. 749

Bowe, .l. ('. 45.)

Bowers, K. D). 607

Boved, 336

Bozorth, R. 107

Braced, A. sol, 804

Braden, C. H. 260, 336

Bradt, H. L. 219, 506, $646,657,675,764$

Brady, E. L. 533

Braid, T. H. 798, 801.802, S0) 4

Bramson, H. J. 350

Bramson, ‥ 649, 653

Branyan, C. E. 698, 751, $76:$

Braun, O. 583, 734

Breit, G. 566

Brennan, J. G. 387

Brewer, H. G. 168

Brimberg, S. A. S. 638

Brindley, G. W. 41

Broda, F. 764

Brode, R. B. 16

Brodie, W. D. 751, 759

Brogen, G. 115

Brosi, A. R. 336, 698, 699, 702

Brown, B. II. 490

Brown, B. W. 827

Brown, F. 183

Brown, H. 282, 316

Brown, J. 262, 697

Brown, L. M. 665

Brown, S. C. 693,842

Browne, C. 321, 804

Broyles, A. A. 566

Broyles, C. D. 629, 633

Bruner, J. 74

Brunner, J. 268

Buckingham, R. A. 26

Buechner, IV. 321, 804

Bunker, II. E. 239, 336, 456, 744

Bunyan, D. 507

Burcham, W. E. 375
Burhop, E. H. S. 624, 631, 632

Burtt, B. P. 175, 829

Busch, H. it

Butt, D. K. 272, 751

Caianiello, E. 356

('aircl, R. S. 483, 720, 763

Cameron, A. 797

Campbell, C. G. 742

Campbell, w. C. 564, 764

C'ampbell, I. G. 843

Camnon, C. V. 847, 849

('arver, .J. H. 194

Casimir, H. B. G. 557

Cassidy, J. M. 332, 333, $336,657,698,742$

Cassie, A. M. 628

Cauchois, Y. 101, 103, $107,335,627,919,927$

Cavanagh, P. E. 54, 660, 665,702

Chadwick, J. 14, 26, 100, 224, 269

Chang, C. H. 272

Chang, C. S. W. 650

Chanson, P. 166

Charpak, G. 175, 833

Chen, J. J. L. 12

Childs, W. J. 698, 751

Christy, R. F. 275, 483, 491, 572

Chu, K. Y. 218

Church, E. L. 598, 915

Clark, A. F. 213

Clark, R. K. 850

Class, C. M. 483, 490, 493

Clay, F. P. 674

Cleland, M. R. 644

Cockcroft, A. L. 14, 166, $167,168,170,174,182$, $183,317,318,326,362$, 853

Coester, F. 534, 539, 545

Cohen, E. 98, 103, 116, 227, 915

Cohen, R. 831

Cohen, S. G. 178, 751

Cohen, V. W. 461 
Colgate. 5. A. 47

Collie. ('. H. 1!2, 1!4. 195,196

Collins, 'T. I. $8(1) 4$

Compton, A. H. 4U, 176 , 660

Compton, H. H. 14

Condon, E. U. 402, 479, 535

Cong, C. R. 601

Conmore, D. J. 653

Cook, C. F. 490, 493

Cook, C. S. 73, 183, 237 , 260, 262, 271, 272, 315, $316,326,697$

Cook, G. B. 828

Cooke-Yarborough, E. H. 826

Cooper, E. P. 591

Coor, T. 778

Cork, J. M. 256, 314, 698, $751,759,762$

Corson, D. R. 16, 503, 765

Coryell, C. D. 260

Cowan, C. 50, 676, 685, 689

Cowie, 646

Cox, C. 73, 257

Cox, J. A. M. 605, 613, $614,619,623$

Cox, S. A. 493

Craggs, J. D. 165, 825

Craig, H. 97

Cramer, H. 354

Cranberg, L. 751

Crane, W. T. 766

Cranshaw, T. E. 168

Craven, J. H. 832

Craythorn, A. R. 842

Crenshaw, C. M. 195

Cross, L. 362

Cross, W. G. 661

Curie, P. 847

Curran, S. C. 14, 133, 165 , $166,167,168,173,174$, $178,180,182,183,210$, $317,318,326,362,751$, 825,853

Curtiss, L. F. 823, 827

Dabbs, J. W. T. 798, 807
d'Ag(s)tino, (). 79:5

Dalitz, R. H. 63:3s, 648

Dancoff, .'. M. 375, 797

Danill, I. A. 7(1).2

Daniel, H. 79,793

Daniel, R. R. 648

Daniels, J. II. 603, 621

Danysz, J. 58, 224

Darbord, R. 103

Darby, E. K. 571, 669

Davey, C. N. 826

Davey, W. G. 524, 530

Davidson, J. P. Jr. 319, 333,739

Davidson, Z. S. 32

Davies, J. H. 648

Davies, W. T. 647

Davis, L. H. 356

Davis, R. 366, 742

Davisson, C.M. 24, 31, 50, 857

Dawson, J. K. 176, 183, 742

De Benedetti, S. 501, 507, 567, 672, 676, 677, 678, $679,680,685,687,689$, 691,716

De Broglie, M. 103

Debye, P. 589

Dee, P. I. 210

De Groot, S. R. 312, 367, $534,607,613,614,616$, $621,622,623$

De Haan, E. 73

Dehmelt, H. G. 573

Delbruck, M. 529

Delsasso, L. A. 780

Demichelis F., 748

Dennison, D. 67

DePasquali, G. 455, 579, 585

Der Mateosian, E.137,192, $345,456,659,798,804$

De Shalit, A. 65, 394, 455, $483,732,735,738$

Detouef, J. F. 669

Deutsch, M. 74, 78, 192, $234,252,336,458,533$, 534, 553, 563, 571, 588,
6117, 664, 67\%. 689. (i9):, (i!13. 6isti, 7:21)

J)(xamev, J. . . . Ifi., 74!), $7.5 \%$

D(ग)1, . 4 .5.), 451, 51x, 646

Dt. IVaard, H. 86

Dewar, J. 847

Dellire, J. 11.45

De Witt, T. W. 699

Diamond, H. 766

Dicke, R. H. 500, 6\$6, 692

Dirac, P. A. M. 668, 689

Dismuke, ․ M. 2.2., 303, $341,434,447$

Dixon, D. 180, 183

Dodson, R. IV. 263

Dohne, C. F. 664

Donelly, R. J. 257

Dougherty, 642

Douglas, D. G. 260, 263

Downing, I. R. 696, 720

Drell, S. D. 517

Dreyfus, B. 175

Driscoll, D. 96

Drisko, R. MI. 679

Dubbs, J. IV. J. 604

Dudjiak, IV. 354

Dulit, E. 692

DuMond, J. IV. MI. 79, 84, $98,99,100,103,105$, $109,111,116,118,120$, $121,123,131,227,483$, $491,674,676,697,743$, 791,915

Duncan, J. F. 828

Dunlavey, D. C. 743

Dunworth, J. V. 202, 208, $219,501,506,836$

Durand, H. 602

Eby, F. S. 461

Eckart, C. 537

Edvarson, K. 95, 227, 920,927

Eggler, C. 332, 336

Eickman, C. 653

Eisinger, J. T. 483,490 , 493 
Elliott, L. 78, 252, 336, $4 \times 1,486,518,553,720$, 759, 790, 800, 804

Fillis, ('. D. 14, 26, 100, $224,269,628,749,920$

Elsasser, W. II. $\mathbf{4 1 9}$

Emery, E. W. 698

Emigh, C. R. 45

Fndt, P. 804

Fngelkemeir, A. G. 842

Engelkemeir, D. W. 260, 756

English, W. N. 172

Evans, R. 31, 50, 78, 208, 825,857

Ewan, G. T. 7.5l

Facchini, L. 801, 804

Fagg, L. W. 560

Failla, G. 850

Fainberg, J. 648

Falk-Vairant, P. 483, 566

Falkoff, D. L. 299, 535, $559,569,622,650$

Famularo, K. F. 489

Fano, L. 517

Fano, U. 170, 534, 614, 617

Farago, 500

Faust, W. R. 664

Feather, N. 21, 86, 202, 208, 501, 565, 740, 743, $744,745,757,759,760$, $763,764,765,766$

Feenberg, E. 246, 284, 333, $335,340,375,419,545$

Feinberg, E. L. 340 '•

Feingold, A. M. 287, 598

Feister, I. 282, 698

Felber, F. 262

Feld, B. T. 566

Feldman, L. 86, 191, 259, $332,333,336,345$

Ference, M. 633

Ferentz, H. 550, 910, 912

Ferguson, A. V. 691

Fermi, E. 225, 276, 314, 422,795

Ferrell, R. A. 690
Feshbach, H. 2

Feynman, R. P. 665

Fields, P. R. 766

Fields, R. 192

Fierz, II. Z. 319

Finkelstrein, R. 356

Fireman, F. L. 669

Fischer, J. 32, 499

Flammersfeld, A. 22, 921

Fleischmann, R. 795, 796

Florida, C. D. 826

Flowers, B. H. 375, 426, 475

Fluharty, R. E. 192

Foldy, L. L. 470

Foley, H. M. 476

Forafontov, N. V. 745

Ford, K. W. 376, 483, 484, 726,727

Forro, M. 836

Foster, B. P. 788

Foster, J. S. 627

Fowler, C. 86

Fowler, R. H. 26

Fowler, W. 71, 774, 780

Francis, J. E. 501, 742

Frank, S. G. F. 174

Frankel, S. 78, 563, 598

Franklin, E. 825

Franz, W. 41, 376, 528

Franzen, W. 169

Fraser, J. S. 561, 568, 585

Frauenfelder, H. 265, 375, 455, 456, 485, 531, 533, $553,557,563,571,575$, $576,579,585,732,734$, 910

Fred, M. 343, 752

Freedman, M. S. 742, 756, 760,786

Freeman, B. E. 566

Freese, E. 3

Freier, H. 422

Fremlin, J. H. 669

French, A. P. 561, 565, 568, 788

Friedlander, G. 455, 579, $580,707,761,764$

Friedlander, H. N. 21
Friedman, A. M. 766

Frilley, M. 100, 756

Frisch, O. R. 171, 193, 503

Froman, D. K. 263

Fröman, P. O. 480, 491

Frost, B. R. T. 589

Frost, R. H. 14

Fry, W. F. 363

Fuchs, M. 569

Fujita, J. 344, 349, 570

Fulbright, H. W. 184, $332,336,345,668,669$

Fulton, T. 690

Funt, B. L. 149

Furnas, Th. C. 115

Furry, W. H. 669

Gaerttner, E. R. 527

Gardner, J. W. 534

Gardner, W. 76, 353

Garth, R. 808

Garwin, R. L. 685

Geiger, H. 201, 205

Gell-Mann, M. 395

Gellman, H. 374, 398, 460,632

Gentner, W. 47

Geoffrian, C. 54

Gerber, H. J. 582

Gerbes, W. 14, 190

Gerhart, J. B. 320

Gerholm, T. 88, 920, 926

Germain, L. S. 630

Ghiorso, A. 517, 669, 759, $760,763,766$

Gie, 643

Gilbert, D. A. 461

Gillon, L. 65

Gilvarg, A. B. 115

Gimmi, F. 563, 582, 590

Glascock, R. F. 842

Glasser, R. G. 648

Glauber, R. J. 659

Gleason, G. I. 20

Glendenin, L. E. 20, 208

Glicksman, M. 797

Gluckstern, R. L. 529

Goeppert-Mayer, M. 246, $287,375,383,393,415$, 
$420,426,428,433,622$, 669, 705, 737

Goertzel, G. 132, 374, 397, $398,399,457,460,533$, $632,704,724,750,905$

Goes, M. L. 563

Goldberg, N. 563

Goldberger, M. L. 456

Goldburg, W. I. 483, 493, 572

Golgate, S. A. 857

Goldhaber, G. S. 607

Goldhaber, M. 86, 137, $192,374,375,394,406$, $453,454,455,456,457$, $459,461,481,483,484$, 487, 558, 579, 704, 706, $714,735,737,738,743$, 762, 764, 798, 804

Goldring, G. 455, 638, 646

Goldwasser, E. L. 11

Good, R. H. 347

Goodman, C. 493, 769

Goodman, L. S. 461

Goodrich, M. 764

Gopalakrishnan, K. 65

Gordy, W. 574

Gorter, C. J. 601, 602

Gouy, M. 103

Goward, F. K. 199

Grace, M. A. 600, 602, $603,621,743$

Graham, R. L. 338, 481, 486, 493, 504, 524, 680, 687, 700,701, 705, 757

Graves, A. C. 263

Graves, G. A. 254, 460

Gray, L. H. 167, 236, 850, 853

Grebe, L. 263

Green, P. 321, 653

Greuling, E. 300, 308, 884

Griffith, B. A. 374, 398, 460,632

Griffiths, G. M. 677

Griffiths, J. H. E. 796

Grivet, P. 54

Gross, L. 317

Grosskurth, K. 48
Groven, L. 627

Gugelot, 219

Guillesen, J. 653

Guillot, M. 756

Guinier, A. 107

Gunning, L. II. 849

Gunst, S. B. 123

Gurney, R. W. 593

Haffner, J. 804

Hafstad, L. R. 193

Hagstrum, H. D. 593

Hahn, O. 58, 373, 764

Hain, K. 3

Halban,'H. 193, 194, 195, $196,600,602,603,604$, $621,644,646,743$

Hall, H. 10, 26, 29

Hall, T. 686

Halliday, D. 691

Halpern, J. 807

Halpern, O. 10, 538, 566, 674

Halter, J. 262, 268, 646

Hamermesh, B. 197, 797, $798,804,807$

Hamilton, D. R. 317, 362, $533,551,559,561$

Hammack, K. C. 419

Hanna, G. C. 168, 172, $182,183,317,318,326$, $362,659,675$

Hanna, S. S. 559, 560

Hänni, F. 533

Hanson, A. O. 3, 6, 11

Harker, D. 115, 264

Harkins, W. D. 744

Harper, A. 96

Harr, J. 132, 374, 397, 457, 704, 750

Harries, G. 647

Harris, S. 808

Hartogh, Chr. D. 616, 621

Hartree, D. 39, 40, 97

Harvey, J. A. 168, 719, 807,819

Haskin, D. M. 648

Havens, W. W. 350

Hawkings, R. C. 832, 842
Haworth, F. E. 107

Haxel. O. 375, 419, 420, 831

Haynes, ‥ 629, 633

Hayward, R. 54

Hedgran, A. 68, 71, 93, $96,123,129,227,234$, 264, 676, 708, 749

Heer, E. 533, 539, 553, 563, 564, 572, 576, 579, $582,583,584,585,590$, 734

Heerschap, M. 746

Heine, H. G. 646

Heisenberg, IV. 41, 362

Heitler, W. 13, 25, 41, $237,373,377,382,537$, $637,664,665,673$

Heller, R. B. 269

Hellund, E. J. 551

Helm, R. H. 743

Helmholtz, A. C. 375

Helmholz, L. 263

Hemming, P. B. 590

Henderson, W. J. 742

Henri, Y. P. 554

Hereford, F. L. 18, 560, 674,675

Hereward, H. G. 518, 646

Herrlander, C. J. 634

Heusinkveld, M. 422

Heydenberg, 646

Heydenburg, N. P. 483, 489, 492, 493

Hibdon, C. T. 799, 807

Hill, D. L. 376, 468

Hill, R. 86, 375, 455, 459, $460,461,465,487,579$, $627,632,706,762,914$, 915

Hincks, E. 349, 350, 507

Hine, G. J. 851

Hinman, G. 704

Hipple, J. 96

Hoffman, J. G. 799

Hofstadter, R. 120, 663, 786

Hok, Ong Ping 73

Hole, N. 826 
Hollander, J. M. 219, 357, 434

Holmes, D. K. 283, 340

Hoover, J. I. 664

Hopkins, J. I. 137

Hopkins, N. J. 764

Horie, H. 483, 484

Hornyak, IV. 81, 235, 319, $518,642,774,778,779$, 786,792

Horton, G. K. 636,637

Hough, P. V. C. 44

Houtermans, F. G. 831, S34

Howland, J. J. 764

Hoyt, H. 99, 120, 121 , $491,674,743$

Hubbard, H. 76, 353, 354

Hubbard, T. P. 559

Huber, O. 219, 268, 459, $563,629,633,732$

Huber, P. 508

Hubert, P. 234

Huddleston, C. M. 234, 335

Hufford, D. L. 263

Hughes, A. L. 644

Hughes, D. J. 332, 336, $765,808,819$

Hughes, F. J. 491

Huizenga, J. R. 756, 766

Hull, M. M.' 566

Hulme, H. R. 26, 29, 44, 638

Hulubei, C. R. 627

Hulubei, H. 919, 927

Hulvey, J. H. 648

Humbel, F. 459, 629, 732

Hummel, V. 798, 804, 807

Hunter, R. F. 842

Hupfeld, H. H. 522

Husimi, K. 796

Hutchinson, G. W. 177

Huus, T. $375,477,483$, $490,492,493,769$

Hyde, E. K. 763

Ilakovac, K. 527

Inglis, D. 475

Ingraham, M. G. 669
Insch, G. II. 14, 168, 174, $178,318,751,853$

Isford, G. 797, 804

Israel, H. I. 762

Itoh, J. 252, 796

Jackson, J. L. 291

Jacobs, J. A. 559

Jacobsen, J. C. 47, 365, 500, 507

Jaeger, J. C. 44, 638

Jaffé, A. A. 178, 751

Jaffey, A. H. 742, 760

James, R. 41, 760

Jarvis, C. J. D. 743

Jastram, P. S. 559

Jauch, J. M. 534, 551, 601,657

Jean, M. 471

Jelley, J. V. 507

Jenks, G. H. 847, 849

Jennings, J. 77

Jensen, E. 79, 81, 86, 230, 336,491

Jensen, J. H. D. 375, 393, 414, 415, 419, 420, 481

Jentschke,W. K. 364, 455, 461, 485, 576, 579, 585

Jesse, W. P. 190

Johann, H. H. 103

Johanson, A. 214

Johansson, S. 702, 731, 766, 783, 786, 799

Johansson, T. 103, 107, 335

Johns, M. 73, 257, 503

Johnson, C. 602

Johnson, F. A. 627

Johnson, G. W. 137

Johnson, W. H. 804

Joliot, F. 673

Joly, R. 268

Jones, G. A. 559

Jones, W. M. 847

Jordan, W. H. 133

Jurney, E. T. 213, 219

Justus, K. 8, 175, 829

Kageyama, S. 261, 749

Kahn, H. 31
Kahn, J. H. 177

Kalckar, F. 468

Kalkstein, M. I. 669

Kallmann, H. 133, 501

Kanei, T. 493

Kapitza, P. 78

Karplus, R. 690

Karraker, D. G. 761

Katz, J. J. 263

Katz, L. 20

Katz, R. 86, 459

Kaus, P. 356

Keim, C. P. 332

Keister, G. L. 345, 553

Keller, H. B. 751, 759

Keller, L. B. 398

Kelley, G. G. 698

Kelman, V. 74

Kennard, E. H. 627

Kern, B. D. 214, 232, 335, 698

Kerst, D. 66

Ketelle, B. H. 135, 336, 698, 702

Keuper, J. P. 560

Kikuchi, S. 796

King, R. W. 339, 343, $375,434,447,766$

Kinsey, B. B. 781, 782, 795, 799, 801, 802, 804, $805,807,810,811,813$, 820

Kirkpatrick, H. A. 103, 105

Kirkwood, D. H. W. 168, 183, 318, 659

Kistemaker, J. 74

Kittel, C. 574

Klein, A. 690

Klein, D. 99, 120, 121, $674,697,743$

Klein, O. 33, 350, 663

Klema, E. D. 171, 553, 555

Klemperer, O. 55, 78, 673

Klinkenberg, P. F. A. 375

Kluyver, J. C. 588

Knight, J. D. 744

Knipp, J. K. 650 
Knop, G. 11

Kobayashi, Y. 90

Koch, J. 193, 268

Kofoed-Hansen, O. 75, $182,286,321,326,327$, $341,357,359,362,365$, $366,368,372,591$

Kohl, J. 84, 131

Konneker, W. R. 676, 685

Konopinski, E. J. 73, 225, 281, 292, 300, 308, 311, $319,324,339,341,356$, 884

Kopfermann, H. 574

Korff, S. A. 165

Korsunsky, M. 74, 77

Kovaric, A. F. 8

Kowarski, L. 415

Kramers, H. A. 660

Kraushaar, J. 455, 461, $558,559,585,598$

Kristensen, P. 365

Krohn, V. 485, 576, 579

Krone, R. 797, 804, 807

Kubitschek, H. E. 797

Kuhn, W. 522

Kulchitsky, L. A. 6

Kulp, J. L. 180

Kurath, D. 426

Kurie, F. 67, 72

Kurti, N. 601, 602, 603, 621

Kyles, J. 86, 742

Laberrigue-Frolow, J. 234

Laborde, A. 847

Lagarrigue, A. 353, 354

Lamb, W. E. 45

Landau, L. 11

Lane, A. M. 466

Lane, R. O. 270

Lang, L. G. 704

Langer, L. 73, 202, 214, $237,254,260,261,263$, $271,281,315,316,317$, $324,326,330,335,336$, $342,345,460,845$

Lanzl, L. H. 6

Laslett, L. 81, 230, 336
Latter, R. 31

Latyshev, G. D. 6, 32, $517,642,643,749$

Laubert, s. 48

Laughlin, J. S. 16

Lauritsen, C. 71, 235, 518, $642,774,779,780$

Lauritsen, T. 81, 235, 319 , $375,518,642,770,774$, 775,779

Lavatelli, L. 74

Lawson, J. L. 45, 256, $314,553,579$

Lawson, J. S. 455, 485, 576,585

Lea, D. E. 795

Le Blanc, J. M. 751, 762

Lecoin, M. 745, 759

Lederman, 354

Lee, D. 77

Leger, P. 262

Leighton, R. B. 349,356

Leininger, R. F. 765

Leipuner, L. 679

Leisegang, S. 11

Lemmer, H. R. 602

Lemonick, A. 561

Lenard, P. 7

Levi-Setti, R. 356

Levin, J. 808

Levine, C. 669

Levy, H. B. 765, 766

Lewis, G. M. 691

Lewis, M. N. 26

Lewis, W. B. 749

$\mathrm{Li}, \mathrm{K} .61$

Libby, W. 77, 180, 669, 842,844

Lidofsky, L. 319, 331, $338,336,345,763$

Lind, D. A. 93, 96, 111, $116,118,123,129,227$, $266,676,697$

Lindenfeld, P. 648

Lindhard, J. 75

Lindsey, G. R. 455, 518, 646

Lindström, G. 64, 95, 96, $123,227,674,920$
Ling. I). S. 534

Lippmann, B. A. $53 \mathrm{x}$

Lipps, F. 650

Littler, J). J. ×우

Livingood, .J. .J. 6946

Lloyd, S. P. 534, 540, 545, 565,575

Lock, II. O. 356

Longmire, ( . 191, 282, 301,316

Loosemore, W. 1R. 82.5

Lord, J. J. 648

Low, W. 476

Lundby, A. 503, 507, 575

Lundén, A. 483, 492

Lyman, E. M. 3, 6

Mack, J. E. 490, 492

MacKenzie, K. R. 765

Mackin, R. J. 642

Macklin, P. 331, 336, 338, 763

Madansky, L. 554, 561, $566,571,650,654,658$, 676,685

Maeder, D. 268, 630, 657

Magnusson, L. B. 766

Mahmoud, H. II. 281, $311,319,356$

Maienschein, F. 211, 218, $219,244,336,786$

Maier-Leibnitz, H. 201, 661,677

Majorana, E. 668

Malmfors, K. G. 521, 525

Malvano, R. 748

Malvicino, A. 801, 804

Man, K. C. 749

Mandeville, C. F. 202

Mandl, F. 666

Mandleberg, C. J. 176, 742

Mann, A. 807

Mann, I. 787, 797, 804

Mann, W. 13. 84?

Manning, W. M. .263

Manov, G. G. 823

Marinelli, L. D. 851

Mark, H. 493 
Marmier, P. E. 131, 483, 491

Narshak, R. E. 191, 340, 746

'Narshall, J. 17, 499

Martin, D. C. E. 339, 517, 746

Martin Jr., D. S. 491

Martin, D. W. 762

Martin, P. C. 659,690

Marty, C. 175

Marty, N. 754

Massey, H. 1, 632

May, J. 760, 788

May, II. MI. 560

Maximom, L. C. 45

MeAskill, D. 73

MeCarthy, J. A. 669

MeClelland, C. L. 493, 769

NIcCord, R. V. 365

MeDaniel, B. 781

McDaniel, P. W. 202, 214

MeDougall, J. 26

MeGinnis, C. L. 557, 731, 733

MeGowan, F. K. 459, 487, $490,501,503,507,553$, $555,558,563,564$

McIntyre, J. A. 120, 663, 786

McKeown, II. 137, 483, 559

McKinley, W. A. 2

McLernon, F. 554

MeNaster, W. H. 541

MeMillan, E. 67, 653

Mc.Iullen, C. C. 257

McNair, A. 183

McNally, J. R. 489

McPherson, A. J. 263

Mech, J. F. 766

Medicus, 219

Meem, J. L. 211, 218

Mei, J. Y. 244, 256, 335, 336

Meitner, L. 58, 522, 744

Merritt, W. F. 832

Messiah, A. M. L. 301
Metzger, F. 345, 5:23, 5:6, $533,558,559,664,696$

Neyer, K. 500, 508

Meyer-Schützmeister, L. 834

Meyerhof, W. E. 455, 787, 797,804

Michel, L. 349, 350, 352, 355

Migdal, A. 340

Mihelich, J. W. 65, 374, $455,460,461,483,487$, $488,559,628,742,915$

Mileikowsky, C. 72

Milford, F. J. 470

Millar, C. 797

Miller, L. C. 365

Miller, W. W. 842

Millington, G. 12

Mills, F. S. 11

Milsted, J. 483, 491

Milton, J. C. D. 184, 186, $332,336,345,561,568$, 585

Mims, W. 644, 646

Minton, G. H. 567, 716

Miskel, J. A. 358, 591

Mitchell, A. C. G. 201, $202,214,224,232,234$, $244,254,256,335,336$, $345,483,698,720,763$

Miwa, M. 749

Miyamoto, G. 76

Mize, J. P. 183, 318, 456

Madjenović, M. 268, 627, 749,766

Moak, C. K. 798, 807

Moch, R. 669

Moe, D. 697

Moffat, R. J. D. 237, 254, 261, 263, 281, 316, 326, $336,345,460$

Mohorovićić, S. 690

Molière, G. 3, 4

Moljk, A. 182, 318

Montgomery, C. G. 673

Moon, M. L. 251, 458, 553

Moon, P. B. 41, 515, 523, 524,527
Moreau, J. 91, 262

Morette, C. 32

Morita, M. 570

Morlet, J. 627

Morrison, P. 651

Morton, G. A. 145, 160, 500

Moszkowski, S. A. 246, 287, 373, 375, 376, 433, $455,478,622,705,737$

llott, N. F. 1, 2, 593

Mottelson, B. R. 132, 324, $376,426,454,468,477$, $479,480,483,488,491$, $587,725,752,769$

Motz, H. T. 317, 798, 804

Moussa, A. 73

Muehlhause, C. O. 456, $799,807,808,809$

Muether, H. R. 338

Mugele, R. A. 825

Muirhead, H. 356

Muller, D. 84, 99, 120, $121,131,674,697,743$

Müller, I. 48

Nurakawa, K. 493

Murray, J. J. 131, 491

Myers, O. E. 847

Myers, V. 192

Nagel, E. 178, 343, 751

Nageotte, E. 166

Nakagawa, S. 801,804

Nakamura, S. 76, 344

Natof, R. 321

Nedelski, L. 636

Neher, H. V. 8

Nelms, A. T. 38

Nelson, C. E. 14

Nelson, E. B. 336,559

Nemirovsky, P. 45

Neumann, H. M. 761, 764,765

Newton, J. O. 177, 483, 561,756

Newton, T. D. 508

Nichols, R. 79, 80, 86, 338,491 
Nicodemus, D. 190

Nielsen, O. 75, 792

Nier, A. O. 804

Nilsson, S. G. 474

Nishina, Y. 33, 663

Nogami, Y. 76

Nordheim, L. 246, 287, $308,375,433,434,447$, $480,622,665,705,725$, 737

Norling, F. 202, 213, 219

Novey, T. B. 260, 533, 539 , $568,571,572,576,583$, $584,585,590,654,734$

Nybö, K. 460

Nijgh, G. J. 630,698

Occhialini, G. P. S. 673

O'Ceallaigh, C. 356

O'Kelley, G. D. 262, 747, $760,761,766$

O'Neal, R. D. 193

O'Neill, 16

Ong, P. H. 746, 760, 764, 766

Opechowski, W. 571

Oppenheimer, J. R. 636, 637

Qre, A. 677, 689

Ornstein, L. Th. M. 630

Orth, D. A. 747,760

Osborn, R. K. 341, 766

Osoba, J. 67, 72

Ovadia, J. 16

Owen, G. E. 190, 237, 260, $262,283,316,697$

Owen, P. H. 262

Ozeroff, M. J. 749

Page, L. A. 123

Pake, G. E. 545, 574

Palevsky, H. 765

Palluel, P. 8

Papp, G. 500, 508

Parry, G. 339

Pasechnik, 643

Passel, T. O. 633

Paul, W. 1, 3, 11, 595
Pauli, W. 541

Payne, P. R. 843

Peacock, C. L. 242, 244, 254, 336, 345, 720

Peacock, W. C. 849

Pearce, R. M. 669

Peaslee, D. C. 319, 339, $343,356,368,766$

Penfold, A. S. 20

Perey, M. 745, 756, 759

Perez-Mendez, V. 319

Perkins, D. H. 648

Perkins, J. F. 633

Perlman, I. 219, 357, 434, $483,484,485,491,517$, $568,743,754,756,757$, $758,761,764,765,766$

Perlman, M. L. 358, 591

Perrin, F. 32

Perry, C. L. 282, 303, 341, $398,724,884$

Perry, W. E. 852

Persico, E. 54, 79

Petch, H. E. 503, 504, 662,701

Peterson, S. 759

Petrov, P. 74

Petrzilka, V. 210

Petschek, A. 340

Peyrou, C. 353

Phibbs, E. 637

Philips, 646

Phillipp, K. 54

Phillips, K. 199

Pieper, G. F. 804

Pincherle, L. 631

Pipkin, F. M. 561

Pirenne, J. 690

Pirenne, M. H. 39

Plassman, E. 633

Pleasonton, F. 365

Pocza, 500

Pohm, A. 86

Pollard, E. 501, 522, 774

Pomeranchuk, I. 312, 689

Pond, T. A. 686, 687, 690, 692

Pontecorvo, B. 167, 168, $182,183,317,318,326$,
$349,350,362,659,795$

Poppema, O. J. 603, 604

Porter, F. T. 591

Post, R. F. 509

Potter, R. M. 263

Pound, R. V. 539, 567, $572,573,574,585,586$, 589, 602

Povelites, J. G. 263

Powell, J. L. 677, 689

Pratt, G. W. 40

Pratt, W. 80, 81, 230

Preiswerk, P. 219, 256, 466, 483, 484, 486, 657, 730, 735, 743

Prescott, J. R. 766

Preston, W. M. 658

Price, B. T. 166

Price, H. C. 237, 254, 260, $263,281,316,317,326$, 330,336

Primakoff, H. 190, 283, $340,591,669,676,685$

Pringle, R. 86, 149, 797, 804, 807

Pruett, C. H. 762

Pruett, J. R. 326

Prugne, P. 262

Pryce, M. H. L. 481, 601, $707,708,763,766$

Purcell, E. 90, 589

Pursey, D. L. 302, 308, $347,356,884$

Putman, J. L. 823, 826, 829,839

Quimby, E. H. 851

Raboy, S. 485, 576, 579

Racah, G. 389, 403, 455,

$534,540,614,615$

Radicati, L. A. 395,466

Rae, E. 77, 642

Raether, H. 572

Rainwater, J. 469, 470, 476

Rall, W. 788 
Ramberg, E. (i. 5y:2, 6:7, 631

liamser, X. F. 5it, 661

Rarita, IV. 26

lisitti, F. $566,571,654$, 6.5. 656, 685, 795, 799

liasmussen, E. 4!!:3

Ratimussen, J. O. 4s:3, $491,567,568,766$

Rasmussen, V. 81, 23.5, iss, 642, 77.5, 779

Riardon, W. 797,804 , 807

Reed, (i. W. 74?

Roich, H. 3

Reid, J. II. 166

Reitz, J. R. 282, 316, 3i4, 398,460

Renard, G. 654

Reynolds, F. L. 756, 761

Reymolds, S. H. 669

Kich, J. A. 677

Richards, J. 262

Richardson, H. 74,79 , $517,744,746$

Richardson, J. R. 321

Richings, H. J. 680

Richtmeyer, F. K. 592, 631

Ridgeway, S. L. 335, 338

Rinehart, 354

Riou, II. 743, 745, 759

Roberts, A. 251, 252, 458, $553,696,720$

Roberts, L. D. 604

Roberts, N. 96

Robinson, B. L. 554, 561

Robinson, F. N. H. 603, 621

Robinson, H. 224, 628

Robson, J. M. 365

Rodeback, G. W. 358

Roderick, H. 797, 804

Rogers, F. Jr. 90, 318, 354

Rohrlich, F. 529

Roos, ('. E. 629

Rose, B. 177, 483, 756

Rose, D. 704

Rose, H. 571

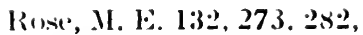
283, :91, 303, 31:. 316. $340,341,374,375,396$. $397,395,399.402,4(133$, $4.57,460$. 334 . 540 , 5.51. 55:, 56ะ. $597,601,602$. 632, 636, 7(14, 724, 75). 766, 875. 884, 905, 910, 91.

Rosenblum, E. S. 45

Rosenblum, s. 483, tst, 491; 756

Rosenzweig. ․ 550, 910. 912

Russ, II. 496, $75 \mathrm{I}$

Rossi, B. 165, 190. 497, 8.5.5

Russi, H. H. 850

Rotblat, J. 501, 506

Rothwell, P. 166, 16s, 173. 174

Roulston, K. 797,807

Rowland, 'T. .J. 586

Rowlands, S. 507

Ruy, R. R. 563

Ruark, E. 690

Rubenstein, R. 592

Rubin, S. 71

Rubinson, IV. 168

Ruby, S. L. 363

Ruderman, M. 344

Rudnick, N. 51, 85i

Rüetschi, R. 553, 585

Russell, A. S. 765

Russell, B. 192

Rustad, B. M. 319,363

Rutheman, G. 74

Rutherford, F. 14, 26, 100, 224, 269, 749

Rutledge, W. C. 698

Rytz, A. 746

Sachs, A. 648

Sachs, D. 192

Sachs, R. G. 383, 387, 393,456

Sadauskis, J. 190

Sagana, R. 76, 353, 354
Siailor, V. L. 804

Sakai, M. 76

Sula, 0.455

Salınon, J. 669

vian-'Tsiung, 'T. 17.5

Siarat. 13. 6.5

Siarl. R. 350)

Sargent. 354

satchler, C. R. 551, 552

Siater, F. 1,29

Sixиm. D. 262, 269

scarrott, G. C. 177

Schaefer, V. J. 264

scharff-Goldhaber, G.

$137,455,462,466,483$,

$484,486,488,559,579$,

743,764

Schein, MI. 648

Scherrer, P. 219, 506, 563, $572,580,582,584,646$, 734,764

Schiff, D. 558, 705

Schiff, L. 509, 527, 651

Schmiclt, F. 83, 345, 553

Schmidt, H. W. 8

Schmidt, T. 490

Schneider, H. 732

Schonland, B. F. J. 8, 269

Schreffler, R. 86

Schultz, H. 501, 508

Schulz, K. 48

Schwartz, C. 475

Schwartzer, D. 669

Schwinger, J. 3

Scott, B. F. 263

Scott, F. 74, 633

Scott, M. B. 3, 6

Scott, M. R. 517

Scott, W. T. 4, 16

Scovil, M. E. D. 493

Seaborg, G. T. 219, 263, $357,434,517,669,696$, 743,766

Seed, J. 565, 568, 788

Segall, B. 471, 568

Segrè, E. 10, 42, 375, 765, 795

Seifert, A. H. 350

Seiler, J. A. 260 
Seliger, H. H. \&

Serber, R. 66

Seren, L. 21

Seriff, A. J. 349,356

Shafroth, S. M. 559

Shaknov, I. 571, 675

Shankland, R. S. 660

Sharpe, J. 137

Shaw, P. F. D. 192

Shearer, J. W. 689

Sher, R. 807

Sherb, M. W. 202

Sherr, B. 320

Shipinel, V. 76, 745

Shortley, G. H. 402, 479, 535

Shrader, E. F. 45, 500

Shull, F. B. 67, 260, 335, 336

Siday, R. 77

Siegbahn, K. XviII, 52, $58,66,68,72,77,78$, $79,85,93,95,96,97$, $99,102,214,227,234$, 252, 266, 335, 458, 563, $642,708,710,712,748$, $783,791,920,927$

Siegbahn, M. 914

Siegel, R. 677, 678, 679, 687,691

Sigurgeirsson. 'Th. 507

Silverston, D. 77,745

Simmons, B. E. 489

Simon, A. 550, 601, 660

Simon, F. F. 601, 603

Simpson, J. A. 8.26, 8:31

Sinclair, W. K. \$.52

Singer, G. 852

Sipple, R. F. 785

Sizoo, G. 73, 653, 760, 764,766

Skyrme, T. H. R. 666

Slack, L. 67, 72, 336

Slater, J. C. 26, 40, 660)

Slätis. H. 63, 65, 77, 79, 85, 95, 259, 266, 26s, 627. 642, 712, 766

Sliv, L. A. 643, 669

Sluis, K. I. I. 4\$?
Smith, A. 302, 308, 340

Smith, A. B. 238

Simith, D. B. 263, 266, 720,831

smith, F. M. 354

Simith, K. 343

Simith, W. V. 574

Snelgrove, P. 491

snell, A. H. 365

inell, A. K. 604

Snyder, C. 71

snyder, H. S. 4, 169

Snyder, J. N. 592

Sobering, S. 149

söderman, II. 102

Sommerfeld, A. 26

iperduto, A. 321, S114

ipiers, J. A. 604, 614

spiess, F. ․ 461, 765

ipinrad, B. I. 132, 374, $397,398,45 \overline{7}, 704,750$

Stafford, G. H. 137, 196

Stahel, E. 653

Stähelin, P. 321, 466, 483, 484, 486, 735, 743

Stanford, C. P. 604

stanley, J. P. 374, 398, 460,632

itarkiewics, J. 47

Starner, J. W. 456, 744

Ntaub, H. 165, 190, 566, 825

Stech, B. 376, 737

iteenberg, N. R. 560, :5:2, $605,614,617,621$

Steenland, II. J. 603

Steffen, R. II. 459, .553, 5.5. 579, 585, .590, .795, $6: 29,633$

itchle, P. 1:3

Strinberg. E. P. 260

steinberger, J. 349, 648

steinwedel, H. 1. 481

Stelsom, P. H. 65s

stepluns, F. 483. stik, 743

Stephenson, 'J. 1..604

sternheimer. R. II. I1)

stroms, ('. M. itit
Stevenson, D. T. 336, 563, 571

Stewart, A. 'T. 657

Stobbe, MI. 26

Stoddard, A. E. 698, 751

Stoker, P. 73, 746, 760

storruste, A. 41, 524, 529

Stout, J. W. 847

itrachan, C. 746

itrait, E. 804

street, K. 766

Strohmaier, K. 263

Strong, P. 132, 374, 397, $45 \overline{7}, 704,750$.

stuart, R. V. $4 \$ 9$

Studier, H. 11.766

Stump, R. 561, 7! ד. S04, 807

Sturcken, E. F. :26!

Suess, H. E. 37.; 415 , 419,420

Sugarman, $x . \quad 260,345$

Sunyar. A. W. 3it. 453, $454,4.55,459,461,463$, 454, 489, 579, 580, 704, $714,737,743,764$

siurugue. I. 920

Sишн. ‥ 493

Sizz) F. 175, 833

sintholm, ․ $66,67,68$, 708

Swan, J. B. 460, 632

Swam, Ch. P. 18

Swietoslawski, W. 847

siwift, C. J. 460

sziland, L. 796

Talern, D. L. 20

Takerhi, 76

Takrola, F. 796

Talbeg, J. 86

Tilmi, I. 475

Tarrant, (i. T. I. il

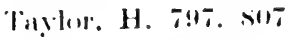

Paylor, 1. 1). $\because 11$

Taylor, 1. . . s.iz

Tivillac. . 1. 483, ititi, 745

int. $75 !$ 
Telegdi, V. L. 199, 395, 481,790

Teller, E. 481

T'emmer, G. II. 483, 489, $492,493,566$

Templeton, D. H. 761

Terrell, J. 781

Thesis, MI. S. 51

Thew, K. 517

Thibaud, J. 100, 673

Thirion, J. 481, 790

Thomas, D. A. 629

Thomas, G. 808

Thomas, H. 96

Thomas, R. 235, 642

Thompson, S. G. 483, 757

Thornton, W. 798, 804

Thulin, S. 91, 268, 460, $633,635,698$

Ticho, H. K. 500

Tiomno, J. 274,350

Todd, W. B. 526, 558

Tolhoek, H. A. 312, 367, $603,605,607,613,614$, $616,619,621,622,623$

Tolman, R. C. 541

Tomasino, G. 356

Tomboulian, D. H. 490

Tomkins, F. S. 343, 752

Tomlinson, 335

Townes, C. H. 191, 476

Townsend; J. 183, 260, 644

Tralli, N. 399

Trambarulo, R. F. 574

Treille, P. 166

Tricker, R. 78

Trigg, G. 246, 284, 287, 321,333

Trott, N. G. 852

Trump, J. G. 8

Tryon, L. E. 180

Tunnicliffe, P. R. 168

Turkel, R. 456

Turkel, S. H. 21

Tuve, M. A. 193

Tyler, A. 314

Tyrén, F. 102
C'hlenbeck, G. E. 225, 299, 300, 308, 339, 534. $569,622,636,650,884$

Uimeda, K. 406

Umezawa, M. 483, 484

Underhill, A. B. 759

Valadares, M. 483, 484, 491,756

Vaientine, J. MI. 14, 167, 853

Valladas, G. 483, 566

Van de Graaff, R. J. 8

Van der Merve, J. $H$. 572

Van der Sluis, J. H. 550

Van Lieshout, R. 698

Van Patter, D. 489, 804

Van Rennes, A. B. 177

Varder, Z. W. 18

Varma, J. 766

Vedder, J. 354

Verster, N. 54, 81, 698

Victoreen, J. A. 49

Vilain, J. H. 354

Vincent, D. H. 834

Vlasov, N. A. 675

Voges, H. 74

Von Baeyer, O. 58

-Von Bayer, H. J. 201

Von Dardel, G. 781

Von Droste, G. 219, 653

Von Weizsäcker, C. F. 373

Votruba, V. 45

Waggoner, M. A. 251, 254, 458, 553

Wagner, F. 742, 756, 760

Walker, D. 507

Walker, J. 481, 486

Walker, R. 45, 781

Walker, W. 781, 801, 804, 807,810

Waller, I. 39

Walter, A. 318

Walter, M. 533, 556, 557, 563,732

Walters, M. C. 669

Wang, M. H. 638
Wapstra, A. 96, 227, 460, $484,630,635,748,766$

Ward, A. G. 17, 168, 506

Warner, R. M. 45

Warren, B. 744

Warren, J. B. 677

Warshaw, S. D. 12

Waterman, H. 73

Watkins, G. D. 586

Watson, K. M. 45

Watson, IV. W. 111, 123, 659,676

Wattenberg, A. 192

Way, K. 447, 517

Weaver, B. 333, 336

Weber, A. H. 269

Weisskopf, V. F. 373, 376, $389,534,538,737,768$, 814,816

Weneser, J. 462, 551

Wentzel, G. 629

West, D. $165,168,173$, $174,176,183,742,743$

West, H. J. 787

West, W. W. 111,118

Wexler, S. 461, 591

Weyl, P. 90

Wheatley, J. 691

Wheeler, G. W. 659

Wheeler, J. A. 45, 274, $350,376,468,690$

White, D. F. 843

White, G. R. 30, 39, 41, 857

White, P. 12

Whittle, C. E. 559

Widdowson, W. P. 765

Wiedenbeck, M. L. 214, 218, 839

Wightman, A. 355, 560, 664

Wigner, E. P. 288, 419, 452, 537

Wildblood, A. M. 266, 845

Wilkins, J. J. 199

Wilkinson, D. H. 165, $167,194,198,455,464$, $466,559,560,816,825$

Wilkinson, R. G. 242, 762

Williams, E. J. 4, 11 
Williams, R. W. 354, 636

Williamson, R. M. 483, 493, 572

Wilson, C. T. R. 15

Wilson, E. 406, 457

Wilson, H. W. 178, 180

Wilson, R. R. 10, 192, $194,196,530,644,646$, 798

Wilson, W. 7, 192

Wilts, J. 84, 131

Winsberg, L. 260

Winter, R. G. 669

Winther, A. 286, 321, 341, 365,477

Witcher, C. 78

Wolfe, H. C. 627
Wolfson, J. L. 260, 338, 481, 486, 704

Woster, W. 61

Wright, B. 336

Wu, C. S. 178, 191, 237, 259, 261, 281, 314, 315, $319,324,331,332,333$, $336,338,339,343,345$, $622,649,653,654,675$, 751, 763

Wykoff, J. M. 566

Yaffé, L. 8, 175, 183, 829

Yamada, M. 341, 570, 766 Yamaguchi, Y. 483, 484 Yang, C. N. 548, 675

Yeater, M. L. 527
Yoshida, S. 483, 484

Yusa, T. 633

Zaffarano, D. J. 183, 214, $232,256,270,318,335$, 336, 698

Zaffarano, J. 268

Zavelski, A. S. 339

Zeldes, H. 336, 698, 699, 702

Zinnes, I. 559

Zobel, W. 579

Zuber, K. 522

Zumwalt, L. R. 829, 849

Zünti,W. 86, 208, 533, 563

Zupančič, C. 375, 477, $483,490,493,769$ 


\section{SUBJECT INDEX}

Aberrations

geometrical - of crystal spectrometer, 107

Absorption

coincidence - 774

- methods, 49

- of $\beta$-rays, 20,208

- of $\gamma$-rays, 25

- of Compton-electrons, 209

- of electrons in matter, 16

self - in source, 829

window - correction, 269, 828

Absorption Coefficient, 24, 25

Compton -, 34

$\gamma$-ray -, table, 857

- of various substances, table, 871

photoelectric -, 28

total -, 25, 47

Acceleration

electron - method, 272

Activity

optimum - of source, 840

specific,- 56

Additivity

non - of nuclear forces, 414

Aligned

- nuclei, 572

Alignment nuclear,- 614

Allowed

- spectra, 241, 279, 314 ; Kurie plots, 283

- transitions, $277,279,319,435$

super - transitions, 288, 324, 347, 435

Alpha

$\gamma$-radiations of - active species; ( (e $\left.\begin{array}{l}e \\ e\end{array}\right)$

740,$752 ;\left(\begin{array}{l}0 \\ 0\end{array}\right) 755 ;\left(\begin{array}{l}e \\ 0\end{array}\right),\left(\begin{array}{l}0 \\ e\end{array}\right) 756$

Amplifier

pulse shape and - design, 172

An-astigmatic, 67

Angle

shielding,- 3

Angular

- correlation (see Correlation)
- distribution; of pair effect, 780; of nuclear radiation : 531 ; from oriented nuclei, 604, 614; of internal bremsstrahlung, 655

- momentum (a); of nuclear states, 374 ; orbital and parity, 423 ; $\gamma$-ray, 534

Anisotropy

- of angular correlation, 550

Annihilating

momentum of - pairs, 675

Annihilation

- of positrons, 673; in liquids and solids, 680 ; in gases, 689

- operator, 377

- radiation and internal pair forma. tion, 644

three-quantum $-, 677,679$; of positronium, 687

Anomalous

- coupling, 426

Anthracene, 147

Aquadag, 238, 260

Atomic

- constants, 98

- structure factor, 40

Attenuation

- coefficient, 588

- factors, 581

Auger

- electrons, 624; classification, 625; energies, 626 ; intensities, 628

- transition probabilities, 630

- yield, 624, 628

Axial

- source, 598

- vector interaction, $\mathbf{2 7 7}$

Background

- rate of $\beta$-counter, 837

Backings

- for sources, 259 ; foils for, 260

Backscattering, 2, 260

- coefficient, 8 
Baffle, 111

Bessel

spherical - functions, 379

\section{Beta}

- decay; theory, 273 ; 292; law of, 311 ; double, 668 ; tables of forbidden, 884; of nuclei with odd $A, 433,437$, 759 ; of nuclei with even $A, 447,449$

- disintegrations; paired, 744; of heavy radinelements, 740

- groups, 240, 242, 243

- interaction, 274, 275, 320

- radiation; directional distrib., 621

- rays; measurement of, 208, 236; absorption of, 20, 208

- spectrometer (see Spectrometer)

- spectrum (see Spectrum)

- transitions; classification, 433; of oriented nuclei: allowed, 621 , forbidden, 622

\section{Bethe-Bloch}

\section{B@}

- formula, 9

measurement of - values, 96

- value, 54; table, 926

\section{$\mathrm{Bi}^{208}$}

decay of,- 707

Bohr-Mottelson

- theory, 132, 468, 588, 725, 752, 754

Bombardment

$\boldsymbol{\gamma}$-radiation from charged particle -, 768

\section{Bothe}

- coincidence circuit, 499

\section{Branching}

- ratios to rotational states, 479

series -, 762

Bremsstrahlung

energy loss due to,- 12

internal -, 649; energy distrib. 650; exper. results, 653; angular distrib. 655 ; and electron capture, 656

Broglie, de

finite - wave length effect, 342

\section{Calibration}

- of $\beta$-spectrographs, 628

- of instruments, 226, 231

- of proportional counter, 168, 189
- and sources, 844

Calorimeter

- for radioactivity; isothermal, 847 ; differential, 849

Capture

- active species of large $Z, 760$

electron -; orbital, 289 ; and internal

Bremsst rahlung, 656: measurement, $\$ 46$

$K$ - (see $K$-cupture)

$L$ - (see $L$-capture)

$\mu$-meson -, 362

multiplicity of - radiation, sos

neutron - and internal conversion electrons, 799

neutron- $\gamma$-rays, 795 ; exper. methods. 783,795 ; exper. results, 799 ; of light elements, 800 ; of heavy elements, 802; of low energy, 798; of high energy, 799; transition probabilities, 813; intensities, 811 , 813; emission probabilities. 815

radiative,- 770

Carbon

- age measurements, 844

Cascades

$$
\text { mixed -, } 556
$$

$\mathrm{Cd}^{111}$

radioactive decays leading to,- 730

Chamber

cloud-and internal pair formation, 647

free air -, 852

ionization -; $\gamma$-ray, $851 ; \beta$-particle, 853

thimble -, 853

Charge

- independence of nuclear forces, 321 , 324,414

measurement of - lost by source, 849 space - limitation, 146

Circuit

coincidence - (see Coincidence)

delay-line clipping -, 503

$\mathrm{RC}$ differentiating -, 501

standard pulse shaping -, 502

univibrator -, 507

Clebsch-Gordon

- coefficients, 300, 536

Clipping 


$$
\text { delay-line -, } 503
$$

Cloud

- chamber and internal pair formation, 647

Coefficient(s)

Clebsch-Gordon -, 300, 536

conversion - (see Conversion)

Greuling -, 303

Coincidence(s)

- absorption, 774

- circuit, 496, 504, 836 ; resolving time, 202, 496; Rossi, 497, 498; Bothe, 499; bridge, 500; differential, 501

- counting, 204, 219; error in, 206; with magnetic spectrometer, 221

delayed - and angular correlation, 575,595

delayed - method, 494, 505; integral, 505; differential, 506

external -, 178

fast-slow - assembly, 503

fast - techniques, 496

high-speed - elements, 500

internal -, 178

- ineasuring apparatus, 202

- method, 201 ; analysis, $211 ; \beta-\gamma, 835$, 841 ; particle- $\gamma, 787$

random,- 837

- ratio, 597

- spectrometry, 159

validity of - method, 839

Collective

- model (see Bohr-Mottelson theory)

Collisions

energy loss due to inelastic -, 9

Collodium

- films, 261

Comparison

- methods for lifetime measurement, 516

self - method in coincidence work, 514

Complex

- spectra, 233

Component, 535

Compound

- nucleus; decay of, 771

Compton

- absorption coefficient, 34, 35

double - effect, 665
- effect, 24, 32, 528, 660

- electrons; range, 209

- scattering, coefficient, 34

Conversion

internal - coefficient, 374, 406; determination, 218, 246, 251, 458; theory, 396; of mixed transition, $557 ; K, 635 ; K, L$ tables, 397,905 internal - electrons; measurement, 216; energy, 217; directional correlation of, 561

photoelectron - measurements, 777 total - correction, 460

Converter, 228

Correction

finite radius,- 342

mirror -, 122

solid angle - factors, 598

Correlation

angular - (see also Directional correlation), 531 ; function, 541 ; theory, 539 ; measurements, 532 ; .between recoil- and $\beta$-particle, 363; between electron and neutrino, 367 ; between particle and $\gamma$-ray, 787; exper. methods, 596; and spin precession, 621; with polycrystalline sources, 585 ; and crystal symmetry, 585; and source conditions, 595; and $K$-capture: in free atom, 591 , in solid or liquid, 592 ; method compared, 574

determination of true -, 595

differential -, 570

directional -, 531, 548; measurement, $532,554,563$; for free nuclei, 534; $\gamma-\gamma, 534,549$, tables 910,$912 ; \alpha-\gamma$, $564 ; \beta-\gamma, 568$; of conversion electrons, 561 ; function, 538 ; perturbed, 581 ; and extranuclear fields, 557,572

- function, 531

polarization -, 531, 559

- time in liquids, 558, 590

Coulomb

- excitation, 477, 485, 490, 768

- field factor, 281

phase shift of - potential, 566

Counter(s), 165

dead time of,- 826 
efficiency of $\gamma-, 2 i 9$

filling mixtures of,- 842

- for activity measurements, 825

- for radioactive gases, 842

Geiger-Müller -, 202, 238, 825, 840,

$842 ; 4 \pi, 831$; window correction, 269

geometries of source and -, 173

halogen quenched,- 825

$4 \pi-, 830$

- precautions, 236

proportional -, 165, 826; recent applications, 182; at low intensity, 179; cylindrical, $199 ; 4 \pi, 832$

proportional - with high pressure, 184,191 ; potential in, 186 ; spectrum in, 187 ; calibration, 189 ; resolving power corrections, 190

scintillation -, 133, 508, 826, 840; $4 \pi, 834$; spectrometer, 668

sensitivity of a gas -, 843

- telescopes, 646

\section{Counting}

coincidence -, 204; error in, 206

- methods, 827, 846

defined solid angle -, 827

\section{Coupling}

anomalous,- 426

- of $\beta$ - and $\gamma$-components, 224

- rules in shell-model, 425, 426

- scheme for closed shells, 470; for strongly deformed nuclei, 470

spin-orbit - in nucleus, $420,422,425$

\section{Creation}

- operator, 377

\section{Crystal}

- diffraction methods, 100; and mag. netic spectrometers, 101

- efficiency, 151

- linearity, 151

- mounting, 147

- preparation, 147

- symmetry and angular correlation, 585

Cross-section, 24; see also absorption coefficient

Compton - -, 38

- for pair production, 43, 44, 46

- for resonant scattering of $\gamma$-rays, $521,523,527$
Klein-Nishina -, 33

photoelectric - - , 28, 228, 233

Rutherford - -, 2

$\mathrm{Cs}^{130}$

disintegration of,- 728

Darwin

- diffraction pattern width, 118

Decay(s) (see also Disintegration)

$\beta$ - (see Beta)

- by electron capture, 846

- constant; for allowed transitions, 284; for forbidden transitions, 303 correction for source -, 838

neutron -, 365

two-body -, 357

$\pi-\mu$ meson,- 362

Decoupling

magnetic - of nucleus, 595

Deflection

direct - method, 58

Delay-line

- clipping, 503

Delayed

- coincidences (see Coincidences)

Delbruck

- scattering, 529

Density

- matrix, 541

Detector

neutron -, 192

- sensitivity, 546; efficiency, 546

Deuteron

photodisintegration of -, 198

Differentiating

RC - circuits, 501

Diffraction

crystal - method, 100; and magnetic spectrometers, 101

Diffusion, 2

back -, 2, 6

Directional

- correlation (see Correlation)

- distribution of $\gamma$-radiation, 616; 619 ; of $\beta$-radiation, 621

Disintegration

measurement of - rate, 823, 824; methods, 824

- schemes, 224, 251, 696 
Dispersion, is

Distribution (see Angular or Directional)

Dose', 851

- rate, 850

\section{Efficiency}

crystal -, 151

light collection,- 143

- of $\gamma$-counters, 219

- of detector, 546

phosphor fluorescent,- 143

photocathode -, 144

photoelectron collection -, 145

Electrodeposition

- procedures, 265

Electron(s)

- acceleration method, 272

- capture (see Capture)

elastic scattering of,- 1

- in matter; range, 16 ; energy loss, 8 positive electron (see Positron)

very low energy -, 315

Electroplating, 265

Emission double quantum -, 383

End

- points, 240

Energy(ies)

- expenditure per ion pair, 167

- of $\beta$ - and $\gamma$-components, 224

pairing,- 427

Ensemble

- of nuclei, 541, 613

Escape

- peak, 176, 459

Evaporation

- of droplets, 263

Ewald

- diffraction pattern width, 118

Excitation

Coulomb -, (see Coulomb)

Excited

measurement of short lifetimes of states, 494

- states of $\left(\begin{array}{l}e \\ \mathrm{e}\end{array}\right)$ nuclei, 484, 740, 752

Extranuclear

influence of - fields, 572
Factor(s)

geometrical -, 536, 545

nuclear,- 536

unfavoured,- 478

Faltung, 118

Fatigue

- effects, 147

Favoured

- transitions, 435

Fermi

- distribution, 225

- function, 281; modified, 282; tables of, 875

- intoraction, $279,320,347,351$

- plot, 240, 241

Field(s)

- adjusting tube, 166

influence of extranuclear -, 572

Fierz

- interference terms, 319,337

- plot, 319

Filling

- mixtures of counters, 842

Films (see Foils)

Fluctuation(s)

statistical - in pulse size, 168

Fluorescence

- yield, 176, 251, 624, 628

Focusing

intermediate image $-, 79,793$

Foil(s)

aluminium -, 262

aluminium oxide -, 263

collodium -, 261

crystal -; single, 572 ; in $\beta$-spectroscopy, 259

- for backings, 260

formvar -, 262

- for windows, 260

mica,- 260

nylon -, 262

other plastic -, 262

- preparation methods, 263

- thickness, 263

zapon lacquer -, 261

Fold, 118

Foldy-Wouthuysen

- transformation, 340 
Forces, nuclear

(see Nuclear forces)

\section{Forbidden}

- spectra, see spectra

- transitions, see transitions

\section{Formvar}

ft

- films, 262

- values, 284; determination, 245

\section{$g$}

determination of nuclear - factors, 533,576

\section{Gamma}

- coefficients, 546

- counters; efficiency of, 219 (see also Counters)

- disintegration of heavy radioelements, 740

- radiation; interaction with matter, 24; from charged particle reactions, 767, 768; directional distribution, 616,619 ; polarization distribution, 617 ; of $\alpha$-active species, $740,755,756$

- ray(s); absorption, 24, 857; polarization, 198; energy measurement, 209,228 ; relative intensities, 234 ; resonant scattering, 521; nonresonant scattering, 528; from neutron capture, see capture

- ray spectroscopy; 100, 767; and scintillation method, 192

- transitions; selection rules, 373, 454; multipolarities, 373; short lifetime measurement, 494; collective, 481

Gamow-Teller

Gas

- interaction, 279, 320, 347

- gain, 166

\section{Gauge}

conventional,- 401

solenoidal -, 400

- transformation, 376

Geiger-Müller counters (see Counters)

Geometrical

- factor, 536, 545

Greuling

- coefficients, 303
Group(s)

$\beta$-ray -, 240, 242, 243

Half-life(ves) (see also Life and Life. times)

comparative - (see $f t$-value)

- of allowed transitions, 286

- of forbidden transitions, 303; unique, 305 , 333 ; once, 308 ; twice, 310

Hamiltonian

- of radiation field, 378

spin - in crystal, 601

Hartmann

- test, 117

Hartree

- model, 414

Heavimet, 114

Helmholtz

- equation; vector, 377,380 ; scalar. 378

Hidden

- transition, 460

$\mathrm{I}^{130}$

experiments on,- 720

$I^{131}$

disintegration of,- 696

Impure

- state, 541

Incompressible

- model of nucleus, 469

Independence

charge - of nuclear forces, see charge

Inelastic

energy loss due to - collisions, 9

Inertia

moment of - of deformed nucleus, 472

Intensity(ies)

- of $\beta$. and $\gamma$-components, 224

Interaction(s)

axial vector,- 277

$\beta-, 274,275$

Fermi -, 279, 320, 347; universal, 351

Gamow-Teller -, 279, 320, 347

pseudoscalar -, 277, 339, 343; effect on shape of allowed spectra, 344 


$$
\begin{aligned}
& \text { scalar -, } 277,452 \\
& \text { static -, } 573 \\
& \text { tensor -, } 348,452 \\
& \text { time-dependent -, 573, } 588 \\
& \text { vector -, } 276 \\
& \text { velocity-dependent }-, 393 \\
& \text { Interference(s) } \\
& \text { Fierz - terms, } 319,337 \\
& \text { - in } \beta-\gamma \text { correlation, } 569 \\
& \text { Smith - formula, } 340 \\
& \text { Internal }
\end{aligned}
$$

\section{Internal}

- conversion (see Conversion)

- bremsstrahlung (see Bremsstrahlung)

- pair formation (see Pair)

Ionization

- chambers, 798, 850; $\gamma$-ray, 851; $\beta$-ray, 853

Isomers, 453

classification of nuclear -, 453

Isomerism, 373,764

core -, 461

islands of,- 375

Isotope

electromagnetic - separator, 267

- separation, 76

Isotopic

- spin, 395

K

- capture, 290; and angular correlation, 591 ; in free atom, 591 ; in solid or liquid, 592; and positron emis. sion, 634

$K / L$

- ratios, 397, 409; determination of, 246,635

Klein-Nishina

- cross section, 33

Konopinski-Uhlenbeck distribution, 225

Kurie

- plots, 241; for allowed spectra, 241, 283

\section{$L$}

- capture, 290

$L_{\mathrm{I}} / L_{\mathrm{II}}$ ratio, 410

$L_{\mathrm{III}} / L_{\mathrm{I}}$ ratio, 411
Lens(es)

magnetic,- 77

- spectrometers (see Spectrometers)

Level

- occupation scheme in nucleus, $\mathbf{4 2 7}$

- sequence in nucleus, 418

Life(ves)

half,- 494

mean -, 494; determination, 527; of positrons, 682

Lifetime(s)

- measurements; of excited states, 494 ; by delayed coincidence method, 507; comparison methods for, 516 ; of positron, 680

- of nuclear states excited by nuclear reactions, 789

Line(s), 535

random sum,- 163

Linearity

crystal -, 151

pre-differentiator non -, 146

Linewidth

- in spectrometer; semicircular, 782; $\gamma$-ray crystal, 117

natural,- 93

Luminosity, 58

Magic

- numbers, 415, 419

Magnetic spectrometer (see Spectrometer)

- decoupling of nucleus, 595

- moments in unified model, 477

Majorana particle (see Particle)

Mass

rest - of neutrino, 182,325

Matrix

density -, 541

efficiency -, 546

- element; reduced, 545; for forbidden transitions, 296

Meson

$\mu$-; $\beta$-spectrum, 349 ; capture, 362

$\pi-\mu$ - decay, 362

Mirror

- correction, 122

- nuclei, 416

- transitions, 288, 322 
Mixed

- transitions, 551; tables, 912

Mixing

- ratio, 551

\section{Model}

harmonic oscillator -, 418, 422

Hartree -, 414

incompressible -, 469

- of individual orbits, 414

shell -, 414

square well $-, 418,422$

unified nuclear -, 469 (see Bohr-

Mottelson theory)

\section{Moments}

nuclear -, 475

\section{Mounting}

crystal,- 147

Muller

- method, 119

Multipolarity(ies), 373, 534

experimental determination of,- 92 , 457

- of $\gamma$-transition, 373

\section{Multipole}

purity of - character, 458

- radiation field, 376; parity of, 385

theory of - radiation, 373

\section{Neutrino, 274, 668}

- as a Majorana particle, 669

- recoil experiments, 357

rest mass of $-, 182,325$

\section{Neutron}

- capture (see Capture)

- decay, 365 ; recoil experiment, 365

- detector, 192

electromagnetic transitions of,- 392

- resonances; radiation width of, 815 , 819

\section{Noise}

amplifier -, 146

photomultiplier -, 146

\section{Nordheim}

- rules; weak, 447; strong, 447

\section{Nuclear}

- alignement, 614

- factor, 536, 545

- forces; exchange character, 414; non additivity, 414; charge independence, 414; potential, 418

- orientation, 614

- polarization, 614

unified - model, 469

Nucleus(i)

aligned -, 572

compound -; decay, 771

$\left(\begin{array}{l}e \\ e\end{array}\right), 484$; excited states, 484, 740, 752;

higher rotational states, 486; in. trinsic quadrupole moment, 484, 489; transition probabilities, 488

level sequence in,- 418

light -, 452

mirror -, 416 ; transition between, 322 orientation of,- 613

oriented -; experiments, 600, 607; production, 600; detection, 604; angular distribution of radiation, $604,613,614$; directional distr. of rad., 616,621 ; polarisation of rad., $606,617,621$

strongly deformed,- 470

- with even $A$; $\beta$-decay of, 447, 449

- with incompletely filled shells, 424

- with odd $A ; \beta$-decay of, 433, 437; rotational transitions, 490

Nylon

- films, 262

Orbital

- angular momentum, 423

- electron capture, 289

Orientation

- of nuclei, 613,614

Oriented

- nuclei (see Nuclei)

Oscillations

collective -, 468

Oscillator

harmonic - model, 418, 422

\section{Pair(s)}

angular distribution of - effect, 780 energy distribution of - electrons, 44 internal - formation, 636 ; coefficient, 636,638 ; at high energies, 647 ; and annihilation radiation, 644; and cloud chamber, 647 
mumentum of annihilating,- 675

- production, 25, 41, 138; cross section, 44,46 ; for $\gamma$-energy meas. urement, Tru

- spectrouncters (see spectrometers)

Pairing

- energy, 4.27

Parameter(s)

particle - , 5ti:

radiation -, $5+6$

Parity(ir's), 4:3

- favoured spectra, 328

- forbidden spectra, 328

- of a multipole field, 385

- of nuclear states, $3 \overline{7} 4,37 \bar{\jmath}$

relative-, 532 ; determination, 559,561

Particle

$\gamma$-radiation from charged - bombard. ment, 767,768

neutrino as Majorana -, 669

- parameter, 562

- transitions in nucleus, 478, 480

$\mathrm{Pb}^{208}$

energy levels of,- 707

Peak

escape $-, 176,459$

Phase

- shift of Coulomb potential, 566

Phenomena

saturation,- 414

Phonon(s), 469

- transitions, 481

Phosphor(s), 133, 138, 143, 148, 149, 164

Photoabsorption, see photoelectric effect

Photocathode

- efficiency, 144

Photodisintegration

- for determination of $\gamma$-ray polarization, 199

- of deuteron, 198

Photoelectric

- absorption, 176; coefficient, 28, 29, $30,228,233$

- effect, 24, 26; nuclear, 481

Photoelectron

- conversion measurements, 777

spectrum of -, 229

Photographic

- plates, 197
Phot omult iplier(s)

- noise, 146

photocathode of,- 146

Photoneutron

measurement of - energies, 193

- threshold reactions, 192

Photoproton mensurement of - enorgies, 194

Plast ic

- films, 26:

Plates

photographic -, 197

Plot

Fermi -, 240, 241

Fierz -, 319

Kurie -, 241

Polarimeter

- for angular correlation, 560

Polarization

- correlation (see Correlation)

degree of,- 617

nuclear,- 614

- of $\gamma$-rays, 198, 559, 617

- of nucleus by particle transitions, 480

- of radiation from oriented nuclei, 606

two-quanta,- 674

- vector, 559

way of,- 617

Polycrystalline

- source, 573

Population

- of states, 772

relative,- 535

Positron(s)

annihilation of,$- 673 ;$ in liquids and solids, 680 ; in gases, 689

cross section for creation of $-, 42,43$

- emitters, 215, 249

$K$-capture and - emission, 634

magnetic spectrometer and - spectrum, 641

mean life of -, 680, 682

- spectrum of excited nuclei, 641

Positive electron (see Positron)

Positronium, 677, 689

formation of,- 686

ortho $-, 691,694$; stability, 695 
paral -., 691

three-quantum annihilation of -, 687

Zeeman effect of -, 692

\section{Potential}

isotropic harmonic oscillator -, 418

square well -, 418, 42.2

\section{Precession}

- frequency, 574

Probability(ies)

absolute emission - of neutron capture $\gamma$-rays, 815

transition -; relative, 535; absolute, 536; reduced, 394; Auger, 630; of particle(s): single, 382, several, 393 ; in unified model, 477; in $\left(\begin{array}{l}\mathrm{e} \\ \mathrm{e}\end{array}\right)$ nuclei,

\section{Profiles} 488; of neutron capture $\gamma$-rays, 813

super-position of -, 119

Proportional

- counters (see Counters)

\section{Proton}

- in central velocity-independent potential, 382

single - units, 456

Pseudoscalar

- interaction, $277,339,343,344$

\section{Pulse}

- amplitude analysis, 177

fluctuations in - size, 168

- shape and amplifier design, 172

\section{Purifier, 171}

\section{Quadrupole}

determination of - coupling, 532, 581

- moments, 476, 532 ; in ( $\left(\begin{array}{l}\mathrm{e} \\ \mathrm{e}\end{array}\right)$ nuclei, 484, 489

\section{Quantization}

- of radiation field, 376

\section{Quantum}

angular momentum - number, 534

double - emission, 383

magnetic - number, 534

three - annihilation, $67 \overline{7}$; polarization of, 679

two - polarization, 674

\section{Racah}

- algebra, 540

- coefficients, 403, 546, 548
Radiation

annihilation - and internal pair for. mation, 644

$\beta-($ see Beta)

$\gamma$ - (see Gamma)

multipole - field; theory, 373; quantization, 376; Hamiltonian, 378; spin, 381

- parameters, 546

- width; of neutron resonances, 815, 819

Radiative

- capture, 770

Radioactive

Auger electrons emitted from - atoms, 624

$\beta$ - and $\gamma$-disintegrations of heavy elements, 740

counters for - gases, 842

Radioactivity

calorimetric measurements of,- 847

differential calorimeter for -, 849

isothermal calorimeters for,- 847

RaE

spectrum of,- 339

spin of,- 343

Radioelements

disintegration of heavy -; $\beta, 740 ; \gamma$, 740

Random

- coincidences, 837

\section{Range}

- of Compton electrons, 209

Rayleigh

- scattering, 528

Reactions

lifetimes of nuclear states excited by nuclear,- 789

nuclear -, 572

photoneutron threshold -, 192

Reactor

nuclear -, 797

Recoil experiments

neutrino -, 357

$\beta-, 363$; interpretation, 367

neutron,$- 3 \dot{6} 5$

two-body-decay -, 357, 362

Relativistic

- effects in nucleus, $\mathbf{4 2 3}$ 
Relaxation

- (onstant, 574, 588

Representation(s)

$-D^{L}, 5+4$

spherical -, 298

Resolution, 57

fluctuations in Pulse size and --, 168

high - spectroscopy, 52

- of magnetic spectrometers, 56

- of scintillation spectrometer, 143 , 145

prompt (coincidence) - curve, 509, 511

Resolving

- power (see Resolution)

- time of coincidence circuit, 202, 496, 837 ; in delayed coincidence work, 509

Resonance

$\gamma$-ray - condition, 522, 523

Resonant scattering

- and thermal motion, 524

non - - processes, 528

- of $\gamma$-rays, 521 ; cross-section, 521, 523; measurement, 527 ; with continuous $\gamma$-spectrum, 527

Retardation

- effects, 294, 336

- expansion, 278

Rhm, 851

Röntgen, 851

Rossi

- coincidence circuit, 497; modified, 498; fast, 498

Rotational

- levels, 769

- states in even-even nuclei, 486

systematics of nuclear - structure, 483

- transitions, 465,482

Rutherford

- cross-section, 2

\section{Saturation}

- phenomena, 414

Scalar

- interaction, 277,452

\section{Scattering}

- and angular correlation work, 596 back - coefficient, 8

coherent -, 38

Compton - (see Compton effect)

Delbruck -, 529

elastic - of electrons by atomic nu. clei, 1

electronic - factor, 39

incoherent -, 39

- in counters, 829

multiple,- 4

- of $\gamma$-rays; resonant, 521, 523, 527; non-resonant, 528

plural -, 1, 4

Rayleigh -, 528

self - in source, 829

single $-, 1,2$

Thomson -, 529

Scintillation

- counters (see Counters)

- crystals (see Phosphor(s))

- method, 133; at ordinary pressures, 165 ; at high pressures, 184 ; in $\gamma$-ray spectroscopy, 192; apṕlications, 660

- phosphors, 133, 138, 148, 149; large, 164

- spectrometer (see Spectrometer)

Screening

- constants, 26,27

- corrections, 282

- function, 406

Screw

calibration of - errors, 121

Selection rules

- for allowed $\beta$-transitions, 279 ; for forbidden $\beta$-transitions, 296 ; for $\gamma$ transitions, $373,395,454,480$; in unified model, 480

Self-conjugate

- nucleus, 395

Seniority

- of states, 394

Sensitivity

- of a gas counter, 843

- of detector, 546

Separation

isotope,- 76

Separator

electromagnetic isotope,- 267 
Shape(s)

- of $\beta$-spectra; experiments, 314 ; al. lowed, 314 ; forbidden, 244, 327 ; unique forbidden, 328 ; 'first forbidden, 336 ; twice forbidden, 345

Shape-factor, 301, 302, 312

- for allowed spectra, 241, 314

- for first forbidden spectra, 336

- for once forbidden spectra, 306

- for twice forbidden spectra, 309, 347

Shell

unique -, 304, 329

nuclear - model, 414

nuclear - systematics, 433

- numbers, 415

Size

finite nuclear - corrections, 283

Slurry

Smith

- technique, 264

- interference formula, 340

Source(s)

backings for,- 259

centered,- 597

- conditions and angular correlation, 595

- geometry, 173

max. - disintegration rate, 163

liquid $\gamma--, 527$

- precautions, 236

preparation of - material, 226

- size correction, 598

- strength, 851, 853

- technique, 259

thin -, 259, 563; preparation, 237

Space

- velocity effects, 293, 336

- charge limitation, 146

Spectrogoniometer, 87, 710

Spectrometer

$\beta-, 52$; measurements, 224; and directional correlations, 563

coincidence -, 86 ; short lens, 88; double lens, 88

crystal -; flat, 100; bent, 100, 109; reflection, 100, 103; curved, 101, 103; transmission, 101, 103, 109; geometrical aberrations, 107 ; and linebreadth, 117 ; precision $\hat{\imath}, 108$, 123

double focusing -, 53, 66, 71, 72,73 electrostatic -, 90

flat $-, 53,73,74$

helical -, 53

intermediate-image -, 79

lens -, 77; short, 79; long, 81; pair, 783 , 784; magnetic for $\beta \cdot \gamma$ coin. cidences, 221 ; double, 710

magnetic -; and crystal diffraction, 101 ; and rèaction $\gamma$-spectra, 774 , 775 ; lens for $\beta$ - $\gamma$ coincidences, 221 ; and coincidence counting, 221 ; axial focussing, 131; and internal pairs, 641

pair -; magnetic, 781 ; uniform field, 781,783 , 792; axial focussing $\beta$, 783 ; lens, 783 , 784 ; lens scintillation crystal, 785, 786; three crystal, 786; orange, 792; intermediate-image, 793

photo disintegration - compared, 197 scintillation -; for reaction $\gamma$-rays, 778 ; resolution, 143 ; and directional correlations, 554, 563; split crystal, 134, 135; hollow crystal, 136

semicircular -, 58, 73, 782

solid angle of,- 57

spiral-orbit -, 76

total absorption $\gamma-, 155$

- transmission, $5 i$

X-ray - , 100

Spectroscopy

$\beta-$, basic relations, 54 ; with high press. proportional counter, $1 \mathrm{si}$

$\gamma \cdot-, 767$; and scintillation method, 192; and crystal diffraction method, 1(10), 13:

high resolution -, 52

mass,- 76

precision -, 90

Spectrum (a), sue also transitions

allowed $\beta-, 241,279,314$; kurie plot, 283

$\beta$-; measurement, 23s; exper. on shape of, 314: of $\mu-n$ from rointillence methed, 21:216:

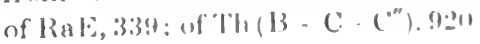


complex -, 233

allowed $\beta$ - -, 279

forbiclden $\beta--, 244,301$; first, 336; mce, 306 ; unique, 304, 328; twice, 309, 345; functions (tables), 884

- in proportional counter, 187

random coincidence -, 163

Splerical

- harmonics, 299

- representations, 298

$\operatorname{spin}(\mathrm{s})$

- difference from internal conversion, 460

- Hamiltonian in crystal, 601

isotopic -, 395

nuclear $-, 475,532$

- of radiation, 381

- of RaE, 343

- orbit coupling in nucleus, 420,422, 425

Pauli - angle functions, 402

- precession and angular correlation, $6: 1$

State

impure -, 541

Statistical

- factor, 389

- weight of $\beta$-transition, 280

Stilbene, 148

Superallowed (see Allowed)

Surface

- oscillations in nucleus, 468

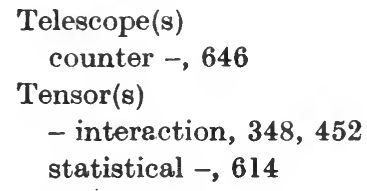

Tetraethylene

- glycol spreading technique, 266

Th $\left(\mathrm{B}+\mathrm{C}+\mathrm{C}^{\prime \prime}\right)$ spectrum. of, 920

Thermal

- motion and resonant scattering, 524

Thimble

- chambers, 853

Thomson

- scattering, 529

Threshold

photoneutron - reactions, 192
Time

dead - of counter, 826

Tolhock-de (iroot symmetry principle, 312

Transformation

Foldy-Wouthuysen -, 340

Transitions (see also spectrum (a))

allowed -, 277, 279, 319; super -, 288, $324,347,435$

$\beta-$, see beta

$\gamma-$, see gainma

electric - in nucleus, 480

favoured -, 435

forbidden -, 292, 327 ; first, 339 ; once, 294,295 ; unique, 295, 297, 304,

333 ; twice, 295; selection rules, 295, 296; matrix elements, 296; parity, 328

hidden -, 460

mirror -, 288, 322

mixed,- 551 ; conversion coefficient of, 557

- of neutron, 392

particle - in nucleus, 478, 480

phonon -, 481

- probability, see probability

rotational -, 482, 465

vibrational -, 481

Transmission

spectrometer,- 57

Unfavoured

- factor, 478

Units

single proton -, 456

Univibrator

- circuit, 507

Vacuum

- methods, 264

Vector

- interaction, 276; axial, 277

Vibrational

- transitions, 481

Wall

- effect in counter, 173,196

- losses in counter, 843 
Wavefunctions

nuclear,- 390

Wavelength

precision - measurements, 109, 119

Waves

surface,- 468

Weight

statistical - of $\beta$-transition, 280

Well

square - potential with finite walls, 418,422

Width

base -, 57

half,- 57

natural line- -, 93

Window(s)

- absorption correction, 269

foils for,- 260
- technique, 259

X-Ray

- absorption energies, table, 914

$\mathrm{X}^{130}$

low states of,- 726

Yield

Auger -, 624, 628

fluorescence -, 176, 251, 624, 628

Zapon

- lacquer foils, 261

- spreading technique, 267

Zeeman

- effect of positronium, 692 


$\therefore 9$

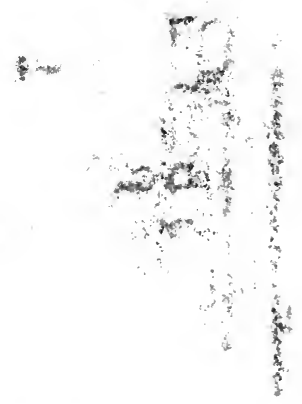


BINDNA SECT. FEB2019/5

ET

QC

Siegbahn, Kai

(ed.)

771

Beta- and gamma-ray

S5 spectroscopy

Engineering 
